\title{
Data Summary Report D-Area Burning/Rubble Pits
}

by

E. R. Palmer

Westinghouse Savannah River Company

Savannah River Site

Aiken, South Carolina 29808

This paper was prepared in connection with work done under the above contract number with the U.S. Department of Energy. By acceptance of this paper, the publisher and/or recipient acknowledges the U.S. Government's right to retain a nonexclusive, royalty-free license in and to any copyright covering this paper, along with the right to reproduce and to authorize others to reproduce all or part of the copyrighted paper. 


\section{DISCLAIMER}

This report was prepared as an account of work sponsored by an agency of the United States Government. Neither the United States Government nor any agency thereof, nor any of their employees, makes any warranty, express or implied, or assumes any legal liability or responsibility for the accuracy, completeness, or usefulness of any information, apparatus, product, or process disclosed, or represents that its use would not infringe privately owned rights. Reference herein to any specific commercial product, process, or service by trade name, trademark, manufacturer, or otherwise does not necessarily constitute or imply its endorsement, recommendation, or favoring by the United States Government or any agency thereof. The views and opinions of authors expressed herein do not necessarily state or reflect those of the United States Government or any agency thereof.

This report has been reproduced directly from the best available copy.

Available to DOE and DOE contractors from the Office of Scientific and Technical Information, P. O. Box 62, Oak Ridge, TN 37831; prices available from (423) 576-8401.

Available to the public from the National Technical Information Service, U. S. Department of Commerce, 5285 Port Royal Road, Springfield, VA 22161. 


\section{DISCLAIMER}

Portions of this document may be illegible electronic image products. Images are produced from the best available original docúment. 


\title{
DATA SUMMARY REPORT
}

\section{D-AREA BURNING/RUBBLE PITS}

\author{
FINAL
}

Prepared For:

Westinghouse Savannah River Company

Savannah River Site

Aiken, South Carolina

Prepared By:

RUST Environment \& Infrastructure Inc.

955 Millbrook Avenue

Aiken, South Carolina

OCTOBER 1994

SAVANMAH PIVER SITE

ENV...: $: \quad-\ldots$-RATION

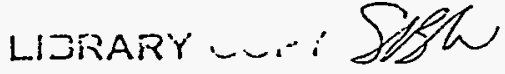




\section{TABLE OF CONTENTS}

1.0 INTRODUCTION

2.0 PROJECT DESCRIPTION

2.1 Site History

2.2 Project Objectives

3.0 SAMPLE COLLECTION 4

3.1 Sampling Procedures 4

3.1.1 Soil

3.1.2 Groundwater

3.1.3 Surface Water/Sediment

3.1.4 Additional Sampling

3.2 Sample Identification

6

3.3 Sample Packing and Transportation

3.4 Decontamination Procedures

4.0 ANALYTICAL METHODS 8

5.0 DATA VALDATION 9

5.1 Assessment of Data Quality Indicators 9

5.2. Evaluation of Data Quality Indicators 11

5.2.1 Precision 11

5.2.2 Accuracy 13

5.2.3 Representativeness 14

5.2.4 Completeness 15

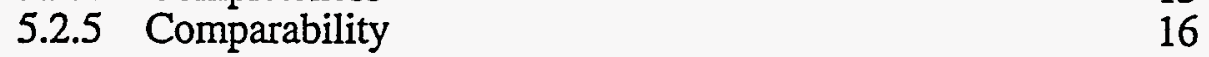

$\begin{array}{lll}6.0 & \text { DATA SUMMARY } & 17\end{array}$

6.1 Soil Data Summary $\quad 17$

6.1.1 Rejected Data 21

6.1.2 Additional Data Qualifiers 22

6.2 Groundwater Data Summary 22

6.2.1 Rejected Data 24

6.2.2 Additional Data Qualifiers 25 
6.3 Surface Water Data Summary 25

6.3.1 Rejected Data 27

6.3.2 Additional Data Qualifiers 27

6.4 Sediment Data Summary 27

6.4.1 Rejected Data 28

6.4.2 Additional Data Qualifiers 28

$\begin{array}{lll}7.0 & \text { SUMMARY } & 29\end{array}$ 


\section{LIST OF FIGURES}

Figure 2-1 Location of the Savannah River Site

Figure 2-2 Location of D-Area Burning/Rubble Pits at the Savannah River Site

Figure 2-3 Location of D-Area Burning/Rubble Pits in Relation to Other Savannah River Site Facilities

Figure 3-1 D-Area Burning/Rubble Pits Unit Assessment Sampling Locations 


\section{LIST OF TABLES}

Table 5.1 Qualifier Codes

Table 5.2 Precision and Accuracy Validation

Table 6.1 Volatile Organic Compounds in Soil

Table 6.2 Semi-Volatile Organic Compounds in Soil

Table 6.3 Pesticides and PCBs in Soil

Table 6.4 PCDD and PCDF Compounds in Soil

Table 6.5 Inorganics and Organic Indicators in Soil

Table 6.6 Radionuclide Parameters in Soil

Table 6.7 TCLP Analyses in Soil

Table 6.8 Volatile Organic Compounds in Groundwater - Monitoring Wells

Table 6.9 Volatile Organic Compounds in Groundwater Hydropunch ${ }^{\text {TM }}$ Samples

Table 6.10 Semi-Volatile Organic Compounds in Groundwater

Table 6.11 Pesticides and PCBs in Groundwater

Table 6.12 PCDD and PCDF Compounds in Groundwater

Table 6.13 Inorganics and Organic Indicators in Groundwater

Table 6.14 Radionuclide Parameters in Groundwater

Table 6.15

Table 6.16

Table 6.17

Table 6.18

Table 6.19

Table 6.20

Table 6.21

Table 6.22

Table 6.23

Volatile Organic Compounds in Surface Water - 8240

Volatile Organic Compounds in Surface Water - 8260

Semi-Volatile Organic Compounds in Surface Water

Inorganics, Organic Indicators, and Radionuclide Parameters in Surface Water

Volatile Organic Compounds in Sediment - 8240

Volatile Organic Compounds in Sediment - 8260

Semi-Volatile Organic Compounds in Sediment

Inorganic and Organic Indicators in Sediment

Radionuclide Parameters in Sediment 


\section{LIST OF APPENDICES}

APPENDIX A Quality Control Summary Report for the D-Area Burning/Rubble Pits RFI/RI Unit Assessment (Exploration Resources Validation)

APPENDIX B Correspondence Between Environmental Monitoring Section and Roy F. Weston, Inc.

(Environmental Monitoring Section Validation)

APPENDIX C Quality Control Summary Report for Surface Water and Sediments Collected during 1994 for the D-Area Burning/Rubble Pits RFI/RI Unit Assessment

(RUST Environment \& Infrastructure Data Validation) 


\section{LIST OF ACRONYMS}

$\begin{array}{ll}\text { ASTM } & \text { American Society of Testing and Materials } \\ \text { bgs } & \text { Below Ground Surface } \\ \text { BRPs } & \text { Burning/Rubble Pits } \\ \text { CEC } & \text { Cation Exchange Capacity } \\ \text { CERCLA } & \text { Comprehensive Environmental Response, Compensation, and Liability Act } \\ \text { CLP } & \text { Contract Laboratory Program } \\ \text { CRDL } & \text { Contract Required Detection Limit } \\ \text { DOE } & \text { U.S. Department of Energy } \\ \text { EMS } & \text { Environmental Monitoring Section } \\ \text { EPA } & \text { U.S. Environmental Protection Agency } \\ \text { EPD } & \text { Environmental Protection Division } \\ \text { FFA } & \text { Federal Facility Agreement } \\ \text { GC } & \text { Gas Chromatograph } \\ \text { HNu } & \text { Trace gas analyzer } \\ \text { NPL } & \text { National Priorities List } \\ \text { OVA } & \text { Organic Vapor Analyzer } \\ \text { PAH } & \text { Polyaromatic Hydrocarbon } \\ \text { PARCC } & \text { Precision, Accuracy, Representativeness, Completeness, and Comparability } \\ \text { PCB } & \text { Polychlorinated Biphenyl } \\ \text { PCDD } & \text { Polychlorinated Dibenzodioxine } \\ \text { PCDF } & \text { Polychlorinated Dibenzofuran } \\ \text { QA } & \text { Quality Assurance } \\ \text { QC } & \text { Quality Control } \\ \text { QCSR } & \text { Quality Control Summary Report } \\ \text { RCRA } & \text { Resource Conservation and Recovery Act } \\ \text { RFI/RI } & \text { RCRA Facility Investigation/Remedial Investigation } \\ \text { RPD } & \text { Relative Percent Difference } \\ \text { RUST E\&I } & \text { RUST Environment \& Infrastructure } \\ \text { SCDHEC } & \text { South Carolina Department of Health and Environmental Control } \\ \text { SCS } & \text { U.S. Soil Conservation Service } \\ \text { SRS } & \text { Savannah River Site } \\ \text { SVOC } & \text { Semivolatile Organic Compound } \\ \text { TAL } & \text { Target Analyte List } \\ \text { TDS } & \text { Total Dissolved Solids } \\ \text { TCLP } & \text { Toxicity Characteristic Leaching Procedure } \\ \text { TOC } & \text { Total Organic Carbon } \\ \text { VOC } & \text { Volatile Organic Compound } \\ \text { WSRC } & \text { Westinghouse Savannah River Company } \\ & \end{array}$




\subsection{INTRODUCTION}

The purpose of this report is to verify that all analytical data collected at the D-Area Burning/Rubble Pits (BRPs) (431-D and 431-1D) at the Savannah River Site (SRS) for use in developing risk assessment and potential remediation procedures have been validated at the appropriate level. Any discrepancies or reasons why the data should be rejected for this purpose will be addressed. This report documents the data validation procedures used by Environmental Monitoring Section (EMS), Exploration Resources, and RUST Environment \& Infrastructure (RUST E\&I) for assigning qualifiers. 


\subsection{PROJECT DESCRIPTION}

The data collected in this investigation are intended to further characterize the nature and extent of chemical residue in groundwater, surface water, soil, and sediment. In addition, the data supplement existing geochemical data for the purpose of developing risk evaluation and appropriate remedial activities at the D-Area BRPs, if necessary.

\subsection{Site History}

SRS is located principally in Barnwell and Aiken Counties near the city of Aiken, South Carolina (Figure 2-1). SRS is owned by the U.S. Department of Energy (DOE). Management and operating services are provided by Westinghouse Savannah River Company (WSRC). SRS has historically produced tritium, plutonium, and other special nuclear materials for national defense. SRS also has provided nuclear materials for the space program and for medical, industrial, and research efforts. Chemical and radioactive wastes are by-products of nuclear material production processes. Hazardous substances, as defined by the Comprehensive Environmental Response, Compensation, and Liability Act (CERCLA) and hazardous waste as defined by Resource Conservation and Recovery Act (RCRA), are currently present in the environment at SRS.

SRS manages waste materials that are regulated under RCRA. Certain SRS activities have required operating or post-closure permits under RCRA. SRS received a RCRA hazardous waste permit from the South Carolina Department of Health and Environmental Control (SCDHEC) on September 30, 1987. On December 21, 1989, SRS was placed on the National Priorities List (NPL). In accordance with Section 120 of CERCLA, DOE has entered into a Federal Facility Agreement (FFA) with the U.S. Environmental Protection Agency (EPA) - Region IV and SCDHEC to coordinate cleanup activities at SRS into one comprehensive strategy that fulfills RCRA Section 3004(u) and CERCLA assessment, investigation, and response action requirements. Appendix $\mathrm{C}$ of the FFA lists the D-Area BRPs as a RCRA/CERCLA unit.

The D-Area BRPs 431-D and 431-1D are located approximately 0.8 kilometer ( 0.5 mile) west of D Area (Figures 2-2 and 2-3). D-Area BRPs 431-D and 431-1D are contiguous pits separated by a clay berm. A shallow run-off ditch is located within 15.2 meters ( 50 feet) west of the pits. The 
Savannah River and the associated swamps are approximately 0.8 kilometer ( 0.5 mile) from the BRP.s.

Burning/rubble pits at SRS were constructed in 1951 for disposal of burnable waste such as paper, plastics, rubber, wood, waste oil, degreasers, and drummed organic solvents. The waste was burned periodically, typically once a month. The chemical composition and volume of waste disposed of are unknown.

In 1973, SRS burning/rubble pits were covered with a soil layer and continued to be used for rubble only. During this time until 1983, these pits were used as rubble pits to dispose of paper, wood, concrete, empty galvanized-steel barrels and drums, and cans. When the pits were filled in 1983, they were covered with a clay layer, compacted, mounded, and closed.

\subsection{Project Objectives}

The purpose of assessment sampling was to further delineate the extent and magnitude of hazardous substance release to surrounding environmental media. 


\subsection{SAMPLE COLLECTION}

Field activities conducted between May and December, 1993 included the calibration of field screening equipment [(i.e., pH/conductivity meters, $\mathrm{HNu}$ and Organic Vapor Analyzers (OVA)], sample equipment decontamination, monitoring well installation, the collection of soil, groundwater, and surface water samples, and waste management. Field activities conducted during May 1994 included the collection of surface water and sediment samples that could not be collected during the 1993 investigation due to lack of surface water.

Bound field logs were maintained for each sampling date. The field logs provide information including date, weather conditions, work performed, sampling performed, field analyses completed, problems, corrective actions, Quality Control (QC) activities, and signature of the sampler. No unusual or problematic situations occurred during these activities.

\subsection{Sampling Procedures}

\subsubsection{Soil}

Soil sampling involved continuous sampling at eight locations within the pit confines and through the bottom of the pit boundary (four random sample locations in the quartered sections of each pit). Four additional sample locations were also selected adjacent to pit confines, including one background. location, with regard to groundwater gradients. Soil sampling locations were chosen to adequately characterize and define the lateral and vertical extent of constituent impact on soils from the RCRA Facility Investigation/Remedial Investigation (RFI/RD) units.

Soil sampling locations are shown on Figure 3-1. Sampling locations 07, 08, 09, and 10 were completed within BRP 431-1D. Locations 11, 12, 13, and 14 were located within BRP 431-D. Four locations outside the pit boundaries were selected. Soil sampling location 15 was completed upgradient from the BRPs, location 16 was completed side-gradient of BRP 431-1D, and locations 17 and 18 were completed downgradient of BRP 431-D. All sample points are shown on Figure 3-1. 
Soil sampling followed requirements and procedures specified by the RFI Program Plan (WSRC, 1989). Surface soil samples [0 to $0.6 \mathrm{~m}$ bgs ( 0 to $2.0 \mathrm{ft}$ )] were collected using a properly decontaminated stainless steel hand auger, as specified in the RFI/RI Plan. Subsurface soil samples were collected continuously [in $0.6-\mathrm{m}(2-\mathrm{ft})$ intervals] starting at $0.6 \mathrm{~m}(2.0 \mathrm{ft})$ by driving a split spoon sampling apparatus into the undisturbed soil below the hollow stem augers.

Sampling and handling procedures and associated decontamination and QC procedures were adhered to as specified in Chapter 4.6 of the RFI Program Plan. Soil cuttings from the augered boreholes were not removed from the waste unit area but were either placed back into the borehole or dispersed about the unit area.

\subsubsection{Groundwater}

Groundwater assessment included the collection of groundwater samples from temporary locations using Direct Push Technology (Hydropunch ${ }^{\mathrm{TM}}$ or piezocone methods). At soil sample locations 15, 16, and 17 (Figure 3-1), groundwater samples were collected via Hydropunch ${ }^{\mathrm{TM}}$ at the current water table and just above the confining unit. In addition, four existing wells and one newly installed well were sampled (Figure 3-1).

\subsubsection{Surface Water/Sediment}

Eleven surface water were originally proposed at locations $19,20,21,22,23,24,25,26$, and 27 and seven sediment samples were originally proposed at locations 21, 22, 23, 24, 25, 26 and 27 as shown on Figure 3-1. However, stations 22, 23, 24, 25, and 27 were dry. For this reason, surface water samples and the corresponding QC samples could not be collected. Locations 19 and 20 required collection of surface water both upstream and downstream of the sample point. The remaining sample locations 21 and 26 consisted of one sediment and one surface water sample collected downstream of the sample point. All sediment samples were collected as proposed. These samples were intended to address the potential for surface water/sediment contamination in the wetland area to the west of the BRPs.

The revised Addendum Surface Water/Sediment Sampling Plan dated April 22, 1994 (included in Appendix C) called for an additional ten surface water/sediment sample locations. These locations are 28, 29, 30, 31, 32, 33, 34, 35, 36, and 37 and are depicted on Figure 3-1. The 
surface water sample was collected prior to the sediment sample so that no interference from the sediment would be detected in the surface water sample.

\subsubsection{Additional Sampling}

Two water tanks located upstream of the BRPs were also sampled (Figure 3-1). The tank at location 38 is approximately 134 meters (440 feet) upstream of the confluence of the tributary and the drainage way. The second tank at location 39 is approximately 268 meters ( 880 feet) upstream of the confluence on the drainage way. The results and discussion of validation for the water tank samples is presented along with the surface water data due to the proximity to the stream.

\subsection{Sample Identification}

Sample identification nomenclature includes the specific unit designation, the location number, and a number corresponding to the depth at which the sample was collected. For example, sample D43111504 was collected from outside the boundaries of D-Area Rubble Pit 431-1D at sample location 15 designated on Figure 3-1 at a depth of 3.1 to 3.7 meters bgs (10.0 to 12.0 feet). Information regarding depth of each sample is found on Table 4-1 of the Phase II RFI/RI Plan (WSRC, 1992). Similar nomenclature was used for groundwater, surface water, and sediment samples.

\subsection{Sampling Packing and Transportation}

Each sample container submitted to the laboratory was labeled with the sample identification, date and time of sample collection, and analyses required. The samples were then immediately placed on ice [approximately $4^{\circ} \mathrm{C}\left(39^{\circ} \mathrm{F}\right)$ ] in a cooler for transport to the laboratory. Chain-ofcustody forms were maintained and included in each sample cooler. A copy of each chain-of custody was retained by the field crew.

\subsection{Decontamination Procedures}

Equipment used for sampling was decontaminated prior to each use. Equipment used for the collection of water or soil samples for analytical analysis were steam cleaned or thoroughly scrubbed with a tap water/phosphate laboratory detergent solution. All equipment was 
subsequently rinsed with de-ionized water. Clean, disposable gloves were worn while handling sampling equipment during the final stages of decontamination.

Prior to arrival on site, all paint and excessive rust were removed from the downhole equipment (split-spoon samplers, augers, drill rods, drill bits, etc.). All downhole equipment and working areas of the drill rig were thoroughly steam cleaned before drilling at each location. In addition, all well screen and riser pipe were steam cleaned prior to installation. Decontaminated well materials were wrapped in plastic sheeting for storage and transportation to the well site. 


\subsection{ANALYTICAL METHODS}

The analytical program for groundwater samples contains both non-radionuclide and radionuclide parameters. Non-radionuclide parameters included volatile organic compounds (VOCs; EPA Method 8240); semivolatile organic compounds (SVOCs; EPA Method 8270); polynuclear aromatic hydrocarbons (PAHs; EPA Method 8100 or 8310); RCRA and other target metals [arsenic (EPA Method 7060), barium, cadmium, chromium, copper, manganese, nickel, silver (EPA Method 6010), lead (EPA Method 7421), mercury (EPA Method 7470), and selenium (EPA Method 7740)]; Toxicity Characteristic Leaching Potential (TCLP) for the above metals (excluding copper, manganese, and nickel); pesticides/polychlorinated biphenyls (PCBs) (EPA Method 8080); polychlorinated dibenzodioxine (PCDD); polychlorinated dibenzo-furans (PCDF; EPA Methods 8280 or 8290); 2,3,7,8-TCDD and PCB 1254; inorganic compounds including chloride, fluoride, nitrate, sulfate (EPA Method 300.0), iron and sodium (EPA Method 6010 or 200.7); baseline physical properties of soil [e.g., soil pH (SW 846 Method 9045); cation exchange capacity (CEC); and total organic carbon (TOC; U.S. Soil Conservation Service (SCS) or American Society of Testing and Materials (ASTM) procedures)].

Radionuclide parameters analyzed included radionuclide indicators (gross alpha, and non-volatile beta (EPA Method 900.0 for water and soil), total alpha-emitting radium (EPA Method 903.0), tritium (EPA Method 906.0).

All analyses were performed by Roy F. Weston, Inc. located in Lionville, Pennsylvania; WestonGulf Coast Laboratories, Inc. located in University Park, Ilinois; Roy F. Weston, Inc. located in Stockton, California; TMA/Eberline located in Oak Ridge, Tennessee; and General Engineering Laboratories, Inc. located in Charleston, South Carolina. 


\subsection{DATA VALIDATION}

All data released by this program are considered to be of Quality Assurance (QA) Level 3 (EPA, 1987, 1990). The groundwater verification and validation program, evaluated and approved by DOE, EPA, SCDHEC, and Environmental Protection Department (EPD), surpasses QA Level 3 requirements. Qualification policy is based on the Contract Laboratory Program Statement of Work for Organic Analysis (EPA, 1988a), the Functional Guidelines for Organics Analyses (EPA, 1988b), Functional Guidelines for Inorganics Analyses (EPA, 1991), Test Methods for Evaluating Solid Waste, SW-846 (EPA, 1986), and Westinghouse Hanford Company' Data Validation Procedures for Radiochemical Analyses. The groundwater QA/QC program is nationally recognized and has been used as a model for quality in the new ANSI N42.2 standard.

The purpose of this section is to provide a data validation summary of the analytical procedures performed by the contract laboratories. The $\mathrm{QC}$ procedures performed at the laboratory included but were not limited to method blanks, laboratory control samples, matrix spikes, matrix spike duplicates, and surrogate recoveries. Data validation were conducted by Exploration Resources located in Athens, Georgia for soil, surface water, and sediment matrices (Appendix A), by EPD/EMS Groundwater Program at SRS for all groundwater analyses (Appendix B), and by RUST E \& I for surface water and sediment from the May 1994 Addendum sampling event (Appendix C).

Three types of qualifier codes are used in the validation process. For example, the result for benzo(a)pyrene in soil sample $\mathrm{D} 43111501$ has been flagged $\mathrm{J} / \mathrm{LH} / \mathrm{L}$. The first flag, "J", is the result flag. By definition (Table 5.1), this flag indicates the analytical result is an estimated quantity. The second set of flags, "LH", are both analysis qualifiers. In thišcase, "L" refers to failed calibration criteria and " $\mathrm{H}$ " refers to failed internal standards performance criteria. Both " $\mathrm{L}$ " and " $\mathrm{H}$ " have a different meaning when found in the third set of flags, the bias codes. In the third set of flags, " $L$ " indicates the associated result may underestimate the true value. Refer to Table 5.1 for additional data qualifier codes.

\subsection{Data Quality Categories}

Different data uses require different levels of data quality. Data quality is defined as the degree of certainty of a data set with respect to PARCC. EPA has defined five levels of qualitative and 
quantitative statements specifying the required quality of data necessary to support the investigative activities and evaluation of remedial activities (EPA, 1987). Data quality Levels 1 , 2 , and 3 were used for the investigation and evaluation of the D-Area BRPs. These three levels of data quality are as follows:

Level 1 Screening: Screening provides the lowest data quality by the most rapid results through the use of OVA and other field screening equipment. It is used for health and safety monitoring in the field, preliminary site characterization, and engineering screening (bench-scale tests). Results are often not compound specific and not quantitative, but rapid or instantaneous results are available. Screening sample results are not applicable to this data summary report.

Level 2 Field Analysis: This level is designed to provide rapidly-generated data for ongoing field activities or to provide the basis for seeking laboratory analytical support. Level 2 analysis is also used for field screening, baseline data development, extent of contamination determination, and onsite remedial activities. Field analysis involves the use of portable or transportable instruments (e.g., portable Gas Chromatograph (GC)). Samples analyzed by field analyses are not applicable to this data summary report.

Level 3 Confirmational: Level 3 analytical support provides data for site characterizations, environmental monitoring, engineering studies (e. g., design, modeling), and risk assessment. This level utilizes EPA-approved procedures, such as those in SW-846 Test Methods for Evaluating Solid Waste. Some Level 3 procedures may be equivalent to the EPA Contract Laboratory Program Routine Analytical Service with the exception of the extensive Contract Laboratory Program (CLP) required documentation.

Level 3 analytical support included the analysis of groundwater, sediment, and soil samples by EPA-approved procedures. 'Data validation for the D-Area BRP analytical data set was based on Quality Assurance/Quality Control Guidance for Removal Activities and Data Quality Objectives for Remedial Response Activities for achieving Quality Assurance Objective 3 (Quality Level 3). This Data Quality Summary Report addresses the Level 3 analytical data.

Ten technical issues were addressed in this CERCLA data validation program:

- Sample documentation

- Chain of custody

- Sample holding times 
- Analytical instrument calibration

- Analyte identification

- Analyte quantitation

- Analytical error

- Blanks

- Performance evaluation samples

- Detection limit

Qualification criteria and policies are based on the Function Guidelines (EPA, 1988b, 1991) and are described in the Quality Control Summary Reports (QCSRs) (Appendices A and C). Samples not conforming to these criteria have been flagged according to the qualifier codes found on Table 5.1 .

\subsection{Evaluation of Data Quality Indicators}

The assessment of the data quality indicators determines the data usability. The assessment of data quality indicators for either sampling or analysis involves the evaluation of five indicators: precision, accuracy, representativeness, completeness, and comparability. The indicators are commonly referred to as the PARCC parameters. The major activity in determining the usability of data based on sampling is assessing the effectiveness of the sampling operations performed. Determining the usability of analytical results begins with the review of QC samples and qualifiers.

The review is used to determine an overall assessment of analytical performance as determined by the laboratory and method performance. A summary of the precision and accuracy validation by each of the validating agencies can be found in Table 5.2. A description of the validation processes may be found in the appropriate documents located in Appendices A, B, and C.

\subsubsection{Precision}

Precision measures the reproducibility of measurements under a given set of conditions. Specifically, it is quantitative measure of the variability of a group of measurements compared to the average value. The overall precision of a measurement data is a mixture of sampling and analytical factors. Analytical precision may be determined by collecting and analyzing replicate 
field samples. The analytical results from laboratory replicates provide data on analytical precision.

Precision is a measure of the repeatability of a single measurement and is evaluated from the results of duplicate samples and splits. Low precision can be caused by poor instrument performance, inconsistent application of method protocols, or by a difficult, heterogeneous -sample matrix. The analytical laboratory performed the analysis of duplicate laboratory control samples to evaluate the precision of the analytical method.

The precision of each analytical method is evaluated by reviewing the results of the analysis of laboratory blanks, laboratory control samples, matrix spikes, and matrix spikes duplicates. The magnitude of the standard deviation in relation to the magnitude of the mean reflects the precision of the results. Five percent of the total wells to be sampled each quarter were selected as blind duplicate samples. A blind blank is sent to the laboratory with each sample and blind sample. Site-specific "critical" samples are used as QA duplicates or blinds. According to the -EMS contract, every 20 samples must be accompanied by a laboratory blank, a laboratory control sample, and a sample replicate.

Duplicate samples were collected in the field to provide precision information for the entire measurement system including samples acquisition, homogeneity, handling, shipping, storage, preparation, and analysis. EPA Data Quality Objectives for Remedial Response Activities (1987) dictates that duplicate samples be collected at a rate of approximately 1 per 20 samples, or five percent. The comparison is performed by computing a relative percent difference (RPD). The RPD is a measure of precision and is determined by the following formula:

$$
\mathrm{RPD}=[(\mathrm{A}-\mathrm{B}) /((\mathrm{A}+\mathrm{B}) / 2)] * 100
$$

Where: $\quad \mathrm{A}=$ Result of Primary Sample

- $\mathrm{B}=$ Result of Duplicate Sample

EMS determined the RPD for each duplicate and determined that the RPD of several groundwater QC samples exceeded historical results by more than 10 times. The laboratory, Weston Analytics, was asked to review all results in question. As noted in the correspondence in Appendix B, Weston responded by verifying the results against the original chromatographs. 
Field duplicate RPDs for soil and sediment samples were not calculated by Exploration Resources. The primary samples (i.e., not a QC sample) will be used in evaluating risk.

A split sample is a replicate of a primary site sample that is submitted to a QA laboratory and subjected to the same environmental conditions and steps in the measurement process. This sample serves as an oversight function in assessing the analytical portion of the measurement system. Methods of sample collection, handling, preservation, and transportation were the same as those followed for the primary site samples. The RPD control limit established by the EPA is \pm 20 percent $( \pm 35$ percent for soil) for sample values $>5$ times the contract required detection limit (CRDL) and \pm CRDL ( \pm 2 times $C R D L$ for soil) for sample values $<5$ times CRDL.

A comparison of inter- and intralaboratory QA samples, such as replicates, duplicates, and blind blanks has been made. EMS determined the RPD for each split sample (analyzed by General Engineering) and determined that the RPD of several groundwater QC samples exceeded 10 percent. The primary laboratory, Weston Analytics, was asked to verify all results in question. As noted in the correspondence in Appendix B, Weston responded by verifying the results. However, EMS did not flag any of the data as questionable, despite the calculated RPD. Soil split samples were not sent to an outside QC laboratory, but to the primary laboratory, Weston Analytics, and are thus considered duplicate samples. RPDs for split soil and sediment samples were not calculated by Exploration Resources.

\subsubsection{Accuracy}

Accuracy measures the closeness of a reported concentration to the true value, and is usually expressed as bias. Accuracy is difficult to measure for the entire data collection activity. Sources of error are the sampling process, cross contamination, preservation, sample handling, sample matrix, sample preparation, and analysis techniques. Analytical accuracy is assessed through use of standard and blind QC samples and spike samples. Accuracy determinations by standard samples include single control and duplicate control samples, commonly referred to as laboratory control samples. These are samples made up of reagent grade water that is spiked with standard amounts of target compounds. Percent recovery and percent difference parameters are determined from these samples. Analytical accuracy determinations by blind samples include the evaluation of matrix interferences in the environmental samples. These samples also provide percent recovery and percent difference parameters through the use of surrogate and matrix spikes in the environmental samples. 
Accuracy may be affected by contamination introduced in the field, during shipping, or at the laboratory. Field and trip blanks are used to monitor accuracy during sample collection and shipment. Field blanks are filled with deionized water the morning samples are collected, and left open in the field during sampling. The field blanks are shipped with the other samples. Positive results from field blanks can result from analytical bias, contaminated sample bottles, contaminated deionized water, vapors in the air during sampling, or contamination during shipping. Rinsate blanks are generated prior to sample collection by pouring deionized water over the sampling equipment, and provide data for potential contamination from the sampling equipment. Trip blanks are used to detect contamination caused during shipping, and are only used for VOCs. Data qualifiers (Table 5-1) are utilized to annotate bias determined from field, trip, and rinsate blanks.

EMS did not utilized trip, field, or rinsate blank data in validating groundwater data. Likewise, Explorations did not consider field or rinsate blanks in the validation of soil and sediment data. However, the Addendum surface water and sediment data were validated in accordance with field accuracy QC data.

Laboratory control samples are water samples that are spiked with the target analyte, digested, and analyzed along with the regular samples for inorganic analyses. They monitor the overall performance of all steps in the analysis, including sample preparation. Blank spikes are sodium powder spiked with selected target analytes, extracted, and analyzed along with the regular samples for organic analyses. Laboratory blanks are portions of the water used for dilutions that are analyzed along with the samples. Laboratory blanks are used to determine the existence and magnitude of contamination problems.

The matrix of a sample can interfere with the accuracy of the analysis. All samples for organic analyses are spiked prior to sample preparation to examine the laboratory's performance. Selected samples are spiked to provide information about the effect of each sample matrix on the digestion and measurement methodology. Matrix spikes are rejected if the concentration of the analyte in the sample is more than four times the concentration of the spike. 


\subsubsection{Representativeness}

Reprè̀sentativeness expresses the degree to which sample data accurately and precisely represent a characteristic of a population, parameter variations at a sampling point, or an environmental condition. Representativeness is a qualitative parameter which is most concerned with the proper design of the sampling program. The representativeness criterion is best satisfied by making certain that sampling locations are selected properly and a sufficient number of samples are collected. Samples must be collected to reflect the site characteristics and sample analyses must represent the properties of the field sample. The homogeneity of the sample, use of appropriate handling, storage, preservation procedures, and the detection of any laboratory blank artifacts are critical to sample representativeness.

The QCSR in Appendix A details the handling of all soil and sediment samples during the 1993 unit assessment. Samples collected during the 1994 Addendum surface water/sediment sampling event are said to be representative (Appendix C). The representativeness of groundwater sample could not be determined from the information supplied by EPD/EMS. Field QC measures include the evaluation of trip, field, and equipment rinsate blanks, laboratory QC measures include the evaluation of method blanks.

\subsubsection{Completeness}

Completeness is defined as the percentage of measurements made which are judged to be valid measurements. The completeness goal is essentially the same for all data uses: that a sufficient amount of valid data be generated. It is important that critical samples are collected and valid data achieved for them.

The measure of completeness is useful for data collection and analysis management. Any decrease in the number of samples from that specified in the work plan may affect the final results. The completeness of the field screening and confirmatory samples for this sampling event was determined to be greater than 99 percent. The completeness of critical sample is 100 percent.

The completeness for analytical data is defined as the percentage of chemical-specific data results that are determined acceptable after data review. All analytical data have been compared against the sampling schedule to verify that all analyses in the schedule have been reported. 
With the exception of surface water samples D431 2201, 2301, 2401, 2501, and 2701 and soil sample D431 1103, all samples were collected and analyzed as proposed. The 1994 addendum surface water/sediment sampling event was performed to compensate for the dry surface water locations in 1993. Insufficient volume precluded collection of sample for Target Analyte List/Target Compound List (TALTCL) VOCs; however, TCLP analyses was performed.

\subsubsection{Comparability}

Comparability is a very important qualitative data indicator for analytical assessment and is a critical parameter when considering the combination of data sets from different analyses for the same chemicals of potential concern. The analytical methods used had common analytical parameters, the same units of measure, and similar detection limits.

Comparability is a qualitative parameter expressing the confidence with which one data set can be compared with another. Sample data should be comparable with other measurement data for - similar samples and sample conditions. This goal is achieved through using standard techniques to collect and analyze representative samples and reporting analytical results in appropriate units. Comparability is limited to the other PARCC parameters because only when precision and accuracy are known can data sets be compared with confidence.

Comparability issues have little impact on performance measures associated with sampling provided that the sample design is unbiased and the sample design or analytical methods have not changed over time. Sample design within the pits was random within each quartered section of the pits. Sample locations outside the pits were selected according to gradient (i.e., downgradient, upgradient, or sidegradient. Comparability was achieved by conforming to the sample design in the Scope of Work and documenting all sample locations. All primary samples proposed in the Phase II RFI/RI were conducted as specified.

In general, results from the 1993 Unit Assessment were comparable with soil data obtained during the 1992 Unit Screening (WSRC, 1992). Analytical data for SVOCs indicated slightly higher results in 1992 than indicate in the more recent event. 


\subsection{DATA SUMMARY}

Summaries of chemical data are provided in Tables 6.1 through 6.23. Summary tables report detected values down to 10 percent of the reporting limits. Estimated values less that the reporting limit are flagged " $\mathrm{J}$ ". The " $\mathrm{J}$ " flag is also used in conjunction with the "V" flag (indicating the analyte was also detected in the method blank) when the concentration in the sample is less than five times the concentration detected in the associated laboratory blank, or less than ten times for common laboratory artifacts acetone, methylene chloride, 2-butanone, and phthalate esters.

\subsection{Soil Data Summary}

.Five soil samples were received at the laboratory at a temperature exceeding four degrees required for proper preservation. Subsequently, all results associated with these samples have been flagged "Y". Analytical results indicating the presence of an analyte below the method rquantitation limit have been flagged " $\mathrm{J}$ " to indicate the concentration is estimated.

\section{$\underline{\text { Volatile Organic Compounds }}$}

Volatile organic compounds (VOCs) in soil were analyzed by EPA Method 8240 . Samples D431 1701 and D431 1703 were held beyond the normal holding time; all results have been flagged "Q" to indicate exceeded holding times and " $\mathrm{J}$ " to indicate the results are estimated.

Common laboratory artifacts acetone and methylene chloride were detected in the method blanks associated with several samples. In addition, benzene and chloroform were also detected in the blanks associated with some of the soil samples. All results for these compounds have been flagged "V" to indicate possible laboratory contamination.

Calibration criteria (initial or continuing) were not met for vinyl chloride in samples D43110807 and D431 1401 and the results have been bias flagged "L". The "L" flag is also used in conjunction with analysis qualifier "O" on all results associated with samples D431 1305 and D431 1702 due to surrogate spike recoveries out of specification. Internal standards performance criteria were not met for sample D431 1401. Subsequently, all results have been flagged " $H$ " to indicate failed performance criteria and " $\mathrm{J}$ " to indicate the results are considered estimated. The "J/H/" flags are also used for certain compounds associated with sample D43111501. 
Analytical data are presented in Table 6.1.

\section{Semi-Volatile Organic Compounds}

Semi-volatile organic compounds (SVOCs) in soil were analyzed by EPA Method 8270 . Samples D431 1204 and D431 1204 were held beyond the normal holding time; all results have been flagged "Q" to indicate exceeded holding times and "J" to indicate the results are estimated.

Common laboratory artifacts di-n-butyl phthalate and bis(2-ethylhexyl) phthalate were detected in the method blanks associated with several samples. In addition, 2,4-dinitrophenol was also detected in the blanks associated with soil sample D431 1204. All results for these compounds have been flagged "V" to indicate possible laboratory contamination.

The laboratory control sample criteria were not met for certain SVOC analytes. For these reason, results for $\mathrm{N}$-nitrosodipropylamine, pentachlorophenol, phenol, and 1,2,4-trichlorobenzene in -some samples have been flagged " $\mathrm{C}$ " and "J" to indicate the results are estimated.

Calibration criteria (initial or continuing) were not met for di-n-octyl phthalate and pentachlorophenol in samples D43110806 and D43110906; therefore, the results have been bias -flagged "L". The "L" flag is also used in conjunction with arialysis qualifier "O" on all results - associated with samples D431 1205, D431 1304, D431 1305, and D431 1306 due to surrogate - spike recoveries out of specification. When " $L$ " is used as an analysis qualifier, as for samples D43111501, D43111502, D43111504, D43111504, D431 1704, and D431 1705, calibration criteria were not met for the analysis. Subsequently, all results flagged with analysis qualifier " $L$ " have also been flagged " $J$ " to indicate the results are estimated.

-Matrix spike recoveries were not within control limits on results flagged "I". Internal standards -performance criteria were not met for several samples. Subsequently, all results have been flagged with the analysis qualifier " $\mathrm{H}$ " to indicate failed performance criteria and " $\mathrm{J}$ " to indicate the results are considered estimated. In some cases, the bias code " $\mathrm{H}$ " is used to qualify the data, indicating an analytical factor has caused bias and the associated result may overestimate the true value.

Analytical data are presented in Table 6.2. 


\section{Pesticides and PCBs}

Soil samples were analyzed for pesticides and PCBs using EPA Method 8080. Surrogate spike recoveries were out of specification on samples D43110706, D43110804, D43110902, D431 1107, D431 1206, D431 1207, D431 1301, D431 1401, D431 1402, D431 1406, D43111501, and D431 1803. Subsequently, all pesticide and PCB data associated with these samples has been flagged with analysis qualifier "O". In addition, bias qualifier " $L$ " has been used on all results to indicate the associated result may underestimate the true value.

The results for alpha-chlordane, gamma-chlordane, p,p-DDE, and p,p-DDT in sample D43111501 and p,p-DDE, and p,p-DDT in sample D43111503 have been flagged " $A$ " to indicate the values reported are the mean of two or more determinations. The results for endrin in sample D43110806 and p,p-DDT in D43110906 have been flagged " $G$ " to indicate the values reported are the maximum of two or more determinations.

Analytical data are presented in Table 6.3.

\section{$\underline{\text { PCDD/PCDF Compounds }}$}

Polychlorinated dibenzo-dioxins (PCDD) and polychlorinated dibenzo-furans (PCDF) in soil have been analyzed using EPA Method 8280. Internal standards performance criteria were not met for several analytes in samples D43111504 and D431 1705. The results associated with failed performance criteria have been flagged " $\mathrm{H}$ ". In addition, these results have also been flagged " $\mathrm{J}$ " to indicate the results are estimated.

Matrix spike recoveries were not within control limits for octachlorodibenzo-p-dioxin in sample D43111504 and hexachlorodibenzo-p-furan in sample D431 1705. These results have been flagged "I". The bias flag "L" was also used on the D43111504 result to indicate the result may underestimate the true value. " $L$ " was not used on the D431 1705 sample. Result qualifier " $L$ " was used for octachlorodibenzo-p-dioxin furan in sample D431 1207 to indicate the result was off-scale high. The actual value for this analyte is not known, but is greater than the value shown. 
The peak height, instead of peak area, was used to quantitate heptachlorodibenzo-p-dioxin and octachlorodibenzo-p-dioxin furan in several samples. For this reason, the associated results have been flagged " $T$ ".

Analytical data are presented in Table 6.4.

\section{Inorganics and Organic Indicators}

Inorganics, including RCRA metals, were analyzed as proposed. Matrix spike recoveries were not within control limits for several soil samples. The results associated with failed matrix spike recoveries are denoted with the "I" analysis qualifier. In addition, some of these samples also have the bias flag " $\mathrm{H}$ ", which indicates the result may overestimate the true value, or the bias flag " $L$ ", which indicates the result may underestimate the true value. The " $\mathrm{X}$ " flag is used to indicate when the duplicate analysis relative percent difference (RPD) was not within control limits.

The analysis qualifier " $\mathrm{L}$ " is used to indicate calibration criteria, initial or continuing, are not met. This flag is used with the " $\mathrm{J}$ " flag to indicate the results are estimated.

Analytical data are presented in Table 6.5.

\section{Radionuclide Parameters}

Radionuclide parameters were analyzed as proposed. Non-volatile beta and total alpha-emitting radium were detected in the method blanks associated with many of the soil samples collected. For this reason, the results have been flagged " $V$ ". However, the five times rule did not come into effect for any of the results.

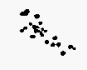

Radium results in all samples collected from pit D4311 location 16 have been flagged "C" to indicate the control sample criteria were not met.

Non-volatile beta in sample D431 1401 and tritium in sample D43111503 have been flagged "X" to indicate the duplicate analysis RPD was not within control limits.

Analytical data are presented in Table 6.6. 


\section{TCLP Data}

Soil sámples D43110705, D43110706, D43110905, and D431 1205 were analyzed for TCLP parameters as proposed. Sample D43110705 arrived at the laboratory at a temperature exceeding four degrees required for proper preservation. All results associated with this sample have been flagged "Y".

Surrogate spike recoveries for SVOCs in samples D43110705 and D43110905 and for pesticides and PCBs in sample D431 1205 were out of specification. For this reason, all associated data have been flagged "O". In addition, the results have been flagged " $\mathrm{J}$ " to indicate the results are estimated and " $\mathrm{L}$ " to indicate the result may underestimate the true value.

Matrix spike recovery for TCLP mercury in sample D431 1205 was not within control limits. The result has been flagged "I". Subsequently, the result has also been flagged "J" and bias "L". The continuing calibration criteria were not met for TCLP cadmium and chromium in sample D43110905. These results have been flagged with the analysis qualifier " $\mathrm{L}$ ", plus " $\mathrm{J}$ " to indicate the results are estimated.

Analytical data are presented in Table 6.7.

\subsubsection{Rejected Data}

No analytical data were rejected by Exploration Resources. Due to re-extraction and re-analysis by the laboratory, multiple analyses were reported. Since no data were rejected during validation, two or more sets of data were presented. The data set representing the most accurate results based on the data qualifiers are used in the development of the risk asséssment.

Data omitted from the data set for development of risk included duplicate analysis for common laboratory artifacts. In the event that two equally defensible results were produced, the higher of the two results was used and the lower results were rejected. Surrogate spike recoveries were out of specification on the original analysis of samples D431 1701 and D431 1703. Subsequently, these results were flagged "O" to indicate out of control surrogate recover, " $\mathrm{H}$ " to indicate the results were biased high, and " $\mathrm{J"}$ to indicate the results were estimated. Both of these samples were re-analyzed out of holding time. However, acceptable surrogate recoveries were achieved. For this reason, the re-analysis results have been used and the original results have been rejected. 
All data with a result qualifier " $L$ " indicate that results were off-scale high were rejected over data not off-scale. Surrogate spike recoveries were out of specification on sample D431 1305 in both the original and re-analysis. The higher of the two results was used for the purpose of developing risk. All remaining results for this sample were rejected.

\subsubsection{Additional Data Qualifiers}

No additional data qualifiers are necessary.

\section{-6.2 Groundwater Data Summary}

All groundwater samples were collected and analyzed in accordance with the work plan. An explanation of the data qualifiers assigned to groundwater analytical results during the validation process is given below.

\section{Volatile Organic Compounds}

VOCs in groundwater monitoring wells were analyzed by EPA Method 8240. All samples were collected and analyzed as proposed. Only one result required data qualification. The result for 1,1-dichloroethane in sample DBP 2 was flagged " $\mathrm{J}$ " to indicate that this compound was detected at a concentration below the quantitation limit for that sample. Results for monitoring well samples may be found in Table 6.8 .

Groundwater samples collected by Hydropunch ${ }^{\mathrm{TM}}$ were also analyzed for VOCs by EPA Method 8240. Sample D43111506 was held beyond the normal holding time. For these reason, all results associated with this sample have been flagged "Y". Common laboratory artifact methylene chloride was detected in three of the four Hydropunch ${ }^{\mathrm{TM}}$ samples; results for methylene chloride have been flagged " $\mathrm{V}$ ". The results have also been flagged " $\mathrm{J}$ " to indicate the results are estimated due to the presence of this analyte in the associated method blank.

Laboratory control sample exceeded control limits for benzene in sample D43111505. For this reason, this result has been flagged " $\mathrm{C}$ ", and subsequently flagged " $\mathrm{J}$ " to indicate the result is estimated. 
Analytical data for Hydropunch ${ }^{\mathrm{TM}}$ samples can be found in Table 6.9.

\section{$\underline{\text { Semi-Volatile Organic Compounds }}$}

SVOC in groundwater were analyzed by EPA Method 8270. Result qualifier "J1" was used on all results for samples DBP 3 collected 3/18/93 and DBP 4 collected 6/5/93. This flag indicates an analytical factor causing bias. The associated results may underestimate the true values. None of the analytes, however, were reported at concentrations above the method quantitation limits and the data are not affected.

Analytical data are presented in Table 6.10.

\section{Pesticides and PCBs}

Pesticides and PCBs in groundwater were analyzed by EPA Method 8080 . In addition, 2,4,5-T, Silvex, and 2,4-Dichlorophenoxyacetic acid were analyzed by EPA Method 8150. All samples collected in March 1993 were held beyond normal holding time and have been qualified with the result qualifier "JQ".

Analytical data are presented in Table 6.11.

\section{$\underline{\text { PCDD/PCDF Compounds }}$}

PCDD and PCDF compounds were analyzed by EPA Method 8280. No data qualifiers were necessary for groundwater samples collected during this effort.

Analytical data are presented in Table 6.12.

$\underline{\text { Inorganics and Organic Indicators }}$

RCRA metals and target inorganic compounds were analyzed as proposed. Several data qualifiers were necessary throughout the metals data.

Samples DBP 1, DBP 2, DBP 4, and DBP 5 collected March 19, 1993 for chloride and sulfate were held beyond normal holding times. Subsequently, the results associated with these 
parameters have been flagged " $Q$ " to indicate analysis out of holding time and " $\mathrm{J}$ " to indicate the results obtained from these analyses are estimated. The "JQ" flags have also been used to qualify the total organic halogens result in sample DBP 5 collected December 11, 1993 and the total dissolved solids (TDS) result in sample DBP 5 collected December 26, 1993. The March 1993 results for TDS in samples DBP 1, DBP 2, DBP 3, and DBP 4 have been flagged " $V$ " due to the presence of this analyte in the associated method blank. However, the concentrations found in the samples were greater that five times the concentration detected in the method blank. For this reason, the results were not flagged "J" and the TDS concentrations are considered to be site related.

Analytical data are presented in Table 6.13.

\section{Radionuclide Parameters}

Radionuclide parameters were analyzed as proposed. No data qualifiers were necessary for groundwater samples collected during this effort.

Analytical data are presented in Table 6.14.

\subsection{Rejected Data}

None of the groundwater analytical data was rejected by EMS, who performed the data validation. However, two sets of data were available for acetone in sample D43111608. Acetone was originally analyzed at a detection limit of $10 \mu \mathrm{g} / \mathrm{L}$. The result of this analysis was off-scale high. For this reason, the result was flagged " $L$ " and the analysis was repeated. The second analysis was within the range of the instrument and therefore the result to be used in development of risk. The original result has been utlized for the development of risk.

\subsubsection{Additional Data Qualifiers}

No additional data requirements were necessary. 


\subsection{Surface Water Data Summary}

Aill surface water samples were collected and analyzed in accordance with the work plan. An explanation of the data qualifiers assigned to surface water analytical results during the validation process is given below.

\section{Volatile Organic Compounds}

VOC analyses were performed using EPA Methods 8240 or 8260 . Common laboratory artifacts acetone and methylene chloride were detected in the method blanks associated with most of the samples. For this reason, the results have been flagged "V". However, since this artifact was not detected in any of the corresponding samples, the data are not affected.

Toluene was detected in two samples, D431-35-01 and D431-39-01. The concentration at which toluene was detected is below the contract required detection limit (CRDL) and the results have been flagged " $\mathrm{B}$ " to indicate this occurrence. In addition to the " $\mathrm{B}$ " qualifier, the results have also been flagged " $\mathrm{J}$ " to indicate that the results are estimated values.

Analytical data for EPA Method 8240 are presented in Table 6.15. Analytical data for EPA Method 8260 are presented in Táble 6.16 .

\section{$\underline{\text { Semi-Volatile Organic Compounds }}$}

SVOC analyses were performed by EPA Method 8270 . Common laboratory artifact di-n-butyl phthalate was detected in the method blank associated with samples D431-37-01 and D431-3801 . Although both results were below the detection limit, the "V" flag has been used to indicate the occurrence of this artifact in the associated method blank.

P-Cresol in sample D431-28-01 and anthracene and butylbenzyl phthalate in sample D431-37-01 were detected at concentrations below the CRDL for these compounds. For this reason, the results for these compounds have been flagged "B". Subsequently, the results have also been flagged " $\mathrm{J}$ " to indicate that the results are estimated quantities.

Analytical data are presented in Table 6.17. 
Inorganics and Organic Indicators

RCRA metals and target analytes iron, manganese, nickel, and sodium were analyzed as proposed. Only one result for sample D431-37-01 required qualification. The iron result has been flagged "I" due to spike recovery outside of control limits. In addition, the result has been flagged " $\mathrm{P}$ " due to post-digestion spike recovery outside of control limits, but sample absorbance was greater than 50 percent of post-digestion spike absorbance. Due to the necessity of the "I" and " $\mathrm{P}$ " qualifiers, the result for iron in this sample has subsequently been flagged " $\mathrm{J}$ " to indicate the result is an estimated quantity.

Several samples for organic indicators were held beyond the normal holding time. For this reason, the results affected have been flagged "J/Q/". Calibration criteria for cadmium and copper were not met for the samples collected in June 1993; samples have been flagged "J/L/" accordingly.

Analytical data are presented in Table 6.18.

\section{Radionuclide Parameters}

Radionuclide parameters were analyzed as proposed. The results for gross alpha in sample D431-37-01, for nonvolatile beta in sample D431-33-01, and for tritium in samples D431-32-01 and D431-36-01 have been flagged "J" to indicate that the result is an estimated quantity below the quantitation limits. Non-volatile beta was detected in the method blank associated with several samples. All corresponding results for non-volatile beta have been flagged "V".

Analytical data are presented in Table 6.18.

\subsubsection{Rejected Data}

Rejected data would have been denoted with the " $R$ " flag. Although some problems were associated with the surface water analytical data, none were serious enough to require rejection of the data. 


\subsubsection{Additional Data Qualifiers}

No additional data qualifiers are necessary.

\subsection{Sediment Data Summary}

All sediment samples were collected and analyzed in accordance with the work plan. An explanation of the data qualifiers assigned to sediment analytical results during the validation process is given below.

\section{Volatile Organic Compounds}

VOCs in sediment were analyzed by EPA Method 8240. Samples D431 2302, D431 2402, D431 2502, and D431 2702 were held beyond normal holding time. All results associated with these samples have been flagged "Y". Common laboratory artifacts acetone and methylene chloride were detected in the method blanks associated with all of the sediment samples collected during this effort. The associated results have been flagged "V". Only acetone was detected in one sample, D431 2402. The concentration detected in the blank could not be determined from the data; however, the ten times rule for common laboratory contaminants has not been applied.

Analytical data are presented in Table 6.19.

\section{$\underline{\text { Semi-Volatile Organic Compounds }}$}

SVOCs in sediment were analyzed by EPA Method 8270. Samples D431 2302, D431 2402, D431 2502, and D431 2702 were held beyond normal holding time. All results associated with these samples have been flagged "Y". Common laboratory artifacts bis(2-ethylhexyl)phthalate and di-n-butyl phthalate were detected in the method blank associated with several samples; corresponding results have been flagged " $\mathrm{V}$ ".

The laboratory control sample was outside control limits; therefore, the results for $n$ nitrosodipropylamine in several sediment samples have been flagged " $C$ ", and subsequently flagged " $\mathrm{J}$ " to indicate the results are estimated.

Analytical data are presented in Table 6.20. 


\section{Inorganic Compounds}

Inorganic compounds, including RCRA metals, were analyzed as proposed. Samples D431 $2302,2402,2502$, and 2702 were held beyond normal holding time. All results associated with these samples have been flagged "Y".

Analytical data are presented in Table 6.21.

$\underline{\text { Radionuclide Parameters }}$

'Radionuclide parameters were analyzed as proposed. Samples D431 2302, 2402, 2502, and 2702 were held beyond normal holding time. All results associated with these samples have been flagged "Y". Radium was detected in the method blank associated with all sediment samples; corresponding results have been flagged " $V$ ".

Analytical data are presented in Table 6.22.

\subsubsection{Rejected Data}

No analytical data for sediment samples have been rejected.

\subsubsection{Additional Data Qualifiers}

No additional qualifiers are necessary. 


\subsection{SUMMARY}

This Data Summary Report consolidates the chemical data that was analyzed and validated for the risk assessment. Data validation was performed by Exploration Resources, Inc., the Environmental Monitoring Section of SRS, and RUST E\&I. The results of the entire validation program demonstrate that the data quality objectives have been met and the data are appropriate for a risk assessment with the exceptions noted.

Data validation for the D-Area Burning/Rubble Pits analytical data set was based on Quality Assurance/Quality Control Guidance for Removal Activities (1990) and Data Quality Objectives for Remedial Response Activities (1987) for achieving Quality Assurance Objective 3 (Quality Level 3). The QA/QC Control Guidance specifies ten technical issues which were addressed in the data validation program: sample documentation, chain of custody, sample holding times, analytical instrument calibration, analyte identification, analyte quantitation, analyte error, blanks, performance evaluation samples, and detection limits. The data for the D-Area Burning/Rubble Pits meet Quality Assurance Objective 3 (Quality Level 3) with the exceptions noted in this report. 


\section{REFERENCES}

EPA (U.S. Environmental Protection Agency), 1986. Test Methods for Evaluating Solid Waste, Volume 1, Methods for Analytes and Properties. Third Edition EPA SW-846, Office of Solid Waste and Emergency Response, Washington, DC.

EPA (U.S. Environmental Protection Agency), 1987. Data Quality Objectives for Remedial Response Activities: Development Process. EPA 540/G-87/003. U.S. Environmental Protection Agency, Washington, DC.

EPA (U.S. Environmental Protection Agency), 1988a. Contract Laboratory Program Statement of Work for Organic Analysis. U.S. Environmental Protection Agency, Washington, DC.

EPA (U.S. Environmental Protection Agency), 1988b. Laboratory Data Validation Functional Guidelines for Evaluating Organics Analysis. U.S. Environmental Protection Agency, Washington, DC.

EPA (U.S. Environmental Protection Agency), 1989. RCRA Facility Investigation (RFI) Guidance, Volume 1, Interim Final. EPA 530/SW-89-001, U.S. Environmental Protection Agency, Washington, DC.

EPA (U.S. Environmental Protection Agency), 1990. Quality Assurance/Quality Control Guidance for Removal Activities. U.S. Environmental Protection Agency, Washington, DC.

EPA (U.S. Environmental Protection Agency), 1991. Laboratory Data Validation Functional Guidelines for Evaluating Inorganics Analysis. U.S. Environmental Protection Agency, Washington, DC.

EPA (U.S. Environmental Protection Agency), 1993. Data Quality Objectives Process for Superfund. EPA 540-R-93-071, U.S. Environmental Protection Agency, Washington, DC.

EPD/EMS, 1994. Quality Control Summary Report for the D-Area Burning/Rubble Pits RFI/RI Unit Assessment. ESH-EMS-940055, (Draft, April 1994), Westinghouse Savannah River Company, Aiken, SC.

RUST Environment \& Infrastructure, 1994. Quality Control Summary Report for Surface Water and Sediments Collected during 1994.

WSRC (Westinghouse Savannah River Company), 1992. Phase II RFI/RI Plan: D-Area Burning/Rubble Pits. WSRC-RP-90-485, Revision 2, Westinghouse Savannah River Company, Aiken, SC.

WSRC (Westinghouse Savannah River Company), 1989. RCRA Facility Investigation/Remedial Investigation Program Plan. WSRC-RP-89-994, Revision 0, Westinghouse Savannah River Company, Savannah River Site, Aiken, SC. 


\section{FIGURES}




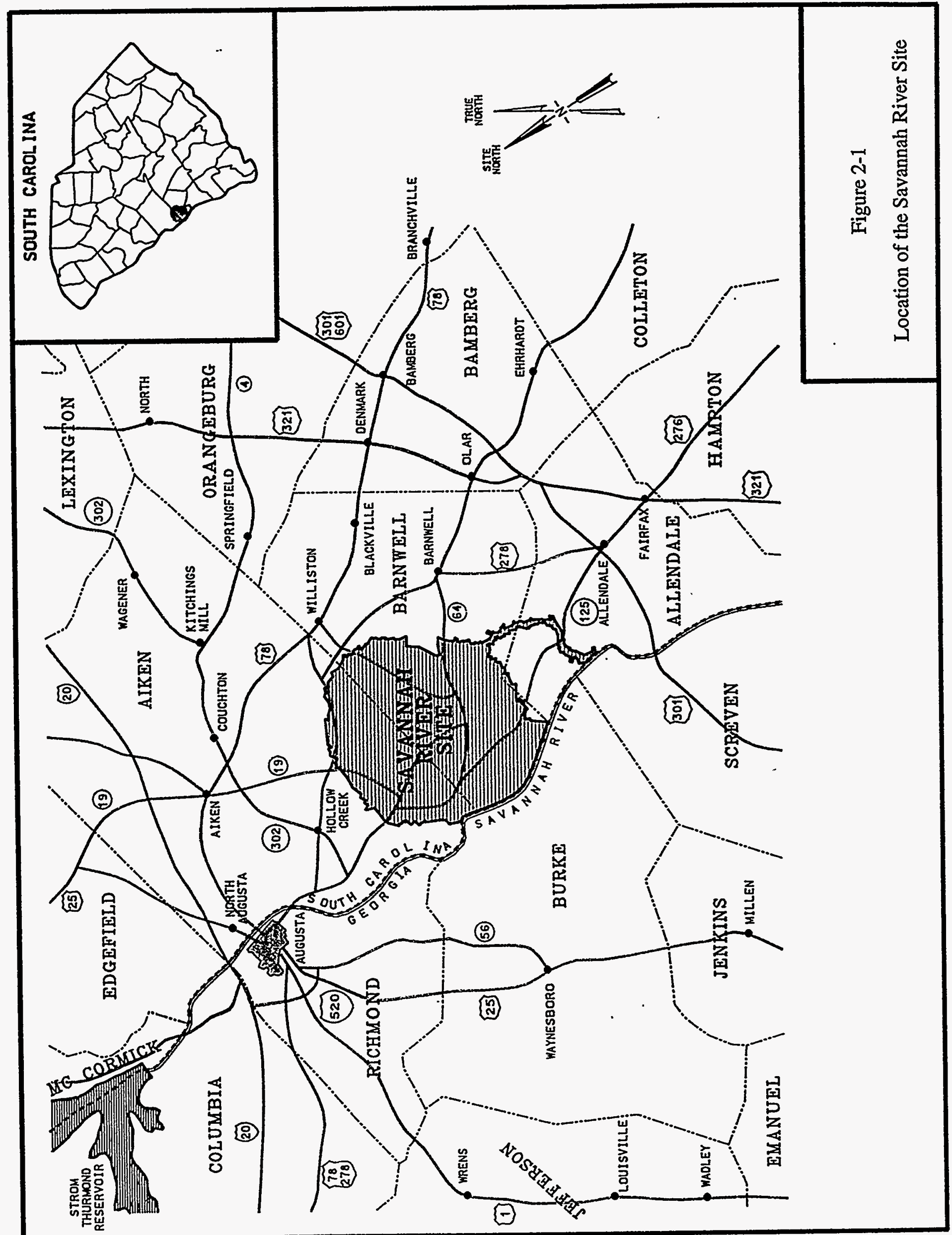




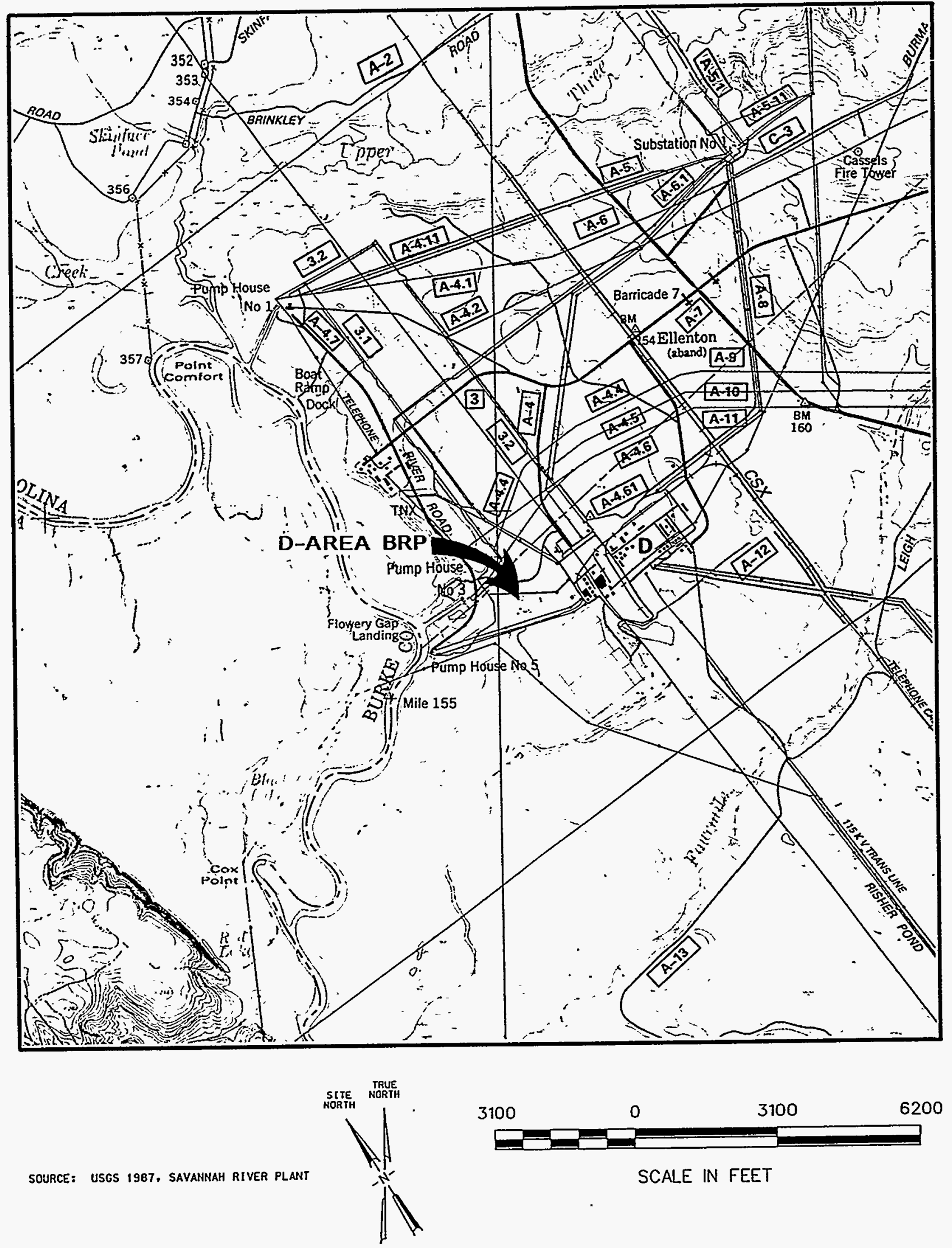

Figure 2-2 Location of D-Area Burning/Rubble Pits at the Savannah River Site 


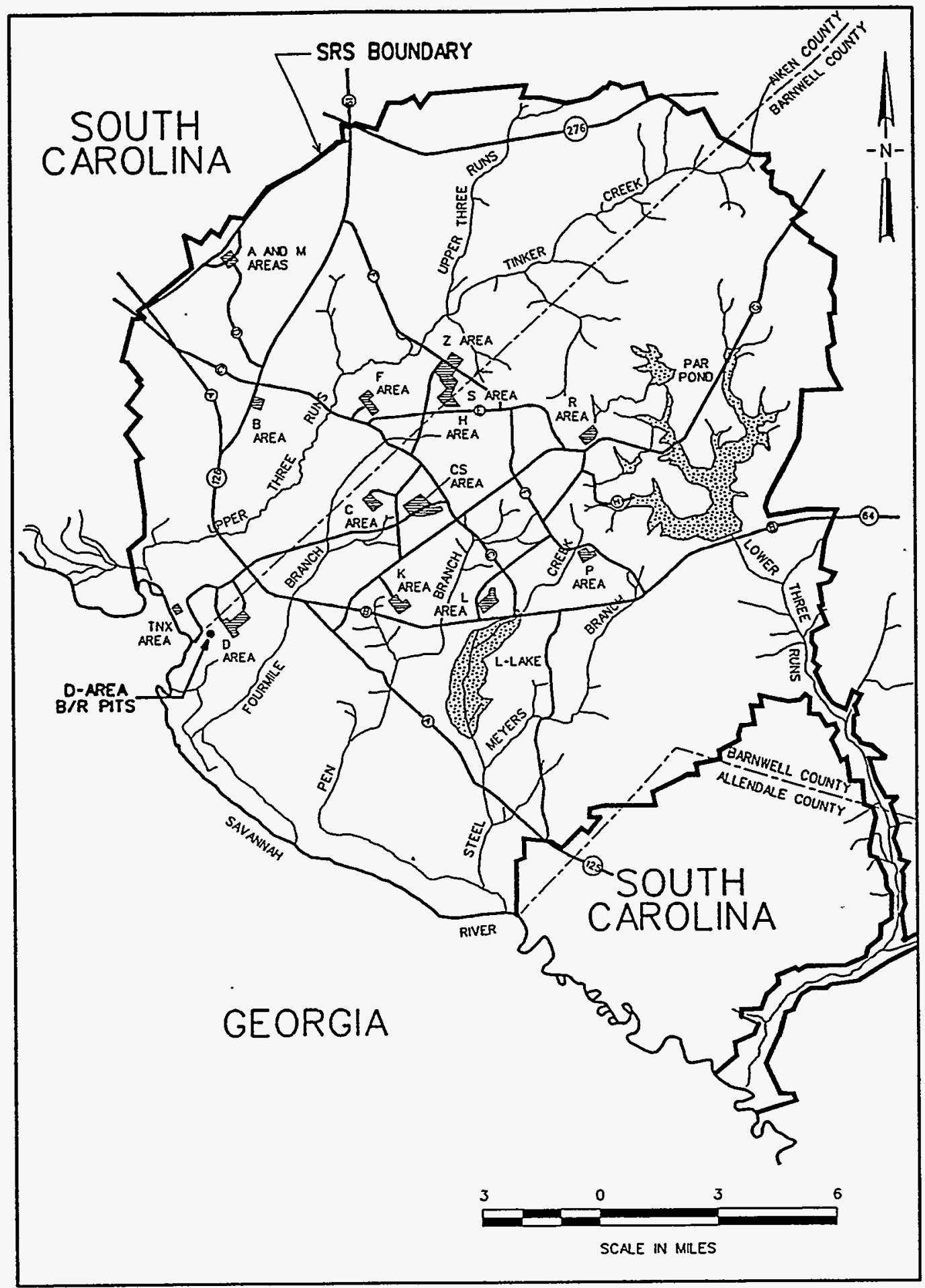

Figure 2-3 Location of D-Area Burning/Rubble Pits in Relation to Other Savannah River Site Facilities 


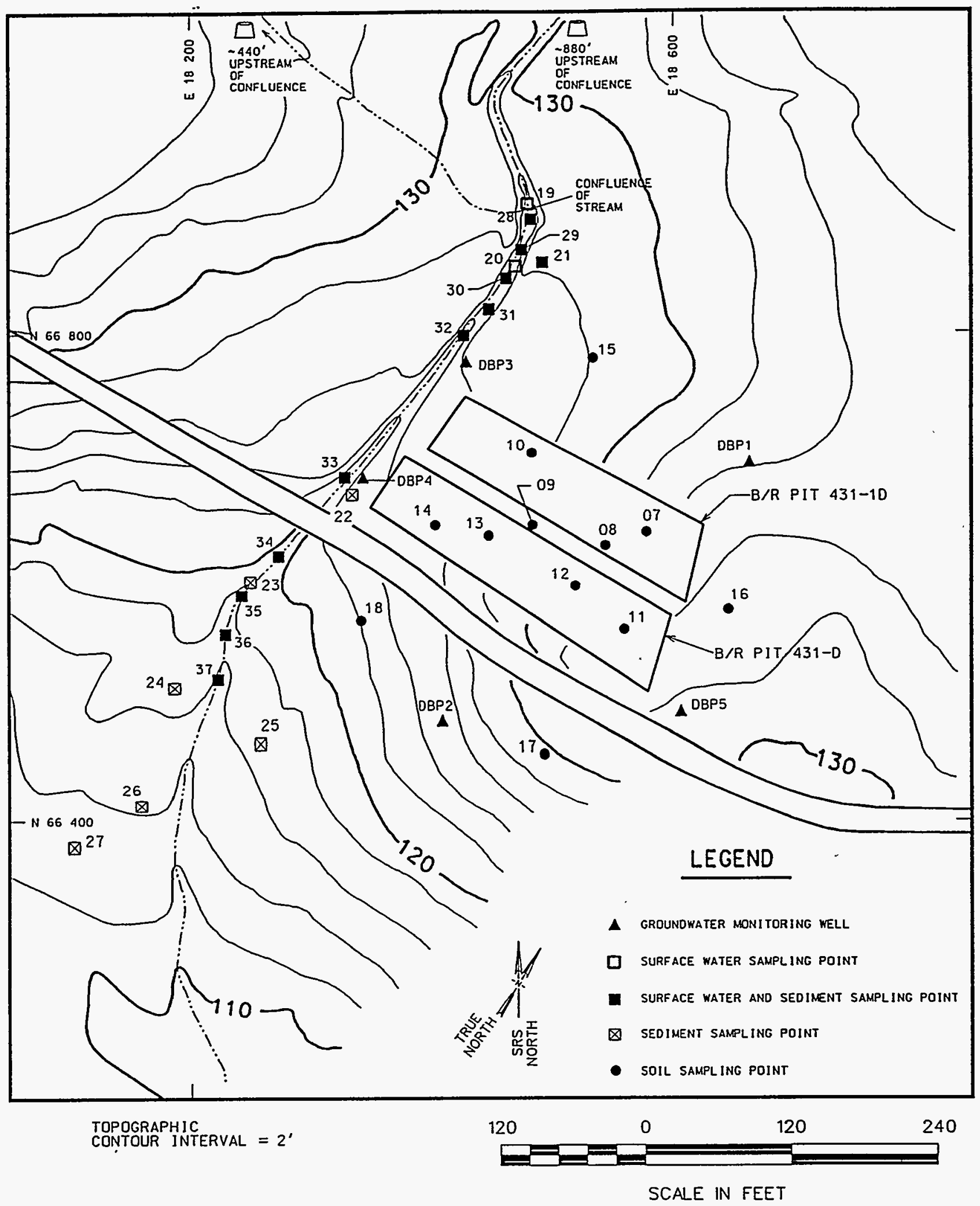

Figure 3-1 D-Area Burning/Rubble Pits Unit Assessment Sampling Locations 
DATA SUMMARY REPORT

D-AREA BURNING/RUBBLE PITS
WSRC-RP-94-709

REV. 0, OCTOBER 1994

\section{TABLES}




\begin{tabular}{|c|c|}
\hline$\overline{\mathrm{A}}$ & $\begin{array}{l}\text { Analysis Qualifier: } \\
\text { Relative Retention Time (RRT) or mass spectra criteria not met for organics compound } \\
\text { identification. Used with "U" or "UI" or " } \mathrm{N} \text { " for all anomalies in pesticides compound } \\
\text { identification. } \\
\text { Bias Codes: } \\
\text { Value reported is the mean of two or more determinations. }\end{array}$ \\
\hline $\bar{B}$ & $\begin{array}{l}\text { The result is below the contract required detection limit and above or equal to the instrument } \\
\text { detection limit. Used with "J". }\end{array}$ \\
\hline $\mathrm{C}$ & Laboratory Control sample exceeded control limits. \\
\hline$\overline{\mathbf{G}}$ & $\begin{array}{l}\text { Bias Codes: } \\
\text { Value reported is the maximum of two or more determinations. }\end{array}$ \\
\hline$\overline{\mathrm{H}}$ & $\begin{array}{l}\text { Analysis Qualifier: } \\
\text { Internal standards performance criteria not met. } \\
\text { Bias Code: } \\
\text { Analytical factor causing bias. The associated result may overestimate the true value (i.e., is } \\
\text { bias high). }\end{array}$ \\
\hline $\bar{I}$ & $\begin{array}{l}\text { Analysis Qualifier: } \\
\text { Matrix spike recovery is not within control limits. }\end{array}$ \\
\hline $\bar{J}$ & $\begin{array}{l}\text { Result Qualifier: } \\
\text { The analytical result is an estimated quantity. }\end{array}$ \\
\hline$\overline{\mathrm{L}}$ & $\begin{array}{l}\text { Result Qualifier: } \\
\text { Off-scale high. The actual value is not known but is known to be greater than the value shown. } \\
\text { Analysis Qualifier: } \\
\text { Calibration criteria (initial or continuing) were not met. Used with "J" or "R". } \\
\text { Bias Codes: } \\
\text { Analytical factor causing bias. The associated result may underestimate the true value (i.e., is } \\
\text { biased low). }\end{array}$ \\
\hline$\overline{\mathrm{M}}$ & $\begin{array}{l}\text { Presence of material is verified but not quantified. The analytical result field should be blank. } \\
\text { For analyses yielding a presence/absence result, the analyte is present. }\end{array}$ \\
\hline$\overline{\mathrm{NA}}$ & Analyte not analyzed. \\
\hline $\mathrm{O}$ & salifier: \\
\hline $\mathrm{Q}$ & $\begin{array}{l}\text { Analysis qualifier: } \\
\text { Sample held beyond normal holding time. }\end{array}$ \\
\hline $\bar{R}$ & $\begin{array}{l}\text { Result Qualifier: } \\
\text { Rejected because performance requirements in the sample or associated quality control analysis } \\
\text { were not met. The analyte may or may not be present. } \\
\text { Analysis Qualifier: } \\
\text { ICP interference check sample criteria are not met. Used with "J", "UJ", or "R". }\end{array}$ \\
\hline $\mathrm{S}$ & $\begin{array}{l}\text { Analysis Qualifier: } \\
\text { The reported value was determined by the method of standard additions. }\end{array}$ \\
\hline$T$ & $\begin{array}{l}\text { Analysis Qualifier: } \\
\text { Peak height (instead of peak area) was used to quantitate the analyte, and all other identification } \\
\text { criteria were met; OR, signal to noise ratio of the confirmation ion is below } 2.5 \text {, but the GC/MS } \\
\text { analyst determined that the peak was positive. }\end{array}$ \\
\hline $\mathrm{U}$ & $\begin{array}{l}\text { Result Qualifier: } \\
\text { The analyte was not detected. Value reported is the sample quantification limit. }\end{array}$ \\
\hline
\end{tabular}


Table 5.1 (Continued)

Qualifier Codes

\begin{tabular}{||ll||}
\hline V. & $\begin{array}{l}\text { Analysis Qualifier: } \\
\text { The analyte was detected in an associated method blank. Used with "J" for non radionuclide } \\
\text { analyses when the result is less than five times the amount in the associated blank (less than ten } \\
\text { times for the common laboratory contaminants acetone, methylene chloride, 2-butanone, and } \\
\text { phthalates). For radionuclide analyses, used with "J" when the result is less than five times the } \\
\text { amount in the associated blank or "UJ" when the result is less than the detection limit. }\end{array}$ \\
\hline $\mathrm{X}$ & Duplicate analysis relative percent difference (RPD) was not within control limits. \\
\hline $\mathrm{Y}$ & $\begin{array}{l}\text { Analysis Qualifier: } \\
\text { Sample was unpreserved or improperly preserved. }\end{array}$ \\
\hline 1 & Analytical factor causing bias-the associated result may underestimate the true value. \\
\hline 2 & Analytical factor causing bias-the associated result may overestimate the true value. \\
\hline 3 & Analytical factor causing bias-the associated result may be of poor precision (high variability). \\
\hline$<$ & Less than the detection limit specified in the results field. \\
\hline$/ I$ & $\begin{array}{l}\text { Separation fields for the three types of data flags: result qualifiers, analysis qualifiers, and bias } \\
\text { codes. In the event no data qualifiers are assigned to validated data, "//" will be displayed in the } \\
\text { data qualifier field. Data which have not been validated will not display "Ir". }\end{array}$ \\
\hline
\end{tabular}


Table 5.2

Precision and Accuracy Validation

\begin{tabular}{|l|l|l|l||}
\hline & $\begin{array}{l}\text { Environmental } \\
\text { Monitoring Section }\end{array}$ & $\begin{array}{l}\text { Exploration } \\
\text { Resources }\end{array}$ & $\begin{array}{l}\text { RUST Environment } \\
\text { \& Infrastructure }\end{array}$ \\
\hline Groundwater & $\begin{array}{l}\text { Soils and } \\
\text { Sediments }\end{array}$ & $\begin{array}{l}\text { Surface Water and } \\
\text { Sediments }\end{array}$ \\
\hline Accuracy & & & \\
\hline Single control samples & YES & YES & YES \\
\hline Surrogate recovery & YES & YES & YES \\
\hline Matrix spike recovery & YES & YES & YES \\
\hline Method blank & YES & YES & YES \\
\hline Trip blank & NO & YES & YES \\
\hline Field blank & NO & NO & YES \\
\hline Rinsate blank & NO & NO & YES \\
\hline Precision & & & \\
\hline Duplicate control sample & YES & YES & YES \\
\hline Matrix spike duplicate & YES & YES & YES \\
\hline Laboratory duplicate & YES & YES & YES \\
\hline Split duplicate & YES & NO & YES \\
\hline Field duplicate & YES & NO & YES \\
\hline \hline
\end{tabular}

YES = Validation regarding this issue was conducted.

NO = Validation regarding this issue was not conducted. 
TABLE 6.1

VOLATILE OROANIC COMPOUNDS

I IN SOIL

D.AREA BURNING/RUBBLE PITS

SAVANNAH RIVER SITE

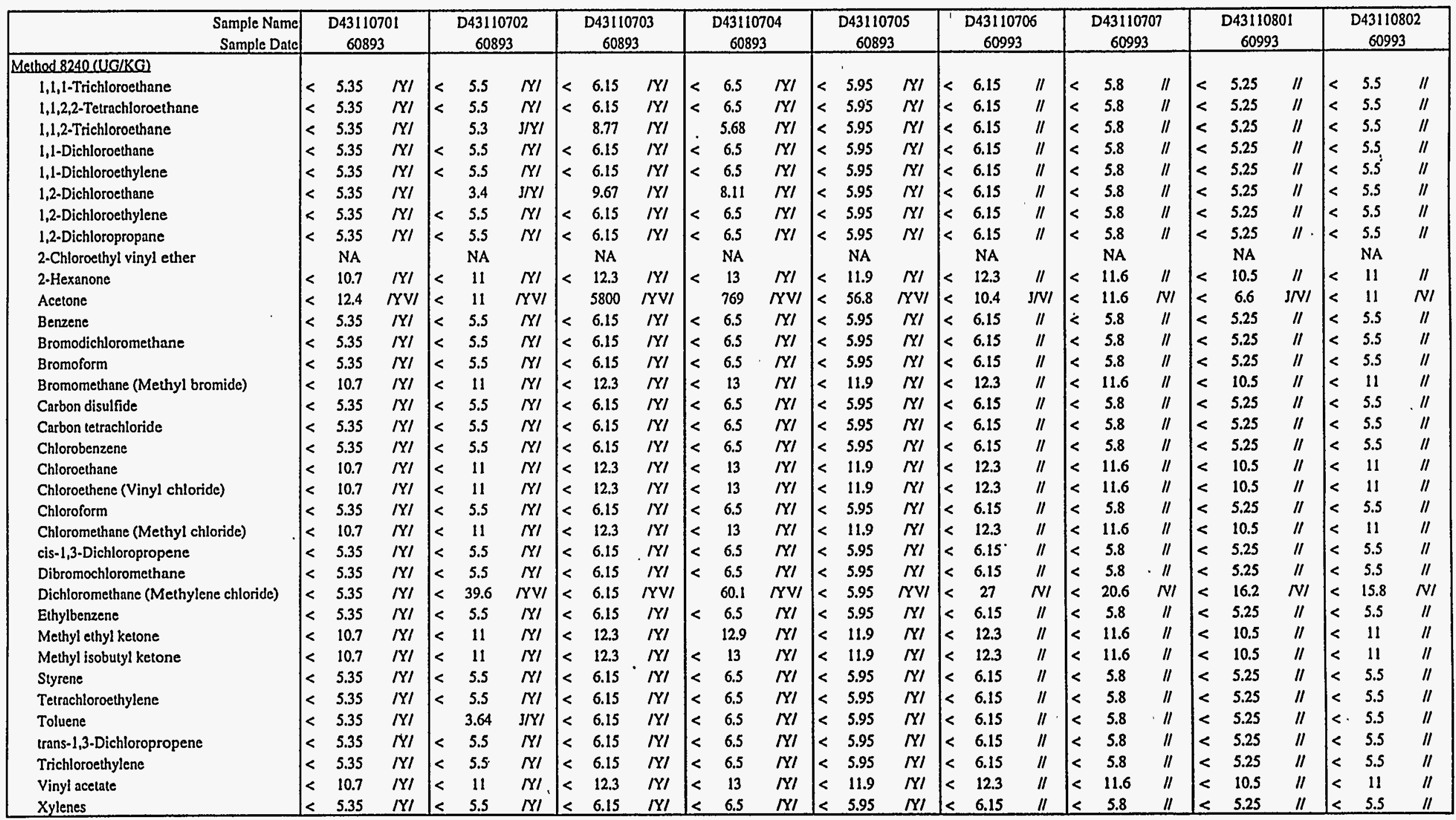


TABLE 6.1

VOLATILE ORGANIC COMPOUNDS

D-AREA IN SOIL

SAVANNAH RIVER SITE

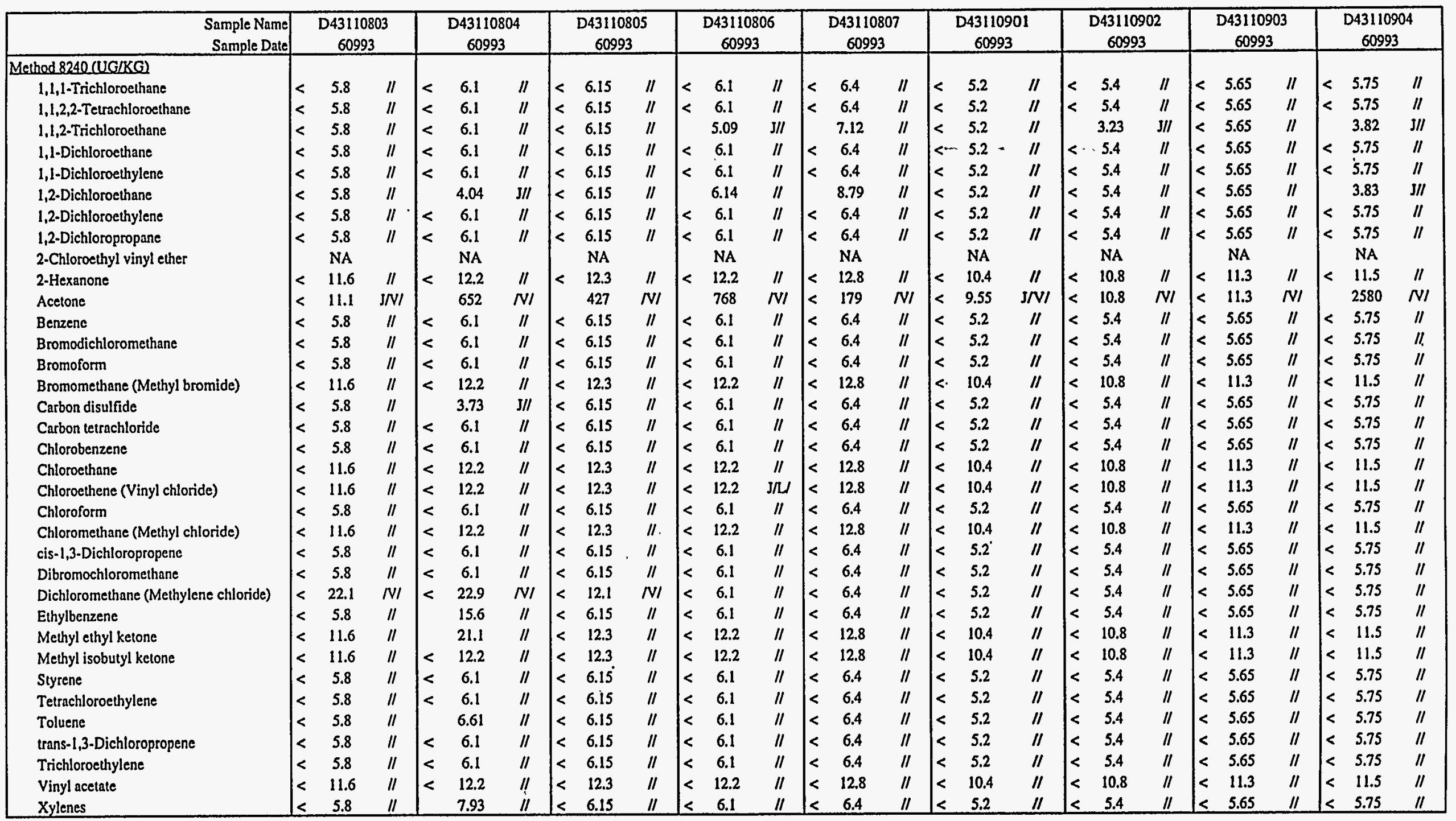


TABLE 6.1

VOLATILE ORGANIC COMPOUNDS

IN SOIL

AREA BURNING/RUBBLE PITS

SAVANNAH RIVER SITE

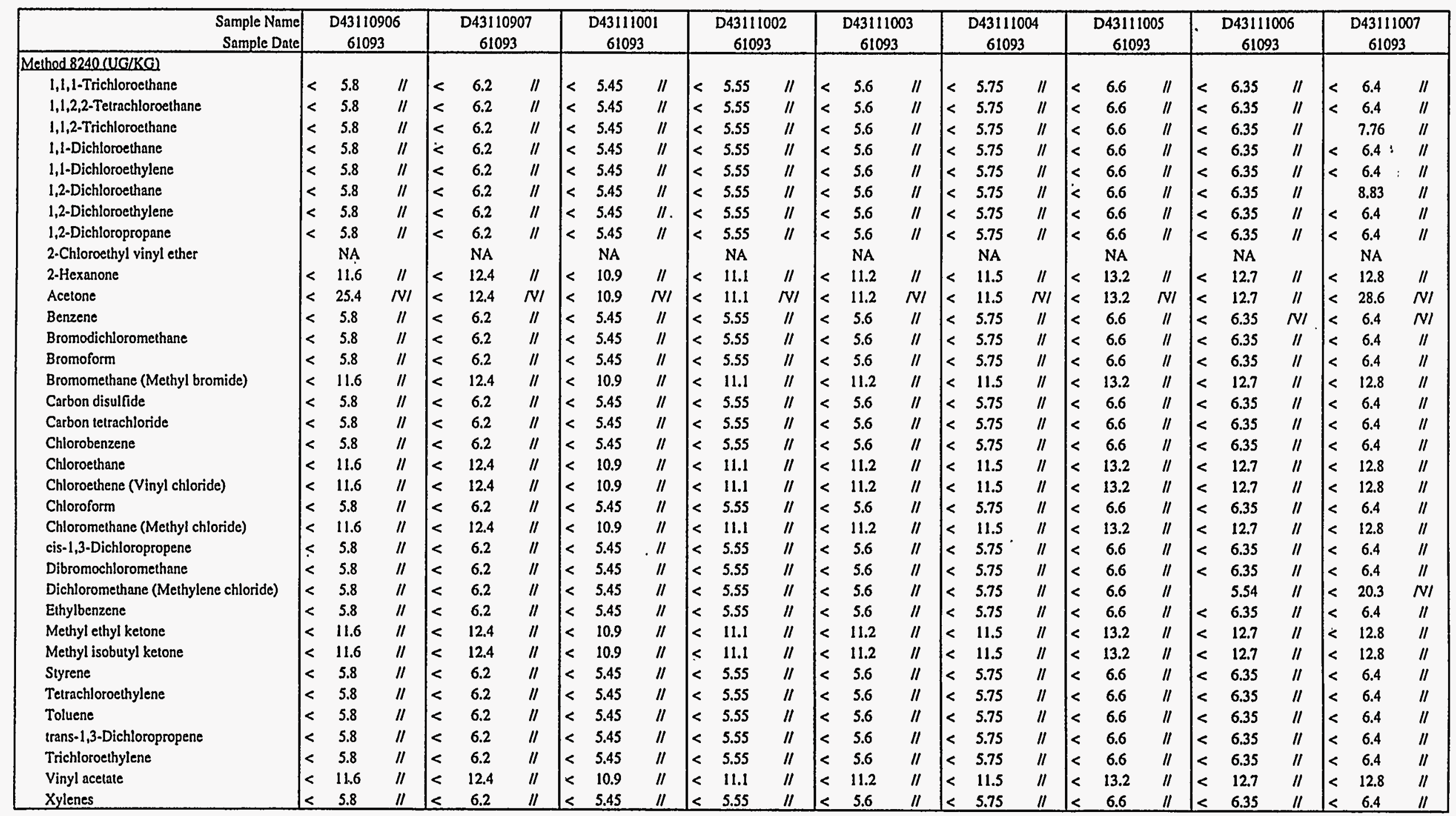


TABLE 6.1

RGANIC COMPOUNDS

IN SOIL

D-AREA BURNING/RUBBLE PITS

SAVANNAH RIVER SITE

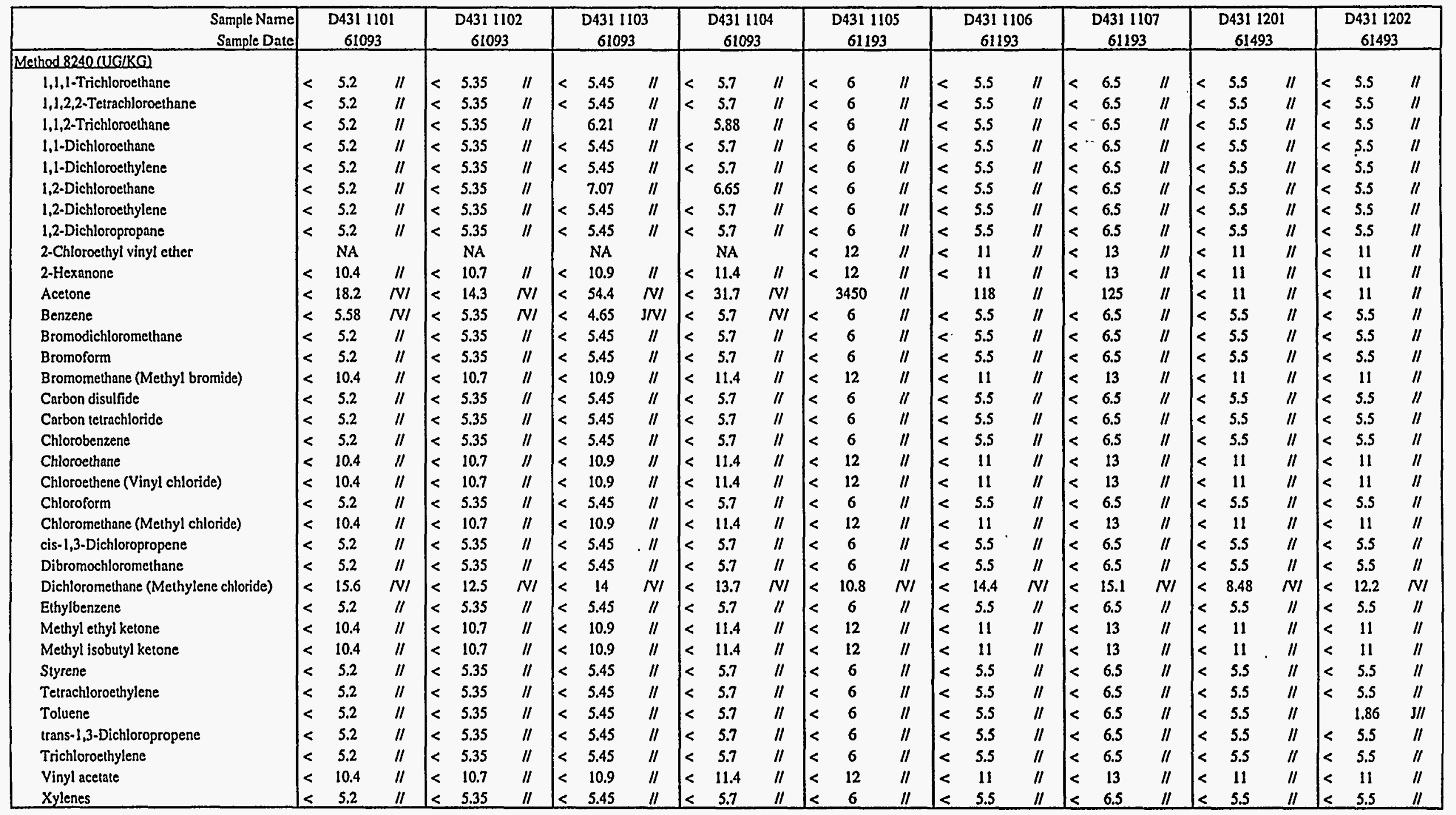




\begin{tabular}{|c|c|c|}
\hline \multirow{10}{*}{ 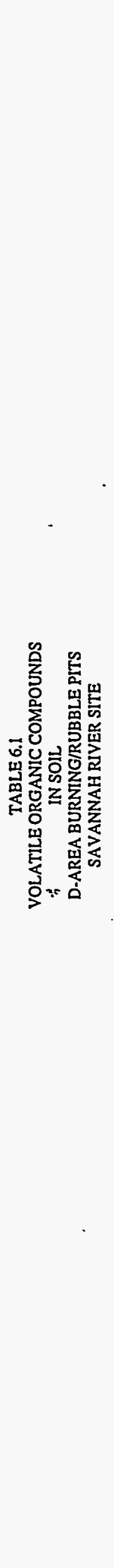 } & $\frac{2}{\frac{2}{3}} \frac{2}{0}$ & 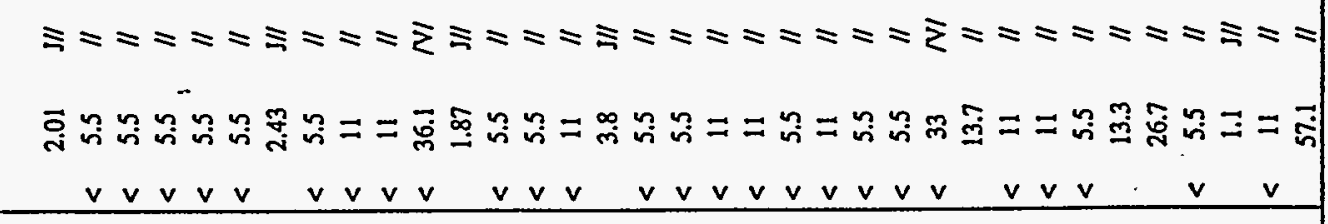 \\
\hline & 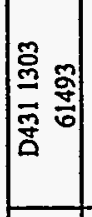 & 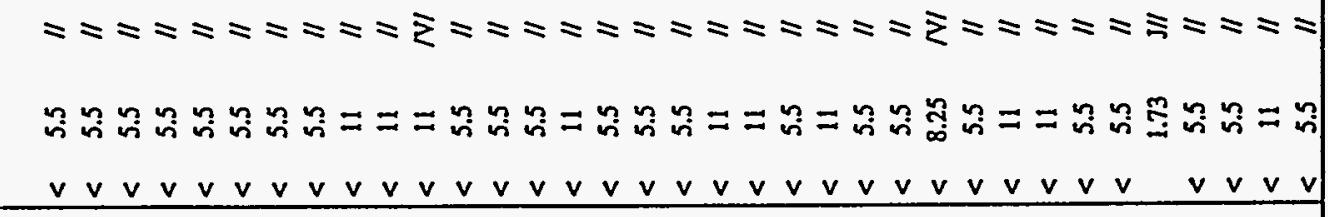 \\
\hline & 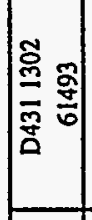 & 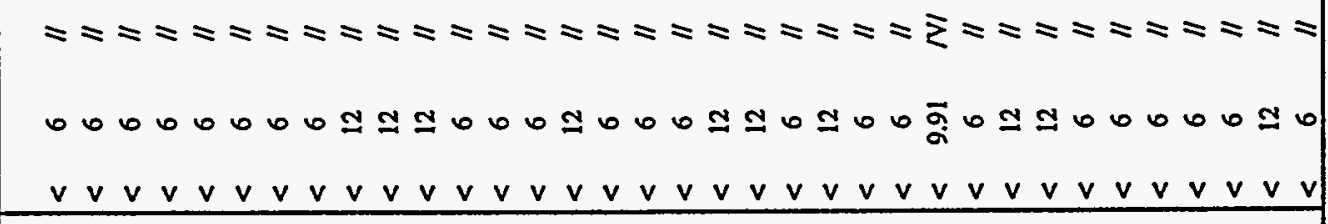 \\
\hline & 兽 & 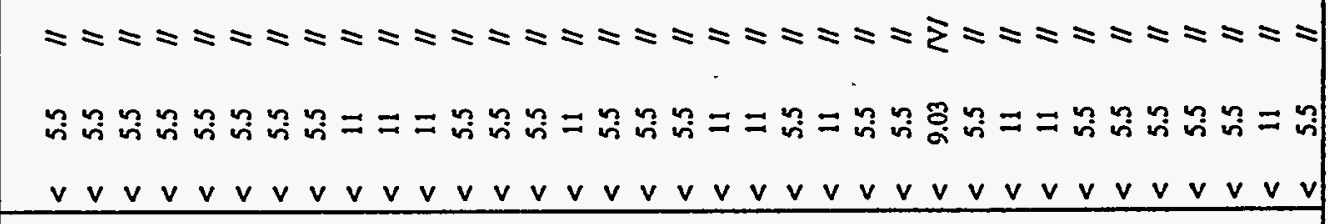 \\
\hline & $\mid$ & 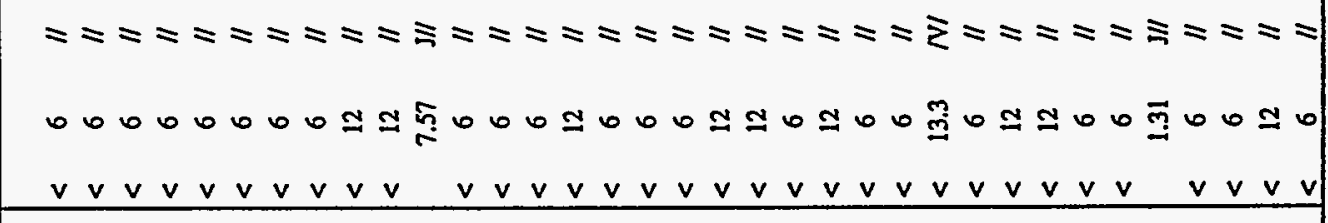 \\
\hline & 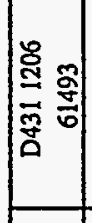 & 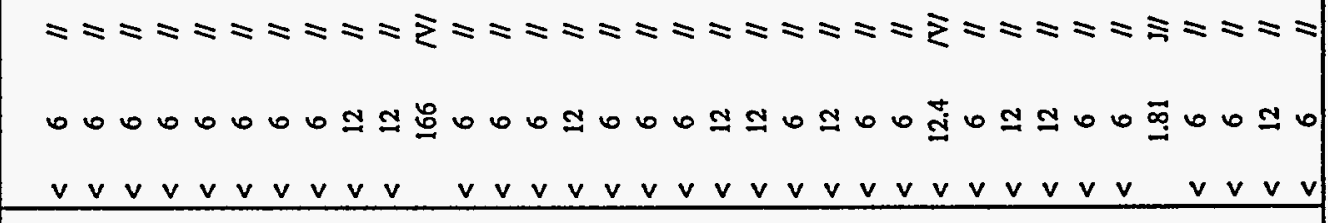 \\
\hline & 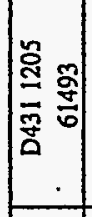 & 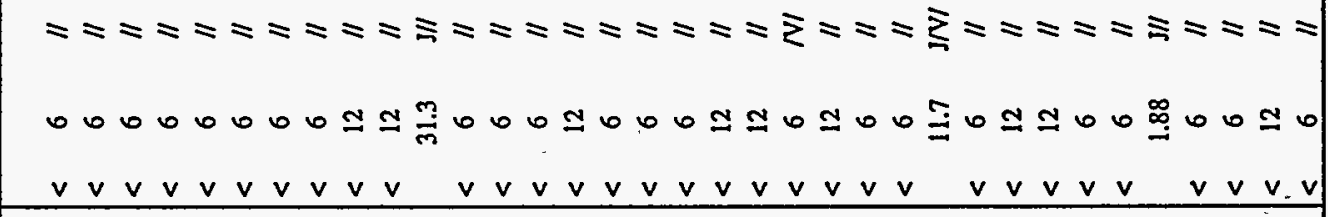 \\
\hline & 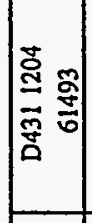 & 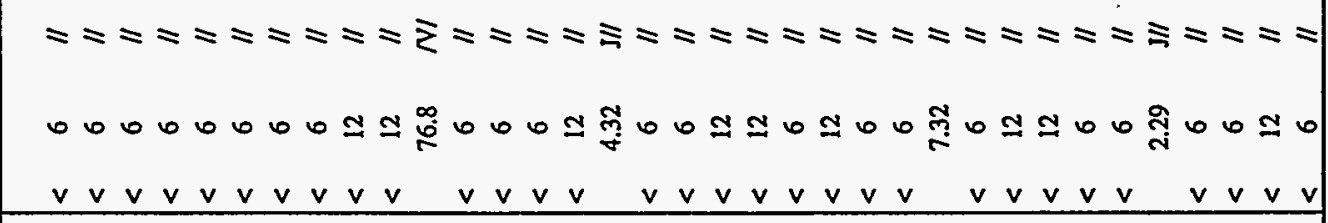 \\
\hline & 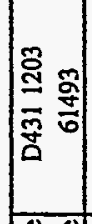 & 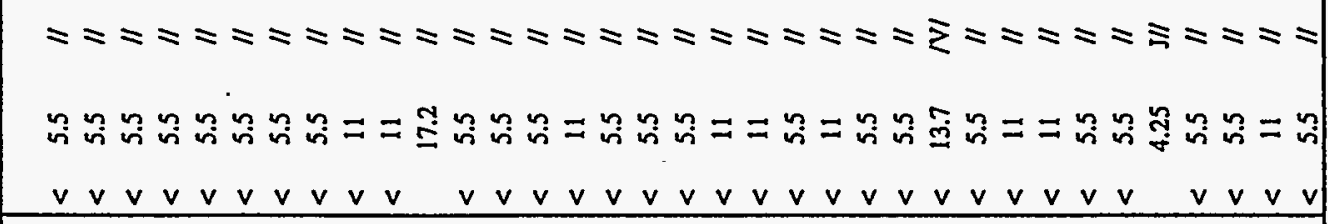 \\
\hline & 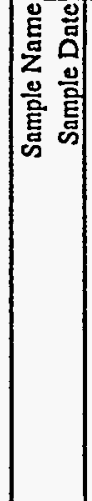 & 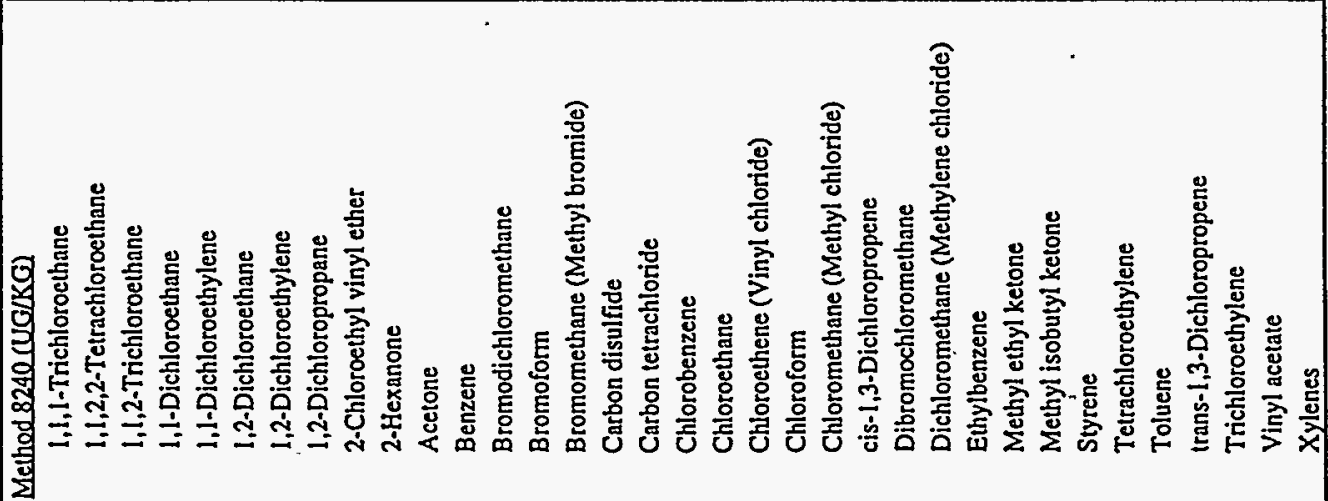 \\
\hline
\end{tabular}


TABLE 6.1

VOLATILE ORGANIC COMPOUNDS IN SOIL

D-AREA BURNING/RUBBLE PITS SAVANNAH RIVER SITE

\begin{tabular}{|c|c|c|c|c|c|c|c|c|c|c|c|c|c|c|c|c|c|c|c|c|c|c|c|c|c|c|c|}
\hline \multirow{3}{*}{$\begin{array}{l}\text { Method } 8240 \text { (UG/KG) } \\
\text { 1,1,1-Trichloroethane }\end{array}$} & \multicolumn{3}{|c|}{$\begin{array}{c}\text { D431 1305 } \\
61493 \\
\end{array}$} & \multicolumn{3}{|c|}{$\begin{array}{c}\text { D431 } 1306 \\
61493 \\
\end{array}$} & \multicolumn{3}{|c|}{$\begin{array}{c}D 4311307 \\
61493 \\
\end{array}$} & \multicolumn{3}{|c|}{$\begin{array}{c}\text { D431 1401 } \\
61593 \\
\end{array}$} & \multicolumn{3}{|c|}{$\begin{array}{c}\text { D431 } 1402 \\
61593 \\
\end{array}$} & \multicolumn{3}{|c|}{$\begin{array}{c}\text { D4311403 } \\
61593 \\
\end{array}$} & \multicolumn{3}{|c|}{$\begin{array}{c}\mathrm{D} 4311404 \\
61593 \\
\end{array}$} & \multicolumn{3}{|c|}{$\begin{array}{c}\text { D431 } 1405 \\
61593 \\
\end{array}$} & \multicolumn{3}{|c|}{$\begin{array}{c}\text { D431 } 1406 \\
61593 \\
\end{array}$} \\
\hline & & & & & & & & & & & & & & & & & & & & & & & & & & & \\
\hline & & 1.9 & $\mathrm{~J} / \mathrm{O} / \mathrm{L}$ & $\mid<$ & 5.5 & $\|$ & $1<$ & 6 & 11 & $<$ & 5.7 & $\cdot \mathrm{J} / \mathrm{H} /$ & $\mid<$ & 5.65 & $/ /$ & $<$ & 5.7 & II & | & 5.45 & $\|$ & $1<$ & 5.7 & "I & |< & 6.5 & 11 \\
\hline 1,1,2,2-Tetrachloroethane & $<$ & 5.5 & $\mathrm{~J} / \mathrm{O} / \mathrm{L}$ & $<$ & 5.5 & $\|$ & $<$ & 6 & $/ 1$ & $<$ & 5.7 & $\mathrm{~J} / \mathrm{H} /$ & $1<$ & 5.65 & $\|$ & |< & 5.7 & $\|$ & $<$ & 5.45 & $/ /$ & $<$ & 5.7 & "I & $<$ & 6.5 & $\|$ \\
\hline 1,1,2-Trichloroethane & $<$ & 5.5 & J/OR & $<$ & 5.5 & $\|$ & $<$ & 6 & $\|$ & $<$ & 5.7 & $\mathrm{~J} / \mathrm{H} /$ & $<$ & 5.65 & $\|$ & $<$ & 5.7 . & $\|$ & $<$ & 5.45 & $\|$ & $<$ & 5.7 & $\|$ & $<$ & 6.5 & $\|$ \\
\hline 1,1-Dichloroethane & $<$ & 5.5 & IIOR & $<$ & 5.5 & $\|$ & $<$ & 6 & $\|$ & $<$ & 5.7 & $\mathrm{~J} / \mathrm{H} /$ & $1<$ & 5.65 & $\|$ & $<$ & 5.7 & $\|$ & $<$ & 5.45 & $\|$ & $<$ & 5.7 & "I & $<$ & 6.5 & /I \\
\hline 1,1-Dichloroethylene & $<$ & 5.5 & $\mathrm{~J} / \mathrm{O} / \mathrm{L}$ & $<$ & 5.5 & $\|$ & $<$ & 6 & $\|$ & $<$ & 5.7 & $\mathrm{~J} / \mathrm{H} /$ & $1<$ & 5.65 & $/ I$ & |< & 5.7 & II & $<$ & 5.45 & II & $<$ & 5.7 & II & $<$ & 6.5 & $\|$ \\
\hline 1,2-Dichloroethane & $<$ & 5.5 & J/OR & $<$ & 5.5 & $\|$ & $<$ & 6 & $\|$ & $<$ & 5.7 & $\mathrm{~J} / \mathrm{H} /$ & $<$ & 5.65 & $\|$ & & 6.05 & $\|$ & & 4.95 & $\|$ & & 6.69 & $\|$ & $<$ & 6.5 & $\|$ \\
\hline 1,2-Dichloroethylene & & 7.19 & $\mathrm{~J} / \mathrm{O} / \mathrm{L}$ & $<$ & 5.5 & $\|$ & $<$ & 6 & $\|$ & $<$ & 5.7 & $\mathrm{~J} / \mathrm{t} / \mathrm{I}$ & $1<$ & 5.65 & $\|$ & $<$ & 5.7 & $\|$ & $<$ & 5.45 & $\|$ & $<$ & 5.7 & $\|$ & $<$ & 6.5 & $\|$ \\
\hline 1,2-Dichloropropane & $<$ & 5.5 & $\mathrm{~J} / \mathrm{O} / \mathrm{L}$ & $<$ & 5.5 & $\|$ & $<$ & 6 & /I & $<$ & 5.7 & $\mathrm{~J} / \mathrm{H} /$ & $<$ & 5.65 & $\|$ & $<$ & 5.7 & $\|$ & $<$ & 5.45 & $\|$ & $<$ & 5.7 & $\|$ & $<$ & 6.5 & $\|$ \\
\hline 2-Chloroethyl vinyl ether & $<$ & 11 & s/OR & $<$ & 11 & $\|$ & $<$ & 12 & $\|$ & & NA & & & NA & & & NA & & & NA & & & NA & & & NA & \\
\hline 2-Hexanone & $<$ & 11 & $\mathrm{~J} / \mathrm{O} \Omega$ & $<$ & 11 & $\|$ & $<$ & 12 & $\|$ & $<$ & 11.4 & $\mathrm{~J} / \mathrm{H} /$ & $<$ & 11.3 & $\|$ & $<$ & 11.4 & $\|$ & $<$ & 10.9 & $\|$ & $<$ & 11.4 & $\|$ & $<$ & 13 & $\|$ \\
\hline Acetone & & 156 & J/VO/L & $<$ & 49 & $N I$ & $<$ & 16.2 & $N I$ & $<$ & 11.4 & $\mathrm{~J} / \mathrm{H} /$ & $<$ & 11.3 & NI & & 14.6 & "I & $<$ & 10.9 & $N I$ & $<$ & 37.8 & $r V I$ & & 740 & $\|$ \\
\hline Benzene & & 5.21 & $\mathrm{~J} / \mathrm{O} / \mathrm{L}$ & $<$ & 5.5 & $\|$ & $<$ & 6 & $\|$ & $<$ & 5.7 & $\mathrm{~J} / \mathrm{H} /$ & $<$ & 5.65 & $\|$ & $<$ & 5.7 & "l & $<$ & 5.45 & $\|$ & $<$ & 5.7 & $\|$ & $<$ & 6.5 & "I \\
\hline Bromodichloromethane & $<$ & 5.5 & $\mathrm{~J} / \mathrm{O} / \mathrm{L}$ & $<$ & 5.5 & $\|$ & $<$ & 6 & $\|$ & $<$ & 5.7 & $\mathrm{~J} / \mathrm{H} /$ & $<$ & 5.65 & $/ 1$ & $<$ & 5.7 & $\|$ & $<$ & 5.45 & $\|$ & $<$ & 5.7 & $\|$ & $<$ & 6.5 & $\|$ \\
\hline Bromoform & $<$ & 5.5 & $\mathrm{~J} / \mathrm{O} / \mathrm{L}$ & $<$ & 5.5 & $\|$ & $<$ & 6 & $\|$ & $<$ & 5.7 & $\mathrm{~J} / \mathrm{H} /$ & $<$ & 5.65 & $\|$ & $<$ & 5.7 & $\|$ & $<$ & 5.45 & $\|$ & $<$ & 5.7 & $\|$ & $<$ & 6.5 & $\|$ \\
\hline Bromomethane (Methyl bromide) & $<$ & 11 & $\mathrm{~J} / \mathrm{O} / \mathrm{L}$ & $<$ & 11 & $\|$ & $<$ & 12 & $\|$ & $<$ & 11.4 & $\mathrm{~J} / \mathrm{H} /$ & $<$ & 11.3 & $\|$ & |< & 11.4 & $\|$ & $<$ & 10.9 & $\|$ & $<$ & 11.4 & $\|$ & $<$ & 13 & $\|$ \\
\hline Carbon disulfide & & 11.5 & $\mathrm{~s} / \mathrm{O} / \mathrm{L}$ & & 1.53 & $\mathrm{~s} / 1$ & $<$ & 6 & $\|$ & $<$ & 5.7 & $\mathrm{~J} / \mathrm{H} /$ & $<$ & 5.65 & $\|$ & $<$ & 5.7 & $\|$ & $<$ & 5.45 & $\|$ & $<$ & 5.7 & $\|$ & $<$ & 6.5 & $\|$ \\
\hline Carbon tetrachloride & $1<$ & 5.5 & $\mathrm{~J} / \mathrm{O} / \mathrm{L}$ & $<$ & 5.5 & $\|$ & $<$ & 6 & $\|$ & $<$ & 5.7 & $\mathrm{~J} / \mathrm{H} /$ & $1<$ & 5.65 & $/ 1$ & $1<$ & 5.7 & $\|$ & $<$ & 5.45 & $\|$ & $<$ & 5.7 & $\|$ & $<$ & 6.5 & $\|$ \\
\hline Chlorobenzene & $<$ & 5.5 & $\mathrm{~J} / \mathrm{O} / \mathrm{L}$ & $<$ & 5.5 & II & $<$ & 6 & $\|$ & $<$ & 5.7 & $\mathrm{~J} / \mathrm{H} /$ & $<$ & 5.65 & $\|$ & $<$ & 5.7 & $\|$ & $<$ & 5.45 & $\|$ & $<$ & 5.7 & $\|$ & $<$ & 6.5 & $\|$ \\
\hline Chloroethane & $<$ & 11 & $\mathrm{~J} / \mathrm{O} / \mathrm{L}$ & $<$ & 11 & $\|$ & $<$ & 12 & $\|$ & $<$ & 11.4 & $\mathrm{~J} / \mathrm{H} /$ & $<$ & 11.3 & $\|$ & $<$ & 11.4 & $\|$ & $<$ & 10.9 & $\|$ & $<$ & 11.4 & II & $<$ & 13 & $\|$ \\
\hline Chlorocthene (Vinyl chloride) & $<$ & 11 & $\mathrm{~J} / \mathrm{O} / \mathrm{L}$ & $<$ & 11 & $\|$ & $<$ & 12 & $\|$ & $<$ & 11.4 & $\mathrm{~J} / \mathrm{HL}$ & $<$ & 11.3 & $/ 1$ & $<$ & 11.4 & $\|$ & $<$ & 10.9 & $/ /$ & $<$ & 11.4 & $\|$ & $<$ & 13 & $\|$ \\
\hline Chloroform & $<$ & 5.5 & $\mathrm{~J} / \mathrm{O} / \mathrm{L}$ & $<$ & 5.5 & $\|$ & $<$ & 6 & $\|$ & $<$ & 5.7 & $\mathrm{~J} / \mathrm{H} /$ & $<$ & 5.65 & $\|$ & $1<$ & 5.7 & $\|$ & k & 5.45 & $\|$ & $<$ & 5.7 & 'll & $<$ & 6.5 & $/ 1$ \\
\hline Chloromethane (Methyl chloride) & $<$ & 11 & $\mathrm{~J} / \mathrm{O} / \mathrm{L}$ & $<$ & 11 & $\|$ & $<$ & 12 & $\|$ & $<$ & 11.4 & $\mathrm{~J} / \mathrm{H} /$ & $<$ & 11.3 & $\|$ & $<$ & 11.4 & $\|$ & $<$ & 10.9 & $\|$ & $<$ & 11.4 & /I & $<$ & 13 & $\|$ \\
\hline cis-1,3-Dichloropropene & $<$ & 5.5 & s/OR & $<$ & 5.5 & $\|$ & $<$ & 6 & $\|$ & $<$ & 5.7 & $\mathrm{~J} / \mathrm{H} /$ & $<$ & 5.65 & $\|$ & $<$ & $5.7^{\circ}$ & $\|$ & $1<$ & 5.45 & $\|$ & $<$ & 5.7 & $\|$ & $<$ & 6.5 & $\|$ \\
\hline Dibromochloromethane & $<$ & 5.5 & $\mathrm{~J} / \mathrm{O} / \mathrm{L}$ & $<$ & 5.5 & $\|$ & $<$ & 6 & $\|$ & $<$ & 5.7 & $\mathrm{~J} / \mathrm{H} /$ & $<$ & 5.65 & $\|$ & $<$ & 5.7 & $\|$ & $<$ & 5.45 & $\|$ & $<$ & 5.7 & $\|$ & $<$ & 6.5 & $\|$ \\
\hline Dichloromethane (Methylene chloride) & $<$ & 27.1 & $\mathrm{~J} / \mathrm{NOR}$ & $<$ & 26 & $N I$ & $<$ & 18.6 & NI & $<$ & 5.7 . & $\mathrm{J} / \mathrm{H} /$ & $<$ & 5.65 & $\|$ & $<$ & 7.32 & IVI & $<$ & 5.45 & $\|$ & $<$ & 5.7 & $\|$ & $<$ & 9.67 & $N I$ \\
\hline Ethylbenzene & & 15.3 & $\mathrm{~J} / \mathrm{O} / \mathrm{L}$ & & 1.14 & $\mathrm{~J} / 1$ & $<$ & 6 & $\|$ & $<$ & 5.7 & $\mathrm{~J} / \mathrm{H} /$ & $<$ & 5.65 & $\|$ & $<$ & 5.7 & $\|$ & $<$ & 5.45 & "I & $<$ & 5.7 & $\|$ & $<$ & 6.5 & $/ 1$ \\
\hline Methyl ethyl ketone & & 28.9 & $\mathrm{~J} / \mathrm{O} / \mathrm{L}$ & & 7.18 & $\mathrm{~J} / 1$ & $<$ & 12 & $\|$ & $<$ & 11.4 & $\mathrm{~J} / \mathrm{H} /$ & $<$ & 11.3 & $\|$ & $<$ & 11.4 & "I & $<$ & 10.9 & $\|$ & $<$ & 11.4 & $\|$ & $<$ & 13 & $\|$ \\
\hline Methyl isobutyl ketone & $1<$ & 11 & $\mathrm{~J} / \mathrm{O} / \mathrm{L}$ & $<$ & 11 & $\|$ & $<$ & 12 & $/ 1$ & $<$ & 11.4 & $\mathrm{~J} / \mathrm{H} /$ & $<$ & 11.3 & $\|$ & $<$ & 11.4 & $\|$ & $<$ & 10.9 & $\|$ & $<$ & 11.4 & $\|$ & $<$ & 13 & $\|$ \\
\hline Styrene & $<$ & 5.5 & $\mathrm{~J} / \mathrm{O} / \mathrm{L}$ & $<$ & 5.5 & $\|$ & $<$ & 6 & $\|$ & $<$ & 5.7 & $\mathrm{~J} / \mathrm{H} /$ & $<$ & 5.65 & $\|$ & |< & 5.7 & "I & $<$ & 5.45 & "I & $<$ & 5.7 & 11 & $<$ & 6.5 & $\|$ \\
\hline Tetrachloroethylene & & 12.5 & $\mathrm{~J} / \mathrm{O} / \mathrm{L}$ & & 2.51 & $\mathrm{~J} / 1$ & $<$ & 6 & $/ 1$ & $<$ & 5.7 & $\mathrm{~J} / \mathrm{H} /$ & |< & 5.65 & $\|$ & |< & 5.7 & 11 & $<$ <. & 5.45 & "l & $<<$ & 5.7 & $\|$ & $\mid<$ & 6.5 & II \\
\hline Toluene & & 22.9 & $\mathrm{~J} / \mathrm{O} / \mathrm{L}$ & & 8.8 & $\|$ & $<$ & 6 & $\|$ & $<$ & 5.7 & $\mathrm{~J} / \mathrm{H} /$ & $<$ & 5.65 & $\|$ & $<$ & 5.7 & $\|$ & $<$ & 5.45 & $\|$ & $<$ & 5.7 & $\|$ & $<$ & 6.5 & $\|$ \\
\hline trans-1,3-Dichloropropene & $<$ & 5.5 & $\mathrm{~J} / \mathrm{O} / \mathrm{L}$ & $<$ & 5.5 & $\|$ & $<$ & 6 & $\|$ & $<$ & 5.7 & $\mathrm{~J} / \mathrm{H} /$ & $<$ & 5.65 & $\|$ & $<$ & 5.7 & II & $<$ & 5.45 & 71 & $<$ & 5.7 & $\|$ & $<$ & 6.5 & $\|$ \\
\hline Trichloroethylene & & 1.58 & $\mathrm{~s} / \mathrm{O} / \mathrm{L}$ & $<$ & 5.5 & $\|$ & $<$ & 6 & $\|$ & $<$ & 5.7 & $\mathrm{~J} / \mathrm{H} /$ & $<$ & 5.65 & $\|$ & $<$ & 5.7 & II & $<$ & 5.45 & $\|$ & $<$ & 5.7 & $\|$ & $<$ & 6.5 & /I \\
\hline Vinyl acetate & $<$ & 11 & $\mathrm{~J} / \mathrm{O} / \mathrm{L}$ & $<$ & 11 & ll & $<$ & 12 & $\|$ & $<$ & 11.4 & $\mathrm{~J} / \mathrm{H} /$ & $<$ & 11.3 & II & $<$ & 11.4 & /I & $<$ & 10.9 & II & $<$ & 11.4 & /I & $<$ & 13 & /I \\
\hline Xylenes & & 39 & $\mathrm{~J} / \mathrm{O} / \mathrm{L}$ & & 5.19 & $\mathrm{~J} / 1$ & $<$ & 6 & 11 & $<$ & 5.7 & $\mathrm{~J} / \mathrm{H} /$ & $<$ & 5.65 & II & $1<$ & 5.7 & II & $1<$ & 5.45 & 11 & $<$ & 5.7 & 11 & $1<$ & 6.5 & 11 \\
\hline
\end{tabular}

$\because$ 
TABLE 6.1

VOLATILE ORGANIC COMPOUNDS

IN SOIL

AREA BURNING/RUBBLE PITS

SAVANNAH RIVER SITE

\begin{tabular}{|c|c|c|c|c|c|c|c|c|c|c|c|c|c|c|c|c|c|c|c|c|c|c|c|c|c|c|c|}
\hline \multirow{3}{*}{$\begin{array}{|cr|} & \begin{array}{r}\text { Sample Name } \\
\text { Sample Date }\end{array} \\
\text { Mechod 8240(UG/KG) } & \\
1,1,1-\text { Trichloroethane } & \end{array}$} & \multicolumn{3}{|c|}{$\begin{array}{c}\text { D4311407 } \\
61593 \\
\end{array}$} & \multicolumn{3}{|c|}{$\begin{array}{c}D 43111501 \\
61693 \\
\end{array}$} & \multicolumn{3}{|c|}{$\begin{array}{c}\text { D43111502 } \\
61693 \\
\end{array}$} & \multicolumn{3}{|c|}{$\begin{array}{c}\text { D43!11503 } \\
61693 \\
\end{array}$} & \multicolumn{3}{|c|}{$\begin{array}{c}\text { D431111504 } \\
61693 \\
\end{array}$} & \multicolumn{3}{|c|}{$\begin{array}{c}43111601 \\
62193 \\
\end{array}$} & \multicolumn{3}{|c|}{$\begin{array}{c}\text { D43111602 } \\
62193 \\
\end{array}$} & \multicolumn{3}{|c|}{$\begin{array}{c}D 43111603 \\
62293\end{array}$} & \multicolumn{3}{|c|}{$\begin{array}{c}43111604 \\
62293 \\
\end{array}$} \\
\hline & & & & & & & & & & & & & & & & & & & & & & & & & & & \\
\hline & $<$ & 6.45 & 11 & $<$ & 5.7 & $\mathrm{~J} / \mathrm{H} /$ & $<$ & 5.55 & $\|$ & $<$ & 5.8 & $\|$ & $<$ & 6.15 & $\|$ & $<$ & 5 & $\prime \prime$ & $<$ & 5.5 & "l & $<$ & 6 & $\|$ & $<$ & 5.5 & $\|$ \\
\hline 1,1,2,2-Tetrachloroethane & $<$ & 6.45 & $\|$ & $<$ & 5.7 & $\mathrm{~J} / \mathrm{H} /$ & $<$ & 5.55 & $\|$ & $<$ & 5.8 & $\|$ & $<$ & 6.15 & $\|$ & $<$ & 5 & $\|$ & $<$ & 5.5 & $\|$ & $<$ & 6 & $\|$ & $<$ & 5.5 & $\|$ \\
\hline 1,1,2-Trichloroethane & $<$ & 6.45 & $/ 1$ & $<$ & 5.7 & $\mathrm{~J} / \mathrm{H} /$ & $<$ & 5.55 & $\|$ & $<$ & 5.8 & $\|$ & $<$ & 6.15 & II & $<$ & 5 & "I & $<$ & 5.5 & 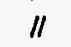 & $<$ & 6 & $\|$ & $<$ & 5.5 & $\|$ \\
\hline 1,1-Dichloroethane & $<$ & 6.45 & $\|$ & $<$ & 5.7 & $\|$ & $<$ & 5.55 & $\|$ & $<$ & 5.8 & $\|$ & $<$ & 6.15 & $\|$ & $<$ & 5 & $\|$ & $<$ & 5.5 & $\|$ & $<$ & 6 & $\|$ & $<$ & 5.5 & $\|$ \\
\hline 1,1-Dichloroethylene & $<$ & 6.45 & $\|$ & $<$ & 5.7 & $\|$ & $<$ & 5.55 & "l & $<$ & 5.8 & $\|$ & $<$ & 6.15 & $\|$ & $<$ & 5 & "I & $<$ & 5.5 & "l & $<$ & 6 & $\|$ & $<$ & 5.5 & $\|$ \\
\hline 1,2-Dichloroethane & $<$ & 6.45 & $\|$ & $<$ & 5.7 & $\|$ & $<$ & 5.55 & $\|$ & $<$ & 5.8 & $\|$ & $<$ & 6.15 & $\|$ & $<$ & 5 & "I & $<$ & 5.5 & /l & $<$ & 6 & $\|$ & $<$ & 5.5 & $\|$ \\
\hline 1,2-Dichloroethylene & $<$ & 6.45 & $\|$ & $<$ & 5.7 & $\|$ & $<$ & 5.55 & /l & $<$ & 5.8 & $\|$ & $<$ & 6.15 & $\|$ & $<$ & 5 & /l & $<$ & 5.5 & /l & $<$ & 6 & $\|$ & $<$ & 5.5 & $\|$ \\
\hline 1,2-Dichloropropane & $<$ & 6.45 & $/ /$ & $<$ & 5.7 & $\mathrm{~J} / \mathrm{H} /$ & $<$ & 5.55 & $\|$ & $<$ & 5.8 & $\|$ & $<$ & 6.15 & $\|$ & $<$ & 5 & " & $<$ & 5.5 & $\|$ & $<$ & 6 & $\|$ & $<$ & 5.5 & $\|$ \\
\hline 2-Chloroethyl vinyl ether & & NA & & & NA & & & NA & & & NA & & & NA & & $<$ & 10 & "I & $<$ & 11 & "l & $<$ & 12 & $\|$ & $<$ & 11 & $/ 1$ \\
\hline 2-Hexanone & $<$ & 12.9 & $\|$ & $<$ & 11.4 & $\mathrm{~J} / \mathrm{H} /$ & $<$ & 11.1 & /I & $<$ & 11.6 & $\|$ & $<$ & 12.3 & $\|$ & $<$ & 10 & $\|$ & $<$ & 11 & $\|$ & $<$ & 12 & $\|$ & $<$ & 11 & $\|$ \\
\hline Acetone & & 53.9 & /l & $<$ & 11.4 & $\|$ & $<$ & 11.1 & $\|$ & $<$ & 11.6 & $\|$ & & 41 & $\|$ & $<$ & 6.21 & $\mathrm{~J} / \mathrm{V} /$ & $<$ & 10.1 & $\mathrm{~J} / \mathrm{VI}$ & $<$ & 11.2 & $\mathrm{~J} / \mathrm{V} /$ & $<$ & 12.8 & $N I$ \\
\hline Benzene & $<$ & 6.45 & $\|$ & $<$ & 5.7 & $\mathrm{~J} / \mathrm{H} /$ & $<$ & 5.55 & $\|$ & $<$ & 5.8 & $\|$ & $<$ & 6.15 & $\|$ & $<$ & 5 & $\|$ & $<$ & 5.5 & $\|$ & $<$ & 6 & $\|$ & $<$ & 5.5 & $\|$ \\
\hline Bromodichloromethane & $<$ & 6.45 & II & $<$ & 5.7 & $\mathrm{~J} / \mathrm{H} /$ & $<$ & 5.55 & $\|$ & $<$ & 5.8 & $\|$ & $<$ & 6.15 & $\|$ & $<$ & 5 & "l & $<$ & 5.5 & $\|$ & $<$ & 6 & $\|$ & $<$ & 5.5 & $\|$ \\
\hline Bromoform & $<$ & 6.45 & $\|$ & $<$ & 5.7 & $\mathrm{~J} / \mathrm{H} /$ & $<$ & 5.55 & $\|$ & $<$ & 5.8 & $\|$ & $<$ & 6.15 & $\|$ & $<$ & 5 & /l & $<$ & 5.5 & $\|$ & $<$ & 6 & $\|$ & $<$ & 5.5 & $\|$ \\
\hline Bromomethane (Methyl bromide) & $<$ & 12.9 & $\|$ & $<$ & 11.4 & $\|$ & $<$ & 11.1 & $\|$ & $<$ & 11.6 & $\|$ & $<$ & 12.3 & $\|$ & $<$ & 10 & /l & $<$ & 11 & $\|$ & $<$ & 12 & "I & $<$ & 11 & $\|$ \\
\hline Carbon disulfide & $<$ & 6.45 & /I & $<$ & 5.7 & $\|$ & $<$ & 5.55 & $\|$ & $<$ & 5.8 & $\|$ & $<$ & 6.15 & $\|$ & $<$ & 5 & "I & $<$ & 5.5 & $\|$ & & 3.48 & $\mathrm{~s} / 1$ & $<$ & 5.5 & II. \\
\hline Carbon tetrachloride & $<$ & 6.45 & $/ 1$ & $<$ & 5.7 & $\mathbf{J} / \mathbf{H} /$ & $<$ & 5.55 & $\|$ & $<$ & 5.8 & $\|$ & $<$ & 6.15 & $\|$ & $<$ & 5 & $\|$ & $<$ & 5.5 & $\|$ & $<$ & 6 & $\|$ & $<$ & 5.5 & $\|$ \\
\hline Chlorobenzene & $<$ & 6.45 & $\|$ & $<$ & 5.7 & $\mathrm{~J} / \mathrm{H} /$ & $<$ & 5.55 & $\|$ & $<$ & 5.8 & $\|$ & $<$ & 6.15 & $\|$ & $<$ & 5 & $\|$ & $<$ & 5.5 & "I & $<$ & 6 & $\|$ & $<$ & 5.5 & $\|$ \\
\hline Chloroethane & $<$ & 12.9 & /I & $<$ & 11.4 & II & $<$ & 11.1 & $\|$ & $<$ & 11.6 & $\|$ & $<$ & 12.3 & $\|$ & $<$ & 10 & $\|$ & $<$ & 11 & $\|$ & $<$ & 12 & $\|$ & $<$ & 11 & $\|$ \\
\hline Chloroethene (Vinyl chloride) & $<$ & 12.9 & $\|$ & $<$ & 11.4 & $\|$ & $<$ & 11.1 & $\|$ & $<$ & 11.6 & $\|$ & $<$ & 12.3 & $\|$ & $<$ & 10 & $\|$ & $<$ & 11 & $\|$ & $<$ & 12 & $\|$ & $<$ & 11 & $\|$ \\
\hline Chloroform & $<$ & 6.45 & "I & $<$ & 5.7 & $/ /$ & $<$ & 5.55 & $\|$ & $<$ & 5.8 & $\|$ & $<$ & 6.15 & $\|$ & $<$ & 5 & $\|$ & $<$ & 5.5 & $\|$ & $<$ & 6 & $\|$ & $<$ & 5.5 & $\|$ \\
\hline Chloromethane (Methyl chloride) & $<$ & 12.9 & $\|$ & $<$ & 11.4 & $\|$ & $<$ & 11.1 & $\|$ & $<$ & 11.6 & $\|$ & $<$ & 12.3 & $\|$ & $<$ & 10 & "I & $<$ & 11 & $\|$ & $<$ & 12 & $\|$ & $<$ & 11 & $\|$ \\
\hline cis-1,3-Dichloropropene & $<$ & 6.45 & $\|$ & $<$ & 5.7 & $\mathrm{~J} / \mathrm{H} /$ & $<$ & 5.55 & $\|$ & $<$ & 5.8 & II & $<$ & 6.15 & $\|$ & $<$ & 5. & $\|$ & $<$ & 5.5 & $\|$ & $<$ & 6 & $\|$ & $<$ & 5.5 & $\|$ \\
\hline Dibromochloromethane & $1<$ & 6.45 & $\|$ & $<$ & 5.7 & $\mathrm{~J} / \mathrm{H} /$ & $<$ & 5.55 & $\|$ & $<$ & 5.8 & $\|$ & $<$ & 6.15 & $\|$ & $<$ & 5 & 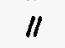 & $<$ & 5.5 & $\|$ & $<$ & 6 & $\|$ & $<$ & 5.5 & $\|$ \\
\hline Dichloromethane (Methylene chloride) & $<$ & 15.2 & $I V I$ & $<$ & 5.7 & $\|$ & $<$ & 5.55 & $\|$ & $<$ & 5.8 & $\|$ & $<$ & 6.15 & $\|$ & $1<$ & 13 & $N I$ & $<$ & 14.2 & $r V I$ & $<$ & 17.4 & $I V I$ & $<$ & 11.9 & $r v I$ \\
\hline Ethylbenzene & $<$ & 6.45 & $\|$ & $<$ & 5.7 & $\mathrm{~J} / \mathrm{H} /$ & $<$ & 5.55 & $\|$ & $<$ & 5.8 & $\|$ & $<$ & 6.15 & $\|$ & $<$ & 5 & $\|$ & $<$ & 5.5 & $\|$ & $<$ & 6 & $\|$ & $<$ & 5.5 & $\|$ \\
\hline Methyl ethyl ketone & $<$ & 12.9 . & $\|$ & $<$ & 11.4 & /I & $<$ & 11.1 & $\|$ & $<$ & 11.6 & "l & $<$ & 12.3 & $\|$ & $<$ & 10 & "l & $<$ & 11 & "I & $<$ & 12 & $\|$ & $<$ & 11 & $\|$ \\
\hline Methyl isobutyl ketone & $<$ & $12.9^{\circ}$ & /I & $<$ & 11.4 & $\mathrm{~J} / \mathrm{H} /$ & $<$ & 11.1 & $\|$ & $<$ & 11.6 & $\|$ & $<$ & 12.3 & $\|$ & $<$ & 10 & II & $<$ & 11 & /l & $<$ & 12 & $\|$ & $<$ & 11 & $/ l$ \\
\hline Styrene & $<$ & 6.45 & "I & $<$ & 5.7 & $\mathrm{~s} / \mathrm{H} /$ & $<$ & 5.55 & $\|$ & $<$ & 5.8 & "I & $<$ & 6.15 & $\|$ & $<$ & 5 & "I & $<$ & 5.5 & $\|$ & $<$ & 6 & $\|$ & $<$ & 5.5 & $\|$ \\
\hline Tetrachloroethylene & $1<$ & 6.45 & /I & $<$ & 5.7 & $J / H /$ & $<$ & 5.55 & $\|$ & $<$ & 5.8 & $\|$ & $<$ & 6.15 & 11 & $<$ & 5 & $\|$ & $<$ & 5.5 & "I & $<$ & 6 & $\|$ & $<$ & 5.5 & $\|$ \\
\hline Toluene & $<$ & 6.45 & $\|$ & $<$ & 5.7 & $\mathrm{~J} / \mathrm{H} /$ & $<$ & 5.55 & $\|$ & $<$ & 5.8 & $\|$ & $<$ & 6.15 & $\|$ & & 1.64 & $\mathrm{~s} / 1$ & $<$ & 5.5 & $\|$ & $<$ & 6 & "I & $<$ & 5.5 & $\|$ \\
\hline trans-1,3-Dichloropropene & $1<$ & 6.45 & $\|$ & $<$ & 5.7 & $\mathrm{~J} / \mathrm{H} /$ & $<$ & 5.55 & $\|$ & $<$ & 5.8 & $\|$ & $<$ & 6.15 & $\|$ & $<$ & 5 & $\|$ & $<$ & 5.5 & "I & $<$ & 6 & "I & $<$ & 5.5 & $\|$ \\
\hline Trichloroethylene & $<$ & 6.45 & $\|$ & $<$ & 5.7 & $\mathrm{~J} / \mathrm{H} /$ & $<$ & 5.55 & $\|$ & $<$ & 5.8 & $\|$ & $<$ & 6.15 & $\|$ & $<$ & 5 & $\|$ & $<$ & 5.5 & $\|$ & $<$ & 6 & "I & $<$ & 5.5 & $\|$ \\
\hline Vinyl acetate & $<$ & 12.9 & /I & $<$ & 11.4 & $\mathrm{~J} / \mathrm{H} /$ & $<$ & 11.1 & $\|$ & $<$ & 11.6 & $\|$ & $<$ & 12.3 & $\|$ & $<$ & 10 & "l & $<$ & 11 & $\|$ & $<$ & 12 & $\|$ & $<$ & 11 & $\|$ \\
\hline Xylenes & $1<$ & 6.45 & 11 & $1<$ & 5.7 & $\mathrm{~J} / \mathrm{H} /$ & $L$ & 5.55 & 11 & $1<$ & 5.8 & II & $<$ & 6.15 & 11 & $1<$ & 5 & II & $1<$ & 5.5 & 11 & $1<$ & 6 & II & $1<$ & 5.5 & II \\
\hline
\end{tabular}


TAOLE 6.1

VOLATILE ORGANIC COMPOUNDS IN SOIL

D.AREA BURNING/RUBBLE PITS SAVANNAH RIVER SITE

\begin{tabular}{|c|c|c|c|c|c|c|c|c|c|c|c|c|c|c|c|c|c|c|c|c|c|c|c|c|c|c|c|}
\hline $\begin{array}{l}\text { Sample Name } \\
\text { Sample Date }\end{array}$ & & $\begin{array}{r}4311 \\
622 \\
\end{array}$ & & & $\begin{array}{r}\text { D4311 } \\
622 \\
\end{array}$ & & & $\begin{array}{r}4311 \\
6239 \\
\end{array}$ & & & $\begin{array}{r}431 \\
623 \\
\end{array}$ & & & $\begin{array}{r}\text { D431 } \\
62 \\
\end{array}$ & & & $\begin{array}{r}\text { D431170 } \\
62393 \\
\end{array}$ & & & $\begin{array}{r}\text { D4311 } \\
6239 \\
\end{array}$ & & & $\begin{array}{r}4311 \\
6239 \\
\end{array}$ & & & $\begin{array}{r}4311 \\
6169 \\
\end{array}$ & \\
\hline Meched 8240 (UG/KG) & & & & & & & & & & & & & & & & & & & & & & & & & & & \\
\hline 1,1,1-Trichloroethane & $<$ & 5.5 & /I & $<$ & 6 & II & $<$ & 5.15 & $\mathrm{~J} / \mathrm{Q} /$ & $<$ & 5.5 & $\mathrm{~J} / \mathrm{O} / \mathrm{L}$ & $<$ & 6.45 & $J / Q /$ & $<$ & 6.25 & II & $<$ & 6.35 & $\|$ & $<$ & 6.25 & $\|$ & $<$ & 5.55 & $\|$ \\
\hline 1,1,2,2-Tetrachloroethane & $<$ & 5.5 & "I & $<$ & 6 & II & $<$ & 5.15 & $\mathrm{~J} / \mathrm{Q} /$ & $<$ & 5.5 & J/OR & $<$ & 6.45 & $J / Q I$ & $<$ & 6.25 & $\|$ & $<$ & 6.35 & $\|$ & $<$ & 6.25 & $\|$ & $<$ & 5.55 & $\|$ \\
\hline 1,1,2-Trichloroethane & $<$ & 5.5 & $\|$ & $<$ & 6 & $\|$ & $<$ & 5.15 & $\mathrm{~J} / \mathrm{Q} /$ & $<$ & 5.5 & $\mathrm{~J} / \mathrm{O} / \mathrm{L}$ & $<$ & 6.45 & $J / Q /$ & & 5.75 & $\mathrm{~J} / /$ & $<$ & 6.35 & $\|$ & $<$ & 6.25 & $\|$ & & 3.32 & $\mathrm{~s} / 1$ \\
\hline 1,1-Dichloroethane & $<$ & 5.5 & $\|$ & $<$ & 6 & /I & $<$ & 5.15 & $\mathrm{~J} / \mathrm{Q} /$ & $<$ & 5.5 & $\mathrm{~J} / \mathrm{O} / \mathrm{L}$ & $<$ & 6.45 & $\mathrm{~J} / \mathrm{Q} /$ & $<$ & $6.25:-$ & $\|$ & $<$ & $\approx 6.35$ & $\|$ & $<$ & 6.25 & $\|$ & $<$ & 5.55 & $\|$ \\
\hline 1,1-Dichloroethylene & $<$ & 5.5 & II & $<$ & 6 & $\|$ & $<$ & 5.15 & $\mathrm{~J} / \mathrm{Q} /$ & $<$ & 5.5 & $\mathrm{~J} / \mathrm{O} / \mathrm{L}$ & $<$ & 6.45 & $J / Q /$ & $<$ & 6.25 & $\|$ & $<$ & 6.35 & $\|$ & $<$ & 6.25 & $\|$ & $<$ & 5.55 & $\|$ \\
\hline 1,2-Dichloroethane & $<$ & 5.5 & "I & $<$ & 6 & $\|$ & $<$ & 5.15 & $J / Q I$ & $<$ & 5.5 & $\mathrm{~J} / \mathrm{O} / \mathrm{L}$ & $<$ & 6.45 & $\mathrm{~J} / \mathrm{Q} /$ & & 6.15 & $\|$ & $<$ & 6.35 & $\|$ & $<$ & 6.25 & $\|$ & & 4.3 & $\mathrm{~s} / 1$ \\
\hline 1,2-Dichloropropane & $<$ & 5.5 & $\|$ & $<$ & 6 & $\|$ & $<$ & 5.15 & $\mathrm{~J} / \mathrm{Q} /$ & $<$ & 5.5 & $\mathrm{~J} / \mathrm{O} \Omega$ & $<$ & 6.45 & $\mathrm{~J} / \mathrm{Q} /$ & $<$ & 6.25 & $\|$ & $<$ & 6.35 & $\|$ & $<$ & 6.25 & $\|$ & $<$ & 5.55 & $\|$ \\
\hline 2-Chloroethyl vinyl ether & $<$ & 11 & "I & $<$ & 12 & $\|$ & & NA & & & NA & & & NA & & & NA & & & NA & & & NA & & & $\mathrm{NA}$ & \\
\hline 2-Hexanone & $<$ & 11 & $\|$ & $<$ & 12 & $\|$ & $<$ & 10.3 & $\mathrm{~J} / \mathrm{Q} /$ & $<$ & 11 & $\mathrm{~J} / \mathrm{O} / \mathrm{L}$ & $<$ & 12.9 & $\mathrm{~J} / \mathrm{Q} /$ & $<$ & 12.5 & $\|$ & $<$ & 12.7 & $\|$ & $<$ & 12.5 & $\|$ & $<$ & 11.1 & $\|$ \\
\hline Acetone & $<$ & 34.2 & $N I$ & $<$ & 25.3 & $/ \mathrm{VI}$ & $<$ & 10 & J/QVI & $<$ & 10.4 & JNOL & $<$ & 26.7 & RQVI & $<$ & 12.8 & $r \mathrm{VI}$ & $<$ & 13.5 & IVI & $<$ & 13.2 & $N I$ & $<$ & 11.1 & $\|$ \\
\hline Benzene & $<$ & 5.5 & /I & $<$ & 6 & $\|$ & $<$ & 5.15 & $3 / Q /$ & $<$ & 7.45 & INOR & $<$ & 6.45 & $\mathrm{~J} / \mathrm{Q}$ & $<$ & 6.25 & $\|$ & $<$ & 6.35 & $\|$ & & 5.64 & $\mathrm{~J} / I$ & $<$ & 5.55 & $\|$ \\
\hline Bromodichloromethane & $<$ & 5.5 & II & $<$ & 6 & /I & $<$ & 5.15 & $J / Q /$ & $<$ & 5.5 & J/OR & $<$ & 6.45 & $\mathrm{~J} / \mathrm{Q} /$ & $<$ & 6.25 & /l & $<$ & 6.35 & $\|$ & $<$ & 6.25 & $\|$ & $<$ & 5.55 & $\|$ \\
\hline Bromoform & $<$ & 5.5 & " & $<$ & 6 & /I & $<$ & 5.15 & $\mathrm{~J} / \mathrm{Q} /$ & $<$ & 5.5 & $3 / 0 / 2$ & $<$ & 6.45 & $\mathrm{~J} / \mathrm{Q} /$ & $<$ & 6.25 & $\|$ & $<$ & 6.35 & $\|$ & $<$ & 6.25 & $\|$ & $<$ & 5.55 & $\|$ \\
\hline Bromomethane (Methyl bromide) & $<$ & 11 & $\|$ & $<$ & 12 & /I & $<$ & 10.3 & $\mathrm{~J} / \mathrm{Q} /$ & $<$ & 11 & $\mathrm{~J} / \mathrm{O} / \mathrm{L}$ & $<$ & 12.9 & $\mathrm{~J} / \mathrm{Q} /$ & $<$ & 12.5 & $\|$ & $<$ & 12.7 & $\|$ & $<$ & 12.5 & $\|$ & $<$ & 11.1 & $\|$ \\
\hline ide & $<$ & 5.5 & II & $<$ & 6 & $\|$ & $<$ & 5.15 & $\mathrm{~J} / \mathrm{Q} /$ & $<$ & 5.5 & $\mathrm{~J} / \mathrm{O} / \mathrm{L}$ & $<$ & 6.45 & $\mathrm{~J} / \mathrm{Q} /$ & $<$ & 6.25 & 11 & $<$ & 6.35 & $\|$ & $<$ & 6.25 & $\|$ & $<$ & 5.55 & $\|$ \\
\hline Chlorobenzene & $<$ & 5.5 & $\|$ & $<$ & 6 & $\|$ & $<$ & 5.15 & $\mathrm{~J} / \mathrm{Q} /$ & $<$ & 5.5 & $\mathrm{~J} / \mathrm{O} / \mathrm{L}$ & $<$ & 6.45 & $J / Q /$ & $<$ & 6.25 & $\|$ & $<$ & 6.35 & $\|$ & $<$ & 6.25 & $\|$ & $<$ & 5.55 & $\|$ \\
\hline Chloroethane & $<$ & 11 & "I & $<$ & 12 & /I & $<$ & 10.3 & $\mathrm{~J} / \mathrm{Q} /$ & $<$ & 11 & $\mathrm{~J} / \mathrm{O} / \mathrm{L}$ & $<$ & $12.9^{\circ}$ & $\mathrm{J} / \mathrm{Q} /$ & $<$ & 12.5 & $\|$ & $<$ & 12.7 & $\|$ & $<$ & 12.5 & $\|$ & $<$ & 11.1 & $\|$ \\
\hline Chloroethene (Vinyl chloride) & $<$ & 11 & $\|$ & $<$ & 12 & $\|$ & $<$ & 10.3 & $J / Q I$ & $<$ & 11 & $\mathrm{~J} / \mathrm{O} / \mathrm{L}$ & $<$ & 12.9 & $\mathrm{~J} / \mathrm{Q} /$ & $<$ & 12.5 & $\|$ & $<$ & 12.7 & $\|$ & $<$ & 12.5 & $\|$ & $<$ & 11.1 & $\|$ \\
\hline Chloroform & $<$ & 5.5 & $\|$ & $<$ & 6 & $\|$ & $<$ & 5.15 & $J / Q /$ & $<$ & 5.5 & $\mathrm{~J} / \mathrm{O} / \mathrm{L}$ & $<$ & 6.45 & $\mathrm{~J} / \mathrm{Q} /$ & $<$ & 6.25 & $\|$ & $<$ & 6.35 & $\|$ & $<$ & 6.25 & "I & $<$ & 5.55 & $\|$ \\
\hline chloride) & $<$ & 11 & / & $<$ & 12 & $\|$ & $<$ & 10.3 & $\mathrm{~J} / \mathrm{Q} /$ & $<$ & 11 & 3/O/L & $<$ & 12.9 & $\mathrm{~J} / \mathrm{Q} /$ & $<$ & 12.5 & $\|$ & $<$ & 12.7 & $\|$ & $<$ & 12.5 & $\|$ & $<$ & 11.1 & $\|$ \\
\hline cis-1, & $<$ & 5.5 & $\|$ & $<$ & 6 & /I & $<$ & 5.15 & $\mathrm{~J} / \mathrm{Q} /$ & $<$ & 5.5 & $\mathrm{~J} / \mathrm{O} / \mathrm{L}$ & $<$ & 6.45 & $\mathrm{~J} / \mathrm{Q} /$ & $<$ & $6.25^{\prime}$ & $\|$ & $<$ & 6.35 & $\|$ & $<$ & 6.25 & $\|$ & $<$ & 5.55 & $\|$ \\
\hline Dibromochloromethane & $<$ & 5.5 & /l & $<$ & 6 & $\|$ & $<$ & 5.15 & $J / Q I$ & $<$ & 5.5 & J/OR & $<$ & 6.45 & $J / Q I$ & $<$ & 6.25 & II. & $<$ & 6.35 & $\|$ & $<$ & 6.25 & $\|$ & $<$ & 5.55 & $\|$ \\
\hline Dichloromethane (Methylene chloride) & $<$ & 12.1 & $N I$ & $<$ & 13.8 & $N I$ & $<$ & 5.15 & $\mathrm{~J} / \mathrm{Q} /$ & $<$ & 5.5 & $\mathrm{~J} / \mathrm{O} / \mathrm{L}$ & $<$ & 6.45 & $J / Q I$ & $<$ & 6.25 & $\|$ & $<$ & 6.35 & $\|$ & $<$ & 6.25 & $\|$ & $<$ & 10.8 & NI \\
\hline Elhylbenzene & $<$ & 5.5 & $\|$ & $<$ & 6 & $\|$ & $<$ & 5.15 & $\mathrm{~J} / \mathrm{Q} /$ & $<$ & 5.5 & $\mathrm{~J} / \mathrm{O} / \mathrm{L}$ & $<$ & 6.45 & $\mathrm{~J} / \mathrm{Q} /$ & $<$ & 6.25 & $/ 1$ & $<$ & 6.35 & $\|$ & $<$ & 6.25 & $\|$ & $<$ & 5.55 & $\|$ \\
\hline Methyl ethyl ketone & $<$ & 11 & "I & $<$ & 12 & $\|$ & $<$ & 10.3 & $J / Q /$ & $<$ & 11 & $\mathrm{~J} / \mathrm{O} / \mathrm{L}$ & $<$ & 12.9 & $\mathrm{~J} / \mathrm{Q} /$ & $<$ & 12.5 & $\|$ & $<$ & 12.7 & $\|$ & $<$ & 12.5 & $\|$ & $<$ & 11.1 & $\|$ \\
\hline Methyl isobutyl ketone & $<$ & 11 & "I & $<$ & 12 & $\|$ & $<$ & 10.3 & $\mathrm{~J} / \mathrm{Q} /$ & $<$ & 11 & $\mathrm{~J} / \mathrm{O} / \mathrm{L}$ & $<$ & 12.9 & $\mathrm{~J} / \mathrm{Q} /$ & $<$ & 12.5 & 11 & $<$ & 12.7 & $\|$ & $<$ & 12.5 & $\|$ & $<$ & 11.1 & "I \\
\hline Styrene & $<$ & 5.5 & $\|$ & $<$ & 6 & /I & $<$ & 5.15 & $\mathrm{~J} / \mathrm{Q} /$ & $<$ & 5.5 & J/OR & $<$ & 6.45 & $J / Q /$ & $<$ & 6.25 & "I & $<$ & 6.35 & $\|$ & $<$ & 6.25 & $\|$ & $<$ & 5.55 & $\|$ \\
\hline Tetrachloroethylene & $<$ & 5.5 & $\|$ & $<$ & 6 & $\|$ & $<$ & 5.15 & $\mathrm{~J} / \mathrm{Q} /$ & $<$ & 5.5 & $\mathrm{~J} / \mathrm{O} / \mathrm{L}$ & $<$ & 6.45 & $J / Q I$ & $<$ & 6.25 & $\|$ & $<$ & 6.35 & $\|$ & $<$ & 6.25 & $\|$ & $<$ & 5.55 & $\|$ \\
\hline Toluene & $<$ & 5.5 & II & $<$ & 6 & $\|$ & $<$ & 5.15 & $\mathrm{~J} / \mathrm{Q} /$ & $<$ & 5.5 & $\mathrm{~J} / \mathrm{O} / \mathrm{L}$ & $<$ & 6.45 & $J / Q /$ & $<$ & 6.25 & $\|$ & $<$ & 6.35 & $\|$ & $<$ & 6.25 & 11 & $<$ & 5.55 & $\|$ \\
\hline trans-1,3-Dichloropropene & $<$ & 5.5 & II & $<$ & 6 & $\|$ & $<$ & 5.15 & $\mathrm{~J} / \mathrm{Q} /$ & $<$ & 5.5 & J/OR & $<$ & 6.45 & $\mathrm{~J} / \mathrm{Q} /$ & $<$ & 6.25 & $\|$ & $<$ & 6.35 & $/ 1$ & $<$ & 6.25 & /I & $<$ & 5.55 & $\|$ \\
\hline Trichloroethylene & $1<$ & 5.5 & $\|$ & $<$ & 6 & $\|$ & $<$ & 5.15 & $J / Q /$ & $<$ & 5.5 & $\mathrm{~J} / \mathrm{O} / \mathrm{L}$ & $<$ & 6.45 & $\mathrm{~J} / \mathrm{Q} /$ & $<$ & 6.25 & $\|$ & $<$. & 6.35 & $\|$ & $<$ & 6.25 & $\|$ & $<$ & 5.55 & $\|$ \\
\hline Vinyl acelate & $<$ & 11 & $\|$ & $<$ & 12 & $\|$ & $<$ & 10.3 & $J / Q /$ & $<$ & 11 & J/OR & $<$ & 12.9 & $\mathrm{~J} / \mathrm{Q} /$ & $<$ & 12.5 & $\|$ & $<$ & 12.7 & $\|$ & $<$ & 12.5 & $\|$ & $<$ & 11.1 & II \\
\hline Xylenes & $<$ & 5.5 & 11 & $1<$ & 6 & 11 & $<$ & 5.15 & $\mathrm{~J} / \mathrm{Q}$ & $1<$ & 5.5 & $\mathrm{~J} / \mathrm{O} / \mathrm{L}$ & $1<$ & 6.45 & $\mathrm{~J} / \mathrm{Q}$ & $1<$ & 6.25 & 11 & $1<$ & 6.35 & II & $1<$ & 6.25 & 11 & $<$ & 5.55 & 11 \\
\hline
\end{tabular}


TABLE 6.1

VOLATILE ORGANIC COMPOUNDS IN SOIL

D-AREA BURNING/RUBBLE PITS SAVANNAH RIVER SITE

\begin{tabular}{|c|c|c|c|c|c|c|c|c|}
\hline $\begin{array}{l}\text { Sample Name } \\
\text { Sample Date }\end{array}$ & $\begin{array}{r}\text { D431 } \\
\quad 616 \\
\end{array}$ & & & $\begin{array}{r}\text { D431 } \\
616 \\
\end{array}$ & & & $\begin{array}{r}D 431 \\
616 \\
\end{array}$ & \\
\hline Method 8240 (UG/KG) & & & & & & & & \\
\hline 1,1,1-Trichloroethane & $<5.55$ & $\|$ & $<$ & 6.8 & II & $<$ & 5.85 & /I \\
\hline 1,1,2,2-Tetrachloroethane & < 5.55 & $\|$ & $<$ & 6.8 & /I & $k$ & 5.85 & /I \\
\hline 1,1,2-Trichloroethane & $<\quad 5.55$ & $\|$ & $<$ & 6.8 & 11 & $<$ & 5.85 & $\|$ \\
\hline 1,1-Dichloroethane & $<\quad 5.55$ & $\|$ & $<$ & 6.8 & $\|$ & $<$ & 5.85 & $\|$ \\
\hline 1,1-Dichloroethylene & $<\quad 5.55$ & $\|$ & $<$ & 6.8 & $\|$ & & 6.11 & $\|$ \\
\hline 1,2-Dichloroethane & $<5.55$ & $\|$ & & 4.2 & $\mathrm{~J} / /$ & $<$ & 5.85 & $\|$ \\
\hline 1,2-Dichloroethylene & $<\quad 5.55$ & $/ 1$ & $<$ & 6.8 & "I & $<$ & 5.85 & "I \\
\hline 1,2-Dichloropropane & $<\quad 5.55$ & $\|$ & $<$ & 6.8 & II & $<$ & 5.85 & II \\
\hline 2-Chloroethyl vinyl ether & NA & & & NA & & & NA & \\
\hline 2-Hexanone & $<11.1$ & $\|$ & $<$ & 13.6 & $\|$ & $<$ & 11.7 & /I \\
\hline Acetone & $<\quad 11.1$ & $/ 1$ & & 12.2 & $3 / /$ & & 398 & $\|$ \\
\hline Benzene & $<5.55$ & $\|$ & $<$ & 6.8 & $\|$ & & 4.31 & $\mathrm{~J} / /$ \\
\hline Bromodichloromethane & $<5.55$ & $/ 1$ & $<$ & 6.8 & $\|$ & $<$ & 5.85 & $\|$ \\
\hline Bromoform & $<5.55$ & /I & $<$ & 6.8 & II & $<$ & 5.85 & "I \\
\hline Bromomethane (Methyl bromide) & $<\quad 11.1$ & $\|$ & $<$ & 13.6 & $\|$ & $<$ & 11.7 & $\|$ \\
\hline Carbon disulfide & $<5.55$ & $\|$ & $<$ & 6.8 & $/ /$ & $<$ & 5.85 & $\|$ \\
\hline Carbon tetrachloride & $<5.55$ & $\|$ & $<$ & 6.8 & $\|$ & $<$ & 5.85 & /I \\
\hline Chlorobenzene & $<5.55$ & $\|$ & $<$ & 6.8 & $\|$ & & 3.54 & $\mathrm{~J} / /$ \\
\hline Chloroethane & $<\quad 11.1$ & $/ 1$ & $<$ & 13.6 & $\|$ & $<$ & 11.7 & $\|$ \\
\hline Chloroethene (Vinyl chloride) & $<\quad 11.1$ & $\|$ & $<$ & 13.6 & $\|$ & $<$ & 11.7 & $\|$ \\
\hline Chloroform & $<\quad 5.55$ & $/ /$ & $<$ & 6.8 & $\|$ & $<$ & 5.85 & $\|$ \\
\hline Chloromethane (Methyl chloride) & $<\quad 11.1$ & $\|$ & $<$ & 13.6 & $\|$ & $<$ & 11.7 & $\|$ \\
\hline cis-1,3-Dichloropropene & $<\quad 5.55$ & $\|$ & $<$ & 6.8 & $\|$ & $<$ & $5.85^{\circ}$ & $\|$ \\
\hline Dibromochloromethane & $<\quad 5.55$ & $\|$ & $<$ & 6.8 & $\|$ & $<$ & 5.85 & $\|$ \\
\hline Dichloromethane (Methylene chloride) & $<13.1$ & IVI & $<$ & 6.8 & $\|$ & $<$ & 5.85 & $\|$ \\
\hline Ethylbenzene & $<\quad 5.55$ & $\|$ & $<$ & 6.8 & $\|$ & $<$ & 5.85 & $\prime \prime$ \\
\hline Methyl ethyl ketone & $<\quad 11.1$ & $\|$ & $<$ & 13.6 & $\|$ & $<$ & 11.7 & $\|$ \\
\hline Methyl isobutyl ketone & $<\quad 11.1$ & $1 /$ & $<$ & 13.6 & $\|$ & $<$ & 11.7 & $\|$ \\
\hline Styrene & $<\quad 5.55$ & $\|$ & $<$ & 6.8 & $\|$ & $<$ & 5.85 & /l \\
\hline Têtirachloroethylene & $<\quad 5.55$ & $/ /$ & $<$ & 6.8 & $\|$ & $<$ & 5.85 & /l \\
\hline Toluene & $<\quad 5.55$ & $\|$ & $<$ & 6.8 & $\|$ & & 4.94 & $\mathrm{~J} / 1$ \\
\hline trans-1,3-Dichloropropene & $<\quad 5.55$ & "I & $<$ & 6.8 & II & $<$ & 5.85 & $\|$ \\
\hline Trichloroethylene & $<\quad 5.55$ & "I & $<$ & 6.8 & $\|$ & & 4.73 & $\mathrm{~J} / /$ \\
\hline Vinyl acetale . & $<\quad 11.1$ & $\|$ & $<$ & 13.6 & $\|$ & $<$ & 11.7 & $\|$ \\
\hline Xylenes & $<\quad 5.55$ & II & $<$ & 6.8 & 11 & $1<$ & 5.85 & II \\
\hline
\end{tabular}


TABLE 6.2

SEMI-VOLATILE ORGANIC COMPOUNDS

IN SOIL

D-AREA BURNING/RUBBLE PITS

SAVANNAH RIVER SITE

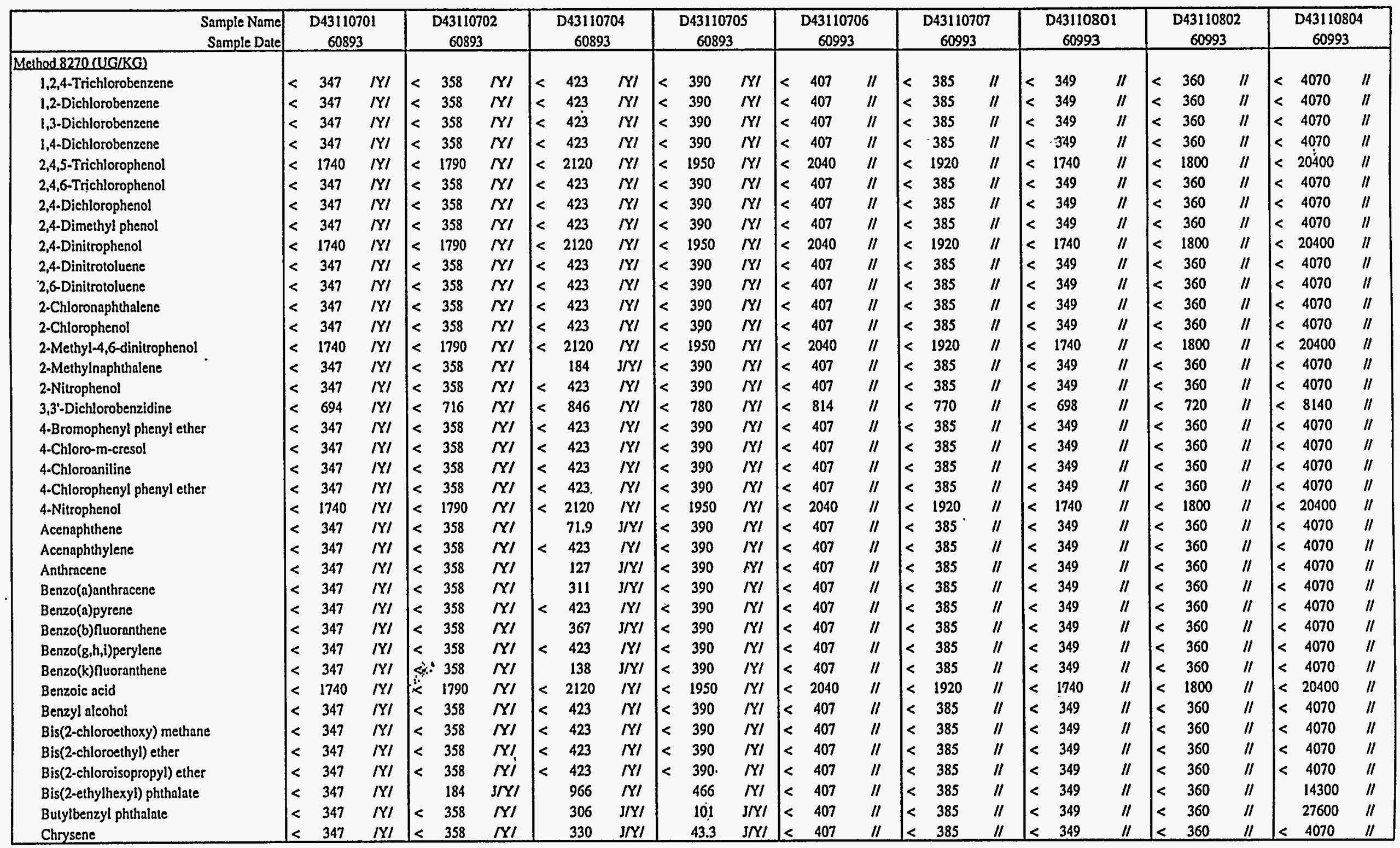


TABLE 6.2

SEM!; VOLATILE ORGANIC COMPOUNDS IN SOIL

D.AREA BURNING/RUBBLE PITS

SAVANNAH RIVER SITE

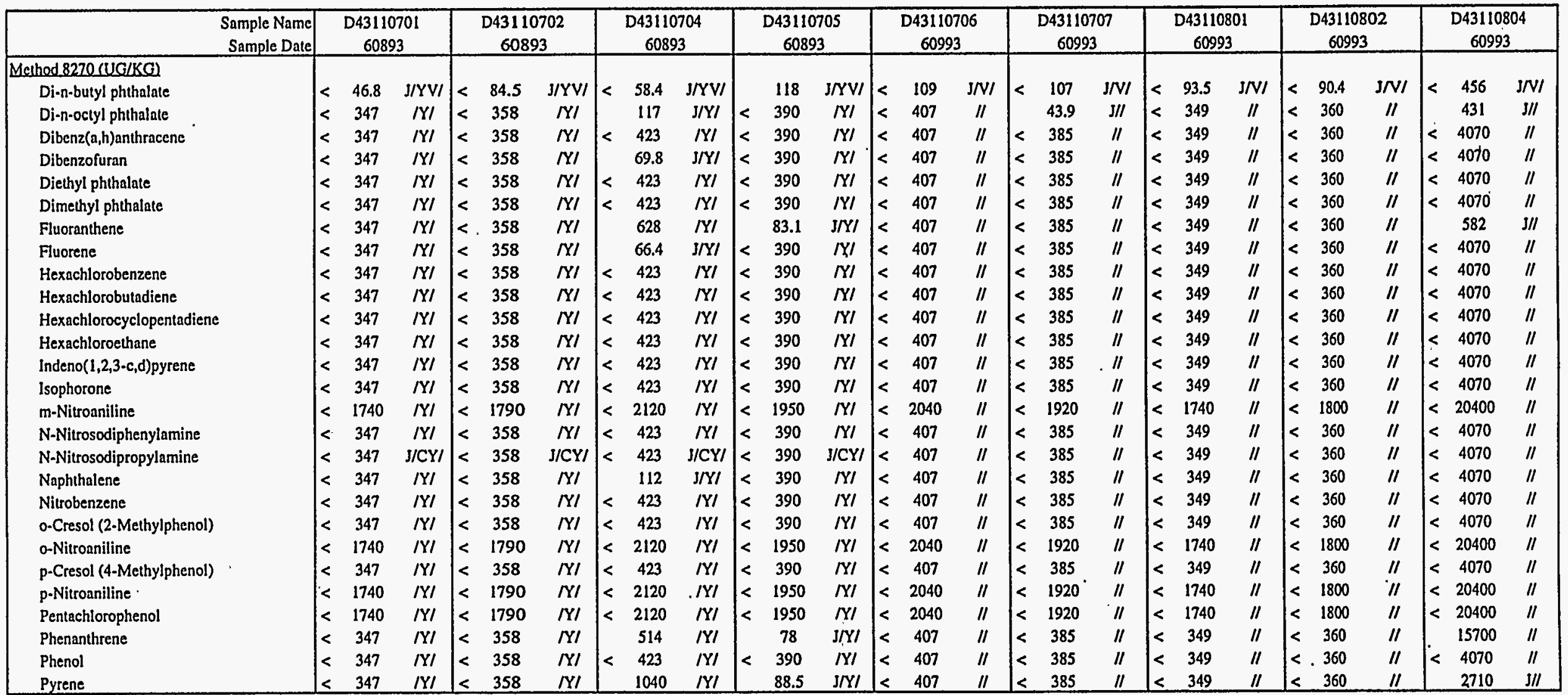


TABLE 6.2

SEMI-VOLATILE ORGANIC COMPOUNDS IN SOIL

D.AREA BURNING/RUBBLE PITS

SAVANNAH RIVER SITE

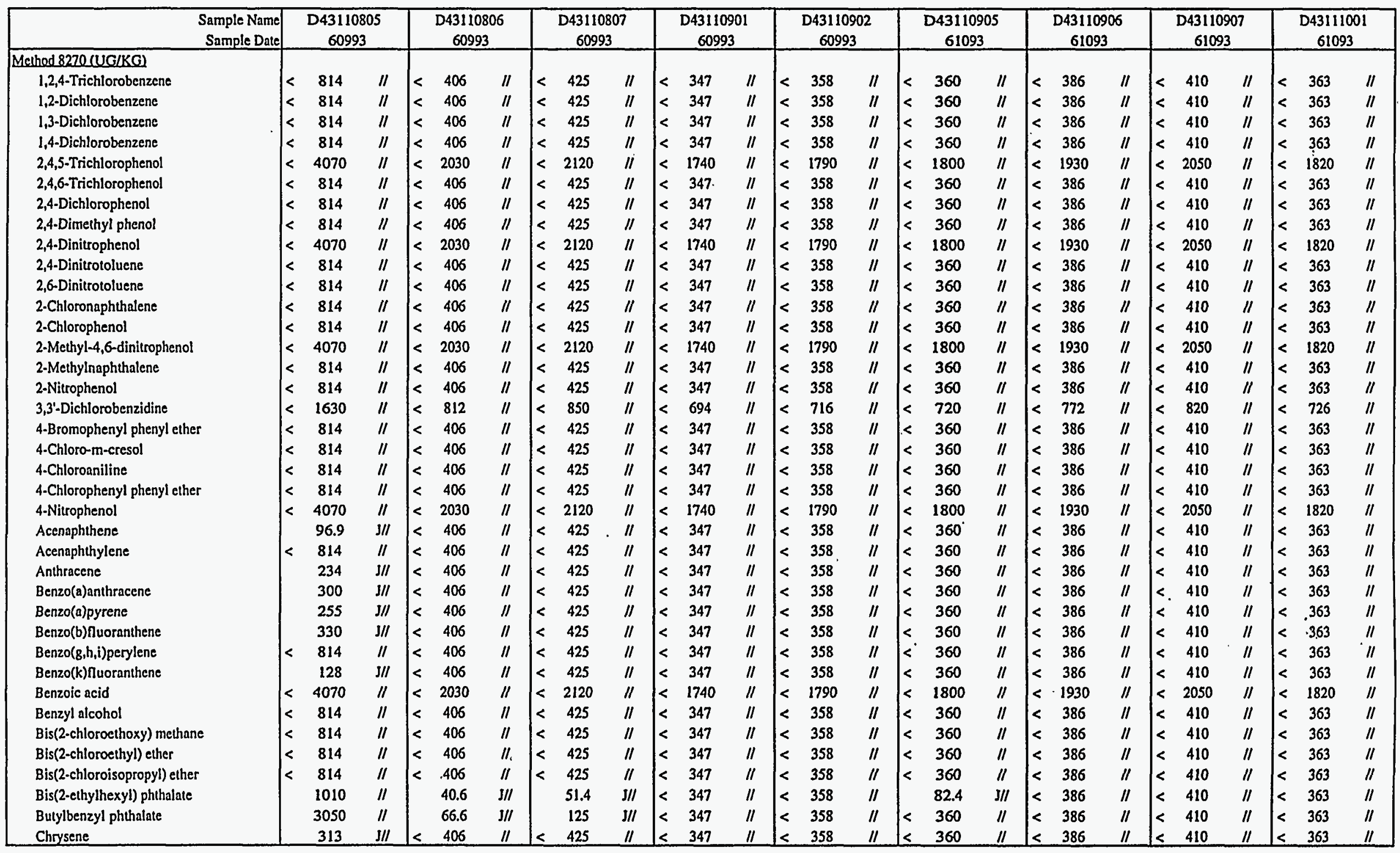


TABLE 6.2

SEMI-VOLATILE ORGANIC COMPOUNDS

IN SOIL

D-AREA BURNING/RUBBLE PITS

SAVANNAH RIVER SITE

\begin{tabular}{|c|c|c|c|c|c|c|c|c|c|c|c|c|c|c|c|c|c|c|c|c|c|c|c|c|c|c|c|}
\hline \multirow{3}{*}{\multicolumn{2}{|c|}{$\begin{array}{|lr|} & \begin{array}{r}\text { Sample Name } \\
\text { Sample Date }\end{array} \\
\text { Method 8270(UG/KG) } & \\
\end{array}$}} & \multicolumn{2}{|c|}{$\begin{array}{c}D 43110805 \\
60993 \\
\end{array}$} & \multicolumn{3}{|c|}{$\begin{array}{c}\mathrm{D} 43110806 \\
60993 \\
\end{array}$} & \multicolumn{3}{|c|}{$\begin{array}{c}D 43110807 \\
60993 \\
\end{array}$} & \multicolumn{3}{|c|}{$\begin{array}{c}D 43110901 \\
60993 \\
\end{array}$} & \multicolumn{3}{|c|}{$\begin{array}{c}43110902 \\
60993 \\
\end{array}$} & \multicolumn{3}{|c|}{$\begin{array}{c}D 43110905 \\
61093 \\
\end{array}$} & \multicolumn{3}{|c|}{$\begin{array}{c}43110906 \\
61093 \\
\end{array}$} & \multicolumn{3}{|c|}{$\begin{array}{c}\text { D43110907 } \\
61093 \\
\end{array}$} & \multicolumn{3}{|c|}{$\begin{array}{c}D 43111001 \\
61093\end{array}$} \\
\hline & & & & & & & & & & & & & & & & & & & & & & & & & & & \\
\hline & & 138 & $\mathrm{~J} / \mathrm{VI}$ & $<$ & 78.8 & $\mathrm{~J} / \mathrm{N} /$ & $<$ & 130 & $\mathrm{~J} / \mathrm{V} /$ & $<$ & 48.9 & $\mathrm{~J} / \mathrm{V} /$ & $<$ & 61.9 & $\mathrm{~J} / \mathrm{VI}$ & $<$ & 233 & $\mathrm{~J} / \mathrm{V} \mathrm{I}$ & $<$ & 288 & $\mathrm{~J} / \mathrm{VI}$ & $<$ & 206 & $\mathrm{~J} / \mathrm{V} /$ & $<$ & 248 & $\mathrm{~J} / \mathrm{VI}$ \\
\hline Di-n-octyl phthalate & & 814 & "l & $<$ & 406 & $M$ & $<$ & 425 & "I & $<$ & 347 & $\|$ & $<$ & 358 & $\|$ & $<$ & 360 & $\|$ & $<$ & 386 & $\|$ & $<$ & 410 & $\|$ & $<$ & 363 & $\|$ \\
\hline Dibenz $(a, h)$ anthracene & & $<814$ & $\|$ & $<$ & 406 & $\|$ & $<$ & 425 & /I & $<$ & 347. & II & $<$ & 358 & $\|$ & $<$ & 360 & $\|$ & $<-$ & 386 & $\|$ & $<$ & 410 & $\|$ & $<$ & 363 & $\|$ \\
\hline Dibenzofuran & & $<\quad 814$ & $/ 1$ & $<$ & 406 & /I & $<$ & 425 & /I & $1<$ & 347 & II & $<$ & 358 & $\|$ & $<$ & 360 & $\|$ & $<$ & 386 & "I & $<$ & 410 & /I & $<$. & 363 & "I \\
\hline Diethyl phthalate & & 814 & "I & k & 406 & "I & $<$ & 425 & /I & |< & 347 & /I & $<$ & 358 & "I & $<$ & 360 & $\|$ & $<$ & 386 & II & $<$ & 410 & $\|$ & $<$ & 363 & II \\
\hline Dimethyl phtthalate & & $<\quad 814$ & "I & $<$ & 406 & $\|$ & $<$ & 425 & /I & $<$ & 347 & $/ 1$ & $<$ & 358 & $\|$ & $<$ & 360 & $\|$ & $<$ & 386 & /I & $<$ & 410 & /l & $<$ & $363^{\prime \prime}$ & /I \\
\hline Fluoranthene & & 882 & $\|$ & $<$ & 406 & $\|$ & $<$ & 425 & /I & $<$ & 347 & $\|$ & $<$ & 358 & /I & $<$ & 360 & $\|$ & $<$ & 386 & "I & $<$ & 410 & "l & $<$ & 363 & "I \\
\hline Fluorene & & 103 & $\mathrm{~J} / 1$ & $<$ & 406 & II & $<$ & 425 & $\|$ & $<$ & 347 & $\|$ & $<$ & 358 & 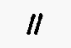 & $<$ & 360 & $\|$ & $<$ & 386 & $\|$ & $<$ & 410 & $\|$ & $<$ & 363 & /I \\
\hline Hexachlorobenzene & & $<\quad 814$ & $\|$ & $<$ & 406 & $\|$ & $<$ & 425 & "I & $<$ & 347 & "l & $<$ & 358 & II & $<$ & 360 & $\|$ & $<$ & 386 & "I & $<$ & 410 & $\|$ & $<$ & 363 & "I \\
\hline Hexachlorobutadiene & & $<814$ & $\|$ & $<$ & 406 & $\|$ & $<$ & 425 & $\|$ & $<$ & 347 & $\|$ & $<$ & 358 & $\|$ & $<$ & 360 & $\|$ & $<$ & 386 & $\|$ & $<$ & 410 & $\|$ & $<$ & 363 & $\|$ \\
\hline Hexachlorocyclopentadiene & & 814 & $\|$ & $<$ & 406 & $\|$ & $<$ & 425 & "I & $<$ & 347 & "l & $<$ & 358 & $\|$ & $<$ & 360 & $\|$ & $<$ & 386 & $\|$ & $<$ & 410 & "I & $<$ & 363 & "I \\
\hline Hexachloroethane & & 814 & /I & $<$ & 406 & "l & $<$ & 425 & /I & $<$ & 347 & $\|$ & $<$ & 358 & $\|$ & $<$ & 360 & $\|$ & $<$ & 386 & "l & $<$ & 410 & "I & $<$ & 363 & $\|$ \\
\hline Indeno $(1,2,3-c, d)$ pyrene & & 83 & $\mathrm{~J} / 1$ & $<$ & 406 & /I & $<$ & 425 & "l & $<$ & 347 & "I & $<$ & 358 & $\|$ & $<$ & 360 & $\|$ & $<$ & 386 & "l & $<$ & 410 & $\|$ & $<$ & 363 & "I \\
\hline Isophorone & & 814 & "I & $<$ & 406 & "l & $<$ & 425 & /I & $<$ & 347 & II & $<$ & 358 & ॥ & $<$ & 360 & $\|$ & $<$ & 386 & "I & $<$ & 410 & "l & $<$ & 363 & $\|$ \\
\hline m-Nitroaniline & & $<4070$ & $\|$ & $<$ & 2030 & 11 & $<$ & 2120 & $\|$ & $<$ & 1740 & $\|$ & $<$ & 1790 & $\|$ & $<$ & 1800. & $\|$ & $<$ & 1930 & $\|$ & $<$ & 2050 & $\|$ & $<$ & 1820 & $/ 1$ \\
\hline N-Nitrosodiphenylaminge & & $<.814$ & "I & $<$ & 406 & $\prime \prime$ & $<$ & 425 & $\|$ & $<$ & 347 & /I & $<$ & 358 & "I & $<$ & $360^{\circ}$ & $\|$ & $<$ & 386 & "I & $<$ & 410 & "I & $\leq$ & 363 & 11. \\
\hline N-Nitrosodipropylamine & & $<814$ & "I & $<$ & 406 & $\prime \prime$ & $<$ & 425 & "I & $<$ & 347 & $\|$ & $<$ & 358 & $\|$ & $<$ & 360 & "I & $<$ & 386 & $\|$ & $<$ & 410 & "I & $\dot{<}$ & 363 & "I \\
\hline Naphthalene & & $<814$ & "I & $<$ & 406 & "I & $<$ & 425 & $\|$ & $<$ & 347 & /I & $<$ & 358 & $\|$ & $<$ & 360 & $\|$ & $<$ & 386 & $\|$ & $<$ & 410 & $\|$ & $<$ & 363 & /I \\
\hline Nitrobenzene & & 814 & "I & $<$ & 406 & $\|$ & $<$ & 425 & $\|$ & $<$ & 347 & $\|$ & $<$ & 358 & $\|$ & $<$ & 360 & $\|$ & $<$ & 386 & "I & $<$ & 410 & $\|$ & $<$ & 363 & "I \\
\hline o-Cresol (2-Methylphenol) & & 814 & "I & $<$ & 406 & /l & $<$ & 425 & /I & $<$ & 347 & II & $<$ & 358 & "l & $<$ & 360 & /l & $<$ & 386 & /I & $<$ & 410 & "I & $<$ & 363 & $\|$ \\
\hline o-Nitroaniline & & $<4070$ & "I & $<$ & 2030 & $\|$ & $<$ & 2120 & "l & $<$ & 1740 & /I & $<$ & 1790 & $\|$ & $<$ & 1800 & $\|$ & $<$ & 1930 & $\|$ & $<$ & 2050 & "l & $<$ & 1820 & $\|$ \\
\hline p-Cresol (4-Methylphenol) & & $<814$ & "I & $<$ & 406 & /I & $<$ & 425 & /I & $<$ & 347 & II & $<$ & 358 & /I & $<$ & 360 & $\|$ & $<$ & 386 & ॥ & $<$ & 410 & /I & $<$ & 363 & "I \\
\hline p-Nitroaniline & & $<4070$ & "I & $<$ & 2030 & "l & $<$ & 2120 & $\|$ & $<$ & 1740 & "I & $<$ & 1790 & $\|$ & $<$ & 1800 & $\|$ & $<$ & 1930 & $\|$ & $<$ & 2050 & /I & $<$ & 1820 & $\|$ \\
\hline Pentachlorophenol & & $<4070$ & $\|$ & $<$ & 2030 & $\|$ & $<$ & 2120 & /I & $<$ & $\$ 740$ & "l & $<$ & 1790 & $\prime \prime$ & $<$ & 1800 & $J / C$ & $<$ & 1930 & $\mathrm{~J} / \mathrm{CL}$ & $<$ & 2050 & $\mathrm{~J} / \mathrm{Cl}$ & $<$ & 1820 & $\mathrm{~J} / \mathrm{C} / \mathrm{H}$ \\
\hline Phenanthrene & & 1630 & "I & & 95 & $\mathrm{~J} / /$ & & 58.2 & $\mathrm{~J} / /$ & $<$ & 347 & $\|$ & $<$ & 358 & $\|$ & $<$ & 360 & $\|$ & $<$ & 386 & $\|$ & $<$ & 410 & $\|$ & $<$ & 363 & $\|$ \\
\hline Phenol & & $<814$ & $\|$ & $<$ & 406 & $\|$ & $<$ & 425 & II & $<$ & 347 & $\|$ & $<$ & 358 & $\|$ & $<$ & 360 & $\mathrm{~J} / \mathrm{Cl}$ & $<$ & 386 & $\mathrm{~J} / \mathrm{Cl}$ & $<$ & 410 & $\mathrm{~J} / \mathrm{Cl}$ & $<$ & 363 & $\mathrm{~J} / \mathrm{Cl}$ \\
\hline Pyrene & & 825 & II & L & 406 & II & $<$ & 425 & 11 & $<$ & 347 & II & $<$ & 358 & II & $<$ & 360 & 11 & $\leq$ & 386 & 11 & $<$ & 410 & II & $<$ & 363 & II \\
\hline
\end{tabular}


TABLE 6.2

SEMI-VOLATILE ORGANIC COMPOUNDS
IN SOIL

D-AREA BURNING/RUBBLE PITS

SAVANNAH RIVER SITE

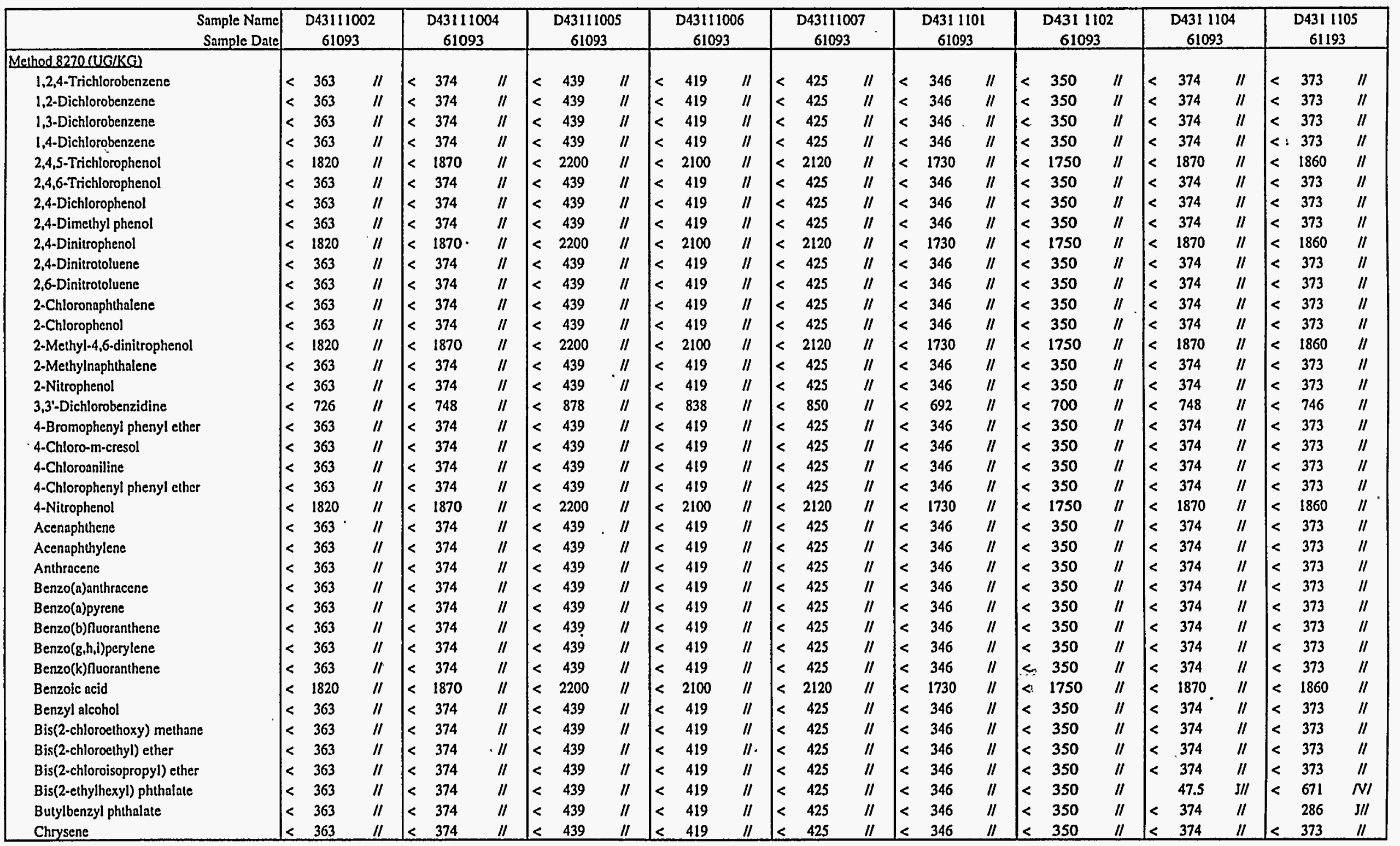


TABLE 6.2

SEMI-VOLATILE ORGANIC COMPOUNDS IN SOIL

D-AREA BURNING/RUBBLE PITS

SAVANNAH RIVER SITE

\begin{tabular}{|c|c|c|c|c|c|c|c|c|c|c|c|c|c|c|c|c|c|c|c|c|c|c|c|c|c|c|c|}
\hline \multirow{3}{*}{$\begin{array}{|cc|} & \begin{array}{r}\text { Sample Name } \\
\text { Sample Date }\end{array} \\
\text { Method 8270 (UG/KG) } & \\
\text { 1,2,4-Trichlorobenzene } & \\
\end{array}$} & \multicolumn{3}{|c|}{$\begin{array}{c}\text { D4311106 } \\
61193 \\
\end{array}$} & \multicolumn{3}{|c|}{$\begin{array}{c}\text { D431 } 1107 \\
61193 \\
\end{array}$} & \multicolumn{3}{|c|}{$\begin{array}{c}\text { D431 1201 } \\
61493 \\
\end{array}$} & \multicolumn{3}{|c|}{$\begin{array}{c}D 4311202 \\
61493 \\
\end{array}$} & \multicolumn{3}{|c|}{$\begin{array}{c}4311204 \\
61493 \\
\end{array}$} & \multicolumn{3}{|c|}{$\begin{array}{c}4311205 \\
61493 \\
\end{array}$} & \multicolumn{3}{|c|}{$\begin{array}{c}4311205 \\
61493 \\
\end{array}$} & \multicolumn{3}{|c|}{$\begin{array}{c}\text { D431 } 1207 \\
61493 \\
\end{array}$} & \multicolumn{3}{|c|}{$\begin{array}{c}D 4311301 \\
61493 \\
\end{array}$} \\
\hline & & & & & & & & & & & & & & & & & & & & & & & & & & & \\
\hline & $<$ & 381 & /I & $<$ & 424 & "I & $<$ & 361 & $\mathrm{~J} / \mathrm{Cl}$ & $<$ & 368 & $\mathrm{~J} / \mathrm{Cl}$ & $<$ & 390 & $\mathrm{~J} / \mathrm{QCl}$ & $<$ & 389 & $\mathrm{~J} / \mathrm{CO} / \mathrm{L}$ & $<$ & 391 & $\mathrm{~J} / \mathrm{Cl}$ & $<$ & 408 & $\mathrm{~J} / \mathrm{Cl}$ & $<$ & 350 & $\mathrm{~J} / \mathrm{Cl}$ \\
\hline 1,2-Dichlorobenzene & $<$ & 381 & "I & $<$ & 424 & II & $<$ & 361 & $\|$ & $<$ & 368 & $\|$ & $<$ & 390 & $\mathrm{~J} / \mathrm{Q} /$ & $<$ & 389 & $\mathrm{~J} / \mathrm{O} / \mathrm{L}$ & $<$ & 391 & $\|$ & $<$ & 408 & /I & $<$ & 350 & $\|$ \\
\hline 1,3-Dichlorobenzene & $<$ & 381 & II & $<$ & 424 & $\|$ & $<$ & 361 & "I & $<$ & 368 & $\|$ & $<$ & 390 & J/QI & $<$ & 389 & $\mathrm{~s} / \mathrm{O} / \mathrm{L}$ & $<$ & 391 & $\|$ & $<$ & 408 & "I & $<$ & 350 & $\|$ \\
\hline 1.4-Dichlorobenzene & $<$ & 381 & "I & $<$ & 424 & $\|$ & $<$ & 361 & "I & $<$ & 368 & $\|$ & $<$ & 390 & $\mathrm{~J} / \mathrm{Q} /$ & $<$ & 389 & $\mathrm{~J} / \mathrm{O} / \mathrm{L}$ & $<$ & 391 & II & $<$ & 408 & II & $<$ & 350 & $\|$ \\
\hline 2,4,5-Trichlorophenol & $<$ & 1900 & 11 & $<$ & 2120 & "I & $<$ & 1800 & $\|$ & $<$ & 1840 & $\|$ & $<$ & 1950 & $J / Q I$ & $<$ & 1940 & $\mathrm{~J} / \mathrm{O} / \mathrm{L}$ & $<$ & 1960 & $\|$ & $<$ & 2040 & $\|$ & $<$ & 1750 & $\|$ \\
\hline 2,4,6-Trichlorophenol & $<$ & 381 & "I & $<$ & 424 & "I & $<$ & 361 & $\|$ & $<$ & 368 & $\|$ & $<$ & 390 & $J / Q I$ & $<$ & 389 & $\mathrm{~J} / \mathrm{O} / \mathrm{L}$ & $<$ & 391 & $\|$ & $<$ & 408 & $\|$ & $<$ & 350 & $\|$ \\
\hline 2,4-Dichlorophenol & $<$ & 381 & "I & $<$ & 424 & "I & $<$ & 361 & $\|$ & $<$ & 368 & $\|$ & $<$ & 390 & $\mathrm{~J} / \mathrm{Q} /$ & $<$ & 389 & $\mathrm{~J} / \mathrm{O} / \mathrm{L}$ & $<$ & 391 & $\|$ & $<$ & 408 & $\|$ & $<$ & 350 & $\|$ \\
\hline 2,4-Dimethyl phenol & $<$ & 381 & $\|$ & $<$ & 424 & /l & $<$ & 361 & /l & $<$ & 368 & $\|$ & $<$ & 390 & $J / Q I$ & $<$ & 389 & $\mathrm{~J} / \mathrm{O} / \mathrm{L}$ & $<$ & 391 & $\|$ & $<$ & 408 & II & $<$ & 350 & $\|$ \\
\hline 2,4-Dinitrophenol & $<$ & 1900 & "I & $<$ & 2120 & "l & $<$ & 1800 & $\|$ & $<$ & 1840 & $\|$ & $<$ & 1950 & $\mathrm{~J} / \mathrm{QV} /$ & $<$ & .1940 & $\mathrm{~J} / \mathrm{O} / \mathrm{L}$ & $<$ & 1960 & $\|$ & $<$ & 2040 & $\|$ & $<$ & 1750 & $\|$ \\
\hline 2,4-Dinitrotoluene & $<$ & 381 & $\|$ & $<$ & 424 & "I & $<$ & 361 & $\|$ & $<$ & 368 & $\|$ & $<$ & 390 & $\mathrm{~J} / \mathrm{QC}$ & $<$ & 389 & $\mathrm{~J} / \mathrm{O} / \mathrm{L}$ & $<$ & 391 & $\|$ & $<$ & 408 & $\|$ & $<$ & 350 & $\|$ \\
\hline 2,6-Dinitrotoluene & $<$ & 381 & /I & $<$ & 424 & "I & $<$ & 361 & II & $<$ & 368 & $\|$ & $<$ & 390 & $\mathrm{~J} / \mathrm{Q} /$ & $<$ & 389 & $\mathrm{~J} / \mathrm{O} / \mathrm{L}$ & $<$ & 391 & $\|$ & $<$ & 408 & $\|$ & $<$ & 350 & $\|$ \\
\hline 2.Chloronaphthalene & $<$ & 381 & "I & $<$ & 424 & "I & $<$ & 361 & $\|$ & $<$ & 368 & $\|$ & $<$ & 390 & $J / Q /$ & $<$ & 389 & $\mathrm{~J} / \mathrm{O} / \mathrm{L}$ & $<$ & 391 & $\|$ & $<$ & 408 & II & $<$ & 350 & $\|$ \\
\hline 2-Chlorophenol & $<$ & 381 & $\|$ & $<$ & 424 & 11 & $<$ & 361 & $\|$ & $<$ & 368 & $\|$ & $<$ & 390 & $J / Q I$ & $<$ & 389 & $\mathrm{~s} / \mathrm{O} / \mathrm{L}$ & $<$ & 391 & $\|$ & $<$ & 408 & $\|$ & $<$ & 350 & $\|$ \\
\hline 2-Methyl-4,6-dinitrophenol & $<$ & 1900 & $\|$ & $<$ & 2120 & "I & $<$ & 1800 & $\|$ & $<$ & 1840 & $\|$ & $<$ & 1950 & $J / Q /$ & $<$ & 1940 & $\mathrm{~J} / \mathrm{O} / \mathrm{L}$ & $<$ & 1960 & $\|$ & $<$ & 2040 & "I & $<$ & 1750 & $\|$ \\
\hline 2-Methylnaphthalene & $<$ & 381 & $\|$ & $<$ & 424 & "I & $<$ & 361 & $\|$ & $<$ & 368 & $\|$ & $<$ & 390 & $3 / Q /$ & $<$ & 389 & J/O/L & $<$ & 391 & $\|$ & $<$ & 408 & "I & $<$ & 350 & $\|$ \\
\hline 2-Nitrophenol & $<$ & 381 & $\|$ & $<$ & 424 & $\|$ & $<$ & 361 & $\|$ & $<$ & 368 & $\|$ & $<$ & 390 & $\mathrm{~J} / \mathrm{Q}$ & $<$ & 389 & $\mathrm{~J} / \mathrm{O} / \mathrm{L}$ & $<$ & 391 & $\|$ & $<$ & 408 & $\|$ & $<$ & 350 & $\|$ \\
\hline 4-Bromophenyl phenyl ether & $<$ & 381 & $\|$ & $<$ & 424 & "l & $<$ & 361 & $\|$ & $<$ & 368 & $\|$ & $<$ & 390 & $\mathrm{~J} / \mathrm{Q} /$ & $<$ & 389 & $\mathrm{~J} / \mathrm{O} / \mathrm{L}$ & $<$ & 391 & $\|$ & $<$ & 408 & /I & $<$ & 350 & $\|$ \\
\hline 4.Chloro-m-cresol & $<$ & 381 & /I & $<$ & 424 & $\|$ & $<$ & 361 & $\|$ & $<$ & 368 & $\|$ & $<$ & 390 & $J / Q /$ & $<$ & 389 & $\mathrm{~J} / \mathrm{O} / \mathrm{L}$ & $<$ & 391 & $\|$ & $<$ & 408 & $\|$ & $<$ & 350 & $\|$ \\
\hline 4-Chloroaniline & $<$ & 381 & /I & $<$ & 424 & II & $<$ & 361 & $\|$ & $1<$ & 368 & $\|$ & $<$ & 390 & $\mathrm{~J} / \mathrm{Q} /$ & $<$ & 389 & $\mathrm{~J} / \mathrm{O} / \mathrm{L}$ & $<$ & 391 & $\|$ & $<$ & 408 & $\|$ & $<$ & 350 & $\|$ \\
\hline 4-Chlorophenyl phenyl ether & $<$ & 381 & /I & $<$ & 424 & "l & $<$ & 361 & $\|$ & $<$ & 368 & $\|$ & $<$ & 390 & $\mathrm{~J} / \mathrm{Q} /$ & $<$ & 389 & J/OR & $<$ & 391 & $\|$ & $<$ & 408 & "I & $<$ & 350 & $\|$ \\
\hline 4-Nitrophenol & $<$ & 1900 & $\|$ & $<$ & 2120 & "I & $<$ & 1800 & $\|$ & $<$ & 1840 & $\|$ & $<$ & 1950 & $J / Q I$ & $<$ & 1940 & $\mathrm{~J} / \mathrm{O} / \mathrm{L}$ & $<$ & 1960 & $\|$ & $<$ & 2040 & $\|$ & $<$ & 1750 & $\|$ \\
\hline Acenaphthene & $<$ & 381 & II & $<$ & 424 & II & $<$ & 361 & $\|$ & $<$ & 368 & $\|$ & $<$ & 390 & $J / Q /$ & $<$ & $389^{\circ}$ & $\mathrm{J} / \mathrm{O} / \mathrm{L}$ & $<$ & 391 & /I & $<$ & 408 & $\|$ & $<$ & 350 & $\|$ \\
\hline Acenaphthylene & $<$ & 381 & "I & $<$ & 424 & $\|$ & $<$ & 361 & 11 & $<$ & 368 & $\|$ & $<$ & 390 & $J / Q /$ & $<$ & 389 & $\mathrm{~J} / \mathrm{O} / \mathrm{L}$ & $<$ & 391 & $\|$ & $<$ & 408 & /I & $<$ & 350 & $/ 1$ \\
\hline Anthracene & $<$ & 381 & /I & $<$ & 424 & "I & $<$ & 361 & $\|$ & $<$ & 368 & $\|$ & $<$ & 390 & $J / Q I$ & $<$ & 389 & $\mathrm{~J} / \mathrm{O} / \mathrm{L}$ & $<$ & 391 & $\|$ & $<$ & 408 & $\|$ & $<$ & 350 & $\|$ \\
\hline Benzo(a)anthracene & $<$ & 381 & $\|$ & $<$ & 424 & $\|$ & $<$ & 361 & $\|$ & & 70.7 & $\mathrm{~J} / 1$ & $<$ & 390 & $\mathrm{~J} / \mathrm{Q} /$ & $<$ & 389 & $\mathrm{~J} / \mathrm{O} / \mathrm{L}$ & & 52.4 & $3 / l$ & $<$ & 408 & $\|$ & $<$ & 350 & $\|$ \\
\hline Benzo(a)pyrene & $<$ & 381 & $/ 1$ & $<$ & 424 & "I & $<$ & 361 & $/ 1$ & & 84.6 & $\mathrm{~J} / 1$ & $<$ & 390 & $\mathrm{~J} / \mathrm{Q} /$ & $<$ & 389 & $\mathrm{~J} / \mathrm{O} / \mathrm{L}$ & $<$ & 391 & $\|$ & $<$ & 408 & $\|$ & $<$ & 350 & $\|$ \\
\hline Benzo(b) nuoranthene & $<$ & 381 & $\|$ & $<$ & 424 & $\|$ & $<$ & 361 & $\|$ & & 108 & $\mathrm{~J} / I$ & $<$ & 390 & $\mathrm{~J} / \mathrm{Q} /$ & $<$ & 389 & $\mathrm{~J} / \mathrm{O} / \mathrm{L}$ & & 56.3 & $\mathrm{~J} / \prime$ & $<$ & 408 & $\|$ & $<$ & 350 & $\|$ \\
\hline Benzo(g,h,i)perylene & $<$ & 381 & $\therefore / 1$ & $<$ & 424 & "I & $<$ & 361 & "I & $<$ & 368 & II & $<$ & 390 & $J / Q /$ & $<$ & 389 & $\mathrm{~J} / \mathrm{O} / \mathrm{L}$ & $<$ & 391 & $\|$ & $<$ & 408 & II & $<$ & 350 & $\|$ \\
\hline Benzo(k)nuoranthene & $<$ & 381 & II & $<$ & 424 & II & $<$ & $36 !$ & $\|$ & & 43.1 & $\mathrm{~J} / \mathrm{I}$ & $<$ & 390 & $\mathrm{~J} / \mathrm{Q} /$ & 1< & 389 & $\mathrm{~J} / \mathrm{O} / \mathrm{L}$ & $<$ & 391 & $\|$ & $<$ & 408 & "I & $\mid<$ & 350 & $/ I$ \\
\hline Benzoic acid & $<$ & 1900 & "I & $<$ & 2120 & "I & $<$ & 1800 & II & $<$ & 1840 & $\|$ & $<$ & 1950 & $J / Q /$ & $<$ & 1940 & $\mathrm{~J} / \mathrm{O} / \mathrm{L}$ & $<$ & 1960 & $\|$ & $<$ & 2040 & $\|$ & $<$ & 1750 & $\|$ \\
\hline Benzyl alcohol & $<$ & 381 & "I & $<$ & 424 & $\|$ & $<$ & 361 & $\|$ & $<$ & 368 & $\|$ & $<$ & 390 & $J / Q /$ & $<$ & 389 & $\mathrm{~J} / \mathrm{O} / \mathrm{L}$ & $<$ & 391 & $\|$ & $<$ & 408 & "I & $<$ & 350 & $\|$ \\
\hline Bis(2-chloroethoxy) methane & $<$ & 381 & "I & $<$ & 424 & "I & $<$ & 361 & $\|$ & $<$ & 368 & $\|$ & $<$ & 390 & $\mathrm{~J} / \mathrm{Q} /$ & $<$ & 389 & J/OR & $<$ & 391 & $\|$ & $<$ & 408 & $\|$ & $<$ & 350 & $\|$ \\
\hline Bis(2-chloroethyl) ether & $<$ & 381 & $\|$ & $<$ & 424 &.$\prime \prime$ & $<$ & 361 & $\|$ & $<$ & 368 & $\|$ & $<$ & 390 & $\mathrm{~J} / \mathrm{Q} /$ & $<$ & 389 & $\mathrm{~J} / \mathrm{O} / \mathrm{L}$ & $<$ & 391 & $\|$ & $<$ & 408 & $\|$ & $<$ & 350 & $\|$ \\
\hline Bis(2-chloroisopropyl) ether & $<$ & 381 & $\|$ & $<$ & 424 & $\|$ & $<$ & 361 & $\|$ & $<$ & 368 & $\|$ & $<$ & 390 & $\mathrm{~J} / \mathrm{Q} /$ & $<$ & 389 & $\mathrm{~J} / \mathrm{O} / \mathrm{h}$ & $<$ & 391 & II & $<$ & 408 & II & $<$ & 350 & $\|$ \\
\hline
\end{tabular}


TABLE 6.2

SEMI-VOLATTLE ORGANIC COMPOUNDS IN SOIL

D-AREA BURNING/RUBBLE PITS

SAVANNAH RIVER SITE

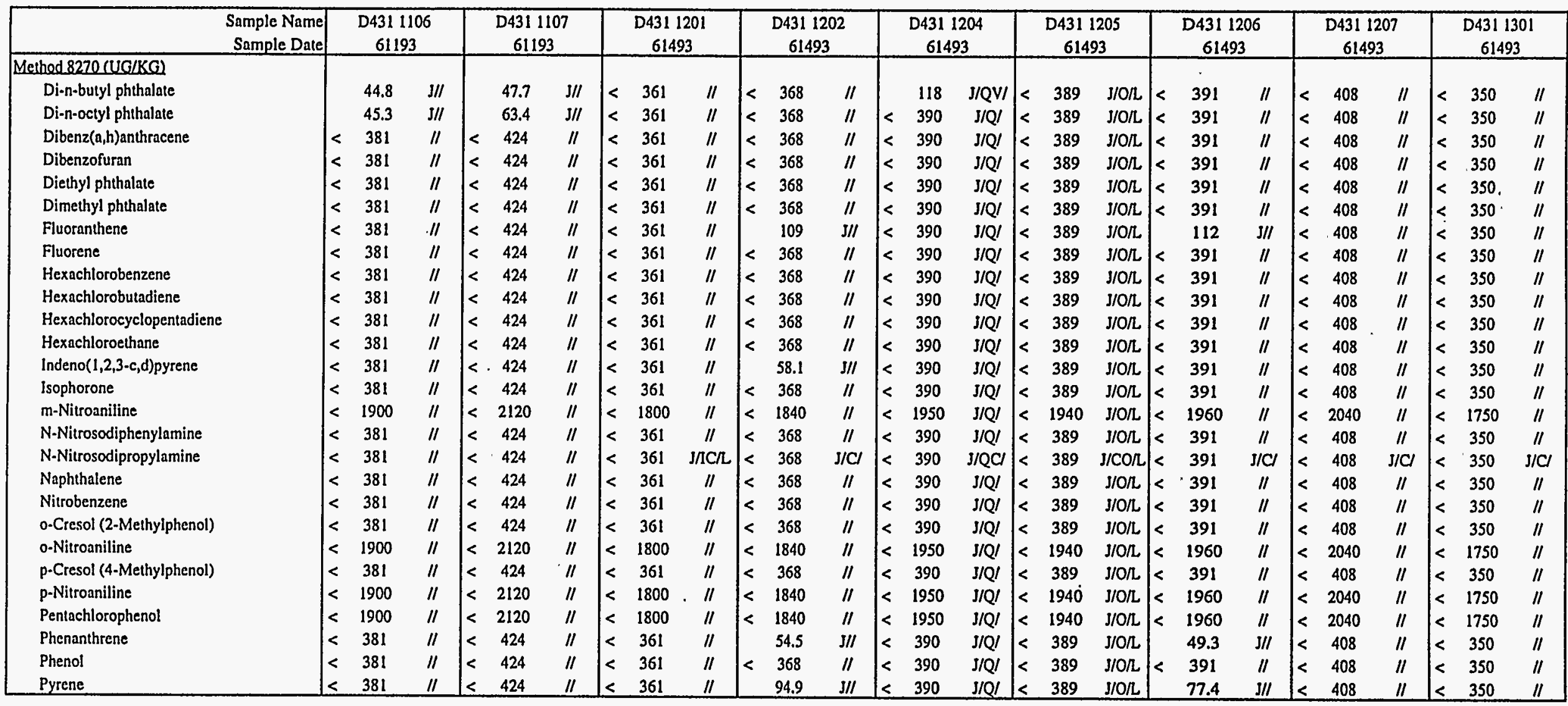


TABLE 6.2

SEMI-VOLATILE ORGANIC COMPOUNDS IN SOIL

D-AREA BURNING/RUBBLE PITS

SAVANNAH RIVER SITE

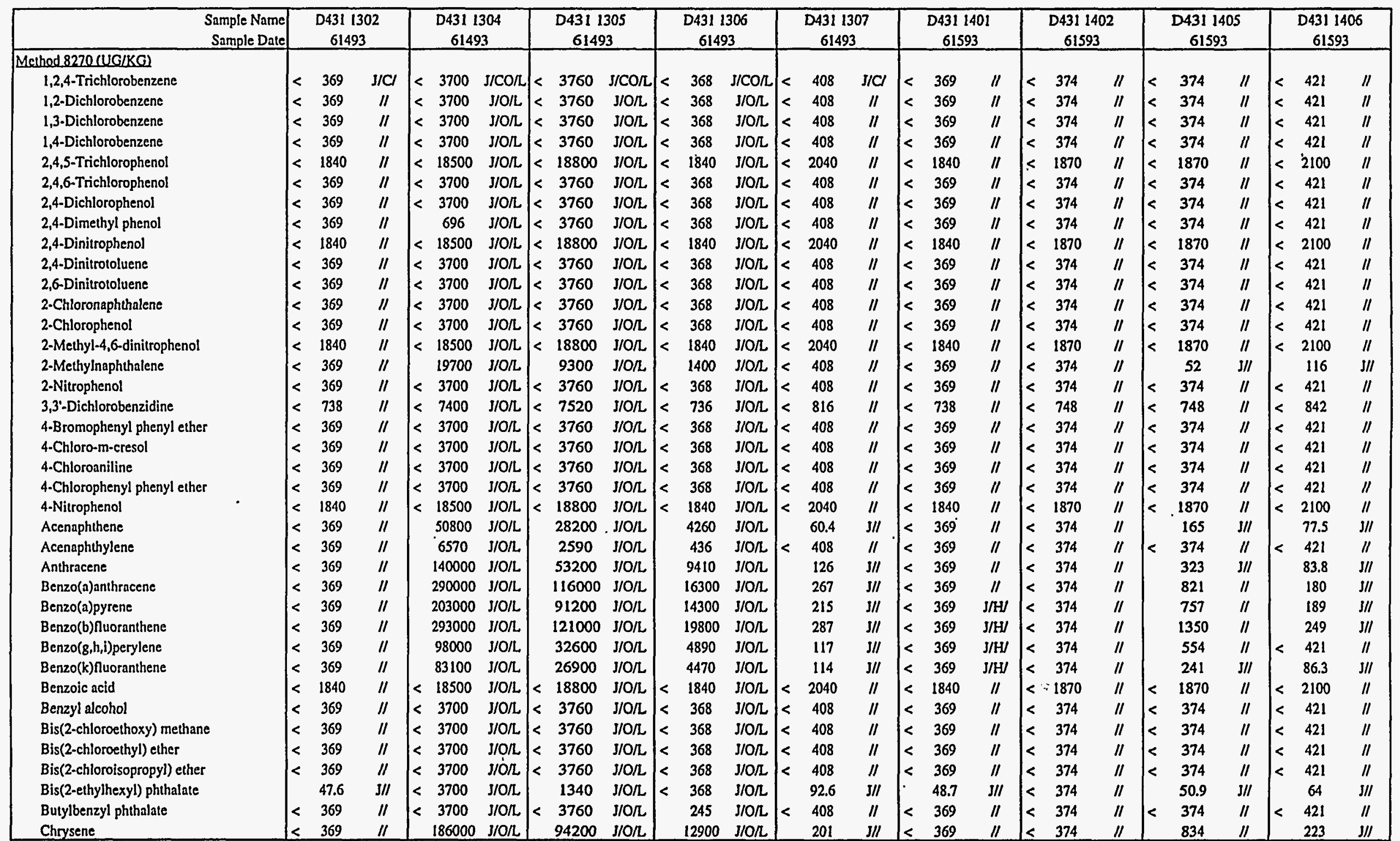


TABLE 6.2

SEMI-VOLATILE ORGANIC COMPOUNDS IN SOIL

D-AREA BURNING/RUBBLE PITS

SAVANNAH RIVER SITE

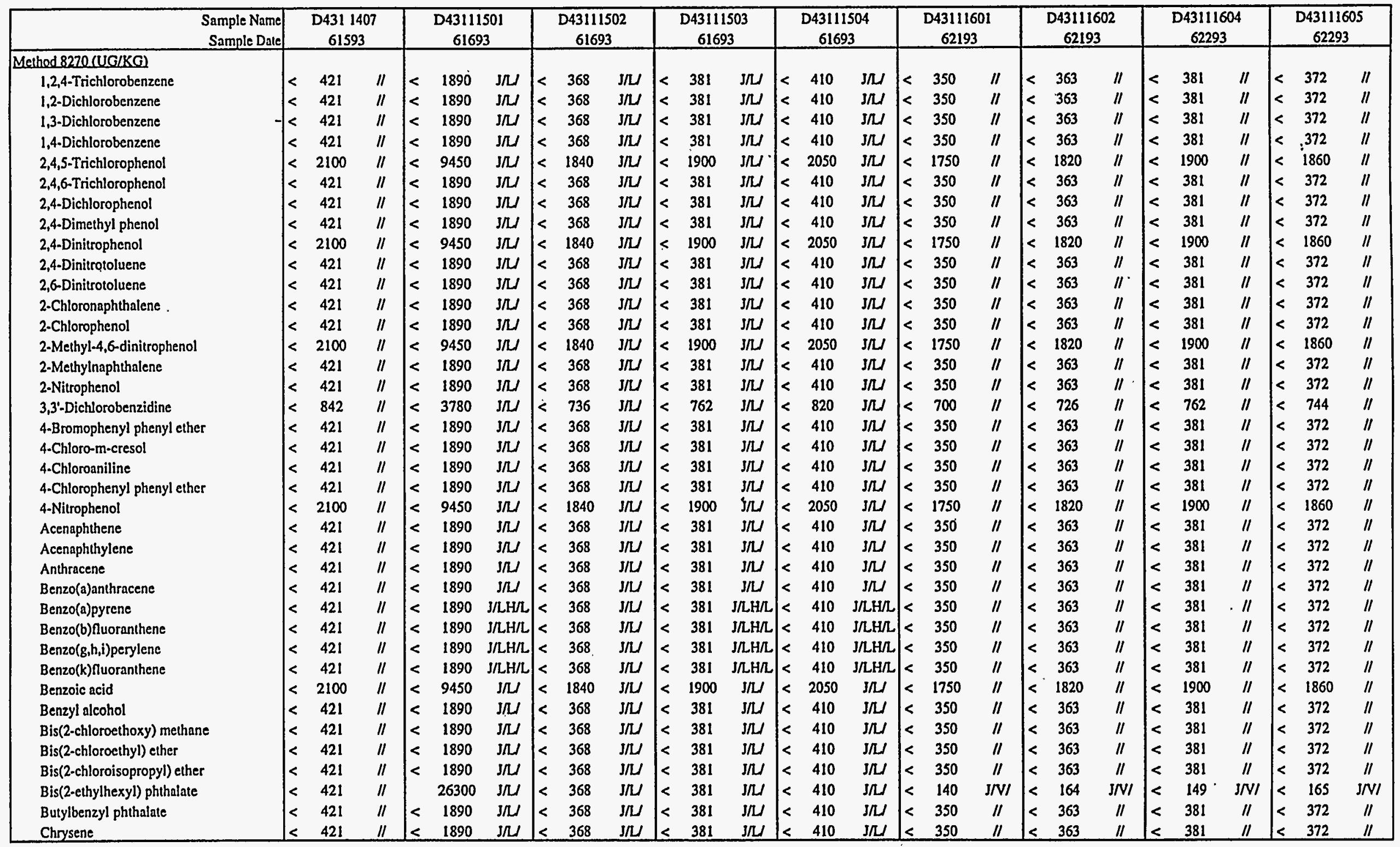


TABLE 6.2

SEMI-VOLATILE ORGANIC COMPOUNDS IN SOIL

D-AREA BURNING/RUBBLE PITS

SAVANNAH RIVER SITE

\begin{tabular}{|c|c|c|c|c|c|c|c|c|c|c|c|c|c|c|c|c|c|c|c|c|c|c|c|c|c|c|}
\hline \multirow{3}{*}{$\begin{array}{|lr|} & \begin{array}{r}\text { Sample Name } \\
\text { Sample Date }\end{array} \\
\text { Method } 8270(U G / K G) & \\
\end{array}$} & \multicolumn{2}{|c|}{$\begin{array}{c}\text { D4311407 } \\
61593 \\
\end{array}$} & \multicolumn{3}{|c|}{$\begin{array}{c}\text { D43111501 } \\
61693 \\
\end{array}$} & \multicolumn{3}{|c|}{$\begin{array}{c}\text { D43111502 } \\
61693 \\
\end{array}$} & \multicolumn{3}{|c|}{$\begin{array}{c}\text { D43111503 } \\
61693 \\
\end{array}$} & \multicolumn{3}{|c|}{$\begin{array}{c}43111504 \\
61693 \\
\end{array}$} & \multicolumn{3}{|c|}{$\begin{array}{c}43111601 \\
62193 \\
\end{array}$} & \multicolumn{3}{|c|}{$\begin{array}{c}\text { D43111602 } \\
62193\end{array}$} & \multicolumn{3}{|c|}{$\begin{array}{c}\text { D43111604 } \\
62293 \\
\end{array}$} & \multicolumn{3}{|c|}{$\begin{array}{c}\mathrm{D} 43111605 \\
62293 \\
\end{array}$} \\
\hline & & & & & & & & & & & & & & & & & & & & & & & & & & \\
\hline & $<\quad 99.8$ & $\mathrm{~J} / \mathrm{VI}$ & $<$ & 1890 & $\mathrm{~J} / \mathrm{L} /$ & $<$ & 40.5 & $\mathrm{~J} / \mathrm{VL}$ & $<$ & 59.4 & J/VU & $<$ & 72.2 & $\mathrm{~J} / \mathrm{VL}$ & $<$ & 88.2 & $J / V I$ & $<$ & 93.3 & $\mathrm{~J} / \mathrm{NI}$ & $<$ & 96.4 & $\mathrm{~J} / \mathrm{V} /$ & $<$ & 104 & $J / N /$ \\
\hline Di-n-octyl phthalate & $<\quad 421$ & $\|$ & $<$ & 1890 & $\mathrm{~J} / \mathrm{LH} / \mathrm{L}$ & $<$ & 368 & $\mathrm{~J} / \mathrm{L}$ & $<$ & 381 & $\mathrm{~J} / \mathrm{LH} / \mathrm{L}$ & $<$ & 410 & $\mathrm{~J} / \mathrm{LH} / \mathrm{L}$ & $<$ & 350 & $\|$ & $<$ & 363 & "I & $<$ & 381 & "l & $<$ & 372 & $\|$ \\
\hline Dibenz(a,h)anthracene & $<\quad 421$ & $\|$ & $<$ & 1890 & $\mathrm{~J} / \mathrm{LH} / \mathrm{L}$ & $<$ & 368 & $\mathrm{~J} / \mathrm{U}$ & $<$ & 381 & $\mathrm{~J} / \mathrm{LH} / \mathrm{L}$ & $<$ & 410 & $\mathrm{~J} / \mathrm{LH} / \mathrm{L}$ & $<$ & 350 & $\|$ & $<$ & 363 & $" 1$ & $<$ & 381 & $\|$ & $<$ & 372 & $\|$ \\
\hline Dibenzofuran & $<\quad 421$ & /I & $<$ & 1890 & $\mathrm{~J} / \mathrm{L}$ & $<$ & 368 & $\mathrm{~J} / \mathrm{M}$ & $<$ & 381 & $\mathrm{~J} / \mathrm{L}$ & $<$ & 410 & $\mathrm{~J} / \mathrm{L}$ & $<$ & 350 & $\|$ & $<$ & 363 & $\|$ & $<$ & 381 & $\|$ & $<$ & $372:$ & " \\
\hline Diethyl phthalate & $<421$ & $\|$ & |< & 1890 & $\mathrm{~J} / \mathrm{L} /$ & $<$ & 368 & $\mathrm{~J} / \mathrm{U}$ & $<$ & 381 & $\mathrm{~J} / \mathrm{L} /$ & 1< & 410 & $3 / \mathrm{L}$ & $<$ & 350 & $\|$ & $k$ & 363 & "I & $<$ & 381 & $\|$ & $<$ & 372 . & $\|$ \\
\hline Dimethyl phthalate & $<\quad 421$ & 11 & $<$ & 1890 & $\mathrm{~J} / \mathrm{LJ}$ & $<$ & 368 & $\mathrm{~J} / \mathrm{L}$ & $<$ & 381 & $\mathrm{~s} / \mathrm{L}$ & $<$ &, 410 & $\mathrm{~J} / \mathrm{L} /$ & $<$ & 350 & $\|$ & $<$ & 363 & $\|$ & $<$ & 381 & 11 & $<$ & 372 & $\|$ \\
\hline Fluoranthene & $<\quad 421$ & /I & $<$ & 1890 & $\mathrm{~J} / \mathrm{L}$ & $<$ & 368 & $\mathrm{~J} / \mathrm{N}$ & $<$ & 381 & $\mathrm{~J} / \mathrm{L}$ & $1<$ & 410 & $\mathrm{~J} / \mathrm{L}$ & $<$ & 350 & $\|$ & $<$ & 363 & $\|$ & $<$ & 381 & $\|$ & $\mid<$ & 372 & /I \\
\hline Fluorene & $<\quad 421$ & /I & $<$ & 1890 & $\mathrm{~J} / \mathrm{L}$ & $<$ & 368 & $\mathrm{~J} / \mathrm{N}$ & $<$ & 381 & $\mathrm{~J} / \mathrm{L}$ & $<$ & 410 & $\mathrm{~J} / \mathrm{L}$ & $<$ & 350 & $\|$ & $<$ & 363 & /I & $<$ & 381 & 11 & $<$ & 372 & "I \\
\hline Hexachlorobenzene & $<\quad 421$ & $\|$ & $<$ & 1890 & $\mathrm{~J} / \mathrm{L}$ & $<$ & 368 & $\mathrm{~J} / \mathrm{L}$ & $<$ & 381 & $\mathrm{~s} / \mathrm{L} /$ & $<$ & 410 & $\mathrm{~J} / \mathrm{L}$ & $<$ & 350 & $\|$ & $<$ & 363 & $\|$ & $<$ & 381 & $\|$ & $<$ & 372 & $\|$ \\
\hline Hexachlorobutadiene & $<\quad 421$ & $\|$ & $<$ & 1890 & $\mathrm{~J} / \mathrm{L}$ & $<$ & 368 & $\mathrm{~J} / \mathrm{H}$ & $<$ & 381 & $3 / \mathrm{L} /$ & $<$ & 410 & $\mathrm{~J} / \mathrm{L}$ & $<$ & 350 & $\|$ & $<$ & 363 & $\|$ & $<$ & 381 & $\|$ & $<$ & 372 & $\|$ \\
\hline Hexachlorocyclopentadiene & $<421$ & $\|$ & $<$ & 1890 & $\mathrm{~J} / \mathrm{L}$ & $<$ & 368 & $\mathrm{~s} / \mathrm{N}$ & $<$ & 381 & $\mathrm{~J} / \mathrm{L}$ & $<$ & 410 & $\mathrm{~J} / \mathrm{L}$ & $<$ & 350 & $\|$ & $<$ & 363 & $\|$ & $<$ & 381 & $\|$ & $<$ & 372 & "I \\
\hline Hexachloroethane & $<\quad 421$ & $\|$ & $<$ & 1890 & $\mathrm{~J} / \mathrm{L}$ & $<$ & 368 & $\mathrm{~J} / \mathrm{U}$ & $<$ & 381 & $\mathrm{~J} / \mathrm{U}$ & $<$ & 410 & $\mathrm{~J} / \mathrm{L}$ & $<$ & 350 & $\|$ & $<$ & 363 & $\|$ & $<$ & 381 & $\|$ & $<$ & 372 & "I \\
\hline Indeno $(1,2,3-c, d)$ pysene & $<421$ & $/ 1$ & $<$ & 1890 & $\mathrm{~J} / \mathrm{LH} / \mathrm{L}$ & $<$ & 368 & $\mathrm{~J} / \mathrm{M}$ & $<$ & 381 & $\mathrm{~J} / \mathrm{LH} / \mathrm{L}$ & $<$ & 410 & $\mathrm{~J} / \mathrm{LH} / \mathrm{L}$ & $<$ & 350 & $\|$ & $<$ & 363 & $\|$ & $<$ & 381 & II & $<$ & 372 & $\|$ \\
\hline Isophorone & $<\quad 421$ & $\|$ & $<$ & 1890 & $3 / \mathrm{J}$ & $<$ & 368 & $\mathrm{~J} / \mathrm{L}$ & $<$ & 381 & $\mathrm{~J} / \mathrm{L}$ & $<$ & 410 & $\mathrm{~J} / \mathrm{L}$ & $<$ & 350 & $\|$ & $<$ & 363 & $\|$ & $<$ & 381 & /l & $<$ & 372 & "l \\
\hline line & $<2100$ & $\|$ & $<$ & 9450 & $3 / 2$ & $<$ & 1840 & $\mathrm{~J} / \mathrm{L}$ & $<$ & 1900 & $\mathrm{~J} / \mathrm{L}$ & $<$ & 2050 & $3 / \mathrm{L}$ & $<$ & 1750 & $\|$ & $<$ & 1820 & $\|$ & $<$ & 1900 & /I & $<$ & 1860 & $\|$ \\
\hline enylamine & $<\quad 421$ & $\|$ & $<$ & 1890 & $\mathrm{~J} / \mathrm{L}$ & $<$ & 368 & $\mathrm{~J} / \mathrm{U}$ & $<$ & 381 & $\mathrm{~J} / \mathrm{L}$ & $<$ & 410 & $\mathrm{~J} / \mathrm{L}$ & $<$ & 350 & "I & $<$ & 363 & $\|$ & $<$ & 381 & "I & $<$ & 372 & $\|$ \\
\hline N-Nitrosodipropylamine & $<\quad 421$ & "l & $<$ & 1890 & $\mathrm{~J} / \mathrm{L}$ & $<$ & 368 & $\mathrm{~J} / \mathrm{U}$ & $<$ & 381 & $\mathrm{~J} / \mathrm{L}$ & $<$ & 410 & $\mathrm{~J} / \mathrm{L}$ & $<$ & 350 & "I & $<$ & 363 & $\|$ & $<$ & 381 & $\|$ & $<$ & 372 & $\|$ \\
\hline Naphthalene & $<\quad 421$ & $\|$ & $<$ & 1890 & $\mathrm{~J} / \mathrm{L} /$ & $<$ & 368 & $\mathrm{~J} / \mathrm{L}$ & $<$ & 381 & $\mathrm{~s} / \mathrm{L}$ & $<$ & 410 & $3 / \mathrm{L}$ & $<$ & 350 & $\|$ & $<$ & 363 & $\|$ & $<$ & 381 & $\|$ & $<$ & 372 & $\|$ \\
\hline Nitrobenzene & $<\quad 421$ & $\|$ & $<$ & 1890 & $\mathrm{~J} / \mathrm{L}$ & $<$ & 368 & $3 / L$ & $<$ & 381 & $\mathrm{~J} / \mathrm{L}$ & $<$ & 410 & $\mathrm{~J} / \mathrm{L}$ & $<$ & 350 & "I & $<$ & 363 & "I & $<$ & 381 & $\|$ & $<$ & 372 & $\|$ \\
\hline o-Cresol (2-Methylphenol) & $<\quad 421$ & $\|$ & $<$ & 1890 & $\mathrm{~J} / \mathrm{L}$ & $<$ & 368 & $\mathrm{~J} / \mathrm{L}$ & $<$ & 381 & $\mathrm{~J} / \mathrm{L}$ & $<$ & 410 & $\mathrm{~J} / \mathrm{L}$ & $<$ & 350 & $\|$ & $<$ & 363 & $\|$ & $<$ & 381 & $\|$ & $<$ & 372 & $\|$ \\
\hline o-Nitroaniline & $<2100$ & /I & $<$ & 9450 & $\mathrm{~J} / \mathrm{L}$ & $<$ & 1840 & $\mathrm{I} / \mathrm{M}$ & $<$ & 1900 & $\mathrm{~J} / \mathrm{L}$ & $<$ & 2050 & $\mathrm{~J} / \mathrm{L}$ & $<$ & 1750 & $\|$ & $<$ & 1820 & $\|$ & $<$. & 1900 & 11 & $<$ & 1860 & $\|$ \\
\hline p-Cresol (4-Methyiphenol) & $<\quad 421$ & $\|$ & $<$ & 1890 & $\mathrm{~J} / \mathrm{L}$ & $<$ & 368 & $\mathrm{~J} / \mathrm{L}$ & $<$ & 381 & $\mathrm{~J} / \mathrm{L} /$ & $<$ & 410 & $\mathrm{~s} / \mathrm{L}$ & $<$ & 350 & $\|$ & $<$ & 363 & $\|$ & $<$ & 381 & $\|$ & $\leqslant$ & 372 & $\|$ \\
\hline p-Nitroaniline & $<2100$ & "I & $<$ & 9450 & $\mathrm{~J} / \mathrm{L}$ & $<$ & 1840 & $\mathrm{~J} / \mathrm{L}$ & $<$ & 1900 & $\mathrm{~J} / \mathrm{L}$ & $<$ & 2050 & $\mathrm{~J} / \mathrm{L}$ & $<$ & $1750^{\circ}$ & "I & $<$ & 1820 & $\|$ & $<$ & 1900 & $\|$ & $i<$ & 1860 & "I \\
\hline Pentachlorophenol & $<2100$ & $\|$ & $<$ & 9450 & $\mathrm{~J} / \mathrm{L}$ & $<$ & 1840 & $\mathrm{~J} / \mathrm{L}$ & $<$ & 1900 & $3 / 2$ & $<$ & 2050 & $\mathrm{~J} / \mathrm{M}$ & $<$ & 1750 & $\|$ & $<$ & 1820 & "I & $<$ & 1900 & $\|$ & $<$ & 1860 & "I \\
\hline Phenanthrene & $<\quad 421$ & 11 & $<$ & 1890 & $\mathrm{~J} / \mathrm{L} /$ & $<$ & 368 & $\mathrm{~J} / \mathrm{L}$ & $<$ & 381 & $\mathrm{~J} / \mathrm{L}$ & $1<$ & 410 & $\mathrm{~J} / \mathrm{L}$ & $<$ & 350 & $\|$ & $<$ & 363 & $\|$ & $<$ & 381 & $\|$ & $<$ & 372 & "I \\
\hline Phenol & $<\quad 421$ & $\mathrm{~J} / \mathrm{Cl}$ & $<$ & 1890 & $\mathrm{~J} / \mathrm{L}$ & $<$ & 368 & $\mathrm{~J} / \mathrm{L}$ & $<$ & 381 & $3 / L$ & $<$ & 410 & $\mathrm{~s} / \mathrm{M}$ & $<$ & 350 & $\|$ & $<$ & 363 & 11 & $<$ & 381 & 11 & $<$ & 372 & "I \\
\hline Pyrene & $<421$ & 11 & $<$ & 1890 & $\mathrm{~J} / \mathrm{L}$ & $<$ & 368 & $\mathrm{~J} / \mathrm{M}$ & < & 381 & $\mathrm{~J} / \mathrm{L}$ & 1< & 410 & $\mathrm{~J} / \mathrm{L}$ & $<$ & 350 & II & |< & 363 & $\|$ & $1<$ & 381 & II & $1<$ & 372 & II \\
\hline
\end{tabular}


TABLE 6.2

SEMI-VOLATILE ORGANIC COMPOUNDS IN SOIL

D.AREA BURNING/RUBBLE PITS

SAVANNAH RIVER SITE

\begin{tabular}{|c|c|c|c|c|c|c|c|c|c|c|c|c|c|c|c|c|c|c|c|c|c|c|c|c|c|c|c|}
\hline \multirow{2}{*}{\begin{tabular}{|lr} 
& $\begin{array}{r}\text { Sample Name } \\
\text { Sample Date }\end{array}$ \\
Mechod 8270(UG/KG) & \\
\end{tabular}} & \multicolumn{3}{|c|}{$\begin{array}{c}\mathrm{D} 43111606 \\
62293 \\
\end{array}$} & \multicolumn{3}{|c|}{$\begin{array}{c}D 4311701 \\
62393 \\
\end{array}$} & \multicolumn{3}{|c|}{$\begin{array}{c}\text { D431 } 1702 \\
62393 \\
\end{array}$} & \multicolumn{3}{|c|}{$\begin{array}{c}\text { D431 } 1704 \\
62393\end{array}$} & \multicolumn{3}{|c|}{$\begin{array}{c}D 4311705 \\
62393\end{array}$} & \multicolumn{3}{|c|}{$\begin{array}{c}\text { D4311706 } \\
62393 \\
\end{array}$} & \multicolumn{3}{|c|}{$\begin{array}{c}\text { D431 } 1801 \\
61693 \\
\end{array}$} & \multicolumn{3}{|c|}{$\begin{array}{c}\text { D431 } 1802 \\
61693 \\
\end{array}$} & \multicolumn{3}{|c|}{$\begin{array}{c}\text { D431 } 1803 \\
61693\end{array}$} \\
\hline & & & & & & & & & & & & & & & & & & & & & & & & & & & \\
\hline 1,2,4-Trichlorobenzene & $<$ & 389 & /1 & $<$ & 344 & $\|$ & $<$ & 364 & $\|$ & $<$ & 403 & $\mathrm{~J} / \mathrm{L}$ & $<$ & 419 & $\mathrm{~J} / \mathrm{L}$ & $<$ & 414 & $\|$ & $<$ & 365 & $\|$ & $<$ & 368 & $\|$ & < & 441 & $\|$ \\
\hline 1,2-Dichlorobenzene & $<$ & 389 & /I & $<$ & 344 & $\|$ & $<$ & 364 & "l & $<$ & 403 & $\mathrm{~J} / \mathrm{L}$ & $<$ & 419 & $\mathrm{~J} / \mathrm{M}$ & $<$ & 414 & $\|$ & $<$ & 365 & $\|$ & $<$ & 368 & $\|$ & $<$ & 441 & $\|$ \\
\hline 1,3-Dichlorobenzene & $<$ & 389 & $\|$ & $<$ & 344 & "I & $<$ & 364 & 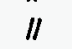 & $<$ & 403 & $\mathrm{~J} / \mathrm{L}$ & $<$ & 419 & $\mathrm{~J} / \mathrm{L}$ & $<$ & 414 & II & $<$ & 365 & $\|$ & $<$ & 368 & $\|$ & $<$ & $44 !$ & $\|$ \\
\hline 1,4-Dichlorobenzene & $<$ & 389 & /I & $<$ & 344 & $\|$ & $<$ & 364 & $\|$ & $<$ & 403 & $\mathrm{~J} / \mathrm{L}$ & $<$ & 419 & $\mathrm{~J} / \mathrm{M}$ & $<$ & 414 & $\|$ & $<$ & 365 & $\|$ & $<$ & 368 & $\|$ & $<$ & 441 & $\|$ \\
\hline 2,4,5-Trichlorophenol & $<$ & 1940 & $\|$ & $<$ & 1720 & $\|$ & $<$ & 1820 & $\|$ & < & 2020 & $3 \pi$ & $<$ & 2100 & $\mathrm{~J} / \mathrm{L}$ & $<$ & 2070 & $\|$ & $<$ & 1820 & $\|$ & $<$ & 1840 & $\|$ & $<$ & 2200 & $\|$ \\
\hline 2,4,6-Trichlorophenol & $<$ & 389 & $\|$ & $<$ & 344 & $\|$ & $<$ & 364 & 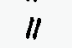 & $<$ & 403 & $\mathrm{~J} / \mathrm{L}$ & $<$ & 419 & $\mathrm{~J} / \mathrm{L}$ & $<$ & 414 & $\|$ & $<$ & 365 & $\|$ & $<$ & 368 & $/ /$ & $<$ & 441 & $\|$ \\
\hline 2,4-Dichlorophenol & $<$ & 389 & $\|$ & $<$ & 344 & $\|$ & $<$ & 364 & $\|$ & $<$ & 403 & $3 / L$ & $<$ & 419 & $\mathrm{~s} / \mathrm{L}$ & $<$ & 414 & $\|$ & $<$ & 365 & $\|$ & $<$ & 368 & $\|$ & $<$ & 441 & II \\
\hline 2,4-Dimethyl phenol & $<$ & 389 & II & $<$ & 344 & $\|$ & $<$ & 364 & $\|$ & $<$ & 403 & $J / L$ & $<$ & 419 & $\mathrm{~J} / \mathrm{M}$ & $<$ & 414 & $\|$ & $<$ & 365 & $\|$ & $<$ & 368 & $\|$ & $<$ & 441 & $\|$ \\
\hline 2,4-Dinitrophenol & $<$ & 1940 & $\|$ & $<$ & 1720 & 11 & $<$ & 1820 & $\|$ & $<$ & 2020 & $\mathrm{~J} / \mathrm{L}$ & $<$ & 2100 & $\mathrm{~J} / \mathrm{L}$ & $<$ & 2070 & II & $<$ & 1820 & $\|$ & $<$ & 1840 & $/ /$ & $<$ & 2200 & $\|$ \\
\hline 2,4-Dinitrotoluene & $<$ & 389 & $\|$ & $<$ & 344 & $" I$ & $<$ & 364 & $" \prime$ & $<$ & 403 & $\mathrm{~J} / \mathrm{L}$ & $<$ & 419 & $\mathrm{~J} / \mathrm{L}$ & $<$ & 414 & $\|$ & $<$ & 365 & $\|$ & $<$ & 368 & "I & $<$ & 441 & $\|$ \\
\hline 2,6.Dinitrotoluene & $<$ & 389 & "l & $<$ & 344 & $\|$ & $<$ & 364 & $\|$ & $<$ & 403 & $\mathrm{~J} / \mathrm{L}$ & $<$ & 419 & $\mathrm{~J} / \mathrm{M}$ & $<$ & 414 & $\|$ & $<$ & 365 & $\|$ & $<$ & 368 & $\|$ & $<$ & 441 & $\|$ \\
\hline 2-Chloronaphthalene & $<$ & 389 & $\|$ & |< & 344 & $\|$ & $<$ & 364 & $\|$ & $<$ & 403 & $\mathrm{~J} / \mathrm{L}$ & $<$ & 419 & $J \pi$ & $<$ & 414 & $\|$ & $<$ & 365 & $\|$ & $<$ & 368 & $\|$ & $<$ & 441 & $\|$ \\
\hline 2.Chlorophenol & $<$ & 389 & $\mathrm{~J} / 1 / \mathrm{H}$ & $<$ & 344 & $\|$ & $<$ & 364 & $\|$ & $<$ & 403 & $\mathrm{~J} / \mathrm{L}$ & $<$ & 419 & $\mathrm{~J} / \mathrm{M}$ & $<$ & 414 & $\|$ & $<$ & 365 & $\|$ & $<$ & 368 & $\|$ & $<$ & 441 & $\|$ \\
\hline 2-Methyl-4,6-dinitrophenol & $<$ & 1940 & $\|$ & $<$ & 1720 & $\|$ & $<$ & 1820 & $\|$ & $<$ & 2020 & $\mathrm{~J} / \mathrm{L}$ & $<$ & 2100 & $\mathrm{~J} / \mathrm{M}$ & $<$ & 2070 & $\|$ & $<$ & 1820 & $\|$ & $<$ & 1840 & "I & $<$ & 2200 & $\|$ \\
\hline 2-Methylnaphthalene & $<$ & 389 & $\|$ & $<$ & 344 & / & $<$ & 364 & $\|$ & $<$ & 403 & $J / L$ & $<$ & 419 & $\mathrm{~J} / \mathrm{L}$ & $<$ & 414 & /I & $<$ & 365 & $\|$ & $<$ & 368 & $\|$ & $<$ & 441 & $\|$ \\
\hline 2-Nitrophenol & $<$ & 389 & $/ 1$ & $<$ & 344 & $\|$ & $<$ & 364 & $\|$ & $<$ & 403 & JUL & $<$ & 419 & $\mathrm{~J} / \mathrm{L}$ & $<$ & 414 & II & $<$ & 365 & $\|$ & $<$ & 368 & II & $<$ & 441 & $\|$ \\
\hline 3,3'-Dichlorobenzidine & $<$ & 778 & $\|$ & $<$ & 688 & $\|$ & $<$ & 728 & $\|$ & $<$ & 806 & $\mathrm{~J} / \mathrm{L}$ & $<$ & 838 & $\mathrm{~J} / \mathrm{L} /$ & $<$ & 828 & $\|$ & $<$ & 730 & $\|$ & $<$ & 736 & $\|$ & $<$ & 882 & $\|$ \\
\hline 4-Bromophenyl phenyl ether & $<$ & 389 & II & $<$ & 344 & $\|$ & $<$ & 364 & $\|$ & $<$ & 403 & JIL & $<$ & 419 & $\mathrm{~J} / \mathrm{L}$ & $<$ & 414 & $/ /$ & $<$ & 365 & $\|$ & $<$ & 368 & $\|$ & $<$ & 441 & $\|$ \\
\hline 4-Chloro-n & $<$ & 389 & $\|$ & $<$ & 344 & "I & $<$ & 364 & $\|$ & $<$ & 403 & $\mathrm{~J} / \mathrm{L} /$ & $<$ & 419 & $\mathrm{~J} / \mathrm{L}$ & $<$ & 414 & $\|$ & $<$ & 365 & $\|$ & $<$ & 368 & /l & $<$ & 441 & $\|$ \\
\hline 4.Chloroaniline & $<$ & 389 & 11 & $<$ & 344 & $\|$ & $<$ & 364 & $\|$ & $<$ & 403 & $\mathrm{~J} / \mathrm{L}$ & $<$ & 419 & $\mathrm{~J} / \mathrm{L}$ & $<$ & 414 & $\|$ & $<$ & 365 & $\|$ & $<$ & 368 & $\|$ & $<$ & 441 & $" 1$ \\
\hline 4-Chlorophenyl phenyl ether & $<$ & 389 & /l & $<$ & 344 & $\|$ & $<$ & 364 & $\|$ & $<$ & 403 & $J / L$ & $<$ & 419 & $\mathrm{~J} / \mathrm{L}$ & $<$ & 414 & II & $<$ & 365 & $\|$ & $<$ & 368 & $\|$ & $\mid<$ & 441 & $\|$ \\
\hline 4.Nitrophenol & $<$ & 1940 & $\|$ & $<$ & 1720 & $\|$ & $<<$ & 1820 & 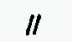 & $<<$ & 2.020 & $\mathrm{~J} / \mathrm{L}$ & $<$ & 2100 & $\mathrm{~s} / \mathrm{M}$ & $<$ & 2070 & $\|$ & $<$ & 1820 & $\|$ & $<$ & 1840 & $\|$ & $<$ & 2200 & $\|$ \\
\hline Acenaphthene & $<$ & 389 & /I & $<$ & 344 & $\|$ & $<$ & 364 & $\|$ & $<$ & 403 & $\mathrm{~J} / \mathrm{U}$ & $<$ & 419 & $\mathrm{~J} / \mathrm{L}$ & $<$ & 414 & $\|$ & $1<$ & 365 & $\|$ & $<$ & 368 & "I & $<$ & 441 & 11 \\
\hline Acenaphthylene & $<$ & 389 & "I & $<$ & 344 & $/ 1$ & $<$ & 364 & $\|$ & $<$ & 403 & $J / L$ & $<$ & 419 & $\mathrm{~J} / \mathrm{M}$ & $<$ & 414 & $\|$ & $<$ & 365 & $\|$ & $<$ & 368 & $\|$ & $<$ & 441 & II \\
\hline Anthracene & $<$ & 389 & 11 & $<$ & 344 & $\|$ & $<$ & 364 & $\|$ & $<$ & 403 & $\mathrm{~J} / \mathrm{L}$ & $<$ & 419 & $\mathrm{~J} / \mathrm{L}$ & $<$ & 414 & II & $1<$ & 365 & II & $<$ & 368 & /I & $<$ & 441 & II \\
\hline Benzo(a)anthracene & $<$ & 389 & "I & $<$ & 344 & $\|$ & $<$ & 364 & $\|$ & $<$ & 403 & $\mathrm{~s} / \mathrm{U}$ & $<$ & 419 & $\mathrm{~J} / \mathrm{L}$ & $<$ & 414 & II & $<$ & 365 & "I & $<$ & 368 & $\|$ & $<$ & 441 & II \\
\hline Benzo(a)pytene & $<$ & 389 & 11 & $<$ & 344 & $\|$ & $<<$ & 364 & $\|$ & $<$ & 403 & $\mathrm{~J} / \mathrm{LH} / \mathrm{H}$ & $<$ & 419 & $\mathrm{~J} / \mathrm{LH} / \mathrm{H}$ & $<$ & 414 & "I & $<$ & 365 & $\|$ & $<$ & 368 & $\|$ & $<$ & 441 & $\|$ \\
\hline Benzo(b)fluoranthene & $<$ & 389 & "I & $<$ & 344 & $\|$ & $<$ & 364 & $\|$ & $<$ & 403 & $\mathrm{~J} / \mathrm{LH} / \mathrm{H}$ & $<$ & 419 & $\mathrm{~J} / \mathrm{LH} / \mathrm{H}$ & $<$ & 414 & II & $<$ & 365 & II & $<$ & 368 & $\|$ & $<$ & 441 & II \\
\hline Benzo $(g, h, i)$ perylene & $<$ & 389 & $" 1$ & $\leqslant$ & 344 & $\|$ & $<$ & 364 & $\|$ & $<$ & 403 & $\mathrm{~J} / \mathrm{LH} / \mathrm{H}$ & $<$ & 419 & $\mathrm{~J} / \mathrm{H} / \mathrm{H}$ & $<$ & 414 & $\|$ & $<$ & 365 & II & $<$ & 368 & "I & $<$ & 441 & $\ddot{\prime \prime}$ \\
\hline Benzo(k)fluoranthene & $<$ & 389 & 11 & : & 344 & $\|$ & $<$ & 364 & 11 & $<$ & 403 & $\mathrm{~J} / \mathrm{LH} / \mathrm{H}$ & $<$ & 419 & $\mathrm{~J} / \mathrm{LH} / \mathrm{H}$ & $<$ & 414 & $\|$ & $<$ & 365 & "I & $<$ & 368 & 11 & $<$ & 441 & $\|$ \\
\hline Benzoic acid & $<$ & 1940 & 11 & $<$ & 1720 & $\|$ & $<$ & 1820 & $\|$ & $<$ & 2020 & $\mathrm{~J} / \mathrm{L}$ & $<$ & 2100 & $\mathrm{~J} / \mathrm{L}$ & $<$ & 2070 & II & & 247 & $\mathrm{~s} / \mathrm{I}$ & $<$ & 1840 & $\|$ & $<$ & 2200 & II \\
\hline Benzyl alcohol & $<$ & 389 & /I & $<$ & 344 & / & $<$ & 364 & $\|$ & $<$ & 403 & $s \pi$ & $<$ & 419 & $3 / L$ & $<$ & 414 & $\|$ & $<$ & 365 & /I & $<$ & 368 & /I & $<$ & 441 & / \\
\hline Bis(2-chloroethoxy) methane & $<$ & 389 & $\|$ & $<$ & 344 & $\|$ & $<$ & 364 & $\|$ & $<$ & 403 & $\mathrm{~J} / \mathrm{L}$ & $<$ & 419 & $\mathrm{~J} / \mathrm{L}$ & i< & 414 & 11 & $<$ & 365 & $\|$ & $<$ & 368 & $\|$ & $<$ & 441 & $\|$ \\
\hline Bis(2-chloroethyl) ether & $<$ & 389 & $\|$ & $<$ & 344 & $\|$ & $<$ & 364 & $\|$ & $<$ & 403 & $\mathrm{~J} / \mathrm{L}$ & $<$ & 419 & $\mathrm{~J} / \mathrm{L}$ & $<$ & 414 & II & $<$ & 365 & $\|$ & $<$ & 368 & $\|$ & $<$ & 441 & $\|$ \\
\hline Bis(2-chloroisopropyl) ether & $<$ & 389 & 11 & $<$ & 344 & $\|$ & $<$ & 364 & 11 & $<$ & 403 & $3 / 4$ & $<$ & 419 & $\mathrm{~s} / \mathrm{N}$ & $<$ & 414 & $\|$ & $<$ & 365 & $\|$ & $<$ & $368^{\circ}$ & $\|$ & $<$ & 441 & $" 1$ \\
\hline Bis(2-ethylhexyl) phthalate & $<$ & 135 & $\mathrm{~J} / \mathrm{N} /$ & $<$ & 344 & $N I$ & $<$ & 364 & J/VI & $<$ & 403 & $\mathrm{~J} / \mathrm{NU}$ & $<$ & 419 & IVUL & $<$ & 414 & $N I$ & $<$ & 365 & II & $1<$ & 368 & $\|$ & $<$ & 441 & $\|$ \\
\hline Butylbenzyl phthalate & $<$ & 389 & $\|$ & $<$ & 344 & $\|$ & $<$ & 364 & II & $<$ & $4 \ddot{03}$ & $\mathrm{~J} / \mathrm{U}$ & $<$ & 419 & $\mathrm{~J} / \mathrm{L}$ & $1<$ & 414 & $\|$ & $<$ & 365 & $\|$ & $<$ & 368 & 11 & $<$ & 441 & /I \\
\hline Chrysene & $<$ & 389 & II & $<$ & 344 & $/ 1$ & $1<$ & 364 & II & $1<$ & 403 & $\Omega$ & $<$ & 419 & $\mathrm{~J} / \mathrm{L}$ & $1<$ & 414 & II & $<$ & 365 & II & $<$ & 368 & 11 & $<$ & 441 & $\|$ \\
\hline
\end{tabular}


TABLE 6.2

SEMI-VOLATILE ORGANIC COMPOUNDS IN SOIL

D.AREA BURNING/RUBBLE PITS

SAVANNAH RIVER SITE

\begin{tabular}{|c|c|c|c|c|c|c|c|c|c|c|c|c|c|c|c|c|c|c|c|c|c|c|c|c|c|c|c|}
\hline \multirow{3}{*}{\multicolumn{2}{|c|}{ Method 8270 (U)/KG) }} & \multicolumn{2}{|c|}{$\begin{array}{c}D 43111606 \\
62293 \\
\end{array}$} & \multicolumn{3}{|c|}{$\begin{array}{c}44311701 \\
62393 \\
\end{array}$} & \multicolumn{3}{|c|}{$\begin{array}{c}4311702 \\
62393 \\
\end{array}$} & \multicolumn{3}{|c|}{$\begin{array}{c}\text { D431 } 1704 \\
62393 \\
\end{array}$} & \multicolumn{3}{|c|}{$\begin{array}{c}\text { D431 } 1705 \\
62393 \\
\end{array}$} & \multicolumn{3}{|c|}{$\begin{array}{c}\text { D431 } 1706 \\
62393 \\
\end{array}$} & \multicolumn{3}{|c|}{$\begin{array}{c}4311801 \\
61693 \\
\end{array}$} & \multicolumn{3}{|c|}{$\begin{array}{c}\text { D431 } 1802 \\
61693 \\
\end{array}$} & \multicolumn{3}{|c|}{$\begin{array}{c}4311803 \\
61693 \\
\end{array}$} \\
\hline & & & & & & & & & & & & & & & & & & & & & & & & & & & \\
\hline & & 103 & $\mathrm{~J} / \mathrm{VI}$ & $<$ & 145 & $\mathrm{~J} / \mathrm{VI}$ & $<$ & 136 & $3 / \mathrm{VI}$ & $<$ & 201 & $\mathrm{~J} / \mathrm{NU}$ & $<$ & 118 & J/NL & $<$ & 105 & $\mathrm{~J} / \mathrm{VI}$ & $<$ & 136 & $\mathrm{~J} / \mathrm{NI}$ & < & 368 & $N I$ & $<$ & 58.2 & $\mathrm{~J} / \mathrm{VI}$ \\
\hline Di-n-octyl phthalate & & 389 & II & $<$ & 344 & $/ 1$ & $<$ & 364 & $\|$ & $<$ & 403 & $\mathrm{~J} / \mathrm{LH} / \mathrm{H}$ & $<$ & 419 & $\mathrm{~J} / \mathrm{LH} / \mathrm{H}$ & $<$ & 414 & /I & $<$ & 365 & $\|$ & $<$ & 368 & $\|$ & $<$ & 441 & 11 \\
\hline Dibenz(a,h)anthracene & & 389 & II & $<$ & 344 & $\|$ & $<$ & 364 & $\|$ & $<$ & 403 & $\mathrm{~J} / \mathrm{LH} / \mathrm{H}$ & $<$ & 419 & $\mathrm{~J} / \mathrm{LH} / \mathrm{H}$ & $<$ & 414 & /I & $<$ & 365 & $\|$ & $<$ & 368 & $\|$ & $<$ & 441 & $\|$ \\
\hline Dibenzofuran & & $<\quad 389$ & /I & $<$ & 344 & $\|$ & $<$ & 364 & $\|$ & $<$ & 403 & $\mathrm{~s} / \mathrm{N}$ & $<$ & 419 & $\mathrm{~s} / \mathrm{N}$ & $<$ & 414 & $\prime \prime$ & $<$ & 365 & $\|$ & $<$ & 368 & $\|$ & $<$ & $44 ! 1$ & $\|$ \\
\hline Diethyl phthalate & & $<\quad 389$ & /I & $<$ & 344 & $\|$ & $<$ & 364 & /1 & $<$ & 403 & $\mathrm{~J} / \mathrm{W}$ & $<$ & 419 & $3 / 2 /$ & $<$ & 414 & $\|$ & $<$ & 365 & /I & $<$ & 368 & $\|$ & $<$ & 441 & $\|$ \\
\hline Dimethyl phthalate & & 389 & II & $<$ & 344 & 11 & $<$ & 364 & $\|$ & |< & 403 & $\mathrm{~J} / \mathrm{L}$ & $1<$ & 419 & $\mathrm{~J} / \mathrm{L}$ & $<<$ & 414 & "I & $<$ & 365 & $\|$ & $<$ & 368 & /I & $<$ & 441 & $\|$ \\
\hline Fluoranthene & & 389 & /I & |< & 344 & $\|$ & $<$ & 364 & $\|$ & $<$ & 403 & $3 / 2 /$ & $<$ & 419 & $3 / \mathrm{U}$ & $<$ & 414 & $\|$ & $<$ & 365 & $\|$ & < & 368 & $\|$ & $<$ & $44 !$ & "I \\
\hline Fluorene & & $<\quad 389$ & /I & $<$ & 344 & /I & $<$ & 364 & "I & $<$ & 403 & $\mathrm{~J} / \mathrm{L}$ & $<$ & 419 & $J / L$ & $<$ & 414 & / & $<$ & 365 & $\|$ & $<$ & 368 & $/ /$ & $<$ & 441 & $\|$ \\
\hline Hexachlorobenzene & & 389 & $1 /$ & $<$ & 344 & $\|$ & $<$ & 364 & "I & $<$ & 403 & $\mathrm{~J} / \mathrm{L} /$ & $1<$ & 419 & $\mathrm{~J} / \mathrm{L}$ & $<$ & 414 & $\|$ & $<$ & 365 & $\|$ & $<$ & 368 & $\|$ & $<$ & 441 & $\|$ \\
\hline Hexachlorobutadiene & & $<\quad 389$ & $\|$ & $<$ & 344 & $/ /$ & $<$ & 364 & $\|$ & $<$ & 403 & $\mathrm{~J} / \mathrm{L}$ & $<$ & 419 & $3 \pi$ & $<$ & 414 & $\|$ & $<$ & 365 & $\|$ & $<$ & 368 & $\|$ & $<$ & 441 & $\|$ \\
\hline Hexachlorocyclopentadiene & & $<\quad 389$ & "I & $<$ & 344 & $\|$ & $<$ & 364 & "l & $<$ & 403 & $\mathrm{~s} / \mathrm{L} /$ & $<$ & 419 & $\mathrm{~J} / \mathrm{L}$ & $<$ & 414 & $\|$ & $<$ & 365 & $\|$ & $<$ & 368 & $\|$ & $<$ & 441 & $\|$ \\
\hline Hexachloroethane & & $<\quad 389$ & $\|$ & $<$ & 344 & $\|$ & $<$ & 364 & $\|$ & $<$ & 403 & $\mathrm{~J} / \mathrm{L}$ & $<$ & 419 & $3 / \mathrm{L}$ & $<$ & 414 & $\|$ & $<$ & 365 & $\|$ & $<$ & 368 & $\|$ & $<$ & 441 & $\|$ \\
\hline Indeno(1,2,3-c,d)pyrene & & $<\quad 389$ & /I & $<$ & 344 & $\|$ & $<$ & 364 & /I & $<$ & 403 & $\mathrm{~J} / \mathrm{LH} / \mathrm{H}$ & $<$ & 419 & $\mathrm{~J} / \mathrm{LH} / \mathrm{H}$ & $<$ & 414 & $\|$ & $<$ & 365 & $\|$ & $<$ & 368 & $\|$ & $<$ & 441 & $\|$ \\
\hline Isophorone & & $<\quad 389$ & /I & $<$ & 344 & $/ l$ & $<$ & 364 & /I & $<$ & 403 & $\mathrm{~J} / \mathrm{L}$ & $<$ & 419 & $\mathrm{~J} / \mathrm{L}$ & $<$ & 414 & "I & $<$ & 365 & /I & k & 368 & $\|$ & $<$ & 441 & $/ 1$ \\
\hline m-Nitroaniline & & $<1940$ & 11 & $<$ & 1720 & $\|$ & $<$ & 1820 & $\|$ & $<$ & 2020 & $\mathrm{~J} / \mathrm{L}$ & $<$ & 2100 & $\mathrm{~J} / \mathrm{L}$ & $<$ & 2070 & $\|$ & $<$ & 1820 & $\|$ & < & 1840 & $\|$ & $<$ & 2200 & $\|$ \\
\hline N-Nitrosodiphenylamine & & $<\quad 389$ & /I & $<$ & 344 & "I & $<$ & 364 & /I & $<$ & 403 & $\mathrm{~J} / \mathrm{L} /$ & $<$ & 419 & $\mathrm{~J} / \mathrm{L}$ & $<$ & 414 & // & $<$. & 365 & $\|$ & $<$ & 368 & $\|$ & $<$ & $44 !$ & $\|$ \\
\hline N-Nitrosodipropylamine & & $<\quad 389$ & $\|$ & $<$ & 344 & /I & $<$ & 364 & /l & $<$ & 403 & $J / L /$ & $<$ & 419 & $\mathrm{~J} / \mathrm{L} /$ & $<$ & 414 & $\|$ & $<$ & 365 & $\|$ & $<$ & 368 & $\|$ & $<$ & 441 & $/ 1$ \\
\hline Naphthalene & & $<\quad 389$ & $\|$ & $<$ & 344 & $\|$ & $<$ & 364 & $\|$ & $<$ & 403 & $\mathrm{~J} / \mathrm{H}$ & $<$ & 419 & $\mathrm{~J} / \mathrm{L}$ & $<$ & 414 & $\|$ & $<$ & 365 & $\|$ & $<$ & 368 & $\|$ & $<$ & 441 & $\|$ \\
\hline Nitrobenzene & & $<\quad 389$ & /I & $<$ & 344 & $\|$ & $<$ & 364 & "I & $<$ & 403 & $\mathrm{~J} / \mathrm{L}$ & $<$ & 419 & $\mathrm{~J} / \mathrm{L}$ & $<$ & 414 & "I & $<$ & 365 & II & $<$ & 368 & $\|$ & $<$ & 441 & $\|$ \\
\hline o-Cresol (2-Methylphenol) & & $<\quad 389$ & $\|$ & $<$ & 344 & $\|$ & $<$ & 364 & "I & $<$ & 403 & $\mathrm{~J} / \mathrm{L}$ & $<$ & 419 & $\mathrm{~J} / \mathrm{M}$ & $<$ & 414 & 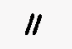 & $<$ & 365 & $\|$ & $<$ & 368 & $\|$ & $<$ & 441 & $\|$ \\
\hline o-Nitroaniline & & $<1940$ & $\|$ & $<$ & 1720 & /I & $<$ & 1820 & $\|$ & $<$ & 2020 & J/LS. & $<$ & 2100 & $\mathrm{~s} / \mathrm{L}$ & $<$ & 2070 & II & $<$ & 1820 & $\|$ & $<$ & 1840 & $\|$ & $<$ & 2200 & $\|$ \\
\hline p-Cresol (4-Methylphenol) & & $<\quad 389$ & $\|$ & $<$ & 344 & $\|$ & $<$ & 364 & $\|$ & $<$ & 403 & $\mathrm{~J} / \mathrm{L}$ & $<$ & 419 & $\mathrm{~J} / \mathrm{L}$ & $<$ & 414 & $\|$ & $<$ & 365 & $\|$ & $<$ & 368 & $/ /$ & $<$ & 441 & $\|$ \\
\hline p-Nitroaniline & & $<\quad 1940$ & $\|$ & $<$ & 1720 & $\|$ & $<$ & 1820 & 11 & $<$ & 2020 & $\mathrm{~J} / \mathrm{N}$ & $<$ & 2100 & $s / 2$ & $1<$ & 2070 & II & $<$ & 1820 & $\|$ & |< & 1840 & $\|$ & $<$ & 2200 & $\|$ \\
\hline Pentachlorophenol & & $<1940$ & /I & $<$ & 1720 & $/ /$ & $<$ & 1820 & II & $<$ & 2020 & $\mathrm{~J} / \mathrm{L}$ & $<$ & 2100 & $\mathrm{~J} / \mathrm{M}$ & $<$ & 2070 & $\|$ & $<$ & 1820 & З///H & $<$ & 1840 & $\|$ & $<$ & 2200 & $n$ \\
\hline Phenanthrene & & $<\quad 389$ & /I & $<$ & 344 & $\|$ & < & 364 & $\|$ & $<$ & 403 & $\mathrm{~J} / \mathrm{L}$ & $<$ & 419 & $J / L$ & $<$ & 414 & $\|$ & $<$ & 365 & /I & $<$ & 368 & $\|$ & $<$ & 441 & $\|$ \\
\hline Phenol & & $<\quad 389$ & "I & $<$ & 344 & $\|$ & $<$ & 364 & $\|$ & $<$ & 403 & $\mathrm{~J} / \mathrm{L}$ & $<$ & 419 & $3 / \mathrm{N}$ & $<$ & 414 & $\|$ & $<$ & 365 & $\|$ & $<$ & 368 & $\|$ & $<$ & 441 & $\|$ \\
\hline Pyrene & & $<\quad 389$ & II & $1<$ & 344 & II & $<$ & 364 & II & $<$ & 403 & $\mathrm{~J} / \mathrm{LI}$ & $1<$ & 419 & $\mathrm{~J} / \mathrm{L}$ & $1<$ & 414 & II & $<$ & 365 & 11 & $1<$ & 368 & $\|$ & $<$ & 441 & II \\
\hline
\end{tabular}


TABLE 6.2

SEMI-VOLATILE ORGANIC COMPOUNDS

IN SOIL

D-AREA BURNING/RUBBLE PITS

SAVANNAH RIVER SITE

\begin{tabular}{|c|c|c|}
\hline \multirow{3}{*}{\begin{tabular}{|lr} 
& $\begin{array}{r}\text { Sample Name } \\
\text { Sample Date }\end{array}$ \\
Method 8270 (UG/KG) & \\
\end{tabular}} & \multicolumn{2}{|c|}{$\begin{array}{c}\text { D431 } 1804 \\
61693\end{array}$} \\
\hline & & \\
\hline & $<\quad 383$ & "I \\
\hline 1,2-Dichlorobenzene & $<\quad 383$ & $\|$ \\
\hline 1,3-Dichlorobenzene & $<\quad 383$ & /l \\
\hline 1,4-Dichlorobenzene & $<383$ & $\|$ \\
\hline 2,4,5-Trichlorophenol & $<\quad 1920$ & $\|$ \\
\hline 2,4,6-Trichlorophenol & $<\quad 383$ & $\|$ \\
\hline 2,4-Dichlorophenol & $<383$ & $\|$ \\
\hline 2,4-Dimethyl phenol & $<\quad 383$ & $\|$ \\
\hline 2,4-Dinitrophenol & $<1920$ & $\|$ \\
\hline 2,4-Dinitrotoluene & $<383$ & 11 \\
\hline 2,6-Dinitrotoluene & $<\quad 383$ & $\prime \prime$ \\
\hline 2-Chloronaphthalene & $<\quad 383$ & /l \\
\hline 2-Chlorophenol & $<\quad 383$ & $\|$ \\
\hline 2-Methyl-4,6-dinitrophenol & $<\quad 1920$ & II \\
\hline 2-Methylnaphthalene & $<\quad 383$ & $\|$ \\
\hline 2-Nitrophenol & $<\quad 383$ & $/ 1$ \\
\hline 3,3'-Dichlorobenzidine & $<766$ & $\|$ \\
\hline 4-Bromophenyl phenyl ether & $<\quad 383$ & $\|$ \\
\hline 4-Chloro-m-cresol & $<383$ & $\|$ \\
\hline 4-Chloroaniline & $<\quad 383$ & $\|$ \\
\hline 4-Chlorophenyl phenyl ether & $<\quad 383$ & /l \\
\hline 4-Nitrophenol & $<1920$ & $\|$ \\
\hline Acenaphthene & $<\quad 383$ & $\ddot{~}$ \\
\hline Acenaphthylene & $<\quad 383$ & $\|$ \\
\hline Anthracene & $<\quad 383$ & $\|$ \\
\hline Benzo(a)anthracene & $<\quad 383$ & " \\
\hline Benzo(a)pyrene & $<\quad 383$ & $/ l$ \\
\hline Benzo(b)fluoranthene & $<\quad 383$ & /l \\
\hline Benzo(g,h,i)perylene & $<383$ & $\|$ \\
\hline Benzo(k)fluoranthene & $<\quad 383$ & $\|$ \\
\hline Benzoic acid & $<1920$ & "l \\
\hline Benzyl alcohol & $<\quad 383$ & $\| 1$ \\
\hline Bis(2-chloroethoxy) methane & $<\quad 383$ & / \\
\hline Bis(2-chloroethyl) ether & $<\quad 383$ & $\|$ \\
\hline Bis(2-chloroisopropyl) ether & $<\quad 383$ & $\|$ \\
\hline Bis(2-ethylhexyl) phthalate & $<\quad 383$ & $\|$ \\
\hline Butylbenzyl phthalate & $<\quad 383$ & $\|$ \\
\hline Chrysene & $<\quad 383$ & 11 \\
\hline
\end{tabular}

Page 15 of 16 
TABLE 6.2

SEMI-VOLATILE ORGANIC COMPOUNDS

$$
\text { , IN SOIL }
$$

D-AREA BURNING/RUBBLE PITS

SAVANNAH RIVER SITE

\begin{tabular}{|c|c|c|c|c|}
\hline & $\begin{array}{r}\text { Sample Name } \\
\text { Sample Date }\end{array}$ & & $\begin{array}{r}D 431 \\
616 \\
\end{array}$ & \\
\hline Method 8270 (UG $/ K G)$ & & & & \\
\hline Di-n-butyl phthalate & & $<$ & 49 & $\mathrm{~J} / \mathrm{V} /$ \\
\hline Di-n-octyl phthalate & & $<$ & 383 & $\|$ \\
\hline Dibenz $(\mathrm{a}, \mathrm{h})$ anthracene & & $<$ & 383 & $\|$ \\
\hline Dibenzofuran & & $<$ & 383 & $\|$ \\
\hline Diethyl phthalate & & $<$ & 383 & $\|$ \\
\hline Dimethyl phthalate & & $<$ & 383 & 11 \\
\hline Fluoranthene & & $<$ & 383 & /I \\
\hline Fluorene & & $<$ & 383 & $\|$ \\
\hline Hexachlorobenzene & & $<$ & 383 & $/ /$ \\
\hline Hexachlorobutadiene & & $<$ & 383 & $\|$ \\
\hline Hexachlorocyclopentadiene & & $<$ & 383 & $\|$ \\
\hline Hexachloroethane & & $<$ & 383 & $\|$ \\
\hline Indeno $(1,2,3 \cdot c, d)$ pyrene & & k & 383 & $\|$ \\
\hline Isophorone & & $<$ & 383 & $\|$ \\
\hline m-Nitroaniline & & $<$ & 1920 & $\|$ \\
\hline N-Nitrosodiphenylamine & & $<$ & 383 & $\|$ \\
\hline N-Nitrosodipropylamine & - & $<$ & 383 & $/ 1$ \\
\hline Naphthalene & & $<$ & 383 & $\|$ \\
\hline Nitrobenzene & & $<$ & 383 & $\|$ \\
\hline o-Cresol (2-Methylphenol) & & $<$ & 383 & $\|$ \\
\hline o-Nitroaniline & & $<$ & 1920 & $\|$ \\
\hline p-Cresol (4-Methylphenol) & & $<$ & 383 & $\|$ \\
\hline p-Nitroaniliṇe & & $<$ & 1920 & $\|$ \\
\hline Pentachlorophenol & & $<$ & 1920 & $\|$ \\
\hline Phenanthrene & & < & 383 & $1 /$ \\
\hline Phenol & & $<<$ & 383 & /I \\
\hline Pyrene & & $<$ & 383 & II \\
\hline
\end{tabular}




\section{TABLE 6.3}

PESTICIDES AND PCBS

$$
\text { IN SOIL }
$$

\section{D-AREA BURNING/RUBBLE PITS}

SAVANNAH RIVER SITE

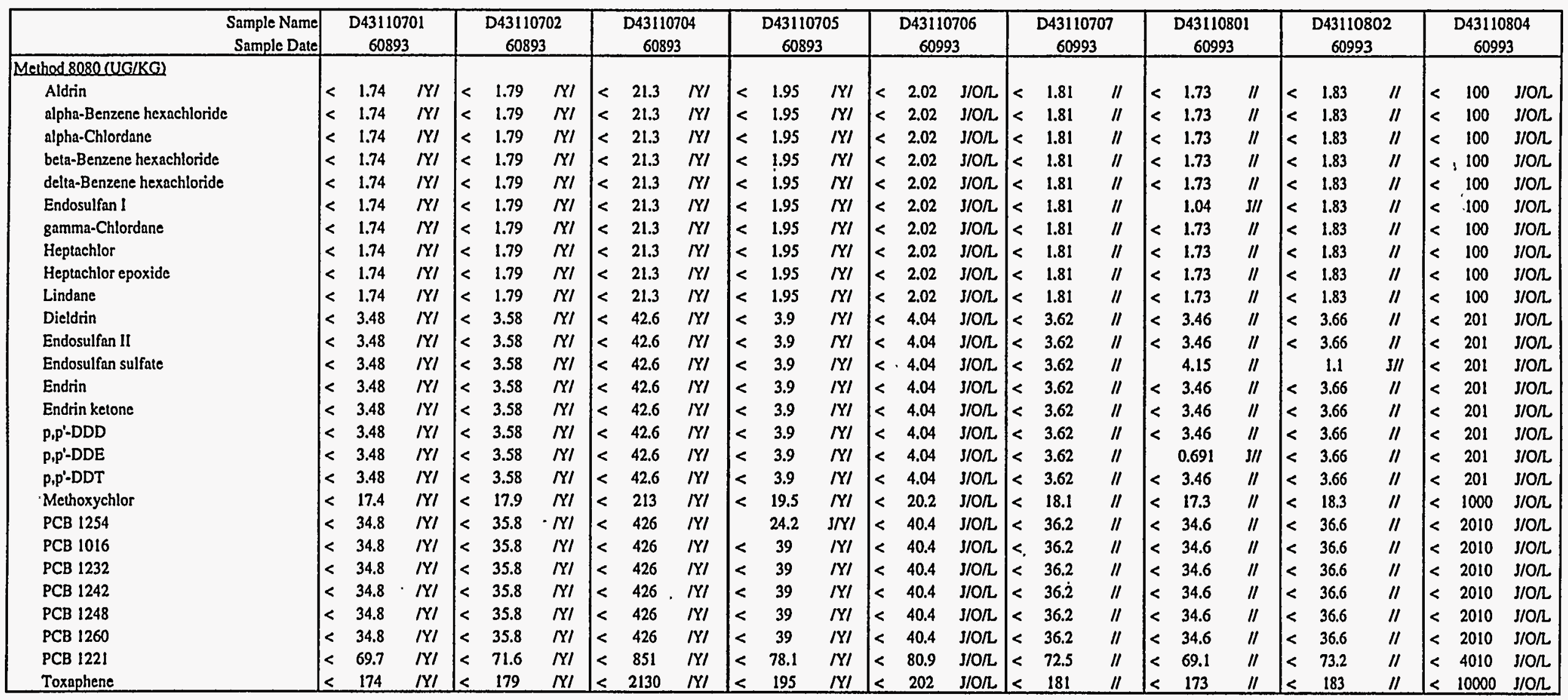


TABLE 6.3

PESTICIDES AND PCBS , *

D-AREA BURNING/RUBBLE PITS

SAVANNAH RIVER SITE

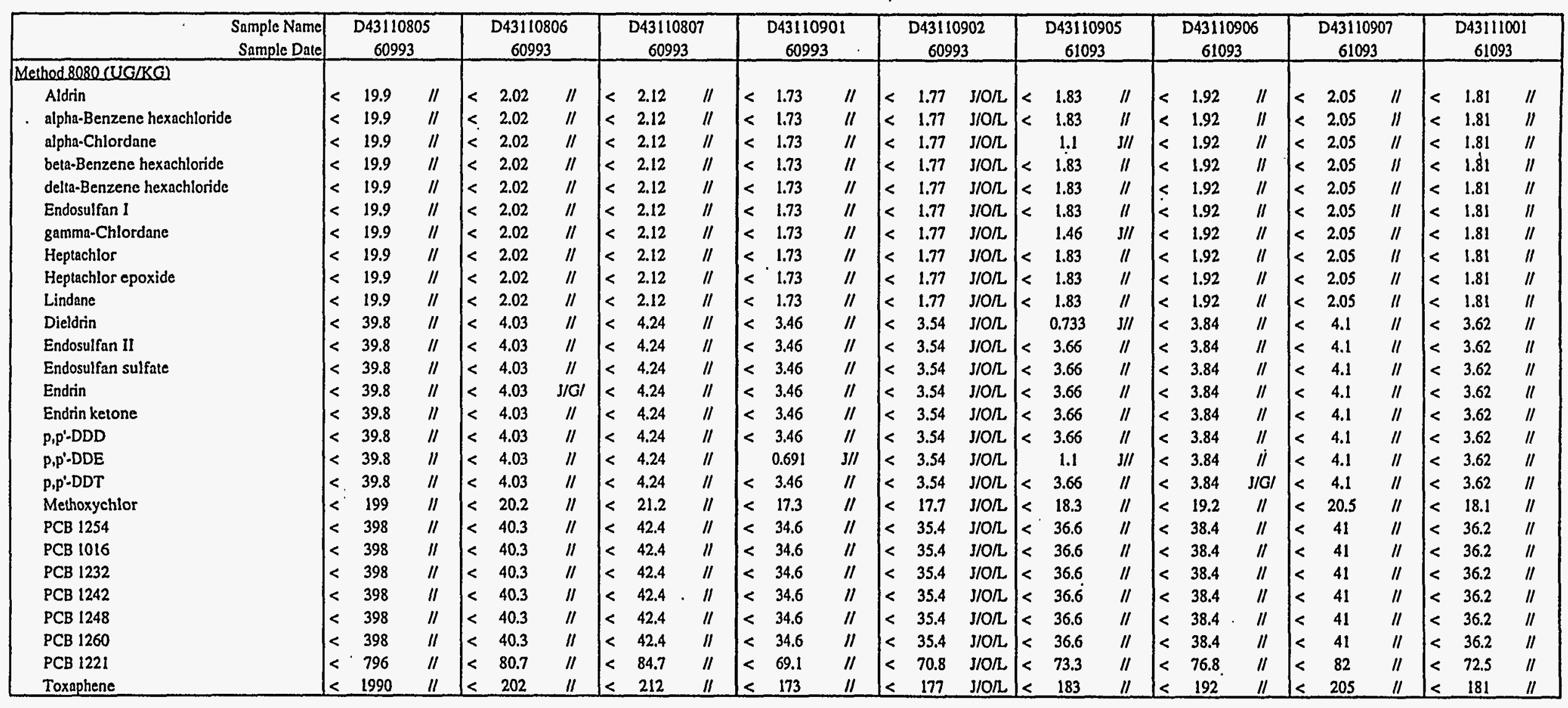


TABLE 6.3

PESTICIDES AND PCBS

IN SOIL

D-AREA BURNING/RUBBLE PITS

SAVANNAH RIVER SITE

\begin{tabular}{|c|c|c|c|c|c|c|c|c|c|c|c|c|c|c|c|c|c|c|c|c|c|c|c|c|c|c|c|}
\hline \begin{tabular}{|r|}
$\begin{array}{r}\text { Sample Name } \\
\text { Sample Date }\end{array}$ \\
\end{tabular} & \multicolumn{3}{|c|}{$\begin{array}{c}\mathrm{D} 43111002 \\
61093\end{array}$} & \multicolumn{3}{|c|}{$\begin{array}{c}\text { D43111005 } \\
61093\end{array}$} & \multicolumn{3}{|c|}{$\begin{array}{c}\text { D43111006 } \\
61093\end{array}$} & \multicolumn{3}{|c|}{$\begin{array}{c}\text { D43111007 } \\
61093\end{array}$} & \multicolumn{3}{|c|}{$\begin{array}{c}\text { D4311101 } \\
61093\end{array}$} & \multicolumn{3}{|c|}{$\begin{array}{c}\text { D4311102 } \\
61093\end{array}$} & \multicolumn{3}{|c|}{$\begin{array}{c}\text { D4311104 } \\
61093\end{array}$} & \multicolumn{3}{|c|}{$\begin{array}{c}\text { D4311105 } \\
61193\end{array}$} & \multicolumn{3}{|c|}{$\begin{array}{c}\text { D4311106 } \\
61193\end{array}$} \\
\hline Mechod 8080 (UG/KG) & & & & & & & & & & & & & & & & & & & & & & & & & & & \\
\hline Aldrin & $<$ & 1.81 & $\|$ & $<$ & 2.18 & $\|$ & $<$ & 2.1 & $\|$ & $<$ & 2.13 & $\|$ & $<$ & 1.71 & $\|$ & $<$ & 1.77 & $\|$ & $<$ & 18.8 & $\|$ & $<$ & 1.91 & $\|$ & $<$ & 1.93 & $\|$ \\
\hline alpha-Benzene hexachloride & $<$ & 1.81 & $\|$ & $<$ & 2.18 & $\|$ & $<$ & 2.1 & $\|$ & $<$ & 2.13 & $\|$ & $<$ & 1.71 & $\|$ & $<$ & 1.77 & $\|$ & $<$ & 18.8 & $\|$ & $<$ & 1.91 & $\|$ & $<$ & 1.93 & $\|$ \\
\hline alpha-Chlordane & $<$ & 1.81 & $\|$ & $<$ & 2.18 & 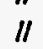 & e & 2.1 & II & $<$ & 2.13 & $\|$ & < & 1.71 & II & & 0.353 & $\mathrm{~J} / I$ & & 131 & $\|$ & & 6.88 & $\|$ & & 1.16 & $\mathrm{~s} / /$ \\
\hline beta-Benzene hexachloride & $<$ & 1.81 & $/ 1$ & $<$ & 2.18 & $\|$ & $<$ & 2.1 & $\|$ & $<$ & 2.13 & 11 & $<$ & 1.71 & II & $<$ & 1.77 & $\|$ & $<$ & 18.8 & $\|$ & $<$ & 1.91 & $\|$ & $<$ & 1.93 & II \\
\hline delta-Benzene hexachloride & $<$ & 1.81 & $\|$ & $<$ & 2.18 & $\|$ & $<$ & 2.1 & /I & 1< & 2.13 & /I & $<$ & 1.71 & 11 & $<$ & 1.77 & $\|$ & $<$ & 18.8 & "I & & 4.59 & "I & le & 1.93 & $\|$ \\
\hline Endosulfan I & $<$ & 1.81 & $\|$ & $k$ & 2.18 & $\|$ & $<$ & 2.1 & $/ /$ & $<$ & 2.13 & $/ /$ & $<$ & 1.71 & /I & $<$ & 1.77 & /I & $<$ & 18.8 & /I & $<$ & 1.91 & II & $<$ & 1.93 & $\|$ \\
\hline gamma-Chlordane & $<$ & 1.81 & 11 & $<$ & 2.18 & $\|$ & $<$ & 2.1 & II & $<$ & 2.13 & $\|$ & $<$ & 1.71 & II & & 0.707 & $3 / I$ & & 234 & $\|$ & & 13.8 & 11 & & 2.7 & II \\
\hline Heptachlor & $<$ & 1.81 & 11 & $<$ & 2.18 & /I & $<$ & 2.1 & /I & $<$ & 2.13 & $/ /$ & $<$ & 1.71 & 11 & $<$ & 1.77 & $\|$ & $<$ & 18.8 & /I & $<$ & 1.91 & II & $<$ & 1.93 & /1 \\
\hline Heptachlor epoxide & $<$ & 1.81 & $\|$ & $<$ & 2.18 & $\|$ & $<$ & 2.1 & "I & $<$ & 2.13 & II & $<$ & 1.71 & II & $<$ & 1.77 & $\|$ & $<$ & 18.8 & II & $<$ & 1.91 & $\|$ & $<$ & 1.93 & II \\
\hline Lindane & $<$ & 1.81 & $\|$ & $<$ & 2.18 & $\|$ & $<$ & 2.1 & II & $<$ & 2.13 & II & $<$ & 1.71 & $\|$ & $<$ & 1.77 & /I & $<$ & 18.8 & $\|$ & $<$ & 1.91 & II & $<$ & 1.93 & /I \\
\hline Dicldrin & $<$ & 3.62 & /I & $<$ & 4.36 & /I & $<$ & 4.2 & II & $<$ & 4.26 & $\|$ & $<$ & 3.43 & $\|$ & $<$ & 3.53 & $\ddot{\prime \prime}$ & & 16.5 & $\mathrm{~J} / /$ & $<$ & 3.82 & II & le & 3.86 & $\|$ \\
\hline Endosulfan II & $<$ & 3.62 & /I & $<$ & 4.36 & $\|$ & $<$ & 4.2 & /I & $<$ & 4.26 & $\|$ & $<$ & 3.43 & $\|$ & & 1.77 & $\mathrm{~J} / /$ & $<$ & 37.5 & /I & $<$ & 3.82 & $\|$ & $<$ & 3.86 & $\|$ \\
\hline Endosulfan sulfate & $<$ & 3.62 & $\|$ & $<$ & 4.36 & 11 & $<$ & 4.2 & "I & $<$ & 4.26 & 11 & $<$ & 3.43 & "I & $<$ & 3.53 & /I & & 4.5 & $\mathrm{~J} / 1$ & $<$ & 3.82 & $\ddot{\prime \prime}$ & $<$ & 3.86 & $\ddot{\|}$ \\
\hline Endrin & $<$ & 3.62 & $/ 1$ & $<<$ & 4.36 & "I & $<$ & 4.2 & "I & $<$ & 4.26 & $\|$ & $<$ & 3.43 & II & & 4.95 & $\ddot{\prime \prime}$ & & 6.38 & $\mathrm{~J} / I$ & $<$ & 3.82 & II & k & 3.86 & $\|$ \\
\hline Endrin ketone & $<$ & 3.62 & $\|$ & $<$ & 4.36 & II & $<$ & 4.2 & /I & $<$ & 4.26 & II & $<$ & 3.43 & $\|$ & & 1.41 & $\mathrm{~J} / I$ & $<$ & 37.5 & II & $<$ & 3.82 & /l & $<$ & 3.86 & II \\
\hline$p, p^{\prime}-D D D$ & $<$ & 3.62 & $/ 1$ & $<$ & 4.36 & $\|$ & $<$ & 4.2 & /I & $<$ & 4.26 & /I & l< & 3.43 & 11 & & 1.41 & $\mathrm{~J} / 1$ & & 15 & $\mathrm{~s} / /$ & $<$ & 3.82 & 11 & & 3.08 & $\mathrm{~s} / \mathrm{I}$ \\
\hline$p, p^{\prime}-D D E$ & $<$ & 3.62 & $\|$ & $<$ & 4.36 & $\|$ & $<$ & 4.2 & /I & $<$ & 4.26 & $\|$ & & 0.685 & $\mathrm{~J} / /$ & & 2.83 & $\mathrm{~J} / /$ & & 15.4 & $\mathrm{~s} / I$ & & 2.29 & $3 / I$ & & 3.47 & $J / l$ \\
\hline$p, p^{\prime}-D D T$ & $<$ & 3.62 & $/ 1$ & $<$ & 4.36 & II & $<$ & 4.2 & II & $<$ & 4.26 & II & $<$ & 3.43 & II & & 4.95 & II & $<$ & 37.5 & 11 & $<$ & 3.82 & 11 & & 2.7 & $\mathrm{~J} / I$ \\
\hline Methoxychlor & $<$ & 18.1 & $\|$ & $<$ & 21.8 & $\|$ & $<$ & 21 & /I & $<$ & 21.3 & $\|$ & $<$ & 17.1 & II & $<$ & 17.7 & $\|$ & $<$ & 188 & 11 & $<$ & 19.1 & /1 & $<$ & 19.3 & $\|$ \\
\hline PCB 1254 & $<$ & 36.2 & /I & |< & 43.6 & /I & $<$ & 42 & $\|$ & $<$ & 42.6 & /I & $<$ & 34.3 & II & $<$ & 35.3 & /I & $<$ & 375 & II & $<$ & 38.2 & II & $<$ & 38.6 & 11 \\
\hline PCB 1016 & $<$ & 36.2 & $\|$ & $<$ & 43.6 & "I & $<$ & 42 & II & $<$ & 42.6 & II & $<$ & 34.3 & II & $<$ & 35.3 & II & $<$ & 375 & II & $<$ & 38.2 & $\|$ & $<$ & 38.6 & 11 \\
\hline PCB 1232 & $<$ & 36.2 & 11 & $<$ & 43.6 & II & $<$ & 42 & "I & $<$ & 42.6 & 11 & $<$ & 34.3 & 11 & $<$ & 35.3 & $\ddot{\prime \prime}$ & $<$ & 375 & $\|$ & $<$ & 38.2 & /I & $<$ & 38.6 & II \\
\hline РCB 1242 & $<$ & 36.2 & $\|$ & |< & 43.6 & $\|$ & $<$ & 42 & $\|$ & $<$ & 42.6 & II & $<$ & 34.3 & II & $<$ & 35.3 & II & $<$ & 375 & II & $k$ & 38.2 & /l & $<$ & 38.6 & II \\
\hline PCB 1248 & $<$ & 36.2 & $\|$ & $k$ & 43.6 & $\|$ & $<$ & 42 & /I & $<$ & 42.6 & $\|$ & $<$ & 34.3 & II & $<$ & 35.3 & $\|$ & $<$ & 375 & II & $k$ & 38.2 & II & $<$ & 38.6 & $\|$ \\
\hline PCB 1260 & $<$ & 36.2 & $/ 1$ & i & 43.6 & $\|$ & $<$ & 42 & $\|$ & $<$ & 42.6 & /I & $<$ & 34.3 & 11 & $<$ & 35.3 & $\|$ & $1<$ & 375 & II & $<$ & 38.2 & II & $<$ & 38.6 & $\ddot{~}$ \\
\hline PCB 1221 & $<$ & 72.3 & $\|$ & $<$ & 87.2 & $\|$ & $<$ & 84 & II & $<$ & 85.3 & II & $1<$ & 68.5 & ll & $<$ & 70.7 & II & $<$ & 750 & II & $<$ & 76.4 & /I & $<<$ & 77.1 & II \\
\hline Toxaphene & $<$ & 181 & II & l< & 218 & /I & $<$ & 210 & II & $k$ & 213 & "1 & $<$ & 171 & 11 & $\mid<$ & 177 & $\|$ & $<$ & 1880 & II & $<$ & 191 & II & $<$ & 193 & II \\
\hline
\end{tabular}


TABLE 6.3

PESTICIDES AND PCBS

IN SOIL

D-AREA BURNING/RUBBLE PITS

SAVANNAH RIVER SITE

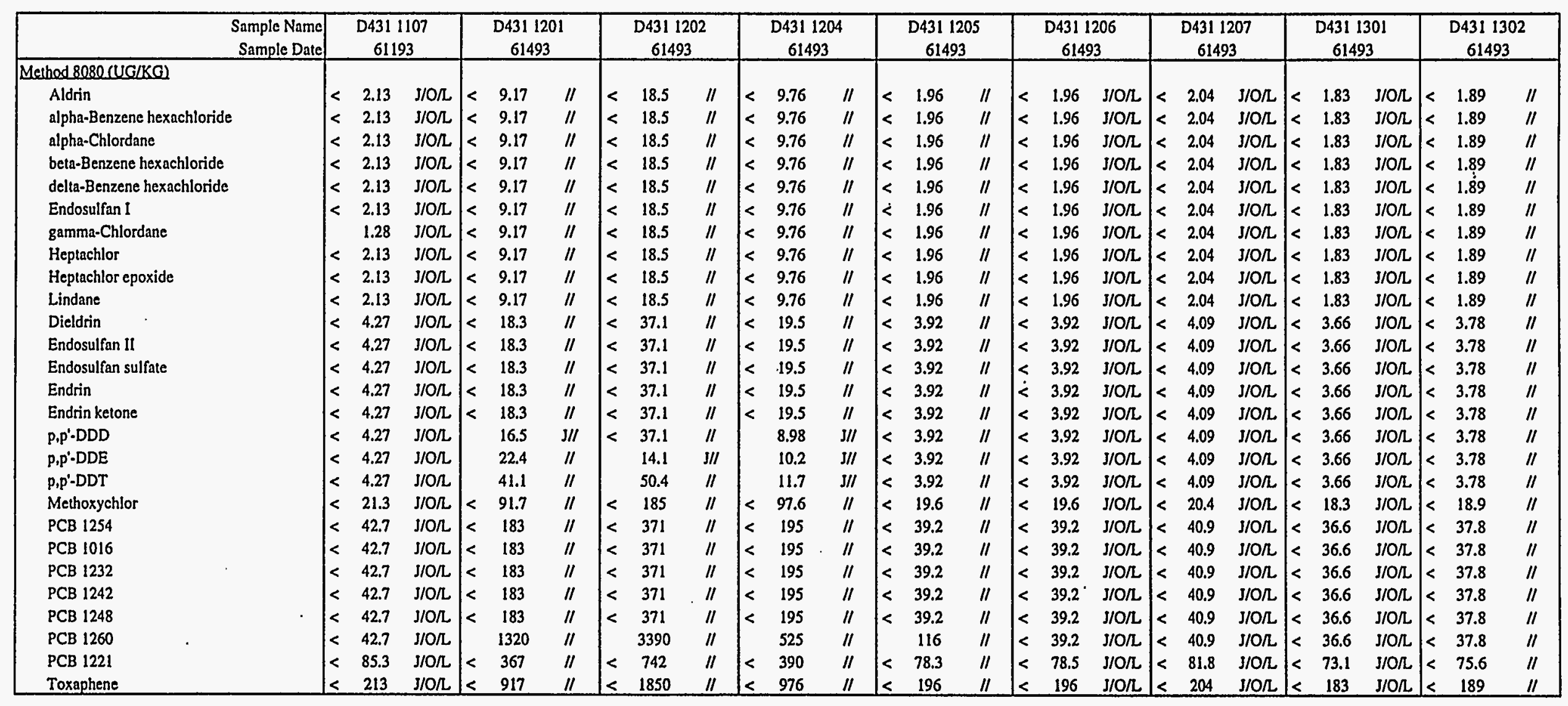


TABLE 6.3

PESTICIDES AND PCBS

IN SOIL

D-AREA BURNING/RUBBLE PITS

SAVANNAH RIVER SITE

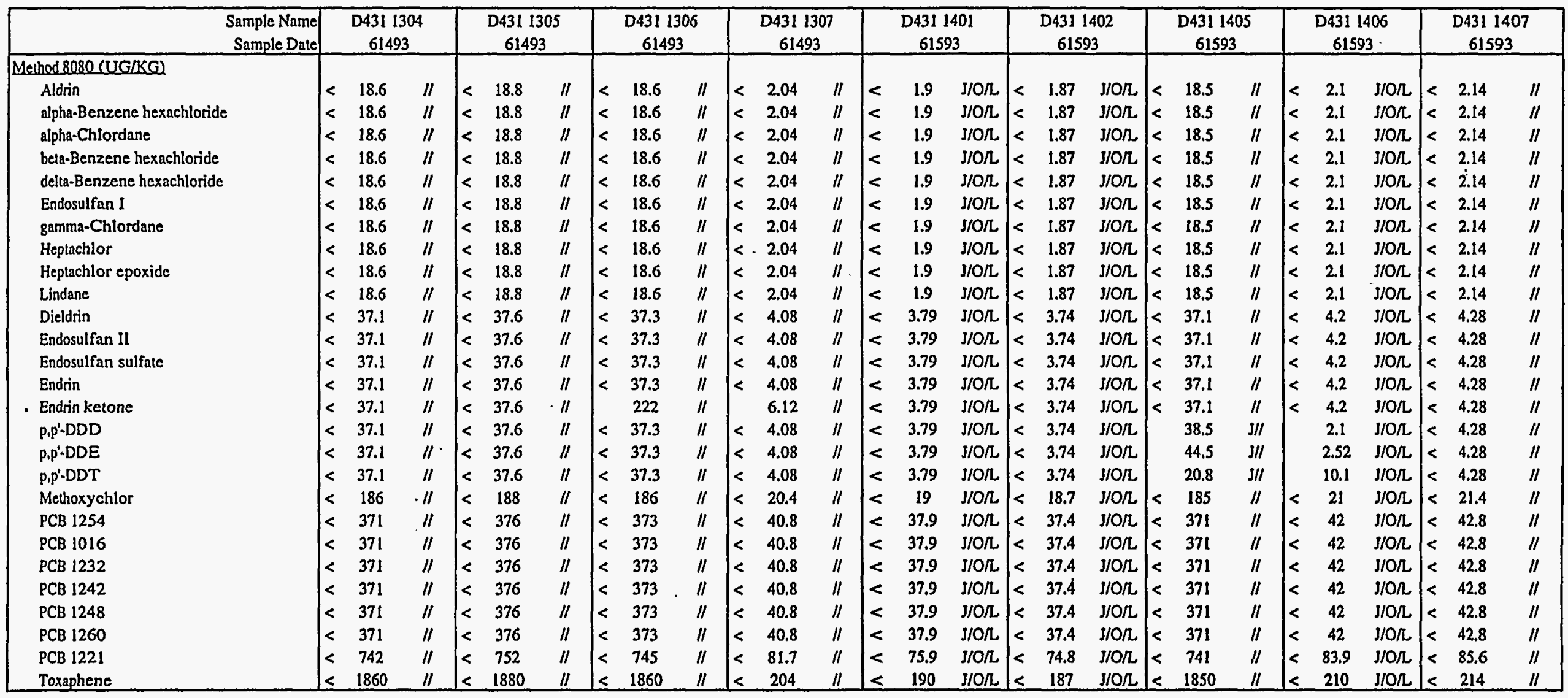


TABLE 6.3

PESTICIDES AND PCBS

IN SOIL

D-AREA BURNING/RUBBLE PITS

SAVANNAH RIVER SITE

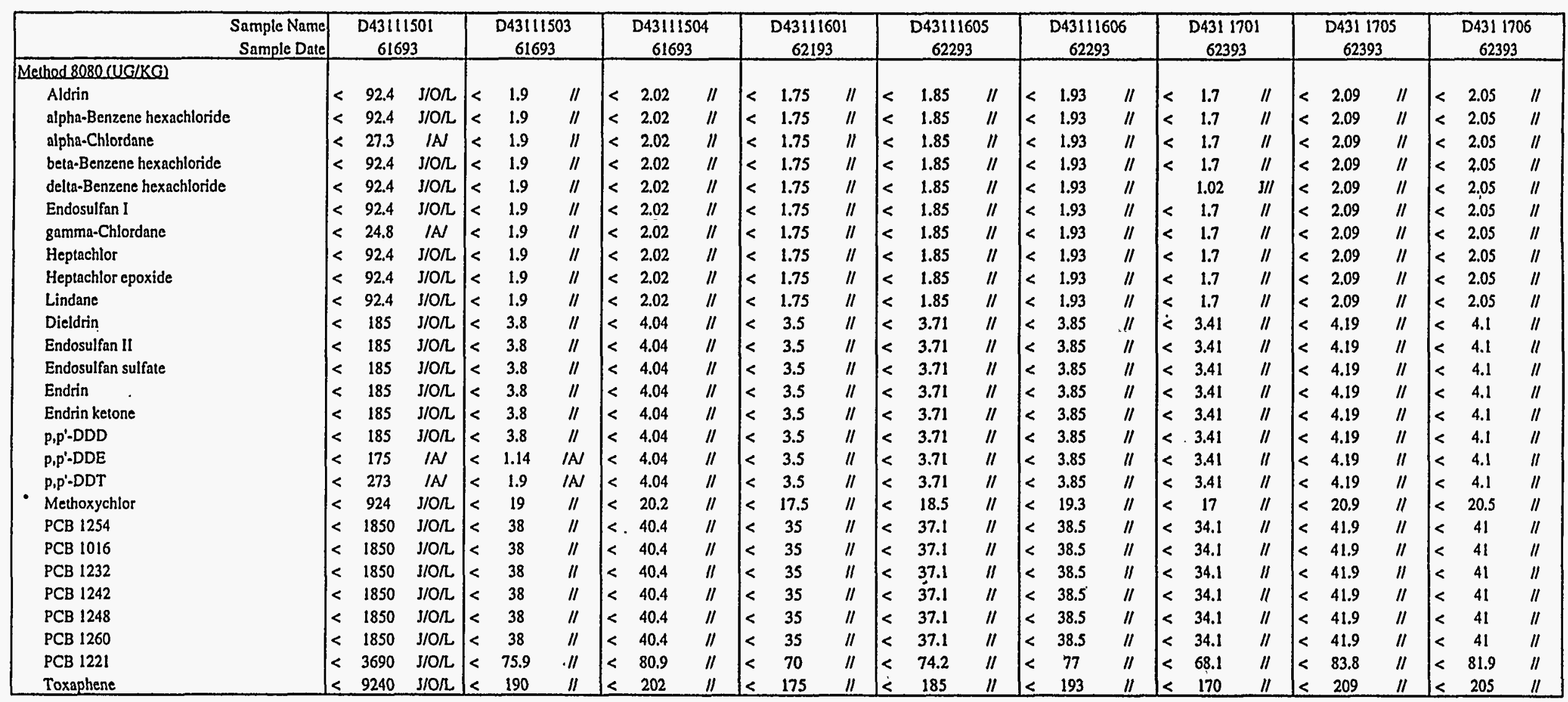


TABLE 6.3

PESTICIDES AND PCBS
IN SOIL

D-AREA BURNING/RUBBLE PITS

SAVANNAH RIVER SITE

\begin{tabular}{|c|c|c|c|c|c|c|c|c|c|c|}
\hline \multirow{2}{*}{ Method 8080(UG/KG) } & $\begin{array}{r}\text { Sample Name } \\
\text { Sample Dare }\end{array}$ & \multicolumn{3}{|c|}{$\begin{array}{c}\text { D431 } 1801 \\
61693 \\
\end{array}$} & \multicolumn{3}{|c|}{$\begin{array}{c}\text { D431 } 1803 \\
61693 \\
\end{array}$} & \multicolumn{3}{|c|}{$\begin{array}{c}\text { D431 } 1804 \\
61693 \\
\end{array}$} \\
\hline & & & & & & & & & & \\
\hline \multirow{2}{*}{\multicolumn{2}{|c|}{$\begin{array}{l}\text { Aldrin } \\
\text { alpha-Benzene hexachloride }\end{array}$}} & $<$ & 1.79 & $\|$ & $1<$ & 2.25 & $\mathrm{~J} / \mathrm{O} / \mathrm{L}$ & $<$ & 1.93 & $\|$ \\
\hline & & $<$ & 1.79 & $\|$ & $<$ & 2.25 & $\mathrm{~J} / \mathrm{O} / \mathrm{L}$ & $<$ & 1.93 & /I \\
\hline \multicolumn{2}{|l|}{ alpha-Chlordane } & $<$ & 1.79 & $\|$ & $<$ & 2.25 & $\mathrm{~J} / \mathrm{O} / \mathrm{L}$ & $<$ & 1.93 & II \\
\hline \multicolumn{2}{|l|}{ beta-Benzene hexachloride } & $<$ & 1.79 & $\|$ & $<$ & 2.25 & $\mathrm{~J} / \mathrm{O} / \mathrm{L}$ & $<$ & 1.93 & /I \\
\hline \multicolumn{2}{|l|}{ delta-Benzene hexachloride } & $<$ & 1.79 & $\|$ & $<$ & 2.25 & $\mathrm{~J} / \mathrm{O} / \mathrm{L}$ & $<$ & 1.93 & "I \\
\hline \multicolumn{2}{|l|}{ Endosulfan I } & $<$ & 1.79 & $\|$ & $<$ & 2.25 & $\mathrm{~J} / \mathrm{O} / \mathrm{L}$ & $<$ & 1.93 & "I \\
\hline \multicolumn{2}{|l|}{ gamma-Chlordane } & $<$ & 1.79 & $\|$ & $<$ & 2.25 & $\mathrm{~J} / \mathrm{O} / \mathrm{L}$ & $<$ & 1.93 & /I \\
\hline \multicolumn{2}{|l|}{ Heptachlor } & $<$ & 1.79 & "l & $<$ & 2.25 & $\mathrm{~J} / \mathrm{O} / \mathrm{L}$ & $<$ & 1.93 & "I \\
\hline \multicolumn{2}{|l|}{ Heptachlor epoxide } & $<$ & 1.79 & $\|$ & $<$ & 2.25 & $\mathrm{~J} / \mathrm{O} / \mathrm{L}$ & $<$ & 1.93 & "I \\
\hline \multicolumn{2}{|l|}{ Lindane } & $<$ & 1.79 & $\|$ & < & 2.25 & $\mathrm{~J} / \mathrm{O} / \mathrm{L}$ & $<$ & 1.93 & /I \\
\hline \multicolumn{2}{|l|}{ Dieldrin } & $<$ & 3.59 & $\|$ & $<$ & 4.5 & $\mathrm{~J} / \mathrm{O} / \mathrm{L}$ & $<$ & 3.85 & /I \\
\hline \multicolumn{2}{|l|}{ Endosulfan II } & $<$ & 3.59 & /I & $<$ & 4.5 & $\mathrm{~J} / \mathrm{O} / \mathrm{L}$ & $<$ & 3.85 & "I \\
\hline \multicolumn{2}{|l|}{ Endosulfan sulfate } & $<$ & 3.59 & $\|$ & $<$ & 4.5 & $\mathrm{~J} / \mathrm{O} / \mathrm{L}$ & $<$ & 3.85 & $\|$ \\
\hline \multicolumn{2}{|l|}{ Endrin } & $<$ & 3.59 & II & $<$ & 4.5 & $\mathrm{~J} / \mathrm{O} / \mathrm{L}$ & $<$ & 3.85 & $\|$ \\
\hline \multicolumn{2}{|l|}{ Endrin ketone } & $<$ & 3.59 & $\|$ & $<$ & 4.5 & $\mathrm{~J} / \mathrm{O} / \mathrm{L}$ & $<$ & 3.85 & $\|$ \\
\hline \multicolumn{2}{|l|}{$p, p^{\prime}-D D D$} & $<$ & 3.59 & 11 & $<$ & 4.5 & $\mathrm{~J} / \mathrm{O} / \mathrm{L}$ & $<$ & 3.85 & II \\
\hline \multicolumn{2}{|l|}{$p, p^{\prime}-D D E$} & & 1.08 & $\mathrm{~J} / /$ & $<$ & 4.5 & $\mathrm{~J} / \mathrm{O} / \mathrm{L}$ & $<$ & 3.85 & "I \\
\hline \multicolumn{2}{|l|}{ p.p'-DDT } & & 1.08 & $\mathrm{~J} / I$ & $<$ & 4.5 & $\mathrm{~J} / \mathrm{O} / \mathrm{L}$ & $<$ & 3.85 & 11 \\
\hline \multicolumn{2}{|l|}{ Methoxychlor } & $<$ & 17.9 & II & $<$ & 22.5 & $\mathrm{~J} / \mathrm{O} / \mathrm{L}$ & $<$ & 19.3 & $\|$ \\
\hline \multicolumn{2}{|l|}{ PCB 1254} & $<$ & 35.9 & 11 & $<$ & 45 & $\mathrm{~J} / \mathrm{O} / \mathrm{L}$ & $<$ & 38.5 & $\|$ \\
\hline \multicolumn{2}{|l|}{ PCB 1016} & $<$ & 35.9 & $\|$ & $<$ & 45 & $\mathrm{~J} / \mathrm{O} / \mathrm{L}$ & $<$ & 38.5 & "I \\
\hline \multicolumn{2}{|l|}{ PCB 1232} & $<$ & 35.9 & 11 & $<$ & 45 & $\mathrm{~J} / \mathrm{O} / \mathrm{L}$ & $<$ & 38.5 & II \\
\hline \multicolumn{2}{|l|}{ PCB 1242} & $<$ & 35.9 & 11 & $<$ & 45 & $\mathrm{~J} / \mathrm{O} / \mathrm{L}$ & $<$ & 38.5 & "I \\
\hline \multicolumn{2}{|l|}{ PCB 1248} & $<$ & 35.9 & $\|$ & $<$ & 45 & $\mathrm{~J} / \mathrm{O} / \mathrm{L}$ & $<$ & 38.5 & "I \\
\hline \multicolumn{2}{|l|}{ PCB 1260} & $<$ & 35.9 & 11 & $<$ & 45 & $\mathrm{~J} / \mathrm{O} / \mathrm{L}$ & $<$ & 38.5 & "I \\
\hline РСB 1221 & & $<$ & 71.8 & II & $<$ & 90 & $\mathrm{~J} / \mathrm{O} / \mathrm{L}$ & $<$ & 77 & "I \\
\hline Toxaphene & & $<$ & 179 & 11 & L & 225 & $\mathrm{~J} / \mathrm{O} / \mathrm{L}$ & $<$ & 193 & 11 \\
\hline
\end{tabular}


TABLE 6.4

PCDD AND PCDF COMPOUNDS

IN SOIL

D-AREA BURNING/RUBBLE PITS

SAVANNAH RIVER SITE

\begin{tabular}{|c|c|c|c|c|c|c|c|c|c|c|c|c|c|c|c|c|c|c|c|c|c|c|c|c|c|c|}
\hline \multirow{3}{*}{$\begin{array}{|cr|} & \begin{array}{r}\text { Sample Name } \\
\text { Sample Date }\end{array} \\
\text { Method } 8280 \text { (NG/G) } \\
2,3,7,8-\mathrm{TCDD}\end{array}$} & \multicolumn{2}{|c|}{$\begin{array}{c}\text { D43110701 } \\
60893 \\
\end{array}$} & \multicolumn{3}{|c|}{$\begin{array}{c}\mathrm{D} 43110702 \\
60893 \\
\end{array}$} & \multicolumn{3}{|c|}{$\begin{array}{c}\text { D43110704 } \\
60893 \\
\end{array}$} & \multicolumn{3}{|c|}{$\begin{array}{c}\text { D43110705 } \\
60893 \\
\end{array}$} & \multicolumn{3}{|c|}{$\begin{array}{c}\text { D43110706 } \\
60993 \\
\end{array}$} & \multicolumn{3}{|c|}{$\begin{array}{c}\text { D43110707 } \\
60993 \\
\end{array}$} & \multicolumn{3}{|c|}{$\begin{array}{c}D 43110801 \\
60993 \\
\end{array}$} & \multicolumn{3}{|c|}{$\begin{array}{c}\text { D43110802 } \\
60993 \\
\end{array}$} & \multicolumn{3}{|c|}{$\begin{array}{c}\text { D43110804 } \\
60993\end{array}$} \\
\hline & & & & & & & & & & & & & & & & & & & & & & & & & & \\
\hline & 0.1 & $/ Y I$ & $<$ & 0.1 & $r Y I$ & $<$ & 0.1 & $/ \mathrm{YI}$ & $<$ & 0.1 & $/ \mathrm{YI}$ & $<$ & 0.1 & $\|$ & $<$ & 0.1 & $/ 1$ & $<$ & 0.1 & $\|$ & $<$ & 0.1 & $\|$ & $<$ & 0.1 & $\|$ \\
\hline $2,3,7,8-\mathrm{TCDF}$ & 0.1 & $/ \mathrm{XI}$ & $<$ & 0.1 & $r Y I$ & $<$ & 0.1 & $/ Y I$ & $<$ & 0.1 & IYI & $<$ & 0.1 & $\|$ & $<$ & 0.1 & $\|$ & $<$ & 0.1 & $\|$ & $<$ & 0.1 & II & $<$ & 0.1 & $\|$ \\
\hline Heptachlorodibenzo-p-dioxin isomers & 0.1 & $/ \mathrm{Y} /$ & $<$ & 0.1 & $\Gamma \mathrm{YI}$ & & 0.8 & $\mathrm{~J} / \mathrm{Y} /$ & $<$ & 0.3 & $/ Y I$ & & 0.3 & $\mathrm{~J} / l$ & & 0.1 & $\mathrm{~J} / /$ & $<$ & 0.1 & $\|$ & $<$ & 0.2 & II & & 0.3 & $\mathrm{~s} / /$ \\
\hline Heptachlorodibenzo-p-furan isomers & 0.1 & $\mid \mathrm{X} I$ & $<$ & 0.1 & $/ Y I$ & & 0.3 & $\mathrm{~J} / \mathrm{Y} /$ & $<$ & 0.1 & $/ Y I$ & $<$ & 0.1 & $\|$ & $<$ & 0.1 & $\|$ & $<$ & 0.2 & $/ 1$ & $<$ & 0.1 & "I & $<$ & 0.1 & $\|$ \\
\hline Hexachlorodibenzo-p-dioxin isomers & 0.1 & $/ Y I$ & $<$ & 0.1 & $r I$ & $<$ & 0.3 & $/ Y I$ & $<$ & 0.4 & $/ \mathrm{XI}$ & $<$ & 0.1 & $\|$ & $<$ & 0.1 & $\|$ & $<$ & 0.1 & $\|$ & $<$ & 0.2 & $\|$ & $<$ & $0 . i$ & $\|$ \\
\hline Hexachlorodibenzo-p-furan isomers & 0.1 & $\mid \mathrm{Y} I$ & $<$ & 0.1 & $/ Y I$ & $<$ & 0.1 & $I Y I$ & $<$ & 0.1 & IYI & $<$ & 0.1 & $\|$ & $<$ & 0.1 & $\|$ & $<$ & 0.1 & $\|$ & $<$ & 0.1 & $\|$ & $<$ & $0.1^{\prime}$ & $\|$ \\
\hline Octachlorodibenzo-p-furan isomers & 0.1 & $/ \mathrm{Y} /$ & $<$ & 0.1 & $/ Y I$ & & 0.2 & $\mathrm{~J} / \mathrm{Y} /$ & $<$ & 0.2 & $/ Y I$ & $<$ & 0.1 & $\|$ & $<$ & 0.6 & $\|$ & $<$ & 0.1 & $\|$ & $<$ & 0.1 & $\|$ & $<$ & 0.2 & $\|$ \\
\hline Pentachlorodibenzo-p-dioxin isomers & 0.1 & $\mid \mathrm{Y} I$ & $<$ & 0.2 & $M I$ & $<$ & 0.1 & $I Y I$ & $<$ & 0.1 & IYI & $<$ & 0.1 & $\|$ & $<$ & 0.1 & $\|$ & $<$ & 0.1 & /I & $<$ & 0.3 & $\|$ & $<$ & 0.2 & $\|$ \\
\hline Pentachlorodibenzo-p-furan isomers & 0.1 & $/ \mathrm{Y} I$ & $<$ & 0.1 & $\mid Y I$ & $<$ & 0.1 & $/ Y I$ & $<$ & 0.1 & $I \mathrm{YI}$ & $<$ & 0.1 & $\|$ & $<$ & 0.1 & $\|$ & $<$ & 0.1 & $\|$ & $<$ & 0.2 & $\|$ & $<$ & 0.1 & $\|$ \\
\hline Tetrachlorodibenzo-p-dioxin isomers & 0.1 & $I Y I$ & $<$ & 0.1 & $\Gamma I$ & $<$ & 0.1 & $I Y I$ & $<$ & 0.1 & $/ \mathrm{Y} I$ & $<$ & 0.1 & $\|$ & $<$ & 0.1 & $\|$ & $<$ & 0.1 & II & $<$ & 0.2 & /l & $<$ & 0.1 & $\|$ \\
\hline Tetrachlorodibenzo-p-furan isomers & 0.1 & $r Y I$ & $<$ & 0.1 & $M I$ & $<$ & 0.1 & $\mid Y I$ & $<$ & 0.1 & $/ Y I$ & $<$ & 0.1 & $\|$ & $<$ & 0.1 & $\|$ & $<$ & 0.1 & $\|$ & $<$ & 0.1 & $\|$ & $<$ & 0.1 & $\|$ \\
\hline Octachlorodibenzo-p-dioxin isomers & 1.6 & $\mathrm{~J} / \mathrm{Y} /$ & & 0.7 & $\mathrm{~J} / \mathrm{Y} /$ & & 3.9 & $\Gamma Y I$ & & 10.5 & $/ \mathrm{YI}$ & & 26 & 11 & & 22 & II & & 0.7 & $\mathrm{JII}$ & & 2.3 & II & & 2.1 & 11 \\
\hline
\end{tabular}


TABLE 6.4

PCDD AND PCDF COMPOUNDS

IN SOIL

D.AREA BURNING/RUBBLE PITS

SAVANNAH RIVER SITE

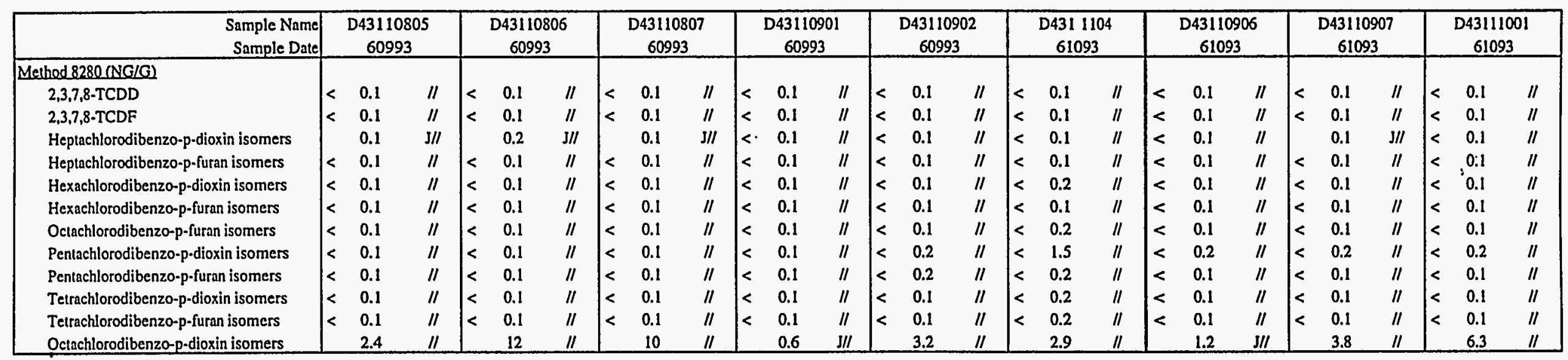


TABLE 6.4

PCDD AND PCDF COMPOUNDS

IN SOIL

D-AREA BURNINO/RUBBLE PITS

SAVANNAH RIVER SITE

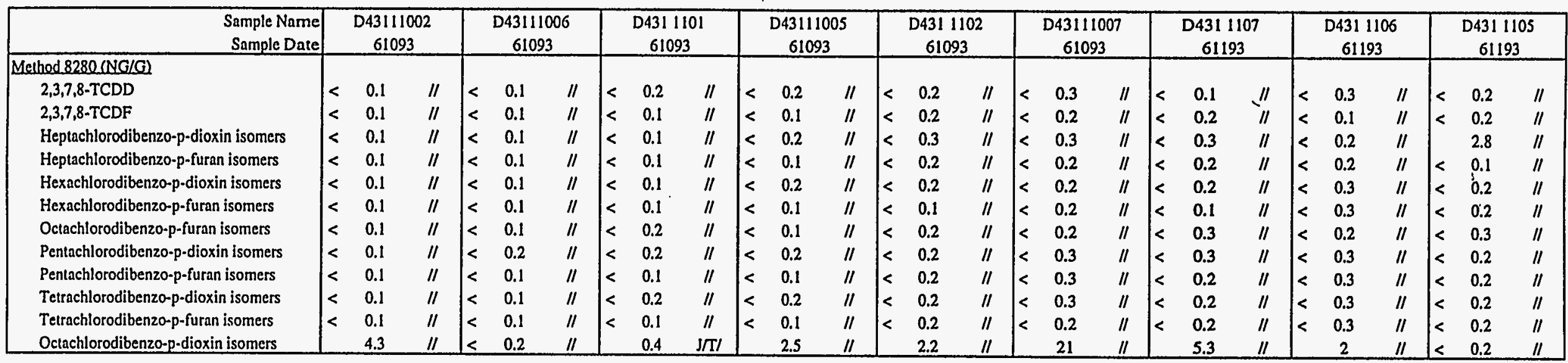


TABLE 6.4

PCDD AND PCDF COMPOUNDS

IN SOIL

D-AREA BURNING/RUBBLE PITS

SAVANNAH RIVER SITE

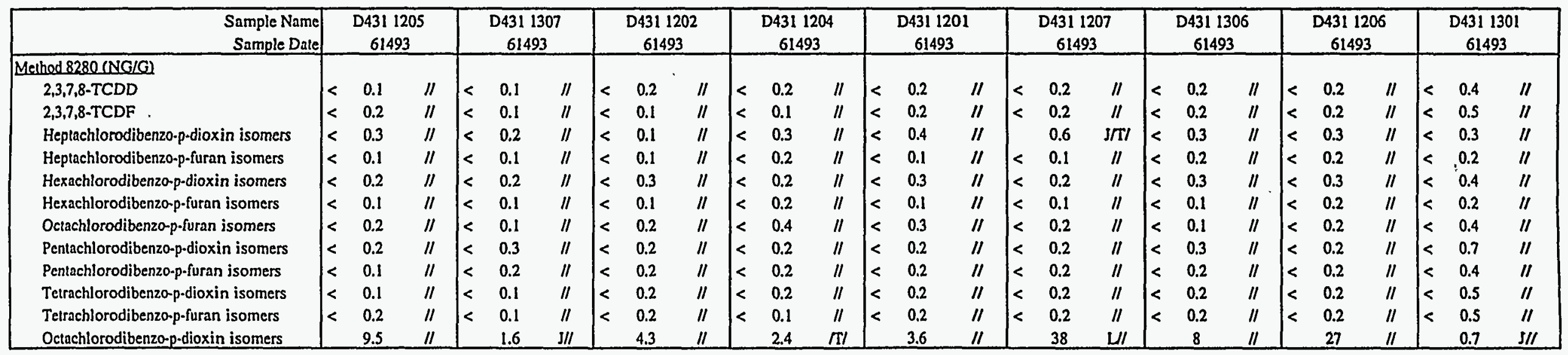


TABLE 6.4

PCDD AND PCDF COMPOUNDS

IN SOIL

D-AREA BURNING/RUBBLE PITS

SAVANNAH RIVER SITE

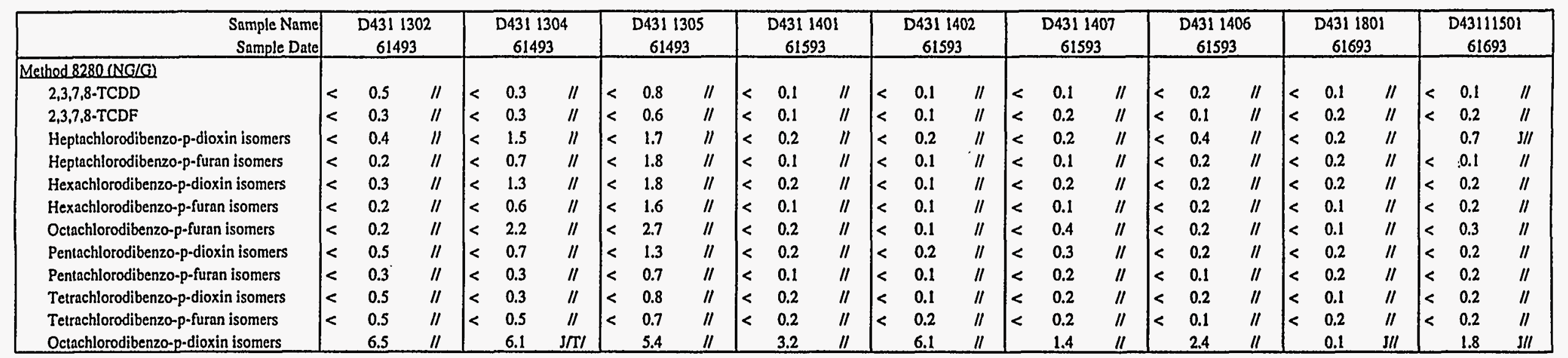


TABLE 6.4

PCDD AND PCDF COMPOUNDS

IN SOIL

D-AREA BURNING/RUBBLE PITS

SAVANNAH RIVER SITE

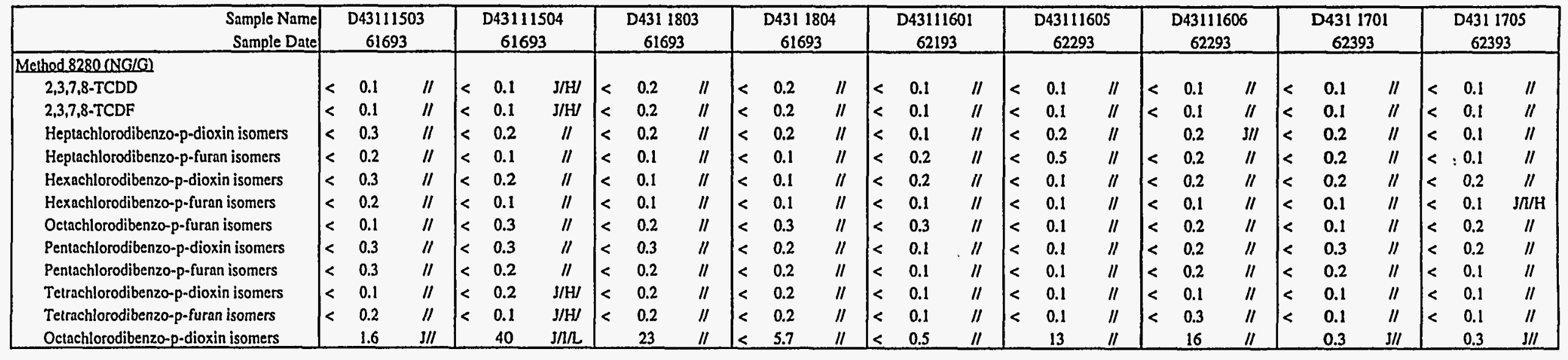


TABLE 6.4

PCDD AND PCDF COMPOUNDS

IN SOIL

D.AREA BURNING/RUBBLE PITS

SAVANNAH RIVER SITE

\begin{tabular}{|l|lll|}
\hline $\begin{array}{r}\text { Sample Name } \\
\text { Sample Date }\end{array}$ & \multicolumn{2}{|c|}{ D431 62393} \\
\hline Melhod 8280(NG/G) & & & \\
\hline $2,3,7,8-T C D D$ & $<$ & 0.1 & $\prime \prime$ \\
$2,3,7,8-T C D F$ & $<$ & 0.1 & $\prime \prime$ \\
Heptachlorodibenzo-p-dioxin isomers. & $<$ & 0.2 & $\prime \prime$ \\
Heptachlorodibenzo-p-furan isomers & $<$ & 0.1 & $\prime \prime$ \\
Hexachlorodibenzo-p-dioxin isomers & $<$ & 0.2 & $\prime \prime$ \\
Hexachlorodibenzo-p-furan isomers & $<$ & 0.1 & $\prime \prime$ \\
Octachlorodibenzo-p-furan isomers & $<$ & 0.1 & $\prime \prime$ \\
Pentachlorodibenzo-p-dioxin isomers & $<$ & 0.2 & $\prime \prime$ \\
Pentachlorodibenzo-p-furan isomers & $<$ & 0.1 & $\prime \prime$ \\
Tetrachlorodibenzo-p-dioxin isomers & $<$ & 0.1 & $\prime \prime$ \\
Tetrachlorodibenzo-p-furan isomers & $<$ & 0.1 & $\prime \prime$ \\
Octachlorodibenzo-p-dioxin isomers & & 1.4 & $\prime \prime$ \\
\hline
\end{tabular}


TABLE 6.5

INORGANICS AND ORGANIC INDICATORS

D-AREA BURNING/RUBBLE PITS

SAVANNAH RIVER SITE

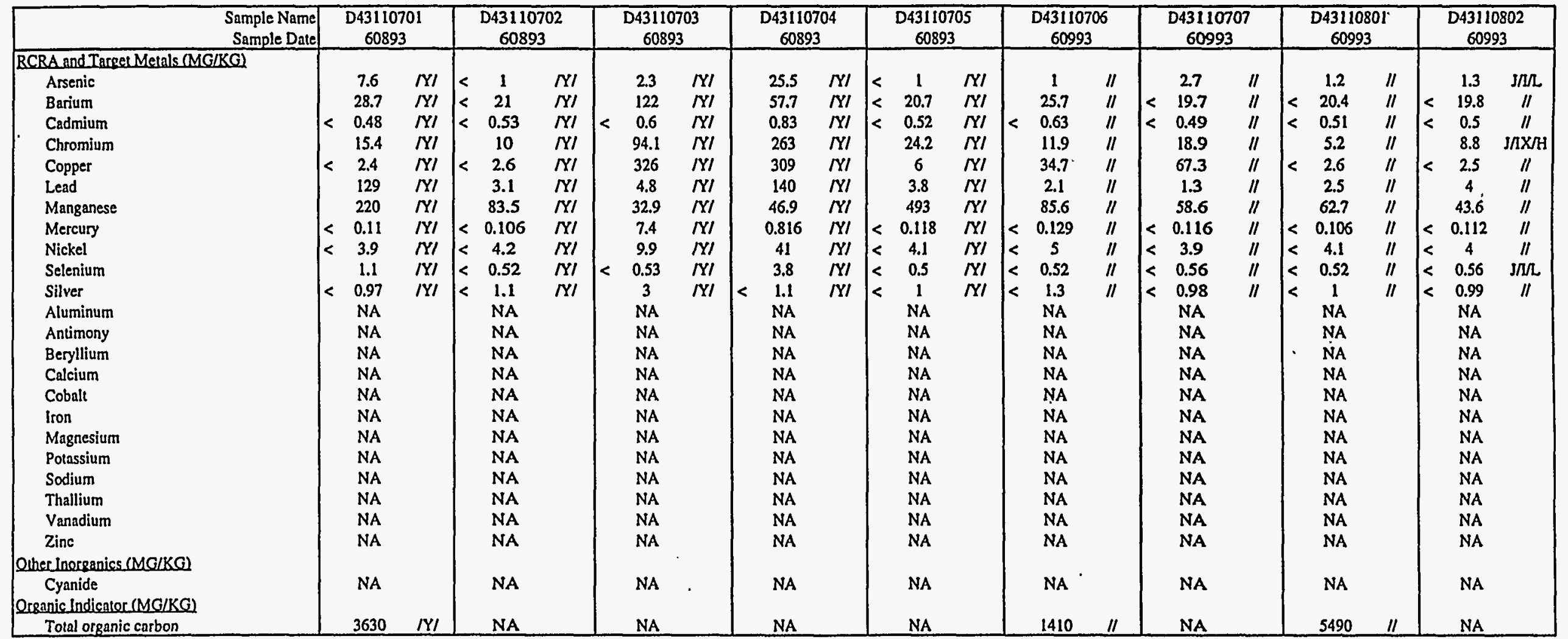


TABLE 6.5

INORGANICS AND ORGANIC INDICATORS IN SOIL

D-AREA BURNING/RUBBLE PITS SAVANNAH RIVER SITE

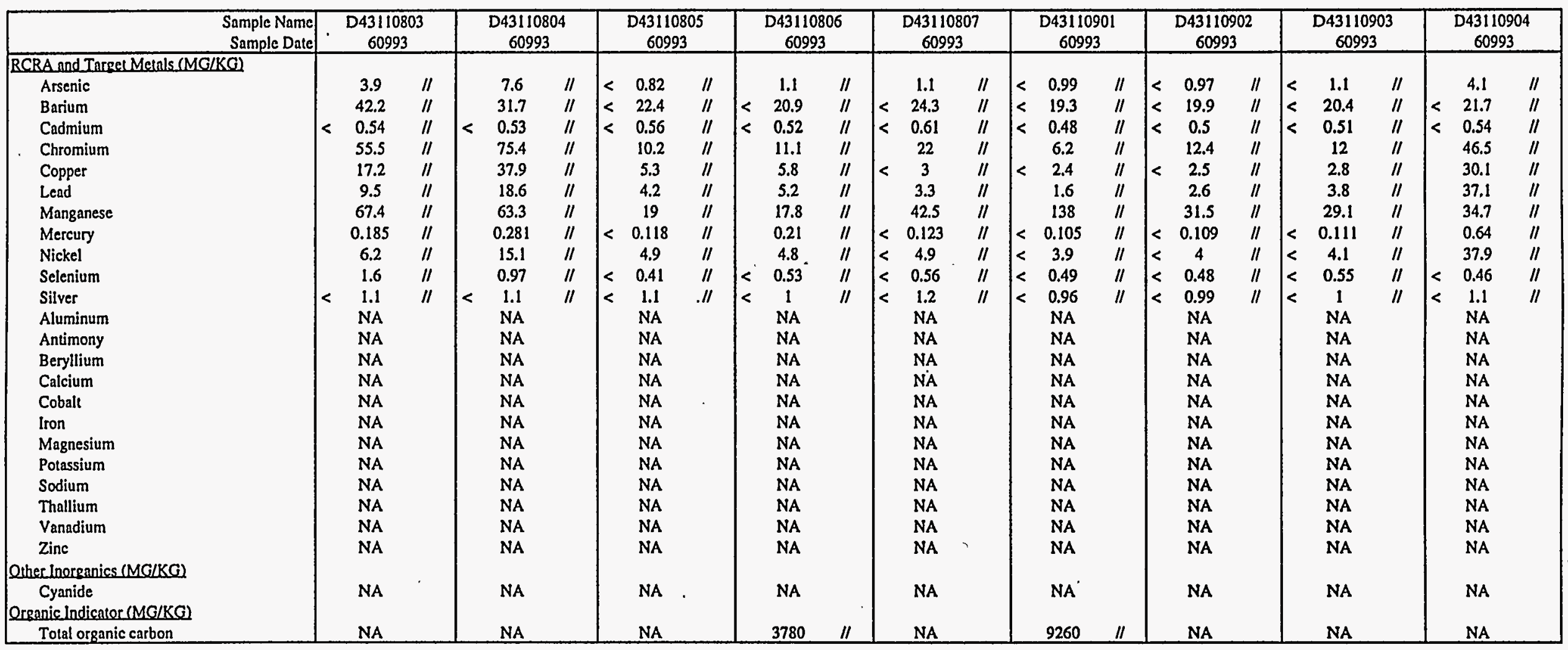


TABLE 6.5

INORGANICS AND ORGANICINDICATORS IN SOIL

D.AREA BURNING/RUBBLE PITS

SAVANNAH RIVER SITE

\begin{tabular}{|c|c|c|c|c|c|c|c|c|c|c|c|c|c|c|c|c|c|c|c|c|c|c|c|c|c|c|}
\hline \multirow{2}{*}{\begin{tabular}{|r|}
$\begin{array}{r}\text { Sample Name } \\
\text { Sample Date }\end{array}$ \\
RCRA and Target Metals (MG/KG)
\end{tabular}} & \multicolumn{2}{|c|}{$\begin{array}{c}\text { D43110905 } \\
61093 \\
\end{array}$} & \multirow{2}{*}{\multicolumn{3}{|c|}{$\begin{array}{c}43110906 \\
61093\end{array}$}} & \multicolumn{3}{|c|}{$\begin{array}{c}43110907 \\
61093 \\
\end{array}$} & \multicolumn{3}{|c|}{$\begin{array}{c}\text { D43111001 } \\
61093 \\
\end{array}$} & \multirow{2}{*}{\multicolumn{3}{|c|}{$\begin{array}{c}\text { D43111002 } \\
61093 \\
\end{array}$}} & \multicolumn{3}{|c|}{$\begin{array}{c}\text { D43111003 } \\
61093\end{array}$} & \multicolumn{3}{|c|}{$\begin{array}{c}43111004 \\
61093 \\
\end{array}$} & \multicolumn{3}{|c|}{$\begin{array}{c}43111005 \\
61093\end{array}$} & \multicolumn{3}{|c|}{$\begin{array}{c}\mathrm{D} 43111006 \\
61093\end{array}$} \\
\hline & & & & & & & & & & & & & & & & & & & & & & & & & & \\
\hline Arsenic & 3.2 & $/ 1$ & & 1.1 & "I & $<$ & 1.1 & $\|$ & $<$ & 0.95 & $\|$ & $<$ & 1 & $\|$ & & 1.1 & /1 & $<$ & 1 & /I & $<$ & 1.1 & $\mathrm{~J} / \Omega$ & & 1.3 & $\|$ \\
\hline Barium & $<\quad 17.9$ & II & & 68.5 & $\|$ & & 22.1 & /I & $<$ & 20.9 & $\|$ & $<$ & 19.1 & $\|$ & $<$ & 21.4 & /I & & 29.6 & $\|$ & & 26 & J///H & & 25.5 & $\|$ \\
\hline Chromium & $<\quad 8.9$ & $\mathrm{~J} / \mathrm{M}$ & & 44 & $\mathrm{~J} / \mathrm{L}$ & $<$ & 7.8 & $\mathrm{~J} / \mathrm{L}$ & & 12.4 & $\mathrm{~J} / \mathrm{L}$ & & 12.2 & $\mathrm{~J} / \mathrm{L}$ & & 11.6 & $\mathrm{~J} / \mathrm{L}$ & & 26.7 & $\mathrm{~J} / \mathrm{M}$ & & 24.2 & $\mathrm{~J} / \mathrm{LI} / \mathrm{H}$ & & 18.7 & $\mathrm{~J} / \mathrm{L}$ \\
\hline Copper & 3.9 & $/ 1$ & $<$ & 3 & $I I$ & $<$ & 2.1 & 11 & $<$ & 2.6 & $\|$ & $<$ & 2.4 & $/ /$ & & 5.6 & $/ 1$ & & 5 & $\|$ & $<$ & 3 & II & $<$ & 2.9 & /I \\
\hline Lead & 22 & II & & 5.4 & $\|$ & & 1.7 & "I & & 3.5 & $\|$ & & 3.7 & $\|$ & & 4.1 & "l & & 2.3 & "I & & 6.5 & $\mathrm{~J} / \mathrm{X} \mathrm{J}$ & & 2.9 & $\|$ \\
\hline Manganese & 13 & $\|$ & & 20.7 & $\|$ & & 32.3 & $\|$ & & 92.3 & 11 & & 41.6 & $\|$ & & 45 & /I & & 252 & II & & 266 & $J / X J$ & & 405 & /I \\
\hline Nickel & $<3.6$ & /I & $<$ & 4.9 & $\|$ & $<$ & 3.3 & /I & $<$ & 4.2 & // & $<$ & 3.8 & $\|$ & $<$ & 4.3 & /I & $<$ & 4.1 & "I & $<$ & 4.9 & "I & & 5.8 & $\|$ \\
\hline Selenium & $<\quad 0.52$ & $\|$ & $<$ & 0.56 & $\|$ & $<$ & 0.57 & "I & $<$ & 0.48 & $\|$ & $<$ & 0.5 & $\|$ & $<$ & 0.5 & $\|$ & $<$ & 0.52 & "l & $<$ & 0.56 & $\mathrm{~J} / \mathrm{L} / \mathrm{L}$ & $<$ & 0.62 & "I \\
\hline Silver & $<\quad 0.9$ & $\|$ & $<$ & 1.2 & II & $<$ & 0.83 & "I & $<$ & 1 & "I & $<$ & 0.96 & $\|$ & $<$ & 1.1 & II & $<$ & 1 & /I & $<$ & 1.2 & $J / X J$ & $<$ & 1.1 & $\|$ \\
\hline Aluminum & 1320 & 11 & & NA & & & NA & & & NA & & & NA & & & $\mathrm{NA}$ & & & NA & & & NA & & & NA & \\
\hline Antimony & $<\quad 5.4$ & $\|$ & & NA & & & NA & & & NA & & & NA & & & NA & & & NA & & & NA & & & $\mathrm{NA}$ & \\
\hline Beryllium & $<\quad 0.45$ & $\|$ & & NA & & & NA & & & NA & & & NA & & & NA & & & NA & & & NA & & & $\mathrm{NA}$ & \\
\hline Calcium & $<\quad 448$ & /I & & $\mathrm{NA}$ & & & NA & & & NA & & & NA & & & NA & & & NA & & & NA & & & NA & \\
\hline Cobalt & $<4.5$ & $\|$ & & $\mathrm{NA}$ & & & NA & & & NA & & & $\mathrm{NA}$ & & & NA & & & NA & & & NA & & & NA & \\
\hline Iron & 5850 & $/ 1$ & & NA & & & $\mathrm{NA}$ & & & NA & & & NA & & & NA & & & NA & & & NA & & & $\mathrm{NA}$ & \\
\hline Magnesium & $<448$ & $\|$ & & NA & & & NA & & & NA & & & NA & & & NA & & & NA & & & NA & & & NA & \\
\hline Sodium & $<448$ & $\|$ & & NA & & & NA & & & NA & & & NA & & & NA & & & NA & & & NA & & & NA & \\
\hline Thallium & $<$ & $\|$ & & NA & & & $\mathrm{NA}$ & & & NA & & & NA & & & NA & & & NA & & & NA & & & NA & \\
\hline Vanadium & 13.9 & $\|$ & & NA & & & NA & & & NA & & & NA & & & NA & & & NA & & & NA & & & NA & \\
\hline Zine & 7.7 & $\|$ & & NA & & & NA & & & NA & & & NA & & & NA & & & NA & & & NA & & & NA & \\
\hline Qther Inoreanics (MG/KG) & & & & & & & & & & & & & & & & & & & & & & & & & & \\
\hline $\begin{array}{l}\text { Cyanide } \\
\text { Oranic Indicator }(M G / K G)\end{array}$ & $<\quad 1.13$ & $\|$ & & NA & & & NA & & & NA & & & NA & & & $\mathrm{NA}^{\prime}$ & & & $\mathrm{NA}$ & & & NA & & & $\mathrm{NA}$ & \\
\hline Total organic carbon & 4890 & $\|$ & & NA & & & $\mathrm{NA}$ & & & 14100 & "I & & NA & & & NA & & & NA & & & NA & & & 1620 & II \\
\hline
\end{tabular}


TABLE 6.5

INORGANICS AND ORGANIC INDICATORS IN SOIL

D-AREA BURNING/RUBBLE PITS SAVANNAH RIVER SITE

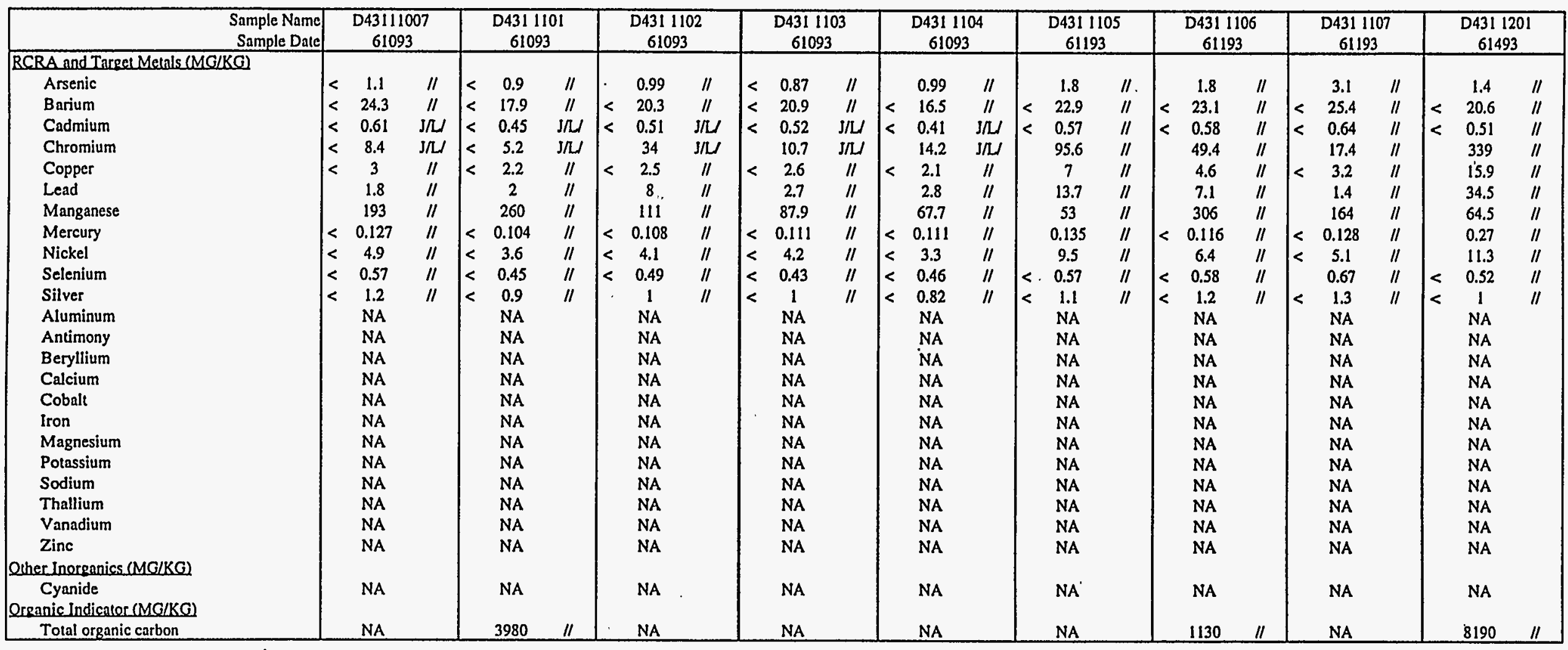


TABLE 6.5 INORGANICS AND ORGANIC INDICATORS

D-AREA BURNING/RUBBLE PITS

SAVANNAH RIVER SITE

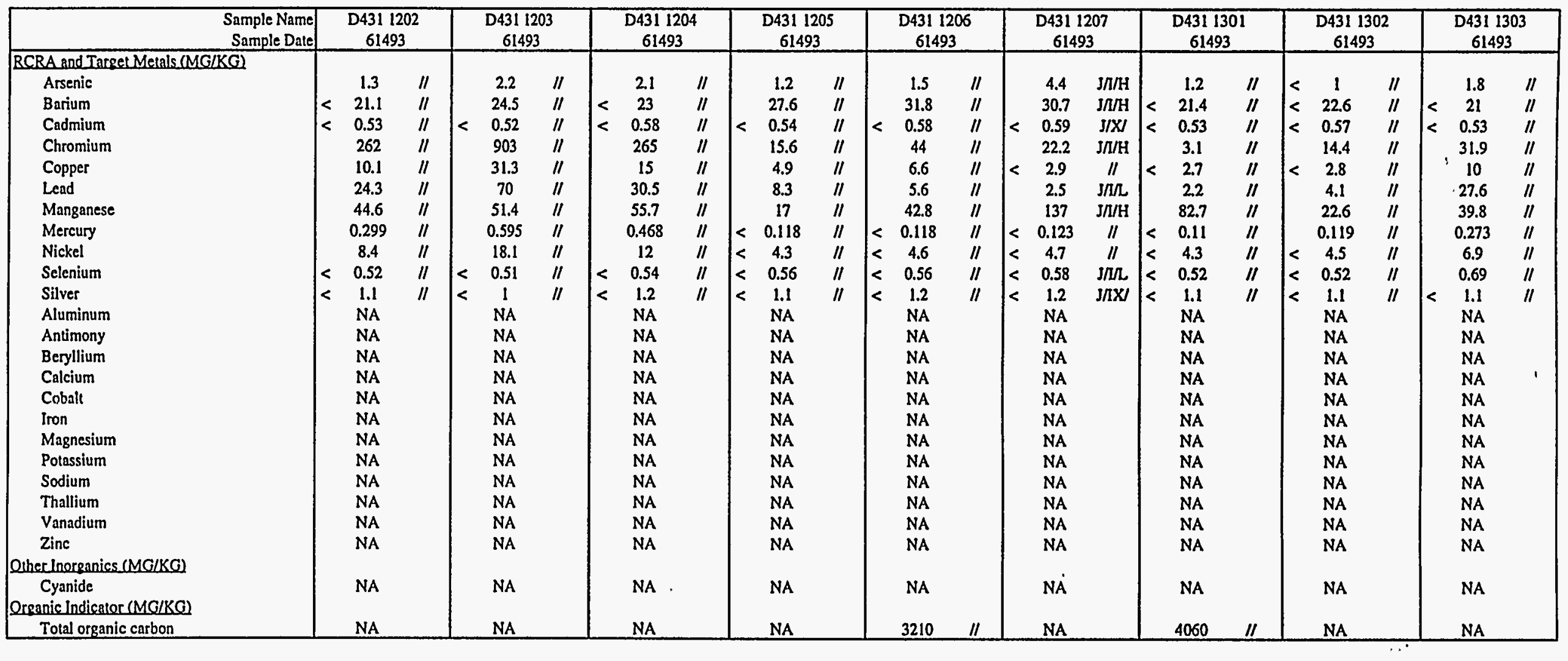


TABLE 6.5

INORGANICS AND ORGANIC INDICATORS

" $"$ IN SOIL

D-AREA BURNING/RUBBLE PITS

SAVANNAH RIVER SITE

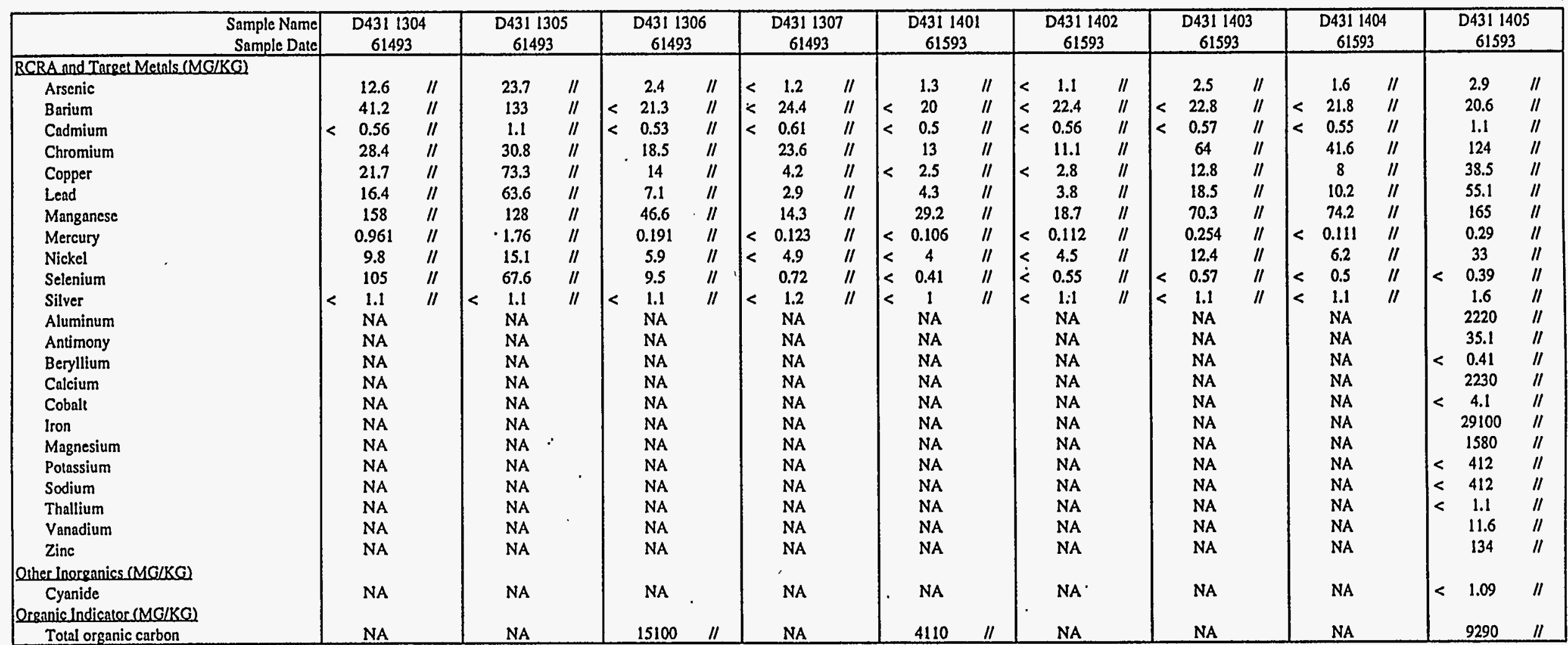


TABLE 6.5

INORGANICS AND ORGANIC INDICATORS IN SOIL

D.AREA BURNING/RUBBLE PITS SAVANNAH RIVER SITE

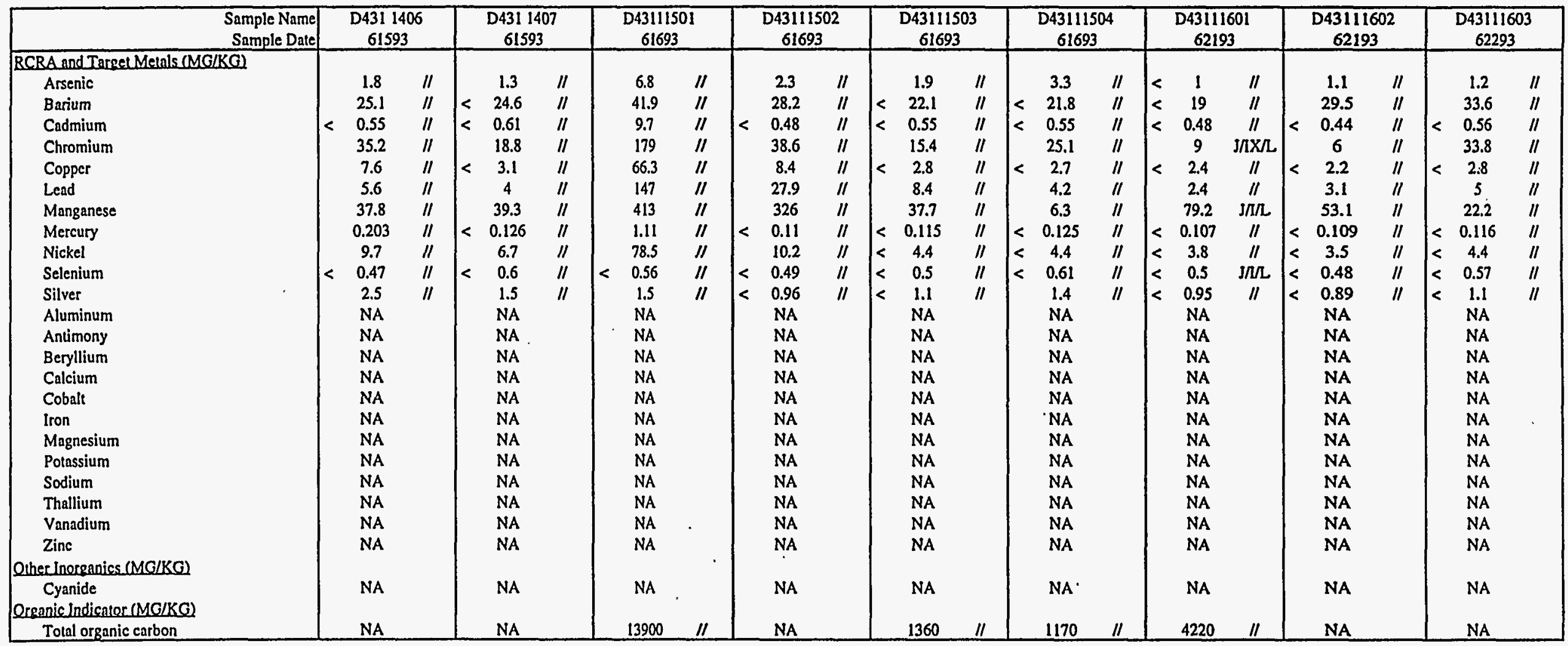


TABLE 6.5

INORGANICS AND ORGANIC INDICATORS

IN SOIL

SAVANNAH RIVER SITE

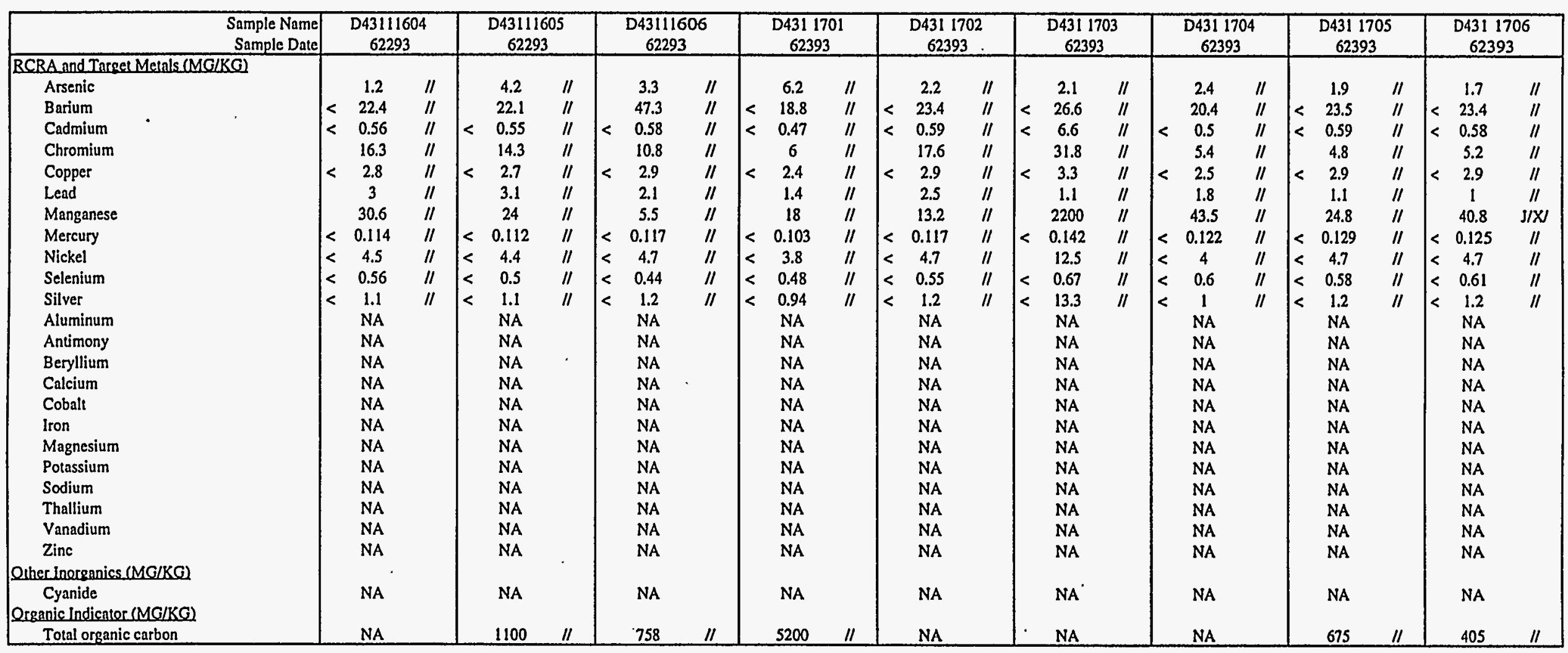




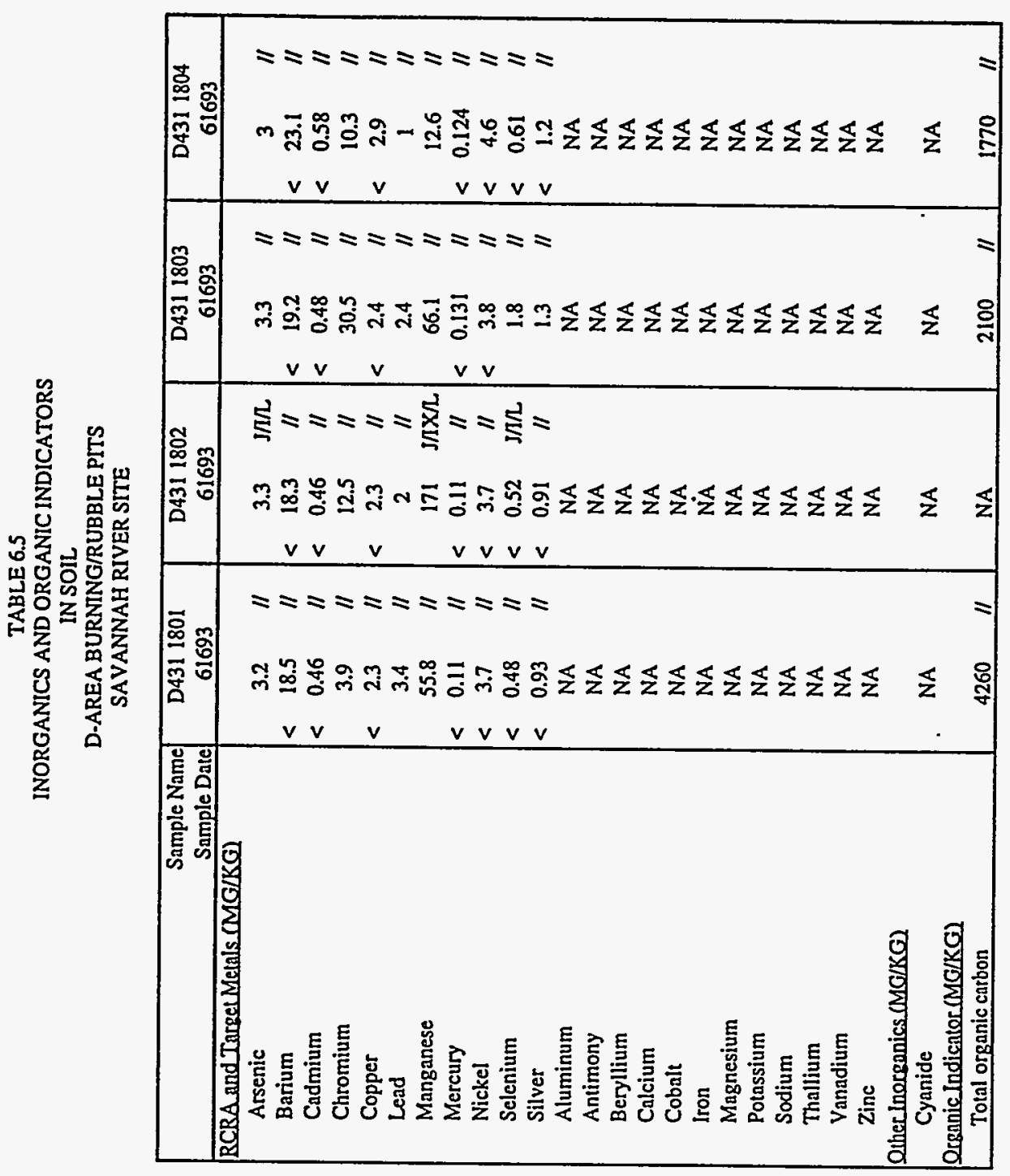




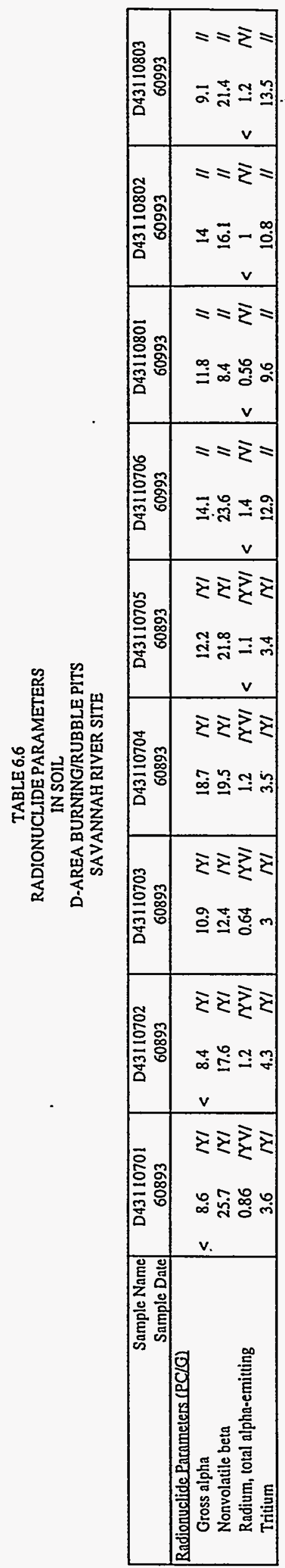


TABLE 6.6

RADIONUCLIDE PARAMETERS

$$
\text { IN SOIL }
$$

D-AREA BURNING/RUBBLE PITS

SAVANNAH RIVER SITE

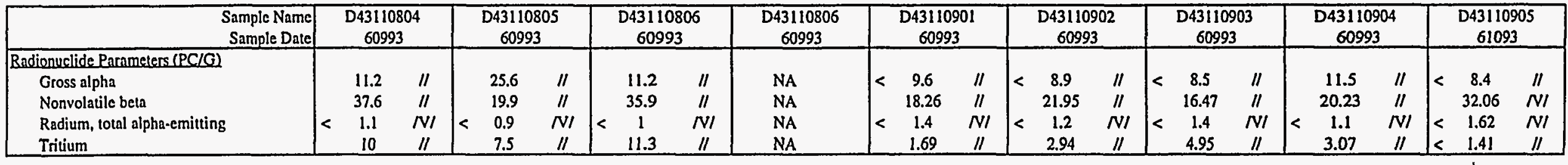


TABLE 6.6

RADIONUCLIDE PARAMETERS IN SOIL

D.AREA BURNING/RUBBLE PITS

SAVANNAH RIVER SITE

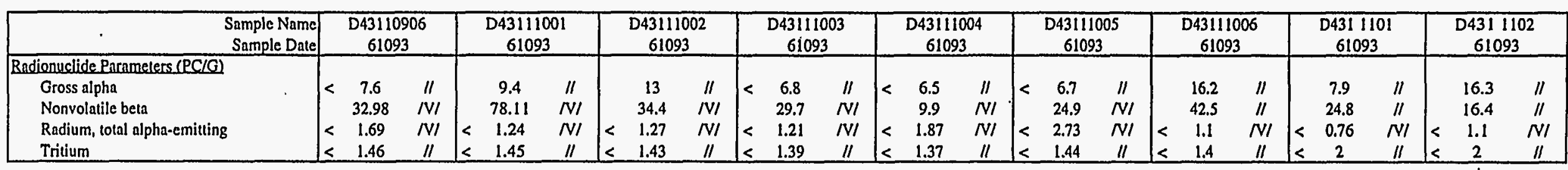


TABLE 6.6

RADIONUCLIDE PARAMETERS

$$
\text { IN SOIL }
$$

D-AREA BURNING/RUBBLE PITS

SAVANNAH RIVER SITE

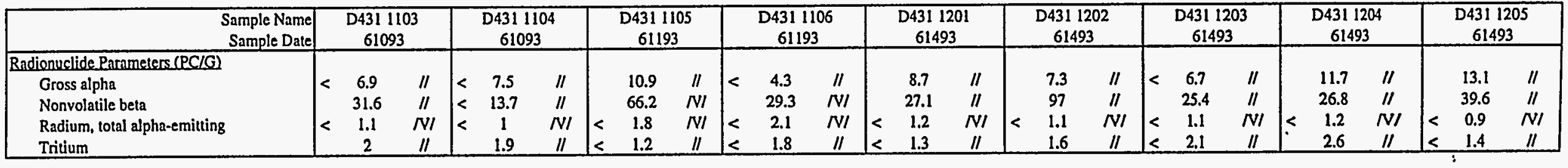


TABLE 6.6

RADIONUCLIDE PARAMETERS

$$
\text { IN SOIL }
$$

D-AREA BURNING/RUBBLE PITS

SAVANNAH RIVER SITE

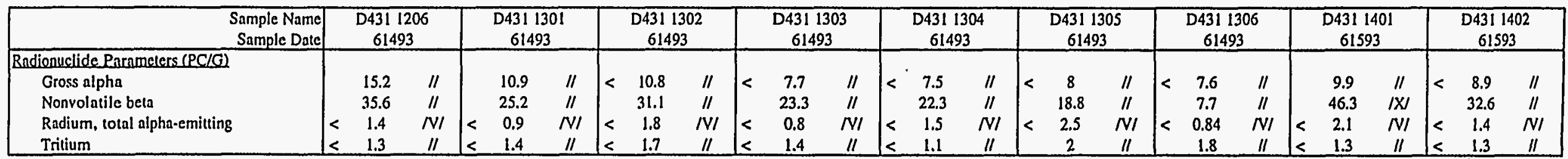


TABLE 6.6

RADIONUCLIDE PARAMETERS

IN SOIL

D-AREA BURNING/RUBBLE PITS

SAVANNAH RIVER SITE

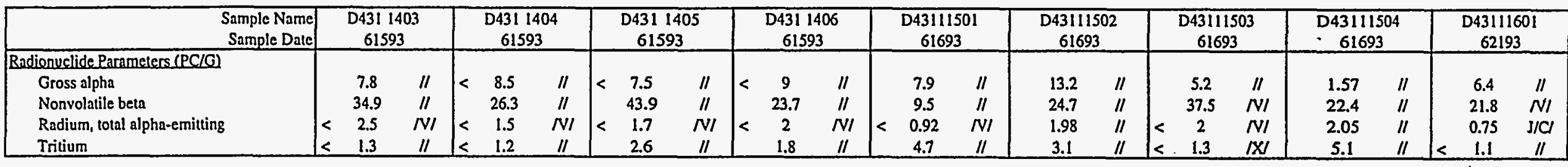


TABLE 6.6

RADIONUCLIDE PARAMETERS

IN SOIL

D.AREA BURNING/RUBBLE PITS

SAVANNAH RIVER SITE

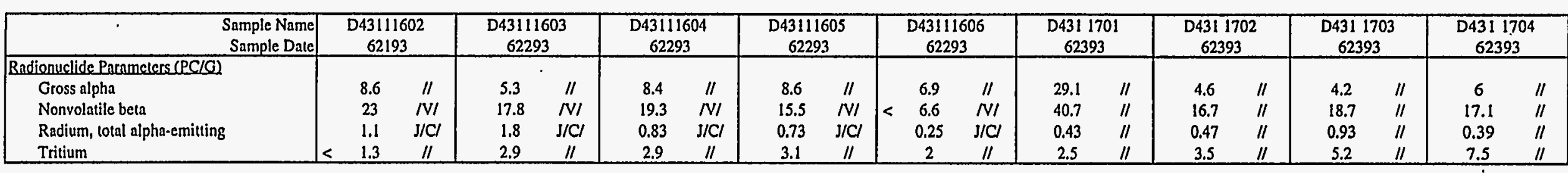


TABLE 6.6

RADIONUCLIDE PARAMETERS

IN SOIL

D-AREA BURNING/RUBBLE PITS

SAVANNAH RIVER SITE

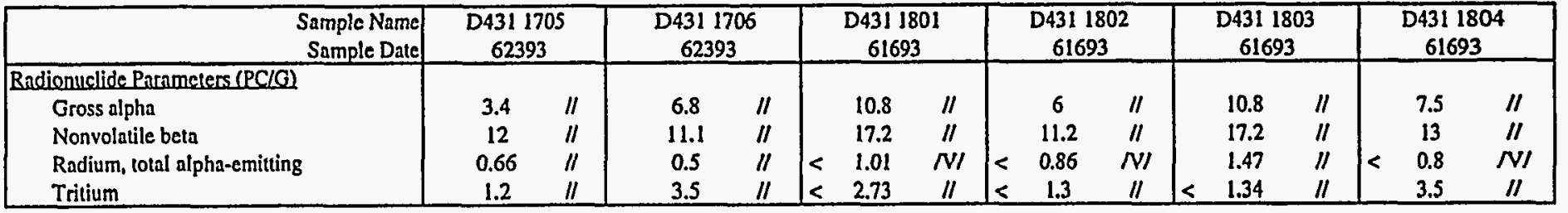


TABLE 6.7

TCLP ANALYSES

IN SOIL

D-AREA BURNING/RUBBLE PITS

SAVANNAH RIVER SITE

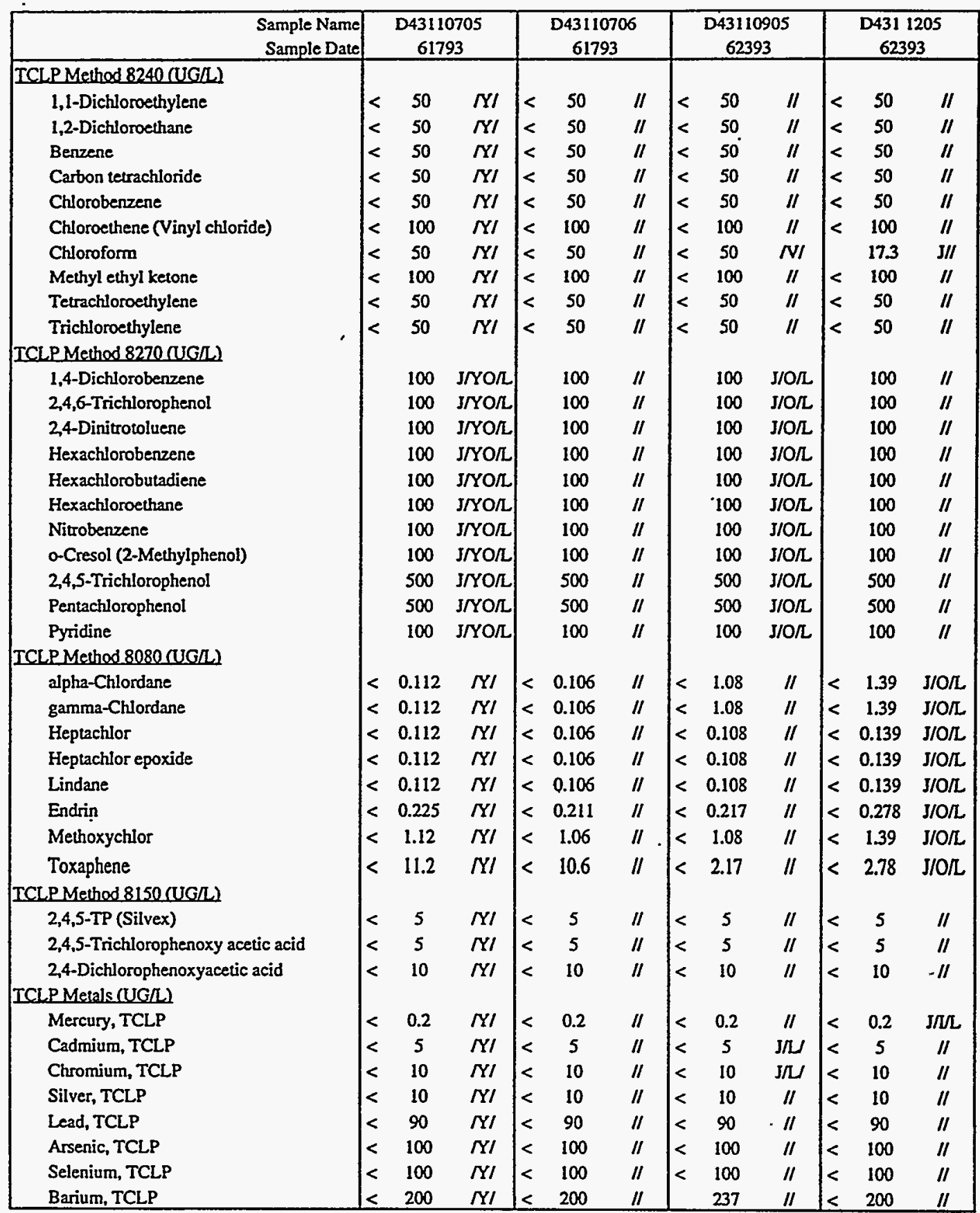


TABLE 6.8

VOLATILE ORGANIC COMPOUNDS

IN GROUNDWATER

D-AREA BURNING/RUBBLE PITS

SAVANNAH RIVER SITE

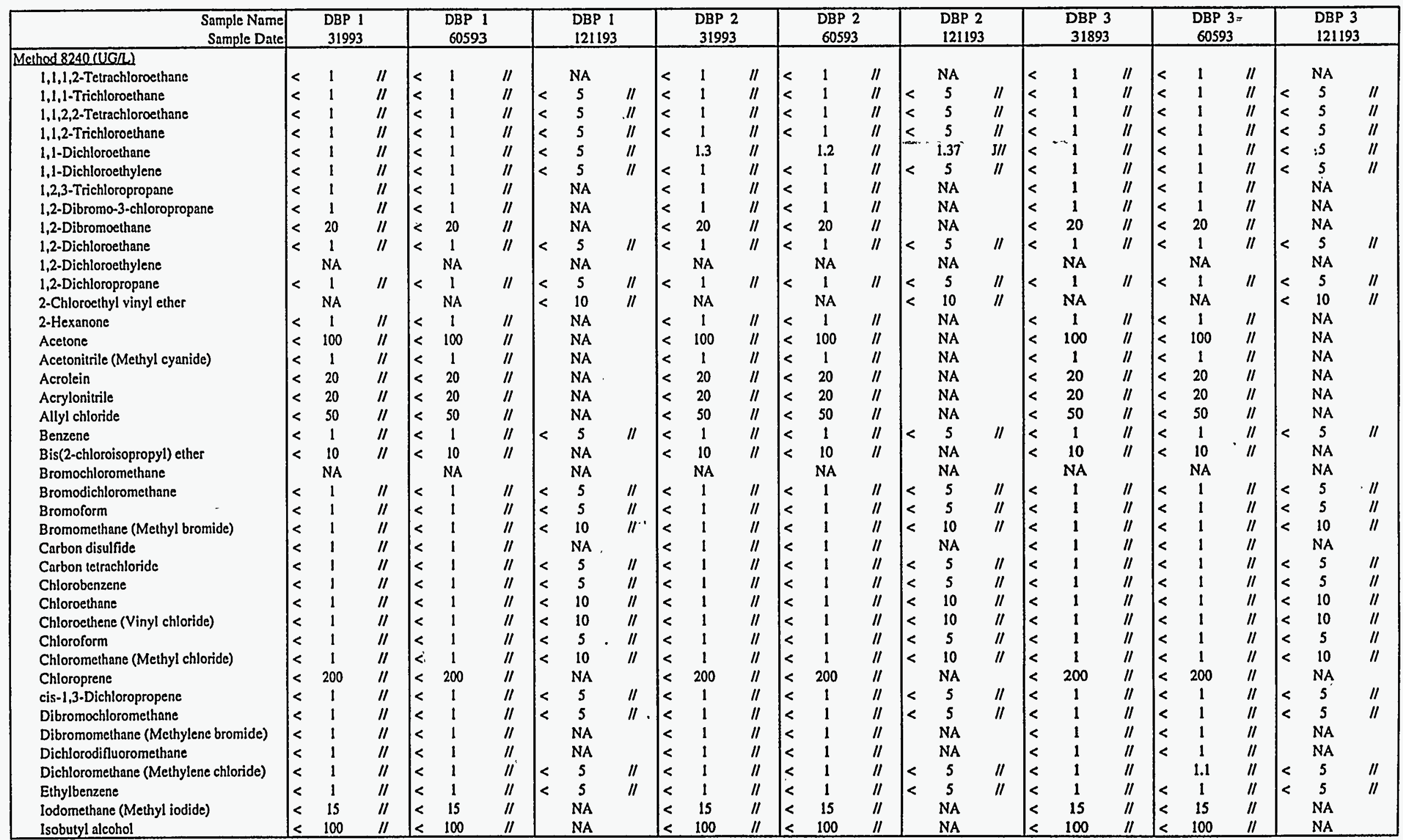


TABLE 6.8

VOLATILE ORGANIC COMPOUNDS IN GROUNDWATER

D-AREA BURNING/RUBBLE PITS SAVANNAH RIVER SITE

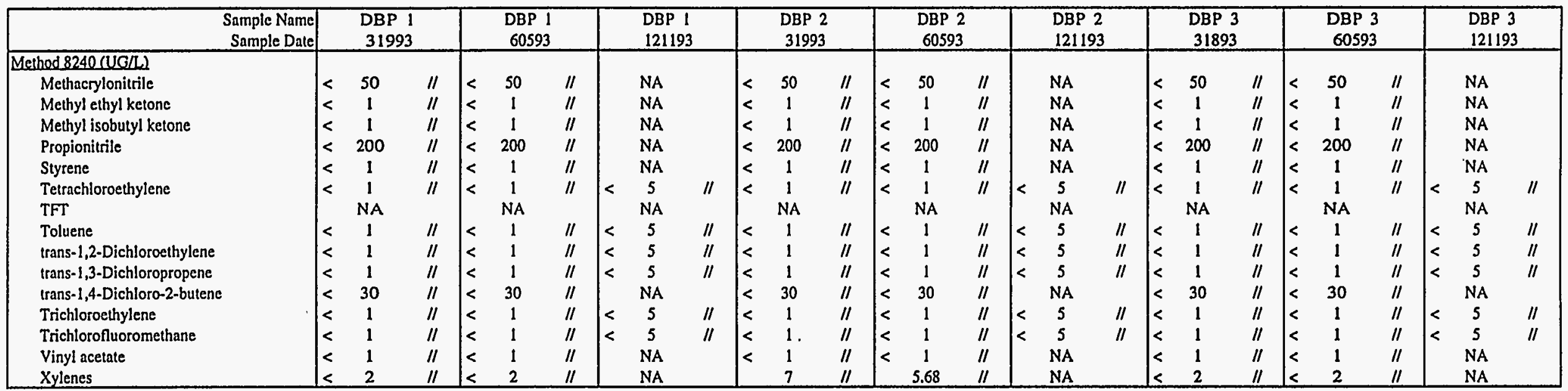


TABLE 6.8

VOLATILE ORGANIC COMPOUNDS

IN GROUNDWATER:

D-AREA BURNING/RUBBLE PITS

SAVANNAH RIVER SITE

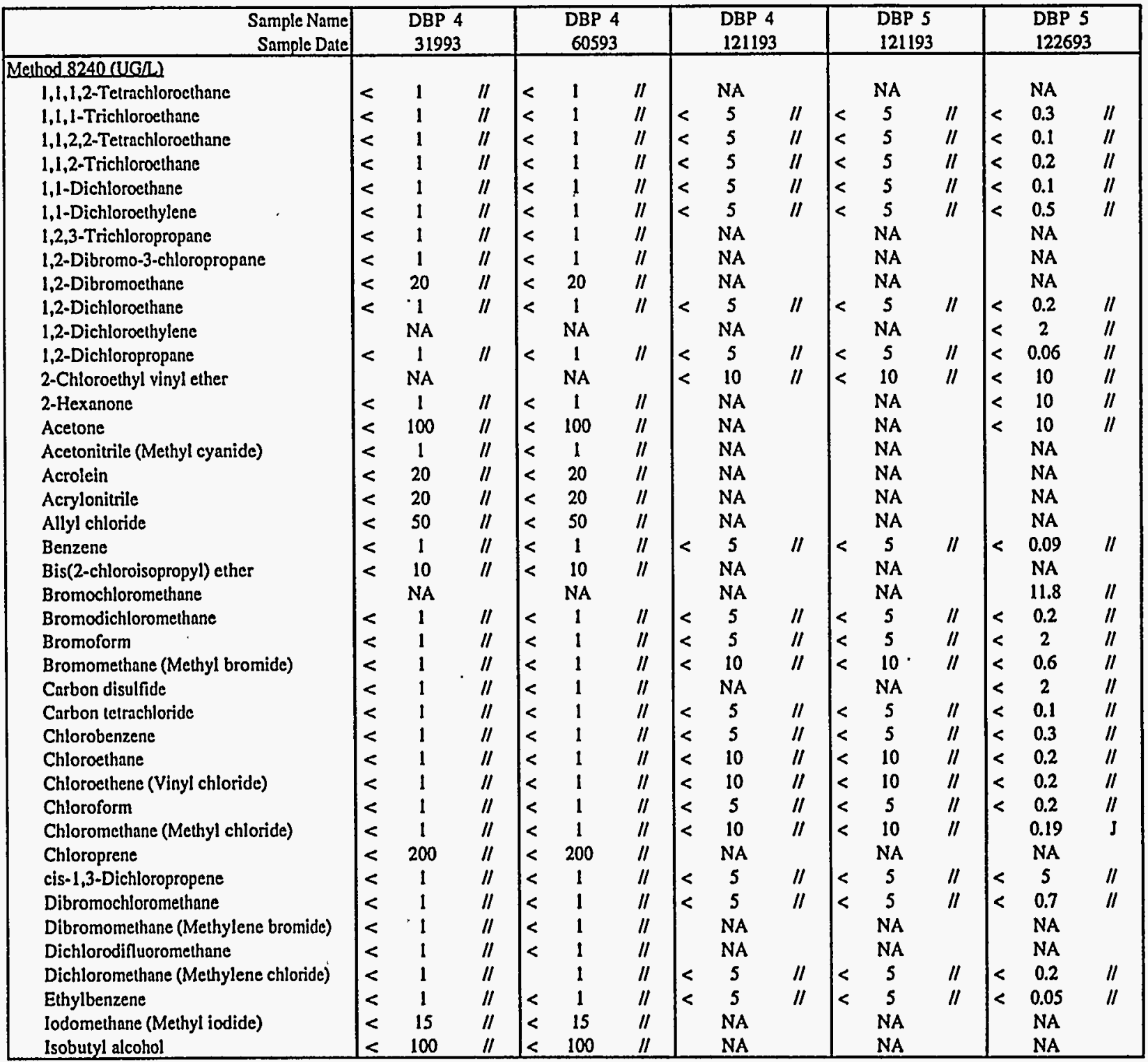


TABLE 6.8

VOLATILE ORGANIC COMPOUNDS

IN GROUNDWATER

D-AREA BURNING/RUBBLE PITS

SAVANNAH RIVER SITE

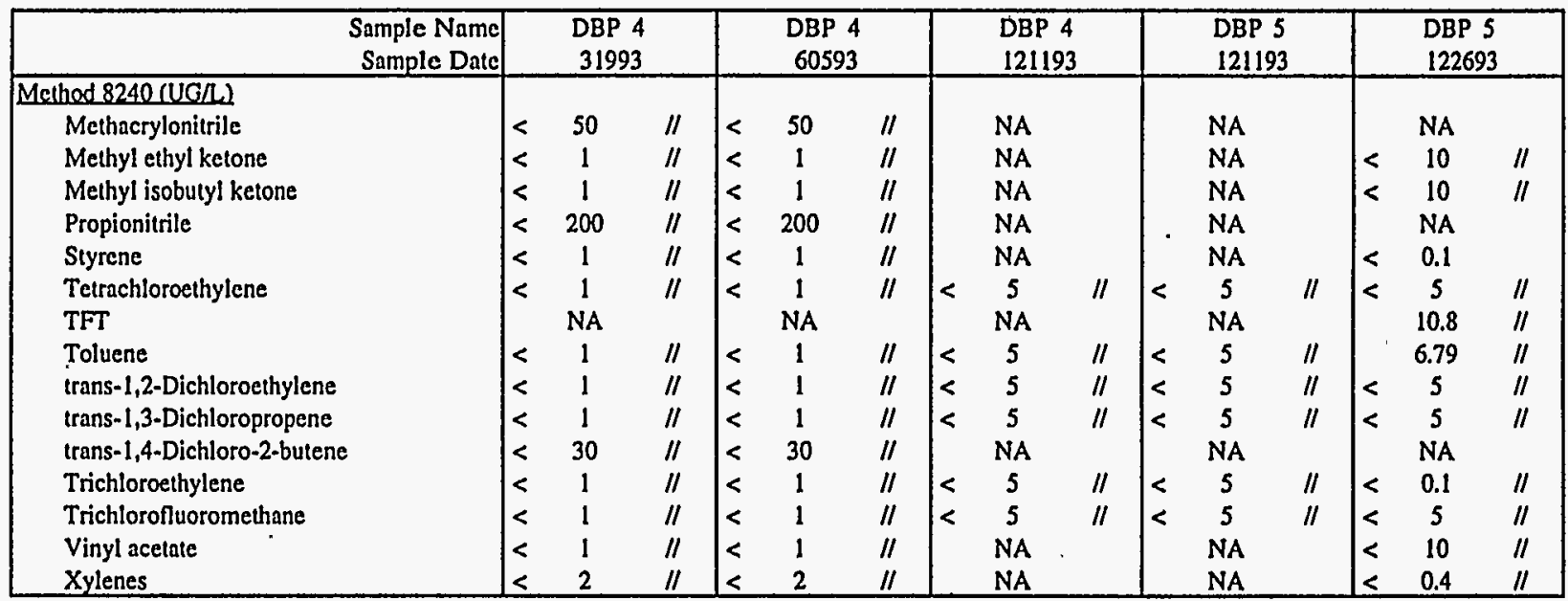




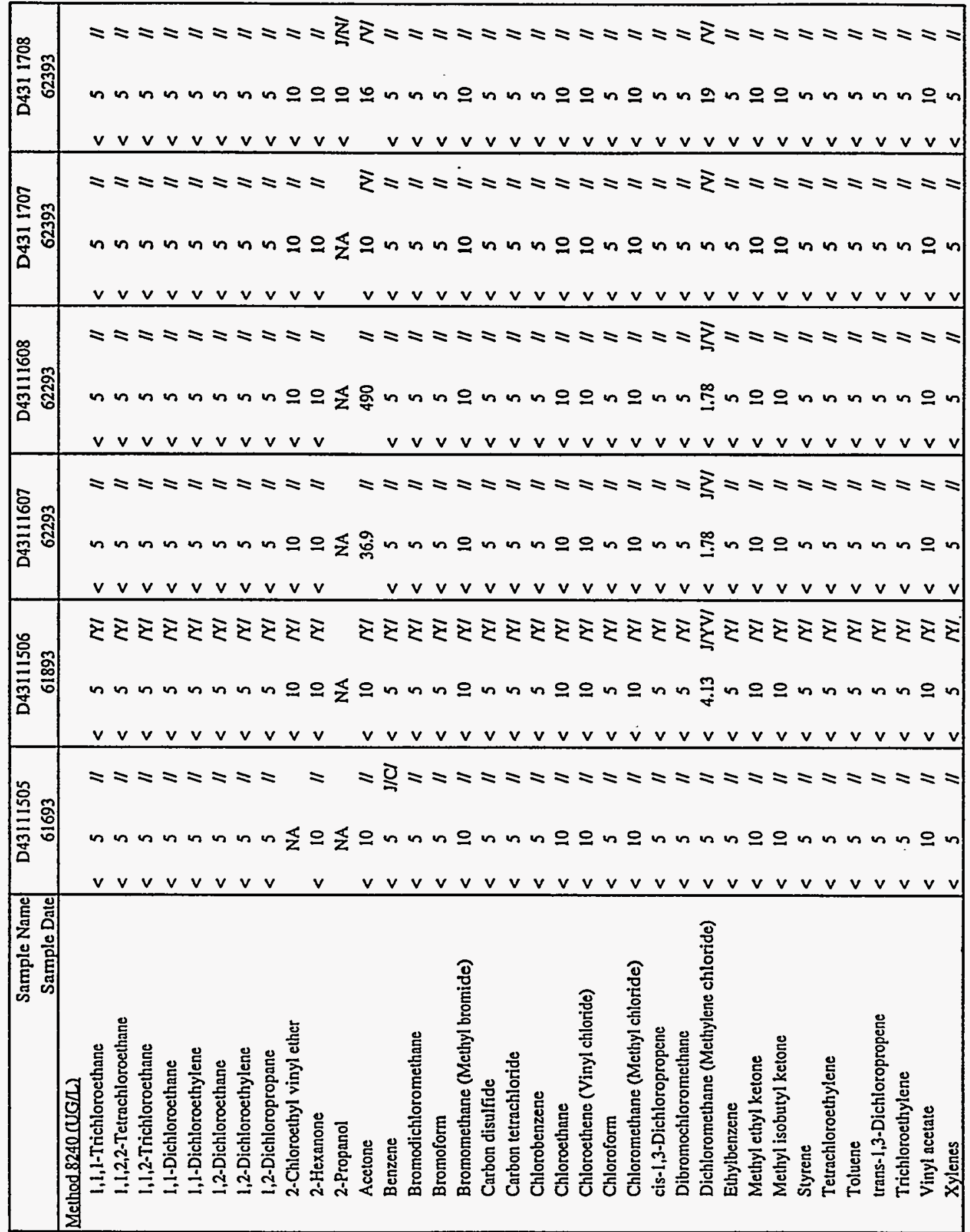


TABLE 6.10

SEMI-VOLATILE ORGANIC COMPOUNDS

IN GROUNDWATER

D-AREA BURNING/RUBBLE PITS

SAVANNAH RIVER SITE

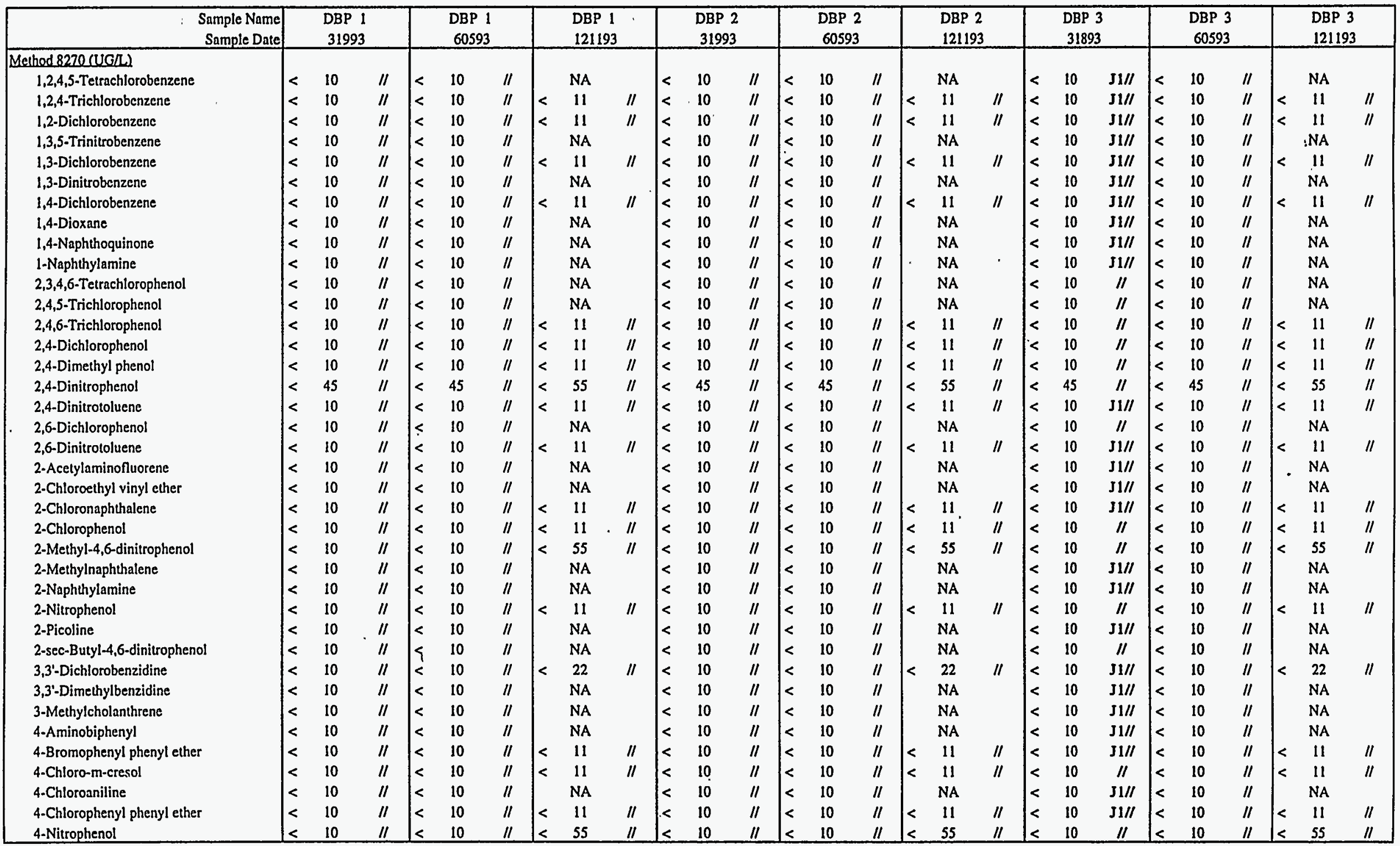


TABLE 6.10

SEMI-VOLATILE ORGANIC COMPOUNDS IN GROUNDWATER

D-AREA BURNING/RUBBLE PITS

SAVANNAH RIVER SITE

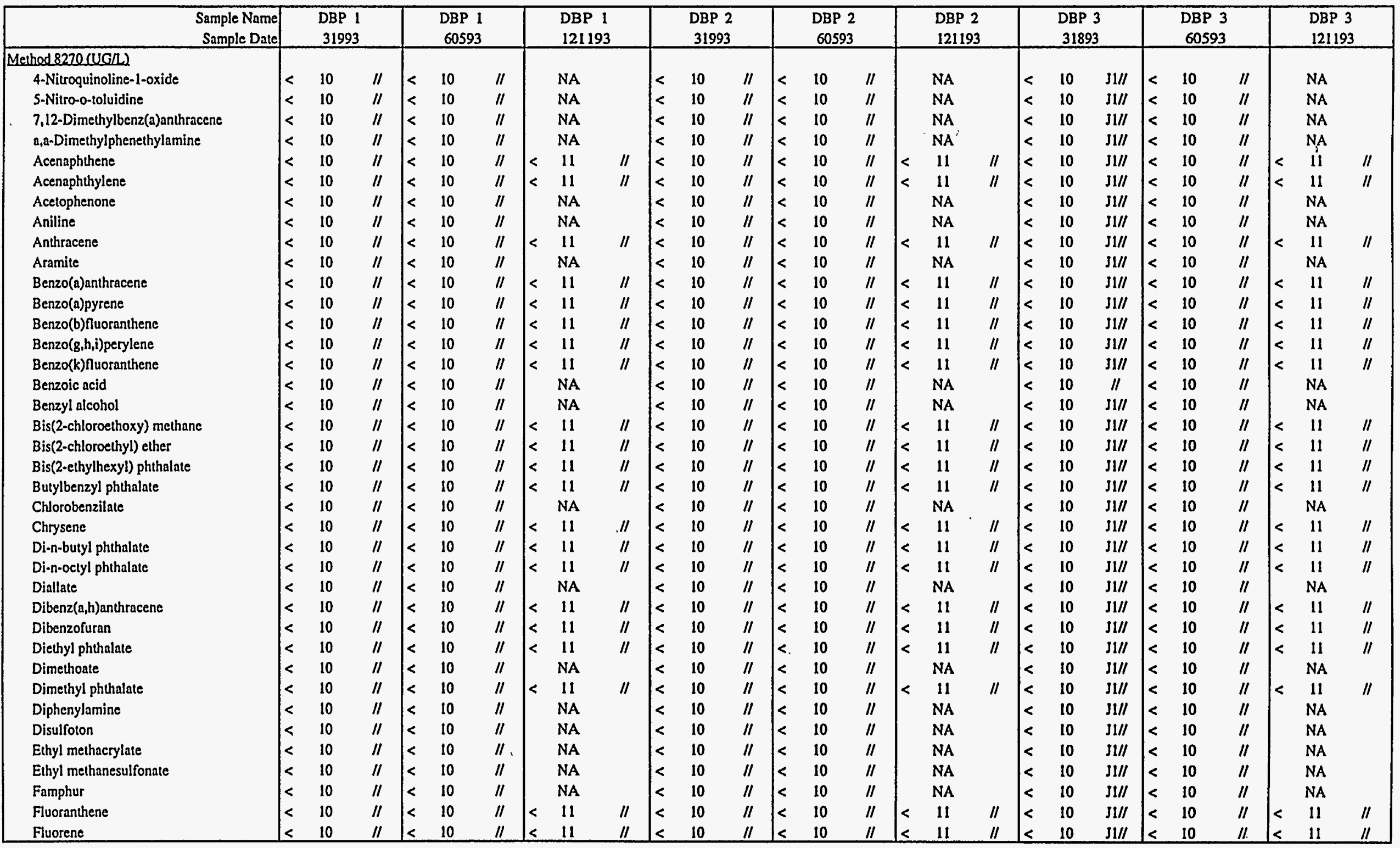


TABLE 6.10

SEMI-VOLATILE ORGANIC COMPOUNDS IN GROUNDWATER

D-AREA BURNING/RUBBLE PITS SAVANNAH RIVER SITE

\begin{tabular}{|c|c|c|c|c|c|c|c|c|c|c|c|c|c|c|c|c|c|c|c|c|c|c|c|c|c|c|c|c|}
\hline \multirow{2}{*}{\multicolumn{2}{|c|}{\begin{tabular}{r|} 
Sample Name \\
Sample Date
\end{tabular}}} & \multicolumn{3}{|c|}{$\begin{array}{r}\text { DBP 1 } \\
31993 \\
\end{array}$} & \multicolumn{3}{|c|}{$\begin{array}{c}\text { DBP 1 } \\
60593\end{array}$} & \multicolumn{3}{|c|}{$\begin{array}{l}\text { DBP 1 } \\
121193 \\
\end{array}$} & \multicolumn{3}{|c|}{$\begin{array}{c}\text { DBP } 2 \\
31993\end{array}$} & \multicolumn{3}{|c|}{$\begin{array}{c}\text { DBP } 2 \\
60593\end{array}$} & \multicolumn{3}{|c|}{$\begin{array}{l}\text { DBP } 2 \\
121193 \\
\end{array}$} & \multicolumn{3}{|c|}{$\begin{array}{r}\text { DBP } 3 \\
31893\end{array}$} & \multicolumn{3}{|c|}{$\begin{array}{c}\text { DBP } 3 \\
60593\end{array}$} & \multicolumn{3}{|c|}{$\begin{array}{l}\text { DBP } 3 \\
121193\end{array}$} \\
\hline & & & & & & & & & & & & & & & & & & & & & & & & & & & & \\
\hline Hexachlorobenzene & & $<$ & 10 & $/ 1$ & $<$ & 10 & $\|$ & $<$ & 11 & $\|$ & $<$ & 10 & $\|$ & $<$ & 10 & $\|$ & $<$ & 11 & "l & $<$ & 10 & $\mathrm{JI} / /$ & $<$ & 10 & $\|$ & $<$ & 11 & $\|$ \\
\hline Hexachlorobutadiene & & $<$ & 10 & 11 & $<$ & 10 & II & $<$ & 11 & /I & $<$ & 10 & 11 & $<$ & 10 & $\|$ & $<$ & 11 & II & $<$ & 10 & $\mathrm{~s} 1 / /$ & $<$ & 10 & $\|$ & $<$ & 11 & /1 \\
\hline Hexachlorocyclopentadiene & & $<$ & 10 & /l & $<$ & 10 & $\|$ & $<$ & 11 & $\|$ & $<$ & 10 & $/ 1$ & $<$ & 10 & 11 & $<$ & 11 & $\|$ & $<$ & 10 & $31 / 1$ & $<$ & 10 & $\|$ & $<$ & 11 & /l \\
\hline Hexachloroethane & & $<<$ & 10 & $/ 1$ & $<$ & 10 & /I & $<$ & 11 & $\|$ & $<$ & 10 & /I & $<$ & 10 & $\|$ & $<$ & 11 & /I & $<$ & 10 & $\mathrm{~J} 1 / /$ & $<$ & 10 & $\|$ & $<$ & 11 & /I \\
\hline Hexachlorophene & & $<$ & 10 & $\|$ & $<$ & 10 & $\|$ & & NA & & $<$ & 10 & $\|$ & $<$ & 10 & $\|$ & & NA & & $<$ & 10 & $\|$ & $<$ & 10 & II & & NA & \\
\hline Hexachloropropene & & $<$ & 10 & $/ 1$ & $<$ & 10 & $\|$ & & $\mathrm{NA}$ & & $<$ & 10 & $\|$ & $<$ & 10 & $\|$ & & NA & & $<$ & 10 & $\mathrm{sill}$ & $<$ & 10 & $\|$ & & NA & \\
\hline Indeno( $(1,2,3-c, d)$ pyrene & & $<$ & 10 & $/ l$ & $<$ & 10 & "I & $<$ & 11 & $\|$ & $<$ & 10 & 11 & $<$ & 10 & $\|$ & $<$ & 11 & 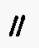 & $<$ & 10 & $\mathrm{~J} 1 / /$ & $<$ & 10 & $\|$ & $<$ & 11 & $\|$ \\
\hline Isodrin & & $<$ & 10 & $/ 1$ & $<$ & 10 & $\|$ & & NA & & $<$ & 10 & $\|$ & $<$ & 10 & $/ /$ & & NA & & $<$ & 10 & $\mathrm{~J} 1 / /$ & $<$ & 10 & $\|$ & & NA & \\
\hline Isophorone & & $<$ & 10 & /I & $<$ & 10 & /I & $<$ & 11 & $/ /$ & $<$ & 10 & /I & $<$ & 10 & /I & $<$ & 11 & "I & $<$ & 10 & $\mathrm{~s} 1 / /$ & $<$ & 10 & $\|$ & $<$ & 11 & $\|$ \\
\hline Isosafrole & & $<$ & 10 & $\|$ & $<$ & 10 & $\|$ & & $\mathrm{NA}$ & & $<$ & .10 & $\|$ & $<$ & 10 & $\|$ & & NA & & $<$ & 10 & $\mathrm{sl} / /$ & $<$ & 10 & $\|$ & & NA & \\
\hline Kepone & & $<$ & 10 & $\|$ & $<$ & 10 & $\|$ & & NA & & $<$ & 10 & $/ 1$ & $<$ & 10 & $\|$ & & NA & & $<$ & 10 & $\mathrm{~J} 1 / /$ & $<$ & 10 & $\|$ & & NA & \\
\hline m-Cresol (3-Methylphenol) & & $<$ & 10 & $/ 1$ & $<$ & 10 & $\|$ & & NA & & $<$ & 10 & $\|$ & $<$ & 10 & /I & & NA & & $<$ & 10 & $\|$ & $<$ & 10 & $\|$ & & NA & \\
\hline m-Nitroaniline & & $<$ & 10 & $\|$ & $<$ & 10 & $\|$ & & NA & & $<$ & 10 & $\|$ & $<$ & 10 & $\|$ & & NA & & $<$ & 10 & $\mathrm{~J} 1 / /$ & $<$ & 10 & $\|$ & & NA & \\
\hline Methapyrilene & & $<$ & 10 & $\|$ & $<$ & 10 & $\|$ & & NA & & $<$ & 10 & $\|$ & $<$ & 10 & /I & & NA & & $<$ & 10 & $\mathrm{JI} / 1$ & $<$ & 10 & $\|$ & & $\mathrm{NA}$ & \\
\hline Methyl methacrylate & & $<$ & 10 & $\|$ & $<$ & 10 & 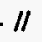 & & NA & & $<$ & 10 & $/ 1$ & $<$ & 10 & $/ /$ & & NA & & $<$ & 10 & $\mathrm{~J} 1 / /$ & $<$ & 10 & $\|$ & & NA & \\
\hline Methyl methanesulfonate & & $<$ & 10 & $/ l$ & $<$ & 10 & $/ /$ & & NA & & $<$ & 10 & $/ /$ & $<$ & 10 & $/ /$ & & NA & & $<$ & 10 & $\mathrm{~s} 1 / /$ & $<$ & 10 & $\|$ & & NA & \\
\hline N-Nitrosodiethylamine & & $<$ & 10 & $\|$ & $<$ & 10 & $\|$ & & NA & & $<$ & 10 & $\|$ & $<$ & 10 & $\|$ & & $\mathrm{NA}$ & & $<$ & 10 & $\mathrm{JI} / 1$ & $<$ & 10 & $\|$ & & NA & \\
\hline N-Nitrosodimethylamine & & $<$ & 10 & $/ I$ & $<$ & 10 & $\|$ & $<$ & 11 & $\|$ & $<$ & 10 & $\|$ & $<$ & 10 & $\|$ & $<$ & 11 & $\|$ & $<$ & 10 & $\mathrm{~J} 1 / 1$ & $<$ & 10 & $\|$ & $<$ & 11 & $\|$ \\
\hline N-Nitrosodiphenylamine & & $<$ & 10 & $\|$ & $<$ & 10 & $\|$ & $<$ & 11 & $\|$ & $<$ & 10 & $\|$ & $<$ & 10 & "I & $<$ & 11 & $\|$ & $<$ & 10 & $31 / 1$ & $<$ & 10 & $\|$ & $<$ & 11 & /I \\
\hline N-Nitrosodipropylamine & . & $<$ & 10 & $\|$ & $<$ & 10 & $\|$ & $<$ & 11 & $\|$ & $<$ & 10 & $/ 1$ & $<$ & 10 & $\|$ & $<$ & 11 & $\|$ & $<$ & 10 & $\mathbf{J} 1 / /$ & $<$ & 10 & $\|$ & $<$ & 11 & $\|$ \\
\hline N-Nitrosomethylethylamine & & $<$ & 10 & $\|$ & $<$ & 10 & $\|$ & & NA & & $<$ & 10 & $\|$ & $<$ & 10 & $/ /$ & & NA & & $<$ & 10 & $\mathrm{~J} 1 / 1$ & $<$ & 10 & $\|$ & & NA & \\
\hline N-Nitrosomorpholine & & $<$ & 10 & $\|$ & $<$ & 10 & $\|$ & & NA & & $<$ & 10 & $\|$ & $<$ & 10 & $\|$ & & $\mathrm{NA}^{\circ}$ & & $<$ & 10 & $\mathrm{~J} \mathbf{1} / /$ & $<$ & 10 & II & & NA & \\
\hline N-Nitrosopiperidine & & $<$ & 10 & $/ /$ & $<$ & 10 & $\|$ & & NA & & $<$ & 10 & 11 & $<$ & 10 & $/ /$ & & NA & & $<$ & 10 & $\mathrm{~J} 1 / /$ & $<$ & 10 & $\|$ & & NA & \\
\hline N-Nitrosopyrrolidine & - & $<$ & 10 & $\|$ & $<$ & 10 & 11 & & $\mathrm{NA}$ & & $<$ & 10 & $\|$ & $<$ & 10 & "I & & $\mathrm{NA}$ & & $<$ & 10 & $\mathrm{~J} 1 / /$ & $<$ & 10 & $\|$ & & NA & \\
\hline Naphthalene & & $<$ & 10 & $\|$ & $<$ & 10 & $\|$ & $<$ & 11 & $\|$ & $<$ & 10 & $\|$ & $<$ & 10 & $/ /$ & $<$ & 11 & II & $<$ & 10 & $\mathrm{J1} / 1$ & $<$ & 10 & $\|$ & $<$ & 11 & $\prime \prime$ \\
\hline Nitrobenzene & & $<$ & 10 & $\|$ & $<$ & 10 & $\|$ & $<$ & 11 & $\|$ & $<$ & 10 & $\|$ & $<$ & 10 & $\|$ & $<$ & 11 & $\|$ & $<$ & 10 & $31 / 1$ & $<$ & 10 & $\|$ & $<$ & 11 & $\|$ \\
\hline $0,0,0$-Triethyl phosphorothioate & & $<$ & 10 & $/ 1$ & $<$ & 10 & $\|$ & & NA & & $<$ & 10 & 11 & $<$ & 10 & $\|$ & & NA & & $<$ & 10 & $\mathrm{~J} 1 / 1$ & $<$ & 10 & $\|$ & & NA & \\
\hline o-Cresol (2-Methylphenol) & & $<$ & 10 & /I & $<$ & 10 & $\|$ & & NA & & $<$ & 10 & $\|$ & $<$ & 10 & $/ /$ & & NA & & $<$ & 10 & $\|$ & $<$ & 10 & $\|$ & & NA & \\
\hline o-Nitroaniline & & $<$ & 10 & $\|$ & $<$ & 10 & $\|$ & & NA & & $<$ & 10 & $\|$ & $<$ & 10 & $/ /$ & & NA & & $<$ & 10 & $\mathrm{~J} \mathbf{l} / /$ & $<$ & 10 & $\|$ & & NA & \\
\hline o-Toluidine & & $<$ & 10 & /I & $<$ & 10 & $\|$ & & NA & & $<$ & 10 & $\|$ & $<$ & 10 & $/ 1$ & & NA & & $<$ & 10 & $\mathrm{~J} \mathbf{1} / \mathrm{l}$ & $<$ & 10 & $\|$ & & NA & \\
\hline p-Cresol (4-Methylphenol) & & $<$ & 10 & $\|$ & $<$ & 10 & $\|$ & & NA & & $<$ & 10 & $\|$ & $<$ & 10 & $\|$ & & NA & & $<$ & 10 & $/ 1$ & $<$ & 10 & $\|$ & & $\mathrm{NA}$ & \\
\hline p-Dimethylaminoazobenzene & & $<$ & 10 & $\|$ & $<$ & 10 & $\|$ & & NA & & $<$ & 10 & $\|$ & $<$ & 10 & $\|$ & & NA & & $<$ & 10 & $\mathrm{~J} 1 / 1$ & $<$ & 10 & $\|$ & & NA & \\
\hline p-Nitroaniline & & $<$ & 10 & $\|$ & $<$ & 10 & $\|$ & & $\mathrm{NA}$ & & $<$ & 10 & $\|$ & $<$ & 10 & II & & NA & & $<$ & 10 & $\mathrm{~J} 1 / l$ & $<$ & 10 & 11 & & NA & \\
\hline p-Phenylenediamine & & $<$ & 10 & $\|$ & $<$ & 10 & $\|$ & & NA & & $<$ & 10 & $\|$ & $<$ & 10 & $\|$ & & NA & & $<$ & 10 & $\mathrm{~J} 1 / 1$ & $<$ & 10 & $\|$ & & NA & \\
\hline
\end{tabular}


TABLE 6.10

SEMI-VOLATILE ORGANIC COMPOUNDS

IN GROUNDWATER

D-AREA BURNING/RUBBLE PIT

SAVANNAH RIVER SITE

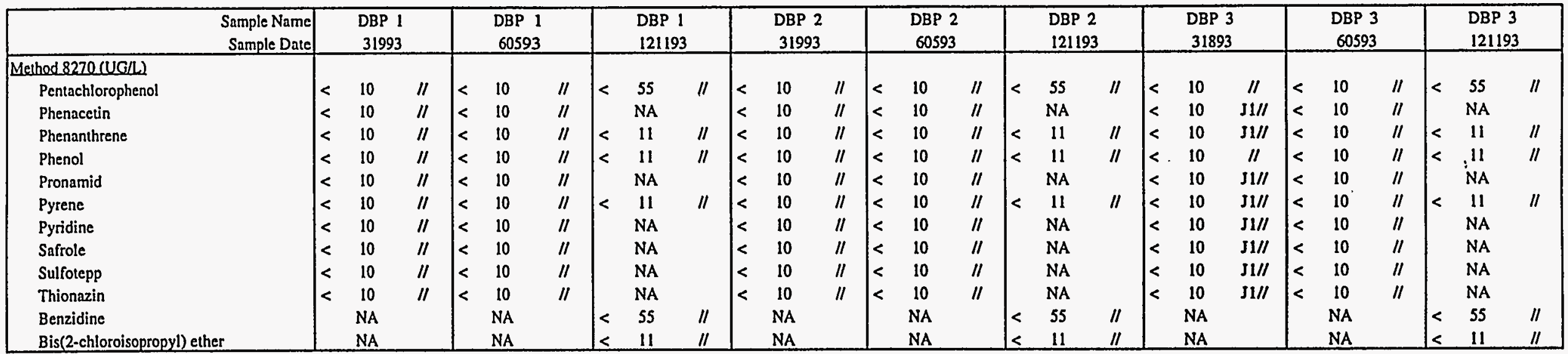


TABLE 6.10

SEMI-VOLATILE ORGANIC COMPOUNDS

IIN GROUNDWATER

D-AREA BURNING/RUBBLE PITS

SAVANNAH RIVER SITE

\begin{tabular}{|c|c|c|c|c|c|c|c|c|c|c|c|}
\hline $\begin{array}{l}\text { Sample Name } \\
\text { Sample Date }\end{array}$ & & & & $\begin{array}{r}\mathrm{DB} \\
60\end{array}$ & & & $\begin{array}{l}\mathrm{DB} \\
12\end{array}$ & & & $\begin{array}{l}\text { DBP 5 } \\
12269\end{array}$ & \\
\hline Method 8270 (UG/L) & & & & & & & & & & & \\
\hline 1,2,4,5-Tetrachlorobenzene & 10 & $/ 1$ & $<$ & 10 & $\mathrm{~J} 1 / /$ & & NA & & & NA & \\
\hline 1,2,4-Trichlorobenzene & 10 & $/ /$ & $<$ & 10 & $\mathrm{~J} 1 / /$ & $<$ & 11 & $\|$ & & 5.5 & II \\
\hline 1,2-Dichlorobenzene & 10 & $\|$ & $<$ & 10 & $J 1 / /$ & $<$ & 11 & $\|$ & $<$ & 5.6 & $\|$ \\
\hline 1,3,5-Trinitrobenzene & 10 & $\|$ & $<$ & 10 & $\mathrm{J1} / /$ & & NA & & & NA & \\
\hline 1,3-Dichlorobenzene & 10 & $\|$ & $<$ & 10 & $\mathrm{~s} 1 / /$ & $<$ & 11 & /I & $<$ & 5.6 & II \\
\hline 1,3-Dinitrobenzene & 10 & $\|$ & $<$ & 10 & $\mathrm{~J} ! / /$ & & NA & & & NA & \\
\hline 1,4-Dichlorobenzene & 10 & $\|$ & $<$ & 10 & $\mathrm{~J} 1 / /$ & $<$ & 11 & $\|$ & & 5.5 & /I \\
\hline 1,4-Dioxane & 10 & $\|$ & $<$ & 10 & $s 1 / /$ & & NA & & & NA & \\
\hline 1,4-Naphthoquinone & 10 & $\|$ & $<$ & 10 & $\mathrm{Jl} / /$. & & NA & & & NA & \\
\hline 1-Naphthylamine & 10 & $\|$ & $<$ & 10 & $\mathrm{~J} 1 / /$ & & NA & & & NA & \\
\hline 2,3,4,6-Tetrachlorophenol & 10 & $\|$ & $\mid<$ & 10 & $\|$ & & NA & & ' & NA & \\
\hline 2,4,5-Trichlorophenol & 10 & $\|$ & $<$ & 10 & // & & NA & & $<$ & 28 & /I \\
\hline 2,4,6-Trichlorophenol & 10 & $\|$ & $<$ & 10 & /I & $<$ & 11 & /I & $<$ & 5.6 & "I \\
\hline 2,4-Dichlorophenol & 10 & $\|$ & $<$ & 10 & $\|$ & $<$ & 11 & $\|$ & $<$ & 5.6 & /I \\
\hline 2,4-Dimethyl phenol & 10 & /I & $<$ & 10 & II & $<$ & 11 & /l & $<$ & 5.6 & "I \\
\hline 2,4-Dinitrophenol & 45 & II & $<$ & 45 & $\|$ & $<$ & 55 & /l & $<$ & 28 & $\|$ \\
\hline 2,4-Dinitrotoluene & 10 & $\|$ & $<$ & 10 & $\mathrm{~J} ! / /$ & $<$ & 11 & /I & & 5.5 & /I \\
\hline 2,6-Dichlorophenol & 10 & $\|$ & $<$ & 10 & /1 & & NA & & & $\mathrm{NA}$ & \\
\hline 2,6-Dinitrotoluene & 10 & $\|$ & $<$ & 10 & $\mathrm{~J} 1 / /$ & $<$ & 11 & $\|$ & $<$ & 5.6 & /l \\
\hline 2-Acelylaminofluorene & 10 & $\|$ & $<$ & 10 & $\mathrm{~s} 1 / /$ & & NA & & & NA & \\
\hline 2-Chloroethyl vinyl ether & 10 & $\|$ & $<$ & 10 & $\mathrm{~J} 1 / /$ & & NA & & & NA & \\
\hline 2-Chloronaphthalene & 10 & $\|$ & $<$ & 10 & $31 / /$ & $<$ & 11 & /I & $<$ & 5.6 & $\|$ \\
\hline 2-Chlorophenol & 10 & $\|$ & $<$ & 10 & $\|$ & $<$ & 11 & /l & & 5.5 & $\|$ \\
\hline 2-Methyl-4,6-dinitrophenol & 10 & $\|$ & $<$ & 10 & /I & $<$ & 55 & /l & $<$ & 28 & /I \\
\hline 2-Methylnaphthalene & 10 & $\|$ & $<$ & 10 & $\mathrm{~s} 1 / /$ & & NA & & $<$ & 5.6 & /l \\
\hline 2-Naphthylamine & 10 & $\|$ & $<$ & 10 & $\mathrm{~J} 1 / /$ & & NA & & & NA & \\
\hline 2-Nitrophenol & 10 & $\|$ & $<$ & 10 & $\|$ & $<$ & 11 & /I & $<$ & 5.6 & /l \\
\hline 2-Picoline & 10 & $\|$ & $<$ & 10 & $\mathrm{~J} 1 / /$ & & NA & & & NA & \\
\hline 2-sec-Butyl-4,6-dinitrophenol & 10 & $\|$ & $<$ & 10 & $\|$ & & NA & & & NA & \\
\hline 3,3'-Dichlorobenzizidine & 10 & $\|$ & $<$ & 10 & $\mathrm{~J} 1 / /$ & $<$ & 22 & /I & $<$ & 11.2 & $\|$ \\
\hline 3,3'-Dimethylbenzidine & 10 & $\|$ & $<$ & 10 & $\mathrm{Jl} / /$ & & NA & & & NA & \\
\hline 3-Methylcholanthrene & 10 & $\|$ & $<$ & 10 & $\mathrm{~J} 1 / /$ & & NA & & & $\mathrm{NA}$ & \\
\hline 4-Aminobiphenyl & 10 & $\|$ & $<$ & 10 & $\mathrm{~J} 1 / /$ & & NA & & & NA & \\
\hline 4-Bromophenyl phenyl ether & 10 & $\|$ & $<$ & 10 & $\mathrm{~J} 1 / /$ & $<$ & 11 & "I & $<$ & 5.6 & "I \\
\hline 4-Chloro-m-cresol & 10 & $\|$ & $<$ & 10 & $\|$ & $<$ & 11 & /I & & 5.5 & /I \\
\hline 4-Chloroaniline & 10 & $\|$ & $<$ & 10 & $\mathrm{~s} 1 / /$ & & NA & & $<$ & 5.6 & /I \\
\hline 4-Chlorophenyl phenyl ether & 10 & $\|$ & $<$ & 10 & $\mathrm{~s} \mathrm{l} / \mathrm{l}$ & $<$ & 11 & II & $<$ & 5.6 & $\|$ \\
\hline 4-Nitrophenol & 10 & $\|$ & $<$ & 10 & 11 & $<$ & 55 & II & & 27.5 & "I \\
\hline
\end{tabular}


TABLE 6.10

SEMI-VOLATILE ORGANIC COMPOUNDS

IN GROUNDWATER

D-AREA BURNING/RUBBLE PITS

SAVANNAH RIVER SITE

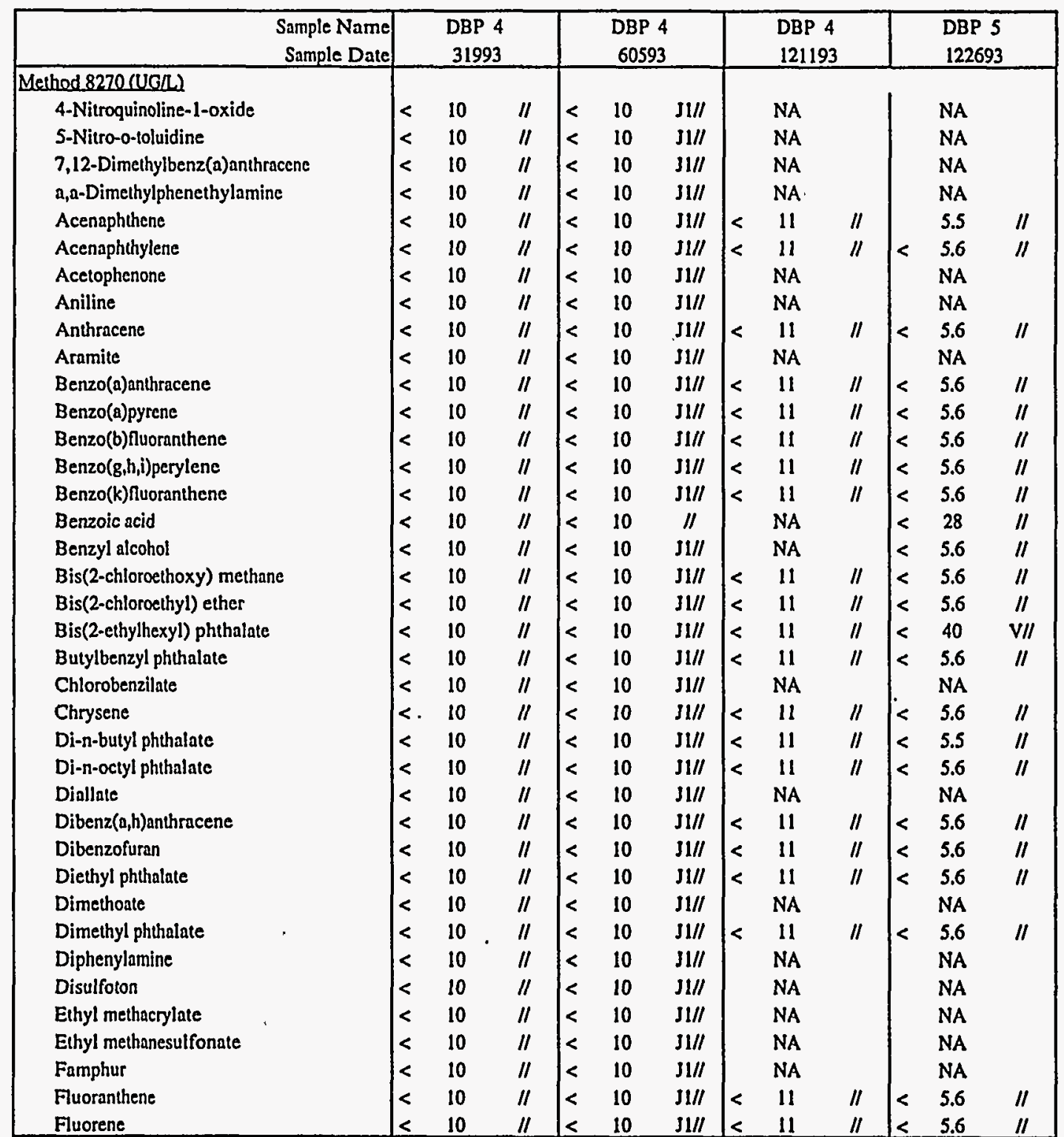




\begin{tabular}{|c|c|c|}
\hline \multirow{5}{*}{ 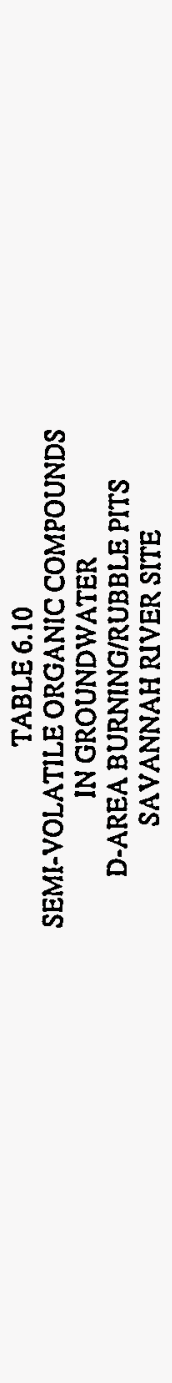 } & & 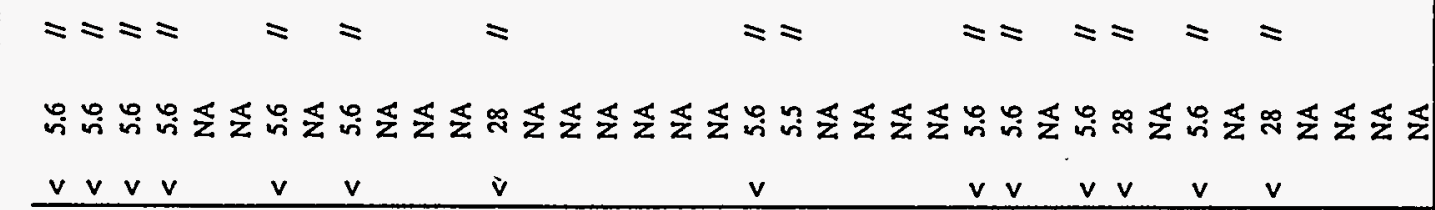 \\
\hline & 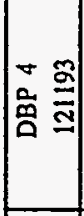 & 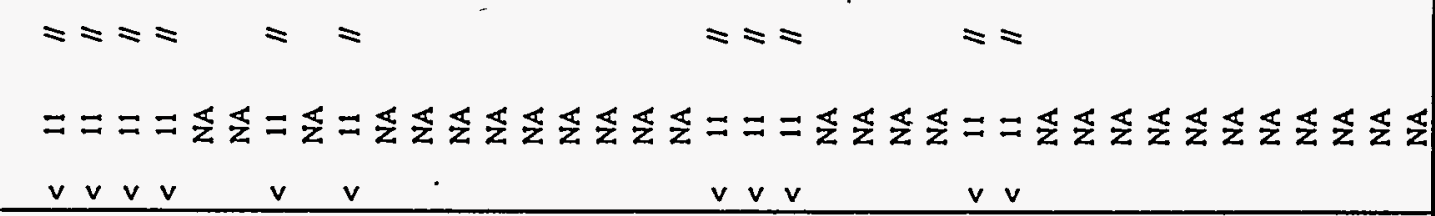 \\
\hline & $\mid \begin{array}{ll}0 & 0 \\
0.0 \\
0 \\
0\end{array}$ & 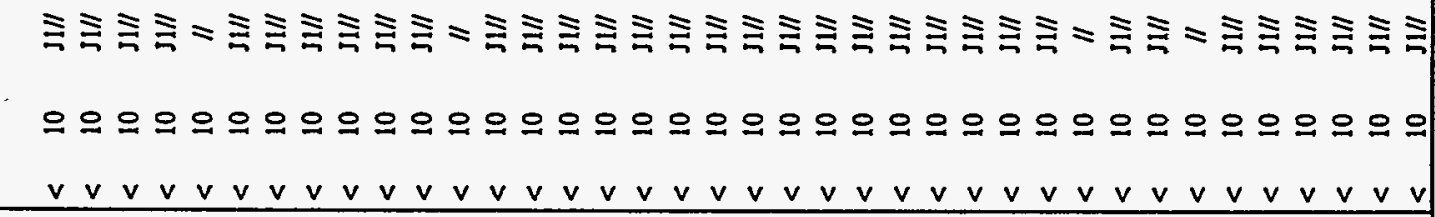 \\
\hline & 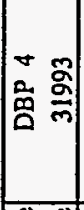 & 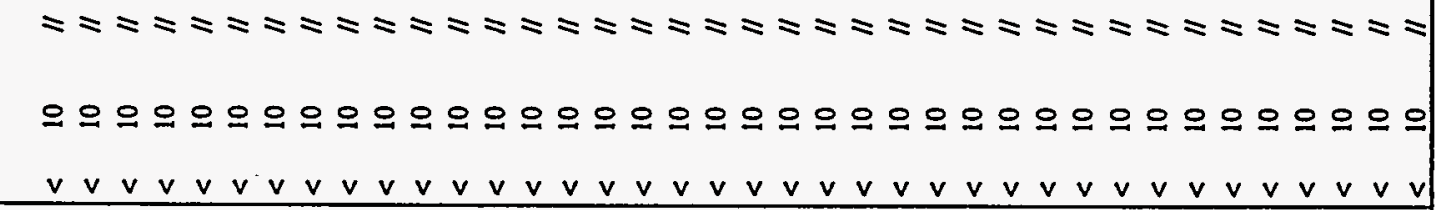 \\
\hline & 吾 & 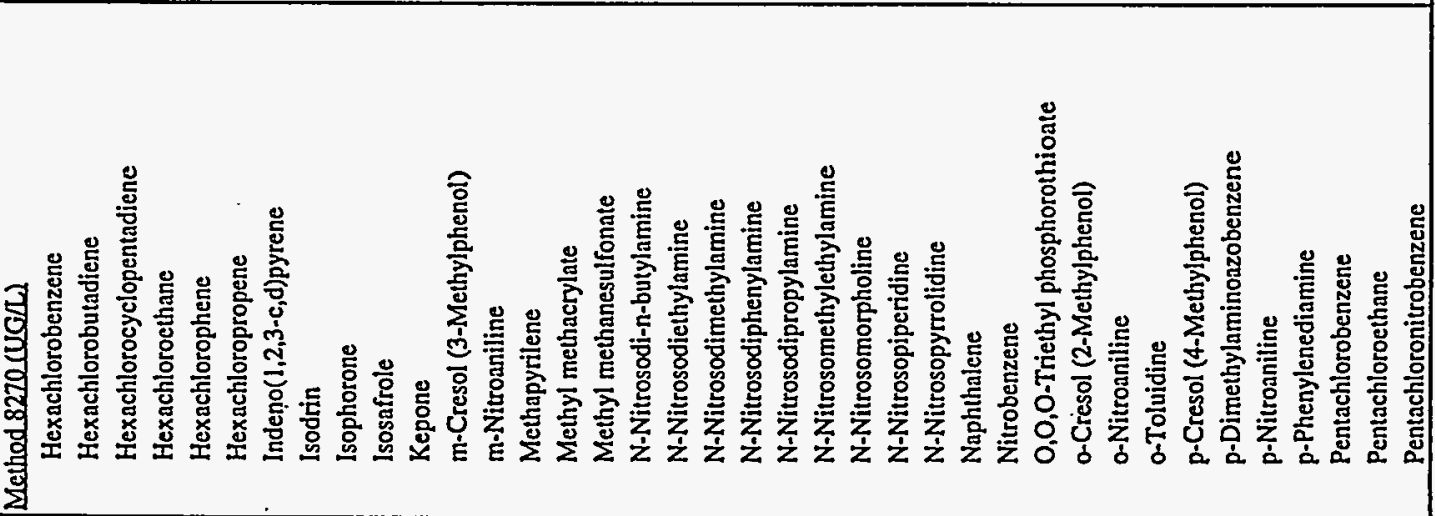 \\
\hline
\end{tabular}


TABLE 6.10

SEMI-VOLATILE ORGANIC COMPOUNDS

IN GROUNDWATER

D-AREA BURNING/RUBBLE PITS

SAVANNAH RIVER SITE

\begin{tabular}{|c|c|c|c|c|c|c|c|c|c|c|c|}
\hline \multirow{2}{*}{$\begin{array}{|lr|} & \begin{array}{r}\text { Sample Name } \\
\text { Sample Date }\end{array} \\
\text { Method 8270(UG(L) } & \\
\end{array}$} & \multicolumn{2}{|c|}{$\begin{array}{r}\text { DBP } 4 \\
31993 \\
\end{array}$} & \multicolumn{3}{|c|}{$\begin{array}{l}\text { DBP } 4 \\
60593 \\
\end{array}$} & \multicolumn{3}{|c|}{$\begin{array}{l}\text { DBP } 4 \\
121193\end{array}$} & \multicolumn{3}{|c|}{$\begin{array}{l}\text { DBP 5 } \\
122693 \\
\end{array}$} \\
\hline & & & & & & & & & & & \\
\hline Pentachlorophenol & 10 & $\|$ & $<$ & 10 & /I & $<$ & 55 & "I & & 27.5 & 11 \\
\hline Phenacetin & 10 & $\|$ & $<$ & 10 & $\mathrm{J1} / /$ & & NA & & & NA & \\
\hline Phenanthrene & 10 & /I & $<$ & 10 & $\mathrm{J1} / \mathrm{I}$ & $<$ & 11 & $\|$ & $<$ & 5.6 & 11 \\
\hline Phenol & 10 & II & $<$ & 10 & $/ 1$ & $<$ & 11 & 11 & & 5.5 & $/ 1$ \\
\hline Pronamid & 10 & "I & $<$ & 10 & $\mathrm{~J} 1 / /$ & & NA & & & NA & \\
\hline Pyrene & 10 & /I & $<$ & 10 & $\mathrm{~J} 1 / /$ & |< & 11 & II & & 5.5 & /I \\
\hline Pyridine & 10 & "I & $<$ & 10 & $\mathrm{~J} 1 / /$ & & NA & & & NA & \\
\hline Safrole & 10 & 11 & $<$ & 10 & $\mathrm{~J} 1 / /$ & & NA & & & NA & \\
\hline Sulfotepp & 10 & $\|$ & $<$ & 10 & $\mathrm{J1} / \mathrm{I}$ & & NA & & & NA & \\
\hline Thionazin & 10 & $\|$ & $<$ & 10 & $\mathrm{~J} 1 / /$ & & NA & & & NA & \\
\hline Benzidine & NA & & & NA & & $<$ & 55 & II & & NA & \\
\hline Bis (2-chloroisopropyl) ether & NA & & & NA & & L $<$ & 11 & II & $<$ & 5.6 & 11 \\
\hline
\end{tabular}


TABLE 6.11

PESTTCIDES AND PCBS

IN GROUNDWATER

F-AREA BURNING/RUBBLE PITS

SAVANNAH RIVER SITE

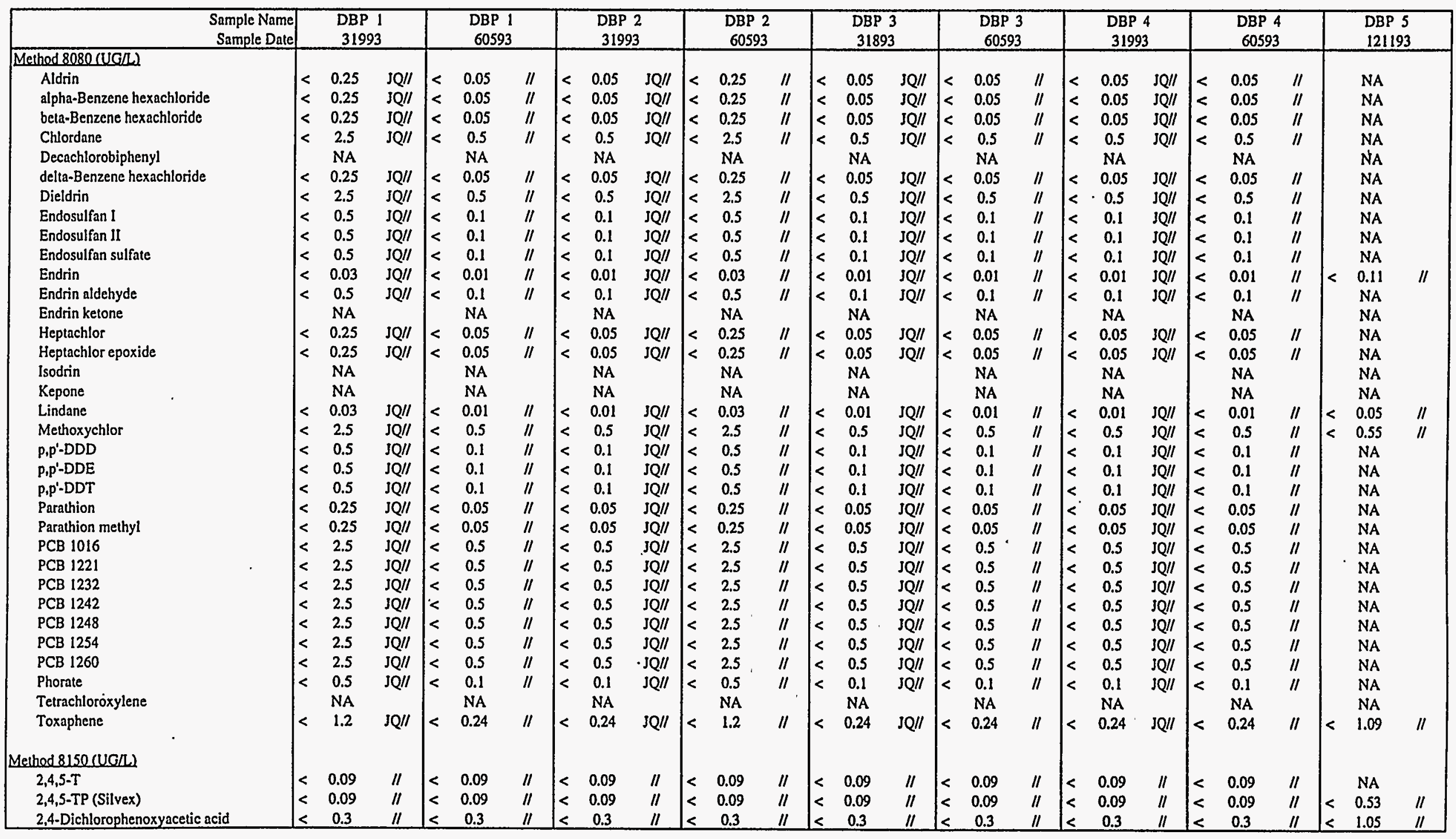


TABLE 6.11

ESTICIDES AND PCBS

IN GROUNDWATER

F-AREA BURNING/RUBBLE PITS

SAVANNAH RIVER SITE

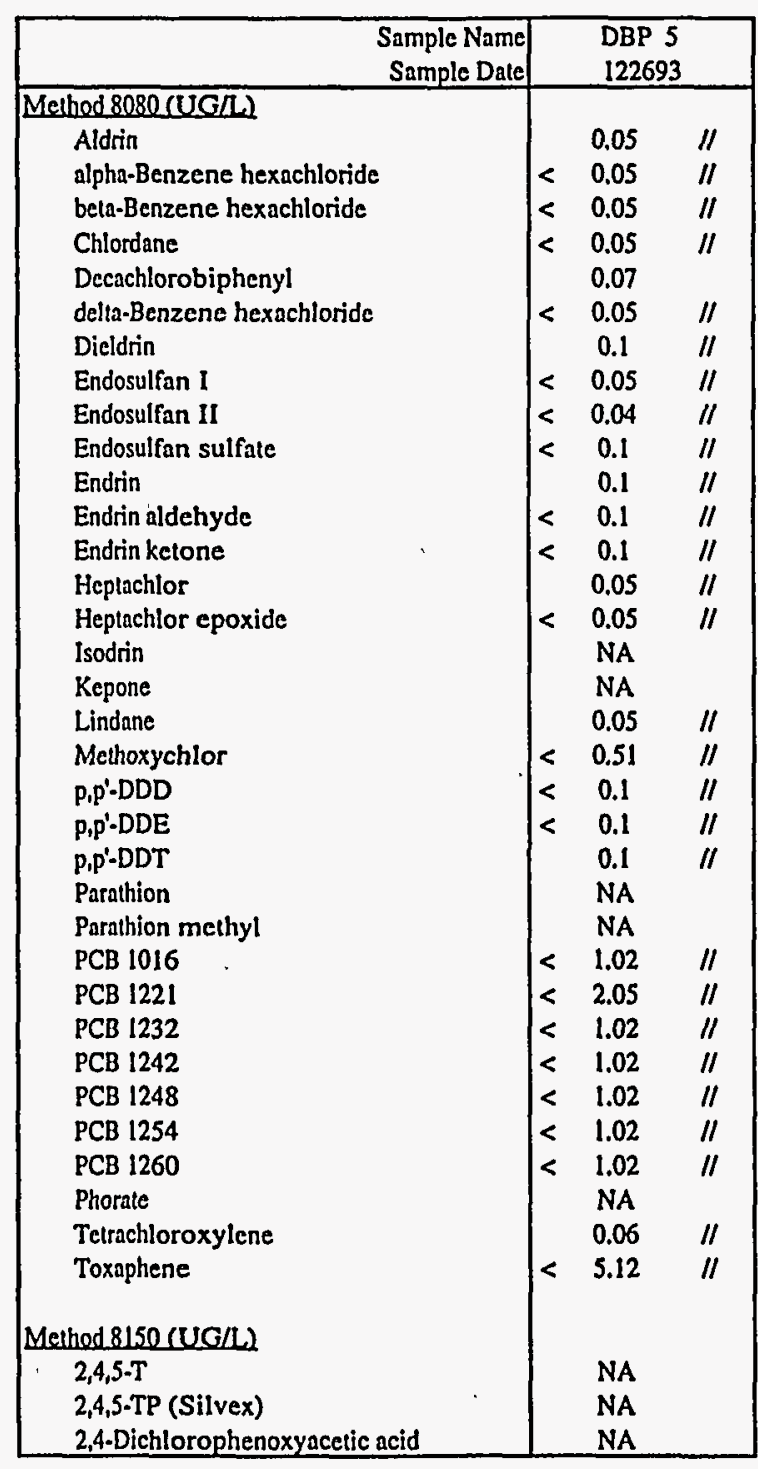

Page 2 of 2 
TABLE 6.12

PCDD AND PCDF COMPOUNDS

IN GROUNDWATER

D.AREA BURNING/RUBBLE PITS

SAVANNAH RIVER SITE

\begin{tabular}{|c|c|c|c|c|c|c|c|c|c|c|c|c|c|c|c|c|c|c|c|c|c|c|c|}
\hline \multirow[b]{2}{*}{ Method 8280 (NG/L) } & \multicolumn{2}{|c|}{$\begin{array}{c}\text { DBP I } \\
31993 \\
\end{array}$} & \multicolumn{3}{|c|}{$\begin{array}{l}\text { DBP I } \\
60593 \\
\end{array}$} & \multicolumn{3}{|c|}{$\begin{array}{l}\text { DBP 2 } \\
31993 \\
\end{array}$} & \multicolumn{3}{|c|}{$\begin{array}{r}\text { DBP } 2 \\
60593\end{array}$} & \multicolumn{3}{|c|}{$\begin{array}{c}\text { DBP } 3 \\
31893\end{array}$} & \multicolumn{3}{|c|}{$\begin{array}{c}\text { DBP } 3 \\
60593\end{array}$} & \multicolumn{3}{|c|}{$\begin{array}{c}\text { DBP } 4 \\
31993\end{array}$} & \multicolumn{3}{|c|}{$\begin{array}{c}\text { DBP 4 } \\
60593\end{array}$} \\
\hline & & & & & & & & & & & & & & & & & & & & & & & \\
\hline $1,2,3,4,6,7,8-\mathrm{HPCDD}$ & 0.65 & $/ /$ & $<$ & 0.65 & $/ /$ & $<$ & 0.65 & /I & $<$ & 0.65 & $\|$ & $<$ & 0.65 & /I & $<$ & 0.65 & $\|$ & $<$ & 0.65 & $/ 1$ & $<$ & 0.65 & /I \\
\hline $1,2,3,4,6,7,8-\mathrm{HPCDF}$ & 0.45 & $\|$ & $<$ & 0.45 & $\|$ & $<$ & 0.45 & /I & $<$ & 0.45 & $\|$ & $<$ & 0.45 & $/ 1$ & $<$ & 0.45 & $\|$ & $<$ & 0.45 & $/ l$ & $<$ & 0.45 & /I \\
\hline $1,2,3,4,7,8 \cdot \mathrm{HXCDD}$ & 0.45 & $\|$ & $\mid<$ & 0.45 & $/ /$ & $<$ & 0.45 & $\|$ & $<$ & 0.45 & /I & $<$ & 0.45 & $\|$ & $<$ & 0.45 & $\|$ & $<$ & 0.45 & /I & $<$ & 0.45 & II \\
\hline $1,2,3,4,7,8-\mathrm{HXCDF}$ & 0.4 & $\|$ & $\mid<$ & 0.4 & /I & $1<$ & 0.4 & $\|$ & $\mid<$ & 0.4 & $\|$ & $<$ & 0.4 & $\|$ & $<$ & 0.4 & $\|$ & < & 0.4 & $\|$ & $<$ & 0.4 & $\|$ \\
\hline $1,2,3,7,8-\mathrm{PCDD}$ & $<0.55$ & /I & $<$ & 0.55 & $/ 1$ & $<$ & 0.55 & $\|$ & $<$ & 0.55 & $\|$ & $<$ & 0.55 & $\|$ & $<$ & $0.55^{\circ}$ & 11 & $<$ & 0.55 & $\|$ & $<$ & 0.55 & /I \\
\hline $1,2,3,7,8 \cdot \mathrm{PCDF}$ & $<0.55$ & $\|$ & $<$ & 0.55 & /I & $<$ & 0.55 & /I & $<<$ & 0.55 & $\|$ & $<<$ & 0.55 & $/ 1$ & $<$ & 0.55 & $\|$ & $<<$ & 0.55 & "I & $<$ & 0.55 & "I \\
\hline $2,3,7,8-\mathrm{TCDD}$ & $<0.45$ & $\|$ & $<$ & 0.45 & /I & $<$ & 0.45 & $\|$ & $<$ & 0.45 & $\|$ & $<$ & 0.45 & $\|$ & $<$ & 0.45 & $\|$ & k & 0.45 & $\|$ & $<$ & 0.45 & II \\
\hline $2,3,7,8-\mathrm{TCDF}$ & 0.4 & $/ 1$ & $<$ & 0.4 & $/ /$ & $<$ & 0.4 & /I & 1< & 0.4 & $\|$ & $<$ & 0.4 & /I & $<$ & 0.4 & $\|$ & $<$ & 0.4 & $\|$ & $<$ & 0.4 & II \\
\hline Heptachlorodibenzo-p-dioxin isomers & 0.65 & $\|$ & $<$ & 0.65 & $/ /$ & | & 0.65 & $\|$ & $<$ & 0.65 & /I & $<<$ & 0.65 & $\|$ & $<$ & 0.65 & $\|$ & $<$ & 0.65 & II & $<$ & 0.65 & II \\
\hline Heptachlorodibenzo-p-furan isomers & 0.45 & $\|$ & $<$ & 0.45 & /I & $<$ & 0.45 & $\|$ & $<<$ & 0.45 & /I & $<<$ & 0.45 & /I & < & 0.45 & $\|$ & $<$ & 0.45 & $\|$ & $<$ & 0.45 & /I \\
\hline Hexachlorodibenzo-p-dioxin isomers & 0.45 & $\|$ & $<$ & 0.45 & /I & $<$ & 0.45 & /I & $<<$ & 0.45 & /I & $<<$ & 0.45 & /I & $<$ & 0.45 & II & $1<$ & 0.45 & II & $<$ & 0.45 & II \\
\hline Hexachlorodibenzo-p-furan isomers & 0.4 & /I & $<<$ & 0.4 & /I & $<$ & 0.4 & /I & 1< & 0.4 & $\|$ & $<$ & 0.4 & /I & $<$ & 0.4 & $\|$ & 1< & 0.4 & "I & $<$ & 0.4 & II \\
\hline Octachlorodibenzo-p-dioxin isomers & $<$ & $\|$ & $<$ & 1 & /I & $<$ & 1 & $\|$ & $<$ & 1 & /I & $<<$ & 1 & /I & $<$ & 1 & II & $<$ & 1 & /I & $<$ & 1 & II \\
\hline Octachlorodibenzo-p-furan isomers & $<$ & $\|$ & |< & 1 & $\|$ & $<$ & 1 & II & | $<$ & 1 & $\|$ & |< & 1 & II & $<$ & 1 & "I & e & 1 & II & |< & 1 & II \\
\hline Pentachlorodibenzo-p-dioxin isomers & $<0.55$ & $\|$ & $<$ & 0.55 & $\|$ & $<$ & 0.55 & $\|$ & $<<$ & 0.55 & $\|$ & $<$ & 0.55 & /I & $<$ & 0.55 & $\|$ & k & 0.55 & "I & $\mid<$ & 0.55 & /I \\
\hline Pentachlorodibenzo-p-furan isomers & $<0.55$ & $\|$ & $<$ & 0.55 & $/ /$ & $<$ & 0.55 & /I & $<$ & 0.55 & $\|$ & 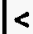 & 0.55 & $\|$ & $<$ & 0.55 & $\|$ & $<$ & 0.55 & /I & $<$ & 0.55 & II \\
\hline Tetrachlorodibenzo-p-dioxin isomers & 0.45 & /I & $<$ & 0.45 & $/ 1$ & $<$ & 0.45 & $\|$ & $<$ & 0.45 & $\|$ & $<$ & 0.45 & /I & $<$ & 0.45 & $\|$ & | & 0.45 & $\|$ & |< & 0.45 & II \\
\hline Tetrachlorodibenzo-p-furan isomers & 0.4 & /I & l< & 0.4 & II & 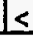 & 0.4 & $\|$ & $\leq$ & 0.4 & 11 & $1<$ & 0.4 & 11 & $1<$ & 0.4 & 11 & $1<$ & 0.4 & II & $1<$ & 0.4 & II \\
\hline
\end{tabular}


TABLE 6.13

INORGANICS AND ORGANIC INDICATORS

IN GROUNDWATER

D-AREA BURNING/RUBBLE PITS

SAVANNAH RIVER SITE

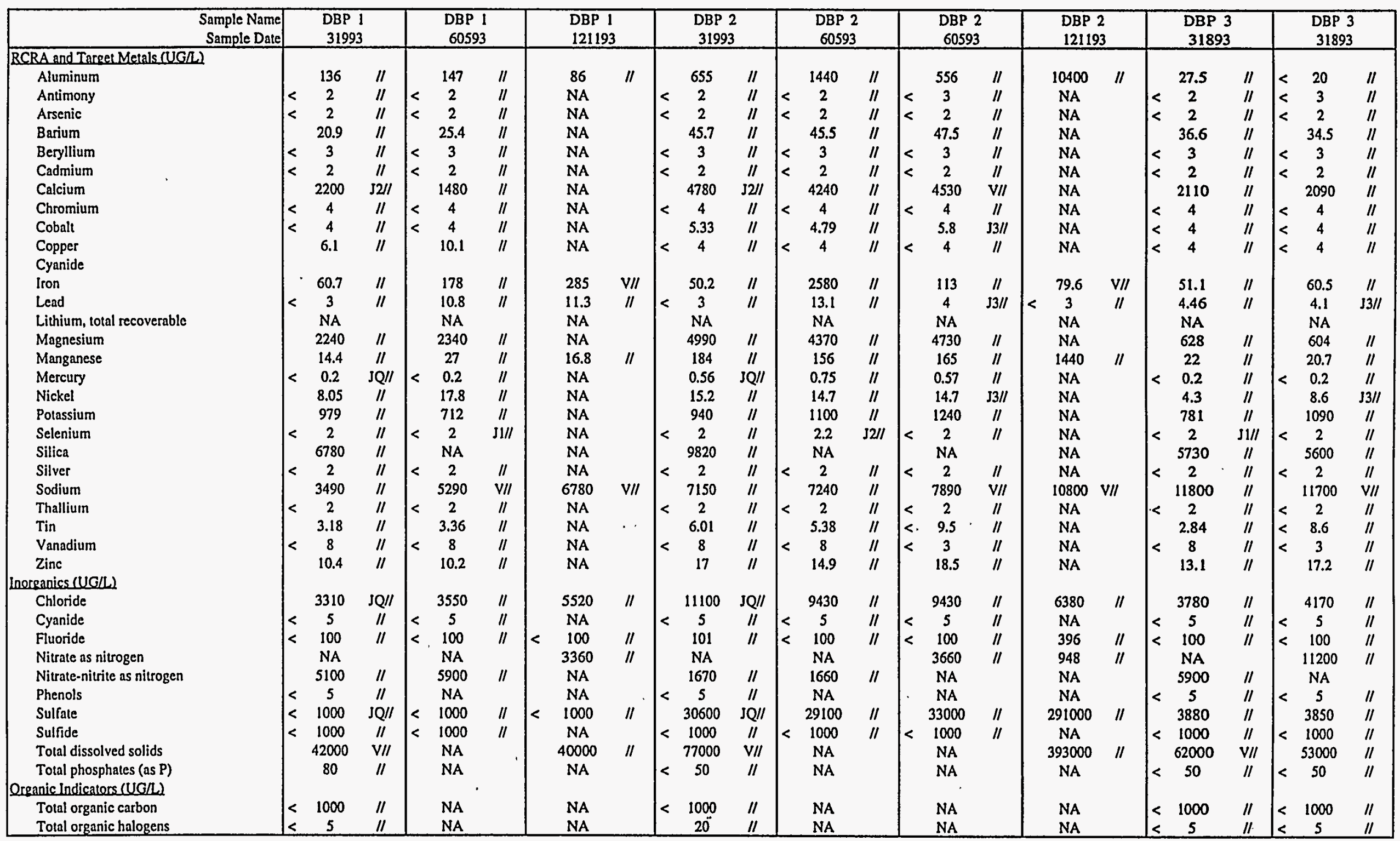


TABLE 6.13

INORGANICS AND ORGANICINDICATORS

IN GROUNDWATER

D-AREA BURNING/RUBBLE PITS

SAVANNAH RIVER SITE

\begin{tabular}{|c|c|c|c|c|c|c|c|c|c|c|c|c|c|c|c|c|c|c|c|c|}
\hline $\begin{array}{|lr|}1 & \begin{array}{r}\text { Sample Name } \\
\text { Sample Date }\end{array} \\
\end{array}$ & \multicolumn{2}{|c|}{$\begin{array}{r}\text { DBP } 3 \\
60593 \\
\end{array}$} & \multicolumn{3}{|c|}{$\begin{array}{l}\text { DBP 3 } \\
12 ! 193\end{array}$} & \multicolumn{3}{|c|}{$\begin{array}{r}\text { DBP } 4 \\
31993\end{array}$} & \multicolumn{3}{|c|}{$\begin{array}{r}\text { DBP } 4 \\
60593\end{array}$} & \multicolumn{3}{|c|}{$\begin{array}{l}\text { DBP 4 } \\
121193\end{array}$} & \multicolumn{3}{|c|}{$\begin{array}{l}\text { DBP 5 } \\
121193\end{array}$} & \multicolumn{3}{|c|}{$\begin{array}{l}\text { DBP } 5 \\
122693\end{array}$} \\
\hline BCBA and Targel Metals (UG/) & & & & & & & & & & & & & & & & & & & & \\
\hline Aluminum & 35.1 & $\|$ & & 36.2 & $\mathrm{~J} 3 / /$ & & 694 & $\|$ & & 597 & /I & & 505 & /I & & 96.5 & / & & 86.4 & /l \\
\hline Antimony & $<$ & "I & & NA & & $<$ & 2 & $\|$ & 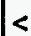 & 2 & "I & & NA & & & NA & & $<$ & 3 & $\|$ \\
\hline Arsenic & $<$ & $\|$ & & NA & & $<$ & 2 & $\|$ & $<$ & 2 & "I & & NA & & & 43.6 & $\|$ & $<$ & 2 & $\|$ \\
\hline Barium & 15.1 & II & & NA & & & 42.4 & /I & & 42.6 & "I & & NA & & & 26.5 & II & & 30.8 & $\|$ \\
\hline Beryllium & $<$ & "I & & NA & & $<$ & 3 & /I & $<$ & 3 & "I & & NA & & & NA & & $<$ & 3 & II \\
\hline Cadmium & $<<$ & /I & & NA & & $<<$ & 2 & $\|$ & $<$ & 2 & /I & & NA & & $\mid<$ & 2 & /I & $<$ & 2 & II \\
\hline Calcium & 727 & "I & & $\mathrm{NA}$ & & & 3740 & $32 / 1$ & & 3190 & "I & & NA & & & 877 & "I & & 940 & VII \\
\hline Chromium & $<$ & /I & & $\mathrm{NA}$ & & $<$ & 4 & "I & $<$ & 4 & II & & NA & & & 4 & II & $<<$ & 4 & /I \\
\hline Cobalt & $<<$ & $\|$ & & NA & & $<$ & 4 & $\|$ & $1<$ & 4 & $\|$ & & NA & & & NA & & |< & 4 & /I \\
\hline Copper & $<$ & $\|$ & & $\mathrm{NA}$ & & $<$ & 4 & "I & $<$ & 4 & "I & & NA & & & NA & & |< & 4 & II \\
\hline Cyanide & & & & & ' & & & & & & & & & & & & & $<$ & 5 & II \\
\hline Iron & 289 & $\|$ & & 674 & $\mathrm{~V} / \mathrm{I}$ & & 122 & /I & & 178 & "I & & 502 & $\mathrm{~V} / \mathrm{I}$ & & 54.7 & "I & & 16.5 & II \\
\hline Lead & 3.29 & "I & $<$ & 3 & ll & $<<$ & 3 & $\|$ & $<$ & 3 & /I & $<$ & 3 & $\|$ & $<$ & 3 & $\|$ & & 3.7 & /I \\
\hline Lithium, total recoverable & NA & & & NA & & & NA & & & NA & & & NA & & & 5.3 & $\|$ & | $<$ & 3 & "I \\
\hline Magnesium & 240 & 11 & & NA & & & 1430 & $\|$ & & 1200 & "I & & NA & & & 1370 & /I & & 1450 & /I \\
\hline Manganese & 25.8 & /I & & 189 & $\|$ & & 734 & "I & & 625 & "I & & 588 & /I & & 7.7 & $\|$ & & 7.2 & /I \\
\hline Mercury & $<\quad 0.2$ & "I & & NA & & $<<$ & 0.2 & $\mathrm{JQ} / \mathrm{I}$ & $<$ & 0.2 & "I & & NA & & |< & 0.2 & $\mathrm{JQ} / \mathrm{I}$ & & 0.97 & /I \\
\hline Nickel & $<$ & $\|$ & & NA & & & 4.67 & II & & 5.98 & /I & & NA & & & NA & & $<$ & 4 & $/ 1$ \\
\hline Potassium & 582 & $/ 1$ & & NA & & & 1160 & 11 & & 1320 & $\|$ & & NA & & & 667 & $\mathrm{~J} 3 / /$ & & 717 & /1 \\
\hline Selenium & $<$ & $\mathrm{J} \mathrm{I} / /$ & & NA & & $<<$ & 2 & $/ 1$ & $<$ & 2. & $\mathrm{~J} ! / /$ & & NA & & & 9 & $\|$ & $<$ & 2 & /I \\
\hline Silica & NA & & & NA & & & 8770 & $\|$ & & NA & & & NA & & & 8390 & $\|$ & & NA & \\
\hline Silver & 2 & /I & & NA & & $<<$ & 2 & $\|$ & $<<$ & 2 & "I & & NA & & $1<$ & 2 & $\|$ & $<$ & 2 & II \\
\hline Sodium & 8700 & $\|$ & & 9380 & $\mathrm{~V} / I$ & & 7910 & "I & & 7840 & "I & & 7650 & $\mathrm{~V} / \mathrm{I}$ & & 5690 & $\|$ & & 5920 & /I \\
\hline Thallium & 2 & "I & & $\mathrm{NA}$ & & $<$ & 2 & "I & $<$ & 2 & "I & & NA & & & NA & & $<$ & 2 & "I \\
\hline Tin & 3.8 & $\|$ & & $\mathrm{NA}$ & & & 6.3 & $\|$ & & 6.18 & "I & & NA & & & NA & & & NA & \\
\hline Vanadium & $<$ & /I & & $\mathrm{NA}$ & & $<$ & 8 & $\|$ & $<$ & 8 & II & & NA & & & NA & & < & 3 & "I \\
\hline $\begin{array}{c}\text { Zinc } \\
\text { Inorganics (UG/) }\end{array}$ & 14.8 & $\|$ & & NA & & & 21.3 & "I & & 21.5 & II & & NA & & & NA & & & 7.4 & $\mathrm{~V} / I$ \\
\hline Chloride & 4740 & "I & & 10900 & $\|$ & & 5890 & JQ/I & & 5860 & "I & & 8490 & $\|$ & & 5660 & $\|$ & & 5730 & JQ/I \\
\hline Cyanide & 5 & / & & NA & & $<$ & 5 & 11 & $<$ & 5 & II & & NA & & & NA & & & NA & \\
\hline Fiuoride & 100 & II & $<$ & 100 & II & & 104 & "I & $<$ & 100 & "I & & 105 & /I & $<$ & 100 & /1 & & 105 & /I \\
\hline Nitrate as nitrogen & NA & & & 116 & II & & NA & & & NA & & & 262 & II & & 3480 & "I & & 3760 & "I \\
\hline Nitrate-nitrite as nitrogen & 1010 & $\|$ & & NA & & & 170 & $\|$ & & 60 & "I & & NA & & & NA & & & NA & \\
\hline Phenols & NA & & & NA & & $<$ & 5 & II & & NA & & & NA & & & NA & & & NA & \\
\hline Sulfate & 7030 & $\|$ & & 5910 & /I & & 29800 & JQ/I & & 29300 & /I & & 25600 & $\|$ & | & 1000 & II & $<<$ & 1000 & 11 \\
\hline Sulfide & $<\quad 1000$ & $\|$ & & NA & & $<<$ & 1000 & II & $<<$ & 1000 & $\|$ & & NA & & & NA & & & NA & \\
\hline Total dissolved solids & NA & & & 36000 & "I & & 66000 & $\mathrm{~V} / \mathrm{I}$ & & NA & & & 55000 & $\|$ & & 86000 & II & & 46000 & $\mathrm{JQ} / /$ \\
\hline Total phosphates (as P) & NA & & & NA & & $<$ & 50 & $\|$ & & NA & & & NA & & $<$ & 50 & /I & & NA & \\
\hline Orennic Indicaters (UG $/ L)$ & & & & & & & & & & & & & & & & & & & & \\
\hline Total organic carbon & NA & & & NA & & $<$ & 1000 & "I & & NA & & & NA & & & 6000 & II & & NA & \\
\hline Total organic halogens & NA & & & NA & & $<<$ & 5 & II & & NA & & & NA & & $<<$ & 5 & $\mathrm{JQ} / \mathrm{I}$ & & NA & \\
\hline
\end{tabular}


TABLE 6.14

RADIONUCLIDE PARAMETERS

IN GROUNDWATER

D-AREA BURNING/RUBBLE PITS

SAVANNAH RIVER SITE

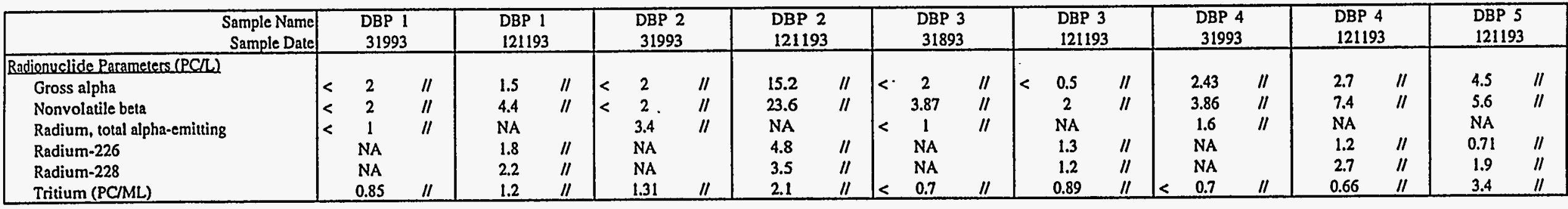



TABLE 6.16

VOLATILE ORGANIC COMPOUNDS

IN SURFACE WATER

D-AREA BURNING/RUBBLE PITS

SAVANNAH RIVER SITE

\begin{tabular}{|c|c|c|c|c|c|c|c|c|c|c|c|c|c|c|c|c|c|c|c|c|c|c|c|c|c|c|}
\hline \multirow{3}{*}{ 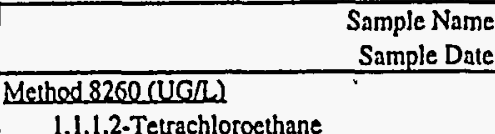 } & \multicolumn{2}{|c|}{$\begin{array}{c}\text { D4312801 } \\
50594 \\
\end{array}$} & \multicolumn{3}{|c|}{$\begin{array}{c}\text { D4312901 } \\
50594\end{array}$} & \multicolumn{3}{|c|}{$\begin{array}{c}\text { D4313001 } \\
50594\end{array}$} & \multicolumn{3}{|c|}{$\begin{array}{c}\text { D4313101 } \\
50594\end{array}$} & \multicolumn{3}{|c|}{$\begin{array}{c}\text { D4313201 } \\
50594\end{array}$} & \multicolumn{3}{|c|}{$\begin{array}{c}\text { D4313301 } \\
50594\end{array}$} & \multicolumn{3}{|c|}{$\begin{array}{c}\text { D4313401 } \\
50594\end{array}$} & \multicolumn{3}{|c|}{$\begin{array}{c}\text { D4313501 } \\
50594\end{array}$} & \multicolumn{3}{|c|}{$\begin{array}{c}\text { D4313601 } \\
50594\end{array}$} \\
\hline & & & & & & & & & & & & & & & & & & & & & & & & & & \\
\hline & 0.4 & /I & $<$ & 0.4 & "I & $<$ & 0.4 & $\|$ & $<$ & 0.4 & II & | & 0.4 & /I & i & 0.4 & "I & $<$ & 0.4 & $\|$ & |< & 0.4 & /I & $<$ & 0.4 & "I \\
\hline 1,1,1-Trichloroethane & 0.4 & /I & $<$ & 0.4 & $\|$ & $<$ & 0.4 & 11 & $<$ & 0.4 & $\|$ & $<$ & 0.4 & "I & $<$ & 0.4 & $\|$ & $<$ & 0.4 & $\|$ & < & 0.4 & // & $<$ & 0.4 & $\|$ \\
\hline 1,1-Dichloroethane & 0.3 & $\|$ & $<$ & 0.3 & $\|$ & $<$ & 0.3 & $\|$ & $<<$ & 0.3 & $\|$ & $<$ & 0.3 & /I & k & 0.3 & II & $<$ & 0.3 & $\|$ & $1<$ & 0.3 & $\|$ & $<$ & 0.3 & /I \\
\hline 1,1-Dichloroethylene & 0.5 & II & $<$ & 0.5 & II & $<$ & 0.5 & /I & $<$ & 0.5 & /I & $<$ & 0.5 & /I & $1<$ & 0.5 & II & $<$ & 0.5 & $\|$ & |< & 0.5 & $\|$ & $<$ & 0.5 & $\|$ \\
\hline 1,2-Dichloroethane & $<0.07$ & II & $<$ & 0.07 & /I & $<$ & 0.07 & II & $<$ & 0.07 & II & $<$ & 0.07 & "I & $<$ & 0.07 & II & 1< & 0.07 & /I & $<$ & 0.07 & $\|$ & $<$ & 0.07 & $\|$ \\
\hline 1,2-Dichloroethylene & 0.3 & $\|$ & $<$ & 0.3 & /I & $<$ & 0.3 & "I & $<$ & 0.3 & II & $<$ & 0.3 & "I & $<$ & 0.3 & "I & $<$ & 0.3 & $\|$ & |< & 0.3 & /I & $<$ & 0.3 & /I \\
\hline 2-Chloroethyl vinyl ether & 10 & $\|$ & $<$ & 10 & II & $<$ & 10 & /I & $<$ & 10 & $\|$ & |< & 10 & /I & $<$ & 10 & II & $<$ & 10 & "I & $<$ & 10 & /I & $<$ & 10 & II \\
\hline Benzene & 0.3 & "I & $<$ & 0.3 & /I & $<$ & 0.3 & $\|$ & $<$ & 0.3 & II & $<$ & 0.3 & "I & $<$ & 0.3 & "I & $<$ & 0.3 & $\|$ & $\mid<$ & 0.3 & /I & $<$ & 0.3 & II \\
\hline Bromoform & $<$ & /I & $<$ & 2 & 11 & $<$ & 2 & $\|$ & $<$ & 2 & /I & $<$ & 2 & $\|$ & $<$ & 2 & "I & $<$ & 2 & "I & $<$ & 2 & II & $<$ & 2 & 11 \\
\hline Bromomethane (Methyl bromide) & 0.6 & /I & $<$ & 0.6 & /I & $<$ & 0.6 & "I & $<<$ & 0.6 & /I & $<$ & 0.6 & /I & $<<$ & 0.6 & "I & | $<$ & 0.6 & /I & $<$ & 0.6 & /I & $<$ & 0.6 & $\|$ \\
\hline Carbon tetrachloride & 0.2 & II & $<$ & 0.2 & II & $<$ & 0.2 & "I & $<$ & 0.2 & "I & $<$ & 0.2 & "I & $<$ & 0.2 & II & $<$ & 0.2 & II & $\mid<$ & 0.2 & "I & $<$ & 0.2 & $\|$ \\
\hline Chlorobenzene & 0.2 & II & $<$ & 0.2 & /I & $<$ & 0.2 & $\|$ & $1<$ & 0.2 & II & $<$ & 0.2 & "I & $<$ & 0.2 & "I & $<$ & 0.2 & II & $<$ & 0.2 & "I & $<$ & 0.2 & $\|$ \\
\hline Chloroethane & 0.2 & /I & $<$ & 0.2 & "I & $<$ & 0.2 & "I & $<$ & 0.2 & /I & $<$ & 0.2 & /I & $<$ & 0.2 & $\|$ & $<$ & 0.2 & II & $<$ & 0.2 & "I & $<$ & 0.2 & /I \\
\hline Chloroethene (Vinyl chloride) & 0.4 & $\|$ & $<$ & 0.4 & /I & $<$ & 0.4 & /I & $<$ & 0.4 & /I & $<$ & 0.4 & "I & $<$ & 0.4 & "I & $<$ & 0.4 & $\|$ & $1<$ & 0.4 & "I & $<$ & 0.4 & "I \\
\hline Chloroform & 0.3 & "I & $<$ & 0.3 & /I & $<$ & 0.3 & II & $<$ & 0.3 & "I & |< & 0.3 & II & $<$ & 0.3 & $\|$ & $<$ & 0.3 & "I & $<$ & 0.3 & /I & $<$ & 0.3 & $\|$ \\
\hline Chloromethane (Methyl chloride) & 0.5 & /I & $<$ & 0.3 & IVI & $<$ & 0.3 & $r \mathrm{VI}$ & $<$ & 0.3 & $\mathrm{NII}$ & $<$ & 0.3 & $N I$ & $<$ & 0.3 & $N I$ & $<$ & 0.3 & IVI & $<$ & 0.3 & $N I$ & $<$ & 0.3 & $N /$ \\
\hline Dibromochloromethane & 0.5 & /I & $<$ & 0.5 & /I & $<$ & 0.5 & "I & $<$ & 0.5 & II & $<$ & 0.5 & /I & $<$ & 0.5 & $\|$ & $<$ & 0.5 & "I & $\mid<$ & 0.5 & $\|$ & $<$ & 0.5 & "I \\
\hline Ethylbenzene & 0.3 & $/ 1$ & $<$ & 0.3 & $\|$ & $<$ & 0.3 & II & $<$ & 0.3 & II & $<$ & 0.3 & /I & $1<$ & 0.3 & II & $<$ & 0.3 & II & $<$ & 0.3 & "I & $<$ & 0.3 & 11 \\
\hline Tetrachloroethylene & 0.5 & "I & $<$ & 0.5 & II & $<$ & 0.5 & II & $<$ & 0.5 & II & $<$ & 0.5 & /I & $<$ & 0.5 & $\|$ & $<$ & 0.5 & /I & $<$ & 0.5 & "I & $<$ & 0.5 & II \\
\hline Toluene & 0.8 & 11 & $<$ & 0.8 & II & $<$ & 0.8 & "I & $<$ & 0.8 & $\|$ & $<$ & 0.8 & /I & $<$ & 0.8 & "I & $<$ & 0.8 & /I & & 0.15 & $\mathrm{~J} / \mathrm{B} /$ & $<$ & 0.8 & II \\
\hline Irans-1,3-Dichloropropene & $<$ & II & $<$ & 2 & $1 /$ & $<$ & 2 & II & $<$ & 2 & "I & $<$ & 2 & /I & $<$ & 2 & II & $<$ & 2 & "I & $<$ & 2 & "I & $<$ & 2 & II \\
\hline Trichloroethylene & 0.2 & /I & $<$ & 0.2 & /I & $<$ & 0.2 & "I & $<$ & 0.2 & /I & $<$ & 0.2 & II & $<$ & 0.2 & "I & $<<$ & 0.2 & $\|$ & $<$ & 0.2 & "I & $<$ & 0.2 & "I \\
\hline Trichlorofluoromethane & 0.7 & II & L & 0.7 & 11 & $<$ & 0.7 & II & $1<$ & 0.7 & II & $\leq$ & 0.7 & II & $1<$ & 0.7 & ii & $1<$ & 0.7 & 11 & $1<$ & 0.7 & 11 & $<$ & 0.7 & II \\
\hline
\end{tabular}


TABLE 6.16

VOLATILE ORGANIC COMPOUNDS

IN SURFACE WATER

D-AREA BURNING/RUBBLE PITS

SAVANNAH RIVER SITE

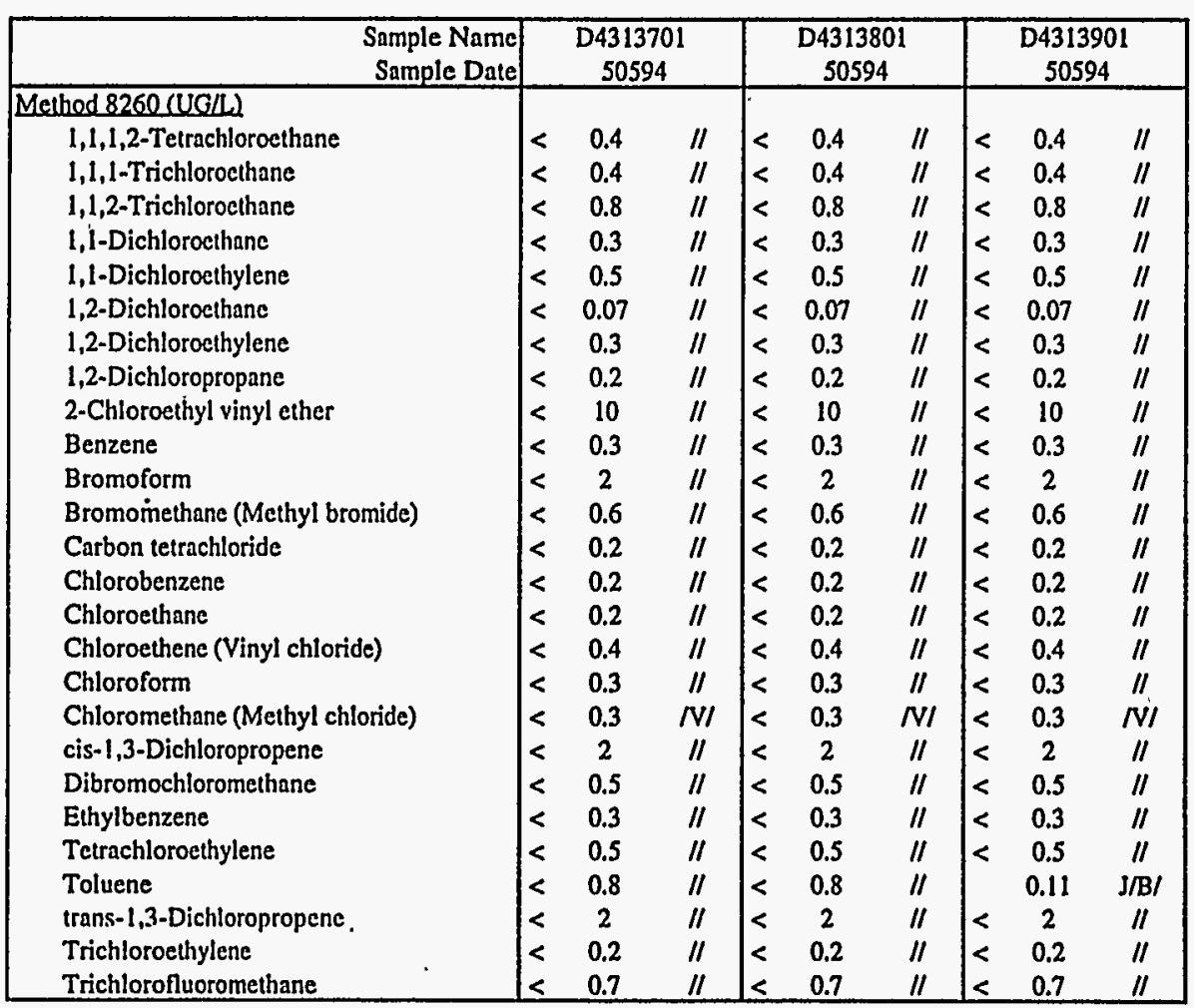

$i^{*}$ 
TABLE 6.17

SEMI-VOLATTLE ORGANIC COMPOUNDS

IN SURFACE WATER

D-AREA BURNING/RUBBLE PITS

SAVANNAH RIVER SITE

\begin{tabular}{|c|c|c|c|c|c|c|c|c|c|c|c|c|c|c|c|c|c|c|c|c|c|c|c|c|c|c|c|}
\hline \multirow{2}{*}{ Method 8270 (UG/N) } & \multicolumn{3}{|c|}{$\begin{array}{c}\text { D431111901 } \\
60793 \\
\end{array}$} & \multicolumn{3}{|c|}{$\begin{array}{c}43111902 \\
60793 \\
\end{array}$} & \multicolumn{3}{|c|}{$\begin{array}{c}43112001 \\
60793 \\
\end{array}$} & \multicolumn{3}{|c|}{$\begin{array}{c}43112002 \\
60793 \\
\end{array}$} & \multicolumn{3}{|c|}{$\begin{array}{c}43112101 \\
60793 \\
\end{array}$} & \multicolumn{3}{|c|}{$\begin{array}{c}\text { D431 2601 } \\
60793\end{array}$} & \multicolumn{3}{|c|}{$\begin{array}{c}\mathrm{D} 4312801 \\
50594\end{array}$} & \multicolumn{3}{|c|}{$\begin{array}{c}\text { D4312901 } \\
50594\end{array}$} & \multicolumn{3}{|c|}{$\begin{array}{c}\text { D4313001 } \\
50594\end{array}$} \\
\hline & & & & & & & & & & & & & & & & & & & & & & & & & & & \\
\hline 1,2,4-Trichlorobenzene & $<$ & 12 & II & $<$ & 12 & $\|$ & $<$ & 12 & $\|$ & $<$ & 9.8 & "I & $<$ & 14 & $\|$ & $<$ & 11 & $\mathrm{~J} / \mathrm{O} / \mathrm{L}$ & $<$ & 11 & $\|$ & $<$ & 10 & $\|$ & $<$ & 10 & $\|$ \\
\hline 1,2-Dichlorobenzene & $<$ & 12 & "I & $<$ & 12 & /I & $<$ & 12 & /I & $<$ & 9.8 & "I & $<$ & 14 & $\|$ & $<$ & 11 & $\mathrm{~J} / \mathrm{O} / \mathrm{L}$ & $<$ & 11 & ॥ & $<$ & 10 & "I & $<$ & 10 & $\|$ \\
\hline 1,4-Dichlorobenzene & $<$ & 12 & II & $<$ & 12 & /I & $<$ & 12 & /I & $<$ & 9.8 & "I & $<$ & 14 & /I & $<$ & 11 & $\mathrm{~J} / \mathrm{O} / \mathrm{L}$ & $<$ & 11 & $\|$ & $<$ & 10 & "I & $<$ & 10 & "I \\
\hline 2,4,5-Trichlorophenol & $<$ & 60 & "I & $<$ & 60 & "I & $<$ & 60 & $\|$ & $<$ & 49 & II & $<$ & 70 & /I & $<$ & 55 & $\mathrm{~J} / \mathrm{O} / \mathrm{L}$ & $<$ & 55 & /I & $<$ & 50 & "I & $<$ & 50 & /I \\
\hline 2,4,6-Trichlorophenol & $1<$ & 12 & /I & $<$ & 12 & /I & $<$ & 12 & /I & $<$ & 9.8 & II & $<$ & 14 & "I & $<$ & 11 & $\mathrm{~J} / \mathrm{O} / \mathrm{L}$ & $<$ & 11 & $\|$ & $<$ & 10 & II & $<$ & 10 & /I \\
\hline 2,4-Dichlorophenol & $<$ & 12 & II & $<$ & 12 & "I & $<$ & 12 & "I & $<$ & 9.8 & $\|$ & $<$ & 14 & "I & $<$ & 11 & $\mathrm{~J} / \mathrm{O} / \mathrm{L}$ & $<$ & 11 & II & $<$ & 10 & "I & $<$ & 10 & /l \\
\hline 2,4-Dinitrophenol & $<$ & 60 & "I & $<$ & 60 & "I & $<$ & 60 & $\|$ & $<$ & 49 & $\|$ & $<$ & 70 & "I & $<$ & 55 & $\mathrm{~J} / \mathrm{O} / \mathrm{L}$ & $<$ & 55 & /I & $<$ & 50 & "I & $<$ & 50 & "I \\
\hline 2,4-Dinitrotoluene & $<$ & 12 & "I & $<$ & 12 & /I & $<$ & 12 & "I & $<$ & 9.8 & "I & $<$ & 14 & $\|$ & $<$ & 11 & $\mathrm{~J} / \mathrm{O} / \mathrm{L}$ & $<$ & 11 & /I & $<$ & 10 & "I & $<$ & 10 & /l \\
\hline 2,6-Dinitrotoluene & $<$ & 12 & $/ 1$ & $<$ & 12 & "I & $<$ & 12 & "I & $<$ & 9.8 & "I & $<$ & 14 & "I & $<$ & 11 & $\mathrm{~J} / \mathrm{O} / \mathrm{L}$ & $<$ & 11 & $\|$ & $<$ & 10 & "I & $<$ & 10 & $\|$ \\
\hline 2.Chloronaphthalene & $<$ & 12 & "I & $<$ & 12 & $\|$ & $<$ & 12 & $\|$ & $<$ & 9.8 & $\|$ & $<$ & 14 & "I & $<$ & 11 & $\mathrm{~J} / \mathrm{O} / \mathrm{h}$ & $<$ & 11 & $\|$ & $<$ & 10 & II & $<$ & 10 & II \\
\hline 2-Chlorophenol & $<$ & 12 & "I & $<$ & 12 & "I & $<$ & 12 & $\|$ & $<$ & 9.8 & "I & $<$ & 14 & $\|$ & $<$ & 11 & $\mathrm{~J} / \mathrm{O} / \mathrm{L}$ & $<$ & 11 & $\|$ & $<$ & 10 & II & $<$ & 10 & "l \\
\hline 2-Methyl-4,6-dinitrophenol & $<$ & 60 & II & $<$ & 60 & /I & $<$ & 60 & /I & $<$ & 49 & $\|$ & $<$ & 70 & $\|$ & $<$ & 55 & $\mathrm{~J} / \mathrm{O} / \mathrm{L}$ & $<$ & 55 & $\|$ & $<$ & 50 & $\|$ & $<$ & 50 & $\|$ \\
\hline 2-Methylnaphthalene & $<$ & 12 & "l & $<$ & 12 & /I & $<$ & 12 & $\|$ & $<$ & 9.8 & $\|$ & $<$ & 14 & II & $<$ & 11 & $\mathrm{~J} / \mathrm{O} / \mathrm{L}$ & $<$ & 11 & $\|$ & $<$ & 10 & $\|$ & $<$ & 10 & /l \\
\hline 2-Nitrophenol & $<$ & 12 & $\|$ & $<$ & 12 & /I & $<$ & 12 & $\|$ & $<$ & 9.8 & $\|$ & $<$ & 14 & $\|$ & $<$ & 11 & $\mathrm{~J} / \mathrm{O} / \mathrm{L}$ & $<$ & 11 & $\|$ & $<$ & 10 & /I & $<$ & 10 & /I \\
\hline 3,3'-Dichlorobenzidine & $<$ & 24 & "I & $<$ & 24 & /I & $<$ & 24 & /I & $<$ & 19.6 & "I & $<$ & 28 & $\|$ & $<$ & 22 & $\mathrm{~J} / \mathrm{OL}$ & $<$ & 22 & $\|$ & $<$ & 20 & II & $<$ & 20 & /l \\
\hline 4-Bromophenyl phenyl ether & $<$ & 12 & $\|$ & $<$ & 12 & /I & $<$ & 12 & "I & $<$ & 9.8 & II & $<$ & 14 & "I & $<$ & 11 & $\mathrm{~J} / \mathrm{O} / \mathrm{L}$ & $<$ & 11 & /I & $<$ & 10 & "I & $<$ & 10 & /I \\
\hline 4-Chloroaniline & $<$ & 12 & 11 & $<$ & 12 & "I & $<$ & 12 & "I & $1<$ & 9.8 & $\|$ & $<$ & 14 & "I & $<$ & 11 & $\mathrm{~J} / \mathrm{O} / \mathrm{L}$ & $<$ & 11 & $\|$ & $<$ & 10 & II & $<$ & 10 & /I \\
\hline 4.Chlorophenyl phenyl ether & $<$ & 12 & "I & $<$ & 12 & /I & $<$ & 12 & $\|$ & $<$ & 9.8 & $\|$ & $<$ & 14 & $\|$ & $<$ & 11 & $\mathrm{~J} / \mathrm{O} / \mathrm{L}$ & < & 11 & /I & $<$ & 10 & $\|$ & $<$ & 10 & /I \\
\hline 4-Nitrophenol & $<$ & 60 & II. & $<<$ & 60 & "I & | & 60 & /I & $<$ & 49 & $\|$ & $<$ & 70 & $\|$ & $<$ & 55 & $\mathrm{~J} / \mathrm{OL}$ & $<$ & 55 & $\|$ & $<$ & 50 & "I & $<$ & 50 & /I \\
\hline Acenaphthene & $<$ & 12 & "I & |< & 12 & /I & $<$ & 12 & "I & $<$ & 9.8 & "I & $<$ & 14 & $\|$ & $<$ & 11 & $\mathrm{~J} / \mathrm{O} / \mathrm{L}$ & $<$ & 11 & /I & $<$ & 10 & "I & $<$ & 10 & /I \\
\hline Acenaphthylene & $<$ & 12 & "l & $<$ & 12 & /I & $<$ & 12 & "l & $<$ & 9.8 & $\|$ & $<$ & 14 & 11 & $<$ & 11 & $\mathrm{~J} / \mathrm{O} / \mathrm{h}$ & $<$ & 11 & $\|$ & $<$ & 10 & $\|$ & $<$ & 10 & $\|$ \\
\hline Anthracene & $<$ & 12 & $\|$ & |< & 12 & /I & $<$ & 12 & /I & $<$ & 9.8 & "I & $<$ & 14 & $\|$ & $<$ & 11 & $\mathrm{~J} / \mathrm{O} / \mathrm{L}$ & $<$ & 11 & $\|$ & $<$ & 10 & "I & $<$ & 10 & /I \\
\hline Benzo(a)anthracene & $<$ & 12 & $\|$ & $<$ & 12 & /I & $<$ & 12 & "I & $<$ & 9.8 & $\|$ & $<$ & 14 & $\|$ & $<$ & 11 & $\mathrm{~J} / \mathrm{O} / \mathrm{L}$ & $<$ & 11 & /I & $<$ & 10 & /I & $<$ & 10 & /I \\
\hline Benzo(a)pyrene & $<$ & 12 & $\|$ & $<$ & 12 & II & $<$ & 12 & /I & $<$ & 9.8 & 11 & $<$ & 14 & $\|$ & $<$ & 11. & $\mathrm{~J} / \mathrm{O} / \mathrm{L}$ & $<$ & 11 & $\|$ & $<$ & 10 & 11 & $<$ & 10 & /l \\
\hline Benzo(b)fluotanthene & $<$ & 12 & 11 & $<$ & 12 & $/ /$ & $<$ & 12 & /I & $<$ & 9.8 & "I & $<$ & 14 & 11 & $<$ & 11 & $\mathrm{~J} / \mathrm{O} / \mathrm{L}$ & $<$ & 11 & $\|$ & $<$ & 10 & "I & $<$ & 10 & /l \\
\hline Benzo $(g, h, i)$ perylene & $<$ & 12 & "I & $<$ & 12 & "I & $<$ & 12 & "I & $<$ & 9.8 & /I & $<$ & 14 & $\|$ & $<$ & 11 & $\mathrm{~J} / \mathrm{O} / \mathrm{L}$ & $<$ & 11 & /I & $<$ & 10 & "I & $<$ & 10 & /I \\
\hline Benzo(k)fluoranthene & $<$ & 12 & II & $<$ & 12 & II & $<$ & 12 & "I & $<$ & 9.8 & "I & $<$ & 14 & "I & |< & 11 & J/O/L & $<$ & 11 & $\|$ & $<$ & 10 & II & $<$ & .10 & $\|$ \\
\hline Benzoic acid & $1<$ & 60 & II & $<$ & 60 & /I & $<$ & 60 & "I & 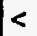 & 49 & /I & $<$ & 70 & $\|$ & $<$ & 55 & $\mathrm{~J} / \mathrm{O} / \mathrm{L}$ & $<$ & 55 & $\|$ & $<$ & 50 & /l & $<$ & 50 & /I \\
\hline Benzyl alcohol & $<$ & 12 & $\|$ & $<$ & 12 & /I & $<$ & 12 & "I & $<$ & 9.8 & 11 & $<$ & 14 & $\|$ & $<$ & 11 & $\mathrm{~J} / \mathrm{O} / \mathrm{L}$ & $<$ & 11 & $\|$ & $<$ & 10 & II & $<$ & 10 & /l \\
\hline Bis(2-chloroethoxy) methane & $<$ & 12 & "I & $<$ & 12 & "I & $<$ & 12 & /I & |< & 9.8 & II & $<$ & 14 & /I & $<$ & 11 & $\mathrm{~J} / \mathrm{O} / \mathrm{L}$ & $<$ & 11 & /I & $<$ & 10 & II & $<$ & 10 & /I \\
\hline Bis(2-chloroethyl) ether & $<$ & 12 & II & $<$ & 12 & /1 & $<$ & 12 & "l & $<$ & 9.8 & $\|$ & $<$ & 14 & $\|$ & $<$ & 11 & $\mathrm{~J} / \mathrm{O} / \mathrm{L}$ & $<$ & 11 & $\|$ & $<$ & 10 & $/ 1$ & $<$ & 10 & $\|$ \\
\hline Bis(2-chloroisopropyl) ether & $<$ & 12 & $\|$ & $<$ & 12 & "I & $<$ & 12 & II & $<$ & 9.8 & II & $<$ & 14 & II & $<$ & 11 & $\mathrm{~J} / \mathrm{O} / \mathrm{L}$ & $<$ & 11 & $\|$ & $<$ & 10 & $\|$ & $<$ & 10 & /l \\
\hline Bis(2-ethylhexyl) phthalate & $<$ & 2.54 & $\mathrm{~J} / \mathrm{VI}$ & $<$ & 2.54 & $\mathrm{~J} / \mathrm{V} /$ & $<$ & 2.69 & $\mathrm{~J} / \mathrm{V} /$ & $<$ & 2.38 & $\mathrm{~J} / \mathrm{V} /$ & $<$ & 3.23 & $\mathrm{~J} / \mathrm{VI}$ & $<$ & 2.77 & J/VOR & $<$ & 11 & $\|$ & $<$ & 10 & $\|$ & $<$ & 10 & /l \\
\hline Butylbenzyl phthalate & $<$ & 12 & $\|$ & $<$ & 12 & $\|$ & $<$ & 12 & /I & $<$ & 9.8 & $\|$ & $<$ & 14 & $\|$ & $<$ & 11 & $\mathrm{~J} / \mathrm{O} / \mathrm{L}$ & $<$ & 11 & $\|$ & $<$ & 10 & /I & $<$ & to & /I \\
\hline Chrysene & $<$ & 12 & $\|$ & $<$ & 12 & II & $<$ & 12 & /I & $<$ & 9.8 & II & $<$ & 14 & $\|$ & $<$ & 11 & $\mathrm{~J} / \mathrm{O} / \mathrm{L}$ & $<$ & 11 & $\|$ & $<$ & 10 & /I & $<$ & 10 & /I \\
\hline Di-n-butyl phthalate & ie & 3.45 & $\mathrm{~J} / \mathrm{V} /$ & |< & 3.39 & $\mathrm{~J} / \mathrm{V} /$ & $<$ & 3.98 & $\mathrm{~J} / \mathrm{V} \mathrm{I}$ & $<$ & 2.75 & $\mathrm{~J} / \mathrm{V} /$ & $<$ & 5.24 & $\mathrm{~J} / \mathrm{VI}$ & $<$ & 11 & $\mathrm{~J} / \mathrm{O} / \mathrm{L}$ & $<$ & 11 & $\|$ & $<$ & 10 & "I & $<$ & 10 & /I \\
\hline
\end{tabular}

Page 1 of 4 
TABLE 6.17

SEMI-VOLATILE ORGANIC COMPOUNDS $\because$ IN SURFACE WATER

D-AREA BURNING/RUBBLE PITS

SAVANNAH RIVER SITE

\begin{tabular}{|c|c|c|c|c|c|c|c|c|c|c|c|c|c|c|c|c|c|c|c|c|c|c|c|c|c|c|c|}
\hline \multirow[b]{2}{*}{ Melhed 8270 (UGa) } & \multicolumn{3}{|c|}{$\begin{array}{c}\text { D43111901 } \\
60793 \\
\end{array}$} & \multicolumn{3}{|c|}{$\begin{array}{c}43111902 \\
60793 \\
\end{array}$} & \multicolumn{3}{|c|}{$\begin{array}{c}\text { D43112001 } \\
60793 \\
\end{array}$} & \multicolumn{3}{|c|}{$\begin{array}{c}43112002 \\
60793 \\
\end{array}$} & \multicolumn{3}{|c|}{$\begin{array}{c}\text { D43112101 } \\
60793 \\
\end{array}$} & \multicolumn{3}{|c|}{$\begin{array}{c}\text { D431 } 2601 \\
60793 \\
\end{array}$} & \multicolumn{3}{|c|}{$\begin{array}{c}\text { D4312801 } \\
50594 \\
\end{array}$} & \multicolumn{3}{|c|}{$\begin{array}{c}\text { D4312901 } \\
50594 \\
\end{array}$} & \multicolumn{3}{|c|}{$\begin{array}{c}\text { D4313001 } \\
50594\end{array}$} \\
\hline & & & & & & & & & & & & & & & & & & & & & & & & & & & \\
\hline Dibenzofuran & $<$ & 12 & $\|$ & $<$ & 12 & $/ /$ & $<$ & 12 & $\|$ & $<$ & 9.8 & /I & $<$ & 14 & /I & $<$ & 11 & $\mathrm{~J} / \mathrm{O} / \mathrm{L}$ & $<$ & 11 & "I & $<$ & 10 & $\|$ & $<$ & 10 & $\|$ \\
\hline Diethyl phthalate & $<$ & 12 & $\|$ & $<$ & 12 & $\|$ & $<$ & 12 & $\|$ & $<$ & 9.8 & $\|$ & $<$ & 14 & $\|$ & $<$ & 11 & $\mathrm{~J} / \mathrm{O} / \mathrm{L}$ & $<$ & 11 & $\|$ & $<$ & 10 & $l l$ & $<$ & 10 & $\|$ \\
\hline Dimethyl phthalate & $<$ & 12 & /l & $<$ & 12 & II & $<$ & 12 & II & $<$ & 9.8 & $\|$ & $<$ & 14 & 11 & $<$ & 11 & $\mathrm{~J} / \mathrm{O} / \mathrm{L}$ & $<$ & 11 & $\|$ & $<$ & 10 & $\|$ & $<$ & 10 & II \\
\hline Fluoranthene & $<$ & 12 & $\|$ & $<$ & 12 & $\|$ & $<$ & 12 & $\|$ & $<$ & 9.8 & II & $<$ & 14 & II & $<$ & 11 & $\mathrm{~J} / \mathrm{O} / \mathrm{L}$ & $<$ & 11 & II & $<$ & 10 & /I & $<$ & 10 & 11 \\
\hline Fluorene & $<$ & 12 & /I & $<$ & 12 & /I & k & 12 & $\|$ & k & 9.8 & /I & $<$ & 14 & "I & $<$ & 11 & $\mathrm{~J} / \mathrm{O} / \mathrm{L}$ & |< & 11 & /I & | & 10 & /I & $<$ & 10 & /I \\
\hline Hexachlorobenzene & $<$ & 12 & $\|$ & $<$ & 12 & $/ 1$ & < & 12 & $\|$ & $<$ & 9.8 & $\|$ & $1<$ & 14 & /I & $1<$ & 11 & J/OR & $<$ & 11 & 11 & $<$ & 10 & /I & $<$ & 10. & /I \\
\hline Hexachlorobutadiene & $<<$ & 12 & II & $<$ & 12 & II & < & 12 & II & |< & 9.8 & $\|$ & $\mid<$ & 14 & II & $<$ & 11 & $\mathrm{~J} / \mathrm{O} / \mathrm{L}$ & $<$ & 11 & /I & |< & 10 & /I & $<$ & 10 & $\|$ \\
\hline Hexachlorocyclopentadiene & $<$ & 12 & 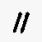 & $<$ & 12 & $/ /$ & $<$ & 12 & $\|$ & $<$ & 9.8 & $\|$ & $<$ & 14 & /I & $<$ & 11 & $\mathrm{~J} / \mathrm{O} / \mathrm{L}$ & $<$ & 11 & "l & $<$ & 10 & /I & $<$ & 10 & "I \\
\hline Hexachloroethane & $<<$ & 12 & $\|$ & $<<$ & 12 & /I & $<<$ & 12 & $\|$ & $<<$ & 9.8 & /I & $<$ & 14 & /I & $<$ & 11 & J/O/L & $<$ & 11 & $\|$ & $<<$ & 10 & "I & $<$ & 10 & "I \\
\hline Indeno(1,2,3-c,d)pyrene & $<$ & 12 & $\|$ & $<$ & 12 & /I & $<$ & 12 & $\|$ & < & 9.8 & $\|$ & $1<$ & 14 & $\|$ & $<$ & 11 & $\mathrm{~J} / \mathrm{O} / \mathrm{L}$ & $<$ & 11 & /I & $<<$ & 10 & $\|$ & $<$ & 10 & $\|$ \\
\hline Isophorone & $<$ & 12 & $/ 1$ & $<$ & 12 & /I & $1<$ & 12 & $/ /$ & $<$ & 9.8 & $\|$ & $<$ & 14 & /I & $<$ & 11 & $\mathrm{~J} / \mathrm{O} / \mathrm{L}$ & $<<$ & 11 & /I & $<<$ & 10 & $\|$ & $<$ & 10 & "l \\
\hline m-Nitroaniline & $<$ & 60 & $\|$ & $<$ & 60 & $\|$ & $<$ & 60 & $\|$ & k & 49 & $\|$ & $<$ & 70 & $\|$ & $<$ & 55 & $\mathrm{~J} / \mathrm{O} / \mathrm{L}$ & $<$ & 55 & /I & |< & 50 & $\|$ & $<$ & 50 & $\|$ \\
\hline N-Nitrosodiphenylamine & $<$ & 12 & $\|$ & $<$ & 12 & $\|$ & $<$ & 12 & $/ 1$ & $<$ & 9.8 & $\|$ & $<$ & 14 & $\|$ & $<$ & 11 & $\mathrm{~J} / \mathrm{O} / \mathrm{L}$ & $<$ & 11 & $\|$ & $<$ & 10 & $\|$ & $<$ & 10 & "I \\
\hline N-Nitrosodipropylamine & $<$ & 12 & $\|$ & $<$ & 12 & $\|$ & $<$ & 12 & $\|$ & $<$ & 9.8 & $\|$ & $<$ & 14 & /I & $<$ & 11 & $\mathrm{~J} / \mathrm{O} / \mathrm{L}$ & $<$ & 11 & "I & $<<$ & 10 & $\|$ & $<$ & 10 & $\|$ \\
\hline Naphthalene & < & 12 & $\|$ & $<$ & 12 & /I & $<$ & 12 & $\|$ & $<<$ & 9.8 & $\|$ & |< & 14 & II & $<$ & 11 & $\mathrm{~J} / \mathrm{O} / \mathrm{L}$ & $<$ & 11 & /I & $<$ & 10 & $/ 1$ & $<$ & 10 & $\|$ \\
\hline Nitrobenzene & $<$ & 12 & /I & $<$ & 12 & II & 1< & 12 & $\|$ & k $<$ & 9.8 & /I & $<$ & 14 & II & $<$ & 11 & $\mathrm{~J} / \mathrm{O} / \mathrm{R}$ & $<$ & 11 & $\|$ & $<<$ & 10 & $\|$ & $<$ & 10 & II \\
\hline o-Cresol (2-Methylphenol) & $<$ & 12 & $\|$ & $<$ & 12 & $\|$ & $\mid<$ & 12 & $\|$ & |< & 9.8 & $\|$ & $<$ & 14 & $\|$ & $<$ & 11 & $\mathrm{~J} / \mathrm{O} / \mathrm{L}$ & $<$ & 11 & $\|$ & $<$ & 10 & $\|$ & $<$ & 10 & /l \\
\hline o-Nitroaniline & $<$ & 60 & $/ /$ & $<$ & 60 & $\|$ & $<$ & 60 & $\|$ & $<$ & 49 & $\|$ & $<$ & 70 & $\|$ & $<$ & 55 & J/O/L & $<$ & 55 & $\|$ & $<$ & 50 & $\|$ & $<$ & 50 & $\|$ \\
\hline p-Cresol (4-Methylphenol) & |< & 12 & $\|$ & $<$ & 12 & /I & $<$ & 12 & /I & 1< & 9.8 & $\|$ & $<<$ & 14 & /I & $<$ & 11 & $\mathrm{~J} / \mathrm{O} / \mathrm{L}$ & & 3.84 & $\mathrm{~J} / \mathrm{B} /$ & | & 10 & $\|$ & $<$ & 10 & /I \\
\hline p-Nitroaniline & $<$ & 60 & $\|$ & $<$ & 60 & $\|$ & $<$ & 60 & $\|$ & $<$ & 49 & /I & $<$ & 70 & $\|$ & $<$ & 55 & $\mathrm{~J} / \mathrm{O} / \mathrm{L}$ & $<$ & 55 & $\|$ & $<$ & 50 & $\|$ & $<$ & 50 & "I \\
\hline Pentachlorophenol & $<$ & 60 & $\|$ & $<$ & 60 & $\|$ & $<$ & 60 & $\|$ & $<$ & 49 & $\|$ & $<$ & 70 & $\|$ & $<$ & 55 & 3/OR & $<$ & 55 & II & $<$ & 50 & II & $<$ & 50 & "I \\
\hline Phenanthrene & | $<$ & 12 & $\|$ & $<$ & 12 & $\|$ & $<$ & 12 & /I & $<$ & 9.8 & /I & $<<$ & 14 & "I & $<$ & 11 & $\mathrm{~J} / \mathrm{O} / \mathrm{L}$ & $<$ & 11 & $\|$ & $<$ & 10 & $\|$ & $<$ & 10 & "I \\
\hline Phenol & $<$ & 12 & $\|$ & $<$ & 12 & $\|$ & $<$ & 12 & $\|$ & $<$ <. & 9.8 & $\|$ & $<<$ & 14 & /I & $<$ & 11 & $\mathrm{~J} / \mathrm{O} / \mathrm{L}$ & $<$ & 11 & $\|$ & l< & 10 & $\|$ & $<$ & 10 & 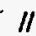 \\
\hline Pyrene & $1<$ & 12 & II & $<<$ & 12 & II & $<<$ & 12 & II & $<<$ & 9.8 & II & L & 14 & II & L & 11 & $\mathrm{~J} / \mathrm{O} / \mathrm{L}$ & $<$ & 11 & $\|$ & $<$ & 10 & 11 & $<$ & 10 & II \\
\hline
\end{tabular}


TABLE 6.17

SEMI-VOLATILE ORGANIC COMPOUNDS

IN SURFACE WATER

D-AREA BURNING/RUBBLE PITS

SAVANNAH RIVER SITE

\begin{tabular}{|c|c|c|c|c|c|c|c|c|c|c|c|c|c|c|c|c|c|c|c|c|c|c|c|c|c|c|c|}
\hline \multirow[b]{2}{*}{ Method 8270 (UG/L) } & \multicolumn{3}{|c|}{$\begin{array}{c}\text { D4313101 } \\
50594 \\
\end{array}$} & \multicolumn{3}{|c|}{$\begin{array}{c}\text { D4313201 } \\
50594 \\
\end{array}$} & \multicolumn{3}{|c|}{$\begin{array}{c}\mathrm{D} 4313301 \\
50594 \\
\end{array}$} & \multicolumn{3}{|c|}{$\begin{array}{c}\mathrm{D} 4313401 \\
50594 \\
\end{array}$} & \multicolumn{3}{|c|}{$\begin{array}{c}\mathrm{D} 4313501 \\
50594 \\
\end{array}$} & \multicolumn{3}{|c|}{$\begin{array}{c}\mathrm{D} 4313601 \\
50594 \\
\end{array}$} & \multirow{2}{*}{\multicolumn{3}{|c|}{$\begin{array}{c}\text { D4313701 } \\
50594 \\
\end{array}$}} & \multirow{2}{*}{\multicolumn{3}{|c|}{$\begin{array}{c}\mathrm{D} 4313801 \\
50594 \\
\end{array}$}} & \multirow{2}{*}{\multicolumn{3}{|c|}{$\begin{array}{c}\mathrm{D} 4313901 \\
50594 \\
\end{array}$}} \\
\hline & & & & & & & & & & & & & & & & & & & & & & & & & & & \\
\hline 1,2,4-Trichlorobenzene & $<$ & 10 & /I & $<$ & 11 & $\|$ & $<$ & 11 & II & $<$ & 11 & $\|$ & $<$ & 11 & /l & $<$ & 10 & $\|$ & k & 10 & $\|$ & $<$ & 10 & $/ /$ & $<$ & 11 & $\|$ \\
\hline 1.2-Dichlorobenzene & $<$ & 10 & II & $<$ & 11 & $\|$ & $<$ & 11 & II & $<$ & 11 & $\|$ & $<$ & 11 & $\|$ & $<$ & 10 & "l & $<$ & 10 & $\|$ & $<$ & 10 & $\|$ & $<$ & 11 & $\|$ \\
\hline 1,3-Dichlorobenzene & $<$ & 10 & $\|$ & $<$ & 11 & /l & $<$ & 11 & "I & $<$ & 11 & $\|$ & $<$ & 11 & $\|$ & $<$ & 10 & $\|$ & $<$ & 10 & "I & $<$ & 10 & $\|$ & $<$ & 11 & $\|$ \\
\hline 1,4-Dichlorobenzene & $<$ & 10 & $\|$ & $<$ & 11 & $\|$ & $<$ & 11 & II & $<$ & 11 & $\|$ & $<$ & 11 & /I & $<$ & 10 & $\|$ & $<$ & 10 & $\|$ & $<$ & 10 & $\|$ & $<$ & 11 & $\|$ \\
\hline 2,4,5-Trichlorophenol & $<$ & so & $\|$ & $<$ & 55 & $\|$ & $<$ & 55 & "I & $<$ & 55 & $\|$ & $<$ & 55 & $\|$ & $<$ & 50 & $\|$ & $<$ & 50 & $\|$ & $<$ & 50 & $\|$ & $<$ & .55 & $\|$ \\
\hline 2,4,6-Trichlorophenol & $<$ & 10 & $\|$ & $<$ & 11 & $\|$ & $<$ & 11 & $\|$ & $<$ & 11 & $\|$ & $<$ & 11 & $\|$ & $<$ & 10 & $\|$ & $<$ & 10 & $\|$ & $<$ & 10 & $\|$ & $<$ & 11 & $\|$ \\
\hline 2,4-Dichlorophenol & $<$ & 10 & $\|$ & $<$ & 11 & $\|$ & $<$ & 11 & $\|$ & $<$ & 11 & $\|$ & $<$ & 11 & $\|$ & $<$ & 10 & $\|$ & $<$ & 10 & /I & $<$ & 10 & $\|$ & $<$ & 11 & /l \\
\hline 2,4-Dimethyl phenol & $<$ & 10 & $\|$ & $<$ & 11 & $\|$ & $<$ & 11 & $\|$ & $<$ & 11 & $\|$ & $<$ & 11 & II & $<$ & 10 & II & $<$ & 10 & "l & $<$ & 10 & $\|$ & $<$ & 11 & $\|$ \\
\hline 2,4-Dinitrophenol & $<$ & 50 & $\|$ & $<$ & 55 & $\|$ & $<$ & 55 & $\|$ & $<$ & 55 & $\|$ & $<$ & 55 & $\|$ & $<$ & 50 & $\|$ & $<$ & 50 & "I & $<$ & so & "I & $<$ & 55 & II \\
\hline 2,4-Dinitrotoluene & $<$ & 10 & $\|$ & $<$ & 11 & /I & $<$ & 11 & $\|$ & $<$ & 11 & $\|$ & $<$ & 11 & II & $<$ & 10 & $\|$ & $<$ & 10 & "l & $<$ & 10 & "l & $<$ & 11 & "l \\
\hline 2,6-Dinitrotoluene & $<$ & 10 & $\|$ & $<$ & 11 & $\|$ & $<$ & 11 & $\|$ & $<$ & 11 & $\|$ & $<$ & 11 & $\|$ & $<$ & 10 & $\|$ & $<$ & 10 & $\|$ & $<$ & 10 & /I & $<$ & 11 & $\|$ \\
\hline 2.Chloronaphthalene & $<$ & 10 & 11 & $<$ & 11 & $\|$ & $<$ & 11 & II & $<$ & 11 & $\|$ & $<$ & 11 & II & $<$ & 10 & /I & $<$ & 10 & II & $<$ & 10 & $\|$ & $<$ & 11 & $\|$ \\
\hline 2-Chlorophenol & $<$ & 10 & $/ /$ & $<$ & 11 & $\|$ & $<$ & 11 & 11 & $<$ & 11 & $\|$ & $<$ & 11 & $\|$ & $<$ & 10 & $\|$ & $<$ & 10 & $\|$ & $<$ & 10 & $\|$ & $<$ & 11 & II \\
\hline 2-Methyl-4,6-dinitrophenol & $<$ & 50 & /l & $<$ & 55 & $\|$ & $<$ & 55 & $\|$ & $<$ & 55 & $\|$ & $<$ & 55 & $\|$ & $<$ & 50 & $\|$ & $<$ & 50 & "I & $<$ & 50 & "l & $<$ & 55 & $\|$ \\
\hline 2-Methylnaphthalene & $<$ & 10 & $\|$ & $<$ & 11 & II & $<$ & 11 & 11 & $<$ & 11 & $\|$ & $<$ & 11 & $\|$ & $<$ & 10 & $\|$ & $<$ & 10 & $\|$ & $<$ & 10 & II & $<$ & 11 & $\|$ \\
\hline 2-Nitrophenol & $<$ & 10 & $\|$ & $<$ & 11 & $\|$ & $<$ & 11 & $\|$ & $<$ & 11 & 11 & $<$ & 11 & 11 & $<$ & 10 & $\|$ & $<$ & 10 & II & $<$ & 10 & $\|$ & $<$ & 11 & $\|$ \\
\hline 3,3'-Dichlorobenzidine & $<$ & 20 & $\|$ & $<$ & 22 & $\|$ & $<$ & 22 & $\|$ & $<$ & 22 & $\|$ & $<$ & 22 & $\|$ & $<$ & 20 & $\|$ & $<$ & 20 & II & $<$ & 20 & $\|$ & $<$ & 22 & $\|$ \\
\hline 4-Bromophenyl phenyl ether & $<$ & 10 & $\|$ & $<$ & 11 & II & $<$ & 11 & II & $<$ & 11 & $\|$ & $<$ & 11 & $\|$ & $<$ & 10 & $\|$ & $<$ & 10 & "I & $<$ & 10 & $\|$ & |< & 11 & II \\
\hline 4-Chloro-m-cresol & $<$ & 10 & II & $<$ & 11 & $\|$ & $<$ & 11 & $\|$ & $<$ & 11 & $\|$ & $<$ & 11 & $\|$ & $<$ & 10 & $\|$ & $<$ & 10 & II & $<$ & 10 & $\|$ & |< & 11 & $\|$ \\
\hline 4-Chloroaniline & $<$ & 10 & $\|$ & $<$ & 11 & $\|$ & $<$ & 11 & $\|$ & $<$ & 11 & 11 & $<$ & 11 & $\| 1$ & $<$ & 10 & $\|$ & $<$ & 10 & II & $<$ & 10 & $\|$ & $<$ & 11 & $\|$ \\
\hline 4-Chlorophenyl phenyl ether & $<$ & 10 & $\|$ & $<$ & 11 & $\|$ & $<$ & 11 & $\|$ & $<$ & 11 & $\|$ & $<$ & 11 & $\|$ & $<$ & 10 & $\|$ & $<$ & 10 & $\|$ & $<$ & 10 & II & $<$ & 11 & $\|$ \\
\hline 4-Nitrophenol & $<$ & 50 & $\|$ & $<$ & 55 & $\|$ & $<$ & 55 & "I & $<$ & 55 & $\|$ & $<$ & 55 & $\|$ & |< & 50 & $\|$ & $<$ & 50 & "I & $<$ & 50 & $\|$ & $<$ & 55 & $\|$ \\
\hline Acenaphthene & $<$ & 10 & $\|$ & $<$ & 11 & $\|$ & $<$ & 11 & $\|$ & $<$ & 11 & II & $<$ & 11 & $\|$ & $<$ & 10 & $\|$ & $<$ & 10 & $\|$ & $<$ & 10 & $\|$ & $<$ & 11 & $\|$ \\
\hline Acenaphthylene & $<$ & 10 & $\|$ & $<$ & 11 & II & $<$ & 11 & "I & $<$ & 11 & $\|$ & $<$ & 11 & /l & $<$ & 10 & "I & $<$ & 10 & /I & $<$ & 10 & $\|$ & $<$ & 11 & II \\
\hline Anthracene & $<$ & 10 & $\|$ & $<$ & 11 & $\|$ & $<$ & 11 & II & $<$ & 11 & $\|$ & $<$ & 11 & $\|$ & $<$ & $10^{\circ}$ & $\|$ & & 1.19 & $\mathrm{~J} / \mathrm{B} /$ & $<$ & 10 & $\|$ & $<$ & 11 & $\|$ \\
\hline Benzo(a)anthracene & $<$ & 10 & II & $<$ & 11 & II & $<$ & 11 & $\|$ & $<$ & 11 & /I & $<$ & 11 & $\|$ & $<$ & 10 & $\|$ & $<$ & 10 & $\|$ & $<$ & 10 & $\|$ & $<$ & 11 & $\|$ \\
\hline Benzo(a)pyrene & $<$ & 10 & 11 & $<$ & 11 & II & $<$ & 11 & II & $<$ & 11 & $\|$ & $<$ & 11 & 11 & $<$ & 10 & 11 & $<$ & 10 & "I & $<$ & 10 & II & $<$ & 11 & $\|$ \\
\hline Benzo(b) fluoranthene & $<$ & 10 & II & $<$ & 11 & II & $<$ & 11 & " & $<$ & 11 & $/ 1$ & $<$ & 11 & $\|$ & $<$ & 10 & $\|$ & $<$ & 10 & II & $<$ & 10 & /l & $<$ & 11 & $\|$ \\
\hline Benzo $(g, h, i)$ perylene & $<$ & 10 & II & $<$ & 11 & II & $<$ & 11 & II & $<$ & 11 & /I & $<$ & 11 & $\|$ & $<$ & 10 & /I & $<$ & 10 & II & $<$ & 10 & $\|$ & $<$ & 11 & $\|$ \\
\hline Benzo(k)nuoranthene & $<$ & 10 & $\|$ & $<$ & 11 & II & $<$ & 11 & "I & $<$ & 11 & $\|$ & $<$ & 11 & $\|$ & $<$ & 10 & II & e & 10 & "I & $<$ & 10 & $\|$ & |< & 11 & $\|$ \\
\hline Benzoic acid & $<$ & 50 & II & $<$ & 55 & $\|$ & $<$ & 55 & $\|$ & $<$ & 55 & $\|$ & $<$ & 55 & $\|$ & $<$ & 50 & $\|$ & $<$ & 50 & II & $<$ & 50 & $\|$ & $<$ & 55 & $\|$ \\
\hline Benzyl alcohol & $<$ & 10 & II & $<$ & 11 & $\|$ & $<$ & 11 & $\|$ & $<$ & 11 & /I & $<$ & 11 & II & $<$ & 10 & II & $<$ & 10 & "I & $<$ & 10 & $\|$ & $<$ & 11 & $\|$ \\
\hline Bis(2-chloroethoxy) methane & $<$ & 10 & $\|$ & $<$ & 11 & $\|$ & $<$ & 11 & $\|$ & $<$ & 11 & $\|$ & $<$ & 11 & $\|$ & $<$ & 10 & $\|$ & $<$ & 10 & $\|$ & $<$ & 10 & $\|$ & $<$ & 11 & /I \\
\hline Bis(2-chloroethyl) ether & $<$ & 10 & $\|$ & $<$ & 11 & /I & $<$ & 11 & II & $<$ & 11 & $\|$ & $<$ & 11 & $\|$ & $<$ & 10 & $\|$ & $<$ & 10 & "I & $<$ & 10 & $\|$ & $<$ & 11 & "I \\
\hline Bis(2-chloroisopropyl) ether & $<$ & 10 & /I & $<$ & 11 & $\|$ & $<$ & 11 & II & $<$ & 11 & $\|$ & $<$ & 11 & $\|$ & $<$ & 10 & $\|$ & $<$ & 10 & $\|$ & $<$ & 10 & $\|$ & $<$ & 11 & /I \\
\hline Bis(2-ethylhexyl) phthalate & $<$ & 10 & $/ 1$ & $<$ & 11 & $\|$ & $<$ & 11 & II & $<$ & 11 & $1 /$ & $<$ & $\mathbb{1 1}$ & $\|$ & $<$ & 10 & $\|$ & $<$ & 10 & II & & 10 & /l & $<$ & 11 & $\|$ \\
\hline Butylbenzyl phthalate & $<$ & 10 & /I & $<$ & 11 & $\|$ & $<$ & 11 & II & $<$ & 11 & $/ /$ & $<$ & 11 & $\|$ & $<$ & 10 & $\|$ & & 1.42 & $\mathrm{~J} / \mathrm{B} /$ & $<$ & 10 & /I & $<$ & 11 & $\|$ \\
\hline Chrysene & $<$ & 10 & $\|$ & $<$ & 11 & $\|{ }^{\circ}$ & $<$ & 11 & II & $<$ & 11 & $\|$ & $<$ & il & $\|$ & $<$ & 10 & $\|$ & $<$ & 10 & $\|$ & $<$ & 10 & "I & $<$ & 11 & $\|$ \\
\hline Di-n-butyl phthalate & $<$ & 10 & 11 & $<$ & 11 & /I & $<$ & 11 & /I & $<$ & 11 & $\|$ & $<$ & 11 & $\|$ & $<$ & 10 & $\|$ & $<$ & 10 & $I V I$ & $<$ & 10 & $N I$ & $<$ & 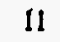 & $\|$ \\
\hline Di-n-octyl phthalate & $<$ & 10 & II & $<$ & 11 & $\|$ & $<$ & 11 & /I & $<$ & 11 & $\|$ & $<$ & 11 & $\|$ & $<$ & 10 & $\|$ & $<$ & 10 & II & $<$ & 10 & $/ 1$ & $<$ & 11 & $\|$ \\
\hline Dibenz(a,h)anthracene & $<$ & 10 & II & $<<$ & 11 & 11 & $<$ & 11 & II & $1 \leq$ & il & 11 & $1<$ & 11 & 11 & $<$ & 10 & II & $<$ & 10 & II & $<$ & 10 & 11 & $<$ & 11 & II \\
\hline
\end{tabular}


TABLE 6.17

SEMI-VOLATILE ORGANIC COMPOUNDS

IN SURFACE WATER

D-AREA BURNING/RUBBLE PITS

SAVANNAH RIVER SITE

\begin{tabular}{|c|c|c|c|c|c|c|c|c|c|c|c|c|c|c|c|c|c|c|c|c|c|c|c|c|c|c|c|}
\hline \multirow[b]{2}{*}{ Melhod 8270(UG/L) } & \multirow{2}{*}{\multicolumn{3}{|c|}{$\begin{array}{c}\text { D4313101 } \\
50594 \\
\end{array}$}} & \multicolumn{3}{|c|}{$\begin{array}{c}\text { D4313201 } \\
50594 \\
\end{array}$} & \multicolumn{3}{|c|}{$\begin{array}{c}\text { D4313301 } \\
50594 \\
\end{array}$} & \multicolumn{3}{|c|}{$\begin{array}{c}\mathrm{D} 4313401 \\
50594 \\
\end{array}$} & \multicolumn{3}{|c|}{$\begin{array}{c}\mathrm{D} 4313501 \\
50594 \\
\end{array}$} & \multicolumn{3}{|c|}{$\begin{array}{c}\mathrm{D} 4313601 \\
50594 \\
\end{array}$} & \multirow{2}{*}{\multicolumn{3}{|c|}{$\begin{array}{c}\mathrm{D} 4313701 \\
50594 \\
\end{array}$}} & \multicolumn{3}{|c|}{$\begin{array}{c}\mathrm{D} 4313801 \\
50594 \\
\end{array}$} & \multicolumn{3}{|c|}{$\begin{array}{c}\mathrm{D} 4313901 \\
50594\end{array}$} \\
\hline & & & & & & & & & & & & & & & & & & & & & & & & & & & \\
\hline Dibenzofuran & $<$ & 10 & $\|$ & $<$ & 11 & $\|$ & $<$ & 11 & $\|$ & $<$ & 11 & $\|$ & $<$ & 11 & $\|$ & $<$ & 10 & $\|$ & k & 10 & $\|$ & $<$ & 10 & $\|$ & $<$ & 11 & $\|$ \\
\hline Diethyl phthalate & |< & 10 & $\|$ & |< & 11 & $\|$ & $<$ & 11 & $\|$ & $<$ & 11 & $\|$ & $<$ & 11 & $\|$ & $<$ & 10 & $\|$ & $<$ & 10 & $\|$ & $<$ & 10 & II & $<$ & 11 & /l \\
\hline Dimethyl phthalate & $<$ & 10 & $\|$ & $<$ & 11 & $\|$ & $<$ & 11 & 11 & $<$ & 11 & $\|$ & $<$ & 11 & $\|$ & $<$ & 10 & $\|$ & $<$ & 10 & $\|$ & $<$ & 10 & $\|$ & $<$ & 11 & $\|$ \\
\hline Fluoranthene & $<$ & 10 & $\|$ & $<$ & 11 & $\|$ & $<$ & 11 & /I & $<$ & 11 & $\|$ & $<$ & 11 & 11 & $<$ & 10 & $\|$ & $<$ & 10 & $\|$ & $<$ & 10 & $\|$ & $<$ & 11 & $\|$ \\
\hline Fluorene & $<$ & 10 & $\|$ & $<$ & II & $\|$ & $<$ & 11 & $\|$ & $<$ & 11 & $\|$ & $<$ & 11 & $\|$ & $<$. & 10 & $\|$ & $<$ & 10 & $\|$ & $<$ & 10 & $\|$ & $<$ & $: 11$ & $\|$ \\
\hline Hexachlorobenzene & $<$ & 10 & $/ /$ & $<$ & 11 & $\|$ & $<$ & 11 & $\|$ & $<$ & 11 & /l & $<$ & 11 & $\|$ & $<$ & 10 & $\|$ & $<$ & 10 & $\|$ & $<$ & 10 & $\|$ & $<$ & 11 & /I \\
\hline Hexachlorobutadiene & $<$ & 10 & $\|$ & $<$ & 11 & $\|$ & $<$ & 11 & $\|$ & $<$ & 11 & $\|$ & $<$ & 11 & $\|$ & $<$ & 10 & $\|$ & $<$ & 10 & $\|$ & $<$ & 10 & "I & $<$ & 11 & $\|$ \\
\hline Hexachlorocyclopentadiene & $<$ & 10 & $\|$ & $<$ & 11 & $/ /$ & $<$ & 11 & /I & $<$ & 11 & $\|$ & $<$ & 11 & $\|$ & $<$ & 10 & $\|$ & $<$ & 10 & $\|$ & $<$ & 10 & $\|$ & $<$ & 11 & $\|$ \\
\hline Hexachloroethane & $<$ & 10 & $\|$ & $<$ & 11 & $\|$ & $<$ & 11 & $\|$ & $<$ & 11 & $\|$ & $<$ & 11 & $\|$ & $<$ & 10 & $\|$ & $<$ & 10 & $\ddot{\|}$ & $<$ & 10 & $\|$ & $<$ & 11 & $\|$ \\
\hline Indeno( $(1,2,3 \cdot c, d)$ pyrene & $<$ & 10 & $/ /$ & $<$ & 11 & $\|$ & $<$ & 11 & $/ /$ & $<$ & 11 & $\|$ & $<$ & 11 & $\ddot{\|}$ & $<$ & 10 & 11 & $<$ & 10 & $\|$ & $<$ & 10 & $\|$ & $<$ & ii & "I \\
\hline Isophorone & $<$ & 10 & $\|$ & $<$ & 11 & $\|$ & $<$ & 11 & $\|$ & $<$ & 11 & /I & $<$ & 11 & $\|$ & $<$ & 10 & /l & $<$ & 10 & $\|$ & $<$ & 10 & $\|$ & $<$ & $i 1$ & 11 \\
\hline m-Nitroaniline & $<$ & 50 & $/ 1$ & $<$ & 55 & $\|$ & $<$ & 55 & $\|$ & $<$ & 55 & $/ 1$ & $<$ & 55 & $\|$ & $<$ & 50 & $\|$ & $<$ & 50 & $\|$ & $<$ & 50 & $\|$ & $<$ & 55 & "I \\
\hline N-Nitrosodiphenylamine & $<$ & 10 & $\|$ & $<$ & 11 & $\|$ & $<$ & 11 & $\|$ & $<$ & 11 & $\|$ & $<$ & 11 & $\|$ & $<$ & 10 & $\|$ & $<$ & 10 & $\|$ & $<$ & 10 & $\|$ & $<$ & 11 & /I \\
\hline N-Nitrosodipropylamine & $<$ & 10 & $\|$ & $<$ & 11 & $\|$ & $<$ & 11 & $/ 1$ & $<$ & 11 & $\|$ & $<$ & 11 & $\|$ & $<$ & 10 & $\|$ & $<$ & 10 & $\|$ & $<$ & 10 & $\|$ & $<$ & 11 & $\|$ \\
\hline Naphthalene & $<$ & 10 & $\|$ & $<$ & 11 & $\|$ & $<$ & 11 & $\|$ & $<$ & 11 & $\|$ & $<$ & 11 & $\|$ & $<$ & 10 & $\|$ & $<$ & 10 & $\|$ & $<$ & 10 & $\|$ & $<$ & 11 & $\|$ \\
\hline Nitrobenzene & $<$ & 10 & $\|$ & $<$ & 11 & $\|$ & $<$ & 11 & $\|$ & $<$ & 11 & $\|$ & $<$ & 11 & $\|$ & $<$ & 10 & $\|$ & $<$ & 10 & $\|$ & $<$ & 10 & /I & $<$ & 11 & $\|$ \\
\hline o-Cresol (2-Methylphenol) & $<$ & 10 & $\|$ & $<$ & 11 & $\|$ & $<$ & 11 & /l & $<$ & 11 & $\|$ & $<$ & 11 & $/ 1$ & $<$ & 10 & $\|$ & $<$ & 10 & $\|$ & $<$ & 10 & $\|$ & $<$ & 11 & $\|$ \\
\hline o-Nitroaniline & $<$ & 50 & $\|$ & $<$ & 55 & $\|$ & $<$ & 55 & $/ l$ & $<$ & 55 & /I & $<$ & 55 & $/ /$ & $<$ & 50 & $\|$ & $<$ & 50 & $\|$ & k & 50 & $\|$ & $<$ & 55 & $\ddot{~}$ \\
\hline p-Cresol (4-Methylphenol) & $<$ & 10 & $\|$ & $<$ & 11 & $\|$ & $<$ & 11 & $\|$ & $<$ & 11 & $\|$ & $<$ & 11 & $/ l$ & $<$ & 10 & $\|$ & $<$ & 10 & II & $<$ & 10 & $\|$ & $<$ & 11 & /l \\
\hline p-Nitroaniline & $<$ & 50 & $\|$ & $<$ & 55 & $\|$ & $<$ & 55 & $\|$ & $<$ & 55 & $/ /$ & $<$ & 55 & $\|$ & $<$ & 50 & $\|$ & $<$ & 50 & $\|$ & $<$ & 50 & "I & $<$ & 55 & $\|$ \\
\hline Pentachlorophenol & $<$ & 50 & $\|$ & $<$ & 55 & $\|$ & $<$ & 55 & $\|$ & $<$ & 55 & $\|$ & $<$ & 55 & $\|$ & $<$ & 50 & $\|$ & $<$ & 50 & $\|$ & < & 50 & II & $<$ & 55 & 11 \\
\hline Phenanthrene & $<$ & 10 & $\|$ & $<$ & 11 & $\|$ & $<$ & 11 & $\|$ & $<$ & 11 & $\|$ & $<$ & 11 & $/ I$ & $<$ & 10 & "I & $<$ & 10 & $\|$ & $1<$ & 10 & II & $<$ & 11 & II \\
\hline Phenol & $<$ & 10 & $\|$ & $<$ & 11 & $\|$ & $<$ & 11 & $/ 1$ & $<$ & 11 & $\|$ & $<$ & 11 & $/ I$ & $<$ & 10 & II & $<$ & 10 & $\|$ & $<$ & 10 & 11 & $<$ & 11 & /I \\
\hline Pyrene & $<$ & 10 & II & $<$ & 11 & II & $<$ & 11 & II & $<<$ & 11 & $\|$ & L & 11 & $\|$ & $<<$ & 10 & II & < & 10 & 11 & < & 10 & II & $<$ & 11 & II \\
\hline
\end{tabular}


TABLE 6.18

INORGANICS, ORGANIC INDICATORS, AND RADIONUCLIDE PARAMETERS

IN SURFACE WATER

D-AREA BURNING/RUBBLE PITS

SAVANNAH RIVER SITE

\begin{tabular}{|c|c|c|c|c|c|c|c|c|c|c|c|c|c|c|c|c|c|c|c|c|c|c|c|c|c|}
\hline $\begin{array}{l}\text { Sample Name } \\
\text { Sample Date }\end{array}$ & $\begin{array}{r}4311 \\
607 \\
\end{array}$ & & & $\begin{array}{r}4311 \\
607 \\
\end{array}$ & & & $\begin{array}{r}4311 \\
607 \\
\end{array}$ & & & $\begin{array}{r}4311 \\
607 \\
\end{array}$ & & & $\begin{array}{r}4311 \\
607 \\
\end{array}$ & & $\begin{array}{r}4312 \\
6079 \\
\end{array}$ & & & $\begin{array}{r}4312 \\
505 \\
\end{array}$ & & & $\begin{array}{r}\text { D4312 } \\
505 \\
\end{array}$ & & & $\begin{array}{r}4313 \\
505 \\
\end{array}$ & \\
\hline BCRA and Targel Metals (UGR) & & & & & & & & & & & & & & & & & & & & & & & & & \\
\hline Arsenic, total recoverable & 10 & /l & $<$ & 10 & "l & $<$ & 10 & $\|$ & $<$ & 10 & /l & $<$ & 10 & $\|$ & 144 & $\|$ & k & 1.7 & $\|$ & k & 1.7 & $/ /$ & | $<$ & 1.7 & $\|$ \\
\hline Barium, total recoverable & $<200$ & $/ /$ & $<$ & 200 & $\|$ & $<$ & 200 & "l & $<$ & 200 & "I & $<$ & 200 & $\|$ & $<\quad 200$ & $\ddot{\prime \prime}$ & & 9.8 & $\ddot{~}$ & & 14.3 & $\|$ & & 15.1 & $\ddot{l}$ \\
\hline Cadmium, total recoverable & $<$ & J/L & $<$ & 5 & $\mathrm{~J} / \mathrm{J}$ & $<$ & 5 & $\mathrm{~s} / \mathrm{L}$ & $<$ & 5 & $\mathrm{~J} / \mathrm{L}$ & |< & 5 & $\mathrm{~J} / \mathrm{L}$ & 1< & $\mathrm{J} / \mathrm{L}$ & k & 3.3 & "I & $<<$ & 3.3 & /I & |< & 3.3 & "l \\
\hline Chromium, total recoverable & 10 & $\|$ & $<$ & 10 & $\|$ & $<$ & 10 & /I & $1<$ & 10 & II & $<$ & 10 & $\|$ & 14.2 & /I & $<<$ & 3.3 & /I & $<$ & 3.3 & /I & $<$ & 3.3 & II \\
\hline Copper, total recoverable & 25 & $\mathrm{~J} / \mathrm{U}$ & $<$ & 25 & $\mathrm{~J} / \mathrm{J}$ & $<$ & 25 & $\mathrm{~J} / \mathrm{L}$ & $<$ & 25 & $\mathrm{~J} / \mathrm{L}$ & $<$ & 25 & $\mathrm{~J} / \mathrm{L}$ & $<25$ & $\mathrm{~J} / \mathrm{W}$ & $<$ & 3.3 & $\|$ & $<$ & 3.3 & $\|$ & & $: 3.3$ & $\|$ \\
\hline Iron, total recoverable & 531 & II & & 292 & II & & 292 & II & & 617 & II & & 398 & II & 269000 & $\|$ & & 116 & $\|$ & & 137 & $\|$ & & .184 & $\|$ \\
\hline Lead, total recoverable & 3 & $\|$ & $<$ & 3 & $\|$ & $<$ & 3 & /l & $<$ & 3 & /I & & 5.3 & $\|$ & 17 & $\|$ & $<$ & 0.5 & $\|$ & $<$ & 0.5 & "I & |< & 0.5 & II \\
\hline Manganese, total recoverable & 1380 & "I & & 533 & "l & & 317 & /I & & 760 & /I & & 254 & $\|$ & 1160 & "l & & 56 & II & & 72.7 & /I & & 67 & II \\
\hline Mercury, total & 0.2 & $\|$ & $<$ & 0.2 & II & $<$ & 0.2 & /I & $<$ & 0.2 & II & $<$ & 0.2 & $\|$ & 0.823 & "I & $<$ & 0.067 & $\|$ & |< & 0.067 & /I & $<$ & 0.067 & $\|$ \\
\hline Nickel, total recoverable & 40 & $\|$ & $<$ & 40 & $\|$ & $<$ & 40 & /I & $<$ & 40 & "I & $<$ & 40 & $\|$ & 40 & $\|$ & $<$ & 11.7 & $\|$ & $<$ & 11.7 & $\|$ & l< & 11.7 & $\|$ \\
\hline Selenium, total recoverable & $<$ & "I & $<$ & 5 & "I & $<$ & 5 & /I & $<$ & 5 & II & | & 5 & II & $<<$ & $\|$ & $k<$ & 1.7 & $\|$ & l< & 1.7 & $\|$ & | $<$ & 1.7 & $\|$ \\
\hline Silver, total recoverable & 11 & $\|$ & $<$ & 10 & "I & $<$ & 10 & II & $<$ & 10 & "I & $<$ & 10 & "I & 10 & $\|$ & $<$ & 0.7 & $\|$ & $<$ & 0.7 & $\|$ & $<$ & 0.7 & $\|$ \\
\hline Inorennic Paramelers (UG/) & " & & & & & & & & & & & & & & & & & & & & & & & & \\
\hline Chloride & 5.12 & $\|$ & & NA & & & 5.11 & $\|$ & & $\mathrm{NA}$ & & & 5.09 & /I & 10.1 & /I & & 10800 & $\|$ & & 10600 & $\|$ & & 10600 & $\|$ \\
\hline Fluoride & 0.1 & $\|$ & & NA & & $<$ & 0.1 & II & & NA & & $\mid<$ & 0.1 & /I & 0.137 & "I & 1< & 72.7 & $\|$ & $<<$ & 72.7 & $\|$ & $1<$ & 72.7 & II \\
\hline Nitrate as nitrogen & 0.209 & /I & & NA & & & 0.21 & /I & & NA & & & 1.05 & "I & $<0.02$ & $N I$ & $<$ & 83.3 & $\|$ & $<<$ & 83.3 & $\|$ & & 212 & "I \\
\hline Sodium, total recoverable & 7990 & /l & & 7950 & /l & & 7860 & /I & & 7870 & $\|$ & & 7690 & /I & 8430 & /I & & 6930 & "I & & 9930 & /I & & 10300 & "II \\
\hline Sulfate & 6.42 & $\|$ & & NA & & & 6.99 & "I & & NA & & & 7.04 & $\|$ & 177 & /l & & 9740 & 11 & & 9650 & $\|$ & & 9450 & "I \\
\hline Oreanic Indic & & & & & & & & & & & & & & & & & & & & & & & & & \\
\hline Total dissolved solids & 56 & 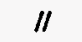 & & 65 & $J / Q /$ & & 54 & /I & & 53 & /l & & 68 & $\|$ & 638 & /I & & 47000 & $\|$ & & 47000 & $\|$ & & 57000 & $\|$ \\
\hline Total organic carbon & 1.94 & /I & & 1.55 & $\|$ & & 2.03 & "I & & 2.33 & /I & & 2.23 & /I & 166 & /I & & 2030 & $\|$ & & 1840 & $\|$ & & 2220 & $\|$ \\
\hline Total organic halogens & 7.17 & $\mathrm{~J} / \mathrm{Q} /$ & & NA & & & 10.9 & $\mathrm{~J} / \mathrm{Q} /$ & & NA & & & 29.2 & $\mathrm{~J} / \mathrm{Q} /$ & 365 & $\mathrm{~J} / \mathrm{Q} /$ & $<$ & 3330 & $\|$ & $<$ & 3330 & $\|$ & $<$ & 3330 & $\|$ \\
\hline Total suspended solids & 10 & $\|$ & $<$ & 5 & $J / Q /$ & & 8 & II & & 9 & II & & 9 & II & 2810 & II & $<$ & 1670 & $\|$ & $<$ & 1670 & $\|$ & $<$ & 1670 & $\prime \prime$ \\
\hline Radionuclide Parnme & & & & & & & & & & & & & & & & & & & & & & & & & \\
\hline Gross alpha & 0.8 & /I & $<$ & 0.7 & $/ 1$ & $<$ & 0.8 & $\|$ & & 4 & $\|$ & & 2.4 & $\|$ & 30.4 & $\|$ & k & 0.33 & $/ 1$ & $<$ & 0.33 & $\|$ & $<$ & 0.4 & $\|$ \\
\hline Nonvolatile beta & $<$ & NI & $1<$ & 0.8 & NII & & 3.6 & $I \mathrm{~V} I$ & & 5.5 & NII & $<$ & 1.1 & $N I$ & 54 & $N I$ & & 1.7 & $\|$ & & 1.8 & $\|$ & $<$ & 1.3 & $\|$ \\
\hline Radium, total alpha-emitting & $<\quad 0.1$ & $\|$ & $<$ & 0.1 & $\|$ & $<$ & 0.1 & 11 & $<$ & 0.1 & 11 & & 0.5 & 11 & 0.6 & 11 & $<$ & 0.09 & $\|$ & $<$ & 0.13 & $\|$ & $<$ & 0.2 & $\|$ \\
\hline Tritium (PC/ML) & 680.2 & II & & 1105 & II & & 467.1 & II & & 806 & "I & & 832.5 & /I & 1488 & /I & & 0.62 & II & & 0.4 & /l & & 0.54 & $\|$ \\
\hline
\end{tabular}


TABLE 6.18

INORGANICS, ORGANIC INDICATORS, AND RADIONUCLIDE PARAMETERS

1 IN SURFACE WATER

REA BURNING/RUBBLE PITS

SAVANNAH RIVER SITE

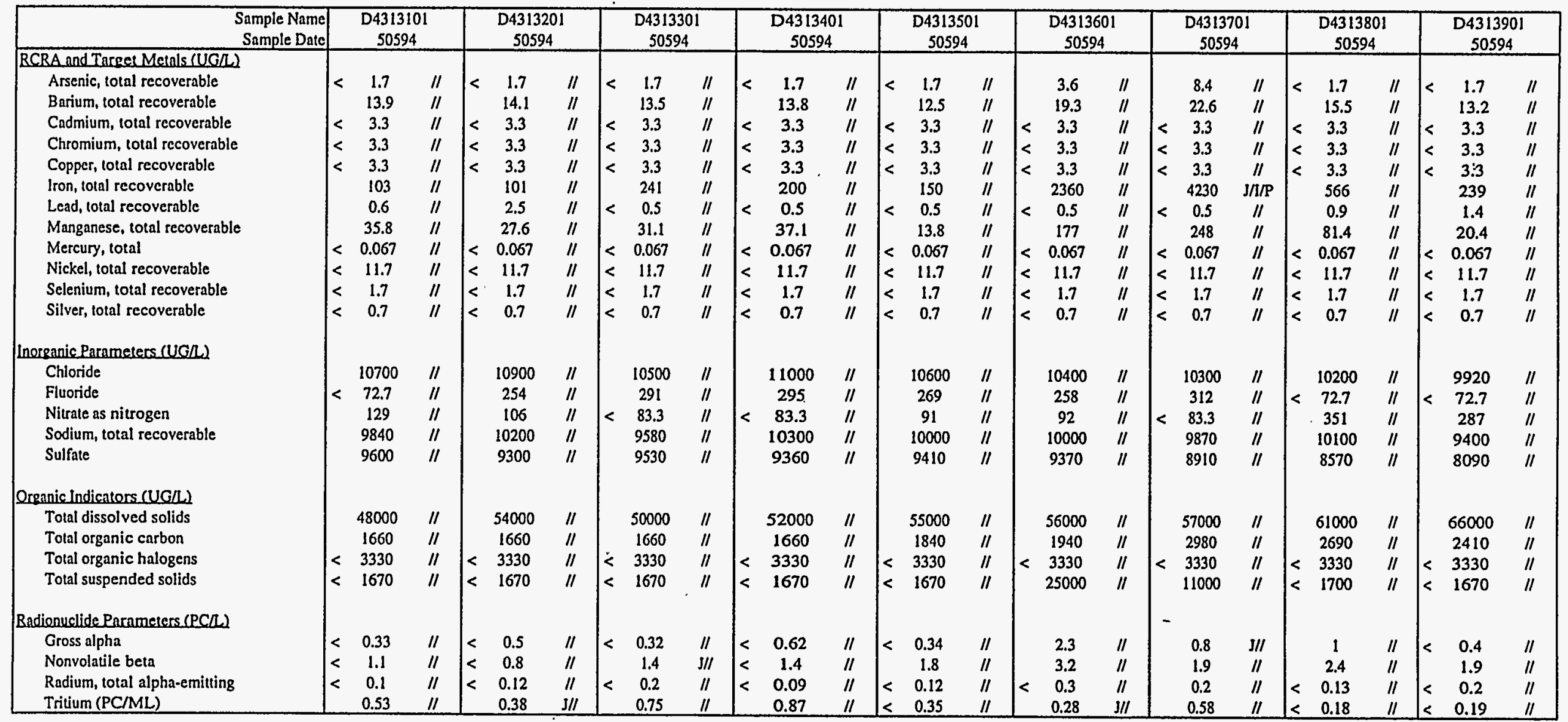


TABLE 6.19

VOLATILE ORGANIC COMPOUNDS
- IN SEDIMENT

D-AREA BURNING/RUBBLE PITS

SAVANNAH RIVER SITE

\begin{tabular}{|c|c|c|c|c|c|c|c|c|c|c|c|c|c|c|c|c|c|c|c|c|}
\hline \multirow{3}{*}{\begin{tabular}{|cr} 
& $\begin{array}{r}\text { Sample Name } \\
\text { Sample Date }\end{array}$ \\
Meihod 8240(UG/KG) \\
$1,1,1-$ Trichloroethane
\end{tabular}} & \multicolumn{2}{|c|}{$\begin{array}{c}\text { D43112102/WA } \\
60793 \\
\end{array}$} & \multicolumn{3}{|c|}{$\begin{array}{c}\text { D431 2202/WA } \\
60793 \\
\end{array}$} & \multicolumn{3}{|c|}{$\begin{array}{c}\text { D431 2302/WA } \\
60893 \\
\end{array}$} & \multicolumn{3}{|c|}{\begin{tabular}{|c|} 
D431 2402/WA \\
60893 \\
\end{tabular}} & \multicolumn{3}{|c|}{$\begin{array}{c}\text { D431 2502NWA } \\
60893 \\
\end{array}$} & \multicolumn{3}{|c|}{$\begin{array}{c}\text { D431 2602/WA } \\
60793 \\
\end{array}$} & \multicolumn{3}{|c|}{$\begin{array}{c}\text { D431 2702/WA } \\
60893\end{array}$} \\
\hline & & & & & & & & & & & & & & & & & & & & \\
\hline & 6.5 & II & $<$ & 6.5 & $\|$ & $<$ & 6.2 & $/ Y I$ & $<$ & 6.1 & $\Gamma \mathrm{Y}$ & $<$ & 5.95 & $r I$ & $<$ & 7 & il & $<$ & 6.4 & $I Y I$ \\
\hline 1,1,2,2-Tetrachlorocthane & 6.5 & $\|$ & $<$ & 6.5 & $\|$ & $<$ & 6.2 & $/ Y I$ & $<$ & 6.1 & $/ Y I$ & $<$ & 5.95 & $r Y I$ & $<$ & 7 & $\|$ & $<$ & 6.4 & $I Y I$ \\
\hline 1,1,2-Trichlorocthane & 6.5 & II & $<$ & 6.5 & $\|$ & $<$ & 6.2 & $\mid Y I$ & & 9.05 & $|Y|$ & $<$ & 5.95 & $r Y I$ & $<$ & 7 & "I & $<$ & 6.4 & $I Y I$ \\
\hline 1,!-Dichlorocthane & 6.5 & $\|$ & $<$ & 6.5 & II & $<$ & 6.2 & $\mid Y I$ & $<$ & 6.1 & $r Y I$ & $<$ & 5.95 & $\Gamma \mathrm{Yl}$ & $<$ & 7 & $\|$ & $<$ & 6.4 & $/ Y I$ \\
\hline 1,1-Dichloroethylene & 6.5 & $\|$ & $<$ & 6.5 & $\|$ & $<$ & 6.2 & $|Y|$ & $<$ & 6.1 & $\Gamma \mathrm{Yl}$ & $<$ & 5.95 & $r Y I$ & $<$ & 7 & $\|$ & $<$ & 6.4 & $/ \mathrm{YI}$ \\
\hline 1,2-Dichloroethane & 6.5 & "I & $<$ & 6.5 & /I & & 6.45 & $I Y I$ & & 6.5 & $\mid Y I$ & $<$ & 5.95 & IYI & $<$ & 7 & II & $<$ & 6.4 & $M I$ \\
\hline 1,2-Dichloroethylene & 6.5 & $\|$ & $<$ & 6.5 & $/ /$ & $<$ & 6.2 & $/ Y I$ & $<$ & 6.1 & $r I$ & $<$ & 5.95 & $\Gamma \mathrm{YI}$ & $<$ & 7 & $\|$ & $<$ & 6.4 & $I Y I$ \\
\hline 1,2-Dichloropropane & 6.5 & II & $<$ & 6.5 & /I & $<$ & 6.2 & $/ Y I$ & $<$ & 6.1 & $N I$ & $<$ & 5.95 & $I Y I$ & $<$ & 7 & II & $<$ & 6.4 & $\pi Y$ \\
\hline 2-Chloroethyl vinyl ether & 13 & "I & $<$ & 13 & "I & & NA & & & NA & & & NA & & $<$ & 14 & $\|$ & & NA & \\
\hline 2-Hexanone & 13 & $\|$ & $<$ & 13 & /I & $<$ & 12.4 & IYI & $<$ & 12.2 & $\Gamma Y$ & $<$ & 11.9 & $r Y$ & $<$ & 14 & /I & $<$ & 12.8 & $/ \mathrm{YI}$ \\
\hline Acetone & $<\quad 8.21$ & $\mathrm{~J} / \mathrm{N} /$ & $<$ & 15.2 & $N I$ & $<$ & 12.6 & /YVI & & 25.6 & IYVI & $<$ & 11.9 & IYVI & $<$ & 14 & $I N I$ & $<$ & 12.8 & IYVI \\
\hline Benzene & 6.5 & /I & $<$ & 6.5 & II & $<$ & 6.2 & $/ Y I$ & $<$ & 6.1 & $\Gamma I$ & $<$ & 5.95 & $\Gamma \mathrm{Y} \mid$ & $<$ & 7 & 11 & $<$ & 6.4 & IYI \\
\hline Bromodichloromethane & 6.5 & II & $<$ & 6.5 & $\|$ & $<$ & 6.2 & $\Gamma I$ & $<$ & 6.1 & $\Gamma I$ & $<$ & 5.95 & $\Gamma I$ & $<$ & 7 & "I & $<$ & 6.4 & $M I$ \\
\hline Bromoform & 6.5 & $\|$ & $<$ & 6.5 & II & $<$ & 6.2 & $/ \mathrm{YI}$ & $<$ & 6.1 & 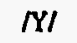 & $<$ & 5.95 & $I Y I$ & $1<$ & 7 & $\|$ & $<$ & 6.4 & $\mid Y I$ \\
\hline Bromomethane (Methy & 13 & II & $<$ & 13 & $\|$ & $<$ & 12.4 & IYI & $<$ & 12.2 & $r Y I$ & $<$ & 11.9 & $r y$ & 15 & 14 & $\|$ & $<$ & 12.8 & $/ Y I$ \\
\hline Carbon disulfide & 6.5 & $\|$ & $<$ & 6.5 & $/ l$ & $<$ & 6.2 & $I Y I$ & $<$ & 6.1 & $r Y I$ & $<$ & 5.95 & IYI & $<$ & 7 & $\|$ & $<$ & 6.4 & $I Y I$ \\
\hline Carbon tetrachloride & 6.5 & II & $<$ & 6.5 & "I & $<$ & 6.2 & $|Y|$ & $<$ & 6.1 & $\Gamma I^{\prime}$ & $<$ & 5.95 & MYI & $<$ & 7 & II & |< & 6.4 & $/ Y I$ \\
\hline Chlorobenzene & 6.5 & II & $<$ & 6.5 & $/ l$ & $<$ & 6.2 & $I \mathrm{Y} I$ & $<$ & 6.1 & $\kappa I$ & $<$ & 5.95 & IYI & $<$ & 7 & $\|$ & $<$ & 6.4 & $/ Y I$ \\
\hline Chloroethane & 13 & II & $<$ & 13 & $/ l$ & $<$ & 12.4 & IYI & $<$ & 12.2 & $N I$ & $<$ & 11.9 & IYI & $<$ & 14 & II & $<$ & 12.8 & $/ Y I$ \\
\hline Chloroethene (Vinyl chloride) & 13 & $\|$ & $<$ & 13 & $/ /$ & $<$ & 12.4 & /YI & $<$ & 12.2 & $M I$ & $<$ & 11.9 & $r Y I$ & $\mid<$ & 14 & $\|$ & $<$ & 12.8 & $/ Y I$ \\
\hline Chloroform & 6.5 & "I & $<$ & 6.5 & $\|$ & $<$ & 6.2 & $/ Y \prime$ & $<$ & 6.1 & $\mid Y I$ & $<$ & 5.95 & IYI & $<$ & 7 & $\|$ & $1<$ & 6.4 & $/ \mathrm{Y} I$ \\
\hline Chloromethane (Methyl chloride) & 13 & $\|$ & $<$ & 13 & $\|$ & $<$ & 12.4 & $I Y I$ & $<$ & 12.2 & $I Y I$ & $<$ & 11.9 & $r Y I$ & $<$ & 14 & $\|$ & $<$ & 12.8 & $\mid \mathrm{YI}$ \\
\hline cis-1,3-Dichloropropene & 6.5 & II & $<$ & 6.5 & $\|$ & $<$ & 6.2 & IYI & $<$ & 6.1 & $\Gamma Y$ & $<$ & 5.95 & $r Y I$ & $<$ & 7 & II & $1<$ & 6.4 & $/ \mathrm{Y} /$ \\
\hline Dibromochloromethane & 6.5 & "I & $<$ & 6.5 & /l & $<$ & 6.2 & $/ Y I$ & $<$ & 6.1 & $\Gamma /$ & $<$ & 5.95 & $r Y I$ & $<$ & 7 & $\|$ & $<$ & 6.4 & $|Y|$ \\
\hline Dichloromethane (Methylene chloride) & $\mid<48.5$ & NI & $<$ & 39.2 & $N I$ & $<$ & 8.42 & IYVI & $<$ & 6.1 & $\Gamma Y I$ & $<$ & 5.95 & rYVI & $<$ & 39.7 & $\mathrm{NI}$ & $<$ & 6.55 & $\Gamma Y V I$ \\
\hline Ethylbenzene & 6.5 & II & $<$ & 6.5 & /I & $<$ & 6.2 & IYI & $<$ & 6.1 & $r Y$ & $<$ & 5.95 & rYI & $<$ & 7 & $\|$ & $<$ & 6.4 & IYI \\
\hline Methy! ethyl ketone & 13 & II & $<$ & 13 & $\|$ & $<$ & 12.4 & IYI & $<$ & 12.2 & $/ Y I$ & $<$ & 11.9 & $/ Y I$ & $<$ & 14 & $\|$ & $<$ & 12.8 & $/ Y I$ \\
\hline Methyl isobutyl ketone & 13 & /I & $<$ & 13 & $/ l$ & $<$ & 12.4 & $/ Y I$ & $<$ & 12.2 & $M I$ & $<$ & 11.9 & /YI & $<$ & 14 & $\|$ & $<$ & 12.8 & MI \\
\hline Styrene & 6.5 & II & $<$ & 6.5 & $\|$ & $<$ & 6.2 & IYI & $<$ & 6.1 & $r Y I$ & $<$ & 5.95 & $r Y I$ & $<$ & 7 & $\|$ & $<$ & 6.4 & $/ Y I$ \\
\hline Tetrachloroethylene & 6.5 & II & $<$ & 6.5 & $\prime \prime$ & $<$ & 6.2 & $/ Y I$ & $<$ & 6.1 & NI & $<$ & 5.95 & IYI & $<$ & 7 & /l & $<$ & 6.4 & $|Y|$ \\
\hline Toluene & 2.55 & $\mathrm{~J} / /$ & & 12.2 & $\|$ & $<$ & 6.2 & $I \mathrm{YI}$ & $<$ & 6.1 & $\Gamma Y$ & $<$ & 5.95 & $\Pi \mathrm{Y}$ & $<$ & 7 & II & $<$ & 6.4 & $r Y I$ \\
\hline trans-1,3-Dichloropropene & 6.5 & "I & | & 6.5 & II & $<$ & 6.2 & $/ Y I$ & $<$ & 6.1 & $/ \mathrm{KI}$ & $<$ & 5.95 & $\mid \mathrm{XI}$ & $<$ & 7 & "I & | $<$ & 6.4 & $|Y|$ \\
\hline Trichloroethylene & 6.5 & /I & $<$ & 6.5 & $/ /$ & $<$ & 6.2 & $/ Y I$ & $<$ & 6.1 & YII & $<$ & 5.95 & IYI & $<$ & 7 & II & $<$ & 6.4 & IYI \\
\hline Vinyl acetate & 13 & II & $<$ & 13 & $\|$ & $<$ & 12.4 & $/ Y I$ & $<$ & 12.2 & $M I$ & $<$ & 11.9 & $\Gamma /$ & k & 14 & $\|$ & $<$ & 12.8 & $I Y I$ \\
\hline Xylenes & 6.5 & 11 & $<$ & 6.5 & II & $1<$ & 6.2 & $I Y I$ & $1<$ & 6.1 & $M I$ & $1<$ & 5.95 & $r$ & $1<$ & 7 & II & $1<$ & 6.4 & $r Y I$ \\
\hline
\end{tabular}


TABLE 6.20

VOLATILE ORGANIC COMPOUNDS

IN SEDIMENT

D-AREA BURNING/RUBBLE PITS

SAVANNAH RIVER SITE

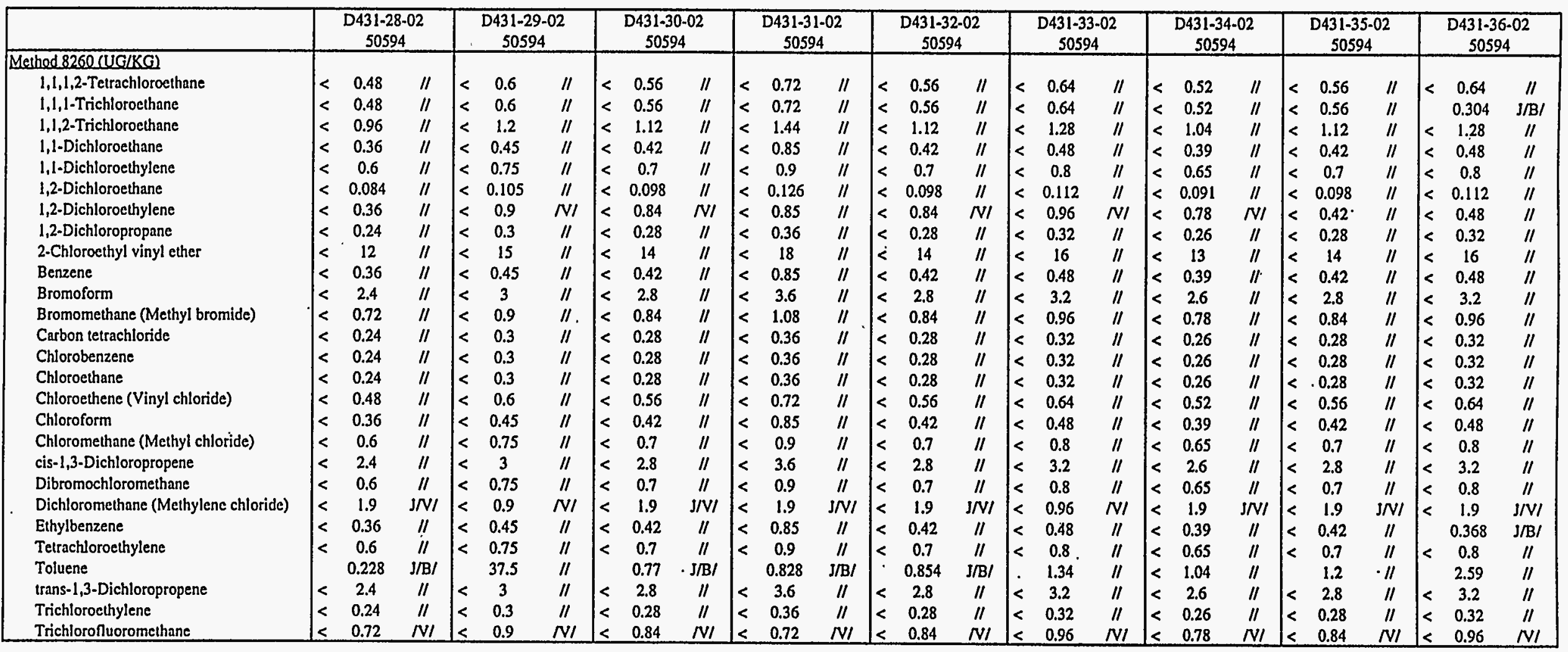


TABLE 6.20

VOLATILE ORGANIC COMPOUNDS -

IN SEDIMENT

D-AREA BURNING/RUBBLE PITS

SAVANNAH RIVER SITE

\begin{tabular}{|c|c|c|c|}
\hline \multirow[b]{2}{*}{ Method 8260 (UG/KG) } & \multicolumn{3}{|c|}{$\begin{array}{c}\text { D431-37-02 } \\
50594\end{array}$} \\
\hline & & & \\
\hline 1,1,1,2-Tetrachloroethane & $<$ & 0.56 & /I \\
\hline 1,1,1-Trichloroethane & $\mid<$ & 0.56 & $\|$ \\
\hline 1,1,2-Trichloroethane & $<$ & 1.12 & $\|$ \\
\hline 1,1-Dichloroethane & $<$ & 0.42 & $\|$ \\
\hline 1,1-Dichloroethylene & $<$ & 0.7 & $\|$ \\
\hline 1,2-Dichlorocthane & $<$ & 0.098 & $\|$ \\
\hline 1,2-Dichloroethylene & $<$ & 0.84 & $\mathrm{NI}$ \\
\hline 1,2-Dichloropropane & $<$ & 0.28 & II \\
\hline 2-Chloroethyl vinyl ether & $<$ & 14 & $/ 1$ \\
\hline Benzene & $<$ & 0.42 & $\|$ \\
\hline Bromoform & $<$ & 2.8 & $/ /$ \\
\hline Bromomethane (Methyl bromide) & $<$ & 0.84 & $\|$ \\
\hline Carbon tetrachloride & $<$ & 0.28 & /I \\
\hline Chlorobenzene & $<$ & 0.28 & $\|$ \\
\hline Chloroethane & $<$ & 0.28 & $\|$ \\
\hline Chloroethene (Vinyl chloride) & $<$ & 0.56 & $\|$ \\
\hline Chloroform & |< & 0.42 & "I \\
\hline Chloromethane (Methyl chloride) & $<$ & 0.7 & 11 \\
\hline cis-1,3-Dichloropropene & $<$ & 2.8 & II \\
\hline Dibromochloromethane & $<$ & 0.7 & II \\
\hline Dichloromethane (Methylene chloride) & |< & 1.9 & $\mathrm{~J} / \mathrm{NI}$ \\
\hline Ethylbenzene & & 0.364 & $\|$ \\
\hline Tetrachloroethylene & $<$ & 0.7 & /I \\
\hline Toluene & & 0.588 & $\mathrm{~J} / \mathrm{B} /$ \\
\hline trans-1,3-Dichloropropene & $<$ & 2.8 & "I \\
\hline Trichloroethylene & $<$ & 0.28 & /I \\
\hline Trichlorofluoromethane & $<$ & 0.84 & NI \\
\hline
\end{tabular}


TABLE 6.21

SEMI-VOLATILE ORGANIC COMPOUNDS

IN SEDIMENT

D-AREA BURNING/RUBBLE PITS

SAVANNAH RIVER SITE

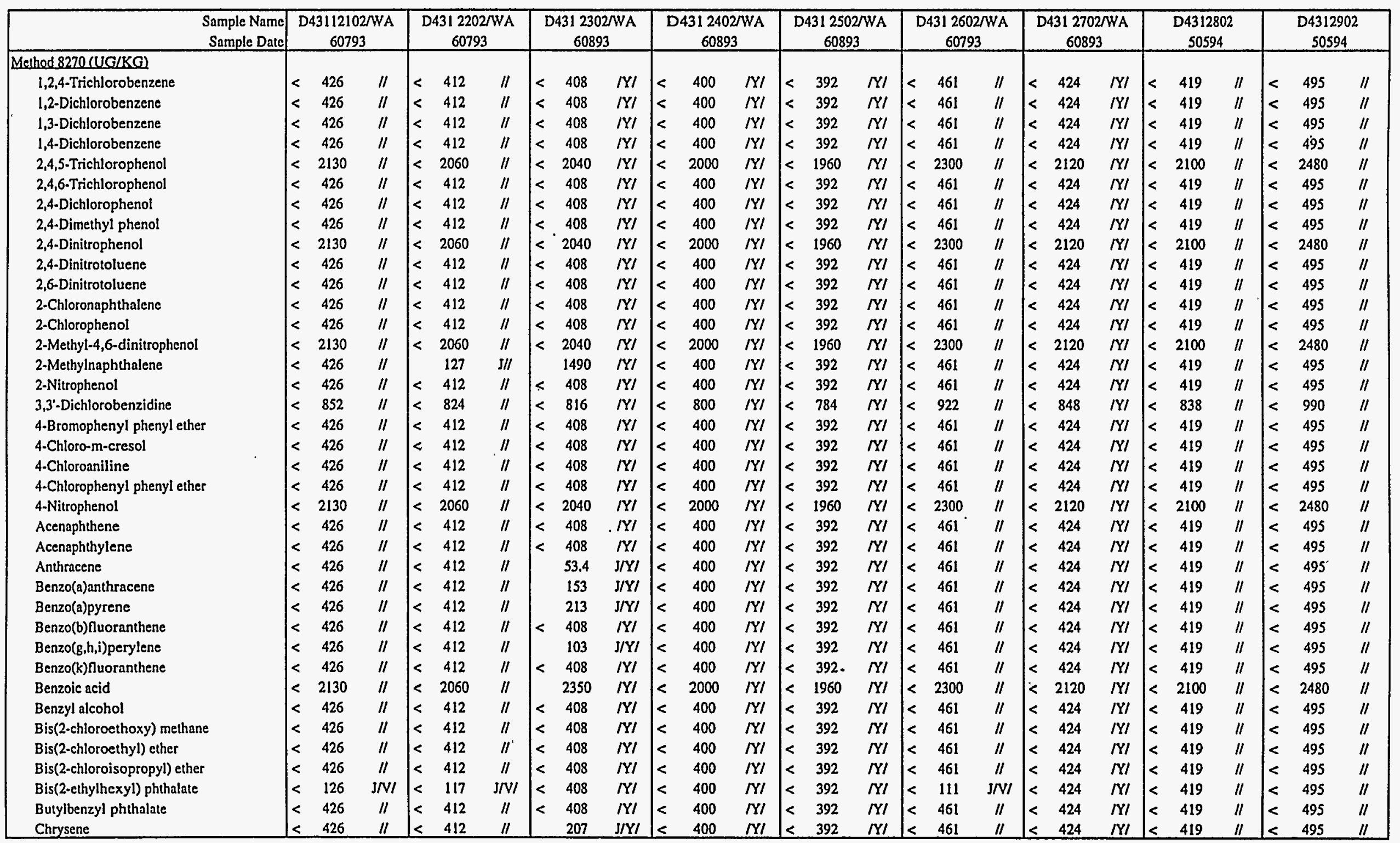


TABLE 6.21

SEMI-VOLATILE ORGANIC COMPOUNDS

IN SEDIMENT

D-AREA BURNING/RUBBLE PITS

SAVANNAH RIVER SITE

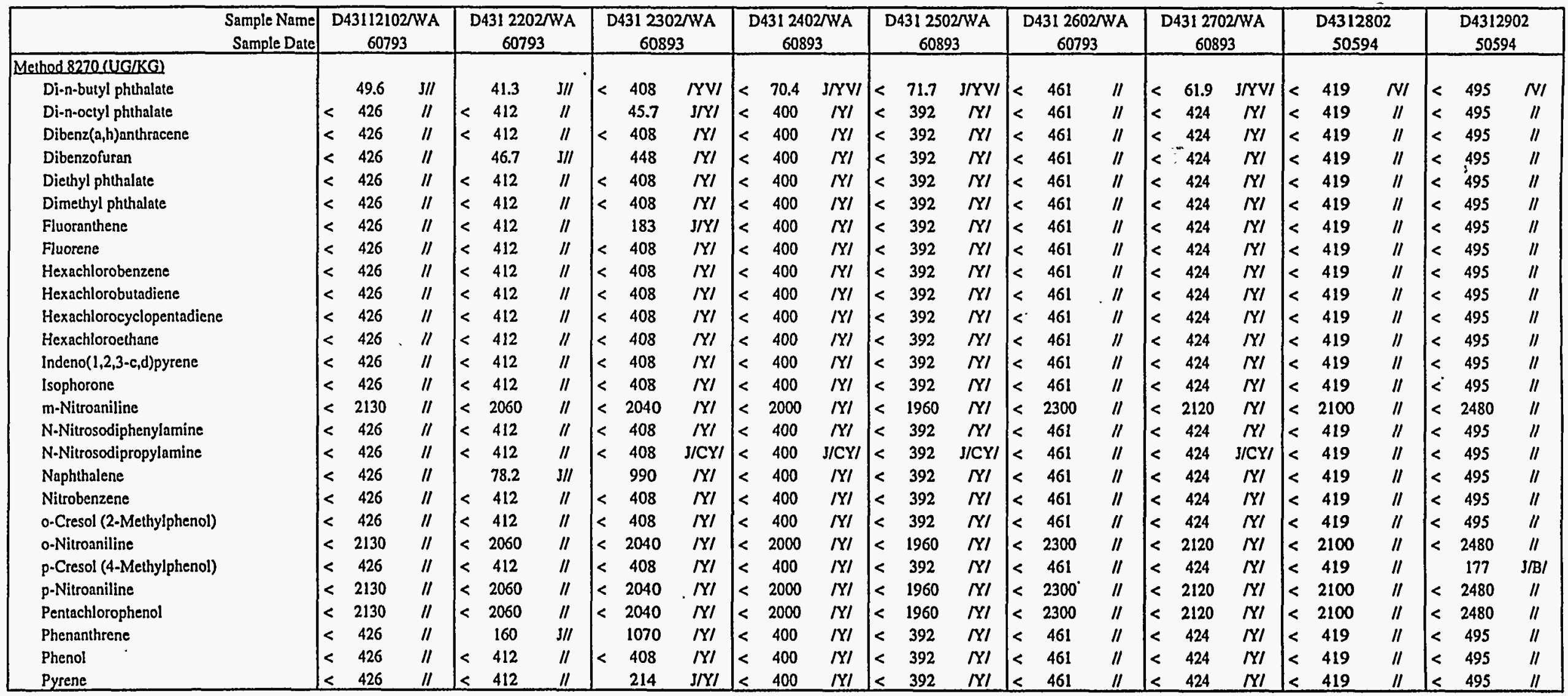


TABLE 6.21

SEMI-VOLATILE ORGANIC COMPOUNDS

IN SEDIMENT

D-AREA BURNING/RUBBLE PITS

SAVANNAH RIVER SITE

\begin{tabular}{|c|c|c|c|c|c|c|c|c|c|c|c|c|c|c|c|c|c|c|c|c|c|c|c|c|}
\hline $\begin{array}{r}\text { Sample Name } \\
\text { Sample Date }\end{array}$ & & $\begin{array}{r}\mathrm{D} 43130 \\
5059 \\
\end{array}$ & & & $\begin{array}{r}\mathrm{D} 4313 \\
5055 \\
\end{array}$ & & & $\begin{array}{r}\mathrm{D} 4313 \\
505 ! \\
\end{array}$ & & & $\begin{array}{r}\mathrm{D} 4313 \\
5059 \\
\end{array}$ & & & $\begin{array}{r}\text { D4313 } \\
5055 \\
\end{array}$ & & & $\begin{array}{r}\text { D4313 } \\
5059 \\
\end{array}$ & & & $\begin{array}{r}\mathrm{D} 4313 \\
505 \\
\end{array}$ & & & $\begin{array}{r}\mathrm{D} 43137 \\
5059 \\
\end{array}$ & \\
\hline Methed 8270 (UG/KG) & & & & & & & & & & & & & & & & & & & & & & & & \\
\hline 1,2,4-Trichlorobenzene & $<$ & 457 & $\|$ & $<$ & 626 & $/ /$ & $<$ & 483 & $\|$ & $<$ & 536 & $\|$ & $<$ & 416 & "l & $<$ & 477 & $\|$ & $<$ & 537 & $\|$ & $<$ & 466 & /l \\
\hline 1,2-Dichlorobenzene & |< & 457 & /I & $<$ & 626 & II & $<$ & 483 & "I & | & 536 & "I & $<$ & 416 & /I & $<$ & 477 & II & $<$ & 537 & II & $<$ & 466 & /I \\
\hline 1,3-Dichlorobenzene & $<$ & 457 & $\|$ & $<$ & 626 & /I & $<$ & 483 & /I & $<$ & 536 & /l & $<$ & 416 & II & $<$ & 477 & II & $<$ & 537 & II & $<$ & 466 & /l \\
\hline 1,4-Dichlorobenzene & $<$ & 457 & $\|$ & $<$ & 626 & /I & $<$ & 483 & $\|$ & $<$ & 536 & / & $<$ & 416 & $\|$ & $<$ & 477 & II & $<$ & 537 & "I & $<$ & 466 & $\| 1$ \\
\hline 2,4,5-Trichlorophenol & $<$ & 2280 & $\|$ & $<$ & 3130 & $\|$ & $<$ & 2420 & $\|$ & $<$ & 2680 & "I & $<$ & 2080 & $\prime \prime$ & $<$ & 2380 & "I & $<$ & 2680 & "I & $<$ & 2330 & /l \\
\hline 2,4,6-Trichlorophenol & $<$ & 457 & $\|$ & $<$ & 626 & $\|$ & $<$ & 483 & $\|$ & $<$ & 536 & $\|$ & $<$ & 416 & "I & $<$ & 477 & II & $<$ & 537 & II & $<$ & 466 & /I \\
\hline 2,4-Dichlorophenol & $<$ & 457 & $/ 1$ & $<$ & 626 & /I & $<$ & 483 & $\|$ & $<$ & 536 & $\|$ & $<$ & 416 & "I & $<$ & 477 & "I & $<$ & 537 & /I & $<$ & 466 & /l \\
\hline 2,4-Dimethyl phenol & $<$ & 457 & $\|$ & $<$ & 626 & /l & $<$ & 483 & $\|$ & $<$ & 536 & II & $<$ & 416 & "l & $<$ & 477 & "I & $<$ & 537 & "I & $<$ & 466 & "l \\
\hline 2,4-Dinitrophenol & $<$ & 2280 & $\|$ & $<$ & 3130 & /l & $<$ & 2420 & $\|$ & $<$ & 2680 & "I & $<$. & 2080 & II & $<$ & 2380 & "l & $<$ & 2680 & "I & $<$ & 2330 & /I \\
\hline 2,4-Dinitrotoluene & $1<$ & 457 & $\|$ & $<$ & 626 & $\|$ & $<$ & 483 & $\|$ & $<$ & 536 & $\|$ & $<$ & 416 & /l & $<$ & 477 & "I & $1<$ & 537 & "I & $<$ & 466 & "l \\
\hline 2,6-Dinitrotoluene & $<$ & 457 & $\|$ & $<$ & 626 & $\|$ & $<$ & 483 & $\|$ & $<$ & 536 & "I & $<$ & 416 & $\|$ & $<$ & 477 & $\|$ & $<$ & 537 & $\|$ & $<$ & 466 & $\|$ \\
\hline 2-Chloronaphthalene & $<$ & 457 & $\|$ & $<$ & 626 & II & $<$ & 483 & $\|$ & $<$ & 536 & $\|$ & $<$ & 416 & "I & $<$ & 477 & II & $<$ & 537 & "I & $<$ & 466 & "I \\
\hline 2-Chlorophenol & $<$ & 457 & $\|$ & $<$ & 626 & $/ /$ & $<$ & 483 & $\|$ & $<$ & 536 & "I & $<$ & 416 & $\|$ & $<$ & 477 & $\|$ & $<$ & 537 & $\|$ & $<$ & 466 & $\|$ \\
\hline 2-Methyl-4,6-dinitrophenol & $<$ & 2280 & $\|$ & $<$ & 3130 & /I & $<$ & 2420 & $\|$ & $<$ & 2680 & $\|$ & $<$ & 2080 & /l & $<$ & 2380 & "I & $<$ & 2680 & "I & $<$ & 2330 & /l \\
\hline 2-Methylnaphthalene & $<$ & 457 & $\|$ & $<$ & 626 & /I & $<$ & 483 & $\|$ & & 452 & $\mathrm{~J} / \mathrm{B} /$ & & 153 & $\mathrm{~J} / \mathrm{B} /$ & . & 1330 & $\|$ & & 324 & $\mathrm{I} / \mathrm{B} /$ & $<$ & 466 & /l \\
\hline 2-Nitrophenol & $<$ & 457 & $\|$ & $<$ & 626 & $! !$ & $<$ & 483 & II & $<$ & 536 & "l & $<$ & 416 & /l & $<$ & 477 & ll & $<$ & 537 & /l & $<$ & 466 & /l \\
\hline 3,3'-Dichlorobenzidine & $<$ & 914 & $\|$ & $<$ & 1250 & il & $<$ & 966 & $/ /$ & $<$ & 1070 & $\|$ & $<$ & 832 & "l & $<$ & 954 & "I & $<$ & 1070 & $\|$ & $<$ & 932 & "l \\
\hline 4-Bromophenyl phenyl ether & $<$ & 457 & 11 & $<$ & 626 & $\|$ & $<$ & 483 & II & $<$ & 536 & $\|$ & $<$ & 416 & $\|$ & $<$ & 477 & $\|$ & $<$ & 537 & $\|$ & $<$ & 466 & /l \\
\hline 4-Chloro-m-cresol & $<$ & 457 & $\|$ & $<$ & 626 & $\|$ & $<$ & 483 & II & $<$ & 536 & $\|$ & $<$ & 416 & /l & $<$ & 477 & $\|$ & $<$ & 537 & "I & $<$ & 466 & $\|$ \\
\hline 4-Chloroaniline & $<$ & 457 & $\|$ & $<$ & 626 & $\|$ & $<$ & 483 & "I & $<$ & 536 & $\prime \prime$ & $<$ & 416 & $\|$ & $<$ & 477 & $\|$ & $<$ & 537 & $\|$ & $<$ & 466 & 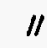 \\
\hline 4-Chlorophenyl phenyl ether & $<$ & 457 & $\|$ & $<$ & 626 & $\|$ & $<$ & 483 & 11 & $<$ & 536 & $\|$ & $<$ & 416 & "l & $<$ & 477 & $\|$ & $<$ & 537 & "l & $<$ & 466 & $\|$ \\
\hline 4-Nitrophenol & $<$ & 2280 & $\|$ & $<$ & 3130 & $\|$ & $<$ & 2420 & /I & $<$ & 2680 & "l & $<$ & 2080 & "l & $<$ & 2380 & $\|$ & $<$ & 2680 & /l & $<$ & 2330 & $\|$ \\
\hline Acenaphthene & $<$ & 457 & $\|$ & $<$ & 626 & $\|$ & $<$. & 483 & $\|$ & $<$ & 536 & $\|$ & $<$ & 416 & II & $<$ & .477 & "I & $<$ & 537 & "I & $<$ & 466 & $\|$ \\
\hline Acenaphthylene & $<$ & 457 & $1 /$ & $<$ & 626 & /I & $<$ & 483 & II & $<$ & 536 & "l & $<$ & 416 & /l & $<$ & 477 & "l & $<$ & 537 & /l & $<$ & 466 & $\|$ \\
\hline Anthracene & $<$ & 457 & /I & $<$ & 626 & II & $<$ & 483 & II & $<$ & 536 & II & $<$ & 416 & "I & & 53.4 & $\mathrm{~J} / \mathrm{B} /$ & |< & 537 & $\|$ & $<$ & 466 & $\|$ \\
\hline Benzo(a)anthracene & $<$ & 457 & $\|$ & $<$ & 626 & $\|$ & $<$ & 483 & "I & & 75.2 & $\mathrm{~J} / \mathrm{B} /$ & $<$ & 416 & "l & & 224 & $\mathrm{~J} / \mathrm{B} /$ & & 65.4 & $J / B /$ & $<$ & 466 & $\|$ \\
\hline Benzo(a)pyrene & $<$ & 457 & $\|$ & i & 626 & II & $<$ & 483 & "I & $<$ & 536 & II & $<$ & 416 & $\cdot \|$ & & 90.1 & $\mathrm{~J} / \mathrm{B} /$ & $<$ & 537 & $\|$ & $<$ & 466 & $\|$ \\
\hline Benzo(b) fluoranthene & $<$ & 457 & $\|$ & $<$ & 626 & $\|$ & $<$ & 483 & "I & & 49.2 & $\mathrm{~J} / \mathrm{B} /$ & $<$ & 416 & $\|$ & & 137 & $\mathrm{~J} / \mathrm{B} /$ & & 68 & $\mathrm{~J} / \mathrm{B} /$ & $<$ & 466 & $\|$ \\
\hline Benzo $(g, h, i)$ perylene & $<$ & 457 & .11 & $<$ & 626 & /l & $<$ & 483 & /l & $<$ & 536 & /l & $<$ & 416 & "I & & 129 & $\mathrm{~J} / \mathrm{B} /$ & $<$ & 537 & "I & $<$ & 466 & $\|$ \\
\hline Benzo(k)fluoranthene & $<$ & 457 & $" / 1$ & $<$ & 626 & /l & $<$ & 483 & $\|$ & & 54.1 & $\mathrm{~J} / \mathrm{B} /$ & $<$ & 416 & $\|$ & & 93.3 & $\mathrm{~J} / \mathrm{B} /$ & & 43.4 & $\mathrm{~J} / \mathrm{B} /$ & $<$ & 466 & $\|$ \\
\hline Benzoic acid & $<$ & $2280^{\circ}$ & $\|$ & $<$ & 3130 & $\|$ & $<$ & 2420 & $\|$ & $<$ & 2680 & $\|$ & $<$ & 2080 & "I & & 300 & $\mathrm{~J} / \mathrm{B} /$ & & 817 & $\mathrm{~J} / \mathrm{B} /$ & $<$ & 2330 & $\|$ \\
\hline Benzyl alcohol & $<$ & 457 & $\|$ & $<$ & 626 & $\|$ & $<$ & 483 & $\|$ & $<$ & 536 & $\|$ & $<$ & 416 & /I & $<$ & 477 & II & $<$ & 537 & $\|$ & $<$ & 466 & $\|$ \\
\hline Bis(2-chloroethoxy) methane & $<$ & 457 & $\|$ & $<$ & 626 & II & $<$ & 483 & II & $<$ & 536 & $\|$ & $<$ & 416 & $\|$ & $<$ & 477 & /l & $<$ & 537 & "I & $<$ & 466 & $\|$ \\
\hline Bis(2-chloroethyl) ether & $<$ & 457 & "I & $<$ & 626 & $\|$ & $<$ & 483 & "I & $<$ & 536 & /I & $<$ & 416 & "I & $<$ & 477 & "I & $<$ & 537 & "l & $<$ & 466 & $\|$ \\
\hline Bis(2-chloroisopropyl) ether & $<$ & 457 & $\|$ & $<$ & 626 & II & $<$ & 483 & II & $<$ & 536 & $\|$ & $<$ & 416 & "I & $<$ & 477 & $\|$ & $<$ & 537 & $\|$ & $<$ & 466 & $\|$ \\
\hline Bis(2-ethylhexyl) phthalate & $<$ & 457 & $\|$ & $<$ & 626 & /I & $<$ & 483 & II & $<$ & 536 & "I & $<$ & 416 & IVI & $<$ & 477 & II & $<$ & 537 & II & $1<$ & 466 & $/ /$ \\
\hline Butylbenzyl phthalate & $<$ & 457 & $\|$ & $<$ & 626 & $\|$ & $<$ & 483 & $\|$ & $<$ & 536 & $\|$ & $<$ & 416 & $\|$ & $<$ & 477 & $\|$ & $<$ & 537 & $\|$ & $<$ & 466 & $\|$ \\
\hline Chrysene & $1<$ & 457 & II & $1<$ & 626 & II & $1<$ & 483 & 11 & & 175 & $\mathrm{~J} / \mathrm{B} /$ & $1<$ & 416 & 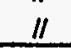 & & 256 & $\mathrm{~J} / \mathrm{B} /$ & & 234 & $\mathrm{~J} / \mathrm{B} /$ & $1<$ & 466 & II \\
\hline
\end{tabular}


TABLE 6.21

SEMI-VOLATILE ORGANIC COMPOUNDS

IN SEDIMENT

D-AREA BURNING/RUBBLE PITS

SAVANNAH RIVER SITE

\begin{tabular}{|c|c|c|c|c|c|c|c|c|c|c|c|c|c|c|c|c|c|c|c|c|c|c|c|c|c|}
\hline \multirow{3}{*}{\multicolumn{2}{|c|}{\begin{tabular}{|r}
$\begin{array}{r}\text { Sample Name } \\
\text { Sample Date }\end{array}$ \\
$\begin{array}{l}\text { Method 8270(UG/KG) } \\
\text { Di-n-butyl phthalate }\end{array}$
\end{tabular}}} & \multicolumn{3}{|c|}{$\begin{array}{c}\text { D4313002 } \\
50594 \\
\end{array}$} & \multicolumn{3}{|c|}{$\begin{array}{c}\text { D4313102 } \\
50594 \\
\end{array}$} & \multicolumn{3}{|c|}{$\begin{array}{c}\mathrm{D} 4313202 \\
50594\end{array}$} & \multicolumn{3}{|c|}{$\begin{array}{c}D 4313302 \\
50594\end{array}$} & \multicolumn{3}{|c|}{$\begin{array}{c}D 4313402 \\
50594\end{array}$} & \multicolumn{3}{|c|}{$\begin{array}{c}\text { D4313502 } \\
50594\end{array}$} & \multicolumn{3}{|c|}{$\begin{array}{c}D 4313602 \\
50594\end{array}$} & \multicolumn{3}{|c|}{$\begin{array}{c}D 4313702 \\
50594 \\
\end{array}$} \\
\hline & & & & & & & & & & & & & & & & & & & & & & & & & \\
\hline & & $<$ & 457 & $N I$ & $<$ & 626 & IVI & $<$ & 483 & $N I$ & $<$ & 536 & $N I$ & $<$ & 416 & $N I$ & $<$ & 477 & $N I$ & $<$ & 537 & $N I$ & $<$ & 466 & $N I$ \\
\hline Di-n-octyl phthalate & & $<$ & 457 & II & $<$ & 626 & 11 & $<$ & 483 & II & $<$ & 536 & "I & k & 416 & "I & k & 477 & II & $<$ & 537 & /I & $<$ & 466 & II \\
\hline Dibenz(a,h)anthracene & & $<$ & 457 & $\|$ & $<$ & 626 & $\|$ & $<$ & 483 & /I & |< & 536 & II & |< & 416 & $\|$ & $<$ & 477 & /I & $<$ & 537 & /I & $<$ & 466 & $\|$ \\
\hline Dibenzofuran & & $<$ & 457 & $\|$ & $<$ & 626 & $\|$ & $<$ & 483 & II & & 137 & $\mathrm{~J} / \mathrm{B} /$ & & 49.8 & $\mathrm{~J} / \mathrm{B} /$ & & 407 & $\mathrm{~J} / \mathrm{B} /$ & & 113 & $\mathrm{~J} / \mathrm{B} /$ & $<$ & 466 & !! \\
\hline Diethyl phthalate & & $<$ & 457 & $\|$ & $<$ & 626 & "I & $<$ & 483 & 11 & $<$ & 536 & "I & $<$ & 416 & II & $<$ & 477 & $\|$ & $<$ & 537 & $\|$ & $<$ & 466 & il \\
\hline Dimethyl phthalate & & $<$ & 457 & II & $<$ & 626 & $\|$ & $<$ & 483 & $\|$ & $<$ & 536 & "I & $<$ & 416 & $\|$ & $<$ & 477 & $\|$ & $<$ & 537 &.$\prime \prime$ & $<$ & 466 & $\|$ \\
\hline Fluoranthene & & $<$ & 457 & $\|$ & $<$ & 626 & $\|$ & $<$ & 483 & $\|$ & & 82.2 & $\mathrm{~J} / \mathrm{B} /$ & $<$ & 416 & $\|$ & & 142 & $\mathrm{~J} / \mathrm{B} /$ & & 75 & $\mathrm{~J} / \mathrm{B} /$ & $<$ & 466 & "I \\
\hline Fluorene & & $<$ & 457 & 11 & $<$ & 626 & $\|$ & $<$ & 483 & "I & $<$ & 536 & /I & $<$ & 416 & $\|$ & $<$ & 477 & II & $<$ & 537 & /I & $<$ & 466 & II \\
\hline Hexachlorobenzene & & $<$ & 457 & "I & $<$ & 626 & $\|$ & $<$ & 483 & II & |< & 536 & /I & $<$ & 416 & $\|$ & $<$ & 477 & II & $<$ & 537 & $\|$ & $<$ & 466 & II \\
\hline Hexachlorobutadiene & & $<$ & 457 & $\|$ & $<$ & 626 & $\|$ & $<$ & 483 & $\|$ & $\mid<$ & 536 & II & $<$ & 416 & $\|$ & $<$ & 477 & II & $<$ & 537 & $\|$ & $<$ & 466 & $\|$ \\
\hline Hexachlorocyclopentadiene & & $<$ & 457 & $\|$ & $<$ & 626 & $\|$ & $<$ & 483 & $\|$ & $<$ & 536 & $\|$ & $<$ & 416 & $\|$ & $<$ & 477 & $\|$ & $<$ & 537 & $\|$ & $<$ & 466 & $\|$ \\
\hline Hexachloroethane & & $<$ & 457 & $\|$ & $<$ & 626 & $\|$ & $<$ & 483 & $\|$ & $<$ & 536 & "I & $<$ & 416 & "I & $<$ & 477 & $\|$ & $<$ & 537 & .11 & $<$ & 466 & $\|$ \\
\hline Indeno( $(1,2,3 \cdot c, d)$ pyrene & & $<$ & 457 & $/ 1$ & $<$ & 626 & $\|$ & $<$ & $483^{\circ}$ & $\|$ & $<$ & 536 & II & $<$ & 416 & $\|$ & $<$ & 477 & $\|$ & $<$ & 537 & $\prime \prime$ & $<$ & 466 & $\|$ \\
\hline Isophorone & & $<$ & 457 & 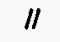 & $<$ & 626 & 11 & $<$ & 483 & $\|$ & $<$ & 536 & "I & $<$ & 416 & $\|$ & $<$ & 477 & $\|$ & $<$ & 537 & II & $<$ & 466 & $\|$ \\
\hline m-Nitroaniline & & $<$ & 2280 & $\|$ & $<$ & 3130 & $\|$ & $<$ & 2420 & $\|$ & $<$ & 2680 & $\|$ & $<$ & 2080 & II & $<$ & 2380 & $\|$ & $<$ & 2680 & $\|$ & $<$ & 2330 & $\|$ \\
\hline N-Nitrosodiphenylamine & & $<$ & 457 & $\|$ & $<$ & 626 & $\|$ & $<$ & 483 & $\|$ & $<$ & 536 & /I & $<$ & 416 & II & $<$ & 477 & $\|$ & $<$ & 537 & $\|$ & $<$ & 466 & II \\
\hline N-Nitrosodipropylamine & & $<$ & 457 & $\|$ & $<$ & 626 & $\|$ & $<$ & 483 & $\|$ & $<$ & 536 & II & $<$ & 416 & "I & $<$ & 477 & $\|$ & $<$ & 537 & $\|$ & $<$ & 466 & "I \\
\hline Naphthalene & & $<$ & 457 & $\|$ & $<$ & 626 & $\|$ & $<$ & 483 & 11 & & 259 & $\mathrm{~J} / \mathrm{B} /$ & & 98.2 & $\mathrm{~J} / \mathrm{B} /$ & & 735 & $\|$ & & 174 & $\mathrm{~J} / \mathrm{B} /$ & $<$ & 466 & $\|$ \\
\hline Nitrobenzene & & $<$ & 457 & $\|$ & $<$ & 626 & $\|$ & $<$ & 483 & $\|$ & $<$ & 536 & $\|$ & $1<$ & 416 & $\|$ & $<$ & 477 & II & $<$ & 537 & $\|$ & $<$ & 466 & $\|$ \\
\hline o-Cresol (2-Methylphenol) & & $<$ & 457 & $\|$ & $<$ & 626 & $\|$ & $<$ & 483 & $\|$ & $<$ & 536 & $\|$ & $<$ & 416 & $\|$ & $<$ & 477 & $\|$ & $<$ & 537 & /I & $<$ & 466 & $\|$ \\
\hline o-Nitroaniline & & $<$ & 2280 & $\|$ & $<$ & 3130 & $\|$ & $<$ & 2420 & /I & $<$ & 2680 & "I & $<$ & 2080 & /I & $<$ & 2380 & $\|$ & |< & 2680 & II & |< & 2330 & $\|$ \\
\hline p-Cresol (4-Methylphenol) & & $<$ & 457 & $\|$ & $<$ & 626 & II & $<$ & 483 & $\|$ & $\mid<$ & 536 & /1 & $<$ & 416 & II & $<$ & 477 & $\|$ & $<$ & 537 & $\|$ & $<$ & 466 & $\|$ \\
\hline p-Nitroaniline & & $<$ & 2280 & $\|$ & $<$ & 3130 & II & $<$ & 2420 & II & $\mid<$ & 2680 & II & k & 2080 & II & $<$ & 2380 & II & $<$ & 2680 & $\|$ & $<$ & 2330 & II \\
\hline Pentachlorophenol & & $<$ & 2280 & $\|$ & $<$ & 3130 & $\|$ & $<$ & 2420 & $\|$ & $1<$ & 2680 & /l & $<$ & 2080 & $\|$ & $<$ & 2380 & $\|$ & $<$ & 2680 & $\|$ & $<$ & 2330 & $\|$ \\
\hline Phenanthrene & & $<$ & 457 & $\|$ & $<$ & 626 & $\|$ & $<$ & 483 & $/ 1$ & & 416 & $\mathrm{~J} / \mathrm{B} /$ & & 117 & $\mathrm{~J} / \mathrm{B} /$ & & 1050 & $\|$ & & 362 & $\mathrm{~J} / \mathrm{B} /$ & $<$ & 466 & "I \\
\hline Phenol & & $<$ & 457 & $\|$ & $<$ & 626 & $\|$ & $<$ & 483 & /I & $<$ & 536 & $\|$ & $<$ & 416 & $\|$ & $<$ & 477 & $\|$ & $<$ & 537 & II & $<$ & 460 & $\|$ \\
\hline Pyrene & & $<$ & 457 & 11 & $<$ & 626 & 11 & $1<$ & 483 & 11 & & 128 & $\mathrm{~J} / \mathrm{B} /$ & $1<$ & 416 & II & & 234 & $\mathrm{~J} / \mathrm{B} /$ & & 172 & $\mathrm{~J} / \mathrm{B} /$ & $1<$ & 466 & $\|$ \\
\hline
\end{tabular}


TABLE 6.22

INORGANIC AND ORGANIC INDICATORS

IN SEDIMENT

D.AREA BURNING/RUBBLE PITS

SAVANNAH RIVER SITE

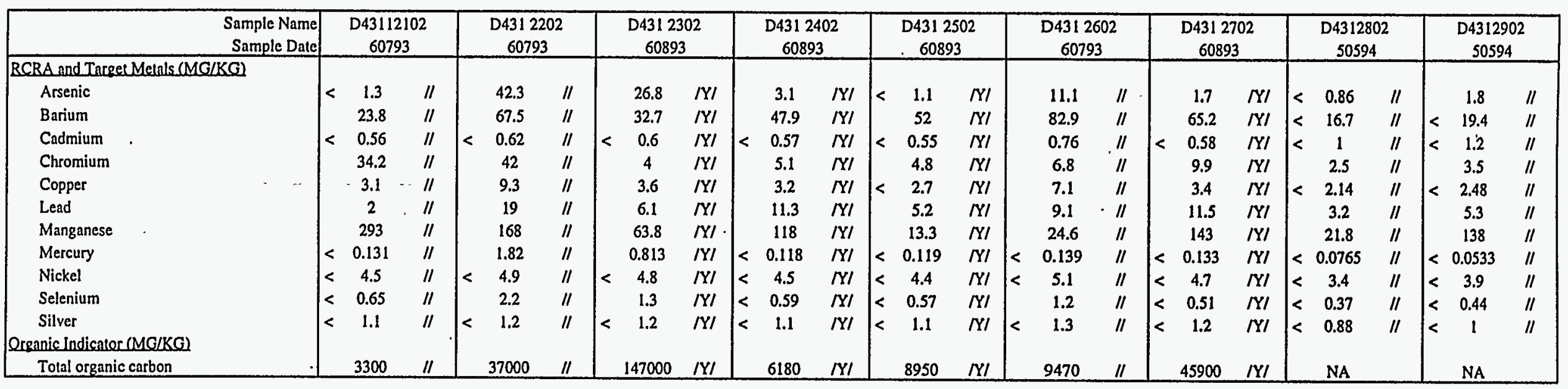


TABLE 6.22

INORGANIC AND ORGANIC INDICATORS

IN SEDIMENT

D.AREA BURNING/RUBBLE PITS

SAVANNAH RIVER SITE

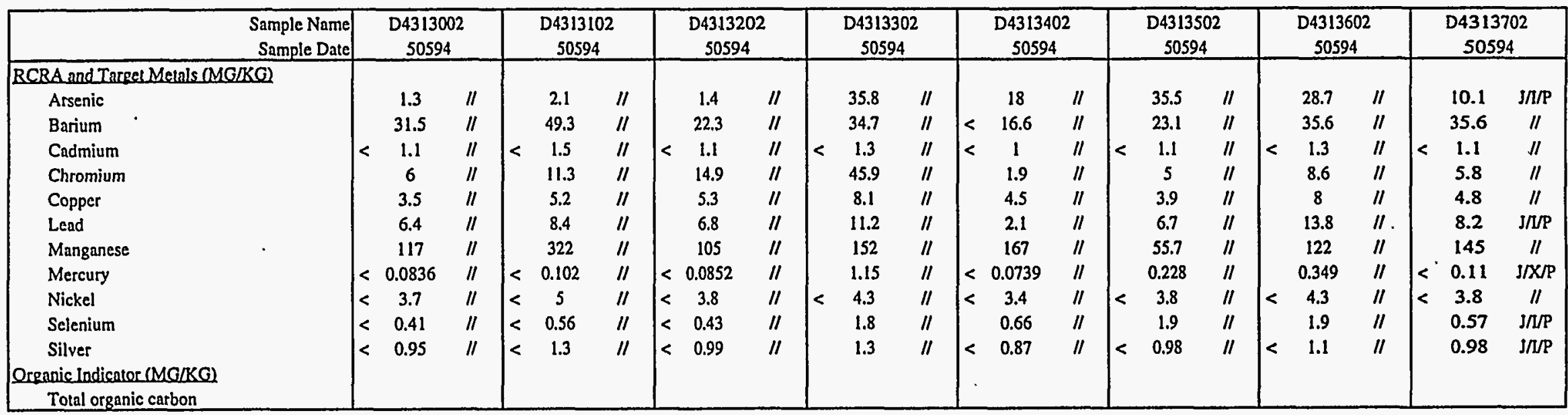


TABLE 6.23

RADIONUCLIDEPARAMETERS IN SEDIMENT MEDIA:

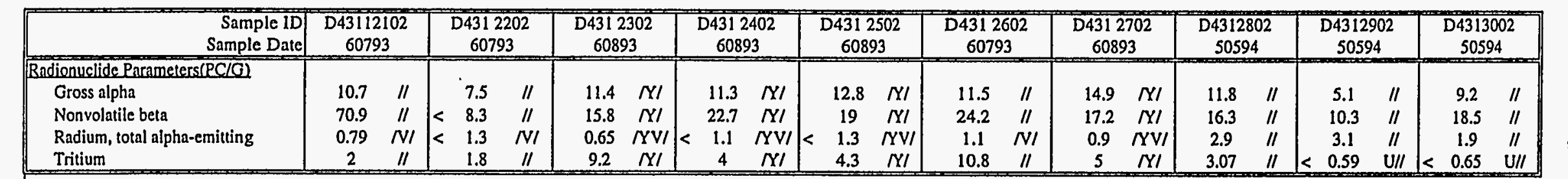

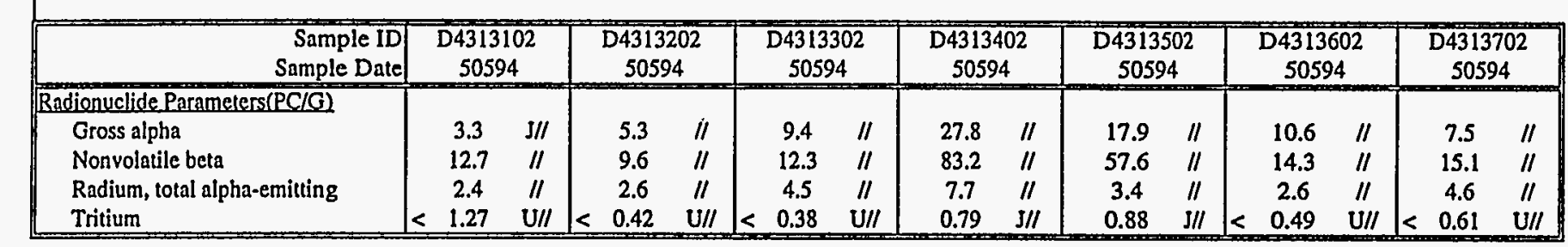




\title{
DATA SUMMARY REPORT
}

FOR THE

D-AREA BURNING/RUBBLE PITS

\author{
APPENDICES
}

\author{
FINA \\ Prepared For: \\ Westinghouse Savannah River Company \\ Savannah River Site \\ Aiken, South Carolina \\ Prepared By: \\ RUST Environment \& Infrastructure Inc. \\ 955 Millbrook Avenue \\ Aiken, South Carolina
}

OCTOBER 1994 
APPENDIX A

QUALTTY CONTROL SUMMARY REPORT

FOR THE D-AREA BURNING/RUBBLE PITS

RFI/RI UNIT ASSESSMENT

(Exploration Resources Validation) 


\section{Quality Control Summary Report for the D-Area Burning/Rubble Pits RFI/RI Unit Assessment}

May 1994 
List of Tables ................................................................................................................

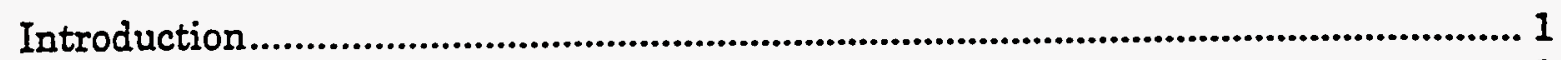

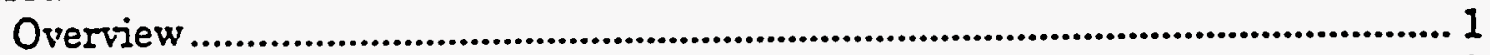

Sample Set and Analyses ....................................................................................... 2

List of Analytical Suites ..................................................................................... 2

Numbers of Records..................................................................................... 2

Discrepancies from the Work Plan ...................................................................... 3

D-Area Burning/Rubble Pits Data Quality Objectives................................................ 3

Validation Status of the Data ................................................................................. 4

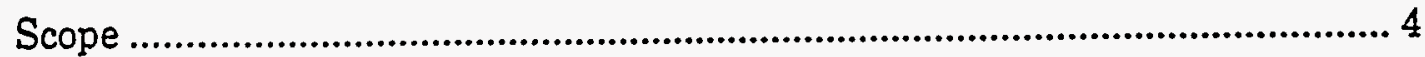

Achierement of Objective .................................................................................... 4

Anticipated Additions............................................................................................. 5

Summary of Data Quality ................................................................................ 5

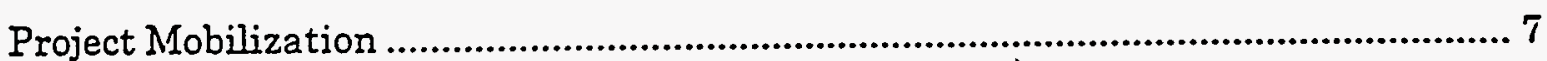

Work Plan Assessment......................................................................................

Changes Made to Sampling Instructions........................................................... 7

Constituent Lists and Methods....................................................................... 8

Production of Chain-of-Custody Forms ................................................................ 9

Chains Created in the Field ............................................................................ 9

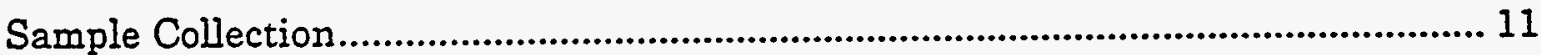

Notations of Insufficient Sample ....................................................................... 11

Field QC Samples..................................................................................................... 11

Missing or Incorrect Field Data ............................................................................... 13

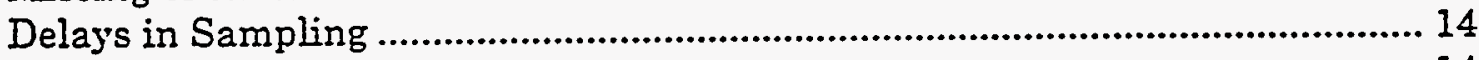

Field Observations .......................................................................................... 14

Chain-of-Custody Review .................................................................................... 16

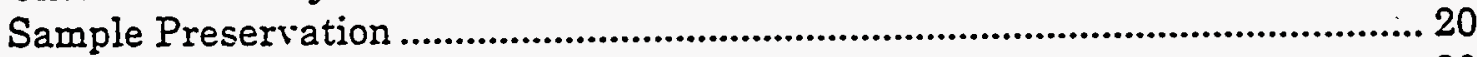

Preservation Requirements........................................................................ 20

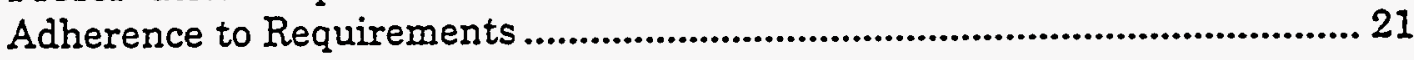

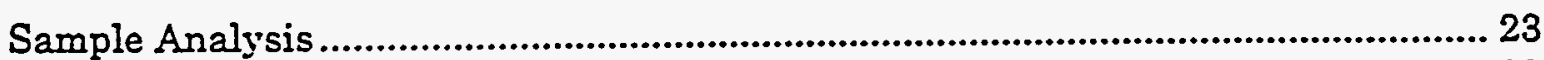

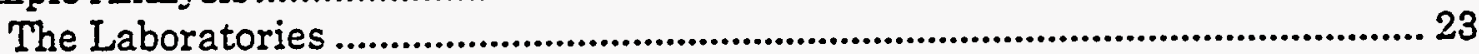

TMA Eberline, Inc., Performance Evaluations................................................... 23

Roy F. Weston, Inc., Performance Evaluations ............................................... 23

Quality Control Standards.................................................................................... 24

Analytical Methods and Detection Iimits............................................................... 24

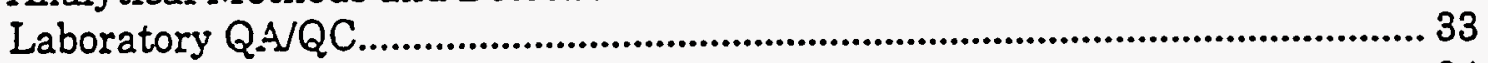

Performance Reviews .......................................................................................... 34

Roy F. Weston Analytical Laboratories .............................................................. 34

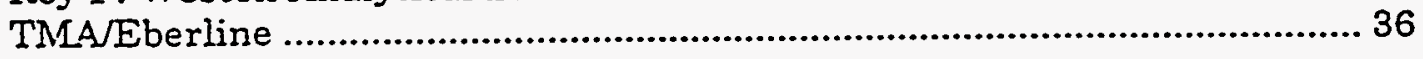

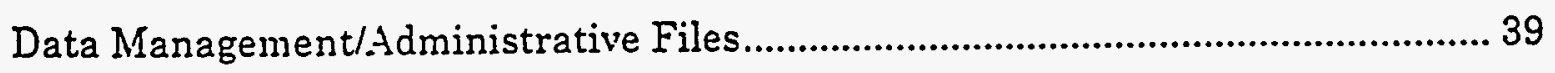


Data Validation and Verification: Scope and Policy ................................................... 41

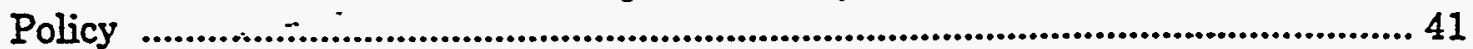

Definitions of Qualifier Codes .............................................................................. 41

Qualification Policy ........................................................................................ 43

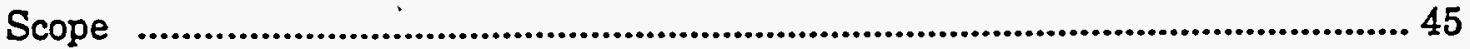

Review of the Electronic Data Deliverables ............................................................. 46

Checking of QA/QC Samples

Review of the Analytical Narratives...................................................................... 48

Laboratory Data Records Review ................................................................................. 48

Data Validation and Verification: Findings .................................................................... 51

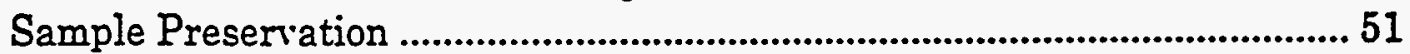

Qualifiers Applied as a Result of EDD Review ..................................................... 51

Rejected Results.................................................................................................... 51

Holding Time ................................................................................................... 51

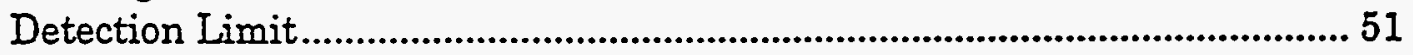

Matrix Spikes ............................................................................................. 51

Method Blanks................................................................................................ 52

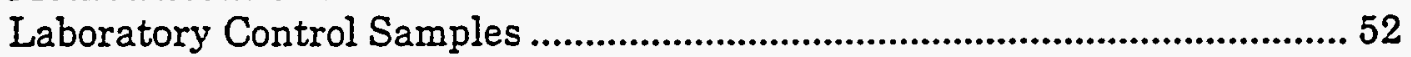

Surrogate Recoveries ........................................................................................ 52

Sample Preservation .................................................................................... 52

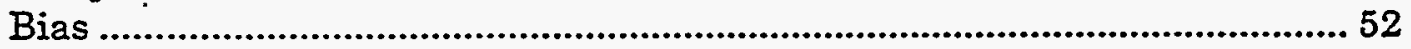

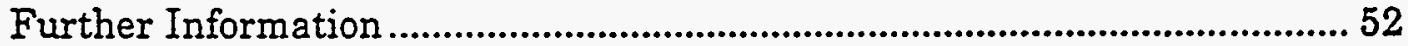

Results of Checking QA/QC Samples .................................................................... 52

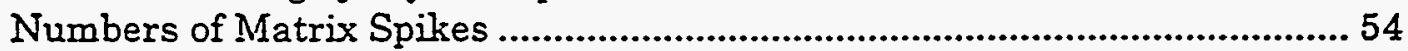

Contamination in QC Samples........................................................................... 55

Review of Analytical Narratives .......................................................................... 57

Laboratory Data Records Reviews......................................................................6 67

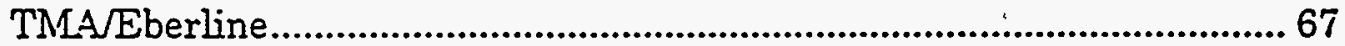

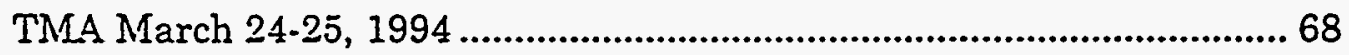

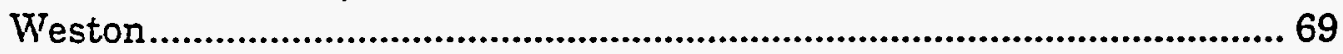

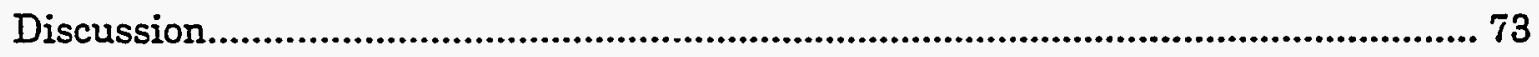

Presentation of Qualified Data .................................................................. 73

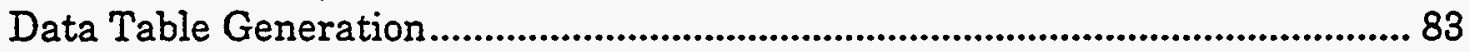

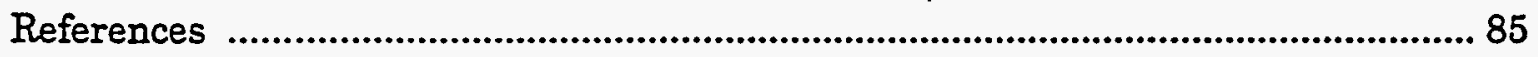

Appendix A: Constituent Lists ......................................................................................

Appendix B: Tables of Laboratory Performance Evaluations.....................................

Appendix C: Formats of the Data Files.........................................................................

Appendix D: Data Tables ..................................................................................... D-1 
Table 1. Changes to the Sample List (Table 4-1 of Work Plan)

Table 2. Changes to the Sample List (Table 4-2 of Work Plan)............................... 8

Table 3. Field-Generated Blanks..................................................................... 12

Table 4. Surface Water Field Data ...................................................................... 14

Table 5. Field Volatiles Readings ................................................................... 14

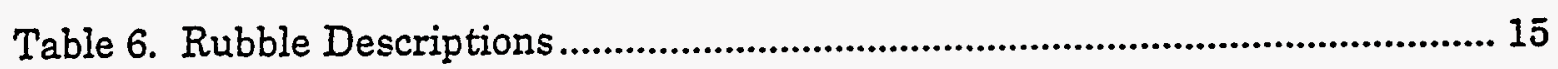

Table 7. Chain of Custody Anomalies and Notations ........................................... 17

Table 8. Analytical Methods and Detection Limits............................................... 25

Table 9. Number of Analytical Records, by. Method and Type of Record .................. 53

Table 10. Matrix Spikes Performed................................................................. 54

Table 11. Contaminated Blanks ............................................................... 56

Table 12. Numbers of Qualified Samples...................................................... 74 


\section{Introduction}

\section{Overview}

This report presents a summary of activities performed at the D-Area Burning/Rubble Pits, 431-D and 431-1D, under the direction of the Environmental Monitoring Section of the Environmental Protection Department (EPD/EMS) at the Savannah River Site (SRS) in support of the Resource Conservation and Recovery Act (RCRA) Facility Investigation/ Remedial Investigation (RFI/RI) Unit Assessment.

The D-Area Burning/Rubble Pits (DBRP) are located approximately $1 / 2$ mile west of D Area and 1-1/2 miles west of State Highway 125. The topography of the area is flat, with a shallow run-off ditch within 50 feet west of the pits. The two pits are contiguous, separated by a center dividing berm. The Savannah River and associated swamps are approximately $1 / 2$ mile from the rubble pits.

The burning/rubble pits were constructed in 1951 for disposal of spent organic solvents, waste oils, paper, plastics, rubber, wood, rags, cardboard, and degreasers. The waste was burned periodically, usually monthly. The chemical composition and volume of waste disposed of in the burning/rubble pits are unknown. In 1973, the pits were covered with a layer of soil, and afterward used only as rubble pits. During this time, they were filled with waste which included concrete, metal, lumber, bricks, tile, asphalt, plastic, empty drums, rubber, telephone poles and other wood products. When the pits were full, a layer of clay was put over the rubble and the pits were compacted, mounded, and closed.

Records indicate that all use of the burning/rubble pits was discontinued by 1983.1

SRS has received a RCRA permit from the South Carolina Department of Health and Environmental Control (SCDHEC). Part V of the permit mandates that SRS establish and implement a RCRA RFI program in order to fulfill requirements of RCRA Section 3004(u). The D.Area Burning/Rubble Pits are two of the solid waste management units identified for further investigation.

As part of the RFI process, a Preliminary Unit Evaluation was done. In 1989, a Unit Screening Program was conducted. Hazardous substances were detected in the soil profile and also in the groundwater during unit screening. In earlier groundwater monitoring, iron, lead, and manganese have exceeded the EPA Maximum Contaminant Levels at least once in at least one well. Soil gas data indicate the presence of benzene, 1,1,1trichloroethane, and tetrachloroethylene. Because of these results, a Unit Assessment was required. This report describes the sampling, analysis, and data validation done as part of the Unit Assessment.

Sampling took place in June 1993 and consisted of hand-augered soil samples, split-spoon samples, groundwater samples from up-and downgradient wells, surface water samples from up-and downstream locations, and sediment samples. Samples were collected by Rust Environmental and Infrastructure and analyzed by Roy F. Weston, Inc., laboratories and TMA/Eberline.

'WSRC-RP-90-485, Rev. 2. See the References section for full citation. 


\section{Sample Set and Analyses}

\section{List of Analytical Suites}

Soil samples were analyzed for one or more of the following groups of constituents: semivolatile organic compounds, including polycyclic aromatic hydrocarbons (PAHs); volatile organic compounds; total metals; radionuclide indicators; physical parameters; pesticides, PCBs, and dioxins and furans (PCDD/PCDF); and Toxicity Characteristic Leaching Procedures (TCLP) constituents.

Sediment samples were analyzed for one or more of the following groups of constituents: metals; semi-volatile organic constituents, including polycyclic aromatic hydrocarbons (PAHs); volatile organic constituents; radionuclide indicators; and TOC.

Water samples, including all sampling blanks, were analyzed for one or more of the following groups of constituents: total organic halogens; metals; semi-volatile organic constituents, including polycyclic aromatic hydrocarbons PAHs; volatile organic constituents; radionuclide indicators; TOC, TSS and TDS; and inorganics.

Field measurements of surface water temperature, $\mathrm{pH}$, conductivity, and dissolved oxygen were taken by the samplers as scheduled for four surface water samples. Water depth and flow rate were not recorded, although they were scheduled in the work plan.

\section{Numbers of Records}

Included in the data for this project are 138 sample logbook records and 138 chain-ofcustody records.

There are 22 station records, one for each of the planned stations in the work plan, including the one new well required by the work plan.

Excluding tentatively identified compounds (TICs), there are 28,960 analytical records. Of these, 28,260 are non-radionuclide analytical records, of which 19,941 are sample records. The remainder are laboratory blanks, blank spikes, and other laboratory-generated QA/QC samples. Of the sample records, 16,621 are for soil samples and 3,320 are for water samples, including blanks generated in the field.<smiles>[13CH]</smiles>

There are 700 radionuclide analytical records, of which 154 are laboratory blank records and 546 are sample records. Of the sample records, 409 are soil sample records and 137 are water sample records.

Total metals analyses were requested for 127 samples; additional target analyte list (TAI) metals were scheduled for two samples as part of a TAL/TCL (target compound list) with TIC request. Semivolatiles (BNA, including polynuclear aromatic hydrocarbons) were scheduled for 101 samples, and volatile organic analyses (VOA) for 148. The pesticides/ $\mathrm{PCBs} /$ dioxins/ furans suite was requested for 68 samples. The Toxicity Characteristic Leaching Procedure (TCLP) was requested for 5 samples. The numbers of scheduled 
analyses for semivolatiles, volatiles, and pesticides/PCBs/dioxins/furans include two samples requesting TICs.

Physical parameters were requested for 35 soil samples. Inorganics were requested for 12 water samples, total organic halogens for 13 water samples, and total organic carbon for eight water samples. For 129 samples, radionuclide indicators were scheduled. See Appendix $A$ of this report for full constituent lists for each of the analytical suites.

\section{Discrepancies from the Work Plan}

Of the 151 samples scheduled in the work plan, thirteen samples were not collected. One sample, D43110905, could not receive TCLP analyses as part of a TAL/TCL request because there was insufficient soil to fill all sample bottles. See the "Sample Collection" section of this report for further information on scheduled analyses that were not performed.

Samples referred to as splits in Table 4-1 and Table 4-2 of the work plan are in fact duplicates because all the validated samples were sent to one laboratory.

A number of changes were made to the constituent groups scheduled for particular samples. See the "Work Plan Assessment" section of this report for details.

Although TICs were scheduled for only two samples, the laboratory provided TIC data for a number of samples. These data are included in the data files and in the data tables of this report. TIC data, by definition, does not meet the same data quality objective as the rest of the data for this project.

\section{D-Area Burning/Rubble Pits Data Quality Objectives}

The purpose of the soil sampling program is to confirm the presence or absence of hazardous substances in the soil within the pits and/or the area surrounding the pits to identify the extent of possible chemical contamination. No hazardous substances were found in the soil outside the pits during the unit screening. Therefore, if hazardous substances are not found significantly above background levels in the soil downgradient of the pits, it will be concluded that hazardous substances are not present. The soil pathway will then be eliminated as a possible route of exposure.

The surface water/sediment sampling plan is designed to confirm the presence or absence of hazardous substances in the surface water/sediment surrounding the unit. If hazardous substances are not found in the surface water/sediment downgradient of the unit significantly above background levels, it will be concluded that hazardous substances are not present. The surface water/sediment pathway will then be eliminated as a possible route of exposure.

The key decisions associated with the data quality objectives relate to the ongoing data collection. Therefore, objectives are continually being refined as more information becomes available. This includes investigating the magnitude and extent of hazardous substance release, risk assessment, and evaluation of remediation alternatives. The tolerance for 
uncertainty was not predetermined in the work plan. Based on the purposes of the soil and surface water/sediment sampling plans, data quality level three (confirmational) is required.

\section{Validation Status of the Data}

\section{Scope}

Data collection, analysis, and reporting for the project were validated and/or verified. The process began with project mobilization, and continued to the delivery of electronic data. deliverables (EDDs) and this Quality Control Summary Report to the group responsible for risk assessment. Data validation was based on achieving Quality Assurance Objective 3 (QA3) (as defined in Quality Assurance/Quality Control (QA/QC) Guidance for Removal Activities) with analytical quality level 3 data (as defined in Data Quality Objectives for Remedial Response Activities).

\section{Achievement of Objective}

In general, sample collection was adequately documented. Some surface water samples were not collected because of dry weather. See the "Sample Collection" and "Work Plan Assessment" sections of this report for more information. Chain-of-custody forms were reviewed. In some cases, the analytical laboratory did not sign the chain-of-custody form as received. In another, instance, a few hours elapsed between the time the samples were relinquished by one party and received by the other. See the "Chain of Custody Review" section of this report for more information.

Sample holding times were usually met; exceptions have been qualified. Some criteria for $\mathrm{QA3}$, such as analytical instrument calibration, analyte identification, and analyte quantitation, were verified during the laboratory data record review; records not meeting criteria have been qualified. Method blanks were performed in ratios of more than one for every twenty samples. Percent recoveries for QA/QC samples have been reviewed, and recoveries outside limits and the associated sample record have been qualified as described in the "Qualification Policy" section of this report. Relative percent differences between duplicate sample analyses and between matrix spike and matrix spike duplicate analyses have not been calculated, the sample results have not been adjusted for bias, and the calculations for determination of precision have not been done. Only one sample analytical data record was rejected.

Contamination of field-generated blanks was reviewed; see Table 11 in the "Discussion" section of this report. Of the positive field blank results, roughly two-thirds were common laboratory contaminants, and one-third were radionuclide indicators. Associated samples were evaulated and qualified on the basis of trip blank results if necessary. 


\section{Anticipated Additions}

The data provided with this report are final and complete. Any resampling of surface water samples that were dry during initial (1993) sampling is not included within the scope of this data validation project.

\section{Summary of Data Quality}

The data for this project meet the data quality objective requirements for the project with the exceptions noted in this report and in the qualification of the data per se. Tentatively identified compounds, qualified with the result qualifier $J$ and the analysis qualifier $N$, by definition do not meet the data quality objective.

During data verification of radiological analyses, many data entry errors were observed and resolved. The analytical laboratory, TMA, employs manual data entry to report results, so many errors can be expected. The vast majority of identified errors have been resolved satisfactorily. 


\section{Project Mobilization}

\section{Work Plan Assessment}

Modifications were made to the work plan for a number of reasons as the investigation progressed. The Project Technical Manager provided the Project Manager a work plan assessment (a preliminary presentation of expected discrepancies from the work plan) in an attachment to the EPD/EMS Soil-Investigation Work Request Form dated 5/28/93. The work plan assessment was based on information from the work plan and the laboratory; some information available at that time was proven incorrect as data were received and validation proceeded. Discrepancies from the work plan are discussed in detail throughout this report; see especially "Sample Collection" and "Constituent Lists and Methods."

\section{Changes Made to Sampling Instructions}

The following table lists changes made from the sample list in Table 4-1 of the work plan, based on the work plan assessment.

Table 1. Changes to Sample List (Table 4-1 of Work Plan)

$\begin{array}{lll}\text { WSRC Identifier } & \begin{array}{l}\text { Constituents for Analysis } \\ \text { Changed From }\end{array} & \begin{array}{l}\text { Constituents for Analysis } \\ \text { Changed To }\end{array} \\ \text { D4311 07 05E } & 134 & 234 \\ \text { D4311 10 07C } & 1236 & 234 \\ \text { D431 11 06A } & 234 & 123456 \\ \text { D431 } 1205 A & 12346 & 123467 \\ \text { D431 14 07A } & 1234 & 1236 \\ \text { D4311 08 07D } & 1234 & 1236 \\ \text { D4311 16 05 } & 24 & 123456\end{array}$

Reviewers changed the instrument listed for sample $D 431$ 11 02D from split spoon to hand auger and changed the sample numbers D431 $1802 \mathrm{~A}$ to D431 $1802 \mathrm{D}$ and D431 $1802 \mathrm{~B}$ to D431 18 02C. An inconsistency for sampling points 17 and 18 mentioned in the work plan assessment between the facility identifier (D431, which indicates Pit 431:D) and the sample location (outside pit 431-1D) was never addressed. The site map, which follows the table of contents in this report, shows the actual sampling location as being south of the pits.

The following table lists changes made from the sample list in Table 4-2 of the work plan. 
Table 2. Changes to Sample List (Table 4-2 of Work Plan)

WSRCIdentifier

D431 22 01A

D431 22 02A

D431 23 01D

D431 2501A

D4311 1902

D4311 2002

\author{
Constituents for Analysis \\ Changed Erom
}

12346

236

12346

12346

23468

23468
Constituents for Analysis Changed To

1234679

23468

1234679

1234679

23467

23467

The work plan's Table 4-2 indicates that samples 100306 and 100307 (D43112001 and D43112002) were to be upstream and downstream surface water samples, respectively. However, the work plan's figure 4-1, Proposed Unit Assessment Sampling Locations, shows only one location. The samplers took the two samples approximately $3 \mathrm{ft}$ apart. The same is true for samples 100308 and 100309 (D43111901 and D43111902). Both of these stations (D4311 19 and D4311 20) actually are upstream of the pits.

\section{Constituent Lists and Methods}

The work plan constituent lists and stipulated methods could not be followed exactly because of the limitations of the analytical laboratory contract used for this project.

Polvnuclear aromatic hvdrocarbons (PAHs) were analyzed as semivolatile organics by method 8270 as described in the work plan. All of the PAHs in the target compound list (TCL) are analyzed by method 8270 , which already was being used to analyze semivolatile organic compounds.

Volatiles were analyzed as described; however, when the work plan assessment was written, Weston had said that it would not test for 2-chloroethyl vinyl ether in water samples preserved to $\mathrm{pH}<2$. When the data was received, however, 2 -chloroethyl vinyl ether was reported in the electronic data deliverables (EDDs) for some samples and not for others; this is true for both soil and water. Instead of trans-1,2-dichloroethylene, which is requested in the TCL list of the work plan, Weston reported total 1,2-dichloroethylene. Ethylbenzene was reported as part of this analytical suite even though it was not requested.

Semivolatiles (BNA): Di-n-butyl phthalate, 1,3-dichlorobenzene, 4-chloro-3-methylphenol, dimethyl phthalate, and hexachloroethane were reported as part of this analytical suite even though they were not requested in the TCL list.

Metals were analyzed as described in the work plan. When TAL/TCL with TIC (ibrary scan) was requested for soil samples, an additional 12 metals were reported: aluminum, antimony, beryllium, calcium, cobalt, iron, magnesium, potassium, sodium, thallium, vanadium and zinc. Analyses for tin, requested as part of TAL/TCL, were not performed. In water, the following 13 metals were tested for: arsenic, barium, cadmium, chromium, copper, iron; lead, manganese, mercury, nickel, selenium, silver, and sodium as requested in Table $4-2$ of the work plan (as constituents 2 and 9). 
Herbicides: Two constituents on the TALTCL list in the work plan, 2,4,5-T and 2-4dichlorophenoxyacetic acid, were analyzed only as part of TCLP analyses. An additional herbicide, silvex, also was analyzed as part of TCLP analyses.

Pesticides: Chlordane, requested as part of TAL/TCL, was reported as the isomers alphaand gamma-chlordane.

Dioxins and furans were analyzed according to the work plan assessment.

Phvsical propertiës/inorganic parameters: When the work plan assessmênit was written, the method to be used for total organic carbon was not known. Weston used EPA415.1. Instead of using EPA Method 9081 for cation exchange capacity as stated in the work plan assessment, Weston used EPA Method 9080. Cyanide was analyzed by EPA Method 335.3 when the TAL/TCL list of analytes was requested.

In water, total dissolved solids and total suspended solids were analyzed by EPA Method 160.2, total organic halogens by EPA Method 9020, and chloride, fluoride, and nitrate as nitrogen by EPA Method 300.0. Dissolved oxygen and water temperature were measured in the field; see the "Sample Collection" section of this report for reported results. Water depth and flow measurements were not recorded.

Radionuclide indicators: Radionuclide indicators were analyzed as indicated in the work plan assessment.

\section{Production of Chain-of-Custody Forms}

Chain-of-custody forms containing the list of requested analyses for each planned sample were printed on waterproof paper during mobilization. A printed tear-off stub to the chainof-custody form stipulated the sample location. Information to be filled in on the chain-ofcustody form included the following: the sample identification number from the sample logbook page for that sample, the laboratory sample ID (to be completed by the analytical laboratory), the sample collection date, shipping information including dates and signatures of each person handling the sample, and cooler number and temperature upon arrival at the analytical laboratory. In addition, a peel-off section of each bottle label printed with the bottle number was affixed to the line requesting the analysis.

\section{Chains Created in the Field}

No additional chains were created in the field for this project. 


\section{Sample Collection}

Field activities for this project were guided by the work plan and by WISRC procedures as contained in manual $3 Q 5$. Sampling was conducted by Rust Environmental and Infrastructure personnel.

\section{Notations of Insufficient Sample}

Several of the scheduled surface water samples and their associated QA/QC samples were not taken because the stations were dry. No field log entries were made. They are listed below:

D4312201

D431 2201A

D431 2201C

D431 2201E
D431 2301

D431 2301D

D431 2401

D431 2501
D431 2501A

D431 2701

D431 2701D

D431 2701E

There was insufficient sample from 100346 (D43110905) to fill all bottles. The sampler noted that "due to the large amount of gravel in the sample, two VOA bottles could not be filled. Followed procedure on chain-of-custody for the filling of bottles when not enough sample is present." This sample requested TCLP VOA, which was filled, and TAL/TCL VOA, which was not.

For sample 100364 (D431 1103), the sampler noted, "Rad sample not filled before bulk of sample was disposed of." There is no bottle number on the chain-of-custody form.

However, there is a chain-of-custody form from Weston to TMA for this sample, and TMA reported results for the scheduled radionuclide indicators, tritium. .

\section{Field QC Samples}

For this project, as is general practice, trip blanks (deionized [DI] water) were prepared by EMS-Weston. The trip blanks were filled in the morning, taken to the field, and placed in the appropriate coolers in the field. The work plan's sampling tables associate trip blanks with a specific associated sample, rather than specifying one trip blank per cooler. Because the samplers followed the work plan's instructions, not every cooler had a trip blank.

Field blanks (also DI water) were prepared by Rust personnel. They were filled in the morning and opened in the field.

A number of the field-generated blanks were coded by the samplers as "other" rather than "blank." This error has been corrected. A sample time was not logged for trip blanks 100303 (D43112102B) and 100316 (D431 2302B).

Sample bottle 10001079, used for field blank 100327 (D43110706C), had HCl preservative present on outside labels. The laboratories did not report any resulting analytical problem. 
The table below lists field-generated blanks, the number of the cooler in which trip blanks were shipped, and the analyses performed on each of the blanks.

Table 3. Field-Generated Blanks

\begin{tabular}{|c|c|c|c|c|}
\hline Scheduled & Type & $\begin{array}{l}\text { Sample } \\
\text { Number }\end{array}$ & $\begin{array}{l}\text { Cooler } \\
\text { Number }\end{array}$ & Analvses \\
\hline $\mathrm{D} 4311204 \mathrm{C}$ & Field blank & 100374 & $\mathrm{NA}^{1}$ & $\begin{array}{l}\text { Volatiles, metals, radionuclide } \\
\text { indicators, tritium }\end{array}$ \\
\hline D431 1301B & Trip blank & 100380 & 511 & $\begin{array}{l}\text { Volatiles, radionuclide indicators, } \\
\text { tritium }\end{array}$ \\
\hline D431 1304E & Rinsate & 100384 & $N A$ & $\begin{array}{l}\text { Volatiles, metals, radionuclide } \\
\text { indicators, tritium }\end{array}$ \\
\hline D431 1406C & Field blank & 100397 & $N A$ & $\begin{array}{l}\text { Volatiles, metals, radionuclide } \\
\text { indicators, tritium }\end{array}$ \\
\hline D431 1708C & Field blank & 100437 & NA & Volatiles \\
\hline D431 1802C & Field blank & 100403 & NA & $\begin{array}{l}\text { Volatiles, metals, radionuclide } \\
\text { indicators, tritium }\end{array}$ \\
\hline D431 2201C & Field blank & $\mathrm{NT}^{2}$ & $\mathrm{NA}$ & NA \\
\hline D431 2201E & Rinsate & NT & NA & NA \\
\hline D431 2302B & Trip blank & 100316 & $\lambda R^{3}$ & $\begin{array}{l}\text { Volatiles, radionuclide indicators, } \\
\text { tritium }\end{array}$ \\
\hline D431 2302E & Rinsate & 100315 & NA & $\begin{array}{l}\text { Metals, volatiles, radionuclide } \\
\text { indicators, tritium }\end{array}$ \\
\hline D431 2601B & Trip blank & NT & NA & NA \\
\hline $\mathrm{D} 4312602 \mathrm{C}$ & Field blank & 100302 & NA & $\begin{array}{l}\text { Volatiles, radionuclide indicators, } \\
\text { tritium }\end{array}$ \\
\hline D431 2701E & Rinsate & NT & NA & NA \\
\hline D43110705E & Rinsate & 100325 & NA & $\begin{array}{l}\text { Volatiles, metals, radionuclide } \\
\text { indicators, tritium }\end{array}$ \\
\hline $\mathrm{D} 43110706 \mathrm{C}$ & Field blank & 100327 & $\mathrm{NA}$ & $\begin{array}{l}\text { Volatiles, metals, radionuclide } \\
\text { indicators, tritium }\end{array}$ \\
\hline D43110804C & Field blank & 100333 & $N A$ & $\begin{array}{l}\text { Volatiles, metals, radionuclide } \\
\text { indicators, tritium }\end{array}$ \\
\hline D43110807B & Trip blank & 100339 & 563 & $\begin{array}{l}\text { Volatiles, radionuclide indicators, } \\
\text { tritium }\end{array}$ \\
\hline D43110901E & Rinsate & 100342 & NA & $\begin{array}{l}\text { Volatiles, metals, radionuclide } \\
\text { indicators, tritium }\end{array}$ \\
\hline D43110906C & Field blank & 100348 & $\mathrm{NA}$ & $\begin{array}{l}\text { Volatiles, metals, radionuclide } \\
\text { indicators, tritium }\end{array}$ \\
\hline D43111001B & Trip blank & 100351 & NR & $\begin{array}{l}\text { Volatiles, radionuclide indicators, } \\
\text { tritium }\end{array}$ \\
\hline D43111004E & Rinsate & 100355 & NA & $\begin{array}{l}\text { Volatiles, metals, radionuclide } \\
\text { indicators, tritium }\end{array}$ \\
\hline D43111007C & Field blank & 100360 & $\mathrm{NA}$ & $\begin{array}{l}\text { Volatiles, metals, radionuclide } \\
\text { indicators, tritium }\end{array}$ \\
\hline
\end{tabular}

1 NA - Not applicable.

${ }^{2}$ NT - Not taken. No reason that D431 2601B was not taken was given in the field logs. All the others were scheduled to be associated with surface water samples precluded by dry weather conditions.

${ }^{3}$ NR - Not recorded. 
Table 3. Field-Generated Blanks, Continued

\begin{tabular}{|c|c|c|c|c|}
\hline Scheduled & Type & $\begin{array}{l}\text { Sample } \\
\text { Number }\end{array}$ & $\begin{array}{l}\text { Cooler } \\
\text { Number }\end{array}$ & Analyses \\
\hline $\mathrm{D} 43111502 \mathrm{~B}$ & Trip blank & 100409 & 413 & $\begin{array}{l}\text { Volatiles, radionuclide indicators, } \\
\text { tritium }\end{array}$ \\
\hline $\mathrm{D} 43111603 \mathrm{C}$ & Field blank & 100420 & NA & $\begin{array}{l}\text { Volatiles, metals, radionuclide } \\
\text { indicators, tritium }\end{array}$ \\
\hline $\mathrm{D} 43111505 \mathrm{~B}$ & Trip blank & 100413 & NR & Volatiles \\
\hline $\mathrm{D} 43111506 \mathrm{E}$ & Rinsate & 100416 & $\mathrm{NA}$ & Volatiles \\
\hline $\mathrm{D} 43112102 \mathrm{~B}$ & Trip blank & 100303 & $\mathrm{NR}$ & $\begin{array}{l}\text { Volatiles, radionuclide indicators, } \\
\text { tritium }\end{array}$ \\
\hline
\end{tabular}

The analyses listed are those scheduled in the work plan or its modification (see the "Mobilization" section of this report). All the analyses in the table were performed, and the results are reported in the data and in the data tables of this report. See the "Discussion" section of this report for a table of field-generated blanks having constituents above . detection limit.

\section{Missing or Incorrect Field Data}

Samples 100301 (D431 2601), 100305 (D43112101), 100310 (D431 2202), 100322

(D43110705), 100326 (D43110706), 100332 (D43110804), and 100334 (D43110805) specify an associated sample ID despite the fact that they were coded as "Normal" sample types.

Sample 100305 (D43112101) was coded by the samplers as "Sediment" with a soil moisture of "Liquid." No Munsell Color or Unified Soil Classification was specified. Because it was scheduled as a surface water sample, and field data scheduled only for surface water samples were taken, the sample type was changed to "Water."

Sample 100319 (D43110703) and its duplicate, 100320 (D43110703D), do not specify a Unified Soil Classification, probably because of much rubble. See the table of rubble descriptions below.

Samples 100335 (D43110805A), 100388 (D431 1307), 100389 (D431 1307A), 100406 (D43111501), 100407 (D43111502), 100408 (D43111502A), and 100424 (D43111606) were not coded for soil moisture.

Sample 100385 (D431 1305) does not specify a soil moisture, Munsell Color, or Unified Soil Classification. See below for field observations of this sample.

Samples 100386 (D431 1306), 100387 (D431 1306D), 100388 (D431 1307), and 100389 (D431 1307A) do not specify Munsell Color; the sampler noted that the mottled color of the samples prevented identification of a predominant color. Sample 100411 (D43111504) also does not specify a Munsell Color 


\section{Delays in Sampling}

The sampler checked the "unusual delay" box in the log for sample 100375 (D431 1205) and its duplicate; 100376 (D431 1205A), and noted, "Had split-spoon refusal. Had to move rig and burn down to attempt taking sample again. Sample taken at 12:04; because of large amount of sample needed, three split spoons were taken at this interval. Each spoon was $50 \%$ full."

Sample 100434 (D431 1707) and its duplicate, 100435 (D431 1707A), were delayed because the hydropunch was dry on the first attempt in borehole SB-17. The sampler drilled deeper and took samples an hour later.

\section{Field Observations}

The samplers recorded field measurements for $\mathrm{pH}$, conductivity, water temperature, and dissolved oxygen for four surface water samples. The other surface water samples scheduled for these data were not taken due to dry conditions at the sampling stations. The results follow.

Table 4. Surface Water Field Data

$\begin{array}{llllll}\text { Sample ID } & \text { pH } & \text { Conductivity } & & \begin{array}{l}\text { Water } \\ \text { Temperature }\end{array} & \begin{array}{l}\text { Dissolved } \\ \text { Oxvgen }\end{array} \\ 100301 \text { (D431 2601) } & 5.8 & 370 \mu \mathrm{S} / \mathrm{cm} & & 30.6^{\circ} \mathrm{C} & \\ 100305(\mathrm{D} 43112101) & 8.1 & 64 \mu \mathrm{S} / \mathrm{cm} & & 30.2^{\circ} \mathrm{C} & 7.0 \mathrm{mg} / \mathrm{L} \\ 100306 \text { (D43112001) } & 8.1 & 64 \mu \mathrm{S} / \mathrm{cm} & 27.9^{\circ} \mathrm{C} & 7.4 \mathrm{mg} / \mathrm{L} \\ 100308 \text { (D43111901) } & 8.0 & 64 \mu \mathrm{S} / \mathrm{cm} & 27.0^{\circ} \mathrm{C} & 6.0 \mathrm{mg} / \mathrm{L}\end{array}$

The following table lists field volatiles readings recorded in the sample logbook.

Table 5. Field Volatiles Readings

$\begin{array}{lll}\text { Sample ID } & \begin{array}{l}\text { Instrumentl } \\ \text { method }\end{array} & \text { Reading } \\ 100322(\mathrm{D} 43110705) & \text { OVA } & 5-10 \mathrm{ppm} \\ 100326 \text { (D43110706) } & \text { OVA } & 5-10 \mathrm{ppm} \\ 100330 \text { (D43110802) } & \text { OVA } & 2 \mathrm{ppm} \\ 100331 \text { (D43110803) } & \text { HNu } & 0.5-1.0 \mathrm{ppm} \\ 100385 \text { (D431 1305) } & \text { OVA } & >1,000 \mathrm{ppm} \\ 100395 \text { (D431 1405) } & \text { OVA } & 40 \mathrm{ppm} \\ 100396 \text { (D431 1406) } & \text { OVA } & 7 \mathrm{ppm} \\ 100398(\mathrm{D} 431 \text { 1407) } & \text { OVA } & 3 \mathrm{ppm} \\ 100399 \text { (D431 1407A) } & \text { OVA } & 3 \mathrm{ppm}\end{array}$

For sediment sample 100310 (D431 2202) and its duplicate, 100311 (D431 2202A), the sampler noted, "Sample taken approx. $10^{\prime}$ west of monitor well DBP4. No surface water present." 
For sediment sample 100312 (D431 2702), the sampler noted, "Sample collected from south end of small wetland located approx. 100 yds south of the BRP's. No surface water at this location."

For sediment sample 100313 (D431 2302), the sampler noted, "Sample taken in middle of drainage swale approximately. $60 \mathrm{ft}$ south of road."

For sample 100319 (D43110703) and its split, sample 100320 (D43110703D), the actual borehole sampled was $3 \mathrm{ft}$ east of the original borehole.

Sample 100334 (D43110805) and its duplicate, 100335 (D43110805A), were noted as saturated with hydrocarbon from 6.0-6.5' and having a strong hydrocarbon odor. Samples 100396 (D431 1406) and 100398 (D431 1407) and its duplicate, 100399 (D431 1407A), had a hydrocarbon odor and stain.

For sample 100344 (D43110903), the sampler noted, "Material appears to be backfill into the trench excavated during initial phase of the investigation."

The sampler noted that sample 100400 (D431 1801) is located in a disturbed area due to grading of the land surface and runoff from the adjacent dirt road. Also, it was noted that the land surface in the vicinity of sample 100406 (D43111501) had been reworked by a grader.

The samplers recorded the following descriptions of rubble.

Table 6. Rubble Descriptions

Sample ID

100319 (D43110703)

and its split, 100320

(D43110703D)

100321 (D43110704)

100331 (D43110803) Wood and plastic

100332 (D43110804)

100364 (D431 1103)

100365 (D431 1104)

\section{Rubble}

Wood, glass, plastic, and rubber hose

Wood, glass, plastic, and concrete

Wood and plastic, heavy fuel oil stains

Traces of charred wood and fabric

Traces of charred roofing tile

\section{Further observations}

Silty sand matrix

Slight fuel oil smell; silty-clayey sand matrix. Alcohol mist from decon area detected during sample container filling.

"Abundant" rubble from 3.0-3.5 $\mathrm{ft}$

Fine-medium grain sand; fuel oil odor 
Table 6. Rubble Descriptions, Continued

\begin{tabular}{|c|c|c|}
\hline Sample ID & Rubble & Further observations \\
\hline $\begin{array}{l}100367 \text { (D431 1106), its } \\
\text { duplicate, } 100368 \\
\text { (D431 1106A), and } \\
100369 \text { (D431 1107) }\end{array}$ & Plastic sheeting & . \\
\hline $\begin{array}{l}100375 \text { (D431 1205) } \\
\text { and its duplicate, } \\
100376 \text { (D431 1205A) }\end{array}$ & Pebbles, ash, and wood & \\
\hline 100377 (D431 1206) & Ash, wood, and plastic & \\
\hline The lower half of & Fill (wood, asphalt, and ash) & Very coarse soil matrix \\
\hline
\end{tabular}
sample 100382 (D431 1303)

The top half of 100386 Fill (wood, ash, and plastic) (D431 1306) and its split, 100387 (D431 1306D)

The bottom half of sample 100393 (D431 1403)

100394 (D431 1404)

100366 (D431 1105)

Plastic and ash Markedly different in Munsell color (5YR3/4) from the top (10R4/6).

Concrete, glass, plastic, and insulated wire

Plastic sheeting, wood, and slate roofing tile

Because of heavy rain the night before sampling, the sample was "slush," with no remaining structure.

100383 (D431 1304)

Wood and plastic

Creosote smell

100385 (D431 1305)

Wood, ash, and plastic

Coarse-grained, moist soil matrix; strong odor

100395 (D431 1405)

Concrete and plastic

Slight $\mathrm{H}_{2} \mathrm{~S}$ odor

The following sample IDs were specifically noted as hydropunch samples: 100412 (D43111505) (water table), 100414 (D43111506), 100415 (D43111506D), 100426 (D43111608), 100434 (D431 1707), 100435 (D431 1707A), and 100436 (D431 1708).

\section{Chain-of-Custody Review}

The chain-of-custody forms were reviewed for comments by the samplers or laboratory personnel and were audited for completeness and an unbroken chain of custody. Notations 
made by the samplers or the laboratory on the forms and discrepancies noted from the audit are recorded below.

\section{Table 7. Chain-of-Custody Anomalies and Notations}

\begin{tabular}{|c|c|c|}
\hline Sample number & Work Plan Number & Comments \\
\hline 100301 & D431 2601 & $\begin{array}{l}\text { Cooler number missing } \\
\text { Cooler temperature missing }\end{array}$ \\
\hline 100303 & D43112102B & $\begin{array}{l}\text { Cooler number missing } \\
\text { Cooler temperature missing } \\
\text { Sample time blank }\end{array}$ \\
\hline 100304 & D43112102 & $\begin{array}{l}\text { Cooler number missing } \\
\text { Cooler temperature missing }\end{array}$ \\
\hline 100309 & D43111902 & $\begin{array}{l}\text { Cooler number missing } \\
\text { Cooler temperature missing }\end{array}$ \\
\hline 100310 & D431 2202 & $\begin{array}{l}\text { Cooler number missing } \\
\text { Cooler temperature missing }\end{array}$ \\
\hline 100311 & D431 2202A & $\begin{array}{l}\text { Cooler number missing } \\
\text { Cooler temperature missing }\end{array}$ \\
\hline 100312 & D431 2702 & $\begin{array}{l}\text { Cooler temperature }=14.6^{\circ} \mathrm{C} \\
\text { Lab left time blank in custody transfer record }\end{array}$ \\
\hline 100313 & D431 2302 & Cooler temperature $=14.6^{\circ} \mathrm{C}$ \\
\hline 100314 & D431 2302D & Cooler temperature $=14.6^{\circ} \mathrm{C}$ \\
\hline 100315 & D431 2302E & $\begin{array}{l}\text { Cooler number missing } \\
\text { Cooler temperature }=14.5^{\circ} \mathrm{C}\end{array}$ \\
\hline 100316 & D431 2302B & $\begin{array}{l}\text { Cooler number missing } \\
\text { Cooler temperature }=14.5^{\circ} \mathrm{C} \\
\text { Sample time blank }\end{array}$ \\
\hline 100317 & D43110701 & Cooler temperature $=14.6^{\circ} \mathrm{C}$ \\
\hline 100318 & D43110702 & Cooler temperature $=14.6^{\circ} \mathrm{C}$ \\
\hline 100319 & D43110703 & $\begin{array}{l}\text { Cooler temperature }=14.6^{\circ} \mathrm{C} \\
\text { Lab did not sign COC }\end{array}$ \\
\hline 100320 & D43110703D & $\begin{array}{l}\text { Cooler temperature }=14.6^{\circ} \mathrm{C} \\
\text { Lab did not sign COC } \\
\text { Only one VOA vial filled }\end{array}$ \\
\hline 100321 & D43110704 & $\begin{array}{l}\text { Cooler temperature }=14.6^{\circ} \mathrm{C} \\
\text { Lab did not sign COC } \\
\text { Sample time blank }\end{array}$ \\
\hline 100322 & D43110705 & $\begin{array}{l}\text { Cooler temperature }=14.6^{\circ} \mathrm{C} \\
\text { Lab did not sign COC }\end{array}$ \\
\hline 100323 & D431 2402 & $\begin{array}{l}\text { Cooler temperature }=14.6^{\circ} \mathrm{C} \\
\text { Lab did not sign COC }\end{array}$ \\
\hline 100324 & D431 2502 & $\begin{array}{l}\text { Cooler temperature }=14.6^{\circ} \mathrm{C} \\
\text { Lab did not sign COC }\end{array}$ \\
\hline 100325 & D43110705E & $\begin{array}{l}\text { Cooler number missing } \\
\text { Cooler temperature }=14.5^{\circ} \mathrm{C} \\
\text { Lab did not sign } \mathrm{COC}\end{array}$ \\
\hline 100326 & D43110706 & $\begin{array}{l}\text { Cooler number missing } \\
\text { Cooler temperature missing } \\
\text { Bottle \#10002198 was a } 500 \mathrm{~mL} \text { (instead of a } 250 \mathrm{~mL} \text { ) } \\
\text { amber bottle. }\end{array}$ \\
\hline
\end{tabular}


Table 7. Chain-of-Custody Anomalies and Notations, Continued

Sample number Work Plan Number Comments

100327

100328

100329

100330

100331

100332

100333

100334

100335

100336

100338

100340

100341

100342

100343

100344

100345

100346

100347

100348

100349
D43110706C

D43110707

D43110801

D43110802

D43110803

D43110804

D43110804C

D43110805

D43110805A

D43110806

D43110807D

D43110901

D43110901A

D43110901E

D43110902

D43110903

D43110904

D43110905

D43110906

D43110906C

D43110907
Cooler number missing

Cooler temperature missing

Cooler number missing

Cooler temperature missing

Cooler number missing

Cooler temperature missing

Cooler number missing

Cooler temperature missing

Cooler number missing

Cooler temperature missing

Cooler number missing

Cooler temperature missing

Missing one VOA sample

Cooler number missing

Cooler temperature missing

Cooler number missing

Cooler temperature missing

Missing one VOA sample

Cooler number missing

Cooler temperature missing

Missing one VOA sample

Cooler number missing

Cooler temperature missing

Cooler number missing

Cooler temperature missing

Cooler number missing

Cooler temperature missing

Bottle \#10002139 was a $125 \mathrm{~mL}$ (instead of a $250 \mathrm{~mL}$ )

amber bottle.

Cooler number missing

Cooler temperature missing

Cooler number missing

Cooler temperature missing

Cooler number missing

Cooler temperature missing

Cooler number missing

Cooler temperature missing

Cooler number missing

Cooler temperature missing

Cooler number missing

Cooler temperature missing

No bottle numbers on target compound list (TCL) VOA

samples

Cooler number missing

Cooler temperature missing

Cooler number missing

Cooler temperature missing

Cooler number missing

Cooler temperature missing 


\section{Table 7. Chain-of-Custody Anomalies and Notations, Continued \\ Sample number comments}

100351

Cooler number missing

Cooler temperature missing

100352

Cooler number missing

Cooler temperature missing

100353

Cooler number missing

Cooler temperature missing

100354

Cooler number missing

Cooler temperature missing

100355

Cooler number missing

Cooler temperature missing

100356

Cooler number missing

Cooler temperature missing

100357

Cooler number missing

100358

Cooler temperature missing

Cooler number missing

100359

Cooler temperature missing

Cooler number missing

Cooler temperature missing

100360

100361

100362

100363

Cooler number missing

Cooler temperature missing

Cooler number missing

Cooler temperature missing

Cooler number missing

Cooler temperature missing

Cooler number missing

Cooler temperature missing

100364

Cooler number missing

Cooler temperature missing

The metals bottle was broken when received by the laboratory. They did

however perform the metals analyses.

100365

No bottle number for $40 \mathrm{~mL}$ VOA vial

100366

100367

Cooler number missing

Cooler temperature missing

Cooler number missing

Relinquished by N.E.U.P. 6/14/93 at 8:45; received by J.E. Williams $6 / 14 / 93$ at $11: 00$

Cooler number missing

Relinquished by N.E.U.P. 6/14/93 at 8:45; received by J.E. Williams

6/14/93 at 11:00

$100368 \quad$ Cooler number missing

Relinquished by N.E.U.P. 6/14/93 at 8:45; received by J.E. Williams

6/14/93 at $11: 00$

100369

100377

Cooler number missing

Relinquished by N.E.U.P. 6/14/93 at 8:45; received by J.E. Williams

6/14/93 at 11:00

100379

Cooler number missing

Cooler number missing

100381

Cooler number missing 


\section{Table 7. Chain-of-Custody Anomalies and Notations, Continued}

Sample number Comments

$100384 \quad$ Cooler number missing

"No ID\# on bottle" in comments; however, all bottles have numbers on

100387 the chain. The meaning of the comment is unclear.

100397

Cooler number missing

100402

Cooler number missing

100412

Bottle \#10002601 was a $250 \mathrm{~mL}$ (instead of $125 \mathrm{~mL}$ ) amber bottle.

Cooler number missing

Cooler temperature missing

100413

Cooler number missing

Cooler temperature missing

100414

Cooler number missing

Cooler temperature $=8.2^{\circ} \mathrm{C}$

$100415 \quad$ Cooler number missing

Cooler temperature $=8.2^{\circ} \mathrm{C}$

$100416 \quad$ Cooler number missing

Cooler temperature $=8.2^{\circ} \mathrm{C}$

100422

Lab did not sign COC.

Analysis qualifiers of $Y$ were applied to all data where the cooler temperature was above $6^{\circ} \mathrm{C}$ when received by the laboratory.

\section{Sample Preservation}

\section{Preservation Requirements}

Sample preservation requirements are determined by regulatory guidance, method specifications, and laboratory protocols prior to field sampling events. General requirements follow.

Soil:

Inorganics: Inorganic analyses for metals require that soil samples be kept at $4^{\circ} \mathrm{C}$.

Organics: Organic analyses for benzidines, chlorinated hydrocarbons, haloethers, PCBs, pesticides, phenols, phthalate esters, purgeable aromatic hydrocarbons, purgeable halocarbons, tetrachlorodibenzo-p-dioxin isomers, and total organic carbon also require that soil samples be kept at $4^{\circ} \mathrm{C}$. Soil samples to be analyzed for nitroaromatics and isophorone, nitrosamines, or polynuclear aromatic hydrocarbons must be kept at $4^{\circ} \mathrm{C}$ and stored in the dark.

\section{TCLP:}

Inorganics: TCLP samples for inorganic analyses must be kept at $4^{\circ} \mathrm{C}$ and have nitric acid added so that the $\mathrm{pH}$ is less than 2 .

Organics: TCLP samples for semivolatile or pesticide/herbicide analyses must be kept at $4^{\circ} \mathrm{C}$. TCLP samples for volatile analyses must be kept at $4^{\circ} \mathrm{C}$ and have hydrochloric acid added so that the $\mathrm{pH}$ is less than 2 . 


\section{Water:}

Inorganics: Water samples to be tested for nitrate must be kept at $4^{\circ} \mathrm{C}$. Water samples collected for metals analyses should have nitric acid added so that the $\mathrm{pH}$ is less than 2 .

Organics: Water samples for all organic analyses should be kept at $4^{\circ} \mathrm{C}$. Samples for benzidines should have the $\mathrm{pH}$ adjusted to 2-7. Purgeable aromatic hydrocarbons analyses require that hydrochloric acid be added so that the $\mathrm{pH}$ is less than 2 , and total organic carbon analysis requires that hydrochloric acid or sulfuric acid be added so that the $\mathrm{pH}$ is less than 2. Samples for nitroaromatics and isophorone, nitrosamines, or polynuclear aromatic hydrocarbons must be stored in the dark. Samples for total organic halogens analysis must have $1 \mathrm{~mL}$ of $0.1 \mathrm{M}$ sodium sulfite added. Samples for pesticide analyses should have the $\mathrm{pH}$ adjusted to $5-9$.

\section{Adherence to Requirements}

The laboratory recorded the temperature of the samples as they were received. All temperatures above $6^{\circ} \mathrm{C}$ are noted in Table 7 .

All base/neutral/acid extractables, including polynuclear aromatic hydrocarbons, for both soil and water samples are shipped in amber bottles.

The $\mathrm{pH}$ of samples received by the laboratory, any adjustment of $\mathrm{pH}$ done by the laboratory, and any other preservatives added by the laboratory after sample receipt are not reported. 


\section{Sample Analysis}

\section{The Laboratories}

Analyses were performed by Roy F. Weston, Inc.-Lionrille (PA); Weston-Gulf Coast Laboratories, Inc., of University Park, IL; Roy F. Weston, Inc.-Stockton (CA); and TMA/Eberline of Oak Ridge, TN. All of the laboratories performing analyses for this project have been certified, or have applied for certification, by SCDHEC.

\section{TMA/Eberline, Inc., (TMA), Performance Evaluations}

TMA participated in two types of national performance evaluation programs during 1993. One study, sponsored by EPA's Environmental Monitoring Systems Laboratory (EMSL) of Las Vegas, NV, was conducted from January through August 1993. EMSL provided samples spiked with known concentrations of constituents to laboratories seeking certification to analyze those constituents. TMA submitted results to EPA for certification. One TMA result for gross alpha in water was outside the normalized deviation range of +3.0 to -3.0 ; however, TMA stated that thorium.230, which was used by EPA, has a $50 \%$ lower counting efficiency than americium-241, the EPA-recommended isotope for alpha-absorption efficiency curves. According to TMA, correction for the $50 \%$ difference gives a value within range. TMA's result for nonvolatile beta in air filters also was outside of range. Table B-1 in Appendix B contains EPA/EMSL Intercomparison Studies Program data for TMA.

The other program was conducted by the Department of Energy (DOE) and used water, soil, air, and vegetation matrices. Results were reported for March and September 1993. The DOE program does not include a range of acceptable values; instead, the value obtained by the lab is divided by the DOE's known value to give a ratio. Tables B-2 and -3 in Appendix B contain results for March 1993 and September 1993, respectively. For the water and soil samples, one analysis for strontium-90 (Table B-2) was outside the ratio range of 0.5 to 1.5. Results for one sample each of americium-241, cesium-137, potassium40 , strontium-90, and uranium mass concentration, although acceptable, fell within the low advisory range of 0.5-0.8. Results for one sample each of americium-241, cesium-137, and plutonium-239, although acceptable, fell within the high advisory range of 1.2-1.5.

Roy F. Weston, Inc. (WA), Performance Evaluations

Roy F. Weston, Inc.-Lionville participated in the EPA Water Pollution Laboratory Performance Evaluation Study (WP030), for which results were reported in June 1993. EPA conducts the water pollution study biannually to certify laboratories for specific analyses. EPA's Environmental Monitoring Systems Laboratory (EMSL) of Cincinnati, $\mathrm{OH}$, prepares water samples spiked with known concentrations of constituents found in polluted waters and submits them to all laboratories seeking certification to analyze wastewater. EMSL evaluates the results, using limits statistically based on the performance of approximately 100 top-rated laboratories that analyze each constituent by the same procedure as the laboratory being evaluated. Table B-4 in Appendix B contains the results of the June study. Results for two samples of mercury and one sample each of 
beryllium, orthophosphate, total phosphorous, and non-filterable residue were outside the acceptable range. Results for two samples of selenium and one'sample.each of chloride, non-filterable residue, orthophosphate, total phosphorous, and silver, although within the acceptable range, were near the limits; the laboratory was instructed to check them for error.

Weston Laboratories also participated in two EPA Water Supply Laboratory Performance Evaluation Studies, WS031 and WS032, for which results were reported in February and August of 1993. Tables B-5 and -6 in Appendix B contain results for WSO31 and WS032, respectively. In the former study, results for one sample each of bromodichloromethane, cadmium, chlorodibromomethane, fluorotrichloroethane, styrene, and zinc were outside the acceptable range. In the latter study, results for one sample each of beryllium, hexachlorobenzene, 1,1,1-trichloroethane, and trifluralin were outside the acceptable range.

\section{Quality Control Standards}

During April and June 1993, EPD/EMS conducted a quality assessment of Weston laboratory by sending them a set of certified environmental quality control standards from Environmental Resource Associates (ERA) of Arvada, CO (Lot Numbers 418, 549, 3205, 3405,8910 , and 9950). The results are listed in Table B-7.

Weston's results were compared with the ERA-certified values and advisory ranges. Weston did not analyze alachlor, alkalinity (as $\mathrm{CaCO}_{3}$ ), ammonia nitrogen, atrazine, biological oxygen demand, boron, chemical oxygen demand, dalapon, dinoseb, molybdenum, nitrate-nitrite as nitrogen, nitrogen by Kjeldahl method, pentachlorophenol (by method 8150), pichloram, simazine, or strontium because they are not in the current contract.

Of 96 analyses reported by Weston, 92 , or $95.8 \%$, fell within the advisory range.

\section{Analytical Methods and Detection Limits}

Generally, the analytical methods used for this project were those specified in the work plan. See the "Work Plan Assessment" section of this report for a discussion of some differences between methods specified in the work plan and methods actually used.

Initially, Weston used EPA 200-series methods for metals analyses. These methods do not meet the data quality requirements of this project because there are no matrix spikes in the 200-series methods. (There are, however, lab blank spikes.) When the subcontract technical representative (STR) was told of this problem, he arranged for the lab to run the additional QC samples required by the equivalent SW-846 methods and to resubmit the data using the SW-846 method codes. Holding time for the samples had not been exceeded. Thereafter, under that STR's contract, the lab used SW-846 methods.

Several radionuclide methods specified in the work plan were not used. The most important difference is that instead of using Standard Method 706 to analyze total radium, the radionuclide laboratory (TMA/Eberline) used EPA903.0, which analyzes only alpha emitters, and thus does not measure the presence of radium-228. 
A table of methods and detection limits follows. The column headed Lab $D L$ contains the . detection limit the laboratories expected to be able to achieve. The column headed Reference $D L$ contains the detection limit given in the method description, the source for which is in the column headed Reference. The method reference is provided even if it does not include a detection limit for a particular constituent. See the "References" section of this report for further information on the sources.

Contract-required detection limits generally were met throughout the project. The detection limits reported in the data tables are the sample quantitation limits. Dilution factor, aliquot size, soil moisture, and method detection limit are used to calculate the sample quantitation limits. In addition, there are recommended dilutions in the method, such as those for medium level analyses, which are not reported as dilution factors in the electronic data deliverable. Because the sample aliquot size was not reported, it was not possible to reproduce the calculation of the sample quantitation limit; however, the sample quantitation limits were checked for reasonableness.

Table 8. Analytical Methods and Detection Limits

\begin{tabular}{|c|c|c|c|c|c|}
\hline Analute & Method & $\underline{\text { Units }}$ & $\begin{array}{l}\text { Lab } \\
\underline{\mathrm{DL}}\end{array}$ & $\begin{array}{l}\text { Nominal } \\
\underline{\text { DL }}\end{array}$ & Reference \\
\hline \multicolumn{6}{|l|}{ Soil } \\
\hline Percent solids & ASTM2216 & $\%$ & 0.1 & NA & ASTM \\
\hline Cyanide & EPA335.3 & $\mathrm{mg} / \mathrm{kg}$ & 1 & NA & EPA-600/4-79-020 \\
\hline Total organic carbon & EPA415.1 & $\mathrm{mg} / \mathrm{kg}$ & 20 & NA & EPA-600/4-79-020 \\
\hline Gross alpha & EPA900.0 & $\mathrm{pCi} / \mathrm{g}$ & 10 & NA & EPA-600/4-80-032 \\
\hline Nonvolatile beta & EPA900.0 & $\mathrm{pCi} / \mathrm{g}$ & 1.0 & $\mathrm{NA}$ & EPA-600/4-80-032 \\
\hline Total alpha-emitting radium & EPA903.0 & $\mathrm{pCi} / \mathrm{g}$ & 1.0 & $\mathrm{NA}$ & EPA-600/4-80-032 \\
\hline Tritium & EPA906.0M & $\mathrm{pCi} / \mathrm{g}$ & 5 & NA & $\mathrm{EPA}-600 / 4 \cdot 80 \cdot 032$ \\
\hline Copper & EPA6010 & $\mathrm{mg} / \mathrm{kg}$ & 5.0 & NA & SW-846 \\
\hline Iron & EPA6010 & $\mathrm{mg} / \mathrm{kg}$ & 10 & NA & $\mathrm{SW} \cdot 846$ \\
\hline Magnesium & EPA6010 & $\mathrm{mg} / \mathrm{kg}$ & 500.00 & $\mathrm{NA}$ & SW-816 \\
\hline Manganese & EPA6010 & $\mathrm{mg} / \mathrm{kg}$ & 3.0 & $\mathrm{NA}$ & SW-846 \\
\hline Nickel & EPA6010 & $\mathrm{mg} / \mathrm{kg}$ & 8.0 & $\mathrm{NA}$ & SW-846 \\
\hline Potassium & EPA6010 & $\mathrm{mg} / \mathrm{kg}$ & 500.0 & NA & SW-846 \\
\hline Silver & EPA6010 & $\mathrm{mg} / \mathrm{kg}$ & 2.0 & $\mathrm{NA}$ & SW-846 \\
\hline Sodium & EPA6010 & $\mathrm{mg} / \mathrm{kg}$ & 500.0 & $\mathrm{NA}$ & SW-846 \\
\hline Vanadium & EPA6010 & $\mathrm{mg} / \mathrm{kg}$ & 5.0 & $\mathrm{NA}$ & SW-846 \\
\hline Zinc & EPA6010 & $\mathrm{mg} / \mathrm{kg}$ & 2.0 & NA & SW-846 \\
\hline Arsenic & EPA7060 & $\mathrm{mg} / \mathrm{kg}$ & 2.0 & $\mathrm{NA}$ & SW-846 \\
\hline Lead & EPA7421 & $\mathrm{mg} / \mathrm{kg}$ & 0.60 & $\mathrm{NA}$ & SW-846 \\
\hline Mercury & EPA7470 & $\mathrm{mg} / \mathrm{kg}$ & 0.10 & $\mathrm{NA}$ & SW-846 \\
\hline Selenium & EPA7740 & $\mathrm{mg} / \mathrm{kg}$ & 1.0 & $\mathrm{NA}$ & SW-846 \\
\hline Thallium & EPA7841 & $\mathrm{mg} / \mathrm{kg}$ & 1.0 & $\mathrm{NA}$ & SW-846 \\
\hline Aldrin & EPA8080 & $\mu \mathrm{g} / \mathrm{kg}$ & 1.7 & 2.68 & SW-846 \\
\hline alpha-BHC & EPA8080 & $\mu \mathrm{g} / \mathrm{kg}$ & 1.7 & 2.01 & SW-846 \\
\hline beta-BHC & EPA8080 & $\mu \mathrm{g} / \mathrm{kg}$ & 1.7 & 4.02 & SW-846 \\
\hline
\end{tabular}

NA - Not available.

$M$ - The method was modified by the laboratory. 
Table 8. Analytical Methods and Detection Limits, Continued

\begin{tabular}{|c|c|c|c|c|c|}
\hline Analyte & Method & Units & $\begin{array}{l}\text { Lab } \\
\text { DL }\end{array}$ & $\begin{array}{l}\text { Nominal } \\
\text { DL }\end{array}$ & Reference \\
\hline delta-BHC & EPA8080 & $\mu g / \mathrm{hg}$ & .1 .7 & 6.03 & SW-846 \\
\hline gamma-BHC & EPA8080 & $\mu \mathrm{g} / \mathrm{kg}$ & 1.7 & 2.68 & SW-846 \\
\hline alpha-Chlordane & EPA8080 & $\mu \mathrm{g} / \mathrm{kg}$ & 1.7 & & \\
\hline gamma-Chlordane & EPA8080 & $\mu g / k g$ & 1.7 & & \\
\hline$p, p^{\prime}-D D D$ & EPA8080 & $\mu g / \mathrm{kg}$ & 3.3 & 7.37 & SW-846 \\
\hline p,p'-DDE & EPA8080 & $\mu \mathrm{g} / \mathrm{kg}$ & 3.3 & 2.68 & SW-846 \\
\hline p,p'-DDT & EPA8080 & $\mu g / k g$ & 3.3 & 8.04 & SW-846 \\
\hline Dieldrin & EPA8080 & $\mu g / \mathrm{hg}$ & 3.3 & 1.34 & SW-846 \\
\hline Endosulfan I & EPA8080 & $\mu g / \mathrm{kg}$ & 1.7 & 9.38 & SW-846 \\
\hline Endosulfan II & EPA8080 & $\mu \mathrm{g} / \mathrm{hg}$ & 3.3 & 2.68 & SW-846 \\
\hline Endosulfan sulfate & EPA8080 & $\mu \mathrm{g} / \mathrm{kg}$ & 3.3 & 44.22 & SW-846 \\
\hline Endrin & EPA8080 & $\mu \mathrm{g} / \mathrm{kg}$ & 3.3 & 4.02 & SW-846 \\
\hline Endrin ketone & EPA8080 & $\mu g / 2 g$ & 3.3 & & \\
\hline Heptachlor & EPA8080 & $\mu g / \mathrm{kg}$ & 1.7 & 2.01 & SW-846 \\
\hline Heptachlor epoxide & EPA8080 & $\mu g / \mathrm{kg}$ & 1.7 & 55.61 & SW-846 \\
\hline Methoxychlor & EPA8080 & $\mu g / h g$ & 17 & 117.92 & SW.846 \\
\hline PCB 1016 & EPA8080 & $\mu g / \mathrm{kg}$ & 33 & $\mathrm{NA}$ & SWi-846 \\
\hline PCB 1221 & EPA8080 & $\mu g / \mathrm{kg}$ & 67 & NA & SW-846 \\
\hline PCB 1232 & EPA8080 & $\mu \mathrm{g} / \mathrm{kg}$ & 33 & NA & SW.846 \\
\hline PCB 1242 & EPA8080 & $\mu \mathrm{g} / \mathrm{kg}$ & 33 & 43.55 & SW-846 \\
\hline PCB 1248 & EPA8080 & $\mu \mathrm{g} / \mathrm{kg}$ & 33 & NA & SW. 846 \\
\hline PCB 1254 & EPA8080 & $\mu \mathrm{g} / \mathrm{kg}$ & 33 & $\mathrm{NA}$ & SW.846 \\
\hline PCB 1260 & EPA8080 & $\mu \mathrm{g} / \mathrm{kg}$ & 33 & NA & SW-846 \\
\hline Toxaphene & EPA8080 & $\mu g / \mathrm{kg}$ & 170 & 160.80 & SW-846 \\
\hline Acetone & EPA8240 & $\mu \mathrm{g} / \mathrm{kg}$ & 10 & 100 & SW.846 \\
\hline Benzene & EPA8240 & $\mu g / 2 g$ & 5 & 5 & SW-846 \\
\hline Bromodichloromethane & EPA8240 & $\mu \mathrm{g} / \mathrm{kg}$ & 5 & 5 & SW-846 \\
\hline Bromoform & EPA8240 & $\mu \mathrm{g} / \mathrm{kg}$ & 5 & 5 & SW-846 \\
\hline Bromomethane & EPA8240 & $\mu \mathrm{g} / \mathrm{kg}$ & 10 & 10 & SW.846 \\
\hline 2-Butanone & EPA8240 & $\mu g / k g$ & 10 & 100 & SW.846 \\
\hline Carbon disulfide & EPA8240 & $\mu \mathrm{g} / \mathrm{kg}$ & 5 & 5 & SW-846 \\
\hline Carbon tetrachloride & EPA8240 & $\mu \mathrm{g} / \mathrm{kg}$ & 5 & 5 & SW-846 \\
\hline Chlorobenzene & EPA8240 & $\mu \mathrm{g} / \mathrm{kg}$ & 5 & 5 & SW-846 \\
\hline Chloroethane & EPA8240 & $\mu \mathrm{g} / \mathrm{kg}$ & 10 & 10 & S.W.846 \\
\hline 2-Chloroethyl vinyl ether & EPA8240 & $\mu \mathrm{g} / \mathrm{kg}$ & 10 & 10 & $S \hbar$ S.846 \\
\hline Chloroform & EPA8240 & $\mu \mathrm{g} / \mathrm{kg}$ & 5 & 5 & SW.846 \\
\hline Chloromethane & EPA8240 & $\mu \mathrm{g} / \mathrm{kg}$ & 10 & 10 & SW-846 \\
\hline Dibromochloromethane & EPA8240 & $\mu \mathrm{g} / \mathrm{kg}$ & 5 & 5 & SW-846 \\
\hline 1,1-Dichloroethane & EPA8240 & $\mu \mathrm{g} / \mathrm{kg}$ & 5 & 5 & SW-846 \\
\hline 1,2-Dichloroethane & EPA8240 & $\mu g / k g$ & 5 & 5 & SW.846 \\
\hline 1,1-Dichloroethene & EPA8240 & $\mu g / \mathrm{kg}$ & 5 & 5 & SW-846 \\
\hline 1,2-Dichloroethylene (total) & EPA8240 & $\mu g / k g$ & 5 & & \\
\hline trans-1,2-Dichloroethylene & EPA8240 & $\mu g / k g$ & 5 & 5 & SW.846 \\
\hline 1,2-Dichloropropane & EPA8240 & $\mu g / \mathrm{kg}$ & 5 & 5 & SW.846 \\
\hline cis-1,3-Dichloropropene & EPA8240 & $\mu \mathrm{g} / \mathrm{kg}$ & 5 & 5 & SW-846 \\
\hline trans-1,3-Dichloropropene & EPA8240 & $\mu g / k g$ & 5 & 5 & SW-846 \\
\hline Ethyl benzene & EPA8240 & $\mu \mathrm{g} / \mathrm{kg}$ & 5 & 5 & SW.846 \\
\hline 2-Hexanone & EPA8240 & $\mu \mathrm{g} / \mathrm{kg}$ & 10 & 50 & SW-846 \\
\hline Methylene chloride & EPA8240 & $\mu \mathrm{g} / \mathrm{kg}$ & 5 & 5 & SW-846 \\
\hline
\end{tabular}


Table 8. Analytical Methods and Detection Limits, Continued

\begin{tabular}{|c|c|c|c|c|c|}
\hline Analve & Method & Units & $\begin{array}{l}\text { Lab } \\
\underline{\mathrm{DL}}\end{array}$ & $\begin{array}{l}\text { Nominal } \\
\underline{\mathrm{DL}}\end{array}$ & $\underline{\text { Reference }}$ \\
\hline 4-Methyl-2-pentanone & EPA8240 & $\mu g / \mathrm{hg}$ & 10 & 50 & SW-846 \\
\hline Styrene & EPA8240 & $\mu \mathrm{g} / \mathrm{kg}$ & 5 & 5 & SW-846 \\
\hline 1,1,2,2-Tetrachloroethane & EPA8240 & $\mu \mathrm{g} / \mathrm{kg}$ & 5 & 5 & SW-846 \\
\hline Tetrachloroethene & EPA8240 & $\mu \mathrm{g} / \mathrm{kg}$ & 5 & 5 & SW-846 \\
\hline Toluene & EPA8240 & $\mu g / \mathrm{kg}$ & 5 & 5 & SW-846 \\
\hline 1,1,1-Trichloroethane & EPA8240 & $\mu g / \mathrm{kg}$ & 5 & 5 & SW-846 \\
\hline 1,1,2-Trichloroethane & EPA8240 & $\mu \mathrm{g} / \mathrm{kg}$ & 5 & 5 & SW-846 \\
\hline Trichloroethene & EPA8240 & $\mu g / \mathrm{hg}$ & 5 & 5 & SW-846 \\
\hline Vinyl acetate & EPA8240 & $\mu \mathrm{g} / \mathrm{kg}$ & 10 & 50 & SW-846 \\
\hline Vinyl chloride & EPA8240 & $\mu \mathrm{g} / \mathrm{kg}$ & 10 & 10 & $\mathrm{SW}^{\top}-846$ \\
\hline Xylenes (total) & EPA8240 & $\mu \mathrm{g} / \mathrm{kg}$ & 5 & 5 & SWT-846 \\
\hline Acenaphthene & EPA8270 & $\mu \mathrm{g} / \mathrm{kg}$ & 330 & 660 & SW-846 \\
\hline Acenaphthylene & EPA8270 & $\mu \mathrm{g} / \mathrm{kg}$ & 330 & 660 & SW-846 \\
\hline Anthracene & EPA8270 & $\mu \mathrm{g} / \mathrm{kg}$ & 330 & 660 & $S W^{\top}-846$ \\
\hline Benzo(a)anthracene & EPA8270 & $\mu \mathrm{g} / \mathrm{hg}$ & 330 & 660 & SW -846 \\
\hline Benzo(a)pyrene & EPA8270 & $\mu \mathrm{g} / \mathrm{kg}$ & 330 & 660 & SW-846 \\
\hline Benzo(b)fluoranthene & EPA8270 & $\mu \mathrm{g} / \mathrm{kg}$ & 330 & 660 & SW-846 \\
\hline Benzo(g,h,i)perylene & EPA8270 & $\mu \mathrm{g} / \mathrm{kg}$ & 330 & 660 & SW -846 \\
\hline Benzoic acid & EPA8270 & $\mu \mathrm{g} / \mathrm{kg}$ & 1600 & 3300 & SW-846 \\
\hline Benzo(k)fluoranthene & EPA8270 & $\mu \mathrm{g} / \mathrm{kg}$ & 330 & 660 & SW-846 \\
\hline Benzyl alcohol & EPA8270 & $\mu \mathrm{g} / \mathrm{kg}$ & 330 & 1300 & SW-846 \\
\hline bis(2.Chloroethoxy)methane & EPA8270 & $\mu \mathrm{g} / \mathrm{hg}$ & 330 & 660 & SW-846 \\
\hline bis(2-Chloroethyl) ether & EPA8270 & $\mu \mathrm{g} / \mathrm{kg}$ & 330 & 660 & SW-846 \\
\hline bis(2-Chloroisopropyl) ether & EPA8270 & $\mu \mathrm{g} / \mathrm{kg}$ & 330 & 660 & SW-846 \\
\hline bis(2-Ethylhexyl) phthalate & EPA8270 & $\mu \mathrm{g} / \mathrm{kg}$ & 330 & 660 & SW-846 \\
\hline 4-Bromophenyl phenyl ether & EPA8270 & $\mu \mathrm{g} / \mathrm{kg}$ & 330 & 660 & SW.846 \\
\hline Butyl benzyl phthalate & EPA8270 & $\mu \mathrm{g} / \mathrm{kg}$ & 330 & 660 & SW-846 \\
\hline 4-Chloroaniline & EPA8270 & $\mu \mathrm{g} / \mathrm{kg}$ & 330 & 1300 & SW-846 \\
\hline $\begin{array}{l}\text { 4-Chloro-3-methylphenol } \\
\text { (para-Chloro-meta-cresol) }\end{array}$ & EPA8270 & $\mu \mathrm{g} / \mathrm{kg}$ & 330 & 1300 & SW.846 \\
\hline 2-Chloronaphthalene & EPA8270 & $\mu g / \mathrm{kg}$ & 330 & 660 & SW-846 \\
\hline 2.Chlorophenol & EPA8270 & $\mu \mathrm{g} / \mathrm{kg}$ & 330 & 660 & SW-846 \\
\hline 4-Chlorophenyl phenyl ether & EPA8270 & $\mu \mathrm{g} / \mathrm{kg}$ & 330 & 660 & SW.846 \\
\hline Chrysene & EPA8270 & $\mu \mathrm{g} / \mathrm{kg}$ & 330 & 660 & SW-846 \\
\hline Dibenz(a,h)anthracene & EPA8270 & $\mu \mathrm{g} / \mathrm{kg}$ & 330 & 660 & SW-846 \\
\hline Dibenzofuran & EPA8270 & $\mu \mathrm{g} / \mathrm{kg}$ & 330 & 660 & SW-846 \\
\hline 1,2-Dichlorobenzene & EPA8270 & $\mu \mathrm{g} / \mathrm{kg}$ & 330 & 660 & SW.846 \\
\hline 1,3-Dichlorobenzene & EPA8270 & $\mu \mathrm{g} / \mathrm{kg}$ & 330 & 660 & SW-846 \\
\hline 1,4-Dichlorobenzene & EPA8270 & $\mu \mathrm{g} / \mathrm{kg}$ & 330 & 660 & SW.846 \\
\hline 3,3'-Dichlorobenzidine & EPA8270 & $\mu \mathrm{g} / \mathrm{kg}$ & 660 & 1300 & SW.846 \\
\hline 2,4-Dichlorophenol & EPA8270 & $\mu \mathrm{g} / \mathrm{kg}$ & 330 & 660 & SW-846 \\
\hline Diethylphthalate & EPA8270 & $\mu \mathrm{g} / \mathrm{kg}$ & 330 & 660 & SW-846 \\
\hline 2,4-Dimethylphenol & EPA8270 & $\mu \mathrm{g} / \mathrm{kg}$ & 330 & 660 & SW-846 \\
\hline Dimethyl phthalate & EPA8270 & $\mu g / k g$ & 330 & 660 & SW-846 \\
\hline Di-n-butyl phthalate & EPA8270 & $\mu \mathrm{g} / \mathrm{kg}$ & 330 & 660 & SW.846 \\
\hline $\begin{array}{l}\text { 4,6-Dinitro-2-methylphenol } \\
\text { (4,6-Dinitro-ortho-cresol) }\end{array}$ & EPA8270 & $\mu \mathrm{g} / \mathrm{kg}$ & 1600 & 3300 & SW-846 \\
\hline 2,4-Dinitrophenol & 270 & $\mu \mathrm{g} \Omega$ & 1600 & 3300 & SW-846 \\
\hline 2,4-Dinitrotoluene & EPA8270 & $\mu g / \mathrm{kg}$ & 330 & 660 & SW.846 \\
\hline
\end{tabular}


Table 8. Analytical Methods and Detection Limits, Continued

\begin{tabular}{|c|c|c|c|c|c|}
\hline Analyte & Method & Units & $\begin{array}{l}\text { Lab } \\
\underline{\mathrm{DL}}\end{array}$ & $\begin{array}{l}\text { Nominal } \\
\text { DL. }\end{array}$ & Referenc \\
\hline 2,6-Dinitrotoluene & EPA8270 & $\mu \mathrm{g} / \mathrm{kg}$ & 330 & 660 & SW.846 \\
\hline Di-n-octyl phthalate & EPA8270 & $\mu \mathrm{g} / \mathrm{kg}$ & 330 & 660 & SW.846 \\
\hline Fluoranthene & EPA8270 & $\mu \mathrm{g} / \mathrm{kg}$ & 330 & 660 & SW-846 \\
\hline Fluorene & EPA8270 & $\mu \mathrm{g} / \mathrm{kg}$ & 330 & 660 & SW.846 \\
\hline Hexachlorobenzene & EPA8270 & $\mu \mathrm{g} / \mathrm{kg}$ & 330 & 660 & SW.846 \\
\hline Hexachlorobutadiene & EPA8270 & $\mu \mathrm{g} / \mathrm{kg}$ & 330 & 660 & SW-846 \\
\hline Hexachlorocyclopentadiene & EPA8270 & $\mu \mathrm{g} / \mathrm{kg}$ & 330 & 660 & SW.846 \\
\hline Hexachloroethane & EPA8270 & $\mu \mathrm{g} / \mathrm{kg}$ & 330 & 660 & SW-846 \\
\hline Indeno(1,2,3-cd)pyrene & EPA8270 & $\mu \mathrm{g} / \mathrm{kg}$ & 330 & 660 & SW-846 \\
\hline Isophorone & EPA8270 & $\mu \mathrm{g} / \mathrm{kg}$ & 330 & 660 & SW-846 \\
\hline 2-Methylnaphthalene & EPA8270 & $\mu \mathrm{g} / \mathrm{kg}$ & 330 & 660 & SW-846 \\
\hline 2-Methylphenol (o-Cresol) & EPA8270 & $\mu \mathrm{g} / \mathrm{kg}$ & 330 & 660 & SW-846 \\
\hline 4-Methylphenol (p-Cresol) & EPA8270 & $\mu \mathrm{g} / \mathrm{kg}$ & 330 & 660 & SW-846 \\
\hline Naphthalene & EPA8270 & $\mu \mathrm{g} / \mathrm{kg}$ & 330 & 660 & SW.846 \\
\hline 2-Nitroaniline & EPA8270 & $\mu \mathrm{g} / \mathrm{kg}$ & 1600 & 3300 & SW-846 \\
\hline 3-Nitroaniline & EPA8270 & $\mu \mathrm{g} / \mathrm{kg}$ & 1600 & 3300 & SW.846 \\
\hline 4-Nitroaniline & EPA8270 & $\mu \mathrm{g} / \mathrm{kg}$ & 1600 & 3300 & $\mathrm{SW}-846$ \\
\hline Nitrobenzene & EPA8270 & $\mu \mathrm{g} / \mathrm{kg}$ & 330 & 660 & SW-846 \\
\hline 2-Nitrophenol & EPA8270 & $\mu \mathrm{g} / \mathrm{kg}$ & 330 & 660 & $\mathrm{SW}-846$ \\
\hline 4-Nitrophenol & EPA8270 & $\mu \mathrm{g} / \mathrm{kg}$ & 1600 & 3300 & SW-846 \\
\hline N-Nitroso-di-n-propylamine & EPA8270 & $\mu \mathrm{g} / \mathrm{kg}$ & 330 & 660 & $\mathrm{SW}-846$ \\
\hline N-Nitrosodiphenylamine & EPA8270 & $\mu \mathrm{g} / \mathrm{kg}$ & 330 & 660 & SW-846 \\
\hline Pentachlorophenol & EPA8270 & $\mu \mathrm{g} / \mathrm{kg}$ & 1600. & 3300 & SW.846 \\
\hline Phenanthrene & EPA8270 & $\mu \mathrm{g} / \mathrm{kg}$ & 330 & 660 & SW-846 \\
\hline Phenol & EPA8270 & $\mu \mathrm{g} / \mathrm{kg}$ & 330 & 660 & SW-846 \\
\hline Pyrene & EPA8270 & $\mu \mathrm{g} / \mathrm{kg}$ & 330 & 660 & SW-846 \\
\hline 1,2,4-Trichlorobenzene & EPA8270 & $\mu \mathrm{g} / \mathrm{kg}$ & 330 & 660 & SW-846 \\
\hline 2,4,5-Trichlorophenol & EPA8270 & $\mu \mathrm{g} / \mathrm{kg}$ & 1600 & 660 & SW-846 \\
\hline 2,4,6-Trichlorophenol & EPA8270 & $\mu \mathrm{g} / \mathrm{kg}$ & 330 & 660 & SW-846 \\
\hline OCDD & EPA8280 & $\mathrm{ng} / \mathrm{g}$ & 2 & 2.35 & SW-846 \\
\hline OCDF & EPA8280 & $\mathrm{ng} / \mathrm{g}$. & 2 & & SW-846 \\
\hline $2,3,7,8-\mathrm{TCDD}$ & EPA8280 & $\mathrm{ng} / \mathrm{g}$ & 1 & 0.17 & SW-846 \\
\hline $2,3,7,8-\mathrm{TCDF}$ & EPA8280 & $\mathrm{ng} / \mathrm{g}$ & 1 & 0.11 & SW.846 \\
\hline Total HPCDD & EPA8280 & $\mathrm{ng} / \mathrm{g}$ & 1 & & SW-846 \\
\hline Total HPCDF & EPA8280 & $\mathrm{ng} / \mathrm{g}$ & 1 & & SW-846 \\
\hline Total HXCDD & EPA8280 & $\mathrm{ng} / \mathrm{g}$ & $\overline{1}$ & & SW.846 \\
\hline Total HXCDF & EPA8280 & $\mathrm{ng} / \mathrm{g}$ & $\overline{1}$ & & SW.846 \\
\hline Total PECDD & EPA8280 & $\mathrm{ng} / \mathrm{g}$ & $i$ & & SW-846 \\
\hline Total PECDF & EPA8280 & $\mathrm{ng} / \mathrm{g}$ & 1 & & SW.846 \\
\hline Total TCDD & EPA8280 & $\mathrm{ng} / \mathrm{g}$ & 1 & & SW. 846 \\
\hline Total TCDF & EPA8280 & $\mathrm{ng} / \mathrm{g}$ & 1 & & SW-846 \\
\hline $\mathrm{pH}$ & EPA9045 & $\mathrm{pH}$ & & NA & SW-846 \\
\hline Specific condu & EPA9050M & $\mu \mathrm{S} / \mathrm{cm}$ & & NA & SW.846 \\
\hline Cation exchange capacity & EPA9080 & meq $/ \mathrm{L}$ & 0.01 & $\mathrm{NA}$ & SW-846 \\
\hline
\end{tabular}


Table 8. Analytical Methods and Detection Limits, Continued

\begin{tabular}{|c|c|c|}
\hline Analute & Method & Units \\
\hline
\end{tabular}

Water

Total dissolved solids

Total suspended solids

Chloride

Fluoride

Nitrate as nitrogen

Sulfate

Cyanide

Total organic carbon

Total organic halogens

Gross alpha

Nonvolatile beta

Total alpha-emitting radium

Tritium

Aluminum

Antimony

Barium

Beryllium

Cadmium

Calcium

Chromium

Cobalt

Copper

Iron

Magnesium

Manganese

Nickel

Potassium

Silver

Sodium

Vanadium

Zinc

Arsenic

Lead

Mercury

Selenium

Thallium

Aldrin

alpha-BHC

beta-BHC

delta-BHC

gamma-BHC

alpha-Chlordane

gamma-Chlordane

$p, p^{\prime}-D D D$

p,p'-DDE

p.p'-DDT

EPA160.1

$\mathrm{mg} / \mathrm{L}$

EPA160.2 mg/L

EPA300.0

$\mathrm{mg} / \mathrm{L}$

EPA300.0

$\mathrm{mg} \pi$

EPA300.0

$m g / L$

EPA300.0

$m g / L$

EPA335.3

EPA415.1

$\mu g / L$

EPA450.1

$\mathrm{mg} / \mathrm{L}$

EPA900.0

$\mu g / L$

$\mathrm{pCi} / 2.0 \quad 3.00$

NA

EPA-600/4-79-020

EPA900.0

$\mathrm{pCi} / 2 \quad 1.0 \quad 4.00$

EPA903.0

$\mathrm{pCi} / 2 \quad 1.0 \quad 1.00$

EPA906.0M

$\mathrm{pCj} / \mathrm{mL} \quad 1.0$

NA

$\mu \mathrm{g} / \mathrm{L} \quad 200.0$

45.00

ug/L $\quad 60.0$

32.00

EPA6010

$\mu \mathrm{g} / \mathrm{L} \quad 200$

2.00

$\mu g / L \quad 5$

0.30

$\mu g / L \quad 5.0 \quad 4.00$

$\mu \mathrm{g} / \mathrm{L} \quad 5000.0 \quad 10.00$

$\mu g / L \quad 10 \quad 7.00$

$\mu g / L \quad 50 \quad 7.00$

$\mu \mathrm{g} / \mathrm{L} \quad 25$

6.00

$\begin{array}{lll}\mathrm{mg} / \mathrm{L} & 20 \quad 0.007\end{array}$

$\mu \mathrm{g} / \mathrm{L} \quad 5000.0 \quad 30.00$

$\mu \mathrm{g} / \mathrm{L} \quad 15 \quad 2.00$

$\mu \mathrm{g} / \mathrm{L} \quad 40 \quad 15.00$

$\mu \mathrm{g} / \mathrm{L} \quad 5000 \quad \mathrm{NA}$

$\mu \mathrm{g} / \mathrm{L} \quad 10 \quad 7.00$

$\mu \mathrm{g} / \mathrm{L} \quad 5000.0 \quad 29.00$

$\mu \mathrm{g} / \mathrm{L} \quad 50.0 \quad 8.00$

$\mu \mathrm{g} / \mathrm{L} \quad 20.0 \quad 2.00$

$\mu \mathrm{g} / \mathrm{L} \quad 10 \quad 1.00$

$\mu \mathrm{g} / \mathrm{L} \quad 3.0 \quad 1.00$

$\mu \mathrm{g} / \mathrm{L} \quad 0.20 \quad 0.20$

$\mu \mathrm{g} / \mathrm{L} \quad 5.0 \quad 2.00$

$\mu \mathrm{g} / \mathrm{L} \quad 10.0 \quad 1.00$

$\mu \mathrm{g} / \mathrm{L} \quad 0.05$

0.04

$\mu \mathrm{g} / \mathrm{L} \quad 0.05$

0.03

$\mu \mathrm{g} / \mathrm{L} \quad 0.05$

0.06

$\mu \mathrm{g} / \mathrm{L}$

0.05

0.09

$\mu \mathrm{g} / \mathrm{L}$

$\mu \mathrm{g} / \mathrm{L}$

0.05

0.04

EPA8080

$\mu \mathrm{g} / \mathrm{L}$

EPA8080

EPA8080

EPA8080

$\mu \mathrm{g} / \mathrm{L}$

$\mu \mathrm{g} / \mathrm{L}$

EPA8080

Dieldrin

EPA8080

$\mu \mathrm{g} / \mathrm{L}$

$\mu \mathrm{g} / \mathrm{L}$

0.10

0.11

0.10

0.04

0.10

0.12

0.10

0.02

Keith

Keith

Pfaff

Keith

EPA-600/4-79-020

EPA-600/4-79-020

Keith

EPA-600/4-80-032

EPA-600/4-80-032

EPA-600/4-80-032

EPA-600/4-80-032

SW-846

SW-846

SW-846

SW.846

SW-846

SW-846

SW-846

SW-846

SW-846

SW-846

SW-846

SW-846

SW-846

SW-846

SW-846

SW-846

SW-846

SW.846

SW-846

SW-846

SW.846

SW-846

SW.-846

SW-846

SW-846

SW-846

SW-846

SW.846

SW-846

SW-846

SW-846

SW-846 
Table 8. Analytical Methods and Detection Limits, Continued

\begin{tabular}{|c|c|c|c|c|c|}
\hline Analyte & Method & Units & $\begin{array}{l}\mathrm{Lab} \\
\underline{\mathrm{DL}}\end{array}$ & $\begin{array}{l}\text { Nominal } \\
\text { DL }\end{array}$ & Reference \\
\hline Endosulfan I & EPA8080 & $\mu \mathrm{g} / \mathrm{L}$ & 0.05 & 0.14 & SW-846 \\
\hline Endosulfan II & EPA8080 & $\mu g / L$ & 0.10 & 0.04 & SW.846 \\
\hline Endosulfan sulfate & EPA8080 & $\mu \mathrm{g} / \mathrm{L}$ & 0.10 & 0.66 & SW-846 \\
\hline Endrin & EPA8080 & $\mu \mathrm{g} / \mathrm{L}$ & 0.10 & 0.06 & SW-846 \\
\hline Endrin ketone & EPA8080 & $\mu \mathrm{g} / \mathrm{L}$ & 0.10 & & \\
\hline Heptachlor & EPA8080 & $\mu \mathrm{g} / \mathrm{L}$ & 0.05 & 0.03 & SW-846 \\
\hline Heptachlor epoxide & EPA8080 & $\mu g / L$ & 0.05 & 0.83 & SW-846 \\
\hline Methoxychlor & EPA8080 & $\mu \mathrm{g} / \mathrm{L}$ & 0.50 & 1.76 & SW-846 \\
\hline PCB 1016 & EPA8080 & $\mu \mathrm{g} / \mathrm{L}$ & 1.0 & NA & SW-846 \\
\hline РCB 1221 & EPA8080 & $\mu \mathrm{g} / \mathrm{L}$ & 2.0 & $\mathrm{NA}$ & SW-846 \\
\hline PCB 1232 & EPA8080 & $\mu g / L$ & 1.0 & NA & SW-846 \\
\hline PCB 1242 & EPA8080 & $\mu \mathrm{g} / \mathrm{L}$ & 1.0 & 0.65 & SW-846 \\
\hline PCB 1248 & EPA8080 & $\mu \mathrm{g} / \mathrm{L}$ & 1.0 & NA & SW-846 \\
\hline PCB 1254 & EPA8080 & $\mu \mathrm{g} / \mathrm{L}$ & 1.0 & $\mathrm{NA}$ & SW-846 \\
\hline PCB 1260 & EPA8080 & $\mu \mathrm{g} / \mathrm{L}$ & 1.0 & NA & SW-846 \\
\hline Toxaphene & EPA8080 & $\mu \mathrm{g} / \mathrm{L}$ & 5.0 & 2.40 & SW-846 \\
\hline Acetone & EPA8240 & $\mu \mathrm{g} / \mathrm{L}$ & 10 & 100 & SW-846 \\
\hline Benzene & EPA8240 & $\mu \mathrm{g} / \mathrm{L}$ & 5 & 5 & SW'-846 \\
\hline Bromodichloromethane & EPA8240 & $\mu g / L$ & 5 & 5 & SW-846 \\
\hline Bromoform & EPA8240 & $\mu g / L$ & 5 & 5 & SW-846 \\
\hline Bromomethane & EPA8240 & $\mu \mathrm{g} / \mathrm{L}$ & 10 & 10 & SW-846 \\
\hline 2-Butanone & EPA8240 & $\mu \mathrm{g} / \mathrm{L}$ & 10 & 100 & SW-846 \\
\hline Carbon disulfide & EPA8240 & $\mu \mathrm{g} / \mathrm{L}$ & 5 & 5 & SW-846 \\
\hline Carbon tetrachloride & EPA8240 & $\mu \mathrm{g} / \mathrm{L}$ & 5 & 5 & SWW-846 \\
\hline Chlorobenzene & EPA8240 & $\mu g / L$ & 5 & 5 & $\dot{S W} \cdot 846$ \\
\hline Chloroethane & EPA8240 & $\mu \mathrm{g} / \mathrm{L}$ & 10 & 10 & SW-846 \\
\hline 2-Chloroethyl vinyl ether & EPA8240 & $\mu \mathrm{g} / \mathrm{L}$ & 10 & 10 & SW-846 \\
\hline Chloroform & EPA8240 & $\mu \mathrm{g} / \mathrm{L}$ & 5 & 5 & SW-846 \\
\hline Chloromethane & EPA8240 & $\mu \mathrm{g} / \mathrm{L}$ & 10 & 10 & SW-846 \\
\hline Dibromochloromethane & EPA8240 & $\mu g / L$ & 5 & 5 & SW-846 \\
\hline 1,1-Dichloroethane & EPA8240 & $\mu \mathrm{g} / \mathrm{L}$ & 5 & 5 & SW-846 \\
\hline 1,2-Dichloroethane & EPA8240 & $\mu \mathrm{g} / \mathrm{L}$ & 5 & 5 & SW-846 \\
\hline 1,1-Dichloroethene & EPA8240 & $\mu \mathrm{g} / \mathrm{L}$ & 5 & 5 & SW-846 \\
\hline 1,2-Dichloroethene (total) & EPA8240 & $\mu \mathrm{g} / \mathrm{L}$ & 5 & & \\
\hline trans-1,2-Dichloroethene & EPA8240 & $\mu \mathrm{g} / \mathrm{L}$ & 5 & 5 & SW-846 \\
\hline 1,2-Dichloropropane & EPA8240 & $\mu \mathrm{g} / \mathrm{L}$ & 5 & 5 & SW-846 \\
\hline cis-1,3-Dichloropropene & EPA8240 & $\mu \mathrm{g} / \mathrm{L}$ & 5 & 5 & SW-846 \\
\hline trans-1,3-Dichloropropene & EPA8240 & $\mu g / L$ & 5 & 5 & SW.846 \\
\hline Ethyl benzene & EPA8240 & $\mu \mathrm{g} / \mathrm{L}$ & 5 & 5 & SW-846 \\
\hline 2-Hexanone & EPA8240 & $\mu g / L$ & 10 & 50 & SW-846 \\
\hline Methylene chloride & EPA8240 & $\mu \mathrm{g} / \mathrm{L}$ & 5 & 5 & SW-846 \\
\hline 4-Methyl-2-pentanone & EPA8240 & $\mu \mathrm{g} / \mathrm{L}$ & 10 & 50 & SW-846 \\
\hline Styrene & EPA8240 & $\mu g / L$ & 5 & 5 & SW-846 \\
\hline 1,1,2,2-Tetrachloroethane & EPA8240 & $\mu g / L$ & 5 & 5 & SW.846 \\
\hline Tetrachloroethene & EPA8240 & $\mu g / L$ & 5 & 5 & SW-846 \\
\hline Toluene & EPA8240 & $\mu \mathrm{g} / \mathrm{L}$ & 5 & 5 & SW-846 \\
\hline 1,1,1-Trichloroethane & EPA8240 & $\mu \mathrm{g} / \mathrm{L}$ & 5 & 5 & SW-846 \\
\hline 1,1,2-Trichloroethane & EPA8240 & $\mu g / L$ & 5 & 5 & $\mathrm{SW} \cdot 846$ \\
\hline Trichloroethene & EPA8240 & $\mu \mathrm{g} / \mathrm{L}$ & 5 & 5 & SW.846 \\
\hline
\end{tabular}


Table 8. Analytical Methods and Detection Limits, Continued

\begin{tabular}{|c|c|c|c|c|c|}
\hline Analite & Method & Units & $\begin{array}{l}\text { Lab } \\
\text { DL }\end{array}$ & $\begin{array}{l}\text { Nominal } \\
\text { DL }\end{array}$ & Reference \\
\hline Vinyl acetate & EPA8240 & $\mu \mathrm{g} / \mathrm{L}$ & 10 & 50 & SW-846 \\
\hline Vinyl chloride & EPA8240 & $\mu \mathrm{g} / \mathrm{L}$ & 10 & 10 & SW'846 \\
\hline Xylenes (total) & EPA8240 & $\mu \mathrm{g} / \mathrm{L}$ & 5 & 5 & SW-846 \\
\hline Acenaphthene & EPA8270 & $\mu \mathrm{g} / \mathrm{L}$ & 10 & 10 & SW-846 \\
\hline Acenaphthylene & EPA8270 & $\mu \mathrm{g} / \mathrm{L}$ & 10 & 10 & SW-846 \\
\hline Anthracene & EPA8270 & $\mu g / L$ & 10 & 10 & SW-846 \\
\hline Benzo(a)anthracene & EPA8270 & $\mu \mathrm{g} / \mathrm{L}$ & 10 & 10 & SW-846 \\
\hline Benzo(a)pyrene & EPA8270 & $\mu \mathrm{g} / \mathrm{L}$ & 10 & 10 & $\mathrm{SW}-846$ \\
\hline Benzo(b)fluoranthene & EPA8270 & $\mu \mathrm{g} / \mathrm{L}$ & 10 & 10 & SW-846 \\
\hline Benzo(g,h,i)perylene & EPA8270 & $\mu \mathrm{g} / \mathrm{L}$ & 10 & 10 & $S W \cdot 846$ \\
\hline Benzoic acid & EPA8270 & $\mu \mathrm{g} / \mathrm{L}$ & 50 & 50 & $S W^{\top}-846$ \\
\hline Benzo(k)fluoranthene & EPA8270 & $\mu \mathrm{g} / \mathrm{L}$ & 10 & 10 & SW.846 \\
\hline Benzyl alcohol & EPA8270 & $\mu g / L$ & 10 & 20 & SW -846 \\
\hline bis(2-Chloroethoxy) methane & EPA8270 & $\mu g / L$ & 10 & 10 & SW-846 \\
\hline bis(2-Chloroethyl) ether & EPA8270 & $\mu \mathrm{g} / \mathrm{L}$ & 10 & 10 & STi-846 \\
\hline bis(2-Chloroisopropyl) ether & EPA8270 & $\mu \mathrm{g} / \mathrm{L}$ & 10 & 10 & $S W-846$ \\
\hline bis(2-Ethylhexyl) phthalate & EPA8270 & $\mu \mathrm{g} / \mathrm{L}$ & 10 & 10 & SW-846 \\
\hline 4-Bromophenyl phenyl ether & EPA8270 & $\mu g / L$ & 10 & 10 & SW.846 \\
\hline Butyl benzyl phthalate & EPA8270 & $\mu \mathrm{g} / \mathrm{L}$ & 10 & 10 & $S W-846$ \\
\hline 4-Chloroaniline & EPA8270 & $\mu \mathrm{g} / \mathrm{L}$ & 10 & 20 & SW-846 \\
\hline $\begin{array}{l}\text { 4-Chloro-3-methylphenol } \\
\text { (para-Chloro-meta-cresol) }\end{array}$ & EPA8270 & $\mu \mathrm{g} / \mathrm{L}$ & 10 & 20 & SW-846 \\
\hline 2-Chloronaphthalene & EPA8270 & $\mu g / L$ & 10 & 10 & SW-846 \\
\hline 2.Chlorophenol & EPA 8270 & ug/L & 10 & 10 & SW.846 \\
\hline 4-Chlorophenyl phenyl ether & EPA8270 & $\mu \mathrm{g} / \mathrm{L}$ & 10 & 10 & SW.846 \\
\hline Chrysene & EPA8270 & $\mu \mathrm{g} / \mathrm{L}$ & 10 & 10 & SW.846 \\
\hline Dibenz $(\mathrm{a}, \mathrm{h})$ anthracene & EPA8270 & $\mu g / L$ & 10 & 10 & SW-846 \\
\hline Dibenzofuran & EPA8270 & $\mu \mathrm{g} / \mathrm{L}$ & 10 & 10 & SW.846 \\
\hline 1,2-Dichlorobenzene & EPA8270 & $\mu \mathrm{g} / \mathrm{L}$ & 10 & 10 & SW-846 \\
\hline 1,3-Dichlorobenzene & EPA8270 & $\mu g / L$ & 10 & 10 & SW-846 \\
\hline 1,4-Dichlorobenzene & EPA8270 & $\mu g / L$ & 10 & 10 & SW.846 \\
\hline 3,3'-Dichlorobenzidine & EPA8270 & $\mu g / L$ & 20 & 20 & SW-846 \\
\hline 2,4-Dichlorophenol & EPA8270 & $\mu \mathrm{g} / \mathrm{L}$ & 10 & 10 & SW.846 \\
\hline Diethylphthalate & EPA8270 & $\mu \mathrm{g} / \mathrm{L}$ & 10 & .10 & SW-846 \\
\hline 2,4-Dimethylphenol & EPA8270 & $\mu \mathrm{g} / \mathrm{L}$ & 10 & 10 & SW!. 846 \\
\hline Dimethyl phthalate & EPA8270 & $\mu \mathrm{g} / \mathrm{L}$ & 10 & 10 & SW-846 \\
\hline Di-n-butylphthalate & EPA8270 & $\mu \mathrm{g} / \mathrm{L}$ & 10 & 10 & SW.846 \\
\hline $\begin{array}{l}\text { 4,6-Dinitro-2-methylphenol } \\
\text { (4,6-Dinitro-ortho-cresol) }\end{array}$ & EPA8270 & $\mu \mathrm{g} / \mathrm{L}$ & 50 & 50 & SW-846 \\
\hline 2,4-Dinitrophenol & EPA8270 & $\mu g / L$ & 50 & 50 & SW.846 \\
\hline 2,4-Dinitrotoluene & EPA8270 & $\mu \mathrm{g} / \mathrm{L}$ & 10 & 10 & SW-846 \\
\hline 2,6-Dinitrotoluene & EPA8270 & $\mu \mathrm{g} / \mathrm{L}$ & 10 & 10 & SW -846 \\
\hline Di-n-octyl phthalate & EPA8270 & $\mu \mathrm{g} / \mathrm{L}$ & 10 & 10 & SW-846 \\
\hline Fluoranthene & EPA8270 & $\mu \mathrm{g} / \mathrm{L}$ & 10 & 10 & SW-846 \\
\hline Fluorene & EPA8270 & $\mu \mathrm{g} / \mathrm{L}$ & 10 & 10 & SW-846 \\
\hline Hexachlorobenzene & ЕРA8270 & $\mu \mathrm{g} / \mathrm{L}$ & 10 & 10 & SW.846 \\
\hline Hexachlorobutadiene & EPA 8270 & $\mu \mathrm{g} / \mathrm{L}$ & 10 & 10 & SW-846 \\
\hline Hexachlorocyclo- & EPA8270 & $\mu \mathrm{g} / \mathrm{L}$ & 10 & 10 & SW-846 \\
\hline
\end{tabular}


Table 8. Analytical Methods and Detection Limits, Continued

\begin{tabular}{|c|c|c|c|c|c|}
\hline Analyte & Method & Units & $\begin{array}{l}\text { Lab } \\
\underline{\mathrm{DL}}\end{array}$ & $\begin{array}{l}\text { Nominal } \\
\text { DL }\end{array}$ & Referenc \\
\hline Hexachloroethane & EPA8270 & $\mu \mathrm{g} / \mathrm{L}$ & 10 & 10 & SW.846 \\
\hline Indeno(1,2,3-cd)pyrene & EPA8270 & $\mu \mathrm{g} / \mathrm{L}$ & 10 & 10 & SW-846 \\
\hline Isophorone & EPA8270 & $\mu g / L$ & 10 & 10 & SW.846 \\
\hline 2-Methylnaphthalene & EPA8270 & $\mu \mathrm{g} / \mathrm{L}$ & 10 & 10 & SW.846 \\
\hline 2-Methylphenol (o-Cresol) & EPA8270 & $\mu \mathrm{g} / \mathrm{L}$ & 10 & 10 & SW-846 \\
\hline 4-Methylphenol (p-Cresol) & EPA8270 & $\mu \mathrm{g} / \mathrm{L}$ & 10 & 10 & SW-846 \\
\hline Naphthalene & EPA8270 & $\mu g / L$ & 10 & 10 & SW.846 \\
\hline 2-Nitroaniline & EPA8270 & $\mu g / L$ & 50 & 50 & SW-846 \\
\hline 3-Nitroaniline & EPA8270 & ug $/ \mathrm{L}$ & 50 & 50 & SW-846 \\
\hline 4-Nitroaniline & EPA8270 & $\mu g / L$ & 50 & 50 & SW-846 \\
\hline Nitrobenzene & EPA8270 & $\mu \mathrm{g} / \mathrm{L}$ & 10 & 10 & SW.846 \\
\hline 2-Nitrophenol & EPA8270 & $\mu \mathrm{g} / \mathrm{L}$ & 10 & 10 & SW-846 \\
\hline 4-Nitrophenol & EPA8270 & $\mu g / L$ & 50 & 50 & SW.846 \\
\hline N-Nitroso-di-n-propylamine & EPA8270 & $\mu g / L$ & 10 & 10 & SW.846 \\
\hline N-Nitrosodiphenylamine & EPA8270 & $\mu g / L$ & 10 & 10 & SW-846 \\
\hline Pentachlorophenol & EPA8270 & $\mu g / L$ & 50 & 50 & SW-846 \\
\hline Phenanthrene & EPA8270 & $\mu g / L$ & 10 & 10 & SW-846 \\
\hline Phenol & EPA8270 & $\mu g / L$ & 10 & 10 & SW-846 \\
\hline Pyrene & EPA8270 & $\mu g / L$ & 10 & 10 & SW.846 \\
\hline 1,2,4-Trichlorobenzene & EPA8270 & $\mu \mathrm{g} / \mathrm{L}$ & 10 & 10 & SW.846 \\
\hline 2,4,5-Trichlorophenol & EPA8270 & $\mu g / L$ & 50 & 10 & SW.846 \\
\hline $2,4,6$-Trichlorophenol & EPA8270 & $\mu g / L$ & 10 & 10 & SW.846 \\
\hline OCDD & EPA8280 & $\mathrm{ng} / \mathrm{L}$ & 10 & 3.93 & SW.846 \\
\hline OCDF & EPA8280 & $\mathrm{ng} / \mathrm{L}$ & 10 & & \\
\hline $2,3,7,8-\mathrm{TCDD}$ & EPA8280 & $\mathrm{ng} / \mathrm{L}$ & 5.0 & 0.44 & SW-846 \\
\hline $2,3,7,8-\mathrm{TCDF}$ & EPA8280 & $\mathrm{ng} / \mathrm{L}$ & 5.0 & 0.63 & SW.846 \\
\hline Total HPCDD & EPA8280 & $\mathrm{ng} / \mathrm{L}$ & 5 & & SW-846 \\
\hline Total HPCDF & EPA8280 & $\mathrm{ng} / \mathrm{L}$ & 5 & & SW.846 \\
\hline Total HXCDD & EPA8280 & $\mathrm{ng} / \mathrm{L}$ & 5 & & SW-846 \\
\hline Total HXCDF & EPA8280 & $\mathrm{ng} / \mathrm{L}$ & 5 & & SW-846 \\
\hline Total PECDD & EPA8280 & $\mathrm{ng} / \mathrm{L}$ & 5 & & SW-846 \\
\hline Total PECDF & EPA8280 & $\mathrm{ng} / \mathrm{L}$ & 5 & & SW-846 \\
\hline Total TCDD & EPA8280 & $\mathrm{ng} / \mathrm{L}$ & 5 & & SW-846 \\
\hline Total TCDF & EPA8280 & $\mathrm{ng} / \mathrm{L}$ & 5 & & SW.846 \\
\hline $\mathrm{pH}$ & EPA9045 & $\mathrm{pH}$ & & $\mathrm{NA}$ & SW. 846 \\
\hline Specific conductance & EPA9050M & $\mu \mathrm{S} / \mathrm{cm}$ & & $\mathrm{NA}$ & SW.846 \\
\hline TCLP & & & & & . \\
\hline Arsenic, TCLP & EPA6010 & $\mu g / L$ & 10 & 53.00 & SW-846 \\
\hline Barium, TCLP & EPA6010 & $\mu \mathrm{g} / \mathrm{L}$ & 200 & 2.00 & SW-846 \\
\hline Cadmium, TCLP & EPA6010 & $\mu \mathrm{g} / \mathrm{L}$ & 5 & 4.00 & SW.846 \\
\hline Chromium, TCLP & EPA6010 & $\mu \mathrm{g} / \mathrm{L}$ & 10 & 7.00 & SW-846 \\
\hline Lead, TCLP & EPA6010 & $\mu \mathrm{g} / \mathrm{L}$ & 3 & 42.00 & SW-846 \\
\hline Selenium, TCLP & EPA6010 & $\mu \mathrm{g} / \mathrm{L}$ & 5 & 75.00 & SW-846 \\
\hline Mercury, TCLP & EPA7470 & $\mu \mathrm{g} / \mathrm{L}$ & 0.2 & 0.20 & SW-846 \\
\hline gamma-BHC & EPA8080 & $\mu \mathrm{g} / \mathrm{L}$ & 0.05 & 0.04 & SW-846 \\
\hline alpha-Chlordane & EPA8080 & $\mu g / L$ & 0.05 & & \\
\hline gamma-Chlordane & EPA8080 & $\mu \mathrm{g} / \mathrm{L}$ & 0.05 & & \\
\hline
\end{tabular}


Table 8. Analytical Methods and Detection Limits, Continued

\begin{tabular}{|c|c|c|c|c|c|}
\hline Analute & Method & Units & $\begin{array}{l}\text { Lab } \\
\underline{\mathrm{DL}}\end{array}$ & $\begin{array}{l}\text { Nominal } \\
\text { DL }\end{array}$ & Reference \\
\hline Endrin & EPA 8080 & $\mu \mathrm{g} / \mathrm{L}$ & 0.1 & 0.06 & SW-846 \\
\hline $\begin{array}{l}\text { Heptachlor (and its } \\
\text { hydroxide) }\end{array}$ & EPA8080 & $\mu g / L$ & 0.05 & 0.03 & SW-846 \\
\hline Heptachlor epoxide & EPA8080 & $\mu g / L$ & & 0.83 & SW-846 \\
\hline Methoxychlor & EPA8080 & $\mu \mathrm{g} / \mathrm{L}$ & 0.5 & 1.76 & SW-846 \\
\hline Toxaphene & EPA8080 & $\mu g / L$ & 5 & 2.40 & SW-846 \\
\hline $2,4-\mathrm{D}$ & EPA8150 & $\mu g / L$ & 10 & 12.0 & SW-846 \\
\hline $2,4,5-T$ & EPA8150 & $\mu g / L$ & 5 & 2.0 & SW-846 \\
\hline 2,4,5-TP (Silvex) & EPA8150 & $\mu \mathrm{g} / \mathrm{L}$ & 5 & 1.70 & SW.846 \\
\hline Benzene & EPA8240 & $\mu \mathrm{g} / \mathrm{L}$ & 5 & 5.00 & SW-846 \\
\hline Carbon tetrachloride & EPA8240 & $\mu \mathrm{g} / \mathrm{L}$ & 5 & 5.00 & SW-846 \\
\hline Chlorobenzene & EPA8240 & $\mu \mathrm{g} / \mathrm{L}$ & 5 & 5.00 & SW-846 \\
\hline Chloroform & EPA8240 & $\mu \mathrm{g} / \mathrm{L}$ & 5 & 5.00 & SW.846 \\
\hline m-Cresol (3-Methylphenol) & EPA8270 & $\mu \mathrm{g} / \mathrm{L}$ & 10 & & \\
\hline o-Cresol (2-Methylphenol) & EPA8270 & $\mu \mathrm{g} / \mathrm{L}$ & 10 & 10.00 & SW.846 \\
\hline p-Cresol (4-Methylphenol) & EPA8270 & $\mu \mathrm{g} / \mathrm{L}$ & 10 & 10.00 & SW-846 \\
\hline 1,2-Dichloroethane & EPA8240 & $\mu \mathrm{g} / \mathrm{L}$ & 5 & & \\
\hline 1,1-Dichloroethylene & EPA8240 & $\mu g / L$ & 5 & 5.00 & SW.846 \\
\hline Methyl ethyl ketone & EPA8240 & $\mu g / L$ & 10 & 100.00 & SW.846 \\
\hline Tetrachloroethylene & EPA8240 & $\mu \mathrm{g} / \mathrm{L}$ & 5 & 5.00 & SW-846 \\
\hline Trichloroethylene & EPA8240 & $\mu g / L$ & 5 & 5.00 & $S W^{\prime} \cdot 846$ \\
\hline Vinyl chloride & EPA8240 & $\mu \mathrm{g} / \mathrm{L}$ & 10 & 10.00 & SW-846 \\
\hline 1,4-Dichlorobenzene & EPA8270 & $\mu g / L$ & 10 & 10.00 & SW-846 \\
\hline 2,4-Dinitrotoluene & EPA8270 & $\mu \mathrm{g} / \mathrm{L}$ & 10 & 10.00 & SW-846 \\
\hline Hexachlorobenzene & EPA8270 & $\mu \mathrm{g} / \mathrm{L}$ & & 10.00 & SW-846 \\
\hline Hexachlorobutadiene & EPA8270 & $\mu \mathrm{g} / \mathrm{L}$ & 10 & 10.00 & SW-846 \\
\hline Hexachloroethane & EPA8270 & $\mu \mathrm{g} / \mathrm{L}$ & 10 & 10.00 & SW.846 \\
\hline Nitrobenzene & EPA8270 & $\mu \mathrm{g} / \mathrm{L}$ & 10 & 10.00 & SW-846 \\
\hline Pentachlorophenol & EPA8270 & $\mu \mathrm{g} / \mathrm{L}$ & 50 & 50.00 & SW-846 \\
\hline Pyridine & EPA8270 & $\mu \mathrm{g} / \mathrm{L}$ & 10 & & \\
\hline 2,4,5-Trichlorophenol & EPA8270 & $\mu \mathrm{g} / \mathrm{L}$ & 50 & 10.00 & SW-846 \\
\hline 2,4,6-Trichlorophenol & EPA8270 & $\mu \mathrm{g} / \mathrm{L}$ & 10 & 10.00 & SW-846 \\
\hline
\end{tabular}

\section{Laboratory Quality Assurance/Quality Control (QA/OC)}

The laboratories follow their own Quality Assurance plans, which are designed to measure and document the accuracy and precision of the laboratory for the various analytical methods it performs. The laboratories also are guided by published method-specific QA/QC requirements as well as other regulatory and contract-required quality assurance procedures. Their adherence to QA/QC practices is evaluated in a number of ways noted in this report. One general measure is examination of the laboratory's achievement in analyses of performance evaluation samples and quality control standards, as discussed above. The performance evaluations discussed below are another.

The sections on validation and verification in this report provide information on evaluation of the laboratories with respect to the specific analyses performed for this project. 


\section{Performance Reviews}

The laboratories providing analytical services to EMS are periodically evaluated for adherence to their Quality Assurance plans and to ensure that the analytical work is being performed in the contract-prescribed manner with respect to proper laboratory procedure, method execution, documentation, record-keeping, and data reporting. The performance reviews, or annual evaluations, are undertaken to identify potential problems at the laboratory or with the contract; as part of continuing efforts to maintain high-quality defensible data for environmental monitoring and assessment at SRS.

\section{Roy F. Weston Analytical Laboratories}

Roy F. Weston, the contracted laboratory, was visited by EMS personnel on June 14-15, 1993. The performance review produced no findings of nonconformance to written requirements. The review team made several observations of conditions or practices which could, in its opinion, affect safety, productivity, or reliability. The team made a recommendation corresponding to each observation, as follow's.

Housekeeping.

The air-circulating vent above the metals preparation bench was very dusty. This could result in contamination of samples during preparation, which in turn could affect the level of metals detected in blanks and WSRC samples.

\section{Recommendation.}

Improve housekeeping in this area by cleaning the vent and beginning a routine of cleaning and inspecting air vents.

Pipette calibration.

There is no evidence of an adjustable pipette calibration program. Inaccurate pipette calibrations could drastically affect the accuracy and precision of the data produced.

Recommendation.

Weston should establish an adjustable pipette calibration program and document the program. It should include a control chart program based on the weight of the volume used during pipetting.

\section{Documentation.}

Original entries, and some corrections to the original entries, in the maintenance log for the mercury analyzer are inconsistent. Analyst initials are not routinely written next to each entry in the log. Overall documentation does not adhere to the guidelines established by EPA quality assurance standards or to Weston QA/QC protocol.

\section{Recommendation.}

Analysts should routinely place initials next to each entry in all logs. Additional training for Weston personnel on basic documentation is essential if the lab is to adhere to its QA/QC protocol. 


\section{Analvzer maintenance.}

Maintenance of the mercury analyzer does not adhere to EPA QC protocol. Specifically, the wash receptacle of the mercury analyzer is never cleaned out. The analyst simply adds water as needed, which could result in contamination during the analysis of mercury in WSRC samples.

Recommendation.

The wash receptacle should be cleaned out once a week to prevent build-up of contaminants. All maintenance operations should be performed according to EPA procedures to eliminate any imprecision in the mercury determinations.

ICP instrument maintenance. .

A deficiency was encountered in the maintenance of ICP instruments used for metal determination: The required monthly change-out of the air filter was not performed in May 1993.

Recommendation.

Perform all maintenance operations as required by procedure and according to EPA recommendations to eliminate any imprecision during metal determinations by ICP.

Data entrv.

As with any lab new to the EPD/EMS monitoring program, there were the usual problems with data entry and format.

Recommendation.

If there are any questions about the EDD or invoice formats, or about the way an item should be entered into the EDD, then the analyst or project manager should contact EPD/EMS personnel for clarification.

Alpha source decav.

The alpha source (Polonium-210) used for daily source checks for the monitors that perform the initial radiological screen of samples showed significant decay from.month to month in the log books. However, there was no clear policy for when the source should be replaced.

Recommendation.

There should be an activity-based policy for replacing a daily source check standard.

Chain-of-custodv $(\mathrm{COC})$ data entrv.

The manual re-entry of information from the WSRC COC to the Weston COC allows for transcription errors. This is especially a problem for samples sent to Weston's subcontractors.

Recommendation. A copy of the original WSRC COC should be forwarded to Weston's subcontractors with the Weston $\mathrm{COC}$ for an independent review of the information. 
A noteworthy practice remarked by the reviewers is Weston's use of EPA contract laboratory program (CLP) standards for WSRC samples. This is not required by the contract, but is an added QA practice performed by Weston to improve the quality of WSRC results.

During the visit, evaluation checklists were filled out covering many specific QA/QC criteria. Comments made on the basis of those checklists include these issues: The organics laboratory solvent storage cabinets are not properly vented for the prevention of possible laboratory contamination: data handling and review computer programs are not validated before use; and the $\mathrm{pH}$ of samples is not checked and recorded on the $\mathrm{COC}$ upon receipt.

\section{TMA/Eberline}

TMA/Eberline, the radiological subcontractor to Roy F. Weston, Inc., the contracted laboratory, was visited by Weston and EMS personnel on September 21-22, 1993. The evalutation report contained both findings and observations. As defined by Weston, findings are items that can, if not corrected, negatively affect the quality of data provided to clients, and observations are items considered not in compliance with best management or good laboratory practices.

\section{Findings}

Several findings that could affect data quality were noted.

Expired Standards.

Several expired standards were found throughout the laboratory. Timely removal of expired standards from storage is necessary to prevent inadvertent use of such solutions.

Corrective Actions Not Implemented.

As a result of comments from previous reviews of TMA, Standard Operating Procedures on water quality checks and documentation were to be written and implemented. These operating procedures were still in draft form after the deadline provided by TMA:

Records Retention.

TMA's policy for long-term record storage is not clear. This presents a problem regarding regulatory compliance with record retention requirements.

\section{Observations}

Observations that could affect data quality also were noted.

Variable Strontium-90 Results.

Performance evaluation results reviewed during the evaluation demonstrated a greater degree of variability than is expected due to method, matrix, or analyst variabilities. The source of the variability needs to be investigated and a corrective action taken to ensure the method does not drift out of control. 
National Institute of Standards and Technology (NIST) Traceability of Standards. Some standard certificates do not contain NIST standard traceability. Traceability of standards is a major concern for Department of Energy contracts. TMA must provide evidence of NIST traceability or the equivalent for standard solutions.

Performance Evaluation (PE) Trend Analysis.

TMA currently does not perform trend analysis of $\mathrm{PE}$ results. Charting PE results can be a valuable tool to identify nonconforming situations before they become systemic errors.

Accuracv of $\mathrm{pH}$ Readings.

The $\mathrm{pH}$ readings recorded as a result of preservation verification have more accuracy than can be provided with $\mathrm{pH}$ paper. The best possible reading with a $\mathrm{pH}$ paper is whole number $\mathrm{pH}$ units. 


\section{Data Management/Administrative Files}

This section discusses the generation, processing, and storage of paper and computer records produced as part of this project. The processing of the materials follows methodology prescribed by the Environmental Geochemistry Group (EGG) of EPD/EMS.

EGG establishes administrative files for each project that are maintained as permanent SRS records. The following materials are placed in the administrative files:

- The bound sample collection logbooks

- The original copies of the chain-of-custody records

- The case narratives from the laboratories

- The data validation logbooks

- Copies of all correspondence between the data validation personnel and the laboratories

- Data entry cover sheets for data files created by EGG

EGG generates computer data files from paper records using double-entry methods to ensure a very low transcription error rate. Computer data files are also received from the laboratories for analytical data. Proposed changes to the data are recorded in the data validation logbook, made to a copy of the data, approved by the Project Technical Manager, then confirmed by running a computer comparison of the modified and original data files. EGG stores the final computer data files, the original source files, and computer listings of all changes made to the original files in the administrative files.

Access to this material is available through the manager of EGG. The administrative files will be maintained within EGG control until June 30, 1995. The material will then be transferred to long-term box storage for permanent storage. EGG plans to have the final data files available for SRS computer users indefinitely. Please contact the manager of EGG to obtain access to this data. 
Policy

Data of Quality Objective 3 (as defined in Quality Assurance/Quality Control Guidance for Removal Activities) and analytical quality level 3 (as defined in Data Quality Objectives for Remedial Response Activities) have been determined most appropriate to meet the data quality objectives of this investigation. Validation and verification activities were undertaken to assess the data against these standards, especially with regard to the following issues:

Sample documentation

Maintenance of chain of custody

Adherence to sample holding time requirements

Achievement of contract-required detection limit

Analytical instrument calibration

Analyte identification

Analyte quantitation

Evaluation of the laboratories' performance in analyte identification and quantitation included examinations of the following:

Analytical error

Contamination of blanks

Laboratory performance in the analysis of independent standards

Definitions of Qualifier Codes

Each analytical record in the computer data files contains three qualifier fields: the result qualifier, the analysis qualifier, and the bias code. The result qualifier describes the analytical result. The analysis qualifier describes why result qualifiers are present, and the bias code describes whether the result is biased high or low or has poor precision. The laboratory uses these fields in reporting the data. During validation and verification of analytical data, additional qualifiers may be applied to provide information about data quality.

Definitions of the qualifiers used in the data files follow. See the "Qualificiation Policy" subsection of this report for details on application of these qualifiers during validation and verification.

Result Qualifier J. The $J$ qualifier indicates that the result is an estimated value.

A specific example of the use of the $J$ qualifier is its application (with the analysis qualifier . Q) when the required holding time from sample date to analysis date was exceeded.

Result Qualifier $\mathrm{L}$. The result qualifier $L$ is assigned to indicate that the reported result is off-scale high. The actual value is not known, but is greater than the value reported. When the laboratory uses this qualifier, it generally also re-analyzes the sample after dilution to provide a quantitative result. See, e.g., acetone for $D 43111608$ (sample 100426). 
Résult Qualifier $\mathrm{P}$. The result qualifier $P$, indicating that precision cannot be determined, is applied to sample results in batches for which the laboratory did not perform matrix spike analyses.

Result Qualifier $R$. The result qualifier $R$ indicates that the result has been rejected. It is applied during validation and verification when the result has exceeded the recommended quality control limits for level 3 acceptability criteria. These results may meet the less rigorous requirements of level 1 or 2 .

Result Qualifier $U$. The result qualifier $U$ is assigned by the laboratory to an analytical result below the sample quantification limit.

Analvsis Qualifier A. Relative retention times or mass spectra criteria were not met. (Assigned based on the laboratory data records review [LDRR].)

Analysis Qualifier C. A Laboratory Control Sample (LCS) was outside the specified recovery limits.

Analysis Qualifier G. Instrument performance for pesticides was outside criteria for any condition. (Assigned based on LDRR.)

Anavsis Qualifier H. Internal standards criteria were not met. (Assigned based on the LDRR.)

Analvsis Qualifier I. Matrix spike results did not meet criteria.

Analysis Qualifier L. Initial or continuing calibration checks were unacceptable. (Assigned based on the LDRR.)

Analysis Qualifier O. Surrogate criteria were not met.

Analysis Qualifier Q. Holding time was exceeded.

Analysis Qualifier $T$. The $T$ analysis qualifier is specific for dioxin/furan analyses and was used to replace $H, S$, and $X$ qualifiers assigned by the laboratories that are invalid in the current data format. The analytical laboratory assigned an $H$ qualifier if peak height instead of peak area was used to quantitate the analyte. An $S$ qualifier was assigned when the signal-to-noise ratio of the confirmation ion did not meet requirements, but the peak was determined to be positive by the analyst. An $X$ qualifier was used by the laboratory when isotope ratio criteria were not met using either peak height or peak area, but the peak was thought to be positive by the analyst. The result qualifier $J$ is now applied with the $T$ analysis qualifier to replace $H, S$, or $X$.

Analysis Qualifier V. Method blank criteria were not met.

Analysis Qualifier X. The relative percent difference (RPD) calculated from duplicate analyses or matrix spike/matrix spike duplicates was not within control limits.

Analysis Qualifier $Y$. Samples were unpreserved or improperly preserved. 
Bias Code $\mathrm{H}$. The result is believed to be biased high.

Bias Code $\mathrm{L}$. The result is believed to be biased low.

\section{Qualification Policy}

This section describes the EMS data validation policy for assigning qualifiers to RFI/RI analytical data. Qualification policy is based on EPA's Contract Laboratory Program Statement of Work (CLP SOW), Functional Guidelines for Organics Analyses, Functional Guidelines for Inorganics Analy'ses (Functional Guidelines 7/88), SW-846, and Westinghouse Hanford Company's Data Validation Procedures for Radiochemical Analyses.

Qualification criteria for instrument calibration, analyte identification, and analyte quantitation are given in the EMS informal "Procedures for Qualifjing Data Based on LDRRs."

$\mathrm{RQ}=$ result qualifier $\quad \mathrm{AQ}=$ analysis qualifier $\quad \mathrm{BQ}=$ bias qualifier

\section{Holding Times}

Criteria: EPA holding times for extraction and analysis

Action: Assign $\mathrm{RQ}=J, \mathrm{AQ}=Q$ to all analyses for which holding time has been exceeded.

\section{Sample Preservation}

Criteria: Temperature $<6^{\circ} \mathrm{C}$ for organics and inorganics; $\mathrm{pH} \leq 2$ for water samples

Action: Assign $\mathrm{AQ}=Y$ for all exceedances.

\section{Instrument Calibration, Analyte Identification, Analyte Quantitation}

Criteria: See LDRR checklists for each analytical method.

Action: See "Procedures for Qualifying Data Based on LDRRs."

\section{Trip Blanks}

Criteria: CLP $5 \times / 10 \times$ rule

Action: Add $R Q=J$ to the failed analyte(s) in every sample shipped in the same cooler as the trip blank.

\section{Field Blanks}

No qualification is done on the basis of field blank results. 


\section{Rinsate Blanks}

No qualification is done on the basis of rinsate blank results.

\section{Method Blanks}

Criteria: CLP $5 \times / 10 \times$ rule

Action: If an analyte is detected in a method blank, assign $\mathrm{AQ}=V$ to that analyte in the blank and in every sample of the preparation batch. If the analyte concentration in a sample is $<5 \times$ that in the blank ( $<10 \times$ for common lab contaminants), assign $R Q=U$ and $\mathrm{AQ}=V$ to the analyte in the sample.

\section{Field Duplicates}

No qualification is done based on field duplicate results.

\section{Lab Duplicates}

Criteria:

Organics: No qualification is done on this basis. Inorganics: Functional Guidelines T/88, page 10

Rads: Hanford Data Validation Procedures for Radiochemical Analyses

Action: For soil samples, if duplicate analysis results for a particular analyte fall outside the control limits for the Relative Percent Difference (RPD), assign $A Q=X$ to that analyte in the parent and duplicate samples.

For water samples, if the RPD for a particular analyte is outside the appropriate control windows, assign $R Q=J$ and $A Q=X$ to that analyte in the parent and duplicate samples, and $\mathrm{AQ}=X$ to the failed analyte in every sample in the same preparation batch.

\section{Matrix Spikes}

\section{Criteria:}

Volatiles: CLP SOW, Table 7, page D-55 VOA

Semivolatiles: CLP SOW, Table 7, page D-59 SV

Pesticides: CLP SOW, page D.61 PEST

Dioxins: acceptable recovery $=50-110 \%$

Inorganics: Functional Guidelines 7/88, page 11

Action: If a matrix spike fails criteria, qualifiers are added only to (1) the failed analyte in the matrix spike, and (2) the failed analyte in the parent sample of the matrix spike. For organics, assign $\mathrm{RQ}=J, \mathrm{AQ}=I, \mathrm{BQ}=L$ or $H$. For inorganics, assign $\mathrm{RQ}$ according to criteria, $\mathrm{AQ}=I, \mathrm{BQ}=L$ or $H$.

For non-radiological analyses, if the laboratory fails to supply matrix spike data, flag all analytes in all samples in the appropriate preparation batch. Assign $\mathrm{RQ}=P, \mathrm{AQ}=I$. 


\section{Laboratory Control Samples \& Blank Spikes (LCS/BS)}

Criteria:

- Organics: same as matrix spike criteria

Inorganics: $80-120 \%$ recovery

Rads: $80-120 \%$ recovery (following Hanford)

Action: Assign $\mathrm{AQ}=C$ to the failed analyte(s) in the LCS/BS. Assign $\mathrm{RQ}=J, \mathrm{AQ}=C$ to the failed analyte(s) in every sample in the same preparation batch as the LCS/BS.

\section{Surrogate Recovery}

\section{Criteria:}

Volatiles by Method 8240: SW.846, Table 8, page 8240.38

Volatiles by Method 8260: SW-846, Table 9, page 8260.44

Pesticides by Method 8080: 60-150\% (from CLP SOW, page D-56 PEST) ${ }^{1}$

Semivolatiles by Method 8270: SW.846, Table 8, page 8270-31

Dioxins: not applicable

Action:

Volatiles \& Pesticides: If one or more surrogates fail acceptance criteria, assign $R Q=J$ or $U J, A Q=O$ to all analytes in the sample. If all the failed surrogates have low recoveries, assign $\mathrm{BQ}=L$. If all the failed surrogates have high recoveries, assign $\mathrm{BQ}=H$. If one surrogate is high and another is low, do not assign a bias qualifier. Also, assign $A Q=O$ to the failed surrogate(s).

Semivolatiles: If only one surrogate is bad, and its recovery is $>10 \%$, take no action. If more than one fails, assign $\mathrm{RQ}=J$ or $U J, \mathrm{AQ}=O$ to all analytes in the sample. If a recovery is negative, assign $R$. If all the failed surrogates have low (high) recoveries, assign $\mathrm{BQ}=L(H)$. If one surrogate is high and another is low, do not assign a bias qualifier. Also, assign $\mathrm{AQ}=O$ to the failed surrogate(s).

\section{Scope}

The validation and verification process conducted by EGG consists of several independent operations. The changes and data qualification identified by these operations are incorporated into the data validation logbook and are made to the electronic data files. The project technical manager is responsible for overseeing and controlling this process.

The operations conducted include examining the electronic data files and paper records and conducting on-site reviews of the laboratory records. The validation and verification operations are summarized in this section.

\footnotetext{
'During this investigation, the laboratory was still using a surrogate (DBC) that is not included in the current SOW, but is listed in the February 1988 organics SOW as having limits of 20-150\%. Those limits were used in evaluating results with that surrogate.
} 


\section{Review of the Electronic Data Files}

Data reviewers used computer programs and lookup tables to review the computer data files of sample collection, shipping, and analytical data. The computer programs performed the following types of examinations of the data:

The following fields were checked to ensure they were not blank: analyte code, sample identification number, sample collection date, lab receipt date, analysis date, analysis batch, laboratory code, laboratory sample identification number, analytical method, instrument, analyst's initials, and EPD/EMS receipt date.

The following fields were checked to ensure the data was within a range of values: sample ;; identification number (between 100,000 and 999,999); sample collection date (after January 1, 1993 and before the date the program was run); lab receipt date (between sample collection date and the program run date except for laboratory blanks and TCLP samples; for TCLP samples, identified by a $T$ in the sample fraction field, the lab receipt date must be equal to or before the sample collection date); analysis date (same as or later than lab receipt date and before the date the program was run); analysis time (an integer between 1 and 2,400 or blank); detection limit (greater than 0); analytical result (a real number if result qualifier is not $M$ or $T$; blank if result qualifier contains $M$ or $T$ ); accuracy (a real number or blank); residual weight (a positive number for radionuclide analyses; blank for others); number of dilutions ( 0 or a positive integer); dilution factor, number of dilutions (if dilution factor is 1 , then number of dilutions must be 0 ; if number of dilutions is greater than 0 , dilution factor cannot equal 1); nominal concentration (a positive number or blank; cannot equal 0 if result units indicate a percent recovery); percent solids (less than or equal to 100 or blank); bottle number (an integer equal to or between 10,000,000 and $99,999,999$ ); material (if percent solid greater than 0 , material must equal $S$ [soil]; if less than 0 , material must equal $W$ [water]).

The following fields were checked against a lookup table or a list of valid codes: laboratory code, laboratory duplicate code, analyte code (testname), analytical method, result qualifier, analysis qualifier, bias of analysis, result units, and sample fraction.

\section{Specific Examinations.}

The computer review of the analytical data also included the following examinations:

Sample holding times were checked from the electronic data deliverable sent by the analytical laboratory. The sample collection date was subtracted from the date the analysis was performed and the result was checked against a lookup table of holding times. Any analyses performed after the holding time was exceeded were qualified with a $Q$ in the analysis qualifier field and $\mathrm{a} J$ in the result qualifier field. Holding times to extraction were not checked because the extraction date was not reported by the analytical laboratory.

Reported detection limits were checked against the method detection limits to determine if they were correctly adjusted to reflect dilution factors, including soil moisture. For soils, sample aliquot size varied, but the sample weight was not reported; therefore, fluctuations due to sample size could not be checked. Fluctuations of two to three times the detection 
limit were considered acceptable for soils. Greater fluctuations were queried in laboratory letters.

Relationships between analytical, sample, and station records were checked to ensure that the station field in the station data records was identical to the first 8 characters of the planned sample name in the planned analyses data records. The planned sample name is a concatenation of facility identifier, sampling point, and sample number.

Additionally, the computer program checked for duplicate records, and for matching records as follows:

There must be a station record for each sample record. The station and sample records must have the same entry in the station field.

There must be at least one planned analyses record for each sample record. The planned analyses record and the sample record must have the same entries in the planned station, planned top of interval, and planned bottom of interval fields.

There must be a bottle receipt record for each sample bottle record. The bottle receipt and sample bottle records must have the same bottle label number.

There must be a sample record for each sample bottle record. The sample and sample bottle records must have the same sample identification number.

The number of sample bottle records for each sample identification number must match the number of bottles field on the matching sample record.

There must be a sample record for each chain-of-custody record. The sample and chain-ofcustody records must have the same sample identification number and sample collection date.

There must be a sample record for each analytical record except for laboratory standards and blanks. The sample and analytical records must have the same sample identification number and sample collection date.

There must be a chain-of-custody record for each analytical record except for laboratory blanks and laboratory-generated QA/QC samples. The chain-of-custody and analytical records must have the same sample identification number, sample collection date, and laboratory sample ID.

There must be a planned analyses record for each analysis record except laboratory standards and blanks. Each analytical record should match a sample record by sample identification number. The analyte from the analysis record and the planned sample name, planned top of interval, and planned bottom of interval fields from the sample record should match a planned analyses record.

There must be a chain-of-custody record for each sample record and for each analytical record except for laboratory standards and blanks. The sample, chain-of-custody, and 
analytical records must have the same sample identification number and sample collection date.

There must be a sample record for each planned analyses record. The planned analyses record and the sample record must have the same entries in the planned station, planned top of interval, and planned bottom of interval fields.

There must be at least one analytical record for each planned analyses record. The analytical record and the planned analyses record combined with the sample record must have the same entries in the analyte and sample identification number fields.

The data validation team resolved anomalies wherever possible. An anomaly might also result in qualification of the data, or a determination and recording of it as unresolvable (e.g., missing Munsell colors from field data).

\section{Checking of QA/QC Samples}

QA/QC samples include field-generated blanks and duplicates and laboratory-generated blanks, matrix spikes, surrogates, internal standards, and others. Both field- and laboratory-generated blanks are checked for contamination which may affect sample results, and environmental samples are qualified when contamination is above established levels.

The recoveries obtained by the laboratory for matrix spikes and surrogates are examined, and the number of matrix spikes is checked for adherence to QA 3 requirements. Internal standards and calibration samples for a portion of the samples are examined during the laboratory data records review. Qualification of environmental samples is done when any of these QA/QC samples exceed criteria.

\section{Review of the Analytical Narratives}

The cover letters and analysis summaries, or analytical narratives, that accompany the EDDs from the laboratories contain information about the data that does not appear elsewhere. The narratives are reviewed and used as a reference during computerized checking of the EDDs.

\section{Laboratory Data Records Reviews}

On September 21-22, 1993, a laboratory data records review (LDRR) was conducted at TMA/Eberline in Oak Ridge, TN, for tritium, total alpha-emitting radium, gross alpha, and nonvolatile beta analyses associated with the D-Area Burning/Rubble Pits RFI/RI waste unit. The review was conducted by C.R. Williamson, T.P. Killeen, W. Frazier, K.J. Kuelske, B.S. Crandall, and S.T. Nichols.

On December 6-10, 1993, data records were reviewed at Weston-Lionville (PA) for organics and inorganics analyses associated with the D-Area Burning/Rubble Pits RFI/RI waste unit. The review was conducted by W. Kubilius, K. Ward, J. Hammock, D. Mohon, L. DeWịtt, S. Stinson, and R. Hunter. 
Because an unusually large number of errors were detected during validation and verification of TMA data, Science Applications International Corporation (SAIC) and Walt Kubilius of EMS conducted a second LDRR at TMA on March 24-25, 1994, to check the laboratory's EDDs against the laboratory's analytical data and records. Only five fields were checked: sample results, minimum detectable activity (MDA or detection limit), accuracy, units, and sample identification.

The purpose of the reviews was to investigate technical validation issues discussed in Quality Assurance/Quality Control Guidance for Removal Activities that are not adequately addressed by computer checking of the EDD, review of analytical narratives, or review of COC forms. These technical issues include instrument calibration, analyte identification, and analyte quantitation. Validation checllists were used to assess the instrument printouts associated with particular analyses. Each checklist consists of 12 to 20 questions. For nonradionuclide analyses, the checklists were prepared from five sources: Q.A/QC Guidance for Removal Activities, SW-846, Functional Guidelines for Organics Analy'ses, Functional Guidelines for Inorganics Analyses, and discussions with laboratory personnel. For radiological analyses, the checklists were prepared from two sources: Westinghouse-Hanford's Data Validation Procedures for Radiochemical Analysis (WHC-SD-EN-SPP-001, Rev. 1) and discussions with laboratory personnel.

For the findings from the reviews, see the "Laboratory Data Records Reviews" subsections in the "Data Validation and Verification: Findings" section of this report. 


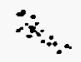


The data provided with this report are final and complete.

Many of the problems detected during validation and verification were resolved during the process, as a result of communication with the samplers and laboratories. There was one issue which could not be verified:

\section{Sample Preservation}

Sample $\mathrm{pH}$ was not recorded by the laboratories, so conformance to the requirement was not verifiable.

\section{Qualifiers Applied as a Result of EDD Review}

Discrepancies found in the data following computer checking of the EDDs were qualified as follows:

\section{Rejected Results}

Only one result for this project was rejected: the pentachlorophenol spike associated with sample 100400.

\section{Holding Time}

The holding times for all analyses were validated based on EPA guidance. When EPArecommended holding times were exceeded, these samples were given the $J$ result qualifier and the $Q$ analysis qualifier.

All laboratory $\mathrm{pH}$ analyses are qualified with $\mathrm{J}$ and $\mathrm{Q}$ because $\mathrm{pH}$ holding time is exceeded before the samples leave the field.

\section{Detection Limit}

Dioxin and furan results between method detection limit and sample quantitation limit were qualified with the $J$ result qualifier.

\section{Matrix Spikes}

The analysis qualifier $I$ and a bias qualifier of $L$ or $H$ were added to individual matrix spike results and results for the parent sample when the matrix spike results were outside the control limits listed in the "Qualification Policy" section of this report. Associated sample results (from the same batch) were not qualified. 


\section{Method Blanks}

Detected concentrations of target analytes in method blanks caused the assignment of $V$ qualifiers to associated data. For water samples, results for the analyte for all samples in the same preparation batch were qualified with $V$ if the sample result was less than 5 times the result in the associated blank, except that for common laboratory contaminants, the $V$ analysis qualifier was assigned if the sample result was less than 10 times the result in the associated blank

\section{Laboratory Control Samples}

Samples associated with Laboratory Control Samples outside recovery limits were qualified with $J$ as well as $C$.

\section{Surrogate Recoveries}

The analysis qualifier $O$ and a bias qualifier of $L$ or $H$ was added to surrogate results with recoveries outside the control limits listed in the "Qualification Policy" section of this report. Associated samples were qualified as described in that section.

\section{Sample Preservation}

The analysis qualifier $Y$ was assigned if the temperature of the cooler in which the sample was shipped was above $6^{\circ} \mathrm{C}$ upon receipt at the laboratory.

\section{Bias}

The laboratory used the qualifier 1 from an older data format along with the $R$ result qualifier to indicate recovery for matrix spikes and surrogates below control limits. This was replaced with the bias qualifier $L$. The laboratory used the qualifier 2 from an older data format along with the $R$ result qualifier to indicate recovery for matrix spikes and surrogates above control limits. This was replaced with the bias qualifier $H$. The laboratory used two other qualifiers, 3 and 4 , to indicate recovery of poor precision and recovery outside control limits because of matrix interference, respectively. These qualifiers were removed from the data and not replaced.

\section{Further Information}

The "Discussion" section of this report contains a table showing the total number of samples having various types of qualifiers.

\section{Results of Checking QA/QC Samples}

EPA-published methods, used for most of the analyses for this project, require specific types and numbers of quality control analyses. The number of each type of analysis performed for this project is presented in Table 9 . 
Table 9. Number of Analytical Records, by Method and Type of Record

\begin{tabular}{|c|c|c|c|c|c|}
\hline Sample Records & $\begin{array}{l}\text { Laboratory } \\
\text { Duplicates }\end{array}$ & $\begin{array}{l}\text { Blank Spikes or } \\
\text { Laboratory Control } \\
\text { Samples (Mctals) }\end{array}$ & Laboralory Blanks & Surrogates & $\begin{array}{l}\text { Malrix } \\
\text { Spikesl } \\
\text { Malrix Spike }\end{array}$ \\
\hline & & & & purtogalces & Duplicales \\
\hline
\end{tabular}

\begin{tabular}{|c|c|c|c|c|c|c|c|c|c|c|c|c|}
\hline Mcthod & Soil & Water & Soil & Water & $\underline{\text { Soil }}$ & Walcr & $\underline{\text { Soil }}$ & Watcr & Śoil & Walcr & Soil & Walce \\
\hline$\triangle S^{\prime} T M 2216$ & 170 & 0 & 14 & 0 & & & 1 & 8 & & & & \\
\hline EPA160.2 & 0 & 12 & 0 & 3 & & & 0 & 6 & & & & \\
\hline LPA300.0 & 0 & $16^{\circ}$ & 0 & 1 & 0 & 16 & 0 & 10 & & & 0 & 3 \\
\hline EPA335.3 & 2 & 0 & & & 4 & 0 & 2 & 0 & & & & \\
\hline EPA353.1 & & & & & 0 & 3 & 0 & 2 & & & & \\
\hline EPA115.1 & 43 & 6 & 4 & 0 & 38 & 17 & 29 & 12 & & & & \\
\hline EPA6010 & 729 & 180 & 42 & 14 & 321 & 310 & 162 & 163 & & & 84 & 56 \\
\hline EPA7060 & 101 & 19 & 6 & 2 & 40 & 38 & 20 & 19 & & & 12 & 4 \\
\hline ЕРА7121 & 101 & 19 & $\mathbf{6}$ & 2 & 40 & 10 & 20 & 19 & & & 12 & 4 \\
\hline EP^7170 & 101 & 21 & 6 & 1 & 40 & 10 & 30 & 30 & & & 7 & 5 \\
\hline$E P \wedge 7710$ & 101 & 19 & 6 & 2 & 10 & 38 & 20 & 19) & & & 12 & 1 \\
\hline EP $\Lambda 7811$ & 2 & 0 & & & 4 & 0 & 2 & 0 & & & & \\
\hline ЕРА 8080 & 1,894 & 10 & 223 & 1 & 154 & 52 & 726 & 36 & 103 & 16 & 47 & 30 \\
\hline EPA8150 & 0 & 15 & & & 0 & 23 & 0 & 12 & & & 0 & 21 \\
\hline EPA8210 & 3,511 & 1,395 & 468 & 236 & 253 & 146 & 1,616 & 768 & 387 & 168 & 80 & 60 \\
\hline LPA8270 & 5,891 & 150 & 432 & 108 & 372 & 177 & 1,681 & 310 & 621 & 111 & 88 & 94 \\
\hline EP $\Lambda 8280$ & 820 & 0 & 90 & 0 & 132 & 0 & 168 & 0 & 234 & 0 & 12 & 0 \\
\hline ЕРАО00.0 & 180 & 55 & 26 & 11 & 0 & 37 & 23 & 15 & & & & \\
\hline ЕРА9020 & 0 & 1 & & & 0 & 10 & 0 & 7 & & & & \\
\hline LPA903.0 & 90 & 27 & 13 & 7 & 0 & 20 & 13 & 7 & & & & \\
\hline ЕРА9015 & 13 & 6 & 1 & 1 & & & & & & & & \\
\hline LPР 9050 & 35 & 0 & 3. & 0 & 18 & 9 & 12 & 6 & & & & \\
\hline EP $\Lambda 906.0$ & 89 & 27 & 11 & 7 & 0 & 20 & 11 & 7 & & & & \\
\hline EPA9080 & 35 & 0 & 6 & 0 & & & & & & & & \\
\hline
\end{tabular}




\section{Numbers of Matrix Spikes}

Analysis of eight.matrix spikes is required for QA 3 validation. As shown in table 10, this requirement was generally met. With the exceptions of chloride and fluoride (which are not QA 3 constituents), dioxins and furans, compounds spiked only for TCLP analyses, and p, $p^{\circ}$-DDT for method EPA8080, this requirement was met for all methods used.

Table 10. Matrix Spikes Performed

\begin{tabular}{|c|c|c|c|c|c|}
\hline Method & sinalute & $\begin{array}{l}\text { Spikes in } \\
\text { W'ater }\end{array}$ & $\begin{array}{l}\text { Spikes in } \\
\text { Soil }\end{array}$ & $\begin{array}{l}\text { TCLP } \\
\text { Spikes }\end{array}$ & $\begin{array}{l}\text { Total } \\
\text { Spikes }\end{array}$ \\
\hline \multirow[t]{2}{*}{ EPA 300.0} & fluoride & 1 & & & 1 \\
\hline & sulfate & 1 & & & 1 \\
\hline \multirow[t]{10}{*}{ EPA 6010} & silver & 2 & 6. & 4 & 12 \\
\hline & arsenic & & & 4 & 4 \\
\hline & barium & 2 & 6 & 4 & 12 \\
\hline & cadmium & 2 & 6 & 4 & 12 \\
\hline & chromium & 2 & 6 & 4 & 12 \\
\hline & copper & 2 & 6 & & 8 \\
\hline & manganese & 2 & 6 & & 8 \\
\hline & nickel & 2 & 6 & & 8 \\
\hline & lead & & & 4 & 4 \\
\hline & selenium & & & 4 & 4 \\
\hline EPA 7060 & arsenic & 2 & 6 & & 8 \\
\hline EPA 7421 & lead & 2 & 6 & & .8 \\
\hline EPA 7470 & mercury & 1 & 6 & 4 & 11 \\
\hline EPA 7740 & selenium & 2 & 6 & & 8 \\
\hline \multirow[t]{11}{*}{ EPA 8080} & alpha-chlordane & & & 4 & 4 \\
\hline & aldrin & & 8 & & 8 \\
\hline & dieldrin & & 8 & & 8 \\
\hline & endrin & & 8 & 4 & 12 \\
\hline & decafluorobiphenyl & & & 3 & 3 \\
\hline & gamma-chlordane & & & 4 & 4 \\
\hline & heptachlor & & 8 & 4 & 12 \\
\hline & heptachlor epoxide & & & 4 & 4 \\
\hline & lindane & & 8 & 4 & 12 \\
\hline & methoxychlor & & & 4 & 4 \\
\hline & $\mathrm{p}, \mathrm{p}-\mathrm{DDT}$ & & 7 & & 7 \\
\hline \multirow[t]{4}{*}{ EPA 8150} & 2,4-dichlorophenylacetic acid & & & 8 & 8 \\
\hline & silvex & & & 4 & 4 \\
\hline & $2,4,5-\mathrm{T}$ & & & 4 & 4 \\
\hline & 2,4-dichlorophenoxyacetic acid & & & 4 & 4 \\
\hline \multirow[t]{10}{*}{ EPA 8240} & vinyl chloride & & & 2 & 2 \\
\hline & benzene & 8 & 16 & 2 & 26 \\
\hline & carbon tetrachloride & & & 2 & 2 \\
\hline & chloroform & & & 2 & 2 \\
\hline & chlorobenzene & 8 & 16 & 2 & 26 \\
\hline & toluene & 8 & 16 & & 24 \\
\hline & methyl ethyl ketone & & & 2 & 2 \\
\hline & tetrachloroethylene & & & 2 & 2 \\
\hline & trichloroethylene & 8 & 16 & 2 & 26 \\
\hline & 1,1-dichloroethylene & 8 & 16 & 2 & 26 \\
\hline
\end{tabular}


Table 10. Matrix Spikes Performed, Continued

Method

EPA 8240, cont.

EPA 8270

EPA 8280
Analute

1,2-dichloroethane

acenaphthene

hexachlorobenzene

hexachloroethane

hexachlorobutadiene

nitrobenzene

n-nitrosodipropylamine

pentachlorophenol

phenol

pyrene

pyridine

1,2,4-trichlorobenzene

1,4-dichlorobenzene

2,4,5-trichlorophenol

2,4,6-trichlorophenol

2,4-dinitrotoluene

2-chlorophenol

o.cresol

m-cresol

4-chloro-m-cresol

4-nitrophenol

hexachlorodibenzo-p-dioxin

isomers

hexachlorodibenzo-p-furan

isomers

octachlorodibenzo-p-dioxin

isomers

octachlorodibenzo-p-furan

isomers

tetrachlorodibenzo-p-dioxin

isomers

tetrachlorodibenzo-p-furan

isomers

2,3,7,8-TCDD

2,3,7,8-TCDF
Spikes in Spikes in TCLP Total

Water Soil Spikes Spikes

$2 \quad 2$

2

8

$\begin{array}{ll}6 & 10 \\ 6 & 6 \\ 6 & 6 \\ 6 & 6 \\ & 6\end{array}$

$\begin{array}{lll}2 & 8 & 10\end{array}$

$\begin{array}{llll}2 & 8 & 6 & 16\end{array}$

2810

$\begin{array}{lll}2 & 8 & 10\end{array}$

$2 \quad 8 \quad 10$

$\begin{array}{llll}2 & 8 & 6 & 16\end{array}$

$\begin{array}{lll}6 & 6 \\ 6 & 6\end{array}$

$\begin{array}{llll}2 & 8 & 6 & 16\end{array}$

$\begin{array}{lll}2 & 8 & 10\end{array}$

$6 \quad 6$

$6 \quad 6$

$\begin{array}{lll}2 & 8 & 10\end{array}$

2

8

6

10

6

$6 \quad 6$

$6 \quad 6$

$6 \quad 6$

$3 \quad 3$

33

$\begin{array}{lll}6 & 6 & 6 \\ 6 & - & 6\end{array}$

\section{Contamination in QC Samples}

Table 11 presents the field-generated blanks that had analytical results above detection limit. The greatest amount of contamination in field-generated blanks was with the common laboratory contaminant acetone, reported above detection limit in 22 of these blanks; only six of these samples were qualified with $V$, indicating that contamination also was found in associated method blanks. Other constituents with positive results in fieldgenerated blanks included methyl ethyl ketone, 2-hexanone, dichloromethane (methylene chloride), and copper. Positive results also were reported for radionuclides: nonvolatile beta in eight, tritium, and total alpha-emitting radium. A pair of duplicate tritium 
analyses was additionally qualified with $\mathrm{X}$, indicating poor correspondence between the duplicate results.

Table 11. Field-Generated Blanks Having Positive Results

\begin{tabular}{|c|c|c|c|c|c|c|c|}
\hline $\begin{array}{l}\text { Sample } \\
\text { ID }\end{array}$ & $\begin{array}{l}\text { Sample } \\
\text { Trpe }\end{array}$ & Analite & $\begin{array}{l}\text { Detection } \\
\text { Limit }\end{array}$ & Results & Units & $\begin{array}{l}\text { Result } \\
\text { Qual. }\end{array}$ & $\begin{array}{l}\text { Analysis } \\
\text { Qualifier }\end{array}$ \\
\hline 100302 & Field blank & Acetone & 10 & 190 & $\mu g / L$ & & $\mathrm{~V}$ \\
\hline 100315 & Rinsate & Acetone & 10 & 64 & $\mu g / L$ & $J$ & $\mathrm{Y}$ \\
\hline 100315 & Rinsate* & Acetone & 10 & 60 & $\mu \mathrm{g} / \mathrm{L}$ & $\mathbf{J}$ & $Y$ \\
\hline 100315 & Rinsate* & Acetone & 10 & 63 & $\mu g / L$ & $J$ & $Y$ \\
\hline 100316 & Trip blank & Acetone & 10 & 20 & $\mu g / L$ & & $\mathrm{Y}$ \\
\hline 100325 & Rinsate & Acetone & 10 & 122 & $\mu g / L$ & $\mathrm{~J}$ & $\mathrm{Y}$ \\
\hline 100333 & Field blank & Acetone & 10 & 28 & $\mu g / L$ & $\mathrm{~J}$ & 0 \\
\hline 100333 & Field blank* & Acetone & 10 & 28 & $\mu g / L$ & $\mathrm{~J}$ & 0 \\
\hline 100339 & Trip blank & Acetone & 10 & 11 & $\mu g / L$ & $\mathrm{~J}$ & 0 \\
\hline 100342 & Rinsate & Acetone & 10 & 42 & $\mu g / L$ & & \\
\hline 100348 & Trip blank & Acetone & 10 & 50 & $\mu \mathrm{g} / \mathrm{L}$ & & \\
\hline 100355 & Rinsate & Acetone & 10 & 54 & $\mu \mathrm{g} / \mathrm{L}$ & & \\
\hline 100374 & Field blank & Acetone & 10 & 54 & $\mu \mathrm{g} / \mathrm{L}$ & $\mathrm{U}$ & V \\
\hline 100384 & Rinsate & Acetone & 10 & 39 & $\mu g / L$ & & $\mathrm{~V}$ \\
\hline 100403 & Field blank & Acetone & 10 & 110 & $\mu \mathrm{g} / \mathrm{L}$ & & \\
\hline 100403 & Field blank* & Acetone & 10 & 110 & $\mu g / L$. & & \\
\hline 100403 & Field blank* & Acetone & 10 & 130 & $\mu g / L$ & & \\
\hline 100413 & Trip blank & Acetone & 10 & 15 & $\mu \mathrm{g} / \mathrm{L}$ & & \\
\hline 100416 & Rinsate & Acetone & 10 & 6,840 & $\mu g / L$ & $\mathrm{~L}$ & YV \\
\hline 100416 & Rinsate & Acetone & 5,000 & 44,700 & $\mu \mathrm{g} / \mathrm{L}$ & - & YV \\
\hline 100420 & Field blank & Acetone & 10 & 135 & $\mu g / L$ & & \\
\hline 100437 & Field blank & Acetone & 10 & 134 & $\mu g / L$ & & $\mathrm{~V}$ \\
\hline 100348 & Field blank & Copper & 25 & 32 & $\mu g / L$ & & \\
\hline 100355 & Rinsate & Copper & 25.0 & 33.1 & $\mu \mathrm{g} / \mathrm{L}$ & & \\
\hline 100416 & Rinsate & Dichloromethane & 5.0 & 7 & $\mu g / L$ & $\mathrm{U}$ & YV \\
\hline 100437 & Field blank & Dichloromethane & 5.0 & 15 & $\mu g / L$ & $U$ & $\mathrm{~V}$ \\
\hline 100302 & Field blank & Dichloromethane & 5.0 & 32 & $\mu g / L$ & $\mathrm{U}$ & $\mathrm{V}$ \\
\hline 100302 & Field blank & 2-Hexanone & 10 & 24 & $\mu \mathrm{g} / \mathrm{L}$ & & - \\
\hline 100403 & Field blank & 2-Hexanone & 10 & 8.1 & $\mu g / L$ & $J$ & \\
\hline 100403 & Field blank* & 2-Hexanone & 10 & 8.7 & $\mu g / L$ & $J$ & \\
\hline 100403 & Field blank* & 2-Hexanone & 10 & 9.7 & $\mu g / L$ & & \\
\hline 100437 & Field blank & 2-Hexanone & 10.0 & 13.9 & $\mu g / L$ & & \\
\hline 100302 & Field blank & $\begin{array}{l}\text { Methyl ethyl } \\
\text { ketone }\end{array}$ & 10 & 99 & $\mu g / L$ & & \\
\hline 100348 & Field blank & $\begin{array}{l}\text { Methyl ethyl } \\
\text { ketone }\end{array}$ & 10 & 21 & $\mu g / L$ & $\cdot$ & \\
\hline 100355 & Rinsate & $\begin{array}{l}\text { Methyl ethyl } \\
\text { ketone }\end{array}$ & 10 & 27 & $\mu g / L$ & & \\
\hline 100360 & Field blank & $\begin{array}{l}\text { Methyl ethyl } \\
\text { ketone }\end{array}$ & 10 & 12 & $\mu \mathrm{g} / \mathrm{L}$ & & \\
\hline 100384 & Rinsate & $\begin{array}{l}\text { Methyl ethyl } \\
\text { ketone }\end{array}$ & 10 & 22 & $\mu \mathrm{g} / \mathrm{L}$ & & \\
\hline
\end{tabular}

* - Lab duplicate. 
Table 11. Field-Generated Blanks Having Positive Results, Continued

\begin{tabular}{|c|c|c|c|c|c|c|c|}
\hline $\begin{array}{l}\text { Sample } \\
\text { ID }\end{array}$ & $\begin{array}{l}\text { Sample } \\
\text { Tupe }\end{array}$ & Analite & $\begin{array}{l}\text { Detection } \\
\text { Limit }\end{array}$ & Resuits & Iinits : & $\begin{array}{l}\text { Result } \\
\text { Qual. }\end{array}$ & $\begin{array}{l}\text { Analysis } \\
\text { Qualifier }\end{array}$ \\
\hline 100397 & Field blank & $\begin{array}{l}\text { Methyl ethyl } \\
\text { betone }\end{array}$ & 10 & 27 & $\mu g / L$ & & \\
\hline 100403 & Field blank & $\begin{array}{l}\text { Methyl ethyl } \\
\text { betone }\end{array}$ & 10 & 36 & $\mu g / L$ & & \\
\hline 100403 & Field blank & $\begin{array}{l}\text { Methyl ethyl } \\
\text { betone }\end{array}$ & 10 & 35 & $\mu g / L$ & & \\
\hline 100403 & Field blank & $\begin{array}{l}\text { Methyl ethyl } \\
\text { betone }\end{array}$ & 10 & 42 & $\mu \mathrm{g} / \mathrm{L}$ & & \\
\hline 100420 & Field blank & $\begin{array}{l}\text { Methyl ethyl } \\
\text { betone }\end{array}$ & 10 & 35 & $\mu \mathrm{g} / \mathrm{L}$ & & \\
\hline 100437 & Field blank & $\begin{array}{l}\text { Methyl ethy-1 } \\
\text { betone }\end{array}$ & 10 & 55 & $\mu \mathrm{g} / \mathrm{L}$ & & \\
\hline 100302 & Field blank & Nonvolatile beta & 0.8 & 1.4 & $\mathrm{pCi} / \mathrm{L}$ & $\mathrm{U}$ & $\mathrm{V}$ \\
\hline 100315 & Rinsate & Nonvolatile beta & 0.5 & 1.0 & $\mathrm{pCi} / \mathrm{L}$ & & $Y$ \\
\hline 100315 & Rinsate* & Nonvolatile beta & 0.5 & 4.2 & $\mathrm{pCi} / \mathrm{L}$ & & $\bar{Y}$ \\
\hline 100327 & Field blank & Nonvolatile beta & 1.0 & 4.9 & $\mathrm{pCi} / \mathrm{L}$ & & \\
\hline 100333 & Field blank & Nonvolatile beta & 1.0 & 2.3 & $\mathrm{pCi} / \mathrm{L}$ & & \\
\hline 100374 & Field blank & Nonvolatile beta & 0.7 & 2.3 & $\mathrm{pCi} / \mathrm{L}$ & $\mathrm{J}$ & $\mathrm{x}$ \\
\hline 100374 & Field blank* & Nonvolatile beta & 0.7 & 2.3 & $\mathrm{pCi} / \mathrm{L}$ & $\mathrm{J}$ & $\mathrm{X}$ \\
\hline 100384 & Rinsate & Nonvolatile beta & 0.7 & 0.8 & $\mathrm{p} \mathrm{Ci} / \mathrm{L}$ & & \\
\hline 100355 & Rinsate & $\begin{array}{l}\text { Radium, total } \\
\text { alpha-emitting }\end{array}$ & 0.2 & 0.3 & $\mathrm{pCi} / \mathrm{L}$ & U & $\mathrm{V}$ \\
\hline 100420 & Field blank & $\begin{array}{l}\text { Radium, total } \\
\text { alpha-emitting }\end{array}$ & 0.1 & 0.2 & $\mathrm{pCi} / \mathrm{L}$ & & \\
\hline 100333 & Field blank & Tritium & 150 & 270 & $\mathrm{pCi} / \mathrm{L}$ & & \\
\hline 100351 & Trip blank & Tritium & 154 & 260 & $\mathrm{pCi} / \mathrm{L}$ & & \\
\hline 100355 , & Rinsate & Tritium & 151 & 163 & $\mathrm{pCi} / \mathrm{L}$ & & \\
\hline 100409 & Trip blank & Tritium & 160 & 130 & $\mathrm{pCi} / \mathrm{L}$ & & \\
\hline 100420 & Field blank & Tritium & 139 & 195 & $\mathrm{pCi} / \mathrm{L}$ & $J$ & $\mathrm{X}$ \\
\hline 100420 & Field blank* & Tritium & 139 & 255 & $\mathrm{pCi} / \mathrm{L}$ & $\mathrm{J}$ & $\ddot{\mathrm{x}}$ \\
\hline
\end{tabular}

\section{Review of the Analytical Narratives}

The cover letters and analysis summaries that accompanied the EDDs from the laboratories were reviewed. This section is organized according to analytical batch; the numbered headings are the first digits of the laboratory sample identification numbers.

The following comment was made by Weston regarding all of the project's dioxin data packages:

\section{Dioxins/Furans}

- The traces of heptachlorodibenzofuran found in the blank spikes and spiked sample were due to a known impurity in the spiking solution.

The following comments made by Weston concern specific batches of data: 


\section{L043}

\section{Inorganics}

- The replicate result for cation exchange capacity was outside the $20 \%$ Relative Percent Differences (RPD) guidance limit. .

\section{Metals}

- A post-digestion matrix spike and serial dilution were performed for analytes where the matrix spike was out of control.

- Manganese in sample 100433 was outside the $20 \%$ RPD control limits for sample values greater than five times reportung limit, or \pm the reporting limits for sample values less than five times reporting limit.

\section{Semivolatiles}

- Samples 100428, 100430, 100431, and 100432 exhibited areas for the last internal standard (perilene-d12) below the $50 \%$ acceptance criteria. The samples xere not reanalyzed because there were no target compounds detected in the samples that used the internal standard perylene-dl2 to quantitate.

\section{GC/MS Volatiles}

- Tro of the laboratory control standard (LCS) samples were analyzed beyond the 12-hour tune limit.

- The matrix spike duplicate for sample 100428 was analyzed just beyond the 12-hour tune limit.

- The surrogate recoveries for samples 100427 and 100429 were outside USEPA QC limits during their volatile analyses. They were reanalyzed, and the surrogates were good. Both sets of data were included.

\section{Dioxins}

- The internal standard C13-OCDD recovery for the laboratory blank 93LS0085-MBI (37\%) was outside SW 846 limits (40-120\%). Internal standard recovery criteria were not met for this blank. Re-extraction requirements were satisfied by the blank spike 93LS0085-MB1, which met all criteria. None of the samples associated with this blank were affected. Internal standard signal to noise ratio is greater than $10: 1$ and the quantitation is valid. (EPA CLP QC allows recoveries as low as $25 \%$. All samples met EPA QC requirements.) These criteria were used to assess method performance. However, when properly applied, results obtained by isotope dilution techniques are independent of internal standard recovery.

- One of six matrix spike recoveries was outside EPA QC limits (50-150\%).

- One of 12 blank spike recoveries was outside EPA QC limits (50-150\%).

- The soil laboratory blank 93LS0085-MBI contained significant contamination in the HxCDD range; how'ever, none of the associated samples contained these peaks and the data is not affected.

\section{$\underline{9306 L 817}$}

Inorganics

- All sample holding times were met with the exception of $\mathrm{pH}$ (received past hold); total organic halides (analyzed past hold); and total suspended solids and total dissolved solids for sample 100309 (analyzed past hold).

- The preparation blank result for nitrate exceeded the required detection limits. 
Metals

- The mercury soil LCS had control limits of $67-134 \%$, exceeding the S0-120\% control limits.

- Mercury exceeded the 20\% RPD control limits (at 20.1\%) in duplicate LCS 93C0170.

- A post-digestion matrix spike and serial dilution were performed for analytes where the matrix spike was out of control.

\section{Semivolatiles}

- Non-target compounds were detected.

- One of 120 surrogate recoveries was outside EPA QC limits; however, EPA CLP surrogate recovery criteria were met (i.e., no more than one outlier per fraction [acid and base neutral] and no recoveries less than $10 \%$ ).

- One of 33 water blank spile recoveries nas outside EPA QC limits.

- The laboratorj blank 95LE0916-MBI contained the common contaminants di-nbutylphthalate and bis(2-ethylhexyl)phthalate at levels less than the CRQL. The laboratory blanks 93LE0934-MBI and 93LE1002-MBI contained the common contaminant bis(2. ethylhexy-1)phthalate at levels less than the CRQL.

- The level of bis(2-ethylhexyl)phthalate detected in the matrix spibe duplicate for sample 100306 exceeded the calibration range due to probable lab contamination. Levels less than the CRQL were reported for sample 100306 and its matrix spile.

\section{GC/MS Volatiles}

- Non-target compounds were detected in these samples.

- The laboratory blants contained the common contaminants methylene chloride and/or : acetone at levels less than three times the CRQL.

\section{Radiological indicators}

- Results for gross alpha/beta analysis for samples 100310 and 100311 were subjective due to low sample aliquots and high self-absorption factors.

\section{L825}

Metals

- Mercury exceeded the 20\% RPD control limits (at 20.1\%) for duplicate LCS 93C0170.LC1. However, it was within in-house control limits (30\%).

- The leachate matrix spite recovery for silver for sample 100322 fell below the $75-125 \%$ control limits (exception allored when sample concentration exceeds the spike-added concentration by a factor of four or more).

- A post-digestion matrix spike and serial dilution were performed for analytes where the matrix spike was out of control.

\section{Semivolatiles}

- The spike recovery was below QC limits for N-nitroso-di-n-propylamine in blank spike 93GB0299-MBI.

\section{GC/MS Volatiles}

- Three LCS were analyzed beyond the 12-hour tune limit.

- Sample 100324, its matrix spike, and its matrix spike duplicate were analyzed just beyond the 12-hour tune limit.

- The secondary dilution of sample 100320 had a slightly high recovery in the volatile analyses.

\section{Pesticides}

- These samples receired a sulfur cleanup. 
- Confirmation was not required because target compounds were not detected in any of the samples.

- Due to matrix interferences, sample 100321 was analyzed at a 1/10 dilution. Reporting limits were adjusted to reflect the necessary dilution.

Herbicides

- Confirmation was not required because target compounds were not detected in any of the samples.

TCLP Semivolatiles

- One of 36 surrogate recoveries was outside EPA QC limits. However, EPA CLP surrogate recovery criteria were met (i.e., no more than one outlier per fraction [acid and base neutral] and no recoveries less than $10 \%$ ).

- One of 24 matrix spike recoveries was outside EPA QC limits.

- One of 12 blank spike recoreries was outside EPA QC limits.

\section{Radiological indicators}

- Results for gross alpha/beta analysis for samples 100312, 100313, 100314, 100317, 100318, $100319,100320,100321,100322,100323$, and 100324 kere subjective due to low sample aliquots and high self-absorption factors.

\section{L849}

Metals

- Arsenic, chromium, and selenium were outside the $75-125 \%$ control limits in the matrix spike for sample 100330.

- A post-digestion matrix spite and serial dilution were performed for analytes where the matrix spike was out of control.

- Arsenic, chromium, and manganese were outside the 20\% RPD control limits for sample values greater than five times reporting limit, or \pm the reporting limits for sample values less than five times reporting limit, in the duplicate sample 100330.

- The silver matrix spile was below $50 \%$ recovery at $25.1 \%$. Silver recovery in the TCLP leachate was less than $80-100 \%$ of the threshold limit so standard addition was not performed per Federal Register Vol. 5i, No. 227, Nov. 24, 1992, page 55117 (57FR:55117).

\section{Semivolatiles}

- The method blank spike duplicate sample 93GB0307-MB1 was analyzed outside the 12-hour tune limit.

- Matrix spike analyses were not conducted on a sample in this batch.

\section{GC/MS Volatiles}

- LCS GVE162 was analyzed past the 12-hour tune limit.

- Surrogate recoveries in the volatile analyses were outside limits for samples 100333 and 100339 and for the matrix spike for 100345 . Samples 100333 and 100339 were reanalyzed; surrogates were outside limits both times.

- Samples 100332, 100341, and 100345 were slightly lower than limits for internal standard areas and retention times.

- Samples 100332, 100334, 100335, 100336, and 100345 were re-analyzed at dilutions to accurately quantitate acetone and dichloromethane.

Pesticides

- These samples received a sulfur cleanup. 
- Confirmation was not required because target compounds were not detected in any of the samples.

- Samples which had the surrogate diluted out were outside control limits for surrogate recoveries. Samples 100326 and 100343 were outside control limits; they had recoveries of $15 \%$ and $12 \%$, respectively.

- Due to matrix interferences, sample 100332, its matrix spike, and its matrix spike duplicate were analyzed at $1 / 50$ dilutions. Reporting limits were adjusted to reflect these necessary dilutions. All spike recoveries were diluted out.

\section{Herbicides}

- Confirmation was not required because target compounds were not detected in any of the samples.

\section{TCLP Semivolatiles}

- One of 24 surrogate recoveries was outside EPA QC limits. However, EP.A CLP surrogate recorery criteria were met (i.e., no more than one outlier per fraction [acid and base neutral] and no recoveries less than $10 \%$ ).

- One of 12 blank spile recoveries was outside EPA QC limits.

\section{Radiological indicators}

- Gross alpha/beta results for samples 100326, 100329, 100330, 100331, 100332, 100334, $100335,100336,100340,100341,100343,100344$, and 100345 kere subjective due to small sample aliquots and large self-absorption factors.

\section{$\underline{9306 \mathrm{~L} 874}$}

\section{Inorganics}

- The cation exchange capacity replicate result was outside the $20 \%$ RPD guidance limit.

\section{Metals}

- The matrix spile recovery for sample 100356 was outside the $75-125 \%$ control limit for silver, arsenic, barium, cadmium, chromium, lead, and selenium.

- A post-digestion matrix spike and serial dilution were performed for analytes where the matrix spike was out of control.

- The matrix spike duplicate for sample 100356 was outside the $20 \%$ RPD control-limits for arsenic, barium, and lead.

- Barium, manganese, and lead were outside the 20\% RPD control limits for sample values greater than five times the reporting limit, or \pm the reporting limits for sample values less than five times the reporting limit, for the duplicate sample 100346 . :

- The matrix spike, matrix spike duplicate, and duplicate for sample 100356 for silver and cadmium were reported with a tenfold dilution due to high iron levels. Iron is used as an interelement correction factor for these analytes.

Semivolatiles

- The spile recovery was above the QC limit for phenol in the blank spike for LCS 93GB0311. MB1. The spike recoveries also were above the QC limit for pentachlorophenol in the blank spike and its duplicate. The blank spike duplicate was analyzed outside the 12-hour tune limit.

- The spile recoveries were above the $Q C$ limit for pentachlorophenol in the matrix spike and matrix spike duplicate for sample 100350. 
GC/MS Volatiles

- Two of the LCS rere analyzed beyond the 12-hour tune limit.

Pesticides

- For continuing calibration standards analyzed prior to the sample extracts, the $15 \%$ criteria for calibration factors were exceeded for methoxychlor. The data reflected an increase in instrument.response for these parameters so the ability to identify target compounds was not impaired. Since quantitation was not required, the data should not be affected.

TCLP Semivolatiles

- One of 24 matrix spike recoveries was outside EPA QC limits.

Radiological indicators

- Results for gross alpha/beta for samples 100346, 100347, 100350, 100352, 100353, 100354, and 100356 were subjective due to small aliquots and high self-absorption factors.

\section{L888}

Metals

- A post-digestion matrix spike and serial dilution were performed for analytes where the matrix spike was out of control.

Semivolatiles

- Non-target compounds were detected in these samples.

- The laboratory blant 93LEI002-MBI contained the common contaminant bis(2ethylhexyl)phthalate at levels less than the CRQL.

\section{GC/MS Volatiles}

- Non-target compounds were detected in these samples.

- Sample 100366 required a medium level analysis because it contained a high level of target compound acetone.

- The laboratory blanks 93LVX131-MBI and 93LVX132-MB1 contained the common contaminant methylene chloride at levels less than three times the CRQL.

Pesticides/PCBs

- These samples received a sulfur cleanup.

- None of the samples contained target compounds in sufficient concentration to be confirmed by GC/MS.

\section{Radiological indicators}

- Gross alpha/beta results for samples 100366, 100367, 100368, 100410, ;and 100411 were subjective due to small aliquots and large self-absorption factors. The replicate results for tritium for the same samples were slightly outside limits; however, there was no additional sample for a reanalysis.

\section{$\underline{9306 \mathrm{~L} 915}$}

\section{Metals}

- Matrix spike recoveries for sample 100378 were outside the $75-125 \%$ control limits for silver, arsenic, barium, chromium, manganese, lead, and selenium.

- A post-digestion matrix spike and serial dilution were performed for analytes where the matrix spike was out of control.

- Matrix spike duplicates were outside the $20 \%$ RPD control limits for selenium in sample 100374 and for barium, cadmium, chromium, manganese, and lead in sample 100378. 
- Duplicate analyses for sample 100378 were outside the $20 \%$ RPD control limits for sample values greater than five times the reporting limit, or \pm the reporting limits for sample values less than five times the reporting limit, for arsenic, barium, and chromium.

- The matrix spike for mercury was below $50 \%$ recovery at $46.3 \%$. The mercury recovery in the TCLP leachate was below S0-100\% of the action level, so standard addition was not required per 5iFR:55117.

\section{Semivolatiles}

- Spike recoveries for N-nitroso-di-n-propylamine and 1,2,4-trichlorobenzene kere below QC limits in the blank spike for LCS 93LE1003-MB1. This sample was analyzed outside the 12 hour tune limit.

- Spike recoveries for N'nitroso-di-n-propylamine were below QC limits in the blant spike and its duplicate for LCS 93LE1043-MB1. Spile recoreries for 1,2,4-trichlorobenzene and 2,4dinitrotoluene tere below $Q C$ limits in the blank spile duplicate. The percentage RPD value was above the QC limit for acenaphthene. The blank spike and blank spike duplicate were analyzed outside the 12-hour tune limit.

- Spike recovery for N-nitroso-di-n-propylamine was below the QC limit in the matrix spike duplicate for sample 100370 .

- Sample 100375 exhibited four low surrogate recoveries. The sample was reanalyzed for low internal standard areas and exhibited five low surrogate recoveries. The surrogate recoveries were not reported, due to the dilutions performed, for the folloring: sample 100383 and its secondary dilution; 100385 and its secondary dilution; two secondary dilutions of 100386; and one secondary dilution of 100387 .

- Samples 100373 and 100375 had internal standard areas below the $50 \%$ acceptance criteria.

The reanalysis samples also had low internal standard areas, indicating a matrix interference. Samples 100383 and 100386 had perylene-d12 below the $50 \%$ acceptance limit. Both samples required secondary dilutions to quantitate target compounds accurately. These samples were not reanalyzed.

\section{GC/MS Volatiles}

- Non-target compounds were detected in these samples.

- Two of 93 surrogate recoveries rere outside EPA QC limits. Sample 100385 was reanalyzed on $6 / 24 / 93$ and reported.

- The laboratory blanks contained the common contaminants methylene chloride and/or acetone at levels less than three times the CRQL.

\section{Pesticides/PCBs}

- These samples received a GPC and a sulfur cleanup.

- The matrix spile recovery for 4,4'-DDT was outside acceptance criteria.

- The following samples required dilutions due to matrix interference and high concentrations of analytes: sample 100370, its replicate and matrix spike, and sample 100373 required dilution factors of five; samples 100371, 100383, 100385, 100386, and 100387 required dilution factors of ten.

- Toxaphene exceeded the $15 \%$ criteria for calibration factors. The data reflected an increase * in instrument response for this parameter, so the ability to identify the target compound was not impaired. Since quantitation was not required, the data should not be affected.

- The 4,4'-DDT series in sample 100370, its replicate and matrix spike, and samples 100371 , 100373 , and 100376 were reported without confirmation due to interferences with AR1260; therefore, their actual existence is suspect. Endrin betone results in samples 100386 , 100387 , and 100388 were reported without confirmation due to matrix interference, and their existence is also suspect. 
- None of the samples contained target compounds in sufficient concentration to be confirmed by GC/MS. -

Pesticides

- These samples received a sulfur cleanup.

Dioxins

- Five of 63 internal standard recoveries were outside the SW-846 QC limits of $40-120 \%$. Internal standard recovery criteria were not satisfied for samples 100383 and 100385 . These samples were re-extracted with similar (slightly worse) results, which are available on request. All re-extraction criteria were satisfied.

- Internal standard signal to noise ratio was greater than 10:1 and the quantitation rias valid. (EPA CLP QC allows recoveries as low as $25 \%$.) These criteria were used to assess method performance. However, when properly applied, results obtained by isotope dilution techniques are independent of internal standard recovery. The only impact on the data is to raise the detection limits of analytes quantitated using the effected internal standards.

- One of six matrix spile recoveries was outside the EPA QC limit of 50-150\%. The out-oflimit recovery was due to the concentration of OCDD native to the sample and the usual nonhomogenous nature of soil samples. This was confirmed by the good blank spike recoreries.

TCLP Semivolatiles

- Two of 24 matrix spite recoveries were outside EPA QC limits.

- One of 12 blank spile recoveries was outside EPA QC limits.

9306L938

Semivolatiles

- The spike recovery for phenol exceeded the QC limits for the blank spite duplicate of LCS . 93GB0316-MBI.

- . Samples 100390 and 100392 , and the blank spike and blank spike duplicate of LCS 93GB0316-MBI, exhibited areas for perylene-d12 below the $50 \%$ acceptance criteria. The samples were not reanalyzed because there were no target compounds detected in the samples that used the internal standard perjlene-d12 to quantitate.

\section{GC/MS Volatiles}

- The method blank spike recovery for benzene exceeded the recommended QC limits. One of the LCS was analyzed beyond the 12-hour tune limit.

\section{Pesticides}

- The surrogate recoveries for di-n-butyl-chlorendate for samples 100390, 100391, 100392, 100396, and 100399 were outside control limits. The low surrogate recoveries may be attributed to the sample matrix.

\section{Dioxins}

- The internal standard C13-OCDD recovery for the laboratory blank spike 93LS0069-MB1 was outside SW 846 QC limits of 40-120\%. Re-extraction requirements were satisfied by its associated unspiked blank 93LS0069-MB1. The samples were not affected.

\section{$\underline{9306 L 957}$}

Inorganics

- The replicate results for cation exchange capacity and total organic carbon were outside the $20 \%$ RPD guidance limit. 


\section{Metals}

- The matrix spike recoveries for arsenic, manganese, and selenium were outside the $75.125 \%$ control limits for sample 100401.

- A post-digestion matrix spike and serial dilution were performed for analytes with matrix spikes out of control.

- The matrix spilie duplicates for arsenic and manganese were outside the $20 \%$ RPD control limits for sample 100400 .

- The duplicate analyses for chromium, manganese, and lead for sample 100401 were outside the $20 \%$ RPD control limits for sample values greater than five times the reporting limit, or \pm the reporting limits for sampie values less than five times the reporting limit.

\section{Semivolatiles}

- The blank spike and blank spike duplicate of LCS 93GB0323-MB1 were analyzed outside the 12-hour tune limit.

- The matrix spike recovery for the matrix spike duplicate of sample 100400 was above the QC limits for pentachlorophenol.

- The following samples exhibited areas for the last internal standard (perylene-d12) below the 50\% acceptance criteria: samples 100406, 100408, 100410, and 100411; the matrix spike duplicate of sample 100400; and the blank spite and blank spite duplicate of LCS 93GB0323MB1. The samples were not reanalyzed because there were no target compounds detected in the samples that used the internal standard perylene-dl2 to quantitate.

- Sample 100406, extracted as low-level soil, had a $5.0 \mathrm{~mL}$ final extract volume and was analyzed without furcher dilution. The results and detection limits have been adjusted to reflect the final extract volume in this sample.

\section{GC/MS Volatiles}

- The method blank spike recovery for benzene was outside recommended QC limits. One of the LCS was analyzed beyond the 12-hour tune limit.

- The matrix spile and matrix spite duplicate of sample 100403 had two recoveries that exceeded QC limits for benzene.

- Sample 100405 required a secondary dilution in order to quantitate acetone accurately.

\section{Pesticides}

- Surrogate recoveries for di-n-butyl-chlorendate were outside control limits for samples 100404, which had a low recovery, and 100406, which had the surrogate diluted out.

\section{Dioxins}

- The internal standard C13-TCDD recovery for sample 100411 was outside the $40-120 \%$ QC limits. The ion ratio for this peak was also outside $Q C$ limits, indicating an interference. As there were no "hits" to be quantified from this internal standard, the data was not affected. Re-extraction requirements were satisfied by the analysis of the matrix spite of sample 100411.

- The internal standard of C13-OCDD recovery for the laboratory blank spike 93LS0069-MB1. was outside the $40-120 \%$ QC limits. Re-extraction requirements teere satisfied by its associated unspiled blank 93LS0069-MB1. The samples were not affected.

- One of six matrix spike recoveries was outside QC limits. This was due to the large amount of the spike compound (OCDD) native to the sample (approximately two times the spilie level) and normal sample non-homogeneity. The good blank spike recoveries confirm the matrix effect. 
Radiological indicators

- The results for gross alpha/beta for samples 100410 and 100411 were subjective due to small aliquots and large self-absorption factors. The replicate results for tritium for these samples were slightly outside limits; however, there was no additional sample for a reanalysis.

9306L966

GC/MS Volatiles

- Non-target compounds were detected in these samples.

- Two of 27 surrogate recoveries were outside EPA QC limits. The analyses of sample 100415 and its matrix spike duplicate fulfilled the reanalysis requirement for the matrix spike of sample 100415. Sample 100416 was diluted and reanalyzed for acetone on $7 / 01 / 93$; both values are reported.

- The laboratory blank 93LVB152-MBI contained the common contaminant methylene chloride at a level less than three times the CRQL. The laboratory blank 93LVR113-MB1 contained the common contaminant acetone at a level less than the CRQL.

\section{L997}

\section{Inorganics}

- The replicate result for cation exchange capacity was outside the $20 \%$ RPD guidance limit. Metals

- The matrix spike recoveries for chromium, manganese, and selenium in sample 100417 were outside the $75-125 \%$ control limits.

- A post-digestion matrix spite and serial dilution were performed for analytes where the matrix spike ras out of control.

- The matrix spile duplicate for manganese in sample 100417 was outside the $20 \%$ RPD control limits.

- The duplicate analysis for chromium in sample 100417 was outside the $20 \%$ RPD control limits for sample values greater than five times the reporting limit, or \pm the reporting limits for sample values less than five times the reporting limit.

\section{Semivolatiles}

- Non-target compounds were detected in these samples.

- One of 22 matrix spike recoveries was outside EPA QC limits.

- The laboratory blank contained the common contaminants di-n-butylphthalate and bis(2ethylhexyl)phthalate at levels less than the CRQL.

\section{GC/MS Volatiles}

- Non-target compounds were detected in these samples.

- Sample 100426 required a five-fold dilution because it contained a high level of the target compound acetone.

- The laboratory blanks contained the common contaminants methylene chloride andor acetone at levels less than three times the CRQL.

\section{Pesticides/PCBs}

- These samples received GPC and sulfur cleanups. 


\section{Laboratory Data Records Reviews \\ TMA/Eberline, September 21-22, 1993}

During the data records review at TMA/Eberline on Septèmber 21-22, 1993, 97 worksheets were filled out for the D.Area Burning Rubble Pit (DBP) RFI/RI waste unit. Each worksheet represents one sample revieked for one analysis.

There were 24 worksheets each for gross alpha, nonvolatile beta, and total alpha-emitting radium, using methods $900.0,900.0$ and 903.0 , respectively. For tritium, analyzed by a minor modification of method 906.0, 25 worksheets were completed.

\section{Major Issues}

Several laboratory practices potentially of concern to validators were identified:

Method blank QC for 903.0 :

Method blanks of low activity levels near the minimum detectable actirity (MDA) are important for the QC process. However, 13 of 24 samples reviewed had method blank results greater than the sample-specific MDA. Two of the method blank results exceeded 1.0 picocurie per gram ( $\mathrm{pC} i \mathrm{~g})$.

\section{Analvtical results:}

For 1 of the 96 samples reviewed, the analytical result on the instrument printout did not match the analytical result in the WSRC database. See also the results of the March 24. 25,1994 , review below.

Initial calibration $Q C$ for 900.0 and 903.0 :

All of the worksheets for methods 900.0 and 903.0 indicated failure of initial calibration in that the counting system efficiency was not calibrated within one year before analysis. However, discussions with several radiochemists and instrument specialists determined that counting system efficiency is adequately verified with proper continuing calibration techniques. Three questions on the methods checklist verify continuing calibration and thus verify the counting system efficiency. Thus none of the samples reviewed were tallied as failing because of initial calibration criteria.

\section{Scoring}

Note: Failure of an analysis for one question in the LDRR means only that it does not meet one criterion, not that the result need be rejected.

900.0 Gross Alpha

Sample 93-06-096-06 (100429) failed because the relative percent difference (RPD) was greater than $35 \%(68.2 \%)$ for duplicates. The result was not qualified.

900.0 Nonvolatile Beta

Sample 93.06-082.04 (100410) failed in that the analytical result did not match the WSRC database (32.5 vs. 37.5). See also the results of the March 24-25, 1994, review below. 
For 2 of 24 samples reviewed (93-06-065-05 [100323] and 93-06-082-04 [100410]), the analysis date/time did not match the W:SRC database.

Samples 93.06.061.09 (100307) and 93.06.082.04 (100410), both qualified with Vonly, failed because the method blank was greater than the sample-specific MDA.

Water sample 93-06-060-05 (100316) failed because the RPD was 100\% (greater than 35\%) . for duplicates in the same preparation batch; the sample was qualified with $X$.

903.0 Total Radium

Because the method blank was greater than the sample-specific MDA, 12 of 24 samples reviewed were qualified with the result qualifier $U$ and the analysis qualifier $V$. The samples are:

93.06-067-09 (100364)

93.06-068-04 (100346)

93.06.066-06 (100330)

93-06-064-05 (100304)
93.06.065-05 (100323)

93-06-080-07 (100373)

93-06-078-09 (100406)

93-06-069-04 (100340)
93.06-082-04 (100410)

93-06-082-03 (100410)

93-06-079-06 (100392)

93-06-081-08 (100386)

Because the lab control spike recovery was not in the range of $80 \%-120 \%, 3$ of 24 samples reviewed failed the record review:

- 93-06-079-06 (100392), $76.5 \%$ (Because the value in the narrative and EDD is within range, this sample was not qualified.)

- 93-06-081-08 (100386), 34\% (Because the value in the narrative and EDD is within range, this sample was not qualified.)

- 93-06-098-05 (100418), $76.2 \%$ (qualified with $J, C$ )

906.0 Tritium

Because the RPD was greater than $35 \%$ for duplicates, one pair of the 25 samples reviewed failed:

- 93-06-082-03 and 93-06-082-04 (100410), with an RPD of 119\% (qualified with $X$ because the result were greater than twice the contract-required detection limit)

TMA/Eberline, March 24-25, 1994

A number of errors, apparently created during hand transcription of the data, were found in this review. These and other errors of this type usually cannot be detected during offsite data validation and verification. The errors found in DBP data during this review were: 4 cases in which the sample results in the EDDs did not match the TMA raw data; 8 cases in which the accuracy results in the EDDs did not match the TMA raw data; raw data for one sample (4 analytes) for which no data had been delivered; and an inconsistent application of TMA's policy to report no accuracy for results below MDA. 


\section{Weston, December 6-10, 1993}

During the data records reriew at Weston-Lionrille on December 6.10, 1993, 204 worksheets were filled out for the D-Area Burning/Rubble Pits RFI/RI waste unit. Each worksheet represents one sample reviered for one analytical method. There were 26 worksheets each for the following methods: 6010, ICP metals; 7060, arsenic by atomic absorption (AA); 7421, lead by AA; 7470, mercury by $A A ; 7740$, and selenium by $A A$ The review team also filled out 25 worksheets for volatiles by method 8240,19 for semivolatiles by 8270,14 for pesticides by 8080,13 for dioxins/furans by 8280 , and one each for chlorine by method 325.2, nitrate as nitrogen by 353.2 , and herbicides by 8150 .

\section{Major Issue}

A laboratory practice potentially of concern to ralidators was identified:

Failure of Electronic Data Deliverable (EDD) to match laboratorv instrument printout: For two of the samples reviewed (both alpha-benzene hexachloride by method 8080), the analytical result on the instrument printout did not match the analytical result in the WSRC data.

\section{Failures by Method}

Note: Failure of an analysis for one question in the LDRR means only that it does not meet that criterion, not that the result need be rejected.

\section{2/Chloride}

The sample reviewed failed because the analysis date/time in the instrument printout differed by more than five minutes from the analysis date/time in the WiSRC database.

\section{$\underline{353.2}$}

No failures were observed in the sample revieured.

\section{$6010 / \mathrm{CP}$ metals}

For two samples, metals concentrations in the initial calibration blank (ICB) failed criteria: cadmium and copper in sample 100301 and chromium in sample 100346. The analyses were qualified with the result qualifiers $U$ and $J$ and the analysis qualifier $L$.

Sample 100321 failed because a continuing calibration blank $(\mathrm{CCB})$ was not performed at the end of a 10-sample run.

The $C C B$ concentrations failed to meet the criteria of $C C B \pm C R D L$ (contract-required detection limit) for cadmium in sample 100346. The analysis was qualified with the result qualifiers $U$ and $J$ and the analysis qualifier $L$.

Samples 100314 and 100321 failed because the required interference check sample (ICS) was not performed at the end of the day. 
7060/Arsenic

Samples 100301, 100310, and 100314 failed because the analysis date/time in the instrument printout differed by more than five minutes from the analy'sis date/time in the WSRC database.

The rerun with dilution of sample 100321 failed because no laboratory control sample (LCS) was performed for the analysis batch. However, the preparation batch for these samples contained an LCS; no qualification of the analysis was required.

\section{$7421 /$ Lead}

Sample 100373 failed because the analysis date/time in the instrument printout differed by more than five minutes from the analysis date/time in the W'SRC database.

Rerun samples 100310,100314 , and 100321 failed because no LCS was performed for the analysis batch. However, the preparation batch for these samples contained an LCS; no qualification of the analysis was required.

\section{Mercurv}

All 26 DBP samples failed because the analysis date/time in the instrument printout differed by more than five minutes from the analysis date/time in the WTRC database; all have analysis times of 1200 in the WSRC database.

The following eight samples failed because LCS samples were performed less often than every 20th sample: 100314 and 100321 (one pair in 30 samples); 100360 (one pair in more than samples); 100406, 100407, 100410, and 100411 (one pair in 32 samples); and 100431 (no LCS performed for the analysis batch. However, the preparation batch for these samples contained an LCS; no qualification of the analysis was required).

Sample 100310 failed because the LCS recovery was outside the $80-120 \%$ limits at $124 \%$. The analysis was qualified with the result qualifier $J$ and the analysis qualifier $C$.

\section{0/Selenium}

Four samples failed because LCS samples were performed less often than once every 20th sample: 100346 (one pair in 25 samples) and 100370, 100371, and 100373 (no LCS performed for the analysis batch. However, the preparation batch for these samples contained an LCS; no qualification of the analysis was required).

\section{0/Pesticide}

Samples 100390 and 100411 failed because the analytical results in the printout did not match the results in the WSRC database for alpha-benzene hexachloride.

Samples 100336 and 100347 failed because breakdown of endrin or DDT, respectively, exceeded $20 \%$. Those analyses were qualified with the result qualifiers $U$ and $J$ and the analysis qualifier $G$.

Sample 100347 failed because the percent difference (\%D) of the continuing calibration standards was greater than $15 \%$ for one or more analytes. Continuing calibration documentation was not provided for sample 100431. 
Two samples failed because a detected compound fell outside rtention time windows during analysis: 100406 (DDE, DDT, alpha-chlordane, and gamma-chlordane) and 100410 (DDE and DDT). The analyses were qualified with the result qualifier $U$ and the analysis qualifier $A$.

Sample 100390 failed because the recovery for surrogate dibutyl chlorendate (15\%) was outside limits. All pesticides for this sample were qualified with the result qualifiers $U$ and $J$, the analysis qualifier $O$, and the bias qualifier $L$.

\section{0/Herbicides}

No failures were observed in the sample reviewed.

\section{Nolatiles}

Samples 100336 (as originally analyzed and as diluted) and 100390 failed because \%D of the continuing calibration check standard (CCC) was greater than $25 \%$ for vinyl chloride. These analyses were qualified with the result qualifiers $U$ and $J$ and analysis qualifier $L$.

Internal standard (IS) peak areas failed criteria in samples 100390 (all three IS were greater than $50 \%$, but surrogates were acceptable) and 100406 (chlorobenzene- $d_{5}$ and 1,4difluorobenzene were lss than 50\%). All compounds for sample 100390, and the compounds quantitated by the two out-of-control standards for sample 100406, were qualified with the result qualifiers $J$ or $U J$ and the analysis qualifier $H$.

\section{0/Semivolatiles}

Sample 100370 failed because the analysis date/time in the instrument printout differed by more than five minutes from the analysis date/time in the WSRC database.

The system performance check compound (SPCC) 2,4-dinitrophenol exceeded limits during continuing calibration for samples 100406, 100407, 100410, 100411, 100430, and 100431. All the compounds in the suite were qualified with the result qualifiers $J$ or $U J$ and the analysis qualifier $L$.

Four samples failed because $\% D$ was greater than $25 \%$ for one or more CCCs: 100314 (din-octyl phthalate=33.2\%), 100336 (di-n-octyl phthalate=36.0\%), 100347 (pentachlorophenol=61.2\%), and 100407 (di-n-octyl phthalate=177\%). The named analysis was qualified in each case with the result qualifiers $J, U$, or $U J$ and the analysis qualifier $L$.

Six samples failed because the peak area for IS perylene-d 12 failed criteria: 100390 , 100406, 100410,100411, 100430, and 100431. The compounds quantitated by that standard were qualified with the result qualifiers $J$ or $U J$, the analysis qualifier $H$, and the bias qualifiers $L$ for samples 100406, 100410, and 100411, and $H$ for samples 100430 and 100431.

Surrogate 2-fluorobiphenyl failed for sample 100301. 


\section{0/Dioxins/furans}

Samples 100411, 100424, and 100431 failed because the analysis date/time in the instrument printout differed by more than five minutes from the analy'sis date/time in the WSRC database.

Sample 100411 failed because the IS recovery of $126 \%$ for $2,3,7,8-T C D D$ was outside limits of $40-120 \%$. The dioxins and furans quantitated by that standard were qualified with the result qualifiers $U$ and $J$ and the analysis qualifier $H$. 


\section{Presentation of qualified data}

Table 12 presents the numbers of project samples for which each analyte was requested, and for which analytical results were receired. It also presents the total number of analytical results reported and rejected and the numbers of results for which holding times were missed or problems were encountered with associated quality assurance samples, preservation, and instrument calibration. Surrogates required by the method are listed like target analytes.

The numbers in the "Analyses Requested" column are the total number of each analyte listed on chain-of-custody forms for enrironmental samples, field-generated replicates, field blanks, and trip blanks. The numbers in the "Received" column are the total number of samples for which at least one analytical record was reported. Missing analyses are discussed in the "Sample Collection" and "Data Validation and Verification" sections of this report. Percent solids was analyzed routinely for all soil samples, but was not requested on the chain-of-custody forms.

The column labeled "Analyses Reported" includes laboratory-initiated replicate analyses and reanalysis results. These ralues do not include analyses of associated laboratory control samples such as blanks or spikes.

The remaining columns in the table list the number of analyses that meet specified conditions.

The column labeled "Rejected" contains the number of records for which the result qualifier was $R$. Because these samples may have an acceptable reanalysis result and appear again in another column in the table, the count in this column is not the number of samples for which only rejected results were reported.

The number of analyses shown in the column labeled "Out of Holding" are those that exceeded the maximum time between sample collection and analysis allowed by the analytical method and have an analysis qualifier of $Q$. All laboratory $\mathrm{pH}$ measurements exceed holding time, regardless of the laboratory's efficiency.

The analyses listed in the column labeled "QA Problems" are those whose records contained an analysis qualifier of $V, O, I$, or $T$ because at least one associated laboratory quality control sample did not meet the required standards.

The analyses listed in the "Preservation" column are those with an analysis qualifier of $Y$ because of preservation problems. In most of these cases, the samples were not maintained cold enough.

The number of analyses with calibration problems is listed in the column labeled "Calibration." The records for these analyses contain an analysis qualifier of $L$. The numbers represent samples from analytical batches that did not meet all the calibration criteria. 
Table 12. Numbers of Qualified Samples

\section{Physical Parameters}

Analyte

Cation exchange capacity

Chloride

Specific conductance

Cyanide

Fluoride

Nitrate as nitrogen

pH

Sulfate

Percent solids

Total dissolved solids

Total organic carbon

Total organic halogens

Total suspended solids

Total Metals

Silver
Aluminum
Arsenic
Barium
Beryllium
Calcium
Cadmium
Cobalt
Chromium
Copper
Iron
Mercury
Potassium
Magnesium
Manganese
Sodium

Samples Analyses $\quad$ QA

Requested Received Reported Rejected Holding Samples Rreservation Calibration

$\begin{array}{llllllll}35 & 35 & 11 & 0 & 0 & 0 & 2 & 0 \\ 12 & 4 & 1 & 0 & 0 & 0 & 0 & 0 \\ 35 & 35 & 38 & 0 & 0 & 0 & 1 & 0 \\ 0 & 2 & 2 & 0 & 0 & 0 & 0 & 0 \\ 12 & 4 & 5 & 0 & 0 & 0 & 0 & 0 \\ 12 & 4 & 4 & 0 & 0 & 1 & 0 & 0 \\ 58 & 49 & 54 & 0 & 53 & 0 & 5 & 0 \\ 12 & 4 & 7 & 0 & 0 & 0 & 0 & 0 \\ 14 & 101 & 184 & 0 & 0 & 0 & 24 & 0 \\ 57 & 6 & 7 & 0 & 1 & 0 & 0 & 0 \\ 13 & 49 & 53 & 0 & 0 & 0 & 6 & 0 \\ 14 & 4 & 4 & 0 & 4 & 0 & 0 & 0 \\ & 6 & 8 & 0 & 1 & 0 & 0 & 0\end{array}$

$\begin{array}{llllllll}129 & 120 & 144 & 0 & 0 & 6 & 13 & 0 \\ 2 & 2 & 2 & 0 & 0 & 0 & 0 & 0 \\ 129 & 120 & 144 & 0 & 0 & 14 & 13 & 0 \\ 129 & 120 & 144 & 0 & 0 & 6 & 13 & 0 \\ 2 & 2 & 2 & 0 & 0 & 0 & 0 & 0 \\ 2 & 2 & 2 & 0 & 0 & 0 & 0 & 0 \\ 129 & 120 & 144 & 0 & 0 & 6 & 13 & 25 \\ 2 & 2 & 2 & 0 & 0 & 0 & 0 & 0 \\ 129 & 120 & 144 & 0 & 0 & 14 & 13 & 19 \\ 129 & 120 & 144 & 0 & 0 & 0 & 13 & 6 \\ 14 & 8 & 8 & 0 & 0 & 0 & 0 & 0 \\ 129 & 120 & 135 & 0 & 10 & 0 & 13 & 0 \\ 2 & 2 & 2 & 0 & 0 & 0 & 0 & 0 \\ 2 & 2 & 2 & 0 & 0 & 0 & 0 & 0 \\ 129 & 120 & 144 & 0 & 0 & 17 & 13 & 0 \\ 14 & 8 & 8 & 0 & 0 & 0 & 0 & 0\end{array}$


Table 12. Numbers of Qualified Samples, Continued

\begin{tabular}{|c|c|c|c|c|c|c|c|c|}
\hline & Samples & & Analysce & & & $\mathrm{Q} \Lambda$ & & \\
\hline Analyte & Requested & Reccived & Reported & Rejected & Holding & Samples & P'rescrvalion & Calibration \\
\hline Nickcl & 129 & 120 & 141 & 0 & 0 & 0 & 13 & 0 \\
\hline Lcad & 129 & $120^{\circ}$ & 111 & 0 & 0 & 9 & 13 & 0 \\
\hline Antimony & 2 & 2 & 2 & 0 & 0 & 0 & 0 & 0 \\
\hline Selenium & 129 & 120 & 111 & 0 & 0 & 20 & 13 & 0 \\
\hline Thallium & 2 & 2 & 2 & 0 & 0 & 0 & 0 & 0 \\
\hline Vanadium & 2 & 2 & 2 & 0 & 0 & 0 & 0 & 0 \\
\hline Zinc & 2 & 2 & 2 & 0 & 0 & 0 & 0 & 0 \\
\hline
\end{tabular}

B/N/A Extractables (including PAH and Phenols)

\begin{tabular}{|c|c|c|c|c|c|c|c|}
\hline Acenaphthene & 103 & 94 & 106 & 0 & 2 & 7 & ? \\
\hline Acenaphthylene & 103 & 94 & 106 & 0 & 2 & 7 & !) \\
\hline Anthracene & 103 & 94 & 109 & 0 & 2 & 10 & ? \\
\hline Bis(2-chlorocthoxy) methane & 103 & 94 & 106 & 0 & 2 & 7 & !) \\
\hline Bis(2-chloroisopropyl) ether & 103 & 94 & 106 & 0 & 2 & 7 & $\mathbf{9}$ \\
\hline Bis(2-chloroethyl) ether & 103 & 94 & 106 & 0 & $\mathbf{2}$ & 7 & !) \\
\hline Bis(2-cthylhexyl) phthalate & 103 & 94 & 106 & 0 & 2 & 36 & !) \\
\hline Benzo(a)anthracene & 103 & 94 & 111 & 0 & 2 & 12 & $\mathbf{9}$ \\
\hline Benzo(a)pyrene & 103 & 94 & 110 & 0 & 2 & 17 & $\mathbf{9}$ \\
\hline Benzo(b) Iluorantliene & 103 & 94 & 111 & 0 & 2 & 18 & $\mathbf{9}$ \\
\hline Butylbenzyl phthalate & 103 & 94 & 106 & $\mathbf{0}$ & 2 & 7 & 9 \\
\hline Benzoic acid & 103 & 94 & 106 & 0 & 2 & 7 & 9 \\
\hline Benzo $(\mathrm{g}, \mathrm{h}, \mathrm{i})$ perylene & 103 & 94 & 107 & 0 & 2 & 11 & ?) \\
\hline Ben\%o(k) nuoranthene & 103 & 94 & 107 & $\mathbf{0}$ & 2 & 11 & !) \\
\hline Benzyl alcohol & 103 & 94 & 106 & $\mathbf{0}$ & 2 & 7 & !) \\
\hline Chrysene & 103 & 94 & 110 & $\mathbf{0}$ & 2 & 11 & $\mathbf{9}$ \\
\hline Hexachlorobenzene & 103 & 94 & 106 & $\mathbf{0}$ & $\mathbf{2}$ & 7 & 9 \\
\hline Hexachlorocyclopenladiene & 103 & 91 & 106 & 0 & 2 & 7 & ) \\
\hline Hexachloroethane & 103 & 94 & 106 & 0 & 2 & 7 & $\mathbf{9}$ \\
\hline Dibenz(a,h)anthracene & 103 & 94 & 106 & $\mathbf{0}$ & 2 & 13 & 9) \\
\hline Diethyl phthalate & 103 & 94 & 106 & $\mathbf{0}$ & 2 & 7 & $\mathbf{9}$ \\
\hline Dibenzofuran & 103 & 94 & 106 & $\mathbf{0}$ & 2 & 7 & $\boldsymbol{9}$ \\
\hline Dimethyl phthalale & 103 & 94 & 106 & 0 & 2 & 7 & 9 \\
\hline
\end{tabular}


Table 12. Numbers of Qualified Samples, Continued

\begin{tabular}{|c|c|c|c|c|c|c|c|c|}
\hline Analyte & $\begin{array}{l}\text { Samples } \\
\text { Requested }\end{array}$ & Reccived & $\begin{array}{l}\text { Analyses } \\
\text { Reported }\end{array}$ & Reiccted & Holding & $\begin{array}{l}\text { QA } \\
\text { Samples }\end{array}$ & Prescrvation & Calibration \\
\hline Di-n-butyl phthalate & 103 & 94 & $10 G$ & 0 & 2 & 86 & 9 & G \\
\hline Di-n-octyl phthalate & 103 & 94 & 106 & 0 & 2 & 13 & 9 & 8 \\
\hline Fluoranthene & 103 & 94 & 112 & 0 & 2 & 13 & 9 & G \\
\hline Fluorene & 103 & 94 & 107 & 0 & 2 & 8 & 9 & 6 \\
\hline Hexachlorobutadiene & 103 & 91 & 106 & 0 & 2 & 7 & ? & $\mathbf{G}$ \\
\hline Indeno $(1,2,3-c, d)$ pyrene & 103 & 91 & 107 & 0 & 2 & 14 & 9 & 6 \\
\hline Isophorone & 103 & 94 & 106 & 0 & 2 & 7 & 9 & 6 \\
\hline Naphthalene & 103 & 94 & 106 & 0 & 2 & 7 & 9 & 6 \\
\hline Nitrobenzene & 103 & 94 & 106 & 0 & 2 & 7 & 9 & $6^{*}$ \\
\hline Nitrobenzene-d5 & 103 & 94 & 112 & 0 & 2 & 1 & $\mathbf{0}$ & G \\
\hline N-Nitrosodipropylamine & 103 & 94 & 106 & 0 & 2 & 29 & $\mathbf{9}$ & 6 \\
\hline N-Nitrosodiphenylamine & 103 & 94 & 106 & 0 & 2 & 7 & 9 & 6 \\
\hline Pentachlorophenol & 103 & 94 & 106 & 1 & 2 & 25 & 9 & 7 \\
\hline Phenanthrene & 103 & 94 & 111 & 0 & 2 & 12 & 9 & 6 \\
\hline Phenol-d5 & 103 & 94 & 112 & 0 & 2 & 1 & 9 & 6 \\
\hline Phenol & 103 & 94 & 106 & 0 & 2 & 30 & 0 & 6 \\
\hline p-Terphenyl-d14 & 103 & 94 & 112 & 0 & 2 & 2 & 9 & 6 \\
\hline Pyrene & 103 & 94 & 111 & 0 & 2 & 12 & $\mathbf{9}$ & 6 \\
\hline $1,2,1$-Trichlorobenzenc & 103 & 94 & 106 & 0 & 2 & 20 & 9 & G \\
\hline 1,2-Dichlorobenzene & 103 & 94 & 106 & 0 & 2 & 7 & 9 & 6 \\
\hline 1,3-Dichlorobenzene & 103 & 94 & 106 & 0 & 2 & 7 & 9 & 6 \\
\hline 1,A-Dichlorobenzene & 103 & 94 & 106 & 0 & 2 & 7 & 9 & 6 \\
\hline 2,4,5-'Trichlorophenol & 103 & 94 & 100 & $\mathbf{0}$ & 2 & 7 & 9 & 6 \\
\hline 2,1,6-Tribromophenol (surr) & 103 & 94 & 112 & 0 & 2 & 1 & 9 & 6 \\
\hline 2,1,6-Trichlorophenol & 103 & 94 & 106 & 0 & 2 & 7 & 9 & 6 \\
\hline 2,4-Dichlorophenol & 103 & 94 & 106 & $\mathbf{0}$ & 2 & 7 & 9 & 6 \\
\hline 2,4-Dimethyl phenol & 103 & 94 & 106 & $\mathbf{0}$ & 2 & 7 & 9 & 6 \\
\hline 2,4-Dinitrophenol & 103 & 94 & 106 & 0 & 2 & 9 & 9 & 6 \\
\hline 2,4-Dinitrotoluene & 103 & 91 & 106 & 0 & 2 & 9 & 9 & $\mathbf{6}$ \\
\hline 2,6-Dinitrotoluene & 103 & 94 & 106 & $\mathbf{0}$ & 2 & 7 & 9 & $\mathbf{6}$ \\
\hline 2-Chlorophenol & 103 & 94 & 106 & 0 & 2 & $\mathbf{9}$ & $\mathbf{9}$ & 6 \\
\hline 2-Chloronaphthalene & 103 & 94 & 106 & $\mathbf{0}$ & 2 & 7 & 9 & 6 \\
\hline 2-Pluorobiphenyl & 103 & 94 & 112 & 0 & 2 & 2 & 9 & $\mathbf{6}$ \\
\hline 2-Fluorophenol & 103 & 94 & 112 & $\mathbf{0}$ & 2 & 1 & 9 & $\mathbf{6}$ \\
\hline
\end{tabular}


Table 12. Numbers of Qualified Samples, Continued

\begin{tabular}{|c|c|c|c|c|c|c|c|c|}
\hline & Samples & & Analyses & & & $\mathrm{Q} \Lambda$ & & \\
\hline Analyte & Requested & Received & Reported & Rejected & Holding & Simpleg & Proscrvalion & Cal \\
\hline 2-Mcthylnaphthalene & 103 & 94 & 106 & 0 & 2 & 7 & ?) & $\mathbf{6}$ \\
\hline o-Cresol (2-Methylphenol) & 103 & 94 & 106 & 0 & 2 & 7 & 9 & $\mathbf{G}$ \\
\hline 0. Nitroaniline & 103 & 94 & 106 & 0 & 2 & 7 & 9 & 6 \\
\hline 2-Nitrophenol & 103 & 91 & 106 & 0 & 2 & 7 & 9 & 6 \\
\hline 3,3'-Dichlorobenzidine & 103 & 91 & 106 & 0 & 2 & 7 & 9 & 6 \\
\hline m-Nitroaniline & 103 & 94 & 106 & $\mathbf{0}$ & 2 & 7 & 9 & 6 \\
\hline 2-Methyl-4,6-dinitrophenol & 103 & 94 & 106 & $\mathbf{0}$ & 2 & 7 & 9 & $\mathbf{6}$ \\
\hline 4-Bromophenyl phenyl ether & 103 & 94 & 106 & 0 & 2 & 7 & $\mathbf{9}$ & 6 \\
\hline 4-Chloroaniline & 103 & 94 & 106 & 0 & 2 & 7 & ) & 6 \\
\hline 4-Chloro-m-cresol & 103 & 94 & 106 & $\mathbf{0}$ & 2 & 7 & $\mathbf{0}$ & G \\
\hline A-Chlorophenyl phenyl ether & 103 & 94 & 106 & 0 & 2 & 7 & 9 & $\mathbf{6}$ \\
\hline p-Cresol (4-Methylphenol) & 103 & 94 & 106 & $\mathbf{0}$ & 2 & 7 & 9 & 6 \\
\hline p-Nitroaniline & 103 & 94 & 106 & $\mathbf{0}$ & 2 & 7 & $\mathbf{9}$ & 6 \\
\hline 4-Nitrophenol & 103 & 94 & 106 & $\mathbf{0}$ & 2 & 7 & ) & 6 \\
\hline \multicolumn{9}{|l|}{ Volatile Organic Compounds } \\
\hline Acetone & 150 & 136 & 178 & $\mathbf{0}$ & 2 & 121 & 26 & $\mathbf{0}$ \\
\hline Bromodichloromethane & 150 & 136 & 165 & 0 & 2 & 17 & 22 & $\mathbf{0}$ \\
\hline Chloroethene (Vinyl chloride) & 150 & 136 & 165 & $\mathbf{0}$ & 2 & 16 & 22 & 2 \\
\hline Chloroethane & 160 & 136 & 165 & $\mathbf{0}$ & 2 & 16 & 22 & $\mathbf{0}$ \\
\hline Benzene & 150 & 136 & 165 & 0 & 2 & 29 & 22 & $\mathbf{0}$ \\
\hline Carbon tetrachloride & 150 & 136 & 165 & 0 & 2 & 17 & 22 & $\mathbf{0}$ \\
\hline $\begin{array}{l}\text { Dichloromethane (Methylene } \\
\text { chloride) }\end{array}$ & 150 & 136 & 165 & 0 & 2 & 101 & 22 & 0 \\
\hline $\begin{array}{l}\text { Bromomethane (Methyl } \\
\text { bromide) }\end{array}$ & 150 & 136 & 165 & 0 & 2 & 16 & 22 & 0 \\
\hline $\begin{array}{l}\text { Chloromethane (Methyl } \\
\text { chloride) }\end{array}$ & 150 & 136 & 165 & 0 & 2 & 16 & 22 & $\mathbf{0}$ \\
\hline Bromoform & 150 & 136 & 165 & 0 & 2 & 17 & 22 & $\mathbf{0}$ \\
\hline Chloroform & 150 & 136 & 165 & $\mathbf{0}$ & 2 & 18 & 22 & $\mathbf{0}$ \\
\hline Chlorobenzene & 150 & 136 & 165 & 0 & 2 & 15 & 22 & $\mathbf{0}$ \\
\hline Carbon disulfide & 150 & 136 & 165 & 0 & 2 & 16 & 22 & $\mathbf{0}$ \\
\hline
\end{tabular}


Table 12. Numbers of Qualified Samples, Continued

\begin{tabular}{|c|c|c|c|c|c|c|c|c|}
\hline & Samples & & Analyses & . & & QA & & \\
\hline Analyte & Requested & Received & Reported & Rejected & Holding & Samples & Prcservation & Calibration \\
\hline Dibromochloromethane & 150 & 136 & 165 & 0 & 2 & 17 & 22 & 0 \\
\hline Ethylbenzene & 150 & 136 & 165 & 0 & 2 & 17 & 22 & 0 \\
\hline Toluene-d8 & 150 & 136 & 178 & 0 & 2 & 2 & 26 & 0 \\
\hline Toluene & 150 & 136 & 165 & 0 & 2 & 15 & 22 & $\mathbf{0}$ \\
\hline Methyl ethyl ketone & 150 & 136 & 165 & 0 & 2 & 16 & 22 & 0 \\
\hline p-lBromofluorobenzene & 150 & 136 & 178 & 0 & 2 & 2 & 26 & 0 \\
\hline Styrene & 150 & 136 & 165 & 0 & 2 & 17 & 22 & 0 \\
\hline 1,1,2,2-Tetrachloroethane & 150 & 136 & 165 & 0 & 2 & 17 & 22 & 0 \\
\hline Tetrachloroethylene & 160 & 136 & 165 & 0 & 2 & 17 & 22 & $\mathbf{0}$ \\
\hline Trichloroethylene & 150 & 136 & 165 & 0 & 2 & 15 & 22 & 0 \\
\hline Vinyl acetate & 150 & 136 & 165 & 0 & 2 & 17 & 22 & 0 \\
\hline 1,1,1-Trichloroethane & 150 & 136 & 165 & 0 & 2 & 17 & 22 & $\mathbf{0}$ \\
\hline 1,1,2-Trichloroethane & 160 & 136 & 165 & 0 & 2 & 17 & 22 & $\mathbf{0}$ \\
\hline 1,1-Dichloroethylene & 150 & 136 & 165 & 0 & 2 & 14 & 22 & $\mathbf{0}$ \\
\hline 1,1-Dichloroethane & 150 & 136 & 165 & 0 & 2 & 16 & 22 & 0 \\
\hline 1,2-Dichloroethane-d4 & 150 & 136 & 178 & 0 & 2 & 2 & 26 & 0 \\
\hline 1,2-Dichloroethylene & . & 136 & 165 & 0 & 2 & 16 & 22 & 0 \\
\hline 1,2-Dichloroethane & 150 & 136 & 165 & 0 & 2 & 16 & 22 & 0 \\
\hline 1,2-Dichloropropane & 150 & 136 & 165 & 0 & 2 & 17 & 22 & 0 \\
\hline cis-1,3-Dichloropropene & 150 & 136 & 165 & 0 & 2 & 17 & 22 & $\mathbf{0}$ \\
\hline trans-1,3-Dichloropropene & 150 & 136 & 165 & 0 & 2 & 17 & 22 & $\mathbf{0}$ \\
\hline 2-Chloroethyl vinyl ether & 100 & 49 & 58 & 0 & 0 & 5 & 5 & 0 \\
\hline 2-Hexanone & 150 & 136 & 165 & 0 & 2 & 17 & 22 & 0 \\
\hline Methyl isobutyl ketone & 160 & 136 & 165 & 0 & 2 & 17 & 22 & 0 \\
\hline Xylencs & 150 & 135 & 164 & 0 & 2 & 17 & 22 & 0 \\
\hline \multicolumn{9}{|c|}{ Pesticides/PCBs and Dioxins/Turans } \\
\hline alpha-Benzene hexachloride & 70 & 70 & 80 & $\mathbf{0}$ & 0 & 16 & 4 & 0 \\
\hline alpha-Chlordane & 70 & $70^{\prime}$ & 80 & $\mathbf{0}$ & 0 & 15 & 4 & 0 \\
\hline Aldrin & 70 & 70 & 80 & 0 & 0 & 14 & 1 & 0 \\
\hline beta-Benzene hexachloride & 70 & 70 & 80 & 0 & 0 & 16 & 1 & 0 \\
\hline Carbon 13-labeled & 70 & 68 & 79 & $\mathbf{0}$ & 0 & 0 & 1 & 0 \\
\hline
\end{tabular}


Table 12. Numbers of Qualified Samples, Continued

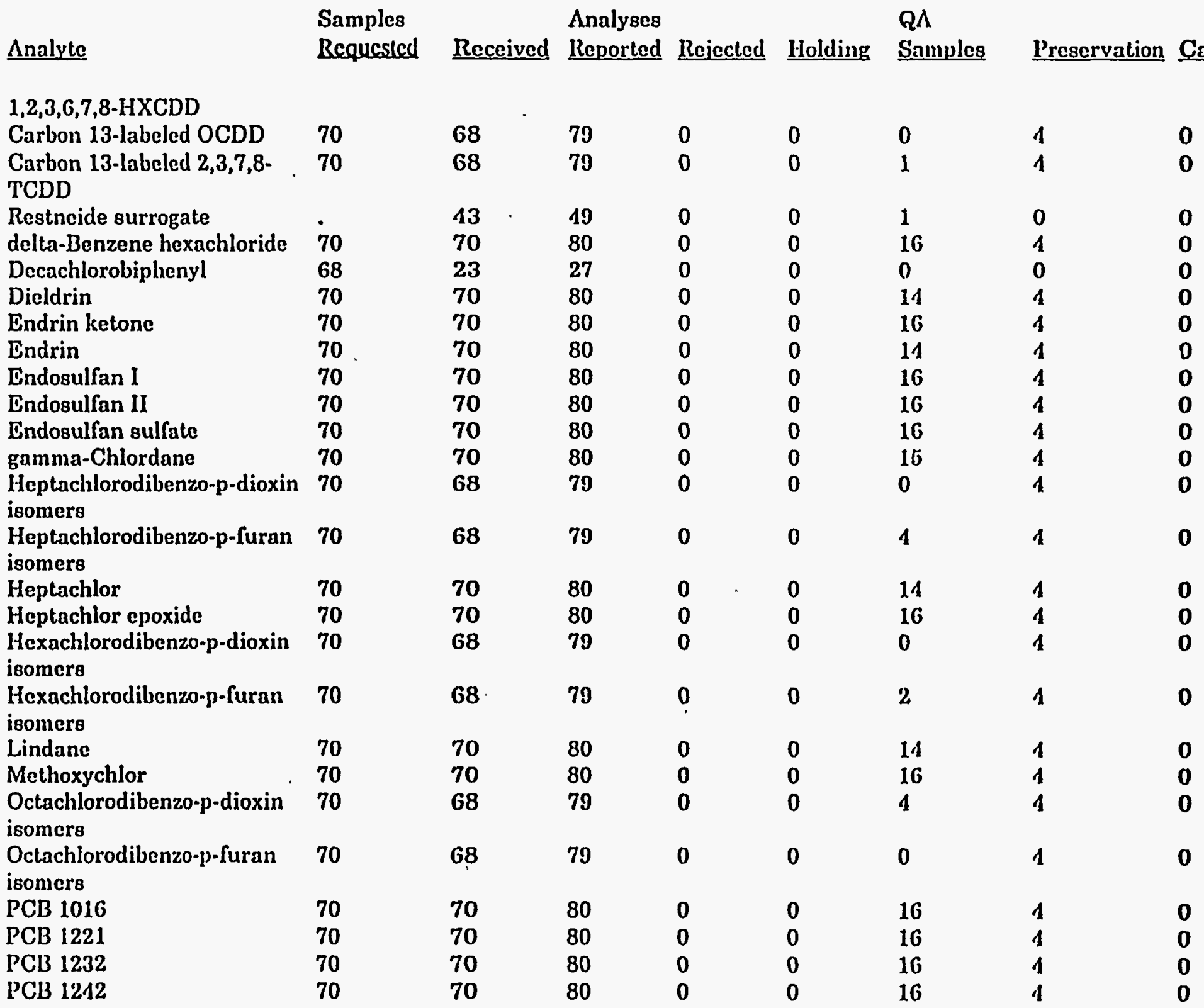


Table 12. Numbers of Qualified Samples, Continued

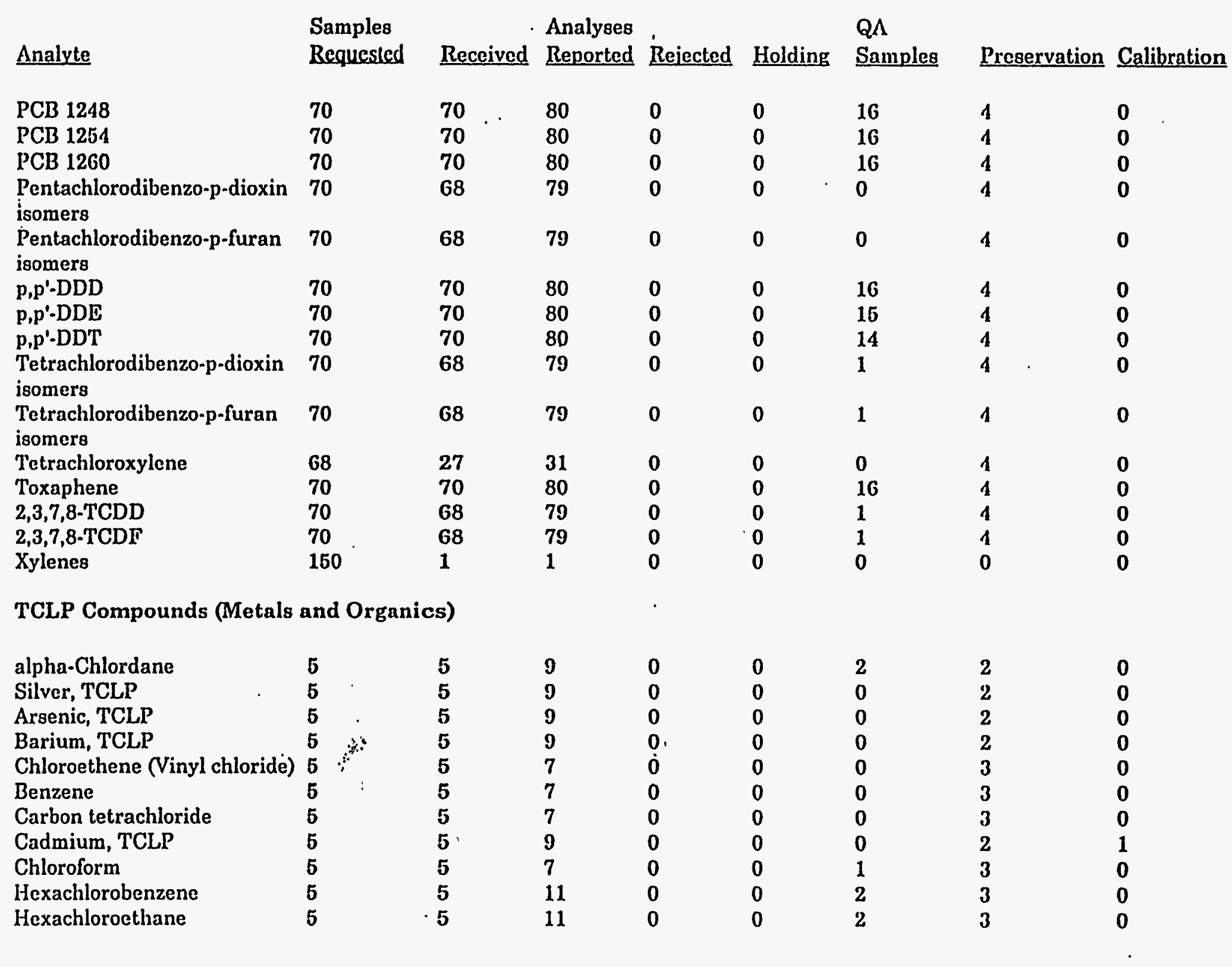


Table 12. Numbers of Qualified Samples, Continued

\begin{tabular}{|c|c|c|c|c|c|c|c|c|}
\hline & Samples & & Analyses & & & $\mathrm{Q} \Lambda$ & & \\
\hline Analyte & Requested & Received & Reported & Reiceted & Iolding & Samules & Prescrvation & Calibration \\
\hline Chlorobenzene & 5 & 5 & 7 & 0 & 0 & 0 & 3 & $\mathbf{0}$ \\
\hline Chromium, TCLP & 5 & 5 & 9 & 0 & 0 & 0 & 2 & 1 \\
\hline 2,4-Dichlorophenylacetic acid & 5 & 5 & 9 & 0 & 0 & 0 & 2 & 0 \\
\hline Decachlorobiphenyl & 5 & 1 & 7 & 0 & 0 & 0 & 2 & $\mathbf{0}$ \\
\hline Endrin & 5 & 5 & 9 & 0 & $\mathbf{0}$ & 2 & 2 & 0 \\
\hline Decalluorobiphenyl & 5 & 1 & 2 & 0 & $\mathbf{0}$ & 0 & $\mathbf{0}$ & 0 \\
\hline gamma-Chlordane & $\mathbf{5}$ & 5 & $\mathbf{9}$ & 0 & $\mathbf{0}$ & 2 & 2 & $\mathbf{0}$ \\
\hline Hexachlorobutadiene & 5 & 5 & 11 & 0 & 0 & 2 & 3 & $\mathbf{0}$ \\
\hline Mercury, TCLP' & 5 & 5 & 9 & 0 & $\mathbf{0}$ & 2 & 2 & $\mathbf{0}$ \\
\hline Heptachlor & 5 & 5 & 9 & 0 & 0 & 2 & 2 & $\mathbf{0}$ \\
\hline Heptachlor epoxide & $\mathbf{5}$ & 5 & 9 & 0 & $\mathbf{0}$ & 2 & 2 & $\mathbf{0}$ \\
\hline Lindanc & 5 & 5 & 9 & 0 & $\mathbf{0}$ & 2 & $\mathbf{2}$ & $\mathbf{0}$ \\
\hline Tolucne-d8 & 5 & 5 & 7 & 0 & $\mathbf{0}$ & 0 & 3 & 0 \\
\hline Methyl ethyl ketone & 5 & 5 & 7 & 0 & 0 & 0 & 3 & $\mathbf{0}$ \\
\hline Methoxychlor & 5 & 5 & 9 & 0 & 0 & 2 & 2 & 0 \\
\hline Nitrobenzene & 5 & 5 & 11 & 0 & 0 & 2 & 3 & $\mathbf{0}$ \\
\hline Nitrobenzenc-d5 & 5 & 5 & 11 & 0 & 0 & 0 & 3 & 0 \\
\hline p-Bromofluorobenzene & 5 & 5 & 7 & 0 & $\mathbf{0}$ & 0 & 3 & 0 \\
\hline Lead, TCLP & 5 & 5 & 9 & 0 & 0 & 0 & 2 & 0 \\
\hline Pentachlorophenol & 5 & 6 & 11 & 0 & $\mathbf{0}$ & 2 & 3 & $\mathbf{0}$ \\
\hline Phenol-d5 & 5 & 5 & 11 & 0 & 0 & 0 & 3 & $\mathbf{0}$ \\
\hline p-Terphenyl-d11 & 5 & 5 & 11 & 0 & 0 & 0 & $\mathbf{3}$ & 0 \\
\hline Pyridine & 6 & 5 & 11 & 0 & 0 & 2 & 3 & 0 \\
\hline Sclenium, TCI,P & 5 & 5 & 9 & 0 & 0 & 0 & 2 & $\mathbf{0}$ \\
\hline 2,4,5.'Tl (Silvex) & 5 & 5 & $\mathbf{9}$ & 0 & 0 & 0 & 2 & 0 \\
\hline Tetrachloroethylene & 5 & 5 & 7 & 0 & 0 & 0 & 3 & 0 \\
\hline Tetrachloroxylene & 5 & 5 & 9 & 0 & 0 & 0 & 2 & 0 \\
\hline Trichlorocthylene & 5 & 5 & 7 & 0 & 0 & 0 & 3 & 0 \\
\hline Toxaphene & 5 & 5 & $\mathbf{9}$ & 0 & 0 & 3 & 2 & 0 \\
\hline 1,1-Dichloroctliylene & 5 & 5 & 7 & 0 & $\mathbf{0}$ & 0 & 3 & 0 \\
\hline 1,2-Dichloroethane-d 4 & 5 & 5 & 7 & 0 & $\mathbf{0}$ & 0 & 3 & $\mathbf{0}$ \\
\hline 1,2-Dichlorocthane & 5 & 5 & 7 & 0 & $\mathbf{0}$ & 0 & 3 & 0 \\
\hline 1,4-Dichlorobenzene & 5 & 5 & 11 & 0 & $\mathbf{0}$ & 2 & 3 & 0 \\
\hline $2,1,5-T$ & 5 & 5 & $\boldsymbol{9}$ & 0 & $\mathbf{0}$ & 0 & 2 & $\mathbf{0}$ \\
\hline
\end{tabular}


Table 12. Numbers of Qualified Samples, Continued

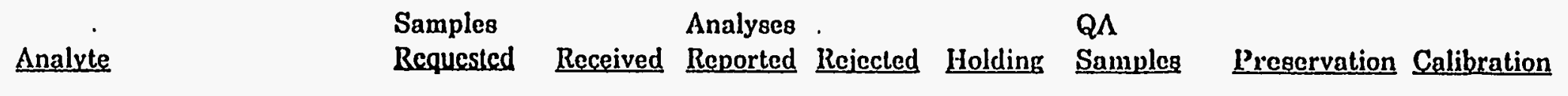

$\begin{array}{lr}\text { 2,4,5-Trichlorophenol } & 5 \\ \text { 2,4,6-Tribromophenol (surr) } & 5 \\ \text { 2,4,6-Trichlorophenol } & 6 \\ \text { 2,4-Dichlorophenoxyacetic } & 6 \\ \text { acid } & \\ \text { 2,4-Dinitrotoluene } & 5 \\ \text { 2-Fluorobiphenyl } & 5 \\ \text { 2-Fluorophenol } & 5 \\ \text { o-Cresol (2-Methylphenol) } & 5 \\ \text { X34MP } & 5\end{array}$

$\begin{array}{lll}\mathbf{5} & 11 & 0 \\ \mathbf{5} & 11 & 0 \\ \mathbf{5} & 11 & 0 \\ \mathbf{5} & \mathbf{9} & \mathbf{0} \\ & & \\ \mathbf{5} & 11 & 0 \\ \mathbf{5} & 11 & 0 \\ \mathbf{5} & 11 & \mathbf{0} \\ \mathbf{5} & 11 & \mathbf{0} \\ \mathbf{5} & \mathbf{1 1} & \mathbf{0}\end{array}$

$\begin{array}{ll}0 & 2 \\ 0 & 0 \\ 0 & 2 \\ 0 & 0 \\ & \\ 0 & 2 \\ 0 & 0 \\ 0 & 0 \\ 0 & 2 \\ 0 & 2\end{array}$

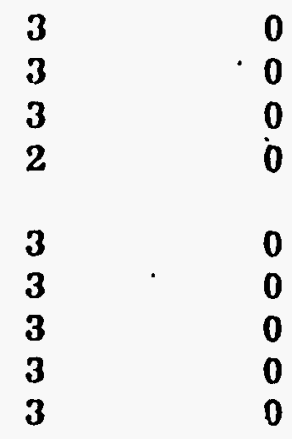

Radionuclide Indicators and Radionuclides

$\begin{array}{lll}\text { Gross alpha } & 129 & 116 \\ \text { Nonvolatile beta } & 129 & 116 \\ \text { Radium, total alpha-enuitting } & 129 & 116\end{array}$

116

Radium, total alpha-enuitting 129

Tritium

$\begin{array}{lll}0 & 0 & 0 \\ 0 & 0 & 43 \\ 0 & 0 & 106 \\ 0 & 0 & 1\end{array}$

$\begin{array}{ll}16 & 0 \\ 16 & 0 \\ 16 & 0 \\ 16 & 0\end{array}$




\section{Data Table Generation}

The printed tables in Appendix $\mathrm{D}$ of this report are an adjunct to the electronic data files, providing a convenient hard-copy compilation of the data collected for this project. They include, for all samples, selected fields of sampling, station, and analytical data. The tables are ordered by station number as specified in Tables 4-1 and 4-2 of the work plan, - with all of the soil samples presented first, followed by the environmental water samples, and finally, the blanks specified in those tables. Results for laboratory blanks, surrogates, matrix spikes, and other laboratory QA/QC samples are not presented in the printed data tables.

Table generation does not in any way alter the data as it exists in the data files.

The printed tables were generated from the validated data files by a SAS program that combines and processes the data into a form suitable for word processing software. The SAS program's output is a combination of station, sampling, and analytical data, which are otherwise maintained as separate data files. For presentation in the tables, analytical data was rounded to two significant figures.

For each sample analyzed, a header prorides the sample name and sample identification number. If the sample is a normal entironmental sample, the header further provides the sampling location in Savannah River Site coordinates, the ground elevation in feet above mean sea level, the depth to the top and bottom of the sampled core interval in feet below ground level, the sample type, matrix, moisture, Munsell color, and Unified Soil Classification, as well as percentage of solids in the sample used for analysis. If the sample is other than a normal sample (duplicate, blank, etc.), the header specifies the sample type and identifies the normal or primary sample with which this sample is associated.

The table includes columns for analyte names, result qualifiers, analytical qualifiers, bias, results, units of measurement, detection limits, and analytical method codes. The constituents are sorted first by analytical suite, and then within each suite according to accepted chemical nomenclature as specified by the American Chemical Society. Analytical suites are set off by identifing headers. 
American Society for Testing and Materials, 1988. ASTM Standards on Precision and Bias for Various Applications, 3rd edition. Philadelphia, PA.

Keith, L. H., ed., 1991. Compilation of EPA's Sampling \& Analysis Methods. Boca Raton, FL.

Krieger, H.L., and E.L. Whitaker, 1980. Prescribed Procedures for Measurement of Radioactivity in Drinking Water, EPA-600/4-80-032. Environmental Monitoring and Support Laboratory, U.S. Environmental Protection Agency, Cincinnati, OH.

Pfaff, J.D., C.A. Brockhoff, and J.W. O'Dell, 1991. Test Method: The Determination of Inorganic Anions in Water by Ion Chromatography-Method 300.0. Environmental Monitoring and Systems Laboratory, U.S. Enrironmental Protection Agency, Cincinnati, $\mathrm{OH}$.

U.S. Department of Energy, Environmental Measurements Laboratory, 1992. Environmental Measurements Laboratory Procedures Manual, 27th edition, HASL-300. New Fork, $\lambda I$.

U.S. Environmental Protection Agency, 1986. Test Methods for Evaluating Solid Waste (STW-846), Volume I. Washington, D.C.

U.S. Environmental Protection Agency, 1987. Data Quality Objectives for Remedial Response Activities: Development Process, EPA 540/G-87/003. Washington, D.C.

U.S. Environmental Protection Agency, 1990. Quality Assurance/Quality Control Guidance for Removal Activities, Sampling QA/QC Plan and Data Validation Procedures, EPA 540/G-90/004.

U.S. Environmental Protection Agency, 1991. USEPA Contract Laboratory Program, Statement of Work for Organics Analysis, Multi-Media, Multi-Concentration, OlMO1.0.

U.S. Environmental Protection Agency, 1992. 40 CFR Parts 261 and 271, Federal Register, Volume 57, Number 227, November 24, 1992, p. 55117.

U.S. Environmental Protection Agency, Data Review Work Group, 1988. Laboratory Data Validation Functional Guidelines for Evaluating Inorganics Analyses.

U.S. Environmental Protection Agency, Data Review Work Group, 1991. Laboratory Data Validation Functional Guidelines for Evaluating Organics Analyses, Draft.

U.S. Environmental Protection Agency, Environmental Monitoring and Support Laboratory, 1983. Methods for Chemical Analysis of Water and Wastes, EPA-600/4. 79-020. Cincinnati, $\mathrm{OH}$. 
U.S. Environmental Protection Agency, Hazardous Site Evaluation Division, 1988.

Laboratory Data Validation Functional Guidelines for Evaluating Inorganics Analyses.

U.S. Environmental Protection Agency, Hazardous Site Evaluation Division, 1988. Laboratory Data Validation Functional Guidelines for Evaluating Organics Analyses.

Westinghouse Hanford Company, Data Validation Procedures for Radiochemical Analyses, WHC-SD-EN.SPP-001, Rev. 1. Richland, WA.

Westinghouse Savannah River Company, 1992. 1992 Renewal Application for a RCRA Part B Permit, Volume I, Appendix 6, WSRC-IM-91-53. Savannah River Site, Aiken, SC.

Westinghouse Savannah River Company, 1992a. Phase II, Revision 2, RCRA Facility Investigation/ Remedial Investigation Plan for the D-Area Burning/Rubble Pits. (431-D and 431-1D), WSRC-RP-90-485, Rev. 2. Savannah River Site, Aiken, SC.

Westinghouse Savannah River Company, 1992b. Hydrogeologic Data Collection, Manual 3Q5. Savannah River Site, Aiken, SC. 


\section{Table A-1: Soil Constituent List}

Base/neutral/acid extractables, including polycyclic aromatic hydrocarbons (PAHs):

Acenaphthene
Acenaphthylene
Anthracene
Benzo(a)anthracene
Benzo(b)fluoranthene
Benzo(k)fluoranthene
Benzoic acid
Benzo(g,h,i)perylene
Benzo(a)pyrene
Benzyl alcohol
Bis(2-chloroethoxy)
methane
Bis(2-chloroethyl) ether
Bis(2-chloroisopropyl) ether
Bis(2-ethylhexyl) phthalate
4-Bromophenyl phenyl
ether
Butylbenzyl phthalate
4-Chloroaniline
4-Chloro-m-cresol
2-Chloronaphthalene
2-Chlorophenol

Acenaphthene

Acenaphthylene

Anthracene

Benzo(a)anthracene

Benzo(b)fluoranthene

Benzo(k)fluoranthene

Benzoic acid

Benzo( $g, h, i)$ perylene

Benzo(a)pyrene

Benzyl alcohol

methane

Bis(2-chloroethyl) ether

Bis(2.ethylhexyl) phthalate

4-Bromophenyl phenyl

Butylbenzyl phthalate

4-Chloroaniline

4-Chloro-m-cresol

2-Chloronaphthalene

2-Chlorophenol

\author{
4.Chlorophenyl-phenyl \\ ether \\ Chrysene \\ - o-Cresol (2-Methylphenol) \\ p-Cresol (4-Methylphenol) \\ Dibenz $(a, h)$ anthracene \\ Dibenzofuran \\ Di-n-butyl phthalate \\ 1,2-Dichlorobenzene \\ 1,3-Dichlorobenzene \\ 1,4-Dichlorobenzene \\ 3,3'-Dichlorobenzidine \\ 2,4-Dichlorophenol \\ Diethyl phthalate \\ 2,4-Dimethyl phenol \\ Dimethyl phthalate \\ 2,4-Dinitrophenol \\ 2,4-Dinitrotoluene \\ 2,6-Dinitrotoluene \\ Di-n-octyl phthalate \\ Fluoranthene \\ Fluorene \\ Hexachlorobenzene
}

Hexachlorobutadiene

Hexachlorocyclopentadiene

Hexachloroethane

Indeno(1,2,3-c,d)pyrene

Isophorone

2-Methyl-4,6-dinitrophenol

2-Methylnaphthalene

Naphthalene

o-Nitroaniline

m-Nitroaniline

p-Nitroaniline

Nitrobenzene

2-Nitrophenol

4-Nitrophenol

$\mathrm{N}$-Nitrosodiphenylamine

N-Nitrosodipropylamine

Pentachlorophenol

Phenanthrene

Phenol

Pyrene

1,2,4-Trichlorobenzene

2,4,5-Trichlorophenol

2,4,6-Trichlorophenol

Volatile organic compounds

Acetone

Benzene

Bromodichloromethane

Bromoform

Bromomethane (Methyl

bromide)

Carbon disulfide

Carbon tetrachloride

Chlorobenzene

Chloroethane

Chloroethene (Vinyl

chloride)

2-Chloroethyl vinyl ether
Chloroform

Chloromethane (Methyl

chloride)

Dibromochloromethane

1,I-Dichloroethane

1,2-Dichloroethane

1,2-Dichloroethylene (Total)

1,1-Dichloroethylene

Methylene chloride

1,2-Dichloropropane

cis-1,3-Dichloropropene

trans-1,3-Dichloropropene

Ethylbenzene
2-Hexanone

Methyl ethyl ketone

Methyl isobutyl ketone

Styrene

1,1,2,2-Tetrachloroethane

Tetrachloroethylene

Toluene

1,1,1-Trichloroethane

1,1,2-Trichloroethane

Trichloroethylene

Vinyl acetate

Xylenes (Total)
Total metals

Arsenic

Barium

Cadmium

Chromium

Copper
Lead

Manganese

Mercury

Nickel

Selenium

Silver
Physical parameters

Cation exchange capacity

(CEC)

Soil pH

Specific conductance

Total organic carbon 


\section{Pesticides/PCBs and Dioxins/Furans}

Aldrin

alpha-Benzene hexachloride

beta-Benzene hexachloride

delta-Benzene hexachloride

gamma-Benzene

hexachloride

alpha-Chlordane

gamma.Chlordane

p,p'-DDD

p,p'-DDE

p,p'-DDT

Dieldrin

Endosulfan I

Endosulfan II

Endosulfan sulfate

Endrin

Endrin ketone

Radionuclide indicators

TCLP

Arsenic

Barium

Benzene

Cadmium

Carbon tetrachloride

Chlordane (alpha and gamma)

Chlorobenzene

Chloroform

Chromium

o-Cresol

m,p-Cresol

2,4-D

TCL/TAL with TIC

TALTCL Inorganic:

Same as total metals with

the addition of:

Aluminum

Antimony

Beryllium

Calcium

Cobalt

Cyanide

Iron
Heptachlor

Heptachlor epoxide

Heptachlorodibenzo-p-

dioxin

isomers

Heptachlorodibenzo-p-furan

isomers

Hexachlorodibenzo-p-dioxin

isomers

Hexachlorodibenzo-p-furan

isomers

Methoxychlor

Octachlorodibenzo-p-dioxin

isomers

Octachlorodibenzo-p-furan

isomers

PCB 1016

Gross alpha

Nonvolatile beta

1,4-Dichlorobenzene

1,2-Dichloroethane

1,1-Dichloroethylene

2,4-Dinitrotoluene

Endrin

Heptachlor

Heptachlor epoxide

Hexachlorobenzene

Hexachlorobutadiene

Hexachloroethane

Lead

Lindane

Mercury

Methoxychlor

Methyl ethyl ketone

Magnesium

Potassium

Sodium

Thallium

Vanadium

Zinc

TALTCL

Pesticides/PCBs:

Same as Pesticides/PCB,

with the addition of
PCB 1221

PCB 1232

PCB 1242

PCB 1248

PCB 1254

PCB 1260

Pentachlorodibenzo-p-dioxin isomers

Pentachlorodibenzo-p-furan isomers

2,3,7,8-TCDD

2,3,7,8-TCDF

Tetrachlorodibenzo-p-dioxin isomers

Tetrachlorodibenzo-p-furan isomers

Toxaphene

Total radium

Tritium

Nitrobenzene

Pentachlorophenol

Pyridine

Selenium

Silver

Tetrachloroethylene

Toxaphene

Trichloroethylene

2,4,5-Trichlorophenol

2,4,6-Trichlorophenol

2,4,5-TP (Silvex)

2,4,5-T

Vinyl chloride

Lindane; does not include dioxins/furans

TAL/TCL Semivolatiles:

Same as semivolatile

organic compounds

TALTCL Volatiles

Same as volatile organic compounds 


\section{Table A-2: Water Constituent List}

Base/neutral/acid extractables, including polycyclic aromatic hydrocarbons (PAHs):

Same as soil semivolatile organic compounds

Volatile organic compounds

Same as soil volatile organic compounds

Total metals

Same as soil total metals

\section{Radionuclide indicators}

Same as soil radionuclide indicators

\section{Inorganics}

Chloride

Fluoride

Iron

Nitrate as nitrogen

$\mathrm{pH}$

Sulfate

Total dissolved solids

Total organic carbon

Total organic halogens

Total suspended solids 
Table B-1. EPAJEMSL Intercomparison Studies Program Data for TMA/Eberline

\begin{tabular}{|c|c|c|c|c|}
\hline Analyte & Matrix & $\begin{array}{l}\text { Known } \\
\text { Value }(\mathrm{pC} \text { iL) }\end{array}$ & $\begin{array}{l}\text { TMA } \\
\text { Result (oCi/L) }\end{array}$ & $\begin{array}{l}\text { Norm. } \\
\text { Deviation }\end{array}$ \\
\hline Cesium-137 & Air filter & $9.0 \pm 5.0$ & $10.0 \pm 1.0$ & +0.4 \\
\hline Gross alpha & Air filter & $19.0 \pm 5.0$ & $24.7 \pm 1.2$ & +2.0 \\
\hline Nonvolatile beta & Air filter & $47.0 \pm 5.0$ & $58.0 \pm 1.7^{1}$ & +3.81 \\
\hline Strontium-90 & Air filter & $19.0 \pm 5.0$ & $22.7 \pm 1.5$ & +1.3 \\
\hline Gross alpha & P.E. "A" & $96.0 \pm 24.0$ & $115.3 \pm 3.8$ & +1.5 \\
\hline Radium-226 & P.E. "A" & $24.9 \pm 3.7$ & $22.5 \pm 0.7$ & -1.1 \\
\hline Radium-228 & P.E. "A" & $19.0 \pm 4.8$ & $21.2 \pm 0.7$ & +0.8 \\
\hline Uranium (natural) & P.E. "A" & $28.9 \pm 3.0$ & $30.9 \pm 2.4$ & +1.2 \\
\hline Cesium-134 & P.E. "B" & $27.0 \pm 5.0$ & $26.7 \pm 0.6$ & -0.1 \\
\hline Cesium-137 & P.E. "B" & $32.0 \pm 5.0$ & $27.0 \pm 1.0$ & -1.7 \\
\hline Cobalt-60 & P.E. "B" & $39.0 \pm 5.0$ & $39.0 \pm 0.0$ & 0.0 \\
\hline Nonvolatile beta & P.E. " $B^{n}$ & $117.0 \pm 27.0$ & $172.7 \pm 4.9$ & -0.3 \\
\hline Strontium.89 & P.E. "B" & $41.0 \pm 5.0$ & $39.7 \pm 1.5$ & .0 .5 \\
\hline Strontium-90 & P.E. "B" & $29.0 \pm 5.0$ & $23.7 \pm 1.1$ & -1.9 \\
\hline Gross alpha & Water & $34.0 \pm 9.0$ & $14.3 \pm 1.5^{2}$ & -3.82 \\
\hline Gross alpha & Water & $15.0 \pm 5.0$ & $9.3 \pm 1.5$ & .2 .0 \\
\hline Nonvolatile beta & Water & $44.0 \pm 5.0$ & $44.7 \pm 5.1$ & +0.2 \\
\hline Nonvolatile beta & Water & $43.0 \pm 6.9$ & $50.3 \pm 3.1$ & +1.8 \\
\hline Plutonium-239 & Water & $20.0 \pm 2.0$ & $18.8 \pm 0.5$ & -0.1 \\
\hline Radium-226 & Water & $9.8 \pm 0.5$ & $9.6 \pm 0.5$ & -0.2 \\
\hline Radium-228 & Water & $18.5 \pm 4.6$ & $19.7 \pm 0.8$ & +0.5 \\
\hline Strontium-89 & Water & $34.0 \pm 5.0$ & $33.7 \pm 1.2$ & .0 .5 \\
\hline Strontium-89 & Water & $15.0 \pm 5.0$ & $16.7 \pm 2.5$ & +0.1 \\
\hline Strontium-90 & Water & $10.0 \pm 5.0$ & $10.3 \pm 1.2$ & +0.1 \\
\hline Strontium-90 & Water & $25.0 \pm 5.0$ & $25.0 \pm 1.7$ & 0.0 \\
\hline Tritium & Water & $9,844 \pm 984$ & $10,427 \pm 211$ & +1.0 \\
\hline Uranium (natural) & Water & $7.6 \pm 3.0$ & $8.7 \pm 0.2$ & -0.5 \\
\hline Uranium (natural) & Water & $25.3 \pm 3.0$ & $23.9 \pm 0.3$ & -0.8 \\
\hline Barium-133 & Water $(y)$ & $99.0 \pm 10.0$ & $87.0 \pm 7.6$ & -2.1 \\
\hline Cesium-134 & Water $(y)$ & $5.0 \pm 5.0$ & $4.7 \pm 0.6$ & .0 .1 \\
\hline Cesium-137 & Water $(\gamma)$ & $5.0 \pm 5.0$ & $5.3 \pm 0.6$ & +0.1 \\
\hline Cobalt- 60 & Water $(y)$ & $15.0 \pm 5.0$ & $14.0 \pm 1.0$ & -0.4 \\
\hline Ruthenium-106 & Water $(\gamma)$ & $119.0 \pm 12.0$ & $96.3 \pm 8.1$ & -2.9 \\
\hline Zinc-65 & Water $(\gamma)$ & $103.0 \pm 10.0$ & $106.0 \pm 3.5$ & +0.5 \\
\hline
\end{tabular}

'A Corrective Action Report was issued.

2TMA stated that thorium-230, which was used by EPA, has a $50 \%$ lower counting efficiency than americium-241, the EPA-recommended isotope for alpha-absorption efficiency curves. According to TMA, correction for the $50 \%$ difference gives an acceptable value. 
Table B-2. TMA Performance Evaluation, DOE Study 93-03

\begin{tabular}{|c|c|c|c|c|}
\hline Analyte & Matrix & $\begin{array}{l}\text { Known } \\
\text { Value }\end{array}$ & $\begin{array}{l}\text { TMA } \\
\text { Result }\end{array}$ & $\begin{array}{l}\text { Ratio } \\
\text { TMA/Known }\end{array}$ \\
\hline Americium-241 & Air & $0.414 \mathrm{E}-01$ & $0.218 \mathrm{E}-01$ & 0.53 \\
\hline Beryllium-7 & Air & $0.274 E+02$ & $0.368 \mathrm{E}+02$ & 1.34 \\
\hline Cerium-144 & Air & $0.193 E+02$ & $0.433 \mathrm{E}+02$ & 2.24 \\
\hline Cesium-134 & Air & $0.196 E+01$ & $0.213 E+01$ & 1.09 \\
\hline Cesium-137 & $\overline{A i r}$ & $0.307 E+01$ & $0.363 E+01$ & 1.18 \\
\hline Cobalt-57 & Air & $0.271 E+01$ & $0.533 E+01$ & 1.97 \\
\hline Cobalt- 60 & Air & $0.170 \mathrm{E}+01$ & $0.213 E+01$ & 1.25 \\
\hline Manganese-54 & Air & $0.117 E+02$ & $0.134 E+02$ & 1.15 \\
\hline Plutonium-238 & Air & $0.363 E-01$ & $0.252 E-01$ & 0.69 \\
\hline Plutonium-239 & Air & $0.234 \mathrm{E}-01$ & $0.198 \mathrm{E}-01$ & 0.85 \\
\hline Strontium-90 & Air & $0.152 E+00$ & $0.229 \mathrm{E}+00$ & 1.51 \\
\hline Uranium-Bq & Air & $0.460 E-01$ & $0.560 \mathrm{E} \cdot 01$ & 1.22 \\
\hline Americium-241 & Soil & $0.650 \mathrm{E}+01$ & $0.420 \mathrm{E}+01$ & 0.65 \\
\hline Cesium-137 & Soil & $0.923 E+03$ & $0.722 E+03$ & 0.78 \\
\hline Plutonium-239 & Soil & $0.116 E+02$ & $0.107 \mathrm{E}+02$ & 0.92 \\
\hline Potassium- 40 & Soil & $0.321 E+03$ & $0.253 E+03$ & 0.79 \\
\hline Strontium-90 & Soil & $0.417 E+02$ & $0.266 \mathrm{E}+02$ & 0.64 \\
\hline Uranium-Bq & Soil & $0.769 E+02$ & $0.733 E+02$ & 0.95 \\
\hline Americium-241 & Vegetation & $0.231 E+00$ & $0.395 E+00$ & 1.71 \\
\hline Cesium-137 & Vegetation & $0.246 \mathrm{E}+02$ & $0.197 E+02$ & 0.80 \\
\hline Plutonium-238 & Vegetation & $0.114 E+01$ & $0.130 E+01$ & 1.14 \\
\hline Plutonium-239 & Vegetation & $0.323 E+00$ & $0.340 E+00$ & 1.05 \\
\hline Potassium-40 & Vegetation & $0.383 E+03$ & $0.330 E+03$ & 0.86 \\
\hline Strontium-90 & Vegetation & $0.237 E+3$ & $0.140 E+03$ & 0.59 \\
\hline Americium-241 & Water & $0.440 E+00$ & $0.520 \mathrm{E}+00$ & 1.18 \\
\hline Cerium-144 & Water & $0.836 \mathrm{E}+02$ & $0.929 E+02$ & 1.11 \\
\hline Cesium-134 & Water & $0.424 \mathrm{E}+02$ & $0.490 \mathrm{E}+02$ & 1.16 \\
\hline Cesium-137 & Water & $0.508 E+02$ & $0.598 \mathrm{E}+02$ & 1.18 \\
\hline Cobalt-60 & Water & $0.453 E+02$ & $0.509 E+02$ & 1.12 \\
\hline Manganese-54 & Water & $0.105 E+03$ & $0.118 E+03$ & 1.12 \\
\hline Plutonium-238 & Water & $0.494 E+00$ & $0.480 E+00$ & 0.97 \\
\hline Plutonium-239 & Water & $0.828 \mathrm{E}+00$ & $0.810 \mathrm{E}+00$ & 0.98 \\
\hline Strontium-90 & Water & $0.103 E+01$ & $0.170 E+01$ & 1.65 \\
\hline Tritium & Water & $0.970 \mathrm{E}+02$ & $0.109 E+03$ & 1.12 \\
\hline Uranium-Bq & Water & $0.289 \mathrm{E}+00$ & $0.260 \mathrm{E}+00$ & 0.90 \\
\hline
\end{tabular}


Table B-3. TMA Performance Evaluation, DOE Study 93-09

\begin{tabular}{|c|c|c|c|c|}
\hline Analyte & Matrix & $\begin{array}{l}\text { Known } \\
\text { Value }\end{array}$ & $\begin{array}{l}\text { TMA } \\
\text { Result }\end{array}$ & $\begin{array}{l}\text { Ratio } \\
\text { TMA/Known }\end{array}$ \\
\hline Americium-241 & Air & $0.654 \mathrm{E}-01$ & $0.650 \mathrm{E}-01$ & 0.99 \\
\hline Antimony-125 & Air & $0.174 \mathrm{E}+02$ & $0.219 \mathrm{E}+02$ & 1.26 \\
\hline Cesium-134 & Air & $0.122 E+02$ & $0.132 E+02$ & 1.08 \\
\hline Cesium-137 & $\overline{A i r}$ & $0.188 \mathrm{E}+02$ & $0.224 E+02$ & 1.19 \\
\hline Cerium-144 & Air & $0.403 E+02$ & Not Reported & $\mathrm{NA}$ \\
\hline Cobalt-57 & Air & $0.173 E+02$ & $0.330 \mathrm{E}+02$ & 1.91 \\
\hline Cobalt. 60 & Air & $0.205 E+02$ & $0.251 E+02$ & 1.22 \\
\hline Manganese-54 & Air & $0.154 E+02$ & $0.183 \mathrm{E}+02$ & 1.19 \\
\hline Plutonium-238 & Air & $0.129 E+00$ & $0.110 \mathrm{E}+00$ & 0.85 \\
\hline Plutonium-239 & Air & $0.800 \mathrm{E}-01$ & $0.740 \mathrm{E}-01$ & 0.93 \\
\hline Strontium-90 & Air & $0.762 \mathrm{E}+00$ & $0.740 \mathrm{E}+00$ & 0.97 \\
\hline Uranium-234 & Air & $0.650 \mathrm{E}-01$ & Not Reported & NA \\
\hline Uranium-238 & Air & $0.650 \mathrm{E}-01$ & Not Reported & NA \\
\hline Uranium-Bq & Air & $0.137 \mathrm{E}+00$ & Not Reported & $\mathrm{NA}$ \\
\hline Uranium- $\mu \mathrm{g}$ & Air & $0.541 E+01$ & $0.520 E+01$ & 0.96 \\
\hline Americium-241 & Soil & $0.248 \mathrm{E}+00$ & $0.360 \mathrm{E}+00$ & 1.45 \\
\hline Cesium-137 & Soil & $0.114 \mathrm{E}+02$ & $0.139 E+02$ & 1.22 \\
\hline Potassium-40 & Soil & $0.286 \mathrm{E}+02$ & $0.337 E+02$ & 1.18 \\
\hline Plutonium-239 & Soil & $0.152 E+01$ & $0.222 E+01$ & 1.46 \\
\hline Strontium-90 & Soil & $0.540 \mathrm{E}+01$ & $0.519 E+01$ & 0.96 \\
\hline Uranium-234 & Soil & $0.248 \mathrm{E}+02$ & Not Reported & $\mathrm{NA}$ \\
\hline Uranium-238 & Soil & $0.255 \mathrm{E}+02$ & Not Reported & NA \\
\hline Uranium-Bq & Soil & $0.503 E+02$ & Not Reported & $\mathrm{NA}$ \\
\hline Uranium- $\mu \mathrm{g}$ & Soil & $0.197 \mathrm{E}+1$ & $0.153 \mathrm{E}+01$ & 0.78 \\
\hline Americium-241 & Vegetation & $0.465 E+00$ & Not Reported & $\mathrm{NA}$ \\
\hline Cesium-137 & Vegetation & $0.892 E+02$ & $0.845 \mathrm{E}+02$ & 0.95 \\
\hline Cobalt- 60 & Vegetation & $0.645 E+01$ & $0.780 \mathrm{E}+01$ & 1.21 \\
\hline Potassium-40 & Vegetation & $0.842 \mathrm{E}+03$ & $0.862 \mathrm{E}+03$ & 1.02 \\
\hline Plutonium-238 & Vegetation & $0.463 \mathrm{E}+00$ & $0.870 E+00$ & 1.88 \\
\hline Plutonium-239 & Vegetation & $0.965 E+00$ & $0.149 E+01$ & 1.54 \\
\hline Strontium-90 & Vegetation & $0.221 E+03$ & $0.275 E+03$ & 1.24 \\
\hline Americium-241 & Water & $0.139 \mathrm{E}+01$ & $0.142 E+01$ & 1.02 \\
\hline Cesium-134 & Water & $0.561 E+02$ & $0.519 \mathrm{E}+02$ & 0.93. \\
\hline Cesium-137 & Water & $0.755 E+02$ & $0.723 E+02$ & 0.96 \\
\hline Cerium-144 & Water & $0.173 E+03$ & $0.155 E+03$ & 0.90 \\
\hline Cobalt- 60 & Water & $0.996 \mathrm{E}+02$ & $0.950 \mathrm{E}+02$ & 0.95 \\
\hline Iron-55 & Water & $0.388 \mathrm{E}+02$ & Not Reported & NA \\
\hline Manganese-54 & Water & $0.109 E+03$ & $0.104 . E+03$ & 0.95 \\
\hline Plutonium-238 & Water & $0.144 E+01$ & $0.110 E+01$ & 0.96 \\
\hline Plutonium-239 & Water & $0.338 E+00$ & $0.300 E+00$ & 0.89 \\
\hline Strontium-90 & Water & $0.252 E+01$ & $0.221 E+01$ & 0.88 \\
\hline Tritium & Water & $0.270 E+03$ & $0.272 E+03$ & 1.01 \\
\hline Uranium-234 & Water & $0.106 \mathrm{E}+01$ & Not Reported & $N A$ \\
\hline Uranium-238 & Water & $0.108 E+01$ & Not Reported & NA \\
\hline Uranium-Bq & Water & $0.214 E+01$ & Not Reported & NA \\
\hline Uranium- $\mu \mathrm{g}$ & Water & $0.842 E-01$ & $0.910 \mathrm{E}-01$ & 1.08 \\
\hline
\end{tabular}

NA Not applicable 
Table B-4. Weston Performance Evaluation, Water Pollution Study WP030

Analyte

Trace Metals ( $\mu g / \mathrm{L})$

Aluminum

Antimony

Arsenic

Beryllium

Cadmium

Chromium

Cobalt

Copper

Iron

Lead

Mariganese

Mercury

Molybdenum

Nickel

Selenium

Silver

Strontium

Thallium

Titanium

Vanadium

Zinc

\section{Reported} Value

\section{1,168}

4,208

107

16.6

259

91.5*

9.35

103

68.5

485

507

254

64.6

429

3,974

910

79.2

422

2,294

231

$1.70^{\star}$

3.00 *

6.19

42.5

142

1,390

$15.0^{\star \star *}$

$53.5^{\star \star}$

$2.90^{\star \star}$

10.5

3.91

53.2

81.6

8.2

65.8

183

8,468

16,045

1,179

263
True

Value

1,100

4,000

116

14.0

280

63.0

8.12

93.9

62.0

460

480

240

62.0

410

3,800

860

79.2

450

2,200

221

0.983

2.10

6.48

39.0

130

1,300

23.0

78.1

2.39

9.75

4.01

51.0

90.2

9.00

66.0

182

8,000

15,000

1,100

240
Acceptance

Limit

$898-1,300$

$3,370-4,570$

79.4-140

7.01-20.4

225-334

51.1-74.5

6.30-10.3

78.3-110

49.2-73.7

378-533

416-536

206.275

53.5.69.8

365-462

$3,350-4,230$

755-963

62.7-97.1

393-513

$1,950-2,450$

196-244

$0.620-1.42$

1.57-2.75

3.34-8.82

30.3-47.9

$111-150$

$1,160-1,450$

14.3-29.1

52.8.94.6

1.80-2.96

7.80-11.5

3.01-4.99

41.4-60.1

70.7.108

6.71-11.3

51.5-76.5

149-205

$7,110 \cdot 8,830$

$12,700-17,400$

961-1,220

209-271

- Not acceptable.

** Acceptable, but near limits. The laboratory was warned to check for error. 
Table B-4. Weston Performance Evaluation, Water Pollution Study WP030, Continued

\begin{tabular}{|c|c|c|c|}
\hline Analyte & $\begin{array}{l}\text { Reported } \\
\text { Value }\end{array}$ & $\begin{array}{l}\text { True } \\
\text { Value }\end{array}$ & $\begin{array}{l}\text { Acceptance } \\
\text { Limit }\end{array}$ \\
\hline \multicolumn{4}{|c|}{ Minerals (mg/L, except as noted) } \\
\hline \multirow[t]{2}{*}{ Calcium } & 4.03 & 3.69 & $3.00-4.47$ \\
\hline & 87.6 & 80.9 & $73.4-90.2$ \\
\hline \multirow[t]{2}{*}{ Chioride } & 52.4 & 54.3 & $48.2-59.1$ \\
\hline & $169^{\star \star}$ & 178 & $166-191$ \\
\hline \multirow{2}{*}{ Fluoride } & 2.38 & 2.40 & $2.09-2.65$ \\
\hline & 0.224 & 0.230 & $0.175-0.286$ \\
\hline \multirow[t]{2}{*}{ Magnesium } & 17.4 & 16.1 & $14.0-18.1$ \\
\hline & 6.00 & 5.64 & 4.84-6.32 \\
\hline \multirow{2}{*}{$\mathrm{pH}$ (pH units) } & 8.64 & 8.70 & $8.31-9.05$ \\
\hline & 6.17 & 6.10 & $5.96-6.22$ \\
\hline \multirow[t]{2}{*}{ Potassium } & 2.71 & 2.60 & $2.05-3.22$ \\
\hline & 19.9 & 19.0 & $16.2-21.9$ \\
\hline \multirow{2}{*}{ Sodium } & 17.1 & 15.9 & $14.0-17.9$ \\
\hline & 70.1 & 65.6 & $59.6-72.0$ \\
\hline \multirow{2}{*}{ Specific conductance $(\mu \mathrm{S} / \mathrm{cm})$} & 263 & 253 & $226-274$ \\
\hline & 889 & 868 & $819-916$ \\
\hline \multirow[t]{2}{*}{ Sulfate } & 9.52 & 9.10 & $6.48-11.4$ \\
\hline & 44.2 & 42.0 & $35.2-48.1$ \\
\hline \multirow{2}{*}{ Total alkalinity (as $\mathrm{CaCO}_{3}$ ) } & 24.0 & 21.1 & $17.2-26.1$ \\
\hline & 96.0 & 97.0 & $85.0-109$ \\
\hline \multirow[t]{2}{*}{ Total dissolved solids } & 131 & 116 & $76.2 \cdot 166$ \\
\hline & 514 & 480 & $385-617$ \\
\hline \multirow{2}{*}{ Total hardness (as $\mathrm{CaCO}_{3}$ ) } & 73.5 & 75.6 & $66.7-84.1$ \\
\hline & 224 & 225 & $209-242$ \\
\hline
\end{tabular}

Nutrients (mg/L)

Ammonia-nitrogen

$\begin{array}{lll}5.55 & 5.50 & 4.35-6.65 \\ 9.83 & 9.80 & 7.80-11.6 \\ 9.71 & 9.30 & 6.67-11.5 \\ 19.2 & 19.0 & 13.9-23.1 \\ 35.3 & 34.0 & 27.5-40.2 \\ 7.61 & 7.10 & 5.70-8.43 \\ 0.941^{\star \star} & 0.830 & 0.692-0.961 \\ 0.125^{\star} & 0.090 & .0617-0.122 \\ 3.55^{\star \star} & 3.20 & 2.35-3.57 \\ 2.01^{\star} & 1.60 & 1.25-1.94\end{array}$

Demands (mg/L)

5-day BOD

\begin{tabular}{|c|c|c|}
\hline 11.0 & 14.0 & $7.36-20.7$ \\
\hline 18.0 & 21.8 & $11.8-31.9$ \\
\hline 21.5 & 21.8 & $12.6-30.5$ \\
\hline 34.0 & 35.4 & $23.6-43.0$ \\
\hline
\end{tabular}


Table B-4. Weston Performance Evaluation, Water Pollution Study WP030, Continued

\begin{tabular}{|c|c|c|c|}
\hline Analyte & $\begin{array}{l}\text { Reported } \\
\text { Value }\end{array}$ & $\begin{array}{l}\text { True } \\
\text { Value }\end{array}$ & $\begin{array}{l}\text { Acceptance } \\
\text { Limit }\end{array}$ \\
\hline Total organic carbon & $\begin{array}{l}8.16 \\
13.5\end{array}$ & $\begin{array}{l}8.61 \\
14.0\end{array}$ & $\begin{array}{l}7.22-10.2 \\
11.9-16.3\end{array}$ \\
\hline \multicolumn{4}{|l|}{ PCBs $(\mu g / L)$} \\
\hline PCB-Aroclor 1016/1242 & $\begin{array}{l}0.64^{\star} \\
3.11\end{array}$ & 4.29 & $\begin{array}{l}\text { D.L.-D.L. } \\
\text { 1.86-5.71 }\end{array}$ \\
\hline \multicolumn{4}{|l|}{ PCBs in Oil $(\mathrm{mg} / \mathrm{kg})$} \\
\hline $\begin{array}{l}\text { PCB in oil } 1016 / 1242 \\
\text { PCB in oil } 1260\end{array}$ & $\begin{array}{l}16.0 \\
31.4\end{array}$ & $\begin{array}{l}21.5 \\
36.6\end{array}$ & $\begin{array}{l}5.04-27.6 \\
7.82-57.8\end{array}$ \\
\hline Pesticides $(\mu \mathrm{g} / \mathrm{L})$ & & & \\
\hline Aldrin & $\begin{array}{l}0.14 \\
0.38\end{array}$ & $\begin{array}{l}0.159 \\
0.444\end{array}$ & $\begin{array}{c}0.0344-0.214 \\
0.0957-0.577\end{array}$ \\
\hline Chlordane & $\begin{array}{l}0.88 \\
8.44\end{array}$ & $\begin{array}{l}0.964 \\
9.48\end{array}$ & $\begin{array}{l}0.469-1.32 \\
5.38-12.4\end{array}$ \\
\hline DDD & $\begin{array}{r}0.22 \\
0.55\end{array}$ & $\begin{array}{l}0.216 \\
0.626\end{array}$ & $\begin{array}{l}0.0863-0.317 \\
0.334-0.865\end{array}$ \\
\hline DDE & $\begin{array}{l}0.15 \\
0.45\end{array}$ & $\begin{array}{l}0.131 \\
0.495\end{array}$ & $\begin{array}{l}0.0562 \cdot 0.171 \\
0.228 \cdot 0.699\end{array}$ \\
\hline DDT & $\begin{array}{l}0.18 \\
0.57\end{array}$ & $\begin{array}{l}0.186 \\
0.576\end{array}$ & $\begin{array}{l}0.0628-0.284 \\
0.252-0.844\end{array}$ \\
\hline Dieldrin & $\begin{array}{l}0.12 \\
0.48\end{array}$ & $\begin{array}{l}0.121 \\
0.553\end{array}$ & $\begin{array}{l}0.0572 \cdot 0.163 \\
0.270-0.750\end{array}$ \\
\hline Heptachlor & $\begin{array}{l}0.15 \\
0.48\end{array}$ & $\begin{array}{l}0.157 \\
0.514\end{array}$ & $\begin{array}{l}0.0488-0.221 \\
0.180 \cdot 0.715\end{array}$ \\
\hline Heptachlor epoxide & $\begin{array}{l}0.09 \\
0.33\end{array}$ & $\begin{array}{l}0.087 \\
0.375\end{array}$ & $\begin{array}{l}0.0427-0.120 \\
0.189-0.513\end{array}$ \\
\hline
\end{tabular}

Volatile Halocarbons ( $\mu \mathrm{g} / \mathrm{L})$

Bromodichloromethane $\quad 51.0$

49.5

7.75

53.7

Bromoform

11.9

$53.8 \quad 46.3$

Carbon tetrachloride

13.6

Chlorobenzene

40.5

11.7

Chloroform

46.6

11.6

Dibromodichloromethane

38.2

14.3

1,2-Dichloroethane

52.3

9.87

13.5

43.2

12.9

45.7

13.8

42.2

16.4

48.6

10.2

$34.2-64.3$
$4.88-10.6$
$33.5-73.7$
$6.48-17.1$
$29.8-63.7$
$8.93-18.2$
$30.8-55.0$
$8.99-16.7$
$31.6-59.5$
$9.42-18.0$
$30.0-54.4$
$10.9-21.5$
$34.5-62.8$
$7.28-13.8$ 
Table B-4. Weston Performance Evaluation, Water Pollution Study WP030, Continued

$\begin{array}{llll}\text { Analvte } & \begin{array}{l}\text { Reported } \\ \text { Value }\end{array} & \begin{array}{l}\text { True } \\ \text { Value }\end{array} & \begin{array}{l}\text { Acceptance } \\ \text { Limit }\end{array} \\ \text { Methylene chloride } & 41.9 & 37.3 & \\ & 8.50 & 8.77 & 24.1-53.2 \\ \text { Tetrachloroethylene } & 53.5 & 57.2 & 3.21-13.5 \\ & 14.2 & 16.2 & 39.7-71.9 \\ \text { 1,1,1-Trichloroethane } & 53.2 & 52.0 & 30.9-21.1 \\ \text { Trichloroethylene } & 7.12 & 9.34 & 5.56-12.9 \\ & 35.2 & 38.8 & 26.8-49.1 \\ & 8.53 & 9.91 & 6.52-13.0\end{array}$

Volatile Aromatics $(\mu g / \mathrm{L})$

Benzene

Ethylbenzene

1,2-Dichlorobenzene

1,3-Dichlorobenzene

1,4-Dichlorobenzene

Toluene
9.45

46.6

11.2

49.9

10.2

45.2

8.39

43.5

10.4

53.2

12.3

30.5
10.3

54.1

11.3

52.4

12.1

52.0

9.43

47.8

11.7

58.1

13.9

33.9
7.34-13.4

37.7-70.8

7.71-14.7

36.2-68.5

$8.47 \cdot 15.4$

$36.1-66.4$

6.17-12.1

$33.6-61.2$

7.95-15.2

39.1-76.5

9.86-17.7

24.0-43.1

Miscellaneous Parameters (mg/L)

Cyanide, total

Non-filterable residue

Oil and grease

Total phenolics

Total residual chlorine

$\begin{array}{ll}0.249 & 0.250 \\ 0.123 & 0.130 \\ 44.3^{\star \star} & 43.2 \\ 35.3^{\star} & 33.0 \\ 16.0 & 15.0 \\ 23.1 & 23.0 \\ 0.031 & .0271 \\ 0.364 & 0.291 \\ 0.675 & 0.729 \\ 0.175 & 0.240\end{array}$

$0.138-0.341$

$0.0821 \cdot 0.169$

$34.7-45.2$

24.0-34.6

8.13-19.4

14.1-28.1

$0.0093-.0449$

$0.125-0.457$

$0.469 \cdot 0.912$

$0.0866-0.280$ 
Table B-5. Weston Performance Evaluation, Water Supply Study WS031

Analyte

Trace Metals $(\mu g / \mathrm{L})$

Aluminum

Antimony

Arsenic

Barium

Beryllium

Boron

Cadmium

Chromium

Copper

Lead

Manganese

Mercury

Molybdenum

Nickel

Selenium

Silver

Thallium

Vanadium

Zinc

Nitrate/Nitrite/Fluoride (mg/L)

Fluoride

Nitrate as nitrogen

Nitrite as nitrogen

Insecticides $(\mu \mathrm{g} / \mathrm{L})$

Alachlor

Atrazine

Bromacil .

Chlordane

Endrin

Heptachlor

Heptachlor epoxide

Hexachlorobenzene

Hexachlorocyclopentadiene

Lindane

Metribuzin

Prometon

Simazine
Reported True

Value Value

74.2

5.0

71.4

676

3.40

723

9.29 *

83.8

117

12.6

$18.2^{\star \star}$

0.82

35.9

67.1

22.1

114

$<2.0 \dagger$

21.5

$191^{*}$

5.51

6.76

0.409

2.12

7.73

$19.4^{\star \star}$

4.49

0.64

1.17

1.63

$2.32^{\star *}$

$0.95^{\star \star}$

0.87

$5.45^{\star \star}$

11.5

12.9
76.3

4.69

70.2

681

3.27

720

12.8

81.6

110

12.4

17.0

0.908

42.3

68.0

22.9

109

1.48

24.2

179

5.70

6.50

0.430

2.50

9.39

22.4

5.16

0.699

1.44

1.92

2.40

1.11

0.971

6.30

12.7

12.5
Acceptance

Limits
62.6-93.2

3.28-6.10

58.2-80.2

579.783

2.78-3.76

652-814

10.2-15.4

69.4-93.8

99.0-121

8.68-16.1

13.8.18.7

$0.636-1.18$

29.2-54.3

57.8-78.2

18.3-27.5

92.4-123

$0.822-2.26$

20.2-27.5

$161-190$.

$5.13 \cdot 6.27$

5.85-7.15

$0.366-0.494$
1.38-3.62

5.16-13.6

D.L.-36.1

2.84-7.48

$0.489 \cdot 0.909$

$0.792-2.09$

1.06-2.78

$0.518-2.95$

$0.517-1.67$

$0.534-1.41$

$0.819-9.25$

1.12-20.6

$0.540-22.5$

- Not acceptable.

** Significant general method bias is expected for this result.

$\dagger$ Unuseable data.

D.L. Detection limit. 
Table B-5. Weston Performance Evaluation, Water Supply Study WS031, Continued

\begin{tabular}{llll} 
Analyte & $\begin{array}{l}\text { Reported } \\
\text { Value }\end{array}$ & $\begin{array}{l}\text { True } \\
\text { Value }\end{array}$ & $\begin{array}{l}\text { Acceptance } \\
\text { Limits }\end{array}$ \\
\cline { 2 - 3 } $\begin{array}{l}\text { Toxaphene } \\
\text { Trifluralin }\end{array}$ & 3.14 & 3.31 & $1.82-4.80$ \\
Herbicides $(\mu \mathrm{g} / \mathrm{L})$ & $0.67^{\star \star}$ & 0.857 & $0.176-1.01$ \\
& & & \\
2,4-D & & & \\
2,4,5-TP (Silvex) & 17.5 & 20.3 & $10.2-30.4$ \\
Bentazon & 7.60 & 8.66 & $4.33-13.0$ \\
Dalapon & 13.1 & 12.7 & D.L.-21.4 \\
Dicamba & $18.0^{\star \star}$ & 22.3 & D.L.-31.5 \\
Dinoseb & $8.80^{\star \star}$ & 9.43 & 0.778-14.2 \\
Pentachlorophenol & $15.0^{\star \star}$ & 18.3 & D.L.-26.1 \\
Picloram & 9.60 & 11.4 & $5.70-17.1$ \\
Total DCPA & $30.9^{\star \star}$ & 26.7 & D.L.-42.5 \\
& $9.90^{\star \star}$ & 11.3 & D.L.- 17.9
\end{tabular}

Polychlorinated Biphenyls ( $\mu \mathrm{g} / \mathrm{L})$

Decachlorobiphenyl

0.84

0.445

D.L. -0.890

Trihalomethanes $(\mu \mathrm{g} / \mathrm{L})$

Bromodichloromethane

$28.2^{*}$

Bromoform

39.6

Chlorodibromomethane

$25.2^{\star}$

Chloroform

40.3

Total trihalomethane

133.3

36.9

43.7

31.8

48.4

160.8

29.5-44.3

35.0-52.4

25.4-38.2

38.7-58.1

129-193

Volatile Organic Compounds $(\mu \mathrm{g} / \mathrm{L})$

Benzene

Carbon tetrachloride

Chlorobenzene

1,2-Dibromochloropropane

1,2-Dichlorobenzene

1,4-Dichlorobenzene

1,2-Dichloroethane

1,1-Dichloroethylene

cis-1,2-Dichloroethylene

trans-1,2-Dichloroethylene

1,2-Dichloropropane

2,2-Dichloropropane

1,1-Dichloropropene

Ethylbenzene

Ethylene dibromide

Fluorotrichloromethane

n-Propylbenzene

Styrene

Tetrachloroethylene
14.1

7.30

6.50

2.28

13.4

8.51

9.77 .

9.69

15.1

8.38

10.3

12.7

6.16

8.62

0.577

$9.81^{*}$

11.5

7.73*

10.1
12.6

8.69

7.68

2.65

16.3

9.40

9.25

7.02

14.5

10.1

12.7

15.7

7.31

9.27

0.637

12.6

11.7

13.4

11.6
10.1-15.1

5.21-12.2

4.61-10.8

$1.59 \cdot 3.71$

13.0-19.6

5.64-13.2

5.55-12.9

$4.21-9.83$

11.6-17.4

8.08-12.1

$10.2-15.2$

12.6-18.8

$4.39 \cdot 10.2$

$5.56-13.0$

$0.382-0.892$

10.1-15.1

9.36-14.0

$9.12 \cdot 13.7$

9.28-13.9 
Table B-5. Weston Performance Evaluation, Water Supply Study WS031, Continued

\begin{tabular}{|c|c|c|c|}
\hline Analvte & $\begin{array}{l}\text { Reported } \\
\text { Value }\end{array}$ & $\begin{array}{l}\text { True } \\
\text { Value }\end{array}$ & $\begin{array}{l}\text { Acceptance } \\
\text { Limits }\end{array}$ \\
\hline Toluene & 14.5 & 15.3 & $12.2-18.4$ \\
\hline Total xylenes & 11.0 & 13.2 & $10.6-15.8$ \\
\hline 1,1,1-Trichloroethane & 12.4 & 13.0 & $10.4-15.6$ \\
\hline Trichloroethylene & 6.98 & 7.46 & $4.48-10.4$ \\
\hline 1,3,5-Trimethylbenzene & 7.46 & 8.60 & $5.16-12.0$ \\
\hline Vinyl chloride & 14.9 & 11.9 & $7.14-16.7$ \\
\hline \multicolumn{4}{|l|}{ Miscellaneous Analytes } \\
\hline Alkalinity (as mg CaCO $3 /$ ) & $49.7^{\star \star}$ & 46.0 & $43.1-52.0$ \\
\hline Calcium (as mg CaCO $3 / \mathrm{L}$ ) & 229 & 230 & $214-244$ \\
\hline $\begin{array}{l}\text { Corrosivity (Langelier ind. at } \\
20^{\circ} \mathrm{C} \text { ) }\end{array}$ & 1.12 & 1.19 & $0.794-1.49$ \\
\hline Cyanide, total (mg/L) & 0.287 & 0.270 & $0.202-0.337$ \\
\hline $\mathrm{pH}(\mathrm{pH})$ & 9.02 & 9.13 & $8.84-9.34$ \\
\hline Residual free chlorine (mg/L) & $0.275^{\star \star}$ & 0.360 & $0.160 \cdot 0.451$ \\
\hline Sodium (mg/L) & 21.1 & 21.1 & $19.2-23.0$ \\
\hline Sulfate (mg/L) & 7.82 & 8.60 & $6.44-10.6$ \\
\hline Total filterable residue (mg/L) & $569^{2 * \star}$ & 406 & $283-618$ \\
\hline Turbidity (NTU) & 2.99 & 3.00 & 2.55-3.57 \\
\hline
\end{tabular}


Table B-6. Weston Performance Evaluation, Water Supply Study WS032

Analyte

$\begin{array}{lll}\text { Reported } & \text { True } & \text { Acceptance } \\ \text { Value } & \text { Value } & \text { Limits }\end{array}$

Trace Metals $(\mu \mathrm{g} / \mathrm{L})$

$\begin{array}{llll}\text { Aluminum } & 328 & 309 & \mathbf{2 7 4 - 3 3 5} \\ \text { Antimony } & 4.6 & 5.58 & \mathbf{3 . 3 4 - 7 . 4 1} \\ \text { Arsenic } & 86.0 & 91.4 & \mathbf{7 6 . 5 - 1 0 3} \\ \text { Barium } & \mathbf{5 1 5} & \mathbf{5 1 1} & \mathbf{4 3 4 - 5 8 8} \\ \text { Beryllium } & 1.89^{\star} & 0.933 & 0.699-1.17 \\ \text { Boron } & 874^{* *} & 864 & 810-1.010 \\ \text { Cadmium } & 4.97 & 4.80 & 3.84-5.76 \\ \text { Chromium } & 67.6 & 68.1 & \mathbf{5 7 . 9 - 7 8 . 3} \\ \text { Copper } & 835 & 820 & \mathbf{7 3 8 - 9 0 2} \\ \text { Lead } & 5.50 & 5.16 & \mathbf{3 . 6 1 - 6 . 7 1} \\ \text { Manganese } & 231 & 221 & 203-234 \\ \text { Mercury } & 6.37 & 6.23 & 4.36-8.10 \\ \text { Molybdenum } & 6.05 & 6.58 & 4.80-8.58 \\ \text { Nickel } & 98.1 & 95.2 & 80.9-109 \\ \text { Selenium } & 64.5 & 65.9 & 52.7-79.1 \\ \text { Silver } & 27.4 & 25.1 & 21.2-29.4 \\ \text { Thallium } & 2.00 & 2.56 & 1.79-3.33 \\ \text { Vanadium } & 163 & 160 & 143-174 \\ \text { Zinc } & 86.8 & 83.8 & 73.7-91.1\end{array}$

Nitrate/Nitrite/Fluoride (mg/L)

Fluoride

Nitrate as nitrogen

Nitrite as nitrogen

Insecticides $(\mu \mathrm{g} / \mathrm{L})$

Alachlor

Atrazine

Bromacil

Chlordane (total)

Heptachlor

Heptachlor epoxide

Hexachlorobenzene

Hexachlorocyclopentadiene

Lindane

Methoxychlor

Metolachlor

Metribuzin

Prometon

Simazine
2.01

3.38

2.15

2.460

4.30

13.0

4.42

0.30

0.35

$1.19^{*}$

$0.84^{\star \star}$

0.19

16.5

11.70

5.32

$9.480^{\star \star}$

4.680
2.00

3.60

2.30

1.80-2.20

3.24-3.96

$1.95 \cdot 2.64$

* Not acceptable.

* Considerable bias is expected for this result.

D.L. Detection limit.

$\begin{array}{ll}2.33 & 1.28-3.38 \\ 7.20 & 3.96-10.4 \\ 13.6 & 0.741-23.0 \\ 5.33 & 2.93-7.73 \\ 0.443 & 0.244-0.642 \\ 0.346 & 0.130-0.502 \\ 0.857 & 0.392-1.17 \\ 0.823 & .0847-1.18 \\ 0.214 & 0.118-0.310 \\ 17.4 & 9.57-25.2 \\ 11.8 & 5.83-17.0 \\ 4.73 & 0.938-7.42 \\ 8.53 & 1.30-12.9 \\ 4.43 & 0.682-8.54\end{array}$


Table B-6. Weston Performance Evaluation, Water Supply Study WS032, Continued

\begin{tabular}{|c|c|c|c|}
\hline Analyte & $\begin{array}{l}\text { Reported } \\
\text { Value }\end{array}$ & $\begin{array}{l}\text { True } \\
\text { Value }\end{array}$ & $\begin{array}{l}\text { Acceptance } \\
\text { Limits }\end{array}$ \\
\hline $\begin{array}{l}\text { Toxaphene } \\
\text { Trifluralin }\end{array}$ & $\begin{array}{l}3.01 \\
1.08^{\star \star * \pi}\end{array}$ & $\begin{array}{l}3.71 \\
0.771\end{array}$ & $\begin{array}{l}2.04-5.38 \\
0.300-1.02\end{array}$ \\
\hline \multicolumn{4}{|l|}{ Herbicides $(\mu \mathrm{g} / \mathrm{L})$} \\
\hline $\begin{array}{l}2,4-\mathrm{D} \\
\text { 2,4,5-TP (Silvex) } \\
\text { Dalapon } \\
\text { Dicamba } \\
\text { Dinoseb } \\
\text { Pentachlorophenol } \\
\text { Picloram }\end{array}$ & $\begin{array}{l}17.0 \\
7.85 \\
4.62^{\star \star} \\
4.53 \\
5.38^{\star \star} \\
6.92 \\
10.6\end{array}$ & $\begin{array}{l}18.6 \\
9.81 \\
12.5 \\
4.73 \\
6.52 \\
10.7 \\
10.6\end{array}$ & $\begin{array}{l}9.30-27.9 \\
4.16-12.5 \\
\text { D.L.-20.8 } \\
2.28-7.05 \\
\text { D.L.-9.19 } \\
\text { 5.35-16.0 } \\
\text { D.L.-20.0 }\end{array}$ \\
\hline
\end{tabular}

Polychlorinated Biphenyls $(\mu g / \mathrm{L})$

Decachlorobiphenyl

0.888

0.959

D.L.-1.92

Trihalomethanes $(\mu g / L)$

Bromodichloromethane

Bromoform

Chlorodibromomethane

Chloroform

Total trihalomethane
22.9

23.0

15.4

17.2

78.50
22.4

26.4

17.9

17.1

83.8
17.9.26.9

21.1.31.7

14.3-21.5

$13.7 \cdot 20.5$

67.0.101

Volatile Organic Compounds ( $\mu \mathrm{g} / \mathrm{L})$

$\begin{array}{llll}\text { 1,2-Dichlorobenzene } & 12.5 & 13.5 & 10.8-16.2 \\ \text { 1,4-Dichlorobenzene } & 12.4 & 13.6 & 10.9-16.3 \\ \text { 1,2-Dichloroethane } & 12.1 & 13.3 & 10.6-16.0 \\ \text { 1,1-Dichloroethylene } & 8.74 & 9.13 & 5.48-12.8 \\ \text { 1,2-Dichloropropane } & 6.50 & 6.46 & 3.88-9.04 \\ \text { 1,1,1-Trichloroethane } & 7.60^{\star} & 10.1 & 8.08-12.1 \\ \text { 1,2-Dibromochloropropane } & 1.08 & 1.78 & 1.07-2.49 \\ \text { 1,2,4-Trichlorobenzene } & 12.1 & 14.1 & 9.80-17.1 \\ \text { 1,1,2-Trichloroethane } & 11.5 & 13.2 & 9.54-15.7 \\ \text { 1,2,3-Trichloropropane } & 7.45 & 9.85 & 5.96-12.4 \\ \text { Benzene } & 16.1 & 16.5 & 13.2-19.8 \\ \text { Bromomethane } & 12.9 & 16.0 & 7.98-22.3 \\ \text { Carbon tetrachloride } & 13.9 & 14.5 & 11.6-17.4 \\ \text { Chlorobenzene } & 12.5 & 13.5 & 10.8-16.2 \\ \text { cis-1,2-Dichloroethylene } & 12.8 & 12.3 & 9.84-14.8 \\ \text { Dichloromethane } & 6.94 & 7.77 & 5.45-10.2 \\ \text { Ethylbenzene } & 10.7 & 11.8 & 9.44-14.2 \\ \text { Ethylene } & 10.7 & 11.8 & 4.44-14.2 \\ \text { Ethylene dibromide (EDB) } & 2.03 & 2.29 & 1.37-3.21 \\ \text { Hexachlorobutadiene } & 17.6 & 19.3 & 14.1-23.8\end{array}$


Table B-6. Weston Performance Evaluation, Water Supply Study WS032, Continued

\begin{tabular}{llll} 
& $\begin{array}{l}\text { Réported } \\
\text { Analue }\end{array}$ & $\begin{array}{l}\text { True } \\
\text { Value }\end{array}$ & $\begin{array}{l}\text { Acceptance } \\
\text { Limits }\end{array}$ \\
\cline { 2 - 3 } & & & \\
Styrene & 7.98 & 8.66 & $5.20-12.1$ \\
Tetrachloroethylene & 6.32 & 7.43 & $4.46-10.4$ \\
Toluene & 6.21 & 6.54 & $3.92-9.16$ \\
trans-1,2-Dichloroethylene & 16.0 & 16.9 & $13.5-20.3$ \\
Trichloroethylene & 9.34 & 11.2 & $8.96-13.4$ \\
Vinyl chloride & 2.45 & 2.57 & $1.54-3.60$ \\
Xylenes, total & 8.14 & 7.54 & $4.52-10.6$ \\
& & & \\
Miscellaneous Analytes & & & \\
& & & \\
Alkalinity (as mg CaCO $3 / \mathrm{L})$ & $29.3^{\star \star}$ & 29.0 & $27.0-33.6$ \\
Calcium (as mg CaCO $/ \mathrm{L})$ & 157 & 160 & $149-172$ \\
Corrosivity (Langelier ind. at & 0.52 & 0.846 & $0.465-1.13$ \\
20 & & & \\
pH (pH) & 9.00 & 9.12 & $8.85-9.34$ \\
Residual free chlorine (mg/L) & $1.03^{\star \star}$ & 1.09 & $0.805-1.21$ \\
Sodium (mg/L) & 14.0 & 13.3 & $11.9-14.8$ \\
Sulfate (mg/L) & 28.2 & 27.0 & $24.7-29.4$ \\
Total cyanide (mg/L) & 0.434 & 0.400 & $0.300-0.500$ \\
Total filterable residue & $455^{\star \star}$ & 275 & $201-463$ \\
(mg/L) & & & \\
Turbidity (NTU) & $4.12^{\star \star}$ & 4.13 & $3.76-4.93$ \\
& & &
\end{tabular}


Table B-7. Quality Control Standards for Selected Analyses

Analvte

$\begin{array}{lll}\text { Certified } & \text { Advisory } & \text { Weston } \\ \text { Value } & \underline{\text { Range }} & \text { Result }\end{array}$

Cyanide \& Phenol (Lot 9950)

Cyanide $(\mu \mathrm{g} / \mathrm{L})$

Phenol $(\mu \mathrm{g} / \mathrm{L})$

Minerals (Lot 9950)

Alkalinity (as $\left.\mathrm{CaCO}_{3}\right)(\mu \mathrm{g} / \mathrm{L})$

Chloride $(\mu g / L)$

Fluoride $(\mu g / \mathrm{L})$

$\mathrm{pH}$

Potassium $(\mu \mathrm{g} / \mathrm{L})$

Specific conductance $(\mu \mathrm{S} / \mathrm{cm})$

Sodium $(\mu \mathrm{g} / \mathrm{L})$

Sulfate $(\mu \mathrm{g} / \mathrm{L})$

Total dissolved solids $(\mu \mathrm{g} / \mathrm{L})$

Grease and Oil (Lot 9950)

Grease and oil (mg/bottle)

Base/Neutrals (Lot 549)

Anthracene $(\mu \mathrm{g} / \mathrm{L})$

Bis(2-ethylhexyl) phthalate $(\mu \mathrm{g} / \mathrm{L})$

4-Bromophenyl phenyl ether $(\mu \mathrm{g} / \mathrm{L})$

Chrysene $(\mu \mathrm{g} / \mathrm{L})$

Dibenzofuran $(\mu \mathrm{g} / \mathrm{L})$

1,2-Dichlorobenzene $(\mu \mathrm{g} / \mathrm{L})$

1,4-Dichlorobenzene $(\mu \mathrm{g} / \mathrm{L})$

2,4-Dinitrotoluene ( $\mu \mathrm{g} / \mathrm{L})$

2,6-Dinitrotoluene $(\mu \mathrm{g} / \mathrm{L})$

Fluorene $(\mu \mathrm{g} / \mathrm{L})$

Indeno[1,2,3-cd] pyrene ( $\mu \mathrm{g} / \mathrm{L})$

2-Methylnaphthalene $(\mu \mathrm{g} / \mathrm{L})$

Naphthalene $(\mu g / L)$

Pyrene $(\mu \mathrm{g} / \mathrm{L})$

1,2,4-Trichlorobenzene ( $\mu \mathrm{g} / \mathrm{L}$ )

$\begin{array}{lll}342 & 250-430 & 321 \\ 335 & 250-420 & 334\end{array}$

$\begin{array}{lll}223,000 & 198,000-248,000 & - \\ 258,000 & 240,000-276,000 & 251,000 \\ 12,100 & 9,800-15,000 & 12,600 \\ 9.10 & 8.90-9.30 & 9.05 \\ 216,000 & 184,000-248,000 & 222,000 \\ 1,800 & 1,530-2,070 & 1,960 \\ 270,000 & 208,000-311,000 & 272,000 \\ 235,000 & 202,000-268,000 & 239,000 \\ 1,420,000 & 1,240,000- & 1,380,000 \\ & 1,600,000 & \end{array}$

73.5

$44.0-91.0$

60.6

$\begin{array}{llll}40.2 & 11.0-53.0 & 27.7 & \\ 80.2 & 13.0-127 & 69.0 & \\ 31.2 & 17.0-40.0 & 21.5 & \\ 89.5 & 15.0-150 & 63.7 & \\ 56.5 & 32.0-64.0 & 38.4 & \\ 169 & 54.0-218 & 83.9 & \\ 58.1 & 25.0-72.0 & 29.6 & \\ 90.0 & 35.0-125 & 63.3 & \ddots . . \\ 118 & 59.0-186 & 79.4 & . \\ 77.7 & 46.0-94.0 & 52.4 & \\ 67.2 & 10.0-115 & 47.2 & . \\ 87.1 & 44.0-98.0 & 44.3 & \\ 50.7 & 19.0-67.0 & 29.0 & \\ 115 & 60.0-132 & 76.3 & \\ 29.2 & 12.0-41.0 & 17.5 & \end{array}$

- Not reported.

- Out of range. 
Table B-7. Quality Control Standards for Selected Analyses, Continued

Analyte

Acids (Lot 549)

2,4-Dimethylphenol $(\mu \mathrm{g} / \mathrm{L})$

2-Methylphenol $(\mu \mathrm{g} / \mathrm{L})$

4-Methylphenol $(\mu \mathrm{g} / \mathrm{L})$

Pentachlorophenol ( $\mu \mathrm{g} / \mathrm{L})$

2,4,6-Trichlorophenol $(\mu \mathrm{g} / \mathrm{L})$

Nutrients (Lot 9950)

Ammonia nitrogen $(\mu g / L)$

Nitrate-nitrite as nitrogen $(\mu \mathrm{g} / \mathrm{L})$

Nitrate as nitrogen $(\mu \mathrm{g} / \mathrm{L})$

Total phosphates (as P) ( $\mu$ g/L)

Trace Metals (Lot 9950)

Aluminum $(\mu \mathrm{g} / \mathrm{L})$

232

Antimony $(\mu \mathrm{g} / \mathrm{L})$

Arsenic $(\mu \mathrm{g} / \mathrm{L})$

Barium $(\mu g / L)$

Beryllium ( $\mu g / L)$

Boron $(\mu \mathrm{g} / \mathrm{L})$

Cadmium $(\mu g / L)$

Chromium ( $\mu \mathrm{g} / \mathrm{L})$

Cobalt $(\mu \mathrm{g} / \mathrm{L})$

Copper $(\mu \mathrm{g} / \mathrm{L})$

Iron $(\mu \mathrm{g} / \mathrm{L})$

Lead $(\mu \mathrm{g} / \mathrm{L})$

Manganese $(\mu \mathrm{g} / \mathrm{L})$

Mercury $(\mu \mathrm{g} / \mathrm{L})$

Molybdenum $(\mu \mathrm{g} / \mathrm{L})$

Nickel $(\mu g / L)$

Selenium $(\mu \mathrm{g} / \mathrm{L})$

Silver $(\mu \mathrm{g} / \mathrm{L})$

Strontium $(\mu \mathrm{g} / \mathrm{L})$

Thallium $(\mu \mathrm{g} / \mathrm{L})$

Vanadium $(\mu g / L)$

Zinc $(\mu \mathrm{g} / \mathrm{L})$
117

207

341

211

505

95.5

610

317

209

330

246

203

18.7

149

178

192

56.8

141

170

203

307

$\begin{array}{lll}\text { Certified } & \text { Advisory } & \text { Weston } \\ \text { Value } & \text { Range } & \text { Result }\end{array}$

98.3

59.5

49.6

79.3

166

31.0-117

55.7

21.0-68.0

32.7

$22.0-54.0$

29.2

$\begin{array}{ll}12.0-140 & 55.3 \\ 61.0-239 & 104\end{array}$

$\begin{array}{lll}13,200 & 11,000-15,000 & - \\ 8,800 & 7,200-10,000 & - \\ 8,800 & 7,200-10,000 & 9,740 \\ 7,180 & 6,100-8,300 & 7,240\end{array}$

Total Petroleum Hydrocarbons (Lot 8910)

Sample 1, no fatty acids (mg/bottle)

Sample 2, containing fatty acids (mg/bottle)
118

98.3
165-278

$88.0-157$

155-244

280-402

173-249

414-596

78.0-113

500-720

260-374

178-240

271-389

202-290

166-240

10.0-23.0

$122-176$

146-210

144-227

$47.0-67.0$

110-166

127-201

166-240

252-362
236

111

213

355

211

-

101

636

336

228

350

238

203

16.7

$-\because$

183

196

58.4 .

$-$

167

207

316 
Table B-7. Quality Control Standards for Selected Analyses, Continued

Analvte

Certified

Advisory

Weston

Value

$\underline{\text { Range }}$

Result

PCBs (Lot 549)

PCB $1254(\mu g / L)$

6.18

$1.80-8.10$

5.98

Volatiles (Lot 549)

Benzene $(\mu g / L)$

Bromodichloromethane $(\mu \mathrm{g} / \mathrm{L})$

Bromoform $(\mu \mathrm{g} / \mathrm{L})$

Carbon tetrachloride $(\mu \mathrm{g} / \mathrm{L})$

Chlorobenzene ( $\mu \mathrm{g} / \mathrm{L})$

1,2-Dichlorobenzene $(\mu \mathrm{g} / \mathrm{L})$

1,4-Dichlorobenzene ( $\mu g / \mathrm{L})$

1,1-Dichloroethane $(\mu \mathrm{g} / \mathrm{L})$

1,2-Dichloroethane $(\mu \mathrm{g} / \mathrm{L})$

Dichloromethane (Methylene chloride) $(\mu \mathrm{g} / \mathrm{L})$

Ethyl benzene $(\mu \mathrm{g} / \mathrm{L})$

2-Hexanone $(\mu \mathrm{g} / \mathrm{L})$

4-Methyl-2-pentanone ( $\mu \mathrm{g} / \mathrm{L})$

Tetrachloroethylene $(\mu \mathrm{g} / \mathrm{L})$

Toluene $(\mu \mathrm{g} / \mathrm{L})$

1,1,1-Trichloroethane $(\mu g / L)$

1,1,2-Trichloroethane ( $\mu \mathrm{g} / \mathrm{L})$

Trichloroethylene ( $\mu \mathrm{g} / \mathrm{L})$

Xylenes, total ( $\mu g / \mathrm{L})$

Cations (Lot 418)

Calcium $(\mu g / \mathrm{L})$

Magnesium $(\mu \mathrm{g} / \mathrm{L})$

Potassium $(\mu \mathrm{g} / \mathrm{L})$

Sodium $(\mu \mathrm{g} / \mathrm{L})$

Toxaphene (Lot 3205)

Toxaphene $(\mu g / L)$

Turbidity (Lot 3405)

Turbidity (NTU)

Pesticides/Herbicides (Lot 3205)

Alachlor $(\mu \mathrm{g} / \mathrm{L})$

Aldrin $(\mu \mathrm{g} / \mathrm{L})$
44.3

29.3

103

69.8

50.5

139

51.3

84.8

111

44.4

64.8

37.5

170

88.1

20.6

55.3

90.4

73.8

60.3

28,600

83,300

46,000

157,000

4.30

$3.50-5.90$

$1.90^{*}$

$16.0-67.0$

40.3

$10.0-45.0$

28.2

91.3

49.0-98.0

66.2

47.7

126

42.6

76.8

106

47.1

60.9

33.1

-

82.4

19.6

50.8

86.5

67.4

53.6
29,900

88,700

46,700

155,000
3.50
$3.0-4.0$
3.36
8.15
1.05
5.10-10.0
0.44-1.30
$<0.25^{*}$ 
Table B-7. Quality Control Standards for Selected Analyses, Continued

Analyte

Atrazine $(\mu \mathrm{g} / \mathrm{L})$

$2,4 \cdot D(\mu g / L)$

Endrin $(\mu \mathrm{g} / \mathrm{L})$

Heptachlor $(\mu \mathrm{g} / \mathrm{L})$

Heptachlor epoxide $(\mu g / L)$

Lindane (gamma-Benzene

hexachloride) $(\mu \mathrm{g} / \mathrm{L})$

Methoxychlor $(\mu \mathrm{g} / \mathrm{L})$

2,4,5-TP (Silvex) $(\mu \mathrm{g} / \mathrm{L})$

Inorganics (Lot 3405)

Alkalinity as $\mathrm{CaCO}_{3}(\mu \mathrm{g} / \mathrm{L})$

Chloride $(\mu \mathrm{g} / \mathrm{L})$

Fluoride $(\mu \mathrm{g} / \mathrm{L})$

Nitrate-nitrite as nitrogen $(\mu \mathrm{g} / \mathrm{L})$

Nitrate as nitrogen $(\mu \mathrm{g} / \mathrm{L})$

$\mathrm{pH}$

Potassium $(\mu \mathrm{g} / \mathrm{L})$

Sodium $(\mu \mathrm{g} / \mathrm{L})$

Sulfate $(\mu \mathrm{g} / \mathrm{L})$

Total dissolved solids $(\mu \mathrm{g} / \mathrm{L})$

Demand (Lot 9950)

Biological oxygen demand $(\mu \mathrm{g} / \mathrm{L})$

Chemical oxygen demand $(\mu \mathrm{g} / \mathrm{L})$

Total organic carbon $(\mu \mathrm{g} / \mathrm{L})$

Total phosphates (as P) ( $\mu \mathrm{g} / \mathrm{L}$ )

Nitrogen by Kjeldahl method $(\mu \mathrm{g} / \mathrm{L})$
Certified

Value

1.54

0.327 。

5.75

4.98

1.38

3.68

7.41

9.13

78,900

249,000

8,960

6,980

6,980

9.0

37,900

278,000

167,000

922,000

135,000

226,000

86,800

8,840

6,030
Advisory

Range

1.0-1.80

$0.16-0.43$

$3.60-6.60$

$3.10-6.60$

0.84-1.60

$2.20-4.30$

4.70-11.0

$3.80-12.0$
7.85

9.93

Weston

Result

0.40

4.75

3.10

1.10

2.61

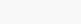

-
0.40
4.75
3.10
1.10
2.61

7.85
9.93




\section{Appendix C: Formats of the Data Files}

\section{Sample Data Format SAM93}

This is the description of the SAM93 format for coding and transmitting sampling data records.

Name

Record format

Sample identi-

fication number

Sample collection date

Sample collection

time

Sample type

Sample matrix

Delay check

Sampler's initials

Associated sample

Planned sample

name

Sample name
Columns Type

1-8

$9-14$

$15-20$

21-24

25

26

27

28-30

$31-36$

$37-46$

$47-56$
Alphanumeric

Integer

Numeric

Integer

Alphanumeric

$$
\begin{aligned}
& \text { Sample type coded as: } \\
& 1=\text { normal } \\
& 2=\text { blank or standard } \\
& 3=\text { split } \\
& 4=\text { rinsate } \\
& 5=\text { duplicate } \\
& 6=\text { other. }
\end{aligned}
$$

Alphanumeric

Sample matrix coded as:

$$
\begin{aligned}
& 1=\text { soil } \\
& 2=\text { rock } \\
& 3=\text { sludge } \\
& 4=\text { water } \\
& 5=\text { sediment } \\
& 6=\text { other. }
\end{aligned}
$$

Alphanumeric

Enter a " $Y$ " if there was an unusual delay in bottling samples.

Sampler's initials.

Sample identification of associated sample if this sample is a duplicate, split, rinsate, etc.

Identification of the sample as it appears in the site work plan.

Identification of the actual sample (includes any modifications made in the field).

Alpha and alphanumeric entries must be left justified in the records; numeric entries must be right justified in the records. There will be no leading zeros except for dates and times. 


\section{Sample Data Format SAM93, Continued}

\begin{tabular}{|c|c|c|c|}
\hline Name & Columns & Iype & Description \\
\hline $\begin{array}{l}\text { Planned top of } \\
\text { interval }\end{array}$ & $57-62$ & Numeric & $\begin{array}{l}\text { Depth from the ground to the top of the planned } \\
\text { sampling interval to the nearest } 0.01 \mathrm{ft} \text {. }\end{array}$ \\
\hline Top of interval & $63-68$ & Numeric & $\begin{array}{l}\text { Depth from the ground to the top of the actual sampling } \\
\text { interval to the nearest } 0.01 \mathrm{ft} \text {. }\end{array}$ \\
\hline $\begin{array}{l}\text { Planned bottom of } \\
\text { interval }\end{array}$ & $69-74$ & Numeric & $\begin{array}{l}\text { Depth from the ground to the bottom of the planned } \\
\text { sampling interval to the nearest } 0.01 \mathrm{ft} \text {. }\end{array}$ \\
\hline Bottom of interval & $75-80$ & Numeric & $\begin{array}{l}\text { Depth from the ground to the bottom of the actual } \\
\text { sampling interval to the nearest } 0.01 \mathrm{ft} \text {. }\end{array}$ \\
\hline Soil moisture & 81 & Alphanumeric & $\begin{array}{l}\text { Soil moisture coded as: } \\
1=\text { dry } \\
2=\text { damp } \\
3=\text { wet } \\
4=\text { sludge } \\
5=\text { liquid. }\end{array}$ \\
\hline Munsell color & $82-91$ & Alphanumeric & Munsell Color. \\
\hline Soil classification & $92-93$ & Alphanumeric & Unified Soil Classification. \\
\hline Number of bottles & $94-96$ & Integer & $\begin{array}{l}\text { The number of bottle label numbers used during } \\
\text { collection. }\end{array}$ \\
\hline $\begin{array}{l}\text { Insufficient } \\
\text { recovery check }\end{array}$ & 97 & Alpha & $\begin{array}{l}\text { Enter an " } R \text { " if insufficient material was recovered to } \\
\text { perform all analyses. }\end{array}$ \\
\hline $\begin{array}{l}\text { Improper procedure } \\
\text { check }\end{array}$ & 98 & Alpha & $\begin{array}{l}\text { Enter a " } P^{n} \text { if an improper sampling procedure was } \\
\text { followed. }\end{array}$ \\
\hline
\end{tabular}




\section{Sample Bottle Data Format SAM93A}

This is the description of the SAM93A format for coding and transmitting sample bottle data records.

Name Columns Type Description

\begin{tabular}{|c|c|c|c|}
\hline Record format & $1-8$ & Alphanumeric & SAM93A (the format of the data record). \\
\hline $\begin{array}{l}\text { Sample identification } \\
\text { number }\end{array}$ & 9-14. & Integer & $\begin{array}{l}\text { The number of the sample printed on the } \\
\text { page of the sample logbook. }\end{array}$ \\
\hline Bottle label number & $15-22$ & Integer & $\begin{array}{l}\text { The number of the bottle label attached to } \\
\text { the bottle. }\end{array}$ \\
\hline
\end{tabular}

Alpha and alphanumeric entries must be left justified in the records; numeric entries must be right justified in the records. There will be no leading zeros except for dates and times. 


\section{Station Data Format STA93}

This is the description of the STA93 format for coding and transmitting station data records.

\begin{tabular}{|c|c|c|c|}
\hline Name & Columns & Type & Description \\
\hline Record format & $1-8$ & Alphanumeric & STA93 (The format of the data record). \\
\hline Station & $10-16$ & Alphanumeric & $\begin{array}{l}\text { The identification of the station (the facility } \\
\text { identification and sampling point). }\end{array}$ \\
\hline SRS north coordinate & $21-30$ & Numeric & $\begin{array}{l}\text { The SRS Grid north coordinate of the } \\
\text { station reported in feet. }\end{array}$ \\
\hline SRS east coordinate & $32-41$ & Numeric & $\begin{array}{l}\text { The SRS Grid east coordinate of the station } \\
\text { reported in feet. }\end{array}$ \\
\hline Ground elevation & $44-49$ & Numeric & $\begin{array}{l}\text { Ground elevation reported in feet above } \\
\text { median sea level (MSL). }\end{array}$ \\
\hline
\end{tabular}

There will be no leading zeros. 


\section{Bottle Receipt Data Format BOT93}

This is the description of the BOT93 format for coding and transmitting bottle receipt data records. This format has been established by the Environmental Monitoring Section of the Environmental Protection Department of the Westinghouse Savannah River Company.

\begin{tabular}{|c|c|c|c|}
\hline Name & Columns & Type & Description \\
\hline Record format & $1-8$ & Alphanumeric & BOT93 (the format of the data record). \\
\hline $\begin{array}{l}\text { Borte label } \\
\text { number }\end{array}$ & $9-16$ & Integer & $\begin{array}{l}\text { The number of the bottle label attached to the } \\
\text { bottle. }\end{array}$ \\
\hline Bottle receipt date & $17-22$ & Numeric & $\begin{array}{l}\text { Date that the bottle receipt is recorded in the bottle } \\
\text { receipt logbook. Use the MMDDYY format. }\end{array}$ \\
\hline $\begin{array}{l}\text { Bottle batch } \\
\text { number }\end{array}$ & $23-33$ & Alphanumeric & $\begin{array}{l}\text { The identification number of the box the bottles are } \\
\text { shipped in. }\end{array}$ \\
\hline
\end{tabular}

Alpha and alphanumeric entries must be left justified in the records; numeric entries must be right justified in the records. There will be no leading zeros except for dates and times. 


\section{Chain Of Custody Data Format COC93}

This is the description of the COC93 format for coding and transmitting chain-of-custody data records. This format has been established by the Environmental Monitoring Section of the Environmental Protection Department of the Westinghouse Savannah River Company.

\begin{tabular}{|c|c|c|c|}
\hline Name & Columns & Type & Description \\
\hline Record format & $1-8$ & Alphanumeric & COC93 (the format of the data record). \\
\hline $\begin{array}{l}\text { Sample identification } \\
\text { number }\end{array}$ & $9-18$ & Integer & $\begin{array}{l}\text { The number of the sample printed on the page of } \\
\text { the sample logbook. }\end{array}$ \\
\hline Laboratory sample ID & $19-33$ & Alphanumeric & $\begin{array}{l}\text { Identifier used for the sample by the analytical } \\
\text { laboratory. }\end{array}$ \\
\hline Sample collection date & $34-39$ & Numeric & $\begin{array}{l}\text { Sample collection date recorded using the } \\
\text { MMDDYY format. }\end{array}$ \\
\hline Shipping date & $40-45$ & Numeric & $\begin{array}{l}\text { Sample shipping date recorded using the } \\
\text { MMDDYY format. }\end{array}$ \\
\hline Cooler identification & $46-53$ & Alphanumeric & $\begin{array}{l}\text { The number assigned to the cooler in which the } \\
\text { samples are shipped to the analytical laboratory. }\end{array}$ \\
\hline $\begin{array}{l}\text { Sample temperanure } \\
\text { on receipt }\end{array}$ & $54-57$ & Numeric & $\begin{array}{l}\text { The temperature of the samples upon receipt at the } \\
\text { analytical laboratory in degrees Centigrade. }\end{array}$ \\
\hline
\end{tabular}




\section{Analytical Data Format AN92}

This is the description of the AN92 format for coding and transminting analytical data records. This format has been established by the Environmental Monitoring Section of the Environmental Protection Department of the Westinghouse Savannah River Company.

\begin{tabular}{|c|c|c|c|}
\hline Name & Columns & Type & Description \\
\hline WSRC identifier & $1-12$ & Alphanumeric & WSRC identifier for the sample \\
\hline COC number & $13-17$ & Alphanumeric & $\begin{array}{l}\text { Identifier of the sample's chain-of-custody form. } \\
\text { Required field for groundwater samples; not } \\
\text { used for soil samples. }\end{array}$ \\
\hline $\begin{array}{l}\text { Sample collection } \\
\text { date** }\end{array}$ & $18-23$ & Numeric & Date that the sample was collected. \\
\hline Lab receipt date ${ }^{* *}$ & $24-29$ & Numeric & $\begin{array}{l}\text { Date that the sample was received by the } \\
\text { laboratory. }\end{array}$ \\
\hline Extraction date & $30-35$ & Numeric & $\begin{array}{l}\text { Date that the sample was extracted; leave blank } \\
\text { unless the holding time is dependent upon the } \\
\text { extraction time. }\end{array}$ \\
\hline
\end{tabular}

Extraction time $\quad 36-39 \quad$ Numeric

Time that the sample was extracted; leave blank unless the holding time is dependent upon the extraction time.

\begin{tabular}{|c|c|c|c|}
\hline Analysis date ${ }^{* *}$ & $40-45$ & Numeric & Date that the analysis was performed. \\
\hline Analysis time $\mathrm{e}^{* *}$ & $46-49$ & Numeric & $\begin{array}{l}\text { Time that the analyte concentration was deter- } \\
\text { mined. }\end{array}$ \\
\hline Analysis batch ${ }^{* *}$ & $50-55$ & Alphanumeric & $\begin{array}{l}\text { The number assigned by the analytical laborator } \\
\text { to the analysis batch (group of samples analyzed } \\
\text { together in a single instrument run along with th } \\
\text { associated QC samples). }\end{array}$ \\
\hline $\begin{array}{l}\text { Laboratory }{ }^{* *} \\
\text {. }\end{array}$ & $56-57$ & Alphanumeric & $\begin{array}{l}\text { Laboratory code assigned by EPD/EMS to } \\
\text { identify the laboratory performing the analysis. }\end{array}$ \\
\hline $\begin{array}{l}\text { Laboratory sample } \\
\text { ID }^{* * *}\end{array}$ & $58-72$ & Alphanumeric & $\begin{array}{l}\text { Identifier used for the sample by the analytical } \\
\text { laboratory. }\end{array}$ \\
\hline
\end{tabular}

Alpha and alphanumeric entries must be left justified in the records; numeric entries must be right justified in the records. There will be no leading zeros except for dates and times. Dates should be in MMDDYY format, times in HHMM format.

- This field must have an entry for every record. 


\section{Analytical Data Format AN92, Continued}

Name Columns Type Description

$\begin{array}{lll}\text { Analyte }^{* *} & 74-83 & \text { Alphanumeric } \\ \begin{array}{c}\text { Extraction/digestion } \\ \text { method }\end{array} & 84-96 & \text { Alphanumeric }\end{array}$

$\begin{array}{lll}\text { Analytical method }^{* *} & 97-109 & \text { Alphanumeric } \\ \text { Detection limit } & 110-117 & \text { Numeric }\end{array}$

Result qualifier

118-120 Alphanumeric

The coded identifier (provided by EPD/EMS) for the analyte.

The coded identifier for the method used to perform the extraction or digestion (e.g., EPA\#\#\#.\#).

The coded identifier for the method used to perform the analysis (e.g., EPA\#\#\#.\#).

The nominal method detection limit used for the analysis and reported in the same units as the analytical result. This field MUST have an entry for every record EXCEPT leave blank for spiked samples reported as percent recovery.

The coded field used to qualify the analytical result (reason codes attached to this document).

Analysis qualifier

121-123 Alphanumeric

The coded field used to qualify the analysis (reason codes attached to this document).

Bias of analysis

124

Alphanumeric

The coded field for data having result qualifiers of "J" or " $R$ " specifying the expected bias (reason codes attached to this document).
Analytical result ${ }^{* *}$
125-134 Numeric
The concentration of the analyte or, for spiked samples, the percent recovery ( 3 significant digits).
Result units**
Accuracy
Residual weight
Number of dilutions ${ }^{* *}$
Dilution factor ${ }^{* *}$
135-138 Alpha
139-146 Numeric
147-150 Numeric
$151 \quad$ Numeric
152-157 Numeric
The coded identifier (provided by EPD/EMS) of the units in which the analytical result is expressed.
The statistically determined value representing a plus or minus value for the result ( 3 significant digits) and reported in the same units as the analytical result.
The weight of material on the planchet after evaporation; used for radionuclide determinations.
The number of steps required to reach the final dilution factor; 0 if the sample was not diluted.
The dilution used in the analysis; a value of 1 indicates the sample was not diluted. 


\section{Analytical Data Format AN92, Continued}

Name

Instrument **

Analyst's initials**

Nominal

concentration ${ }^{* *}$

EPD/EMS receipt date

EPA quality level

Sample fraction

Percent solids

Bottle label number
Columns Type

158-159 Alphanumeric

160-162 Alpha

163-170 Numeric

171-176 Numeric

177-178 Alphanumeric

179-182 Alphanumeric

183-187 Numeric

188-197 Integer

\section{Description}

The coded identifier for the instrument used to perform the analysis; each laboratory develops its own codes.

The initials of the analyst.

The concentration of analyte added to the sample prior to analysis; reported in the same units as the normal reporting units for that analyte; the value of zero must be entered if the sample was not spiked.

The laboratory will leave this blank; date that the analytical data is received by EPD/EMS from the laboratory, in MMDDYY format.

The first character is 1,2 , or 3 , and states the quality objective of the data (Quality Assurance/Quality Control Guidance for Removal Activities, EPA/540/G90/004).

The coded identifier (provided by EPD/EMS) for the fraction of the sample analyzed.

The percentage of the sample that is dry solids.

The number on the bottle label. Required field if bottle label is numbered. 


\begin{tabular}{|c|c|c|c|}
\hline \multicolumn{4}{|c|}{ Analytical Data Format AN92, Continued } \\
\hline \multicolumn{4}{|c|}{ WSRC Identifier for Groundwater Samples } \\
\hline Name & Columns & Type & Description \\
\hline \multicolumn{4}{|l|}{ Well name } \\
\hline Series name & $1-3$ & Alpha & $\begin{array}{l}\text { Name of the well series (except } 241-\mathrm{H} \text { ) from } \\
\text { which the sample was collected; QA/QC } \\
\text { laboratory deionized water samples will have LB } \\
\text { (the first character is blank) as well series } \\
\text { designation. }\end{array}$ \\
\hline Cluster number & $4-6$ & Numeric & Number of the cluster (except 241-H). \\
\hline Well within cluster & $\begin{array}{l}7-8 \\
9-10\end{array}$ & $\begin{array}{l}\text { Alphanumeric } \\
\text { Blank }\end{array}$ & $\begin{array}{l}\text { Can be blank. } \\
\text { Unused. }\end{array}$ \\
\hline Duplicate ID & 11 & Alphanumeric & $\begin{array}{l}\text { Assigned by field personnel to identify different } \\
\text { sampling events occurring in one day. }\end{array}$ \\
\hline Secondary identifier & 12 & Alphanumeric & $\begin{array}{l}\text { Used as an alternate identifier by the } \\
\text { Groundwater Monitoring (GM) group. }\end{array}$ \\
\hline \multicolumn{4}{|c|}{ WSRC Identifier for Sediment, Core, and Surface Water Samples } \\
\hline $\begin{array}{l}\text { Sample identification } \\
\text { number }\end{array}$ & $1-6$ & Numeric & The sample logbook page number \\
\hline
\end{tabular}




Abbreviations Used in the Tables
$\begin{array}{ll}\text { RQ } & \text { Result qualifier } \\ \text { AQ } & \text { Analysis qualifier } \\ \text { B } & \text { Bias code } \\ \text { D. Limit } & \text { Detection limit (sample quantitation limit) } \\ \text { B/N/A Extractables } & \text { Base/neutralacid extractable semivolatile organic compounds } \\ \text { PAH } & \text { Polycyclic aromatic hydrocarbon } \\ \text { arom. } & \text { aromatic } \\ \text { polyarom. } & \text { polyaromatic } \\ \text { sub., subst. } & \text { substituted }\end{array}$

\section{Qualifier Codes}

\section{Result Qualifiers}
J The analytical result is an estimated quantity.
L Off-scale high. The actual value is not bnown but is known to be greater than the value shorn.
P Precision cannot be determined because matrix spikes were not performed.
$R \quad$ Rejected because performance requirements in the sample or associated quality control analysis were not met. The analyte may or may not be present. The analyte was not detected. Value reported is the sample quantification limit.

Analysis Qualifiers

A Relative Retention Time (RRT) or mass spectra criteria were not met for organics compound identification. Used with $U, U J$, or $N$ for all anomalies in pesticides compound identification.

Laboratory control sample (LCS) or blank spike criteria were not met. Pesticides instrument performance was outside criteria. Internal standards performance criteria were not met. Matrix spike recovery is not within control limits. A tentatively identified compound is a suspected aldol-condensation product. Calibration criteria (initial or continuing) were not met. Used with $J$ or $R$. GC/MS tuning is out of specification. Tentatively identified compound; does not meet Quality Objective 3 criteria. Surrogate spike recoveries are out of specification. Sample held beyond normal holding time. Used with result qualifier $J$. ICP interference check sample criteria were not met. Used with $J, U J$, or $R$. The reported value was determined by the method of standard additions. Peak height (instead of peak area) was used to quantitate the analyte, and all other identification criteria were met; OR, signal-to-noise ratio of the confirmation ion is below 2.5 , but the GC/MS analyst determined that the peak was positive. 
V The analyte was detected in an associated method blank. Used with $J$ for nonradiological analyses when the result is less than 5 times the amount in the associated blank (less than 10 times for the common laboratory contaminants acetone, dichloromethane, 2-butanone, and phthalates). Used with $J$ or $U J$ for radiological analy'ses: $J$ when result is less than 5 times the amount in the associated blank; $U J$ when the result is less than the detection limit.

X The relative percent difference (RPD) calculated from duplicate analyses or matrix spike/matrix spike duplicates was not within control limits.

$Y$ Sample was unpreserved or improperly preserved.

\section{Bias Codes}

H QAVC sample results indicate a high bias. The associated result may overestimate the true value.

L QA/QC sample results indicate a low bias. The associated result may underestimate the true value.

\section{Color Notation}

Munsell notation describes three distinct components of color: hue, value, and chroma in an abbreviated unified color designation.

Hue is designated by a letter abbreviation ( $R$ for red, $Y R$ for yellow-red, $Y$ for yellow) preceded by a number between 1 and 10 that signifies the position of the hue between red and yellow. Lower numbers are closer to red and higher numbers are closer to yellow. The hues overlap at 0 and 10 with OYR being $10 \mathrm{R}$.

The notation for value ranges from 0 to 10 , with 0 being absolute black and 10, absolute white.

The notation for chroma begins at 0 for neutral grays and increases in standard intervals to 20 . Twenty is never approached in soils; the highest working number is 8 . For colors with no hue and 0 chroma (pure grays, white, and black), the letter $N$ (neutral) is used in place of a number.

An example of Munsell notation is: 10 YR $5 / 6$, which indicates a yellow-red hue of 10 , a value of 5 , and a chroma of 6 . This corresponds to a yellowish-brown color.

Any of these numbers can be a decimal, indicating a color between two defined points, although for practical field purposes only a .5 for a color halfway between two defined color points would be used. 


\section{Unified Soil Classification System}

\begin{tabular}{|c|c|c|c|c|}
\hline \multicolumn{3}{|c|}{ Major Divisions } & Group & Typical Names \\
\hline \multirow{8}{*}{$\begin{array}{l}\text { COARSE. } \\
\text { GRAINED SOILS } \\
\text { More than half of } \\
\text { material is larger } \\
\text { than no. } 200 \text { sieve } \\
\text { size. }\end{array}$} & \multirow{2}{*}{$\begin{array}{l}\text { GRAVELS } \\
\text { More than half of } \\
\text { coarse fraction is } \\
\text { larger than no. } 4 \\
\text { sieve size. }\end{array}$} & Clean gravels & GW & $\begin{array}{l}\text { Well-graded gravels, gravel- } \\
\text { sand mixtures, little or no } \\
\text { fines. }\end{array}$ \\
\hline & & & GP & $\begin{array}{l}\text { Poorly graded gravels, } \\
\text { gravel-sand mixtures, little } \\
\text { or no fines. }\end{array}$ \\
\hline & & Gravels with fines & GM & $\begin{array}{l}\text { Silty gravels, gravel-sand-silt } \\
\text { mixtures. }\end{array}$ \\
\hline & . & & GC & $\begin{array}{l}\text { Clayey gravels, gravel-sand- } \\
\text { clay mixtures. }\end{array}$ \\
\hline & \multirow{2}{*}{$\begin{array}{l}\text { SANDS } \\
\text { More than half of } \\
\text { coarse fraction is } \\
\text { smaller than no. } 4 \\
\text { sieve size. }\end{array}$} & Clean sands & SW & $\begin{array}{l}\text { Well-graded sands, gravelly } \\
\text { sands, little or no fines. }\end{array}$ \\
\hline & & & $\overline{S P}$ & $\begin{array}{l}\text { Poorly graded sands, } \\
\text { gravelly sands, little or no } \\
\text { Gnes. }\end{array}$ \\
\hline & 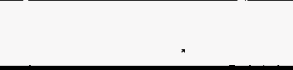 & Sands with fines & SM & $\begin{array}{l}\text { Silty sands, sand-silt } \\
\text { mixtures. }\end{array}$ \\
\hline & & & SC & $\begin{array}{l}\text { Clayey sands, sand-clay } \\
\text { mixtures. }\end{array}$ \\
\hline \multirow{6}{*}{$\begin{array}{l}\text { FINE-GRAINED } \\
\text { sOIIS } \\
\text { More than half of } \\
\text { material is smaller } \\
\text { than no. } 200 \text { sieve } \\
\text { size. }\end{array}$} & SILTS AND CLAYS & Low liquid limit & ML & $\begin{array}{l}\text { Inorganic silts and very fine } \\
\text { sands, rock flour, silty or } \\
\text { clayey fine sands, or clayey } \\
\text { silts. of slight plasticity. }\end{array}$ \\
\hline & & & $\mathrm{CL}$ & $\begin{array}{l}\text { Inorganic clays of low to } \\
\text { medium plasticity, gravelly } \\
\text { clays, sand clays, silty clays, } \\
\text { lean clavs. }\end{array}$ \\
\hline & & & OL & $\begin{array}{l}\text { Organic silts and organic } \\
\text { silty clays of low plasticity. }\end{array}$ \\
\hline & & High liquid limit & MH & $\begin{array}{l}\text { Inorganic silts, micaceous or } \\
\text { diatomaceous fine sandy or } \\
\text { silty soils, elastic silts. }\end{array}$ \\
\hline & & & $\overline{\mathrm{CH}}$ & $\begin{array}{l}\text { Inorganic clays of high } \\
\text { plasticiti. fat clavs. }\end{array}$ \\
\hline & & & $\mathrm{OH}$ & $\begin{array}{l}\text { Organic clay's of medium to } \\
\text { high plasticity. organic silts. }\end{array}$ \\
\hline & \multicolumn{2}{|l|}{ Highly Organic Soils } & $\mathbf{P t}$ & $\begin{array}{l}\text { Peat and other highly } \\
\text { organic silts. }\end{array}$ \\
\hline
\end{tabular}

Notes:

1. Boundary Classification: Soils possessing characteristics of two groups are designated by combinations of group symbols. For example, GW.GC means well-graded gravel-sand mixture with clay binder.

2. All sieve sizes on this chart are U.S. Standard.

3. The terms "silt" and "clay" are used respectively to distinguish materials exhibiting lower plasticity from those urith higher plasticity.

4. For a complete description of the Unified Soil Classification System, see "Technical Memorandum No. 3-357," prepared for Office, Chief of Engineers, by Waterways Equipment Station, Vicksburg, Mississippi, March 1953. 
SAMPLE NAME: D431 1101

Sample ID: 100361

Location (SRS Coordinates): 18558.54E 66554.39N

Ground Elevation Above MSL: $134.6 \mathrm{ft}$

Depth of Core Interval: 0.00 to $0.50 \mathrm{ft}$

Sample Type: Normal

Sample Color: 5YR 4/4

\section{Constituent}

Physical Parameters

Cation exchange capacity

$\mathrm{pH}$

Specific conductance

Total organic carbon

Total organic carbon

Total Metals

Arsenic

Barium

Cadmium

Chromium

Copper

Lead

Manganese

Mercury

Nickel

Selenium

Silver

$\begin{array}{lll}\text { U } & & 0.90 \\ \mathrm{U} & & 18 \\ \mathrm{UJ} & \mathrm{L} & 0.45 \\ \mathrm{UJ} & \mathrm{L} & 5.2 \\ \mathrm{U} & & 2.2 \\ & & 2.0 \\ & & 260 \\ \mathrm{U} & & 0.10 \\ \mathrm{U} & & 3.6 \\ \mathrm{U} & & 0.45 \\ \mathrm{U} & & 0.90\end{array}$

6.7
4.6
30
4.000
4,200



0.90
18
0.45
5.2
2.2
2.0
260
0.10
3.6
0.45
0.90

$\mathrm{meq} / 100 \mathrm{~g}$
$\mathrm{pH}$
$\mu \mathrm{S} / \mathrm{cm}$
$\mathrm{mg} / \mathrm{kg}$
$\mathrm{mg} / \mathrm{kg}$

2.0

EPA9080

0.010

EPA9045

1.0

180

EPA9050

EPA415.1

220

EPA415.1

B/N/A Extractables (including PAH and phenols)

\begin{abstract}
Acenaphthene
Acenaphthylene

Anthracene

Benzo(a)anthracene

Benzo(b)fluoranthene

Benzo(k)fluoranthene

Benzoic acid

Benzo(g,h,i)perylene

Benzo(a)pyrene

Benzyl alcohol

Bis(2-chloroethoxy) methane

Bis(2-chloroethyl) ether

Bis(2-chloroisopropyl) ether

Bis(2-ethylhexyl) phthalate

4-Bromophenyl phenyl ether

Butylbenzyl phthalate

4-Chloroaniline

4-Chloro-m-cresol

2-Chloronaphthalene

2-Chlorophenol

4-Chlorophenyl phenyl ether

Chrysene
\end{abstract}

$\begin{array}{lllll}\mathrm{U} & 350 & \mu \mathrm{g} / \mathrm{kg} & 350 & \text { EPA8270 } \\ \mathrm{U} & 350 & \mu \mathrm{g} / \mathrm{kg} & 350 & \text { EPA8270 } \\ \mathrm{U} & 350 & \mu \mathrm{g} / \mathrm{kg} & 350 & \text { EPA8270 } \\ \mathrm{U} & 350 & \mu \mathrm{g} / \mathrm{kg} & 350 & \text { EPA8270 } \\ \mathrm{U} & 350 & \mu \mathrm{g} / \mathrm{kg} & 350 & \text { EPA8270 } \\ \mathrm{U} & 350 & \mu \mathrm{g} / \mathrm{kg} & 350 & \text { EPA8270 } \\ \mathrm{U} & 1,700 & \mu \mathrm{g} / \mathrm{kg} & 1,700 & \text { EPA8270 } \\ \mathrm{U} & 350 & \mu \mathrm{g} / \mathrm{kg} & 350 & \text { EPA8270 } \\ \mathrm{U} & 350 & \mu \mathrm{g} / \mathrm{kg} & 350 & \text { EPA8270 } \\ \mathrm{U} & 350 & \mu \mathrm{g} / \mathrm{kg} & 350 & \text { EPA8270 } \\ \mathrm{U} & 350 & \mu \mathrm{g} / \mathrm{kg} & 350 & \text { EPA8270 } \\ \mathrm{U} & 350 & \mu \mathrm{g} / \mathrm{kg} & 350 & \text { EPA8270 } \\ \mathrm{U} & 350 & \mu \mathrm{g} / \mathrm{kg} & 350 & \text { EPA8270 } \\ \mathrm{U} & 350 & \mu \mathrm{g} / \mathrm{kg} & 350 & \text { EPA8270 } \\ \mathrm{U} & 350 & \mu \mathrm{g} / \mathrm{kg} & 350 & \text { EPA8270 } \\ \mathrm{U} & 350 & \mu \mathrm{g} / \mathrm{kg} & 350 & \text { EPA8270 } \\ \mathrm{U} & 350 & \mu \mathrm{g} / \mathrm{kg} & 350 & \text { EPA8270 } \\ \mathrm{U} & 350 & \mu \mathrm{g} / \mathrm{kg} & 350 & \text { EPA8270 } \\ \mathrm{U} & 350 & \mu \mathrm{g} / \mathrm{kg} & 350 & \text { EPA8270 } \\ \mathrm{U} & 350 & \mu \mathrm{g} / \mathrm{kg} & 350 & \text { EPA8270 } \\ \mathrm{U} & 350 & \mu \mathrm{g} / \mathrm{kg} & 350 & \text { EPA8270 } \\ \mathrm{U} & 350 & \mu \mathrm{g} / \mathrm{kg} & 350 & \text { EPA8270 }\end{array}$

$\begin{array}{lll}\mathrm{mg} / \mathrm{kg} & 0.90 & \text { EPA7060 } \\ \mathrm{mg} / \mathrm{kg} & 18 & \text { EPA6010 } \\ \mathrm{mg} / \mathrm{kg} & 0.45 & \text { EPA6010 } \\ \mathrm{mg} / \mathrm{kg} & 0.90 & \text { EPA6010 } \\ \mathrm{mg} / \mathrm{kg} & 2.2 & \text { EPA6010 } \\ \mathrm{mg} / \mathrm{kg} & 0.27 & \text { EPA7421 } \\ \mathrm{mg} / \mathrm{kg} & 1.3 & \text { EPA6010 } \\ \mathrm{mg} / \mathrm{kg} & 0.10 & \text { EPA7470 } \\ \mathrm{mg} / \mathrm{kg} & 3.6 & \text { EPA6010 } \\ \mathrm{mg} / \mathrm{kg} & 0.45 & \text { EPA7740 } \\ \mathrm{mg} / \mathrm{kg} & 0.90 & \text { EPA6010 }\end{array}$


SAMPLE NAME: D43İ 1101 (Continued)

Sample ID: 100361

Constituent

RQ AQ B Result

Unit

D. Limit Method

$B / N / A$ Extractables (including PAH and phenols)

o-Cresol (2-Methylphenol)

p-Cresol (4-Methylphenol)

Dibenz $(a, h)$ anthracene

Dibenzofuran

Di-n-butyl phthalate

1,2-Dichlorobenzene

1,3-Dichlorobenzene

1,4-Dichlorobenzene

3,3'-Dichlorobenzidine

2,4-Dichlorophenol

Diethyl phthalate

2,4-Dimethyl phenol

Dimethyl phthalate

2,4-Dinitrophenol

2,4-Dinitrotoluene

2,6-Dinitrotoluene

Di-n-octyl phthalate

Fluoranthene

Fluorene

Hexachlorobenzene

Hexachlorobutadiene

Hexachlorocyclopentadiene

Hexachloroethane

Indeno(1,2,3-c,d)pyrene

Isophorone

2-Methyl-4,6-dinitrophenol

2-Methylnaphthalene

Naphthalene

o-Nitroaniline

m-Nitroaniline

p-Nitroaniline

Nitrobenzene

2-Nitrophenol

4-Nitrophenol

N-Nitrosodiphenylamine

N-Nitrosodipropylamine

Péntachlorophenol

Phenanthrene

Phenol

Pyrene

1,2,4-Trichlorobenzene

2,4,5-Trichlorophenol

2,4,6-Trichlorophenol

Unknown hydrocarbon

Unknown ketone

\begin{tabular}{|c|c|c|}
\hline $\mathbf{U}$ & & 350 \\
\hline $\mathbf{U}$ & & 350 \\
\hline$U$ & & 350 \\
\hline $\mathbf{U}$ & & 350 \\
\hline U & $v$ & 98 \\
\hline $\mathbf{U}$ & & 350 \\
\hline $\mathbf{U}$ & & 350 \\
\hline $\mathrm{U}$ & & 350 \\
\hline $\mathbf{U}$ & & 690 \\
\hline U & & 350 \\
\hline$U$ & & 350 \\
\hline$U$ & & 350 \\
\hline U & & 350 \\
\hline $\mathbf{U}$ & & 1,700 \\
\hline U & & 350 \\
\hline U & & 350 \\
\hline $\mathrm{U}$ & & 350 \\
\hline $\mathrm{U}$ & & 350 \\
\hline $\mathbf{U}$ & & 350 \\
\hline $\mathbf{U}$ & & 350 \\
\hline $\mathbf{U}$ & & 350 \\
\hline U & & 350 \\
\hline U & & 350 \\
\hline U & & 350 \\
\hline $\mathrm{U}$ & & 350 \\
\hline U & & 1,700 \\
\hline$U$ & & 350 \\
\hline U & & 350 \\
\hline $\mathbf{U}$ & & 1,700 \\
\hline U & & 1,700 \\
\hline $\mathrm{U}$ & & 1,700 \\
\hline $\mathrm{U}$ & & 350 \\
\hline $\mathrm{U}$ & & 350 \\
\hline $\mathrm{U}$ & & 1,700 \\
\hline $\mathbf{U}$ & & 350 \\
\hline$U$ & & 350 \\
\hline UJ & C & 1,700 \\
\hline U & & 350 \\
\hline UJ & C & 350 \\
\hline U & & 350 \\
\hline U & & 350 \\
\hline $\mathbf{U}$ & & 1,700 \\
\hline U & & 350 \\
\hline I & $\mathbf{N}$ & 100 \\
\hline 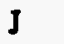 & NV & 6,000 \\
\hline
\end{tabular}

$\begin{array}{lll}\mu \mathrm{g} / \mathrm{kg} & 350 & \text { EPA8270 } \\ \mu \mathrm{g} / \mathrm{kg} & 350 & \text { EPA8270 } \\ \mu \mathrm{g} / \mathrm{kg} & 350 & \text { EPA8270 } \\ \mu \mathrm{g} / \mathrm{kg} & 350 & \text { EPA8270 } \\ \mu \mathrm{g} / \mathrm{kg} & 350 & \text { EPA8270 } \\ \mu \mathrm{g} / \mathrm{kg} & 350 & \text { EPA8270 } \\ \mu \mathrm{g} / \mathrm{kg} & 350 & \text { EPA8270 } \\ \mu \mathrm{g} / \mathrm{kg} & 350 & \text { EPA8270 } \\ \mu \mathrm{g} / \mathrm{kg} & 690 & \text { EPA8270 } \\ \mu \mathrm{g} / \mathrm{kg} & 350 & \text { EPA8270 } \\ \mu \mathrm{g} / \mathrm{kg} & 350 & \text { EPA8270 } \\ \mu \mathrm{g} / \mathrm{kg} & 350 & \text { EPA8270 } \\ \mu \mathrm{g} / \mathrm{kg} & 350 & \text { EPA8270 } \\ \mu \mathrm{g} / \mathrm{kg} & 1,700 & \text { EPA8270 } \\ \mu \mathrm{g} / \mathrm{kg} & 350 & \text { EPA8270 } \\ \mu \mathrm{g} / \mathrm{kg} & 350 & \text { EPA8270 } \\ \mu \mathrm{g} / \mathrm{kg} & 350 & \text { EPA8270 } \\ \mu \mathrm{g} / \mathrm{kg} & 350 & \text { EPA8270 } \\ \mu \mathrm{gg} / \mathrm{kg} & 350 & \text { EPA8270 } \\ \mu \mathrm{g} / \mathrm{kg} & 350 & \text { EPA8270 } \\ \mu \mathrm{g} / \mathrm{kg} & 350 & \text { EPA8270 } \\ \mu \mu / \mathrm{kg} & 350 & \text { EPA8270 } \\ \mu \mathrm{g} / \mathrm{kg} & 350 & \text { EPA8270 } \\ \mu \mathrm{g} / \mathrm{kg} & 350 & \text { EPA8270 } \\ \mu \mathrm{g} / \mathrm{kg} & 350 & \text { EPA8270 } \\ \mu \mathrm{g} / \mathrm{kg} & 1,700 & \text { EPA8270 } \\ \mu \mathrm{g} / \mathrm{kg} & 350 & \text { EPA8270 } \\ \mu \mathrm{g} / \mathrm{kg} & 350 & \text { EPA8270 } \\ \mu \mathrm{g} / \mathrm{kg} & 1,700 & \text { EPA8270 } \\ \mu \mathrm{g} / \mathrm{kg} & 1,700 & \text { EPA8270 } \\ \mu \mathrm{g} / \mathrm{kg} & 1,700 & \text { EPA8270 } \\ \mu \mathrm{g} / \mathrm{kg} & 350 & \text { EPA8270 } \\ \mu \mathrm{g} / \mathrm{kg} & 350 & \text { EPA8270 } \\ \mu \mathrm{g} / \mathrm{kg} & 1,700 & \text { EPA8270 } \\ \mu \mathrm{g} / \mathrm{kg} & 350 & \text { EPA8270 } \\ \mu \mathrm{g} / \mathrm{kg} & 350 & \text { EPA8270 } \\ \mu \mathrm{g} / \mathrm{kg} & 1,700 & \text { EPA8270 } \\ \mu \mathrm{g} / \mathrm{kg} & 350 & \text { EPA8270 } \\ \mu \mathrm{g} / \mathrm{kg} & 350 & \text { EPA8270 } \\ \mu \mathrm{g} / \mathrm{kg} & 350 & \text { EPA8270 } \\ \mu \mathrm{g} / \mathrm{kg} & 350 & \text { EPA8270 } \\ \mu \mathrm{g} / \mathrm{kg} & 1,700 & \text { EPA8270 } \\ \mu \mathrm{g} / \mathrm{kg} & 350 & \text { EPA8270 } \\ \mu \mathrm{g} / \mathrm{kg} & & \text { EPA8270 } \\ \mu \mathrm{g} / \mathrm{kg} & & \text { EPA8270 } \\ & & \end{array}$


SAMPLE NAME: D431-1101 (Continued)

Constituent

Volatile Organic Compounds

Acetone
Benzene
Bromodichloromethane
Bromoform
Bromomethane (Methyl bromide)
Carbon disulfide
Carbon tetrachloride
Chlorobenzene
Chloroethane
Chloroethene (Vinyl chloride)
Chloroform
Chloromethane (Methyl chloride)
Dibromochloromethane
1,1-Dichloroethane
1.2-Dichloroethane
1,1-Dichloroethylene
1,2-Dichloroethylene
Dichloromethane (Methylene chloride)
1,2-Dichloropropane
cis-1,3-Dichloropropene
trans-1,3-Dichloropropene
Ethylbenzene
2-Hexanone
Methyl ethyl ketone
Methyl isobutyl ketone
Styrene
1,1,2,2-Tetrachloroethane
Tetrachloroethylene
Toluene
1,1,1-Trichloroethane
1,1.2-Trichloroethane
Trichloroethylene
Vinyl acetate
Xylenes

Sample ID: 100361

RQ AQ B Result Unit D. Limit Method

\begin{tabular}{|c|c|c|c|}
\hline 18 & $\mu \mathrm{g} / \mathrm{kg}$ & 10 & EPA8240 \\
\hline 5.6 & $\mu g / \mathrm{kg}$ & 5.2 & EPA8240 \\
\hline 5.2 & $\mu \mathrm{g} / \mathrm{kg}$ & 5.2 & EPA8240 \\
\hline 5.2 & $\mu g / k g$ & 5.2 & EPA8240 \\
\hline 10 & $\mu \mathrm{g} / \mathrm{kg}$ & 10 & EPA8240 \\
\hline 5.2 & $\mu g / \mathrm{kg}$ & 5.2 & EPA8240 \\
\hline 5.2 & $\mu \mathrm{g} / \mathrm{kg}$ & 5.2 & EPA8240 \\
\hline 5.2 & $\mu \mathrm{g} / \mathrm{kg}$ & 5.2 & EPA8240 \\
\hline 10 & $\mu g / \mathrm{kg}$ & 10 & EPA 8240 \\
\hline 10 & $\mu \mathrm{g} / \mathrm{kg}$ & 10 & EPA8240 \\
\hline 5.2 & $\mu g / \mathrm{kg}$ & 5.2 & EPA8240 \\
\hline 10 & $\mu g / \mathrm{kg}$ & 10 & EPA8240 \\
\hline 5.2 & $\mu \mathrm{g} / \mathrm{kg}$ & 5.2 & EPA8240 \\
\hline 5.2 & $\mu \mathrm{g} / \mathrm{kg}$ & 5.2 & EPA8240 \\
\hline 5.2 & $\mu g / \mathrm{kg}$ & 5.2 & EPA8240 \\
\hline 5.2 & $\mu \mathrm{g} / \mathrm{kg}$ & 5.2 & EPA8240 \\
\hline 5.2 & $\mu g / \mathrm{kg}$ & 5.2 & EPA8240 \\
\hline 16 & $\mu \mathrm{g} / \mathrm{kg}$ & 5.2 & EPA8240 \\
\hline 5.2 & $\mu \mathrm{g} / \mathrm{kg}$ & 5.2 & EPA8240 \\
\hline 5.2 & $\mu \mathrm{g} / \mathrm{kg}$ & 5.2 & EPA8240 \\
\hline 5.2 & $\mu g / \mathrm{kg}$ & 5.2 & EPA8240 \\
\hline 5.2 & $\mu \mathrm{g} / \mathrm{kg}$ & 5.2 & EPA8240 \\
\hline 10 & $\mu g / \mathrm{kg}$ & 10 & EPA8240 \\
\hline 10 & $\mu \mathrm{g} / \mathrm{kg}$ & 10 & EPA8240 \\
\hline 10 & $\mu \mathrm{g} / \mathrm{kg}$ & 10 & EPA8240 \\
\hline 5.2 & $\mu \mathrm{g} / \mathrm{kg}$ & 5.2 & EPA8240 \\
\hline 5.2 & $\mu g / \mathrm{kg}$ & 5.2 & EPA8240 \\
\hline 5.2 & $\mu g / \mathrm{kg}$ & 5.2 & EPA8240 \\
\hline 5.2 & $\mu g / \mathrm{kg}$ & 5.2 & EPA8240 \\
\hline 5.2 & $\mu g / \mathrm{kg}$ & 5.2 & EPA8240 \\
\hline 5.2 & $\mu \mathrm{g} / \mathrm{kg}$ & 5.2 & EPA8240 \\
\hline 5.2 & $\mu \mathrm{g} / \mathrm{kg}$ & 5.2 & EPA8240 \\
\hline 10 & $\mu \mathrm{g} / \mathrm{kg}$ & 10 & EPA8240 \\
\hline 5.2 & $\mu g / \mathrm{kg}$ & 5.2 & EPA8240 \\
\hline
\end{tabular}

Pesticides/PCBs and Dioxins/Furans

Aldrin

U

alpha-Benzene hexachloride

1.7

1.7

1.7

beta-Benzene hexachloride

delta-Benzene hexachloride

alpha-Chlordane

1.7

1.7

1.7

3.4

0.69

3.4

3.4

1.7

3.4

3.4 $\mu \mathrm{g} / \mathrm{kg}$
$\mu \mathrm{g} / \mathrm{kg}$
$\mu \mathrm{g} / \mathrm{kg}$
$\mu \mathrm{g} / \mathrm{kg}$
$\mu \mathrm{g} / \mathrm{kg}$
$\mu \mathrm{g} / \mathrm{kg}$
$\mu \mathrm{g} / \mathrm{kg}$
$\mu \mathrm{g} / \mathrm{kg}$
$\mu \mathrm{g} / \mathrm{kg}$
$\mu \mathrm{g} / \mathrm{kg}$
$\mu \mathrm{g} / \mathrm{kg}$
$\mu \mathrm{g} / \mathrm{kg}$
$\mu \mathrm{g} / \mathrm{kg}$
1.7

1.7 EPA8080

1.7 EPA8080

1.7 . EPA8080

1.7 . EPA8080

3.4 EPA8080

3.4 EPA8080

3.4 EPA8080

$3.4 \quad$ EPA8080

1.7 EPA8080

3.4 EPA8080

$3.4 \quad$ EPA8080
1.7 EPA8080 
SAMPLE NAME: D431 1101 (Continued)

Constituent
Sample ID: 100361

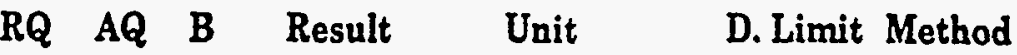

Pesticides/PCBs and Dioxins/Furans

Endrin

Endrin ketone

Heptachlor

Heptachlor epoxide

Heptachlorodibenzo-p-dioxin isomers

Heptachlorodibenzo-p-furan isomers

Hexachlorodibenzo-p-dioxin isomers

Hexachlorodibenzo-p-furan isomers

Lindane

Octachlorodibenzo-p-dioxin

Octachlorodibenzo-p-furan

PCB 1016

PCB 1221

PCB 1232

PCB 1242

PCB 1248

PCB 1254

PCB 1260

Pentachlorodibenzo-p-dioxin isomers

Pentachlorodibenzo-p-furan isomers

2,3.7,8-TCDD

2,3,7,8-TCDF

Tetrachlorodibenzo-p-dioxin isomers

Tetrachlorodibenzo-p-furan isomers

Toxaphene
Methoxychlor

$U$
$U$
$U$
$U$
$U$
$U$
$U$
$U$
$U$
$U$
$J$
$U$
$U$
$U$
$U$
$U$
$U$
$U$
$U$
$U$
$U$
$U$
$U$
$U$
$U$
$U$

Radionuclide Indicators and Radionuclides

Gross alpha

Nonvolatile beta

Radium, total alpha-emitting

Tritium

$\begin{array}{llll} & 7.9 \pm 11 & \mathrm{pCi} / g \\ & & 25 \pm 18 & \mathrm{pCi} / \mathrm{g} \\ \mathrm{U} & \mathrm{v} & 0.76 \pm 0.50 & \mathrm{pCi} / g \\ \mathrm{U} & & 2.0 & \mathrm{pCi} / \mathrm{g}\end{array}$

Sample ID: 100362

\section{SAMPLE NAME: D431 1102}

Location (SRS Coordinates): 18558.54E 66554.39N

Ground Elevation Above MSL: $134.6 \mathrm{ft}$

Depth of Core Interval: 0.50 to $2.00 \mathrm{ft}$

Sample Type: Normal

Sample Color: 5YR 4/4

Sample Matrix: Soil

USC Soil Classification: SM

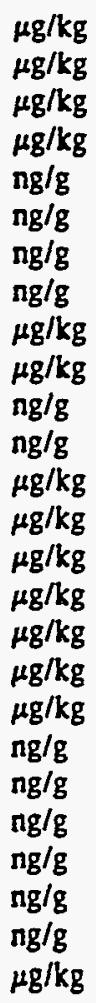

3.4

3.4

1.7

1.7

0.10

0.10

0.10

0.10

1.7

17

0.30

0.20

34

69

34

34

34

34

34

0.20

0.10

0.20

0.10

0.20

0.10

170

EPA8080

EPA8080

EPA8080

EPA8080

EPA8280

EPA8280

EPA8280

EPA8280

EPA8080

EPA8080

EPA8280

EPA8280

EPA8080

EPA8080

EPA8080

EPA8080

EPA8080

EPA8080

EPA8080

EPA8280

EPA8280

EPA8280

EPA8280

EPA8280

EPA8280

EPA8080

Constituent

$\mathbf{R Q} \mathbf{A Q}$

B Result

Unit

$\begin{array}{lr}6.5 & \text { EPA900.0 } \\ 12 & \text { EPA900.0 } \\ 0.12 & \text { EPA903.0 } \\ 2.0 & \text { - EPA906.0 }\end{array}$

Total Metals

Arsenic

Barium

Cadmium

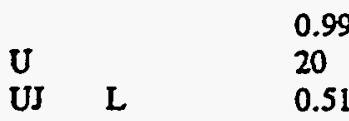

Sample Moisture: Dry

Percent Solids: 92.50

D. Limit Method 
SAMPLE NAME: D4311102 (Continued)

Sample ID: 100362

Constituent

RQ AQ B Result

Total Metals

$\mathbf{J}$
$\mathbf{U}$
$\mathbf{U}$
$\mathbf{U}$

Silver

$\begin{array}{llll}34 & \mathrm{mg} / \mathrm{kg} & 1.0 & \text { EPA6010 } \\ 2.5 & \mathrm{mg} / \mathrm{kg} & 2.5 & \text { EPA6010 } \\ 8.0 & \mathrm{mg} / \mathrm{kg} & 0.59 & \text { EPA7421 } \\ 110 & \mathrm{mg} / \mathrm{kg} & 1.5 & \text { EPA6010 } \\ 0.11 & \mathrm{mg} / \mathrm{kg} & 0.11 & \text { EPA7470 } \\ 4.1 & \mathrm{mg} / \mathrm{kg} & 4.1 & \text { EPA6010 } \\ 0.49 & \mathrm{mg} / \mathrm{kg} & 0.49 & \text { EPA7740 } \\ 1.0 & \mathrm{mg} / \mathrm{kg} & 1.0 & \text { EPA6010 }\end{array}$

$B / N / A$ Extractables (including PAH and phenols)

Acenaphthene
Acenaphthylene
Anthracene
Benzo(a)anthracene
Benzo(b)fluoranthene
Benzo(k)fluoranthene
Benzoic acid
Benzo(g,h.i)perylene
Benzo(a)pyrene
Benzyl alcohol
Bis(2-chloroethoxy) methane
Bis(2-chloroethyl) ether
Bis(2-chloroisopropyl) ether
Bis(2-ethylhexyl) phthalate
4-Bromophenyl phenyl ether
Butylbenzyl phthalate
4-Chloroaniline
4-Chloro-m-cresol
2-Chloronaphthalene
2-Chlorophenol
4-Chlorophenyl phenyl ether
Chrysene
o-Cresol (2-Methylphenol)
p-Cresol (4-Methylphenol)
Dibenz(a,h)anthracene
Dibenzofuran
Di-n-butyl phthalate
1,2-Dichlorobenzene
1,3-Dichlorobenzene
1,4-Dichlorobenzene
3,3'-Dichlorobenzidine
2.4-Dichlorophenol
Diethyl phthalate
2,4-Dimethyl phenol
Dimethyl phthalate
2,4-Dinitrophenol
2,4-Dinitrotoluene
2,6-Dinitrotoluene
Di-n-octyl phthalate

350
Unit

D. Limit Method 
SAMPLE NAME: D431 1102 (Continued)

Sample ID: 100362
Constituent
RQ AQ
B Result
Unit
D. Limit Method.

$B / N / A$ Extractables (including PAH and phenols)

Fluoranthene
Fiuorene
Hexachlorobenzene
Hexachlorobutadiene
Hexachlorocyclopentadiene
Hexachloroethane
Indeno(1,2,3-c,d)pyrene
Isophorone
2-Methyl-4,6-dinitrophenol
2-Methylnaphthalene
Naphthalene
o-Nitroaniline
m-Nitroaniline
p-Nitroaniline
Nitrobenzene
2-Nitrophenol
4-Nitrophenol
N-Nitrosodiphenylamine
N-Nitrosodipropylamine
Pentachlorophenol
Phenanthrene
Phenol
Pyrene
1,2,4-Trichlorobenzene
2,4,5-Trichlorophenol
2,4,6-Trichlorophenol
Unknown arom. hydrocarbon
Unknown ketone

$\mathrm{U}$

$\mathrm{U}-350$

$\mathrm{U} \quad 350$

U 350

U 350

$\mathrm{U}-350$

$\mathrm{U} \quad 350$

U $\quad \cdot 350$

U 1,800

U 350

U 350

U $\quad 1,800$

U $\quad 1,800$

U $\quad 1,800$

$\mathrm{U} \quad 350$

$\mathrm{U} \quad 350$

U $\quad 1,800$

$\mathrm{U} \quad 350$

$\mathrm{U} \quad 350$

UJ C $\quad 1,800$

U 350

UJ C 350

U $\quad 350$

U $\quad 350$

$\mathrm{U} \quad 1,800$

$\mathrm{U} \quad 350$

J $\quad \mathbf{N} 200$

J NV 7,000

Volatile Organic Compounds

Acetone

Benzene

Bromodichloromethane

Bromoform

Bromomethane (Methyl bromide)

Carbon disulfide

Carbon tetrachloride

Chlorobenzene -

Chloroethane

Chloroethene (Vinyl chloride)

Chloroform

Chloromethane (Methyl chloride)

Dibromochloromethane

1,1-Dichloroethane

1,2-Dichloroethane

1,1-Dichloroethylene

1,2-Dichloroethylene

Dichloromethane (Methylene chloride)

1,2-Dichloropropane

\begin{tabular}{|c|c|c|c|c|}
\hline $\mathrm{v}$ & 14 & $\mu \mathrm{g} / \mathrm{kg}$ & 11 & EPA8240 \\
\hline V & 5.4 & $\mu \mathrm{g} / \mathrm{kg}$ & 5.4 & EPA8240 \\
\hline U & 5.4 & $\mu \mathrm{g} / \mathrm{kg}$ & 5.4 & EPA8240 \\
\hline $\mathrm{U}$ & 5.4 & $\mu \mathrm{g} / \mathrm{kg}$ & 5.4 & EPA8240 \\
\hline $\mathrm{U}$ & 11 & $\mu \mathrm{g} / \mathrm{kg}$ & 11 & EPA8240 \\
\hline$U$ & 5.4 & $\mu \mathrm{g} / \mathrm{kg}$ & 5.4 & EPA8240 \\
\hline$U$ & 5.4 & $\mu \mathrm{g} / \mathrm{kg}$ & 5.4 & EPA8240 \\
\hline $\mathbf{U}$ & 5.4 & $\mu \mathrm{g} / \mathrm{kg}$ & 5.4 & EPA8240 \\
\hline $\mathbb{U}$ & 11 & $\mu g / k g$ & 11 & EPA8240 \\
\hline U & 11 & $\mu \mathrm{g} / \mathrm{kg}$ & 11 & EPA8240 \\
\hline $\mathbf{U}$ & 5.4 & $\mu \mathrm{g} / \mathrm{kg}$ & 5.4 & EPA8240 \\
\hline I & 11 & $\mu \mathrm{g} / \mathrm{kg}$ & 11 & EPA8240 \\
\hline 0 & 5.4 & $\mu \mathrm{g} / \mathrm{kg}$ & 5.4 & EPA8240 \\
\hline $\mathbf{U}$ & 5.4 & $\mu \mathrm{g} / \mathrm{kg}$ & 5.4 & EPA8240 \\
\hline U & 5.4 & $\mu \mathrm{g} / \mathrm{kg}$ & 5.4 & EPA8240 \\
\hline & 5.4 & $\mu \mathrm{g} / \mathrm{kg}$ & 5.4 & EPA8240 \\
\hline & 5.4 & $\mu \mathrm{g} / \mathrm{kg}$ & 5.4 & EPA8240 \\
\hline v & 13 & $\mu \mathrm{g} / \mathrm{kg}$ & 5.4 & EPA8240 \\
\hline & 5.4 & & 5 & EPA8240 \\
\hline
\end{tabular}

$\begin{array}{lll}\mu \mathrm{g} / \mathrm{kg} & 350 & \text { EPA8270 } \\ \mu \mathrm{g} / \mathrm{kg} & 350 & \text { EPA8270 } \\ \mu \mathrm{g} / \mathrm{kg} & 350 & \text { EPA8270 } \\ \mu \mathrm{g} / \mathrm{kg} & 350 & \text { EPA8270 } \\ \mu \mathrm{g} / \mathrm{kg} & 350 & \text { EPA8270 } \\ \mu \mathrm{g} / \mathrm{kg} & 350 & \text { EPA8270 } \\ \mu \mathrm{g} / \mathrm{kg} & 350 & \text { EPA8270 } \\ \mu \mathrm{g} / \mathrm{kg} & 350 & \text { EPA8270 } \\ \mu \mathrm{g} / \mathrm{kg} & 1,800 & \text { EPA8270 } \\ \mu \mathrm{g} / \mathrm{kg} & 350 & \text { EPA8270 } \\ \mu \mathrm{g} / \mathrm{kg} & 350 & \text { EPA8270 } \\ \mu \mathrm{g} / \mathrm{kg} & 1,800 & \text { EPA8270 } \\ \mu \mathrm{g} / \mathrm{kg} & 1,800 & \text { EPA8270 } \\ \mu \mathrm{g} / \mathrm{kg} & 1,800 & \text { EPA8270 } \\ \mu \mathrm{g} / \mathrm{kg} & 350 & \text { EPA8270 } \\ \mu \mathrm{g} / \mathrm{kg} & 350 & \text { EPA8270 } \\ \mu \mathrm{g} / \mathrm{kg} & 1,800 & \text { EPA8270 } \\ \mu \mathrm{g} / \mathrm{kg} & 350 & \text { EPA8270 } \\ \mu \mathrm{g} / \mathrm{kg} & 350 & \text { EPA8270 } \\ \mu \mathrm{g} / \mathrm{kg} & 1,800 & \text { EPA8270 } \\ \mu \mathrm{g} / \mathrm{kg} & 350 & \text { EPA8270 } \\ \mu \mathrm{g} / \mathrm{kg} & 350 & \text { EPA8270 } \\ \mu \mathrm{g} / \mathrm{kg} & 350 & \text { EPA8270 } \\ \mu \mathrm{g} / \mathrm{kg} & 350 & \text { EPA8270 } \\ \mu \mathrm{g} / \mathrm{kg} & 1,800 & \text { EPA8270 } \\ \mu \mathrm{g} / \mathrm{kg} & 350 & \text { EPA8270 } \\ \mu \mathrm{g} / \mathrm{kg} & & \text { EPA8270 } \\ \mu \mathrm{g} / \mathrm{kg} & & \text { EPA8270 }\end{array}$


SAMPLE NAME: D431-1102 (Continued)

\section{Constituent}

Volatile Organic Compounds

cis-1,3-Dichloropropene

trans-1,3-Dichloropropene

Ethylbenzene

2-Hexanone

Methyl ethyl ketone

Methyl isobutyl ketone

Styrene

1,1,2,2-Tetrachloroethane

Tetrachloroethylene

Tolvene

1,1,1-Trichloroethane

1.1.2-Trichloroethane

Trichloroethylene

Vinyl acetate

Xylenes
Sample D: 100362

RQ AQ B Result

5.4

5.4

5.4

11

11

11

5.4

5.4

5.4

5.4

5.4

5.4

5.4

11

5.4

Pesticides/PCBs and Dioxins/Furans

Aldrin

alpha-Benzene hexachloride

beta-Benzene hexachloride

delta-Benzene hexachloride

alpha-Chlordane

gamma-Chlordane

P.P'-DDD

p. $\mathrm{P}^{\prime}-\mathrm{DDE}$

p.p'-DDT

Dieldrin

Endosulfan I

Endosulfan II

Endosulfan sulfate

Endrin

Endrin ketone

Heptachlor

Heptachlor epoxide

Heptachlorodibenzo-p-dioxin isomers

Heptachlorodibenzo-p-furan isomers

Hexachloradibenzo-p-dioxin isomers

Hexachlorodibenzo-p-furan isomers

Lindane

Methoxychlor

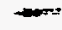

Octachlorodibenzo-p-dioxin

Octachlorodibenzo-p-furan

PCB 1016

PCB 1221

PCB 1232

PCB 1242

PCB 1248

PCB 1254

PCB 1260
$\mathrm{U}$

U

U

U

I

J

J

J

$\mathrm{U}$

U

J

U

J

U

U

U

U

U

$\mathrm{U}$

U

U

U

U

U

$\mathrm{U}$

$\mathrm{U}$

U

U
1.8

1.8

1.8

1.8

0.35

0.71

1.4

2.8

5.0

3.5

1.8

1.8

3.5

5.0

1.4

1.8

1.8

0.30

0.20

0.20

0.10

1.8

18

2.2

0.20

35

71

35

35

35

35

35
Unit

D. Limit Method
5.4

5.4

5.4

11

11

11

5.4

5.4

5.4

5.4

5.4

5.4

5.4

11

5.4
EPA8240

EPA8240

EPA8240

EPA8240

EPA8240

EPA8240

EPA8240

EPA8240

EPA8240

EPA8240

EPA8240

EPA8240

EPA8240

EPA8240

EPA8240

$\mu \mathrm{g} / \mathrm{kg}$

$\begin{array}{lll}\mu \mathrm{g} / \mathrm{kg} & 1.8 & \text { EPA8080 } \\ \mu \mathrm{g} / \mathrm{kg} & 1.8 & \text { EPA8080 } \\ \mu \mathrm{g} / \mathrm{kg} & 1.8 & \text { EPA8080 } \\ \mu \mathrm{g} / \mathrm{kg} & 1.8 & \text { EPA8080 } \\ \mu \mathrm{g} / \mathrm{kg} & 1.8 & \text { EPA8080 } \\ \mu \mathrm{g} / \mathrm{kg} & 1.8 & \text { EPA8080 } \\ \mu \mathrm{g} / \mathrm{kg} & 3.5 & \text { EPA8080 } \\ \mu \mathrm{g} / \mathrm{kg} & 3.5 & \text { EPA8080 } \\ \mu \mathrm{g} / \mathrm{kg} & 3.5 & \text { EPA8080 } \\ \mu \mathrm{g} / \mathrm{kg} & 3.5 & \text { EPA8080 } \\ \mu \mathrm{g} / \mathrm{kg} & 1.8 & \text { EPA8080 } \\ \mu \mathrm{g} / \mathrm{kg} & 3.5 & \text { EPA8080 } \\ \mu \mathrm{g} / \mathrm{kg} & 3.5 & \text { EPA8080 } \\ \mu \mathrm{g} / \mathrm{kg} & 3.5 & \text { EPA8080 } \\ \mu \mathrm{g} / \mathrm{kg} & 3.5 & \text { EPA8080 } \\ \mu \mathrm{g} / \mathrm{kg} & 1.8 & \text { EPA8080 } \\ \mu \mathrm{g} / \mathrm{kg} & 1.8 & \text { EPA8080 } \\ \mathrm{ng} / \mathrm{g} & 0.30 & \text { EPA8280 } \\ \mathrm{ng} / \mathrm{g} & 0.20 & \text { EPA8280 } \\ \mathrm{ng} / \mathrm{g} & 0.20 & \text { EPA8280 } \\ \mathrm{ng} / \mathrm{g} & 0.10 & \text { EPA8280 } \\ \mu \mathrm{g} / \mathrm{kg} & 1.8 & \text { EPA8080 } \\ \mu \mathrm{g} / \mathrm{kg} & 18 & \text { EPA8080 } \\ \mathrm{ng} / \mathrm{g} & 0.10 & \text { EPA8280 } \\ \mathrm{ng} / \mathrm{g} & 0.20 & \text { EPA8280 } \\ \mu \mathrm{g} / \mathrm{kg} & 35 & \text { EPA8080 } \\ \mu \mathrm{g} / \mathrm{kg} & 71 & \text { EPA8080 } \\ \mu \mathrm{g} / \mathrm{kg} & 35 & \text { EPA8080 } \\ \mu \mathrm{g} / \mathrm{kg} & 35 & \text { EPA8080 } \\ \mu \mathrm{g} / \mathrm{kg} & 35 & \text { EPA8080 } \\ \mu \mathrm{g} / \mathrm{kg} & 35 & \text { EPA8080 } \\ \mu \mathrm{g} / \mathrm{kg} & 35 & \text { EPA8080 }\end{array}$


SAMPLE NAME: D431"1102 (Continued)

Constituent

$$
*
$$

Pesticides/PCBs and Dioxins/Furans

Pentachlorodibenzo-p-dioxin isomers U

Pentachlorodibenzo-p-furan isomers U

2,3,7,8-TCDD

2,3,7,8-TCDF

Tetrachlorodibenzo-p-dioxin isomers

Tetrachlorodibenzo-p-furan isomers

Toxaphene
Sample ID: 100362

RQ AQ B Result Unit D. Limit Method

\section{Radionuclide Indicators and Radionuclides}

Gross alpha

Nonvolatile beta

Radium, cotal alpha-emitting

Tritium

$\begin{array}{lllll} & 16 \pm 14 & \mathrm{pCi} / \mathrm{g} & 7.3 & \text { EPA900.0 } \\ & 16 \pm 19 & \mathrm{pCi} / \mathrm{g} & 13 & \text { EPA900.0 } \\ \mathrm{U} \cdot \mathrm{V} & 1.1 \pm 0.62 & \mathrm{pCi} / \mathrm{g} & 0.13 & \text { EPA903.0 } \\ \mathrm{U} & 2.0 & \mathrm{pCi} / \mathrm{g} & 2.0 & \text { EPA906.0 }\end{array}$

SAMPLE NAME: D431 1102D

Sample Type: Duplicate

\section{Constituent}

Total Metals

Arsenic
Barium
Cadmium
Chromium
Copper
Lead
Manganese
Mercury
Nickel
Selenium
Silver

$B / N / A$ Extractables (including $P A H$ and phenols)

$\begin{array}{llll}\text { U } & & 0.81 & \mathrm{mg} / \mathrm{kg} \\ \mathrm{U} & & 21 & \mathrm{mg} / \mathrm{kg} \\ \mathrm{UJ} & \mathrm{L} & 0.51 & \mathrm{mg} / \mathrm{kg} \\ \mathrm{J} & \mathrm{L} & 39 & \mathrm{mg} / \mathrm{kg} \\ \mathrm{U} & & 2.6 & \mathrm{mg} / \mathrm{kg} \\ & & 2.5 & \mathrm{mg} / \mathrm{kg} \\ & & 130 & \mathrm{mg} / \mathrm{kg} \\ \mathrm{U} & & 0.11 & \mathrm{mg} / \mathrm{kg} \\ \mathrm{U} & & 4.1 & \mathrm{mg} / \mathrm{kg} \\ \mathrm{U} & & 0.41 & \mathrm{mg} / \mathrm{kg} \\ \mathrm{U} & & 1.0 & \mathrm{mg} / \mathrm{kg}\end{array}$

U

U

U

U

U

U

U

U

U

$\mathbf{U}$

U

360
360
360
360
360
360
1,800
360
360
360
360
360

$\mu g / \mathrm{kg}$ $\mu \mathrm{g} / \mathrm{kg}$ $\mu \mathrm{g} / \mathrm{kg}$ $\mu \mathrm{g} / \mathrm{kg}$ $\mu \mathrm{g} / \mathrm{kg}$ $\mu g / k g$ $\mu g / k g$ $\mu \mathrm{g} / \mathrm{kg}$ $\mu g / \mathrm{kg}$ $\mu \mathrm{g} / \mathrm{kg}$ $\mu \mathrm{g} / \mathrm{kg}$ $\mu \mathrm{g} / \mathrm{kg}$
Sample ID: 100363

Associated Sample: 100362
EPA8280
EPA8280
EPA8280
EPA8280
EPA8280
EPA8280
EPA8080
0.20

0.20

0.20

0.20

180

EPA906.0

Acenaphthene
Acenaphthylene
Anthracene
Benzo(a)anthracene
Benzo(b)fluoranthene
Benzo(k)fluoranthene
Benzoic acid
Benzo(g,h,i)perylene
Benzo(a)pyrene
Benzyl alcohol
Bis(2-chloroethoxy) methane
Bis(2-chloroethyl) ether

Bis(2-chloroethyl) ether
D. Limit Method

$\begin{array}{ll}0.81 & \text { EPA7060 } \\ 21 & \text { EPA6010 } \\ 0.51 & \text { EPA6010 } \\ 1.0 & \text { EPA6010 } \\ 2.6 & \text { EPA6010 } \\ 0.24 & \text { EPA7421 } \\ 1.5 & \text { EPA6010 } \\ 0.11 & \text { EPA7470 } \\ 4.1 & \text { EPA6010 } \\ 0.41 & \text { EPA7740 } \\ 1.0 & \text { EPA6010 }\end{array}$<smiles>[13CH]</smiles>

360

360

360

360

360

360

1,800

360

360

360

360

360
EPA8270

EPA8270

EPA8270

EPA 8270

EPA8270

EPA8270

EPA8270

EPA8270

EPA8270

EPA8270

EPA8270

EPA8270 
Constituent

$\mathbf{R Q} \mathbf{A Q} \mathbf{B} \quad$ Result

Unit

D. Limit Method

$B / N / A$ Extractables (including $P A H$ and phenols)

Bis(2-chloroisopropyl) ether

Bis(2-ethylhexyl) phthalate

4-Bromophenyl phenyl ether

Butylbenzyl phthalate :

4-Chloroaniline

4-Chloro-m-cresol

2-Chloronaphthalene

2-Chlorophenol

4-Chlorophenyl phenyl ether

Chrysene

o-Cresol (2-Methylphenol)

p-Cresol (4-Methylphenol)

Dibenz(a.h)anthracene

Dibenzofuran

Di-n-butyl phthalate

1,2-Dichlorobenzene

1,3-Dichlorobenzene

1,4-Dichlorobenzene

3.3'-Dichlorobenzidine

2.4-Dichlorophenol

Diethyl phthalate

2,4-Dimethyl phenol

Dimethyl phthalate

2,4-Dinitrophenol

2,4-Dinitrotoluene

2,6-Dinitrotoluene

Di-n-octyl phthalate

Fiuoranthene

Fluorene

Hexachlorobenzene

Hexachlorobutadiene

Hexachlorocyclopentadiene

Hexachloroethane

Indeno(1,2,3-c,d)pyrene

Isophorone

2-Methyl-4,6-dinitrophenol

2-Methylnaphthalene

Naphthalene

o-Nitroaniline

m-Nitroaniline

p-Nitroaniline

Nitrobenzene

2-Nitrophenol

4-Nitrophenol

N-Nitrosodiphenylamine

$\mathrm{N}$-Nitrosodipropylamine

Pentachlorophenol

Phenanthrene

Phenol

Pyrene
$\mathrm{U}$

360

U 360

U 360

U 360

U 360

$U \quad 360$

360

U 360

U 360

U 360

$U \quad 360$

U 360

U 360

UJ $\quad \mathrm{V} \quad 120$

U 360

U 360

$\mathrm{U} \quad 360$

U $\quad 710$

$\mathrm{U}$

$\mathrm{U}$

$\mathrm{U} \quad 360$

U 360

$\mathrm{U} \quad 1,800$

$U \quad 360$

U 360

$\mathrm{U}$

$\mathrm{U} \quad 360$

$\mathrm{U} \quad 360$

U 360

U 360

U 360

$U \quad 360$

$\mathrm{U} \quad 360$

U 360

$\mathrm{U} \quad 1.800$

U 360

$\mathrm{U} \quad 360$

$U \quad 1,800$

1,800

1,800

360

360

1,800

360

360

UJ C $\quad 1,800$

U C 360

UJ C 360

U 360

\begin{tabular}{|c|c|c|}
\hline$\mu g / k g$ & 360 & EPA8270 \\
\hline$\mu \mathrm{g} / \mathrm{kg}$ & 360 & EPA8270 \\
\hline$\mu g / k g$ & 360 & EPA8270 \\
\hline$\mu g / k g$ & 360 & EPA8270 \\
\hline$\mu \mathrm{g} / \mathrm{kg}$ & 360 & EPA8270 \\
\hline$\mu \mathrm{g} / \mathrm{kg}$ & 360 & EPA8270 \\
\hline$\mu \mathrm{g} / \mathrm{kg}$ & 360 & EPA8270 \\
\hline$\mu \mathrm{g} / \mathrm{kg}$ & 360 & EPA8270 \\
\hline$\mu \mathrm{g} / \mathrm{kg}$ & 360 & EPA8270 \\
\hline$\mu g / k g$ & 360 & EPA8270 \\
\hline $\mathrm{Hg} / \mathrm{kg}$ & 360 & EPA8270 \\
\hline$\mu g / k g$ & 360 & EPA8270 \\
\hline$\mu g / k g$ & 360 & EPA8270 \\
\hline$\mu \mathrm{g} / \mathrm{kg}$ & 360 & EPA8270 \\
\hline$\mu \mathrm{g} / \mathrm{kg}$ & 360 & EPA 8270 \\
\hline$\mu \mathrm{g} / \mathrm{kg}$ & 360 & EPA8270 \\
\hline$\mu \mathrm{g} / \mathrm{kg}$ & 360 & EPA 8270 \\
\hline$\mu \mathrm{g} / \mathrm{kg}$ & 360 & EPA 8270 \\
\hline$\mu \mathrm{g} / \mathrm{kg}$ & 710 & EPA8270 \\
\hline$\mu g / k g$ & 360 & EPA8270 \\
\hline$\mu \mathrm{g} / \mathrm{kg}$ & 360 & EPA8270 \\
\hline$\mu \mathrm{g} / \mathrm{kg}$ & 360 & EPA 8270 \\
\hline$\mu \mathrm{g} / \mathrm{kg}$ & 360 & EPA 8270 \\
\hline$\mu \mathrm{g} / \mathrm{kg}$ & 1.800 & EPA 8270 \\
\hline$\mu \mathrm{g} / \mathrm{kg}$ & 360 & EPA8270 \\
\hline$\mu \mathrm{g} / \mathrm{kg}$ & 360 & EPA8270 \\
\hline$\mu g / k g$ & 360 & EPA8270 \\
\hline$\mu g / k g$ & 360 & EPA 8270 \\
\hline$\mu g / \mathrm{kg}$ & 360 & EPA8270 \\
\hline$\mu g / k g$ & 360 & EPA 8270 \\
\hline$\mu \mathrm{g} / \mathrm{kg}$ & 360 & EPA 8270 \\
\hline$\mu \mathrm{g} / \mathrm{kg}$ & 360 & EPA8270 \\
\hline$\mu \mathrm{g} / \mathrm{kg}$ & 360 & EPA 8270 \\
\hline$\mu g / \mathrm{kg}$ & 360 & EPA8270 \\
\hline$\mu \mathrm{g} / \mathrm{kg}$ & 360 & EPA8270 \\
\hline$\mu g / \mathrm{kg}$ & 1,800 & EPA 8270 \\
\hline$\mu \mathrm{g} / \mathrm{kg}$ & 360 & EPA8270 \\
\hline$\mu \mathrm{g} / \mathrm{kg}$ & 360 & EPA8270 \\
\hline$\mu \mathrm{g} / \mathrm{kg}$ & 1,800 & EPA8270 \\
\hline$\mu g / k g$ & 1.800 & EPA8270 \\
\hline$\mu g / k g$ & 1,800 & EPA8270 \\
\hline$\mu g / \mathrm{kg}$ & 360 & EPA8270 \\
\hline$\mu \mathrm{g} / \mathrm{kg}$ & 360 & EPA8270 \\
\hline$\mu \mathrm{g} / \mathrm{kg}$ & 1,800 & EPA8270 \\
\hline$\mu \mathrm{g} / \mathrm{kg}$ & 360 & EPA 8270 \\
\hline$\mu \mathrm{g} / \mathrm{kg}$ & 360 & EPA8270 \\
\hline$\mu \mathrm{g} / \mathrm{kg}$ & 1,800 & EPA 8270 \\
\hline$\mu \mathrm{g} / \mathrm{kg}$ & 360 & EPA 8270 \\
\hline$\mu \mathrm{g} / \mathrm{kg}$ & 360 & EPA8270 \\
\hline$\mu g / k g$ & 360 & EPA 8270 \\
\hline
\end{tabular}




\section{Constituent}

RQ AQ B Result . Unit

D. Limit Method

$B / N / A$ Extractables (including $P A H$ and phenols)

1,2,4-Trichlorobenzene

2,4,5-Trichlorophenol

2,4,6-Trichlorophenol .

Unknown hydrocarbon

Unknown ketone

$\begin{array}{lll}\mathbf{U} & & 360 \\ \mathrm{U} & & 1,800 \\ \mathbf{U} & & 360 \\ \mathrm{~J} & \mathrm{~N} & 200 \\ \mathrm{~J} & \mathrm{NV} & 2,000\end{array}$

$\begin{array}{lll}\mu g / \mathrm{kg} & 360 & \text { EPA8270 } \\ \mu \mathrm{g} / \mathrm{kg} & 1,800 & \text { EPA8270 } \\ \mu \mathrm{g} / \mathrm{kg} & 360 & \text { EPA8270 } \\ \mu \mathrm{g} / \mathrm{kg} & & \text { EPA8270 } \\ \mu \mathrm{g} \cdot \mathrm{kg} & & \text { EPA8270 }\end{array}$

Volatile Organic Compounds

Ácetone

Benzene

Bromodichloromethane

Bromoform

Bromomethane (Methyl bromide)

Carbon disulfide

Carbon tetrachloride

Chlorobenzene

Chloroethane

Chloroethene (Vinyl chloride)

Chloroform

Chloromethane (Methyl chloride)

Dibromochloromethane

1,1-Dichloroethane

1,2-Dichloroethane

1,1-Dichloroethylene

1,2-Dichloroethylene

Dichloromethane (Methylene chloride)

1,2-Dichloropropane

cis-1,3-Dichloropropene

trans-1,3-Dichloropropene

Ethylbenzene

2-Hexanone

Methyl ethyl ketone

Methyl isobutyl ketone

Styrene

1,1,2,2-Tetrachloroethane

Tetrachloroethylene

Toluene

1,1,1-Trichloroethane

1,1,2-Trichloroethane_...

Trichloroethylene -

Vinyl acetate

v

Xylenes

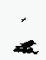

\section{3}

5.4

5.4

5.4

11

5.4

5.4

5.4

11

11

5.4

11

5.4

5.4

5.4

5.4

5.4

12

5.4

5.4

5.4

5.4

11

11

11

5.4

5.4

5.4

5.4

5.5

5.4

11

5.4

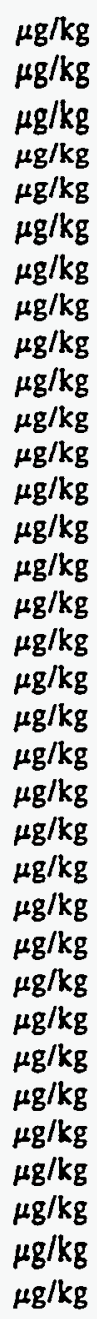

$\mu \mathrm{g} / \mathrm{kg}$

$\mu \mathrm{g} / \mathrm{kg}$

$\mu \mathrm{g} / \mathrm{kg}$

$\mu \mathrm{g} / \mathrm{kg}$

$\mu \mathrm{g} / \mathrm{kg}$

$\mu \mathrm{g} / \mathrm{kg}$

$\mu \mathrm{g} / \mathrm{kg}$

$\mu \mathrm{g} / \mathrm{kg}$

$\mu \mathrm{g} / \mathrm{kg}$

$\mu \mathrm{g} / \mathrm{kg}$

$\mu \mathrm{g} / \mathrm{kg}$

$\mu \mathrm{g} / \mathrm{kg}$

$\mu \mathrm{g} / \mathrm{kg}$

$\mu \mathrm{g} / \mathrm{kg}$

$\mu g / k g$

$\mu \mathrm{g} / \mathrm{kg}$

$\mu \mathrm{g} / \mathrm{kg}$

$\mu \mathrm{g} / \mathrm{kg}$

$\mu \mathrm{g} / \mathrm{kg}$

$\mu \mathrm{g} / \mathrm{kg}$

$\mu \mathrm{g} / \mathrm{kg}$

$\mu \mathrm{g} / \mathrm{kg}$

$\mu \mathrm{g} / \mathrm{kg}$

$\mu g / \mathrm{kg}$

$\mu \mathrm{g} / \mathrm{kg}$

$\mu \mathrm{g} / \mathrm{kg}$

$\mu \mathrm{g} / \mathrm{kg}$

$\mu g / k g$

$\mu \mathrm{g} / \mathrm{kg}$

$\mu \mathrm{g} / \mathrm{kg}$

$\mu \mathrm{g} / \mathrm{kg}$

$\mu \mathrm{g} / \mathrm{kg}$

$\mu \mathrm{g} / \mathrm{kg}$
11

5.4

5.4

5.4

11

5.4

5.4

5.4

11

11

5.4

11

5.4

5.4

5.4

5.4

5.4

5.4

5.4

5.4

5.4

5.4

11

11

11

5.4

5.4

5.4

5.4

5.4

5.4

5.4

11

5.4
EPA8240

EPA8240

EPA8240

EPA8240

EPA8240

EPA8240

EPA8240

EPA8240

EPA8240

EPA8240

EPA8240

EPA8240

EPA8240

EPA8240

EPA8240

EPA8240

EPA8240

EPA8240

EPA8240

EPA8240

EPA8240

EPA8240

EPA8240

EPA8240

EPA8240

EPA8240

EPA8240

EPA8240

EPA8240

EPA8240

EPA8240

EPA8240

EPA8240

EPA8240

Pesticides/PCBs and Dioxins/Furans

Aldrin

alpha-Benzene hexachloride

beta-Benzene hexachloride

delta-Benzene hexachloride

alpha-Chlordane
$\mathrm{U}$

U

U

U

J
1.8

1.8

1.8

1.8

0.35 $\mu g / k g$

$\mu \mathrm{g} / \mathrm{kg}$

$\mu \mathrm{g} / \mathrm{kg}$

$\mu g / k g$

$\mu \mathrm{g} / \mathrm{kg}$

\section{8}

1.8

1.8

1.8

1.8
EPA8080

EPA8080

EPA8080

EPA8080

EPA8080 
SAMPLE NAME: D43i 1102D (Continued)

Sample ID: 100363

\section{Constituent}

RQ AQ B Result Unit

D. Limit Method

Pesticides/PCBs and Dioxins/Furans

gamma-Chlordane

p.p'-DDD

p.p'-DDE

p.p'-DDT

Dieldrin

Endosulfan I

Endosulfan II

Endosulfan sulfate

Endrin

Endrin ketone

Heptachlor

Heptachlor epoxide

Heptachlorodibenzo-p-dioxin isomers

Heptachlorodibenzo-p-dioxin isomers

Heptachlorodibenzo-p-furan isomers

Heptachlorodibenzo-p-furan isomers

Hexachlorodibenzo-p-dioxin isomers

Hexachlorodibenzo-p-dioxin isomers

Hexachlorodibenzo-p-furan isomers

Hexachlorodibenzo-p-furan isomers

Lindane

Methoxychlor

Octachlorodibenzo-p-dioxin

Octachlorodibenzo-p-dioxin

Octachlorodibenzo-p-furan

Octachlorodibenzo-p-furan

PCB 1016

PCB 1221

PCB 1232

PCB 1242

PCB 1248

PCB 1254

PCB 1260

Pentachlorodibenzo-p-dioxin isomers

Pentachlorodibenzo-p-furan isomers

Pentachlorodibenzo-p-dioxin isomers

Pentachlorodibenzo-p-furan isomers

2,3,7,8-TCDD

2,3,7,8-TCDD

2,3,7,8-TCDF

2,3,7,8-TCDF

Tetrachlorodibenzo-p-dioxin isomers

Tetrachlorodibenzo-p-dioxin isomers

Terrachlorodibenzo-p-furan isomers

Tetrachlorodibenzo-p-furan isomers

Toxaphene
J

s

U

U

$\mathrm{U}$

$\mathrm{U}$

J

U

U

U

U

U

$\mathrm{U}$

U

$\mathrm{U}$

U

U

U

J

U

U

U

U

U

$U$

U

U

U

U

U

U

U

U

U

U

U

U

U

U

U

U

\begin{tabular}{llll} 
& & & \\
0.70 & $\mu \mathrm{g} / \mathrm{kg}$ & 1.8 & EPA8080 \\
2.1 & $\mu \mathrm{g} / \mathrm{kg}$ & 3.5 & EPA8080 \\
2.5 & $\mu \mathrm{g} / \mathrm{kg}$ & 3.5 & EPA8080 \\
4.9 & $\mu \mathrm{g} / \mathrm{kg}$ & 3.5 & EPA8080 \\
3.5 & $\mu \mathrm{g} / \mathrm{kg}$ & 3.5 & EPA8080 \\
1.8 & $\mu \mathrm{g} / \mathrm{kg}$ & 1.8 & EPA8080 \\
3.5 & $\mu \mathrm{g} / \mathrm{kg}$ & 3.5 & EPA8080 \\
3.5 & $\mu \mathrm{g} / \mathrm{kg}$ & 3.5 & EPA8080 \\
4.2 & $\mu \mathrm{g} / \mathrm{kg}$ & 3.5 & EPA8080 \\
1.8 & $\mu \mathrm{g} / \mathrm{kg}$ & 3.5 & EPA8080 \\
1.8 & $\mu \mathrm{g} / \mathrm{kg}$ & 1.8 & EPA8080 \\
1.8 & $\mu \mathrm{g} / \mathrm{kg}$ & 1.8 & EPA8080 \\
0.20 & $\mathrm{ng} / \mathrm{g}$ & 0.20 & EPA8280 \\
0.10 & $\mathrm{ng} / \mathrm{g}$ & 0.10 & EPA8280 \\
0.10 & $\mathrm{ng} / \mathrm{g}$ & 0.10 & EPA8280 \\
0.10 & $\mathrm{ng} / \mathrm{g}$ & 0.10 & EPA8280 \\
0.10 & $\mathrm{ng} / \mathrm{g}$ & 0.10 & EPA8280 \\
0.10 & $\mathrm{ng} / \mathrm{g}$ & 0.10 & EPA8280 \\
0.10 & $\mathrm{ng} / \mathrm{g}$ & 0.10 & EPA8280 \\
0.10 & $\mathrm{ng} / \mathrm{g}$ & 0.10 & EPA8280 \\
1.8 & $\mu \mathrm{g} / \mathrm{kg}$ & 1.8 & EPA8080 \\
18 & $\mu \mathrm{g} / \mathrm{kg}$ & 18 & EPA8080 \\
0.70 & $\mathrm{ng} / \mathrm{g}$ & 0.10 & EPA8280 \\
0.60 & $\mathrm{ng} / \mathrm{g}$ & 0.20 & EPA8280 \\
0.10 & $\mathrm{ng} / \mathrm{g}$ & 0.10 & EPA8280 \\
0.10 & $\mathrm{ng} / \mathrm{g}$ & 0.10 & EPA8280 \\
35 & $\mu \mathrm{g} / \mathrm{kg}$ & 35 & EPA8080 \\
70 & $\mu \mathrm{g} / \mathrm{kg}$ & 70 & EPA8080 \\
35 & $\mu \mathrm{g} / \mathrm{kg}$ & 35 & EPA8080 \\
35 & $\mu \mathrm{g} / \mathrm{kg}$ & 35 & EPA8080 \\
35 & $\mu \mathrm{g} / \mathrm{kg}$ & 35 & EPA8080 \\
35 & $\mu \mathrm{g} / \mathrm{kg}$ & 35 & EPA8080 \\
35 & $\mu \mathrm{g} / \mathrm{kg}$ & 35 & EPA8080 \\
0.10 & $\mathrm{ng} / \mathrm{g}$ & 0.10 & EPA8280 \\
0.10 & $\mathrm{ng} / \mathrm{g}$ & 0.10 & EPA8280 \\
0.10 & $\mathrm{ng} / \mathrm{g}$ & 0.10 & EPA8280 \\
0.10 & $\mathrm{ng} / \mathrm{g}$ & 0.10 & EPA8280 \\
0.10 & $\mathrm{ng} / \mathrm{g}$ & 0.10 & EPA8280 \\
0.10 & $\mathrm{ng} / \mathrm{g}$ & 0.10 & EPA8280 \\
0.10 & $\mathrm{ng} / \mathrm{g}$ & 0.10 & EPA8280 \\
0.10 & $\mathrm{ng} / \mathrm{g}$ & 0.10 & EPA8280 \\
0.10 & $\mathrm{ng} / \mathrm{g}$ & 0.10 & EPA8280 \\
0.10 & $\mathrm{ng} / \mathrm{g}$ & 0.10 & EPA8280 \\
0.10 & $\mathrm{ng} / \mathrm{g}$ & 0.10 & EPA8280 \\
$\mathrm{ng} / \mathrm{g}$ & 0.10 & EPA8280 \\
\hline 10 & $\mu \mathrm{g} / \mathrm{kg}$ & 180 & EPA8080
\end{tabular}


SAMPLE NAME: D431 1102D (Continued)

\section{Constituent}

\section{$\mathbf{R Q}$}

Sample ID: 100363

$\begin{array}{llllll}\text { RQ AQ } & \text { B } & \text { Result } & \text { Unit } & \text { D. Limit } & \text { Method } \\ \text { clides } & & & & & \\ & & & & & \\ & & 27 \pm 17 & \mathrm{pCi} / \mathrm{g} & 7.6 & \text { EPA990.0 } \\ \mathrm{U} & \mathrm{V} & 54 \pm 24 & \mathrm{pCi} / \mathrm{g} & 14 & \text { EPA900.0 } \\ \mathrm{U} & & 1.2 \pm 0.70 & \mathrm{pCi} / \mathrm{g} & 0.15 & \text { EPA903.0 } \\ & & 1.0 & \mathrm{pCi} / \mathrm{g} & 1.0 & \text { EPA906.0 }\end{array}$

Radionuclide Indicators and Radionuclides

Gross alpha

Nonvolatile beta

Radium, total alpha-emitting

Tritium

Sample ID: 100364

Sample Matrix: Soil

USC Soil Classification: SM

RQ AQ B Result Unit

0.87

21
Barium

Cadmium

Chromium

Copper

Lead

Manganese

Mercury

Nickel

Selenium

Silver

Volatile Organic Compounds

U

U

UJ L

J L

U

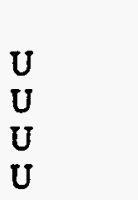

U

Benzene

Bromodichloromethane

Bromoform

Bromomethane (Methyl bromide)

Carbon disulfide

Carbon tetrachloride

Chlorobenzene

Chloroethane

Chloroethene (Vinyl chloride)

Chloroform

Chloromethane (Methyl chloride)

Dibromochloromethane

1,1-Dichloroethane

1,2-Dichloroethane

1,1-Dichloroethylene

1,2-Dichloroethylene

Dichloromethane (Methylene chloride)
U

U

U

U

U

U

$\mathrm{U}$

U

U

U

U

U

U

U

U V

\section{$\mathrm{V} \quad 54$}

$\mathrm{V} \quad 4.7$

4.7
5.5

5.5

11

5.5

5.5

5.5

11

11

5.5

11

5.5

5.5

7.1

5.5

5.5

14
Sample Moisture: Dry Percent Solids: 90.40

D. Limit Method

\begin{tabular}{|c|c|c|}
\hline $\mathrm{mg} / \mathrm{kg}$ & 0.87 & EPA7060 \\
\hline $\mathrm{mg} / \mathrm{kg}$ & 21 & EPA 6010 \\
\hline $\mathrm{mg} / \mathrm{kg}$ & 0.52 & EPA6010 \\
\hline $\mathrm{mg} / \mathrm{kg}$ & 1.0 & EPA6010 \\
\hline $\mathrm{mg} / \mathrm{kg}$ & 2.6 & EPA6010 \\
\hline $\mathrm{mg} / \mathrm{kg}$ & 0.26 & EPA7421 \\
\hline $\mathrm{mg} / \mathrm{kg}$ & . 1.6 & EPA6010 \\
\hline $\mathrm{mg} / \mathrm{kg}$ & 0.11 & EPA7470 \\
\hline $\mathrm{mg} / \mathrm{kg}$ & 4.2 & EPA6010 \\
\hline $\mathrm{mg} / \mathrm{kg}$ & 0.43 & EPA7740 \\
\hline $\mathrm{mg} / \mathrm{kg}$ & 1.0 & EPA6010 \\
\hline
\end{tabular}

$\mu \mathrm{g} / \mathrm{kg}$

$\mu g / \mathrm{kg}$

$\mu \mathrm{g} / \mathrm{kg}$

$\mu g / \mathrm{kg}$

$\mu \mathrm{g} / \mathrm{kg}$

$\mu \mathrm{g} / \mathrm{kg}$

$\mu \mathrm{g} / \mathrm{kg}$

$\mu \mathrm{g} / \mathrm{kg}$

$\mu \mathrm{g} / \mathrm{kg}$

$\mu \mathrm{g} / \mathrm{kg}$

$\mu \mathrm{g} / \mathrm{kg}$

$\mu \mathrm{g} / \mathrm{kg}$

$\mu \mathrm{g} / \mathrm{kg}$

$\mu \mathrm{g} / \mathrm{kg}$

$\mu g / \mathrm{kg}$

$\mu g / \mathrm{kg}$

$\mu \mathrm{g} / \mathrm{kg}$

$\mu \mathrm{g} / \mathrm{kg}$

$\begin{array}{ll}11 & \text { EPA8240 } \\ 5.5 & \text { EPA8240 } \\ 5.5 & \text { EPA8240 } \\ 5.5 & \text { EPA8240 } \\ 11 & \text { EPA8240 } \\ 5.5 & \text { EPA8240 } \\ 5.5 & \text { EPA8240 } \\ 5.5 & \text { EPA8240 } \\ 11 & \text { EPA8240 } \\ 11 & \text { EPA8240 } \\ 5.5 & \text { EPA8240 } \\ 11 & \text { EPA8240 } \\ 5.5 & \text { EPA8240 } \\ 5.5 & \text { EPA8240 } \\ 5.5 & \text { EPA8240 } \\ 5.5 & \text { EPA8240 } \\ 5.5 & \text { EPA8240 } \\ 5.5 & \text { EPA8240 }\end{array}$


SAMPLE NAME: D431-1103 (Continued)

Sample ID: 100364

Constituent

RQ AQ B Result

Unit

D. Limit Method

Volatile Organic Compounds

$\begin{array}{ll}\text { 1,2-Dichloropropane } & \\ \text { cis-1,3-Dichloropropene } & \\ \text { trans-1,3-Dichloropropene } & \\ \text { Ethylbenzene } & \\ \text { 2-Hexanone } & \\ \text { Methyl ethyl ketone } & \\ \text { Methyl isobutyl ketone } & \\ \text { Styrene } & \\ \text { 1,1,2,2-Tetrachloroethane } & \\ \text { Tetrachloroethylene } & \\ \text { Toluene } & \\ \text { 1,1,1-Trichloroethane } & \\ \text { 1,1,2-Trichloroethane } & \\ \text { Trichloroethylene } & U \\ \text { Vinyl acetate } & \\ \text { Xylenes } & U\end{array}$

Radionuclide Indicators and Radionuclides

\begin{tabular}{|c|c|c|c|c|c|c|}
\hline $\begin{array}{l}\text { Gross alpha } \\
\text { Nonvolatile beta } \\
\text { Radium, total alpha-emitting } \\
\text { Tritium }\end{array}$ & $\mathrm{U}$ & V & $\begin{array}{l}6.9 \\
32 \pm 19 \\
1.1 \pm 0.65 \\
2.0 \pm 2.5\end{array}$ & $\begin{array}{l}\mathrm{pCi} / \mathrm{g} \\
\mathrm{PCi} / \mathrm{g} \\
\mathrm{PCi} / \mathrm{g} \\
\mathrm{pCi} / \mathrm{g}\end{array}$ & $\begin{array}{l}6.9 \\
13 \\
0.13 \\
1.4\end{array}$ & $\begin{array}{l}\text { EPA900.0 } \\
\text { EPA900.0 } \\
\text { EPA903.0 } \\
\text { EPA906.0 }\end{array}$ \\
\hline
\end{tabular}

SAMPLE NAME: D431 1104

Location (SRS Coordinates): $18558.54 \mathrm{E}$ 66554.39N

Ground Elevation Above MSL: $134.6 \mathrm{ft}$

Depth of Core Interval: 4.00 to $6.00 \mathrm{ft}$

Sample Type: Normal

Sample Color: 10YR 6/2

Constituent

Total Metals

Arsenic

Barium

Cadmium

Chromium

Copper

Lead

Manganese

Mercury

Nickel

Selenium

Silver

\begin{tabular}{|c|c|c|c|}
\hline 5.5 & $\mu g / k g$ & 5.5 & EPA8240 \\
\hline 5.5 & $\mu g / k g$ & 5.5 & EPA8240 \\
\hline 5.5 & $\mu g / k g$ & 5.5 & EPA8240 \\
\hline 5.5 & $\mu g / k g$ & 5.5 & EPA8240 \\
\hline 11 & $\mu g / \mathrm{kg}$ & 11 & EPA8240 \\
\hline 11 & $\mu \mathrm{g} / \mathrm{kg}$ & 11 & EPA8240 \\
\hline 11 & $\mu \mathrm{g} / \mathrm{kg}$ & 11 & EPA8240 \\
\hline 5.5 & $\mu \mathrm{g} / \mathrm{kg}$ & 5.5 & EPA8240 \\
\hline 5.5 & $\mu g / k g$ & 5.5 & EPA8240 \\
\hline 5.5 & $\mu g / k g$ & 5.5 & EPA8240 \\
\hline 5.5 & $\mu g / \mathrm{kg}$ & 5.5 & EPA8240 \\
\hline 5.5 & $\mu g / k g$ & 5.5 & EPA8240 \\
\hline 6.2 & $\mu g / \mathrm{kg}$ & 5.5 & EPA8240 \\
\hline 5.5 & $\mu g / \mathrm{kg}$ & 5.5 & EPA8240 \\
\hline 11 & $\mu g / \mathrm{kg}$ & 11 & EPA8240 \\
\hline 5.5 & $\mu g / \mathrm{kg}$ & 5.5 & EPA 8240 \\
\hline
\end{tabular}

$\begin{array}{lll}U & 5.5 & \mu \mathrm{g} / \mathrm{kg} \\ \mathrm{U} & 5.5 & \mu \mathrm{g} / \mathrm{kg} \\ \mathrm{U} & 5.5 & \mu \mathrm{g} / \mathrm{kg} \\ \mathrm{U} & 5.5 & \mu \mathrm{g} / \mathrm{kg} \\ \mathrm{U} & 11 & \mu \mathrm{g} / \mathrm{kg} \\ \mathrm{U} & 11 & \mu \mathrm{g} / \mathrm{kg} \\ \mathrm{U} & 11 & \mu \mathrm{g} / \mathrm{kg} \\ \mathrm{U} & 5.5 & \mu \mathrm{g} / \mathrm{kg} \\ \mathrm{U} & 5.5 & \mu \mathrm{g} / \mathrm{kg} \\ \mathrm{U} & 5.5 & \mu \mathrm{g} / \mathrm{kg} \\ \mathrm{U} & 5.5 & \mu \mathrm{g} / \mathrm{kg} \\ \mathrm{U} & 5.5 & \mu \mathrm{g} / \mathrm{kg} \\ & 6.2 & \mu \mathrm{g} / \mathrm{kg} \\ \mathrm{U} & 5.5 & \mu \mathrm{g} / \mathrm{kg} \\ \mathrm{U} & 11 & \mu \mathrm{g} / \mathrm{kg} \\ \mathrm{U} & 5.5 & \mu \mathrm{g} / \mathrm{kg}\end{array}$

Sample ID: 100365

Sample Matrix: Soil

USC Soil Classification: SM

Sample Moisture: Damp

Percent Solids: 90.00

RQ AQ B Result Unit

D. Limit Method

$\begin{array}{lll}\mathrm{mg} / \mathrm{kg} & 0.92 & \text { EPA7060 } \\ \mathrm{mg} / \mathrm{kg} & 17 & \text { EPA6010 } \\ \mathrm{mg} / \mathrm{kg} & 0.41 & \text { EPA6010 } \\ \mathrm{mg} / \mathrm{kg} & 0.82 & \text { EPA6010 } \\ \mathrm{mg} / \mathrm{kg} & 2.1 & \text { EPA6010 } \\ \mathrm{mg} / \mathrm{kg} & 0.28 & \text { EPA7421 } \\ \mathrm{mg} / \mathrm{kg} & 1.2 & \text { EPA6010 } \\ \mathrm{mg} / \mathrm{kg} & 0.11 & \text { EPA7470 } \\ \mathrm{mg} / \mathrm{kg} & 3.3 & \text { EPA6010 } \\ \mathrm{mg} / \mathrm{kg} & 0.46 & \text { EPA7740 } \\ \mathrm{mg} / \mathrm{kg} & 0.82 & \text { EPA6010 }\end{array}$


SAMPLE NAME: D43ï 1104 (Continued)

Sample ID: 100365

$\begin{array}{lllll}\text { Constituent } & \text { RQ AQ B Result Unit - D. Limit Method }\end{array}$

$B / N / A$ Extractables (including $P A H$ and phenols)

\begin{tabular}{|c|c|c|c|c|c|c|}
\hline Acenaphthene & $\mathrm{U}$ & & 370 & $\mu g / k g$ & 370 & EPA8270 \\
\hline Acenaphthylene & $\mathbf{U}$ & & 370 & $\mu \mathrm{g} / \mathrm{kg}$ & 370 & EPA8270 \\
\hline Anthracene & $\mathbf{U}$ & & 370 & $\mu g / \mathrm{kg}$ & 370 & EPA8270 \\
\hline Benzo(a)anthracene & $\mathbf{U}$ & . & 370 & $\mu g / k g$ & 370 & EPA8270 \\
\hline Benzo(b)fluoranthene & $\mathbf{U}$ & & 370 & $\mu g / \mathrm{kg}$ & 370 & EPA8270 \\
\hline Benzo(k)fluoranthene & $U$ & & 370 & $\mu g / \mathrm{kg}$ & 370 & EPA8270 \\
\hline Benzoic acid & $\mathbf{U}$ & & 1,900 & $\mu \mathrm{g} / \mathrm{kg}$ & 1,900 & EPA8270 \\
\hline Benzo(g,h,i)perylene & $\mathbf{U}$ & & 370 & $\mu \mathrm{g} / \mathrm{kg}$ & 370 & EPA8270 \\
\hline Benzo(a)pyrene & $\mathrm{U}$ & & 370 & $\mu g / k g$ & 370 & EPA8270 \\
\hline Benzyl alcohol & $\mathbf{U}$ & & 370 & $\mu \mathrm{g} / \mathrm{kg}$ & 370 & EPA8270 \\
\hline Bis(2-chloroethoxy) methane & U & & 370 & $\mu g / \mathrm{kg}$ & 370 & EPA8270 \\
\hline Bis(2-chloroethyl) ether & $\mathbf{U}$ & & 370 & $\mu \mathrm{g} / \mathrm{kg}$ & 370 & EPA8270 \\
\hline Bis(2-chloroisopropyl) ether & $\mathbf{U}$ & & 370 & $\mu g / k g$ & 370 & EPA8270 \\
\hline Bis(2-ethylhexyl) phthalate & $\mathbf{J}$ & & 48 & $\mu \mathrm{g} / \mathrm{kg}$ & 370 & EPA8270 \\
\hline 4-Bromophenyl phenyl ether & $\mathrm{U}$ & & 370 & $\mu \mathrm{g} / \mathrm{kg}$ & 370 & EPA8270 \\
\hline Butylbenzyl phthalate & $\mathbf{U}$ & & 370 & $\mu \mathrm{g} / \mathrm{kg}$ & 370 & EPA8270 \\
\hline Alkylbenzene $\mathrm{C} 10 \mathrm{H} 14$ & $\mathbf{J}$ & $\mathbf{N}$ & 200 & $\mu \mathrm{g} / \mathrm{kg}$ & & EPA8270 \\
\hline Alkylbenzene $\mathrm{ClOH} 14$ & J & $\mathrm{N}$ & 200 & $\mu g / k g$ & & EPA8270 \\
\hline Hydrocarbon $\mathrm{C} 13 \mathrm{H} 28$ & J & $\mathbf{N}$ & 300 & $\mu g / \mathrm{kg}$ & & EPA8270 \\
\hline Hydrocarbon $\mathrm{C} 16 \mathrm{H} 34$ & J & $\mathbf{N}$ & 100 & $\mu \mathrm{g} / \mathrm{kg}$ & & EPA8270 \\
\hline Hydrocarbon $\mathrm{C} 2 \mathrm{OH} 42$ & J & $\mathbf{N}$ & 200 & $\mu g / \mathrm{kg}$ & & EPA8270 \\
\hline 4-Chloroaniline & $\mathbf{U}$ & & 370 & $\mu \mathrm{g} / \mathrm{kg}$ & 370 & EPA8270 \\
\hline 4-Chloro-m-cresol & $\mathbf{U}$ & & 370 & $\mu \mathrm{g} / \mathrm{kg}$ & 370 & EPA8270 \\
\hline 2-Chloronaphthalene & $\mathrm{U}$ & & 370 & $\mu g / \mathrm{kg}$ & 370 & EPA8270 \\
\hline 2-Chlorophenol & $\mathrm{U}$ & & 370 & $\mu \mathrm{g} / \mathrm{kg}$ & 370 & EPA8270 \\
\hline 4-Chlorophenyl phenyl ether & $\boldsymbol{U}$ & & 370 & $\mu \mathrm{g} / \mathrm{kg}$ & 370 & EPA8270 \\
\hline Chrysene & U & & 370 & $\mu \mathrm{g} / \mathrm{kg}$ & 370 & EPA8270 \\
\hline o-Cresol (2-Methylphenol) & U & & 370 & $\mu \mathrm{g} / \mathrm{kg}$ & 370 & EPA8270 \\
\hline p-Cresol (4-Methylphenol) & $\mathrm{U}$ & & 370 & $\mu g / \mathrm{kg}$ & 370 & EPA8270 \\
\hline Dibenz $(a, h)$ anthracene & $\mathrm{U}$ & & 370 & $\mu \mathrm{g} / \mathrm{kg}$ & 370 & EPA8270 \\
\hline Dibenzofuran ${ }^{\circ}$ & $\mathbf{U}$ & & 370 & $\mu \mathrm{g} / \mathrm{kg}$ & 370 & EPA8270 \\
\hline Di-n-butyl phthalate & UJ & $\mathrm{V}$ & 150 & $\mu \mathrm{g} / \mathrm{kg}$ & 370 & EPA8270 \\
\hline 1,2-Dichlorobenzene & $\mathrm{U}$ & & 370 & $\mu \mathrm{g} / \mathrm{kg}$ & 370 & EPA8270 \\
\hline 1,3-Dichlorobenzene & U & & 370 & $\mu \mathrm{g} / \mathrm{kg}$ & 370 & EPA8270 \\
\hline 1.4-Dichlorobenzene & $\mathrm{U}$ & & 370 & $\mu \mathrm{g} / \mathrm{kg}$ & 370 & EPA8270 \\
\hline 3,3'-Dichlorobenzidine & $\mathbf{U}$ & & 750 & $\mu \mathrm{g} / \mathrm{kg}$ & 750 & EPA8270 \\
\hline 2,4-Dichlorophenol & $\mathbf{U}$ & & 370 & $\mu \mathrm{g} / \mathrm{kg}$ & 370 & EPA8270 \\
\hline Diethyl phthalate & $\mathbf{U}$ & & 370 & $\mu \mathrm{g} / \mathrm{kg}$ & 370 & EPA8270 \\
\hline 2.4-Dimethyl phenol & $\mathrm{U}$ & & 370 & $\mu \mathrm{g} / \mathrm{kg}$ & 370 & EPA8270 \\
\hline Dimethyl phthalate - & $\mathrm{U}$ & & 370 & $\mu g / k g$ & 370 & EPA8270 \\
\hline 2,4-Dinitrophenol - & $\mathbf{U}$ & & 1,900 & $\mu \mathrm{g} / \mathrm{kg}$ & 1,900 & EPA8270 \\
\hline 2,4-Dinitrotoluene & $\mathbf{U}$ & & 370 & $\mu \mathrm{g} / \mathrm{kg}$ & 370 & EPA8270 \\
\hline 2,6-Dinitrotoluene & $\mathbf{U}$ & & 370 & $\mu \mathrm{g} / \mathrm{kg}$ & 370 & EPA8270 \\
\hline Di-n-octyl phthalate & $\mathbf{U}$ & & 370 & $\mu \mathrm{g} / \mathrm{kg}$ & 370 & EPA8270 \\
\hline Fluoranthene & $\mathrm{U}$ & & 370 & $\mu \mathrm{g} / \mathrm{kg}$ & 370 & EPA8270 \\
\hline Fluorene & $\mathrm{U}$ & & 370 & $\mu \mathrm{g} / \mathrm{kg}$ & 370 & EPA8270 \\
\hline Hexachlorobenzene & $\mathrm{U}$ & & 370 & $\mu \mathrm{g} / \mathrm{kg}$ & 370 & EPA8270 \\
\hline Hexachlorobutadiene & $\mathbf{U}$ & & 370 & $\mu \mathrm{g} / \mathrm{kg}$ & 370 & EPA8270 \\
\hline Hexachlorocyclopentadiene & $\mathbf{U}$ & & 370 & $\mu \mathrm{g} / \mathrm{kg}$ & 370 & EPA8270 \\
\hline Hexachloroethane & $\mathbf{U}$ & & 370 & $\mu \mathrm{g} / \mathrm{kg}$ & 370 & EPA8270 \\
\hline
\end{tabular}


SAMPLE NAME: D431 1104 (Continued)

Constituent

RQ AQ B Result

$B / N / A$ Extractables (including $P A H$ and phenols)
Sample ID: 100365

Unit

D. Limit Method

Indeno(1,2,3-c,d)pyrene
Isophorone
2-Methyl-4,6-dinitrophenol
2-Methylnaphthalene
Naphthalene
o-Nitroaniline
m-Nitroaniline
p-Nitroaniline
Nitrobenzene
2-Nitrophenol
4-Nitrophenol
N-Nitrosodiphenylamine
N-Nitrosodipropylamine
Pentachlorophenol
Phenanthrene
Phenol
Pyrene
1,2.4-Trichlorobenzene
2.4.5-Trichlorophenol
2.4.6-Trichlorophenol
Unknown arom. ketone
Unknown hydrocarbon
Unknown hydrocarbon
Unknown hydrocarbon
Unknown hydrocarbon
Unknown hydrocarbon
Unknown
Unknown
Unknown
Unknown
Unknown
Unknown ketone

Volatile Organic Compounds

\begin{tabular}{|c|c|c|}
\hline $\mathbf{U}$ & & 370 \\
\hline U & & 370 \\
\hline U & & 1,900 \\
\hline $\mathbf{U}$ & & 370 \\
\hline $\mathbf{U}$ & & 370 \\
\hline $\mathbf{U}$ & & 1,900 \\
\hline $\mathbf{U}$ & & 1,900 \\
\hline U & & 1,900 \\
\hline $\mathbf{U}$ & & 370 \\
\hline U & & 370 \\
\hline U & & 1,900 \\
\hline U & & 370 \\
\hline $\mathrm{U}$ & & 370 \\
\hline UJ & c & 1,900 \\
\hline $\mathrm{J}$ & & 40 \\
\hline UJ & C & 370 \\
\hline $\mathbf{U}$ & & 370 \\
\hline$U$ & & 370 \\
\hline $\mathrm{U}$ & & 1,900 \\
\hline $\mathbf{U}$ & & 370 \\
\hline J & $N$ & 200 \\
\hline $\mathrm{J}$ & $\mathrm{N}$ & 200 \\
\hline J & $N$ & 200 \\
\hline$J$ & $N$ & 400 \\
\hline$J$ & $\mathrm{~N}$ & 200 \\
\hline J & $\mathrm{N}$ & 600 \\
\hline J & $\mathrm{N}$ & 100 \\
\hline$J$ & $N$ & 400 \\
\hline$J$ & $\mathrm{~N}$ & 300 \\
\hline J & $\mathrm{N}$ & 500 \\
\hline $\mathrm{J}$ & $N$ & 600 \\
\hline J & NV & 8,000 \\
\hline
\end{tabular}

EPA8270

EPA8270

EPA8270

EPA8270

EPA8270

EPA8270

EPA8270

EPA8270

EPA8270

EPA8270

EPA8270

EPA8270

EPA.8270

EPA8270

EPA8270

EPA8270

EPA8270

EPA8270

EPA8270

EPA8270

EPA8270

EPA8270

EPA8270

EPA8270

EPA8270

EPA8270

EPA8270

EPA8270

EPA8270

EPA8270

EPA8270

EPA8270

Acetone
Subst. alkylbenzene
Subst. alkylbenzene
Subst. alkylbenzene
Subst. alkylbenzene
Subst. alkylbenzene
Benzene
Bromodichloromethane
Bromoform
Bromomethane (Methyl bromide)
Carbon disulfide
Carbon tetrachloride
Chlorobenzene
Chloroethane
Chloroethene (Vinyl chloride)

Acetone

Subst, alkylbenzene

ylbenzene

Benzene

Bromodichloromethane

Bromomethane (Methyl bromide)

Chlorobenzene

Chloroethene (Vinyl chloride)
32

$\begin{array}{lll}\mu \mathrm{g} / \mathrm{kg} & 11 & \begin{array}{l}\text { EPA8240 } \\ \mu \mathrm{g} / \mathrm{kg}\end{array} \\ \mu \mathrm{g} / \mathrm{kg} & & \text { EPA8240 } \\ \mu \mathrm{g} / \mathrm{kg} & & \text { EPA8240 } \\ \mu \mathrm{g} / \mathrm{kg} & & \text { EPA8240 } \\ \mu \mathrm{g} / \mathrm{kg} & . & \text { EPA8240 } \\ \mu \mathrm{g} / \mathrm{kg} & 5.7 & \text { EPA8240 } \\ \mu \mathrm{g} / \mathrm{kg} & 5.7 & \text { EPA8240 } \\ \mu \mathrm{g} / \mathrm{kg} & 5.7 & \text { EPA8240 } \\ \mu \mathrm{g} / \mathrm{kg} & 11 & \text { EPA8240 } \\ \mu \mathrm{g} / \mathrm{kg} & 5.7 & \text { EPA8240 } \\ \mu \mathrm{g} / \mathrm{kg} & 5.7 & \text { EPA8240 } \\ \mu \mathrm{g} / \mathrm{kg} & 5.7 & \text { EPA8240 } \\ \mu \mathrm{g} / \mathrm{kg} & 11 & \text { EPA8240 } \\ \mu \mathrm{g} / \mathrm{kg} & 11 & \text { EPA8240 }\end{array}$


SAMPLE NAME: D431 1104 (Continued)

Constituent

Volatile Organic Compounds

Chloroform

Chloromethane (Methyl chloride)

Dibromochloromethane

1,1-Dichloroethane

1,2-Dichloroethane

1,1-Dichlorocthylene .

1,2-Dichloroethylene

Dichloromethane (Methylene chloride)

1,2-Dichloropropane

cis-1,3-Dichloropropene

trans-1,3-Dichloropropene

Diethylbenzene

Diethylbenzene

Ethylbenzene

2-Hexanone

Trimethyibenzene

Trimethylbenzene

Methylpropylbenzene

Methyl ethyl ketone

Methyl isobutyl ketone

Styrene

1,1,2,2-Tetrachloroethane

Tetrachloroethylene

Toluene

1,1,1-Trichloroethane

1,1,2-Trichloroethane

Trichloroethylene

Vinyl acetate

Xylenes

U

U

U

U

U

$\mathrm{U}$

$\mathrm{U}$

U

U

U

U

U

U

U

U

U

U

U

U

U
Sample ID: 100365

RQ AQ B Result Unit $\quad$ D. Limit Method

\section{Pesticides/PCBs and Dioxins/Furans}

Aldrin

alpha-Benzene hexachloride

beta-Benzene hexachloride

delta-Benzene hexachloride

alpha-Chlordane

gamma-Chlordane

p.p'-DDD

p.p'-DDE

p.p'-DDT

Dieldrin

Endosulfan I

Endosulfan II

Endosulfan sulfate

Endrin

Endrin ketone

Heptachlor

Heptachlor epoxide

Heprachlorodibenzo-p-dioxin isomers

U

J $\quad \mathbf{N}$

J $\quad N$

U

J $\quad \mathbf{N}$

J $N$

$v$

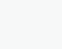

N

N

\section{7}

11

5.7

5.7

6.7

5.7

5.7

14

5.7

5.7

5.7

20

20

5.7

11

20

10

10

11

11

5.7

5.7

5.7

5.7

5.7

5.9

5.7

11

5.7

$$
\begin{aligned}
& \mu g / k g \\
& \mu g / k g \\
& \mu g / k g \\
& \mu g / k g \\
& \mu g / k g \\
& \mu g / k g \\
& \mu g / k g \\
& \mu g / k g \\
& \mu g / k g \\
& \mu g / k g \\
& \mu g / k g \\
& \mu g / k g \\
& \mu g / k g \\
& \mu g / k g \\
& \mu g / k g \\
& \mu g / k g \\
& \mu g / k g \\
& \mu g / k g \\
& \mu g / k g \\
& \mu g / k g \\
& \mu g / k g \\
& \mu g / k g \\
& \mu g / k g \\
& \mu g / k g \\
& \mu g / k g \\
& \mu g / k g \\
& \mu g / k g \\
& \mu g / k g \\
& \mu g / k g
\end{aligned}
$$

$\mu \mathrm{g} / \mathrm{kg}$

$\mu \mathrm{g} / \mathrm{kg}$

$\mu \mathrm{g} / \mathrm{kg}$

$\mu \mathrm{g} / \mathrm{kg}$

$\mu \mathrm{g} / \mathrm{kg}$

$\mu g / \mathrm{kg}$

$\mu \mathrm{g} / \mathrm{kg}$

$\mu \mathrm{g} / \mathrm{kg}$

$\mu \mathrm{g} / \mathrm{kg}$

$\mu \mathrm{g} / \mathrm{kg}$

$\mu \mathrm{g} / \mathrm{kg}$

$\mu \mathrm{g} / \mathrm{kg}$

$\mu \mathrm{g} / \mathrm{kg}$

$\mu \mathrm{g} / \mathrm{kg}$

$\mu \mathrm{g} / \mathrm{kg}$

$\mu g / k g$

$\mu \mathrm{g} / \mathrm{kg}$

$\mathrm{ng} / \mathrm{g}$
5.7

5.7

5.7 EPA8240

5.7 EPA8240

5.7 EPA8240

5.7 EPA8240

5.7 EPA8240

5.7 EPA8240

5.7 EPA8240

5.7 EPA8240

EPA8240

EPA8240

5.7 EPA8240

11 EPA8240

EPA8240

EPA8240

EPA8240

11 EPA8240

11 EPA8240

5.7 EPA8240

5.7 EPA8240

5.7 EPA8240

5.7 EPA8240

5.7 EPA8240

5.7 EPA8240

5.7 EPA8240

11 EPA8240

5.7 EPA8240

$\begin{array}{ll}19 & \text { EPA8080 } \\ 19 & \text { EPA8080 } \\ 19 & \text { EPA8080 } \\ 19 & \text { EPA8080 } \\ 19 & \text { EPA8080 } \\ 19 & \text { EPA8080 } \\ 38 & \text { EPA8080 } \\ 38 & \text { EPA8080 } \\ 38 & \text { EPA8080 } \\ 38 & \text { EPA8080 } \\ 19 & \text { EPA8080 } \\ 38 & \text { EPA8080 } \\ 38 & \text { EPA8080 } \\ 38 & \text { EPA8080 } \\ 38 & \text { EPA8080 } \\ 19 & \text { EPA8080 } \\ 19 & \text { EPA8080 } \\ 0.10 & \text { EPA8280 }\end{array}$


SAMPLE NAME: D431-1104 (Continued)

Constituent

$\mathbf{R Q} \quad \mathbf{A Q}$

Sample ID: 100365

Pesticides/PCBs and Dioxins/Furans

$\begin{array}{ll}\text { Heptachlorodibenzo-p-dioxin isomers } & \\ \text { Heptachlorodibenzo-p-furan isomers } & \\ \text { Heptachlorodibenzo-p-furan isomers } & \mathrm{J} \\ \text { Hexachlorodibenzo-p-dioxin isomers } & \\ \text { Hexachlorodibenzo-p-furan isomers } & \\ \text { Lindane } & \\ \text { Methoxychlor } & \\ \text { Octachlorodibenzo-p-dioxin } & \\ \text { Octachlorodibenzo-p-furan } & \\ \text { PCB 1016 } & \\ \text { PCB 1221 } & \\ \text { PCB 1232 } & \\ \text { PCB 1242 } & \mathrm{U} \\ \text { PCB 1248 } & \mathrm{U} \\ \text { PCB 1254 } & \mathrm{U} \\ \text { PCB 1260 } & \mathrm{U} \\ \text { Pentachlorodibenzo-p-dioxin isomers } & \mathrm{U} \\ \text { Pentachlorodibenzo-p-furan isomers } & \mathrm{U} \\ \text { Pentachlorodibenzo-p-dioxin isomers } & \mathrm{U} \\ \text { Pentachlorodibenzo-p-furan isomers } & \mathrm{U} \\ \text { 2,3,7,8-TCDD } & \mathrm{U} \\ \text { 2.3.7.8-TCDF } & \mathrm{U} \\ \text { Tetrachlorodibenzo-p-dioxin isomers } & \text { Tetrachlorodibenzo-p-furan isomers } \\ \text { Toxaphene } & \mathrm{U}\end{array}$

Radionuclide Indicators and Radionuclides

Gross alpha

Nonvolatile beta

Radium, total alpha-emitting

Tritium

\begin{tabular}{|c|c|}
\hline $\mathbf{U}$ & 0.20 \\
\hline U & 0.10 \\
\hline J & 0.60 \\
\hline $\mathbf{U}$ & 0.20 \\
\hline $\mathbf{U}$ & 0.10 \\
\hline U & 19 \\
\hline $\mathbf{U}$ & $\begin{array}{l}190 \\
2.9\end{array}$ \\
\hline $\mathbf{U}$ & 0.20 \\
\hline $\mathbf{U}$ & 380 \\
\hline$U$ & 750 \\
\hline $\mathbf{U}$ & 380 \\
\hline U & 380 \\
\hline $\mathrm{U}$ & 380 \\
\hline $\mathbf{U}$ & 380 \\
\hline $\mathrm{U}$ & 380 \\
\hline U & 1.5 \\
\hline $\mathbf{U}$ & 0.20 \\
\hline U & 0.60 \\
\hline $\mathbf{U}$ & 0.20 \\
\hline $\mathrm{U}$ & 0.10 \\
\hline $\mathbf{U}$ & 0.10 \\
\hline U & 0.20 \\
\hline $\mathbf{U}$ & 0.20 \\
\hline U & 1,900 \\
\hline
\end{tabular}

Unit

D. Limit Method

$\begin{array}{llll}0.20 & \mathrm{ng} / \mathrm{g} & 0.20 & \text { EPA8280 } \\ 0.10 & \mathrm{ng} / \mathrm{g} & 0.10 & \text { EPA8280 } \\ 0.60 & \mathrm{ng} / \mathrm{g} & 0.10 & \text { EPA8280 } \\ 0.20 & \mathrm{ng} / \mathrm{g} & 0.20 & \text { EPA8280 } \\ 0.10 & \mathrm{ng} / \mathrm{g} & 0.10 & \text { EPA8280 } \\ 19 & \mu \mathrm{g} / \mathrm{kg} & 19 & \text { EPA8080 } \\ 190 & \mu \mathrm{g} / \mathrm{kg} & 190 & \text { EPA8080 } \\ 2.9 & \mathrm{ng} / \mathrm{g} & 0.10 & \text { EPA8280 } \\ 0.20 & \mathrm{ng} / \mathrm{g} & 0.20 & \text { EPA8280 } \\ 380 & \mu \mathrm{g} / \mathrm{kg} & 380 & \text { EPA8080 } \\ 750 & \mu \mathrm{g} / \mathrm{kg} & 750 & \text { EPA8080 } \\ 380 & \mu \mathrm{g} / \mathrm{kg} & 380 & \text { EPA8080 } \\ 380 & \mu \mathrm{g} / \mathrm{kg} & 380 & \text { EPA8080 } \\ 380 & \mu \mathrm{g} / \mathrm{kg} & 380 & \text { EPA8080 } \\ 380 & \mu \mathrm{g} / \mathrm{kg} & 380 & \text { EPA8080 } \\ 380 & \mu \mathrm{g} / \mathrm{kg} & 380 & \text { EPA8080 } \\ 1.5 & \mathrm{ng} / \mathrm{g} & 1.5 & \text { EPA8280 } \\ 0.20 & \mathrm{ng} / \mathrm{g} & 0.20 & \text { EPA8280 } \\ 0.60 & \mathrm{ng} / \mathrm{g} & 0.60 & \text { EPA8280 } \\ 0.20 & \mathrm{ng} / \mathrm{g} & 0.20 & \text { EPA8280 } \\ 0.10 & \mathrm{ng} / \mathrm{g} & 0.10 & \text { EPA8280 } \\ 0.10 & \mathrm{ng} / \mathrm{g} & 0.10 & \text { EPA8280 } \\ 0.20 & \mathrm{ng} / \mathrm{g} & 0.20 & \text { EPA8280 } \\ 0.20 & \mathrm{ng} / \mathrm{g} & 0.20 & \text { EPA8280 } \\ 1.900 & \mu \mathrm{g} / \mathrm{kg} & 1,900 & \text { EPA8080 }\end{array}$

SAMPLE NAME: D431 1105

Location (SRS Coordinates): 18558.54E 66554.39N

Ground Elevation Above MSL: $134.6 \mathrm{ft}$

Depth of Core Interval: 6.00 to $8.00 \mathrm{ft}$

Sample Type: Normal

Sample Color: 10YR 42 .

Constituent

Total Metals

Arsenic

Barium

Cadmium

Chromium

$\mathrm{U}$
$\mathrm{U}$
$\mathrm{U}$

$\begin{array}{ll}7.5 & \mathrm{pCi} / \mathrm{g} \\ 14 & \mathrm{pCi} / \mathrm{g} \\ 1.0 \pm 0.62 & \mathrm{pCi} / \mathrm{g} \\ 1.9 \pm 2.4 & \mathrm{pCi} / \mathrm{g}\end{array}$

Sample ID: 100366

Sample Matrix: Soil

USC Soil Classification: SM

RQ AQ B Result Unit

$\begin{array}{ll}1.8 & \mathrm{mg} / \mathrm{kg} \\ 23 & \mathrm{mg} / \mathrm{kg} \\ 0.57 & \mathrm{mg} / \mathrm{kg} \\ 96 & \mathrm{mg} / \mathrm{kg}\end{array}$

$\begin{array}{ll}7.5 & \text { EPA900.0 } \\ 14 & \text { EPA900.0 } \\ 0.14 & \text { EPA903.0 } \\ 1.3 & \text { EPA906.0 }\end{array}$

$\therefore$

Sample Moisture: Wet Percent Solids: 87.20

D. Limit Method

$\begin{array}{ll}1.1 & \text { EPA7060 } \\ 23 & \text { EPA6010 } \\ 0.57 & \text { EPA6010 } \\ 1.1 & \text { EPA6010 }\end{array}$


SAMPLE NAME: D4311105 (Continued)

Constituent

Total Metals

Copper

Lead

Manganese

Mercury

Nickel

Selenium

Silver
Sample ID: 100366

RQ AQ B Result Unit $\quad$ D. Limit Method

$B / N / A$ Extractables (including $P A H$ and phenols)

\begin{tabular}{|c|c|c|c|c|c|c|}
\hline Acenaphthene & $\mathbf{U}$ & & 370 & $\mu g / \mathrm{kg}$ & 370 & EPA8270 \\
\hline Acenaphthylene & U & & 370 & $\mu g / \mathrm{kg}$ & 370 & EPA8270 \\
\hline Adipate & J & $\mathrm{N}$ & 2,000 & $\mu \mathrm{g} / \mathrm{kg}$ & & EPA8270 \\
\hline Aldol condensate & J & NVK & 2,000 & $\mu \mathrm{g} / \mathrm{kg}$ & & EPA8270 \\
\hline Anchracene & $\mathrm{U}$ & & 370 & $\mu g / k g$ & 370 & EPA8270 \\
\hline Aromatic & J & $\mathrm{N}$ & 200 & $\mu \mathrm{g} / \mathrm{kg}$ & & EPA8270 \\
\hline Benzo(a)anthracene & U & & 370 & $\mu \mathrm{g} / \mathrm{kg}$ & 370 & EPA8270 \\
\hline Benzo(b)fluoranthene & U & & 370 & $\mu \mathrm{g} / \mathrm{kg}$ & 370 & EPA8270 \\
\hline Benzo(k)fluoranthene & $\mathrm{U}$ & & 370 & $\mu g / \mathrm{kg}$ & 370 & EPA8270 \\
\hline Benzoic acid & $\mathbf{U}$ & & 1.900 & $\mu \mathrm{g} / \mathrm{kg}$ & 1,900 & EPA8270 \\
\hline Benzo(g,h,i)perylene & $\mathrm{U}$ & & 370 & $\mu \mathrm{g} / \mathrm{kg}$ & 370 & EPA8270 \\
\hline Benzo(a)pyrene & $\mathbf{U}$ & & 370 & $\mu \mathrm{g} / \mathrm{kg}$ & 370 & EPA8270 \\
\hline Benzyl alcohol & $\mathbf{U}$ & & 370 & $\mu \mathrm{g} / \mathrm{kg}$ & 370 & EPA8270 \\
\hline Bis(2-chloroethoxy) methane & $U$ & & 370 & $\mu \mathrm{g} / \mathrm{kg}$ & 370 & EPA8270 \\
\hline Bis(2-chloroethyl) ether & $\mathrm{U}$ & & 370 & $\mu \mathrm{g} / \mathrm{kg}$ & 370 & EPA8270 \\
\hline Bis(2-chloroisopropyl) ether & U & & 370 & $\mu g / \mathrm{kg}$ & 370 & EPA8270 \\
\hline Bis(2-ethylhexyl) phthalate & $\mathrm{U}$ & $\mathrm{v}$ & 670 & $\mu \mathrm{g} / \mathrm{kg}$ & 370 & EPA8270 \\
\hline 4-Bromophenyl phenyl ether & $\mathrm{U}$ & & 370 & $\mu \mathrm{g} / \mathrm{kg}$ & 370 & EPA8270 \\
\hline Butylbenzyl phthalate & $\mathrm{J}$ & & 290 & $\mu \mathrm{g} / \mathrm{kg}$ & 370 & EPA8270 \\
\hline 4-Chloroaniline & $\mathrm{U}$ & & 370 & $\mu \mathrm{g} / \mathrm{kg}$ & 370 & EPA8270 \\
\hline 4-Chloro-m-cresol & $\mathrm{U}$ & & 370 & $\mu \mathrm{g} / \mathrm{kg}$ & 370 & EPA8270 \\
\hline 2-Chloronaphthalene & U & & 370 & $\mu \mathrm{g} / \mathrm{kg}$ & 370 & EPA8270 \\
\hline 2-Chlorophenol & $U$ & & 370 & $\mu \mathrm{g} / \mathrm{kg}$ & 370 & EPA8270 \\
\hline 4-Chlorophenyl phenyl ether & $\mathrm{U}$ & & 370 & $\mu \mathrm{g} / \mathrm{kg}$ & 370 & EPA8270 \\
\hline Chrysene & $\mathbf{U}$ & & 370 & $\mu g / k g$ & 370 & EPA8270 \\
\hline o-Cresol (2-Mechylphenol) & $\mathrm{U}$ & & 370 & $\mu \mathrm{g} / \mathrm{kg}$ & 370 & EPA8270 \\
\hline p-Cresol (4-Methylphenol) & $\mathrm{U}$ & & 370 & $\mu g / \mathrm{kg}$ & 370 & EPA8270 \\
\hline Dibenz $(\mathrm{a}, \mathrm{h})$ anthracene & $\mathrm{U}$ & & 370 & $\mu \mathrm{g} / \mathrm{kg}$ & 370 & EPA8270 \\
\hline Dibenzofuran & $\mathbf{U}$ & & 370 & $\mu \mathrm{g} / \mathrm{kg}$ & 370 & EPA8270 \\
\hline Di-n-butyl phthalate & J & & 62 & $\mu \mathrm{g} / \mathrm{kg}$ & 370 & EPA8270 \\
\hline 1.2-Dichlorobenzene - & $\mathbf{U}$ & & 370 & $\mu g / \mathrm{kg}$ & 370 & EPA8270 \\
\hline 1,3-Dichlorobenzene & $\mathbf{U}$ & & 370 & $\mu \mathrm{g} / \mathrm{kg}$ & 370 & EPA8270 \\
\hline 1,4-Dichlorobenzene & U & & 370 & $\mu \mathrm{g} / \mathrm{kg}$ & 370 & EPA8270 \\
\hline 3,3'-Dichlorobenzidine & $\mathrm{U}$ & & 750 & $\mu g / k g$ & 750 & EPA8270 \\
\hline 2,4-Dichlorophenol & $\mathrm{U}$ & & 370 & $\mu g / \mathrm{kg}$ & 370 & EPA8270 \\
\hline Diethyl phthalate & U & & 370 & $\mu \mathrm{g} / \mathrm{kg}$ & 370 & EPA8270 \\
\hline 2,4-Dimethyl phenol & $\mathrm{U}$ & & 370 & $\mu g / k g$ & 370 & EPA8270 \\
\hline Dimethyl phthalate & $\mathrm{U}$ & & 370 & $\mu \mathrm{g} / \mathrm{kg}$ & 370 & EPA8270 \\
\hline 2,4-Dinitrophenol & U & & 1,900 & $\mu \mathrm{g} / \mathrm{kg}$ & 1,900 & EPA8270 \\
\hline 2,4-Dinitrotoluene & $\mathbf{U}$ & & 370 & $\mu \mathrm{g} / \mathrm{kg}$ & 370 & EPA8270 \\
\hline
\end{tabular}

$\begin{array}{lll}\mathrm{mg} / \mathrm{kg} & 2.9 & \text { EPA6010 } \\ \mathrm{mg} / \mathrm{kg} & 1.7 & \text { EPA7421 } \\ \mathrm{mg} / \mathrm{kg} & 1.7 & \text { EPA6010 } \\ \mathrm{mg} / \mathrm{kg} & 0.12 & \text { EPA7470 } \\ \mathrm{mg} / \mathrm{kg} & 4.6 & \text { EPA6010 } \\ \mathrm{mg} / \mathrm{kg} & 0.57 & \text { EPA7740 } \\ \mathrm{mg} / \mathrm{kg} & 1.1 & \text { EPA6010 }\end{array}$


SAMPLE NAME: D431 1105 (Continued)

Sample ID: 100366

Constituent

RQ AQ B Result

Unit

D. Limit Method

$B / N / A$ Extractables (including $P A H$ and phenols)

2,6-Dinitrotoluene

Di-n-octyl phthalate

Fluoranthene

Fluorene

Hexachlorobenzene

Hexachlorobutadiene

Hexachlorocyclopentadiene

Hexachloroethane

Indeno $(1,2,3-c, d)$ pyrene

Isophorone

2-Methyl-4.6-dinitrophenol

2-Methylnaphthalene

Naphthalene

o-Nitroaniline

m-Nitroaniline

p-Nitroaniline

Nitrobenzene

2-Nitrophenol

4-Nitrophenol

$\mathrm{N}$-Nitrosodiphenylamine

$\mathrm{N}$-Nitrosodipropylamine

Organic acid

Pentachlorophenol

Phenanthrene

Phenol

Pyrene

Sulfur

1,2,4-Trichlorobenzene

2,4,5-Trichlorophenol

2,4,6-Trichlorophenol

Unknown

Unknown

Unknown

\begin{tabular}{|c|c|c|}
\hline U & & 370 \\
\hline $\mathbf{J}$ & & 120 \\
\hline $\mathbf{U}$ & & 370 \\
\hline $\mathbf{U}$ & & 370 \\
\hline $\mathbf{U}$ & & 370 \\
\hline $\mathbf{U}$ & & 370 \\
\hline $\mathrm{U}$ & & 370 \\
\hline U & & 370 \\
\hline U & & 370 \\
\hline U & & 370 \\
\hline $\mathbf{U}$ & & 1,900 \\
\hline $\mathbf{U}$ & & 370 \\
\hline $\mathbf{U}$ & & 370 \\
\hline $\mathbf{U}$ & & 1,900 \\
\hline $\mathrm{U}$ & & 1,900 \\
\hline $\mathrm{U}$ & & 1,900 \\
\hline U & & 370 \\
\hline $\mathrm{U}$ & & 370 \\
\hline $\mathrm{U}$ & & 1,900 \\
\hline $\mathrm{U}$ & & 370 \\
\hline U & & 370 \\
\hline J & $\mathbf{N}$ & 200 \\
\hline $\mathbf{U}$ & & 1,900 \\
\hline $\mathrm{U}$ & & 370 \\
\hline $\mathrm{U}$ & . & 370 \\
\hline $\mathrm{U}$ & & 370 \\
\hline $\mathrm{J}$ & $\mathbf{N}$ & 3,000 \\
\hline $\mathrm{U}$ & & 370 \\
\hline U & & 1,900 \\
\hline$U$ & & 370 \\
\hline J & $\mathrm{N}$ & 300 \\
\hline $\mathrm{J}$ & $\mathrm{N}$ & 200 \\
\hline J & $N$ & 300 \\
\hline
\end{tabular}

$$
\begin{aligned}
& \mu g / k g \\
& \mu g / k g \\
& \mu g / k g \\
& \mu g / k g \\
& \mu g / k g \\
& \mu g / k g \\
& \mu g / k g \\
& \mu g / k g \\
& \mu g / k g \\
& \mu g / k g \\
& \mu g / k g \\
& \mu g / k g \\
& \mu g / k g \\
& \mu g / k g \\
& \mu g / k g \\
& \mu g / k g \\
& \mu g / k g \\
& \mu g / k g \\
& \mu g / k g \\
& \mu g / k g \\
& \mu g / k g \\
& \mu g / k g \\
& \mu g / k g \\
& \mu g / k g \\
& \mu g / k g \\
& \mu g / k g \\
& \mu g / k g \\
& \mu g / k g \\
& \mu g / k g \\
& \mu g / k g \\
& \mu g / k g \\
& \mu g / k g \\
& \mu g / k g
\end{aligned}
$$

\begin{tabular}{ll}
370 & EPA8270 \\
370 & EPA8270 \\
370 & EPA8270 \\
370 & EPA8270 \\
370 & EPA8270 \\
370 & EPA8270 \\
370 & EPA8270 \\
370 & EPA8270 \\
370 & EPA8270 \\
370 & EPA8270 \\
1,900 & EPA8270 \\
370 & EPA8270 \\
370 & EPA8270 \\
1,900 & EPA8270 \\
1,900 & EPA8270 \\
1,900 & EPA8270 \\
370 & EPA8270 \\
370 & EPA8270 \\
1,900 & EPA8270 \\
370 & EPA8270 \\
370 & EPA8270 \\
& EPA8270 \\
1,900 & EPA8270 \\
370 & EPA8270 \\
370 & EPA8270 \\
370 & EPA8270 \\
370 & EPA8270 \\
1,900 & EPA8270 \\
370 & EPA8270 \\
& EPA8270 \\
& EPA8270 \\
\hline & EPA8270
\end{tabular}

Volatile Organic Compounds

Acetone
Acetone
Benzene
Bromodichloromethane.-
Bromoform
Bromomethane (Methyl bromide)
Carbon disulfide
Carbon tetrachloride
Chlorobenzene
Chloroethane
Chloroethene (Vinyl chloride)
2-Chloroethyl vinyl ether
Chloroform
Chloromethane (Methyl chloride)

L

400
3,500
6.0
6.0
6.0
12
6.0
6.0
6.0
12
12
12
6.0
12

12

1,400

6.0

6.0

6:0

12

6.0

6.0

6.0

12

12

12

6.0

12
EPA8240

EPA8240

EPA8240

EPA8240

EPA8240

EPA8240

EPA8240

EPA8240

EPA8240

EPA8240

EPA8240

EPA8240

EPA8240

EPA8240 
SAMPLE NAME: D43i1105 (Continued)

Sample ID: 100366

Constituent

Volatile Organic Compounds

Dibromochloromethane

1,1-Dichloroethane

1,2-Dichloroethane

1,1-Dichloroethylene

1,2-Dichloroethylene

Dichloromethane (Methylene chloride)

1,2-Dichloropropane

cis-1,3-Dichloropropene

trans-1,3-Dichloropropene

Ethylbenzene

2-Hexanone

Methyl ethyl ketone

Methyl isobutyl ketone

Styrene

1,1,2,2-Tetrachloroethane

Tetrachloroethylene

Toluene

1,1,1-Trichloroethane

1,1,2-Trichloroethane

Trichloroethylene

Unknown

Vinyl acetate

Xylenes

Pesticides/PCBs and Dioxins/Furans

Aldrin

alpha-Benzene hexachloride

beta-Benzene hexachloride

delta-Benzene hexachloride

alpha-Chlordane

gamma-Chlordane

p.p'-DDD

p.p'-DDE

p,p'-DDT

Dieldrin

Endosulfan I

Endosulfan II

Endosulfan sulfate

Endrin

Endrin ketone

Heptachlor

Heptachlor epoxide

Heptachlorodibenzo-p-dioxin isomers

Heptachlorodibenzo-p-furan isomers

Hexachlorodibenzo-p-dioxin isomers

Hexachlorodibenzo-p-furan isomers

Lindane

Methoxychlor

Octachlorodibenzo-p-dioxin
RQ AQ B Result

Unit

D. Limit Method

$\begin{array}{llll}6.0 & \mu \mathrm{g} / \mathrm{kg} & 6.0 & \text { EPA8240 } \\ 6.0 & \mu \mathrm{g} / \mathrm{kg} & 6.0 & \text { EPA8240 } \\ 6.0 & \mu \mathrm{ggg} & 6.0 & \text { EPA8240 } \\ 6.0 & \mu \mathrm{g} / \mathrm{kg} & 6.0 & \text { EPA8240 } \\ 6.0 & \mu \mathrm{g} / \mathrm{kg} & 6.0 & \text { EPA8240 } \\ 11 & \mu \mathrm{g} / \mathrm{kg} & 6.0 & \text { EPA8240 } \\ 6.0 & \mu \mathrm{gg} / \mathrm{kg} & 6.0 & \text { EPA8240 } \\ 6.0 & \mu \mu / \mathrm{kg} & 6.0 & \text { EPA8240 } \\ 6.0 & \mu \mathrm{g} / \mathrm{kg} & 6.0 & \text { EPA8240 } \\ 6.0 & \mu \mathrm{g} / \mathrm{kg} & 6.0 & \text { EPA8240 } \\ 12 & \mu \mathrm{g} / \mathrm{kg} & 12 & \text { EPA8240 } \\ 12 & \mu \mathrm{g} / \mathrm{kg} & 12 & \text { EPA8240 } \\ 12 & \mu \mathrm{g} / \mathrm{kg} & 12 & \text { EPA8240 } \\ 6.0 & \mu \mathrm{gg} / \mathrm{kg} & 6.0 & \text { EPA8240 } \\ 6.0 & \mu \mathrm{g} / \mathrm{kg} & 6.0 & \text { EPA8240 } \\ 6.0 & \mu \mathrm{g} / \mathrm{kg} & 6.0 & \text { EPA8240 } \\ 6.0 & \mu \mathrm{g} / \mathrm{kg} & 6.0 & \text { EPA8240 } \\ 6.0 & \mu \mathrm{g} / \mathrm{kg} & 6.0 & \text { EPA8240 } \\ 6.0 & \mu \mathrm{g} / \mathrm{kg} & 6.0 & \text { EPA8240 } \\ 6.0 & \mu \mathrm{g} / \mathrm{kg} & 6.0 & \text { EPA8240 } \\ 100 & \mu \mathrm{g} / \mathrm{kg} & & \text { EPA8240 } \\ 12 & \mu \mathrm{g} / \mathrm{kg} & 12 & \text { EPA8240 } \\ 6.0 & \mu \mathrm{g} / \mathrm{kg} & 6.0 & \text { EPA8240 }\end{array}$

1.9

1.9

1.9

4.6

6.9

14

3.8

2.3

3.8

3.8

1.9

3.8

3.8

3.8

3.8

1.9

1.9

2.8

0.10

0.20

0.20

1.9

19

0.20 $\mu \mathrm{g} / \mathrm{kg}$

$\mu \mathrm{g} / \mathrm{kg}$

$\mu \mathrm{g} / \mathrm{kg}$

$\mu \mathrm{g} / \mathrm{kg}$

$\mu \mathrm{g} / \mathrm{kg}$

$\mu \mathrm{g} / \mathrm{kg}$

$\mu \mathrm{g} / \mathrm{kg}$

$\mu \mathrm{g} / \mathrm{kg}$

$\mu g / k g$

$\mu \mathrm{g} / \mathrm{kg}$

$\mu \mathrm{g} / \mathrm{kg}$

$\mu \mathrm{g} / \mathrm{kg}$

$\mu \mathrm{g} / \mathrm{kg}$

$\mu \mathrm{g} / \mathrm{kg}$

$\mu \mathrm{g} / \mathrm{kg}$

$\mu g / \mathrm{kg}$

$\mu \mathrm{g} / \mathrm{kg}$

$\mathrm{ng} / \mathrm{g}$

$\mathrm{ng} / \mathrm{g}$

$\mathrm{ng} / \mathrm{g}$

$\mathrm{ng} / \mathrm{g}$

$\mu \mathrm{g} / \mathrm{kg}$

$\mu \mathrm{g} / \mathrm{kg}$

$\mathrm{ng} / \mathrm{g}$
1.9

EPA8080

EPA8080

EPA8080

EPA8080

EPA8080

EPA8080

EPA8080

EPA8080

EPA8080

EPA8080

EPA8080

EPA8080

EPA8080

EPA8080

EPA8080

EPA8080

EPA8080

EPA8280

EPA8280

EPA8280

EPA8280

EPA8080

EPA8080

EPA8280 
SAMPLE NAME: D43I 1105 (Continued)

Constituent
Pesticides/PCBs and Dioxins/Furans
Octachlorodibenzo-p-furan
PCB 1016
PCB 1221
PCB 1232
PCB 1242
PCB 1248
PCB 1254
PCB 1260
Pentachlorodibenzo-p-dioxin isomers
Pentachlorodibenzo-p-furan isomers
2,3,7,8-TCDD
2,3,7.8-TCDF
Tetrachlorodibenzo-p-dioxin isomers
Tetrachlorodibenzo-p-furan isomers
Toxaphene

Radionuclide Indicators and Radionuclides

Gross alpha

Nonvolatile beta

Radium, total alpha-emitting

Tritium

$\begin{array}{ll} & \mathrm{V} \\ \mathrm{U} & \mathrm{V} \\ \mathrm{U} & \end{array}$

SAMPLE NAME: D431 1106

Location (SRS Coordinates): $18558.54 \mathrm{E} 66554.39 \mathrm{~N}$

Ground Elevation Above MSL: $134.6 \mathrm{ft}$

Depth of Core Interval: 8.00 to $10.00 \mathrm{ft}$

Sample Type: Normal

Sample Color: 10YR 3/4

Constituent

RQ AQ

Sample Matrix: Soil

USC Soil Classification: SC

$\mathrm{pCi} / \mathrm{g}$

$\mathrm{pCi} / \mathrm{g}$

$\mathrm{pCi} / \mathrm{g}$

$\mathrm{pCi} / \mathrm{g}$

Sample ID: 100367

B Result Unit

Physical Parameters

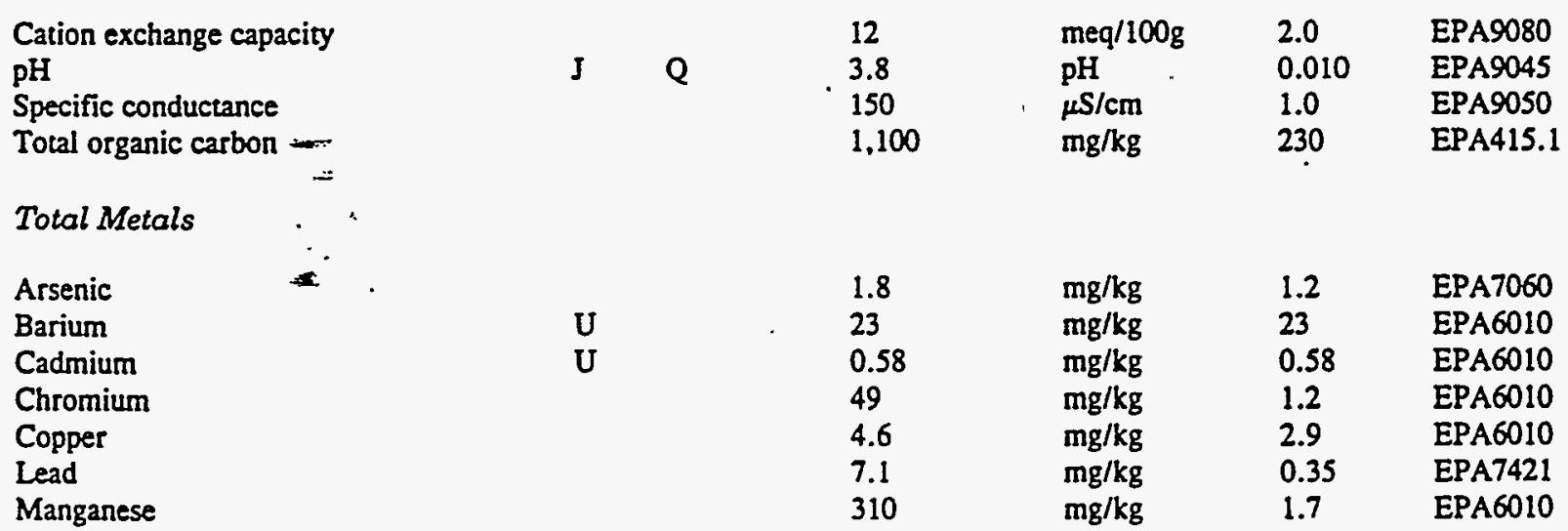


SAMPLE NAME: D431 1106 (Continued)

Sample ID: 100367

$\begin{array}{llllll}\text { Constituent } & \text { RQ AQ B Result Unit Dimit Method }\end{array}$

Total Metals

$\begin{array}{llllll}\text { Mercury } & \mathrm{U} & 0.12 & \mathrm{mg} / \mathrm{kg} & 0.12 & \text { EPA7470 } \\ \text { Nickel } & & 6.4 & \mathrm{mg} / \mathrm{kg} & 4.6 & \text { EPA6010 } \\ \text { Selenium } & \mathrm{U} & 0.58 & \mathrm{mg} / \mathrm{kg} & 0.58 & \text { EPA7740 } \\ \text { Silver } & \mathrm{U} & 1.2 & \mathrm{mg} / \mathrm{kg} & 1.2 & \text { EPA6010 }\end{array}$

$B / N / A$ Extractables (including $P A H$ and phenols)

Acenaphthene
Acenaphthylene
Adipate
Aldol condensate
Aldol condensate
Anthracene
Benzo(a)anthracene
Benzo(b)fluoranthene
Benzo(k)fluoranthene
Benzoic acid
Benzo(g,h,i)perylene
Benzo(a)pyrene
Benzyl alcohol
Bis(2-chloroethoxy) methane
Bis(2-chloroethyl) ether
Bis(2-chloroisopropyl) ether
Bis(2-ethylhexyl) phthalate
4-Bromophenyl phenyl ether
Butylbenzyl phthalate
4-Chloroaniline
4-Chloro-m-cresol
2-Chloronaphthalene
2-Chlorophenol
4-Chlorophenyl phenyl ether
Chrysene
0-Cresol (2-Methylphenol)
p-Cresol (4-Methylphenol)
Dibenz(a,h)anthracene
Dibenzofuran
Di-n-butyl phthalate
1,2-Dichlorobenzene
1,3-Dichlorobenzene
1,4-Dichlorobenzene
3,3'-Dichlorobenzidine
2,4-Dichlorophenol
Diethyl phthalate
2,4-Dimethyl phenol
Dimethyl phthalate
2,4-Dinitrophenol
2,4-Dinitrotoluene
2,6-Dinitrotoluene
Di-n-octyl phthalate
Fluoranthene

\begin{tabular}{|c|c|c|}
\hline $\mathbf{U}$ & & 380 \\
\hline$U$ & & 380 \\
\hline $\mathbf{J}$ & $\mathbf{N}$ & 20,000 \\
\hline $\mathbf{J}$ & NVK & 2,000 \\
\hline J & NK & 300 \\
\hline $\mathbf{U}$ & & 380 \\
\hline $\mathbf{U}$ & & 380 \\
\hline $\mathbf{U}$ & & 380 \\
\hline $\mathbf{U}$ & & 380 \\
\hline $\mathbf{U}$ & & 1,900 \\
\hline $\mathbf{U}$ & & 380 \\
\hline U & & 380 \\
\hline $\mathbf{U}$ & & 380 \\
\hline $\mathbf{U}$ & & 380 \\
\hline $\mathbf{U}$ & & 380 \\
\hline $\mathbf{U}$ & & 380 \\
\hline UJ & $\mathrm{V}$ & 140 \\
\hline $\mathbf{U}$ & & 380 \\
\hline$U$ & & 380 \\
\hline $\mathrm{U}$ & & 380 \\
\hline $\mathrm{U}$ & & 380 \\
\hline $\mathrm{U}$ & & 380 \\
\hline $\mathbf{U}$ & & 380 \\
\hline$U$ & & 380 \\
\hline U & & 380 \\
\hline $\mathbf{U}$ & & 380 \\
\hline $\mathbf{U}$ & & 380 \\
\hline $\mathbf{U}$ & & 380 \\
\hline $\mathbf{U}$ & & 380 \\
\hline J & & 45 \\
\hline $\mathbf{U}$ & & 380 \\
\hline $\mathbf{U}$ & & 380 \\
\hline $\mathbf{U}$ & . & 380 \\
\hline $\mathbf{U}$ & & 760 \\
\hline $\mathbf{U}$ & & 380 \\
\hline $\mathbf{U}$ & & 380 \\
\hline $\mathbf{U}$ & & 380 \\
\hline $\mathbf{U}$ & & 380 \\
\hline U & & 1,900 \\
\hline $\mathbf{U}$ & & 380 \\
\hline $\mathbf{U}$ & & 380 \\
\hline J & & 45 \\
\hline $\mathrm{U}$ & & 380 \\
\hline
\end{tabular}

\begin{tabular}{|c|c|c|}
\hline$\mu g / k g$ & 380 & EPA 8270 \\
\hline$\mu g / \mathrm{kg}$ & 380 & EPA 8270 \\
\hline$\mu \mathrm{g} / \mathrm{kg}$ & & EPA8270 \\
\hline$\mu \mathrm{g} / \mathrm{kg}$ & & EPA 8270 \\
\hline$\mu \mathrm{g} / \mathrm{kg}$ & & EPA8270 \\
\hline$\mu \mathrm{g} / \mathrm{kg}$ & 380 & EPA8270 \\
\hline$\mu \mathrm{g} / \mathrm{kg}$ & 380 & EPA 8270 \\
\hline$\mu g / \mathrm{kg}$ & 380 & EPA8270 \\
\hline$\mu \mathrm{g} / \mathrm{kg}$ & 380 & EPA8270 \\
\hline$\mu g / k g$ & 1,900 & EPA8270 \\
\hline$\mu \mathrm{g} / \mathrm{kg}$ & 380 & EPA8270 \\
\hline$\mu \mathrm{g} / \mathrm{kg}$ & 380 & EPA8270 \\
\hline$\mu g / k g$ & 380 & EPA8270 \\
\hline$\mu \mathrm{g} / \mathrm{kg}$ & 380 & EPA8270 \\
\hline$\mu g / k g$ & 380 & EPA8270 \\
\hline$\mu \mathrm{g} / \mathrm{kg}$ & 380 & EPA8270 \\
\hline$\mu \mathrm{g} / \mathrm{kg}$ & 380 & EPA8270 \\
\hline$\mu \mathrm{g} / \mathrm{kg}$ & 380 & EPA8270 \\
\hline$\mu \mathrm{g} / \mathrm{kg}$ & 380 & EPA8270 \\
\hline$\mu \mathrm{g} / \mathrm{kg}$ & 380 & EPA8270 \\
\hline$\mu \mathrm{g} / \mathrm{kg}$ & 380 & EPA8270 \\
\hline$\mu \mathrm{g} / \mathrm{kg}$ & 380 & EPA8270 \\
\hline$\mu \mathrm{g} / \mathrm{kg}$ & 380 & EPA8270 \\
\hline$\mu \mathrm{g} / \mathrm{kg}$ & 380 & EPA8270 \\
\hline$\mu \mathrm{g} / \mathrm{kg}$ & 380 & EPA8270 \\
\hline$\mu \mathrm{g} / \mathrm{kg}$ & 380 & EPA8270 \\
\hline$\mu \mathrm{g} / \mathrm{kg}$ & 380 & EPA8270 \\
\hline$\mu \mathrm{g} / \mathrm{kg}$ & 380 & EPA8270 \\
\hline$\mu \mathrm{g} / \mathrm{kg}$ & 380 & EPA8270 \\
\hline$\mu g / k g$ & 380 & EPA8270 \\
\hline$\mu \mathrm{g} / \mathrm{kg}$ & 380 & EPA8270 \\
\hline$\mu g / \mathrm{kg}$ & 380 & EPA8270 \\
\hline$\mu g / k g$ & 380 & EPA8270 \\
\hline$\mu g / \mathrm{kg}$ & 760 & EPA8270 \\
\hline$\mu \mathrm{g} / \mathrm{kg}$ & 380 & EPA 8270 \\
\hline$\mu \mathrm{g} / \mathrm{kg}$ & 380 & EPA8270 \\
\hline$\mu \mathrm{g} / \mathrm{kg}$ & 380 & EPA8270 \\
\hline$\mu g / \mathrm{kg}$ & 380 & EPA8270 \\
\hline$\mu g / \mathrm{kg}$ & 1,900 & EPA8270 \\
\hline$\mu g / \mathrm{kg}$ & 380 & EPA8270 \\
\hline$\mu g / \mathrm{kg}$ & 380 & EPA8270 \\
\hline$\mu \mathrm{g} / \mathrm{kg}$ & 380 & EPA8270 \\
\hline$\mu \mathrm{g} / \mathrm{kg}$ & 380 & EPA 8270 \\
\hline
\end{tabular}


SAMPLE NAME: D431-1106 (Continued)

Sample ID: 100367

Constituent

RQ AQ B Result Unit

D. Limit Method

$B / N / A$ Extractables (including $P A H$ and phenols)

Fluorene

Hexachlorobenzene

Hexachlorobutadiene

Hexachlorocyclopentadiene

Hexachloroethane

Indeno(1,2,3-c,d)pyrene

Isophorone

2-Methyl-4,6-dinitrophenol

2-Methylnaphthalene

Naphthalene

o-Nitroaniline

m-Nitroaniline

p-Nitroaniline

Nitrobenzene

2-Nitrophenol

4-Nitrophenol

N-Nitrosodiphenylamine

N-Nitrosodipropylamine

Pentachlorophenol

Phenanthrene

Phenol

Pyrene

1,2,4-Trichlorobenzene

2,4,5-Trichlorophenol

2,4,6-Trichlorophenol

Unknown

Unknown

Volatile Organic Compounds

Acetone

Benzene

Bromodichloromethane

Bromoform

Bromomethane (Methyl bromide)

Carbon disulfide

Carbon tetrachloride

Chlorobenzene

Chloroethane

Chloroethene (Vinyl chloride)

2-Chloroethyl vinyl ether

Chloroform

Chloromethane (Methyl chloride)

Dibromochloromethane

1,1-Dichloroethane

1,2-Dichloroethane

1,1-Dichloroethylene

1,2-Dichloroethylene

Dichloromethane (Methylene chloride)

1,2-Dichloropropane
U $\quad 380$

380

380

380

380

380

380

1,900

380

380

1,900

1,900

1,900

380

380

1,900

380

380

1,900

380

380

380

380

1,900

380

900

500

N

120

5.5

5.5

5.5

11

5.5

5.5

5.5

11

11

11

5.5

11

5.5

5.5

5.5

5.5

5.5

14

5.5

$\begin{array}{lll}\mu \mathrm{g} / \mathrm{kg} & 380 & \text { EPA8270 } \\ \mu \mathrm{g} / \mathrm{kg} & 380 & \text { EPA8270 } \\ \mu \mathrm{g} / \mathrm{kg} & 380 & \text { EPA8270 } \\ \mu \mathrm{g} / \mathrm{kg} & 380 & \text { EPA8270 } \\ \mu \mathrm{g} / \mathrm{kg} & 380 & \text { EPA8270 } \\ \mu \mathrm{g} / \mathrm{kg} & 380 & \text { EPA8270 } \\ \mu \mathrm{g} / \mathrm{kg} & 380 & \text { EPA8270 } \\ \mu \mathrm{g} / \mathrm{kg} & 1,900 & \text { EPA8270 } \\ \mu \mathrm{g} / \mathrm{kg} & 380 & \text { EPA8270 } \\ \mu \mathrm{g} / \mathrm{kg} & 380 & \text { EPA8270 } \\ \mu \mathrm{g} / \mathrm{kg} & 1,900 & \text { EPA8270 } \\ \mu \mathrm{g} / \mathrm{kg} & 1,900 & \text { EPA8270 } \\ \mu \mathrm{g} / \mathrm{kg} & 1,900 & \text { EPA8270 } \\ \mu \mathrm{g} / \mathrm{kg} & 380 & \text { EPA8270 } \\ \mu \mathrm{g} / \mathrm{kg} & 380 & \text { EPA8270 } \\ \mu \mathrm{g} / \mathrm{kg} & 1,900 & \text { EPA8270 } \\ \mu \mathrm{g} / \mathrm{kg} & 380 & \text { EPA8270 } \\ \mu \mathrm{g} / \mathrm{kg} & 380 & \text { EPA8270 } \\ \mu \mathrm{g} / \mathrm{kg} & 1,900 & \text { EPA8270 } \\ \mu \mathrm{g} / \mathrm{kg} & 380 & \text { EPA8270 } \\ \mu \mathrm{g} / \mathrm{kg} & 380 & \text { EPA8270 } \\ \mu \mathrm{g} / \mathrm{kg} & 380 & \text { EPA8270 } \\ \mu \mathrm{g} / \mathrm{kg} & 380 & \text { EPA8270 } \\ \mu \mathrm{g} / \mathrm{kg} & 1,900 & \text { EPA8270 } \\ \mu \mathrm{g} / \mathrm{kg} & 380 & \text { EPA8270 } \\ \mu \mathrm{g} / \mathrm{kg} & & \text { EPA8270 } \\ \mu \mathrm{g} / \mathrm{kg} & & \text { EPA8270 }\end{array}$

$\mu g / k g$

$\mu \mathrm{g} / \mathrm{kg}$

$\mu \mathrm{g} / \mathrm{kg}$

$\mu \mathrm{g} / \mathrm{kg}$

$\mu \mathrm{g} / \mathrm{kg}$

$\mu \mathrm{g} / \mathrm{kg}$

$\mu \mathrm{g} / \mathrm{kg}$

$\mu \mathrm{g} / \mathrm{kg}$

$\mu g / \mathrm{kg}$

$\mu \mathrm{g} / \mathrm{kg}$

$\mu \mathrm{g} / \mathrm{kg}$

$\mu \mathrm{g} / \mathrm{kg}$

$\mu g / k g$

$\mu \mathrm{g} / \mathrm{kg}$

$\mu \mathrm{g} / \mathrm{kg}$

$\mu \mathrm{g} / \mathrm{kg}$

$\mu \mathrm{g} / \mathrm{kg}$

$\mu \mathrm{g} / \mathrm{kg}$

$\mu \mathrm{g} / \mathrm{kg}$

$\mu \mathrm{g} / \mathrm{kg}$
EPA8240

EPA8240

EPA8240

EPA8240

EPA8240

EPA8240

EPA 8240

EPA8240

EPA8240

EPA8240

EPA8240

EPA8240

EPA8240

EPA8240

EPA8240

EPA8240

EPA8240

EPA8240

EPA8240

EPA8240 
SAMPLE NAME: D431 1106 (Continued)

Sample ID: 100367

Constituent

Volatile Organic Compounds

\section{$\mathbf{R}$}

AQ
Result

5.5

5.5

5.5

11

11

11

5.5

5.5

5.5

5.5

5.5

5.5

5.5

10

11

5.5
Unit

D. Limit Method
Unknown

Vinyl acetate

Xylenes
J

U

Pesticides/PCBs and Dioxins/Furans

Aldrin
alpha-Benzene hexachloride
beta-Benzene hexachloride
delta-Benzene hexachloride
alpha-Chlordane
gamma-Chlordane
p,p'-DDD
p,p'-DDE
p.p'-DDT
Dieldrin
Endosulfan I
Endosulfan II
Endosulfan sulfate
Endrin
Endrin ketone
Heptachlor
Heptachlor epoxide
Heptachlorodibenzo-p-dioxin isomers
Heptachlorodibenzo-p-furan isomers
Hexachlorodibenzo-p-dioxin isomers
Hexachlorodibenzo-p-furan isomers
Lindane
Methoxychlor
Octachlorodibenzo-p-dioxin
Octachlorodibenzo-p-fưfan
PCB 1016
PCB 1221
PCB 1232 -
PCB 1242
PCB 1248 -
PCB 1254

$$
\begin{aligned}
& \mu \mathrm{g} / \mathrm{kg} \\
& \mu \mathrm{g} / \mathrm{kg} \\
& \mu \mathrm{g} / \mathrm{kg} \\
& \mu \mathrm{g} / \mathrm{kg} \\
& \mu \mathrm{g} / \mathrm{kg} \\
& \mu \mathrm{g} / \mathrm{kg} \\
& \mu \mathrm{g} / \mathrm{kg} \\
& \mu \mathrm{g} / \mathrm{kg} \\
& \mu \mathrm{g} / \mathrm{kg} \\
& \mu \mathrm{g} / \mathrm{kg} \\
& \mu \mathrm{g} / \mathrm{kg} \\
& \mu \mathrm{g} / \mathrm{kg} \\
& \mu \mathrm{g} / \mathrm{kg} \\
& \mu \mathrm{g} / \mathrm{kg} \\
& \mu \mathrm{g} / \mathrm{kg} \\
& \mu \mathrm{g} / \mathrm{kg}
\end{aligned}
$$

\section{5}

5.5

5.5

11

11

11

5.5

5.5

5.5

5.5

5.5

5.5

5.5

11

5.5
EPA8240
EPA8240
EPA8240
EPA8240
EPA8240
EPA8240
EPA8240
EPA8240
EPA8240
EPA8240
EPA8240
EPA8240
EPA8240
EPA8240
EPA8240
EPA8240
U

$\mathrm{U}$

$\mathrm{U}$

U

J

J

J

$\mathrm{J}$

$\mathbf{U}$

U

U

U

U

U

U

U

$U$

$U$

U

U

1.9
1.9
1.9
1.9
1.2
2.7
3.1
3.5
2.7
3.9
1.9
3.9
3.9
3.9
3.9
1.9
1.9
0.20
0.20
0.30
0.30
1.9
19
2.0
0.20
39
77
39
39
39
39

$\begin{array}{lll}\mu \mathrm{g} / \mathrm{kg} & 1.9 & \text { EPA8080 } \\ \mu \mathrm{g} / \mathrm{kg} & 1.9 & \text { EPA8080 } \\ \mu \mathrm{g} / \mathrm{kg} & 1.9 & \text { EPA8080 } \\ \mu \mathrm{g} / \mathrm{kg} & 1.9 & \text { EPA8080 } \\ \mu \mathrm{g} / \mathrm{kg} & 1.9 & \text { EPA8080 } \\ \mu \mathrm{g} / \mathrm{kg} & 1.9 & \text { EPA8080 } \\ \mu \mathrm{g} / \mathrm{kg} & 3.9 & \text { EPA8080 } \\ \mu \mathrm{g} / \mathrm{kg} & 3.9 & \text { EPA8080 } \\ \mu \mathrm{g} / \mathrm{kg} & 3.9 & \text { EPA8080 } \\ \mu \mathrm{g} / \mathrm{kg} & 3.9 & \text { EPA8080 } \\ \mu \mathrm{g} / \mathrm{kg} & 1.9 & \text { EPA8080 } \\ \mu \mathrm{g} / \mathrm{kg} & 3.9 & \text { EPA8080 } \\ \mu \mathrm{g} / \mathrm{kg} & 3.9 & \text { EPA8080 } \\ \mu \mathrm{g} / \mathrm{kg} & 3.9 & \text { EPA8080 } \\ \mu \mathrm{g} / \mathrm{kg} & 3.9 & \text { EPA8080 } \\ \mu \mathrm{g} / \mathrm{kg} & 1.9 & \text { EPA8080 } \\ \mu \mathrm{g} / \mathrm{kg} & 1.9 & \text { EPA8080 } \\ \mathrm{ng} / \mathrm{g} & 0.20 & \text { EPA8280 } \\ \mathrm{ng} / \mathrm{g} & 0.20 & \text { EPA8280 } \\ \mathrm{ng} / \mathrm{g} & 0.30 & \text { EPA8280 } \\ \mathrm{ng} / \mathrm{g} & 0.30 & \text { EPA8280 } \\ \mu \mathrm{g} / \mathrm{kg} & 1.9 & \text { EPA8080 } \\ \mu \mathrm{g} / \mathrm{kg} & 19 & \text { EPA8080 } \\ \mathrm{ng} / \mathrm{g} & 0.20 & \text { EPA8280 } \\ \mathrm{ng} / \mathrm{g} & 0.20 & \text { EPA8280 } \\ \mu \mathrm{g} / \mathrm{kg} & 39 & \text { EPA8080 } \\ \mu \mathrm{g} / \mathrm{kg} & 77 & \text { EPA8080 } \\ \mu \mathrm{g} / \mathrm{kg} & 39 & \text { EPA8080 } \\ \mu \mathrm{g} / \mathrm{kg} & 39 & \text { EPA8080 } \\ \mu \mathrm{g} / \mathrm{kg} & 39 & \text { EPA8080 } \\ \mu \mathrm{g} / \mathrm{kg} & 39 & \text { EPA8080 }\end{array}$


SAMPLE NAME: D43I 1106 (Continued) Sample D: 100367

Constituent

RQ AQ B Result

Unit

D. Limit Method

Pesticides/PCBs and Dioxins/Furans

PCB 1260

Pentachlorodibenzo-p-dioxin isomers

Pentachlorodibenzo-p-furan isomers

2,3,7,8-TCDD

2,3.7,8-TCDF

Tetrachlorodibenzo-p-dioxin isomers

Tetrachlorodibenzo-p-furan isomers

Toxaphene

$\begin{array}{ll}\mathbf{U} & 39 \\ \mathbf{U} & 0.30 \\ \mathbf{U} & 0.30 \\ \mathbf{U} & 0.30 \\ \mathbf{U} & 0.10 \\ \mathbf{U} & 0.30 \\ \mathbf{U} & 0.30 \\ \mathbf{U} & 190\end{array}$

Radionuclide Indicators and Radionuclides

Gross alpha

Nonvolatile beta

Radium, total alpha-emitting

Tritium

$\begin{array}{ll}\text { U } & \\ & \mathrm{V} \\ \mathrm{U} & \mathrm{V}\end{array}$

SAMPLE NAME: D431 1106A

Sample Type: Duplicate

Constituent

Physical Parameters

Cation exchange capacity

$\mathrm{pH}$

Specific conductance

Total organic carbon

Total organic carbon

Total Metals

\section{Arsenic}

Barium

Cadmium

Chromium

Copper

Lead

Manganese

Mercury

Nickel

. Selenium

Silver

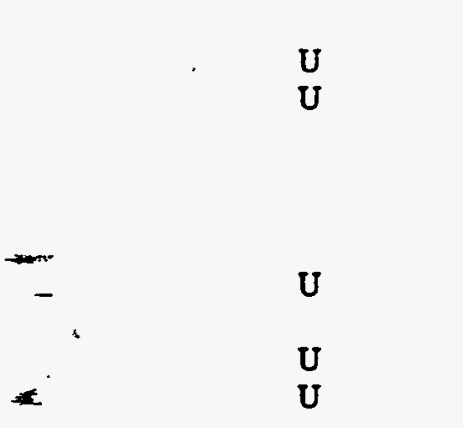

$B / N / A$ Extractables (including $P A H$ and phenols)

Acenaphthene

Acenaphthylene

Adipate

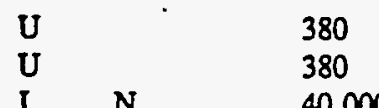

40,000

$\begin{array}{ll}4.3 & \mathrm{pCi} / \mathrm{g} \\ 29 \pm 17 & \mathrm{pCi} / \mathrm{g} \\ 2.1 \pm 0.96 & \mathrm{pCi} / \mathrm{g} \\ 1.8 & \mathrm{pCi} / \mathrm{g}\end{array}$

$\mu \mathrm{g} / \mathrm{kg}$

$\mathrm{ng} / \mathrm{g}$

$\mathrm{ng} / \mathrm{g}$

$\mathrm{ng} / \mathrm{g}$

$\mathrm{ng} / \mathrm{g}$

$\mathrm{ng} / \mathrm{g}$

$\mathrm{ng} / \mathrm{g}$

$\mu \mathrm{g} / \mathrm{kg}$

39

0.30

0.30

0.30

0.10

0.30

0.30

190

EPA8080
EPA8280
EPA8280
EPA8280
EPA8280
EPA8280
EPA8280
EPA8080

Sample ID: 100368

Associated Sample: 100367
$\mathbf{R Q}$
$\mathbf{A Q}$
Result
Unit
D. Limit Method

11

3.9
150

1,400

1,700
meq/100g
$\mathrm{pH}$
$\mu \mathrm{S} / \mathrm{cm}$
$\mathrm{mg} / \mathrm{kg}$
$\mathrm{mg} / \mathrm{kg}$

\section{0}

0.010

EPA9080

EPA9045

EPA9050

1.0

EPA415.1

130

EPA415.1

$\begin{array}{llll}2.8 & \mathrm{mg} / \mathrm{kg} & 1.1 & \text { EPA7060 } \\ 23 & \mathrm{mg} / \mathrm{kg} & 23 & \text { EPA6010 } \\ 0.57 & \mathrm{mg} / \mathrm{kg} & 0.57 & \text { EPA6010 } \\ 59 & \mathrm{mg} / \mathrm{kg} & 1.1 & \text { EPA6010 } \\ 4.6 & \mathrm{mg} / \mathrm{kg} & 2.9 & \text { EPA6010 } \\ 9.1 & \mathrm{mg} / \mathrm{kg} & 0.69 & \text { EPA7421 } \\ 330 & \mathrm{mg} / \mathrm{kg} & 1.7 & \text { EPA6010 } \\ 0.12 & \mathrm{mg} / \mathrm{kg} & 0.12 & \text { EPA7470 } \\ 7.3 & \mathrm{mg} / \mathrm{kg} & 4.6 & \text { EPA6010 } \\ 0.57 & \mathrm{mg} / \mathrm{kg} & 0.57 & \text { EPA7740 } \\ 1.1 & \mathrm{mg} / \mathrm{kg} & 1.1 & \text { EPA6010 }\end{array}$

$\mu \mathrm{g} / \mathrm{kg}$

$\mu \mathrm{g} / \mathrm{kg}$

$\mu \mathrm{g} / \mathrm{kg}$ 
SAMPLE NAME: D431 1106A (Continued)

Sample D: 100368
Constituent
RQ AQ B Result
Unit
D. Limit Method

$B / N / A$ Extractables (including $P A H$ and phenols)

\begin{tabular}{|c|c|c|c|c|c|c|}
\hline Aldol condensate & $J$ & NVK & 2,000 & $\mu \mathrm{g} / \mathrm{kg}$ & & EPA8270 \\
\hline Aldol condensate & J & NK & 300 & $\mu \mathrm{g} / \mathrm{kg}$ & & EPA8270 \\
\hline Anthracene & $U$ & & 380 & $\mu g / k g$ & 380 & EPA8270 \\
\hline Benzo(a)anthracene & $\mathbf{U}$ & & 380 & $\mu g / \mathrm{kg}$ & 380 & EPA8270 \\
\hline Benzo(b)fluoranthene & $\mathbf{U}$ & & 380 & $\mu g / \mathrm{kg}^{\prime}$ & 380 & EPA8270 \\
\hline Benzo(k)fluoranthene & $\mathbf{U}$ & & 380 & $\mu g / \mathrm{kg}$ & 380 & EPA8270 \\
\hline Benzoic acid & $U$ & & 1,900 & $\mu g / \mathrm{kg}$ & 1,900 & EPA8270 \\
\hline Benzo $(g, h, i)$ perylene & $U$ & & 380 & $\mu g / \mathrm{kg}$ & 380 & EPA8270 \\
\hline Benzo(a)pyrene & $\mathrm{U}$ & & 380 & $\mu \mathrm{g} / \mathrm{kg}$ & 380 & EPA8270 \\
\hline Benzyl alcohol & $\mathrm{U}$ & & 380 & $\mu \mathrm{g} / \mathrm{kg}$ & 380 & EPA8270 \\
\hline Bis(2-chloroethoxy) methane & $\mathbf{U}$ & & 380 & $\mu \mathrm{g} / \mathrm{kg}$ & 380 & EPA8270 \\
\hline Bis(2-chloroethyl) ether & $U$ & & 380 & $\mu g / \mathrm{kg}$ & 380 & EPA8270 \\
\hline Bis(2-chloroisopropyl) ether & $U$ & & 380 & $\mu \mathrm{g} / \mathrm{kg}$ & 380 & EPA8270 \\
\hline Bis(2-ethylhexyl) phthalate & UJ & $\mathrm{v}$ & 140 & $\mu g / k g$ & 380 & EPA8270 \\
\hline 4-Bromophenyl phenyl ether & $\mathrm{U}$ & & 380 & $\mu \mathrm{g} / \mathrm{kg}$ & 380 & EPA8270 \\
\hline Butylbenzyl phthalate & U & & 380 & $\mu \mathrm{g} / \mathrm{kg}$ & 380 & EPA8270 \\
\hline 4-Chloroaniline & $\mathbf{U}$ & & 380 & $\mu \mathrm{g} / \mathrm{kg}$ & 380 & EPA8270 \\
\hline 4-Chloro-m-cresol & $\mathrm{U}$ & & 380 & $\mu \mathrm{g} / \mathrm{kg}$ & 380 & EPA8270 \\
\hline 2-Chloronaphthalene & $\mathrm{U}$ & & 380 & $\mu \mathrm{g} / \mathrm{kg}$ & 380 & EPA8270 \\
\hline 2-Chlorophenol & $\mathbf{U}$ & & 380 & $\mu \mathrm{g} / \mathrm{kg}$ & 380 & EPA8270 \\
\hline 4-Chlorophenyl phenyl ether & $\mathbf{U}$ & & 380 & $\mu \mathrm{g} / \mathrm{kg}$ & 380 & EPA8270 \\
\hline Chrysene & U & - & 380 & $\mu \mathrm{g} / \mathrm{kg}$ & 380 & EPA8270 \\
\hline o-Cresol (2-Methylphenol) & $\mathbf{U}$ & & 380 & $\mu \mathrm{g} / \mathrm{kg}$ & 380 & EPA8270 \\
\hline p-Cresol (4-Methylphenol) & $\mathbf{U}$ & & 380 & $\mu \mathrm{g} / \mathrm{kg}$ & 380 & EPA8270 \\
\hline Dibenz(a,h)anthracene & $\mathbf{U}$ & & 380 & $\mu \mathrm{g} / \mathrm{kg}$ & 380 & EPA8270 \\
\hline Dibenzofuran & U & & 380 & $\mu \mathrm{g} / \mathrm{kg}$ & 380 & EPA8270 \\
\hline Di-n-butyl phthalate & $\mathbf{J}$ & & 40 & $\mu \mathrm{g} / \mathrm{kg}$ & 380 & EPA8270 \\
\hline 1,2-Dichlorobenzene & $\mathrm{U}$ & & 380 & $\mu \mathrm{g} / \mathrm{kg}$ & 380 & EPA8270 \\
\hline 1,3-Dichlorobenzene & $\mathbf{U}$ & & 380 & $\mu \mathrm{g} / \mathrm{kg}$ & 380 & EPA8270 \\
\hline 1,4-Dichlorobenzene & $\mathbf{U}$ & & 380 & $\mu g / k g$ & 380 & EPA8270 \\
\hline 3,3'-Dichlorobenzidine & $\mathbf{U}$ & & 760 & $\mu \mathrm{g} / \mathrm{kg}$ & 760 & EPA8270 \\
\hline 2,4-Dichlorophenol & $\mathbf{U}$ & & 380 & $\mu g / k g$ & 380 & EPA8270 \\
\hline Diethyl phthalate & $\mathrm{U}$ & & 380 & $\mu \mathrm{g} / \mathrm{kg}$ & 380 & EPA8270 \\
\hline 2,4-Dimethyl phenol & $\mathrm{U}$ & & 380 & $\mu g / \mathrm{kg}$ & 380 & EPA8270 \\
\hline Dimethyl phthalate & $\mathbf{U}$ & & 380 & $\mu g / \mathrm{kg}$ & 380 & EPA8270 \\
\hline 2,4-Dinitrophenol & $\mathbf{U}$ & & 1.900 & $\mu \mathrm{g} / \mathrm{kg}$ & 1,900 & EPA8270 \\
\hline 2,4-Dinitrotoluene & U & & 380 & $\mu g / k g$ & 380 & EPA8270 \\
\hline 2,6-Dinitrotoluene & U & & 380 & $\mu g / \mathrm{kg}$ & 380 & EPA8270 \\
\hline Di-n-octyl phthalate & $\mathrm{U}$ & & 380 & $\mu g / \mathrm{kg}$ & 380 & EPA8270 \\
\hline Fluoranthene & U & & 380 & $\mu g / \mathrm{kg}$ & 380 & EPA8270 \\
\hline Fluorene & $\mathbf{U}$ & & 380 & $\mu g / \mathrm{kg}$ & 380 & EPA8270 \\
\hline Hexachlorobenzene & $\mathbf{U}$ & & 380 & $\mu g / \mathrm{kg}$ & 380 & EPA8270 \\
\hline Hexachlorobutadiene ${ }^{2}$ & $\mathbf{U}$ & & 380 & $\mu g / \mathrm{kg}$ & 380 & EPA8270 \\
\hline Hexachlorocyclopentadiene & $\mathrm{U}$ & & 380 & $\mu g / \mathrm{kg}$ & 380 & EPA8270 \\
\hline Hexachloroethane & $\mathbf{U}$ & & 380 & $\mu g / k g$ & 380 & EPA8270 \\
\hline Indeno(1,2,3-c,d)pyrene & U & & 380 & $\mu g / k g$ & 380 & EPA8270 \\
\hline Isophorone & $\mathbf{U}$ & & 380 & $\mu \mathrm{g} / \mathrm{kg}$ & 380 & EPA8270 \\
\hline 2-Methyl-4,6-dinitrophenol & $\mathbf{U}$ & & 1,900 & $\mu g / \mathrm{kg}$ & 1.900 & EPA8270 \\
\hline 2-Methylnaphthalene & $\mathbf{U}$ & & 380 & $\mu \mathrm{g} / \mathrm{kg}$ & 380 & EPA8270 \\
\hline Naphthalene & $\mathbf{U}$ & & 380 & $\mu \mathrm{g} / \mathrm{kg}$ & 380 & EPA8270 \\
\hline
\end{tabular}


$B / N / A$ Extractables (including $P A H$ and phenols)

o-Nitroaniline
m-Nitroaniline
p-Nitroaniline
Nitrobenzene
2-Nitrophenol
4-Nitrophenol
N-Nitrosodiphenylamine
N-Nitrosodipropylamine
Pentachlorophenol
Phenanthrene
Phenol
Pyrene
1.2.4-Trichlorobenzene
2.4,5-Trichlorophenol
2.4,6-Trichlorophenol
Unknown
Unknown

Volatile Organic Compounds

Acetone
Benzene
Bromodichloromethane
Bromoform
Bromomethane (Methyl bromide)
Carbon disulfide
Carbon tetrachloride
Chlorobenzene
Chloroethane
Chloroethene (Vinyl chloride)
2-Chloroethyl vinyl ether
Chloroform
Chloromethane (Methyl chloride)
Dibromochloromethane
1,1-Dichloroethane
1,2-Dichloroethane
1,1-Dichloroethylene
1,2-Dichloroethylene
Dichloromethane (Methylene chloride)
1,2-Dichloropropane
cis-1,3-Dichloropropente
trans-1,3-Dichloropropene
Ethylbenzene
2-Hexanone
Methyl ethyl ketone
Methyl isobutyl ketone
Styrene
1,1,2,2-Tetrachloroethane
Tetrachloroethylene
Toluene

$\begin{array}{ll}\mathbf{U} \\ \mathbf{U} \\ \mathbf{U} \\ \mathbf{U} \\ \mathbf{U} \\ \mathbf{U} \\ \mathbf{U} \\ \mathbf{U} \\ \mathbf{U} & \\ \mathbf{U} & \\ \mathbf{U} & \\ \mathbf{U} & \\ \mathbf{U} & \\ \mathbf{U} & \\ \mathbf{U} & \\ \mathrm{J} & \mathbf{N} \\ \mathrm{J} & \mathrm{N}\end{array}$

1,900
1,900
1,900
380
380
1,900
380
380
1,900
380
380
380
380
1,900
380
400
400

$$
\begin{aligned}
& \mu \mathrm{g} / \mathrm{kg} \\
& \mu \mathrm{g} / \mathrm{kg} \\
& \mu \mathrm{g} / \mathrm{kg} \\
& \mu \mathrm{g} / \mathrm{kg} \\
& \mu \mathrm{g} / \mathrm{kg} \\
& \mu \mathrm{g} / \mathrm{kg} \\
& \mu \mathrm{g} / \mathbf{k g} \\
& \mu \mathrm{g} / \mathrm{kg} \\
& \mu \mathrm{g} / \mathbf{k g} \\
& \mu \mathrm{g} / \mathbf{k g} \\
& \mu \mathrm{g} / \mathbf{k g} \\
& \mu \mathrm{g} / \mathrm{kg} \\
& \mu \mathrm{g} / \mathbf{k g} \\
& \mu \mathrm{g} / \mathbf{k g} \\
& \mu \mathrm{g} / \mathrm{kg} \\
& \mu \mathrm{g} / \mathrm{kg} \\
& \mu \mathrm{g} / \mathrm{kg}
\end{aligned}
$$

$\begin{array}{ll}1,900 & \text { EPA8270 } \\ 1,900 & \text { EPA8270 } \\ 1,900 & \text { EPA8270 } \\ 380 & \text { EPA8270 } \\ 380 & \text { EPA8270 } \\ 1,900 & \text { EPA8270 } \\ 380 & \text { EPA8270 } \\ 380 & \text { EPA8270 } \\ 1,900 & \text { EPA8270 } \\ 380 & \text { EPA8270 } \\ 380 & \text { EPA8270 } \\ 380 & \text { EPA8270 } \\ 380 & \text { EPA8270 } \\ 1,900 & \text { EPA8270 } \\ 380 & \text { EPA8270 } \\ & \text { EPA8270 } \\ & \text { EPA8270 }\end{array}$

EPA8240
EPA8240
EPA8240
EPA8240
EPA8240
EPA8240
EPA8240
EPA8240
EPA8240
EPA8240
EPA8240
EPA8240
EPA8240
EPA8240
EPA8240
EPA8240
EPA8240
EPA8240
EPA8240
EPA8240
EPA8240
EPA8240
EPA8240
EPA8240
EPA8240
EPA8240
EPA8240
EPA8240
EPA8240
EPA8240 
SAMPLE NAME: D431 1106A (Continued)

Constituent

Volatile Organic Compounds

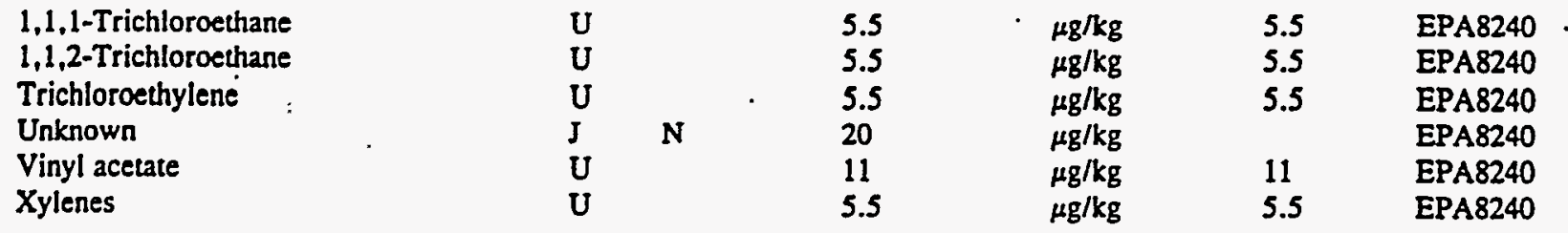

Pesticides/PCBs and Dioxins/Furans

Aldrin
alpha-Benzene hexachloride
beta-Benzene hexachloride
delta-Benzene hexachloride
alpha-Chlordane
gamma-Chlordane
p.p'-DDD
p.p'-DDE
p.p'-DDT
Dieldrin
Endosulfan I
Endosulfan II
Endosulfan sulfate
Endrin
Endrin ketone
Heptachlor
Heptachlor epoxide
Heptachlorodibenzo-p-dioxin isomers
Heptachlorodibenzo-p-furan isomers
Hexachlorodibenzo-p-dioxin isomers
Hexachlorodibenzo-p-furan isomers
Lindane
Methoxychlor
Octachlorodibenzo-p-dioxin
Octachlorodibenzo-p-furan
PCB 1016
PCB 1221
PCB 1232
PCB 1242
PCB 1248
PCB 1254
PCB 1260
Pentachlorodibenzo-p-dioxin isomers
Pentachlorodibenzo-p-furan isomers
$2,3,7,8-T C D D$
$2,3,7,8-$ TCDF
Tetrachlorodibenzo-p-dioxin isomers
Tetrachlorodibenzo-p-furan isomers
Toxaphene

Sample ID: 100368

Unit

D. Limit Method

\begin{tabular}{|c|c|c|c|c|}
\hline U & 1.9 & $\mu \mathrm{g} / \mathrm{kg}$ & 1.9 & EPA8080 \\
\hline U & 1.9 & $\mu \mathrm{g} / \mathrm{kg}$ & 1.9 & EPA8080 \\
\hline U & 1.9 & $\mu \mathrm{g} / \mathrm{kg}$ & 1.9 & EPA8080 \\
\hline J & 1.2 & $\mu \mathrm{g} / \mathrm{kg}$ & 1.9 & EPA8080 \\
\hline & 1.5 & $\mu g / k g$ & 1.9 & EPA8080 \\
\hline & 3.1 & $\mu \mathrm{g} / \mathrm{kg}$ & 1.9 & EPA8080 \\
\hline J & 3.1 & $\mu \mathrm{g} / \mathrm{kg}$ & 3.8 & EPA8080 \\
\hline & 3.1 & $\mu g / \mathrm{kg}$ & 3.8 & EPA8080 \\
\hline & 2.7 & $\mu g / \mathrm{kg}$ & 3.8 & EPA8080 \\
\hline J & 3.8 & $\mu \mathrm{g} / \mathrm{kg}$ & 3.8 & EPA8080 \\
\hline$U$ & 1.9 & $\mu \mathrm{g} / \mathrm{kg}$ & 1.9 & EPA8080 \\
\hline U & 3.8 & $\mu g / \mathrm{kg}$ & 3.8 & EPA8080 \\
\hline$U$ & 3.8 & $\mu \mathrm{g} / \mathrm{kg}$ & 3.8 & EPA8080 \\
\hline$U$ & 3.8 & $\mu \mathrm{g} / \mathrm{kg}$ & 3.8 & EPA8080 \\
\hline $\mathrm{J}$ & 3.8 & $\mu \mathrm{g} / \mathrm{kg}$ & 3.8 & EPA8080 \\
\hline U & 1.9 & $\mu \mathrm{g} / \mathrm{kg}$ & 1.9 & EPA8080 \\
\hline & 1.9 & $\mu \mathrm{g} / \mathrm{kg}$ & 1.9 & EPA8080 \\
\hline & 0.40 & $\mathrm{ng} / \mathrm{g}$ & 0.40 & EPA8280 \\
\hline & 0.20 & $\mathrm{ng} / \mathrm{g}$ & 0.20 & EPA8280 \\
\hline & 0.30 & $\mathrm{ng} / \mathrm{g}$ & 0.30 & EPA8280 \\
\hline & 0.20 & $\mathrm{ng} / \mathrm{g}$ & $0: 20$ & EPA8280 \\
\hline & 1.9 & $\mu \mathrm{g} / \mathrm{kg}$ & 1.9 & EPA8080 \\
\hline & 19 & $\mu \mathrm{g} / \mathrm{kg}$ & 19 & EPA8080 \\
\hline & 4.3 & $\mathrm{ng} / \mathrm{g}$ & 0.20 & EPA 8280 \\
\hline & 0.20 & $\mathrm{ng} / \mathrm{g}$ & 0.20 & EPA8280 \\
\hline & 38 & $\mu \mathrm{g} / \mathrm{kg}$ & 38 & EPA8080 \\
\hline & 77 & $\mu g / \mathrm{kg}$ & 77 & EPA8080 \\
\hline & 38 & $\mu \mathrm{g} / \mathrm{kg}$ & 38 & EPA8080 \\
\hline & 38 & $\mu \mathrm{g} / \mathrm{kg}$ & 38 & EPA8080 \\
\hline & 38 & $\mu \mathrm{g} / \mathrm{kg}$ & 38 & EPA8080 \\
\hline & 38 & $\mu \mathrm{g} / \mathrm{kg}$ & 38 & EPA8080 \\
\hline & 38 & $\mu \mathrm{g} / \mathrm{kg}$ & $-\quad 38$ & EPA8080 \\
\hline & 0.30 & $\mathrm{ng} / \mathrm{g}$ & 0.30 & EPA8280 \\
\hline & 0.30 & $\mathrm{ng} / \mathrm{g}$ & 0.30 & EPA8280 \\
\hline & 0.20 & $\mathrm{ng} / \mathrm{g}$ & 0.20 & EPA8280 \\
\hline & 0.20 & $\mathrm{ng} / \mathrm{g}$ & 0.20 & EPA8280 \\
\hline & 0.20 & $\mathrm{ng} / \mathrm{g}$ & 0.20 & EPA8280 \\
\hline & 0.30 & $\mathrm{ng} / \mathrm{g}$ & 0.30 & EPA8280 \\
\hline & 190 & $\mu \mathrm{g} / \mathrm{kg}$ & 190 & EPA8080 \\
\hline
\end{tabular}


SAMPLE NAME: D431 1106A (Continued)

Constituent

Radionuclide Indicators and Radionuclides

Gross alpha

Nonvolatile beta

Radium, total alpha-emitting

Tritium

Sample ID: 100368

AQ B Result Unit

D. Limit Method

SAMPLE NAME: D431 1107

Location (SRS Coordinates): 18558.54E 66554.39N

Ground Elevation Above MSL: $134.6 \mathrm{ft}$

Depth of Core Interval: 10.00 to $12.00 \mathrm{ft}$

Sample Type: Normal

Sample Color: 10YR 4/2

Constituent

Total Metals

Arsenic

Barium

Cadmium

Chromium

Copper

Lead

Manganese

Mercury

Nickel

Selenium

Silver

$\begin{array}{ll}5.0 \pm 13 & \mathrm{pCi} / \mathrm{g} \\ 48 \pm 20 & \mathrm{pCi} / \mathrm{g} \\ 1.8 \pm 0.91 & \mathrm{pCi} / \mathrm{g} \\ 1.8 & \mathrm{pCi} / \mathrm{g}\end{array}$

Sample ID: 100369

Sample Matrix: Soil

USC Soil Classification: SC

RQ AQ B Result

Unit

4.6

8.4

0.090

1.8

EPA900.0

EPA900.0

EPA903.0

EPA906.0

Sample Moisture: Damp Percent Solids: 78.10

D. Limit Method

$B / N / A$ Extractables (including PAH and phenols)

Acenaphthene

Acenaphthylene

Adipate

Aldol condensate

Aldol condensate

Anthracene

Benzo(a)anthracene

Benzo(b)fluoranthene

Benzo(k)fluoranthene -

Benzoic acid

Benzo(g,h,i)perylene .

Benzo(a)pyrene

Benzyl alcohol

Bis(2-chloroethoxy) methane

Bis(2-chloroethyl) ether

Bis(2-chloroisopropyl) ether

Bis(2-ethylhexyl) phthalate

4-Bromophenyl phenyl ether

$\begin{array}{lllll}\mathrm{U} & 3.1 & \mathrm{mg} / \mathrm{kg} & 1.3 & \text { EPA7060 } \\ \mathrm{U} & 25 & \mathrm{mg} / \mathrm{kg} & 25 & \text { EPA6010 } \\ & 0.64 & \mathrm{mg} / \mathrm{kg} & 0.64 & \text { EPA6010 } \\ \mathrm{U} & 17 & \mathrm{mg} / \mathrm{kg} & 1.3 & \text { EPA6010 } \\ & 3.2 & \mathrm{mg} / \mathrm{kg} & 3.2 & \text { EPA6010 } \\ & 1.4 & \mathrm{mg} / \mathrm{kg} & 0.38 & \text { EPA7421 } \\ \mathrm{U} & 160 & \mathrm{mg} / \mathrm{kg} & 1.9 & \text { EPA6010 } \\ \mathrm{U} & 0.13 & \mathrm{mg} / \mathrm{kg} & 0.13 & \text { EPA7470 } \\ & 5.1 & \mathrm{mg} / \mathrm{kg} & 5.1 & \text { EPA6010 } \\ \mathrm{U} & 0.67 & \mathrm{mg} / \mathrm{kg} & 0.64 & \text { EPA7740 } \\ & 1.3 & \mathrm{mg} / \mathrm{kg} & 1.3 & \text { EPA6010 }\end{array}$


SAMPLE NAME: D431 1107 (Continued)

Sample ID: 100369

$\begin{array}{lllllll}\text { Constituent } & \text { RQ AQ } & \text { B } & \text { Result } & \text { Unit } & \text { D. Limit Method }\end{array}$

$B / N / A$ Extractables (including $P A H$ and phenols)

\begin{tabular}{|c|c|c|c|c|c|}
\hline Butylbenzyl phthalate & $\mathbf{U}$ & 420 & $\mu \mathrm{g} / \mathrm{kg}$ & 420 & EPA8270 \\
\hline 4-Chloroaniline & U & 420 & $\mu \mathrm{g} / \mathrm{kg}$ & 420 & EPA8270 \\
\hline 4-Chloro-m-cresol & $\mathbf{U}$ & 420 & $\mu \mathrm{g} / \mathrm{kg}$ & 420 & EPA8270 \\
\hline 2-Chloronaphthalene & $\mathbf{U}$ & 420 & $\mu \mathrm{g} / \mathrm{kg}$ & 420 & EPA8270 \\
\hline 2-Chlorophenol & $\mathbf{U}$. & 420 & $\mu \mathrm{g} / \mathrm{kg}$ & 420 & EPA8270 \\
\hline 4-Chlorophenyl phenyl ether & U & 420 & $\mu \mathrm{g} / \mathrm{kg}$ & 420 & EPA8270 \\
\hline Chrysene & $\mathbf{U}$ & 420 & $\mu \mathrm{g} / \mathrm{kg}$ & 420 & EPA8270 \\
\hline a-Cresol (2-Mechylphenol) & $\mathbf{U}$ & 420 & $\mu \mathrm{g} / \mathrm{kg}$ & 420 & EPA8270 \\
\hline p-Cresol (4-Methylphenol) & $\mathbf{U}$ & 420 & $\mu \mathrm{g} / \mathrm{kg}$ & 420 & EPA8270 \\
\hline Dibenz $(a, h)$ anthracene & U & 420 & $\mu \mathrm{g} / \mathrm{kg}$ & 420 & EPA8270 \\
\hline Dibenzofuran & U & 420 & $\mu \mathrm{g} / \mathrm{kg}$ & 420 & EPA8270 \\
\hline Di-n-butyl phthalate & $J$ & 48 & $\mu \mathrm{g} / \mathrm{kg}$ & 420 & EPA8270 \\
\hline 1,2-Dichlorobenzene & $\mathbf{U}$ & 420 & $\mu \mathrm{g} / \mathrm{kg}$ & 420 & EPA8270 \\
\hline 1,3-Dichlorobenzene & $\mathbf{U}$ & 420 & $\mu \mathrm{g} / \mathrm{kg}$ & 420 & EPA8270 \\
\hline 1,4-Dichlorobenzene & $\mathbf{U}$ & 420 & $\mu \mathrm{g} / \mathrm{kg}$ & 420 & EPA8270 \\
\hline 3.3'-Dichlorobenzidine & $\mathbf{U}$ & 850 & $\mu \mathrm{g} / \mathrm{kg}$ & 850 & EPA8270 \\
\hline 2,4-Dichlorophenol & $\mathbf{U}$ & 420 & $\mu \mathrm{g} / \mathrm{kg}$ & 420 & EPA8270 \\
\hline Diethyl phthalate & $\mathbf{U}$ & 420 & $\mu \mathrm{g} / \mathrm{kg}$ & 420 & EPA8270 \\
\hline 2,4-Dimethyl phenol & $\mathbf{U}$ & 420 & $\mu \mathrm{g} / \mathrm{kg}$ & 420 & EPA8270 \\
\hline Dimethyl phthalate & $\mathbf{U}$ & 420 & $\mu \mathrm{g} / \mathrm{kg}$ & 420 & EPA8270 \\
\hline 2,4-Dinitrophenol & $\mathbf{U}$ & 2,100 & $\mu \mathrm{g} / \mathrm{kg}$ & 2,100 & EPA8270 \\
\hline 2,4-Dinitsotoluene & $\mathbf{U}$ & 420 & $\mu \mathrm{g} / \mathrm{kg}$ & 420 & EPA8270 \\
\hline 2,6-Dinitrotoluene & $\mathbf{U}$ & 420 & $\mu \mathrm{g} / \mathrm{kg}$ & 420 & EPA8270 \\
\hline Di-n-octyl phthalate & J & 63 & $\mu \mathrm{g} / \mathrm{kg}$ & 420 & EPA8270 \\
\hline Fluoranthene & $\mathbf{U}$ & 420 & $\mu \mathrm{g} / \mathrm{kg}$ & 420 & EPA8270 \\
\hline Fluorene & $\mathbf{U}$ & 420 & $\mu \mathrm{g} / \mathrm{kg}$ & 420 & EPA8270 \\
\hline Hexachlorobenzene & $\mathrm{U}$ & 420 & $\mu \mathrm{g} / \mathrm{kg}$ & 420 & EPA8270 \\
\hline Hexachlorobutadiene & U & 420 & $\mu \mathrm{g} / \mathrm{kg}$ & 420 & EPA8270 \\
\hline Hexachlorocyclopentadiene & $\mathrm{U}$ & 420 & $\mu \mathrm{g} / \mathrm{kg}$ & 420 & EPA8270 \\
\hline Hexachloroethane & $\mathbf{U}$ & 420 & $\mu \mathrm{g} / \mathrm{kg}$ & 420 & EPA8270 \\
\hline Indeno(1,2,3-c,d)pyrene & $\mathrm{U}$ & 420 & $\mu \mathrm{g} / \mathrm{kg}$ & 420 & EPA8270 \\
\hline Isophorone & $\mathbf{U}$ & 420 & $\mu \mathrm{g} / \mathrm{kg}$ & 420 & EPA8270 \\
\hline 2-Methyl-4,6-dinitrophenol & $\mathbf{U}$ & 2,100 & $\mu g / \mathrm{kg}$ & 2,100 & EPA8270 \\
\hline 2-Methylnaphthalene & $\mathbf{U}$ & 420 & $\mu \mathrm{g} / \mathrm{kg}$ & 420 & EPA8270 \\
\hline Naphthalene & $\mathbf{U}$ & 420 & $\mu \mathrm{g} / \mathrm{kg}$ & 420 & EPA8270 \\
\hline o-Nitroaniline & $\mathbf{U}$ & 2,100 & $\mu g / \mathrm{kg}$ & 2,100 & EPA8270 \\
\hline m-Nitroaniline & $\mathbf{U}$ & 2,100 & $\mu \mathrm{g} / \mathrm{kg}$ & 2.100 & EPA8270 \\
\hline p-Nitroaniline & $\mathbf{U}$ & 2,100 & $\mu \mathrm{g} / \mathrm{kg}$ & 2,100 & EPA8270 \\
\hline Nitrobenzene & $\mathbf{U}$ & 420 & $\mu \mathrm{g} / \mathrm{kg}$ & 420 & EPA8270 \\
\hline 2-Nitrophenol & $\mathbf{U}$ & 420 & $\mu \mathrm{g} / \mathrm{kg}$ & 420 & EPA8270 \\
\hline 4-Nitrophenol & $\mathbf{U}$ & 2,100 & $\mu \mathrm{g} / \mathrm{kg}$ & 2,100 & EPA8270 \\
\hline N-Nitrosodiphenylamine & $\mathbf{U}$ & 420 & $\mu \mathrm{g} / \mathrm{kg}$ & 420 & EPA8270 \\
\hline N-Nitrosodipropylamige & $\mathbf{U}$ & 420 & $\mu g / \mathrm{kg}$ & 420 & EPA8270 \\
\hline Pentachlorophenol & $\mathbf{U}$ & 2,100 & $\mu \mathrm{g} / \mathrm{kg}$ & 2.100 & EPA8270 \\
\hline Phenanthrene & $U$ & 420 & $\mu \mathrm{g} / \mathrm{kg}$ & 420 & EPA8270 \\
\hline Phenol & $\mathbf{U}$ & 420 & $\mu \mathrm{g} / \mathrm{kg}$ & 420 & EPA8270 \\
\hline Pyrene & U & 420 & $\mu \mathrm{g} / \mathrm{kg}$ & 420 & EPA8270 \\
\hline 1,2,4-Trichlorobenzene & $\mathbf{U}$ & 420 & $\mu \mathrm{g} / \mathrm{kg}$ & 420 & EPA8270 \\
\hline 2,4,5-Trichlorophenol & $\mathbf{U}$ & 2.100 & $\mu \mathrm{g} / \mathrm{kg}$ & 2,100 & EPA8270 \\
\hline 2,4,6-Trichlorophenol & $\mathrm{U}$ & 420 & $\mu \mathrm{g} / \mathrm{kg}$ & 420 & EPA8270 \\
\hline
\end{tabular}


SAMPLE NAME: D431 1107 (Continued)

Sample D: 100369

$\begin{array}{lllll}\text { Constituent } & \text { RQ AQ } & \text { Result Unit : D. Limit Method }\end{array}$

$B / N / A$ Extractables (including PAH and phenols)

$\begin{array}{llllll}\text { Unknown } & \mathrm{J} & \mathrm{N} & 200 & \mu \mathrm{g} / \mathrm{kg} & \text { EPA8270 } \\ \text { Unknown } & \mathrm{J} & \mathrm{N} & 600 & \mu \mathrm{g} / \mathrm{kg} & \text { EPA8270 } \\ \text { Unknown } & \mathrm{J} & \mathrm{N} & 400 & \mu \mathrm{g} / \mathrm{kg} & \text { EPA8270 }\end{array}$

Volatile Organic Compounds

Acetone

Benzene

Bromodichloromethane

Bromoform

Bromomethane (Methyl bromide)

Carbon disulfide

Carbon tetrachloride

Chlorobenzene

Chloroethane

Chloroethene (Vinyl chloride)

2-Chloroethyl vinyl ether

Chloroform

Chloromethane (Methyl chloride)

Dibromochloromethane

1,1-Dichloroethane

1,2-Dichloroethane

1,1-Dichloroethylene

1,2-Dichloroethylene

Dichloromethane (Methylene chloride)

1,2-Dichloropropane

cis-1,3-Dichloropropene

trans-1,3-Dichloropropene

Ethylbenzene

2-Hexanone

Methyl ethyl ketone

Methyl isobutyl ketone

Styrene

1,1,2,2-Tetrachlorocthane

Tetrachloroethylene

Toluene

1,1,1-Trichloroethane

1,1,2-Trichlorocthane

Trichloroethylene

Unknown

Vinyl acetate

Xylenes

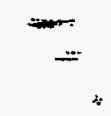

130

6.5

6.5

6.5

13

6.5

6.5

6.5

13

13

13

6.5

13

6.5

6.5

6.5

6.5

6.5

15

6.5

6.5

6.5

6.5

13

13

13

6.5

6.5

6.5

6.5

6.5

6.5

6.5

60

13

6.5

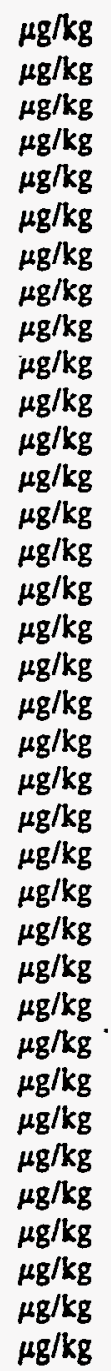

13

6.5

6.5

6.5

13

6.5

6.5

6.5

13

13

13

6.5

13

6.5

6.5

6.5

6.5

6.5

6.5

6.5

6.5

6.5

6.5

13

13

13

6.5

6.5

6.5

6.5

6.5

6.5

6.5

13 .

6.5

EPA8240

EPA8240

EPA8240

EPA8240

EPA8240

EPA8240

EPA8240

EPA8240

EPA8240

EPA8240

EPA8240

EPA8240

EPA8240

EPA8240

EPA8240

EPA8240

EPA8240

EPA8240

EPA8240

EPA8240

EPA8240

EPA8240

EPA8240

EPA8240

EPA8240

EPA8240

EPA 8240

EPA8240

EPA8240

EPA8240

EPA8240

EPA8240

EPA8240

EPA8240

EPA8240

EPA 8240

Pesticides/PCBs and Dioxins/Furans

\section{Aldrin}

alpha-Benzene hexachloride

beta-Benzene hexachloride

delta-Benzene hexachloride

alpha-Chlordane

$\begin{array}{llll}\text { UJ } & \text { O } & \text { L } & 2.1 \\ \text { UJ } & \text { O } & \text { L } & 2.1 \\ \text { UJ } & \text { O } & \text { L } & 2.1 \\ \text { UJ } & \text { O } & \text { L } & 2.1 \\ \text { UJ } & \text { O } & \text { L } & 2.1\end{array}$

$\mu g / \mathrm{kg}$
$\mu g / \mathrm{kg}$
$\mu g / \mathrm{kg}$
$\mu g / \mathrm{kg}$
$\mu g / \mathrm{kg}$

\begin{tabular}{|c|c|}
\hline 2.1 & EPA8080 \\
\hline 2.1 & EPA808 \\
\hline 2.1 & EPA808 \\
\hline 2.1 & EPA 808 \\
\hline 2.1 & EP \\
\hline
\end{tabular}


SAMPLE NAME: D431 1107 (Continued)

Sample ID: 100369

$\begin{array}{llllll}\text { Constituent } & \text { RQ AQ B Result Dit Limit Method }\end{array}$

Pesticides/PCBs and Dioxins/Furans

\begin{tabular}{|c|c|c|c|c|c|c|c|}
\hline gamma-Chlordane & J & 0 & L & 1.3 & $\mu g / \mathrm{kg}$ & 2.1 & EPA8080 \\
\hline$p, p^{\circ}-D D D$ & UJ & 0 & L & 4.3 & $\mu g / k g$ & 4.3 & EPA8080 \\
\hline$p, p^{\prime}-D D E$ & UJ & 0 & $\mathbf{L}$ & 4.3 & $\mu g / k g$ & 4.3 & EPA8080 \\
\hline$p, p^{\prime}-D D T$ & US & 0 & L & 4.3 & $\mu g / \mathrm{kg}$ & 4.3 & EPA8080 \\
\hline Dieldrin & UJ & 0 & L & 4.3 & $\mu \mathrm{g} / \mathrm{kg}$ & 4.3 & EPA8080 \\
\hline Endosulfan I & UJ & 0 & $\mathbf{L}$ & 2.1 & $\mu \mathrm{g} / \mathrm{kg}$ & 2.1 & EPA8080 \\
\hline Endosulfan II & UJ & 0 & $\mathbf{L}$ & 4.3 & $\mu g / \mathrm{kg}$ & 4.3 & EPA8080 \\
\hline Endosulfan sulfate & UJ & 0 & L & 4.3 & $\mu \mathrm{g} / \mathrm{kg}$ & 4.3 & EPA8080 \\
\hline Endrin & UJ & 0 & $\mathbf{L}$ & 4.3 & $\mu g / \mathrm{kg}$ & 4.3 & EPA8080 \\
\hline Endrin ketone & UJ & 0 & L & 4.3 & $\mu \mathrm{g} / \mathrm{kg}$ & 4.3 & EPA8080 \\
\hline Heptachlor & UJ & 0 & L & 2.1 & $\mu \mathrm{g} / \mathrm{kg}$ & 2.1 & EPA8080 \\
\hline Heprachlor epoxide & UJ & 0 & L & 2.1 & $\mu \mathrm{g} / \mathrm{kg}$ & 2.1 & EPA8080 \\
\hline Heptachlorodibenzo-p-dioxin isomers & $\mathrm{U}$ & & & 0.30 & $\mathrm{ng} / \mathrm{g}$ & 0.30 & EPA8280 \\
\hline Heptachlorodibenzo-p-furan isomers & $\mathrm{U}$ & & & 0.20 & $\mathrm{ng} / \mathrm{g}$ & 0.20 & EPA8280 \\
\hline Hexachlorodibenzo-p-dioxin isomers & $\mathrm{U}$ & & & 0.20 & $\mathrm{ng} / \mathrm{g}$ & 0.20 & EPA8280 \\
\hline Hexachlorodibenzo-p-furan isomers & $\mathbf{U}$ & & & 0.10 & $\mathrm{ng} / \mathrm{g}$ & $0.10^{\circ}$ & EPA8280 \\
\hline Lindane & UJ & 0 & L & 2.1 & $\mu g / \mathrm{kg}$ & 2.1 & EPA8080 \\
\hline Methoxychlor & UJ & 0 & L & 21 & $\mu g / \mathrm{kg}$ & 21 & EPA8080 \\
\hline Octachlorodibenzo-p-dioxin & & & & 5.3 & $\mathrm{ng} / \mathrm{g}$ & 0.20 & EPA8280 \\
\hline Octachlorodibenzo-p-furan & $\mathbf{U}$ & & & 0.30 & $\mathrm{ng} / \mathrm{g}$ & 0.30 & EPA8280 \\
\hline PCB 1016 & UJ & 0 & $L$ & 43 & $\mu \mathrm{g} / \mathrm{kg}$ & 43 & EPA8080 \\
\hline PCB 1221 & UJ & $\mathbf{0}$ & L & 85 & $\mu \mathrm{g} / \mathrm{kg}$ & 85 & EPA8080 \\
\hline PCB 1232 & UJ & $\mathbf{0}$ & L & 43 & $\mu \mathrm{g} / \mathrm{kg}$ & 43 & EPA8080 \\
\hline PCB 1242 & UJ & 0 & L & 43 & $\mu \mathrm{g} / \mathrm{kg}$ & 43 & EPA8080 \\
\hline PCB 1248 & UJ & $\mathbf{0}$ & $\mathbf{L}$ & 43 & $\mu \mathrm{g} / \mathrm{kg}$ & 43 & EPA8080 \\
\hline PCB 1254 & UJ & 0 & L & 43 & $\mu \mathrm{g} / \mathrm{kg}$ & 43 & EPA8080 \\
\hline PCB 1260 & UJ & 0 & L & 43 & $\mu \mathrm{g} / \mathrm{kg}$ & 43 & EPA8080 \\
\hline Pentachlorodibenzo-p-dioxin isomers & $\mathrm{U}$ & & & 0.30 & $\mathrm{ng} / \mathrm{g}$ & 0.30 & EPA8280 \\
\hline Pentachlorodibenzo-p-furan isomers & $\mathbf{U}$ & & & 0.20 & $\mathrm{ng} / \mathrm{g}$ & 0.20 & EPA8280 \\
\hline $2,3,7,8-\mathrm{TCDD}$ & U & & & 0.10 & $\mathrm{ng} / \mathrm{g}$ & 0.10 & EPA8280 \\
\hline $2,3,7,8-\mathrm{TCDF}$ & $\mathrm{U}$ & & & 0.20 & $\mathrm{ng} / \mathrm{g}$ & 0.20 & EPA8280 \\
\hline Tetrachlorodibenzo-p-dioxin isomers & $\mathbf{U}$ & & & 0.20 & $\mathrm{ng} / \mathrm{g}$ & 0.20 & EPA8280 \\
\hline Tetrachlorodibenzo-p-furan isomers & U & & & 0.20 & $\mathrm{ng} / \mathrm{g}$ & 0.20 & EPA8280 \\
\hline Toxaphene & UJ & 0 & L & 210 & $\mu \mathrm{g} / \mathrm{kg}$ & 210 & EPA8080 \\
\hline SAMPLE NAME: D431 1201 & & & San & W: & & & \\
\hline
\end{tabular}

Location (SRS Coordinates): 18518.11E 66590.27N

Ground Elevation Above MSL: $127.7 \mathrm{ft}$

Depth of Core Interval: 0.00 to $0.50 \mathrm{ft}$

Sample Type: Normal

Sample Color: 10R 3/4

Sample Matrix: Soil

USC Soil Classification: SM

Sample Moisture: Dry

Percent Solids: 90.90

D. Limit Method

\section{Constituent}

RQ AQ B Result Unit

$\begin{array}{ll}\text { meq/100g } & 2.0 \\ \mathrm{pH} & 0.010 \\ \mu \mathrm{S} / \mathrm{cm} & 1.0 \\ \mathrm{mg} / \mathrm{kg} & 400\end{array}$

EPA9080

EPA9045

4.7

55

Specific conductance

J $\quad$ Q

8.200

$\mathrm{mg} / \mathrm{kg} \quad 400$

EPA9050

EPA415.1 
SAMPLE NAME: D431. 1201 (Continued)

Sample ID: 100370

\section{Constituent}

Total Metals

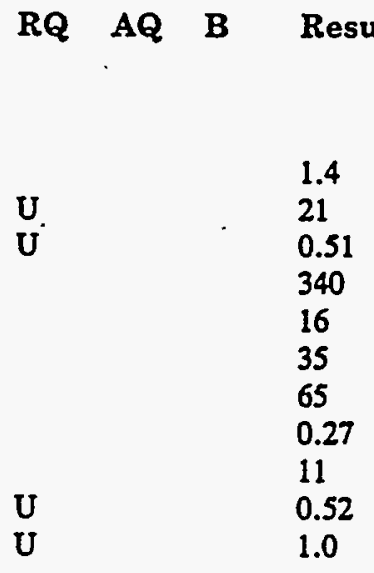

$B / N / A$ Extractables (including PAH and phenols)

\begin{tabular}{|c|c|c|c|c|c|}
\hline Acenaphthene & $\mathrm{U}$ & 360 & $\mu g / \mathrm{kg}$ & 360 & EPA8270 \\
\hline Acenaphthylene & $\mathbf{U}$ & 360 & $\mu g / k g$ & 360 & EPA8270 \\
\hline Acenaphthylene & $\mathbf{U}$ & 370 & $\mu \mathrm{g} / \mathrm{kg}$ & 370 & EPA8270 \\
\hline Acenaphthylene & $\mathrm{U}$ & 360 & $\mu g / \mathrm{kg}$ & 360 & EPA8270 \\
\hline Anthracene & $\mathrm{U}$ & 360 & $\mu \mathrm{g} / \mathrm{kg}$ & 360 & EPA8270 \\
\hline Anthracene & $\mathrm{U}$ & 370 & $\mu \mathrm{g} / \mathrm{kg}$ & 370 & EPA8270 \\
\hline Anthracene & $\mathbf{U}$ & 360 & $\mu \mathrm{g} / \mathrm{kg}$ & 360 & EPA8270 \\
\hline Benzo(a)anthracene & $\mathbf{U}$ & 360 & $\mu \mathrm{g} / \mathrm{kg}$ & 360 & EPA8270 \\
\hline Benzo(a)anthracene & $\mathbf{U}$ & 370 & $\mu g / \mathrm{kg}$ & 370 & EPA8270 \\
\hline Benzo(a)anthracene & $\mathrm{U}$ & 360 & $\mu \mathrm{g} / \mathrm{kg}$ & 360 & EPA8270 \\
\hline Benzo(b)fluoranthene & $\mathbf{U}$ & 360 & $\mu \mathrm{g} / \mathrm{kg}$ & 360 & EPA8270 \\
\hline Benzo(b)fluoranthene & $\mathbf{U}$ & 370 & $\mu \mathrm{g} / \mathrm{kg}$ & 370 & EPA8270 \\
\hline Benzo(b)fluoranthene & $\mathbf{U}$ & 360 & $\mu \mathrm{g} / \mathrm{kg}$ & 360 & EPA8270 \\
\hline Benzo(k)fluoranthene & $\mathrm{U}$ & 360 & $\mu \mathrm{g} / \mathrm{kg}$ & 360 & EPA8270 \\
\hline Benzo(k)fluoranthene & $U$ & 370 & $\mu \mathrm{g} / \mathrm{kg}$ & 370 & EPA8270 \\
\hline Benzo(k)fluoranthene & $U$ & 360 & $\mu \mathrm{g} / \mathrm{kg}$ & 360 & EPA8270 \\
\hline Benzoic acid & $\mathrm{U}$ & 1,800 & $\mu \mathrm{g} / \mathrm{kg}$ & 1.800 & EPA8270 \\
\hline Benzoic acid & $\mathrm{U}$ & 1,800 & $\mu g / \mathrm{kg}$ & 1,800 & EPA8270 \\
\hline Benzoic acid & $\mathrm{U}$ & 1,800 & $\mu \mathrm{g} / \mathrm{kg}$ & 1,800 & EPA8270 \\
\hline Benzo(g,h,i)perylene & $\mathrm{U}$ & 360 & $\mu \mathrm{g} / \mathrm{kg}$ & 360 & ÉPA8270 \\
\hline Benzo $(\mathrm{g}, \mathrm{h}, \mathrm{i})$ perylene & $\mathrm{U}$ & 370 & $\mu \mathrm{g} / \mathrm{kg}$ & 370 & EPA8270 \\
\hline Benzo $(\mathrm{g}, \mathrm{h}, \mathrm{i})$ perylene & $\mathrm{U}$ & 360 & $\mu \mathrm{g} / \mathrm{kg}$ & 360 & EPA8270 \\
\hline Benzo(a)pyrene & U & 360 & $\mu \mathrm{g} / \mathrm{kg}$ & 360 & EPA8270 \\
\hline Benzo(a)pyrene & $\mathrm{U}$ & 370 & $\mu \mathrm{g} / \mathrm{kg}$ & 370 & EPA8270 \\
\hline Benzo(a)pyrene & $\mathrm{U}$ & 360 & $\mu g / \mathrm{kg}$ & 360 & EPA8270 \\
\hline Benzyl alcohol & $\mathrm{U}$ & 360 & $\mu \mathrm{g} / \mathrm{kg}$ & 360 & EPA8270 \\
\hline Benzyl alcohol & U & 370 & $\mu g / \mathrm{kg}$ & 370. & EPA8270 \\
\hline Benzyl alcohol & $\mathrm{U}$ & 360 & $\mu g / \mathrm{kg}$ & 360 & EPA8270 \\
\hline Bis(2-chloroethoxy) methane & $\mathrm{U}$ & 360 & $\mu \mathrm{g} / \mathrm{kg}$ & 360 & EPA8270 \\
\hline Bis(2-chloroethoxy) metthane & $\mathbf{U}$ & 370 & $\mu \mathrm{g} / \mathrm{kg}$ & 370 & EPA8270 \\
\hline Bis(2-chloroethoxy) methane & $\mathrm{U}$ & 360 & $\mu \mathrm{g} / \mathrm{kg}$ & 360 & EPA8270 \\
\hline Bis(2-chloroethyl) ether & $\mathbf{U}$ & 360 & $\mu \mathrm{g} / \mathrm{kg}$ & 360 & EPA8270 \\
\hline Bis(2-chloroisopropyl) ether & $\mathbf{U}$ & 360 & $\mu \mathrm{g} / \mathrm{kg}$ & 360 & EPA8270 \\
\hline Bis(2-chloroethyl) ether & $\mathbf{U}$ & 370 & $\mu \mathrm{g} / \mathrm{kg}$ & 370 & EPA8270 \\
\hline Bis(2-chloroisopropyl) ether & $\mathrm{U}$ & 370 & $\mu g / \mathrm{kg}$ & 370 & EPA8270 \\
\hline Bis(2-chloroethyl) ether & $\mathrm{U}$ & 360 & $\mu \mathrm{g} / \mathrm{kg}$ & 360 & EPA8270 \\
\hline Bis(2-chloroisopropyl) ether & $\mathrm{U}$ & 360 & $\mu \mathrm{g} / \mathrm{kg}$ & 360 & EPA8270 \\
\hline Bis(2-ethylhexyl) phthalate & $\mathrm{J}$ & 79 & $\mu \mathrm{g} / \mathrm{kg}$ & 360 & EPA8270 \\
\hline Bis(2-ethylhexyl) phthalate & $\mathrm{J}$ & 81 & $\mu \mathrm{g} / \mathrm{kg}$ & 370 & EPA8270 \\
\hline
\end{tabular}

EPA7060
EPA6010
EPA6010
EPA6010
EPA7421
EPA6010
EPA7470
EPA6010
EPA7740
EPA6010

$\begin{array}{lll}\mathrm{mg} / \mathrm{kg} & 1.0 & \text { EPA7060 } \\ \mathrm{mg} / \mathrm{kg} & 21 \text { EPA6010 } & \\ \mathrm{mg} / \mathrm{kg} & 0.51 & \text { EPA6010 } \\ \mathrm{mg} / \mathrm{kg} & 1.0 & \text { EPA6010 } \\ \mathrm{mg} / \mathrm{kg} & 2.6 & \text { EPA6010 } \\ \mathrm{mg} / \mathrm{kg} & 6.2 & \text { EPA7421 } \\ \mathrm{mg} / \mathrm{kg} & 1.5 & \text { EPA6010 } \\ \mathrm{mg} / \mathrm{kg} & 0.11 & \text { EPA7470 } \\ \mathrm{mg} / \mathrm{kg} & 4.1 & \text { EPA6010 } \\ \mathrm{mg} / \mathrm{kg} & 0.52 & \text { EPA7740 } \\ \mathrm{mg} / \mathrm{kg} & 1.0 & \text { EPA6010 }\end{array}$


SAMPLE NAME: D43ï 1201 (Continued)

Sample ID: 100370

$\begin{array}{lllll}\text { Constituent } & \text { RQ AQ B Result Unit D. Limit Method }\end{array}$

$B / N / A$ Extractables (including $P A H$ and phenols)

Bis(2-ethylhexyl) phthalate

4-Bromophenyl phenyl ether

4-Bromophenyl phenyl ether

4-Bromophenyl phenyl ether

Butylbenzyl phthalate

Butylbenzyl phthalate

Butylbenzyl phthalate.

4.Chloroaniline

4-Chloroaniline

4-Chloroaniline

4-Chloro-m-cresol

2.Chloronaphthalene

2-Chloronaphthalene

2-Chloronaphthalene

2-Chlorophenol

4-Chlorophenyl phenyl ether

4-Chlorophenyl phenyl ether

4-Chlorophenyl phenyl ether

Chrysene

Chrysene

Chrysene

0-Cresol (2-Methylphenol)

o-Cresol (2-Methylphenol)

o-Cresol (2-Methylphenol)

p-Cresol (4-Methylphenol)

p-Cresol (4-Methylphenol)

p-Cresol (4-Methylphenol)

Hexadecanoic acid

Dibenz(a,h)anthracene

Dibenz $(a, h) a n t h r a c e n e$

Dibenz(a,h)anthracene

Dibenzofuran

Dibenzofuran

Dibenzofuran

Di-n-butyl phthalate

Di-n-butyl phthalate

Di-n-butyl phthalate

1,2-Dichlorobenzene

1,2-Dichlorobenzene

1,2-Dichlorobenzene -

1,3-Dichlorobenzene

1,3-Dichlorobenzene

1,3-Dichlorobenzene

1.4-Dichlorobenzene

3,3'-Dichlorobenzidine

3,3'-Dichlorobenzidine

3,3'-Dichlorobenzidine

2,4-Dichlorophenol

2,4-Dichlorophenol

2,4-Dichlorophenol
J

U

U

U

U

U

U

U

U

U

$U$

U

U

U

U

U

U

U

U

U

U

U

U

U

U

U

U

U

U

U

U

U

U

U

U

U

U

U

U

U

U

U

U

U

U

U

U

U

U
65

360

370

360

360

370

360

360

370

360

360

360

370

360

360

360

370

360

360

370

360

360

370

360

360

370

360

200

360

370

360

360

370

360

360

370

360

360

370

360

360

370

360

360

720

730

710

360

.370

360

\begin{tabular}{|c|c|c|}
\hline $\begin{array}{l}\mu \mathrm{g} / \mathrm{kg} \\
\mu \mathrm{g} / \mathrm{kg}\end{array}$ & $\begin{array}{l}360 \\
360\end{array}$ & $\begin{array}{l}\text { EPA8270 } \\
\text { EPA8270 }\end{array}$ \\
\hline $\begin{array}{l}\mu \mathrm{g} / \mathrm{kg} \\
\mu \mathrm{g} / \mathrm{kg}\end{array}$ & 370 & EPA8270 \\
\hline$\mu \mathrm{g} / \mathrm{kg}$ & 360 & EPA8270 \\
\hline$\mu \mathrm{g} / \mathrm{kg}$ & 360 & EPA8270 \\
\hline$\mu \mathrm{g} / \mathrm{kg}$ & 370 & EPA8270 \\
\hline$\mu g / \mathrm{kg}$ & 360 & EPA8270 \\
\hline$\mu g / k g$ & 360 & EPA8270 \\
\hline$\mu \mathrm{g} / \mathrm{kg}$ & 370 & EPA8270 \\
\hline$\mu g / \mathrm{kg}$ & 360 & EPA8270 \\
\hline$\mu \mathrm{g} / \mathrm{kg}$ & 360 & EPA8270 \\
\hline$\mu \mathrm{g} / \mathrm{kg}$ & 360 & EPA8270 \\
\hline$\mu \mathrm{g} / \mathrm{kg}$ & 370 & EPA8270 \\
\hline$\mu \mathrm{g} / \mathrm{kg}$ & 360 & EPA8270 \\
\hline$\mu \mathrm{g} / \mathrm{kg}$ & 360 & EPA8270 \\
\hline$\mu \mathrm{g} / \mathrm{kg}$ & 360 & EPA8270 \\
\hline$\mu \mathrm{g} / \mathrm{kg}$ & 370 & EPA8270 \\
\hline$\mu \mathrm{g} / \mathrm{kg}$ & 360 & EPA8270 \\
\hline$\mu \mathrm{g} / \mathrm{kg}$ & 360 & EPA8270 \\
\hline$\mu \mathrm{g} / \mathrm{kg}$ & 370 & EPA8270 \\
\hline$\mu \mathrm{g} / \mathrm{kg}$ & 360 & EPA8270 \\
\hline$\mu \mathrm{g} / \mathrm{kg}$ & 360 & EPA8270 \\
\hline$\mu g / \mathrm{kg}$ & 370 & EPA8270 \\
\hline$\mu g / \mathrm{kg}$ & 360 & EPA8270 \\
\hline$\mu \mathrm{g} / \mathrm{kg}$ & 360 & EPA8270 \\
\hline$\mu \mathrm{g} / \mathrm{kg}$ & 370 & EPA8270 \\
\hline$\mu \mathrm{g} / \mathrm{kg}$ & 360 & EPA8270 \\
\hline$\mu \mathrm{g} / \mathrm{kg}$ & & EPA8270 \\
\hline$\mu \mathrm{g} / \mathrm{kg}$ & 360 & EPA8270 \\
\hline$\mu \mathrm{g} / \mathrm{kg}$ & 370 & EPA8270 \\
\hline$\mu \mathrm{g} / \mathrm{kg}$ & 360 & EPA8270 \\
\hline$\mu \mathrm{g} \mathrm{kg}$ & 360 & EPA8270 \\
\hline$\mu \mathrm{g} / \mathrm{kg}$ & 370 & EPA8270 \\
\hline$\mu g / \mathrm{kg}$ & 360 & EPA8270 \\
\hline$\mu g / \mathrm{kg}$ & 360 & EPA8270 \\
\hline$\mu \mathrm{g} / \mathrm{kg}$ & 370 & EPA8270 \\
\hline$\mu g / k g$ & 360 & EPA8270 \\
\hline$\mu \mathrm{g} / \mathrm{kg}$ & 360 & EPA8270 \\
\hline$\mu g / \mathrm{kg}$ & 370 & EPA8270 \\
\hline$\mu \mathrm{g} / \mathrm{kg}$ & 360 & EPA8270 \\
\hline$\mu \mathrm{g} / \mathrm{kg}$ & 360 & EPA8270 \\
\hline$\mu \mathrm{g} / \mathrm{kg}$ & 370 & EPA8270 \\
\hline$\mu g / \mathrm{kg}$ & 360 & EPA8270 \\
\hline$\mu \mathrm{g} / \mathrm{kg}$ & 360 & EPA8270 \\
\hline$\mu \mathrm{g} / \mathrm{kg}$ & 720 & EPA8270 \\
\hline$\mu \mathrm{g} / \mathrm{kg}$ & 730 & EPA8270 \\
\hline$\mu g / \mathrm{kg}$ & 710 & EPA8270 \\
\hline$\mu \mathrm{g} / \mathrm{kg}$ & 360 & EPA8270 \\
\hline$\mu \mathrm{g} / \mathrm{kg}$ & 370 & EPA8270 \\
\hline$\mu \mathrm{g} / \mathrm{kg}$ & 360 & EPA8270 \\
\hline
\end{tabular}


SAMPLE NAME: D431 1201 (Continued)

Sample ID: 100370

$\begin{array}{llllll}\text { Constituent } & \text { RQ AQ B Result Unit Limit Method }\end{array}$

$B / N / A$ Extractables (including $P A H$ and phenols)

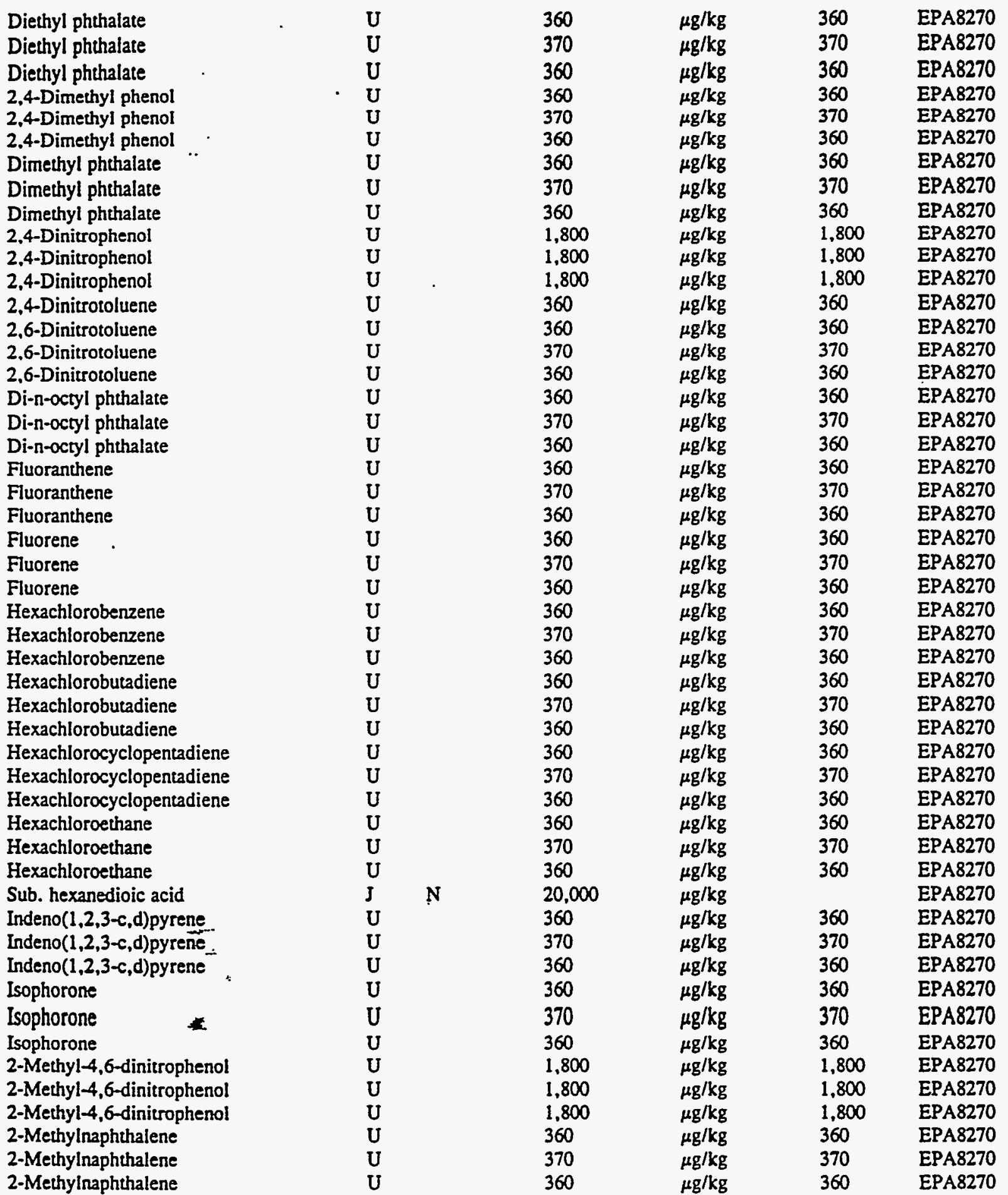


SAMPLE NAME: D431 1201 (Continued)

Sample ID: 100370

Constituent RQ AQ B Result Unit D. Limit Method

$B / N / A$ Extractables (including PAH and phenols)

Naphthalene

Naphthalene

Naphthalene

o-Nitroaniline

o-Nitroaniline

o-Nitroaniline

m-Nitroaniline

m-Nitroaniline

m-Nitroaniline

p-Nitroaniline

Nitrobenzene

Nitrobenzene

Nitrobenzene

2-Nitrophenol

2-Nitrophenol

2-Nitrophenol

4-Nitrophenol

N-Nitrosodiphenylamine

N-Nitrosodiphenylamine

N-Nitrosodiphenylamine

$\mathrm{N}$-Nitrosodipropylamine

Pentachlorophenol

Phenanthrene

Phenanthrene

Phenanthrene

Phenol

Pyrene

1,2,4-Trichlorobenzene

2,4,5-Trichlorophenol

2,4,5-Trichlorophenol

2,4,5-Trichlorophenol

2,4,6-Trichlorophenol

2,4,6-Trichlorophenol

2,4,6-Trichlorophenol

Unknown

Unknown

Unknown

Unknown

Unknown

Unknown ketone

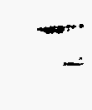

$+$
p-Nitroaniline

p-Nitroaniline
U 360

360
370

360

1,800

1,800

1,800

1,800

1,800

1,800

1,800

1,800

1,800

360

370

360

360

370

360

1,800

360

370

360

360

1,800

360

370

360

360

360

360

1,800

1,800

1,800

360

370

360

NV $\quad 3,000$

NV $\quad 700$

N 300

$N \quad 200$

$N \quad 200$

NV

40,000

\section{$\mu \mathrm{g} / \mathrm{kg}$}

$\mu \mathrm{g} / \mathrm{kg}$

$\mu \mathrm{g} / \mathrm{kg}$

$\mu g / \mathrm{kg}$

$\mu \mathrm{g} / \mathrm{kg}$

$\mu \mathrm{g} / \mathrm{kg}$

$\mu \mathrm{g} / \mathrm{kg}$

$\mu \mathrm{g} / \mathrm{kg}$

$\mu \mathrm{g} / \mathrm{kg}$

$\mu g / k g$

$\mu \mathrm{g} / \mathrm{kg}$

$\mu \mathrm{g} / \mathrm{kg}$

$\mu g / \mathrm{kg}$

$\mu \mathrm{g} / \mathrm{kg}$

$\mu \mathrm{g} / \mathrm{kg}$

$\mu \mathrm{g} / \mathrm{kg}$

$\mu \mathrm{g} / \mathrm{kg}$

$\mu g / k g$

$\mu \mathrm{g} / \mathrm{kg}$

$\mu \mathrm{g} / \mathrm{kg}$

$\mu \mathrm{g} / \mathrm{kg}$

$\mu g / \mathrm{kg}$

$\mu \mathrm{g} / \mathrm{kg}$

$\mu \mathrm{g} / \mathrm{kg}$

$\mu \mathrm{g} / \mathrm{kg}$

$\mu \mathrm{g} / \mathrm{kg}$

$\mu \mathrm{g} / \mathrm{kg}$

$\mu \mathrm{g} / \mathrm{kg}$

$\mu \mathrm{g} / \mathrm{kg}$

$\mu \mathrm{g} / \mathrm{kg}$

$\mu \mathrm{g} / \mathrm{kg}$

$\mu \mathrm{g} / \mathrm{kg}$

$\mu \mathrm{g} / \mathrm{kg}$

$\mu \mathrm{g} / \mathrm{kg}$

$\mu \mathrm{g} / \mathrm{kg}$

$\mu \mathrm{g} / \mathrm{kg}$

$\mu \mathrm{g} / \mathrm{kg}$

$\mu \mathrm{g} / \mathrm{kg}$

$\mu g / \mathrm{kg}$

$\mu g / \mathrm{kg}$

$\mu \mathrm{g} / \mathrm{kg}$

$\mu \mathrm{g} / \mathrm{kg}$
EPA8270

EPA8270

EPA8270

EPA8270

EPA8270

EPA8270

EPA8270

EPA8270

EPA8270

EPA8270

EPA8270

EPA8270

EPA8270

EPA8270

EPA8270

EPA8270

EPA8270

EPA8270

EPA8270

EPA8270

EPA8270

EPA8270

EPA8270

EPA8270

EPA8270

EPA8270

EPA8270

EPA8270

EPA8270

EPA8270

EPA8270

EPA8270

EPA8270

EPA8270

EPA8270

EPA8270

EPA8270

EPA8270

EPA8270

EPA8270

EPA8270

EPA8270

Volatile Organic Compounds

Acetone

U

11

5.5

5.5

Bromodichloromethane

Bromoform

Bromomethane (Methyl bromide)
U

U

U

U
5.5

11 $\mu \mathrm{g} / \mathrm{kg}$

$\mu \mathrm{g} / \mathrm{kg}$

$\mu \mathrm{g} / \mathrm{kg}$

$\mu \mathrm{g} / \mathrm{kg}$

$\mu \mathrm{g} / \mathrm{kg}$

$\begin{array}{ll}11 & \text { EPA8240 } \\ 5.5 & \text { EPA8240 } \\ 5.5 & \text { EPA8240 } \\ 5.5 & \text { EPA8240 } \\ 11 & \text { EPA8240 }\end{array}$


SAMPLE NAME: D43I 1201 (Continued)

Sample ID: 100370

\section{Constituent}

Volatile Organic Compounds
RQ AQ B Result

Unit

D. Limit Method

Carbon disulfide
Carbon tetrachloride
Chlorobenzene
Chloroethane :
Chloroethene (Vinyl chloride)
2-Chloroethyl vinyl ether
Chloroform
Chloromethane (Methyl chloride)
Dibromochloromethane
1,1-Dichloroethane
1.2-Dichloroethane
1,1-Dichloroethylene
1,2-Dichloroethylene
Dichloromethane (Methylene chloride)
1,2-Dichloropropane
cis-1,3-Dichloropropene
trans-1,3-Dichloropropene
Ethylbenzene
2-Hexanone
Methyl ethyl ketone
Methyl isobutyl ketone
Styrene
1,1,2,2-Tetrachloroethane
Tetrachloroethylene
Toluene
1,1,1-Trichloroethane
1,1,2-Trichloroethane
Trichloroethylene
Vinyl acetate
Xylenes

Pesticides/PCBs and Dioxins/Furans

5.5
5.5
5.5
11
11
11
5.5
11
5.5
5.5
5.5
5.5
5.5
8.5
5.5
5.5
5.5
5.5
11
11
11
5.5
5.5
5.5
5.5
5.5
5.5
5.5
11
5.5

$\mu \mathrm{g} / \mathrm{kg}$
$\mu \mathrm{g} / \mathrm{kg}$
$\mu \mathrm{g} / \mathrm{kg}$
$\mu \mathrm{g} / \mathrm{kg}$
$\mu \mathrm{kg} / \mathrm{kg}$
$\mu \mathrm{g} / \mathrm{kg}$
$\mu \mathrm{g} / \mathrm{kg}$
$\mu \mathrm{g} / \mathrm{kg}$
$\mu \mathrm{g} / \mathrm{kg}$
$\mu \mathrm{g} / \mathrm{kg}$
$\mu \mathrm{g} / \mathrm{kg}$
$\mu \mathrm{g} / \mathrm{kg}$
$\mu \mathrm{g} / \mathrm{kg}$
$\mu \mathrm{g} / \mathrm{kg}$
$\mu \mathrm{g} / \mathrm{kg}$
$\mu \mathrm{g} / \mathrm{kg}$
$\mu \mathrm{g} / \mathrm{kg}$
$\mu \mathrm{g} / \mathrm{kg}$
$\mu \mathrm{g} / \mathrm{kg}$
$\mu \mathrm{g} / \mathrm{kg}$
$\mu \mathrm{g} / \mathrm{kg}$
$\mu \mathrm{g} / \mathrm{kg}$
$\mu \mathrm{g} / \mathrm{kg}$
$\mu \mathrm{g} / \mathrm{kg}$
$\mu \mathrm{g} / \mathrm{kg}$
$\mu \mathrm{g} / \mathrm{kg}$
$\mu \mathrm{g} / \mathrm{kg}$
$\mu \mathrm{g} / \mathrm{kg}$
$\mu \mathrm{g} / \mathrm{kg}$
$\mu \mathrm{g} / \mathrm{kg}$

5.5

5.5

5.5

11

11

11

5.5

11

5.5

5.5

5.5

5.5

5.5

5.5

5.5

5.5

5.5

5.5

11

11

5.5

5.5

5.5

5.5

5.5

5.5

5.5

11

5.5

\begin{abstract}
Aldrin
Aldrin

alpha-Benzene hexachloride

alpha-Benzene hexachloride

alpha-Benzene hexachloride

beta-Benzene hexachloride

beta-Benzene hexachloride

beta-Benzene hexachloride

delta-Benzene hexachloride

delta-Benzene hexachloride

delta-Benzene hexachloride

alpha-Chlordane

alpha-Chlordane

alpha-Chlordane

gamma-Chlordane

gamma-Chlordane

gamma-Chlordane
\end{abstract}

9.2

9.2

9.2

9.2

9.2

9.2

9.2

9.2

9.2

9.2

9.2

9.2

9.2

9.2

.9 .2

9.2

9.2 $\mu \mathrm{g} / \mathrm{kg}$
$\mu \mathrm{g} / \mathrm{kg}$
$\mu \mathrm{g} / \mathrm{kg}$
$\mu \mathrm{g} / \mathrm{kg}$
$\mu \mathrm{g} / \mathrm{kg}$
$\mu \mathrm{g} / \mathrm{kg}$
$\mu \mathrm{g} / \mathrm{kg}$
$\mu \mathrm{g} / \mathrm{kg}$
$\mu \mathrm{g} / \mathrm{kg}$
$\mu \mathrm{g} / \mathrm{kg}$
$\mu \mathrm{g} / \mathrm{kg}$
$\mu \mathrm{g} / \mathrm{kg}$
$\mu \mathrm{g} / \mathrm{kg}$
$\mu \mathrm{g} / \mathrm{kg}$
$\mu \mathrm{g} / \mathrm{kg}$
$\mu \mathrm{g} / \mathrm{kg}$
$\mu \mathrm{g} / \mathrm{kg}$

9.2

9.2

9.2

9.2

9.2

9.2

9.2

9.2

9.2

9.2

9.2

9.2

9.2

9.2

9.2

9.2

9.2
EPA8240

EPA8240

EPA8240

EPA8240

EPA8240

EPA8240

EPA8240

EPA8240

EPA8240

EPA8240

EPA8240

EPA8240

EPA8240

EPA8240

EPA8240

EPA8240

EPA8240

EPA8240

EPA8240

EPA8240

EPA8240

EPA8240

EPA8240

EPA8240

EPA8240

EPA8240

EPA8240

EPA8240

EPA8240

EPA8240

EPA8080

EPA8080

EPA8080

EPA8080

EPA8080

EPA8080

EPA8080

EPA8080

EPA.8080

EPA8080

EPA8080

EPA8080

EPA8080

EPA8080

EPA8080

EPA8080

EPA8080 
SAMPLE NAME: D431 1201 (Continued).

RQ AQ B Result

Constituent

Pesticides/PCBs and Dioxins/Furans

\begin{tabular}{|c|c|c|c|c|c|}
\hline p.p'-DDD & 了 & 17 & $\mu g / k g$ & 18 & EPA8080 \\
\hline p.p'-DDD & & 25 & $\mu \mathrm{g} / \mathrm{kg}$ & 18 & EPA8080 \\
\hline P.P'-DDD & $\mathbf{J}$ & 8.8 & $\mu \mathrm{g} / \mathrm{kg}$ & 18 & EPA8080 \\
\hline p, $p^{\prime}-D D E$ & & 22 & $\mu \mathrm{g} / \mathrm{kg}$ & 18 & EPA8080 \\
\hline p.p'-DDE & & 29 & $\mu g / k g$ & 18 & EPA8080 \\
\hline p.p'-DDE & s & 13 & $\mu \mathrm{g} / \mathrm{kg}$ & 18 & EPA8080 \\
\hline p.p'-DDT & & 41 & $\mu \mathrm{g} / \mathrm{kg}$ & 18 & EPA8080 \\
\hline p.p'-DDT & & 61 & $\mu \mathrm{g} / \mathrm{kg}$ & 18 & EPA8080 \\
\hline Dieldrin & $\mathbf{U}$ & 18 & $\mu \mathrm{g} / \mathrm{kg}$ & 18 & EPA8080 \\
\hline Dieldrin & $\mathbf{U}$ & 18 & $\mu g / k g$ & 18 & EPA8080 \\
\hline Endosulfan I & $\mathbf{U}$ & 9.2 & $\mu \mathrm{g} / \mathrm{kg}$ & 9.2 & EPA8080 \\
\hline Endosulfan I & $\mathbf{U}$ & 9.2 & $\mu \mathrm{g} / \mathrm{kg}$ & 9.2 & EPA 8080 \\
\hline Endosulfan I & $\mathbf{U}$ & 9.2 & $\mu \mathrm{g} / \mathrm{kg}$ & 9.2 & EPA8080 \\
\hline Endosulfan II & U & 18 & $\mu \mathrm{g} / \mathrm{kg}$ & 18 & EPA8080 \\
\hline Endosulfan II & $\mathrm{U}$ & 18 & $\mu \mathrm{g} / \mathrm{kg}$ & 18 & EPA8080 \\
\hline Endosulfan II & U & 18 & $\mu \mathrm{g} / \mathrm{kg}$ & 18 & EPA8080 \\
\hline Endosulfan sulfate & $\mathbf{U}$ & 18 & $\mu g / k g$ & 18 & EPA8080 \\
\hline Endosulfan sulfate & $\mathbf{U}$ & 18 & $\mu g / \mathrm{kg}$ & 18 & EPA8080 \\
\hline Endosulfan sulfate & $\mathrm{U}$ & 18 & $\mu g / \mathrm{kg}$ & 18 & EPA8080 \\
\hline Endrin & $\mathbf{U}$ & 18 & $\mu \mathrm{g} / \mathrm{kg}$ & 18 & EPA8080 \\
\hline Endrin & $\mathrm{U}$ & 18 & $\mu \mathrm{g} / \mathrm{kg}$ & 18 & EPA8080 \\
\hline Endrin ketone & $\mathrm{U}$ & 18 & $\mu \mathrm{g} / \mathrm{kg}$ & 18 & EPA8080 \\
\hline Endrin ketone & $\mathrm{U}$ & 18 & $\mu \mathrm{g} / \mathrm{kg}$ & 18 & EPA8080 \\
\hline Endrin ketone & $\mathrm{U}$ & 18 & $\mu \mathrm{g} / \mathrm{kg}$ & 18 & EPA8080 \\
\hline Heptachlor & U & 9.2 & $\mu \mathrm{g} / \mathrm{kg}$ & 9.2 & EPA8080 \\
\hline Heptachlor & $\mathbf{U}$ & 9.2 & $\mu \mathrm{g} / \mathrm{kg}$ & 9.2 & EPA8080 \\
\hline Heptachlor epoxide & $\mathrm{U}$ & 9.2 & $\mu \mathrm{g} / \mathrm{kg}$ & 9.2 & EPA8080 \\
\hline Heptachlor epoxide & $\mathrm{U}$ & 9.2 & $\mu \mathrm{g} / \mathrm{kg}$ & 9.2 & EPA8080 \\
\hline Heptachlor epoxide & $\mathrm{U}$ & 9.2 & $\mu \mathrm{g} / \mathrm{kg}$ & 9.2 & EPA8080 \\
\hline Heptachlorodibenzo-p-dioxị isomers & $\mathbf{U}$ & 0.40 & $\mathrm{ng} / \mathrm{g}$ & 0.40 & EPA8280 \\
\hline Heptachlorodibenzo-p-furan isomers & $\mathbf{U}$ & 0.10 & $\mathrm{ng} / \mathrm{g}$ & 0.10 & EPA8280 \\
\hline Hexachlorodibenzo-p-dioxin isomers & $\mathrm{U}$ & 0.30 & $\mathrm{ng} / \mathrm{g}$ & 0.30 & EPA8280 \\
\hline Hexachlorodibenzo-p-furan isomers & U & 0.10 & $\mathrm{ng} / \mathrm{g}$ & 0.10 & EPA8280 \\
\hline Lindane & $\mathrm{U}$ & 9.2 & $\mu \mathrm{g} / \mathrm{kg}$ & 9.2 & EPA8080 \\
\hline Lindane & $\mathrm{U}$ & 9.2 & $\mu \mathrm{g} / \mathrm{kg}$ & 9.2 & EPA8080 \\
\hline Methoxychlor & $\mathrm{U}$ & 92 & $\mu \mathrm{g} / \mathrm{kg}$ & 92 & EPA8080 \\
\hline Methoxychlor & $\mathrm{U}$ & 92 & $\mu \mathrm{g} / \mathrm{kg}$ & 92 & EPA8080 \\
\hline Methoxychlor & $\mathbf{U}$ & 92 & $\mu g / \mathrm{kg}$ & 92 & EPA8080 \\
\hline Octachlorodibenzo-p-dioxin & & 3.6 & $\mathrm{ng} / \mathrm{g}$ & 0.10 & EPA8280 \\
\hline Octachlorodibenzo-p-furan & $\mathbf{U}$ & 0.30 & $\mathrm{ng} / \mathrm{g}$ & 0.30 & EPA8280 \\
\hline PCB 1016 & $\mathrm{U}$ & 180 & $\mu \mathrm{g} / \mathrm{kg}$ & 180 & EPA8080 \\
\hline PCB 1016 & $\mathbf{U}$ & 180 & $\mu \mathrm{g} / \mathrm{kg}$ & 180 & EPA8080 \\
\hline PCB 1016 & $\mathbf{U}$ & 180 & $\mu g / \mathrm{kg}$ & 180 & EPA8080 \\
\hline PCB 1221 & $\mathrm{U}$ & 370 & $\mu g / \mathrm{kg}$ & 370 & EPA8080 \\
\hline РCB 1221 & $\mathbf{U}$ & 370 & $\mu g / \mathrm{kg}$ & 370 & EPA8080 \\
\hline РСB 1221 & $\mathbf{U}$ & 370 & $\mu \mathrm{g} / \mathrm{kg}$ & 370 & EPA8080 \\
\hline PCB 1232 & $\mathbf{U}$ & 180 & $\mu \mathrm{g} / \mathrm{kg}$ & 180 & EPA8080 \\
\hline PCB 1232 & $\mathbf{U}$ & 180 & $\mu g / \mathrm{kg}$ & 180 & EPA8080 \\
\hline PCB 1232 & $\mathbf{U}$ & 180 & $\mu \mathrm{g} / \mathrm{kg}$ & 180 & EPA8080 \\
\hline РCB 1242 & $U$ & 180 & $\mu \mathrm{g} / \mathrm{kg}$ & 180 & EPA8080 \\
\hline
\end{tabular}

Sample ID: 100370

Unit D. Limit Method 
SAMPLE NAME: D431 1201 (Continued)

\section{Constituent}

Sample D: 100370

RQ AQ B Result Unit D. Limit Method

Pesticides/PCBs and Dioxins/Furans

PCB 1242
PCB 1242
PCB 1248
PCB 1248
PCB 1248
PCB 1254
PCB 1254
PCB 1254
PCB 1260
PCB 1260

PCB 1260

Pentachlorodibenzo-p-dioxin isomers

Pentachlorodibenzo-p-furan isomers

2.3,7,8-TCDD

2,3,7,8-TCDF

U 180

180

180

180

180

180

180

180

1,300

1,300

1,200

0.20

0.20

0.20

0.20

Tetrachlorodibenzo-p-dioxin isomers

0.20

0.20

920

Toxaphene

Toxaphene

Toxaphene

920

920 .

$\begin{array}{lll}\mu \mathrm{g} / \mathrm{kg} & 180 & \text { EPA8080 } \\ \mu \mathrm{g} / \mathrm{kg} & 180 & \text { EPA8080 } \\ \mu \mathrm{g} / \mathrm{kg} & 180 & \text { EPA8080 } \\ \mu \mathrm{g} / \mathrm{kg} & 180 & \text { EPA8080 } \\ \mu \mathrm{g} / \mathrm{kg} & 180 & \text { EPA8080 } \\ \mu \mathrm{g} / \mathrm{kg} & 180 & \text { EPA8080 } \\ \mu \mathrm{g} / \mathrm{kg} & 180 & \text { EPA8080 } \\ \mu \mathrm{g} / \mathrm{kg} & 180 & \text { EPA8080 } \\ \mu \mathrm{g} / \mathrm{kg} & 180 & \text { EPA8080 } \\ \mu \mathrm{g} / \mathrm{kg} & 180 & \text { EPA8080 } \\ \mu \mathrm{g} / \mathrm{kg} & 180 & \text { EPA8080 } \\ \mathbf{n g} / \mathrm{g} & 0.20 & \text { EPA8280 } \\ \mathbf{n g} / \mathrm{g} & 0.20 & \text { EPA8280 } \\ \mathrm{ng} / \mathrm{g} & 0.20 & \text { EPA8280 } \\ \mathrm{ng} / \mathrm{g} & 0.20 & \text { EPA8280 } \\ \mathrm{ng} / \mathrm{g} & 0.20 & \text { EPA8280 } \\ \mathrm{ng} / \mathrm{g} & 0.20 & \text { EPA8280 } \\ \mu \mathrm{g} / \mathrm{kg} & 920 & \text { EPA8080 } \\ \mu \mathrm{g} / \mathrm{kg} & 920 & \text { EPA8080 } \\ \mu \mathrm{g} / \mathrm{kg} & 920 & \text { EPA8080 }\end{array}$

Radionuclide Indicators and Radionuclides

Gross alpha

Gross alpha

$8.7 \pm 12$

$11 \pm 12$

$\mathrm{pCi} / \mathrm{g}$

6.7

EPA900.0

Nonvolatile beta

$27 \pm 21$

Nonvolatile beta

Radium, total alpha-emitting

$33 \pm 19$

Radium, total alpha-emitting

Tritium

$1.2 \pm 0.86$

$\mathrm{pCi} / \mathrm{g}$

$\mathrm{pCi} / \mathrm{g}$

5.9

EPA900.0

9.3 EPA900.0

8.2 EPA900.0

0.16 EPA903.0

Tritium

$1.1 \pm 0.78$

$\mathrm{pCl} / \mathrm{g}$

0.15

EPA903.0

1.3

1.4

$\mathrm{pCi} / \mathrm{g}$

$\mathrm{pCi} / \mathrm{g}$

1.3

EPA906.0

1.4 EPA906.0

SAMPLE NAME: D431 1202

Location (SRS Coordinates): 18518.11E 66590.27N

Ground Elevation Above MSL: $127.7 \mathrm{ft}$

Depth of Core Interval: 0.50 to $2.00 \mathrm{ft}$

Sample Type: Normal

Sample Color: 10R 3/4

Constituent :

Total Metals

Arsenic

Barium

Cadmium

Chromium

Copper
Sample Matrix: Soil USC Soil Classification: SM

RQ AQ B Result Unit

$+$
Sample ID: 100371

Sample Moisture: Dry

Percent Solids: 89.90

D. Limit Method
1.3

U 21

U $\quad 0.53$

260

10 $\mathrm{mg} / \mathrm{kg}$
$\mathrm{mg} / \mathrm{kg}$
$\mathrm{mg} / \mathrm{kg}$
$\mathrm{mg} / \mathrm{kg}$
$\mathrm{mg} / \mathrm{kg}$
1.0

21

0.53

1.1

2.6
EPA7060

EPA6010

EPA6010

EPA6010

EPA6010 
SAMPLE NAME: D431 1202 (Continued)

Sample ID: 100371

$\begin{array}{lllllll}\text { Constituent } & \text { RQ } & \text { AQ } & \text { B } & \text { Result } & \text { Unit } & \text { D. Limit Method }\end{array}$

Total Metals

Lead
Manganese
Mercury
Nickel
Selenium
Silver

24

45

0.30

8.4

$\mathrm{U} \quad 0.52$

Silver

\section{$B / N / A$ Extractables (including PAH and phenols)}

\begin{tabular}{|c|c|}
\hline U & 370 \\
\hline U & 370 \\
\hline$U$ & 370 \\
\hline J & 71 \\
\hline J & 110 \\
\hline J & 43 \\
\hline $\mathrm{U}$ & 1,800 \\
\hline $\mathbf{U}$ & 370 \\
\hline J & 85 \\
\hline $\mathbf{U}$ & 370 \\
\hline U & 370 \\
\hline U & 370 \\
\hline U & 370 \\
\hline J & 66 \\
\hline $\mathrm{U}$ & 370 \\
\hline $\mathrm{U}$ & 370 \\
\hline U & 370 \\
\hline $\mathrm{U}$ & 370 \\
\hline $\mathbf{U}$ & 370 \\
\hline $\mathbf{U}$ & 370 \\
\hline U & 370 \\
\hline J & 54 \\
\hline $\mathbf{U}$ & 370 \\
\hline $\mathbf{U}$ & 370 \\
\hline $\mathrm{U}$ & 370 \\
\hline $\mathbf{U}$ & 370 \\
\hline $\mathbf{U}$ & 370 \\
\hline $\mathrm{U}$ & 370 \\
\hline $\mathrm{U}$ & 370 \\
\hline $\mathbf{U}$ & 370 \\
\hline $\mathrm{U}$ & 740 \\
\hline $\mathbf{U}$ & 370 \\
\hline U & 370 \\
\hline $\mathbf{U}$ & 370 \\
\hline U & 370 \\
\hline $\mathrm{U}$ & 1,800 \\
\hline $\mathbf{U}$ & 370 \\
\hline $\mathbf{U}$ & 370 \\
\hline $\mathbf{U}$ & 370 \\
\hline J & 110 \\
\hline$U$ & 370 \\
\hline
\end{tabular}

$\mathrm{mg} / \mathrm{kg}$
$\mathrm{mg} / \mathrm{kg}$
$\mathrm{mg} / \mathrm{kg}$
$\mathrm{mg} / \mathrm{kg}$
$\mathrm{mg} / \mathrm{kg}$
$\mathrm{mg} / \mathrm{kg}$

6.2

1.6

0.11

4.2

0.52

1.1

\author{
EPA7421 \\ EPA6010 \\ EPA7470 \\ EPA6010 \\ EPA7740 \\ EPA6010
}

\begin{tabular}{|c|c|c|}
\hline$\mu g / \mathrm{kg}$ & 370 & EPA8270 \\
\hline$\mu \mathrm{g} / \mathrm{kg}$ & 370 & EPA8270 \\
\hline$\mu \mathrm{g} / \mathrm{kg}$ & 370 & EPA8270 \\
\hline$\mu \mathrm{g} / \mathrm{kg}$ & 370 & EPA8270 \\
\hline$\mu \mathrm{g} / \mathrm{kg}$ & 370 & EPA8270 \\
\hline$\mu \mathrm{g} / \mathrm{kg}$ & 370 & EPA8270 \\
\hline$\mu \mathrm{g} / \mathrm{kg}$ & 1.800 & EPA8270 \\
\hline$\mu \mathrm{g} / \mathrm{kg}$ & 370 & EPA8270 \\
\hline$\mu \mathrm{g} / \mathrm{kg}$ & 370 & EPA8270 \\
\hline$\mu \mathrm{g} / \mathrm{kg}$ & 370 & EPA8270 \\
\hline$\mu \mathrm{g} / \mathrm{kg}$ & 370 & EPA8270 \\
\hline$\mu \mathrm{g} / \mathrm{kg}$ & 370 & EPA8270 \\
\hline$\mu \mathrm{g} / \mathrm{kg}$ & 370 & EPA8270 \\
\hline$\mu \mathrm{g} / \mathrm{kg}$ & 370 & EPA8270 \\
\hline$\mu \mathrm{g} / \mathrm{kg}$ & 370 & EPA8270 \\
\hline$\mu \mathrm{g} / \mathrm{kg}$ & 370 & EPA8270 \\
\hline$\mu \mathrm{g} / \mathrm{kg}$ & 370 & EPA8270 \\
\hline$\mu \mathrm{g} / \mathrm{kg}$ & 370 & EPA8270 \\
\hline$\mu \mathrm{g} / \mathrm{kg}$ & 370 & EPA8270 \\
\hline$\mu \mathrm{g} / \mathrm{kg}$ & 370 & EPA8270 \\
\hline$\mu \mathrm{g} / \mathrm{kg}$ & 370 & EPA8270 \\
\hline$\mu \mathrm{g} / \mathrm{kg}$ & 370 & EPA8270 \\
\hline$\mu \mathrm{g} / \mathrm{kg}$ & 370 & EPA8270 \\
\hline$\mu \mathrm{g} / \mathrm{kg}$ & 370 & EPA8270 \\
\hline$\mu \mathrm{g} / \mathrm{kg}$ & 370 & EPA8270 \\
\hline$\mu \mathrm{g} / \mathrm{kg}$ & 370 & EPA8270 \\
\hline$\mu \mathrm{g} / \mathrm{kg}$ & 370 & EPA8270 \\
\hline$\mu \mathrm{g} / \mathrm{kg}$ & 370 & EPA8270 \\
\hline$\mu \mathrm{g} / \mathrm{kg}$ & 370 & EPA8270 \\
\hline$\mu \mathrm{g} / \mathrm{kg}$ & 370 & EPA8270 \\
\hline$\mu \mathrm{g} / \mathrm{kg}$ & 740 & EPA 8270 \\
\hline$\mu \mathrm{g} / \mathrm{kg}$ & 370 & EPA8270 \\
\hline$\mu \mathrm{g} / \mathrm{kg}$ & 370 & EPA8270 \\
\hline$\mu \mathrm{g} / \mathrm{kg}$ & 370 & EPA8270 \\
\hline$\mu \mathrm{g} / \mathrm{kg}$ & 370 & EPA8270 \\
\hline$\mu \mathrm{g} / \mathrm{kg}$ & 1,800 & EPA8270 \\
\hline$\mu \mathrm{g} / \mathrm{kg}$ & 370 & EPA8270 \\
\hline$\mu \mathrm{g} / \mathrm{kg}$ & 370 & EPA8270 \\
\hline$\mu \mathrm{g} / \mathrm{kg}$ & 370 & EPA8270 \\
\hline$\mu \mathrm{g} / \mathrm{kg}$ & 370 & ËPA8270 \\
\hline$\mu \mathrm{g} / \mathrm{kg}$ & 370 & EPA8270 \\
\hline
\end{tabular}


SAMPLE NAME: D43I 1202 (Continued)

Constituent
Sample D: 100371

RQ AQ B Result Unit D. Limit Method

$B / N / A$ Extractables (including $P A H$ and phenols)

\author{
Hexachlorobenzene \\ Hexachlorobutadiene \\ Hexachlorocyclopentadiene \\ Hexachloroethane \\ Sub. hexanedioic acid \\ Indeno(1,2,3-c,d)pyrene \\ Isophorone \\ 2-Methyl-4.6-dinitrophenol \\ 2-Methyinaphthalene \\ Naphthalene \\ o-Nitroaniline \\ m-Nitroaniline \\ p-Nitroaniline \\ Nitrobenzene \\ 2-Nitrophenol \\ 4-Nitrophenol \\ N-Nitrosodiphenylamine \\ N-Nitrosodipropylamine \\ Pentachlorophenol \\ Phenanthrene \\ Phenol \\ Pyrene \\ 1,2,4-Trichlorobenzene \\ 2.4,5-Trichlorophenol \\ 2,4,6-Trichlorophenol \\ Unknown \\ Unknown \\ Unknown \\ Unknown \\ Unknown \\ Unknown \\ Unknown ketone
}

Volatile Organic Compounds
$U$
$U$
$U$
$U$
$U$
$U$
$U$
$U$
$U$
$U$
$U$
$U$
$U$
$U$

370
370
370
370
20,000
58
370
1,800
370
370
1,800
1,800
1,800
370
370
1,800
370
370
1,800
55
370
95
370
1,800
370
3,000
900
200
100
300
300
50,000

EPA8270

EPA8270

EPA8270

EPA8270

EPA8270

EPA8270

EPA8270

EPA8270

EPA8270

EPA8270

EPA8270

EPA8270

EPA8270

EPA8270

EPA8270

EPA8270

EPA8270

EPA8270

EPA8270

EPA8270

EPA8270

EPA8270

EPA8270

EPA8270

EPA8270

EPA8270

EPA8270

EPA8270

EPA8270

EPA8270

EPA8270

EPA8270

Acetone

Benzene

11

5.5

Bromodichloromethane

5.5

Bromoform

Bromomethane (Methyl bromide)

5.5

11

Carbon disulfide

5.5

Carbon tetrachloride -

Chlorobenzene

5.5

5.5

Chloroethane

11

11

(Vinyl chloride)

2-Chloroethyl vinyl ether

Chloroform

Chloromethane (Methyl chloride)

Dibromochloromethane

11

5.5

11

5.5

1,1-Dichloroethane

\begin{abstract}
$\mu \mathrm{g} / \mathrm{kg}$
$\mu \mathrm{g} / \mathrm{kg}$

$\mu \mathrm{g} / \mathrm{kg}$

$\mu \mathrm{g} / \mathrm{kg}$

$\mu \mathrm{g} / \mathrm{kg}$

$\mu \mathrm{g} / \mathrm{kg}$

$\mu \mathrm{g} / \mathrm{kg}$

$\mu \mathrm{g} / \mathrm{kg}$

$\mu \mathrm{g} / \mathrm{kg}$

$\mu \mathrm{g} / \mathrm{kg}$

$\mu \mathrm{g} / \mathrm{kg}$

$\mu \mathrm{g} / \mathrm{kg}$

$\mu \mathrm{g} / \mathrm{kg}$

$\mu \mathrm{g} / \mathrm{kg}$

$\mu \mathrm{g} / \mathrm{kg}$
\end{abstract}

EPA8240

EPA8240

EPA8240

EPA8240

EPA8240

EPA8240

EPA8240

EPA8240

EPA8240

EPA8240

EPA8240

EPA8240

EPA8240

EPA8240

EPA8240 
SAMPLE NAME: D43i 1202 (Continued)

Constituent

$\mathbf{R Q}$

AQ

Q

Sample ID: 100371

B Result Unit D. Limit Method

Volatile Organic Compounds

1,2-Dichloroethane
1,1-Dichloroethylene
1,2-Dichloroethylene
Dichloromethane (Methylene chloride)
1,2-Dichloropropane
cis-1,3-Dichloropropene
trans-1,3-Dichloropropene
Ethylbenzene
2-Hexanone
Methyl ethyl ketone
Methyl isobutyl ketone
Styrene
1,1,2,2-Tetrachloroethane
Tetrachloroethylene
Toluene
1,1,1-Trichloroethane
1,1,2-Trichloroethane
Trichloroethylene
Vinyl acetate
Xylenes

U

U

U

$U$

V

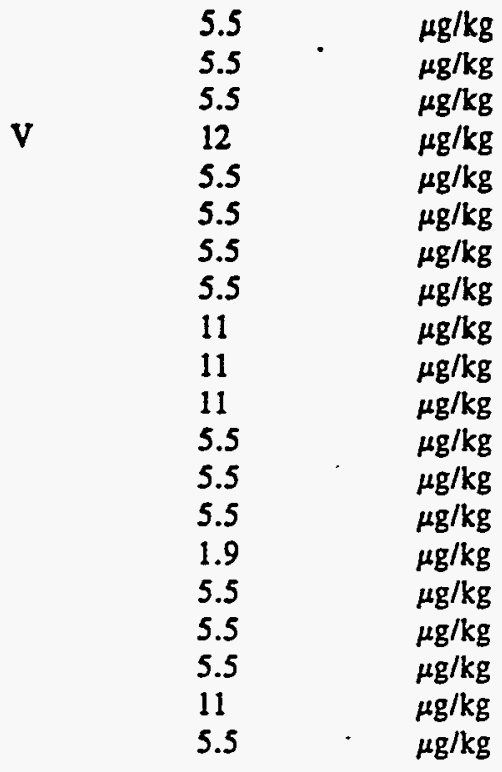

Pesticides/PCBs and Dioxins/Furans

Aldrin

alpha-Benzene hexachloride

beta-Benzene hexachloride

delta-Benzene hexachloride

alpha-Chlordane

gamma-Chlordane

p.p'-DDD

P,P'-DDE

P.P'-DDT

Dieldrin

Endosulfan I

Endosulfan II

Endosulfan sulfate

Endrin

Endrin ketone

Heptachlor

Heptachlor epoxide

Heptachlorodibenzo-p-dioxin isomers

Heplachlorodibenzo-p-furan isomers

Hexachlorodibenzo-p-dioxin isomers

Hexachlorodibenzo-p-furan isomers

Lindane

Methoxychlor

Octachlorodibenzo-p-dioxin

Octachlorodibenzo-p-furan

PCB 1016

PCB 1221

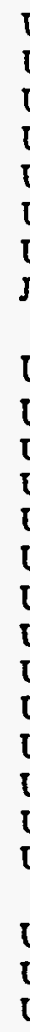

U
5.5

5.5

5.5

5.5

5.5

5.5

5.5

5.5

11

11

11

5.5

5.5

5.5

5.5

5.5

5.5

5.5

11

5.5
EPA8240
EPA8240
EPA8240
EPA8240
EPA8240
EPA8240
EPA8240
EPA8240
EPA8240
EPA8240
EPA8240
EPA8240
EPA8240
EPA8240
EPA8240
EPA8240
EPA8240
EPA8240
EPA8240
EPA8240

EPA8080

EPA8080

EPA8080

EPA8080

EPA8080

EPA8080

EPA8080

EPA8080

EPA8080

EPA8080

EPA8080

EPA8080

EPA8080

EPA8080

EPA8080

EPA8080

EPA8080

EPA8280

EPA8280

EPA8280

EPA8280

EPA8080

EPA8080

EPA8280

EPA8280

EPA8080

EPA8080 
SAMPLE NAME: D43r 1202 (Continued)

Sample ID: 100371

Constituent

RQ AQ B Result

Unit

D. Limit Method

Pesticides/PCBs and Dioxins/Furans

PCB 1232

PCB 1242

PCB 1248

PCB 1254

PCB 1260

Pentachlorodibenzo-p-dioxin isomers

Pentachlorodibenzo-p-furan isomers

2,3,7,8-TCDD

2,3,7,8-TCDF

Tetrachlorodibenzo-p-dioxin isomers

Tetrachlorodibenzo-p-furan isomers

Toxaphene

$\begin{array}{ll}\mathbf{U} & 370 \\ \mathbf{U} & 370 \\ \mathrm{U} & 370 \\ \mathbf{U} & 370 \\ & 3.400 \\ \mathbf{U} & 0.20 \\ \mathbf{U} & 0.20 \\ \mathrm{U} & 0.20 \\ \mathbf{U} & 0.10 \\ \mathrm{U} & 0.20 \\ \mathrm{U} & 0.20 \\ \mathrm{U} & 1,900\end{array}$

$$
\mu \mathrm{g} / \mathbf{k g}
$$

$\mu \mathrm{g} / \mathrm{kg}$

$\mu \mathrm{g} / \mathrm{kg}$

$\mu g / k g$

$\mathrm{ng} / \mathrm{g}$

$\mathrm{ng} / \mathrm{g}$

$\mathrm{ng} / \mathrm{g}$

$\mathrm{ng} / \mathrm{g}$

$\mathrm{ng} / \mathrm{g}$

$\mathrm{ng} / \mathrm{g}$

\begin{tabular}{|c|c|}
\hline 370 & EPA8080 \\
\hline 370 & EPA8080 \\
\hline 370 & EPA8080 \\
\hline 370 & EPA8080 \\
\hline 370 & EPA8080 \\
\hline 0.20 & EPA8280 \\
\hline 0.20 & EPA8280 \\
\hline 0.20 & EPA8280 \\
\hline 0.10 & EPA8280 \\
\hline 0.20 & EPA8280 \\
\hline 0.20 & EPA8280 \\
\hline 1,900 & EPA8080 \\
\hline
\end{tabular}

$\mu g / \mathrm{kg}$

Radionuclide Indicators and Radionuclides

Gross alpha

Nonvolatile beta

Radium, total alpha-emitting

U v

$\begin{array}{ll}7.3 \pm 12 & \mathrm{pCi} / \mathrm{g} \\ 97 \pm 29 & \mathrm{pCi} / \mathrm{g} \\ 1.1 \pm 0.81 & \mathrm{pCi} / \mathrm{g} \\ 1.6 \pm 0.32 & \mathrm{pCi} / \mathrm{g}\end{array}$

7.2

EPA900.0

Tritium

SAMPLE NAME: D431 1203

Location (SRS Coordinates): 18518.11E 66590.27N

Ground Elevation Above MSL: $127.7 \mathrm{ft}$

Depth of Core Interval: 2.00 to $4.00 \mathrm{ft}$

Sample Type: Normal

Sample Color: 5YR 3/4
Sample Moisture: Damp

Percent Solids: 90.40

D. Limit Method

\section{Constituent}

Total Metals

Arsenic

Barium

Cadmium

Chromium

Copper

Lead

Manganese

Mercury

Nickel

Selenium

Silver

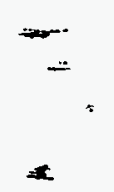

Volatile Organic Compounds

Acetone

Benzene

Bromodichloromethane
Sample ID: 100372
Sample Matrix: Soil

USC Soil Classification: SC
RQ AQ B Result Unit

0.14

1.4

\section{Sample ID: 100372}

EPA906.0 
SAMPLE NAME: D431 1203 (Continued)

Sample ID: 100372

Constituent

Volatile Organic Compounds

Bromoform
Bromomethane (Methyl bromide)
Carbon disulfide
Carbon tetrachloride
Chlorobenzene
Chloroethane
Chlorothene (Vinyl chloride)
2-Chloroethyl vinyl ether
Chloroform
Chloromethane (Methyl chloride)
Dibromochloromethane
1,1-Dichloroethane
1,2-Dichloroethane
1,1-Dichloroethylene
1,2-Dichloroethylene
Dichloromethane (Methylene chloride)
1,2-Dichloropropane
cis-1,3-Dichloropropene
trans-1,3-Dichloropropene
Ethylbenzene
2-Hexanone
Methyl ethyl ketone
Methyl isobutyl ketone
Styrene
1,1,2,2-Tetrachloroethane
Tetrachloroethylene
Toluene
1,1,1-Trichloroethane
1,1,2-Trichloroethane
Trichloroethylene
Vinyl acetate
Xylenes

RQ AQ B Result Unit D. Limit Method

\begin{tabular}{|c|c|c|c|c|}
\hline U & 5.5 & $\mu \mathrm{g} / \mathrm{kg}$ & 5.5 & EPA8240 \\
\hline $\mathbf{U}$ & 11 & $\mu g / \mathbf{k g}$ & 11 & EPA8240 \\
\hline U & 5.5 & $\mu g / k g$ & 5.5 & EPA8240 \\
\hline $\mathbf{U}$ & 5.5 & $\mu \mathrm{g} / \mathrm{kg}$ & 5.5 & EPA8240 \\
\hline U & 5.5 & $\mu \mathrm{g} / \mathrm{kg}$ & 5.5 & EPA8240 \\
\hline $\mathbf{U}$ & 11 & $\mu g / k g$ & 11 & EPA8240 \\
\hline U & 11 & $\mu \mathrm{g} / \mathrm{kg}$ & 11 & EPA8240 \\
\hline U & 11 & $\mu \mathrm{g} / \mathrm{kg}$ & 11 & EPA8240 \\
\hline $\mathrm{U}$ & 5.5 & $\mu \mathrm{g} / \mathrm{kg}$ & 5.5 & EPA8240 \\
\hline $\mathrm{U}$ & 11 & $\mu \mathrm{g} / \mathrm{kg}$ & 11 & EPA8240 \\
\hline $\mathrm{U}$ & 5.5 & $\mu \mathrm{g} / \mathrm{kg}$ & 5.5 & EPA8240 \\
\hline $\mathbf{U}$ & 5.5 & $\mu g / k g$ & 5.5 & EPA8240 \\
\hline U & 5.5 & $\mu \mathrm{g} / \mathrm{kg}$ & 5.5 & EPA8240 \\
\hline U & 5.5 & $\mu \mathrm{g} / \mathrm{kg}$ & 5.5 & EPA8240 \\
\hline U & 5.5 & $\mu g / \mathrm{kg}$ & 5.5 & EPA 8240 \\
\hline v & 14 & $\mu g / k g$ & 5.5 & EPA 8240 \\
\hline $\mathrm{U}$ & 5.5 & $\mu g / \mathrm{kg}$ & 5.5 & EPA8240 \\
\hline $\mathrm{U}$ & 5.5 & $\mu \mathrm{g} / \mathrm{kg}$ & 5.5 & EPA8240 \\
\hline U & 5.5 & $\mu \mathrm{g} / \mathrm{kg}$ & 5.5 & EPA8240 \\
\hline U & 5.5 & $\mu \mathrm{g} / \mathrm{kg}$ & 5.5 & EPA8240 \\
\hline$U$ & 11 & $\mu g / \mathrm{kg}$ & 11 & EPA8240 \\
\hline U & 11 & $\mu \mathrm{g} / \mathrm{kg}$ & 11 & EPA8240 \\
\hline U & 11 & $\mu \mathrm{g} / \mathrm{kg}$ & 11 & EPA8240 \\
\hline$U$ & 5.5 & $\mu \mathrm{g} / \mathrm{kg}$ & 5.5 & EPA8240 \\
\hline U & 5.5 & $\mu \mathrm{g} / \mathrm{kg}$ & 5.5 & EPA8240 \\
\hline U & 5.5 & $\mu \mathrm{g} / \mathrm{kg}$ & 5.5 & EPA8240 \\
\hline & 4.3 & $\mu \mathrm{g} / \mathrm{kg}$ & 5.5 & EPA8240 \\
\hline 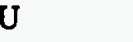 & 5.5 & $\mu \mathrm{g} / \mathrm{kg}$ & 5.5 & EPA8240 \\
\hline U & 5.5 & $\mu \mathrm{g} / \mathrm{kg}$ & 5.5 & EPA8240 \\
\hline U & 5.5 & $\mu \mathrm{g} / \mathrm{kg}$ & 5.5 & EPA8240 \\
\hline$U$ & 11 & $\mu \mathrm{g} / \mathrm{kg}$ & 11 & EPA8240 \\
\hline$U$ & 5.5 & $\mu \mathrm{g} / \mathrm{kg}$ & 5.5 & EPA8240 \\
\hline
\end{tabular}

Radionuclide Indicators and Radionuclides

\begin{tabular}{|c|c|c|c|c|c|c|}
\hline Gross alpha & U & & 6.7 & $\mathrm{pCi} / \mathrm{g}$ & 6.7 & EPA900.0 \\
\hline Nonvolatile beta & & & $25 \pm 21$ & $\mathrm{pCi} / \mathrm{g}$ & $\therefore 9$ & EPA900.0 \\
\hline Radium, total alpha-emitting & U & $\mathrm{v}$ & $1.1 \pm 0.78$ & $\mathrm{pCi} / \mathrm{g}$ & 0.14 & EPA903.0 \\
\hline Tritium & $\mathbf{U}$ & & 2.1 & $\mathrm{pCi} / \mathrm{g}$ & 2.1 & EPA906.0 \\
\hline
\end{tabular}


SAMPLE NAME: D431 1204

Location (SRS Coordinates): 18518.11E 66590.27N

Ground Elevation Above MSL: $127.7 \mathrm{ft}$

Depth of Core Interval: 4.00 to $6.00 \mathrm{ft}$

Sample Type: Normal

Sample Color: 5YR 2/2

\section{Constituent}

RQ AQ B Result Unit

Total Metals

Arsenic

Barium

Cadmium

Chromium

Copper

Lead

Manganese

Mercury

Nickel

Selenium

Silver
Sample ID: 100373

Sample Matrix: Soil

USC Soil Classification: SC
Sample Moisture: Damp

Percent Solids: 85.40

D. Limit Method

\section{$B / N / A$ Extractables (including $P A H$ and phenols)}

\begin{tabular}{|c|c|c|c|c|c|c|}
\hline Acenaphthene & UJ & $Q$ & 390 & $\mu \mathrm{g} / \mathrm{kg}$ & 390 & EPA8270 \\
\hline Acenaphthene & UJ & $Q$ & 390 & $\mu \mathrm{g} / \mathrm{kg}$ & 390 & EPA8270 \\
\hline Acenaphthylene & UJ & Q & 390 & $\mu \mathrm{g} / \mathrm{kg}$ & 390 & EPA8270 \\
\hline Acenaphthylene & UJ & $\mathbf{Q}$ & 390 & $\mu \mathrm{g} / \mathrm{kg}$ & 390 & EPA8270 \\
\hline Anthracene & UJ & $\mathbf{Q}$ & 390 & $\mu \mathrm{g} / \mathrm{kg}$ & 390 & EPA8270 \\
\hline Anthracene & UJ & $\mathbf{Q}$ & 390 & $\mu g / k g$ & 390 & EPA8270 \\
\hline Benzo(a)anthracene & UJ & $Q$ & 390 & $\mu \mathrm{g} / \mathrm{kg}$ & 390 & EPA8270 \\
\hline Benzo(a)anthracene & UJ & $\mathbf{Q}$ & 390 & $\mu \mathrm{g} / \mathrm{kg}$ & 390 & EPA8270 \\
\hline Benzo(b)fluoranthene & UJ & $\mathbf{Q}$ & 390 & $\mu \mathrm{g} / \mathrm{kg}$ & 390 & EPA8270 \\
\hline Benzo(b)fluoranthene & UJ & $Q$ & 390 & $\mu g / k g$ & 390 & EPA8270 \\
\hline Benzo(k)fluoranthene & UJ & Q & 390 & $\mu \mathrm{g} / \mathrm{kg}$ & 390 & EPA8270 \\
\hline Benzo(k)fluoranthene & UJ & $Q$ & 390 & $\mu \mathrm{g} / \mathrm{kg}$ & 390 & EPA8270 \\
\hline Benzoic acid & UI & $\mathbf{Q}$ & 2,000 & $\mu g / \mathrm{kg}$ & 2,000 & EPA8270 \\
\hline Benzoic acid & UJ & $\dot{Q}$ & 2,000 & $\mu \mathrm{g} / \mathrm{kg}$ & 2,000 & EPA8270 \\
\hline Benzo(g,h,i)perylene & UJ & $Q$ & 390 & $\mu \mathrm{g} / \mathrm{kg}$ & 390 & EPA8270 \\
\hline Benzo $(g, h, i)$ perylene & UJ & $\mathbf{Q}$ & 390 & $\mu g / \mathrm{kg}$ & 390 & EPA8270 \\
\hline Benzo(a)pyrene & UU & $\mathbf{Q}$ & 390 & $\mu g / \mathrm{kg}$ & 390 & EPA8270 \\
\hline Berzo(a)pyrene & UJ & $Q$ & 390 & $\mu g / \mathrm{kg}$ & 390 & EPA8270 \\
\hline Benzyl alcohol & UJ & $Q$ & 390 & $\mu \mathrm{g} / \mathrm{kg}$ & 390 & EPA8270 \\
\hline Benzyl alcohol & UJ & $\mathbf{Q}$ & 390 & $\mu \mathrm{g} / \mathrm{kg}$ & 390 & EPA8270 \\
\hline Bis(2-chloroethoxy) methane & UJ & $\mathbf{Q}$ & 390 & $\mu g / k g$ & 390 & EPA8270 \\
\hline Bis(2-chloroethoxy) methane & UJ & $\mathbf{Q}$ & 390 & $\mu g / k g$ & 390 & EPA8270 \\
\hline Bis(2-chloroethyl) ether & UJ & $\mathbf{Q}$ & 390 & $\mu \mathrm{g} / \mathrm{kg}$ & 390 & EPA8270 \\
\hline Bis(2-chloroisopropyl) ether & UJ & $\mathbf{Q}$ & 390 & $\mu g / \mathrm{kg}$ & 390 & EPA 8270 \\
\hline Bis(2-chloroethyl) ether & UJ & $\mathbf{Q}$ & 390 & $\mu g / \mathrm{kg}$ & 390 & EPA8270 \\
\hline Bis(2-chloroisopropyl) ether & UJ & $\mathbf{Q}$ & 390 & $\mu \mathrm{g} / \mathrm{kg}$ & 390 & EPA8270 \\
\hline Bis(2-ethylhexyl) phthalate & J & $\mathbf{Q}$ & 100 & $\mu g / \mathrm{kg}$ & 390 & EPA8270 \\
\hline Bis(2-ethylhexyl) phthalate & J & $Q$ & 68 & $\mu \mathrm{g} / \mathrm{kg}$ & 390 & EPA8270 \\
\hline 4-Bromophenyl phenyl ether & UJ & $\mathbf{Q}$ & 390 & $\mu \mathrm{g} / \mathrm{kg}$ & 390 & EPA8270 \\
\hline 4-Bromophenyl phenyl ether & UJ & $\mathbf{Q}$ & 390 & $\mu g / k g$ & 390 & EPA8270 \\
\hline
\end{tabular}


SAMPLE NAME: D431 1204 (Continued)

Sample ID: 100373

$\begin{array}{llllll}\text { Constituent } & \text { RQ } & \text { B } & \text { Result } & \text { Unit } & \text { D. Limit Method }\end{array}$

$B / N / A$ Extractables (including $P A H$ and phenols)

\begin{tabular}{|c|c|c|c|c|c|c|}
\hline Butylbenzyl phthalate & UJ & $Q$ & 390 & $\mu g / \mathrm{kg}$. & 390 & EPA8270 \\
\hline Butylbenzyl phthalate & UJ & $\mathbf{Q}$ & 390 & $\mu g / k g$ & 390 & EPA8270 \\
\hline 4-Chloroaniline & UJ & $\mathbf{Q}$ & 390 & $\mu g / \mathrm{kg}$ & 390 & EPA8270 \\
\hline 4-Chloroaniline & UJ & $\mathbf{Q}$ & 390 & $\mu \mathrm{g} / \mathrm{kg}$ & 390 & EPA8270 \\
\hline 4-Chloro-m-cresol & UJ & $Q$ & 390 & $\mu \mathrm{g} / \mathrm{kg}$ & 390 & EPA8270 \\
\hline 4-Chloro-m-cresol & UJ & $\mathbf{Q}$ & 390 & $\mu \mathrm{g} / \mathrm{kg}$ & 390 & EPA8270 \\
\hline 2-Chloronaphthalene ." & UJ & $\mathbf{Q}$ & 390 & $\mu \mathrm{g} / \mathrm{kg}$ & 390 & EPA8270 \\
\hline 2-Chloronaghthalene & UJ & $\mathbf{Q}$ & 390 & $\mu \mathrm{g} / \mathrm{kg}$ & 390 & EPA8270 \\
\hline 2-Chlorophenul & UJ & $\mathbf{Q}$ & 390 & $\mu \mathrm{g} / \mathrm{kg}$ & 390 & EPA8270 \\
\hline 2-Chlorophenol & UJ & Q & 390 & $\mu \mathrm{g} / \mathrm{kg}$ & 390 & EPA8270 \\
\hline 4-Chlorophenyl phenyl ether & UJ & Q & 390 & $\mu \mathrm{g} / \mathrm{kg}$ & 390 & EPA8270 \\
\hline 4-Chlorophenyl phenyl ether & UJ & $Q$ & 390 & $\mu g / \mathrm{kg}$ & 390 & EPA8270 \\
\hline Chrysene & UJ & $\mathbf{Q}$ & 390 & $\mu \mathrm{g} / \mathrm{kg}$ & 390 & EPA8270 \\
\hline Chrysene & UJ & Q & 390 & $\mu g / k g$ & 390 & EPA8270 \\
\hline o-Cresol (2-Methylphenol) & UJ & Q & 390 & $\mu \mathrm{g} / \mathrm{kg}$ & 390 & EPA8270 \\
\hline o-Cresol (2-Methylphenol) & UJ & $Q$ & 390 & $\mu \mathrm{g} / \mathrm{kg}$ & 390 & EPA8270 \\
\hline p-Cresol (4-Methylphenol) & UJ & Q & 390 & $\mu \mathrm{g} / \mathrm{kg}$ & 390 & EPA8270 \\
\hline p-Cresol (4-Methylphenol) & UJ & Q & 390 & $\mu \mathrm{g} / \mathrm{kg}$ & 390 & EPA8270 \\
\hline Dibenz(a,h)anthracene & UJ & Q & 390 & $\mu \mathrm{g} / \mathrm{kg}$ & 390 & EPA8270 \\
\hline Dibenz $(a, h)$ anthracene & UJ . & Q & 390 & $\mu g / k g$ & 390 & EPA8270 \\
\hline Dibenzofuran & UJ & Q & 390 & $\mu \mathrm{g} / \mathrm{kg}$ & 390 & EPA8270 \\
\hline Dibenzofuran & UJ & Q & 390 & $\mu \mathrm{g} / \mathrm{kg}$ & 390 & EPA8270 \\
\hline Di-n-butyl phthalate & $\mathrm{J}$ & QV & 120 & $\mu \mathrm{g} / \mathrm{kg}$ & 390 & EPA8270 \\
\hline Di-n-butyl phthalate & J & QV & 100 & $\mu \mathrm{g} / \mathrm{kg}$ & 390 & EPA8270 \\
\hline 1,2-Dichlorobenzene & UJ & Q & 390 & $\mu \mathrm{g} / \mathrm{kg}$ & 390 & EPA8270 \\
\hline 1,2-Dichlorobenzene & UJ & Q & 390 & $\mu \mathrm{g} / \mathrm{kg}$ & 390 & EPA8270 \\
\hline 1,3-Dichlorobenzene & UJ & Q & 390 & $\mu \mathrm{g} / \mathrm{kg}$ & 390 & EPA8270 \\
\hline 1.3-Dichlorobenzene & UJ & Q & 390 & $\mu \mathrm{g} / \mathrm{kg}$ & 390 & EPA8270 \\
\hline 1,4-Dichlorobenzene & UJ & Q & 390 & $\mu \mathrm{g} / \mathrm{kg}$ & 390 & EPA8270 \\
\hline 1,4-Dichlorobenzene & UJ & Q & 390 & $\mu g / k g$ & 390 & EPA8270 \\
\hline 3.3'-Dichlorobenzidine & UJ & Q & 780 & $\mu \mathrm{g} / \mathrm{kg}$ & 780 & EPA8270 \\
\hline 3.3'-Dichlorobenzidine & UJ & Q & 780 & $\mu \mathrm{g} / \mathrm{kg}$ & 780 & EPA8270 \\
\hline 2,4-Dichlorophenol & UJ & Q & 390 & $\mu \mathrm{g} / \mathrm{kg}$ & 390 & EPA8270 \\
\hline 2,4-Dichlorophenol & UJ & Q & 390 & $\mu \mathrm{g} / \mathrm{kg}$ & 390 & EPA8270 \\
\hline Diethyl phthalate & UJ & Q & 390 & $\mu \mathrm{g} / \mathrm{kg}$ & 390 & EPA8270 \\
\hline Diethyl phthalate & UJ & Q & 390 & $\mu \mathrm{g} / \mathrm{kg}$ & 390 & EPA8270 \\
\hline 2,4-Dimethyl phenol & UJ & Q & 390 & $\mu \mathrm{g} / \mathrm{kg}$ & 390 & EPA8270 \\
\hline 2,4-Dimethyl phenol & UJ & Q & 390 & $\mu g / k g$ & 390 & EPA8270 \\
\hline Dimethyl phthalate & UJ & Q & 390 & $\mu \mathrm{g} / \mathrm{kg}$ & 390 & EPA8270 \\
\hline Dimethyl phthalate & UJ & $\mathbf{Q}$ & 390 & $\mu \mathrm{g} / \mathrm{kg}$ & 390 & EPA8270 \\
\hline 2,4-Dinitrophenol & UJ & QV & 2,000 & $\mu g / \mathrm{kg}$ & 2,000 & EPA8270 \\
\hline 2,4-Dinitrophenol & UJ & QV & 2,000 & $\mu \mathrm{g} / \mathrm{kg}$ & 2,000 & EPA8270 \\
\hline 2.4-Dinitrotoluene & UJ & $Q C$ & 390 & $\mu \mathrm{g} / \mathrm{kg}$ & 390 & EPA8270 \\
\hline 2.4-Dinitrotoluene & UJ & QC & 390 & $\mu \mathrm{g} / \mathrm{kg}$ & 390 & EPA8270 \\
\hline 2,6-Dinitrotoluene & UJ & $\mathbf{Q}$ & 390 & $\mu \mathrm{g} / \mathrm{kg}$ & 390 & EPA8270 \\
\hline 2.6-Dinitrotoluene & UJ & Q & 390 & $\mu \mathrm{g} / \mathrm{kg}$ & 390 & EPA8270 \\
\hline Di-n-octyl phthalate & UJ & $\mathbf{Q}$ & 390 & $\mu \mathrm{g} / \mathrm{kg}$ & 390 & EPA8270 \\
\hline Di-n-octyl phthalate & UJ & Q & 390 & $\mu \mathrm{g} / \mathrm{kg}$ & 390 & EPA8270 \\
\hline Fluoranthene & UJ & $\mathbf{Q}$ & 390 & $\mu \mathrm{g} / \mathrm{kg}$ & 390 & EPA8270 \\
\hline Fluoranthene & UJ & Q & 390 & $\mu \mathrm{g} / \mathrm{kg}$ & 390 & EPA8270 \\
\hline
\end{tabular}


SAMPLE NAME: D43i 1204 (Continued)

Sample ID: 100373

Constituent RQ AQ B Result Unit D. Limit Method .

$B / N / A$ Extractables (including $P A H$ and phenols)

\begin{tabular}{|c|c|c|c|c|c|c|}
\hline Fluorene & UJ & $Q$ & 390 & $\mu \mathrm{g} / \mathrm{kg}$ & 390 & EPA8270 \\
\hline Fluorene & UJ & $\mathbf{Q}$ & 390 & $\mu \mathrm{g} / \mathrm{kg}$ & 390 & EPA8270 \\
\hline Hexachlorobenzene & UJ & $\mathbf{Q}$ & 390 & $\mu \mathrm{g} / \mathrm{kg}$ & 390 & EPA8270 \\
\hline Hexachlorobenzene & UJ & $\mathbf{Q}$ & 390 & $\mu \mathrm{g} / \mathrm{kg}$ & 390 & EPA8270 \\
\hline Hexachlorobutadiene & UJ & $\mathbf{Q}$ & 390 & $\mu \mathrm{g} / \mathrm{kg}$ & 390 & EPA8270 \\
\hline Hexachlorobutadiene & UJ & $\mathbf{Q}$ & 390 & $\mu \mathrm{g} / \mathrm{kg}$ & 390 & EPA8270 \\
\hline Hexachlorocyclopentadiene & UJ & $\mathbf{Q}$ & 390 & $\mu \mathrm{g} / \mathrm{kg}$ & 390 & EPA8270 \\
\hline Hexachlorocyclopentadiene & UJ & $Q$ & 390 & $\mu \mathrm{g} / \mathrm{kg}$ & 390 & EPA8270 \\
\hline Hexachloroethane & UJ & $\mathbf{Q}$ & 390 & $\mu \mathrm{g} / \mathrm{kg}$ & 390 & EPA8270 \\
\hline Hexachloroethane & UJ & $\mathbf{Q}$ & 390 & $\mu \mathrm{g} / \mathrm{kg}$ & 390 & EPA8270 \\
\hline Sub. hexanedioic acid & J & $\mathbf{N}$ & 30,000 & $\mu \mathrm{g} / \mathrm{kg}$ & & EPA8270 \\
\hline Indeno(1,2,3-c,d)pyrene & UJ & $\mathbf{Q}$ & 390 & $\mu \mathrm{g} / \mathrm{kg}$ & 390 & EPA.8270 \\
\hline Indeno( $1,2,3-c, d)$ pyrene & UJ & $\mathbf{Q}$ & 390 & $\mu \mathrm{g} / \mathrm{kg}$ & 390 & EPA8270 \\
\hline Isophorone & UJ & $Q$ & 390 & $\mu \mathrm{g} / \mathrm{kg}$ & 390 & EPA8270 \\
\hline Isophorone & UJ & $\mathbf{Q}$ & 390 & $\mu \mathrm{g} / \mathrm{kg}$ & 390 & EPA8270 \\
\hline 2-Methyl-4,6-dinitrophenol & UJ & $\mathbf{Q}$ & 2,000 & $\mu \mathrm{g} / \mathrm{kg}$ & 2,000 & EPA8270 \\
\hline 2-Methyl-4,6-dinitrophenol & UJ & $\mathbf{Q}$ & 2,000 & $\mu \mathrm{g} / \mathrm{kg}$ & 2,000 & EPA8270 \\
\hline 2-Methylnaphthalene & UJ & $Q$ & 390 & $\mu \mathrm{g} / \mathrm{kg}$ & 390 & EPA8270 \\
\hline 2-Methylnaphthalene & UJ & $Q$ & 390 & $\mu \mathrm{g} / \mathrm{kg}$ & 390 & EPA8270 \\
\hline Naphthalene & UJ & $\mathrm{Q}$ & 390 & $\mu \mathrm{g} / \mathrm{kg}$ & 390 & EPA8270 \\
\hline Naphthalene & UJ & $\mathbf{Q}$ & 390 & $\mu \mathrm{g} / \mathrm{kg}$ & 390 & EPA8270 \\
\hline o-Nitroaniline & UJ & $Q$ & 2,000 & $\mu \mathrm{g} / \mathrm{kg}$ & 2,000 & EPA8270 \\
\hline o-Nitroaniline & UJ & 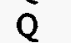 & 2,000 & $\mu \mathrm{g} / \mathrm{kg}$ & 2,000 & EPA8270 \\
\hline m-Nitroaniline & UJ & $Q$ & 2.000 & $\mu \mathrm{g} / \mathrm{kg}$ & 2,000 & EPA8270 \\
\hline m-Nitroaniline & UJ & $\mathbf{Q}$ & 2,000 & $\mu \mathrm{g} / \mathrm{kg}$ & 2,000 & EPA8270 \\
\hline p-Nitroaniline & UJ & $Q$ & 2.000 & $\mu \mathrm{g} / \mathrm{kg}$ & 2,000 & EPA8270 \\
\hline p-Nitroaniline & UJ & $Q$ & 2,000 & $\mu \mathrm{g} / \mathrm{kg}$ & 2,000 & EPA8270 \\
\hline Nitrobenzene & UJ & $Q$ & 390 & $\mu \mathrm{g} / \mathrm{kg}$ & 390 & EPA8270 \\
\hline Nitrobenzene & UJ & $Q$ & 390 & $\mu \mathrm{g} / \mathrm{kg}$ & 390 & EPA8270 \\
\hline 2-Nitrophenol & UJ & $Q$ & 390 & $\mu \mathrm{g} / \mathrm{kg}$ & 390 & EPA8270 \\
\hline 2-Nitrophenol & UJ & $Q$ & 390 & $\mu \mathrm{g} / \mathrm{kg}$ & 390 & EPA8270 \\
\hline 4-Nitrophenol & UJ & $Q$ & 2,000 & $\mu \mathrm{g} / \mathrm{kg}$ & 2,000 & EPA8270 \\
\hline 4-Nitrophenol & UJ & $Q$ & 2,000 & $\mu \mathrm{g} / \mathrm{kg}$ & 2,000 & EPA8270 \\
\hline N-Nitrosodiphenylamine & UJ & $Q$ & 390 & $\mu \mathrm{g} / \mathrm{kg}$ & 390 & EPA8270 \\
\hline N-Nitrosodiphenylamine & UJ & $\mathbf{Q}$ & 390 & $\mu \mathrm{g} / \mathrm{kg}$ & 390 & EPA8270 \\
\hline N-Nitrosodipropylamine & UJ & QC & 390 & $\mu \mathrm{g} / \mathrm{kg}$ & 390 & EPA8270 \\
\hline N-Nitrosodipropylamine & UJ & QC & 390 & $\mu \mathrm{g} / \mathrm{kg}$ & 390 & EPA8270 \\
\hline Pentachlorophenol & UJ & $\mathbf{Q}$ & 2,000 & $\mu \mathrm{g} / \mathrm{kg}$ & 2,000 & EPA8270 \\
\hline Pentachlorophenol & UJ & Q & 2,000 & $\mu \mathrm{g} / \mathrm{kg}$ & 2,000 & EPA8270 \\
\hline Phenanthrene & UJ & $Q$ & 390 & $\mu \mathrm{g} / \mathrm{kg}$ & 390 & EPA8270 \\
\hline Phenanthrene & UJ & $Q$ & 390 & $\mu \mathrm{g} / \mathrm{kg}$ & 390 & EPA8270 \\
\hline Phenol & UJ & $Q$ & 390 & $\mu \mathrm{g} / \mathrm{kg}$ & 390 & EPA8270 \\
\hline Phenol & UJ & $Q$ & 390 & $\mu \mathrm{g} / \mathrm{kg}$ & 390 & EPA8270 \\
\hline Pyrene & UJ & $\mathbf{Q}$ & 390 & $\mu \mathrm{g} / \mathrm{kg}$ & 390 & EPA8270 \\
\hline Pyrene & UJ & $\mathbf{Q}$ & 390 & $\mu \mathrm{g} / \mathrm{kg}$ & 390 & EPA8270 \\
\hline 1,2,4-Trichlorobenzene & UJ & $Q \mathbf{C}$ & 390 & $\mu \mathrm{g} / \mathrm{kg}$ & 390 & EPA8270 \\
\hline 1,2,4-Trichlorobenzene & UJ & $Q C$ & 390 & $\mu \mathrm{g} / \mathrm{kg}$ & 390 & EPA8270 \\
\hline 2,4,5-Trichlorophenol & UJ & $\mathbf{Q}$ & 2,000 & $\mu \mathrm{g} / \mathrm{kg}$ & 2,000 & EPA8270 \\
\hline 2,4,5-Trichlorophenol & UJ & $\mathbf{Q}$ & 2,000 & $\mu \mathrm{g} / \mathrm{kg}$ & 2,000 & EPA8270 \\
\hline 2,4,6-Trichlorophenol & UJ & $Q$ & 390 & $\mu \mathrm{g} / \mathrm{kg}$ & 390 & EPA8270 \\
\hline
\end{tabular}


SAMPLE NAME: D43i 1204 (Continued)

Sampie ID: 100373

$\begin{array}{llllll}\text { Constituent } & \text { RQ AQ } & \text { Unit Result D. Limit Method }\end{array}$

$B / N / A$ Extractables (including PAH and phenols)

2,4,6-Trichlorophenol
Unknown
Unknown
Unknown
Unknown
Unknown ketone
Unknown alcohol -

$\begin{array}{llllll}\mathrm{UJ} & \mathrm{Q} & 390 & \mu \mathrm{g} / \mathrm{kg} & 390 & \text { EPA8270 } \\ \mathrm{J} & \mathrm{NV} & 300 & \mu \mathrm{g} / \mathrm{kg} & & \text { EPA8270 } \\ \mathrm{J} & \mathrm{N} & 200 & \mu \mathrm{g} / \mathrm{kg} & & \text { EPA8270 } \\ \mathrm{J} & \mathrm{N} & 200 & \mu \mathrm{g} / \mathrm{kg} & & \text { EPA8270 } \\ \mathrm{J} & \mathrm{N} & 200 & \mu \mathrm{g} / \mathrm{kg} & & \text { EPA8270 } \\ \mathrm{J} & \mathrm{NV} & 3,000 & \mu \mathrm{g} / \mathrm{kg} & & \text { EPA8270 } \\ \mathrm{J} & \mathrm{N} & 400 & \mu \mathrm{g} / \mathrm{kg} & & \text { EPA8270 }\end{array}$

Volatile Organic Compounds

Acetone

Benzene

Bromodichloromethane

Bromoform

Bromomethane (Methyl bromide)

Carbon disulfide

Carbon tetrachloride

Chlorobenzene

Chloroethane

Chloroethene (Vinyl chloride)

2-Chioroethyl vinyl ether

Chloroform

Chloromethane (Methyl chloride)

Dibromochloromethane

1,1-Dichloroethane

1,2-Dichloroethane

1,1-Dichloroethylene

1,2-Dichloroethylene

Dichloromethane (Methylene chloride)

1,2-Dichloropropane

cis-1.3-Dichloropropene

trans-1,3-Dichloropropene

Ethylbenzene

2-Hexanone

Methyl ethyl ketone

Methyl isobutyl ketone

Styrene

1,1,2,2-Tetrachloroethane

Tetrachloroethylene

Toluene

1,1,1-Trichloroethane -

1,1,2-Trichloroethane *

Trichloroethylene

Vinyl acetate

Xylenes
V $\quad 77$

6.0

6.0

6.0

12

4.3

6.0

6.0

12

12

12

6.0

12

6.0

6.0

6.0

6.0

6.0

7.3

6.0

6.0

6.0

6.0

12

12

12

6.0

6.0

6.0

2.3

6.0

6.0

6.0

12

6.0

$\begin{array}{lll}\mu \mathrm{g} / \mathrm{kg} & 12 & \text { EPA8240 } \\ \mu \mathrm{g} / \mathrm{kg} & 6.0 & \text { EPA8240 } \\ \mu \mathrm{g} / \mathrm{kg} & 6.0 & \text { EPA8240 } \\ \mu \mathrm{g} / \mathrm{kg} & 6.0 & \text { EPA8240 } \\ \mu \mathrm{g} / \mathrm{kg} & 12 & \text { EPA8240 } \\ \mu \mathrm{g} / \mathrm{kg} & 6.0 & \text { EPA8240 } \\ \mu \mathrm{g} / \mathrm{kg} & 6.0 & \text { EPA8240 } \\ \mu \mathrm{g} / \mathrm{kg} & 6.0 & \text { EPA8240 } \\ \mu \mathrm{g} / \mathrm{kg} & 12 & \text { EPA8240 } \\ \mu \mathrm{g} / \mathrm{kg} & 12 & \text { EPA8240 } \\ \mu \mathrm{g} / \mathrm{kg} & 12 & \text { EPA8240 } \\ \mu \mathrm{g} / \mathrm{kg} & 6.0 & \text { EPA8240 } \\ \mu \mathrm{g} / \mathrm{kg} & 12 & \text { EPA8240 } \\ \mu \mathrm{g} / \mathrm{kg} & 6.0 & \text { EPA8240 } \\ \mu \mathrm{g} / \mathrm{kg} & 6.0 & \text { EPA8240 } \\ \mu \mathrm{g} / \mathrm{kg} & 6.0 & \text { EPA8240 } \\ \mu \mathrm{g} / \mathrm{kg} & 6.0 & \text { EPA8240 } \\ \mu \mathrm{g} / \mathrm{kg} & 6.0 & \text { EPA8240 } \\ \mu \mathrm{g} / \mathrm{kg} & 6.0 & \text { EPA8240 } \\ \mu \mathrm{g} / \mathrm{kg} & 6.0 & \text { EPA8240 } \\ \mu \mathrm{g} / \mathrm{kg} & 6.0 & \text { EPA8240 } \\ \mu \mathrm{g} / \mathrm{kg} & 6.0 & \text { EPA8240 } \\ \mu \mathrm{g} / \mathrm{kg} & 6.0 & \text { EPA8240 } \\ \mu \mathrm{g} / \mathrm{kg} & 12 & \text { EPA8240 } \\ \mu \mathrm{g} / \mathrm{kg} & 12 & \text { EPA8240 } \\ \mu \mathrm{g} / \mathrm{kg} & 12 & \text { EPA8240 } \\ \mu \mathrm{g} / \mathrm{kg} & 6.0 & \text { EPA8240 } \\ \mu \mathrm{g} / \mathrm{kg} & 6.0 & \text { EPA8240 } \\ \mu \mathrm{g} / \mathrm{kg} & 6.0 & \text { EPA8240 } \\ \mu \mathrm{g} / \mathrm{kg} & 6.0 & \text { EPA8240 } \\ \mu \mathrm{g} / \mathrm{kg} & 6.0 & \text { EPA8240 } \\ \mu \mathrm{g} / \mathrm{kg} & 6.0 & \text { EPA8240 } \\ \mu \mathrm{g} / \mathrm{kg} & 6.0 & \text { EPA8240 } \\ \mu \mathrm{g} / \mathrm{kg} & 12 & \text { EPA8240 } \\ \mu \mathrm{g} / \mathrm{kg} & 6.0 & \text { EPA8240 }\end{array}$


SAMPLE NAME: D431. 1204 (Continued)

Sample ID: 100373

$\begin{array}{llllll}\text { Constituent } & \text { RQ AQ } & \text { Result } & \text { Unit Limit Method }\end{array}$

Pesticides/PCBs and Dioxins/Furans

Aldrin

alpha-Benzene hexachloride

beta-Benzene hexachloride

delta-Benzene hexachloride

alpha-Chlordane

gamma-Chlordane

p.p'-DDD

p.p'-DDE

p.p'-DDT

Dieldrin

Endosulfan I

Endosulfan II

Endosulfan sulfate

Endrin

Endrin ketone

Heptachlor

Heptachlor epoxide

Heptachlorodibenzo-p-dioxin isomers

Heptachlorodibenzo-p-furan isomers

Hexachlorodibenzo-p-dioxin isomers

Hexachlorodibenzo-p-furan isomers

Lindane

Methoxychlor

Octachlorodibenzo-p-dioxin

Octachlorodibenzo-p-furan

PCB 1016

PCB 1221

PCB 1232

PCB 1242

PCB 1248

PCB 1254

PCB 1260

Pentachlorodibenzo-p-dioxin isomers

Pentachlorodibenzo-p-furan isomers

2,3,7,8-TCDD

2.3,7,8-TCDF

Tetrachlorodibenzo-p-dioxin isomers

Tetrachlorodibenzo-p-furan isomers

Toxaphene

\section{U}

U

U

U

$\mathrm{U}$

U

J

J

J

U

U

U

U

U

U

U

U

U

U

U

U

$\begin{array}{lll} & T & 2.4 \\ \mathrm{U} & & 0.40\end{array}$

$\mathbf{U}$

$\mathrm{U}$

U

U

U

U

. 530

U

U

U

U

$\mathrm{U}$

U

U
9.8

\section{8}

9.8

9.8

9.8

9.0

10

12

20

9.8

20

20

20

20

9.8

9.8

0.30

0.20

0.20

0.20

9.8

98

0.40

200

390

200

200

200

200

0.20

0.20

0.20

0.10

0.20

0.10

980 $\mu \mathrm{g} / \mathrm{kg}$

$$
\mu \mathrm{g} / \mathrm{kg}
$$

$\mu g / k g$

$\mu g / \mathrm{kg}$

$\mu g / k g$

$\mu \mathrm{g} / \mathrm{kg}$

$\mu \mathrm{g} / \mathrm{kg}$

$\mu \mathrm{g} / \mathrm{kg}$

$\mu g / k g$

$\mu g / \mathrm{kg}$

$\mu \mathrm{g} / \mathrm{kg}$

$\mu \mathrm{g} / \mathrm{kg}$

$\mu g / \mathrm{kg}$

$\mu \mathrm{g} / \mathrm{kg}$

$\mu g / k g$

$\mu \mathrm{g} / \mathrm{kg}$

$\mu \mathrm{g} / \mathrm{kg}$

$\mathrm{ng} / \mathrm{g}$

$\mathrm{ng} / \mathrm{g}$

$\mathrm{ng} / \mathrm{g}$

$\mathrm{ng} / \mathrm{g}$

$\mu \mathrm{g} / \mathrm{kg}$

$\mu \mathrm{g} / \mathrm{kg}$

ng/g

$\mathrm{ng} / \mathrm{g}$

$\mu \mathrm{g} / \mathrm{kg}$

$\mu \mathrm{g} / \mathrm{kg}$

$\mu \mathrm{g} / \mathrm{kg}$

$\mu \mathrm{g} / \mathrm{kg}$

$\mu \mathrm{g} / \mathrm{kg}$

$\mu \mathrm{g} / \mathrm{kg}$

$\mu \mathrm{g} / \mathrm{kg}$

$\mathrm{ng} / \mathrm{g}$

$\mathrm{ng} / \mathrm{g}$

$\mathrm{ng} / \mathrm{g}$

$\mathrm{ng} / \mathrm{g}$

$\mathrm{ng} / \mathrm{g}$.

ng/g

$\mu \mathrm{g} / \mathrm{kg}$

\section{8}

9.8

9.8

9.8

9.8

9.8

20

20

\section{0}

\section{0}

9.8

\section{0}

$$
20
$$

$$
20 \text {. }
$$

$$
20
$$

\section{8}

9.8

0.30

0.20

.0 .20

0.20

9.8

98

0.10

0.40

200

390

200

200

200

200

200

0.20

0.20

0.20

0.10

0.20

0.10

980

Radionuclide Indicätörs and Radionuclides

Gross alpha -

Nonvolatile beta

Radium, total alpha-emitting

Tritium

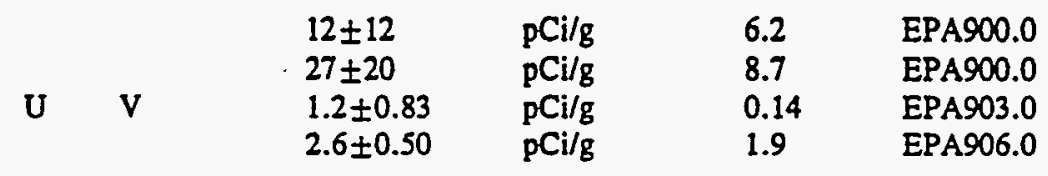

EPA8080

EPA8080

EPA8080

EPA8080

EPA8080

EPA8080

EPA8080

EPA8080

EPA8080

EPA8080

EPA8080

EPA8080

EPA8080

EPA8080

EPA8080

EPA8080

EPA8080

EPA8280

EPA8280

EPA8280

EPA8280

EPA8080

EPA8080

EPA8280

EPA8280

EPA8080

EPA8080

EPA8080

EPA8080

EPA8080

EPA8080

EPA8080

EPA8280

EPA8280

EPA8280

EPA8280

EPA8280

EPA8280

EPA8080 
SAMPLE NAME: D431 1205

Location (SRS Coordinates): 18518.11E 66590.27N

Ground Elevation Above MSL: $127.7 \mathrm{ft}$

Depth of Core Interval: 6.00 to $8.00 \mathrm{ft}$

Sample Type: Normal

Sample Color: SYR 2/2

\section{Constituent}

Total Metals
Sample ID: 100375

Sample Matrix: Soil

USC Soil Classification: SW
Sample Moisture: Damp

Percent Solids: 85.10

D. Limit Method

$\begin{array}{llllll}\text { Arsenic } & & 1.2 & \mathrm{mg} / \mathrm{kg} & 1.1 & \text { EPA7060 } \\ \text { Barium } & & 28 & \mathrm{mg} / \mathrm{kg} & 22 & \text { EPA6010 } \\ \text { Cadmium } & \mathrm{U} & 0.54 & \mathrm{mg} / \mathrm{kg} & 0.54 & \text { EPA6010 } \\ \text { Chromium } & & 16 & \mathrm{mg} / \mathrm{kg} & 1.1 & \text { EPA6010 } \\ \text { Copper } & & 4.9 & \mathrm{mg} / \mathrm{kg} & 2.7 & \text { EPA6010 } \\ \text { Lead } & & 8.3 & \mathrm{mg} / \mathrm{kg} & 0.67 & \text { EPA7421 } \\ \text { Manganese } & & 17 & \mathrm{mg} / \mathrm{kg} & 1.6 & \text { EPA6010 } \\ \text { Mercury } & \mathrm{U} & 0.12 & \mathrm{mg} / \mathrm{kg} & 0.12 & \text { EPA7470 } \\ \text { Nicke! } & \mathrm{U} & 4.3 & \mathrm{mg} / \mathrm{kg} & 4.3 & \text { EPA6010 } \\ \text { Selenium } & \mathrm{U} & 0.56 & \mathrm{mg} / \mathrm{kg} & 0.56 & \text { EPA7740 } \\ \text { Silver } & \mathrm{U} & 1.1 & \mathrm{mg} / \mathrm{kg} & 1.1 & \text { EPA6010 }\end{array}$

$B / N / A$ Extractables (including $P A H$ and phenols)

\begin{tabular}{|c|c|c|c|c|c|c|c|}
\hline Acenaphthene & UJ & 0 & L & 390 & $\mu g / k g$ & 390 & EPA8270 \\
\hline Acenaphthene & UJ & 0 & L & 390 & $\mu \mathrm{g} / \mathrm{kg}$ & 390 & EPA8270 \\
\hline Acenaphthylene & UJ & 0 & L & 390 & $\mu \mathrm{g} / \mathrm{kg}$ & 390 & EPA8270 \\
\hline Acenaphthylene & UJ & 0 & L & 390 & $\mu g / k g$ & 390 & EPA8270 \\
\hline Anthracene & UJ & 0 & L & 390 & $\mu \mathrm{g} / \mathrm{kg}$ & 390 & EPA8270 \\
\hline Anthracene & UJ & 0 & L & 390 & $\mu \mathrm{g} / \mathrm{kg}$ & 390 & EPA8270 \\
\hline Benzo(a)anthracene & UJ & 0 & L & 390 & $\mu \mathrm{g} / \mathrm{kg}$ & 390 & EPA8270 \\
\hline Benzo(a)anthracene & UJ & 0 & L & 390 & $\mu \mathrm{g} / \mathrm{kg}$ & 390 & EPA8270 \\
\hline Benzo(b)fluoranthene & UJ & 0 & L & 390 & $\mu g / k g$ & 390 & EPA8270 \\
\hline Benzo(b)fluoranthene & UJ & 0 & L & 390 & $\mu g / \mathrm{kg}$ & 390 & EPA8270 \\
\hline Benzo(k)fluoranthene & UJ & 0 & L & 390 & $\mu g / \mathrm{kg}$ & 390 & EPA8270 \\
\hline Benzo(k)fluoranthene & UJ & 0 & L & 390 & $\mu g / \mathrm{kg}$ & 390 & EPA8270 \\
\hline Benzoic acid & UJ & 0 & L & 1,900 & $\mu g / \mathrm{kg}$ & 1.900 & EPA8270 \\
\hline Benzoic acid & UJ & 0 & L & 1,900 & $\mu \mathrm{g} / \mathrm{kg}$ & 1,900 & EPA8270 \\
\hline Benzo $(\mathrm{g}, \mathrm{h}, \mathrm{i})$ perylene & UJ & 0 & L & 390 & $\mu \mathrm{g} / \mathrm{kg}$ & 390 & EPA8270 \\
\hline Benzo $(g, h, i)$ perylene & UJ & 0 & L & 390 & $\mu g / k g$ & 390 & EPA8270 \\
\hline Benzo(a)pyrene & UJ & 0 & L & 390 & $\mu \mathrm{g} / \mathrm{kg}$ & 390 & EPA8270 \\
\hline Benzo(a)pyrene & UJ & 0 & L & 390 & $\mu \mathrm{g} / \mathrm{kg}$ & 390 & EPA8270 \\
\hline Benzyl alcohol & UJ & 0 & L & 390 & $\mu g / \mathrm{kg}$ & 390 & EPA8270 \\
\hline Benzyl alcohol & UJ & 0 & L & 390 & $\mu \mathrm{g} / \mathrm{kg}$ & 390 & EPA8270 \\
\hline Bis(2-chloroethoxy) methane & UJ & 0 & L & 390 & $\mu g / \mathrm{kg}$ & 390 & EPA8270 \\
\hline Bis(2-chloroethoxy) methane & UJ & 0 & L & 390 & $\mu g / \mathrm{kg}$ & 390 & EPA8270 \\
\hline Bis(2-chloroethyl) ether & UJ & 0 & L & 390 & $\mu \mathrm{g} / \mathrm{kg}$ & 390 & EPA8270 \\
\hline Bis(2-chloroisopropyl) etther & UJ & 0 & L & 390 & $\mu \mathrm{g} / \mathrm{kg}$ & 390 & EPA8270 \\
\hline Bis(2-chloroethyl) ether & UJ & 0 & L & 390 & $\mu \mathrm{g} / \mathrm{kg}$ & 390 & EPA8270 \\
\hline Bis(2-chloroisopropyl) ether & UJ & 0 & L & 390 & $\mu \mathrm{g} / \mathrm{kg}$ & 390 & EPA8270 \\
\hline Bis(2-ethylhexyl) phthalate & UJ & 0 & L & 390 & $\mu g / \mathrm{kg}$ & 390 & EPA8270 \\
\hline Bis(2-ethylhexyl) phthalate & UJ & 0 & L & 390 & $\mu \mathrm{g} / \mathrm{kg}$ & 390 & EPA8270 \\
\hline 4-Bromophenyl phenyl ether & UJ & 0 & L & 390 & $\mu \mathrm{g} / \mathrm{kg}$ & 390 & EPA8270 \\
\hline 4-Bromophenyl phenyl ether & UJ & 0 & L & 390 & $\mu g / \mathrm{kg}$ & 390 & EPA8270 \\
\hline
\end{tabular}


SAMPLE NAME: D4311205 (Continued)

Sample D: 100375

\section{Constituent}

RQ AQ B Result Unit.

D. Limit Method

$B / N / A$ Extractables (including $P A H$ and phenols)

\begin{tabular}{|c|c|c|c|c|c|c|c|}
\hline Butylbenzyl phthalate & UJ & 0 & L & 390 & $\mu g / \mathrm{kg}$ & 390 & EPA8270 \\
\hline Butylbenzyl phthalate & UJ. & 0 & L & 390 & $\mu \mathrm{g} / \mathrm{kg}$ & 390 & EPA8270 \\
\hline 4-Chloroaniline & UJ & 0 & L & 390 & $\mu \mathrm{g} / \mathrm{kg}$ & 390 & EPA8270 \\
\hline 4-Chloroaniline & UJ & o & $\vec{L}$ & 390 & $\mu \mathrm{g} / \mathrm{kg}$ & 390 & EPA8270 \\
\hline 4-Chloro-m-cresol & UJ & 0 & $\bar{L}$ & 390 & $\mu \mathrm{g} / \mathrm{kg}$ & 390 & EPA8270 \\
\hline 4-Chloro-m-cresol & UJ & 0 & $\mathrm{~L}$ & 390 & $\mu \mathrm{g} / \mathrm{kg}$ & 390 & EPA8270 \\
\hline 2-Chloronaphthalene & UJ & 0 & L & 390 & $\mu \mathrm{g} / \mathrm{kg}$ & 390 & EPA8270 \\
\hline 2-Chloronaphthalene & UJ & 0 & L & 390 & $\mu \mathrm{g} / \mathrm{kg}$ & 390 & EPA8270 \\
\hline 2-Chlorophenol & UJ & 0 & L & 390 & $\mu \mathrm{g} / \mathrm{kg}$ & 390 & EPA8270 \\
\hline 2-Chlorophenol & UJ & 0 & L & 390 & $\mu \mathrm{g} / \mathrm{kg}$ & 390 & EPA8270 \\
\hline 4-Chlorophenyl phenyl ether & UJ & 0 & L & 390 & $\mu \mathrm{g} / \mathrm{kg}$ & 390 & EPA8270 \\
\hline 4-Chlorophenyl phenyl ether & UJ & 0 & L & 390 & $\mu \mathrm{g} / \mathrm{kg}$ & 390 & EPA8270 \\
\hline Chrysene & UJ & 0 & L & 390 & $\mu \mathrm{g} / \mathrm{kg}$ & 390 & EPA8270 \\
\hline Chrysene & UJ & 0 & L & 390 & $\mu \mathrm{g} / \mathrm{kg}$ & 390 & EPA8270 \\
\hline o-Cresol (2-Methylphenol) & UJ & 0 & L & 390 & $\mu \mathrm{g} / \mathrm{kg}$ & 390 & EPA8270 \\
\hline o-Cresol (2-Methylphenol) & UJ & 0 & I & 390 & $\mu \mathrm{g} / \mathrm{kg}$ & 390 & EPA8270 \\
\hline p-Cresol (4-Methylphenol) & UJ & 0 & $\mathrm{~L}$ & 390 & $\mu \mathrm{g} / \mathrm{kg}$ & 390 & EPA8270 \\
\hline p-Cresol (4-Methylphenol) & UJ & 0 & L & 390 & $\mu \mathrm{g} / \mathrm{kg}$ & 390 & EPA8270 \\
\hline Dibenz $(a, h)$ anthracene & UJ & 0 & L & 390 & $\mu \mathrm{g} / \mathrm{kg}$ & 390 & EPA8270 \\
\hline Dibenz(a,h)anthracene & UJ & 0 & L & 390 & $\mu \mathrm{g} / \mathrm{kg}$ & 390 & EPA8270 \\
\hline Dibenzofuran & UJ & 0 & L & 390 & $\mu \mathrm{g} / \mathrm{kg}$ & 390 & EPA8270 \\
\hline Dibenzofuran & UJ & 0 & L & 390 & $\mu \mathrm{g} / \mathrm{kg}$ & 390 & EPA8270 \\
\hline Di-n-butyl phthalate & UI & 0 & $\mathrm{~L}$ & 390 & $\mu g / \mathrm{kg}$ & 390 & EPA8270 \\
\hline Di-n-butyl phthalate & UJ & 0 & L & 390 & $\mu \mathrm{g} / \mathrm{kg}$ & 390 & EPA8270 \\
\hline 1,2-Dichlorobenzene & UJ & 0 & L & 390 & $\mu \mathrm{g} / \mathrm{kg}$ & 390 & EPA8270 \\
\hline 1,2-Dichlorobenzene & UJ & 0 & L. & 390 & $\mu \mathrm{g} / \mathrm{kg}$ & 390 & EPA8270 \\
\hline 1,3-Dichlorobenzene & UJ & 0 & $\mathrm{~L}$ & 390 & $\mu \mathrm{g} / \mathrm{kg}$ & 390 & EPA8270 \\
\hline 1,3-Dichlorobenzene & UJ & 0 & L & 390 & $\mu \mathrm{g} / \mathrm{kg}$ & 390 & EPA8270 \\
\hline 1,4-Dichlorobenzene & UJ & 0 & $\mathrm{~L}$ & 390 & $\mu \mathrm{g} / \mathrm{kg}$ & 390 & EPA8270 \\
\hline 1,4-Dichlorobenzene & UJ & 0 & L & 390 & $\mu \mathrm{g} / \mathrm{kg}$ & 390 & EPA8270 \\
\hline 3,3'-Dichlorobenzidine & UJ & 0 & L & 780 & $\mu \mathrm{g} / \mathrm{kg}$ & 780 & EPA8270 \\
\hline 3,3'-Dichlorobenzidine & UJ & 0 & L & 780 & $\mu \mathrm{g} / \mathrm{kg}$ & 780 & EPA8270 \\
\hline 2,4-Dichlorophenol & UJ & 0 & L & 390 & $\mu \mathrm{g} / \mathrm{kg}$ & 390 & EPA8270 \\
\hline 2.4-Dichlorophenol & UJ & 0 & L & 390 & $\mu \mathrm{g} / \mathrm{kg}$ & 390 & EPA8270 \\
\hline Diethyl phthalate & UJ & 0 & L & 390 & $\mu \mathrm{g} / \mathrm{kg}$ & 390 & EPA8270 \\
\hline Diethyl phthalate & UJ & 0 & $\bar{L}$ & 390 & $\mu \mathrm{g} / \mathrm{kg}$ & 390 & EPA8270 \\
\hline 2,4-Dimethyl phenol & UJ & 0 & L & 390 & $\mu g / \mathrm{kg}$ & 390 & EPA8270 \\
\hline 2,4-Dimethyl phenol & UJ & 0 & L & 390 & $\mu \mathrm{g} / \mathrm{kg}$ & 390 & EPA 8270 \\
\hline Dimethyl phthalate & UJ & 0 & L & 390 & $\mu \mathrm{g} / \mathrm{kg}$ & 390 & EPA8270 \\
\hline Dimethyl phthalate & UJ & 0 & L & 390 & $\mu \mathrm{g} / \mathrm{kg}$ & 390 & EPA8270 \\
\hline 2,4-Dinitrophenol & UJ_ & 0 & L & 1,900 & $\mu g / \mathrm{kg}$ & 1,900 & EPA8270 \\
\hline 2,4-Dinitrophenol & UJ & 0 & L & 1,900 & $\mu \mathrm{g} / \mathrm{kg}$ & 1,900 & EPA8270 \\
\hline 2,4-Dinitrotoluene & UJ & $\mathbf{o}$ & L & 390 & $\mu \mathrm{g} / \mathrm{kg}$ & 390 & EPA8270 \\
\hline 2,4-Dinitrotoluene & UJ & 0 & L & 390 & $\mu \mathrm{g} / \mathrm{kg}$ & 390 & EPA8270 \\
\hline 2,6-Dinitrotoluene & UJ & 0 & L & 390 & $\mu \mathrm{g} / \mathrm{kg}$ & 390 & EPA8270 \\
\hline 2,6-Dinitrotoluene & UJ & 0 & L & 390 & $\mu \mathrm{g} / \mathrm{kg}$ & 390 & EPA8270 \\
\hline Di-n-octyl phthalate & UJ & 0 & L & 390 & $\mu \mathrm{g} / \mathrm{kg}$ & 390 & EPA8270 \\
\hline Di-n-octyl phthalate & UJ & $\mathbf{0}$ & L & 390 & $\mu \mathrm{g} / \mathrm{kg}$ & 390 & EPA8270 \\
\hline Fluoranthene & UJ & 0 & L & 390 & $\mu \mathrm{g} / \mathrm{kg}$ & 390 & EPA8270 \\
\hline Fluoranthene & UJ & 0 & L & 390 & $\mu \mathrm{g} / \mathrm{kg}$ & 390 & EPA8270 \\
\hline
\end{tabular}




$\begin{array}{llllll}\text { Constituent } & \text { RQ AQ B Result Dimit Method }\end{array}$

$B / N / A$ Extractables (including $P A H$ and phenols)

\begin{tabular}{|c|c|c|c|c|c|c|c|}
\hline Fluorene & UJ & 0 & L & 390 & $\mu \mathrm{g} / \mathrm{kg}$ & 390 & EPA8270 \\
\hline Fluorene & Uנ & 0 & L & 390 & $\mu g / k g$ & 390 & EPA8270 \\
\hline Hexachlorobenzene & UJ & 0 & L & 390 & $\mu \mathrm{g} / \mathrm{kg}$ & 390 & EPA8270 \\
\hline Hexachlorobenzene & UJ & 0 & L & 390 & $\mu \mathrm{g} / \mathrm{kg}$ & 390 & EPA8270 \\
\hline Hexachlorobutadiene & UJ & 0 & L & 390 & $\mu \mathrm{g} / \mathrm{kg}$ & 390 & EPA8270 \\
\hline Hexachlorobutadiene & UJ & 0 & L & 390 & $\mu \mathrm{g} / \mathrm{kg}$ & 390 & EPA8270 \\
\hline Hexachlorocyclopentadiene & UJ & 0 & L & 390 & $\mu g / \mathrm{kg}$ & 390 & EPA8270 \\
\hline Hexachlorocyclopentadiene & UJ & 0 & L & 390 & $\mu \mathrm{g} / \mathrm{kg}$ & 390 & EPA8270 \\
\hline Hexachloroethane & UJ & 0 & L & 390 & $\mu \mathrm{g} / \mathrm{kg}$ & 390 & EPA8270 \\
\hline Hexachloroethane & UJ & 0 & L & 390 & $\mu g / \mathrm{kg}$ & 390 & EPA8270 \\
\hline Sub. hexanedioic acid & 3 & $\mathbf{N}$ & & 30,000 & $\mu \mathrm{g} / \mathrm{kg}$ & & EPA8270 \\
\hline Indeno(1,2,3-c,d)pyrene & UJ & 0 & L & 390 & $\mu g / \mathrm{kg}$ & 390 & EPA8270 \\
\hline Indeno(1,2,3-c,d)pyrene & UJ & 0 & L & 390 & $\mu \mathrm{g} / \mathrm{kg}$ & 390 & EPA8270 \\
\hline Isophorone & UJ & 0 & L & 390 & $\mu \mathrm{g} / \mathrm{kg}$ & 390 & EPA8270 \\
\hline Isophorone & UJ & 0 & $\mathrm{~L}$ & 390 & $\mu g / k g$ & 390 & EPA 8270 \\
\hline 2-Methyl-4,6-dinitrophenol & UJ & 0 & L & 1,900 & $\mu g / \mathrm{kg}$ & 1,900 & EPA8270 \\
\hline 2-Methyl-4,6-dinitrophenol & UJ & 0 & L & 1.900 & $\mu \mathrm{g} / \mathrm{kg}$ & 1.900 & EPA8270 \\
\hline 2-Methylnaphthalene & UJ & 0 & L & 390 & $\mu \mathrm{g} / \mathrm{kg}$ & 390 & EPA8270 \\
\hline 2-Methylnaphthalene & UJ & 0 & L & 390 & $\mu \mathrm{g} / \mathrm{kg}$ & 390 & EPA8270 \\
\hline Naphthalene & UJ & 0 & L & 390 & $\mu \mathrm{g} / \mathrm{kg}$ & 390 & EPA8270 \\
\hline Naphthalene & UJ & 0 & L & 390 & $\mu g / \mathrm{kg}$ & 390 & EPA8270 \\
\hline o-Nitroaniline & UJ & 0 & L & 1,900 & $\mu \mathrm{g} / \mathrm{kg}$ & 1,900 & EPA8270 \\
\hline o-Nitroaniline & UJ & 0 & L & 1,900 & $\mu \mathrm{g} / \mathrm{kg}$ & 1.900 & EPA8270 \\
\hline m-Nitroaniline & UJ & 0 & L & 1,900 & $\mu \mathrm{g} / \mathrm{kg}$ & 1,900 & EPA8270 \\
\hline m-Nitroaniline & UJ & 0 & L & 1,900 & $\mu \mathrm{g} / \mathrm{kg}$ & 1,900 & EPA8270 \\
\hline p-Nitroaniline & UJ & 0 & L & 1,900 & $\mu \mathrm{g} / \mathrm{kg}$ & 1,900 & EPA8270 \\
\hline p-Nitroaniline & UJ & 0 & L & 1,900 & $\mu \mathrm{g} / \mathrm{kg}$ & 1,900 & EPA8270 \\
\hline Nitrobenzene & UJ & 0 & L & 390 & $\mu \mathrm{g} / \mathrm{kg}$ & 390 & EPA8270 \\
\hline Nitrobenzene & UJ & 0 & L & 390 & $\mu \mathrm{g} / \mathrm{kg}$ & 390 & EPA8270 \\
\hline 2-Nitrophenol & UJ & 0 & L & 390 & $\mu \mathrm{g} / \mathrm{kg}$ & 390 & EPA8270 \\
\hline 2-Nitrophenol & UJ & 0 & L & 390 & $\mu g / \mathrm{kg}$ & 390 & EPA8270 \\
\hline 4-Nitrophenol & UJ & 0 & L & 1,900 & $\mu \mathrm{g} / \mathrm{kg}$ & 1,900 & EPA8270 \\
\hline 4-Nitrophenol & UJ & 0 & L & 1,900 & $\mu g / k g$ & 1,900 & EPA8270 \\
\hline N-Nitrosodiphenylamine & UJ & 0 & L & 390 & $\mu \mathrm{g} / \mathrm{kg}$ & 390 & EPA8270 \\
\hline N-Nitrosodiphenylamine & UJ & 0 & L & 390 & $\mu \mathrm{g} / \mathrm{kg}$ & 390 & EPA8270 \\
\hline N-Nitrosodipropylamine & UI & $\mathrm{CO}$ & L & 390 & $\mu \mathrm{g} / \mathrm{kg}$ & 390 & EPA8270 \\
\hline N-Nitrosodipropylamine & UI & $\mathrm{CO}$ & L & 390 & $\mu \mathrm{g} / \mathrm{kg}$ & 390 & EPA8270 \\
\hline Pentachlorophenol & UJ & 0 & L & 1,900 & $\mu g / k g$ & 1.900 & EPA8270 \\
\hline Pentachlorophenol & UJ & 0 & L & 1,900 & $\mu \mathrm{g} / \mathrm{kg}$ & 1.900 & EPA8270 \\
\hline Phenanthrene & UJ & 0 & L & 390 & $\mu \mathrm{g} / \mathrm{kg}$ & 390 & EPA8270 \\
\hline Phenanthrene & UJ & 0 & L & 390 & $\mu \mathrm{g} / \mathrm{kg}$ & 390 & EPA8270 \\
\hline Phenol & UJ & 0 & $\mathbf{L}$ & 390 & $\mu g / k g$ & 390 & EPA827 \\
\hline Phenol & UJ & 0 & L & 390 & $\mu \mathrm{g} / \mathrm{kg}$ & 390 & EPA827 \\
\hline Pyrene & UJ & 0 & L & 390 & $\mu \mathrm{g} / \mathrm{kg}$ & 390 & EPA8270 \\
\hline Pyrene & UJ & 0 & L & 390 & $\mu g / \mathrm{kg}$ & 390 & EPA827 \\
\hline 1.2,4-Trichlorobenzene & UJ & CO & L & 390 & $\mu \mathrm{g} / \mathrm{kg}$ & 390 & EPA827 \\
\hline 1,2,4-Trichlorobenzene & UJ & CO & L & 390 & $\mu \mathrm{g} / \mathrm{kg}$ & 390 & EPA827 \\
\hline 2,4,5-Trichlorophenol & UJ & 0 & L & 1,900 & $\mu g / \mathrm{kg}$ & 1,900 & EPA8270 \\
\hline 2,4,5-Trichlorophenol & UJ & 0 & L & 1,900 & $\mu \mathrm{g} / \mathrm{kg}$ & 1,900 & EPA8270 \\
\hline 2,4,6-Trichlorophenol & UJ & 0 & L & 390 & $\mu g / \mathrm{kg}$ & 390 & EPA 8270 \\
\hline
\end{tabular}


SAMPLE NAME: D431 1205 (Continued) Sample ID: 100375

$\begin{array}{llllll}\text { Constituent } & \text { RQ AQ B Result Unit Dimit Method }\end{array}$

$B / N / A$ Extractables (including $P A H$ and phenols)

\begin{tabular}{|c|c|c|c|c|c|c|c|}
\hline $\begin{array}{l}\text { 2,4,6-Trichlorophenol } \\
\text { Unknown }\end{array}$ & UJ & $\begin{array}{l}\mathrm{O} \\
\text { NV }\end{array}$ & $L$ & $\begin{array}{l}390 \\
1,000\end{array}$ & $\begin{array}{l}\mu \mathrm{g} / \mathrm{kg} \\
\mu \mathrm{g} / \mathrm{kg}\end{array}$ & 390 & $\begin{array}{l}\text { EPA8270 } \\
\text { EPA8270 }\end{array}$ \\
\hline Unknown & J & $\mathrm{N}$ & & 300 & $\mu \mathrm{g} / \mathrm{kg}$ & & EPA8270 \\
\hline Unknown & J & $\mathbf{N}$ & & 300 & $\mu \mathrm{g} / \mathrm{kg}$ & & EPA8270 \\
\hline Unknown & J & $\mathbf{N}$ & & 200 & $\mu \mathrm{g} / \mathrm{kg}$ & & EPA8270 \\
\hline Unknown & j & $N$ & & 400 & $\mu g / \mathrm{kg}$ & & EPA8270 \\
\hline Unknown & $\mathbf{J}$ & $\mathbf{N}$ & & 200 & $\mu \mathrm{g} / \mathrm{kg}$ & & EPA8270 \\
\hline Unknown ketone & J & NV & & 200,000 & $\mu \mathrm{g} / \mathrm{kg}$ & & EPA8270 \\
\hline
\end{tabular}

\section{Volatile Organic Compounds}

Acetone
Benzene
Bromodichloromethane
Bromoform
Bromomethane (Methyl bromide)
Carbon disulfide
Carbon tetrachloride
Chlorobenzene
Chloroethane
Chloroethene (Vinyl chloride)
2-Chloroethyl vinyl ether
Chloroform
Chloromethane (Methyl chloride)
Dibromochloromethane
1,1-Dichloroethane
1,2-Dichloroethane
1,1-Dichloroethylene
1,2-Dichloroethylene
Dichloromethane (Methylene chloride)
1,2-Dichloropropane
cis-1,3-Dichloropropene
trans-1,3-Dichloropropene
Ethylbenzene
2-Hexanone
Methyl ethyl ketone
Methyl isobutyl ketone
Styrene
1,1,2,2-Tetrachloroethane
Tetrachloroethylene
Toluene
1,1,1-Trichloroethane
1,1,2-Trichloroethane
Trichloroethylene
Vinyl acetate
Xylenes

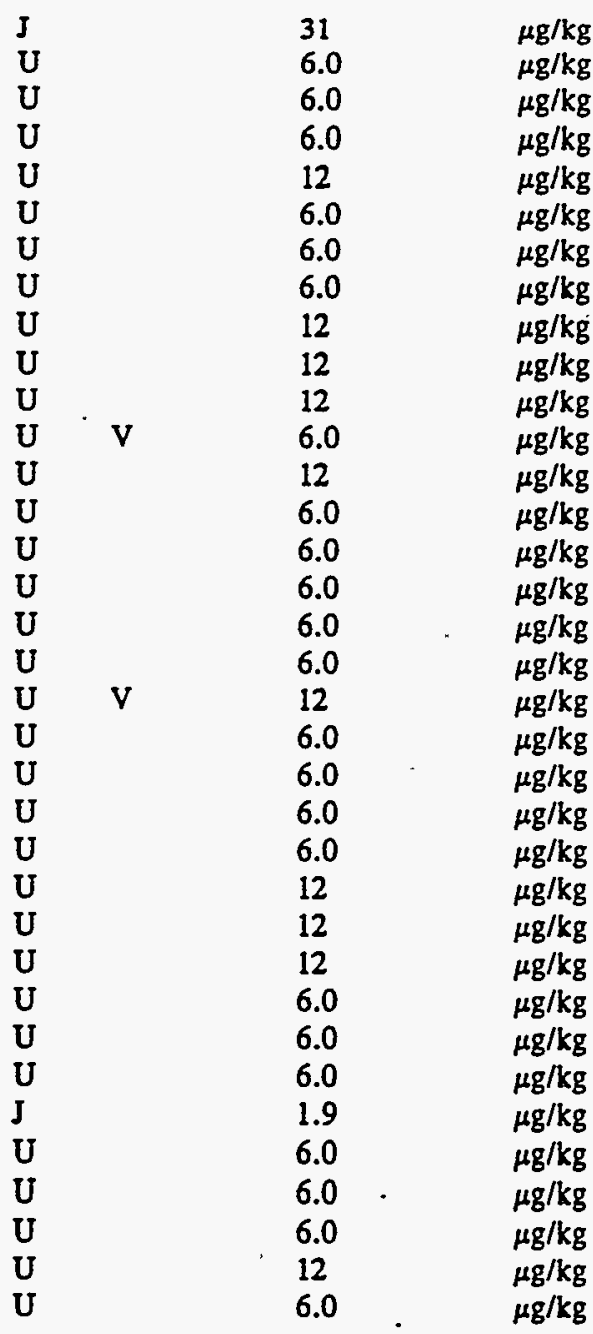

$\begin{array}{ll}12 & \text { EPA8240 } \\ 6.0 & \text { EPA8240 } \\ 6.0 & \text { EPA8240 } \\ 6.0 & \text { EPA8240 } \\ 12 & \text { EPA8240 } \\ 6.0 & \text { EPA8240 } \\ 6.0 & \text { EPA8240 } \\ 6.0 & \text { EPA8240 } \\ 12 & \text { EPA8240 } \\ 12 & \text { EPA8240 } \\ 12 & \text { EPA8240 } \\ 6.0 & \text { EPA8240 } \\ 12 & \text { EPA8240 } \\ 6.0 & \text { EPA8240 } \\ 6.0 & \text { EPA8240 } \\ 6.0 & \text { EPA8240 } \\ 6.0 & \text { EPA8240 } \\ 6.0 & \text { EPA8240 } \\ 6.0 & \text { EPA8240 } \\ 6.0 & \text { EPA8240 } \\ 6.0 & \text { EPA8240 } \\ 6.0 & \text { EPA8240 } \\ 6.0 & \text { EPA8240 } \\ 12 & \text { EPA8240 } \\ 12 & \text { EPA8240 } \\ 12 & \text { EPA8240 } \\ 6.0 . & \text { EPA8240 } \\ 6.0 & \text { EPA8240 } \\ 6.0 & \text { EPA8240 } \\ 6.0 & \text { EPA8240 } \\ 6.0 & \text { EPA8240 } \\ 6.0 & \text { EPA8240 } \\ 6.0 & \text { EPA8240 } \\ 12 & \text { EPA8240 } \\ 6.0 & \text { EPA8240 }\end{array}$




\section{Constituent}

\section{$\mathrm{RQ}$}

AQ B Result

Unit

D. Limit Method

Pesticides/PCBs and Dioxins/Furans

Aldrin

alpha-Benzene hexachloride

beta-Benzene hexachloride

delta-Benzene hexachloride

alpha-Chlordane

gamma-Chlordane .

p.p'-DDD

P.p'-DDE

$p, p^{\prime}-D D T$

Dieldrin

Endosulfan I

Endosulfan II

Endosulfan sulfate

Endrin

Endrin ketone

Heptachlor

Heptachlor epoxide

Heptachlorodibenzo-p-dioxin isomers

Heptachlorodibenzo-p-furan isomers

Hexachlorodibenzo-p-dioxin isomers

Hexachlorodibenzo-p-furan isomers

Lindane

Methoxychlor

Octachlorodibenzo-p-dioxin

Octachlorodibenzo-p-furan

PCB 1016

PCB 1221

PCB 1232

PCB 1242

PCB 1248

PCB 1254

PCB 1260

Pentachlorodibenzo-p-dioxin isomers U

Pentachlorodibenzo-p-furan isomers

2,3,7,8-TCDD

2,3,7,8-TCDF

Tetrachlorodibenzo-p-dioxin isomers

Tetrachlorodibenzo-p-furan isomers

Toxaphene

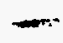

Radionuclide Indicators and Radionuclides

Gross alpha . $\rightarrow$

Nonvolatile beta

Radium, total alpha-emitting

Tritium
U

2.0

2.0

2.0

2.0

2.0

3.9

3.9

3.9

3.9

2.0

3.9

3.9

3.9

3.9

2.0

2.0

0.30

0.10

0.20

0.10

2.0

20

9.5

0.20

39

78

39

39

39

39

120

0.20

0.10

0.10

0.20

0.10

0.20

200

$\begin{array}{ll}13 \pm 14 & \mathrm{pCi} / \mathrm{g} \\ 40 \pm 24 & \mathrm{pCi} / \mathrm{g} \\ 0.90 \pm 0.72 & \mathrm{pCi} / \mathrm{g} \\ 1.4 & \mathrm{pCi} / \mathrm{g}\end{array}$

$\mu g / k g$
$\mu g / k g$
$\mu g / k g$
$\mu g / k g$
$\mu g / k g$
$\mu g / k g$
$\mu g / k g$
$\mu g / k g$
$\mu g / k g$
$\mu g / k g$
$\mu g / k g$
$\mu g / k g$
$\mu g / k g$
$\mu g / k g$
$\mu g / k g$
$\mu g / k g$
$\mu g / k g$
$n g / g$
$n g / g$
$n g / g$
$n g / g$
$\mu g / k g$
$\mu g / k g$
$n g / g$
$n g / g$
$\mu g / k g$
$\mu g / k g$
$\mu g / k g$
$\mu g / k g$
$\mu g / k g$
$\mu g / k g$
$\mu g / k g$
$n g / g$
$n g / g$
$n g / g$
$n g / g$
$n g / g$
$n g / g$
$\mu g / k g$

$\mu \mathrm{g} / \mathrm{kg}$

$\mu \mathrm{g} / \mathrm{kg}$

$\mu g / k g$

$\mu \mathrm{g} / \mathrm{kg}$

$\mu g / \mathrm{kg}$

$\mu g / k g$

$\mu \mathrm{g} / \mathrm{kg}$

$\mu \mathrm{g} / \mathrm{kg}$

$\mu \mathrm{g} / \mathrm{kg}$

$\mu \mathrm{g} / \mathrm{kg}$

$\mu \mathrm{g} / \mathrm{kg}$

$\mu \mathrm{g} / \mathrm{kg}$

$\mathrm{ng} / \mathrm{g}$

ng/g

$\mathrm{g} / \mathrm{g}$

$\mu \mathrm{g} / \mathrm{kg}$

$\mu \mathrm{g} / \mathrm{kg}$

$\mathrm{ng} / \mathrm{g}$

$\mu \mathrm{g} / \mathrm{kg}$

$\mu g / \mathrm{kg}$

$\mu \mathrm{g} / \mathrm{kg}$

$\mu \mathrm{g} / \mathrm{kg}$

$\mu \mathrm{g} / \mathrm{kg}$

$\mu \mathrm{g} / \mathrm{kg}$

ng/g

$\mathrm{ng} / \mathrm{g}$

$\mathrm{ng} / \mathrm{g}$

$\mathrm{ng} / \mathrm{g}$

$\mathrm{ng} / \mathrm{g}$

\section{0}

2.0

2.0

2.0

2.0

2.0

3.9

3.9

3.9

3.9

2.0

3.9

3.9

3.9

3.9

2.0

2.0

0.30

0.10

0.20

0.10

2.0

20

0.10

0.20

39

78

39

39

39
39

39

39

0.20

0.10

0.10

0.20

0.10

0.20

200

$\begin{array}{ll}7.1 & \text { EPA900.0 } \\ 10 & \text { EPA900.0 } \\ 0.15 & \text { EPA903.0 } \\ 1.4 & \text { EPA906.0 }\end{array}$

EPA8080

EPA8080

EPA8080

EPA8080

EPA8080

EPA8080

EPA8080

EPA8080

EPA8080

EPA8080

EPA8080

EPA8080

EPA8080

EPA8080

EPA8080

EPA8080

EPA8080

EPA8280

EPA8280

EPA8280

EPA8280

EPA8080

EPA8080

EPA8280

EPA8280

EPA8080

EPA8080

EPA8080

EPA8080

EPA8080

EPA8080

EPA8080

EPA8280

EPA8280

EPA8280

EPA8280

EPA8280

EPA8280

EPA8080 
SAMPLE NAME: D43II 1205 (Continued)

Constituent

RQ

TCLP Compounds (Metals and Organics)
Sample ID: 100375

AQ B Result Unit
D. Limit Method

\begin{tabular}{|c|c|c|c|c|c|c|c|}
\hline Arsenic, TCLP & U & & & 100 & $\mu \mathrm{g} / \mathrm{L}$ & 100 & EPA6010 \\
\hline Barium. TCLP & U & & & 200 & $\mu g / L$ & 200 & EPA6010 \\
\hline Benzene & $\mathbf{U}$ & & & 50 & $\mu \mathrm{g} / \mathrm{L}$ & 50 & EPA8240 \\
\hline Cadmium, TCLP & $\mathbf{U}$ & & & 5.0 & $\mu \mathrm{g} / \mathrm{L}$ & 5.0 & EPA6010 \\
\hline Carbon tetrachloride & $U$ & & & 50 & $\mu \mathrm{g} / \mathrm{L}$ & 50 & EPA8240 \\
\hline alpha-Chlordane & UJ & 0 & $L$ & 1.4 & $\mu \mathrm{g} / \mathrm{L}$ & 1.4 & EPA8080 \\
\hline gamma-Chlordane .. & UJ & 0 & L & 1.4 & $\mu \mathrm{g} / \mathrm{L}$ & 1.4 & EPA8080 \\
\hline Chlorobenzene & $\mathrm{U}$ & & & 50 & $\mu g / L$ & 50 & EPA8240 \\
\hline Chloroethene (Vinyl chloride) & $\mathbf{U}$ & & & 100 & $\mu \mathrm{g} / \mathrm{L}$ & 100 & EPA8240 \\
\hline Chloroform & $\mathrm{J}$ & & & 17 & $\mu \mathrm{g} / \mathrm{L}$ & 50 & EPA8240 \\
\hline Chromium, TCLP & $\mathbf{U}$ & & & 10 & $\mu \mathrm{g} / \mathrm{L}$ & 10 & EPA6010 \\
\hline o-Cresol (2-Methylphenol) & $\mathrm{U}$ & & & 100 & $\mu \mathrm{g} / \mathrm{L}$ & 100 & EPA8270 \\
\hline 1,4-Dichlorobenzene & $\mathrm{U}$ & & & 100 & $\mu \mathrm{g} / \mathrm{L}$ & 100 & EPA8270 \\
\hline 1,2-Dichloroethane & $\mathrm{U}$ & & & 50 & $\mu \mathrm{g} / \mathrm{L}$ & 50 & EPA8240 \\
\hline 1,1-Dichloroethylene & $\mathrm{U}$ & & & 50 & $\mu \mathrm{g} / \mathrm{L}$ & 50 & EPA8240 \\
\hline 2,4-Dichlorophenoxyacetic acid & $\mathrm{U}$ & & & 10 & $\mu \mathrm{g} / \mathrm{L}$ & 10 & EPA8150 \\
\hline 2.4-Dinitrotoluene & $\mathrm{U}$ & & & 100 & $\mu \mathrm{g} / \mathrm{L}$ & 100 & EPA8270 \\
\hline Endrin & UJ & 0 & L & 0.28 & $\mu \mathrm{g} / \mathrm{L}$ & 0.28 & EPA8080 \\
\hline Heptachlor & UJ & 0 & L & 0.14 & $\mu \mathrm{g} / \mathrm{L}$ & 0.14 & EPA8080 \\
\hline Heptachlor epoxide & UJ & 0 & L & 0.14 & $\mu \mathrm{g} / \mathrm{L}$ & 0.14 & EPA8080 \\
\hline Hexachlorobenzene & $\mathrm{U}$ & & & 100 & $\mu \mathrm{g} / \mathrm{L}$ & 100 & EPA8270 \\
\hline Hexachlorobutadiene & $\mathrm{U}$ & & & 100 & $\mu \mathrm{g} / \mathrm{L}$ & 100 & EPA8270 \\
\hline Hexachloroethane & $\mathrm{U}$ & & & 100 & $\mu \mathrm{g} / \mathrm{L}$ & 100 & EPA8270 \\
\hline Lead, TCLP & $U$ & & & 90 & $\mu \mathrm{g} / \mathrm{L}$ & 90 & EPA6010 \\
\hline Lindane & UJ & 0 & $\mathrm{~L}$ & 0.14 & $\mu \mathrm{g} / \mathrm{L}$ & 0.14 & EPA8080 \\
\hline Mercury, TCLP & UJ & I & L & 0.20 & $\mu \mathrm{g} / \mathrm{L}$ & 0.20 & EPA7470 \\
\hline Methoxychlor & UJ & 0 & L & 1.4 & $\mu \mathrm{g} / \mathrm{L}$ & 1.4 & EPA8080 \\
\hline Methyl ethyl ketone & $U$ & & & 100 & $\mu \mathrm{g} / \mathrm{L}$ & 100 & EPA8240 \\
\hline Nitrobenzene & $\mathrm{U}$ & & & 100 & $\mu \mathrm{g} / \mathrm{L}$ & 100 & EPA8270 \\
\hline Pentachlorophenol & $\mathrm{U}$ & & & 500 & $\mu \mathrm{g} / \mathrm{L}$ & 500 & EPA8270 \\
\hline Pyridine & $\mathrm{U}$ & & & 100 & $\mu \mathrm{g} / \mathrm{L}$ & 100 & EPA8270 \\
\hline Selenium, TCLP & $U$ & & & 100 & $\mu \mathrm{g} / \mathrm{L}$ & 100 & EPA6010 \\
\hline Silver, TCLP & $\mathrm{U}$ & & & 10 & $\mu \mathrm{g} / \mathrm{L}$ & 10 & EPA6010 \\
\hline Tetrachloroethylene & U & & & so & $\mu \mathrm{g} / \mathrm{L}$ & 50 & EPA8240 \\
\hline Toxaphene & UJ & 0 & L & 2.8 & $\mu \mathrm{g} / \mathrm{L}$ & 2.8 & EPA8080 \\
\hline Toxaphene & UJ & 0 & L & 2.6 & $\mu \mathrm{g} / \mathrm{L}$ & 2.6 & EPA8080 \\
\hline 2,4,5-TP (Silvex) & $\mathbf{U}$ & & & 5.0 & $\mu \mathrm{g} / \mathrm{L}$ & 5.0 & EPA8150 \\
\hline Trichloroethylene & $\mathbf{U}$ & & & 50 & $\mu \mathrm{g} / \mathrm{L}$ & 50 & EPA8240 \\
\hline 2,4,5-Trichlorophenol & $\mathbf{U}$ & & & 500 & $\mu \mathrm{g} / \mathrm{L}$ & 500 & EPA8270 \\
\hline 2,4,6-Trichlorophenol_... & $\mathrm{U}$ & & & 100 & $\mu \mathrm{g} / \mathrm{L}$ & 100 & EPA8270 \\
\hline $2,4,5-\mathrm{T}$ & $\mathrm{U}$ & & & 5.0 & $\mu \mathrm{g} / \mathrm{L}$ & 5.0 & EPA8150 \\
\hline $\mathrm{m}, \mathrm{p}$-Cresol & $\mathbf{U}$ & & & 100 & $\mu \mathrm{g} / \mathrm{L}$ & 100 & EPA8270 \\
\hline
\end{tabular}


SAMPLE NAME: D431 1205A

Sample Type: Duplicate

Constituent

Total Metals
Sample ID: 100376

Associated Sample: 100375

RQ AQ B Result Unit D. Limit Metbod
1.1

39

$\begin{array}{ll}\mathrm{U} & 0.57\end{array}$

20

5.3

4.5

35

0.12

4.6

0.56

1.1 $\mathrm{mg} / \mathrm{kg}$

$\mathrm{mg} / \mathrm{kg}$

$\mathrm{mg} / \mathrm{kg}$

$\mathrm{mg} / \mathrm{kg}$

$\mathrm{mg} / \mathrm{kg}$

$\mathrm{mg} / \mathrm{kg}$

$\mathrm{mg} / \mathrm{kg}$

$\mathrm{mg} / \mathrm{kg}$

$\mathrm{mg} / \mathrm{kg}$

$\mathrm{mg} / \mathrm{kg}$

$\mathrm{mg} / \mathrm{kg}$

$\begin{array}{ll}1.1 & \text { EPA7060 } \\ 23 & \text { EPA6010 } \\ 0.57 & \text { EPA6010 } \\ 1.1 & \text { EPA6010 } \\ 2.9 & \text { EPA6010 } \\ 0.33 & \text { EPA7421 } \\ 1.7 & \text { EPA6010 } \\ 0.12 & \text { EPA7470 } \\ 4.6 & \text { EPA6010 } \\ 0.56 & \text { EPA7740 } \\ 1.1 & \text { EPA6010 }\end{array}$

$B / N / A$ Extractables (including PAH and phenols)

\begin{abstract}
Acenaphthene
Acenaphchylene

Anthracene

Benzo(a)anthracene

Benzo(b)fluoranthene

Benzo(k) fluoranthene

Benzoic acid

Benzo(g,h,i)perylene

Benzo(a)pyrene

Benzyl alcohol

Bis(2-chloroethoxy) methane

Bis(2-chloroethyl) ether

Bis(2-chloroisopropyl) ether

Bis(2-ethylhexyl) phthalate

4-Bromophenyl phenyl ether

Butylbenzyl phthalate

4-Chloroaniline

4-Chloro-m-cresol

2-Chloronaphthalene

2-Chlorophenol

4-Chlorophenyl phenyl echer

Chrysene

0-Cresol (2-Methylphenol)

p-Cresol (4-Methylpȟ̉̌nol)

Dibenz(a,h)anthracene -

Dibenzofuran

Di-n-butyl phthalate

1,2-Dichlorobenzene

1,3-Dichlorobenzene

1.4-Dichlorobenzene

3,3'-Dichlorobenzidine

2,4-Dichlorophenol

Diethyl phthalate

2,4-Dimethyl phenol
\end{abstract}

390

390

390

390

390

390

1,900

390

390

390

390

390

390

60

390

390

390

390

390

390

390

390

390

390

390

390

390

390

390

390

770

390

390

390 .

$\begin{array}{lcc}\mu \mathrm{g} / \mathrm{kg} & 390 & \text { EPA8270 } \\ \mu \mathrm{g} / \mathrm{kg} & 390 & \text { EPA8270 } \\ \mu \mathrm{g} / \mathrm{kg} & 390 & \text { EPA8270 } \\ \mu \mathrm{g} / \mathrm{kg} & 390 & \text { EPA8270 } \\ \mu \mathrm{g} / \mathrm{kg} & 390 & \text { EPA8270 } \\ \mu \mathrm{g} / \mathrm{kg} & 390 & \text { EPA8270 } \\ \mu \mathrm{g} / \mathrm{kg} & 1,900 & \text { EPA8270 } \\ \mu \mathrm{g} / \mathrm{kg} & 390 & \text { EPA8270 } \\ \mu \mathrm{g} / \mathrm{kg} & 390 & \text { EPA8270 } \\ \mu \mathrm{g} / \mathrm{kg} & 390 & \text { EPA8270 } \\ \mu \mathrm{g} / \mathrm{kg} & 390 & \text { EPA8270 } \\ \mu \mathrm{g} / \mathrm{kg} & 390 & \text { EPA8270 } \\ \mu \mathrm{g} / \mathrm{kg} & 390 & \text { EPA8270 } \\ \mu \mathrm{g} / \mathrm{kg} & 390 & \text { EPA8270 } \\ \mu \mathrm{g} / \mathrm{kg} & 390 & \text { EPA8270 } \\ \mu \mathrm{g} / \mathrm{kg} & 390 & \text { EPA8270 } \\ \mu \mathrm{g} / \mathrm{kg} & 390 & \text { EPA8270 } \\ \mu \mathrm{g} / \mathrm{kg} & 390 & \text { EPA8270 } \\ \mu \mathrm{g} / \mathrm{kg} & 390 & \text { EPA8270 } \\ \mu \mathrm{g} / \mathrm{kg} & 390 & \text { EPA8270 } \\ \mu \mathrm{g} / \mathrm{kg} & 3390 & \text { EPA8270 } \\ \mu \mathrm{g} / \mathrm{kg} & 390 & \text { EPA8270 } \\ \mu \mathrm{g} / \mathrm{kg} & 390 & \text { EPA8270 } \\ \mu \mathrm{g} / \mathrm{kg} & 390 & \text { EPA8270 } \\ \mu \mathrm{g} / \mathrm{kg} & 390 & \text { EPA8270 } \\ \mu \mathrm{g} / \mathrm{kg} & 390 & \text { EPA8270 } \\ \mu \mathrm{g} / \mathrm{kg} & 390 & \text { EPA8270 } \\ \mu \mathrm{g} / \mathrm{kg} & 390 & \text { EPA8270 } \\ \mu \mathrm{g} / \mathrm{kg} & 390 & \text { EPA8270 } \\ \mu \mathrm{g} / \mathrm{kg} & 390 & \text { EPA8270 } \\ \mu \mathrm{g} / \mathrm{kg} & 770 & \text { EPA8270 } \\ \mu \mathrm{g} / \mathrm{kg} & 390 & \text { EPA8270 } \\ \mu \mathrm{g} / \mathrm{kg} & 390 & \text { EPA8270 } \\ \mu \mathrm{g} / \mathrm{kg} & 390 & \text { EPA8270 }\end{array}$


SAMPLE NAME: D43I 1205A (Continued)

Sample ID: 100376

$\begin{array}{llllll}\text { Constituent } & \text { RQ AQ B Result Unit D. Limit Method }\end{array}$

$B / N / A$ Extractables (including $P A H$ and phenols)

\begin{tabular}{|c|c|c|c|c|c|c|}
\hline Dimethyl phthalate & $\mathrm{U}$ & & 390 & $\mu \mathrm{g} / \mathrm{kg}$ & 390 & EPA8270 \\
\hline 2,4-Dinitrophenol & $\mathrm{U}$ & & 1,900 & $\mu \mathrm{g} / \mathrm{kg}$ & 1,900 & EPA8270 \\
\hline 2.4-Dinitrotoluene & $U$ & & 390 & $\mu \mathrm{g} / \mathrm{kg}$ & 390 & EPA8270 \\
\hline 2,6-Dinitrotoluene & $\mathrm{U}$ & & 390 & $\mu g / \mathrm{kg}$ & 390 & EPA8270 \\
\hline Di-n-octyl phthalate & $U$ & & 390 & $\mu g / \mathrm{kg}$ & 390 & EPA8270 \\
\hline Fluoranthene & $\mathbf{U}$ & & 390 & $\mu \mathrm{g} / \mathrm{kg}$ & 390 & EPA8270 \\
\hline Fluorene & $U$ & & 390 & $\mu \mathrm{g} / \mathrm{kg}$ & 390 & EPA8270 \\
\hline Hexachlorobenzene & U & & 390 & $\mu \mathrm{g} / \mathrm{kg}$ & 390 & EPA8270 \\
\hline Hexachlorobutadiene & $\mathbf{U}$ & & 390 & $\mu g / \mathrm{kg}$ & 390 & EPA8270 \\
\hline Hexachlorocyclopentadiene & $\mathbf{U}$ & & 390 & $\mu \mathrm{g} / \mathrm{kg}$ & 390 & EPA8270 \\
\hline Hexachloroethane & $\mathbf{U}$ & & 390 & $\mu \mathrm{g} / \mathrm{kg}$ & 390 & EPA8270 \\
\hline Sub. hexanedioic acid & $\mathrm{J}$ & $\mathbf{N}$ & 10,000 & $\mu \mathrm{g} / \mathrm{kg}$ & & EPA8270 \\
\hline Indeno(1.2.3-c,d)pyrene & $\mathrm{U}$ & & 390 & $\mu g / k g$ & 390 & EPA8270 \\
\hline Isophorone & $\mathrm{U}$ & & 390 & $\mu \mathrm{g} / \mathrm{kg}$ & 390 & EPA8270 \\
\hline 2-Methyl-4.6-dinitrophenol & $\mathrm{U}$ & & 1,900 & $\mu \mathrm{g} / \mathrm{kg}$ & 1,900 & EPA8270 \\
\hline 2-Methyinaphthalene & $\mathrm{U}$ & & 390 & $\mu \mathrm{g} / \mathrm{kg}$ & 390 & EPA8270 \\
\hline Naphthalene & $\mathrm{U}$ & & 390 & $\mu \mathrm{g} / \mathrm{kg}$ & 390 & EPA8270 \\
\hline o-Nitroaniline & $\mathrm{U}$ & & 1,900 & $\mu \mathrm{g} / \mathrm{kg}$ & 1,900 & EPA8270 \\
\hline m-Nitroaniline & $\mathrm{U}$ & & 1,900 & $\mu \mathrm{g} / \mathrm{kg}$ & 1,900 & EPA8270 \\
\hline p-Nitroaniline & $\mathrm{U}$ & . & 1,900 & $\mu g / \mathrm{kg}$ & 1,900 & EPA8270 \\
\hline Nitrobenzene & $\mathrm{U}$ & & 390 & $\mu \mathrm{g} / \mathrm{kg}$ & 390 & EPA8270 \\
\hline 2-Nitrophenol & $U$ & & 390 & $\mu \mathrm{g} / \mathrm{kg}$ & 390 & EPA8270 \\
\hline 4-Nitrophenol & $\mathrm{U}$ & & 1.900 & $\mu \mathrm{g} / \mathrm{kg}$ & 1,900 & EPA8270 \\
\hline N-Nitrosodiphenylamine & $\mathrm{U}$ & & 390 & $\mu \mathrm{g} / \mathrm{kg}$ & 390 & EPA8270 \\
\hline N-Nitrosodipropylamine & UJ & $\mathrm{C}$ & 390 & $\mu g / \mathrm{kg}$ & 390 & EPA8270 \\
\hline Pentachlorophenol & $\mathrm{U}$ & & 1,900 & $\mu \mathrm{g} / \mathrm{kg}$ & 1.900 & EPA8270 \\
\hline Phenanthrene & $\mathrm{U}$ & & 390 & $\mu \mathrm{g} / \mathrm{kg}$ & 390 & EPA8270 \\
\hline Phenol & $\mathrm{U}$ & & 390 & $\mu \mathrm{g} / \mathrm{kg}$ & 390 & EPA8270 \\
\hline Pyrene & $\mathrm{U}$ & & 390 & $\mu \mathrm{g} / \mathrm{kg}$ & 390 & EPA8270 \\
\hline 1,2,4-Trichlorobenzene & UJ & C & 390 & $\mu \mathrm{g} / \mathrm{kg}$ & 390 & EPA8270 \\
\hline 2,4,5-Trichlorophenol & $\mathrm{U}$ & & 1.900 & $\mu \mathrm{g} / \mathrm{kg}$ & 1,900 & EPA8270 \\
\hline 2,4,6-Trichlorophenol & $U$ & & 390 & $\mu \mathrm{g} / \mathrm{kg}$ & 390 & EPA8270 \\
\hline Unknown & J & NV & 2,000 & $\mu \mathrm{g} / \mathrm{kg}$ & & EPA8270 \\
\hline Unknown & $\mathrm{J}$ & NV & 500 & $\mu \mathrm{g} / \mathrm{kg}$ & & EPA8270 \\
\hline Unknown & $\mathrm{J}$ & $\mathrm{N}$ & 200 & $\mu \mathrm{g} / \mathrm{kg}$ & & EPA8270 \\
\hline Unknown & J & $\mathbf{N}$ & 200 & $\mu \mathrm{g} / \mathrm{kg}$ & & EPA8270 \\
\hline Unknown ketone & $J$ & NV & 30,000 & $\mu \mathrm{g} / \mathrm{kg}$ & & EPA8270 \\
\hline \multicolumn{7}{|l|}{ Volatile Organic Compounds } \\
\hline Acetone & & & 200 & $\mu \mathrm{g} / \mathrm{kg}$ & 12 & EPA8240 \\
\hline Benzene & $\mathbf{U}$ & & 6.0 & $\mu g / \mathrm{kg}$ & 6.0 & EPA8240 \\
\hline Bromodichloromethane & $\mathbf{U}$ & & 6.0 & $\mu \mathrm{g} / \mathrm{kg}$ & 6.0 & EPA8240 \\
\hline Bromoform & $\mathrm{U}$ & & 6.0 & $\mu \mathrm{g} / \mathrm{kg}$ & 6.0 & EPA8240 \\
\hline Bromomethane (Methyl bromide) & $\mathbf{U}$ & & 12 & $\mu g / \mathrm{kg}$ & 12 & EPA8240 \\
\hline Carbon disulfide & $\overrightarrow{\mathrm{U}}$ & & 6.0 & $\mu \mathrm{g} / \mathrm{kg}$ & 6.0 & EPA8240 \\
\hline Carbon tetrachloride & $\mathrm{U}$ & & 6.0 & $\mu \mathrm{g} / \mathrm{kg}$ & 6.0 & EPA8240 \\
\hline Chlorobenzene & $\mathrm{U}$ & & 6.0 & $\mu \mathrm{g} / \mathrm{kg}$ & 6.0 & EPA8240 \\
\hline Chloroethane & U & & 12 & $\mu \mathrm{g} / \mathrm{kg}$ & 12 & EPA8240 \\
\hline Chloroethene (Vinyl chloride) & $\mathrm{U}$ & & 12 & $\mu \mathrm{g} / \mathrm{kg}$ & 12 & EPA8240 \\
\hline
\end{tabular}


SAMPLE NAME: D431 1205A (Continued)

\section{Constituent}

Volatile Organic Compounds

2-Chloroethyl vinyl ether

Chloroform

Chloromethane (Methyl chloride)

Dibromochloromethane

1,1-Dichloroethane

1,2-Dichloroethane

1,1-Dichloroethylene

1,2-Dichloroethylene

Dichloromethane (Methylene chloride)

1,2-Dichloropropane

cis-1,3-Dichloropropene

trans-1,3-Dichloropropene

Ethylbenzene

2-Hexanone

Methyl ethyl ketone

Methyl isoburyl ketone

Styrene

1,1.2,2-Tetrachloroethane

Tetrachloroethylene

Toluene

1,1,1-Trichloroethane

1,1,2-Trichloroethane

Trichloroethylene

Vinyl acetate

Xylenes
Sample ID: 100376

RQ AQ B Result Unit

D. Limit Method

Pesticides/PCBs and Dioxins/Furans

Aldrin

alpha-Benzene hexachloride

beta-Benzene hexachloride

delta-Benzene hexachloride

alpha-Chlordane

gamma-Chlordane

p,p'-DDD

P,p'-DDE

p.p'-DDT

Dieldrin

Endosulfan I

Endosulfan II

Endosulfan sulfate -

Endrin

Endrin ketone

Heptachlor

Heptachlor epoxide

Heptachlorodibenzo-p-dioxin isomers

Heptachlorodibenzo-p-furan isomers

Hexachlorodibenzo-p-dioxin isomers

Hexachlorodibenzo-p-furan isomers

Lindane

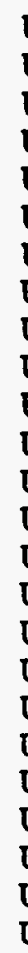

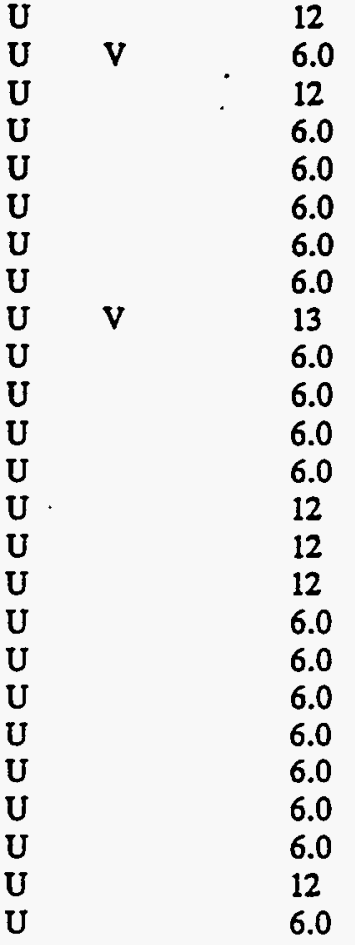

$\begin{array}{lll}\mu \mathrm{g} / \mathrm{kg} & 12 & \text { EPA8240 } \\ \mu \mathrm{g} / \mathrm{kg} & 6.0 & \text { EPA8240 } \\ \mu \mathrm{g} / \mathrm{kg} & 12 & \text { EPA8240 } \\ \mu \mathrm{g} / \mathrm{kg} & 6.0 & \text { EPA8240 } \\ \mu \mathrm{g} / \mathrm{kg} & 6.0 & \text { EPA8240 } \\ \mu \mathrm{g} / \mathrm{kg} & 6.0 & \text { EPA8240 } \\ \mu \mathrm{g} / \mathrm{kg} & 6.0 & \text { EPA8240 } \\ \mu \mathrm{g} / \mathrm{kg} & 6.0 & \text { EPA8240 } \\ \mu \mathrm{g} / \mathrm{kg} & 6.0 & \text { EPA8240 } \\ \mu \mathrm{g} / \mathrm{kg} & 6.0 & \text { EPA8240 } \\ \mu \mathrm{g} / \mathrm{kg} & 6.0 & \text { EPA8240 } \\ \mu \mathrm{g} / \mathrm{kg} & 6.0 & \text { EPA8240 } \\ \mu \mathrm{g} / \mathrm{kg} & 6.0 & \text { EPA8240 } \\ \mu \mathrm{g} / \mathrm{kg} & 12 & \text { EPA8240 } \\ \mu \mathrm{g} / \mathrm{kg} & 12 & \text { EPA8240 } \\ \mu \mathrm{g} / \mathrm{kg} & 12 & \text { EPA8240 } \\ \mu \mathrm{g} / \mathrm{kg} & 6.0 & \text { EPA8240 } \\ \mu \mathrm{g} / \mathrm{kg} & 6.0 & \text { EPA8240 } \\ \mu \mathrm{g} / \mathrm{kg} & 6.0 & \text { EPA8240 } \\ \mu \mathrm{g} / \mathrm{kg} & 6.0 & \text { EPA8240 } \\ \mu \mathrm{g} / \mathrm{kg} & 6.0 & \text { EPA8240 } \\ \mu \mathrm{g} / \mathrm{kg} & 6.0 & \text { EPA8240 } \\ \mu \mathrm{g} / \mathrm{kg} & 6.0 & \text { EPA8240 } \\ \mu \mathrm{g} / \mathrm{kg} & 12 & \text { EPA8240 } \\ \mu \mathrm{g} / \mathrm{kg} & 6.0 & \text { EPA8240 }\end{array}$

$\mu \mathrm{g} / \mathrm{kg}$

$\mu \mathrm{g} / \mathrm{kg}$

$\mu \mathrm{g} / \mathrm{kg}$

$\mu \mathrm{g} / \mathrm{kg}$

$\mu \mathrm{g} / \mathrm{kg}$

$\mu \mathrm{g} / \mathrm{kg}$

$\mu \mathrm{g} / \mathrm{kg}$

$\mu \mathrm{g} / \mathrm{kg}$

$\mu \mathrm{g} / \mathrm{kg}$

$\mu \mathrm{g} / \mathrm{kg}$

$\mu \mathrm{g} / \mathrm{kg}$

$\mu \mathrm{g} / \mathrm{kg}$

$\mu \mathrm{g} / \mathrm{kg}$

$\mu \mathrm{g} / \mathrm{kg}$

$\mu \mathrm{g} / \mathrm{kg}$

$\mu \mathrm{g} / \mathrm{kg}$

$\mu \mathrm{g} / \mathrm{kg}$

ng/g

$\mathrm{ng} / \mathrm{g}$

ng/g

$\mathrm{ng} / \mathrm{g}$

$\mu \mathrm{g} / \mathrm{kg}$
2.0

0.20

0.10

0.20

0.10

2.0
EPA8080

EPA8080

EPA8080

EPA8080

EPA8080

EPA8080

EPA8080

EPA8080

EPA8080

EPA8080

EPA8080

EPA8080

EPA8080

EPA8080

EPA8080

EPA8080

EPA8080

EPA8280

EPA8280

EPA8280

EPA8280

EPA8080 


\section{Constituent}

\section{RQ AQ B Result Unit}

D. Limit Method

Pesticides/PCBs and Dioxins/Furans

Methoxychlor

Octachlorodibenzo-p-dioxin

Octachlorodibenzo-p-furan

PCB 1016

PCB 1221

PCB 1232

PCB 1242

PCB 1248

PCB 1254

PCB 1260

Pentachlorodibenzo-p-dioxin isomers

Pentachlorodibenzo-p-furan isomers

2,3,7,8-TCDD

2,3,7,8-TCDF

Tetrachlorodibenzo-p-dioxin isomers

Tetrachlorodibenzo-p-furan isomers

Toxaphene
U

$\mathrm{U}$

U

$\mathrm{U}$

U

U

$\mathrm{U}$

U

$\mathrm{U}$

$\mathrm{U}$

U

$\mathrm{U}$

$\mathrm{U}$

$\mathrm{U}$

$\mathrm{U}$
20

10

0.20

39

78

39

39

39 .

39

170

0.30

0.20

0.20

0.20

0.20

0.20

200

$\mu \mathrm{g} / \mathrm{kg}$
$\mathrm{ng} / \mathrm{g}$
$\mathrm{ng} / \mathrm{g}$
$\mu \mathrm{g} / \mathrm{kg}$
$\mu \mathrm{g} / \mathrm{kg}$
$\mu \mathrm{g} / \mathrm{kg}$
$\mu \mathrm{g} / \mathrm{kg}$
$\mu \mathrm{g} / \mathrm{kg}$
$\mu \mathrm{g} / \mathrm{kg}$
$\mu \mathrm{g} / \mathrm{kg}$
$\mathrm{ng} / \mathrm{g}$
$\mathrm{ng} / \mathrm{g}$
$\mathrm{ng} / \mathrm{g}$
$\mathrm{ng} / \mathrm{g}$
$\mathrm{ng} / \mathrm{g}$
$\mathrm{ng} / \mathrm{g}$
$\mu \mathrm{g} / \mathrm{kg}$

20

0.10

0.20

39

78

39

39

39

39

39

0.30

0.20

0.20

0.20

0.20

0.20

200
EPA8080

EPA8280

EPA8280

EPA8080

EPA8080

EPA8080

EPA8080

EPA8080

EPA8080

EPA8080

EPA8280

EPA8280

EPA8280

EPA8280

EPA8280

EPA8280

EPA8080

Radionuclide Indicators and Radionuclides

Gross alpha

Nonvolatile beta

Radium, total alpha-emitting

Tritium
U

$\mathrm{U}$

U V

7.3

10

$0.79 \pm 0.69$

1.4
$\mathrm{pCi} / \mathrm{g}$

$\mathrm{pCi} / \mathrm{g}$

$\mathrm{pCi} / \mathrm{g}$

$\mathrm{pCi} / \mathrm{g}$
7.3

10

0.15

1.4
EPA900.0

EPA900.0

EPA903.0

EPA906.0

TCLP Compounds (Metals and Organics)

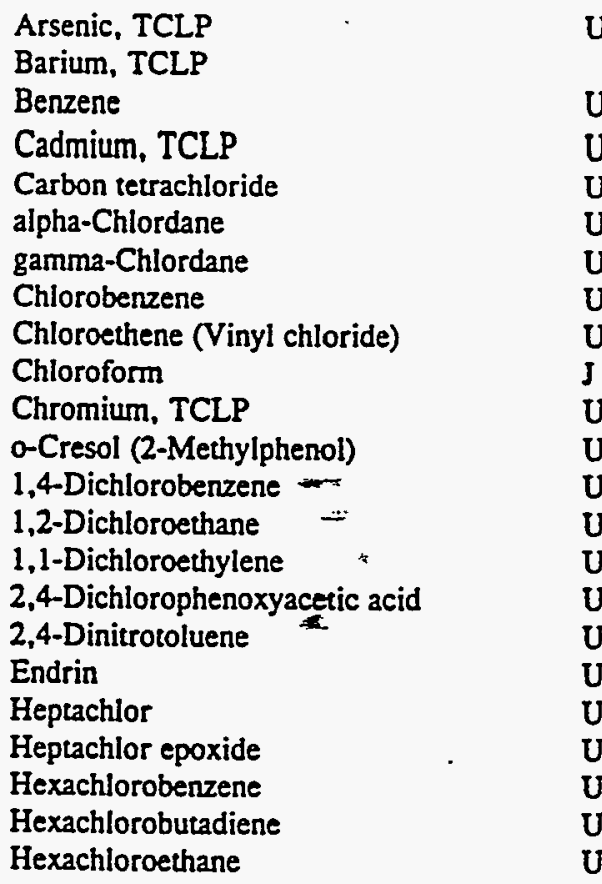

$\begin{array}{llll}\text { U } & & & 100 \\ \text { U } & & & 520 \\ \text { U } & & & 50 \\ \text { U } & & & 5.0 \\ \text { UJ } & \text { O } & \text { L } & 50 \\ \text { UJ } & 0 & \text { L } & 1.2 \\ \text { U } & & & 50 \\ \text { U } & & & 100 \\ \text { J } & & & 26 \\ \text { U } & & & 10 \\ \text { U } & & & 100 \\ \text { U } & & & 100 \\ \text { U } & & & 50 \\ \text { U } & & & 50 \\ \text { U } & & & 10 \\ \text { U } & & & 100 \\ \text { UJ } & 0 & \text { L } & 0.24 \\ \text { UJ } & 0 & \text { L } & 0.12 \\ \text { UJ } & 0 & \text { L } & 0.12 \\ \text { U } & & & 100 \\ \text { U } & & & 100 \\ \text { U } & & & 100\end{array}$

$\mu \mathrm{g} / \mathrm{L}$
$\mu \mathrm{g} / \mathrm{L}$
$\mu \mathrm{g} / \mathrm{L}$
$\mu \mathrm{g} / \mathrm{L}$
$\mu \mathrm{g} / \mathrm{L}$
$\mu \mathrm{g} / \mathrm{L}$
$\mu \mathrm{g} / \mathrm{L}$
$\mu \mathrm{g} / \mathrm{L}$
$\mu \mathrm{g} / \mathrm{L}$
$\mu \mathrm{g} / \mathrm{L}$
$\mu \mathrm{g} / \mathrm{L}$
$\mu \mathrm{g} / \mathrm{L}$
$\mu \mathrm{g} / \mathrm{L}$
$\mu \mathrm{g} / \mathrm{L}$
$\mu \mathrm{g} / \mathrm{L}$
$\mu \mathrm{g} / \mathrm{L}$
$\mu \mathrm{g} / \mathrm{L}$
$\mu \mathrm{g} / \mathrm{L}$
$\mu \mathrm{g} / \mathrm{L}$
$\mu \mathrm{g} / \mathrm{L}$
$\mu \mathrm{g} / \mathrm{L}$
$\mu \mathrm{g} / \mathrm{L}$
$\mu \mathrm{g} / \mathrm{L}$

100

200

50

5.0

50

1.2

1.2

50

100

50

10

100

100

50

50

10

100

0.24

0.12

0.12

100

100

100
EPA6010

EPA6010

EPA8240

EPA6010

EPA8240

EPA8080

EPA8080

EPA8240

EPA8240

EPA8240

EPA6010

EPA8270

EPA8270

EPA8240

EPA8240

EPA8150

EPA8270

EPA8080

EPA8080

EPA8080

EPA8270

EPA8270

EPA8270 
SAMPLE NAME: D431 I205A (Continued)

Sample ID: 100376

\begin{tabular}{|c|c|c|c|c|c|c|c|}
\hline Constituent & $\mathbf{R Q}$ & $\mathbf{A Q}$ & $\mathbf{B}$ & Result & Unit & D. Limit & Method \\
\hline \multicolumn{8}{|c|}{ TCLP Compounds (Metals and Organics) } \\
\hline Lead, TCLP & $\mathbf{U}$ & & & 90 & $\mu g / L$ & 90 & EPA6010 \\
\hline Lindane & UJ & 0 & $\mathrm{~L}$ & 0.12 & $\mu g / L$ & 0.12 & EPA8080 \\
\hline Mercury, TCLP & $\mathrm{U}$ & & & 0.20 & $\mu \mathrm{g} / \mathrm{L}$ & 0.20 & EPA7470 \\
\hline Methoxychlor & UJ & 0 & L & 1.2 & $\mu \mathrm{g} / \mathrm{L}$ & 1.2 & EPA8080 \\
\hline Methyl ethyl ketone & $\mathbf{U}$ & & & 100 & $\mu g / L$ & 100 & EPA8240 \\
\hline Nitrobenzene & $\mathbf{U}$ & & & 100 & $\mu g / L$ & 100 & EPA8270 \\
\hline Pentachlorophenol & $\mathbf{U}$ & & & 500 & $\mu g / L$ & 500 & EPA8270 \\
\hline Pyridine & $\mathbf{U}$ & & & 100 & $\mu \mathrm{g} / \mathrm{L}$ & 100 & EPA8270 \\
\hline Selenium, TCLP & $\mathbf{U}$ & & & 100 & $\mu \mathrm{g} / \mathrm{L}$ & 100 & EPA6010 \\
\hline Silver, TCLP & $\mathbf{U}$ & & & 10 & $\mu g / L$ & 10 & EPA6010 \\
\hline Tetrachloroethylene & $\mathbf{U}$ & & & 50 & $\mu \mathrm{g} / \mathrm{L}$ & 50 & EPA8240 \\
\hline Toxaphene & UJ & 0 & L & 2.4 & $\mu \mathrm{g} / \mathrm{L}$ & 2.4 & EPA8080 \\
\hline $2,4,5-\mathrm{TP}$ (Silvex) & $\mathbf{U}$ & & & 5.0 & $\mu \mathrm{g} / \mathrm{L}$ & 5.0 & EPA8150 \\
\hline Trichloroethylene & $\mathbf{U}$ & & & 50 & $\mu \mathrm{g} / \mathrm{L}$ & 50 & EPA8240 \\
\hline 2,4,5-Trichlorophenol & U & & & 500 & $\mu g / L$ & 500 & EPA8270 \\
\hline 2,4,6-Trichlorophenol & $\mathbf{U}$ & & & 100 & $\mu \mathrm{g} / \mathrm{L}$ & 100 & EPA8270 \\
\hline $2,4,5-\mathrm{T}$ & U & & & 5.0 & $\mu g / L$ & 5.0 & EPA8150 \\
\hline m.p-Cresol & $\mathbf{U}$ & & & 100 & $\mu \mathrm{g} / \mathrm{L}$ & 100 & EPA8270 \\
\hline
\end{tabular}

SAMPLE NAME: D431 1206

Location (SRS Coordinates): 18518.11E 66590.27N

Ground Elevation Above MSL: $127.7 \mathrm{ft}$

Depth of Core Interval: 8.00 to $10.00 \mathrm{ft}$

Sample Type: Normal

Sample Color: 5YR 3/2

Constituent

Physical Parameters

Cation exchange capacity

Cation exchange capacity

$\mathrm{pH}$

Specific conductance

Total organic carbon

Total Metals

\begin{tabular}{|c|c|c|c|}
\hline & & & \\
\hline Arsenic & - & & 1.5 \\
\hline Barium & & & 32 \\
\hline Cadmium & - & $\mathbf{U}$ & 0.58 \\
\hline Chromium & $\alpha$ & & 44 \\
\hline Copper & & & 6.6 \\
\hline Lead & & & 5.6 \\
\hline Manganese & & & 43 \\
\hline Mercury & & $\mathbf{U}$ & 0.12 \\
\hline Nickel & & $\mathbf{U}$ & 4.6 \\
\hline Selenium & & $\mathbf{U}$ & 0.56 \\
\hline
\end{tabular}

Sample ID: 100377
Sample Matrix: Soil

USC Soil Classification: SC
Sample Moisture: Damp

Percent Solids: $\mathbf{8 4 . 9 0}$
RQ AQ B Result Unit D. Limit Method
10

12

5.6

230

3,200

$\begin{array}{lll}\mathrm{meq} / 100 \mathrm{~g} & 2.0 & \text { EPA9080 } \\ \mathrm{meq} / 100 \mathrm{~g} & 2.0 & \text { EPA9080 } \\ \mathrm{pH} & 0.010 & \text { EPA9045 } \\ \mu \mathrm{S} / \mathrm{cm} & 1.0 & \text { EPA9050 } \\ \mathrm{mg} / \mathrm{kg} & 400 & \text { EPA415.1 }\end{array}$

$\begin{array}{lll}\mathrm{mg} / \mathrm{kg} & 1 . \dot{1} & \text { EPA7060 } \\ \mathrm{mg} / \mathrm{kg} & 23 & \text { EPA6010 } \\ \mathrm{mg} / \mathrm{kg} & 0.58 & \text { EPA6010 } \\ \mathrm{mg} / \mathrm{kg} & 1.2 & \text { EPA6010 } \\ \mathrm{mg} / \mathrm{kg} & 2.9 & \text { EPA6010 } \\ \mathrm{mg} / \mathrm{kg} & 0.34 & \text { EPA7421 } \\ \mathrm{mg} / \mathrm{kg} & 1.7 & \text { EPA6010 } \\ \mathrm{mg} / \mathrm{kg} & 0.12 & \text { EPA7470 } \\ \mathrm{mg} / \mathrm{kg} & 4.6 & \text { EPA6010 } \\ \mathrm{mg} / \mathrm{kg} & 0.56 & \text { EPA7740 }\end{array}$


SAMPLE NAME: D431 1206 (Continued)

\section{Constituent}

Sample ID: 100377

RQ AQ B Result Unit $\quad$ D. Limit Method

Total Metals

Silver

U

1.2

$\mathrm{mg} / \mathrm{kg}$

1.2

EPA6010

\section{$B / N / A$ Extractables (including $P A H$ and ghenols)}

\begin{tabular}{|c|c|c|}
\hline Acenaphthene & $U$ & 390 \\
\hline Acenaphthylene & $\mathrm{U}$ & 390 \\
\hline Anthracene & $\mathrm{U}$ & 390 \\
\hline Benzo(a)anthracene & $\mathbf{J}$ & 52 \\
\hline Benzo(b)fluoranthene & $J$ & 56 \\
\hline Benzo(k)fluoranthene & $U$ & 390 \\
\hline Benzoic acid & $\mathrm{U}$ & 2,000 \\
\hline Benzo(g,h,i)perylene & $\mathrm{U}$ & 390 \\
\hline Benzo(a)pyrene & $\mathrm{U}$ & 390 \\
\hline Benzyl alcohol & $\mathrm{U}$ & 390 \\
\hline Bis(2-chloroethoxy) methane & U & 390 \\
\hline Bis(2-chloroethyl) ether & $U$ & 390 \\
\hline Bis(2-chloroisopropyl) ether & $\mathbf{U}$ & 390 \\
\hline Bis(2-ethylhexyl) phthalate & J & 82 \\
\hline 4-Bromophenyl phenyl ether & $\mathrm{U}$ & 390 \\
\hline Butylbenzyl phthalate & $\mathrm{U}$ & 390 \\
\hline .4-Chloroaniline & $\mathrm{U}$ & 390 \\
\hline 4-Chloro-m-cresol & $\mathrm{U}$ & 390 \\
\hline 2-Chloronaphthalene & $U$ & 390 \\
\hline 2-Chlorophenol & $\mathrm{U}$ & 390 \\
\hline 4-Chlorophenyl phenyl ether & $\mathrm{U}$ & 390 \\
\hline Chrysene & J & 40 \\
\hline 0-Cresol (2-Methylphenol) & $\mathrm{U}$ & 390 \\
\hline p-Cresol (4-Methylphenol) & U & 390 \\
\hline Dibenz(a,h)anthracene & $\mathrm{U}$ & 390 \\
\hline Dibenzofuran & $\mathrm{U}$ & 390 \\
\hline Di-n-butyl phthalate & $\mathrm{U}$ & 390 \\
\hline 1,2-Dichlorobenzene & $\mathrm{U}$ & 390 \\
\hline 1,3-Dichlorobenzene & $\mathrm{U}$ & 390 \\
\hline 1,4-Dichlorobenzene & $\mathrm{U}$ & 390 \\
\hline 3,3'-Dichlorobenzidine & $\mathbf{U}$ & 780 \\
\hline 2,4-Dichlorophenol & $\mathbf{U}$ & 390 \\
\hline Diethyl phthalate & U & 390 \\
\hline 2,4-Dimethyl phenol & $\mathrm{U}$ & 390 \\
\hline Dimethyl phthalate & $\mathbf{U}$ & 390 \\
\hline 2,4-Dinitrophenol & $\mathrm{U}$ & 2,000 \\
\hline 2,4-Dinitrotoluene & U & 390 \\
\hline 2,6-Dinitrotoluene & $U$ & 390 \\
\hline Di-n-octyl phthalate & U & 390 \\
\hline Fluoranthene & $\mathbf{J}$ & 110 \\
\hline Fluorene & $\mathrm{U}$ & 390 \\
\hline Hexachlorobenzene & $\mathrm{U}$ & 390 \\
\hline Hexachlorobutadiene & U & 390 \\
\hline Hexachlorocyclopentadiene & $\mathbf{U}$ & 390 \\
\hline Hexachloroethane & U & 390 \\
\hline Sub. hexanedioic acid & $\mathbf{N}$ & 20.000 \\
\hline
\end{tabular}

\begin{tabular}{|c|c|c|}
\hline$\mu \mathrm{g} / \mathrm{kg}$ & 390 & EPA8270 \\
\hline$\mu g / \mathrm{kg}$ & 390 & EPA8270 \\
\hline$\mu \mathrm{g} / \mathrm{kg}$ & 390 & EPA8270 \\
\hline$\mu \mathrm{g} / \mathrm{kg}$ & 390 & EPA8270 \\
\hline$\mu \mathrm{g} / \mathrm{kg}$ & 390 & EPA8270 \\
\hline$\mu \mathrm{g} / \mathrm{kg}$ & 390 & EPA8270 \\
\hline$\mu g / \mathrm{kg}$ & 2,000 & EPA8270 \\
\hline$\mu \mathrm{g} / \mathrm{kg}$ & 390 & EPA8270 \\
\hline$\mu \mathrm{g} / \mathrm{kg}$ & 390 & EPA8270 \\
\hline$\mu \mathrm{g} / \mathrm{kg}$ & 390 & EPA8270 \\
\hline$\mu \mathrm{g} / \mathrm{kg}$ & 390 & EPA8270 \\
\hline$\mu \mathrm{g} / \mathrm{kg}$ & 390 & EPA8270 \\
\hline$\mu \mathrm{g} / \mathrm{kg}$ & 390 & EPA8270 \\
\hline$\mu \mathrm{g} / \mathrm{kg}$ & 390 & EPA8270 \\
\hline$\mu \mathrm{g} / \mathrm{kg}$ & 390 & EPA8270 \\
\hline$\mu \mathrm{g} / \mathrm{kg}$ & 390 & EPA8270 \\
\hline$\mu \mathrm{g} / \mathrm{kg}$ & 390 & EPA8270 \\
\hline$\mu g / \mathrm{kg}$ & 390 & EPA8270 \\
\hline$\mu \mathrm{g} / \mathrm{kg}$ & 390 & EPA8270 \\
\hline$\mu \mathrm{g} / \mathrm{kg}$ & 390 & EPA8270 \\
\hline$\mu \mathrm{g} / \mathrm{kg}$ & 390 & EPA8270 \\
\hline$\mu \mathrm{g} / \mathrm{kg}$ & 390 & EPA8270 \\
\hline$\mu \mathrm{g} / \mathrm{kg}$ & 390 & EPA8270 \\
\hline$\mu \mathrm{g} / \mathrm{kg}$ & 390 & EPA8270 \\
\hline$\mu \mathrm{g} / \mathrm{kg}$ & 390 & EPA8270 \\
\hline$\mu \mathrm{g} / \mathrm{kg}$ & 390 & EPA8270 \\
\hline$\mu \mathrm{g} / \mathrm{kg}$ & 390 & EPA8270 \\
\hline$\mu \mathrm{g} / \mathrm{kg}$ & 390 & EPA8270 \\
\hline$\mu g / k g$ & 390 & EPA8270 \\
\hline$\mu \mathrm{g} / \mathrm{kg}$ & 390 & EPA8270 \\
\hline$\mu \mathrm{g} / \mathrm{kg}$ & 780 & EPA 8270 \\
\hline$\mu \mathrm{g} / \mathrm{kg}$ & 390 & EPA8270 \\
\hline$\mu \mathrm{g} / \mathrm{kg}$ & 390 & EPA8270 \\
\hline$\mu \mathrm{g} / \mathrm{kg}$ & 390 & EPA8270 \\
\hline$\mu \mathrm{g} / \mathrm{kg}$ & 390 & EPA8270 \\
\hline$\mu g / \mathrm{kg}$ & 2,000 & EPA8270 \\
\hline$\mu \mathrm{g} / \mathrm{kg}$ & 390 & EPA8270 \\
\hline$\mu \mathrm{g} / \mathrm{kg}$ & 390 & EPA8270 \\
\hline$\mu g / \mathrm{kg}$ & 390 & EPA8270 \\
\hline$\mu \mathrm{g} / \mathrm{kg}$ & 390 & EPA8270 \\
\hline$\mu \mathrm{g} / \mathrm{kg}$ & 390 & EPA8270 \\
\hline$\mu g / \mathrm{kg}$ & 390 & EPA8270 \\
\hline$\mu \mathrm{g} / \mathrm{kg}$ & 390 & EPA8270 \\
\hline$\mu \mathrm{g} / \mathrm{kg}$ & 390 & EPA8270 \\
\hline$\mu g / \mathrm{kg}$ & 390 & EPA8270 \\
\hline$\mu \mathrm{g} / \mathrm{k}$ & & EPA8270 \\
\hline
\end{tabular}


SAMPLE NAME: D43Г 1206 (Continued)

Constituent

Sample ID: 100377

$B / N / A$ Extractables (including $P A H$ and phenols)

Indeno(1,2,3-c,d)pyrene
Isophorone
2-Methyl-4,6-dinitrophenol
2-Methylnaphthalene
Naphthalene
o-Nitroaniline
m-Nitroaniline
p-Nitroaniline
Nitrobenzene
2-Nitrophenol
4-Nitrophenol
N-Nitrosodiphenylamine
N-Nitrosodipropylamine
Pentachlorophenol
Phenanthrene
Phenol
Pyrene
1,2,4-Trichlorobenzene
2,4,5-Trichlorophenol
2,4,6-Trichlorophenol
Unknown
Unknown
Unknown
Unknown
Unknown
Unknown
Unknown ketone

Volatile Organic Compounds

\author{
RQ AQ
nd phenols)
}

J

$U$
$U$
$U$
$U$
$U$
$U$
$U$
$U$
$U$
$U$
$U$
$U$
$U J$
$U$
$J$
$U$
$J$
$U J$
$U$
$U$
$J$
$J$
$J$
$J$
$J$
$J$
$J$

C

$\mathrm{N}$

N

NV
390

390

2,000

390

390

2,000

2,000

2,000

390

390

2,000

390

390

2.000

49

390

77

390

2,000

390

4,000

1,000

200

500

300

300

50,000

Unit

D. Limit Method

$\mu \mathrm{g} / \mathrm{kg}$
$\mu \mathrm{g} / \mathrm{kg}$
$\mu \mathrm{g} / \mathrm{kg}$
$\mu \mathrm{g} / \mathrm{kg}$
$\mu \mathrm{g} / \mathrm{kg}$
$\mu \mathrm{g} / \mathrm{kg}$
$\mu \mathrm{g} / \mathrm{kg}$
$\mu \mathrm{g} / \mathrm{kg}$
$\mu \mathrm{g} / \mathrm{kg}$
$\mu \mathrm{g} / \mathrm{kg}$
$\mu \mathrm{g} / \mathrm{kg}$
$\mu \mathrm{g} / \mathrm{kg}$
$\mu \mathrm{g} / \mathrm{kg}$
$\mu \mathrm{g} / \mathrm{kg}$
$\mu \mathrm{g} / \mathrm{kg}$
$\mu \mathrm{g} / \mathrm{kg}$
$\mu \mathrm{g} / \mathrm{kg}$
$\mu \mathrm{g} / \mathrm{kg}$
$\mu \mathrm{g} / \mathrm{kg}$
$\mu \mathrm{g} / \mathrm{kg}$
$\mu \mathrm{g} / \mathrm{kg}$
$\mu \mathrm{g} / \mathrm{kg}$
$\mu \mathrm{g} / \mathrm{kg}$
$\mu \mathrm{g} / \mathrm{kg}$
$\mu \mathrm{g} / \mathrm{kg}$
$\mu \mathrm{g} / \mathrm{kg}$
$\mu \mathrm{g} / \mathrm{kg}$

390

390

2,000

390

390

2,000

2.000

2.000

390

390

2,000

390

390

2,000

390

390

390

390

2,000

390
EPA8270

EPA8270

EPA8270

EPA8270

EPA8270

EPA8270

EPA8270

EPA8270

EPA8270

EPA8270

EPA8270

EPA8270

EPA8270

EPA8270

EPA8270

EPA8270

EPA8270

EPA8270

EPA8270

EPA8270

EPA8270

EPA8270

EPA8270

EPA8270

EPA8270

EPA8270

EPA8270

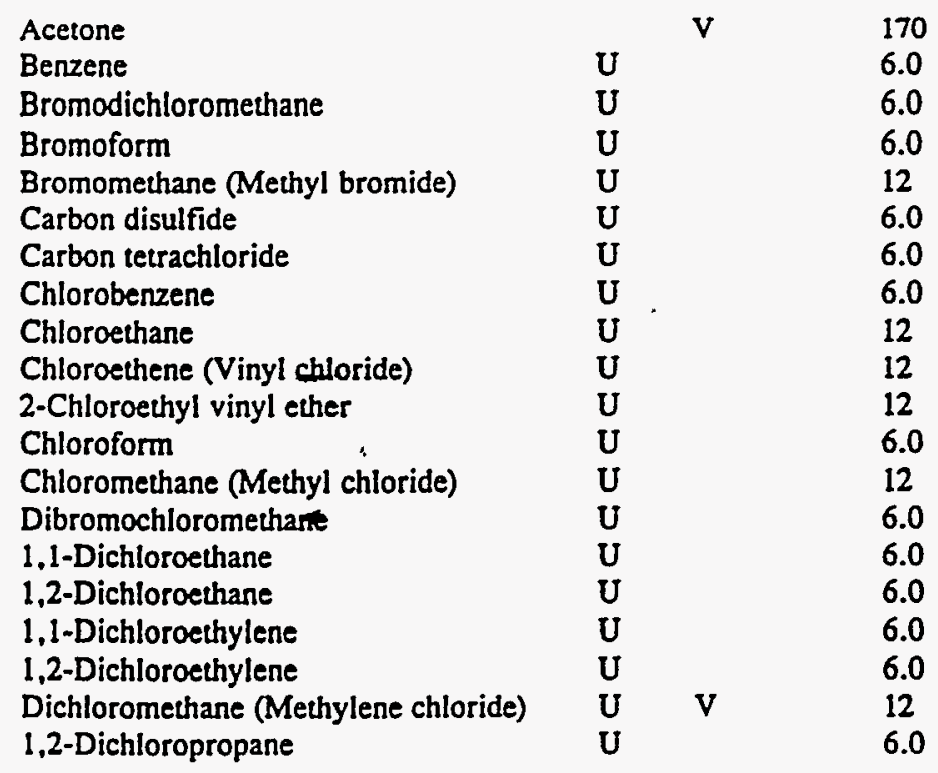

$\begin{array}{lll}\mu \mathrm{g} / \mathrm{kg} & 12 & \text { EPA8240 } \\ \mu \mathrm{g} / \mathrm{kg} & 6.0 & \text { EPA8240 } \\ \mu \mathrm{g} / \mathrm{kg} & 6.0 & \text { EPA8240 } \\ \mu \mathrm{g} / \mathrm{kg} & 6.0 & \text { EPA8240 } \\ \mu \mathrm{g} / \mathrm{kg} & 12 & \text { EPA8240 } \\ \mu \mathrm{g} / \mathrm{kg} & 6.0 & \text { EPA8240 } \\ \mu \mathrm{g} / \mathrm{kg} & 6.0 & \text { EPA8240 } \\ \mu \mathrm{g} / \mathrm{kg} & 6.0 & \text { EPA8240 } \\ \mu \mathrm{g} / \mathrm{kg} & 12 & \text { EPA8240 } \\ \mu \mathrm{g} / \mathrm{kg} & 12 & \text { EPA8240 } \\ \mu \mathrm{g} / \mathrm{kg} & 12 & \text { EPA8240 } \\ \mu \mathrm{g} / \mathrm{kg} & 6.0 & \text { EPA8240 } \\ \mu \mathrm{g} / \mathrm{kg} & 12 & \text { EPA8240 } \\ \mu \mathrm{g} / \mathrm{kg} & 6.0 & \text { EPA8240 } \\ \mu \mathrm{g} / \mathrm{kg} & 6.0 & \text { EPA8240 } \\ \mu \mathrm{g} / \mathrm{kg} & 6.0 & \text { EPA8240 } \\ \mu \mathrm{g} / \mathrm{kg} & 6.0 & \text { EPA8240 } \\ \mu \mathrm{g} / \mathrm{kg} & 6.0 & \text { EPA8240 } \\ \mu \mathrm{g} / \mathrm{kg} & 6.0 & \text { EPA8240 } \\ \mu \mathrm{g} / \mathrm{kg} & 6.0 & \text { EPA8240 }\end{array}$


SAMPLE NAME: D431 1206 (Continued) Sample ID: 100377

Constituent

Volatile Organic Compounds $\begin{array}{lllll}\text { RQ AQ B Result Unit } & \text { D. Limit Method }\end{array}$

$\begin{array}{ll}\text { cis-1,3-Dichloropropene } & \\ \text { trans-1,3-Dichloropropene } & \text { : } \\ \text { Ethylbenzene } & \\ \text { 2-Hexanone } & \\ \text { Methyl ethyl ketone } & \\ \text { Methyl isobutyl ketone } & \\ \text { Styrene } & \\ \text { 1,1,2,2-Tetrachloroethane } & \\ \text { Tetrachloroethylene } & \\ \text { Toluene } & \text { J } \\ \text { 1,1,1-Trichloroethane } & \text { U } \\ \text { 1,1,2-Trichloroethane } & \text { U } \\ \text { Trichloroethylene } & \text { U } \\ \text { Unknown } & \text { J } \\ \text { Vinyl acetate } & \text { U } \\ \text { Xylenes } & \text { U }\end{array}$

Pesticides/PCBs and Dioxins/Furans

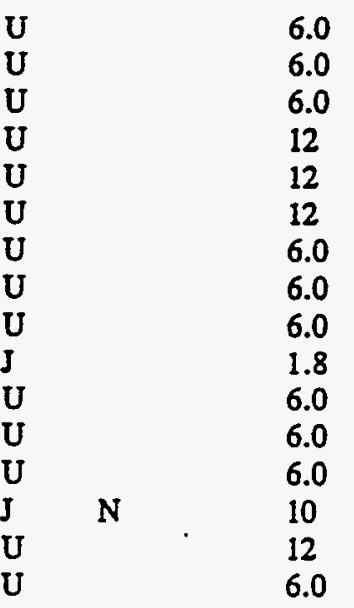

\section{Aldrin}

alpha-Benzene hexachloride

beta-Benzene hexachloride

delta-Benzene hexachloride

alpha-Chlordane

gamma-Chlordane

p. $\mathrm{p}^{\circ}-\mathrm{DDD}$

$p, p^{\prime}-D D E$

p.p'-DDT

Dieldrin

Endosulfan I

Endosulfan II

Endosulfan sulfate

Endrin

Endrin ketone

Heptachlor

Heptachlor epoxide

Heptachlorodibenzo-p-dioxin isomers

Heptachlorodibenzo-p-furan isomers

Hexachlorodibenzo-p-dioxin isomers

Hexachlorodibenzo-p-furan isomers

Lindane

Methoxychlor

Octachlorodibenzo-p-dioxin

Octachlorodibenzo-p-firan

PCB 1016

PCB 1221

PCB 1232

PCB 1242

PCB 1248

PCB 1254

$$
\begin{aligned}
& \mu g / \mathrm{kg} \\
& \mu g / \mathrm{kg} \\
& \mu g / \mathrm{kg} \\
& \mu g / \mathrm{kg} \\
& \mu g / \mathrm{kg} \\
& \mu g / \mathrm{kg} \\
& \mu g / \mathrm{kg} \\
& \mu g / \mathrm{kg} \\
& \mu \mathrm{g} / \mathrm{kg} \\
& \mu g / \mathrm{kg} \\
& \mu \mathrm{g} / \mathrm{kg} \\
& \mu g / \mathrm{kg} \\
& \mu \mathrm{g} / \mathrm{kg} \\
& \mu g / \mathrm{kg} \\
& \mu \mathrm{g} / \mathrm{kg} \\
& \mu g / \mathrm{kg}
\end{aligned}
$$

\begin{tabular}{|c|c|c|c|c|c|c|}
\hline UJ & 0 & L & 2.0 & $\mu \mathrm{g} / \mathrm{kg}$ & 2.0 & EPA 8080 \\
\hline $\mathrm{UJ}$ & 0 & L & 2.0 & $\mu g / \mathrm{kg}$ & 2.0 & EPA8080 \\
\hline UJ & 0 & L & 2.0 & $\mu \mathrm{g} / \mathrm{kg}$ & 2.0 & EPA 8080 \\
\hline UJ & 0 & L & 2.0 & $\mu \mathrm{g} / \mathrm{kg}$ & 2.0 & EPA8080 \\
\hline UJ & 0 & L & 2.0 & $\mu g / \mathrm{kg}$ & 2.0 & EPA8080 \\
\hline UJ & 0 & $L$ & 2.0 & $\mu g / \mathrm{kg}$ & 2.0 & EPA8080 \\
\hline UJ & 0 & L & 3.9 & $\mu \mathrm{g} / \mathrm{kg}$ & 3.9 & EPA8080 \\
\hline UJ & 0 & $\mathrm{~L}$ & 3.9 & $\mu g / k g$ & 3.9 & EPA8080 \\
\hline UJ & 0 & L & 3.9 & $\mu g / \mathrm{kg}$ & 3.9 & EPA8080 \\
\hline UJ & 0 & $\mathbf{L}$ & 3.9 & $\mu \mathrm{g} / \mathrm{kg}$ & 3.9 & EPA8080 \\
\hline UJ & 0 & L & 2.0 & $\mu \mathrm{g} / \mathrm{kg}$ & 2.0 & EPA8080 \\
\hline UJ & 0 & L & 3.9 & $\mu \mathrm{g} / \mathrm{kg}$ & 3.9 & EPA8080 \\
\hline UJ & 0 & L & 3.9 & $\mu \mathrm{g} / \mathrm{kg}$ & 3.9 & EPA 8080 \\
\hline UJ & 0 & $\mathbf{L}$ & 3.9 & $\mu g / \mathrm{kg}$ & 3.9 & EPA8080 \\
\hline UJ & 0 & $\mathbf{L}$ & 3.9 & $\mu \mathrm{g} / \mathrm{kg}$ & 3.9 & EPA8080 \\
\hline UJ & 0 & $\mathrm{~L}$ & 2.0 & $\mu \mathrm{g} / \mathrm{kg}$ & 2.0 & EPA 8080 \\
\hline UJ & 0 & $L$ & 2.0 & $\mu \mathrm{g} / \mathrm{kg}$ & 2.0 & EPA8080 \\
\hline $\mathbf{U}$ & & & 0.30 & $\mathrm{ng} / \mathrm{g}$ & 0.30 & EPA8280 \\
\hline $\mathbf{U}$ & & & 0.20 & $\mathrm{ng} / \mathrm{g}$ & 0.20 & EPA8280 \\
\hline $\mathbf{U}$ & & & 0.30 & $\mathrm{ng} / \mathrm{g}$ & 0.30 & EPA8280 \\
\hline $\mathrm{U}$ & & & 0.20 & ng/g & 0.20 & EPA8280 \\
\hline UJ & 0 & L & 2.0 & $\mu \mathrm{g} / \mathrm{kg}$ & 2.0 & EPA8080 \\
\hline UJ & 0 & $I$ & 20 & $\mu \mathrm{g} / \mathrm{kg}$ & 20 & EPA8080 \\
\hline & & & 27 & $\mathrm{ng} / \mathrm{g}$ & 0.20 & EPA8280 \\
\hline$U$ & & & 0.20 & $\mathrm{ng} / \mathrm{g}$ & 0.20 & EPA8280 \\
\hline UJ & 0 & L & 39 & $\mu \mathrm{g} / \mathrm{kg}$ & 39 & EPA8080 \\
\hline UJ & 0 & L & 79 & $\mu \mathrm{g} / \mathrm{kg}$ & 79 & EPA8080 \\
\hline UJ & 0 & L & 39 & $\mu \mathrm{g} / \mathrm{kg}$ & 39 & EPA8080 \\
\hline UI & 0 & L & 39 & $\mu \mathrm{g} / \mathrm{kg}$ & 39 & EPA8080 \\
\hline UJ & 0 & L & 39 & $\mu \mathrm{g} / \mathrm{kg}$ & 39 & EPA8080 \\
\hline UJ & 0 & L & 39 & $\mu \mathrm{g} / \mathrm{kg}$ & 39 & EPA8080 \\
\hline
\end{tabular}

EPA8240

EPA8240

EPA8240

EPA8240

EPA8240

EPA8240

EPA8240

EPA8240

EPA8240

EPA8240

EPA8240

EPA8240

EPA8240

EPA8240

EPA8240
EPA8240 
SAMPLE NAME: D431 1206 (Continued)

Sample ID: 100377

Constituent

RQ AQ B Result Unit

D. Limit Method

Pesticides/PCBs and Dioxins/Furans

\begin{tabular}{|c|c|c|c|c|c|c|c|}
\hline PCB 1260 & UJ & 0 & $\mathcal{L}$ & 39 & $\mu g / \mathrm{kg}$ & 39 & $\begin{array}{l}\text { EPA8080 } \\
\text { EPA8280 }\end{array}$ \\
\hline Pentachlorodibenzo-p-dioxin isomers & $\mathrm{U}$ & & & 0.20 & $\mathrm{ng} / \mathrm{g}$ & 0.20 & $\begin{array}{l}\text { EPA8280 } \\
\text { EPA8280 }\end{array}$ \\
\hline Pentachlorodibenzo-p-furan isomers & $\mathrm{U}$ & & & 0.20 & $\mathrm{ng} / \mathrm{g}$ & 0.20 & $\begin{array}{l}\text { EPA82 } \\
\text { EPA82 }\end{array}$ \\
\hline 2,3,7,8-TCDD & U & & & $\begin{array}{l}0.20 \\
0.20\end{array}$ & $\mathrm{ng} / \mathrm{g}$ & $\begin{array}{l}0.20 \\
0.20\end{array}$ & $\begin{array}{l}\text { EPA82 } \\
\text { EPA82 }\end{array}$ \\
\hline $\begin{array}{l}\text { 2,3,7,8-TCDF } \\
\text { Tetrachlorodibenzo-p-dioxin isomers }\end{array}$ & $\mathbf{U}$ & & & $\begin{array}{l}0.20 \\
0.20\end{array}$ & $\begin{array}{l}\text { ng/g } \\
\mathrm{ng} / \mathrm{g}\end{array}$ & 0.20 & PA8280 \\
\hline Tetrachlorodibenzo-p-furan isomers & $\mathbf{U}$ & & & 0.20 & $\mathrm{ng} / \mathrm{g}$ & 0.20 & PA82 \\
\hline & UJ & 0 & L & 200 & $\mu \mathrm{g} / \mathrm{kg}$ & 200 & \\
\hline
\end{tabular}

Radionuclide Indicators and Radionuclides

Gross alpha

Nonvolatile beta

Radium, total alpha-emitting

Tritium

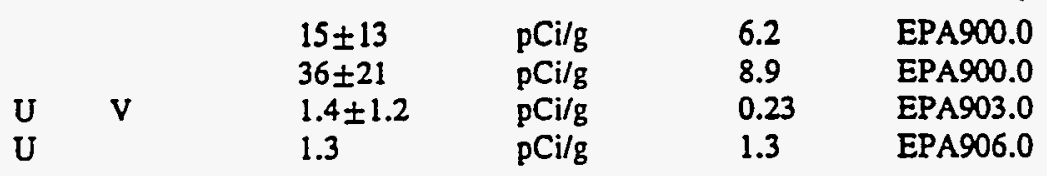

SAMPLE NAME: D431 1207

Location (SRS Coordinates): $18518.11 \mathrm{E} 66590.27 \mathrm{~N}$

Ground Elevation Above MSL: $127.7 \mathrm{ft}$

Depth of Core Interval: 10.00 to $12.00 \mathrm{ft}$

Sample Type: Normal

Sample Color: 8YR 4/4

Constituent

Total Metals

Arsenic
Arsenic
Barium
Barium
Cadmium
Cadmium
Chromium
Chromium
Copper
Copper
Lead
Lead
Manganese
Manganese
Mercury
Mercury
Nickel
Nickel
Selenium
Selenium
Silver

Sample Matrix: Soil

USC Soil Classification: SC

RQ AQ B Result Unit
Sample Moisture: Damp Percent Solids: $\mathbf{8 1 . 5 0}$

D. Limit Method

Sample ID: 100378

$\begin{array}{llll}\text { J } & \text { I } & \text { H } & 4.4 \\ \text { J } & \text { I } & \text { H } & 7.8 \\ \text { J } & \text { I } & \text { H } & 31 \\ \text { J } & \text { I } & \text { H } & 80 \\ \text { UJ } & \text { X } & & 0.59 \\ \text { UJ } & \text { X } & & 2.7 \\ \text { J } & \text { I } & \text { H } & 22 \\ \text { J } & \text { I } & \text { H } & 28 \\ \text { U } & & & 2.9 \\ \text { U } & & & 2.7 \\ \text { J } & \text { I } & \text { L } & 2.5 \\ \text { J } & \text { I } & \text { L } & 3.0 \\ \text { J } & \text { I } & \text { H } & 140 \\ \text { J } & \text { I } & \text { H } & 140 \\ \text { U } & & & 0.12 \\ \text { UJ } & \text { Q } & & 0.12 \\ \text { U } & & & 4.7 \\ \text { U } & & & 4.3 \\ \text { UJ } & \text { I } & \text { L } & 0.58 \\ \text { UJ } & \text { I } & \text { L } & 0.61 \\ \text { UJ } & \text { IX } & & 1.2\end{array}$

$$
\begin{aligned}
& \mathrm{mg} / \mathrm{kg} \\
& \mathrm{mg} / \mathrm{kg} \\
& \mathrm{mg} / \mathrm{kg} \\
& \mathrm{mg} / \mathrm{kg} \\
& \mathrm{mg} / \mathrm{kg} \\
& \mathrm{mg} / \mathrm{kg} \\
& \mathrm{mg} / \mathrm{kg} \\
& \mathrm{mg} / \mathrm{kg} \\
& \mathrm{mg} / \mathrm{kg} \\
& \mathrm{mg} / \mathrm{kg} \\
& \mathrm{mg} / \mathrm{kg} \\
& \mathrm{mg} / \mathrm{kg} \\
& \mathrm{mg} / \mathrm{kg} \\
& \mathrm{mg} / \mathrm{kg} \\
& \mathrm{mg} / \mathrm{kg} \\
& \mathrm{mg} / \mathrm{kg} \\
& \mathrm{mg} / \mathrm{kg} \\
& \mathrm{mg} / \mathrm{kg} \\
& \mathrm{mg} / \mathrm{kg} \\
& \mathrm{mg} / \mathrm{kg} \\
& \mathrm{mg} / \mathrm{kg}
\end{aligned}
$$

$\begin{array}{ll}1.2 & \text { EPA7060 } \\ 2.4 & \text { EPA7060 } \\ 24 & \text { EPA6010 } \\ 22 & \text { EPA6010 } \\ 0.59 & \text { EPA6010 } \\ 2.7 & \text { EPA6010 } \\ 1.2 & \text { EPA6010 } \\ 1.1 & \text { EPA6010 } \\ 2.9 & \text { EPA6010 } \\ 2.7 & \text { EPA6010 } \\ 0.35 & \text { EPA7421 } \\ 0.36 & \text { EPA7421 } \\ 1.8 & \text { EPA6010 } \\ 1.6 & \text { EPA6010 } \\ 0.12 & \text { EPA7470 } \\ 0.12 & \text { EPA7470 } \\ 4.7 & \text { EPA6010 } \\ 4.3 & \text { EPA6010 } \\ 0.58 & \text { EPA7740 } \\ 0.61 & \text { EPA7740 } \\ 1.2 & \text { EPA6010 }\end{array}$

EPA7060
EPA7060
EPA6010
EPA6010
EPA6010
EPA610
EPA6010
EPA6010
EPA6010
EPA6010
EPA7421
EPA7421
EPA6010
EPA6010
EPA7470
EPA7470
EPA6010
EPA6010
EPA7740
EPA7740
EPA6010 
SAMPLE NAME: D431-1207 (Continued) Sample ID: 100378

Constituent

RQ AQ B Result

Unit

D. Limit Method

Total Metals

Silver

UJ $\quad[X$

5.4

$\mathrm{mg} / \mathrm{kg}$

5.4

EPA6010

$B / N / A$ Extractables (including PAH and phenols)

\begin{abstract}
Acenaphthene
Acenaphthylene

Anthracene

Benzo(a)anthracene -

Benzo(b)fluoranthene

Benzo(k)fluoranthene

Benzoic acid

Benzo(g,h.i)perylene

Benzo(a)pyrene

Benzyl alcohol

Bis(2-chloroethoxy) methane

Bis(2-chloroethyl) ether

Bis(2-chloroisopropyl) ether

Bis(2-ethylhexyl) phthalate

4-Bromophenyl phenyl ether

Butylbenzyl phthalate

4-Chloroaniline

4-Chloro-m-cresol

2-Chloronaphthalene

2-Chiorophenol

4-Chlorophenyl phenyl ether

Chrysene

0-Cresol (2-Methylphenol)

p-Cresol (4-Methylphenol)

Dibenz(a.h)anthracene

Dibenzofuran

Di-n-butyl phthalate

1,2-Dichlorobenzene

1,3-Dichlorobenzene

1,4-Dichlorobenzene

3.3'-Dichlorobenzidine

2.4-Dichlorophenol

Diethyl phthalate

2,4-Dimethyl phenol

Dimethyl phthalate

2,4-Dinitrophenol

2,4-Dinitrotoluene -

2,6-Dinitrotoluene .-

Di-n-octyl phthalate

Fluoranthene

Fluorene

Hexachlorobenzene

Hexachlorobutadiene

Hexachlorocyclopentadiene

Hexachloroethane

Sub. hexanedioic acid
\end{abstract}

\section{$\mathrm{U}$}

U 410

410

410

410

2,000

410

410

410

410

410

410

90

410

410

410

410

410

410

410

410

410

410

410

410

410

410

410

410

820

410

410

410

410

2,000

410

410

410

410

410

410

410

410

410

$\mathbf{N}$ $\mu \mathrm{g} / \mathrm{kg}$

$\mu \mathrm{g} / \mathrm{kg}$

$\mu \mathrm{g} / \mathrm{kg}$

$\mu \mathrm{g} / \mathrm{kg}$

$\mu \mathrm{g} / \mathrm{kg}$

$\mu \mathrm{g} / \mathrm{kg}$

$\mu \mathrm{g} / \mathrm{kg}$

$\mu \mathrm{g} / \mathrm{kg}$

$\mu \mathrm{g} / \mathrm{kg}$

$\mu \mathrm{g} / \mathrm{kg}$

$\mu \mathrm{g} / \mathrm{kg}$

$\mu \mathrm{g} / \mathrm{kg}$

$\mu \mathrm{g} / \mathrm{kg}$

$\mu \mathrm{g} / \mathrm{kg}$

$\mu \mathrm{g} / \mathrm{kg}$

$\mu \mathrm{g} / \mathrm{kg}$

$\mu \mathrm{g} / \mathrm{kg}$

$\mu \mathrm{g} / \mathrm{kg}$

$\mu \mathrm{g} / \mathrm{kg}$

$\mu \mathrm{g} / \mathrm{kg}$

$\mu \mathrm{g} / \mathrm{kg}$

$\mu \mathrm{g} / \mathrm{kg}$

$\mu \mathrm{g} / \mathrm{kg}$

$\mu \mathrm{g} / \mathrm{kg}$

$\mu \mathrm{g} / \mathrm{kg}$

$\mu \mathrm{g} / \mathrm{kg}$

$\mu \mathrm{g} / \mathrm{kg}$

$\mu \mathrm{g} / \mathrm{kg}$

$\mu \mathrm{g} / \mathrm{kg}$

$\mu \mathrm{g} / \mathrm{kg}$

$\mu \mathrm{g} / \mathrm{kg}$

$\mu \mathrm{g} / \mathrm{kg}$

$\mu \mathrm{g} / \mathrm{kg}$

$\mu \mathrm{g} / \mathrm{kg}$

$\mu \mathrm{g} / \mathrm{kg}$

$\mu \mathrm{g} / \mathrm{kg}$

$\mu \mathrm{g} / \mathrm{kg}$

$\mu g / \mathrm{kg}$

$\mu \mathrm{g} / \mathrm{kg}$

$\mu \mathrm{g} / \mathrm{kg}$

$\mu \mathrm{g} / \mathrm{kg}$

$\mu \mathrm{g} / \mathrm{kg}$

$\mu g / \mathrm{kg}$

$\mu \mathrm{g} / \mathrm{kg}$

$\mu g / \mathrm{kg}$

$\mu \mathrm{g} / \mathrm{kg}$
410

410

410

410

410

410

2,000

410

410

410

410

410

410

410

410

410

410

410

410

410

410

410

410

410

410

410

410

410

410

410

820

410

410

410
410

2.000

410

410

410

410

410

410

410

410

410
EPA8270

EPA8270

EPA8270

EPA8270

EPA8270

EPA8270

EPA8270

EPA8270

EPA8270

EPA8270

EPA8270

EPA8270

EPA8270

EPA8270

EPA8270

EPA8270

EPA8270

EPA8270

EPA8270

EPA8270

EPA8270

EPA8270

EPA8270

EPA8270

EPA8270

EPA8270

EPA8270

EPA8270

EPA8270

EPA8270

EPA8270

EPA8270

EPA8270

EPA8270

EPA8270

EPA8270

EPA8270

EPA8270

EPA8270

EPA8270

EPA8270

EPA8270

EPA8270

EPA8270

EPA8270

EPA8270 
SAMPLE NAME: D431 1207 (Continued)

RQ AQ B Result
Unit
D. Limit Method
Constituent

Sample ID: 100378

$B / N / A$ Extractables (including $P A H$ and phenols)

Indeno(1,2,3-c,d)pyrene
Isophorone
2-Methyl-4,6-dinitrophenol
2-Methylnaphthalene
Naphthalene
o-Nitroaniline
m-Nitroaniline
p-Nitroaniline
Nitrobenzene
2-Nitrophenol
4-Nitrophenol
N-Nitrosodiphenylamine
N-Nitrosodipropylamine
Pentachlorophenol
Phenanthrene
Phenol
Pyrene
1,2,4-Trichlorobenzene
2,4.5-Trichlorophenol
2,4.6-Trichlorophenol
Unknown
Unknown
Unknown
Unknown
Unknown
Unknown
Unknown ketone

Volatile Organic Compounds

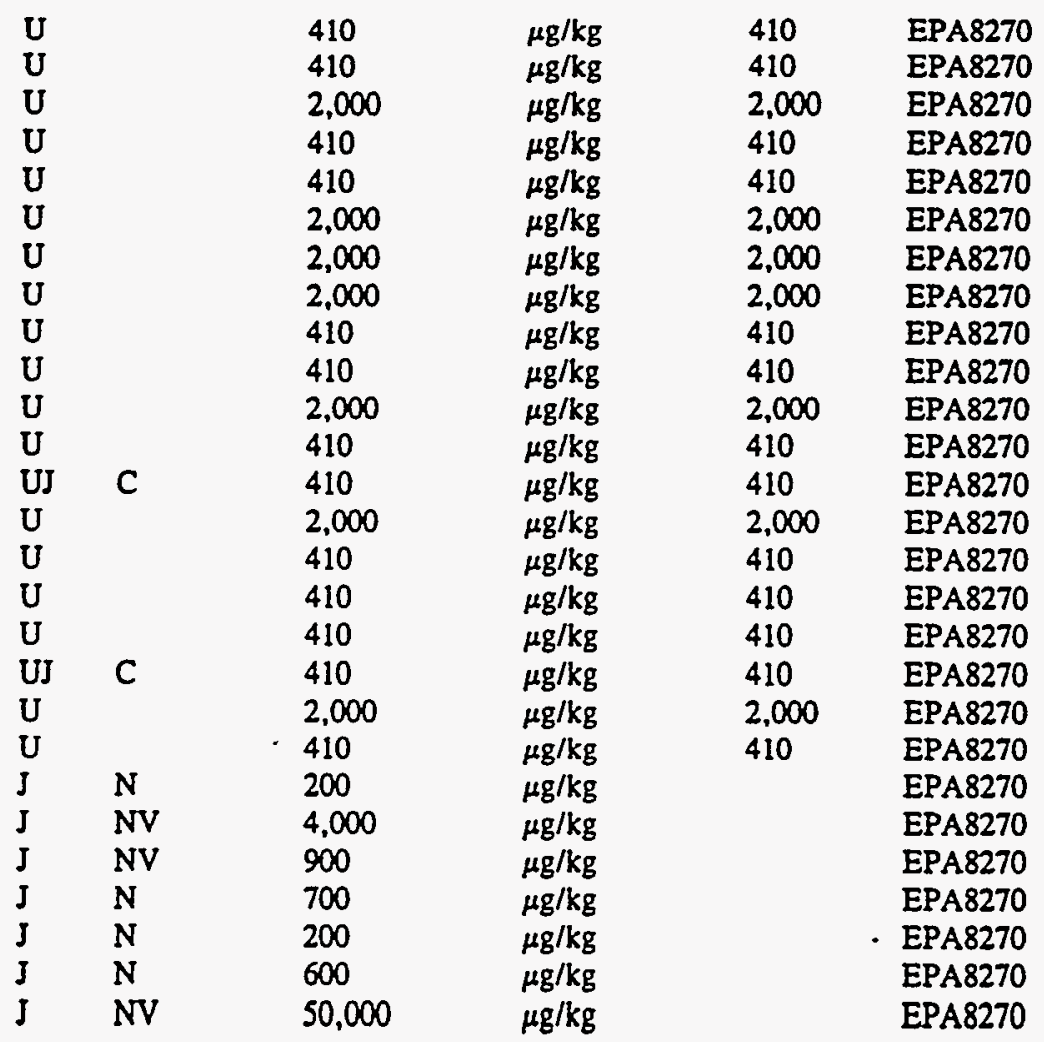

\begin{tabular}{|c|c|c|c|c|c|}
\hline Acetone & J & 7.6 & $\mu \mathrm{g} / \mathrm{kg}$ & 12 & EPA8240 \\
\hline Benzene & $\mathbf{U}$ & 6.0 & $\mu \mathrm{g} / \mathrm{kg}$ & 6.0 & EPA8240 \\
\hline Bromodichloromethane & $\mathrm{U}$ & 6.0 & $\mu g / \mathrm{kg}$ & 6.0 & EPA8240 \\
\hline Bromoform & $\mathrm{U}$ & 6.0 & $\mu g / \mathrm{kg}$ & 6.0 & EPA8240 \\
\hline Bromomethane (Methyl bromide) & U & 12 & $\mu \mathrm{g} / \mathrm{kg}$ & 12 & EPA8240 \\
\hline Carbon disulfide & $\mathrm{U}$ & 6.0 & $\mu \mathrm{g} / \mathrm{kg}$ & 6.0 & EPA8240 \\
\hline Carbon tetrachloride & $\mathbf{U}$ & 6.0 & $\mu \mathrm{g} / \mathrm{kg}$ & 6.0 & EPA8240 \\
\hline Chlorobenzene & $\mathbf{U}$ & 6.0 & $\mu \mathrm{g} / \mathrm{kg}$ & 6.0 & EPA8240 \\
\hline Chloroethane & $\mathbf{U}$ & 12 & $\mu \mathrm{g} / \mathrm{kg}$ & 12 & EPA8240 \\
\hline Chloroethene (Vinyl chtoride) & $\mathbf{U}$ & 12 & $\mu \mathrm{g} / \mathrm{kg}$ & 12 & EPA8240 \\
\hline 2-Chloroethyl vinyl ethet & U & 12 & $\mu \mathrm{g} / \mathrm{kg}$ & 12 & EPA8240 \\
\hline Chloroform & $\mathrm{U}$ & 6.0 & $\mu \mathrm{g} / \mathrm{kg}$ & 6.0 & EPA8240 \\
\hline Chloromethane (Methyl chloride) & U & 12 & $\mu \mathrm{g} / \mathrm{kg}$ & 12 & EPA8240 \\
\hline Dibromochloromethane & U & 6.0 & $\mu \mathrm{g} / \mathrm{kg}$ & 6.0 & EPA8240 \\
\hline 1,1-Dichloroethane & $\mathbf{U}$ & 6.0 & $\mu g / k g$ & 6.0 & EPA8240 \\
\hline 1,2-Dichloroethane & $\mathbf{U}$ & 6.0 & $\mu \mathrm{g} / \mathrm{kg}$ & 6.0 & EPA8240 \\
\hline 1,1-Dichloroethylene & U & 6.0 & $\mu \mathrm{g} / \mathrm{kg}$ & 6.0 & EPA8240 \\
\hline 1,2-Dichloroethylene & $\mathbf{U}$ & 6.0 & $\mu \mathrm{g} / \mathrm{kg}$ & 6.0 & EPA8240 \\
\hline Dichloromethane (Methylene chloride) & $\mathbf{U}$ & 13 & $\mu \mathrm{g} / \mathrm{kg}$ & 6.0 & EPA8240 \\
\hline 1,2-Dichloropropane & $\mathrm{U}$ & 6.0 & $\mu \mathrm{g} / \mathrm{kg}$ & 6.0 & EPA8240 \\
\hline
\end{tabular}


SAMPLE NAME: D431 1207 (Continued)

Sample D: 100378

Constituent

RQ AQ B Result

Unit

D. Limit Method

Volatile Organic Compounds

cis-1,3-Dichloropropene
trans-1,3-Dichloropropene
Ethylbenzene
2-Hexanone
Methyl ethyl ketone
Methyl isobutyl ketone
Styrene
1,1,2,2-Tetrachloroethane
Tetrachloroethylene
Toluene
1,1,1-Trichloroethane
1,1,2-Trichloroethane
Trichloroethylene
Vinyl acetate
Xylenes

Pesticides/PCBs and Dioxins/Furans

\begin{tabular}{|c|c|c|c|c|}
\hline $\mathrm{U}$ & 6.0 & $\mu \mathrm{g} / \mathrm{kg}$ & 6.0 & EPA8240 \\
\hline $\mathbf{U}$ & 6.0 & $\mu g / \mathrm{kg}$ & 6.0 & EPA8240 \\
\hline $\mathbf{U}$ & 6.0 & $\mu g / \mathrm{kg}$ & 6.0 & EPA8240 \\
\hline $\mathbf{U}$ & 12 & $\mu \mathrm{g} / \mathrm{kg}$ & 12 & EPA8240 \\
\hline $\mathbf{U}$ & 12 & $\mu g / k g$ & 12 & EPA8240 \\
\hline $\mathbf{U}$ & 12 & $\mu \mathrm{g} / \mathrm{kg}$ & 12 & EPA8240 \\
\hline $\mathbf{U}$ & 6.0 & $\mu \mathrm{g} / \mathrm{kg}$ & 6.0 & EPA8240 \\
\hline $\mathbf{U}$ & 6.0 & $\mu g / \mathrm{kg}$ & 6.0 & EPA8240 \\
\hline $\mathbf{U}$ & 6.0 & $\mu \mathrm{g} / \mathrm{kg}$ & 6.0 & EPA8240 \\
\hline J & 1.3 & $\mu g / k g$ & 6.0 & EPA8240 \\
\hline $\mathbf{U}$ & 6.0 & $\mu \mathrm{g} / \mathrm{kg}$ & 6.0 & EPA8240 \\
\hline U & 6.0 & $\mu \mathrm{g} / \mathrm{kg}$ & 6.0 & EPA8240 \\
\hline U & 6.0 & $\mu \mathrm{g} / \mathrm{kg}$ & 6.0 & EPA8240 \\
\hline $\mathrm{U}$ & 12 & $\mu / \mathrm{gg}$ & 12 & EPA8240 \\
\hline U & 6.0 & $\mu \mathrm{g} / \mathrm{kg}$ & 6.0 & EPA8240 \\
\hline
\end{tabular}

\begin{tabular}{|c|c|c|c|c|c|c|c|}
\hline Aldrin & UJ & 0 & L & 2.0 & $\mu g / \mathrm{kg}$ & 2.0 & EPA8080 \\
\hline alpha-Benzene hexachloride & UJ & 0 & L & 2.0 & $\mu \mathrm{g} / \mathrm{kg}$ & 2.0 & EPA8080 \\
\hline beta-Benzene hexachloride & UJ & 0 & L & 2.0 & $\mu \mathrm{g} / \mathrm{kg}$ & 2.0 & EPA8080 \\
\hline delta-Benzene hexachloride & UJ & 0 & L & 2.0 & $\mu \mathrm{g} / \mathrm{kg}$ & 2.0 & EPA8080 \\
\hline alpha-Chlordane & UJ & 0 & $\bar{L}$ & 2.0 & $\mu \mathrm{g} / \mathrm{kg}$ & 2.0 & EPA8080 \\
\hline gamma-Chlordane & UJ & 0 & $\bar{L}$ & 2.0 & $\mu \mathrm{g} / \mathrm{kg}$ & 2.0 & EPA8080 \\
\hline p.p'-DDD & UJ & 0 & $\bar{L}$ & 4.1 & $\mu \mathrm{g} / \mathrm{kg}$ & 4.1 & EPA8080 \\
\hline p.p'-DDE & UJ & 0 & L & 4.1 & $\mu \mathrm{g} / \mathrm{kg}$ & 4.1 & EPA8080 \\
\hline p.p'-DDT & UJ & 0 & $\bar{L}$ & 4.1 & $\mu \mathrm{g} / \mathrm{kg}$ & 4.1 & EPA8080 \\
\hline Dieldrin & UJ & 0 & $\bar{L}$ & 4.1 & $\mu g / \mathrm{kg}$ & 4.1 & EPA8080 \\
\hline Endosulfan I & UJ & 0 & L & 2.0 & $\mu \mathrm{g} / \mathrm{kg}$ & 2.0 & EPA8080 \\
\hline Endosulfan II & UJ & 0 & L & 4.1 & $\mu \mathrm{g} / \mathrm{kg}$ & 4.1 & EPA8080 \\
\hline Endosulfan sulfate & UJ & 0 & L & 4.1 & $\mu \mathrm{g} / \mathrm{kg}$ & 4.1 & EPA 8080 \\
\hline Endrin & UJ & 0 & L & 4.1 & $\mu \mathrm{g} / \mathrm{kg}$ & 4.1 & EPA8080 \\
\hline Endrin ketone & UJ & 0 & L & 4.1 & $\mu \mathrm{g} / \mathrm{kg}$ & 4.1 & EPA8080 \\
\hline Heptachlor & UJ & 0 & $\bar{L}$ & 2.0 & $\mu \mathrm{g} / \mathrm{kg}$ & 2.0 & EPA8080 \\
\hline Heptachlor epoxide & UJ & 0 & $\bar{L}$ & 2.0 & $\mu \mathrm{g} / \mathrm{kg}$ & 2.0 & EPA8080 \\
\hline Heptachlorodibenzo-p-dioxin isomers & J & $\mathrm{T}$ & & 0.60 & $\mathrm{ng} / \mathrm{g}$ & 0.20 & EPA8280 \\
\hline Heptachlorodibenzo-p-furan isomers & U & & & 0.10 & $\mathrm{ng} / \mathrm{g}$ & 0.10 & EPA8280 \\
\hline Hexachlorodibenzo-p-dioxin isomers & $\mathrm{U}$ & & & 0.20 & $\mathrm{ng} / \mathrm{g}$ & 0.20 & EPA8280 \\
\hline Hexachlorodibenzo-p-furan isomers & $\mathrm{U}$ & & & 0.10 & $\mathrm{ng} / \mathrm{g}$ & 0.10 & EPA8280 \\
\hline Lindane & UJ & 0 & L & 2.0 & $\mu \mathrm{g} / \mathrm{kg}$ & 2.0 & EPA8080 \\
\hline Methoxychlor & UJ & 0 & L & 20 & $\mu \mathrm{g} / \mathrm{kg}$ & 20 & EPA8080 \\
\hline Octachlorodibenzo-p-dioxin & L & & & 38 & $\mathrm{ng} / \mathrm{g}$ & 0.20 & EPA8280 \\
\hline Octachlorodibenzo-p-furan & $\mathrm{U}$ & & & 0.20 & $\mathrm{ng} / \mathrm{g}$ & 0.20 & EPA8280 \\
\hline PCB 1016 & UJ & 0 & L & 41 & $\mu \mathrm{g} / \mathrm{kg}$ & 41 & EPA8080 \\
\hline PCB 1221 & UJ & 0 & L & 82 & $\mu \mathrm{g} / \mathrm{kg}$ & 82 & EPA8080 \\
\hline РCB 1232 & UJ & 0 & L & 41 & $\mu \mathrm{g} / \mathrm{kg}$ & 41 & EPA8080 \\
\hline РCB 1242 & UJ & 0 & L & 41 & $\mu g / \mathrm{kg}$ & 41 & EPA8080 \\
\hline РCB 1248 & UJ & 0 & L & 41 & $\mu \mathrm{g} / \mathrm{kg}$ & 41 & EPA8080 \\
\hline PCB 1254 & UJ & 0 & L & 41 & $\mu \mathrm{g} / \mathrm{kg}$ & 41 & EPA8080 \\
\hline PCB 1260 & UJ & 0 & L & 41 & $\mu \mathrm{g} / \mathrm{kg}$ & 41 & EPA8080 \\
\hline
\end{tabular}


SAMPLE NAME: D431 1207 (Continued)

Sample ID: 100378

Constituent

RQ AQ B Result

Unit

D. Limit Method

Pesticides/PCBs and Dioxins/Furans

Pentachlorodibenzo-p-dioxin isomers

Pentachlorodibenzo-p-furan isomers

2,3,7,8-TCDD

2,3,7,8-TCDF

Tetrachlorodibenzo-p-dioxin isomers

Tetrachlorodibenzo-p-furan isomers .

Toxaphene

$\begin{array}{llll}\text { U } & & & 0.20 \\ U & & & 0.20 \\ U & & & 0.20 \\ U & & & 0.20 \\ U & & & 0.20 \\ \text { U } & & & 0.20 \\ \text { UJ } & \text { O } & \text { L } & 200\end{array}$

Sample ID: 100379

SAMPLE NAME: D431 1301

Sample Matrix: Soil

USC Soil Classification: SP

Sample Color: 10R 3/4

Constituent

RQ AQ B Result

Unit

Sample Moisture: Dry

Percent Solids: 91.20

D. Limit Method

Physical Parameters

Cation exchange capacity

$\mathrm{pH}$

Specific conductance

Total organic carbon

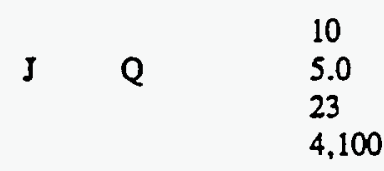

Total Metals

Arsenic

Barium

Cadmium

Chromium

Copper

Lead

Manganese

Mercury

Nickel

Selenium

Silver

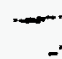

$\begin{array}{lll} & 1.2 & \mathrm{mg} / \mathrm{kg} \\ \mathrm{U} & 21 & \mathrm{mg} / \mathrm{kg} \\ \mathrm{U} & 0.53 & \mathrm{mg} / \mathrm{kg} \\ \mathrm{U} & 3.1 & \mathrm{mg} / \mathrm{kg} \\ & 2.7 & \mathrm{mg} / \mathrm{kg} \\ & 2.2 & \mathrm{mg} / \mathrm{kg} \\ \mathrm{U} & 83 & \mathrm{mg} / \mathrm{kg} \\ \mathrm{U} & 0.11 & \mathrm{mg} / \mathrm{kg} \\ \mathrm{U} & 4.3 & \mathrm{mg} / \mathrm{kg} \\ \mathrm{U} & 0.52 & \mathrm{mg} / \mathrm{kg} \\ & 1.1 & \mathrm{mg} / \mathrm{kg}\end{array}$

$B / N / A$ Extractables (including $P A H$ and phenols)

$\mathrm{meq} / 100 \mathrm{~g}$
$\mathrm{pH}$
$\mu \mathrm{S} / \mathrm{cm}$
$\mathrm{mg} / \mathrm{kg}$

2.0

0.010

1.0

440

EPA9080

EPA9045

EPA9050

EPA415.1
EPA7060

EPA6010

$0.53 \quad$ EPA6010

1.1 EPA6010

2.7 EPA6010

0.31 EPA7421

$1.6 \quad$ EPA6010

0.11 EPA7470

4:3. EPA6010

0.52 EPA7740

$1.1 \quad$ EPA6010

$\mathbf{U}$
$\mathbf{U}$
$\mathbf{U}$
$\mathbf{U}$
$\mathbf{U}$
$\mathbf{U}$
$\mathbf{U}$

350
350
350
350
350
350
1.800
350

\begin{abstract}
Acenaphthene
Acenaphthylene

Benzo(a)anthracene

Benzo(b)fluoranthene

Benzoic acid

Benzo(g,h,i)perylene
\end{abstract}

$\begin{array}{lll}\mu \mathrm{g} / \mathrm{kg} & 350 & \text { EPA8270 } \\ \mu \mathrm{g} / \mathrm{kg} & 350 & \text { EPA8270 } \\ \mu \mathrm{g} / \mathrm{kg} & 350 & \text { EPA8270 } \\ \mu \mathrm{g} / \mathrm{kg} & 350 & \text { EPA8270 } \\ \mu \mathrm{g} / \mathrm{kg} & 350 & \text { EPA8270 } \\ \mu \mathrm{g} / \mathrm{kg} & -350 & \text { EPA8270 } \\ \mu \mathrm{g} / \mathrm{kg} & 1,800 & \text { EPA8270 } \\ \mu \mathrm{g} / \mathrm{kg} & 350 & \text { EPA8270 }\end{array}$


SAMPLE NAME: D431¹301 (Continued)

Sample ID: 100379

$\begin{array}{lllllll}\text { Constituent } & \text { RQ AQ } & \text { R } & \text { Result } & \text { D. Limit Method }\end{array}$

$B / N / A$ Extractables (including $P A H$ and phenols)

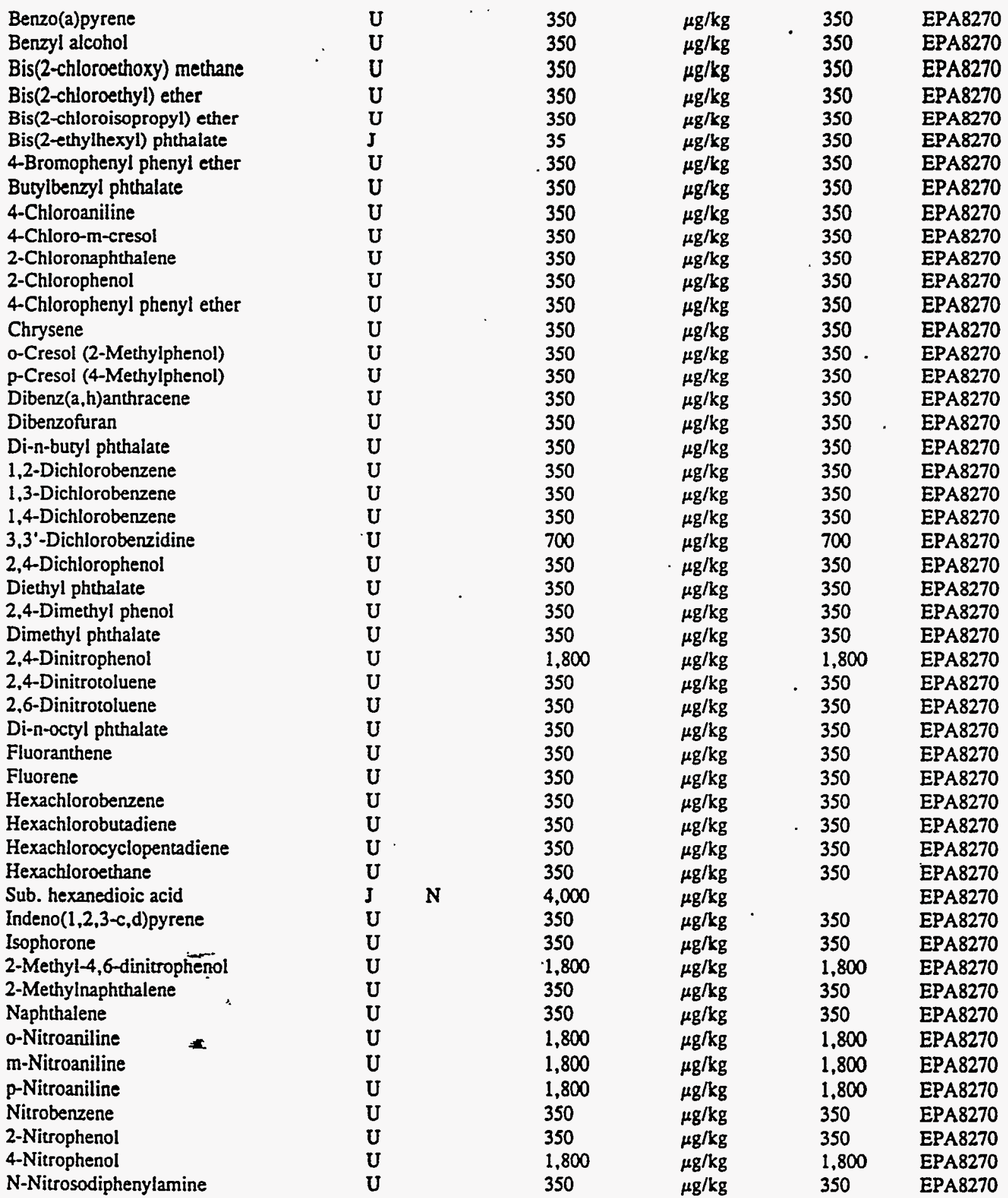




$\begin{array}{llllll}\text { Constituent } & \text { RQ AQ B Result D. Limit Method }\end{array}$

$B / N / A$ Extractables (including PAH and phenols)

$\begin{array}{lllllll}\text { N-Nitrosodipropylamine } & \mathrm{UJ} & \mathrm{C} & 350 & \mu \mathrm{g} / \mathrm{kg} & 350 & \text { EPA8270 } \\ \text { Pentachlorophenol } & \mathrm{U} & & 1,800 & \mu \mathrm{g} / \mathrm{kg} & 1,800 & \text { EPA8270 } \\ \text { Phenanthrene } & \mathrm{U} & & 350 & \mu \mathrm{g} / \mathrm{kg} & 350 & \text { EPA8270 } \\ \text { Phenol } & \mathrm{U} & & 350 & \mu \mathrm{g} / \mathrm{kg} & 350 & \text { EPA8270 } \\ \text { Pyrene } & \mathrm{U} & & 350 & \mu \mathrm{g} / \mathrm{kg} & 350 & \text { EPA8270 } \\ \text { 1,2,4-Trichlorobenzene } & \mathrm{UJ} & \mathrm{C} & 350 & \mu \mathrm{kg} & 350 & \text { EPA8270 } \\ 2,4,5-\text { Trichlorophenol } & \mathrm{U} & & 1,800 & \mu \mathrm{kg} / \mathrm{kg} & 1,800 & \text { EPA8270 } \\ 2,4,6-\text { Trichlorophenol } & \mathrm{U} & & 350 & \mu \mathrm{g} / \mathrm{kg} & 350 & \text { EPA8270 } \\ \text { Unknown } & \mathrm{J} & \mathrm{NV} & 400 & \mu \mathrm{g} / \mathrm{kg} & & \text { EPA8270 } \\ \text { Unknown } & \mathrm{J} & \mathrm{NV} & 1,000 & \mu \mathrm{g} / \mathrm{kg} & & \text { EPA8270 } \\ \text { Unknown } & \mathrm{J} & \mathrm{N} & 200 & \mu \mathrm{g} / \mathrm{kg} & & \text { EPA8270 } \\ \text { Unknown } & \mathrm{J} & \mathrm{N} & 300 & \mu \mathrm{g} / \mathrm{kg} & & \text { EPA8270 } \\ \text { Unknown } & \mathrm{J} & \mathrm{N} & 200 & \mu \mathrm{g} / \mathrm{kg} & & \text { EPA8270 } \\ \text { Unknown } & \mathrm{J} & \mathrm{N} & 200 & \mu \mathrm{g} / \mathrm{kg} & & \text { EPA8270 } \\ \text { Unknown ketone } & \mathrm{J} & \mathrm{NV} & 60,000 & \mu \mathrm{g} / \mathrm{kg} & & \text { EPA8270 }\end{array}$

Volatile Organic Compounds

Acetone
Benzene
Bromodichloromethane
Bromoform
Bromomethane (Methyl bromide)
Carbon disulfide
Carbon tetrachloride
Chlorobenzene
Chloroethane
Chloroethene (Vinyl chloride)
2-Chloroethyl vinyl ether
Chloroform
Chloromethane (Methyl chloride)
Dibromochloromethane
1,1-Dichloroethane
1,2-Dichloroethane
1,1-Dichloroethylene
1,2-Dichloroethylene
Dichloromethane (Methylene chloride)
1,2-Dichloropropane
cis-1,3-Dichloropropene..
trans-1,3-Dichloropropene
Ethylbenzene
2-Hexanone
Methyl ethyl ketone
Methyl isobutyl ketone
Styrene
1,1,2,2-Tetrachloroethane
Tetrachloroethylene
Toluene
1,1,1-Trichloroethane
1,1,2-Trichloroethane

U

U

U

U

U

U

U

U

U

U

U

U

U

U

U

U

U

U

U

U

$\mathrm{U}$

U

U

U

U

U

U

U

$\mathrm{U}$

$\mathrm{U}$

U
11

5.5

5.5 .

5.5

11

5.5

5.5

5.5

11

11

11

5.5

11

5.5

5.5

5.5

5.5

5.5

9.0

5.5

5.5

5.5

5.5

11

11

11

5.5

5.5

5.5

5.5

5.5

5.5

$\begin{array}{lll}\mu \mathrm{g} / \mathrm{kg} & 11 & \text { EPA8240 } \\ \mu \mathrm{g} / \mathrm{kg} & 5.5 & \text { EPA8240 } \\ \mu \mathrm{g} / \mathrm{kg} & 5.5 & \text { EPA8240 } \\ \mu \mathrm{g} / \mathrm{kg} & 5.5 & \text { EPA8240 } \\ \mu \mathrm{g} / \mathrm{kg} & 11 & \text { EPA8240 } \\ \mu \mathrm{g} / \mathrm{kg} & 5.5 & \text { EPA8240 } \\ \mu \mathrm{g} / \mathrm{kg} & 5.5 & \text { EPA8240 } \\ \mu \mathrm{g} / \mathrm{kg} & 5.5 & \text { EPA8240 } \\ \mu \mathrm{g} / \mathrm{kg} & 11 & \text { EPA8240 } \\ \mu \mathrm{g} / \mathrm{kg} & 11 & \text { EPA8240 } \\ \mu \mathrm{g} / \mathrm{kg} & 11 & \text { EPA8240 } \\ \mu \mathrm{g} / \mathrm{kg} & 5.5 & \text { EPA8240 } \\ \mu \mathrm{g} / \mathrm{kg} & 11 & \text { EPA8240 } \\ \mu \mathrm{g} / \mathrm{kg} & 5.5 & \text { EPA8240 } \\ \mu \mathrm{g} / \mathrm{kg} & 5.5 & \text { EPA8240 } \\ \mu \mathrm{g} / \mathrm{kg} & 5.5 & \text { EPA8240 } \\ \mu \mathrm{g} / \mathrm{kg} & 5.5 & \text { EPA8240 } \\ \mu \mathrm{g} / \mathrm{kg} & 5.5 & \text { EPA8240 } \\ \mu \mathrm{g} / \mathrm{kg} & 5.5 & \text { EPA8240 } \\ \mu \mathrm{g} / \mathrm{kg} & 5.5 & \text { EPA8240 } \\ \mu \mathrm{g} / \mathrm{kg} & 5.5 & \text { EPA8240 } \\ \mu \mathrm{g} / \mathrm{kg} & 5.5 & \text { EPA8240 } \\ \mu \mathrm{g} / \mathrm{kg} & 5.5 & \text { EPA8240 } \\ \mu \mathrm{g} / \mathrm{kg} & 11 & \text { EPA8240 } \\ \mu \mathrm{g} / \mathrm{kg} & 11 & \text { EPA8240 } \\ \mu \mathrm{g} / \mathrm{kg} & 11 & \text { EPA8240 } \\ \mu \mathrm{g} / \mathrm{kg} & 5.5 & \text { EPA8240 } \\ \mu \mathrm{g} / \mathrm{kg} & 5.5 & \text { EPA8240 } \\ \mu \mathrm{g} / \mathrm{kg} & 5.5 & \text { EPA8240 } \\ \mu \mathrm{g} / \mathrm{kg} & 5.5 & \text { EPA8240 } \\ \mu \mathrm{g} / \mathrm{kg} & 5.5 & \text { EPA8240 } \\ \mu \mathrm{g} / \mathrm{kg} & 5.5 & \text { EPA8240 } \\ & & \end{array}$


SAMPLE NAME: D43ї 1301 (Continued)

Sample ID: 100379

Constituent

Volatile Organic Compounds

Trichloroethylene

Vinyl acetate

Xylenes
RQ AQ B Result

U

U

Pesticides/PCBs and Dioxins/Furans

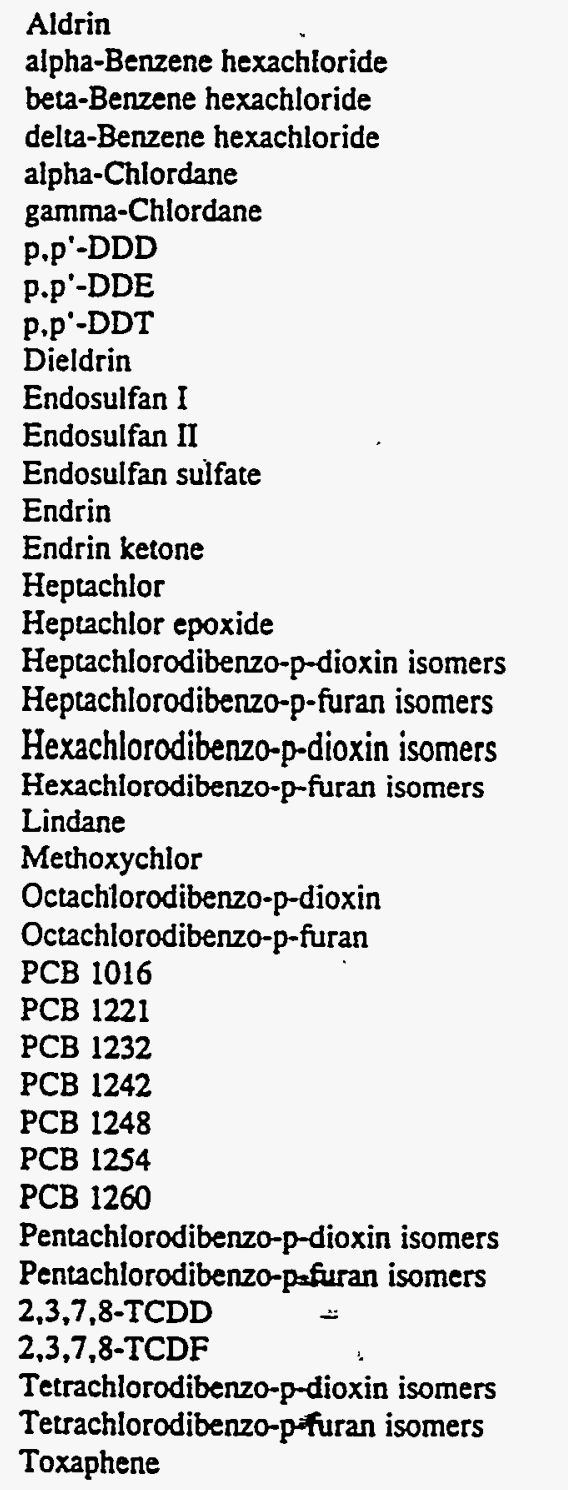

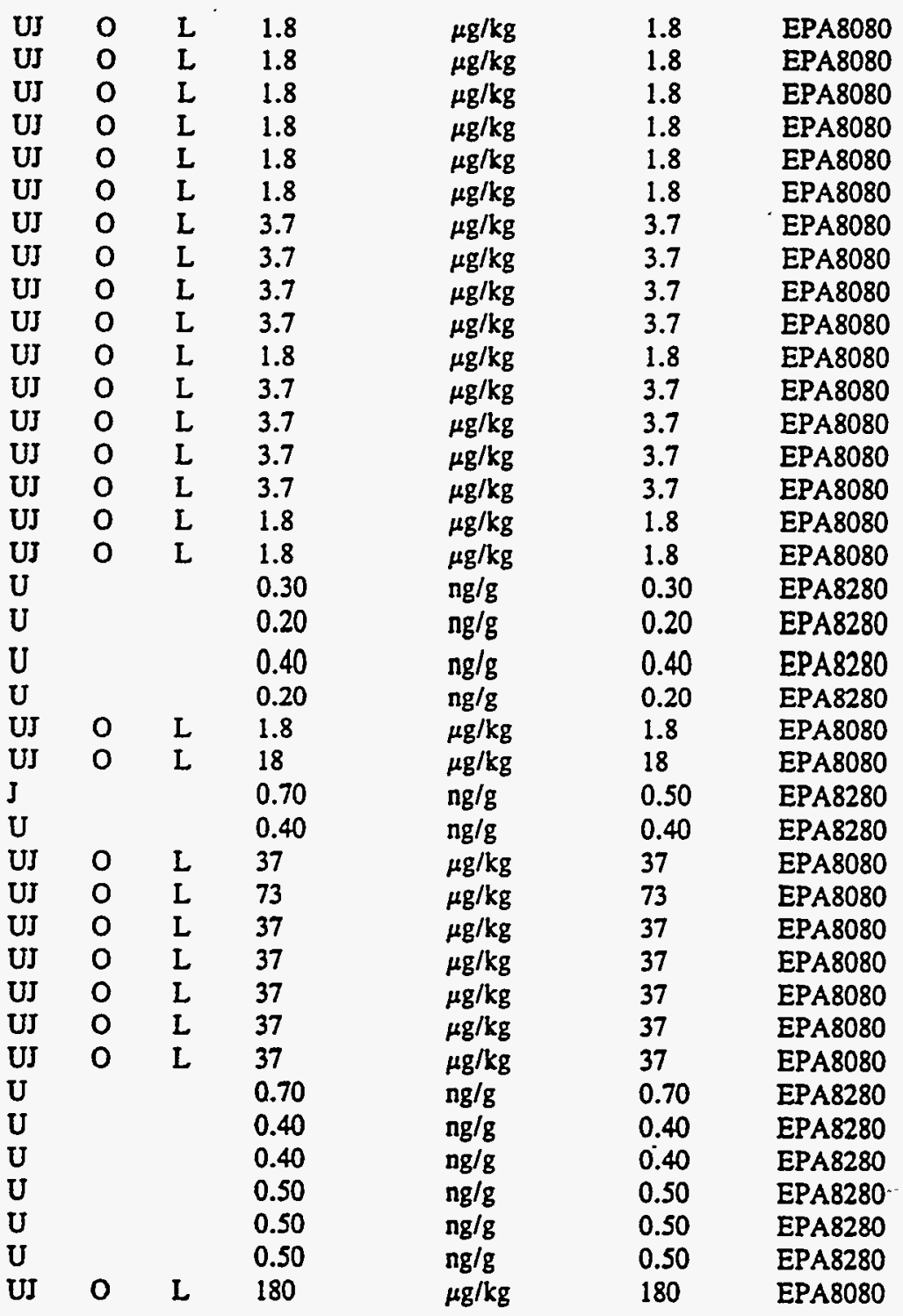


SAMPLE NAME: D43Ī 1301 (Continued)

Constituent

RQ AQ B Result

Unit

D. Limit Method

Radionuclide Indicators and Radionuclides

Gross alpha

Nonvolatile beta

Radium, total alpha-emitting

Tritium

$5.8 \quad$ EPA900.0

$8.0 \quad$ EPA900.0

0.14 EPA903.0

$1.4 \quad$ EPA906.0

$\begin{array}{ll}11 \pm 11 & \mathrm{pCi} / \mathrm{g} \\ 25 \pm 18 & \mathrm{pCi} / \mathrm{g} \\ 0.90 \pm 0.69 & \mathrm{pCi} / \mathrm{g} \\ 1.4 & \mathrm{pCi} / \mathrm{g}\end{array}$

Sample ID: 100381
SAMPLE NAME: D431 1302

Location (SRS Coordinates): 18446.1E 66631.93N

Ground Elevation Above MSL: $131.4 \mathrm{ft}$

Depth of Core Interval: 0.50 to $2.00 \mathrm{ft}$

Sample Type: Normal

Sample Color: 10R 3/4

Constituent

Total Metals

Arsenic
Barium
Cadmium
Chromium
Copper
Lead
Manganese
Mercury
Nickel
Selenium
Silver

$B / N / A$ Extractables (including $P A H$ and phenols)
RQ AQ B Result Unit

U $\quad 1.0$

U 23

U $\quad 0.57$

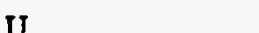

2.8

4.1

23

0.12

U $\quad 4.5$

$\mathrm{U} \quad 0.52$

$\mathrm{U}$

1.1

\begin{tabular}{|c|c|c|}
\hline $\mathrm{mg} / \mathrm{kg}$ & 1.0 & EPA7060 \\
\hline $\mathrm{mg} / \mathrm{kg}$ & 23 & EPA6010 \\
\hline $\mathrm{mg} / \mathrm{kg}$ & 0.57 & EPA6010 \\
\hline $\mathrm{mg} / \mathrm{kg}$ & 1.1 & EPA6010 \\
\hline $\mathrm{mg} / \mathrm{kg}$ & 2.8 & EPA6010 \\
\hline $\mathrm{mg} / \mathrm{kg}$ & 0.31 & EPA7421 \\
\hline $\mathrm{mg} / \mathrm{kg}$ & 1.7 & EPA6010 \\
\hline $\mathrm{mg} / \mathrm{kg}$ & 0.11 & EPA7470 \\
\hline $\mathrm{mg} / \mathrm{kg}$ & 4.5 & EPA6010 \\
\hline $\mathrm{mg} / \mathrm{kg}$ & 0.52 & EPA7740 \\
\hline $\mathrm{mg} / \mathrm{k}$ & 1.1 & EPA6010 \\
\hline
\end{tabular}

Sample Moisture: Dry

Percent Solids: $\mathbf{8 8 . 2 0}$

D. Limit Method

\begin{tabular}{|c|c|c|c|c|c|}
\hline Acenaphthene & $\mathbf{U}$ & 370 & $\mu \mathrm{g} / \mathrm{kg}$ & 370 & EPA8270 \\
\hline Acenaphthylene & $\mathrm{U}$ & 370 & $\mu g / k g$ & 370 & EPA8270 \\
\hline Anthracene & $\mathbf{U}$ & 370 & $\mu g / k g$ & 370 & EPA8270 \\
\hline Benzo(a)anthracene & $\mathbf{U}$ & 370 & $\mu g / k g$ & 370 & EPA8270 \\
\hline Benzo(b)fluoranthene & U & 370 & $\mu \mathrm{g} / \mathrm{kg}$ & 370 & EPA8270 \\
\hline Benzo(k)fluoranthene & $\mathbf{U}$ & 370 & $\mu \mathrm{g} / \mathrm{kg}$ & 370 & EPA8270 \\
\hline Benzoic acid & $\mathbf{U}$ & 1,800 & $\mu \mathrm{g} / \mathrm{kg}$ & 1,800 & EPA8270 \\
\hline Benzo $(g, h, i)$ perylene $\rightarrow$ & $\mathbf{U}$ & 370 & $\mu g / \mathrm{kg}$ & 370 & EPA8270 \\
\hline Benzo(a)pyrene & $\mathbf{U}$ & 370 & $\mu \mathrm{g} / \mathrm{kg}$ & 370 & EPA8270 \\
\hline Benzyl alcohol & $\mathbf{U}$ & 370 & $\mu \mathrm{g} / \mathrm{kg}$ & 370 & EPA8270 \\
\hline Bis(2-chloroethoxy) methane & $\mathbf{U}$ & 370 & $\mu \mathrm{g} / \mathrm{kg}$ & 370 & EPA8270 \\
\hline Bis(2-chloroethy!) ethitet & $\mathbf{U}$ & 370 & $\mu \mathrm{g} / \mathrm{kg}$ & 370 & EPA8270 \\
\hline Bis(2-chloroisopropyl) ether & $\mathbf{U}$ & 370 & $\mu \mathrm{g} / \mathrm{kg}$ & 370 & EPA8270 \\
\hline Bis(2-ethylhexyl) phthalate & $\mathbf{J}$ & 48 & $\mu \mathrm{g} / \mathrm{kg}$ & 370 & EPA8270 \\
\hline 4-Bromophenyl phenyl ether & $\mathbf{U}$ & 370 & $\mu g / \mathrm{kg}$ & 370 & EPA8270 \\
\hline Butylbenzyl phthalate & $\mathbf{U}$ & 370 & $\mu \mathrm{g} / \mathrm{kg}$ & 370 & EPA8270 \\
\hline 4-Chloroaniline & $\mathbf{U}$ & 370 & $\mu g / \mathrm{kg}$ & 370 & EPA8270 \\
\hline 4-Chloro-m-cresol & $\mathbf{U}$ & 370 & $\mu \mathrm{g} / \mathrm{kg}$ & 370 & EPA8270 \\
\hline
\end{tabular}




$\begin{array}{llllll}\text { Constituent } & \text { RQ AQ B Result Unit D. Limit Method }\end{array}$

$B / N / A$ Extractables (including $P A H$ and phenols)

2-Chloronaphthalene
2-Chlorophenol
4-Chlorophenyl phenyl ether
Chrysene
o-Cresol (2-Methylphenol)
p-Cresol (4-Methylphenol)
Dibenz(a,h)anthracene
Dibenzofuran
Di-n-butyl phthalate
1,2-Dichlorobenzene
1,3-Dichlorobenzene
1,4-Dichlorobenzene
3,3-Dichlorobenzidine
2,4-Dichlorophenol
Diethyl phthalate
2,4-Dimethyl phenol
Dimethyl phthalate
2,4-Dinitrophenol
2.4-Dinitrotoluene
2,6-Dinitrotoluene
Di-n-octyl phthalate
Fluoranthene
Fluorene
Hexachlorobenzene
Hexachlorobutadiene
Hexachlorocyclopentadiene
Hexachloroethane
Sub. hexanedioic acid
Indeno(1,2,3-c,d)pyrene
Isophorone
2-Methyl-4,6-dinitrophenol
2-Methylnaphthalene
Naphthalene
o-Nitroaniline
m-Nitroaniline
p-Nitroaniline
Nitrobenzene
2-Nitrophenol
4-Nitrophenol
N-Nitrosodiphenylamine
N-Nitrosodipropylamine
Pentachlorophenol
Phenanthrene
Phenol
Pyrene
1,2,4-Trichlorobenzene
2,4,5-Trichlorophenol
2,4,6-Trichlorophenol
Unknown
Unknown

\begin{tabular}{|c|c|c|c|c|c|}
\hline$U$ & & 370 & $\mu g / k g$ & 370 & EPA8270 \\
\hline U & & 370 & $\mu \mathrm{g} / \mathrm{kg}$ & 370 & EPA8270 \\
\hline$U$ & & 370 & $\mu g / k g$ & 370 & EPA8270 \\
\hline U & & 370 & $\mu g / k g$ & 370 & EPA8270 \\
\hline$U$ & & 370 & $\mu g / k g$ & 370 & EPA8270 \\
\hline$U$ & & 370 & $\mu \mathrm{g} / \mathrm{kg}$ & 370 & EPA8270 \\
\hline U & & 370 & $\mu \mathrm{g} / \mathrm{kg}$ & 370 & EPA8270 \\
\hline $\mathrm{U}$ & & 370 & $\mu \mathrm{g} / \mathrm{kg}$ & 370 & EPA8270 \\
\hline U & & 370 & $\mu \mathrm{g} / \mathrm{kg}$ & 370 & EPA8270 \\
\hline $\mathrm{U}$ & & 370 & $\mu \mathrm{g} / \mathrm{kg}$ & 370 & EPA8270 \\
\hline U. & & 370 & $\mu \mathrm{g} / \mathrm{kg}$ & 370 & EPA8270 \\
\hline $\mathrm{U}$ & & 370 & $\mu \mathrm{g} / \mathrm{kg}$ & 370 & EPA8270 \\
\hline U & & 740 & $\mu \mathrm{g} / \mathrm{kg}$ & 740 & EPA8270 \\
\hline $\mathrm{U}$ & & 370 & $\mu \mathrm{g} / \mathrm{kg}$ & 370 & EPA8270 \\
\hline U & & 370 & $\mu \mathrm{g} / \mathrm{kg}$ & 370 & EPA8270 \\
\hline $\mathbf{U}$ & & 370 & $\mu \mathrm{g} / \mathrm{kg}$ & 370 & EPA8270 \\
\hline U & & 370 & $\mu \mathrm{g} / \mathrm{kg}$ & 370 & EPA8270 \\
\hline $\mathbf{U}$ & & 1,800 & $\mu \mathrm{g} / \mathrm{kg}$ & 1.800 & EPA8270 \\
\hline $\mathbf{U}$ & & 370 & $\mu \mathrm{g} / \mathrm{kg}$ & 370 & EPA8270 \\
\hline U & & 370 & $\mu \mathrm{g} / \mathrm{kg}$ & 370 & EPA8270 \\
\hline $\mathrm{U}$ & & 370 & $\mu \mathrm{g} / \mathrm{kg}$ & 370 & EPA8270 \\
\hline$U$ & & 370 & $\mu \mathrm{g} / \mathrm{kg}$ & 370 & EPA8270 \\
\hline $\mathrm{U}$ & & 370 & $\mu \mathrm{g} / \mathrm{kg}$ & 370 & EPA8270 \\
\hline $\mathrm{U}$ & & 370 & $\mu g / k g$ & 370 & EPA8270 \\
\hline$U$ & & 370 & $\mu g / \mathrm{kg}$ & 370 & EPA8270 \\
\hline$U$ & & 370 & $\mu \mathrm{g} / \mathrm{kg}$ & 370 & EPA8270 \\
\hline $\mathbf{U}$ & & 370 & $\mu g / \mathrm{kg}$ & $370^{\circ}$ & EPA8270 \\
\hline $\mathrm{J}$ & $\mathrm{N}$ & 1,000 & $\mu \mathrm{g} / \mathrm{kg}$ & & EPA8270 \\
\hline$U$ & & 370 & $\mu \mathrm{g} / \mathrm{kg}$ & 370 & EPA8270 \\
\hline $\mathrm{U}$ & & 370 & $\mu \mathrm{g} / \mathrm{kg}$ & 370 & EPA8270 \\
\hline$U$ & & 1,800 & $\mu \mathrm{g} / \mathrm{kg}$ & 1,800 & EPA8270 \\
\hline $\mathrm{U}$ & & 370 & $\mu \mathrm{g} / \mathrm{kg}$ & 370 & EPA8270 \\
\hline $\mathbf{U}$ & & 370 & $\mu \mathrm{g} / \mathrm{kg}$ & 370 & EPA8270 \\
\hline $\mathrm{U}$ & & 1,800 & $\mu \mathrm{g} / \mathrm{kg}$ & 1,800 & EPA8270 \\
\hline U. & & 1,800 & $\mu g / k g$ & 1,800 & EPA8270 \\
\hline $\mathrm{U}$ & & 1,800 & $\mu \mathrm{g} / \mathrm{kg}$ & 1,800 & EPA8270 \\
\hline $\mathrm{U}$ & & 370 & $\mu \mathrm{g} / \mathrm{kg}$ & 370 & EPA8270 \\
\hline U & & 370 & $\mu \mathrm{g} / \mathrm{kg}$ & 370 & EPA8270 \\
\hline U & & 1,800 & $\mu \mathrm{g} / \mathrm{kg}$ & 1,800 & EPA8270 \\
\hline $\mathrm{U}$ & & 370 & $\mu \mathrm{g} / \mathrm{kg}$ & 370 & EPA8270 \\
\hline UI & C & 370 & $\mu g / \mathrm{kg}$ & 370 & EPA8270 \\
\hline $\mathbf{U}$ & & 1,800 & $\mu \mathrm{g} / \mathrm{kg}$ & 1.800 & EPA8270 \\
\hline U & & 370 & $\mu \mathrm{g} / \mathrm{kg}$ & 370 & EPA8270 \\
\hline $\mathrm{U}$ & & 370 & $\mu \mathrm{g} / \mathrm{kg}$ & 370 & EPA8270 \\
\hline$U$ & & 370 & $\mu g / k g$ & 370 & EPA8270 \\
\hline UJ & C & 370 & $\mu \mathrm{g} / \mathrm{kg}$ & 370 & EPA8270 \\
\hline U & & 1,800 & $\mu \mathrm{g} / \mathrm{kg}$ & 1,800 & EPA8270 \\
\hline U & & 370 & $\mu \mathrm{g} / \mathrm{kg}$ & 370 & EPA8270 \\
\hline J & NV & 4,000 & $\mu \mathrm{g} / \mathrm{kg}$ & & EPA8270 \\
\hline - & NV & 1,000 & $\mu g / \mathrm{kg}$ & & EPA8270 \\
\hline
\end{tabular}


SAMPLE NAME: D43r 1302 (Continued)

Sample ID: 100381

Constituent

RQ AQ B Result

Unit

D. Limit Method

$B / N / A$ Extractables (including $P A H$ and phenols)

Unknown
Unknown
Unknown
Unknown keton

Volatile Organic Compounds

$\begin{array}{llll}\mathrm{J} & \mathrm{N} & 200 & \mu \mathrm{g} / \mathrm{k} \\ \mathrm{J} & \mathrm{N} & 200 & \mu \mathrm{g} / \mathrm{kg} \\ \mathrm{J} & \mathrm{N} & 300 & \mu \mathrm{g} / \mathrm{k} \\ \mathrm{J} & \mathrm{NV} & 50,000 & \mu \mathrm{g} / \mathrm{kg}\end{array}$

$\mu \mathrm{g} / \mathrm{kg}$
$\mu \mathrm{g} / \mathrm{kg}$
$\mu \mathrm{g} / \mathrm{kg}$
$\mu \mathrm{g} / \mathrm{kg}$

EPA8270

EPA8270

EPA8270

EPA8270

\section{2}

6.0

6.0

6.0

12

6.0

6.0

6.0

12

12

12

6.0

12

6.0

6.0

6.0

6.0

6.0

10

6.0

6.0

6.0

6.0

12

12

12

6.0

6.0

6.0

6.0

6.0

6.0

6.0

12

6.0 $\mu \mathrm{g} / \mathrm{kg}$
$\mu \mathrm{kg} / \mathrm{kg}$
$\mu \mathrm{g} / \mathrm{kg}$
$\mu \mathrm{g} / \mathrm{kg}$
$\mu \mathrm{g} / \mathrm{kg}$
$\mu \mathrm{g} / \mathrm{kg}$
$\mu \mathrm{g} / \mathrm{kg}$
$\mu \mathrm{g} / \mathrm{kg}$
$\mu \mathrm{g} / \mathrm{kg}$
$\mu \mathrm{g} / \mathrm{kg}$
$\mu \mathrm{kg} / \mathrm{kg}$
$\mu \mathrm{g} / \mathrm{kg}$
$\mu \mathrm{g} / \mathrm{kg}$
$\mu \mathrm{g} / \mathrm{kg}$
$\mu \mathrm{g} / \mathrm{kg}$
$\mu \mathrm{g} / \mathrm{kg}$
$\mu \mathrm{g} / \mathrm{kg}$
$\mu \mathrm{g} / \mathrm{kg}$
$\mu \mathrm{g} / \mathrm{kg}$
$\mu \mathrm{g} / \mathrm{kg}$
$\mu \mathrm{kg}$
$\mu \mathrm{g} / \mathrm{kg}$
$\mu \mathrm{g} / \mathrm{kg}$
$\mu \mathrm{g} / \mathrm{kg}$
$\mu \mathrm{g} / \mathrm{kg}$
$\mu \mathrm{g} / \mathrm{kg}$
$\mu \mathrm{g} / \mathrm{kg}$
$\mu \mathrm{g} / \mathrm{kg}$
$\mu \mathrm{g} / \mathrm{kg}$
$\mu \mathrm{g} / \mathrm{kg}$
$\mu \mathrm{g} / \mathrm{kg}$
$\mu \mathrm{g} / \mathrm{kg}$
$\mu \mathrm{g} / \mathrm{kg}$
$\mu \mathrm{g} / \mathrm{kg}$
$\mu \mathrm{g} / \mathrm{kg}$
$12 \quad$ EPA8240

6.0 EPA8240

6.0 EPA8240

6.0 EPA8240

12 EPA8240

6.0 EPA8240

6.0 EPA8240

6.0 EPA8240

12 EPA8240

$12 \quad$ EPA8240

12 EPA8240

6.0 EPA8240

12 EPA8240

6.0 EPA8240

6.0 EPA8240

6.0 EPA8240

6.0 EPA8240

6.0 EPA8240

6.0 EPA8240

6.0 EPA8240

6.0 EPA8240

6.0 EPA8240

$6.0 \quad$ EPA8240

12 EPA8240

12 EPA8240

12 EPA8240

6.0 EPA8240

$6.0 \quad$ EPA8240

6.0 EPA8240

6.0 EPA8240

$6.0 \quad$ EPA8240

$6.0 \quad$ EPA8240

$12 \quad$ EPA8240

$6.0 \quad$ EPA8240
$6.0 \quad$ EPA8240

Pesticides/PCBs and Dioxins/Furans

Aldrin

alpha-Benzene hexachloride

beta-Benzene hexachloride

delta-Benzene hexachloride

alpha-Chlordane
1.9

1.9

1.9

1.9

1.9 $\mu \mathrm{g} / \mathrm{kg}$

$\mu \mathrm{g} / \mathrm{kg}$

$\mu \mathrm{g} / \mathrm{kg}$

$\mu \mathrm{g} / \mathrm{kg}$

$\mu \mathrm{g} / \mathrm{kg}$
1.9

1.9

1.9

1.9

1.9
EPA8080

EPA8080

EPA8080

EPA8080

EPA8080 
SAMPLE NAME: D431 1302 (Concinued)

Constituent

$\mathbf{R Q}$

AQ B

B Result

1.9

3.8

3.8

3.8

3.8

1.9

3.8

3.8

3.8

3.8

1.9

1.9

0.40

0.20

0.30

0.20

1.9

19

6.5

0.20

38

76

38

38

38

38

38

0.50

0.30

0.50

0.30

0.50

0.50

190

Pentachlorodibenzo-p-furan isomers U

2,3,7,8-TCDD

Tetrachlorodibenzo-p-dioxin isomers

Tetrachlorodibenzo-p-furan isomers

Toxaphene

$\mathrm{U}$

Unit

D. Limit Method

Radionuclide Indicators and Radionuclides

\begin{tabular}{|c|c|c|c|c|c|c|}
\hline Gross alpha & $\mathrm{U}$ & & 11 & $\mathrm{pCi} / \mathrm{g}$ & 11 & EPA9900.0 \\
\hline Gross alpha & $\mathbf{U}$ & & 11 & $\mathrm{pCi} / \mathrm{g}$ & 11 & EPA990.0 \\
\hline Nonvolatile beta & & & $31 \pm 24$ & $\mathrm{pCi} / \mathrm{g}$ & 11 & EPA990.0 \\
\hline Nonvolatile beta & & & $28 \pm 24$ & $\mathrm{pCi} / \mathrm{g}$ & $11^{\circ}$ & EPA990.0 \\
\hline Radium, total alpha-emitting & $\mathbf{U}$ & v & $1.8 \pm 1.1$ & $\mathrm{pCi} / \mathrm{g}$ & 0.20 & EPA903. \\
\hline Radjum, total alpha-emitting & $\mathrm{U}$ & v & $1.6 \pm 0.98$ & $\mathrm{pCi} / \mathrm{g}$ & 0.18 & EPA903. \\
\hline Tritium $=0$ & $\mathrm{U}$ & & 1.7 & $\mathrm{pCi} / \mathrm{g}$ & 1.7 & EPA906.0 \\
\hline Tritium & $\mathrm{U}$ & & 1.3 & $\mathrm{pCi} / \mathrm{g}$ & 1.3 & EPA906.0 \\
\hline
\end{tabular}

EPA8080

EPA8080

EPA8080

EPA8080

EPA8080

EPA8080

EPA8080

EPA8080

EPA8080

EPA8080

EPA8080

EPA8280

EPA8280

EPA8280

EPA8280

EPA8080

EPA8080

EPA8280

EPA8280

EPA8080

EPA8080

EPA8080

EPA8080

EPA8080

EPA8080

EPA8080

EPA8280

EPA8280

EPA8280

EPA8280

EPA8280

EPA8280

EPA8080 
SAMPLE NAME:D431 1303

Location (SRS Coordinates): 18446.1E 66631.93N

Ground Elevation Above MSL: $131.4 \mathrm{ft}$

Depth of Core Interval: 2.00 to $4.00 \mathrm{ft}$

Sample Type: Normal

Sample Color: 10R 3/4

Constituent

Total Metals

Arsenic

Barium

Cadmium

Chromium

Copper

Lead

Manganese

Mercury

Nickel

Selenium

Silver

Volatile Organic Compounds
Sample ID: 100382

Sample Matrix: Soil

USC Soil Classification: SW

RQ AQ B Result Unit

$\begin{array}{lll}\mathrm{mg} / \mathrm{kg} & 1.0 & \text { EPA7060 } \\ \mathrm{mg} / \mathrm{kg} & 21 & \text { EPA6010 } \\ \mathrm{mg} / \mathrm{kg} & 0.53 & \text { EPA6010 } \\ \mathrm{mg} / \mathrm{kg} & 1.1 & \text { EPA6010 } \\ \mathrm{mg} / \mathrm{kg} & 2.6 & \text { EPA6010 } \\ \mathrm{mg} / \mathrm{kg} & 6.2 & \text { EPA7421 } \\ \mathrm{mg} / \mathrm{kg} & 1.6 & \text { EPA6010 } \\ \mathrm{mg} / \mathrm{kg} & 0.11 & \text { EPA7470 } \\ \mathrm{mg} / \mathrm{kg} & 4.2 & \text { EPA6010 } \\ \mathrm{mg} / \mathrm{kg} & 0.51 & \text { EPA7740 } \\ \mathrm{mg} / \mathrm{kg} & 1.1 & \text { EPA6010 }\end{array}$

1.8

21

0.53

32

10

28

40

0.27

6.9

0.69

1.1

1.1

EPA 6010

Sample Moisture: Damp

Percent Solids: 91.40

D. Limit Method

Acetone
Benzene
Bromodichloromethane
Bromoform
Bromomethane (Methyl bromide)
Carbon disulfide
Carbon tetrachloride
Chlorobenzene
Chloroethane
Chloroethene (Vinyl chloride)
2-Chloroethyl vinyl ether
Chloroform
Chloromethane (Methyl chloride)
Dibromochloromethane
1,1-Dichloroethane
1,2-Dichloroethane
1,1-Dichloroethylene
1,2-Dichloroethylene
Dichloromethane (Methylene chloride)
1,2-Dichloropropane
cis-1,3-Dichloropropene
trans-1,3-Dichloropropene
Ethylbenzene
2-Hexanone
Methyl ethyl ketone
Methyl isobutyl ketone
Styrene
1,1,2,2-Tetrachloroethane
Tetrachlorvethylene
Toluene

$\begin{array}{ll}\mathrm{U} & \mathrm{V} \\ \mathrm{U} & \\ \mathrm{U} & \\ \mathrm{U} & \\ \mathrm{U} \\ \mathrm{U} \\ \mathrm{U} \\ \mathrm{U} \\ \mathrm{U} \\ \mathrm{U} \\ \mathrm{U} \\ \mathrm{U} \\ \mathrm{U} \\ \mathrm{U} \\ \mathrm{U} \\ \mathrm{U} \\ \mathrm{U} \\ \mathrm{U} \\ \mathrm{U} \\ \mathrm{U} \\ \mathrm{U} \\ \mathrm{U} \\ \mathrm{U} \\ \mathrm{U} \\ \mathrm{U} \\ \mathrm{U} \\ \mathrm{U} \\ \mathrm{U} \\ \mathrm{J}\end{array}$

$\begin{array}{lll}\mu \mathrm{g} / \mathrm{kg} & 11 & \text { EPA8240 } \\ \mu \mathrm{g} / \mathrm{kg} & 5.5 & \text { EPA8240 } \\ \mu \mathrm{g} / \mathrm{kg} & 5.5 & \text { EPA8240 } \\ \mu \mathrm{g} / \mathrm{kg} & 5.5 & \text { EPA8240 } \\ \mu \mathrm{g} / \mathrm{kg} & 11 & \text { EPA8240 } \\ \mu \mathrm{g} / \mathrm{kg} & 5.5 & \text { EPA8240 } \\ \mu \mathrm{g} / \mathrm{kg} & 5.5 & \text { EPA8240 } \\ \mu \mathrm{g} / \mathrm{kg} & 5.5 & \text { EPA8240 } \\ \mu \mathrm{g} / \mathrm{kg} & 11 & \text { EPA8240 } \\ \mu \mathrm{g} / \mathrm{kg} & 11 & \text { EPA8240 } \\ \mu \mathrm{g} / \mathrm{kg} & 11 & \text { EPA8240 } \\ \mu \mathrm{g} / \mathrm{kg} & 5.5 & \text { EPA8240 } \\ \mu \mathrm{g} / \mathrm{kg} & 11 & \text { EPA8240 } \\ \mu \mathrm{g} / \mathrm{kg} & 5.5 & \text { EPA8240 } \\ \mu \mathrm{g} / \mathrm{kg} & 5.5 & \text { EPA8240 } \\ \mu \mathrm{g} / \mathrm{kg} & 5.5 & \text { EPA8240 } \\ \mu \mathrm{g} / \mathrm{kg} & 5.5 & \text { EPA8240 } \\ \mu \mathrm{g} / \mathrm{kg} & 5.5 & \text { EPA8240 } \\ \mu \mathrm{g} / \mathrm{kg} & 5.5 & \text { EPA8240 } \\ \mu \mathrm{g} / \mathrm{kg} & 5.5 & \text { EPA8240 } \\ \mu \mathrm{g} / \mathrm{kg} & 5.5 & \text { EPA8240 } \\ \mu \mathrm{g} / \mathrm{kg} & 5.5 & \text { EPA8240 } \\ \mu \mathrm{g} / \mathrm{kg} & 5.5 & \text { EPA8240 } \\ \mu \mathrm{g} / \mathrm{kg} & 11 & \text { EPA8240 } \\ \mu \mathrm{g} / \mathrm{kg} & 11 & \text { EPA8240 } \\ \mu \mathrm{g} / \mathrm{kg} & 11 & \text { EPA8240 } \\ \mu \mathrm{g} / \mathrm{kg} & 5.5 & \text { EPA8240 } \\ \mu \mathrm{g} / \mathrm{kg} & 5.5 & \text { EPA8240 } \\ \mu \mathrm{g} / \mathrm{kg} & 5.5 & \text { EPA8240 } \\ \mu \mathrm{g} / \mathrm{kg} & 5.5 & \text { EPA8240 }\end{array}$


SAMPLE NAME: D431 1303 (Continued)

Constituent

Volatile Organic Compounds

1,1,1-Trichloroethane

1,1,2-Trichloroethane

Trichloroethylene

Vinyl acetate

Xylenes
Sample ID: 100382

RQ AQ B Result Unit

$\begin{array}{ll}5.5 & \mu \mathrm{g} / \mathrm{kg} \\ 5.5 & \mu \mathrm{g} / \mathrm{kg} \\ 5.5 & \mu \mathrm{g} / \mathrm{kg} \\ 11 & \mu \mathrm{g} / \mathrm{kg} \\ 5.5 & \mu \mathrm{g} / \mathrm{kg}\end{array}$

5.5

5.5

5.5

11

5.5

Radionuclide Indicators and Radionuclides

Gross alpha .

Nonvolatile beta

Radium, total alpha-emitting

Tritium
SAMPLE NAME: D431 1304

Location (SRS Coordinates): 18446.1E 66631.93N

Ground Elevation Above MSL: $131.4 \mathrm{ft}$

Depth of Core Interval: 4.00 to $6.00 \mathrm{ft}$

Sample Type: Normal

Sample Color: 10R 4/6

Constituent

Total Metals

Arsenic

Barium
Cadmium

Chromium

Copper

Lead

Manganese

Mercury

Nickel

Selenium

Silver

\section{U}

U

$B / N / A$ Extractables_(including $P A H$ and phenols)

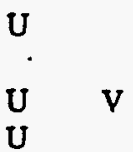

\section{RQ AQ B Result Unit}

Sample Matrix: Soil

USC Soil Classification: SW

$\begin{array}{ll}7.7 & \mathrm{pCi} / \mathrm{g} \\ 23 \pm 17 & \mathrm{pCi} / \mathrm{g} \\ 0.80 \pm 0.72 & \mathrm{pCi} / \mathrm{g} \\ 1.4 & \mathrm{pCi} / \mathrm{g}\end{array}$

Sample ID: 100383

$\begin{array}{ll}7.7 & \text { EPA900.0 } \\ 7.3 & \text { EPA900.0 } \\ 0.19 & \text { EPA903.0 } \\ 1.4 & \text { EPA906.0 }\end{array}$

Sample Moisture: Damp Percent Solids: 89.80

D. Limit Method

D. Limit Method

EPA8240

EPA8240

EPA8240

EPA8240

EPA8240

$\begin{array}{ll}5.5 & \text { EPA7060 } \\ 22 & \text { EPA6010 } \\ 0.56 & \text { EPA6010 } \\ 1.1 & \text { EPA6010 } \\ 2.8 & \text { EPA6010 } \\ 3.3 & \text { EPA7421 } \\ 1.7 & \text { EPA6010 } \\ 0.11 & \text { EPA7470 } \\ 4.4 & \text { EPA6010 } \\ 22 & \text { EPA7740 } \\ -2.1 & \text { EPA6010 }\end{array}$

$\mathrm{mg} / \mathrm{kg}$

$\mathrm{mg} / \mathrm{kg}$

$\mathrm{mg} / \mathrm{kg}$

$\mathrm{mg} / \mathrm{kg}$

$\mathrm{mg} / \mathrm{kg}$

$\mathrm{mg} / \mathrm{kg}$

$\mathrm{mg} / \mathrm{kg}$

$\mathrm{mg} / \mathrm{kg}$

$\mathrm{mg} / \mathrm{kg}$

$\mathrm{mg} / \mathrm{kg}$

$\mathrm{mg} / \mathrm{kg}$

EPA6010

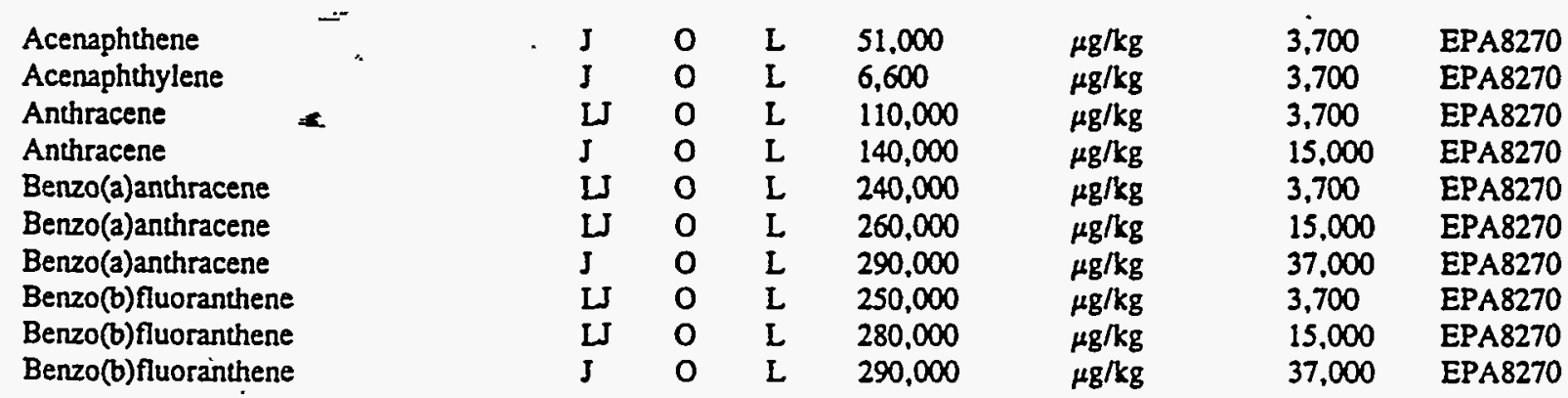


SAMPLE NAME: D431 1304 (Continued)

Sample ID: 100383

Constituent

RQ AQ B Result

Unit

D. Limit Method

$B / N / A$ Extractables (including $P A H$ and phenols)

\begin{tabular}{|c|c|c|c|c|c|c|c|}
\hline Benzo(k)fluoranthene & $\mathrm{U}$ & 0 & L & 66,000 & $\mu g / k g$ & 3,700 & EPA8270 \\
\hline Benzo(k)fluoranthene & J & 0 & L & 83,000 & $\mu \mathrm{g} / \mathrm{kg}$ & 15,000 & EPA8270 \\
\hline Benzoic acid & UJ & 0 & L & 19,000 & $\mu \mathrm{g} / \mathrm{kg}$ & 19.000 & EPA8270 \\
\hline Benzo(g,h,i)perylene & $\mathrm{U}$ & 0 & L & 67,000 & $\mu \mathrm{g} / \mathrm{kg}$ & 3,700 & EPA8270 \\
\hline Benzo(g,h,i)perylene & $\mathbf{J}$ & 0 & L & 98,000 & $\mu \mathrm{g} / \mathrm{kg}$ & 15,000 & EPA8270 \\
\hline Benzo(a)pyrene & $\mathrm{LJ}$ & 0 & L & 170,000 & $\mu \mathrm{g} / \mathrm{kg}$ & 3,700 & EPA8270 \\
\hline Benzo(a)pyrene & $\mathbf{J}$ & 0 & $\bar{L}$ & 200.000 & $\mu \mathrm{g} / \mathrm{kg}$ & 15,000 & EPA8270 \\
\hline Benzyl alcohol & UJ & 0 & L & 3,700 & $\mu \mathrm{g} / \mathrm{kg}$ & 3,700 & EPA8270 \\
\hline Bis(2-chloroethoxy) methane & UJ & 0 & L & 3,700 & $\mu \mathrm{g} / \mathrm{kg}$ & 3,700 & EPA8270 \\
\hline Bis(2-chloroethyl) ether & UJ & 0 & L & 3,700 & $\mu \mathrm{g} / \mathrm{kg}$ & 3,700 & EPA8270 \\
\hline Bis(2-chloroisopropyl) ether & UJ & 0 & L & 3,700 & $\mu \mathrm{g} / \mathrm{kg}$ & 3,700 & EPA8270 \\
\hline Bis(2-ethylhexyl) phthalate & UJ & 0 & L & 3.700 & $\mu \mathrm{g} / \mathrm{kg}$ & 3,700 & EPA8270 \\
\hline 4-Bromophenyl phenyl ether & UJ & 0 & L & 3,700 & $\mu \mathrm{g} / \mathrm{kg}$ & 3,700 & EPA8270 \\
\hline Butylbenzyl phthalate & UJ & 0 & L & 3,700 & $\mu g / k g$ & 3,700 & EPA8270 \\
\hline Benzonapthothiophene & $\mathrm{J}$ & $\mathbf{N}$ & & 200,000 & $\mu \mathrm{g} / \mathrm{kg}$ & & EPA8270 \\
\hline Benzofluorene isomer & $J$ & $\mathbf{N}$ & & 500,000 & $\mu g / k g$ & & EPA8270 \\
\hline Benzofluorene isomer & $\mathrm{J}$ & $\mathrm{N}$ & & 300,000 & $\mu \mathrm{g} / \mathrm{kg}$ & & EPA8270 \\
\hline Benzonapthofuran isomer & J & $\mathbf{N}$ & & 200,000 & $\mu \mathrm{g} / \mathrm{kg}$ & & EPA8270 \\
\hline Benzo-pyrene isomer & $\mathrm{J}$ & $\mathbf{N}$ & & 100,000 & $\mu g / \mathrm{kg}$ & & EPA8270 \\
\hline Benzo-pyrene isomer & $\mathrm{J}$ & $N$ & & 300,000 & $\mu g / \mathrm{kg}$ & & EPA8270 \\
\hline Carbazole & $\mathrm{J}$ & $\mathbf{N}$ & & 50,000 & $\mu \mathrm{g} / \mathrm{kg}$ & & EPA8270 \\
\hline 4-Chloroaniline & UJ & 0 & L & 3,700 & $\mu \mathrm{g} / \mathrm{kg}$ & 3.700 & EPA8270 \\
\hline 4-Chloro-m-cresol & UJ & 0 & L & 3,700 & $\mu \mathrm{g} / \mathrm{kg}$ & 3.700 & EPA8270 \\
\hline 2-Chloronaphthalene & UJ & 0 & L & 3,700 & $\mu g / \mathrm{kg}$ & 3,700 & EPA8270 \\
\hline 2-Chlorophenol & UJ & 0 & L & 3,700 & $\mu g / \mathrm{kg}$ & 3,700 & EPA8270 \\
\hline 4-Chlorophenyl phenyl ether & UJ & 0 & $\mathbf{L}$ & 3,700 & $\mu \mathrm{g} / \mathrm{kg}$ & 3,700 & EPA8270 \\
\hline Chrysene & $\mathbf{L}$ & 0 & L. & 130.000 & $\mu \mathrm{g} / \mathrm{kg}$ & 3,700 & EPA8270 \\
\hline Chrysene & J & 0 & $\mathbf{L}^{\circ}$ & 190,000 & $\mu \mathrm{g} / \mathrm{kg}$ & 15,000 & EPA8270 \\
\hline 0.Cresol (2-Methylphenol) & $\mathrm{UJ}$ & 0 & L & 3.700 & $\mu g / k g$ & 3,700 & EPA8270 \\
\hline p-Cresol (4-Methylphenol) & UJ & 0 & $L$ & 3,700 & $\mu g / \mathrm{kg}$ & 3,700 & EPA8270 \\
\hline Dibenz $(a, h)$ anthracene & $\mathrm{J}$ & 0 & L & 18,000 & $\mu \mathrm{g} / \mathrm{kg}$ & 3,700 & EPA8270 \\
\hline Dibenzofuran & $\mathbf{J}$ & 0 & L & 42,000 & $\mu \mathrm{g} / \mathrm{kg}$ & 3.700 & EPA8270 \\
\hline Di-n-butyl phthalate & UJ & 0 & L & 3.700 & $\mu g / \mathrm{kg}$ & 3,700 & EPA8270 \\
\hline Dibenzothiophene & $\mathbf{J}$ & $\mathrm{N}$ & & 30,000 & $\mu \mathrm{g} / \mathrm{kg}$ & & EPA8270 \\
\hline 1,2-Dichlorobenzene & UJ & 0 & L & 3,700 & $\mu \mathrm{g} / \mathrm{kg}$ & 3.700 & EPA8270 \\
\hline 1,3-Dichlorobenzene & UJ & 0 & L & 3,700 & $\mu g / \mathrm{kg}$ & 3.700 & EPA8270 \\
\hline 1,4-Dichlorobenzene & UJ & 0 & L & 3.700 & $\mu \mathrm{g} / \mathrm{kg}$ & 3.700 & EPA8270 \\
\hline 3,3'-Dichlorobenzidine & UJ & 0 & L & 7,400 & $\mu \mathrm{g} / \mathrm{kg}$ & 7,400 & EPA8270 \\
\hline 2,4-Dichlorophenol & UJ & 0 & L & 3,700 & $\mu g / k g$ & 3,700 & EPA8270 \\
\hline Diethyl phthalate & U & 0 & L & 3.700 & $\mu \mathrm{g} / \mathrm{kg}$ & 3,700 & EPA8270 \\
\hline 2,4-Dimethyl phenol & J & 0 & L & 700 & $\mu \mathrm{g} / \mathrm{kg}$ & 3,700 & EPA8270 \\
\hline Dimethyl phchalate & U & 0 & L & 3,700 & $\mu \mathrm{g} / \mathrm{kg}$ & 3,700 & EPA8270 \\
\hline 2,4-Dinitrophenol & UJ & 0 & L & 19,000 & $\mu \mathrm{g} / \mathrm{kg}$ & 19,000 & EPA8270 \\
\hline 2,4-Dinitrotoluene & UJ & 0 & L & 3.700 & $\mu \mathrm{g} / \mathrm{kg}$ & 3,700 & EPA8270 \\
\hline 2,6-Dinitrotoluene & UJ & 0 & L & 3,700 & $\mu g / k g$ & 3,700 & EPA8270 \\
\hline Di-n-octyl phehalate & UJ & 0 & L & 3.700 & $\mu \mathrm{g} / \mathrm{kg}$ & 3,700 & EPA8270 \\
\hline Fluoranthene & $\mathbf{L}$ & 0 & L & 310,000 & $\mu g / k g$ & 3.700 & EPA8270 \\
\hline Fluoranthene & LJ & 0 & L & 450,000 & $\mu \mathrm{g} / \mathrm{kg}$ & 15,000 & EPA8270 \\
\hline Fluoranthene & $\mathbf{J}$ & 0 & L & 570,000 & $\mu g / k g$. & 37.000 & EPA8270 \\
\hline Fluorene & LJ & 0 & L & 64,000 & $\mu \mathrm{g} / \mathrm{kg}$ & 3,700 & EPA8270 \\
\hline
\end{tabular}


SAMPLE NAME: D431 1304 (Continued)

Sample ID: 100383

Constituent

RQ AQ B Result Unit

D. Limit Method

$B / N / A$ Extractables (including PAH and phenols)

\begin{tabular}{|c|c|c|c|c|c|c|c|}
\hline Fluorene & $\mathrm{J}$ & 0 & $L$ & 91,000 & $\mu \mathrm{g} / \mathrm{kg}$ & 15,000 & $\begin{array}{l}\text { EPA8270 } \\
\text { EPA8270 }\end{array}$ \\
\hline Hexachlorobenzene & UJ & 0 & L & 3,700 & $\mu \mathrm{g} / \mathrm{kg}$ & 3,700 & EPA8270 \\
\hline Hexachlorobutadiene & UJ & 0 & L & 3,700 & $\mu \mathrm{g} / \mathrm{kg}$ & 3,700 & EPA8270 \\
\hline Hexachlorocyclopentadiene & UJ & 0 & L & 3,700 & $\mu \mathrm{g} / \mathrm{kg}$ & 3,700 & EPA8270 \\
\hline Hexachloroethane & UJ & 0 & L & 3,700 & $\mu \mathrm{g} / \mathrm{kg}$ & 3,700 & EPA8270 \\
\hline Indeno(1,2,3-c,d)pyrene & LJ & 0 & L & 79,000 & $\mu \mathrm{g} / \mathrm{kg}$ & 3,700 & EPA8270 \\
\hline Indeno( $1,2,3-c, d)$ pyrenge & $\mathbf{J}$ & 0 & L & 110,000 & $\mu \mathrm{g} / \mathrm{kg}$ & 15,000 & EPA8270 \\
\hline Isophorone & UJ & 0 & L & 3,700 & $\mu \mathrm{g} / \mathrm{kg}$ & 3,700 & EPA8270 \\
\hline Methylanthracene isomer & $\mathbf{J}$ & $\mathbf{N}$ & & 30,000 & $\mu \mathrm{g} / \mathrm{kg}$ & & EPA8270 \\
\hline Methyl-benz-anthracene isomer & $\mathbf{J}$ & $\mathrm{N}$ & & 200,000 & $\mu \mathrm{g} / \mathrm{kg}$ & & EPA8270 \\
\hline Methylphenanthrene isomer & $\mathrm{J}$ & $\mathbf{N}$ & & 30,000 & $\mu \mathrm{g} / \mathrm{kg}$ & & EPA8270 \\
\hline Methylpyrene isomer & $\mathrm{J}$ & $\mathbf{N}$ & & 300,000 & $\mu \mathrm{g} / \mathrm{kg}$ & & EPA8270 \\
\hline 2-Methyl-4,6-dinitrophenol & UJ & 0 & L & 19,000 & $\mu \mathrm{g} / \mathrm{kg}$ & 19,000 & EPA8270 \\
\hline 2-Methylnaphthalene & $\mathbf{J}$ & 0 & $\mathbf{L}$ & 20,000 & $\mu \mathrm{g} / \mathrm{kg}$ & 3,700 & EPA8270 \\
\hline Naphthalene & $\mathbf{J}$ & 0 & L & 52,000 & $\mu \mathrm{g} / \mathrm{kg}$ & 3,700 & EPA8270 \\
\hline o-Nitroaniline & UJ & 0 & L & 19,000 & $\mu \mathrm{g} / \mathrm{kg}$ & 19,000 & EPA8270 \\
\hline m-Nitroaniline & UJ & 0 & L & 19,000 & $\mu \mathrm{g} / \mathrm{kg}$ & 19,000 & EPA8270 \\
\hline p-Nitroaniline & UJ & 0 & L & 19,000 & $\mu \mathrm{g} / \mathrm{kg}$ & 19,000 & EPA8270 \\
\hline Nitrobenzene & UJ & 0 & L & 3,700 & $\mu \mathrm{g} / \mathrm{kg}$ & 3,700 & EPA8270 \\
\hline 2-Nitrophenol & UJ & 0 & L & 3.700 & $\mu \mathrm{g} / \mathrm{kg}$ & 3,700 & EPA8270 \\
\hline 4-Nitrophenol & UJ & 0 & L & 19,000 & $\mu \mathrm{g} / \mathrm{kg}$ & 19,000 & EPA8270 \\
\hline N-Nitrosodiphenylamine & UJ & 0 & L & 3,700 & $\mu \mathrm{g} / \mathrm{kg}$ & 3,700 & EPA8270 \\
\hline N-Nitrosodipropylamine & UJ & Co & L & 3,700 & $\mu \mathrm{g} / \mathrm{kg}$ & 3,700 & EPA8270 \\
\hline Pentachlorophenol & UJ & 0 & L & 19,000 & $\mu \mathrm{g} / \mathrm{kg}$ & 19,000 & EPA8270 \\
\hline Phenanthrene & $\mathrm{LJ}$ & 0 & $\mathrm{~L}$ & 310,000 & $/ \mu g / k g$ & 3,700 & EPA8270 \\
\hline Phenanthrene & U & 0 & L & 400,000 & $\mu g / k g$ & 15,000 & EPA8270 \\
\hline Phenanthrene & $\mathbf{J}$ & 0 & L & 510,000 & $\mu \mathrm{g} / \mathrm{kg}$ & 37,000 & EPA8270 \\
\hline Phenol & UJ & 0 & L & 3,700 & $\mu \mathrm{g} / \mathrm{kg}$ & 3,700 & EPA8270 \\
\hline Phenyl-napthalene isomer & $\mathrm{J}$ & $\mathbf{N}$ & & 20,000 & $\mu \mathrm{g} / \mathrm{kg}$ & & EPA8270 \\
\hline Pyrene & $\mathrm{LJ}$ & 0 & L & 310,000 & $\mu \mathrm{g} / \mathrm{kg}$ & 3,700 & EPA8270 \\
\hline Pyrene & U & 0 & L & 350,000 & $\mu \mathrm{g} / \mathrm{kg}$ & 15,000 & EPA8270 \\
\hline Pyrene & $\mathrm{J}$ & 0 & L & 420,000 & $\mu \mathrm{g} / \mathrm{kg}$ & 37,000 & EPA8270 \\
\hline 1,2.4-Trichlorobenzene & UJ & $\mathrm{CO}$ & $\mathbf{L}$ & 3,700 & $\mu \mathrm{g} / \mathrm{kg}$ & 3,700 & EPA8270 \\
\hline 2,4,5-Trichlorophenol & UJ & 0 & L & 19,000 & $\mu \mathrm{g} / \mathrm{kg}$ & 19.000 & EPA8270 \\
\hline 2,4,6-Trichlorophenol & UJ & 0 & L & 3,700 & $\mu \mathrm{g} / \mathrm{kg}$ & 3,700 & EPA8270 \\
\hline Unknown arom. hydrocarbon & $\mathbf{J}$ & $\mathbf{N}$ & & 70,000 & $\mu \mathrm{g} / \mathrm{kg}$ & & EPA8270 \\
\hline Unknown arom. hydrocarbon & $\mathrm{J}$ & $\mathbf{N}$ & & 200,000 & $\mu \mathrm{g} / \mathrm{kg}$ & & EPA8270 \\
\hline Unknown & $\mathbf{J}$ & $\mathbf{N}$ & & 200,000 & $\mu \mathrm{g} / \mathrm{kg}$ & & EPA8270 \\
\hline Unknown & $\mathrm{J}$ & $\mathbf{N}$ & & 200,000 & $\mu \mathrm{g} / \mathrm{kg}$ & & EPA8270 \\
\hline Unknown ketone & $\mathrm{J}$ & NV & & 90,000 & $\mu \mathrm{g} / \mathrm{kg}$ & & EPA8270 \\
\hline Unknown polyarom. hydrocarbon & $\mathbf{J}$ & $\mathbf{N}$ & & 200,000 & $\mu g / \mathrm{kg}$ & & EPA8270 \\
\hline Unknown polyarom. hydrọcarbon & J & $\mathrm{N}$ & & 200,000 & $\mu \mathrm{g} / \mathrm{kg}$ & & EPA8270 \\
\hline \multicolumn{8}{|l|}{ Volatile Organic Compounds } \\
\hline Acetone & $\mathbf{U}$ & $\mathbf{v}$ & & 36 & $\mu \mathrm{g} / \mathrm{kg}$ & 11 & EPA8240 \\
\hline Aromatic & $\mathbf{J}$ & $\mathrm{N}$ & & 100 & $\mu \mathrm{g} / \mathrm{kg}$ & & EPA8240 \\
\hline Aromatic & J & $\mathbf{N}$ & & 70 & $\mu \mathrm{g} / \mathrm{kg}$ & & EPA8240 \\
\hline Benzene & J & & & 1.9 & $\mu \mathrm{g} / \mathrm{kg}$ & 5.5 & EPA8240 \\
\hline Bromodichloromethane & $\mathrm{U}$ & & & 5.5 & $\mu \mathrm{g} / \mathrm{kg}$ & 5.5 & EPA8240 \\
\hline
\end{tabular}


SAMPLE NAME: D43ï 1304 (Continued)

Constituent

Volatile Organic Compounds
RQ AQ B Result Unit D. Limit Method
Sample ID: 100383

Bromoform
Bromomethane (Methyl bromide)
C3-benzene
C3-benzene
Carbon disulfide
Carbon tetrachloride
Chlorobenzene
Chloroethane
Chloroethene (Vinyl chloride)
2-Chloroethyl vinyl ether
Chloroform
Chloromethane (Methyl chloride)
Dibromochloromethane
1,1-Dichloroethane
1,2-Dichloroethane
1,1-Dichloroethylene
1,2-Dichloroethylene
Dichloromethane (Methylene chloride)
1,2-Dichloropropane
cis-1,3-Dichloropropene
trans-1,3-Dichloropropene
Ethylbenzene
2-Hexanone
Methyl ethyl ketone
Methyl isobutyl ketone
Styrene
1,1,2,2-Tetrachloroethane
Tetrachloroethylene
Toluene
1,1,1-Trichloroethane
1,1,2-Trichloroethane
Trichloroethylene
Unknown
Vinyl acetate
Xylenes

Pesticides/PCBs and Dioxins/Furans

5.5
11
30
40
3.8
5.5
5.5
11
11
11
5.5
11
5.5
5.5
5.5
5.5
2.4
33
5.5
5.5
5.5
14
11
11
11
5.5
5.5
13
27
2.0
5.5
1.1
40
11
57

5.5

$\mathrm{U}$

J $\quad \mathbf{N}$

J $\quad N$

$\mathrm{J}$

U

U

U

U

U

U

U

$\mathrm{U}$

U

J

U V

U

$U$

U

$\mathrm{U}$

$\mathrm{U}$

U

U

J

U

J N

U $\mu g / k g$
$\mu g / k g$
$\mu g / k g$
$\mu g / k g$
$\mu g / k g$
$\mu g / k g$
$\mu g / k g$
$\mu g / k g$
$\mu g / k g$
$\mu g / k g$
$\mu g / k g$
$\mu g / k g$
$\mu g / k g$
$\mu g / k g$
$\mu g / k g$
$\mu g / k g$
$\mu g / k g$
$\mu g / k g$
$\mu g / k g$
$\mu g / k g$
$\mu g / k g$
$\mu g / k g$
$\mu g / k g$
$\mu g / k g$
$\mu g / k g$
$\mu g / k g$
$\mu g / k g$
$\mu g / k g$
$\mu g / k g$
$\mu g / k g$
$\mu g / k g$
$\mu g / k g$
$\mu g / k g$
$\mu g / k g$
$\mu g / k g$

$\mu \mathrm{g} / \mathrm{kg}$

$\mu \mathrm{g} / \mathrm{kg}$

$\mu \mathrm{g} / \mathrm{kg}$

$\mu \mathrm{g} / \mathrm{kg}$

$\mu \mathrm{g} / \mathrm{kg}$

$\mu \mathrm{g} / \mathrm{kg}$

$\mu \mathrm{g} / \mathrm{kg}$

$\mu \mathrm{g} / \mathrm{kg}$

$\mu g / k g$

$\mu \mathrm{g} / \mathrm{kg}$

$\mu \mathrm{g} / \mathrm{kg}$

$\mu \mathrm{g} / \mathrm{kg}$
5.5

11

EPA8240

EPA8240

EPA8240

EPA8240

EPA8240

EPA8240

EPA8240

EPA8240

EPA8240

EPA8240

EPA8240

EPA8240

EPA8240

EPA8240

EPA8240

EPA8240

EPA8240

EPA8240

EPA8240

EPA8240

EPA8240

EPA8240

EPA8240

EPA8240

EPA8240

EPA8240

EPA8240

EPA8240

EPA8240

EPA8240

EPA8240

EPA8240

EPA8240

EPA8240

EPA8240

$\therefore$

EPA8080

EPA8080

EPA8080

EPA8080

EPA8080

EPA8080

EPA8080

EPA8080

EPA8080

EPA8080

EPA8080

EPA8080 
SAMPLE NAME: D4311304 (Continued)

Constituent

Pesticides/PCBs and Dioxins/Furans

Endosulfan sulfate

Endrin

Endrin ketone

Heptachlor

Heptachlor epoxide

Heptachlorodibenzo-p-dioxin isomers

Heptachlorodibenzo-p-furan isomers

Hexachlorodibenzo-p-dioxin isomers

Hexachlorodibenzo-p-furan isomers . U

Lindane

Methoxychlor

Octachlorodibenzo-p-dioxin

Octachlorodibenzo-p-furan

PCB 1016

PCB 1221

PCB 1232

PCB 1242

PCB 1248

PCB 1254

PCB 1260

Pentachlorodibenzo-p-dioxin isomers

Pentachlorodibenzo-p-furan isomers

2,3,7,8-TCDD

2,3,7,8-TCDF

Tetrachlorodibenzo-p-dioxin isomers

Tetrachlorodibenzo-p-furan isomers

Toxaphene

Radionuclide Indicators and Radionuclides

Gross alpha

Nonvolatile beta

Radium, total alpha-emitting

Tritium

\author{
RQ AQ
}

Sample ID: 100383
D. Limit Method

37

$\mu \mathrm{gg} / \mathrm{kg}$

$37 \quad \mu g / \mathrm{kg}$

$37 \quad \mu \mathrm{g} / \mathrm{kg}$

$19 \mu \mathrm{g} / \mathrm{kg}$

$19 \mu \mathrm{g} / \mathrm{kg}$

1.5

.0 .70

1.3

0.60

19

190

6.1

2.2

370

740

370

370

370

370

370

0.70

0.30

0.30

0.30

0.30

0.50

1,900

$\mathrm{ng} / \mathrm{g}$

$\mathrm{ng} / \mathrm{g}$

$\mathrm{ng} / \mathrm{g}$

$\mathrm{ng} / \mathrm{g}$

$\mu \mathrm{g} / \mathrm{kg}$

$\mu \mathrm{g} / \mathrm{kg}$

$\mathrm{ng} / \mathrm{g}$

$\mathrm{ng} / \mathrm{g}$

$\mu \mathrm{g} / \mathrm{kg}$

$\mu \mathrm{g} / \mathrm{kg}$

$\mu \mathrm{g} / \mathrm{kg}$

$\mu \mathrm{g} / \mathrm{kg}$

$\mu \mathrm{g} / \mathrm{kg}$

$\mu \mathrm{g} / \mathrm{kg}$

$\mu \mathrm{g} / \mathrm{kg}$

$\mathrm{ng} / \mathrm{g}$

$\mathrm{ng} / \mathrm{g}$

$\mathrm{ng} / \mathrm{g}$

$\mathrm{ng} / \mathrm{g}$

ng/g

$\mathrm{ng} / \mathrm{g}$

$\mu \mathrm{g} / \mathrm{kg}$

$37 \quad$ EPA8080

$37 \quad$ EPA8080

37 EPA8080

19 EPA8080

19 EPA8080

1.5 EPA8280

0.70 EPA8280

1.3 EPA8280

0.60 EPA8280

19 EPA8080

$190 \quad$ EPA8080

1.7 EPA8280

2.2 EPA8280

370 EPA8080

740 . EPA8080

370 EPA8080

$370 \quad$ EPA8080

370 EPA8080

370 EPA8080

370 EPA8080

0.70 EPA8280

0.30 EPA8280

0.30 EPA8280

0.30 EPA8280

0.30 EPA8280

0.50 EPA8280

1,900 EPA8080

$7.5 \quad \mathrm{pCi} / \mathrm{g}$

$\mathrm{pCi} / \mathrm{g}$

$\mathrm{pCi} / \mathrm{g}$

$\mathrm{pCi} / \mathrm{g}$
7.5

7.2

0.20

1.1
EPA900.0

EPA900.0

EPA903.0

EPA906.0

\section{SAMPLE NAME: D431 1305}

Location (SRS Coordinates): 18446.1E 66631.93N

Ground Elevation Above MSL: $131.4 \mathrm{ft}$

Depth of Core Interval:- 6.00 to $8.00 \mathrm{ft}$

Sample Type: Normal

Sample Color:

$$
=
$$

Total Metals

Arsenic

Barium

Cadmium

Chromium
Sample Moisture:

Percent Solids: $\mathbf{8 8 . 6 0}$
Sample Matrix: Soil

USC Soil Classification:
Sample ID: 100385

24
130
1.1
31

\begin{abstract}
$\mathrm{mg} / \mathrm{kg}$ $\mathrm{mg} / \mathrm{kg}$ $\mathrm{mg} / \mathrm{kg}$ $\mathrm{mg} / \mathrm{kg}$
\end{abstract}

11

22

0.56

1.1
EPA7060

EPA6010

EPA6010

EPA6010 
SAMPLE NAME: D431 1305 (Continued)

Sample ID: 100385

Constituent

RQ AQ B Result Unit

D. Limit Method

Total Metals

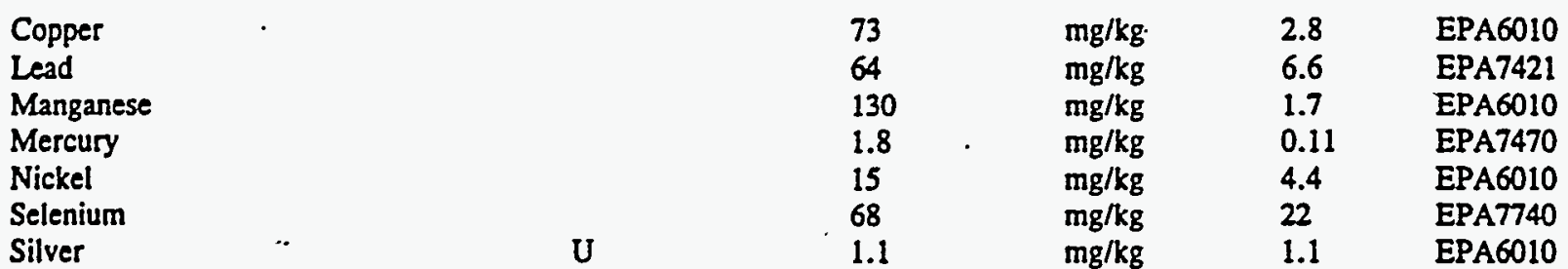

$B / N / A$ Extractables (including $P A H$ and phenols)

\begin{tabular}{|c|c|c|c|c|c|c|c|}
\hline $\begin{array}{l}\text { Acenaphthene } \\
\text { Acenaphthylene }\end{array}$ & $\begin{array}{l}\mathrm{J} \\
\mathrm{J}\end{array}$ & 0 & L & $\begin{array}{l}28,000 \\
2,600\end{array}$ & $\mu g / \mathrm{kg}$ & 3,800 & EPA8270 \\
\hline Anthracene & $\mathrm{J}$ & 0 & $\begin{array}{l}L \\
L\end{array}$ & $\begin{array}{l}2.600 \\
53,000\end{array}$ & $\begin{array}{l}\mu \mathrm{g} / \mathrm{kg} \\
\mu \mathrm{g} / \mathrm{kg}\end{array}$ & $\begin{array}{l}3,800 \\
3,800\end{array}$ & $\begin{array}{l}\text { EPA8270 } \\
\text { EPA8270 }\end{array}$ \\
\hline Benzo(a)anthracene & L & 0 & L & 100,000 & $\mu \mathrm{g} / \mathrm{kg}$ & 3,800 & EPA8270 \\
\hline Benzo(a)anthracene & $\mathbf{J}$ & 0 & L & 120,000 & $\mu \mathrm{g} / \mathrm{kg}$ & 15,000 & EPA8270 \\
\hline Benzo(b)fluoranthene & $\mathrm{LJ}$ & 0 & L & 110,000 & $\mu \mathrm{g} / \mathrm{kg}$ & 3.800 & EPA8270 \\
\hline Benzo(b)fluoranthene & $\mathbf{J}$ & 0 & L & 120,000 & $\mu \mathrm{g} / \mathrm{kg}$ & 15,000 & EPA8270 \\
\hline Benzo(k)fluoranthene & $\mathbf{J}$ & 0 & L & 27,000 & $\mu \mathrm{g} / \mathrm{kg}$ & 3,800 & EPA8270 \\
\hline Benzoic acid & UJ & 0 & L & 19,000 & $\mu \mathrm{g} / \mathrm{kg}$ & 19,000 & EPA8270 \\
\hline Benzo $(g, h, i)$ perylene & $\mathbf{J}$ & 0 & L & 33,000 & $\mu \mathrm{g} / \mathrm{kg}$ & 3.800 & EPA8270 \\
\hline Benzo(a)pyrene & $\mathrm{L}$ & 0 & $\mathrm{~L}$ & 80,000 & $\mu \mathrm{g} / \mathrm{kg}$ & 3,800 & EPA8270 \\
\hline Benzo(a)pyrene & J & 0 & $\mathrm{~L}$ & 91,000 & $\mu \mathrm{g} / \mathrm{kg}$ & 15,000 & EPA8270 \\
\hline Benzyl alcohol & $\mathrm{UJ}$ & 0 & $\bar{L}$ & 3,800 & $\mu \mathrm{g} / \mathrm{kg}$ & 3,800 & EPA8270 \\
\hline Bis(2-chloroethoxy) methane & UJ & $\mathbf{o}$ & $\bar{L}$ & 3,800 & $\mu \mathrm{g} / \mathrm{kg}$ & 3,800 & EPA8270 \\
\hline Bis(2-chloroethyl) ether & UJ & 0 & L & 3,800 & $\mu \mathrm{g} / \mathrm{kg}$ & 3.800 & EPA8270 \\
\hline Bis(2-chloroisopropyl) ether & UJ & 0 & L & 3,800 & $\mu \mathrm{g} / \mathrm{kg}$ & 3,800 & EPA8270 \\
\hline Bis(2-ethylhexyl) phthalate & $\mathbf{J}$ & 0 & L & 1,300 & $\mu g / k g$ & 3,800 & EPA8270 \\
\hline 4-Bromophenyl phenyl ether & UJ & 0 & L & 3,800 & $\mu \mathrm{g} / \mathrm{kg}$ & 3,800 & EPA8270 \\
\hline Butylbenzyl phthalate & $\mathrm{UJ}$ & 0 & $\bar{L}$ & 3,800 & $\mu \mathrm{g} / \mathrm{kg}$ & 3,800 & EPA8270 \\
\hline Benzo-anthracene isomer & J & $\mathbf{N}$ & & 30,000 & $\mu \mathrm{g} / \mathrm{kg}$ & & EPA8270 \\
\hline Benz-anthracenone isomer & $\mathbf{J}$ & $\mathbf{N}$ & & 40,000 & $\mu \mathrm{g} / \mathrm{kg}$ & & EPA8270 \\
\hline Benzonapthothiophene & J & $\mathrm{N}$ & & 50,000 & $\mu \mathrm{g} / \mathrm{kg}$ & & EPA8270 \\
\hline Benzofluorene isomer & $\mathbf{J}$ & $\mathbf{N}$ & & 100,000 & $\mu \mathrm{g} / \mathrm{kg}$ & & EPA8270 \\
\hline Benzofluorene isomer & J & $\mathbf{N}$ & & 70,000 & $\mu g / k g$ & & EPA8270 \\
\hline Benzonapthofuran isomer & J & $\mathrm{N}$ & & 30,000 & $\mu \mathrm{g} / \mathrm{kg}$ & & EPA8270 \\
\hline Benzo-pyrene isomer & $\mathrm{J}$ & $\mathrm{N}$ & & 60,000 & $\mu \mathrm{g} / \mathrm{kg}$ & & EPA8270 \\
\hline Benzophenanthrene isomer & J & $\mathbf{N}$ & & 40,000 & $\mu g / \mathrm{kg}$ & & EPA8270 \\
\hline Carbazole & J & $\mathbf{N}$ & & 20,000 & $\mu \mathrm{g} / \mathrm{kg}$ & & EPA8270 \\
\hline 4-Chloroaniline & UJ & 0 & L & 3,800 & $\mu \mathrm{g} / \mathrm{kg}$ & 3,800 & EPA8270 \\
\hline 4-Chloro-m-cresol & UJ & 0 & L & 3,800 & $\mu \mathrm{g} / \mathrm{kg}$ & 3,800 & EPA8270 \\
\hline 2-Chloronaphthalene - & UJ & 0 & L & 3,800 & $\mu \mathrm{g} / \mathrm{kg}$ & 3,800 & EPA8270 \\
\hline 2-Chlorophenol & UJ & 0 & L & 3,800 & $\mu \mathrm{g} / \mathrm{kg}$ & 3,800 & EPA8270 \\
\hline 4-Chlorophenyl phenyl ether & UJ & 0 & L & 3,800 & $\mu \mathrm{g} / \mathrm{kg}$ & 3,800 & EPA8270 \\
\hline Chrysene & LJ & 0 & L & 65,000 & $\mu \mathrm{g} / \mathrm{kg}$ & 3,800 & EPA8270 \\
\hline Chrysene & $\mathbf{J}$ & 0 & L & 94,000 & $\mu \mathrm{g} / \mathrm{kg}$ & 15,000 & EPA8270 \\
\hline O-Cresol (2-Methylphenol) & UJ & 0 & $\bar{L}$ & 3,800 & $\mu \mathrm{g} / \mathrm{kg}$ & 3,800 & EPA8270 \\
\hline p-Cresol (4-Methylphenol) & UJ & 0 & L & 3,800 & $\mu \mathrm{g} / \mathrm{kg}$ & 3,800 & EPA8270 \\
\hline Dibenz $(a, h)$ anthracene & $\mathrm{J}$ & 0 & L & 9,200 & $\mu \mathrm{g} / \mathrm{kg}$ & 3,800 & EPA8270 \\
\hline Dibenzofuran & $\mathrm{J}$ & 0 & $\mathbf{L}$ & 20,000 & $\mu g / \mathrm{kg}$ & 3,800 & EPA8270 \\
\hline Di-n-butyl phthalate & UJ & 0 & L & 3,800 & $\mu \mathrm{g} / \mathrm{kg}$. & 3,800 & EPA8270 \\
\hline
\end{tabular}


SAMPLE NAME: D431 1305 (Continued)

Sample D: 100385

$\begin{array}{llllll}\text { Constituent } & \text { RQ AQ B Result Unit Dimit Method }\end{array}$

$B / N / A$ Extractables (including $P A H$ and phenols)

\begin{tabular}{|c|c|c|c|c|c|c|c|}
\hline 1,2-Dichlorobenzene & UJ & 0 & L & 3,800 & $\mu g / \mathrm{kg}$ & 3,800 & EPA8270 \\
\hline 1.3-Dichlorobenzene & UJ & 0 & L & 3,800 & $\mu \mathrm{g} / \mathrm{kg}$ & 3,800 & EPA8270 \\
\hline 1,4-Dichlorobenzene & UJ & 0 & L & 3,800 & $\mu \mathrm{g} / \mathrm{kg}$ & 3,800 & EPA8270 \\
\hline 3,3'-Dichlorobenzidine & UJ & $\mathbf{0}$ & L & 7.500 & $\mu g / \mathrm{kg}$ & 7,500 & EPA8270 \\
\hline 2,4-Dichlorophenol & UJ & 0 & L & 3,800 & $\mu g / \mathrm{kg}$ & 3,800 & EPA8270 \\
\hline Diethyl phthalate & UJ & 0 & L & 3.800 & $\mu g / k g$ & 3,800 & EPA8270 \\
\hline 2,4-Dimethyl phenol .. & UJ & 0 & L & 3,800 & $\mu \mathrm{g} / \mathrm{kg}$ & 3,800 & EPA8270 \\
\hline Dimethyl phthalate & UJ & 0 & L & 3.800 & $\mu g / k g$ & 3,800 & EPA8270 \\
\hline 2,4-Dinitrophenol & UJ & 0 & L & 19,000 & $\mu g / \mathrm{kg}$ & 19,000 & EPA8270 \\
\hline 2,4-Dinitrotoluene & UJ & 0 & L & 3,800 & $\mu \mathrm{g} / \mathrm{kg}$ & 3,800 & EPA8270 \\
\hline 2,6-Dinitrotoluene & UJ & 0 & L & 3,800 & $\mu \mathrm{g} / \mathrm{kg}$ & 3,800 & EPA8270 \\
\hline Di-n-octyl phthalate & UJ & 0 & L & 3,800 & $\mu g / \mathrm{kg}$ & 3,800 & EPA8270 \\
\hline Fluoranthene & $L$ & 0 & L & 160,000 & $\mu \mathrm{g} / \mathrm{kg}$ & 3,800 & EPA8270 \\
\hline Fluoranthene & $J$ & 0 & $\mathbf{L}$ & 230.000 & $\mu \mathrm{g} / \mathrm{kg}$ & 15,000 & EPA8270 \\
\hline Fiuorene & $\mathrm{J}$ & 0 & L & 33,000 & $\mu \mathrm{g} / \mathrm{kg}$ & 3,800 & EPA8270 \\
\hline Hexachlorobenzene & UJ & 0 & L & 3,800 & $\mu \mathrm{g} / \mathrm{kg}$ & 3,800 & EPA8270 \\
\hline Hexachlorobutadiene & UJ & 0 & $\mathbf{L}$ & 3.800 & $\mu \mathrm{g} / \mathrm{kg}$ & 3,800 & EPA8270 \\
\hline Hexachlorocyclopentadiene & UJ & 0 & L & 3,800 & $\mu \mathrm{g} / \mathrm{kg}$ & 3,800 & EPA8270 \\
\hline Hexachloroethane & UJ & 0 & L & 3.800 & $\mu g / \mathrm{kg}$ & 3,800 & EPA8270 \\
\hline Indeno(1,2,3-c,d)pyrene & $\mathrm{J}$ & 0 & $\mathbf{L}$ & 40,000 & $\mu \mathrm{g} / \mathrm{kg}$ & 3,800 & EPA8270 \\
\hline Isophorone & UJ & 0 & L & 3,800 & $\mu g / \mathrm{kg}$ & 3,800 & EPA8270 \\
\hline Methylanthracene isomer & J & $\mathbf{N}$ & & 20,000 & $\mu \mathrm{g} / \mathrm{kg}$ & & EPA8270 \\
\hline Methylphenanthrene isomer & $\mathrm{J}$ & $\mathbf{N}$ & & 10,000 & $\mu \mathrm{g} / \mathrm{kg}$ & & EPA8270 \\
\hline 2-Methyl-4,6-dinitrophenol & UJ & 0 & L & 19,000 & $\mu \mathrm{g} / \mathrm{kg}$ & 19,000 & EPA8270 \\
\hline 2-Methylnaphthalene & $\mathrm{J}$ & 0 & L & 9,300 & $\mu \mathrm{g} / \mathrm{kg}$ & 3,800 & EPA8270 \\
\hline Naphthalene & $\mathrm{J}$ & $\mathbf{0}$ & $\mathbf{L}$ & 28,000 & $\mu \mathrm{g} / \mathrm{kg}$ & 3,800 & EPA8270 \\
\hline o-Nitroaniline & UJ & 0 & L & 19,000 & $\mu \mathrm{g} / \mathrm{kg}$ & 19,000 & EPA8270 \\
\hline m-Nitroaniline & UJ & 0 & L & 19,000 & $\mu \mathrm{g} / \mathrm{kg}$ & 19,000 & EPA8270 \\
\hline p-Nitroaniline & UJ & $\mathbf{0}$ & L & 19,000 & $\mu \mathrm{g} / \mathrm{kg}$ & 19.000 & EPA8270 \\
\hline Nitrobenzene & UJ & 0 & L & 3.800 & $\mu \mathrm{g} / \mathrm{kg}$ & 3,800 & EPA8270 \\
\hline 2-Nitrophenol & UJ & 0 & L & 3.800 & $\mu \mathrm{g} / \mathrm{kg}$ & 3,800 & EPA8270 \\
\hline 4-Nitrophenol & UJ & 0 & L & 19,000 & $\mu \mathrm{g} / \mathrm{kg}$ & 19,000 & EPA8270 \\
\hline N-Nitrosodiphenylamine & UJ & 0 & L & 3,800 & $\mu \mathrm{g} / \mathrm{kg}$ & 3,800 & EPA8270 \\
\hline N-Nitrosodipropylamine & UJ & $\mathrm{CO}$ & L & 3,800 & $\mu \mathrm{g} / \mathrm{kg}$ & 3,800 & EPA8270 \\
\hline Pentachlorophenol & UJ & 0 & L & 19,000 & $\mu \mathrm{g} / \mathrm{kg}$ & 19,000 & EPA8270 \\
\hline Phenanthrene & LJ & 0 & L & 160,000 & $\mu \mathrm{g} / \mathrm{kg}$ & 3,800 & EPA8270 \\
\hline Phenanthrene & J & 0 & L & 210,000 & $\mu \mathrm{g} / \mathrm{kg}$ & 15,000 & EPA8270 \\
\hline Phenol & UJ & 0 & L & 3,800 & $\mu \mathrm{g} / \mathrm{kg}$ & 3,800 & EPA8270 \\
\hline Phenyl-napthalene isomer & $\mathbf{J}$ & $\mathbf{N}$ & & 20,000 & $\mu \mathrm{g} / \mathrm{kg}$ & & EPA8270 \\
\hline Pyrene & LJ & 0 & L & 160,000 & $\mu \mathrm{g} / \mathrm{kg}$ & 3,800 & EPA8270 \\
\hline Pyrene & $\mathbf{J}$ & 0 & L & 170,000 & $\mu \mathrm{g} / \mathrm{kg}$ & 15,000 & EPA8270 \\
\hline 1,2,4-Trichlorobenzene & UJ & $\mathrm{CO}$ & $\mathbf{L}$ & 3,800 & $\mu g / k g$ & 3,800 & EPA8270 \\
\hline 2,4,5-Trichlorophenol & UJ & 0 & L & 19,000 & $\mu \mathrm{g} / \mathrm{kg}$ & 19,000 & EPA8270 \\
\hline 2,4,6-Trichlorophenol & UJ & 0 & L & 3,800 & $\mu \mathrm{g} / \mathrm{kg}$ & 3,800 & EPA8270 \\
\hline Unknown & J & $\mathbf{N}$ & & 30,000 & $\mu \mathrm{g} / \mathrm{kg}$ & & EPA8270 \\
\hline Unknown & $\mathrm{J}$ & $\mathbf{N}$ & & 30,000 & $\mu \mathrm{g} / \mathrm{kg}$ & & EPA8270 \\
\hline Unknown ketone & $J$ & NV & & 90,000 & $\mu \mathrm{g} / \mathrm{kg}$ & & EPA8270 \\
\hline Unknown polyarom. hydrocarbon & $\mathbf{J}$ & $\mathbf{N}$ & . & 30,000 & $\mu g / k g$ & & EPA8270 \\
\hline Unknown polyarom. hydrocarbon & $J$ & $\mathbf{N}$ & & 50,000 & $\mu \mathrm{g} / \mathrm{kg}$ & & EPA8270 \\
\hline Unknown polyarom. hydrocarbon & $\mathrm{J}$ & $\mathbf{N}$ & & 60,000 & $\mu \mathrm{g} / \mathrm{kg}$ & & EPA8270 \\
\hline
\end{tabular}


SAMPLE NAME: D43i 1305 (Continued)

Sample ID: 100385

$\begin{array}{llllll}\text { Constituent } & \text { RQ } & \text { AQ } & \text { Result } & \text { Unit Limit Method }\end{array}$ i';

$B / N / A$ Extractables (including $P A H$ and phenols)

Dimethylpyrene isomer

Di-n-benzothiophene

Volatile Organic Compounds

$\begin{array}{llllll}\mathrm{J} & \mathrm{N} & 30,000 & \mu \mathrm{g} / \mathrm{kg} & & \text { EPA8270 } \\ \mathrm{J} & \mathrm{N} & 20,000 & \mu \mathrm{g} / \mathrm{kg} & \text { EPA8270 }\end{array}$

\begin{tabular}{|c|c|c|c|c|c|c|c|}
\hline Acetone & $\mathrm{J}$ & vo & L & 160 & $\mu \mathrm{g} / \mathrm{kg}$ & 11 & EPA8240 \\
\hline Acetone & UJ & vo & L & 100 & $\mu \mathrm{g} / \mathrm{kg}$ & 11 & EPA8240 \\
\hline Benzene & $\mathbf{J}$ & 0 & $\mathbf{L}$ & 1.7 & $\mu \mathrm{g} / \mathrm{kg}$ & 5.5 & EPA8240 \\
\hline Benzene & $\mathbf{J}$ & 0 & $\mathbf{L}$ & 5.2 & $\mu g / \mathrm{kg}$ & 5.5 & EPA8240 \\
\hline Bromodichloromethane & UJ & 0 & $\mathbf{L}$ & 5.5 & $\mu \mathrm{g} / \mathrm{kg}$ & 5.5 & EPA8240 \\
\hline Bromodichloromethane & UJ & 0 & L & 5.5 & $\mu \mathrm{g} / \mathrm{kg}$ & 5.5 & EPA8240 \\
\hline Bromoform & UJ & 0 & L & 5.5 & $\mu \mathrm{g} / \mathrm{kg}$ & 5.5 & EPA8240 \\
\hline Bromoform & UJ & 0 & L & 5.5 & $\mu \mathrm{g} / \mathrm{kg}$ & 5.5 & EPA8240 \\
\hline Bromomethane (Methyl bromide) & UJ & 0 & L & 11 & $\mu g / \mathrm{kg}$ & 11 & EPA8240 \\
\hline Bromomethane (Methyl bromide) & UJ & 0 & L & 11 & $\mu \mathrm{g} / \mathrm{kg}$ & 11 & EPA8240 \\
\hline Carbon disulfide & $\mathbf{J}$ & 0 & $\mathbf{L}$ & 5.1 & $\mu \mathrm{g} / \mathrm{kg}$ & 5.5 & EPA8240 \\
\hline Carbon disulfide & $\mathbf{J}$ & 0 & L & 12 & $\mu \mathrm{g} / \mathrm{kg}$ & 5.5 & EPA8240 \\
\hline Carbon tetrachloride & UJ & 0 & L & 5.5 & $\mu \mathrm{g} / \mathrm{kg}$ & 5.5 & EPA8240 \\
\hline Carbon tetrachloride & UJ & 0 & L & 5.5 & $\mu \mathrm{g} / \mathrm{kg}$ & 5.5 & EPA8240 \\
\hline Chlorobenzene & UJ & 0 & L & 5.5 & $\mu \mathrm{g} / \mathrm{kg}$ & 5.5 & EPA8240 \\
\hline Chlorobenzene & UJ & 0 & L & 5.5 & $\mu \mathrm{g} / \mathrm{kg}$ & 5.5 & EPA8240 \\
\hline Chloroethane & UJ & 0 & L & 11 & $\mu \mathrm{g} / \mathrm{kg}$ & 11 & EPA8240 \\
\hline Chloroethane & UJ & 0 & L & 11 & $\mu \mathrm{g} / \mathrm{kg}$ & 11 & EPA8240 \\
\hline Chloroethene (Vinyl chloride) & UJ & 0 & L & 11 & $\mu \mathrm{g} / \mathrm{kg}$ & 11 & EPA8240 \\
\hline Chloroethene (Vinyl chloride) & UJ & 0 & L & 11 & $\mu \mathrm{g} / \mathrm{kg}$ & 11 & EPA8240 \\
\hline 2-Chloroethyl vinyl ether & UJ & 0 & L & 11 & $\mu g / \mathrm{kg}$ & 11 & EPA8240 \\
\hline 2-Chloroethyl vinyl ether & UJ & 0 & L & 11 & $\mu \mathrm{g} / \mathrm{kg}$ & 11 & EPA8240 \\
\hline Chloroform & UJ & 0 & L & 5.5 & $\mu \mathrm{g} / \mathrm{kg}$ & 5.5 & EPA8240 \\
\hline Chloroform & UJ & 0 & L & 5.5 & $\mu \mathrm{g} / \mathrm{kg}$ & 5.5 & EPA8240 \\
\hline Chloromethane (Methyl chloride) & UJ & 0 & L & 11 & $\mu \mathrm{g} / \mathrm{kg}$ & 11 & EPA8240 \\
\hline Chloromethane (Methyl chloride) & UJ & 0 & L & 11 & $\mu g / \mathrm{kg}$ & 11 & EPA8240 \\
\hline Dibromochloromethane & UJ & 0 & L & 5.5 & $\mu g / k g$ & 5.5 & EPA8240 \\
\hline Dibromochloromethane & UJ & 0 & L & 5.5 & $\mu \mathrm{g} / \mathrm{kg}$ & 5.5 & EPA8240 \\
\hline 1.1-Dichloroethane & UJ & 0 & L & 5.5 & $\mu \mathrm{g} / \mathrm{kg}$ & 5.5 & EPA8240 \\
\hline 1,1-Dichloroethane & UJ & 0 & L & 5.5 & $\mu g / \mathrm{kg}$ & 5.5 & EPA8240 \\
\hline 1,2-Dichloroethane & UJ & 0 & L & 5.5 & $\mu \mathrm{g} / \mathrm{kg}$ & 5.5 & EPA8240 \\
\hline 1,2-Dichloroethane & UJ & 0 & $\mathrm{~L}$ & 5.5 & $\mu \mathrm{g} / \mathrm{kg}$ & 5.5 & EPA8240 \\
\hline 1,1-Dichloroethylene & UJ & $\mathbf{0}$ & L & 5.5 & $\mu \mathrm{g} / \mathrm{kg}$ & 5.5 & EPA8240 \\
\hline 1,1-Dichloroethylene & UJ & 0 & L & 5.5 & $\mu \mathrm{g} / \mathrm{kg}$ & 5.5 & EPA8240 \\
\hline 1,2-Dichloroethylene & $\mathrm{J}$ & 0 & L & 3.7 & $\mu \mathrm{g} / \mathrm{kg}$ & 5.5 & EPA8240 \\
\hline 1,2-Dichloroethylene " & $\mathbf{J}$ & 0 & L & 7.2 & $\mu g / \mathrm{kg}$ & 5.5 & EPA8240 \\
\hline Dichloromethane (Methylene chloride) & UJ & vo & $\mathrm{L}$ & 21 & $\mu \mathrm{g} / \mathrm{kg}$ & 5.5 & EPA8240 \\
\hline Dichloromethane (Methylene chloride) & UJ & vo & L & 27 & $\mu \mathrm{g} / \mathrm{kg}$ & 5.5 & EPA8240 \\
\hline 1,2-Dichloropropane & UJ & 0 & $\mathbf{L}$ & 5.5 & $\mu \mathrm{g} / \mathrm{kg}$ & 5.5 & EPA8240 \\
\hline 1,2-Dichloropropane & UJ & 0 & L & 5.5 & $\mu \mathrm{g} / \mathrm{kg}$ & 5.5 & EPA8240 \\
\hline cis-1,3-Dichloropropene & UJ & 0 & L & 5.5 & $\mu \mathrm{g} / \mathrm{kg}$ & 5.5 & EPA8240 \\
\hline cis-1,3-Dichloropropene & UJ & 0 & $\mathbf{L}$ & 5.5 & $\mu \mathrm{g} / \mathrm{kg}$ & 5.5 & EPA8240 \\
\hline trans-1,3-Dichloropropene & UJ & 0 & L & 5.5 & $\mu \mathrm{g} / \mathrm{kg}$ & 5.5 & EPA8240 \\
\hline trans-1,3-Dichloropropene & UI & 0 & L & 5.5 & $\mu \mathrm{g} / \mathrm{kg}$ & 5.5 & EPA8240 \\
\hline Ethylbenzene & $\mathrm{J}$ & 0 & L & 6.5 & $\mu \mathrm{g} / \mathrm{kg}$ & 5.5 & EPA8240 \\
\hline
\end{tabular}


SAMPLE NAME: D43r 1305 (Continued)

Sample ID: 100385

Constituent

Volatile Organic Compounds

$\begin{array}{lllll}\text { Ethylbenzene } & \text { J } & \text { O } & \text { L } & 15 \\ \text { 2-Hexanone } & \text { UJ } & \text { O } & \text { L } & 11 \\ \text { 2-Hexanone } & \text { UJ } & \text { O } & \text { L } & 11 \\ \text { Methyl ethyl ketone } & \text { J } & \text { O } & \text { L } & 29 \\ \text { Methyl ethyl ketone } & \text { J } & \text { O } & \text { L } & 27 \\ \text { Methyl isobutyl ketone } & \text { UJ } & \text { O } & \text { L } & 11 \\ \text { Methyl isobutyl ketone } & \text { UJ } & \text { O } & \text { L } & 11 \\ \text { Styrene } & \text { UJ } & \text { O } & \text { L } & 5.5 \\ \text { Styrene } & \text { UJ } & \text { O } & \text { L } & 5.5 \\ \text { l,1,2,2-Tetrachloroethane } & \text { UJ } & \text { O } & \text { L } & 5.5 \\ \text { 1,1,2,2-Tetrachloroethane } & \text { UJ } & \text { O } & \text { L } & 5.5 \\ \text { Tetrachloroethylene } & \text { J } & \text { O } & \text { L } & 5.2 \\ \text { Tetrachloroethylene } & \text { J } & \text { O } & \text { L } & 13 \\ \text { Toluene } & \text { J } & \text { O } & \text { L } & 14 \\ \text { Toluene } & \text { J } & \text { O } & \text { L } & 23 \\ \text { 1,1,1-Trichloroethane } & \text { UJ } & \text { O } & \text { L } & 5.5 \\ \text { 1,1,1-Trichloroethane } & \text { J } & \text { O } & \text { L } & 1.9 \\ \text { l,1,2-Trichloroethane } & \text { UJ } & \text { O } & \text { L } & 5.5 \\ \text { 1,1,2-Trichloroethane } & \text { UJ } & \text { O } & \text { L } & 5.5 \\ \text { Trichloroethylene } & \text { UJ } & \text { O } & \text { L } & 5.5 \\ \text { Trichloroethylene } & \text { J } & \text { O } & \text { L } & 1.6 \\ \text { Unknown } & \text { J } & \text { N } & & 10 \\ \text { Unknown } & \text { J } & \text { N } & & 10 \\ \text { Unknown } & \text { J } & \text { N } & & 40 \\ \text { Vinyl acetate } & \text { UJ } & \text { O } & \text { L } & 11 \\ \text { Vinyl acetate } & \text { UJ } & 0 & \text { L } & 11 \\ \text { Xylenes } & \text { J } & 0 & \text { L } & 20 \\ \text { Xylenes } & \text { J } & \text { O } & \text { L } & 39\end{array}$

Pesticides/PCBs and Dioxins/Furans
RQ AQ B Result Unit D. Limit Method

$\begin{array}{llll}\mu \mathrm{g} / \mathrm{kg} & 5.5 & \text { EPA8240 } \\ \mu \mathrm{g} / \mathrm{kg} & 11 & \text { EPA8240 } \\ \mu \mathrm{g} / \mathrm{kg} & 11 & \text { EPA8240 } \\ \mu \mathrm{g} / \mathrm{kg} & 11 & \text { EPA8240 } \\ \mu \mathrm{g} / \mathrm{kg} & 11 & \text { EPA8240 } \\ \mu \mathrm{g} / \mathrm{kg} & 11 & \text { EPA8240 } \\ \mu \mathrm{g} / \mathrm{kg} & 11 & \text { EPA8240 } \\ \mu \mathrm{g} / \mathrm{kg} & 5.5 & \text { EPA8240 } \\ \mu \mathrm{g} / \mathrm{kg} & 5.5 & \text { EPA8240 } \\ \mu \mathrm{g} / \mathrm{kg} & 5.5 & \text { EPA8240 } \\ \mu \mathrm{g} / \mathrm{kg} & 5.5 & \text { EPA8240 } \\ \mu \mathrm{g} / \mathrm{kg} & 5.5 & \text { EPA8240 } \\ \mu \mathrm{g} / \mathrm{kg} & 5.5 & \text { EPA8240 } \\ \mu \mathrm{g} / \mathrm{kg} & 5.5 & \text { EPA8240 } \\ \mu \mathrm{g} / \mathrm{kg} & 5.5 & \text { EPA8240 } \\ \mu \mathrm{g} / \mathrm{kg} & 5.5 & \text { EPA8240 } \\ \mu \mathrm{g} / \mathrm{kg} & 5.5 & \text { EPA8240 } \\ \mu \mathrm{g} / \mathrm{kg} & 5.5 & \text { EPA8240 } \\ \mu \mathrm{g} / \mathrm{kg} & 5.5 & \text { EPA8240 } \\ \mu \mathrm{g} / \mathrm{kg} & 5.5 & \text { EPA8240 } \\ \mu \mathrm{g} / \mathrm{kg} & 5.5 & \text { EPA8240 } \\ \mu \mathrm{g} / \mathrm{kg} & & \text { EPA8240 } \\ \mu \mathrm{g} / \mathrm{kg} & & \text { EPA8240 } \\ \mu \mathrm{g} / \mathrm{kg} & & \text { EPA8240 } \\ \mu \mathrm{g} / \mathrm{kg} & 11 & \text { EPA8240 } \\ \mu \mathrm{g} / \mathrm{kg} & 11 & \text { EPA8240 } \\ \mu \mathrm{g} / \mathrm{kg} & 5.5 & \text { EPA8240 } \\ \mu \mathrm{g} / \mathrm{kg} & 5.5 & \text { EPA8240 }\end{array}$

Aldrin

alpha-Benzene hexachloride

beta-Benzene hexachloride

delta-Benzene hexachloride

alpha-Chlordane

gamma-Chlordane

p.p'-DDD

p.p'-DDE

p. $\mathrm{p}^{\circ}-\mathrm{DDT}$

Dieldrin

Endosulfan I

Endosulfan II

Endosulfan sulfate

Endrin

Endrin ketone

Heptachlor

Heptachlor epoxide

Heptachlorodibenzo-p-dioxin isomers

Heptachlorodibenzo-p-furan isomers

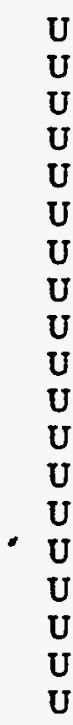

19

$\mu \mathrm{g} / \mathrm{kg}$

$\mu \mathrm{g} / \mathrm{kg}$

$\mu \mathrm{g} / \mathrm{kg}$

$\mu \mathrm{g} / \mathrm{kg}$

$\mu \mathrm{g} / \mathrm{kg}$

$\mu \mathrm{g} / \mathrm{kg}$

$\mu \mathrm{g} / \mathrm{kg}$

$\mu \mathrm{g} / \mathrm{kg}$

$\mu \mathrm{g} / \mathrm{kg}$

$\mu \mathrm{g} / \mathrm{kg}$

$\mu g / \mathrm{kg}$

$\mu \mathrm{g} / \mathrm{kg}$

$\mu \mathrm{g} / \mathrm{kg}$

$\mu \mathrm{g} / \mathrm{kg}$

$\mu g / \mathrm{kg}$

$\mu \mathrm{g} / \mathrm{kg}$

$\mu g / k g$ $\begin{array}{llll}1.7 & \mathrm{ng} / \mathrm{g} & 1.7 & \text { EPA8280 } \\ 1.8 & \mathrm{ng} / \mathrm{g} & 1.8 & \text { EPA8280 }\end{array}$

$\begin{array}{llll}1.7 & \mathrm{ng} / \mathrm{g} & 1.7 & \text { EPA8280 } \\ 1.8 & \mathrm{ng} / \mathrm{g} & 1.8 & \text { EPA8280 }\end{array}$

EPA8080

EPA8080

EPA8080

EPA8080

EPA8080

EPA8080

EPA8080

EPA8080

EPA8080

EPA8080

EPA8080

EPA8080

EPA8080

EPA8080

EPA8080

EPA8080

EPA8080

$\begin{array}{llll}1.7 & \mathrm{ng} / \mathrm{g} & 1.7 & \text { EPA8280 } \\ 1.8 & \mathrm{ng} / \mathrm{g} & 1.8 & \text { EPA8280 }\end{array}$ 
SAMPLE NAME: D431 1305 (Continued)

Constituent
Pesticides/PCBs and Dioxins/Furans
Hexachlorodibenzo-p-dioxin isomers
Hexachlorodibenzo-p-furan isomers
Lindane
Methoxychlor
Octachlorodibenzo-p-dioxin
Octachlorodibenzo-p-furan
PCB 1016
PCB 1221
PCB 1232
PCB 1242
PCB 1248
PCB 1254
PCB 1260
Pentachlorodibenzo-p-dioxin isomers
Pentachlorodibenzo-p-furan isomers
2.3.7,8-TCDD
2,3,7,8-TCDF
Tetrachlorodibenzo-p-dioxin isomers
Tetrachlorodibenzo-p-furan isomers
Toxaphene

Radionuclide Indicators and Radionuclides
Sample ID: 100385

RQ AQ B Result Unit $\quad$ D. Limit Method

$\begin{array}{llll}1.8 & \mathrm{ng} / \mathrm{g} & 1.8 & \text { EPA8280 } \\ 1.6 & \mathrm{ng} / \mathrm{g} & 1.6 & \text { EPA8280 } \\ 19 & \mu \mathrm{g} / \mathrm{kg} & 19 & \text { EPA8080 } \\ 190 & \mu \mathrm{g} / \mathrm{kg} & 190 & \text { EPA8080 } \\ 5.4 & \mathrm{ng} / \mathrm{g} & 2.2 & \text { EPA8280 } \\ 2.7 & \mathrm{ng} / \mathrm{g} & 2.7 & \text { EPA8280 } \\ 380 & \mu \mathrm{g} / \mathrm{kg} & 380 & \text { EPA8080 } \\ 750 & \mu \mathrm{g} / \mathrm{kg} & 750 & \text { EPA8080 } \\ 380 & \mu \mathrm{g} / \mathrm{kg} & 380 & \text { EPA8080 } \\ 380 & \mu \mathrm{g} / \mathrm{kg} & 380 & \text { EPA8080 } \\ 380 & \mu \mathrm{g} / \mathrm{kg} & 380 & \text { EPA8080 } \\ 380 & \mu \mathrm{g} / \mathrm{kg} & 380 & \text { EPA8080 } \\ 380 & \mu \mathrm{g} / \mathrm{kg} & 380 & \text { EPA8080 } \\ 1.3 & \mathrm{ng} / \mathrm{g} & 1.3 & \text { EPA8280 } \\ 0.70 & \mathrm{ng} / \mathrm{g} & 0.70 & \text { EPA8280 } \\ 0.80 & \mathrm{ng} / \mathrm{g} & 0.80 & \text { EPA8280 } \\ 0.60 & \mathrm{ng} / \mathrm{g} & 0.60 & \text { EPA8280 } \\ 0.80 & \mathrm{ng} / \mathrm{g} & 0.80 & \text { EPA8280 } \\ 0.70 & \mathrm{ng} / \mathrm{g} & 0.70 & \text { EPA8280 } \\ 1.900 & \mu \mathrm{g} / \mathrm{kg} & 1.900 & \text { EPA8080 }\end{array}$

\begin{tabular}{|c|c|c|c|c|c|c|}
\hline $\begin{array}{l}\text { Gross alpha } \\
\text { Nonvolatile beta } \\
\text { Radium, cotal alpha-emitting }\end{array}$ & $\mathrm{U}$ & $\mathbf{v}$ & $\begin{array}{l}8.0 \\
19 \pm 17 \\
2.5 \pm 1.4\end{array}$ & $\begin{array}{l}\mathrm{pCi} / \mathrm{g} \\
\mathrm{pCi} / \mathrm{g} \\
\mathrm{pCi} / \mathrm{g}\end{array}$ & $\begin{array}{l}8.0 \\
7.7 \\
0.22\end{array}$ & $\begin{array}{l}\text { EPA900.0 } \\
\text { EPA900.0 } \\
\text { EPA903.0 }\end{array}$ \\
\hline Tritium & & & $2.0 \pm 0.48$ & & & \\
\hline
\end{tabular}

Sample ID: 100386

Sample Matrix: Soil

USC Soil Classification: SW
Sample Moisture: Damp

Percent Solids: 89.50

D. Limit Method

\section{Constituent}

Physical Parameters.

Cation exchange capacity

pH

Specific conductance

Total organic carbon

\section{RQ AQ B Result Unit}

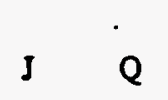

$\begin{array}{llll}6.5 & \mathrm{meq} / 100 \mathrm{~g} & 2.0 & \text { EPA9080 } \\ 7.0 & \mathrm{pH} & 0.010 & \text { EPA9045 } \\ 140 & \mu \mathrm{s} / \mathrm{cm} & 1.0 & \text { EPA9050 } \\ 15,000 & \mathrm{mg} / \mathrm{kg} & 390 & \text { EPA415.1 }\end{array}$


SAMPLE NAME: D431 1306 (Continued)

Sample ID: 100386

Constituent

RQ
B Result Unit

$\begin{array}{llllll}\text { Arsenic } & & 2.4 & \mathrm{mg} / \mathrm{kg} & 1.0 & \text { EPA7060 } \\ \text { Barium } & \mathrm{U} & 21 & \mathrm{mg} / \mathrm{kg} & 21 & \text { EPA6010 } \\ \text { Cadmium } & \mathrm{U} & 0.53 & \mathrm{mg} / \mathrm{kg} & 0.53 & \text { EPA6010 } \\ \text { Chromium } & & 19 & \mathrm{mg} / \mathrm{kg} & 1.1 & \text { EPA6010 } \\ \text { Copper } & & 14 & \mathrm{mg} / \mathrm{kg} & 2.7 & \text { EPA6010 } \\ \text { Lead } & & 7.1 & \mathrm{mg} / \mathrm{kg} & 0.62 & \text { EPA7421 } \\ \text { Manganese } & & 47 & \mathrm{mg} / \mathrm{kg} & 1.6 & \text { EPA6010 } \\ \text { Mercury } & & 0.19 & \mathrm{mg} / \mathrm{kg} & 0.11 & \text { EPA7470 } \\ \text { Nickel } & & 5.9 & \mathrm{mg} / \mathrm{kg} & 4.3 & \text { EPA6010 } \\ \text { Selenium } & & 9.5 & \mathrm{mg} / \mathrm{kg} & 1.0 & \text { EPA7740 } \\ \text { Silver } & \mathrm{U} & 1.1 & \mathrm{mg} / \mathrm{kg} & 1.1 & \text { EPA6010 }\end{array}$

Total Metals

$B / N / A$ Extractables (including $P A H$ and phenols)

$\begin{array}{lllll}\text { Acenaphthene } & \text { J } & \text { O } & \text { L } & 4,300 \\ \text { Acenaphthylene } & \text { J } & \text { O } & \text { L } & 440 \\ \text { Anthracene } & \text { LJ } & \text { O } & \text { L } & 7,700 \\ \text { Anthracene } & \text { J } & \text { O } & \text { L } & 9,400 \\ \text { Benzo(a)anthracene } & \text { UJ } & \text { O } & \text { L } & 18,000 \\ \text { Benzo(a)anthracene } & \text { J } & \text { O } & \text { L } & 16,000 \\ \text { Benzo(b)fluoranthene } & \text { UJ } & \text { O } & \text { L } & 20,000 \\ \text { Benzo(b)fluoranthene } & \text { J } & \text { O } & \text { L } & 20,000 \\ \text { Benzo(k)fluoranthene } & \text { J } & \text { O } & \text { L } & 4,500 \\ \text { Benzoic acid } & \text { UJ } & \text { O } & \text { L } & 1,800 \\ \text { Benzo(g,h,i)perylene } & \text { J } & \text { O } & \text { L } & 4,900 \\ \text { Benzo(a)pyrene } & \text { UJ } & \text { O } & \text { L } & 13,000 \\ \text { Benzo(a)pyrene } & \text { J } & \text { O } & \text { L } & 14,000 \\ \text { Benzyl alcohol } & \text { UJ } & \text { O } & \text { L } & 370 \\ \text { Bis(2-chloroethoxy) methane } & \text { UJ } & \text { O } & \text { L } & 370 \\ \text { Bis(2-chloroethyl) ether } & \text { UJ } & \text { O } & \text { L } & 370 \\ \text { Bis(2-chloroisopropyl) ether } & \text { UJ } & \text { O } & \text { L } & 370 \\ \text { Bis(2-ethylhexyl) phthalate } & \text { UJ } & \text { O } & \text { L } & 370 \\ \text { 4-Bromophenyl phenyl ether } & \text { UJ } & \text { O } & \text { L } & 370 \\ \text { Butylbenzyl phthalate } & \text { J } & \text { O } & \text { L } & 250 \\ \text { Benz-anthracenone isomer } & \text { J } & \text { N } & & 10,000 \\ \text { Benzonapthothiophene } & \text { J } & \text { N } & & 10,000 \\ \text { Benzofluorene isomer } & \text { J } & \text { N } & & 30,000 \\ \text { Benzofluorene isomer } & \text { J } & \text { N } & & 20,000 \\ \text { Benzonapthofuran isomer } & \text { J } & \text { N } & & 9,000 \\ \text { Benzo-pyrene isomer } & \text { J } & \text { N } & & 10,000 \\ \text { Benzophenanthrene isomer } & \text { J } & \text { N- } & & 10,000 \\ \text { Carbazole } & \text { J } & \text { N } & & 3,000 \\ \text { 4-Chloroaniline } & \text { UJ } & \text { O } & \text { L } & 370 \\ \text { 4-Chloro-m-cresol } & \text { UJ } & \text { O } & \text { L } & 370 \\ \text { 2-Chloronaphthalere } & \text { UJ } & \text { O } & \text { L } & 370 \\ \text { 2-Chlorophenol } & \text { UJ } & \text { O } & \text { L } & 370 \\ \text { 4-Chlorophenyl phenyl ether } & \text { UJ } & \text { O } & \text { L } & 370 \\ \text { Chrysene } & \text { LJ } & \text { O } & \text { L } & 10,000 \\ \text { Chrysene } & \text { J } & \text { O } & \text { L } & 13,000 \\ \text { 0-Cresol (2-Methylphenol) } & \text { UJ } & \text { O } & \text { L } & 370 \\ & & & & \end{array}$

$\begin{array}{lll}\mu \mathrm{g} / \mathrm{kg} & 370 & \text { EPA8270 } \\ \mu \mathrm{g} / \mathrm{kg} & 370 & \text { EPA8270 } \\ \mu \mathrm{g} / \mathrm{kg} & 370 & \text { EPA8270 } \\ \mu \mathrm{g} / \mathrm{kg} & 1,800 & \text { EPA8270 } \\ \mu \mathrm{g} / \mathrm{kg} & 370 & \text { EPA8270 } \\ \mu \mathrm{g} / \mathrm{kg} & 1,800 & \text { EPA8270 } \\ \mu \mathrm{g} / \mathrm{kg} & 370 & \text { EPA8270 } \\ \mu \mathrm{g} / \mathrm{kg} & 1,800 & \text { EPA8270 } \\ \mu \mathrm{g} / \mathrm{kg} & 370 & \text { EPA8270 } \\ \mu \mathrm{g} / \mathrm{kg} & 1,800 & \text { EPA8270 } \\ \mu \mathrm{g} / \mathrm{kg} & 370 & \text { EPA8270 } \\ \mu \mathrm{g} / \mathrm{kg} & 370 & \text { EPA8270 } \\ \mu \mathrm{g} / \mathrm{kg} & 1,800 & \text { EPA8270 } \\ \mu \mathrm{g} / \mathrm{kg} & 370 & \text { EPA8270 } \\ \mu \mathrm{g} / \mathrm{kg} & 370 & \text { EPA8270 } \\ \mu \mathrm{g} / \mathrm{kg} & 370 & \text { EPA8270 } \\ \mu \mathrm{g} / \mathrm{kg} & 370 & \text { EPA8270 } \\ \mu \mathrm{g} / \mathrm{kg} & 370 & \text { EPA8270 } \\ \mu \mathrm{g} / \mathrm{kg} & 370 & \text { EPA8270 } \\ \mu \mathrm{g} / \mathrm{kg} & 370 & \text { EPA8270 } \\ \mu \mathrm{g} / \mathrm{kg} & & \text { EPA8270 } \\ \mu \mathrm{g} / \mathrm{kg} & & \text { EPA8270 } \\ \mu \mathrm{g} / \mathrm{kg} & & \text { EPA8270 } \\ \mu \mathrm{g} / \mathrm{kg} & & \text { EPA8270 } \\ \mu \mathrm{g} / \mathrm{kg} & & \text { EPA8270 } \\ \mu \mathrm{g} / \mathrm{kg} & . & \text { EPA8270 } \\ \mu \mathrm{g} / \mathrm{kg} & & \text { EPA8270 } \\ \mu \mathrm{g} / \mathrm{kg} & & \text { EPA8270 } \\ \mu \mathrm{g} / \mathrm{kg} & 370 & \text { EPA8270 } \\ \mu \mathrm{g} / \mathrm{kg} & 370 & \text { EPA8270 } \\ \mu \mathrm{g} / \mathrm{kg} & 370 & \text { EPA8270 } \\ \mu \mathrm{g} / \mathrm{kg} & 370 & \text { EPA8270 } \\ \mu \mathrm{g} / \mathrm{kg} & 370 & \text { EPA8270 } \\ \mu \mathrm{g} / \mathrm{kg} & 370 & \text { EPA8270 } \\ \mu \mathrm{g} / \mathrm{kg} & 1,800 & \text { EPA8270 } \\ \mu \mathrm{g} / \mathrm{kg} & 370 & \text { EPA8270 }\end{array}$


SAMPLE NAME: D431 1306 (Continued)

Sample ID: 100386

$\begin{array}{lllllll}\text { Constituent } & \text { RQ AQ } & \text { B } & \text { Result } & \text { Unit } & \text { D. Limit Method }\end{array}$

$B / N / A$ Extractables (including $P A H$ and phenols)

\begin{tabular}{|c|c|c|c|c|c|c|c|}
\hline p-Cresol (4-Mechylphenol) & UJ & 0 & L & 370 & $\mu g / k g$ & 370 & EPA8270 \\
\hline Dibenz(a,h)anthracene & $\mathbf{J}$ & 0 & L & 1,300 & $\mu \mathrm{g} / \mathrm{kg}$ & 370 & EPA8270 \\
\hline Dibenzofuran & J & 0 & $\mathbf{L}$ & 3,100 & $\mu \mathrm{g} / \mathrm{kg}$ & 370 & EPA8270 \\
\hline Di-n-butyl phthalate & UJ & 0 & L & 370 & $\mu g / k g$ & 370 & EPA8270 \\
\hline Dibenzothiophene & $\mathrm{J}$ & $\mathbf{N}$ & & 2,000 & $\mu \mathrm{g} / \mathrm{kg}$ & & EPA8270 \\
\hline 1,2-Dichlorobenzene.. & UJ & 0 & L & 370 & $\mu g / \mathbf{k g}$ & 370 & EPA8270 \\
\hline 1,3-Dichlorobenzene & UJ & 0 & L & 370 & $\mu \mathrm{g} / \mathrm{kg}$ & 370 & EPA8270 \\
\hline 1.4-Dichlorobenzene & UJ & 0 & L & 370 & $\mu g / k g$ & 370 & EPA8270 \\
\hline 3,3'-Dichlorobenzidine & UJ & 0 & I & 740 & $\mu \mathrm{g} / \mathrm{kg}$ & 740 & EPA8270 \\
\hline 2,4-Dichlorophenol & UJ & 0 & L & 370 & $\mu \mathrm{g} / \mathrm{kg}$ & 370 & EPA8270 \\
\hline Diethyl phthalate & UJ & 0 & L & 370 & $\mu g / k g$ & 370 & EPA8270 \\
\hline 2,4-Dimethyl phenol & UJ & 0 & L & 370 & $\mu g / k g$ & 370 & EPA8270 \\
\hline Dimethyl phthalate & UJ & 0 & L & 370 & $\mu g / \mathrm{kg}$ & 370 & EPA8270 \\
\hline 2,4-Dinitrophenol & UJ & 0 & L & 1,800 & $\mu \mathrm{g} / \mathrm{kg}$ & 1,800 & EPA8270 \\
\hline 2,4-Dinitrotoluene & UJ & 0 & L & 370 & $\mu \mathrm{g} / \mathrm{kg}$ & 370 & EPA8270 \\
\hline 2,6-Dinitrotoluene & UJ & 0 & L & 370 & $\mu g / \mathrm{kg}$ & 370 & EPA8270 \\
\hline Di-n-octyl phthalate & UJ & 0 & L & 370 & $\mu \mathrm{g} / \mathrm{kg}$ & 370 & EPA8270 \\
\hline Fluoranthene & $\mathrm{LJ}$ & 0 & L & 23,000 & $\mu \mathrm{g} / \mathrm{kg}$ & 370 & EPA8270 \\
\hline Fluoranthene & LJ & 0 & L & 31,000 & $\mu \mathrm{g} / \mathrm{kg}$ & 1,800 & EPA8270 \\
\hline Fluoranthene & $\mathrm{J}$ & 0 & $\mathrm{~L}$ & 37,000 & $\mu \mathrm{g} / \mathrm{kg}$ & 3,700 & EPA8270 \\
\hline Fluorene & $\mathbf{J}$ & .0 & L & 4,900 & $\mu g / k g$ & 370 & EPA8270 \\
\hline Hexachlorobenzene & UJ & 0 & L & 370 & $\mu \mathrm{g} / \mathrm{kg}$ & 370 & EPA8270 \\
\hline Hexachlorobutadiene & UJ & 0 & $\mathbf{L}$ & 370 & $\mu \mathrm{g} / \mathrm{kg}$ & 370 & EPA8270 \\
\hline Hexachlorocyclopentadiene & UJ & 0 & $L$ & 370 & $\mu \mathrm{g} / \mathrm{kg}$ & 370 & EPA8270 \\
\hline Hexachloroethane & UJ & 0 & L & 370 & $\mu \mathrm{g} / \mathrm{kg}$ & 370 & EPA8270 \\
\hline Indeno(1,2,3-c,d)pyrene & $\mathbf{J}$ & 0 & L & 5,800 & $\mu g / \mathrm{kg}$ & 370 & EPA8270 \\
\hline Isophorone & UJ & 0 & L & 370 & $\mu \mathrm{g} / \mathrm{kg}$ & 370 & EPA8270 \\
\hline Methylanthracene isomer & $\mathbf{J}$ & $\mathbf{N}$ & & 2,000 & $\mu g / \mathrm{kg}$ & & EPA8270 \\
\hline Methyl-benz-anthracene isomer & $\mathbf{J}$ & $\mathbf{N}$ & & 8,000 & $\mu \mathrm{g} / \mathrm{kg}$ & & EPA8270 \\
\hline Methylphenanthrene isomer & J & $\mathbf{N}$ & & 2,000 & $\mu \mathrm{g} / \mathrm{kg}$ & & EPA8270 \\
\hline 2-Methyl-4,6-dinitrophenol & UJ & 0 & $L$ & 1.800 & $\mu \mathrm{g} / \mathrm{kg}$ & 1.800 & EPA8270 \\
\hline 2-Methylnaphthalene & $\mathbf{J}$ & 0 & L & 1,400 & $\mu \mathrm{g} / \mathrm{kg}$ & 370 & EPA8270 \\
\hline Naphthalene & J & 0 & L & 3,800 & $\mu \mathrm{g} / \mathrm{kg}$ & 370 & EPA8270 \\
\hline o-Nitroaniline & UJ & 0 & L & 1,800 & $\mu \mathrm{g} / \mathrm{kg}$ & 1,800 & EPA8270 \\
\hline m-Nitroaniline & UJ & 0 & L & 1,800 & $\mu \mathrm{g} / \mathrm{kg}$ & 1,800 & EPA8270 \\
\hline p-Nitroaniline & UJ & 0 & L & 1,800 & $\mu \mathrm{g} / \mathrm{kg}$ & 1,800 & EPA8270 \\
\hline Nitrobenzene & UJ & 0 & L & 370 & $\mu g / \mathrm{kg}$ & 370 & EPA8270 \\
\hline 2-Nitrophenol & UJ & 0 & L & 370 & $\mu \mathrm{g} / \mathrm{kg}$ & 370 & EPA8270 \\
\hline 4Nitrophenol & UJ & 0 & L & 1,800 & $\mu g / k g$ & 1,800 & EPA8270 \\
\hline N-Nitrosodiphenylamine & UJ & 0 & L & 370 & $\mu g / \mathrm{kg}$ & 370 & EPA8270 \\
\hline N-Nitrosodipropylamine & UJ & $\mathrm{CO}$ & L & 370 & $\mu g / k g$ & 370 & EPA8270 \\
\hline Pentachlorophenol & UJ & 0 & L & 1.800 & $\mu \mathrm{g} / \mathrm{kg}$ & 1,800 & EPA8270 \\
\hline Phenanthrene & LJ & 0 & L & 24,000 & $\mu g / \mathrm{kg}$ & 370 & EPA8270 \\
\hline Phenanthrene & J & 0 & L & 28,000 & $\mu g / k g$ & 1,800 & EPA8270 \\
\hline Phenol & UJ & 0 & L & 370 & $\mu g / \mathrm{kg}$ & 370 & EPA8270 \\
\hline Phenyl-napthalene isomer & J & $\mathbf{N}$ & & 1,000 & $\mu \mathrm{g} / \mathrm{kg}$ & & EPA8270 \\
\hline Pyrene & L & 0 & L & 24,000 & $\mu g / \mathrm{kg}$ & 370 & EPA8270 \\
\hline Pyrene & $\mathbf{J}$ & 0 & L & 25,000 & $\mu g / k g$ & 1,800 & EPA8270 \\
\hline 1,2,4-Trichlorobenzene & UJ & $\mathrm{CO}$ & L & 370 & $\mu \mathrm{g} / \mathrm{kg}$ & 370 & EPA8270 \\
\hline 2,4,5-Trichlorophenol & UJ & 0 & L & 1,800 & $\mu \mathrm{g} / \mathrm{kg}$ & 1,800 & EPA8270 \\
\hline
\end{tabular}


SAMPLE NAME: D431 1306 (Continued)

Sample ID: 100386

Constituent

RQ AQ B Result Unit

D. Limit Method

$B / N / A$ Extractables (including $P A H$ and phenols)

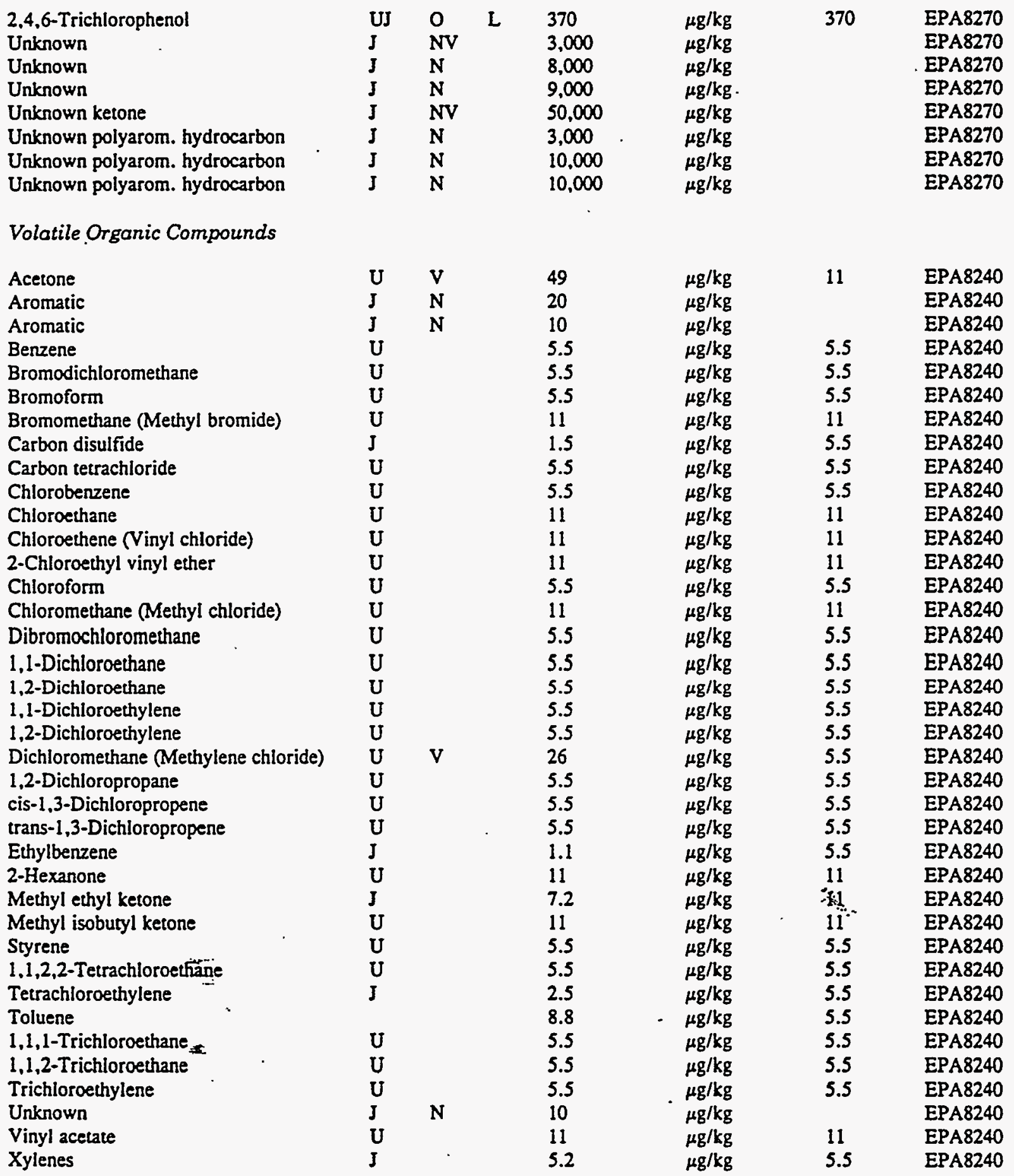


SAMPLE NAME: D431 1306 (Continued)

Sample D: 100386

Constituent

RQ

Pesticides/PCBs and Dioxins/Furans

Aldrin

alpha-Benzene hexachloride

beta-Benzene hexachloride

delta-Benzene hexachloride

alpha-Chlordane

gamma-Chlordane

P.p'-DDD

$p, p^{\prime}-D D E$

p.p'-DDT

Dieldrin

Endosulfan I

Endosulfan $\Pi$

Endosulfan sulfate

Endrin

Endrin ketone

Heptachlor

Heptachlor epoxide

Heptachlorodibenzo-p-dioxin isomers

Heptachlorodibenzo-p-furan isomers

Hexachlorodibenzo-p-dioxin isomers

Hexachlorodibenzo-p-furan isomers

Lindane

Methoxychlor

Octachlorodibenzo-p-dioxin

Octachlorodibenzo-p-furan

PCB 1016

PCB 1221

PCB 1232

PCB 1242

PCB 1248

PCB 1254

PCB 1260

Pentachlorodibenzo-p-dioxin isomers

Pentachlorodibenzo-p-furan isomers

2,3,7,8-TCDD

2,3,7,8-TCDF

Tetrachlorodibenzo-p-dioxin isomers

Tetrachlorodibenzo-p-furan isomers

Toxaphene

$\mathrm{U}$

U

$\mathrm{U}$

U

U

U

U

U

U

U

U

$\mathrm{U}$

U

$\mathrm{U}$

$\mathrm{U}$

U

U

U

U

U

$\mathrm{U}$

Radionuclide Indicators and Radionuclides

Gross alpha $\quad U$

Nonvolatile beta

Radium, total alpha-emitting

Tritium
Q AQ B Result

Unit

D. Limit Method
19
19
19
19
19
19
37
37
37
37
19
37
37
37

220

19

19

0.30

0.20

0.30

0.10

19

190

8.0

0.10

370

750

370

370

370

370

370

0.30

0.20

0.20

0.20

0.20

0.20

1,900
,

$\mu \mathrm{g} / \mathrm{kg}$

$\mu g / \mathrm{kg}$

$\mu \mathrm{g} / \mathrm{kg}$

$\mu \mathrm{g} / \mathrm{kg}$

$\mu \mathrm{g} / \mathrm{kg}$

$\mu \mathrm{g} / \mathrm{kg}$

$\mu \mathrm{g} / \mathrm{kg}$

$\mu \mathrm{g} / \mathrm{kg}$

$\mu \mathrm{g} / \mathrm{kg}$

- $\mu \mathrm{g} / \mathrm{kg}$

$\mu \mathrm{g} / \mathrm{kg}$

$\mu \mathrm{g} / \mathrm{kg}$

$\mu \mathrm{g} / \mathrm{kg}$

$\mu \mathrm{g} / \mathrm{kg}$

$\mu \mathrm{g} / \mathrm{kg}$

$\mu \mathrm{g} / \mathrm{kg}$

$\mu \mathrm{g} / \mathrm{kg}$

ng/g

$\mathrm{ng} / \mathrm{g}$

$\mathrm{ng} / \mathrm{g}$

$\mathrm{ng} / \mathrm{g}$

$\mu \mathrm{g} / \mathrm{kg}$

$\mu \mathrm{g} / \mathrm{kg}$

$\mathrm{ng} / \mathrm{g}$

$\mathrm{ng} / \mathrm{g}$

$\mu \mathrm{g} / \mathrm{kg}$

$\mu \mathrm{g} / \mathrm{kg}$

$\mu \mathrm{g} / \mathrm{kg}$

$\mu \mathrm{g} / \mathrm{kg}$

$\mu \mathrm{g} / \mathrm{kg}$

$\mu \mathrm{g} / \mathrm{kg}$

$\mu \mathrm{g} / \mathrm{kg}$

$\mathrm{ng} / \mathrm{g}$

$\mathrm{ng} / \mathrm{g}$

$\mathrm{ng} / \mathrm{g}$

$\mathrm{ng} / \mathrm{g}$

$\mathrm{ng} / \mathrm{g}$

$\mathrm{ng} / \mathrm{g}$

$\mu \mathrm{g} / \mathrm{kg}$
19

19

19

19

19

37

37

37

37

19

37

37

37

37

19

19

0.30

0.20

0.30

0.10

19

190

0.10

0.10

370

750

370

370

370

370

370

0.30

0.20

0.20

0.20

0.20

0.20

1,900

7.6

7.4

0.38

1.2
EPA8080

EPA8080

EPA8080

EPA8080

EPA8080

EPA8080

EPA8080

EPA8080

EPA8080

EPA8080

EPA8080

EPA8080

EPA8080

EPA8080

EPA8080

EPA8080

EPA8080

EPA8280

EPA8280

EPA8280

EPA8280

EPA8080

EPA8080

EPA8280

EPA8280

EPA8080

EPA8080

EPA8080

EPA8080

EPA8080

EPA8080

EPA8080

EPA8280

EPA8280

EPA8280

EPA8280

EPA8280

EPA8280

EPA8080

$\begin{array}{ll}7.6 & \mathrm{pCi} / \mathrm{g} \\ 7.7 \pm 15 & \mathrm{pCi} / \mathrm{g} \\ 0.84 \pm 1.1 & \mathrm{pCi} / \mathrm{g} \\ 1.8 \pm 0.43 & \mathrm{pCi} / \mathrm{g}\end{array}$

EPA900.0

EPA900.0

EPA903.0

EPA906.0 
SAMPLE NAME: D431'1306D

Sample Type: Split

Constituent

Physical Parameters

Cation exchange capacity

$\mathrm{pH}$

Specific conductance

Total organic carbon

Total Metals

Arsenic

Barium

Cadmium

Chromium

Copper

Lead

Manganese

Mercury

Nickel

Selenium

Silver
Sample ID: 100387

Associated Sample: 100386 .

\section{$B / N / A$ Extractables (including $P A H$ and phenols)}

\begin{tabular}{|c|c|c|c|c|c|c|c|}
\hline Acenaphthene & J & 0 & L & 3,400 & $\mu \mathrm{g} / \mathrm{kg}$ & 370 & EPA8270 \\
\hline Acenaphthylene & $\mathrm{J}$ & 0 & L & 370 & $\mu \mathrm{g} / \mathrm{kg}$ & 370 & EPA8270 \\
\hline Anthracene & L & 0 & L & 6,100 & $\mu \mathrm{g} / \mathrm{kg}$ & 370 & EPA8270 \\
\hline Anthracene & $\mathbf{J}$ & 0 & L & 6,500 & $\mu g / \mathrm{kg}$ & 1,500 & EPA8270 \\
\hline Benzo(a)anthracene & $\mathrm{U}$ & 0 & L & 14,000 & $\mu \mathrm{g} / \mathrm{kg}$ & 370 & EPA8270 \\
\hline Benzo(a)anthracene & $\mathbf{J}$ & 0 & L & 11,000 & $\mu \mathrm{g} / \mathrm{kg}$ & 1,500 & EPA8270 \\
\hline Benzo(b)fluoranthene & $\mathrm{LJ}$ & 0 & $\bar{L}$ & 15,000 & $\mu \mathrm{g} / \mathrm{kg}$ & 370 & EPA8270 \\
\hline Benzo(b)fluoranthene & $\mathbf{J}$ & 0 & $\bar{L}$ & 13,000 & $\mu \mathrm{g} / \mathrm{kg}$ & 1,500 & EPA8270 \\
\hline Benzo(k)fluoranthene & $\mathbf{J}$ & 0 & L & 4,900 & $\mu \mathrm{g} / \mathrm{kg}$ & 370 & EPA8270 \\
\hline Benzoic acid & UJ & 0 & L & 1,900 & $\mu \mathrm{g} / \mathrm{kg}$ & 1,900 & EPA8270 \\
\hline Benzo $(g, h, i)$ perylene & J & 0 & L & 3,900 & $\mu \mathrm{g} / \mathrm{kg}$ & 370 & EPA8270 \\
\hline Benzo(a)pyrene & LJ & 0 & L & 10,000 & $\mu \mathrm{g} / \mathrm{kg}$ & 370 & EPA8270 \\
\hline Benzo(a)pyrene & $\mathbf{J}$ & 0 & L & 9,200 & $\mu \mathrm{g} / \mathrm{kg}$ & 1,500 & EPA8270 \\
\hline Benzyl alcohol & UJ & 0 & L & 370 & $\mu \mathrm{g} / \mathrm{kg}$ & 370 & EPA8270 \\
\hline Bis(2-chloroethoxy) methane & UJ & 0 & L & 370 & $\mu \mathrm{g} / \mathrm{kg}$ & $370^{\circ}$ & EPA8270 \\
\hline Bis(2-chloroethyl) ether & UJ & 0 & L & 370 & $\mu \mathrm{g} / \mathrm{kg}$ & 370 & EPA8270 \\
\hline Bis(2-chloroisopropyl) ether & UJ & 0 & L & 370 & $\mu \mathrm{g} / \mathrm{kg}$ & 370 & EPA8270 \\
\hline Bis(2-ethylhexyl) phthăläte & J & 0 & $\bar{L}$ & 120 & $\mu g / \mathrm{kg}$ & 370 & EPA 8270 \\
\hline 4-Bromophenyl phenyl ether & UI & 0 & L & 370 & $\mu \mathrm{g} / \mathrm{kg}$ & 370 & EPA8270 \\
\hline Butylbenzyl phthalate & UJ & 0 & L & 370 & $\mu \mathrm{g} / \mathrm{kg}$ & 370 & EPA8270 \\
\hline Benzonapthothiophene & J & $\mathbf{N}$ & & 300 & $\mu \mathrm{g} / \mathrm{kg}$ & & EPA 8270 \\
\hline Benzofluorene isomer & J & $\mathbf{N}$ & & 600 & $\mu \mathrm{g} / \mathrm{kg}$ & & EPA8270 \\
\hline Benzofluorene isomer & J & $\mathbf{N}$ & & 400 & $\mu \mathrm{g} / \mathrm{kg}$ & & EPA8270 \\
\hline Benzo-pyrene isomer & J & $\mathbf{N}$ & & 9,000 & $\mu \mathrm{g} / \mathrm{kg}$ & & EPA8270 \\
\hline Benzophenanthrene isomer & J & $\mathbf{N}$ & & 300 & $\mu \mathrm{g} / \mathrm{kg}$ & & EPA8270 \\
\hline Benzophenanthrene isomer & J & $\mathbf{N}$ & & 300 & $\mu \mathrm{g} / \mathrm{kg}$ & & EPA8270 \\
\hline Carbazole & J & $\mathrm{N}$ & & 3,000 & $\mu \mathrm{g} / \mathrm{kg}$ & & EPA8270 \\
\hline
\end{tabular}

$\begin{array}{llll}4.5 & \mathrm{mg} / \mathrm{kg} & 1.1 & \text { EPA7060 } \\ 22 & \mathrm{mg} / \mathrm{kg} & 22 & \text { EPA6010 } \\ 0.55 & \mathrm{mg} / \mathrm{kg} & 0.55 & \text { EPA6010 } \\ 17 & \mathrm{mg} / \mathrm{kg} & 1.1 & \text { EPA6010 } \\ 17 & \mathrm{mg} / \mathrm{kg} & 2.7 & \text { EPA6010 } \\ 10 & \mathrm{mg} / \mathrm{kg} & 0.64 & \text { EPA7421 } \\ 57 & \mathrm{mg} / \mathrm{kg} & 1.6 & \text { EPA6010 } \\ 0.36 & \mathrm{mg} / \mathrm{kg} & 0.11 & \text { EPA7470 } \\ 6.3 & \mathrm{mg} / \mathrm{kg} & 4.4 & \text { EPA6010 } \\ 26 & \mathrm{mg} / \mathrm{kg} & 5.3 & \text { EPA7740 } \\ 1.1 & \mathrm{mg} / \mathrm{kg} & 1.1 & \text { EPA6010 }\end{array}$

$\begin{array}{llll}6.7 & \mathrm{meq} / 100 \mathrm{~g} & 2.0 & \text { EPA9080 } \\ 7.3 & \mathrm{pH} & 0.010 & \text { EPA9045 } \\ 160 & \mu \mathrm{S} / \mathrm{cm} & 1.0 & \text { EPA9050 } \\ 10.000 & \mathrm{mg} / \mathrm{kg} & 230 & \text { EPA415.1 }\end{array}$

U

17

6.3

1.1
Unit.

D. Limit Method 
SAMPLE NAME: D4331 1306D (Continued)

Constituent

RQ

AQ, $\mathbf{B}$

$B / N / A$ Extractables (including $P A H$ and phenols)
Sample ID: 100387

Unit

D. Limit Method

\begin{tabular}{|c|c|c|c|c|c|c|c|}
\hline 4-Chloroaniline & UJ & 0 & L & 370 & $\mu g / \mathrm{kg}$ & 370 & EPA8270 \\
\hline 4-Chloro-m-cresol & UJ & 0 & L & 370 & $\mu \mathrm{g} / \mathrm{kg}$ & 370 & EPA8270 \\
\hline 2-Chloronaphthalene & UI & 0 & L & 370 & $\mu \mathrm{g} / \mathrm{kg}$ & 370 & EPA8270 \\
\hline 2-Chlorophenol & UJ & 0 & L & 370 & $\mu \mathrm{g} / \mathrm{kg}$ & 370 & EPA8270 \\
\hline 4-Chlorophenyl phenyl ether & UJ & 0 & L & 370 & $\mu \mathrm{g} / \mathrm{kg}$ & 370 & EPA8270 \\
\hline Chrysene & LI & 0 & $L$ & 9.300 & $\mu g / \mathrm{kg}$ & 370 & EPA8270 \\
\hline Chrysene & J & 0 & L & 8,600 & $\mu \mathrm{g} / \mathrm{kg}$ & 1,500 & EPA8270 \\
\hline o-Cresol (2-Methylphenol) & UJ & $\mathbf{0}$ & L & 370 & $\mu \mathrm{g} / \mathrm{kg}$ & 370 & EPA8270 \\
\hline p-Cresol (4-Methylphenol) & UJ & 0 & $\mathbf{L}$ & 370 & $\mu g / \mathrm{kg}$ & 370 & EPA8270 \\
\hline Dibenz(a,h)anthracene & I & 0 & $\mathrm{~L}$ & 1,100 & $\mu \mathrm{g} / \mathrm{kg}$ & 370 & EPA8270 \\
\hline Dibenzofuran & J & 0 & L & 2,500 & $\mu g / k g$ & 370 & EPA8270 \\
\hline Di-n-butyl phthalate & UJ & 0 & $\mathbf{L}$ & 370 & $\mu \mathrm{g} / \mathrm{kg}$ & 370 & EPA8270 \\
\hline Dibenzothiophene & $\mathrm{s}$ & $\mathbf{N}$ & & 2,000 & $\mu g / k g$ & & EPA8270 \\
\hline 1,2-Dichlorobenzene & UJ & 0 & L & 370 & $\mu \mathrm{g} / \mathrm{kg}$ & 370 & EPA8270 \\
\hline 1,3-Dichlorobenzene & UJ & 0 & L & 370 & $\mu \mathrm{g} / \mathrm{kg}$ & 370 & EPA8270 \\
\hline 1,4-Dichlorobenzene & UJ & 0 & L & 370 & $\mu \mathrm{g} / \mathrm{kg}$ & 370 & EPA8270 \\
\hline 3,3'-Dichlorobenzidine & UJ & 0 & $\mathrm{~L}$ & 750 & $\mu \mathrm{g} / \mathrm{kg}$ & 750 & EPA8270 \\
\hline 2,4-Dichlorophenol & UJ & 0 & L & 370 & $\mu \mathrm{g} / \mathrm{kg}$ & 370 & EPA8270 \\
\hline Diecthyl phthalate & UJ & 0 & L & 370 & $\mu \mathrm{g} / \mathrm{kg}$ & 370 & EPA8270 \\
\hline 2,4-Dimethyl phenol & UJ & 0 & $\mathbf{L}$ & 370 & $\mu g / k g$ & 370 & EPA8270 \\
\hline Dimethyl phthalate & UJ & 0 & L & 370 & $\mu \mathrm{g} / \mathrm{kg}$ & 370 & EPA8270 \\
\hline 2,4-Dinitrophenol & UJ & 0 & L & 1,900 & $\mu \mathrm{g} / \mathrm{kg}$ & 1,900 & EPA8270 \\
\hline 2,4-Dinitrotoluene & UJ & 0 & $\mathbf{L}$ & 370 & $\mu g / k g$ & 370 & EPA8270 \\
\hline 2,6-Dinitrotoluene & UJ & 0 & $L$ & 370 & $\mu \mathrm{g} / \mathrm{kg}$ & 370 & EPA8270 \\
\hline Di-n-octy! phthalate & UJ & 0 & L & 370 & $\mu \mathrm{g} / \mathrm{kg}$ & 370 & EPA8270 \\
\hline Fluoranthene & LJ & 0 & $L$ & 19,000 & $\mu \mathrm{g} / \mathrm{kg}$ & 370 & EPA8270 \\
\hline Fluoranthene & $\mathbf{J}$ & 0 & L & 23.000 & $\mu \mathrm{g} / \mathrm{kg}$ & 1,500 & EPA8270 \\
\hline Fluorene & $\mathbf{J}$ & 0 & $\mathrm{~L}$ & 4.100 & $\mu \mathrm{g} / \mathrm{kg}$ & 370 & EPA8270 \\
\hline Hexachlorobenzene & UJ & 0 & L & 370 & $\mu \mathrm{g} / \mathrm{kg}$ & 370 & EPA8270 \\
\hline Hexachlorobutadiene & UJ & 0 & L & 370 & $\mu \mathrm{g} / \mathrm{kg}$ & 370 & EPA8270 \\
\hline Hexachlorocyclopentadiene & UJ & 0 & L & 370 & $\mu \mathrm{g} / \mathrm{kg}$ & 370 & EPA8270 \\
\hline Hexachloroethane & UJ & 0 & $L$ & 370 & $\mu \mathrm{g} / \mathrm{kg}$ & 370 & EPA8270 \\
\hline Indeno(1,2,3-c,d)pyrene & J & 0 & L & 4,600 & $\mu \mathrm{g} / \mathrm{kg}$ & 370 & EPA8270 \\
\hline Isophorone & UJ & 0 & L & 370 & $\mu g / k g$ & 370 & EPA8270 \\
\hline Methylanthracene isomer & J & $\mathbf{N}$ & & 2.000 & $\mu g / k g$ & & EPA8270 \\
\hline Methyl-benz-anthracene isomer & J & $\mathbf{N}$ & & 300 & $\mu \mathrm{g} / \mathrm{kg}$ & & EPA8270 \\
\hline Methylphenanthrene isomer & $\mathrm{J}$ & $\mathbf{N}$ & & 2,000 & $\mu g / \mathrm{kg}$ & , & EPA8270 \\
\hline Methylpyrene isomer & $\mathbf{J}$ & $\mathbf{N}$ & & 200 & $\mu \mathrm{g} / \mathrm{kg}$ & & EPA8270 \\
\hline 2-Methyl-4,6-dinitrophenol & UJ & 0 & L & 1,900 & $\mu g / \mathrm{kg}$ & 1,900 & EPA8270 \\
\hline 2-Methy!naphthalene & $\mathbf{J}$ & 0 & L & 1,000 & $\mu \mathrm{g} / \mathrm{kg}$ & 370 & EPA8270 \\
\hline Naphthalene & $\mathbf{J}$ & 0 & L & 2,800 & $\mu \mathrm{g} / \mathrm{kg}$ & 370 & EPA8270 \\
\hline o-Nitroaniline & US & 0 & L & 1,900 & $\mu g / k g$ & 1,900 & EPA8270 \\
\hline m-Nitroaniline & UJ & 0 & L & 1,900 & $\mu \mathrm{g} / \mathrm{kg}$ & 1,900 & EPA8270 \\
\hline p-Nitroaniline & UJ & 0 & L & 1,900 & $\mu g / k g$ & 1,900 & EPA8270 \\
\hline Nitrobenzene & UJ & 0 & L & 370 & $\mu \mathrm{g} / \mathrm{kg}$ & 370 & EPA8270 \\
\hline 2-Nitrophenol & UJ & 0 & L & 370 & $\mu \mathrm{g} / \mathrm{kg}$ & 370 & EPA8270 \\
\hline 4-Nitrophenol & UJ & 0 & L & 1,900 & $\mu g / k g$ & 1,900 & EPA8270 \\
\hline N-Nitrosodiphenylamine & UJ & 0 & L & 370 & $\mu g / k g$ & 370 & EPA8270 \\
\hline N-Nitrosodipropylamine & UJ & $\mathrm{CO}$ & L & 370 & $\mu \mathrm{g} / \mathrm{kg}$ & 370 & EPA8270 \\
\hline Pentachlorophenol & UJ & 0 & L & 1,900 & $\mu g / k g$ & 1,900 & EPA8270 \\
\hline
\end{tabular}


SAMPLE NAME: D431 1306D (Continued)

Sample D: 100387
Constituent
RQ AQ B Result
Unit
D. Limit Method

$B / N / A$ Extractables (including PAH and phenols)

\begin{tabular}{|c|c|c|c|c|c|c|}
\hline Phenanthrene & LJ & 0 & 19,000 & $\mu g / \mathrm{kg}$ & 370 & EPA8270 \\
\hline Phenanthrene & J & 0 & 20,000 & $\mu g / \mathrm{kg}$ & 1,500 & EPA8270 \\
\hline Phenol & UJ & 0 & 370 & $\mu \mathrm{g} / \mathrm{kg}$ & 370 & EPA8270 \\
\hline Phenyl-napthalene isomer & $\mathrm{J}$ & $\mathbf{N}$ & 200 & $\mu g / k g$ & & EPA8270 \\
\hline Pyrene & $\mathrm{LJ}$ & 0 & 20,000 & $\mu g / \mathrm{kg}$ & 370 & EPA8270 \\
\hline Pyrene & J & o & 17,000 & $\mu \mathrm{g} / \mathrm{kg}$ & 1,500 & EPA8270 \\
\hline 1,2,4-Trichlorobenzene & UJ & CO & 370 & $\mu \mathrm{g} / \mathrm{kg}$ & 370 & EPA8270 \\
\hline 2.4,5-Trichlorophenol & UJ & 0 & 1,900 & $\mu \mathrm{g} / \mathrm{kg}$ & 1.900 & EPA8270 \\
\hline 2,4.6-Trichlorophenol & UJ & 0 & 370 & $\mu \mathrm{g} / \mathrm{kg}$ & 370 & EPA8270 \\
\hline Unknown & $\mathbf{J}$ & NV & 4,000 & $\mu \mathrm{g} / \mathrm{kg}$ & & EPA8270 \\
\hline Unknown & $\mathbf{J}$ & $\mathbf{N}$ & 300 & $\mu \mathrm{g} / \mathrm{kg}$ & & EPA8270 \\
\hline Unknown & J & $\mathbf{N}$ & 200 & $\mu \mathrm{g} / \mathrm{kg}$ & & EPA8270 \\
\hline Unknown & $\mathrm{J}$ & $\mathbf{N}$ & 200 & $\mu \mathrm{g} / \mathrm{kg}$ & & EPA8270 \\
\hline Unknown ketone & $\mathrm{J}$ & NV & 50,000 & $\mu \mathrm{g} / \mathrm{kg}$ & & EPA8270 \\
\hline Unknown polyarom. hydrocarbon & $\mathrm{J}$ & $\mathbf{N}$ & 600 & $\mu \mathrm{g} / \mathrm{kg}$ & & EPA8270 \\
\hline Unknown polyarom. hydrocarbon & J & N & 300 & $\mu \mathrm{g} / \mathrm{kg}$ & & EPA8270 \\
\hline \multicolumn{7}{|l|}{ Volatile Organic Compounds } \\
\hline Acetone & & $\mathrm{v}$ & 100 & $\mu \mathrm{g} / \mathrm{kg}$ & 11 & EPA8240 \\
\hline Aromatic & J & $\mathrm{N}$ & 30 & $\mu \mathrm{g} / \mathrm{kg}$ & & EPA8240 \\
\hline Aromatic & $\mathrm{J}$ & $\mathrm{N}$ & 10 & $\mu \mathrm{g} / \mathrm{kg}$ & & EPA8240 \\
\hline Benzene & $\mathrm{U}$ & & 5.5 & $\mu \mathrm{g} / \mathrm{kg}$ & 5.5 & EPA8240 \\
\hline Bromodichloromethane & $\mathrm{U}$ & & 5.5 & $\mu \mathrm{g} / \mathrm{kg}$ & 5.5 & EPA8240 \\
\hline Bromoform & $U$ & & 5.5 & $\mu \mathrm{g} / \mathrm{kg}$ & 5.5 & EPA8240 \\
\hline Bromomethane (Methyl bromide) & $\mathrm{U}$ & & 11 & $\mu \mathrm{g} / \mathrm{kg}$ & 11 & EPA8240 \\
\hline Carbon disulfide & J & & 1.2 & $\mu g / \mathrm{kg}$ & 5.5 & EPA8240 \\
\hline Carbon tetrachloride & $\mathrm{U}$ & & 5.5 & $\mu g / \mathrm{kg}$ & 5.5 & EPA8240 \\
\hline Chlorobenzene & $\mathrm{U}$ & & 5.5 & $\mu g / \mathrm{kg}$ & 5.5 & EPA 8240 \\
\hline Chloroethane & $\mathrm{U}$ & & 11 & $\mu \mathrm{g} / \mathrm{kg}$ & 11 & EPA8240 \\
\hline Chloroethene (Vinyl chloride) & $\mathrm{U}$ & & 11 & $\mu \mathrm{g} / \mathrm{kg}$ & 11 & EPA8240 \\
\hline 2-Chloroethyl vinyl ether & $\mathrm{U}$ & & 11 & $\mu \mathrm{g} / \mathrm{kg}$ & 11 & EPA8240 \\
\hline Chloroform & $\mathrm{U}$ & & 5.5 & $\mu g / \mathrm{kg}$ & 5.5 & EPA8240 \\
\hline Chloromethane (Methyl chloride) & $\mathrm{U}$ & & 11 & $\mu \mathrm{g} / \mathrm{kg}$ & 11 & EPA8240 \\
\hline Dibromochloromethane & $\mathbf{U}$ & & 5.5 & $\mu g / \mathrm{kg}$ & 5.5 & EPA8240 \\
\hline 1,1-Dichloroethane & $\mathbf{U}$ & & 5.5 & $\mu \mathrm{g} / \mathrm{kg}$ & 5.5 & EPA8240 \\
\hline 1,2-Dichloroethane & $\mathbf{U}$ & & 5.5 & $\mu g / \mathrm{kg}$ & 5.5 & EPA8240 \\
\hline 1,1-Dichloroethylene & $\mathbf{U}$ & & 5.5 & $\mu g / \mathrm{kg}$ & 5.5 & EPA8240 \\
\hline 1,2-Dichloroethylene & $\mathbf{U}$ & & 5.5 & $\mu \mathrm{g} / \mathrm{kg}$ & 5.5 & EPA8240 \\
\hline Dichloromethane (Methylene chloride) & $\mathrm{U}$ & $\mathbf{v}$ & 30 & $\mu \mathrm{g} / \mathrm{kg}$ & 5.5 & EPA8240 \\
\hline 1,2-Dichloropropane & $\mathrm{U}$ & & 5.5 & $\mu \mathrm{g} / \mathrm{kg}$ & 5.5 & EPA8240 \\
\hline cis-1,3-Dichloropropene & $\mathrm{U}$ & & 5.5 & $\mu \mathrm{g} / \mathrm{kg}$ & 5.5 & EPA8240 \\
\hline trans-1,3-Dichloropropene & $\mathrm{U}$ & & 5.5 & $\mu g / \mathrm{kg}$ & 5.5 & EPA 8240 \\
\hline Ethylbenzene & J & & 2.0 & $\mu g / k g$ & 5.5 & EPA8240 \\
\hline 2-Hexanone & U & & 11 & $\mu g / \mathrm{kg}$ & 11 & EPA8240 \\
\hline Methyl ethyl ketone & $\mathbf{U}$ & & 11 & $\mu g / k g$ & 11 & EPA8240 \\
\hline Methyl isobutyl ketone & $\mathbf{U}$ & & 11 & $\mu \mathrm{g} / \mathrm{kg}$ & 11 & EPA8240 \\
\hline Styrene & $\mathrm{U}$ & & 5.5 & $\mu \mathrm{g} / \mathrm{kg}$ & 5.5 & EPA8240 \\
\hline 1,1,2,2-Tetrachloroethane & $\mathrm{U}$ & & 5.5 & $\mu \mathrm{g} / \mathrm{kg}$ & 5.5 & EPA8240 \\
\hline Tetrachloroethylene & $\mathrm{J}$ & & 2.9 & $\mu g / \mathrm{kg}$ & 5.5 & EPA8240 \\
\hline
\end{tabular}


SAMPLE NAME: D431 1306D (Continued)

Constituent

$\mathbf{R Q}$
Sample ID: 100387

B Result Unit D. Limit Method

Volatile Organic Compounds

Toluene

1,1,1-Trichloroethane

1,1,2-Trichloroethane

Trichloroethylene

Vinyl acetate
3.1

1.4

5.5

5.5

11

$$
\begin{aligned}
& \mu g / \mathrm{kg} \\
& \mu g / \mathrm{kg} \\
& \mu g / \mathrm{kg} \\
& \mu \mathrm{g} / \mathrm{kg} \\
& \mu \mathrm{g} / \mathrm{kg}
\end{aligned}
$$

5.5
5.5
5.5
5.5
11

EPA8240 EPA8240

EPA8240

EPA8240

EPA8240

Pesticides/PCBs and Dioxins/Furans

Aldrin
alpha-Benzene hexachloride
beta-Benzene hexachloride
delta-Benzene hexachloride
alpha-Chlordane
gamma-Chlordane
p.p'-DDD
p.p'-DDE
p.p'-DDT
Dieldrin
Endosulfan I
Endosulfan II
Endosulfan sulfate
Endrin
Endrin ketone
Heptachlor
Heptachlor epoxide
Heptachlorodibenzo-p-dioxin isomers
Heptachlorodibenzo-p-furan isomers
Hexachlorodibenzo-p-dioxin isomers
Hexachlorodibenzo-p-furan isomers
Lindane
Methoxychlor
Octachlorodibenzo-p-dioxin
Octachlorodibenzo-p-furan
PCB 1016
PCB 1221
PCB 1232
PCB 1242
PCB 1248
PCB 1254
PCB 1260
Pentachlorodibenzo-p-dioxin isomers
Pentachlorodibenzo-p-furan isomers
$2,3,7,8-T C D D$
Tet3,7,8-TCDF
Tetrachlorodibenzo-p-furan isomers
Toxaphene
Xylenes

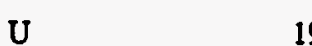

19

19

19

19

19

38

38

38

38

19

38

38

38

190

19

19

0.20

0.10

0.10

0.10

19

190

6.2

0.10

380

760

380

380

380

380

380

0.20

0.20

0.10

0.10

0.10

0.10

1,900

6.5

$\mu g / k g$
$\mu g / k g$
$\mu g / k g$
$\mu g / k g$
$\mu g / k g$
$\mu g / k g$
$\mu g / k g$
$\mu g / k g$
$\mu g / k g$
$\mu g / k g$
$\mu g / k g$
$\mu g / k g$
$\mu g / k g$
$\mu g / k g$
$\mu g / k g$
$\mu g / k g$
$\mu g / k g$
$n g / g$
$n g / g$
$n g / g$
$n g / g$
$\mu g / k g$
$\mu g / k g$
$n g / g$
$n g / g$
$\mu g / k g$
$\mu g / k g$
$\mu g / k g$
$\mu g / k g$
$\mu g / k g$

$\mu \mathrm{g} / \mathrm{kg}$

$\mu \mathrm{g} / \mathrm{kg}$

$\mu \mathrm{g} / \mathrm{kg}$

$\mu \mathrm{g} / \mathrm{kg}$

$\mu g / k g$

$\mu \mathrm{g} / \mathrm{kg}$

$\mu \mathrm{g} / \mathrm{kg}$

$\mu \mathrm{g} / \mathrm{kg}$

$\mu \mathrm{g} / \mathrm{kg}$

$\mu \mathrm{g} / \mathrm{kg}$

$\mu \mathrm{g} / \mathrm{kg}$

$\mu \mathrm{g} / \mathrm{kg}$

$\mu \mathrm{g} / \mathrm{kg}$

$\mu \mathrm{g} / \mathrm{kg}$

$\mathrm{ng} / \mathrm{g}$

ng/g

$\mathrm{ng} / \mathrm{g}$

$\mu \mathrm{g} / \mathrm{kg}$

$\mu \mathrm{g} / \mathrm{kg}$

$\mathrm{ng} / \mathrm{g}$

$\mu \mathrm{g} / \mathrm{kg}$

$\mu \mathrm{g} / \mathrm{kg}$

$\mu g / \mathrm{kg}$

$\mu \mathrm{g} / \mathrm{kg}$

$\mu \mathrm{g} / \mathrm{kg}$

$\mu \mathrm{g} / \mathrm{kg}$

$\mu \mathrm{g} / \mathrm{k}$

$\mathrm{ng} / \mathrm{g}$

$\mathrm{ng} / \mathrm{g}$

$\mathrm{ng} / \mathrm{g}$

$\mathrm{ng} / \mathrm{g}$

$\mu g / \mathrm{kg}$

$\mathrm{kg} / \mathrm{kg}$
EPA8080

EPA8080

EPA8080

EPA8080

EPA8080

EPA8080

EPA8080

EPA8080

EPA8080

EPA8080

EPA8080

EPA8080

EPA8080

EPA8080

EPA8080

EPA8080

EPA8080

EPA8280

EPA8280

EPA8280

EPA8280

EPA8080

EPA8080

EPA8280

EPA 8280

EPA8080

EPA8080

EPA8080

EPA8080

EPA8080

EPA8080

EPA8080

EPA8280

EPA8280

EPA8280

EPA8280

EPA8280

EPA8280

EPA8080

EPA8280 
SAMPLE NAME: D431 1306D (Continued)

Constituent

$\mathbf{R Q}$

RQ

Radionuclide Indicators and Radionuclides

Gross alpha

Nonvolatile beta

Radium, total alpha-emitting

Tritium
Sample ID: 100387 .

$\begin{array}{llll}\text { B Result } & \text { Unit } & \text { D. Limit Method } \\ & & & \\ & & & \\ 10 \pm 11 & \mathrm{pCi} / \mathrm{g} & 7.4 & \text { EPA900.0 } \\ 37 \pm 17 & \mathrm{pCi} / \mathrm{g} & 7.1 & \text { EPA900.0 } \\ 2.3 \pm 1.2 & \mathrm{pCi} / \mathrm{g} & 0.20 & \text { EPA903.0 } \\ 2.2 \pm 0.52 & \mathrm{pCi} / \mathrm{g} & 1.1 & \text { EPA906.0 }\end{array}$

\section{SAMPLE NAME: D431 1307}

Location (SRS Coordinates): 18446.1E 66631.93N

Ground Elevation Above MSL: $131.4 \mathrm{ft}$

Depth of Core Interval: 10.00 to $12.00 \mathrm{ft}$ Sample Type: Normal

Sample Color:

\section{Constituent}

\section{Total Metals}

Arsenic
Barium

$B / N / A$ Extractables (including $P A H$ and phenols)

Acenaphthene

Acenaphthylene

Anthracene

Benzo(a)anthracene

Benzo(b)fluoranthene

Benzo(k)fluoranthene

Benzoic acid

Benzo(g,h,i)perylene

Benzo(a)pyrene

Benzyl alcohol

Bis(2-chloroethoxy) methane

Bis(2-chloroethyl) ether

Bis(2-chloroisopropyl) ether

Bis(2-ethylhexyl) phthalate

4-Bromophenyl phenyl ether

Butylbenzyl phthalate

4-Chloroaniline

4-Chloro-m-cresol
Sample ID: 100388

Sample Matrix: Soil

USC Soil Classification: SC

RQ AQ B Result Unit

Sample Moisture: Damp

Percent Solids: 81.60

D. Limit Method

$\begin{array}{lllll}\mathrm{U} & 1.2 & \mathrm{mg} / \mathrm{kg} & 1.2 & \text { EPA7060 } \\ \mathrm{U} & 24 & \mathrm{mg} / \mathrm{kg} & 24 & \text { EPA6010 } \\ \mathrm{U} & 0.61 & \mathrm{mg} / \mathrm{kg} & 0.61 & \text { EPA6010 } \\ & 24 & \mathrm{mg} / \mathrm{kg} & 1.2 & \text { EPA6010 } \\ & 4.2 & \mathrm{mg} / \mathrm{kg} & 3.0 & \text { EPA6010 } \\ & 2.9 & \mathrm{mg} / \mathrm{kg} & 0.35 & \text { EPA7421 } \\ & 14 & \mathrm{mg} / \mathrm{kg} & 1.8 & \text { EPA6010 } \\ \mathrm{U} & 0.12 & \mathrm{mg} / \mathrm{kg} & 0.12 & \text { EPA7470 } \\ \mathrm{U} & 4.9 & \mathrm{mg} / \mathrm{kg} & 4.9 & \text { EPA6010 } \\ & 0.72 & \mathrm{mg} / \mathrm{kg} & 0.59 & \text { EPA7740 } \\ \mathrm{U} & 1.2 & \mathrm{mg} / \mathrm{kg} & 1.2 & \text { EPA6010 }\end{array}$

$\begin{array}{ll}J & 60 \\ U & 410 \\ J & 130 \\ J & 270 \\ J & 290 \\ J & 110 \\ U & 2,000 \\ J & 120 \\ J & 220 \\ U & 410 \\ U & 410 \\ U & 410 \\ U & 410 \\ J & 93 \\ U & 410 \\ U & 410 \\ U & 410 \\ U & 410\end{array}$

EPA 8270

EPA8270

EPA8270

EPA8270

EPA 8270

EPA8270

EPA8270

EPA8270

EPA8270

EPA8270

EPA8270

EPA8270

EPA8270

EPA8270

EPA8270

EPA8270

EPA 8270

EPA8270 
Constituent

RQ AQ B Result

Unit

D. Limit Method

$B / N / A$ Extractables (including PAH and phenols)

2-Chloronaphthalene

2-Chlorophenol

4-Chlorophenyl phenyl ether

Chrysene

o-Cresol (2-Methylphenol)

p-Cresol (4-Methylphenol)

Dibenz $(a, h)$ anthracene

Dibenzofuran

Di-n-butyl phthalate

1,2-Dichlorobenzene

1,3-Dichlorobenzene

1,4-Dichlorobenzene

3,3'-Dichlorobenzidine

2,4-Dichlorophenol

Diethyl phthalate

2,4-Dimethyl phenol

Dimethyl phthalate

2,4-Dinitrophenol

2,4-Dinitrotoluene

2,6-Dinitrotoluene

Di-n-octyl phthalate

Fluoranthene

Fluorene

Hexachlorobenzene

Hexachlorobutadiene

Hexachlorocyclopentadiene

Hexachloroethane

Indeno(1,2,3-c,d)pyrene

Isophorone

2-Methyl-4,6-dinitrophenol

2-Methyinaphthalene

Naphthalene

o-Nitroaniline

m-Nitroaniline

p-Nitroaniline

Nitrobenzene

2-Nitrophenol

4-Nitrophenol

N-Nitrosodiphenylamine

N-Nitrosodipropylamine

Pentachlorophenol

Phenanthrene

Phenol

Pyrene

1,2,4-Trichlorobenzene

2,4,5-Trichlorophenol

2,4,6-Trichlorophenol

Unknown

Unknown

Unknown
U

U

U

J

U

U

U

J

U

U

U

U

U

U

U

U

U

U

U

U

J

U

U

$U$

U

J

U

U

U

J

U

U

U

U

U

U

UJ

U

U

$\begin{array}{lll}\text { UJ } & \mathrm{C} & 410 \\ \mathrm{U} & & 2,000 \\ \mathrm{U} & & 410 \\ \mathrm{~J} & \mathrm{NV} & 3,000 \\ \mathrm{~J} & \mathrm{NV} & 900 \\ \mathrm{~J} & \mathrm{~N} & 600\end{array}$

410

410

410

200

410

410

410

45

410

410

410

410

820

410

410

410

410

2.000

410

410

410

610

79

410

410

410

410

140

410

2,000

410

45

2,000

2,000

2,000

410

410

2,000

410

c

410

2,000

530

410

450

410

410

600 $\mu g / \mathrm{kg}$
$\mu g / \mathrm{kg}$
$\mu g / \mathrm{kg}$
$\mu g / \mathrm{kg}$
$\mu g / \mathrm{kg}$
$\mu g / \mathrm{kg}$
$\mu g / \mathrm{kg}$
$\mu g / \mathrm{kg}$
$\mu g / \mathrm{kg}$
$\mu g / \mathrm{kg}$
$\mu g / \mathrm{kg}$
$\mu g / \mathrm{kg}$
$\mu g / \mathrm{kg}$
$\mu g / \mathrm{kg}$
$\mu g / \mathrm{kg}$
$\mu g / \mathrm{kg}$
$\mu g / \mathrm{kg}$
$\mu \mathrm{g} / \mathrm{kg}$
$\mu g / \mathrm{kg}$
$\mu g / \mathrm{kg}$
$\mu g / \mathrm{kg}$
$\mu g / \mathrm{kg}$
$\mu g / \mathrm{kg}$
$\mu g / \mathrm{kg}$
$\mu g / \mathrm{kg}$

$\mu \mathrm{g} / \mathrm{kg}$

$\mu \mathrm{g} / \mathrm{kg}$

$\mu \mathrm{g} / \mathrm{kg}$

$\mu \mathrm{g} / \mathrm{kg}$

$\mu \mathrm{g} / \mathrm{kg}$

$\mu \mathrm{g} / \mathrm{kg}$

$\mu \mathrm{g} / \mathrm{kg}$

$\mu \mathrm{g} / \mathrm{kg}$

$\mu \mathrm{g} / \mathrm{kg}$

$\mu \mathrm{g} / \mathrm{kg}$

$\mu \mathrm{g} / \mathrm{kg}$

$\mu \mathrm{g} / \mathrm{kg}$

$\mu \mathrm{g} / \mathrm{kg}$

$\mu g / \mathrm{kg}$

$\mu \mathrm{g} / \mathrm{kg}$

$\mu \mathrm{g} / \mathrm{kg}$

$\mu \mathrm{g} / \mathrm{kg}$

$\mu \mathrm{g} / \mathrm{kg}$

$\mu \mathrm{g} / \mathrm{kg}$

$\mu \mathrm{g} / \mathrm{kg}$

$\mu g / \mathrm{kg}$

$\mu \mathrm{g} / \mathrm{kg}$

$\mu g / \mathrm{kg}$

$\mu \mathrm{g} / \mathrm{kg}$

$\mu g / k g$
EPA8270

EPA8270

EPA8270

EPA8270

EPA8270.

EPA8270

EPA8270

EPA8270

EPA8270

EPA8270

EPA8270

EPA8270

EPA8270

EPA8270

EPA8270

EPA8270

EPA8270

EPA8270

EPA8270

EPA8270

EPA8270

EPA8270

EPA8270

EPA8270

EPA8270

EPA8270

EPA8270

EPA8270

EPA8270

EPA8270

EPA8270

EPA8270

EPA8270

EPA8270

EPA 8270

EPA8270

EPA8270

EPA8270

EPA8270

EPA8270

EPA8270

EPA8270

EPA8270

EPA8270

EPA8270

EPA8270

EPA8270

EPA8270

EPA8270

EPA8270 
SAMPLE NAME: D43ï 1307 (Continued)

Sample D: 100388

$\begin{array}{lllllll}\text { Constituent } & \text { RQ AQ } & \text { R } & \text { Result } & \text { Unit Limit Method }\end{array}$

$B / N / A$ Extractables (including $P A H$ and phenols)

Unknown ketone

J NV

50,000

$\mu g / k g$

EPA8270

Volatile Organic Compounds

Acetone
Benzene
Bromodichloromethane
Bromoform
Bromomethane (Methyl bromide)
Carbon disulfide
Carbon tetrachloride
Chlorobenzene
Chloroethane
Chloroethene (Vinyl chloride)
2-Chloroethyl vinyl ether
Chloroform
Chloromethane (Methyl chloride)
Dibromochloromethane
1,1-Dichloroethane
1,2-Dichloroethane
1,1-Dichloroethylene
1,2-Dichlorothylene
Dichloromethane (Methylene chloride)
1.2-Dichloropropane
cis-1,3-Dichloropropene
trans-1,3-Dichloropropene
Ethylbenzene
2-Hexanone
Methyl ethyl ketone
Methyl isobutyl ketone
Styrene
1,1,2,2-Tetrachloroethane
Tetrachloroethylene
Toluene
1,1,1-Trichloroethane
1,1,2-Trichloroethane
Trichloroethylene
Vinyl acetate
Xylenes

$\mathrm{U}$
$\mathrm{U}$
$\mathrm{U}$
$\mathrm{U}$
$\mathrm{U}$
$\mathrm{U}$
$\mathrm{U}$
$\mathrm{U}$
$\mathrm{U}$
$\mathrm{U}$
$\mathrm{U}$
$\mathrm{U}$
$\mathrm{U}$
$\mathrm{U}$
$\mathrm{U}$
$\mathrm{U}$
$\mathrm{U}$
$\mathrm{U}$
$\mathrm{U}$
$\mathrm{U}$
$\mathrm{U}$
$\mathrm{U}$
$\mathrm{U}$
$\mathrm{U}$
$\mathrm{U}$
$\mathrm{U}$
$\mathrm{U}$
$\mathrm{U}$
$\mathrm{U}$
$\mathrm{U}$
$\mathrm{U}$
$\mathrm{U}$
$\mathrm{U}$
$\mathrm{U}$

\begin{tabular}{|c|c|c|c|}
\hline 16 & $\mu g / \mathbf{k g}$ & 12 & EPA8240 \\
\hline 6.0 & $\mu g / k g$ & 6.0 & EPA 8240 \\
\hline 6.0 & $\mu \mathrm{g} / \mathrm{kg}$ & 6.0 & EPA8240 \\
\hline 6.0 & $\mu \mathrm{g} / \mathrm{kg}$ & 6.0 & EPA8240 \\
\hline 12 & $\mu g / \mathrm{kg}$ & 12 & EPA8240 \\
\hline 6.0 & $\mu g / \mathrm{kg}$ & 6.0 & EPA8240 \\
\hline 6.0 & $\mu g / \mathrm{kg}$ & 6.0 & EPA8240 \\
\hline 6.0 & $\mu \mathrm{g} / \mathrm{kg}$ & 6.0 & EPA 8240 \\
\hline 12 & $\mu g / \mathrm{kg}$ & 12 & EPA 8240 \\
\hline 12 & $\mu \mathrm{g} / \mathrm{kg}$ & 12 & EPA8240 \\
\hline 12 & $\mu \mathrm{g} / \mathrm{kg}$ & 12 & EPA8240 \\
\hline 6.0 & $\mu g / k g$ & 6.0 & EPA8240 \\
\hline 12. & $\mu \mathrm{g} / \mathrm{kg}$ & 12 & EPA8240 \\
\hline 6.0 & $\mu \mathrm{g} / \mathrm{kg}$ & 6.0 & EPA8240 \\
\hline 6.0 & $\mu \mathrm{g} / \mathrm{kg}$ & 6.0 & EPA8240 \\
\hline 6.0 & $\mu \mathrm{g} / \mathrm{kg}$ & 6.0 & EPA8240 \\
\hline 6.0 & $\mu \mathrm{g} / \mathrm{kg}$ & 6.0 & EPA8240 \\
\hline 6.0 & $\mu \mathrm{g} / \mathrm{kg}$ & 6.0 & EPA8240 \\
\hline 19 & $\mu \mathrm{g} / \mathrm{kg}$ & 6.0 & EPA8240 \\
\hline 6.0 & $\mu \mathrm{g} / \mathrm{kg}$ & 6.0 & EPA8240 \\
\hline 6.0 & $\mu g / \mathrm{kg}$ & 6.0 & EPA8240 \\
\hline 6.0 & $\mu \mathrm{g} / \mathrm{kg}$ & 6.0 & EPA8240 \\
\hline 6.0 & $\mu \mathrm{g} / \mathrm{kg}$ & 6.0 & EPA8240 \\
\hline 12 & $\mu g / \mathrm{kg}$ & 12 & EPA8240 \\
\hline 12 & $\mu \mathrm{g} / \mathrm{kg}$ & 12 & EPA8240 \\
\hline 12 & $\mu \mathrm{g} / \mathrm{kg}$ & 12 & EPA8240 \\
\hline 6.0 & $\mu \mathrm{g} / \mathrm{kg}$ & 6.0 & EPA8240 \\
\hline 6.0 & $\mu \mathrm{g} / \mathrm{kg}$ & 6.0 & EPA8240 \\
\hline 6.0 & $\mu \mathrm{g} / \mathrm{kg}$ & 6.0 & EPA8240 \\
\hline 6.0 & $\mu \mathrm{g} / \mathrm{kg}$ & 6.0 & EPA8240 \\
\hline 6.0 & $\mu \mathrm{g} / \mathrm{kg}$ & 6.0 & EPA8240 \\
\hline 6.0 & $\mu \mathrm{g} / \mathrm{kg}$ & 6.0 & EPA8240 \\
\hline 5.0 & $\mu g / \mathrm{kg}$ & 6.0 & EPA8240 \\
\hline & $\mu \mathrm{g} / \mathrm{kg}$ & 12 & EPA8240 \\
\hline 5.0 & $\mu \mathrm{g} / \mathrm{kg}$ & 6.0 & EPA8240 \\
\hline
\end{tabular}

Pesticides/PCBs and-Dioxins/Furans

Aldrin

alpha-Benzene hexachforide

beta-Benzene hexachloride

delta-Benzene hexachloride

alpha-Chlordane

gamma-Chiordane

p.p'-DDD

$p, p^{\prime}-D D E$

\section{0}

2.0

2.0

2.0

2.0

2.0

4.1

4.1 $\mu g / \mathrm{kg}$

$\mu g / \mathrm{kg}$

$\mu \mathrm{g} / \mathrm{kg}$

$\mu \mathrm{g} / \mathrm{kg}$

$\mu g / \mathrm{kg}$

$\mu \mathrm{g} / \mathrm{kg}$

$\mu \mathrm{g} / \mathrm{kg}$

$\mu \mathrm{g} / \mathrm{kg}$

\section{0}

EPA8080

EPA8080

EPA8080

EPA8080

EPA8080

EPA8080

EPA8080

EPA8080 


Constituent RQ AQ. B Result Unit D. Limit Method

Pesticides/PCBs and Dioxins/Furans

P.P'-DDT
Dieldrin
Endosulfan I
Endosulfan II
Endosulfan sulfate
Endrin

Endrin

Heptachlor

Heptachlor epoxide

Heptachlorodibenzo-p-dioxin isomers

Heptachlorodibenzo-p-dioxin isomers

Heptachlorodibenzo-p-furan isomers

Heptachlorodibenzo-p-furan isomers

Hexachlorodibenzo-p-dioxin isomers

Hexachlorodibenzo-p-dioxin isomers

Hexachlorodibenzo-p-furan isomers

Hexachlorodibenzo-p-furan isomers

Lindane

Methoxychlor

Octachlorodibenzo-p-dioxin

Octachlorodibenzo-p-dioxin

Octachlorodibenzo-p-furan

Octachlorodibenzo-p-furan

PCB 1016

PCB 1221

PCB 1232

PCB 1242

PCB 1248

PCB 1254

PCB 1260

Pentachlorodibenzo-p-dioxin isomers

Pentachlorodibenzo-p-furan isomers

Pentachlorodibenzo-p-dioxin isomers

Pentachlorodibenzo-p-furan isomers

2,3,7,8-TCDD

2,3,7,8-TCDD

$2,3,7,8-\mathrm{TCDF}$

2,3,7,8-TCDF

Tetrachlorodibenzo-podioxin isomers

Tetrachlorodibenzo-p-dioxin isomers

Tetrachlorodibenzo-p-furan isomers

Tetrachlorodibenzo-p-furan isomers

Toxaphene
$U$
$U$
$U$
$U$
$U$
$U$

$\mathrm{U}$

U

U

U

$\mathrm{U}$

U

$\mathrm{U}$

U

U

U

U

J

U

U

U

U

J

U

U

.

$\mathrm{U}$

U

U

U

U

U
4.1

4.1

2.0

4.1

4.1

4.1

6.1

2.0

2.0

0.20

0.20

0.10

0.10

0.20

0.10

0.10

0.10

2.0

20

1.6

10

0.10

0.10

41

82

41

41

41

41

41

0.30

0.20

0.30

0.20

0.10

0.10

0.10

0.10

0.10

0.10

0.10

0.10

200 $\mu g / k g$

$\mu \mathrm{g} / \mathrm{kg}$

$\mu \mathrm{g} / \mathrm{kg}$

$\mu \mathrm{g} / \mathrm{kg}$

$\mu g / \mathrm{kg}$

$\mu \mathrm{g} / \mathrm{kg}$

$\mu \mathrm{g} / \mathrm{kg}$

$\mu \mathrm{g} / \mathrm{kg}$

$\mu \mathrm{g} / \mathrm{kg}$

ng/g

ng/g

$\mathrm{ng} / \mathrm{g}$

$\mathrm{ng} / \mathrm{g}$

$\mathrm{ng} / \mathrm{g}$

$\mathrm{ng} / \mathrm{g}$

$\mathrm{ng} / \mathrm{g}$

$\mathrm{ng} / \mathrm{g}$

$\mu \mathrm{g} / \mathrm{kg}$

$\mu \mathrm{g} / \mathrm{kg}$

$\mathrm{ng} / \mathrm{g}$

$\mathrm{ng} / \mathrm{g}$

$\mathrm{ng} / \mathrm{g}$

ng/g

$\mu \mathrm{g} / \mathrm{kg}$

$\mu \mathrm{g} / \mathrm{kg}$

$\mu \mathrm{g} / \mathrm{kg}$

$\mu \mathrm{g} / \mathrm{kg}$

$\mu \mathrm{g} / \mathrm{kg}$

$\mu \mathrm{g} / \mathrm{kg}$

$\mu \mathrm{g} / \mathrm{kg}$

$\mathrm{ng} / \mathrm{g}$

$\mathrm{ng} / \mathrm{g}$

$\mathrm{ng} / \mathrm{g}$

ng/g

$\mathrm{ng} / \mathrm{g}$

$\mathrm{ng} / \mathrm{g}$

$\mathrm{ng} / \mathrm{g}$

$\mathrm{ng} / \mathrm{g}$

$\mathrm{ng} / \mathrm{g}$

$\mathrm{ng} / \mathrm{g}$

$\mathrm{ng} / \mathrm{g}$

$\mathrm{ng} / \mathrm{g}$

$\mu \mathrm{g} / \mathrm{kg}$
4.1

4.1

2.0

4.1

4.1

4.1

4.1

$2.0^{\circ}$

2.0

0.20

0.20

0.10

0.10

0.20

0.10

0.10

0.10

2.0

20

0.30

0.20

0.10

0.10

41

82

41

41

41

41

41

0.30

0.20

0.30

0.20

0.10

0.10

0.10

0.10

0.10

0.10

0.10

0.10

200
EPA8080

EPA8080

EPA8080

EPA8080

EPA8080

EPA8080

EPA8080

EPA8080

EPA8080

EPA8280

EPA8280

EPA8280

EPA8280

EPA8280

EPA8280

EPA8280

EPA8280

EPA8080

EPA8080

EPA8280

EPA8280

EPA8280

EPA8280

EPA8080

EPA8080

EPA8080

EPA8080

EPA8080

EPA8080

EPA8080

EPA8280

- EPA8280

EPA8280

EPA8280

EPA8280

EPA8280

EPA8280

EPA8280

EPA8280

EPA8280

EPA8280

EPA8280

EPA8080 
SAMPLE NAME: D431 1307A

Sample Type: Duplicate

Constituent

Total Metals
Sample ID: 100389

Associated Sample: 100388

RQ AQ B Result Unit D. Limit Method

Arsenic
Barium
Cadmium
Chromium
Copper
Lead
Manganese
Mercury
Nickel
Selenium
Silver

$B / N / A$ Extractables (including $P A H$ and phenols)

$\begin{array}{lllll}\mathrm{U} & 1.1 & \mathrm{mg} / \mathrm{kg} & 1.1 & \text { EPA7060 } \\ \mathrm{U} & 23 & \mathrm{mg} / \mathrm{kg} & 23 & \text { EPA6010 } \\ \mathrm{U} & 0.57 & \mathrm{mg} / \mathrm{kg} & 0.57 & \text { EPA6010 } \\ & 21 & \mathrm{mg} / \mathrm{kg} & 1.1 & \text { EPA6010 } \\ & 3.7 & \mathrm{mg} / \mathrm{kg} & 2.8 & \text { EPA6010 } \\ & 2.3 & \mathrm{mg} / \mathrm{kg} & 0.32 & \text { EPA7421 } \\ & 12 & \mathrm{mg} / \mathrm{kg} & 1.7 & \text { EPA6010 } \\ \mathrm{U} & 0.12 & \mathrm{mg} / \mathrm{kg} & 0.12 & \text { EPA7470 } \\ \mathrm{U} & 4.5 & \mathrm{mg} / \mathrm{kg} & 4.5 & \text { EPA6010 } \\ \mathrm{U} & 0.54 & \mathrm{mg} / \mathrm{kg} & 0.54 & \text { EPA7740 } \\ \mathrm{U} & 1.1 & \mathrm{mg} / \mathrm{kg} & 1.1 & \text { EPA6010 }\end{array}$

Acenaphthene
Acenaphthylene
Anthracene
Benzo(a)anthracene
Benzo(b)fluoranthene
Benzo(k)fluoranthene
Benzoic acid
Benzo(g,h,i)perylene
Benzo(a)pyrene
Benzyl alcohol
Bis(2-chloroethoxy) methane
Bis(2-chloroethyl) ether
Bis(2-chloroisopropyl) ether
Bis(2-ethylhexyl) phthalate
4-Bromophenyl phenyl ether
Butylbenzyl phthalate
4-Chloroaniline
4-Chloro-m-cresol
2-Chloronaphthalene
2-Chlorophenol
4-Chlorophenyl phenyl ether
Chrysene
0-Cresol (2-Methylphenol)
p-Cresol (4-Methylphenol)
Dibenz(a,h)anthracene --
Dibenzofuran
Di-n-butyl phthalate
1,2-Dichlorobenzene
1,3-Dichlorobenzene
1,4-Dichlorobenzene
3,3'-Dichlorobenzidine
2,4-Dichlorophenol
Diethyl phthalate
2,4-Dimethyl phenol

$\begin{array}{ll}\text { U } & 380 \\ U & 380 \\ J & 64 \\ J & 130 \\ J & 170 \\ J & 38 \\ U & 1,900 \\ U & 380 \\ J & 110 \\ U & 380 \\ U & 380 \\ U & 380 \\ U & 380 \\ J & 65 \\ U & 380 \\ U & 380 \\ U & 380 \\ U & 380 \\ U & 380 \\ U & 380 \\ U & 380 \\ J & 110 \\ U & 380 \\ U & 380 \\ U & 380 \\ U & 380 \\ U & 380 \\ U & 380 \\ U & 380 \\ U & 380 \\ U & 770 \\ U & 380 \\ U & 380 \\ U & 380 \\ & \\ U & \\ U & \\ U & \\ U & 3\end{array}$

\begin{tabular}{|c|c|c|}
\hline$\mu g / \mathrm{kg}$ & 380 & EPA8270 \\
\hline$\mu \mathrm{g} / \mathrm{kg}$ & 380 & EPA 8270 \\
\hline$\mu \mathrm{g} / \mathrm{kg}$ & 380 & EPA8270 \\
\hline$\mu \mathrm{g} / \mathrm{kg}$ & 380 & EPA8270 \\
\hline$\mu g / \mathrm{kg}$ & 380 & EPA 827 \\
\hline$\mu g / k g$ & 380 & EPA827 \\
\hline$\mu g / \mathrm{kg}$ & 1,900 & EPA 827 \\
\hline$\mu \mathrm{g} / \mathrm{kg}$ & 380 & EPA827 \\
\hline$\mu g / \mathrm{kg}$ & 380 & EPA8270 \\
\hline$\mu \mathrm{g} / \mathrm{kg}$ & 380 & EPA 827 \\
\hline$\mu \mathrm{g} / \mathrm{kg}$ & 380 & EPA827 \\
\hline$\mu \mathrm{g} / \mathrm{kg}$ & 380 & EPA827 \\
\hline$\mu \mathrm{g} / \mathrm{kg}$ & 380 & EPA827 \\
\hline$\mu \mathrm{g} / \mathrm{kg}$ & 380 & EPA8270 \\
\hline$\mu \mathrm{g} / \mathrm{kg}$ & 380 & EPA827 \\
\hline$\mu \mathrm{g} / \mathrm{kg}$ & 380 & EPA 8270 \\
\hline$\mu \mathrm{g} / \mathrm{kg}$ & 380 & EPA8270 \\
\hline$\mu \mathrm{g} / \mathrm{kg}$ & 380 & EPA8270 \\
\hline$\mu g / \mathrm{kg}$ & 380 & EPA8270 \\
\hline$\mu \mathrm{g} / \mathrm{kg}$ & 380 & EPA827C \\
\hline$\mu \mathrm{g} / \mathrm{kg}$ & 380 & EPA8270 \\
\hline$\mu \mathrm{g} / \mathrm{kg}$ & 380 & EPA8270 \\
\hline$\mu \mathrm{g} / \mathrm{kg}$ & 380 & EPA8270 \\
\hline$\mu \mathrm{g} / \mathrm{kg}$ & 380 & EPA 8270 \\
\hline$\mu \mathrm{g} / \mathrm{kg}$ & 380 & EPA 8270 \\
\hline$\mu \mathrm{g} / \mathrm{kg}$ & 380 & EPA8270 \\
\hline$\mu g / k g$ & 380 & EPA8270 \\
\hline$\mu g / k g$ & 380 & EPA8270 \\
\hline$\mu \mathrm{g} / \mathrm{kg}$ & 380 & EPA8270 \\
\hline$\mu \mathrm{g} / \mathrm{kg}$ & 380 & EPA8270 \\
\hline$\mu g / k g$ & 770 & EPA8270 \\
\hline$\mu g / k g$ & 380 & EPA 8270 \\
\hline$\mu \mathrm{g} / \mathrm{kg}$ & 380 & EPA8270 \\
\hline$\mu g / k g$ & 380 & EPA8270 \\
\hline
\end{tabular}


SAMPLE NAME: D43i 1307 A (Continued)

Constituent

\section{RQ AQ B Result}

$B / N / A$ Extractables (including $P A H$ and phenols)
Sample ID: 100389

Unit

D. Limit Method

U

U

2,4-Dinitrophenol

2,4-Dinitrotoluene :

2,6-Dinitrotoluene

Di-n-octyl phthalate

Fluoranthene

Fluorene

Hexachlorobenzene

Hexachlorobutadiene

Hexachlorocyclopentadiene

Hexachloroethane

Indeno(1,2,3-c,d)pyrene

Isophorone

2-Methyl-4,6-dinitrophenol

2-Methylnaphthalene

Naphthalene

o-Nitroaniline

m-Nitroaniline

p-Nitroaniline

Nitrobenzene

2-Nitrophenol

4-Nitrophenol

$\mathrm{N}$-Nitrosodiphenylamine

$\mathrm{N}$-Nitrosodipropylamine

Pentachlorophenol

Phenanthrene

Phenol

Pyrene

1,2,4-Trichlorobenzene

2,4,5-Trichlorophenol

2,4,6-Trichlorophenol

Unknown

Unknown

Unknown

Unknown ketone
U

U

U

J

J

U

U

U

U

J

U

$\cdot \mathrm{U}$

$\mathrm{U}$

U

U

U

U

U

$\mathrm{U}$

U

U

UJ

U

J

U

$\mathrm{J}$

US

U

U

J

J

Volatile Organic Compounds

Acetone
Acetone
Acetone
Benzene
Bromodichloromethane
Bromodichloromethane
Bromodichloromethane
Bromoform
Bromoform
Bromoform
Bromomethane (Methyl bromide)
Bromomethane (Methyl bromide)

$\begin{array}{ll}\mathbf{U} & \mathrm{V} \\ \mathbf{U} & \mathrm{V} \\ \mathbf{U} & \mathrm{V} \\ \mathbf{U} & \\ \mathbf{U} & \\ \mathbf{U} & \\ \mathbf{U} & \\ \mathbf{U} & \\ \mathbf{U} & \\ \mathbf{U} & \\ \mathbf{U} & \\ \mathbf{U} & \end{array}$

380
1,900
380
380
380
310
38
380
380
380
380
64
380
1,900
380
380
1,900
1,900
1,900
380
380
1,900
380
380
1,900
270
380
240
380
1,900
380
3,000
800
500
50,000

$\mu \mathrm{g} / \mathrm{kg}$

380

EPA8270

$\mu g / \mathrm{kg}$

$\mu \mathrm{g} / \mathrm{kg}$

$\mu \mathrm{g} / \mathrm{kg}$

$\mu g / k g$

$\mu \mathrm{g} / \mathrm{kg}$

$\mu g / k g$

$\mu \mathrm{g} / \mathrm{kg}$

$\mu \mathrm{g} / \mathrm{kg}$

$\mu \mathrm{g} / \mathrm{kg}$

$\mu \mathrm{g} / \mathrm{kg}$

$\mu \mathrm{g} / \mathrm{kg}$

$\mu \mathrm{g} / \mathrm{kg}$

$\mu \mathrm{g} / \mathrm{kg}$

$\mu \mathrm{g} / \mathrm{kg}$

$\mu \mathrm{g} / \mathrm{kg}$

$\mu \mathrm{g} / \mathrm{kg}$

$\mu \mathrm{g} / \mathrm{kg}$

$\mu \mathrm{g} / \mathrm{kg}$

$\mu g / k g$

$\mu \mathrm{g} / \mathrm{kg}$

$\mu \mathrm{g} / \mathrm{kg}$

$\mu \mathrm{g} / \mathrm{kg}$

$\mu \mathrm{g} / \mathrm{kg}$

$\mu \mathrm{g} / \mathrm{kg}$

$\mu \mathrm{g} / \mathrm{kg}$

$\mu g / k g$

$\mu \mathrm{g} / \mathrm{kg}$

$\mu g / \mathrm{kg}$

$\mu \mathrm{g} / \mathrm{kg}$

$\mu \mathrm{g} / \mathrm{kg}$

$\mu \mathrm{g} / \mathrm{kg}$

$\mu \mathrm{g} / \mathrm{kg}$

$\mu g / \mathrm{kg}$

$\mu \mathrm{g} / \mathrm{kg}$

1,900

380

380

380

380

380

380

380

380

380

380

380

1,900

380

380

1,900

1,900

1,900

380

380

1,900

380

380

1.900

380

380

380

380

1,900

380

NV

NV

500
50,000

19

29

28

6.0

6.0

6.0

6.0

6.0

6.0

6.0

12

12

$\begin{array}{lll}\mu \mathrm{g} / \mathrm{kg} & 12 & \text { EPA8240 } \\ \mu \mathrm{g} / \mathrm{kg} & 12 & \text { EPA8240 } \\ \mu \mathrm{g} / \mathrm{kg} & 12 & \text { EPA8240 } \\ \mu \mathrm{g} / \mathrm{kg} & 6.0 & \text { EPA8240 } \\ \mu \mathrm{g} / \mathrm{kg} & 6.0 & \text { EPA8240 } \\ \mu \mathrm{g} / \mathrm{kg} & 6.0 & \text { EPA8240 } \\ \mu \mathrm{g} / \mathrm{kg} & 6.0 & \text { EPA8240 } \\ \mu \mathrm{g} / \mathrm{kg} & 6.0 & \text { EPA8240 } \\ \mu \mathrm{g} / \mathrm{kg} & 6.0 & \text { EPA8240 } \\ \mu \mathrm{g} / \mathrm{kg} & 6.0 & \text { EPA8240 } \\ \mu \mathrm{g} / \mathrm{kg} & 12 & \text { EPA8240 } \\ \mu \mathrm{g} / \mathrm{kg} & 12 & \text { EPA8240 }\end{array}$

EPA8270

EPA8270

EPA8270

EPA8270

EPA8270

EPA8270

EPA8270

EPA8270

EPA8270

EPA8270

EPA8270

EPA8270

EPA8270

EPA8270

EPA8270

EPA8270

EPA8270

EPA8270

EPA8270

EPA8270

EPA8270

EPA8270

EPA8270

EPA8270

EPA8270

EPA8270

EPA8270

EPA8270

EPA8270

EPA8270

EPA8270

EPA8270

EPA8270

EPA8240

EPA8240

EPA8240

EPA8240

EPA8240 
SAMPLE NAME: D43ī 1307A (Continued)

Sample ID: 100389

Constituent

Volatile Organic Compounds

Bromomethane (Methyl bromide)

Carbon disulfide

Carbon disulfide

Carbon disulfide

Carbon tetrachloride

Carbon tetrachloride

Carbon tetrachloride

Chlorobenzene

Chloroethane

Chloroethane

Chloroethane

Chloroethene (Vinyl chloride)

Chloroethene (Vinyl chloride)

Chloroethene (Vinyl chloride)

2-Chloroethyl vinyl ether

2-Chloroethyl vinyl ether

2-Chloroethyl vinyl ether

Chloroform

Chloroform

Chloroform

Chloromethane (Methyl chloride)

Chloromethane (Methyl chloride)

Chloromethane (Methyl chloride)

Dibromochloromethane

Dibromochloromethane

Dibromochloromethane

1,1-Dichloroethane

1.1-Dichloroethane

1.1-Dichloroethane

1,2-Dichloroethane

1,2-Dichloroethane

1,2-Dichloroethane

1,1-Dichloroethylene

1,2-Dichloroethylene

1,2-Dichloroethylene

1.2-Dichloroethylene

Dichloromethane (Methylene chloride)

Dichloromethane (Methylene chloride)

Dichloromethane (Methylene chloride)

1.2-Dichloropropane - -

1.2-Dichloropropane --

1,2-Dichloropropane

cis-1,3-Dichloropropeñe

cis-1,3-Dichloropropene

trans-1,3-Dichloropropene

trans-1,3-Dichloropropene

trans-1,3-Dichloropropene

Ethylbenzene

Ethylbenzene

\section{RQ AQ B Result}

Unit

D. Limit Method

\begin{tabular}{|c|c|c|c|}
\hline 12 & $\mu \mathrm{g} / \mathrm{kg}$ & 12 & EPA8240 \\
\hline 6.0 & $\mu \mathrm{g} / \mathrm{kg}$ & 6.0 & EPA8240 \\
\hline 6.0 & $\mu g / \mathrm{kg}$ & 6.0 & EPA8240 \\
\hline 6.0 & $\mu \mathrm{g} / \mathrm{kg}$ & 6.0 & EPA8240 \\
\hline 6.0 & $\mu \mathrm{g} / \mathrm{kg}$ & 6.0 & EPA8240 \\
\hline 6.0 & $\mu \mathrm{g} / \mathrm{kg}$ & 6.0 & EPA8240 \\
\hline 6.0 & $\mu \mathrm{g} / \mathrm{kg}$ & 6.0 & EPA8240 \\
\hline 6.0 & $\mu \mathrm{g} / \mathrm{kg}$ & 6.0 & EPA8240 \\
\hline 12 & $\mu \mathrm{g} / \mathrm{kg}$ & 12 & EPA8240 \\
\hline 12 & $\mu \mathrm{g} / \mathrm{kg}$ & 12 & EPA8240 \\
\hline 12 & $\mu \mathrm{g} / \mathrm{kg}$ & 12 & EPA8240 \\
\hline 12 & $\mu \mathrm{g} / \mathrm{kg}$ & 12 & EPA8240 \\
\hline 12 & $\mu g / \mathrm{kg}$ & 12 & EPA8240 \\
\hline 12 & $\mu \mathrm{g} / \mathrm{kg}$ & 12 & EPA8240 \\
\hline 12 & $\mu \mathrm{g} / \mathrm{kg}$ & 12 & EPA8240 \\
\hline 12 & $\mu \mathrm{g} / \mathrm{kg}$ & 12 & EPA8240 \\
\hline 12 & $\mu \mathrm{g} / \mathrm{kg}$ & 12 & EPA8240 \\
\hline 6.0 & $\mu \mathrm{g} / \mathrm{kg}$ & 6.0 & EPA8240 \\
\hline 6.0 & $\mu \mathrm{g} / \mathrm{kg}$ & 6.0 & EPA8240 \\
\hline 6.0 & $\mu \mathrm{g} / \mathrm{kg}$ & 6.0 & EPA8240 \\
\hline 12 & $\mu \mathrm{g} / \mathrm{kg}$ & 12 & EPA8240 \\
\hline 12 & $\mu \mathrm{g} / \mathrm{kg}$ & 12 & EPA8240 \\
\hline 12 & $\mu \mathrm{g} / \mathrm{kg}$ & 12 & EPA8240 \\
\hline 6.0 & $\mu \mathrm{g} / \mathrm{kg}$ & 6.0 & EPA8240 \\
\hline 6.0 & $\mu \mathrm{g} / \mathrm{kg}$ & 6.0 & EPA8240 \\
\hline 6.0 & $\mu \mathrm{g} / \mathrm{kg}$ & 6.0 & EPA8240 \\
\hline 6.0 & $\mu \mathrm{g} / \mathrm{kg}$ & 6.0 & EPA8240 \\
\hline 6.0 & $\mu \mathrm{g} / \mathrm{kg}$ & 6.0 & EPA8240 \\
\hline 6.0 & $\mu \mathrm{g} / \mathrm{kg}$ & 6.0 & EPA8240 \\
\hline 6.0 & $\mu \mathrm{g} / \mathrm{kg}$ & 6.0 & EPA8240 \\
\hline 6.0 & $\mu \mathrm{g} / \mathrm{kg}$ & 6.0 & EPA8240 \\
\hline 6.0 & $\mu \mathrm{g} / \mathrm{kg}$ & 6.0 & EPA8240 \\
\hline 6.0 & $\mu \mathrm{g} / \mathrm{kg}$ & 6.0 & EPA8240 \\
\hline 6.0 & $\mu \mathrm{g} / \mathrm{kg}$ & 6.0 & EPA8240 \\
\hline 6.0 & $\mu g / \mathrm{kg}$ & 6.0 & EPA8240 \\
\hline 6.0 & $\mu g / k g$ & 6.0 & EPA8240 \\
\hline 19 & $\mu \mathrm{g} / \mathrm{kg}$ & $: 6.0$ & EPA8240 \\
\hline 22 & $\mu \mathrm{g} / \mathrm{kg}$ & $6: 0$ & EPA8240 \\
\hline 19 & $\mu \mathrm{g} / \mathrm{kg}$ & 6.0 & EPA8240 \\
\hline 6.0 & $\mu g / \mathrm{kg}$ & 6.0 & EPA8240 \\
\hline 6.0 & $\mu \mathrm{g} / \mathrm{kg}$ & 6.0 & EPA8240 \\
\hline 6.0 & $\mu \mathrm{g} / \mathrm{kg}$ & 6.0 & EPA8240 \\
\hline 6.0 & $\mu \mathrm{g} / \mathrm{kg}$ & 6.0 & EPA8240 \\
\hline 6.0 & $\mu g / \mathrm{kg}$ & 6.0 & EPA8240 \\
\hline 6.0 & $\mu \mathrm{g} / \mathrm{kg}$ & 6.0 & EPA8240 \\
\hline 6.0 & $\mu \mathrm{g} / \mathrm{kg}$ & 6.0 & EPA8240 \\
\hline 6.0 & $\mu g / \mathrm{kg}$ & 6.0 & EPA8240 \\
\hline 6.0 & $\mu g / \mathrm{kg}$ & 6.0 & EPA8240 \\
\hline 6.0 & $\mu \mathrm{g} / \mathrm{kg}$ & 6.0 & EPA8240 \\
\hline 6.0 & $\mu \mathrm{g} / \mathrm{kg}$ & 6.0 & EPA8240 \\
\hline
\end{tabular}


Constituent

Volatile Organic Compounds
RQ AQ B Result Unit D. Limit Method

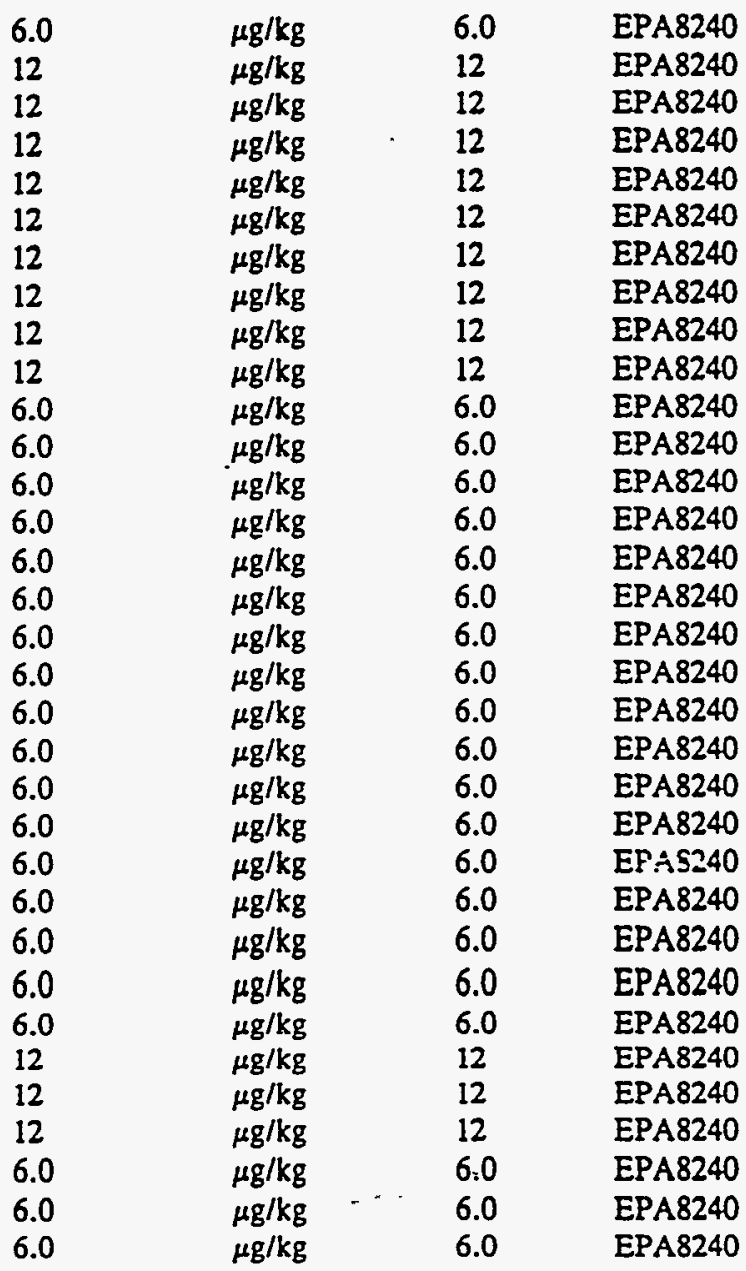

Pesticides/PCBs and Dioxins/Furans

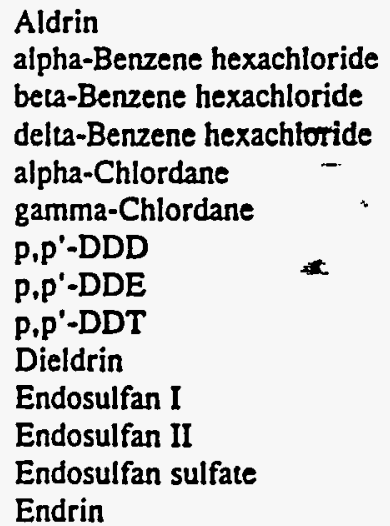

Aldrin

alpha-Benzene hexachloride

delta-Benzene hexachloride

alpha-Chlordane

p.p'-DDD

p.p'-DDE

P,P'-DDT

Endosulfan II

Endrin

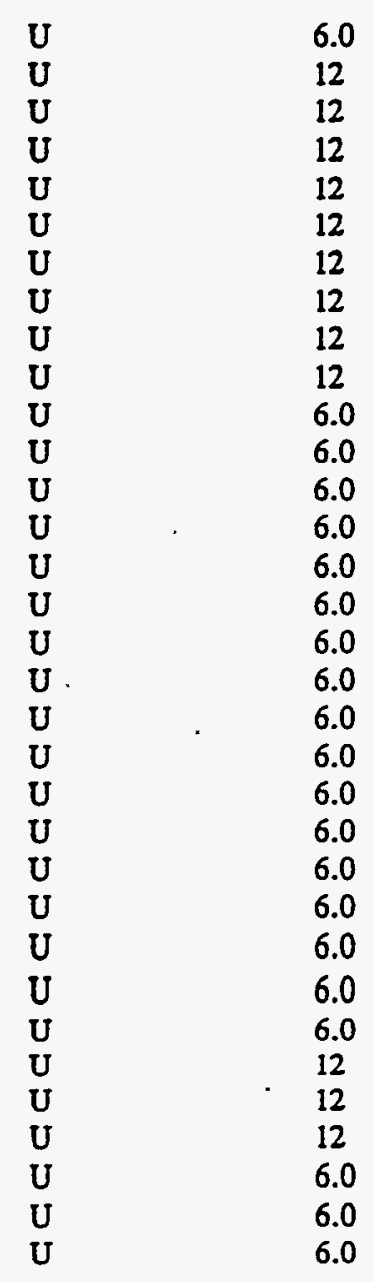

\section{9}

1.9

1.9

1.9

1.9

1.9

3.9

3.9

3.9

3.9

1.9

3.9

3.9

3.9 $\mu g / \mathrm{kg}$
$\mu \mathrm{g} / \mathrm{kg}$
$\mu \mathrm{g} / \mathrm{kg}$
$\mu \mathrm{g} / \mathrm{kg}$
$\mu \mathrm{g} / \mathrm{kg}$
$\mu \mathrm{g} / \mathrm{kg}$
$\mu \mathrm{g} / \mathrm{kg}$
$\mu \mathrm{g} / \mathrm{kg}$
$\mu \mathrm{g} / \mathrm{kg}$
$\mu g / \mathrm{kg}$
$\mu \mathrm{g} / \mathrm{kg}$
$\mu \mathrm{g} / \mathrm{kg}$
$\mu \mathrm{g} / \mathrm{kg}$
$\mu \mathrm{g} / \mathrm{kg}$

1.9
1.9
1.9
1.9
1.9
1.9
3.9
3.9
3.9
3.9
1.9
3.9
3.9
3.9

EPA8080

EPA8080

EPA8080

EPA8080

EPA8080

EPA8080

EPA8080

EPA8080

EPA8080

EPA8080

EPA8080

EPA8080

EPA8080

EPA8080 
SAMPLE NAME: D431 1307A (Continued)

Constituent

$\mathbf{R Q}$

Pesticides/PCBs and Dioxins/Furans

Endrin ketone
Heptachlor
Heptachlor epoxide
Heptachlorodibenzo-p-dioxin isomers
Heptachlorodibenzo-p-dioxin isomers
Heptachlorodibenzo-p-furan isomers
Heptachlorodibenzo-p-furan isomers
Hexachlorodibenzo-p-dioxin isomers
Hexachlorodibenzo-p-furan isomers
Lindane
Methoxychlor
Octachlorodibenzo-p-dioxin
Octachlorodibenzo-p-furan
PCB 1016
PCB 1221
PCB 1232
PCB 1242
PCB 1248
PCB 1254
PCB 1260
Pentachlorodibenzo-p-dioxin isomers
Pentachlorodibenzo-p-furan isomers
Pentachlorodibenzo-p-dioxin isomers
Pentachlorodibenzo-p-furan isomers
2,3,7,8-TCDD
2,3,7,8-TCDF
Tetrachlorodibenzo-p-dioxin isomers
Tetrachlorodibenzo-p-furan isomers
Toxaphene

SAMPLE NAME: D431 1401

Location (SRS Coordinates): 18401.83E $66640.68 \mathrm{~N}$

Ground Elevation Above MSL: $129.4 \mathrm{ft}$

Depth of Core Interval: 0.00 to $0.50 \mathrm{ft}$

Sample Type: Normal

Sample Color: 10R 3/4

\begin{tabular}{|c|c|c|}
\hline 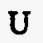 & & 3.9 \\
\hline$U$ & & 1.9 \\
\hline U & & 1.9 \\
\hline U & & 0.50 \\
\hline$U$ & & 0.20 \\
\hline U & & 0.20 \\
\hline UJ & V & 0.70 \\
\hline U & & 0.30 \\
\hline & & 0.30 \\
\hline U & & 1.9 \\
\hline U & & 19 \\
\hline & I & 9.7 \\
\hline$U$ & & 0.60 \\
\hline U & & 39 \\
\hline & & 77 \\
\hline$U$ & & 39 \\
\hline U & & 39 \\
\hline & & 39 \\
\hline & & 39 \\
\hline U & & 39 \\
\hline & & 0.50 \\
\hline & & 0.30 \\
\hline 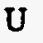 & & 0.30 \\
\hline 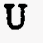 & & 0.20 \\
\hline & & 0.30 \\
\hline & & 0.20 \\
\hline & & 0.30 \\
\hline & & 0.20 \\
\hline & & 190 \\
\hline
\end{tabular}

Sample ID: 100390
Sample Matrix: Soil USC Soil Classification: SP

$\begin{array}{lll}\mu \mathrm{g} / \mathrm{kg} & 3.9 & \text { EPA8080 } \\ \mu \mathrm{g} / \mathrm{kg} & 1.9 & \text { EPA8080 } \\ \mu \mathrm{g} / \mathrm{kg} & 1.9 & \text { EPA8080 } \\ \mathrm{ng} / \mathrm{g} & 0.50 & \text { EPA8280 } \\ \mathrm{ng} / \mathrm{g} & 0.20 & \text { EPA8280 } \\ \mathrm{ng} / \mathrm{g} & 0.20 & \text { EPA8280 } \\ \mathrm{ng} / \mathrm{g} & 0.10 & \text { EPA8280 } \\ \mathrm{ng} / \mathrm{g} & 0.30 & \text { EPA8280 } \\ \mathrm{ng} / \mathrm{g} & 0.30 & \text { EPA8280 } \\ \mu \mathrm{g} / \mathrm{kg} & 1.9 & \text { EPA8080 } \\ \mu \mathrm{g} / \mathrm{kg} & 19 & \text { EPA8080 } \\ \mathrm{ng} / \mathrm{g} & 0.40 & \text { EPA8280 } \\ \mathrm{ng} / \mathrm{g} & 0.60 & \text { EPA8280 } \\ \mu \mathrm{g} / \mathrm{kg} & 39 & \text { EPA8080 } \\ \mu \mathrm{g} / \mathrm{kg} & 77 & \text { EPA8080 } \\ \mu \mathrm{g} / \mathrm{kg} & 39 & \text { EPA8080 } \\ \mu \mathrm{g} / \mathrm{kg} & 39 & \text { EPA8080 } \\ \mu \mathrm{g} / \mathrm{kg} & 39 & \text { EPA8080 } \\ \mu \mathrm{g} / \mathrm{kg} & 39 & \text { EPA8080 } \\ \mu \mathrm{g} / \mathrm{kg} & 39 & \text { EPA8080 } \\ \mathrm{ng} / \mathrm{g} & 0.50 & \text { EPA8280 } \\ \mathrm{ng} / \mathrm{g} & 0.30 & \text { EPA8280 } \\ \mathrm{ng} / \mathrm{g} & 0.30 & \text { EPA8280 } \\ \mathrm{ng} / \mathrm{g} & 0.20 & \text { EPA8280 } \\ \mathrm{ng} / \mathrm{g} & 0.30 & \text { EPA8280 } \\ \mathrm{ng} / \mathrm{g} & 0.20 & \text { EPA8280 } \\ \mathrm{ng} / \mathrm{g} & 0.30 & \text { EPA8280 } \\ \mathrm{ng} / \mathrm{g} & 0.20 & \text { EPA8280 } \\ \mu \mathrm{g} / \mathrm{kg} & 190 & \text { EPA8080 } \\ & & \end{array}$

\begin{tabular}{|c|c|c|c|c|c|c|c|}
\hline Constituent & $\mathbf{R Q}$ & $\mathbf{A Q}$ & $\mathbf{B}$ & Result & Unit & D. Limit & Method \\
\hline Physical Parameters & & & & & & & \\
\hline $\begin{array}{l}\text { Cation exchange capacioy } \\
\text { pH } \\
\text { Specific conductance } \\
\text { Total organic carbon }\end{array}$ & $\mathbf{J}$ & $Q$ & & $\begin{array}{l}9.4 \\
4.9 \\
32 \\
4,100\end{array}$ & $\begin{array}{l}\mathrm{meq} / 100 \mathrm{~g} \\
\mathrm{pH} \\
\mu \mathrm{S} / \mathrm{cm} \\
\mathrm{mg} / \mathrm{kg}\end{array}$ & $\begin{array}{l}2.0 \\
0.010 \\
1.0 \\
250\end{array}$ & $\begin{array}{l}\text { EPA9080 } \\
\text { EPA9045 } \\
\text { EPA9050 } \\
\text { EPA415.1 }\end{array}$ \\
\hline
\end{tabular}

Sample Moisture: Dry Percent Solids: 94.10

D. Limit Method

EPA415.1 
SAMPLE NAME: D4311401 (Continued)

Sample D: 100390

\section{Constituent}

Total Metals

Arsenic

Barium

Cadmium

Chromium

Copper

Lead

Manganese

Mercury

Nickel

Selenium

Silver
RQ AQ B Result Unit

$\begin{array}{lll}\mathrm{mg} / \mathrm{kg} & 0.82 & \text { EPA7060 } \\ \mathrm{mg} / \mathrm{kg} & 20 & \text { EPA6010 } \\ \mathrm{mg} / \mathrm{kg} & 0.50 & \text { EPA6010 } \\ \mathrm{mg} / \mathrm{kg} & 1.0 & \text { EPA6010 } \\ \mathrm{mg} / \mathrm{kg} & 2.5 & \text { EPA6010 } \\ \mathrm{mg} / \mathrm{kg} & 0.25 & \text { EPA7421 } \\ \mathrm{mg} / \mathrm{kg} & 1.5 & \text { EPA6010 } \\ \mathrm{mg} / \mathrm{kg} & 0.11 & \text { EPA7470 } \\ \mathrm{mg} / \mathrm{kg} & 4.0 & \text { EPA6010 } \\ \mathrm{mg} / \mathrm{kg} & 0.41 & \text { EPA7740 } \\ \mathrm{mg} / \mathrm{kg} & 1.0 & \text { EPA6010 }\end{array}$

$\begin{array}{ll}\mathrm{U} & 1.3 \\ \mathrm{U} & 20 \\ & 0.50 \\ \mathrm{U} & 13 \\ & 2.5 \\ & 4.3 \\ \mathrm{U} & 29 \\ \mathrm{U} & 0.11 \\ \mathrm{U} & 4.0 \\ \mathrm{U} & 0.41\end{array}$

1.3

0.50

2.5

29

0.11

0.41

1.0
D. Limit Method

$B / N / A$ Extractables (including PAH and phenols)

Acenaphthene

Acenaphthylene

Anthracene

Benzo(a)anthracene

Benzo(b)fluoranthene

Benzo(k)fluoranthene

Benzoic acid

Benzo(g,h,i)perylene

Benzo(a)pyrene

Benzyl alcohol

Bis(2-chloroethoxy) methane

Bis(2-chloroethyl) ether

Bis(2-chloroisopropyl) ether

Bis(2-ethylhexyl) phthalate

4-Bromophenyl phenyl ether

Butylbenzyl phthalate

4-Chloroaniline

4-Chloro-m-cresol

2-Chloronaphthalene

2-Chlorophenol

4-Chlorophenyl phenyl ether

Chrysene

o-Cresol (2-Methylphenol)

p-Cresol (4-Methylphenol)

Dibenz $(\mathrm{a}, \mathrm{h})$ anthracene

Dibenzofuran

Di-n-butyl phthalate -

1,2-Dichlorobenzene

1,3-Dichlorobenzene

1,4-Dichlorobenzene

3,3'-Dichlorobenzidine

2,4-Dichlorophenol

Diethyl phthalate

2,4-Dimethyl phenol

Dimethyl phthalate

2,4-Dinitrophenol
U 370

370

370

370

370

370

1,800

UJ $\mathrm{H}$

UJ $\mathrm{H}$

UJ $\mathrm{H}$

U

U

U

J

U

U

U

U

U

U

$\begin{array}{ll}\mathbf{U} & 370 \\ \mathrm{U} & 370\end{array}$

UJ $\mathrm{H} \quad 370$

U $\quad 370$

$\mathrm{V} \quad 370$

370

370

370

740

370

370

370

370

1,800

\begin{tabular}{|c|c|c|}
\hline$\mu \mathrm{g} / \mathrm{kg}$ & 370 & EPA8270 \\
\hline$\mu g / k g$ & 370 & EPA8270 \\
\hline$\mu \mathrm{g} / \mathrm{kg}$ & 370 & EPA8270 \\
\hline$\mu \mathrm{g} / \mathrm{kg}$ & 370 & EPA8270 \\
\hline$\mu \mathrm{g} / \mathrm{kg}$ & 370 & EPA 8270 \\
\hline$\mu \mathrm{g} / \mathrm{kg}$ & 370 & EPA 8270 \\
\hline$\mu \mathrm{g} / \mathrm{kg}$ & 1,800 & EPA8270 \\
\hline$\mu \mathrm{g} / \mathrm{kg}$ & 370 & EPA 8270 \\
\hline$\mu \mathrm{g} / \mathrm{kg}$ & 370 & EPA8270 \\
\hline$\mu \mathrm{g} / \mathrm{kg}$ & 370 & EPA 8270 \\
\hline$\mu \mathrm{g} / \mathrm{kg}$ & 370 & EPA8270 \\
\hline$\mu g / \mathrm{kg}$ & 370 & EPA8270 \\
\hline$\mu \mathrm{g} / \mathrm{kg}$ & 370 & EPA8270 \\
\hline$\mu \mathrm{g} / \mathrm{kg}$ & 370 & EPA8270 \\
\hline$\mu \mathrm{g} / \mathrm{kg}$ & 370 & EPA8270 \\
\hline$\mu \mathrm{g} / \mathrm{kg}$ & 370 & EPA8270 \\
\hline$\mu \mathrm{g} / \mathrm{kg}$ & 370 & EPA8270 \\
\hline$\mu \mathrm{g} / \mathrm{kg}$ & 370 & EPA 8270 \\
\hline$\mu g / k g$ & 370 & EPA8270 \\
\hline$\mu \mathrm{g} / \mathrm{kg}$ & 370 & EPA8270 \\
\hline$\mu \mathrm{g} / \mathrm{kg}$ & 370 & EPA8270 \\
\hline$\mu \mathrm{g} / \mathrm{kg}$ & 370 & EPA8270 \\
\hline$\mu \mathrm{g} / \mathrm{kg}$ & 370 & EPA8270 \\
\hline$\mu \mathrm{g} / \mathrm{kg}$ & 370 & EPA8270 \\
\hline$\mu \mathrm{g} / \mathrm{kg}$ & 370 & EPA8270 \\
\hline$\mu \mathrm{g} / \mathrm{kg}$ & 370 & EPA8270 \\
\hline$\mu \mathrm{g} / \mathrm{kg}$ & 370 & EPA8270 \\
\hline$\mu g / k g$ & 370 & EPA8270 \\
\hline$\mu \mathrm{g} / \mathrm{kg}$ & 370 & EPA 8270 \\
\hline$\mu \mathrm{g} / \mathrm{kg}$ & 370 & EPA8270 \\
\hline$\mu \mathrm{g} / \mathrm{kg}$ & 740 & EPA8270 \\
\hline$\mu \mathrm{g} / \mathrm{kg}$ & 370 & EPA8270 \\
\hline$\mu \mathrm{g} / \mathrm{kg}$ & 370 & EPA8270 \\
\hline$\mu \mathrm{g} / \mathrm{kg}$ & 370 & EPA8270 \\
\hline$\mu g / k g$ & 370 & EPA8270 \\
\hline$\mu \mathrm{g} / \mathrm{kg}$ & 1,800 & EPA827 \\
\hline
\end{tabular}


SAMPLE NAME: D43ï 1401 (Continued)

Constituent
Sample ID: 100390
RQ AQ B Result
Unit
D. Limit Method

$B / N / A$ Extractables (including PAH and phenols)

\begin{tabular}{|c|c|c|c|c|c|}
\hline U & & 370 & $\mu \mathrm{g} / \mathrm{kg}$ & 370 & EPA8270 \\
\hline $\mathbf{U}$ & & 370 & $\mu \mathrm{g} / \mathrm{kg}$ & 370 & EPA8270 \\
\hline UJ & H & 370 & $\mu \mathrm{g} / \mathrm{kg}$ & 370 & EPA8270 \\
\hline $\mathbf{U}$ & & 370 & $\mu \mathrm{g} / \mathrm{kg}$ & 370 & EPA8270 \\
\hline U & & 370 & $\mu g / \mathrm{kg}$ & 370 & EPA8270 \\
\hline $\mathbf{U}$ & & 370 & $\mu g / \mathrm{kg}$ & 370 & EPA8270 \\
\hline $\mathbf{U}$ & & 370 & $\mu g / \mathrm{kg}$ & 370 & EPA8270 \\
\hline$U$ & & 370 & $\mu \mathrm{g} / \mathrm{kg}$ & 370 & EPA8270 \\
\hline $\mathbf{U}$ & & 370 & $\mu \mathrm{g} / \mathrm{kg}$ & 370 & EPA8270 \\
\hline J & $N$ & 20,000 & $\mu \mathrm{g} / \mathrm{kg}$ & & EPA8270 \\
\hline UJ & H & 370 & $\mu \mathrm{g} / \mathrm{kg}$ & 370 & EPA8270 \\
\hline $\mathbf{U}$ & & 370 & $\mu \mathrm{g} / \mathrm{kg}$ & 370 & EPA8270 \\
\hline$U$ & & 1,800 & $\mu \mathrm{g} / \mathrm{kg}$ & 1,800 & EPA8270 \\
\hline U & & 370 & $\mu g / \mathrm{kg}$ & 370 & EPA8270 \\
\hline $\mathrm{U}$ & & 370 & $\mu \mathrm{g} / \mathrm{kg}$ & 370 & EPA.8270 \\
\hline $\mathbf{U}$ & & 1,800 & $\mu \mathrm{g} / \mathrm{kg}$ & 1,800 & EPA8270 \\
\hline$U$ & & 1,800 & $\mu \mathrm{g} / \mathrm{kg}$ & 1,800 & EPA8270 \\
\hline U & & 1,800 & $\mu \mathrm{g} / \mathrm{kg}$ & 1,800 & EPA8270 \\
\hline U & & 370 & $\mu \mathrm{g} / \mathrm{kg}$ & 370 & EPA8270 \\
\hline U & & 370 & $\mu g / k g$ & 370 & EPA8270 \\
\hline $\mathbf{U}$ & & 1,800 & $\mu \mathrm{g} / \mathrm{kg}$ & 1.800 & EPA8270 \\
\hline $\mathbf{U}$ & & 370 & $\mu \mathrm{g} / \mathrm{kg}$ & 370 & EPA8270 \\
\hline $\mathbf{U}$ & & 370 & $\mu \mathrm{g} / \mathrm{kg}$ & 370 & EPA8270 \\
\hline $\mathrm{U}$ & & 1,800 & $\mu \mathrm{g} / \mathrm{kg}$ & 1,800 & EPA8270 \\
\hline U & & 370 & $\mu \mathrm{g} / \mathrm{kg}$ & 370 & EPA8270 \\
\hline UJ & C & 370 & $\mathrm{\mu g} / \mathrm{kg}$ & 370 & EPA8270 \\
\hline$U$ & & 370 & $\mu g / k g$ & 370 & EPA8270 \\
\hline $\mathbf{U}$ & & 370 & $\mu \mathrm{g} / \mathrm{kg}$ & 370 & EPA8270 \\
\hline U & & 1,800 & $\mu \mathrm{g} / \mathrm{kg}$ & 1,800 & EPA8270 \\
\hline U & & 370 & $\mu g / \mathrm{kg}$ & 370 & EPA8270 \\
\hline J & $\mathrm{N}$ & 200 & $\mu \mathrm{g} / \mathrm{kg}$ & & EPA8270 \\
\hline J & $\mathbf{N}$ & 200 & $\mu \mathrm{g} / \mathrm{kg}$ & & EPA8270 \\
\hline J & $\mathrm{N}$ & 200 & $\mu \mathrm{g} / \mathrm{kg}$ & & EPA8270 \\
\hline J & $\mathrm{N}$ & 2,000 & $\mu g / k g$ & & EPA8270 \\
\hline J & $\mathbf{N}$ & 200 & $\mu g / \mathrm{kg}$ & & EPA8270 \\
\hline J & $\mathrm{N}$ & 600 & $\mu g / \mathrm{kg}$ & & EPA8270 \\
\hline J & $\mathrm{N}$ & 6,000 & $\mu \mathrm{g} / \mathrm{kg}$ & & EPA8270 \\
\hline
\end{tabular}

Volatile Organic Compounds,

2.4-Dinitrotoluene
2,6-Dinitrotoluene
Di-n-octyl phthalate
Fluoranthene
Fluorene
Hexachlorobenzene
Hexachlorobutadiene
Hexachlorocyclopentadiene
Hexachloroethane
Hexanedioic acid ester
Indeno(1,2,3-c,d)pyrene
Isophorone
2-Methyl-4,6-dinitrophenol
2-Methylnaphthalene
Naphthalene
o-Nitroaniline
m-Nitroaniline
p-Nitroaniline
Nitrobenzene
2-Nitrophenol
4-Nitrophenol
N-Nitrosodiphenylamine
N-Nitrosodipropylamine
Pentachlorophenol
Phenanthrene
Phenol
Pyrene
1,2,4-Trichlorobenzene
2,4,5-Trichlorophenol
2,4,6-Trichlorophenol
Unknown hydrocarbon
Unknown hydrocarbon
Unknown hydrocarbon
Unknown hydrocarbon
Unknown hydrocarbon
Unknown
Unknown ketone
Volatile Organic Compounds.
Acetone
Benzene
Bromodichloromethane
Bromoform
Bromomethane (Methyl bromide)
Carbon disulfide
Carbon tetrachloride
Chlorobenzene
Chloroethane
Chloroethene (Vinyl chloride)

$\begin{array}{lll}\mathrm{UJ} & \mathrm{H} & 11 \\ \mathrm{UJ} & \mathrm{H} & 5.7 \\ \mathrm{UJ} & \mathrm{H} & 5.7 \\ \mathrm{UJ} & \mathrm{H} & 5.7 \\ \mathrm{UJ} & \mathrm{H} & 11 \\ \mathrm{UJ} & \mathrm{H} & 5.7 \\ \mathrm{UJ} & \mathrm{H} & 5.7 \\ \mathrm{UJ} & \mathrm{H} & 5.7 \\ \mathrm{UJ} & \mathrm{H} & 11 \\ \mathrm{UJ} & \mathrm{HL} & 11\end{array}$

$\begin{array}{llll}11 & \mu \mathrm{g} / \mathrm{kg} & 11 & \text { EPA8240 } \\ 5.7 & \mu \mathrm{g} / \mathrm{kg} & 5.7 & \text { EPA8240 } \\ 5.7 & \mu \mathrm{g} / \mathrm{kg} & 5.7 & \text { EPA8240 } \\ 5.7 & \mu \mathrm{gg} & 5.7 & \text { EPA8240 } \\ 11 & \mu \mathrm{kg} / \mathrm{kg} & 11 & \text { EPA8240 } \\ 5.7 & \mu \mathrm{g} / \mathrm{kg} & 5.7 & \text { EPA8240 } \\ 5.7 & \mu \mathrm{g} / \mathrm{kg} & 5.7 & \text { EPA8240 } \\ 5.7 & \mu \mathrm{gg} & 5.7 & \text { EPA8240 } \\ 11 & \mu \mathrm{kg} / \mathrm{kg} & 11 & \text { EPA8240 } \\ 11 & \mu \mathrm{g} / \mathrm{kg} & 11 & \text { EPA8240 }\end{array}$


SAMPLE NAME: D43î 1401 (Continued)

\section{Constituent}

Volatile Organic Compounds

Chloroform
Chloromethane (Methyt chloride)
Dibromochloromethane
1,1-Dichloroethane
1,2-Dichloroethane
1,1-Dichloroethylene
1,2-Dichloroethylene
Dichloromethane (Methylene chloride)
1,2-Dichloropropane
cis-1,3-Dichloropropene
trans-1,3-Dichloropropene
Ethylbenzene
2-Hexanone
Methyl ethyl ketone
Methyl isobutyl ketone
Styrene
1,1,2,2-Tetrachloroethane
Tetrachloroethylene
Toluene
1,1,1-Trichloroethane
1,1,2-Trichloroethane
Trichloroethylene
Vinyl acetate
Xylenes

Sample ID: 100390

RQ AQ B Result Unit $\quad$ D. Limit Method

\section{Pesticides/PCBs and Dioxins/Furans}

\begin{tabular}{|c|c|c|c|c|c|c|c|}
\hline Aldrin & UJ & 0 & L & 1.9 & $\mu \mathrm{g} / \mathrm{kg}$ & 1.9 & EPA8080 \\
\hline alpha-Benzene hexachloride & UJ & 0 & L & 1.9 & $\mu \mathrm{g} / \mathrm{kg}$ & 1.9 & EPA8080 \\
\hline beta-Benzene hexachloride & UJ & 0 & L & 1.9 & $\mu \mathrm{g} / \mathrm{kg}$ & 1.9 & EPA8080 \\
\hline delta-Benzene hexachloride & UJ & 0 & L & 1.9 & $\mu \mathrm{g} / \mathrm{kg}$ & 1.9 & EPA8080 \\
\hline alpha-Chlordane & UJ & 0 & L & 1.9 & $\mu g / \mathrm{kg}$ & 1.9 & EPA8080 \\
\hline gamma-Chlordane & UJ & 0 & L & 1.9 & $\mu g / \mathrm{kg}$ & 1.9 & EPA8080 \\
\hline p.p'-DDD & UJ & 0 & L & 3.8 & $\mu \mathrm{g} / \mathrm{kg}$ & 3.8 & EPA8080 \\
\hline$p, p^{\prime}-D D E$ & UJ & 0 & L & 3.8 & $\mu \mathrm{g} / \mathrm{kg}$ & 3.8 & EPA8080 \\
\hline $\mathrm{p}, \mathrm{p}^{\prime}-\mathrm{DDT}$ & UJ & 0 & L & 3.8 & $\mu \mathrm{g} / \mathrm{kg}$ & 3.8 & EPA8080 \\
\hline Dieldrin & UJ & 0 & L & 3.8 & $\mu g / \mathrm{kg}$ & 3.8 & EPA8080 \\
\hline Endosulfan I & UJ & 0 & L & 1.9 & $\mu g / \mathrm{kg}$ & 1.9 & EPA8080 \\
\hline Endosulfan II & UJ & 0 & L & 3.8 & $\mu \mathrm{g} / \mathrm{kg}$ & 3.8 & EPA8080 \\
\hline Endosulfan sulfate & UJ & 0 & $L$ & 3.8 & $\mu \mathrm{g} / \mathrm{kg}$ & 3.8 & EPA8080 \\
\hline Endrin & UJ & 0 & L & 3.8 & $\mu \mathrm{g} / \mathrm{kg}$ & 3.8 & EPA8080 \\
\hline Endrin ketone & UJ & 0 & L & 3.8 & $\mu g / \mathrm{kg}$ & 3.8 & EPA8080 \\
\hline Heptachlor & UJ & 0 & L & 1.9 & $\mu g / \mathrm{kg}$ & 1.9 & EPA8080 \\
\hline Heptachlor epoxide & UJ & 0 & L & 1.9 & $\mu \mathrm{g} / \mathrm{kg}$ & 1.9 & EPA8080 \\
\hline Heptachlorodibenzo-p-dioxin isomers & $\mathbf{U}$ & & & 0.20 & $\mathrm{ng} / \mathrm{g}$ & 0.20 & EPA8280 \\
\hline Heptachlorodibenzo-p-furan isomers & $\mathrm{U}$ & & & 0.10 & $\mathrm{ng} / \mathrm{g}$ & 0.10 & EPA8280 \\
\hline Hexachlorodibenzo-p-dioxin isomers & $\mathbf{U}$ & & & 0.20 & $\mathrm{ng} / \mathrm{g}$ & 0.20 & EPA8280 \\
\hline Hexachlorodibenzo-p-furan isomers & $\mathbf{U}$ & & & 0.10 & $\mathrm{ng} / \mathrm{g}$ & 0.10 & EPA8280 \\
\hline Lindane & UJ & 0 & L & 1.9 & $\mu \mathrm{g} / \mathrm{kg}$ & 1.9 & EPA8080 \\
\hline Methoxychlor & UJ & 0 & L & 19 & $\mu \mathrm{g} / \mathrm{kg}$ & 19 & EPA8080 \\
\hline
\end{tabular}

\begin{tabular}{|c|c|c|c|c|}
\hline UJ & 5.7 & $\mu g / \mathrm{kg}$. & 5.7 & EPA8240 \\
\hline UJ & 11 & $\mu \mathrm{g} / \mathrm{kg}$ & 11 & EPA8240 \\
\hline U! & 5.7 & $\mu \mathrm{g} / \mathrm{kg}$ & 5.7 & EPA8240 \\
\hline UJ & 5.7 & $\mu \mathrm{g} / \mathrm{kg}$ & 5.7 & EPA8240 \\
\hline US & 5.7 & $\mu g / k g$ & 5.7 & EPA8240 \\
\hline UJ & 5.7 & $\mu \mathrm{g} / \mathrm{kg}$ & 5.7 & EPA8240 \\
\hline UJ & 5.7 & $\mu \mathrm{g} / \mathrm{kg}$ & 5.7 & EPA8240 \\
\hline UJ & 5.7 & $\mu \mathrm{g} / \mathrm{kg}$ & 5.7 & EPA8240 \\
\hline UJ & 5.7 & $\mu \mathrm{g} / \mathrm{kg}$ & 5.7 & EPA8240 \\
\hline UJ & 5.7 & $\mu \mathrm{g} / \mathrm{kg}$ & 5.7 & EPA8240 \\
\hline UJ & 5.7 & $\mu \mathrm{g} / \mathrm{kg}$ & 5.7 & EPA8240 \\
\hline UJ & 5.7 & $\mu \mathrm{g} / \mathrm{kg}$ & 5.7 & EPA8240 \\
\hline UJ & 11 & $\mu \mathrm{g} / \mathrm{kg}$ & 11 & EPA8240 \\
\hline UJ & 11 & $\mu \mathrm{g} / \mathrm{kg}$ & 11 & EPA8240 \\
\hline UJ & 11 & $\mu \mathrm{g} / \mathrm{kg}$ & 11 & EPA8240 \\
\hline UJ & 5.7 & $\mu \mathrm{g} / \mathrm{kg}$ & 5.7 & EPA8240 \\
\hline UJ & 5.7 & $\mu \mathrm{g} / \mathrm{kg}$ & 5.7 & EPA8240 \\
\hline UJ & 5.7 & $\mu \mathrm{g} / \mathrm{kg}$ & 5.7 & EPA8240 \\
\hline UJ & 5.7 & $\mu \mathrm{g} / \mathrm{kg}$ & 5.7 & EPA8240 \\
\hline UJ & 5.7 & $\mu \mathrm{g} / \mathrm{kg}$ & 5.7 & EPA8240 \\
\hline UI & 5.7 & $\mu \mathrm{g} / \mathrm{kg}$ & 5.7 & EPA8240 \\
\hline UJ & 5.7 & $\mu \mathrm{g} / \mathrm{kg}$ & 5.7 & EPA8240 \\
\hline UJ & 11 & $\mu \mathrm{g} / \mathrm{kg}$ & 11. & EPA8240 \\
\hline $\mathrm{UJ}$ & 5.7 & $\mu \mathrm{g} / \mathrm{kg}$ & 5.7 & EPA8240 \\
\hline
\end{tabular}


SAMPLE NAME: D431̈ 1401 (Continued) Sample ID: 100390

Constituent

RQ AQ B Result

Pesticides/PCBs and Dioxins/Furans

Octachlorodibenzo-p-dioxin

Octachlorodibenzo-p-furan

PCB 1016

PCB 1221

PCB 1232

PCB 1242

PCB 1248

PCB 1254

PCB 1260

Pentachlorodibenzo-p-dioxin isomers

Pentachlorodibenzo-p-furan isomers

\subsubsection{8-TCDD}

$2,3,7,8-\mathrm{TCDF}$

Tetrachlorodibenzo-p-dioxin isomers

Tetrachlorodibenzo-p-furan isomers

Toxaphene

\begin{tabular}{|c|c|c|c|c|c|c|}
\hline & & & 3.2 & $\mathrm{ng} / \mathrm{g}$ & 0.10 & EPA8280 \\
\hline $\mathrm{U}$ & & & 0.20 & $\mathrm{ng} / \mathrm{g}$ & 0.20 & EPA8280 \\
\hline UJ & 0 & L & 38 & $\mu \mathrm{g} / \mathrm{kg}$ & 38 & EPA8080 \\
\hline UJ & 0 & L & 76 & $\mu \mathrm{g} / \mathrm{kg}$ & 76 & EPA8080 \\
\hline UJ & 0 & L & 38 & $\mu \mathrm{g} / \mathrm{kg}$ & 38 & EPA8080 \\
\hline UJ & 0 & L & 38 & $\mu \mathrm{g} / \mathrm{kg}$ & 38 & EPA8080 \\
\hline UJ & 0 & L & 38 & $\mu \mathrm{g} / \mathrm{kg}$ & 38 & EPA8080 \\
\hline UJ & 0 & $\bar{L}$ & 38 & $\mu \mathrm{g} / \mathrm{kg}$ & 38 & EPA8080 \\
\hline UJ & 0 & L & 38 & $\mu \mathrm{g} / \mathrm{kg}$ & 38 & EPA8080 \\
\hline $\mathbf{U}$ & & & 0.20 & $\mathrm{ng} / \mathrm{g}$ & 0.20 & EPA8280 \\
\hline $\mathrm{U}$ & & & 0.10 & $\mathrm{ng} / \mathrm{g}$ & 0.10 & EPA8280 \\
\hline $\mathbf{U}$ & & & 0.10 & $\mathrm{ng} / \mathrm{g}$ & 0.10 & EPA8280 \\
\hline $\mathrm{U}$ & & & 0.10 & $\mathrm{ng} / \mathrm{g}$ & 0.10 & EPA8280 \\
\hline$U$ & & & 0.20 & $\mathrm{ng} / \mathrm{g}$ & 0.20 & EPA8280 \\
\hline $\mathrm{U}$ & & & 0.20 & $\mathrm{ng} / \mathrm{g}$ & 0.20 & EPA8280 \\
\hline UJ & 0 & L & 190 & $\mu \mathrm{g} / \mathrm{kg}$ & 190 & EPA8080 \\
\hline
\end{tabular}

Radionuclide Indicators and Radionuclides

Gross alpha

Gross alpha

Nonvolatile beta

Nonvolatile beta

Radium, total alpha-emitting

Radium, total alpha-emitting

Tritium

Tritium

SAMPLE NAME: D431 1401D

Sample Type: Split

Constituent

Physical Parameters

Cation exchange capacity

$\mathrm{pH}$

$\mathrm{pH}$

Specific conductance --

Specific conductance

Total organic carbon

Total Metals

Arsenic

Barium

Cadmium

Chromium

$\begin{array}{lll} & & \\ & X \\ U & \mathrm{X} \\ \mathrm{U} & \mathrm{V} \\ \mathrm{U} & & \\ \mathrm{U} & & \end{array}$

RQ AQ B Result

Unit

D. Limit Method

$\begin{array}{llll}12 \pm 19 & \mathrm{pCi} / \mathrm{g} & 9.7 & \text { EPA900.0 } \\ 9.9 \pm 17 & \mathrm{pCi} / \mathrm{g} & 9.3 & \text { EPA900.0 } \\ 74 \pm 29 & \mathrm{pCi} / \mathrm{g} & 17 & \text { EPA900.0 } \\ 46 \pm 25 & \mathrm{pCi} / \mathrm{g} & 16 & \text { EPA900.0 } \\ 1.4 \pm 1.1 & \mathrm{pCi} / \mathrm{g} & 0.20 & \text { EPA903.0. } \\ 2.1 \pm 1.2 & \mathrm{pCi} / \mathrm{g} & 0.17 & \text { EPA903.0 } \\ 1.2 & \mathrm{pCi} / \mathrm{g} & 1.2 & \text { EPA906.0 } \\ 1.3 & \mathrm{pCi} / \mathrm{g} & 1.3 & \text { EPA906.0 }\end{array}$

Sample ID: 100391

Associated Sample: 100390

RQ AQ B Result Unit $\quad$ D. Limit Method

$\begin{array}{llllll} & & 12 & \mathrm{meq} / 100 \mathrm{~g} & 2.0 & \text { EPA9080 } \\ \mathrm{J} & \mathrm{Q} & 4.8 & \mathrm{pH} & 0.010 & \text { EPA9045 } \\ \mathrm{J} & \mathrm{Q} & 4.7 & \mathrm{pH} & 0.010 & \text { EPA9045 } \\ & & 35 & \mu \mathrm{S} / \mathrm{cm} & 1.0 & \text { EPA9050 } \\ & & 35 & \mu \mathrm{cm} & 1.0 & \text { EPA9050 } \\ & & 5,700 & \mathrm{mg} / \mathrm{kg} & 280 & \text { EPA415.1 }\end{array}$

$\begin{array}{llll}2.4 & \mathrm{mg} / \mathrm{kg} & 2.0 & \text { EPA7060 } \\ 33 & \mathrm{mg} / \mathrm{kg} & 33 & \text { EPA6010 } \\ 0.83 & \mathrm{mg} / \mathrm{kg} & 0.83 & \text { EPA6010 } \\ 25 & \mathrm{mg} / \mathrm{kg} & 1.7 & \text { EPA6010 }\end{array}$


SAMPLE NAME: D431 1401D (Continued)
Sample ID: 100391

\section{Constituent}

Total Metals

RQ AQ B Result

$\begin{array}{ll}\mathrm{U} & 4.2 \\ & 7.9 \\ \mathrm{U} & 54 \\ \mathrm{U} & 0.21 \\ \mathrm{U} & 6.7 \\ & 0.98 \\ & 1.8\end{array}$

4.2

U

Lead

Manganese

Mercury

Nickel

Selenium

Silver

$B / N / A$ Extractables (including PAH and phenols)

Acenaphthene

Acenaphthylene

Anthracene

Benzo(a)anthracene

Benzo(b)fluoranthene

Benzo(k)fluoranthene

Benzoic acid

Benzo(g,h,i)perylene

Benzo(a)pyrene

Benzyl alcohol

Bis(2-chloroethoxy) methane

Bis(2-chloroethyl) ether

Bis(2-chloroisopropyl) ether

Bis(2-ethylhexyl) phthalate

4-Bromophenyl phenyl ether

Butanoic acid ester

Butylbenzyl phthalate

4-Chloroaniline

4-Chloro-m-cresol

2-Chloronaphthalene

2-Chlorophenol

4-Chlorophenyl phenyl ether

Chrysene

o-Cresol (2-Methylphenol)

p-Cresol (4-Methylphenol)

Hexadecanoic acid ester

Dibenz $(a, h)$ anthracene

Dibenzofuran

Di-n-butyl phthalate

1,2-Dichlorobenzene -

1,3-Dichlorobenzene

1,4-Dichlorobenzene

3,3'-Dichlorobenzidine

2.4-Dichlorophenol

Diethyl phthalate

2.4-Dimethyl phenol

Dimethyl phethalate

2,4-Dinitrophenol

2,4-Dinitrotoluene

2,6-Dinitrotoluene
U .

U

U

U

U

U

U

U

U

U

U

U

U

U

U

J

U

U

U

U

U

U

U

U

J

U

U

UJ

U

- U

U

U

U

U

U

U

U

U
370

370

370

370

370

370

1,900

370

370

370

370

370

370

370

370

NV

$$
200
$$$$
370
$$

370

370

370

370

370

370

370

370

200

370

370

94

370

370

370

750

370

370

370

370

1,900

$\cdot 370$

370 $\mathrm{mg} / \mathrm{kg}$
$\mathrm{mg} / \mathrm{kg}$
$\mathrm{mg} / \mathrm{kg}$
$\mathrm{mg} / \mathrm{kg}$
$\mathrm{mg} / \mathrm{kg}$
$\mathrm{mg} / \mathrm{kg}$
$\mathrm{mg} / \mathrm{kg}$

4.2

0.59

2.5

0.21

6.7

0.98

1.7

D. Limit Method
EPA6010
EPA7421
EPA6010
EPA7470
EPA6010
EPA7740
EPA6010 
$B / N / A$ Extractables (including $P A H$ and phenols)

Di-n-octyl phthalate

Fluoranthene

Fluorene

Hexachlorobenzene

Hexachlorobutadiene

Hexachlorocyclopentadiene

Hexachloroethane

Hexanedioic acid ester

Indeno(1.2.3-c,d)pyrene

Isophorone

2-Methyl-4,6-dinitrophenol

2-Methyinaphthalene

Naphthalene

o-Nitroaniline

m-Nitroaniline

p-Nitroaniline

Nitrobenzene

2-Nitrophenol

4-Nitrophenol

N-Nitrosodiphenylamine

$\mathrm{N}$-Nitrosodipropylamine

Pentachlorophenol

Phenanthrene

Phenol

Pyrene

1,2,4-Trichlorobenzene

2,4,5-Trichlorophenol

2,4,6-Trichlorophenol

Unknown hydrocarbon

Unknown hydrocarbon

Unknown

Unknown ketone

Volatile Organic Compounds

\section{Acetone}

Benzene

Bromodichloromethane

Bromoform

Bromomethane (Methyl bromide)

Carbon disulfide

Carbon tetrachloride

Chlorobenzene

Chloroethane

Chloroethene (Vinyl chloride)

Chloroform

Chloromethane (Methyl chloride)

Dibromochloromethane

1,1-Dichloroethane

1,2-Dichloroethane

\begin{tabular}{|c|c|c|}
\hline $\mathrm{U}$ & & 370 \\
\hline $\mathbf{U}$ & & 370 \\
\hline $\mathbf{U}$ & & 370 \\
\hline$U$ & & 370 \\
\hline $\mathbf{U}$ & & 370 \\
\hline U & & 370 \\
\hline U & & 370 \\
\hline $\mathbf{J}$ & $\mathbf{N}$ & 2,000 \\
\hline $\mathrm{U}$ & & 370 \\
\hline $\mathbf{U}$ & & 370 \\
\hline $\mathbf{U}$ & & 1,900 \\
\hline $\mathrm{U}$ & & 370 \\
\hline$J$ & & 40 \\
\hline $\mathrm{U}$ & & 1,900 \\
\hline $\mathrm{U}$ & & 1,900 \\
\hline$U$ & & 1,900 \\
\hline $\mathrm{U}$ & & 370 \\
\hline $\mathrm{U}$ & & 370 \\
\hline $\mathrm{U}$ & & 1,900 \\
\hline $\mathbf{U}$ & & 370 \\
\hline $\mathbf{U}$ & & 370 \\
\hline $\mathbf{U}$ & & 1,900 \\
\hline J & & 40 \\
\hline UJ & C & 370 \\
\hline $\mathbf{U}$ & & 370 \\
\hline $\mathbf{U}$ & & 370 \\
\hline $\mathrm{U}$ & & 1,900 \\
\hline $\mathbf{U}$ & & 370 \\
\hline $\mathbf{J}$ & $N$ & 300 \\
\hline $\mathbf{J}$ & $\mathbf{N}$ & 400 \\
\hline $\mathbf{J}$ & $\mathbf{N}$ & 200 \\
\hline $\mathrm{J}$ & $\mathrm{N}$ & 5,000 \\
\hline
\end{tabular}

$\mu g / \mathrm{kg}$
$\mu \mathrm{g} / \mathrm{kg}$
$\mu \mathrm{g} / \mathrm{kg}$
$\mu \mathrm{g} / \mathrm{kg}$
$\mu \mathrm{g} / \mathrm{kg}$
$\mu \mathrm{g} / \mathrm{kg}$
$\mu \mathrm{g} / \mathrm{kg}$
$\mu \mathrm{g} / \mathrm{kg}$
$\mu \mathrm{g} / \mathrm{kg}$
$\mu \mathrm{g} / \mathrm{kg}$
$\mu \mathrm{g} / \mathrm{kg}$
$\mu \mathrm{g} / \mathrm{kg}$
$\mu \mathrm{g} / \mathrm{kg}$
$\mu \mathrm{g} / \mathrm{kg}$
$\mu \mathrm{g} / \mathrm{kg}$
$\mu \mathrm{g} / \mathrm{kg}$
$\mu \mathrm{g} / \mathrm{kg}$
$\mu \mathrm{g} / \mathrm{kg}$
$\mu \mathrm{g} / \mathrm{kg}$
$\mu \mathrm{g} / \mathrm{kg}$
$\mu \mathrm{g} / \mathrm{kg}$
$\mu \mathrm{g} / \mathrm{kg}$
$\mu \mathrm{g} / \mathrm{kg}$
$\mu \mathrm{g} / \mathrm{kg}$
$\mu \mathrm{g} / \mathrm{kg}$
$\mu \mathrm{g} / \mathrm{kg}$
$\mu \mathrm{g} / \mathrm{kg}$
$\mu g / \mathrm{kg}$
$\mu \mathrm{g} / \mathrm{kg}$
$\mu \mathrm{g} / \mathrm{kg}$
$\mu \mathrm{g} / \mathrm{kg}$
$\mu \mathrm{g} / \mathrm{kg}$

370

EPA8270

EPA8270

EPA8270

EPA8270

EPA8270

EPA8270

EPA8270

EPA8270

EPA8270

EPA8270

EPA8270

EPA8270

EPA8270

EPA8270

EPA8270

EPA8270

EPA8270

EPA8270

EPA8270

EPA8270

EPA8270

EPA8270

EPA8270

EPA8270

EPA8270

EPA8270

EPA8270

EPA8270

EPA8270

EPA8270

EPA8270

EPA8270

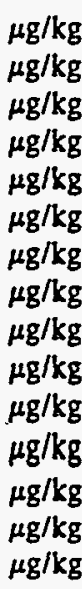

11

5.6

EPA8240

EPA8240

EPA8240

EPA 8240

5.6

EPA8240

EPA8240

5.6

EPA8240

EPA8240

EPA8240

EPA8240

EPA8240

EPA8240

EPA8240

EPA8240

EPA8240 
SAMPLE NAME: D431 1401D (Continued)

Sample ID: 100391

\section{Constituent}

RQ

AQ

B Result

Volatile Organic Compounds
Pesticides/PCBs and Dioxins/Furans

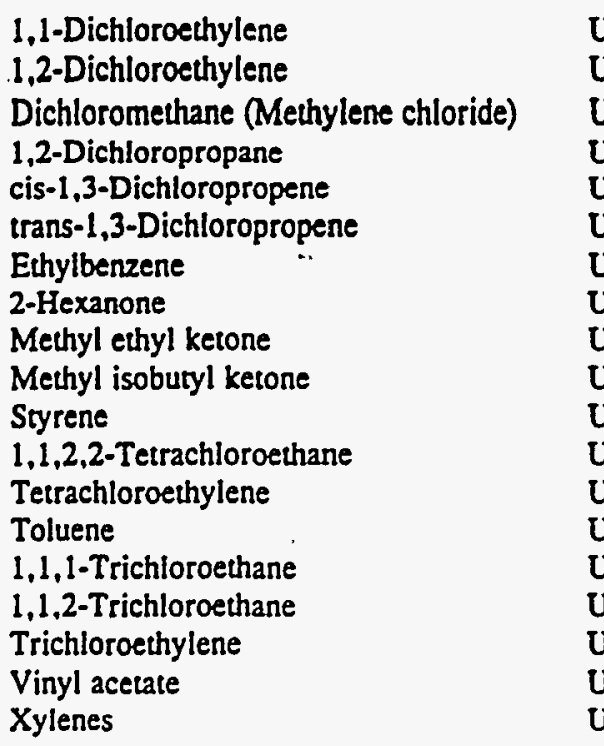

$\begin{array}{ll}\mathrm{U} & 5.6 \\ \mathrm{U} & 5.6 \\ \mathrm{U} & 5.6 \\ \mathrm{U} & 5.6 \\ \mathrm{U} & 5.6 \\ \mathrm{U} & 5.6 \\ \mathrm{U} & 5.6 \\ \mathrm{U} & 11 \\ \mathrm{U} & 11 \\ \mathrm{U} & 11 \\ \mathrm{U} & 5.6 \\ \mathrm{U} & 5.6 \\ \mathrm{U} & 5.6 \\ \mathrm{U} & 5.6 \\ \mathrm{U} & 5.6 \\ \mathrm{U} & 5.6 \\ \mathrm{U} & 5.6 \\ \mathrm{U} & 11 \\ \mathrm{U} & 5.6\end{array}$

\begin{tabular}{|c|c|c|c|}
\hline UJ & 0 & L & 1.9 \\
\hline UJ & 0 & L & 1.9 \\
\hline UJ & 0 & L & 1.9 \\
\hline UJ & 0 & L & 1.9 \\
\hline UJ & 0 & $\bar{L}$ & 1.9 \\
\hline UJ & 0 & L & 1.9 \\
\hline U & 0 & L & 3.7 \\
\hline UJ & 0 & L & 3.7 \\
\hline UJ & 0 & L & 3.7 \\
\hline UI & 0 & L & 3.7 \\
\hline UJ & 0 & L & 1.9 \\
\hline UJ & 0 & $\bar{L}$ & 3.7 \\
\hline UI & 0 & L & 3.7 \\
\hline UJ & 0 & L & 3.7 \\
\hline UJ & 0 & L & 3.7 \\
\hline UI & 0 & L. & 1.9 \\
\hline UJ & 0 & L & 1.9 \\
\hline $\mathbf{U}$ & & & 0.20 \\
\hline$U$ & & & 0.10 \\
\hline U & & & 0.20 \\
\hline $\mathrm{U}$ & & & 0.10 \\
\hline UI & 0 & L & 1.9 \\
\hline UJ & 0 & L & 19 \\
\hline & & & 4,7 \\
\hline $\mathbf{U}$ & & & 0.30 \\
\hline UJ & 0 & $L$ & 37 \\
\hline UJ & 0 & $\bar{L}$ & 75 \\
\hline UJ & 0 & L & 37 \\
\hline
\end{tabular}

Unit

D. Limit Method
Aldrin

alpha-Benzene hexachloride

beta-Benzene hexachloride

delta-Benzene hexachloride

alpha-Chlordane

gamma-Chlordane

p.p'-DDD

p.p'-DDE

p.p'-DDT

Dieldrin

Endosulfan I

Endosulfan II

Endosulfan sulfate

Endrin

Endrin ketone

Heptachlor

Heptachlor epoxide

Heptachlorodibenzo-p-dioxin isomers

Heptachlorodibenzo-p-füran isomers

Hexachlorodibenzo-p-dioxin isomers

Hexachlorodibenzo-p-furan isomers

Lindane

Methoxychlor

Octachlorodibenzo-p-dioxin

Octachlorodibenzo-p-furan

PCB 1016

PCB 1221

PCB 1232

$\begin{array}{ll}5.6 & \text { EPA8240 } \\ 5.6 & \text { EPA8240 } \\ 5.6 & \text { EPA8240 } \\ 5.6 & \text { EPA8240 } \\ 5.6 & \text { EPA8240 } \\ 5.6 & \text { EPA8240 } \\ 5.6 & \text { EPA8240 } \\ 11 & \text { EPA8240 } \\ 11 & \text { EPA8240 } \\ 11 & \text { EPA8240 } \\ 5.6 & \text { EPA8240 } \\ 5.6 & \text { EPA8240 } \\ 5.6 & \text { EPA8240 } \\ 5.6 & \text { EPA8240 } \\ 5.6 & \text { EPA8240 } \\ 5.6 & \text { EPA8240 } \\ 5.6 & \text { EPA8240 } \\ 11 & \text { EPA8240 } \\ 5.6 & \text { EPA8240 }\end{array}$

$$
\begin{aligned}
& \mu \mathrm{g} / \mathrm{kg} \\
& \mu \mathrm{g} / \mathrm{kg} \\
& \mu \mathrm{g} / \mathrm{kg} \\
& \mu \mathrm{g} / \mathrm{kg} \\
& \mu \mathrm{g} / \mathrm{kg} \\
& \mu \mathrm{g} / \mathrm{kg} \\
& \mu \mathrm{g} / \mathrm{kg} \\
& \mu \mathrm{gg} \\
& \mu \mathrm{g} / \mathrm{kg} \\
& \mu \mathrm{g} / \mathrm{kg} \\
& \mu \mathrm{g} / \mathrm{kg} \\
& \mu \mathrm{g} / \mathrm{kg} \\
& \mu \mathrm{kg} \\
& \mu \mathrm{g} / \mathrm{kg} \\
& \mu \mathrm{g} / \mathrm{kg} \\
& \mu \mathrm{g} / \mathrm{kg} \\
& \mu \mathrm{g} / \mathrm{kg} \\
& \mu \mathrm{g} / \mathrm{kg} \\
& \mu \mathrm{g} / \mathrm{kg}
\end{aligned}
$$

5.6

$\begin{array}{lll}\mu \mathrm{g} / \mathrm{kg} & 1.9 & \text { EPA8080 } \\ \mu \mathrm{g} / \mathrm{kg} & 1.9 & \text { EPA8080 } \\ \mu \mathrm{g} / \mathrm{kg} & 1.9 & \text { EPA8080 } \\ \mu \mathrm{g} / \mathrm{kg} & 1.9 & \text { EPA8080 } \\ \mu \mathrm{g} / \mathrm{kg} & 1.9 & \text { EPA8080 } \\ \mu \mathrm{g} / \mathrm{kg} & 1.9 & \text { EPA8080 } \\ \mu \mathrm{g} / \mathrm{kg} & 3.7 & \text { EPA8080 } \\ \mu \mathrm{g} / \mathrm{kg} & 3.7 & \text { EPA8080 } \\ \mu \mathrm{g} / \mathrm{kg} & 3.7 & \text { EPA8080 } \\ \mu \mathrm{g} / \mathrm{kg} & 3.7 & \text { EPA8080 } \\ \mu \mathrm{g} / \mathrm{kg} & 1.9 & \text { EPA8080 } \\ \mu \mathrm{g} / \mathrm{kg} & 3.7 & \text { EPA8080 } \\ \mu \mathrm{g} / \mathrm{kg} & 3.7 & \text { EPA8080 } \\ \mu \mathrm{g} / \mathrm{kg} & 3.7 & \text { EPA8080 } \\ \mu \mathrm{g} / \mathrm{kg} & 3.7 & \text { EPA8080 } \\ \mu \mathrm{g} / \mathrm{kg} & 1.9 & \text { EPA8080 } \\ \mu \mathrm{g} / \mathrm{kg} & 1.9 & \text { EPA8080 } \\ \mathrm{ng} / \mathrm{g} & 0.20 & \text { EPA8280 } \\ \mathrm{ng} / \mathrm{g} & 0.10 & \text { EPA8280 } \\ \mathrm{ng} / \mathrm{g} & 0.20 & \text { EPA8280 } \\ \mathrm{ng} / \mathrm{g} & 0.10 & \text { EPA8280 } \\ \mu \mathrm{g} / \mathrm{kg} & 1.9 & \text { EPA8080 } \\ \mu \mathrm{g} / \mathrm{kg} & 19 & \text { EPA8080 } \\ \mathrm{ng} / \mathrm{g} & 0.10 & \text { EPA8280 } \\ \mathrm{ng} / \mathrm{g} & 0.30 & \text { EPA8280 } \\ \mu \mathrm{g} / \mathrm{kg} & 37 & \text { EPA8080 } \\ \mu \mathrm{g} / \mathrm{kg} & 75 & \text { EPA8080 } \\ \mu \mathrm{gg} / \mathrm{kg} & 37 & \text { EPA8080 }\end{array}$


SAMPLE NAME: D431-1401D (Continued) Sample ID: 100391

Constituent

RQ AQ B Result

Unit

D. Limit Method

Pesticides/PCBs and Dioxins/Furans

PCB 1242
PCB 1248
PCB 1254
PCB 1260

UJ 0 L 37

$\begin{array}{llll}\text { UJ } & 0 & \text { L } & 37\end{array}$

UJ $\begin{array}{lll}0 & \mathrm{~L} & 37\end{array}$

UJ $\quad 0 \quad$ L 37

Pentorodibenzo-p-dioxin isomers

U $\quad 0.20$

Pentachlorodibenzo-p-furan isomers

2,3,7,8-TCDD

$\mathrm{U} \quad 0.20$

0.20
0.10

$\begin{array}{ll}\mathrm{U} & 0.10 \\ \mathrm{U} & 0.10\end{array}$

2,3,7,8-TCDF

Tetrachlorodibenzo-p-dioxin isomers

0.20

Tetrachlorodibenzo-p-furan isomers

$\begin{array}{llll}U & & & 0.20 \\ \text { UJ } & 0 & L & 190\end{array}$

Toxaphene

$\mu \mathrm{g} / \mathrm{kg}$

$\mu g / k g$

$\mu g / \mathrm{kg}$

$\mu g / \mathrm{kg}$

$\mathrm{ng} / \mathrm{g}$

$\mathrm{ng} / \mathrm{g}$

$\mathrm{ng} / \mathrm{g}$

$\mathrm{ng} / \mathrm{g}$

$\mathrm{ng} / \mathrm{g}$

$\mathrm{ng} / \mathrm{g}$

Radionuclide Indicators and Radionuclides

Gross alpha

Nonvolatile beta

Radium, total alpha-emitting

Tritium

$\begin{array}{llll} & & 9.6 & \mathrm{pCi} / \mathrm{g} \\ & & 29 \pm 23 & \mathrm{pCi} / \mathrm{g} \\ \mathrm{U} & \mathrm{V} & 1.2 \pm 0.96 & \mathrm{pCi} / \mathrm{g} \\ & & 1.6 \pm 0.39 & \mathrm{pCi} / \mathrm{g}\end{array}$

Sample ID: 100392

Sample Matrix: Soil

USC Soil Classification: SC
Sample Moisture: Dry Percent Solids: 89.10

D. Limit Method

Constituent

RQ AQ B Result

Unit

9.6

EPA900.0

16

EPA900.0

1.4

EPA906.0

Total Metals

Arsenic

Barium

Cadmium

Chromium

Copper

Lead

Manganese

Mercury

Nickel

Selenium

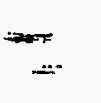

$U$
$U$
$U$

Silver

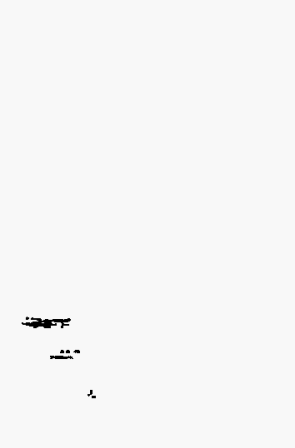

U 1.1

22

0.56

11

U

2.8

3.8

19

U $\quad 0.11$

4.5

$\begin{array}{ll}\mathrm{U} & 4.5 \\ \mathrm{U} & 0.55 \\ & 1.1\end{array}$

$+$

$B / N / A$ Extractables (including PAH and phenols)

Acenaphthene

Acenaphthylene

U

Anthracene

U

Benzo(a)anchracene

U

U

370
370
370
370

$\mu \mathrm{g} / \mathrm{kg}$

$\mu g / \mathrm{kg}$

$\mu \mathrm{g} / \mathrm{kg}$

$$
\begin{aligned}
& \mathrm{mg} / \mathrm{kg} \\
& \mathrm{mg} / \mathrm{kg} \\
& \mathrm{mg} / \mathrm{kg} \\
& \mathrm{mg} / \mathrm{kg} \\
& \mathrm{mg} / \mathrm{kg} \\
& \mathrm{mg} / \mathrm{kg} \\
& \mathrm{mg} / \mathrm{kg} \\
& \mathrm{mg} / \mathrm{kg} \\
& \mathrm{mg} / \mathrm{kg} \\
& \mathrm{mg} / \mathrm{kg} \\
& \mathrm{mg} / \mathrm{kg}
\end{aligned}
$$

$\begin{array}{ll}1.1 & \text { EPA7060 } \\ 22 & \text { EPA6010 } \\ 0.56 & \text { EPA6010 } \\ 1.1 & \text { EPA6010 } \\ 2.8 & \text { EPA6010 } \\ 0.33 & \text { EPA7421 } \\ 1.7 & \text { EPA6010 } \\ 0.11 & \text { EPA7470 } \\ 4.5 & \text { EPA6010 } \\ 0.55 & \text { EPA7740 } \\ 1.1 & \text { EPA6010 }\end{array}$

1.1 EPA6010

$\mu \mathrm{g} / \mathrm{kg}$

370

EPA8270

370

EPA8270

EPA8270

EPA8270 
SAMPLE NAME: D43T 1402 (Continued)

Constituent

RQ AQ B Result

$B / N / A$ Extractables (including $P A H$ and phenols)
Sample ID: 100392

Unit

D. Limit Method

\begin{tabular}{|c|c|c|c|c|c|c|}
\hline Benzo(b)fluoranthene & $\mathbf{U}$ & & 370 & $\mu \mathrm{g} / \mathrm{kg}$ & 370 & EPA8270 \\
\hline Benzo(k)fluoranthene & $\mathbf{U}$ & & 370 & $\mu g / \mathrm{kg}$ & 370 & EPA8270 \\
\hline Benzoic acid & $\mathbf{U}$ & & 1,900 & $\mu \mathrm{g} / \mathrm{kg}$ & 1,900 & EPA8270 \\
\hline Benzo $(g, h, i)$ perylene & $\mathrm{U}$ & & 370 & $\mu g / \mathrm{kg}$ & 370 & EPA8270 \\
\hline Benzo(2)pyrene & $\mathbf{U}$ & & 370 & $\mu \mathrm{g} / \mathrm{kg}$ & 370 & EPA8270 \\
\hline Benzyl alcohol & $\mathbf{U}$ & & 370 & $\mu \mathrm{g} / \mathrm{kg}$ & 370 & EPA8270 \\
\hline Bis(2-chloroethoxy) methane & $\mathbf{U}$ & & 370 & $\mu \mathrm{g} / \mathrm{kg}$ & 370 & EPA8270 \\
\hline Bis(2-chloroethyl) ether & U & & 370 & $\mu \mathrm{g} / \mathrm{kg}$ & 370 & EPA8270 \\
\hline Bis(2-chloroisopropyl) ether & $\mathrm{U}$ & & 370 & $\mu \mathrm{g} / \mathrm{kg}$ & 370 & EPA8270 \\
\hline Bis(2-ethylhexyi) phthalate & $\mathbf{U}$ & & 370 & $\mu g / k g$ & 370 & EPA8270 \\
\hline 4-Bromophenyl phenyl ether & U & & 370 & $\mu g / \mathrm{kg}$ & 370 & EPA8270 \\
\hline Butanoic acid ester & J & NV & 300 & $\mu \mathrm{g} / \mathrm{kg}$ & & EPA8270 \\
\hline Butylbenzyl phthalate & U & & 370 & $\mu \mathrm{g} / \mathrm{kg}$ & 370 & EPA8270 \\
\hline 4-Chloroaniline & $\mathrm{U}$ & & 370 & $\mu g / \mathrm{kg}$ & 370 & EPA8270 \\
\hline 4-Chloro-m-cresol & U & & 370 & $\mu \mathrm{g} / \mathrm{kg}$ & 370 & EPA8270 \\
\hline 2-Chloronaphthalene & $\mathbf{U}$ & & 370 & $\mu \mathrm{g} / \mathrm{kg}$ & 370 & EPA8270 \\
\hline 2-Chlorophenol & $\mathrm{U}$ & & 370 & $\mu \mathrm{g} / \mathrm{kg}$ & 370 & EPA8270 \\
\hline 4-Chlorophenyl phenyl echer & U & & 370 & $\mu \mathrm{g} / \mathrm{kg}$ & 370 & EPA8270 \\
\hline Chrysene & U & & 370 & $\mu \mathrm{g} / \mathrm{kg}$ & 370 & EPA8270 \\
\hline o-Cresol (2-Methylphenol) & U & & 370 & $\mu \mathrm{g} / \mathrm{kg}$ & 370 & EPA8270 \\
\hline p-Cresol (4-Methylphenol) & $\mathbf{U}$ & & 370 & $\mu \mathrm{g} / \mathrm{kg}$ & 370 & EPA8270 \\
\hline Dibenz(a,h)anthracene & $\mathbf{U}$ & & 370 & $\mu \mathrm{g} / \mathrm{kg}$ & 370 & EPA8270 \\
\hline Dibenzofuran & U & & 370 & $\mu \mathrm{g} / \mathrm{kg}$ & 370 & EPA8270 \\
\hline Di-n-butyl phthalate & UJ & $\mathrm{v}$ & 82 & $\mu \mathrm{g} / \mathrm{kg}$ & 370 & EPA8270 \\
\hline 1,2-Dichlorobenzene & $\mathrm{U}$ & & 370 & $\mu \mathrm{g} / \mathrm{kg}$ & 370 & EPA8270 \\
\hline 1,3-Dichlorobenzene & $\mathbf{U}$ & & 370 & $\mu g / k g$ & 370 & EPA8270 \\
\hline 1,4-Dichlorobenzene & $\mathbf{U}$ & & 370 & $\mu \mathrm{g} / \mathrm{kg}$ & 370 & EPA8270 \\
\hline 3,3'-Dichlorobenzidine & U & & 750 & $\mu \mathrm{g} / \mathrm{kg}$ & 750 & EPA8270 \\
\hline 2.4-Dichlorophenol & $\mathrm{U}$ & & 370 & $\mu \mathrm{g} / \mathrm{kg}$ & 370 & EPA8270 \\
\hline Diethyl phthalate & $\mathrm{U}$ & & 370 & $\mu \mathrm{g} / \mathrm{kg}$ & 370 & EPA8270 \\
\hline 2,4-Dimethyl phenol & U & & 370 & $\mu \mathrm{g} / \mathrm{kg}$ & 370 & EPA8270 \\
\hline Dimethyl phthalate & $\mathrm{U}$ & & 370 & $\mu g / k g$ & 370 & EPA8270 \\
\hline 2,4-Dinitrophenol & $\mathrm{U}$ & & 1.900 & $\mu \mathrm{g} / \mathrm{kg}$ & 1,900 & EPA8270 \\
\hline 2,4-Dinitrotoluene & $\mathrm{U}$ & & 370 & $\mu g / \mathrm{kg}$ & 370 & EPA8270 \\
\hline 2,6-Dinitrotoluene & $U$ & & 370 & $\mu \mathrm{g} / \mathrm{kg}$ & 370 & EPA8270 \\
\hline Di-n-octyl phthalate & $\mathrm{U}$ & & 370 & $\mu \mathrm{g} / \mathrm{kg}$ & 370 & EPA8270 \\
\hline Fluoranthene & $\mathrm{U}$ & & 370 & $\mu \mathrm{g} / \mathrm{kg}$ & 370 & EPA8270 \\
\hline Fluorene & $U$ & & 370 & $\mu \mathrm{g} / \mathrm{kg}$ & 370 & EPA8270 \\
\hline Hexachlorobenzene & $\mathrm{U}$ & & 370 & $\mu g / k g$ & 370 & EPA8270 \\
\hline Hexachlorobutadiene - & U & & 370 & $\mu \mathrm{g} / \mathrm{kg}$ & 370 & EPA8270 \\
\hline Hexachlorocyclopentadiene & $\mathrm{U}$ & & 370 & $\mu \mathrm{g} / \mathrm{kg}$ & 370 & EPA8270 \\
\hline Hexachloroethane & $\mathbf{U}$ & & 370 & $\mu \mathrm{g} / \mathrm{kg}$ & 370 & EPA8270 \\
\hline Indeno(1,2,3-c,d)pyrene & $\mathrm{U}$ & & 370 & $\mu g / k g$ & 370 & EPA8270 \\
\hline Isophorone & $U$ & & 370 & $\mu \mathrm{g} / \mathrm{kg}$ & 370 & EPA8270 \\
\hline 2-Methyl-4,6-dinitrophenol & U & & 1,900 & $\mu \mathrm{g} / \mathrm{kg}$ & 1,900 & EPA8270 \\
\hline 2-Methylnaphthalene & $\mathbf{U}$ & & 370 & $\mu g / \mathrm{kg}$ & 370 & EPA8270 \\
\hline Naphthalene & U & & 370 & $\mu \mathrm{g} / \mathrm{kg}$ & 370 & EPA8270 \\
\hline o-Nitroaniline & U & & 1.900 & $\mu \mathrm{g} / \mathrm{kg}$ & 1.900 & EPA8270 \\
\hline m-Nitroaniline & U & & 1,900 & $\mu \mathrm{g} / \mathrm{kg}$ & 1.900 & EPA8270 \\
\hline p-Nitroaniline & $\mathrm{U}$ & & 1.900 & $\mu \mathrm{g} / \mathrm{kg}$ & 1,900 & EPA8270 \\
\hline
\end{tabular}


SAMPLE NAME: D43i 1402 (Continued)

Sample ID: 100392

$\begin{array}{lllll}\text { Constituent } & \text { RQ AQ B Result Unit D. Limit Method }\end{array}$

$B / N / A$ Extractables (including $P A H$ and phenols)

Nitrobenzene
2-Nitrophenol
4-Nitrophenol
N-Nitrosodiphenylamine
N-Nitrosodipropylamine
Pentachlorophenol
Phenanthrene
Phenol
Pyrene
1.2,4-Trichlorobenzene
2,4,S-Trichlorophenol
2,4,6-Trichlorophenol
Unknown
Unknown
Unknown
Unknown ketone

\begin{tabular}{|c|c|c|c|c|}
\hline U & 370 & $\mu g / \mathrm{kg}$ & 370 & EPA8270 \\
\hline & 370 & $\mu \mathrm{g} / \mathrm{kg}$ & 370 & EPA8270 \\
\hline $\mathrm{U}$ & 1,900 & $\mu g / \mathrm{kg}$ & 1,900 & EPA8270 \\
\hline$U$ & 370 & $\mu \mathrm{g} / \mathrm{kg}$ & 370 & EPA8270 \\
\hline $\mathbf{U}$ & 370 & $\mu \mathrm{g} / \mathrm{kg}$ & 370 & EPA8270 \\
\hline $\mathrm{U}$ & 1.900 & $\mu g / \mathrm{kg}$ & 1,900 & EPA8270 \\
\hline \multirow{2}{*}{ UJ } & 370 & $\mu \mathrm{g} / \mathrm{kg}$ & 370 & EPA8270 \\
\hline & 370 & $\mu \mathrm{g} / \mathrm{kg}$ & 370 & EPA8270 \\
\hline U & 370 & $\mu g / \mathrm{kg}$ & 370 & EPA8270 \\
\hline$U$ & 370 & $\mu \mathrm{g} / \mathrm{kg}$ & 370 & EPA8270 \\
\hline U & 1,900 & $\mu \mathrm{g} / \mathrm{kg}$ & 1,900 & EPA8270 \\
\hline $\mathrm{U}$ & 370 & $\mu \mathrm{g} / \mathrm{kg}$ & 370 & EPA8270 \\
\hline$N$ & 300 . & $\mu \mathrm{g} / \mathrm{kg}$ & & EPA8270 \\
\hline $\mathbf{N}$ & 1,000 & $\mu \mathrm{g} / \mathrm{kg}$ & & EPA8270 \\
\hline $\mathbf{N}$ & 700 & $\mu \mathrm{g} / \mathrm{kg}$ & & EPA8270 \\
\hline $\mathbf{N}$ & 5,000 & $\mu \mathrm{g} / \mathrm{kg}$ & & EPA8270 \\
\hline
\end{tabular}

Volatile Organic Compounds

Acetone
Benzene
Bromodichloromethane
Bromoform
Bromomethane (Methyl bromide)
Carbon disulfide
Carbon tetrachloride
Chlorobenzene
Chloroethane
Chloroethene (Vinyl chloride)
Chloroform
Chloromethane (Methyl chloride)
Dibromochloromethane
1,1-Dichloroethane
1,2-Dichloroethane
1,1-Dichloroethylene
1,2-Dichloroethylene
Dichloromethane (Methylene chloride)
1,2-Dichloropropane
cis-1,3-Dichloropropene
trans-1,3-Dichloropropene
Ethylbenzene
2-Hexanone
Methyl ethyl ketone
Methyl isobutyl ketone
Styrene
1,1,2,2-Tetrachloroethane
Tetrachloroethylene
Toluene
1,1,1-Trichloroethane
1,1,2-Trichloroethane

\begin{tabular}{|c|c|c|c|c|}
\hline $\mathbf{v}$ & 11 & $\mu g / \mathrm{kg}$ & 11 & EPA8240 \\
\hline U & 5.7 & $\mu \mathrm{g} / \mathrm{kg}$ & 5.7 & EPA8240 \\
\hline U & 5.7 & $\mu \mathrm{g} / \mathrm{kg}$ & 5.7 & EPA8240 \\
\hline $\mathrm{U}$ & 5.7 & $\mu \mathrm{g} / \mathrm{kg}$ & 5.7 & EPA8240 \\
\hline $\mathbf{U}$ & 11 & $\mu \mathrm{g} / \mathrm{kg}$ & 11 & EPA8240 \\
\hline $\mathbf{U}$ & 5.7 & $\mu \mathrm{g} / \mathrm{kg}$ & 5.7 & EPA8240 \\
\hline $\mathbf{U}$ & 5.7 & $\mu \mathrm{g} / \mathrm{kg}$ & 5.7 & EPA8240 \\
\hline U & 5.7 & $\mu \mathrm{g} / \mathrm{kg}$ & 5.7 & EPA 8240 \\
\hline $\mathrm{U}$ & 11 & $\mu g / \mathrm{kg}$ & 11 & EPA8240 \\
\hline $\mathbf{U}$ & 11 & $\mu \mathrm{g} / \mathrm{kg}$ & 11 & EPA8240 \\
\hline $\mathbf{U}$ & 5.7 & $\mu \mathrm{g} / \mathrm{kg}$ & 5.7 & EPA8240 \\
\hline U & 11 & $\mu \mathrm{g} / \mathrm{kg}$ & 11 & EPA8240 \\
\hline $\mathbf{U}$ & 5.7 & $\mu \mathrm{g} / \mathrm{kg}$ & 5.7 & EPA8240 \\
\hline $\mathrm{U}$ & 5.7 & $\mu \mathrm{g} / \mathrm{kg}$ & 5.7 & EPA8240 \\
\hline $\mathbf{U}$ & 5.7 & $\mu \mathrm{g} / \mathrm{kg}$ & 5.7 & EPA8240 \\
\hline $\mathbf{U}$ & 5.7 & $\mu \mathrm{g} / \mathrm{kg}$ & 5.7 & EPA8240 \\
\hline $\mathbf{U}$ & 5.7 & $\mu \mathrm{g} / \mathrm{kg}$ & 5.7 & EPA8240 \\
\hline$U$ & 5.7 & $\mu g / k g$ & $\because 5.7$ & EPA8240 \\
\hline $\mathrm{U}$ & 5.7 & $\mu g / k g$ & 5.7 & EPA8240 \\
\hline $\mathbf{U}$ & 5.7 & $\mu \mathrm{g} / \mathrm{kg}$ & 5.7 & EPA8240 \\
\hline$U$ & 5.7 & $\mu \mathrm{g} / \mathrm{kg}$ & 5.7 & EPA8240 \\
\hline$U$ & 5.7 & $\mu g / \mathrm{kg}$ & 5.7 & EPA8240 \\
\hline $\mathbf{U}$ & 11 & $\mu g / k g$ & 11 & EPA8240 \\
\hline U & 11 & $\mu g / \mathrm{kg}$ & 11 & EPA8240 \\
\hline$U$ & 11 & $\mu g / \mathrm{kg}$ & 11 & EPA8240 \\
\hline $\mathbf{U}$ & 5.7 & $\mu g / \mathrm{kg}$ & 5.7 & EPA8240 \\
\hline $\mathbf{U}$ & 5.7 & $\mu g / \mathrm{kg}$ & 5.7 & EPA8240 \\
\hline $\mathbf{U}$ & 5.7 & $\mu \mathrm{g} / \mathrm{kg}$ & 5.7 & EPA8240 \\
\hline $\mathbf{U}$ & 5.7 & $\mu g / \mathrm{kg}$ & 5.7 & EPA8240 \\
\hline$U$ & 5.7 & $\mu g / \mathrm{kg}$ & 5.7 & EPA8240 \\
\hline $\mathbf{U}$ & 5.7 & $\mu \mathrm{g} / \mathrm{kg}$ & 5.7 & EPA 8240 \\
\hline
\end{tabular}


SAMPLE NAME: D431 1402 (Continued)

Sample ID: 100392

Constituent

RQ AQ B Result Unit

D. Limit Method

Volatile Organic Compounds

Trichloroethylene

Vinyl acetate

Xylenes
U

U

U
5.7

11

5.7 $\mu \mathrm{g} / \mathrm{kg}$

$\mu g / k g$

$\mu \mathrm{g} / \mathrm{kg}$
5.7

11

5.7
EPA8240

EPA8240

EPA8240

\section{Pesticides/PCBs and Dioxins/Furans}

\begin{tabular}{|c|c|c|c|c|c|c|c|}
\hline Aldrin & UJ & 0 & L & 1.9 & $\mu \mathrm{g} / \mathrm{kg}$ & 1.9 & EPA8080 \\
\hline alpha-Benzene hexachloride & UJ & 0 & L & 1.9 & $\mu \mathrm{g} / \mathrm{kg}$ & 1.9 & EPA8080 \\
\hline beta-Benzene hexachloride & UJ & 0 & $\bar{L}$ & 1.9 & $\mu \mathrm{g} / \mathrm{kg}$ & 1.9 & EPA8080 \\
\hline delta-Benzene hexachloride & UJ & 0 & L & 1.9 & $\mu \mathrm{g} / \mathrm{kg}$ & 1.9 & EPA8080 \\
\hline alpha-Chlordane & UJ & 0 & L & 1.9 & $\mu \mathrm{g} / \mathrm{kg}$ & 1.9 & EPA8080 \\
\hline gamma-Chlordane & UJ & 0 & L & 1.9 & $\mu \mathrm{g} / \mathrm{kg}$ & 1.9 & EPA8080 \\
\hline p,p'-DDD & UJ & 0 & $\bar{L}$ & 3.7 & $\mu \mathrm{g} / \mathrm{kg}$ & 3.7 & EPA8080 \\
\hline p.p'-DDE & UJ & 0 & L & 3.7 & $\mu \mathrm{g} / \mathrm{kg}$ & 3.7 & EPA8080 \\
\hline p,p'-DDT & UJ & 0 & L & 3.7 & $\mu \mathrm{g} / \mathrm{kg}$ & 3.7 & EPA8080 \\
\hline Dieldrin & UJ & 0 & L & 3.7 & $\mu \mathrm{g} / \mathrm{kg}$ & 3.7 & EPA8080 \\
\hline Endosulfan I & UJ & 0 & L & 1.9 & $\mu \mathrm{g} / \mathrm{kg}$ & 1.9 & EPA8080 \\
\hline Endosulfan II & UJ & 0 & $\bar{L}$ & 3.7 & $\mu \mathrm{g} / \mathrm{kg}$ & 3.7 & EPA8080 \\
\hline Endosulfan sulfate & UJ & 0 & $\bar{L}$ & 3.7 & $\mu \mathrm{g} / \mathrm{kg}$ & 3.7 & EPA8080 \\
\hline Endrin & UJ & $\mathbf{o}$ & $\mathbf{L}$ & 3.7 & $\mu \mathrm{g} / \mathrm{kg}$ & 3.7 & EPA8080 \\
\hline Endrin ketone & UJ & 0 & $\bar{L}$ & 3.7 & $\mu \mathrm{g} / \mathrm{kg}$ & 3.7 & EPA8080 \\
\hline Heptachlor & UJ & 0 & L & 1.9 & $\mu \mathrm{g} / \mathrm{kg}$ & 1.9 & EPA8080 \\
\hline Heptachlor epoxide & UJ & 0 & $\mathrm{~L}$ & 1.9 & $\mu \mathrm{g} / \mathrm{kg}$ & 1.9 & EPA8080 \\
\hline Heptachlorodibenzo-p-dioxin isomers & $\mathbf{U}$ & & & 0.20 & $\mathrm{ng} / \mathrm{g}$ & 0.20 & EPA8280 \\
\hline Hepcachlorodibenzo-p-furan isomers & $\mathbf{U}$ & & & 0.10 & $\mathrm{ng} / \mathrm{g}$ & 0.10 & EPA8280 \\
\hline Hexachlorodibenzo-p-dioxin isomers & $\mathbf{U}$ & & & 0.10 & $\mathrm{ng} / \mathrm{g}$ & 0.10 & EPA8280 \\
\hline Hexachlorodibenzo-p-furan isomers & $\mathrm{U}$ & & & 0.10 & $\mathrm{ng} / \mathrm{g}$ & 0.10 & EPA8280 \\
\hline Lindane & UJ & 0 & L & 1.9 & $\mu \mathrm{g} / \mathrm{kg}$ & 1.9 & EPA8080 \\
\hline Methoxychlor & UJ & 0 & $\mathrm{~L}$ & 19 & $\mu \mathrm{g} / \mathrm{kg}$ & 19 & EPA8080 \\
\hline Octachlorodibenzo-p-dioxin & & & & 6.1 & $\mathrm{ng} / \mathrm{g}$ & 0.10 & EPA8280 \\
\hline Octachlorodibenzo-p-furan & $\mathbf{U}$ & & & 0.10 & $\mathrm{ng} / \mathrm{g}$ & 0.10 & EPA8280 \\
\hline РCB 1016 & UJ & 0 & L & 37 & $\mu \mathrm{g} / \mathrm{kg}$ & 37 & EPA8080 \\
\hline PCB 1221 & $\mathrm{UJ}$ & $\mathbf{o}$ & $\bar{L}$ & 75 & $\mu \mathrm{g} / \mathrm{kg}$ & 75 & EPA8080 \\
\hline PCB 1232 & UJ & 0 & L & 37 & $\mu \mathrm{g} / \mathrm{kg}$ & 37 & EPA8080 \\
\hline PCB 1242 & UJ & 0 & L & 37 & $\mu \mathrm{g} / \mathrm{kg}$ & 37 & EPA8080 \\
\hline PCB 1248 & UJ & 0 & L & 37 & $\mu \mathrm{g} / \mathrm{kg}$ & 37 & EPA8080 \\
\hline PCB 1254 & UJ & 0 & $\bar{L}$ & 37 & $\mu \mathrm{g} / \mathrm{kg}$ & 37 & EPA8080 \\
\hline PCB 1260 & UJ & 0 & L & 37 & $\mu \mathrm{g} / \mathrm{kg}$ & 37 & EPA8080 \\
\hline Pentachlorodibenzo-p=dioxin isomers & $\mathbf{U}$ & & & 0.20 & $\mathrm{ng} / \mathrm{g}$ & 0.20 & EPA8280 \\
\hline Pentachlorodibenzo-p-füran isomers & U & & & 0.10 & $\mathrm{ng} / \mathrm{g}$ & $0: 10$ & EPA8280 \\
\hline $2,3.7 .8-T C D D$ & $\mathrm{U}$ & & & 0.10 & $\mathrm{ng} / \mathrm{g}$ & 0.10 & EPA8280 \\
\hline $2,3,7,8-T C D F$ & $\mathrm{U}$ & & & 0.10 & $\mathrm{ng} / \mathrm{g}$ & 0.10 & EPA8280 \\
\hline Tetrachlorodibenzo-p-dioxin isomers & $\mathrm{U}$ & & & 0.10 & $\mathrm{ng} / \mathrm{g}$ & 0.10 & EPA8280 \\
\hline Tetrachlorodibenzo-p-furan isomers & $\mathbf{U}$ & & & 0.20 & $\mathrm{ng} / \mathrm{g}$ & 0.20 & EPA8280 \\
\hline Toxaphene & UJ & 0 & L & 190 & $\mu \mathrm{g} / \mathrm{kg}$ & 190 & EPA8080 \\
\hline
\end{tabular}


SAMPLE NAME: D43Ii 1402 (Continued)

Constituent
Sample ID: 100392

RQ AQ B Result Unit

D. Limit Method

Radionuclide Indicators and Radionuclides

Gross alpha

Nonvolatile beta

Radium, total alpha-emitting

Tritium

$\begin{array}{ll}\mathbf{U} \\ \mathbf{U} & \mathbf{V}\end{array}$

SAMPLE NAME: D431 1403

Location (SRS Coordinates): 18401.83E 66640.68N

Ground Elevation Above MSL: $129.4 \mathrm{ft}$

Depth of Core Interval: 2.00 to $4.00 \mathrm{ft}$

Sample Type: Normal

Sample Color: IOR 4/6

Constituent

Total Metals

Arsenic

Barium

Cadmium

Chromium

Copper

Lead

Manganese

Mercury

Nickel

Selenium

Silver

Volatile Organic Compounds

Acetone

Benzene

Bromodichloromethane

Bromoform

Bromomethane (Methyl bromide)

Carbon disulfide

Carbon tetrachloride

Chlorobenzene =nt

Chloroethane -

Chloroethene (Vinyl chloride)

Chloroform

Chloromethane (Methyt chloride)

Dibromochloromethane

1,1-Dichloroethane

1,2-Dichloroethane

1,1-Dichloroethylene

1,2-Dichloroethylene

Dichloromethane (Methylene chloride)
RQ AQ

Sample Matrix: Soil

USC Soil Classification: SW

Unit

8.9

15

0.18

1.3

EPA900.0

$33 \pm 22$

1.3

$\mathrm{pCi} / \mathrm{g}$

$\mathrm{pCi} / \mathrm{g}$

Sample ID: 100393

B Result

D. Limit Method
Sample Moisture: Damp Percent Solids: 87.40
EPA900.0

EPA903.0

EPA906.0

$\begin{array}{ll} & 2.5 \\ U & 23 \\ & 0.57 \\ & 64 \\ & 13 \\ & 19 \\ & 70 \\ & 0.25 \\ U & 12 \\ U & 0.57\end{array}$

$$
\begin{aligned}
& \mathrm{mg} / \mathrm{kg} \\
& \mathrm{mg} / \mathrm{kg} \\
& \mathrm{mg} / \mathrm{kg} \\
& \mathrm{mg} / \mathrm{kg} \\
& \mathrm{mg} / \mathrm{kg} \\
& \mathrm{mg} / \mathrm{kg} \\
& \mathrm{mg} / \mathrm{kg} \\
& \mathrm{mg} / \mathrm{kg} \\
& \mathrm{mg} / \mathrm{kg} \\
& \mathrm{mg} / \mathrm{kg} \\
& \mathrm{mg} / \mathrm{kg}
\end{aligned}
$$

1.1

$$
23
$$

0.57

1.1

2.9

3.4

1.7

0.11

1


.0
.0
6.0


SAMPLE NAME: D43i 1403 (Continued)

\section{Constituent}

Volatile Organic Compounds

\section{RQ AQ B Result}

$\begin{array}{lll}\mathrm{U} & 5.7 & \mu \mathrm{g} / \mathrm{kg} \\ \mathrm{U} & 5.7 & \mu \mathrm{kg} \\ \mathrm{U} & 5.7 & \mu \mathrm{kg} / \mathrm{kg} \\ \mathrm{U} & 5.7 & \mu \mathrm{g} / \mathrm{kg} \\ \mathrm{U} & 11 & \mu \mathrm{g} / \mathrm{kg} \\ \mathrm{U} & 11 & \mu \mathrm{g} / \mathrm{kg} \\ \mathrm{U} & 11 & \mu \mathrm{g} / \mathrm{kg} \\ \mathrm{U} & 5.7 & \mu \mathrm{g} / \mathrm{kg} \\ \mathrm{U} & 5.7 & \mu \mathrm{g} / \mathrm{kg} \\ \mathrm{U} & 5.7 & \mu \mathrm{g} / \mathrm{kg} \\ \mathrm{U} & 5.7 & \mu \mathrm{g} / \mathrm{kg} \\ \mathrm{U} & 5.7 & \mu \mathrm{g} / \mathrm{kg} \\ \mathrm{U} & 5.7 & \mu \mathrm{g} / \mathrm{kg} \\ \mathrm{U} & 5.7 & \mu \mathrm{g} / \mathrm{kg} \\ \mathrm{U} & 11 & \mu \mathrm{g} / \mathrm{kg} \\ \mathrm{U} & 5.7 & \mu \mathrm{g} / \mathrm{kg}\end{array}$

Unit

D. Limit Method

$\begin{array}{ll}5.7 & \text { EPA8240 } \\ 5.7 & \text { EPA8240 } \\ 5.7 & \text { EPA8240 } \\ 5.7 & \text { EPA8240 } \\ 11 & \text { EPA8240 } \\ 11 & \text { EPA8240 } \\ 11 & \text { EPA8240 } \\ 5.7 & \text { EPA8240 } \\ 5.7 & \text { EPA8240 } \\ 5.7 & \text { EPA8240 } \\ 5.7 & \text { EPA8240 } \\ 5.7 & \text { EPA8240 } \\ 5.7 & \text { EPA8240 } \\ 5.7 & \text { EPA8240 } \\ 11 & \text { EPA8240 } \\ 5.7 & \text { EPA8240 }\end{array}$

Radionuclide Indicators and Radionuclides

Gross alpha

$\begin{array}{ll}7.8 \pm 13 & \text { pCi } / g \\ 35 \pm 17 & \text { PCi } / g \\ 2.5 \pm 1.4 & \text { pCi } / g \\ 1.3 & \text { pCi/g }\end{array}$

$\begin{array}{ll}6.8 & \text { EPA900.0 } \\ 11 & \text { EPA900.0 } \\ 0.20 & \text { EPA903.0 } \\ 1.3 & \text { EPA906.0 }\end{array}$

Radium, total alpha-emitting

U. V

\section{SAMPLE NAME: D431 1404}

Location (SRS Coordinates): 18401.83E 66640.68N

Ground Elevation Above MSL: $129.4 \mathrm{ft}$

Depth of Core Interval: 4.00 to $6.00 \mathrm{ft}$

Sample Type: Normal

Sample Color: 10R $4 / 6$

\section{Constituent}

$\mathbf{R Q} \mathbf{A Q}$
Sample Matrix: Soil

USC Soil Classification: SW
Sample Moisture: Damp Percent Solids: 89.90

D. Limit Method
Total Metals

Arsenic
Barium
Cadmium
Chromium
Copper
Lead
Manganese
Mercury
Nickel
Selenium
Silver

$\begin{array}{ll} & 1.6 \\ \mathrm{U} & 22 \\ & 0.55 \\ & 42 \\ & 8.0 \\ & 10 \\ \mathrm{U} & 74 \\ \mathrm{U} & 0.11 \\ \mathrm{U} & 6.2 \\ & 0.50\end{array}$

$\begin{array}{ll} & \\ 1.0 & \text { EPA7060 } \\ 22 & \text { EPA6010 } \\ 0.55 & \text { EPA6010 } \\ 1.1 & \text { EPA6010 } \\ 2.7 & \text { EPA6010 } \\ 1.5 & \text { EPA7421 } \\ 1.6 & \text { EPA6010 } \\ 0.11 & \text { EPA7470 } \\ 4.4 & \text { EPA6010 } \\ 0.50 & \text { EPA7740 } \\ 1.1 & \text { EPA6010 }\end{array}$


SAMPLE NAME: D431-1404 (Continued)

Sample ID: 100394

Constituent

Volatile Organic Compounds

Acetone
Benzene
Bromodichloromethane
Bromoform
Bromomethane (Methyl bromide)
Carbon disulfide
Carbon tetrachloride
Chlorobenzene
Chloroethane
Chloroethene (Vinyl chloride)
Chloroform
Chloromethane (Methyl chloride)
Dibromochloromethane
1.1-Dichloroethane
1,2-Dichloroethane
1,1-Dichloroethylene
1,2-Dichloroethylene
Dichloromethane (Methylene chloride)
1,2-Dichloropropane
cis-1,3-Dichloropropene
trans-1,3-Dichloropropene
Ethylbenzene
2-Hexanone
Methyl ethyl ketone
Methyl isobutyl ketone
Styrene
1,1,2,2-Tetrachloroethane
Tetrachloroethylene
Toluene
1,1,1-Trichloroethane
1,1,2-Trichloroethane
Trichloroethylene
Vinyl acetate
Xylenes

RQ AQ B Result

Unit

D. Limit Method

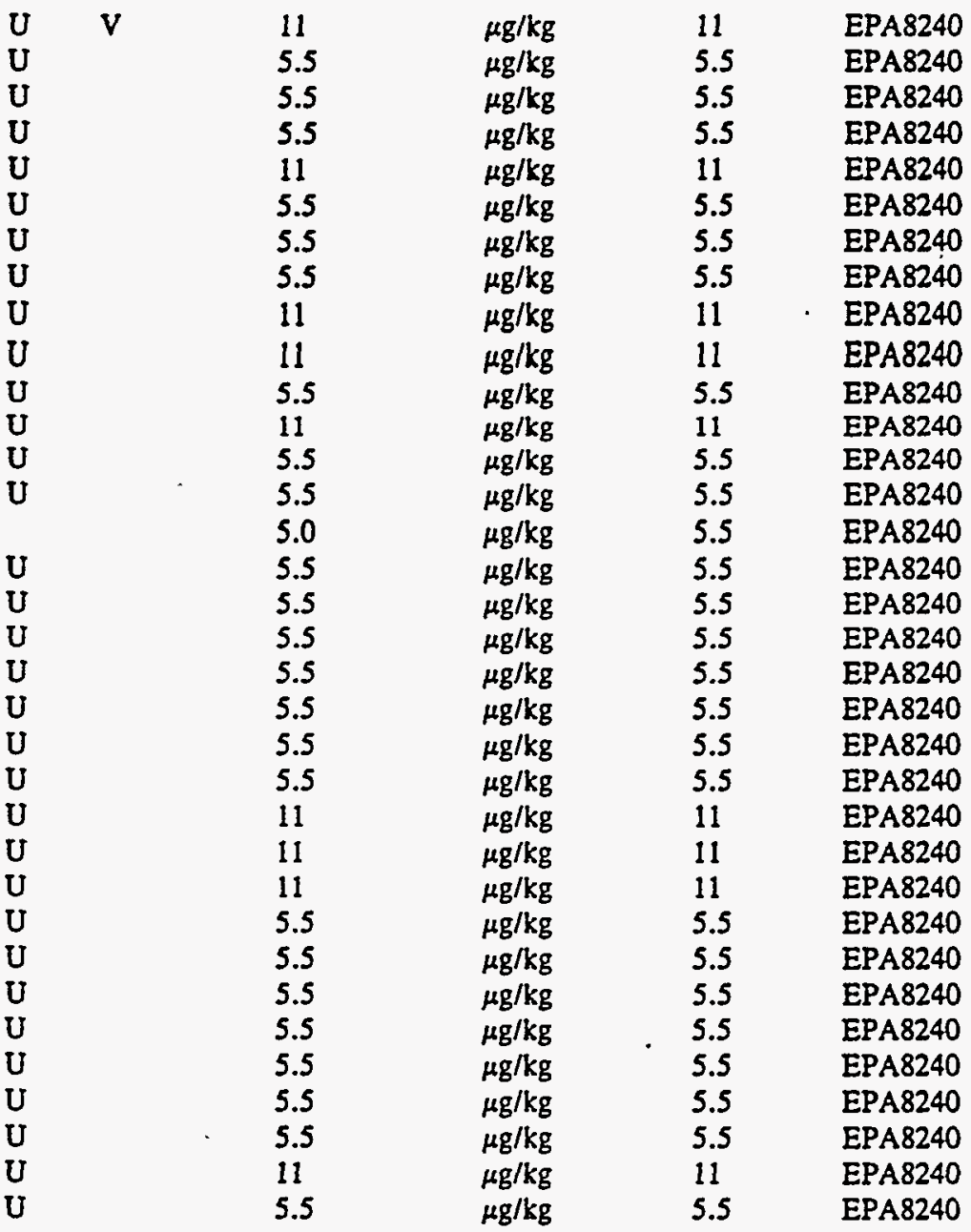

Radionuclide Indicators and Radionuclides

Gross alpha

Nonvolatile beta

Radium, total alpha-emitting

Tritium

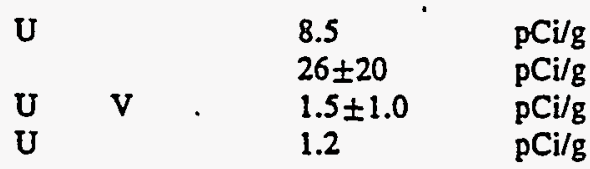

$\begin{array}{ll}8.5 & \text { EPA900.0 } \\ 14 & \text { EPA900.0 } \\ 0.17 & \text { EPA903.0 } \\ 1.2 & \text { EPA906.0 }\end{array}$


SAMPLE NAME: D431 1405

Location (SRS Coordinates): 18401.83E 66640.68N

Ground Elevation Above MSL: $129.4 \mathrm{ft}$

Depth of Core Interval: 6.00 to $8.00 \mathrm{ft}$

Sample Type: Normal

Sample Color: 10R 3/4

Constituent

Physical Parameters

Cation exchange capacity

Cyanide

$\mathrm{pH}$

Specific conductance

Total organic carbon

Total Metals

Aluminum
Antimony
Arsenic
Barium
Beryllium
Cadmium
Calcium
Chromium
Cobalt
Copper
Iron
Lead
Magnesium
Manganese
Mercury
Nickel
Potassium
Selenium
Silver
Sodium
Thallium
Vanadium
Zinc

$B / N / A$ Extractables (including PAH and phenols)

Acenaphthene

Acenaphthylene

Anthracene

Benzo(a)anthracene

Benzo(b)fluoranthene

Benzo(k)fluoranthene

Benzoic acid

Benzo(g,h,i)perylene

Benzo(a)pyrene

Benzyl alcohol
Sample ID: 100395
Sample Matrix: Soil
USC Soil Classification: SW
Sample Moisture: Damp

Percent Solids: 91.50

D. Limit Method

$\begin{array}{lll}\mathrm{meq} / 100 \mathrm{~g} & 2.0 & \text { EPA9080 } \\ \mathrm{mg} / \mathrm{kg} & 1.1 & \text { EPA335.3 } \\ \mathrm{pH} & 0.010 & \text { EPA9045 } \\ \mu \mathrm{S} / \mathrm{cm} & 1.0 & \text { EPA9050 } \\ \mathrm{mg} / \mathrm{kg} & 350 & \text { EPA415.1 }\end{array}$

$17 \quad$ EPA6010

$4.9 \quad$ EPA6010

0.79 EPA7060

$17 \quad$ EPA6010

0.41 EPA6010

0.41 EPA6010

$410^{\circ} \quad$ EPA6010

0.82 EPA6010

$4.1 \quad$ EPA6010

2.1 EPA6010

8.2 EPA6010

4.7 EPA7421

$410 \quad$ EPA6010

1.2 EPA6010

$0.11 \quad$ EPA7470

$3.3 \quad$ EPA6010

410 EPA6010

$0.39 \quad$ EPA7740

0.82 EPA6010

$410 \quad$ EPA6010

1.1 EPA7841

$4.1 \quad$ EPA6010

1.6 EPA6010
J 170

U 370

J 320

820

1.400

$\begin{array}{ll}\mathrm{J} & 240 \\ \mathrm{U} & 1,900\end{array}$

550

760

370 $\mu g / k g$

$\mu \mathrm{g} / \mathrm{kg}$

$\mu \mathrm{g} / \mathrm{kg}$

$\mu \mathrm{g} / \mathrm{kg}$

$\mu \mathrm{g} / \mathrm{kg}$

$\mu \mathrm{g} / \mathrm{kg}$

$\mu \mathrm{g} / \mathrm{kg}$

$\mu \mathrm{g} / \mathrm{kg}$

$\mu \mathrm{g} / \mathrm{kg}$

$\mu g / k g$
EPA8270

EPA8270

EPA8270

EPA8270

EPA8270

EPA8270

EPA8270

EPA8270

EPA8270

EPA8270 
SAMPLE NAME: D431 1405 (Continued)

\author{
Constituent
}

RQ AQ B Result

$B / N / A$ Extractables (including $P A H$ and phenols)

Bis(2-chloroethoxy) methane

Bis(2-chloroethyl) ether

Bis(2-chloroisopropyl) ether

Bis(2-ethylhexyl) phthalate

4-Bromophenyl phenyl ether

Butanoic acid ester

Butylbenzyl phthalate

4-Chloroaniline

4-Chloro-m-cresol

2-Chloronaphthalene

2-Chlorophenol

4-Chlorophenyl phenyl ether

Chrysene

o-Cresol (2-Methylphenol)

p-Cresol (4-Methylphenol)

Dibenz $(a, h)$ anthracene

Dibenzofuran

Di-n-butyl phthalate

1,2-Dichlorobenzene

1,3-Dichlorobenzene

1.4-Dichlorobenzene

3,3'-Dichlorobenzidine

2,4-Dichlorophenol

Diethyl phthalate

2,4-Dimethyl phenol

Dimethyl phthalate

2,4-Dinitrophenol

2,4-Dinitrotoluene

2,6-Dinitrotoluene

Di-n-octyl phthalate

Fluoranthene

Fluorene

Hexachlorobenzene

Hexachlorobutadiene

Hexachlorocyclopentadiene

Hexachloroethane

Indeno(1,2,3-c,d)pyrene

Isophorone

2-Methyl-4,6-dinitrophenol

2-Methylnaphthalene :

Naphthalene

o-Nitroaniline

m-Nitroaniline

p-Nitroaniline

Nitrobenzene

2-Nitrophenol

4-Nitrophenol

N-Nitrosodiphenylamine

$\mathrm{N}$-Nitrosodipropylamine

Pentachlorophenol
Sample ID: 100395

Unit

D. Limit Method

\begin{tabular}{|c|c|c|}
\hline$\mu \mathrm{g} / \mathrm{kg}$ & 370 & EPA8270 \\
\hline$\mu \mathrm{g} / \mathrm{kg}$ & 370 & EPA8270 \\
\hline$\mu \mathrm{g} / \mathrm{kg}$ & 370 & EPA8270 \\
\hline$\mu \mathrm{g} / \mathrm{kg}$ & 370 & EPA8270 \\
\hline$\mu \mathrm{g} / \mathrm{kg}$ & 370 & EPA8270 \\
\hline$\mu \mathrm{g} / \mathrm{kg}$ & 370 & $\begin{array}{l}\text { EPA8270 } \\
\text { EPA8270 }\end{array}$ \\
\hline $\begin{array}{l}\mu \mathrm{g} / \mathrm{kg} \\
\mu \mathrm{g} / \mathrm{kg}\end{array}$ & 370 & EPA8270 \\
\hline$\mu \mathrm{g} / \mathrm{kg}$ & 370 & EPA8270 \\
\hline$\mu \mathrm{g} / \mathrm{kg}$ & 370 & EPA8270 \\
\hline$\mu \mathrm{g} / \mathrm{kg}$ & 370 & EPA8270 \\
\hline$\mu \mathrm{g} / \mathrm{kg}$ & 370 & EPA8270 \\
\hline$\mu \mathrm{g} / \mathrm{kg}$ & 370 & EPA8270 \\
\hline$\mu \mathrm{g} / \mathrm{kg}$ & 370 & EPA8270 \\
\hline$\mu \mathrm{g} / \mathrm{kg}$ & 370 & EPA8270 \\
\hline$\mu \mathrm{g} / \mathrm{kg}$ & 370 & EPA8270 \\
\hline$\mu \mathrm{g} / \mathrm{kg}$ & 370 & EPA8270 \\
\hline$\mu \mathrm{g} / \mathrm{kg}$ & 370 & EPA8270 \\
\hline$\mu \mathrm{g} / \mathrm{kg}$ & 370 & EPA8270 \\
\hline$\mu \mathrm{g} / \mathrm{kg}$ & 370 & EPA8270 \\
\hline$\mu \mathrm{g} / \mathrm{kg}$ & 370 & EPA8270 \\
\hline$\mu \mathrm{g} / \mathrm{kg}$ & 750 & EPA8270 \\
\hline$\mu \mathrm{g} / \mathrm{kg}$ & 370 & EPA8270 \\
\hline$\mu \mathrm{g} / \mathrm{kg}$ & 370 & EPA8270 \\
\hline$\mu \mathrm{g} / \mathrm{kg}$ & 370 & EPA8270 \\
\hline$\mu \mathrm{g} / \mathrm{kg}$ & 370 & EPA8270 \\
\hline$\mu \mathrm{g} / \mathrm{kg}$ & 1,900 & EPA8270 \\
\hline$\mu \mathrm{g} / \mathrm{kg}$ & 370 & EPA8270 \\
\hline$\mu \mathrm{g} / \mathrm{kg}$ & 370 & EPA8270 \\
\hline$\mu \mathrm{g} / \mathrm{kg}$ & 370 & EPA8270 \\
\hline$\mu \mathrm{g} / \mathrm{kg}$ & 370 & EPA8270 \\
\hline$\mu \mathrm{g} / \mathrm{kg}$ & 370 & EPA8270 \\
\hline$\mu \mathrm{g} / \mathrm{kg}$ & 370 & EPA 8270 \\
\hline$\mu \mathrm{g} / \mathrm{kg}$ & 370 & EPA8270 \\
\hline$\mu \mathrm{g} / \mathrm{kg}$ & 370 & EPA8270 \\
\hline$\mu \mathrm{g} / \mathrm{kg}$ & 370 & EPA8270 \\
\hline$\mu \mathrm{g} / \mathrm{kg}$ & 370 & EPA.8270 \\
\hline$\mu \mathrm{g} / \mathrm{kg}$ & 370 & EPA8270 \\
\hline$\mu g / \mathrm{kg}$ & 1,900 & EPA8270 \\
\hline$\mu \mathrm{g} / \mathrm{kg}$ & 370 & EPA8270 \\
\hline$\mu \mathrm{g} / \mathrm{kg}$ & 370 & EPA8270 \\
\hline$\mu \mathrm{g} / \mathrm{kg}$ & 1,900 & EPA827 \\
\hline$\mu \mathrm{g} / \mathrm{kg}$ & 1,900 & EPA8270 \\
\hline$\mu \mathrm{g} / \mathrm{kg}$ & 1,900 & EPA827 \\
\hline$\mu \mathrm{g} / \mathrm{kg}$ & 370 & EPA8270 \\
\hline$\mu \mathrm{g} / \mathrm{kg}$ & 370 & EPA8270 \\
\hline$\mu \mathrm{g} / \mathrm{kg}$ & 1,900 & EPA8270 \\
\hline$\mu \mathrm{g} / \mathrm{kg}$ & 370 & EPA8270 \\
\hline$\mu \mathrm{g} / \mathrm{kg}$ & 370 & EPA8270 \\
\hline$\mu \mathrm{g} / \mathrm{kg}$ & 1.900 & EPA8270 \\
\hline
\end{tabular}

370

370

370

51

370

5,000

370

370

370

370

370

830

370

370

370

70

100

370

370

370

750

370

370

370

370

1.900

370

370

370

1.800

160

370

370

370

370

370

370

1,900

52

92

1,900

1.900

1,900

370

370

1,900

370

370

1,900 
SAMPLE NAME: D431 1405 (Continued)

RQ AQ B Result

Constituent

Unit

D. Limit Method

$B / N / A$ Extractables (including $P A H$ and phenols)

Phenanthrene

Phenol

Pyrene

1,2,4-Trichlorobenzene

2,4,5-Trichlorophenol

2,4,6-Trichlorophenol

Unknown hydrocarbon

Unknown hydrocarbon

Unknown

Unknown acid

Unknown acid

Unknown ketone

Unknown phthalate

Unknown phthalate

Volatile Organic Compounds

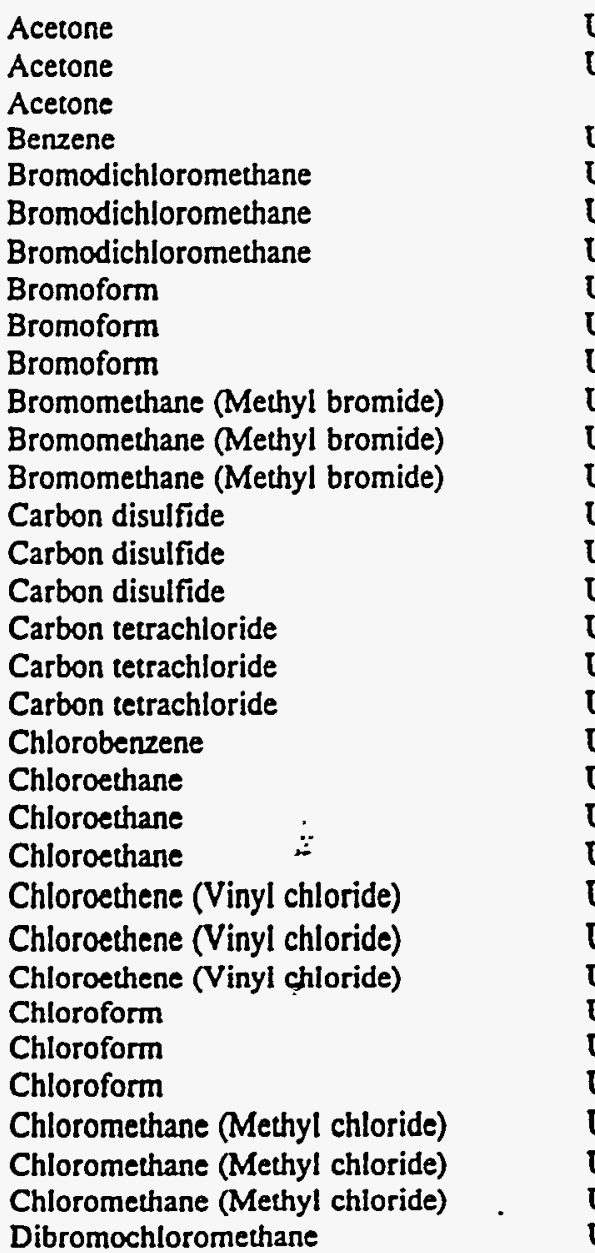

$\begin{array}{llllll} & & 1,200 & \mu \mathrm{g} / \mathrm{kg} & 370 & \text { EPA8270 } \\ \mathrm{UJ} & \mathrm{C} & 370 & \mu \mathrm{g} / \mathrm{kg} & 370 & \text { EPA8270 } \\ & & 1,600 & \mu \mathrm{g} / \mathrm{kg} & 370 & \text { EPA8270 } \\ \mathrm{U} & & 370 & \mu \mathrm{g} / \mathrm{kg} & 370 & \text { EPA8270 } \\ \mathrm{U} & & 1,900 & \mu \mathrm{g} / \mathrm{kg} & 1,900 & \text { EPA8270 } \\ \mathrm{U} & & 370 & \mu \mathrm{g} / \mathrm{kg} & 370 & \text { EPA8270 } \\ \mathrm{J} & \mathrm{N} & 300 & \mu \mathrm{g} / \mathrm{kg} & & \text { EPA8270 } \\ \mathrm{J} & \mathrm{N} & 200 & \mu \mathrm{g} / \mathrm{kg} & & \text { EPA8270 } \\ \mathrm{J} & \mathrm{N} & 300 & \mu \mathrm{g} / \mathrm{kg} & & \text { EPA8270 } \\ \mathrm{J} & \mathrm{N} & 500 & \mu \mathrm{g} / \mathrm{kg} & & \text { EPA8270 } \\ \mathrm{J} & \mathrm{N} & 200 & \mu \mathrm{g} / \mathrm{kg} & & \text { EPA8270 } \\ \mathrm{J} & \mathrm{N} & 6,000 & \mu \mathrm{g} / \mathrm{kg} & & \text { EPA8270 } \\ \mathrm{J} & \mathrm{N} & 200 & \mu \mathrm{g} / \mathrm{kg} & & \text { EPA8270 } \\ \mathrm{J} & \mathrm{N} & 500 & \mu \mathrm{g} / \mathrm{kg} & & \text { EPA8270 }\end{array}$

$38 \mu \mathrm{g} / \mathrm{kg}$

$\mu \mathrm{g} / \mathrm{kg}$

$\mu \mathrm{g} / \mathrm{kg}$

$\mu \mathrm{g} / \mathrm{kg}$

$\mu \mathrm{g} / \mathrm{kg}$

$\mu \mathrm{g} / \mathrm{kg}$

$\mu \mathrm{g} / \mathrm{kg}$

$\mu \mathrm{g} / \mathrm{kg}$

$\mu \mathrm{g} / \mathrm{kg}$

$\mu \mathrm{g} / \mathrm{kg}$

$\mu \mathrm{g} / \mathrm{kg}$

$\mu \mathrm{g} / \mathrm{kg}$

$\mu \mathrm{g} / \mathrm{kg}$

$\mu g / \mathrm{kg}$

$\mu \mathrm{g} / \mathrm{kg}$

$\mu \mathrm{g} / \mathrm{kg}$

$\mu g / \mathrm{kg}$

$\mu \mathrm{g} / \mathrm{kg}$

$\mu \mathrm{g} / \mathrm{kg}$

$\mu g / k g$

$\mu \mathrm{g} / \mathrm{kg}$

$\mu g / \mathrm{kg}$

$\mu g / \mathrm{kg}$

$\mu g / \mathrm{kg}$

$\mu \mathrm{g} / \mathrm{kg}$

$\mu \mathrm{g} / \mathrm{kg}$

$\mu \mathrm{g} / \mathrm{kg}$

$\mu \mathrm{g} / \mathrm{kg}$

$\mu \mathrm{g} / \mathrm{kg}$

$\mu \mathrm{g} / \mathrm{kg}$

$\mu \mathrm{g} / \mathrm{kg}$

$\mu \mathrm{g} / \mathrm{kg}$

$\mu \mathrm{g} / \mathrm{kg}$
EPA8240

EPA8240

EPA8240

EPA8240

EPA8240

EPA8240

EPA8240

EPA8240

EPA8240

EPA8240

EPA8240

EPA8240

EPA8240

EPA8240

EPA8240

EPA8240

EPA8240

EPA8240

EPA8240

EPA8240

EPA8240

EPA8240

EPA8240

EPA8240

EPA8240

EPA8240

EPA8240

EPA8240

EPA8240

EPA8240

EPA8240

EPA8240

EPA8240 
SAMPLE NAME: D431:1405 (Continued)

Constituent

Volatile Organic Compounds

\section{Dibromochloromethane}

Dibromochloromethane

1,1-Dichloroethane

1,1-Dichloroethane

1.1-Dichloroethane

1.2-Dichloroethane

1,2-Dichloroethane

1,2-Dichloroethane

1,1-Dichloroethylene

1,2-Dichloroethylene

1,2-Dichloroethylene

1.2-Dichloroethylene

Dichloromethane (Methylene chloride)

Dichloromethane (Methylene chloride)

Dichloromethane (Methylene chloride)

1,2-Dichloropropane

1,2-Dichloropropane

1,2-Dichloropropane

cis-1,3-Dichloropropene

cis-1,3-Dichloropropene

cis-1,3-Dichloropropene

trans-1,3-Dichloropropene

trans-1,3-Dichloropropene

trans-1,3-Dichloropropene

Ethylbenzene

Ethylbenzene

Ethylbenzene

2-Hexanone

2-Hexanone

2-Hexanone

Methyl ethyl ketone

Methyl ethyl ketone

Methyl ethyl ketone

Methyl isobutyl ketone

Methyl isobutyl ketone

Methyl isobutyl ketone

Styrene

Styrene

Styrene

1,1,2,2-Tetrachloroethäne

1,1,2,2-Tetrachloroethane

1,1,2,2-Tetrachloroethane

Tetrachloroethylene

Tetrachloroethylene

Tetrachloroethylene

Toluene

1.1,1-Trichloroethane

1,1,1-Trichloroethane

1,1,1-Trichloroethane

1,1,2-Trichloroethane
RQ

$A Q$

Sample ID: 100395

Unit

D. Limit Method
$\mathrm{U}$
$\mathrm{U}$
$\mathrm{U}$
$\mathrm{U}$
$\mathrm{U}$

U

U

U

U

U

U

U

U

U

U

U

U

U

U

U

U

U

U

$\mathrm{U}$

U

U

U

U

U

U

U

U

U

U

U

U

U

U

U

U

U

U

U

U

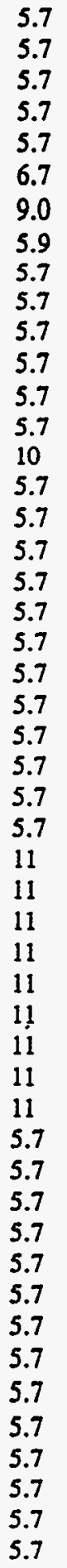

5.7

5.7

5.7

5.7

6.7

9.0

5.9

7

.7

.7

5

5.7

.7

v
5.7

5.7

5.7

5.7

5.7

5.7

5.7

5.7

5.7

7

5.7

1

11

11

11

$1 !$

11

11

5.7

5.7

5.7

5.7

5.7

5.7

5.7

5.7

5.7

5.7

5.7

$$
\begin{aligned}
& \mu \mathrm{g} / \mathrm{kg} \\
& \mu \mathrm{g} / \mathrm{kg} \\
& \mu \mathrm{g} / \mathrm{kg} \\
& \mu \mathrm{g} / \mathrm{kg} \\
& \mu \mathrm{g} / \mathrm{kg} \\
& \mu \mathrm{g} / \mathrm{kg} \\
& \mu \mathrm{g} / \mathrm{kg} \\
& \mu \mathrm{g} / \mathrm{kg} \\
& \mu \mathrm{g} / \mathrm{kg} \\
& \mu \mathrm{g} / \mathrm{kg} \\
& \mu \mathrm{g} / \mathrm{kg} \\
& \mu \mathrm{g} / \mathrm{kg} \\
& \mu \mathrm{g} / \mathrm{kg} \\
& \mu \mathrm{g} / \mathrm{kg} \\
& \mu \mathrm{g} / \mathrm{kg}
\end{aligned}
$$

$\mu \mathrm{g} / \mathrm{kg}$

$\mu \mathrm{g} / \mathrm{kg}$

$\mu \mathrm{g} / \mathrm{kg}$

$\mu \mathrm{g} / \mathrm{kg}$

$\mu \mathrm{g} / \mathrm{kg}$

$\mu \mathrm{g} / \mathrm{kg}$

$\mu \mathrm{g} / \mathrm{kg}$

$\mu \mathrm{g} / \mathrm{kg}$

$\mu \mathrm{g} / \mathrm{kg}$

$\mu \mathrm{g} / \mathrm{kg}$

$\mu \mathrm{g} / \mathrm{kg}$

$\mu \mathrm{g} / \mathrm{kg}$

$\mu \mathrm{g} / \mathrm{kg}$

$\mu \mathrm{g} / \mathrm{kg}$

$\mu \mathrm{g} / \mathrm{kg}$

$\mu \mathrm{g} / \mathrm{kg}$

$\mu g / k g$

$\mu \mathrm{g} / \mathrm{kg}$

$\mu \mathrm{g} / \mathrm{kg}$

$\mu \mathrm{g} / \mathrm{kg}$

$\mu g / k g$

$\mu \mathrm{g} / \mathrm{kg}$

$\mu \mathrm{g} / \mathrm{kg}$

$\mu \mathrm{g} / \mathrm{kg}$

$\mu \mathrm{g} / \mathrm{kg}$

$\mu \mathrm{g} / \mathrm{kg}$

$\mu g / k g$

$\mu g / \mathrm{kg}$

$\mu \mathrm{g} / \mathrm{kg}$

$\mu g / \mathrm{kg}$

$\mu \mathrm{g} / \mathrm{kg}$

$\mu \mathrm{g} / \mathrm{kg}$

$\mu \mathrm{g} / \mathrm{kg}$

$\mu \mathrm{g} / \mathrm{kg}$

$\mu \mathrm{g} / \mathrm{kg}$
5.7

5.7
5.7

5.7

5.7

5.7

5.7

5.7

5.7

5.7

5.7

5.7

5.7

5.7

5.7

5.7

5.7

5.7

5.7

5.7

5.7

5.7

5.7

5.7

5.7

5.7

5.7

5.7

11

11

11

11

11

11

11

11

5.7

5.7

5.7

5.7

5.7

5.7

5.7

5.7

5.7

5.7

5.7

5.7

5.7

5.7

EPA8240

EPA8240

EPA8240

EPA8240

EPA8240

EPA8240

EPA8240

EPA8240

EPA8240

EPA8240

EPA8240

EPA8240

EPA8240

EPA8240

EPA8240

EPA8240

EPA8240

EPA8240

EPA8240

EPA8240

EPA8240

EPA8240

EPA8240

EPA8240

EPA8240

EPA8240

EPA8240

EPA8240

EPA8240

EPA8240

EPA8240

EPA8240 
SAMPLE NAME: D431־1405 (Continued)

RQ AQ B Result

5.7

5.7

5.7

11

11

11

5.7

5.7

5.7
Xylenes

Xylenes

U

Pesticides/PCBs and Dioxins/Furans
alpha-Benzene hexachloride beta-Benzene hexachloride

delta-Benzene hexachloride

alpha-Chlordane

gamma-Chlordane

p,p'-DDD

p,p'-DDE

p, $p^{\prime}-$ DDT

Dieldrin

Endosulfan I

Endosulfan II

Endosulfan sulfate

Endrin

Endrin ketone

Heptachlor

Heptachlor epoxide

Lindane

Methoxychlor

PCB 1016

PCB 1221

PCB 1232

PCB 1242

PCB 1254

PCB 1260

Toxaphene
PCB 1248

U

U

U

U

U

U

J

J

0

U

U

U

U

U

$\mathrm{U}$

$\mathrm{U}$

U

U

U

U

U

U.

$\because$

Radionuclide Indicators and Radionuclides

Gross alpha

Nonvolatile beta

Radium, total alpha-emitring

Tritium
U

U V
19

19

19

19

19

19

39

45

21

37

19

37

37

37

37

19

19

19

190

370

740

370

370

370

370

370

1,900 $\mu g / \mathrm{kg}$

$\mu g / \mathrm{kg}$

$\mu \mathrm{g} / \mathrm{kg}$

$\mu g / \mathrm{kg}$

$\mu \mathrm{g} / \mathrm{kg}$

$\mu g / \mathrm{kg}$

$\mu g / \mathrm{kg}$

$\mu \mathrm{g} / \mathrm{kg}$

$\mu \mathrm{g} / \mathrm{kg}$

$\mu \mathrm{g} / \mathrm{kg}$

$\mu g / \mathrm{kg}$

$\mu g / \mathrm{kg}$

$\mu \mathrm{g} / \mathrm{kg}$

$\mu g / k g$

$\mu g / \mathrm{kg}$

$\mu \mathrm{g} / \mathrm{kg}$

$\mu \mathrm{g} / \mathrm{kg}$

$\mu g / \mathrm{kg}$

$\mu g / \mathrm{kg}$

$\mu \mathrm{g} / \mathrm{kg}$

$\mu \mathrm{g} / \mathrm{kg}$

$\mu g / \mathrm{kg}$

$\mu g / \mathrm{kg}$

$\mu g / \mathrm{kg}$

$\mu \mathrm{g} / \mathrm{kg}$

$\mu \mathrm{g} / \mathrm{kg}$

$\mu \mathrm{g} / \mathrm{kg}$
5.7

5.7

5.7

11

11

11

5.7

5.7

5.7
D. Limit Method

7 EPA8240

EPA8240

EPA8240

EPA8240

EPA8240

EPA8240

EPA8240

EPA8240

EPA8240

EPA8080

EPA8080

EPA8080

EPA8080

EPA8080

EPA8080

EPA8080

EPA8080

EPA8080

EPA8080

EPA8080

EPA8080

EPA8080

EPA8080

EPA8080

EPA8080

EPA8080

EPA8080

EPA8080

EPA8080

EPA8080

EPA8080

EPA8080

EPA8080

EPA8080

EPA8080

370

EPA8080

1,900

7.5

$\mathrm{PCi} / \mathrm{g}$

7.5

EPA 900.0

$44 \pm 20$

$\mathrm{pCi} / \mathrm{g}$

13

EPA900.0

$1.7 \pm 1.1$

$\mathrm{pCi} / \mathrm{g}$

$2.6 \pm 0.60$

$\mathrm{pCi} / \mathrm{g}$

EPA903.0

1.3 EPA906.0 
SAMPLE NAME: D431 1406

Location (SRS Coordinates): 18401.83E 66640.68N

Ground Elevation Above MSL: $129.4 \mathrm{ft}$

Depth of Core Interval: 8.00 to $9.75 \mathrm{ft}$

Sample Type: Normal

Sample Color: 10R 4/6

\section{Constituent}

RQ AQ B Result
Sample Matrix: Soil

USC Soil Classification: SC
Sample Moisture: Damp Percent Solids: 89.60

D. Limit Method
Total Metals

Arsenic
Barium
Cadmium
Chromium
Copper
Lead
Manganese
Mercury
Nickel
Selenium
Silver

$B / N / A$ Extractables (including $P A H$ and pherols)

Acenaphthene

Acenaphthylene

Anthracene

Benzo(a)anthracene

Benzo(b)fluoranthene

Benzo(k)fluoranthene

Benzoic acid

Benzo(g,h,i)perylene

Benzo(a)pyrene

Benzyl alcohol

Bis(2-chloroethoxy) methane

Bis(2-chloroethyl) ether

Bis(2-chloroisopropyl) ether

Bis(2-ethylhexyl) phthalate

4-Bromophenyl phenyl ether

Butylbenzyl phthalate

4-Chloroaniline

4-Chloro-m-cresol

2-Chloronaphthalene

2-Chlorophenol

4-Chlorophenyl phenyl ether

Chrysene

o-Cresol (2-Methylphenol)

p-Cresol (4-Methyiphenol)

Dibenz $(\mathrm{a}, \mathrm{h})$ anthracene

Dibenzofuran

Di-n-butyl phthalate

1,2-Dichlorobenzene

1,3-Dichlorobenzene

1,4-Dichlorobenzene
U

1.8
25
0.55
35
7.6
5.6
38
0.20
9.7
0.47
2.5

1.8

0.55

7.6

5.6

0.20

2.5
Unit

$\begin{array}{ll}0.95 & \text { EPA7060 } \\ 22 & \text { EPA6010 } \\ 0.55 & \text { EPA6010 } \\ 1.1 & \text { EPA6010 } \\ 2.8 & \text { EPA6010 } \\ 0.28 & \text { EPA7421 } \\ 1.7 & \text { EPA6010 } \\ 0.11 & \text { EPA7470 } \\ 4.4 & \text { EPA6010 } \\ 0.47 & \text { EPA7740 } \\ 1.1 & \text { EPA6010 }\end{array}$

$\mathrm{mg} / \mathrm{kg}$
$\mathrm{mg} / \mathrm{kg}$
$\mathrm{mg} / \mathrm{kg}$
$\mathrm{mg} / \mathrm{kg}$
$\mathrm{mg} / \mathrm{kg}$
$\mathrm{mg} / \mathrm{kg}$
$\mathrm{mg} / \mathrm{kg}$
$\mathrm{mg} / \mathrm{kg}$
$\mathrm{mg} / \mathrm{kg}$
$\mathrm{mg} / \mathrm{kg}$
$\mathrm{mg} / \mathrm{kg}$
420

84

180

250

86

2,100

420

190

420

420

420

420

64

420

420

420

420

420

420

420

220

420

420

420

58

95

420

420

420 $\mu \mathrm{g} / \mathrm{kg}$

$\mu \mathrm{g} / \mathrm{kg}$

$\mu \mathrm{g} / \mathrm{kg}$

$\mu \mathrm{g} / \mathrm{kg}$

$\mu \mathrm{g} / \mathrm{kg}$

$\mu g / \mathrm{kg}$

$\mu \mathrm{g} / \mathrm{kg}$

$\mu \mathrm{g} / \mathrm{kg}$

$\mu \mathrm{g} / \mathrm{kg}$

$\mu \mathrm{g} / \mathrm{kg}$

$\mu \mathrm{g} / \mathrm{kg}$

$\mu \mathrm{g} / \mathrm{kg}$

$\mu \mathrm{g} / \mathrm{kg}$

$\mu \mathrm{g} / \mathrm{kg}$

$\mu \mathrm{g} / \mathrm{kg}$

$\mu \mathrm{g} / \mathrm{kg}$

$\mu \mathrm{g} / \mathrm{kg}$

$\mu \mathrm{g} / \mathrm{kg}$

$\mu \mathrm{g} / \mathrm{kg}$

$\mu \mathrm{g} / \mathrm{kg}$

$\mu \mathrm{g} / \mathrm{kg}$

$\mu g / \mathrm{kg}$

$\mu \mathrm{g} / \mathrm{kg}$

$\mu \mathrm{g} / \mathrm{kg}$

$\mu \mathrm{g} / \mathrm{kg}$

$\mu \mathrm{g} / \mathrm{kg}$

$\mu \mathrm{g} / \mathrm{kg}$

$\mu \mathrm{g} / \mathrm{kg}$

$\mu g / \mathrm{kg}$

$\mu \mathrm{g} / \mathrm{kg}$
EPA8270

EPA8270

EPA8270

EPA8270

EPA8270

EPA8270

EPA8270

EPA8270

EPA8270

EPA8270

EPA8270

EPA8270

EPA8270

EPA8270

EPA8270

EPA8270

EPA8270

EPA8270

EPA8270

EPA8270

EPA8270

EPA8270

EPA8270

EPA8270

EPA8270

EPA8270

EPA8270

EPA8270

EPA8270

EPA8270 
SAMPLE NAME: D43Ï 1406 (Continued)

Sample ID: 100396

$\begin{array}{llllll}\text { Constituent } & \text { RQ AQ B Result Unit Dimit Method }\end{array}$

$B / N / A$ Extractables (including $P A H$ and phenols)

3.3'-Dichlorobenzidine

2,4-Dichlorophenol

Diechyl phthalate

2,4-Dimethyl phenol

Dimethyl phthalate

2,4-Dinitrophenol

2,4-Dinitrotoluene

2,6-Dinitrotoluene

Di-n-octyl phthalate

Fluoranthene

Fluorene

Hexachlorobenzene

Hexachlorobutadiene

Hexachlorocyclopentadiene

Hexachloroethane

Indeno(1,2,3-c,d)pyrene

Isophorone

2-Methyl-4,6-dinitrophenol

2-Methylnaphthalene

Naphthalene

o-Nitroaniline

m-Nitroaniline

p-Nitroaniline

Nitrobenzene

2-Nitrophenol

4-Nitrophenol

$\mathrm{N}$-Nitrosodiphenylamine

$\mathrm{N}$-Nitrosodipropylamine

Pentachlorophenol

Phenanthrene

Phenol

Propanoic acid ester

Pyrene

1,2,4-Trichlorobenzene

2,4,5-Trichlorophenol

2,4,6-Trichlorophenol

Unknown hydrocarbon

Unknown hydrocarbon

Unknown hydrocarbon

Unknown hydrocarbon:

Unknown

Unknown ketone

Unknown PAH

Unknown PAH

Volatile Organic Compounds

Acetone

Acetone

Benzene

\section{$\mathrm{U}$}

U

U

U

U

U

U

U

U

J

U

U

U

U

U

U

U

J

J

U

U

U

J

U

U

U

U

U

UJ $\quad C$

J N

J

U

U

U

J $N$

J $N$

J $\mathbf{N}$

J $N \quad 200$

J $N \quad 300$

J $N \quad 500$

J $\mathbf{N} \quad 600$

J $\quad \mathbf{N} \quad 500$

\begin{tabular}{|c|c|c|}
\hline$\mu g / \mathrm{kg}$. & 840 & EPA827 \\
\hline$\mu \mathrm{g} / \mathrm{kg}$ & 420 & EPA8270 \\
\hline$\mu \mathrm{g} / \mathrm{kg}$ & 420 & EPA8270 \\
\hline$\mu g / k g$ & 420 & EPA8270 \\
\hline$\mu \mathrm{g} / \mathrm{kg}$ & 420 & EPA8270 \\
\hline$\mu \mathrm{g} / \mathrm{kg}$ & 2,100 & EPA 827 \\
\hline$\mu \mathrm{g} / \mathrm{kg}$ & 420 & EPA8270 \\
\hline$\mu g / k g$ & 420 & EPA827 \\
\hline$\mu \mathrm{g} / \mathrm{kg}$ & 420 & EPA827 \\
\hline$\mu g / k g$ & 420 & EPA8270 \\
\hline$\mu \mathrm{g} / \mathrm{kg}$ & 420 & EPA8270 \\
\hline$\mu \mathrm{g} / \mathrm{kg}$ & 420 & EPA8270 \\
\hline$\mu \mathrm{g} / \mathrm{kg}$ & 420 & EPA8270 \\
\hline$\mu \mathrm{g} / \mathrm{kg}$ & 420 & EPA8270 \\
\hline$\mu \mathrm{g} / \mathrm{kg}$ & 420 & EPA8270 \\
\hline$\mu \mathrm{g} / \mathrm{kg}$ & 420 & EPA8270 \\
\hline$\mu \mathrm{g} / \mathrm{kg}$ & 420 & EPA827 \\
\hline$\mu g / \mathrm{kg}$ & 2,100 & EPA8270 \\
\hline$\mu \mathrm{g} / \mathrm{kg}$ & 420 & EPA8270 \\
\hline$\mu g / \mathrm{kg}$ & 420 & EPA827 \\
\hline$\mu \mathrm{g} / \mathrm{kg}$ & 2,100 & EPA 827 \\
\hline$\mu \mathrm{g} / \mathrm{kg}$ & 2,100 & EPA8270 \\
\hline$\mu \mathrm{g} / \mathrm{kg}$ & 2,100 & EPA8270 \\
\hline$\mu \mathrm{g} / \mathrm{kg}$ & 420 & EPA 8270 \\
\hline$\mu \mathrm{g} / \mathrm{kg}$ & 420 & EPA 8270 \\
\hline$\mu \mathrm{g} / \mathrm{kg}$ & 2,100 & EPA8270 \\
\hline$\mu \mathrm{g} / \mathrm{kg}$ & 420 & EPA8270 \\
\hline$\mu \mathrm{g} / \mathrm{kg}$ & 420 & EPA 8270 \\
\hline$\mu \mathrm{g} / \mathrm{kg}$ & 2,100 & EPA 8270 \\
\hline$\mu \mathrm{g} / \mathrm{kg}$ & 420 & EPA 8270 \\
\hline$\mu \mathrm{g} / \mathrm{kg}$ & 420 & EPA8270 \\
\hline$\mu \mathrm{g} / \mathrm{kg}$ & & EPA8270 \\
\hline$\mu \mathrm{g} / \mathrm{kg}$ & 420 & EPA827 \\
\hline$\mu \mathrm{g} / \mathrm{kg}$ & 420 & EPA827C \\
\hline$\mu \mathrm{g} / \mathrm{kg}$ & 2,100 & EPA8270 \\
\hline$\mu \mathrm{g} / \mathrm{kg}$ & 420 & EPA8270 \\
\hline$\mu g / \mathrm{kg}$ & & EPA827C \\
\hline$\mu g / \mathrm{kg}$ & & EPA8270 \\
\hline$\mu \mathrm{g} / \mathrm{kg}$ & & EPA8270 \\
\hline$\mu \mathrm{g} / \mathrm{kg}$ & • & EPA8270 \\
\hline$\mu g / k g$ & & EPA 8270 \\
\hline$\mu g / k g$ & & EPA827 \\
\hline$\mu g / k g$ & & EPA827C \\
\hline$\mu \mathrm{g} / \mathrm{kg}$ & & EPA827 \\
\hline
\end{tabular}

$\begin{array}{ll}\text { L } & 510 \\ & 740 \\ \text { U } & 6.5\end{array}$

510
740

6.5

$\begin{array}{ll}13 & \text { EPA8240 } \\ 65 & \text { EPA8240 } \\ 6.5 & \text { EPA8240 }\end{array}$


SAMPLE NAME: D431 1406 (Continued)

Constituent

Volatile Organic Compounds
Sample ID: 100396

RQ AQ B Result Unit D. Limit Method

Bromodichloromethane
Bromoform
Bromomethane (Methyl bromide)
Carbon disulfide
Carbon tetrachloride
Chlorobenzene
Chloroethane
Chloroethene (Vinyl chloride)
Chloroform
Chloromethane (Methyl chloride)
Dibromochloromethane
1,1-Dichloroethane
1,2-Dichloroethane
1.1-Dichloroethylene
1,2-Dichloroethylene
Dichloromethane (Methylene chloride)
1,2-Dichloropropane
cis-1,3-Dichloropropene
trans-1,3-Dichloropropene
Ethylbenzene
2-Hexanone .
Methyl ethyl ketone
Methyl isobutyl ketone
Styrene
1,1,2,2-Tetrachloroethane
Tetrachloroethylene
Toluene
1.1.1-Trichloroethane
1,1,2-Trichloroethane
Trichloroethylene
Vinyl acetate
Xylenes

$\begin{array}{llll}6.5 & \mu \mathrm{g} / \mathrm{kg} & 6.5 & \text { EPA8240 } \\ 6.5 & \mu \mathrm{g} / \mathrm{kg} & 6.5 & \text { EPA8240 } \\ 13 & \mu \mathrm{g} / \mathrm{kg} & 13 & \text { EPA8240 } \\ 6.5 & \mu \mathrm{g} / \mathrm{kg} & 6.5 & \text { EPA8240 } \\ 6.5 & \mu \mathrm{g} / \mathrm{kg} & 6.5 & \text { EPA8240 } \\ 6.5 & \mu \mathrm{g} / \mathrm{kg} & 6.5 & \text { EPA8240 } \\ 13 & \mu \mathrm{g} / \mathrm{kg} & 13 & \text { EPA8240 } \\ 13 & \mu \mathrm{g} / \mathrm{kg} & 13 & \text { EPA8240 } \\ 6.5 & \mu \mathrm{g} / \mathrm{kg} & 6.5 & \text { EPA8240 } \\ 13 & \mu \mathrm{g} / \mathrm{kg} & 13 & \text { EPA8240 } \\ 6.5 & \mu \mathrm{kg} & 6.5 & \text { EPA8240 } \\ 6.5 & \mu \mathrm{g} / \mathrm{kg} & 6.5 & \text { EPA8240 } \\ 6.5 & \mu \mathrm{g} / \mathrm{kg} & 6.5 & \text { EPA8240 } \\ 6.5 & \mu \mathrm{g} / \mathrm{kg} & 6.5 & \text { EPA8240 } \\ 6.5 & \mu \mathrm{g} / \mathrm{kg} & 6.5 & \text { EPA8240 } \\ 9.7 & \mu \mathrm{g} / \mathrm{kg} & 6.5 & \text { EPA8240 } \\ 6.5 & \mu \mathrm{g} / \mathrm{kg} & 6.5 & \text { EPA8240 } \\ 6.5 & \mu \mathrm{g} / \mathrm{kg} & 6.5 & \text { EPA8240 } \\ 6.5 & \mu \mathrm{g} / \mathrm{kg} & 6.5 & \text { EPA8240 } \\ 6.5 & \mu \mathrm{g} / \mathrm{kg} & 6.5 & \text { EPA8240 } \\ 13 & \mu \mathrm{g} / \mathrm{kg} & 13 & \text { EPA8240 } \\ 13 & \mu \mathrm{g} / \mathrm{kg} & 13 & \text { EPA8240 } \\ 13 & \mu \mathrm{g} / \mathrm{kg} & 13 & \text { EPA8240 } \\ 6.5 & \mu \mathrm{g} / \mathrm{kg} & 6.5 & \text { EPA8240 } \\ 6.5 & \mu \mathrm{g} / \mathrm{kg} & 6.5 & \text { EPA8240 } \\ 6.5 & \mu \mathrm{g} / \mathrm{kg} & 6.5 & \text { EPA8240 } \\ 6.5 & \mu \mathrm{g} / \mathrm{kg} & 6.5 & \text { EPA8240 } \\ 6.5 & \mu \mathrm{g} / \mathrm{kg} & 6.5 & \text { EPA8240 } \\ 6.5 & \mu \mathrm{g} / \mathrm{kg} & 6.5 & \text { EPA8240 } \\ 6.5 & \mu \mathrm{g} / \mathrm{kg} & 6.5 & \text { EPA8240 } \\ 13 & \mu \mathrm{g} / \mathrm{kg} & 13 & \text { EPA8240 } \\ 6.5 & \mu \mathrm{g} / \mathrm{kg} & 6.5 & \text { EPA8240 }\end{array}$

Pesticides/PCBs and Dioxins/Furans

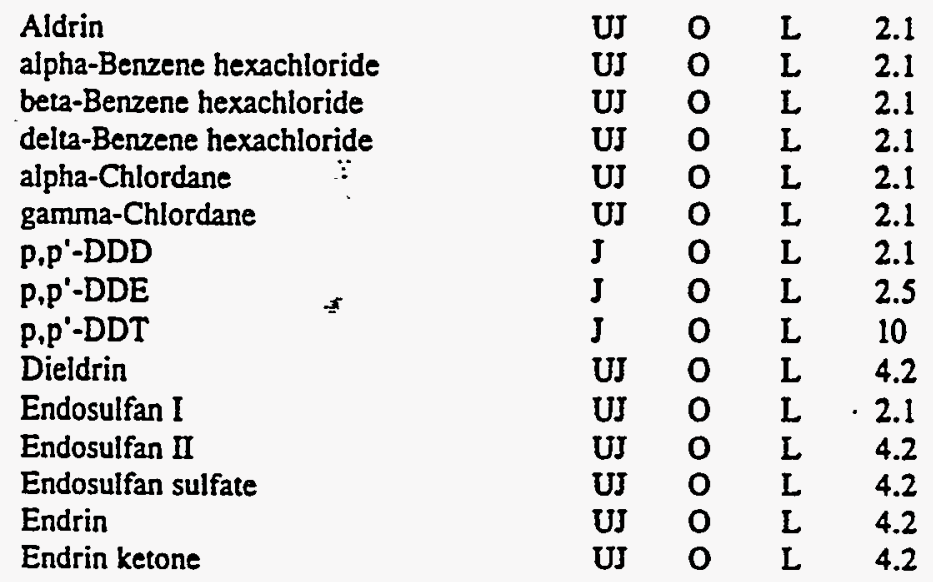

$\begin{array}{lll}\mu \mathrm{g} / \mathrm{kg} & 2.1 & \text { EPA8080 } \\ \mu \mathrm{g} / \mathrm{kg} & 2 . & \text { EPA8080 } \\ \mu \mathrm{g} / \mathrm{kg} & 2.1 & \text { EPA8080 } \\ \mu \mathrm{g} / \mathrm{kg} & 2.1 & \text { EPA8080 } \\ \mu \mathrm{g} / \mathrm{kg} & 2.1 & \text { EPA8080 } \\ \mu \mathrm{g} / \mathrm{kg} & 2.1 & \text { EPA8080 } \\ \mu \mathrm{g} / \mathrm{kg} & 4.2 & \text { EPA8080 } \\ \mu \mathrm{g} / \mathrm{kg} & 4.2 & \text { EPA8080 } \\ \mu \mathrm{g} / \mathrm{kg} & 4.2 & \text { EPA8080 } \\ \mu \mathrm{g} / \mathrm{kg} & 4.2 & \text { EPA8080 } \\ \mu \mathrm{g} / \mathrm{kg} & 2.1 & \text { EPA8080 } \\ \mu \mathrm{g} / \mathrm{kg} & 4.2 & \text { EPA8080 } \\ \mu \mathrm{g} / \mathrm{kg} & 4.2 & \text { EPA8080 } \\ \mu \mathrm{g} / \mathrm{kg} & 4.2 & \text { EPA8080 } \\ \mu \mathrm{g} / \mathrm{kg} & 4.2 & \text { EPA8080 }\end{array}$


SAMPLE NAME: D431 1406 (Continued)

Sample ID: 100396

$\begin{array}{llllll}\text { Constituent } & \text { RQ AQ } & \text { U Result } & \text { D. Limit Method }\end{array}$

Pesticides/PCBs and Dioxins/Furans

\begin{tabular}{|c|c|c|c|c|c|c|c|}
\hline Heptachlor & UJ & 0 & $\mathrm{~L}$ & 2.1 & $\mu g / \mathrm{kg}$ & 2.1 & EPA8080 \\
\hline Heptachlor epoxide & UJ & 0 & $L$ & 2.1 & $\mu g / \mathrm{kg}$ & 2.1 & EPA8080 \\
\hline Heptachlorodibenzo-p-dioxin isomers & $\mathbf{U}$ & & & 0.40 & $\mathrm{ng} / \mathrm{g}$ & 0.40 & EPA8280 \\
\hline Heptachlorodibenzo-p-furan isomers & $\mathrm{U}$ & & & 0.20 & $\mathrm{ng} / \mathrm{g}$ & 0.20 & EPA8280 \\
\hline Hexachlorodibenzo-p-dioxin isomers & $\mathbf{U}$ & & & 0.20 & $\mathrm{ng} / \mathrm{g}$ & 0.20 & EPA8280 \\
\hline Hexachlorodibenzo-p-furan isomers & $\mathrm{U}$ & & & 0.20 & ng/g & 0.20 & EPA8280 \\
\hline Lindane & UJ & 0 & $\mathbf{L}$ & 2.1 & $\mu \mathrm{g} / \mathrm{kg}$ & 2.1 & EPA8080 \\
\hline Methoxychlor & UJ & 0 & $\mathrm{~L}$ & 21 & $\mu \mathrm{g} / \mathrm{kg}$ & 21 & EPA8080 \\
\hline Octachlorodibenzo-p-dioxin & & & & 2.4 & $\mathrm{ng} / \mathrm{g}$ & 0.10 & EPA8280 \\
\hline Octachlorodibenzo-p-furan & U & & & 0.20 & $\mathrm{ng} / \mathrm{g}$ & 0.20 & EPA8280 \\
\hline PCB 1016 & UJ & 0 & L & 42 & $\mu \mathrm{g} / \mathrm{kg}$ & 42 & EPA8080 \\
\hline PCB 1221 & UJ & 0 & L & 84 & $\mu \mathrm{g} / \mathrm{kg}$ & 84 & EPA8080 \\
\hline PCB 1232 & UJ & 0 & $\bar{L}$ & 42 & $\mu \mathrm{g} / \mathrm{kg}$ & 42 & EPA8080 \\
\hline PCB 1242 & UJ & 0 & $\bar{L}$ & 42 & $\mu \mathrm{g} / \mathrm{kg}$ & 42 & EPA8080 \\
\hline PCB 1248 & UJ & 0 & L & 42 & $\mu \mathrm{g} / \mathrm{kg}$ & 42 & EPA8080 \\
\hline PCB 1254 & UJ & 0 & $\bar{L}$ & 42 & $\mu \mathrm{g} / \mathrm{kg}$ & 42 & EPA8080 \\
\hline PCB 1260 & UJ & 0 & $\vec{L}$ & 42 & $\mu \mathrm{g} / \mathrm{kg}$ & 42 & EPA8080 \\
\hline Pentachlorodibenzo-p-dioxin isomers & $\mathbf{U}$ & & & 0.20 & $\mathrm{ng} / \mathrm{g}$ & 0.20 & EPA8280 \\
\hline Pentachlorodibenzo-p-furan isomers & $\mathbf{U}$ & & & 0.10 & $\mathrm{ng} / \mathrm{g}$ & 0.10 & EPA8280 \\
\hline $2,3,7,8-\mathrm{TCDD}$ & $\mathbf{U}$ & & & 0.20 & $\mathrm{ng} / \mathrm{g}$ & 0.20 & EPA8280 \\
\hline $2,3,7,8-\mathrm{TCDF}$ & $\mathbf{U}$ & & & 0.10 & $\mathrm{ng} / \mathrm{g}$ & 0.10 & EPA8280 \\
\hline Tetrachlorodibenzo-p-dioxin isomers & U & & & 0.20 & $\mathrm{ng} / \mathrm{g}$ & 0.20 & EPA8280 \\
\hline Tetrachlorodibenzo-p-furan isomers & $\mathbf{U}$ & & & 0.10 & $\mathrm{ng} / \mathrm{g}$ & 0.10 & EPA8280 \\
\hline Toxaphene & UJ & 0 & $\mathbf{L}$ & 210 & $\mu g / \mathrm{kg}$ & 210 & EPA8080 \\
\hline \multicolumn{8}{|c|}{ Radionuclide Indicators and Radionuclides } \\
\hline Gross alpha & U & & & 9.0 & $\mathrm{pCi} / \mathrm{g}$ & 9.0 & EPA900.0 \\
\hline Nonvolatile beta & & & & $24 \pm 21$ & $\mathrm{pCi} / \mathrm{g}$ & 15 & EPA900.0 \\
\hline Radium, total alpha-emitting & $\mathbf{U}$ & $\mathrm{v}$ & & $2.0 \pm 1.4$ & $\mathrm{pCi} / \mathrm{g}$ & 0.25 & EPA903.0 \\
\hline Tritium & & & & $1.8+0.43$ & $\mathrm{pCi} / \mathrm{g}$ & 1.3 & EPA906.0 \\
\hline
\end{tabular}

SAMPLE NAME: D431 1407

Sample ID: 100398

Location (SRS Coordinates): 18401.83E $66640.68 \mathrm{~N}$

Ground Elevation Above MSL: $129.4 \mathrm{ft}$

Depth of Core Interval: 10.00 to $11.75 \mathrm{ft}$

Sample Type: Normal :.

Sample Color: 10R $4 / 6$

Sample Matrix: Soil

USC Soil Classification: CL
Sample Moisture: Wet Perçent Solids: 79.20

D. Limit Method

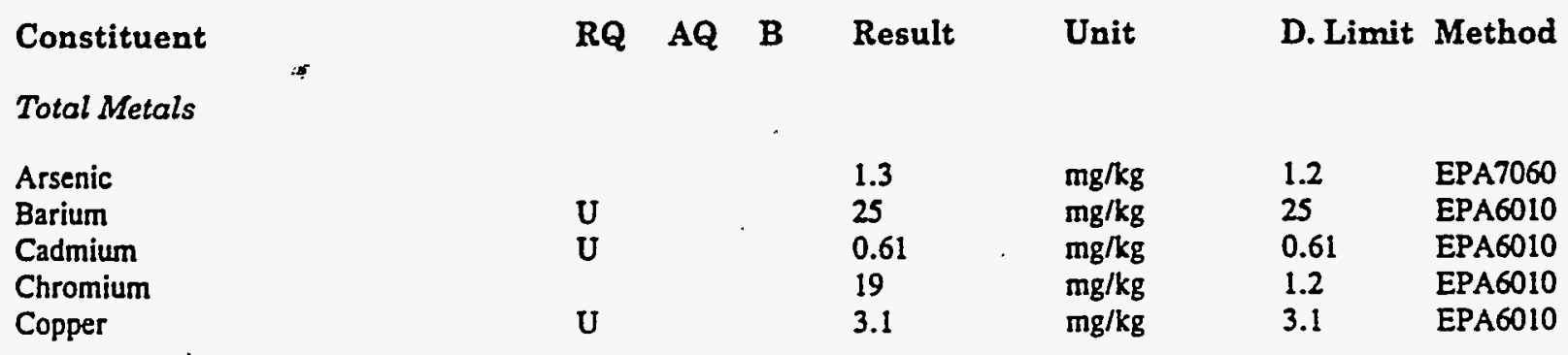


SAMPLE NAME: D4311407 (Continued)

\section{Constituent}

Total Metals

Lead

Manganese

Mercury

Nickel

Selenium

Silver
Sample ID: 100398

RQ AQ B Result

4.0

39

0.13

6.7

0.60

1.5

Unit

D. Limit Method

$B / N / A$ Extractables (including PAH and phenols)

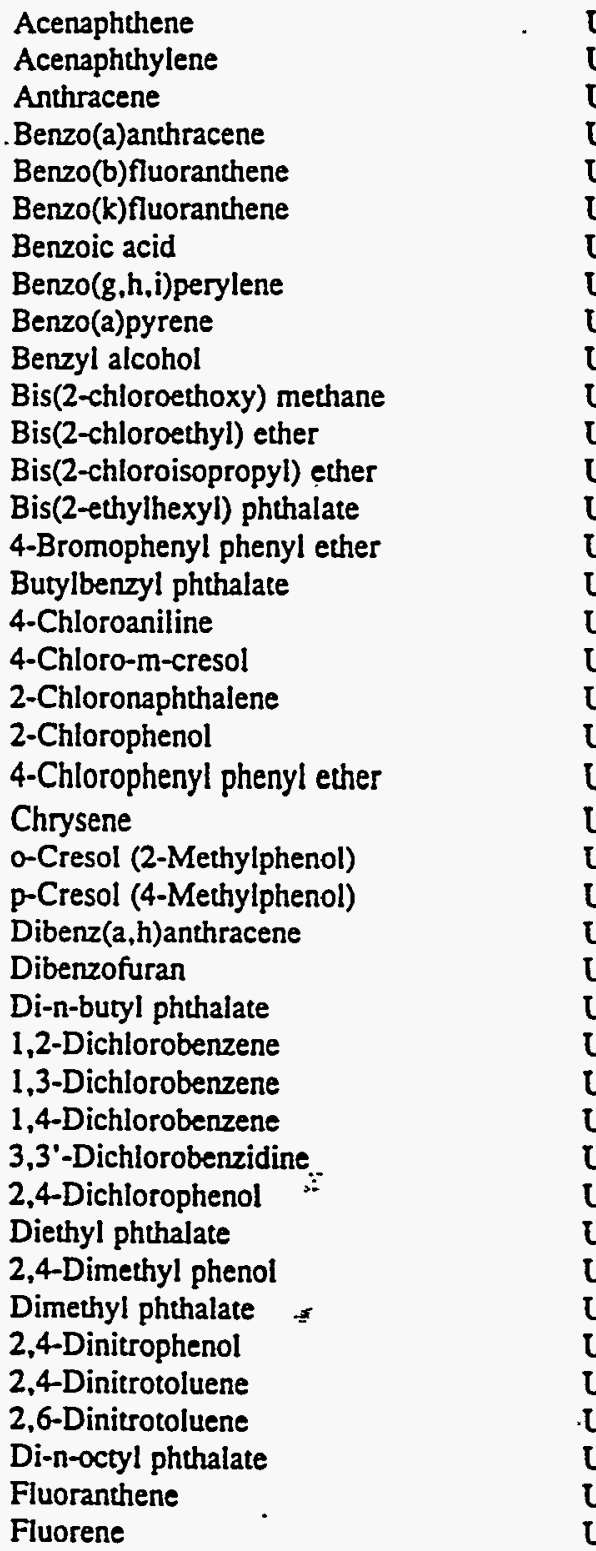

420

420

420

420

420

420

2,100

420

420

420

420

420

420

420

420

420

420

420

420

420

420

420

420

420

420

420

100

420

420

420

840

420

420

420

420

2,100

420

420

420

420

420

$\begin{array}{ll}0.36 & \text { EPA7421 } \\ 1.8 & \text { EPA6010 } \\ 0.13 & \text { EPA7470 } \\ 4.9 & \text { EPA6010 } \\ 0.60 & \text { EPA7740 } \\ 1.2 & \text { EPA6010 }\end{array}$


SAMPLE NAME: D43111407 (Continued)

Constituent
BQ
B/N/A Extractables (including $P A H$ and phenols)

Hexachlorobenzene

Hexachlorobutadiene

Hexachlorocyclopentadiene

Hexachloroethane

Hexanedioic acid ester

Indeno(1,2,3-c,d)pyrene

Isophorone

2-Methyl-4,6-dinitrophenol

2-MethyInaphthalene

Naphthalene

o-Nitroaniline

m-Nitroaniline

p-Nitroaniline

Nitrobenzene.

2-Nitrophenol

4-Nitrophenol

N-Nitrosodiphenylamine

N-Nitrosodipropylamine .

Pentachlorophenol

Phenanthrene

Phenol

Pyrene

1,2,4-Trichlorobenzene

2,4,5-Trichlorophenol

2,4,6-Trichlorophenol

Unknown ketone

Unknown PAH

Unknown PAH

Unknown phthalate

Volatile Organic Compounds

Acetone

Benzene

Bromodichloromethane

Bromoform

Bromomethane (Methyl bromide)

Carbon disulfide

Carbon tetrachloride

Chlorobenzene

Chloroethane

Chloroethene (Vinyl chloride)

Chloroform

Chloromethane (Methyl chloride)

Dibromochloromethane

1,1-Dichloroethane

1,2-Dichloroethane

1,1-Dichloroethylene

1,2-Dichloroethylene

Dichloromethane (Methylene chioride)
Sample ID: 100398

Unit

D. Limit Method

$\begin{array}{llll}420 & \mu \mathrm{g} / \mathrm{kg} & 420 & \text { EPA8270 } \\ 420 & \mu \mathrm{g} / \mathrm{kg} & 420 & \text { EPA8270 } \\ 420 & \mu \mathrm{g} / \mathrm{kg} & 420 & \text { EPA8270 } \\ 420 & \mu \mathrm{g} / \mathrm{kg} & 420 & \text { EPA8270 } \\ 10,000 & \mu \mathrm{g} / \mathrm{kg} & & \text { EPA8270 } \\ 420 & \mu \mathrm{gg} & 420 & \text { EPA8270 } \\ 420 & \mu \mathrm{kg} & 420 & \text { EPA8270 } \\ .2,100 & \mu \mathrm{kg} & 2,100 & \text { EPA8270 } \\ 420 & \mu \mathrm{kg} & 420 & \text { EPA8270 } \\ 420 & \mu \mathrm{kg} & 420 & \text { EPA8270 } \\ 2,100 & \mu \mathrm{kg} & 2,100 & \text { EPA8270 } \\ 2,100 & \mu \mathrm{kg} / \mathrm{kg} & 2,100 & \text { EPA8270 } \\ 2,100 & \mu \mathrm{g} / \mathrm{kg} & 2,100 & \text { EPA8270 } \\ 420 & \mu \mathrm{g} / \mathrm{kg} & 420 & \text { EPA8270 } \\ 420 & \mu \mathrm{g} / \mathrm{kg} & 420 & \text { EPA8270 } \\ 2,100 & \mu \mathrm{g} / \mathrm{kg} & 2,100 & \text { EPA8270 } \\ 420 & \mu \mathrm{g} / \mathrm{kg} & 420 & \text { EPA8270 } \\ 420 & \mu \mathrm{g} / \mathrm{kg} & 420 & \text { EPA8270 } \\ 2,100 & \mu \mathrm{g} / \mathrm{kg} & 2,100 & \text { EPA8270 } \\ 420 & \mu \mathrm{gg} / \mathrm{kg} & 420 & \text { EPA8270 } \\ 420 & \mu \mathrm{g} / \mathrm{kg} & 420 & \text { EPA8270 } \\ 420 & \mu \mathrm{g} / \mathrm{kg} & 420 & \text { EPA8270 } \\ 420 & \mu \mathrm{g} / \mathrm{kg} & 420 & \text { EPA8270 } \\ 2,100 & \mu \mathrm{g} / \mathrm{kg} & 2,100 & \text { EPA8270 } \\ 420 & \mu \mathrm{g} / \mathrm{kg} & 420 & \text { EPA8270 } \\ 6,000 & \mu \mathrm{g} / \mathrm{kg} & & \text { EPA8270 } \\ 200 & \mu \mathrm{g} / \mathrm{kg} & & \text { EPA8270 } \\ 200 & \mu \mathrm{g} / \mathrm{kg} & & \text { EPA8270 } \\ 200 & \mu \mathrm{g} / \mathrm{kg} & & \text { EPA8270 }\end{array}$

$\begin{array}{llll}54 & \mu \mathrm{g} / \mathrm{kg} & 13 & \text { EPA8240 } \\ 6.5 & \mu \mathrm{g} / \mathrm{kg} & 6.5 & \text { EPA8240 } \\ 6.5 & \mu \mathrm{g} / \mathrm{kg} & 6.5 & \text { EPA8240 } \\ 6.5 & \mu \mathrm{g} / \mathrm{kg} & 6.5 & \text { EPA8240 } \\ 13 & \mu \mathrm{g} / \mathrm{kg} & 13 & \text { EPA8240 } \\ 6.5 & \mu \mathrm{g} / \mathrm{kg} & 6.5 & \text { EPA8240 } \\ 6.5 & \mu \mathrm{gg} & 6.5 & \text { EPA8240 } \\ 6.5 & \mu \mathrm{g} / \mathrm{kg} & 6.5 & \text { EPA8240 } \\ 13 & \mu \mathrm{g} / \mathrm{kg} & 13 & \text { EPA8240 } \\ 13 & \mu \mathrm{g} / \mathrm{kg} & 13 & \text { EPA8240 } \\ 6.5 & \mu \mathrm{g} / \mathrm{kg} & 6.5 & \text { EPA8240 } \\ 13 & \mu \mathrm{g} / \mathrm{kg} & 13 & \text { EPA8240 } \\ 6.5 & \mu \mathrm{g} / \mathrm{kg} & 6.5 & \text { EPA8240 } \\ 6.5 & \mu \mathrm{g} / \mathrm{kg} & 6.5 & \text { EPA8240 } \\ 6.5 & \mu \mathrm{g} / \mathrm{kg} & 6.5 & \text { EPA8240 } \\ 6.5 & \mu \mathrm{g} / \mathrm{kg} & 6.5 & \text { EPA8240 } \\ 6.5 & \mu \mathrm{g} / \mathrm{kg} & 6.5 & \text { EPA8240 } \\ 15 & \mu \mathrm{g} / \mathrm{kg} & 6.5 & \text { EPA8240 }\end{array}$


SAMPLE NAME: D431 1407 (Continued)

Sample ID: 100398

\section{Constituent}

Volatile Organic Compounds
RQ

AQ B Result
Unit

$\begin{array}{ll}6.5 & \mu g / \mathrm{kg} \\ 6.5 & \mu g / \mathrm{kg} \\ 6.5 & \mu g / \mathrm{kg} \\ 6.5 & \mu g / \mathrm{kg} \\ 13 & \mu g / \mathrm{kg} \\ 13 & \mu \mathrm{g} / \mathrm{kg} \\ 13 & \mu \mathrm{g} / \mathrm{kg} \\ 6.5 & \mu g / \mathrm{kg} \\ 6.5 & \mu \mathrm{g} / \mathrm{kg} \\ 6.5 & \mu g / \mathrm{kg} \\ 6.5 & \mu \mathrm{g} / \mathrm{kg} \\ 6.5 & \mu \mathrm{g} / \mathrm{kg} \\ 6.5 & \mu \mathrm{g} / \mathrm{kg} \\ 6.5 & \mu \mathrm{g} / \mathrm{kg} \\ 13 & \mu \mathrm{g} / \mathrm{kg} \\ 6.5 & \mu \mathrm{g} / \mathrm{kg}\end{array}$

$U$
$U$
$U$
$U$
$U$
$U$
$U$
$U$
$U$
$U$
$U$
$U$
$U$
$U$
$U$
$U$

Pesticides/PCBs and Dioxins/Furans

Aldrin
alpha-Benzene hexachloride
beta-Benzene hexachloride
delta-Benzene hexachloride
alpha-Chlordane
gamma-Chlordane
p,p'-DDD
p.p'-DDE
p,p'-DDT
Dieldrin
Endosulfan I
Endosulfan II
Endosulfan sulfate
Endrin
Endrin ketone
Heptachlor
Heptachlor epoxide
Heptachlorodibenzo-p-dioxin isomers
Heptachlorodibenzo-p-furan isomers
Hexachlorodibenzo-p-dioxin isomers
Hexachlorodibenzo-p-furan isomers
Lindane
Methoxychlor
Octachlorodibenzo-p-dioxin
Octachlorodibenzo-p-furan
PCB 1016
PCB 1221
PCB 1232
PCB 1242
PCB 1248
PCB 1254

\begin{tabular}{|c|c|c|c|}
\hline 2.1 & $\mu \mathrm{g} / \mathrm{kg}$ & 2.1 & EPA8080 \\
\hline 2.1 & $\mu \mathrm{g} / \mathrm{kg}$ & 2.1 & EPA8080 \\
\hline 2.1 & $\mu \mathrm{g} / \mathrm{kg}$ & 2.1 & EPA8080 \\
\hline 2.1 & $\mu \mathrm{g} / \mathrm{kg}$ & 2.1 & EPA8080 \\
\hline 2.1 & $\mu \mathrm{g} / \mathrm{kg}$ & 2.1 & EPA8080 \\
\hline 2.1 & $\mu \mathrm{g} / \mathrm{kg}$ & 2.1 & EPA8080 \\
\hline 4.3 & $\mu \mathrm{g} / \mathrm{kg}$ & 4.3 & EPA8080 \\
\hline 4.3 & $\mu \mathrm{g} / \mathrm{kg}$ & 4.3 & EPA8080 \\
\hline 4.3 & $\mu \mathrm{g} / \mathrm{kg}$ & 4.3 & EPA8080 \\
\hline 4.3 & $\mu \mathrm{g} / \mathrm{kg}$ & 4.3 & EPA8080 \\
\hline 2.1 & $\mu \mathrm{g} / \mathrm{kg}$ & 2.1 & EPA8080 \\
\hline 4.3 & $\mu \mathrm{g} / \mathrm{kg}$ & 4.3 & EPA8080 \\
\hline 4.3 & $\mu \mathrm{g} / \mathrm{kg}$ & 4.3 & EPA8080 \\
\hline 4.3 & $\mu \mathrm{g} / \mathrm{kg}$ & 4.3 & EPA8080 \\
\hline 4.3 & $\mu \mathrm{g} / \mathrm{kg}$ & 4.3 & EPA8080 \\
\hline 2.1 & $\mu \mathrm{g} / \mathrm{kg}$ & 2.1 & EPA8080 \\
\hline 2.1 & $\mu \mathrm{g} / \mathrm{kg}$ & 2.1 & EPA8080 \\
\hline 0.20 & $\mathrm{ng} / \mathrm{g}$ & 0.20 & EPA8280 \\
\hline 0.10 & $\mathrm{ng} / \mathrm{g}$ & 0.10 & EPA8280 \\
\hline 0.20 & $\mathrm{ng} / \mathrm{g}$ & 0.20 & EPA8280 \\
\hline 0.10 & $\mathrm{ng} / \mathrm{g}$ & 0.10 & EPA8280 \\
\hline 2.1 & $\mu g / \mathrm{kg}$ & 2.1 & EPA8080 \\
\hline 21 & $\mu g / \mathrm{kg}$ & 21 & EPA8080 \\
\hline 1.4 & $\mathrm{ng} / \mathrm{g}$ & 0.20 & EPA8280 \\
\hline 0.40 & $\mathrm{ng} / \mathrm{g}$ & 0.40 & EPA8280 \\
\hline 43 & $\mu \mathrm{g} / \mathrm{kg}$ & 43 & EPA 8080 \\
\hline 86 & $\mu g / \mathrm{kg}$ & 86 & EPA8080 \\
\hline 43 & $\mu g / \mathrm{kg}$ & 43 & EPA8080 \\
\hline 43 & $\mu g / k g$ & 43 & EPA8080 \\
\hline 43 & $\mu \mathrm{g} / \mathrm{kg}$ & 43 & EPA8080 \\
\hline 43 & $\mu g / \mathrm{kg}$ & 43 & EPA808C \\
\hline
\end{tabular}

U

U

U

$\mathrm{U}$

U

U

U

U

U

U

U

U

U

U

U

U

U

U

U

U

U

U

U

U

U

U

U
D. Limit Method 
SAMPLE NAME: D431 1407 (Continued)

Sample ID: 100398

Constituent

RQ AQ B Result "Unit

D. Limit Method

Pesticides/PCBs and Dioxins/Furans

PCB 1260

Pentachlorodibenzo-p-dioxin isomers

Pentachlorodibenzo-p-furan isomers

2,3,7,8-TCDD

$2,3,7,8-\mathrm{TCDF}$

Tetrachlorodibenzo-p-dioxin isomers

Tetrachlorodibenzo-p-furan isomers

Toxaphene

$\begin{array}{ll}U & 43 \\ U & 0.30 \\ U & 0.20 \\ U & 0.10 \\ U & 0.20 \\ U & 0.20 \\ U & 0.20 \\ U & 210\end{array}$

Sample ID: 100399

Associated Sample: 100398

Sample Type: Duplicate

Constituent

RQ AQ B Result

Unit
43

0.30

0.20

0.10

0.20

0.20

0.20

210
EPA8080

EPA8280

EPA8280

EPA8280

EPA8280

EPA8280

EPA8280

EPA8080

Total Metals

Arsenic
Barium
Cadmium
Chromium
Copper
Lead
Manganese
Mercury
Nickel
Selenium
Silver

Arsenic

Barium

Chromium

Copper

Lead

Mercury

Selenium

$B / N / A$ Extractables (including $P A H$ and phenols)

$\begin{array}{lll}\mathrm{U} & 1.2 & \mathrm{mg} / \mathrm{kg} \\ \mathrm{U} & 18 & \mathrm{mg} / \mathrm{kg} \\ \mathrm{U} & 0.46 & \mathrm{mg} / \mathrm{kg} \\ & 29 & \mathrm{mg} / \mathrm{kg} \\ & 5.6 & \mathrm{mg} / \mathrm{kg} \\ & 5.1 & \mathrm{mg} / \mathrm{kg} \\ \mathrm{U} & 130 & \mathrm{mg} / \mathrm{kg} \\ & 0.12 & \mathrm{mg} / \mathrm{kg} \\ \mathrm{U} & 9.0 & \mathrm{mg} / \mathrm{kg} \\ & 0.59 & \mathrm{mg} / \mathrm{kg} \\ & 1.1 & \mathrm{mg} / \mathrm{kg}\end{array}$

440

440

440

440

440

440

2.200

440

440

440

440

440

440

440

440

440

440

440

$\begin{array}{lll}\mu \mathrm{g} / \mathrm{kg} & 440 & \text { EPA8270 } \\ \mu \mathrm{g} / \mathrm{kg} & 440 & \text { EPA8270 } \\ \mu \mathrm{g} / \mathrm{kg} & 440 & \text { EPA8270 } \\ \mu \mathrm{g} / \mathrm{kg} & 440 & \text { EPA8270 } \\ \mu \mathrm{g} / \mathrm{kg} & 440 & \text { EPA8270 } \\ \mu \mathrm{g} / \mathrm{kg} & 440 & \text { EPA8270 } \\ \mu \mathrm{g} / \mathrm{kg} & 2,200 & \text { EPA8270 } \\ \mu \mathrm{g} / \mathrm{kg} & 440 & \text { EPA8270 } \\ \mu \mathrm{g} / \mathrm{kg} & 440 & \text { EPA8270 } \\ \mu \mathrm{g} / \mathrm{kg} & 440 & \text { EPA8270 } \\ \mu \mathrm{g} / \mathrm{kg} . & 440 & \text { EPA8270 } \\ \mu \mathrm{g} / \mathrm{kg} & 440 & \text { EPA8270 } \\ \mu \mathrm{g} / \mathrm{kg} & 440 & \text { EPA8270 } \\ \mu \mathrm{g} / \mathrm{kg} & 440 & \text { EPA8270 } \\ \mu \mathrm{g} / \mathrm{kg} & 440 & \text { EPA8270 } \\ \mu \mathrm{g} / \mathrm{kg} & 440 & \text { EPA8270 } \\ \mu \mathrm{g} / \mathrm{kg} & 440 & \text { EPA8270 } \\ \mu \mathrm{g} / \mathrm{kg} & 440 & \text { EPA8270 }\end{array}$


SAMPLE NAME: D43「 1407A (Continued)

RQ AQ B Result Unit

Sample ID: 100399
D. Limit Method

$B / N / A$ Extractables (including $P A H$ and phenols)

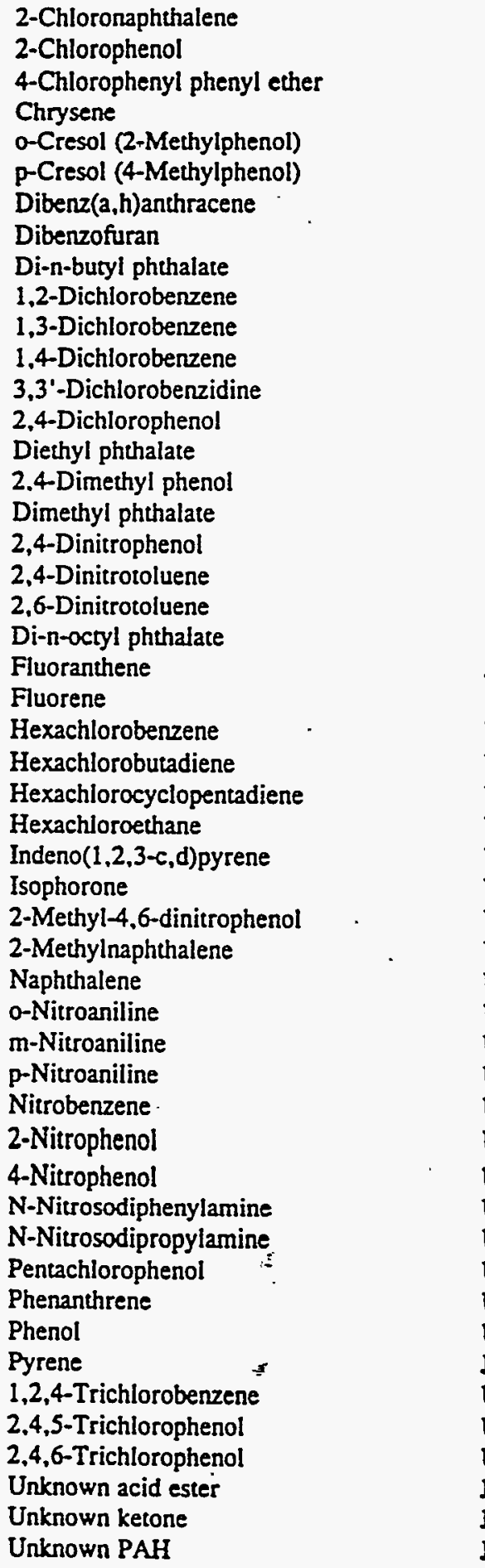

$\begin{array}{lllllll}\text { Constituent } & \text { RQ } & \text { AQ } & \text { B } & \text { Result } & \text { Unit } & \text { D. Limit Method }\end{array}$

U

U

440

U $\quad 440$

440

U $\quad 440$

$\mathrm{U} \quad 440$

UJ V $\quad 100$

$U$

U 440

U 440

U $\quad 870$

U 440

U 440

U 440

U 440

U 2.200

U 440

U 440

U 440

J 66

U 440

U 440

U 440

U 440

U 440

440

440

2,200

440

440

2,200

2,200

2,200

440

440

2,200

440

440

2,200

440

C

440

61

440

2,200

440

400

6,000

200 $\mu \mathrm{g} / \mathrm{kg}$

$\mu \mathrm{g} / \mathrm{kg}$

$\mu g / k g$

$\mu \mathrm{g} / \mathrm{kg}$

$\mu \mathrm{g} / \mathrm{kg}$

$\mu \mathrm{g} / \mathrm{kg}$

$\mu \mathrm{g} / \mathrm{kg}$

$\mu \mathrm{g} / \mathrm{kg}$

$\mu \mathrm{g} / \mathrm{kg}$

$\mu \mathrm{g} / \mathrm{kg}$

$\mu \mathrm{g} / \mathrm{kg}$

$\mu \mathrm{g} / \mathrm{kg}$

$\mu \mathrm{g} / \mathrm{kg}$

$\mu \mathrm{g} / \mathrm{kg}$

$\mu \mathrm{g} / \mathrm{kg}$

$\mu \mathrm{g} / \mathrm{kg}$

$\mu \mathrm{g} / \mathrm{kg}$

$\mu \mathrm{g} / \mathrm{kg}$

$\mu \mathrm{g} / \mathrm{kg}$

$\mu \mathrm{g} / \mathrm{kg}$

$\mu \mathrm{g} / \mathrm{kg}$

$\mu \mathrm{g} / \mathrm{kg}$

$\mu \mathrm{g} / \mathrm{kg}$

$\mu \mathrm{g} / \mathrm{kg}$

$\mu g / k g$

$\mu \mathrm{g} / \mathrm{kg}$

$\mu \mathrm{g} / \mathrm{kg}$

$\mu \mathrm{g} / \mathrm{kg}$

$\mu \mathrm{g} / \mathrm{kg}$

$\mu \mathrm{g} / \mathrm{kg}$

$\mu \mathrm{g} / \mathrm{kg}$

$\mu \mathrm{g} / \mathrm{kg}$

$\mu \mathrm{g} / \mathrm{kg}$

$\mu \mathrm{g} / \mathrm{kg}$

$\mu \mathrm{g} / \mathrm{kg}$

$\mu \mathrm{g} / \mathrm{kg}$

$\mu g / k g$

$\mu \mathrm{g} / \mathrm{kg}$

$\mu \mathrm{g} / \mathrm{kg}$

$\mu \mathrm{g} / \mathrm{kg}$

$\mu \mathrm{g} / \mathrm{kg}$

$\mu g / k g$

$\mu g / \mathrm{kg}$

$\mu g / \mathrm{kg}$

$\mu g / \mathrm{kg}$

$\mu g / \mathrm{kg}$

$\mu \mathrm{g} / \mathrm{kg}$

$\mu g / k g$

$\mu \mathrm{g} / \mathrm{kg}$

$\mu g / \mathrm{kg}$
EPA8270

EPA8270

EPA8270

EPA8270

EPA8270

EPA8270

EPA8270

EPA8270

EPA8270

EPA8270

EPA8270

EPA8270

EPA8270

EPA8270

EPA8270

EPA8270

EPA8270

EPA8270

EPA8270

EPA8270

EPA8270

EPA8270

EPA8270

EPA8270

EPA8270

EPA8270

EPA8270

EPA8270

EPA8270

EPA8270

EPA8270

EPA8270

EPA8270

EPA8270

EPA8270

EPA8270

EPA8270

EPA8270

EPA8270

EPA8270

EPA8270

EPA8270

EPA8270

EPA8270

EPA8270

EPA8270

EPA8270

EPA8270

EPA8270

EPA8270 
SAMPLE NAME: D431 1407A (Continued)

Sample ID: 100399

Constituent RQ AQ B Result Unit D. Limit Method

$B / N / A$ Extractables (including $P A H$ and phenols)

Unknown PAH

Unknown PAH

Volatile Organic Compounds

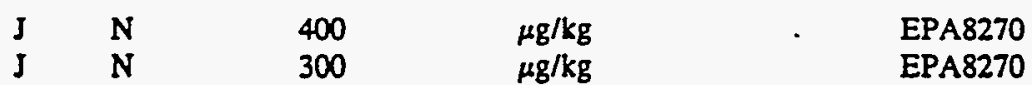

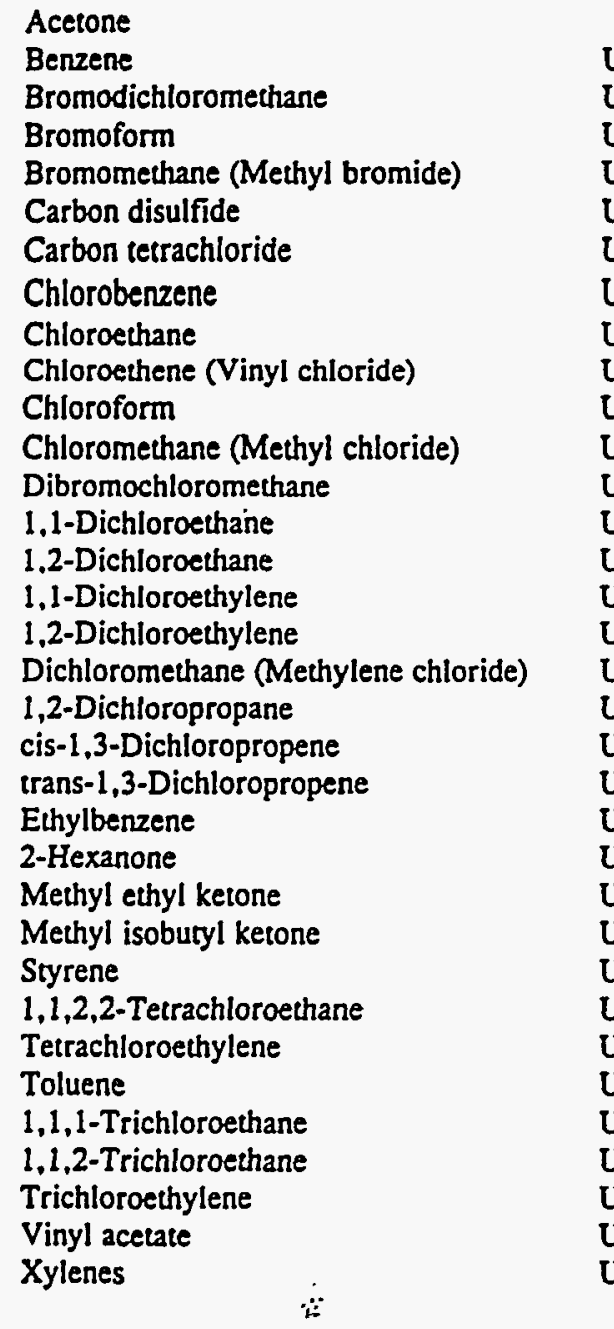

Pesticides/PCBs and Dioxins/Furans

Aldrin

alpha-Benzene hexachloride

beta-Benzene hexachloride

delta-Benzene hexachloride

alpha-Chlordane

gamma-Chlordane

$p, p^{\prime}-D D D$

$p, p^{\prime}-D D E$

$\begin{array}{ll}75 & \mu \mathrm{g} / \mathrm{kg} \\ 6.6 & \mu \mathrm{g} / \mathrm{kg} \\ 6.6 & \mu \mathrm{g} / \mathrm{kg} \\ 6.6 & \mu \mathrm{g} / \mathrm{kg} \\ 13 & \mu \mathrm{g} / \mathrm{kg} \\ 6.6 & \mu \mathrm{g} / \mathrm{kg} \\ 6.6 & \mu \mathrm{g} / \mathrm{kg} \\ 6.6 & \mu \mathrm{g} / \mathrm{kg} \\ 13 & \mu \mathrm{g} / \mathrm{kg} \\ 13 & \mu \mathrm{g} / \mathrm{kg} \\ 6.6 & \mu \mathrm{g} / \mathrm{kg} \\ 13 & \mu \mathrm{g} / \mathrm{kg} \\ 6.6 & \mu \mathrm{g} / \mathrm{kg} \\ 6.6 & \mu \mathrm{g} / \mathrm{kg} \\ 6.6 & \mu \mathrm{g} / \mathrm{kg} \\ 6.6 & \mu \mathrm{g} / \mathrm{kg} \\ 6.6 & \mu \mathrm{g} / \mathrm{kg} \\ 15 & \mu \mathrm{g} / \mathrm{kg} \\ 6.6 & \mu \mathrm{g} / \mathrm{kg} \\ 6.6 & \mu \mathrm{g} / \mathrm{kg} \\ 6.6 & \mu \mathrm{g} / \mathrm{kg} \\ 6.6 & \mu \mathrm{g} / \mathrm{kg} \\ 13 & \mu \mathrm{g} / \mathrm{kg} \\ 13 & \mu \mathrm{g} / \mathrm{kg} \\ 13 & \mu \mathrm{g} / \mathrm{kg} \\ 6.6 & \mu \mathrm{g} / \mathrm{kg} \\ 6.6 & \mu \mathrm{g} / \mathrm{kg} \\ 6.6 & \mu \mathrm{g} / \mathrm{kg} \\ 6.6 & \mu \mathrm{g} / \mathrm{kg} \\ 6.6 & \mu \mathrm{g} / \mathrm{kg} \\ 6.6 & \mu \mathrm{g} / \mathrm{kg} \\ 6.6 & \mu \mathrm{g} / \mathrm{kg} \\ 13 & \mu \mathrm{g} / \mathrm{kg} \\ 6.6 & \mu \mathrm{g} / \mathrm{kg} \\ & \end{array}$

$\mu g / \mathrm{kg}$
$\mu g / \mathrm{kg}$
$\mu g / \mathrm{kg}$
$\mu g / \mathrm{kg}$
$\mu g / \mathrm{kg}$
$\mu g / \mathrm{kg}$
$\mu g / \mathrm{kg}$
$\mu g / \mathrm{kg}$
$\mu g / \mathrm{kg}$
$\mu g / \mathrm{kg}$
$\mu g / \mathrm{kg}$
$\mu g / \mathrm{kg}$
$\mu g / \mathrm{kg}$
$\mu g / \mathrm{kg}$
$\mu g / \mathrm{kg}$
$\mu g / \mathrm{kg}$
$\mu g / \mathrm{kg}$
$\mu g / \mathrm{kg}$
$\mu g / \mathrm{kg}$
$\mu \mathrm{g} / \mathrm{kg}$
$\mu g / \mathrm{kg}$
$\mu g / \mathrm{kg}$
$\mu g / \mathrm{kg}$
$\mu g / \mathrm{kg}$
$\mu g / \mathrm{kg}$
$\mu g / \mathrm{kg}$
$\mu g / \mathrm{kg}$
$\mu g / \mathrm{kg}$
$\mu g / \mathrm{kg}$
$\mu g / \mathrm{kg}$
$\mu g / \mathrm{kg}$
$\mu \mathrm{g} / \mathrm{kg}$
$\mu g / \mathrm{kg}$
$\mu g / \mathrm{kg}$

13

6.6

EPA8240

EPA8240

$6.6 \quad$ EPA8240

6.6 EPA8240

13 EPA8240

6.6 EPA8240

6.6 EPA8240

6.6 EPA8240

13 EPA8240

13 EPA8240

$6.6 \quad$ EPA8240

13 EPA8240

6.6 EPA8240

6.6 EPA8240

6.6 EPA8240

6.6 EPA8240

6.6 EPA8240

6.6 EPA8240

6.6 EPA8240

6.6 EPA8240

$6.6 \quad$ EPA8240

$6.6 \quad$ EPA8240

13 EPA8240

13 EPA8240

13 EPA8240

6.6 EPA8240

6.6 EPA8240

6.6 EPA8240

6.6 EPA8240

$6.6 \quad$ EPA8240

6.6 EPA8240

6.6 EPA8240

13 EPA8240

6.6 EPA8240

$\begin{array}{lll}\mu \mathrm{g} / \mathrm{kg} & 2.1 & \text { EPA8080 } \\ \mu \mathrm{g} / \mathrm{kg} & 2.1 & \text { EPA8080 } \\ \mu \mathrm{g} / \mathrm{kg} & 2.1 & \text { EPA8080 } \\ \mu \mathrm{g} / \mathrm{kg} & 2.1 & \text { EPA8080 } \\ \mu \mathrm{g} / \mathrm{kg} & 2.1 & \text { EPA8080 } \\ \mu \mathrm{g} / \mathrm{kg} & 2.1 & \text { EPA8080 } \\ \mu \mathrm{g} / \mathrm{kg} & 4.3 & \text { EPA8080 } \\ \mu \mathrm{g} / \mathrm{kg} & 4.3 & \text { EPA8080 }\end{array}$


SAMPLE NAME: D431 1407A (Continued)

Sample ID: 100399

Constituent RQ AQ B Result Unit D. Limit Method

Pesticides/PCBs and Dioxins/Furans

\begin{tabular}{|c|c|c|c|c|c|c|c|}
\hline p.p'-DDT & UJ & 0 & L & 4.3 & $\mu \mathrm{g} / \mathrm{kg}$ & 4.3 & EPA8080 \\
\hline Dieldrin & UJ & 0 & L & 4.3 & $\mu \mathrm{g} / \mathrm{kg}$ & 4.3 & EPA8080 \\
\hline Endosulfan I & UJ & 0 & L & 2.1 & $\mu \mathrm{g} / \mathrm{kg}$ & 2.1 & EPA8080 \\
\hline Endosulfan II & UJ & 0 & $\bar{L}$ & 4.3 & $\mu \mathrm{g} / \mathrm{kg}$ & 4.3 & EPA8080 \\
\hline Endosulfan sulfate & UJ & 0 & L & 4.3 & $\mu \mathrm{g} / \mathrm{kg}$ & 4.3 & EPA8080 \\
\hline Endrin & UJ & 0 & L & 4.3 & $\mu \mathrm{g} / \mathrm{kg}$ & 4.3 & EPA8080 \\
\hline Endrin ketone & UJ & 0 & L & 4.3 & $\mu \mathrm{g} / \mathrm{kg}$ & 4.3 & EPA8080 \\
\hline Heptachlor & UJ & 0 & L & 2.1 & $\mu \mathrm{g} / \mathrm{kg}$ & 2.1 & EPA8080 \\
\hline Heptachlor epoxide & UJ & 0 & L & 2.1 & $\mu g / \mathrm{kg}$ & 2.1 & EPA8080 \\
\hline Heptachlorodibenzo-p-dioxin isomers & U & & & 0.30 & $\mathrm{ng} / \mathrm{g}$ & 0.30 & EPA8280 \\
\hline Heptachlorodibenzo-p-furan isomers & $\mathrm{U}$ & & & 0.10 & ng/g & 0.10 & EPA8280 \\
\hline Hexachlorodibenzo-p-dioxin isomers & $\mathbf{U}$ & & & 0.20 & $\mathrm{ng} / \mathrm{g}$ & 0.20 & EPA8280 \\
\hline Hexachlorodibenzo-p-furan isomers & $\mathbf{U}$ & & & 0.20 & $\mathrm{ng} / \mathrm{g}$ & 0.20 & EPA8280 \\
\hline Lindane & Uנ & 0 & L & 2.1 & $\mu \mathrm{g} / \mathrm{kg}$ & 2.1 & EPA8080 \\
\hline Methoxychlor & UJ & 0 & $\mathrm{~L}$ & 21 & $\mu \mathrm{g} / \mathrm{kg}$ & 21 & EPA8080 \\
\hline Octachlorodibenzo-p-dioxin & & & & 3.6 & $\mathrm{ng} / \mathrm{g}$ & 0.20 & EPA8280 \\
\hline Octachlorodibenzo-p-furan & $\mathrm{U}$ & & & 0.20 & $\mathrm{ng} / \mathrm{g}$ & 0.20 & EPA8280 \\
\hline PCB 1016 & UJ & 0 & L & 43 & $\mu \mathrm{g} / \mathrm{kg}$ & 43 & EPA8080 \\
\hline PCB 1221 & UJ & 0 & L & 85 & $\mu \mathrm{g} / \mathrm{kg}$ & 85 & EPA8080 \\
\hline PCB 1232 & UJ & 0 & L & 43 & $\mu \mathrm{g} / \mathrm{kg}$ & 43 & EPA8080 \\
\hline PCB 1242 & UJ & 0 & L & 43 & $\mu \mathrm{g} / \mathrm{kg}$ & 43 & EPA8080 \\
\hline PCB 1248 & UJ & 0 & $\mathrm{~L}$ & 43 & $\mu \mathrm{g} / \mathrm{kg}$ & 43 & EPA8080 \\
\hline РCB 1254 & UJ & 0 & $\bar{L}$ & 43 & $\mu \mathrm{g} / \mathrm{kg}$ & 43 & EPA8080 \\
\hline PCB 1260 & UJ & 0 & L & 43 & $\mu \mathrm{g} / \mathrm{kg}$ & 43 & EPA8080 \\
\hline Pentachlorodibenzo-p-dioxin isomers & $\mathrm{U}$ & & & 0.30 & $\mathrm{ng} / \mathrm{g}$ & 0.30 & EPA8280 \\
\hline Pentachlorodibenzo-p-furan isomers & $\mathrm{U}$ & & & 0.20 & $\mathrm{ng} / \mathrm{g}$ & 0.20 & EPA8280 \\
\hline $2,3,7,8-\mathrm{TCDD}$ & U & & & 0.20 & $\mathrm{ng} / \mathrm{g}$ & 0.20 & EPA8280 \\
\hline $2,3.7 .8-\mathrm{TCDF}$ & $\mathbf{U}$ & & & 0.20 & $\mathrm{ng} / \mathrm{g}$ & 0.20 & EPA8280 \\
\hline Tetrachlorodibenzo-p-dioxin isomers & $\mathbf{U}$ & & & 0.20 & $\mathrm{ng} / \mathrm{g}$ & 0.20 & EPA8280 \\
\hline Tetrachlorodibenzo-p-furan isomers & $\mathbf{U}$ & & & 0.20 & $\mathrm{ng} / \mathrm{g}$ & 0.20 & EPA8280 \\
\hline Toxaphene & UJ & 0 & L & 210 & $\mu \mathrm{g} / \mathrm{kg}$ & 210 & EPA8080 \\
\hline
\end{tabular}

SAMPLE NAME: D431 1701

Location (SRS Coordinates): 18492.14E 66454.17N

Ground Elevation Above MSL: $124.9 \mathrm{ft}$

Depth of Core Interval: 0.00 to $0.50 \mathrm{ft}$

Sample Type: Normal

Sample Color: 5YR 4/4

Constituent

Physical Parameters

Cation exchange capacity

Cation exchange capacity

$\mathrm{pH}$

Specific conductance

Total organic carbon
Sample Matrix: Soil

USC Soil Classification: SW

RQ AQ B Result

Unit

Sample Moisture: Dry

Percent Solids: 96.70

D. Limit Method

$\begin{array}{llll}6.8 & \mathrm{meq} / 100 \mathrm{~g} & 2.1 & \text { EPA9080 } \\ 4.7 & \mathrm{meq} / 100 \mathrm{~g} & 2.1 & \text { EPA9080 } \\ 4.0 & \mathrm{pH} & 0.010 & \text { EPA9045 } \\ 48 & \mu \mathrm{S} / \mathrm{cm} & 1.0 & \text { EPA9050 } \\ 5,200 & \mathrm{mg} / \mathrm{kg} & 260 & \text { EPA415.1 }\end{array}$


Total Metals

Arsenic
Barium
Cadmium
Chromium
Copper
Lead
Manganese
Mercury
Mercury
Nickel
Selenium
Silver

\begin{tabular}{|c|c|c|c|c|}
\hline & 6.2 & $\mathrm{mg} / \mathrm{kg}$ & 1.9 & EPA7060 \\
\hline $\mathbf{U}$ & 19 & $\mathrm{mg} / \mathrm{kg}$ & 19 & EPA6010 \\
\hline & 0.47 & $\mathrm{mg} / \mathrm{kg}$ & 0.47 & EPA6010 \\
\hline & 6.0 & $\mathrm{mg} / \mathrm{kg}$ & 0.94 & EPA6010 \\
\hline & 2.4 & $\mathrm{mg} / \mathrm{kg}$ & 2.4 & EPA6010 \\
\hline & 1.4 & $\mathrm{mg} / \mathrm{kg}$ & 0.29 & EPA7421 \\
\hline & 18 & $\mathrm{mg} / \mathrm{kg}$ & 1.4 & EPA6010 \\
\hline & 0.10 & $\mathrm{mg} / \mathrm{kg}$ & 0.10 & EPA7470 \\
\hline & 0.10 & $\mathrm{mg} / \mathrm{kg}$ & 0.10 & EPA7470 \\
\hline & 3.8 & $\mathrm{mg} / \mathrm{kg}$ & 3.8 & EPA6010 \\
\hline & 0.48 & $\mathrm{mg} / \mathrm{kg}$ & 0.48 & EPA7740 \\
\hline & 0.94 & $\mathrm{mg} / \mathrm{kg}$ & 0.94 & EPA601 \\
\hline
\end{tabular}

$B / N / A$ Extractables (including $P A H$ and phenols)

\begin{tabular}{|c|c|c|c|c|c|}
\hline Acenaphthene &. $\mathrm{U}$ & 340 & $\mu g / \mathrm{kg}$ & 340 & EPA8270 \\
\hline Acenaphthylene & $\mathbf{U}$ & 340 & $\mu g / \mathrm{kg}$ & 340 & EPA8270 \\
\hline Anthracene & $\mathrm{U}$ & 340 & $\mu \mathrm{g} / \mathrm{kg}$ & 340 & EPA8270 \\
\hline Benzo(a)anthracene & $\mathbf{U}$ & 340 & $\mu g / \mathrm{kg}$ & 340 & EPA8270 \\
\hline Benzo(b)fluoranthene & $\mathbf{U}$ & 340 & $\mu g / \mathrm{kg}$ & 340 & EPA8270 \\
\hline Benzo(k)fluoranthene & $\mathrm{U}$ & 340 & $\mu g / \mathrm{kg}$ & 340 & EPA8270 \\
\hline Benzoic acid & $\mathrm{U}$ & 1,700 & $\mu g / \mathrm{kg}$ & 1.700 & EPA8270 \\
\hline Benzo(g,h,i)perylene & $\mathbf{U}$ & 340 & $\mu g / \mathrm{kg}$ & 340 & EPA8270 \\
\hline Benzo(a)pyrene & $\mathbf{U}$ & 340 & $\mu \mathrm{g} / \mathrm{kg}$ & 340 & EPA8270 \\
\hline Benzyl alcohol & $\mathbf{U}$ & 340 & $\mu g / \mathrm{kg}$ & 340 & EPA8270 \\
\hline Bis(2-chloroethoxy) methane & U & 340 & $\mu \mathrm{g} / \mathrm{kg}$ & 340 & EPA8270 \\
\hline Bis(2-chloroethyl) ether & $\mathbf{U}$ & 340 & $\mu g / \mathrm{kg}$ & 340 & EPA8270 \\
\hline Bis(2-chloroisopropyl) ether & $\mathbf{U}$ & 340 & $\mu g / \mathrm{kg}$ & 340 & EPA8270 \\
\hline Bis(2-ethylhexyl) phthalate & $\mathbf{U}$ & 340 & $\mu \mathrm{g} / \mathrm{kg}$ & 340 & EPA8270 \\
\hline 4-Bromophenyl phenyl ether & U & 340 & $\mu \mathrm{g} / \mathrm{kg}$ & 340 & EPA8270 \\
\hline Butylbenzyl phthalate & $\mathbf{U}$ & 340 & $\mu g / k g$ & 340 & EPA8270 \\
\hline 4-Chloroaniline & $\mathbf{U}$ & 340 & $\mu \mathrm{g} / \mathrm{kg}$ & 340 & EPA8270 \\
\hline 4-Chloro-m-cresol & $\mathbf{U}$ & 340 & $\mu g / \mathbf{k g}$ & 340 & EPA8270 \\
\hline 2-Chloronaphthalene & $\mathbf{U}$ & 340 & $\mu g / k g$ & 340 & EPA8270 \\
\hline 2-Chlorophenol & $\mathbf{U}$ & 340 & $\mu g / k g$ & 340 & EPA8270 \\
\hline 4-Chlorophenyl phenyl ether & $\mathbf{U}$ & 340 & $\mu g / \mathrm{kg}$ & 340 & EPA8270 \\
\hline Chrysene & $\mathrm{U}$ & 340 & $\mu g / \mathrm{kg}$ & 340 & EPA8270 \\
\hline o-Cresol (2-Methylphenol) & $\mathrm{U}$ & 340 & $\mu g / k g$ & 340 & EPA8270 \\
\hline p-Cresol (4-Methylphenol) & $U$ & 340 & $\mu g / \mathrm{kg}$ & 340 & EPA8270 \\
\hline Dibenz(a,h)anthracene & $\mathbf{U}$ & 340 & $\mu g / \mathbf{k g}$ & 340 & EPA8270 - \\
\hline Dibenzofuran & $\mathbf{U}$ & 340 & $\mu g / \mathrm{kg}$ & 340 & EPA8270 \\
\hline Di-n-butyl phthalate & UJ & 150 & $\mu \mathrm{g} / \mathrm{kg}$ & 340 & EPA8270 \\
\hline 1,2-Dichlorobenzene & $\mathbf{U}$ & 340 & $\mu g / \mathbf{k g}$ & 340 & EPA8270 \\
\hline 1,3-Dichlorobenzene & $\mathbf{U}$ & 340 & $\mu \mathrm{g} / \mathrm{kg}$ & 340 & EPA8270 \\
\hline 1,4-Dichlorobenzene & $\mathbf{U}$ & 340 & $\mu g / \mathbf{k g}$ & 340 & EPA8270 \\
\hline 3,3'-Dichlorobenzidine & $\mathbf{U}$ & 690 & $\mu g / k g$ & 690 & EPA8270 \\
\hline 2,4-Dichlorophenol & $\mathbf{U}$ & 340 & $\mu g / k g$ & 340 & EPA8270 \\
\hline Diethyl phthalate & $\mathbf{U}$ & 340 & $\mu g / \mathrm{kg}$ & 340 & EPA8270 \\
\hline 2,4-Dimethyl phenol & $\mathbf{U}$ & 340 & $\mu g / \mathrm{kg}$ & 340 & EPA8270 \\
\hline Dimethyl phthalate & $\mathbf{U}$ & 340 & $\mu g / k g$ & 340 & EPA8270 \\
\hline 2,4-Dinitrophenol & U & 1,700 & $\mu \mathrm{g} / \mathrm{kg}$ & 1.700 & EPA8270 \\
\hline 2,4-Dinitrotoluene & $\mathbf{U}$ & 340 & $\mu g / k g$ & 340 & EPA8270 \\
\hline 2,6-Dinitrotoluene & $\mathbf{U}$ & 340 & $\mu g / \mathrm{kg}$ & 340 & EPA8270 \\
\hline
\end{tabular}


SAMPLE NAME: D4311701 (Continued)

Sample ID: 100427

$\begin{array}{llllll}\text { Constituent } & \text { RQ AQ } & \text { B Result Unit D. Limit Method }\end{array}$

$B / N / A$ Extractables (including $P A H$ and phenols)

\begin{tabular}{|c|c|c|c|c|c|c|c|}
\hline Di-n-octyl phthalate & $\mathrm{U}$ & & & 340 & $\mu \mathrm{g} / \mathrm{kg}$ & 340 & EPA8270 \\
\hline Fluoranthene & $\mathbf{U}$ & & & 340 & $\mu \mathrm{g} / \mathrm{kg}$ & 340 & EPA8270 \\
\hline Fluorene & $\mathrm{U}$ & & & 340 & $\mu g / k g$ & 340 & EPA8270 \\
\hline Hexachlorobenzene & $\mathrm{U}$ & & & 340 & $\mu g / k g$ & 340 & EPA8270 \\
\hline Hexachlorobutadiene & $\mathrm{U}$ & & & 340 & $\mu \mathrm{g} / \mathrm{kg}$ & 340 & EPA8270 \\
\hline Hexachlorocyclopentadiene & $\mathrm{U}$ & & & 340 & $\mu \mathrm{g} / \mathrm{kg}$ & 340 & EPA8270 \\
\hline Hexachloroethane & $\bar{U}$ & & & 340 & $\mu g / k g$ & 340 & EPA8270 \\
\hline Indeno(1,2,3-c,d)pyrene & $\mathbf{U}$ & & & 340 & $\mu \mathrm{g} / \mathrm{kg}$ & 340 & EPA8270 \\
\hline Isophorone & $\mathrm{U}$ & & & 340 & $\mu g / \mathrm{kg}$ & 340 & EPA8270 \\
\hline 2-Methyl-4,6-dinitrophenol & $U$ & & & 1.700 & $\mu g / \mathrm{kg}$ & 1,700 & EPA8270 \\
\hline 2-Methylnaphthalene & $\mathbf{U}$ & & & 340 & $\mu g / \mathrm{kg}$ & 340 & EPA8270 \\
\hline Naphthalene & $\mathrm{U}$ & & & 340 & $\mu \mathrm{g} / \mathrm{kg}$ & 340 & EPA8270 \\
\hline o-Nitroaniline & $\mathrm{U}$ & & & 1,700 & $\mu \mathrm{g} / \mathrm{kg}$ & 1,700 & EPA 8270 \\
\hline m-Nitroaniline & $\mathrm{U}$ & & & 1,700 & $\mu \mathrm{g} / \mathrm{kg}$ & 1,700 & EPA8270 \\
\hline p-Nitroaniline & $\mathrm{U}$ & & & 1,700 & $\mu \mathrm{g} / \mathrm{kg}$ & 1,700 & EPA8270 \\
\hline Nitrobenzene & $\mathrm{U}$ & & & 340 & $\mu \mathrm{g} / \mathrm{kg}$ & 340 & EPA8270 \\
\hline 2-Nitrophenol & U & & & 340 & $\mu \mathrm{g} / \mathrm{kg}$ & 340 & EPA8270 \\
\hline 4-Nitrophenol & $U$ & & & 1,700 & $\mu \mathrm{g} / \mathrm{kg}$ & 1,700 & EPA8270 \\
\hline N-Nitrosodiphenylamine & U & & & 340 & $\mu \mathrm{g} / \mathrm{kg}$ & 340 & EPA8270 \\
\hline N-Nitrosodipropylamine & U & & & 340 & $\mu \mathrm{g} / \mathrm{kg}$ & 340 & EPA8270 \\
\hline Pentachlorophenol & $\mathrm{U}$ & & & 1.700 & $\mu \mathrm{g} / \mathrm{kg}$ & 1.700 & EPA8270 \\
\hline Phenanthrene & $\mathbf{U}$ & & & 340 & $\mu \mathrm{g} / \mathrm{kg}$ & 340 & EPA8270 \\
\hline Phenol & $U$ & & & 340 & $\mu \mathrm{g} / \mathrm{kg}$ & 340 & EPA8270 \\
\hline Pyrene & $\mathbf{U}$ & & & 340 & $\mu \mathrm{g} / \mathrm{kg}$ & 340 & EPA8270 \\
\hline 1,2,4-Trichlorobenzene & $\mathrm{U}$ & & & 340 & $\mu \mathrm{g} / \mathrm{kg}$ & 340 & EPA8270 \\
\hline 2,4,5-Trichlorophenol & $\mathbf{U}$ & & & 1,700 & $\mu \mathrm{g} / \mathrm{kg}$ & 1,700 & EPA8270 \\
\hline 2,4,6-Trichlorophenol & $\mathrm{U}$ & & & 340 & $\mu g / \mathrm{kg}$ & 340 & EPA8270 \\
\hline Unknown & J & $N$ & & 1,000 & $\mu \mathrm{g} / \mathrm{kg}$ & & EPA8270 \\
\hline Unknown ketone & J & $\mathrm{N}$ & & 8,000 & $\mu \mathrm{g} / \mathrm{kg}$ & & EPA8270 \\
\hline \multicolumn{8}{|l|}{ Volatile Organic Compounds } \\
\hline Acetone & $\mathrm{U}$ & vo & $\mathrm{H}$ & 13 & $\mu \mathrm{g} / \mathrm{kg}$ & 10 & EPA8240 \\
\hline Acetone & UJ & QV & & 10 & $\mu g / \mathrm{kg}$ & 10 & EPA8240 \\
\hline Benzene & UJ & 0 & $\mathbf{H}$ & 5.2 & $\mu \mathrm{g} / \mathrm{kg}$ & 5.2 & EPA8240 \\
\hline Benzene & UJ & $Q$ & & 5.2 & $\mu \mathrm{g} / \mathrm{kg}$ & 5.2 & EPA8240 \\
\hline Bromodichloromethane & UJ & 0 & $\mathrm{H}$ & 5.2 & $\mu \mathrm{g} / \mathrm{kg}$ & 5.2 & EPA8240 \\
\hline Bromodichloromethane & UJ & $\mathbf{Q}$ & & 5.2 & $\mu \mathrm{g} / \mathrm{kg}$ & 5.2 & EPA8240 \\
\hline Bromoform & UJ & 0 & $\mathrm{H}$ & 5.2 & $\mu g / \mathrm{kg}$ & 5.2 & EPA8240 \\
\hline Bromoform & UJ & $\mathbf{Q}$ & & 5.2 & $\mu \mathrm{g} / \mathrm{kg}$ & 5.2 & EPA8240 \\
\hline Bromomethane (Methyl b̈romide) & UI & 0 & H & 10 & $\mu \mathrm{g} / \mathrm{kg}$ & 10 & EPA8240 \\
\hline Bromomethane (Methyl bromide) & UJ & $\mathbf{Q}$ & & 10 & $\mu g / k g$ & 10 & EPA8240 \\
\hline Carbon disulfide & UJ & 0 & H & 5.2 & $\mu g / k g$ & 5.2 & EPA8240 \\
\hline Carbon disulfide & UJ & $Q$ & & 5.2 & $\mu g / k g$ & 5.2 & EPA8240 \\
\hline Carbon tetrachloride & UJ & 0 & H & 5.2 & $\mu g / \mathrm{kg}$ & 5.2 & EPA8240 \\
\hline Carbon tetrachloride & UJ & $\mathbf{Q}$ & & 5.2 & $\mu \mathrm{g} / \mathrm{kg}$ & 5.2 & EPA8240 \\
\hline Chlorobenzene & UJ & 0 & H & 5.2 & $\mu \mathrm{g} / \mathrm{kg}$ & 5.2 & EPA8240 \\
\hline Chlorobenzene & UJ & $\mathbf{Q}$ & & 5.2 & $\mu g / k g$ & 5.2 & EPA8240 \\
\hline Chloroethane & UJ & 0 & H & 10 & $\mu g / \mathrm{kg}$ & 10 & EPA8240 \\
\hline Chloroethane & UJ & $\mathbf{Q}$ & & 10 & $\mu g / \mathrm{kg}$ & 10 & EPA8240 \\
\hline
\end{tabular}


SAMPLE NAME: D43ï 1701 (Continued)

\section{Constituent}

Volatile Organic Compounds
Sample ID: 100427

$\begin{array}{lllll}\text { RQ AQ B } & \text { Result Unit } & \text { D. Limit Method }\end{array}$

\begin{tabular}{|c|c|c|c|c|c|c|c|}
\hline Chloroethene (Vinyl chloride) & UJ & 0 & H & 10 & $\mu \mathrm{g} / \mathrm{kg} \cdot$ & 10 & EPA8240 \\
\hline Chloroethene (Vinyl chloride) & UJ & $\mathbf{Q}$ & & 10 & $\mu \mathrm{g} / \mathrm{kg}$ & 10 & EPA8240 \\
\hline Chloroform & UJ & 0 & $\mathrm{H}$ & 5.2 & $\mu \mathrm{g} / \mathrm{kg}$ & 5.2 & EPA8240 \\
\hline Chloroform & UJ & $\mathbf{Q}$ & & 5.2 & $\mu g / \mathrm{kg}$ & 5.2 & EPA8240 \\
\hline Chloromethane (Methyl chloride) & UJ & 0 & $\mathbf{H}$ & 10 & $\mu \mathrm{g} / \mathrm{kg}$ & 10 & EPA8240 \\
\hline Chloromethane (Methyl chloride) & UJ & $\mathbf{Q}$ & & 10 & $\mu \mathrm{g} / \mathrm{kg}$ & 10 & EPA8240 \\
\hline Dibromochloromethane & UJ & 0 & H & 5.2 & $\mu \mathrm{g} / \mathrm{kg}$ & 5.2 & EPA8240 \\
\hline Dibromochloromethane & UJ & $\mathbf{Q}$ & & 5.2 & $\mu \mathrm{g} / \mathrm{kg}$ & 5.2 & EPA8240 \\
\hline 1,1-Dichloroethane & UJ & 0 & H & 5.2 & $\mu \mathrm{g} / \mathrm{kg}$ & 5.2 & EPA8240 \\
\hline 1,1-Dichloroethane & UJ & $\mathbf{Q}$ & & 5.2 & $\mu g / \mathrm{kg}$ & 5.2 & EPA8240 \\
\hline 1.2-Dichloroethane & U\} & 0 & H & 5.2 & $\mu \mathrm{g} / \mathrm{kg}$ & 5.2 & EPA8240 \\
\hline 1,2-Dichloroethane & UJ & Q & & 5.2 & $\mu \mathrm{g} / \mathrm{kg}$ & 5.2 & EPA8240 \\
\hline 1,1-Dichloroethylene & UJ & 0 & H & 5.2 & $\mu \mathrm{g} / \mathrm{kg}$ & 5.2 & EPA8240 \\
\hline 1.1-Dichloroethylene & UJ & Q & & 5.2 & $\mu \mathrm{g} / \mathrm{kg}$ & 5.2 & EPA8240 \\
\hline 1,2-Dichloroethylene & UJ & 0 & H & 5.2 & $\mu g / k g$ & 5.2 & EPA8240 \\
\hline 1,2-Dichloroethylene & UJ & $\mathbf{Q}$ & & 5.2 & $\mu \mathrm{g} / \mathrm{kg}$ & 5.2 & EPA8240 \\
\hline Dichloromethane (Methylene chloride) & J & 0 & H & 7.0 & $\mu \mathrm{g} / \mathrm{kg}$ & 5.2 & EPA8240 \\
\hline Dichloromethane (Methylene chloride) & UJ & Q & & 5.2 & $\mu \mathrm{g} / \mathrm{kg}$ & 5.2 & EPA8240 \\
\hline 1,2-Dichloropropane & UJ & 0 & H & 5.2 & $\mu \mathrm{g} / \mathrm{kg}$ & 5.2 & EPA8240 \\
\hline 1.2-Dichloropropane & UJ & $\mathbf{Q}$ & & 5.2 & $\mu g / \mathrm{kg}$ & 5.2 & EPA8240 \\
\hline cis-1,3-Dichloropropene & UJ & 0 & H & 5.2 & $\mu \mathrm{g} / \mathrm{kg}$ & 5.2 & EPA8240 \\
\hline cis-1,3-Dichloropropene & UJ & Q & & 5.2 & $\mu \mathrm{g} / \mathrm{kg}$ & 5.2 & EPA8240 \\
\hline trans-1,3-Dichloropropene & UJ & 0 & $\mathrm{H}$ & 5.2 & $\mu \mathrm{g} / \mathrm{kg}$ & 5.2 & EPA8240 \\
\hline trans-1,3-Dichloropropene & UJ & Q & & 5.2 & $\mu \mathrm{g} / \mathrm{kg}$ & 5.2 & EPA8240 \\
\hline Ethylbenzene & UJ & 0 & H & 5.2 & $\mu \mathrm{g} / \mathrm{kg}$ & 5.2 & EPA8240 \\
\hline Ethylbenzene & UJ & $\mathbf{Q}$ & & 5.2 & $\mu \mathrm{g} / \mathrm{kg}$ & 5.2 & EPA8240 \\
\hline 2-Hexanone & UJ & 0 & $\mathrm{H}$ & 10 & $\mu \mathrm{g} / \mathrm{kg}$ & 10 & EPA8240 \\
\hline 2-Hexanone & UJ & $\mathbf{Q}$ & & 10 & $\mu g / k g$ & 10 & EPA8240 \\
\hline Methyl ethyl ketone & UJ & 0 & $\mathrm{H}$ & 10 & $\mu \mathrm{g} / \mathrm{kg}$ & 10 & EPA8240 \\
\hline Methyl ethyl ketone & UJ & Q & & 10 & $\mu \mathrm{g} / \mathrm{kg}$ & 10 & EPA8240 \\
\hline Methyl isobutyl ketone & UJ & 0 & H & 10 & $\mu \mathrm{g} / \mathrm{kg}$ & 10 & EPA8240 \\
\hline Methyl isobutyl ketone & UJ & Q & & 10 & $\mu \mathrm{g} / \mathrm{kg}$ & 10 & EPA8240 \\
\hline Styrene & UJ & 0 & H & 5.2 & $\mu g / \mathrm{kg}$ & 5.2 & EPA8240 \\
\hline Siyrene & UJ & $\mathbf{Q}$ & & 5.2 & $\mu g / k g$ & 5.2 & EPA8240 \\
\hline 1,1,2,2-Tetrachloroethane & UJ & 0 & H & 5.2 & $\mu \mathrm{g} / \mathrm{kg}$ & 5.2 & EPA8240 \\
\hline 1,1,2,2-Tetrachloroethane & UI & Q & & 5.2 & $\mu g / \mathrm{kg}$ & 5.2 & EPA8240 \\
\hline Tetrachloroethylene & UJ & 0 & H & 5.2 & $\mu g / k g$ & 5.2 & EPA8240 \\
\hline Tetrachloroethylene & UJ & $\mathbf{Q}$ & & 5.2 & $\mu \mathrm{g} / \mathrm{kg}$ & 5.2 & EPA8240 \\
\hline Toluene & UJ & 0 & $\mathrm{H}$ & 5.2 & $\mu \mathrm{g} / \mathrm{kg}$ & 5.2 & EPA8240 \\
\hline Toluene & UJ & $\mathbf{Q}$ & & 5.2 & $\mu g / k g$ & 5.2 & EPA8240 \\
\hline 1,1,1-Trichloroethane & UJ & 0 & $\mathrm{H}$ & 5.2 & $\mu \mathrm{g} / \mathrm{kg}$ & 5.2 & EPA8240 \\
\hline 1,1,1-Trichloroethane & UJ & $\mathbf{Q}$ & & 5.2 & $\mu \mathrm{g} / \mathrm{kg}$ & 5.2 & EPA8240 \\
\hline 1,1,2-Trichloroethane & U̇ & 0 & $\mathrm{H}$ & 5.2 & $\mu \mathrm{g} / \mathrm{kg}$ & 5.2 & EPA8240 \\
\hline 1,1,2-Trichloroethane & UI & $\mathbf{Q}$ & & 5.2 & $\mu \mathrm{g} / \mathrm{kg}$ & 5.2 & EPA8240 \\
\hline Trichloroethylene & UJ & 0 & H & 5.2 & $\mu \mathrm{g} / \mathrm{kg}$ & 5.2 & EPA8240 \\
\hline Trichloroethylene & UJ & $\mathbf{Q}$ & & 5.2 & $\mu \mathrm{g} / \mathrm{kg}$ & 5.2 & EPA8240 \\
\hline Vinyl acetate & UJ & 0 & H & 10 & $\mu g / k g$ & 10 & EPA8240 \\
\hline Vinyl acetate & UJ & $\mathbf{Q}$ & & 10 & $\mu g / \mathrm{kg}$ & 10 & EPA8240 \\
\hline Xylenes & UJ & 0 & $\mathbf{H}$ & 5.2 & $\mu \mathrm{g} / \mathrm{kg}$ & 5.2 & EPA8240 \\
\hline Xylenes & UJ & $\mathbf{Q}$ & & 5.2 & $\mu \mathrm{g} / \mathrm{kg}$ & 5.2 & EPA8240 \\
\hline
\end{tabular}


Pesticides/PCBs and Dioxins/Furans

Aldrin
alpha-Benzene hexachloride
beta-Benzene hexachloride
delta-Benzene hexachloride
alpha-Chlordane
gamma-Chlordane
p.p'-DDD
p.p'-DDE
p.p'-DDT
Dieldrin
Endosulfan I
Endosulfan II
Endosulfan sulfate
Endrin
Endrin ketone
Heptachlor
Heptachlor epoxide
Heptachlorodibenzo-p-dioxin isomers
Heptachlorodibenzo-p-furan isomers
Hexachlorodibenzo-p-dioxin isomers
Hexachlorodibenzo-p-furan isomers
Lindane
Methoxychlor
Octachlorodibenzo-p-dioxin
Octachlorodibenzo-p-furan
PCB 1016
PCB 1221
PCB 1232
PCB 1242
PCB 1248
PCB 1254
PCB 1260
Pentachlorodibenzo-p-dioxin isomers
Pentachlorodibenzo-p-furan isomers
$2.3,7,8-T C D D$
$2.3,7,8-T C D F$
Tetrachlorodibenzo-p-dioxin isomers
Tetrachlorodibenzo-p-furan isomers
Toxaphene

$\mathrm{U}$

U 1.7

$\begin{array}{ll}\mathrm{U} & 1.7\end{array}$

J 1.0

U 1.7

U $\quad 1.7$

$\mathrm{U}$

U

$\mathrm{U}$

U

U

U

U

$\mathrm{U}$

$U$

$\mathrm{U}$

$\mathrm{U}$

$\mathrm{U}$

U

U

U

U

U

$\mathrm{U}$

U

$\mathrm{U}$

$U$

U

$\mathrm{U}$

$\mathrm{U}$

$\mathrm{U}$

$\mathrm{U}$

U

U

U

Radionuclide Indicators and Radionuclides

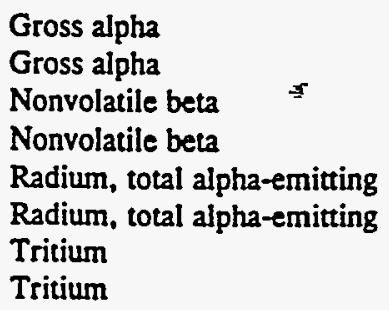

$\begin{array}{lll}\mu \mathrm{g} / \mathrm{kg} & 1.7 & \text { EPA8080 } \\ \mu \mathrm{g} / \mathrm{kg} & 1.7 & \text { EPA8080 } \\ \mu \mathrm{g} / \mathrm{kg} & 1.7 & \text { EPA8080 } \\ \mu \mathrm{g} / \mathrm{kg} & 1.7 & \text { EPA8080 } \\ \mu \mathrm{g} / \mathrm{kg} & 1.7 & \text { EPA8080 } \\ \mu \mathrm{g} / \mathrm{kg} & 1.7 & \text { EPA8080 } \\ \mu \mathrm{g} / \mathrm{kg} & 3.4 & \text { EPA8080 } \\ \mu \mathrm{g} / \mathrm{kg} & 3.4 & \text { EPA8080 } \\ \mu \mathrm{g} / \mathrm{kg} & 3.4 & \text { EPA8080 } \\ \mu \mathrm{g} / \mathrm{kg} & 3.4 & \text { EPA8080 } \\ \mu \mathrm{g} / \mathrm{kg} & 1.7 & \text { EPA8080 } \\ \mu \mathrm{g} / \mathrm{kg} & 3.4 & \text { EPA8080 } \\ \mu \mathrm{g} / \mathrm{kg} & 3.4 & \text { EPA8080 } \\ \mu \mathrm{g} / \mathrm{kg} & 3.4 & \text { EPA8080 } \\ \mu \mathrm{g} / \mathrm{kg} & 3.4 & \text { EPA8080 } \\ \mu \mathrm{g} / \mathrm{kg} & 1.7 & \text { EPA8080 } \\ \mu \mathrm{g} / \mathrm{kg} & 1.7 & \text { EPA8080 } \\ \mathrm{ng} / \mathrm{g} & 0.20 & \text { EPA8280 } \\ \mathrm{ng} / \mathrm{g} & 0.20 & \text { EPA8280 } \\ \mathrm{ng} / \mathrm{g} & 0.20 & \text { EPA8280 } \\ \mathrm{ng} / \mathrm{g} & 0.10 & \text { EPA8280 } \\ \mu \mathrm{g} / \mathrm{kg} & 1.7 & \text { EPA8080 } \\ \mu \mathrm{g} / \mathrm{kg} & 17 & \text { EPA8080 } \\ \mathrm{ng} / \mathrm{g} & 0.10 & \text { EPA8280 } \\ \mathrm{ng} / \mathrm{g} & 0.10 & \text { EPA8280 } \\ \mu \mathrm{g} / \mathrm{kg} & 34 & \text { EPA8080 } \\ \mu \mathrm{g} / \mathrm{kg} & 68 & \text { EPA8080 } \\ \mu \mathrm{g} / \mathrm{kg} & 34 & \text { EPA8080 } \\ \mu \mathrm{gg} / \mathrm{kg} & 34 & \text { EPA8080 } \\ \mu \mathrm{g} / \mathrm{kg} & 34 & \text { EPA8080 } \\ \mu \mathrm{g} / \mathrm{kg} & 34 & \text { EPA8080 } \\ \mu \mathrm{g} / \mathrm{kg} & 34 & \text { EPA8080 } \\ \mathrm{ng} / \mathrm{g} & 0.30 & \text { EPA8280 } \\ \mathrm{ng} / \mathrm{g} & 0.20 & \text { EPA8280 } \\ \mathrm{ng} / \mathrm{g} & 0.10 & \text { EPA8280 } \\ \mathrm{ng} / \mathrm{g} & 0.10 & \text { EPA8280 } \\ \mathrm{ng} / \mathrm{g} & 0.10 & \text { EPA8280 } \\ \mathrm{ng} / \mathrm{g} & 0.10 & \text { EPA8280 } \\ \mu \mathrm{g} / \mathrm{kg} & 170 & \text { EPA8080 } \\ & & \\ & 14 & \end{array}$

$\begin{array}{ll}29 \pm 12 & \mathrm{pCi} / \mathrm{g} \\ 14 \pm 9.1 & \mathrm{pCi} / \mathrm{g} \\ 41 \pm 14 & \mathrm{pCi} / \mathrm{g} \\ 34 \pm 14 & \mathrm{pCi} / \mathrm{g} \\ 0.43 \pm 0.41 & \mathrm{pCi} / \mathrm{g} \\ 0.55 \pm 0.46 & \mathrm{pCi} / \mathrm{g} \\ 2.5 \pm 0.69 & \mathrm{pCi} / \mathrm{g} \\ 2.6 \pm 0.73 & \mathrm{pCi} / \mathrm{g}\end{array}$

$-$

4.6

4.8

5.1

5.3

0.12

0.12

0.92

1.0

EPA900.0
EPA900.0
EPA900.0
EPA900.0
EPA903.0
EPA903.0
EPA906.0
EPA906.0


SAMPLE NAME: D431 1702

Location (SRS Coordinates): 18492.14E 66454.17N

Ground Elevation Above MSL: $124.9 \mathrm{ft}$

Depth of Core Interval: 0.50 to $2.00 \mathrm{ft}$

Sample Type: Normal

Sample Color: 10YR 4/6

Constituent

RQ AQ B Result

Sample Matrix: Soil

USC Soil Classification: SW

Sample ID: 100428

Total Metals

Arsenic

Barium

Cadmium

Chromium

Copper

Lead

Manganese

Mercury

Nickel

Selenium

Silver

$\begin{array}{ll} & 2.2 \\ \mathrm{U} & 23 \\ \mathrm{U} & 0.59 \\ \mathrm{U} & 18 \\ & 2.9 \\ & 2.5 \\ \mathrm{U} & 13 \\ \mathrm{U} & 0.12 \\ \mathrm{U} & 4.7 \\ \mathrm{U} & 0.55 \\ & 1.2\end{array}$

$B / N / A$ Extractables (including $P A H$ and phenols)

\begin{tabular}{|c|c|c|c|c|c|c|}
\hline Acenaphthene & $\mathbf{U}$ & & 360 & $\mu g / \mathrm{kg}$ & 360 & EPA8270 \\
\hline Acenaphthylene & U & & 360 & $\mu \mathrm{g} / \mathrm{kg}$ & 360 & EPA8270 \\
\hline Anthracene & $\mathrm{U}$ & & 360 & $\mu \mathrm{g} / \mathrm{kg}$ & 360 & EPA8270 \\
\hline Benzo(a)anthracene & U & & 360 & $\mu g / k g$ & 360 & EPA8270 \\
\hline Benzo(b)fluoranthene & $U$ & & 360 & $\mu g / k g$ & 360 & EPA8270 \\
\hline Benzo(k)fluoranthene & $\mathrm{U}$ & & 360 & $\mu \mathrm{g} / \mathrm{kg}$ & 360 & EPA8270 \\
\hline Benzoic acid & $\mathbf{U}$ & & 1,800 & $\mu \mathrm{g} / \mathrm{kg}$ & 1,800 & EPA8270 \\
\hline Benzo(g,h,i)perylene & $\mathrm{U}$ & & 360 & $\mu \mathrm{g} / \mathrm{kg}$ & 360 & EPA8270 \\
\hline Benzo(a)pyrene & $\mathbf{U}$ & & 360 & $\mu \mathrm{g} / \mathrm{kg}$ & 360 & EPA8270 \\
\hline Benzyl alcohol & U & & 360 & $\mu \mathrm{g} / \mathrm{kg}$ & 360 & EPA8270 \\
\hline Bis(2-chloroethoxy) methane & U & & 360 & $\mu g / \mathrm{kg}$ & 360 & EPA8270 \\
\hline Bis(2-chloroethyl) ether & $\mathrm{U}$ & & 360 & $\mu \mathrm{g} / \mathrm{kg}$ & 360 & EPA8270 \\
\hline Bis(2-chloroisopropyl) ether & $\mathbf{U}$ & & 360 & $\mu \mathrm{g} / \mathrm{kg}$ & 360 & EPA8270 \\
\hline Bis(2-ethylhexyl) phthalate & UJ & $\mathrm{v}$ & 360 & $\mu \mathrm{g} / \mathrm{kg}$ & 360 & EPA8270 \\
\hline 4-Bromophenyl phenyl ether & $\mathbf{U}$ & & 360 & $\mu \mathrm{g} / \mathrm{kg}$ & 360 & EPA8270 \\
\hline Butylbenzyl phthalate & $\mathbf{U}$ & & 360 & $\mu \mathrm{g} / \mathrm{kg}$ & 360 & EPA8270 \\
\hline 4-Chloroaniline & $\mathrm{U}$ & & 360 & $\mu \mathrm{g} / \mathrm{kg}$ & 360 & EPA8270 \\
\hline 4-Chloro-m-cresol & U & & 360 & $\mu \mathrm{g} / \mathrm{kg}$ & 360 & EPA8270 \\
\hline 2-Chloronaphthalene & $\mathrm{U}$ & & 360 & $\mu \mathrm{g} / \mathrm{kg}$ & 360 & EPA8270 \\
\hline 2-Chlorophenol & $\mathbf{U}$ & & 360 & $\mu \mathrm{g} / \mathrm{kg}$ & 360 & EPA8270 \\
\hline 4-Chlorophenyl phenyl ether & $\mathbf{U}$ & & 360 & $\mu \mathrm{g} / \mathrm{kg}$ & 360 & EPA8270 \\
\hline Chrysene & $\mathrm{U}$ & & 360 & $\mu \mathrm{g} / \mathrm{kg}$ & 360 & EPA8270 \\
\hline o-Cresol (2-Methylphenol) & $\mathrm{U}$ & & 360 & $\mu \mathrm{g} / \mathrm{kg}$ & 360 & EPA8270 \\
\hline p-Cresol (4-Methylphenol) & $\mathrm{U}$ & & 360 & $\mu g / \mathrm{kg}$ & 360 & EPA8270 \\
\hline Dibenz $(\mathrm{a}, \mathrm{h})$ anthracene & $\mathbf{U}$ & & 360 & $\mu \mathrm{g} / \mathrm{kg}$ & 360 & EPA8270 \\
\hline Dibenzofuran & $\mathbf{U}$ & & 360 & $\mu g / \mathrm{kg}$ & 360 & EPA8270 \\
\hline Di-n-butyl phthalate & UJ & $\mathrm{v}$ & 140 & $\mu \mathrm{g} / \mathrm{kg}$ & 360 & EPA8270 \\
\hline 1,2-Dichlorobenzene & $\mathrm{U}$ & & 360 & $\mu \mathrm{g} / \mathrm{kg}$ & 360 & EPA8270 \\
\hline 1.3-Dichlorobenzene & $\mathbf{U}$ & & 360 & $\mu \mathrm{g} / \mathrm{kg}$ & 360 & EPA8270 \\
\hline 1,4-Dichlorobenzene. & $\mathrm{U}$ & & 360 & $\mu \mathrm{g} / \mathrm{kg}$ & 360 & EPA8270 \\
\hline
\end{tabular}

Sample Moisture: Dry

Percent Solids: 85.40

D. Limit Method

$\begin{array}{lll}\mathrm{mg} / \mathrm{kg} & 1.1 & \text { EPA7060 } \\ \mathrm{mg} / \mathrm{kg} & 23 & \text { EPA6010 } \\ \mathrm{mg} / \mathrm{kg} & 0.59 & \text { EPA6010 } \\ \mathrm{mg} / \mathrm{kg} & 1.2 & \text { EPA6010 } \\ \mathrm{mg} / \mathrm{kg} & 2.9 & \text { EPA6010 } \\ \mathrm{mg} / \mathrm{kg} & 0.33 & \text { EPA7421 } \\ \mathrm{mg} / \mathrm{kg} & 1.8 & \text { EPA6010 } \\ \mathrm{mg} / \mathrm{kg} & 0.12 & \text { EPA7470 } \\ \mathrm{mg} / \mathrm{kg} & 4.7 & \text { EPA6010 } \\ \mathrm{mg} / \mathrm{kg} & 0.55 & \text { EPA7740 } \\ \mathrm{mg} / \mathrm{kg} & 1.2 & \text { EPA6010 }\end{array}$


SAMPLE NAME: D431 1702 (Continued)

Sample ID: 100428

\section{Constituent}

Unit

D. Limit Method

\section{$B / N / A$ Extractables (including $P A H$ and phenols)}

3,3'-Dichlorobenzidine

2,4-Dichlorophenol

Diethyl phthalate

2,4-Dimethyl phenol

Dimethyl phthalate

2,4-Dinitrophenol

2,4-Dinitrotoluene

2,6-Dinitrotoluene

Di-n-octyl phthalate

Fluoranthene

Fluorene

Hexachlorobenzene

Hexachlorobutadiene

Hexachlorocyclopentadiene

Hexachloroethane

Indeno(1,2,3-c,d)pyrene

Isophorone

2-Methyl-4,6-dinitrophenol

2-Methylnaphthalene

Naphthalene

o-Nitroaniline

m-Nitroaniline

- p-Nitroaniline

Nitrobenzene

2-Nitrophenol

4-Nitrophenol

$\mathrm{N}$-Nitrosodiphenylamine

N-Nitrosodipropylamine

Pentachlorophenol

Phenanthrene

Phenol

Pyrene

1,2,4-Trichlorobenzene

2,4,5-Trichlorophenol

2,4,6-Trichlorophenol

Unknown ketone

Volatile Organic Compounds

$\begin{array}{lllll}\text { Acetone } & \text { UJ } & \text { VO } & \text { L } & 10 \\ \text { Acetone } & \text { UJ } & \text { VO } & \text { L } & 11 \\ \text { Acetone } & \text { U } & \text { VO } & \text { L } & 12 \\ \text { Benzene } & \text { UJ } & \text { VO } & \text { L } & 7.5 \\ \text { Bromodichloromethane } & \text { UJ } & \text { O } & \text { L } & 5.5 \\ \text { Bromodichloromethane } & \text { UJ } & \text { O } & \text { L } & 5.5 \\ \text { Bromodichloromethane } & \text { UJ } & \text { O } & \text { L } & 5.5 \\ \text { Bromoform } & \text { UJ } & \text { O } & \text { L } & 5.5 \\ \text { Bromoform } & \text { UJ } & \text { O } & \text { L } & 5.5 \\ \text { Bromoform } & \text { UJ } & \text { O } & \text { L } & 5.5 \\ \text { Bromomethane (Methyl bromide) } & \text { UJ } & \text { O } & \text { L } & 11\end{array}$

730
360
360
360
360
1,800
360
360
360
360
360
360
360
360
360
360
360
1,800
360
360
1,800
1,800
1,800
360
360
1,800
360
360
1,800
360
360
360
360
1,800
360
6,000

$\mu \mathrm{g} / \mathrm{kg}$.

$\mu \mathrm{g} / \mathrm{kg}$

$\mu g / k g$

$\mu g / k g$

$\mu \mathrm{g} / \mathrm{kg}$

$\mu \mathrm{g} / \mathrm{kg}$

$\mu g / k g$

$\mu g / k g$

$\mu \mathrm{g} / \mathrm{kg}$

$\mu \mathrm{g} / \mathrm{kg}$

$\mu \mathrm{g} / \mathrm{kg}$

$\mu \mathrm{g} / \mathrm{kg}$

$\mu g / k g$

$\mu \mathrm{g} / \mathrm{kg}$

$\mu \mathrm{g} / \mathrm{kg}$

$\mu \mathrm{g} / \mathrm{kg}$

$\mu \mathrm{g} / \mathrm{kg}$

$\mu \mathrm{g} / \mathrm{kg}$

$\mu \mathrm{g} / \mathrm{kg}$

$\mu \mathrm{g} / \mathrm{kg}$

$\mu \mathrm{g} / \mathrm{kg}$

$\mu \mathrm{g} / \mathrm{kg}$

$\mu g / k g$

$\mu \mathrm{g} / \mathrm{kg}$

$\mu \mathrm{g} / \mathrm{kg}$

$\mu \mathrm{g} / \mathrm{kg}$

$\mu \mathrm{g} / \mathrm{kg}$

$\mu \mathrm{g} / \mathrm{kg}$

$\mu g / \mathrm{kg}$

$\mu \mathrm{g} / \mathrm{kg}$

$\mu \mathrm{g} / \mathrm{kg}$

$\mu \mathrm{g} / \mathrm{kg}$

$\mu \mathrm{g} / \mathrm{kg}$

EPA8270

EPA8270

EPA8270

EPA8270

EPA8270

EPA8270

EPA8270

EPA8270

EPA8270

EPA8270

EPA8270

EPA8270

EPA8270

EPA8270

EPA8270

EPA8270

EPA8270

EPA8270

EPA8270

EPA8270

EPA8270

EPA8270

EPA8270

EPA8270

EPA8270

EPA8270

EPA8270

EPA8270

EPA8270

EPA8270

EPA8270

EPA8270

EPA8270

EPA8270

EPA8270

EPA8270

1.800

360

$\begin{array}{lll}\mu \mathrm{g} / \mathrm{kg} & 11 & \text { EPA8240 } \\ \mu \mathrm{g} / \mathrm{kg} & 11 & \text { EPA8240 } \\ \mu \mathrm{g} / \mathrm{kg} & 11 & \text { EPA8240 } \\ \mu \mathrm{g} / \mathrm{kg} & 5.5 & \text { EPA8240 } \\ \mu \mathrm{g} / \mathrm{kg} & 5.5 & \text { EPA8240 } \\ \mu \mathrm{g} / \mathrm{kg} & 5.5 & \text { EPA8240 } \\ \mu \mathrm{g} / \mathrm{kg} & 5.5 & \text { EPA8240 } \\ \mu \mathrm{g} / \mathrm{kg} & 5.5 & \text { EPA8240 } \\ \mu \mathrm{g} / \mathrm{kg} & 5.5 & \text { EPA8240 } \\ \mu \mathrm{g} / \mathrm{kg} & 5.5 & \text { EPA8240 } \\ \mu \mathrm{g} / \mathrm{kg} & 11 & \text { EPA8240 }\end{array}$


Constituent

Volatile Organic Compounds

Bromomethane (Methyl bromide)

Bromomethane (Methyl bromide)

Carbon disulfide

Carbon disulfide

Carbon disulfide

Carbon tetrachloride

Carbon tetrachloride

Carbon tetrachloride

Chlorobenzene

Chloroethane

Chloroethane

Chloroethane

Chloroethene (Vinyl chloride)

Chloroethene (Vinyl chloride)

Chloroethene (Vinyl chloride)

Chloroform

Chloroform

Chloroform

Chloromethane (Methyl chloride)

Chloromethane (Methyl chloride)

Chloromethane (Methyl chloride)

Dibromochloromethane

Dibromochloromethane

Dibromochloromethane

1,1-Dichloroethane

1,1-Dichloroethane

1,1-Dichloroethane

1,2-Dichloroethane

1,2-Dichloroethane

1,2-Dichloroethane

1,1-Dichloroethylene

1,2-Dichloroethylene

1,2-Dichloroethylene

1,2-Dichloroethylene

Dichloromethane (Methylene chloride)

Dichloromethane (Methylene chloride)

Dichloromethane (Methylene chloride)

1,2-Dichloropropane

1,2-Dichloropropane

1,2-Dichloropropane :

cis-1,3-Dichloropropene

cis-1,3-Dichloropropene

cis-1,3-Dichloropropene

trans-1,3-Dichloropropene

trans-1,3-Dichloropropene

trans-1,3-Dichloropropene

Ethylbenzene

Ethylbenzene

Ethylbenzene

2-Hexanone
RQ AQ B Result Unit D. Limit Method

\begin{tabular}{|c|c|c|c|c|c|}
\hline UJ & 0 & 11 & $\mu \mathrm{g} / \mathrm{kg}$ & 11 & EPA8240 \\
\hline UJ & 0 & 11 & $\mu \mathrm{g} / \mathrm{kg}$ & 11 & EPA 8240 \\
\hline U & 0 & 5.5 & $\mu \mathrm{g} / \mathrm{kg}$ & 5.5 & EPA8240 \\
\hline UJ & 0 & 5.5 & $\mu \mathrm{g} / \mathrm{kg}$ & 5.5 & EPA8240 \\
\hline UJ & 0 & 5.5 & $\mu \mathrm{g} / \mathrm{kg}$ & 5.5 & EPA8240 \\
\hline UJ & 0 & 5.5 & $\mu \mathrm{g} / \mathrm{kg}$ & 5.5 & EPA8240 \\
\hline UJ & 0 & 5.5 & $\mu \mathrm{g} / \mathrm{kg}$ & 5.5 & EPA8240 \\
\hline UJ & 0 & 5.5 & $\mu g / k g$ & 5.5 & EPA8240 \\
\hline UJ & 0 & 5.5 & $\mu \mathrm{g} / \mathrm{kg}$ & 5.5 & EPA8240 \\
\hline UJ & 0 & 11 & $\mu \mathrm{g} / \mathrm{kg}$ & 11 & EPA8240 \\
\hline UJ & 0 & 11 & $\mu \mathrm{g} / \mathrm{kg}$ & 11 & EPA8240 \\
\hline UJ & 0 & 11 & $\mu \mathrm{g} / \mathrm{kg}$ & 11 & EPA8240 \\
\hline UJ & 0 & 11 & $\mu \mathrm{g} / \mathrm{kg}$ & 11 & EPA8240 \\
\hline UJ & 0 & 11 & $\mu \mathrm{g} / \mathrm{kg}$ & 11 & EPA8240 \\
\hline UJ & 0 & 11 & $\mu g / \mathrm{kg}$ & 11 & EPA8240 \\
\hline UJ & 0 & 5.5 & $\mu \mathrm{g} / \mathrm{kg}$ & 5.5 & EPA8240 \\
\hline UJ & 0 & 5.5 & $\mu g / \mathrm{kg}$ & 5.5 & EPA8240 \\
\hline UJ & 0 & 5.5 & $\mu g / \mathrm{kg}$ & 5.5 & EPA8240 \\
\hline UJ & 0 & 11 & $\mu g / \mathrm{kg}$ & 11 & EPA8240 \\
\hline UJ & 0 & 11 & $\mu \mathrm{g} / \mathrm{kg}$ & 11 & EPA8240 \\
\hline UJ & 0 & 11 & $\mu \mathrm{g} / \mathrm{kg}$ & 11 & EPA8240 \\
\hline UJ & 0 & 5.5 & $\mu \mathrm{g} / \mathrm{kg}$ & 5.5 & EPA8240 \\
\hline UJ & 0 & 5.5 & $\mu \mathrm{g} / \mathrm{kg}$ & 5.5 & EPA8240 \\
\hline US & 0 & 5.5 & $\mu \mathrm{g} / \mathrm{kg}$ & 5.5 & EPA8240 \\
\hline UJ & 0 & 5.5 & $\mu \mathrm{g} / \mathrm{kg}$ & 5.5 & EPA8240 \\
\hline UI & 0 & 5.5 & $\mu \mathrm{g} / \mathrm{kg}$ & 5.5 & EPA8240 \\
\hline UJ & 0 & 5.5 & $\mu \mathrm{g} / \mathrm{kg}$ & 5.5 & EPA8240 \\
\hline UJ & 0 & 5.5 & $\mu \mathrm{g} / \mathrm{kg}$ & 5.5 & EPA8240 \\
\hline UJ & 0 & 5.5 & $\mu \mathrm{g} / \mathrm{kg}$ & 5.5 & EPA8240 \\
\hline UJ & 0 & 5.5 & $\mu \mathrm{g} / \mathrm{kg}$ & 5.5 & EPA8240 \\
\hline UJ & 0 & 5.5 & $\mu \mathrm{g} / \mathrm{kg}$ & 5.5 & EPA8240 \\
\hline UJ & 0 & 5.5 & $\mu g / \mathrm{kg}$ & 5.5 & EPA8240 \\
\hline UJ & 0 & 5.5 & $\mu \mathrm{g} / \mathrm{kg}$ & 5.5 & EPA8240 \\
\hline UJ & 0 & 5.5 & $\mu \mathrm{g} / \mathrm{kg}$ & 5.5 & EPA8240 \\
\hline UJ & 0 & 5.5 & $\mu g / \mathrm{kg}$ & 5.5 & EPA8240 \\
\hline UJ & 0 & 5.5 & $\mu \mathrm{g} / \mathrm{kg}$ & 5.5 & EPA8240 \\
\hline UJ & 0 & 5.5 & $\mu \mathrm{g} / \mathrm{kg}$ & 5.5 & EPA8240 \\
\hline Uנ & 0 & 5.5 & $\mu \mathrm{g} / \mathrm{kg}$ & 5.5 & EPA8240 \\
\hline UJ & 0 & 5.5 & $\mu g / \mathrm{kg}$ & 5.5 & EPA8240 \\
\hline UJ & 0 & 5.5 & $\mu g / \mathrm{kg}$ & 5.5 & EPA8240 \\
\hline UJ & 0 & 5.5 & $\mu \mathrm{g} / \mathrm{kg}$ & 5.5 & EPA8240 \\
\hline UJ & 0 & 5.5 & $\mu \mathrm{g} / \mathrm{kg}$ & 5.5 & EPA8240 \\
\hline UJ & 0 & 5.5 & $\mu \mathrm{g} / \mathrm{kg}$ & 5.5 & EPA8240 \\
\hline UJ & 0 & 5.5 & $\mu \mathrm{g} / \mathrm{kg}$ & 5.5 & EPA8240 \\
\hline UJ & 0 & 5.5 & $\mu g / \mathrm{kg}$ & 5.5 & EPA8240 \\
\hline UJ & 0 & 5.5 & $\mu \mathrm{g} / \mathrm{kg}$ & 5.5 & EPA8240 \\
\hline UJ & 0 & 5.5 & $\mu g / \mathrm{kg}$ & 5.5 & EPA8240 \\
\hline US & 0 & 5.5 & $\mu g / k g$ & 5.5 & EPA8240 \\
\hline UJ & 0 & 5.5 & $\mu \mathrm{g} / \mathrm{kg}$ & 5.5 & EPA8240 \\
\hline UI & 0 & 11 & $\mu \mathrm{g} / \mathrm{kg}$ & 11 & EPA8240 \\
\hline
\end{tabular}


SAMPLE NAME: D431 1702 (Continued)

Sample ID: 100428

Constituent

Volatile Organic Compounds
Re

$\mathbf{R Q}$

\author{
Sample ID: 100428
}

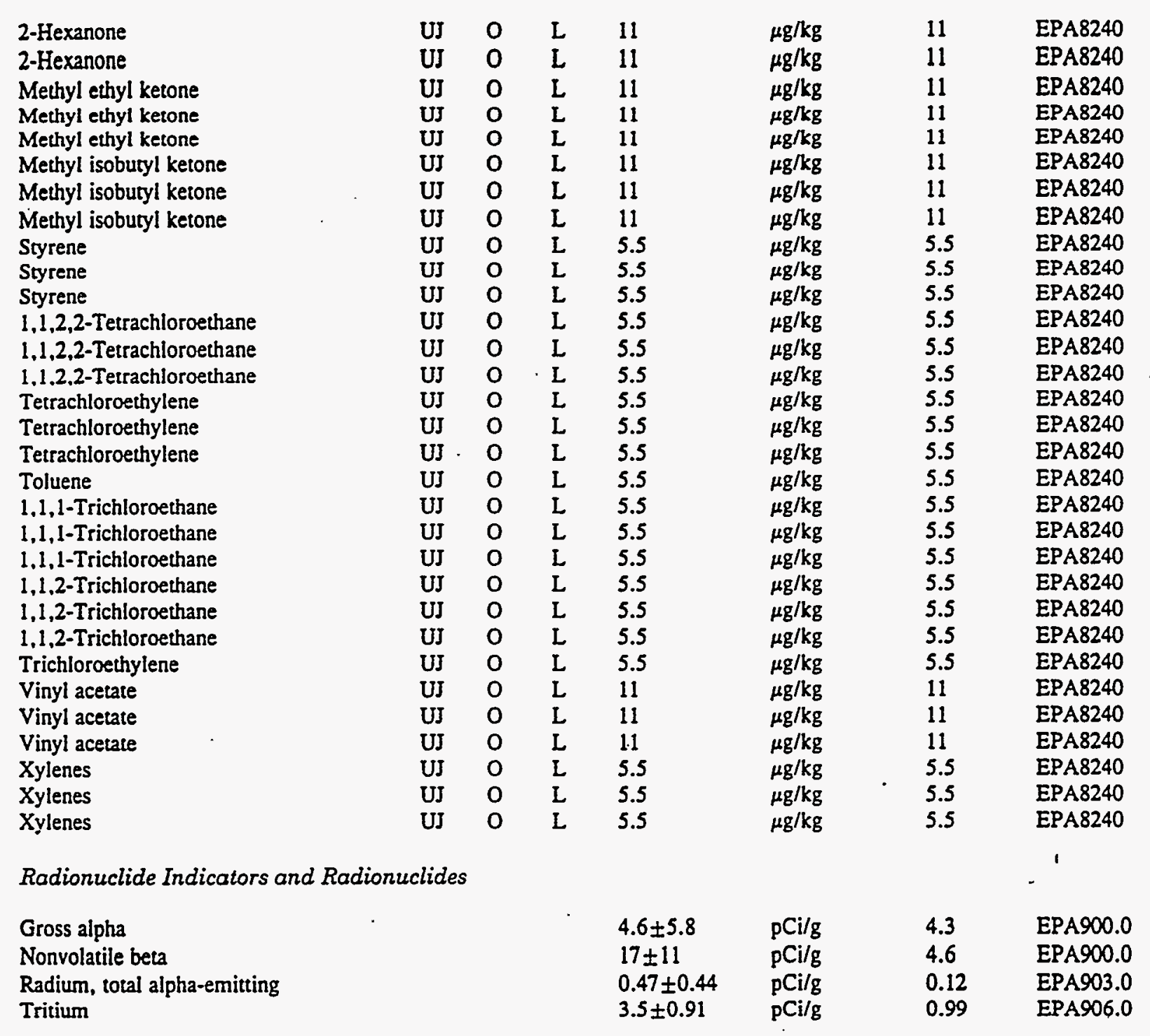


SAMPLE NAME: D431 1703

Location (SRS Coordinates): 18492.14E 66454.17N

Ground Elevation Above MSL: $124.9 \mathrm{ft}$

Depth of Core Interval: 5.00 to $7.00 \mathrm{ft}$

Sample Type: Normal

Sample Color: 5YR 4/4

Constituent

Total Metals

Arsenic

Barium

Cadmium

Chromium

Copper

Lead

Manganese

Mercury

Nickel

Selenium

Silver

Volatile Organic Compounds

$\mathrm{U}$
Sample ID: 100429
Sample Matrix: Soil

USC Soil Classification: SW
Sample Moisture: Damp

Percent Solids: 70.50

D. Limit Method
EPA7060

EPA6010

EPA6010

EPA6010

EPA6010

EPA7421

EPA6010

EPA7470

EPA6010

EPA7740

0.67

13

0.14

5.3

$\mathrm{mg} / \mathrm{kg}$

$\mathrm{mg} / \mathrm{kg}$

0.67

EPA6010

$\begin{array}{llll}\text { U } & \text { VO } & \text { H } & 59 \\ \text { U } & \text { QV } & & 27 \\ \text { UJ } & \text { O } & \text { H } & 6.5 \\ \text { UJ } & \text { Q } & & 6.5 \\ \text { UJ } & \text { O } & \text { H } & 6.5 \\ \text { UJ } & \text { Q } & & 6.5 \\ \text { UJ } & \text { O } & \text { H } & 6.5 \\ \text { UJ } & \text { Q } & & 6.5 \\ \text { UJ } & \text { O } & \text { H } & 13 \\ \text { UJ } & \text { Q } & & 13 \\ \text { UJ } & \text { O } & \text { H } & 6.5 \\ \text { UJ } & \text { Q } & & 6.5 \\ \text { UJ } & \text { O } & \text { H } & 6.5 \\ \text { UJ } & \text { Q } & & 6.5 \\ \text { UJ } & \text { O } & \text { H } & 6.5 \\ \text { UJ } & \text { Q } & & 6.5 \\ \text { UJ } & \text { O } & \text { H } & 13 \\ \text { UJ } & \text { Q } & & 13 \\ \text { UJ } & \text { O } & \text { H } & 13 \\ \text { UJ } & \text { Q } & & 13 \\ \text { UJ } & \text { O } & \text { H } & 6.5 \\ \text { UJ } & \text { Q } & & 6.5 \\ \text { UJ } & \text { O } & \text { H } & 13 \\ \text { UJ } & \text { Q } & & 13 \\ \text { UJ } & \text { O } & \text { H } & 6.5 \\ \text { UJ } & \text { Q } & & 6.5 \\ \text { UJ } & \text { O } & \text { H } & 6.5 \\ \text { UJ } & \text { Q } & & 6.5 \\ \text { UJ } & \text { O } & \text { H } & 6.5 \\ \text { UJ } & \text { Q } & & 6.5 \\ & & & \end{array}$

$\begin{array}{lll}\mu \mathrm{g} / \mathrm{kg} & 13 & \text { EPA8240 } \\ \mu \mathrm{g} / \mathrm{kg} & 13 & \text { EPA8240 } \\ \mu \mathrm{g} / \mathrm{kg} & 6.5 & \text { EPA8240 } \\ \mu \mathrm{g} / \mathrm{kg} & 6.5 & \text { EPA8240 } \\ \mu \mathrm{g} / \mathrm{kg} & 6.5 & \text { EPA8240 } \\ \mu \mathrm{g} / \mathrm{kg} & 6.5 & \text { EPA8240 } \\ \mu \mathrm{g} / \mathrm{kg} & 6.5 & \text { EPA8240 } \\ \mu \mathrm{g} / \mathrm{kg} & 6.5 & \text { EPA8240 } \\ \mu \mathrm{g} / \mathrm{kg} & 13 & \text { EPA8240 } \\ \mu \mathrm{g} / \mathrm{kg} & 13 & \text { EPA8240 } \\ \mu \mathrm{g} / \mathrm{kg} & 6.5 & \text { EPA8240 } \\ \mu \mathrm{g} / \mathrm{kg} & 6.5 & \text { EPA8240 } \\ \mu \mathrm{g} / \mathrm{kg} & 6.5 & \text { EPA8240 } \\ \mu \mathrm{g} / \mathrm{kg} & 6.5 & \text { EPA8240 } \\ \mu \mathrm{g} / \mathrm{kg} & 6.5 & \text { EPA8240 } \\ \mu \mathrm{g} / \mathrm{kg} & 6.5 & \text { EPA8240 } \\ \mu \mathrm{g} / \mathrm{kg} & 13 & \text { EPA8240 } \\ \mu \mathrm{g} / \mathrm{kg} & 13 & \text { EPA8240 } \\ \mu \mathrm{g} / \mathrm{kg} & 13 & \text { EPA8240 } \\ \mu \mathrm{g} / \mathrm{kg} & 13 & \text { EPA8240 } \\ \mu \mathrm{g} / \mathrm{kg} & 6.5 & \text { EPA8240 } \\ \mu \mathrm{g} / \mathrm{kg} & 6.5 & \text { EPA8240 } \\ \mu \mathrm{g} / \mathrm{kg} & 13 & \text { EPA8240 } \\ \mu \mathrm{g} / \mathrm{kg} & 13 & \text { EPA8240 } \\ \mu \mathrm{g} / \mathrm{kg} & 6.5 & \text { EPA8240 } \\ \mu \mathrm{g} / \mathrm{kg} & 6.5 & \text { EPA8240 } \\ \mu \mathrm{g} / \mathrm{kg} & 6.5 & \text { EPA8240 } \\ \mu \mathrm{gg} / \mathrm{kg} & 6.5 & \text { EPA8240 } \\ \mu \mathrm{gg} / \mathrm{kg} & 6.5 & \text { EPA8240 } \\ \mu \mathrm{g} / \mathrm{kg} & 6.5 & \text { EPA8240 }\end{array}$


SAMPLE NAME: D431 1703 (Continued)

Sample ID: 100429

\section{Constituent}

Volatile Organic Compounds

\section{RQ AQ B Result Unit}

\begin{tabular}{|c|c|c|c|c|}
\hline UJ & 0 & H & 6.5 & $\mu g / \mathrm{kg}$ \\
\hline UJ & $\mathbf{Q}$ & & 6.5 & $\mu \mathrm{g} / \mathrm{kg}$ \\
\hline UJ & 0 & H & 6.5 & $\mu \mathrm{g} / \mathrm{kg}$ \\
\hline UJ & $\mathbf{Q}$ & & 6.5 & $\mu \mathrm{g} / \mathrm{kg}$ \\
\hline J & 0 & H & 16 & $\mu \mathrm{g} / \mathrm{kg}$ \\
\hline UJ & $\mathbf{Q}$ & & 6.5 & $\mu g / k g$ \\
\hline UJ & 0 & $\mathbf{H}$ & 6.5 & $\mu \mathrm{g} / \mathrm{kg}$ \\
\hline UJ & $\mathbf{Q}$ & & 6.5 & $\mu \mathrm{g} / \mathrm{kg}$ \\
\hline UJ & 0 & H & 6.5 & $\mu g / \mathrm{kg}$ \\
\hline UJ & $\mathbf{Q}$ & & 6.5 & $\mu g / \mathrm{kg}$ \\
\hline UJ & 0 & $\mathrm{H}$ & 6.5 & $\mu \mathrm{g} / \mathrm{kg}$ \\
\hline UJ & Q & & 6.5 & $\mu \mathrm{g} / \mathrm{kg}$ \\
\hline UJ & 0 & H & 6.5 & $\mu \mathrm{g} / \mathrm{kg}$ \\
\hline UJ & $\mathbf{Q}$ & & 6.5 & $\mu g / \mathrm{kg}$ \\
\hline UJ & 0 & $\mathrm{H}$ & 13 & $\mu \mathrm{g} / \mathrm{kg}$ \\
\hline UJ & $\mathbf{Q}$ & & 13 & $\mu \mathrm{g} / \mathrm{kg}$ \\
\hline UJ & 0 & $\mathrm{H}$ & 13 & $\mu \mathrm{g} / \mathrm{kg}$ \\
\hline UJ & $\mathrm{Q}$ & & 13 & $\mu \mathrm{g} / \mathrm{kg}$ \\
\hline UJ & 0 & $\mathrm{H}$ & 13 & $\mu \mathrm{g} / \mathrm{kg}$ \\
\hline UJ & Q & & 13 & $\mu \mathrm{g} / \mathrm{kg}$ \\
\hline UJ & 0 & H & 6.5 & $\mu \mathrm{g} / \mathrm{kg}$ \\
\hline UJ & $Q$ & & 6.5 & $\mu \mathrm{g} / \mathrm{kg}$ \\
\hline UJ & 0 & H & 6.5 & $\mu \mathrm{g} / \mathrm{kg}$ \\
\hline UJ & $Q$ & & 6.5 & $\mu \mathrm{g} / \mathrm{kg}$ \\
\hline UJ & 0 & $\mathrm{H}$ & 6.5 & $\mu \mathrm{g} / \mathrm{kg}$ \\
\hline UJ & $Q$ & & 6.5 & $\mu \mathrm{g} / \mathrm{kg}$ \\
\hline UJ & 0 & $\mathrm{H}$ & 6.5 & $\mu \mathrm{g} / \mathrm{kg}$ \\
\hline UJ & $Q$ & & 6.5 & $\mu g / k g$ \\
\hline UJ & 0 & $\mathrm{H}$ & 6.5 & $\mu \mathrm{g} / \mathrm{kg}$ \\
\hline UJ & $\mathbf{Q}$ & & 6.5 & $\mu \mathrm{g} / \mathrm{kg}$ \\
\hline UJ & 0 & $\mathrm{H}$ & 6.5 & $\mu \mathrm{g} / \mathrm{kg}$ \\
\hline UJ & $Q$ & & 6.5 & $\mu \mathrm{g} / \mathrm{kg}$ \\
\hline UJ & 0 & $\mathrm{H}$ & 6.5 & $\mu \mathrm{g} / \mathrm{kg}$ \\
\hline UJ & $Q$ & & 6.5 & $\mu g / \mathrm{kg}$ \\
\hline UJ & 0 & H & 13 & $\mu g / \mathrm{kg}$ \\
\hline UJ & $\mathrm{Q}$ & & 13 & $\mu \mathrm{g} / \mathrm{kg}$ \\
\hline UJ & 0 & $\mathrm{H}$ & 6.5 & $\mu g / \mathrm{kg}$ \\
\hline UJ & $\mathbf{Q}$ & & 6.5 & $\mu \mathrm{g} / \mathrm{kg}$ \\
\hline
\end{tabular}

\author{
D. Limit Method
}

1.1-Dichloroethylene
1,1-Dichloroethylene
1,2-Dichloroethylene
1,2-Dichloroethylene
Dichloromethane (Methylene chloride)
Dichloromethane (Methylene chloride)
1,2-Dichloropropane
1,2-Dichloropropane
cis-1,3-Dichloropropene
cis-1,3-Dichloropropene
trans-1,3-Dichloropropene
trans-1,3-Dichloropropene
Ethylbenzene
Ethylbenzene
2-Hexanone
2-Hexanone
Methyl ethyl ketone
Methyl ethyl ketone
Methyl isobutyl ketone
Methyl isoburyl ketone
Styrene
Styrene
1,1,2,2-Tetrachloroethane
1,1,2,2-Tetrachloroethane
Tetrachloroethylene
Tetrachloroethylene
Toluene
Toluene
1,1,1-Trichloroethane
1,1,1-Trichloroethane
1,1,2-Trichloroethane
1,1,2-Trichloroethane
Trichloroethylene
Trichloroethylene
Vinyl acetate
Vinyl acetate
Xylenes
Xylenes

Radionuclide Indicators and Radionuclides

Gross alpha

Nonvolatile beta

Radium, total alpha-emitting

Tritium

$\begin{array}{llll}4.2 \pm 5.2 & \mathrm{pCi} / \mathrm{g} & 3.8 & \text { EPA900.0 } \\ 19 \pm 10 & \mathrm{pCi} / \mathrm{g} & 4.1 & \text { EPA900.0 } \\ 0.93 \pm 0.59 & \mathrm{pCi} / \mathrm{g} & 0.12 & \text { EPA903.0 } \\ 5.2 \pm 1.2 & \mathrm{pCi} / \mathrm{g} & 1.1 & \text { EPA906.0 }\end{array}$

EPA8240

EPA8240

EPA8240

EPA8240

EPA8240

EPA8240

EPA8240

EPA8240

EPA8240

EPA8240

EPA8240

EPA8240

EPA8240

EPA8240

EPA8240

EPA8240.

EPA8240

EPA8240

EPA8240

EPA8240

EPA8240

EPA8240

EPA8240

EPA8240

EPA8240

EPA8240

EPA8240

EPA8240

EPA8240

EPA8240

EPA8240

EPA8240

EPA8240

EPA8240

EPA8240

EPA8240

EPA 8240

EPA8240 
SAMPLE NAME: D431 1704

Location (SRS Coordinates): $18492.14 \mathrm{E}$ 66454.17N

Ground Elevation Above MSL: $124.9 \mathrm{ft}$

Depth of Core Interval: 10.00 to $12.00 \mathrm{ft}$ Sample Type: Normal

Sample Color: 5YR 5/6

Constituent

Sample ID: 100430

Sample Matrix: Soil USC Soil Classification: SW
Sample Moisture: Wet Percent Solids: 82.20

D. Limit Method

Total Metals

Arsenic
Barium
Cadmium
Chromium
Copper
Lead
Manganese
Mercury
Nickel
Selenium
Silver

$\begin{array}{ll} & 2.4 \\ \mathrm{U} & 20 \\ \mathrm{U} & 0.50 \\ & 5.4 \\ & 2.5 \\ \mathrm{U} & 1.8 \\ \mathrm{U} & 44 \\ \mathrm{U} & 0.12 \\ \mathrm{U} & 4.0 \\ & 0.60 \\ & 1.0\end{array}$

$B / N / A$ Extractables (including $P A H$ and phenols)

\begin{tabular}{|c|c|c|c|c|c|c|c|}
\hline Acenaphthene & UJ & L & & 400 & $\mu \mathrm{g} / \mathrm{kg}$ & 400 & EPA8270 \\
\hline Acenaphthylene & UJ & L & & 400 & $\mu \mathrm{g} / \mathrm{kg}$ & 400 & EPA8270 \\
\hline Anthracene & UJ & L & & 400 & $\mu \mathrm{g} / \mathrm{kg}$ & 400 & EPA8270 \\
\hline Benzo(a)anthracene & UJ & $\bar{L}$ & & 400 & $\mu \mathrm{g} / \mathrm{kg}$ & 400 & EPA8270 \\
\hline Benzo(b)fluoranthene & UJ & LH & $\mathrm{H}$ & 400 & $\mu \mathrm{g} / \mathrm{kg}$ & 400 & EPA8270 \\
\hline Benzo(k)fluoranthene & UJ & $\mathrm{LH}$ & $\mathbf{H}$ & 400 & $\mu \mathrm{g} / \mathrm{kg}$ & 400 & EPA8270 \\
\hline Benzoic acid & $\mathrm{UJ}$ & L & & 2,000 & $\mu \mathrm{g} / \mathrm{kg}$ & 2,000 & EPA8270 \\
\hline Benzo $(\mathrm{g}, \mathrm{h}, \mathrm{i})$ perylene & $\mathrm{UJ}$ & $\mathrm{LH}$ & $\mathbf{H}$ & 400 & $\mu \mathrm{g} / \mathrm{kg}$ & 400 & EPA8270 \\
\hline Benzo(a)pyrene & UJ & LH & $\mathrm{H}$ & 400 & $\mu \mathrm{g} / \mathrm{kg}$ & 400 & EPA8270 \\
\hline Benzyl alcohol & UJ & L & & 400 & $\mu \mathrm{g} / \mathrm{kg}$ & 400 & EPA8270 \\
\hline Bis(2-chloroethoxy) methane & UJ & $\vec{L}$ & & 400 & $\mu \mathrm{g} / \mathrm{kg}$ & 400 & EPA8270 \\
\hline Bis(2-chloroethyl) ether & UJ & L & & 400 & $\mu \mathrm{g} / \mathrm{kg}$ & 400 & EPA8270 \\
\hline Bis(2-chloroisopropyl) ether & UJ & L & & 400 & $\mu g / \mathrm{kg}$ & 400 & EPA8270 \\
\hline Bis(2-ethylhexyl) phthalate & UJ & VL & & 400 & $\mu \mathrm{g} / \mathrm{kg}$ & 400 & EPA8270 \\
\hline 4-Bromophenyl phenyl ether & UJ & L & & 400 & $\mu \mathrm{g} / \mathrm{kg}$ & 400 & EPA8270 \\
\hline Butanoic acid ester & $\mathbf{J}$ & $\mathbf{N}$ & & 200 & $\mu \mathrm{g} / \mathrm{kg}$ & & EPA8270 \\
\hline Butylbenzyl phthalate & U & L & & 400 & $\mu \mathrm{g} / \mathrm{kg}$ & 400 & EPA8270 \\
\hline 4-Chloroaniline & UJ & L & & 400 & $\mu \mathrm{g} / \mathrm{kg}$ & 400 & EPA8270 \\
\hline 4-Chloro-m-cresol & UJ & $\bar{L}$ & & 400 & $\mu g / \mathrm{kg}$ & 400 & EPA8270 \\
\hline 2-Chloronaphthalene & UJ & $\vec{L}$ & & 400 & $\mu g / \mathrm{kg}$ & 400 & EPA8270 \\
\hline 2.Chlorophenol & UJ & L & & 400 & $\mu \mathrm{g} / \mathrm{kg}$ & 400 & EPA8270 \\
\hline 4-Chlorophenyl phenyl ether & UJ & L & & 400 & $\mu g / \mathrm{kg}$ & 400 & EPA8270 \\
\hline Chrysene & UJ & L & & 400 & $\mu g / \mathrm{kg}$ & 400 & EPA8270 \\
\hline a-Cresol (2-Methylphenol) & $\mathrm{UJ}$ & $\bar{L}$ & & 400 & $\mu g / \mathrm{kg}$ & 400 & EPA8270 \\
\hline p-Cresol (4-Methylphenol) & UJ & $\bar{L}$ & & 400 & $\mu \mathrm{g} / \mathrm{kg}$ & 400 & EPA8270 \\
\hline Dibenz(a,h)anthracene & UJ & $\mathrm{LH}$ & $\mathbf{H}$ & 400 & $\mu g / \mathrm{kg}$ & 400 & EPA8270 \\
\hline Dibenzofuran & UJ & L & & 400 & $\mu \mathrm{g} / \mathrm{kg}$ & 400 & EPA8270 \\
\hline Di-n-butyl phthalate & UJ & VL & & 200 & $\mu \mathrm{g} / \mathrm{kg}$ & 400 & EPA8270 \\
\hline 1.2-Dichlorobenzene & UJ & L & & 400 & $\mu \mathrm{g} / \mathrm{kg}$ & 400 & EPA8270 \\
\hline 1,3-Dichlorobenzene & UJ & L & & 400 & $\mu g / \mathrm{kg}$ & 400 & EPA8270 \\
\hline
\end{tabular}


SAMPLE NAME: D431 1704 (Continued)

Constituent
RQ AQ B Result

$B / N / A$ Extractables (including PAH and phenols)
Sample ID: 100430

Unit

D. Limit Method

\begin{tabular}{|c|c|c|c|c|}
\hline 1.4-Dichlorobenzene & UJ & $\mathbf{L}$ & & 400 \\
\hline 3.3'-Dichlorobenzidine & UJ & $\mathbf{L}$ & & 810 \\
\hline 2,4-Dichlorophenol & UJ & $\mathbf{L}$ & & 400 \\
\hline Diethyl phthalate & UJ & $\mathbf{L}$ & & 400 \\
\hline 2,4-Dimethyl phenol & UJ & L & & 400 \\
\hline Dimethyl phthalate & UJ & $\mathbf{L}$ & & 400 \\
\hline 2,4-Dinitrophenol & UJ & L & & 2,000 \\
\hline 2,4-Dinitrotoluene & UJ & L & & 400 \\
\hline 2.6-Dinitrotoluene & UJ & L & & 400 \\
\hline Di-n-octyl phthalate & UJ & LH & H & 400 \\
\hline Fluoranthene & UJ & L & & 400 \\
\hline Fluorene & UJ & L & & 400 \\
\hline Hexachlorobenzene & UJ & L & & 400 \\
\hline Hexachlorobutadiene & UJ & L & & 400 \\
\hline Hexachlorocyclopentadiene & UJ & L & & 400 \\
\hline Hexachloroethane & UJ & L & & 400 \\
\hline Indeno(1,2,3-c,d)pyrene & UJ & LH & $\mathrm{H}$ & 400 \\
\hline Isophorone . & UJ & L & & 400 \\
\hline 2-Methyl-4,6-dinitrophenol & UJ & L & & 2,000 \\
\hline 2-Methylnaphthalene & UJ & L & & 400 \\
\hline Naphthalene & UJ & L & & 400 \\
\hline o-Nitroaniline & UJ & L & & 2,000 \\
\hline m-Nitroaniline & UJ & L & & 2,000 \\
\hline p-Nitroaniline & UJ & L & & 2,000 \\
\hline Nitrobenzene & UJ & $\mathrm{L}$ & & 400 \\
\hline 2-Nitrophenol & UJ & L & & 400 \\
\hline 4-Nitrophenol & UJ & L & & 2,000 \\
\hline $\mathrm{N}$-Nitrosodiphenylamine & UJ & L & & 400 \\
\hline N-Nitrosodipropylamine & UJ & L & & 400 \\
\hline Pentachlorophenol & UJ & L & & 2,000 \\
\hline Phenanthrene & UJ & L & & 400 \\
\hline Phenol & UJ & L & & 400 \\
\hline Pyrene & UJ & L & & 400 \\
\hline 1,2,4-Trichlorobenzene & UJ & L & & 400 \\
\hline 2,4,5-Trichlorophenol & UJ & L & & 2,000 \\
\hline 2,4,6-Trichlorophenol & UJ & L & & 400 \\
\hline Unknown ketone & J & $\mathrm{N}$ & & 7,000 \\
\hline
\end{tabular}

$\begin{array}{lll}\mu \mathrm{g} / \mathrm{kg} & 400 & \text { EPA8270 } \\ \mu \mathrm{g} / \mathrm{kg} & 810 & \text { EPA8270 } \\ \mu \mathrm{g} / \mathrm{kg} & 400 & \text { EPA8270 } \\ \mu \mathrm{g} / \mathrm{kg} & 400 & \text { EPA8270 } \\ \mu \mathrm{g} / \mathrm{kg} & 400 & \text { EPA8270 } \\ \mu \mathrm{g} / \mathrm{kg} & 400 & \text { EPA8270 } \\ \mu \mathrm{g} / \mathrm{kg} & 2,000 & \text { EPA8270 } \\ \mu \mathrm{g} / \mathrm{kg} & 400 & \text { EPA8270 } \\ \mu \mathrm{g} / \mathrm{kg} & 400 & \text { EPA8270 } \\ \mu \mathrm{g} / \mathrm{kg} & 400 & \text { EPA8270 } \\ \mu \mathrm{g} / \mathrm{kg} & 400 & \text { EPA8270 } \\ \mu \mathrm{g} / \mathrm{kg} & 400 & \text { EPA8270 } \\ \mu \mathrm{g} / \mathrm{kg} & 400 & \text { EPA8270 } \\ \mu \mathrm{g} / \mathrm{kg} & 400 & \text { EPA8270 } \\ \mu \mathrm{g} / \mathrm{kg} & 400 & \text { EPA8270 } \\ \mu \mathrm{g} / \mathrm{kg} & 400 & \text { EPA8270 } \\ \mu \mathrm{g} / \mathrm{kg} & 400 & \text { EPA8270 } \\ \mu \mathrm{g} / \mathrm{kg} & 400 & \text { EPA8270 } \\ \mu \mathrm{g} / \mathrm{kg} & 2,000 & \text { EPA8270 } \\ \mu \mathrm{g} / \mathrm{kg} & 400 & \text { EPA8270 } \\ \mu \mathrm{g} / \mathrm{kg} & 400 & \text { EPA8270 } \\ \mu \mathrm{g} / \mathrm{kg} & 2,000 & \text { EPA8270 } \\ \mu \mathrm{g} / \mathrm{kg} & 2,000 & \text { EPA8270 } \\ \mu \mathrm{g} / \mathrm{kg} & 2,000 & \text { EPA8270 } \\ \mu \mathrm{g} / \mathrm{kg} & 400 & \text { EPA8270 } \\ \mu \mathrm{g} / \mathrm{kg} & 400 & \text { EPA8270 } \\ \mu \mathrm{g} / \mathrm{kg} & 2,000 & \text { EPA8270 } \\ \mu \mathrm{g} / \mathrm{kg} & 400 & \text { EPA8270 } \\ \mu \mathrm{g} / \mathrm{kg} & 400 & \text { EPA8270 } \\ \mu \mathrm{g} / \mathrm{kg} & 2,000 & \text { EPA8270 } \\ \mu \mathrm{g} / \mathrm{kg} & 400 & \text { EPA8270 } \\ \mu \mathrm{g} / \mathrm{kg} & 400 & \text { EPA8270 } \\ \mu \mathrm{g} / \mathrm{kg} & 400 & \text { EPA8270 } \\ \mu \mathrm{g} / \mathrm{kg} & 400 & \text { EPA8270 } \\ \mu \mathrm{g} / \mathrm{kg} & 2,000 & \text { EPA8270 } \\ \mu \mathrm{g} / \mathrm{kg} & 400 & \text { EPA8270 } \\ \mu \mathrm{g} / \mathrm{kg} & & \text { EPA8270 } \\ & & \\ & & \end{array}$

Volatile Organic Compounds

\begin{tabular}{|c|c|c|c|c|c|c|}
\hline Acetone & $\mathbf{U}$ & $\mathbf{v}$ & 13 & $\mu \mathrm{g} / \mathrm{kg}$ & $13^{\circ}$ & EPA8240 \\
\hline Benzene & $\mathbf{U}$ & & 6.3 & $\mu \mathrm{g} / \mathrm{kg}$ & 6.3 & EPA8240 \\
\hline Bromodichloromethane & $\mathrm{U}$ & & 6.3 & $\mu \mathrm{g} / \mathrm{kg}$ & 6.3 & EPA8240 \\
\hline Bromoform & $\mathbf{U}$ & & 6.3 & $\mu \mathrm{g} / \mathrm{kg}$ & 6.3 & EPA8240 \\
\hline Bromomethane (Methyl bromide) & U & & 13 & $\mu \mathrm{g} / \mathrm{kg}$ & 13 & EPA8240 \\
\hline Carbon disulfide & $\mathbf{U}$ & & 6.3 & $\mu \mathrm{g} / \mathrm{kg}$ & 6.3 & EPA8240 \\
\hline Carbon tetrachloride & $\mathbf{U}$ & & 6.3 & $\mu \mathrm{g} / \mathrm{kg}$ & 6.3 & EPA8240 \\
\hline Chlorobenzene & $\mathbf{U}$ & & 6.3 & $\mu \mathrm{g} / \mathrm{kg}$ & 6.3 & EPA8240 \\
\hline Chloroethane & $\mathbf{U}$ & & 13 & $\mu \mathrm{g} / \mathrm{kg}$ & 13 & EPA8240 \\
\hline Chloroethene (Vinyl chloride) & U & & 13 & $\mu \mathrm{g} / \mathrm{kg}$ & 13 & EPA8240 \\
\hline
\end{tabular}


SAMPLE NAME: D431 1704 (Continued)

Constituent

RQ

$A Q$

Sample ID: 100430

Volatile Organic Compounds

Chloroform
Chloromethane (Methyl chloride)
Dibromochloromethane
1,1-Dichloroethane
1,2-Dichloroethane
1,1-Dichloroethylene
1,2-Dichloroethylene
Dichloromethane (Methylene chloride)
1,2-Dichloropropane
cis-1,3-Dichloropropene
trans-1.3-Dichloropropene
Ethylbenzene
2-Hexanone
Methyl ethyl ketone
Methyl isobutyl ketone
Styrene
$1,1,2,2-$ Tetrachloroethane
Tetrachloroethylene
Toluene
1,1,1-Trichloroethane
1,1,2-Trichloroethane
Trichloroethylene
Vinyl acetate
Xylenes

\begin{tabular}{ll}
$U$ & 6.3 \\
$U$ & 13 \\
$U$ & 6.3 \\
$U$ & 6.3 \\
$U$ & 6.2 \\
$U$ & 6.3 \\
$U$ & 6.3 \\
$U$ & 6.3 \\
$U$ & 6.3 \\
$U$ & 6.3 \\
$U$ & 6.3 \\
$U$ & 6.3 \\
$U$ & 13 \\
$U$ & 13 \\
$U$ & 13 \\
$U$ & 6.3 \\
$U$ & 6.3 \\
$U$ & 6.3 \\
$U$ & 6.3 \\
$J$ & 6.3 \\
$U$ & 5.8 \\
$U$ & 6.3 \\
$U$ & 13 \\
\hline & 6.3
\end{tabular}

Radionuclide Indicators and Radionuclides

Gross alpha

Nonvolatile beta

Radium, total alpha-emitting

Tritium

$\begin{array}{ll}6.0 \pm 5.8 & \mathrm{pCi} / \mathrm{g} \\ 17 \pm 10 & \mathrm{pCi} / \mathrm{g} \\ 0.39 \pm 0.39 & \mathrm{pCi} / \mathrm{g} \\ 7.5 \pm 1.5 & \mathrm{pCi} / \mathrm{g}\end{array}$

Sample ID: 100431

\section{SAMPLE NAME: D431 1705}

Location (SRS Coordinates): 18492.14E 66454.17N

Ground Elevation Above MSL: $124.9 \mathrm{ft}$

Depth of Core Interval: 15.00 to $17.00 \mathrm{ft}$

Sample Type: Normal :

Sample Color: SYR 5/6

Sample Matrix: Soil

USC Soil Classification: SW

\section{Constituent \\ Physical Parameters \\ Cation exchange capacity \\ $\mathrm{pH}$ \\ pH \\ Specific conductance}

3.8

EPA900.0

4.1

EPA900.0

0.11

EPA903.0

1.0

EPA906.0

Total organic carbon
RQ AQ B Result Unit

RQ AQ B Result Unit

$\begin{array}{lllll}\text { RQ } & \text { AQ } & B & \text { Result } & \text { Unit }\end{array}$

$\mathrm{meq} / 100 \mathrm{~g}$
$\mathrm{pH}$
$\mathrm{pH}$
$\mu \mathrm{S} / \mathrm{cm}$
$\mathrm{mg} / \mathrm{kg}$

$$
\therefore
$$

Sample Moisture: Wet Percent Solids: $\mathbf{7 7 . 3 0}$

D. Limit Method 
Total Metals

\begin{tabular}{|c|c|c|c|c|c|}
\hline Arsenic & & 1.9 & $\mathrm{mg} / \mathrm{kg}$ & 1.2 & $\begin{array}{l}\text { EPA7060 } \\
\text { EPA6010 }\end{array}$ \\
\hline Barium & $\mathrm{U}$ & 24 & $\mathrm{mg} / \mathrm{kg}$ & 24 & EPA6010 \\
\hline Cadmium & $\mathrm{U}$ & 0.59 & $\mathrm{mg} / \mathrm{kg}$ & 0.59 & EPA6010 \\
\hline Chromium & & 4.8 & $\mathrm{mg} / \mathrm{kg}$ & 1.2 & EPA6010 \\
\hline Copper & $\mathrm{U}$ & 2.9 & $\mathrm{mg} / \mathrm{kg}$ & 2.9 & EPA6010 \\
\hline Lead & & 1.1 & $\mathrm{mg} / \mathrm{kg}$ & 0.35 & EPA7421 \\
\hline Manganese & & 25 & $\mathrm{mg} / \mathrm{kg}$ & 1.8 & EPA6010 \\
\hline Mercury & $\dot{U}$ & 0.13 & $\mathrm{mg} / \mathrm{kg}$ & 0.13 & EPA7470 \\
\hline Nickel & $\mathrm{U}$ & 4.7 & $\mathrm{mg} / \mathrm{kg}$ & 4.7 & EPA6010 \\
\hline Selenium & U & 0.58 & $\mathrm{mg} / \mathrm{kg}$ & 0.58 & EPA7740 \\
\hline Silver & $\mathrm{U}$ & 1.2 & $\mathrm{mg} / \mathrm{kg}$ & 1.2 & EPA6010 \\
\hline
\end{tabular}

$B / N / A$ Extractables (including PAH and phenols)

\begin{tabular}{|c|c|c|c|c|c|c|c|}
\hline Acenaphthene & UJ & L & & 420 & $\mu \mathrm{g} / \mathrm{kg}$ & 420 & EPA8270 \\
\hline Acenaphthylene & UJ & $\mathrm{L}$ & & 420 & $\mu g / \mathrm{kg}$ & 420 & EPA8270 \\
\hline Anthracene & UJ' & L & & 420 & $\mu \mathrm{g} / \mathrm{kg}$ & 420 & EPA8270 \\
\hline Benzo(a)anthracene & UJ & $\mathrm{L}$ & & 420 & $\mu g / k g$ & 420 & EPA8270 \\
\hline Benzo(b)fluoranthene & UJ & $\mathrm{LH}$ & $\mathrm{H}$ & 420 & $\mu \mathrm{g} / \mathrm{kg}$ & 420 & EPA8270 \\
\hline Benzo(k)fluoranthene & UJ & LH & $\mathrm{H}$ & 420 & $\mu \mathrm{g} / \mathrm{kg}$ & 420 & EPA8270 \\
\hline Benzoic acid & UJ & L & & 2,100 & $\mu \mathrm{g} / \mathrm{kg}$ & 2,100 & EPA8270 \\
\hline Benzo(g,h,i)perylene & UJ & LH & $\mathrm{H}$ & 420 & $\mu g / k g$ & 420 & EPA8270 \\
\hline Benzo(a)pyrene & UJ & $\mathrm{LH}$ & $\mathrm{H}$ & 420 & $\mu g / \mathrm{kg}$ & 420 & EPA8270 \\
\hline Benzyl alcohol & UJ & L & & 420 & $\mu g / \mathrm{kg}$ & 420 & EPA8270 \\
\hline Bis(2-chloroethoxy) methane & UJ & L & & 420 & $\mu \mathrm{g} / \mathrm{kg}$ & 420 & EPA8270 \\
\hline Bis(2-chloroethyl) ether & UJ & L & & 420 & $\mu \mathrm{g} / \mathrm{kg}$ & 420 & EPA8270 \\
\hline Bis(2-chloroisopropyl) ether & UJ & L & & 420 & $\mu \mathrm{g} / \mathrm{kg}$ & 420 & EPA8270 \\
\hline Bis(2-ethylhexyl) phthalate & UJ & VL & & 420 & $\mu \mathrm{g} / \mathrm{kg}$ & 420 & EPA8270 \\
\hline 4-Bromophenyl phenyl ether & UJ & L & & 420 & $\mu \mathrm{g} / \mathrm{kg}$ & 420 & EPA8270 \\
\hline Burylbenzyl phthalate & UJ & $\mathbf{L}$ & & 420 & $\mu \mathrm{g} / \mathrm{kg}$ & 420 & EPA8270 \\
\hline 4-Chloroaniline & UJ & L & & 420 & $\mu \mathrm{g} / \mathrm{kg}$ & 420 & EPA8270 \\
\hline 4-Chloro-m-cresol & UJ & $\mathrm{L}$ & & 420 & $\mu \mathrm{g} / \mathrm{kg}$ & 420 & EPA 8270 \\
\hline 2-Chloronaphthalene & UJ & $\mathrm{L}$ & & 420 & $\mu \mathrm{g} / \mathrm{kg}$ & 420 & EPA8270 \\
\hline 2-Chlorophenol & UJ & L & & 420 & $\mu \mathrm{g} / \mathrm{kg}$ & 420 & EPA8270 \\
\hline 4-Chlorophenyl phenyl ether & UJ & L & & 420 & $\mu \mathrm{g} / \mathrm{kg}$ & 420 & EPA8270 \\
\hline Chrysene & UJ & $\mathrm{L}$ & & 420 & $\mu \mathrm{g} / \mathrm{kg}$ & 420 & EPA8270 \\
\hline 0-Cresol (2-Methylphenol) & UJ & L & & 420 & $\mu g / \mathrm{kg}$ & 420 & EPA8270 \\
\hline p-Cresol (4-Methylphenol) & UJ & $\bar{L}$ & & 420 & $\mu \mathrm{g} / \mathrm{kg}$ & $420^{\circ}$ & EPA8270 \\
\hline Dibenz(a,h)anthracene & UJ & $\mathrm{LH}$ & H & 420 & $\mu g / k g$ & 420 & EPA8270 \\
\hline Dibenzofuran & UJ & L & & 420 & $\mu \mathrm{g} / \mathrm{kg}$ & 420 & EPA8270 \\
\hline Di-n-buryl phthalate $\because$ & UJ & $\mathrm{VL}$ & & 120 & $\mu g / \mathrm{kg}$ & 420 & EPA8270 \\
\hline 1,2-Dichlorobenzene & UJ & L & & 420 & $\mu \mathrm{g} / \mathrm{kg}$ & 420 & EPA8270 \\
\hline 1,3-Dichlorobenzene & UJ & L & & 420 & $\mu \mathrm{g} / \mathrm{kg}$ & 420 & EPA8270 \\
\hline 1,4-Dichlorobenzene & UJ & L & & 420 & $\mu \mathrm{g} / \mathrm{kg}$ & 420 & EPA8270 \\
\hline 3,3'-Dichlorobenzidinế & UJ & $\mathrm{L}$ & & 840 & $\mu \mathrm{g} / \mathrm{kg}$ & 840 & EPA8270 \\
\hline 2,4-Dichlorophenol & UJ & L & & 420 & $\mu \mathrm{g} / \mathrm{kg}$ & 420 & EPA8270 \\
\hline Diethyl phthalate & UJ & L & & 420 & $\mu \mathrm{g} / \mathrm{kg}$ & 420 & EPA8270 \\
\hline 2,4-Dimethyl phenol & UJ & L & & 420 & $\mu \mathrm{g} / \mathrm{kg}$ & 420 & EPA8270 \\
\hline Dimethyl phthalate & UJ & L & & 420 & $\mu \mathrm{g} / \mathrm{kg}$ & 420 & EPA8270 \\
\hline 2,4-Dinitrophenol & UJ & $\bar{L}$ & & 2,100 & $\mu \mathrm{g} / \mathrm{kg}$ & 2,100 & EPA8270 \\
\hline 2,4-Dinitrotoluene & UJ & L & & 420 & $\mu \mathrm{g} / \mathrm{kg}$ & 420 & EPA 8270 \\
\hline 2.6-Dinitrotoluene & UJ & L & & 420 & $\mu \mathrm{g} / \mathrm{kg}$ & 420 & EPA 8270 \\
\hline Di-n-octyl phthalate & UJ & LH & $\mathrm{H}$ & 420 & $\mu \mathrm{g} / \mathrm{kg}$ & 420 & EPA8270 \\
\hline
\end{tabular}


$B / N / A$ Extractables (including $P A H$ and phenols)

Fluoranthene
Fluorene
Hexachlorobenzene
Hexachlorobutadiene
Hexachlorocyclopentadiene
Hexachloroethane
Indeno(1,2,3-c,d)pyrene
Isophorone
2-Methyl-4,6-dinitrophenol
2-Methylnaphthalene
Naphthalene
o-Nitroaniline
m-Nitroaniline
p-Nitroaniline
Nitrobenzene
2-Nitrophenol
4-Nitrophenol
N-Nitrosodiphenylamine
N-Nitrosodipropylamine
Pentachlorophenol
Phenanthrene
Phenol
Pyrene
1,2,4-Trichlorobenzene
2,4,5-Trichlorophenol
2,4,6-Trichlorophenol
Unknown acid ester
Unknown ketone

Volatile Organic Compounds

\begin{tabular}{|c|c|c|}
\hline UJ & $L$ & 420 \\
\hline UJ & L & 420 \\
\hline UJ & L & 420 \\
\hline UJ & $L$ & 420 \\
\hline UJ & L & 420 \\
\hline UJ & L & 420 \\
\hline UJ & LH $\quad H$ & 420 \\
\hline UJ & L & 420 \\
\hline UJ & L & 2,100 \\
\hline UJ & L & 420 \\
\hline UJ & L & 420 \\
\hline UJ & $\bar{L}$ & 2,100 \\
\hline UJ & L & 2,100 \\
\hline UJ & L & 2,100 \\
\hline UJ & L & 420 \\
\hline UJ & $\mathrm{L}$ & 420 \\
\hline UJ & $\mathbf{L}$ & 2,100 \\
\hline UJ & L & 420 \\
\hline UJ & L & 420 \\
\hline UJ & L & 2,100 \\
\hline UJ & $\bar{L}$ & 420 \\
\hline UJ & L & 420 \\
\hline UJ & L & 420 \\
\hline UJ & $\mathrm{L}$ & 420 \\
\hline UJ & L & 2,100 \\
\hline UJ & L & 420 \\
\hline 7 & $\mathbf{N}$ & 300 \\
\hline $\mathrm{J}$ & $\mathbf{N}$ & 9,000 \\
\hline
\end{tabular}

$$
\begin{aligned}
& \mu g / \mathbf{k g} \\
& \mu g / k g \\
& \mu g / k g \\
& \mu g / k g \\
& \mu g / k g \\
& \mu g / k g \\
& \mu g / k g \\
& \mu g / k g \\
& \mu g / k g \\
& \mu g / k g \\
& \mu g / k g \\
& \mu g / k g \\
& \mu g / k g \\
& \mu g / k g \\
& \mu g / k g \\
& \mu g / k g \\
& \mu g / k g \\
& \mu g / k g \\
& \mu g / k g \\
& \mu g / k g \\
& \mu g / k g \\
& \mu g / k g \\
& \mu g / k g \\
& \mu g / k g \\
& \mu g / k g \\
& \mu g / k g \\
& \mu g / k g \\
& \mu g / k g
\end{aligned}
$$$$
\cdot 420
$$

EPA8270

EPA8270

EPA8270

EPA8270

EPA8270

EPA8270

EPA8270

EPA8270

EPA8270

EPA8270

EPA8270

EPA8270

EPA8270

EPA8270

EPA8270

EPA8270

EPA8270

EPA8270

EPA8270

EPA8270

EPA8270

EPA8270

EPA8270

EPA8270

EPA8270

EPA8270

EPA8270

EPA8270

EPA8240

EPA8240

EPA8240

EPA8240

EPA8240

EPA8240

EPA8240

EPA8240

EPA8240

EPA8240

EPA 8240

EPA8240

EPA8240

EPA8240

EPA8240

EPA8240

EPA8240

EPA8240

EPA 8240 
SAMPLE NAME: D43̈1 1705 (Continued)

Sample ID: 100431

Constituent

Volatile Organic Compounds

trans-1,3-Dichloropropene

Ethylbenzene

2-Hexanone

Methyl ethyl ketone

Methyl isobutyl ketone

Styrene

1,1,2,2-Tetrachloroethane

Tetrachloroethylene

Toluene

1,1,1-Trichloroethane

1.1.2-Trichloroethane

Trichloroethylene

Vinyl acetate

Xylenes

\section{RQ AQ B Result Unit}

6.4

6.4

6.4

13

13

13

6.4

6.4

6.4

6.4

6.4

6.4

6.4

13

6.4
EPA8240

EPA8240

EPA8240

EPA8240

EPA8240

EPA8240

EPA8240.

EPA8240

EPA8240

EPA8240

EPA8240

EPA8240

EPA8240

EPA8240

EPA8240

Pesticides/PCBs and Dioxins/Furans

$\begin{array}{ll}\text { Aldrin } & \\ \text { alpha-Benzene hexachloride } & \\ \text { beta-Benzene hexachloride } & \\ \text { delta-Benzene hexachloride } & \\ \text { alpha-Chlordane } & \\ \text { gamma-Chlordane } & \\ \text { p.p'-DDD } & \\ \text { p.p'-DDE } & \\ \text { p,p'-DDT } & \\ \text { Dieldrin } & U \\ \text { Endosulfan I } & U \\ \text { Endosulfan II } & \\ \text { Endosulfan sulfate } & \\ \text { Endrin } & U \\ \text { Endrin ketone } & U \\ \text { Heptachlor } & U \\ \text { Heptachlor epoxide } & \\ \text { Heptachlorodibenzo-p-dioxin isomers } & U \\ \text { Heptachlorodibenzo-p-dioxin isomers } & U \\ \text { Heptachlorodibenzo-p-furan isomers } & U \\ \text { Heptachlorodibenzo-p-furan isomers } & \text { J } \\ \text { Hexachlorodibenzo-p-dioxin isomers } & U \\ \text { Hexachlorodibenzo-p-furan isomers } & U \\ \text { Lindane } & U \\ \text { Methoxychlor } & U \\ \text { Octachlorodibenzo-p-dioxin } & J \\ \text { Octachlorodibenzo-p-furan } & U \\ \text { PCB 1016 } & U \\ \text { PCB 1221 } & U \\ \text { PCB 1232 } & U \\ \text { PCB 1242 } & U \\ \text { PCB 1248 } & U \\ & \end{array}$

$U$
$U$
$U$
$U$
$U$
$U$
$U$
$U$
$U$
$U$
$U$
$U$
$U$
$U$
$U$
$U$
$U$
$U$
$U$
$J$
$U$
$U$
$U$
$U$
$J$
$U$
$U$
$U$
$U$
$U$
$U$

$\begin{array}{ll}\mathrm{U} & 2.1 \\ \mathrm{U} & 2.1\end{array}$

2.1

2.1

2.1

4.2

4.2

4.2

4.2

2.1

4.2

4.2

4.2

4.2

2.1

2.1

0.10

0.30

0.10

0.60

0.20

0.10

2.1

21

0.30

0.20

42

84

42

42

42

$\begin{array}{lcc}\mu \mathrm{g} / \mathrm{kg} & 2.1 & \text { EPA8080 } \\ \mu \mathrm{g} / \mathrm{kg} & 2.1 & \text { EPA8080 } \\ \mu \mathrm{g} / \mathrm{kg} & 2.1 & \text { EPA8080 } \\ \mu \mathrm{g} / \mathrm{kg} & 2.1 & \text { EPA8080 } \\ \mu \mathrm{g} / \mathrm{kg} & 2.1 & \text { EPA8080 } \\ \mu \mathrm{g} / \mathrm{kg} & 2.1 & \text { EPA8080 } \\ \mu \mathrm{g} / \mathrm{kg} & 4.2 & \text { EPA8080 } \\ \mu \mathrm{g} / \mathrm{kg} & 4.2 & \text { EPA8080 } \\ \mu \mathrm{g} / \mathrm{kg} & 4.2 & \text { EPA8080 } \\ \mu \mathrm{g} / \mathrm{kg} & 4.2 & \text { EPA8080 } \\ \mu \mathrm{g} / \mathrm{kg} & 2.1 & \text { EPA8080 } \\ \mu \mathrm{g} / \mathrm{kg} & 4.2 & \text { EPA8080 } \\ \mu \mathrm{g} / \mathrm{kg} & 4.2 & \text { EPA8080 } \\ \mu \mathrm{g} / \mathrm{kg} & 4.2 & \text { EPA8080 } \\ \mu \mathrm{g} / \mathrm{kg} & 4.2 & \text { EPA8080 } \\ \mu \mathrm{g} / \mathrm{kg} & 2.1 & \text { EPA8080 } \\ \mu \mathrm{g} / \mathrm{kg} & 2.1 & \text { EPA8080 } \\ \mathrm{ng} / \mathrm{g} & 0.10 & \text { EPA8280 } \\ \mathrm{ng} / \mathrm{g} & 0.30 & \text { EPA8280 } \\ \mathrm{ng} / \mathrm{g} & 0.10 & \text { EPA8280 } \\ \mathrm{ng} / \mathrm{g} & 0.10 & \text { EPA8280 } \\ \mathrm{ng} / \mathrm{g} & 0.20 & \text { EPA8280 } \\ \mathrm{ng} / \mathrm{g} & 0.10 & \text { EPA8280 } \\ \mu \mathrm{g} / \mathrm{kg} & 2.1 & \text { EPA8080 } \\ \mu \mathrm{g} / \mathrm{kg} & 21 & \text { EPA8080 } \\ \mathrm{ng} / \mathrm{g} & 0.10 & \text { EPA8280 } \\ \mathrm{ng} / \mathrm{g} & 0.20 & \text { EPA8280 } \\ \mu \mathrm{g} / \mathrm{kg} & 42 & \text { EPA8080 } \\ \mu \mathrm{g} / \mathrm{kg} & 84 & \text { EPA8080 } \\ \mu \mathrm{g} / \mathrm{kg} & 42 & \text { EPA8080 } \\ \mu \mathrm{g} / \mathrm{kg} & 42 & \text { EPA8080 } \\ \mu \mathrm{g} / \mathrm{kg} & 42 & \text { EPA8080 } \\ & & \end{array}$


SAMPLE NAME: D43̈1 1705 (Continued)

Sample ID: 100431

$\begin{array}{llllll}\text { Constituent } & \text { RQ AQ B Result D. Limit Method }\end{array}$

Pesticides/PCBs and Dioxins/Furans

PCB 1254

PCB 1260

Pentachlorodibenzo-p-dioxin isomers

Pentachlorodibenzo-p-furan isomers

Pentachlorodibenzo-p-dioxin isomers

Pentachlorodibenzo-p-furan isomers

2,3,7,8-TCDD

2,3,7,8-TCDF

Tetrachlorodibenzo-p-dioxin isomers

Tetrachlorodibenzo-p-furan isomers

Toxaphene

$\begin{array}{ll}U & 42 \\ U & 42 \\ U & 0.20 \\ U & 0.10 \\ U & 0.20 \\ U & 0.10 \\ U & 0.10 \\ U & 0.10 \\ U & 0.10 \\ U & 0.10 \\ U & 210\end{array}$

Radionuclide Indicators and Radionuclides

Gross alpha

Nonvolatile beta

Radium, total alpha-emitting

Tritium

SAMPLE NAME: D431 1705A

Sample Type: Duplicate

Constituent

Physical Parameters

Cation exchange capacity

pH

Specific conductance

Total organic carbon

Total Metals

Arsenic

Barium

Cadmium

Chromium

Copper

Lead

Manganese

Mercury

Nickel

Selenium

Silver $\mu g / \mathrm{kg}$

$\mu \mathrm{g} / \mathrm{kg}$

$\mathrm{ng} / \mathrm{g}$

$\mathrm{ng} / \mathrm{g}$

$\mathrm{ng} / \mathrm{g}$

$\mathrm{ng} / \mathrm{g}$

$\mathrm{ng} / \mathrm{g}$

$\mathrm{ng} / \mathrm{g}$

$\mathrm{ng} / \mathrm{g}$

$\mathrm{ng} / \mathrm{g}$

$\mu \mathrm{g} / \mathrm{kg}$

42

42

0.20

EPA8080

0.10

EPA8080

$0.20 \quad$ EPA8280

0.10 EPA8280

$0.10 \quad$ EPA8280

0.10 EPA8280

$0.10 \quad$ EPA8280

$0.10 \quad$ EPA8280

$210 \quad$ EPA8080

$\begin{array}{ll}3.4 \pm 5.1 & \mathrm{pCi} / \mathrm{g} \\ 12 \pm 10 & \mathrm{pCi} / \mathrm{g} \\ 0.66 \pm 0.50 & \mathrm{pCi} / \mathrm{g} \\ 1.2 \pm 0.40 & \mathrm{pCi} / \mathrm{g}\end{array}$

Sample ID: 100432

Associated Sample: 100431

RQ AQ B Result Unit

$\begin{array}{ll}4.0 & \text { EPA900.0 } \\ 4.2 & \text { EPA900.0 } \\ 0.12 & \text { EPA903.0 } \\ 0.95 & \text { EPA906.0 }\end{array}$

$\begin{array}{lllll}\mathrm{J} \quad \mathrm{Q} & 11 & \mathrm{meq} / 100 \mathrm{~g} & 2.5 & \text { EPA9080 } \\ & 4.5 & \mathrm{pH} & 0.010 & \text { EPA9045 } \\ & 44 & \mu \mathrm{S} / \mathrm{cm} & 1.0 & \text { EPA9050 } \\ & 690 & \mathrm{mg} / \mathrm{kg} & 150 & \text { EPA415.1 }\end{array}$

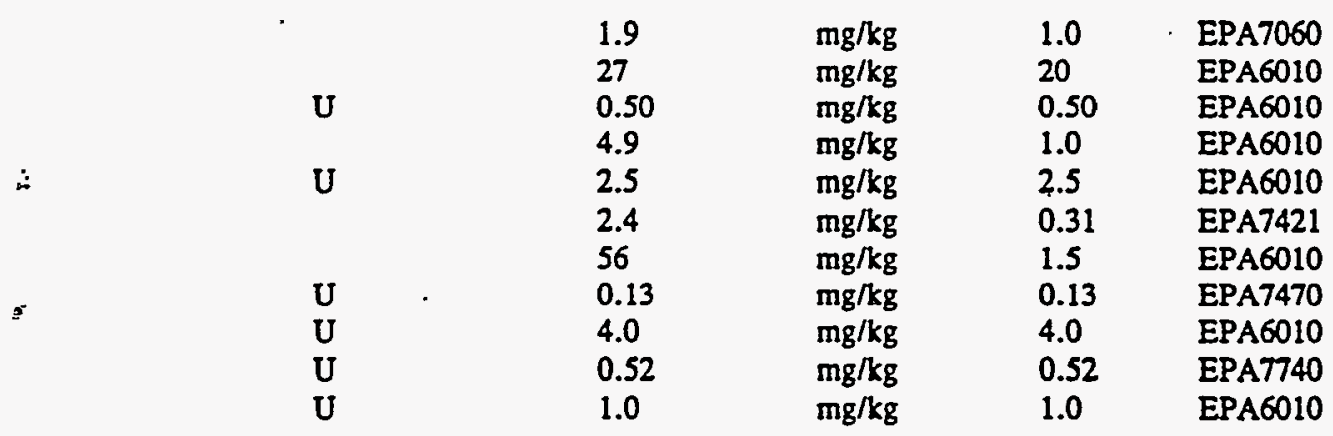


SAMPLE NAME: D431 1705A (Continued)

Sample ID: 100432

$\begin{array}{llllll}\text { Constituent Un AQ } & \text { R Result D. Limit Method }\end{array}$

$B / N / A$ Extractables (including PAH and phenols)

\begin{tabular}{|c|c|c|c|c|c|c|}
\hline Acenaphthene & $\mathbf{U}$ & & 410 & $\mu g / \mathrm{kg}$ & 410 & EPA8270 \\
\hline Acenaphthylene & $\mathbf{U}$ & . & 410 & $\mu \mathrm{g} / \mathrm{kg}$ & 410 & EPA8270 \\
\hline Anthracene & $\mathbf{U}$ & & 410 & $\mu \mathrm{g} / \mathrm{kg}$ & 410 & EPA8270 \\
\hline Benzo(a)anthracene & $\mathbf{U}$ & & 410 & $\mu \mathrm{g} / \mathrm{kg}$ & 410 & EPA8270 \\
\hline Benzo(b)fluoranthene & $\mathbf{U}$ & & 410 & $\mu \mathrm{g} / \mathrm{kg}$ & 410 & EPA8270 \\
\hline Benzo(k)fluoranthene & $\mathbf{U}$ & & 410 & $\mu \mathrm{g} / \mathrm{kg}$ & 410 & EPA8270 \\
\hline Benzoic acid & $\mathbf{U}$ & & 2,100 & $\mu \mathrm{g} / \mathrm{kg}$ & 2.100 & EPA8270 \\
\hline Benzo(g,h.i)perylene & $\mathbf{U}$ & & 410 & $\mu \mathrm{g} / \mathrm{kg}$ & 410 & EPA8270 \\
\hline Benzo(a)pyrene & $\mathbf{U}$ & & 410 & $\mu \mathrm{g} / \mathrm{kg}$ & 410 & EPA8270 \\
\hline Benzyl alcohol & $\mathrm{U}$ & & 410 & $\mu \mathrm{g} / \mathrm{kg}$ & 410 & EPA8270 \\
\hline Bis(2-chloroethoxy) methane & $\mathbf{U}$ & & 410 & $\mu \mathrm{g} / \mathrm{kg}$ & 410 & EPA8270 \\
\hline Bis(2-chloroethyl) ether & $\mathrm{U}$ & & 410 & $\mu \mathrm{g} / \mathrm{kg}$ & 410 & EPA8270 \\
\hline Bis(2-chloroisopropyl) ether & $\mathbf{U}$ & & 410 & $\mu \mathrm{g} / \mathrm{kg}$ & 410 & EPA8270 \\
\hline Bis(2-ethylhexyl) phthalate & $\mathrm{U}$ & $\mathrm{V}$ & 410 & $\mu \mathrm{g} / \mathrm{kg}$ & 410 & EPA8270 \\
\hline 4-Bromophenyl phenyl ether & $\mathbf{U}$ & & 410 & $\mu g / k g$ & 410 & EPA8270 \\
\hline Butanoic acid ester & $\mathbf{J}$ & $\mathbf{N}$ & 300 & $\mu \mathrm{g} / \mathrm{kg}$ & & EPA.8270 \\
\hline Butylbenzyl phthalate & $\mathrm{U}$ & & 410 & $\mu \mathrm{g} / \mathrm{kg}$ & 410 & EPA8270 \\
\hline 4-Chloroaniline & $U$ & & 410 & $\mu \mathrm{g} / \mathrm{kg}$ & 410 & EPA8270 \\
\hline 4-Chloro-m-cresol & $U$ & & 410 & $\mu \mathrm{g} / \mathrm{kg}$ & 410 & EPA8270 \\
\hline 2-Chloronaphthalene & $\mathrm{U}$ & & 410 & $\mu \mathrm{g} / \mathrm{kg}$ & 410 & EPA8270 \\
\hline 2-Chlorophenol & $\mathrm{U}$ & & 410 & $\mu \mathrm{g} / \mathrm{kg}$ & 410 & EPA8270 \\
\hline 4-Chlorophenyl phenyl echer & $\mathrm{U}$ & & 410 & $\mu \mathrm{g} / \mathrm{kg}$ & 410 & EPA8270 \\
\hline Chrysene & $\mathrm{U}$ & . & 410 & $\mu \mathrm{g} / \mathrm{kg}$ & 410 & EPA8270 \\
\hline o-Cresol (2-Methylphenol) & $\mathrm{U}$ & & 410 & $\mu \mathrm{g} / \mathrm{kg}$ & 410 & EPA8270 \\
\hline p-Cresol (4-Methylphenol) & $\mathbf{U}$ & & 410 & $\mu \mathrm{g} / \mathrm{kg}$ & 410 & EPA8270 \\
\hline Dibenz $(a, h)$ anthracene & $\mathrm{U}$ & & 410 & $\mu \mathrm{g} / \mathrm{kg}$ & 410 & EPA8270 \\
\hline Dibenzofuran & $\mathrm{U}$ & & 410 & $\mu \mathrm{g} / \mathrm{kg}$ & 410 & EPA8270 \\
\hline Di-n-butyl phthalate & UJ & $\mathrm{v}$ & 120 & $\mu \mathrm{g} / \mathrm{kg}$ & 410 & EPA8270 \\
\hline 1,2-Dichlorobenzene & $\mathbf{U}$ & & 410 & $\mu \mathrm{g} / \mathrm{kg}$ & 410 & EPA8270 \\
\hline 1,3-Dichlorobenzene & $\mathrm{U}$ & & 410 & $\mu \mathrm{g} / \mathrm{kg}$ & 410 & EPA8270 \\
\hline 1,4-Dichlorobenzene & $\mathbf{U}$ & & 410 & $\mu \mathrm{g} / \mathrm{kg}$ & 410 & EPA8270 \\
\hline 3,3'-Dichlorobenzidine & $\mathbf{U}$ & & 820 & $\mu \mathrm{g} / \mathrm{kg}$ & 820 & EPA8270 \\
\hline 2,4-Dichlorophenol & $U$ & & 410 & $\mu \mathrm{g} / \mathrm{kg}$ & 410 & EPA8270 \\
\hline Diethyl phthalate & $\mathbf{U}$ & & 410 & $\mu \mathrm{g} / \mathrm{kg}$ & 410 & EPA8270 \\
\hline 2,4-Dimethyl phenol & $\mathrm{U}$ & & 410 & $\mu \mathrm{g} / \mathrm{kg}$ & 410 & EPA8270 \\
\hline Dimechyl phthalate & $\mathbf{U}$ & & 410 & $\mu \mathrm{g} / \mathrm{kg}$ & 410 & EPA8270 \\
\hline 2,4-Dinitrophenol & $\mathrm{U}$ & & 2,100 & $\mu \mathrm{g} / \mathrm{kg}$ & 2,100 & EPA8270 \\
\hline 2,4-Dinitrotoluene & $\mathbf{U}$ & & 410 & $\mu \mathrm{g} / \mathrm{kg}$ & 410 & EPA8270 \\
\hline 2,6-Dinitrotoluene & $\mathbf{U}$ & & 410 & $\mu \mathrm{g} / \mathrm{kg}$ & 410 & EPA8270 \\
\hline Di-n-octyl phthalate $\dddot{t}$ & $\mathbf{U}$ & & 410 & $\mu \mathrm{g} / \mathrm{kg}$ & 410 & EPA8270 \\
\hline Fluoranthene & $\mathbf{U}$ & & 410 & $\mu \mathrm{g} / \mathrm{kg}$ & 410 & EPA8270 \\
\hline Fluorene & $\mathbf{U}$ & & 410 & $\mu \mathrm{g} / \mathrm{kg}$ & 410 & EPA8270 \\
\hline Hexachlorobenzene & $\mathbf{U}$ & & 410 & $\mu \mathrm{g} / \mathrm{kg}$ & 410 & EPA8270 \\
\hline Hexachlorobutadiene & $\mathbf{U}$ & & 410 & $\mu \mathrm{g} / \mathrm{kg}$ & 410 & EPA8270 \\
\hline Hexachlorocyclopentadiene & $\mathbf{U}$ & & 410 & $\mu \mathrm{g} / \mathrm{kg}$ & 410 & EPA8270 \\
\hline Hexachloroethane & $\mathbf{U}$ & & 410 & $\mu \mathrm{g} / \mathrm{kg}$ & 410 & EPA8270 \\
\hline Indeno( $1,2,3-c, d)$ pyrene & $\mathbf{U}$ & & 410 & $\mu \mathrm{g} / \mathrm{kg}$ & 410 & EPA8270 \\
\hline Isophorone & $\mathrm{U}$ & & 410 & $\mu \mathrm{g} / \mathrm{kg}$ & $410^{\circ}$ & EPA8270 \\
\hline 2-Methyl-4,6-dinitrophenol & $\mathrm{U}$ & & 2,100 & $\mu \mathrm{g} / \mathrm{kg}$ & 2,100 & EPA8270 \\
\hline 2-Methylnaphthalene & U & . & 410 & $\mu \mathrm{g} / \mathrm{kg}$ & 410 & EPA8270 \\
\hline
\end{tabular}


SAMPLE NAME: D431 1705A (Continued)

Constituent

RQ AQ B Result

$B / N / A$ Extractables (including PAH and phenols)

\begin{tabular}{|c|c|c|c|c|}
\hline$U$ & 410 & $\mu g / \mathrm{kg}$ & 410 & EPA8270 \\
\hline$U$ & 2,100 & $\mu g / \mathrm{kg}$ & 2,100 & EPA8270 \\
\hline $\mathbf{U}$ & 2,100 & $\mu \mathrm{g} / \mathrm{kg}$ & 2,100 & EPA8270 \\
\hline $\mathrm{U}$ & 2,100 & $\mu \mathrm{g} / \mathrm{kg}$ & 2,100 & EPA8270 \\
\hline U & 410 & $\mu \mathrm{g} / \mathrm{kg}$ & 410 & EPA8270 \\
\hline $\mathrm{U}$ & 410 & $\mu \mathrm{g} / \mathrm{kg}$ & 410 & EPA8270 \\
\hline$U$ & 2,100 & $\mu \mathrm{g} / \mathrm{kg}$ & 2,100 & EPA8270 \\
\hline $\mathbf{U}$ & 410 & $\mu \mathrm{g} / \mathrm{kg}$ & 410 & EPA8270 \\
\hline $\mathbf{U}$ & 410 & $\mu \mathrm{g} / \mathrm{kg}$ & 410 & EPA8270 \\
\hline U & 2,100 & $\mu \mathrm{g} / \mathrm{kg}$ & 2,100 & EPA8270 \\
\hline U & 410 & $\mu \mathrm{g} / \mathrm{kg}$ & 410 & EPA8270 \\
\hline$U$ & 410 & $\mu \mathrm{g} / \mathrm{kg}$ & 410 & EPA8270 \\
\hline $\mathrm{U}$ & 410 & $\mu \mathrm{g} / \mathrm{kg}$ & 410 & EPA8270 \\
\hline$U$ & 410 & $\mu \mathrm{g} / \mathrm{kg}$ & 410 & EPA8270 \\
\hline U & 2.100 & $\mu \mathrm{g} / \mathrm{kg}$ & 2,100 & EPA8270 \\
\hline $\mathbf{U}$ & 410 & $\mu \mathrm{g} / \mathrm{kg}$ & 410 & EPA8270 \\
\hline$N$ & 8,000 & $\mu \mathrm{g} / \mathrm{kg}$ & & EPA8270 \\
\hline $\mathbf{N}$ & 900 & $\mu \mathrm{g} / \mathrm{kg}$ & & EPA8270 \\
\hline $\mathrm{N}$ & 600 & $\mu \mathrm{g} / \mathrm{kg}$ & & EPA8270 \\
\hline
\end{tabular}

$U$
$U$
$U$
$U$
$U$
$U$
$U$
$U$
$U$
$U$
$U$
$U$
$U$
$U$
$U$
$U$
$U$
$U$
$U$
$U$
$U$
$U$
$U$
$U$
$U$
$U$
$U$

16

6.3

6.3

6.3

13

6.3

6.3

6.3

13

13

6.3

13

6.3

6.3

8.7

6.3

6.3

6.3

6.3

6.3

6.3

6.3

13

13

13

6.3

6.3

6.3
Unit

D. Limit Method
Volatile Organic Compounds

Naphthalene
o-Nitroaniline
m-Nitroaniline
p-Nitroaniline
Nitrobenzene
2-Nitrophenol
4-Nitrophenol
N-Nitrosodiphenylamine
N-Nitrosodipropylamine
Pentachlorophenol
Phenanthrene
Phenol
Pyrene
1,2,4-Trichlorobenzene
2,4,5-Trichlorophenol
2,4,6-Trichlorophenol
Unknown ketone
Unknown phthalate
Unknown phthalate
Volatile Organic Compounds
Acetone
Benzene
Bromodichloromethane
Bromoform
Bromomethane (Methyl bromide)
Carbon disulfide
Carbon tetrachloride
Chlorobenzene
Chloroethane
Chloroethene (Vinyl chloride)
Chloroform
Chloromethane (Methyl chloride)
Dibromochloromethane
1,1-Dichloroethane
1,2-Dichloroethane
1,1-Dichloroethylene
1,2-Dichloroethylene
Dichloromethane (Methylene chloride)
1,2-Dichloropropane
cis-1,3-Dichloropropene
trans-1,3-Dichloropropene
Ethylbenzene
2-Hexanone
Methyl ethyl ketone
Methyl isobutyl ketone
Styrene
1,1,2,2-Tetrachloroethane
Tetrachloroethylene

$\mu \mathrm{g} / \mathrm{kg}$
$\mu \mathrm{g} / \mathrm{kg}$
$\mu \mathrm{g} / \mathrm{kg}$
$\mu \mathrm{g} / \mathrm{kg}$
$\mu \mathrm{g} / \mathrm{kg}$
$\mu \mathrm{g} / \mathrm{kg}$
$\mu \mathrm{g} / \mathrm{kg}$
$\mu \mathrm{g} / \mathrm{kg}$
$\mu \mathrm{g} / \mathrm{kg}$
$\mu \mathrm{g} / \mathrm{kg}$
$\mu \mathrm{g} / \mathrm{kg}$
$\mu \mathrm{g} / \mathrm{kg}$
$\mu \mathrm{g} / \mathrm{kg}$
$\mu \mathrm{g} / \mathrm{kg}$
$\mu \mathrm{g} / \mathrm{kg}$
$\mu \mathrm{g} / \mathrm{kg}$
$\mu \mathrm{g} / \mathrm{kg}$
$\mu \mathrm{g} / \mathrm{kg}$
$\mu \mathrm{g} / \mathrm{kg}$
$\mu \mathrm{g} / \mathrm{kg}$
$\mu \mathrm{g} / \mathrm{kg}$
$\mu \mathrm{g} / \mathrm{kg}$
$\mu \mathrm{g} / \mathrm{kg}$
$\mu \mathrm{g} / \mathrm{kg}$
$\mu \mathrm{g} / \mathrm{kg}$
$\mu \mathrm{kg} / \mathrm{kg}$
$\mu \mathrm{g} / \mathrm{kg}$
$\mu \mathrm{g} / \mathrm{kg}$

EPA8240

EPA8240

EPA8240

EPA8240

EPA8240

EPA8240

EPA8240

EPA8240

EPA8240

EPA8240

EPA8240

EPA8240

EPA8240

EPA8240

EPA8240

EPA8240

EPA8240

EPA8240

EPA8240

EPA8240

EPA8240

EPA8240

EPA8240

EPA8240

EPA8240

EPA8240

EPA8240

EPA8240 
SAMPLE NAME: D431 1705A (Continued)

Constituent

Volatile Organic Compounds

Toluene

1.1.1-Trichloroethane

1,1,2-Trichloroethane

Trichloroethylene

Vinyl acetate

Xylenes
U

U

U

U

$\mathrm{U}$
Sample ID: 100432

RQ AQ B Result Unit D. Limit Method

Pesticides/PCBs and Dioxins/Furans

Aldrin

alpha-Benzene hexachloride

alpha-Benzene hexachloride

alpha-Benzene hexachloride

beta-Benzene hexachloride

beta-Benzene hexachloride

beta-Benzene hexachloride

delta-Benzene hexachloride

delta-Benzene hexachloride

delta-Benzene hexachloride

alpha-Chiordane

alpha-Chlordane

alpha-Chlordane

gamma-Chlordane

gamma-Chlordane

gamma-Chlordane

p.p'-DDD

P.P'-DDD

p, $p^{\prime}-D D D$

p.p'-DDE

$p, p^{\prime}-D D E$

p. $p^{\prime}-D D E$

p.p'-DDT

Dieldrin

Endosulfan I

Endosulfan I

Endosulfan I

Endosulfan II

Endosulfan II

Endosulfan II

Endosulfan sulfate

Endosulfan sulfate

Endosulfan sulfate

Endrin

Endrin ketone $\leq$

Endrin ketone

Endrin ketone

Heptachlor

Heptachlor epoxide

Heptachlor epoxide

Heptachlor epoxide
6.3

6.3

9.4

6.3

13

6.3 $\mu \mathrm{g} / \mathrm{kg}$
$\mu \mathrm{g} / \mathrm{kg}$
$\mu \mathrm{g} / \mathrm{kg}$
$\mu \mathrm{g} / \mathrm{kg}$
$\mu \mathrm{g} / \mathrm{kg}$
$\mu \mathrm{g} / \mathrm{kg}$

6.3

6.3

6.3

6.3

13

6.3
EPA8240
EPA8240
EPA8240
EPA8240
EPA8240
EPA8240
2.1

2.1

2.1

2.1

2.1

2.1

2.1

2.1

2.1

2.1

2.1

2.1

2.1

2.1

2.1

2.1

4.1

\section{1}

4.1

\section{1}

4.1

4.1

4.1

$\mu \mathrm{g} / \mathrm{kg}$
$\mu \mathrm{g} / \mathrm{kg}$
$\mu \mathrm{g} / \mathrm{kg}$
$\mu \mathrm{g} / \mathrm{kg}$
$\mu \mathrm{g} / \mathrm{kg}$
$\mu \mathrm{g} / \mathrm{kg}$
$\mu \mathrm{g} / \mathrm{kg}$
$\mu \mathrm{g} / \mathrm{kg}$
$\mu \mathrm{g} / \mathrm{kg}$
$\mu \mathrm{g} / \mathrm{kg}$
$\mu \mathrm{g} / \mathrm{kg}$
$\mu \mathrm{g} / \mathrm{kg}$
$\mu \mathrm{g} / \mathrm{kg}$
$\mu \mathrm{g} / \mathrm{kg}$
$\mu \mathrm{g} / \mathrm{kg}$
$\mu \mathrm{g} / \mathrm{kg}$
$\mu \mathrm{g} / \mathrm{kg}$
$\mu \mathrm{g} / \mathrm{kg}$
$\mu \mathrm{g} / \mathrm{kg}$
$\mu \mathrm{g} / \mathrm{kg}$
$\mu \mathrm{g} / \mathrm{kg}$
$\mu \mathrm{kg}$
$\mu \mathrm{g} / \mathrm{kg}$
$\mu \mathrm{g} / \mathrm{kg}$
$\mu \mathrm{g} / \mathrm{kg}$
$\mu \mathrm{g} / \mathrm{kg}$
$\mu \mathrm{g} / \mathrm{kg}$
$\mu \mathrm{g} / \mathrm{kg}$
$\mu \mathrm{g} / \mathrm{kg}$
$\mu \mathrm{g} / \mathrm{kg}$
$\mu \mathrm{g} / \mathrm{kg}$
$\mu \mathrm{g} / \mathrm{kg}$
$\mu \mathrm{g} / \mathrm{kg}$
$\mu \mathrm{g} / \mathrm{kg}$
$\mu \mathrm{g} / \mathrm{kg}$
$\mu \mathrm{g} / \mathrm{kg}$
$\mu \mathrm{g} / \mathrm{kg}$
$\mu \mathrm{g} / \mathrm{kg}$
$\mu \mathrm{g} / \mathrm{kg}$
$\mu \mathrm{g} / \mathrm{kg}$
$\mu \mathrm{g} / \mathrm{kg}$

2.1

2.1

2.1

2.1

2.1

2.1

2.1

2.1

2.1

2.1

2.1

2.1

2.1

2.1

2.1

2.1

4.1

4.1

4.1

- 4.1

4.1

4.1

4.1

4.1

2.1

2.1

2.1

4.1

4.1

4.1

4.1

4.1 .

4.1

4.1

4.1

4.1

4.1

2.1

2.1

2.1

2.1
EPA8080

EPA8080

EPA8080

EPA8080

EPA8080

EPA8080

EPA8080

EPA8080

EPA8080

EPA8080

EPA8080

EPA8080

EPA8080

EPA8080

EPA8080

EPA8080

EPA8080

EPA8080

EPA8080

EPA8080

EPA8080

EPA8080

EPA8080

EPA8080

EPA8080

EPA8080

EPA8080

EPA8080

EPA8080

EPA8080

EPA8080

EPA8080

EPA8080

EPA8080

EPA8080

EPA8080

EPA8080

EPA8080

EPA8080

EPA8080

EPA8080 
SAMPLE NAME: D431 1705A (Continued)

Constituent

RQ

Sample ID: 100432

Pesticides/PCBs and Dioxins/Furans

Heptachlorodibenzo-p-dioxin isomers
Heptachlorodibenzo-p-furan isomers
Hexachlorodibenzo-p-dioxin isomers
Hexachlorodibenzo-p-furan isomers
Lindane
Methoxychlor
Methoxychlor
Methoxychlor
Octachlorodibenzo-p-dioxin
Octachlorodibenzo-p-furan
PCB 1016
PCB 1016
PCB 1016
PCB 1221
PCB 1221
PCB 1221
PCB 1232
PCB 1232
PCB 1232
PCB 1242
PCB 1242
PCB 1242
PCB 1248
PCB 1248
PCB 1248
PCB 1254
PCB 1254
PCB 1254
PCB 1260
PCB 1260
PCB 1260
Pentachlorodibenzo-p-dioxin isomers
Pentachlorodibenzo-p-furan isomers
$2,3,7,8-T C D D$
$2,377,8-T C D F$
Tetrachlorodibenzo-p-dioxin isomers
Tetrachlorodibenzo-p-furan isomers
Toxaphene
Toxaphene
Toxaphene

0.20
0.10
0.10
0.10
2.1
21
21

$\mathrm{ng} / \mathrm{g}$

0.20

EPA8280

$\mathrm{ng} / \mathrm{g}$

EPA8280

$\mathrm{ng} / \mathrm{g}$

0.10

EPA8280

$\mathrm{ng} / \mathrm{g}$

$\mu \mathrm{g} / \mathrm{kg}$

$\mu \mathrm{g} / \mathrm{kg}$

0.10

EPA8280

U 2.1

21

2.9

0.30

41

$\mu \mathrm{g} / \mathrm{kg}$

$\mu \mathrm{g} / \mathrm{kg}$

$\mathrm{ng} / \mathrm{g}$

ng/g

$\mu \mathrm{g} / \mathrm{kg}$

$\mu \mathrm{g} / \mathrm{kg}$

$\mu \mathrm{g} / \mathrm{kg}$

$\mu \mathrm{g} / \mathrm{kg}$

$\mu \mathrm{g} / \mathrm{kg}$

$\mu \mathrm{g} / \mathrm{kg}$

$\mu \mathrm{g} / \mathrm{kg}$

$\mu \mathrm{g} / \mathrm{kg}$

$\mu \mathrm{g} / \mathrm{kg}$

$\mu \mathrm{g} / \mathrm{kg}$

$\mu \mathrm{g} / \mathrm{kg}$

$\mu \mathrm{g} / \mathrm{kg}$

$\mu \mathrm{g} / \mathrm{kg}$

$\mu \mathrm{g} / \mathrm{kg}$

$\mu \mathrm{g} / \mathrm{kg}$

$\mu \mathrm{g} / \mathrm{kg}$

$\mu \mathrm{g} / \mathrm{kg}$

$\mu \mathrm{g} / \mathrm{kg}$

$\mu \mathrm{g} / \mathrm{kg}$

$\mu g / \mathrm{kg}$

$\mu \mathrm{g} / \mathrm{kg}$

$\mathrm{ng} / \mathrm{g}$

$\mathrm{ng} / \mathrm{g}$

$\mathrm{ng} / \mathrm{g}$

$\mathrm{ng} / \mathrm{g}$

$\mathrm{ng} / \mathrm{g}$

$\mathrm{ng} / \mathrm{g}$

$\mu g / \mathrm{kg}$

$\mu \mathrm{g} / \mathrm{kg}$

0.10

EPA8080

EPA8080

EPA8080

EPA8080

EPA8280

EPA8280

EPA8080

EPA8080

EPA8080

EPA8080

EPA8080

EPA8080

EPA8080

EPA8080

EPA8080

EPA8080

EPA8080

EPA8080

EPA8080

EPA8080

EPA8080

EPA8080

EPA8080

EPA8080

EPA8080

EPA8080

EPA8080

EPA8280

0.20

0.10

0.20

0.10

210

210

210

$\mu \mathrm{g} / \mathrm{kg}$

EPA8280

EPA8280

EPA8280

EPA8280

EPA8280

EPA8080

EPA8080

EPA8080

Radionuclide Indicators and Radionuclides

Gross alpha

Nonvolatile beta

Radium, total alpha-emitting

Tritium

$\begin{array}{llll}6.8 \pm 6.0 & \mathrm{pCi} / \mathrm{g} & 3.8 & \text { EPA990.0 } \\ 12 \pm 9.5 & \mathrm{pCi} / \mathrm{g} & 4.0 & \text { EPA900.0 } \\ 0.46 \pm 0.42 & \mathrm{pCi} / \mathrm{g} & 0.12 & \text { EPA903.0 } \\ 5.6 \pm 1.2 & \mathrm{pCi} / \mathrm{g} & 0.91 & \text { EPA906.0 }\end{array}$


SAMPLE NAME: D431 1706

Location (SRS Coordinates): 18492.14E 66454.17N

Ground Elevation Above MSL: $124.9 \mathrm{ft}$

Depth of Core Interval: 20.00 to $22.00 \mathrm{ft}$

Sample Type: Normal

Sample Color: SYR S/6

\section{Constituent}

Physical Parameters

Cation exchange capacity .

$\mathrm{pH}$

Specific conductance

Total organic carbon

Total Metals

Arsenic

Arsenic

Barium

Barium

Cadmium

Cadmium

Chromium

Chromium

Copper

Copper

Lead

Lead

Manganese

Manganese

Mercury

Nickel

Nickel

Selenium

Selenium

Silver

Silver
Sample ID: 100433

Sample Matrix: Soil

USC Soil Classification: SW

Sample Moisture: Wet

Percent Solids: 80.00

D. Limit Method

RQ AQ B Result Unit D. Limit Method

$\begin{array}{llllll} & & 9.5 & \mathrm{meq} / 100 \mathrm{~g} & 2.5 & \text { EPA9080 } \\ \mathrm{J} \quad \mathrm{Q} & 4.4 & \mathrm{pH} & 0.010 & \text { EPA9045 } \\ & 44 & \mu \mathrm{S} / \mathrm{cm} & 1.0 & \text { EPA9050 } \\ & & 410 & \mathrm{mg} / \mathrm{kg} & 120 & \text { EPA415.1 }\end{array}$

$B / N / A$ Extractables (including $P A H$ and phenols)

Acenaphthene

Acenaphthylene

Anthracene

Benzo(a)anthracene ::

Benzo(b)fluoranthene

Benzo(k)fluoranthene

Benzoic acid

Benzo(g,h,i)perylene

Benzo(a)pyrene

Benzyl alcohol

Bis(2-chloroethoxy) methane

Bis(2-chloroethyl) ether

Bis(2-chloroisopropyl) ether
U 410

U $\quad 410$

U 410

$U^{*} \quad 410$

U 410

U 410

U 2,100

U 410

U 410

U 410

U 410

U 410

U $\quad 410$
1.7

$\mathrm{mg} / \mathrm{kg}$

$\mathrm{mg} / \mathrm{kg}$

$\mathrm{mg} / \mathrm{kg}$

$\mathrm{mg} / \mathrm{kg}$

$\mathrm{mg} / \mathrm{kg}$

$\mathrm{mg} / \mathrm{kg}$

$\mathrm{mg} / \mathrm{kg}$

$\mathrm{mg} / \mathrm{kg}$

$\mathrm{mg} / \mathrm{kg}$

$\mathrm{mg} / \mathrm{kg}$

$\mathrm{mg} / \mathrm{kg}$

$\mathrm{mg} / \mathrm{kg}$

$\mathrm{mg} / \mathrm{kg}$

$\mathrm{mg} / \mathrm{kg}$

$\mathrm{mg} / \mathrm{kg}$

$\mathrm{mg} / \mathrm{kg}$

$\mathrm{mg} / \mathrm{kg}$

$\mathrm{mg} / \mathrm{kg}$

$\mathrm{mg} / \mathrm{kg}$

$\mathrm{mg} / \mathrm{kg}$
1.2

1.2

23

23

0.58

0.58

1.2

1.2

2.9

2.9

0.36

0.36

1.8

1.8

0.13

4.7

4.7

0.61

0.61

1.2

1.2

410

410

410

410

410

410

2,100

410

410

410

410

410

410
EPA7060

EPA7060

EPA6010

EPA6010

EPA6010

EPA6010

EPA6010

EPA 6010

EPA6010

EPA6010

EPA7421

EPA7421

EPA6010

EPA6010

EPA7470

EPA6010

EPA6010

EPA7740

EPA7740

EPA6010

EPA6010

EPA8270

EPA8270

EPA8270

EPA8270

EPA8270

EPA8270

EPA8270

EPA8270

EPA8270

EPA8270

EPA8270

EPA8270

$\mu \mathrm{g} / \mathrm{kg}$

$\mu \mathrm{g} / \mathrm{kg}$

EPA8270 
SAMPLE NAME: D431 1706 (Continued)

Sample ID: 100433

$\begin{array}{llllll}\text { Constituent } & \text { RQ AQ B Result Unit D. Limit Method }\end{array}$

$B / N / A$ Extractables (including $P A H$ and phenols)

\begin{tabular}{|c|c|c|c|c|c|c|}
\hline Bis(2-ethylhexyl) phthalate & $\mathbf{U}$ & $\mathrm{v}$ & 410 & $\mu g / k g$ & 410 & EPA8270 \\
\hline 4-Bromophenyl phenyl ether & $U$ & & 410 & $\mu g / k g$ & 410 & EPA8270 \\
\hline Butylbenzyl phthalate & $\mathrm{U}$ & & 410 & $\mu g / k g$ & 410 & EPA8270 \\
\hline 4-Chloroaniline & $\mathbf{U}$ & & 410 & $\mu \mathrm{g} / \mathrm{kg}$ & 410 & EPA8270 \\
\hline 4-Chloro-m-cresol & $\mathbf{U}$ & & 410 & $\mu \mathrm{g} / \mathrm{kg}$ & 410 & EPA8270 \\
\hline 2-Chloronaphthalene & $\mathrm{U}$ & & 410 & $\mu \mathrm{g} / \mathrm{kg}$ & 410 & EPA8270 \\
\hline 2-Chlorophenol & $\mathrm{U}$ & & 410 & $\mu g / \mathrm{kg}$ & 410 & EPA8270 \\
\hline 4-Chlorophenyl phenyl ether & $\mathrm{U}$ & & 410 & $\mu \mathrm{g} / \mathrm{kg}$ & 410 & EPA8270 \\
\hline Chrysene & $\mathrm{U}$ & & 410 & $\mu g / \mathrm{kg}$ & 410 & EPA8270 \\
\hline 0-Cresol (2-Methyiphenol) & $\mathrm{U}$ & & 410 & $\mu \mathrm{g} / \mathrm{kg}$ & 410 & EPA8270 \\
\hline p-Cresol (4-Methylphenol) & $\mathrm{U}$ & & 410 & $\mu \mathrm{g} / \mathrm{kg}$ & 410 & EPA8270 \\
\hline Dibenz(a,h)anthracene & $\mathrm{U}$ & & 410 & $\mu \mathrm{g} / \mathrm{kg}$ & 410 & EPA8270 \\
\hline Dibenzofuran & $U$ & & 410 & $\mu \mathrm{g} / \mathrm{kg}$ & 410 & EPA8270 \\
\hline Di-n-butyl phthalate & UJ & $\mathrm{v}$ & 110 & $\mu \mathrm{g} / \mathrm{kg}$ & 410 & EPA8270 \\
\hline 1.2-Dichlorobenzene & $U$ & & 410 & $\mu \mathrm{g} / \mathrm{kg}$ & 410 & EPA8270 \\
\hline 1,3-Dichlorobenzene & $\mathrm{U}$ & & 410 & $\mu \mathrm{g} / \mathrm{kg}$ & 410 & EPA8270 \\
\hline 1,4-Dichlorobenzene & U & & 410 & $\mu \mathrm{g} / \mathrm{kg}$ & 410 & EPA8270 \\
\hline 3,3'-Dichlorobenzidine & $\mathrm{U}$ & & 830 & $\mu \mathrm{g} / \mathrm{kg}$ & 830 & EPA8270 \\
\hline 2.4-Dichlorophenol & $U$ & & 410 & $\mu \mathrm{g} / \mathrm{kg}$ & $410^{\circ}$ & EPA8270 \\
\hline Diethyl phthalate & U & & 410 & $\mu \mathrm{g} / \mathrm{kg}$ & 410 & EPA8270 \\
\hline 2.4-Dimethy! phenol & U & & 410 & $\mu \mathrm{g} / \mathrm{kg}$ & 410 & EPA8270 \\
\hline Dimethyl phthalate & $\mathbf{U}$ & & 410 & $\mu g / \mathrm{kg}$ & 410 & EPA8270 \\
\hline 2,4-Dinitrophenol & U & & 2,100 & $\mu \mathrm{g} / \mathrm{kg}$ & 2,100 & EPA8270 \\
\hline 2.4-Dinitrotoluene & $\mathbf{U}$ & & 410 & $\mu g / \mathrm{kg}$ & 410 & EPA8270 \\
\hline 2,6-Dinitrotoluene & $U$ & & 410 & $\mu \mathrm{g} / \mathrm{kg}$ & 410 & EPA8270 \\
\hline Di-n-octyl phthalate & $\mathbf{U}$ & & 410 & $\mu \mathrm{g} / \mathrm{kg}$ & 410 & EPA8270 \\
\hline Fluoranthene & $U$ & & 410 & $\mu \mathrm{g} / \mathrm{kg}$ & 410 & EPA8270 \\
\hline Fluorene & $\mathrm{U}$ & & 410 & $\mu \mathrm{g} / \mathrm{kg}$ & 410 & EPA8270 \\
\hline Hexachlorobenzene & $\mathbf{U}$ & & 410 & $\mu \mathrm{g} / \mathrm{kg}$ & 410 & EPA8270 \\
\hline Hexachlorobutadiene & $\mathrm{U}$ & & 410 & $\mu \mathrm{g} / \mathrm{kg}$ & 410 & EPA8270 \\
\hline Hexachlorocyclopentadiene & $\mathrm{U}$ & & 410 & $\mu \mathrm{g} / \mathrm{kg}$ & 410 & EPA8270 \\
\hline Hexachloroethane & $\mathrm{U}$ & & 410 & $\mu \mathrm{g} / \mathrm{kg}$ & 410 & EPA8270 \\
\hline Indeno(1,2,3-c,d)pyrene & $\mathrm{U}$ & & 410 & $\mu \mathrm{g} / \mathrm{kg}$ & 410 & EPA8270 \\
\hline Isophorone & $\mathrm{U}$ & & 410 & $\mu \mathrm{g} / \mathrm{kg}$ & 410 & EPA8270 \\
\hline 2-Methyl-4,6-dinitrophenol & $\mathbf{U}$ & & 2,100 & $\mu \mathrm{g} / \mathrm{kg}$ & 2,100 & EPA8270 \\
\hline 2-Methylnaphthalene & $\mathbf{U}$ & & 410 & $\mu \mathrm{g} / \mathrm{kg}$ & 410 & EPA8270 \\
\hline Naphthalene & $\mathbf{U}$ & & 410 & $\mu \mathrm{g} / \mathrm{kg}$ & 410 & EPA8270 \\
\hline o-Nitroaniline & $\mathbf{U}$ & & 2,100 & $\mu \mathrm{g} / \mathrm{kg}$ & 2.100 & EPA8270 \\
\hline m-Nitroaniline & $\mathbf{U}$ & & 2.100 & $\mu \mathrm{g} / \mathrm{kg}$ & 2.100 & EPA8270 \\
\hline p-Nitroaniline & $\mathbf{U}$ & & 2,100 & $\mu \mathrm{g} / \mathrm{kg}$ & $2 ; 100$ & EPA8270 \\
\hline Nitrobenzene & $U$ & & 410 & $\mu \mathrm{g} / \mathrm{kg}$ & 410 & EPA8270 \\
\hline 2-Nitrophenol & $\mathrm{U}$ & & 410 & $\mu \mathrm{g} / \mathrm{kg}$ & 410 & EPA8270 \\
\hline 4-Nitrophenol & U & & 2.100 & $\mu \mathrm{g} / \mathrm{kg}$ & 2.100 & EPA8270 \\
\hline N-Nitrosodiphenylamine & $\mathbf{U}$ & & 410 & $\mu \mathrm{g} / \mathrm{kg}$ & $410^{\circ}$ & EPA8270 \\
\hline N-Nitrosodipropylamine & $\mathbf{U}$ & & 410 & $\mu g / \mathrm{kg}$ & 410 & EPA8270 \\
\hline Pentachlorophenol & $\mathrm{U}$ & & 2.100 & $\mu g / \mathbf{k g}$ & 2,100 & EPA8270 \\
\hline Phenanthrene & $\mathbf{U}$ & & 410 & $\mu \mathrm{g} / \mathrm{kg}$ & 410 & EPA8270 \\
\hline Phenol & $\mathbf{U}$ & & 410 & $\mu g / \mathrm{kg}$ & 410 & EPA8270 \\
\hline Pyrene & U & & 410 & $\mu \mathrm{g} / \mathrm{kg}$ & 410 & EPA8270 \\
\hline 1,2,4-Trichlorobenzene & $\mathrm{U}$ & & 410 & $\mu \mathrm{g} / \mathrm{kg}$ & 410 & EPA8270 \\
\hline
\end{tabular}




\section{Constituent \\ RQ AQ B Result Unit \\ D. Limit Method}

$B / N / A$ Extractables (including PAH and phenols)

2,4,5-Trichlorophenol
2,4,6-Trichlorophenol
Unknown ketone

Volatile Organic Compounds

$\begin{array}{llllll}\mathrm{U} & & 2,100 & \mu \mathrm{g} / \mathrm{kg} & 2,100 & \text { EPA8270 } \\ \mathrm{U} & & 410 & \mu \mathrm{g} / \mathrm{kg} & 410 & \text { EPA8270 } \\ \mathrm{J} & \mathrm{NV} & 8,000 & \mu \mathrm{g} / \mathrm{kg} & & \text { EPA8270 }\end{array}$

13

5.6

6.3

6.3

13

6.3

6.3

6.3

13

13

6.3

13

6.3

6.3

6.3

6.3

6.3

6.3

6.3

6.3

6.3

6.3

13

13

13

6.3

6.3

6.3

6.3

6.3

6.3

6.3

13

6.3
EPA8240

EPA8240

EPA8240

EPA8240

EPA8240

EPA8240

EPA8240

EPA8240

EPA8240

EPA8240

EPA8240

EPA8240

EPA8240

EPA8240

EPA8240

EPA8240

EPA8240

EPA8240

EPA8240

EPA8240

EPA8240

EPA8240

EPA8240

EPA8240

- EPA8240

EPA8240

EPA8240

EPA8240

EPA8240

EPA8240

EPA8240

EPA8240

ERA8240
EPA8240

Pesticides/PCBs and Dioxins/Furans

Aldrin

alpha-Benzene hexachloride

beta-Benzene hexachloride

delta-Benzene hexachloride

alpha-Chlordane

gamma-Chlordane

p.p'-DDD
2.1

2.1

2.1

2.1

2.1

2.1

4.1 $\mu g / \mathrm{kg}$

$\mu \mathrm{g} / \mathrm{kg}$

$\mu \mathrm{g} / \mathrm{kg}$

$\mu \mathrm{g} / \mathrm{kg}$

$\mu \mathrm{g} / \mathrm{kg}$

$\mu \mathrm{g} / \mathrm{kg}$

$\mu \mathrm{g} / \mathrm{kg}$
2.1

EPA8080

EPA8080

EPA8080

EPA8080

EPA8080

EPA8080

EPA8080 
SAMPLE NAME: D431 1706 (Continued)

Sample ID: 100433

Constituent

RQ AQ B Result

Unit

D. Limit Method

Pesticides/PCBs and Dioxins/Furans

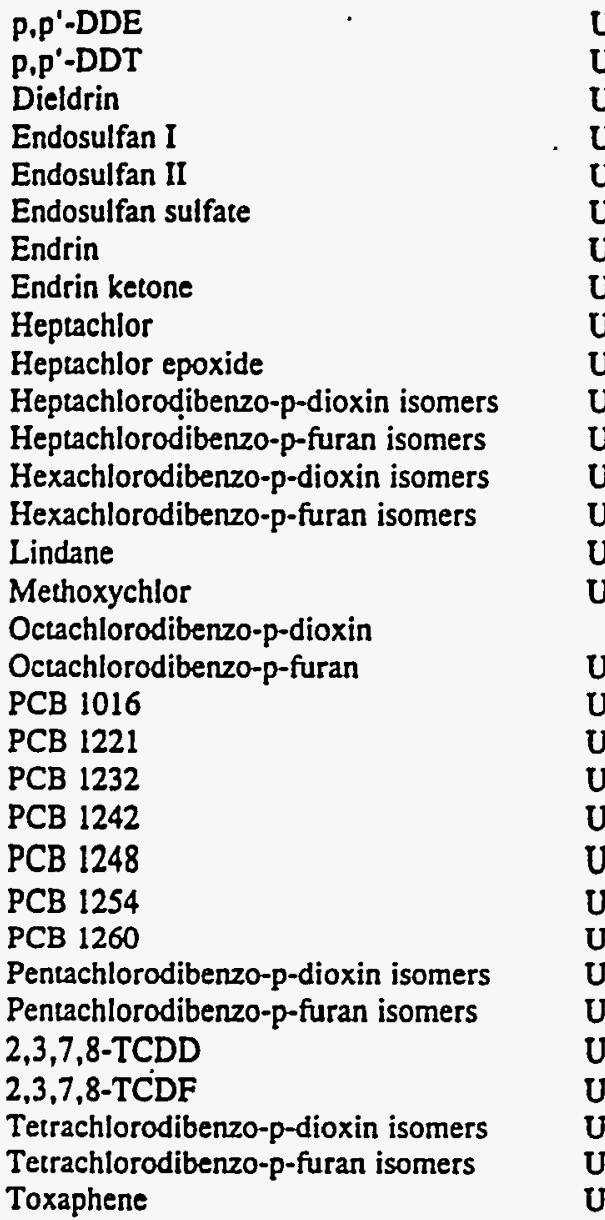

$\begin{array}{llll}4.1 & \mu \mathrm{g} / \mathrm{kg} & 4.1 & \text { EPA8080 } \\ 4.1 & \mu \mathrm{g} / \mathrm{kg} & 4.1 & \text { EPA8080 } \\ 4.1 & \mu \mathrm{g} / \mathrm{kg} & 4.1 & \text { EPA8080 } \\ 2.1 & \mu \mathrm{g} / \mathrm{kg} & 2.1 & \text { EPA8080 } \\ 4.1 & \mu \mathrm{g} / \mathrm{kg} & 4.1 & \text { EPA8080 } \\ 4.1 & \mu \mathrm{g} / \mathrm{kg} & 4.1 & \text { EPA8080 } \\ 4.1 & \mu \mathrm{g} / \mathrm{kg} & 4.1 & \text { EPA8080 } \\ 4.1 & \mu \mathrm{g} / \mathrm{kg} & 4.1 & \text { EPA8080 } \\ 2.1 & \mu \mathrm{g} / \mathrm{kg} & 2.1 & \text { EPA8080 } \\ 2.1 & \mu \mathrm{g} / \mathrm{kg} & 2.1 & \text { EPA8080 } \\ 0.20 & \mathrm{ng} / \mathrm{g} & 0.20 & \text { EPA8280 } \\ 0.10 & \mathrm{ng} / \mathrm{g} & 0.10 & \text { EPA8280 } \\ 0.20 & \mathrm{ng} / \mathrm{g} & 0.20 & \text { EPA8280 } \\ 0.10 & \mathrm{ng} / \mathrm{g} & 0.10 & \text { EPA8280 } \\ 2.1 & \mu \mathrm{g} / \mathrm{kg} & 2.1 & \text { EPA8080 } \\ 21 & \mu \mathrm{g} / \mathrm{kg} & 21 & \text { EPA8080 } \\ 1.4 & \mathrm{ng} / \mathrm{g} & 0.20 & \text { EPA8280 } \\ 0.10 & \mathrm{ng} / \mathrm{g} & 0.10 & \text { EPA8280 } \\ 41 & \mu \mathrm{g} / \mathrm{kg} & 41 & \text { EPA8080 } \\ 82 & \mu \mathrm{g} / \mathrm{kg} & 82 & \text { EPA8080 } \\ 41 & \mu \mathrm{g} / \mathrm{kg} & 41 & \text { EPA8080 } \\ 41 & \mu \mathrm{g} / \mathrm{kg} & 41 & \text { EPA8080 } \\ 41 & \mu \mathrm{g} / \mathrm{kg} & 41 & \text { EPA8080 } \\ 41 & \mu \mathrm{g} / \mathrm{kg} & 41 & \text { EPA8080 } \\ 41 & \mu \mathrm{g} / \mathrm{kg} & 41 & \text { EPA8080 } \\ 0.20 & \mathrm{ng} / \mathrm{g} & 0.20 & \text { EPA8280 } \\ 0.10 & \mathrm{ng} / \mathrm{g} & 0.10 & \text { EPA8280 } \\ 0.10 & \mathrm{ng} / \mathrm{g} & 0.10 & \text { EPA8280 } \\ 0.10 & \mathrm{ng} / \mathrm{g} & 0.10 & \text { EPA8280 } \\ 0.10 & \mathrm{ng} / \mathrm{g} & 0.10 & \text { EPA8280 } \\ 0.10 & \mathrm{ng} / \mathrm{g} & 0.10 & \text { EPA8280 } \\ 210 & \mu \mathrm{g} / \mathrm{kg} & 210 & \text { EPA8080 }\end{array}$

Radionuclide Indicators and Radionuclides

Gross alpha

Nonvolatile beta

Radium, total alpha-emitting

Tritium

$\begin{array}{llcl}6.8 \pm 5.9 & \mathrm{pCi} / \mathrm{g} & \$ 3.7 & \text { EPA900.0 } \\ 11 \pm 9.3 & \mathrm{pCi} / \mathrm{g} & 4.0 & \text { EPA900.0 } \\ 0.50 \pm 0.44 & \mathrm{pCi} / \mathrm{g} & 0.12 & \text { EPA903.0 } \\ 3.5 \pm 0.90 & \mathrm{pCi} / \mathrm{g} & 0.94 & \text { EPA906.0 }\end{array}$


SAMPLE NAME: D431 1801

. Location (SRS Coordinates): 18340.39E 66562.05N

Ground Elevation Above MSL: $122.4 \mathrm{ft}$

Depth of Core Interval: 0.00 to $0.50 \mathrm{ft}$

Sample Type: Normal

Sample Color: 5YR 4/4

Constituent

Physical Parameters

Cation exchange capacity

$\mathrm{pH}$

Specific conductance

Total organic carbon

Total Metals

Arsenic

Barium

Cadmium

Chromium

Copper

Lead

Manganese

Mercury

Nickel

Selenium

Silver
Sample ID: 100400

Sample Matrix: Soil

USC Soil Classification: SP

RQ AQ B Result Unit

$\begin{array}{llll}3.0 & \mathrm{meq} / 100 \mathrm{~g} & 2.0 & \text { EPA9080 } \\ 4.0 & \mathrm{pH} & 0.010 & \text { EPA9045 } \\ 40 & \mu \mathrm{S} / \mathrm{cm} & 1.0 & \text { EPA9050 } \\ 4.300 & \mathrm{mg} / \mathrm{kg} & 150 & \text { EPA415.1 }\end{array}$

Sample Moisture: Dry

Percent Solids: 91.20

D. Limit Method

$B / N / A$ Extractables (ịncluding $P A H$ and phenols)

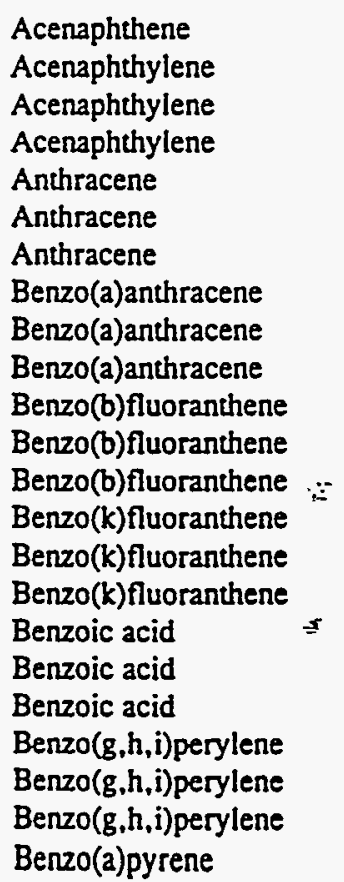

Acenaphthene

Acenaphthylene

Acenaphthylene

Acenaphthylene

Anthracene

Benzo(a)anthracene

Benzo(a)anthracene

Benzo(a)anthracene

Benzo(b)fluoranthene

Benzo(b)fluoranthen

Benzo(k)fluoranthene

Benzo(k)fluoranthene

Benzoic acid

Benzoic acid

Benzo(g,h,i)perylene

Benzo(a)pyrene

$\begin{array}{ll}U & 370 \\ U & 370 \\ U & 360 \\ U & 360 \\ U & 370 \\ U & 360 \\ U & 360 \\ U & 370 \\ U & 360 \\ U & 360 \\ U & 370 \\ U & 360 \\ U & 360 \\ U & 370- \\ U & 360 \\ U & 360 \\ J & 250 \\ J & 280 \\ J & 370 \\ U & 370 \\ U & 360 \\ U & 360 \\ U & 370\end{array}$

$\begin{array}{ll}0.97 & \text { EPA7060 } \\ 19 & \text { EPA6010 } \\ 0.46 & \text { EPA6010 } \\ 0.93 & \text { EPA6010 } \\ 2.3 & \text { EPA6010 } \\ 0.29 & \text { EPA7421 } \\ 1.4 & \text { EPA6010 } \\ 0.11 & \text { EPA7470 } \\ 3.7 & \text { EPA6010 } \\ 0.48 & \text { EPA7740 } \\ 0.93 & \text { EPA6010 }\end{array}$

3.2

0.46

3.9

2.3

3.4

56

0.11

3.7

0.48

0.93 $\mathrm{mg} / \mathrm{kg}$
$\mathrm{mg} / \mathrm{kg}$
$\mathrm{mg} / \mathrm{kg}$
$\mathrm{mg} / \mathrm{kg}$
$\mathrm{mg} / \mathrm{kg}$
$\mathrm{mg} / \mathrm{kg}$
$\mathrm{mg} / \mathrm{kg}$
$\mathrm{mg} / \mathrm{kg}$
$\mathrm{mg} / \mathrm{kg}$
$\mathrm{mg} / \mathrm{kg}$
$\mathrm{mg} / \mathrm{kg}$

$\mu \mathrm{g} / \mathrm{kg}$

$\mu \mathrm{g} / \mathrm{kg}$

$\mu \mathrm{g} / \mathrm{kg}$

$\mu \mathrm{g} / \mathrm{kg}$

$\mu \mathrm{g} / \mathrm{kg}$

$\mu \mathrm{g} / \mathrm{kg}$

$\mu \mathrm{g} / \mathrm{kg}$

$\mu \mathrm{g} / \mathrm{kg}$

$\mu \mathrm{g} / \mathrm{kg}$

$\mu \mathrm{g} / \mathrm{kg}$

$\mu g / \mathrm{kg}$

$\mu \mathrm{g} / \mathrm{kg}$

$\mu g / \mathrm{kg}$

$\mu \mathrm{g} / \mathrm{kg}$

$\mu g / \mathrm{kg}$

$\mu \mathrm{g} / \mathrm{kg}$

$\mu g / \mathrm{kg}$

$\mu g / \mathrm{kg}$

$\mu \mathrm{g} / \mathrm{kg}$

$\mu \mathrm{g} / \mathrm{kg}$

$\mu g / k g$

$\mu \mathrm{g} / \mathrm{kg}$

$\mu g / k g$
370

370

360

360

370

360

360

370

360

360

370

360

360

370

360

360

1,800

1,800

1,800

370 .

360

360

370
EPA8270

EPA8270

EPA8270

EPA8270

EPA8270

EPA8270

EPA8270

EPA8270

EPA8270

EPA8270

EPA8270

EPA8270

EPA8270

EPA8270

EPA8270

EPA8270

EPA8270

EPA8270

EPA8270

EPA8270

EPA8270

EPA8270

EPA8270 
SAMPLE NAME: D431 1801 (Continued)

Sample ID: 100400

Constituent

RQ AQ B Result

Unit

D. Limit Method

$B / N / A$ Extractables (including $P A H$ and phenols)

\begin{tabular}{|c|c|c|c|c|c|}
\hline Benzo(a)pyrene & $\mathrm{U}$ & 360 & $\mu g / \mathrm{kg}$. & 360 & EPA8270 \\
\hline Benzo(a)pyrene & $\mathbf{U}$ & 360 & $\mu \mathrm{g} / \mathrm{kg}$ & 360 & EPA8270 \\
\hline Benzyl alcohol & $\mathbf{U}$ & 370 & $\mu g / k g$ & 370 & EPA8270 \\
\hline Benzyl alcohol & $\mathbf{U}$ & 360 & $\mu g / \mathrm{kg}$ & 360 & EPA8270 \\
\hline Benzyl alcohol & $\mathrm{U}$ & 360 & $\mu g / k g$ & 360 & EPA8270 \\
\hline Bis(2-chloroethoxy) methane & $\mathbf{U}$ & 370 & $\mu \mathrm{g} / \mathrm{kg}$ & 370 & EPA8270 \\
\hline Bis(2-chloroethoxy) methane & $\mathbf{U}$ & 360 & $\mu \mathrm{g} / \mathrm{kg}$ & 360 & EPA8270 \\
\hline Bis(2-chloroethoxy) methane & U & 360 & $\mu \mathrm{g} / \mathrm{kg}$ & 360 & EPA8270 \\
\hline Bis(2-chloroethyl) ether & $\mathbf{U}$ & 370 & $\mu \mathrm{g} / \mathrm{kg}$ & 370 & EPA8270 \\
\hline Bis(2-chloroisopropyl) ether & U & 370 & $\mu \mathrm{g} / \mathrm{kg}$ & 370 & EPA8270 \\
\hline Bis(2-chloroethyl) ether & $\mathbf{U}$ & 360 & $\mu \mathrm{g} / \mathrm{kg}$ & 360 & EPA8270 \\
\hline Bis(2-chloroisopropyl) ether & $U$ & 360 & $\mu \mathrm{g} / \mathrm{kg}$ & 360 & EPA8270 \\
\hline Bis(2-chloroethyl) ether & $\boldsymbol{U}$ & 360 & $\mu \mathrm{g} / \mathrm{kg}$ & 360 & EPA8270 \\
\hline Bis(2-chloroisopropyl) ether & $\mathrm{U}$ & 360 & $\mu g / \mathrm{kg}$ & 360 & EPA8270 \\
\hline Bis(2-ethylhexyl) phthalate & $\mathbf{U}$ & 370 & $\mu \mathrm{g} / \mathrm{kg}$ & 370 & EPA8270 \\
\hline Bis(2-ethylhexyl) phthalate & U & 360 & $\mu \mathrm{g} / \mathrm{kg}$ & 360 & EPA8270 \\
\hline Bis(2-ethylhexyl) phthalate & $U$ & 360 & $\mu \mathrm{g} / \mathrm{kg}$ & 360 & EPA8270 \\
\hline 4-Bromophenyl phenyl ether & $\mathrm{U}$ & 370 & $\mu \mathrm{g} / \mathrm{kg}$ & 370 & EPA8270 \\
\hline 4-Bromophenyl phenyl ether & $\mathbf{U}$ & 360 & $\mu \mathrm{g} / \mathrm{kg}$ & 360 & EPA8270 \\
\hline 4-Bromophenyl phenyl ether & $\mathrm{U}$ & 360 & $\mu \mathrm{g} / \mathrm{kg}$ & 360 & EPA8270 \\
\hline Butylbenzyl phthalate & $\mathbf{U}$ & 370 & $\mu \mathrm{g} / \mathrm{kg}$ & 370 & EPA8270 \\
\hline Butylbenzyl phthalate & $\mathbf{U}$ & 360 & $\mu g / \mathrm{kg}$ & 360 & EPA8270 \\
\hline Burylbenzyl phthalate & $\mathbf{U}$ & 360 & $\mu \mathrm{g} / \mathrm{kg}$ & 360 & EPA8270 \\
\hline 4-Chloroaniline & $\mathrm{U}$ & 370 & $\mu \mathrm{g} / \mathrm{kg}$ & 370 & EPA8270 \\
\hline 4-Chloroaniline & $\mathbf{U}$ & 360 & $\mu \mathrm{g} / \mathrm{kg}$ & 360 & EPA8270 \\
\hline 4-Chloroaniline & $\mathbf{U}$ & 360 & $\mu \mathrm{g} / \mathrm{kg}$ & 360 & EPA8270 \\
\hline 4-Chloro-m-cresol & $\mathrm{U}$ & 370 & $\mu \mathrm{g} / \mathrm{kg}$ & 370 & EPA8270 \\
\hline 2-Chloronaphthalene & $\mathbf{U}$ & 370 & $\mu \mathrm{g} / \mathrm{kg}$ & 370 & EPA8270 \\
\hline 2-Chloronaphthalene & $\mathrm{U}$ & 360 & $\mu \mathrm{g} / \mathrm{kg}$ & 360 & EPA8270 \\
\hline 2-Chloronaphthalene & $\mathbf{U}$ & 360 & $\mu \mathrm{g} / \mathrm{kg}$ & 360 & EPA8270 \\
\hline 2-Chlorophenol & $\mathrm{U}$ & 370 & $\mu g / \mathrm{kg}$ & 370 & EPA8270 \\
\hline 4-Chlorophenyl phenyl ether & $\mathrm{U}$ & 370 & $\mu g / k g$ & 370 & EPA8270 \\
\hline 4-Chlorophenyl phenyl ether & $\mathbf{U}$ & 360 & $\mu g / k g$ & 360 & EPA8270 \\
\hline 4-Chlorophenyl phenyl ether & $\mathrm{U}$ & 360 & $\mu \mathrm{g} / \mathrm{kg}$ & 360 & EPA8270 \\
\hline Chrysene & $\mathbf{U}$ & 370 & $\mu \mathrm{g} / \mathrm{kg}$ & 370 & EPA8270 \\
\hline Chrysene & $\mathbf{U}$ & 360 & $\mu g / k g$ & 360 & EPA8270 \\
\hline Chrysene & $\mathbf{U}$ & 360 & $\mu \mathrm{g} / \mathrm{kg}$ & 360 & EPA8270 \\
\hline o-Cresol (2-Mechylphenol) & $U$ & 370 & $\mu g / k g$ & 370 & EPA8270 \\
\hline o-Cresol (2-Methylphenol) & $\mathbf{U}$ & 360 & $\mu \mathrm{g} / \mathrm{kg}$ & 360 & EPA8270 \\
\hline o-Cresol (2-Methylpheriol) & $\mathrm{U}$ & 360 & $\mu \mathrm{g} / \mathrm{kg}$ & 360 & EPA8270 \\
\hline p-Cresol (4-Methylphenol) & $\mathbf{U}$ & 370 & $\mu g / k g$ & 370 & EPA8270 \\
\hline p-Cresol (4-Methylphenol) & $\mathbf{U}$ & 360 & $\mu \mathrm{g} / \mathrm{kg}$ & 360 & EPA8270 \\
\hline p-Cresol (4-Methylphenol) & $U$ & 360 & $\mu \mathrm{g} / \mathrm{kg}$ & 360 & EPA8270 \\
\hline Dibenz $(\mathrm{a}, \mathrm{h})$ anthracene & $\mathbf{U}$ & 370 & $\mu \mathrm{g} / \mathrm{kg}$ & $370_{2}$ & EPA8270 \\
\hline Dibenz $(a, h)$ anthracene & $\mathbf{U}$ & 360 & $\mu \mathrm{g} / \mathrm{kg}$ & 360 & EPA8270 \\
\hline Dibenz $(a, h)$ anthracene & $\mathbf{U}$ & 360 & $\mu \mathrm{g} / \mathrm{kg}$ & 360 & EPA8270 \\
\hline Dibenzofuran & $\mathbf{U}$ & 370 & $\mu \mathrm{g} / \mathrm{kg}$ & 370 & EPA8270 \\
\hline Dibenzofuran & $\mathbf{U}$ & 360 & $\mu \mathrm{g} / \mathrm{kg}$ & 360 & EPA8270 \\
\hline Dibenzofuran & $\mathbf{U}$ & 360 & $\mu \mathrm{g} / \mathrm{kg}$ & 360 & EPA8270 \\
\hline Di-n-butyl phthalate & UJ $\quad$ V & 140 & $\mu \mathrm{g} / \mathrm{kg}$ & 370 & EPA8270 \\
\hline
\end{tabular}


SAMPLE NAME: D431 1801 (Continued)

Sample D: 100400

\section{Constituent}

RQ AQ B Result

Unit

D. Limit Method

$B / N / A$ Extractables (including $P A H$ and phenols)

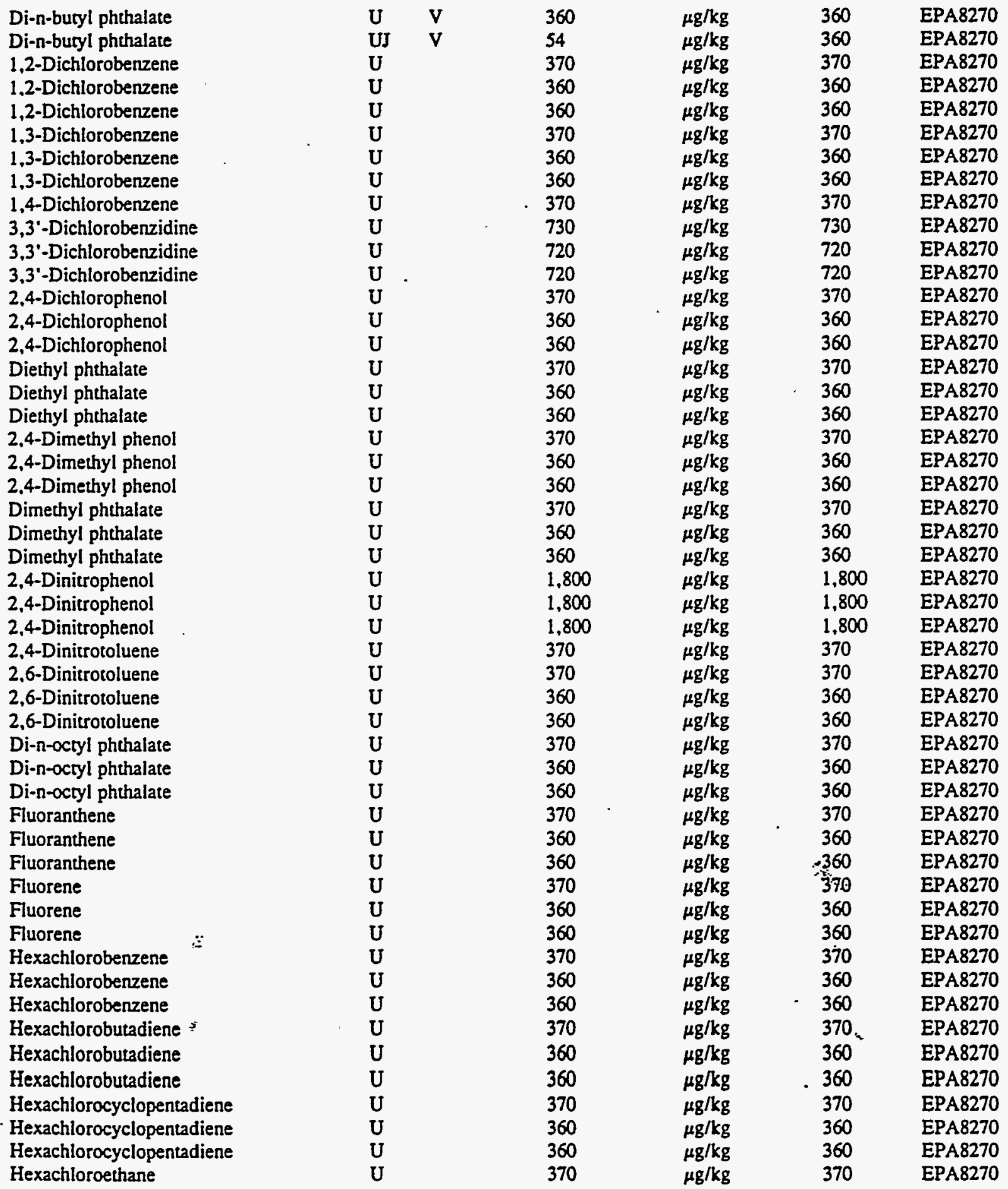


SAMPLE NAME: D431 1801 (Continued)

RQ AQ B Result

Constituent

RQ AQ

$B / N / A$ Extractables (including $P A H$ and phenols)
Sample ID: 100400

Unit

D. Limit Method

\begin{tabular}{|c|c|c|c|}
\hline Hexachloroethane & $\mathbf{U}$ & & 360 \\
\hline Hexachloroecthane & $\mathbf{U}$ & & 360 \\
\hline Indeno(1,2,3-c,d)pyrene & $\mathrm{U}$ & & 370 \\
\hline Indeno(1.2,3-c,d)pyrene & U & & 360 \\
\hline Indeno(1,2,3-c,d)pyrene & U & & 360 \\
\hline Isophorone & $\mathbf{U}$ & & 370 \\
\hline Isophorone & $\mathrm{U}$ & & 360 \\
\hline Isophorone & $\mathbf{U}$ & & 360 \\
\hline 2-Methyl-4.6-dinitrophenol & $\mathrm{U}$ & & 1.800 \\
\hline 2-Methyl-4,6-dinitrophenol & $\mathbf{U}$ & & 1,800 \\
\hline 2-Methyl-4,6-dinitrophenol & $\mathrm{U}$ & & 1,800 \\
\hline 2-Methylnaphthalene & U & & 370 \\
\hline 2-Methylnaphthalene & $\mathrm{U}$ & & 360 \\
\hline 2-Methylnaphthalene & $\mathrm{U}$ & & 360 \\
\hline Naphthalene & $\mathrm{U}$ & & 370 \\
\hline Naphthalene & $\mathrm{U}$ & & 360 \\
\hline Naphthalene & U & & 360 \\
\hline 0 -Nitroaniline & $U$ & & 1,800 \\
\hline o-Nitroaniline & $\mathbf{U}$ & & 1,800 \\
\hline o-Nitroaniline & $U$ & & 1,800 \\
\hline m-Nitroaniline & U & & 1,800 \\
\hline m-Nitroaniline & $\mathbf{U}$ & & 1,800 \\
\hline m-Nitroaniline & $\mathbf{U}$ & & 1,800 \\
\hline p-Nitroaniline & $\mathbf{U}$ & & 1,800 \\
\hline p-Nitroaniline & U & & 1,800 \\
\hline p-Nitroaniline & U & & 1,800 \\
\hline Nitrobenzene & $\mathrm{U}$ & & 370 \\
\hline Nitrobenzene & $\mathrm{U}$ & & 360 \\
\hline Nitrobenzene & $\mathrm{U}$ & & 360 \\
\hline 2-Nitrophenol & $\mathrm{U}$ & & 370 \\
\hline 2-Nitrophenol & $\mathbf{U}$ & & 360 \\
\hline 2-Nitrophenol & $\mathbf{U}$ & & 360 \\
\hline 4-Nitrophenol & $\mathbf{U}$ & & 1,800 \\
\hline N-Nitrosodiphenylamine & U & & 370 \\
\hline N-Nitrosodiphenylamine & $\mathbf{U}$ & & 360 \\
\hline N-Nitrosodiphenylamine & $\mathbf{U}$ & & 360 \\
\hline N-Nitrosodipropylamine & $\mathbf{U}$ & & 370 \\
\hline Pentachlorophenol & UJ & $\mathrm{H}$ & 1.800 \\
\hline Phenanthrene & $\mathbf{U}$ & & 370 \\
\hline Phenanthrene & $\vec{U}$ & & 360 \\
\hline Phenanthrene & $\mathbf{U}$ & & 360 \\
\hline Phenol & $\mathbf{U}$ & & 370 \\
\hline Pyrene & $\mathrm{U}$ & & 370 \\
\hline 1,2,4-Trichlorobenzene & $\mathbf{U}$ & & 370 \\
\hline 2,4,5-Trichlorophenol & $\mathbf{U}$ & & 1,800 \\
\hline 2,4,5-Trichlorophenol & $\mathbf{U}$ & & 1,800 \\
\hline 2,4,5-Trichlorophenol & $U$ & & 1,800 \\
\hline 2,4,6-Trichlorophenol & $\mathbf{U}$ & & 370 \\
\hline 2,4,6-Trichlorophenol & $\mathbf{U}$ & & 360 \\
\hline 2,4,6-Trichlorophenol & $\mathbf{U}$ & & 360 \\
\hline
\end{tabular}

\begin{tabular}{|c|c|c|}
\hline$\mu g / \mathrm{kg}$ & 360 & EPA8270 \\
\hline$\mu \mathrm{g} / \mathrm{kg}$ & 360 & EPA8270 \\
\hline$\mu g / k g$ & 370 & EPA8270 \\
\hline$\mu \mathrm{g} / \mathrm{kg}$ & 360 & EPA8270 \\
\hline$\mu \mathrm{g} / \mathrm{kg}$ & 360 & EPA8270 \\
\hline$\mu g / k g$ & 370 & EPA8270 \\
\hline$\mu \mathrm{g} / \mathrm{kg}$ & 360 & EPA8270 \\
\hline$\mu \mathrm{g} / \mathrm{kg}$ & 360 & EPA8270 \\
\hline$\mu g / \mathrm{kg}$ & 1,800 & EPA8270 \\
\hline$\mu \mathrm{g} / \mathrm{kg}$ & 1,800 & EPA8270 \\
\hline$\mu \mathrm{g} / \mathrm{kg}$ & 1.800 & EPA8270 \\
\hline$\mu \mathrm{g} / \mathrm{kg}$ & 370 & EPA8270 \\
\hline$\mu \mathrm{g} / \mathrm{kg}$ & 360 & EPA8270 \\
\hline$\mu g / \mathrm{kg}$ & 360 & EPA8270 \\
\hline$\mu \mathrm{g} / \mathrm{kg}$ & 370 & EPA8270 \\
\hline$\mu \mathrm{g} / \mathrm{kg}$ & 360 & EPA8270 \\
\hline$\mu g / \mathrm{kg}$ & 360 & EPA8270 \\
\hline$\mu \mathrm{g} / \mathrm{kg}$ & 1,800 & EPA8270 \\
\hline$\mu \mathrm{g} / \mathrm{kg}$ & 1,800 & EPA8270 \\
\hline$\mu \mathrm{g} / \mathrm{kg}$ & 1,800 & EPA8270 \\
\hline$\mu g / \mathrm{kg}$ & 1.800 & EPA8270 \\
\hline$\mu \mathrm{g} / \mathrm{kg}$ & 1,800 & EPA8270 \\
\hline$\mu \mathrm{g} / \mathrm{kg}$ & 1,800 & EPA8270 \\
\hline$\mu \mathrm{g} / \mathrm{kg}$ & 1,800 & EPA8270 \\
\hline$\mu \mathrm{g} / \mathrm{kg}$ & 1,800 & EPA8270 \\
\hline$\mu g / \mathrm{kg}$ & 1,800 & EPA8270 \\
\hline$\mu \mathrm{g} / \mathrm{kg}$ & 370 & EPA8270 \\
\hline$\mu \mathrm{g} / \mathrm{kg}$ & 360 & EPA8270 \\
\hline$\mu \mathrm{g} / \mathrm{kg}$ & 360 & EPA8270 \\
\hline$\mu \mathrm{g} / \mathrm{kg}$ & 370 & EPA8270 \\
\hline$\mu g / \mathrm{kg}$ & 360 & EPA8270 \\
\hline$\mu \mathrm{g} / \mathrm{kg}$ & 360 & EPA8270 \\
\hline$\mu g / \mathrm{kg}$ & 1.800 & EPA8270 \\
\hline$\mu \mathrm{g} / \mathrm{kg}$ & 370 & EPA8270 \\
\hline$\mu \mathrm{g} / \mathrm{kg}$ & 360 & EPA8270 \\
\hline$\mu \mathrm{g} / \mathrm{kg}$ & 360 & EPA8270 \\
\hline$\mu \mathrm{g} / \mathrm{kg}$ & 370 & EPA8270 \\
\hline$\mu \mathrm{g} / \mathrm{kg}$ & 1,800 & EPA8270 \\
\hline$\mu g / \mathrm{kg}$ & 370 & EPA8270 \\
\hline$\mu \mathrm{g} / \mathrm{kg}$ & 360 & EPA8270 \\
\hline$\mu \mathrm{g} / \mathrm{kg}$ & 360 & EPA8270 \\
\hline$\mu g / \mathrm{kg}$ & 370 & EPA8270 \\
\hline$\mu g / \mathrm{kg}$ & 370 & EPA8270 \\
\hline$\mu \mathrm{g} / \mathrm{kg}$ & $370^{\prime}$ & EPA8270 \\
\hline$\mu \mathrm{g} / \mathrm{kg}$ & 1,800 & EPA8270 \\
\hline$\mu \mathrm{g} / \mathrm{kg}$ & 1,800 & EPA8270 \\
\hline$\mu g / \mathrm{kg}$ & 1.800 & EPA8270 \\
\hline$\mu \mathrm{g} / \mathrm{kg}$ & 370 & EPA8270 \\
\hline$\mu \mathrm{g} / \mathrm{kg}$ & 360 & EPA8270 \\
\hline$\mu \mathrm{g} / \mathrm{kg}$ & 360 & EPA8270 \\
\hline
\end{tabular}




\section{Constituent \\ RQ AQ B Result Unit \\ D. Limit Method}

$B / N / A$ Extractables (including $P A H$ and phenols)

Unknown adipate

Unknown hydrocarbon

Unknown hydrocarbon

Unknown hydrocarbon

Unknown

Unknown

Unknown ester

Unknown ketone

Volatile Organic Compounds

$\begin{array}{lll}\mathbf{J} & \mathbf{N} & 300 \\ \mathrm{~J} & \mathbf{N} & 400 \\ \mathrm{~J} & \mathbf{N} & 1,000 \\ \mathrm{~J} & \mathbf{N} & 2,000 \\ \mathrm{~J} & \mathbf{N} & 200 \\ \mathrm{~J} & \mathbf{N} & 200 \\ \mathrm{~J} & \mathrm{NV} & 300 \\ \mathrm{~J} & \mathrm{NV} & 6,000\end{array}$

$\mu \mathrm{g} / \mathrm{kg}$.

$\mu \mathrm{g} / \mathrm{kg}$

$\mu \mathrm{g} / \mathrm{kg}$

$\mu \mathrm{g} / \mathrm{kg}$

$\mu \mathrm{g} / \mathrm{kg}$

$\mu g / k g$

$\mu \mathrm{g} / \mathrm{kg}$

$\mu \mathrm{g} / \mathrm{kg}$

$\mu \mathrm{g} / \mathrm{kg}$

$\mu \mathrm{g} / \mathrm{kg}$

$\mu \mathrm{g} / \mathrm{kg}$

$\mu \mathrm{g} / \mathrm{kg}$

$\mu \mathrm{g} / \mathrm{kg}$

$\mu g / k g$

$\mu \mathrm{g} / \mathrm{kg}$

$\mu \mathrm{g} / \mathrm{kg}$

$\mu g / \mathrm{kg}$

$\mu \mathrm{g} / \mathrm{kg}$

$\mu \mathrm{g} / \mathrm{kg}$

$\mu \mathrm{g} / \mathrm{kg}$

$\mu \mathrm{g} / \mathrm{kg}$

$\mu \mathrm{g} / \mathrm{kg}$

$\mu \mathrm{g} / \mathrm{kg}$

$\mu \mathrm{g} / \mathrm{kg}$

$\mu \mathrm{g} / \mathrm{kg}$

$\mu \mathrm{g} / \mathrm{kg}$

$\mu \mathrm{g} / \mathrm{kg}$

$\mu \mathrm{g} / \mathrm{kg}$

$\mu \mathrm{g} / \mathrm{kg}$

$\mu \mathrm{g} / \mathrm{kg}$

$\mu \mathrm{g} / \mathrm{kg}$

$\mu \mathrm{g} / \mathrm{kg}$

$\mu g / k g$

$\mu \mathrm{g} / \mathrm{kg}$

$\mu \mathrm{g} / \mathrm{kg}$

$\mu \mathrm{g} / \mathrm{kg}$

$\mu \mathrm{g} / \mathrm{kg}$

$\mu \mathrm{g} / \mathrm{kg}$

$\mu \mathrm{g} / \mathrm{kg}$

$\mu \mathrm{g} / \mathrm{kg}$

$\mu \mathrm{g} / \mathrm{kg}$

$\mu \mathrm{g} / \mathrm{kg}$

11

5.6
EPA8270

EPA8270

EPA8270

EPA8270

EPA8270

EPA8270

EPA8270

EPA8270

1,1,1-Trichloroethane

Trichloroethylene

Vinyl acetate

Xylenes

$\begin{array}{ll}11 & \text { EPA8240 } \\ 5.6 & \text { EPA8240 } \\ 5.6 & \text { EPA8240 } \\ 5.6 & \text { EPA8240 } \\ 11 & \text { EPA8240 } \\ 5.6 & \text { EPA8240 } \\ 5.6 & \text { EPA8240 } \\ 5.6 & \text { EPA8240 } \\ 11 & \text { EPA8240 } \\ 11 & \text { EPA8240 } \\ 5.6 & \text { EPA8240 } \\ 11 & \text { EPA8240 } \\ 5.6 & \text { EPA8240 } \\ 5.6 & \text { EPA8240 } \\ 5.6 & \text { EPA8240 } \\ 5.6 & \text { EPA8240 } \\ 5.6 & \text { EPA8240 } \\ 5.6 & \text { EPA8240 } \\ 5.6 & \text { EPA8240 } \\ 5.6 & \text { EPA8240 } \\ 5.6 & \text { EPA8240 } \\ 5.6 & \text { EPA8240 } \\ 11 & \text { EPA8240 } \\ 11 & \text { EPA8240 } \\ 11 & \text { EPA8240 } \\ 5.6 & \text { EPA8240 } \\ 5.6 & \text { EPA8240 } \\ 5.6 & \text { EPA8240 } \\ 5.6 & \text { EPA8240 } \\ 5.6 & \text { EPA8240 } \\ 5.6 & \text { EPA8240 } \\ 5.6 & \text { EPA8240 } \\ 11 & \text { EPA8240 } \\ 5.6 & \text { EPA8240 }\end{array}$


Pesticides/PCBs and Dioxins/Furans

$\begin{array}{ll}\text { Aldrin } & \mathrm{U} \\ \text { alpha-Benzene hexachloride } & \mathrm{U} \\ \text { beta-Benzene hexachloride } & \mathrm{U} \\ \text { delta-Benzene hexachloride } & \mathrm{U} \\ \text { alpha-Chlordane } & \mathrm{U} \\ \text { gamma-Chlordane } & \mathrm{U} \\ \text { p,p'-DDD } & \mathrm{U} \\ \text { p,p'-DDE } & \mathrm{J} \\ \text { p,p'-DDT } & \mathrm{J} \\ \text { Dieldrin } & \mathrm{U} \\ \text { Endosulfan I } & \mathrm{U} \\ \text { Endosulfan II } & \mathrm{U} \\ \text { Endosulfan sulfate } & \mathrm{U} \\ \text { Endrin } & \mathrm{U} \\ \text { Endrin ketone } & \mathrm{U} \\ \text { Heptachlor } & \mathrm{U} \\ \text { Heptachlor epoxide } & \mathrm{U} \\ \text { Heptachlorodibenzo-p-dioxin isomers } & \mathrm{U} \\ \text { Heptachlorodibenzo-p-furan isomers } & \mathrm{U} \\ \text { Hexachlorodibenzo-p-dioxin isomers } & \mathrm{U} \\ \text { Hexachlorodibenzo-p-furan isomers } & \mathrm{U} \\ \text { Lindane } & \mathrm{U} \\ \text { Methoxychlor } & \mathrm{U} \\ \text { Octachlorodibenzo-p-dioxin } & \mathrm{J} \\ \text { Octachlorodibenzo-p-furan } & \mathrm{U} \\ \text { PCB 1016 } & \mathrm{U} \\ \text { PCB 1221 } & \mathrm{U} \\ \text { PCB 1232 } & \mathrm{U} \\ \text { PCB 1242 } & \mathrm{U} \\ \text { PCB 1248 } & \mathrm{U} \\ \text { PCB 1254 } & \mathrm{U} \\ \text { PCB 1260 } & \mathrm{U} \\ \text { Pentachlorodibenzo-p-dioxin isomers } & \mathrm{U} \\ \text { Pentachlorodibenzo-p-furan isomers } & \mathrm{U} \\ \text { 2,3,7,8-TCDD } & \mathrm{U} \\ \text { 2,3,7,8-TCDF } & \mathrm{U} \\ \text { Tetrachlorodibenzo-p-dioxin isomers } & \mathrm{U} \\ \text { Tetrachlorodibenzo-p-furan isomers } & \mathrm{U} \\ \text { Toxaphene } & \mathrm{U} \\ & \\ & \end{array}$

$\begin{array}{ll}U & 1.8 \\ U & 1.8 \\ U & 1.8 \\ U & 1.8 \\ U & 1.8 \\ U & 1.8 \\ U & 3.6 \\ J & 1.1 \\ J & 1.1 \\ U & 3.6 \\ U & 1.8 \\ U & 3.6 \\ U & 3.6 \\ U & 3.6 \\ U & 3.6 \\ U & 1.8 \\ U & 1.8 \\ U & 0.20 \\ U & 0.20 \\ U & 0.20 \\ U & 0.10 \\ U & 1.8 \\ U & 18 \\ J & 0.10 \\ U & 0.10 \\ U & 36 \\ U & 72 \\ U & 36 \\ U & 36 \\ U & 36 \\ U & 36 \\ U & 36 \\ U & 0.20 \\ U & 0.20 \\ U & 0.10 \\ U & 0.20 \\ U & 0.10 \\ U & 180 \\ U & \\ U & \end{array}$

Radionuclide Indicators and Radionuclides

$\begin{array}{ll}7.8 \pm 6.9 & \mathrm{pCi} / g \\ 11 \pm 7.6 & \mathrm{pCi} / \mathrm{g} \\ 17 \pm 11 & \mathrm{pCi} / \mathrm{g} \\ 17 \pm 12 & \mathrm{pCi} / \mathrm{g} \\ 1.3 \pm 1.2 & \mathrm{pCi} / \mathrm{g} \\ 1.0 \pm 0.91 & \mathrm{pCi} / \mathrm{g} \\ 1.7 & \mathrm{pCi} / \mathrm{g} \\ 2.7 & \mathrm{pCi} / \mathrm{g}\end{array}$

\begin{tabular}{|c|c|c|}
\hline$\mu \mathrm{g} / \mathrm{kg}$ & 1.8 & EPA8080 \\
\hline$\mu \mathrm{g} / \mathrm{kg}$ & 1.8 & EPA8080 \\
\hline$\mu \mathrm{g} / \mathrm{kg}$ & 1.8 & EPA8080 \\
\hline$\mu \mathrm{g} / \mathrm{kg}$ & 1.8 & EPA8080 \\
\hline$\mu \mathrm{g} / \mathrm{kg}$ & 1.8 & EPA8080 \\
\hline$\mu \mathrm{g} / \mathrm{kg}$ & 1.8 & EPA8080 \\
\hline$\mu \mathrm{g} / \mathrm{kg}$ & 3.6 & EPA 8080 \\
\hline$\mu g / k g$ & 3.6 & EPA8080 \\
\hline$\mu \mathrm{g} / \mathrm{kg}$ & 3.6 & EPA8080 \\
\hline$\mu g / k g$ & 3.6 & EPA8080 \\
\hline$\mu \mathrm{g} / \mathrm{kg}$ & 1.8 & EPA8080 \\
\hline$\mu \mathrm{g} / \mathrm{kg}$ & 3.6 & EPA8080 \\
\hline$\mu \mathrm{g} / \mathrm{kg}$ & 3.6 & EPA8080 \\
\hline$\mu g / \mathrm{kg}$ & 3.6 & EPA8080 \\
\hline$\mu g / \mathrm{kg}$ & 3.6 & EPA8080 \\
\hline$\mu \mathrm{g} / \mathrm{kg}$ & 1.8 & EPA8080 \\
\hline$\mu \mathrm{g} / \mathrm{kg}$ & 1.8 & EPA8080 \\
\hline $\mathrm{ng} / \mathrm{g}$ & 0.20 & EPA8280 \\
\hline $\mathrm{ng} / \mathrm{g}$ & 0.20 & EPA8280 \\
\hline $\mathrm{ng} / \mathrm{g}$ & 0.20 & EPA8280 \\
\hline $\mathrm{ng} / \mathrm{g}$ & 0.10 & EPA8280 \\
\hline$\mu \mathrm{g} / \mathrm{kg}$ & 1.8 & EPA8080 \\
\hline$\mu \mathrm{g} / \mathrm{kg}$ & 18 & EPA8080 \\
\hline $\mathrm{ng} / \mathrm{g}$ & 0.10 & EPA8280 \\
\hline $\mathrm{ng} / \mathrm{g}$ & 0.10 & EPA8280 \\
\hline$\mu \mathrm{g} / \mathrm{kg}$ & 36 & EPA8080 \\
\hline$\mu \mathrm{g} / \mathrm{kg}$ & 72 & EPA 8080 \\
\hline$\mu \mathrm{g} / \mathrm{kg}$ & 36 & EPA8080 \\
\hline$\mu \mathrm{g} / \mathrm{kg}$ & 36 & EPA8080 \\
\hline$\mu \mathrm{g} / \mathrm{kg}$ & 36 & EPA8080 \\
\hline$\mu \mathrm{g} / \mathrm{kg}$ & 36 & EPA8080 \\
\hline$\mu g / \mathrm{kg}$ & 36 & EPA8080 \\
\hline $\mathrm{ng} / \mathrm{g}$ & 0.20 & EPA8280 \\
\hline $\mathrm{ng} / \mathrm{g}$ & 0.20 & EPA8280 \\
\hline $\mathrm{ng} / \mathrm{g}$ & 0.10 & EPA8280 \\
\hline$n g / g$ & 0.20 & EPA8280 \\
\hline $\mathrm{ng} / \mathrm{g}$ & 0.10 & EPA8280 \\
\hline $\mathrm{ng} / \mathrm{g}$ & 0.20 & EPA8280 \\
\hline$\mu \mathrm{g} / \mathrm{kg}$ & 180 & EPA8080 \\
\hline
\end{tabular}

$\mu \mathrm{g} / \mathrm{kg}$
EPA8080

EPA8080

EPA8080

EPA8080

EPA8080

PA8080

EPA8080

PA8080

EPA8080

EPA8280

EPA8280

EPA8080

EPA8280

EPA8280

EPA8080

EPA8080

EPA8080

EPA8280

EPA8280

EPA8280

EPA8280

PA8080
EPA8080
Gross alpha

Gross alpha

Nonvolatile beta

Nonvolatile beta

Radium, total alpha-emitting

Radium, total alpha-emitting

Tritium

Tritium

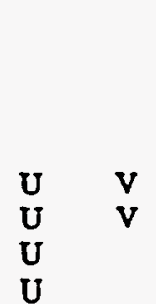

$\mathrm{v}$

v 
SAMPLE NAME: D43ī 1802

Sample ID: 100401

Location (SRS Coordinates): $18340.39 \mathrm{E}$ 66562.05N

Ground Elevation Above MSL: $122.4 \mathrm{ft}$

Depth of Core Interval: 0.50 to $2.00 \mathrm{ft}$

Sample Type: Normal

Sample Color: SYR 4/4

Constituent

RQ AQ B Result Unit

Sample Matrix: Soil

USC Soil Classification: SP

Sample Moisture: Damp

Percent Solids: 90.90

D. Limit Method

Total Metals

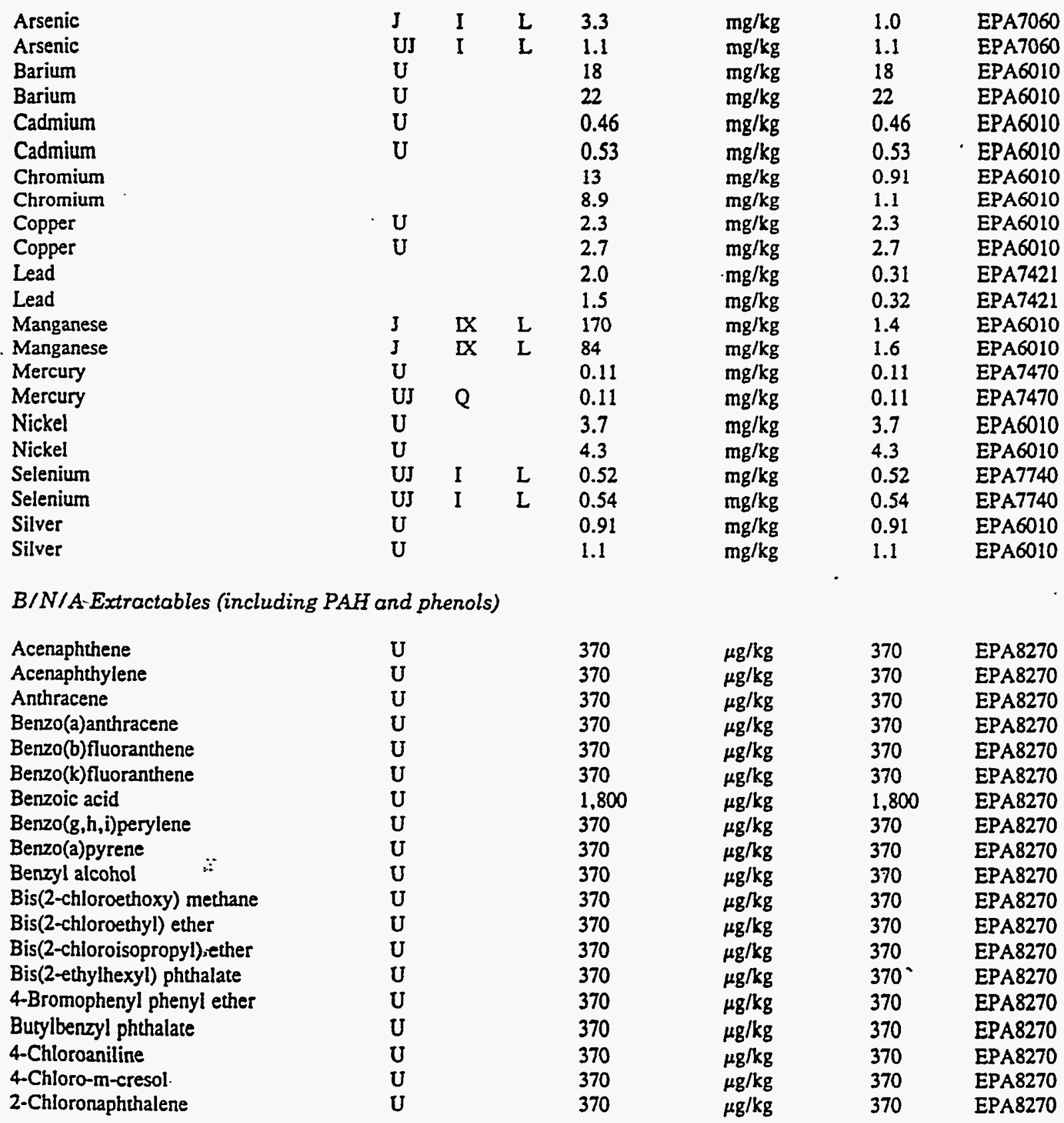


SAMPLE NAME: D431 1802 (Continued)

Sample ID: 100401

Constituent RQ AQ B Result Unit D. Limit Method

$B / N / A$ Extractables (including $P A H$ and phenols)

\begin{tabular}{|c|c|c|c|c|c|c|}
\hline 2-Chlorophenol & U & & 370 & $\mu g / \mathrm{kg}$ & 370 & EPA8270 \\
\hline 4.Chlorophenyl phenyl ether & $\mathbf{U}$ & & 370 & $\mu g / \mathrm{kg}$ & 370 & EPA8270 \\
\hline Chrysene & $\mathbf{U}$ & & 370 & $\mu \mathrm{g} / \mathrm{kg}$ & 370 & EPA8270 \\
\hline a-Cresol (2-Methylphenol) & $\mathbf{U}$ & & 370 & $\mu \mathrm{g} / \mathrm{kg}$ & 370 & EPA8270 \\
\hline p-Cresol (4-Methylphenol) & 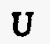 & & 370 & $\mu \mathrm{g} / \mathrm{kg}$ & 370 & EPA8270 \\
\hline Dibenz(a.h)anthracene & $\mathbf{U}$ & & 370 & $\mu \mathrm{g} / \mathrm{kg}$ & 370 & EPA8270 \\
\hline Dibenzofuran & U & & 370 & $\mu \mathrm{g} / \mathrm{kg}$ & 370 & EPA8270 \\
\hline Di-n-buryl phthalate & $\mathrm{U}$ & $\mathbf{v}$ & 370 & $\mu \mathrm{g} / \mathrm{kg}$ & 370 & EPA8270 \\
\hline 1,2-Dichlorobenzene & U & & 370 & $\mu \mathrm{g} / \mathrm{kg}$ & 370 & EPA8270 \\
\hline 1,3-Dichlorobenzene & U & & 370 & $\mu \mathrm{g} / \mathrm{kg}$ & 370 & EPA8270 \\
\hline 1,4-Dichlorobenzene & $\mathbf{U}$ & & 370 & $\mu \mathrm{g} / \mathrm{kg}$ & 370 & EPA8270 \\
\hline 3,3'-Dichlorobenzidine & $\mathrm{U}$ & & 740 & $\mu \mathrm{g} / \mathrm{kg}$ & 740 & EPA8270 \\
\hline 2,4-Dichlorophenol & $\mathrm{U}$ & & 370 & $\mu \mathrm{g} / \mathrm{kg}$ & 370 & EPA8270 \\
\hline Diethyl phthalate & U & & 370 & $\mu \mathrm{g} / \mathrm{kg}$ & 370 & EPA8270 \\
\hline 2,4-Dimethyl phenol & $\mathrm{U}$ & & 370 & $\mu \mathrm{g} / \mathrm{kg}$ & 370 & EPA8270 \\
\hline Dimethyl phthalate & $U$ & & 370 & $\mu \mathrm{g} / \mathrm{kg}$ & 370 & EPA8270 \\
\hline 2.4-Dinitrophenol & $\mathrm{U}$ & & 1.800 & $\mu \mathrm{g} / \mathrm{kg}$ & 1,800 & EPA8270 \\
\hline 2,4-Dinitrotoluene & $U$ & & 370 & $\mu \mathrm{g} / \mathrm{kg}$ & 370 & EPA8270 \\
\hline 2.6-Dinitrotoluene & $U$ & & 370 & $\mu \mathrm{g} / \mathrm{kg}$ & 370 & EPA8270 \\
\hline Di-n-ocryl phthalate & $\mathbf{U}$ & & 370 & $\mu \mathrm{g} / \mathrm{kg}$ & 370 & EPA8270 \\
\hline Fluoranthene & $U$ & & 370 & $\mu \mathrm{g} / \mathrm{kg}$ & 370 & EPA8270 \\
\hline Fluorene & $U$ & & 370 & $\mu \mathrm{g} / \mathrm{kg}$ & 370 & EPA8270 \\
\hline Hexachlorobenzene & $\mathbf{U}$ & & 370 & $\mu g / \mathrm{kg}$ & 370 & EPA8270 \\
\hline Hexachlorobutadiene & U & & 370 & $\mu \mathrm{g} / \mathrm{kg}$ & 370 & EPA8270 \\
\hline Hexachlorocyclopentadiene & $\mathbf{U}$ & & 370 & $\mu g / \mathrm{kg}$ & 370 & EPA8270 \\
\hline Hexachloroethane & $\mathbf{U}$ & & 370 & $\mu \mathrm{g} / \mathrm{kg}$ & 370 & EPA8270 \\
\hline Indeno(1,2,3-c,d)pyrene & $\boldsymbol{U}$ & & 370 & $\mu g / \mathrm{kg}$ & 370 & EPA8270 \\
\hline Isophorone & $\mathrm{U}$ & & 370 & $\mu \mathrm{g} / \mathrm{kg}$ & 370 & EPA8270 \\
\hline 2-Methyl-4,6-dinitrophenol & $\mathrm{U}$ & & 1,800 & $\mu g / \mathrm{kg}$ & 1,800 & EPA8270 \\
\hline 2-Methylnaphthalene & $\mathrm{U}$ & & 370 & $\mu \mathrm{g} / \mathrm{kg}$ & 370 & EPA8270 \\
\hline Naphthalene & $\mathrm{U}$ & & 370 & $\mu g / \mathrm{kg}$ & 370 & EPA8270 \\
\hline o-Nitroaniline & $U^{\circ}$ & & 1,800 & $\mu g / \mathrm{kg}$ & 1,800 & EPA8270 \\
\hline m-Nitroaniline & $\mathrm{U}$ & & 1,800 & $\mu g / \mathrm{kg}$ & 1,800 & EPA8270 \\
\hline p-Nitroaniline & $\mathbf{U}$ & & 1,800 & $\mu g / \mathrm{kg}$ & 1,800 & EPA8270 \\
\hline Nitrobenzene & $\mathrm{U}$ & & 370 & $\mu \mathrm{g} / \mathrm{kg}$ & 370 & EPA8270 \\
\hline 2-Nitrophenol & $\mathbf{U}$ & & 370 & $\mu g / \mathrm{kg}$ & 370 & EPA8270 \\
\hline 4-Nitrophenol & $\mathrm{U}$ & & 1,800 & $\mu g / \mathrm{kg}$ & 1,800 & EPA8270 \\
\hline N-Nitrosodiphenylamine & $\mathbf{U}$ & & 370 & $\mu \mathrm{g} / \mathrm{kg}$ & 370 & EPA8270 \\
\hline N-Nitrosodipropylamine & $\mathbf{U}$ & & 370 & $\mu \mathrm{g} / \mathrm{kg}$ & 370 & EPA8270 \\
\hline Pentachlorophenol & $\mathrm{U}$ & & 1,800 & $\mu g / \mathrm{kg}$ & 1,800 & EPA8270 \\
\hline Phenanthrene & $\mathbf{U}$ & & 370 & $\mu \mathrm{g} / \mathrm{kg}$ & 370 & EPA8270 \\
\hline Phenol & $\mathrm{U}$ & & 370 & $\mu \mathrm{g} / \mathrm{kg}$ & 370 & EPA8270 \\
\hline Pyrene & $\boldsymbol{U}$ & & 370 & $\mu \mathrm{g} / \mathrm{kg}$ & 370 & EPA8270 \\
\hline 1,2,4-Trichlorobenzene & $\mathbf{U}$ & & 370 & $\mu \mathrm{g} / \mathrm{kg}$ & $370<$ & EPA8270 \\
\hline 2,4,5-Trichlorophenol & $\mathbf{U}$ & & 1.800 & $\mu g / \mathrm{kg}$ & 1,800 & EPA8270 \\
\hline 2,4,6-Trichlorophenol & $\mathbf{U}$ & & 370 & $\mu g / \mathrm{kg}$ & 370 & EPA8270 \\
\hline Unknown adipate & $\mathbf{J}$ & $\mathbf{N}$ & 10,000 & $\mu g / \mathrm{kg}$ & & EPA8270 \\
\hline Unknown adipate & $\mathbf{J}$ & $\mathbf{N}$ & 200 & $\mu g / \mathrm{kg}$ & & EPA8270 \\
\hline Unknown ketone & $\mathbf{J}$ & NV & 5.000 & $\mu \mathrm{g} / \mathrm{kg}$ & & EPA8270 \\
\hline Unknown ketone & $\mathbf{J}$ & $\mathbf{N}$ & 300 & $\mu \mathrm{g} / \mathrm{kg}$ & & EPA8270 \\
\hline
\end{tabular}


Volatile Organic Compounds

Acetone
Benzene
Bromodichloromethane
Bromoform
Bromomethane (Methyl bromide)
Carbon disulfide
Carbon tetrachloride
Chlorobenzene
Chloroethane
Chloroethene (Vinyl chloride)
Chloroform
Chloromethane (Methyl chloride)
Dibromochloromethane
1,1-Dichloroethane
1,2-Dichloroethane
1,1-Dichloroethylene
1,2-Dichloroethylene
Dichloromethane (Methylene chloride)
1,2-Dichloropropane
cis-1,3-Dichloropropene
trans-1,3-Dichloropropene
Ethylbenzene
2-Hexanone
Methyl ethyl ketone
Methyl isobutyl ketone
Styrene
1,1,2,2-Tetrachloroethane
Tetrachloroethylene
Toluene
1,1,1-Trichloroethane
1,1,2-Trichloroethane
Trichloroethylene
Vinyl acetate
Xylenes

$U$
$U$
$U$
$U$
$U$
$U$
$U$
$U$
$U$
$U$
$U$
$U$
$U$
$U$
$U$
$U$
$U$
$U$
$U$
$U$
$U$
$U$
$U$
$U$
$U$
$U$
$U$
$U$
$U$
$U$
$U$
$U$
$U$
$U$

Radionuclide Indicators and Radionuclides

Gross alpha

Nonvolatile beta

Radium, total alpha-emitting

Tritium

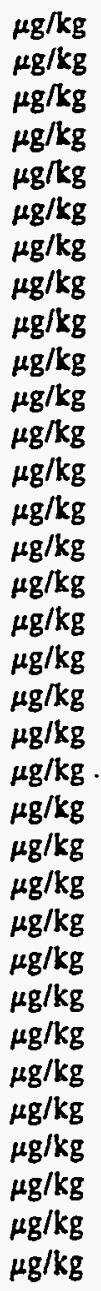

11

5.6

5.6

5.6

5.6 .

11

5.6

5.6

5.6

11

11

5.6

11

5.6

5.6

5.6

5.6

5.6

13

5.6

5.6

5.6

5.6

11

11

11

5.6

5.6

5.6

5.6

5.6

5.6

5.6

11

5.6
EPA 8240

EPA8240

EPA8240

EPA8240

EPA8240

EPA8240

EPA8240

EPA8240

EPA8240

EPA8240

EPA8240

EPA8240

EPA 8240

EPA8240

EPA8240

EPA8240

EPA 8240

EPA8240

EPA8240

EPA8240

EPA8240

EPA8240

EPA8240

EPA8240

EPA8240

EPA8240

EPA8240

EPA8240

EPA8240

EPA8240

EPA8240

EPA8240

EPA 8240

EPA8240
SAMPLE NAME: D431 1802A

Sample Type: Duplicate

Total Metals

Arsenic

Barium

Cadmium

Chromium
U V

U V

$6.0 \pm 5.8$

$11 \pm 9.6$

$0.86 \pm 0.82$

1.3
3.5

3.5

0.16

1.3

EPA900.0

EPA900.0

EPA903.0

EPA906.0

Sample ID: 100402

Associated Sample: 100401 
SAMPLE NAME: D431 1802A (Continued)

RQ AQ B Result

Constituent

Total Metals
Sample D: 100402

Unit

D. Limit Method

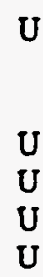

$B / N / A$ Extractables (including $P A H$ and phenols)

\section{Copper \\ Lead \\ Selenium \\ Silver}

$\begin{array}{ll}\mathrm{U} & 2.7 \\ & 1.9 \\ \mathrm{U} & 160 \\ \mathrm{U} & 0.11 \\ \mathrm{U} & 4.3 \\ \mathrm{U} & 0.39\end{array}$

Acenaphthene

Acenaphthylene

Anthracene

Benzo(a)anthracene

Benzo(b)fluoranthene

Benzo(k)fluoranthene

Benzoic acid

Benzo(g.h.i)perylene

Benzo(a)pyrene

Benzyl alcohol

Bis(2-chloroethoxy) methane

Bis(2-chloroethyl) ether

Bis(2-chloroisopropyl) ether

Bis(2-ethylhexyl) phthalate

4-Bromophenyl phenyl ether

Butylbenzyl phthalate

4-Chioroaniline

4-Chloro-m-cresol

2-Chloronaphthalene

2-Chlorophenol

4-Chlorophenyl phenyl ether

Chrysene

o-Cresol (2-Methylphenol)

p-Cresol (4-Methylphenol)

Dibenz $(\mathrm{a}, \mathrm{h})$ anthracene

Dibenzofuran

Di-n-butyl phthalate

1,2-Dichlorobenzene

1,3-Dichlorobenzene

1,4-Dichlorobenzene

3,3'-Dichlorobenzidine

2,4-Dichlorophenol

Diethyl phthalate

2,4-Dimethyl phenol

Dimethyl phthalate

2,4-Dinitrophenol

2,4-Dinitrotoluene

2,6-Dinitrotoluene

Di-n-octyl phthalate

Fluoranthene
U

U

U

U

U

U

U

U

U

U

U

U

U

U

U

U

U

U

U

U

U

U

U

U

U

U

U

U

U

U

U

U

U

U

U

U

U

U

U
370

370

370

370

370

370

370

370

370

370

370

370

740

370

370

370

370

1,900

370

370

370

370

\begin{tabular}{lll}
$\mu \mathrm{g} / \mathrm{kg}$ & 370 & EPA8270 \\
$\mu \mathrm{g} / \mathrm{kg}$ & 370 & EPA8270 \\
$\mu \mathrm{g} / \mathrm{kg}$ & 370 & EPA8270 \\
$\mu \mathrm{g} / \mathrm{kg}$ & 370 & EPA8270 \\
$\mu \mathrm{g} / \mathrm{kg}$ & 370 & EPA8270 \\
$\mu \mathrm{g} / \mathrm{kg}$ & 370 & EPA8270 \\
$\mu \mathrm{g} / \mathrm{kg}$ & 1,900 & EPA8270 \\
$\mu \mathrm{g} / \mathrm{kg}$ & 370 & EPA8270 \\
$\mu \mathrm{g} / \mathrm{kg}$ & 370 & EPA8270 \\
$\mu \mathrm{g} / \mathrm{kg}$ & 370 & EPA8270 \\
$\mu \mathrm{gg} / \mathrm{kg}$ & 370 & EPA8270 \\
$\mu \mathrm{g} / \mathrm{kg}$ & 370 & EPA8270 \\
$\mu \mathrm{g} / \mathrm{kg}$ & 370 & EPA8270 \\
$\mu \mathrm{g} / \mathrm{kg}$ & 370 & EPA8270 \\
$\mu \mathrm{g} / \mathrm{kg}$ & 370 & EPA8270 \\
$\mu \mathrm{g} / \mathrm{kg}$ & 370 & EPA8270 \\
$\mu \mathrm{g} / \mathrm{kg}$ & 370 & EPA8270 \\
$\mu \mathrm{g} / \mathrm{kg}$ & 370 & EPA8270 \\
$\mu \mathrm{g} / \mathrm{kg}$ & 370 & EPA8270 \\
$\mu \mathrm{g} / \mathrm{kg}$ & 370 & EPA8270 \\
$\mu \mathrm{g} / \mathrm{kg}$ & 370 & EPA8270 \\
$\mu \mathrm{g} / \mathrm{kg}$ & 370 & EPA8270 \\
$\mu \mathrm{g} / \mathrm{kg}$ & 370 & EPA8270 \\
$\mu \mathrm{g} / \mathrm{kg}$ & 370 & EPA8270 \\
$\mu \mathrm{g} / \mathrm{kg}$ & 370 & EPA8270 \\
$\mu \mathrm{g} / \mathrm{kg}$ & 370 & EPA8270 \\
$\mu \mathrm{g} / \mathrm{kg}$ & 370 & EPA8270 \\
$\mu \mathrm{gg} / \mathrm{kg}$ & 370 & EPA8270 \\
$\mu \mathrm{g} / \mathrm{kg}$ & 370 & EPA8270 \\
$\mu \mathrm{g} / \mathrm{kg}$ & 370 & EPA8270 \\
$\mu \mathrm{g} / \mathrm{kg}$ & 740 & EPA8270 \\
$\mu \mathrm{g} / \mathrm{kg}$ & 370 & EPA8270 \\
$\mu \mathrm{g} / \mathrm{kg}$ & 370 & EPA8270 \\
$\mu \mathrm{g} / \mathrm{kg}$ & 370 & EPA8270 \\
$\mu \mathrm{g} / \mathrm{kg}$ & 370 & EPA8270 \\
$\mu \mathrm{g} / \mathrm{kg}$ & 1,900 & EPA8270 \\
$\mu \mathrm{g} / \mathrm{kg}$ & 370 & EPA8270 \\
$\mu \mathrm{g} / \mathrm{kg}$ & -370 & EPA8270 \\
$\mu \mathrm{g} / \mathrm{kg}$ & 370 & EPA8270 \\
$\mu \mathrm{g} / \mathrm{kg}$ & 370 & EPA8270 \\
& & \\
\hline
\end{tabular}

EPA6010

EPA7421

$\mathrm{mg} / \mathrm{kg}$

$\mathrm{mg} / \mathrm{kg}$

$\mathrm{mg} / \mathrm{kg}$

$\mathrm{mg} / \mathrm{kg}$

$\mathrm{mg} / \mathrm{kg}$

$\mathrm{mg} / \mathrm{kg}$

0.39 EPA7740

1.1 EPA6010

$\begin{array}{ll}0.24 & \text { EPA7421 } \\ 1.6 & \text { EPA6010 } \\ 0.11 & \text { EPA7470 } \\ 4.3 & \text { EPA6010 } \\ 0.39 & \text { EPA7740 } \\ 1.1 & \text { EPA6010 }\end{array}$


SAMPLE NAME: D431 1802A (Continued)

Sample D: 100402

\section{RQ AQ B Result}

Unit

$B / N / A$ Extractables (including PAH and phenols)

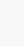

Fluorene

Hexachlorobenzene

Hexachlorobutadiene

Hexachlorocyclopentadiene

Hexachloroethane

Indeno(1,2,3-c,d)pyrene

Isophorone

2-Methyl-4.6-dinitrophenol

2-Methyinaphthalene

Naphthaiene

o-Nitroaniline

m-Nitroaniline

p-Nitroaniline

Nitrobenzene

2-Nitrophenol

4-Nitrophenol

$\mathrm{N}$-Nitrosodiphenylamine

$\mathrm{N}$-Nitrosodipropylamine

Pentachlorophenol

Phenanthrene

Phenol

Pyrene

1,2,4-Trichlorobenzene

2,4,5-Trichlorophenol

2,4,6-Trichlorophenol

Unknown adipate

Unknown adipate

Unknown hydrocarbon

Unknown hydrocarbon

Unknown ketone
U

U

U

U

U

U

U

U

U

U

U

U

U

U

U

U

U

U

U

U

U

U

U

U

U

J $\mathrm{N}$

J N

J $N$

J N
370

370

370

370

370

370

370

1,900

370

370

1.900

1,900

1.900

370

370

1,900

370

370

1,900

370

370

370

370

1,900

370

20,000

300

500

500

7,000

\begin{tabular}{|c|c|c|}
\hline$\mu g / k g$ & 370 & EPA8270 \\
\hline$\mu \mathrm{g} / \mathrm{kg}$ & 370 & EPA8270 \\
\hline$\mu \mathrm{g} / \mathrm{kg}$ & 370 & EPA8270 \\
\hline$\mu \mathrm{g} / \mathrm{kg}$ & 370 & EPA8270 \\
\hline$\mu g / k g$ & 370 & EPA8270 \\
\hline$\mu g / k g$ & 370 & EPA8270 \\
\hline$\mu \mathrm{g} / \mathrm{kg}$ & 370 & EPA8270 \\
\hline$\mu g / \mathrm{kg}$ & 1,900 & EPA8270 \\
\hline$\mu g / k g$ & 370 & EPA8270 \\
\hline$\mu \mathrm{g} / \mathrm{kg}$ & 370 & EPA8270 \\
\hline$\mu \mathrm{g} / \mathrm{kg}$ & 1,900 & EPA8270 \\
\hline$\mu \mathrm{g} / \mathrm{kg}$ & 1,900 & EPA8270 \\
\hline$\mu g / k g$ & 1.900 & EPA8270 \\
\hline$\mu \mathrm{g} / \mathrm{kg}$ & 370 & EPA8270 \\
\hline$\mu \mathrm{g} / \mathrm{kg}$ & 370 & EPA8270 \\
\hline$\mu \mathrm{g} / \mathrm{kg}$ & 1,900 & EPA8270 \\
\hline$\mu \mathrm{g} / \mathrm{kg}$ & 370 & EPA8270 \\
\hline$\mu g / k g$ & 370 & EPA8270 \\
\hline$\mu \mathrm{g} / \mathrm{kg}$ & 1,900 & EPA8270 \\
\hline$\mu \mathrm{g} / \mathrm{kg}$ & 370 & EPA8270 \\
\hline$\mu g / \mathrm{kg}$ & 370 & EPA8270 \\
\hline$\mu \mathrm{g} / \mathrm{kg}$ & 370 & EPA8270 \\
\hline$\mu \mathrm{g} / \mathrm{kg}$ & 370 & EPA8270 \\
\hline$\mu g / k g$ & 1,900 & EPA8270 \\
\hline$\mu \mathrm{g} / \mathrm{kg}$ & 370 & EPA8270 \\
\hline$\mu \mathrm{g} / \mathrm{kg}$ & & EPA8270 \\
\hline$\mu \mathrm{g} / \mathrm{kg}$ & & EPA8270 \\
\hline$\mu g / k g$ & & EPA8270 \\
\hline$\mu g / \mathrm{kg}$ & & EPA8270 \\
\hline$\mu \mathrm{g} / \mathrm{kg}$ & & EPA8270 \\
\hline
\end{tabular}

Volatile Organic Compounds

Acetone
Acetone
Acetone
Benzene
Bromodichloromethane
Bromodichloromethane:
Bromodichloromethane:
Bromoform
Bromoform
Bromoform
Bromomethane (Methyl bromide)
Bromomethane (Methyl bromide)
Bromomethane (Methyl bromide)
Carbon disulfide
Carbon disulfide
Carbon disulfide
Carbon tetrachloride

\section{1}

41

41

U

U

U

$\mathrm{U}$

$\mathrm{U}$

$\mathrm{U}$

U

U

$\mathrm{U}$

$\mathrm{U}$

U

$\mathrm{U}$

Carbon tetrachloride

D. Limit Method 
SAMPLE NAME: D431 1802A (Continued)

Constituent

Volatile Organic Compounds

Carbon tetrachloride

Carbon tetrachloride

Chlorobenzene

Chloroethane

Chloroethane

Chloroethane

Chloroethene (Vinyl chloride)

Chloroethene (Vinyl chloride)

Chloroethene (Vinyl chloride)

Chloroform

Chloroform

Chloroform

Chloromethane (Methyl chloride)

Chloromethane (Methyl chloride)

Chloromethane (Methyl chloride)

Dibromochloromethane

Dibromochloromethane

Dibromochloromethane

1,1-Dichloroethane

1,1-Dichloroethane

1,1-Dichloroethane

1,2-Dichloroethane

1.2-Dichloroethane

1,2-Dichloroethane

1,1-Dichloroethylene

1,2-Dichloroethylene

1,2-Dichloroethylene

1,2-Dichloroethylene

Dichloromethane (Methylene chloride)

Dichloromethane (Methylene chloride)

Dichloromethane (Methylene chloride)

1.2-Dichloropropane

1.2-Dichloropropane

1,2-Dichloropropane

cis-1,3-Dichloropropene

cis-1,3-Dichloropropene

cis-1,3-Dichloropropene

trans-1,3-Dichloropropene

trans-1,3-Dichloropropene

trans-1,3-Dichloropropene

Ethylbenzene

Ethylbenzene

Ethylbenzene

2-Hexanone

2-Hexanone

2-Hexanone

Methyl ethyl ketone

Methyl ethyl ketone

Methyl ethyl ketone

Methyl isoburyl ketone

\author{
RQ AQ B Result \\ $\mathbf{R Q}$
}

Sample ID: 100402

Unit

D. Limit Method

$\mu g / \mathrm{kg}$
$\mu \mathrm{g} / \mathrm{kg}$
$\mu \mathrm{g} / \mathrm{kg}$
$\mu \mathrm{g} / \mathrm{kg}$
$\mu \mathrm{g} / \mathrm{kg}$
$\mu \mathrm{g} / \mathrm{kg}$
$\mu \mathrm{g} / \mathrm{kg}$
$\mu \mathrm{g} / \mathrm{kg}$
$\mu \mathrm{g} / \mathrm{kg}$
$\mu \mathrm{g} / \mathrm{kg}$
$\mu \mathrm{g} / \mathrm{kg}$
$\mu \mathrm{g} / \mathrm{kg}$
$\mu \mathrm{g} / \mathrm{kg}$
$\mu \mathrm{g} / \mathrm{kg}$
$\mu \mathrm{g} / \mathrm{kg}$
$\mu \mathrm{g} / \mathrm{kg}$

$\mu \mathrm{g} / \mathrm{kg}$

$\mu \mathrm{g} / \mathrm{kg}$

$\mu \mathrm{g} / \mathrm{kg}$

$\mu \mathrm{g} / \mathrm{kg}$

$\mu \mathrm{g} / \mathrm{kg}$

$\mu \mathrm{g} / \mathrm{kg}$

$\mu \mathrm{g} / \mathrm{kg}$

$\mu \mathrm{g} / \mathrm{kg}$

$\mu \mathrm{g} / \mathrm{kg}$

$\mu \mathrm{g} / \mathrm{kg}$

$\mu \mathrm{g} / \mathrm{kg}$

$\mu \mathrm{g} / \mathrm{kg}$

$\mu g / \mathrm{kg}$

$\mu g / k g$

$\mu \mathrm{g} / \mathrm{kg}$

$\mu \mathrm{g} / \mathrm{kg}$

$\mu \mathrm{g} / \mathrm{kg}$

$\mu \mathrm{g} / \mathrm{kg}$

$\mu \mathrm{g} / \mathrm{kg}$

$\mu \mathrm{g} / \mathrm{kg}$

$\mu g / \mathrm{kg}$

$\mu \mathrm{g} / \mathrm{kg}$

$\mu \mathrm{g} / \mathrm{kg}$

$\mu \mathrm{g} / \mathrm{kg}$

$\mu \mathrm{g} / \mathrm{kg}$

$\mu \mathrm{g} / \mathrm{kg}$

$\mu \mathrm{g} / \mathrm{kg}$

$\mu \mathrm{g} / \mathrm{kg}$

$\mu \mathrm{g} / \mathrm{kg}$

$\mu \mathrm{g} / \mathrm{kg}$

$\mu \mathrm{g} / \mathrm{kg}$

$\mu \mathrm{g} / \mathrm{kg}$

$\mu \mathrm{g} / \mathrm{kg}$

$\mu \mathrm{g} / \mathrm{kg}$

$\begin{array}{cc}5.7 & \text { EPA8240 } \\ 5.7 & \text { EPA8240 } \\ 5.7 & \text { EPA8240 } \\ 11 & \text { EPA8240 } \\ 11 & \text { EPA8240 } \\ 11 & \text { EPA8240 } \\ 11 & \text { EPA8240 } \\ 11 & \text { EPA8240 } \\ 11 & \text { EPA8240 } \\ 5.7 & \text { EPA8240 } \\ 5.7 & \text { EPA8240 } \\ 5.7 & \text { EPA8240 } \\ 11 & \text { EPA8240 } \\ 11 & \text { EPA8240 } \\ 11 & \text { EPA8240 } \\ 5.7 & \text { EPA8240 } \\ 5.7 & \text { EPA8240 } \\ 5.7 & \text { EPA8240 } \\ 5.7 & \text { EPA8240 } \\ 5.7 & \text { EPA8240 } \\ 5.7 & \text { EPA8240 } \\ 5.7 & \text { EPA8240 } \\ 5.7 & \text { EPA8240 } \\ 5.7 & \text { EPA8240 } \\ 5.7 & \text { EPA8240 } \\ 5.7 & \text { EPA8240 } \\ 5.7 & \text { EPA8240 } \\ 5.7 & \text { EPA8240 } \\ 5.7 & \text { EPA8240 } \\ 5.7 & \text { EPA8240 } \\ 5.7 & \text { EPA8240 } \\ 5.7 & \text { EPA8240 } \\ 5.7 & \text { EPA8240 } \\ 5.7 & \text { EPA8240 } \\ 5.7 & \text { EPA8240 } \\ .5 .7 & \text { EPA8240 } \\ 5.7 & \text { EPA8240 } \\ 5.7 & \text { EPA8240 } \\ 5.7 & \text { EPA8240 } \\ 5.7 & \text { EPA8240 } \\ 5.7 & \text { EPA8240 } \\ 5.7 & \text { EPA8240 } \\ 5.7 & \text { EPA8240 } \\ 11 & \text { EPA8240 } \\ 11 & \text { EPA8240 } \\ 11 & \text { EPA8240 } \\ 11 & \text { EPA8240 } \\ 11 & \text { EPA8240 } \\ 11 & \text { EPA8240 } \\ 11 & \text { EPA8240 }\end{array}$


SAMPLE NAME: D43I $1802 \mathrm{~A}$ (Continued)

\section{Constituent}

$\mathbf{R Q}$

$A Q$

Sample ID: 100402

Volatile Organic Compounds

Methyl isobutyl ketone

Methyl isobutyl ketone

Styrene

Styrene

Styrene

1,1,2,2-Tetrachloroethane

1,1,2,2-Tetrachloroethane

1,1,2,2-Tetrachloroechane

Tetrachloroethylene

Tetrachloroethylene

Tetrachloroethylene

Toluene

1,1.1-Trichloroethane

1,1.1-Trichloroethane

1,1,1-Trichloroethane

1,1.2-Trichloroethane

1.1.2-Trichloroethane

1,1,2-Trichloroethane

Trichloroethylene

Vinyl acetate

Vinyl acetate

Vinyl acetate

Xylenes

Xylenes

Xylenes

$\begin{array}{lll}U & 11 & \mu g / k g \\ U & 11 & \mu g / k g \\ U & 5.7 & \mu g / k g \\ U & 5.7 & \mu g / k g \\ U & 5.7 & \mu g / k g \\ U & 5.7 & \mu g / k g \\ U & 5.7 & \mu g / k g \\ U & 5.7 & \mu g / k g \\ U & 5.7 & \mu g / k g \\ U & 5.7 & \mu g / k g \\ U & 5.7 & \mu g / k g \\ U & 5.7 & \mu g / k g \\ U & 5.7 & \mu g / k g \\ U & 5.7 & \mu g / k g \\ U & 5.7 & \mu g / k g \\ U & 5.7 & \mu g / k g \\ U & 5.7 & \mu g / k g \\ U & 5.7 & \mu g / k g \\ U & 5.7 & \mu g / k g \\ U & 11 & \mu g / k g \\ U & 11 & \mu g / k g \\ U & 11 & \mu g / k g \\ U & 5.7 & \mu g / k g \\ U & 5.7 & \mu g / k g \\ U & 5.7 & \mu g / k g \\ U & & \end{array}$

Radionuclide Indicators and Radionuclides

Gross alpha

Nonvolatile beta

Radium, total alpha-emitting

Tritium

$\begin{array}{llllll} & & 23 \pm 10 & \mathrm{PCi} / \mathrm{g} & 3.9 & \text { EPA990.0 } \\ & & 39 \pm 13 & \mathrm{pCi} / \mathrm{g} & 3.9 & \text { EPA900.0 } \\ \mathrm{U} & \mathrm{V} & 0.84 \pm 1.2 & \mathrm{pCi} / \mathrm{g} & 0.32 & \text { EPA903.0 } \\ \mathrm{U} & & 1.9 & \mathrm{pCi} / \mathrm{g} & 1.9 & \text { EPA906.0 }\end{array}$

SAMPLE NAME: D431 1803

Sample ID: 100404

Location (SRS Coordinates): $18340.39 \mathrm{E}$ 66562.05N

Ground Elevation Above MSL: $122.4 \mathrm{ft}$

Depth of Core Interval: 5.00 to $7.00 \mathrm{ft}$

Sample Type: Normal

Sample Color: 10R 6/6

Sample Matrix: Soil

USC Soil Classification: CL

RQ AQ B Result

Unit

meq $/ 100 \mathrm{~g}$

$\mathrm{pH}$

$\mu \mathrm{S} / \mathrm{cm}$

$\mathrm{mg} / \mathrm{kg}$
10
3.4

170

2,100
Sample Moisture: Wet

Percent Solids: 76.50

D. Limit Method

Physical Parameters

Cation exchange capacity

$\mathrm{pH}$

Specific conductance

Total organic carbon
2.0

0.010

1.0

170
EPA8240

EPA8240

EPA8240

EPA8240

EPA8240

EPA8240

EPA8240

EPA8240

EPA8240

EPA8240

EPA8240

EPA8240

EPA8240

EPA8240

EPA8240

EPA8240

EPA8240

EPA8240

EPA8240

EPA8240

EPA8240

EPA8240

EPA8240

EPA8240

EPA8240 
SAMPLE NAME: D431 1803 (Continued)

Sample D: 100404

$\begin{array}{llllll}\text { Constituent RQ AQ } & \text { U Result Unit Limit Method }\end{array}$

Physical Parameters

Total organic carbon

1,700

$\mathrm{mg} / \mathrm{kg}$ •

130

EPA415.1

Total Metals

Arsenic

Barium

Cadmium

Chromium

Copper

Lead

Manganese

Mercury

Selenium

Silver
Nickel

3.3

19

0.48

31

U

$\mathrm{U}$

$B / N / A$ Extractables (including $P A H$ and phenols)

Acenaphthene

Acenaphthylene

Anthracene

Benzo(a)anthracene

Benzo(b)fluoranthene

Benzo(k)fluoranthene

Benzoic acid

Benzo(g,h,i)perylene

Benzo(a)pyrene

Benzyl alcohol

Bis(2-chloroethoxy) methane

Bis(2-chloroethyl) ether

Bis(2-chloroisopropyl) ether

Bis(2-ethylhexyl) phthalate

4-Bromophenyl phenyl ether

Butylbenzyl phthalate

4-Chloroaniline

4-Chloro-m-cresol

2-Chloronaphthalene

2-Chlorophenol

4-Chlorophenyl phenyl ether

Chrysene

o-Cresol (2-Methylphenol)

p-Cresol (4-Methylphenol)

Dibenz $(a, h)$ anthracene,

Dibenzofuran

Di-n-butyl phthalate

1,2-Dichlorobenzene

1,3-Dichlorobenzene

1,4-Dichlorobenzene

3,3'-Dichlorobenzidine

2,4-Dichlorophenol

$U$
$U$
$U$
$U$
$U$
$U$
$U$
$U$
$U$
$U$
$U$
$U$
$U$
$U$
$U$
$U$
$U$
$U$
$U$
$U$
$U$
$U$
$U$
$U$
$U$
$U$
$U J$
$U$
$U$
$U$
$U$
$U$

440

440

440

440

440

440

2,200

440

440

440

440

440

440

440

440

440

440

440

440

440

440

440

440

440

$440^{\circ}$

440

58

440

440

440

880

440 $\mathrm{mg} / \mathrm{kg}$
$\mathrm{mg} / \mathrm{kg}$
$\mathrm{mg} / \mathrm{kg}$
$\mathrm{mg} / \mathrm{kg}$
$\mathrm{mg} / \mathrm{kg}$
$\mathrm{mg} / \mathrm{kg}$
$\mathrm{mg} / \mathrm{kg}$
$\mathrm{mg} / \mathrm{kg}$
$\mathrm{mg} / \mathrm{kg}$
$\mathrm{mg} / \mathrm{kg}$
$\mathrm{mg} / \mathrm{kg}$

1.2

19 EPA6010

$0.48 \quad$ EPA6010

0.96 EPA6010

2.4 EPA6010

0.37 EPA7421

$1.4 \quad$ EPA6010

0.13 EPA7470

3.8 EPA6010

0.61 EPA7740

0.96 EPA6010

.


SAMPLE NAME: D431 1803 (Continued)

Constituent
Sample ID: 100404

RQ AQ B Result Unit D. Limit Method

$B / N / A$ Extractables (including $P A H$ and phenols)

Diethyl phthalate

2.4-Dimethyl phenol

Dimethyl phthalate

2,4-Dinitrophenol

2,4-Dinitrotoluene

2,6-Dinitrotoluene

Di-n-octyl phthalate

Fluoranthene

Fluorene

Hexachlorobenzene

Hexachlorobutadiene

Hexachlorocyclopentadiene

Hexachloroethane

Indeno(1.2,3-c,d)pyrene

Isophorone

2-Methyl-4,6-dinitrophenol

2-Methylnaphthalene

Naphthalene

o-Nitroaniline

m-Nitroaniline

p-Nitroaniline

Nitrobenzene

2-Nitrophenol

4-Nitrophenol

N-Nitrosodiphenylamine

$\mathrm{N}$-Nitrosodipropylamine

Pentachlorophenol

Phenanthrene

Phenol

Pyrene

1,2.4-Trichlorobenzene

2,4,5-Trichlorophenol

2,4,6-Trichlorophenol

Unknown ester

Unknown ketone

Unknown phthalate

Unknown phthalate

Unknown phthalate
U

U

U

U

U

U

U

U

U

U

U

U

U

U

U

$\mathrm{U}$

U

U

U

U

U

U

U

U

U

U

U

U

U

U

U

J NV $\quad 400$

J NV $\quad 8,000$

J $N \quad 300$

J $\quad N \quad 300$

J $N \quad 400$
440

440

2,200

440

440

440

440

440

440

440

440

440

440

2,200

440

440

2,200

2,200

2,200

440

2,200

440

440

2,200

440

440

440

440

2.200 $\mu g / \mathrm{kg}$
$\mu \mathrm{g} / \mathrm{kg}$
$\mu \mathrm{g} / \mathrm{kg}$
$\mu \mathrm{g} / \mathrm{kg}$
$\mu \mathrm{g} / \mathrm{kg}$
$\mu \mathrm{g} / \mathrm{kg}$
$\mu \mathrm{g} / \mathrm{kg}$
$\mu \mathrm{g} / \mathrm{kg}$
$\mu \mathrm{g} / \mathrm{kg}$
$\mu \mathrm{g} / \mathrm{kg}$
$\mu \mathrm{g} / \mathrm{kg}$
$\mu \mathrm{g} / \mathrm{kg}$
$\mu \mathrm{g} / \mathrm{kg}$
$\mu \mathrm{g} / \mathrm{kg}$
$\mu \mathrm{g} / \mathrm{kg}$
$\mu \mathrm{g} / \mathrm{kg}$
$\mu \mathrm{g} / \mathrm{kg}$
$\mu \mathrm{g} / \mathrm{kg}$
$\mu g / \mathrm{kg}$
$\mu \mathrm{g} / \mathrm{kg}$

$\mu \mathrm{g} / \mathrm{kg}$

$\mu \mathrm{g} / \mathrm{kg}$

$\mu \mathrm{g} / \mathrm{kg}$

$\mu \mathrm{g} / \mathrm{kg}$

$\mu \mathrm{g} / \mathrm{kg}$

$\mu \mathrm{g} / \mathrm{kg}$

$\mu \mathrm{g} / \mathrm{kg}$

$\mu \mathrm{g} / \mathrm{kg}$

$\mu \mathrm{g} / \mathrm{kg}$

$\mu \mathrm{g} / \mathrm{kg}$

$\mu \mathrm{g} / \mathrm{kg}$

$\mu \mathrm{g} / \mathrm{kg}$

$\mu \mathrm{g} / \mathrm{kg}$

$\mu \mathrm{g} / \mathrm{kg}$

$\mu \mathrm{g} / \mathrm{kg}$

$\mu \mathrm{g} / \mathrm{kg}$

$\mu \mathrm{g} / \mathrm{kg}$

$\mu \mathrm{g} / \mathrm{kg}$
$440 \quad$ EPA8270

440 EPA8270

$440 \quad$ EPA8270

2,200 EPA8270

$440 \quad$ EPA8270

$440 \quad$ EPA8270

440 EPA8270

440 EPA8270

$440 \quad$ EPA8270

$440 \quad$ EPA8270

$440 \quad$ EPA8270

$440 \quad$ EPA8270

$440 \quad$ EPA8270

$440 \quad$ EPA8270

$440 \quad$ EPA8270

$2,200 \quad$ EPA8270

$440 \quad$ EPA8270

$440 \quad$ EPA8270

$2,200 \quad$ EPA8270

2,200 EPA8270

2,200 EPA8270

$440 \quad$ EPA8270

440 EPA8270

2,200 EPA8270

440 EPA8270

$440 \quad$ EPA8270

$2,200 \quad$ EPA8270

440 EPA8270

$440 \quad$ EPA8270

440 EPA8270

$440 \quad$ EPA8270

$2,200 \quad$ EPA8270

440 EPA8270

EPA8270

EPA8270

EPA8270

$\therefore \quad$ EPA8270

EPA8270

Volatile Organic Compounds

Acetone

Benzene

Bromodichloromethane

Bromoform

Bromomethane (Methyl bromide)

Carbon disulfide:

Carbon tetrachloride

Chlorobenzene

Chloroethane
J

U

U

U

U

U

U

U

U

\section{2}

6.8

6.8

6.8

14

6.8

6.8

6.8

14 $\mu \mathrm{g} / \mathrm{kg}$

$\mu \mathrm{g} / \mathrm{kg}$

$\mu g / k g$

$\mu \mathrm{g} / \mathrm{kg}$

$\mu \mathrm{g} / \mathrm{kg}$

$\mu \mathrm{g} / \mathrm{kg}$

$\mu g / k g$

$\mu \mathrm{g} / \mathrm{kg}$

$\mu \mathrm{g} / \mathrm{kg}$

\section{4}

6.8

6.8 、

6.8

14

6.8

6.8

6.8

14
EPA8240

EPA8240

EPA8240

EPA8240

EPA8240

EPA8240

EPA8240

EPA8240

EPA8240 
SAMPLE NAME: D431 1803 (Continued)

Constituent

Volatile Organic Compounds

Chloroethene (Vinyl chloride)
Chloroform
Chloromethane (Methyl chloride)
Dibromochloromethane
1,1-Dichloroethane
1,2-Dichloroethane
1,1-Dichloroethylene
1,2-Dichloroethylene
Dichloromethane (Methylene chloride)
1,2-Dichloropropane
cis-1,3-Dichloropropene
trans-1,3-Dichloropropene
Ethylbenzene
2-Hexanone
Methyl ethyl ketone
Methyl isoburyl ketone
Styrene
1,1,2.2-Tetrachloroethane
Tetrachloroethylene
Toluene
1,1,1-Trichloroethane
1,1,2-Trichloroethane
Trichloroethylene
Vinyl acetate
Xylenes

$\begin{array}{ll}. U & 14 \\ U & 6.8 \\ U & 14 \\ U & 6.8 \\ U & 6.8 \\ J & 4.2 \\ U & 6.8 \\ U & 6.8 \\ U & 6.8 \\ U & 6.8 \\ U & 6.8 \\ U & 6.8 \\ U & 6.8 \\ U & 14 \\ U & 14 \\ U & 14 \\ U & 6.8 \\ U & 6.8 \\ U & 6.8 \\ U & 6.8 \\ U & 6.8 \\ U & 6.8 \\ U & 6.8 \\ U & 14 \\ U & 6.8\end{array}$

Pesticides/PCBs and Dioxins/Furans
RQ AQ B Result Unit $\quad$ D. Limit Method

$\begin{array}{lll}\mu \mathrm{g} / \mathrm{kg} & 14 & \text { EPA8240 } \\ \mu \mathrm{gg} / \mathrm{kg} & 6.8 & \text { EPA8240 } \\ \mu \mathrm{g} / \mathrm{kg} & 14 & \text { EPA8240 } \\ \mu \mathrm{gg} / \mathrm{kg} & 6.8 & \text { EPA8240 } \\ \mu \mathrm{g} / \mathrm{kg} & 6.8 & \text { EPA8240 } \\ \mu \mathrm{g} / \mathrm{kg} & 6.8 & \text { EPA8240 } \\ \mu \mathrm{g} / \mathrm{kg} & 6.8 & \text { EPA8240 } \\ \mu \mathrm{g} / \mathrm{kg} & 6.8 & \text { EPA8240 } \\ \mu \mathrm{g} / \mathrm{kg} & 6.8 & \text { EPA8240 } \\ \mu \mathrm{g} / \mathrm{kg} & 6.8 & \text { EPA8240 } \\ \mu \mathrm{g} / \mathrm{kg} & 6.8 & \text { EPA8240 } \\ \mu \mathrm{g} / \mathrm{kg} & 6.8 & \text { EPA8240 } \\ \mu \mathrm{g} / \mathrm{kg} & 6.8 & \text { EPA8240 } \\ \mu \mathrm{g} / \mathrm{kg} & 14 & \text { EPA8240 } \\ \mu \mathrm{g} / \mathrm{kg} & 14 & \text { EPA8240 } \\ \mu \mathrm{g} / \mathrm{kg} & 14 & \text { EPA8240 } \\ \mu \mathrm{g} / \mathrm{kg} & 6.8 & \text { EPA8240 } \\ \mu \mathrm{g} / \mathrm{kg} & 6.8 & \text { EPA8240 } \\ \mu \mathrm{g} / \mathrm{kg} & 6.8 & \text { EPA8240 } \\ \mu \mathrm{gg} / \mathrm{kg} & 6.8 & \text { EPA8240 } \\ \mu \mathrm{g} / \mathrm{kg} & 6.8 & \text { EPA8240 } \\ \mu \mathrm{g} / \mathrm{kg} & 6.8 & \text { EPA8240 } \\ \mu \mathrm{g} / \mathrm{kg} & 6.8 & \text { EPA8240 } \\ \mu \mathrm{g} / \mathrm{kg} & 14 & \text { EPA8240 } \\ \mu \mathrm{g} / \mathrm{kg} & 6.8 & \text { EPA8240 }\end{array}$

$\begin{array}{llll}\text { UJ } & 0 & L & 2.3\end{array}$

UJ $\quad 0 \quad$ L 2.3

$\begin{array}{llll}\text { UJ } & O & \mathrm{~L} & 2.3\end{array}$

UJ $\quad O \quad L \quad 2.3$

$\begin{array}{llll}\text { UJ } & 0 & \text { L } & 2.3\end{array}$

$\begin{array}{llll}\text { UJ } & O & \mathrm{~L} & 2.3\end{array}$

$\begin{array}{llll}\text { UJ } & O & \mathrm{~L} & 4.5\end{array}$

UJ $O \quad$ L 4.5

UI $\quad 0 \quad$ L 4.5

$\begin{array}{llll}\text { UJ } & O & L & 4.5\end{array}$

UJ $O$ L $2.3^{\circ}$

UJ 0 L 4.5

UJ $\quad \mathrm{O} \quad \mathrm{L} \quad 4.5$

UJ $\begin{array}{lll}\text { UJ } & \text { L } & 4.5\end{array}$

UJ 0 L 4.5

UJ $\quad 0 \quad$ L 2.3

$\begin{array}{llll}\text { UJ } & O & \mathrm{~L} & 2.3\end{array}$

U 0.20

U $\quad 0.10$

$\mathrm{U} \quad 0.10$

U $\quad 0.10$

$\begin{array}{lll}\mu \mathrm{g} / \mathrm{kg} & 2.3 & \text { EPA8080 } \\ \mu \mathrm{g} / \mathrm{kg} & 2.3 & \text { EPA8080 } \\ \mu \mathrm{g} / \mathrm{kg} & 2.3 & \text { EPA8080 } \\ \mu \mathrm{g} / \mathrm{kg} & 2.3 & \text { EPA8080 } \\ \mu \mathrm{g} / \mathrm{kg} & 2.3 & \text { EPA8080 } \\ \mu \mathrm{g} / \mathrm{kg} & 2.3 & \text { EPA8080 } \\ \mu \mathrm{g} / \mathrm{kg} & 4.5 & \text { EPA8080 } \\ \mu \mathrm{g} / \mathrm{kg} & 4.5 & \text { EPA8080 } \\ \mu \mathrm{g} / \mathrm{kg} & 4.5 & \text { EPA8080 } \\ \mu \mathrm{g} / \mathrm{kg} & 4.5 & \text { EPA8080 } \\ \mu \mathrm{g} / \mathrm{kg} & 2.3 & \text { EPA8080 } \\ \mu \mathrm{g} / \mathrm{kg} & 4.5 & \text { EPA8080 } \\ \mu \mathrm{g} / \mathrm{kg} & 4.5 & \text { EPA8080 } \\ \mu \mathrm{g} / \mathrm{kg} & 4.5 & \text { EPA8080 } \\ \mu \mathrm{g} / \mathrm{kg} & 4.5 & \text { EPA8080 } \\ \mu \mathrm{g} / \mathrm{kg} & 2.3 & \text { EPA8080 } \\ \mu \mathrm{g} / \mathrm{kg} & 2.3 & \text { EPA8080 } \\ \mathrm{ng} / \mathrm{g} & 0.20 & \text { EPA8280 } \\ \mathrm{ng} / \mathrm{g} & 0.10 & \text { EPA8280 } \\ \mathrm{ng} / \mathrm{g} & 0.10 & \text { EPA8280 } \\ \mathrm{ng} / \mathrm{g} & 0.10 & \text { EPA8280 } \\ \mu \mathrm{g} / \mathrm{kg} & 2.3 & \text { EPA8080 }\end{array}$


SAMPLE NAME: D431 1803 (Continued)

Sample ID: 100404

$\begin{array}{lllll}\text { Constituent RQ AQ B Result Unit } & \text { D. Limit Method }\end{array}$

Pesticides/PCBs and Dioxins/Furans

\begin{tabular}{|c|c|c|c|c|c|c|c|}
\hline $\begin{array}{l}\text { Methoxychlor } \\
\text { Octachlorodibenzo-p-dioxin }\end{array}$ & UI & 0 & L & $\begin{array}{l}23 \\
23\end{array}$ & $\begin{array}{l}\mu \mathrm{g} / \mathrm{kg} \\
\mathrm{ng} / \mathrm{g}\end{array}$ & $\begin{array}{l}23 \\
0.10\end{array}$ & $\begin{array}{l}\text { EPA8080 } \\
\text { EPA8280 }\end{array}$ \\
\hline Octachlorodibenzo-p-furan & $\mathbf{U}$ & & & 0.20 & $\mathrm{ng} / \mathrm{g}$ & 0.20 & EPA8280 \\
\hline PCB 1016 & UJ & 0 & L & 45 & $\mu \mathrm{g} / \mathrm{kg}$ & 45 & EPA8080 \\
\hline PCB 1221 & UJ & 0 & L & 90 & $\mu \mathrm{g} / \mathrm{kg}$ & 90 & EPA8080 \\
\hline PCB 1232 & UJ & 0 & L & 45 & $\mu \mathrm{g} / \mathrm{kg}$ & 45 & EPA8080 \\
\hline PCB 1242 & UJ & 0 & L & 45 & $\mu \mathrm{g} / \mathrm{kg}$ & 45 & EPA8080 \\
\hline РCB 1248 & UJ & 0 & L & 45 & $\mu g / k g$ & 45 & EPA8080 \\
\hline PCB 1254 & UJ & 0 & L & 45 & $\mu \mathrm{g} / \mathrm{kg}$ & 45 & EPA8080 \\
\hline PCB 1260 & UJ & 0 & L & 45 & $\mu \mathrm{g} / \mathrm{kg}$ & 45 & EPA8080 \\
\hline Pentachlorodibenzo-p-dioxin isomers & $\mathbf{U}$ & & & 0.30 & $\mathrm{ng} / \mathrm{g}$ & 0.30 & EPA8280 \\
\hline Pentachlorodibenzo-p-furan isomers & $\mathbf{U}$ & & & 0.20 & $\mathrm{ng} / \mathrm{g}$ & 0.20 & EPA8280 \\
\hline $2,3,7,8-\mathrm{TCDD}$ & $\mathrm{U}$ & & & 0.20 & $\mathrm{ng} / \mathrm{g}$ & 0.20 & EPA8280 \\
\hline $2,3,7,8-\mathrm{TCDF}$ & $\mathbf{U}$ & & & 0.20 & $\mathrm{ng} / \mathrm{g}$ & 0.20 & EPA8280 \\
\hline Tetrachlorodibenzo-p-dioxin isomers & U & & & 0.20 & $\mathrm{ng} / \mathrm{g}$ & 0.20 & EPA8280 \\
\hline Tetrachlorodibenzo-p-furan isomers & $\mathbf{U}$ & & & 0.20 & $\mathrm{ng} / \mathrm{g}$ & 0.20 & EPA8280 \\
\hline Toxaphene & UJ & 0 & L & 230 & $\mu g / \mathrm{kg}$ & 230 & EPA8080 \\
\hline \multicolumn{8}{|c|}{ Radionuclide Indicators and Radionuclides } \\
\hline Gross alpha & & & - & $11 \pm 8.2$ & $\mathrm{pCi} / \mathrm{g}$ & 4.4 & EPA900.0 \\
\hline Nonvolatile beta & & & & $17 \pm 13$ & $\mathrm{pCi} / \mathrm{g}$ & 4.5 & EPA900.0 \\
\hline Radium, total alpha-emitting & & & & $1.5 \pm 1.4$ & $\mathrm{pCi} / \mathrm{g}$ & 0.25 & EPA903.0 \\
\hline Tritium & $\mathbf{U}$ & & & 1.3 & $\mathrm{pCi} / \mathrm{g}$ & 1.3 & EPA906.0 \\
\hline
\end{tabular}

SAMPLE NAME: D431 1804

Location (SRS Coordinates): 18340.39E 66562.05N

Ground Elevation Above MSL: $122.4 \mathrm{ft}$

Depth of Core Interval: 10.00 to $12.00 \mathrm{ft}$

Sample Type: Normal

Sample Color: 10R 6/6

Constituent

Physical Parameters

Cation exchange capacity

$\mathrm{pH}$

Specific conductance

Total organic carbon

Total Metals

Arsenic

Barium

Cadmium

Chromium

Copper

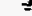

Sample ID: 100405

Sample Matrix: Soil

USC Soil Classification: CL

Sample Moisturé: Wet

Percent Solids: 80.40

RQ AQ B Result Unit $\quad$ D. Limit Method

$12 \quad$ meq $/ 100 \mathrm{~g} \quad 2.0 \quad$ EPA9080

$\begin{array}{llll}3.3 & \mathrm{pH} & 0.010 & \text { EPA9045 }\end{array}$

$\begin{array}{llll}150 & \mu \mathrm{S} / \mathrm{cm} & 1.0 & \text { EPA9050 }\end{array}$

$1,800 \quad \mathrm{mg} / \mathrm{kg} \quad 240 \quad$ EPA415.1

J $Q$

150
1,800

EPA4ls.

$\begin{array}{ll} & 3.0 \\ \mathrm{U} & 23 \\ \mathrm{U} & 0.58 \\ \mathrm{U} & 10 \\ & 2.9\end{array}$

$\mathrm{mg} / \mathrm{kg}$

$\mathrm{mg} / \mathrm{kg}$

$\mathrm{mg} / \mathrm{kg}$

$\mathrm{mg} / \mathrm{kg}$

$\mathrm{mg} / \mathrm{kg}$

1.2

EPA7060

23 EPA6010

0.58 EPA6010

1.2 EPA6010

2.9 EPA6010 
SAMPLE NAME: D431"1804 (Continued)

Sample ID: 100405

$\begin{array}{llllll}\text { Constituent } & \text { RQ AQ B Result Unit Dimit Method }\end{array}$

Total Metals

Lead

Manganese

1.0

Mercury

Nickel

13

Selenium

0.12

4.6

Silver

$\begin{array}{ll}U & 4.6 \\ U & 0.61\end{array}$

1.2

$\begin{array}{lll}\mathrm{mg} / \mathrm{kg} & 0.37 & \text { EPA7421 } \\ \mathrm{mg} / \mathrm{kg} & 1.7 & \text { EPA6010 } \\ \mathrm{mg} / \mathrm{kg} & 0.12 & \text { EPA7470 } \\ \mathrm{mg} / \mathrm{kg} & 4.6 & \text { EPA6010 } \\ \mathrm{mg} / \mathrm{kg} & 0.61 & \text { EPA7740 } \\ \mathrm{mg} / \mathrm{kg} & 1.2 & \text { EPA6010 }\end{array}$

$B / N / A$ Extractables (including $P A H$ and phenols)

Acenaphthene

Acenaphthylene

Anthracene

Benzo(a)anthracene

Benzo(b)fluoranthene

Benzo(k)fluoranthene

Benzoic acid

Benzo(g,h,i)perylene

Benzo(a)pyrene

Benzyl alcohol

Bis(2-chloroethoxy) methane

Bis(2-chloroethyl) ether

Bis(2-chloroisopropyl) ether

Bis(2-ethylhexyl) phthalate

4-Bromophenyl phenyl ether

Butylbenzyl phthalate

4-Chloroaniline

4-Chloro-m-cresol

2-Chloronaphthalene

2-Chlorophenol

4-Chlorophenyl phenyl ether

Chrysene

o-Cresol (2-Methylphenol)

p-Cresol (4-Methylphenol)

Dibenz $(a, h)$ anthracene

Dibenzofuran

Di-n-butyl phthalate

1,2-Dichlorobenzene

1,3-Dichlorobenzene

1,4-Dichlorobenzene

3,3'-Dichlorobenzidine

2,4-Dichlorophenol

Diethyl phthalate

2,4-Dimethyl phenol

Dimethyl phthalate

2,4-Dinitrophenol

2,4-Dinitrotoluene

2,6-Dinitrotoluene

Di-n-octyl phthalate

Fluoranthene

Fluorene
U

U

U

U

U

U

U

U

$\mathrm{U}$

U

U

U

U

U

$\mathrm{U}$

$\mathrm{U}$

U

U

U

U

U

$\mathrm{U}$

U

U

U

U

U

U

U

U

U

U

U

U

U

U

380
380
380
380
380
380
1,900
380
380
380
380
380
380
380
380
380
380
380
3
380
$\mid$
380
380
$\mid$

$\mu$

$\mu \mathrm{g} / \mathrm{kg}$

$\mu \mathrm{g} / \mathrm{kg}$

$\mu \mathrm{g} / \mathrm{kg}$

$\mu \mathrm{g} / \mathrm{kg}$

$\mu \mathrm{g} / \mathrm{kg}$

$\mu \mathrm{g} / \mathrm{kg}$

$\mu \mathrm{g} / \mathrm{kg}$

$\mu \mathrm{g} / \mathrm{kg}$

$\mu \mathrm{g} / \mathrm{kg}$

$\mu \mathrm{g} / \mathrm{kg}$

$\mu \mathrm{g} / \mathrm{kg}$

$\mu g / \mathrm{kg}$

$\mu g / \mathrm{kg}$

$\mu \mathrm{g} / \mathrm{kg}$

$\mu \mathrm{g} / \mathrm{kg}$

$\mu \mathrm{g} / \mathrm{kg}$

$\mu \mathrm{g} / \mathrm{kg}$

$\mu \mathrm{g} / \mathrm{kg}$

$\mu \mathrm{g} / \mathrm{kg}$

$\mu \mathrm{g} / \mathrm{kg}$

$\mu \mathrm{g} / \mathrm{kg}$

$\mu \mathrm{g} / \mathrm{kg}$

$\mu \mathrm{g} / \mathrm{kg}$

$\mu \mathrm{g} / \mathrm{kg}$

$\mu \mathrm{g} / \mathrm{kg}$

$\mu \mathrm{g} / \mathrm{kg}$

$\mu \mathrm{g} / \mathrm{kg}$

$\mu \mathrm{g} / \mathrm{kg}$

$\mu \mathrm{g} / \mathrm{kg}$

$\mu \mathrm{g} / \mathrm{kg}$

$\mu \mathrm{g} / \mathrm{kg}$

$\mu \mathrm{g} / \mathrm{kg}$

$\mu \mathrm{g} / \mathrm{kg}$

$\mu \mathrm{g} / \mathrm{kg}$

$\mu \mathrm{g} / \mathrm{kg}$

$\mu g / k g$

$\mu \mathrm{g} / \mathrm{kg}$

$\mu \mathrm{g} / \mathrm{kg}$

$\mu \mathrm{g} / \mathrm{kg}$

$\mu \mathrm{g} / \mathrm{kg}$
EPA8270

EPA8270

EPA8270

EPA8270

EPA8270

EPA8270

EPA8270

EPA8270

EPA8270

EPA8270

EPA8270

EPA8270

EPA8270

EPA8270

EPA8270

EPA8270

EPA8270

EPA8270

EPA8270

EPA8270

EPA8270

EPA8270

EPA8270

EPA8270

EPA8270

EPA8270

EPA8270

EPA8270

EPA8270

EPA 8270

EPA8270

EPA8270

EPA8270.

EPA8270

EPA8270

EPA8270

EPA8270

EPA8270

EPA 8270

EPA8270

EPA8270 
SAMPLE NAME: D431 1804 (Continued)

Constituent
Sample ID: 100405

RQ AQ B Result Unit

380

380

380

380

380

380

1,900

380

380

1,900

1,900

1,900

380

380

1,900

380

380

1,900

380

380

380

380

1,900

380

NV

200

NV $\quad 6,000$
D. Limit Method

1,2,4-Trichlorobenzene

2,4,6-Trichlorophenol

Unknown ester

Unknown ketone

$\begin{array}{lll}\mu g / \mathrm{kg} & 380 & \text { EPA8270 } \\ \mu \mathrm{g} / \mathrm{kg} & 380 & \text { EPA8270 } \\ \mu \mathrm{g} / \mathrm{kg} & 380 & \text { EPA8270 } \\ \mu \mathrm{g} / \mathrm{kg} & 380 & \text { EPA8270 } \\ \mu \mathrm{g} / \mathrm{kg} & 380 & \text { EPA8270 } \\ \mu \mathrm{g} / \mathrm{kg} & 380 & \text { EPA8270 } \\ \mu \mathrm{g} / \mathrm{kg} & 1,900 & \text { EPA8270 } \\ \mu \mathrm{g} / \mathrm{kg} & 380 & \text { EPA8270 } \\ \mu \mathrm{g} / \mathrm{kg} & 380 & \text { EPA8270 } \\ \mu \mathrm{g} / \mathrm{kg} & 1,900 & \text { EPA8270 } \\ \mu \mathrm{g} / \mathrm{kg} & 1,900 & \text { EPA8270 } \\ \mu \mathrm{g} / \mathrm{kg} & 1,900 & \text { EPA8270 } \\ \mu \mathrm{g} / \mathrm{kg} & 380 & \text { EPA8270 } \\ \mu \mathrm{g} / \mathrm{kg} & 380 & \text { EPA8270 } \\ \mu \mathrm{g} / \mathrm{kg} & 1,900 & \text { EPA8270 } \\ \mu \mathrm{g} / \mathrm{kg} & 380 & \text { EPA8270 } \\ \mu \mathrm{g} / \mathrm{kg} & 380 & \text { EPA8270 } \\ \mu \mathrm{g} / \mathrm{kg} & 1,900 & \text { EPA8270 } \\ \mu \mathrm{g} / \mathrm{kg} & 380 & \text { EPA8270 } \\ \mu \mathrm{g} / \mathrm{kg} & 380 & \text { EPA8270 } \\ \mu \mathrm{g} / \mathrm{kg} & 380 & \text { EPA8270 } \\ \mu \mathrm{g} / \mathrm{kg} & 380 & \text { EPA8270 } \\ \mu \mathrm{g} / \mathrm{kg} & 1,900 & \text { EPA8270 } \\ \mu \mathrm{gg} / \mathrm{kg} & 380 & \text { EPA8270 } \\ \mu \mathrm{g} / \mathrm{kg} & & \text { EPA8270 } \\ \mu \mathrm{g} / \mathrm{kg} & & \text { EPA8270 }\end{array}$

Volatile Organic Compounds

Acetone
Acetone
Benzene
Bromodichloromethane
Bromoform
Bromomethane (Methyl bromide)
Carbon disulfide
Carbon tetrachloride
Chlorobenzene
Chloroethane
Chloroethene (Vinyl chloride)
Chloroform
Chloromethane (Methyl chloride)
Dibromochloromethane
1,1-Dichloroethane
1,2-Dichloroethane
1,1-Dichloroethylene
1,2-Dichloroethylene
Dichloromethane (Methylene chloride)
1,2-Dichloropropane
cis-1,3-Dichloropropene

260
400
4.3
5.9
5.9
12
5.9
5.9
3.5
12
12
5.9
12
5.9
5.9
5.9
6.1
5.9
5.9
5.9
5.9

$\mu g / \mathrm{kg}$
$\mu \mathrm{g} / \mathrm{kg}$
$\mu \mathrm{g} / \mathrm{kg}$
$\mu \mathrm{g} / \mathrm{kg}$
$\mu \mathrm{g} / \mathrm{kg}$
$\mu \mathrm{gg}$
$\mu \mathrm{gg} / \mathrm{kg}$
$\mu \mathrm{g} / \mathrm{kg}$
$\mu \mathrm{g} / \mathrm{kg}$
$\mu \mathrm{g} / \mathrm{kg}$
$\mu \mathrm{g} / \mathrm{kg}$
$\mu \mathrm{g} / \mathrm{kg}$
$\mu \mathrm{g} / \mathrm{kg}$
$\mu \mathrm{g} / \mathrm{kg}$
$\mu \mathrm{g} / \mathrm{kg}$
$\mu \mathrm{g} / \mathrm{kg}$
$\mu \mathrm{g} / \mathrm{kg}$
$\mu \mathrm{g} / \mathrm{kg}$
$\mu \mathrm{g} / \mathrm{kg}$
$\mu \mathrm{gg}$
$\mu \mathrm{gg} / \mathrm{kg}$

$\begin{array}{ll}12 & \text { EPA8240 } \\ 23 & \text { EPA8240 } \\ 5.9 & \text { EPA8240 } \\ 5.9 & \text { EPA8240 } \\ 5.9 & \text { EPA8240 } \\ 12 & \text { EPA8240 } \\ 5.9 & \text { EPA8240 } \\ 5.9 & \text { EPA8240 } \\ 5.9 & \text { EPA8240 } \\ 12 & \text { EPA8240 } \\ 12 & \text { EPA8240 } \\ 5.9 & \text { EPA8240 } \\ 12 & \text { EPA8240 } \\ 5.9 & \text { EPA8240 } \\ 5.9 & \text { EPA8240 } \\ 5.9 & \text { EPA8240 } \\ 5.9 & \text { EPA8240 } \\ 5.9 & \text { EPA8240 } \\ 5.9 & \text { EPA8240 } \\ 5.9 & \text { EPA8240 } \\ 5.9 & \text { EPA8240 }\end{array}$


SAMPLE NAME: D431 1804 (Continued)

Conștituent

RQ

AQ B Result

5.9

trans-1,3-Dichloropropene

Ethylbenzene

2-Hexanone

Methyl ethyl ketone

Methyll isobutyl ketone

Styrene

1,1,2,2-Tetrachloroethane .

Tetrachloroethylene

Toluene

1,1,1-Trichloroethane

1,1,2-Trichloroethane

Trichloroethylene

Vinyl acetate

Xylenes
Sample ID: 100405

Unit D. Limit Method

Pesticides/PCBs and Dioxins/Furans

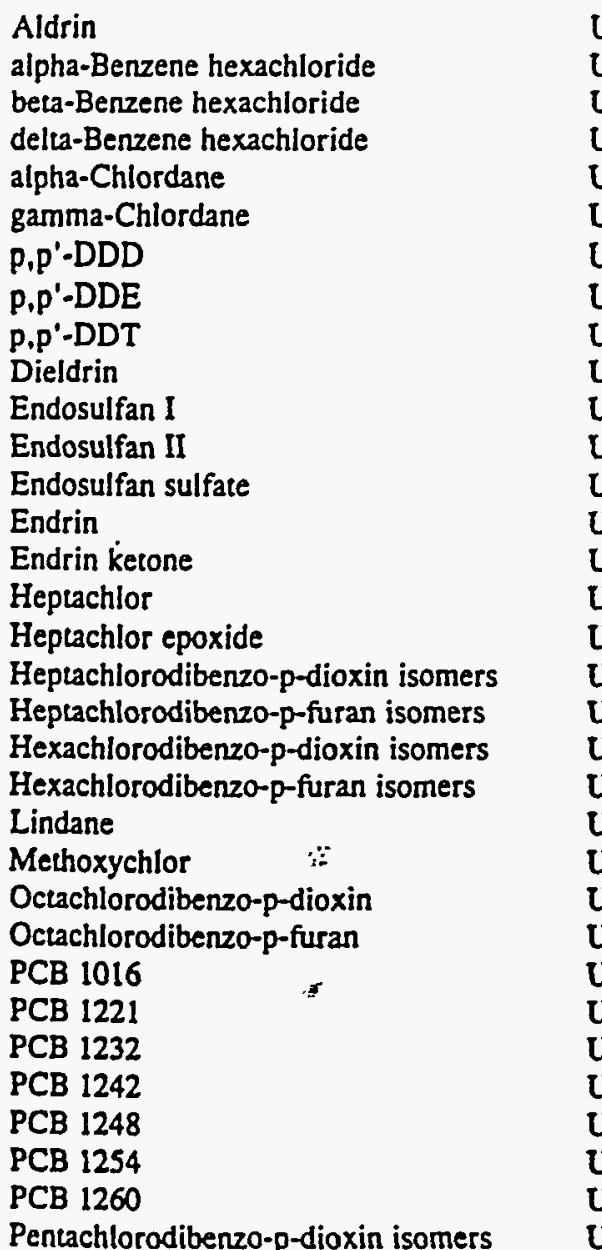

Aldrin

alpha-Benzene hexachloride

dela-Benzene hexachloride

alpha-Chlordane

gamma-Chlordane

P.P'-DDE

p.p'-DDT

Endosulfan II

Endosulfan sulfate

Hepiachlor

Heptachlor epoxide

Hexachlorodibenzo-p-dioxin isomers

Hexachlorodibenzo-p-furan isomers

Lindane

Octachlorodibenzo-p-dioxin

Octachlorodibenzo-p-furan

PCB 1232

PCB 1242

PCB 1248

PCB 1260

Pentachlorodibenzo-p-dioxin isomers

$\begin{array}{lll}\mathrm{U} & 5.9 & \mu \mathrm{g} / \mathrm{kg} \\ \mathrm{U} & 5.9 & \mu \mathrm{g} / \mathrm{kg} \\ \mathrm{U} & 12 & \mu \mathrm{g} / \mathrm{kg} \\ \mathrm{U} & 12 & \mu \mathrm{g} / \mathrm{kg} \\ \mathrm{U} & 12 & \mu \mathrm{g} / \mathrm{kg} \\ \mathrm{U} & 5.9 & \mu \mathrm{g} / \mathrm{kg} \\ \mathrm{U} & 5.9 & \mu \mathrm{g} / \mathrm{kg} \\ \mathrm{U} & 5.9 & \mu \mathrm{g} / \mathrm{kg} \\ \mathrm{J} & 4.9 & \mu \mathrm{g} / \mathrm{kg} \\ \mathrm{U} & 5.9 & \mu \mathrm{g} / \mathrm{kg} \\ \mathrm{U} & 5.9 & \mu \mathrm{g} / \mathrm{kg} \\ \mathrm{J} & 4.7 & \mu \mathrm{g} / \mathrm{kg} \\ \mathrm{U} & 12 & \mu \mathrm{g} / \mathrm{kg} \\ \mathrm{U} & 5.9 & \mu \mathrm{g} / \mathrm{kg}\end{array}$

$\begin{array}{ll}5.9 & \text { EPA8240 } \\ 5.9 & \text { EPA8240 } \\ 12 & \text { EPA8240 } \\ 12 & \text { EPA8240 } \\ 12 & \text { EPA8240 } \\ 5.9 & \text { EPA8240 } \\ 5.9 & \text { EPA8240 } \\ 5.9 & \text { EPA8240 } \\ 5.9 & \text { EPA8240 } \\ 5.9 & \text { EPA8240 } \\ 5.9 & \text { EPA8240 } \\ 5.9 & \text { EPA8240 } \\ 12 & \text { EPA8240 } \\ 5.9 & \text { EPA8240 }\end{array}$

$\mathrm{U}$

U

$U$

U

U

U

U

U

$U$

U

$\mathrm{U}$

$\mathrm{U}$

U

U

U

U

U

U

U

U

U 5.7

$\mathrm{U} \quad 0.30$

$\mathrm{U} \cdot 39$

U 77

U 39

U 39

U 39

U 39

U 39

U

\begin{tabular}{l}
1.9 \\
1.9 \\
1.9 \\
1.9 \\
1.9 \\
1.9 \\
3.9 \\
3.9 \\
3.9 \\
3.9 \\
1.9 \\
3.9 \\
3.9 \\
3.9 \\
3.9 \\
1.9 \\
1.9 \\
0.20 \\
0.10 \\
0.10 \\
0.10 \\
1.9 \\
19 \\
5.7 \\
0.30 \\
39 \\
77 \\
39 \\
39 \\
39 \\
39 \\
39 \\
0.20 \\
\hline
\end{tabular}

$\mu \mathrm{g} / \mathrm{kg}$

$\mu \mathrm{g} / \mathrm{kg}$

$\mu \mathrm{g} / \mathrm{kg}$

$\mu \mathrm{g} / \mathrm{kg}$

$\mu \mathrm{g} / \mathrm{kg}$

$\mu g / k g$

$\mu \mathrm{g} / \mathrm{kg}$

$\mu \mathrm{g} / \mathrm{kg}$

$\mu \mathrm{g} / \mathrm{kg}$

$\mu \mathrm{g} / \mathrm{kg}$

$\mu \mathrm{g} / \mathrm{kg}$

$\mu \mathrm{g} / \mathrm{kg}$

$\mu g / \mathrm{kg}$

$\mu \mathrm{g} / \mathrm{kg}$

$\mu \mathrm{g} / \mathrm{kg}$

$\mu \mathrm{g} / \mathrm{kg}$

$\mu \mathrm{g} / \mathrm{kg}$

$\mathrm{ng} / \mathrm{g}$

$\mathrm{ng} / \mathrm{g}$

$\mathrm{ng} / \mathrm{g}$

$\mathrm{ng} / \mathrm{g}$

$\mu \mathrm{g} / \mathrm{kg}$

$\mu \mathrm{g} / \mathrm{kg}$

$\mathrm{ng} / \mathrm{g}$

$\mathrm{ng} / \mathrm{g}$

$\mu \mathrm{g} / \mathrm{kg}$

$\mu \mathrm{g} / \mathrm{kg}$

$\mu \mathrm{g} / \mathrm{kg}$

$\mu \mathrm{g} / \mathrm{kg}$

$\mu \mathrm{g} / \mathrm{kg}$

$\mu \mathrm{g} / \mathrm{kg}$

$\mu \mathrm{g} / \mathrm{kg}$

$\mathrm{ng} / \mathrm{g}$

$\begin{array}{ll}1.9 & \text { EPA8080 } \\ 1.9 & \text { EPA8080 } \\ 1.9 & \text { EPA8080 } \\ 1.9 & \text { EPA8080 } \\ 1.9 & \text { EPA8080 } \\ 1.9 & \text { EPA8080 } \\ 3.9 & \text { EPA8080 } \\ 3.9 & \text { EPA8080 } \\ 3.9 & \text { EPA8080 } \\ 3.9 & \text { EPA8080 } \\ 1.9 & \text { EPA8080 } \\ 3.9 & \text { EPA8080 } \\ 3.9 & \text { EPA8080 } \\ 3.9 & \text { EPA8080 } \\ 3.9 & \text { EPA8080 } \\ 1.9 & \text { EPA8080 } \\ 1.9 & \text { EPA8080 } \\ 0.20 & \text { EPA8280 } \\ 0.10 & \text { EPA8280 } \\ 0.10 & \text { EPA8280 } \\ 0.10 & \text { EPA8280 } \\ 1.9 & \text { EPA8080 } \\ 19 & \text { EPA8080 } \\ 0.20 & \text { EPA8280 } \\ 0.30 & \text { EPA8280 } \\ 39 & \text { EPA8080 } \\ 77 & \text { EPA8080 } \\ 39 & \text { EPA8080 } \\ 39 & \text { EPA8080 } \\ 39 & \text { EPA8080 } \\ 39 & \text { EPA8080 } \\ 39 & \text { EPA8080 } \\ 0.20 & \text { EPA8280 }\end{array}$


SAMPLE NAME: D431 1804 (Continued)

Constituent
Sample ID: 100405

RQ AQ B Result Unit $\quad$ D. Limit Method

Pesticides/PCBs and Dioxins/Furans

Pentachlorodibenzo-p-furan isomers

2.3.7.8-TCDD

Tetrachlorodibenzo-p-dioxin isomers

Tetrachlorodibenzo-p-furan isomers

Toxaphene
2,3,7.8-TCDF

$\begin{array}{ll}U & 0.20 \\ U & 0.20 \\ U & 0.20 \\ U & 0.20 \\ U & 0.20 \\ U & 190\end{array}$

Radionuclide Indicators and Radionuclides

Gross alpha

Nonvolatile beta

Radium, total alpha-emitting

Tritium

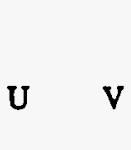

SAMPLE NAME: D431 2202

Location (SRS Coordinates): 18333E 66666N

Ground Elevation Above MSL: . $\mathrm{ft}$

Depth of Core Interval: 0.00 to $0.50 \mathrm{ft}$

Sample Type: Normal

Sample Color: 5YR 3/2

\section{Constituent}

Physical Parameters

$\mathrm{pH}$

Total organic carbon

Total Metals

Arsenic

Barium

Cadmium

Chromium

Copper

Lead

Manganese

Mercury

Nickel

Selenium

Silver $\mathrm{ng} / \mathrm{g}$

$\mathrm{ng} / \mathrm{g}$

$\mathrm{ng} / \mathrm{g}$

$\mathrm{ng} / \mathrm{g}$

$\mathrm{ng} / \mathrm{g}$

$\mu \mathrm{g} / \mathrm{kg}$

$7.5 \pm 6.1$

$13 \pm 9.5$

$0.80 \pm 0.85$

$3.5 \pm 0.68$

$\mathrm{pCi} / \mathrm{g}$

$\mathrm{pCi} / \mathrm{g}$

$\mathrm{pCi} / \mathrm{g}$

$\mathrm{pCi} / \mathrm{g}$

Sample ID: 100310

Sample Matrix: Sediment USC Soil Classification: $\mathrm{OH}$

RQ AQ B Result Unit

4.7 37,000

$\mathrm{pH}$ $\mathrm{mg} / \mathrm{kg}$

0.010

1,200

EPA9045

EPA415.1
Sample Moisture: Damp Percent Solids: 78.60

D. Limit Method
EPA8280

EPA8280

EPA8280

EPA8280

EPA8080 EPA903.0

EPA906.0

$B / N / A$ Extractables (including $P A H$ and phenols)

Acenaphthene

Acenaphthylene

Adipate

Aldol condensate
U 410

U 410

J N 700

J NVK $\quad 1,000$ $\mathrm{mg} / \mathrm{kg}$

$\mathrm{mg} / \mathrm{kg}$

$\mathrm{mg} / \mathrm{kg}$

$\mathrm{mg} / \mathrm{kg}$

$\mathrm{mg} / \mathrm{kg}$

$\mathrm{mg} / \mathrm{kg}$

$\mathrm{mg} / \mathrm{kg}$

$\mathrm{mg} / \mathrm{kg}$

$\mathrm{mg} / \mathrm{kg}$

$\mathrm{mg} / \mathrm{kg}$

$\mathrm{mg} / \mathrm{kg}$ 
SAMPLE NAME: D431 2202 (Continued)

Sample ID: 100310

$\begin{array}{lllll}\text { Constituent } & \text { RQ AQ B Result Unit D. Limit Method }\end{array}$

$B / N / A$ Extractables (including $P A H$ and phenols)

\begin{tabular}{|c|c|c|c|c|c|c|}
\hline Alkane & J & $\mathbf{N}$ & 300 & $\mu g / \mathrm{kg}$ & & EPA8270 \\
\hline Alkane & $J$ & $\mathrm{~N}$ & 900 & $\mu \mathrm{g} / \mathrm{kg}$ & & EPA8270 \\
\hline Alkane & J & $N$ & 300 & $\mu \mathrm{g} / \mathrm{kg}$ & & EPA8270 \\
\hline Alkane & $\mathbf{J}$ & $\mathbf{N}$ & 400 & $\mu \mathrm{g} / \mathrm{kg}$ & & EPA8270 \\
\hline Alkane & $\mathrm{J}$ & $\mathbf{N}$ & 500 & $\mu \mathrm{g} / \mathrm{kg}$ & & EPA8270 \\
\hline Anthracene & $\mathrm{U}$ & & 410 & $\mu g / k g$ & 410 & EPA8270 \\
\hline Benzo(a)anthracene & $\mathbf{U}$ & & 410 & $\mu \mathrm{g} / \mathrm{kg}$ & 410 & EPA8270 \\
\hline Benzo(b)fluoranthene & $\mathbf{U}$ & & 410 & $\mu \mathrm{g} / \mathrm{kg}$ & 410 & EPA8270 \\
\hline Benzo(k)fluoranthene & $\mathbf{U}$ & & 410 & $\mu \mathrm{g} / \mathrm{kg}$ & 410 & EPA8270 \\
\hline Benzoic acid & $\mathbf{U}$ & & 2,100 & $\mu g / \mathrm{kg}$ & 2,100 & EPA8270 \\
\hline Benzo(g,h,i)perylene & $\mathrm{U}$ & & 410 & $\mu \mathrm{g} / \mathrm{kg}$ & 410 & EPA8270 \\
\hline Benzo(a)pyrene & $\mathrm{U}$ & & 410 & $\mu g / \mathrm{kg}$ & 410 & EPA8270 \\
\hline Benzyl alcohol & $\mathrm{U}$ & & 410 & $\mu g / k g$ & 410 & EPA8270 \\
\hline Bis(2-chloroethoxy) methane & $\mathrm{U}$ & & 410 & $\mu g / k g$ & 410 & EPA8270 \\
\hline Bis(2-chloroethyl) ether & $\mathbf{U}$ & & 410 & $\mu \mathrm{g} / \mathrm{kg}$ & 410 & EPA8270 \\
\hline Bis(2-chloroisopropyl) ether & $\mathbf{U}$ & & 410 & $\mu \mathrm{g} / \mathrm{kg}$ & 410 & EPA8270 \\
\hline Bis(2-ethylhexyl) phthalate & UJ & $\mathrm{v}$ & 120 & $\mu \mathrm{g} / \mathrm{kg}$ & 410 & EPA8270 \\
\hline 4-Bromophenyl phenyl ether & $U$ & & 410 & $\mu \mathrm{g} / \mathrm{kg}$ & 410 & EPA8270 \\
\hline Burylbenzyl phthalate & U & & 410 & $\mu \mathrm{g} / \mathrm{kg}$ & 410 & EPA8270 \\
\hline 4-Chloroaniline & $\mathrm{U}$ & & 410 & $\mu \mathrm{g} / \mathrm{kg}$ & 410 & EPA8270 \\
\hline 4-Chloro-m-cresol & $\mathrm{U}$ & & 410 & $\mu \mathrm{g} / \mathrm{kg}$ & 410 & EPA8270 \\
\hline 2-Chloronaphthalene & $\mathrm{U}$ & & 410 & $\mu \mathrm{g} / \mathrm{kg}$ & 410 & EPA8270 \\
\hline 2-Chlorophenol & $\mathrm{U}$ & & 410 & $\mu \mathrm{g} / \mathrm{kg}$ & 410 & EPA8270 \\
\hline 4-Chlorophenyl phenyl ether & $\mathrm{U}$ & & 410 & $\mu \mathrm{g} / \mathrm{kg}$ & 410 & EPA8270 \\
\hline Chrysene & U & & 410 & $\mu \mathrm{g} / \mathrm{kg}$ & 410 & EPA8270 \\
\hline o-Cresol (2-Methylphenol) & $\mathrm{U}$ & & 410 & $\mu \mathrm{g} / \mathrm{kg}$ & 410 & EPA8270 \\
\hline p-Cresol (4-Methylphenol) & U & & 410 & $\mu \mathrm{g} / \mathrm{kg}$ & 410 & EPA8270 \\
\hline Dibenz(a,h)anthracene & $\mathrm{U}$ & & 410 & $\mu \mathrm{g} / \mathrm{kg}$ & 410 & EPA8270 \\
\hline Dibenzofuran & $\mathrm{J}$ & & 47 & $\mu \mathrm{g} / \mathrm{kg}$ & 410 & EPA8270 \\
\hline Di-n-butyl phthalate & J & & 41 & $\mu \mathrm{g} / \mathrm{kg}$ & 410 & EPA8270 \\
\hline 1,2-Dichlorobenzene & $\mathrm{U}$ & & 410 & $\mu \mathrm{g} / \mathrm{kg}$ & 410 & EPA8270 \\
\hline 1,3-Dichlorobenzene & U & & 410 & $\mu \mathrm{g} / \mathrm{kg}$ & 410 & EPA8270 \\
\hline 1,4-Dichlorobenzene & $\mathrm{U}$ & & 410 & $\mu \mathrm{g} / \mathrm{kg}$ & 410 & EPA8270 \\
\hline 3,3'-Dichlorobenzidine & U & & 820 & $\mu \mathrm{g} / \mathrm{kg}$ & 820 & EPA8270 \\
\hline 2,4-Dichlorophenol & $\mathrm{U}$ & & 410 & $\mu \mathrm{g} / \mathrm{kg}$ & 410 & EPA8270 \\
\hline Diechyl phthalate & $\mathrm{U}$ & & 410 & $\mu \mathrm{g} / \mathrm{kg}$ & 410 & EPA8270 \\
\hline 2,4-Dimethyl phenol & $\mathbf{U}$ & & 410 & $\mu \mathrm{g} / \mathrm{kg}$ & 410 & EPA8270 \\
\hline Dimethyl phthalate & U & & 410 & $\mu \mathrm{g} / \mathrm{kg}$ & 410 & EPA8270 \\
\hline 2,4-Dinitrophenol & $\mathbf{U}$ & & 2,100 & $\mu \mathrm{g} / \mathrm{kg}$ & 2,100 & EPA8270 \\
\hline 2,4-Dinitrotoluene & $\mathbf{U}$ & & 410 & $\mu g / \mathrm{kg}$ & 410 & EPA8270 \\
\hline 2,6-Dinitrotoluene & $\mathbf{U}$ & - & 410 & $\mu \mathrm{g} / \mathrm{kg}$ & 410 & EPA8270 \\
\hline Di-n-octyl phthalate & . U & & 410 & $\mu g / \mathrm{kg}$ & 410 & EPA8270 \\
\hline Fluoranthene & $\mathbf{U}$ & & 410 & $\mu g / k g$ & 410 & EPA8270 \\
\hline Fluorene & $\mathrm{U}$ & & 410 & $\mu g / k g$ & 410 & EPA8270 \\
\hline Hexachlorobenzene & $\mathbf{U}$ & & 410 & $\mu g / \mathrm{kg}$ & 410 & EPA8270 \\
\hline Hexachlorobutadiene & $\mathrm{U}$ & & 410 & $\mu \mathrm{g} / \mathrm{kg}$ & 410 & EPA8270 \\
\hline Hexachlorocyclopentadiene & U & & 410 & $\mu \mathrm{g} / \mathrm{kg}$ & 410 & EPA8270 \\
\hline Hexachloroethane & $\boldsymbol{U}$ & & 410 & $\mu \mathrm{g} / \mathrm{kg}$ & 410 & EPA8270 \\
\hline Indeno(1,2,3-c,d)pyrene & $\mathbf{U}$ & & 410 & $\mu \mathrm{g} / \mathrm{kg}$ & 410 & EPA8270 \\
\hline Isophorone & $U$ & & 410 & $\mu g / k g$ & 410 & EPA8270 \\
\hline
\end{tabular}


SAMPLE NAME: D431 2202 (Continued)

Sample ID: 100310

Constituent

RQ AQ B Result

Unit

D. Limit Method

$B / N / A$ Extractables (including $P A H$ and phenols)

2-Methyl-4.6-dinitrophenol

2-Methyinaphthalene

Naphthalene

o-Nitroaniline

m-Nitroaniline

p-Nitroaniline

Nitrobenzene

2-Nitrophenol

4-Nitrophenol

N-Nitrosodiphenylamine

N-Nitrosodipropylamine

Pentachlorophenol

Phenanthrene

Phenol

Pyrene

Terpene

1,2.4-Trichlorobenzene

2,4.5-Trichlorophenol

2.4.6-Trichlorophenol

Unknown

Volatile Organic Compounds

$\begin{array}{lll}\mathbf{U} & 2,100 \\ \mathrm{~J} & 130 \\ \mathrm{~J} & 78 \\ \mathrm{U} & 2,100 \\ \mathrm{U} & 2,100 \\ \mathrm{U} & 2,100 \\ \mathrm{U} & 410 \\ \mathrm{U} & 410 \\ \mathrm{U} & & 2,100 \\ \mathrm{U} & & 410 \\ \mathrm{U} & & 410 \\ \mathrm{U} & & 2,100 \\ \mathbf{J} & & 160 \\ \mathrm{U} & & 410 \\ \mathrm{U} & & 410 \\ \mathrm{~J} & \mathrm{~N} & 400 \\ \mathrm{U} & & 410 \\ \mathrm{U} & & 2,100 \\ \mathrm{U} & & 410 \\ \mathrm{~J} & \mathrm{~N} & 400\end{array}$

$\begin{array}{ll}2,100 & \text { EPA8270 } \\ 410 & \text { EPA8270 } \\ 410 & \text { EPA8270 } \\ 2,100 & \text { EPA8270 } \\ 2,100 & \text { EPA8270 } \\ 2,100 & \text { EPA8270 } \\ 410 & \text { EPA8270 } \\ 410 & \text { EPA8270 } \\ 2,100 & \text { EPA8270 } \\ 410 & \text { EPA8270 } \\ 410 & \text { EPA8270 } \\ 2,100 & \text { EPA8270 } \\ 410 & \text { EPA8270 } \\ 410 & \text { EPA8270 } \\ 410 & \text { EPA8270 } \\ & \text { EPA8270 } \\ 410 & \text { EPA8270 } \\ 2,100 & \text { EPA8270 } \\ 410 & \text { EPA8270 } \\ & \text { EPA8270 }\end{array}$

$\mu g / \mathrm{kg}$
$\mu g / \mathrm{kg}$
$\mu g / \mathrm{kg}$
$\mu g / \mathrm{kg}$
$\mu g / \mathrm{kg}$
$\mu g / \mathrm{kg}$
$\mu g / \mathrm{kg}$
$\mu g / \mathrm{kg}$
$\mu g / \mathrm{kg}$
$\mu g / \mathrm{kg}$
$\mu g / \mathrm{kg}$
$\mu g / \mathrm{kg}$
$\mu g / \mathrm{kg}$
$\mu g / \mathrm{kg}$
$\mu g / \mathrm{kg}$
$\mu g / \mathrm{kg}$
$\mu g / \mathrm{kg}$
$\mu g / \mathrm{kg}$
$\mu g / \mathrm{kg}$
$\mu g / \mathrm{kg}$

\section{5}

1,1 -Dichloroethylene

Dichloromethane (Methylene chloride)

EPA8240

EPA8240

EPA8240

EPA8240

EPA8240

EPA8240

EPA8240

EPA8240

EPA8240

EPA8240

EPA8240

EPA8240

EPA8240

EPA8240

EPA8240

EPA8240

EPA8240

EPA8240

EPA8240

EPA8240

EPA8240

EPA8240

EPA8240

EPA8240

EPA8240

EPA8240

EPA8240 
SAMPLE NAME: D431 2202 (Continued)

Constituent

Volatile Organic Compounds

1,1,2,2-Tetrachloroethane

Tetrachloroechylene

Toluene

1,1,1-Trichloroethane

1,1,2-Trichloroethane

Trichloroethylene

Unknown

Vinyl acetate

Xylenes
RQ AQ B Result

U

U

U

$\mathrm{U}$

U

J $N$

U

U
Sample D: 100310

Unit

D. Limit Method

Radionuclide Indicators and Radionuclides

Gross alpha

Nonvolatile beta

Radium, total alpha-emitting

Tritium

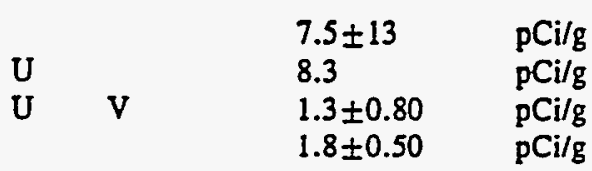

Sample ID: 100311

Ássociated Sample: 100310

RQ AQ B Result Unit

D. Limit Method

$7.4 \quad$ EPA900.0

$8.3 \quad$ EPA900.0

$0.10 \quad$ EPA903.0

$1.8 \quad$ EPA906.0
SAMPLE NAME: D431 2202A

Sample Type: Duplicate

Constituent

Physical Parameters

$\mathrm{pH}$

$\mathrm{pH}$

Total organic carbon
4.7

4.8

7,900

\section{Total Metals}

Arsenic
Barium
Cadmium
Chromium
Copper
Lead
Manganese
Mercury
Nickel
Selenium
Silver

$\begin{array}{ccc} & 11 & \mathrm{mg} / \mathrm{kg} \\ \mathrm{U} & 22 & \mathrm{mg} / \mathrm{kg} \\ & 0.51 & \mathrm{mg} / \mathrm{kg} \\ & 44 & \mathrm{mg} / \mathrm{kg} \\ & 5.2 & \mathrm{mg} / \mathrm{kg} \\ & 12 & \mathrm{mg} / \mathrm{kg} \\ & 94 & \mathrm{mg} / \mathrm{kg} \\ \mathrm{U} & .0 .25 & \mathrm{mg} / \mathrm{kg} \\ & 4.1 & \mathrm{mg} / \mathrm{kg} \\ \mathrm{U} & 0.74 & \mathrm{mg} / \mathrm{kg} \\ & 1.0 & \mathrm{mg} / \mathrm{kg}\end{array}$

$B / N / A$ Extractables (including $P A H$ and phenols)

Acenaphthene

Acenaphthylene

Adipate

Aldol condensate

$\begin{array}{lll}\text { U } & & 380 \\ \mathrm{U} & & 380 \\ \mathrm{~J} & \mathrm{~N} & 900 \\ \mathrm{~J} & \mathrm{NVK} & 1000\end{array}$

J NVK $\quad 1.000$

$\begin{array}{lll}\mathrm{pH} & 0.010 & \text { EPA9045 } \\ \mathrm{pH} & 0.010 & \text { EPA9045 } \\ \mathrm{mg} / \mathrm{kg} & 230 & \text { EPA415.1 }\end{array}$

EPA415.1 
SAMPLE NAME: D431 2202A (Continued)

Constituent
Sample ID: 100311

RQ AQ B Result Unit D. Limit Method

$B / N / A$ Extractables (including $P A H$ and phenols)

\begin{tabular}{|c|c|c|c|c|c|c|}
\hline Aldol condensate & $\mathrm{J}$ & NK & 300 & $\mu \mathrm{g} / \mathrm{kg}$ & & EPA8270 \\
\hline Alkane & J & $\mathrm{N}$ & 300 & $\mu \mathrm{g} / \mathrm{kg}$ & & EPA8270 \\
\hline Alkane & J & $N$ & 200 & $\mu g / k g$ & & EPA8270 \\
\hline Alkane & J & $\mathbf{N}$ & 200 & $\mu \mathrm{g} / \mathrm{kg}$ & & EPA8270 \\
\hline Anthracene & $\mathrm{U}$ & & 380 & $\mu \mathrm{g} / \mathrm{kg}$ & 380 & EPA8270 \\
\hline Benzo(a)anthracene & $\mathrm{U}$ & & 380 & $\mu \mathrm{g} / \mathrm{kg}$ & 380 & EPA8270 \\
\hline Benzo(b)fluoranthene & $\mathbf{U}$ & & 380 & $\mu \mathrm{g} / \mathrm{kg}$ & 380 & EPA8270 \\
\hline Benzo(k)fluoranthene & $\mathrm{U}$ & & 380 & $\mu \mathrm{g} / \mathrm{kg}$ & 380 & EPA8270 \\
\hline Benzoic acid & $\mathrm{U}$ & & 1,900 & $\mu \mathrm{g} / \mathrm{kg}$ & 1,900 & EPA8270 \\
\hline Benzo(g,h.i)perylene & $\mathrm{U}$ & & 380 & $\mu \mathrm{g} / \mathrm{kg}$ & 380 & EPA8270 \\
\hline Benzo(a)pyrene & U & & 380 & $\mu \mathrm{g} / \mathrm{kg}$ & 380 & EPA8270 \\
\hline Benzyl alcohol & $\mathrm{U}$ & & 380 & $\mu \mathrm{g} / \mathrm{kg}$ & 380 & EPA8270 \\
\hline Bis(2-chioroethoxy) methane & $\mathrm{U}$ & & 380 & $\mu \mathrm{g} / \mathrm{kg}$ & 380 & EPA8270 \\
\hline Bis(2-chloroethyl) ether & $\mathbf{U}$ & & 380 & $\mu \mathrm{g} / \mathrm{kg}$ & 380 & EPA8270 \\
\hline Bis(2-chloroisopropyl) ether & U & & 380 & $\mu \mathrm{g} / \mathrm{kg}$ & 380 & EPA8270 \\
\hline Bis(2-ethylhexyl) phthalate & UJ & $\mathrm{v}$ & 130 & $\mu \mathrm{g} / \mathrm{kg}$ & 380 & EPA8270 \\
\hline 4-Bromophenyl phenyl ether & $U$ & & 380 & $\mu \mathrm{g} / \mathrm{kg}$ & 380 & EPA8270 \\
\hline Butylbenzyl phthalate & $\mathrm{U}$ & & 380 & $\mu \mathrm{g} / \mathrm{kg}$ & 380 & EPA8270 \\
\hline 4-Chloroaniline & $\mathrm{U}$ & & 380 & $\mu \mathrm{g} / \mathrm{kg}$ & 380 & EPA8270 \\
\hline 4-Chloro-m-cresol & $\mathbf{U}$ & & 380 & $\mu \mathrm{g} / \mathrm{kg}$ & 380 & EPA8270 \\
\hline 2-Chloronaphthalene & $\mathbf{U}$ & & 380 & $\mu \mathrm{g} / \mathrm{kg}$ & 380 & EPA8270 \\
\hline 2-Chlorophenol & $\mathbf{U}$ & & 380 & $\mu \mathrm{g} / \mathrm{kg}$ & 380 & EPA8270 \\
\hline 4-Chlorophenyl phenyl ether & $\mathbf{U}$ & & 380 & $\mu \mathrm{g} / \mathrm{kg}$ & 380 & EPA8270 \\
\hline Chrysene & $\mathrm{U}$ & & 380 & $\mu \mathrm{g} / \mathrm{kg}$ & 380 & EPA8270 \\
\hline o-Cresol (2-Methylphenol) & $\mathbf{U}$ & & 380 & $\mu \mathrm{g} / \mathrm{kg}$ & 380 & EPA8270 \\
\hline p-Cresol (4-Methylphenol) & $\mathrm{U}$ & & 380 & $\mu \mathrm{g} / \mathrm{kg}$ & 380 & EPA8270 \\
\hline Dibenz $(\mathrm{a}, \mathrm{h}$ )anthracene & $\mathbf{U}$ & & 380 & $\mu \mathrm{g} / \mathrm{kg}$ & 380 & EPA8270 \\
\hline Dibenzofuran & $\mathbf{U}$ & & 380 & $\mu \mathrm{g} / \mathrm{kg}$ & 380 & EPA8270 \\
\hline Di-n-butyl phthalate & $\mathbf{U}$ & & 380 & $\mu \mathrm{g} / \mathrm{kg}$ & 380 & EPA8270 \\
\hline 1,2-Dichlorobenzene & $\mathrm{U}$ & & 380 & $\mu \mathrm{g} / \mathrm{kg}$ & 380 & EPA8270 \\
\hline 1,3-Dichlorobenzene & $\mathrm{U}$ & & 380 & $\mu \mathrm{g} / \mathrm{kg}$ & 380 & EPA8270 \\
\hline 1.4-Dichlorobenzene & $\mathrm{U}$ & & 380 & $\mu \mathrm{g} / \mathrm{kg}$ & 380 & EPA8270 \\
\hline 3,3'-Dichlorobenzidine & $\mathrm{U}$ & & 760 & $\mu \mathrm{g} / \mathrm{kg}$ & 760 & EPA8270 \\
\hline 2.4-Dichlorophenol & $\mathbf{U}$ & & 380 & $\mu \mathrm{g} / \mathrm{kg}$ & 380. & EPA8270 \\
\hline Diethyl phthalate & $\mathrm{U}$ & & 380 & $\mu \mathrm{g} / \mathrm{kg}$ & 380 & EPA8270 \\
\hline 2.4-Dimethyl phenol & $\mathbf{U}$ & & 380 & $\mu \mathrm{g} / \mathrm{kg}$ & 380 & EPA8270 \\
\hline Dimethyl phthalate & $\mathbf{U}$ & & 380 & $\mu \mathrm{g} / \mathrm{kg}$ & 380 & EPA8270 \\
\hline 2,4-Dinitrophenol & $U$ & & 1,900 & $\mu \mathrm{g} / \mathrm{kg}$ & 1,900 & EPA8270 \\
\hline 2,4-Dinitrotoluene & $\mathbf{U}$ & & 380 & $\mu \mathrm{g} / \mathrm{kg}$ & 380 & EPA8270 \\
\hline 2,6-Dinitrotoluene & $U$ & & 380 & $\mu g / \mathrm{kg}$ & 380 & EPA8270 \\
\hline Di-n-octyl phthalate & J & & 100 & $\mu g / \mathrm{kg}$ & 380 & EPA8270 \\
\hline Fluoranthene & U & & 380 & $\mu \mathrm{g} / \mathrm{kg}$ & 380 & EPA8270 \\
\hline Fuorene & $\mathbf{U}$ & & 380 & $\mu g / \mathrm{kg}$ & 380 & EPA8270 \\
\hline Hexachlorobenzene & $U$ & & 380 & $\mu g / \mathrm{kg}$ & 380 & EPA8270 \\
\hline Hexachlorobutadiene & $\mathbf{U}$ & & 380 & $\mu g / \mathrm{kg}$ & $380^{\circ}$ & EPA8270 \\
\hline Hexachlorocyclopentadiene & $U$ & & 380 & $\mu \mathrm{g} / \mathrm{kg}$ & 380 & EPA8270 \\
\hline Hexachloroethane & $\mathrm{U}$ & & 380 & $\mu g / \mathrm{kg}$ & 380 & EPA8270 \\
\hline Indeno(1,2,3-c,d)pyrene & $U$ & & 380 & $\mu g / \mathrm{kg}$ & 380 & EPA8270 \\
\hline Isophorone & $\mathrm{U}$ & & 380 & $\mu \mathrm{g} / \mathrm{kg}$ & 380 & EPA8270 \\
\hline 2-Methyl-4,6-dinitrophenol & $\mathbf{U}$ & & 1,900 & $\mu g / k g$ & 1,900 & EPA8270 \\
\hline
\end{tabular}


SAMPLE NAME: D43ï 2202A (Continued)

Sample ID: 100311

Constituent

RQ AQ B Result

Unit

D. Limit Method

$B / N j / A$ Extractables (including $P A H$ and phenols)

2-Methylnaphthalene

Naphthalene

o-Nitroaniline

m-Nitroaniline

p-Nitroaniline

Nitrobenzene

2-Nitrophenol

4-Nitrophenol

N-Nitrosodiphenylamine

N-Nitrosodipropylamine

Pentachlorophenol

Phenanthrene

Phenol

Pyrene

1,2,4-Trichlorobenzene

2,4,5-Trichlorophenol

2,4.6-Trichlorophenol

Unknown

\begin{tabular}{|c|c|}
\hline $\mathbf{U}$ & 380 \\
\hline $\mathbf{U}$ & 380 \\
\hline $\mathbf{U}$ & 1,900 \\
\hline $\mathbf{U}$ & 1,900 \\
\hline $\mathbf{U}$ & 1,900 \\
\hline $\mathbf{U}$ & 380 \\
\hline $\mathbf{U}$ & 380 \\
\hline $\mathbf{U}$ & 1,900 \\
\hline $\mathrm{U}$ & 380 \\
\hline $\mathrm{U}$ & 380 \\
\hline $\mathbf{U}$ & 1,900 \\
\hline $\mathrm{J}$ & 71 \\
\hline$U$ & 380 \\
\hline $\mathrm{U}$ & 380 \\
\hline $\mathrm{U}$ & 380 \\
\hline $\mathrm{U}$ & 1,900 \\
\hline $\mathrm{U}$ & 380 \\
\hline NV & 200 \\
\hline
\end{tabular}

Volatile Organic Compounds
12

6.0

6.0

6.0

12

6.0

6.0

6.0

12

12

12

6.0

12

6.0

6.0

6.0

6.0

6.0

14

6.0

6.0

6.0

6.0

12

12

12

6.0

6.0

6.0

$\begin{array}{lll}\mu \mathrm{g} / \mathrm{kg} . & 380 & \text { EPA8270 } \\ \mu \mathrm{g} / \mathrm{kg} & 380 & \text { EPA8270 } \\ \mu \mathrm{g} / \mathrm{kg} & 1,900 & \text { EPA8270 } \\ \mu \mathrm{g} / \mathrm{kg} & 1,900 & \text { EPA8270 } \\ \mu \mathrm{g} / \mathrm{kg} & 1,900 & \text { EPA8270 } \\ \mu \mathrm{g} / \mathrm{kg} & 380 & \text { EPA8270 } \\ \mu \mathrm{g} / \mathrm{kg} & 380 & \text { EPA8270 } \\ \mu \mathrm{g} / \mathrm{kg} & 1,900 & \text { EPA8270 } \\ \mu \mathrm{g} / \mathrm{kg} & 380 & \text { EPA8270 } \\ \mu \mathrm{g} / \mathrm{kg} & 380 & \text { EPA8270 } \\ \mu \mathrm{g} / \mathrm{kg} & 1,900 & \text { EPA8270 } \\ \mu \mathrm{g} / \mathrm{kg} & 380 & \text { EPA8270 } \\ \mu \mathrm{g} / \mathrm{kg} & 380 & \text { EPA8270 } \\ \mu \mathrm{g} / \mathrm{kg} & 380 & \text { EPA8270 } \\ \mu \mathrm{g} / \mathrm{kg} & 380 & \text { EPA8270 } \\ \mu \mathrm{g} / \mathrm{kg} & 1,900 & \text { EPA8270 } \\ \mu \mathrm{g} / \mathrm{kg} & 380 & \text { EPA8270 } \\ \mu \mathrm{g} / \mathrm{kg} & & \text { EPA8270 }\end{array}$

\begin{tabular}{|c|c|c|}
\hline$\mu g / \mathrm{kg}$ & 12 & EPA 8240 \\
\hline$\mu \mathrm{g} / \mathrm{kg}$ & 6.0 & EPA 8240 \\
\hline$\mu \mathrm{g} / \mathrm{kg}$ & 6.0 & EPA8240 \\
\hline$\mu g / \mathrm{kg}$ & 6.0 & EPA8240 \\
\hline$\mu g / k g$ & 12 & EPA 8240 \\
\hline$\mu \mathrm{g} / \mathrm{kg}$ & 6.0 & EPA8240 \\
\hline$\mu \mathrm{g} / \mathrm{kg}$ & 6.0 & EPA 8240 \\
\hline$\mu \mathrm{g} / \mathrm{kg}$ & 6.0 & EPA 8240 \\
\hline$\mu g / \mathrm{kg}$ & 12 & EPA8240 \\
\hline$\mu \mathrm{g} / \mathrm{kg}$ & 12 & EPA8240 \\
\hline$\mu g / \mathrm{kg}$ & 12 & EPA8240 \\
\hline$\mu \mathrm{g} / \mathrm{kg}$ & 6.0 & EPA 8240 \\
\hline$\mu \mathrm{g} / \mathrm{kg}$ & 12 & EPA 8240 \\
\hline$\mu \mathrm{g} / \mathrm{kg}$ & 6.0 & EPA8240 \\
\hline$\mu \mathrm{g} / \mathrm{kg}$ & 6.0 & EPA8240 \\
\hline$\mu g / \mathrm{kg}$ & 6.0 & EPA8240 \\
\hline$\mu \mathrm{g} / \mathrm{kg}$ & 6.0 & EPA8240 \\
\hline$\mu g / k g$ & 6.0 & EPA 8240 \\
\hline$\mu \mathrm{g} / \mathrm{kg}$ & 6.0 & EPA 8240 \\
\hline$\mu \mathrm{g} / \mathrm{kg}$ & 6.0 & EPA8240 \\
\hline$\mu \mathrm{g} / \mathrm{kg}$ & 6.0 & EPA8240 \\
\hline$\mu \mathrm{g} / \mathrm{kg}$ & 6.0 & EPA8240 \\
\hline$\mu \mathrm{g} / \mathrm{kg}$ & 6.0 & EPA 8240 \\
\hline$\mu \mathrm{g} / \mathrm{kg}$ & 12 & EPA 8240 \\
\hline$\mu \mathrm{g} / \mathrm{kg}$ & 12 & EPA 8240 \\
\hline$\mu \mathrm{g} / \mathrm{kg}$ & 12 & EPA8240 \\
\hline$\mu \mathrm{g} / \mathrm{kg}$ & 6.0 & EPA8240 \\
\hline$\mu g / \mathrm{kg}$ & 6.0 & EPA8240 \\
\hline$\mu \mathrm{g} / \mathrm{kg}$ & 6.0 & EPA8240 \\
\hline
\end{tabular}


SAMPLE NAME: D431 2202A (Continued)

Constituent

RQ AQ B Result

Volatile Organic Compounds

Toluene

1,1,1-Trichloroethane

1,1,2-Trichloroethane

Trichloroethylene

Unknown

Vinyl acetate

Xylenes

Sample ID: 100311

Unit D. Limit Method

$\begin{array}{llll}6.0 & \mu \mathrm{g} / \mathrm{kg} & 6.0 & \text { EPA8240 } \\ 6.0 & \mu \mathrm{g} / \mathrm{kg} & 6.0 & \text { EPA8240 } \\ 6.0 & \mu \mathrm{g} / \mathrm{kg} & 6.0 & \text { EPA8240 } \\ 6.0 & \mu \mathrm{g} / \mathrm{kg} & 6.0 & \text { EPA8240 } \\ 10 & \mu \mathrm{g} / \mathrm{kg} & & \text { EPA8240 } \\ 12 & \mu \mathrm{g} / \mathrm{kg} & 12 & \text { EPA8240 } \\ 6.0 & \mu \mathrm{g} / \mathrm{kg} & 6.0 & \text { EPA8240 }\end{array}$

Radionuclide Indicators and Radionuclides

Gross alpha

Nonvolatile beta

Radium, total alpha-emitting

Tritium

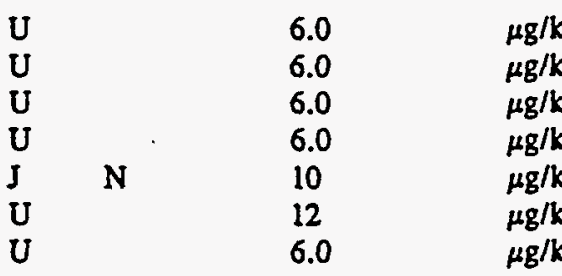

6.7

$8.7 \pm 12 \quad \mathrm{pCi} / \mathrm{g}$

$16 \pm 19 \quad \mathrm{pCi} / \mathrm{g}$

$1.2 \pm 0.80 \quad \mathrm{pCi} / \mathrm{g}$

$1.7 \pm 0.45 \quad \mathrm{pCi} / \mathrm{g}$

Sample ID: 100313

Sample Matrix: Sediment USC Soil Classification: SM

RQ AQ B Result Unit
Sample Moisture: Damp Percent Solids: 81.10

D. Limit Method

\section{Constituent}

Physical Parameters

$\mathrm{pH}$

Total organic carbon

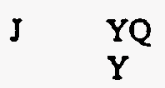

4.8

150,000

$\mathrm{pH}$

$\mathrm{mg} / \mathrm{kg}$

0.010

3.600

EPA9045

EPA415.1

Total Metals

Arsenic
Barium
Cadmium
Chromium
Copper
Lead
Manganese
Mercury
Nickel
Selenium
Silver

$\begin{array}{lll} & \mathbf{Y} & 27 \\ & \mathbf{Y} & 33 \\ \mathrm{U} & \mathbf{Y} & 0.60 \\ & \mathbf{Y} & 4.0 \\ & \mathbf{Y} & 3.6 \\ & \mathbf{Y} & 6.1 \\ & \mathbf{Y} & 64 \\ & \mathbf{Y} & 0.81 \\ \mathrm{U} & \mathbf{Y} & 4.8 \\ & \mathbf{Y} & 1.3 \\ \mathrm{U} & \mathbf{Y} & 1.2\end{array}$

$\mathrm{mg} / \mathrm{kg}$
$\mathrm{mg} / \mathrm{kg}$
$\mathrm{mg} / \mathrm{kg}$
$\mathrm{mg} / \mathrm{kg}$
$\mathrm{mg} / \mathrm{kg}$
$\mathrm{mg} / \mathrm{kg}$
$\mathrm{mg} / \mathrm{kg}$
$\mathrm{mg} / \mathrm{kg}$
$\mathrm{mg} / \mathrm{kg}$
$\mathrm{mg} / \mathrm{kg}$
$\mathrm{mg} / \mathrm{kg}$

6.0

EPA7060

1:2

$3.0^{-}$

0.36

1.8

$0.12 \quad$ EPA7470

EPA6010

EPA6010

EPA6010

EPA6010

EPA7421

EPA6010

4.8 EPA6010

$0.60 \quad$ EPA7740

1.2 EPA6010

$B / N / A$ Extractables (including PAH and phenols)

Acenaphthene

Acenaphthylene

Anthracene

$\begin{array}{lll}U & Y & 410 \\ U & Y & 410\end{array}$

J $Y$ $\mu g / \mathrm{kg}$
$\mu \mathrm{g} / \mathrm{kg}$
$\mu \mathrm{g} / \mathrm{kg}$
410

410

410
EPA8270

EPA8270

EPA8270 
SAMPLE NAME: D431 2302 (Continued)

Constituent

RQ AQ B Result

Unit

D. Limit Method

\section{$B / N / A$ Extractables (including PAH and phenols)}

\begin{tabular}{|c|c|c|c|c|c|c|}
\hline $\begin{array}{l}\text { Benzo(a)anthracene } \\
\text { Benzo(b)fluoranthene }\end{array}$ & $\mathrm{J}$ & $Y$ & 150 & $\mu g / k g$ & 410 & EPA8270 \\
\hline $\begin{array}{l}\text { Benzo(b)fluoranthene } \\
\text { Benzo(k)fluoranthene }\end{array}$ & U & $\mathbf{Y}$ & 410 & $\mu g / \mathrm{kg}$ & 410 & EPA8270 \\
\hline $\begin{array}{l}\text { Benzo(k)fluoranthene } \\
\text { Benzoic acid }\end{array}$ & $\mathbf{U}$ & $\mathbf{Y}$ & 410 & $\mu \mathrm{g} / \mathrm{kg}$ & 410 & EPA8270 \\
\hline $\begin{array}{l}\text { Benzoic acid } \\
\text { Benzo(g,h,i)perylene }\end{array}$ & & $\mathbf{Y}$ & 2,400 & $\mu g / \mathrm{kg}$ & 2,000 & EPA8270 \\
\hline $\begin{array}{l}\text { Benzo(g,h,i)perylene } \\
\text { Benzo(a)pyrene }\end{array}$ & J & $\mathbf{Y}$ & 100 & $\mu \mathrm{g} / \mathrm{kg}$ & 410 & EPA8270 \\
\hline $\begin{array}{l}\text { Benzo(a)pyrene } \\
\text { Benzyl alcohol }\end{array}$ & J & $\mathbf{Y}$ & 210 & $\mu \mathrm{g} / \mathrm{kg}$ & 410 & EPA8270 \\
\hline $\begin{array}{l}\text { Benzyl alcohol } \\
\text { Bis(2-chloroethoxy) methane }\end{array}$ & $\mathbf{U}$ & $\mathbf{Y}$ & 410 & $\mu \mathrm{g} / \mathrm{kg}$ & $410^{\circ}$ & EPA8270 \\
\hline $\begin{array}{l}\text { Bis(2-chloroethoxy) methane } \\
\text { Bis(2-chloroethyl) ether }\end{array}$ & $\mathrm{U}$ & $\mathbf{Y}$ & 410 & $\mu \mathrm{g} / \mathrm{kg}$ & 410 & EPA8270 \\
\hline & $\mathrm{U}$ & $\mathbf{Y}$ & 410 & $\mu g / k g$ & 410 & EPA8270 \\
\hline $\begin{array}{l}\text { Bis(2-chloroisopropyl) ether } \\
\text { Bis(2-ethylhexyl) phthalate }\end{array}$ & $\mathbf{U}$ & $\mathbf{Y}$ & 410 & $\mu \mathrm{g} / \mathrm{kg}$ & 410 & EPA8270 \\
\hline $\begin{array}{l}\text { Bis(2-ethylhexyl) phthalate } \\
\text { 4-Bromophenyl phenyl ether }\end{array}$ & $\mathrm{U}$ & $Y$ & 410 & $\mu g / k g$ & 410 & EPA8270 \\
\hline $\begin{array}{l}\text { 4-Bromophenyl phenyl ether } \\
\text { Butylbenzyl phthalate }\end{array}$ & U & $Y$ & 410 & $\mu g / k g$ & 410 & EPA8270 \\
\hline $\begin{array}{l}\text { Butylbenzyl phthalate } \\
\text { Hydrocarbon } \mathrm{Cl} 4 \mathrm{H} 30\end{array}$ & U & $Y$ & 410 & $\mu \mathrm{g} / \mathrm{kg}$ & 410 & EPA8270 \\
\hline $\begin{array}{l}\text { Hydrocarbon } \mathrm{C} 14 \mathrm{H} 30 \\
\text { Hydrocarbon } \mathrm{Cl} 6 \mathrm{H} 34\end{array}$ & S & YN & 1.000 & $\boldsymbol{\mu g} / \mathbf{k g}$. & & EPA8270 \\
\hline $\begin{array}{l}\text { Hydrocaroon CloH34 } \\
\text { Hydrocarbon } \mathrm{Cl} 19 \mathrm{H} 40\end{array}$ & the & IN & 4,000 & $\mu \mathrm{g} / \mathrm{kg}$ & & EPA8270 \\
\hline $\begin{array}{l}\text { 4yarocaroon C } 19 \mathrm{H} 40 \\
\text {-Chloroaniline }\end{array}$ & u & 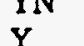 & 1,000 & $\mu \mathrm{g} / \mathrm{kg}$ & & EPA8270 \\
\hline 4-Chloro-m-cresol & $\mathrm{U}$ & $\mathrm{Y}$ & $\begin{array}{l}410 \\
410\end{array}$ & $\mu \mathrm{g} / \mathrm{kg}$ & $\begin{array}{l}410 \\
410\end{array}$ & $\begin{array}{l}\text { EPA8270 } \\
\text { EPA8270 }\end{array}$ \\
\hline 2-Chloronaphthalene & U & $\mathbf{Y}$ & 410 & $\mu \mathrm{g} / \mathrm{kg}$ & 410 & EPA8270 \\
\hline 2-Chlorophenol & $\mathbf{U}$ & $\mathbf{Y}$ & 410 & $\mu \mathrm{g} / \mathrm{kg}$ & 410 & EPA8270 \\
\hline 4-Chlorophenyl phenyl ether & $\mathbf{U}$ & $Y$ & 410 & $\mu \mathrm{g} / \mathrm{kg}$ & 410 & EPA8270 \\
\hline Chrysene & J & $\mathbf{Y}$ & 210 & $\mu g / k g$ & 410 & EPA8270 \\
\hline o-Cresol (2-Methylphenol) & $\mathrm{U}$ & $\mathbf{Y}$ & 410 & $\mu \mathrm{g} / \mathrm{kg}$ & 410 & EPA8270 \\
\hline p-Cresol (4-Methylphenol) & $\mathbf{U}$ & $\mathbf{Y}$ & 410 & $\mu \mathrm{g} / \mathrm{kg}$ & 410 & EPA8270 \\
\hline Dibenz $(a, h)$ anthracene & $U$ & $\mathbf{Y}$ & 410 & $\mu \mathrm{g} / \mathrm{kg}$ & 410 & EPA8270 \\
\hline Dibenzofuran & & $\mathbf{Y}$ & 450 & $\mu \mathrm{g} / \mathrm{kg}$ & 410 & EPA8270 \\
\hline Di-n-butyl phthalate & $\mathbf{U}$ & YV & 410 & $\mu \mathrm{g} / \mathrm{kg}$ & 410 & EPA8270 \\
\hline 1,2-Dichlorobenzene & U & $\mathbf{Y}$ & 410 & $\mu \mathrm{g} / \mathrm{kg}$ & 410 & EPA8270 \\
\hline 1,3-Dichlorobenzene & $\mathrm{U}$ & $Y$ & 410 & $\mu \mathrm{g} / \mathrm{kg}$ & 410 & EPA8270 \\
\hline 1,4-Dichlorobenzene & $U$ & $\mathbf{Y}$ & 410 & $\mu \mathrm{g} / \mathrm{kg}$ & 410 & EPA8270 \\
\hline 3,3'-Dichlorobenzidine & $\mathbf{U}$ & $\mathbf{Y}$ & 820 & $\mu \mathrm{g} / \mathrm{kg}$ & 820 & EPA8270 \\
\hline 2.4-Dichlorophenol & $\mathrm{U}$ & $\mathbf{Y}$ & 410 & $\mu \mathrm{g} / \mathrm{kg}$ & 410 & EPA8270 \\
\hline Diethyl phthalate & $\mathrm{U}$ & $\mathbf{Y}$ & 410 & $\mu g / \mathrm{kg}$ & 410 & EPA8270 \\
\hline 2,4-Dimethyl phenol & $\mathrm{U}$ & $\mathbf{Y}$ & 410 & $\mu g / \mathrm{kg}$ & 410 & EPA8270 \\
\hline Dimethyl phthalate & $\mathbf{U}$ & $\mathbf{Y}$ & 410 & $\mu \mathrm{g} / \mathrm{kg}$ & 410 & EPA8270 \\
\hline 2,4-Dinitrophenol & $\mathbf{U}$ & $\mathbf{Y}$ & 2,000 & $\mu \mathrm{g} / \mathrm{kg}$ & 2,000 & EPA8270 \\
\hline 2,4-Dinitrotoluene & U & $\mathbf{Y}$ & 410 & $\mu g / \mathrm{kg}$ & 410 & EPA8270 \\
\hline 2,6-Dinitrotoluene & $\mathbf{U}$ & $\mathbf{Y}$ & 410 & $\mu g / k g$ & 410 & EPA8270 \\
\hline Di-n-octyl phthalate $\because$ & J & $\mathbf{Y}$ & 46 & $\mu \mathrm{g} / \mathrm{kg}$ & 410 & EPA8270 \\
\hline Fluoranthene & J & $Y$ & 180 & $\mu g / \mathrm{kg}$ & 410 & EPA8270 \\
\hline Fluorene & $\mathrm{U}$ & $\mathbf{Y}$ & 410 & $\mu \mathrm{g} / \mathrm{kg}$ & 410 & EPA8270 \\
\hline Hexachlorobenzene & $\mathbf{U}$ & $\mathbf{Y}$ & 410 & $\mu \mathrm{g} / \mathrm{kg}$ & 410 & EPA8270 \\
\hline Hexachlorobutadiene & U & $\mathbf{Y}$ & 410 & $\mu \mathrm{g} / \mathrm{kg}$ & 410 & EPA8270 \\
\hline Hexachlorocyclopentadiene & $\mathrm{U}$ & $\mathbf{Y}$ & 410 & $\mu \mathrm{g} / \mathrm{kg}$ & $410^{4}$ & EPA8270 \\
\hline Hexachloroethane & $\mathbf{U}$ & $\mathbf{Y}$ & 410 & $\mu g / k g$ & 410 & EPA8270 \\
\hline Sub. hexanedioic acid & J & $\mathbf{Y N}$ & 10,000 & $\mu \mathrm{g} / \mathrm{kg}$ & & EPA8270 \\
\hline Indeno(1,2,3-c,d)pyrene & $\mathbf{U}$ & $\mathbf{Y}$ & 410 & $\mu \mathrm{g} / \mathrm{kg}$ & 410 & EPA8270 \\
\hline Isophorone & $\mathrm{U}$ & $\mathbf{Y}$ & 410 & $\mu \mathrm{g} / \mathrm{kg}$ & 410 & EPA8270 \\
\hline Dimethylnaphthalene + unknown & J & YN & 4,000 & $\mu \mathrm{g} / \mathrm{kg}$ & & EPA8270 \\
\hline Trimethylnaphthiene & J & YN & 2,000 & $\mu \mathrm{g} / \mathrm{kg}$ & & EPA8270 \\
\hline
\end{tabular}


SAMPLE NAME: D43i 2302 (Continued)

\author{
Constituent
}

RQ

AQ

B

$B / N / A$ Extractables (including $P A H$ and phenols)
Sample ID: 100313

\begin{tabular}{|c|c|c|}
\hline $\mathrm{J}$ & $\mathbf{Y}$ & 2.000 \\
\hline & $Y$ & 1,500 \\
\hline & $Y$ & 990 \\
\hline $\mathrm{U}$ & $\mathbf{Y}$ & 2,000 \\
\hline $\mathrm{U}$ & $\mathbf{Y}$ & 2,000 \\
\hline $\mathrm{U}$ & $\mathbf{Y}$ & 2,000 \\
\hline $\mathbf{U}$ & $\mathbf{Y}$ & 410 \\
\hline $\mathrm{U}$ & $\mathrm{Y}$ & 410 \\
\hline $\mathrm{U}$ & $Y$ & 2,000 \\
\hline $\mathrm{U}$ & $Y$ & 410 \\
\hline U. & CY & 410 \\
\hline $\mathrm{U}$ & & 2,000 \\
\hline & $\mathbf{Y}$ & 1,100 \\
\hline $\mathrm{U}$ & $Y$ & 410 \\
\hline J & $Y$ & 210 \\
\hline$U$ & $Y$ & 410 \\
\hline $\mathrm{U}$ & $Y$ & 2,000 \\
\hline U & $Y$ & 410 \\
\hline J & YN & 2.000 \\
\hline J & YN & 1,000 \\
\hline J & YN & 2,000 \\
\hline $\mathrm{J}$ & YN & 2,000 \\
\hline J & $Y N$ & 2,000 \\
\hline J & YN & 2,000 \\
\hline $\mathrm{J}$ & $Y N$ & 2,000 \\
\hline $\mathbf{J}$ & $\mathrm{YN}$ & 3,000 \\
\hline J & YN & 3,000 \\
\hline J & YN & 4,000 \\
\hline J & YN & 2,000 \\
\hline J & YN & 5,000 \\
\hline $\mathbf{J}$ & YNV & 5,000 \\
\hline T. & YN & 1,000 \\
\hline
\end{tabular}

\section{2,000}

2,000

2,000

410

410

2,000

410

2,000

1,100

210

410

410

2.000

1,000

2,000

2,000

2,000

2,000

3,000

4,000

2,000

5,000

1,000
Unit.

D. Limit Method
2-Methyl-4,6-dinitrophenol

2-Methylnaphthalene

Naphthalene

o-Nitroaniline

m-Nitroaniline

p-Nitroaniline

Nitrobenzene

2-Nitrophenol

4-Nitrophenol

N-Nitrosodiphenylamine

N-Nitrosodipropylamine

Pentachlorophenol

Phenanthrene

Phenol

Pyrene

1,2.4-Trichlorobenzene

2,4,5-Trichlorophenol

2,4,6-Trichlorophenol

Unknown hydrocarbon

Unknown hydrocarbon

Unknown hydrocarbon

Unknown hydrocarbon

Unknown hydrocarbon

Unknown hydrocarbon

Unknown hydrocarbon

Unknown hydrocarbon

Unknown hydrocarbon

Unknown hydrocarion

Unknown

Unknown

Unknown ketone

1-Methylnapthalene

$\begin{array}{lll}\mu \mathrm{g} / \mathrm{kg} & 2,000 & \text { EPA8270 } \\ \mu \mathrm{g} / \mathrm{kg} & 410 & \text { EPA8270 } \\ \mu \mathrm{g} / \mathrm{kg} & 410 & \text { EPA8270 } \\ \mu \mathrm{g} / \mathrm{kg} & 2,000 & \text { EPA8270 } \\ \mu \mathrm{g} / \mathrm{kg} & 2,000 & \text { EPA8270 } \\ \mu \mathrm{g} / \mathrm{kg} & 2,000 & \text { EPA8270 } \\ \mu \mathrm{g} / \mathrm{kg} & 410 & \text { EPA8270 } \\ \mu \mathrm{g} / \mathrm{kg} & 410 & \text { EPA8270 } \\ \mu \mathrm{g} / \mathrm{kg} & 2,000 & \text { EPA8270 } \\ \mu \mathrm{g} / \mathrm{kg} & 410 & \text { EPA8270 } \\ \mu \mathrm{g} / \mathrm{kg} & 410 & \text { EPA8270 } \\ \mu \mathrm{g} / \mathrm{kg} & 2,000 & \text { EPA8270 } \\ \mu \mathrm{g} / \mathrm{kg} & 410 & \text { EPA8270 } \\ \mu \mathrm{g} / \mathrm{kg} & 410 & \text { EPA8270 } \\ \mu \mathrm{g} / \mathrm{kg} & 410 & \text { EPA8270 } \\ \mu \mathrm{g} / \mathrm{kg} & 410 & \text { EPA8270 } \\ \mu \mathrm{g} / \mathrm{kg} & 2,000 & \text { EPA8270 } \\ \mu \mathrm{g} / \mathrm{kg} & 410 & \text { EPA8270 } \\ \mu \mathrm{g} / \mathrm{kg} & \cdot & \text { EPA8270 } \\ \mu \mathrm{g} / \mathrm{kg} & & \text { EPA8270 } \\ \mu \mathrm{g} / \mathrm{kg} & & \text { EPA8270 } \\ \mu \mathrm{g} / \mathrm{kg} & & \text { EPA8270 } \\ \mu \mathrm{g} / \mathrm{kg} & & \text { EPA8270 } \\ \mu \mathrm{g} / \mathrm{kg} & & \text { EPA8270 } \\ \mu \mathrm{g} / \mathrm{kg} & & \text { EPA8270 } \\ \mu \mathrm{\mu g} / \mathrm{kg} & & \text { EPA8270 } \\ \mu \mathrm{g} / \mathrm{kg} & & \text { EPA8270 } \\ \mu \mathrm{g} / \mathrm{kg} & & \text { EPA8270 } \\ \mu \mathrm{g} / \mathrm{kg} & & \text { EPA8270 } \\ \mu \mathrm{g} / \mathrm{kg} & & \text { EPA8270 } \\ \mu \mathrm{g} / \mathrm{kg} & & \text { EPA8270 } \\ \mu \mathrm{g} / \mathrm{kg} & & \text { EPA8270 } \\ & & -\end{array}$

Volatile Organic Compounds

Acetone
Benzene
Bromodichloromethane
Bromoform
Bromomethane (Methyl bromide)
Carbon disulfide
Carbon tetrachloride
Chlorobenzene
Chloroethane
Chloroethene (Vinyl chloride)
Chloroform
Chloromethane (Methyl chloride)
Dibromochloromethane
1,1-Dichloroethane
1,2-Dichloroethane

$\begin{array}{lll}U & Y V & 13 \\ U & Y & 6.2 \\ U & Y & 6.2 \\ U & Y & 6.2 \\ U & Y & 12 \\ U & Y & 6.2 \\ U & Y & 6.2 \\ U & Y & 6.2 \\ U & Y & 12 \\ U & Y & 12 \\ U & Y & 6.2 \\ U & Y & 12 \\ U & Y & 6.2 \\ U & Y & 6.2 \\ & Y & 6.5\end{array}$

$\because$

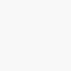


SAMPLE NAME: D431 2302 (Continued)

Constituent

\section{RQ}

AQ

Volatile Organic Compounds

1,1-Dichloroethylene
1,2-Dichloroethylene
Dichloromethane (Methylene chloride)
1,2-Dichloropropane
cis-1,3-Dichloropropene
trans-1,3-Dichloropropene
Ethylbenzene
2-Hexanone
Methyl ethyl ketone
Methyl isobutyl ketone
Styrene
1,1,2,2-Tetrachloroethane
Tetrachloroethylene
Toluene
1,1,1-Trichloroethane
1.1,2-Trichloroethane
Trichloroethylene
Vinyl acetate
Xylenes

Sample ID: 100313
Unit
D. Limit Method

Radionuclide Indicators and Radionuclides

\section{Gross alpha}

Nonvolatile beta

Radium, total alpha-emitting

Tritium

\begin{tabular}{|c|c|c|c|}
\hline $\mathbf{U}$ & $\mathbf{Y}$ & 6.2 & $\mu \mathrm{g} / \mathrm{kg}$ \\
\hline $\mathrm{U}$ & $\mathbf{Y}$ & 6.2 & $\mu \mathrm{g} / \mathrm{kg}$ \\
\hline $\mathbf{U}$ & YV & 8.4 & $\mu \mathrm{g} / \mathrm{k}$ \\
\hline $\mathbf{U}$ & $\mathbf{Y}$ & 6.2 & $\mu \mathrm{g} / \mathrm{kg}$ \\
\hline $\mathbf{U}$ & $\mathbf{Y}$ & 6.2 & $\mu \mathrm{g} / \mathrm{kg}$ \\
\hline $\mathbf{U}$ & $Y$ & 6.2 & $\mu \mathrm{g} / \mathrm{k}$ \\
\hline $\mathbf{U}$ & $\mathbf{Y}$ & 6.2 & $\mu \mathrm{g} / \mathrm{kg}$ \\
\hline $\mathrm{U}$ & $\mathbf{Y}$ & 12 & $\mu \mathrm{g} / \mathrm{kg}$ \\
\hline U & $\mathbf{Y}$ & 12 & $\mu \mathrm{g} / \mathrm{k}$ \\
\hline $\mathbf{U}$ & $\mathbf{Y}$ & 12 & $\mu \mathrm{g} / \mathrm{kg}$ \\
\hline $\mathbf{U}$ & $\mathbf{Y}$ & 6.2 & $\mu \mathrm{g} / \mathrm{kg}$ \\
\hline $\mathbf{U}$ & $Y$ & 6.2 & $\mu \mathrm{g} / \mathrm{k}$ \\
\hline $\mathbf{U}$ & $\mathbf{Y}$ & 6.2 & $\mu \mathrm{g} / \mathrm{kg}$ \\
\hline $\mathbf{U}$ & $\mathbf{Y}$ & 6.2 & $\mu g / k$ \\
\hline $\mathbf{U}$ & $\mathbf{Y}$ & 6.2 & $\mu \mathrm{g} / \mathrm{k}$ \\
\hline U & $Y$ & 6.2 & $\mu \mathrm{g} / \mathrm{k}$ \\
\hline $\mathbf{U}$ & $Y$ & 6.2 & $\mu g / k$ \\
\hline $\mathbf{U}$ & $Y$ & 12 & $\mu \mathrm{g} / \mathrm{kg}$ \\
\hline $\mathbf{U}$ & $\mathbf{Y}$ & 6.2 & \\
\hline
\end{tabular}

$\begin{array}{ll}6.2 & \text { EPA8240 } \\ 6.2 & \text { EPA8240 } \\ 6.2 & \text { EPA8240 } \\ 6.2 & \text { EPA8240 } \\ 6.2 & \text { EPA8240 } \\ 6.2 & \text { EPA8240 } \\ 6.2 & \text { EPA8240 } \\ 12 & \text { EPA8240 } \\ 12 & \text { EPA8240 } \\ 12 & \text { EPA8240 } \\ 6.2 & \text { EPA8240 } \\ 6.2 & \text { EPA8240 } \\ 6.2 & \text { EPA8240 } \\ 6.2 & \text { EPA8240 } \\ 6.2 & \text { EPA8240 } \\ 6.2 & \text { EPA8240 } \\ 6.2 & \text { EPA8240 } \\ 12 & \text { EPA8240 } \\ 6.2 & \text { EPA8240 }\end{array}$

SAMPLE NAME: D431 2302D

Sample Type: Split

Constituent

Total Metals

Arsenic

Barium

Cadmium

Chromium

Copper

Lead

Manganese

Mercury

Nickel

Selenium

Silver

$\begin{array}{lll}\mathrm{Y} & 11 \pm 14 & \mathrm{pCi} / \mathrm{g} \\ \mathrm{Y} & 16 \pm 21 & \mathrm{pCi} / \mathrm{g} \\ \mathrm{YV} & 0.65 \pm 0.65 & \mathrm{pCi} / \mathrm{g} \\ \mathrm{Y} & 9.2 \pm 1.6 & \mathrm{pCi} / \mathrm{g}\end{array}$

Sample ID: 100314

Associated Sample: 100313

RQ AQ B Result Unit D. Limit Method

$\begin{array}{ll}8.1 & \text { EPA900.0 } \\ 9.9 & \text { EPA900.0 } \\ 0.070 & \text { EPA903.0 } \\ 1.9 & \text { EPA906.0 }\end{array}$

$\begin{array}{clllll} & \mathrm{Y} & 110 & \mathrm{mg} / \mathrm{kg} & .62 & \text { EPA7060 } \\ & \mathrm{Y} & 84 & \mathrm{mg} / \mathrm{kg} & 24 & \text { EPA6010 } \\ \mathrm{U} & \mathrm{Y} & 0.60 & \mathrm{mg} / \mathrm{kg} & 0.60 & \text { EPA6010 } \\ & \mathrm{Y} & 32 & \mathrm{mg} / \mathrm{kg} & 1.2 & \text { EPA6010 } \\ & \mathrm{Y} & 13 & \mathrm{mg} / \mathrm{kg} & 3.0 & \text { EPA6010 } \\ & \mathrm{Y} & 23 & \mathrm{mg} / \mathrm{kg} & 1.9 & \text { EPA7421 } \\ & \mathrm{Y} & 160 & \mathrm{mg} / \mathrm{kg} & 1.8 & \text { EPA6010 } \\ & \mathrm{Y} & 1.2 & \mathrm{mg} / \mathrm{kg} & 0.12 \sim & \text { EPA7470 } \\ \mathrm{U} & \mathrm{Y} & 4.8 & \mathrm{mg} / \mathrm{kg} & 4.8 & \text { EPA6010 } \\ & \mathrm{Y} & 3.5 & \mathrm{mg} / \mathrm{kg} & 0.62 & \text { EPA7740 } \\ \mathrm{U} & \mathrm{Y} & 1.2 & \mathrm{mg} / \mathrm{kg} & 1.2 & \text { EPA6010 }\end{array}$


SAMPLE NAME: D431 2302D (Continued)

RQ AQ B Result Unit

Sample ID: 100314

\section{Constituent}

\author{
D. Limit Method
}

$B / N / A$ Extractables (including $P A H$ and phenols)

Acenaphthene
Acenaphthylene
Anthracene
Benzo(a)anthracene
Benzo(b)fluoranthene
Benzo(k)fluoranthene
Benzoic acid
Benzo(g,h,i)perylene
Benzo(a)pyrene
Benzyl alcohol
Bis(2-chloroethoxy) methane
Bis(2-chloroethyl) ether
Bis(2-chloroisopropyl) ether
Bis(2-ethylhexyl) phthalate
4-Bromophenyl phenyl ether
Butylbenzyl phthalate
Hydrocarbon C14H30 + arom.
Hydrocarbon C16H34
Hydrocarbon C17H36
Hydrocarbon C19H40
4-Chloroaniline
4-Chloro-m-cresol
2-Chloronaphthàlene
2-Chlorophenol
4-Chlorophenyl phenyl ether
Chrysene
o-Cresol (2-Methylphenol)
p-Cresol (4-Methylphenol)
Dibenz(a.h)anthracene
Dibenzofuran
Di-n-butyl phthalate
1.2-Dichlorobenzene
1.3-Dichlorobenzene
1,4-Dichlorobenzene
3,3-Dichlorobenzidine
2.4-Dichlorophenol
Diethyl phthalate
2,4-Dimethyl phenol
Dimethyl phthalate
2.4-Dinitrophenol
2.4-Dinitrotoluene
2.6-Dinitrotoluene
Di-n-octyl phthalate
Fluoranthene
Fluorene
Hexachlorobenzene
Hexachlorobutadiene
Hexachlorocyclopentadiene
Hexachloroethane
Sub. hexanedioic acid

\begin{tabular}{|c|c|c|}
\hline $\mathbf{U}$ & $\mathbf{Y}$ & 380 \\
\hline $\mathbf{U}$ & $\mathbf{Y}$ & 380 \\
\hline $\mathbf{U}$ & $\mathbf{Y}$ & 380 \\
\hline $\mathbf{J}$ & $\mathbf{Y}$ & 370 \\
\hline $\mathbf{J}$ & $\mathbf{Y}$ & 300 \\
\hline $\mathbf{U}$ & $\mathbf{Y}$ & 380 \\
\hline $\mathrm{J}$ & $\mathbf{Y}$ & 1,200 \\
\hline J & $Y$ & 190 \\
\hline $\mathrm{J}$ & $Y$ & 350 \\
\hline $\mathrm{U}$ & $\bar{Y}$ & 380 \\
\hline $\mathrm{U}$ & $Y$ & 380 \\
\hline $\mathrm{U}$ & $Y$ & 380 \\
\hline $\mathrm{U}$ & $\mathrm{Y}$ & 380 \\
\hline J & $Y$ & 200 \\
\hline $\mathbf{U}$ & $Y$ & 380 \\
\hline $\mathrm{U}$ & $Y$ & 380 \\
\hline J & $Y N$ & 3,000 \\
\hline J & $Y N$ & 3,000 \\
\hline J & YN & 10,000 \\
\hline $\mathrm{J}$ & $\mathrm{YN}$ & 3,000 \\
\hline $\mathrm{U}$ & $Y$ & 380 \\
\hline U & $\mathbf{Y}$ & 380 \\
\hline $\mathrm{U}$ & $Y$ & 380 \\
\hline $\mathrm{U}$ & $Y$ & 380 \\
\hline \multirow[t]{2}{*}{$\mathbf{U}$} & $\mathbf{Y}$ & 380 \\
\hline & $\mathbf{Y}$ & 460 \\
\hline $\mathbf{U}$ & $\bar{Y}$ & 380 \\
\hline $\mathbf{U}$ & $\mathbf{Y}$ & 380 \\
\hline \multirow[t]{2}{*}{$\mathrm{U}$} & $Y$ & 380 \\
\hline & $Y$ & 1,300 \\
\hline $\mathrm{U}$ & YV & 380 \\
\hline $\mathrm{U}$ & $\mathbf{Y}$ & 380 \\
\hline$U$ & $Y$ & 380 \\
\hline$U$ & $\mathbf{Y}$ & 380 \\
\hline $\mathrm{U}$ & $Y$ & 770 \\
\hline $\mathrm{U}$ & $\mathbf{Y}$ & 380 \\
\hline $\mathrm{U}$ & $\mathbf{Y}$ & 380 \\
\hline $\mathrm{U}$ & $\mathbf{Y}$ & 380 \\
\hline 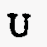 & $\mathbf{Y}$ & 380 \\
\hline $\mathrm{U}$ & $\mathbf{Y}$ & 1,900 \\
\hline $\mathbf{U}$ & $\mathbf{Y}$ & 380 \\
\hline $\mathbf{U}$ & $\mathbf{Y}$ & 380 \\
\hline \multirow[t]{2}{*}{ J } & YL & 90 \\
\hline & $Y$ & 450 \\
\hline$U$ & $\mathbf{Y}$ & 380 \\
\hline $\mathrm{U}$ & $\mathbf{Y}$ & 380 \\
\hline $\mathbf{U}$ & $Y$ & 380 \\
\hline $\mathbf{U}$ & $\bar{Y}$ & 380 \\
\hline $\mathbf{U}$ & $\mathbf{Y}$ & 380 \\
\hline J & $\mathrm{YN}$ & 10,000 \\
\hline
\end{tabular}

\begin{tabular}{|c|c|c|}
\hline$\mu g / \mathrm{kg}$ & 380 & EPA8270 \\
\hline$\mu \mathrm{g} / \mathrm{kg}$ & 380 & EPA 8270 \\
\hline$\mu g / \mathrm{kg}$ & 380 & EPA8270 \\
\hline$\mu g / \mathrm{kg}$ & 380 & EPA8270 \\
\hline$\mu \mathrm{g} / \mathrm{kg}$ & 380 & EPA8270 \\
\hline$\mu g / \mathrm{kg}$ & 380 & EPA8270 \\
\hline$\mu \mathrm{g} / \mathrm{kg}$ & 1,900 & EPA8270 \\
\hline$\mu \mathrm{g} / \mathrm{kg}$ & 380 & EPA8270 \\
\hline$\mu g / \mathrm{kg}$ & 380 & EPA8270 \\
\hline$\mu g / \mathrm{kg}$ & 380 & EPA8270 \\
\hline$\mu \mathrm{g} / \mathrm{kg}$ & 380 & EPA8270 \\
\hline$\mu g / k g$ & 380 & EPA8270 \\
\hline$\mu g / \mathrm{kg}$ & 380 & EPA8270 \\
\hline$\mu \mathrm{g} / \mathrm{kg}$ & 380 & EPA8270 \\
\hline$\mu \mathrm{g} / \mathrm{kg}$ & 380 & EPA8270 \\
\hline$\mu \mathrm{g} / \mathrm{kg}$ & 380 & EPA8270 \\
\hline$\mu \mathrm{g} / \mathrm{kg}$ & & EPA8270 \\
\hline$\mu \mathrm{g} / \mathrm{kg}$ & & EPA8270 \\
\hline$\mu g / k g$ & & EPA8270 \\
\hline$\mu \mathrm{g} / \mathrm{kg}$ & & EPA8270 \\
\hline$\mu \mathrm{g} / \mathrm{kg}$ & 380 & EPA8270 \\
\hline$\mu \mathrm{g} / \mathrm{kg}$ & 380 & EPA 8270 \\
\hline$\mu \mathrm{g} / \mathrm{kg}$ & 380 & EPA8270 \\
\hline$\mu \mathrm{g} / \mathrm{kg}$. & 380 & EPA8270 \\
\hline$\mu g / k g$ & 380 & EPA8270 \\
\hline$\mu \mathrm{g} / \mathrm{kg}$ & 380 & EPA 8270 \\
\hline$\mu \mathrm{g} / \mathrm{kg}$ & 380 & EPA8270 \\
\hline$\mu \mathrm{g} / \mathrm{kg}$ & 380 & EPA8270 \\
\hline$\mu \mathrm{g} / \mathrm{kg}$ & 380 & EPA8270 \\
\hline$\mu \mathrm{g} / \mathrm{kg}$ & 380 & EPA8270 \\
\hline$\mu \mathrm{g} / \mathrm{kg}$ & 380 & EPA8270 \\
\hline$\mu \mathrm{g} / \mathrm{kg}$ & 380 & EPA8270 \\
\hline$\mu \mathrm{g} / \mathrm{kg}$ & 380 & EPA8270 \\
\hline$\mu \mathrm{g} / \mathrm{kg}$ & 380 & EPA8270 \\
\hline$\mu \mathrm{g} / \mathrm{kg}$ & 770 & EPA 8270 \\
\hline$\mu g / \mathrm{kg}$ & 380 & EPA 8270 \\
\hline$\mu \mathrm{g} / \mathrm{kg}$ & 380 & EPA 8270 \\
\hline$\mu \mathrm{g} / \mathrm{kg}$ & 380 & EPA 8270 \\
\hline$\mu \mathrm{g} / \mathrm{kg}$ & 380 & EPA 8270 \\
\hline$\mu \mathrm{g} / \mathrm{kg}$ & 1,900 & EPA 8270 \\
\hline$\mu \mathrm{g} / \mathrm{kg}$ & 380 & EPA8270 \\
\hline$\mu \mathrm{g} / \mathrm{kg}$ & 380 & EPA 8270 \\
\hline$\mu g / k g$ & 380 & EPA8270 \\
\hline$\mu \mathrm{g} / \mathrm{kg}$ & 380 & EPA8270 \\
\hline$\mu \mathrm{g} / \mathrm{kg}$ & $380^{-}$ & EPA8270 \\
\hline$\mu \mathrm{g} / \mathrm{kg}$ & 380 & EPA8270 \\
\hline$\mu \mathrm{g} / \mathrm{kg}$ & 380 & EPA8270 \\
\hline$\mu \mathrm{g} / \mathrm{kg}$ & 380 & EPA8270 \\
\hline$\mu \mathrm{g} / \mathrm{kg}$ & 380 . & EPA8270 \\
\hline$\mu \mathrm{g} / \mathrm{kg}$ & & EPA8270 \\
\hline
\end{tabular}


SAMPLE NAME: D431 2302D (Continued)

\section{Constituent}

\section{RQ AQ B Result}

$B / N / A$ Extractables (including $P A H$ and phenols)
Sample ID: 100314

Unit

D. Limit Method

\begin{tabular}{|c|c|c|}
\hline U & $Y$ & 380 \\
\hline U & $\mathbf{Y}$ & 380 \\
\hline J & YN & 3,000 \\
\hline J & YN & 3,000 \\
\hline J & YN & 2,000 \\
\hline U & $Y$ & 1,900 \\
\hline & $Y$ & 3,600 \\
\hline & $Y$ & 2,700 \\
\hline U & $Y$ & 1,900 \\
\hline U & $Y$ & 1,900 \\
\hline U & $Y$ & 1,900 \\
\hline U & $Y$ & 380 \\
\hline U & $Y$ & 380 \\
\hline U & $Y$ & 1,900 \\
\hline J & $Y$ & 210 \\
\hline UJ & CY & 380 \\
\hline U & $Y$ & 1.900 \\
\hline & $Y$ & 2,700 \\
\hline U & $Y$ & 380 \\
\hline & $Y$ & 540 \\
\hline U & $Y$ & 380 \\
\hline U & $Y$ & 1,900 \\
\hline U & $Y$ & 380 \\
\hline J & YN & 3,000 \\
\hline J & $\mathbf{Y N}$ & 3,000 \\
\hline J & YN & 4,000 \\
\hline J & $Y N$ & 3,000 \\
\hline J & YN & 3,000 \\
\hline J & YN & 3,000 \\
\hline J & YN & 3,000 \\
\hline J & YN & 7,000 \\
\hline J & $Y N$ & 6,000 \\
\hline J & YN & 5,000 \\
\hline J & YNV & 7,000 \\
\hline J & YN & 3,000 \\
\hline
\end{tabular}

\section{Radionuclide Indicators and Radionuclides}

\section{Gross alpha}

Nonvolatile beta $\ddot{i}$

Radium, total alpha-emitting

Tritium

$\begin{array}{llll}\mathrm{U} & \mathrm{Y} & 7.0 & \mathrm{pCi} / \mathrm{g} \\ \mathrm{Y} & 32 \pm 20 & \mathrm{pCi} / \mathrm{g} \\ \mathrm{YV} & 0.99 \pm 0.82 & \mathrm{pCi} / \mathrm{g} \\ \mathrm{Y} & 5.9 \pm 1.2 & \mathrm{pCi} / \mathrm{g}\end{array}$

$\begin{array}{ll}7.0 & \text { EPA900.0 } \\ 8.3 & \text { EPA900.0 } \\ 0.070 & \text { EPA903.0 } \\ 1.9 & \text { EPA906.0 }\end{array}$

EPA906.0

$\begin{array}{lll}\mu \mathrm{g} / \mathrm{kg} & 380 & \text { EPA8270 } \\ \mu \mathrm{g} / \mathrm{kg} & 380 & \text { EPA8270 } \\ \mu \mathrm{g} / \mathrm{kg} & & \text { EPA8270 } \\ \mu \mathrm{g} / \mathrm{kg} & & \text { EPA8270 } \\ \mu \mathrm{g} / \mathrm{kg} & & \text { EPA8270 } \\ \mu \mathrm{g} / \mathrm{kg} & 1,900 & \text { EPA8270 } \\ \mu \mathrm{g} / \mathrm{kg} & 380 & \text { EPA8270 } \\ \mu \mathrm{g} / \mathrm{kg} & 380 & \text { EPA8270 } \\ \mu \mathrm{g} / \mathrm{kg} & 1,900 & \text { EPA8270 } \\ \mu \mathrm{g} / \mathrm{kg} & 1,900 & \text { EPA8270 } \\ \mu \mathrm{g} / \mathrm{kg} & 1,900 & \text { EPA8270 } \\ \mu \mathrm{g} / \mathrm{kg} & 380 & \text { EPA8270 } \\ \mu \mathrm{g} / \mathrm{kg} & 380 & \text { EPA8270 } \\ \mu \mathrm{g} / \mathrm{kg} & 1,900 & \text { EPA8270 } \\ \mu \mathrm{g} / \mathrm{kg} & 380 & \text { EPA8270 } \\ \mu \mathrm{g} / \mathrm{kg} & 380 & \text { EPA8270 } \\ \mu \mathrm{g} / \mathrm{kg} & 1,900 & \text { EPA8270 } \\ \mu \mathrm{g} / \mathrm{kg} & 380 & \text { EPA8270 } \\ \mu \mathrm{g} / \mathrm{kg} & 380 & \text { EPA8270 } \\ \mu \mathrm{g} / \mathrm{kg} & 380 & \text { EPA8270 } \\ \mu \mathrm{g} / \mathrm{kg} & 380 & \text { EPA8270 } \\ \mu \mathrm{g} / \mathrm{kg} & 1,900 & \text { EPA8270 } \\ \mu \mathrm{g} / \mathrm{kg} & 380 & \text { EPA8270 } \\ \mu \mathrm{g} / \mathrm{kg} & & \text { EPA8270 } \\ \mu \mathrm{g} / \mathrm{kg} & & \text { EPA8270 } \\ \mu \mathrm{g} / \mathrm{kg} & & \text { EPA8270 } \\ \mu \mathrm{g} / \mathrm{kg} & & \text { EPA8270 } \\ \mu \mathrm{g} / \mathrm{kg} & & \text { EPA8270 } \\ \mu \mathrm{g} / \mathrm{kg} & & \text { EPA8270 } \\ \mu \mathrm{g} / \mathrm{kg} & & \text { EPA8270 } \\ \mu \mathrm{g} / \mathrm{kg} & & \text { EPA8270 } \\ \mu \mathrm{g} / \mathrm{kg} & & \text { EPA8270 } \\ \mu \mathrm{g} / \mathrm{kg} & & \text { EPA8270 } \\ \mu \mathrm{g} / \mathrm{kg} & & \text { EPA8270 } \\ \mu \mathrm{g} / \mathrm{kg} & & \text { EPA8270 } \\ & & \\ & & \end{array}$


SAMPLE NAME: D43i 2402

Location (SRS Coordinates): 18187E 66506N

Ground Elevation Above MSL: . ft

Depth of Core Interval: 0.00 to $0.50 \mathrm{ft}$

Sample Type: Normal

Sample Color: 5YR 3/2

Constituent

RQ AQ B Result Unit

Sample Matrix: Sediment

USC Soil Classification: SM

Sample ID: 100323

Physical Parameters

$\mathrm{pH}$

Total organic carbon

J YQ

$\mathrm{Y}$

4.6

6,200

$\mathrm{pH}$

$\mathrm{mg} / \mathrm{kg}$

0.010

EPA9045

Total Metals

Arsenic

Barium

Cadmium

Chromium

Copper

Lead

Manganese

Mercury

Nickel

Selenium

Silver

$\begin{array}{ll}3.1 & \mathrm{mg} / \mathrm{kg} \\ 48 & \mathrm{mg} / \mathrm{kg} \\ 0.57 & \mathrm{mg} / \mathrm{kg} \\ 5.1 & \mathrm{mg} / \mathrm{kg} \\ 3.2 & \mathrm{mg} / \mathrm{kg} \\ 11 & \mathrm{mg} / \mathrm{kg} \\ 120 & \mathrm{mg} / \mathrm{kg} \\ 0.12 & \mathrm{mg} / \mathrm{kg} \\ 4.5 & \mathrm{mg} / \mathrm{kg} \\ 0.59 & \mathrm{mg} / \mathrm{kg} \\ 1.1 & \mathrm{mg} / \mathrm{kg}\end{array}$

$\mathrm{mg} / \mathrm{kg}$

$\mathrm{mg} / \mathrm{kg}$

$\mathrm{mg} / \mathrm{kg}$

$\mathrm{mg} / \mathrm{kg}$

$\mathrm{mg} / \mathrm{kg}$

$\mathrm{mg} / \mathrm{kg}$

$\mathrm{mg} / \mathrm{kg}$

$\mathrm{mg} / \mathrm{kg}$
Sample Moisture: Dry

Percent Solids: 84.50

D. Limit Method
EPA415.1

$\begin{array}{ll}1.2 & \text { EPA7060 } \\ 23 & \text { EPA6010 } \\ 0.57 & \text { EPA6010 } \\ 1.1 & \text { EPA6010 } \\ 2.8 & \text { EPA6010 } \\ 0.71 & \text { EPA7421 } \\ 1.7 & \text { EPA6010 } \\ 0.12 & \text { EPA7470 } \\ 4.5 & \text { EPA6010 } \\ 0.59 & \text { EPA7740 } \\ 1.1 & \text { EPA6010 }\end{array}$

1.1 EPA6010

$B / N / A$ Extractables (including PAH and phenols)

Acenaphthene

Acenaphthylene

Anthracene

Benzo(a)anthracene

Benzo(b)fluoranthene

Benzo(k)fluoranthene

Benzoic acid

Benzo(g,h,i)perylene

Benzo(a)pyrene

Benzyl alcohol

Bis(2-chloroethoxy) methane

Bis(2-chloroethyl) ether

Bis(2-chloroisopropyl) ether

Bis(2-ethylhexyl) phthalate

4-Bromophenyl phenyl ether

Butylbenzyl phthalate :

Sub. carboxylic acid

4-Chloroaniline

4-Chloro-m-cresol

2-Chloronaphthalene

2-Chlorophenol

4-Chlorophenyl phenyl ether

Chrysene

o-Cresol (2-Methylphenol)

p-Cresol (4-Methylphenol)

$\begin{array}{lll}\text { U } & Y & 400 \\ U & Y & 400 \\ U & Y & 400 \\ U & Y & 400 \\ U & Y & 400 \\ U & Y & 400 \\ U & Y & 2,000 \\ \text { U } & Y & 400 \\ U & Y & 400 \\ U & Y & 400 \\ U & Y & 400 \\ U & Y & 400 \\ U & Y & 400 \\ U & Y & 400 \\ U & Y & 400 \\ U & Y & 400 \\ J & Y N & 200 \\ U & Y & 400 \\ U & Y & 400 \\ U & Y & 400 \\ U & Y & 400 \\ U & Y & 400 \\ U & Y & 400 \\ U & Y & 400 \\ U & Y & 400\end{array}$

$\mu g / \mathrm{kg}$
$\mu g / \mathrm{kg}$
$\mu g / \mathrm{kg}$
$\mu g / \mathrm{kg}$
$\mu g / \mathrm{kg}$
$\mu g / \mathrm{kg}$
$\mu g / \mathrm{kg}$
$\mu g / \mathrm{kg}$
$\mu g / \mathrm{kg}$
$\mu g / \mathrm{kg}$
$\mu g / \mathrm{kg}$
$\mu g / \mathrm{kg}$
$\mu g / \mathrm{kg}$
$\mu g / \mathrm{kg}$
$\mu g / \mathrm{kg}$
$\mu g / \mathrm{kg}$
$\mu g / \mathrm{kg}$
$\mu g / \mathrm{kg}$
$\mu g / \mathrm{kg}$
$\mu g / \mathrm{kg}$
$\mu g / \mathrm{kg}$
$\mu g / \mathrm{kg}$
$\mu g / \mathrm{kg}$
$\mu g / \mathrm{kg}$
$\mu g / \mathrm{kg}$ 


$\begin{array}{llllll}\text { Constituent } & \text { RQ AQ B Result Dit Limit Method }\end{array}$

$B / N / A$ Extractables (including $P A H$ and phenols)

\begin{tabular}{|c|c|c|c|c|c|c|}
\hline Hexadecanoic acid & J & YN & 300 & $\mu g / \mathrm{kg}$ & & EPA8270 \\
\hline Dibenz(a,h)anthracene & $U$ & $\mathbf{Y}$ & 400 & $\mu g / k g$ & 400 & EPA8270 \\
\hline Dibenzofuran & $\mathrm{U}$ & $\mathbf{Y}$ & 400 & $\mu g / \mathrm{kg}$ & 400 & EPA8270 \\
\hline Di-n-butyl phthalate & UJ & YV & 70 & $\mu g / \mathrm{kg}$ & 400 & EPA8270 \\
\hline 1,2-Dichlorobenzene & $U$ & $Y$ & 400 & $\mu g / \mathrm{kg}$ & 400 & EPA8270 \\
\hline 1,3-Dichlorobenzene & U & $\mathrm{Y}$ & 400 & $\mu \mathrm{g} / \mathrm{kg}$ & 400 & EPA8270 \\
\hline 1,4-Dichlorobenzene & U & $Y$ & 400 & $\mu g / \mathrm{kg}$ & 400 & EPA8270 \\
\hline 3,3'-Dichlorobenzidine & $U$ & $\mathbf{Y}$ & 800 & $\mu \mathrm{g} / \mathrm{kg}$ & 800 & EPA8270 \\
\hline 2,4-Dichlorophenol & $\mathbf{U}$ & $\mathrm{Y}$ & 400 & $\mu \mathrm{g} / \mathrm{kg}$ & 400 & EPA8270 \\
\hline Diethyl phthalate & $\mathrm{U}$ & $\mathbf{Y}$ & 400 & $\mu \mathrm{g} / \mathrm{kg}$ & 400 & EPA8270 \\
\hline 2.4-Dimethyl phenol & $U$ & $Y$ & 400 & $\mu \mathrm{g} / \mathrm{kg}$ & 400 & EPA8270 \\
\hline Dimethyl phthalate & $\mathbf{U}$ & $Y$ & 400 & $\mu \mathrm{g} / \mathrm{kg}$ & 400 & EPA8270 \\
\hline 2,4-Dinitrophenol & $\mathrm{U}$ & $\mathbf{Y}$ & 2,000 & $\mu g / \mathrm{kg}$ & 2,000 & EPA 8270 \\
\hline 2,4-Dinitrotoluene & U & $\mathrm{Y}$ & 400 & $\mu g / \mathrm{kg}$ & 400 & EPA8270 \\
\hline 2,6-Dinitrotoluene & $\mathrm{U}$ & $Y$ & 400 & $\mu g / \mathrm{kg}$ & 400 & EPA8270 \\
\hline Di-n-octyl phthalate & $\mathrm{U}$ & $\mathbf{Y}$ & 400 & $\mu g / \mathrm{kg}$ & 400 & EPA8270 \\
\hline Fluoranthene & $\mathbf{U}$ & $Y$ & 400 & $\mu \mathrm{g} / \mathrm{kg}$ & 400 & EPA8270 \\
\hline Fluorene & $\mathrm{U}$ & $Y$ & 400 & $\mu \mathrm{g} / \mathrm{kg}$ & 400 & EPA8270 \\
\hline Hexachlorobenzene & $\mathrm{U}$ & $\mathrm{Y}$ & 400 & $\mu g / \mathrm{kg}$ & 400 & EPA8270 \\
\hline Hexachlorobutadiene & $\mathbf{U}$ & $\mathbf{Y}$ & 400 & $\mu \mathrm{g} / \mathrm{kg}$ & 400 & EPA8270 \\
\hline Hexachlorocyclopentadiene & $\mathrm{U}$ & $\mathbf{Y}$ & 400 & $\mu g / \mathrm{kg}$ & 400 & EPA8270 \\
\hline Hexachloroethane & $\mathrm{U}$ & $\mathrm{Y}$ & 400 & $\mu \mathrm{g} / \mathrm{kg}$ & 400 & EPA8270 \\
\hline Indenio(1,2,3-c,d)pyrene & U & $Y$ & 400 & $\mu \mathrm{g} / \mathrm{kg}$ & 400 & EPA8270 \\
\hline Isophorone & $\mathrm{U}$ & $Y$ & 400 & $\mu \mathrm{g} / \mathrm{kg}$ & 400 & EPA8270 \\
\hline 2-Methyl-4,6-dinitrophenol & $\mathbf{U}$ & $\mathbf{Y}$ & 2,000 & $\mu \mathrm{g} / \mathrm{kg}$ & 2,000 & EPA8270 \\
\hline 2-Methylnaphthalene & U & $Y$ & 400 & $\mu \mathrm{g} / \mathrm{kg}$ & 400 & EPA8270 \\
\hline Naphthalene & U & $\mathbf{Y}$ & 400 & $\mu \mathrm{g} / \mathrm{kg}$ & 400 & EPA8270 \\
\hline o-Nitroaniline & $U$ & $Y$ & 2,000 & $\mu \mathrm{g} / \mathrm{kg}$ & 2,000 & EPA8270 \\
\hline m-Nitroaniline & U & $Y$ & 2,000 & $\mu \mathrm{g} / \mathrm{kg}$ & 2,000 & EPA8270 \\
\hline p-Nitroaniline & $U$ & $\mathbf{Y}$ & 2,000 & $\mu \mathrm{g} / \mathrm{kg}$ & 2.000 & EPA8270 \\
\hline Nitrobenzene & $\mathbf{U}$ & $\mathbf{Y}$ & 400 & $\mu \mathrm{g} / \mathrm{kg}$ & 400 & EPA8270 \\
\hline 2-Nitrophenol & $\mathrm{U}$ & $\mathrm{Y}$ & 400 & $\mu \mathrm{g} / \mathrm{kg}$ & 400 & EPA8270 \\
\hline 4-Nitrophenol & $U$ & $\mathbf{Y}$ & 2.000 & $\mu g / \mathrm{kg}$ & 2.000 & EPA8270 \\
\hline N-Nitrosodiphenylamine & $\mathrm{U}$ & $Y$ & 400 & $\mu g / k g$ & 400 & EPA8270 \\
\hline N-Nitrosodipropylamine & UJ & CY & 400 & $\mu \mathrm{g} / \mathrm{kg}$ & 400 & EPA8270 \\
\hline Pentachlorophenol & $\mathbf{U}$ & $\mathbf{Y}$ & 2,000 & $\mu \mathrm{g} / \mathrm{kg}$ & 2.000 & EPA8270 \\
\hline Phenanthrene & $U$ & $\mathbf{Y}$ & 400 & $\mu \mathrm{g} / \mathrm{kg}$ & 400 & EPA8270 \\
\hline Phenol & $\mathbf{U}$ & $Y$ & 400 & $\mu g / k g$ & 400 & EPA8270 \\
\hline Sub. propanoic acid & J & YN & 200 & $\mu g / \mathrm{kg}$ & & EPA8270 \\
\hline Pyrene & $\mathbf{U}$ & $\mathbf{Y}$ & 400 & $\mu g / k g$ & 400 & EPA8270 \\
\hline 1,2,4-Trichlorobenzene & U & $\mathbf{Y}$ & 400 & $\mu \mathrm{g} / \mathrm{kg}$ & 400 & EPA8270 \\
\hline 2,4,5-Trichlorophenol & $\mathbf{U}$ & $\mathbf{Y}$ & 2,000 & $\mu \mathrm{g} / \mathrm{kg}$ & 2,000 & EPA8270 \\
\hline 2,4,6-Trichlorophenol. & $\mathbf{U}$ & $\mathbf{Y}$ & 400 & $\mu g / \mathbf{k g}$ & 400 & EPA8270 \\
\hline Unknown hydrocarbon & J & YN & 800 & $\mu \mathrm{g} / \mathrm{kg}$ & $"$ & EPA8270 \\
\hline Unknown hydrocarbon & J & YN & 1,000 & $\mu g / \mathrm{kg}$ & & EPA8270 \\
\hline Unknown hydrocarbon & J & YN & 600 & $\mu g / \mathrm{kg}$ & & EPA8270 \\
\hline Unknown & J & $\mathbf{Y N}$ & 200 & $\mu g / \mathrm{kg}$ & & EPA8270 \\
\hline Unknown & J & YN & 200 & $\mu \mathrm{g} / \mathrm{kg}$ & & EPA8270 \\
\hline Unknown & J & YN & 200 & $\mu g / \mathrm{kg}$ & & EPA8270 \\
\hline Unknown & $\mathrm{J}$ & YN & 1,000 & $\mu \mathrm{g} / \mathrm{kg}$ & & EPA8270 \\
\hline
\end{tabular}


SAMPLE NAME: D431 2402 (Continued)

Sample ID: 100323

Constituent

RQ AQ B Result

Unit

D. Limit Method

$B / N / A$ Extractables (including PAH and phenols)

Unknown

Unknown

Unknown

Unknown

Unknown

Unknown

Unknown

Unknown

Unknown

Unknown ketone
J $Y N$

J YN

J YN

J YN

J YN

J $Y N$

J $\mathrm{YN}$

J $Y N$

J YN

J YNV $\mu g / \mathrm{kg}$
$\mu \mathrm{g} / \mathrm{kg}$
$\mu \mathrm{g} / \mathrm{kg}$
$\mu \mathrm{g} / \mathrm{kg}$
$\mu \mathrm{g} / \mathrm{kg}$
$\mu \mathrm{g} / \mathrm{kg}$
$\mu \mathrm{g} / \mathrm{kg}$
$\mu \mathrm{g} / \mathrm{kg}$
$\mu \mathrm{g} / \mathrm{kg}$
$\mu \mathrm{g} / \mathrm{kg}$

1,000
EPA8270

EPA8270

EPA8270

EPA8270

EPA8270

EPA8270

EPA8270

EPA8270

EPA8270

EPA8270

Volatile Organic Compounds

Acetone

Benzene

Bromodichloromethane

Bromoform

Bromomethane (Methyl bromide)

Carbon disulfide

Carbon tetrachloride

Chlorobenzene

Chloroethane

Chloroethene (Vinyl chloride)

Chloroform

Chloromethane (Methyl chloride)

Dibromochloromethane

1,1-Dichloroethane

1,2-Dichloroethane

1,1-Dichloroethylene

1,2-Dichloroethylene

Dichloromethane (Methylene chloride)

1,2-Dichloropropane

cis-1,3-Dichloropropene

trans-1,3-Dichloropropene

Ethylbenzene

2-Hexanone

Methyl ethyl ketone

Methyl isobutyl ketone

Styrene

1,1,2,2-Tetrachloroethane

Tetrachloroethylene"

Toluene

1,1,1-Trichloroethane

1,1,2-Trichloroethane'

Trichloroethylene

Vinyl acetate

Xylenes

\begin{tabular}{|c|c|c|c|}
\hline & YV & 26 & $\mu g / \mathrm{kg}$ \\
\hline $\mathrm{U}$ & $Y$ & 6.1 & $\mu \mathrm{g} / \mathrm{kg}$ \\
\hline$U$ & $Y$ & 6.1 & $\mu \mathrm{g} / \mathrm{kg}$ \\
\hline $\mathrm{U}$ & $\mathbf{Y}$ & 6.1 & $\mu \mathrm{g} / \mathrm{kg}$ \\
\hline $\mathrm{U}$ & $\mathbf{Y}$ & 12 & $\mu \mathrm{g} / \mathrm{kg}$ \\
\hline $\mathrm{U}$ & $\mathbf{Y}$ & 6.1 & $\mu \mathrm{g} / \mathrm{kg}$ \\
\hline U & $\mathbf{Y}$ & 6.1 & $\mu g / \mathrm{kg}$ \\
\hline $\mathrm{U}$ & $\mathbf{Y}$ & 6.1 & $\mu \mathrm{g} / \mathrm{kg}$ \\
\hline $\mathrm{U}$ & $Y$ & 12 & $\mu \mathrm{g} / \mathrm{kg}$ \\
\hline $\mathrm{U}$ & $\mathbf{Y}$ & 12 & $\mu \mathrm{g} / \mathrm{kg}$ \\
\hline $\mathbf{U}$ & $\mathbf{Y}$ & 6.1 & $\mu g / \mathrm{kg}$ \\
\hline U & $\mathbf{Y}$ & 12 & $\mu g / k g$ \\
\hline $\mathbf{U}$ & $\mathbf{Y}$ & 6.1 & $\mu \mathrm{g} / \mathrm{kg}$ \\
\hline $\mathbf{U}$ & $\mathbf{Y}$ & 6.1 & $\mu \mathrm{g} / \mathrm{kg}$ \\
\hline & $\mathbf{Y}$ & 6.5 & $\mu \mathrm{g} / \mathrm{kg}$ \\
\hline U & $Y$ & 6.1 & $\mu \mathrm{g} / \mathrm{kg}$ \\
\hline $\mathrm{U}$ & $\mathbf{Y}$ & 6.1 & $\mu g / \mathrm{kg}$ \\
\hline U & $\mathrm{Y}$ & 6.1 & $\mu g / \mathrm{kg}$ \\
\hline$U$ & $\mathbf{Y}$ & 6.1 & $\mu \mathrm{g} / \mathrm{kg}$ \\
\hline $\mathrm{U}$ & $\mathbf{Y}$ & 6.1 & $\mu g / k g$ \\
\hline U & $\mathbf{Y}$ & 6.1 & $\mu g / \mathrm{kg}$ \\
\hline $\mathbf{U}$ & $\mathbf{Y}$ & 6.1 & $\mu \mathrm{g} / \mathrm{kg}$ \\
\hline $\mathrm{U}$ & $\mathbf{Y}$ & 12 & $\mu g / \mathrm{kg}$ \\
\hline U & $\mathbf{Y}$ & 12 & $\mu g / \mathrm{kg}$ \\
\hline $\mathrm{U}$ & $\mathbf{Y}$ & 12 & $\mu g / k g$ \\
\hline $\mathbf{U}$ & $\mathbf{Y}$ & 6.1 & $\mu \mathrm{g} / \mathrm{kg}$ \\
\hline U & $\mathbf{Y}$ & 6.1 & $\mu g / k g$ \\
\hline $\mathbf{U}$ & $\mathbf{Y}$ & 6.1 & $\mu \mathrm{g} / \mathrm{kg}$ \\
\hline $\mathbf{U}$ & $\mathbf{Y}$ & 6.1 & $\mu \mathrm{g} / \mathrm{kg}$ \\
\hline $\mathrm{U}$ & $\mathbf{Y}$ & 6.1 & $\mu \mathrm{g} / \mathrm{kg}$ \\
\hline & $\mathbf{Y}$ & 9.1 & $\mu \mathrm{g} / \mathrm{kg}$ \\
\hline $\mathbf{U}$ & $\mathbf{Y}$ & 6.1 & $\mu g / k$ \\
\hline$U$ & $\mathbf{Y}$ & 12 & $\mu \mathrm{g} / \mathrm{kg}$ \\
\hline U & $\mathbf{Y}$ & 6.1 & $\mu g / k$ \\
\hline
\end{tabular}

12

6.1

6.1

6.1

12

6.1

6.1

6.1

12

12

6.1

12

6.1

6.1

6.1

6.1

6.1

6.1

6.1

6.1

6.1

6.1

12

12

12

6.1

6.1

6.1

6.1

6.1

6.1

6.1

12

6.1
EPA8240

EPA8240

EPA8240

EPA8240

EPA8240

EPA8240

EPA8240

EPA8240

EPA8240

EPA8240

EPA8240

EPA8240

EPA8240

EPA8240

EPA8240

EPA8240

EPA8240

EPA8240

EPA8240

EPA8240

EPA8240

EPA8240

EPA8240

EPA8240

EPA8240

EPA8240

EPA8240

EPA8240

EPA8240

EPA8240

EPA8240

EPA8240

EPA8240

EPA8240 
SAMPLE NAME: D431 2402 (Continued)

Constituent

RQ AQ

Radionuclide Indicators and Radionuclides

Gross alpha

Nonvolatile beta

Radium, total alpha-emitting

Tritium

Sample ID: 100323

B Result Unit D. Limit Method

Sample Matrix: Sediment

USC Soil Classification: SM
Sample Moisture: Damp

Percent Solids: 84.20

D. Limit Method

\section{Constituent}

RQ AQ

B Result Unit

7.4

EPA900.0

$23 \pm 22$

$1.1 \pm 0.70$

$\mathrm{pCi} / \mathrm{g}$

$\mathrm{pCi} / \mathrm{g}$

8.3

EPA900.0

1.9

EPA906.0

Physical Parameters

$\mathrm{pH}$

Total organic carbon

Total Metals

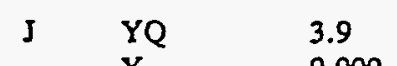

$Y$

9,000
$\mathrm{pH}$

$\mathrm{mg} / \mathrm{kg}$
0.010

440

$\mathrm{mg} / \mathrm{kg}$

$\mathrm{mg} / \mathrm{kg}$

$\mathrm{mg} / \mathrm{kg}$

$\mathrm{mg} / \mathrm{kg}$

$\mathrm{mg} / \mathrm{kg}$

$\mathrm{mg} / \mathrm{kg}$

$\mathrm{mg} / \mathrm{kg}$

$\mathrm{mg} / \mathrm{kg}$

$\mathrm{mg} / \mathrm{kg}$

$\mathrm{mg} / \mathrm{kg}$

$\mathrm{mg} / \mathrm{kg}$

$\begin{array}{ll}1.1 & \text { EPA7060 } \\ 22 & \text { EPA6010 } \\ 0.55 & \text { EPA6010 } \\ 1.1 & \text { EPA6010 } \\ 2.7 & \text { EPA6010 } \\ 0.34 & \text { EPA7421 } \\ 1.6 & \text { EPA6010 } \\ 0.12 & \text { EPA7470 } \\ 4.4 & \text { EPA6010 } \\ 0.57 & \text { EPA7740 } \\ 1.1 & \text { EPA6010 }\end{array}$

$B / N / A$ Extractables (including $P A H$ and phenols)

Acenaphthene

Acenaphthylene $\quad \because:$

Anthracene

Benzo(a)anthracene

Benzo(b)fluoranthene

Benzo(k)fluoranthene :

Benzoic acid

Benzo(g,h,i)perylene

Benzo(a)pyrene

Benzyl alcohol

Bis(2-chloroethoxy) methane

Bis(2-chloroethyl) ether

Bis(2-chloroisopropyl) ether

$\begin{array}{lll}U & Y & 390 \\ U & Y & 390 \\ U & Y & 390 \\ U & Y & 390 \\ U & Y & 390 \\ U & Y & 390 \\ U & Y & 2,000 \\ U & Y & 390 \\ U & Y & 390 \\ U & Y & 390 \\ U & Y & 390 \\ U & Y & 390 \\ U & Y & 390\end{array}$

$\therefore$

$390 \quad$ ERA8270

$390 \quad$ EPA8270

$390 \quad$ EPA8270

$390 \quad$ EPA8270

$390 \quad$ EPA8270

$390 \quad$ EPA8270

$2,000 \quad$ EPA8270

$390 \quad$ EPA8270

$390 \quad$ EPA8270

$390 \quad$ EPA8270

$390 \quad$ EPA8270

$390 \quad$ EPA8270

$390 \quad$ EPA8270 
SAMPLE NAME: D431 2502 (Continued)

Sample ID: 100324

Constituent RQ AQ B Result Unit D. Limit Method

$B / N / A$ Extractables (including $P A H$ and phenols)

Bis(2-ethylhexyl) phthalate 4-Bromophenyl phenyl ether Butylbenzyl phthalate Hydrocarbon $\mathrm{Cl} 2 \mathrm{H} 18$ + arom.

Sub. carboxylic acid

Sub. carboxylic acid

4-Chloroaniline

4-Chloro-m-cresol

2-Chloronaphthalene

2-Chlorophenol

4-Chlorophenyl phenyl ether

Chrysene

o-Cresol (2-Methylphenol)

p-Cresol (4-Methylphenol)

Hexadecanoic acid ester

Dibenz $(a, h)$ anthracene

Dibenzofuran

Di-n-butyl phthalate

1,2-Dichlorobenzene

1,3-Dichlorobenzene

1,4-Dichlorobenzene

3,3'-Dichlorobenzidine

2,4-Dichlorophenol

Diethyl phthalate

2,4-Dimethyl phenol

Dimethyl phthalate

2,4-Dinitrophenol

2,4-Dinitrotoluene

2,6-Dinitrotoluene

Di-n-octyl phthalate

Fluoranthene

Fluorene

Hexachlorobenzene

Hexachlorobutadiene

Hexachlorocyclopentadiene

Hexachloroethane

Indeno(1,2,3-c,d)pyrene

Isophorone

2-Methyl-4,6-dinitrophenol

2-Methylnaphthalene

Naphthalene

o-Nitroaniline

m-Nitroaniline

p-Nitroaniline

Nitrobenzene

2-Nitrophenol

4-Nitrophenol

N-Nitrosodiphenylamine

N-Nitrosodipropylamine

Pentachlorophenol

\begin{tabular}{|c|c|c|}
\hline $\mathrm{U}$ & $\mathbf{Y}$ & 390 \\
\hline U & $Y$ & 390 \\
\hline $\mathbf{U}$ & $\mathbf{Y}$ & 390 \\
\hline $\mathbf{J}$ & YN & 200 \\
\hline J & $\mathrm{YN}$ & 400 \\
\hline J & YN & 300 \\
\hline$U$ & $\mathbf{Y}$ & 390 \\
\hline $\mathbf{U}$ & $\mathbf{Y}$ & 390 \\
\hline U & $\mathbf{Y}$ & 390 \\
\hline $\mathbf{U}$ & $Y$ & 390 \\
\hline U & $Y$ & 390 \\
\hline$U$ & $Y$ & 390 \\
\hline U & $\mathbf{Y}$ & 390 \\
\hline $\mathbf{U}$ & $\mathbf{Y}$ & 390 \\
\hline J & YN & 200 \\
\hline $\mathbf{U}$ & $\mathbf{Y}$ & 390 \\
\hline$U$ & $\mathbf{Y}$ & 390 \\
\hline UJ & YV & 72 \\
\hline $\mathrm{U}$ & $Y$ & 390 \\
\hline $\mathrm{U}$ & $Y$ & 390 \\
\hline U & $\mathbf{Y}$ & 390 \\
\hline U & $Y$ & 780 \\
\hline$U$ & $Y$ & 390 \\
\hline $\mathrm{U}$ & $\mathrm{Y}$ & 390 \\
\hline $\mathrm{U}$ & $Y$ & 390 \\
\hline$U$ & $\mathbf{Y}$ & 390 \\
\hline $\mathbf{U}$ & $Y$ & 2,000 \\
\hline$U$ & $Y$ & 390 \\
\hline $\mathbf{U}$ & $Y$ & 390 \\
\hline $\mathbf{U}$ & $Y$ & 390 \\
\hline $\mathrm{U}$ & $\mathbf{Y}$ & 390 \\
\hline U & $Y$ & 390 \\
\hline U & $Y$ & 390 \\
\hline U & $Y$ & 390 \\
\hline U & $\mathbf{Y}$ & 390 \\
\hline$U$ & $Y$ & 390 \\
\hline$U$ & $\mathbf{Y}$ & 390 \\
\hline $\mathbf{U}$ & $\mathbf{Y}$ & 390 \\
\hline $\mathbf{U}$ & $\mathbf{Y}$ & 2,000 \\
\hline $\mathbf{U}$ & $\mathbf{Y}$ & 390 \\
\hline U & $\mathbf{Y}$ & 390 \\
\hline $\mathrm{U}$ & $\mathbf{Y}$ & 2,000 \\
\hline $\mathbf{U}$ & $Y$ & 2,000 \\
\hline U & $\mathbf{Y}$ & 2,000 \\
\hline U & $Y$ & 390 \\
\hline$U$ & $\mathbf{Y}$ & 390 \\
\hline $\mathbf{U}$ & $\mathbf{Y}$ & 2,000 \\
\hline $\mathbf{U}$ & $\mathbf{Y}$ & 390 \\
\hline UJ & CY & 390 \\
\hline$U$ & $\mathbf{Y}$ & 2,000 \\
\hline
\end{tabular}

\begin{tabular}{|c|c|c|}
\hline$\mu \mathrm{g} / \mathrm{kg}$ & 390 & EPA8270 \\
\hline$\mu \mathrm{g} / \mathrm{kg}$ & 390 & EPA8270 \\
\hline$\mu \mathrm{g} / \mathrm{kg}$ & 390 & EPA8270 \\
\hline$\mu \mathrm{g} / \mathrm{kg}$ & & EPA8270 \\
\hline$\mu \mathrm{g} / \mathrm{kg}$ & & EPA8270 \\
\hline$\mu g / k g$ & & EPA8270 \\
\hline$\mu \mathrm{g} / \mathrm{kg}$ & 390 & EPA8270 \\
\hline$\mu g / k g$ & 390 & EPA8270 \\
\hline$\mu g / k g$ & 390 & EPA8270 \\
\hline$\mu \mathrm{g} / \mathrm{kg}$ & 390 & EPA8270 \\
\hline$\mu \mathrm{g} / \mathrm{kg}$ & 390 & EPA8270 \\
\hline$\mu g / k g$ & 390 & EPA8270 \\
\hline$\mu \mathrm{g} / \mathrm{kg}$ & 390 & EPA8270 \\
\hline$\mu g / k g$ & 390 & EPA8270 \\
\hline$\mu g / k g$ & & EPA8270 \\
\hline$\mu \mathrm{g} / \mathrm{kg}$ & 390 & EPA8270 \\
\hline$\mu g / \mathrm{kg}$ & 390 & EPA8270 \\
\hline$\mu \mathrm{g} / \mathrm{kg}$ & 390 & EPA8270 \\
\hline$\mu \mathrm{g} / \mathrm{kg}$ & 390 & EPA8270 \\
\hline$\mu \mathrm{g} / \mathrm{kg}$ & 390 & EPA8270 \\
\hline$\mu g / k g$ & 390 & EPA8270 \\
\hline$\mu g / \mathrm{kg}$ & 780 & EPA8270 \\
\hline$\mu g / \mathrm{kg}$ & 390 & EPA8270 \\
\hline$\mu g / \mathrm{kg}$ & 390 & EPA8270 \\
\hline$\mu \mathrm{g} / \mathrm{kg}$ & 390 & EPA8270 \\
\hline$\mu g / k g$ & 390 & EPA8270 \\
\hline$\mu g / \mathrm{kg}$ & 2,000 & EPA8270 \\
\hline$\mu \mathrm{g} / \mathrm{kg}$ & 390 & EPA8270 \\
\hline$\mu g / \mathrm{kg}$ & 390 & EPA8270 \\
\hline$\mu \mathrm{g} / \mathrm{kg}$ & 390 & EPA8270 \\
\hline$\mu \mathrm{g} / \mathrm{kg}$ & 390 & EPA8270 \\
\hline$\mu \mathrm{g} / \mathrm{kg}$ & 390 & EPA8270 \\
\hline$\mu g / \mathrm{kg}$ & 390 & EPA8270 \\
\hline$\mu \mathrm{g} / \mathrm{kg}$ & 390 & EPA8270 \\
\hline$\mu g / \mathrm{kg}$ & 390 & EPA8270 \\
\hline$\mu g / \mathrm{kg}$ & 390 & EPA8270 \\
\hline$\mu g / k g$ & 390 & EPA8270 \\
\hline$\mu g / \mathrm{kg}$ & 390 & EPA8270 \\
\hline$\mu g / \mathrm{kg}$ & 2,000 & EPA8270 \\
\hline$\mu g / \mathrm{kg}$ & 390 & EPA8270 \\
\hline$\mu \mathrm{g} / \mathrm{kg}$ & 390 & EPA8270 \\
\hline$\mu g / \mathrm{kg}$ & 2,000 & EPA8270 \\
\hline$\mu g / \mathrm{kg}$ & 2,000 & EPA8270 \\
\hline$\mu g / \mathrm{kg}$ & 2,000 & EPA8270 \\
\hline$\mu g / \mathrm{kg}$ & 390 a & EPA8270 \\
\hline$\mu g / \mathrm{kg}$ & 390 & EPA8270 \\
\hline$\mu g / \mathrm{kg}$ & 2,000 & EPA8270 \\
\hline$\mu g / \mathrm{kg}$ & 390 & EPA8270 \\
\hline$\mu g / k g$ & 390 & EPA8270 \\
\hline$\mu g / k g$ & 2,000 & EPA8270 \\
\hline
\end{tabular}


SAMPLE NAME: D4312502 (Continued)

Sample ID: 100324

Constituent

RQ AQ B Result Unit

D. Limit Method

$B / N / A$ Extractables (including $P A H$ and phenols)

Phenanthrene

Phenol

Sub. propanoic acid

Pyrene

1,2,4-Trichlorobenzene

2,4,5-Trichlorophenol

2,4,6-Trichlorophenol

Unknown hydrocarbon

Unknown hydrocarbon

Unknown hydrocarbon

Unknown hydrocarbon + acid

Unknown

Unknown

Unknown

Unknown

Unknown

Unknown

Unknown

Unknown ketone

Volatile Organic Compounds

$\begin{array}{lll}\text { U } & Y & 390 \\ U & Y & 390 \\ J & Y N & 200 \\ U & Y & 390 \\ U & Y & 390 \\ U & Y & 2,000 \\ U & Y & 390 \\ J & Y N & 500 \\ J & Y N & 700 \\ J & Y N & 400 \\ J & Y N & 200 \\ J & Y N & 200 \\ J & Y N & 400 \\ J & Y N & 500 \\ J & Y N & 200 \\ J & Y N & 200 \\ J & Y N & 300 \\ J & Y N & 2,000 \\ J & Y N V & 50,000\end{array}$

$$
\begin{aligned}
& \mu g / \mathbf{k g} . \\
& \mu g / k g \\
& \mu g / k g \\
& \mu g / k g \\
& \mu g / k g \\
& \mu g / k g \\
& \mu g / k g \\
& \mu g / k g \\
& \mu g / k g \\
& \mu g / k g \\
& \mu g / k g \\
& \mu g / k g \\
& \mu g / k g \\
& \mu g / k g \\
& \mu g / k g \\
& \mu g / k g \\
& \mu g / k g \\
& \mu g / k g \\
& \mu g / k g
\end{aligned}
$$

$\begin{array}{ll}390 & \text { EPA8270 } \\ 390 & \text { EPA8270 } \\ & \text { EPA8270 } \\ 390 & \text { EPA8270 } \\ 390 & \text { EPA8270 } \\ 2,000 & \text { EPA8270 } \\ 390 & \text { EPA8270 } \\ & \text { EPA8270 } \\ & \text { EPA8270 } \\ & \text { EPA8270 } \\ & \text { EPA8270 } \\ & \text { EPA8270 } \\ & \text { EPA8270 } \\ & \text { EPA8270 } \\ & \text { EPA8270 } \\ & \text { EPA8270 } \\ & \text { EPA8270 } \\ & \text { EPA8270 } \\ \text { EPA8270 }\end{array}$

\begin{tabular}{lll}
$\mu \mathrm{g} / \mathrm{kg}$ & 12 & EPA8240 \\
$\mu \mathrm{g} / \mathrm{kg}$ & 12 & EPA8240 \\
$\mu \mathrm{g} / \mathrm{kg}$ & 12 & EPA8240 \\
$\mu \mathrm{g} / \mathrm{kg}$ & 6.0 & EPA8240 \\
$\mu \mathrm{g} / \mathrm{kg}$ & 6.0 & EPA8240 \\
$\mu \mathrm{g} / \mathrm{kg}$ & 6.0 & EPA8240 \\
$\mu \mathrm{g} / \mathrm{kg}$ & 6.0 & EPA8240 \\
$\mu \mathrm{g} / \mathrm{kg}$ & 6.0 & EPA8240 \\
$\mu \mathrm{g} / \mathrm{kg}$ & 6.0 & EPA8240 \\
$\mu \mathrm{g} / \mathrm{kg}$ & 6.0 & EPA8240 \\
$\mu \mathrm{g} / \mathrm{kg}$ & 12 & EPA8240 \\
$\mu \mathrm{g} / \mathrm{kg}$ & 12 & EPA8240 \\
$\mu \mathrm{g} / \mathrm{kg}$ & 12 & EPA8240 \\
$\mu \mathrm{g} / \mathrm{kg}$ & 6.0 & EPA8240 \\
$\mu \mathrm{g} / \mathrm{kg}$ & 6.0 & EPA8240 \\
$\mu \mathrm{g} / \mathrm{kg}$ & 6.0 & EPA8240 \\
$\mu \mathrm{g} / \mathrm{kg}$ & 6.0 & EPA8240 \\
$\mu \mathrm{g} / \mathrm{kg}$ & 6.0 & EPA8240 \\
$\mu \mathrm{g} / \mathrm{kg}$ & 6.0 & EPA8240 \\
$\mu \mathrm{g} / \mathrm{kg}$ & 6.0 & EPA8240 \\
$\mu \mathrm{g} / \mathrm{kg}$ & 12 & EPA8240 \\
$\mu \mathrm{g} / \mathrm{kg}$ & 12 & EPA8240 \\
$\mu \mathrm{g} / \mathrm{kg}$ & 12 & EPA8240 \\
$\mu \mathrm{g} / \mathrm{kg}$ & 12 & EPA8240 \\
$\mu \mathrm{g} / \mathrm{kg}$ & 12 & EPA8240 \\
$\mu \mathrm{g} / \mathrm{kg}$ & 12 & EPA8240 \\
$\mu \mathrm{g} / \mathrm{kg}$ & 6.0 & EPA8240 \\
$\mu \mathrm{g} / \mathrm{kg}$ & 6.0 & EPA8240 \\
\hline & &
\end{tabular}


SAMPLE NAME: D43r 2502 (Continued)

Constituent

$\mathbf{R Q}$

RQ AQ B Result

Unit

D. Limit Method

Volatile Organic Compounds

Chloroform

Chloromethane (Methyl chloride)

Chloromethane (Methyl chloride)

Chloromethane (Methyl chloride)

Dibromochloromethane

Dibromochloromethane

Dibromochloromethane

1,1-Dichloroethane

1,1-Dichloroethane

1,1-Dichloroethane

1,2-Dichloroethane

1,2-Dichloroethane

1.2-Dichloroethane

1.1-Dichloroethylene

1.2-Dichloroethylene

1,2-Dichloroethylene

1,2-Dichloroethylene

Dichloromethane (Methylene chloride)

Dichloromethane (Methylene chloride)

Dichloromethane (Methylene chloride)

1,2-Dichloropropane

1,2-Dichloropropane

1,2-Dichloropropane

cis-1,3-Dichloropropene

cis-1,3-Dichloropropene

cis-1,3-Dichloropropene

trans-1,3-Dichloropropene

trans-1,3-Dichloropropene

trans-1,3-Dichloropropene

Ethylbenzene

Ethylbenzene

Ethylbenzene

2-Hexanone

2-Hexanone

2-Hexanone

Methyl ethyl ketone

Methyi ethyl ketone

Methyl ethyl ketone

Methyl isobutyl ketone

Methyl isobutyl ketone

Methyl isobutyl ketone :

Styrene .

Styrene

Styrene

1,1,2,2-Tetrachloroethắne

1,1,2.2-Tetrachloroethane

1,1,2,2-Tetrachloroethane

Tetrachloroethylene

Tetrachloroethylene

Tetrachloroethylene

\begin{tabular}{|c|c|c|c|}
\hline $\mathbf{U}$ & $\mathbf{Y}$ & 6.0 & \\
\hline $\mathbf{U}$ & $\mathbf{Y}$ & 12 & \\
\hline $\mathbf{U}$ & $\mathbf{Y}$ & 12 & \\
\hline $\mathbf{U}$ & $\mathbf{Y}$ & 12 & \\
\hline $\mathbf{U}$ & $\mathbf{Y}$ & 6.0 & \\
\hline $\mathrm{U}$ & $\mathbf{Y}$ & 6.0 & \\
\hline $\mathrm{U}$ & $\mathbf{Y}$ & 6.0 & \\
\hline $\mathbf{U}$ & $\mathbf{Y}$ & 6.0 & \\
\hline $\mathbf{U}$ & $\mathbf{Y}$ & 6.0 & \\
\hline $\mathrm{U}$ & $\mathbf{Y}$ & 6.0 & \\
\hline $\mathrm{U}$ & $\mathbf{Y}$ & 6.0 & \\
\hline $\mathbf{U}$ & $\mathbf{Y}$ & 6.0 & \\
\hline $\mathbf{U}$ & $\mathbf{Y}$ & 6.0 & \\
\hline $\mathrm{U}$ & $\mathbf{Y}$ & 6.0 & \\
\hline $\mathrm{U}$ & $\mathbf{Y}$ & 6.0 & \\
\hline$U$ & $\mathrm{Y}$ & 6.0 & \\
\hline $\mathrm{U}$ & $\mathrm{Y}$ & 6.0 & \\
\hline$U$ & YV & 6.0 & \\
\hline $\mathrm{U}$ & YV & 6.0 & \\
\hline $\mathbf{U}$ & YV & 6.0 & \\
\hline $\mathrm{U}$ & $Y$ & 6.0 & \\
\hline$U$ & $Y$ & 6.0 & \\
\hline $\mathrm{U}$ & $\mathbf{Y}$ & 6.0 & \\
\hline$U$ & $\mathbf{Y}$ & 6.0 & . \\
\hline $\mathbf{U}$ & $\mathbf{Y}$ & 6.0 & " \\
\hline$U$ & $Y$ & 6.0 & \\
\hline $\mathbf{U}$ & $Y$ & 6.0 & \\
\hline$U$ & $Y$ & 6.0 & \\
\hline $\mathbf{U}$ & $Y$ & 6.0 & \\
\hline $\mathbf{U}$ & $Y$ & 6.0 & \\
\hline U & $Y$ & 6.0 & \\
\hline $\mathbf{U}$ & $Y$ & 6.0 & \\
\hline $\mathbf{U}$ & $\mathbf{Y}$ & 12 & \\
\hline $\mathrm{U}$ & $Y$ & 12 & \\
\hline $\mathrm{U}$ & $Y$ & 12 & \\
\hline $\mathbf{U}$ & $Y$ & 12 & \\
\hline $\mathbf{U}$ & $\mathbf{Y}$ & 12 & - \\
\hline $\mathbf{U}$ & $\mathbf{Y}$ & 12 & \\
\hline $\mathbf{U}$ & $\mathbf{Y}$ & 12 & \\
\hline $\mathbf{U}$ & $\mathbf{Y}$ & 12 & \\
\hline U & $Y$ & 12 & \\
\hline $\mathrm{U}$ & $Y$ & 6.0 & \\
\hline $\mathbf{U}$ & $\mathbf{Y}$ & 6.0 & \\
\hline $\mathbf{U}$ & $Y$ & 6.0 & \\
\hline $\mathrm{U}$ & $Y$ & 6.0 & \\
\hline $\mathbf{U}$ & $Y$ & 6.0 & \\
\hline $\mathrm{U}$ & $Y$ & 6.0 & \\
\hline $\mathbf{U}$ & $Y$ & 6.0 & \\
\hline $\mathrm{U}$ & $\mathbf{Y}$ & 6.0 & \\
\hline $\mathbf{U}$ & $\mathbf{Y}$ & 6.0 & \\
\hline
\end{tabular}

\begin{tabular}{|c|c|}
\hline 6.0 & EPA 8240 \\
\hline 12 & EPA 8240 \\
\hline 12 & EPA8240 \\
\hline 12 & EPA8240 \\
\hline 6.0 & EPA8240 \\
\hline 6.0 & EPA8240 \\
\hline 6.0 & EPA8240 \\
\hline 6.0 & EPA8240 \\
\hline 6.0 & EPA8240 \\
\hline 6.0 & EPA 8240 \\
\hline 6.0 & EPA824 \\
\hline 6.0 & EPA8240 \\
\hline 6.0 & EPA8240 \\
\hline 6.0 & EPA824 \\
\hline 6.0 & EPA8240 \\
\hline 6.0 & EPA8240 \\
\hline 6.0 & EPA824C \\
\hline 6.0 & EPA8240 \\
\hline 6.0 & EPA8240 \\
\hline 6.0 & EPA8240 \\
\hline 6.0 & EPA8240 \\
\hline 6.0 & EPA8240 \\
\hline 6.0 & EPA 8240 \\
\hline 6.0 & EPA824 \\
\hline 6.0 & EPA 8240 \\
\hline 6.0 & EPA824C \\
\hline 6.0 & EPA8240 \\
\hline 6.0 & EPA 8240 \\
\hline 6.0 & EPA 8240 \\
\hline 6.0 & EPA8240 \\
\hline 6.0 & EPA 8240 \\
\hline 6.0 & EPA 8240 \\
\hline 12 & EPA8240 \\
\hline 12 & EPA8240 \\
\hline 12 & EPA824C \\
\hline 12 & EPA824 \\
\hline 12 & EPA8240 \\
\hline 12 & EPA8240 \\
\hline 12 & EPA 8240 \\
\hline 12 & EPA8240 \\
\hline 12. & EPA8240 \\
\hline 6.0 & EPA 8240 \\
\hline 6.0 & EPA 8240 \\
\hline 6.0 & EPA8240 \\
\hline 6.0 & EPA 8240 \\
\hline 6.0 & EPA824 \\
\hline 6.0 & EPA 8240 \\
\hline 6.0 & EPA 8240 \\
\hline 6.0 & EPA824 \\
\hline 6.0 & EPA824 \\
\hline
\end{tabular}


SAMPLE NAME: D431 2502 (Continued)

Constituent

Volatile Organic Compounds

Toluene

1,1,1-Trichloroethane

1,1,1-Trichloroethane

1,1,1-Trichloroethane

1,1,2-Trichloroethane

1,1,2-Trichloroethane

1,1,2-Trichloroethane

Trichloroethylene

Vinyl acetate

Vinyl acetate

Vinyl acetate

Xylenes

Xylenes

Xylenes
RQ AQ

B Result

Unit

6.0

6.0

6.0

6.0

6.0

6.0

6.0

6.0

12

12

12

6.0

6.0

6.0 $\mu \mathrm{g} / \mathrm{kg}$

$\mu g / \mathrm{kg}$

$\mu \mathrm{g} / \mathrm{kg}$

$\mu \mathrm{g} / \mathrm{kg}$

$\mu g / \mathrm{kg}$

$\mu \mathrm{g} / \mathrm{kg}$

$\mu \mathrm{g} / \mathrm{kg}$

$\mu g / \mathrm{kg}$

$\mu \mathrm{g} / \mathrm{kg}$

$\mu \mathrm{g} / \mathrm{kg}$

$\mu g / \mathrm{kg}$

$\mu \mathrm{g} / \mathrm{kg}$

$\mu \mathrm{g} / \mathrm{kg}$

$\mu \mathrm{g} / \mathrm{kg}$
D. Limit Method

6.0

6.0

6.0

6.0

6.0

6.0

6.0

6.0

12

12

12

6.0

6.0

6.0

6.1

6.7

0.10

1.8
EPA8240

EPA8240

EPA8240

EPA8240

EPA8240

EPA8240

EPA8240

EPA8240

EPA8240

EPA8240

EPA8240

EPA8240

EPA8240

EPA8240
EPA990.0

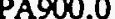

EPA906.0

Radionuclide Indicators and Radionuclides

Gross alpha

Nonvolatile beta

Radium, total alpha-emitting

Tritium

$\begin{array}{llll} & \mathrm{Y} & 13 \pm 12 & \mathrm{pCi} / \mathrm{g} \\ & \mathrm{Y} & 19 \pm 17 & \mathrm{pCi} / \mathrm{g} \\ \mathrm{Y} & \mathrm{YV} & 1.3 \pm 0.80 & \mathrm{pCi} / \mathrm{g} \\ & \mathrm{Y} & 4.3 \pm 1.0 & \mathrm{pCi} / \mathrm{g}\end{array}$

Sample ID: 100300

SAMPLE NAME: D431 2602

Location (SRS Coordinates): $18160 \mathrm{E}$ 66410N

Ground Elevation Above MSL: . fit

Depth of Core Interval: 0.50 to $0.50 \mathrm{ft}$

Sample Type: Normal

Sample Color: 5YR 3/2

Sample Matrix: Sediment USC Soil Classification: SM
RQ

$A Q$

Result

Unit

Sample Moisnure: Wet Percent Solids: 71.80

D. Limit Method

Physical Parameters

$\mathrm{pH}$

Total organic carbon

Total Metals

Arsenic

Barium

Cadmium

Chromium

Copper

Lead

Manganese

Mercury

Nicke!

Selenium
4.7

9,500

$\mathrm{pH}$

$\mathrm{mg} / \mathrm{kg}$

0.010

240

EPA9045

EPA415.1

$\begin{array}{ll} & \\ & 11 \\ & 83 \\ & 0.76 \\ & 6.8 \\ & 7.1 \\ & 9.1 \\ \mathrm{U} & 25 \\ \mathrm{U} & 0.14 \\ & 5.1 \\ & 1.2\end{array}$

$\mathrm{mg} / \mathrm{kg}$

$\mathrm{mg} / \mathrm{kg}$

$\mathrm{mg} / \mathrm{kg}$

$\mathrm{mg} / \mathrm{kg}$

$\mathrm{mg} / \mathrm{kg}$

$\mathrm{mg} / \mathrm{kg}$

$\mathrm{mg} / \mathrm{kg}$

$\mathrm{mg} / \mathrm{kg}$

$\mathrm{mg} / \mathrm{kg}$

$\mathrm{mg} / \mathrm{kg}$

\section{5}

26

0.64

$1.3 \mathrm{~m}$

3.2

0.74

1.9

0.14

5.1

0.62
EPA7060

EPA6010

EPA6010

EPA6010

EPA6010

EPA7421

EPA6010

EPA7470

EPA6010

EPA7740 
SAMPLE NAME: D431 2602 (Continued) Sample ID: 100300

Constituent

RQ AQ B Result

Unit

D. Limit Method

Total Metals

Silver

$\mathrm{U}$

1.3

$\mathrm{mg} / \mathrm{kg}$.

1.3

EPA6010

$B / N / A$ Extractables (including $P A H$ and phenols)

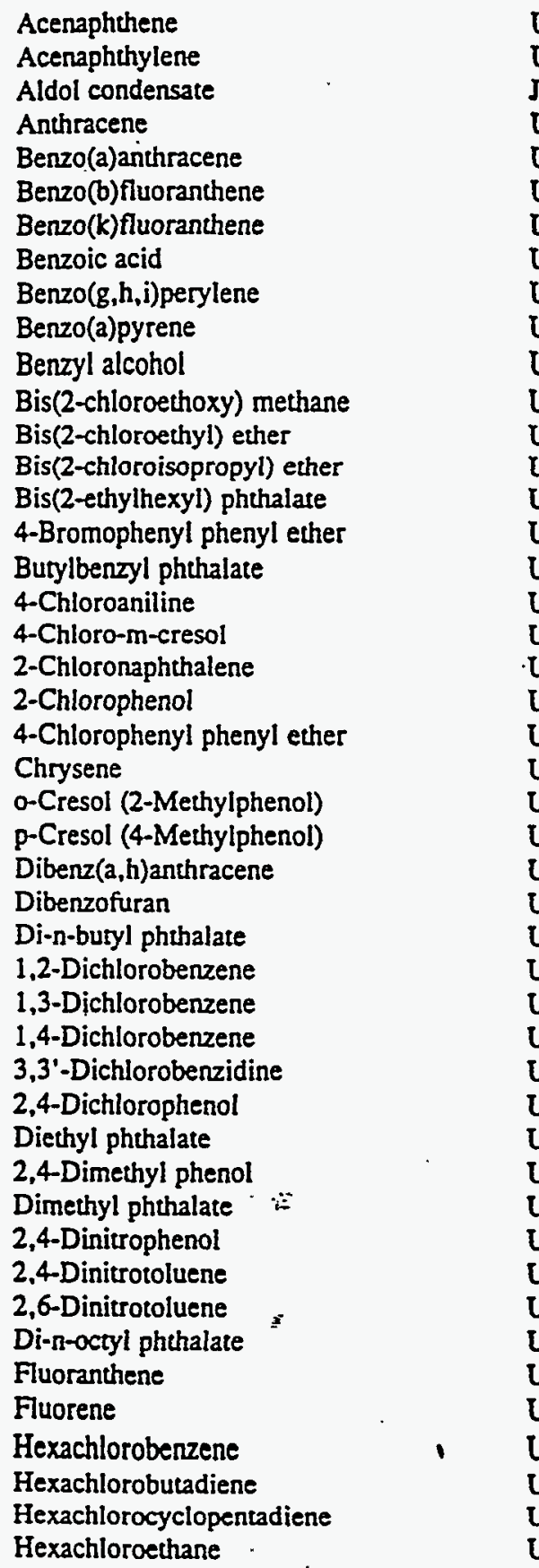

\begin{tabular}{|c|c|c|c|c|}
\hline & 460 & $\mu g / \mathrm{kg}$ & 460 & EPA8270 \\
\hline & 460 & $\mu \mathrm{g} / \mathrm{kg}$ & 460 & EPA8270 \\
\hline \multirow[t]{12}{*}{ NVK } & 300 & $\mu g / \mathrm{kg}$ & & EPA8270 \\
\hline & 460 & $\mu \mathrm{g} / \mathrm{kg}$ & 460 & EPA8270 \\
\hline & 460 & $\mu \mathrm{g} / \mathrm{kg}$ & 460 & EPA8270 \\
\hline & 460 & $\mu \mathrm{g} / \mathrm{kg}$ & 460 & EPA8270 \\
\hline & 460 & $\mu g / \mathrm{kg}$ & 460 & EPA8270 \\
\hline & 2,300 & $\mu g / \mathrm{kg}$ & 2,300 & EPA8270 \\
\hline & 460 & $\mu g / \mathrm{kg}$ & 460 & EPA8270 \\
\hline & 460 & $\mu \mathrm{g} / \mathrm{kg}$ & 460 & EPA8270 \\
\hline & 460 & $\mu g / k g$ & 460 & EPA8270 \\
\hline & 460 & $\mu g / \mathbf{k g}$ & 460 & EPA8270 \\
\hline & 460 & $\mu \mathrm{g} / \mathrm{kg}$ & 460 & EPA8270 \\
\hline & 460 & $\mu g / k g$ & 460 & EPA8270 \\
\hline \multirow[t]{32}{*}{ v } & 110 & $\mu \mathrm{g} / \mathrm{kg}$ & 460 & EPA8270 \\
\hline & 460 & $\mu \mathrm{g} / \mathrm{kg}$ & 460 & EPA8270 \\
\hline & 460 & $\mu \mathrm{g} / \mathrm{kg}$ & 460 & EPA8270 \\
\hline & 460 & $\mu \mathrm{g} / \mathrm{kg}$ & 460 & EPA8270 \\
\hline & 460 & $\mu \mathrm{g} / \mathrm{kg}$ & 460 & EPA8270 \\
\hline & 460 & $\mu \mathrm{g} / \mathrm{kg}$ & 460 & EPA8270 \\
\hline & 460 & $\mu \mathrm{g} / \mathrm{kg}$ & 460 & EPA8270 \\
\hline & 460 & $\mu \mathrm{g} / \mathrm{kg}$ & 460 & EPA8270 \\
\hline & 460 & $\mu \mathrm{g} / \mathrm{kg}$ & 460 & EPA8270 \\
\hline & 460 & $\mu \mathrm{g} / \mathrm{kg}$ & 460 & EPA8270 \\
\hline & 460 & $\mu \mathrm{g} / \mathrm{kg}$ & 460 & EPA8270 \\
\hline & 460 & $\mu \mathrm{g} / \mathrm{kg}$ & 460 & EPA8270 \\
\hline & 460 & $\mu \mathrm{g} / \mathrm{kg}$ & 460 & EPA8270 \\
\hline & 460 & $\mu \mathrm{g} / \mathrm{kg}$ & 460 & EPA8270 \\
\hline & 460 & $\mu \mathrm{g} / \mathrm{kg}$ & 460 & EPA8270 \\
\hline & 460 & $\mu \mathrm{g} / \mathrm{kg}$ & 460 & EPA8270 \\
\hline & 460 & $\mu \mathrm{g} / \mathrm{kg}$ & 460 & EPA8270 \\
\hline & 920 & $\mu \mathrm{g} / \mathrm{kg}$ & 920 & EPA8270 \\
\hline & 460 & $\mu \mathrm{g} / \mathrm{kg}$ & 460 & EPA8270 \\
\hline & 460 & $\mu \mathrm{g} / \mathrm{kg}$ & 460 & EPA8270 \\
\hline & 460 & $\mu \mathrm{g} / \mathrm{kg}$ & 460 & EPA8270 \\
\hline & 460 & $\mu \mathrm{g} / \mathrm{kg}$ & 460 & EPA8270 \\
\hline & 2,300 & $\mu \mathrm{g} / \mathrm{kg}$ & 2,300 & EPA8270 \\
\hline & 460 & $\mu g / k g$ & 460 & EPA8270 \\
\hline & 460 & $\mu \mathrm{g} / \mathrm{kg}$ & 460 & EPA8270 \\
\hline & 460 & $\mu g / \mathrm{kg}$ & 460 & EPA8270 \\
\hline & 460 & $\mu \mathrm{g} / \mathrm{kg}$ & 460 & EPA8270 \\
\hline & 460 & $\mu g / \mathrm{kg}$ & 460 & EPA8270 \\
\hline & 460 & $\mu g / k g$ & 460 & EPA8270 \\
\hline & 460 & $\mu \mathrm{g} / \mathrm{kg}$ & 460 & EPA8270 \\
\hline & 460 & $\mu \mathrm{g} / \mathrm{kg}$ & 460 & EPA8270 \\
\hline & 460 & $\mu \mathrm{g} / \mathrm{kg}$ & 460 & EPA8270 \\
\hline
\end{tabular}


SAMPLE NAME: D431 2602 (Continued)

Sample ID: 100300

Constituent

RQ AQ B Result

Unit

D. Limit Method

$B / N / A$ Extractables (including $P A H$ and phenols)

Indeno(1,2,3-c.d)pyrene

isophorone

2-Methyl-4,6-dinitrophenol

2-Methylnaphthalene

Naphthalene

o-Nitroaniline

m-Nitroaniline

p-Nitroaniline

Nitrobenzene

2-Nitrophenol

4-Nitrophenol

N-Nitrosodiphenylamine

$\mathrm{N}$-Nitrosodipropylamine

Pentachlorophenol

Phenanthrene

Phenol

Pyrene

1,2,4-Trichlorobenzene

2,4,5-Trichlorophenol

2,4,6-Trichlorophenol

Volatile Organic Compounds

Acetone

Acetone

Acetone

Benzene

Bromodichloromethane

Bromodichloromethane

Bromodichloromethane

Bromoform

Bromoform

Bromoform

Bromomethane (Methyl bromide)

Bromomethane (Methyl bromide)

Brrmomethane (Methyl bromide)

Carcon disulfide

Carbon disulfide

Carbon disulfide

Carbon tetrachloride

Carbon tetrachloride

Carbon tetrachloride

Chlorobenzene

Chloroethane

Chloroethane

Chloroethane

Chloroethene (Vinyl chloride)

Chloroethene (Vinyl chloride)

Chloroethene (Vinyl chloride)

2-Chloroethyl vinyl ether

\begin{tabular}{|c|c|c|}
\hline $\mathbf{U}$ & 460 & $\mu g / \mathrm{kg}$ \\
\hline $\mathbf{U}$ & 460 & $\mu \mathrm{g} / \mathrm{kg}$ \\
\hline $\mathbf{U}$ & 2,300 & $\mu g / k g$ \\
\hline $\mathbf{U}$ & 460 & $\mu g / \mathrm{kg}$ \\
\hline $\mathrm{U}$ & 460 & $\mu g / \mathrm{kg}$ \\
\hline U & 2,300 & $\mu g / \mathrm{kg}$ \\
\hline $\mathrm{U}$ & 2,300 & $\mu g / k g$ \\
\hline$U$ & 2,300 & $\mu \mathrm{g} / \mathrm{kg}$ \\
\hline $\mathrm{U}$ & 460 & $\mu \mathrm{g} / \mathrm{kg}$ \\
\hline U & 460 & $\mu \mathrm{g} / \mathrm{kg}$ \\
\hline $\mathbf{U}$ & 2.300 & $\mu \mathrm{g} / \mathrm{kg}$ \\
\hline $\mathrm{U}$ & 460 & $\mu g / k g$ \\
\hline $\mathbf{U}$ & 460 & $\mu \mathrm{g} / \mathrm{kg}$ \\
\hline $\mathbf{U}$ & 2,300 & $\mu g / \mathrm{kg}$ \\
\hline$U$ & 460 & $\mu \mathrm{g} / \mathrm{kg}$ \\
\hline $\mathrm{U}$ & 460 & $\mu \mathrm{g} / \mathrm{kg}$ \\
\hline $\mathbf{U}$ & 460 & $\mu \mathrm{g} / \mathrm{kg}$ \\
\hline U & 460 & $\mu \mathrm{g} / \mathrm{kg}$ \\
\hline U & 2.300 & $\mu \mathrm{g} / \mathrm{kg}$ \\
\hline $\mathbf{U}$ & 460 & \\
\hline
\end{tabular}

$\mu g / \mathrm{kg}$
$\mu g / \mathrm{kg}$
$\mu g / \mathrm{kg}$
$\mu g / \mathrm{kg}$
$\mu g / \mathrm{kg}$
$\mu g / \mathrm{kg}$
$\mu \mathrm{g} / \mathrm{kg}$
$\mu \mathrm{g} / \mathrm{kg}$
$\mu \mathrm{g} / \mathrm{kg}$
$\mu \mathrm{g} / \mathrm{kg}$
$\mu \mathrm{g} / \mathrm{kg}$
$\mu g / \mathrm{kg}$
$\mu g / \mathrm{kg}$
$\mu \mathrm{g} / \mathrm{kg}$
$\mu g / \mathrm{kg}$
$\mu \mathrm{g} / \mathrm{kg}$
$\mu g / \mathrm{kg}$
$\mu g / \mathrm{kg}$
$\mu \mathrm{g} / \mathrm{kg}$
$\mu g / \mathrm{kg}$

460

EPA8270

460

2.300

460

460

2,300

2,300

2,300

460

460

2,300

460

460

2,300

460

460

460

460

2.300 。

460

EPA8270

EPA8270

EPA8270

EPA8270

EPA8270

EPA8270

EPA8270

EPA8270

EPA8270

EPA8270

EPA8270

EPA8270

EPA8270

EPA8270

EPA8270

EPA8270

EPA8270

EPA8270

EPA8270

$\mu \mathrm{g} / \mathrm{kg}$

$\mu \mathrm{g} / \mathrm{kg}$

$\mu \mathrm{g} / \mathrm{kg}$

$\mu \mathrm{g} / \mathrm{kg}$

$\mu \mathrm{g} / \mathrm{kg}$

$\mu g / \mathrm{kg}$

$\mu \mathrm{g} / \mathrm{kg}$

$\mu \mathrm{g} / \mathrm{kg}$

$\mu \mathrm{g} / \mathrm{kg}$

$\mu \mathrm{g} / \mathrm{kg}$

$\mu g / k g$

$\mu \mathrm{g} / \mathrm{kg}$

$\mu g / \mathrm{kg}$

$\mu \mathrm{g} / \mathrm{kg}$

$\mu g / \mathrm{kg}$

$\mu g / \mathrm{kg}$

$\mu g / \mathrm{kg}$

$\mu g / \mathrm{kg}$

$\mu g / \mathrm{kg}$

$\mu g / \mathrm{kg}$

$\mu g / \mathrm{kg}$

$\mu \mathrm{g} / \mathrm{kg}$

$\mu g / \mathbf{k g}$

$\mu g / \mathbf{k g}$

$\mu g / \mathrm{kg}$

$\mu g / \mathrm{kg}$

$\mu \mathrm{g} / \mathrm{kg}$
EPA8240

EPA8240

EPA 8240

EPA8240

EPA8240

EPA8240

EPA8240

EPA 8240

EPA8240

EPA8240

EPA8240

EPA8240

EPA8240

EPA8240

EPA8240

EPA8240

EPA8240

EPA8240

EPA8240

EPA8240

EPA8240

EPA8240

EPA8240

EPA8240

EPA8240

EPA8240

EPA8240 
SAMPLE NAME: D431 2602 (Continued)

\section{Constituent}

Volatile Organic Compounds

2-Chloroethyl vinyl ether

2-Chloroethyl vinyl ether

Chloroform

Chloroform

Chloroform

Chloromethane (Methyl chloride)

Chloromethane (Methyl chloride)

Chloromethane (Methyl chloride)

Dibromochloromethane

Dibromochloromethane

Dibromochloromethane

1,1-Dichloroethane

1,1-Dichloroethane

1,1-Dichloroethane

1,2-Dichloroethane

1,2-Dichloroethane

1,2-Dichloroethane

1,1-Dichloroethylene

1,2-Dichloroethylene

1,2-Dichloroethyleñe

1,2-Dichloroethylene

Dichloromethane (Methylene chloride)

Dichloromethane (Methylene chloride)

Dichloromethane (Methylene chloride)

1.2-Dichloropropane

1,2-Dichloropropane

1,2-Dichloropropane .

cis-1.3-Dichloropropene

cis-1,3-Dichloropropene

cis-1.3-Dichloropropene

trans-1,3-Dichloropropene

trans-1,3-Dichloropropene

trans-1,3-Dichloropropene

Ethylbenzene

Ethylbenzene

Ethylbenzene

2-Hexanone

2-Hexanone

2-Hexanone

Methyl ethyl ketone

Methyl ethyl ketone is

Methyl ethyl'ketone

Methyl isobutyl ketone

Methyl isobutyl ketone :

Methyl isoburyl ketone

Styrene

Styrene

Styrene

1,1,2,2-Tetrachloroethane

1,1,2,2-Tetrachloroethane
Sample ID: 100300

$\begin{array}{lllll}\text { RQ AQ B Result Unit } & \text { D. Limit Method }\end{array}$

U

U

U

$\mathrm{U}$

$\mathrm{U}$

U

U

U

U

U

U

U

U

U

U

U

U

U

U

U

U

$\mathrm{U}$

U

U

U

U

U

U

U

U

U

U

U

U

U

U

U

U

U

U

U

U

U

U

U

$\mathrm{U}$
14

14

7.0

7.0

7.0

14

14

14

7.0

7.0

7.0

7.0

7.0

7.0

7.0

7.0

7.0

7.0

7.0

7.0

7.0

40

23

24

7.0

7.0

7.0

7.0

7.0

7.0

7.0

7.0

7.0

7.0

7.0

7.0

14

14

14

14

14

14

14

14

14

7.0

7.0

7.0

7.0

7.0 $\mu \mathrm{g} / \mathrm{kg}$
$\mu \mathrm{g} / \mathrm{kg}$
$\mu \mathrm{g} / \mathrm{kg}$
$\mu \mathrm{g} / \mathrm{kg}$
$\mu \mathrm{g} / \mathrm{kg}$
$\mu \mathrm{g} / \mathrm{kg}$
$\mu \mathrm{g} / \mathrm{kg}$
$\mu \mathrm{g} / \mathrm{kg}$
$\mu \mathrm{g} / \mathrm{kg}$
$\mu \mathrm{g} / \mathrm{kg}$
$\mu \mathrm{g} / \mathrm{kg}$
$\mu \mathrm{g} / \mathrm{kg}$
$\mu \mathrm{g} / \mathrm{kg}$
$\mu \mathrm{g} / \mathrm{kg}$
$\mu \mathrm{g} / \mathrm{kg}$
$\mu \mathrm{g} / \mathrm{kg}$

$\mu \mathrm{g} / \mathrm{kg}$

$\mu \mathrm{g} / \mathrm{kg}$

$\mu \mathrm{g} / \mathrm{kg}$

$\mu \mathrm{g} / \mathrm{kg}$

$\mu \mathrm{g} / \mathrm{kg}$

$\mu g / \mathrm{kg}$

$\mu \mathrm{g} / \mathrm{kg}$

$\mu g / k g$

$\mu \mathrm{g} / \mathrm{kg}$

$\mu g / k g$

$\mu \mathrm{g} / \mathrm{kg}$

$\mu \mathrm{g} / \mathrm{kg}$

$\mu g / \mathrm{kg}$

$\mu \mathrm{g} / \mathrm{kg}$

$\mu g / \mathrm{kg}$

$\mu \mathrm{g} / \mathrm{kg}$

$\mu \mathrm{g} / \mathrm{kg}$

$\mu \mathrm{g} / \mathrm{kg}$

$\mu \mathrm{g} / \mathrm{kg}$

$\mu g / \mathrm{kg}$

$\mu g / k g$

$\mu \mathrm{g} / \mathrm{kg}$

$\mu \mathrm{g} / \mathrm{kg}$

$\mu \mathrm{g} / \mathrm{kg}$

$\mu \mathrm{g} / \mathrm{kg}$

$\mu \mathrm{g} / \mathrm{kg}$

$\mu \mathrm{g} / \mathrm{kg}$

$\mu \mathrm{g} / \mathrm{kg}$

$\mu \mathrm{g} / \mathrm{kg}$

$\mu \mathrm{g} / \mathrm{kg}$

$\mu \mathrm{g} / \mathrm{kg}$

$\mu \mathrm{g} / \mathrm{kg}$

$\mu \mathrm{g} / \mathrm{kg}$

$\mu g / \mathrm{kg}$
14

14

7.0

7.0

7.0

14

14

14

7.0

7.0

7.0

7.0

7.0

7.0

7.0

7.0

7.0

7.0

7.0

7.0

7.0

7.0

7.0

7.0

7.0

7.0

7.0

7.0

7.0

7.0

7.0

7.0

7.0

7.0

7.0

7.0

14

14
14

14

14

14

14

14

14

7.0

7.0

7.0

7.0

7.0

EPA8240

EPA8240

EPA8240

EPA8240

EPA8240

EPA8240

EPA8240

EPA8240

EPA8240

EPA8240

EPA8240

EPA8240

EPA8240

EPA8240

EPA8240

EPA8240

EPA8240

EPA8240

EPA8240

EPA8240

EPA8240

EPA8240

EPA8240

EPA8240

EPA8240

EPA8240

EPA8240

EPA8240

EPA8240

EPA8240 
SAMPLE NAME: D431 2602 (Continued)

Constituent

Volatile Organic Compounds
$\mathbf{R Q}$

AQ

Sample D: 100300

B Result Unit D. Limit Method

\section{1,1.2,2-Tetrachloroethane \\ Tetrachloroethylene \\ Tetrachloroethylene \\ Tetrachloroethylene \\ Toluene \\ 1,1,1-Trichloroethane \\ 1.1,1-Trichloroethane \\ 1,1,1-Trichloroethane \\ 1,1,2-Trichloroethane \\ 1.1,2-Trichloroethane \\ 1,1,2-Trichloroethane \\ Trichloroethylene \\ Vinyl acetate \\ Vinyl acetate \\ Vinyl acetate \\ Xylenes \\ Xylenes \\ Xylenes}

$\begin{array}{ll}U & 7.0 \\ U & 7.0 \\ U & 7.0 \\ U & 7.0 \\ U & 7.0 \\ U & 7.0 \\ U & 7.0 \\ U & 7.0 \\ U & 7.0 \\ U & 7.0 \\ U & 7.0 \\ U & 7.0 \\ U & 14 \\ U & 14 \\ U & 14 \\ U & 7.0 \\ U & 7.0 \\ U & 7.0\end{array}$

$$
\begin{aligned}
& \mu g / \mathrm{kg} \\
& \mu g / \mathrm{kg} \\
& \mu g / \mathrm{kg} \\
& \mu g / \mathrm{kg} \\
& \mu \mathrm{g} / \mathrm{kg} \\
& \mu \mathrm{g} / \mathrm{kg} \\
& \mu \mathrm{g} / \mathrm{kg} \\
& \mu \mathrm{g} / \mathrm{kg} \\
& \mu \mathrm{g} / \mathrm{kg} \\
& \mu \mathrm{g} / \mathrm{kg} \\
& \mu \mathrm{g} / \mathrm{kg} \\
& \mu \mathrm{g} / \mathrm{kg} \\
& \mu \mathrm{g} / \mathrm{kg} \\
& \mu \mathrm{g} / \mathrm{kg} \\
& \mu \mathrm{g} / \mathrm{kg} \\
& \mu \mathrm{g} / \mathrm{kg} \\
& \mu \mathrm{g} / \mathrm{kg} \\
& \mu \mathrm{g} / \mathrm{kg}
\end{aligned}
$$

7.0
7.0
7.0
7.0
7.0
7.0
7.0
7.0
7.0
7.0
7.0
7.0
14
14
14
7.0
7.0
7.0

EPA8240

EPA8240

EPA8240

EPA8240

EPA8240

EPA8240

EP.48240

EPA8240

EPA8240

EPA8240

EPA8240

EPA8240

EPA8240

EPA8240

EPA8240

EPA8240

EPA8240

EPA8240

Radionuclide Indicators and Radionuclides

\author{
Gross alpha \\ Gross alpha \\ Nonvolatile beta \\ Nonvolatile beta \\ Radium, total alpha-emitting \\ Radium, total alpha-emitting \\ Tritium
}

U

$\begin{array}{ll} & 8.1 \\ & 12 \pm 14 \\ & 9.9 \pm 20 \\ & 24 \pm 22 \\ \mathrm{~V} \quad & 0.64 \pm 0.67 \\ \mathrm{~V}, \quad & 1.1 \pm 0.85 \\ & 11 \pm 1.9\end{array}$

SAMPLE NAME: D431 2702

Location (SRS Coordinates): 18105E 66376N

Ground Elevation Above MSL: . ft

Depth of Core Interval: 0.00 to $0.50 \mathrm{ft}$

Sample Type: Normal

Sample Color: $5 Y R 3 / 2$

Constituent $\quad:$

Physical Parameters

$\mathrm{pH}$

Total organic carbon

Total Metals

Arsenic

Barium

Cadmium

$\begin{array}{ll}8.1 & \text { EPA900.0 } \\ 8.2 & \text { EPA900.0 } \\ 9.8 & \text { EPA900.0 } \\ 9.9 & \text { EPA900.0 } \\ 0.070 & \text { EPA903.0 } \\ 0.070 & \text { EPA903.0 } \\ 2.0 & \text { EPA906.0 }\end{array}$$$
\text { EPA906.0 }
$$

Sample ID: 100312

Sample Matrix: Sediment USC Soil Classification: $\mathrm{OH}$

$\mathrm{pCi} / \mathrm{g}$
$\mathrm{pCi} / \mathrm{g}$
$\mathrm{pCi} / \mathrm{g}$
$\mathrm{pCi} / \mathrm{g}$
$\mathrm{pCi} / \mathrm{g}$
$\mathrm{pCi} / \mathrm{g}$
$\mathrm{pCi} / \mathrm{g}$
Sample Moisture: Wet Percent Solids: $\mathbf{7 4 . 9 0}$

D. Limit Method

RQ AQ $B$ Result Unit

4.7 46,000

$\mathrm{pH}$

$\mathrm{mg} / \mathrm{kg}$

$\begin{array}{ll}0.010 & \text { EPA9045 } \\ 1.900 & \text { EPA415.1 }\end{array}$

$\begin{array}{ll}0.010 & \text { EPA9045 } \\ 1.900 & \text { EPA415.1 }\end{array}$

1.7

65

0.58 $\mathrm{mg} / \mathrm{kg}$
$\mathrm{mg} / \mathrm{kg}$
$\mathrm{mg} / \mathrm{kg}$
1.0
23

EPA7060

EPA6010

$0.58 \quad$ EPA6010 
SAMPLE NAME: D43ï 2702 (Continued)

Constituent

RQ AQ B Result

Unit

D. Limit Method

Total Metals

Chromium
Copper
Lead
Manganese
Mercury
Nickel
Selenium
Silver

$B / N / A$ Extractables (including $P A H$ and phenols)

\begin{tabular}{|c|c|c|c|}
\hline Acenaphthene & $\mathbf{U}$ & $\mathbf{Y}$ & 420 \\
\hline Acenaphthylene & $\mathrm{U}$ & $\mathrm{Y}$ & 420 \\
\hline Anthracene & $\mathrm{U}$ & $\mathrm{Y}$ & 420 \\
\hline Benzo(a)anthracene & $\mathbf{U}$ & $\mathbf{Y}$ & 420 \\
\hline Benzo(b)fluoranthene & $\mathrm{U}$ & $\mathbf{Y}$ & 420 \\
\hline Benzo(k)fluoranthene & $\mathrm{U}$ & $\mathbf{Y}$ & 420 \\
\hline Benzoic acid & $\mathrm{U}$ & $\mathrm{Y}$ & 2,100 \\
\hline Benzo(g.h.i)perylene & $\mathrm{U}$ & $\mathrm{Y}$ & 420 \\
\hline Benzo(a)pyrene & U & $\mathbf{Y}$ & 420 \\
\hline Benzyl alcohol & $\mathrm{U}$ & $\mathbf{Y}$ & 420 \\
\hline Bis(2-chloroethoxy) methane & $\mathbf{U}$ & $\mathbf{Y}$ & 420 \\
\hline Bis(2-chloroethyl) ether & $\mathbf{U}$ & $\mathbf{Y}$ & 420 \\
\hline Bis(2-chloroisopropyl) ether & $\mathbf{U}$ & $\mathbf{Y}$ & 420 \\
\hline Bis(2-ethylhexyl) phthalate & $\mathrm{U}$ & $\mathbf{Y}$ & 420 \\
\hline 4-Bromophenyl phenyl ether & $\mathbf{U}$ & $\mathbf{Y}$ & 420 \\
\hline Butylbenzyl phthalate & $\mathbf{U}$ & $\mathbf{Y}$ & 420 \\
\hline Arom. hydrocarbon $\mathrm{C} 18 \mathrm{H} 18$ & J & YN & 200 \\
\hline Sub. carboxylic acid & J & $\mathrm{YN}$ & 400 \\
\hline Sub. carboxylic acid & $\mathrm{J}$ & YN & 300 \\
\hline 4-Chloroaniline & $\mathbf{U}$ & $\mathbf{Y}$ & 420 \\
\hline 4-Chloro-m-cresol & $\mathrm{U}$ & $\mathbf{Y}$ & 420 \\
\hline 2-Chloronaphthalene & $\mathbf{U}$ & $\mathbf{Y}$ & 420 \\
\hline 2-Chlorophenol & $\mathrm{U}$ & $\mathbf{Y}$ & 420 \\
\hline 4-Chlorophenyl phenyl ether & $\mathbf{U}$ & $\mathbf{Y}$ & 420 \\
\hline Chrysene & $\mathrm{U}$ & $\mathbf{Y}$ & 420 \\
\hline o-Cresol (2-Methylphenol) & $\mathbf{U}$ & $\mathbf{Y}$ & 420 \\
\hline p-Cresol (4-Methylphenol) & $\mathbf{U}$ & $\mathbf{Y}$ & 420 \\
\hline Dibenz $(a, h)$ anthracene & $\mathbf{U}$ & $\mathbf{Y}$ & 420 \\
\hline Dibenzofuran & U & $\mathbf{Y}$ & 420 \\
\hline Di-n-butyl phthaiate & UJ & YV & 62 \\
\hline 1.2-Dichlorobenzene & $\mathbf{U}$ & $\mathbf{Y}$ & 420 \\
\hline 1,3-Dichlorobenzene & $\mathbf{U}$ & $\mathbf{Y}$ & 420 \\
\hline 1,4-Dichlorobenzene & $\mathbf{U}$ & $\mathbf{Y}$ & 420 \\
\hline 3,3'-Dichlorobenzidine & $\mathbf{U}$ & $\mathbf{Y}$ & 850 \\
\hline 2,4-Dichlorophenol & $\mathrm{U}$ & $\mathbf{Y}$ & 420 \\
\hline Diethyl phthalate & $\mathbf{U}$ & $\mathbf{Y}$ & 420 \\
\hline 2,4-Dimethyl phenol & $\mathrm{U}$ & $\mathbf{Y}$ & 420 \\
\hline Dimethyl phthalate & $\mathrm{U}$ & $\mathbf{Y}$ & 420 \\
\hline 2,4-Dinitrophenol & $\mathrm{U}$ & $\mathrm{Y}$ & 2.100 \\
\hline
\end{tabular}

420

420

420

420

2,100

420

420

420

420

420

200

400

300

420

420

20

420

420

20

420

20

420

420

20

850

420

420

2,100

$\begin{array}{llll}9.9 & \mathrm{mg} / \mathrm{kg} & 1.2 & \text { EPA6010 } \\ 3.4 & \mathrm{mg} / \mathrm{kg} & 2.9 & \text { EPA6010 } \\ 12 & \mathrm{mg} / \mathrm{kg} & 0.61 & \text { EPA7421 } \\ 140 & \mathrm{mg} / \mathrm{kg} & 1.8 & \text { EPA6010 } \\ 0.13 & \mathrm{mg} / \mathrm{kg} & 0.13 & \text { EPA7470 } \\ 4.7 & \mathrm{mg} / \mathrm{kg} & 4.7 & \text { EPA6010 } \\ 0.51 & \mathrm{mg} / \mathrm{kg} & 0.51 & \text { EPA7740 } \\ 1.2 & \mathrm{mg} / \mathrm{kg} & 1.2 & \text { EPA6010 }\end{array}$

$\begin{array}{lll}\mu \mathrm{g} / \mathrm{kg} & 420 & \text { EPA8270 } \\ \mu \mathrm{g} / \mathrm{kg} & 420 & \text { EPA8270 } \\ \mu \mathrm{g} / \mathrm{kg} & 420 & \text { EPA8270 } \\ \mu \mathrm{g} / \mathrm{kg} & 420 & \text { EPA8270 } \\ \mu \mathrm{g} / \mathrm{kg} & 420 & \text { EPA8270 } \\ \mu \mathrm{g} / \mathrm{kg} & 420 & \text { EPA8270 } \\ \mu \mathrm{g} / \mathrm{kg} & 2,100 & \text { EPA8270 } \\ \mu \mathrm{g} / \mathrm{kg} & 420 & \text { EPA8270 } \\ \mu \mathrm{g} / \mathrm{kg} & 420 & \text { EPA8270 } \\ \mu \mathrm{g} / \mathrm{kg} & 420 & \text { EPA8270 } \\ \mu \mathrm{g} / \mathrm{kg} & 420 & \text { EPA8270 } \\ \mu \mathrm{g} / \mathrm{kg} & 420 & \text { EPA8270 } \\ \mu \mathrm{g} / \mathrm{kg} & 420 & \text { EPA8270 } \\ \mu \mathrm{g} / \mathrm{kg} & 420 & \text { EPA8270 } \\ \mu \mathrm{g} / \mathrm{kg} & 420 & \text { EPA8270 } \\ \mu \mathrm{g} / \mathrm{kg} & 420 & \text { EPA8270 } \\ \mu \mathrm{g} / \mathrm{kg} & & \text { EPA8270 } \\ \mu \mathrm{g} / \mathrm{kg} & & \text { EPA8270 } \\ \mu \mathrm{g} / \mathrm{kg} & & \text { EPA8270 } \\ \mu \mathrm{g} / \mathrm{kg} & 420 & \text { EPA8270 } \\ \mu \mathrm{g} / \mathrm{kg} & 420 & \text { EPA8270 } \\ \mu \mathrm{g} / \mathrm{kg} & 420 & \text { EPA8270 } \\ \mu \mathrm{g} / \mathrm{kg} & 420 & \text { EPA8270 } \\ \mu \mathrm{g} / \mathrm{kg} & 420 & \text { EPA8270 } \\ \mu \mathrm{g} / \mathrm{kg} & 420 & \text { EPA8270 } \\ \mu \mathrm{g} / \mathrm{kg} & 420 & \text { EPA8270 } \\ \mu \mathrm{g} / \mathrm{kg} & 420 & \text { EPA8270 } \\ \mu \mathrm{g} / \mathrm{kg} & 420 & \text { EPA8270 } \\ \mu \mathrm{g} / \mathrm{kg} & 420 & \text { EPA8270 } \\ \mu \mathrm{g} / \mathrm{kg} & 420 & \text { EPA8270 } \\ \mu \mathrm{g} / \mathrm{kg} & 420 & \text { EPA8270 } \\ \mu \mathrm{g} / \mathrm{kg} & 420 & \text { EPA8270 } \\ \mu \mathrm{g} / \mathrm{kg} & 420 & \text { EPA8270 } \\ \mu \mathrm{g} / \mathrm{kg} & 850 & \text { EPA8270 } \\ \mu \mathrm{g} / \mathrm{kg} & 420 & \text { EPA8270 } \\ \mu \mathrm{g} / \mathrm{kg} & 420 & \text { EPA8270 } \\ \mu \mathrm{g} / \mathrm{kg} & 420 & \text { EPA8270 } \\ \mu \mathrm{g} / \mathrm{kg} & 420 & \text { EPA8270 } \\ \mu \mathrm{g} / \mathrm{kg} & 2,100 & \text { EPA8270 } \\ & & \\ & & \end{array}$


SAMPLE NAME: D431 2702 (Continued)

Sample ID: 100312

$\begin{array}{lllllll}\text { Constituent } & \text { RQ AQ } & \text { B Result } & \text { Unit } & \text { D. Limit Method }\end{array}$

$B / N / A$ Extractables (including $P A H$ and phenols)

\begin{tabular}{|c|c|c|c|c|c|c|}
\hline 2.4-Dinitrotoluene & 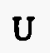 & $Y$ & 420 & $\mu g / k g$ & 420 & EPA8270 \\
\hline 2,6-Dinitrotoluene & $\mathrm{U}$ & $\mathbf{Y}$ & 420 & $\mu \mathrm{g} / \mathrm{kg}$ & 420 & EPA8270 \\
\hline Di-n-octyl phthalate & $\mathrm{U}$ & $\mathbf{Y}$ & 420 & $\mu \mathrm{g} / \mathrm{kg}$ & 420 & EPA8270 \\
\hline Fluoranthene & $\mathrm{U}$ & $\mathrm{Y}$ & 420 & $\mu g / k g$ & 420 & EPA8270 \\
\hline Fluorene . & $\mathrm{U}$ & $Y$ & 420 & $\mu g / k g$ & 420 & EPA8270 \\
\hline Hexachlorobenzene & $\mathrm{U}$ & $\mathbf{Y}$ & 420 & $\mu \mathrm{g} / \mathrm{kg}$ & 420 & EPA8270 \\
\hline Hexachlorobutadiene & $\mathbf{U}$ & $\mathbf{Y}$ & 420 & $\mu \mathrm{g} / \mathrm{kg}$ & 420 & EPA8270 \\
\hline Hexachlorocyclopentadiene & $\mathbf{U}$ & $\mathbf{Y}$ & 420 & $\mu g / k g$ & 420 & EPA8270 \\
\hline Hexachloroethane & $\mathrm{U}$ & $Y$ & 420 & $\mu \mathrm{g} / \mathrm{kg}$ & 420 & EPA8270 \\
\hline Indeno(1.2.3-c.d)pyrene & $\mathrm{U}$ & $Y$ & 420 & $\mu g / \mathrm{kg}$ & 420 & EPA8270 \\
\hline Isophorone & $\mathrm{U}$ & $\mathbf{Y}$ & 420 & $\mu g / \mathrm{kg}$ & 420 & EPA8270 \\
\hline 2-Methyl-4,6-dinitrophenol & $\mathbf{U}$ & $\mathbf{Y}$ & 2.100 & $\mu \mathrm{g} / \mathrm{kg}$ & 2,100 & EPA8270 \\
\hline 2-Methylnaphthalene & $\mathrm{U}$ & $\mathbf{Y}$ & 420 & $\mu g / \mathrm{kg}$ & 420 & EPA8270 \\
\hline Naphthalene & $\mathrm{U}$ & $\mathbf{Y}$ & 420 & $\mu \mathrm{g} / \mathrm{kg}$ & 420 & EPA8270 \\
\hline 0-Nitroaniline & $\mathrm{U}$ & $\mathbf{Y}$ & 2,100 & $\mu \mathrm{g} / \mathrm{kg}$ & 2,100 & EPA8270 \\
\hline m-Nitroaniline & $\mathbf{U}$ & . $Y$ & 2,100 & $\mu \mathrm{g} / \mathrm{kg}$ & 2,100 & EPA8270 \\
\hline p-Nitroaniline & $\mathbf{U}$ & $\mathrm{Y}$ & 2.100 & $\mu g / \mathrm{kg}$ & 2.100 & EPA8270 \\
\hline Nitrobenzene & $\mathrm{U}$ & $\mathbf{Y}$ & 420 & $\mu g / k g$ & 420 & EPA8270 \\
\hline 2-Nitrophenol & $U$ & $\mathbf{Y}$ & 420 & $\mu g / k g$ & 420 & EPA8270 \\
\hline 4-Nitrophenol & $U$ & $\mathrm{Y}$ & 2,100 & $\mu \mathrm{g} / \mathrm{kg}$ & 2,100 & EPA8270 \\
\hline N-Nitrosodiphenylamine & $\mathrm{U}$ & $\mathbf{Y}$ & 420 & $\mu \mathrm{g} / \mathrm{kg}$ & 420 & EPA8270 \\
\hline N-Nitrosodipropylamine & UJ & CY & 420 & $\mu \mathrm{g} / \mathrm{kg}$ & 420 & EPA8270 \\
\hline Pentachlorophenol & $\mathrm{U}$ & $\mathbf{Y}$ & 2,100 & $\mu \mathrm{g} / \mathrm{kg}$ & 2,100 & EPA8270 \\
\hline Phenanthrene & $\mathrm{U}$ & $\mathbf{Y}$ & 420 & $\mu \mathrm{g} / \mathrm{kg}$ & 420 & EPA8270 \\
\hline Phenol & $\mathbf{U}$ & $\mathbf{Y}$ & 420 & $\mu \mathrm{g} / \mathrm{kg}$ & 420 & EPA8270 \\
\hline Pyrene & U & $\mathbf{Y}$ & 420 & $\mu \mathrm{g} / \mathrm{kg}$ & 420 & EPA8270 \\
\hline 1,2,4-Trichlorobenzene & $\mathrm{U}$ & $\mathbf{Y}$ & 420 & $\mu \mathrm{g} / \mathrm{kg}$ & 420 & EPA8270 \\
\hline 2,4,5-Trichlorophenol & $\mathrm{U}$ & $\mathbf{Y}$ & 2,100 & $\mu g / \mathrm{kg}$ & 2.100 & EPA8270 \\
\hline 2,4.6-Trichlorophenol & U & $Y$ & 420 & $\mu \mathrm{g} / \mathrm{kg}$ & 420 & EPA8270 \\
\hline Unknown hydrocarbon & $\mathrm{J}$ & YN & 200 & $\mu \mathrm{g} / \mathrm{kg}$ & & EPA8270 \\
\hline Unknown & $\mathrm{J}$ & YN & 300 & $\mu \mathrm{g} / \mathrm{kg}$ & & EPA8270 \\
\hline Unknown & J & YN & 500 & $\mu \mathrm{g} / \mathrm{kg}$ & & EPA8270 \\
\hline Unknown ketone & $J$ & YNV & 6,000 & $\mu \mathrm{g} / \mathrm{kg}$ & & EPA8270 \\
\hline \multicolumn{7}{|l|}{ Volatile Organic Compounds } \\
\hline Acetone & $\mathbf{U}$ & YV & 13 & $\mu \mathrm{g} / \mathrm{kg}$ & 13 & EPA8240 \\
\hline Benzene & $\mathbf{U}$ & $\mathbf{Y}$ & 6.4 & $\mu \mathrm{g} / \mathrm{kg}$ & 6.4 & EPA8240 \\
\hline Bromodichloromethane & U & $\mathbf{Y}$ & 6.4 & $\mu g / k g$ & 6.4 & EPA8240 \\
\hline Bromoform & $\mathbf{U}$ & $\mathbf{Y}$ & 6.4 & $\mu g / k g$ & 6.4 & EPA8240 \\
\hline Bromomethane (Methyl bromide) & $\mathbf{U}$ & $\mathbf{Y}$ & 13 & $\mu g / k g$ & 13 & EPA8240 \\
\hline Carbon disulfide & $\mathrm{U}$ & $\mathbf{Y}$ & 6.4 & $\mu g / \mathrm{kg}$ & 6.4 & EPA 8240 \\
\hline Carbon tetrachloride & $\mathbf{U}$ & $\mathbf{Y}$ & 6.4 & $\mu g / \mathrm{kg}$ & 6.4 & EPA8240 \\
\hline Chlorobenzene & $\mathbf{U}$ & $\mathbf{Y}$ & 6.4 & $\mu g / k g$ & 6.4 & EPA8240 \\
\hline Chloroethane & $\mathbf{U}$ & $\mathbf{Y}$ & 13 & $\mu \mathrm{g} / \mathrm{kg}$ & $13=$ & EPA8240 \\
\hline Chloroethene (Vinyl chloride) & $\mathbf{U}$ & $\mathbf{Y}$ & 13 & $\mu g / k g$ & 13 & EPA8240 \\
\hline Chloroform & $\mathbf{U}$ & $\mathbf{Y}$ & 6.4 & $\mu g / k g$ & 6.4 & EPA8240 \\
\hline Chloromethane (Methyl chloride) & $\mathbf{U}$ & $\mathbf{Y}$ & 13 & $\mu g / k g$ & 13 & EPA8240 \\
\hline Dibromochloromethane & $\mathbf{U}$ & $\mathbf{Y}$ & 6.4 & $\mu \mathrm{g} / \mathrm{kg}$ & 6.4 & EPA8240 \\
\hline 1,1-Dichloroethane & $\mathrm{U}$ & $\mathbf{Y}$ & 6.4 & $\mu \mathrm{g} / \mathrm{kg}$ & 6.4 & EPA8240 \\
\hline
\end{tabular}


SAMPLE NAME: D43i 2702 (Continued)

Constituent

RQ AQ B Result

Volatile Organic Compounds

1.2-Dichloroethane
1,1-Dichloroethylene
1,2-Dichloroethylene
Dichloromethane (Methylene chloride)
1,2-Dichloropropane
cis-1,3-Dichloropropene
trans-1,3-Dichloropropene
Ethylbenzene
2-Hexanone
Methyl ethyl ketone
Methyl isobutyl ketone
Styrene
1,1,2,2-Tetrachloroethane
Tetrachloroethylene
Toluene
1,1,1-Trichloroethane
1,1,2-Trichloroethane
Trichloroethylene
Vinyl acetate
Xylenes

$\begin{array}{ll}U & Y \\ U & Y \\ U & Y \\ U & Y V \\ U & Y \\ U & Y \\ U & Y \\ U & Y \\ U & Y \\ U & Y \\ U & Y \\ U & Y \\ U & Y \\ U & Y \\ U & Y \\ U & Y \\ U & Y \\ U & Y \\ U & Y \\ U & Y\end{array}$

Sample ID: 100312
Unit
D. Limit Method

Radionuclide Indicators and Radionuclides

Gross alpha

Nonvolatile beta

Radium, total alpha-emitting

Tritium

$\begin{array}{llll}6.4 & \mu g / k g & 6.4 & \text { EPA8240 } \\ 6.4 & \mu g / k g & 6.4 & \text { EPA8240 } \\ 6.4 & \mu g / k g & 6.4 & \text { EPA8240 } \\ 6.6 & \mu g / k g & 6.4 & \text { EPA8240 } \\ 6.4 & \mu g / k g & 6.4 & \text { EPA8240 } \\ 6.4 & \mu g / k g & 6.4 & \text { EPA8240 } \\ 6.4 & \mu g / k g & 6.4 & \text { EPA8240 } \\ 6.4 & \mu g / k g & 6.4 & \text { EPA8240 } \\ 13 & \mu g / k g & 13 & \text { EPA8240 } \\ 13 & \mu g / k g & 13 & \text { EPA8240 } \\ 13 & \mu g / k g & 13 & \text { EPA8240 } \\ 6.4 & \mu g / k g & 6.4 & \text { EPA8240 } \\ 6.4 & \mu g / k g & 6.4 & \text { EPA8240 } \\ 6.4 & \mu g / k g & 6.4 & \text { EPA8240 } \\ 6.4 & \mu g / k g & 6.4 & \text { EPA8240 } \\ 6.4 & \mu g / k g & 6.4 & \text { EPA8240 } \\ 6.4 & \mu g / k g & 6.4 & \text { EPA8240 } \\ 6.4 & \mu g / k g & 6.4 & \text { EPA8240 } \\ 13 & \mu g / k g & 13 & \text { EPA8240 } \\ 6.4 & \mu g / k g & 6.4 & \text { EPA8240 }\end{array}$

$Y$
$Y$
$Y V$
$Y$

$\begin{array}{ll}15 \pm 13 & \mathrm{pCi} / \mathrm{g} \\ 17 \pm 18 & \mathrm{pCi} / \mathrm{g} \\ 0.90 \pm 0.79 & \mathrm{pCi} / \mathrm{g} \\ 5.0 \pm 0.95 & \mathrm{pCi} / \mathrm{g}\end{array}$

Sample ID: 100317

$\begin{array}{ll}6.9 & \text { EPA900.0 } \\ 8.4 & \text { EPA900.0 } \\ 0.070 & \text { EPA903.0 } \\ 1.4 & \text { EPA906.0 }\end{array}$

\section{SAMPLE NAME: D43110701}

Location (SRS Coordinates): 18576.98E 66634.66N

Ground Elevation Above MSL: $132.8 \mathrm{ft}$

Depth of Core Interval: 0.00 to $0.50 \mathrm{ft}$

Sample Type: Normal

Sample Color: 10R 3/4

\section{Constituent}

Physical Parameters:

Cation exchange capacity

Cation exchange capacity

$\mathrm{pH}$

Specific conductance

Total organic carbon

Total organic carbon
Sample Matrix: Soil USC Soil Classification: SM

RQ AQ B Result Unit

Sample Moisture: Dry

Percent Solids: 91.20

D. Limit Method

\begin{tabular}{|c|c|c|c|c|c|}
\hline \multirow{6}{*}{ J } & $Y$ & 14 & meq $/ 100 \mathrm{~g}$ & 2.0 & EPA9080 \\
\hline & $\mathbf{Y}$ & 12 & $\mathrm{meq} / 100 \mathrm{~g}$ & 2.0 & EPA9080 \\
\hline & YQ & 4.8 & $\mathrm{pH}$ & 0.010 & EPA9045 \\
\hline & $\mathrm{Y}$ & & $\mu \mathrm{S} / \mathrm{cm}$ & $1.0^{\circ}$ & EPA9050 \\
\hline & $\mathbf{Y}$ & 3,600 & $\mathrm{mg} / \mathrm{kg}$ & 390 & EPA415.1 \\
\hline & $\mathbf{Y}$ & 4,200 & $\mathrm{mg} / \mathrm{kg}$ & 320 & EPA415. \\
\hline
\end{tabular}


SAMPLE NAME: D43110701 (Continued)

\begin{tabular}{|c|c|c|c|c|}
\hline Constituent & $\mathbf{R Q}$ & $\mathbf{A Q}$. & B & Resul \\
\hline Total Metals & & & & \\
\hline Arsenic & & $\mathbf{Y}$ & & 7.6 \\
\hline Barium & & $\mathbf{Y}$ & & 29 \\
\hline Cadmium & $\mathbf{U}$ & $\mathbf{Y}$ & & 0.48 \\
\hline Chromium & & $\mathrm{Y}$ & & 15 \\
\hline Copper & $U$ & $\mathbf{Y}$ & & 2.4 \\
\hline Lead & & $Y$ & & 130 \\
\hline Manganese & & $y$ & & 220 \\
\hline Mercury & U & $\mathbf{Y}$ & & 0.11 \\
\hline Nickel & U & $Y$ & & 3.9 \\
\hline Selenium & & $Y$ & & 1.1 \\
\hline Silver & $\mathrm{U}$ & $Y$ & & 0.97 \\
\hline
\end{tabular}

$B / N / A$ Extractables (including $P A H$ and phenols)
Sample ID: 100317

Unit D. Limit Method

\begin{tabular}{|c|c|c|c|c|c|c|}
\hline Acenaphthene & U & $Y$ & 350 & $\mu \mathrm{g} / \mathrm{kg}$ & 350 & EPA8270 \\
\hline Acenaphthylene & U & $\mathbf{Y}$ & 350 & $\mu \mathrm{g} / \mathrm{kg}$ & 350 & EPA8270 \\
\hline Anthracene & $\mathrm{U}$ & $\mathbf{Y}$ & 350 & $\mu \mathrm{g} / \mathrm{kg}$ & 350 & EPA8270 \\
\hline Benzo(a)anthracene & $U$ & $Y$ & 350 & $\mu \mathrm{g} / \mathrm{kg}$ & 350 & EPA8270 \\
\hline Benzo(b)fluoranthene & $\mathrm{U}$ & $Y$ & 350 & $\mu g / \mathrm{kg}$ & 350 & EPA8270 \\
\hline Benzo(k)fluoranthene & $\mathrm{U}$ & $Y$ & 350 & $\mu \mathrm{g} / \mathrm{kg}$ & 350 & EPA8270 \\
\hline Benzoic acid & U & $\mathbf{Y}$ & 1,700 & $\mu \mathrm{g} / \mathrm{kg}$ & 1,700 & EPA8270 \\
\hline Benzo $(g, h, i)$ perylene & $\mathrm{U}$ & $\mathbf{Y}$ & 350 & $\mu \mathrm{g} / \mathrm{kg}$ & 350 & EPA8270 \\
\hline Benzo(a)pyrene & U & $Y$ & 350 & $\mu g / \mathrm{kg}$ & 350 & EP.A8270 \\
\hline Benzyl alcohol & $\mathrm{U}$ & $\mathbf{Y}$ & 350 & $\mu g / k g$ & 350 & EPA8270 \\
\hline Bis(2-chloroethoxy) methane & $\mathrm{U}$ & $\mathbf{Y}$ & 350 & $\mu \mathrm{g} / \mathrm{kg}$ & 350 & EPA8270 \\
\hline Bis(2-chloroethyl) ether & $\mathrm{U}$ & $\mathbf{Y}$ & 350 & $\mu \mathrm{g} / \mathrm{kg}$ & 350 & EPA8270 \\
\hline Bis(2-chloroisopropyl) ether & U & $\mathbf{Y}$ & 350 & $\mu g / \mathrm{kg}$ & 350 & EPA8270 \\
\hline Bis(2-ethylhexyl) phthalate & $\mathbf{U}$ & $\mathbf{Y}$ & 350. & $\mu g / \mathrm{kg}$ & 350 & EPA8270 \\
\hline 4-Bromophenyl phenyl ether & $\mathrm{U}$ & $\mathbf{Y}$ & 350 & $\mu g / \mathrm{kg}$ & 350 & EPA8270 \\
\hline Butylbenzyl phthalate & $U$ & $Y$ & 350 & $\mu g / \mathrm{kg}$ & 350 & EPA8270 \\
\hline 4-Chloroaniline & U & $\mathbf{Y}$ & 350 & $\mu \mathrm{g} / \mathrm{kg}$ & 350 & EPA8270 \\
\hline 4-Chloro-m-cresol & U & $\mathbf{Y}$ & 350 & $\mu \mathrm{g} / \mathrm{kg}$ & 350 & EPA8270 \\
\hline 2-Chloronaphthalene & $U$ & $Y$ & 350 & $\mu \mathrm{g} / \mathrm{kg}$ & 350 & EPA8270 \\
\hline 2-Chlorophenol & U & $\mathbf{Y}$ & 350 & $\mu g / \mathrm{kg}$ & 350 & EPA8270 \\
\hline 4-Chlorophenyl phenyl ether & $\mathrm{U}$ & $\mathbf{Y}$ & 350 & $\mu g / \mathrm{kg}$ & 350 & EPA8270 \\
\hline Chrysene & U & $\mathbf{Y}$ & 350 & $\mu \mathrm{g} / \mathrm{kg}$ & 350 & EPA8270 \\
\hline o-Cresol (2-Methylphenol) & U & $\mathbf{Y}$ & 350 & $\mu \mathrm{g} / \mathrm{kg}$ & 350 & EPA8270 \\
\hline p-Cresol (4-Methylphenol) & $\mathbf{U}$ & $\mathbf{Y}$ & 350 & $\mu \mathrm{g} / \mathrm{kg}$ & 350 & EPA8270 \\
\hline Hexadecanoic acid ester & $\mathbf{J}$ & $\mathrm{YN}$ & 1.000 & $\mu g / \mathrm{kg}$ & & EPA8270 \\
\hline Octadecanoic acid $\ddot{\ddot{~}}$ & J & YN & 2.000 & $\mu \mathrm{g} / \mathrm{kg}$ & & EPA8270 \\
\hline Octadecanoic acid & J & YN & 300 & $\mu \mathrm{g} / \mathrm{kg}$ & & EPA8270 \\
\hline Dibenz(a,h)anthracene & U & $\mathbf{Y}$ & 350 & $\mu g / \mathrm{kg}$ & 350 & EPA8270 \\
\hline Dibenzofuran & U & $\mathbf{Y}$ & 350 & $\mu \mathrm{g} / \mathrm{kg}$ & 350 & EPA8270 \\
\hline Di-n-butyl phthalate & UJ & YV & 47 & $\mu g / \mathrm{kg}$ & 350 & EPA8270 \\
\hline 1,2-Dichlorobenzene & U & $\mathbf{Y}$ & 350 & $\mu g / \mathrm{kg}$ & 350 & EPA8270 \\
\hline 1,3-Dichlorobenzene & U & $\mathbf{Y}$ & 350 & $\mu \mathrm{g} / \mathrm{kg}$ & 350 & EPA8270 \\
\hline 1,4-Dichlorobenzene & U & $\mathbf{Y}$ & 350 & $\mu g / \mathrm{kg}$ & 350 & EPA8270 \\
\hline 3,3'-Dichlorobenzidine & $\mathbf{U}$ & $\mathbf{Y}$ & 690 & $\mu g / \mathrm{kg}$ & 690 & EPA8270 \\
\hline 2,4-Dichlorophenol & $\mathbf{U}$ & $\mathbf{Y}$ & 350 & $\mu \mathrm{g} / \mathrm{kg}$ & 350 & EPA8270 \\
\hline Diethyl phthalate & $\mathbf{U}$ & $\mathbf{Y}$ & 350 & $\mu \mathrm{g} / \mathrm{kg}$ & 350 & EPA8270 \\
\hline
\end{tabular}


Constituent

$B / N / A$ Extractables (including PAH and phenols)

2.4-Dimethyl phenol

Dimethyl phehalate

2,4-Dinitrophenol

2.4-Dinitrotoluene

2,6-Dinitrotoluene

Di-n-octyl phthalate

Fluoranthene

Fluorene

Hexachlorobenzene

Hexachlorobutadiene

Hexachlorocyclopentadiene

Hexachloroethane

Indeno(1.2.3-c.d)pyrene

Isophorone

Cholesterol

2-Methyl-4,6-dinitrophenol

2-Methylnaphthalene

Naphthalene

o-Nitroaniline

$\mathrm{m}$-Nitroaniline

p-Nitroaniline

Nitrobenzene

2-Nitrophenol

4-Nitrophenol

N-Nitrosodiphenylamine

$\mathrm{N}$-Nitrosodipropylamine

Pentachlorophenol

Phenanthrene

Phenol

Sub. propanoic acid

Pyrene

1,2,4-Trichlorobenzene

2,4,5-Trichlorophenol

2,4,6-Trichlorophenol

Unknown hydrocarbon

Unknown hydrocarbon

Unknown hydrocarbon

Unknown

Unknown

Unknown

Unknown

Unknown

Unknown ketone

Volatile Organic Compounds

Acetone

Benzene

Bromodichloromethane

Bromoform
RQ AQ B Result

\begin{tabular}{|c|c|c|}
\hline U & $Y$ & 350 \\
\hline$U$ & $Y$ & 350 \\
\hline $\mathrm{U}$ & $Y$ & 1,700 \\
\hline $\mathrm{U}$ & $\mathbf{Y}$. & 350 \\
\hline$U$ & $Y$ & 350 \\
\hline $\mathrm{U}$ & $Y$ & 350 \\
\hline U & $Y$ & 350 \\
\hline $\mathrm{U}$ & $Y$ & 350 \\
\hline U & $Y$ & 350 \\
\hline $\mathrm{U}$ & $Y$ & 350 \\
\hline $\mathrm{U}$ & $Y$ & 350 \\
\hline $\mathrm{U}$ & $Y$ & 350 \\
\hline $\mathrm{U}$ & $Y$ & 350 \\
\hline $\mathrm{U}$ & $Y$ & 350 \\
\hline J & YN & 2,000 \\
\hline$U$ & $Y$ & 1,700 \\
\hline $\mathrm{U}$ & $Y$ & 350 \\
\hline $\mathrm{U}$ & $Y$ & 350 \\
\hline $\mathrm{U}$ & $Y$ & 1,700 \\
\hline U & $Y$ & 1,700 \\
\hline U & $\mathbf{Y}$ & 1,700 \\
\hline U & $Y$ & 350 \\
\hline$U$ & $\mathbf{Y}$ & 350 \\
\hline U & $Y$ & 1,700 \\
\hline$U$ & $Y$ & 350 \\
\hline U. & $C Y$ & 350 \\
\hline $\mathrm{U}$ & $Y$ & 1,700 \\
\hline U & $Y$ & 350 \\
\hline$U$ & $Y$ & 350 \\
\hline J & YN & 300 \\
\hline$U$ & $Y$ & 350 \\
\hline $\mathrm{U}$ & $Y$ & 350 \\
\hline U & $Y$ & 1,700 \\
\hline $\mathrm{U}$ & $Y$ & 350 \\
\hline J & YN & 400 \\
\hline J & YN & 100 \\
\hline J & YN & 300 \\
\hline J & YN & 300 \\
\hline J & YN & 200 \\
\hline 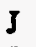 & YN & 100 \\
\hline J & YN & 200 \\
\hline- & YN & 200 \\
\hline J & YNV & 6,000 \\
\hline
\end{tabular}

350

1,700

350

350

350

350

350

350

350

350

350

1,700

350

1,700

1,700

1.700

350

1,700

350

1,700

350

300

350

1.700

350

400

100

300

200

100

200

Unit

D. Limit Method

\begin{tabular}{|c|c|c|}
\hline$\mu \mathrm{g} / \mathrm{kg}$ & 350 & EPA8270 \\
\hline$\mu \mathrm{g} / \mathrm{kg}$ & 350 & EPA8270 \\
\hline$\mu \mathrm{g} / \mathrm{kg}$ & 1,700 & EPA $8270^{\circ}$ \\
\hline$\mu \mathrm{g} / \mathrm{kg}$ & 350 & EPA8270 \\
\hline$\mu \mathrm{g} / \mathrm{kg}$ & 350 & EPA8270 \\
\hline$\mu \mathrm{g} / \mathrm{kg}$ & 350 & EPA8270 \\
\hline$\mu \mathrm{g} / \mathrm{kg}$ & 350 & EPA8270 \\
\hline$\mu \mathrm{g} / \mathrm{kg}$ & 350 & EPA8270 \\
\hline$\mu \mathrm{g} / \mathrm{kg}$ & 350 & EPA8270 \\
\hline$\mu \mathrm{g} / \mathrm{kg}$ & 350 & EPA8270 \\
\hline$\mu \mathrm{g} / \mathrm{kg}$ & 350 & EPA8270 \\
\hline$\mu \mathrm{g} / \mathrm{kg}$ & 350 & EPA8270 \\
\hline$\mu \mathrm{g} / \mathrm{kg}$ & 350 & EPA8270 \\
\hline$\mu \mathrm{g} / \mathrm{kg}$ & 350 & EPA8270 \\
\hline$\mu \mathrm{g} / \mathrm{kg}$ & & EPA8270 \\
\hline$\mu \mathrm{g} / \mathrm{kg}$ & 1,700 & EPA8270 \\
\hline$\mu \mathrm{g} / \mathrm{kg}$ & 350 & EPA8270 \\
\hline$\mu \mathrm{g} / \mathrm{kg}$ & 350 & EPA8270 \\
\hline$\mu \mathrm{g} / \mathrm{kg}$ & 1,700 & EPA8270 \\
\hline$\mu \mathrm{g} / \mathrm{kg}$ & 1.700 & EPA8270 \\
\hline$\mu \mathrm{g} / \mathrm{kg}$ & 1.700 & EPA8270 \\
\hline$\mu \mathrm{g} / \mathrm{kg}$ & 350 & EPA8270 \\
\hline$\mu \mathrm{g} / \mathrm{kg}$ & 350 & EPA8270 \\
\hline$\mu \mathrm{g} / \mathrm{kg}$ & 1,700 & EPA8270 \\
\hline$\mu \mathrm{g} / \mathrm{kg}$ & 350 & EPA8270 \\
\hline$\mu \mathrm{g} / \mathrm{kg}$ & 350 & EPA8270 \\
\hline$\mu \mathrm{g} / \mathrm{kg}$ & 1.700 & EPA8270 \\
\hline$\mu \mathrm{g} / \mathrm{kg}$ & 350 & EPA8270 \\
\hline$\mu \mathrm{g} / \mathrm{kg}$ & 350 & EPA8270 \\
\hline$\mu \mathrm{g} / \mathrm{kg}$ & & EPA8270 \\
\hline$\mu \mathrm{g} / \mathrm{kg}$ & 350 & EPA8270 \\
\hline$\mu \mathrm{g} / \mathrm{kg}$ & 350 & EPA8270 \\
\hline$\mu \mathrm{g} / \mathrm{kg}$ & 1,700 & EPA8270 \\
\hline$\mu \mathrm{g} / \mathrm{kg}$ & 350 & EPA8270 \\
\hline$\mu \mathrm{g} / \mathrm{kg}$ & & EPA8270 \\
\hline$\mu \mathrm{g} / \mathrm{kg}$ & & EPA8270 \\
\hline$\mu \mathrm{g} / \mathrm{kg}$ & & EPA8270 \\
\hline$\mu \mathrm{g} / \mathrm{kg}$ & & EPA8270 \\
\hline$\mu \mathrm{g} / \mathrm{kg}$ & & EPA8270 \\
\hline$\mu \mathrm{g} / \mathrm{kg}$ & & EPA8270 \\
\hline$\mu \mathrm{g} / \mathrm{kg}$ & & EPA8270 \\
\hline$\mu \mathrm{g} / \mathrm{kg}$ & & EPA8270̄ \\
\hline$\mu \mathrm{g} / \mathrm{kg}$ & & EPA8270 \\
\hline
\end{tabular}

$\mu \mathrm{g} / \mathrm{kg}$

$\mu \mathrm{g} / \mathrm{kg}$

$\mu \mathrm{g} / \mathrm{kg}$

$\mu \mathrm{g} / \mathrm{kg}$

$\begin{array}{ll}11 & \text { EPA8240 } \\ 5.4 & \text { EPA8240 } \\ 5.4 & \text { EPA8240 } \\ 5.4 & \text { EPA8240 }\end{array}$

12
5.4
$\begin{array}{lll}\mathrm{U} & \mathrm{Y} & 5.4 \\ \mathrm{U} & \mathrm{Y} & 5.4\end{array}$

$\begin{array}{lll}\mathrm{U} & \mathrm{Y} & 5.4 \\ \mathrm{U} & \mathrm{Y} & 5.4\end{array}$ 
SAMPLE NAME: D43110701 (Continued)

Constituent

Volatíle Organic Compounds

Bromomethane (Methyl bromide)
Carbon disulfide
Carbon tetrachloride
Chlorobenzene
Chloroethane
Chloroethene (Vinyl chloride)
Chloroform
Chloromethane (Methyl chloride)
Dibromochloromethane
1,1-Dichloroethane
1,2-Dichloroethane
1,1-Dichloroethylene
1,2-Dichloroethylene
Dichloromethane (Methylene chloride)
1,2-Dichloropropane
cis-1,3-Dichloropropene
trans-1,3-Dichloropropene
Ethylbenzene
2-Hexanone
Methyl ethyl ketone
Methyl isobutyl ketone
Styrene
1,1,2,2-Tetrachloroethane
Tetrachloroethylene
Toluene
1,1,1-Trichloroethane
1,1,2-Trichloroethane
Trichloroethylene
Vinyl acetate
Xylenes

Sample ID: 100317

RQ AQ B Result

Unit

D. Limit Method

\section{Pesticides/PCBs and Dioxins/Furans}

Aldrin
alpha-Benzene hexachloride
beta-Benzene hexachloride
delta-Benzene hexachloride
alpha-Chlordane
gamma-Chlordane
P,p'-DDD
P.p'-DDE
p.p'-DDT
Dieldrin
Endosulfan I
Endosulfan II
Endosulfan sulfate
Endrin
Endrin ketone
Heptachlor
Heptachlor epoxide

Aldrin

beta-Benzene hexachloride

delta-Benzene hexachloride

P,P'-DDD

P.P'-DDT

Endosulfan II

Endosulfan sulface

Endrin ketone

Heptachlor epoxide

$\begin{array}{lll}\text { U } & Y & 11 \\ U & Y & 5.4 \\ U & Y & 5.4 \\ U & Y & 5.4 \\ U & Y & 11 \\ U & Y & 11 \\ U & Y & 5.4 \\ U & Y & 11 \\ U & Y & 5.4 \\ U & Y & 5.4 \\ U & Y & 5.4 \\ U & Y & 5.4 \\ U & Y & 5.4 \\ U & Y & 5.4 \\ U & Y & 5.4 \\ U & Y & 5.4 \\ U & Y & 5.4 \\ U & Y & 5.4 \\ U & Y & 11 \\ U & Y & 11 \\ U & Y & 11 \\ U & Y & 5.4 \\ U & Y & 5.4 \\ U & Y & 5.4 \\ U & Y & 5.4 \\ U & Y & 5.4 \\ U & Y & 5.4 \\ U & Y & 5.4 \\ U & Y & 11 \\ U & Y & 5.4\end{array}$

11

5.4

5.4

11

5.4

5.4

5.4

5.4

5.4

5.4

5.4

5.4

5.4

5.4

11

11

5.4

5.4

5.4

5.4

5.4

5.4

1.7

1.7

1.7

1.7

1.7

1.7

3.5

3.5

3.5

3.5

1.7

3.5

3.5

3.5

3.5

1.7

1.7

$\begin{array}{lll}\mu \mathrm{g} / \mathrm{kg} & 11 & \text { EPA8240 } \\ \mu \mathrm{g} / \mathrm{kg} & 5.4 & \text { EPA8240 } \\ \mu \mathrm{g} / \mathrm{kg} & 5.4 & \text { EPA8240 } \\ \mu \mathrm{g} / \mathrm{kg} & 5.4 & \text { EPA8240 } \\ \mu \mathrm{g} / \mathrm{kg} & 11 & \text { EPA8240 } \\ \mu \mathrm{g} / \mathrm{kg} & 11 & \text { EPA8240 } \\ \mu \mathrm{g} / \mathrm{kg} & 5.4 & \text { EPA8240 } \\ \mu \mathrm{g} / \mathrm{kg} & 11 & \text { EPA8240 } \\ \mu \mathrm{g} / \mathrm{kg} & 5.4 & \text { EPA8240 } \\ \mu \mathrm{g} / \mathrm{kg} & 5.4 & \text { EPA8240 } \\ \mu \mathrm{g} / \mathrm{kg} & 5.4 & \text { EPA8240 } \\ \mu \mathrm{g} / \mathrm{kg} & 5.4 & \text { EPA8240 } \\ \mu \mathrm{g} / \mathrm{kg} & 5.4 & \text { EPA8240 } \\ \mu \mathrm{g} / \mathrm{kg} & 5.4 & \text { EPA8240 } \\ \mu \mathrm{g} / \mathrm{kg} & 5.4 & \text { EPA8240 } \\ \mu \mathrm{g} / \mathrm{kg} & 5.4 & \text { EPA8240 } \\ \mu \mathrm{g} / \mathrm{kg} & 5.4 & \text { EPA8240 } \\ \mu \mathrm{g} / \mathrm{kg} & 5.4 & \text { EPA8240 } \\ \mu \mathrm{g} / \mathrm{kg} & 11 & \text { EPA8240 } \\ \mu \mathrm{g} / \mathrm{kg} & 11 & \text { EPA8240 } \\ \mu \mathrm{g} / \mathrm{kg} & 11 & \text { EPA8240 } \\ \mu \mathrm{g} / \mathrm{kg} & 5.4 & \text { EPA8240 } \\ \mu \mathrm{g} / \mathrm{kg} & 5.4 & \text { EPA8240 } \\ \mu \mathrm{g} / \mathrm{kg} & 5.4 & \text { EPA8240 } \\ \mu \mathrm{g} / \mathrm{kg} & 5.4 & \text { EPA8240 } \\ \mu \mathrm{g} / \mathrm{kg} & 5.4 & \text { EPA8240 } \\ \mu \mathrm{g} / \mathrm{kg} & 5.4 & \text { EPA8240 } \\ \mu \mathrm{g} / \mathrm{kg} & 5.4 & \text { EPA8240 } \\ \mu \mathrm{g} / \mathrm{kg} & 11 & \text { EPA8240 } \\ \mu \mathrm{g} / \mathrm{kg} & 5.4 & \text { EPA8240 }\end{array}$

$\begin{array}{lll}\mu \mathrm{g} / \mathrm{kg} & 1.7 & \text { EPA8080 } \\ \mu \mathrm{g} / \mathrm{kg} & 1.7 & \text { EPA8080 } \\ \mu \mathrm{g} / \mathrm{kg} & 1.7 & \text { EPA8080 } \\ \mu \mathrm{g} / \mathrm{kg} & 1.7 & \text { EPA8080 } \\ \mu \mathrm{g} / \mathrm{kg} & 1.7 & \text { EPA8080 } \\ \mu \mathrm{g} / \mathrm{kg} & 1.7 & \text { EPA8080 } \\ \mu \mathrm{g} / \mathrm{kg} & 3.5 & \text { EPA8080 } \\ \mu \mathrm{g} / \mathrm{kg} & 3.5 & \text { EPA8080 } \\ \mu \mathrm{g} / \mathrm{kg} & 3.5 & \text { EPA8080 } \\ \mu \mathrm{g} / \mathrm{kg} & 3.5 & \text { EPA8080 } \\ \mu \mathrm{g} / \mathrm{kg} & 1.7 & \text { EPA8080 } \\ \mu \mathrm{g} / \mathrm{kg} & 3.5 & \text { EPA8080 } \\ \mu \mathrm{g} / \mathrm{kg} & 3.5 & \text { EPA8080 } \\ \mu \mathrm{g} / \mathrm{kg} & 3.5 & \text { EPA8080 } \\ \mu \mathrm{g} / \mathrm{kg} & 3.5 & \text { EPA8080 } \\ \mu \mathrm{g} / \mathrm{kg} & 1.7 & \text { EPA8080 } \\ \mu \mathrm{g} / \mathrm{kg} & 1.7 & \text { EPA8080 }\end{array}$


SAMPLE NAME: D43110701 (Continued)

Constituent

RQ AQ

Pesticides/PCBs and Dioxins/Furans

Heptachlorodibenzo-p-dioxin isomers
Heptachlorodibenzo-p-furan isomers
Hexachlorodibenzo-p-dioxin isomers
Hexachlorodibenzo-p-furan isomers
Lindane
Methoxychlor
Octachlorodibenzo-p-dioxin
Octachlorodibenzo-p-furan
PCB 1016
PCB 1221
PCB 1232
PCB 1242
PCB 1248
PCB 1254
PCB 1260
Pentachlorodibenzo-p-dioxin isomers
Pentachlorodibenzo-p-furan isomers
2,3.7,8-TCDD
2.3.7,8-TCDF
Tetrachlorodibenzo-p-dioxin isomers
Tetrachlorodibenzo-p-furan isomers
Toxaphene

Sample ID: 100317

B Result Unit D. Limit Method

$\begin{array}{llllll}\mathrm{U} & \mathrm{Y} & 0.10 & \mathrm{ng} / \mathrm{g} & 0.10 & \text { EPA8280 } \\ \mathrm{U} & \mathrm{Y} & 0.10 & \mathrm{ng} / \mathrm{g} & 0.10 & \text { EPA8280 } \\ \mathrm{U} & \mathrm{Y} & 0.10 & \mathrm{ng} / \mathrm{g} & 0.10 & \text { EPA8280 } \\ \mathrm{U} & \mathrm{Y} & 0.10 & \mathrm{ng} / \mathrm{g} & 0.10 & \text { EPA8280 } \\ \mathrm{U} & \mathrm{Y} & 1.7 & \mu \mathrm{g} / \mathrm{kg} & 1.7 & \text { EPA8080 } \\ \mathrm{U} & \mathrm{Y} & 17 & \mu \mathrm{g} / \mathrm{kg} & 17 & \text { EPA8080 } \\ \mathrm{J} & \mathrm{Y} & 1.6 & \mathrm{ng} / \mathrm{g} & 0.10 & \text { EPA8280 } \\ \mathrm{U} & \mathrm{Y} & 0.10 & \mathrm{ng} / \mathrm{g} & 0.10 & \text { EPA8280 } \\ \mathrm{U} & \mathrm{Y} & 35 & \mu \mathrm{g} / \mathrm{kg} & 35 & \text { EPA8080 } \\ \mathrm{U} & \mathrm{Y} & 70 & \mu \mathrm{g} / \mathrm{kg} & 70 & \text { EPA8080 } \\ \mathrm{U} & \mathrm{Y} & 35 & \mu \mathrm{g} / \mathrm{kg} & 35 & \text { EPA8080 } \\ \mathrm{U} & \mathrm{Y} & 35 & \mu \mathrm{g} / \mathrm{kg} & 35 & \text { EPA8080 } \\ \mathrm{U} & \mathrm{Y} & 35 & \mu \mathrm{g} / \mathrm{kg} & 35 & \text { EPA8080 } \\ \mathrm{U} & \mathrm{Y} & 35 & \mu \mathrm{g} / \mathrm{kg} & 35 & \text { EPA8080 } \\ \mathrm{U} & \mathrm{Y} & 35 & \mu \mathrm{g} / \mathrm{kg} & 35 & \text { EPA8080 } \\ \mathrm{U} & \mathrm{Y} & 0.10 & \mathrm{ng} / \mathrm{g} & 0.10 & \text { EPA8280 } \\ \mathrm{U} & \mathrm{Y} & 0.10 & \mathrm{ng} / \mathrm{g} & 0.10 & \text { EPA8280 } \\ \mathrm{U} & \mathrm{Y} & 0.10 & \mathrm{ng} / \mathrm{g} & 0.10 & \text { EPA8280 } \\ \mathrm{U} & \mathrm{Y} & 0.10 & \mathrm{ng} / \mathrm{g} & 0.10 & \text { EPA8280 } \\ \mathrm{U} & \mathrm{Y} & 0.10 & \mathrm{ng} / \mathrm{g} & 0.10 & \text { EPA8280 } \\ \mathrm{U} & \mathrm{Y} & 0.10 & \mathrm{ng} / \mathrm{g} & 0.10 & \text { EPA8280 } \\ \mathrm{U} & \mathrm{Y} & 170 & \mu \mathrm{g} / \mathrm{kg} & 170 & \text { EPA8080 }\end{array}$

\section{Radionuclide Indicators and Radionuclides}

Gross alpha

Nonvolatile beta

Radium, total alpha-emitting

Tritium

\section{SAMPLE NAME: D43110702}

Location (SRS Coordinates): $18576.98 \mathrm{E}$ 66634.66N

Ground Elevation Above MSL: $132.8 \mathrm{ft}$

Depth of Core Interval: 0.50 to $2.00 \mathrm{ft}$

Sample Type: Normal

Sample Color: 10R 3/4

\section{Constituent $\quad \because$}

Total Metals

$\begin{array}{lllllll}\text { Arsenic } & \mathrm{U} & \mathrm{Y} & 1.0 & \mathrm{mg} / \mathrm{kg} & 1.0 & \text { EPA7060 } \\ \text { Barium } & \mathrm{U} & \mathrm{Y} & 21 & \mathrm{mg} / \mathrm{kg} & 21 & \text { EPA6010 } \\ \text { Cadmium } & \mathrm{U} & \mathrm{Y} & 0.53 & \mathrm{mg} / \mathrm{kg} & 0.53 & \text { EPA6010 } \\ \text { Chromium } & & \mathrm{Y} & 10 & \mathrm{mg} / \mathrm{kg} & 1.1 & \text { EPA6010 } \\ \text { Copper } & \mathrm{U} & \mathrm{Y} & 2.6 & \mathrm{mg} / \mathrm{kg} & 2.6 & \text { EPA6010 } \\ \text { Lead } & & \mathrm{Y} & 3.1 & \mathrm{mg} / \mathrm{kg} & 0.31 & \text { EPA7421 } \\ \text { Manganese } & & \mathrm{Y} & 84 & \mathrm{mg} / \mathrm{kg} & 1.6 & \text { EPA6010 }\end{array}$

$\begin{array}{ll}\mathbf{U} & \mathbf{Y} \\ & \mathrm{Y} \\ & \mathrm{YV} \\ & \mathrm{Y}\end{array}$

$$
\begin{array}{ll}
8.6 & \mathrm{pCi} / \mathrm{g} \\
26 \pm 23 & \mathrm{pCi} / \mathrm{g} \\
0.86 \pm 0.74 & \mathrm{pCi} / \mathrm{g} \\
3.6 \pm 0.74 & \mathrm{pCi} / \mathrm{g}
\end{array}
$$

Sample ID: 100318

Sample Matrix: Soil USC Soil Classification: SM

RQ AQ
B Result Unit
Sample Moisture: Dry Pêrcent Solids: 94.10

D. Limit Method 
SAMPLE NAME: D43110702 (Continued)

Sample D: 100318

Constituent

RQ AQ

B Result

Unit

D. Limit Method

Total Metals

Mercury

Nickel

Selenium

Silver

$\begin{array}{ll}U & Y \\ U & Y \\ U & Y \\ U & Y\end{array}$

$\mathbf{Y}$

$\mathbf{Y}$

$\mathbf{Y}$
0.11

4.2

0.52

1.1 $\mathrm{mg} / \mathrm{kg}$
$\mathrm{mg} / \mathrm{kg}$
$\mathrm{mg} / \mathrm{kg}$
$\mathrm{mg} / \mathrm{kg}$ $0.11^{\circ}$

4.2

0.52

1.1
EPA7470

EPA6010

EPA7740

EPA6010

$B / N / A$ Extractables (including PAH and phenols)

\begin{tabular}{|c|c|c|c|}
\hline Acenaphthene & $\mathbf{U}$ & $Y$ & 360 \\
\hline Acenaphthylene & U & $\mathbf{Y}$ & 360 \\
\hline Anthracene & $\mathbf{U}$ & $\mathrm{Y}$ & 360 \\
\hline Benzo(a)anthracene & $\mathbf{U}$ & $\mathbf{Y}$ & 360 \\
\hline Benzo(b)fluoranthene & $\mathbf{U}$ & $\mathbf{Y}$ & 360 \\
\hline Benzo(k)fluoranthene & $\mathbf{U}$ & $Y$ & 360 \\
\hline Benzoic acid & $\mathbf{U}$ & $\mathbf{Y}$ & 1,800 \\
\hline Benzo(g,h,i)perylene & $U$ & $\mathbf{Y}$ & 360 \\
\hline Benzo(a)pyrene & $\mathrm{U}$ & $\mathrm{Y}$ & 360 \\
\hline Benzyl alcohol & $U$ & $\mathrm{Y}$ & 360 \\
\hline Bis (2-chloroethoxy) methane & $\mathbf{U}$ & $\mathrm{Y}$ & 360 \\
\hline Bis(2-chloroethyl) ether & $\mathbf{U}$ & $\mathbf{Y}$ & 360 \\
\hline Bis(2-chloroisopropyl) ether & $\mathbf{U}$ & $\mathbf{Y}$ & 360 \\
\hline Bis(2-ethylhexyl) phthalate & $\mathbf{J}$ & $\mathbf{Y}$ & 180 \\
\hline 4-Bromophenyl phenyl ether & $\mathrm{U}$ & $Y$ & 360 \\
\hline Butylbenzyl phthalate & $\mathbf{U}$ & $Y$ & 360 \\
\hline 4-Chloroaniline & $\mathbf{U}$ & $\mathbf{Y}$ & 360 \\
\hline 4-Chloro-m-cresol & $\mathbf{U}$ & $\mathbf{Y}$ & 360 \\
\hline 2-Chloronaphthalene & $\mathrm{U}$ & $\mathbf{Y}$ & 360 \\
\hline 2-Chlorophenol & $\mathbf{U}$ & $\mathbf{Y}$ & 360 \\
\hline 4-Chlorophenyl phenyl ether & $\mathbf{U}$ & $\mathbf{Y}$ & 360 \\
\hline Chrysene & $\mathrm{U}$ & $Y$ & 360 \\
\hline o-Cresol (2-Methylphenol) & $\mathbf{U}$ & $\mathbf{Y}$ & 360 \\
\hline p-Cresol (4-Methylphenol) & $U$ & $\mathbf{Y}$ & 360 \\
\hline Tetradecanoic acid & $\mathbf{J}$ & YN & 200 \\
\hline Hexadecanoic acid ester & $\mathbf{J}$ & $\mathrm{YN}$ & 700 \\
\hline Octadecanoic acid & $\mathbf{J}$ & YN & 500 \\
\hline Dibenz(a,h)anthracene & $\mathbf{U}$ & $\mathbf{Y}$ & 360 \\
\hline Dibenzofuran & $\mathbf{U}$ & $\mathbf{Y}$ & 360 \\
\hline Di-n-butyl phthaiate & UJ & $\mathrm{YV}$ & 85 \\
\hline 1,2-Dichlorobenzene & $\mathbf{U}$ & $\mathbf{Y}$ & 360 \\
\hline 1,3-Dichlorobenzene & $\mathbf{U}$ & $\mathbf{Y}$ & 360 \\
\hline 1,4-Dichlorobenzene & $\mathrm{U}$ & $\mathbf{Y}$ & 360 \\
\hline 3,3'-Dichlorobenzidine & $\mathbf{U}$ & $\mathbf{Y}$ & 720 \\
\hline 2,4-Dichlorophenol & U & $\mathbf{Y}$ & 360 \\
\hline Diethyl phthalate & $\mathrm{U}$ & $\mathbf{Y}$ & 360 \\
\hline 2,4-Dimethyl phenol & U & $\mathbf{Y}$ & 360 \\
\hline Dimethyl phthalate & $\mathbf{U}$ & $\mathbf{Y}$ & 360 \\
\hline 2.4-Dinitrophenol & $\mathbf{U}$ & $\mathbf{Y}$ & 1,800 \\
\hline 2,4-Dinitrotoluene & $\mathbf{U}$ & $Y$ & 360 \\
\hline 2,6-Dinitrotoluene & U & $Y$ & 360 \\
\hline Di-n-octyl phthalate & $\mathrm{U}$ & $\mathbf{Y}$ & 360 \\
\hline Fluoranthene & $\mathrm{U}$ & $Y$ & 360 \\
\hline
\end{tabular}

\begin{tabular}{|c|c|c|}
\hline$\mu g / k g$ & 360 & EPA8270 \\
\hline$\mu g / k g$ & 360 & EPA8270 \\
\hline$\mu \mathrm{g} / \mathrm{kg}$ & 360 & EPA8270 \\
\hline$\mu g / \mathrm{kg}$ & 360 & EPA8270 \\
\hline$\mu g / \mathrm{kg}$ & 360 & EPA8270 \\
\hline$\mu \mathrm{g} / \mathrm{kg}$ & 360 & EPA8270 \\
\hline$\mu g / \mathrm{kg}$ & 1,800 & EPA8270 \\
\hline$\mu \mathrm{g} / \mathrm{kg}$ & $360^{\circ}$ & EPA8270 \\
\hline$\mu g / \mathrm{kg}$ & 360 & EPA8270 \\
\hline$\mu g / k g$ & 360 & EPA8270 \\
\hline$\mu \mathrm{g} / \mathrm{kg}$ & 360 & EPA8270 \\
\hline$\mu \mathrm{g} / \mathrm{kg}$ & 360 & EPA8270 \\
\hline$\mu \mathrm{g} / \mathrm{kg}$ & 360 & EPA8270 \\
\hline$\mu \mathrm{g} / \mathrm{kg}$ & 360 & EPA8270 \\
\hline$\mu \mathrm{g} / \mathrm{kg}$ & 360 & EPA8270 \\
\hline$\mu \mathrm{g} / \mathrm{kg}$ & 360 & EPA8270 \\
\hline$\mu \mathrm{g} / \mathrm{kg}$ & 360 & EPA8270 \\
\hline$\mu \mathrm{g} / \mathrm{kg}$ & 360 & EPA8270 \\
\hline$\mu \mathrm{g} / \mathrm{kg}$ & 360 & EPA8270 \\
\hline$\mu \mathrm{g} / \mathrm{kg}$ & 360 & EPA8270 \\
\hline$\mu \mathrm{g} / \mathrm{kg}$ & 360 & EPA8270 \\
\hline$\mu \mathrm{g} / \mathrm{kg}$ & 360 & EPA8270 \\
\hline$\mu \mathrm{g} / \mathrm{kg}$ & 360 & EPA8270 \\
\hline$\mu \mathrm{g} / \mathrm{kg}$ & 360 & EPA8270 \\
\hline$\mu \mathrm{g} / \mathrm{kg}$ & & EPA8270 \\
\hline$\mu \mathrm{g} / \mathrm{kg}$ & & EPA 8270 \\
\hline$\mu \mathrm{g} / \mathrm{kg}$ & & EPA8270 \\
\hline$\mu \mathrm{g} / \mathrm{kg}$ & 360 & EPA8270 \\
\hline$\mu \mathrm{g} / \mathrm{kg}$ & 360 & EPA8270 \\
\hline$\mu \mathrm{g} / \mathrm{kg}$ & 360 & EPA8270 \\
\hline$\mu \mathrm{g} / \mathrm{kg}$ & 360 & EPA8270 \\
\hline$\mu \mathrm{g} / \mathrm{kg}$ & 360 & EPA8270 \\
\hline$\mu \mathrm{g} / \mathrm{kg}$ & 360 & EPA8270 \\
\hline$\mu \mathrm{g} / \mathrm{kg}$ & 720 & EPA8270 \\
\hline$\mu \mathrm{g} / \mathrm{kg}$ & 360 & EPA8270 \\
\hline$\mu \mathrm{g} / \mathrm{kg}$ & 360 & EPA8270 \\
\hline$\mu \mathrm{g} / \mathrm{kg}$ & 360. & EPA8270 \\
\hline$\mu \mathrm{g} / \mathrm{kg}$ & 360 & EPA8270 \\
\hline$\mu \mathrm{g} / \mathrm{kg}$ & 1,800 & EPA8270 \\
\hline$\mu g / k g$ & 360 & EPA8270 \\
\hline$\mu g / \mathrm{kg}$ & 360 & EPA8270 \\
\hline$\mu \mathrm{g} / \mathrm{kg}$ & 360 & EPA8270 \\
\hline$\mu \mathrm{g} / \mathrm{kg}$ & 360 & EPA827 \\
\hline
\end{tabular}


SAMPLE NAME: D43110702 (Continued) Sample ID: 100318

Constituent RQ AQ B Result

$B / N / A$ Extractables (including $P A H$ and phenols)

Fluorene

Hexachlorobenzene.

Hexachlorobutadiene

Hexachlorocyclopentadiene

Hexachloroethane

Indeno(1,2,3-c,d)pyrene

Isophorone

2-Methyl-4,6-dinitrophenol

2-Methylnaphthalene

Naphthalene

o-Nitroaniline

m-Nitroaniline

p-Nitroaniline

Nitrobenzene

2-Nitrophenol

4-Nitrophenol

N-Nitrosodiphenylamine

N-Nitrosodipropylamine

Pentachlorophenol

Phenanthrene

Phenol

Sub. propanoic acid

Pyrene

1,2,4-Trichlorobenzene

2,4,5-Trichlorophenol

2,4,6-Trichlorophenol

Unknown hydrocarbon

Unknown hydrocarbon

Unknown

Unknown

Unknown

Unknown ketone

Volatile Organic Compounds

Acetone

Benzene

Bromodichloromethane

Bromoform

Bromomethane (Methyl bromide)

Carbon disulfide

Carbon tetrachloride $\because$

Chlorobenzene

Chloroethane

Chloroethene (Vinyl chloride)

Chloroform

Chloromethane (Methyl chloride)

Dibromochloromethane

1.1-Dichloroethane

1,2-Dichloroethane

$\begin{array}{lll}U & Y & 360 \\ U & Y & 360 \\ U & Y & 360 \\ U & Y & 360 \\ U & Y & 360 \\ U & Y & 360 \\ U & Y & 360 \\ U & Y & 1.800 \\ U & Y & 360 \\ U & Y & 360 \\ U & Y & 1,800 \\ U & Y & 1,800 \\ U & Y & 1,800 \\ U & Y & 360 \\ U & Y & 360 \\ U & Y & 1,800 \\ U & Y & 360 \\ U J & C Y & 360 \\ U & Y & 1,800 \\ U & Y & 360 \\ U & Y & 360 \\ J & Y N & 500 \\ U & Y & 360 \\ U & Y & 360 \\ U & Y & 1,800 \\ U & Y & 360 \\ J & Y N & 300 \\ J & Y N & 200 \\ J & Y N & 500 \\ J & Y N & 200 \\ J & Y N & 100 \\ J & Y N V & 5,000 \\ & & \\ & Y & \end{array}$

$\begin{array}{lll}\mu \mathrm{g} / \mathrm{kg} & 360 & \text { EPA8270 } \\ \mu \mathrm{g} / \mathrm{kg} & 360 & \text { EPA8270 } \\ \mu \mathrm{g} / \mathrm{kg} & 360 & \text { EPA8270 } \\ \mu \mathrm{g} / \mathrm{kg} & 360 & \text { EPA8270 } \\ \mu \mathrm{g} / \mathrm{kg} & 360 & \text { EPA8270 } \\ \mu \mathrm{g} / \mathrm{kg} & 360 & \text { EPA8270 } \\ \mu \mathrm{g} / \mathrm{kg} & 360 & \text { EPA8270 } \\ \mu \mathrm{g} / \mathrm{kg} & 1.800 & \text { EPA8270 } \\ \mu \mathrm{g} / \mathrm{kg} & 360 & \text { EPA8270 } \\ \mu \mathrm{g} / \mathrm{kg} & 360 & \text { EPA8270 } \\ \mu \mathrm{g} / \mathrm{kg} & 1,800 & \text { EPA8270 } \\ \mu \mathrm{g} / \mathrm{kg} & 1,800 & \text { EPA8270 } \\ \mu \mathrm{g} / \mathrm{kg} & 1.800 & \text { EPA8270 } \\ \mu \mathrm{g} / \mathrm{kg} & 360 & \text { EPA8270 } \\ \mu \mathrm{g} / \mathrm{kg} & 360 & \text { EPA8270 } \\ \mu \mathrm{g} / \mathrm{kg} & 1,800 & \text { EPA8270 } \\ \mu \mathrm{gg} / \mathrm{kg} & 360 & \text { EPA8270 } \\ \mu \mathrm{g} / \mathrm{kg} & 360 & \text { EPA8270 } \\ \mu \mathrm{g} / \mathrm{kg} & 1,800 & \text { EPA8270 } \\ \mu \mathrm{g} / \mathrm{kg} & 360 & \text { EPA8270 } \\ \mu \mathrm{g} / \mathrm{kg} & 360 & \text { EPA8270 } \\ \mu \mathrm{g} / \mathrm{kg} & & \text { EPA8270 } \\ \mu \mathrm{g} / \mathrm{kg} & 360 & \text { EPA8270 } \\ \mu \mathrm{g} / \mathrm{kg} & 360 & \text { EPA8270 } \\ \mu \mathrm{gg} / \mathrm{kg} & 1,800 & \text { EPA8270 } \\ \mu \mathrm{g} / \mathrm{kg} & 360 & \text { EPA8270 } \\ \mu \mathrm{g} / \mathrm{kg} & & \text { EPA8270 } \\ \mu \mathrm{g} / \mathrm{kg} & & \text { EPA8270 } \\ \mu \mathrm{g} / \mathrm{kg} & & \text { EPA8270 } \\ \mu \mathrm{g} / \mathrm{kg} & & \text { EPA8270 } \\ \mu \mathrm{g} / \mathrm{kg} & & \text { EPA8270 } \\ \mu \mathrm{g} / \mathrm{kg} & & \text { EPA8270 } \\ & & \end{array}$

11

5.5

5.5

5.5

11

5.5

5.5

5.5

11

11

5.5

11

5.5

5.5

3.4

60

60

1,800

$\quad \mu \mathrm{g} / \mathrm{kg}$

$\quad \mu \mathrm{g} / \mathrm{kg}$

$\mu g / k$

$\begin{array}{lll}\mu \mathrm{g} / \mathrm{kg} & 11 & \text { EPA8240 } \\ \mu \mathrm{g} / \mathrm{kg} & 5.5 & \text { EPA8240 } \\ \mu \mathrm{g} / \mathrm{kg} & 5.5 & \text { EPA8240 } \\ \mu \mathrm{g} / \mathrm{kg} & 5.5 & \text { EPA8240 } \\ \mu \mathrm{g} / \mathrm{kg} & 11 & \text { EPA8240 } \\ \mu \mathrm{g} / \mathrm{kg} & 5.5 & \text { EPA8240 } \\ \mu \mathrm{g} / \mathrm{kg} & 5.5 & \text { EPA8240 } \\ \mu \mathrm{g} / \mathrm{kg} & 5.5 & \text { EPA8240 } \\ \mu \mathrm{g} / \mathrm{kg} & 11 & \text { EPA8240 } \\ \mu \mathrm{g} / \mathrm{kg} & 11 & \text { EPA8240 } \\ \mu \mathrm{g} / \mathrm{kg} & 5.5 & \text { EPA8240 } \\ \mu \mathrm{g} / \mathrm{kg} & 11 & \text { EPA8240 } \\ \mu \mathrm{g} / \mathrm{kg} & 5.5 & \text { EPA8240 } \\ \mu \mathrm{g} / \mathrm{kg} & 5.5 & \text { EPA8240 } \\ \mu \mathrm{g} / \mathrm{kg} & 5.5 & \text { EPA8240 }\end{array}$

Unit $\therefore$

D. Limit Method 
Constituent

RQ

AQ

B

Result

Unit

D. Limit Method

Volatile Organic Compounds

1,1-Dichloroethylene
1,2-Dichloroethylene
Dichloromethane (Methylene chloride)
1,2-Dichloropropane
cis-1,3-Dichloropropene
trans-1,3-Dichloropropene
Ethylbenzene
2-Hexanone
Methyl ethyl ketone
Methyl isobutyl ketone
Styrene
1,1.2,2-Tetrachloroethane
Tetrachloroethylene
Toluene
1,1.1-Trichloroethane
1,1.2-Trichloroethane
Trichloroethylene
Vinyl acetate
Xylenes

$\begin{array}{lll}U & Y & 5.5 \\ U & Y & 5.5 \\ U & Y V & 40 \\ U & Y & 5.5 \\ U & Y & 5.5 \\ U & Y & 5.5 \\ U & Y & 5.5 \\ U & Y & 11 \\ U & Y & 11 \\ U & Y & 11 \\ U & Y & 5.5 \\ U & Y & 5.5 \\ U & Y & 5.5 \\ J & Y & 3.6 \\ U & Y & 5.5 \\ J & Y & 5.3 \\ U & Y & 5.5 \\ U & Y & 11 \\ U & Y & 5.5\end{array}$

$\mu g / \mathrm{kg}$
$\mu g / \mathrm{kg}$
$\mu g / \mathrm{kg}$
$\mu g / \mathrm{kg}$
$\mu g / \mathrm{kg}$
$\mu g / \mathrm{kg}$
$\mu g / \mathrm{kg}$
$\mu g / \mathrm{kg}$
$\mu g / \mathrm{kg}$
$\mu g / \mathrm{kg}$
$\mu g / \mathrm{kg}$
$\mu g / \mathrm{kg}$
$\mu \mathrm{g} / \mathrm{kg}$
$\mu g / \mathrm{kg}$
$\mu g / \mathrm{kg}$
$\mu g / \mathrm{kg}$
$\mu \mathrm{g} / \mathrm{kg}$
$\mu g / \mathrm{kg}$
$\mu g / \mathrm{kg}$

EPA8240

EPA8240

$5.5 \quad$ EPA8240

5.5 EPA8240

$5.5 \quad$ EPA8240

$5.5 \quad$ EPA8240

$5.5 \quad$ EPA8240

11 EPA8240

11 EPA8240

11 EPA8240

$5.5 \quad$ EPA8240

$5.5 \quad$ EPA8240

$5.5 \quad$ EPA8240

$5.5 \quad$ EPA8240

$5.5 \quad$ EPA8240

$5.5 \quad$ EPA8240

$5.5 \quad$ EPA8240

11 EPA8240

Xylenes

Pesticides/PCBs and Dioxins/Furans

Aldrin
alpha-Benzene hexachloride
beta-Benzene hexachloride
delta-Benzene hexachloride
alpha-Chlordane
gamma-Chlordane
p.p'-DDD
p.p'-DDE
p.p'-DDT
Dieldrin
Endosulfan I
Endosulfan II
Endosulfan sulfate
Endrin
Endrin ketone
Heptachlor
Heptachlor epoxide
Heptachlorodibenzo-p-dioxin isomers
Heptachlorodibenzo-p-furan isomers
Hexachlorodibenzo-p-dioxin isomers
Hexachlorodibenzo-p-furan isomers
Lindane
Methoxychlor
Octachlorodibenzo-p-dioxin
Octachlorodibenzo-p-furan
PCB 1016
PCB 1221
PCB I232

$\begin{array}{lll}U & Y & 1.8 \\ U & Y & 1.8 \\ U & Y & 1.8 \\ U & Y & 1.8 \\ U & Y & 1.8 \\ U & Y & 1.8 \\ U & Y & 3.6 \\ U & Y & 3.6 \\ U & Y & 3.6 \\ U & Y & 3.6 \\ U & Y & 1.8 \\ U & Y & 3.6 \\ U & Y & 3.6 \\ U & Y & 3.6 \\ U & Y & 3.6 \\ U & Y & 1.8 \\ U & Y & 1.8 \\ U & Y & 0.10 \\ U & Y & 0.10 \\ U & Y & 0.10 \\ U & Y & 0.10 \\ U & Y & 1.8 \\ U & Y & 18 \\ J & Y & 0.70 \\ U & Y & 0.10 \\ U & Y & 36 \\ U & Y & 72 \\ U & Y & 36 \\ & & \end{array}$

$\mu g / \mathrm{kg}$
$\mu g / \mathrm{kg}$
$\mu \mathrm{g} / \mathrm{kg}$
$\mu \mathrm{g} / \mathrm{kg}$
$\mu g / \mathrm{kg}$
$\mu \mathrm{g} / \mathrm{kg}$
$\mu g / \mathrm{kg}$
$\mu \mathrm{g} / \mathrm{kg}$
$\mu g / \mathrm{kg}$
$\mu g / \mathrm{kg}$
$\mu g / \mathrm{kg}$
$\mu \mathrm{g} / \mathrm{kg}$
$\mu \mathrm{g} / \mathrm{kg}$
$\mu \mathrm{g} / \mathrm{kg}$
$\mu g / \mathrm{kg}$
$\mu g / \mathrm{kg}$
$\mu \mathrm{g} / \mathrm{kg}$
$\mathrm{ng} / \mathrm{g}$
$\mathrm{ng} / \mathrm{g}$
$\mathrm{ng} / \mathrm{g}$
$\mathrm{ng} / \mathrm{g}$
$\mu g / \mathrm{kg}$
$\mu g / \mathrm{kg}$
$\mathrm{ng} / \mathrm{g}$
$\mathrm{ng} / \mathrm{g}$
$\mu g / \mathrm{kg}$
$\mu g / \mathrm{kg}$
$\mu g / \mathrm{kg}$

$\begin{array}{ll}1.8 & \text { EPA8080 } \\ 1.8 & \text { EPA8080 } \\ 1.8 & \text { EPA8080 } \\ 1.8 & \text { EPA8080 } \\ 1.8 & \text { EPA8080 } \\ 1.8 & \text { EPA8080 } \\ 3.6 & \text { EPA8080 } \\ 3.6 & \text { EPA8080 } \\ 3.6 & \text { EPA8080 } \\ 3.6 & \text { EPA8080 } \\ 1.8 & \text { EPA8080 } \\ 3.6 & \text { EPA8080 } \\ 3.6 & \text { EPA8080 } \\ 3.6 & \text { EPA8080 } \\ 3.6 & \text { EPA8080 } \\ 1.8 & \text { EPA8080 } \\ 1.8 & \text { EPA8080 } \\ 0.10 & \text { EPA8280 } \\ 0.10 & \text { EPA8280 } \\ 0.10 & \text { EPA8280 } \\ 0.10 & \text { EPA8280 } \\ 1.8 & \text { EPA8080 } \\ 18 & \text { EPA8080 } \\ 0.10 & \text { EPA8280 } \\ 0.10 & \text { EPA8280 } \\ 36 & \text { EPA8080 } \\ 72 & \text { EPA8080 } \\ 36 & \text { EPA8080 }\end{array}$


SAMPLE NAME: D43110702 (Continued)

Constituent
Sample ID: 100318

RQ AQ B Result Unit D. Limit Method

Pesticides/PCBs and Dioxins/Furans

PCB 1242

PCB 1248

PCB 1254

PCB 1260

Pentachlorodibenzo-p-dioxin isomers

Pentachlorodibenzo-p-furan isomers

2.3.7.8-TCDD

2.3.7,8-TCDF

Tetrachlorodibenzo-p-dioxin isomers

Tetrachlorodibenzo-p-furan isomers

Toxaphene

$\begin{array}{lll}\mathbf{U} & \mathrm{Y} & \cdot \\ \mathrm{U} & \mathrm{Y} & 36 \\ \mathrm{U} & \mathrm{Y} & 36 \\ \mathrm{U} & \mathrm{Y} & 36 \\ \mathrm{U} & \mathrm{Y} & 36 \\ \mathrm{U} & \mathrm{Y} & 0.20 \\ \mathrm{U} & \mathrm{Y} & 0.10 \\ \mathrm{U} & \mathrm{Y} & 0.10 \\ \mathrm{U} & \mathrm{Y} & 0.10 \\ \mathrm{U} & \mathrm{Y} & 0.10 \\ \mathrm{U} & \mathrm{Y} & 0.10 \\ & & 180\end{array}$

Radionuclide Indicators and Radionuclides

Gross alpha

Nonvolatile beta

Radium, total alpha-emitting

Tritium

$\mathrm{U}$

$$
\begin{aligned}
& Y \\
& Y \\
& Y V
\end{aligned}
$$

SAMPLE NAME: D43110703

Location (SRS Coordinates): $18576.98 \mathrm{E} 66634.66 \mathrm{~N}$

Ground Elevation Above MSL: $132.8 \mathrm{ft}$

Depth of Core Interval: 2.00 to $4.00 \mathrm{ft}$

Sample Type: Normal

Sample Color: 2N2

Constituent

Total Metals

Arsenic

Barium

Cadmium

Chromium

Copper

Lead

Manganese

Mercury

Nickel

Selenium

Silver

$$
\begin{aligned}
& \mu \mathrm{g} / \mathrm{kg} \\
& \mu \mathrm{g} / \mathrm{kg} \\
& \mu \mathrm{g} / \mathrm{kg} \\
& \mu \mathrm{g} / \mathrm{kg} \\
& \mathrm{ng} / \mathrm{g} \\
& \mathrm{ng} / \mathrm{g} \\
& \mathrm{ng} / \mathrm{g} \\
& \mathrm{ng} / \mathrm{g} \\
& \mathrm{ng} / \mathrm{g} \\
& \mathrm{ng} / \mathrm{g} \\
& \mu \mathrm{g} / \mathrm{kg}
\end{aligned}
$$

$\mathrm{pCi} / \mathrm{g}$
$\mathrm{pCi} / \mathrm{g}$
$\mathrm{pCi} / \mathrm{g}$
$\mathrm{pCi} / \mathrm{g}$

Sample ID: 100319

$\begin{array}{ll}36 & \text { EPA8080 } \\ 36 & \text { EPA8080 } \\ 36 & \text { EPA8080 } \\ 36 & \text { EPA8080 } \\ 0.20 & \text { EPA8280 } \\ 0.10 & \text { EPA8280 } \\ 0.10 & \text { EPA8280 } \\ 0.10 & \text { EPA8280 } \\ 0.10 & \text { EPA8280 } \\ 0.10 & \text { EPA8280 } \\ 180 . & \text { EPA8080 }\end{array}$

$\begin{array}{ll}8.4 & \text { EPA900.0 } \\ 10 & \text { EPA900.0 } \\ 0.070 & \text { EPA903.0 } \\ 1.4 & \text { EPA906.0 }\end{array}$

Sample Matrix: Soil

USC Soil Classification:
Sample Moisture: Dry Percent Solids: 83.10

RQ AQ B Result Unit . D. Limit Method

Volatile Organic Compounds

$\begin{array}{lllllll}\text { Acetone } & \mathrm{L} & \mathrm{YV} & 6,600 & \mu \mathrm{g} / \mathrm{kg} & 12 & \text { EPA8240 } \\ \text { Acetone } & & \mathrm{YV} & 5,800 & \mu \mathrm{g} / \mathrm{kg} & 1,200 & \text { EPA8240 } \\ \text { Benzene } & \mathrm{U} & \mathrm{Y} & 6.2 & \mu \mathrm{g} / \mathrm{kg} & 6.2 & \text { EPA8240 } \\ \text { Bromodichloromethane } & \mathrm{U} & \mathrm{Y} & 6.2 & \mu \mathrm{g} / \mathrm{kg} & 6.2 & \text { EPA8240 }\end{array}$


SAMPLE NAME: D431̈10703 (Continued)

Constituent

Volatile Organic Compounds

Bromoform
Bromomethane (Methyl bromide)
Carbon disulfide
Carbon tetrachloride
Chlorobenzene
Chloroethane
Chloroethene (Vinyl chloride)
Chloroform
Chloromethane (Methyl chloride)
Dibromochloromethane
1,1-Dichloroethane
1,2-Dichloroethane
1,1-Dichloroethylene
1,2-Dichloroethylene
Dichloromethane (Methylene chloride)
1,2-Dichloropropane
cis-1,3-Dichloropropene
trans-1,3-Dichloropropene
Ethylbenzene
2-Hexanone
Methyl ethyl ketone
Methy! isobutyl ketone
Styrene
1,1,2,2-Tetrachloroethane
Tetrachloroethylene
Toluene
1,1,1-Trichloroethane
1,1,2-Trichloroethane
Trichloroethylene
Vinyl acetate
Xylenes

Sample D: 100319

RQ AQ B Result Unit $\quad$ D. Limit Method

\begin{tabular}{|c|c|c|c|c|c|}
\hline U & $Y$ & 6.2 & $\mu g / \mathrm{kg}$ & 6.2 & EPA 8240 \\
\hline U & $\mathbf{Y}$ & 12 & $\mu g / k g$ & 12 & EPA8240 \\
\hline$U$ & $\mathbf{Y}$ & 6.2 & $\mu g / \mathrm{kg}$ & 6.2 & EPA8240 \\
\hline $\mathbf{U}$ & $\mathbf{Y}$ & 6.2 & $\mu g / \mathrm{kg}$ & 6.2 & EPA8240 \\
\hline $\mathrm{U}$ & $\mathbf{Y}$ & 6.2 & $\mu g / k g$ & 6.2 & EPA8240 \\
\hline U & $\mathbf{Y}$ & 12 & $\mu \mathrm{g} / \mathrm{kg}$ & 12 & EPA8240 \\
\hline $\mathrm{U}$ & $\mathbf{Y}$ & 12 & $\mu g / \mathrm{kg}$ & 12 & EPA8240 \\
\hline U & $\mathbf{Y}$ & 6.2 & $\mu g / \mathrm{kg}$ & 6.2 & EPA8240 \\
\hline U & $\mathbf{Y}$ & 12 & $\mu \mathrm{g} / \mathrm{kg}$ & 12 & EPA8240 \\
\hline$U$ & $\mathbf{Y}$ & 6.2 & $\mu g / \mathrm{kg}$ & 6.2 & EPA8240 \\
\hline \multirow[t]{2}{*}{ U } & $\mathbf{Y}$ & 6.2 & $\mu g / k g$ & 6.2 & EPA8240 \\
\hline & $\mathbf{Y}$ & 9.7 & $\mu \mathrm{g} / \mathrm{kg}$ & 6.2 & EPA8240 \\
\hline U & $\mathbf{Y}$ & 6.2 & $\mu \mathrm{g} / \mathrm{kg}$ & 6.2 & EPA8240 \\
\hline U & $\mathbf{Y}$ & 6.2 & $\mu \mathrm{g} / \mathrm{kg}$ & 6.2 & EPA8240 \\
\hline$U$ & YV & 6.2 & $\mu g / k g$ & 6.2 & EPA8240 \\
\hline U & $\mathbf{Y}$ & 6.2 & $\mu \mathrm{g} / \mathrm{kg}$ & 6.2 & EPA8240 \\
\hline $\mathrm{U}$ & $\mathbf{Y}$ & 6.2 & $\mu g / k g$ & 6.2 & EPA8240 \\
\hline U & $Y$ & 6.2 & $\mu g / \mathrm{kg}$ & 6.2 & EPA8240 \\
\hline U & $Y$ & 6.2 & $\mu g / \mathrm{kg}$ & 6.2 & EPA8240 \\
\hline U & $\mathbf{Y}$ & 12 & $\mu \mathrm{g} / \mathrm{kg}$ & 12 & EPA8240 \\
\hline U & $Y$ & 12 & $\mu \mathrm{g} / \mathrm{kg}$ & 12 & EPA8240 \\
\hline $\mathrm{U}$ & $\mathbf{Y}$ & 12 & $\mu \mathrm{g} / \mathrm{kg}$ & 12 & EPA8240 \\
\hline $\mathrm{U}$ & $\mathbf{Y}$ & 6.2 & $\mu g / \mathrm{kg}$ & 6.2 & EPA8240 \\
\hline $\mathbf{U}$ & $\mathbf{Y}$ & 6.2 & $\mu \mathrm{g} / \mathrm{kg}$ & 6.2 & EPA8240 \\
\hline $\mathrm{U}$ & $\mathbf{Y}$ & 6.2 & $\mu \mathrm{g} / \mathrm{kg}$ & 6.2 & EPA8240 \\
\hline U & $Y$ & 6.2 & $\mu \mathrm{g} / \mathrm{kg}$ & 6.2 & EPA8240 \\
\hline \multirow[t]{2}{*}{$\mathbf{U}$} & $Y$ & 6.2 & $\mu \mathrm{g} / \mathrm{kg}$ & 6.2 & EPA8240 \\
\hline & $Y$ & 8.8 & $\mu g / k g$ & 6.2 & EPA8240 \\
\hline $\mathbf{U}$ & $\mathbf{Y}$ & 6.2 & $\mu \mathrm{g} / \mathrm{kg}$ & 6.2 & EPA8240 \\
\hline $\mathrm{U}$ & $Y$ & 12 & $\mu g / k g$ & 12 & EPA8240 \\
\hline $\mathbf{U}$ & $\mathbf{Y}$ & 6.2 & $\mu \mathrm{g} / \mathrm{kg}$ & 6.2 & EPA8240 \\
\hline
\end{tabular}

Radionuclide Indicators and Radionuclides

Gross alpha

Nonvolatile beta

Radium, total alpha-emitting

Tritium

$\begin{array}{lll}\mathrm{Y} & 11 \pm 13 & \mathrm{pCi} / \mathrm{g} \\ \mathrm{Y} & 12 \pm 20 & \mathrm{pCi} / \mathrm{g} \\ \mathrm{YV} & 0.64 \pm 0.64 & \mathrm{pCi} / \mathrm{g} \\ \mathrm{Y} & 3.0 \pm 0.63 & \mathrm{pCi} / \mathrm{g}\end{array}$

7.7

EPA900.0

$9.4 \quad$ EPA900.0

$0.070 \quad$ EPA903.0

1.3 EPA906.0
SAMPLE NAME: D43110703D

Sample Type: Split

Total Metals

Arsenic

Barium

Cadmium

Chromium
Sample ID: 100320

Associated Sample: 100319

9.0
71
0.56
120
$\mathrm{mg} / \mathrm{kg}$
$\mathrm{mg} / \mathrm{kg}$
$\mathrm{mg} / \mathrm{kg}$
$\mathrm{mg} / \mathrm{kg}$


SAMPLE NAME: D431 10703D (Continued)

Sample ID: 100320

- Constituent

Total Metals

Copper

Lead

Manganese

Mercury

Nickel

Selenium

Silver

Volatile Organic Compounds

\begin{tabular}{|c|c|c|c|c|c|c|c|}
\hline Acetone & J & YVO & $\mathrm{H}$ & 4,200 & $\mu \mathrm{g} / \mathrm{kg}$ & 13 & EPA8240 \\
\hline Acetone & & $Y V$ & & 8,000 & $\mu \mathrm{g} / \mathrm{kg}$ & 630 & EPA8240 \\
\hline Benzene & UJ & YO & $\mathrm{H}$ & 6.3 & $\mu \mathrm{g} / \mathrm{kg}$ & 6.3 & EPA8240 \\
\hline Bromodichloromethane & UJ & YO & $\mathrm{H}$ & 6.3 & $\mu \mathrm{g} / \mathrm{kg}$ & 6.3 & EPA8240 \\
\hline Bromoform & UJ & YO & $\mathrm{H}$ & 6.3 & $\mu g / \mathrm{kg}$ & 6.3 & EPA8240 \\
\hline Bromomethane (Methyl bromide) & UJ & YO & $\mathbf{H}$ & 13 & $\mu \mathrm{g} / \mathrm{kg}$ & 13 & EPA8240 \\
\hline Carbon disulfide & $\mathrm{UJ}$ & Yo & H & 6.3 & $\mu \mathrm{g} / \mathrm{kg}$ & 6.3 & EPA8240 \\
\hline Carbon tetrachloride & UJ & YO & $\mathbf{H}$ & 6.3 & $\mu \mathrm{g} / \mathrm{kg}$ & 6.3 & EPA8240 \\
\hline Chlorobenzene & UJ & YO & $\mathbf{H}$ & 6.3 & $\mu \mathrm{g} / \mathrm{kg}$ & 6.3 & EPA8240 \\
\hline Chloroethane & UJ & YO & H & 13 & $\mu \mathrm{g} / \mathrm{kg}$ & 13 & EPA8240 \\
\hline Chloroethene (Vinyl chloride) & UJ & YO & $\mathrm{H}$ & 13 & $\mu \mathrm{g} / \mathrm{kg}$ & 13 & EPA8240 \\
\hline Chloroform & UJ & YO & $\mathrm{H}$ & 6.3 & $\mu \mathrm{g} / \mathrm{kg}$ & 6.3 & EPA8240 \\
\hline Chloromethane (Methyl chloride) & UJ & YO & $\mathrm{H}$ & 13 & $\mu \mathrm{g} / \mathrm{kg}$ & 13 & EPA8240 \\
\hline Dibromochloromethane & UJ & YO & $\mathbf{H}$ & 6.3 & $\mu \mathrm{g} / \mathrm{kg}$ & 6.3 & EPA8240 \\
\hline 1,1-Dichloroethane & UJ & YO & $\mathrm{H}$ & 6.3 & $\mu \mathrm{g} / \mathrm{kg}$ & 6.3 & EPA8240 \\
\hline 1,2-Dichloroethane & J & YO & $\mathrm{H}$ & 9.6 & $\mu \mathrm{g} / \mathrm{kg}$ & 6.3 & EPA8240 \\
\hline 1,1-Dichloroethylene & UJ & YO & $\mathrm{H}$ & 6.3 & $\mu \mathrm{g} / \mathrm{kg}$ & 6.3 & EPA8240 \\
\hline 1,2-Dichloroethylene & UJ & Yo & $\mathrm{H}$ & 6.3 & $\mu \mathrm{g} / \mathrm{kg}$ & 6.3 & EPA8240 \\
\hline Dichloromethane (Methylene chloride) & UJ & YVO & $\mathrm{H}$ & 6.6 & $\mu \mathrm{g} / \mathrm{kg}$ & 6.3 & EPA8240 \\
\hline 1.2-Dichloropropane & UJ & YO & $\mathrm{H}$ & 6.3 & $\mu \mathrm{g} / \mathrm{kg}$ & 6.3 & EPA8240 \\
\hline cis-1,3-Dichloropropene & UJ & Yo & $\mathrm{H}$ & 6.3 & $\mu \mathrm{g} / \mathrm{kg}$ & 6.3 & EPA8240 \\
\hline trans-1,3-Dichloropropene & UJ & Yo & $\mathrm{H}$ & 6.3 & $\mu \mathrm{g} / \mathrm{kg}$ & 6.3 & EPA8240 \\
\hline Ethylbenzene & UJ & YO & $\mathrm{H}$ & 6.3 & $\mu \mathrm{g} / \mathrm{kg}$ & 6.3 & EPA8240 \\
\hline 2-Hexanone & UJ & YO & $\mathrm{H}$ & 13 & $\mu \mathrm{g} / \mathrm{kg}$ & 13 & EPA8240 \\
\hline Methyl ethyl ketone & UJ & YO & $\mathrm{H}$ & 13 & $\mu \mathrm{g} / \mathrm{kg}$ & 13 & EPA8240 \\
\hline Methyl isoburyl ketone & UJ & YO & $\mathrm{H}$ & 13 & $\mu \mathrm{g} / \mathrm{kg}$ & 13 & EPA8240 \\
\hline Styrene & UJ & YO & $\mathrm{H}$ & 6.3 & $\mu \mathrm{g} / \mathrm{kg}$ & 6.3 & EPA8240 \\
\hline 1,1,2,2-Tetrachloroethane & UJ & Yo & $\mathrm{H}$ & 6.3 & $\mu \mathrm{g} / \mathrm{kg}$ & 6.3 & EPA8240 \\
\hline Tetrachloroethylene & J & Yo & $\mathrm{H}$ & 4.8 & $\mu \mathrm{g} / \mathrm{kg}$ & 6.3 & EPA8240 \\
\hline Toluene & J & YO & $\mathrm{H}$ & 9.6 & $\mu \mathrm{g} / \mathrm{kg}$ & 6.3 & EPA8240 \\
\hline 1,1,1-Trichloroethane $\because \because$ & UJ & YO & $\mathrm{H}$ & 6.3 & $\mu \mathrm{g} / \mathrm{kg}$ & 6.3 & EPA8240 \\
\hline 1,1,2-Trichloroethane & $\mathbf{J}$ & Yo & $\mathrm{H}$ & 6.6 & $\mu \mathrm{g} / \mathrm{kg}$ & 6.3 & EPA8240 \\
\hline Trichloroethylene & UJ & YO & $\mathrm{H}$ & 6.3 & $\mu \mathrm{g} / \mathrm{kg}$ & 6.3 & EPA8240 \\
\hline Vinyl acetate & UJ & YO & $\mathrm{H}$ & 13 & $\mu \mathrm{g} / \mathrm{kg}$ & 13 & EPA8240 \\
\hline Xylenes & $\mathbf{J}$ & YO & $\mathrm{H}$ & 7.8 & $\mu \mathrm{g} / \mathrm{kg}$ & $6.3=$ & EPA8240 \\
\hline
\end{tabular}

\begin{tabular}{|c|c|c|c|c|c|c|c|}
\hline Acetone & J & YVO & $\mathrm{H}$ & 4,200 & $\mu \mathrm{g} / \mathrm{kg}$ & 13 & EPA8240 \\
\hline Acetone & & $Y V$ & & 8,000 & $\mu \mathrm{g} / \mathrm{kg}$ & 630 & EPA8240 \\
\hline Benzene & UJ & YO & $\mathrm{H}$ & 6.3 & $\mu \mathrm{g} / \mathrm{kg}$ & 6.3 & EPA8240 \\
\hline Bromodichloromethane & UJ & YO & $\mathrm{H}$ & 6.3 & $\mu \mathrm{g} / \mathrm{kg}$ & 6.3 & EPA8240 \\
\hline Bromoform & UJ & YO & $\mathrm{H}$ & 6.3 & $\mu g / \mathrm{kg}$ & 6.3 & EPA8240 \\
\hline Bromomethane (Methyl bromide) & UJ & YO & $\mathbf{H}$ & 13 & $\mu \mathrm{g} / \mathrm{kg}$ & 13 & EPA8240 \\
\hline Carbon disulfide & $\mathrm{UJ}$ & Yo & H & 6.3 & $\mu \mathrm{g} / \mathrm{kg}$ & 6.3 & EPA8240 \\
\hline Carbon tetrachloride & UJ & YO & $\mathbf{H}$ & 6.3 & $\mu \mathrm{g} / \mathrm{kg}$ & 6.3 & EPA8240 \\
\hline Chlorobenzene & UJ & YO & $\mathbf{H}$ & 6.3 & $\mu \mathrm{g} / \mathrm{kg}$ & 6.3 & EPA8240 \\
\hline Chloroethane & UJ & YO & H & 13 & $\mu \mathrm{g} / \mathrm{kg}$ & 13 & EPA8240 \\
\hline Chloroethene (Vinyl chloride) & UJ & YO & $\mathrm{H}$ & 13 & $\mu \mathrm{g} / \mathrm{kg}$ & 13 & EPA8240 \\
\hline Chloroform & UJ & YO & $\mathrm{H}$ & 6.3 & $\mu \mathrm{g} / \mathrm{kg}$ & 6.3 & EPA8240 \\
\hline Chloromethane (Methyl chloride) & UJ & YO & $\mathrm{H}$ & 13 & $\mu \mathrm{g} / \mathrm{kg}$ & 13 & EPA8240 \\
\hline Dibromochloromethane & UJ & YO & $\mathbf{H}$ & 6.3 & $\mu \mathrm{g} / \mathrm{kg}$ & 6.3 & EPA8240 \\
\hline 1,1-Dichloroethane & UJ & YO & $\mathrm{H}$ & 6.3 & $\mu \mathrm{g} / \mathrm{kg}$ & 6.3 & EPA8240 \\
\hline 1,2-Dichloroethane & J & YO & $\mathrm{H}$ & 9.6 & $\mu \mathrm{g} / \mathrm{kg}$ & 6.3 & EPA8240 \\
\hline 1,1-Dichloroethylene & UJ & YO & $\mathrm{H}$ & 6.3 & $\mu \mathrm{g} / \mathrm{kg}$ & 6.3 & EPA8240 \\
\hline 1,2-Dichloroethylene & UJ & Yo & $\mathrm{H}$ & 6.3 & $\mu \mathrm{g} / \mathrm{kg}$ & 6.3 & EPA8240 \\
\hline Dichloromethane (Methylene chloride) & UJ & YVO & $\mathrm{H}$ & 6.6 & $\mu \mathrm{g} / \mathrm{kg}$ & 6.3 & EPA8240 \\
\hline 1.2-Dichloropropane & UJ & YO & $\mathrm{H}$ & 6.3 & $\mu \mathrm{g} / \mathrm{kg}$ & 6.3 & EPA8240 \\
\hline cis-1,3-Dichloropropene & UJ & Yo & $\mathrm{H}$ & 6.3 & $\mu \mathrm{g} / \mathrm{kg}$ & 6.3 & EPA8240 \\
\hline trans-1,3-Dichloropropene & UJ & Yo & $\mathrm{H}$ & 6.3 & $\mu \mathrm{g} / \mathrm{kg}$ & 6.3 & EPA8240 \\
\hline Ethylbenzene & UJ & YO & $\mathrm{H}$ & 6.3 & $\mu \mathrm{g} / \mathrm{kg}$ & 6.3 & EPA8240 \\
\hline 2-Hexanone & UJ & YO & $\mathrm{H}$ & 13 & $\mu \mathrm{g} / \mathrm{kg}$ & 13 & EPA8240 \\
\hline Methyl ethyl ketone & UJ & YO & $\mathrm{H}$ & 13 & $\mu \mathrm{g} / \mathrm{kg}$ & 13 & EPA8240 \\
\hline Methyl isoburyl ketone & UJ & YO & $\mathrm{H}$ & 13 & $\mu \mathrm{g} / \mathrm{kg}$ & 13 & EPA8240 \\
\hline Styrene & UJ & YO & $\mathrm{H}$ & 6.3 & $\mu \mathrm{g} / \mathrm{kg}$ & 6.3 & EPA8240 \\
\hline 1,1,2,2-Tetrachloroethane & UJ & Yo & $\mathrm{H}$ & 6.3 & $\mu \mathrm{g} / \mathrm{kg}$ & 6.3 & EPA8240 \\
\hline Tetrachloroethylene & J & Yo & $\mathrm{H}$ & 4.8 & $\mu \mathrm{g} / \mathrm{kg}$ & 6.3 & EPA8240 \\
\hline Toluene & J & YO & $\mathrm{H}$ & 9.6 & $\mu \mathrm{g} / \mathrm{kg}$ & 6.3 & EPA8240 \\
\hline 1,1,1-Trichloroethane $\because \because$ & UJ & YO & $\mathrm{H}$ & 6.3 & $\mu \mathrm{g} / \mathrm{kg}$ & 6.3 & EPA8240 \\
\hline 1,1,2-Trichloroethane & $\mathbf{J}$ & Yo & $\mathrm{H}$ & 6.6 & $\mu \mathrm{g} / \mathrm{kg}$ & 6.3 & EPA8240 \\
\hline Trichloroethylene & UJ & YO & $\mathrm{H}$ & 6.3 & $\mu \mathrm{g} / \mathrm{kg}$ & 6.3 & EPA8240 \\
\hline Vinyl acetate & UJ & YO & $\mathrm{H}$ & 13 & $\mu \mathrm{g} / \mathrm{kg}$ & 13 & EPA8240 \\
\hline Xylenes & $\mathbf{J}$ & YO & $\mathrm{H}$ & 7.8 & $\mu \mathrm{g} / \mathrm{kg}$ & $6.3=$ & EPA8240 \\
\hline
\end{tabular}

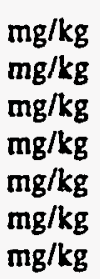

\section{8}

6.5

1.7

0.12

4.5

0.54

1.1

EPA6010

EPA7421

EPA6010

EPA7470

EPA6010

EPA7740

EPA6010 
SAMPLE NAME: D43110703D (Continued)

Constituent

RQ

$A Q$

B

Result

Unit

D. Limit Method

Radionuclide Indicators and Radionuclides

Gross alpha

Nonvolatile beta

Radium, total alpha-emitring

Tritium

Sample ID: 100320

6.7
7.9
0.070
1.9

EPA900.0

EPA900.0

EPA903.0

EPA906.0

\section{SAMPLE NAME: D43110704}

Location (SRS Coordinates): $18576.98 \mathrm{E} 6663 \dot{4} .66 \mathrm{~N}$

Ground Elevation Above MSL: $132.8 \mathrm{ft}$

Depth of Core Interval: 4.00 to $5.50 \mathrm{ft}$

Sample Type: Normal

Sample Color: 10R $4 / 6$

\section{Constituent}

Total Metals
Sample Matrix: Soil

USC Soil Classification: SM

RQ AQ B Result Unit
Sample Moisture: Dry Percent Solids: 84.70

D. Limit Method
Arsenic

Barium.

Cadmium

Chromium

Copper

Lead

Manganese

Mercury

Nickel

Selenium

Silver

$\begin{array}{lll} & \mathrm{Y} & 26 \\ & \mathrm{Y} & 58 \\ \mathrm{Y} & 0.83 \\ \mathrm{Y} & 260 \\ \mathrm{Y} & 310 \\ \mathrm{Y} & 140 \\ & \mathrm{Y} & 47 \\ & \mathrm{Y} & 0.82 \\ & \mathrm{Y} & 41 \\ & \mathrm{Y} & 3.8 \\ \mathrm{U} & \mathrm{Y} & 1.1\end{array}$

$$
\begin{aligned}
& \mathrm{mg} / \mathrm{kg} \\
& \mathrm{mg} / \mathrm{kg} \\
& \mathrm{mg} / \mathrm{kg} \\
& \mathrm{mg} / \mathrm{kg} \\
& \mathrm{mg} / \mathrm{kg} \\
& \mathrm{mg} / \mathrm{kg} \\
& \mathrm{mg} / \mathrm{kg} \\
& \mathrm{mg} / \mathrm{kg} \\
& \mathrm{mg} / \mathrm{kg} \\
& \mathrm{mg} / \mathrm{kg} \\
& \mathrm{mg} / \mathrm{kg}
\end{aligned}
$$

$B / N / A$ Extractables (including PAH and phenols)

Acenaphthene

Acenaphthylene

Anthracene

Benzo(a)anthracene

Benzo(b)fluoranthene

Benzo(k)fluoranthene

Benzoic acid

Benzo(g,h,i)perylene :

Benzo(a)pyrene

Benzyl alcohol

Bis(2-chloroethoxy) methane

Bis(2-chloroethyl) ether

Bis(2-chloroisopropyl) ether

Bis(2-ethylhexyl) phthalate

4-Bromophenyl phenyl ether

Butylbenzyl phthalate

Hydrocarbon $\mathrm{C} 14 \mathrm{H} 30$

Hydrocarbon $\mathrm{Cl} 6 \mathrm{H} 34$

\section{$\begin{array}{lll}\mathrm{J} & \mathrm{Y} & \mathbf{7 2}\end{array}$}

U $\quad Y \quad 420$

J $Y$

J $Y$

J $Y$

J $Y^{\circ}$

U Y

U Y

U Y

U $\quad Y$

U Y

$\begin{array}{ll}U & Y \\ U & Y\end{array}$

$\begin{array}{ll}\mathbf{Y} & \mathbf{Y} \\ & \mathbf{Y}\end{array}$

U $Y$

J $Y$

J YN

J YN
420

130

310

370

140

2,100

420

420

420

420

420

420

970

420

310

200

800 $\mu \mathrm{g} / \mathrm{kg}$

$\mu \mathrm{g} / \mathrm{kg}$

$\mu \mathrm{g} / \mathrm{kg}$

$\mu \mathrm{g} / \mathrm{kg}$

$\mu g / \mathrm{kg}$

$\mu \mathrm{g} / \mathrm{kg}$

$\mu \mathrm{g} / \mathrm{kg}$

$\mu \mathrm{g} / \mathrm{kg}$

$\mu \mathrm{g} / \mathrm{kg}$

$\mu g / \mathrm{kg}$

$\mu \mathrm{g} / \mathrm{kg}$

$\mu \mathrm{g} / \mathrm{kg}$

$\mu g / \mathrm{kg}$

$\mu g / \mathrm{kg}$

$\mu \mathrm{g} / \mathrm{kg}$

$\mu \mathrm{g} / \mathrm{kg}$

$\mu g / \mathrm{kg}$

$\mu \mathrm{g} / \mathrm{kg}$
5.7

$0.56 \quad$ EPA6010

1.1 EPA6010

$2.8 \quad$ EPA6010

17 EPA7421

1.7 EPA6010

$0.12 \quad$ EPA7470

4.5 EPA6010

0.57 EPA7740

1.1 EPA6010
420

420

420

420

420

420

2,100

420

420

420

420

420

420

420

420

420
EPA8270

EPA8270

EPA8270

EPA8270

EPA8270

EPA8270

EPA8270

EPA8270

EPA8270

EPA8270

EPA8270

EPA8270

EPA8270

EPA8270

EPA8270

EPA8270

EPA8270

EPA8270 
SAMPLE NAME: D43110704 (Continued)

Sample ID: 100321

$\begin{array}{llllll}\text { Constituent RQ AQ B Result Unit Dimit Method } & \text { B }\end{array}$

$B / N / A$ Extractables (including $P A H$ and phenols)

Hydrocarbon $\mathrm{Cl} 6 \mathrm{H} 34$ + arom.

Hydrocarbon C17H36

Hydrocarbon $\mathrm{C} 19 \mathrm{H} 40$

4-Chloroaniline

4-Chloro-m-cresol

2-Chloronaphthalene

2-Chlorophenol

4-Chlorophenyl phenyl ether.

Chrysene

o-Cresol (2-Methylphenol)

p-Cresol (4-Methylphenol)

Hexadecanoic acid ester

Dibenz(a,h)anthracene

Dibenzofuran

Di-n-butyl phthalate

1,2-Dichlorobenzene

1,3-Dichlorobenzene

1,4-Dichlorobenzene

3.3'-Dichlorobenzidine

2.4-Dichlorophenol

Diethyl phthalate

2;4-Dimethyl phenol

Dimethyl phthalate

2,4-Dinitrophenol

2,4-Dinitrotoluene

2,6-Dinitrotoluene

Di-n-octyl phthalate

Fluoranthene

Fluorene

Hexachlorobenzene

Hexachlorobutadiene

Hexachlorocyclopentadiene

Hexachloroethane

Indeno(1,2,3-c,d)pyrene

Isophorone

2-Methyl-4,6-dinitrophenol

2-Methylnaphthalene

Naphthalene

o-Nitroaniline

m-Nitroaniline

p-Nitroaniline

Nitrobenzene

2-Nitrophenol

4-Nitrophenol

N-Nitrosodiphenylamine

N-Nitrosodipropylamine

Pentachlorophenol

Phenanthrene

Phenol

Sub. propanoic acid

\begin{tabular}{|c|c|c|}
\hline J & YN & 300 \\
\hline $\mathbf{J}$ & YN & 300 \\
\hline J & YN & 300 \\
\hline $\mathrm{U}$ & $\mathbf{Y}$ & 420 \\
\hline U & $\mathbf{Y}$ & 420 \\
\hline $\mathrm{U}$ & $\mathbf{Y}$ & 420 \\
\hline $\mathrm{U}$ & $\mathbf{Y}$ & 420 \\
\hline $\mathrm{U}$ & $Y$ & 420 \\
\hline J & $\mathbf{Y}$ & 330 \\
\hline $\mathbf{U}$ & $\mathbf{Y}$ & 420 \\
\hline $\mathrm{U}$ & $\mathbf{Y}$ & 420 \\
\hline $\mathrm{J}$ & $\mathbf{Y N}$ & 400 \\
\hline$U$ & $Y$ & 420 \\
\hline $\mathbf{J}$ & $\mathbf{Y}$ & 70 \\
\hline UJ & $\mathrm{YV}$ & 58 \\
\hline $\mathrm{U}$ & $Y$ & 420 \\
\hline$U$ & $Y$ & 420 \\
\hline $\mathrm{U}$ & $Y$ & 420 \\
\hline $\mathbf{U}$ & $Y$ & 850 \\
\hline $\mathrm{U}$ & $Y$ & 420 \\
\hline $\mathbf{U}$ & $Y$ & 420 \\
\hline $\mathrm{U}$ & $Y$ & 420 \\
\hline $\mathbf{U}$ & $Y$ & 420 \\
\hline $\mathbf{U}$ & $Y$ & 2,100 \\
\hline $\mathrm{U}$ & $Y$ & 420 \\
\hline $\mathbf{U}$ & $Y$ & 420 \\
\hline \multirow[t]{2}{*}{$\mathbf{J}$} & $Y$ & 120 \\
\hline & $Y$ & 630 \\
\hline $\mathbf{J}$ & $Y$ & 66 \\
\hline $\mathbf{U}$ & $Y$ & 420 \\
\hline $\mathrm{U}$ & $Y$ & 420 \\
\hline $\mathbf{U}$ & $Y$ & 420 \\
\hline $\mathbf{U}$ & $Y$ & 420 \\
\hline $\mathbf{U}$ & $Y$ & 420 \\
\hline $\mathrm{U}$ & $Y$ & 420 \\
\hline $\mathrm{U}$ & $Y$ & 2,100 \\
\hline J & $Y$ & 180 \\
\hline $\mathbf{J}$ & $Y$ & 110 \\
\hline $\mathbf{U}$ & $\mathbf{Y}$ & 2,100 \\
\hline$U$ & $\mathbf{Y}$ & 2,100 \\
\hline $\mathbf{U}$ & $Y$ & 2,100 \\
\hline $\mathbf{U}$ & $Y$ & 420 \\
\hline U & $Y$ & 420 \\
\hline U & $Y$ & 2,100 \\
\hline $\mathrm{U}$ & $Y$ & 420 \\
\hline UJ & $C Y$ & 420 \\
\hline $\mathbf{U}$ & $\mathbf{Y}$ & 2,100 \\
\hline & $\mathbf{Y}$ & 510 \\
\hline $\mathrm{U}$ & $Y$ & 420 \\
\hline $\mathrm{J}$ & YN & 400 \\
\hline
\end{tabular}

$\mu \mathrm{g} / \mathrm{kg}$

EPA8270

EPA8270

$\mu \mathrm{g} / \mathrm{kg}$

$\mu \mathrm{g} / \mathrm{kg}$

$\mu \mathrm{g} / \mathrm{kg}$

$\mu \mathrm{g} / \mathrm{kg}$

$\mu \mathrm{g} / \mathrm{kg}$

$\mu \mathrm{g} / \mathrm{kg}$

$\mu \mathrm{g} / \mathrm{kg}$

$\mu \mathrm{g} / \mathrm{kg}$

$\mu \mathrm{g} / \mathrm{kg}$

$\mu \mathrm{g} / \mathrm{kg}$

$\mu \mathrm{g} / \mathrm{kg}$

$\mu \mathrm{g} / \mathrm{kg}$

$\mu g / k g$

$\mu \mathrm{g} / \mathrm{kg}$

$\mu \mathrm{g} / \mathrm{kg}$

$\mu \mathrm{g} / \mathrm{kg}$

$\mu \mathrm{g} / \mathrm{kg}$

$\mu \mathrm{g} / \mathrm{kg}$

$\mu \mathrm{g} / \mathrm{kg}$

$\mu \mathrm{g} / \mathrm{kg}$

$\mu \mathrm{g} / \mathrm{kg}$

$\mu \mathrm{g} / \mathrm{kg}$

$\mu \mathrm{g} / \mathrm{kg}$

$\mu \mathrm{g} / \mathrm{kg}$

$\mu \mathrm{g} / \mathrm{kg}$

$\mu \mathrm{g} / \mathrm{kg}$

$\mu \mathrm{g} / \mathrm{kg}$

$\mu \mathrm{g} / \mathrm{kg}$

$\mu \mathrm{g} / \mathrm{kg}$

$\mu \mathrm{g} / \mathrm{kg}$

$\mu \mathrm{g} / \mathrm{kg}$

$\mu \mathrm{g} / \mathrm{kg}$

$\mu g / \mathrm{kg}$

$\mu \mathrm{g} / \mathrm{kg}$

$\mu \mathrm{g} / \mathrm{kg}$

$\mu \mathrm{g} / \mathrm{kg}$

$\mu \mathrm{g} / \mathrm{kg}$

$\mu \mathrm{g} / \mathrm{kg}$

$\mu \mathrm{g} / \mathrm{kg}$

$\mu \mathrm{g} / \mathrm{kg}$

$\mu \mathrm{g} / \mathrm{kg}$

$\mu \mathrm{g} / \mathrm{kg}$

$\mu \mathrm{g} / \mathrm{kg}$

$\mu \mathrm{g} / \mathrm{kg}$

$\mu g / \mathrm{kg}$

$\mu \mathrm{g} / \mathrm{kg}$

$\mu \mathrm{g} / \mathrm{kg}$

$\mu \mathrm{g} / \mathrm{kg}$

$\mu \mathrm{g} / \mathrm{kg}$
EPA8270

EPA8270

EPA8270

EPA8270

EPA8270

EPA8270

EPA8270

EPA8270

EPA8270

EPA8270

EPA8270

EPA8270

EPA8270

EPA8270

EPA8270

EPA8270

EPA8270

EPA8270

EPA8270

EPA8270

EPA8270

EPA8270

EPA8270

EPA8270

EPA8270

EPA8270

EPA8270

EPA8270

EPA8270

EPA8270

EPA8270

EPA8270

EPA 8270

EPA8270

EPA8270

EPA8270

EPA8270

EPA8270

EPA8270

EPA8270

EPA8270

EPA8270

EPA8270

EPA8270

EPA8270

EPA8270

EPA8270

EPA8270 
SAMPLE NAME: D43110704 (Continued)

Sample ID: 100321
Constituent
RQ AQ B Result
Unit
D. Limit Method

$B / N / A$ Extractables (including $P A H$ and phenols)

\section{Pyrene \\ 1,2,4-Trichlorobenzene \\ 2,4,5-Trichlorophenol \\ 2,4,6-Trichloropheno! \\ Unknown hydrocarbon \\ Unknown hydrocarbon \\ Unknown hydrocarbon \\ Unknown hydrocarbon \\ Unknown hydrocarbon \\ Unknown hydrocarbon \\ Unknown hydrocarbon \\ Unknown hydrocarbon \\ Unknown hydrocarbon \\ Unknown \\ Unknown \\ Unknown ketone \\ 1-Methylnapthalene}

\section{Volatile Organic Compounds}

Acetone
Acetone
Benzene
Bromodichloromethane
Bromoform
Bromomethane (Methyl bromide)
Carbon disulfide
Carbon tetrachloride
Chlorobenzene
Chloroethane
Chloroethene (Vinyl chloride)
Chloroform
Chloromethane (Methyl chloride)
Dibromochloromethane
1,1-Dichloroethane
1,2-Dichloroethane
1,1-Dichloroethylene
1,2-Dichloroethylene
Dichloromethane (Methylene chloride)
1,2-Dichloropropane
cis-1,3-Dichloropropene
trans-1,3-Dichloropropene
Ethylbenzene
2-Hexanone
Methyl ethyl ketone
Methyl isobutyl ketone
Styrene
1,1,2,2-Tetrachloroethane
Tetrachloroethylene
Toluene

$\begin{array}{lll} & Y & 1,000 \\ U & Y & 420 \\ U & Y & 2,100 \\ U & Y & 420 \\ J & Y N & 300 \\ J & Y N & 300 \\ J & Y N & 400 \\ J & Y N & 500 \\ J & Y N & 400 \\ J & Y N & 400 \\ J & Y N & 1,000 \\ J & Y N & 1,000 \\ J & Y N & 1,000 \\ J & Y N & 1,000 \\ J & Y N & 1,000 \\ J & Y N V & 5,000 \\ J & Y N & 200\end{array}$

$$
\begin{aligned}
& \mu g / k g \\
& \mu g / k g \\
& \mu g / k g \\
& \mu g / k g \\
& \mu g / k g \\
& \mu g / k g \\
& \mu g / k g \\
& \mu g / k g \\
& \mu g / k g \\
& \mu g / k g \\
& \mu g / k g \\
& \mu g / k g \\
& \mu g / k g \\
& \mu g / k g \\
& \mu g / k g \\
& \mu g / k g \\
& \mu g / k g
\end{aligned}
$$

$\begin{array}{ll}420 & \text { EPA8270 } \\ 420 & \text { EPA8270 } \\ 2,100 & \text { EPA8270 } \\ 420 & \text { EPA8270 } \\ & \text { EPA8270 } \\ & \text { EPA8270 } \\ & \text { EPA8270 } \\ & \text { EPA8270 } \\ & \text { EPA8270 } \\ & \text { EPA8270 } \\ & \text { EPA8270 } \\ & \text { EPA8270 } \\ & \text { EPA8270 } \\ & \text { EPA8270 } \\ & \text { EPA8270 } \\ & \text { EPA8270 } \\ & \text { EPA8270 }\end{array}$

L YV $\quad 790$


SAMPLE NAME: D431-10704 (Continued)

Constituent

RQ AQ B Result

6.5

5.7

6.5

13

6.5

Volatile Organic Compounds

$\begin{array}{ll}\mathbf{U} & \mathbf{Y} \\ & \mathbf{Y} \\ \mathbf{U} & \mathbf{Y} \\ \mathbf{U} & \mathbf{Y} \\ \mathbf{U} & \mathbf{Y}\end{array}$

Unit

D. Limit Method

\author{
1,1.1-Trichloroethane \\ 1,1,2-Trichloroethane \\ Trichloroethylene \\ Vinyl acetate \\ Xylenes
}

Pesticides/PCBs and Dioxins/Furans

Aldrin

alpha-Benzene hexachloride

beta-Benzene hexachloride

delta-Benzene hexachloride

alpha-Chlordane

gamma-Chlordane

p.p'-DDD

p.p-DDE

P.p'-DDT

Dieldrin

Endosulfan I

Endosulfan II

Endosulfan sulfate

Endrin

Endrin ketone

Heptachlor

Heptachlor epoxide

Heptachlorodibenzo-p-dioxin isomers

Heptachlorodibenzo-p-furan isomers

Hexachlorodibenzo-p-dioxin isomers

Hexachlorodibenzo-p-furan isomers

Lindane

Methoxychlor

Octachlorodibenzo-p-dioxin

Octachlorodibenzo-p-furan

PCB 1016

PCB 1221

PCB 1232

PCB 1242

PCB 1248

PCB 1254

PCB 1260

Pentachlorodibenzo-p-dioxin isomers

Pentachlorodibenzo-p-furan isomers

2,3,7,8-TCDD

2,3,7,8-TCDF

Tetrachlorodibenzo-p-dioxin isomers

Tetrachlorodibenzo-p-furan isomers

Toxaphene

\begin{tabular}{|c|c|c|c|c|}
\hline U & 21 & $\mu \mathrm{g} / \mathrm{kg}$ & 21 & EPA8080 \\
\hline $\mathbf{U}$ & 21 & $\mu g / \mathrm{kg}$ & 21 & EPA8080 \\
\hline U & 21 & $\mu \mathrm{g} / \mathrm{kg}$ & 21 & EPA8080 \\
\hline$U$ & 21 & $\mu \mathrm{g} / \mathrm{kg}$ & 21 & EPA8080 \\
\hline U & 21 & $\mu g / k g$ & 21 & EPA8080 \\
\hline$Y$ & 21 & $\mu \mathrm{g} / \mathrm{kg}$ & 21 & EPA8080 \\
\hline$Y$ & 43 & $\mu \mathrm{g} / \mathrm{kg}$ & 43 & EPA8080 \\
\hline$Y$ & 43 & $\mu \mathrm{g} / \mathrm{kg}$ & 43 & EPA8080 \\
\hline$Y$ & 43 & $\mu \mathrm{g} / \mathrm{kg}$ & 43 & EPA8080 \\
\hline$Y$ & 43 & $\mu g / \mathrm{kg}$ & 43 & EPA8080 \\
\hline$Y$ & 21 & $\mu \mathrm{g} / \mathrm{kg}$ & 21 & EPA8080 \\
\hline $\mathbf{U}$ & 43 & $\mu \mathrm{g} / \mathrm{kg}$ & 43 & EPA8080 \\
\hline$Y$ & 43 & $\mu \mathrm{g} / \mathrm{kg}$ & 43 & EPA8080 \\
\hline$Y$ & 43 & $\mu \mathrm{g} / \mathrm{kg}$ & 43 & EPA8080 \\
\hline$Y$ & 43 & $\mu \mathrm{g} / \mathrm{kg}$ & 43 & EPA8080 \\
\hline$Y$ & 21 & $\mu \mathrm{g} / \mathrm{kg}$ & 21 & EPA8080 \\
\hline U & 21 & $\mu \mathrm{g} / \mathrm{kg}$ & 21 & EPA8080 \\
\hline $\mathbf{Y}$ & 0.80 & $\mathrm{ng} / \mathrm{g}$ & 0.10 & EPA8280 \\
\hline$Y$ & 0.30 & $\mathrm{ng} / \mathrm{g}$ & 0.10 & EPA8280 \\
\hline U & 0.30 & $\mathrm{ng} / \mathrm{g}$ & 0.30 & EPA8280 \\
\hline$Y$ & 0.10 & $\mathrm{ng} / \mathrm{g}$ & 0.10 & EPA8280 \\
\hline U & 21 & $\mu \mathrm{g} / \mathrm{kg}$ & 21 & EPA8080 \\
\hline $\mathbf{U}$ & 210 & $\mu \mathrm{g} / \mathrm{kg}$ & 210 & EPA8080 \\
\hline$Y$ & 3.9 & $\mathrm{ng} / \mathrm{g}$ & 0.10 & EPA8280 \\
\hline$Y$ & 0.20 & $\mathrm{ng} / \mathrm{g}$ & 0.10 & EPA8280 \\
\hline $\bar{Y}$ & 430 & $\mu \mathrm{g} / \mathrm{kg}$ & 430 & EPA8080 \\
\hline$Y$ & 850 & $\mu \mathrm{g} / \mathrm{kg}$ & 850 & EPA8080 \\
\hline$Y$ & 430 & $\mu \mathrm{g} / \mathrm{kg}$ & 430 & EPA8080 \\
\hline$U$ & 430 & $\mu \mathrm{g} / \mathrm{kg}$ & 430 & EPA8080 \\
\hline$Y$ & 430 & $\mu \mathrm{g} / \mathrm{kg}$ & 430 & EPA8080 \\
\hline$Y$ & 430 & $\mu \mathrm{g} / \mathrm{kg}$ & 430 & EPA8080 \\
\hline $\mathbf{Y}$ & 430 & $\mu g / \mathrm{kg}$ & 430 & EPA8080 \\
\hline U & 0.10 & $\mathrm{ng} / \mathrm{g}$ & 0.10 & EPA8280 \\
\hline $\mathbf{Y}$ & 0.10 & $\mathrm{ng} / \mathrm{g}$ & 0.10 & EPA8280 \\
\hline $\mathbf{Y}$ & 0.10 & $\mathrm{ng} / \mathrm{g}$ & 0.10 & EPA8280 \\
\hline$Y$ & 0.10 & $\mathrm{ng} / \mathrm{g}$ & 0.10 & EPA8280 \\
\hline$Y$ & 0.10 & $\mathrm{ng} / \mathrm{g}$ & $0.10 n$ & EPA8280 \\
\hline $\mathbf{Y}$ & 0.10 & $\mathrm{ng} / \mathrm{g}$ & 0.10 & EPA8280 \\
\hline$Y$ & 2,100 & $\mu \mathrm{g} / \mathrm{kg}$ & 2,100 & EPA8080 \\
\hline
\end{tabular}

$\begin{array}{lll}\mu \mathrm{g} / \mathrm{kg} & 6.5 & \text { EPA8240 } \\ \mu \mathrm{g} / \mathrm{kg} & 6.5 & \text { EPA8240 } \\ \mu \mathrm{g} / \mathrm{kg} & 6.5 & \text { EPA8240 } \\ \mu \mathrm{g} / \mathrm{kg} & 13 & \text { EPA8240 } \\ \mu \mathrm{g} / \mathrm{kg} & 6.5 & \text { EPA8240 }\end{array}$

EPA8080

EPA8080

EPA8080

EPA8080

EPA8080

EPA8080

EPA8080

4080

EPA8280

EPA8280

EPA8080

EPA8280

EPA8080

EPA8080

EPA8080

EPA8280

PA8280

EPA8080 
SAMPLE NAME: D43110704 (Continued)

Constituent

RQ AQ

Radionuclide Indicators and Radionuclides

Gross alpha

Nonvolatile beta

Radium, total alpha-emitring

Tritium
Sample ID: 100321

B Result Unit

D. Limit Method

\section{SAMPLE NAME: D43110705}

Location (SRS Coordinates): 18576.98E 66634.66N

Ground Elevation Above MSL: $132.8 \mathrm{ft}$

Depth of Core Interval: 6.00 to $8.00 \mathrm{ft}$

Sample Type: Normal

Sample Color: 10R 3/4

Constituent

Total Metals

$Y$
$Y$
$Y Y$
$Y$

$19 \pm 16$

$20 \pm 22$

$1.2 \pm 0.86$

$3.5 \pm 0.71$

Sample ID: 100322

Sample Matrix: Soil

USC Soil Classification: SM

RQ AQ B Result Unit
Arsenic
Barium
Cadmium
Chromium
Copper
Lead
Manganese
Mercury
Nickel
Selenium
Silver

$\begin{array}{lll}\mathrm{U} & \mathrm{Y} & 1.0 \\ \mathrm{U} & \mathrm{Y} & 21 \\ \mathrm{U} & \mathrm{Y} & 0.52 \\ & \mathrm{Y} & 24 \\ & \mathrm{Y} & 6.0 \\ & \mathrm{Y} & 3.8 \\ & \mathrm{Y} & 490 \\ \mathrm{U} & \mathrm{Y} & 0.12 \\ \mathrm{U} & \mathrm{Y} & 4.1 \\ \mathrm{U} & \mathrm{Y} & 0.50 \\ \mathrm{U} & \mathrm{Y} & 1.0\end{array}$

$B / N / A$ Extractables (including $P A H$ and phenols)

Acenaphthene

Acenaphthylene

Anthracene

Benzo(a)anthracene

Benzo(b)fluoranthene

Benzo(k)fluoranthene

Benzoic acid

Benzo(g,h,i)perylene :-

Benzo(a)pyrene

Benzyl alcohol

Bis(2-chloroethoxy) methane

Bis(2-chloroethyl) ether

Bis(2-chloroisopropyl) ether

Bis(2-ethylhexyl) phthalate

4-Bromophenyl phenyl ether

Butylbenzyl phethalate

Sub. carboxylic acid

4-Chloroaniline

$\begin{array}{lll}U & Y & 390 \\ U & Y & 390 \\ U & Y & 390 \\ U & Y & 390 \\ U & Y & 390 \\ U & Y & 390 \\ U & Y & 2,000 \\ U & Y & 390 \\ U & Y & 390 \\ U & Y & 390 \\ U & Y & 390 \\ U & Y & 390 \\ U & Y & 390 \\ & Y & 470 \\ U & Y & 390 \\ J & Y & 100 \\ J & Y N & 200 \\ U & Y & 390\end{array}$

pCi/g. $\mathrm{pCi} / \mathrm{g}$

$\mathrm{pCi} / \mathrm{g}$

$\mathrm{pCi} / \mathrm{g}$

Sample Moisture: Damp Percent Solids: $\mathbf{8 5 . 0 0}$

D. Limit Method

$$
\begin{gathered}
\mathrm{mg} / \mathrm{kg} \\
\mathrm{mg} / \mathrm{kg} \\
\mathrm{mg} / \mathrm{kg} \\
\mathrm{mg} / \mathrm{kg} \\
\mathrm{mg} / \mathrm{kg} \\
\mathrm{mg} / \mathrm{kg} \\
\mathrm{mg} / \mathrm{kg} \\
\mathrm{mg} / \mathrm{kg} \\
\mathrm{mg} / \mathrm{kg} \\
\mathrm{mg} / \mathrm{kg} \\
\mathrm{mg} / \mathrm{kg}
\end{gathered}
$$

8.0

9.9

EPA900.0

0.060

EPA900.0

EPA903.0

1.3

EPA906.0

$\begin{array}{ll}1.0 & \text { EPA7060 } \\ 21 & \text { EPA6010 } \\ 0.52 & \text { EPA6010 } \\ 1.0 & \text { EPA6010 } \\ 2.6 & \text { EPA6010 } \\ 0.30 & \text { EPA7421 } \\ 1.6 & \text { EPA6010 } \\ 0.12 & \text { EPA7470 } \\ 4.1 & \text { EPA6010 } \\ 0.50 & \text { EPA7740 } \\ 1.0 & \text { EPA6010 }\end{array}$

EPA7060

EPA6010

PA6010

PA7421

$0.12 \quad$ EPA7470

$0.50 \quad$ EPA7740

0


SAMPLE NAME: D43110705 (Continued)

RQ AQ B Result

Constituent

$B / N / A$ Extractables (including $P A H$ and phenols)
Sample ID: 100322

Unit

D. Limit Method
4-Chloro-m-cresol

2-Chloronaphthalene

2-Chlorophenol

4-Chlorophenyl phenyl ether

Chrysere

o-Cresol (2-Methylphenol)

p-Cresol (4-Methylphenol)

Dibenz(a.h)anthracene

Dibenzofuran

Di-n-butyl phthalate

1.2-Dichlorobenzene

1,3-Dichlorobenzene

1.4-Dichlorobenzene

3.3'-Dichlorobenzidine

2.4-Dichlorophenol

Diethyl phthalate

2,4-Dimethyl phenol

Dimethyl phthalate

2,4-Dinitrophenol

2,4-Dinitrotoluene

2,6-Dinitrotoluene

Di-n-octyl phthalate

Fluoranthene

Fluorene

Hexachlorobenzene

Hexachlorobutadiene

Hexachlorocyclopentadiene

Hexachloroethane

Indeno(1,2,3-c,d)pyrene

Isophorone

2-Methyl-4,6-dinitrophenol

2-Methylnaphthalene

Naphthalene

o-Nitroaniline

m-Nitroaniline

p-Nitroaniline

Nitrobenzene

2-Nitrophenol

4-Nitrophenol

N-Nitrosodiphenylamine

N-Nitrosodipropylaminè

Pentachlorophenol

Phenanthrene

Phenol

Sub. propanoic acid

Pyrene

1,2,4-Trichlorobenzene

2,4,5-Trichlorophenol

2,4,6-Trichlorophenol

Unknown hydrocarbon

\begin{tabular}{|c|c|c|}
\hline $\mathrm{U}$ & $\mathbf{Y}$ & 390 \\
\hline $\mathbf{U}$ & $\mathbf{Y}$ & 390 \\
\hline $\mathbf{U}$ & $\mathbf{Y}$ & 390 \\
\hline $\mathbf{U}$ & $\mathbf{Y}$ & 390 \\
\hline$J$ & $\mathbf{Y}$ & 43 \\
\hline $\mathbf{U}$ & $\mathbf{Y}$ & 390 \\
\hline $\mathbf{U}$ & $\mathbf{Y}$ & 390 \\
\hline $\mathrm{U}$ & $\mathbf{Y}$ & 390 \\
\hline $\mathbf{U}$ & $\mathbf{Y}$ & 390 \\
\hline $\mathbf{J}$ & YV & 120 \\
\hline U & $\mathbf{Y}$ & 390 \\
\hline $\mathbf{U}$ & $\mathbf{Y}$ & 390 \\
\hline $\mathrm{U}$ & $\mathbf{Y}$ & 390 \\
\hline $\mathrm{U}$ & $\mathbf{Y}$ & 780 \\
\hline $\mathrm{U}$ & $Y$ & 390 \\
\hline $\mathrm{U}$ & $\mathbf{Y}$ & 390 \\
\hline $\mathrm{U}$ & $\mathbf{Y}$ & 390 \\
\hline $\mathrm{U}$ & Y & 390 \\
\hline $\mathrm{U}$ & $Y$ & 2,000 \\
\hline $\mathrm{U}$ & Y & 390 \\
\hline$U$ & $Y$ & 390 \\
\hline $\mathbf{U}$ & $Y$ & 390 \\
\hline J & $Y$ & 83 \\
\hline $\mathrm{U}$ & $Y$ & 390 \\
\hline $\mathbf{U}$ & $Y$ & 390 \\
\hline $\mathrm{U}$ & $Y$ & 390 \\
\hline $\mathrm{U}$ & $Y$ & 390 \\
\hline U & $Y$ & 390 \\
\hline $\mathbf{U}$ & $Y$ & 390 \\
\hline$U$ & $Y$ & 390 \\
\hline U & $Y$ & 2,000 \\
\hline$U$ & $Y$ & 390 \\
\hline$U$ & $Y$ & 390 \\
\hline $\mathbf{U}$ & $Y$ & 2,000 \\
\hline $\mathbf{U}$ & $\mathbf{Y}$ & 2,000 \\
\hline$U$ & $\mathbf{Y}$ & 2,000 \\
\hline $\mathbf{U}$ & $Y$ & 390 \\
\hline $\mathbf{U}$ & $Y$ & 390 \\
\hline U & $Y$ & 2,000 \\
\hline $\mathbf{U}$ & $\mathbf{Y}$ & 390 \\
\hline UJ & CY & 390 \\
\hline $\mathbf{U}$ & $Y$ & 2,000 \\
\hline$J$ & $Y$ & 78 \\
\hline U & $Y$ & 390 \\
\hline J & YN & 600 \\
\hline J & $Y$ & 89 \\
\hline $\mathbf{U}$ & $Y$ & 390 \\
\hline $\mathbf{U}$ & $Y$ & 2,000 \\
\hline $\mathbf{U}$ & $\mathbf{Y}$ & 390 \\
\hline J & $Y N$ & 200 \\
\hline
\end{tabular}

EPA8270

EPA8270

EPA8270

EPA8270

EPA8270

EPA8270

EPA8270

EPA8270

EPA8270

EPA8270

EPA8270

EPA8270

EPA8270

EPA8270

EPA8270

EPA8270

EPA8270

EPA8270

EPA8270

EPA8270

EPA8270

EPA8270

EPA8270

EPA8270

EPA8270

EPA8270

EPA8270

EPA8270

EPA8270

EPA8270

EPA8270

EPA8270

EPA8270

EPA8270

EPA8270

EPA8270

EPA8270

EPA8270

EPA8270

EPA8270

EPA8270

EPA8270

EPA8270

EPA8270

EPA8270

EPA8270

EPA8270

EPA8270

EPA8270

EPA8270 
SAMPLE NAME: D43110705 (Continued)

Constituent

RQ AQ B Result

200

400

200

300

200

400

200

Unknown

Unknown ketone

Unknown hydrocarbon

Unknown hydrocarbon

Unknown hydrocarbon

Unknown

Volatile Organic Compounds

Acetone
Benzene
Bromodichloromethane
Bromoform
Bromomethane (Methyl bromide)
Carbon disulfide
Carbon tetrachloride
Chlorobenzene
Chloroethane
Chloroethene (Vinyl chloride)
Chloroform
Chloromethane (Methyl chloride)
Dibromochloromethane
1,1-Dichloroethane
1,2-Dichloroethane
1,1-Dichloroethylene
1,2-Dichloroethylene
Dichloromethane (Methylene chloride)
1,2-Dichloropropane
cis-1,3-Dichloropropene
trans-1,3-Dichloropropene
Ethylbenzene
2-Hexanone
Methyl ethyl ketone
Methyl isobutyl ketone
Styrene
1,1,2,2-Tetrachloroethane
Tetrachloroethylene
Toluene
1,1,1-Trichloroethane
1,1,2-Trichloroethane
Trichloroethylene
Vinyl acetate
Xylenes

$\begin{array}{llll}\mathrm{U} & \mathrm{YV} & 57 & \mu \mathrm{g} / \mathrm{kg} \\ \mathrm{U} & \mathrm{Y} & 6.0 & \mu \mathrm{g} / \mathrm{kg} \\ \mathrm{U} & \mathrm{Y} & 6.0 & \mu \mathrm{g} / \mathrm{kg} \\ \mathrm{U} & \mathrm{Y} & 6.0 & \mu \mathrm{g} / \mathrm{kg} \\ \mathrm{U} & \mathrm{Y} & 12 & \mu \mathrm{g} / \mathrm{kg} \\ \mathrm{U} & \mathrm{Y} & 6.0 & \mu \mathrm{g} / \mathrm{kg} \\ \mathrm{U} & \mathrm{Y} & 6.0 & \mu \mathrm{g} / \mathrm{kg} \\ \mathrm{U} & \mathrm{Y} & 6.0 & \mu \mathrm{g} / \mathrm{kg} \\ \mathrm{U} & \mathrm{Y} & 12 & \mu \mathrm{g} / \mathrm{kg} \\ \mathrm{U} & \mathrm{Y} & 12 & \mu \mathrm{g} / \mathrm{kg} \\ \mathrm{U} & \mathrm{Y} & 6.0 & \mu \mathrm{g} / \mathrm{kg} \\ \mathrm{U} & \mathrm{Y} & 12 & \mu \mathrm{g} / \mathrm{kg} \\ \mathrm{U} & \mathrm{Y} & 6.0 & \mu \mathrm{g} / \mathrm{kg} \\ \mathrm{U} & \mathrm{Y} & 6.0 & \mu \mathrm{g} / \mathrm{kg} \\ \mathrm{U} & \mathrm{Y} & 6.0 & \mu \mathrm{g} / \mathrm{kg} \\ \mathrm{U} & \mathrm{Y} & 6.0 & \mu \mathrm{g} / \mathrm{kg} \\ \mathrm{U} & \mathrm{Y} & 6.0 & \mu \mathrm{g} / \mathrm{kg} \\ \mathrm{U} & \mathrm{Y} & 6.0 & \mu \mathrm{g} / \mathrm{kg} \\ \mathrm{U} & \mathrm{Y} & 6.0 & \mu \mathrm{g} / \mathrm{kg} \\ \mathrm{U} & \mathrm{Y} & 6.0 & \mu \mathrm{g} / \mathrm{kg} \\ \mathrm{U} & \mathrm{Y} & 6.0 & \mu \mathrm{g} / \mathrm{kg} \\ \mathrm{U} & \mathrm{Y} & 6.0 & \mu \mathrm{g} / \mathrm{kg} \\ \mathrm{U} & \mathrm{Y} & 12 & \mu \mathrm{g} / \mathrm{kg} \\ \mathrm{U} & \mathrm{Y} & 12 & \mu \mathrm{g} / \mathrm{kg} \\ \mathrm{U} & \mathrm{Y} & 12 & \mu \mathrm{g} / \mathrm{kg} \\ \mathrm{U} & \mathrm{Y} & 6.0 & \mu \mathrm{g} / \mathrm{kg} \\ \mathrm{U} & \mathrm{Y} & 6.0 & \mu \mathrm{g} / \mathrm{kg} \\ \mathrm{U} & \mathrm{Y} & 6.0 & \mu \mathrm{g} / \mathrm{kg} \\ \mathrm{U} & \mathrm{Y} & 6.0 & \mu \mathrm{g} / \mathrm{kg} \\ \mathrm{U} & \mathrm{Y} & 6.0 & \mu \mathrm{g} / \mathrm{kg} \\ \mathrm{U} & \mathrm{Y} & 6.0 & \mu \mathrm{g} / \mathrm{kg} \\ \mathrm{U} & \mathrm{Y} & 6.0 & \mu \mathrm{g} / \mathrm{kg} \\ \mathrm{U} & \mathrm{Y} & 12 & \mu \mathrm{g} / \mathrm{kg} \\ \mathrm{U} & \mathrm{Y} & 6.0 & \mu \mathrm{g} / \mathrm{kg} \\ & & & \end{array}$

EPA8270

EPA8270

EPA8270

EPA8270

EPA8270

EPA8270

EPA8270

EPA8270

EPA8240

EPA8240

EPA8240

EPA8240

EPA8240

EPA8240

EPA8240

EPA8240

EPA8240

EPA8240

EPA8240

EPA8240

EPA8240

EPA8240

EPA8240

EPA8240

EPA8240

EPA8240

EPA8240

EPA8240

EPA8240

EPA8240

EPA8240

EPA8240

EPA8240

EPA8240

EPA8240

EPA8240

EPA8240

EPA8240

EPA8240

EPA8240

EPA8240

EPA8240 
SAMPLE NAME: D43110705 (Continued)

\section{Constituent}

Sample ID: 100322

RQ AQ B Result Unit
D. Limit Method

Pesticides/PCBs and Dioxins/Furans

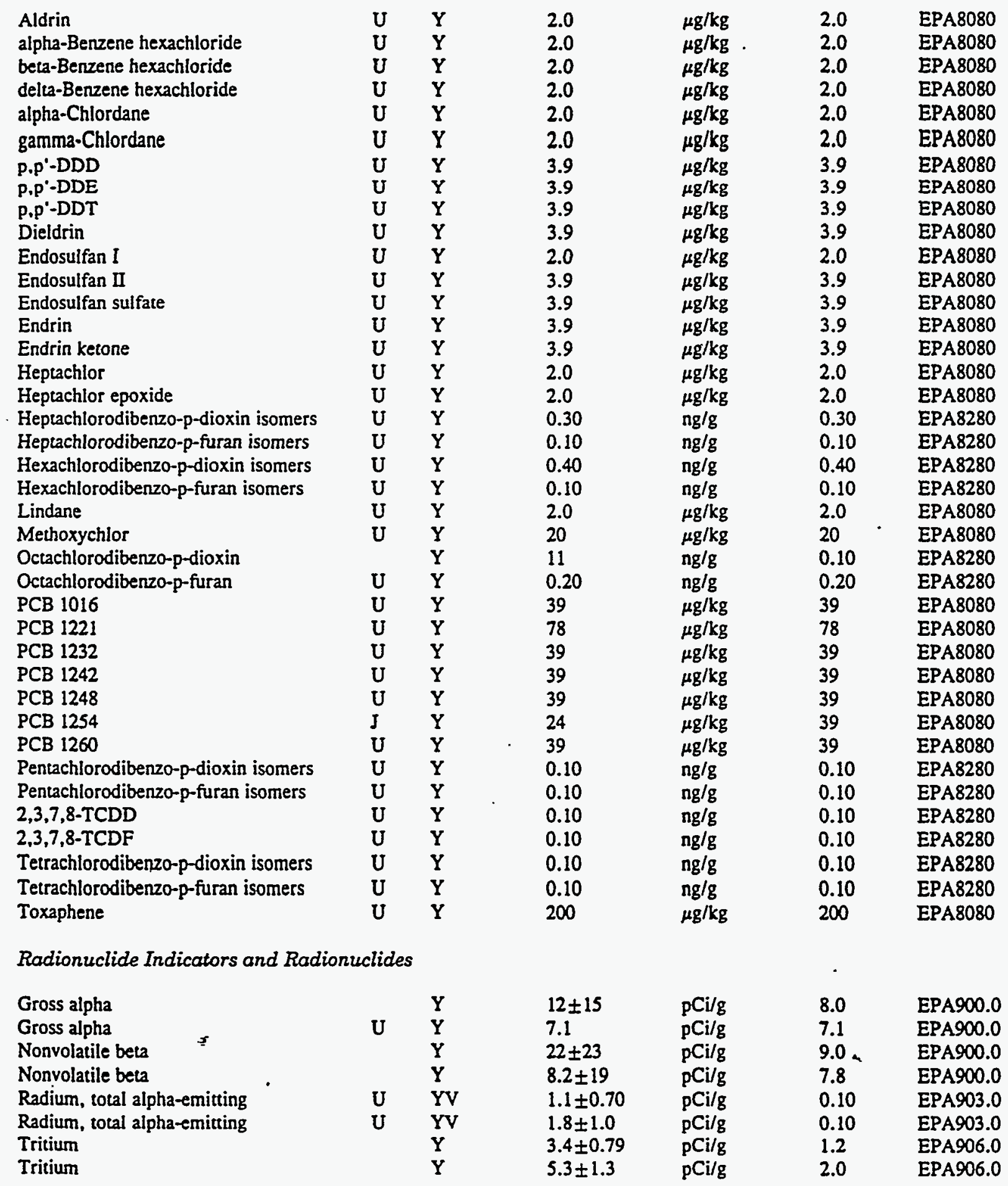


TCLP Compounds (Metals and Organics)

\begin{tabular}{|c|c|c|c|c|c|c|c|}
\hline Arsenic, TCLP & U & $Y$ & & 100 & $\mu g / L$ & 100 & EPA6010 \\
\hline Barium. TCLP & $\mathbf{U}$ & $Y$ & & 200 & $\mu g / L$ & 200 & EPA6010 \\
\hline Benzene & $U$ & $\mathbf{Y}$ & & 50 & $\mu \mathrm{g} / \mathrm{L}$ & 50 & EPA8240 \\
\hline Cadmium, TCLP & $\mathbf{U}$ & $Y$ & & 5.0 & $\mu g / L$ & 5.0 & EPA6010 \\
\hline Carbon tetrachloride & U & $\mathbf{Y}$ & & 50 & $\mu \mathrm{g} / \mathrm{L}$ & 50 & EPA8240 \\
\hline alpha-Chlordane & $\mathbf{U}$ & $\mathbf{Y}$ & & 0.11 & $\mu g / L$ & 0.11 & EPA8080 \\
\hline gamma-Chlordane & $\mathrm{U}$ & $\mathbf{Y}$ & & 0.11 & $\mu g / L$ & 0.11 & EPA8080 \\
\hline Chlorobenzene & $\mathbf{U}$ & $\mathbf{Y}$ & & 50 & $\mu g / L$ & 50 & EPA8240 \\
\hline Chloroethene (Vinyl chloride) & $\mathbf{U}$ & $\mathbf{Y}$ & & 100 & $\mu g / L$ & 100 & EPA8240 \\
\hline Chloroform & $\mathrm{U}$ & $\mathrm{Y}$ & & 50 & $\mu g / L$ & 50 & EPA8240 \\
\hline Chromium. TCLP & $\mathrm{U}$ & $\mathbf{Y}$ & & 10 & $\mu \mathrm{g} / \mathrm{L}$ & 10 & EPA6010 \\
\hline o-Cresol (2-Methylphenol) & UJ & YO & L & 100 & $\mu \mathrm{g} / \mathrm{L}$ & 100 & EPA8270 \\
\hline 1.4-Dichlorobenzene & UJ & YO & $L$ & 100 & $\mu g / L$ & 100 & EPA8270 \\
\hline 1.2-Dichloroethane & $\mathrm{U}$ & $\mathbf{Y}$ & & 50 & $\mu \mathrm{g} / \mathrm{L}$ & so & EPA8240 \\
\hline 1,1-Dichloroethylene & $\mathrm{U}$ & $\mathbf{Y}$ & & 50 & $\mu \mathrm{g} / \mathrm{L}$ & 50 & EPA8240 \\
\hline 2,4-Dichlorophenoxyacetic acid & $\mathrm{U}$ & $\mathbf{Y}$ & & 10 & $\mu \mathrm{g} / \mathrm{L}$ & 10 & EPA8150 \\
\hline 2,4-Dinitrotoluene & UJ & YO & L & 100 & $\mu \mathrm{g} / \mathrm{L}$ & 100 & EPA8270 \\
\hline Endrin & $U$ & $\mathrm{Y}$ & & 0.23 & $\mu g / L$ & 0.23 & EPA8080 \\
\hline Heptachlor & $\mathrm{U}$ & $\mathbf{Y}$ & & 0.11 & $\mu \mathrm{g} / \mathrm{L}$ & 0.11 & EPA8080 \\
\hline Heptachlor epoxide & U & $Y$ & & 0.11 & $\mu \mathrm{g} / \mathrm{L}$ & 0.11 & EPA8080 \\
\hline Hexachlorobenzene & UJ & YO & L & 100 & $\mu \mathrm{g} / \mathrm{L}$ & 100 & EPA8270 \\
\hline Hexachlorobutadiene & UJ & YO & L & 100 & $\mu \mathrm{g} / \mathrm{L}$ & 100 & EPA8270 \\
\hline Hexachloroechane & UJ & YO & L & 100 & $\mu \mathrm{g} / \mathrm{L}$ & 100 & EPA8270 \\
\hline Lead, TCLP & $\mathrm{U}$ & $\mathbf{Y}$ & & 90 & $\mu \mathrm{g} / \mathrm{L}$ & 90 & EPA6010 \\
\hline Lindane & $\mathbf{U}$ & $\mathbf{Y}$ & & 0.11 & $\mu \mathrm{g} / \mathrm{L}$ & 0.11 & EPA8080 \\
\hline Mercury, TCLP & $\mathrm{U}$ & $\mathbf{Y}$ & & 0.20 & $\mu g / L$ & 0.20 & EPA7470 \\
\hline Methoxychlor & $\mathrm{U}$ & $\mathbf{Y}$ & & 1.1 & $\mu \mathrm{g} / \mathrm{L}$ & 1.1 & EPA8080 \\
\hline Methyl ethyl ketone & $\mathrm{U}$ & $\mathbf{Y}$ & & 100 & $\mu \mathrm{g} / \mathrm{L}$ & 100 & EPA8240 \\
\hline Nitrobenzene & UJ & YO & $\mathrm{L}$ & 100 & $\mu \mathrm{g} / \mathrm{L}$ & 100 & EPA8270 \\
\hline Pentachlorophenol & UJ & YO & L & 500 & $\mu \mathrm{g} / \mathrm{L}$ & 500 & EPA8270 \\
\hline Pyridine & UJ & YO & L & 100 & $\mu g / L$ & 100 & EPA8270 \\
\hline Selenium, TCLP & $\mathrm{U}$ & $\mathrm{Y}$ & & 100 & $\mu g / L$ & 100 & EPA6010 \\
\hline Silver, TCLP & $\mathrm{U}$ & $\mathbf{Y}$ & & 10 & $\mu \mathrm{g} / \mathrm{L}$ & 10 & EPA6010 \\
\hline Tetrachloroethylene & $\mathrm{U}$ & $Y$ & & 50 & $\mu g / L$ & 50 & EPA8240 \\
\hline Toxaphene & $\mathbf{U}$ & $\mathbf{Y}$ & & 11 & $\mu \mathrm{g} / \mathrm{L}$ & 11 & EPA8080 \\
\hline Toxaphene & $\mathrm{U}$ & $Y$ & & 11 & $\mu g / L$ & 11 & EPA8080 \\
\hline 2,4,5-TP (Silvex) & $\mathrm{U}$ & $\mathbf{Y}$ & & 5.0 & $\mu \mathrm{g} / \mathrm{L}$ & 5.0 & EPA8150 \\
\hline Trichloroethylene & $\mathrm{U}$ & $Y$ & & 50 & $\mu g / L$ & 50 & EPA8240 \\
\hline 2,4,5-Trichlorophenol . & UJ & YO & L & 500 & $\mu g / L$ & 500 & EPA8270 \\
\hline 2,4,6-Trichlorophenol & UJ & YO & $\mathbf{L}$ & 100 & $\mu \mathrm{g} / \mathrm{L}$ & 100 & EPA8270 \\
\hline $2,4,5-T$ & $\mathrm{U}$ & $\mathbf{Y}$ & & 5.0 & $\mu \mathrm{g} / \mathrm{L}$ & 5.0 & EPA8150 \\
\hline m,p-Cresol & US & YO & L & 100 & $\mu g / L$ & 100 & EPA8270 \\
\hline
\end{tabular}


SAMPLE NAME: D43110706

Sample ID: 100326

Location (SRS Coordinates): 18576.98E 66634.66N

Ground Elevation Above MSL: $132.8 \mathrm{ft}$

Depth of Core Interval: 8.00 to $10.00 \mathrm{ft}$

Sample Type: Normal

Sample Color: 1OR $4 / 6$

\section{Constituent}

Physical Parameters

Cation exchange capacity

$\mathrm{pH}$

Specific conductance

Total organic carbon

Total Metals

\section{Arsenic}

Barium

Cadmium

Chromium

Copper

Lead

Manganese

Mercury

Nickel

Selenium

Silver
Sample Matrix: Soil

USC Soil Classification: SC
Sample Moisture: Damp Percent Solids: $\mathbf{7 7 . 6 0}$
RQ AQ
B Result
Unit
D. Limit Method

$B / N / A$ Extractables (including $P A H$ and phenols)

\begin{abstract}
Acenaphthene
Acenaphthylene

Anthracene

Benzo(a)anthracene

Benzo(b)fluoranthene

Benzo(k)fluoranthene

Benzoic acid

Benzo(g,h,i)perylene

Benzo(a)pyrene

Benzyl alcohol

Bis(2-chloroethoxy) methane

Bis(2-chloroethyl) ether

Bis(2-chloroisopropyl) ether

Bis(2-ethylhexyl) phthalate

4-Bromophenyl phenyl ether

Burylbenzyl phehalate

4-Chloroaniline

4-Chloro-m-cresol

2-Chloronaphthalene

2-Chlorophenol

4-Chlorophenyl phenyl ether

Chrysene

o-Cresol (2-Methylphenol)
\end{abstract}

$\begin{array}{lllll} & 12 & m e q / 100 \mathrm{~g} & 2.0 & \text { EPA9080 } \\ \mathrm{J} \quad \mathrm{Q} & 3.5 & \mathrm{pH} & 0.010 & \text { EPA9045 } \\ & 210 & \mu S / \mathrm{cm} & 1.0 & \text { EPA9050 } \\ & 1.400 & \mathrm{mg} / \mathrm{kg} & 150 & \text { EPA415.1 }\end{array}$

26

26

0.63

12

35

2.1

86

0.13

5.0

0.52

1.3

$\mathrm{mg} / \mathrm{kg}$
$\mathrm{mg} / \mathrm{kg}$
$\mathrm{mg} / \mathrm{kg}$
$\mathrm{mg} / \mathrm{kg}$
$\mathrm{mg} / \mathrm{kg}$
$\mathrm{mg} / \mathrm{kg}$
$\mathrm{mg} / \mathrm{kg}$
$\mathrm{mg} / \mathrm{kg}$
$\mathrm{mg} / \mathrm{kg}$
$\mathrm{mg} / \mathrm{kg}$
$\mathrm{mg} / \mathrm{kg}$

1.0

EPA7060

25

EPA6010

0.63 EPA6010

1.3 EPA6010

3.2 EPA6010

0.31 EPA7421

1.9 EPA6010

0.13 EPA7470

5.0 EPA6010

0.52 EPA7740

1.3 EPA6010
410

410

410

410

410

410

2,000

410

410

410

410

410

410

410

410

410

410

410

410

410

410

410

410

$\begin{array}{lll}\mu g / \mathrm{kg} & 410 & \text { EPA8270 } \\ \mu \mathrm{g} / \mathrm{kg} & 410 & \text { EPA8270 } \\ \mu \mathrm{g} / \mathrm{kg} & 410 & \text { EPA8270 } \\ \mu \mathrm{g} / \mathrm{kg} & 410 & \text { EPA8270 } \\ \mu g / \mathrm{kg} & 410 & \text { EPA8270 } \\ \mu \mathrm{g} / \mathrm{kg} & 410 & \text { EPA8270 } \\ \mu \mathrm{g} / \mathrm{kg} & 2,000 & \text { EPA8270 } \\ \mu \mathrm{g} / \mathrm{kg} & 410 & \text { EPA8270 } \\ \mu \mathrm{g} / \mathrm{kg} & 410 & \text { EPA8270 } \\ \mu \mathrm{g} / \mathrm{kg} & 410 & \text { EPA8270 } \\ \mu g / \mathrm{kg} & 410 & \text { EPA8270 } \\ \mu g / \mathrm{kg} & 410 & \text { EPA8270 } \\ \mu \mathrm{g} / \mathrm{kg} & 410 & \text { EPA8270 } \\ \mu \mathrm{g} / \mathrm{kg} & 410 & \text { EPA8270 } \\ \mu \mathrm{g} / \mathrm{kg} & 410 & \text { EPA8270 } \\ \mu \mathrm{g} / \mathrm{kg} & 410 & \text { EPA8270 } \\ \mu g / \mathrm{kg} & 410 & \text { EPA8270 } \\ \mu g / \mathrm{kg} & 410 . & \text { EPA8270 } \\ \mu g / \mathrm{kg} & 410 & \text { EPA8270 } \\ \mu \mathrm{g} / \mathrm{kg} & 410 & \text { EPA8270 } \\ \mu \mathrm{gg} / \mathrm{kg} & 410 & \text { EPA8270 } \\ \mu \mathrm{g} / \mathrm{kg} & 410 & \text { EPA8270 } \\ \mu \mathrm{g} / \mathrm{kg} & 410 & \text { EPA8270 }\end{array}$


SAMPLE NAME: D431̈̈0706 (Continued)

Sample ID: 100326

$\begin{array}{lllllll}\text { Constituent } & \text { RQ } & \text { AQ } & \text { B } & \text { Result Unit } & \text { D. Limit Method }\end{array}$

$B / N / A$ Extractables (including $P A H$ and phenols)

\begin{tabular}{|c|c|c|c|c|c|c|}
\hline p-Cresol (4-Methylphenol) & $\mathbf{U}$ & & 410 & $\mu g / \mathrm{kg}$ & 410 & EPA 8270 \\
\hline Dibenz $(a, h)$ anthracene & $\mathbf{U}$ & & 410 & $\mu g / \mathrm{kg}$ & 410 & EPA8270 \\
\hline Dibenzofuran & U & & 410 & $\mu g / \mathrm{kg}$ & 410 & EPA8270 \\
\hline Di-n-butyl phthalate & UJ & $\mathbf{V}$ & 110 & $\mu g / \mathrm{kg}$ & 410 & EPA8270 \\
\hline 1.2-Dichlorobenzene & $\mathbf{U}$ & & 410 & $\mu g / k g$ & 410 & EPA8270 \\
\hline 1,3-Dichlorobenzene & $\mathbf{U}$ & & 410 & $\mu \mathrm{g} / \mathrm{kg}$ & 410 & EPA8270 \\
\hline 1,4-Dichlorobenzene & $\mathbf{U}$ & & 410 & $\mu g / k g$ & 410 & EPA8270 \\
\hline 3,3'-Dichlorobenzidine & $U$ & & 810 & $\mu \mathrm{g} / \mathrm{kg}$ & 810 & EPA8270 \\
\hline 2,4-Dichlorophenol & $U$ & & 410 & $\mu \mathrm{g} / \mathrm{kg}$ & 410 & EPA8270 \\
\hline Diethyl phthalate & $U$ & & 410 & $\mu g / \mathrm{kg}$ & 410 & EPA8270 \\
\hline 2,4-Dimethyl phenol & $\mathbf{U}$ & & 410 & $\mu \mathrm{g} / \mathrm{kg}$ & 410 & EPA8270 \\
\hline Dimethyl phthalate & $\mathbf{U}$ & & 410 & $\mu g / k g$ & 410 & EPA8270 \\
\hline 2,4-Dinitrophenol & $\mathbf{U}$ & & 2.000 & $\mu \mathrm{g} / \mathrm{kg}$ & 2,000 & EPA 8270 \\
\hline 2,4-Dinitrotoluene & $\mathbf{U}$ & & 410 & $\mu \mathrm{g} / \mathrm{kg}$ & 410 & EPA8270 \\
\hline 2.6-Dinitrotoluene & $\mathrm{U}$ & & 410 & $\mu \mathrm{g} / \mathrm{kg}$ & 410 & EPA8270 \\
\hline Di-n-octyl phehalate & U & & 410 & $\mu \mathrm{g} / \mathrm{kg}$ & 410 & EPA8270 \\
\hline Fluoranthene & $\mathbf{U}$ & & 410 & $\mu \mathrm{g} / \mathrm{kg}$ & 410 & EPA8270 \\
\hline Fluorene & $\mathbf{U}$ & & 410 & $\mu \mathrm{g} / \mathrm{kg}$ & 410 & EPA8270 \\
\hline Hexachlorobenzene & $\mathbf{U}$ & & 410 & $\mu g / k g$ & 410 & EPA8270 \\
\hline Hexachlorobutadiene & $\mathbf{U}$ & & 410 & $\mu \mathrm{g} / \mathrm{kg}$ & 410 & EPA8270 \\
\hline Hexachlorocyclopentadiene & $\mathbf{U}$ & & 410 & $\mu \mathrm{g} / \mathrm{kg}$ & 410 & EPA8270 \\
\hline Hexachloroethane & $\mathbf{U}$ & & 410 & $\mu g / \mathrm{kg}$ & 410 & EPA8270 \\
\hline Indeno( $(1,2,3-c, d)$ pyrene & $\mathbf{U}$ & & 410 & $\mu \mathrm{g} / \mathrm{kg}$ & 410 & EPA8270 \\
\hline Isophorone & $\mathbf{U}$ & & 410 & $\mu g / \mathrm{kg}$ & 410 & EPA8270 \\
\hline 2-Methyl-4,6-dinitrophenol & $\mathbf{U}$ & & 2,000 & $\mu g / \mathrm{kg}$ & 2,000 & EPA8270 \\
\hline 2-Methylnaphthalene & $\mathbf{U}$ & & 410 & $\mu g / \mathrm{kg}$ & 410 & EPA8270 \\
\hline Naphthalene & $\mathbf{U}$ & & 410 & $\mu \mathrm{g} / \mathrm{kg}$ & 410 & EPA8270 \\
\hline o-Nitroaniline & $\mathbf{U}$ & & 2,000 & $\mu g / \mathrm{kg}$ & 2.000 & EPA8270 \\
\hline m-Nitroaniline & $\mathbf{U}$ & & 2,000 & $\mu g / k g$ & 2,000 & EPA8270 \\
\hline p-Nitroaniline & $\mathbf{U}$ & & 2,000 & $\mu g / k g$ & 2,000 & EPA8270 \\
\hline Nitrobenzene & $\mathbf{U}$ & & 410 & $\mu g / k g$ & 410 & EPA8270 \\
\hline 2-Nitrophenol & $\mathbf{U}$ & & 410 & $\mu \mathrm{g} / \mathrm{kg}$ & 410 & EPA8270 \\
\hline 4-Nitrophenol & $\mathbf{U}$ & & 2,000 & $\mu g / k g$ & 2,000 & EPA8270 \\
\hline N-Nitrosodiphenylamine & $\mathbf{U}$ & & 410 & $\mu \mathrm{g} / \mathrm{kg}$ & 410 & EPA8270 \\
\hline N-Nitrosodipropylamine & $\mathbf{U}$ & & 410 & $\mu \mathrm{g} / \mathrm{kg}$ & 410 & EPA8270 \\
\hline Pentachlorophenol & $\mathbf{U}$ & & 2,000 & $\mu g / k g$ & 2,000 & EPA8270 \\
\hline Phenanthrene & $\mathrm{U}$ & & 410 & $\mu \mathrm{g} / \mathrm{kg}$ & 410 & EPA8270 \\
\hline Phenol & $\mathbf{U}$ & & 410 & $\mu \mathrm{g} / \mathrm{kg}$ & 410 & EPA8270 \\
\hline Pyrene & $\mathbf{U}$ & & 410 & $\mu g / \mathrm{kg}$ & 410 & EPA8270 \\
\hline 1,2,4-Trichlorobenzenë & $\mathbf{U}$ & & 410 & $\mu g / k g$ & 410 & EPA8270 \\
\hline 2,4,5-Trichlorophenol & $\mathbf{U}$ & & 2,000 & $\mu g / \mathrm{kg}$ & 2,000 & EPA8270 \\
\hline 2,4,6-Trichlorophenol & $\mathbf{U}$ & & 410 & $\mu g / k g$ & 410 & EPA8270 \\
\hline Unknown hydrocarbon & J & $\mathbf{N}$ & 500 & $\mu g / \mathbf{k g}$ & & EPA8270 \\
\hline Unknown & $J$ & $\mathbf{N}$ & 900 & $\mu \mathrm{g} / \mathrm{kg}$ & 4 & EPA. 8270 \\
\hline Unknown & J & $\mathbf{N}$ & 400 & $\mu g / \mathrm{kg}$ & & EPA8270 \\
\hline Unknown k & J & NV & 7,000 & $\mu g / k g$ & & EPA8270 \\
\hline
\end{tabular}


SAMPLE NAME: D43110706 (Continued)

RQ AQ B Result

Constituent

Volatile Organic Compounds

Acetone
Benzene
Bromodichloromethane
Bromoform
Bromomethane (Methyl bromide)
Carbon disulfide
Carbon tetrachloride
Chlorobenzene
Chloroethane
Chloroethene (Vinyl chloride)
Chloroform
Chloromethane (Methyl chloride)
Dibromochloromethane
1.1-Dichloroethane
1,2-Dichloroethane
1,1-Dichloroethylene
1,2-Dichloroethylene
Dichloromethane (Methylene chloride)
1,2-Dichloropropane
cis-1,3-Dichloropropene
trans-1,3-Dichloropropene
Ethylbenzene
2-Hexanone
Methyl ethyl ketone
Methyl isobutyl ketone
Styrene
1,1,2,2-Tetrachloroethane
Tetrachloroethylene
Toluene
1,1,1-Trichloroethane
1.1,2-Trichloroethane
Trichloroethylene
Vinyl acetate
Xylenes

Sample ID: 100326

Unit

D. Limit Method

\begin{tabular}{|c|c|c|c|c|}
\hline UJ & 10 & $\mu \mathrm{g} / \mathrm{kg}$ & 12 & EPA8240 \\
\hline U & 6.2 & $\mu \mathrm{g} / \mathrm{kg}$ & 6.2 & EPA8240 \\
\hline U & 6.2 & $\mu \mathrm{g} / \mathrm{kg}$ & 6.2 & EPA8240 \\
\hline U & 6.2 & $\mu \mathrm{g} / \mathrm{kg}$ & 6.2 & EPA8240 \\
\hline $\mathbf{U}$ & 12 & $\mu \mathrm{g} / \mathrm{kg}$ & 12 & EPA8240 \\
\hline U & 6.2 & $\mu \mathrm{g} / \mathrm{kg}$ & 6.2 & EPA8240 \\
\hline$U$ & 6.2 & $\mu \mathrm{g} / \mathrm{kg}$ & 6.2 & EPA8240 \\
\hline$U$ & 6.2 & $\mu \mathrm{g} / \mathrm{kg}$ & 6.2 & EPA8240 \\
\hline $\mathrm{U}$ & 12 & $\mu \mathrm{g} / \mathrm{kg}$ & 12 & EPA8240 \\
\hline $\mathrm{U}$ & 12 & $\mu \mathrm{g} / \mathrm{kg}$ & 12 & EPA8240 \\
\hline $\mathbf{U}$ & 6.2 & $\mu \mathrm{g} / \mathrm{kg}$ & 6.2 & EPA8240 \\
\hline U & 12 & $\mu \mathrm{g} / \mathrm{kg}$ & 12 & EPA8240 \\
\hline$U$ & 6.2 & $\mu \mathrm{g} / \mathrm{kg}$ & 6.2 & EPA8240 \\
\hline$U$ & 6.2 & $\mu \mathrm{g} / \mathrm{kg}$ & 6.2 & EPA 8240 \\
\hline U & 6.2 & $\mu \mathrm{g} / \mathrm{kg}$ & 6.2 & EPA8240 \\
\hline$U$ & 6.2 & $\mu \mathrm{g} / \mathrm{kg}$ & 6.2 & EPA8240 \\
\hline U & 6.2 & $\mu \mathrm{g} / \mathrm{kg}$ & 6.2 & EPA8240 \\
\hline $\mathrm{v}$ & 27 & $\mu \mathrm{g} / \mathrm{kg}$ & 6.2 & EPA8240 \\
\hline$U^{\circ}$ & 6.2 & $\mu \mathrm{g} / \mathrm{kg}$ & 6.2 & EPA8240 \\
\hline U & 6.2 & $\mu \mathrm{g} / \mathrm{kg}$ & 6.2 & EPA8240 \\
\hline 0 & 6.2 & $\mu \mathrm{g} / \mathrm{kg}$ & 6.2 & EPA8240 \\
\hline U & 6.2 & $\mu \mathrm{g} / \mathrm{kg}$ & 6.2 & EPA8240 \\
\hline$U$ & 12 & $\mu \mathrm{g} / \mathrm{kg}$ & 12 & EPA8240 \\
\hline U & 12 & $\mu \mathrm{g} / \mathrm{kg}$ & 12 & EPA8240 \\
\hline U & 12 & $\mu \mathrm{g} / \mathrm{kg}$ & 12 & EPA8240 \\
\hline $\mathbf{U}$ & 6.2 & $\mu \mathrm{g} / \mathrm{kg}$ & 6.2 & EPA8240 \\
\hline U & 6.2 & $\mu \mathrm{g} / \mathrm{kg}$ & 6.2 & EPA8240 \\
\hline U & 6.2 & $\mu \mathrm{g} / \mathrm{kg}$ & 6.2 & EPA8240 \\
\hline $\mathbf{U}$ & 6.2 & $\mu \mathrm{g} / \mathrm{kg}$ & 6.2 & EPA8240 \\
\hline U & 6.2 & $\mu g / \mathrm{kg}$ & 6.2 & EPA8240 \\
\hline $\mathrm{U}$ & 6.2 & $\mu \mathrm{g} / \mathrm{kg}$ & 6.2 & EPA8240 \\
\hline U & 6.2 & $\mu \mathrm{g} / \mathrm{kg}$ & 6.2 & EPA8240 \\
\hline 1 & 12 & $\mu \mathrm{g} / \mathrm{kg}$ & 12 & EPA8240 \\
\hline & 6.2 & $\mu \mathrm{g} / \mathrm{kg}$ & 6.2 & EPA8240 \\
\hline
\end{tabular}

Pesticides/PCBs and Dioxins/Furans

Aldrin

alpha-Benzene hexachloride

beta-Benzene hexachloride

delta-Benzene hexachloride

alpha-Chlordane

gamma-Chlordane

p.p'-DDD

P, $p^{\prime}$-DDE

P.p'-DDT

Dieidrin

Endosulfan I

Endosulfan II

Endosulfan sulfate

$\begin{array}{llll}\text { UJ } & \text { O } & \text { L } & 2.0 \\ \text { UJ } & \text { O } & \text { L } & 2.0 \\ \text { UJ } & \text { O } & \text { L } & 2.0 \\ \text { UJ } & \text { O } & \text { L } & 2.0 \\ \text { UJ } & \text { O } & \text { L } & 2.0 \\ \text { UJ } & \text { O } & \text { L } & 2.0 \\ \text { UJ } & \text { O } & \text { L } & 4.0 \\ \text { UJ } & \text { O } & \text { L } & 4.0 \\ \text { UJ } & 0 & \text { L } & 4.0 \\ \text { UJ } & \text { O } & \text { L } & 4.0 \\ \text { UJ } & \text { O } & \text { L } & 2.0 \\ \text { UJ } & \text { O } & \text { L } & 4.0 \\ \text { UJ } & \text { O } & \text { L } & 4.0\end{array}$

$\begin{array}{llll}\mu \mathrm{g} / \mathrm{kg} & 2.0 & \text { EPA8080 } \\ \mu \mathrm{g} / \mathrm{kg} & 2.0 & \text { EPA8080 } \\ \mu \mathrm{g} / \mathrm{kg} & 2.0 & \text { EPA8080 } \\ \mu \mathrm{g} / \mathrm{kg} & 2.0 & \text { EPA8080 } \\ \mu \mathrm{g} / \mathrm{kg} & 2.0 & \text { EPA8080 } \\ \mu \mathrm{g} / \mathrm{kg} & 2.0 & \text { EPA8080 } \\ \mu \mathrm{g} / \mathrm{kg} & 4.0 & \text { EPA8080 } \\ \mu \mathrm{g} / \mathrm{kg} & 4.0 & \text { EPA8080 } \\ \mu \mathrm{g} / \mathrm{kg} & 4.0 & \text { EPA8080 } \\ \mu \mathrm{g} / \mathrm{kg} & 4.0 & \text { EPA8080 } \\ \mu \mathrm{g} / \mathrm{kg} & 2.0 & \text { EPA8080 } \\ \mu \mathrm{g} / \mathrm{kg} & 4.0 & \text { EPA8080 } \\ \mu \mathrm{g} / \mathrm{kg} & 4.0 & \text { EPA8080 }\end{array}$


SAMPLE NAME: D43110706 (Continued)

Sample ID: 100326

$\begin{array}{llllll}\text { Constituent } & \text { RQ AQ } & \text { B Result Unit D. Limit Method }\end{array}$

Pesticides/PCBs and Dioxins/Furans

\begin{tabular}{|c|c|c|c|c|c|c|c|}
\hline Endrin & UJ & 0 & L & 4.0 & $\mu \mathrm{g} / \mathrm{kg}$ & 4.0 & EPA8080 \\
\hline Endrin ketone & UJ & 0 & L & 4.0 & $\mu \mathrm{g} / \mathrm{kg}$ & 4.0 & EPA8080 \\
\hline Heptachlor & UJ & 0 & L & 2.0 & $\mu \mathrm{g} / \mathrm{kg}$ & 2.0 & EPA8080 \\
\hline Heptachlor epoxide & UJ & 0 & L & 2.0 & $\mu \mathrm{g} / \mathrm{kg}$ & 2.0 & EPA8080 \\
\hline Heptachlorodibenzo-p-dioxin isomers & J & & & 0.30 & $\mathrm{ng} / \mathrm{g}$ & 0.10 & EPA8280 \\
\hline Heptachlorodibenzo-p-furan isomers & $\mathbf{U}$ & & & 0.10 & $\mathrm{ng} / \mathrm{g}$ & 0.10 & EPA8280 \\
\hline Hexachlorodibenzo-p-dioxin isomers & $\mathbf{U}$ & & & 0.10 & $\mathrm{ng} / \mathrm{g}$ & 0.10 & EPA8280 \\
\hline Hexachlorodibenzo-p-furan isomers & $\mathbf{U}$ & & & 0.10 & $\mathbf{n g} / \mathbf{g}$ & 0.10 & EPA8280 \\
\hline Lindane & UJ & 0 & L & 2.0 & $\mu \mathrm{g} / \mathrm{kg}$ & 2.0 & EPA8080 \\
\hline Methoxychlor & UJ & 0 & L & 20 & $\mu \mathrm{g} / \mathrm{kg}$ & 20 & EPA8080 \\
\hline Octachlorodibenzo-p-dioxin & & & & 26 & $\mathrm{ng} / \mathrm{g}$ & 0.10 & EPA8280 \\
\hline Octachlorodibenzo-p-furan & $\mathbf{U}$ & & & 0.10 & $\mathrm{ng} / \mathrm{g}$ & 0.10 & EPA8280 \\
\hline РCB 1016 & UJ & 0 & L & 40 & $\mu \mathrm{g} / \mathrm{kg}$ & 40 & EPA8080 \\
\hline PCB 1221 & UJ & 0 & L & 81 & $\mu \mathrm{g} / \mathrm{kg}$ & 81 & EPA8080 \\
\hline PCB 1232 & UJ & 0 & L & 40 & $\mu \mathrm{g} / \mathrm{kg}$ & 40 & EPA8080 \\
\hline PCB 1242 & UJ & 0 & L & 40 & $\mu \mathrm{g} / \mathrm{kg}$ & 40 & EPA8080 \\
\hline PCB 1248 & UJ & 0 & L & 40 & $\mu \mathrm{g} / \mathrm{kg}$ & 40 & EPA8080 \\
\hline PCB 1254 & UJ & 0 & $\mathbf{L}$ & 40 & $\mu g / \mathrm{kg}$ & 40 & EPA8080 \\
\hline РСB 1260 & UI & 0 & L & 40 & $\mu \mathrm{g} / \mathrm{kg}$ & 40 & EPA8080 \\
\hline Pentachlorodibenzo-p-dioxin isomers & $\mathrm{U}$ & & & 0.10 & $\mathrm{ng} / \mathrm{g}$ & 0.10 & EPA8280 \\
\hline Pentachlorodibenzo-p-furan isomers & $\mathrm{U}$ & & & 0.10 & $\mathrm{ng} / \mathrm{g}$ & 0.10 & EPA8280 \\
\hline $2,3,7,8-\mathrm{TCDD}$ & $\mathbf{U}$ & & & 0.10 & $\mathrm{ng} / \mathrm{g}$ & 0.10 & EPA8280 \\
\hline $2,3,7,8-\mathrm{TCDF}$ & $\mathrm{U}$ & & & 0.10 & $\mathrm{ng} / \mathrm{g}$ & 0.10 & EPA8280 \\
\hline Tetrachlorodibenzo-p-dioxin isomers & U & & & 0.10 & $\mathrm{ng} / \mathrm{g}$ & 0.10 & EPA8280 \\
\hline Tetrachlorodibenzo-p-furan isomers & $\mathbf{U}$ & & & 0.10 & $\mathrm{ng} / \mathrm{g}$ & 0.10 & EPA8280 \\
\hline Toxaphene & UJ & 0 & L & 200 & $\mu \mathrm{g} / \mathrm{kg}$ & 200 & EPA8080 \\
\hline
\end{tabular}

Radionuclide Indicators and Radionuclides

Gross alpha

Gross alpha

Nonvolatile beta

Nonvolatile beta

Radium, total alpha-emitting

Radium, total alpha-emitting

Tritium

Tritium

TCLP Compounds (Metals and Organics)

$\begin{array}{llll}14 \pm 14 & \mathrm{pCi} / \mathrm{g} & 6.9 & \text { EPA900.0 } \\ 25 \pm 16 & \mathrm{pCi} / \mathrm{g} & 6.7 & \text { EPA900.0 } \\ 24 \pm 20 & \mathrm{pCi} / \mathrm{g} & 8.4 & \text { EPA900.0 } \\ 22 \pm 19 & \mathrm{pCi} / \mathrm{g} & 8.1 & \text { EPA900.0 } \\ 1.4 \pm 1.1 & \mathrm{pCi} / \mathrm{g} & 0.090 & \text { EPA903.0 } \\ 1.5 \pm 1.2 & \mathrm{pCi} / \mathrm{g} & 0.10 & \text { EPA903.0 } \\ 13 \pm 2.3 & \mathrm{pCi} / \mathrm{g} & 1.8 & \text { EPA906.0 } \\ 11 \pm 2.0 & \mathrm{pCi} / \mathrm{g} & 1.7 & \text { EPA996.0 }\end{array}$

100

200

50

5.0

so

0.11

0.11

50

100

so $\mu g / L$

$\mu \mathrm{g} / \mathrm{L}$

$\mu g / L$

$\mu g / L$

$\mu \mathrm{g} / \mathrm{L}$

$\mu \mathrm{g} / \mathrm{L}$

$\mu g / L$

$\mu \mathrm{g} / \mathrm{L}$

$\mu g / L$

$\mu g / L$
EPA6010

EPA6010

EPA8240

EPA6010

EPA8240

EPA8080

EPA8240

EPA8240

EPA8240
EPA8080 
SAMPLE NAME: D431:10706 (Continued)

Constituent

\section{RQ}

TCLP Compounds (Metals and Organics)

Chromium. TCLP

o-Cresol (2-Methylphenol)

1.4-Dichlorobenzene

1.2-Dichloroethane

1,1-Dichloroethylene

2,4-Dichlorophenoxyacetic acid

2.4-Dinitrotoluene

Endrin

Heptachlor

Heptachlor epoxide

Hexachlorobenzene

Hexachloroburadiene

Hexachloroethane

Lead, TCLP

Lindane

Mercury, TCLP

Methoxychlor

Methyl ethyl ketone

Nitrobenzene

Pentachlorophenol

Pyridine

Selenium. TCLP

Silver, TCLP

Tetrachloroethylene

Toxaphene

Toxaphene

2,4,5-TP (Silvex)

Trichloroethylene

2,4,5-Trichlorophenol

2,4,6-Trichlorophenol

2.4.5-T

m.p-Cresol
Sample ID: 100326
AQ B Result
Unit
D. Limit Method

$\mu \mathrm{g} / \mathrm{L}$
$\mu \mathrm{g} / \mathrm{L}$
$\mu \mathrm{g} / \mathrm{L}$
$\mu \mathrm{g} / \mathrm{L}$
$\mu \mathrm{g} / \mathrm{L}$
$\mu \mathrm{g} / \mathrm{L}$
$\mu \mathrm{g} / \mathrm{L}$
$\mu \mathrm{g} / \mathrm{L}$
$\mu \mathrm{g} / \mathrm{L}$
$\mu \mathrm{g} / \mathrm{L}$
$\mu \mathrm{g} / \mathrm{L}$
$\mu \mathrm{g} / \mathrm{L}$
$\mu \mathrm{g} / \mathrm{L}$
$\mu \mathrm{g} / \mathrm{L}$
$\mu \mathrm{g} / \mathrm{L}$
$\mu \mathrm{g} / \mathrm{L}$
$\mu \mathrm{g} / \mathrm{L}$
$\mu \mathrm{g} / \mathrm{L}$
$\mu \mathrm{g} / \mathrm{L}$
$\mu \mathrm{g} / \mathrm{L}$
$\mu \mathrm{g} / \mathrm{L}$
$\mu \mathrm{g} / \mathrm{L}$
$\mu \mathrm{g} / \mathrm{L}$
$\mu \mathrm{g} / \mathrm{L}$
$\mu \mathrm{g} / \mathrm{L}$
$\mu \mathrm{g} / \mathrm{L}$
$\mu \mathrm{g} / \mathrm{L}$
$\mu \mathrm{g} / \mathrm{L}$
$\mu \mathrm{g} / \mathrm{L}$
$\mu \mathrm{g} / \mathrm{L}$
$\mu \mathrm{g} / \mathrm{L}$
$\mu \mathrm{g} / \mathrm{L}$

$\mu \mathrm{g} / \mathrm{L}$

$\mu g / L$

$\mu \mathrm{g} / \mathrm{L}$

$\mu g / L$

$\mu \mathrm{g} / \mathrm{L}$

$\mu \mathrm{g} / \mathrm{L}$

$\mu g / L$

$\mu \mathrm{g} / \mathrm{L}$

$\mu g / L$

$\mu g / L$

$\mu g / L$

$\mu \mathrm{g} / \mathrm{L}$

$\mu \mathrm{g} / \mathrm{L}$

$\mu g / L$

$\mu \mathrm{g} / \mathrm{L}$

$\mu \mathrm{g} / \mathrm{L}$

$\mu \mathrm{g} / \mathrm{L}$

$\mu \mathrm{g} / \mathrm{L}$

$\mu g / L$

$\mu g / L$

$\mu \mathrm{g} / \mathrm{L}$

$\mu g / L$

$\mu g / L$

$\mu \mathrm{g} / \mathrm{L}$

$\mu \mathrm{g} / \mathrm{L}$

\begin{tabular}{|c|c|}
\hline 10 & EPA6010 \\
\hline 100 & EPA8270 \\
\hline 100 & EPA8270 \\
\hline 50 & EPA8240 \\
\hline 50 & EPA8240 \\
\hline 10 & EPA8150 \\
\hline 100 & EPA8270 \\
\hline 0.21 & EPA8080 \\
\hline 0.11 & EPA8080 \\
\hline 0.11 & EPA8080 \\
\hline 100 & EPA8270 \\
\hline 100 & EPA8270 \\
\hline 100 & EPA8270 \\
\hline 90 & EPA6010 \\
\hline 0.11 & EPA8080 \\
\hline 0.20 & EPA7470 \\
\hline 1.1 & EPA8080 \\
\hline 100 & EPA824C \\
\hline 100 & EPA8270 \\
\hline 500 & EPA8270 \\
\hline 100 & EPA8270 \\
\hline 100 & EPA6010 \\
\hline 10 & EPA6010 \\
\hline 50 & EPA8240 \\
\hline 11 & EPA8080 \\
\hline 11 & EPA8080 \\
\hline 5.0 & EPA8150 \\
\hline 50 & EPA8240 \\
\hline 500 & EPA8270 \\
\hline 100 & EPA8270 \\
\hline 5.0 & EPA8150 \\
\hline 100 & 5010 \\
\hline
\end{tabular}

Sample D: 100328

\section{SAMPLE NAME: D43110707}

Location (SRS Coordinates): 18576.98E 66634.66N

Ground Elevation Above MSL: $132.8 \mathrm{ft}$

Depth of Core Interval: 10.00 to $12.00 \mathrm{ft}$

Sample Type: Normal

Sample Color: 10R 4/6.

\section{Constituent}

Total Metals

Arsenic

Barium

Cadmium

Chromium
Sample Matrix: Soil

USC Soil Classification: CL
Sample Moisture: Damp Percent Solids: 86.50

D. Limit Method

\section{RQ AQ $\quad$ B Result Unit}

$\begin{array}{ll}\mathrm{U} & 2.7 \\ \mathrm{U} & 20 \\ & 0.49 \\ & 19\end{array}$

$\mathrm{mg} / \mathrm{kg}$
$\mathrm{mg} / \mathrm{kg}$
$\mathrm{mg} / \mathrm{kg}$
$\mathrm{mg} / \mathrm{kg}$

1.1

20

0.49

0.98
EPA7060

EPA6010

EPA6010 
SAMPLE NAME: D43110707 (Continued)

RQ AQ B Result
Unit

D. Limit Method

Total Metals

Copper
Lead
Manganese
Mercury
Nickel
Selenium
Silver

$B / N / A$ Extractables (including PAH and phenols)

Acenaphthene

Acenaphthylene

Anthracene

Benzo(a)anthracene

Benzo(b)fluoranthene

Benzo(k)fluoranthene

Benzoic acid

Benzo(g,h.i)perylene

Benzo(a)pyrene

Benzyl alcohol

Bis(2-chloroethoxy) methane

Bis(2-chloroethyl) ether

Bis(2-chloroisopropyl) ether

Bis(2-ethylhexyl) phthalate

4-Bromophenyl phenyl ether

Butylbenzy! phthalate

4-Chloroaniline

4-Chloro-m-cresol

2-Chloronaphthalene

2-Chlorophenol

4-Chlorophenyl phenyl ether

Chrysene

o-Cresol (2-Methylphenol)

p-Cresol (4-Methylphenol)

Dibenz $(\mathrm{a}, \mathrm{h})$ anthracene

Dibenzofuran

Di-n-butyl phthalate

1,2-Dichlorobenzene

1.3-Dichlorobenzene

1.4-Dichlorobenzene :"

3,3'-Dichlorobenzidine

2,4-Dichlorophenol

Diechyl phthalate

2,4-Dimethyl phenol

Dimethyl phthalate

2,4-Dinitrophenol

2,4-Dinitrotoluene

2,6-Dinitrotoluene

Di-n-octyl phthalate

Fluoranthene
U

U

U

U

U

U

U

U

U

$\mathrm{U}$

U

U

U

U

U

U

U

U

U

U

U

U

U

UJ

$\mathrm{U}$

U

U

U

U

U

U

U

U

U

J

J
390

390

390

390

390

390

1,900

390

390

390

390

390

390

390

390

390

390

390

390

390

390

390

390

390

390

390

110

390

390

390

770

390

390

390

390

1,900

390

390

44

390

$\mathrm{mg} / \mathrm{kg}$
$\mathrm{mg} / \mathrm{kg}$
$\mathrm{mg} / \mathrm{kg}$
$\mathrm{mg} / \mathrm{kg}$
$\mathrm{mg} / \mathrm{kg}$
$\mathrm{mg} / \mathrm{kg}$
$\mathrm{mg} / \mathrm{kg}$

2.5

0.34

1.5

0.12

3.9

0.56

0.98

EPA6010

EPA7421

EPA6010

EPA7470

EPA6010

EPA7740

EPA6010

EPA8270

390

EPA8270

$\mu g / k g$

390

EPA8270

$\mu g / k g$

$\mu \mathrm{g} / \mathrm{kg}$

$\mu \mathrm{g} / \mathrm{kg}$

$\mu \mathrm{g} / \mathrm{kg}$

$\mu \mathrm{g} / \mathrm{kg}$

$\mu \mathrm{g} / \mathrm{kg}$

$\mu \mathrm{g} / \mathrm{kg}$

$\mu \mathrm{g} / \mathrm{kg}$

$\mu \mathrm{g} / \mathrm{kg}$

$\mu \mathrm{g} / \mathrm{kg}$

$\mu \mathrm{g} / \mathrm{kg}$

$\mu \mathrm{g} / \mathrm{kg}$

$\mu g / \mathrm{kg}$

$\mu \mathrm{g} / \mathrm{kg}$

$\mu \mathrm{g} / \mathrm{kg}$

$\mu \mathrm{g} ' \mathrm{~kg}$

$\mu g / \mathrm{kg}$

$\mu \mathrm{g} / \mathrm{kg}$

$\mu \mathrm{g} / \mathrm{kg}$

$\mu \mathrm{g} / \mathrm{kg}$

$\mu \mathrm{g} / \mathrm{kg}$

$\mu \mathrm{g} / \mathrm{kg}$

$\mu \mathrm{g} / \mathrm{kg}$

$\mu \mathrm{g} / \mathrm{kg}$

$\mu \mathrm{g} / \mathrm{kg}$

$\mu g / \mathrm{kg}$

$\mu g / k g$

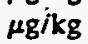

$\mu \mathrm{g} / \mathrm{kg}$

$\mu \mathrm{g} / \mathrm{kg}$

$\mu g / k g$

$\mu \mathrm{g} / \mathrm{kg}$

$\mu \mathrm{g} / \mathrm{kg}$

$\mu \mathrm{g} / \mathrm{kg}$

$\mu g / \mathbf{k g}$

$\mu \mathrm{g} / \mathrm{kg}$

$\mu \mathrm{g} / \mathrm{kg}$

$\mu \mathrm{g} / \mathrm{kg}$

EPA8270

EPA8270

EPA8270

EPA8270

EPA8270

EPA8270

EPA8270

EPA8270

EPA8270

EPA8270

EPA8270

EPA8270

EPA8270

EPA8270

EPA8270

EPA8270

EPA8270

EPA8270

EPA8270

EPA8270

EPA8270

EPA8270

EPA8270

EPA8270

EPA8270

EPA8270

EPA8270

EPA8270

EPA8270

EPA8270

EPA8270

EPA8270

EPA8270

EPA8270

EPA8270

EPA8270

EPA8270 


$\begin{array}{lllll}\text { Constituent RQ AQ B Result Unit D. Limit Method } & \text { B }\end{array}$

$B / N / A$ Extractables (including $P A H$ and phenols)

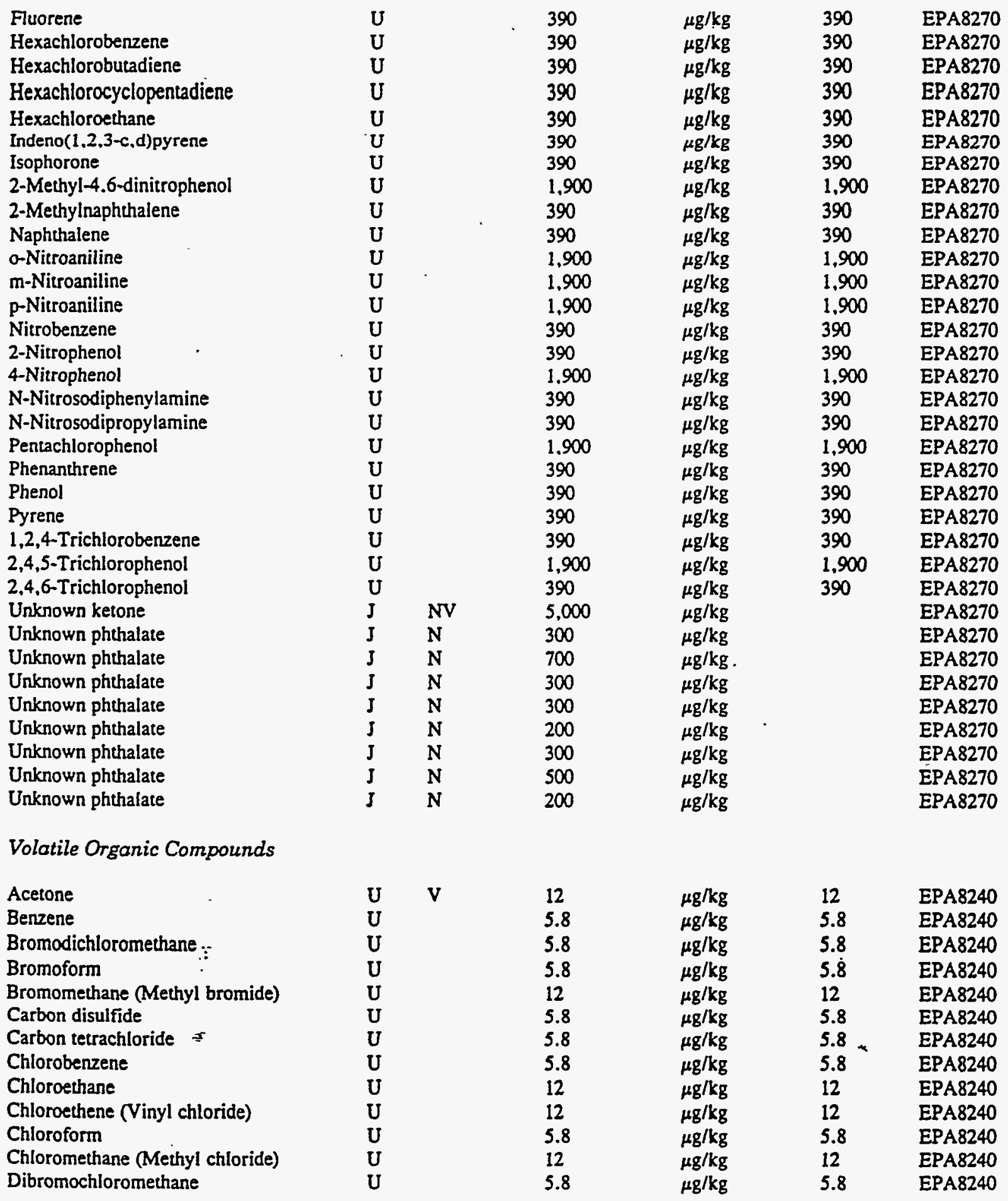


SAMPLE NAME: D43ï10707 (Continued)

\section{Constituent}

Volatile Organic Compounds
Sample ID: 100328

RQ AQ B Result Unit D. Limit Method

1.1-Dichloroethane
1,2-Dichloroethane
1,1-Dichloroethylene
1,2-Dichloroethylene
Dichloromethane (Methylene chloride)
1.2-Dichloropropane
cis-1,3-Dichloropropene
trans-1,3-Dichloropropene
Ethylbenzene
2-Hexanone
Methyl ethyl ketone
Methyl isobutyl ketone
Styrene
1,1,2,2-Tetrachloroethane
Tetrachloroethylene
Toluene
1.1,1-Trichloroethane
1,1,2-Trichloroethane
Trichloroethylene
Vinyl acetate
Xylenes

Pesticides/PCBs and Dioxins/Furans

$\begin{array}{rl}5.8 \\ \\ & 5.8 \\ & 5.8 \\ 5.8 & 21 \\ 5.8 \\ 5.8 \\ 5.8 \\ 5.8 \\ 12 \\ 12 \\ 12 \\ 5.8 \\ 5.8 \\ 5.8 \\ 5.8 \\ 5.8 \\ 5.8 \\ 5.8 \\ 12\end{array}$

$$
\begin{aligned}
& \mu \mathrm{g} / \mathrm{kg} . \\
& \mu \mathrm{g} / \mathrm{kg} \\
& \mu \mathrm{g} / \mathrm{kg} \\
& \mu \mathrm{g} / \mathrm{kg} \\
& \mu \mathrm{g} / \mathrm{kg} \\
& \mu \mathrm{g} / \mathrm{kg} \\
& \mu \mathrm{gg} / \mathrm{kg} \\
& \mu \mathrm{g} / \mathrm{kg} \\
& \mu \mathrm{g} / \mathrm{kg} \\
& \mu \mathrm{g} / \mathrm{kg} \\
& \mu \mathrm{g} / \mathrm{kg} \\
& \mu \mathrm{g} / \mathrm{kg} \\
& \mu \mathrm{g} / \mathrm{kg} \\
& \mu \mathrm{g} / \mathrm{kg} \\
& \mu \mathrm{g} / \mathrm{kg} \\
& \mu \mathrm{g} / \mathrm{kg} \\
& \mu \mathrm{g} / \mathrm{kg} \\
& \mu \mathrm{g} / \mathrm{kg} \\
& \mu \mathrm{g} \\
& \mu \mathrm{kg} \\
& \mu \mathrm{kg} \\
& \mu \mathrm{g} / \mathrm{kg}
\end{aligned}
$$

\section{8}

5.8

5.8

5.8

5.8

5.8

5.8

5.8

5.8

12

12

12

5.8

5.8

5.8

5.8

5.8

5.8

5.8

12

5.8
EPA8240

EPA8240

EPA8240

EPA8240

EPA8240

EPA8240

EPA8240

EPA8240

EPA8240

EPA8240

EPA8240

EPA8240

EPA8240

EPA8240

EPA8240

EPA8240

EPA8240

EPA8240

EPA8240

EPA8240

EPA8240
Aldrin

alpha-Benzene hexachloride

beta-Benzene hexachloride

delta-Benzene hexachloride

alpha-Chlordane

gamma-Chlordane

p.p'-DDD

p.p'-DDE

P.p'-DDT

Dieldrin

Endosulfan I

Endosulfan II

Endosulfan sulfate

Endrin

Endrin ketone

Heptachlor

Heptachlor epoxide

Hepeachlorodibenzo-p-dioxin isomers

Heptachlorodibenzo-p-furan isomers

Hexachlorodibenzo-p-dioxin isomers

Hexachlorodibenzo-p-furan isomers

Lindane

Methoxychlor

Octachlorodibenzo-p-dioxin

Octachlorodibenzo-p-furan

PCB 1016

\section{$\mathrm{U}$}

U

$U$

U

$\mathrm{U}$

$U$

$\mathrm{U}$

U

$\mathrm{U}$

U

U

U

U

U

U

U

U

J

U

U

U

U

U

U

U
1.8

1.8

1.8

1.8

1.8

1.8

3.6

3.6

3.6

3.6

1.8

3.6

3.6

3.6

3.6

1.8

1.8

0.10

0.10

0.10

0.10

1.8

18

22

0.60

36 $\mu \mathrm{g} / \mathrm{kg}$

$\mu \mathrm{g} / \mathrm{kg}$

$\mu g / k g$

$\mu \mathrm{g} / \mathrm{kg}$

$\mu g / \mathrm{kg}$

$\mu \mathrm{g} / \mathrm{kg}$

$\mu \mathrm{g} / \mathrm{kg}$

$\mu \mathrm{g} / \mathrm{kg}$

$\mu g / \mathrm{kg}$

$\mu g / k g$

$\mu g / k g$

$\mu \mathrm{g} / \mathrm{kg}$

$\mu \mathrm{g} / \mathrm{kg}$

$\mu g / k g$

$\mu \mathrm{g} / \mathrm{kg}$

$\mu g / \mathrm{kg}$

$\mu \mathrm{g} / \mathrm{kg}$

$\mathrm{ng} / \mathrm{g}$

$\mathrm{ng} / \mathrm{g}$

$\mathrm{ng} / \mathrm{g}$

$\mathrm{ng} / \mathrm{g}$

$\mu \mathrm{g} / \mathrm{kg}$

$\mu g / \mathrm{kg}$

$\mathrm{ng} / \mathrm{g}$

$\mathrm{ng} / \mathrm{g}$

$\mu g / \mathrm{kg}$
1.8

1.8

1.8

1.8

1.8

1.8

3.6

3.6

3.6

3.6

1.8

3.6

3.6

3.6

3.6

1.8

1.8

0.10

0.10

0.10

0.10

1.8

18

0.90

0.60

36

EPA8080

EPA8080

EPA8080

EPA8080

EPA8080

EPA8080

EPA8080

EPA8080

EPA8080

EPA8080

EPA8080

EPA8080

EPA8080

EPA8080

EPA8080

EPA8080

EPA8080

EPA8280

EPA8280

EPA8280

EPA8280

EPA8080

EPA8080

EPA8280

EPA8280

EPA8080 
SAMPLE NAME: D43i110707 (Continued)

Constituent

RQ AQ B Result

Pesticides/PCBs and Dioxins/Furans

PCB 1221

PCB 1232

PCB 1242

PCB 1248

PCB 1254

PCB 1260

Pentachlorodibenzo-p-dioxin isomers

Pentachlorodibenzo-p-furan isomers

2.3.7,8-TCDD

2,3,7,8-TCDF

Tetrachlorodibenzo-p-dioxin isomers

Tetrachlorodibenzo-p-furan isomers

Toxaphene

$U$
$U$
$U$
$U$
$U$
$U$
$U$
$U$
$U$
$U$
$U$
$U$
$U$

Sample ID: 100328
SAMPLE NAME: D43110801

Location (SRS Coordinates): 18542.87E 66623.81N

Ground Elevation Above MSL: $132.8 \mathrm{ft}$

Depth of Core Interval: 0.00 to $0.50 \mathrm{ft}$

Sample Type: Normal

Sample Color: 10R 3/4

Constituient

Physical Parameters

Cation exchange capacity

$\mathrm{pH}$

Specific conductance

Total organic carbon

Total Metals

Arsenic

Barium

Cadmium

Chromium

Copper

Lead

Manganese

Mercury

Nickel

Selenium

Silver

Unit D. Limit Method

$\begin{array}{llll}73 & \mu \mathrm{g} / \mathrm{kg} & 73 & \text { EPA8080 } \\ 36 & \mu \mathrm{g} / \mathrm{kg} & 36 & \text { EPA8080 } \\ 36 & \mu \mathrm{g} / \mathrm{kg} & 36 & \text { EPA8080 } \\ 36 & \mu \mathrm{g} / \mathrm{kg} & 36 & \text { EPA8080 } \\ 36 & \mu \mathrm{g} / \mathrm{kg} & 36 & \text { EPA8080 } \\ 36 & \mu \mathrm{g} / \mathrm{kg} & 36 & \text { EPA8080 } \\ 0.10 & \mathrm{ng} / \mathrm{g} & 0.10 & \text { EPA8280 } \\ 0.10 & \mathrm{ng} / \mathrm{g} & 0.10 & \text { EPA8280 } \\ 0.10 & \mathrm{ng} / \mathrm{g} & 0.10 & \text { EPA8280 } \\ 0.10 & \mathrm{ng} / \mathrm{g} & 0.10 & \text { EPA8280 } \\ 0.10 & \mathrm{ng} / \mathrm{g} & 0.10 & \text { EPA8280 } \\ 0.10 & \mathrm{ng} / \mathrm{g} & 0.10 & \text { EPA8280 } \\ 180 & \mu \mathrm{g} / \mathrm{kg} & 180 & \text { EPA8080 }\end{array}$

Sample ID: 100329

Sample Matrix: Soil

USC Soil Classification: SC

RQ AQ B Result

Unit

D. Limit Method Percent Solids: 94.30
Sample Moisture: Dry

$\begin{array}{llll}7.9 & \mathrm{meq} / 100 \mathrm{~g} & 2.0 & \text { EPA9080 } \\ 4.6 & \mathrm{pH} & 0.010 & \text { EPA9045 } \\ 33 & \mu \mathrm{S} / \mathrm{cm} & 1.0 & \text { EPA9050 } \\ 5.500 & \mathrm{mg} / \mathrm{kg} & 200 & \text { EPA415.1 }\end{array}$

1.2

$\mathrm{mg} / \mathrm{kg}$

$\mathrm{mg} / \mathrm{kg}$

$\mathrm{mg} / \mathrm{kg}$

$\mathrm{mg} / \mathrm{kg}$

$\mathrm{mg} / \mathrm{kg}$

$\mathrm{mg} / \mathrm{kg}$

$\mathrm{mg} / \mathrm{kg}$

$\mathrm{mg} / \mathrm{kg}$

$\mathrm{mg} / \mathrm{kg}$

$\mathrm{mg} / \mathrm{kg}$

$\mathrm{mg} / \mathrm{kg}$
1.0

EPA7060

20

0.51

1:0

2.6

0.31

1.5

0.11

4.1

0.52

1.0
EPA6010

EPA6010

EPA6010

EPA6010

EPA7421

EPA6010

EPA7470

EPA6010

EPA7740

EPA6010 
SAMPLE NAME: D43̈110801 (Continued)

RQ AQ B Result

350

350

350

350

350

350

1,700

350

350

350

350

350

350

350

350

350

350

350

350

350

350

350

350

350

350

Dibenz(a,h)anthracene

Dibenzofuran

Di-n-butyl phthalate

1.2-Dichlorobenzene

1,3-Dichlorobenzene

1.4-Dichlorobenzene

3,3'-Dichlorobenzidine

2,4-Dichlorophenol

Diethyl phthalate

2,4-Dimethyl phenol

Dimethyl phthalate

2,4-Dinitrophenol

2,4-Dinitrotoluene

2,6-Dinitsotoluene

Di-n-octyl phthalate

Fluoranthene

Fluorene

Hexachlorobenzene

Hexachlorobutadiene,

Hexachlorocyclopentadiene

Hexachloroethane

Indeno(1,2,3-c,d)pyrene

Isophorone

2-Methyl-4,6-dinitrophenol

2-Methylnaphthalene

Naphthalene
350

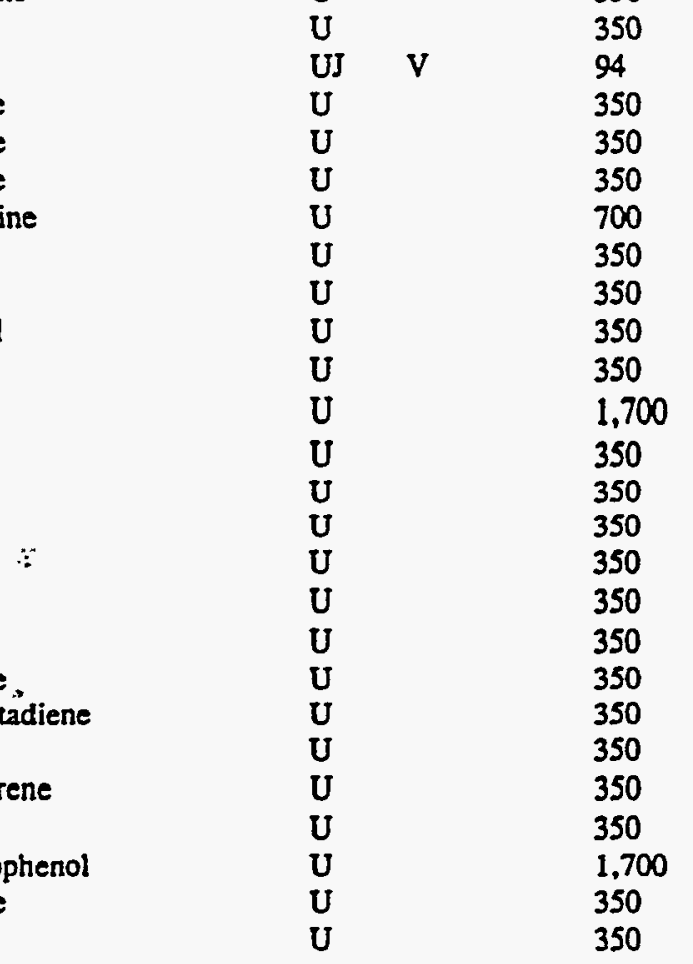

Unit

D. Limit Method

\begin{tabular}{|c|c|c|}
\hline$\mu \mathrm{g} / \mathrm{kg}$ & 350 & EPA8270 \\
\hline$\mu \mathrm{g} / \mathrm{kg}$ & 350 & EPA8270 \\
\hline$\mu \mathrm{g} / \mathrm{kg}$ & 350 & EPA8270 \\
\hline$\mu \mathrm{g} / \mathrm{kg}$ & 350 & EPA8270 \\
\hline$\mu \mathrm{g} / \mathrm{kg}$ & 350 & EPA8270 \\
\hline$\mu \mathrm{g} / \mathrm{kg}$ & 350 & EPA8270 \\
\hline$\mu g / \mathrm{kg}$ & 1,700 & EPA8270 \\
\hline$\mu \mathrm{g} / \mathrm{kg}$ & 350 & EPA8270 \\
\hline$\mu \mathrm{g} / \mathrm{kg}$ & 350 & EPA8270 \\
\hline$\mu g / \mathrm{kg}$ & 350 & EPA8270 \\
\hline$\mu g / \mathrm{kg}$ & 350 & EPA8270 \\
\hline$\mu \mathrm{g} / \mathrm{kg}$ & 350 & EPA8270 \\
\hline$\mu \mathrm{g} / \mathrm{kg}$ & 350 & EPA8270 \\
\hline$\mu \mathrm{g} / \mathrm{kg}$ & 350 & EPA8270 \\
\hline$\mu \mathrm{g} / \mathrm{kg}$ & 350 & EPA8270 \\
\hline$\mu \mathrm{g} / \mathrm{kg}$ & 350 & EPA8270 \\
\hline$\mu \mathrm{g} / \mathrm{kg}$ & 350 & EPA8270 \\
\hline$\mu \mathrm{g} / \mathrm{kg}$ & 350 & EPA8270 \\
\hline$\mu \mathrm{g} / \mathrm{kg}$ & 350 & EPA8270 \\
\hline$\mu g / k g$ & 350 & EPA8270 \\
\hline$\mu \mathrm{g} / \mathrm{kg}$ & 350 & EPA8270 \\
\hline$\mu \mathrm{g} / \mathrm{kg}$ & 350 & EPA8270 \\
\hline$\mu \mathrm{g} / \mathrm{kg}$ & 350 & EPA8270 \\
\hline$\mu g / \mathrm{kg}$ & 350 & EPA8270 \\
\hline$\mu \mathrm{g} / \mathrm{kg}$ & 350 & EPA8270 \\
\hline$\mu g / k g$ & 350 & EPA8270 \\
\hline$\mu \mathrm{g} / \mathrm{kg}$ & 350 & EPA8270 \\
\hline$\mu \mathrm{g} / \mathrm{kg}$ & 350 & EPA8270 \\
\hline$\mu \mathrm{g} / \mathrm{kg}$ & 350 & EPA8270 \\
\hline$\mu \mathrm{g} / \mathrm{kg}$ & 350 & EPA8270 \\
\hline$\mu \mathrm{g} / \mathrm{kg}$ & 700 & EPA8270 \\
\hline$\mu g / k g$ & 350 & EPA8270 \\
\hline$\mu \mathrm{g} / \mathrm{kg}$ & 350 & EPA8270 \\
\hline$\mu \mathrm{g} / \mathrm{kg}$ & 350 & EPA8270 \\
\hline$\mu \mathrm{g} / \mathrm{kg}$ & 350 & EPA8270 \\
\hline$\mu \mathrm{g} / \mathrm{kg}$ & 1,700 & EPA8270 \\
\hline$\mu \mathrm{g} / \mathrm{kg}$ & 350 & EPA8270 \\
\hline$\mu \mathrm{g} / \mathrm{kg}$ & 350 & EPA8270 \\
\hline$\mu \mathrm{g} / \mathrm{kg}$ & 350 & EPA8270 \\
\hline$\mu \mathrm{g} / \mathrm{kg}$ & 350 & EPA8270 \\
\hline$\mu \mathrm{g} / \mathrm{kg}$ & 350 & EPA8270 \\
\hline$\mu \mathrm{g} / \mathrm{kg}$ & 350 & EPA8270 \\
\hline$\mu \mathrm{g} / \mathrm{kg}$ & 350 & EPA8270 \\
\hline$\mu \mathrm{g} / \mathrm{kg}$ & $35 a_{2}$ & EPA8270 \\
\hline$\mu \mathrm{g} / \mathrm{kg}$ & 350 & EPA8270 \\
\hline$\mu \mathrm{g} / \mathrm{kg}$ & 350 & EPA8270 \\
\hline$\mu \mathrm{g} / \mathrm{kg}$ & 350 & EPA8270 \\
\hline$\mu \mathrm{g} / \mathrm{kg}$ & 1,700 & EPA8270 \\
\hline$\mu \mathrm{g} / \mathrm{kg}$ & 350 & EPA8270 \\
\hline & 350 & EPA8270 \\
\hline
\end{tabular}


SAMPLE NAME: D43\%10801 (Continued)

Constituent

RQ

AQ

Sample ID: 100329

$B / N / A$ Extractables (including $P A H$ and phenols)

o-Nitroaniline

m-Nitroaniline

p-Nitroaniline

Nitrobenzene

2-Nitrophenol

4-Nitrophenol

N-Nitrosodiphenylamine

N-Nitrosodipropylamine

Pentachlorophenol

Phenanthrene

Phenol

Pyrene

1,2,4-Trichlorobenzene

2,4,5-Trichlorophenol

2,4,6-Trichlorophenol

Unknown arom. hydrocarbon

Unknown hydrocarbon

Unknown

Unknown

Unknown

Unknown

Unknown

Unknown

Unknown acid

Unknown ketone

Volatile Organic Compounds

Acetone

Benzene

Bromodichloromethane

Bromoform

Bromomethane (Methyl bromide)

Carbon disulfide

Carbon tetrachloride

Chlorobenzene

Chloroethane

Chloroethene (Vinyl chloride)

Chloroform

Chloromethane (Methyl chloride)

Dibromochloromethane:

1,1-Dichloroethane

1,2-Dichloroethane

1,1-Dichloroethylene

1,2-Dichloroechylene "

Dichloromethane (Methylene chloride)

1,2-Dichloropropane

cis-1,3-Dichloropropene

trans-1,3-Dichloropropene

Ethylbenzene

$\begin{array}{lll}\text { U } & & 1,700 \\ \text { U } & & 1,700 \\ \text { U } & & 1,700 \\ \text { U } & & 350 \\ \text { U } & & 350 \\ \text { U } & & 1,700 \\ \text { U } & & 350 \\ \text { U } & & 350 \\ \text { U } & & 1,700 \\ \text { U } & & 350 \\ \text { U } & & 350 \\ \text { U } & & 350 \\ \text { U } & & 350 \\ \text { U } & & 1,700 \\ \text { U } & & 350 \\ \text { J } & N & 100 \\ \text { J } & \text { N } & 300 \\ \text { J } & \text { N } & 400 \\ \text { J } & \text { N } & 200 \\ \text { J } & \text { N } & 600 \\ \text { J } & \text { N } & 200 \\ \text { J } & \text { N } & 200 \\ \text { J } & \text { N } & 300 \\ \text { J } & \text { N } & 200 \\ \text { J } & \text { NV } & 5,000\end{array}$

6.6

5.3

5.3

5.3

11

5.3

5.3

5.3

11

11

5.3

11

5.3

5.3

5.3

5.3

5.3

16

5.3

5.3

5.3

5.3

$$
\begin{aligned}
& \mu \mathrm{g} / \mathrm{kg} \\
& \mu \mathrm{g} / \mathrm{kg} \\
& \mu \mathrm{g} / \mathrm{kg} \\
& \mu \mathrm{g} / \mathrm{kg} \\
& \mu \mathrm{g} / \mathrm{kg} \\
& \mu \mathrm{g} / \mathrm{kg} \\
& \mu \mathrm{g} / \mathrm{kg} \\
& \mu \mathrm{g} / \mathrm{kg} \\
& \mu \mathrm{g} / \mathrm{kg} \\
& \mu \mathrm{g} / \mathrm{kg} \\
& \mu \mathrm{g} / \mathrm{kg} \\
& \mu \mathrm{g} / \mathrm{kg} \\
& \mu \mathrm{g} / \mathrm{kg} \\
& \mu \mathrm{g} / \mathrm{kg} \\
& \mu \mathrm{g} / \mathrm{kg} \\
& \mu \mathrm{g} / \mathrm{kg} \\
& \mu \mathrm{g} / \mathrm{kg} \\
& \mu \mathrm{g} / \mathrm{kg} \\
& \mu \mathrm{g} / \mathrm{kg} \\
& \mu \mathrm{g} / \mathrm{kg} \\
& \mu \mathrm{g} / \mathrm{kg} \\
& \mu \mathrm{g} / \mathrm{kg} \\
& \mu \mathrm{g} / \mathrm{kg} \\
& \mu \mathrm{g} / \mathrm{kg} \\
& \mu \mathrm{g} / \mathrm{kg}
\end{aligned}
$$

1.700

1,700

1,700

350

350

1,700

350

350

1,700

350

350

350

350

1,700

350

EPA8270

EPA8270

EPA8270

EPA8270

EPA8270

EPA8270

EPA8270

EPA8270

EPA8270

EPA8270

EPA8270

EPA8270

EPA8270

EPA8270

EPA8270

EPA8270

EPA8270

EPA8270

EPA8270

EPA8270

EPA8270

EPA8270

EPA8270

EPA8270

EPA8270

$\mu \mathrm{g} / \mathrm{kg}$

$\mu \mathrm{g} / \mathrm{kg}$

$\mu \mathrm{g} / \mathrm{kg}$

$\mu \mathrm{g} / \mathrm{kg}$

$\mu \mathrm{g} / \mathrm{kg}$

$\mu g / \mathrm{kg}$

$\mu \mathrm{g} / \mathrm{kg}$

$\mu \mathrm{g} / \mathrm{kg}$

$\mu \mathrm{g} / \mathrm{kg}$

$\mu \mathrm{g} / \mathrm{kg}$

$\mu \mathrm{g} / \mathrm{kg}$

$\mu \mathrm{g} / \mathrm{kg}$

$\mu \mathrm{g} / \mathrm{kg}$

$\mu \mathrm{g} / \mathrm{kg}$

$\mu \mathrm{g} / \mathrm{kg}$

$\mu \mathrm{g} / \mathrm{kg}$

$\mu \mathrm{g} / \mathrm{kg}$

$\mu \mathrm{g} / \mathrm{kg}$

$\mu \mathrm{g} / \mathrm{kg}$

$\mu g / \mathrm{kg}$

$\mu \mathrm{g} / \mathrm{kg}$

$\mu \mathrm{g} / \mathrm{kg}$
EPA8240

EPA8240

EPA8240

EPA8240

EPA8240

EPA8240

EPA8240

EPA8240

EPA8240

EPA8240

EPA8240

EPA8240

EPA8240

EPA8240

EPA8240

EPA8240

EPA8240

EPA8240

EPA8240

EPA8240

EPA8240

EPA8240 


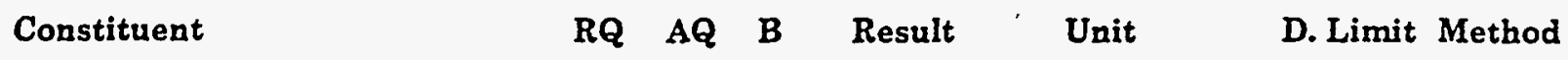

Volatile Organic Compounds

2-Hexanone
Methyl ethyl ketone
Methyl isobutyl ketone
Styrene
$1,1,2,2$-Tetrachloroethane
Tetrachloroethylene
Toluene
1,1,1-Trichloroethane
1,1,2-Trichloroethane
Trichlorothylene
Vinyl acetate
Xylenes

$\begin{array}{lllll}\mathrm{U} & 11 & \mu g / \mathrm{kg} & 11 & \text { EPA8240 } \\ \mathrm{U} & 11 & \mu \mathrm{g} / \mathrm{kg} & 11 & \text { EPA8240 } \\ \mathrm{U} & 11 & \mu g / \mathrm{kg} & 11 & \text { EPA8240 } \\ \mathrm{U} & 5.3 & \mu g / \mathrm{kg} & 5.3 & \text { EPA8240 } \\ \mathrm{U} & 5.3 & \mu g / \mathrm{kg} & 5.3 & \text { EPA8240 } \\ \mathrm{U} & 5.3 & \mu \varepsilon \mathrm{kg} & 5.3 & \text { EPA8240 } \\ \mathrm{U} & 5.3 & \mu \varepsilon \mathrm{kg} & 5.3 & \text { EPA8240 } \\ \mathrm{U} & 5.3 & \mu \mathrm{kg} & 5.3 & \text { EPA8240 } \\ \mathrm{U} & 5.3 & \mu \mathrm{kg} & 5.3 & \text { EPA8240 } \\ \mathrm{U} & 5.3 & \mu g / \mathrm{kg} & 5.3 & \text { EPA8240 } \\ \mathrm{U} & 11 & \mu g / \mathrm{kg} & 11 & \text { EPA8240 } \\ \mathrm{U} & 5.3 & \mu \mathrm{kg} & 5.3 & \text { EPA8240 }\end{array}$

Pesticides/PCBs and Dioxins/Furans

Aldrin
alpha-Benzene hexachloride
beta-Benzene hexachloride
delta-Benzene hexachloride
alpha-Chlordane
gamma-Chlordane
p.p'-DDD
p,p'-DDE
p.p'-DDT
Dieldrin
Endosulfan I
Endosulfan II
Endosulfan sulfate
Endrin
Endrin ketone
Heptachlor
Heptachlor epoxide
Heptachlorodibenzo-p-dioxin isomers
Heptachlorodibenzo-p-furan isomers
Hexachlorodibenzo-p-dioxin isomers
Hexachlorodibenzo-p-furan isomers
Lindane
Methoxychlor
Octachlorodibenzo-p-dioxin
Octachlorodibenzo-p-furan
PCB 1016
PCB 1221
PCB 1232
PCB 1242
PCB 1248
PCB 1254
PCB 1260
Pentachlorodibenzo-p-dioxin isomers
Pentachlorodibenzo-p-furan isomers
$2,3,7,8-T C D D$

$\begin{array}{lllll}\mathrm{U} & 1.7 & \mu \mathrm{g} / \mathrm{kg} & 1.7 & \text { EPA8080 } \\ \mathrm{U} & 1.7 & \mu \mathrm{g} / \mathrm{kg} & 1.7 & \text { EPA8080 } \\ \mathrm{U} & 1.7 & \mu \mathrm{g} / \mathrm{kg} & 1.7 & \text { EPA8080 } \\ \mathrm{U} & 1.7 & \mu \mathrm{g} / \mathrm{kg} & 1.7 & \text { EPA8080 } \\ \mathrm{U} & 1.7 & \mu \mathrm{g} / \mathrm{kg} & 1.7 & \text { EPA8080 } \\ \mathrm{U} & 1.7 & \mu \mathrm{g} / \mathrm{kg} & 1.7 & \text { EPA8080 } \\ \mathrm{U} & 3.5 & \mu \mathrm{g} / \mathrm{kg} & 3.5 & \text { EPA8080 } \\ \mathrm{J} & 0.69 & \mu \mathrm{g} / \mathrm{kg} & 3.5 & \text { EPA8080 } \\ \mathrm{U} & 3.5 & \mu \mathrm{g} / \mathrm{kg} & 3.5 & \text { EPA8080 } \\ \mathrm{U} & 3.5 & \mu \mathrm{g} / \mathrm{kg} & 3.5 & \text { EPA8080 } \\ \mathrm{J} & 1.0 & \mu \mathrm{g} / \mathrm{kg} & 1.7 & \text { EPA8080 } \\ \mathrm{U} & 3.5 & \mu \mathrm{g} / \mathrm{kg} & 3.5 & \text { EPA8080 } \\ & 4.2 & \mu \mathrm{g} / \mathrm{kg} & 3.5 & \text { EPA8080 } \\ \mathrm{U} & 3.5 & \mu \mathrm{g} / \mathrm{kg} & 3.5 & \text { EPA8080 } \\ \mathrm{U} & 3.5 & \mu \mathrm{g} / \mathrm{kg} & 3.5 & \text { EPA8080 } \\ \mathrm{U} & 1.7 & \mu \mathrm{g} / \mathrm{kg} & 1.7 & \text { EPA8080 } \\ \mathrm{U} & 1.7 & \mu \mathrm{g} / \mathrm{kg} & 1.7 & \text { EPA8080 } \\ \mathrm{U} & 0.10 & \mathrm{ng} / \mathrm{g} & 0.10 & \text { EPA8280 } \\ \mathrm{U} & 0.20 & \mathrm{ng} / \mathrm{g} & 0.20 & \text { EPA8280 } \\ \mathrm{U} & 0.10 & \mathrm{ng} / \mathrm{g} & 0.10 & \text { EPA8280 } \\ \mathrm{U} & 0.10 & \mathrm{ng} / \mathrm{g} & 0.10 & \text { EPA8280 } \\ \mathrm{U} & 1.7 & \mu \mathrm{g} / \mathrm{kg} & 1.7 & \text { EPA8080 } \\ \mathrm{U} & 17 & \mu \mathrm{g} / \mathrm{kg} & 17 & \text { EPA8080 } \\ \mathrm{J} & 0.70 & \mathrm{ng} / \mathrm{g} & 0.10 & \text { EPA8280 } \\ \mathrm{U} & 0.10 & \mathrm{ng} / \mathrm{g} & 0.10 & \text { EPA8280 } \\ \mathrm{U} & 35 & \mu \mathrm{g} / \mathrm{kg} & 35 & \text { EPA8080 } \\ \mathrm{U} & 69 & \mu \mathrm{g} / \mathrm{kg} & 69 & \text { EPA8080 } \\ \mathrm{U} & 35 & \mu \mathrm{g} / \mathrm{kg} & 35 & \text { EPA8080 } \\ \mathrm{U} & 35 & \mu \mathrm{g} / \mathrm{kg} & 35 & \text { EPA8080 } \\ \mathrm{U} & 35 & \mu \mathrm{g} / \mathrm{kg} & 35 & \text { EPA8080 } \\ \mathrm{U} & 35 & \mu \mathrm{g} / \mathrm{kg} & 35 & \text { EPA8080 } \\ \mathrm{U} & 35 & \mu \mathrm{g} / \mathrm{kg} & 35 & \text { EPA8080 } \\ \mathrm{U} & 35 & \mathrm{ng} / \mathrm{g} & 0.10 & \text { EPA8280 } \\ \mathrm{U} & 0.10 & \mathrm{ng} / \mathrm{g} & 0.10 & \text { EPA8280 } \\ \mathrm{U} & 0.10 & \mathrm{ng} / \mathrm{g} & 0.10 & \text { EPA8280 } \\ & 0.10 & & & \\ & & & & \end{array}$


SAMPLE NAME: D43110801 (Continued)

Constituent
Sample ID: 100329

RQ AQ B Result Unit D. Limit Method

Pesticides/PCBs and Dioxins/Furans

$\begin{array}{llllll}\text { 2.3.7.8-TCDF } & \mathrm{U} & 0.10 & \mathrm{ng} / \mathrm{g} & 0.10 & \text { EPA8280 } \\ \text { Tetrachlorodibenzo-p-dioxin isomers } & \mathrm{U} & 0.10 & \mathrm{ng} / \mathrm{g} & 0.10 & \text { EPA8280 } \\ \text { Tetrachlorodibenzo-p-furan isomers } & \mathrm{U} & 0.10 & \mathrm{ng} / \mathrm{g} & 0.10 & \text { EPA8280 } \\ \text { Toxaphene } & . \mathrm{U} & 170 & \mathrm{\mu g} / \mathrm{kg} & 170 & \text { EPA8080 }\end{array}$

Radionuclide Indicators and Radionuclides

Gross alpha

Nonvolatile beta

Radium, total alpha-emining

Tritium

SAMPLE NAME: D43110802

Location (SRS Coordinates): 18542.87E 66623.81N

Ground Elevation Above MSL: $132.8 \mathrm{ft}$

Depth of Core Interval: 0.50 to $1.50 \mathrm{ft}$

Sample Type: Normal

Sample Color: 10R 3/4

Constituent

Total Metals

Arsenic
Arsenic
Barium
Barium
Cadmium
Cadmium
Chromium
Chromium
Copper
Copper
Lead
Lead
Manganese
Manganese
Mercury
Mercury
Nickel
Nickel
Selenium
Selenium
Silver
Silver

Sample Matrix: Soil USC Soil Classification: SC

RQ AQ
Unit

$\mathrm{mg} / \mathrm{kg}$

$\mathrm{mg} / \mathrm{kg}$

$\mathrm{mg} / \mathrm{kg}$

$\mathrm{mg} / \mathrm{kg}$

$\mathrm{mg} / \mathrm{kg}$

$\mathrm{mg} / \mathrm{kg}$

$\mathrm{mg} / \mathrm{kg}$

$\mathrm{mg} / \mathrm{kg}$

$\mathrm{mg} / \mathrm{kg}$

$\mathrm{mg} / \mathrm{kg}$

$\mathrm{mg} / \mathrm{kg}$

$\mathrm{mg} / \mathrm{kg}$

$\mathrm{mg} / \mathrm{kg}$

$\mathrm{mg} / \mathrm{kg}$

$\mathrm{mg} / \mathrm{kg}$

$\mathrm{mg} / \mathrm{kg}$

$\mathrm{mg} / \mathrm{kg}$

$\mathrm{mg} / \mathrm{kg}$

$\mathrm{mg} / \mathrm{kg}$

$\mathrm{mg} / \mathrm{kg}$

$\mathrm{mg} / \mathrm{kg}$

$\mathrm{mg} / \mathrm{kg}$
Sample Moisture: Dry

Percent Solids: 89.40

D. Limit Method

$7.9 \quad$ EPA900.0

$0.070 \quad$ EPA903.0

1.7 EPA906.0 
SAMPLE NAME: D431 10802 (Continued)

Sample ID: 100330

Constituent

RQ AQ B Result Unit

D. Limit Method

$B / N / A$ Extractables (including $P A H$ and phenols)

\begin{tabular}{|c|c|c|c|c|c|}
\hline Acenaphthene & $\mathbf{U}$ & 360 & $\mu g / k g$ & 360 & EPA8270 \\
\hline Acenaphthylene & U & 360 & $\mu g / \mathrm{kg}$ & 360 & EPA8270 \\
\hline Anthracene & $\mathbf{U}$ & 360 & $\mu g / \mathrm{kg}$ & 360 & EPA8270 \\
\hline Benzo(a)anthracene & $\mathbf{U}$ & 360 & $\mu g / k g$ & 360 & EPA8270 \\
\hline Benzo(b)fluoranthene & $\mathbf{U}$ & 360 & $\mu \mathrm{g} / \mathrm{kg}$ & 360 & EPA8270 \\
\hline Benzo(k)fluoranthene & $\mathbf{U}$ & 360 & $\mu \mathrm{g} / \mathrm{kg}$ & 360 & EPA8270 \\
\hline Benzoic acid & $\mathbf{U}$ & 1.800 & $\mu g / k g$ & 1,800 & EPA8270 \\
\hline Benzo(g.h,i)perylene & $\mathbf{U}$ & 360 & $\mu g / \mathrm{kg}$ & 360 & EPA8270 \\
\hline Benzo(a)pyrene & $\mathbf{U}$ & 360 & $\mu \mathrm{g} / \mathrm{kg}$ & 360 & EPA8270 \\
\hline Benzyl alcohol & $\mathbf{U}$ & 360 & $\mu \mathrm{g} / \mathrm{kg}$ & 360 & EPA8270 \\
\hline Bis(2-chloroethoxy) methane & $\mathbf{U}$ & 360 & $\mu \mathrm{g} / \mathrm{kg}$ & 360 & EPA8270 \\
\hline Bis(2-chloroethyl) ether & $\mathrm{U}$ & 360 & $\mu \mathrm{g} / \mathrm{kg}$ & 360 & EPA8270 \\
\hline Bis(2-chloroisopropyl) ethèr & $\mathrm{U}$ & 360 & $\mu g / k g$ & 360 & EPA8270 \\
\hline Bis(2-ethylhexyl) phthalate & $\mathbf{U}$ & 360 & $\mu \mathrm{g} / \mathrm{kg}$ & 360 & EPA8270 \\
\hline 4-Bromophenyl phenyl echer & $\mathrm{U}$ & 360 & $\mu g / \mathrm{kg}$ & 360 & EPA8270 \\
\hline Butylbenzyl phthalate & $\mathbf{U}$ & 360 & $\mu \mathrm{g} / \mathrm{kg}$ & 360 & EPA8270 \\
\hline 4-Chloroaniline & $\mathrm{U}$ & 360 & $\mu \mathrm{g} / \mathrm{kg}$ & 360 & EPA8270 \\
\hline 4-Chloro-m-cresol & $\mathrm{U}$ & 360 & $\mu g / k g$ & 360 & EPA8270 \\
\hline 2-Chloronaphthalene & $\mathrm{U}$ & 360 & $\mu \mathrm{g} / \mathrm{kg}$ & 360 & EPA8270 \\
\hline 2-Chlorophenol & $\mathrm{U}$ & 360 & $\mu g / \mathrm{kg}$ & 360 & EPA8270 \\
\hline 4-Chlorophenyl phenyl ether & $\mathrm{U}$ & 360 & $\mu \mathrm{g} / \mathrm{kg}$ & 360 & EPA8270 \\
\hline Chrysene & $\mathrm{U}$ & 360 & $\mu \mathrm{g} / \mathrm{kg}$ & 360 & EPA8270 \\
\hline o-Cresol (2-Methylphenol) & $U$ & 360 & $\mu \mathrm{g} / \mathrm{kg}$ & 360 & EPA8270 \\
\hline p-Cresol (4-Methylphenol) & $\mathrm{U}$ & 360 & $\mu g / k g$ & 360 & EPA8270 \\
\hline Dibenz(a,h)anthracene & U & 360 & $\mu g / k g$ & 360 & EPA8270 \\
\hline Dibenzofuran & $\mathrm{U}$ & 360 & $\mu \mathrm{g} / \mathrm{kg}$ & 360 & EPA8270 \\
\hline Di-n-buryl phthalate & UJ & 90 & $\mu \mathrm{g} / \mathrm{kg}$ & 360 & EPA8270 \\
\hline 1,2-Dichlorobenzene & $U$ & 360 & $\mu g / k g$ & 360 & EPA8270 \\
\hline 1.3-Dichlorobenzene & $\mathrm{U}$ & 360 & $\mu g / \mathrm{kg}$ & 360 & EPA8270 \\
\hline 1,4-Dichlorobenzene & $\mathbf{U}$ & 360 & $\mu g / \mathrm{kg}$ & 360 & EPA8270 \\
\hline 3,3'-Dichlorobenzidine & U & 720 & $\mu \mathrm{g} / \mathrm{kg}$ & 720 & EPA8270 \\
\hline 2,4-Dichlorophenol & $\mathrm{U}$ & 360 & $\mu g / \mathrm{kg}$ & 360 & EPA8270 \\
\hline Diethyl phthalate & $\mathbf{U}$ & 360 & $\mu \mathrm{g} / \mathrm{kg}$ & 360 & EPA8270 \\
\hline 2,4-Dimethyl phenol & U & 360 & $\mu g / \mathrm{kg}$ & 360 & EPA8270 \\
\hline Dimethyl phthalate & $\mathrm{U}$ & 360 & $\mu \mathrm{g} / \mathrm{kg}$ & 360 & EPA8270 \\
\hline 2,4-Dinitrophenol & $\mathrm{U}$ & 1,800 & $\mu g / \mathrm{kg}$ & 1,800 & EPA8270 \\
\hline 2,4-Dinitrotoluene & $\mathbf{U}$ & 360 & $\mu \mathrm{g} / \mathrm{kg}$ & 360. & EPA8270 \\
\hline 2,6-Dinitrotoluene & $\mathbf{U}$ & 360 & $\mu \mathrm{g} / \mathrm{kg}$ & $360^{-}$ & EPA8270 \\
\hline Di-n-octyl phehalate & U & 360 & $\mu g / \mathbf{k g}$ & 360 & EPA8270 \\
\hline Fluoranthene & $\mathrm{U}$ & 360 & $\mu \mathrm{g} / \mathrm{kg}$ & 360 & EPA8270 \\
\hline Fluorene & U & 360 & $\mu g / k g$ & 360 & EPA8270 \\
\hline Hexachlorobenzene & $\mathrm{U}$ & 360 & $\mu \mathrm{g} / \mathrm{kg}$ & 360 & EPA8270 \\
\hline Hexachlorobutadiene & $\mathbf{U}$ & 360 & $\mu \mathrm{g} / \mathrm{kg}$ & 360 & EPA8270 \\
\hline Hexachlorocyclopentadiene & $\mathbf{U}$ & 360 & $\mu \mathrm{g} / \mathrm{kg}$ & $360=$ & EPA8270 \\
\hline Hexachloroethane & $U$ & 360 & $\mu g / k g$ & 360 & EPA8270 \\
\hline Indeno(1,2,3-c,d)pyrene & $U$ & 360 & $\mu \mathrm{g} / \mathrm{kg}$ & 360 & EPA8270 \\
\hline Isophorone & $\mathrm{U}$ & 360 & $\mu \mathrm{g} / \mathrm{kg}$ & 360 & EPA8270 \\
\hline 2-Methyl-4,6-dinitrophenol & U & 1,800 & $\mu \mathrm{g} / \mathrm{kg}$ & 1.800 & EPA8270 \\
\hline 2-Methylnaphthalene & $\mathbf{U}$ & 360 & $\mu \mathrm{g} / \mathrm{kg}$ & 360 & EPA8270 \\
\hline Naphthaiene & $\mathrm{U}$ & 360 & $\mu \mathrm{g} / \mathrm{kg}$ & 360 & EPA8270 \\
\hline
\end{tabular}


SAMPLE NAME: D43110802 (Continued)

Sample ID: 100330

$\begin{array}{llllll}\text { Constituent } & \text { RQ AQ B Result Unit D. Limit Method }\end{array}$

$B / N / A$ Extractables (including PAH and phenols)

o-Nitroaniline

m-Nitroaniline

p-Nitroaniline

Nitrobenzene

2-Nitrophenol

4-Nitrophenol

N-Nitrosodiphenylamine

N-Nitrosodipropylamine

Pentachlorophenol

Phenanthrene

Phenol

Pyrene

1,2.4-Trichlorobenzene

2.4.5-Trichlorophenol

2,4,6-Trichlorophenol

Unknown arom. hydrocarbon

Unknown ketone

Volatile Organic Compounds

Acetone

Benzene

Bromodichloromethane

Bromoform

- Bromomethane (Methyl bromide)

Carbon disulfide

Carbon tetrachloride

Chlorobenzene

Chloroethane

Chloroethene (Vinyl chloride)

Chloroform

Chloromethane (Methyl chloride)

Dibromochloromethane

1,1-Dichloroethane

1,2-Dichloroethane

1,1-Dichloroethylene

1,2-Dichloroethylene

Dichloromethane (Methylene chloride)

1,2-Dichloropropane

cis-1,3-Dichloropropene

trans-1,3-Dichloropropene

Ethylbenzene

2-Hexanone

Methyl ethyl ketone

Methyl isobutyl ketone

Styrene

1,1,2,2-Tetrachloroethane

Tetrachloroethylene

Toluene

1,1,1-Trichloroethane

$\begin{array}{lll}U & & 1,800 \\ U & & 1,800 \\ U & & 1,800 \\ U & & 360 \\ U & & 360 \\ U & & 1,800 \\ U & & 360 \\ U & - & 360 \\ U & & 1,800 \\ U & & 360 \\ U & & 360 \\ U & & 360 \\ U & & 360 \\ U & & 1,800 \\ U & & 360 \\ J & N & 200 \\ J & N V & 7,000\end{array}$

11

5.5

5.5

5.5

11

5.5

5.5

5.5

11

11

5.5

11

5.5

5.5

5.5

5.5

5.5

16

5.5

5.5

5.5

5.5

11

11

11

5.5

5.5

5.5

5.5

5.5 $\mu \mathrm{g} / \mathrm{kg}$

$\mu \mathrm{g} / \mathrm{kg}$

$\mu \mathrm{g} / \mathrm{kg}$

$\mu \mathrm{g} / \mathrm{kg}$

$\mu \mathrm{g} / \mathrm{kg}$

$\mu \mathrm{g} / \mathrm{kg}$

$\mu \mathrm{g} / \mathrm{kg}$

$\mu \mathrm{g} / \mathrm{kg}$

$\mu \mathrm{g} / \mathrm{kg}$

$\mu \mathrm{g} / \mathrm{kg}$

$\mu \mathrm{g} / \mathrm{kg}$

$\mu \mathrm{g} / \mathrm{kg}$

$\mu \mathrm{g} / \mathrm{kg}$

$\mu \mathrm{g} / \mathrm{kg}$

$\mu g / k g$

$\mu \mathrm{g} / \mathrm{kg}$

$\mu \mathrm{g} / \mathrm{kg}$

$\mu g / k g$

$\mu \mathrm{g} / \mathrm{kg}$

$\mu g / k g$

$\mu \mathrm{g} / \mathrm{kg}$

$\mu \mathrm{g} / \mathrm{kg}$

$\mu g / k g$

$\mu \mathrm{g} / \mathrm{kg}$

$\mu \mathrm{g} / \mathrm{kg}$

$\mu \mathrm{g} / \mathrm{kg}$

$\mu \mathrm{g} / \mathrm{kg}$

$\mu g / k g$

$\mu \mathrm{g} / \mathrm{kg}$

$\mu g / \mathrm{kg}$

$\mu \mathrm{g} / \mathrm{kg}$

$\mu \mathrm{g} / \mathrm{kg}$

$\mu \mathrm{g} / \mathrm{kg}$
1,800

1,800

1.800

360

360

1,800

360

360

1,800

360

360

360

360

1,800

360

EPA8270

EPA8270

EPA8270

EPA8270

EPA8270

EPA8270

EPA8270

EPA8270

EPA8270

EPA8270

EPA8270

EPA8270

EPA8270

EPA8270

EPA8270

EPA8270

EPA8270

\section{1}

5.5

EPA8240

EPA8240

EPA8240

EPA8240

EPA8240

EPA8240

EPA8240

EPA8240

EPA8240

EPA8240

EPA8240

EPA8240

EPA8240

EPA8240

EPA8240

EPA8240

EPA8240

EPA8240

EPA8240

EPA8240

EPA8240

EPA8240

EPA8240

EPA8240

EPA8240

EPA8240

EPA8240

EPA8240

EPA8240

EPA8240 
SAMPLE NAME: D43110802 (Continued)

Sample ID: 100330

\section{Constituent}

Volatile Organic Compounds

1,1,2-Trichloroethane

Trichloroethylene

Vinyl acetate

Xylenes
RQ AQ B Result Unit

5.5

5.5

11

5.5

.5
.5
1
.5

Pesticides/PCBs and Dioxins/Furans

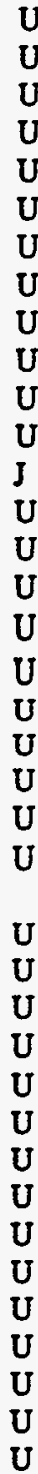

alpha-Benzene hexachloride

beta-Benzene hexachloride

deita-Benzene hexachloride

alpha-Chlordane

gamma-Chlordane

p.p'-DDD

p.p'-DDE

p.p'-DDT

Dieldrin

Endosulfan I

Endosulfan II

Endosulfan sulfate

Endrin

Endrin ketone

Heptachlor

Heptachlor epoxide

Heptachlorodibenzo-p-dioxin isomers

Heptachlorodibenzo-p-furan isomers

Hexachlorodibenzo-p-dioxin isomers

Hexachlorodibenzo-p-furan isomers

Lindane

Methoxychlor

Octachlorodibenzo-p-dioxin

Octachlorodibenzo-p-furan

PCB 1016

PCB 1221

PCB 1232

PCB 1242

PCB 1248

PCB 1254

PCB 1260

Pentachlorodibenzo-p-dioxin isomers

Pentachlorodibenzo-p-furan isomers

2,3,7,8-TCDD

2,3,7,8-TCDF

Tetrachlorodibenzo-p-dioxin isomers

Tetrachlorodibenzo-p-furan isomers

Toxaphene
U

U

U

U

U

U

U

U

U

U

U

U

J

U

U

U

U

U

U

U

U

U

U

U

U

U

U

U

U

U

U

U

U

U

U

U

U
1.8

1.8

1.8

1.8

1.8

1.8

3.7

3.7

3.7

3.7

1.8

3.7

1.1

3.7

3.7

1.8

1.8

0.20

0.10

0.20

0.10

1.8

18

2.3

0.10

37

73

37

37

37

37

37

0.30

0.20

0.10

0.10

0.20

0.10

180

$\begin{array}{lll}\mu g / k g & 5.5 & \text { EPA8240 } \\ \mu g / \mathrm{kg} & 5.5 & \text { EPA8240 } \\ \mu \mathrm{g} / \mathrm{kg} & 11 & \text { EPA8240 } \\ \mu \mathrm{g} / \mathrm{kg} & 5.5 & \text { EPA8240 }\end{array}$

$\mu \mathrm{g} / \mathrm{kg}$

$\mu g / k g$

$\mu g / \mathrm{kg}$

$\mu \mathrm{g} / \mathrm{kg}$

$\mu \mathrm{g} / \mathrm{kg}$

$\mu g / k g$

$\mu \mathrm{g} / \mathrm{kg}$

$\mu \mathrm{g} / \mathrm{kg}$

$\mu \mathrm{g} / \mathrm{kg}$

$\mu \mathrm{g} / \mathrm{kg}$

$\mu \mathrm{g} / \mathrm{kg}$

$\mu \mathrm{g} / \mathrm{kg}$

$\mu \mathrm{g} / \mathrm{kg}$

$\mu \mathrm{g} / \mathrm{kg}$

$\mu \mathrm{g} / \mathrm{kg}$

$\mu g / k g$

$\mu \mathrm{g} / \mathrm{kg}$

$\mathrm{ng} / \mathrm{g}$

$\mathrm{ng} / \mathrm{g}$

$\mathrm{ng} / \mathrm{g}$

$\mathrm{ng} / \mathrm{g}$

$\mu \mathrm{g} / \mathrm{kg}$

$\mu g / k g$

$\mathrm{ng} / \mathrm{g}$

$\mathrm{ng} / \mathrm{g}$

$\mu \mathrm{g} / \mathrm{kg}$

$\mu \mathrm{g} / \mathrm{kg}$

$\mu \mathrm{g} / \mathrm{kg}$

$\mu \mathrm{g} / \mathrm{kg}$

$\mu \mathrm{g} / \mathrm{kg}$

$\mu g / \mathrm{kg}$

$\mu \mathrm{g} / \mathrm{kg}$

$\mathrm{ng} / \mathrm{g}$

$\mathrm{ng} / \mathrm{g}$

$\mathrm{ng} / \mathrm{g}$

$\mathrm{ng} / \mathrm{g}$

$\mathrm{ng} / \mathrm{g}$

$\mathrm{ng} / \mathrm{g}$

$\mu \mathrm{g} / \mathrm{kg}$
D. Limit Method 
SAMPLE NAME: D43110802 (Continued)

Constituent

AQ

Radionuclide Indicators and Radionuclides

Gross alpha

Nonvolatile beta

Radium, total alpha-emitting

Tritium

SAMPLE NAME: D43110803

Location (SRS Coordinates): 18542.87E 66623.81N

Ground Elevation Above MSL: $132.8 \mathrm{ft}$

Depth of Core Interval: 2.00 to $3.50 \mathrm{ft}$

Sample Type: Normal

Sample Color: 10R $3 / 4$

Constituent

RQ AQ B Result Unit

Sample Matrix: Soil

USC Soil Classification: SC

Sample D: 100331

Total Metals

Arsenic

Barium

Cadmium

Chromium

Copper

Lead

Manganese

Mercury

Nickel

Selenium

Silver

$\mathbf{U}$

U

Volatile Organic Compounds

$\begin{array}{ll}\text { Acetone } & \mathrm{UJ} \mathrm{V} \\ \text { Benzene } & \mathrm{U} \\ \text { Bromodichloromethane } & \mathrm{U} \\ \text { Bromoform } & \mathrm{U} \\ \text { Bromomethane (Methyl bromide) } & \mathrm{U} \\ \text { Carbon disulfide } & \mathrm{U} \\ \text { Carbon tetrachloride } & \mathrm{U} \\ \text { Chlorobenzene } & \mathrm{U} \\ \text { Chloroethane } & \mathrm{U} \\ \text { Chloroethene (Vinyl chloride) } & \mathrm{U} \\ \text { Chloroform } & \mathrm{U} \\ \text { Chloromethane (Methyl chloride) } & \mathrm{U} \\ \text { Dibromochloromethane } & \mathrm{U} \\ \text { 1,1-Dichloroethane } & \mathrm{U} \\ \text { 1,2-Dichloroethane } & \mathrm{U} \\ \text { 1,1-Dichloroethylere } & \mathrm{U} \\ \text { 1,2-Dichloroethylene } & \mathrm{U} \\ \text { Dichloromethane (Methylene chloride) } & \mathrm{U} \\ \end{array}$

Sample ID: 100330

B Result Unit D. Limit Method

Sample Moisture: Dry

Percent Solids: 86.30

D. Limit Method

EPA900.0

$\begin{array}{ll}0.070 & \text { EPA903.0 } \\ 1.7 & \text { EPA906.0 }\end{array}$ 
SAMPLE NAME: D431:10803 (Continued)

Constituent

RQ

Volatile Organic Compounds
Sample ID: 100331

RQ AQ B Result Unit D. Limit Method

\begin{tabular}{|c|c|c|c|}
\hline 5.8 & $\mu \mathrm{g} / \mathrm{kg}$ & 5.8 & EPA8240 \\
\hline 5.8 & $\mu g / \mathrm{kg}$ & 5.8 & EPA8240 \\
\hline 5.8 & $\mu \mathrm{g} / \mathrm{kg}$ & 5.8 & EPA8240 \\
\hline 5.8 & $\mu \mathrm{g} / \mathrm{kg}$ & 5.8 & EPA8240 \\
\hline 12 & $\mu \mathrm{g} / \mathrm{kg}$ & 12 & EPA8240 \\
\hline 12 & $\mu g / k g$ & 12 & EPA8240 \\
\hline 12 & $\mu \mathrm{g} / \mathrm{kg}$ & 12 & EPA8240 \\
\hline 5.8 & $\mu \mathrm{g} / \mathrm{kg}$ & 5.8 & EPA8240 \\
\hline 5.8 & $\mu \mathrm{g} / \mathrm{kg}$ & 5.8 & EPA8240 \\
\hline 5.8 & $\mu \mathrm{g} / \mathrm{kg}$ & 5.8 & EPA8240 \\
\hline 5.8 & $\mu g / \mathrm{kg}$ & 5.8 & EPA8240 \\
\hline 5.8 & $\mu g / \mathrm{kg}$ & 5.8 & EPA8240 \\
\hline 5.8 & $\mu \mathrm{g} / \mathrm{kg}$ & 5.8 & EPA8240 \\
\hline 5.8 & $\mu \mathrm{g} / \mathrm{kg}$ & 5.8 & EPA8240 \\
\hline 12 & $\mu \mathrm{g} / \mathrm{kg}$ & 12 & EPA8240 \\
\hline 5.8 & $\mu g / \mathrm{kg}$ & 5.8 & EPA8240 \\
\hline
\end{tabular}

Radionuclide Indicators and Radionuclides

Gross alpha

Nonvolatile beta

Radium, total alpha-emitring

Tritium

$\begin{array}{lll}\mathrm{U} & 5.8 & \mu \mathrm{g} / \mathrm{kg} \\ \mathrm{U} & 5.8 & \mu \mathrm{g} / \mathrm{kg} \\ \mathrm{U} & 5.8 & \mu \mathrm{g} / \mathrm{kg} \\ \mathrm{U} & 5.8 & \mu \mathrm{g} / \mathrm{kg} \\ \mathrm{U} & 12 & \mu \mathrm{g} / \mathrm{kg} \\ \mathrm{U} & 12 & \mu \mathrm{g} / \mathrm{kg} \\ \mathrm{U} & 12 & \mu \mathrm{g} / \mathrm{kg} \\ \mathrm{U} & 5.8 & \mu \mathrm{g} / \mathrm{kg} \\ \mathrm{U} & 5.8 & \mu \mathrm{g} / \mathrm{kg} \\ \mathrm{U} & 5.8 & \mu \mathrm{g} / \mathrm{kg} \\ \mathrm{U} & 5.8 & \mu \mathrm{g} / \mathrm{kg} \\ \mathrm{U} & 5.8 & \mu \mathrm{g} / \mathrm{kg} \\ \mathrm{U} & 5.8 & \mu \mathrm{g} / \mathrm{kg} \\ \mathrm{U} & 5.8 & \mu \mathrm{g} / \mathrm{kg} \\ \mathrm{U} & 12 & \mu \mathrm{g} / \mathrm{kg} \\ \mathrm{U} & 5.8 & \mu \mathrm{g} / \mathrm{kg}\end{array}$

$\begin{array}{llllll} & & 9.1 \pm 13 & \mathrm{pCi} / \mathrm{g} & 7.1 & \text { EPA900.0 } \\ \mathrm{U} & 21 \pm 21 & \mathrm{pCi} / \mathrm{g} & 8.8 & \text { EPA900.0 } \\ & \mathrm{V} & 1.2 \pm 1.0 & \mathrm{pCi} / \mathrm{g} & 0.090 & \text { EPA903.0 } \\ & 14 \pm 2.3 & \mathrm{pCi} / \mathrm{g} & 1.6 & \text { EPA906.0 }\end{array}$

\section{SAMPLE NAME: D43110804}

Location (SRS Coordinates): 18542.87E 66623.81N

Ground Elevation Above MSL: $132.8 \mathrm{ft}$

Depth of Core Interval: 4.00 to $5.00 \mathrm{ft}$

Sample Type: Norma!

Sample Color: 2N2

Constituent

Total Metals

Sample ID: 100332

Sample Matrix: Soil

USC Soil Classification: SM

$\mathbf{R Q} \quad \mathbf{A Q}$
B Result Unit
Sample Moisture: Damp Percent Solids: 80.00

D. Limit Method

$\begin{array}{llllll}\text { Arsenic } & & 7.6 & \mathrm{mg} / \mathrm{kg} & 1.9 & \text { EPA7060 } \\ \text { Barium } & & 32 & \mathrm{mg} / \mathrm{kg} & 21 & \text { EPA6010 } \\ \text { Cadmium } & \mathrm{U} & 0.53 & \mathrm{mg} / \mathrm{kg} & 0.53 & \text { EPA6010 } \\ \text { Chromium } & & 75 & \mathrm{mg} / \mathrm{kg} & 1.1 & \text { EPA6010 } \\ \text { Copper } & & 38 & \mathrm{mg} / \mathrm{kg} & 2.7 & \text { EPA6010 } \\ \text { Lead } & & 19 & \mathrm{mg} / \mathrm{kg} & 1.5 & \text { EPA7421 } \\ \text { Manganese } & & 63 & \mathrm{mg} / \mathrm{kg} & 1.6 & \text { EPA6010 } \\ \text { Mercury } & & 0.28 & \mathrm{mg} / \mathrm{kg} & 0.13 & \text { EPA7470 } \\ \text { Nickel } & & 15 & \mathrm{mg} / \mathrm{kg} & 4.2 & \text { EPA6010 } \\ \text { Selenium } & & 0.97 & \mathrm{mg} / \mathrm{kg} & 0.49 & \text { EPA7740 } \\ \text { Silver } & & 1.1 & \mathrm{mg} / \mathrm{kg} & 1.1 . & \text { EPA6010 }\end{array}$


SAMPLE NAME: D43110804 (Continued)

Sample ID: 100332

Constituent

RQ AQ B Result

Unit

D. Limit Method

$B / N / A$ Extractables (including PAH and phenols)

Acenaphthene

Acenaphthylene

Anthracene

Benzo(a)anthracene

Benzo(b)fluoranthene

Benzo(k)fluoranthene

Benzoic acid

Benzo(g,h,i)perylene

Benzo(a)pyrene

Benzyl alcohol

Bis(2-chloroethoxy) methane

Bis(2-chloroethyl) ether

Bis(2-chloroisopropyl) ether

Bis(2-ethylhexyl) phthalate

4-Bromophenyl phenyl ether

Butylbenzy! phthalate

Alkylbenzene C15HI6

4-Chloroaniline

4-Chloro-m-cresol

2-Chloronaphthalene

2-Chlorophenol

4-Chlorophenyl phenyl ether

Chrysene

o-Cresol (2-Methylphenol)

p-Cresol (4-Methylphenol)

Dibenz $(a, h)$ anthracene

Dibenzofuran

Di-n-butyl phthalate

1,2-Dichlorobenzene

1,3-Dichlorobenzene

1,4-Dichlorobenzene

3,3'-Dichlorobenzidine

2,4-Dichlorophenol

Diethyl phthalate

2,4-Dimethyl phenol

Dimethyl phthalate

2,4-Dinitrophenol

2,4-Dinitrotoluene

2,6-Dinitrotoluene

Di-n-octyl phthalate

Fluoranthene

Fluorene

Hexachlorobenzene

Hexachlorobutadiene

Hexachlorocyclopentadiene

Hexachloroethane

Indeno(1,2,3-c,d)pyrene

Isophorone

Methyldibenzothiophene isomer

Methyldibenzothiophene isomer
U

U

U

U

U

U

U

U

U

U

U

U

U

U

U

U

U

U

U

U.

U

U

U

U

UJ

U

U

U

U

U

U

U

U

U

U

U

J

J

U

U

U

U

U

U

U

J N
4,100

4.100

4,100

4,100

4,100

4,100

20,000

4,100

4,100

4,100

4,100

4,100

4,100

14,000

4,100

28,000

6,000

4,100

4,100

4,100

4,100

4,100

4,100

4,100

4,100

4,100

4,100

460

4,100

4,100

4,100

8,100

4,100

4,100

4,100

4,100

20,000

4,100

4,100

430

580 .

4,100

4,100

4,100

4,100

4,100

4,100

4,100

10,000

5,000 $\mu \mathrm{g} / \mathrm{kg}$

$\mu \mathrm{g} / \mathrm{kg}$

$\mu \mathrm{g} / \mathrm{kg}$

$\mu \mathrm{g} / \mathrm{kg}$

$\mu \mathrm{g} / \mathrm{kg}$

$\mu \mathrm{g} / \mathrm{kg}$

$\mu \mathrm{g} / \mathrm{kg}$

$\mu \mathrm{g} / \mathrm{kg}$

$\mu \mathrm{g} / \mathrm{kg}$

$\mu \mathrm{g} / \mathrm{kg}$

$\mu \mathrm{g} / \mathrm{kg}$

$\mu \mathrm{g} / \mathrm{kg}$

$\mu \mathrm{g} / \mathrm{kg}$

$\mu \mathrm{g} / \mathrm{kg}$

$\mu \mathrm{g} / \mathrm{kg}$

$\mu \mathrm{g} / \mathrm{kg}$

$\mu \mathrm{g} / \mathrm{kg}$

$\mu \mathrm{g} / \mathrm{kg}$

$\mu \mathrm{g} / \mathrm{kg}$

$\mu g / \mathrm{kg}$

$\mu \mathrm{g} / \mathrm{kg}$

$\mu \mathrm{g} / \mathrm{kg}$

$\mu \mathrm{g} / \mathrm{kg}$

$\mu \mathrm{g} / \mathrm{kg}$

$\mu \mathrm{g} / \mathrm{kg}$

$\mu \mathrm{g} / \mathrm{kg}$

$\mu \mathrm{g} / \mathrm{kg}$

$\mu \mathrm{g} / \mathrm{kg}$

$\mu \mathrm{g} / \mathrm{kg}$

$\mu \mathrm{g} / \mathrm{kg}$

$\mu \mathrm{g} / \mathrm{kg}$

$\mu \mathrm{g} / \mathrm{kg}$

$\mu \mathrm{g} / \mathrm{kg}$

$\mu g / k g$

$\mu \mathrm{g} / \mathrm{kg}$

$\mu g / k g$
4.100

4,100

4,100

4,100

4,100

4,100

20,000

4,100

4,100

4,100

4,100

4,100

4,100

4,100

4,100

4,100

EPA8270

EPA8270

EPA8270

EPA8270

EPA 8270

EPA8270

EPA8270

EPA8270

EPA8270

EPA8270

EPA8270

EPA8270

EPA8270

EPA8270

EPA8270

EPA8270

EPA8270

$4,100 \quad$ EPA8270

4,100

4,100

4,100

4,100

4,100

4,100

4,100

4,100

4,100

4,100

4,100

4,100

4,100

8,100

4.100

4,100

4,100

4,100

20,000

4,100

4,100

4,100

4,100

$4 ; 100$

4,100

4,100

4,100

4,100

4,100

4,100

EPA8270

EPA8270

EPA8270

EPA8270

EPA8270

EPA8270

EPA8270

EPA8270

EPA8270

EPA8270

EPA8270

EPA8270

EPA8270

EPA8270

EPA8270

EPA8270

EPA8270

EPA8270

EPA8270

EPA8270

EPA8270

EPA8270

EPA8270

EPA8270

EPA8270

EPA8270

EPA8270

EPA8270

EPA8270

EPA8270

EPA8270

EPA8270 
SAMPLE NAME: D43ï10804 (Continued)

Sample ID: 100332

\section{Constituent}

RQ AQ B Result

$B / N / A$ Extractables (including PAH and phenols)
Dimethyinaphthothiophene isomer

Dimethylphenanthrene isomer

Dimethylphenanthrene isomer

Dimethylphenanthrene isomer

Dimethylphenanthrene isomer

Dimethylphenanthrene isomer

Dimethylphenanthrene isomer

Trimethylphenanthrene isomer

Trimethylphenanthrene isomer

Methylanthracene isomer

Methylanthracene isomer

Methylanthracene isomer

Methylphenanthrene isomer

2-Methyl-4.6-dinitrophenol

2-Methyinaphthalene

Naphthalene

o-Nitroaniline

m-Nitroaniline

p-Nitroaniline

Nitrobenzene

2-Nitrophenol

4-Nitrophenol

N-Nitrosodiphenylamine

N-Nitrosodipropylamine

Pentachlorophenol

Phenanthrene

Phenol

Pyrene

1,2,4-Trichlorobenzene

2,4,5-Trichlorophenol

2,4,6-Trichlorophenol

Unknown arom. hydrocarbon

Unknown arom. hydrocarbon

Unknown arom. hydrocarbon

Unknown ketone

$J$
$J$
$J$
$J$
$J$
$J$
$J$
$J$
$J$
$J$
$J$
$J$
$J$
$U$
$U$
$U$
U
U
U
U
U
U
U
U
U

U

J

U

U

U

J $N$

J $N$

J $N$

NV
7,000

9,000

10,000

5,000

20,000

9,000

8,000

10,000

10,000

20,000

10,000

9,000

20,000

20,000

4,100

4,100

20,000

20.000

20,000

4,100

4,100

20,000

4,100

4,100

20,000

16,000

4,100

2,700

4,100

20,000

4,100

9,000

9,000

7,000

7,000
Unit

D. Limit Method

Volatile Organic Compounds

Acetone
Acetone
Benzene
Bromodichloromethane
Bromoform
Bromomethane (Methyl bromide)
Hydrocarbon C10H16
Subst. benzene C9H12
Carbon disulfide
Carbon tetrachloride
Chlorobenzene
Chloroethane

$\begin{array}{ll}\text { L } & \text { V } \\ & \text { V } \\ \text { U } & \\ \text { U } & \\ \mathbf{U} & \\ \mathbf{U} & \\ \mathbf{J} & \text { N } \\ \mathbf{J} & \text { N } \\ \mathbf{J} & \\ \mathbf{U} & \\ \mathbf{U} & \\ \mathbf{U} & \end{array}$

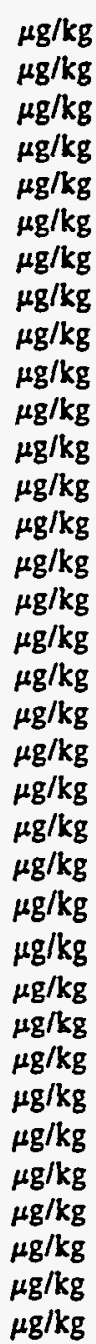

EPA8270

EPA8270

EPA8270

EPA8270

EPA8270

EPA8270

EPA8270

EPA8270

EPA8270

EPA8270

EPA8270

EPA8270

EPA8270

20,000

4,100

4,100

20,000

20,000

20,000

4,100

EPA8270

EPA8270

EPA8270

EPA8270

EPA8270

EPA8270

EPA8270

4,100 EPA8270

20,000

4,100

EPA8270

EPA8270

4,100

EPA8270

20,000

EPA8270

4,100

EPA8270

EPA8270

EPA8270

EPA8270

EPA8270

EPA8270

EPA8270

EPA8270

EPA8270

EPA8270

560

650

6.1

6.1

6.1

12

50

10

3.7

6.1

6.1

12 $\mu g / \mathrm{kg}$

$\mu \mathrm{g} / \mathrm{kg}$

$\mu \mathrm{g} / \mathrm{kg}$

$\mu \mathrm{g} / \mathrm{kg}$

$\mu \mathrm{g} / \mathrm{kg}$

$\mu \mathrm{g} / \mathrm{kg}$

$\mu \mathrm{g} / \mathrm{kg}$

$\mu g / \mathrm{kg}$

$\mu g / k g$

$\mu \mathrm{g} / \mathrm{kg}$

$\mu \mathrm{g} / \mathrm{kg}$

$\mu g / k g$
12

61

6.1

6.1

6.1

12

6.1

6.1

6.1

12
EPA8240

EPA8240

EPA8240

EPA8240

EPA8240

EPA8240

EPA8240

EPA8240

EPA8240

EPA8240

EPA8240

EPA8240 
SAMPLE NAME: D43110804 (Continued)

Sample ID: 100332

Constituent

Volatile Organic Compounds
RQ

AQ B

Result

12

6.1

12

6.1

6.1

4.0

6.1

6.1

23

6.1

6.1

6.1

16

12

21

12

6.1

6.1

6.1

6.6

6.1

6.1

6.1
$\begin{array}{ll}N & 6.0 \\ & 12\end{array}$

$\begin{array}{ll}N & 6.0 \\ & 12\end{array}$

7.9

Xylenes

Pesticides/PCBs and Dioxins/Furans

Aldrin

alpha-Benzene hexachloride

alpha-Benzene hexachloride

alpha-Benzene hexachloride

beta-Benzene hexachloride

beta-Benzene hexachloride

beta-Benzene hexachloride

delta-Benzene hexachioride

delta-Benzene hexachloride

delta-Benzene hexachloride

alpha-Chlordane

alpha-Chlordane

alpha-Chlordane

gamma-Chlordane

gamma-Chlordane

gamma-Chlordane

p.p'-DDD

p.p'-DDD

p,p'-DDD

P.P'-DDE

$p, p^{\prime}-D D E$

$\begin{array}{llll}\text { UJ } & \text { O } & \text { L } & 100 \\ \text { UI } & \text { O } & \text { L } & 100 \\ \text { UJ } & \text { O } & \text { L } & 100 \\ \text { UJ } & 0 & \text { L } & 100 \\ \text { UJ } & \text { O } & \text { L } & 100 \\ \text { UJ } & 0 & \text { L } & 100 \\ \text { UJ } & 0 & \text { L } & 100 \\ \text { UJ } & \text { O } & \text { L } & 100 \\ \text { UJ } & 0 & \text { L } & 100 \\ \text { UJ } & 0 & \text { L } & 100 \\ \text { UJ } & 0 & \text { L } & 100 \\ \text { UJ } & 0 & \text { L } & 100 \\ \text { UJ } & 0 & \text { L } & 100 \\ \text { UJ } & 0 & \text { L } & 100 \\ \text { UJ } & 0 & \text { L } & 100 \\ \text { UJ } & 0 & \text { L } & 100 \\ \text { UJ } & 0 & \text { L } & 200 \\ \text { UJ } & 0 & \text { L } & 200 \\ \text { UJ } & 0 & \text { L } & 200 \\ \text { UJ } & 0 & \text { L } & 200 \\ \text { UJ } & 0 & \text { L } & 200\end{array}$

Unit

\author{
D. Limit Method
}

12

6.1

12

6.1

6.1

6.1

6.1

6.1

6.1

6.1

6.1

6.1

6.1

12

12

12

6.1

6.1

6.1

6.1

6.1

6.1

6.1

12

6.1
EPA8240

EPA8240

EPA8240

EPA8240

EPA8240

EPA8240

EPA8240

EPA8240

EPA8240

EPA8240

EPA8240

EPA8240

EPA8240

EPA8240

EPA8240

EPA8240

EPA8240

EPA8240

EPA8240

EPA8240

EPA8240

EPA8240

EPA8240

EPA8240

EPA8240

EPA8240
EPA8080

EPA8080

EPA8080

EPA8080

EPA8080

EPA8080

EPA8080

EPA8080

EPA8080

EPA8080

EPA8080

EPA8080

EPA8080

EPA8080

EPA8080

EPA8080

EPA8080

EPA8080

EPA8080

EPA8080

EPA8080 
SAMPLE NAME: D43110804 (Continued)

Re

Pesticides/PCBs and Dioxins/Furans
Sample ID: 100332

Unit D. Limit Method
Constituent

AQ
B Result

Hexachlorodibenzo-p-dioxin isomers

Hexachlorodibenzo-p-furan isomers

Lindane

Methoxychlor

Methoxychlor

Methoxychlor

Octachlorodibenzo-p-dioxin

Octachlorodibenzo-p-furan

PCB 1016

PCB 1016

PCB 1016

PCB 1221

PCB 1221

PCB 1221

PCB 1232

PCB 1232

PCB 1232

PCB 1242

PCB 1242

PCB 1242

PCB 1248

PCB 1248

PCB 1248

PCB 1254

PCB 1254

PCB 1254

PCB 1260

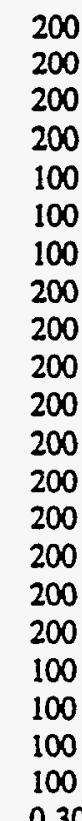

0.30

0.10

0.10

0.10

\begin{tabular}{|c|c|c|c|}
\hline UJ & 0 & L & 200 \\
\hline UJ & 0 & L & 200 \\
\hline UJ & 0 & L & 200 \\
\hline UI & 0 & L & 200 \\
\hline UJ & 0 & L & 100 \\
\hline UJ & 0 & L & 100 \\
\hline UJ & 0 & L & 100 \\
\hline UJ & 0 & L & 200 \\
\hline UJ & 0 & L & 200 \\
\hline UJ & 0 & L & 200 \\
\hline UJ & 0 & L & 200 \\
\hline UJ & 0 & L & 200 \\
\hline UJ & 0 & L & 200 \\
\hline UJ & 0 & L & 200 \\
\hline UJ & 0 & L & 200 \\
\hline UI & 0 & L & 200 \\
\hline UJ & 0 & L & 200 \\
\hline UJ & 0 & L & 100 \\
\hline UJ & 0 & L & 100 \\
\hline UJ & 0 & L & 100 \\
\hline UI & 0 & L & 100 \\
\hline J & & & 0.30 \\
\hline U & & & 0.10 \\
\hline $\mathbf{U}$ & & & 0.10 \\
\hline U & & & 0.10 \\
\hline UJ & 0 & L & 100 \\
\hline UJ & 0 & L & 1,000 \\
\hline UJ & 0 & L & 1,000 \\
\hline UJ & 0 & $\vec{L}$ & 1,000 \\
\hline & & & 2.1 \\
\hline $\mathbf{U}$ & & & 0.20 \\
\hline UJ & 0 & L & 2,000 \\
\hline UJ & 0 & L & 2,000 \\
\hline UJ & 0 & L & 2,000 \\
\hline UJ & 0 & L & 4.000 \\
\hline UJ & 0 & L & 4,000 \\
\hline UJ & 0 & L & 4,000 \\
\hline UJ & 0 & L & 2,000 \\
\hline UJ & 0 & L & 2,000 \\
\hline UJ & 0 & L & 2,000 \\
\hline UJ & .0 & L & 2,000 \\
\hline UJ & 0 & L & 2,000 \\
\hline UJ & 0 & L & 2,000 \\
\hline UI & 0 & L & 2,000 \\
\hline UJ. & 0 & L & 2.000 \\
\hline Uj & 0 & L & 2,000 \\
\hline UJ & 0 & L & 2,000 \\
\hline UJ & 0 & L & 2,000 \\
\hline UJ & 0 & L & 2.000 \\
\hline $\mathrm{U}$ & & L & 2.000 \\
\hline
\end{tabular}

\begin{tabular}{|c|c|c|}
\hline$\mu g / k g$ & 200 & EPA8080 \\
\hline$\mu \mathrm{g} / \mathrm{kg}$ & 200 & EPA8080 \\
\hline$\mu \mathrm{g} / \mathrm{kg}$ & 200 & EPA8080 \\
\hline$\mu \mathrm{g} / \mathrm{kg}$ & 200 & EPA8080 \\
\hline$\mu \mathrm{g} / \mathrm{kg}$ & 100 & EPA8080 \\
\hline$\mu \mathrm{g} / \mathrm{kg}$ & 100 & EPA8080 \\
\hline$\mu g / k g$ & 100 & EPA8080 \\
\hline$\mu \mathrm{g} / \mathrm{kg}$ & 200 & EPA8080 \\
\hline$\mu g / \mathrm{kg}$ & 200 & EPA8080 \\
\hline$\mu g / \mathrm{kg}$ & 200 & EPA8080 \\
\hline$\mu \mathrm{g} / \mathrm{kg}$ & 200 & EPA8080 \\
\hline$\mu \mathrm{g} / \mathrm{kg}$ & 200 & EPA8080 \\
\hline$\mu \mathrm{g} / \mathrm{kg}$ & 200 & EPA8080 \\
\hline$\mu \mathrm{g} / \mathrm{kg}$ & 200 & EPA8080 \\
\hline$\mu \mathrm{g} / \mathrm{kg}$ & 200 & EPA8080 \\
\hline$\mu \mathrm{g} / \mathrm{kg}$ & 200 & EPA8080 \\
\hline$\mu \mathrm{g} / \mathrm{kg}$ & 200 & EPA8080 \\
\hline$\mu \mathrm{g} / \mathrm{kg}$ & 100 & EPA8080 \\
\hline$\mu \mathrm{g} / \mathrm{kg}$ & 100 & EPA8080 \\
\hline$\mu g / \mathrm{kg}$ & 100 & EPA8080 \\
\hline$\mu \mathrm{g} / \mathrm{kg}$ & 100 & EPA8080 \\
\hline $\mathrm{ng} / \mathrm{g}$ & 0.10 & EPA8280 \\
\hline $\mathrm{ng} / \mathrm{g}$ & 0.10 & EPA8280 \\
\hline $\mathrm{ng} / \mathrm{g}$ & 0.10 & EPA8280 \\
\hline $\mathrm{ng} / \mathrm{g}$ & 0.10 & EPA8280 \\
\hline$\mu g / k g$ & 100 & EPA8080 \\
\hline$\mu \mathrm{g} / \mathrm{kg}$ & 1,000 & EPA8080 \\
\hline$\mu \mathrm{g} / \mathrm{kg}$ & 1.000 & EPA8080 \\
\hline$\mu g / \mathrm{kg}$ & 1,000 & EPA8080 \\
\hline $\mathrm{ng} / \mathrm{g}$ & 0.10 & EPA8280 \\
\hline $\mathrm{ng} / \mathrm{g}$ & 0.20 & EPA8280 \\
\hline$\mu g / \mathrm{kg}$ & 2,000 & EPA8080 \\
\hline$\mu \mathrm{g} / \mathrm{kg}$ & 2,000 & EPA8080 \\
\hline$\mu \mathrm{g} / \mathrm{kg}$ & 2,000 & EPA8080 \\
\hline$\mu \mathrm{g} / \mathrm{kg}$ & 4.000 & EPA8080 \\
\hline$\mu \mathrm{g} / \mathrm{kg}$ & 4,000 & EPA8080 \\
\hline$\mu \mathrm{g} / \mathrm{kg}$ & 4,000 & EPA8080 \\
\hline$\mu g / \mathrm{kg}$ & 2,000 & EPA8080 \\
\hline$\mu \mathrm{g} / \mathrm{kg}$ & 2,000 & EPA8080 \\
\hline$\mu g / \mathbf{k g}$ & 2,000 & EPA8080 \\
\hline$\mu g / k g$ & 2,000 & EPA8080 \\
\hline$\mu \mathrm{g} / \mathrm{kg}$ & 2,000 & EPA8080 \\
\hline$\mu g / \mathbf{k g}$ & 2.000 & EPA8080 \\
\hline$\mu g / k g$ & 2,000 & EPA8080 \\
\hline$\mu g / \mathrm{kg}$ & 2,000 & EPA808 \\
\hline$\mu g / k g$ & 2,000 & EPA808 \\
\hline$\mu g / \mathrm{kg}$ & 2,000 & EPA8080 \\
\hline$\mu \mathrm{g} / \mathrm{kg}$ & 2,000 & EPA808 \\
\hline$\mu \mathrm{g} / \mathrm{kg}$ & 2,000 & EPA8080 \\
\hline$\mu g / k g$ & 2,000 & EPA8080 \\
\hline
\end{tabular}


SAMPLE NAME: D431110804 (Continued)

Sample ID: 100332

Constituent

RQ AQ B Result

Unit

D. Limit Method

Pesticides/PCBs and Dioxins/Furans

\begin{tabular}{|c|c|c|c|c|c|c|c|}
\hline PCB 1260 & UJ & o & $\mathbf{L}$ & 2,000 & $\mu g / \mathrm{kg}$ & 2,000 & EPA8080 \\
\hline PCB 1260 & UJ & 0 & L & 2,000 & $\mu \mathrm{g} / \mathrm{kg}$ & 2,000 & EPA8080 \\
\hline Pentachlorodibenzo-p-dioxin isomers & $\mathbf{U}$ & & & 0.20 & $\mathrm{ng} / \mathrm{g}$ & 0.20 & EPA8280 \\
\hline Pentachlorodibenzo-p-furan isomers & $\mathbf{U}$ & & & 0.10 & $\mathrm{ng} / \mathrm{g}$ & 0.10 & EPA8280 \\
\hline $2,3,7,8-T C D D$ & $\mathbf{U}$ & & & 0.10 & $\mathrm{ng} / \mathrm{g}$ & 0.10 & EPA8280 \\
\hline 2,3.7,8-TCDF & $\mathbf{U}$ & & & 0.10 & $\mathrm{ng} / \mathrm{g}$ & 0.10 & EPA8280 \\
\hline Tetrachlorodibenzo-p-dioxin isomers & $\mathbf{U}$ & & & 0.10 & $\mathbf{n g} / \mathbf{g}$ & 0.10 & EPA8280 \\
\hline Tetrachlorodibenzo-p-furan isomers & $\mathrm{U}$ & & & 0.10 & $\mathrm{ng} / \mathrm{g}$ & 0.10 & EPA8280 \\
\hline Toxaphene & UJ & 0 & L & 10,000 & $\mu \mathrm{g} / \mathrm{kg}$ & 10,000 & EPA8080 \\
\hline Toxaphene & UJ & 0 & L & 10,000 & $\mu \mathrm{g} / \mathrm{kg}$ & 10,000 & EPA8080 \\
\hline Toxaphene & UJ & 0 & L & 10,000 & $\mu \mathrm{g} / \mathrm{kg}$ & 10,000 & EPA8080 \\
\hline \multicolumn{8}{|c|}{ Radionuclide Indicators and Radionuclides } \\
\hline Gross alpha & & & & $11 \pm 14$ & $\mathrm{pCi} / \mathrm{g}$ & 7.5 & EPA900.0 \\
\hline Nonvolatile beta & & & & $38 \pm 24$ & $\mathrm{pCi} / \mathrm{g}$ & 9.4 & EPA900.0 \\
\hline Radium, total alpha-emining & $\mathbf{U}$ & $\mathbf{v}$ & & $1.1 \pm 1.1$ & $\mathrm{pCi} / \mathrm{g}$ & 0.10 & EPA903.0 \\
\hline Tritium & & & & $10 \pm 1.9$ & $\mathrm{pCi} / \mathrm{g}$ & 1.5 & EPA906.0 \\
\hline
\end{tabular}

SAMPLE NAME: D43110805

Sample D: 100334

Location (SRS Coordinates): 18542.87E 66623.81N

Ground Elevation Above MSL: $132.8 \mathrm{ft}$

Depth of Core Interval: 6.00 to $7.75 \mathrm{ft}$

Sample Type: Normal

Sample Color: 10R 4.2

Sample Matrix: Soil

USC Soil Classification: SM

RQ AQ B Result Unit

Constituent

Total Metals

Arsenic
Barium
Cadmium
Chromium
Copper
Lead
Manganese
Mercury
Nickel
Selenium
Silver

$B / N / A$ Extractables (including PAH and phenols)

Acenaphthene

Acenaphthylene

Anthracene

Benzo(a)anthracene

$\begin{array}{lll}\mathrm{U} & 0.82 & \mathrm{mg} / \mathrm{kg} \\ \mathrm{U} & 22 & \mathrm{mg} / \mathrm{kg} \\ \mathrm{U} & 0.56 & \mathrm{mg} / \mathrm{kg} \\ & 10 & \mathrm{mg} / \mathrm{kg} \\ & 5.3 & \mathrm{mg} / \mathrm{kg} \\ & 4.2 & \mathrm{mg} / \mathrm{kg} \\ \mathrm{U} & 19 & \mathrm{mg} / \mathrm{kg} \\ \mathrm{U} & 0.12 & \mathrm{mg} / \mathrm{kg} \\ \mathrm{U} & 4.9 & \mathrm{mg} / \mathrm{kg} \\ \mathrm{U} & 0.41 & \mathrm{mg} / \mathrm{kg} \\ & 1.1 & \mathrm{mg} / \mathrm{kg}\end{array}$

J 97

U 810

J 230

J 300

$\mu g / \mathrm{kg}$

$\mu \mathrm{g} / \mathrm{kg}$

$\mu \mathrm{g} / \mathrm{kg}$

$\mu \mathrm{g} / \mathrm{kg}$
Sample Moisture: Damp Percent Solids: 84.50

D. Limit Method

$\begin{array}{ll}0.82 & \text { EPA7060 } \\ 22 & \text { EPA6010 } \\ 0.56 & \text { EPA6010 } \\ 1.1 & \text { EPA6010 } \\ 2.8 & \text { EPA6010 } \\ 0.25 & \text { EPA7421 } \\ 1.7 & \text { EPA6010 } \\ 0.12 & \text { EPA7470 } \\ 4.5 & \text { EPA6010 } \\ 0.41 & \text { EPA7740 } \\ 1.1 & \text { EPA6010 }\end{array}$

810

810
EPA8270

EPA8270

EPA8270

EPA8270 
SAMPLE NAME: D43110805 (Continued)

RQ AQ B Result

Constituent
Sample ID: 100334

Unit

D. Limit Method

$B / N / A$ Extractables (including $P A H$ and phenols)

\begin{tabular}{|c|c|c|c|c|}
\hline Benzo(b)fluoranthene & J & & 330 & $\mu \mathrm{g} / \mathrm{kg}$ \\
\hline Benzo(k)fluoranthene & J & & 130 & $\mu \mathrm{g} / \mathrm{kg}$ \\
\hline Benzoic acid & $\mathbf{U}$ & & 4,100 & \\
\hline Benzo(g,h,i)perylene & $\mathbf{U}$ & & 810 & \\
\hline Benzo(a)pyrene & $J$ & & 260 & \\
\hline Benzyl alcohol & $\mathbf{U}$ & & 810 & \\
\hline Bis(2-chloroethoxy) methane & $\mathbf{U}$ & & 810 & \\
\hline Bis(2-chloroethyl) ether & $\mathbf{U}$ & & 810 & \\
\hline Bis(2-chloroisopropyl) ether & $\mathbf{U}$ & & 810 & \\
\hline Bis(2-ethylhexyl) phthalate & & & 1.000 & \\
\hline 4-Bromophenyl phenyl ether & $\mathbf{U}$ & & 810 & \\
\hline Butylbenzyl phthalate & & & 3,100 & \\
\hline Alkylbenzene C15H16 & J & $\mathbf{N}$ & 400 & \\
\hline Arom. hydrocarbon $\mathrm{C} 24 \mathrm{H} 18$ & $J$ & $\mathrm{~N}$ & 400 & \\
\hline 4-Chloroaniline & $\mathrm{U}$ & & 810 & \\
\hline 4-Chloro-m-cresol & U & & 810 & \\
\hline 2-Chloronaphthalene & U̦ & & 810 & \\
\hline 2-Chlorophenol & U & & 810 & $\mu g / \mathrm{kg}$ \\
\hline 4-Chlorophenyl phenyl ether & $U$ & & 810 & $\mu g$ \\
\hline Chrysene & $J$ & & 310 & $\mu \mathrm{g} / \mathrm{k}$ \\
\hline o-Cresol (2-Methylphenol) & $\mathbf{U}$ & & 810 & \\
\hline p-Cresol (4-Methylphenol) & $\mathbf{U}$ & & 810 & $\mu \mathrm{g} /$ \\
\hline Dibenz $(a, h)$ anthracene & $\mathrm{U}$ & & 810 & $\mu \mathrm{g}$ \\
\hline Dibenzofuran & $\mathbf{U}$ & & 810 & $\mu g / k$ \\
\hline Di-n-butyl phthalate & UJ & V & 140 & $\mu \mathrm{g} / \mathrm{l}$ \\
\hline 1,2-Dichlorobenzene & $\mathrm{U}$ & & 810 & $\mu g /$ \\
\hline 1,3-Dichlorobenzene & U & & 810 & $\mu \mathrm{g} / \mathrm{kg}$ \\
\hline 1,4-Dichlorobenzene & $U$ & & 810 & $\mu g / \mathrm{kg}$ \\
\hline 3,3'-Dichlorobenzidine & $\mathbf{U}$ & & 1,600 & $\mu g / k$ \\
\hline 2,4-Dichlorophenol & U & & 810 & $\mu g /$ \\
\hline Diethyl phthalate & U & & 810 & \\
\hline 2,4-Dimethyl phenol & $\mathbf{U}$ & & 810 & $\mu \mathrm{g} / \mathrm{k}$ \\
\hline Dimethyl phthalate & $\mathrm{U}$ & & 810 & $\mu g /$ \\
\hline 2,4-Dinitrophenol & $\mathbf{U}$ & & 4,100 & $\mu \mathrm{g} /$ \\
\hline 2,4-Dinitrotoluene & $\mathbf{U}$ & & 810 & $\mu g / k$ \\
\hline 2,6-Dinitrotoluene & $\mathbf{U}$ & & 810 & $\mu \mathrm{g} / \mathrm{l}$ \\
\hline Di-n-octyl phthalate & $\mathbf{U}$ & & 810 & $\mu \mathrm{g} / \mathrm{kg}$ \\
\hline Fluoranthene & & & 880 & $\mu g / k$ \\
\hline Fluorene & J & & 100 & $\mu \mathrm{g} / \mathrm{l}$ \\
\hline Hexachlorobenzene & $U$ & & 810 & $\mu g / k$ \\
\hline Hexachlorobutadiene & U & & 810 & $\mu g / k$ \\
\hline Hexachlorocyclopentadiene & $\mathrm{U}$ & & 810 & $\mu g / k$ \\
\hline Hexachloroethane & $\mathbf{U}$ & & 810 & $\mu \mathrm{g} / \mathrm{l}$ \\
\hline Indeno(1,2,3-c,d)pyrene & $\mathrm{J}$ & & 83 & $\mu g /$ \\
\hline Isophorone & U & & 810 & $\mu \mathrm{g} / \mathrm{k}$ \\
\hline Dimethylanthracene isomer & $J$ & $\mathbf{N}$ & 600 & $\mu g / k$ \\
\hline Methyldibenzothiophene isomer & J & $\mathbf{N}$ & 700 & $\mu g / k g$ \\
\hline Methyldibenzothiophene isomer & J & $\mathbf{N}$ & 500 & $\mu g / k$ \\
\hline Dimethylphenanthrene isomer & J & $\mathrm{N}$ & 600 & \\
\hline Dimethylphenanthrene isomer & J & $\mathrm{N}$ & 1.000 & \\
\hline
\end{tabular}

\begin{tabular}{|c|c|}
\hline 810 & EPA8270 \\
\hline 810 & EPA8270 \\
\hline 4,100 & EPA8270 \\
\hline 810 & EPA8270 \\
\hline 810 & EPA8270 \\
\hline 810 & EPA8270 \\
\hline 810 & EPA8270 \\
\hline 810 & EPA8270 \\
\hline 810 & EPA8270 \\
\hline 810 & EPA8270 \\
\hline 810 & EPA8270 \\
\hline \multirow[t]{2}{*}{810} & EPA8270 \\
\hline & $\begin{array}{l}\text { EPA8270 } \\
\text { EPA8270 }\end{array}$ \\
\hline 810 & EPA8270 \\
\hline 810 & EPA8270 \\
\hline 810 & EPA8270 \\
\hline 810 & EPA8270 \\
\hline 810 & EPA8270 \\
\hline 810 & EPA8270 \\
\hline 810 & EPA8270 \\
\hline 810 & EPA8270 \\
\hline 810 & EPA8270 \\
\hline 810 & EPA8270 \\
\hline 810 & EPA8270 \\
\hline 810 & EPA8270 \\
\hline 810 & EPA8270 \\
\hline 810 & EPA8270 \\
\hline 1,600 & EPA8270 \\
\hline 810 & EPA8270 \\
\hline 810 & EPA8270 \\
\hline 810 & EPA8270 \\
\hline 810 & EPA8270 \\
\hline 4,100 & EPA8270 \\
\hline 810 & EPA8270 \\
\hline 810 & EPA8270 \\
\hline 810 & EPA8270 \\
\hline 810 & EPA8270 \\
\hline 810 & EPA8270 \\
\hline 810 & EPA8270 \\
\hline 810 & EPA8270 \\
\hline 810 & EPA8270 \\
\hline 810 & EPA8270 \\
\hline 810 & EPA8270 \\
\hline 810 & EPA8270 \\
\hline & EPA8270 \\
\hline & EPA8270 \\
\hline & EPA8270 \\
\hline & $\begin{array}{l}\text { EPA8270 } \\
\text { EPA8270 }\end{array}$ \\
\hline
\end{tabular}


SAMPLE NAME: D43110805 (Continued)

Sample [D: 100334

$\begin{array}{llllll}\text { Constituent } & \text { RQ AQ } & \text { B Result Unit D. Limit Method }\end{array}$

$B / N / A$ Extractables (including PAH and phenols)

Dimethylphenanthrene isomer

Trimethylphenanthrene isomer

Trimethylphenanthrene isomer

Methylanthracene isomer

Methylanthracene isomer

Methylanthracene isomer

Methylphenanthrene isomer

2-Methyl-4,6-dinitrophenol

2-Methylnaphthalene

Naphthalene

o-Nitroaniline

m-Nitroaniline

p-Nitroaniline

Nitrobenzene

2-Nitrophenol

4-Nitrophenol

N-Nitrosodiphenylamine

N-Nitrosodipropylamine

Pentachlorophenol

Phenanthrene

Phenol

Pyrene

1,2,4-Trichlorobenzene

2,4,5-Trichlorophenol

2,4,6-Trichlorophenol

Unknown arom. hydrocarbon

Unknown arom. hydrocarbon

Unknown hydrocarbon

Unknown hydrocarbon

Unknown ketone

Volatile Organic Compounds

$\begin{array}{llll}\mathrm{J} & \mathrm{N} & 500 & \mu \mathrm{g} / \mathrm{kg} \\ \mathrm{J} & \mathrm{N} & 500 & \mu \mathrm{g} / \mathrm{kg} \\ \mathrm{J} & \mathrm{N} & 600 & \mu \mathrm{g} / \mathrm{kg} \\ \mathrm{J} & \mathrm{N} & 1,000 & \mu \mathrm{g} / \mathrm{kg} \\ \mathrm{J} & \mathrm{N} & 900 & \mu \mathrm{g} / \mathrm{kg} \\ \mathrm{J} & \mathrm{N} & 400 & \mu \mathrm{g} / \mathrm{kg} \\ \mathrm{J} & \mathrm{N} & 1,000 & \mu \mathrm{g} / \mathrm{kg} \\ \mathrm{U} & & 4,100 & \mu \mathrm{g} / \mathrm{kg} \\ \mathrm{U} & & 810 & \mu \mathrm{g} / \mathrm{kg} \\ \mathrm{U} & & 810 & \mu \mathrm{g} / \mathrm{kg} \\ \mathrm{U} & & 4,100 & \mu \mathrm{g} / \mathrm{kg} \\ \mathrm{U} & & 4,100 & \mu \mathrm{g} / \mathrm{kg} \\ \mathrm{U} & & 4,100 & \mu \mathrm{g} / \mathrm{kg} \\ \mathrm{U} & & 810 & \mu \mathrm{g} / \mathrm{kg} \\ \mathrm{U} & & 810 & \mu \mathrm{g} / \mathrm{kg} \\ \mathrm{U} & & 4,100 & \mu \mathrm{g} / \mathrm{kg} \\ \mathrm{U} & & 810 & \mu \mathrm{g} / \mathrm{kg} \\ \mathrm{U} & & 810 & \mu \mathrm{g} / \mathrm{kg} \\ \mathrm{U} & & 4,100 & \mu \mathrm{g} / \mathrm{kg} \\ & & 1,600 & \mu \mathrm{g} / \mathrm{kg} \\ \mathrm{U} & & 810 & \mu \mathrm{g} / \mathrm{kg} \\ & & 830 & \mu \mathrm{g} / \mathrm{kg} \\ \mathrm{U} & & 810 & \mu \mathrm{g} / \mathrm{kg} \\ \mathrm{U} & & 4,100 & \mu \mathrm{g} / \mathrm{kg} \\ \mathrm{U} & & 810 & \mu \mathrm{g} / \mathrm{kg} \\ \mathrm{J} & \mathrm{N} & 500 & \mu \mathrm{g} / \mathrm{kg} \\ \mathrm{J} & \mathrm{N} & 600 & \mu \mathrm{g} / \mathrm{kg} \\ \mathrm{J} & \mathrm{N} & 600 & \mu \mathrm{g} / \mathrm{kg} \\ \mathrm{J} & \mathrm{N} & 400 & \mu \mathrm{g} / \mathrm{kg} \\ \mathrm{J} & \mathrm{NV} & 6,000 & \mu \mathrm{g} / \mathrm{kg} \\ & & & \end{array}$

EPA8270

EPA8270

EPA8270

EPA8270

EPA8270

EPA8270

EPA8270

$4,100 \quad$ EPA8270

$810 \quad$ EPA8270

810 EPA8270

$4.100 \quad$ EPA 8270

$4,100 \quad$ EPA8270

4,100 EPA8270

$810 \quad$ EPA8270

$810 \quad$ EPA8270

$4,100 \quad$ EPA8270

$810 \quad$ EPA8270

$810 \quad$ EPA8270

$4,100 \quad$ EPA8270

810 EPA8270

$810 \quad$ EPA8270

$810 \quad$ EPA8270

810 EPA8270

$4,100 \quad$ EPA8270

$810 \quad$ EPA8270

EPA8270

EPA8270

EPA8270

EPA8270

EPA8270

$\begin{array}{lll}\mu \mathrm{g} / \mathrm{kg} & 12 & \text { EPA8240 } \\ \mu \mathrm{g} / \mathrm{kg} & 25 & \text { EPA8240 } \\ \mu \mathrm{g} / \mathrm{kg} & 6.2 & \text { EPA8240 } \\ \mu \mathrm{g} / \mathrm{kg} & 6.2 & \text { EPA8240 } \\ \mu \mathrm{g} / \mathrm{kg} & 6.2 & \text { EPA8240 } \\ \mu \mathrm{g} / \mathrm{kg} & 12 & \text { EPA8240 } \\ \mu \mathrm{g} / \mathrm{kg} & 6.2 & \text { EPA8240 } \\ \mu \mathrm{g} / \mathrm{kg} & 6: 2 & \text { EPA8240 } \\ \mu \mathrm{g} / \mathrm{kg} & 6.2 & \text { EPA8240 } \\ \mu \mathrm{g} / \mathrm{kg} & 12 & \text { EPA8240 } \\ \mu \mathrm{g} / \mathrm{kg} & 12 & \text { EPA8240 } \\ \mu \mathrm{g} / \mathrm{kg} & 6.2 & \text { EPA8240 } \\ \mu \mathrm{g} / \mathrm{kg} & 12 & \text { EPA8240 } \\ \mu \mathrm{g} / \mathrm{kg} & 6.2 & \text { EPA8240 } \\ \mu \mathrm{g} / \mathrm{kg} & 6.2 & \text { EPA8240 } \\ \mu \mathrm{g} / \mathrm{kg} & 6.2 & \text { EPA8240 } \\ \mu \mathrm{g} / \mathrm{kg} & 6.2 & \text { EPA8240 }\end{array}$


SAMPLE NAME: D43110805 (Continued)

Constituent

Volatile Organic Compounds

1.2-Dichloroethylene

Dichloromethane (Methylene chloride)

1.2-Dichloropropane

cis-1,3-Dichloropropene

trans-1,3-Dichloropropene

Ethylbenzene

2-Hexanone

Methyl ethyl ketone

Methyl isobutyl ketone

Styrene

1,1,2,2-Tetrachloroethane

Tetrachloroethylene

Toluene

1.1.1-Trichloroethane

1,1.2-Trichloroethane

Trichloroethylene

Vinyl acetate

2-Propanol

Xylenes
Sample ID: 100334

RQ AQ B Result

Unit

D. Limit Method

Pesticides/PCBs and Dioxins/Furans

Aldrin

alpha-Benzene hexachloride

beta-Benzene hexachloride

delta-Benzene hexachloride

alpha-Chlordane

gamma-Chlordane

p.p'-DDD

p,p'-DDE

p.p'-DDT

Dieldrin

Endosulfan I

Endosulfan II

Endosulfan sulfate

Endrin

Endrin ketone

Heptachlor

Heptachlor epoxide

Heptachlorodibenzo-p-dioxin isomers

Heptachlorodibenzo-p-furan isomers

Hexachlorodibenzo-p-dioxin isomers

Hexachlorodibenzo-p-furan isomers

Lindane

Methoxychlor

Octachlorodibenzo-p-dioxin

Octachlorodibenzo-p-furan

PCB 1016

PCB 1221

PCB 1232

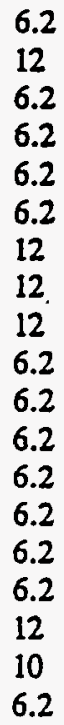

$\mu g / \mathrm{kg}$

$\mu g / \mathrm{kg}$

$\mu g / k g$

$\mu g / \mathrm{kg}$

$\mu \mathrm{g} / \mathrm{kg}$

$\mu \mathrm{g} / \mathrm{kg}$

$\mu g / \mathrm{kg}$

$\mu \mathrm{g} / \mathrm{kg}$

$\mu \mathrm{g} / \mathrm{kg}$

$\mu \mathrm{g} / \mathrm{kg}$

$\mu \mathrm{g} / \mathrm{kg}$

$\mu \mathrm{g} / \mathrm{kg}$

$\mu \mathrm{g} / \mathrm{kg}$

$\mu \mathrm{g} / \mathrm{kg}$

$\mu \mathrm{g} / \mathrm{kg}$

$\mu \mathrm{g} / \mathrm{kg}$

$\mu \mathrm{g} / \mathrm{kg}$

$\mu \mathrm{g} / \mathrm{kg}$

$\mu \mathrm{g} / \mathrm{kg}$

$\mu \mathrm{g} / \mathrm{kg}$

$\mu \mathrm{g} / \mathrm{kg}$

$\mu \mathrm{g} / \mathrm{kg}$

$\mu \mathrm{g} / \mathrm{kg}$

$\mu g / \mathrm{kg}$

$\mu \mathrm{g} / \mathrm{kg}$

$\mu \mathrm{g} / \mathrm{kg}$

$\mu \mathrm{g} / \mathrm{kg}$

$\mu \mathrm{g}^{\prime} \mathrm{kg}$

$\mu \mathrm{g} / \mathrm{kg}$

$\mu \mathrm{g} / \mathrm{kg}$

$\mu \mathrm{g} / \mathrm{kg}$

$\mu \mathrm{g} / \mathrm{kg}$

$\mu \mathrm{g} / \mathrm{kg}$

$\mu \mathrm{g} / \mathrm{kg}$

$\mu g / \mathrm{kg}$

$\mu \mathrm{g} / \mathrm{kg}$

$\mathrm{ng} / \mathrm{g}$

ng'g

$\mathrm{ng} / \mathrm{g}$

$\mathrm{ng} / \mathrm{g}$

$\mu \mathrm{g} / \mathrm{kg}$

$\mu \mathrm{g} / \mathrm{kg}$

$\mathrm{ng} / \mathrm{g}$

$\mathrm{ng} / \mathrm{g}$

$\mu \mathrm{g} / \mathrm{kg}$

$\mu \mathrm{g} / \mathrm{kg}$

$\mu \mathrm{g} / \mathrm{kg}$
6.2

6.2

6.2

6.2

6.2

6.2

12

12

12

6.2

6.2

6.2

6.2

6.2

6.2

6.2

12

6.2

EPA8240

EPA8240

EPA8240

EPA8240

EPA8240

EPA8240

EPA8240

EPA8240

EPA8240

EPA8240

EPA8240

EPA8240

EPA8240

EPA8240

EPA8240

EPA8240

EPA8240

EPA8240

EPA8240

EPA8080

EPA8080

EPA8080

EPA8080

EPA8080

EPA8080

EPA8080

EPA8080

EPA8080

EPA8080

EPA8080

EPA8080

EPA8080

EPA8080

EPA8080

EPA8080

EPA8080

EPA8280

EPA8280

EPA8280

EPA8280

EPA8080

EPA8080

EPA8280

EPA8280

EPA8080

EPA8080

EPA8080 
SAMPLE NAME: D43110805 (Continued) Sample ID: 100334

\section{Constituent}

$\mathbf{R Q}$

Pesticides/PCBs and Dioxins/Furans

PCB 1242

PCB 1248

PCB 1254

PCB 1260

Pentachlorodibenzo-p-dioxin isomers

Pentachlorodibenzo-p-furan isomers

2,3,7,8-TCDD

2,3,7,8-TCDF

Tetrachlorodibenzo-p-dioxin isomers

Tetrachlorodibenzo-p-furan isomers

Toxaphene

$\begin{array}{lll}\mathrm{U} & 400 & \mu \mathrm{g} / \mathrm{kg} \\ \mathrm{U} & 400 & \mu \mathrm{k} / \mathrm{kg} \\ \mathrm{U} & 400 & \mu \mathrm{g} / \mathrm{kg} \\ \mathrm{U} & 400 & \mu \mathrm{g} / \mathrm{kg} \\ \mathrm{U} & 0.10 & \mathrm{ng} / \mathrm{g} \\ \mathrm{U} & 0.10 & \mathrm{ng} / \mathrm{g} \\ \mathrm{U} & 0.10 & \mathrm{ng} / \mathrm{g} \\ \mathrm{U} & 0.10 & \mathrm{ng} / \mathrm{g} \\ \mathrm{U} & 0.10 & \mathrm{ng} / \mathrm{g} \\ \mathrm{U} & 0.10 & \mathrm{ng} / \mathrm{g} \\ \mathrm{U} & 2,000 & \mu \mathrm{g} / \mathrm{kg}\end{array}$

Unit

D. Limit Method

Radionuclide Indicators and Radionuclides

Gross alpha

Nonvolatile beta

Radium, total alpha-emitting

Tritium

SAMPLE NAME: D43!10805A

Sample Type: Duplicate

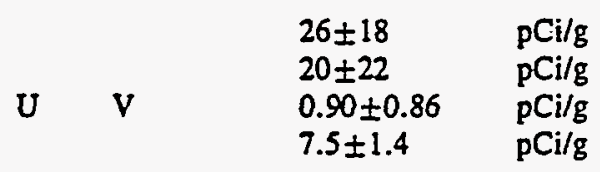

Sample ID: 100335

Associated Sample: 100334

RQ AQ B Result Unit

\section{Total Metals}

Arsenic

Barium

Cadmium

Chromium

Copper

Lead

Manganese

Mercury

Nickel

Selenium

Silver

\section{U}

U

U

U

$\begin{array}{ll}1.1 & \mathrm{mg} / \mathrm{kg} \\ 22 & \mathrm{mg} / \mathrm{kg} \\ 0.79 & \mathrm{mg} / \mathrm{kg} \\ 21 & \mathrm{mg} / \mathrm{kg} \\ 5.9 & \mathrm{mg} / \mathrm{kg} \\ 6.5 & \mathrm{mg} / \mathrm{kg} \\ 17 & \mathrm{mg} / \mathrm{kg} \\ 0.12 & \mathrm{mg} / \mathrm{kg} \\ 4.8 & \mathrm{mg} / \mathrm{kg} \\ 0.54 & \mathrm{mg} / \mathrm{kg} \\ 1.1 & \mathrm{mg} / \mathrm{kg}\end{array}$

$B / N / A$ Extractables (including $P A H$ and phenols)

Acenaphthene

Acenaphthylene

Anthracene

Benzo(a)anthracene

Benzo(b)fluoranthene

Benzo(k)fluoranthene

Benzoic acid

Benzo $(g, h, i)$ perylene

$\begin{array}{ll}\mathbf{U} & 420 \\ \mathbf{U} & 420 \\ \mathrm{U} & 420 \\ \mathrm{U} & 420 \\ \mathrm{U} & 420 \\ \mathrm{U} & 420 \\ \mathrm{U} & 2.100 \\ \mathrm{U} & 420\end{array}$

$\mu g / \mathrm{kg}$
$\mu g / \mathrm{kg}$
$\mu g / \mathrm{kg}$
$\mu g / \mathrm{kg}$
$\mu g / \mathrm{kg}$
$\mu g / \mathrm{kg}$
$\mu g / \mathrm{kg}$
$\mu g / \mathrm{kg}$
7.7

9.6

0.080

1.1
EPA8080

EPA8080

EPA8080

EPA8080

EPA8280

EPA8280

EPA8280

EPA8280

EPA8280

EPA8280

EPA8080 


$\begin{array}{llllll}\text { Constituent } & \text { RQ AQ B Result Unit Limit Method }\end{array}$

$B / N / A$ Extractables (including $P A H$ and phenols)

\begin{tabular}{|c|c|c|c|c|c|c|}
\hline Benzo(a)pyrene & $\mathbf{U}$ & & 420 & $\mu \mathrm{g} / \mathrm{kg}{ }^{\circ}$ & 420 & EPA8270 \\
\hline Benzyl alcohol & $\mathbf{U}$ & & 420 & $\mu g / k g$ & 420 & EPA8270 \\
\hline Bis(2-chloroethoxy) methane & $\mathbf{U}$ & & 420 & $\mu \mathrm{g} / \mathrm{kg}$ & 420 & EPA8270 \\
\hline Bis(2-chloroethyl) ether & $\mathbf{U}$ & & 420 & $\mu g / k g$ & 420 & EPA8270 \\
\hline Bis(2-chloroisopropyl) ether & $\mathbf{U}$ & & 420 & $\mu \mathrm{g} / \mathrm{kg}$ & 420 & EPA8270 \\
\hline Bis(2-ethylhexyl) phthalate & J & & 190 & $\mu \mathrm{g} / \mathrm{kg}$ & 420 & EPA8270 \\
\hline 4-Bromophenyl phenyl ether & $\mathbf{U}$ & & 420 & $\mu \mathrm{g} / \mathrm{kg}$ & 420 & EPA8270 \\
\hline Butylbenzyl phthalate & $\mathrm{J}$ & & 180 & $\mu g / \mathrm{kg}$ & 420 & EPA8270 \\
\hline 4-Chloroaniline & $\mathbf{U}$ & & 420 & $\mu \mathrm{g} / \mathrm{kg}$ & 420 & EPA8270 \\
\hline 4-Chloro-m-cresol & $\mathbf{U}$ & & 420 & $\mu \mathrm{g} / \mathrm{kg}$ & 420 & EPA8270 \\
\hline 2-Chloronaphthalene & $\mathbf{U}$ & & 420 & $\mu \mathrm{g} / \mathrm{kg}$ & 420 & EPA8270 \\
\hline 2-Chlorophenol & $\mathbf{U}$ & & 420 & $\mu \mathrm{g} / \mathrm{kg}$ & 420 & EPA8270 \\
\hline 4-Chlorophenyl phenyl echer & $\mathrm{U}$ & & 420 & $\mu \mathrm{g} / \mathrm{kg}$ & 420 & EPA8270 \\
\hline Chrysene & $\mathbf{U}$ & & 420 & $\mu \mathrm{g} / \mathrm{kg}$ & 420 & EPA8270 \\
\hline o-Cresol (2-Methylphenol) & $\mathbf{U}$ & & 420 & $\mu \mathrm{g} / \mathrm{kg}$ & 420 & EPA8270 \\
\hline p-Cresol (4-Methylphenol) & $U$ & & 420 & $\mu \mathrm{g} / \mathrm{kg}$ & 420 & EPA8270 \\
\hline Dibenz(a,h)anthracene & $\mathbf{U}$ & & 420 & $\mu \mathrm{g} / \mathrm{kg}$ & 420 & EPA8270 \\
\hline Dibenzofuran & $\mathrm{U}$ & & 420 & $\mu \mathrm{g} / \mathrm{kg}$ & 420 & EPA8270 \\
\hline Di-n-butyl phthalate & UJ & V & 93 & $\mu g / \mathrm{kg}$ & 420 & EPA8270 \\
\hline 1,2-Dichlorobenzene & $\mathrm{U}$ & & 420 & $\mu g / \mathrm{kg}$ & 420 & EPA 8270 \\
\hline 1,3-Dichlorobenzene & $U$ & & 420 & $\mu \mathrm{g} / \mathrm{kg}$ & 420 & EPA8270 \\
\hline 1.4-Dichlorobenzene & $\mathrm{U}$ & & 420 & $\mu \mathrm{g} / \mathrm{kg}$ & 420 & EPA8270 \\
\hline 3,3'-Dichlorobenzidine & $\mathbf{U}$ & & 840 & $\mu g / k g$ & 840 & EPA8270 \\
\hline 2,4-Dichlorophenol & $\mathbf{U}$ & & 420 & $\mu \mathrm{g} / \mathrm{kg}$ & 420 & EPA8270 \\
\hline Diethyl phthalate & $\mathbf{U}$ & & 420 & $\mu \mathrm{g} / \mathrm{kg}$ & 420 & EPA8270 \\
\hline 2,4-Dimethyl phenol & $\mathbf{U}$ & & 420 & $\mu \mathrm{g} / \mathrm{kg}$ & 420 & EPA8270 \\
\hline Dimethyl phthalate & U & & 420 & $\mu \mathrm{g} / \mathrm{kg}$ & 420 & EPA8270 \\
\hline 2,4-Dinitrophenol & $\mathrm{U}$ & & 2,100 & $\mu \mathrm{g} / \mathrm{kg}$ & 2,100 & EPA8270 \\
\hline 2.4-Dinitrotoluene & $\mathbf{U}$ & & 420 & $\mu g / k g$ & 420 & EPA8270 \\
\hline 2,6-Dinitrotoluene & $\mathrm{U}$ & & 420 & $\mu \mathrm{g} / \mathrm{kg}$ & 420 & EPA8270 \\
\hline Di-n-octyl phthalate & $\mathrm{U}$ & & 420 & $\mu \mathrm{g} / \mathrm{kg}$ & 420 & EPA8270 \\
\hline Fluoranthene & J & & 110 & $\mu g / \mathrm{kg}$ & 420 & EPA8270 \\
\hline Fluorene & $\mathbf{U}$ & & 420 & $\mu \mathrm{g} / \mathrm{kg}$ & 420 & EPA8270 \\
\hline Hexachlorobenzene & $\mathrm{U}$ & & 420 & $\mu \mathrm{g} / \mathrm{kg}$ & 420 & EPA8270 \\
\hline Hexachlorobutadiene & $\mathbf{U}$ & & 420 & $\mu \mathrm{g} / \mathrm{kg}$ & 420 & EPA8270 \\
\hline Hexachlorocyclopentadiene & $\mathbf{U}$ & & 420 & $\mu g / \mathrm{kg}$ & 420 & EPA8270 \\
\hline Hexachloroethane & $\mathbf{U}$ & & 420 & $\mu \mathrm{g} / \mathrm{kg}$ & 420 & EPA8270 \\
\hline Indeno(1,2,3-c,d)pyrene & $\mathbf{U}$ & & 420 & $\mu g / \mathrm{kg}$ & 420 & EPA8270 \\
\hline Isophorone & $\mathbf{U}$ & & 420 & $\mu \mathrm{g} / \mathrm{kg}$ & 420 & EPA8270 \\
\hline Dimethylphenanthrene isomer & $\mathrm{J}$ & $\mathbf{N}$ & 400 & $\mu g / \mathrm{kg}$ & . & EPA8270 \\
\hline Trimethylanthracene isomer & $\mathbf{J}$ & $\mathbf{N}$ & 200 & $\mu g / k g$ & & EPA8270 \\
\hline Trimethylphenanthrene isomer & $\mathbf{J}$ & $\mathbf{N}$ & 200 & $\mu g / k g$ & & EPA8270 \\
\hline Methylanthracene isomer & $\mathrm{J}$ & $\mathbf{N}$ & 300 & $\mu g / \mathrm{kg}$ & & EPA8270 \\
\hline Methylphenanthrene isomer & J & $\mathbf{N}$ & 300 & $\mu \mathrm{g} / \mathrm{kg}$ & $n$ & EPA8270 \\
\hline 2-Methyl-4,6-dinitrophenol & $\mathbf{U}$ & & 2,100 & $\mu \mathrm{g} / \mathrm{kg}$ & 2,100 & EPA8270 \\
\hline 2-Methylnaphthalene & $\mathrm{U}$ & & 420 & $\mu \mathrm{g} / \mathrm{kg}$ & 420 & EPA8270 \\
\hline Naphthalene & $\mathbf{U}$ & & 420 & $\mu g / \mathrm{kg}$ & 420 & EPA8270 \\
\hline o-Nitroaniline & U & & 2,100 & $\mu g / k g$ & 2,100 & EPA8270 \\
\hline m-Nitroaniline & $\mathrm{U}$ & & 2,100 & $\mu g / \mathrm{kg}$ & 2.100 & EPA8270 \\
\hline $\mathrm{p}$-Nitroaniline & $\mathbf{U}$ & & 2,100 & $\mu \mathrm{g} / \mathrm{kg}$ & 2.100 & EPA8270 \\
\hline
\end{tabular}


SAMPLE NAME: D43110805A (Continued)

Sample ID: 100335

\section{Constituent}

RQ AQ B Result

Unit

D. Limit Method

$B / N / A$ Extractables (including $P A H$ and phenols)

Nitrobenzene

2-Nitrophenol

4-Nitrophenol

N-Nitrosodiphenylamine

N-Nitrosodipropylamine

Pentachlorophenol

Phenanthrene

Phenol

Pyrene

1,2,4-Trichlorobenzene

2,4,5-Trichlorophenol

2,4,6-Trichlorophenol

Unknown arom. hydrocarbon

Unknown hydrocarbon

Unknown ketone

Volatile Organic Compounds

$\begin{array}{lll}U & & 420 \\ U & & 420 \\ U & & 2,100 \\ U & & 420 \\ U & & 420 \\ U & & 2,100 \\ J & & 260 \\ U & & 420 \\ J & & 120 \\ U & & 420 \\ U & & 2,100 \\ U & & 420 \\ J & N & 200 \\ J & N & 200 \\ J & N V & 4,000\end{array}$

$$
\begin{aligned}
& \mu g / k g \\
& \mu g / k g \\
& \mu g / k g \\
& \mu g / k g \\
& \mu g / k g \\
& \mu g / k g \\
& \mu g / k g \\
& \mu g / k g \\
& \mu g / k g \\
& \mu g / k g \\
& \mu g / k g \\
& \mu g / k g \\
& \mu g / k g \\
& \mu g / k g \\
& \mu g / k g
\end{aligned}
$$

420
420
2,100
420
420
2,100
420
420
420
420
2,100
420

EPA8270

EPA8270

EPA8270

EPA8270

EPA8270

EPA8270

EPA8270

EPA8270

EPA8270

EPA8270

EPA8270

EPA8270

EPA8270

EPA8270

EPA8270

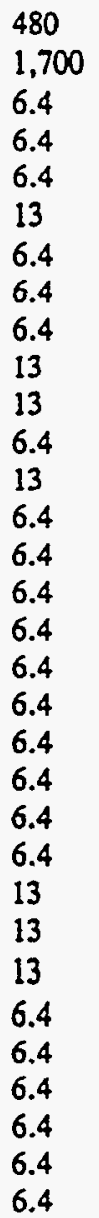

$$
\begin{aligned}
& \mu g / k g \\
& \mu g / k g \\
& \mu g / k g \\
& \mu g / k g \\
& \mu g / k g \\
& \mu g / k g \\
& \mu g / k g \\
& \mu g / k g \\
& \mu g / k g \\
& \mu g / k g \\
& \mu g / k g \\
& \mu g / k g \\
& \mu g / k g \\
& \mu g / k g \\
& \mu g / k g \\
& \mu g / k g \\
& \mu g / k g \\
& \mu g / k g \\
& \mu g / k g \\
& \mu g / k g \\
& \mu g / k g \\
& \mu g / k g \\
& \mu g / k g \\
& \mu g / k g \\
& \mu g / k g \\
& \mu g / k g \\
& \mu g / k g \\
& \mu g / k g \\
& \mu g / k g \\
& \mu g / k g \\
& \mu g / k g \\
& \mu g / k g
\end{aligned}
$$

$\begin{array}{ll}13 & \text { EPA8240 } \\ 130 & \text { EPA8240 } \\ 6.4 & \text { EPA8240 } \\ 6.4 & \text { EPA8240 } \\ 6.4 & \text { EPA8240 } \\ 13 & \text { EPA8240 } \\ 6.4 & \text { EPA8240 } \\ 6.4 & \text { EPA8240 } \\ 6.4 & \text { EPA8240 } \\ 13 & \text { EPA8240 } \\ 13 & \text { EPA8240 } \\ 6.4 & \text { EPA8240 } \\ 13 & \text { EPA8240 } \\ 6.4 & \text { EPA8240 } \\ 6.4 & \text { EPA8240 } \\ 6.4 & \text { EPA8240 } \\ 6.4 & \text { EPA8240 } \\ 6.4 & \text { EPA8240 } \\ 6.4 & \text { EPA8240 } \\ 66.4 . & \text { EPA8240 } \\ 6.4 & \text { EPA8240 } \\ 6.4 & \text { EPA8240 } \\ 6.4 & \text { EPA8240 } \\ 13 & \text { EPA8240 } \\ 13 & \text { EPA8240 } \\ 13 & \text { EPA8240 } \\ 6.4 & \text { EPA8240 } \\ 6.4 & \text { EPA8240 } \\ 6.4 & \text { EPA8240 } \\ 6.4 & \text { EPA8240 } \\ 6.4 & \text { EPA8240 } \\ 6.4 & \text { EPA8240 }\end{array}$


SAMPLE NAME: D43110805A (Continued)

Sample ID: 100335

\section{Constituent}

Volatile Organic Compounds

Trichloroethylene

Vinyl acetate

2-Propanol

Xylenes

\section{RQ AQ B Result}

6.4
13
40
6.4

Pesticides/PCBs and Dioxins/Furans

$\begin{array}{lll}\mathrm{U} & \\ \mathrm{U} & \mathrm{N} \\ \mathrm{U} & & \end{array}$

$\mu g / \mathrm{kg}$
$\mu \mathrm{g} / \mathrm{kg}$
$\mu \mathrm{g} / \mathrm{kg}$
$\mu \mathrm{g} / \mathrm{kg}$

$\mu \mathrm{g} / \mathrm{kg}$

$\mu \mathrm{g} / \mathrm{kg}$

$\mu g / k g$

$\mu \mathrm{g} / \mathrm{kg}$

$\mu \mathrm{g} / \mathrm{kg}$

$\mu \mathrm{g} / \mathrm{kg}$

$\mu \mathrm{g} / \mathrm{kg}$

$\mu g / k g$

$\mu \mathrm{g} / \mathrm{kg}$

$\mu \mathrm{g} / \mathrm{kg}$

$\mu \mathrm{g} / \mathrm{kg}$

$\mu g / \mathrm{kg}$

$\mu \mathrm{g} / \mathrm{kg}$

$\mu g / \mathrm{kg}$

$\mu g / k g$

$\mu g / \mathrm{kg}$

$\mu \mathrm{g} / \mathrm{kg}$

$\mathrm{ng} / \mathrm{g}$

$\mathrm{ng} / \mathrm{g}$

$\mathrm{ng} / \mathrm{g}$

$\mathrm{ng} / \mathrm{g}$

$\mu \mathrm{g} / \mathrm{kg}$

$\mu \mathrm{g} / \mathrm{kg}$

$\mathrm{ng} / \mathrm{g}$

$\mathrm{ng} / \mathrm{g}$

$\mu g / \mathrm{kg}$

$\mu \mathrm{g} / \mathrm{kg}$

$\mu \mathrm{g} / \mathrm{kg}$

$\mu g / \mathrm{kg}$

$\mu \mathrm{g} / \mathrm{kg}$

$\mu g / \mathrm{kg}$

$\mu \mathrm{g} / \mathrm{kg}$

$\mathrm{ng} / \mathrm{g}$

$\mathrm{ng} / \mathrm{g}$

$\mathrm{ng} / \mathrm{g}$

$\mathrm{ng} / \mathrm{g}$

$\mathrm{ng} / \mathrm{g}$

$\mathrm{ng} / \mathrm{g}$

$\mu g / \mathrm{kg}$

$\begin{array}{ll}6.4 & \text { EPA8240 } \\ 13 & \text { EPA8240 } \\ & \text { EPA8240 } \\ 6.4 & \text { EPA8240 }\end{array}$

D. Limit Method
0.10

0.10

0.10

0.10

210
2.1

\section{1}

\section{1}

2.1

2.1

2.1

4.2

4.2

4.2

4.2

2.1

\section{2}

\section{2}

4.2

4.2

2.1

\section{1}

0.10

0.10

0.10

0.10

\section{1}

21

0.10

0.10

42

84

42

42

42

42

42

0.10

0.10

0.10

0.10

$0.10=$

0.20

210
EPA8080

EPA8080

EPA8080

EPA8080

EPA8080

EPA8080

EPA8080

EPA8080

EPA8080

EPA8080

EPA8080

EPA8080

EPA8080

EPA8080

EPA8080

EPA8080

EPA8080

EPA8280

EPA8280

EPA8280

EPA8280

EPA8080

EPA8080

EPA8280

EPA8280

EPA8080

EPA8080

EPA8080

EPA8080

EPA8080

EPA8080

EPA8080

EPA8280

EPA8280

EPA8280

EPA8280

EPA8280

EPA8280

EPA8080 
SAMPLE NAME: D43110̄80SA (Continued)

Constituent
Sample ID: 100335

RQ AQ B Result
Unit.

D. Limit Method

Radionuclide Indicators and Radionuclides

Gross alpha

Nonvolatile beta

Radium, total alpha-emitting

Tritium
8.3

$36 \pm 26$

$0.86 \pm 0.90$

$5.4 \pm 1.1$

$$
\begin{aligned}
& \mathrm{pCi} / \mathrm{g} \\
& \mathrm{pCi} / \mathrm{g} \\
& \mathrm{pCi} / \mathrm{g} \\
& \mathrm{pCi} / \mathrm{g}
\end{aligned}
$$

\section{3}

10

0.090

1.1

EPA900.0

EPA900.0

EPA903.0

EPA906.0

\section{SAMPLE NAME: D43110806}

Location (SRS Coordinates): 18542.87E 66623.81N

Ground Elevation Above MSL: $132.8 \mathrm{ft}$

- Depth of Core Interval: 8.00 to $10.00 \mathrm{ft}$

Sample Type: Normal

Sample Color: 10YR 6/6

\section{Constituent}

Physical Parameters

Cation exchange capacity

$\mathrm{pH}$

Specific conductance

Total organic carbon

Total Metals

Arsenic
Barium
Cadmium
Chromium
Copper
Lead
Manganese
Mercury
Nickel
Selenium
Silver

$B / N / A$ Extractables (including $P A H$ and phenols)

Acenaphthene

Acenaphthylene

Anthracene

Benzo(a)anthracene

Benzo(b)fluoranthene

Benzo(k)fluoranthene

Benzoic acid

Benzo(g,h,i)perylene

Benzo(a)pyrene

Benzyl alcohol

Bis(2-chloroethoxy) methane
Sample ID: 100336
Sample Matrix: Soil

USC Soil Classification: SM
Sample Moisture: Damp Percent Solids: 84.10

D. Limit Method RQ AQ B Result Unit

$\begin{array}{lll}\mathrm{meq} / 100 \mathrm{~g} & 2.0 & \text { EPA9080 } \\ \mathrm{pH} & 0.010 & \text { EPA9045 } \\ \mu \mathrm{S} / \mathrm{cm} & 1.0 & \text { EPA9050 } \\ \mathrm{mg} / \mathrm{kg} & 180 & \text { EPA415.1 }\end{array}$

9.3

3.9

190

3,800

1.1

21

0.52

11

5.8

5.2

18

0.21

4.8

0.53

1.0 $\mathrm{mg} / \mathrm{kg}$

$\mathrm{mg} / \mathrm{kg}$

$\mathrm{mg} / \mathrm{kg}$

$\mathrm{mg} / \mathrm{kg}$

$\mathrm{mg} / \mathrm{kg}$

$\mathrm{mg} / \mathrm{kg}$

$\mathrm{mg} / \mathrm{kg}$

$\mathrm{mg} / \mathrm{kg}$

$\mathrm{mg} / \mathrm{kg}$

$\mathrm{mg} / \mathrm{kg}$

$\mathrm{mg} / \mathrm{kg}$
1.1

21

0.52

1.0

2.6

0.32

1.6

0.12

4.2

0.53

1.0
EPA7060

EPA6010

EPA6010

EPA6010

EPA6010

EPA7421

EPA6010

EPA7470

EPA6010

EPA7740

EPA6010

$\begin{array}{llll}410 & \mu g / k g & 410 & \text { EPA8270 } \\ 410 & \mu g / k g & 410 & \text { EPA8270 } \\ 410 & \mu g / k g & 410 & \text { EPA8270 } \\ 410 & \mu g / k g & 410 & \text { EPA8270 } \\ 410 & \mu g / k g & 410 & \text { EPA8270 } \\ 410 & \mu g / k g & 410 & \text { EPA8270 } \\ 2,000 & \mu g / k g & 2,000 & \text { EPA8270 } \\ 410 & \mu g / k g & 410 & \text { EPA8270 } \\ 410 & \mu g / k g & 410 & \text { EPA8270 } \\ 410 & \mu g / k g & 410 & \text { EPA8270 } \\ 410 & \mu g / k g & 410 & \text { EPA8270 }\end{array}$


SAMPLE NAME: D43110806 (Continued)
Sample ID: 100336

RQ AQ B Result Unit D. Limit Method

\section{$B / N / A$ Extractables (including $P A H$ and phenols)}

Bis(2-chloroethyl) ether

Bis(2-chloroisopropyl) ether

Bis(2-ethylhexyl) phthalate

4-Bromophenyl phenyl ether

Butylbenzyl phthalate

4-Chloroaniline

4-Chloro-m-cresol

2.Chloronaphthalene

2-Chlorophenol

4-Chlorophenyl phenyl ether

Chrysene

o-Cresol (2-Methylphenol)

p-Cresol (4-Methylphenol)

Dibenz $(\mathrm{a}, \mathrm{h})$ anthracene

Dibenzofuran

Di-n-butyl phthalate

1,2-Dichlorobenzene

1,3-Dichlorobenzene

1,4-Dichlorobenzene

3,3'-Dichlorobenzidine

2,4-Dichlorophenol

Diethyl phthalate

2.4-Dimethyl phenol

Dimethyl phthalate

2,4-Dinitrophenol

2,4-Dinitrotoluene

2,6-Dinitrotoluene

Di-n-octyl phthalate

Fluoranthene

Fluorene

Hexachlorobenzene

Hexachlorobutadiene

Hexachlorocyclopentadiene

Hexachloroethane

Indeno(1,2,3-c,d)pyrene

Isophorone

2-Methyl-4,6-dinitrophenol

2-Methylnaphthalene

Naphthalene

o-Nitroaniline

m-Nitroaniline

p-Nitroaniline

Nitrobenzene

2-Nitrophenol

4-Nitrophenol

N-Nitrosodiphenylamine

N-Nitrosodipropylamine

Pentachlorophenol

Phenanthrene

Phenol
U

U

了

U

J

U

$\mathrm{U}$

U

U

U

U

U

U

U

U

U

U

U

U

U

U

U

U

U

U

U

$\mathrm{U}$

U

U

U

U

U

U

U

U

U

U

U

$\mathbf{U}$

U

U

U

U

U

U

U

I

U
410

.410

41

410

67

410

410

410

410

410

410

410

410

410

410

79

410

410

410

810

410

410

410

410

2,000

410

410

L

410

410

410

410

410

410

410

410

410

2,000

410

410

2,000

2,000

2,000

410

410

2,000

410

410

2,000

95

410 $\mu \mathrm{g} / \mathrm{kg}$

$\mu \mathrm{g} / \mathrm{kg}$

$\mu \mathrm{g} / \mathrm{kg}$

$\mu \mathrm{g} / \mathrm{kg}$

$\mu \mathrm{g} / \mathrm{kg}$

$\mu \mathrm{g} / \mathrm{kg}$

$\mu \mathrm{g} / \mathrm{kg}$

$\mu \mathrm{g} / \mathrm{kg}$

$\mu \mathrm{g} / \mathrm{kg}$

$\mu \mathrm{g} / \mathrm{kg}$

$\mu \mathrm{g} / \mathrm{kg}$

$\mu g / k g$

$\mu \mathrm{g} / \mathrm{kg}$

$\mu \mathrm{g} / \mathrm{kg}$

$\mu \mathrm{g} / \mathrm{kg}$

$\mu \mathrm{g} / \mathrm{kg}$

$\mu \mathrm{g} / \mathrm{kg}$

$\mu \mathrm{g} / \mathrm{kg}$

$\mu \mathrm{g} / \mathrm{kg}$

$\mu \mathrm{g} / \mathrm{kg}$

$\mu \mathrm{g} / \mathrm{kg}$

$\mu \mathrm{g} / \mathrm{kg}$

$\mu \mathrm{g} / \mathrm{kg}$

$\mu \mathrm{g} / \mathrm{kg}$

$\mu \mathrm{g} / \mathrm{kg}$

$\mu \mathrm{ig} / \mathrm{kg}$

$\mu \mathrm{g} / \mathrm{kg}$

$\mu \mathrm{g} / \mathrm{kg}$

$\mu \mathrm{g} / \mathrm{kg}$

$\mu \mathrm{g} / \mathrm{kg}$

$\mu \mathrm{g} / \mathrm{kg}$

$\mu \mathrm{g} / \mathrm{kg}$

$\mu \mathrm{g} / \mathrm{kg}$

$\mu \mathrm{g} / \mathrm{kg}$

$\mu \mathrm{g} / \mathrm{kg}$

$\mu \mathrm{g} / \mathrm{kg}$

$\mu \mathrm{g} / \mathrm{kg}$

$\mu \mathrm{g} / \mathrm{kg}$

$\mu \mathrm{g} / \mathrm{kg}$

$\mu \mathrm{g} / \mathrm{kg}$

$\mu \mathrm{g} / \mathrm{kg}$

$\mu \mathrm{g} / \mathrm{kg}$

$\mu \mathrm{g} / \mathrm{kg}$

$\mu \mathrm{g} / \mathrm{kg}$

$\mu \mathrm{g} / \mathrm{kg}$

$\mu \mathrm{g} / \mathrm{kg}$

$\mu \mathrm{g} / \mathrm{kg}$

$\mu \mathrm{g} / \mathrm{kg}$

$\mu \mathrm{g} / \mathrm{kg}$

$\mu \mathrm{g} / \mathrm{kg}$
410

EPA8270

EPA8270

EPA8270

EPA8270

EPA8270

EPA8270

EPA8270

EPA8270

EPA8270

EPA8270

EPA8270

EPA8270

EPA8270

EPA8270

EPA8270

EPA8270

EPA8270

EPA8270

EPA8270

EPA8270

EPA8270

EPA8270

EPA8270

EPA8270

EPA8270

EPA8270

EPA8270

EPA8270

EPA8270

EPA8270

EPA8270

EPA8270

EPA8270

EPA8270

EPA8270

EPA8270

EPA8270

EPA8270

EPA8270

EPA8270

EPA8270

EPA8270

EPA8270

EPA8270

EPA8270

EPA8270

EPA8270

EPA8270

EPA8270

EPA8270 
SAMPLE NAME: D43110806 (Continued) Sample ID: 100336

Constituent

RQ AQ B Result

Unit

D. Limit Method

$B / N / A$ Extractables (including $P A H$ and phenols)

Pyrene

1,2,4-Trichlorobenzene

2,4,5-Trichlorophenol

2,4,6-Trichlorophenol

Unknown ketone
U

U

U

$$
\text { J }
$$

410

410

2,000

410

NV $\mu g / \mathrm{kg}$
$\mu \mathrm{g} / \mathrm{kg}$
$\mu g / \mathrm{kg}$
$\mu \mathrm{g} / \mathrm{kg}$
$\mu g / \mathrm{kg}$

$\mu \mathrm{g} / \mathrm{kg}$

$\mu \mathrm{g} / \mathrm{kg}$

$\mu \mathrm{g} / \mathrm{kg}$

$\mu \mathrm{g} / \mathrm{kg}$

$\mu \mathrm{g} / \mathrm{kg}$

$\mu \mathrm{g} / \mathrm{kg}$

$\mu \mathrm{g} / \mathrm{kg}$

$\mu \mathrm{g} / \mathrm{kg}$

$\mu \mathrm{g} / \mathrm{kg}$

$\mu \mathrm{g} / \mathrm{kg}$

$\mu \mathrm{g} / \mathrm{kg}$

$\mu \mathrm{g} / \mathrm{kg}$

$\mu \mathrm{g} / \mathrm{kg}$

$\mu \mathrm{g} / \mathrm{kg}$

$\mu \mathrm{g} / \mathrm{kg}$

$\mu \mathrm{g} / \mathrm{kg}$

$\mu \mathrm{g} / \mathrm{kg}$

$\mu \mathrm{g} / \mathrm{kg}$

$\mu \mathrm{g} / \mathrm{kg}$

$\mu \mathrm{g} / \mathrm{kg}$

$\mu \mathrm{g} / \mathrm{kg}$

$\mu \mathrm{g} / \mathrm{kg}$

$\mu \mathrm{g} / \mathrm{kg}$

$\mu \mathrm{g} / \mathrm{kg}$

$\mu \mathrm{g} / \mathrm{kg}$

$\mu \mathrm{g} / \mathrm{kg}$

$\mu \mathrm{g} / \mathrm{kg}$

$\mu \mathrm{g} / \mathrm{kg}$

$\mu \mathrm{g} / \mathrm{kg}$

$\mu \mathrm{g} / \mathrm{kg}$

$\mu \mathrm{g} / \mathrm{kg}$

$\mu \mathrm{g} / \mathrm{kg}$

$\mu g / k g$

$\mu \mathrm{g} / \mathrm{kg}$

$\mu \mathrm{g} / \mathrm{kg}$

$\mu \mathrm{g} / \mathrm{kg}$

$\begin{array}{ll}410 & \text { EPA8270 } \\ 410 & \text { EPA8270 } \\ 2,000 & \text { EPA8270 } \\ 410 & \text { EPA8270 } \\ & \text { EPA8270 }\end{array}$

12

EPA8240

EPA8240

EPA8240

EPA8240

EPA8240

EPA8240

EPA8240

EPA8240

EPA8240

EPA8240

EPA8240

EPA8240

EPA8240

EPA8240

EPA8240

EPA8240

EPA8240

EPA8240

EPA8240

EPA 8240

EPA8240

EPA 8240

EPA8240

EPA8240

EPA8240

EPA8240

EPA8240

EPA8240

EPA8240

EPA8240

EPA8240

EPA8240

EPA8240

EPA8240

EPA 8240

EPA8240

Xylenes

6.1

Pesticides/PCBs and Dioxins/Furans

Aldrin

alpha-Benzene hexachloride

beta-Benzene hexachloride
U 2.0

U 2.0

U 2.0 $\mu g / \mathrm{kg}$
$\mu g / \mathrm{kg}$
$\mu g / \mathrm{kg}$

2.0

EPA8080

EPA8080

EPA8080 
SAMPLE NAME: D43ï10806 (Continued)

Constituent

RQ

$A Q$

Pesticides/PCBs and Dioxins/Furans

delta-Benzene hexachloride

alpha-Chlordane

gamma-Chlordane

p.p'-DDD

p.p'-DDE

p.p'-DDT

Dieldrin

Endosulfan I

Endosulfan II

Endosulfan sulfate

Endrin

Endrin ketone

Heptachlor

Heptachlor epoxide

Heptachlorodibenzo-p-dioxin isomers

Heptachlorodibenzo-p-furan isomers

Hexachlorodibenzo-p-dioxin isomers

Hexachlorodibenzo-p-furan isomers

Lindane

Methoxychlor

Octachlorodibenzo-p-dioxin

Octachlorodibenzo-p-furan

PCB 1016

PCB 1221

PCB 1232

PCB 1242

PCB 1248

PCB 1254

PCB 1260

Pentachlorodibenzo-p-dioxin isomers

Pentachlorodibenzo-p-furan isomers

2,3,7,8-TCDD

2,3,7,8-TCDF

Tetrachlorodibenzo-p-dioxin isomers

Tetrachlorodibenzo-p-furan isomers

Toxaphene
Sample ID: 100336

Unit

D. Limit Method

\section{Radionuclide Indicators and Radionuclides}

Gross alpha

Nonvolatile beta

Radium, total alpha-emitting

Tritium

2.0
2.0
2.0
4.0
4.0
4.0
4.0
2.0
4.0
4.0
4.0
4.0
2.0
2.0
0.20
0.10
0.10
0.10
2.0
20
12
0.10
40
81
40
40
40
40
40
0.10
0.10
0.10
0.10
0.10
0.10
200

200

$$
\begin{aligned}
& \mu g / k g \\
& \mu g / k g \\
& \mu g / k g \\
& \mu g / k g \\
& \mu g / k g \\
& \mu g / k g \\
& \mu g / k g \\
& \mu g / k g \\
& \mu g / k g \\
& \mu g / k g \\
& \mu g / k g \\
& \mu g / k g \\
& \mu g / k g \\
& \mu g / k g \\
& n g / g \\
& n g / g \\
& n g / g \\
& n g / g \\
& \mu g / k g \\
& \mu g / k g \\
& \mathrm{ng} / \mathrm{g} \\
& \mathrm{ng} / \mathrm{g} \\
& \mu g / k g \\
& \mu g / k g \\
& \mu g / k g \\
& \mu g / k g \\
& \mu g / k g \\
& \mu g / k g \\
& \mu g / k g \\
& \mathrm{ng} / \mathrm{g} \\
& \mathrm{ng} / \mathrm{g} \\
& \mathrm{ng} / \mathrm{g} \\
& \mathrm{ng} / \mathrm{g} \\
& \mathrm{ng} / \mathrm{g} \\
& \mathrm{ng} / \mathrm{g} \\
& \mu \mathrm{g} / \mathrm{kg}
\end{aligned}
$$

2.0

2.0

2.0

4.0

4.0

4.0

4.0

2.0

4.0

4.0

4.0

4.0

2.0

2.0

0.10

0.10

0.10

0.10

2.0

20

0.10

0.10

40

81

40

40

40

40

40

0.10

0.10

0.10

0.10

0.10

0.10

200
EPA8080

EPA8080

EPA8080

EPA8080

EPA8080

EPA8080

EPA8080

EPA8080

EPA8080

EPA8080

EPA8080

EPA8080

EPA8080

EPA8080

EPA8280

EPA8280

EPA8280

EPA8280

EPA8080

EPA8080

EPA8280

EPA8280

EPA8080

EPA8080

EPA8080

EPA8080

EPA8080

EPA8080

EPA8080

EPA8280

EPA8280

EPA8280

EPA8280

EPA8280

EPA8280

EPA8080
$11 \pm 14$

$36 \pm 23$

$1.0 \pm 1.0$

$11 \pm 1.9$
$\mathrm{pCi} / \mathrm{g}$

$\mathrm{pCi} / \mathrm{g}$

$\mathrm{pCi} / \mathrm{g}$

$\mathrm{pCi} / \mathrm{g}$ $\begin{array}{ll}7.4 & \text { EPA900.0 } \\ 9.1 & \text { EPA900.0 } \\ 0.090 & \text { EPA903.0 } \\ 1.4 & \text { EPA906.0 }\end{array}$ 
SAMPLE NAME: D43100807

Location (SRS Coordinates): 18542.87E 66623.81N

Ground Elevation Above MSL: $132.8 \mathrm{ft}$

Depth of Core Interval: 10.00 to $12.00 \mathrm{ft}$

Sample Type: Normal

Sample Color: 10R $3 / 4$

Constituent

Total Metals

Sample ID: 100337

Sample Matrix: Soil

USC Soil Classification: SM
Sample Moisture: Damp Percent Solids: 81.20

D. Limit Method

\section{Arsenic \\ Barium \\ Cadmium \\ Chromium \\ Copper \\ Lead \\ Manganese \\ Mercury \\ Nickel \\ Selenium \\ Silver}

\section{RQ AQ B Result Unit}

$\begin{array}{ll}1.1 & \mathrm{mg} / \mathrm{kg} \\ 24 & \mathrm{mg} / \mathrm{kg} \\ 0.61 & \mathrm{mg} / \mathrm{kg} \\ 22 & \mathrm{mg} / \mathrm{kg} \\ 3.0 & \mathrm{mg} / \mathrm{kg} \\ 3.3 & \mathrm{mg} / \mathrm{kg} \\ 43 & \mathrm{mg} / \mathrm{kg} \\ 0.12 & \mathrm{mg} / \mathrm{kg} \\ 4.9 & \mathrm{mg} / \mathrm{kg} \\ 0.56 & \mathrm{mg} / \mathrm{kg} \\ 1.2 & \mathrm{mg} / \mathrm{kg}\end{array}$

$\begin{array}{ll}1.1 & \text { EPA7060 } \\ 24 & \text { EPA6010 } \\ 0.61 & \text { EPA6010 } \\ 1.2 & \text { EPA6010 } \\ 3.0 & \text { EPA6010 } \\ 0.34 & \text { EPA7421 } \\ 1.8 & \text { EPA6010 } \\ 0.12 & \text { EPA7470 } \\ 4.9 & \text { EPA6010 } \\ 0.56 & \text { EPA7740 } \\ 1.2 & \text { EPA6010 }\end{array}$

$B / N / A$ Extractables (including $P A H$ and phenols)

\begin{tabular}{|c|c|c|c|c|c|}
\hline Acenaphthene & U & 430 & $\mu g / \mathrm{kg}$ & 430 & EPA8270 \\
\hline Acenaphthylene & $U$ & 430 & $\mu g / \mathrm{kg}$ & 430 & EPA8270 \\
\hline Anthracene & $\mathbf{U}$ & 430 & $\mu g / k g$ & 430 & EPA8270 \\
\hline Benzo(a)anthracene & $\mathbf{U}$ & 430 & $\mu g / \mathrm{kg}$ & 430 & EPA8270 \\
\hline Benzo(b)fluoranthene & $\mathbf{U}$ & 430 & $\mu g / \mathrm{kg}$ & 430 & EPA8270 \\
\hline Benzo(k)fluoranthene & $\mathrm{U}$ & 430 & $\mu \mathrm{g} / \mathrm{kg}$ & 430 & EPA8270 \\
\hline Benzoic acid & U & 2,100 & $\mu g / k g$ & 2,100 & EPA8270 \\
\hline Benzo(g,h,i)perylene & $\mathrm{U}$ & 430 & $\mu g / \mathrm{kg}$ & 430 & EPA8270 \\
\hline Benzo(a)pyrene & $\mathrm{U}$ & 430 & $\mu g / \mathrm{kg}$ & 430 & EPA 8270 \\
\hline Benzyl alcohol & U & 430 & $\mu g / \mathrm{kg}$ & 430 & EPA8270 \\
\hline Bis(2-chloroethoxy) methane & $U$ & 430 & $\mu g / \mathrm{kg}$ & 430 & EPA8270 \\
\hline Bis(2-chloroethyl) ether & $\mathrm{U}$ & 430 & $\mu g / \mathrm{kg}$ & 430 & EPA8270 \\
\hline Bis(2-chloroisopropyl) echer & $U$ & 430 & $\mu g / k g$ & 430 & EPA8270 \\
\hline Bis(2-ethylhexyl) phthalate & $J$ & 51 & $\mu g / k g$ & 430 & EPA8270 \\
\hline 4-Bromophenyl phenyl ether & $\mathrm{U}$ & 430 & $\mu g / \mathrm{kg}$ & 430 & EPA8270 \\
\hline Butylbenzyl phthalate & J & 130 & $\mu g / \mathrm{kg}$ & 430 & EPA8270 \\
\hline 4-Chloroaniline & $\mathbf{U}$ & 430 & $\mu g / k g$ & 430 & EPA8270 \\
\hline 4-Chloro-m-cresol & $\mathrm{U}$ & 430 & $\mu \mathrm{g} / \mathrm{kg}$ & 430 & EPA8270 \\
\hline 2-Chloronaphthalene & U & 430 & $\mu g / k g$ & 430 & EPA8270 \\
\hline 2-Chlorophenol & $\mathbf{U}$ & 430 & $\mu g / \mathrm{kg}$ & 430 & EPA8270 \\
\hline 4-Chlorophenyl phenyl ether & $U$ & 430 & $\mu g / \mathrm{kg}$ & 430 & EPA 8270 \\
\hline Chrysene & $\mathbf{U}$ & 430 & $\mu g / k g$ & 430 & EPA8270 \\
\hline o-Cresol (2-Methylphenol) & $U$ & 430 & $\mu g / k g$ & 430 & EPA8270 \\
\hline p-Cresol (4-Methylphenol) & $\mathrm{U}$ & 430 & $\mu g / \mathrm{kg}$ & 430 & EPA8270 \\
\hline Dibenz $(a, h)$ anthracene & $\mathrm{U}$ & 430 & $\mu g / \mathrm{kg}$ & $430^{2}$ & EPA8270 \\
\hline Dibenzofuran & U & 430 & $\mu g / \mathrm{kg}$ & 430 & EPA8270 \\
\hline Di-n-butyl phthalate & UJ & 130 & $\mu g / k g$ & 430 & EPA8270 \\
\hline 1,2-Dichlorobenzene & $\mathrm{U}$ & 430 & $\mu g / \mathrm{kg}$ & 430 & EPA8270 \\
\hline 1,3-Dichlorobenzene & $\bar{U}$ & 430 & $\mu \mathrm{g} / \mathrm{kg}$ & 430 & EPA8270 \\
\hline 1,4-Dichlorobenzene & $\mathbf{U}$ & 430 & $\mu g / k g$ & 430 & EPA8270 \\
\hline
\end{tabular}


Constituent

RQ AQ B Result

Unit

D. Limit Method

$B / N / A$ Extractables (including PAH and phenols)

3.3'-Dichlorobenzidine

2,4-Dichlorophenol

Diethyl phthalate

2,4-Dimethyl phenol

Dimethyl phthalate

2,4-Dinitrophenol

2.4-Dinitrotoluene

2,6-Dinitrotoluene

Di-n-octyl phthalate

Fluoranthene

Fluorene

Hexachlorobenzene

Hexachlorobutadiene

Hexachlorocyclopentadiene

Hexachloroethane

Indeno(1,2,3-c,d)pyrene

Isophorone

2-Methyl-4,6-dinitrophenol

2-Methylnaphthalene

Naphthalene

o-Nitroaniline

m-Nitroaniline

p-Nitroaniline

Nitrobenzene

2-Nitrophenol

4-Nitrophenol

N-Nitrosodiphenylamine

$\mathrm{N}$-Nitrosodipropylamine

Pentachlorophenol

Phenanthrene

Phenol

Pyrene

1,2,4-Trichlorobenzene

2,4,5-Trichlorophenol

2,4,6-Trichlorophenol

Unknown ketone

Volatile Organic Compounds

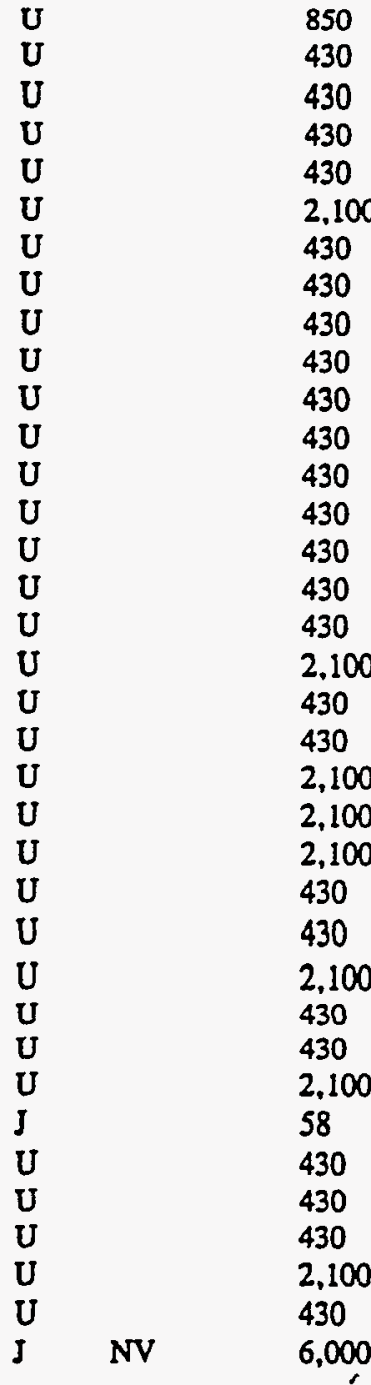

$\mu g / \mathrm{kg}$

$\mu \mathrm{g} / \mathrm{kg}$

$\mu \mathrm{g} / \mathrm{kg}$

$\mu \mathrm{g} / \mathrm{kg}$

$\mu g / \mathrm{kg}$

$\mu g / k g$

$\mu \mathrm{g} i \mathrm{~kg}$

$\mu \mathrm{g} / \mathrm{kg}$

$\mu \mathrm{g} / \mathrm{kg}$

$\mu \mathrm{g} / \mathrm{kg}$

$\mu g / k g$

$\mu \mathrm{g} / \mathrm{kg}$

$\mu \mathrm{g} / \mathrm{kg}$

$\mu \mathrm{g} / \mathrm{kg}$

$\mu \mathrm{g} / \mathrm{kg}$

$\mu g / \mathrm{kg}$

$\mu g / k g$

$\mu \mathrm{g} / \mathrm{kg}$

$\mu \mathrm{g} / \mathrm{kg}$

$\mu g / k g$

$\mu \mathrm{g} / \mathrm{kg}$

$\mu \mathrm{g} / \mathrm{kg}$

$\mu \mathrm{g} / \mathrm{kg}$

$\mu \mathrm{g} / \mathrm{kg}$

$\mu \mathrm{g} / \mathrm{kg}$

$\mu \mathrm{g} / \mathrm{kg}$

$\mu \mathrm{g} / \mathrm{kg}$

$\mu \mathrm{g} / \mathrm{kg}$

$\mu \mathrm{g} / \mathrm{kg}$

$\mu \mathrm{g} / \mathrm{kg}$

$\mu \mathrm{g} / \mathrm{kg}$

$\mu \mathrm{g} / \mathrm{kg}$

$\mu \mathrm{g} / \mathrm{kg}$

$\mu g / \mathrm{kg}$

$\mu \mathrm{g} / \mathrm{kg}$

$\mu g / k g$
850

430

430

430

430

2.100

430

430

430

430

430

430

430

430

430

430

430

2.100

430

430

2,100

2,100

2,100

430

430

2,100

430

430

2,100

430

430

430

430

2,100

430
EPA8270

EPA8270

EPA8270

EPA8270

EPA8270

EPA8270

EPA8270

EPA8270

EPA8270

EPA8270

EPA8270

EPA8270

EPA8270

EPA8270

EPA8270

EPA8270

EPA8270

EPA8270

EPA8270

EPA8270

EPA8270

EPA8270

EPA8270

EPA8270

EPA8270

EPA8270

EPA8270

EPA8270

EPA8270

EPA8270

EPA8270

EPA8270

EPA8270

EPA8270

EPA8270

EPA8270

$\mu g / \mathrm{kg}$
$\mu g / \mathrm{kg}$
$\mu g / \mathrm{kg}$
$\mu \mathrm{g} / \mathrm{kg}$
$\mu \mathrm{g} / \mathrm{kg}$
$\mu g / \mathrm{kg}$
$\mu \mathrm{g} / \mathrm{kg}$
$\mu \mathrm{g} / \mathrm{kg}$
$\mu g / \mathrm{kg}$
$\mu g / \mathrm{kg}$
$\mu \mathrm{g} / \mathrm{kg}$

EPA8240

EPA8240

EPA8240

EPA8240

EPA8240

EPA8240

EPA8240

EPA8240

EPA8240

EPA8240

EPA8240 


\section{Constituent}

Volatile Organic Compounds

Chloromethane (Methyl chloride)

Dibromochloromethane

1,1-Dichloroethane

1,2-Dichloroethane

1,1-Dichloroethylene

1.2-Dichloroethylene

Dichloromethane (Methylene chloride)

1,2-Dichloropropane

cis-1.3-Dichloropropene

trans-1,3-Dichloropropene

Ethylbenzene

2-Hexanone

Methyl ethyl ketone

Methyl isobutyl ketone

Styrene

1,1,2,2-Tetrachloroethane

Tetrachloroethylene

Toluene

1,1,1-Trichloroethane

1,1,2-Trichloroethane

Trichloroethylene

Vinyl acetate

2-Propanol

Xylenes

\section{$\mathbf{R Q}$}

AQ

B

Result

Unit

D. Limit Method

Pesticides/PCBs and Dioxins/Furans

Aldrin
alpha-Benzene hexachloride
beta-Benzene hexachloride
delta-Benzene hexachloride
alpha-Chlordane
gamma-Chlordane
p.,'-DDD
p.p'-DDE
p., -DDT
Dieldrin
Endosulfan I
Endosulfan II
Endosulfan sulfate
Endrin
Endrin ketone
Heptachlor
Heptachlor epoxide
Heptachlorodibenzo-p-dioxin isomers
Heptachlorodibenzo-p-furan isomers
Hexachlorodibenzo-p-dioxin isomers
Hexachlorodibenzo-p-furan isomers
Lindane
Methoxychlor

U

U

U

U

U

U

U

U

U

U

U

U

U

U

U

U

U

U

$\mathrm{N}$

$\mathrm{N} \quad 20$

$\begin{array}{ll}13 & \mu g / k g \\ 6.4 & \mu g / k g \\ 6.4 & \mu g / k g \\ 8.8 & \mu g / k g \\ 6.4 & \mu g / k g \\ 6.4 & \mu g / k g \\ 6.4 & \mu g / k g \\ 6.4 & \mu g / k g \\ 6.4 & \mu g / k g \\ 6.4 & \mu g / k g \\ 6.4 & \mu g / k g \\ 13 & \mu g / k g \\ 13 & \mu g / k g \\ 13 & \mu g / k g \\ 6.4 & \mu g / k g \\ 6.4 & \mu g / k g \\ 6.4 & \mu g / k g \\ 6.4 & \mu g / k g \\ 6.4 & \mu g / k g \\ 7.1 & \mu g / k g \\ 6.4 & \mu g / k g \\ 13 & \mu g / k g \\ 20 & \mu g / k g \\ 6.4 & \mu g / k g\end{array}$

13

6.4

6.4

6.4

6.4

6.4

6.4

6.4

6.4

6.4

6.4

13

13

13

6.4

6.4

6.4

6.4

6.4

6.4

6.4

13

6.4
EPA 8240

EPA8240

EPA8240

EPA8240

EPA8240

EPA8240

EPA8240

EPA8240

EPA8240

EPA8240

EPA8240

EPA8240

EPA8240

EPA8240

EPA8240

EPA8240

EPA8240

EPA8240

EPA8240

EPA8240

EPA8240

EPA8240

EPA8240

EPA8240

\section{1}

$\mu \mathrm{g} / \mathrm{kg}$

$\mu \mathrm{g} / \mathrm{kg}$

$\mu \mathrm{g} / \mathrm{kg}$

$\mu \mathrm{g} / \mathrm{kg}$

$\mu g / \mathrm{kg}$

$\mu \mathrm{g} / \mathrm{kg}$

$\mu \mathrm{g} / \mathrm{kg}$

$\mu \mathrm{g} / \mathrm{kg}$

$\mu \mathrm{g} / \mathrm{kg}$

$\mu \mathrm{g} / \mathrm{kg}$

$\mu \mathrm{g} / \mathrm{kg}$

$\mu \mathrm{g} / \mathrm{kg}$

$\mu \mathrm{g} / \mathrm{kg}$

$\mu \mathrm{g} / \mathrm{kg}$

$\mu \mathrm{g} / \mathrm{kg}$

$\mu \mathrm{g} / \mathrm{kg}$

$\mu \mathrm{g} / \mathrm{kg}$

ng/g

ng/g

$\mathrm{ng} / \mathrm{g}$

$\mathrm{ng} / \mathrm{g}$

$\mu \mathrm{g} / \mathrm{kg}$

$\mu \mathrm{g} / \mathrm{kg}$

$\begin{array}{ll}2.1 & \text { EPA8080 } \\ 2.1 & \text { EPA8080 } \\ 2.1 & \text { EPA8080 } \\ 2.1 & \text { EPA8080 } \\ 2.1 & \text { EPA8080 } \\ 2.1 & \text { EPA8080 } \\ 4.2 & \text { EPA8080 } \\ 4.2 & \text { EPA8080 } \\ 4.2 & \text { EPA8080 } \\ 4.2 & \text { EPA8080 } \\ 2.1 & \text { EPA8080 } \\ 4.2 & \text { EPA8080 } \\ 4.2 & \text { EPA8080 } \\ 4.2 & \text { EPA8080 } \\ 4.2 & \text { EPA8080 } \\ 2.1 & \text { EPA8080 } \\ 2.1 & \text { EPA8080 } \\ 0.10 & \text { EPA8280 } \\ 0.10 & \text { EPA8280 } \\ 0.10 & \text { EPA8280 } \\ 0.10 & \text { EPA8280 } \\ 2.1 & \text { EPA8080 } \\ 21 & \text { EPA8080 }\end{array}$


SAMPLE NAME: D431 10807 (Continued)

Constituent

Pesticides/PCBs and Dioxins/Furans

Octachlorodibenzo-p-dioxin

Octachlorodibenzo-p-furan

PCB 1016

PCB 1221

PCB 1232

PCB 1242

PCB 1248

PCB 1254

PCB 1260

Pentachlorodibenzo-p-dioxin isomers

Pentachlorodibenzo-p-furan isomers

2.3.7.8-TCDD

2,3,7,8-TCDF

Tetrachlorodibenzo-p-dioxin isomers

Tetrachlorodibenzo-p-furan isomers

Toxaphene

SAMPLE NAME: D43110807D

Sample Type: Split

Constituent

Total Metals

Arsenic

Barium

Cadmium

Chromium

Copper

Lead

Manganese

Mercury

Nickel

Selenium

Silver

-

RQ AQ B Result

Sample ID: 100337

$U$
$U$
$U$
$U$
$U$
$U$
$U$
$U$
$U$
$U$
$U$
$U$
$U$
$U$
$U$

$B / N / A$ Extractables (including $P A H$ and phenols)

Acenaphthene

Acenaphuhylene

Anthracene

Benzo(a)anthracene

Benzo(b)fluoranthene

Benzo(k)fluoranthene

Benzoic acid

Benzo(g,h,i)perylene

Benzo(a)pyrene

Benzyl alcohol

$\begin{array}{ll}U & 420 \\ U & 420 \\ U & 420 \\ U & 420 \\ U & 420 \\ U & 420 \\ U & 2,100 \\ U & 420 \\ U & 420 \\ U & 420\end{array}$

RQ AQ B Result Unit

D. Limit Method

Sample ID: 100338

Associated Sample: 100337

$\mathrm{ng} / \mathrm{g}$
$\mathrm{ng} / \mathrm{g}$
$\mu \mathrm{g} / \mathrm{kg}$
$\mu \mathrm{g} / \mathrm{kg}$
$\mu \mathrm{g} / \mathrm{kg}$
$\mu \mathrm{g} / \mathrm{kg}$
$\mu \mathrm{g} / \mathrm{kg}$
$\mu \mathrm{g} / \mathrm{kg}$
$\mu \mathrm{g} / \mathrm{kg}$
$\mathrm{ng} / \mathrm{g}$
$\mathrm{ng} / \mathrm{g}$
$\mathrm{ng} / \mathrm{g}$
$\mathrm{ng} / \mathrm{g}$
$\mathrm{ng} / \mathrm{g}$
$\mathrm{ng} / \mathrm{g}$
$\mu \mathrm{g} / \mathrm{kg}$

0.30

0.10

EPA8280

0.10

42

85

42

42

42

42

42

EPA8280

EPA8080

EPA8080

EPA8080

EPA8080

EPA8080

EPA8080

EPA8080

0.10 EPA8280

$0.10 \quad$ EPA8280

0.10 EPA8280

0.10

EPA8280

0.10

EPA8280

0.10 EPA8280

210 EPA8080

$\begin{array}{lllll} & & \mathrm{mg} / \mathrm{kg} & 1.3 & \text { EPA7060 } \\ \mathrm{U} & 3.1 & \mathrm{mg} / \mathrm{kg} & 23 & \text { EPA6010 } \\ \mathrm{U} & 23 & \mathrm{mg} / \mathrm{kg} & 0.58 & \text { EPA6010 } \\ & 0.58 & \mathrm{mg} / \mathrm{kg} & 1.2 & \text { EPA6010 } \\ & 24 & \mathrm{mg} / \mathrm{kg} & 2.9 & \text { EPA6010 } \\ & 3.7 & \mathrm{mg} / \mathrm{kg} & 0.40 & \text { EPA7421 } \\ & 4.3 & \mathrm{mg} / \mathrm{kg} & 1.7 & \text { EPA6010 } \\ \mathrm{U} & 170 & \mathrm{mg} / \mathrm{kg} & 0.13 & \text { EPA7470 } \\ \mathrm{U} & 0.13 & \mathrm{mg} / \mathrm{kg} & 4.7 & \text { EPA6010 } \\ \mathrm{U} & 6.1 & \mathrm{mg} / \mathrm{kg} & 0.66 & \text { EPA7740 } \\ \mathrm{U} & 0.66 & \mathrm{mg} / \mathrm{kg} & \text { EPA. } & \text { EPA6010 }\end{array}$

$\begin{array}{lll}\mu g / \mathrm{kg} & 420 & \text { EPA8270 } \\ \mu \mathrm{g} / \mathrm{kg} & 420 & \text { EPA8270 } \\ \mu \mathrm{g} / \mathrm{kg} & 420 & \text { EPA8270 } \\ \mu \mathrm{g} / \mathrm{kg} & 420 & \text { EPA8270 } \\ \mu \mathrm{g} / \mathrm{kg} & 420 & \text { EPA8270 } \\ \mu \mathrm{g} / \mathrm{kg} & 420 & \text { EPA8270 } \\ \mu \mathrm{g} / \mathrm{kg} & 2,100 & \text { EPA8270 } \\ \mu \mathrm{g} / \mathrm{kg} & 420 & \text { EPA8270 } \\ \mu \mathrm{g} / \mathrm{kg} & 420 & \text { EPA8270 } \\ \mu g / \mathrm{kg} & 420 & \text { EPA8270 }\end{array}$


SAMPLE NAME: D43110807D (Continued)

Sample ID: 100338

Constituent

RQ AQ B Result.

Unit

D. Limit Method

$B / N / A$ Extractables (including $P A H$ and phenols)

Bis(2-chloroethoxy) methane

Bis(2-chloroethyl) ether

Bis(2-chloroisopropyl) ether

Bis(2-ethylhexyl) phthalate

4-Bromophenyl phenyl ether

Butylbenzyl phthalate

4-Chloroaniline

4-Chloro-m-cresol

2-Chloronaphthalene

2-Chlorophenol

4-Chlorophenyl phenyl ether

Chrysene

o-Cresol (2-Methylphenol)

p-Cresol (4-Methylphenol)

Dibenz $(\mathbf{a}, \mathrm{h})$ anthracene

Dibenzofuran

Di-n-butyl phthalate

1.2-Dichlorobenzene

1,3-Dichlorobenzene

1,4-Dichlorobenzene

3,3'-Dichlorobenzidine

2,4-Dichlorophenol

Diethyl phthalate

2,4-Dimethyl phenol

Dimethyl phthalate

2,4-Dinitrophenol

2,4-Dinitrotoluene

2,6-Dinitrotoluene

Di-n-octyl phthalate

Fluoranthene

Fluorene

Hexachlorobenzene

Hexachlorobutadiene

Hexachlorocyclopentadiene

Hexachloroethane

Indeno $(1,2,3-c, d)$ pyrene

Isophorone

2-Methyl-4,6-dinitrophenol

2-Methylnaphthalene

Naphthalene

o-Nitroaniline

m-Nitroaniline

p-Nitroaniline

Nitrobenzene

2-Nitrophenol

4-Nitrophenol

$\mathrm{N}$-Nitrosodiphenylamine

N-Nitrosodipropylamine

Pentachlorophenol

Phenanthrene
U

420

420

420

49

420

420

420

420

420

420

420

420

420

420

110

420

420

420

840

420

420

420

420

2,100

420

420

420

42

420

420

420

420

420

420

420

2,100

420

420

2,100

2,100

2,100

420

420

2,100

420

420

2,100

56 $\mu \mathrm{g} / \mathrm{kg}$

$\mu \mathrm{g} / \mathrm{kg}$

$\mu g / \mathrm{kg}$

$\mu \mathrm{g} / \mathrm{kg}$

$\mu \mathrm{g} / \mathrm{kg}$

$\mu \mathrm{g} / \mathrm{kg}$

$\mu g / \mathrm{kg}$

$\mu \mathrm{g} / \mathrm{kg}$

$\mu \mathrm{g} / \mathrm{kg}$

$\mu \mathrm{g} / \mathrm{kg}$

$\mu \mathrm{g} / \mathrm{kg}$

$\mu \mathrm{g} / \mathrm{kg}$

$\mu \mathrm{g} / \mathrm{kg}$

$\mu \mathrm{g} / \mathrm{kg}$

$\mu \mathrm{g} / \mathrm{kg}$

$\mu \mathrm{g} / \mathrm{kg}$

$\mu \mathrm{g} / \mathrm{kg}$

$\mu \mathrm{g} / \mathrm{kg}$

$\mu \mathrm{g} / \mathrm{kg}$

$\mu \mathrm{g} / \mathrm{kg}$

$\mu \mathrm{g} / \mathrm{kg}$

$\mu \mathrm{g} / \mathrm{kg}$

$\mu \mathrm{g} / \mathrm{kg}$

$\mu \mathrm{g} / \mathrm{kg}$

$\mu \mathrm{g} / \mathrm{kg}$

$\mu \mathrm{g} / \mathrm{kg}$

$\mu \mathrm{g} / \mathrm{kg}$

$\mu \mathrm{g} / \mathrm{kg}$

$\mu \mathrm{g} / \mathrm{kg}$

$\mu \mathrm{g} / \mathrm{kg}$

$\mu \mathrm{g} / \mathrm{kg}$

$\mu \mathrm{g} / \mathrm{kg}$

$\mu \mathrm{g} / \mathrm{kg}$

$\mu \mathrm{g} / \mathrm{kg}$

$\mu \mathrm{g} / \mathrm{kg}$

$\mu \mathrm{g} / \mathrm{kg}$

$\mu \mathrm{g} / \mathrm{kg}$

$\mu \mathrm{g} / \mathrm{kg}$

$\mu \mathrm{g} / \mathrm{kg}$

$\mu \mathrm{g} / \mathrm{kg}$

$\mu \mathrm{g} / \mathrm{kg}$

$\mu \mathrm{g} / \mathrm{kg}$

$\mu \mathrm{g} / \mathrm{kg}$

$\mu \mathrm{g} / \mathrm{kg}$

$\mu g / \mathrm{kg}$

$\mu g / \mathrm{kg}$

$\mu g / k g$

$\mu g / \mathrm{kg}$

$\mu \mathrm{g} / \mathrm{kg}$

$\mu \mathrm{g} / \mathrm{kg}$

\section{0}

420

420

420

420

420

420

420

420

420

420

420

420

420

420

420

420

420

420

420

840

420

420

420

420

2,100

420

420

420

420

420

420

420

420

420

420

420

2,100

420

420

2,100

2,100

2,100

420

$420^{4}$

2.100

420

420

2,100

420
EPA8270

EPA8270

EPA8270

EPA8270

EPA8270

EPA8270

EPA8270

EPA8270

EPA8270

EPA8270

EPA8270

EPA8270

EPA8270

EPA8270

EPA8270

EPA8270

EPA8270

EPA8270

EPA8270

EPA8270

EPA8270

EPA8270

EPA8270

EPA8270

EPA8270

EPA8270

EPA8270

EPA8270

EPA8270

EPA8270

EPA8270

EPA8270

EPA8270

EPA8270

EPA8270

EPA8270

EPA8270

EPA8270

EPA8270

EPA8270

EPA8270

EPA8270

EPA8270

EPA8270

EPA8270

EPA8270

EPA8270

EPA8270

EPA8270

EPA8270 
SAMPLE NAME: D43110807D (Continued)

Sample ID: 100338

Constituent

RQ AQ B Result

Unit

D. Limit Method

$B / N / A$ Extractables (including $P A H$ and phenols)

Phenol

1,2,4-Trichlorobenzene

2,4,5-Trichlorophenol

2,4,6-Trichlorophenol

Unknown ketone

Volatile Organic Compounds

$\begin{array}{lll}\text { U } & & 420 \\ J & & 43 \\ U & & 420 \\ U & & 2.100 \\ U & & 420 \\ J & \text { NV } & 8,000\end{array}$

140

6.3

6.3

6.3

13

6.3

6.3

6.3

13

13

6.3

13

6.3

6.3

4.1

6.3

6.3

6.3

6.3

6.3

6.3

6.3

13

13

13

6.3

6.3

6.3

6.3

6.3

6.3

6.3

13

10

6.3

$\begin{array}{lll}\mu \mathrm{g} / \mathrm{kg} & 420 & \text { EPA8270 } \\ \mu \mathrm{g} / \mathrm{kg} & 420 & \text { EPA8270 } \\ \mu \mathrm{g} / \mathrm{kg} & 420 & \text { EPA8270 } \\ \mu \mathrm{g} / \mathrm{kg} & 2,100 & \text { EPA8270 } \\ \mu \mathrm{g} / \mathrm{kg} & 420 & \text { EPA8270 } \\ \mu \mathrm{g} / \mathrm{kg} & & \text { EPA8270 }\end{array}$

\begin{tabular}{lll}
$\mu \mathrm{g} / \mathrm{kg}$ & 13 & EPA8240 \\
$\mu \mathrm{g} / \mathrm{kg}$ & 6.3 & EPA8240 \\
$\mu \mathrm{g} / \mathrm{kg}$ & 6.3 & EPA8240 \\
$\mu \mathrm{g} / \mathrm{kg}$ & 6.3 & EPA8240 \\
$\mu \mathrm{g} / \mathrm{kg}$ & 13 & EPA8240 \\
$\mu \mathrm{g} / \mathrm{kg}$ & 6.3 & EPA8240 \\
$\mu \mathrm{g} / \mathrm{kg}$ & 6.3 & EPA8240 \\
$\mu \mathrm{g} / \mathrm{kg}$ & 6.3 & EPA8240 \\
$\mu \mathrm{g} / \mathrm{kg}$ & 13 & EPA8240 \\
$\mu \mathrm{g} / \mathrm{kg}$ & 13 & EPA8240 \\
$\mu \mathrm{g} / \mathrm{kg}$ & 6.3 & EPA8240 \\
$\mu \mathrm{g} / \mathrm{kg}$ & 13 & EPA8240 \\
$\mu \mathrm{g} / \mathrm{kg}$ & 6.3 & EPA8240 \\
$\mu \mathrm{g} / \mathrm{kg}$ & 6.3 & EPA8240 \\
$\mu \mathrm{g} / \mathrm{kg}$ & 6.3 & EPA8240 \\
$\mu \mathrm{g} / \mathrm{kg}$ & 6.3 & EPA8240 \\
$\mu \mathrm{g} / \mathrm{kg}$ & 6.3 & EPA8240 \\
$\mu \mathrm{g} / \mathrm{kg}$ & 6.3 & EPA8240 \\
$\mu \mathrm{g} / \mathrm{kg}$ & 6.3 & EPA8240 \\
$\mu \mathrm{g} / \mathrm{kg}$ & 6.3 & EPA8240 \\
$\mu \mathrm{g} / \mathrm{kg}$ & 6.3 & EPA8240 \\
$\mu \mathrm{g} / \mathrm{kg}$ & 6.3 & EPA8240 \\
$\mu \mathrm{g} / \mathrm{kg}$ & 13 & EPA8240 \\
$\mu \mathrm{g} / \mathrm{kg}$ & 13 & EPA8240 \\
$\mu \mathrm{g} / \mathrm{kg}$ & 13 & EPA8240 \\
$\mu \mathrm{g} / \mathrm{kg}$ & 6.3 & EPA8240 \\
$\mu \mathrm{g} / \mathrm{kg}$ & 6.3 & EPA8240 \\
$\mu \mathrm{g} / \mathrm{kg}$ & 6.3 & EPA8240 \\
$\mu \mathrm{g} / \mathrm{kg}$ & 6.3 & EPA8240 \\
$\mu \mathrm{g} / \mathrm{kg}$ & 6.3 & EPA8240 \\
$\mu \mathrm{g} / \mathrm{kg}$ & 6.3 & EPA8240 \\
$\mu \mathrm{g} / \mathrm{kg}$ & 6.3 & EPA8240 \\
$\mu \mathrm{g} / \mathrm{kg}$ & 13 & EPA8240 \\
$\mu \mathrm{g} / \mathrm{kg}$ & & EPA8240 \\
$\mu \mathrm{g} / \mathrm{kg}$ & 6.3 & EPA8240 \\
& & \\
\hline
\end{tabular}

Pesticides/PCBs and Dioxins/Furans

Aldrin

alpha-Benzene hexachloride

beta-Benzene hexachloride
$\mathbf{U}$
$\mathbf{U}$
$\mathbf{U}$
2.1

2.1

2.1 $\mu \mathrm{g} / \mathrm{kg}$
$\mu \mathrm{g} / \mathrm{kg}$
$\mu \mathrm{g} / \mathrm{kg}$.
2.1

2.1

2.1
EPA8080

EPA8080

EPA8080 
SAMPLE NAME: D43110807D (Continued)

Constituent

AQ
Sample ID: 100338

B Result Unit D. Limit Method

Pesticides/PCBs and Dioxins/Furans

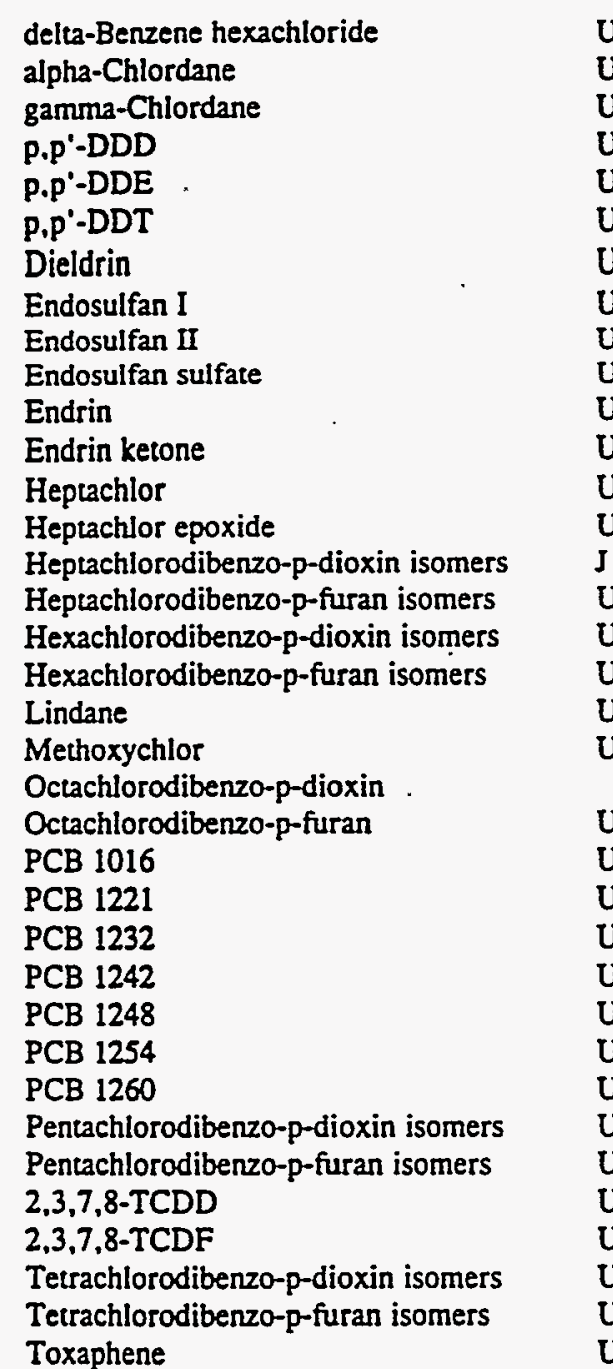

$\begin{array}{llll}2.1 & \mu g / k g & 2.1 & \text { EPA8080 } \\ 2.1 & \mu g / k g & 2.1 & \text { EPA8080 } \\ 2.1 & \mu g / k g & 2.1 & \text { EPA8080 } \\ 4.1 & \mu g / k g & 4.1 & \text { EPA8080 } \\ 4.1 & \mu g / k g & 4.1 & \text { EPA8080 } \\ 4.1 & \mu g / k g & 4.1 & \text { EPA8080 } \\ 4.1 & \mu g / k g & 4.1 & \text { EPA8080 } \\ 2.1 & \mu g / k g & 2.1 & \text { EPA8080 } \\ 4.1 & \mu \mathrm{g} / \mathrm{kg} & 4.1 & \text { EPA8080 } \\ 4.1 & \mu \mathrm{g} / \mathrm{kg} & 4.1 & \text { EPA8080 } \\ 4.1 & \mu \mathrm{g} / \mathrm{kg} & 4.1 & \text { EPA8080 } \\ 4.1 & \mu \mathrm{g} / \mathrm{kg} & 4.1 & \text { EPA8080 } \\ 2.1 & \mu \mathrm{g} / \mathrm{kg} & 2.1 & \text { EPA8080 } \\ 2.1 & \mu \mathrm{g} / \mathrm{kg} & 2.1 & \text { EPA8080 } \\ 0.20 & \mathrm{ng} / \mathrm{g} & 0.10 & \text { EPA8280 } \\ 0.10 & \mathrm{ng} / \mathrm{g} & 0.10 & \text { EPA8280 } \\ 0.10 & \mathrm{ng} / \mathrm{g} & 0.10 & \text { EPA8280 } \\ 0.10 & \mathrm{ng} / \mathrm{g} & 0.10 & \text { EPA8280 } \\ 2.1 & \mu \mathrm{g} / \mathrm{kg} & 2.1 & \text { EPA8080 } \\ 21 & \mu \mathrm{g} / \mathrm{kg} & 21 & \text { EPA8080 } \\ 14 & \mathrm{ng} / \mathrm{g} & 0.20 & \text { EPA8280 } \\ 0.10 & \mathrm{ng} / \mathrm{g} & 0.10 & \text { EPA8280 } \\ 41 & \mu \mathrm{g} / \mathrm{kg} & 41 & \text { EPA8080 } \\ 83 & \mu \mathrm{g} / \mathrm{kg} & 83 & \text { EPA8080 } \\ 41 & \mu \mathrm{g} / \mathrm{kg} & 41 & \text { EPA8080 } \\ 41 & \mu \mathrm{g} / \mathrm{kg} & 41 & \text { EPA8080 } \\ 41 & \mu \mathrm{g} / \mathrm{kg} & 41 & \text { EPA8080 } \\ 41 & \mu \mathrm{g} / \mathrm{kg} & 41 & \text { EPA8080 } \\ 41 & \mu \mathrm{g} / \mathrm{kg} & 41 & \text { EPA8080 } \\ 0.10 & \mathrm{ng} / \mathrm{g} & 0.10 & \text { EPA8280 } \\ 0.10 & \mathrm{ng} / \mathrm{g} & 0.10 & \text { EPA8280 } \\ 0.10 & \mathrm{ng} / \mathrm{g} & 0.10 & \text { EPA8280 } \\ 0.10 & \mathrm{ng} / \mathrm{g} & 0.10 & \text { EPA8280 } \\ 0.10 & \mathrm{ng} / \mathrm{g} & 0.10 & \text { EPA8280 } \\ 0.10 & \mathrm{ng} / \mathrm{g} & 0.10 & \text { EPA8280 } \\ 210 & \mathrm{Hg} / \mathrm{kg} & 210 & \text { EPA8080 }\end{array}$

$\therefore$ 
SAMPLE NAME: D431 10901

Location (SRS Coordinates): 18482.49E 66640.62N

Ground Elevation Above MSL: $132 \mathrm{ft}$

Depth of Core Interval: 0.00 to $0.50 \mathrm{ft}$

Sample Type: Normal

Sample Color: 10R 3/4

\section{Constituent}

Physical Parameters

Cation exchange capacity

$\mathrm{pH}$

$\mathrm{pH}$

Specific conductance

Total organic carbon

Total Metals

Arsenic

Barium

Cadmium

Chromium

Copper

Lead

Manganese

Mercury

Nickel

Selenium

Silver

Sample ID: 100340

Sample Matrix: Soil

USC Soil Classification: SM
Sample Moisture: Dry

Percent Solids: 95.30
D. Limit Method
RQ AQ B Result
Unit

EPA9080

EPA9045

EPA9045

4.7

42

9.300

$\begin{array}{ll}\mathrm{meq} / 100 \mathrm{~g} & 2.0 \\ \mathrm{pH} & 0.010 \\ \mathrm{pH} & 0.010 \\ \mu \mathrm{S} / \mathrm{cm} & 1.0 \\ \mathrm{mg} / \mathrm{kg} & 530\end{array}$

EPA9050

EPA415.1

$B / N / A$ Extractables (including $P A H$ and phenols)

Acenaphthene
Acenaphthylene
Anthracene
Benzo(a)anthracene
Benzo(b)fluoranthene
Benzo(k)fluoranthene
Benzoic acid
Benzo(g,h,i)perylene
Benzo(a)pyrene
Benzyl alcohol
Bis(2-chloroethoxy) methane
Bis(2-chloroethyl) ether
Bis(2-chloroisopropyl) ether
Bis(2-ethylhexyl) phthalare
4-Bromophenyl phenyl ether
Butanoic acid ester
Butylbenzyl phthalate
4-Chloroaniline
4-Chloro-m-cresol
2-Chloronaphthalene
2-Chlorophenol
4-Chlorophenyl phenyl ether

Acenaphthene

Acenaphthylene

Benzo(a)anthracene

Benzo(b)fluoranthene

Benzo $(g, h, i)$ perylene

Benzo(a)pyrene

Bis(2-chloroethoxy) methane

Bis(2-chlorocthyl) ether

Bis(2-chloroisopropyl) ether

4-Bromophenyl phenyl ether

Butanoic acid ester

Butylbenzyl phthalate

2-Chloronaphthalene

4-Chlorophenyl phenyl ether

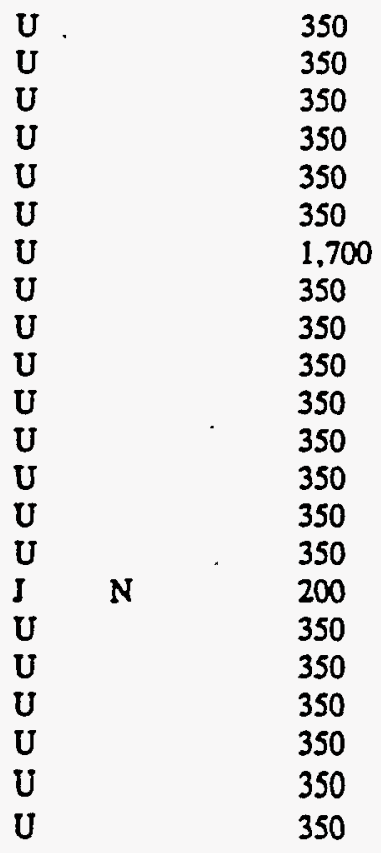

$\mathrm{mg} / \mathrm{kg}$
$\mathrm{mg} / \mathrm{kg}$
$\mathrm{mg} / \mathrm{kg}$
$\mathrm{mg} / \mathrm{kg}$
$\mathrm{mg} / \mathrm{kg}$
$\mathrm{mg} / \mathrm{kg}$
$\mathrm{mg} / \mathrm{kg}$
$\mathrm{mg} / \mathrm{kg}$
$\mathrm{mg} / \mathrm{kg}$
$\mathrm{mg} / \mathrm{kg}$
$\mathrm{mg} / \mathrm{kg}$

0.99

EPA7060

19

0.48

6.2

2.4

1.6

140

0.11

3.9

0.49

0.96
19 EPA6010

$0.48 \quad$ EPA6010

0.96 EPA6010

2.4 EPA6010

0.30 EPA7421

1.4 EPA6010

0.11 EPA7470

3.9 EPA6010

0.49 EPA7740

0.96 EPA6010

and


SAMPLE NAME: D43110901 (Continued)

Constituent

RQ AQ B Result

$B / N / A$ Extractables (including $P A H$ and phenols)

Chrysene

o-Cresol (2-Methylphenol)

p-Cresol (4-Methylphenol)

- Dibenz(a,h)anthracene

Dibenzofuran

Di-n-butyl phthalate

1,2-Dichlorobenzene

1.3-Dichlorobenzene

1.4-Dichlorobenzene

3.3'-Dichlorobenzidine

2,4-Dichlorophenol

Diethyl phthalate

2,4-Dimethyl phenol

Dimethyl phthalate

2.4-Dinitrophenol

2.4-Dinitrotoluene

2.6-Dinitrotoluene

Di-n-octyl phthalate

Fluoranthene

Fluorene

Hexachlorobenzene

Hexachlorobutadiene

Hexachlorocyclopentadiene

Hexachloroethane

Indeno(1.2.3-c,d)pyrene

Isophorone

2-Methyl-4,6-dinitrophenol

2-Mechylnaphthalene

Naphthalene

o-Nitroaniline

m-Nitroaniline

p-Nitroaniline

Nitrobenzene

2-Nitrophenol

4-Nitrophenol

N-Nitrosodiphenylamine

N-Nitrosodipropylamine

Pentachlorophenol

Phenanthrene

Phenol

Pyrene

1,2,4-Trichlorobenzene

2,4,5-Trichlorophenol

2,4,6-Trichlorophenol

Unknown ketone
Sample ID: 100340

Unit:

D. Limit Method

\begin{tabular}{|c|c|c|c|c|c|}
\hline $\mathbf{U}$ & & 350 & $\mu g / k g$ & 350 & EPA8270 \\
\hline U & & 350 & $\mu \mathrm{g} / \mathrm{kg}$ & 350 & EPA8270 \\
\hline $\mathbf{U}$ & & 350 & $\mu \mathrm{g} / \mathrm{kg}$ & 350 & EPA8270 \\
\hline $\mathbf{U}$ & & 350 & $\mu \mathrm{g} / \mathrm{kg}$ & 350 & EPA 8270 \\
\hline $\mathbf{U}$ & & 350 & $\mu \mathrm{g} / \mathrm{kg}$ & 350 & EPA8270 \\
\hline UJ & v & 49 & $\mu \mathrm{g} / \mathrm{kg}$ & 350 & EPA8270 \\
\hline $\mathbf{U}$ & & 350 & $\mu \mathrm{g} / \mathrm{kg}$ & 350 & EPA8270 \\
\hline U & & 350 & $\mu \mathrm{g} / \mathrm{kg}$ & 350 & EPA8270 \\
\hline $\mathbf{U}$ & & 350 & $\mu \mathrm{g} / \mathrm{kg}$ & 350 & EPA8270 \\
\hline $\mathbf{U}$ & & 690 & $\mu \mathrm{g} / \mathrm{kg}$ & 690 & EPA8270 \\
\hline U & & 350 & $\mu \mathrm{g} / \mathrm{kg}$ & 350 & EPA8270 \\
\hline U & & 350 & $\mu \mathrm{g} / \mathrm{kg}$ & 350 & EPA8270 \\
\hline U & & 350 & $\mu \mathrm{g} / \mathrm{kg}$ & 350 & EPA8270 \\
\hline $\mathbf{U}$ & & 350 & $\mu \mathrm{g} / \mathrm{kg}$ & 350 & EPA8270 \\
\hline U & & 1,700 & $\mu g / \mathrm{kg}$ & 1,700 & EPA8270 \\
\hline U & & 350 & $\mu \mathrm{g} / \mathrm{kg}$ & 350 & EPA8270 \\
\hline $\mathbf{U}$ & & 350 & $\mu \mathrm{g} / \mathrm{kg}$ & 350 & EPA8270 \\
\hline $\mathbf{U}$ & & 350 & $\mu \mathrm{g} / \mathrm{kg}$ & 350 & EPA8270 \\
\hline $\mathbf{U}$ & & 350 & $\mu \mathrm{g} / \mathrm{kg}$ & 350 & EPA8270 \\
\hline U & & 350 & $\mu \mathrm{g} / \mathrm{kg}$ & 350 & EPA8270 \\
\hline $\mathbf{U}$ & & 350 & $\mu \mathrm{g} / \mathrm{kg}$ & 350 & EPA8270 \\
\hline U & & 350 & $\mu g / \mathrm{kg}$ & 350 & EPA8270 \\
\hline $\mathbf{U}$ & & 350 & $\mu \mathrm{g} / \mathrm{kg}$ & 350 & EPA8270 \\
\hline $\mathbf{U}$ & & 350 & $\mu \mathrm{g} / \mathrm{kg}$ & 350 & EPA8270 \\
\hline U & & 350 & $\mu \mathrm{g} / \mathrm{kg}$ & 350 & EPA8270 \\
\hline $\mathbf{U}$ & & 350 & $\mu \mathrm{g} / \mathrm{kg}$ & 350 & EPA8270 \\
\hline $\mathbf{U}$ & & 1,700 & $\mu \mathrm{g} / \mathrm{kg}$ & 1,700 & EPA8270 \\
\hline $\mathrm{U}$ & & 350 & $\mu \mathrm{g} / \mathrm{kg}$ & 350 & EPA8270 \\
\hline U & & 350 & $\mu \mathrm{g} / \mathrm{kg}$ & 350 & EPA8270 \\
\hline $\mathrm{U}$ & & 1,700 & $\mu \mathrm{g} / \mathrm{kg}$ & 1.700 & EPA8270 \\
\hline $\mathbf{U}$ & & 1,700 & $\mu \mathrm{g} / \mathrm{kg}$ & 1,700 & EPA8270 \\
\hline $\mathbf{U}$ & & 1,700 & $\mu \mathrm{g} / \mathrm{kg}$ & 1.700 & EPA8270 \\
\hline $\mathbf{U}$ & & 350 & $\mu \mathrm{g} / \mathrm{kg}$ & 350 & EPA8270 \\
\hline $\mathbf{U}$ & & 350 & $\mu \mathrm{g} / \mathrm{kg}$ & 350 & EPA8270 \\
\hline $\mathbf{U}$ & & 1,700 & $\mu \mathrm{g} / \mathrm{kg}$ & 1,700 & EPA8270 \\
\hline $\mathbf{U}$ & & 350 & $\mu \mathrm{g} / \mathrm{kg}$ & 350 & EPA8270 \\
\hline $\mathbf{U}$ & & 350 & $\mu \mathrm{g} / \mathrm{kg}$ & 350 & EPA8270 \\
\hline $\mathbf{U}$ & & 1,700 & $\mu g / k g$ & 1,700 & EPA8270 \\
\hline $\mathbf{U}$ & & 350 & $\mu \mathrm{g} / \mathrm{kg}$ & 350 & EPA8270 \\
\hline $\mathbf{U}$ & & 350 & $\mu g / k g$ & 350 & EPA8270 \\
\hline U & & 350 & $\mu \mathrm{g} / \mathrm{kg}$ & 350 & EPA8270 \\
\hline$U$ & & 350 & $\mu \mathrm{g} / \mathrm{kg}$ & 350 & EPA8270 \\
\hline $\mathbf{U}$ & & 1,700 & $\mu \mathrm{g} / \mathrm{kg}$ & 1,700 & EPA8270 \\
\hline $\mathbf{U}$ & & 350 & $\mu \mathrm{g} / \mathrm{kg}$ & 350 & EPA8270 \\
\hline & NV & 6,000 & $\mu \mathrm{g} / \mathrm{kg}$ & & EPA8270 \\
\hline
\end{tabular}


SAMPLE NAME: D43110901 (Continued)

\section{Constituent}

$\mathbf{R Q}$

$A Q$

Volatile Organic Compounds

Acetone
Benzene
Bromodichloromethane
Bromoform
Bromomethane (Methyl bromide)
Carbon disulfide
Carbon tetrachloride
Chlorobenzene
Chloroethane
Chloroethene (Vinyl chloride)
Chloroform
Chloromethane (Methyl chloride)
Dibromochloromethane
1,1-Dichloroethane
1,2-Dichloroethane
1,1-Dichloroethylene
1,2-Dichloroethylene
Dichloromethane (Methylene chloride)
1,2-Dichloropropane
cis-1,3-Dichloropropene
trans-1,3-Dichloropropene
Ethylbenzene
2-Hexanone
Methyl ethyl ketone
Methyl isobutyl ketone
Styrene
1,1,2,2-Tetrachloroethane
Tetrachloroethylene
Toluene
1,1,1-Trichloroethane
1.1,2-Trichloroethane
Trichloroethylene
Vinyl acetate
Xylenes

Sample ID: 100340

$\mu \mathrm{g} / \mathrm{kg}$

$\mu \mathrm{g} / \mathrm{kg}$

$\mu \mathrm{g} / \mathrm{kg}$

$\mu \mathrm{g} / \mathrm{kg}$

$\mu \mathrm{g} / \mathrm{kg}$

$\mu \mathrm{g} / \mathrm{kg}$

$\mu \mathrm{g} / \mathrm{kg}$

$\mu \mathrm{g} / \mathrm{kg}$

$\mu g / k g$

$\mu \mathrm{g} / \mathrm{kg}$

$\mu \mathrm{g} / \mathrm{kg}$

$\mu \mathrm{g} / \mathrm{kg}$

$\mu \mathrm{g} / \mathrm{kg}$

$\mu \mathrm{g} / \mathrm{kg}$

$\mu \mathrm{g} / \mathrm{kg}$

$\mu \mathrm{g} / \mathrm{kg}$

$\mu \mathrm{g} / \mathrm{kg}$

$\mu \mathrm{g} / \mathrm{kg}$

$\mu \mathrm{g} / \mathrm{kg}$

$\mu g / k g$

$\mu \mathrm{g} / \mathrm{kg}$

$\mu g / \mathrm{kg}$

$\mu \mathrm{g} / \mathrm{kg}$

$\mu \mathrm{g} / \mathrm{kg}$

$\mu \mathrm{g} / \mathrm{kg}$

$\mu g / \mathrm{kg}$

$\mu \mathrm{g} / \mathrm{kg}$

$\mu \mathrm{g} / \mathrm{kg}$

$\mu \mathrm{g} / \mathrm{kg}$

$\mu \mathrm{g} / \mathrm{kg}$

$\mu \mathrm{g} / \mathrm{kg}$

$\mu \mathrm{g} / \mathrm{kg}$

$\mu \mathrm{g} / \mathrm{kg}$

$\mu \mathrm{g} / \mathrm{kg}$
D. Limit Method

$\begin{array}{ll}10 & \text { EPA8240 } \\ 5.2 & \text { EPA8240 } \\ 5.2 & \text { EPA8240 } \\ 5.2 & \text { EPA8240 } \\ 10 & \text { EPA8240 } \\ 5.2 & \text { EPA8240 } \\ 5.2 & \text { EPA8240 } \\ 5.2 & \text { EPA8240 } \\ 10 & \text { EPA8240 } \\ 10 & \text { EPA8240 } \\ 5.2 & \text { EPA8240 } \\ 10 & \text { EPA8240 } \\ 5.2 & \text { EPA8240 } \\ 5.2 & \text { EPA8240 } \\ 5.2 & \text { EPA8240 } \\ 5.2 & \text { EPA8240 } \\ 5.2 & \text { EPA8240 } \\ 5.2 & \text { EPA8240 } \\ 5.2 & \text { EPA8240 } \\ 5.2 & \text { EPA8240 } \\ 5.2 & \text { EPA8240 } \\ 5.2 & \text { EPA8240 } \\ 10 & \text { EPA8240 } \\ 10 & \text { EPA8240 } \\ 10 & \text { EPA8240 } \\ 5.2 & \text { EPA8240 } \\ 5.2 & \text { EPA8240 } \\ 5.2 & \text { EPA8240 } \\ 5.2 & \text { EPA8240 } \\ 5.2 & \text { EPA8240 } \\ 5.2 & \text { EPA8240 } \\ 5.2 & \text { EPA8240 } \\ 10 & \text { EPA8240 } \\ 5.2 & \text { EPA8240 }\end{array}$

Pesticides/PCBs and Dioxins/Furans

Aldrin
alpha-Benzene hexachloride
beta-Benzene hexachloride
delta-Benzene hexachloride
alpha-Chlordane
gamma-Chlordane
p.p'-DDD
p.p'-DDE
P,p'-DDT
Dieldrin
Endosulfan I
Endosulfan II
Endosulfan sulfate

\footnotetext{
$\mathrm{U}$
$\mathrm{U}$
$\mathrm{U}$
$\mathrm{U}$
$\mathrm{U}$
$\mathrm{U}$
$\mathrm{U}$
$\mathrm{J}$
$\mathrm{U}$
$\mathrm{U}$
$\mathrm{U}$
$\mathrm{U}$
$\mathrm{U}$
}

1.7

1.7

1.7

1.7

1.7

1.7

3.5

0.69

3.5

3.5

1.7

3.5

3.5 $\mu \mathrm{g} / \mathrm{kg}$
$\mu \mathrm{g} / \mathrm{kg}$
$\mu \mathrm{g} / \mathrm{kg}$
$\mu \mathrm{g} / \mathrm{kg}$
$\mu \mathrm{g} / \mathrm{kg}$
$\mu \mathrm{g} / \mathrm{kg}$
$\mu \mathrm{g} / \mathrm{kg}$
$\mu \mathrm{g} / \mathrm{kg}$
$\mu \mathrm{g} / \mathrm{kg}$
$\mu \mathrm{g} / \mathrm{kg}$
$\mu \mathrm{g} / \mathrm{kg}$
$\mu \mathrm{g} / \mathrm{kg}$
$\mu \mathrm{g} / \mathrm{kg}$
EPA8080

EPA8080

EPA8080

EPA8080

EPA8080

EPA8080

EPA8080

EPA8080

EPA8080

EPA8080

EPA8080

EPA8080

EPA8080 
SAMPLE NAME: D431.10901 (Continued)

Constituent
$\mathbf{R Q}$

AQ
Sample ID: 100340

B Result Unit D. Limit Method

Pesticides/PCBs and Dioxins/Furans

Endrin
Endrin ketone
Heptachlor
Heptachlor epoxide
Heptachlorodibenzo-p-dioxin isomers
Heptachlorodibenzo-p-furan isomers
Hexachlorodibenzo-p-dioxin isomers.
Hexachlorodibenzo-p-furan isomers
Lindane
Methoxychlor
Octachlorodibenzo-p-dioxin
Octachlorodibenzo-p-furan
PCB 1016
PCB 1221
PCB 1232
PCB 1242 .
PCB 1248
PCB 1254
PCB 1260
Pentachlorodibenzo-p-dioxin isomers
Pentachlorodibenzo-p-furan isomers
$2,3,7,8-T C D D$
$2,3,7,8-T C D F$
Tetrachlorodibenzo-p-dioxin isomers
Tetrachlorodibenzo-p-furan isomers
Toxaphene

$\begin{array}{ll}U & 3.5 \\ U & 3.5 \\ U & 1.7 \\ U & 1.7 \\ U & 0.10 \\ U & 0.10 \\ U & 0.10 \\ U & 0.10 \\ U & 1.7 \\ U & 17 \\ J & 0.60 \\ U & 0.10 \\ U & 35 \\ U & 69 \\ U & 35 \\ U & 35 \\ U & 35 \\ U & 35 \\ U & 35 \\ U & 0.10 \\ U & 0.10 \\ U & 0.10 \\ U & 0.10 \\ U & 0.10 \\ U & 0.10 \\ U & 170\end{array}$

$$
\begin{aligned}
& \mu g / \mathbf{k g} \\
& \mu g / k g \\
& \mu g / k g \\
& \mu g / k g \\
& n g / g \\
& n g / g \\
& n g / g \\
& n g / g \\
& \mu g / k g \\
& \mu g / k g \\
& n g / g \\
& n g / g \\
& \mu g / k g \\
& \mu g / k g \\
& \mu g / k g \\
& \mu g / k g \\
& \mu g / k g \\
& \mu g / k g \\
& \mu g / k g \\
& n g / g \\
& n g / g \\
& n g / g \\
& n g / g \\
& n g / g \\
& n g / g \\
& \mu g / k g
\end{aligned}
$$

$\begin{array}{lr}3.5 & \text { EPA8080 } \\ 3.5 & \text { EPA8080 } \\ 1.7 & \text { EPA8080 } \\ 1.7 & \text { EPA8080 } \\ 0.10 & \text { EPA8280 } \\ 0.10 & \text { EPA8280 } \\ 0.10 & \text { EPA8280 } \\ 0.10 & \text { EPA8280 } \\ 1.7 & \text { EPA8080 } \\ 17 & \text { EPA8080 } \\ 0.10 & \text { EPA8280 } \\ 0.10 & \text { EPA8280 } \\ 35 & \text { EPA8080 } \\ 69 & \text { EPA8080 } \\ 35 & \text { EPA8080 } \\ 35 & \text { EPA8080 } \\ 35 & \text { EPA8080 } \\ 35 & \text { EPA8080 } \\ 35 & \text { EPA8080 } \\ 0.10 & \text { EPA8280 } \\ 0.10 & \text { EPA8280 } \\ 0.10 & \text { EPA8280 } \\ 0.10 & \text { EPA8280 } \\ 0.10 & \text { EPA8280 } \\ 0.10 & \text { EPA8280 } \\ 170 & \text { EPA8080 }\end{array}$

\section{Radionuclide Indicators and Radionuclides}

Gross alpha

Gross alpha

Nonvolatile beta

Nonvolatile beta

Radium, total alpha-emitting

Radium, total alpha-emitting

Tritium

$\begin{array}{llllll}\mathrm{U} & & 9.6 & \mathrm{pCi} / \mathrm{g} & \cdot 9.6 & \text { EPA900.0 } \\ \mathrm{U} & & 9.1 & \mathrm{pCi} / \mathrm{g} & 9.1 & \text { EPA900.0 } \\ & & 18 \pm 21 & \mathrm{pCi} / \mathrm{g} & 9.7 & \text { EPA900.0 } \\ & & 16 \pm 20 & \mathrm{pCi} / \mathrm{g} & 9.1 & \text { EPA900.0 } \\ \mathrm{U} & \mathrm{V} & 1.4 \pm 0.80 & \mathrm{pCi} / \mathrm{g} & 0.20 & \text { EPA903.0 } \\ \mathrm{U} & \mathrm{V} & 1.2 \pm 0.71 & \mathrm{pCi} / \mathrm{g} & 0.20 & \text { EPA903.0 } \\ & & 1.7 \pm 0.38 & \mathrm{pCi} / \mathrm{g} & 1.2 & \text { EPA906.0 }\end{array}$

\section{SAMPLE NAME: D43110901A}

Sample Type: Split

\section{Constituent}

Physical Parameters

Cation exchange capacity

$\mathrm{pH}$

Specific conductance

Total organic carbon
Sample ID: 100341

Associated Sample: 100340

RQ AQ B Result Unit

D. Limit Method

$\begin{array}{llll}13 & \mathrm{meq} / 100 \mathrm{~g} & 2.0 & \text { EPA9080 } \\ 4.4 & \mathrm{pH} & 0.010 & \text { EPA9045 } \\ 44 & \mu \mathrm{s} / \mathrm{cm} & 1.0 & \text { EPA9050 } \\ 7,800 & \mathrm{mg} / \mathrm{kg} & 440 & \text { EPA415.1 }\end{array}$




\section{Total Metals}

Arsenic
Barium
Cadmium
Chromium
Copper
Lead
Manganese
Mercury
Nickel
Selenium
Silver

$\begin{array}{ll}\mathrm{U} & 0.86 \\ \mathrm{U} & 21 \\ \mathrm{U} & 0.51 \\ \mathrm{U} & 6.7 \\ & 2.6 \\ \mathrm{U} & 2.1 \\ \mathrm{U} & 62 \\ \mathrm{U} & 0.11 \\ \mathrm{U} & 4.1 \\ & 0.43 \\ & 1.0\end{array}$

$B / N / A$ Extractables (including $P A H$ and phenols)

\author{
Acenaphthene \\ Acenaphthylene \\ Anthracene \\ Benzo(a)anthracene \\ Benzo(b)fluoranthene \\ Benzo(k)fluoranthene \\ Benzoic acid \\ Benzo(g,h,i)perylene \\ Benzo(a)pyrene \\ Benzyl alcohol \\ Bis(2-chloroethoxy) methane \\ Bis(2-chloroethyl) ether \\ Bis(2-chloroisopropyl) ether \\ Bis(2-ethylhexyl) phthalate \\ 4-Bromophenyl phenyl ether \\ Butylbenzyl phthalate \\ 4-Chloroaniline \\ 4-Chloro-m-cresol \\ 2-Chloronaphthalene \\ 2-Chlorophenol \\ 4-Chlorophenyl phenyl ether \\ Chrysene \\ o-Cresol (2-Methylphenol) \\ p-Cresol (4-Methylphenol) \\ Dibenz(a,h)anthracene \\ Dibenzofuran \\ Di-n-butyl phthalate \\ 1,2-Dichlorobenzene \\ 1,3-Dichlorobenzene \\ 1,4-Dichlorobenzene \\ 3,3'-Dichlorobenzidine \\ 2,4-Dichlorophenol \\ Diethyl phthalate \\ 2,4-Dimethyl phenol \\ Dimethyl phthalate \\ 2,4-Dinitrophenol \\ 2,4-Dinitrotoluene \\ 2,6-Dinitrotoluene \\ Di-n-octyl phthalate
}

\begin{tabular}{|c|c|c|}
\hline U & & 350 \\
\hline U & & 350 \\
\hline $\mathbf{U}$ & & 350 \\
\hline U & & 350 \\
\hline U & & 350 \\
\hline U & & 350 \\
\hline $\mathbf{U}$ & & 1,800 \\
\hline $\mathbf{U}$ & & 350 \\
\hline $\mathrm{U}$ & & 350 \\
\hline $\mathrm{U}$ & & 350 \\
\hline$U$ & & 350 \\
\hline $\mathbf{U}$ & & 350 \\
\hline U & & 350 \\
\hline U & & 350 \\
\hline $\mathbf{U}$ & & 350 \\
\hline U & & 350 \\
\hline $\mathrm{U}$ & & 350 \\
\hline $\mathrm{U}$ & & 350 \\
\hline $\mathrm{U}$ & & 350 \\
\hline $\mathrm{U}$ & & 350 \\
\hline$U$ & & 350 \\
\hline $\mathrm{U}$ & & 350 \\
\hline $\mathbf{U}$ & & 350 \\
\hline $\mathbf{U}$ & & 350 \\
\hline $\mathbf{U}$ & & 350 \\
\hline $\mathbf{U}$ & & 350 \\
\hline UJ & V & 53 \\
\hline $\mathbf{U}$ & & 350 \\
\hline $\mathbf{U}$ & & 350 \\
\hline $\mathbf{U}$ & & 350 \\
\hline $\mathbf{U}$ & & 700 \\
\hline $\mathbf{U}$ & & 350 \\
\hline $\mathbf{U}$ & & 350 \\
\hline $\mathbf{U}$ & & 350 \\
\hline $\mathbf{U}$ & & 350 \\
\hline $\mathbf{U}$ & & 1,800 \\
\hline $\mathbf{U}$ & & 350 \\
\hline $\mathbf{U}$ & & 350 \\
\hline $\mathbf{U}$ & & 350 \\
\hline
\end{tabular}

$\begin{array}{lll}\mathrm{mg} / \mathrm{kg} & 0.86 & \text { EPA7060 } \\ \mathrm{mg} / \mathrm{kg} & 21 & \text { EPA6010 } \\ \mathrm{mg} / \mathrm{kg} & 0.51 & \text { EPA6010 } \\ \mathrm{mg} / \mathrm{kg} & 1.0 & \text { EPA6010 } \\ \mathrm{mg} / \mathrm{kg} & 2.6 & \text { EPA6010 } \\ \mathrm{mg} / \mathrm{kg} & 0.26 & \text { EPA7421 } \\ \mathrm{mg} / \mathrm{kg} & 1.5 & \text { EPA6010 } \\ \mathrm{mg} / \mathrm{kg} & 0.11 & \text { EPA7470 } \\ \mathrm{mg} / \mathrm{kg} & 4.1 & \text { EPA6010 } \\ \mathrm{mg} / \mathrm{kg} & 0.43 & \text { EPA7740 } \\ \mathrm{mg} / \mathrm{kg} & 1.0 & \text { EPA6010 }\end{array}$

$\mu \mathrm{g} / \mathrm{kg}$

350

EPA8270

$\mu \mathrm{g} / \mathrm{kg}$

$\mu g / k g$

$\mu g / k g$

$\mu g / \mathrm{kg}$

$\mu g / k g$

$\mu \mathrm{g} / \mathrm{kg}$

$\mu g / k g$

$\mu \mathrm{g} / \mathrm{kg}$

$\mu \mathrm{g} / \mathrm{kg}$

$\mu g / k g$

$\mu \mathrm{g} / \mathrm{kg}$

$\mu \mathrm{g} / \mathrm{kg}$

$\mu g / k g$

$\mu \mathrm{g} / \mathrm{kg}$

$\mu \mathrm{g} / \mathrm{kg}$

$\mu g / \mathrm{kg}$

$\mu \mathrm{g} / \mathrm{kg}$

$\mu \mathrm{g} / \mathrm{kg}$

$\mu \mathrm{g} / \mathrm{kg}$

$\mu \mathrm{g} / \mathrm{kg}$

$\mu \mathrm{g} / \mathrm{kg}$

$\mu \mathrm{g} / \mathrm{kg}$

$\mu \mathrm{g} / \mathrm{kg}$

$\mu \mathrm{g} / \mathrm{kg}$

$\mu g / \mathbf{k g}$

$\mu g / k g$

$\mu \mathrm{g} / \mathrm{kg}$

$\mu \mathrm{g} / \mathrm{kg}$

$\mu \mathrm{g} / \mathrm{kg}$

$\mu g / \mathrm{kg}$

$\mu \mathrm{g} / \mathrm{kg}$

$\mu \mathrm{g} / \mathrm{kg}$

$\mu \mathrm{g} / \mathrm{kg}$

$\mu \mathrm{g} / \mathrm{kg}$

$\mu \mathrm{g} / \mathrm{kg}$

$\mu \mathrm{g} / \mathrm{kg}$

$\mu \mathrm{g} / \mathrm{kg}$

$\mu \mathrm{g} / \mathrm{kg}$
350

350

350

350

350

1.800

350

350

350

350

350

350

350

350

350

350

350

350

350

350

350

350

350

350

350

350

350

350

350

700 -

350

350

350

350

1,800

350

350

350
EPA8270

EPA8270

EPA8270

EPA8270

EPA8270

EPA8270

EPA8270

EPA8270

EPA8270

EPA8270

EPA8270

EPA8270

EPA8270

EPA8270

EPA8270

EPA8270

EPA8270

EPA8270

EPA8270

EPA8270

EPA8270

EPA8270

EPA8270

EPA8270

EPA8270

EPA8270

EPA8270

EPA8270

EPA8270

EPA8270

EPA8270

EPA8270

EPA8270

EPA8270

EPA8270

EPA8270

EPA8270
EPA8270 
SAMPLE NAME: D43 I10901A (Continued)

Sample ID: 100341

Constituent RQ AQ B Result Unit D. Limit Method

$B / N / A$ Extractables (including $P A H$ and phenols)

Fluoranthene
Fluorene
Hexachlorobenzene
Hexachlorobutadiene
Hexachlorocyclopentadiene
Hexachloroethane
Indeno(1,2,3-c,d)pyrene
Isophorone
2-Methyl-4.6-dinitrophenol
2-Methylnaphthalene
Naphthalene
o-Nitroaniline
m-Nitroaniline
p-Nitroaniline
Nitrobenzene
2-Nitrophenol
4-Nitrophenol
N-Nitrosodiphenylamine
N-Nitrosodipropylamine
Pentachlorophenol
Phenanthrene
Phenol
Pyrene
1,2,4-Trichlorobenzene
2,4,5-Trichlorophenol
2,4,6-Trichlorophenol
Unknown hydrocarbon
Unknown hydrocarbon
Unknown hydrocarbon
Unknown hydrocarbon
Unknown ketone
Unknown alcohol

U $\quad 350$

$\mathrm{U} \quad 350$

U 350

U 350

200

Volatile Organic Compounds

Acetone

Benzene

Bromodichloromethane

Bromoform

Bromomethane (Methyl bromide)

Carbon disulfide

Carbon tetrachloride

Chlorobenzene

Chloroethane

Chloroethene (Vinyl chloride)

Chloroform

Chloromethane (Methyl chloride)

Dibromochloromethane

1,1-Dichloroethane

1,2-Dichloroethane
350

350

350

350

1,800

350

350

1,800

1,800

1,800

350

350

1,800

350

350

1,800

350

350

350

350

1,800

350

200

400

400

6,000

100

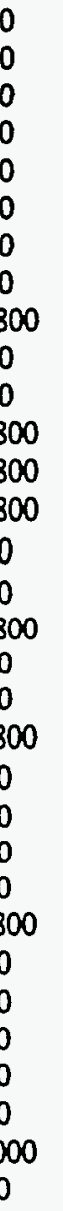

$\mu g / k g$
$\mu g / k g$
$\mu g / k g$
$\mu g / k g$
$\mu g / k g$
$\mu g / k g$
$\mu g / k g$
$\mu g / k g$
$\mu g / k g$
$\mu g / k g$
$\mu g / k g$
$\mu g / k g$
$\mu g / k g$
$\mu g / k g$
$\mu g / k g$
$\mu g / k g$
$\mu g / k g$
$\mu g / k g$
$\mu g / k g$
$\mu g / k g$
$\mu g / k g$
$\mu g / k g$
$\mu g / k g$
$\mu g / k g$
$\mu g / k g$
$\mu g / k g$
$\mu g / k g$
$\mu g / k g$
$\mu g / k g$
$\mu g / k g$
$\mu g / k g$
$\mu g / k g$
$g$

350

EPA8240

$\mu \mathrm{g} / \mathrm{kg}$

$\mu \mathrm{g} / \mathrm{kg}$

$\mu \mathrm{g} / \mathrm{kg}$

$\mu \mathrm{g} / \mathrm{kg}$

$\mu \mathrm{g} / \mathrm{kg}$

$\mu \mathrm{g} / \mathrm{kg}$

$\mu \mathrm{g} / \mathrm{kg}$

$\mu \mathrm{g} / \mathrm{kg}$

$\mu \mathrm{g} / \mathrm{kg}$

$\mu \mathrm{g} / \mathrm{kg}$

$\mu \mathrm{g} / \mathrm{kg}$

$\mu \mathrm{g} / \mathrm{kg}$

$\mu \mathrm{g} / \mathrm{kg}$

$\mu \mathrm{g} / \mathrm{kg}$

$\mu \mathrm{g} / \mathrm{kg}$
11

5.3

5.3

5.3

11

5:3

5.3

5.3

11

11 .

5.3

11

5.3

5.3

5.3
EPA8240

EPA8240

EPA8240

EPA8240

EPA8240

EPA8240

EPA8240

EPA8240

EPA8240

EPA8240

EPA8240

EPA8240

5.3

EPA8240

EPA8240 
SAMPLE NAME: D43110901A (Continued)

Constituent

Volatile Organic Compounds
Sample ID: 100341

RQ AQ B Result Unit $\quad$ D. Limit Method

$\begin{array}{ll}\text { 1.1-Dichloroethylene } & \mathrm{U} \\ \text { 1,2-Dichloroethylene } & \mathrm{U} \\ \text { Dichloromethane (Methylene chloride) } & \mathrm{U} \\ \text { 1,2-Dichloropropane } & \mathrm{U} \\ \text { cis-1,3-Dichloropropene } & \mathrm{U} \\ \text { trans-1,3-Dichloropropene } & \mathrm{U} \\ \text { Ethylbenzene } & \mathrm{U} \\ \text { 2-Hexanone } & \mathrm{U} \\ \text { Methyl ethyl ketone } & \mathrm{U} \\ \text { Methyl isobutyl ketone } & \mathrm{U} \\ \text { Styrene } & \mathrm{U} \\ \text { 1,1,2,2-Tetrachloroethane } & \mathrm{U} \\ \text { Tetrachloroethylene } & \mathrm{U} \\ \text { Toluene } & \mathrm{U} \\ \text { 1,1,1-Trichloroethane } & \mathrm{U} \\ \text { 1,1,2-Trichloroethane } & \mathrm{J} \\ \text { Trichloroethylene } & \mathrm{U} \\ \text { Vinyl acetate } & \mathrm{U} \\ \text { Xylenes } & \mathrm{U}\end{array}$

Pesticides/PCBs and Dioxins/Furans

$\begin{array}{llll}5.3 & \mu \mathrm{g} / \mathrm{kg} & 5.3 & \text { EPA8240 } \\ 5.3 & \mu \mathrm{g} / \mathrm{kg} & 5.3 & \text { EPA8240 } \\ 5.3 & \mu \mathrm{g} / \mathrm{kg} & 5.3 & \text { EPA8240 } \\ 5.3 & \mu \mathrm{g} / \mathrm{kg} & 5.3 & \text { EPA8240 } \\ 5.3 & \mu \mathrm{g} / \mathrm{kg} & 5.3 & \text { EPA8240 } \\ 5.3 & \mu \mathrm{g} / \mathrm{kg} & 5.3 & \text { EPA8240 } \\ 5.3 & \mu \mathrm{g} / \mathrm{kg} & 5.3 & \text { EPA8240 } \\ 11 & \mu \mathrm{g} / \mathrm{kg} & 11 & \text { EPA8240 } \\ 11 & \mu \mathrm{g} / \mathrm{kg} & 11 & \text { EPA8240 } \\ 11 & \mu \mathrm{g} / \mathrm{kg} & 11 & \text { EPA8240 } \\ 5.3 & \mu \mathrm{g} / \mathrm{kg} & 5.3 & \text { EPA8240 } \\ 5.3 & \mu \mathrm{g} / \mathrm{kg} & 5.3 & \text { EPA8240 } \\ 5.3 & \mu \mathrm{g} / \mathrm{kg} & 5.3 & \text { EPA8240 } \\ 5.3 & \mu \mathrm{g} / \mathrm{kg} & 5.3 & \text { EPA8240 } \\ 5.3 & \mu \mathrm{g} / \mathrm{kg} & 5.3 & \text { EPA8240 } \\ 4.0 & \mu \mathrm{g} / \mathrm{kg} & 5.3 & \text { EPA8240 } \\ 5.3 & \mu \mathrm{g} / \mathrm{kg} & 5.3 & \text { EPA8240 } \\ 11 & \mu \mathrm{g} / \mathrm{kg} & 11 & \text { EPA8240 } \\ 5.3 & \mu \mathrm{g} / \mathrm{kg} & 5.3 & \text { EPA8240 }\end{array}$

1.7

1.7

1.7

1.7

1.7

1.7

3.5

3.5

3.5

3.5

1.7

3.5

3.5

3.

3.:

1.:

1.7

0.10

0.10

0.10

0.50

0.10

0.10

1.7

17

1.6

0.10

35

$\mu \mathrm{g} / \mathrm{kg}$
$\mu \mathrm{g} / \mathrm{kg}$
$\mu \mathrm{g} / \mathrm{kg}$
$\mu \mathrm{g} / \mathrm{kg}$
$\mu \mathrm{g} / \mathrm{kg}$
$\mu \mathrm{g} / \mathrm{kg}$
$\mu \mathrm{g} / \mathrm{kg}$
$\mu \mathrm{g} / \mathrm{kg}$
$\mu \mathrm{g} / \mathrm{kg}$
$\mu \mathrm{g} / \mathrm{kg}$
$\mu \mathrm{g} / \mathrm{kg}$
$\mu \mathrm{g} / \mathrm{kg}$
$\mu \mathrm{g} / \mathrm{kg}$
$\mu \mathrm{g} / \mathrm{kg}$
$\mu \mathrm{g} / \mathrm{kg}$
$\mu \mathrm{g} / \mathrm{kg}$
$\mu \mathrm{g} / \mathrm{kg}$
$\mathrm{ng} / \mathrm{g}$
$\mathrm{ng} / \mathrm{g}$
$\mathrm{ng} / \mathrm{g}$
$\mathrm{ng} / \mathrm{g}$
$\mathrm{ng} / \mathrm{g}$
$\mathrm{ng} / \mathrm{g}$
$\mu \mathrm{g} / \mathrm{kg}$
$\mu \mathrm{g} / \mathrm{kg}$
$\mathrm{ng} / \mathrm{g}$
$\mathrm{ng} / \mathrm{g}$
$\mu \mathrm{g} / \mathrm{kg}$

1.7

0.10

0.10

0.10

1.7

17

0.10

0.10

35
EPA8080
EPA8080
EPA8080
EPA8080
EPA8080
EPA8080
EPA8080
EPA8080
EPA8080
EPA8080
EPA8080
EPA8080
EPA8080
EPA8080
EPA8080
EPA8080
EPA8080
EPA8280
EPA8280
EPA8280
EPA8280
EPA8280
EPA8280
EPA8080
EPA8080
EPA8280
EPA8280
EPA8080 
SAMPLE NAME: D43H0901A (Continued)

\section{Constituent}

Sample D: 100341

RQ AQ B Result Unit $\quad$ D. Limit Method

Pesticides/PCBs and Dioxins/Furans

PCB 1221
PCB 1232
PCB 1242
PCB 1248
PCB 1254
PCB 1260
Pentachlorodibenzo-p-dioxin isomers
Pentachlorodibenzo-p-furan isomers
Pentachlorodibenzo-p-dioxin isomers
Pentachlorodibenzo-p-furan isomers
2.3.7.8-TCDD
2.3.7.8-TCDF
Tetrachlorodibenzo-p-dioxin isomers
Tetrachlorodibenzo-p-furan isomers
Tetrachlorodibenzo-p-furan isomers
Toxaphene

Radionuclide Indicators and Radionuclides

Nonvolatile beta

Radium, total alpha-emitting

Tritium

$\begin{array}{ll}\mathrm{U} & 69 \\ \mathrm{U} & 35 \\ \mathrm{U} & 35 \\ \mathrm{U} & 35 \\ \mathrm{U} & 35 \\ \mathrm{U} & 35 \\ \mathrm{U} & 0.10 \\ \mathrm{U} & 0.10 \\ \mathrm{U} & 0.20 \\ \mathrm{U} & 0.10 \\ \mathrm{U} & 0.10 \\ \mathrm{U} & 0.10 \\ \mathrm{U} & 0.10 \\ \mathrm{U} & 0.10 \\ \mathrm{U} & 87 \\ \mathrm{U} & 170\end{array}$

\section{SAMPLE NAME: D43110902}

Location (SRS Coordinates): 18482.49E 66640.62N

Ground Elevation Above MSL: $132 \mathrm{ft}$

Depth of Core Interval: 0.50 to $2.00 \mathrm{ft}$

Sample Type: Normal

Sample Color: 10R 3/4

U

U V

9.5

$32 \pm 23$

$1.3 \pm 0.80$

$3.2 \pm 0.65$

pCi/g
pCi/g
pCi/g
pCi/g

Sample ID: 100343

$\mu \mathrm{g} / \mathrm{kg}$
$\mu \mathrm{g} / \mathrm{kg}$
$\mu \mathrm{g} / \mathrm{kg}$
$\mu \mathrm{g} / \mathrm{kg}$
$\mu \mathrm{g} / \mathrm{kg}$
$\mu \mathrm{g} / \mathrm{kg}$
$\mathrm{ng} / \mathrm{g}$
$\mathrm{ng} / \mathrm{g}$
$\mathrm{ng} / \mathrm{g}$
$\mathrm{ng} / \mathrm{g}$
$\mathrm{ng} / \mathrm{g}$
$\mathrm{ng} / \mathrm{g}$
$\mathrm{ng} / \mathrm{g}$
$\mathrm{ng} / \mathrm{g}$
$\mathrm{ng} / \mathrm{g}$
$\mu \mathrm{g} / \mathrm{kg}$

69

EPA8080

EPA8080

EPA8080

EPA8080

35

35

EPA8080

35

EPA8080

EPA8280

0.10 EPA8280

0.20 EPA8280

$0.10 \quad$ EPA8280

0.10 EPA8280

0.10 EPA8280

0.10 EPA8280

0.10 EPA8280

0.10 EPA8280

170 EPA8080

$\begin{array}{ll}9.5 & \text { EPA900.0 } \\ 9.6 & \text { EPA900.0 } \\ 0.20 & \text { EPA903.0 } \\ 1.2 & \text { EPA906.0 }\end{array}$

Sample Matrix: Soil USC Soil Classification: SM
Sample Moisture: Dry

Percent Solids: 92.00

D. Limit . Method

\section{Constituent}

Total Metals

Arsenic
Barium
Cadmium
Chromium
C̄opper
Lead
Manganese
Mercury
Nickel
Selenium
Silver

\section{RQ AQ B Result Unit}

$\begin{array}{ll}\mathrm{U} & 0.97 \\ \mathrm{U} & 20 \\ \mathrm{U} & 0.50 \\ & 12 \\ \mathrm{U} & 2.5 \\ & 2.6 \\ \mathrm{U} & 32 \\ \mathrm{U} & 0.11 \\ \mathrm{U} & 4.0 \\ \mathrm{U} & 0.48 \\ & 0.99\end{array}$

$\mathrm{mg} / \mathrm{kg}$
$\mathrm{mg} / \mathrm{kg}$
$\mathrm{mg} / \mathrm{kg}$
$\mathrm{mg} / \mathrm{kg}$
$\mathrm{mg} / \mathrm{kg}$
$\mathrm{mg} / \mathrm{kg}$
$\mathrm{mg} / \mathrm{kg}$
$\mathrm{mg} / \mathrm{kg}$
$\mathrm{mg} / \mathrm{kg}$
$\mathrm{mg} / \mathrm{kg}$
$\mathrm{mg} / \mathrm{kg}$

0.97

20

0.50

$0: 99$

2.5

0.29

1.5

0.11

4.0

0.48

0.99
EPA7060

EPA6010

EPA6010

EPA6010

EPA6010

EPA7421

EPA6010

EPA7470

EPA6010

EPA7740

EPA6010 
SAMPLE NAME: D43110902 (Continued)

Sample ID: 100343

Constituent

RQ AQ B Result

Unit

D. Limit Method

$B / N / A$ Extractables (including PAH and phenols)

Acenaphthene
Acenaphthylene
Anthracene
Benzo(a)anthracene
Benzo(b)fluoranthene
Benzo(k)fluoranthene
Benzoic acid
Benzo(g,h,i)perylene
Benzo(a)pyrene
Benzyl alcohol
Bis(2-chloroethoxy) methane
Bis(2-chlorothyl) ether
Bis(2-chloroisopropyl) ether
Bis(2-ethylhexyl) phthalate
4-Bromophenyl phenyl ether
Butylbenzyl phthalate
4-Chloroaniline
4-Chloro-m-cresol
2-Chloronaphthalene
2-Chlorophenol
4-Chlorophenyl phenyl ether
Chrysene
o-Cresol (2-Methylphenol)
p-Cresol (4-Methylphenol)
Dibenz(a,h)anthracene
Dibenzofuran
Di-n-butyl phthalate
1,2-Dichlorobenzene
1,3-Dichlorobenzene
1,4-Dichlorobenzene
3,3'-Dichlorobenzidine
2,4-Dichlorophenol
Diethyl phthalate
2,4-Dimethyl phenol
Dimethyl phthalate
2,4-Dinitrophenol
2,4-Dinitrotoluene
2,6-Dinitrotoluene
Di-n-octyl phthalate
Fluoranthene
Fluorene
Hexachlorobenzene
Hexachlorobutadiene
Hexachlorocyclopentadiene
Hexachloroethane
Indeno(1,2,3-c,d)pyrene
Isophorone
2-Methyl-4,6-dinitrophenol
2-Methylnaphthalene
Naphthalene

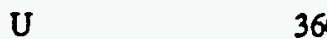

U 360

$\mathrm{U} \quad 360$

$U \quad 360$

U 360

U 360

U

U

U

$\mathrm{U}$

U

U

U

U

U

U

U

U

U

U

U

$\mathrm{U}$

U 360

U 360

U 360

U 360

UJ $\quad V \quad 62$

U 360

U 360

U 360

U $\quad$ : $\quad 720$

$\mathrm{U} \quad 360$

U 360

U 360

U 360

U $\quad 1,800$

U 360

U 360

U 360

U 360

$U \quad 360$

$\mathrm{U} \quad 360$

U 360

U 360

U 360

U 360

U 360

U $\quad 1,800$

U 360

U $\quad 360$

\begin{tabular}{|c|c|c|}
\hline$\mu g / k g$ & 360 & EPA8270 \\
\hline$\mu g / \mathrm{kg}$ & 360 & EPA8270 \\
\hline$\mu \mathrm{g} / \mathrm{kg}$ & 360 & EPA8270 \\
\hline$\mu g / \mathrm{kg}$ & 360 & EPA8270 \\
\hline$\mu \mathrm{g} / \mathrm{kg}$ & 360 & EPA8270 \\
\hline$\mu g / \mathrm{kg}$ & 360 & EPA8270 \\
\hline$\mu \mathrm{g} / \mathrm{kg}$ & 1.800 & EPA8270 \\
\hline$\mu g / \mathrm{kg}$ & 360 & EPA8270 \\
\hline$\mu \mathrm{g} / \mathrm{kg}$ & 360 & EPA8270 \\
\hline$\mu \mathrm{g} / \mathrm{kg}$ & 360 & EPA8270 \\
\hline$\mu g / k g$ & 360 & EPA8270 \\
\hline$\mu g / \mathrm{kg}$ & 360 & EPA8270 \\
\hline$\mu g / k g$ & 360 & EPA8270 \\
\hline$\mu \mathrm{g} / \mathrm{kg}$ & 360 & EPA8270 \\
\hline$\mu g / k g$ & 360 & EPA8270 \\
\hline$\mu \mathrm{g} / \mathrm{kg}$ & 360 & EPA8270 \\
\hline$\mu \mathrm{g} / \mathrm{kg}$ & 360 & EPA8270 \\
\hline$\mu \mathrm{g} / \mathrm{kg}$ & 360 & EPA 8270 \\
\hline$\mu g / k g$ & 360 & EPA8270 \\
\hline$\mu \mathrm{g} / \mathrm{kg}$ & 360 & EPA8270 \\
\hline$\mu \mathrm{g} / \mathrm{kg}$ & 360 & EPA 8270 \\
\hline$\mu \mathrm{g} / \mathrm{kg}$ & 360 & EPA8270 \\
\hline$\mu g / \mathrm{kg}$ & 360 & EPA8270 \\
\hline$\mu \mathrm{g} / \mathrm{kg}$ & 360 & EPA 8270 \\
\hline$\mu \mathrm{g} / \mathrm{kg}$ & 360 & EPA8270 \\
\hline$\mu \mathrm{g} / \mathrm{kg}$ & 360 & EPA8270 \\
\hline$\mu g / k g$ & 360 & EPA8270 \\
\hline$\mu \mathrm{g} / \mathrm{kg}$ & 360 & EPA8270 \\
\hline$\mu g / \mathrm{kg}$ & 360 & EPA8270 \\
\hline$\mu \mathrm{g} / \mathrm{kg}$ & 360 & EPA8270 \\
\hline$\mu \mathrm{g} / \mathrm{kg}$ & 720 & EPA8270 \\
\hline$\mu \mathrm{g} / \mathrm{kg}$ & 360 & EPA8270 \\
\hline$\mu \mathrm{g} / \mathrm{kg}$ & 360 & EPA8270 \\
\hline$\mu g / k g$ & 360 & EPA8270 \\
\hline$\mu \mathrm{g} / \mathrm{kg}$ & 360 & EPA8270 \\
\hline$\mu \mathrm{g} / \mathrm{kg}$ & 1.800 & EPA8270 \\
\hline$\mu g / \mathrm{kg}$ & -360 & EPA8270 \\
\hline$\mu g / k g$ & 360 & EPA8270 \\
\hline$\mu \mathrm{g} / \mathrm{kg}$ & 360 & EPA8270 \\
\hline$\mu \mathrm{g} / \mathrm{kg}$ & 360 & EPA8270 \\
\hline$\mu g / \mathrm{kg}$ & 360 & EPA8270 \\
\hline$\mu g / \mathrm{kg}$ & 360 & EPA8270 \\
\hline$\mu g / k g$ & 360 & EPA8270 \\
\hline$\mu g / k g$ & 360 & EPA8270 \\
\hline$\mu \mathrm{g} / \mathrm{kg}$ & 360 & EPA8270 \\
\hline$\mu g / \mathrm{kg}$ & 360 & EPA8270 \\
\hline$\mu g / \mathrm{kg}$ & 360 & EPA8270 \\
\hline$\mu g / k g$ & 1,800 & EPA8270 \\
\hline$\mu \mathrm{g} / \mathrm{kg}$ & 360 & EPA8270 \\
\hline & 360 & \\
\hline
\end{tabular}


SAMPLE NAME: D43110902 (Continued)

Sample ID: 100343

$\begin{array}{llllll}\text { Constituent } & \text { RQ AQ } & \text { B Result Unit D. Limit Method }\end{array}$

$B / N / A$ Extractables (including PAH and phenols)

o-Nitroaniline

m-Nitroaniline

p-Nitroaniline

Nitrobenzene

2-Nitrophenol

4-Nitrophenol

N-Nitrosodiphenylamine

N-Nitrosodipropylamine

Pentachlorophenol

Phenanthrene

Phenol

Pyrene

1,2,4-Trichlorobenzene

2,4,5-Trichlorophenol

2,4,6-Trichlorophenol

Unknown hydrocarbon

Unknown ketone

$\begin{array}{lll}U & 1,800 \\ U & 1,800 \\ U & 1,800 \\ U & 360 \\ U & 360 \\ U & 1,800 \\ U & 360 \\ U & 360 \\ U & & 1,800 \\ U & & 360 \\ U & & 360 \\ U & & 360 \\ U & & 360 \\ U & & 1,800 \\ U & & 360 \\ J & N & 200 \\ J & N V & 7,000\end{array}$

Volatile Organic Compounds

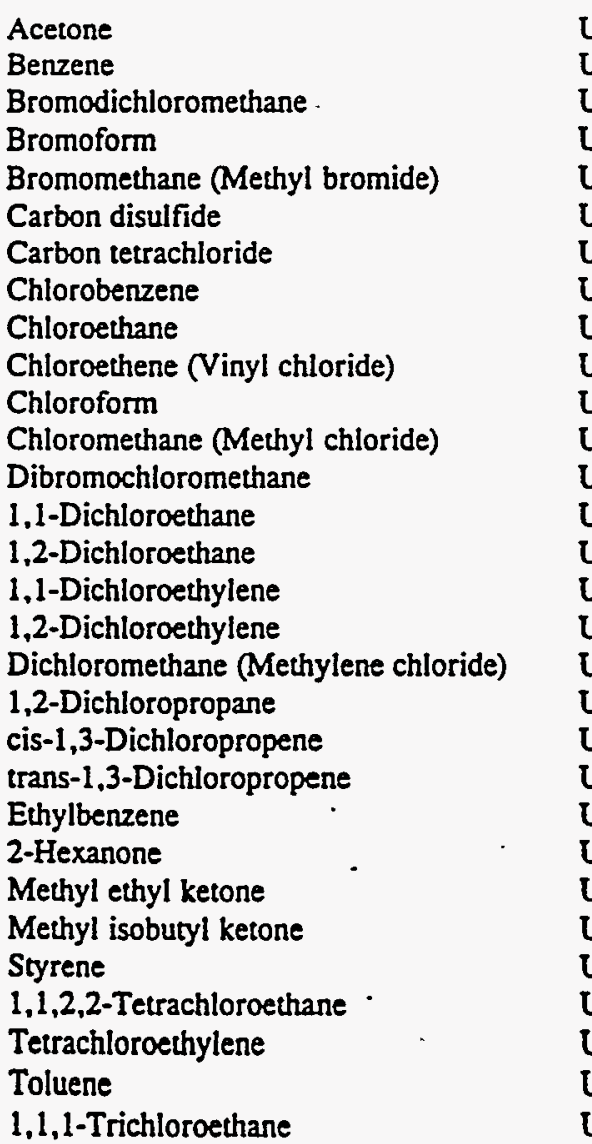

11

5.4

5.4

5.4

11

5.4

5.4

5.4

11

11

5.4

11

5.4

5.4

5.4

5.4

5.4

5.4

5.4

5.4

5.4

5.4

11

11

11

5.4

5.4

5.4

5.4

5.4

$\begin{array}{llll}1,800 & \mu g / \mathrm{kg} & 1,800 & \text { EPA8270 } \\ 1,800 & \mu g / \mathrm{kg} & 1,800 & \text { EPA8270 } \\ 1,800 & \mu \mathrm{g} / \mathrm{kg} & 1,800 & \text { EPA8270 } \\ 360 & \mu \mathrm{g} / \mathrm{kg} & 360 & \text { EPA8270 } \\ 360 & \mu \mathrm{g} / \mathrm{kg} & 360 & \text { EPA8270 } \\ 1,800 & \mu \mathrm{g} / \mathrm{kg} & 1,800 & \text { EPA8270 } \\ 360 & \mu \mathrm{g} / \mathrm{kg} & 360 & \text { EPA8270 } \\ 360 & \mu \mathrm{g} / \mathrm{kg} & 360 & \text { EPA8270 } \\ 1,800 & \mu \mathrm{g} / \mathrm{kg} & 1,800 & \text { EPA8270 } \\ 360 & \mu \mathrm{g} / \mathrm{kg} & 360 & \text { EPA8270 } \\ 360 & \mu \mathrm{g} / \mathrm{kg} & 360 & \text { EPA8270 } \\ 360 & \mu \mathrm{g} / \mathrm{kg} & 360 & \text { EPA8270 } \\ 360 & \mu \mathrm{g} / \mathrm{kg} & 360 & \text { EPA8270 } \\ 1,800 & \mu \mathrm{g} / \mathrm{kg} & 1,800 & \text { EPA8270 } \\ 360 & \mu \mathrm{g} / \mathrm{kg} & 360 & \text { EPA8270 } \\ 200 & \mu \mathrm{g} / \mathrm{kg} & & \text { EPA8270 } \\ 7,000 & \mu \mathrm{g} / \mathrm{kg} & & \text { EPA8270 }\end{array}$

$\mu \mathrm{g} / \mathrm{kg}$

$\mu \mathrm{g} / \mathrm{kg}$

$\mu \mathrm{g} / \mathrm{kg}$

$\mu \mathrm{g} / \mathrm{kg}$

$\mu \mathrm{g} / \mathrm{kg}$

$\mu g / \mathrm{kg}$

$\mu \mathrm{g} / \mathrm{kg}$

$\mu \mathrm{g} / \mathrm{kg}$

$\mu \mathrm{g} / \mathrm{kg}$

$\mu g / k g$

$\mu \mathrm{g} / \mathrm{kg}$

$\mu \mathrm{g} / \mathrm{kg}$

$\mu g / k g$

$\mu \mathrm{g} / \mathrm{kg}$

$\mu \mathrm{g} / \mathrm{kg}$

$\mu \mathrm{g} / \mathrm{kg}$

$\mu \mathrm{g} / \mathrm{kg}$

$\mu \mathrm{g} / \mathrm{kg}$

$\mu \mathrm{g} / \mathrm{kg}$

$\mu \mathrm{g} / \mathrm{kg}$

$\mu g / k g$

$\mu \mathrm{g} / \mathrm{kg}$

$\mu \mathrm{g} / \mathrm{kg}$

$\mu \mathrm{g} / \mathrm{kg}$

$\mu \mathrm{g} / \mathrm{kg}$

$\mu \mathrm{g} / \mathrm{kg}$

$\mu \mathrm{g} / \mathrm{kg}$

$\mu \mathrm{g} / \mathrm{kg}$

$\mu \mathrm{g} / \mathrm{kg}$

$\mu \mathrm{g} / \mathrm{kg}$

$\begin{array}{ll}11 & \text { EPA8240 } \\ 5.4 & \text { EPA8240 } \\ 5.4 & \text { EPA8240 } \\ 5.4 & \text { EPA8240 } \\ 11 & \text { EPA8240 } \\ 5.4 & \text { EPA8240 } \\ 5.4 & \text { EPA8240 } \\ 5.4 & \text { EPA8240 } \\ 11 & \text { EPA8240 } \\ 11 & \text { EPA8240 } \\ 5.4 & \text { EPA8240 } \\ 11 & \text { EPA8240 } \\ 5.4 & \text { EPA8240 } \\ 5.4 & \text { EPA8240 } \\ 5.4 & \text { EPA8240 } \\ 5.4 & \text { EPA8240 } \\ 5.4 & \text { EPA8240 } \\ 5.4 & \text { EPA8240 } \\ 5.4 & \text { EPA8240 } \\ 5.4 & \text { EPA8240 } \\ 5.4 & \text { EPA8240 } \\ 5.4 & \text { EPA8240 } \\ 11 & \text { EPA8240 } \\ 11 & \text { EPA8240 } \\ 11 & \text { EPA8240 } \\ 5.4 & \text { EPA8240 } \\ 5.4 & \text { EPA8240 } \\ 5.4 & \text { EPA8240 } \\ 5.4 & \text { EPA8240 } \\ 5.4 & \text { EPA8240 }\end{array}$


SAMPLE NAME: D43110902 (Continued)

Sample ID: 100343

\section{Constituent}

Volatile Organic Compounds

1,1,2-Trichloroethane
Trichloroethylene
Vinyl acetate
Xylenes

Pesticides/PCBs and Dioxins/Furans

\begin{tabular}{|c|c|c|c|c|c|c|c|}
\hline Aldrin & UJ & o & L & 1.8 & $\mu \mathrm{g} / \mathrm{kg}$ & 1.8 & EPA8080 \\
\hline alpha-Benzene hexachloride & UJ & 0 & L & 1.8 & $\mu \mathrm{g} / \mathrm{kg}$ & 1.8 & EPA8080 \\
\hline beta-Benzene hexachloride & UJ & 0 & L & 1.8 & $\mu \mathrm{g} / \mathrm{kg}$ & 1.8 & EPA8080 \\
\hline delta-Benzene hexachloride & UJ & 0 & L & 1.8 & $\mu \mathrm{g} / \mathrm{kg}$ & 1.8 & EPA8080 \\
\hline alpha-Chlordane & U & 0 & L & 1.8 & $\mu \mathrm{g} / \mathrm{kg}$ & 1.8 & EPA8080 \\
\hline gamma-Chlordane & UJ & 0 & L & 1.8 & $\mu \mathrm{g} / \mathrm{kg}$ & 1.8 & EPA8080 \\
\hline$p, p^{\prime}-D D D$ & UJ & 0 & L & 3.5 & $\mu \mathrm{g} / \mathrm{kg}$ & 3.5 & EPA8080 \\
\hline p.p'-DDE & UJ & 0 & $L$ & 3.5 & $\mu \mathrm{g} / \mathrm{kg}$ & 3.5 & EPA8080 \\
\hline p.p'-DDT & UJ & 0 & L & 3.5 & $\mu \mathrm{g} / \mathrm{kg}$ & 3.5 & EPA8080 \\
\hline Dieldrin & UJ & 0 & L & 3.5 & $\mu \mathrm{g} / \mathrm{kg}$ & 3.5 & EPA8080 \\
\hline Endosulfan I & UJ & 0 & L & 1.8 & $\mu \mathrm{g} / \mathrm{kg}$ & 1.8 & EPA8080 \\
\hline Endosulfan II & UJ & 0 & L & 3.5 & $\mu \mathrm{g} / \mathrm{kg}$ & 3.5 & EPA8080 \\
\hline Endosulfan sulfate & UJ & 0 & L & 3.5 & $\mu \mathrm{g} / \mathrm{kg}$ & 3.5 & EPA8080 \\
\hline Endrin & UJ & 0 & L & 3.5 & $\mu \mathrm{g} / \mathrm{kg}$ & 3.5 & EPA8080 \\
\hline Endrin ketone & UJ & 0 & L & 3.5 & $\mu \mathrm{g} / \mathrm{kg}$ & 3.5 & EPA8080 \\
\hline Heptachlor & UJ & 0 & L & 1.8 & $\mu \mathrm{g} / \mathrm{kg}$ & 1.8 & EPA8080 \\
\hline Heptachlor epoxide & UJ & $\mathbf{0}$ & L & 1.8 & $\mu \mathrm{g} / \mathrm{kg}$ & 1.8 & EPA8080 \\
\hline Heptachlorodibenzo-p-dioxin isomers & U & & & 0.10 & $\mathrm{ng} / \mathrm{g}$ & 0.10 & EPA8280 \\
\hline Heptachlorodibenzo-p-dioxin isomers & $\mathbf{U}$ & & & 0.10 & $\mathrm{ng} / \mathrm{g}$ & 0.10 & EPA8280 \\
\hline Heptachlorodibenzo-p-furan isomers & U & & & 0.10 & $\mathrm{ng} / \mathrm{g}$ & 0.10 & EPA8280 \\
\hline Heptachlorodibenzo-p-furan isomers & $\mathbf{U}$ & & & 0.10 & $\mathrm{ng} / \mathrm{g}$ & 0.10 & EPA8280 \\
\hline Hexachlorodibenzo-p-dioxin isomers & $\mathrm{U}$ & & & 0.10 & $\mathrm{ng} / \mathrm{g}$ & 0.10 & EPA8280 \\
\hline Hexachlorodibenzo-p-dioxin isomers & $\mathbf{U}$ & & & 0.10 & $\mathrm{ng} / \mathrm{g}$ & 0.10 & EPA8280 \\
\hline Hexachlorodibenzo-p-furan isomers & $\mathbf{U}$ & & & 0.10 & $\mathrm{ng} / \mathrm{g}$ & 0.10 & EPA8280 \\
\hline Hexachlorodibenzo-p-furan isomers & U & & & 0.10 & $\mathrm{ng} / \mathrm{g}$ & 0.10 & EPA8280 \\
\hline Lindane & UJ & 0 & L & 1.8 & $\mu \mathrm{g} / \mathrm{kg}$ & 1.8 & EPA8080 \\
\hline Methoxychlor & UJ & 0 & L & 18 & $\mu \mathrm{g} / \mathrm{kg}$ & 18 & EPA8080 \\
\hline Octachlorodibenzo-p-dioxin & & & & 3.2 & $\mathrm{ng} / \mathrm{g}$ & 0.30 & EPA8280 \\
\hline Octachlorodibenzo-p-dioxin & & & & 2.1 & $\mathrm{ng} / \mathrm{g}$ & 0.10 & EPA8280 \\
\hline Octachlorodibenzo-p-furan & $\mathbf{U}$ & & & 0.10 & $\mathrm{ng} / \mathrm{g}$ & 0.10 & EPA8280 \\
\hline Octachlorodibenzo-p-furan & $\mathbf{U}$ & & & 0.10 & $\mathrm{ng} / \mathrm{g}$ & 0.10 & EPA8280 \\
\hline PCB 1016 & UJ & 0 & $L$ & 35 & $\mu \mathrm{g} / \mathrm{kg}$ & 35 & EPA8080 \\
\hline PCB 1221 & UJ & o & $\mathrm{L}$ & 71 & $\mu \mathrm{g} / \mathrm{kg}$ & 71 & EPA8080 \\
\hline PCB 1232 & UJ & $\mathbf{o}$ & $\bar{L}$ & 35 & $\mu \mathrm{g} / \mathrm{kg}$ & 35 & EPA8080 \\
\hline PCB 1242 & UJ & 0 & L & 35 & $\mu \mathrm{g} / \mathrm{kg}$ & 35 & EPA8080 \\
\hline PCB 1248 & UJ & 0 & L & 35 & $\mu \mathrm{g} / \mathrm{kg}$ & 35 & EPA8080 \\
\hline РСB 1254 & UJ & 0 & L & 35 & $\mu \mathrm{g} / \mathrm{kg}$ & 35 & EPA8080 \\
\hline PCB 1260 & UJ & 0 & $\bar{L}$ & 35 & $\mu \mathrm{g} / \mathrm{kg}$ & 35 & EPA8080 \\
\hline Pentachlorodibenzo-p-dioxin isomers & $\mathbf{U}$ & & & 0.20 & $\mathrm{ng} / \mathrm{g}$ & 0.20 & EPA8280 \\
\hline Pentachlorodibenzo-p-furan isomers & $\mathbf{U}$ & & & 0.20 & $\mathrm{ng} / \mathrm{g}$ & 0.20 & EPA8280 \\
\hline Pentachlorodibenzo-p-dioxin isomers & $\mathbf{U}$ & & & 0.10 & $\mathrm{ng} / \mathrm{g}$ & 0.10 & EPA8280 \\
\hline Pentachlorodibenzo-p-furan isomers & $\mathbf{U}$ & & & 0.10 & $\mathrm{ng} / \mathrm{g}$ & 0.10 & EPA8280 \\
\hline $2,3,7,8-\mathrm{TCDD}$ & $\mathbf{U}$ & & & 0.10 & $\mathrm{ng} / \mathrm{g}$ & 0.10 & EPA8280 \\
\hline
\end{tabular}


SAMPLE NAME: D43110902 (Continued)

Constituent

RQ AQ B Result

Sample ID: 100343

Pesticides/PCBs and Dioxins/Furans

2.3.7.8-TCDD

2,3,7,8-TCDF

2,3,7,8-TCDF

U

Tetrachlorodibenzo-p-dioxin isomers

Tetrachlorodibenzo-p-dioxin isomers

Tetrachlorodibenzo-p-furan isomers

Tetrachlorodibenzo-p-furan isomers

Toxaphene

$\begin{array}{llll}\text { U } & & & 0.10 \\ U & & & 0.10 \\ U & & & 0.10 \\ U & & & 0.10 \\ U & & & 0.10 \\ \text { U } & & & 0.10 \\ \text { U } & & & 0.10 \\ \text { UJ } & \text { O } & \text { L } & 180\end{array}$

Radionuclide Indicators and Radionuclides

Gross alpha

U

Nonvolatile beta

Radium, total alpha-emitting

Tritium

U V

\section{9}

$22 \pm 20$

$1.2 \pm 0.70$

$2.9 \pm 0.61$

Unit

D. Limit Method

\section{SAMPLE NAME: D43110903}

Sample ID: 100344

Location (SRS Coordinates): $18482.49 \mathrm{E} 66640.62 \mathrm{~N}$

Ground Elevation Above MSL: $132 \mathrm{ft}$

Depth of Core Interval: 2.00 to $4.00 \mathrm{ft}$

Sample Type: Normal

Sample Color: 10R 4/6

Sample Matrix: Soil

USC Soil Classification: SM

RQ AQ B Result Unit
Sample Moisture: Dry Percent Solids: $\mathbf{9 0 . 2 0}$.

D. Limit Method
Constituent

Total Metals

Arsenic

Barium

Cadmium

Chromium

Copper

Lead

Manganese

Mercury

Nickel

Selenium

Silver

Volatile Organic Compounds

Acetone

Benzene

Bromodichloromethane

Bromoform

Bromomethane (Methyl bromide)

Carbon disulfide

Carbon tetrachloride

$\begin{array}{ll}\mathrm{U} & 1.1 \\ \mathrm{U} & 20 \\ \mathrm{U} & 0.51 \\ & 12 \\ & 2.8 \\ & 3.8 \\ \mathrm{U} & 29 \\ \mathrm{U} & 0.11 \\ \mathrm{U} & 4.1 \\ \mathrm{U} & 0.55\end{array}$

ng/g

$\mathrm{ng} / \mathrm{g}$

$\mathrm{ng} / \mathrm{g}$

$\mathrm{ng} / \mathrm{g}$

$\mathrm{ng} / \mathrm{g}$

$\mathrm{ng} / \mathrm{g}$

$\mathrm{ng} / \mathrm{g}$

$\mu g / \mathrm{kg}$

0.10

0.10

0.10

0.10

0.10

0.10

0.10

180

8.9

EPA900.0

$\mathrm{pCi} / \mathrm{g}$

$\mathrm{pCi} / \mathrm{g}$

$\mathrm{pCi} / \mathrm{g}$

0.20

1.2

EPA903.0

EPA906.0

EPA8280

EPA8280

EPA8280

EPA8280

EPA8280

EPA8080

0


SAMPLE NAME: D431 10903 (Continued)

\section{Constituent}

Volatile Organic Compounds

Chlorobenzene
Chloroethane
Chloroethene (Vinyl chloride)
Chloroform
Chloromethane (Methyl chloride)
Dibromochloromethane
1,1-Dichloroethane
1,2-Dichloroethane
1,1-Dichloroethylene
1,2-Dichloroethylene
Dichloromethane (Methylene chloride)
1,2-Dichloropropane
cis-1,3-Dichloropropene
trans-1,3-Dichloropropene
Ethylbenzene
2-Hexanone
Methyl ethyl ketone
Methyl isobutyl ketone
Styrene
1.1,2,2-Tetrachloroethane
Tetrachloroethylene
Toluene
1,1,1-Trichloroethane
1,1,2-Trichloroethane
Trichloroethylene
Vinyl acetate
Xylenes

Sample ID: 100344

RQ AQ B Result Unit D. Limit Method

\begin{tabular}{|c|c|c|c|c|}
\hline$U$ & 5.7 & $\mu \mathrm{g} / \mathrm{kg}$ & 5.7 & EPA 8240 \\
\hline $\mathbf{U}$ & 11 & $\mu \mathrm{g} / \mathrm{kg}$ & 11 & EPA8240 \\
\hline $\mathbf{U}$ & 11 & $\mu \mathrm{g} / \mathrm{kg}$ & 11 & EPA8240 \\
\hline $\mathbf{U}$ & 5.7 & $\mu \mathrm{g} / \mathrm{kg}$ & 5.7 & EPA8240 \\
\hline $\mathbf{U}$ & 11 & $\mu \mathrm{g} / \mathrm{kg}$ & 11 & EPA8240 \\
\hline $\mathbf{U}$ & 5.7 & $\mu \mathrm{g} / \mathrm{kg}$ & 5.7 & EPA8240 \\
\hline $\mathbf{U}$ & 5.7 & $\mu \mathrm{g} / \mathrm{kg}$ & 5.7 & EPA8240 \\
\hline $\mathrm{U}$ & 5.7 & $\mu \mathrm{g} / \mathrm{kg}$ & 5.7 & EPA8240 \\
\hline U & 5.7 & $\mu \mathrm{g} / \mathrm{kg}$ & 5.7 & EPA8240 \\
\hline U & 5.7 & $\mu g / \mathrm{kg}$ & 5.7 & EPA8240 \\
\hline U & 5.7 & $\mu \mathrm{g} / \mathrm{kg}$ & 5.7 & EPA8240 \\
\hline $\mathrm{U}$ & 5.7 & $\mu g / \mathrm{kg}$ & 5.7 & EPA8240 \\
\hline U & 5.7 & $\mu g / k g$ & 5.7 & EPA8240 \\
\hline $\mathbf{U}$ & 5.7 & $\mu \mathrm{g} / \mathrm{kg}$ & 5.7 & EPA8240 \\
\hline $\mathbf{U}$ & 5.7 & $\mu \mathrm{g} / \mathrm{kg}$ & 5.7 & EPA8240 \\
\hline $\mathbf{U}$ & 11 & $\mu g / k g$ & 11 & EPA8240 \\
\hline U & 11 & $\mu \mathrm{g} / \mathrm{kg}$ & 11 & EPA8240 \\
\hline $\mathrm{U}$ & 11 & $\mu \mathrm{g} / \mathrm{kg}$ & 11 & EPA8240 \\
\hline $\mathbf{U}$ & 5.7 & $\mu \mathrm{g} / \mathrm{kg}$ & 5.7 & EPA8240 \\
\hline $\mathbf{U}$ & 5.7 & $\mu \mathrm{g} / \mathrm{kg}$ & 5.7 & EPA8240 \\
\hline $\mathbf{U}$ & 5.7 & $\mu \mathrm{g} / \mathrm{kg}$ & 5.7 & EPA8240 \\
\hline $\mathbf{U}$ & 5.7 & $\mu \mathrm{g} / \mathrm{kg}$ & 5.7 & EPA8240 \\
\hline $\mathbf{U}$ & 5.7 & $\mu \mathrm{g} / \mathrm{kg}$ & 5.7 & EPA8240 \\
\hline $\mathbf{U}$ & 5.7 & $\mu \mathrm{g} / \mathrm{kg}$ & 5.7 & EPA8240 \\
\hline $\mathbf{U}$ & 5.7 & $\mu \mathrm{g} / \mathrm{kg}$ & 5.7 & EPA8240 \\
\hline $\mathbf{U}$ & 11 & $\mu g / k g$ & 11 & EPA8240 \\
\hline $\mathbf{U}$ & 5.7 & $\mu \mathrm{g} / \mathrm{kg}$ & 5.7 & EPA8240 \\
\hline
\end{tabular}

Radionuclide Indicators and Radionuclides

$\begin{array}{lllllll}\text { Gross alpha } & \mathrm{U} & & 8.5 & \mathrm{pCi} / \mathrm{g} & 8.5 & \text { EPA900.0 } \\ \text { Nonvolatile beta } & & & 16 \pm 19 & \mathrm{pCi} / \mathrm{g} & 8.5 & \text { EPA900.0 } \\ \text { Radium, total alpha-emitting } & \mathrm{U} & \mathrm{V} & 1.4 \pm 0.70 & \mathrm{pCi} / \mathrm{g} & 0.14 & \text { EPA903.0 } \\ \text { Tritium } & & & 5.0 \pm 0.97 & \mathrm{pCi} / \mathrm{g} & 1.5 & \text { EPA906.0 }\end{array}$

\section{SAMPLE NAME: D43110904}

Location (SRS Coordinates): 18482.49E 66640.62N

Ground Elevation Above MSL: $132 \mathrm{ft}$

Depth of Core Interval: 4.00 to $6.00 \mathrm{ft}$

Sample Type: Normal

Sample Color: 10R 4/6
Sample ID: 100345

Sample Matrix: Soil

USC Soil Classification: SM
Sample Moisture: Dry Percent Solids: 88.90

\section{Total Metals}

Arsenic

Barium

Cadmium

Chromium
U

$\mathbf{U}$

4.1
22
0.54
47

4.1

0.54

47 $\mathrm{mg} / \mathrm{kg}$
$\mathrm{mg} / \mathrm{kg}$
$\mathrm{mg} / \mathrm{kg}$
$\mathrm{mg} / \mathrm{kg}$
0.91

22

0.54

1.1
EPA7060

EPA6010

EPA6010

EPA6010 
SAMPLE NAME: D43110904 (Continued)

Constituent

RQ AQ B Result

Total Metals

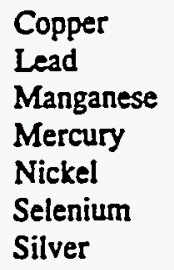

Volatile Organic Compounds

Acetone
Acetone
Acetone
Acetone
Benzene
Bromodichloromethane
Bromodichloromethane
Bromodichloromethane
Bromoform
Bromoform
Bromoform
Bromomethane (Methyl bromide)
Bromomethane (Methyl bromide)
Bromomethane (Methyl bromide)
Carbon disulfide
Carbon disulfide
Carbon disulfide
Carbon tetrachloride
Carbon tetrachloride
Carbon tetrachloride
Chlorobenzene
Chloroethane
Chloroethane
Chloroethane
Chloroethene (Vinyl chloride)
Chloroethene (Vinyl chloride)
Chloroethene (Vinyl chloride)
Chloroform
Chloroform
Chloroform
Chloromethane (Methyl chloride)
Chloromethane (Methyl chloride)
Chloromethane (Methyl chloride)
Dibromochloromethane
Dibromochloromethane
Dibromochloromethane
1.1-Dichloroethane
1,1-Dichloroethane
1,1-Dichloroethane
1,2-Dichloroethane

$\mathrm{U}$

$\mathrm{U}$

L V

U

U

U

$\mathrm{U}$

$\mathrm{U}$

U

U

U

U

U

U

U

U

U

U

$\mathrm{U}$

U

U

U

U

U

U

$\mathrm{U}$

U

U

U

U

U

U

U

U

U

U

U

U $\begin{array}{lll} & \text { V } \\ \text { L } & \text { V }\end{array}$
30

37

35

0.64

38

0.46

1.1

2,400

2,600

2,000

2,000

5.8

5.8

5.8

5.8

5.8

5.8

5.8

12

12

12

5.8

5.8

5.8

5.8

5.8

5.8

5.8

12

12

12

12

12

12

5.8

5.8

5.8

12

12

12

5.8

5.8

5.8

5.8

5.8

5.8

3.8
Sample ID: 100345

Unit

D. Limit Method

$\begin{array}{lll}\mu \mathrm{g} / \mathrm{kg} & 12 & \text { EPA8240 } \\ \mu \mathrm{g} / \mathrm{kg} & 120 & \text { EPA8240 } \\ \mu \mathrm{g} / \mathrm{kg} & 12 & \text { EPA8240 } \\ \mu \mathrm{g} / \mathrm{kg} & 12 & \text { EPA8240 } \\ \mu \mathrm{g} / \mathrm{kg} & 5.8 & \text { EPA8240 } \\ \mu \mathrm{g} / \mathrm{kg} & 5.8 & \text { EPA8240 } \\ \mu \mathrm{g} / \mathrm{kg} & 5.8 & \text { EPA8240 } \\ \mu \mathrm{g} / \mathrm{kg} & 5.8 & \text { EPA8240 } \\ \mu \mathrm{g} / \mathrm{kg} & 5.8 & \text { EPA8240 } \\ \mu \mathrm{g} / \mathrm{kg} & 5.8 & \text { EPA8240 } \\ \mu \mathrm{g} / \mathrm{kg} & 5.8 & \text { EPA8240 } \\ \mu \mathrm{g} / \mathrm{kg} & 12 & \text { EPA8240 } \\ \mu \mathrm{g} / \mathrm{kg} & 12 & \text { EPA8240 } \\ \mu \mathrm{g} / \mathrm{kg} & 12 & \text { EPA8240 } \\ \mu \mathrm{g} / \mathrm{kg} & 5.8 & \text { EPA8240 } \\ \mu \mathrm{g} / \mathrm{kg} & 5.8 & \text { EPA8240 } \\ \mu \mathrm{g} / \mathrm{kg} & 5.8 & \text { EPA8240 } \\ \mu \mathrm{g} / \mathrm{kg} & 5.8 & \text { EPA8240 } \\ \mu \mathrm{g} / \mathrm{kg} & 5.8 & \text { EPA8240 } \\ \mu \mathrm{g} / \mathrm{kg} & 5.8 & \text { EPA8240 } \\ \mu \mathrm{g} / \mathrm{kg} & 5.8 & \text { EPA8240 } \\ \mu \mathrm{g} / \mathrm{kg} & 12 & \text { EPA8240 } \\ \mu \mathrm{g} / \mathrm{kg} & 12 & \text { EPA8240 } \\ \mu \mathrm{g} / \mathrm{kg} & 12 & \text { EPA8240 } \\ \mu \mathrm{g} / \mathrm{kg} & 12 & \text { EPA8240 } \\ \mu \mathrm{g} / \mathrm{kg} & 12 & \text { EPA8240 } \\ \mu \mathrm{g} / \mathrm{kg} & 12 & \text { EPA8240 } \\ \mu \mathrm{g} / \mathrm{kg} & 5.8 & \text { EPA8240 } \\ \mu \mathrm{g} / \mathrm{kg} & 5.8 & \text { EPA8240 } \\ \mu \mathrm{g} / \mathrm{kg} & 5.8 & \text { EPA8240 } \\ \mu \mathrm{g} / \mathrm{kg} & 12 & \text { EPA8240 } \\ \mu \mathrm{g} / \mathrm{kg} & 12 & \text { EPA8240 } \\ \mu \mathrm{g} / \mathrm{kg} & 12 & \text { EPA8240 } \\ \mu \mathrm{g} / \mathrm{kg} & 5.8 & \text { EPA8240 } \\ \mu \mathrm{g} / \mathrm{kg} & 5.8 & \text { EPA8240 } \\ \mu \mathrm{g} / \mathrm{kg} & 5.8 & \text { EPA8240 } \\ \mu \mathrm{g} / \mathrm{kg} & 5.8 & \text { EPA8240 } \\ \mu \mathrm{g} / \mathrm{kg} & 5.8 & \text { EPA8240 } \\ \mu \mathrm{g} / \mathrm{kg} & 5.8 & \text { EPA8240 } \\ \mu \mathrm{g} / \mathrm{kg} & 5.8 & \text { EPA8240 } \\ & & \\ & 12 & \\ & 12 & \end{array}$

$2.7 \quad$ EPA6010

5.5 EPA7421

$1.6 \quad$ EPA6010

$0.11 \quad$ EPA7470

4.3 EPA6010

$0.46 \quad$ EPA7740

1.1 EPA6010 


\section{Constituent}

Volotile Organic Compounds

1,2-Dichloroethane

1,2-Dichloroethane

1,1-Dichloroethylene

1,2-Dichloroethylene

1,2-Dichloroethylene

1,2-Dichloroethylene

Dichloromethane (Methylene chloride)

Dichloromethane (Mechylene chloride)

Dichloromethane (Methylene chloride)

1,2-Dichloropropane

1,2-Dichloropropane

1,2-Dichloropropane

cis-1,3-Dichloropropene

cis-1,3-Dichloropropene

cis-1.3-Dichloropropene

trans-1,3-Dichloropropene

trans-1,3-Dichloropropene

trans-1,3-Dichloropropene

Ethylbenzene

Ethylbenzene

Ethylbenzene

2-Hexanone

2-Hexanone

2-Hexanone

Methyl ethyl ketone

Methyl ethyl ketone

Methyl ethyl ketone

Methyl isobutyl ketone

Mechyl isobutyl ketone

Methyl isobutyl ketone

Styrene

Styrene

Styrene

1,1,2.2-Tetrachloroethane

1,1,2,2-Tetrachloroethane

1,1,2,2-Tetrachloroethane

Tetrachloroethylene

Tetrachloroethylene

Tetrachloroethylene

Toluene

1,1,1-Trichloroethane

1,1,1-Trichloroethane

1,1,1-Trichloroethane

1,1,2-Trichloroethane

1,1,2-Trichloroethane

1,1,2-Trichloroethane

Trichloroethylene

Vinyl acetate

Vinyl acetate

Vinyl acetate
$\mathbf{R Q}$
AQ B Result
Unit
D. Limit Method

4.0

5.8

5.8

5.8

5.8

5.8

5.8

5.8

5.8

5.8

5.8

5.8

5.8

5.8

5.8

5.8

5.8

5.8

5.8

5.8

5.8

12

12

12

12

12

12

12

12

12

5.8

5.8

5.8

5.8

5.8

5.8

5.8

5.8

5.8

5.8

5.8

5.8

5.8

3.8

3.0

5.8

5.8

12

12

12
5.8

5.8

5.8

5.8

5.8

5.8

5.8

5.8

5.8

5.8

5.8

5.8

5.8

5.8

5.8

5.8

5.8

5.8

5.8

5.8

5.8

12

12

12

12

12

12

12

12

12

5.8

5.8

5.8

5.8

5.8

5.8

5.8

5.8

5.8

5.8

5.8

5.8

5.8

5.8

5.8

5.8

5.8

12

12

12
EPA8240

EPA8240

EPA8240

EPA8240

EPA8240

EPA8240

EPA8240

EPA8240

EPA8240

EPA8240

EPA8240

EPA8240

EPA8240

EPA8240

EPA8240

EPA8240

EPA8240

EPA8240

EPA8240

EPA8240

EPA8240

EPA8240

EPA8240

EPA8240

EPA8240

EPA8240

EPA8240

EPA8240

EPA8240

EPA8240

EPA8240

EPA8240

EPA8240

EPA8240

EPA8240

EPA8240

EPA8240

EPA8240

EPA8240

EPA8240

EPA8240

EPA8240

EPA8240

EPA8240

EPA8240

EPA8240

EPA8240

EPA8240

EPA8240
EPA8240 
SAMPLE NAME: D43110904 (Continued) Sample ID: 100345

Constituent

RQ AQ B Result

Unit

D. Limit Method

Volatile Organic Compounds

Xylenes

Xylenes

Xylenes

$\mathbf{U}$
$\mathbf{U}$

Radionuclide Indicators and Radionuclides

Gross alpha

Nonvolatile beta

Radium, total alpha-emitting

Tritium

$\begin{array}{lllll} & 12 \pm 14 & \mathrm{pCi} / \mathrm{g} & 9.5 & \text { EPA900.0 } \\ & 20 \pm 21 & \mathrm{pCi} / \mathrm{g} & 9.5 & \text { EPA900.0 } \\ \mathrm{U} & 1.1 \pm 0.70 & \mathrm{pCi} / \mathrm{g} & 0.20 & \text { EPA903.0 } \\ & 3.1 \pm 0.63 & \mathrm{pCi} / \mathrm{g} & 1.2 & \text { EPA906.0 }\end{array}$

Sample ID: 100346

Sample Matrix: Soil

USC Soil Classification: SW

Sample Moisture: Damp Percent Solids: $\mathbf{8 8 . 4 0}$

D. Limit Method

\section{Constituent}

Physical Parameters

Cation exchange capacity

Cyanide

$\mathrm{pH}$

Specific conductance

Total organic carbon

Total Metals

RQ AQ B Result Unit

meq $/ 100 \mathrm{~g}$

$\mathrm{mg} / \mathrm{kg}$

$\mathrm{pH}$

$\mu \mathrm{S} / \mathrm{cm}$

$\mathrm{mg} / \mathrm{kg}$ $\begin{array}{ll}5.8 & \text { EPA8240 } \\ 5.8 & \text { EPA8240 } \\ 5.8 & \text { EPA8240 }\end{array}$

Location (SRS Coordinates): 18482.49E 66640.62N

Depth of Core Interval: 6.00

Sample Type: Normal

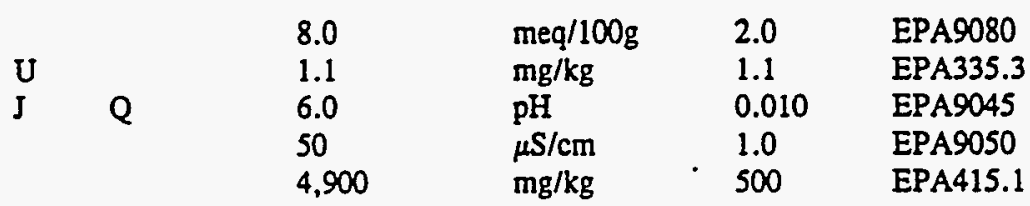

Aluminum

Antimony

Arsenic

Barium

Beryllium

Cadmium

Calcium

Chromium

Cobalt

Copper

Iron

Lead

Magnesium

Manganese

Mercury

Nickel

Potassium

Selenium

\begin{tabular}{|c|c|c|c|c|c|}
\hline \multirow[b]{2}{*}{$\mathrm{U}$} & & 1,300 & $\mathrm{mg} / \mathrm{kg}$ & 18 & EPA6010 \\
\hline & & 5.4 & $\mathrm{mg} / \mathrm{kg}$ & 5.4 & EPA6010 \\
\hline & & 3.2 & $\mathrm{mg} / \mathrm{kg}$ & 1.0 & EPA7060 \\
\hline $\mathrm{U}$ & & 18 & $\mathrm{mg} / \mathrm{kg}$ & 18 & EPA6010 \\
\hline U & & 0.45 & $\mathrm{mg} / \mathrm{kg}$ & 0.45 & EPA6010 \\
\hline UJ & L & 0.45 & $\mathrm{mg} / \mathrm{kg}$ & 0.45 & EPA6010 \\
\hline $\mathbf{U}$ & & 450 & $\mathrm{mg} / \mathrm{kg}$ & 450 & EPA6010 \\
\hline UJ & L & 8.9 & $\mathrm{mg} / \mathrm{kg}$ & 0.90 & EPA6010 \\
\hline $\mathbf{U}$ & & 4.5 & $\mathrm{mg} / \mathrm{kg}$ & 4.5 & EPA6010 \\
\hline & & 3.9 & $\mathrm{mg} / \mathrm{kg}$ & 2.2 & EPA6010 \\
\hline & & 5,900 & $\mathrm{mg} / \mathrm{kg}$ & 9.0 & EPA6010 \\
\hline & & 22 & $\mathrm{mg} / \mathrm{kg}$ & 3.1 & EPA7421 \\
\hline $\mathbf{U}$ & & 450 & $\mathrm{mg} / \mathrm{kg}$ & 450 & EPA6010 \\
\hline & & 13 & $\mathrm{mg} / \mathrm{kg}$ & 1.3 & EPA6010 \\
\hline $\mathbf{U}$ & & 0.11 & $\mathrm{mg} / \mathrm{kg}$ & 0.11 & EPA7470 \\
\hline U & & 3.6 & $\mathrm{mg} / \mathrm{kg}$ & 3.6 & EPA6010 \\
\hline U & & 450 & $\mathrm{mg} / \mathrm{kg}$ & 450 & EPA6010 \\
\hline 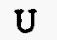 & & 0.52 & $\mathrm{mg} / \mathrm{kg}$ & 0.52 & EPA7740 \\
\hline
\end{tabular}


SAMPLE NAME: D43110905 (Continued)

Constituent

RQ AQ B Result

Total Metals

Silver

Sodium

Thallium

Vanadium

Zine

\section{$B / N / A$ Extractables (including $P A H$ and phenols)}

Acenaphthene

Acenaphthylene

Anthracene

Benzo(a)anthracene

Benzo(b)fluoranthene

Benzo(k)fluoranthene

Benzoic acid

Benzo(g,h,i)perylene

Benzo(a)pyrene

Benzyl alcohol

Bis(2-chloroethoxy) methane

Bis(2-chloroethyl) ether

Bis(2-chloroisopropyl) ether

Bis(2-ethylhexyl) phthalate

4-Bromophenyl phenyl ether

Butylbenzyl phthalate

4-Chloroaniline

4-Chloro-m-cresol

2-Chloronaphthalene

2-Chlorophenol

4-Chlorophenyl phenyl ether

Chrysene

o-Cresol (2-Methylphenol)

p-Cresol (4-Methylphenol)

Dibenz(a,h)anthracene

Dibenzofuran

Di-n-butyl phthalate

1,2-Dichlorobenzene

1,3-Dichlorobenzene

1,4-Dichlorobenzene

3,3'-Dichlorobenzidine

2,4-Dichlorophenol

Diethyl phthalate

2,4-Dimethyl phenol

Dimethyl phthalate

2,4-Dinitrophenol

2,4-Dinitrotoluene

2,6-Dinitrotoluene

Di-n-octyl phthalate

Fluoranthene

Fluorene

Hexachlorobenzene
Sample ID: 100346

Unit

D. Limit Method

$\begin{array}{llll}0.90 & \mathrm{mg} / \mathrm{kg} & 0.90 & \text { EPA6010 } \\ 450 & \mathrm{mg} / \mathrm{kg} & 450 & \text { EPA6010 } \\ 1.0 & \mathrm{mg} / \mathrm{kg} & 1.0 & \text { EPA7841 } \\ 14 & \mathrm{mg} / \mathrm{kg} & 4.5 & \text { EPA6010 } \\ 7.7 & \mathrm{mg} / \mathrm{kg} & 1.8 & \text { EPA6010 }\end{array}$

360

$\mu \mathrm{g} / \mathrm{kg}$

$\mu \mathrm{g} / \mathrm{kg}$

$\mu \mathrm{g} / \mathrm{kg}$

$\mu \mathrm{g} / \mathrm{kg}$

$\mu \mathrm{g} / \mathrm{kg}$

$\mu \mathrm{g} / \mathrm{kg}$

$\mu \mathrm{g} / \mathrm{kg}$

$\mu \mathrm{g} / \mathrm{kg}$

$\mu \mathrm{g} / \mathrm{kg}$

$\mu \mathrm{g} / \mathrm{kg}$

$\mu \mathrm{g} / \mathrm{kg}$

$\mu \mathrm{g} / \mathrm{kg}$

$\mu \mathrm{g} / \mathrm{kg}$

$\mu g / k g$

$\mu \mathrm{g} / \mathrm{kg}$

$\mu \mathrm{g} / \mathrm{kg}$

$\mu \mathrm{g} / \mathrm{kg}$

$\mu \mathrm{g} / \mathrm{kg}$

$\mu \mathrm{g} / \mathrm{kg}$

$\mu \mathrm{g} / \mathrm{kg}$

$\mu g / k g$

$\mu \mathrm{g} / \mathrm{kg}$

$\mu g / k g$

$\mu \mathrm{g} / \mathrm{kg}$

$\mu \mathrm{g} / \mathrm{kg}$

$\mu \mathrm{g} / \mathrm{kg}$

$\mu \mathrm{g} / \mathrm{kg}$

$\mu \mathrm{g} / \mathrm{kg}$

$\mu \mathrm{g} / \mathrm{kg}$

$\mu \mathrm{g} / \mathrm{kg}$

$\mu g / \mathrm{kg}$

$\mu \mathrm{g} / \mathrm{kg}$

$\mu \mathrm{g} / \mathrm{kg}$

$\mu \mathrm{g} / \mathrm{kg}$

$\mu g / \mathrm{kg}$

$\mu \mathrm{g} / \mathrm{kg}$

$\mu g / \mathrm{kg}$

$\mu g / \mathrm{kg}$

$\mu g / \mathrm{kg}$

$\mu g / \mathrm{kg}$

$\mu \mathrm{g} / \mathrm{kg}$

$\mu g / k g$

$\begin{array}{ll}360 & \text { EPA8270 } \\ 360 & \text { EPA8270 } \\ 360 & \text { EPA8270 } \\ 360 & \text { EPA8270 } \\ 360 & \text { EPA8270 } \\ 360 & \text { EPA8270 } \\ 1,800 & \text { EPA8270 } \\ 360 & \text { EPA8270 } \\ 360 & \text { EPA8270 } \\ 360 & \text { EPA8270 } \\ 360 & \text { EPA8270 } \\ 360 & \text { EPA8270 } \\ 360 & \text { EPA8270 } \\ 360 & \text { EPA8270 } \\ 360 & \text { EPA8270 } \\ 360 & \text { EPA8270 } \\ 360 & \text { EPA8270 } \\ 360 & \text { EPA8270 } \\ 360 & \text { EPA8270 } \\ 360 & \text { EPA8270 } \\ 360 & \text { EPA8270 } \\ 360 & \text { EPA8270 } \\ 360 & \text { EPA8270 } \\ 360 & \text { EPA8270 } \\ 360 & \text { EPA8270 } \\ 360 & \text { EPA8270 } \\ 360 & \text { EPA8270 } \\ 360 & \text { EPA8270 } \\ 360 & \text { EPA8270 } \\ 360 & \text { EPA8270 } \\ 720 & \text { EPA8270 } \\ 360 & \text { EPA8270 } \\ 360 & \text { EPA8270 } \\ 360 & \text { EPA8270 } \\ 360 & \text { EPA8270 } \\ 1,800 & \text { EPA8270 } \\ 360 & \text { EPA8270 } \\ 360 & \text { EPA8270 } \\ 360 & \text { EPA8270 } \\ 360 & \text { EPA8270 } \\ 360 & \text { EPA8270 } \\ 360 & \text { EPA8270 }\end{array}$

EPA8270

EPA8270

EPA8270

EPA8270

EPA8270

EPA8270

EPA8270

EPA8270

EPA8270

EPA8270

EPA8270

EPA8270

EPA8270

EPA8270

EPA8270

EPA8270

EPA8270

EPA8270

EPA8270

EPA8270

EPA8270

EPA8270 
SAMPLE NAME: D43+10905 (Continued)

Constituent

Sample ID: 100346

RQ AQ B Result Unit $\quad$ D. Limit Method

$B / N / A$ Extractables (including $P A H$ and phenols)

Hexachlorobutadiene
Hexachlorocyclopentadiene
Hexachloroethane
Indeno(1,2,3-c,d)pyrene
Isophorone
2-Methyl-4,6-dinitrophenol
2-Methylnaphthalene
Naphthalene
o-Nitroaniline
m-Nitroaniline
p-Nitroaniline
Nitrobenzene
2-Nitrophenol
4-Nitrophenol
N-Nitrosodiphenylamine
N-Nitrosodipropylamine
Pentachlorophenol
Phenanthrene
Phenol
Pyrene
1,2,4-Trichlorobenzene
2.4,5-Trichlorophenol
2,4,6-Trichlorophenol
Unknown hydrocarbon
Unknown
Unknown
Unknown ketone
Unknown phthalate

$\begin{array}{llll}\mathrm{U} & 360 & \mu \mathrm{g} / \mathrm{kg} \\ \mathrm{U} & 360 & \mu \mathrm{g} / \mathrm{kg} \\ \mathrm{U} & 360 & \mu \mathrm{g} / \mathrm{kg} \\ \mathrm{U} & 360 & \mu \mathrm{g} / \mathrm{kg} \\ \mathrm{U} & 360 & \mu \mathrm{g} / \mathrm{kg} \\ \mathrm{U} & 1,800 & \mu \mathrm{g} / \mathrm{kg} \\ \mathrm{U} & 360 & \mu \mathrm{g} / \mathrm{kg} \\ \mathrm{U} & 360 & \mu \mathrm{g} / \mathrm{kg} \\ \mathrm{U} & 1,800 & \mu \mathrm{g} / \mathrm{kg} \\ \mathrm{U} & & 1,800 & \mu \mathrm{g} / \mathrm{kg} \\ \mathrm{U} & & 1,800 & \mu \mathrm{g} / \mathrm{kg} \\ \mathrm{U} & & 360 & \mu \mathrm{g} / \mathrm{kg} \\ \mathrm{U} & & 360 & \mu \mathrm{g} / \mathrm{kg} \\ \mathrm{U} & & 1,800 & \mu \mathrm{g} / \mathrm{kg} \\ \mathrm{U} & & 360 & \mu \mathrm{g} / \mathrm{kg} \\ \mathrm{U} & & 360 & \mu \mathrm{g} / \mathrm{kg} \\ \mathrm{UJ} & \mathrm{C} & 1,800 & \mu \mathrm{g} / \mathrm{kg} \\ \mathrm{U} & & 360 & \mu \mathrm{g} / \mathrm{kg} \\ \mathrm{UJ} & \mathrm{C} & 360 & \mu \mathrm{g} / \mathrm{kg} \\ \mathrm{U} & & 360 & \mu \mathrm{g} / \mathrm{kg} \\ \mathrm{U} & & 360 & \mu \mathrm{g} / \mathrm{kg} \\ \mathrm{U} & & 1,800 & \mu \mathrm{g} / \mathrm{kg} \\ \mathrm{U} & & 360 & \mu \mathrm{g} / \mathrm{kg} \\ \mathrm{J} & \mathrm{N} & 200 & \mu \mathrm{g} / \mathrm{kg} \\ \mathrm{J} & \mathrm{N} & 400 & \mu \mathrm{g} / \mathrm{kg} \\ \mathrm{J} & \mathrm{N} & 500 & \mu \mathrm{g} / \mathrm{kg} \\ \mathrm{J} & \mathrm{NV} & 10,000 & \mu \mathrm{g} / \mathrm{kg} \\ \mathrm{J} & \mathrm{N} & 300 & \mu \mathrm{g} / \mathrm{kg} \\ & & & \end{array}$

$\begin{array}{ll}360 & \text { EPA8270 } \\ 360 & \text { EPA8270 } \\ 360 & \text { EPA8270 } \\ 360 & \text { EPA8270 } \\ 360 & \text { EPA8270 } \\ 1,800 & \text { EPA8270 } \\ 360 & \text { EPA8270 } \\ 360 & \text { EPA8270 } \\ 1,800 & \text { EPA8270 } \\ 1,800 & \text { EPA8270 } \\ 1,800 & \text { EPA8270 } \\ 360 & \text { EPA8270 } \\ 360 & \text { EPA8270 } \\ 1,800 & \text { EPA8270 } \\ 360 & \text { EPA8270 } \\ 360 & \text { EPA8270 } \\ 1,800 & \text { EPA8270 } \\ 360 & \text { EPA8270 } \\ 360 & \text { EPA8270 } \\ 360 & \text { EPA8270 } \\ 360 & \text { EPA8270 } \\ 1,800 & \text { EPA8270 } \\ 360 & \text { EPA8270 } \\ & \text { EPA8270 } \\ & \text { EPA8270 } \\ & \text { EPA8270 } \\ & \text { EPA8270 } \\ & \text { EPA8270 }\end{array}$

Pesticides/PCBs and Dioxins/Furans

Aldrin
alpha-Benzene hexachloride
beta-Benzene hexachloride
delta-Benzene hexachloride
alpha-Chlordane
gamma-Chlordane
p.p'-DDD
p,p'-DDE
p,p'-DDT
Dieldrin
Endosulfan I
Endosulfan II
Endosulfan sulfate
Endrin
Endrin ketone
Heptachlor
Heptachlor epoxide
Lindane
Methoxychlor

$\begin{array}{ll}1.8 & \mu \mathrm{g} / \mathrm{kg} \\ 1.8 & \mu \mathrm{g} / \mathrm{kg} \\ 1.8 & \mu \mathrm{g} / \mathrm{kg} \\ 1.8 & \mu \mathrm{g} / \mathrm{kg} \\ 1.1 & \mu \mathrm{g} / \mathrm{kg} \\ 1.5 & \mu \mathrm{g} / \mathrm{kg} \\ 3.7 & \mu \mathrm{gg} / \mathrm{kg} \\ 1.1 & \mu \mathrm{kg} \\ 3.7 & \mu \mathrm{g} / \mathrm{kg} \\ 0.73 & \mu \mathrm{g} / \mathrm{kg} \\ 1.8 & \mu \mathrm{g} / \mathrm{kg} \\ 3.7 & \mu \mathrm{g} / \mathrm{kg} \\ 3.7 & \mu \mathrm{g} / \mathrm{kg} \\ 3.7 & \mu \mathrm{g} / \mathrm{kg} \\ 3.7 & \mu \mathrm{g} / \mathrm{kg} \\ 1.8 & \mu \mathrm{g} / \mathrm{kg} \\ 1.8 & \mu \mathrm{gg} \\ 1.8 & \mu \mathrm{kg} \\ 18 & \mu \mathrm{kg} \\ & \mu \mathrm{gg}\end{array}$

$\begin{array}{ll}1.8 & \text { EPA8080 } \\ 1.8 & \text { EPA8080 } \\ 1.8 & \text { EPA8080 } \\ 1.8 & \text { EPA8080 } \\ 1.8 & \text { EPA8080 } \\ 1.8 & \text { EPA8080 } \\ 3.7 & \text { EPA8080 } \\ 3.7 & \text { EPA8880 } \\ 3.7 & \text { EPA8080 } \\ 3.7 & \text { EPA8080 } \\ 1.8 & \text { EPA8080 } \\ 3.7 & \text { EPA8080 } \\ 3.7 & \text { EPA8080 } \\ 3.7 & \text { EPA8080 } \\ 3.7 & \text { EPA8080 } \\ 1.8 & \text { EPA8080 } \\ 1.8 & \text { EPA8880 } \\ 1.8 & \text { EPA8080 } \\ 18 & \text { EPA8080 }\end{array}$


SAMPLE NAME: D43110905 (Continued)

Sample ID: 100346

$\begin{array}{llllll}\text { Constituent } & \text { RQ AQ B Result Unit Limit Method }\end{array}$

Pesticides/PCBs and Dioxins/Furans

PCB 1016
PCB 1221
PCB 1232
PCB 1242
PCB 1248
PCB 1254
PCB 1260 :
Toxaphene

U $\quad 37$

U $\quad 73$

U 37

U $\quad 37$

U 37

U 37

Toxaphene

U 180

73
37
7
7
37
180

$\mu \mathrm{g} / \mathrm{kg}$

$\mu g / \mathrm{kg}$

$\mu g / \mathrm{kg}$

$\mu g / \mathrm{kg}$

$\mu \mathrm{g} / \mathrm{kg}$

$\mu \mathrm{g} / \mathrm{kg}$

$\mu \mathrm{g} / \mathrm{kg}$

$\mu \mathrm{g} / \mathrm{kg}$

$\begin{array}{ll}37 & \text { EPA8080 } \\ 73 & \text { EPA8080 } \\ 37 & \text { EPA8080 } \\ 37 & \text { EPA8080 } \\ 37 & \text { EPA8080 } \\ 37 & \text { EPA8080 } \\ 37 & \text { EPA8080 } \\ 180 & \text { EPA8080 }\end{array}$

Radionuclide Indicators and Radionuclides

Gross alpha

Gross alpha

Nonvolatile beta

Radium, rotal alpha-emitting

Radium, total alpha-emitting

Tritium

Tritium
Nonvolatile beta

$\mathrm{U}$

$\begin{array}{ll} & \text { V } \\ & \text { V } \\ \text { U } & \text { V } \\ \text { U } & \text { V } \\ \text { U } & \\ \text { U } & \end{array}$

TCLP Compounds (Metals and Organics)

Arsenic, TCLP

Barium, TCLP

Benzene

Cadmium, TCLP

Carbon tetrachloride

alpha-Chlordane

gamma-Chlordane

Chlorobenzene

Chloroethene (Vinyl chloride)

Chloroform

Chromium, TCLP

o-Cresol (2-Methylphenol)

1.4-Dichlorobenzene

1,2-Dichloroethane

1,1-Dichloroethylene

2,4-Dichlorophenoxyacetic acid

2,4-Dinitrotoluene .

Endrin

Heptachlor

Heptachlor epoxide

Hexachlorobenzene

Hexachlorobutadiene

Hexachloroethane

Lead, TCLP

Lindane

Mercury, TCLP

Methoxychlor

Methyl ethyl ketone

$\begin{array}{llll}\text { U } & & & 100 \\ \text { U } & & & 240 \\ \mathrm{UJ} & \mathrm{L} & & 50 \\ \mathrm{U} & & & 5.0 \\ \mathrm{U} & & & 50 \\ \mathrm{U} & & & 1.1 \\ \mathrm{U} & & & 50 \\ \mathrm{U} & & & 100 \\ \mathrm{U} & \mathrm{V} & & 50 \\ \mathrm{UJ} & \mathrm{L} & & 10 \\ \mathrm{UJ} & 0 & \mathrm{~L} & 100 \\ \mathrm{UJ} & 0 & \mathrm{~L} & 100 \\ \mathrm{U} & & & 50 \\ \mathrm{U} & & & 50 \\ \mathrm{U} & & & 10 \\ \mathrm{UJ} & 0 & \mathrm{~L} & 100 \\ \mathrm{U} & & & 0.22 \\ \mathrm{U} & & & 0.11 \\ \mathrm{U} & & & 0.11 \\ \mathrm{UJ} & 0 & \mathrm{~L} & 100 \\ \mathrm{UJ} & 0 & \mathrm{~L} & 100 \\ \mathrm{UJ} & 0 & \mathrm{~L} & 100 \\ \mathrm{U} & & & 90 \\ \mathrm{U} & & & 0.11 \\ \mathrm{U} & & & 0.20 \\ \mathrm{U} & & & 1.1 \\ \mathrm{U} & & & 100\end{array}$

$\begin{array}{llll}8.4 & \mathrm{pCi} / \mathrm{g} & 8.4 & \text { EPA990.0 } \\ 18 \pm 16 & \mathrm{pCi} / \mathrm{g} & 8.1 & \text { EPA900.0 } \\ 32 \pm 24 & \mathrm{pCi} / \mathrm{g} & 11 & \text { EPA900.0 } \\ 46 \pm 24 & \mathrm{pCi} / \mathrm{g} & 11 & \text { EPA990.0 } \\ 1.6 \pm 0.77 & \mathrm{pCi} / \mathrm{g} & 0.20 & \text { EPA903.0 } \\ 1.5 \pm 0.74 & \mathrm{pCi} / \mathrm{g} & 0.21 & \text { EPA903.0 } \\ 1.4 & \mathrm{pCi} / \mathrm{g} & 1.4 & \text { EPA906.0 } \\ 1.4 & \mathrm{pCi} / \mathrm{g} & 1.4 & \text { EPA906.0 }\end{array}$

100

$.0^{\circ}$

s.

.1

.1

50

so

10

100

0.11

0.11

100

100

90

0.20

1.1

100

$\begin{array}{lll}\mu \mathrm{g} / \mathrm{L} & 100 & \text { EPA6010 } \\ \mu \mathrm{g} / \mathrm{L} & 200 & \text { EPA6010 } \\ \mu \mathrm{g} / \mathrm{L} & 50 & \text { EPA8240 } \\ \mu \mathrm{g} / \mathrm{L} & 5.0 & \text { EPA6010 } \\ \mu \mathrm{g} / \mathrm{L} & 50 & \text { EPA8240 } \\ \mu \mathrm{g} / \mathrm{L} & 1.1 & \text { EPA8080 } \\ \mu \mathrm{g} / \mathrm{L} & 1.1 & \text { EPA8080 } \\ \mu \mathrm{g} / \mathrm{L} & 50 & \text { EPA8240 } \\ \mu \mathrm{g} / \mathrm{L} & 100 & \text { EPA8240 } \\ \mu \mathrm{g} / \mathrm{L} & 50 & \text { EPA8240 } \\ \mu \mathrm{g} / \mathrm{L} & 10 & \text { EPA6010 } \\ \mu \mathrm{g} / \mathrm{L} & 100 & \text { EPA8270 } \\ \mu \mathrm{g} / \mathrm{L} & 100 & \text { EPA8270 } \\ \mu \mathrm{g} / \mathrm{L} & 50 & \text { EPA8240 } \\ \mu \mathrm{g} / \mathrm{L} & 50 & \text { EPA8240 } \\ \mu \mathrm{g} / \mathrm{L} & 10 & \text { EPA8150 } \\ \mu \mathrm{g} / \mathrm{L} & 100 & \text { EPA8270 } \\ \mu \mathrm{g} / \mathrm{L} & 0.22 & \text { EPA8080 } \\ \mu \mathrm{g} / \mathrm{L} & 0.11 & \text { EPA8080 } \\ \mu \mathrm{g} / \mathrm{L} & 0.11 & \text { EPA8080 } \\ \mu \mathrm{g} / \mathrm{L} & 100 & \text { EPA8270 } \\ \mu \mathrm{g} / \mathrm{L} & 100 & \text { EPA8270 } \\ \mu \mathrm{g} / \mathrm{L} & 100 & \text { EPA8270 } \\ \mu \mathrm{g} / \mathrm{L} & 90 & \text { EPA6010 } \\ \mu \mathrm{g} / \mathrm{L} & 0.11 & \text { EPA8080 } \\ \mu \mathrm{g} / \mathrm{L} & 0.20 & \text { EPA7470 } \\ \mu \mathrm{g} / \mathrm{L} & 1.1 & \text { EPA8080 } \\ \mu \mathrm{g} / \mathrm{L} & 100 & \text { EPA8240 }\end{array}$


SAMPLE NAME: D43110905 (Continued) Sample ID: 100346

Constituent

RQ AQ B Result

Unit

D. Limit Method

TCLP Compounds (Metals and Organics)

$\begin{array}{llllllll}\text { Nitrobenzene } & \mathrm{UJ} & \mathrm{O} & \mathrm{L} & 100 & \mu \mathrm{g} / \mathrm{L} & 100 & \text { EPA8270 } \\ \text { Pentachlorophenol } & \mathrm{UJ} & \mathrm{O} & \mathrm{L} & 500 & \mu \mathrm{L} / \mathrm{L} & 500 & \text { EPA8270 } \\ \text { Pyridine } & \mathrm{UJ} & \mathrm{O} & \mathrm{L} & 100 & \mu \mathrm{g} / \mathrm{L} & 100 & \text { EPA8270 } \\ \text { Selenium. TCLP } & \mathrm{U} & & & 100 & \mu \mathrm{g} / \mathrm{L} & 100 & \text { EPA6010 } \\ \text { Silver, TCLP } & \mathrm{U} & & & 10 & \mu \mathrm{g} / \mathrm{L} & 10 & \text { EPA6010 } \\ \text { Tetrachloroethylene } & \mathrm{U} & & & 50 & \mu \mathrm{g} / \mathrm{L} & 50 & \text { EPA8240 } \\ \text { Toxaphene } & \mathrm{U} & & & 2.2 & \mu \mathrm{g} / \mathrm{L} & 2.2 & \text { EPA8080 } \\ \text { Toxaphene } & \mathrm{U} & & & 2.3 & \mu \mathrm{g} / \mathrm{L} & 2.3 & \text { EPA8080 } \\ \text { 2.4,5-TP (Silvex) } & \mathrm{U} & & & 5.0 & \mu \mathrm{g} / \mathrm{L} & 5.0 & \text { EPA8150 } \\ \text { Trichloroethylene } & \mathrm{U} & & & 50 & \mu \mathrm{g} / \mathrm{L} & 50 & \text { EPA8240 } \\ \text { 2.4,5-Trichlorophenol } & \mathrm{UJ} & 0 & \mathrm{~L} & 500 & \mu \mathrm{g} / \mathrm{L} & 500 & \text { EPA8270 } \\ 2.4,6-\text { Trichlorophenol } & \mathrm{UJ} & \mathrm{O} & \mathrm{L} & 100 & \mu \mathrm{g} / \mathrm{L} & 100 & \text { EPA8270 } \\ 2.4,5-\mathrm{T} & \mathrm{U} & & & 5.0 & \mu \mathrm{g} / \mathrm{L} & 5.0 & \text { EPA8150 } \\ \text { m.p-Cresol } & \mathrm{UJ} & 0 & \mathrm{~L} & 100 & \mu \mathrm{g} / \mathrm{L} & 100 & \text { EPA8270 }\end{array}$

SAMPLE NAME: D43110906

Sample ID: 100347

Location (SRS Coordinates): $18482.49 \mathrm{E} 66640.62 \mathrm{~N}$

Ground Elevation Above MSL: $132 \mathrm{ft}$

Depth of Core Interval: 8.00 to $10.00 \mathrm{ft}$

Sample Type: Normal

Sample Matrix: Soil .

Sample Color: 1OR 4/6

USC Soil Classification: SW

Sample Moisture: Damp

Percent Solids: 82.00

Constituent

RQ AQ B Result Unit

D. Limit Method

Total Metals

\begin{tabular}{|c|c|c|c|c|c|c|}
\hline \multirow{2}{*}{\multicolumn{3}{|c|}{$\begin{array}{l}\text { Arsenic } \\
\text { Barium }\end{array}$}} & 1.1 & $\mathrm{mg} / \mathrm{kg}$ & 1.1 & EPA7060 \\
\hline & & & 69 & $\mathrm{mg} / \mathrm{kg}$ & 24 & EPA6010 \\
\hline Cadmium & UJ & L & 0.61 & $\mathrm{mg} / \mathrm{kg}$ & 0.61 & EPA6010 \\
\hline Chromium & J & L & 44 & $\mathrm{mg} / \mathrm{kg}$ & 1.2 & EPA6010 \\
\hline Copper & \multicolumn{2}{|l|}{$\mathrm{U}$} & 3.0 & $\mathrm{mg} / \mathrm{kg}$ & 3.0 & EPA6010 \\
\hline Lead & & & 5.4 & $\mathrm{mg} / \mathrm{kg}$ & 0.33 & EPA7421 \\
\hline Manganese & & & 21 & $\mathrm{mg} / \mathrm{kg}$ & 1.8 & EPA6010 \\
\hline Mercury & \multicolumn{2}{|l|}{$\mathrm{U}$} & 0.12 & $\mathrm{mg} / \mathrm{kg}$ & 0.12 & EPA7470 \\
\hline Nickel & \multicolumn{2}{|l|}{$\mathrm{U}$} & 4.9 & $\mathrm{mg} / \mathrm{kg}$ & 4.9 & EPA6010 \\
\hline Selenium & \multicolumn{2}{|l|}{ U } & 0.56 & $\mathrm{mg} / \mathrm{kg}$ & 0.56 & EPA7740 \\
\hline Silver & \multicolumn{2}{|l|}{$\mathbf{U}$} & 1.2 & $\mathrm{mg} / \mathrm{kg}$ & 1.2 & EPA6010 \\
\hline \multicolumn{7}{|c|}{$B / N / A$ Extractables (including $P A H$ and phenols) } \\
\hline Acenaphthene & \multicolumn{2}{|l|}{$\mathrm{U}$} & 390 & $\mu \mathrm{g} / \mathrm{kg}$ & 390 & EPA8270 \\
\hline Acenaphthylene & \multicolumn{2}{|l|}{$\mathbf{U}$} & 390 & $\mu \mathrm{g} / \mathrm{kg}$ & 390 & EPA8270 \\
\hline Anthracene & \multicolumn{2}{|l|}{$\mathbf{U}$} & 390 & $\mu \mathrm{g} / \mathrm{kg}$ & 390 & EPA8270 \\
\hline Benzo(a)anthracene & \multicolumn{2}{|l|}{$\mathrm{U}$} & 390 & $\mu \mathrm{g} / \mathrm{kg}$ & 390 & EPA8270 \\
\hline Benzo(b)fluoranthene & \multicolumn{2}{|l|}{ U } & 390 & $\mu \mathrm{g} / \mathrm{kg}$ & 390 & EPA8270 \\
\hline Benzo(k)fluoranthene & \multicolumn{2}{|l|}{ U } & 390 & $\mu g / \mathrm{kg}$ & 390 & EPA8270 \\
\hline Benzoic acid & \multirow{2}{*}{\multicolumn{2}{|c|}{$\begin{array}{ll}U \\
U\end{array}$}} & 1,900 & $\mu \mathrm{g} / \mathrm{kg}$ & 1,900 & EPA8270 \\
\hline Benzo $(g, h, i)$ perylene & & & 390 & $\mu \mathrm{g} / \mathrm{kg}$ & 390 & EPA8270 \\
\hline
\end{tabular}


SAMPLE NAME: D43ï10906 (Continued)

Constituent
Sample ID: 100347

RQ AQ B Result Unit.
D. Limit Method

$B / N / A$ Extractables (including $P A H$ and phenols)

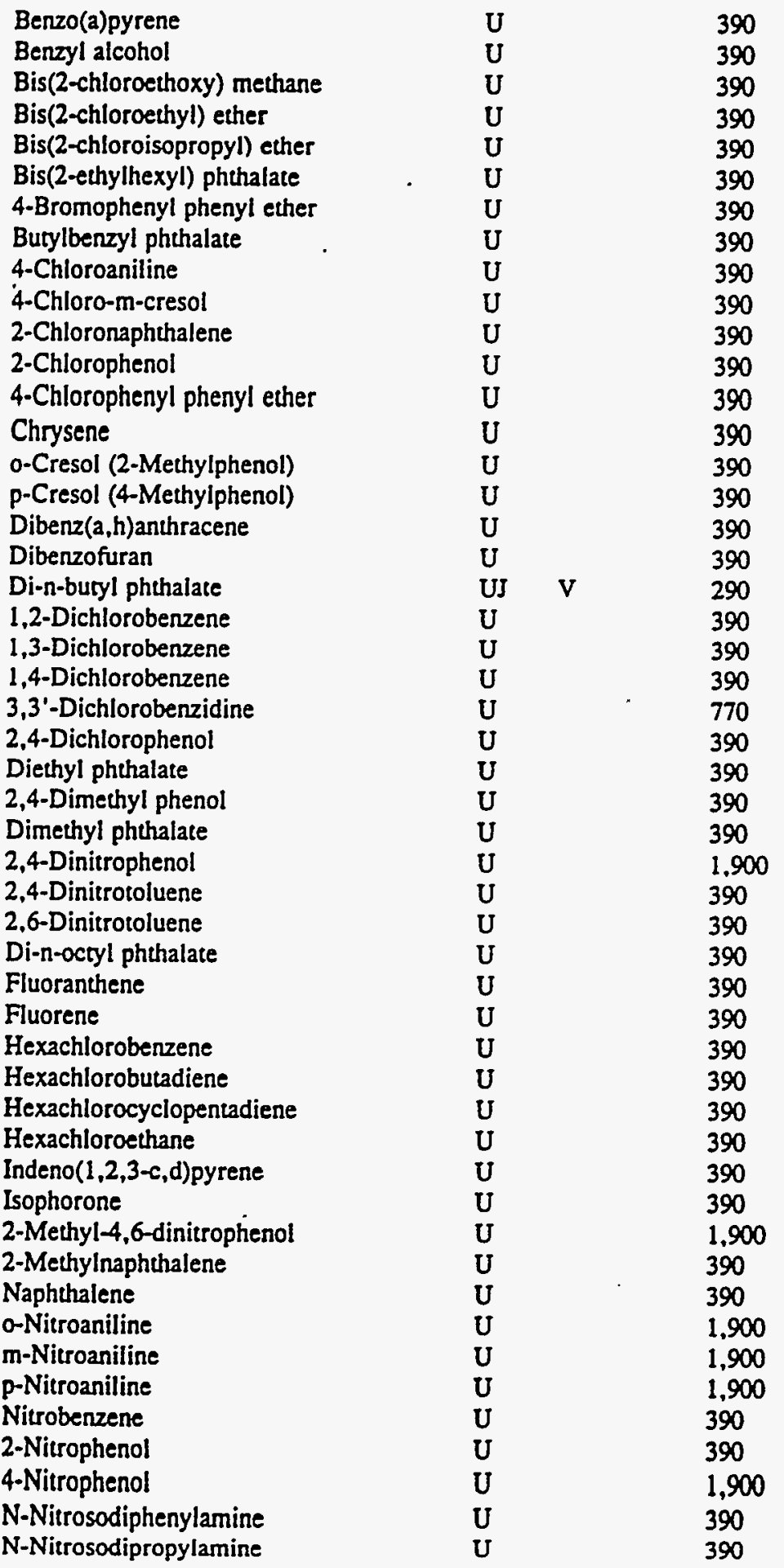

\begin{tabular}{lll}
$\mu \mathrm{g} / \mathrm{kg}$ & 390 & EPA8270 \\
$\mu \mathrm{g} / \mathrm{kg}$ & 390 & EPA8270 \\
$\mu \mathrm{g} / \mathrm{kg}$ & 390 & EPA8270 \\
$\mu \mathrm{g} / \mathrm{kg}$ & 390 & EPA8270 \\
$\mu \mathrm{g} / \mathrm{kg}$ & 390 & EPA8270 \\
$\mu \mathrm{g} / \mathrm{kg}$ & 390 & EPA8270 \\
$\mu \mathrm{g} / \mathrm{kg}$ & 390 & EPA8270 \\
$\mu \mathrm{g} / \mathrm{kg}$ & 390 & EPA8270 \\
$\mu \mathrm{g} / \mathrm{kg}$ & 390 & EPA8270 \\
$\mu \mathrm{g} / \mathrm{kg}$ & 390 & EPA8270 \\
$\mu \mathrm{g} / \mathrm{kg}$ & 390 & EPA8270 \\
$\mu \mathrm{g} / \mathrm{kg}$ & 390 & EPA8270 \\
$\mu \mathrm{g} / \mathrm{kg}$ & 390 & EPA8270 \\
$\mu \mathrm{g} / \mathrm{kg}$ & 390 & EPA8270 \\
$\mu \mathrm{g} / \mathrm{kg}$ & 390 & EPA8270 \\
$\mu \mathrm{g} / \mathrm{kg}$ & 390 & EPA8270 \\
$\mu \mathrm{g} / \mathrm{kg}$ & 390 & EPA8270 \\
$\mu \mathrm{g} / \mathrm{kg}$ & 390 & EPA8270 \\
$\mu \mathrm{g} / \mathrm{kg}$ & 390 & EPA8270 \\
$\mu \mathrm{g} / \mathrm{kg}$ & 390 & EPA8270 \\
$\mu \mathrm{g} / \mathrm{kg}$ & 390 & EPA8270 \\
$\mu \mathrm{g} / \mathrm{kg}$ & 390 & EPA8270 \\
$\mu \mathrm{g} / \mathrm{kg}$ & -770 & EPA8270 \\
$\mu \mathrm{g} / \mathrm{kg}$ & 390 & EPA8270 \\
$\mu \mathrm{g} / \mathrm{kg}$ & 390 & EPA8270 \\
$\mu \mathrm{g} / \mathrm{kg}$ & 390 & EPA8270 \\
$\mu \mathrm{g} / \mathrm{kg}$ & 390 & EPA8270 \\
$\mu \mathrm{g} / \mathrm{kg}$ & 1990 & EPA8270 \\
$\mu \mathrm{g} / \mathrm{kg}$ & 390 & EPA8270 \\
$\mu \mathrm{g} / \mathrm{kg}$ & 390 & EPA8270 \\
$\mu \mathrm{g} / \mathrm{kg}$ & 390 & EPA8270 \\
$\mu \mathrm{g} / \mathrm{kg}$ & 390 & EPA8270 \\
$\mu \mathrm{g} / \mathrm{kg}$ & 390 & EPA8270 \\
$\mu \mathrm{g} / \mathrm{kg}$ & 390 & EPA8270 \\
$\mu \mathrm{g} / \mathrm{kg}$ & 390 & EPA8270 \\
$\mu \mathrm{g} / \mathrm{kg}$ & 3990 & EPA8270 \\
$\mu \mathrm{g} / \mathrm{kg}$ & 390 & EPA8270 \\
$\mu \mathrm{g} / \mathrm{kg}$ & 390 & EPA8270 \\
$\mu \mathrm{g} / \mathrm{kg}$ & 390 & EPA8270 \\
$\mu \mathrm{g} / \mathrm{kg}$ & 1,900 & EPA8270 \\
$\mu \mathrm{g} / \mathrm{kg}$ & 390 & EPA8270 \\
$\mu \mathrm{g} / \mathrm{kg} / \mathrm{kg}$ & 390 & EPA8270 \\
$\mu \mathrm{g} / \mathrm{kg}$ & 1,900 & EPA8270 \\
$\mu \mathrm{g} / \mathrm{kg}$ & 1,900 & EPA8270 \\
$\mu \mathrm{g} / \mathrm{kg}$ & 1,900 & EPA8270 \\
$\mu \mathrm{g} / \mathrm{kg}$ & 390 & EPA8270 \\
$\mu \mathrm{g} / \mathrm{kg}$ & 390 & EPA8270 \\
\hline & 3900 & EPA8270 \\
\hline & 390 & EPA8270 \\
\hline & & EPA8270
\end{tabular}




Constituent RQ AQ B Result Unit D. Limit Method

$B / N / A$ Extractables (including $P A H$ and phenols)

$\begin{array}{llll}\text { Pentachlorophenol } & \text { UJ } & \text { CL } & 1,900 \\ \text { Phenanthrene } & \text { U } & & 390 \\ \text { Phenol } & \text { UJ } & \text { C } & 390 \\ \text { Pyrene } & \text { U } & & 390 \\ \text { 1.2.4-Trichlorobenzene } & \text { U } & & 390 \\ \text { 2,4,5-Trichlorophenol } & \text { U } & & 1,900 \\ \text { 2.4.6-Trichlorophenol } & \text { U } & & 390 \\ \text { Unknown } & \text { J } & \text { N } & 700 \\ \text { Unknown ketone } & \text { J } & \text { NV } & 20.000 \\ \text { Unknown phthalate } & \text { J } & \text { N } & 300\end{array}$

$\begin{array}{lll}\mu \mathrm{g} / \mathrm{kg} & 1,900 & \text { EPA8270 } \\ \mu \mathrm{g} / \mathrm{kg} & 390 & \text { EPA8270 } \\ \mu \mathrm{g} / \mathrm{kg} & 390 & \text { EPA8270 } \\ \mu \mathrm{g} / \mathrm{kg} & 390 & \text { EPA8270 } \\ \mu \mathrm{g} / \mathrm{kg} & 390 & \text { EPA8270 } \\ \mu \mathrm{g} / \mathrm{kg} & 1,900 & \text { EPA8270 } \\ \mu \mathrm{g} / \mathrm{kg} & 390 & \text { EPA8270 } \\ \mu \mathrm{g} / \mathrm{kg} & & \text { EPA8270 } \\ \mu \mathrm{g} / \mathrm{kg} & & \text { EPA8270 } \\ \mu \mathrm{g} / \mathrm{kg} & & \text { EPA8270 }\end{array}$

Volatile Organic Compounds

\begin{tabular}{|c|c|c|c|c|c|c|}
\hline Acetone & $\mathrm{U}$ & $\mathrm{v}$ & 25 & $\mu g / \mathrm{kg}$ & 12 & EPA8240 \\
\hline Benzene & $\mathrm{U}$ & & 5.8 & $\mu \mathrm{g} / \mathrm{kg}$ & 5.8 & EPA8240 \\
\hline Bromodichloromethane & $\mathbf{U}$ & & 5.8 & $\mu \mathrm{g} / \mathrm{kg}$ & 5.8 & EPA8240 \\
\hline Bromoform & $\mathrm{U}$ & & 5.8 & $\mu \mathrm{g} / \mathrm{kg}$ & 5.8 & EPA8240 \\
\hline Bromomethane (Methyl bromide) & $\mathrm{U}$ & & 12 & $\mu \mathrm{g} / \mathrm{kg}$ & 12 & EPA8240 \\
\hline Carbon disulfide & $\mathbf{U}$ & & 5.8 & $\mu \mathrm{g} / \mathrm{kg}$ & 5.8 & EPA8240 \\
\hline Carbon tetrachloride & U & & 5.8 & $\mu \mathrm{g} / \mathrm{kg}$ & 5.8 & EPA8240 \\
\hline Chlorobenzene & $\mathrm{U}$ & & 5.8 & $\mu \mathrm{g} / \mathrm{kg}$ & 5.8 & EPA8240 \\
\hline Chloroethane & $\mathrm{U}$ & & 12 & $\mu g / k g$ & 12 & EPA8240 \\
\hline Chloroethene (Vinyl chloride) & $\mathbf{U}$ & & 12 & $\mu g / \mathrm{kg}$ & 12 & EPA8240 \\
\hline Chloroform & $\mathbf{U}$ & & 5.8 & $\mu \mathrm{g} / \mathrm{kg}$ & 5.8 & EPA8240 \\
\hline Chloromethane (Methyl chloride) & $\mathrm{U}$ & & 12 & $\mu \mathrm{g} / \mathrm{kg}$ & 12 & EPA8240 \\
\hline Dibromochloromethane & $\mathrm{U}$ & & 5.8 & $\mu \mathrm{g} / \mathrm{kg}$ & 5.8 & EPA8240 \\
\hline 1,1-Dichloroethane & $\mathrm{U}$ & & 5.8 & $\mu \mathrm{g} / \mathrm{kg}$ & 5.8 & EPA8240 \\
\hline 1,2-Dichloroechane & $\mathbf{U}$ & & 5.8 & $\mu \mathrm{g} / \mathrm{kg}$ & 5.8 & EPA8240 \\
\hline 1.1-Dichloroethylene & $\mathrm{U}$ & & 5.8 & $\mu \mathrm{g} / \mathrm{kg}$ & 5.8 & EPA8240 \\
\hline 1,2-Dichloroethylene & $\mathrm{U}$ & & 5.8 & $\mu \mathrm{g} / \mathrm{kg}$ & 5.8 & EPA8240 \\
\hline Dichloromethane (Methylene chloride) & U & & 5.8 & $\mu \mathrm{g} / \mathrm{kg}$ & 5.8 & EPA8240 \\
\hline 1,2-Dichloropropane & U & & 5.8 & $\mu \mathrm{g} / \mathrm{kg}$ & 5.8 & EPA8240 \\
\hline cis-1,3-Dichloropropene & $\mathrm{U}$ & & 5.8 & $\mu \mathrm{g} / \mathrm{kg}$ & 5.8 & EPA8240 \\
\hline trans-1,3-Dichloropropene & $\mathrm{U}$ & & 5.8 & $\mu \mathrm{g} / \mathrm{kg}$ & 5.8 & EPA8240 \\
\hline Ethylbenzene & $\mathrm{U}$ & & 5.8 & $\mu \mathrm{g} / \mathrm{kg}$ & 5.8 & EPA8240 \\
\hline 2-Hexanone & $\mathrm{U}$ & & 12 & $\mu \mathrm{g} / \mathrm{kg}$ & 12 & EPA8240 \\
\hline Methyl ethyl ketone & $\mathbf{U}$ & & 12 & $\mu \mathrm{g} / \mathrm{kg}$ & 12 & EPA8240 \\
\hline Methyl isobutyl ketone & $\mathrm{U}$ & & 12 & $\mu g / \mathrm{kg}$ & 12 & EPA8240 \\
\hline Styrene & $\mathbf{U}$ & & 5.8 & $\mu \mathrm{g} / \mathrm{kg}$ & 5.8 & EPA8240 \\
\hline 1,1,2,2-Tetrachloroethane & $\mathbf{U}$ & & 5.8 & $\mu \mathrm{g} / \mathrm{kg}$ & 5.8 & EPA8240 \\
\hline Tetrachloroethylene . & $\mathbf{U}$ & & 5.8 & $\mu g / \mathrm{kg}$ & 5.8 & EPA8240 \\
\hline Toluene & $\mathrm{U}$ & & 5.8 & $\mu g / \mathrm{kg}$ & 5.8 & EPA 8240 \\
\hline 1,1,1-Trichloroethane & $\mathrm{U}$ & & 5.8 & $\mu g / \mathrm{kg}$ & 5.8 & EPA8240 \\
\hline 1,1,2-Trichloroethane & $\mathrm{U}$ & & 5.8 & $\mu \mathrm{g} / \mathrm{kg}$ & 5.8 & EPA8240 \\
\hline Trichloroethylene & U & & 5.8 & $\mu g / k g$ & 5.8 & EPA8240 \\
\hline Vinyl acetate & $U$ & & 12 & $\mu \mathrm{g} / \mathrm{kg}$ & 12 & EPA8240 \\
\hline Tetradecane & $\mathbf{J}$ & $\mathbf{N}$ & 10 & $\mu g / \mathrm{kg}$ & & EPA8240 \\
\hline Xylenes & $\mathbf{U}$ & & 5.8 & $\mu \mathrm{g} / \mathrm{kg}$ & 5.8 & EPA8240 \\
\hline
\end{tabular}


SAMPLE NAME: D43110906 (Continued)

Sample ID: 100347

Constituent

RQ

\begin{abstract}
$A Q$
\end{abstract}
Pesticides/PCBs and Dioxins/Furans

Aldrin

alpha-Benzene hexachloride

beta-Benzene hexachloride

delta-Benzene hexachloride

alpha-Chiordane

gamma-Chlordane

P.P'-DDD

p.p'-DDE

P.P'-DDT

Dieldrin

Endosulfan I

Endosulfan II

Endosulfan sulfate

Endrin

Endrin ketone

Heptachlor

Heptachlor epoxide

Heptachlorodibenzo-p-dioxin isomers

Heptachlorodibenzo-p-furan isomers

Hexachlorodibenzo-p-dioxin isomers

Hexachlorodibenzo-p-furan isomers

Lindane

Methoxychlor

Octachlorodibenzo-p-dioxin

Octachlorodibenzo-p-furan

PCB 1016

PCB 1221

PCB 1232

PCB 1242

PCB 1248

PCB 1254.

PCB 1260

Pentachlorodibenzo-p-dioxin isomers

Pentachlorodibenzo-p-furan isomers

$2,3,7,8$-TCDD

2,3,7,8-TCDF

Tetrachlorodibenzo-p-dioxin isomers

Tetrachlorodibenzo-p-furan isomers

Toxaphene
$\mathrm{U}$

U

U

$\mathrm{U}$

U

U

U

U

UJ

U

U

U

U

U

U

U

U

U

U

U

$\mathrm{U}$

U

U

J

U

U

U

U

U

U

U

U

U

U

U

U

$\mathrm{U}$

U
1.9

1.9

1.9

1.9

1.9

1.9

3.8

3.8

3.8

3.8

1.9

3.8

3.8

3.8

3.8

1.9

1.9

0.10

0.10

0.10

0.10

1.9

19

1.2

0.10

38

77

38

38

38

38

38

0.20

0.10

0.10

0.10

0.10

0.10

190

\section{$\therefore$}

Radionuclide Indicators and Radionuclides

Gross alpha

Nonvolatile beta

Radium, total alpha-emitting

Tritium

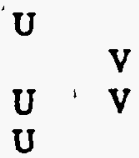

7.6

$33 \pm 21$

$1.7 \pm 0.78$

1.5

$\mu g / \mathrm{kg}$
$\mu g / \mathrm{kg}$
$\mu g / \mathrm{kg}$
$\mu \mathrm{g} / \mathrm{kg}$
$\mu \mathrm{g} / \mathrm{kg}$
$\mu \mathrm{g} / \mathrm{kg}$
$\mu \mathrm{g} / \mathrm{kg}$
$\mu \mathrm{g} / \mathrm{kg}$
$\mu \mathrm{g} / \mathrm{kg}$
$\mu \mathrm{g} / \mathrm{kg}$
$\mu \mathrm{g} / \mathrm{kg}$
$\mu \mathrm{g} / \mathrm{kg}$
$\mu \mathrm{g} / \mathrm{kg}$
$\mu \mathrm{g} / \mathrm{kg}$
$\mu \mathrm{g} / \mathrm{kg}$
$\mu \mathrm{g} / \mathrm{kg}$
$\mu \mathrm{g} / \mathrm{kg}$
$\mathrm{ng} / \mathrm{g}$
$\mathrm{ng} / \mathrm{g}$
$\mathrm{ng} / \mathrm{g}$
$\mathrm{ng} / \mathrm{g}$
$\mu \mathrm{g} / \mathrm{kg}$
$\mu \mathrm{g} / \mathrm{kg}$
$\mathrm{ng} / \mathrm{g}$
$\mathrm{ng} / \mathrm{g}$
$\mu \mathrm{g} / \mathrm{kg}$
$\mu g / \mathrm{kg}$
$\mu \mathrm{g} / \mathrm{kg}$
$\mu \mathrm{g} / \mathrm{kg}$
$\mu g / \mathrm{kg}$
$\mu \mathrm{k} / \mathrm{kg}$
$\mu \mathrm{g} / \mathrm{kg}$
$\mathrm{ng} / \mathrm{g}$
$\mathrm{ng} / \mathrm{g}$
$\mathrm{ng} / \mathrm{g}$
$\mathrm{ng} / \mathrm{g}$
$\mathrm{ng} / \mathrm{g}$
$\mathrm{ng} / \mathrm{g}$
$\mu \mathrm{g} / \mathrm{kg}$

$\mu g / \mathrm{kg}$
D. Limit Method

Unit

38
38
1.9

1.9

1.9

1.9

1.9

1.9

3.8

3.8

3.8

3.8

1.9

3.8

3.8

3.8

3.8

1.9

1.9

0.10

0.10

0.10

0.10

1.9

19

0.10

0.10

38

77

38

.38

38

38

0.20

0.10

$0.10^{\circ}$

0.10

0.10

0.10

190

.

EPA8080

EPA8080

EPA8080

EPA8080

EPA8080

EPA8080

EPA8080

EPA8080

EPA8080

EPA8080

EPA8080

EPA8080

EPA8080

EPA8080

EPA8080

EPA8080

EPA8080

EPA8280

EPA8280

EPA8280

EPA8280

EPA8080

EPA8080

EPA8280

EPA8280

EPA8080

EPA8080

EPA8080

EPA8080

EPA8080

EPA8080

EPA8280

EPA8280

EPA8280

EPA8280

EPA8280

EPA8280

EPA8080

7.6

EPA900.0

9.7 EPA900.0

$0.20 \quad$ EPA903.0

1.5 EPA906.0
EPA8080 
SAMPLE NAME: D4311090̣7.

Location (SRS Coordinates): $18482.49 \mathrm{E} 66640.62 \mathrm{~N}$

Ground Elevation Above MSL: $132 \mathrm{ft}$

Depth of Core Interval: 10.00 to $12.00 \mathrm{ft}$

Sample Type: Normal

Sample Color: 10R 4/6

Constituent

Total Metals

Arsenic
Barium
Cadmium
Chromium
Copper
Lead
Manganese
Mercury
Nickel
Selenium
Silver

$B / N / A$ Extractables (including $P A H$ and phenols)
Sample D: 100349

Sample Matrix: Soil

USC Soil Classification: SP

RQ AQ B Result Unit

$\mathrm{mg} / \mathrm{kg}$

$\mathrm{mg} / \mathrm{kg}$

$\mathrm{mg} / \mathrm{kg}$

$\mathrm{mg} / \mathrm{kg}$

$\mathrm{mg} / \mathrm{kg}$

$\mathrm{mg} / \mathrm{kg}$

$\mathrm{mg} / \mathrm{kg}$

$\mathrm{mg} / \mathrm{kg}$

$\mathrm{mg} / \mathrm{kg}$

$\mathrm{mg} / \mathrm{kg}$

$\mathrm{mg} / \mathrm{kg}$
Sample Moisture: Damp Percent Solids: 83.20

D. Limit Method

\begin{tabular}{|c|c|c|c|c|c|c|}
\hline Acenaphthene & U & & 410 & $\mu \mathrm{g} / \mathrm{kg}$ & 410 & EPA8270 \\
\hline Acenaphthylene & $\mathbf{U}$ & & 410 & $\mu \mathrm{g} / \mathrm{kg}$ & 410 & EPA8270 \\
\hline Anthracene & $\mathbf{U}$ & & 410 & $\mu \mathrm{g} / \mathrm{kg}$ & 410 & EPA8270 \\
\hline Benzo(a)anthracene & $\mathrm{U}$ & 7 & 410 & $\mu g / \mathrm{kg}$ & 410 & EPA8270 \\
\hline Benzo(b)fluoranthene & $\mathbf{U}$ & & 410 & $\mu \mathrm{g} / \mathrm{kg}$ & 410 & EPA8270 \\
\hline Benzo(k)fluoranthene & $\mathbf{U}$ & & 410 & $\mu \mathrm{g} / \mathrm{kg}$ & 410 & EPA8270 \\
\hline Benzoic acid & $\mathbf{U}$ & & 2,100 & $\mu \mathrm{g} / \mathrm{kg}$ & 2,100 & EPA8270 \\
\hline Benzo $(g, h, i)$ perylene & $\mathbf{U}$ & & 410 & $\mu g / k g$ & 410 & EPA8270 \\
\hline Benzo(a)pyrene & $\mathrm{U}$ & & 410 & $\mu \mathrm{g} / \mathrm{kg}$ & 410 & EPA8270 \\
\hline Benzyl alcohol & $\mathrm{U}$ & & 410 & $\mu \mathrm{g} / \mathrm{kg}$ & 410 & EPA8270 \\
\hline Bis(2-chloroethoxy) methane & $U$ & & 410 & $\mu g / k g$ & 410 & EPA8270 \\
\hline Bis(2-chloroethyl) ether & $\mathbf{U}$ & & 410 & $\mu \mathrm{g} / \mathrm{kg}$ & 410 & EPA8270 \\
\hline Bis(2-chloroisopropyl) ether & $\mathrm{U}$ & & 410 & $\mu \mathrm{g} / \mathrm{kg}$ & 410 & EPA8270 \\
\hline Bis(2-ethylhexyl) phthalate & $\mathrm{U}$ & & 410 & $\mu \mathrm{g} / \mathrm{kg}$ & 410 & EPA8270 \\
\hline 4-Bromophenyl phenyl ether & $\mathrm{U}$ & & 410 & $\mu \mathrm{g} / \mathrm{kg}$ & 410 & EPA 8270 \\
\hline Butylbenzyl phthalate & $U$ & & 410 & $\mu g / k g$ & 410 & EPA8270 \\
\hline 4-Chloroaniline & $\mathbf{U}$ & & 410 & $\mu \mathrm{g} / \mathrm{kg}$ & 410 & EPA8270 \\
\hline 4-Chloro-m-cresol & $\mathbf{U}$ & & 410 & $\mu \mathrm{g} / \mathrm{kg}$ & 410 & EPA8270 \\
\hline 2-Chloronaphthalene & $\mathbf{U}$ & & 410 & $\mu \mathrm{g} / \mathrm{kg}$ & 410 & EPA8270 \\
\hline 2-Chlorophenol & $\mathbf{U}$ & & 410 & $\mu \mathrm{g} / \mathrm{kg}$ & $410^{\circ}$ & EPA8270 \\
\hline 4-Chlorophenyl phenyl ether & $\mathbf{U}$ & & 410 & $\mu \mathrm{g} / \mathrm{kg}$ & 410 & EPA8270 \\
\hline Chrysene & $\mathrm{U}$ & & 410 & $\mu \mathrm{g} / \mathrm{kg}$ & 410 & EPA8270 \\
\hline o-Cresol (2-Methylphenol) & $\mathbf{U}$ & & 410 & $\mu \mathrm{g} / \mathrm{kg}$ & 410 & EPA8270 \\
\hline p-Cresol (4-Methylphenol) & $\mathbf{U}$ & & 410 & $\mu \mathrm{g} / \mathrm{kg}$ & 410 & EPA8270 \\
\hline Dibenz $(\mathrm{a}, \mathrm{h})$ anthracene & $\mathbf{U}$ & & 410 & $\mu g / \mathrm{kg}$ & 410 & EPA8270 \\
\hline Dibenzofuran & $\mathbf{U}$ & & 410 & $\mu \mathrm{g} / \mathrm{kg}$ & 410 & EPA8270 \\
\hline Di-n-butyl phthalate & UJ & $\mathrm{v}$ & 210 & $\mu \mathrm{g} / \mathrm{kg}$ & 410 & EPA8270 \\
\hline 1,2-Dichlorobenzene & $\mathbf{U}$ & & 410 & $\mu \mathrm{g} / \mathrm{kg}$ & 410 & EPA8270 \\
\hline 1,3-Dichlorobenzene & $\mathbf{U}$ & & 410 & $\mu g / \mathrm{kg}$ & 410 & EPA8270 \\
\hline 1,4-Dichlorobenzene & $\mathbf{U}$ & & 410 & $\mu g / \mathrm{kg}$ & 410 & EPA8270 \\
\hline
\end{tabular}

EPA7060

EPA6010

EPA6010

EPA6010

EPA6010

EPA7421

EPA6010

EPA7470

EPA6010

EPA7740

EPA6010 
SAMPLE NAME: D43110907 (Continued)

Sample ID: 100349

Constituent

RQ AQ B Result

Unit

D. Limit . Method

$B / N / A$ Extractables (including PAH and phenols)

\begin{tabular}{|c|c|c|c|c|c|}
\hline \multicolumn{2}{|l|}{$\mathbf{U}$} & 820 & $\mu \mathrm{g} / \mathrm{kg}$ & 820 & EPA8270 \\
\hline \multicolumn{2}{|l|}{$\mathrm{U}$} & 410 & $\mu g / k g$ & 410 & EPA8270 \\
\hline \multicolumn{2}{|l|}{ U } & 410 & $\mu \mathrm{g} / \mathrm{kg}$ & 410 & EPA8270 \\
\hline \multicolumn{2}{|l|}{$\mathbf{U}$} & 410 & $\mu g / k g$ & 410 & EPA8270 \\
\hline \multicolumn{2}{|l|}{ U } & 410 & $\mu \mathrm{g} / \mathrm{kg}$ & 410 & EPA8270 \\
\hline \multicolumn{2}{|l|}{$\mathrm{U}$} & 2,100 & $\mu \mathrm{g} / \mathrm{kg}$ & 2,100 & EPA8270 \\
\hline \multicolumn{2}{|l|}{$U$} & 410 & $\mu g / k g$ & 410 & EPA8270 \\
\hline \multicolumn{2}{|l|}{$U$} & 410 & $\mu \mathrm{g} / \mathrm{kg}$ & 410 & EPA8270 \\
\hline \multicolumn{2}{|l|}{ U } & 410 & $\mu \mathrm{g} / \mathrm{kg}$ & 410 & EPA8270 \\
\hline \multicolumn{2}{|l|}{$\mathrm{U}$} & 410 & $\mu g / k g$ & 410 & EPA8270 \\
\hline \multicolumn{2}{|l|}{$\mathbf{U}$} & 410 & $\mu \mathrm{g} / \mathrm{kg}$ & 410 & EPA8270 \\
\hline \multicolumn{2}{|l|}{ U } & 410 & $\mu \mathrm{g} / \mathrm{kg}$ & 410 & EPA8270 \\
\hline \multicolumn{2}{|l|}{ U } & 410 & $\mu \mathrm{g} / \mathrm{kg}$ & 410 & EPA8270 \\
\hline \multicolumn{2}{|l|}{ U } & 410 & $\mu \mathrm{g} / \mathrm{kg}$ & 410 & EPA8270 \\
\hline \multicolumn{2}{|l|}{ U } & 410 & $\mu \mathrm{g} / \mathrm{kg}$ & 410 & EPA8270 \\
\hline \multicolumn{2}{|l|}{ U } & 410 & $\mu \mathrm{g} / \mathrm{kg}$ & 410 & EPA8270 \\
\hline \multicolumn{2}{|l|}{$U$} & 410 & $\mu \mathrm{g} / \mathrm{kg}$ & 410 & EPA8270 \\
\hline \multicolumn{2}{|l|}{$\mathrm{U}$} & 2.100 & $\mu \mathrm{g} / \mathrm{kg}$ & 2.100 & EPA8270 \\
\hline \multicolumn{2}{|l|}{ U } & 410 & $\mu \mathrm{g} / \mathrm{kg}$ & 410 & EPA8270 \\
\hline \multicolumn{2}{|l|}{ U } & 410 & $\mu g / k g$ & 410 & EPA8270 \\
\hline \multicolumn{2}{|l|}{$\mathrm{U}$} & 2,100 & $\mu g / k g$ & 2,100 & EPA8270 \\
\hline \multicolumn{2}{|l|}{$\mathbf{U}$} & 2,100 & $\mu \mathrm{g} / \mathrm{kg}$ & 2.100 & EPA8270 \\
\hline \multicolumn{2}{|l|}{$\mathrm{U}$} & 2,100 & $\mu g / k g$ & 2,100 & EPA8270 \\
\hline \multicolumn{2}{|l|}{ U } & 410 & $\mu \mathrm{g} / \mathrm{kg}$ & 410 & EPA8270 \\
\hline \multicolumn{2}{|l|}{ U } & 410 & $\mu \mathrm{g} / \mathrm{kg}$ & 410 & EPA8270 \\
\hline \multicolumn{2}{|l|}{ U } & 2,100 & $\mu \mathrm{g} / \mathrm{kg}$ & 2,100 & EPA8270 \\
\hline \multicolumn{2}{|l|}{$U$} & 410 & $\mu g / k g$ & 410 & EPA8270 \\
\hline U & & 410 & $\mu \mathrm{g} / \mathrm{kg}$ & 410 & EPA8270 \\
\hline UJ & C & 2.100 & $\mu \mathrm{g} / \mathrm{kg}$ & 2.100 & EPA8270 \\
\hline$U$ & & 410 & $\mu g / \mathrm{kg}$ & 410 & EPA8270 \\
\hline UJ & C & 410 & $\mu \mathrm{g} / \mathrm{kg}$ & 410 & EPA8270 \\
\hline J & N & 300 & $\mu g / k g$ & & EPA8270 \\
\hline $\mathbf{U}$ & & 410 & $\mu \mathrm{g} / \mathrm{kg}$ & 410 & EPA8270 \\
\hline $\mathbf{U}$ & & 410 & $\mu g / k g$ & 410 & EPA8270 \\
\hline U & & 2,100 & $\mu \mathrm{g} / \mathrm{kg}$ & 2.100 & EPA8270 \\
\hline $\mathbf{U}$ & & 410 & $\mu \mathrm{g} / \mathrm{kg}$ & 410 & EPA8270 \\
\hline J & NV & 20,000 & $\mu \mathrm{g} / \mathrm{kg}$ & & EPA827 \\
\hline
\end{tabular}

Volatile Organic Compounds

3.3'-Dichlorobenzidine
2,4-Dichlorophenol
Diethyl phthalate
2.4-Dimethyl phenol
Dimethyl phthalate
2,4-Dinitrophenol
2,4-Dinitrotoluene
2,6-Dinitrotoluene
Di-n-octyl phthalate
Fluoranthene
Fluorene
Hexachlorobenzene
Hexachlorobutadiene
Hexachlorocyclopentadiene
Hexachloroethane
Indeno(1,2.3-c.d)pyrene
Isophorone
2-Methyl-4.6-dinitrophenol
2-Methylnaphthalene
Naphhthalene
a-Nitroaniline
m-Nitroaniline
p-Nitroaniline
Nitrobenzene
2-Nitrophenol
4-Nitrophenol
N-Nitrosodiphenylamine
N-Nitrosodipropylamine
Pentachlorophenol
Phenanthrene
Phenol
Unknown phosphonic ester
Pyrene
1,2.4-Trichlorobenzene
2,4.5-Trichlorophenol
2,4,6-Trichlorophenol
Unknown ketone
Volatile Organic Compounds
Acetone
Benzene
Bromodichloromethane
Bromoform
Bromomethane (Methyl bromide)
Carbon disulfide
Carbon tetrachloride
Chlorobenzene
Chloroethane
Chloroethene (Vinyl chloride)

(1)

\begin{tabular}{|c|c|c|c|c|c|}
\hline $\mathbf{U}$ & $\mathrm{v}$ & 12 & $\mu \mathrm{g} / \mathrm{kg}$ & 12 & EPA8240 \\
\hline $\mathbf{U}$ & & 6.2 & $\mu g / \mathrm{kg}$ & 6.2 & EPA8240 \\
\hline $\mathbf{U}$ & & 6.2 & $\mu \mathrm{g} / \mathrm{kg}$ & 6.2 & EPA8240 \\
\hline $\mathbf{U}$ & & 6.2 & $\mu \mathrm{g} / \mathrm{kg}$ & 6.2 & EPA8240 \\
\hline U & & 12 & $\mu \mathrm{g} / \mathrm{kg}$ & 12 & EPA8240 \\
\hline $\mathbf{U}$ & & 6.2 & $\mu \mathrm{g} / \mathrm{kg}$ & 6.2 & EPA8240 \\
\hline $\mathbf{U}$ & & 6.2 & $\mu \mathrm{g} / \mathrm{kg}$ & 6.2 & EPA8240 \\
\hline $\mathrm{U}$ & & 6.2 & $\mu g / \mathrm{kg}$ & 6.2 & EPA8240 \\
\hline $\mathbf{U}$ & & 12 & $\mu \mathrm{g} / \mathrm{kg}$ & 12 & EPA8240 \\
\hline $\mathbf{U}$ & & 12 & $\mu \mathrm{g} / \mathrm{kg}$ & 12 & EPA8240 \\
\hline
\end{tabular}


SAMPLE NAME: D43Ï10907 (Continued)

\section{Constituent}

Volatile Organic Compounds

Chloroform

Chloromethane (Methyl chloride)

Dibromochloromethane

1.1-Dichloroethane

1.2-Dichloroechane

1,1-Dichloroethylene

1,2-Dichloroethylene

Dichloromethane (Methylene chloride)

1,2-Dichloropropane

cis-1,3-Dichloropropene

trans-1,3-Dichloropropene

Ethylbenzene

2-Hexanone

Methyl ethyl ketone

Methyl isobutyl ketone

Styrene

1,1,2,2-Tetrachloroethane

Tetrachloroethylene

Toluene

1,1,1-Trichloroethane

1,1.2-Trichloroethane

Trichloroethylene

Vinyl acetate

Xylenes
Sample ID: 100349

RQ AQ B Result

6.2

12

6.2

6.2

6.2

6.2

6.2

6.2

6.2

6.2

6.2

6.2

12

12

12

6.2

6.2

6.2

6.2

6.2

6.2

6.2

12

6.2

Pesticides/PCBs and Dioxins/Furans

\begin{abstract}
Aldrin
alpha-Benzene hexachloride

alpha-Benzene hexachloride

alpha-Benzene hexachloride

beta-Benzene hexachloride

beta-Benzene hexachloride

beta-Benzene hexachloride

delta-Benzene hexachloride

delta-Benzene hexachloride

delta-Benzene hexachloride

alpha-Chlordane

alpha-Chlordane

alpha-Chlordane

gamma-Chlordane

gamma-Chlordane

gamma-Chlordane

P.P'-DDD

p,p'-DDD

P.P'-DDD

P.P'-DDE

p,p'-DDE

P.P'-DDE

p,p'-DDT
\end{abstract}

2.1

2.1

2.0

2.0

2.1

2.0

2.0

2.1

2.0

2.0

2.1

2.0

2.0

2.1

2.0

2.0

4.1

4.1

4.1

4.1

4.1

4.1

4.1
Unit

D. Limit Method

$\begin{array}{lll}\mu \mathrm{g} / \mathrm{kg} & 6.2 & \text { EPA8240 } \\ \mu \mathrm{g} / \mathrm{kg} & 12 & \text { EPA8240 } \\ \mu \mathrm{g} / \mathrm{kg} & 6.2 & \text { EPA8240 } \\ \mu \mathrm{g} / \mathrm{kg} & 6.2 & \text { EPA8240 } \\ \mu \mathrm{g} / \mathrm{kg} & 6.2 & \text { EPA8240 } \\ \mu \mathrm{g} / \mathrm{kg} & 6.2 & \text { EPA8240 } \\ \mu \mathrm{g} / \mathrm{kg} & 6.2 & \text { EPA8240 } \\ \mu \mathrm{g} / \mathrm{kg} & 6.2 & \text { EPA8240 } \\ \mu \mathrm{g} / \mathrm{kg} & 6.2 & \text { EPA8240 } \\ \mu \mathrm{g} / \mathrm{kg} & 6.2 & \text { EPA8240 } \\ \mu \mathrm{g} / \mathrm{kg} & 6.2 & \text { EPA8240 } \\ \mu \mathrm{g} / \mathrm{kg} & 6.2 & \text { EPA8240 } \\ \mu \mathrm{g} / \mathrm{kg} & 12 & \text { EPA8240 } \\ \mu \mathrm{g} / \mathrm{kg} & 12 & \text { EPA8240 } \\ \mu \mathrm{g} / \mathrm{kg} & 12 & \text { EPA8240 } \\ \mu \mathrm{g} / \mathrm{kg} & 6.2 & \text { EPA8240 } \\ \mu \mathrm{g} / \mathrm{kg} & 6.2 & \text { EPA8240 } \\ \mu \mathrm{g} / \mathrm{kg} & 6.2 & \text { EPA8240 } \\ \mu \mathrm{g} / \mathrm{kg} & 6.2 & \text { EPA8240 } \\ \mu \mathrm{g} / \mathrm{kg} & 6.2 & \text { EPA8240 } \\ \mu \mathrm{g} / \mathrm{kg} & 6.2 & \text { EPA8240 } \\ \mu \mathrm{g} / \mathrm{kg} & 6.2 & \text { EPA8240 } \\ \mu \mathrm{g} / \mathrm{kg} & 12 & \text { EPA8240 } \\ \mu \mathrm{g} / \mathrm{kg} & 6.2 & \text { EPA8240 }\end{array}$

$\begin{array}{lll}\mu \mathrm{g} / \mathrm{kg} & 2.1 & \text { EPA8080 } \\ \mu \mathrm{g} / \mathrm{kg} & 2.1 & \text { EPA8080 } \\ \mu \mathrm{g} / \mathrm{kg} & 2.0 & \text { EPA8080 } \\ \mu \mathrm{g} / \mathrm{kg} & 2.0 & \text { EPA8080 } \\ \mu \mathrm{g} / \mathrm{kg} & 2.1 & \text { EPA8080 } \\ \mu \mathrm{g} / \mathrm{kg} & 2.0 & \text { EPA8080 } \\ \mu \mathrm{g} / \mathrm{kg} & 2.0 & \text { EPA8080 } \\ \mu \mathrm{g} / \mathrm{kg} & 2.1 & \text { EPA8080 } \\ \mu \mathrm{g} / \mathrm{kg} & 2.0 & \text { EPA8080 } \\ \mu \mathrm{g} / \mathrm{kg} & 2.0 & \text { EPA8080 } \\ \mu \mathrm{g} / \mathrm{kg} & 2.1 & \text { EPA8080 } \\ \mu \mathrm{g} / \mathrm{kg} & 2.0 & \text { EPA8080 } \\ \mu \mathrm{g} / \mathrm{kg} & 2.0 & \text { EPA8080 } \\ \mu \mathrm{g} / \mathrm{kg} & 2: 1 & \text { EPA8080 } \\ \mu \mathrm{g} / \mathrm{kg} & 2.0 & \text { EPA8080 } \\ \mu \mathrm{g} / \mathrm{kg} & 2.0 & \text { EPA8080 } \\ \mu \mathrm{g} / \mathrm{kg} & 4.1 & \text { EPA8080 } \\ \mu \mathrm{g} / \mathrm{kg} & 4.1 & \text { EPA8080 } \\ \mu \mathrm{g} / \mathrm{kg} & 4.1 & \text { EPA8080 } \\ \mu \mathrm{g} / \mathrm{kg} & 4.1 & \text { EPA8080 } \\ \mu \mathrm{g} / \mathrm{kg} & 4.1 & \text { EPA8080 } \\ \mu \mathrm{g} / \mathrm{kg} & 4.1 & \text { EPA8080 } \\ \mu \mathrm{g} / \mathrm{kg} & 4.1 & \text { EPA8080 }\end{array}$


SAMPLE NAME: D43110907 (Continued)

Sample ID: 100349

Constituent RQ AQ B Result Unit D. Limit Method

Pesticides/PCBs and Dioxins/Furans

\begin{tabular}{|c|c|c|c|c|c|}
\hline Dieldrin & U & 4.1 & $\mu g / k g$ & 4.1 & EPA8080 \\
\hline Endosulfan I & U & 2.1 & $\mu \mathrm{g} / \mathrm{kg}$ & 2.1 & EPA8080 \\
\hline Endosulfan I & U & 2.0 & $\mu g / k g$ & 2.0 & EPA8080 \\
\hline Endosulfan I & $\mathrm{U}$ & 2.0 & $\mu \mathrm{g} / \mathrm{kg}$ & 2.0 & EPA8080 \\
\hline Endosulfan II & $\mathbf{U}$ & 4.1 & $\mu g / \mathrm{kg}$ & 4.1 & EPA8080 \\
\hline Endosulfan II & U & 4.1 & $\mu \mathrm{g} / \mathrm{kg}$ & 4.1 & EPA8080 \\
\hline Endosulfan II & $\mathbf{U}$ & 4.1 & $\mu \mathrm{g} / \mathrm{kg}$ & 4.1 & EPA8080 \\
\hline Endosulfan sulfate & U & 4.1 & $\mu \mathrm{g} / \mathrm{kg}$ & 4.1 & EPA8080 \\
\hline Endosulfan sulfate & $\mathbf{U}$ & 4.1 & $\mu \mathrm{g} / \mathrm{kg}$ & 4.1 & EPA8080 \\
\hline Endosulfan sulfate & $\mathrm{U}$ & 4.1 & $\mu \mathrm{g} / \mathrm{kg}$ & 4.1 & EPA8080 \\
\hline Endrin & $\mathrm{U}$ & 4.1 & $\mu \mathrm{g} / \mathrm{kg}$ & 4.1 & EPA8080 \\
\hline Endrin ketone & $\mathbf{U}$ & 4.1 & $\mu \mathrm{g} / \mathrm{kg}$ & 4.1 & EPA8080 \\
\hline Endrin ketone & $\mathrm{U}$ & 4.1 & $\mu \mathrm{g} / \mathrm{kg}$ & 4.1 & EPA8080 \\
\hline Endrin ketone & U & 4.1 & $\mu \mathrm{g} / \mathrm{kg}$ & 4.1 & EPA8080 \\
\hline Heptachlor & $\mathrm{U}$ & 2.1 & $\mu g / k g$ & 2.1 & EPA8080 \\
\hline Heptachlor epoxide & $\mathrm{U}$ & 2.1 & $\mu \mathrm{g} / \mathrm{kg}$ & 2.1 & EPA8080 \\
\hline Heptachlor epoxide & $\mathrm{U}$ & 2.0 & $\mu \mathrm{g} / \mathrm{kg}$ & 2.0 & EPA8080 \\
\hline Heptachlor epoxide & $\mathrm{U}$ & 2.0 & $\mu \mathrm{g} / \mathrm{kg}$ & 2.0 & EPA8080 \\
\hline Heptachlorodibenzo-p-dioxin isomers & J & 0.10 & $\mathrm{ng} / \mathrm{g}$ & 0.10 & EPA8280 \\
\hline Heptachlorodibenzo-p-furan isomers & $\mathrm{U}$ & 0.10 & $\mathrm{ng} / \mathrm{g}$ & 0.10 & EPA8280 \\
\hline Hexachlorodibenzo-p-dioxin isomers & $\mathrm{U}$ & 0.10 & $\mathrm{ng} / \mathrm{g}$ & 0.10 & EPA8280 \\
\hline Hexachlorodibenzo-p-furan isomers & $\mathrm{U}$ & 0.10 & $\mathrm{ng} / \mathrm{g}$ & 0.10 & EPA8280 \\
\hline Lindane & $\mathrm{U}$ & 2.1 & $\mu \mathrm{g} / \mathrm{kg}$ & 2.1 & EPA8080 \\
\hline Methoxychlor & $\mathrm{U}$ & 21 & $\mu \mathrm{g} / \mathrm{kg}$ & 21 & EPA8080 \\
\hline Methoxychlor & $\mathbf{U}$ & 20 & $\mu \mathrm{g} / \mathrm{kg}$ & 20 & EPA8080 \\
\hline Methoxychlor & $\mathbf{U}$ & 20 & $\mu \mathrm{g} / \mathrm{kg}$ & 20 & EPA8080 \\
\hline Octachlorodibenzo-p-dioxin & & 3.8 & $\mathrm{ng} / \mathrm{g}$ & 0.10 & EPA8280 \\
\hline Octachlorodibenzo-p-furan & $\mathrm{U}$ & 0.10 & $\mathrm{ng} / \mathrm{g}$ & 0.10 & EPA8280 \\
\hline PCB 1016 & $U$ & 41 & $\mu g / \mathrm{kg}$ & 41 & EPA8080 \\
\hline PCB 1016 & $\mathrm{U}$ & 41 & $\mu g / \mathrm{kg}$ & 41 & EPA8080 \\
\hline PCB 1016 & $\mathrm{U}$ & 41 & $\mu g / k g$ & 41 & EPA8080 \\
\hline PCB 1221 & $\mathbf{U}$ & 82 & $\mu \mathrm{g} / \mathrm{kg}$ & 82 & EPA8080 \\
\hline PCB 1221 & $\mathrm{U}$ & 81 & $\mu g / k g$ & 81 & EPA8080 \\
\hline PCB 1221 & $\mathrm{U}$ & 81 & $\mu \mathrm{g} / \mathrm{kg}$ & 81 & EPA8080 \\
\hline PCB 1232 & $\mathbf{U}$ & 41 & $\mu g / k g$ & 41 & EPA8080 \\
\hline PCB 1232 & $\mathrm{U}$ & 41 & $\mu \mathrm{g} / \mathrm{kg}$ & 41 & EPA8080 \\
\hline PCB 1232 & $\mathrm{U}$ & 41 & $\mu \mathrm{g} / \mathrm{kg}$ & 41 & EPA8080 \\
\hline PCB 1242 & $\mathbf{U}$ & 41 & $\mu g / \mathrm{kg}$ & 41 & EPA8080 \\
\hline PCB 1242 & U & 41 & $\mu g / \mathrm{kg}$ & 41 & EPA8080 \\
\hline PCB 1242 & $\mathbf{U}$ & 41 & $\mu \mathrm{g} / \mathrm{kg}$ & 41 & EPA8080 \\
\hline PCB 1248 & $\mathbf{U}$ & 41 & $\mu g / k g$ & 41 & EPA8080 \\
\hline PCB 1248 & $\mathbf{U}$ & 41 & $\mu g / k g$ & 41 & EPA8080 \\
\hline PCB 1248 & $\mathbf{U}$ & 41 & $\mu g / \mathrm{kg}$ & 41 & EPA8080 \\
\hline PCB 1254 & $\mathbf{U}$ & 41 & $\mu \mathrm{g} / \mathrm{kg}$ & 41 & EPA8080 \\
\hline PCB 1254 & $\ldots$ & 41 & $\mu g / \mathrm{kg}$ & 41 & EPA8080 \\
\hline PCB 1254 & $\mathbf{U}$ & 41 & $\mu g / k g$ & 41 & EPA8080 \\
\hline РCB 1260 & $\mathrm{U}$ & 41 & $\mu g / k g$ & 41 & EPA8080 \\
\hline PCB 1260 & $\mathbf{U}$ & 41 & $\mu g / \mathrm{kg}$ & 41 & EPA8080 \\
\hline PCB 1260 & $\mathrm{U}$ & 41 & $\mu g / k g$ & 41 & EPA8080 \\
\hline Pentachlorodibenzo-p-dioxin isomers & U & 0.20 & $\mathrm{ng} / \mathrm{g}$ & 0.20 & EPA8280 \\
\hline
\end{tabular}


SAMPLE NAME: D43110907 (Continued)

Sample ID: 100349

Constituent

RQ AQ B Result Unit

D. Limit Method

Pesticides/PCBs and Dioxins/Furans

Pentachlorodibenzo-p-furan isomers
2,3,7,8-TCDD
2,3,7,8-TCDF
Tetrachlorodibenzo-p-dioxin isomers
Tetrachlorodibenzo-p-furan isomers
Toxaphene
Toxaphene

$\begin{array}{lllll}\mathrm{U} & 0.10 & \mathrm{ng} / \mathrm{g} & 0.10 & \text { EPA8280 } \\ \mathrm{U} & 0.10 & \mathrm{ng} / \mathrm{g} & 0.10 & \text { EPA8280 } \\ \mathrm{U} & 0.10 & \mathrm{ng} / \mathrm{g} & 0.10 & \text { EPA8280 } \\ \mathrm{U} & 0.10 & \mathrm{ng} / \mathrm{g} & 0.10 & \text { EPA8280 } \\ \mathrm{U} & 0.10 & \mathrm{ng} / \mathrm{g} & 0.10 & \text { EPA8280 } \\ \mathrm{U} & 210 & \mu \mathrm{g} / \mathrm{kg} & 210 & \text { EPA8080 } \\ \mathrm{U} & 200 & \mu \mathrm{g} / \mathrm{kg} & 200 & \text { EPA8080 } \\ \mathrm{U} & 200 & \mu \mathrm{g} / \mathrm{kg} & 200 & \text { EPA8080 }\end{array}$

SAMPLE NAME: D43111001

Sample ID: 100350

Location (SRS Coordinates): 18482.04E 66700.55N

Ground Elevation Above MSL: $129.5 \mathrm{ft}$

Depth of Core Interval: 0.00 to $0.50 \mathrm{ft}$

Sample Type: Normal

Sample Color: 10R $4 / 6$

Sample Matrix: Soil

USC Soil Classification: SC

RQ AQ B Result

Unit

Sample Moisture: Dry Percent Solids: 90.60

Constituent

Physical Parameters

Cation exchange capacity

Cation exchange capacity

pH

Specific conductance

Total organic carbon

11

$\mathrm{~J} . \mathrm{Q} .6$
4.3
58
14,000

Total Metals

Arsenic

Barium

Cadmium

Chromium

Copper

Lead

Manganese

Mercury

Nickel

Selenium

Silver

$\begin{array}{ll}\text { U } & \\ \text { UJ } & \text { L } \\ \text { U U } & \text { L }\end{array}$

0.95

21

0.52

12

2.6

3.5

92

0.11

4.2

0.48

1.0

$B / N / A$ Extractables (including $P A H$ and phenols)

$\mathrm{meq} / 100 \mathrm{~g}$
$\mathrm{meq} / 100 \mathrm{~g}$
$\mathrm{pH}$
$\mu \mathrm{S} / \mathrm{cm}$
$\mathrm{mg} / \mathrm{kg}$

2.0

2.0

0.010

1.0

390

EPA9080

EPA9080

EPA9045

EPA9050

EPA415.1

$$
\begin{gathered}
\mathrm{mg} / \mathrm{kg} \\
\mathrm{mg} / \mathrm{kg} \\
\mathrm{mg} / \mathrm{kg} \\
\mathrm{mg} / \mathrm{kg} \\
\mathrm{mg} / \mathrm{kg} \\
\mathrm{mg} / \mathrm{kg} \\
\mathrm{mg} / \mathrm{kg} \\
\mathrm{mg} / \mathrm{kg} \\
\mathrm{mg} / \mathrm{kg} \\
\mathrm{mg} / \mathrm{kg} \\
\mathrm{mg} / \mathrm{kg}
\end{gathered}
$$

$0.95 \quad$ EPA7060

$21 \quad$ EPA6010

0.52 EPA6010

$1.0 \quad$ EPA6010

2.6 EPA6010

0.29 EPA7421

$1.6 \quad$ EPA6010

$0.11 \quad$ EPA7470

4.2 EPA6010

$0.48 \quad$ EPA7740

1.0 EPA6010

$\begin{array}{lll}\text { Acenaphthene } & \mathbf{U} & 360 \\ \text { Acenaphthylene } & \mathbf{U}^{-} & 360 \\ \text { Acenaphthylene } & \mathrm{U} & 360 \\ \text { Acenaphthylene } & \mathrm{U} & 360 \\ \text { Anthracene } & \mathrm{U} & 360 \\ \text { Anthracene } & \mathrm{U} & 360\end{array}$

$\begin{array}{llll}360 & \mu g / \mathrm{kg} & 360 & \text { EPA8270 } \\ 360 & \mu g / \mathrm{kg} & 360 & \text { EPA8270 } \\ 360 & \mu \mathrm{g} / \mathrm{kg} & 360 & \text { EPA8270 } \\ 360 & \mu \mathrm{g} / \mathrm{kg} & 360 & \text { EPA8270 } \\ 360 & \mu \mathrm{g} / \mathrm{kg} & 360 & \text { EPA8270 } \\ 360 & \mu \mathrm{g} / \mathrm{kg} & 360 & \text { EPA8270 }\end{array}$


SAMPLE NAME: D43111001 (Continued)

Constituent
Sample ID: 100350

RQ AQ B Result Unit \. Dimit Method

$B / N / A$ Extractables (including $P A H$ and phenols)

\begin{tabular}{|c|c|c|c|c|c|}
\hline Anthracene & $\mathbf{U}$ & 360 & $\mu g / \mathrm{kg}$ & 360 & $\begin{array}{l}\text { EPA8270 } \\
\text { EPA8270 }\end{array}$ \\
\hline Benzo(a)anthracene & $\mathbf{U}$ & 360 & $\mu \mathrm{g} / \mathrm{kg}$ & 360 & EPA8270 \\
\hline Benzo(a)anthracene & $\mathbf{U}$ & 360 & $\mu \mathrm{g} / \mathrm{kg}$ & 360 & EPA8270 \\
\hline Benzo(a)anthracene & $\mathbf{U}$ & 360 & $\mu \mathrm{g} / \mathrm{kg}$ & 360 & EPA8270 \\
\hline Benzo(b)fluoranthene & $\mathrm{U}$ & 360 & $\mu \mathrm{g} / \mathrm{kg}$ & 360 & EPA8270 \\
\hline Benzo(b)fluoranthene & $\mathbf{U}$ & 360 & $\mu g / \mathrm{kg}$ & 360 & EPA8270 \\
\hline Benzo(b)fluoranthene & $\mathbf{U}$ & 360 & $\mu \mathrm{g} / \mathrm{kg}$ & 360 & EPA8270 \\
\hline Benzo(k)fluoranthene & $\mathrm{U}$ & 360 & $\mu g / \mathrm{kg}$ & 360 & EPA8270 \\
\hline Benzo(k)fluoranthene & $\mathrm{U}$ & 360 & $\mu \mathrm{g} / \mathrm{kg}$ & 360 & EPA8270 \\
\hline Benzo(k)fluoranthene & $\mathbf{U}$ & 360 & $\mu \mathrm{g} / \mathrm{kg}$ & 360 & EPA8270 \\
\hline Benzoic acid & $\mathrm{U}$ & 1,800 & $\mu \mathrm{g} / \mathrm{kg}$ & 1,800 & EPA8270 \\
\hline Benzoic acid & $\mathrm{U}$ & 1,800 & $\mu \mathrm{g} / \mathrm{kg}$ & 1,800 & EPA8270 \\
\hline Benzoic acid & $U$ & 1.800 & $\mu g / \mathrm{kg}$ & 1,800 & EPA8270 \\
\hline Benzo $(\mathrm{g}, \mathrm{h}, \mathrm{i})$ perylene & $\mathrm{U}$ & 360 & $\mu \mathrm{g} / \mathrm{kg}$ & 360 & EPA8270 \\
\hline Benzo $(g, h, i)$ perylene & U & 360 & $\mu g / \mathrm{kg}$ & 360 & EPA8270 \\
\hline Benzo $(g, h, i)$ perylene & $\mathrm{U}$ & 360 & $\mu \mathrm{g} / \mathrm{kg}$ & 360 & EPA8270 \\
\hline Benzo(a)pyrene & U & 360 & $\mu \mathrm{g} / \mathrm{kg}$ & 360 & EPA8270 \\
\hline Benzo(a)pyrene & $\mathrm{U}$ & 360 & $\mu \mathrm{g} / \mathrm{kg}$ & 360 & EPA8270 \\
\hline Benzo(a)pyrene & $\mathrm{U}$ & 360 & $\mu \mathrm{g} / \mathrm{kg}$ & 360 & EPA8270 \\
\hline Benzyl alcohol & $\mathrm{U}$ & 360 & $\mu \mathrm{g} / \mathrm{kg}$ & 360 & EPA8270 \\
\hline Benzyl alcohol & $\mathrm{U}$ & 360 & $\mu g / \mathrm{kg}$ & 360 & EPA8270 \\
\hline Benzyl alcohol & $\mathbf{U}$ & 360 & $\mu \mathrm{g} / \mathrm{kg}$ & 360 & EPA8270 \\
\hline Bis(2-chloroethoxy) methane & $\mathbf{U}$ & 360 & $\mu g / \mathrm{kg}$ & 360 & EPA8270 \\
\hline Bis(2-chloroethoxy) methane & $\mathbf{U}$ & 360 & $\mu \mathrm{g} / \mathrm{kg}$ & 360 & EPA8270 \\
\hline Bis(2-chloroethoxy) methane & $\mathbf{U}$ & 360 & $\mu g / \mathrm{kg}$ & 360 & EPA8270 \\
\hline Bis(2-chloroethyl) ether & U & 360 & $\mu g / \mathrm{kg}$ & 360 & EPA8270 \\
\hline Bis(2-chloroisopropyl) ether & $\mathrm{U}$ & 360 & $\mu \mathrm{g} / \mathrm{kg}$ & 360 & EPA8270 \\
\hline Bis(2-chloroethyl) ether & $\mathrm{U}$ & 360 & $\mu g / \mathrm{kg}$ & 360 & EPA8270 \\
\hline Bis(2-chloroisopropyl) ether & $\mathrm{U}$ & 360 & $\mu \mathrm{g} / \mathrm{kg}$ & 360 & EPA8270 \\
\hline Bis(2-chloroethyl) ether & $\mathrm{U}$ & 360 & $\mu g i k g$ & 360 & EPA8270 \\
\hline Bis(2-chloroisopropyl) ether & $\mathrm{U}$ & 360 & $\mu \mathrm{g} / \mathrm{kg}$ & 360 & EPA8270 \\
\hline Bis(2-ethylhexyl) phthalate & $\mathbf{U}$ & 360 & $\mu g / \mathrm{kg}$ & 360 & EPA8270 \\
\hline Bis(2-ethylhexyl) phthalate & $\mathrm{U}$ & 360 & $\mu \mathrm{g} / \mathrm{kg}$ & 360 & EPA8270 \\
\hline Bis(2-ethylhexyl) phthalate & $\mathrm{U}$ & 360 & $\mu \mathrm{g} / \mathrm{kg}$ & 360 & EPA8270 \\
\hline 4-Bromophenyl phenyl ether & $\mathbf{U}$ & 360 & $\mu \mathrm{g} / \mathrm{kg}$ & 360 & EPA8270 \\
\hline 4-Bromophenyl phenyl ether & U & 360 & $\mu \mathrm{g} / \mathrm{kg}$ & 360 & EPA8270 \\
\hline 4-Bromophenyl phenyl ether & $\mathrm{U}$ & 360 & $\mu g / k g$ & 360 & EPA8270 \\
\hline Butylbenzyl phehalate & U & 360 & $\mu \mathrm{g} / \mathrm{kg}$ & 360 & EPA8270 \\
\hline Burylbenzyl phthalate & $\mathrm{u}$ & 360 & $\mu \mathrm{g} / \mathrm{kg}$ & 360 & EPA8270 \\
\hline Butylbenzyl phthalate & U & 360 & $\mu \mathrm{g} / \mathrm{kg}$ & 360 & EPA8270 \\
\hline 4-Chloroaniline & $\mathbf{U}$ & 360 & $\mu \mathrm{g} / \mathrm{kg}$ & 360 & EPA8270 \\
\hline 4-Chloroaniline & $\mathbf{U}$ & 360 & $\mu g / k g$ & 360 & EPA8270 \\
\hline 4-Chloroaniline & $U$ & 360 & $\mu \mathrm{g} / \mathrm{kg}$ & 360 & EPA8270 \\
\hline 4-Chloro-m-cresol & $\mathbf{U}$ & 360 & $\mu g / \mathrm{kg}$ & 360 & EPA8270 \\
\hline 2-Chloronaphthalene & U & 360 & $\mu g / \mathrm{kg}$ & 360 & EPA8270 \\
\hline 2-Chloronaphthalene & $\mathrm{U}$ & 360 & $\mu \mathrm{g} / \mathrm{kg}$ & 360 & EPA8270 \\
\hline 2-Chloronaphthalene & U & 360 & $\mu g / \mathrm{kg}$ & 360 & EPA8270 \\
\hline 2-Chlorophenol & $\mathrm{U}$ & 360 & $\mu \mathrm{g} / \mathrm{kg}$ & 360 & EPA8270 \\
\hline 4-Chlorophenyl phenyl ether & $\mathbf{U}$ & 360 & $\mu g / \mathrm{kg}$ & 360 & EPA8270 \\
\hline 4-Chlorophenyl phenyl ether & $\mathbf{U}$ & 360 & $\mu \mathrm{g} / \mathrm{kg}$ & 360 & EPA8270 \\
\hline
\end{tabular}


SAMPLE NAME: D43111001 (Continued)

RQ AQ B Result

Constituent

$B / N / A$ Extractables (including PAH and phenols)

4-Chiorophenyl phenyl ether

Chrysene

Chrysene

Chrysene

o-Cresol (2-Methylphenol)

o-Cresol (2-Methylphenol)

o-Cresol (2-Methylphenol)

p-Cresol (4-Methylphenol)

p-Cresol (4-Methylphenol)

p-Cresol (4-Methylphenol)

Dibenz $(a, h)$ anthracene

Dibenz(a,h)anthracene

Dibenz(a,h)anthracene

Dibenzofuran

Dibenzofuran

Dibenzofuran

Di-n-butyl phthalate

Di-n-butyl phthalate

Di-n-butyl phthalate

1,2-Dichlorobenzene

1,2-Dichlorobenzene

1,2-Dichlorobenzene

1,3-Dichlorobenzene

1,3-Dichlorobenzene

1,3-Dichlorobenzene

1,4-Dichlorobenzene

3,3'-Dichlorobenzidine

3,3'-Dichlorobenzidine

3.3'-Dichlorobenzidine

2,4-Dichlorophenol

2,4-Dichlorophenol

2,4-Dichlorophenol

Diethyl phthalate

Diethyl phthalate

Diethyl phthalate

2,4-Dimethyl phenol

2.4-Dimethyl phenol

2,4-Dimethyl phenol

Dimethyl phthalate

Dimethyl phthalate

Dimethyl phthalate

2,4-Dinitrophenol

2,4-Dinitrophenol

2,4-Dinitrophenol

2,4-Dinitrotoluene

2,6-Dinitrotoluene

2,6-Dinitrotoluene

2,6-Dinitrotoluene

Di-n-octyl phthalate

Di-n-octyl phthalate
Sample ID: 100350

Unit

D. Limit Method

\begin{tabular}{|c|c|c|c|c|c|}
\hline $\mathbf{U}$ & & 360 & $\mu g / \mathrm{kg}$ & 360 & EPA8270 \\
\hline 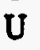 & & 360 & $\mu g / \mathrm{kg}$ & 360 & EPA8270 \\
\hline $\mathrm{U}$ & & 360 & $\mu g / \mathrm{kg}$ & 360 & EPA8270 \\
\hline $\mathbf{U}$ & & 360 & $\mu \mathrm{g} / \mathrm{kg}$ & 360 & EPA8270 \\
\hline $\mathbf{U}$ & & 360 & $\mu \mathrm{g} / \mathrm{kg}$ & 360 & EPA8270 \\
\hline $\mathbf{U}$ & & 360 & $\mu \mathrm{g} / \mathrm{kg}$ & 360 & EPA8270 \\
\hline $\mathbf{U}$ & & 360 & $\mu g / \mathrm{kg}$ & 360 & EPA8270 \\
\hline $\mathrm{U}$ & & 360 & $\mu g / \mathrm{kg}$ & 360 & EPA8270 \\
\hline U & & 360 & $\mu \mathrm{g} / \mathrm{kg}$ & 360 & EPA8270 \\
\hline $\mathbf{U}$ & & 360 & $\mu g / \mathrm{kg}$ & 360 & EPA8270 \\
\hline $\mathbf{U}$ & & 360 & $\mu \mathrm{g} / \mathrm{kg}$ & 360 & EPA8270 \\
\hline$U$ & & 360 & $\mu \mathrm{g} / \mathrm{kg}$ & 360 & EPA8270 \\
\hline $\mathbf{U}$ & & 360 & $\mu g / \mathrm{kg}$ & 360 & EPA8270 \\
\hline $\mathrm{U}$ & & 360 & $\mu g / \mathrm{kg}$ & 360 & EPA8270 \\
\hline U & & 360 & $\mu \mathrm{g} / \mathrm{kg}$ & 360 & EPA8270 \\
\hline$U$ & & 360 & $\mu \mathrm{g} / \mathrm{kg}$ & 360 & EPA8270 \\
\hline UJ & v & 250 & $\mu \mathrm{g} / \mathrm{kg}$ & 360 & EPA8270 \\
\hline 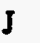 & V & 230 & $\mu g / k g$ & 360 & EPA8270 \\
\hline UJ & V & 170 & $\mu \mathrm{g} / \mathrm{kg}$ & 360 & EPA8270 \\
\hline $\mathbf{U}$ & & 360 & $\mu \mathrm{g} / \mathrm{kg}$ & 360 & EPA8270 \\
\hline $\mathrm{U}$ & & 360 & $\mu \mathrm{g} / \mathrm{kg}$ & 360 & EPA8270 \\
\hline U & & 360 & $\mu g / \mathrm{kg}$ & 360 & EPA8270 \\
\hline$U$ & & 360 & $\mu \mathrm{g} / \mathrm{kg}$ & 360 & EPA8270 \\
\hline $\mathrm{U}$ & & 360 & $\mu \mathrm{g} / \mathrm{kg}$ & 360 & EPA8270 \\
\hline$U$ & & 360 & $\mu g / \mathrm{kg}$ & 360 & EPA8270 \\
\hline $\mathbf{U}$ & & 360 & $\mu g / \mathrm{kg}$ & 360 & EPA8270 \\
\hline $\mathrm{U}$ & & 730 & $\mu g / \mathrm{kg}$ & 730 & EPA8270 \\
\hline$U$ & & 720 & $\mu \mathrm{g} / \mathrm{kg}$ & 720 & EPA8270 \\
\hline $\mathbf{U}$ & & 720 & $\mu g / k g$ & 720 & EPA8270 \\
\hline $\mathrm{U}$ & & 360 & $\mu \mathrm{g} / \mathrm{kg}$ & 360 & EPA8270 \\
\hline $\mathrm{U}$ & & 360 & $\mu \mathrm{g} / \mathrm{kg}$ & 360 & EPA8270 \\
\hline $\mathrm{U}$ & & 360 & $\mu \mathrm{g} / \mathrm{kg}$ & 360 & EPA8270 \\
\hline $\mathrm{U}$ & & 360 & $\mu \mathrm{g} / \mathrm{kg}$ & 360 & EPA8270 \\
\hline $\mathbf{U}$ & & 360 & $\mu \mathrm{g} / \mathrm{kg}$ & 360 & EPA8270 \\
\hline $\mathbf{U}$ & & 360 & $\mu g / \mathrm{kg}$ & 360 & EPA8270 \\
\hline $\mathrm{U}$ & & 360 & $\mu g / \mathrm{kg}$ & 360 & EPA8270 \\
\hline $\mathbf{U}$ & & 360 & $\mu g / k g$ & 360 & EPA8270 \\
\hline $\mathbf{U}$ & & 360 & $\mu g / \mathrm{kg}$ & 360 & EPA8270 \\
\hline $\mathbf{U}$ & & 360 & $\mu g / \mathrm{kg}$ & 360 & EPA8270 \\
\hline $\mathbf{U}$ & & 360 & $\mu g / \mathrm{kg}$ & 360 & EPA8270 \\
\hline $\mathbf{U}$ & & 360 & $\mu g / k g$ & 360 & EPA8270 \\
\hline U & & 1,800 & $\mu g / \mathrm{kg}$ & 1,800 & EPA8270 \\
\hline U & & 1,800 & $\mu g / \mathrm{kg}$ & 1,800 & EPA8270 \\
\hline $\mathbf{U}$ & & 1,800 & $\mu g / \mathrm{kg}$ & 1,800 & EPA8270 \\
\hline U & & 360 & $\mu g / \mathrm{kg}$ & 360 & EPA8270 \\
\hline $\mathrm{U}$ & & 360 & $\mu \mathrm{g} / \mathrm{kg}$ & 360 & EPA8270 \\
\hline U & & 360 & $\mu g / \mathrm{kg}$ & 360 & EPA8270 \\
\hline $\mathrm{I}$ & & 360 & $\mu \mathrm{g} / \mathrm{kg}$ & 360 & EPA8270 \\
\hline $\mathbf{U}$ & & 360 & $\mu \mathrm{g} / \mathrm{kg}$ & 360 & EPA8270 \\
\hline 7 & & 360 & $\mu \mathrm{g} / \mathrm{kg}$ & 360 & EPA8270 \\
\hline
\end{tabular}


SAMPLE NAME: D43111001 (Continued)

Sample ID: 100350

Constituent RQ AQ B Result Unit D. Limit Method

\section{$B / N / A$ Extractables (including $P A H$ and phenols)}

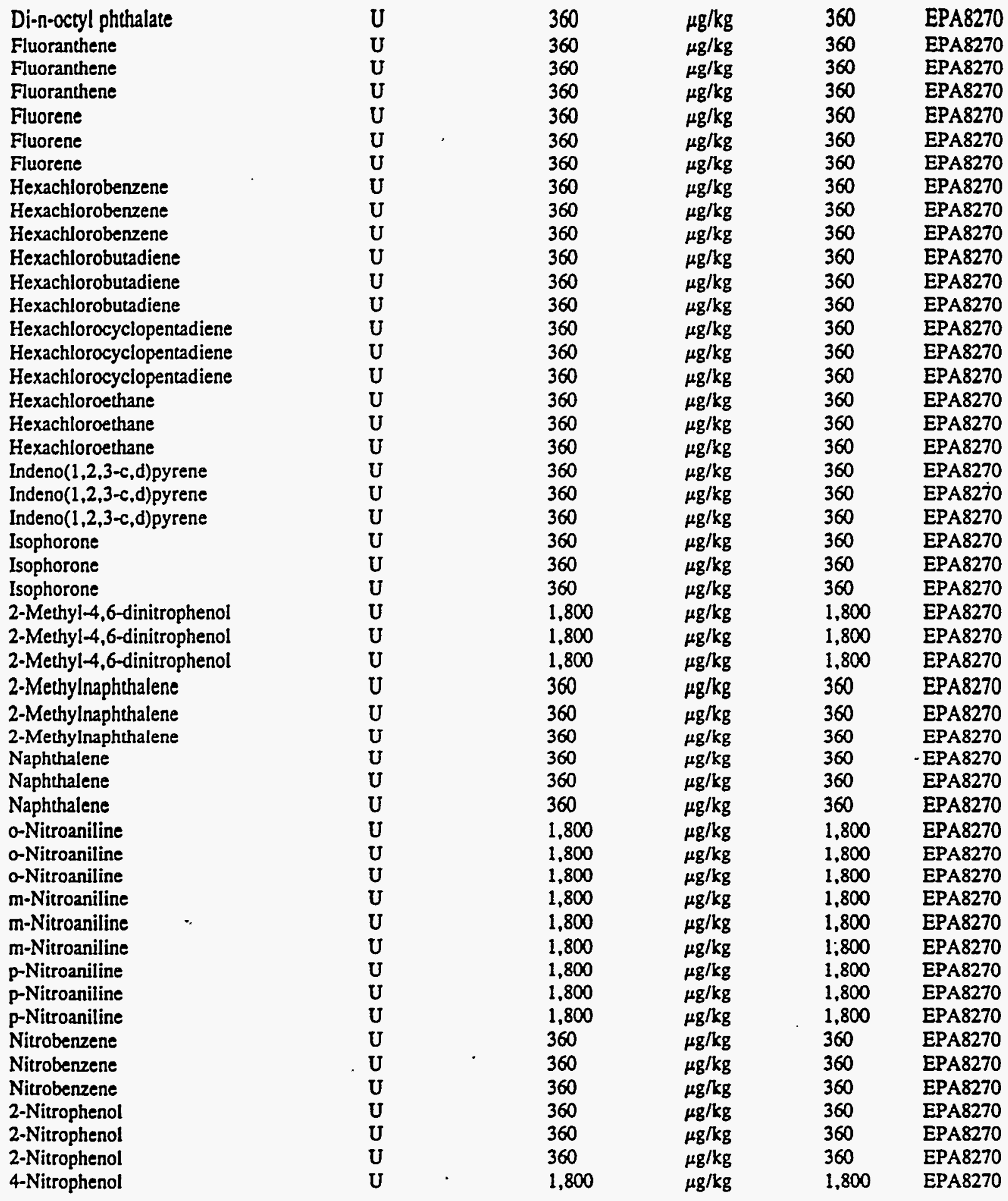


SAMPLE NAME: D43111001 (Continued)

Sample ID: 100350

Constituent

RQ AQ B Result Unit

D. Limit Method

$B / N / A$ Extractables (including $P A H$ and phenols)

N-Nitrosodiphenylamine

N-Nitrosodiphenylamine

N-Nitrosodiphenylamine

N-Nitrosodipropylamine

Pentachlorophenol

Phenanthrene

Phenanthrene

Phenanthrene

Phenol

Pyrene

1,2,4-Trichlorobenzene

2,4,5-Trichlorophenol

2,4,5-Trichlorophenol

2,4,5-Trichlorophenol

2,4,6-Trichlorophenol

2,4,6-Trichlorophenol

2,4,6-Trichlorophenol

Unknown hydrocarbon

Unknown hydrocarbon

Unknown hydrocarbon

Unknown hydrocarbon

Unknown

Unknown

Unknown ketone

Unknown phthalate

$\begin{array}{llll}\mathbf{U} & & & 360 \\ \mathrm{U} & & & 360 \\ \mathrm{U} & & & 360 \\ \mathrm{U} & & & 360 \\ \mathrm{UJ} & \mathrm{IC} & \mathrm{H} & 1,800 \\ \mathrm{U} & & & 360 \\ \mathrm{U} & & & 360 \\ \mathrm{U} & & & 360 \\ \mathrm{UJ} & \mathrm{C} & & 360 \\ \mathrm{U} & & 360 \\ \mathrm{U} & & 360 \\ \mathrm{U} & & 1,800 \\ \mathrm{U} & & 1,800 \\ \mathrm{U} & & 1,800 \\ \mathrm{U} & & 360 \\ \mathrm{U} & & 360 \\ \mathrm{U} & & 360 \\ \mathrm{~J} & \mathrm{~N} & 200 \\ \mathrm{~J} & \mathrm{~N} & 300 \\ \mathrm{~J} & \mathrm{~N} & 300 \\ \mathrm{~J} & \mathrm{~N} & 300 \\ \mathrm{~J} & \mathrm{~N} & 200 \\ \mathrm{~J} & \mathrm{~N} & 400 \\ \mathrm{~J} & \mathrm{NV} & 20,000 \\ \mathrm{~J} & \mathrm{~N} & 200 \\ & & \end{array}$

Volatile Organic Compounds

Acetone

Benzene

Bromodichloromethane

Bromoform

Bromomethane (Methyl bromide)

Carbon disulfide

Carbon tetrachloride

Chiorobenzene

Chloroethane

Chloroethene (Vinyl chloride)

Chloroform

Chloromethane (Methyl chloride)

Dibromochloromethane

1,1-Dichloroethane

1,2-Dichloroethane

1,1-Dichloroethylene

1,2-Dichloroethylene

Dichloromethane (Methylene chloride)

1,2-Dichloropropane

cis-1,3-Dichloropropene

trans-1,3-Dichloropropene

Ethylbenzene
$U$
$U$
$U$
$U$
$U$
$U$
$U$
$U$
$U$
$U$
$U$
$U$
$U$
$U$
$U$
$U$
$U$
$U$
$U$
$U$
$U$

$U$
$U$
$U$
$U$
$U$
$U$
$U$
$U$
$U$
$U$
$U$
$U$
$U$
$U$
$U$
$U$
$U$
$U$

$\begin{array}{lll}\mu \mathrm{g} / \mathrm{kg} & 360 & \text { EPA8270 } \\ \mu \mathrm{g} / \mathrm{kg} & 360 & \text { EPA8270 } \\ \mu \mathrm{g} / \mathrm{kg} & 360 & \text { EPA8270 } \\ \mu \mathrm{g} / \mathrm{kg} & 360 & \text { EPA8270 } \\ \mu \mathrm{g} / \mathrm{kg} & 1,800 & \text { EPA8270 } \\ \mu \mathrm{g} / \mathrm{kg} & 360 & \text { EPA8270 } \\ \mu \mathrm{g} / \mathrm{kg} & 360 & \text { EPA8270 } \\ \mu \mathrm{g} / \mathrm{kg} & 360 & \text { EPA8270 } \\ \mu \mathrm{g} / \mathrm{kg} & 360 & \text { EPA8270 } \\ \mu \mathrm{g} / \mathrm{kg} & 360 & \text { EPA8270 } \\ \mu \mathrm{g} / \mathrm{kg} & 360 & \text { EPA8270 } \\ \mu \mathrm{g} / \mathrm{kg} & 1,800 & \text { EPA8270 } \\ \mu \mathrm{g} / \mathrm{kg} & 1,800 & \text { EPA8270 } \\ \mu \mathrm{g} / \mathrm{kg} & 1,800 & \text { EPA8270 } \\ \mu \mathrm{g} / \mathrm{kg} & 360 & \text { EPA8270 } \\ \mu \mathrm{g} / \mathrm{kg} & 360 & \text { EPA8270 } \\ \mu \mathrm{g} / \mathrm{kg} & 360 & \text { EPA8270 } \\ \mu \mathrm{g} / \mathrm{kg} & & \text { EPA8270 } \\ \mu \mathrm{g} / \mathrm{kg} & & \text { EPA8270 } \\ \mu \mathrm{g} / \mathrm{kg} & & \text { EPA8270 } \\ \mu \mathrm{g} / \mathrm{kg} & & \text { EPA8270 } \\ \mu \mathrm{g} / \mathrm{kg} & & \text { EPA8270 } \\ \mu \mathrm{g} / \mathrm{kg} & & \text { EPA8270 } \\ \mu \mathrm{g} / \mathrm{kg} & & \text { EPA8270 } \\ \mu \mathrm{g} / \mathrm{kg} & & \text { EPA8270 }\end{array}$

$\mu \mathrm{g} / \mathrm{kg}$

$\mu \mathrm{g} / \mathrm{kg}$

$\mu \mathrm{g} / \mathrm{kg}$

$\mu \mathrm{g} / \mathrm{kg}$

$\mu \mathrm{g} / \mathrm{kg}$

$\mu \mathrm{g} / \mathrm{kg}$

$\mu \mathrm{g} / \mathrm{kg}$

$\mu g / k g$

$\mu \mathrm{g} / \mathrm{kg}$

$\mu \mathrm{g} / \mathrm{kg}$

$\mu g / \mathrm{kg}$

$\mu g / \mathrm{kg}$

$\mu g / k g$

$\mu \mathrm{g} / \mathrm{kg}$

$\mu g / \mathrm{kg}$

$\mu \mathrm{g} / \mathrm{kg}$

$\mu \mathrm{g} / \mathrm{kg}$

$\mu g / \mathrm{kg}$

$\mu g / \mathrm{kg}$

$\mu \mathrm{g} / \mathrm{kg}$

$\mu \mathrm{g} / \mathrm{kg}$

$\mu \mathrm{g} / \mathrm{kg}$
11

5.5

5.5

5.5

11

5.5

5.5

5.5

11

11

5.5

11

5.5

5.5

5.5

5.5

5.5

5.5

5.5

5.5

5.5

5.5
EPA8240

EPA8240

EPA8240

EPA8240

EPA 8240

EPA8240

EPA8240

EPA8240

EPA8240

EPA8240

EPA 8240

EPA 8240

EPA8240

EPA8240

EPA8240

EPA8240

EPA8240

EPA8240

EPA8240

EPA8240
EPA 8240

EPA 8240 
Constituent

Volatile Organic Compounds

$\begin{array}{ll}\text { 2-Hexanone } & \\ \text { Methyl ethyl ketone } & \\ \text { Methyl isobutyl ketone } & U \\ \text { Styrene } & \\ \text { 1.1,2,2-Tetrachloroethane } & \\ \text { Tetrachloroethylene } & U \\ \text { Toluene } & U \\ \text { 1,1,1-Trichloroethane } & U \\ \text { 1,1,2-Trichloroethane } & U \\ \text { Trichloroethylene } & U \\ \text { Vinyl acetate } & U \\ \text { Xylenes } & U \\ \end{array}$

Pesticides/PCBs and Dioxins/Furans

Aldrin

alpha-Benzene hexachloride

beta-Benzene hexachloride

delta-Benzene hexachloride

alpha-Chlordane

gamma-Chlordane

p.p'-DDD

p. $p^{\prime}-D D E$

P.P'-DDT

Dieldrin

Endosulfan I

Endosulfan II

Endosulfan sulfate

Endrin

Endrin ketone

Heptachlor

Heptachlor epoxide

Heptachlorodibenzo-p-dioxin isomers

Heptachlorodibenzo-p-furan isomers

Hexachlorodibenzo-p-dioxin isomers

Hexachlorodibenzo-p-furan isomers

Lindane

Methoxychlor

Octachlorodiberzo-p-dioxin

Octachlorodibenzo-p-furan

PCB 1016

PCB 1221

PCB 1232

PCB 1242

PCB 1248

PCB 1254

PCB 1260

Pentachlorodibenzo-p-dioxin isomers

Pentachlorodibenzo-p-furan isomers

2,3,7,8-TCDD
RQ

$A Q$

B

Result

Unit

D. Limit Method
$U$
$U$
$U$
$U$
$U$
$U$
$U$
$U$
$U$
$U$
$U$
$U$

11

11

11

5.5

5.5

5.5

5.5

5.5

5.5

5.5

11

5.5

$$
\begin{aligned}
& \mu \mathrm{g} / \mathrm{kg} \\
& \mu \mathrm{g} / \mathrm{kg} \\
& \mu \mathrm{g} / \mathrm{kg} \\
& \mu \mathrm{g} / \mathrm{kg} \\
& \mu \mathrm{g} / \mathrm{kg} \\
& \mu \mathrm{g} / \mathrm{kg} \\
& \mu \mathrm{g} / \mathrm{kg} \\
& \mu \mathrm{g} / \mathrm{kg} \\
& \mu \mathrm{g} / \mathrm{kg} \\
& \mu \mathrm{g} / \mathrm{kg} \\
& \mu \mathrm{g} / \mathrm{kg} \\
& \mu \mathrm{g} / \mathrm{kg}
\end{aligned}
$$

1.8

1.8

1.8

1.8

1.8

1.8

3.6

3.6

3.6

3.6

1.8

3.6

3.6

3.6

3.6

1.8

1.8

0.10

0.10

0.10

0.10

1.8

18

6.3

0.10

36

73

36

36

36

36

36

0.20

0.10

0.10 $\mu \mathrm{g} / \mathrm{kg}$

$\mu \mathrm{g} / \mathrm{kg}$

$\mu g / \mathrm{kg}$

$\mu g / k g$

$\mu g / \mathrm{kg}$

$\mu g / \mathrm{kg}$

$\mu \mathrm{g} / \mathrm{kg}$

$\mu g / k g$

$\mu g / k g$

$\mu \mathrm{g} / \mathrm{kg}$

$\mu \mathrm{g} / \mathrm{kg}$

$\mu \mathrm{g} / \mathrm{kg}$

$\mu \mathrm{g} / \mathrm{kg}$

$\mu \mathrm{g} / \mathrm{kg}$

$\mu g / \mathrm{kg}$

$\mu \mathrm{g} / \mathrm{kg}$

$\mu \mathrm{g} / \mathrm{kg}$

$\mathrm{ng} / \mathrm{g}$

$\mathrm{ng} / \mathrm{g}$

$\mathrm{ng} / \mathrm{g}$

$\mathrm{ng} / \mathrm{g}$

$\mu \mathrm{g} / \mathrm{kg}$

$\mu \mathrm{g} / \mathrm{kg}$

$\mathrm{ng} / \mathrm{g}$

$\mathrm{ng} / \mathrm{g}$

$\mu g / \mathrm{kg}$

$\mu \mathrm{g} / \mathrm{kg}$

$\mu g / \mathrm{kg}$

$\mu \mathrm{g} / \mathrm{kg}$

$\mu g / k g$

$\mu \mathrm{g} / \mathrm{kg}$

$\mu \mathrm{g} / \mathrm{kg}$

$\mathrm{ng} / \mathrm{g}$

$\mathrm{ng} / \mathrm{g}$

$\mathrm{ng} / \mathrm{g}$
11

11

11

5.5

5.5

5.5

5.5

5.5

5.5

5.5

11

5.5

EPA8240

EPA8240

EPA8240

EPA8240

EPA8240

EPA8240

EPA8240

EPA8240

EPA8240

EPA8240

EPA8240

EPA8240 
SAMPLE NAME: D43111001 (Continued)

\section{Constituent}

Sample ID: 100350

RQ AQ B Result Unit D. Limit Method

Pesticides/PCBs and Dioxins/Furans
2.3,7,8-TCDF

Tetrachlorodibenzo-p-dioxin isomers

Tetrachlorodibenzo-p-furan isomers

Toxaphene
$U$
$U$
$U$
$U$

Radionuclide Indicators and Radionuclides

Gross alpha

Nonvolatile beta

Tritium
Radium, total alpha-emitting
0.10
0.10
0.10
180

$\mathrm{ng} / \mathrm{g}$

$\mathrm{ng} / \mathrm{g}$

$\mathrm{ng} / \mathrm{g}$

$\mu \mathrm{g} / \mathrm{kg}$
0.10

0.10

0.10

180

EPA8280

EPA8280

EPA8280

EPA8080
SAMPLE NAME: D43111002

Location (SRS Coordinates): 18482.04E 66700.55N

Ground Elevation Above MSL: $129.5 \mathrm{ft}$

Depth of Core Interval: 0.50 to $2.00 \mathrm{ft}$

Sample Type: Normal

Sample Color: 10R 3/4

Constituent

Total Metals
Sample Matrix: Soil

USC Soil Classification: SC
6.4

8.0

0.20

1.5
EPA900.0

EPA900.0

EPA.903.0

EPA906.0
Sample ID: 100352

Sample Moisture: Dry Percent Solids: 90.10

RQ AQ B Result Unit

D. Limit Method

$\begin{array}{lll}\mathrm{mg} / \mathrm{kg} & 1.0 & \text { EPA7060 } \\ \mathrm{mg} / \mathrm{kg} & 19 & \text { EPA6010 } \\ \mathrm{mg} / \mathrm{kg} & 0.48 & \text { EPA6010 } \\ \mathrm{mg} / \mathrm{kg} & 0.96 & \text { EPA6010 } \\ \mathrm{mg} / \mathrm{kg} & 2.4 & \text { EPA6010 } \\ \mathrm{mg} / \mathrm{kg} & 0.30 & \text { EPA7421 } \\ \mathrm{mg} / \mathrm{kg} & 1.4 & \text { EPA6010 } \\ \mathrm{mg} / \mathrm{kg} & 0.11 & \text { EPA7470 } \\ \mathrm{mg} / \mathrm{kg} & 3.8 & \text { EPA6010 } \\ \mathrm{mg} / \mathrm{kg} & 0.50 & \text { EPA7740 } \\ \mathrm{mg} / \mathrm{kg} & 0.96 & \text { EPA6010 }\end{array}$

$B / N / A$ Extractables (including $P A H$ and phenols)

Acenaphthene

Acenaphthylene

Anthracene

Benzo(a)anthracene

Benzo(b)fluoranthene

Benzo(k)fluoranthene

Benzoic acid

Benzo $(g, h, i)$ perylene

Benzo(a)pyrene

Benzyl alcohol

Bis(2-chloroethoxy) methane

$\begin{array}{lll}\mathrm{U} & & 1.0 \\ \mathrm{U} & & 19 \\ \mathrm{UJ} & \mathrm{L} & 1.8 \\ \mathrm{~J} & \mathrm{~L} & 12 \\ \mathrm{U} & & 2.4 \\ \cdot & & 3.7 \\ & & 42 \\ \mathrm{U} & & 0.11 \\ \mathrm{U} & & 3.8 \\ \mathrm{U} & & 0.50 \\ \mathrm{U} & & 0.96\end{array}$

360
360
360
360
360
360
1,800
360
360
360
360

EPA8270

EPA8270

EPA8270

EPA8270

EPA8270

EPA8270

EPA8270

EPA8270

EPA8270

$\begin{array}{ll}360 & \text { EPA8270 } \\ 360 & \text { EPA8270 }\end{array}$ 


Constituent RQ AQ B Result Unit

$B / N / A$ Extractables (including PAH and phenols)

\begin{tabular}{|c|c|c|c|c|c|c|}
\hline Bis(2-chloroechyl) ether & $U$ & & 360 & $\mu \mathrm{g} / \mathrm{kg}$ & 360 & EPA8270 \\
\hline Bis(2-chloroisopropyl) ether & $\mathbf{U}$ & & 360 & $\mu \mathrm{g} / \mathrm{kg}$ & 360 & EPA8270 \\
\hline Bis(2-ethylhexyl) phthalate & $\mathbf{U}$ & & 360 & $\mu \mathrm{g} / \mathrm{kg}$ & 360 & EPA8270 \\
\hline 4-Bromophenyl phenyl ether & $\mathbf{U}$ & & 360 & $\mu \mathrm{g} / \mathrm{kg}$ & 360 & EPA8270 \\
\hline Burylbenzyl phthalate & $\mathbf{U}$ & & 360 & $\mu \mathrm{g} / \mathrm{kg}$ & 360 & EPA8270 \\
\hline 4-Chloroaniline & $\mathbf{U}$ & & 360 & $\mu \mathrm{g} / \mathrm{kg}$ & 360 & EPA8270 \\
\hline 4-Chloro-m-cresol & $\mathbf{U}$ & & 360 & $\mu \mathrm{g} / \mathrm{kg}$ & 360 & EPA8270 \\
\hline 2-Chloronaphthalene & $\mathbf{U}$ & & 360 & $\mu \mathrm{g} / \mathrm{kg}$ & 360 & EPA8270 \\
\hline 2-Chlorophenol & U & & 360 & $\mu \mathrm{g} / \mathrm{kg}$ & 360 & EPA8270 \\
\hline 4-Chlorophenyl phenyl ether & $\mathbf{U}$ & & 360 & $\mu g / k g$ & 360 & EPA8270 \\
\hline Chrysene & $\mathbf{U}$ & & 360 & $\mu g / k g$ & 360 & EPA8270 \\
\hline o-Cresol (2-Methylphenol) & $\mathbf{U}$ & & 360 & $\mu \mathrm{g} / \mathrm{kg}$ & 360 & EPA8270 \\
\hline p-Cresol (4-Methylphenol) & $\mathbf{U}$ & & 360 & $\mu g / k g$ & 360 & EPA8270 \\
\hline Dibenz $(\mathrm{a}, \mathrm{h})$ anthracene & U & & 360 & $\mu \mathrm{g} / \mathrm{kg}$ & 360 & EPA8270 \\
\hline Dibenzofuran & $\mathbf{U}$ & & 360 & $\mu \mathrm{g} / \mathrm{kg}$ & 360 & EPA8270 \\
\hline Di-n-butyl phthalate & UJ & v & 190 & $\mu g / \mathrm{kg}$ & 360 & EPA8270 \\
\hline 1,2-Dichlorobenzene & $\mathbf{U}$ & & 360 & $\mu \mathrm{g} / \mathrm{kg}$ & 360 & EPA8270 \\
\hline 1,3-Dichlorobenzene & $\mathbf{U}$ & & 360 & $\mu \mathrm{g} / \mathrm{kg}$ & 360 & EPA8270 \\
\hline 1,4-Dichlorobenzene & $\mathbf{U}$ & & 360 & $\mu \mathrm{g} / \mathrm{kg}$ & 360 & EPA8270 \\
\hline 3,3'-Dichlorobenzidine & U & & 730 & $\mu g / k g$ & 730 & EPA8270 \\
\hline 2,4-Dichlorophenol & $\mathbf{U}$ & & 360 & $\mu \mathrm{g} / \mathrm{kg}$ & 360 & EPA8270 \\
\hline Diethyl phthalate & $\mathbf{U}$ & & 360 & $\mu \mathrm{g} / \mathrm{kg}$ & 360 & EPA8270 \\
\hline 2,4-Dimethyl phenol & $\mathrm{U}$ & & $\cdot 360$ & $\mu \mathrm{g} / \mathrm{kg}$ & 360 & EPA8270 \\
\hline Dimethyl phthalate & U & & 360 & $\mu g / \mathrm{kg}$ & 360 & EPA8270 \\
\hline 2,4-Dinitrophenol & $\mathrm{U}$ & & 1,800 & $\mu g / k g$ & 1,800 & EPA8270 \\
\hline 2,4-Dinitrotoluene & $\mathbf{U}$ & & 360 & $\mu g / \mathrm{kg}$ & 360 & EPA8270 \\
\hline 2,6-Dinitrotoluene & $\mathrm{U}$ & & 360 & $\mu g / k g$ & 360 & EPA8270 \\
\hline Di-n-octyl phthalate & $\mathbf{U}$ & & 360 & $\mu \mathrm{g} / \mathrm{kg}$ & 360 & EPA8270 \\
\hline Fluoranthere & $\mathbf{U}$ & & 360 & $\mu \mathrm{g} / \mathrm{kg}$ & 360 & EPA8270 \\
\hline Fluorene & $\mathbf{U}$ & & 360 & $\mu \mathrm{g} / \mathrm{kg}$ & 360 & EPA8270 \\
\hline Hexachlorobenzene & $\mathbf{U}$ & & 360 & $\mu \mathrm{g} / \mathrm{kg}$ & 360 & EPA8270 \\
\hline Hexachlorobutadiene & $\mathbf{U}$ & & 360 & $\mu \mathrm{g} / \mathrm{kg}$ & 360 & EPA8270 \\
\hline Hexachlorocyclopentadiene & $\mathbf{U}$ & & 360 & $\mu g / k g$ & 360 & EPA8270 \\
\hline Hexachloroethane & $\mathbf{U}$ & & 360 & $\mu g / \mathrm{kg}$ & 360 & EPA8270 \\
\hline Indeno(1,2,3-c,d)pyrene & $\mathbf{U}$ & & 360 & $\mu g / \mathrm{kg}$ & 360 & EPA8270 \\
\hline Isophorone & $\mathbf{U}$ & & 360 & $\mu \mathrm{g} / \mathrm{kg}$ & 360 & EPA8270 \\
\hline 2-Methyl-4,6-dinitrophenol & $\mathbf{U}$ & & 1,800 & $\mu g / k g$ & 1.800 & EPA8270 \\
\hline 2-Methylnaphthalene & $\mathbf{U}$ & & 360 & $\mu \mathrm{g} / \mathrm{kg}$ & 360 & EPA8270 \\
\hline Naphthalene & $\mathbf{U}$ & & 360 & $\mu g / \mathrm{kg}$ & 360 & EPA8270 \\
\hline o-Nitroaniline & $\mathbf{U}$ & & 1.800 & $\mu \mathrm{g} / \mathrm{kg}$ & 1,800 & EPA8270 \\
\hline m-Nitroaniline & $\mathbf{U}$ & & 1,800 & $\mu \mathrm{g} / \mathrm{kg}$ & 1,800 & EPA8270 \\
\hline p-Nitroaniline & $\mathbf{U}$ & & 1,800 & $\mu \mathrm{g} / \mathrm{kg}$ & 1,800 & EPA8270 \\
\hline Nitrobenzene & $\mathbf{U}$ & & 360 & $\mu \mathrm{g} / \mathrm{kg}$ & 360 & EPA8270 \\
\hline 2-Nitrophenol & $\mathbf{U}$ & & 360 & $\mu g / k g$ & 360 & EPA8270 \\
\hline 4-Nitrophenol & $\mathbf{U}$ & & 1,800 & $\mu \mathrm{g} / \mathrm{kg}$ & 1,800 & EPA8270 \\
\hline N-Nitrosodiphenylamine & $\mathbf{U}$ & & 360 & $\mu \mathrm{g} / \mathrm{kg}$ & 360 & EPA8270 \\
\hline N-Nitrosodipropylamine & $\mathbf{U}$ & & 360 & $\mu \mathrm{g} / \mathrm{kg}$ & 360 & EPA8270 \\
\hline Pentachlorophenol & UJ & c & 1.800 & $\mu \mathrm{g} / \mathrm{kg}$ & 1.800 & EPA8270 \\
\hline Phenanthrene & $\mathbf{U}$ & & 360 & $\mu \mathrm{g} / \mathrm{kg}$ & 360 & EPA8270 \\
\hline Phenol & UJ & C & 360 & $\mu \mathrm{g} / \mathrm{kg}$ & 360 & EPA8270 \\
\hline
\end{tabular}


SAMPLE NAME: D43111002 (Continued) Sample ID: 100352

Constituent RQ AQ B Result Unit D. Limit Method

$B / N / A$ Extractables (including PAH and phenols)

Pyrene

1,2,4-Trichlorobenzene

2,4,5-Trichlorophenol

2,4,6-Trichlorophenol

Unknown ketone

Volatile Organic Compounds

Acetone
Benzene
Bromodichloromethane
Bromoform
Bromomethane (Methyl bromide)
Carbon disulfide
Carbon tetrachloride.
Chlorobenzene
Chloroethane
Chloroethene (Vinyl chloride)
Chloroform
Chloromethane (Methyl chloride)
Dibromochloromethane
1,1-Dichloroethane
1,2-Dichloroethane
1,1-Dichloroethylene
1,2-Dichloroethylene
Dichloromethane (Methylene chloride)
1,2-Dichloropropane
cis-1,3-Dichloropropene
trans-1,3-Dichloropropene
Ethylbenzene
2-Hexanone
Methyl ethyl ketone
Methyl isobutyl ketone
Styrene
1,1,2,2-Tetrachloroethane
Tetrachloroethylene
Toluene
1,1,1-Trichloroethane
1,1,2-Trichloroethane
Trichloroethylene
Vinyl acetate
Xylenes

\begin{tabular}{|c|c|c|c|c|}
\hline $\mathbf{U}$ & 360 & $\mu \mathrm{g} / \mathrm{kg}$ & 360 & EPA8270 \\
\hline $\mathbf{U}$ & 360 & $\mu g / \mathrm{kg}$ & 360 & EPA8270 \\
\hline $\mathbf{U}$ & 1,800 & $\mu g / \mathrm{kg}$ & 1,800 & EPA 8270 \\
\hline $\mathbf{U}$ & 360 & $\mu \mathrm{g} / \mathrm{kg}$ & 360 & EPA8270 \\
\hline NV & 10,000 & $\mu \mathrm{g} / \mathrm{kg}$ & & EPA8270 \\
\hline
\end{tabular}

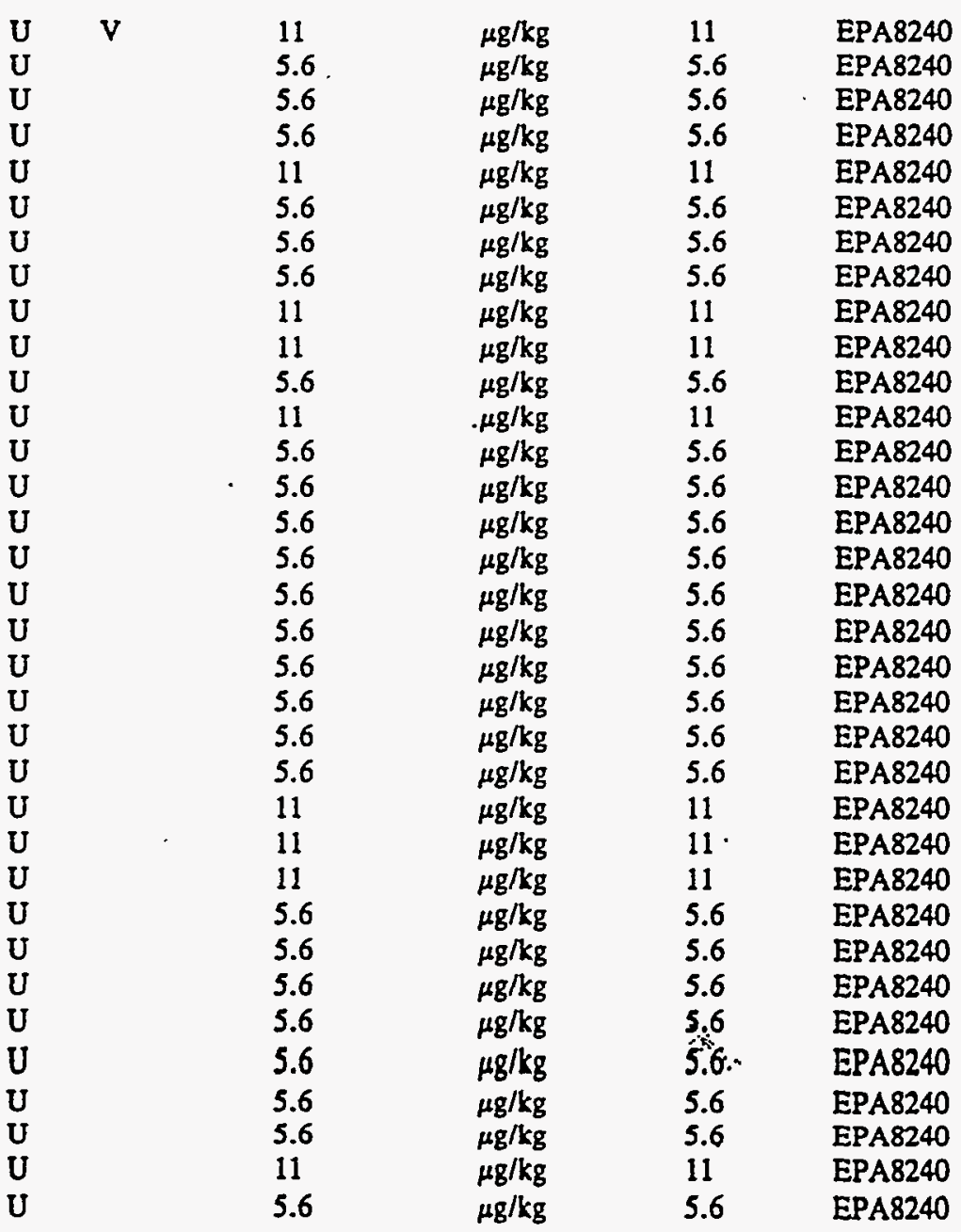

Pesticides/PCBs and Dioxins/Furans

Aldrin

alpha-Benzene hexachloride

beta-Benzene hexachloride

delta-Benzene hexachloride

alpha-Chlordane
U

U

U

U

$\mathrm{U}$
1.8
1.8
1.8
1.8
1.8

$\mu \mathrm{g} / \mathrm{kg}$

$\mu \mathrm{g} / \mathrm{kg}$

$\mu g / \mathrm{kg}$

$\mu \mathrm{g} / \mathrm{kg}$

$\mu g / k g$

$\begin{array}{ll}1.8 & \text { EPA8080 } \\ 1.8 & \text { EPA8080 } \\ 1.8 & \text { EPA8080 } \\ 1.8 & \text { EPA8080 } \\ 1.8 & \text { EPA8080 }\end{array}$


SAMPLE NAME: D43111002 (Continued)

Constituent

$\mathbf{R}$

Pesticides/PCBs and Dioxins/Furans
Sample ID: 100352

RQ AQ B Result Unit D. Limit Method

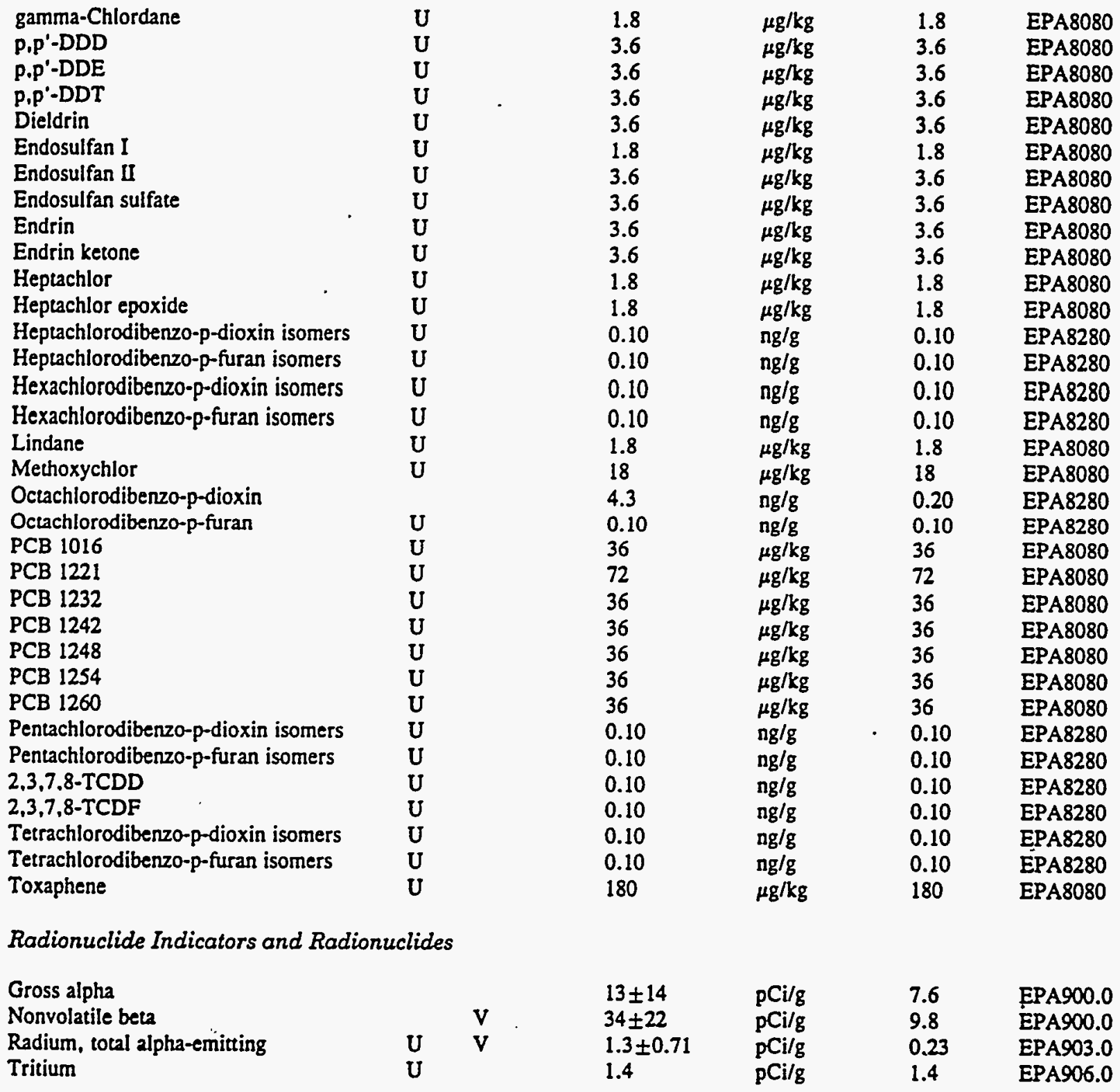


SAMPLE NAME: D43H11003

Location (SRS Coordinates): 18482.04E 66700.55N

Ground Elevation Above MSL: $129.5 \mathrm{ft}$

Depth of Core Interval: 2.00 to $4.00 \mathrm{ft}$

Sample Type: Normal

Sample Color: 10R 3/4

Constituent

Total Metals

Arsenic

Barium

Cadmium

Chromium

Copper

Lead

Manganese

Mercury

Nickel

Selenium

Silver

Volatile Organic Compounds

Acetone

Benzene

Bromodichloromethane

Bromoform

Bromomethane (Methyl bromide)

Carbon disulfide

Carbon tetrachloride

Chlorobenzene

Chloroetharie

Chloroethene (Vinyl chloride)

Chloroform

Chloromethane (Methyl chloride)

Dibromochloromethane

1.1-Dichloroethane

1,2-Dichloroethane

1,1-Dichloroethylene

1,2-Dichloroethylene

Dichloromethane (Methylene chloride)

1,2-Dichloropropane

cis-1,3-Dichloropropene

trans-1,3-Dichloropropene

Ethylbenzene

2-Hexanone

Methyl ethyl ketone

Methyl isobutyl ketone

Styrene

1,1,2,2-Tetrachloroethane

Tetrachloroethylene

Toluene

1,1,1-Trichloroethane
Sample ID: 100353

Sample Matrix: Soil

USC Soil Classification: SC

Sample Moisture: Dry

Percent Solids: $\mathbf{9 0 . 3 0}$

RQ AQ B Result Unit D. Limit Method


SAMPLE NAME: D43Ï11003 (Continued)

\section{Constituent}

Volatile Organic Compounds

\section{RQ AQ}

Sample ID: 100353

B Result Unit

$\mu \mathrm{g} / \mathrm{kg}$

$\mu g / \mathrm{kg}$

$\mu g / k g$

$\mu g / \mathrm{kg}$

$\begin{array}{ll}5.6 & \text { EPA8240 } \\ 5.6 & \text { EPA8240 } \\ 11 & \text { EPA8240 } \\ 5.6 & \text { EPA8240 }\end{array}$

Radionuclide Indicators and Radionuclides

Gross alpha

Nonvolatile beta

Radium, total alpha-emiting

Tritium

$\begin{array}{ll}\text { U } & \\ & \text { V } \\ \text { U } & \text { V }\end{array}$

SAMPLE NAME: D43111004

Location (SRS Coordinates): 18482.04E 66700.55N

Ground Elevation Above MSL: 129.5 ft

Depth of Core Interval: 4.00 to $6.00 \mathrm{ft}$

Sample Type: Normal

Sample Color: 10R 3/4

Constituent

RQ AQ B Result
Sample Moisture: Damp Percent Solids: $\mathbf{8 8 . 3 0}$

D. Limit Method

Total Metals

Arsenic
Barium
Cadmium
Chromium
Copper
Lead
Manganese
Mercury
Nickel
Selenium
Silver

$B / N / A$ Extractables (including $P A H$ and phenols)

$\begin{array}{lll}U & & 1.0 \\ & & 30 \\ \mathrm{UJ} & \mathrm{L} & 0.51 \\ & \mathrm{~L} & 27 \\ & & 5.0 \\ & & 2.3 \\ & & 250 \\ & & 0.13 \\ \mathrm{U} & & 4.1 \\ \mathrm{U} & & 0.52 \\ \mathrm{U} & & 1.0\end{array}$

$\mathrm{pCi} / \mathrm{g}$
$\mathrm{pCi} / \mathrm{g}$
$\mathrm{pCi} / \mathrm{g}$
$\mathrm{pCi} / \mathrm{g}$

$\mathrm{Ci} / \mathrm{g}$

$\begin{array}{lll}6.8 & \text { EPA900.0 } \\ 8.6 & \text { EPA900.0 } \\ 0.20 & \text { EPA903.0 } \\ 1.4 & \text { EPA906.0 }\end{array}$

Sample Matrix: Soil

USC Soil Classification: SC

Sample ID: 100354

Unit

$\mathrm{mg} / \mathrm{kg}$ $\mathrm{mg} / \mathrm{kg}$ $\mathrm{mg} / \mathrm{kg}$ $\mathrm{mg} / \mathrm{kg}$ $\mathrm{mg} / \mathrm{kg}$ $\mathrm{mg} / \mathrm{kg}$ $\mathrm{mg} / \mathrm{kg}$ $\mathrm{mg} / \mathrm{kg}$ $\mathrm{mg} / \mathrm{kg}$ $\mathrm{mg} / \mathrm{kg}$ $\mathrm{mg} / \mathbf{k g}$
1.0

20

0.51

1.0

2.6

0.31

1.5

0.11

4.1

0.52

1.0

\begin{tabular}{|c|c|c|}
\hline$\mu \mathrm{g} / \mathrm{kg}$ & 370 & EPA8270 \\
\hline$\mu \mathrm{g} / \mathrm{kg}$ & 370 & EPA8270 \\
\hline$\mu \mathrm{g} / \mathrm{kg}$ & 370 & EPA8270 \\
\hline$\mu g / \mathrm{kg}$ & 370 & EPA8270 \\
\hline$\mu g / \mathrm{kg}$ & 370 & EPA8270 \\
\hline$\mu \mathrm{g} / \mathrm{kg}$ & 370 & EPA8270 \\
\hline$\mu \mathrm{g} / \mathrm{kg}$ & 1,900 & EPA8270 \\
\hline$\mu g / \mathrm{kg}$ & 370 & EPA8270 \\
\hline$\mu g / \mathbf{k g}$ & 370 & EPA8270 \\
\hline$\mu g / \mathrm{kg}$ & 370 & EPA827C \\
\hline$\mu \mathrm{g} / \mathrm{kg}$ & 370 & EPA8270 \\
\hline
\end{tabular}


SAMPLE NAME: D43i11004 (Continued)

Sample ID: 100354

Constituent

RQ AQ B Result

Unit

D. Limit Method

\section{$B / N / A$ Extractables (including $P A H$ and phenols)}

Bis(2-chloroethyl) ether

Bis(2-chloroisopropyl) ether

Bis(2-ethylhexyl) phthalate

4-Bromophenyl phenyl ether

Butylbenzyl phthalate

4-Chloroaniline

4-Chloro-m-cresol

2-Chloronaphthalene

2-Chlorophenol

4-Chlorophenyl phenyl ether

Chirysene

o-Cresol (2-Methylphenol)

p-Cresol (4-Methylphenol)

Dibenz(a.h)anthracene

Dibenzofuran

Di-n-butyl phthalate

1,2-Dichlorobenzene

1.3-Dichlorobenzene

1,4-Dichlorobenzene

3,3'-Dichlorobenzidine

2.4-Dichlorophenol

Diechyl phthalate

2,4-Dimethyl phenol

Dimethyl phthalate

2,4-Dinitrophenol

2,4-Dinitrotoluene

2,6-Dinitrotoluene

Di-n-octyl phthalate

Fluoranthene

Fluorene

Hexachlorobenzene

Hexachlorobutadiene

Hexachlorocyclopentadiene

Hexachloroethane

Indeno(1,2,3-c,d)pyrene

Isophorone

2-Methyl-4,6-dinitrophenol

2-Methylnaphthalene

Naphthalene

o-Nitroaniline

m-Nitroaniline

p-Nitroaniline

Nitrobenzene

2-Nitrophenol

4-Nitrophenol

N-Nitrosodiphenylamine

N-Nitrosodipropylamine

Pentachlorophenol

Phenanthrene

Phenol
U 37

U 370

U 370

370

370

370

370

370

370

370

370

370

370

370

370

200

370

370

370

750

370

370

370

370

1,900

370

370

370

370

370

370

370

370

370

370

370

1,900

370

370

1,900

1,900

1,900

370

370

1,900

370

370

1,900

370

370

\begin{tabular}{|c|c|c|}
\hline$\mu \mathrm{g} / \mathrm{kg}$ & 370 & EPA8270 \\
\hline$\mu \mathrm{g} / \mathrm{kg}$ & 370 & EPA8270 \\
\hline$\mu \mathrm{g} / \mathrm{kg}$ & 370 & EPA8270 \\
\hline$\mu \mathrm{g} / \mathrm{kg}$ & 370 & EPA8270 \\
\hline$\mu \mathrm{g} / \mathrm{kg}$ & 370 & EPA 8270 \\
\hline$\mu \mathrm{g} / \mathrm{kg}$ & 370 & EPA8270 \\
\hline$\mu g / \mathrm{kg}$ & 370 & EPA8270 \\
\hline$\mu \mathrm{g} / \mathrm{kg}$ & 370 & EPA.8270 \\
\hline$\mu \mathrm{g} / \mathrm{kg}$ & 370 & EPA 8270 \\
\hline$\mu \mathrm{g} / \mathrm{kg}$ & 370 & EPA8270 \\
\hline$\mu \mathrm{g} / \mathrm{kg}$ & 370 & EPA8270 \\
\hline$\mu \mathrm{g} / \mathrm{kg}$ & 370 & EPA8270 \\
\hline$\mu \mathrm{g} / \mathrm{kg}$ & 370 & EPA8270 \\
\hline$\mu \mathrm{g} / \mathrm{kg}$ & 370 & EPA8270 \\
\hline$\mu \mathrm{g} / \mathrm{kg}$ & 370 & EPA8270 \\
\hline$\mu \mathrm{g} / \mathrm{kg}$ & 370 & EPA8270 \\
\hline$\mu \mathrm{g} / \mathrm{kg}$ & 370 & EPA8270 \\
\hline$\mu \mathrm{g} / \mathrm{kg}$ & 370 & EPA8270 \\
\hline$\mu \mathrm{g} / \mathrm{kg}$ & 370 & EPA8270 \\
\hline$\mu \mathrm{g} / \mathrm{kg}$ & 750 & EPA8270 \\
\hline$\mu \mathrm{g} / \mathrm{kg}$ & 370 & EPA8270 \\
\hline$\mu g / \mathrm{kg}$ & 370 & EPA8270 \\
\hline$\mu \mathrm{g} / \mathrm{kg}$ & 370 & EPA8270 \\
\hline$\mu \mathrm{g} / \mathrm{kg}$ & 370 & EPA8270 \\
\hline$\mu \mathrm{g} / \mathrm{kg}$ & 1,900 & EPA8270 \\
\hline$\mu \mathrm{g} / \mathrm{kg}$ & 370 & EPA8270 \\
\hline$\mu \mathrm{g} / \mathrm{kg}$ & 370 & EPA8270 \\
\hline$\mu \mathrm{g} / \mathrm{kg}$ & 370 & EPA8270 \\
\hline$\mu \mathrm{g} / \mathrm{kg}$ & 370 & EPA8270 \\
\hline$\mu \mathrm{g} / \mathrm{kg}$ & 370 & EPA8270 \\
\hline$\mu \mathrm{g} / \mathrm{kg}$ & 370 & EPA8270 \\
\hline$\mu \mathrm{g} / \mathrm{kg}$ & 370 & EPA8270 \\
\hline$\mu \mathrm{g} / \mathrm{kg}$ & 370 & EPA8270 \\
\hline$\mu \mathrm{g} / \mathrm{kg}$ & 370 & EPA8270 \\
\hline$\mu \mathrm{g} / \mathrm{kg}$ & 370 & EPA8270 \\
\hline$\mu \mathrm{g} / \mathrm{kg}$ & 370 & EPA8270 \\
\hline$\mu \mathrm{g} / \mathrm{kg}$ & 1,900 & EPA8270 \\
\hline$\mu \mathrm{g} / \mathrm{kg}$ & 370 & EPA8270 \\
\hline$\mu \mathrm{g} / \mathrm{kg}$ & 370 & EPA8270 \\
\hline$\mu \mathrm{g} / \mathrm{kg}$ & 1,900 & EPA8270 \\
\hline$\mu \mathrm{g} / \mathrm{kg}$ & $1 ; 900$ & EPA8270 \\
\hline$\mu \mathrm{g} / \mathrm{kg}$ & 1,900 & EPA8270 \\
\hline$\mu \mathrm{g} / \mathrm{kg}$ & 370 & EPA8270 \\
\hline$\mu \mathrm{g} / \mathrm{kg}$ & 370 & EPA8270 \\
\hline$\mu \mathrm{g} / \mathrm{kg}$ & 1,900 & EPA8270 \\
\hline$\mu \mathrm{g} / \mathrm{kg}$ & 370 & EPA8270 \\
\hline$\mu \mathrm{g} / \mathrm{kg}$ & 370 & EPA8270 \\
\hline$\mu \mathrm{g} / \mathrm{kg}$ & 1,900 & EPA8270 \\
\hline$\mu \mathrm{g} / \mathrm{kg}$ & 370 & EPA8270 \\
\hline$\mu \mathrm{g} / \mathrm{kg}$ & 370 & EPA8270 \\
\hline
\end{tabular}


SAMPLE NAME: D43111004 (Continued)

Constituent

$\mathbf{R Q}$

AQ B

Result

Unit

D. Limit Method

\section{$B / N / A$ Extractables (including $P A H$ and phenols)}

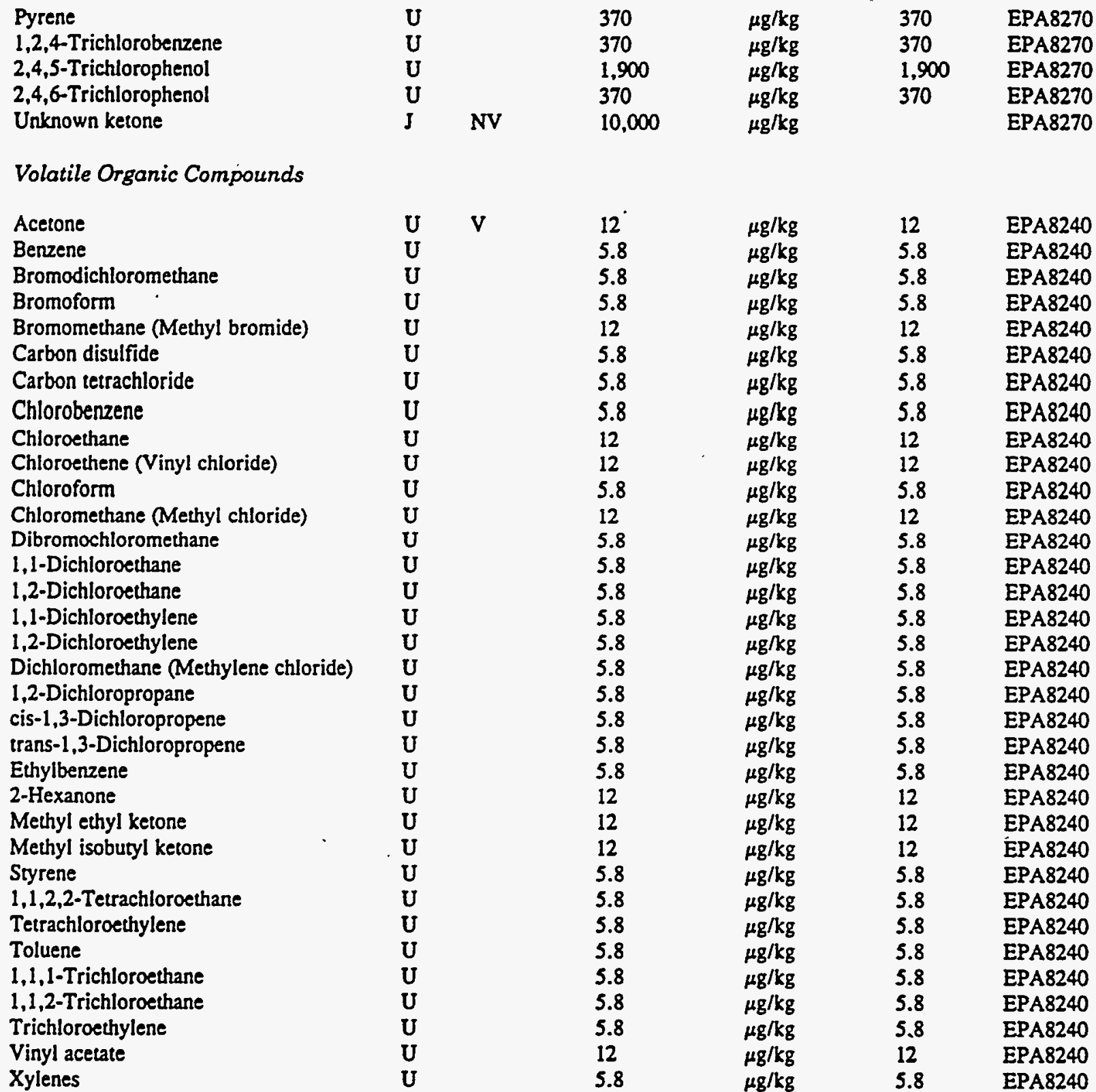

Radionuclide Indicators and Radionuclides

$\begin{array}{lllllll}\text { Gross alpha } & \mathrm{U} & & 6.5 & \mathrm{pCi} / \mathrm{g} & 6.5 & \text { EPA900.0 } \\ \text { Nonvolatile beta } & & \mathrm{V} & 9.9 \pm 16 & \mathrm{pCi} / \mathrm{g} & 8.3 & \text { EPA900.0 } \\ \text { Radium, total alpha-emitting } & \mathrm{U} & \mathrm{V} & 1.9 \pm 0.83 & \mathrm{pCi} / \mathrm{g} & 0.21 & \text { EPA903.0 } \\ \text { Tritium } & \mathrm{U} & & 1.4 & \mathrm{pCi} / \mathrm{g} & 1.4 & \text { EPA906.0 }\end{array}$


SAMPLE NAME: D43111005

Location (SRS Coordinates): 18482.04 E 66700.55N

Ground Elevation Above MSL: $129.5 \mathrm{ft}$

Depth of Core Interval: 6.00 to $8.00 \mathrm{ft}$

Sample Type: Normal

Sample Color: 10R 3/4

Constituent

RQ AQ B Result
Sample ID: 100356

Sample Matrix: Soil

USC Soil Classification: SC

Sample Moisture: Damp

Percent Solids: 78.50

D. Limit Method

Total Metals

\begin{tabular}{|c|c|c|c|c|c|c|c|}
\hline Arsenic & UJ & I & L & 1.1 & $\mathrm{mg} / \mathrm{kg}$ & 1.1 & EPA7060 \\
\hline Arsenic & $\mathbf{J}$ & I & L & 2.7 & $\mathrm{mg} / \mathrm{kg}$ & 1.3 & EPA7060 \\
\hline Barium & $\mathbf{J}$ & I & $\mathrm{H}$ & 26 & $\mathrm{mg} / \mathrm{kg}$ & 24 & EPA6010 \\
\hline Barium & J & I & $\mathrm{H}$ & 35 & $\mathrm{mg} / \mathrm{kg}$ & 25 & EPA6010 \\
\hline Cadmium & UJ & LIX & $\mathrm{H}$ & 0.61 & $\mathrm{mg} / \mathrm{kg}$ & 0.61 & EPA6010 \\
\hline Cadmium & UJ & IX & $\mathrm{H}$ & 3.1 & $\mathrm{mg} / \mathrm{kg}$ & 3.1 & EPA6010 \\
\hline Chromium & J & LI & $\mathbf{H}$ & 24 & $\mathrm{mg} / \mathrm{kg}$ & 1.2 & EPA6010 \\
\hline Chromium & & I & $\mathbf{H}$ & 24 & $\mathrm{mg} / \mathrm{kg}$ & 1.2 & EPA6010 \\
\hline Copper & $\mathbf{U}$ & & & 3.0 & $\mathrm{mg} / \mathrm{kg}$ & 3.0 & EPA6010 \\
\hline Copper & $U$ & & & 3.1 & $\mathrm{mg} / \mathrm{kg}$ & 3.1 & EPA6010 \\
\hline Lead & J & DX & & 6.5 & $\mathrm{mg} / \mathrm{kg}$ & 0.33 & EPA7421 \\
\hline Lead & J & IX & & 16 & $\mathrm{mg} / \mathrm{kg}$ & 3.8 & EPA7421 \\
\hline Manganese & J & $\mathrm{x}$ & & 270 & $\mathrm{mg} / \mathrm{kg}$ & 1.8 & EPA6010 \\
\hline Manganese & $\mathrm{J}$ & $\mathrm{x}$ & & 520 & $\mathrm{mg} / \mathrm{kg}$ & 1.8 & EPA6010 \\
\hline Mercury & & & & 0.16 & $\mathrm{mg} / \mathrm{kg}$ & 0.13 & EPA7470 \\
\hline Mercury & UJ & $\mathbf{Q}$ & & 0.13 & $\mathrm{mg} / \mathrm{kg}$ & 0.13 & EPA7470 \\
\hline Nickel & $\mathbf{U}$ & & & 4.9 & $\mathrm{mg} / \mathrm{kg}$ & 4.9 & EPA6010 \\
\hline Nickel & & & & 5.3 & $\mathrm{mg} / \mathrm{kg}$ & 4.9 & EPA6010 \\
\hline Selenium & UI & I & L & 0.56 & $\mathrm{mg} / \mathrm{kg}$ & 0.56 & EPA7740 \\
\hline Selenium & UJ & I & L & 0.63 & $\mathrm{mg} / \mathrm{kg}$ & 0.63 & EPA7740 \\
\hline Silver & UJ & $\mathrm{x}$ & & 1.2 & $\mathrm{mg} / \mathrm{kg}$ & 1.2 & EPA6010 \\
\hline Silver & UJ & $\mathrm{x}$ & & 6.1 & $\mathrm{mg} / \mathrm{kg}$ & 6.1 & EPA6010 \\
\hline
\end{tabular}

$B / N / A$ Extractables (including PAH and phenols)

Acenaphthene

Acenaphithylene

Anthracene

Benzo(a)anthracene

Benzo(b)fluoranthene

Benzo(k)fluoranthene

Benzoic acid

Benzo $(g, h, i)$ perylene

Benzo(a)pyrene

Benzyl alcohol

Bis(2-chloroethoxy) methane

Bis(2-chloroethyl) ether

Bis(2-chloroisopropyl) ether

Bis(2-ethylhexyl) phthalate

4-Bromophenyl phenyl ether

Butylbenzyl phthalate

4-Chloroaniline

4-Chioro-m-cresol

2-Chloronaphthalene
U

U

U

U

U

U

U

U

U

U

U

U

U

U

U

U

U

U
440

440

440

440

440

440

2,200

440

440

440

440

440

440

440

440

440

440

440

440

$\begin{array}{lll}\mu \mathrm{g} / \mathrm{kg} & 440 & \text { EPA8270 } \\ \mu \mathrm{g} / \mathrm{kg} & 440 & \text { EPA8270 } \\ \mu \mathrm{g} / \mathrm{kg} & 440 & \text { EPA8270 } \\ \mu \mathrm{g} / \mathrm{kg} & 440 & \text { EPA8270 } \\ \mu \mathrm{g} / \mathrm{kg} & 440 & \text { EPA8270 } \\ \mu \mathrm{g} / \mathrm{kg} & 440 & \text { EPA8270 } \\ \mu \mathrm{g} / \mathrm{kg} & 2,200 & \text { EPA8270 } \\ \mu \mathrm{g} / \mathrm{kg} & 440 & \text { EPA8270 } \\ \mu \mathrm{g} / \mathrm{kg} & 440 & \text { EPA8270 } \\ \mu \mathrm{g} / \mathrm{kg} & 440 & \text { EPA8270 } \\ \mu \mathrm{g} / \mathrm{kg} & 440 & \text { EPA8270 } \\ \mu \mathrm{g} / \mathrm{kg} & 440 & \text { EPA8270 } \\ \mu \mathrm{g} / \mathrm{kg} & 440 & \text { EPA8270 } \\ \mu \mathrm{g} / \mathrm{kg} & 440 & \text { EPA8270 } \\ \mu \mathrm{g} / \mathrm{kg} & 440 & \text { EPA8270 } \\ \mu \mathrm{g} / \mathrm{kg} & 440 & \text { EPA8270 } \\ \mu \mathrm{g} / \mathrm{kg} & 440 & \text { EPA8270 } \\ \mu \mathrm{g} / \mathrm{kg} & 440 & \text { EPA8270 } \\ \mu \mathrm{g} / \mathrm{kg} & 440 & \text { EPA8270 }\end{array}$


SAMPLE NAME: D43111005 (Continued)

Sample ID: 100356

$\begin{array}{llllll}\text { Constituent } & \text { RQ AQ } & \text { R } & \text { Resuit } & \text { D. Limit Method }\end{array}$

$B / N / A$ Extractables (including $P A H$ and phenols)

2-Chlorophenol
4-Chlorophenyl phenyl ether
Chrysene
o-Cresol (2-Methylphenol)
p-Cresol (4-Methylphenol)
Dibenz(a,h)anthracene
Dibenzofuran
Di-n-butyl phthalate
1,2-Dichlorobenzene
1,3-Dichlorobenzene
1.4-Dichlorobenzene
3,3'-Dichlorobenzidine
2,4-Dichlorophenol
Diethyl phthalate
2,4-Dimethyl phenol
Dimethyl phthalate
2,4-Dinitrophenol
2,4-Dinitrotoluene
2,6-Dinitrotoluene
Di-n-octyl phthalate
Fluoranthene
Fluorene
Hexachlorobenzene
Hexachlorobutadiene
Hexachlorocyclopentadiene
Hexachloroethane
Indeno(1,2,3-c,d)pyrene
Isophorone
2-Methyl-4,6-dinitrophenol
2-Methylnaphthalene
Naphthalene
o-Nitroaniline
m-Nitroaniline
p-Nitroaniline
Nitrobenzene
2-Nitrophenol
4-Nitrophenol
N-Nitrosodiphenylamine
N-Nitrosodipropylamine
Pentachlorophenol
Phenanthrene
Phenol
Pyrene
1,2,4-Trichlorobenzene
2,4,5-Trichlorophenol
2,4,6-Trichlorophenol
Unknown ketone
(n)

\begin{tabular}{|c|c|c|c|c|c|}
\hline $\mathrm{U}$ & & 440 & $\mu g / k g$ & 440 & EPA8270 \\
\hline $\mathbf{U}$ & & 440 & $\mu \mathrm{g} / \mathrm{kg}$ & 440 & EPA8270 \\
\hline $\mathbf{U}$ & & 440 & $\mu \mathrm{g} / \mathrm{kg}$ & 440 & EPA8270 \\
\hline $\mathrm{U}$ & & 440 & $\mu \mathrm{g} / \mathrm{kg}$ & 440 & EPA8270 \\
\hline U & & 440 & $\mu g / \mathrm{kg}$ & 440 & EPA8270 \\
\hline $\mathrm{U}$ & & 440 & $\mu g / k g$ & 440 & EPA8270 \\
\hline $\mathbf{U}$ & & 440 & $\mu \mathrm{g} / \mathrm{kg}$ & 440 & EPA8270 \\
\hline UJ & v & 200 & $\mu g / k g$ & 440 & EPA8270 \\
\hline U & & 440 & $\mu g / \mathrm{kg}$ & 440 & EPA8270 \\
\hline$U$ & & 440 & $\mu \mathrm{g} / \mathrm{kg}$ & 440 & EPA8270 \\
\hline $\mathbf{U}$ & & 440 & $\mu \mathrm{g} / \mathrm{kg}$ & 440 & EPA8270 \\
\hline U & & 880 & $\mu g / \mathrm{kg}$ & 880 & EPA8270 \\
\hline U & & 440 & $\mu \mathrm{g} / \mathrm{kg}$ & 440 & EPA8270 \\
\hline $\mathbf{U}$ & & 440 & $\mu \mathrm{g} / \mathrm{kg}$ & 440 & EPA8270. \\
\hline U & & 440 & $\mu \mathrm{g} / \mathrm{kg}$ & 440 & EPA8270 \\
\hline $\mathrm{U}$ & & 440 & $\mu \mathrm{g} / \mathrm{kg}$ & 440 & EPA8270 \\
\hline U & & 2,200 & $\mu \mathrm{g} / \mathrm{kg}$ & 2,200 & EPA8270 \\
\hline $\mathrm{U}$ & & 440 & $\mu \mathrm{g} / \mathrm{kg}$ & 440 & EPA8270 \\
\hline U & & 440 & $\mu \mathrm{g} / \mathrm{kg}$ & 440 & EPA8270 \\
\hline U & & 440 & $\mu \mathrm{g} / \mathrm{kg}$ & 440 & EPA8270 \\
\hline$U$ & & 440 & $\mu g / \mathrm{kg}$ & 440 & EPA8270 \\
\hline $\mathrm{U}$ & & 440 & $\mu g / \mathrm{kg}$ & 440 & EPA8270 \\
\hline U & & 440 & $\mu \mathrm{g} / \mathrm{kg}$ & 440 & EPA8270 \\
\hline$U$ & & 440 & $\mu \mathrm{g} / \mathrm{kg}$ & 440 & EPA8270 \\
\hline U & & 440 & $\mu \mathrm{g} / \mathrm{kg}$ & 440 & EPA8270 \\
\hline $\mathbf{U}$ & & 440 & $\mu \mathrm{g} / \mathrm{kg}$ & 440 & EPA8270 \\
\hline U & & 440 & $\mu g / \mathrm{kg}$ & 440 & EPA8270 \\
\hline $\mathrm{U}$ & & 440 & $\mu g / k g$ & 440 & EPA8270 \\
\hline $\mathbf{U}$ & & 2,200 & $\mu \mathrm{g} / \mathrm{kg}$ & 2,200 & EPA8270 \\
\hline$U$ & & 440 & $\mu g / k g$ & 440 & EPA8270 \\
\hline U & & 440 & $\mu \mathrm{g} / \mathrm{kg}$ & 440 & EPA8270 \\
\hline $\mathbf{U}$ & & 2,200 & $\mu g / k g$ & 2,200 & EPA8270 \\
\hline U & & 2,200 & $\mu g / k g$ & 2,200 & EPA8270 \\
\hline U & & 2,200 & $\mu \mathrm{g} / \mathrm{kg}$ & 2.200 & EPA8270 \\
\hline U & & 440 & $\mu g / \mathrm{kg}$ & 440 & EPA8270 \\
\hline $\mathbf{U}$ & & 440 & $\mu \mathrm{g} / \mathrm{kg}$ & 440 & EPA8270 \\
\hline U & & 2,200 & $\mu g / \mathrm{kg}$ & 2,200 & EPA8270 \\
\hline U & & 440 & $\mu \mathrm{g} / \mathrm{kg}$ & 440 & EPA8270 \\
\hline U & & 440 & $\mu g / k g$ & 440 & EPA8270 \\
\hline UJ & C & 2,200 & $\mu g / k g$ & $2 ; 200$ & EPA8270 \\
\hline $\mathrm{U}$ & & 440 & $\mu g / k g$ & 440 & EPA8270 \\
\hline UJ & C & 440 & $\mu g / \mathrm{kg}$ & 440 & EPA8270 \\
\hline $\mathbf{U}$ & & 440 & $\mu \mathrm{g} / \mathrm{kg}$ & 440 & EPA8270 \\
\hline U & & 440 & $\mu \mathrm{g} / \mathrm{kg}$ & 440 & EPA8270 \\
\hline$U$ & & 2,200 & $\mu \mathrm{g} / \mathrm{kg}$ & 2,200 & EPA8270 \\
\hline $\mathbf{U}$ & & 440 & $\mu g / \mathrm{kg}$ & 440 & EPA8270 \\
\hline & NV & 10,000 & $\mu \mathrm{g} / \mathrm{kg}$ & & EPA8270 \\
\hline
\end{tabular}




\section{Constituent}

Volatile Organic Compounds

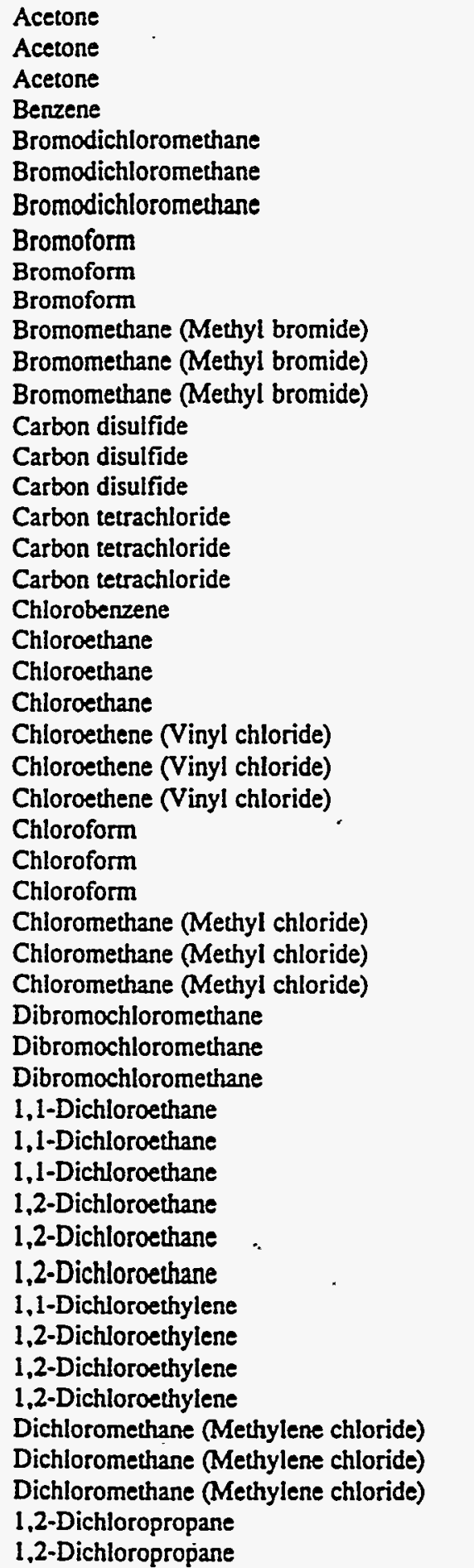

RQ AQ B Result Unit $\quad$ D. Limit Method

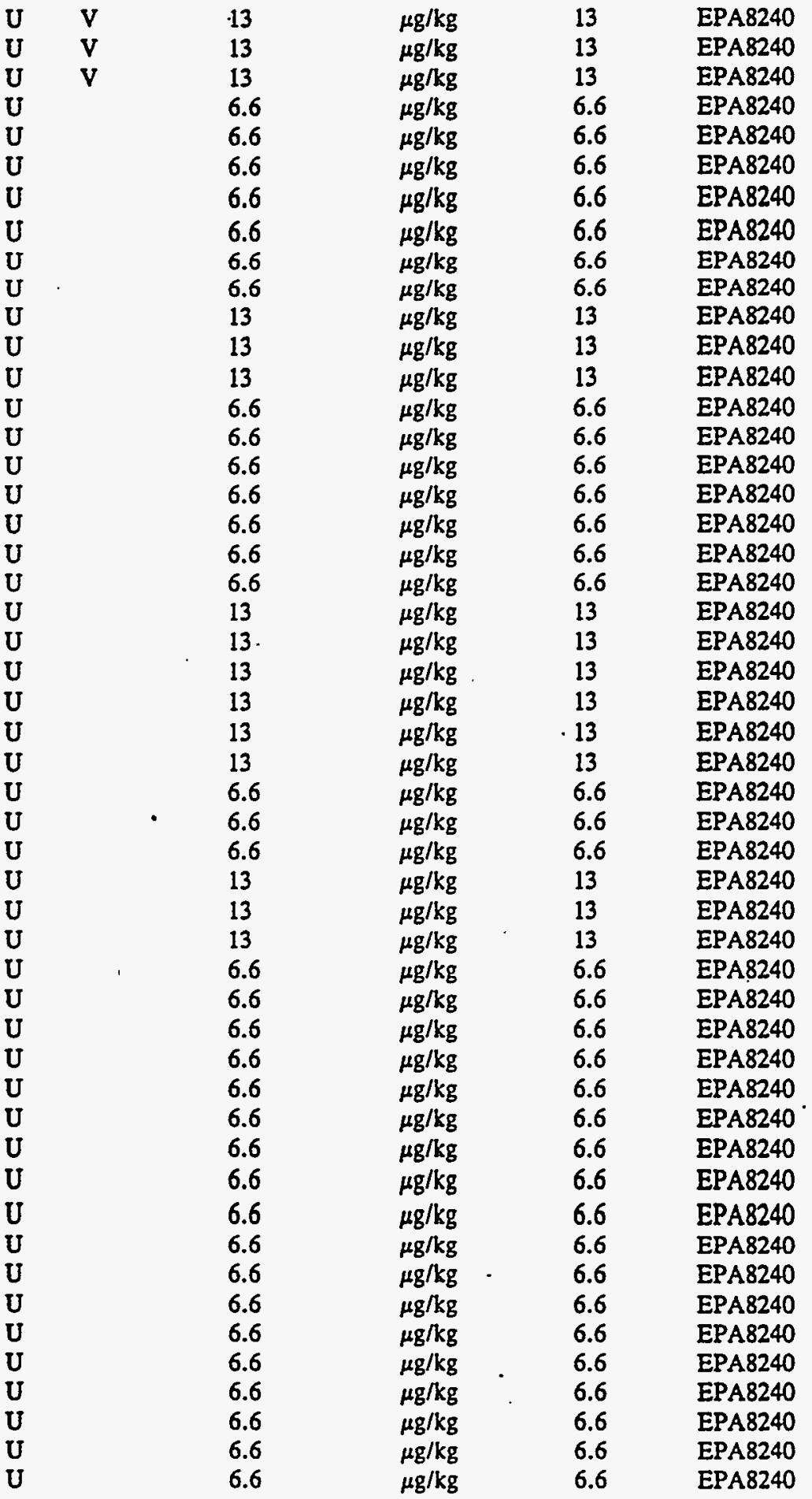


Constituent

Volatile Organic Compounds
RQ AQ B Result

Unit
D. Limit Method

\begin{abstract}
1,2-Dichloropropane
cis-1,3-Dichloropropene

cis-1,3-Dichloropropene

cis-1,3-Dichloropropene

trans-1,3-Dichloropropene

trans-1,3-Dichloropropene

trans-1,3-Dichloropropene

Ethylbenzene

Ethylbenzene

Ethylbenzene

2-Hexanone

2-Hexanone

2-Hexanone

Methyl ethyl ketone

Methyl ethyl ketone

Methyl ethyl ketone

Methyl isobutyl ketone

Methyl isoburyl ketone

Methyl isobutyl ketone

Styrene

Styrene

Styrene

1,1,2,2-Tetrachloroethane

1,1,2,2-Tetrachloroethane

1,1,2,2-Tetrachloroethane

Tetrachloroethylene

Tetrachloroethylene

Tetrachloroethylene

Toluene

1,1,1-Trichloroethane

1,1,1-Trichloroethane

1,1,1-Trichloroethane

1,1,2-Trichloroethane

1,1,2-Trichloroethane

1,1,2-Trichloroethane

Trichloroethylene

Vinyl acetate

Vinyl acetate

Vinyl acetate

Xylenes

Xylenes

Xylenes
\end{abstract}

6.6

6.6

6.6

6.6

6.6

6.6

6.6

6.6

6.6

6.6

13

13

13

13

13

13

13

13

13

6.6

6.6

6.6

6.6

6.6

6.6

6.6

6.6

6.6

6.6

6.6

6.6

6.6

6.6

6.6

6.6

6.6

13

13

13

6.6

6.6

6.6

$\mu \mathrm{g} / \mathrm{kg}$
$\mu \mathrm{g} / \mathrm{kg}$
$\mu \mathrm{g} / \mathrm{kg}$
$\mu \mathrm{g} / \mathrm{kg}$
$\mu \mathrm{g} / \mathrm{kg}$
$\mu \mathrm{g} / \mathrm{kg}$
$\mu \mathrm{g} / \mathrm{kg}$
$\mu \mathrm{g} / \mathrm{kg}$
$\mu \mathrm{g} / \mathrm{kg}$
$\mu \mathrm{g} / \mathrm{kg}$
$\mu \mathrm{g} / \mathrm{kg}$
$\mu \mathrm{g} / \mathrm{kg}$
$\mu \mathrm{g} / \mathrm{kg}$
$\mu \mathrm{g} / \mathrm{kg}$
$\mu \mathrm{g} / \mathrm{kg}$
$\mu \mathrm{g} / \mathrm{kg}$
$\mu \mathrm{g} / \mathrm{kg}$
$\mu \mathrm{g} / \mathrm{kg}$
$\mu \mathrm{g} / \mathrm{kg}$
$\mu \mathrm{g} / \mathrm{kg}$
$\mu \mathrm{g} / \mathrm{kg}$
$\mu \mathrm{g} / \mathrm{kg}$
$\mu \mathrm{g} / \mathrm{kg}$
$\mu \mathrm{g} / \mathrm{kg}$
$\mu \mathrm{g} / \mathrm{kg}$
$\mu \mathrm{g} / \mathrm{kg}$
$\mu \mathrm{g} / \mathrm{kg}$
$\mu \mathrm{g} / \mathrm{kg}$
$\mu \mathrm{g} / \mathrm{kg}$
$\mu \mathrm{g} / \mathrm{kg}$
$\mu \mathrm{g} / \mathrm{kg}$
$\mu \mathrm{g} / \mathrm{kg}$
$\mu \mathrm{g} / \mathrm{kg}$
$\mu \mathrm{g} / \mathrm{kg}$
$\mu \mathrm{g} / \mathrm{kg}$
$\mu \mathrm{g} / \mathrm{kg}$
$\mu \mathrm{g} / \mathrm{kg}$
$\mu \mathrm{g} / \mathrm{kg}$
$\mu \mathrm{g} / \mathrm{kg}$
$\mu \mathrm{g} / \mathrm{kg}$
$\mu \mathrm{g} / \mathrm{kg}$
$\mu \mathrm{g} / \mathrm{kg}$

$\mu g / \mathrm{kg}$

$\mu \mathrm{g} / \mathrm{kg}$

$\mu \mathrm{g} / \mathrm{kg}$

$\mu g / k g$

$\mu \mathrm{g} / \mathrm{kg}$

$\mu \mathrm{g} / \mathrm{kg}$

$\mu \mathrm{g} / \mathrm{kg}$

$\mu \mathrm{g} / \mathrm{kg}$

$\mu \mathrm{g} / \mathrm{kg}$

$\mu g / k g$

$\mu \mathrm{g} / \mathrm{kg}$

$\mu \mathrm{g} / \mathrm{kg}$

$\mu \mathrm{g} / \mathrm{kg}$

$\mu \mathrm{g} / \mathrm{kg}$

$\mu \mathrm{g} / \mathrm{kg}$

$\mu \mathrm{g} / \mathrm{kg}$

$\mu \mathrm{g} / \mathrm{kg}$

$\mu \mathrm{g} / \mathrm{kg}$

$\mu \mathrm{g} / \mathrm{kg}$

$\mu \mathrm{g} / \mathrm{kg}$

$\mu \mathrm{g} / \mathrm{kg}$

$\mu \mathrm{g} / \mathrm{kg}$

$\mu \mathrm{g} / \mathrm{kg}$

$\mu \mathrm{g} / \mathrm{kg}$

$\mu \mathrm{g} / \mathrm{kg}$

$\mu \mathrm{g} / \mathrm{kg}$

$\mu \mathrm{g} / \mathrm{kg}$

$\mu \mathrm{g} / \mathrm{kg}$

$\mu \mathrm{g} / \mathrm{kg}$

$\mu g / k g$

$\mu g / k g$

$\mu \mathrm{g} / \mathrm{kg}$

$\mu \mathrm{g} / \mathrm{kg}$

$\mu \mathrm{g} / \mathrm{kg}$

$\mu \mathrm{g} / \mathrm{kg}$
6.6

6.6

6.6

6.6

6.6

6.6

6.6

6.6

6.6

6.6

13

13

13

13

13

13

13

13

13

6.6

6.6

6.6

6.6

6.6

6.6

6.6

6.6

6.6

6.6

6.6

6.6

6.6

6.6

6.6

6.6

$=6.6$

13.

13

13

6.6

6.6

6.6

$\mu \mathrm{g} / \mathrm{kg}$

$\mu g / \mathrm{kg}$

$\mu \mathrm{g} / \mathrm{kg}$

$\mu \mathrm{g} / \mathrm{kg}$

$\mu \mathrm{g} / \mathrm{kg}$
EPA8240

EPA8240

EPA8240

EPA8240

EPA8240

EPA8240

EPA8240

EPA8240

EPA8240

EPA8240

EPA8240

EPA8240

EPA8240

EPA8240

EPA8240

EPA8240

EPA8240

EPA8240

EPA8240

EPA8240

EPA8240

EPA8240

EPA8240

EPA8240

EPA8240

EPA8240

EPA8240

EPA8240

EPA8240

EPA8240

EPA8240

EPA8240

EPA8240

EPA8240

EPA8240

EPA8240

EPA8240

EPA8240

EPA8240

EPA8240

EPA8240

EPA8240

Pesticides/PCBs and Dioxins/Furans

Aldrin

beta-Benzene hexachloride

delta-Benzene hexachloride

alpha-Chlordane
$U$
$U$
$U$
$U$
$U$
2.2

2.2

2.2

$\begin{array}{ll}2.2 & \text { EPA8080 } \\ 2.2 & \text { EPA8080 } \\ 2.2 & \text { EPA8080 } \\ 2.2 & \text { EPA8080 } \\ 2.2 & \text { EPA8080 }\end{array}$


SAMPLE NAME: D43111005 (Continued)

Constituent

RQ

$A Q$

Sample ID: 100356

Pesticides/PCBs and Dioxins/Furans

\begin{tabular}{|c|c|c|c|}
\hline \\
\hline 2.2 & $\mu \mathrm{g} / \mathrm{kg}$ & 2.2 & EPA8080 \\
\hline 4.4 & $\mu \mathrm{g} / \mathrm{kg}$ & 4.4 & EPA8080 \\
\hline 4.4 & $\mu \mathrm{g} / \mathrm{kg}$ & 4.4 & EPA8080 \\
\hline 4.4 & $\mu \mathrm{g} / \mathrm{kg}$ & 4.4 & EPA8080 \\
\hline 4.4 & $\mu \mathrm{g} / \mathrm{kg}$ & 4.4 & EPA8080 \\
\hline 2.2 & $\mu \mathrm{g} / \mathrm{kg}$ & 2.2 & EPA8080 \\
\hline 4.4 & $\mu \mathrm{g} / \mathrm{kg}$ & 4.4 & EPA8080 \\
\hline 4.4 & $\mu \mathrm{g} / \mathrm{kg}$ & 4.4 & EPA8080 \\
\hline 4.4 & $\mu \mathrm{g} / \mathrm{kg}$ & 4.4 & EPA8080 \\
\hline 4.4 & $\mu \mathrm{g} / \mathrm{kg}$ & 4.4 & EPA8080 \\
\hline 2.2 & $\mu \mathrm{g} / \mathrm{kg}$ & 2.2 & EPA8080 \\
\hline 2.2 & $\mu \mathrm{g} / \mathrm{kg}$ & 2.2 & EPA8080 \\
\hline 0.20 & $\mathrm{ng} / \mathrm{g}$ & 0.20 & EPA8280 \\
\hline 0.10 & $\mathrm{ng} / \mathrm{g}$ & 0.10 & EPA8280 \\
\hline 0.20 & $\mathrm{ng} / \mathrm{g}$ & 0.20 & EPA8280 \\
\hline 0.10 & $\mathrm{ng} / \mathrm{g}$ & 0.10 & EPA8280 \\
\hline 2.2 & $\mu \mathrm{g} / \mathrm{kg}$ & 2.2 & EPA8080 \\
\hline 22 & $\mu \mathrm{g} / \mathrm{kg}$ & 22 & EPA8080 \\
\hline 2.5 & $\mathrm{ng} / \mathrm{g}$ & 0.30 & EPA8280 \\
\hline 0.10 & $\mathrm{ng} / \mathrm{g}$ & 0.10 & EPA8280 \\
\hline 44 & $\mu \mathrm{g} / \mathrm{kg}$ & 44 & EPA8080 \\
\hline 87 & $\mu \mathrm{g} / \mathrm{kg}$ & 87 & EPA8080 \\
\hline 44 & $\mu \mathrm{g} / \mathrm{kg}$ & 44 & EPA8080 \\
\hline 44 & $\mu \mathrm{g} / \mathrm{kg}$ & 44 & EPA8080 \\
\hline 44 & $\mu \mathrm{g} / \mathrm{kg}$ & 44 & EPA8080 \\
\hline 44 & $\mu \mathrm{g} / \mathrm{kg}$ & 44 & EPA8080 \\
\hline 44 & $\mu \mathrm{g} / \mathrm{kg}$ & 44 & EPA8080 \\
\hline 0.20 & $\mathrm{ng} / \mathrm{g}$ & 0.20 & EPA8280 \\
\hline 0.10 & $\mathrm{ng} / \mathrm{g}$ & 0.10 & EPA8280 \\
\hline 0.20 & $\mathrm{ng} / \mathrm{g}$ & 0.20 & EPA8280 \\
\hline 0.10 & $\mathrm{ng} / \mathrm{g}$ & 0.10 & EPA8280 \\
\hline 0.20 & $\mathrm{ng} / \mathrm{g}$ & 0.20 & EPA8280 \\
\hline 0.10 & $\mathrm{ng} / \mathrm{g}$ & 0.10 & EPA8280 \\
\hline 220 & $\mu \mathrm{g} / \mathrm{kg}$ & 220 & EPA8080 \\
\hline
\end{tabular}

Radionuclide Indicators and Radionuclides
Gross alpha

Nonvolatile beta

Radium, total alpha-emitting

Tritium
$\mathrm{U}$

U

U

U

U

Endosulfan I

Endosulfan II

Endosulfan sulfate

Endrin ketone

Heptachlor

Heptachlorodibenzo-p-dioxin isomers

Heptachlorodibenzo-p-furan isomers

Hexachlorodibenzo-p-dioxin isomers

Lindane

Methoxychlor

Octochloribenzo-p-dioxin

PCB 1016

PCB 1221

PCB 1248

CB 1254

Pentachlorodibenzo-p-dioxin isomers

Pentachlorodibenzo-p-furan isomers

2,3,7,8-TCDF

Tetrachlorodibenzo-p-dioxin isomers

Tetrachlorodibenzo-p-furan isomers

Toxaphene

$\mathrm{U}$

U $\quad \mathrm{v}$

Result

Unit

D. Limit Method

$\begin{array}{llll}6.7 & \mathrm{pCi} / \mathrm{g} & 6.7 & \text { EPA990.0 } \\ 25 \pm 18 & \mathrm{pCi} / \mathrm{g} & 8.4 & \text { EPA900.0 } \\ 2.7 \pm 0.99 & \mathrm{pCi} / \mathrm{g} & 0.21 & \text { EPA903.0 } \\ 1.4 & \mathrm{pCi} / \mathrm{g} & 1: 4 & \text { EPA906.0 }\end{array}$


SAMPLE NAME: D43111006

Location (SRS Coordinates): 18482.04E 66700.55N

Ground Elevation Above MSL: $129.5 \mathrm{ft}$

Depth of Core Interval: 8.00 to $10.00 \mathrm{ft}$

Sample Type: Norma!

Sample Color: 10R 3/4

Constituent

Physical Parameters

Cation exchange capacity

$\mathrm{pH}$

Specific conductance

Specific conductance

Total organic carbon

Total Metals

Arsenic

Barium

Cadmium

Chromium

Copper

Lead

Manganese .

Mercury

Nickel

Selenium

Silver
Sample ID: 100357

Sample Matrix: Soil

$\begin{array}{ll}\text { Sample Matrix: Soil } & \text { Sample Moisture: Damp } \\ \text { USC Soil Classification: SM } \quad \text { Percent Solids: } 80.00\end{array}$

RQ AQ B Result Unit D. Limit Method

$\begin{array}{llll}8.6 & \mathrm{meq} / 100 \mathrm{~g} & 2.0 & \text { EPA9080 } \\ 4.7 & \mathrm{pH} & 0.010 & \text { EPA9045 } \\ 28 & \mu \mathrm{S} / \mathrm{cm} & 1.0 & \text { EPA9050 } \\ 28 & \mu \mathrm{S} / \mathrm{cm} & 1.0 & \text { EPA9050 } \\ 1.600 & \mathrm{mg} / \mathrm{kg} & 220 & \text { EPA415.1 }\end{array}$

$B / N / A$ Extractables (including $P A H$ and phenols)

Acenaphthene
Acenaphthylene
Anthracene
Benzo(a)anthracene
Benzo(b)fluoranthene
Benzo(k)fluoranthene
Benzoic acid
Benzo(g,h,i)perylene
Benzo(a)pyrene
Benzyl alcohol
Bis(2-chloroethoxy) methane
Bis(2-chloroethyl) ether
Bis(2-chioroisopropyl) ether
Bis(2-ethylhexyl) phthaiate
4-Bromophenyl phenyl ether
Butylbenzyl phthalate
4-Chloroaniline
4-Chloro-m-cresol
2-Chloronaphthalene
2-Chlorophenol
4-Chlorophenyl phenyl ether
Chrysene

Acenaphthene

Anthracene

Benzo(a)anthracene

Benzo(k)fluoranthene

Benzoic acid

Benzyl alcohol

Bis(2-chloroethoxy) methane

Bis(2-chloroethyl) ether

Bis(2-chloroisopropyl) ether

4-Bromophenyl phenyl ether

Butylbenzyl phthalate

2-Chloronaphthalene

4-Chlorophenyl phenyl ether

Chrysene

$\begin{array}{ll}U & 420 \\ U & 420 \\ U & 420 \\ U & 420 \\ U & 420 \\ U & 420 \\ U & 2,100 \\ U & 420 \\ U & 420 \\ U & 420 \\ U & 420 \\ U & 420 \\ U & 420 \\ U & 420 \\ U & 420 \\ U & 420 \\ U & 420 \\ U & 420 \\ U & 420 \\ U & 420 \\ U & 420 \\ U & 420\end{array}$

1.3

26

0.57

19

2.9

2.9

410

0.13

5.8

0.62

1.1

$\mathrm{mg} / \mathrm{kg}$
$\mathrm{mg} / \mathrm{kg}$
$\mathrm{mg} / \mathrm{kg}$
$\mathrm{mg} / \mathrm{kg}$
$\mathrm{mg} / \mathrm{kg}$
$\mathrm{mg} / \mathrm{kg}$
$\mathrm{mg} / \mathrm{kg}$
$\mathrm{mg} / \mathrm{kg}$
$\mathrm{mg} / \mathrm{kg}$
$\mathrm{mg} / \mathrm{kg}$
$\mathrm{mg} / \mathrm{kg}$

$\begin{array}{ll}1.2 & \text { EPA7060 } \\ 23 & \text { EPA6010 } \\ 0.57 & \text { EPA6010 } \\ 1.1 & \text { EPA6010 } \\ 2.9 & \text { EPA6010 } \\ 0.37 & \text { EPA7421 } \\ 1.7 & \text { EPA6010 } \\ 0.13 & \text { EPA7470 } \\ 4.6 & \text { EPA6010 } \\ 0.62 & \text { EPA7740 } \\ 1.1 & \text { EPA6010 }\end{array}$

20

420

420

420

420

2,100

420

420

420

420

420

420

420

420

20

420

420

20

420 $\mu g / k g$
$\mu g / k g$
$\mu g / k g$
$\mu g / k g$
$\mu g / k g$
$\mu g / k g$
$\mu g / k g$
$\mu g / k g$
$\mu g / k g$
$\mu g / k g$
$\mu g / k g$
$\mu g / k g$
$\mu g / k g$
$\mu g / k g$
$\mu g / k g$
$\mu g / k g$
$\mu g / k g$
$\mu g / k g$
$\mu g / k g$
$\mu g / k g$
$\mu g / k g$
$\mu g / k g$

EPA8270

EPA8270

EPA8270

EPA8270

EPA8270

EPA8270

EPA8270

EPA8270

EPA8270

EPA8270

EPA8270

EPA8270

EPA8270

EPA8270

EPA8270

EPA8270

EPA8270

EPA8270

EPA8270

EPA8270

EPA8270

EPA8270 
SAMPLE NAME: D43111006 (Continued)

RQ AQ B Result

Constituent
Sample ID: 100357

Unit

D. Limit Method

$B / N / A$ Extractables (including $P A H$ and phenols)

o-Cresol (2-Methylphenol)

p-Cresol (4-Methylphenol)

Dibenz(a,h)anthracene

Dibenzofuran

Di-n-butyl phthalate

1.2-Dichlorobenzene

1.3-Dichlorobenzene

1,4-Dichlorobenzene

3.3'-Dichlorobenzidine

2,4-Dichlorophenol

Diethyl phthalate

2.4-Dimethyl phenol

Dimethyl phthalate

2,4-Dinitrophenol

2,4-Dinitrotoluene

2,6-Dinitrotoluene

Di-n-octyl phthalate

Fluoranthene

Fluorene

Hexachlorobenzene

Hexachlorobutadiene

Hexachlorocyclopentadiene

Hexachloroethane

Indeno(1,2,3-c,d)pyrene

Isophorone

2-Methyl-4,6-dinitrophenol

2-Methylnaphthalene

Naphthalene

o-Nitroaniline

$\mathrm{m}$-Nitroaniline

p-Nitroaniline

Nitrobenzene

2-Nitrophenol

4-Nitrophenol

$\mathrm{N}$-Nitrosodiphenylamine

$\mathrm{N}$-Nitrosodipropylamine

Pentachlorophenol

Phenanthrene

Phenol

Pyrene

1,2,4-Trichlorobenzene

2,4,5-Trichlorophenol

2,4,6-Trichlorophenol

Unknown hydrocarbon

Unknown hydrocarbon

Unknown hydrocarbon

Unknown ketone

Unknown phthalate

Unknown phthalate

Unknown phthalate

$$
\text { U }
$$

U 420

$\mathrm{U} \quad 420$

U 420

UJ $\mathrm{V} \quad 190$

U 420

$\mathrm{U} .420$

$\mathrm{U} \quad 420$

U $\quad 840$

U 420

$\mathrm{U}-420$

U 420

$\mathrm{U} \quad 420$

U 2.100

U 420

$\mathrm{U} \quad 420$

520

420

420

420

420

420

420

420

420

2,100

420

420

2,100

2,100

2,100

420

420

2,100

420

420

UJ C 2,100

$\mathrm{U} \quad 420$

UJ C 420

U 420

420
420

420
2,100

420

200

200

200

10,000

200

300

300
500

\begin{tabular}{|c|c|c|}
\hline$\mu \mathrm{g} / \mathrm{kg}$ & 420 & EPA8270 \\
\hline$\mu g / \mathrm{kg}$ & 420 & EPA8270 \\
\hline$\mu \mathrm{g} / \mathrm{kg}$ & 420 & EPA8270 \\
\hline$\mu g / \mathrm{kg}$ & 420 & EPA8270 \\
\hline$\mu g / \mathrm{kg}$ & 420 & EPA8270 \\
\hline$\mu g / \mathrm{kg}$ & 420 & EPA8270 \\
\hline$\mu \mathrm{g} / \mathrm{kg}$ & 420 & EPA8270 \\
\hline$\mu \mathrm{g} / \mathrm{kg}$ & 420 & EPA8270 \\
\hline$\mu \mathrm{g} / \mathrm{kg}$ & 840 & EPA8270 \\
\hline$\mu g / \mathrm{kg}$ & 420 & EPA8270 \\
\hline$\mu g / \mathrm{kg}$ & 420 & EPA8270 \\
\hline$\mu \mathrm{g} / \mathrm{kg}$ & 420 & EPA8270 \\
\hline$\mu \mathrm{g} / \mathrm{kg}$ & 420 & EPA8270 \\
\hline$\mu g / \mathrm{kg}$ & 2.100 & EPA8270 \\
\hline$\mu g / \mathrm{kg}$ & 420 & EPA8270 \\
\hline$\mu \mathrm{g} / \mathrm{kg}$ & 420 & EPA8270 \\
\hline$\mu \mathrm{g} / \mathrm{kg}$ & 420 & EPA8270 \\
\hline$\mu \mathrm{g} / \mathrm{kg}$ & 420 & EPA8270 \\
\hline$\mu \mathrm{g} / \mathrm{kg}$ & 420 & EPA8270 \\
\hline$\mu g / \mathrm{kg}$ & 420 & EPA8270 \\
\hline$\mu \mathrm{g} / \mathrm{kg}$ & 420 & EPA8270 \\
\hline$\mu \mathrm{g} / \mathrm{kg}$ & 420 & EPA8270 \\
\hline$\mu \mathrm{g} / \mathrm{kg}$ & 420 & EPA8270 \\
\hline$\mu \mathrm{g} / \mathrm{kg}$ & 420 & EPA8270 \\
\hline$\mu \mathrm{g} / \mathrm{kg}$ & 420 & EPA8270 \\
\hline$\mu \mathrm{g} / \mathrm{kg}$ & 2,100 & EPA8270 \\
\hline$\mu g / \mathrm{kg}$ & 420 & EPA8270 \\
\hline$\mu g / \mathrm{kg}$ & 420 & EPA8270 \\
\hline$\mu \mathrm{g} / \mathrm{kg}$ & 2,100 & EPA8270 \\
\hline$\mu \mathrm{g} / \mathrm{kg}$ & 2.100 & EPA8270 \\
\hline$\mu g / \mathrm{kg}$ & 2,100 & EPA8270 \\
\hline$\mu \mathrm{g} / \mathrm{kg}$ & 420 & EPA8270 \\
\hline$\mu g / \mathrm{kg}$ & 420 & EPA8270 \\
\hline$\mu g / \mathrm{kg}$ & 2,100 & EPA8270 \\
\hline$\mu g / \mathrm{kg}$ & 420 & EPA8270 \\
\hline$\mu \mathrm{g} / \mathrm{kg}$ & 420 & EPA8270 \\
\hline$\mu g / \mathrm{kg}$ & 2,100 & EPA8270 \\
\hline$\mu \mathrm{g} / \mathrm{kg}$ & 420. & EPA8270 \\
\hline$\mu g / \mathrm{kg}$ & $420^{\circ}$ & EPA8270 \\
\hline$\mu g / \mathrm{kg}$ & 420 & EPA8270 \\
\hline$\mu \mathrm{g} / \mathrm{kg}$ & 420 & EPA8270 \\
\hline$\mu \mathrm{g} / \mathrm{kg}$ & 2,100 & EPA8270 \\
\hline$\mu \mathrm{g} / \mathrm{kg}$ & 420 & EPA8270 \\
\hline$\mu g / \mathrm{kg}$ & & EPA8270 \\
\hline$\mu \mathrm{g} / \mathrm{kg}$ & & EPA8270 \\
\hline$\mu g / \mathrm{kg}$ & & EPA8270 \\
\hline$\mu \mathrm{g} / \mathrm{kg}$ & & EPA8270 \\
\hline$\mu g / k g$ & & EPA8270 \\
\hline$\mu \mathrm{g} / \mathrm{kg}$ & & EPA8270 \\
\hline$\mu \mathrm{g} / \mathrm{kg}$ & & EPA8270 \\
\hline
\end{tabular}


SAMPLE NAME: D43111006 (Continued)

Constituent

Sample ID: 100357

$B / N / A$ Extractables (including $P A H$ and phenols)

Unknown phthalate

Unknown phthalate

Unknown phehalate

Unknown phthalate

Unknown phthalate

Unknown phthalate

Unknown phthalate

Volatile Organic Compounds

Acetone
Benzene
Bromodichloromethane
Bromoform
Bromomethane (Methyl bromide)
Carbon disulfide
Carbon tetrachloride
Chlorobenzene
Chloroethane
Chloroethene (Vinyl chloride)
Chloroform
Chloromethane (Methyl chloride)
Dibromochloromethane
1,1-Dichloroethane
1,2-Dichloroethane
1,1-Dichloroethylene
1,2-Dichloroethylene
Dichloromethane (Methylene chloride)
1,2-Dichloropropane
cis-1,3-Dichloropropene
trans-1,3-Dichloropropene
Ethylbenzene
2-Hexanone
Methyl ethyl ketone
Methyl isobutyl ketone
Styrene
1,1,2,2-Tetrachloroethane
Tetrachloroethylene
Toluene
1,1,1-Trichlorocthane
1,1,2-Trichloroethane
Trichloroethylene
Vinyl acetate
Xylenes

Pesticides/PCBs and Dioxins/Furans

Aldrin

alpha-Benzene hexachloride

beta-Benzene hexachloride
RQ AQ

$\begin{array}{lll}\text { J } & \mathbf{N} & 700 \\ \mathbf{J} & \mathbf{N} & 500 \\ \mathrm{~J} & \mathbf{N} & 200 \\ \mathbf{J} & \mathbf{N} & 500 \\ \mathrm{~J} & \mathbf{N} & 800 \\ \mathbf{J} & \mathbf{N} & 300 \\ \mathrm{~J} & \mathbf{N} & 300\end{array}$

700
500
200
500
800
300
300

$\mu \mathrm{g} / \mathrm{kg}$

$\mu \mathrm{g} / \mathrm{kg}$

$\mu \mathrm{g} / \mathrm{kg}$

$\mu \mathrm{g} / \mathrm{kg}$

$\mu g / \mathrm{kg}$

$\mu \mathrm{g} / \mathrm{kg}$

$\mu g / \mathrm{kg}$

D. Limit Method
EPA8270

EPA8270

EPA8270

EPA8270

EPA8270

EPA8270

EPA8270

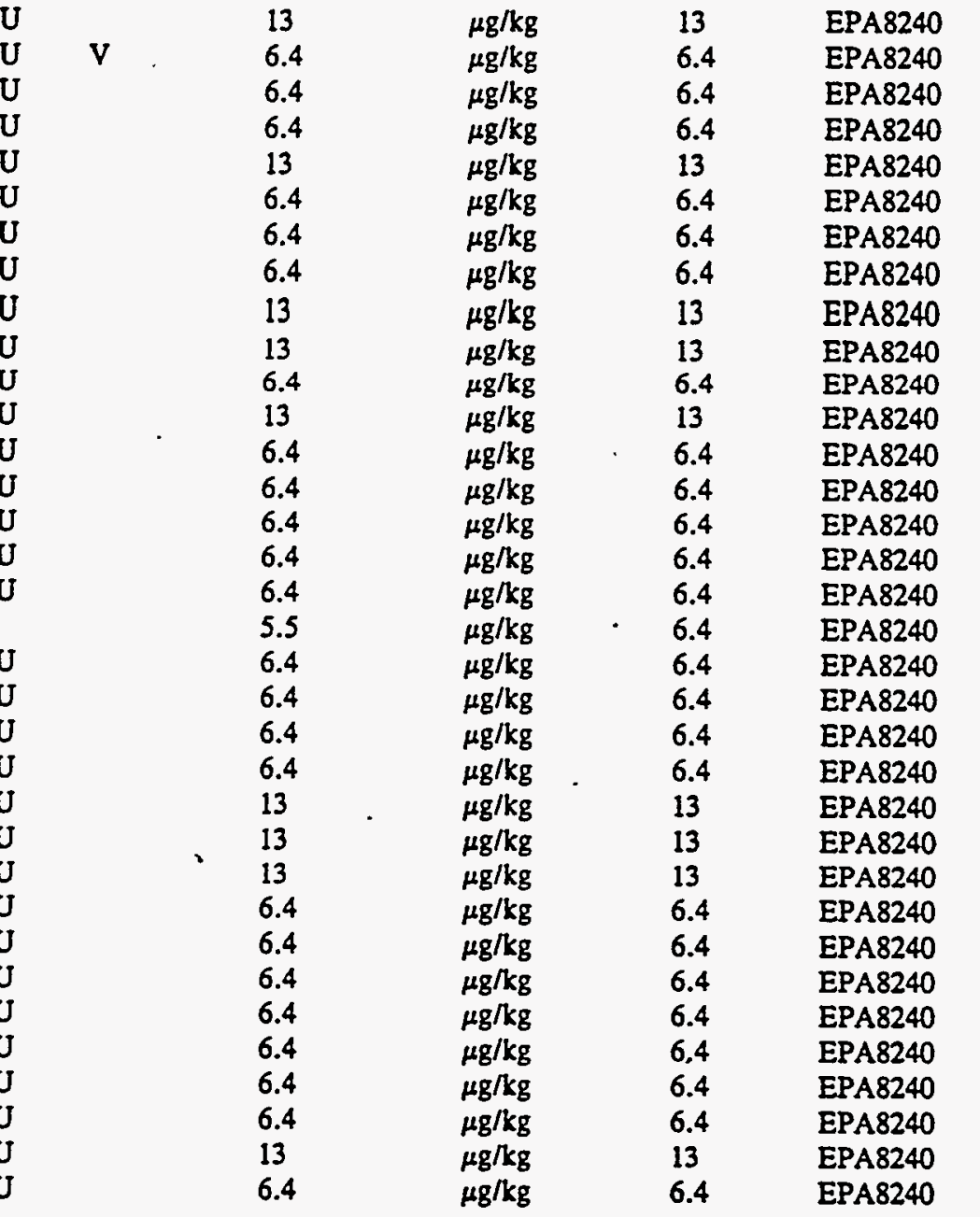


SAMPLE NAME: D43111006 (Continued)

Constituent

RQ AQ

Sample ID: 100357

B Result

2.1

2.1

2.1

4.2

4.2

4.2

4.2

2.1

4.2

4.2

4.2

4.2

2.1

2.1

0.10

0.10

0.10

0.10

2.1

21

0.20

0.10

42

84

42

42

42

42

42

0.20

0.10

0.10

0.10

0.10

0.10

210

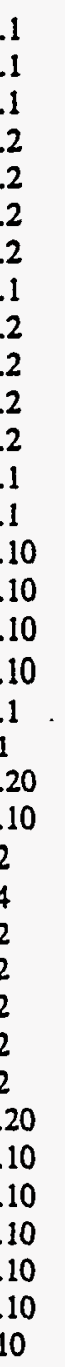

p.p'-DDD
p.p'-DDE
p.p'-DDT
Dieldrin
Endosulfan I
Endosulfan II
Endosulfan sulfate
Endrin
Endrin ketone
Heptachlor
Heptachlor epoxide
Heptachlorodibenzo-p-dioxin isomers
Heptachlorodibenzo-p-furan isomers
Hexachlorodibenzo-p-dioxin isomers
Hexachlorodibenzo-p-furan isomers
Lindane
Methoxychlor
Octachlorodibenzo-p-dioxin
Octachlorodibenzo-p-furan
PCB 1016
PCB 1221
PCB 1232
PCB 1242
PCB 1248
PCB 1254
PCB 1260
Pentachlorodibenzo-p-dioxin isomers
Pentachlorodibenzo-p-furan isomers
$2,3,7,8-T C D D$
$2,3,7,8-T C D F$
Tetrachlorodibenzo-p-dioxin isomers
Tetrachlorodibenzo-p-furan isomers
Toxaphene

Unit:

D. Limit Method

$\because$

Radionuclide Indicators and Radionuclides

Gross alpha

Gross alpha

Nonvolatile beta

Nonvolatile beta

Radium, total alpha-emitting

Radium, total alpha-emitting

Tritium

Tritium

$\begin{array}{llll}16 \pm 14 & \mathrm{pCi} / \mathrm{g} & 7.3 & \text { EPA900.0 } \\ 8.5 & \mathrm{pCi} / \mathrm{g} & 8.5 & \text { EPA900.0 } \\ 43 \pm 21 & \mathrm{pCi} / \mathrm{g} & 13 & \text { EPA900.0 } \\ 60 \pm 27 & \mathrm{pCi} / \mathrm{g} & 16 & \text { EPA900.0 } \\ 1.1 \pm 0.81 & \mathrm{pCi} / \mathrm{g} & 0.21 & \text { EPA903.0 } \\ 0.53 \pm 0.52 & \mathrm{pCi} / \mathrm{g} & 0.18 & \text { EPA903.0 } \\ 1.4 & \mathrm{pCi} / \mathrm{g} & 1.4 & \text { EPA906.0 } \\ 3.1 \pm 2.7 & \mathrm{pCi} / \mathrm{g} & 1.4 & \text { EPA906.0 }\end{array}$

EPA8080

EPA8080

$\begin{array}{lll}\mu \mathrm{g} / \mathrm{kg} \cdot & 2.1 & \text { EPA8080 } \\ \mu \mathrm{g} / \mathrm{kg} & 2.1 & \text { EPA8080 }\end{array}$

$\begin{array}{lll}\mu \mathrm{g} / \mathrm{kg} & 4.2 & \text { EPA8080 }\end{array}$

$\mu g / \mathrm{kg} \quad 4.2 \quad$ EPA8080

$\mu \mathrm{g} / \mathrm{kg} \quad 4.2 \quad$ EPA8080

$\mu \mathrm{g} / \mathrm{kg} \quad 4.2 \quad$ EPA8080

$\mu \mathrm{g} / \mathrm{kg} \quad 2.1 \quad$ EPA8080

$\mu g / \mathrm{kg} \quad 4.2 \quad$ EPA8080

$\mu \mathrm{g} / \mathrm{kg} \quad 4.2 \quad$ EPA8080

$\mu \mathrm{g} / \mathrm{kg} \quad 4.2 \quad$ EPA8080

$\mu \mathrm{g} / \mathrm{kg} \quad 4.2 \quad$ EPA8080

$\mu \mathrm{g} / \mathrm{kg} \quad 2.1 \quad$ EPA8080

$\mu \mathrm{g} / \mathrm{kg} \quad 2.1 \quad$ EPA8080

ng/g $\quad 0.10 \quad$ EPA8280

$\mathrm{ng} / \mathrm{g} \quad 0.10 \quad$ EPA8280

ng/g $\quad 0.10 \quad$ EPA8280

$\mathrm{ng} / \mathrm{g} \quad 0.10 \quad$ EPA8280

$\mu g / \mathrm{kg} \quad 2.1 \quad$ EPA8080

$\mu \mathrm{g} / \mathrm{kg} \quad 21 \quad$ EPA8080

$\begin{array}{lll}\text { ng/g } & 0.20 & \text { EPA8280 }\end{array}$

$\begin{array}{lll}\mathrm{ng} / \mathrm{g} & 0.10 \quad \text { EPA8280 }\end{array}$

$\mu \mathrm{g} / \mathrm{kg} \quad 42 \quad$ EPA8080

$\mu \mathrm{g} / \mathrm{kg} \quad 84 \quad$ EPA8080

$\mu \mathrm{g} / \mathrm{kg} \quad 42 \quad$ EPA8080

$\mu \mathrm{g} / \mathrm{kg} \quad 42 \quad$ EPA8080

$\mu \mathrm{g} / \mathrm{kg} \quad 42 \quad$ EPA8080

$\mu \mathrm{g} / \mathrm{kg} \quad 42 \quad$ EPA8080

$\mu \mathrm{g} / \mathrm{kg} \quad 42 \quad$ EPA8080

ng/g $\quad 0.20 \quad$ EPA8280

$\begin{array}{lll}\mathrm{ng} / \mathrm{g} & 0.10 \quad \text { EPA8280 }\end{array}$

$\begin{array}{lll}\mathrm{ng} / \mathrm{g} & 0.10 \quad \text { EPA8280 }\end{array}$

ng/g $\quad 0.10 \quad$ EPA8280

$\mathrm{ng} / \mathrm{g} \quad 0.10 \quad$ EPA8280

$\begin{array}{lll}\mathrm{ng} / \mathrm{g} & 0.10 \quad \text { EPA8280 }\end{array}$

$\mu \mathrm{g} / \mathrm{kg} \quad 210 \quad$ EPA8080 
SAMPLE NAME: D43111006D

Sample Type: Duplicate

Constituent

Physical Parameters

Cation exchange capacity

$\mathrm{pH}$

Specific conductance

Total organic carbon

\section{Total Metals}

Arsenic
Barium
Cadmium
Chromium
Copper
Lead
Manganese
Mercury
Nickel
Selenium ,
Silver

$B / N / A$ Extractables (including $P A H$ and phenols)
U

UJ L

J L

U

$\mathrm{U}$

U

Sample ID: 100358

Associated Sample: 100357

RQ AQ B Result Unit D. Limit Method

8.4

4.9

33

1,300

$\begin{array}{lll}\text { meq/100g } & 2.0 & \text { EPA9080 } \\ \mathrm{pH} & 0.010 & \text { EPA9045 } \\ \mu \mathrm{S} / \mathrm{cm} & 1.0 & \text { EPA9050 } \\ \mathrm{mg} / \mathrm{kg} & 250 & \text { EPA415.1 }\end{array}$

1.0

36

0.57

22

2.9

3.2

460

0.12

4.6

0.51

1.1 $\mathrm{mg} / \mathrm{kg}$
$\mathrm{mg} / \mathrm{kg}$
$\mathrm{mg} / \mathrm{kg}$
$\mathrm{mg} / \mathrm{kg}$
$\mathrm{mg} / \mathrm{kg}$
$\mathrm{mg} / \mathrm{kg}$
$\mathrm{mg} / \mathrm{kg}$
$\mathrm{mg} / \mathrm{kg}$
$\mathrm{mg} / \mathrm{kg}$
$\mathrm{mg} / \mathrm{kg}$
$\mathrm{mg} / \mathrm{kg}$

$\mu \mathrm{g} / \mathrm{kg}$

$\mu \mathrm{g} / \mathrm{kg}$

$\mu \mathrm{g} / \mathrm{kg}$

$\mu \mathrm{g} / \mathrm{kg}$

$\mu \mathrm{g} / \mathrm{kg}$

$\mu \mathrm{g} / \mathrm{kg}$

$\mu \mathrm{g} / \mathrm{kg}$

$\mu \mathrm{g} / \mathrm{kg}$

$\mu g / k g$

$\mu \mathrm{g} / \mathrm{kg}$

$\mu \mathrm{g} / \mathrm{kg}$

$\mu \mathrm{g} / \mathrm{kg}$

$\mu \mathrm{g} / \mathrm{kg}$

$\mu \mathrm{g} / \mathrm{kg}$

$\mu \mathrm{g} / \mathrm{kg}$

$\mu \mathrm{g} / \mathrm{kg}$

$\mu g / \mathrm{kg}$

$\mu \mathrm{g} / \mathrm{kg}$

$\mu \mathrm{g} / \mathrm{kg}$

$\mu \mathrm{g} / \mathrm{kg}$

$\mu \mathrm{g} / \mathrm{kg}$

$\mu \mathrm{g} / \mathrm{kg}$

$\mu \mathrm{g} / \mathrm{kg}$

$\mu \mathrm{g} / \mathrm{kg}$

$\mu \mathrm{g} / \mathrm{kg}$

$\mu \mathrm{g} / \mathrm{kg}$

$\mu \mathrm{g} / \mathrm{kg}$

$\begin{array}{ll}1.0 & \text { EPA7060 } \\ 23 & \text { EPA6010 } \\ 0.57 & \text { EPA6010 } \\ 1.1 & \text { EPA6010 } \\ 2.9 & \text { EPA6010 } \\ 0.31 & \text { EPA7421 } \\ 1.7 & \text { EPA6010 } \\ 0.12 & \text { EPA7470 } \\ 4.6 & \text { EPA6010 } \\ 0.51 & \text { EPA7740 } \\ 1.1 & \text { EPA6010 }\end{array}$

o-Cresol (2-Methylphenol)

p-Cresol (4-Methylphenol)

Dibenzofuran

Di-n-butyl phthalate
U 420

$U \quad 420$

UJ V. $\quad 170$
420

420

420

420

420

420

2,100

420

420

420

420

420

420

420

420

420

420

420

420

420

420

420

420

420

420

420

420
EPA8270

EPA8270

EPA8270

EPA8270

EPA8270

EPA8270

EPA8270

EPA8270

EPA8270

EPA8270

EPA8270

EPA8270

EPA8270

EPA8270

EPA8270

EPA8270

EPA8270

EPA8270

EPA8270

EPA8270

EPA8270

EPA8270

EPA8270

EPA8270

EPA8270

EPA8270

EPA8270 
SAMPLE NAME: D43111006D (Continued) · Sample ID: 100358

Constituent RQ AQ B Result Unit D. Limit Method

$B / N / A$ Extractables (including $P A H$ and phenols)

1.2-Dichlorobenzene

1,3-Dichlorobenzene

1,4-Dichlorobenzene

3,3'-Dichlorobenzidine

2,4-Dichlorophenol

Diethyl phthalate

2.4-Dimethyl phenol

Dimethyl phthalate

2.4-Dinitrophenol

2,4-Dinitrotoluene

2,6-Dinitrotoluene

Di-n-octyl phthalate

Fluoranthene

Fluorene

Hexachlorobenzene

Hexachlorobutadiene

Hexachlorocyclopentadiene

Hexachloroethane

Indeno(1,2.3-c,d)pyrene

Isophorone

2-Methyl-4,6-dinitrophenol

2-Methylnaphthalene

Naphthalene

o-Nitroaniline

m-Nitroaniline

p-Nitroaniline

Nitrobenzene

2-Nitrophenol

4-Nitrophenol

N-Nitrosodiphenylamine

N-Nitrosodipropylamine

Pentachlorophenol

Phenanthrene

Phenol

Pyrene

1,2,4-Trichlorobenzene

2,4,5-Trichlorophenol

2,4,6-Trichlorophenol

Unknown hydrocarbon

Unknown hydrocarbon

Unknown hydrocarbon

Unknown hydrocarbon

Unknown hydrocarbon

Unknown hydrocarbon

Unknown ketone

Unknown phthalate

Unknown phthalate

Unknown phthalate

Unknown phthalate

Unknown phthalate

\begin{tabular}{|c|c|c|c|}
\hline \multicolumn{2}{|l|}{$\mathbf{U}$} & 420 & $\mu \mathrm{g} / \mathrm{kg}$ \\
\hline \multicolumn{2}{|l|}{$\begin{array}{l}U \\
U\end{array}$} & 420 & $\mu \mathrm{g} / \mathrm{kg}$ \\
\hline \multicolumn{2}{|l|}{$U$} & 420 & $\mu \mathrm{g} / \mathrm{kg}$ \\
\hline \multicolumn{2}{|l|}{$\mathbf{U}$} & 830 & $\mu \mathrm{g} / \mathrm{kg}$ \\
\hline \multicolumn{2}{|l|}{$U$} & 420 & $\mu \mathrm{g} / \mathrm{kg}$ \\
\hline \multicolumn{2}{|l|}{ U } & 420 & $\mu \mathrm{g} / \mathrm{kg}$ \\
\hline \multicolumn{2}{|c|}{$\mathbf{U}$} & 420 & $\mu \mathrm{g} / \mathrm{kg}$ \\
\hline \multicolumn{2}{|l|}{$\mathbf{U}$} & 420 & $\mu \mathrm{g} / \mathrm{kg}$ \\
\hline \multicolumn{2}{|c|}{$\mathrm{U}$} & 2,100 & $\mu \mathrm{g} / \mathrm{kg}$ \\
\hline \multicolumn{2}{|c|}{$\mathrm{U}$} & 420 & $\mu \mathrm{g} / \mathrm{kg}$ \\
\hline \multicolumn{2}{|c|}{$\mathbf{U}$} & 420 & $\mu \mathrm{g} / \mathrm{kg}$ \\
\hline \multicolumn{2}{|l|}{3} & 46 & $\mu \mathrm{g} / \mathrm{kg}$ \\
\hline \multirow{2}{*}{\multicolumn{2}{|c|}{$\begin{array}{l}\mathbf{U} \\
\mathbf{U}\end{array}$}} & 420 & $\mu \mathrm{g} / \mathrm{kg}$ \\
\hline & & 420 & $\mu \mathrm{g} / \mathrm{kg}$ \\
\hline \multicolumn{2}{|l|}{$\mathrm{U}$} & 420 & $\mu \mathrm{g} / \mathrm{kg}$ \\
\hline \multicolumn{2}{|l|}{$\mathbf{U}$} & 420 & $\mu \mathrm{g} / \mathrm{kg}$ \\
\hline \multicolumn{2}{|c|}{$\mathbf{U}$} & 420 & $\mu \mathrm{g} / \mathrm{kg}$ \\
\hline \multicolumn{2}{|l|}{ U } & 420 & $\mu \mathrm{g} / \mathrm{kg}$ \\
\hline \multicolumn{2}{|l|}{$U$} & 420 & $\mu \mathrm{g} / \mathrm{kg}$ \\
\hline \multicolumn{2}{|l|}{$\mathrm{U}$} & 420 & $\mu \mathrm{g} / \mathrm{kg}$ \\
\hline \multicolumn{2}{|l|}{$\mathrm{U}$} & 2,100 & $\mu \mathrm{g} / \mathrm{kg}$ \\
\hline \multicolumn{2}{|l|}{ U } & 420 & $\mu \mathrm{g} / \mathrm{kg}$ \\
\hline \multicolumn{2}{|c|}{$\mathrm{U}$} & 420 & $\mu \mathrm{g} / \mathrm{kg}$ \\
\hline $\mathbf{U}$ & & 2,100 & $\mu g / k g$ \\
\hline $\mathbf{U}$ & & 2,100 & $\mu \mathrm{g} / \mathrm{kg}$ \\
\hline U & & 2,100 & $\mu \mathrm{g} / \mathrm{kg}$ \\
\hline $\mathbf{U}$ & & 420 & $\mu g / k g$ \\
\hline $\mathbf{U}$ & & 420 & $\mu \mathrm{g} / \mathrm{kg}$ \\
\hline $\mathbf{U}$ & & 2,100 & $\mu \mathrm{g} / \mathrm{kg}$ \\
\hline $\mathrm{U}$ & & 420 & $\mu \mathrm{g} / \mathrm{kg}$ \\
\hline $\mathbf{U}$ & & 420 & $\mu \mathrm{g} / \mathrm{kg}$ \\
\hline UJ & C & 2,100 & $\mu \mathrm{g} / \mathrm{kg}$ \\
\hline $\mathrm{U}$ & & 420 & $\mu \mathrm{g} / \mathrm{kg}$ \\
\hline UJ & C & 420 & $\mu \mathrm{g} / \mathrm{kg}$ \\
\hline $\mathbf{U}$ & & 420 & $\mu \mathrm{g} / \mathrm{kg}$ \\
\hline $\mathbf{U}$ & & 420 & $\mu \mathrm{g} / \mathrm{kg}$ \\
\hline $\mathbf{U}$ & & 2,100 & $\mu \mathrm{g} / \mathrm{kg}$ \\
\hline $\mathbf{U}$ & & 420 & $\mu g / \mathrm{kg}$ \\
\hline $\mathbf{J}$ & $\mathbf{N}$ & 200 & $\mu \mathrm{g} / \mathrm{kg}$ \\
\hline $\mathbf{J}$ & $\mathbf{N}$ & 200 & $\mu g / k g$ \\
\hline J & $\mathbf{N}$ & 400 & $\mu g / k g$ \\
\hline J & $\mathbf{N}$ & 300 & $\mu \mathrm{g} / \mathrm{kg}$ \\
\hline $\mathbf{J}$ & $\mathbf{N}$ & 200 & $\mu \mathrm{g} / \mathrm{kg}$ \\
\hline $\mathbf{J}$ & $\mathbf{N}$ & 200 & $\mu g / k g$ \\
\hline J & NV & 10,000 & $\mu \mathrm{g} / \mathrm{kg}$ \\
\hline $\mathbf{J}$ & $\mathbf{N}$ & 400 & $\mu \mathrm{g} / \mathrm{kg}$ \\
\hline $\mathbf{J}$ & $\mathbf{N}$ & 500 & $\mu \mathrm{g} / \mathrm{kg}$ \\
\hline J & $\mathbf{N}$ & 200 & $\mu g / \mathrm{kg}$ \\
\hline $\mathbf{J}$ & $\mathbf{N}$ & 400 & $\mu \mathrm{g} / \mathrm{kg}$ \\
\hline J & $\mathbf{N}$ & 300 & $\mu g / \mathrm{kg}$ \\
\hline
\end{tabular}
EPA8270
EPA8270
EPA8270
EPA8270
EPA8270
EPA8270
EPA8270
EPA8270
EPA8270
EPA8270
EPA8270
EPA8270
EPA8270
EPA8270
EPA8270
EPA8270
EPA8270
EPA8270
EPA8270
EPA8270
EPA8270
EPA8270
EPA8270
EPA8270
EPA8270
EPA8270
EPA8270
EPA8270
EPA8270
EPA8270
EPA8270
EPA8270
EPA8270
EPA8270
EPA8270
EPA8270
EPA8270
EPA8270
EPA8270
EPA8270
EPA8270
EPA8270
EPA8270
EPA8270
EPA8270
EPA8270
EPA8270
EPA8270
EPA8270
EPA8270 
SAMPLE NAME: D43111006D (Continued) ～Sample ID: 100358

$\begin{array}{llllll}\text { Constituent } & \text { RQ AQ B Result Unit Dimit Method }\end{array}$

$B / N / A$ Extractables (including $P A H$ and phenols)

Unknown phthalate

Unknown phthalate

Unknown phthalate

Volatile Organic Compounds

$\begin{array}{lllll}\mathrm{J} & \mathrm{N} & 200 & \mu \mathrm{g} / \mathrm{kg} & \text { EPA8270 } \\ \mathrm{J} & \mathrm{N} & 300 & \mu \mathrm{g} / \mathrm{kg} & \text { EPA8270 } \\ \mathrm{J} & \mathrm{N} & 600 & \mu \mathrm{g} / \mathrm{kg} & \text { EPA8270 }\end{array}$

$\mu \mathrm{g} / \mathrm{kg}$

$\mu \mathrm{g} / \mathrm{kg}$

$\mu \mathrm{g} / \mathrm{kg}$

$\mu \mathrm{g} / \mathrm{kg}$

$\mu \mathrm{g} / \mathrm{kg}$

$\mu \mathrm{g} / \mathrm{kg}$

$\mu \mathrm{g} / \mathrm{kg}$

$\mu g / \mathrm{kg}$

$\mu \mathrm{g} / \mathrm{kg}$

$\mu \mathrm{g} / \mathrm{kg}$

$\mu \mathrm{g} / \mathrm{kg}$

$\mu \mathrm{g} / \mathrm{kg}$

$\mu \mathrm{g} / \mathrm{kg}$

$\mu \mathrm{g} / \mathrm{kg}$

$\mu \mathrm{g} / \mathrm{kg}$

$\mu \mathrm{g} / \mathrm{kg}$

$\mu \mathrm{g} / \mathrm{kg}$

$\mu \mathrm{g} / \mathrm{kg}$

$\mu \mathrm{g} / \mathrm{kg}$

$\mu \mathrm{g} / \mathrm{kg}$

$\mu \mathrm{g} / \mathrm{kg}$

$\mu \mathrm{g} / \mathrm{kg}$

$\mu \mathrm{g} / \mathrm{kg}$

$\mu \mathrm{g} / \mathrm{kg}$

$\mu \mathrm{g} / \mathrm{kg}$

$\mu \mathrm{g} / \mathrm{kg}$

$\mu \mathrm{g} / \mathrm{kg}$

$\mu g / \mathrm{kg}$

$\mu \mathrm{g} / \mathrm{kg}$

$\mu \mathrm{g} / \mathrm{kg}$

$\mu \mathrm{g} / \mathrm{kg}$

$\mu \mathrm{g} / \mathrm{kg}$

$\mu \mathrm{g} / \mathrm{kg}$

$\mu \mathrm{g} / \mathrm{kg}$
EPA8270

EPA8240

EPA8240

EPA8240

EPA8240

EPA8240

EPA8240

EPA8240

EPA8240

EPA8240

EPA8240

EPA8240

EPA8240

EPA8240

EPA8240

EPA8240

EPA8240

EPA8240

EPA8240

EPA8240

EPA8240

EPA8240

EPA8240

EPA8240

EPA8240

EPA8240

EPA8240

EPA8240

EPA8240

EPA8240

EPA8240

EPA8240

EPA8240

EPA8240
EPA8240

Pesticides/PCBs and Dioxins/Furans

\begin{abstract}
Aldrin
alpha-Benzene hexachloride

beta-Benzene hexachloride

delta-Benzene hexachloride

alpha-Chlordane

gamma-Chlordane

p.p'-DDD
\end{abstract}
2.1
2.1
2.1
2.1
2.1
2.1
4.2

\begin{tabular}{|c|c|c|}
\hline$\mu g / \mathrm{kg}$ & 2.1 & EPA8080 \\
\hline$\mu \mathrm{g} / \mathrm{kg}$ & 2.1 & EPA8080 \\
\hline$\mu g / \mathrm{kg}$ & 2.1 & EPA8080 \\
\hline$\mu \mathrm{g} / \mathrm{kg}$ & 2.1 & EPA8080 \\
\hline$\mu g / \mathrm{kg}$ & 2.1 & EPA8080 \\
\hline$\mu \mathrm{g} / \mathrm{kg}$ & 2.1 & EPA8080 \\
\hline$\mu g / \mathrm{kg}$ & 4.2 & EPA8080 \\
\hline
\end{tabular}


SAMPLE NAME: D43.111006D (Continued)

Sample ID: 100358

$\begin{array}{llllll}\text { Constituent } & \text { RQ AQ B Result Unit D. Limit Method }\end{array}$

Pesticides / PCBs arid Dioxins/Furans

p.p'-DDE

P.p'-DDT U

Dieldrin

Endosulfan I

Endosulfan II

Endosulfan sulfate

Endrin

Endrin ketone

Heptachlor

Heptachlor epoxide

4.2

4.2

4.2

2.1

4.2

4.2

4.2

4.2

2.1

2.1

Heptachlorodibenzo-p-dioxin isomers

0.20

Heptachlorodibenzo-p-furan isomers

0.10

Hexachlorodibenzo-p-dioxin isomers

0.10

0.10

Lindane

2.1

Methoxychlor

21

Octachlorodibenzo-p-dioxin

6.2
0.20

Octachlorodibenzo-p-furan

PCB 1016

PCB 1221

PCB 1232

PCB 1242

PCB 1248

PCB 1254

PCB 1260

Pentachlorodibenzo-p-dioxin isomers

0.20

82

42

42

42

42

42

0.30

0.20

0.20
0.10

2,3,7,8-TCDD

2,3,7,8-TCDF

0.20

0.20

Tetrachlorodibenzo-p-furan isomers

210

$\mu \mathrm{g} / \mathrm{kg}$
$\mu \mathrm{g} / \mathrm{kg}$
$\mu \mathrm{g} / \mathrm{kg}$
$\mu \mathrm{g} / \mathrm{kg}$
$\mu \mathrm{g} / \mathrm{kg}$
$\mu \mathrm{g} / \mathrm{kg}$
$\mu \mathrm{g} / \mathrm{kg}$
$\mu \mathrm{g} / \mathrm{kg}$
$\mu \mathrm{g} / \mathrm{kg}$
$\mu \mathrm{g} / \mathrm{kg}$
$\mathrm{ng} / \mathrm{g}$
$\mathrm{ng} / \mathrm{g}$
$\mathrm{ng} / \mathrm{g}$
$\mathrm{ng} / \mathrm{g}$
$\mu \mathrm{g} / \mathrm{kg}$
$\mu \mathrm{g} / \mathrm{kg}$
$\mathrm{ng} / \mathrm{g}$
$\mathrm{ng} / \mathrm{g}$
$\mu \mathrm{g} / \mathrm{kg}$
$\mu \mathrm{g} / \mathrm{kg}$
$\mu \mathrm{g} / \mathrm{kg}$
$\mu \mathrm{g} / \mathrm{kg}$
$\mu \mathrm{g} / \mathrm{kg}$
$\mu \mathrm{g} / \mathrm{kg}$
$\mu \mathrm{g} / \mathrm{kg}$
$\mathrm{ng} / \mathrm{g}$
$\mathrm{ng} / \mathrm{g}$
$\mathrm{ng} / \mathrm{g}$
$\mathrm{ng} / \mathrm{g}$
$\mathrm{ng} / \mathrm{g}$
$\mathrm{ng} / \mathrm{g}$
$\mu \mathrm{g} / \mathrm{kg}$
$\mathrm{g} / \mathrm{g}$

4.2

EPA8080

4.2

EPA8080

4.2 EPA8080

2.1 EPA8080

4.2 EPA8080

4.2 EPA8080

4.2 EPA8080

4.2 EPA8080

2.1 EPA8080

2.1 EPA8080

0.20 EPA8280

0.10 EPA8280

0.10 EPA8280

0.10 EPA8280

2.1 EPA8080

21 EPA8080

0.20 EPA8280

0.20 EPA8280

42 EPA8080

84 EPA8080

42 EPA8080

42 EPA8080

42 EPA8080

42 EPA8080

42 EPA8080

0.30 EPA8280

0.20 EPA8280

0.20 EPA8280

0.10 EPA8280

0.20 EPA8280

0.20 EPA8280

Toxaphene

U

Radionuclide Indicators and Radionuclides

Gross alpha

Nonvolatile beta

Radium, total alpha-emitting

Tritium
U

U V

7.4

$30 \pm 20$

$1.0 \pm 0.65$

1.4
$\mathrm{pCi} / \mathrm{g}$

$\mathrm{pCi} / \mathrm{g}$

$\mathrm{pCi} / \mathrm{g}$

$\mathrm{pCi} / \mathrm{g}$
7.4

13

0.15

1.4
EPA900.0

EPA900.0

EPA903.0

EPA906.0 


\section{SAMPLE NAME: D431111007}

Location (SRS Coordinates): 18482.04E 66700.55N

Ground Elevation Above MSL: $129.5 \mathrm{ft}$

Depth of Core Interval: 10.00 to $12.00 \mathrm{ft}$

Sample Type: Normal

Sample Color: 10R 3/4

Constituent

RQ AQ B Result

Sample Matrix: Soil

USC Soil Classification: SM
Sample Moisture: Damp

Percent Solids: 78.60

D. Limit Method

Total Metals

\begin{tabular}{|c|c|c|c|c|c|c|}
\hline Arsenic & $\mathrm{U}$ & & 1.1 & $\mathrm{mg} / \mathrm{kg}$ & 1.1 & EPA7060 \\
\hline Barium & U & & 24 & $\mathrm{mg} / \mathrm{kg}$ & 24 & EPA6010 \\
\hline Cadmium & UJ & L & 0.61 & $\mathrm{mg} / \mathrm{kg}$ & 0.61 & EPA6010 \\
\hline Chromium & $\mathrm{UJ}$ & L & 8.4 & $\mathrm{mg} / \mathrm{kg}$ & 1.2 & EPA6010 \\
\hline Copper & $U$ & & 3.0 & $\mathrm{mg} / \mathrm{kg}$ & 3.0 & EPA6010 \\
\hline Lead & & & 1.8 & $\mathrm{mg} / \mathrm{kg}$ & 0.34 & EPA7421 \\
\hline Manganese & & & 190 & $\mathrm{mg} / \mathrm{kg}$ & 1.8 & EPA6010 \\
\hline Mercury & $U$ & & 0.13 & $\mathrm{mg} / \mathrm{kg}$ & 0.13 & EPA7470 \\
\hline Nickel & $\mathrm{U}$ & & 4.9 & $\mathrm{mg} / \mathrm{kg}$ & 4.9 & EPA6010 \\
\hline Selenium & $\mathbf{U}$ & & 0.57 & $\mathrm{mg} / \mathrm{kg}$ & 0.57 & EPA7740 \\
\hline Silver & $\mathrm{U}$ & & 1.2 & $\mathrm{mg} / \mathrm{kg}$ & 1.2 & EPA6010 \\
\hline
\end{tabular}

$B / N / A$ Extractables (including PAH and phenols)

Acenaphthene

Acenaphthylene

Anthracene

Benzo(a)anthracene

Benzo(b)fluoranthene

Benzo(k)fluoranthene

Benzoic acid

Benzo(g,h,i)perylene

Benzo(a)pyrene

Benzyl alcohol

Bis(2-chloroethoxy) methane

Bis(2-chloroethyl) ether

Bis(2-chloroisopropyl) ether

Bis(2-ethylhexyl) phthalate

4-Bromophenyl phenyl ether

Butylbenzyl phthalate

4-Chloroaniline

4-Chloro-m-cresol

2-Chloronaphthalene

2-Chlorophenol

4-Chlorophenyl phenyl ether

Chrysene

o-Cresol (2-Methylphenol)

p-Cresol (4-Methylphenol)

Dibenz $(2, h)$ anthracene

Dibenzofuran

Di-n-butyl phthalate

1,2-Dichlorobenzene

1,3-Dichlorobenzene

1,4-Dichlorobenzene

\begin{tabular}{|c|c|c|}
\hline $\mathbf{U}$ & & 430 \\
\hline $\mathrm{U}$ & & 430 \\
\hline $\mathbf{U}$ & & 430 \\
\hline $\mathbf{U}$ & & 430 \\
\hline $\mathbf{U}$ & & 430 \\
\hline $\mathbf{U}$ & & 430 \\
\hline$U$ & & 2,10 \\
\hline $\mathbf{U}$ & & 430 \\
\hline $\mathbf{U}$ & & 430 \\
\hline $\mathrm{U}$ & & 430 \\
\hline $\mathbf{U}$ & & 430 \\
\hline $\mathbf{U}$ & & 430 \\
\hline $\mathbf{U}$ & & 430 \\
\hline $\mathbf{U}$ & & 430 \\
\hline $\mathbf{U}$ & & 430 \\
\hline $\mathbf{U}$ & & 430 \\
\hline $\mathbf{U}$ & & 430 \\
\hline $\mathbf{U}$ & & 430 \\
\hline $\mathbf{U}$ & & 430 \\
\hline $\mathbf{U}$ & & 430 \\
\hline $\mathrm{U}$ & & 430 \\
\hline $\mathbf{U}$ & & 430 \\
\hline $\mathbf{U}$ & & 430 \\
\hline $\mathbf{U}$ & & 430 \\
\hline $\mathbf{U}$ & & 430 \\
\hline $\mathbf{U}$ & & 430 \\
\hline UJ & $\mathbf{V}$ & 200 \\
\hline $\mathbf{U}$ & & 430 \\
\hline $\mathbf{U}$ & & 430 \\
\hline $\mathbf{U}$ & & 430 \\
\hline
\end{tabular}

\begin{tabular}{|c|c|c|}
\hline$\mu g / k g$ & 430 & EPA8270 \\
\hline$\mu \mathrm{g} / \mathrm{kg}$ & 430 & EPA8270 \\
\hline$\mu g / k g$ & 430 & EPA8270 \\
\hline$\mu \mathrm{g} / \mathrm{kg}$ & 430 & EPA8270 \\
\hline$\mu \mathrm{g} / \mathrm{kg}$ & 430 & EPA8270 \\
\hline$\mu \mathrm{g} / \mathrm{kg}$ & 430 & EPA8270 \\
\hline$\mu g / \mathrm{kg}$ & 2,100 & EPA8270 \\
\hline$\mu g / \mathrm{kg}$ & 430 & EPA8270 \\
\hline$\mu g / \mathrm{kg}$ & 430 & EPA8270 \\
\hline$\mu \mathrm{g} / \mathrm{kg}$ & 430 & EPA 8270 \\
\hline$\mu g / \mathrm{kg}$ & 430 & EPA8270 \\
\hline$\mu g / \mathrm{kg}$ & 430 & EPA8270 \\
\hline$\mu g / \mathrm{kg}$ & 430 & EPA8270 \\
\hline$\mu g / \mathrm{kg}$ & 430 & EPA8270 \\
\hline$\mu g / k g$ & 430 & EPA 8270 \\
\hline$\mu g / k g$ & 430 & EPA8270 \\
\hline$\mu \mathrm{g} / \mathrm{kg}$ & 430 & EPA8270 \\
\hline$\mu g / k g$ & 430 & EPA8270 \\
\hline$\mu g / \mathrm{kg}$ & 430 & EPA 8270 \\
\hline$\mu g / \mathrm{kg}$ & 430 & EPA8270 \\
\hline$\mu \mathrm{g} / \mathrm{kg}$ & 430 & EPA8270 \\
\hline$\mu \mathrm{g} / \mathrm{kg}$ & 430 & EPA8270 \\
\hline$\mu \mathrm{g} / \mathrm{kg}$ & 430 & EPA8270 \\
\hline$\mu \mathrm{g} / \mathrm{kg}$ & 430 & EPA8270 \\
\hline$\mu g / \mathrm{kg}$ & 430 & EPA 8270 \\
\hline$\mu g / k g$ & 430 & EPA8270 \\
\hline$\mu g / k g$ & 430 & EPA8270 \\
\hline$\mu \mathrm{g} / \mathrm{kg}$ & 430 & EPA8270 \\
\hline$\mu \mathrm{g} / \mathrm{kg}$ & 430 & EPA8270 \\
\hline$\mu \mathrm{g} / \mathrm{kg}$ & 430 & EPA 8270 \\
\hline
\end{tabular}


SAMPLE NAME: D431I1007 (Continued)

Sample ID: 100359

Constituent

RQ AQ B Result

Unit

D. Limit Method

$B / N / A$ Extractables (including $P A H$ and phenols)

3,3'-Dichlorobenzidine

2,4-Dichlorophenol

Diethyl phthalate

2,4-Dimethyl phenol

Dimethyl phthalate

2.4-Dinitrophenol

2.4-Dinitrotoluene

2.6-Dinitrotoluene

Di-n-octyl phthalate

Fluoranthene

Fluorene

Hexachlorobenzene

Hexachlorobutadiene

Hexachlorocyclopentadiene

Hexachloroethane

Indeno(1,2,3-c,d)pyrene

Isophorone

2-Methyl-4,6-dinitrophenol

2-Methylnaphthalene

Naphthalene

o-Nitroaniline

m-Nitroaniline

p-Nitroaniline

Nitrobenzene

2-Nitrophenol

4-Nitrophenol

N-Nitrosodiphenylamine

N-Nitrosodipropylamine

Pentachlorophenol

Phenanthrene

Phenol

Pyrene

1,2,4-Trichlorobenzene

2,4,5-Trichlorophenol

2,4,6-Trichlorophenol

Unknown ketone

Unknown phthalate

Unknown phthalate

Unknown phthalate

Unknown phthalate

Unknown phthalate

Unknown phthalate
U

$\mathrm{U}$

$\mathrm{U}$

U

$\mathrm{U}$

U

$\mathrm{U}$

U

U

U

U

U

U

U

U

U

U

U

U

U

$\mathrm{U}$

$\mathrm{U}$

U

U

U

U

U

$\mathrm{U}$

$\mathrm{U}$

$\mathrm{U}$

U

U

U

U

U

J

J

J

J

$\mathrm{J}$

J

Volatile Organic Compounds

Acetone

Benzene

Bromodichloromethane

Bromoform

Bromomethane (Methyl bromide)

\begin{tabular}{|c|c|c|c|c|}
\hline & 850 & $\mu \mathrm{g} / \mathrm{kg}$ & 850 & EPA8270 \\
\hline & 430 & $\mu \mathrm{g} / \mathrm{kg}$ & 430 & EPA8270 \\
\hline & 430 & $\mu \mathrm{g} / \mathrm{kg}$ & 430 & EPA8270 \\
\hline & 430 & $\mu g / \mathrm{kg}$ & 430 & EPA8270 \\
\hline & 430 & $\mu \mathrm{g} / \mathrm{kg}$ & 430 & EPA8270 \\
\hline & 2,100 & $\mu \mathrm{g} / \mathrm{kg}$ & 2.100 & EPA8270 \\
\hline & 430 & $\mu g / \mathrm{kg}$ & 430 & EPA8270 \\
\hline & 430 & $\mu \mathrm{g} / \mathrm{kg}$ & 430 & EPA8270 \\
\hline & 430 & $\mu g / k g$ & 430 & EPA8270 \\
\hline & 430 & $\mu \mathrm{g} / \mathrm{kg}$ & 430 & EPA8270 \\
\hline & 430 & $\mu \mathrm{g} / \mathrm{kg}$ & 430 & EPA8270 \\
\hline & 430 & $\mu \mathrm{g} / \mathrm{kg}$ & 430 & EPA8270 \\
\hline & 430 & $\mu \mathrm{g} / \mathrm{kg}$ & 430 & EPA8270 \\
\hline & 430 & $\mu \mathrm{g} / \mathrm{kg}$ & 430 & EPA8270 \\
\hline & 430 & $\mu \mathrm{g} / \mathrm{kg}$ & 430 & EPA8270 \\
\hline & 430 & $\mu \mathrm{g} / \mathrm{kg}$ & 430 & EPA8270 \\
\hline & 430 & $\mu \mathrm{g} / \mathrm{kg}$ & 430 & EPA8270 \\
\hline & 2,100 & $\mu \mathrm{g} / \mathrm{kg}$ & 2,100 & EPA8270 \\
\hline & 430 & $\mu \mathrm{g} / \mathrm{kg}$ & 430 & EPA8270 \\
\hline & 430 & $\mu \mathrm{g} / \mathrm{kg}$ & 430 & EPA8270 \\
\hline & 2,100 & $\mu \mathrm{g} / \mathrm{kg}$ & 2,100 & EPA8270 \\
\hline & 2,100 & $\mu g / k g$ & 2,100 & EPA8270 \\
\hline & 2.100 & $\mu \mathrm{g} / \mathrm{kg}$ & 2,100 & EPA8270 \\
\hline & 430 & $\mu \mathrm{g} / \mathrm{kg}$ & 430 & EPA8270 \\
\hline & 430 & $\mu \mathrm{g} / \mathrm{kg}$ & 430 & EPA8270 \\
\hline & 2,100 & $\mu \mathrm{g} / \mathrm{kg}$ & 2,100 & EPA8270 \\
\hline & 430 & $\mu \mathrm{g} / \mathrm{kg}$ & 430 & EPA8270 \\
\hline & 430 & $\mu \mathrm{g} / \mathrm{kg}$ & 430 & EPA8270 \\
\hline C & 2,100 & $\mu \mathrm{g} / \mathrm{kg}$ & 2,100 & EPA8270 \\
\hline & 430 & $\mu \mathrm{g} / \mathrm{kg}$ & 430 & EPA8270 \\
\hline C & 430 & $\mu \mathrm{g} / \mathrm{kg}$ & 430 & EPA8270 \\
\hline & 430 & $\mu \mathrm{g} / \mathrm{kg}$ & 430 & EPA 8270 \\
\hline & 430 & $\mu g / k g$ & 430 & EPA8270 \\
\hline & 2,100 & $\mu \mathrm{g} / \mathrm{kg}$ & 2,100 & EPA8270 \\
\hline & 430 & $\mu g / \mathrm{kg}$ & 430 & EPA8270 \\
\hline NV & 10,000 & $\mu \mathrm{g} / \mathrm{kg}$ & & EPA8270 \\
\hline$N$ & 200 & $\mu g / \mathrm{kg}$ & & EPA8270 \\
\hline $\mathbf{N}$ & 400 & $\mu \mathrm{g} / \mathrm{kg}$ & & EPA8270 \\
\hline $\mathbf{N}$ & 300 & $\mu \mathrm{g} / \mathrm{kg}$ & & EPA8270 \\
\hline N & 200 & $\mu g / \mathrm{kg}$ & & EPA8270 \\
\hline $\mathrm{N}$ & 200 & $\mu g / k g$ & - & EPA8270 \\
\hline $\mathbf{N}$ & 300 & $\mu \mathrm{g} / \mathrm{kg}$ & & EPA8270 \\
\hline
\end{tabular}

$\begin{array}{lll}\mathrm{U} & \mathrm{V} & 29 \\ \mathrm{U} & \mathrm{V} & 6.4 \\ \mathrm{U} & & 6.4 \\ \mathrm{U} & & 6.4 \\ \mathrm{U} & & 13\end{array}$

$\mu \mathrm{g} / \mathrm{kg}$

$\mu \mathrm{g} / \mathrm{kg}$

$\mu \mathrm{g} / \mathrm{kg}$

$\mu \mathrm{g} / \mathrm{kg}$

$\mu \mathrm{g} / \mathrm{kg}$

$\begin{array}{ll}13 & \text { EPA8240 } \\ 6.4 & \text { EPA8240 } \\ 6.4 & \text { EPA8240 } \\ 6.4 & \text { EPA8240 } \\ 13 & \text { EPA8240 }\end{array}$


SAMPLE NAME: D43111007 (Continued)

Constituent

Volatile Organic Compounds

Carbon disulfide

Carbon tetrachloride

Chlorobenzene

Chloroethane

Chloroethene (Vinyl chloride)

Chloroform

Chloromethane (Methyl chloride)

Dibromochloromethane

1.1-Dichloroethane

1.2-Dichloroethane

1.1-Dichloroethylene

1.2-Dichloroethylene

Dichloromethane (Methylene chloride)

1,2-Dichloropropane

cis-1,3-Dichloropropene

trans-1.3-Dichloropropene

Ethylbenzene

2-Hexanone

Methyl ethyl ketone

Methyl isobutyl ketone

Styrene

1,1,2,2-Tetrachloroethane

Tetrachloroethylene

Toluene

1,1,1-Trichloroethane

1,1,2-Trichloroethane

Trichloroethylene

Vinyl acetate

Xylenes
Sample ID: 100359

RQ AQ B Result Unit D. Limit Method

Pesticides/PCBs and Dioxins/Furans

$\begin{array}{lc}\text { Aldrin } & \mathrm{U} \\ \text { alpha-Benzene hexachloride } & \mathrm{U} \\ \text { beta-Benzene hexachloride } & \mathrm{U} \\ \text { deita-Benzene hexachloride } & \mathrm{U} \\ \text { alpha-Chlordane } & \mathrm{U} \\ \text { gamma-Chlordane } & \mathrm{U} \\ \text { p.p'-DDD } & \mathrm{U} \\ \text { p.p'-DDE } & \mathrm{U} \\ \text { p.p'-DDT } & \mathrm{U} \\ \text { Dieldrin } & \mathrm{U} \\ \text { Endosulfan I } & \mathrm{U} \\ \text { Endosulfan II } & \mathrm{U} \\ \text { Endosulfan sulfate } & \mathrm{U} \\ \text { Endrin } & \mathrm{U} \\ \text { Endrin ketone } & \mathrm{U} \\ \text { Heplachlor } & \mathrm{U} \\ \text { Heptachlor epoxide } & \mathrm{U} \\ \text { Heptachlorodibenzo-p-dioxin isomers } & \mathrm{U}\end{array}$

6.4

6.4

13

13

6.4

13

6.4

6.4

8.8

6.4

6.4

20

6.4

6.4

6.4

6.4

13

13

13

6.4

6.4

6.4

6.4

6.4

7.8

6.4

13

6.4 $\mu \mathrm{g} / \mathrm{kg}$

$$
\mu g / \mathrm{kg}
$$

$\mu \mathrm{g} / \mathrm{kg}$

$\mu \mathrm{g} / \mathrm{kg}$

$\mu \mathrm{g} / \mathrm{kg}$

$\mu \mathrm{g} / \mathrm{kg}$

$\mu \mathrm{g} / \mathrm{kg}$

$\mu \mathrm{g} / \mathrm{kg}$

$\mu g / k g$

$\mu \mathrm{g} / \mathrm{kg}$

$\mu \mathrm{g} / \mathrm{kg}$

$\mu \mathrm{g} / \mathrm{kg}$

$\mu \mathrm{g} / \mathrm{kg}$

$\mu \mathrm{g} / \mathrm{kg}$

$\mu \mathrm{g} / \mathrm{kg}$

$\mu \mathrm{g} / \mathrm{kg}$

$\mu \mathrm{g} / \mathrm{kg}$

$\mu \mathrm{g} / \mathrm{kg}$

$\mu \mathrm{g} / \mathrm{kg}$

$\mu \mathrm{g} / \mathrm{kg}$

$\mu \mathrm{g} / \mathrm{kg}$

$\mu \mathrm{g} / \mathrm{kg}$

$\mu \mathrm{g} / \mathrm{kg}$

$\mu \mathrm{g} / \mathrm{kg}$

$\mu \mathrm{g} / \mathrm{kg}$

$\mu \mathrm{g} / \mathrm{kg}$

$\mu \mathrm{g} / \mathrm{kg}$

$\mu \mathrm{g} / \mathrm{kg}$

$\mu \mathrm{g} / \mathrm{kg}$
6.4

6.4

6.4

13

13

6.4

13

6.4

6.4

6.4

6.4

6.4

6.4

6.4

6.4

6.4

6.4

13

13

13

6.4

6.4

6.4

6.4

6.4

6.4

6.4

13

6.4
EPA8240

EPA8240

EPA8240

EPA8240

EPA8240

EPA8240

EPA8240

EPA8240

EPA8240

EPA8240

EPA8240

EPA8240

EPA8240

EPA8240

EPA8240

EPA8240

EPA8240

EPA8240

EPA8240

EPA8240

EPA8240

EPA8240

EPA8240

EPA8240

EPA8240

EPA8240

EPA8240

EPA8240

EPA8240
2.1

2.1

2.1

2.1

2.1

2.1

4.3

4.3

4.3

4.3

2.1

4.3

4.3

4.3

4.3

2.1

2.1

0.30 $\mu \mathrm{g} / \mathrm{kg}$
$\mu \mathrm{g} / \mathrm{kg}$
$\mu \mathrm{g} / \mathrm{kg}$
$\mu \mathrm{g} / \mathrm{kg}$
$\mu \mathrm{g} / \mathrm{kg}$
$\mu \mathrm{g} / \mathrm{kg}$
$\mu \mathrm{g} / \mathrm{kg}$
$\mu \mathrm{g} / \mathrm{kg}$
$\mu \mathrm{g} / \mathrm{kg}$
$\mu \mathrm{g} / \mathrm{kg}$
$\mu \mathrm{g} / \mathrm{kg}$
$\mu \mathrm{g} / \mathrm{kg}$
$\mu \mathrm{g} / \mathrm{kg}$
$\mu \mathrm{g} / \mathrm{kg}$
$\mu \mathrm{g} / \mathrm{kg}$
$\mu \mathrm{g} / \mathrm{kg}$
$\mu \mathrm{g} / \mathrm{kg}$
$\mathrm{ng} / \mathrm{g}$

2.1

2.1

EPA8080

EPA8080

EPA8080

$\begin{array}{ll}-2.1 & \text { EPA8080 } \\ 2: 1 . & \text { EPA8080 }\end{array}$

2.1 EPA8080

4.3 EPA8080

4.3 EPA8080

4.3 EPA8080

4.3 EPA8080

2.1 EPA8080

4.3 EPA8080

4.3 EPA8080

4.3 EPA8080

4.3 EPA8080

2.1 EPA8080

2.1 EPA8080

0.30 
SAMPLE NAME: D43111007 (Continued)

RQ AQ B Result

Constituent
Sample ID: 100359

Unit

D. Limit Method

Pesticides/PCBs and Dioxins/Furans

$\begin{array}{lc}\text { Heptachlorodibenzo-p-furan isomers } & \mathrm{U} \\ \text { Hexachlorodibenzo-p-dioxin isomers } & \mathrm{U} \\ \text { Hexachlorodibenzo-p-furan isomers } & \mathrm{U} \\ \text { Lindane } & \mathrm{U} \\ \text { Methoxychlor } & \mathrm{U} \\ \text { Octachlorodibenzo-p-dioxin } & \mathrm{U} \\ \text { Octachlorodibenzo-p-furan } & \mathrm{U} \\ \text { PCB 1016 } & \mathrm{U} \\ \text { PCB 1221 } & \mathrm{U} \\ \text { PCB 1232 } & \mathrm{U} \\ \text { PCB 1242 } & \mathrm{U} \\ \text { PCB 1248 } & \mathrm{U} \\ \text { PCB 1254 } & \mathrm{U} \\ \text { PCB 1260 } & \mathrm{U} \\ \text { Pentachlorodibenzo-p-dioxin isomers } & \mathrm{U} \\ \text { Pentachlorodibenzo-p-furan isomers } & \mathrm{U} \\ \text { 2.3,7,8-TCDD } & \mathrm{U} \\ \text { 2.3.7,8-TCDF } & \mathrm{U} \\ \text { Tetrachlorodibenzo-p-dioxin isomers } & \mathrm{U} \\ \text { Tetrachlorodibenzo-p-furan isomers } & \mathrm{U} \\ \text { Toxaphene } & \end{array}$

SAMPLE NAME: D43111501

$\begin{array}{ll}U & 0.20 \\ U & 0.20 \\ U & 0.20 \\ U & 2.1 \\ U & 21 \\ U & 21 \\ U & 0.20 \\ U & 43 \\ U & 85 \\ U & 43 \\ U & 43 \\ U & 43 \\ U & 43 \\ U & 43 \\ U & 0.30 \\ U & 0.30 \\ U & 0.30 \\ U & 0.20 \\ U & 0.30 \\ U & 0.20 \\ & 210\end{array}$

Sample ID: 100406

$\mathrm{ng} / \mathrm{g}$
$\mathrm{ng} / \mathrm{g}$
$\mathrm{ng} / \mathrm{g}$
$\mu \mathrm{g} / \mathrm{kg}$
$\mu \mathrm{g} / \mathrm{kg}$
$\mathrm{ng} / \mathrm{g}$
$\mathrm{ng} / \mathrm{g}$
$\mu \mathrm{g} / \mathrm{kg}$
$\mu \mathrm{g} / \mathrm{kg}$
$\mu \mathrm{g} / \mathrm{kg}$
$\mu \mathrm{g} / \mathrm{kg}$
$\mu \mathrm{g} / \mathrm{kg}$
$\mu \mathrm{g} / \mathrm{kg}$
$\mu \mathrm{g} / \mathrm{kg}$
$\mathrm{ng} / \mathrm{g}$
$\mathrm{ng} / \mathrm{g}$
$\mathrm{ng} / \mathrm{g}$
$\mathrm{ng} / \mathrm{g}$
$\mathrm{ng} / \mathrm{g}$
$\mathrm{ng} / \mathrm{g}$
$\mu \mathrm{g} / \mathrm{kg}$

0.20

0.20

0.20

2.1

21

0.10

0.20

43

85

43

43

43

43

43

0.30

0.30

0.30

0.20

0.30

0.20

210
EPA8280
EPA8280
EPA8280
EPA8080
EPA8080
EPA8280
EPA8280
EPA8080
EPA8080
EPA8080
EPA8080
EPA8080
EPA8080
EPA8080
EPA8280
EPA8280
EPA8280
EPA8280
EPA8280
EPA8280
EPA8080
Location (SRS Coordinates): 18533.07E 66779.25N

Ground Elevation Above MSL: $128.5 \mathrm{ft}$

Depth of Core Interval: 0.00 to $0.50 \mathrm{ft}$

Sample Type: Normal

Sample Color: SYR 4/4

Constituent

Physical Parameters

Cation exchange capacity

Cation exchange capacity

$\mathrm{pH}$

Specific conductance

Total organic carbon

\section{Total Metals}

\section{Arsenic \\ Barium \\ Cadmium \\ Chromium \\ Copper \\ Lead \\ Manganese}

Sample Matrix: Soil

USC Soil Classification: SP

RQ AQ B Result Unit

meq $/ 100 \mathrm{~g}$

$\mathrm{meq} / 100 \mathrm{~g}$

$\mathrm{pH}$

$\mu \mathrm{S} / \mathrm{cm}$

$\mathrm{mg} / \mathrm{kg}$
Sample Moisture: Damp Percent Solids: 87.40

D. Limit Method

$\begin{array}{llllll} & 10 & \mathrm{meq} / 100 \mathrm{~g} & 2.3 & \text { EPA9080 } \\ & & 13 & \mathrm{meq} / 100 \mathrm{~g} & 2.3 & \text { EPA9880 } \\ \mathrm{J} & \mathrm{Q} & 7.7 & \mathrm{pH} & 0.010 & \text { EPA9045 } \\ & 100 & \mu \mathrm{S} / \mathrm{cm} & 1.0 & \text { EPA9050 } \\ & 14,000 & \mathrm{mg} / \mathrm{kg} & 670 & \text { EPA415.1 }\end{array}$

$\begin{array}{llll}6.8 & \mathrm{mg} / \mathrm{kg} & 1.1 & \text { EPA7060 } \\ 42 & \mathrm{mg} / \mathrm{kg} & 21 & \text { EPA6010 } \\ 9.7 & \mathrm{mg} / \mathrm{kg} & 0.53 & \text { EPA6010 } \\ 180 & \mathrm{mg} / \mathrm{kg} & 1.1 & \text { EPA6010 } \\ 66 & \mathrm{mg} / \mathrm{kg} & 2.7 & \text { EPA6010 } \\ 150 & \mathrm{mg} / \mathrm{kg} & 13 & \text { EPA7421 } \\ 410 & \mathrm{mg} / \mathrm{kg} & 1.6 & \text { EPA6010 }\end{array}$


SAMPLE NAME: D43111501 (Continued)

Sample ID: 100406

Constituent

RQ
Q AQ B Result

Total Metals

Mercury

1.1
79
0.56

1.5

1.1
Nickel

Selenium

Silver

U

$B / N / A$ Extroctables (including $P A H$ and phenols)

\begin{tabular}{|c|c|c|c|c|c|c|c|}
\hline Acenaphthene & UJ & L & & 1,900 & $\mu \mathrm{g} / \mathrm{kg}$ & 1,900 & EPA8270 \\
\hline Acenaphthylene & UJ & L & & 1,900 & $\mu \mathrm{g} / \mathrm{kg}$ & 1,900 & EPA8270 \\
\hline Anthracene & UJ & L & & 1,900 & $\mu \mathrm{g} / \mathrm{kg}$ & 1,900 & EPA8270 \\
\hline Benzo(a)anthracene & UJ & $L$ & & 1,900 & $\mu g / \mathrm{kg}$ & 1,900 & EPA8270 \\
\hline Benzo(b)fluoranthene & UJ & LH & L & 1,900 & $\mu \mathrm{g} / \mathrm{kg}$ & 1,900 & EPA8270 \\
\hline Benzo(k)fluoranthene & UJ & LH & $\mathrm{L}$ & 1,900 & $\mu \mathrm{g} / \mathrm{kg}$ & 1,900 & EPA8270 \\
\hline Benzoic acid & UJ & L & & 9.500 & $\mu \mathrm{g} / \mathrm{kg}$ & 9,500 & EPA8270 \\
\hline Benzo(g,h,i)perylene & UJ & LH & L & 1,900 & $\mu \mathrm{g} / \mathrm{kg}$ & 1,900 & EPA8270 \\
\hline Benzo(a)pyrene & UJ & LH & $L$ & 1,900 & $\mu \mathrm{g} / \mathrm{kg}$ & 1,900 & EPA8270 \\
\hline Benzyl alcohol & UJ & L & & 1,900 & $\mu \mathrm{g} / \mathrm{kg}$ & 1,900 & EPA8270 \\
\hline Bis(2-chloroethoxy) methane & UJ & L & & 1,900 & $\mu \mathrm{g} / \mathrm{kg}$ & 1,900 & EPA8270 \\
\hline Bis(2-chloroethyl) ether & UJ & L & & 1,900 & $\mu \mathrm{g} / \mathrm{kg}$ & 1,900 & EPA8270 \\
\hline Bis(2-chloroisopropyl) ether & UJ & L & & 1.900 & $\mu \mathrm{g} / \mathrm{kg}$ & 1.900 & EPA8270 \\
\hline Bis(2-ethylhexyl) phthalate & $\mathrm{J}$ & L & & 26,000 & $\mu \mathrm{g} / \mathrm{kg}$ & 1,900 & EPA8270 \\
\hline 4-Bromophenyl phenyl ether & UJ & L & & 1,900 & $\mu \mathrm{g} / \mathrm{kg}$ & 1,900 & EPA8270 \\
\hline Butylbenzyl phthalate & UJ & L & & 1,900 & $\mu \mathrm{g} / \mathrm{kg}$ & 1,900 & EPA8270 \\
\hline 4-Chloroaniline & UJ & L & & 1,900 & $\mu \mathrm{g} / \mathrm{kg}$ & 1.900 & EPA8270 \\
\hline 4-Chloro-m-cresol & UJ & L & & 1.900 & $\mu \mathrm{g} / \mathrm{kg}$ & 1,900 & EPA8270 \\
\hline 2-Chloronaphthalene & UJ & L & & 1,900 & $\mu \mathrm{g} / \mathrm{kg}$ & 1,900 & EPA8270 \\
\hline 2-Chlorophenol & UJ & $\mathrm{L}$ & & 1,900 & $\mu \mathrm{g} / \mathrm{kg}$ & 1,900 & EPA8270 \\
\hline 4-Chlorophenyl phenyl ether & UJ & L & & 1,900 & - $\mu \mathrm{g} / \mathrm{kg}$ & 1,900 & EPA8270 \\
\hline Chrysene & UJ & L & & 1,900 & $\mu \mathrm{g} / \mathrm{kg}$ & 1,900 & EPA8270 \\
\hline o-Cresol (2-Methylphenol) & UJ & L & & 1,900 & $\mu \mathrm{g} / \mathrm{kg}$ & 1,900 & EPA8270 \\
\hline p-Cresol (4-Methylphenol) & UJ & L & & 1,900 & $\mu g / k g$ & 1,900 & EPA8270 \\
\hline Dibenz(a,h)anthracene & UJ & LH & $\mathrm{L}$ & 1,900 & $\mu \mathrm{g} / \mathrm{kg}$ & 1,900 & EPA8270 \\
\hline Dibenzofuran & $\mathrm{UJ}$ & L & & 1,900 & $\mu \mathrm{g} / \mathrm{kg}$ & 1,900 & EPA8270 \\
\hline Di-n-butyl phthalate & UJ & VL & & 1,900 & $\mu \mathrm{g} / \mathrm{kg}$ & 1,900 & EPA8270 \\
\hline 1,2-Dichlorobenzene & UJ & L & & 1,900 & $\mu \mathrm{g} / \mathrm{kg}$ & 1,900 & EPA8270 \\
\hline 1,3-Dichlorobenzene & UJ & L & & 1,900 & $\mu \mathrm{g} / \mathrm{kg}$ & 1,900 & EPA8270 \\
\hline 1,4-Dichlorobenzene & UJ & L & & 1,900 & $\mu \mathrm{g} / \mathrm{kg}$ & 1,900 & EPA8270 \\
\hline 3,3'-Dichlorobenzidine & UJ & L & & 3,800 & $\mu g / k g$ & 3,800 & EPA8270 \\
\hline 2,4-Dichlorophenol & UJ & L & & 1,900 & $\mu g / k g$ & 1,900 & EPA8270 \\
\hline Diethyl phthalate & UJ & L & & 1,900 & $\mu \mathrm{g} / \mathrm{kg}$ & 1.900 & EPA8270 \\
\hline 2,4-Dimethyl phenol & UJ & L & & 1,900 & $\mu \mathrm{g} / \mathrm{kg}$ & 1,900 & EPA8270 \\
\hline Dimethyl phthalate & $\mathrm{UJ}$ & L & & 1,900 & $\mu \mathrm{g} / \mathrm{kg}$ & 1.900 & EPA8270 \\
\hline 2,4-Dinitrophenol & UJ & L & & 9,500 & $\mu \mathrm{g} / \mathrm{kg}$ & 9,500 & EPA8270 \\
\hline 2,4-Dinitrotoluene & UJ & L & & 1,900 & $\mu \mathrm{g} / \mathrm{kg}$ & 1,900 & EPA8270 \\
\hline 2,6-Dinitrotoluene & UJ & L & & 1,900 & $\mu \mathrm{g} / \mathrm{kg}$ & 1.900 & EPA8270 \\
\hline Di-n-octyl phthalate & Uנ & LH & L & 1,900 & $\mu g / \mathrm{kg}$ & 1,900 & EPA8270 \\
\hline Fluoranthene & UJ & L & & 1,900 & $\mu \mathrm{g} / \mathrm{kg}$ & 1,900 & EPA8270 \\
\hline Fluorene & UJ & L & & 1.900 & $\mu \mathrm{g} / \mathrm{kg}$ & 1,900 & EPA8270 \\
\hline Hexachlorobenzene & UI & $L$ & & 1,900 & $\mu g / \mathrm{kg}$ & 1,900 & EPA8270 \\
\hline Hexachlorobutadiene & UJ & L & & 1,900 & $\mu g / \mathrm{kg}$ & 1.900 & EPA8270 \\
\hline
\end{tabular}


SAMPLE NAME: D43ï11501 (Continued)

Sample ID: 100406
Constituent
RQ AQ B Result
Unit
D. Limit Method

$B / N / A$ Extractables (including $P A H$ and phenols)

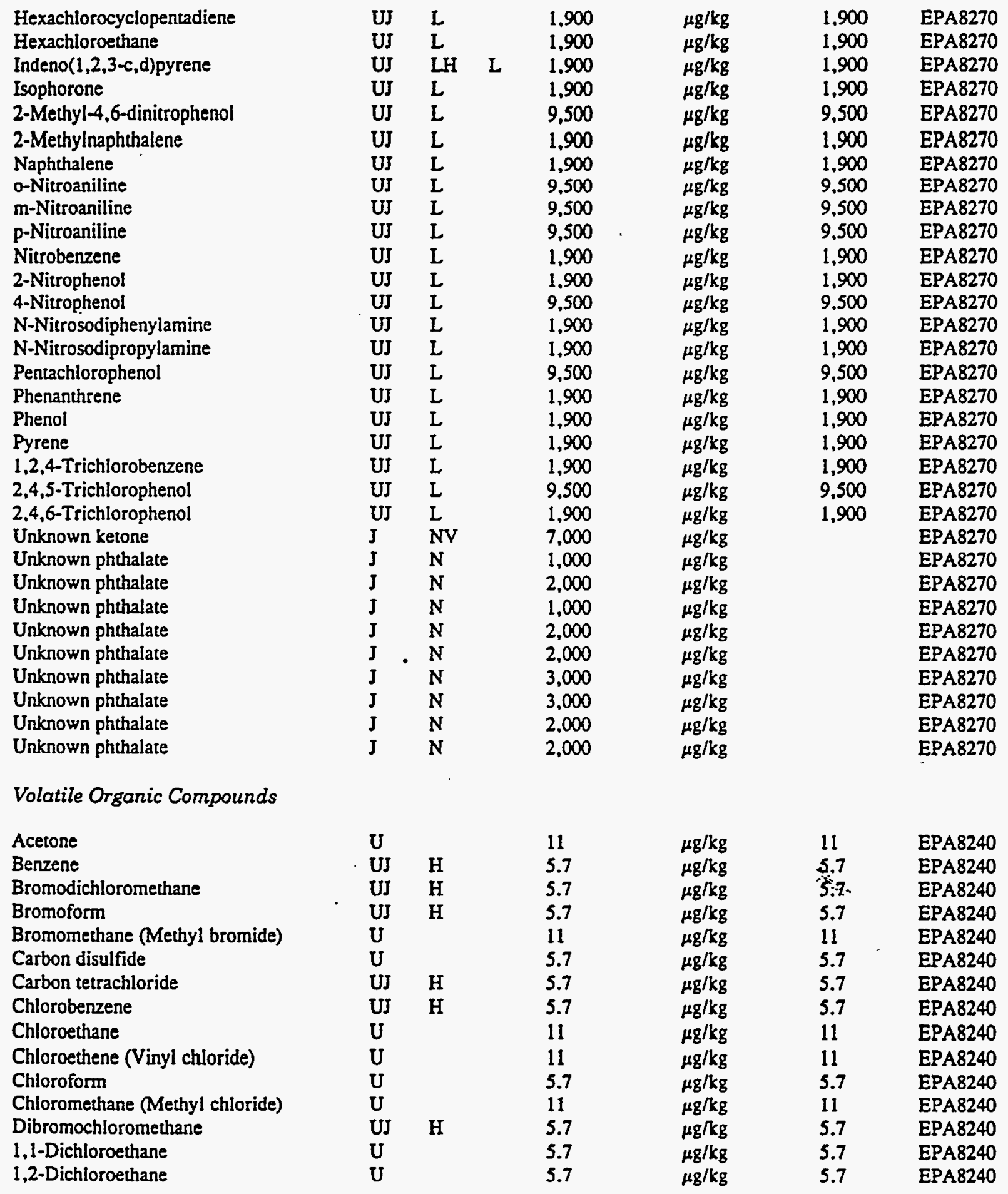


SAMPLE NAME: D43111501 (Continued)

Constituent

Volatile Organic Compounds

1,1-Dichloroethylene
1,2-Dichloroethylene
Dichloromethane (Methylene chloride)
1,2-Dichloropropane
cis-1,3-Dichloropropene
trans-1,3-Dichloropropene
Ethylbenzene
2-Hexanone
Methyl ethyl ketone
Methyl isobutyl ketone
Styrene
1,1,2,2-Tetrachloroethane
Tetrachloroethylene
Toluene
1,1,1-Trichloroethane
1,1,2-Trichloroethane
Trichloroethylene
Vinyl acetate
Xylenes

RQ AQ

B Result

Unit

D. Limit Method
Sample ID: 100406

$\begin{array}{llllll}\mathrm{U} & & 5.7 & \mu \mathrm{g} / \mathrm{kg} & 5.7 & \text { EPA8240 } \\ \mathrm{U} & & 5.7 & \mu \mathrm{g} / \mathrm{kg} & 5.7 & \text { EPA8240 } \\ \mathrm{U} & & 5.7 & \mu \mathrm{g} / \mathrm{kg} & 5.7 & \text { EPA8240 } \\ \mathrm{UJ} & \mathrm{H} & 5.7 & \mu \mathrm{g} / \mathrm{kg} & 5.7 & \text { EPA8240 } \\ \mathrm{UJ} & \mathrm{H} & 5.7 & \mu \mathrm{g} / \mathrm{kg} & 5.7 & \text { EPA8240 } \\ \mathrm{UJ} & \mathrm{H} & 5.7 & \mu \mathrm{g} / \mathrm{kg} & 5.7 & \text { EPA8240 } \\ \mathrm{UJ} & \mathrm{H} & 5.7 & \mu \mathrm{k} / \mathrm{kg} & 5.7 & \text { EPA8240 } \\ \mathrm{UJ} & \mathrm{H} & 11 & \mu \mathrm{g} / \mathrm{kg} & 11 & \text { EPA8240 } \\ \mathrm{U} & & 11 & \mu \mathrm{g} / \mathrm{kg} & 11 & \text { EPA8240 } \\ \mathrm{UJ} & \mathrm{H} & 11 & \mu \mathrm{g} / \mathrm{kg} & 11 & \text { EPA8240 } \\ \mathrm{UJ} & \mathrm{H} & 5.7 & \mu \mathrm{g} / \mathrm{kg} & 5.7 & \text { EPA8240 } \\ \mathrm{UJ} & \mathrm{H} & 5.7 & \mu \mathrm{g} / \mathrm{kg} & 5.7 & \text { EPA8240 } \\ \mathrm{UJ} & \mathrm{H} & 5.7 & \mu \mathrm{kg} & 5.7 & \text { EPA8240 } \\ \mathrm{UJ} & \mathrm{H} & 5.7 & \mu \mathrm{k} / \mathrm{kg} & 5.7 & \text { EPA8240 } \\ \mathrm{UJ} & \mathrm{H} & 5.7 & \mu \mathrm{g} / \mathrm{kg} & 5.7 & \text { EPA8240 } \\ \mathrm{UJ} & \mathrm{H} & 5.7 & \mu \mathrm{g} / \mathrm{kg} & 5.7 & \text { EPA8240 } \\ \mathrm{UJ} & \mathrm{H} & 5.7 & \mu \mathrm{kg} & 5.7 & \text { EPA8240 } \\ \text { UJ } & \mathrm{H} & 11 & \mu \mathrm{g} / \mathrm{kg} & 11 & \text { EPA8240 } \\ \mathrm{UJ} & \mathrm{H} & 5.7 & \mu \mathrm{g} / \mathrm{kg} & 5.7 & \text { EPA8240 }\end{array}$

Pesticides/PCBs and Dioxins/Furans

\begin{tabular}{|c|c|c|c|c|c|c|c|}
\hline Aldrin & UJ & 0 & L & 92 & $\mu \mathrm{g} / \mathrm{kg}$ & 92 & EPA8080 \\
\hline alpha-Benzene hexachloride & UJ & 0 & L & 92 & $\mu \mathrm{g} / \mathrm{kg}$ & 92 & EPA8080 \\
\hline beta-Benzene hexachloride & UJ & 0 & L & 92 & $\mu \mathrm{g} / \mathrm{kg}$ & 92 & EPA8080 \\
\hline delta-Benzene hexachloride & UJ & 0 & L & 92 & $\mu \mathrm{g} / \mathrm{kg}$ & 92 & EPA8080 \\
\hline alpha-Chlordane & $\mathbf{U}$ & A & & 27 & $\mu \mathrm{g} / \mathrm{kg}$ & 92 & EPA8080 \\
\hline gamma-Chlordane & $\mathbf{U}$ & $A$ & & 25 & $\mu \mathrm{g} / \mathrm{kg}$ & 92 & EPA8080 \\
\hline p.p'-DDD & UJ & 0 & L & 190 & $\mu \mathrm{g} / \mathrm{kg}$ & 190 & EPA8080 \\
\hline$p, p^{\prime}-D D E$ & U & A & & 180 & $\mu g / k g$ & 190 & EPA8080 \\
\hline p.p'-DDT & U & A & & 270 & $\mu \mathrm{g} / \mathrm{kg}$ & 190 & EPA8080 \\
\hline Dieldrin & UJ & 0 & L & 190 & $\mu \mathrm{g} / \mathrm{kg}$ & 190 & EPA8080 \\
\hline Endosulfan I & UJ & 0 & L & 92 & $\mu \mathrm{g} / \mathrm{kg}$ & 92 & EPA8080 \\
\hline Endosulfan II & UJ & 0 & $\mathbf{L}$ & 190 & $\mu g / \mathrm{kg}$ & 190 & EPA8080 \\
\hline Endosulfan sulfate & UJ & 0 & L & 190 & $\mu \mathrm{g} / \mathrm{kg}$ & 190 & EPA8080 \\
\hline Endrin & UJ & 0 & $\bar{L}$ & 190 & $\mu g / \mathrm{kg}$ & 190 & EPA8080 \\
\hline Endrin ketone & UJ & 0 & L & 190 & $\mu g / \mathrm{kg}$ & 190 & EPA8080 \\
\hline Heptachlor & UJ & 0 & L & 92 & $\mu \mathrm{g} / \mathrm{kg}$ & 92 & EPA8080 \\
\hline Heptachlor epoxide & UJ & 0 & $\mathrm{~L}$ & 92 & $\mu g / \mathrm{kg}$ & 92 & EPA8080 \\
\hline Heptachlorodibenzo-p-dioxin isomers & J & & & 0.70 & $\mathrm{ng} / \mathrm{g}$ & 0.20 & EPA8280 \\
\hline Heptachlorodibenzo-p-furan isomers & $\mathbf{U}$ & & & 0.10 & $\mathrm{ng} / \mathrm{g}$ & 0.10 & EPA8280 \\
\hline Hexachlorodibenzo-p-dioxin isomers & $\mathbf{U}$ & & & 0.20 & $\mathrm{ng} / \mathrm{g}$ & 0.20 & EPA8280 \\
\hline Hexachlorodibenzo-p-furan isomers & $\mathbf{U}$ & & & 0.20 & $\mathrm{ng} / \mathrm{g}$ & 0.20 & EPA8280 \\
\hline Lindane & UJ & 0 & $\mathbf{L}$ & 92 & $\mu g / \mathrm{kg}$ & 92 & EPA8080 \\
\hline Methoxychlor & UJ & 0 & $\mathbf{L}$ & 920 & $\mu g / \mathrm{kg}$ & 920 & EPA8080 \\
\hline Octachlorodibenzo-p-dioxin & J & & & 1.8 & $\mathrm{ng} / \mathrm{g}$ & 0.10 & EPA8280 \\
\hline Octachlorodibenzo-p-furan & $\mathbf{U}$ & & & 0.30 & $\mathrm{ng} / \mathrm{g}$ & 0.30 & EPA8280 \\
\hline PCB 1016 & UJ & 0 & L & 1,900 & $\mu g / \mathrm{kg}$ & 1,900 & EPA8080 \\
\hline PCB 1221 & UJ & 0 & $\mathbf{L}$ & 3,700 & $\mu \mathrm{g} / \mathrm{kg}$ & 3,700 & EPA8080 \\
\hline PCB 1232 & UJ & 0 & L & 1,900 & $\mu g / \mathrm{kg}$ & 1,900 & EPA8080 \\
\hline
\end{tabular}


SAMPLE NAME: D431!11501 (Continued) Sample ID: 100406

Constituent

RQ AQ B Result

Unit

D. Limit Method

Pesticides/PCBs and Dioxins/Furans

PCB 1242

PCB 1248

UJ $\quad 0 \quad \mathrm{~L} \quad 1,900$

PCB 1254

PCB 1260

UJ $\quad 0 \quad$ L $\quad 1,900$

UJ $\quad 0 \quad$ L $\quad 1,900$

Pentachlorodibenzo-p-dioxin isomers

UJ $\quad 0 \quad$ L $\quad 1,900$

Pentachlorodibenzo-p-furan isomers

$\mathrm{U} \quad 0.20$

2.3.7.8-TCDD

2.3.7,8-TCDF

$\mathrm{U} \quad 0.10^{\circ}$

Tetrachlorodibenzo-p-dioxin isomers

Tetrachlorodibenzo-p-furan isomers

$\mathrm{U} \quad 0.20$

$\mathrm{U} \quad 0.20$

U $\quad 0.20$

Toxaphene

UJ $\quad 0 \quad$ L $\quad 9,200$

Radionuclide Indicators and Radionuclides

Gross alpha

Nonvolatile beta

Radium, total alpha-emitting

Tritium

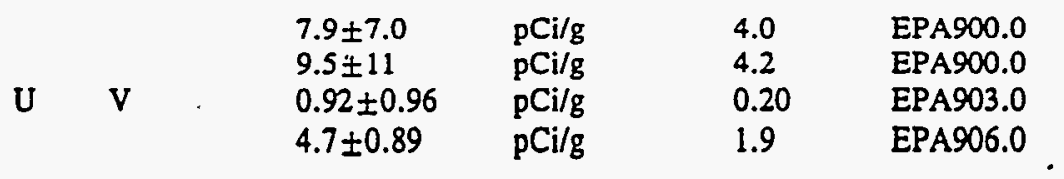

SAMPLE NAME: D43111502

Sample ID: 100407

Location (SRS Coordinates): 18533.07E 66779.25N

Ground Elevation Above MSL: $128.5 \mathrm{ft}$

Depth of Core Interval: 0.50 to $2.00 \mathrm{ft}$

Sample Type: Normal

Sample Color: 5YR 4/4

Sample Matrix: Soil

USC Soil Classification: SP

Constituent

Total Metals

Arsenic

Barium

Cadmium

Chromium

Copper

Lead

Manganese

Mercury

Nickel

Selenium

Silver
RQ AQ B Result Unit

$\mathrm{mg} / \mathrm{kg}$

$\mathrm{mg} / \mathrm{kg}$

$\mathrm{mg} / \mathrm{kg}$

$\mathrm{mg} / \mathrm{kg}$

$\mathrm{mg} / \mathrm{kg}$

$\mathrm{mg} / \mathrm{kg}$

$\mathrm{mg} / \mathrm{kg}$

$\mathrm{mg} / \mathrm{kg}$

$\mathrm{mg} / \mathrm{kg}$

$\mathrm{mg} / \mathrm{kg}$

$\mathrm{U} \quad 0.49$

$\begin{array}{ll}\mathrm{U} & 0.96\end{array}$

$\mathrm{mg} / \mathrm{kg}$
Sample Moisture: Damp Percent Solids: 91.20

D. Limit Method

$B / N / A$ Extractables (including PAH and phenols)

Acenaphthene

Acenaphthylene

Anthracene

Benzo(a)anthracene
UJ L 370

UJ L 370

UJ L 370

UJ L 370

$\begin{array}{ll}0.98 & \text { EPA7060 } \\ 19 & \text { EPA6010 } \\ 0.48 & \text { EPA6010 } \\ 0.96 & \text { EPA6010 } \\ 2.4 & \text { EPA6010 } \\ 5.9 & \text { EPA7421 } \\ 1.4 & \text { EPA6010 } \\ 0.11 & \text { EPA7470 } \\ 3.8 & \text { EPA6010 } \\ 0.49 & \text { EPA7740 } \\ 0.96 & \text { EPA6010 }\end{array}$

EPA8080

EPA8080

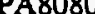

EPA8280

EPA8280

EPA8280

EPA8280

EPA8280

$9,200 \quad$ EPA 8080 
SAMPLE NAME: D43111502 (Continued)

Constituent
Sample ID: 100407

RQ AQ B Result Unit D. Limit Method

$B / N / A$ Extractables (including $P A H$ and phenols)

\begin{tabular}{|c|c|c|c|c|c|c|}
\hline Benzo(b)fluoranthene & UJ & $\mathbf{L}$ & 370 & $\mu \mathrm{g} / \mathrm{kg}$ & 370 & EPA8270 \\
\hline Benzo(k)fluoranthene & UJ & L & 370 & $\mu \mathrm{g} / \mathrm{kg}$ & 370 & EPA8270 \\
\hline Benzoic acid & UJ & L & 1,800 & $\mu g / \mathrm{kg}$ & 1,800 & EPA8270 \\
\hline Benzo(g,h,i)perylene & UJ & L & 370 & $\mu \mathrm{g} / \mathrm{kg}$ & 370 & EPA8270 \\
\hline Benzo(a)pyrene & UJ & L & 370 & $\mu \mathrm{g} / \mathrm{kg}$ & 370 & EPA8270 \\
\hline Benzyl alcohol & UJ & L & 370 & $\mu g / \mathrm{kg}$ & 370 & EPA8270 \\
\hline Bis(2-chloroethoxy) methane & UJ & L & 370 & $\mu \mathrm{g} / \mathrm{kg}$ & 370 & EPA8270 \\
\hline Bis(2-chloroethyl) ether & UJ & L & 370 & $\mu g / k g$ & 370 & EPA8270 \\
\hline Bis(2-chloroisopropyl) ether & UJ & L & 370 & $\mu g / \mathrm{kg}$ & 370 & EPA8270 \\
\hline Bis(2-ethylhexyl) phthalate & UJ & L & 370 & $\mu \mathrm{g} / \mathrm{kg}$ & 370 & EPA8270 \\
\hline 4-Bromophenyl phenyl ether & UJ & L & 370 & $\mu \mathrm{g} / \mathrm{kg}$ & 370 & EPA8270 \\
\hline Butylbenzyl phthalate & UJ & L & 370 & $\mu \mathrm{g} / \mathrm{kg}$ & 370 & EPA8270 \\
\hline 4-Chloroaniline & UJ & L & 370 & $\mu \mathrm{g} / \mathrm{kg}$ & 370 & EPA8270 \\
\hline 4.Chloro-m-cresol & UJ & L & 370 & $\mu g / \mathrm{kg}$ & 370 & EPA8270 \\
\hline 2-Chloronaphthalene & UJ & L & 370 & $\mu \mathrm{g} / \mathrm{kg}$ & 370 & EPA8270 \\
\hline 2-Chlorophenol & UJ & L & 370 & $\mu g / \mathrm{kg}$ & 370 & EPA8270 \\
\hline 4-Chlorophenyl phenyl ether & UI & L & 370 & $\mu \mathrm{g} / \mathrm{kg}$ & 370 & EPA8270 \\
\hline Chrysene & UJ & L & 370 & $\mu \mathrm{g} / \mathrm{kg}$ & 370 & EPA8270 \\
\hline o-Cresol (2-Methylphenol) & UJ & L & 370 & $\mu g / \mathrm{kg}$ & 370 & EPA8270 \\
\hline p-Cresol (4-Methylphenol) & UI & L & 370 & $\mu \mathrm{g} / \mathrm{kg}$ & 370 & EPA8270 \\
\hline Dibenz $(\mathrm{a}, \mathrm{h})$ anthracene & UJ & L & 370 & $\mu \mathrm{g} / \mathrm{kg}$ & 370 & EPA8270 \\
\hline Dibenzofuran & UI & L & 370 & $\mu \mathrm{g} / \mathrm{kg}$ & 370 & EPA8270 \\
\hline Di-n-butyl phthalate & UJ & VL & 41 & $\mu \mathrm{g} / \mathrm{kg}$ & 370 & EPA8270 \\
\hline 1,2-Dichlorobenzene & UJ & L & 370 & $\mu \mathrm{g} / \mathrm{kg}$ & 370 & EPA8270 \\
\hline 1,3-Dichlorobenzene & UJ & L & 370 & $\mu \mathrm{g} / \mathrm{kg}$ & 370 & EPA8270 \\
\hline 1,4-Dichlorobenzene & UJ & L & 370 & $\mu \mathrm{g} / \mathrm{kg}$ & 370 & EPA8270 \\
\hline 3,3'-Dichlorobenzidine & UJ & L & 740 & $\mu \mathrm{g} / \mathrm{kg}$ & 740 & EPA8270 \\
\hline 2,4-Dichlorophenol & UJ & L & 370 & $\mu \mathrm{g} / \mathrm{kg}$ & 370 & EPA8270 \\
\hline Diethyl phthalate & UJ & L & 370 & $\mu \mathrm{g} / \mathrm{kg}$ & 370 & EPA8270 \\
\hline 2.4-Dimethyl phenol & UJ & L & 370 & $\mu \mathrm{g} / \mathrm{kg}$ & 370 & EPA8270 \\
\hline Dimethyl phthalate & UJ & L & 370 & $\mu \mathrm{g} / \mathrm{kg}$ & 370 & EPA8270 \\
\hline 2.4-Dinitrophenol & UJ & $\mathrm{L}$ & 1,800 & $\mu g / \mathrm{kg}$ & 1,800 & EPA8270 \\
\hline 2,4-Dinitrotoluene & UJ & L & 370 & $\mu g / \mathrm{kg}$ & 370 & EPA8270 \\
\hline 2,6-Dinitrotoluene & UJ & L & 370 & $\mu g / k g$ & 370 & EPA8270 \\
\hline Di-n-octyl phthalate & UJ & L & 370 & $\mu \mathrm{g} / \mathrm{kg}$ & 370 & EPA8270 \\
\hline Fluoranthene & UJ & $\mathrm{L}$ & 370 & $\mu \mathrm{g} / \mathrm{kg}$ & 370 & EPA8270 \\
\hline Fluorene & UJ & L & 370 & $\mu g / \mathrm{kg}$ & 370 & EPA8270 \\
\hline Hexachlorobenzene & UJ & L & 370 & $\mu \mathrm{g} / \mathrm{kg}$ & 370 & EPA8270 \\
\hline Hexachlorobutadiene & UJ & L & 370 & $\mu g / \mathrm{kg}$ & 370 & EPA8270 \\
\hline Hexachlorocyclopentadiene & UJ & $\mathbf{L}$ & 370 & $\mu \mathrm{g} / \mathrm{kg}$ & 370 & EPA8270 \\
\hline Hexachloroethane & UJ & L - & 370 & $\mu \mathrm{g} / \mathrm{kg}$ & 370 & EPA8270 \\
\hline Indeno(1,2,3-c,d)pyrene & UJ & L & 370 & $\mu \mathrm{g} / \mathrm{kg}$ & 370 & EPA8270 \\
\hline Isophorone & UJ & L & 370 & $\mu \mathrm{g} / \mathrm{kg}$ & 370 & EPA8270 \\
\hline 2-Methyl-4,6-dinitrophenol & UJ & L & 1.800 & $\mu g / \mathrm{kg}$ & 1,800 & EPA8270 \\
\hline 2-Methyinaphthalene & UJ & $\mathbf{L}$ & 370 & $\mu \mathrm{g} / \mathrm{kg}$ & 370 & EPA8270 \\
\hline Naphthalene & UJ & $\mathbf{L}$ & 370 & $\mu \mathrm{g} / \mathrm{kg}$ & 370 & EPA8270 \\
\hline o-Nitroaniline & UJ & $\mathbf{L}$ & 1,800 & $\mu g / k g$ & 1,800 & EPA8270 \\
\hline m-Nitroaniline & UJ & L & 1,800 & $\mu g / k g$ & 1,800 & EPA8270 \\
\hline p-Nitroaniline & UJ & L & 1,800 & $\mu g / \mathrm{kg}$ & 1,800 & EPA8270 \\
\hline Nitrobenzene & UJ & L & 370 & $\mu \mathrm{g} / \mathrm{kg}$ & 370 & EPA8270 \\
\hline
\end{tabular}


SAMPLE NAME: D43111502 (Continued)

Constituent
RQ AQ

$B / N / A$ Extractables (including PAH and phenols)
Sample ID: 100407

B Result Unit D. Limit Method

$\begin{array}{llll}\text { 2-Nitrophenol } & \text { UJ } & \text { L } & 370 \\ \text { 4-Nitrophenol } & \text { UJ } & \text { L } & 1,800 \\ \text { N-Nitrosodiphenylamine } & \text { UJ } & \text { L } & 370 \\ \text { N-Nitrosodipropylamine } & \text { UJ } & \text { L } & 370 \\ \text { Pentachlorophenol } & \text { UJ } & \text { L } & 1,800 \\ \text { Phenanthrene } & \text { UJ } & \text { L } & 370 \\ \text { Phenol } & \text { UJ } & \text { L } & 370 \\ \text { Pyrene } & \text { UJ } & \text { L } & 370 \\ \text { 1,2,4-Trichlorobenzene } & \text { UJ } & \text { L } & 370 \\ \text { 2,4,5-Trichlorophenol } & \text { UJ } & \text { L } & 1,800 \\ \text { 2,4,6-Trichlorophenol } & \text { UJ } & \text { L } & 370 \\ \text { Unknown ester } & \text { J } & \text { N } & 300 \\ \text { Unknown ketone } & \text { J } & \text { N } & 6,000\end{array}$

$\mu g / k g$
$\mu g / k g$
$\mu g / k g$
$\mu g / k g$
$\mu g / k g$
$\mu g / k g$
$\mu g / k g$
$\mu g / k g$
$\mu g / k g$
$\mu g / k g$
$\mu g / k g$
$\mu g / k g$
$\mu g / k g$

370

1,800

370

370

1,800

370

370

370

370

1,800

370
EPA8270

EPA8270

EPA8270

EPA8270

EPA8270

EPA8270

EPA8270

EPA8270

EPA8270

EPA8270

EPA8270

EPA8270

EPA8270

Volatile Organic Compounds

Acetone
Benzene
Bromodichloromethane
Bromoform
Bromomethane (Methyl bromide)
Carbon disulfide
Carbon tetrachloride
Chlorobenzene
Chloroethane
Chloroethene (Vinyl chloride)
Chloroform
Chloromethane (Methyl chloride)
Dibromochloromethane
1,1-Dichloroethane
1,2-Dichloroethane
1,1-Dichloroethylene
1,2-Dichloroethylene
Dichloromethane (Methylene chloride)
1,2-Dichloropropane
cis-1,3-Dichloropropene
trans-1,3-Dichloropropene
Ethylbenzene
2-Hexanone
Methyl ethyl ketone
Methyl isobutyl ketone
Styrene
1,1,2,2-Tetrachloroethane
Tetrachloroethylene
Toluene
1,1,1-Trichloroethane
1,1,2-Trichloroethane
Trichloroethylere
Vinyl acetate
Xylenes

11

5.6

5.6

5.6

11

5.6

5.6

5.6

11

11

5.6

11

5.6

5.6

5.6

5.6

5.6

5.6

5.6

5.6

5.6

5.6

11

11

11

5.6

5.6

5.6

5.6

5.6

5.6

5.6

11

5.6 $\mu \mathrm{g} / \mathrm{kg}$

$\mu \mathrm{g} / \mathrm{kg}$

$\mu \mathrm{g} / \mathrm{kg}$

$\mu \mathrm{g} / \mathrm{kg}$

$\mu \mathrm{g} / \mathrm{kg}$

$\mu \mathrm{g} / \mathrm{kg}$

$\mu \mathrm{g} / \mathrm{kg}$

$\mu \mathrm{g} / \mathrm{kg}$

$\mu g / \mathrm{kg}$

$\mu \mathrm{g} / \mathrm{kg}$

$\mu \mathrm{g} / \mathrm{kg}$

$\mu \mathrm{g} / \mathrm{kg}$

$\mu \mathrm{g} / \mathrm{kg}$

$\mu \mathrm{g} / \mathrm{kg}$

$\mu \mathrm{g} / \mathrm{kg}$

$\mu \mathrm{g} / \mathrm{kg}$

$\mu \mathrm{g} / \mathrm{kg}$

$\mu \mathrm{g} / \mathrm{kg}$

$\mu \mathrm{g} / \mathrm{kg}$

$\mu \mathrm{g} / \mathrm{kg}$

$\mu \mathrm{g} / \mathrm{kg}$

$\mu \mathrm{g} / \mathrm{kg}$

$\mu g / k g$

$\mu \mathrm{g} / \mathrm{kg}$

$\mu g / k g$

$\mu \mathrm{g} / \mathrm{kg}$

$\mu \mathrm{g} / \mathrm{kg}$

$\mu g / k g$

$\mu \mathrm{g} / \mathrm{kg}$

$\mu g / k g$

$\mu \mathrm{g} / \mathrm{kg}$

$\mu \mathrm{g} / \mathrm{kg}$

$\mu \mathrm{g} / \mathrm{kg}$

$\mu \mathrm{g} / \mathrm{kg}$
11

5.6

5.6

5.6

11

5.6

5.6

5.6

11

11

5.6

11

5.6

5.6

5.6

5.6

5.6

5.6

5.6

5.6

5.6

5.6

11

11

1.1

5.6

5.6

5.6

5.6

5.6

5.6

5.6

11

5.6
EPA8240

EPA8240

EPA8240

EPA8240

EPA8240

EPA8240

EPA8240

EPA8240

EPA8240

EPA8240

EPA8240

EPA8240

EPA8240

EPA8240

EPA8240

EPA8240

EPA8240

EPA8240

EPA8240

EPA8240

EPA8240

EPA8240

EPA8240

EPA8240

EPA8240

EPA8240

EPA8240

EPA8240

EPA8240

EPA8240

EPA8240

EPA8240

EPA8240

EPA 8240 
SAMPLE NAME: D43111502 (Continued)

Radionuclide Indicators and Radionuclides

Gross alpha

Nonvolatile beta

Radium, total alpha-emitting

Tritium

SAMPLE NAME: D43111502A

Sample Type: Duplicate

Constituent

Total Metals

$\begin{array}{llll}13 \pm 8.0 & \mathrm{pCi} / \mathrm{g} & 3.8 & \text { EPA900.0 } \\ 25 \pm 12 & \mathrm{pCi} / \mathrm{g} & 3.8 & \text { EPA900.0 } \\ 2.0 \pm 1.5 & \mathrm{pCi} / \mathrm{g} & 0.22 & \text { EPA903.0 } \\ 3.1 \pm 0.61 & \mathrm{pCi} / \mathrm{g} & 1.8 & \text { EPA906.0 }\end{array}$

Sample ID: 100408

Associated Sample: 100407

Arsenic
Barium
Cadmium
Chromium
Copper
Lead
Manganese
Mercury
Nickel
Selenium
Silver

$B / N / A$ Extractables (including $P A H$ and phenols)
Sample ID: 100407

\begin{tabular}{|c|c|c|c|c|c|}
\hline Acenaphthene & $\mathbf{U}$ & 370 & $\mu \mathrm{g} / \mathrm{kg}$ & 370 & EPA8270 \\
\hline Acenaphthylene & $\mathbf{U}$ & 370 & $\mu \mathrm{g} / \mathrm{kg}$ & 370 & EPA8270 \\
\hline Anthracene & $\mathbf{U}$ & 370 & $\mu \mathrm{g} / \mathrm{kg}$ & 370 & EPA8270 \\
\hline Benzo(a)anthracene & $\mathbf{U}$ & 370 & $\mu g / \mathrm{kg}$ & 370 & EPA8270 \\
\hline Benzo(b)fluoranthene & $\mathbf{U}$ & 370 & $\mu \mathrm{g} / \mathrm{kg}$ & 370 & EPA8270 \\
\hline Benzo(k)fluoranthene & $\mathbf{U}$ & 370 & $\mu g / \mathrm{kg}$ & 370 & EPA8270 \\
\hline Benzoic acid & U & 1,800 & $\mu \mathrm{g} / \mathrm{kg}$ & 1,800 & EPA8270 \\
\hline Benzo(g,h,i)perylene & $\mathbf{U}$ & 370 & $\mu \mathrm{g} / \mathrm{kg}$ & 370 & EPA8270 \\
\hline Benzo(a)pyrene & $\mathbf{U}$ & 370 & $\mu \mathrm{g} / \mathrm{kg}$ & 370 & EPA8270 \\
\hline Benzyl alcohol & $\mathbf{U}$ & 370 & $\mu g / k g$ & 320 & EP.A8270 \\
\hline Bis(2-chloroethoxy) methane & $\mathbf{U}$ & 370 & $\mu \mathrm{g} / \mathrm{kg}$ & 370 & EPA8270 \\
\hline Bis(2-chloroethyl) ether & $\mathbf{U}$ & 370 & $\mu g / \mathrm{kg}$ & 370 & EPA8270 \\
\hline Bis(2-chloroisopropyl) ether & $\mathbf{U}$ & 370 & $\mu \mathrm{g} / \mathrm{kg}$ & 370 & EPA8270 \\
\hline Bis(2-ethylhexyl) phthalate & $\mathbf{U}$ & 370 & $\mu g / \mathbf{k g}$ & 370 & EPA8270 \\
\hline 4-Bromophenyl phenyl ether & $\mathbf{U}$ & 370 & $\mu g / \mathrm{kg}$ & 370 & EPA8270 \\
\hline Butylbenzyl phthalate &. $\mathbf{U}$ & 370 & $\mu g / \mathrm{kg}$ & 370 & EPA8270 \\
\hline 4-Chloroaniline & $\mathbf{U}$ & 370 & $\mu \mathrm{g} / \mathrm{kg}$ & 370 & EPA8270 \\
\hline 4-Chloro-m-cresol & $\mathbf{U}$ & 370 & $\mu \mathrm{g} / \mathrm{kg}$ & 370 & EPA8270 \\
\hline 2-Chloronaphthalene & $\mathbf{U}$ & 370 & $\mathrm{Hg} / \mathrm{kg}$ & 370 & EPA8270 \\
\hline 2-Chlorophenol & $\mathbf{U}$ & 370 & $\mu g / k g$ & 370 & EPA8270 \\
\hline 4-Chlorophenyl phenyl ether & $\mathbf{U}$ & 370 & $\mu \mathrm{g} / \mathrm{kg}$ & 370 & EPA8270 \\
\hline Chrysene & $\mathbf{U}$ & 370 & $\mu \mathrm{g} / \mathrm{kg}$ & 370 & EPA8270 \\
\hline o-Cresol (2-Methylphenol) & $\mathbf{U}$ & 370 & $\mu \mathrm{g} / \mathrm{kg}$ & 370 & EPA8270 \\
\hline p-Cresol (4-Methylphenol) & $\mathbf{U}$ & 370 & $\mu \mathrm{g} / \mathrm{kg}$ & 370 & EPA8270 \\
\hline Dibenz(a,h)anthracene & $\mathbf{U}$ & 370 & $\mu \mathrm{g} / \mathrm{kg}$ & 370 & EPA8270 \\
\hline
\end{tabular}


SAMPLE NAME: D43111502A (Continued) RQ AQ

Constituent
Sample ID: 100408

B Result

Unit

D. Limit Method

\section{$B / N / A$ Extractables (including PAH and phenols)}

Dibenzofuran

Di-n-butyl phthalate

1,2-Dichlorobenzene

1,3-Dichlorobenzene

1,4-Dichlorobenzene

3.3'-Dichlorobenzidine

2,4-Dichlorophenol

Diethyl phthalate

Dimethyl phthalate

2,4-Dinitrophenol

2.4-Dinitrotoluene

2.6-Dinitrotoluene

Di-n-octyl phthalate

Fluoranthene

Fluorene

Hexachlorobenzene

Hexachlorobutadiene

Hexachlorocyclopentadiene

Hexachloroethane

Indeno(1,2,3-c,d)pyrene

Isophorone

2-Methyl-4,6-dinitrophenol

2-Methylnaphthalene

Naphthalene

o-Nitroaniline

$\mathrm{m}$-Nitroaniline

p-Nitroaniline

Nitrobenzene

2-Nitrophenol

4-Nitrophenol

$\mathrm{N}$-Nitrosodiphenylamine

$\mathrm{N}$-Nitrosodipropylamine

Pentachlorophenol

Phenanthrene

Phenol

Pyrene

1,2,4-Trichlorobenzene

2,4,5-Trichlorophenol

2,4,6-Trichlorophenol

Unknown hydrocarbon

Unknown hydrocarbon

Unknown ester

Unknown ketone
2.4-Dimethyl phenol

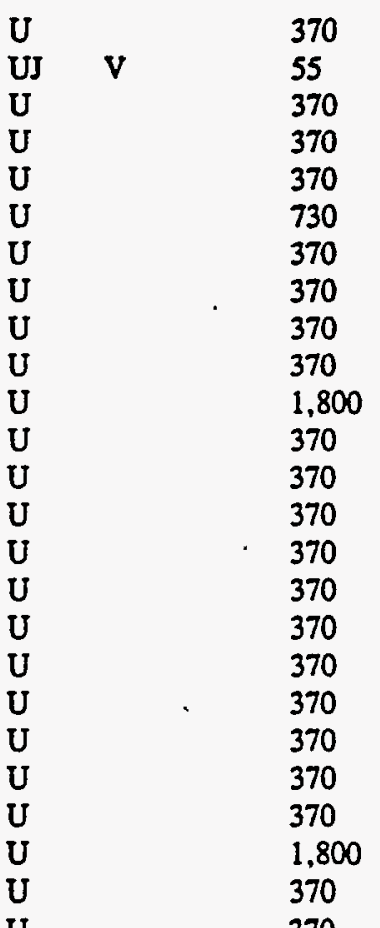

370

1,800

1,800

1.800

370

370

1.800

370

370

1,800

370

370

370

370

1,800

370

200

200

- 400

6,000 $\mu g / k g$

$\mu g / \mathrm{kg}$

$\mu g / \mathrm{kg}$

$\mu \mathrm{g} / \mathrm{kg}$

$\mu g / \mathrm{kg}$

$\mu \mathrm{g} / \mathrm{kg}$

$\mu \mathrm{g} / \mathrm{kg}$

$\mu \mathrm{g} / \mathrm{kg}$

$\mu \mathrm{g} / \mathrm{kg}$

$\mu \mathrm{g} / \mathrm{kg}$

$\mu \mathrm{g} / \mathrm{kg}$

$\mu \mathrm{g} / \mathrm{kg}$

$\mu \mathrm{g} / \mathrm{kg}$

$\mu g / \mathrm{kg}$

$\mu \mathrm{g} / \mathrm{kg}$

$\mu \mathrm{g} / \mathrm{kg}$

$\mu \mathrm{g} / \mathrm{kg}$

$\mu g / k g$

$\mu \mathrm{g} / \mathrm{kg}$

$\mu \mathrm{g} / \mathrm{kg}$

$\mu \mathrm{g} / \mathrm{kg}$

$\mu \mathrm{g} / \mathrm{kg}$

$\mu \mathrm{g} / \mathrm{kg}$

$\mu g / k g$

$\mu \mathrm{g} / \mathrm{kg}$

$\mu \mathrm{g} / \mathrm{kg}$

$\mu \mathrm{g} / \mathrm{kg}$

$\mu \mathrm{g} / \mathrm{kg}$

$\mu \mathrm{g} / \mathrm{kg}$

$\mu g / \mathrm{kg}$

$\mu \mathrm{g} / \mathrm{kg}$

$\mu \mathrm{g} / \mathrm{kg}$

$\mu g / \mathrm{kg}$

$\mu \mathrm{g} / \mathrm{kg}$

ig $/ \mathrm{kg}$

$\mu \mathrm{g} / \mathrm{kg}$

$\mu \mathrm{g} / \mathrm{kg}$

$\mu \mathrm{g} / \mathrm{kg}$

$\mu \mathrm{g} / \mathrm{kg}$

$\mu g / k g$

$\mu \mathrm{g} / \mathrm{kg}$

$\mu g / \mathbf{k g}$

$\mu \mathrm{g} / \mathrm{kg}$

$\mu \mathrm{g} / \mathrm{kg}$
370

370

370

370

370

730

370

370

370

370

1,800

370

370

370

370

370

370

370

370

370

370

370

1,800

370

370

1,800

1,800

1,800

370

370

1.800

370

370

1,800

370

370

370

370

1,800

370
EPA8270

EPA8270

EPA8270

EPA8270

EPA8270

EPA8270

EPA8270

EPA8270

EPA8270

EPA8270

EPA8270

EPA8270

EPA8270

EPA8270

EPA8270

EPA8270

EPA8270

EPA8270

EPA8270

EPA8270

EPA8270

EPA8270

EPA8270

EPA8270

EPA8270

EPA8270

EPA8270

EPA8270

EPA8270

EPA8270

EPA8270

EPA8270

EPA8270

EPA8270

EPA8270

EPA8270

EPA8270

EPA8270

EPA8270

EPA8270

EPA8270

EPA8270

EPA8270

EPA8270

Volatile Organic Compounds

Acetone

Benzene

Bromodichloromethane

\section{1}

5.5

5.5 $\mu \mathrm{g} / \mathrm{kg}$

$\mu \mathrm{g} / \mathrm{kg}$

$\mu \mathrm{g} / \mathrm{kg}$
11
5.5
5.5

EPA8240

EPA8240

EPA8240 
SAMPLE NAME: D43i11502A (Continued)

\section{Constituent}

$\mathbf{R Q}$

AQ

B

Sample D: 100408

Volatile Organic Compounds

Bromoform
Bromomethane (Methyl bromide)
Carbon disulfide
Carbon tetrachloride
Chlorobenzene
Chloroethane
Chloroethene (Vinyl chloride)
Chloroform

$\begin{array}{llll}5.5 & \mu \mathrm{g} / \mathrm{kg} & 5.5 & \text { EPA8240 } \\ 11 & \mu \mathrm{g} / \mathrm{kg} & 11 & \text { EPA8240 } \\ 5.5 & \mu \mathrm{g} / \mathrm{kg} & 5.5 & \text { EPA8240 } \\ 5.5 & \mu \mathrm{g} / \mathrm{kg} & 5.5 & \text { EPA8240 } \\ 5.5 & \mu \mathrm{g} / \mathrm{kg} & 5.5 & \text { EPA8240 } \\ 11 & \mu \mathrm{g} / \mathrm{kg} & 11 & \text { EPA8240 } \\ 11 & \mu \mathrm{g} / \mathrm{kg} & 11 & \text { EPA8240 } \\ 5.5 & \mu \mathrm{g} / \mathrm{kg} & 5.5 & \text { EPA8240 } \\ 11 & \mu \mathrm{g} / \mathrm{kg} & 11 & \text { EPA8240 } \\ 5.5 & \mu \mathrm{g} / \mathrm{kg} & 5.5 & \text { EPA8240 } \\ 5.5 & \mu \mathrm{g} / \mathrm{kg} & 5.5 & \text { EPA8240 } \\ 5.5 & \mu \mathrm{g} / \mathrm{kg} & 5.5 & \text { EPA8240 } \\ 5.5 & \mu \mathrm{g} / \mathrm{kg} & 5.5 & \text { EPA8240 } \\ 5.5 & \mu \mathrm{g} / \mathrm{kg} & 5.5 & \text { EPA8240 } \\ 5.5 & \mu \mathrm{g} / \mathrm{kg} & 5.5 & \text { EPA8240 } \\ 5.5 & \mu \mathrm{g} / \mathrm{kg} & 5.5 & \text { EPA8240 } \\ 5.5 & \mu \mathrm{g} / \mathrm{kg} & 5.5 & \text { EPA8240 } \\ 5.5 & \mu \mathrm{g} / \mathrm{kg} & 5.5 & \text { EPA8240 } \\ 5.5 & \mu \mathrm{g} / \mathrm{kg} & 5.5 & \text { EPA8240 } \\ 11 & \mu \mathrm{g} / \mathrm{kg} & 11 & \text { EPA8240 } \\ 11 & \mu \mathrm{g} / \mathrm{kg} & 11 & \text { EPA8240 } \\ 11 & \mu \mathrm{g} / \mathrm{kg} & 11 & \text { EPA8240 } \\ 5.5 & \mu \mathrm{g} / \mathrm{kg} & 5.5 & \text { EPA8240 } \\ 5.5 & \mu \mathrm{g} / \mathrm{kg} & 5.5 & \text { EPA8240 } \\ 5.5 & \mu \mathrm{g} / \mathrm{kg} & 5.5 & \text { EPA8240 } \\ 5.5 & \mu \mathrm{g} / \mathrm{kg} & 5.5 & \text { EPA8240 } \\ 5.5 & \mu \mathrm{g} / \mathrm{kg} & 5.5 & \text { EPA8240 } \\ 5.5 & \mu \mathrm{g} / \mathrm{kg} & 5.5 & \text { EPA8240 } \\ 5.5 & \mu \mathrm{g} / \mathrm{kg} & 5.5 & \text { EPA8240 } \\ 11 & \mu \mathrm{g} / \mathrm{kg} & 11 & \text { EPA8240 } \\ 5.5 & \mu \mathrm{g} / \mathrm{kg} & 5.5 & \text { EPA8240 }\end{array}$

Gross alpha

U

U

U

U

U

U

U

U

U

U

U

U

U

U

U

U

U

U

U

U

U

U

U

U

י

Result

Unit

D. Limit Method

\section{Radionuclide Indicators and Radionuclides}

Nonvolatile beta

Radium, total alpha-emitting

U V

$14 \pm 8.3$

$\mathrm{pCi} / \mathrm{g}$

$11 \pm 10$

$\mathrm{pCi} / \mathrm{g}$

$1.0 \pm 0.80$

$\mathrm{pCi} / \mathrm{g}$

Tritium

$5.4 \pm 0.97$

$\mathrm{pCi} / \mathrm{g}$

$\begin{array}{ll}3.8 & \text { EPA900.0 } \\ 3.8 & \text { EPA900.0 } \\ 0.13 & \text { EPA903.0 } \\ 1.7 & \text { EPA906.0 }\end{array}$


SAMPLE NAME: D43111503

Location (SRS Coordinates): 18533.07E 66779.25N

Ground Elevation Above MSL: $128.5 \mathrm{ft}$

Depth of Core Interval: 5.00 to $7.00 \mathrm{ft}$

Sample Type: Normal

Sample Color: SYR 5/6

Constituent

Physical Parameters

Cation exchange capacity

$\mathrm{pH}$

Specific conductance

Total organic carbon

Total Metals

Arsenic

Barium

Cadmium

Chromium

Copper

Lead

Manganese

Mercury

Nickel

Selenium

Silver
Sample ID: 100410

Sample Matrix: Soil

USC Soil Classification: SC

Sample Moisture: Damp

Percent Solids: 87.10

RQ AQ B Result Unit $\quad$ D. Limit Method

$\begin{array}{llll}8.2 & \mathrm{meq} / 100 \mathrm{~g} & 2.0 & \text { EPA9080 } \\ 5.6 & \mathrm{pH} & 0.010 & \text { EPA9045 } \\ 28 & \mu \mathrm{S} / \mathrm{cm} & 1.0 & \text { EPA9050 } \\ 1.400 & \mathrm{mg} / \mathrm{kg} & 210 & \text { EPA415.1 }\end{array}$

$B / N / A$ Extractables (including $P A H$ and phenols)

\begin{tabular}{|c|c|c|c|c|c|c|c|}
\hline Acenaphthene & UJ & L & & 380 & $\mu g / \mathrm{kg}$ & 380 & EPA8270 \\
\hline Acenaphthylene & UJ & L & & 380 & $\mu g / \mathrm{kg}$ & 380 & EPA8270 \\
\hline Anthracene & UJ & L & & 380 & $\mu \mathrm{g} / \mathrm{kg}$ & 380 & EPA8270 \\
\hline Benzo(a)anthracene & UJ & $\mathbf{L}$ & & 380 & $\mu \mathrm{g} / \mathrm{kg}$ & 380 & EPA8270 \\
\hline Benzo(b)fluoranthene & UJ & $\mathrm{LH}$ & L & 380 & $\mu \mathrm{g} / \mathrm{kg}$ & 380 & EPA8270 \\
\hline Benzo(k)fluoranthene & UJ & LH & $L$ & 380 & $\mu \mathrm{g} / \mathrm{kg}$ & 380 & EPA8270 \\
\hline Benzoic acid & UJ & L & & 1,900 & $\mu g / \mathrm{kg}$ & 1.900 & EPA8270 \\
\hline Benzo(g,h,i)perylene & UJ & LH & $L$ & 380 & $\mu \mathrm{g} / \mathrm{kg}$ & 380 & EPA8270 \\
\hline Benzo(a)pyrene & UJ & $\mathrm{LH}$ & L & 380 & $\mu \mathrm{g} / \mathrm{kg}$ & 380 & EPA8270 \\
\hline Benzyl alcohol & UJ & $\mathbf{L}$ & & 380 & $\mu g / \mathrm{kg}$ & 380 & EPA8270 \\
\hline Bis(2-chloroethoxy) methane & UJ & $\mathbf{L}$ & & 380 & $\mu \mathrm{g} / \mathrm{kg}$ & 380 & EPA8270 \\
\hline Bis(2-chloroethyl) ether & UJ & $\mathbf{L}$ & & 380 & $\mu \mathrm{g} / \mathrm{kg}$ & 380 & EPA8270 \\
\hline Bis(2-chloroisopropyl) ether & UJ & $\mathbf{L}$ & & 380 & $\mu g / \mathrm{kg}$ & 380 & EPA8270 \\
\hline Bis(2-ethylhexyl) phthalate & UJ & L & & 380 & $\mu g / \mathrm{kg}$ & 380 & EPA8270 \\
\hline 4-Bromophenyl phenyl ether & UJ & $L$ & & 380 & $\mu g / \mathrm{kg}$ & 380 & EPA8270 \\
\hline Butylbenzyl phthalate & UJ & $\mathbf{L}$ & & 380 & $\mu \mathrm{g} / \mathrm{kg}$ & 380 & EPA8270 \\
\hline 4-Chloroaniline & UJ & $\mathbf{L}$ & & 380 & $\mu \mathrm{g} / \mathrm{kg}$ & 380 & EPA8270 \\
\hline 4-Chloro-m-cresol & UJ & $\mathbf{L}$ & & 380 & $\mu g / \mathrm{kg}$ & 380 & EPA8270 \\
\hline 2-Chloronaphthalene & UJ & $\mathbf{L}$ & & 380 & $\mu g / \mathrm{kg}$ & 380 & EPA8270 \\
\hline 2-Chlorophenol & UJ & $\mathbf{L}$ & & 380 & $\mu \mathrm{g} / \mathrm{kg}$ & 380 & EPA8270 \\
\hline 4-Chlorophenyl phenyl ether & UJ & L & & 380 & $\mu \mathrm{g} / \mathrm{kg}$ & 380 & EPA8270 \\
\hline Chrysene & UJ & $\mathbf{L}$ & & 380 & $\mu g / \mathrm{kg}$ & 380 & EPA8270 \\
\hline o-Cresol (2-Methylphenol) & UJ & $\mathbf{L}$ & & 380 & $\mu g / \mathrm{kg}$ & 380 & EPA 8270 \\
\hline
\end{tabular}

$\begin{array}{ll}1.9 & \mathrm{mg} / \mathrm{kg} \\ 22 & \mathrm{mg} / \mathrm{kg} \\ 0.55 & \mathrm{mg} / \mathrm{kg} \\ 15 & \mathrm{mg} / \mathrm{kg} \\ 2.8 & \mathrm{mg} / \mathrm{kg} \\ 8.4 & \mathrm{mg} / \mathrm{kg} \\ 38 & \mathrm{mg} / \mathrm{kg} \\ 0.12 & \mathrm{mg} / \mathrm{kg} \\ 4.4 & \mathrm{mg} / \mathrm{kg} \\ 0.50 & \mathrm{mg} / \mathrm{kg} \\ 1.1 & \mathrm{mg} / \mathrm{kg}\end{array}$

$\begin{array}{ll}1.0 & \text { EPA7060 } \\ 22 & \text { EPA6010 } \\ 0.55 & \text { EPA6010 } \\ 1.1 & \text { EPA6010 } \\ 2.8 & \text { EPA6010 } \\ 1.2 & \text { EPA7421 } \\ 1.7 & \text { EPA6010 } \\ 0.12 & \text { EPA7470 } \\ 4.4 & \text { EPA6010 } \\ 0.50 & \text { EPA7740 } \\ 1.1 & \text { EPA6010 }\end{array}$




$\begin{array}{llllll}\text { Constituent } & \text { RQ } & \text { AQ } & \text { Result } & \text { Unit Limit Method }\end{array}$

$B / N / A$ Extractables (including $P A H$ and phenols)

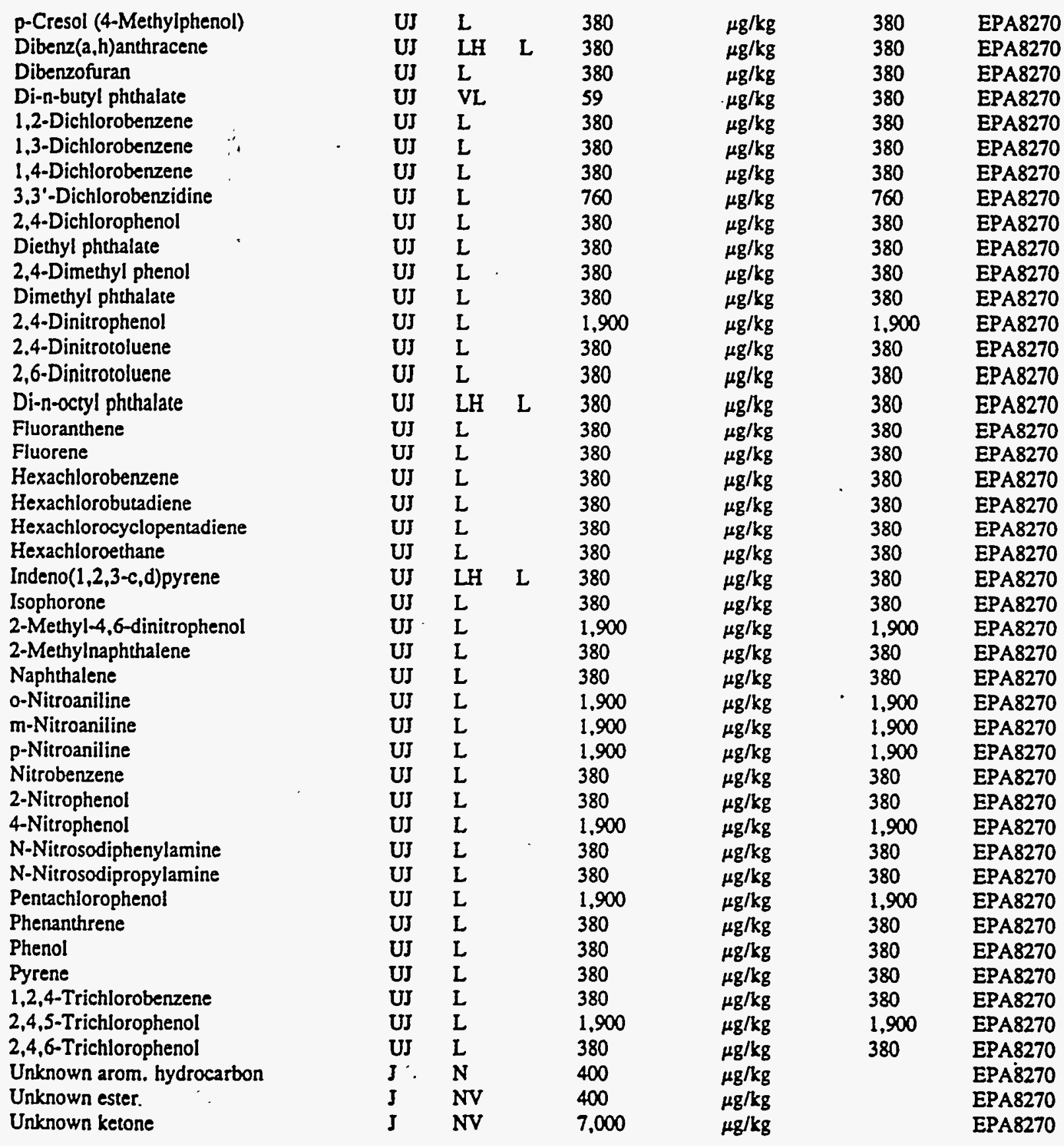


SAMPLE NAME: D43111503 (Continued)

Constituent

RQ AQ B Result

Volatile Organic Compounds
Sample ID: 100410

Unit D. Limit Method

Acetone
Benzene
Bromodichloromethane
Bromoform
Bromomethane (Methyl bromide)
Carbon disulfide
Carbon tetrachloride
Chlorobenzene
Chloroethane
Chloroethene (Vinyl chloride)
Chloroform
Chloromethane (Methyl chloride)
Dibromochloromethane
1.1-Dichloroethane
1.2-Dichloroethane
1,1-Dichloroethylene
1,2-Dichloroethylene
Dichloromethane (Methylene chloride)
1,2-Dichloropropane
cis-1,3-Dichloropropene
trans-1,3-Dichloropropene
Ethylbenzene
2-Hexanone
Methyl ethyl ketone
Methyl isobutyl ketone
Styrene
1.1,2,2-Tetrachloroethane
Tetrachloroethylene
Toluene
1,1.1-Trichloroethane
1,1,2-Trichloroethane
Trichloroethylene
Vinyl acetate
Xylenes

Pesticides/PCBs and Dioxins/Furans

\begin{tabular}{|c|c|c|c|c|}
\hline U & 12 & $\mu \mathrm{g} / \mathrm{kg}$ & 12 & EPA 8240 \\
\hline$U$ & 5.8 & $\mu g / \mathrm{kg}$ & 5.8 & EPA8240 \\
\hline U & 5.8 & $\mu g / \mathrm{kg}$ & 5.8 & EPA8240 \\
\hline $\mathbf{U}$ & 5.8 & $\mu \mathrm{g} / \mathrm{kg}$ & 5.8 & EPA8240 \\
\hline$U$ & 12 & $\mu g / \mathrm{kg}$ & 12 & EPA 8240 \\
\hline $\mathbf{U}$ & 5.8 & $\mu g / \mathrm{kg}$ & 5.8 & EPA8240 \\
\hline U & 5.8 & $\mu g / \mathrm{kg}$ & 5.8 & EPA8240 \\
\hline $\mathrm{U}$ & 5.8 & $\mu \mathrm{g} / \mathrm{kg}$ & 5.8 & EPA8240 \\
\hline$U$ & 12 & $\mu g / \mathrm{kg}$ & 12 & EPA8240 \\
\hline U & 12 & $\mu \mathrm{g} / \mathrm{kg}$ & 12 & EPA8240 \\
\hline$U$ & 5.8 & $\mu \mathrm{g} / \mathrm{kg}$ & 5.8 & EPA8240 \\
\hline U & 12 & $\mu \mathrm{g} / \mathrm{kg}$ & 12 & EPA8240 \\
\hline$U$ & 5.8 & $\mu \mathrm{g} / \mathrm{kg}$ & 5.8 & EPA8240 \\
\hline U & 5.8 & $\mu \mathrm{g} / \mathrm{kg}$ & 5.8 & EPA8240 \\
\hline$U$ & 5.8 & $\mu g / \mathrm{kg}$ & 5.8 & EPA8240 \\
\hline U & 5.8 & $\mu g / \mathrm{kg}$ & 5.8 & EPA8240 \\
\hline $\mathrm{U}$ & 5.8 & $\mu g / \mathrm{kg}$ & 5.8 & EPA8240 \\
\hline U & 5.8 & $\mu \mathrm{g} / \mathrm{kg}$ & 5.8 & EPA8240 \\
\hline U & 5.8 & $\mu \mathrm{g} / \mathrm{kg}$ & 5.8 & EPA8240 \\
\hline$U$ & 5.8 & $\mu \mathrm{g} / \mathrm{kg}$ & 5.8 & EPA8240 \\
\hline$U$ & 5.8 & $\mu \mathrm{g} / \mathrm{kg}$ & 5.8 & EPA8240 \\
\hline$U$ & 5.8 & $\mu \mathrm{g} / \mathrm{kg}$ & 5.8 & EPA8240 \\
\hline $\mathrm{U}$ & 12 & $\mu g / k g$ & 12 & EPA8240 \\
\hline$U$ & 12 & $\mu \mathrm{g} / \mathrm{kg}$ & 12 & EPA8240 \\
\hline $\mathbf{U}$ & 12 & $\mu \mathrm{g} / \mathrm{kg}$ & 12 & EPA8240 \\
\hline $\mathbf{U}$ & 5.8 & $\mu \mathrm{g} / \mathrm{kg}$ & 5.8 & EPA8240 \\
\hline $\mathbf{U}$ & 5.8 & $\mu g / k g$ & 5.8 & EPA8240 \\
\hline $\mathrm{U}$ & 5.8 & $\mu \mathrm{g} / \mathrm{kg}$ & 5.8 & EPA8240 \\
\hline $\mathbf{U}$ & 5.8 & $\mu \mathrm{g} / \mathrm{kg}$ & 5.8 & EPA8240 \\
\hline$U$ & 5.8 & $\mu \mathrm{g} / \mathrm{kg}$ & 5.8 & EPA8240 \\
\hline$U$ & 5.8 & $\mu g / k g$ & 5.8 & EPA8240 \\
\hline U & 5.8 & $\mu g / \mathrm{kg}$ & 5.8 & EPA8240 \\
\hline$U$ & 12 & $\mu \mathrm{g} / \mathrm{kg}$ & 12 & EPA8240 \\
\hline U & 5.8 & $\mu \mathrm{g} / \mathrm{kg}$ & 5.8 & EPA8240 \\
\hline
\end{tabular}

\begin{tabular}{|c|c|c|c|c|c|c|}
\hline Aldrin & $\mathbf{U}$ & & 1.9 & $\mu \mathrm{g} / \mathrm{kg}$ & 1.9 & EPA8080 \\
\hline alpha-Benzene hexachloride & $\mathbf{U}$ & & 1.9 & $\mu \mathrm{g} / \mathrm{kg}$ & 1.9 & EPA8080 \\
\hline beta-Benzene hexachloride & $\mathrm{U}$ & & 1.9 & $\mu \mathrm{g} / \mathrm{kg}$ & 1.9 & EPA8080 \\
\hline delta-Benzene hexachloride & $\mathbf{U}$ & & 1.9 & $\mu \mathrm{g} / \mathrm{kg}$ & 1.9 & EPA8080 \\
\hline alpha-Chlordane & U & & 1.9 & $\mu \mathrm{g} / \mathrm{kg}$ & 1.9 & EPA8080 \\
\hline gamma-Chlordane & $\mathbf{U}$ & & 1.9 & $\mu \mathrm{g} / \mathrm{kg}$ & 1.9 & EPA8080 \\
\hline p,p'-DDD & U & & 3.8 & $\mu \mathrm{g} / \mathrm{kg}$ & 3.8 & EPA8080 \\
\hline p,p'-DDE & $\boldsymbol{U}$ & A & 1.1 & $\mu g / \mathrm{kg}$ & 3.8 & EPA8080 \\
\hline p.p'-DDT & $\mathbf{U}$ & A & 1.9 & $\mu \mathrm{g} / \mathrm{kg}$ & 3.8 & EPA8080 \\
\hline Dieldrin & $\mathbf{U}$ & & 3.8 & $\mu \mathrm{g} / \mathrm{kg}$ & 3.8 & EPA8080 \\
\hline Endosulfan I & U & & 1.9 & $\mu \mathrm{g} / \mathrm{kg}$ & 1.9 & EPA8080 \\
\hline Endosulfan II & $\mathrm{U}$ & & 3.8 & $\mu \mathrm{g} / \mathrm{kg}$ & 3.8 & EPA8080 \\
\hline Endosulfan sulfate & $\mathbf{U}$ & & 3.8 & $\mu \mathrm{g} / \mathrm{kg}$ & 3.8 & EPA8080 \\
\hline
\end{tabular}


SAMPLE NAME: D43111503 (Continued)

Constituent

RQ

$A Q$

Result

Unit

D. Limit Method

Pesticides/PCBs and Dioxins/Furans

Endrin

Endrin ketone

Heptachlor

Heptachlor epoxide

Heptachlorodibenzo-p-dioxin isomers

Heptachlorodibenzo-p-dioxin isomers

Heptachlorodibenzo-p-furan isomers

Heptachlorodibenzo-p-furan isomers

Hexachlorodibenzo-p-dioxin isomers

Hexachlorodibenzo-p-dioxin isomers

Hexachlorodibenzo-p-furan isomers

Hexachlorodibenzo-p-furan isomers

Lindane

Methoxychlor

Octachlorodibenzo-p-dioxin

Octachlorodibenzo-p-dioxin

Octachlorodibenzo-p-furan

Octachlorodibenzo-p-furan

PCB 1016

PCB 1221

PCB 1232

PCB 1242

PCB 1248

PCB 1254

PCB 1260

Pentachlorodibenzo-p-dioxin isomers

Pentachlorodibenzo-p-furan isomers

Pentachlorodibenzo-p-dioxin isomers

Pentachlorodibenzo-p-furan isomers

2,3,7,8-TCDD

$2,3,7,8-\mathrm{TCDD}$

2.3,7,8-TCDF

2,3,7,8-TCDF

Tetrachlorodibenzo-p-dioxin isomers

Tetrachlorodibenzo-p-dioxin isomers

Tetrachlorodibenzo-p-furan isomers

Tetrachlorodibenzo-p-furan isomers

Toxaphene

Sample ID: 100410

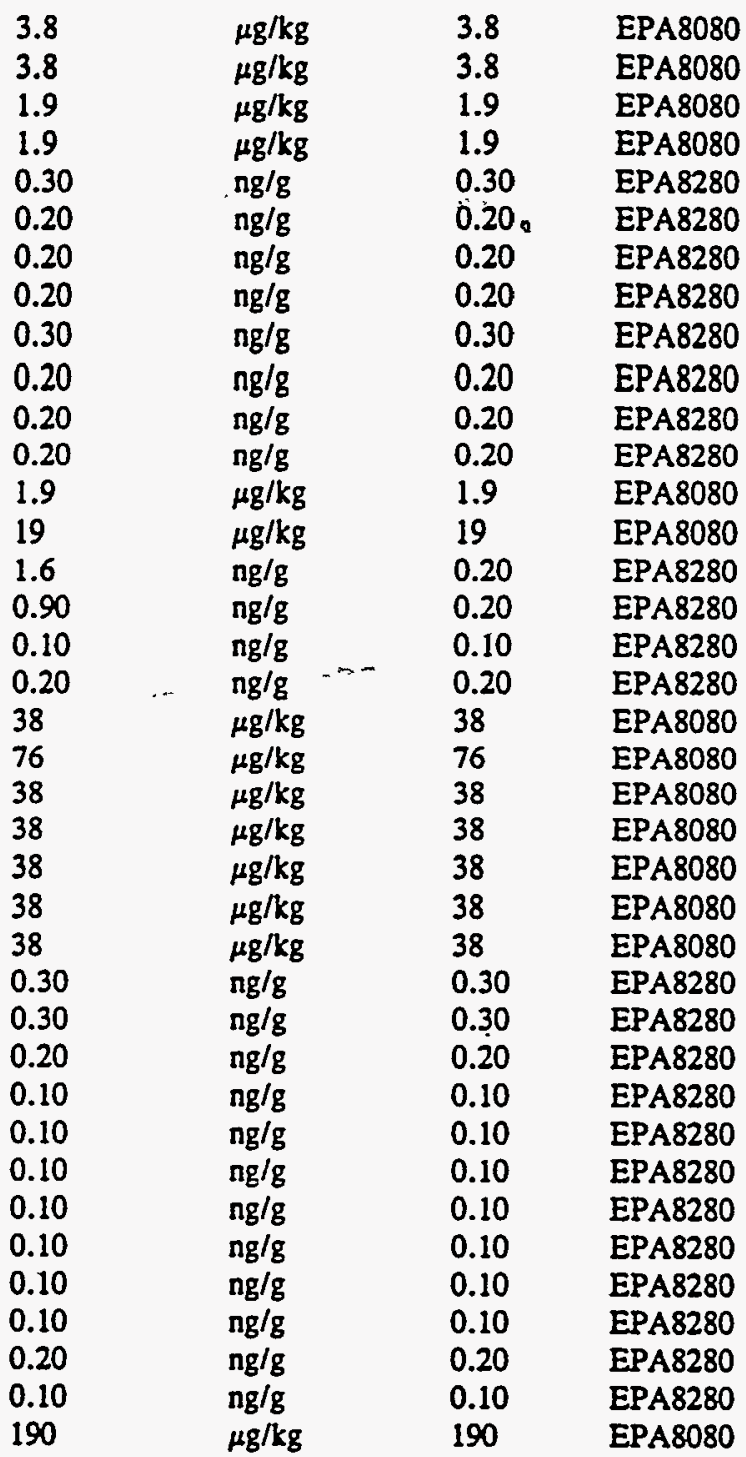

\section{Radionuclide Indicators and Radionuclides}

$\begin{array}{lll}\text { Gross alpha } & \text { U } & \\ \text { Gross alpha } & & \\ \text { Nonvolatile beta } & & \text { V } \\ \text { Nonvolatile beta } & & \text { V } \\ \text { Radium, total alpha-emitting } & \text { U } & \text { V } \\ \text { Radium, total alpha-emitting } & & \text { V } \\ \text { Tritium } & \text { U } & \text { X } \\ \text { Tritium } & & \text { X }\end{array}$

$\begin{array}{ll}5.2 & \mathrm{pCi} / \mathrm{g} \\ 5.2 \pm 14 & \mathrm{pCi} / \mathrm{g} \\ 38 \pm 21 & \mathrm{pCi} / \mathrm{g} \\ 33 \pm 21 & \mathrm{pCi} / \mathrm{g} \\ 2.0 \pm 0.92 & \mathrm{pCi} / \mathrm{g} \\ 2.1 \pm 1.0 & \mathrm{pCi} / \mathrm{g} \\ 1.3 & \mathrm{pCi} / \mathrm{g} \\ 3.8 \pm 0.88 & \mathrm{pCi} / \mathrm{g}\end{array}$

5.2

5.0

9.1

9.7

0.080

0.10

1.3

1.8
EPA900.0

EPA900.0

EPA900.0

EPA900.0

EPA903.0

EPA903.0

EPA906.0 
SAMPLE NAME: D43111504

Location (SRS Coordinates): 18533.07E 66779.25N

Ground Elevation Above MSL: $128.5 \mathrm{ft}$

Depth of Core Interval: 10.00 to $12.00 \mathrm{ft}$

Sample Type: Normal

Sample Color:

Constituent

Physical Parameters

Cation exchange capacity

$\mathrm{pH}$

Specific conductance

Total organic carbon

Total Metals

Arsenic

Barium

Cadmium

Chromium

Copper

Lead

Manganese

Mercury

Nickel

Selenium

Silver

$B / N / A$ Extractables (including $P A H$ and phenols)

Acenaphthene

Acenaphthylene

Anthracene

Benzo(a)anthracene

Benzo(b)fluoranthene

Benzo(k)fluoranthene

Benzoic acid

Benzo $(g, h, i)$ perylene

Benzo(a)pyrene

Benzyl alcohol

Bis(2-chloroethoxy) methane

Bis(2-chloroethyl) ether

Bis(2-chloroisopropyl) ether

Bis(2-ethylhexyl) phthalate

4-Bromophenyl phenyl ether

Butylbenzyl phthalate

4-Chloroaniline

4-Chloro-m-cresol

2-Chloronaphthalene

2-Chlorophenol

4-Chlorophenyl phenyl ether

Chrysene

o-Cresol (2-Methylphenol)
Sample ID: 100411

Sample Matrix: Soil

USC Soil Classification: SW

Sample Moisture: Damp

Percent Solids: 80.30

RQ AQ B Result Unit

D. Limit Method
12

5.0

22

1,200 $\mathrm{mg} / \mathrm{kg}$

$\mathrm{mg} / \mathrm{kg}$

$\mathrm{mg} / \mathrm{kg}$

$\mathrm{mg} / \mathrm{kg}$

$\mathrm{mg} / \mathrm{kg}$

$\mathrm{mg} / \mathrm{kg}$

$\mathrm{mg} / \mathrm{kg}$

$\mathrm{mg} / \mathrm{kg}$

$\mathrm{mg} / \mathrm{kg}$

$\mathrm{mg} / \mathrm{kg}$

$\mathrm{mg} / \mathrm{kg}$

$\begin{array}{ll}1.2 & \text { EPA7060 } \\ 22 & \text { EPA6010 } \\ 0.55 & \text { EPA6010 } \\ 1.1 & \text { EPA6010 } \\ 2.7 & \text { EPA6010 } \\ 0.37 & \text { EPA7421 } \\ 1.6 & \text { EPA6010 } \\ 0.13 & \text { EPA7470 } \\ 4.4 & \text { EPA6010 } \\ 0.61 & \text { EPA7740 } \\ 1.1 & \text { EPA6010 }\end{array}$

$\begin{array}{lll}\mathrm{meq} / 100 \mathrm{~g} & 2.0 & \text { EPA9080 } \\ \mathrm{pH} & 0.010 & \text { EPA9045 } \\ \mu \mathrm{S} / \mathrm{cm} & 1.0 & \text { EPA9050 } \\ \mathrm{mg} / \mathrm{kg} & 190 & \text { EPA415.1 }\end{array}$

$\begin{array}{lll}\mu \mathrm{g} / \mathrm{kg} & 410 & \text { EPA8270 } \\ \mu \mathrm{g} / \mathrm{kg} & 410 & \text { EPA8270 } \\ \mu \mathrm{g} / \mathrm{kg} & 410 & \text { EPA8270 } \\ \mu \mathrm{g} / \mathrm{kg} & 410 & \text { EPA8270 } \\ \mu \mathrm{g} / \mathrm{kg} & 410 & \text { EPA8270 } \\ \mu \mathrm{g} / \mathrm{kg} & 410 & \text { EPA8270 } \\ \mu \mathrm{g} / \mathrm{kg} & 2,100 & \text { EPA8270 } \\ \mu \mathrm{g} / \mathrm{kg} & 410 & \text { EPA8270 } \\ \mu \mathrm{g} / \mathrm{kg} & 410 & \text { EPA8270 } \\ \mu \mathrm{g} / \mathrm{kg} & 410 & \text { EPA8270 } \\ \mu \mathrm{g} / \mathrm{kg} & 410 & \text { EPA8270 } \\ \mu \mathrm{g} / \mathrm{kg} & 410 & \text { EPA8270 } \\ \mu \mathrm{g} / \mathrm{kg} & 410 & \text { EPA8270 } \\ \mu \mathrm{g} / \mathrm{kg} & 410 & \text { EPA8270 } \\ \mu \mathrm{g} / \mathrm{kg} & 410 & \text { EPA8270 } \\ \mu \mathrm{g} / \mathrm{kg} & 410 & \text { EPA8270 } \\ \mu \mathrm{g} / \mathrm{kg} & 410 & \text { EPA8270 } \\ \mu \mathrm{g} / \mathrm{kg} & 410 & \text { EPA8270 } \\ \mu \mathrm{g} / \mathrm{kg} & 410 & \text { EPA8270 } \\ \mu \mathrm{gg} / \mathrm{kg} & 410 & \text { EPA8270 } \\ \mu \mathrm{g} / \mathrm{kg} & 410 & \text { EPA8270 } \\ \mu \mathrm{g} / \mathrm{kg} & 410 & \text { EPA8270 } \\ \mu \mathrm{g} / \mathrm{kg} & 410 & \text { EPA8270 }\end{array}$

UJ L $\quad 410$

L $\quad 410$

410

UJ LH L 410

UJ LH L 410

UJ L $\quad 2,100$

UJ LH L 410

410

410

410

410

410

410

410

410

410

410
EPA8270 
Constituent

RQ AQ B Result

$B / N / A$ Extractables (including $P A H$ and phenols)

p-Cresol (4-Methylphenol)

Dibenz $(a, h)$ anchracene

Dibenzofuran

Di-n-buryl phthalate

1,2-Dichlorobenzene .

1,3-Dichlorobenzene

1.4-Dichlorobenzene

3,3'-Dichlorobenzidine

2,4-Dichlorophenol

Diethyl phthalate

2,4-Dimethyl phenol

Dimethyl phthalate

2,4-Dinitrophenol

2,4-Dinitrotoluene

2,6-Dinitrotoluene

Di-n-octyl phthalate

Fluoranthene

Fluorene

Hexachlorobenzene

Hexachlorobutadiene

Hexachlorocyclopentadiene

Hexachloroethane

Indeno(1,2,3-c,d)pyrene

Isophorone

2-Methyl-4,6-dinitrophenol

2-Methylnaphthalene

Naphthalene

o-Nitroaniline

m-Nitroaniline

p-Nitroaniline

Nitrobenzene

2-Nitrophenol

4-Nitrophenol

$\mathrm{N}$-Nitrosodiphenylamine

N-Nitrosodipropylamine

Pentachlorophenol

Phenanthrene

Phenol

Pyrene

1,2,4-Trichlorobenzene

2,4,5-Trichlorophenol

2,4,6-Trichlorophenol

Unknown ester

Unknown ketone

Unknown phthalate

Unknown phthalate

Unknown phthalate

Unknown phthalate

Unknown phuhalate

Unknown phthalate

\begin{tabular}{|c|c|c|c|c|c|c|}
\hline UJ & L & & 410 & $\mu g / \mathrm{kg}$ & 410 & EPA8270 \\
\hline UJ & LH & L & 410 & $\mu g / k g$ & 410 & EPA8270 \\
\hline UJ & L & & 410 & $\mu \mathrm{g} / \mathrm{kg}$ & 410 & EPA8270 \\
\hline UJ & VL & & 72 & $\mu g / \mathrm{kg}$ & 410 & EPA8270 \\
\hline UJ & L & & 410 & $\mu \mathrm{g} / \mathrm{kg}$ & 410 & EPA8270 \\
\hline UJ & L & & 410 & $\mu \mathrm{g} / \mathrm{kg}$ & 410 & EPA8270 \\
\hline UJ & L & & 410 & $\mu \mathrm{g} / \mathrm{kg}$ & 410 & EPA8270 \\
\hline UJ & L & & 820 & $\mu \mathrm{g} / \mathrm{kg}$ & 820 & EPA8270 \\
\hline UJ & L & & 410 & $\mu \mathrm{g} / \mathrm{kg}$ & 410 & EPA8270 \\
\hline UJ & L & & 410 & $\mu \mathrm{g} / \mathrm{kg}$ & 410 & EPA8270 \\
\hline UJ & L & & 410 & $\mu g / \mathrm{kg}$ & 410 & EPA8270 \\
\hline UJ & L & & 410 & $\mu \mathrm{g} / \mathrm{kg}$ & 410 & EPA8270 \\
\hline UJ & L & & 2,100 & $\mu g / k g$ & 2.100 & EPA8270 \\
\hline UJ & L & & 410 & $\mu g / \mathrm{kg}$ & 410 & EPA8270 \\
\hline UJ & L & & 410 & $\mu g / k g$ & 410 & EPA8270 \\
\hline UJ & $\mathrm{LH}$ & L & 410 & $\mu g / k g$ & 410 & EPA8270 \\
\hline UJ & L & & 410 & $\mu \mathrm{g} / \mathrm{kg}$ & 410 & EPA8270 \\
\hline UJ & L & & 410 & $\mu \mathrm{g} / \mathrm{kg}$ & 410 & EPA8270 \\
\hline UJ & L & & 410 & $\mu g / k g$ & 410 & EPA8270 \\
\hline UJ & L & & 410 & $\mu \mathrm{g} / \mathrm{kg}$ & 410 & EPA8270 \\
\hline UJ & L & & 410 & $\mu g / k g$ & 410 & EPA8270 \\
\hline UJ & L & & 410 & $\mu \mathrm{g} / \mathrm{kg}$ & 410 & EPA8270 \\
\hline UJ & LH & L & 410 & $\mu \mathrm{g} / \mathrm{kg}$ & 410 & EPA8270 \\
\hline UJ & L & & 410 & $\mu \mathrm{g} / \mathrm{kg}$ & 410 & EPA8270 \\
\hline UJ & L & & 2,100 & $\mu g / k g$ & 2,100 & EPA8270 \\
\hline UJ & L & & 410 & $\mu \mathrm{g} / \mathrm{kg}$ & 410 & EPA8270 \\
\hline UJ & L & & 410 & $\mu \mathrm{g} / \mathrm{kg}$ & 410 & EPA8270 \\
\hline UJ & L & & 2,100 & $\mu g / \mathrm{kg}$ & 2,100 & EPA8270 \\
\hline UJ & L & & 2,100 & $\mu \mathrm{g} / \mathrm{kg}$ & 2,100 & EPA8270 \\
\hline UJ & L & & 2,100 & $\mu \mathrm{g} / \mathrm{kg}$ & 2,100 & EPA8270 \\
\hline UJ & L & & 410 & $\mu \mathrm{g} / \mathrm{kg}$ & 410 & EPA8270 \\
\hline UJ & L & & 410 & $\mu \mathrm{g} / \mathrm{kg}$ & 410 & EPA8270 \\
\hline UJ & L & & 2,100 & $\mu \mathrm{g} / \mathrm{kg}$ & 2,100 & EPA8270 \\
\hline UJ & L & & 410 & $\mu \mathrm{g} / \mathrm{kg}$ & 410 & EPA8270 \\
\hline UJ & L & & 410 & $\mu \mathrm{g} / \mathrm{kg}$ & 410 & EPA8270 \\
\hline UJ & L & & 2.100 & $\mu g / k g$ & 2.100 & EPA8270 \\
\hline UJ & L & & 410 & $\mu \mathrm{g} / \mathrm{kg}$ & 410 & EPA8270 \\
\hline UJ & L & & 410 & $\mu g / \mathrm{kg}$ & 410 & EPA8270 \\
\hline UJ & L & & 410 & $\mu g / \mathrm{kg}$ & 410 & EPA8270 \\
\hline UJ & $L$ & & 410 & $\mu g / k g$ & 410 & EPA8270 \\
\hline UJ & L & & 2.100 & $\mu g / \mathrm{kg}$ & 2,100 & EPA8270 \\
\hline UJ & L & & 410 & $\mu g / k g$ & 410 & EPA8270 \\
\hline$J$ & NV & & 500 & $\mu \mathrm{g} / \mathrm{kg}$ & & EPA8270 \\
\hline J & NV & & 9,000 & $\mu g / k g$ & & EPA8270 \\
\hline J & $\mathbf{N}$ & & 500 & $\mu \mathrm{g} / \mathrm{kg}$ & & EPA 8270 \\
\hline J & $\mathbf{N}$ & & 1.000 & $\mu \mathrm{g} / \mathrm{kg}$ & & EPA8270 \\
\hline J & $\mathbf{N}$ & & 800 & $\mu \mathrm{g} / \mathrm{kg}$ & & EPA8270 \\
\hline J & $\mathbf{N}$ & & 300 & $\mu g / k g$ & & EPA8270 \\
\hline J & $\mathbf{N}$ & & 500 & $\mu \mathrm{g} / \mathrm{kg}$ & & EPA8270 \\
\hline J & $\mathbf{N}$ & & 600 & $\mu \mathrm{g} / \mathrm{kg}$ & & EPA8270 \\
\hline
\end{tabular}


SAMPLE NAME: D43111504 (Continued)

Sample ID: 100411

Constituent RQ AQ B Result Unit D. Limit Method

$B / N / A$ Extractables (including $P A H$ and phenols)

Unknown phthalate

J $\quad \mathrm{N}$

300

$\mu \mathrm{g} / \mathrm{kg}$

EPA8270

Volatile Organic Compounds

Acetone

Benzene

Bromodichloromethane

Bromoform

Bromomethane (Methyl bromide)

Carbon disulfide

Carbon tetrachloride

Chlorobenzene

Chloroethane

Chloroethene (Vinyl chloride)

Chloroform

Chloromethane (Methyl chloride)

Dibromochloromethane

1,1-Dichloroethane

1,2-Dichloroethane

1,1-Dichloroethylene

1.2-Dichloroethylene

Dichloromethane (Methylene chloride)

1,2-Dichloropropane

cis-1,3-Dichloropropene

trans-1,3-Dichloropropene

Ethylbenzene

2-Hexanone

Methyl ethyl ketone

Methyl isobutyl ketone

Styrene

1,1,2,2-Tetrachloroethane

Tetrachloroethylene

Toluene

1,1,1-Trichloroethane

1,1,2-Trichloroethane

Trichloroethylene

Vinyl acetate

Xylenes

Pesticides/PCBs and Dioxins/Furans

Aldrin

alpha-Benzene hexachloride

beta-Benzene hexachloride

delta-Benzene hexachloride

alpha-Chlordane

gamma-Chlordane

p, $p^{\prime}-D D D$

P,p'-DDE

p,p'-DDT
41

6.2

6.2

6.2

12

6.2

6.2

6.2

12

12

6.2

12

6.2

6.2

6.2

6.2

6.2

6.2

6.2

6.2

6.2

6.2

12

12

12

6.2

6.2

6.2

6.2

6.2

6.2

6.2

12

6.2

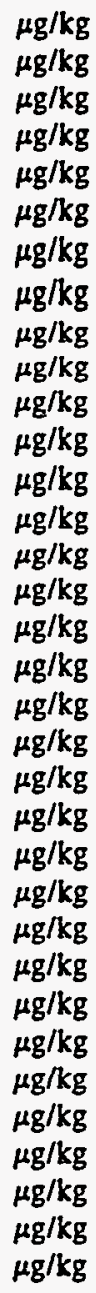

$\mu \mathrm{g} / \mathrm{kg}$

$\mu \mathrm{g} / \mathrm{kg}$

$\mu \mathrm{g} / \mathrm{kg}$

$\mu \mathrm{g} / \mathrm{kg}$

$\mu \mathrm{g} / \mathrm{kg}$

$\mu \mathrm{g} / \mathrm{kg}$

$\mu \mathrm{g} / \mathrm{kg}$

$\mu \mathrm{g} / \mathrm{kg}$

$\mu \mathrm{g} / \mathrm{kg}$

$\mu \mathrm{g} / \mathrm{kg}$

$\mu \mathrm{g} / \mathrm{kg}$

$\mu \mathrm{g} / \mathrm{kg}$

$\mu \mathrm{g} / \mathrm{kg}$

$\mu \mathrm{g} / \mathrm{kg}$

$\mu \mathrm{g} / \mathrm{kg}$

$\mu \mathrm{g} / \mathrm{kg}$

$\mu \mathrm{g} / \mathrm{kg}$

$\mu g / k g$

$\mu \mathrm{g} / \mathrm{kg}$

$\mu \mathrm{g} / \mathrm{kg}$

$\mu \mathrm{g} / \mathrm{kg}$

$\mu \mathrm{g} / \mathrm{kg}$

$\mu g / \mathrm{kg}$

$\mu \mathrm{g} / \mathrm{kg}$

$\mu \mathrm{g} / \mathrm{kg}$

$\mu g / k g$

$\mu \mathrm{g} / \mathrm{kg}$

$\mu \mathrm{g} / \mathrm{kg}$

$\mu \mathrm{g} / \mathrm{kg}$

2.0

2.0

2.0

2.0

2.0

2.0

4.0

4.0

4.0 $\mu g / k g$

$\mu \mathrm{g} / \mathrm{kg}$

$\mu \mathrm{g} / \mathrm{kg}$

$\mu \mathrm{g} / \mathrm{kg}$

$\mu \mathrm{g} / \mathrm{kg}$

$\mu \mathrm{g} / \mathrm{kg}$

$\mu \mathrm{g} / \mathrm{kg}$

$\mu \mathrm{g} / \mathrm{kg}$

$\mu \mathrm{g} / \mathrm{kg}$
12

6.2

6.2

6.2

12

6.2

6.2

6.2

12

12

6.2

12

6.2

6.2

6.2

6.2

6.2

6.2

$-6.2$

6.2

6.2

6.2

12

12

12
12

6.2

6.2

6.2

6.2

6.2

6.2

6.2

12

.6 .2

2.0

2.0

2.0

2.0

2.0

2.0

4.0

4.0

4.0
EPA8240

EPA8240

EPA8240

EPA8240

EPA8240

EPA8240

EPA8240

EPA8240

EPA8240

EPA8240

EPA8240

EPA8240

EPA8240

EPA8240

EPA8240

EPA8240

EPA8240

EPA8240

EPA8240

EPA8240

EPAं8240

EPA8240

EPA8240

EPA8240

EPA8240

EPA8240

EPA8240

EPA8240

EPA8240

EPA8240

EPA8240

EPA8240

EPA8240

EPA8240

EPA8080

EPA8080

EPA8080

EPA8080

EPA8080

EPA8080

EPA8080

EPA8080

EPA8080 
SAMPLE NAME: D43111504 (Continued)

Constituent

Pesticides/PCBs and Dioxins/Furans

Dieldrin

Endosulfan I

Endosulfan II

Endosulfan sulfate

Endrin

Endrin ketone

Heptachlor

Heptachlor epoxide

Heptachlorodibenzo-p-dioxin isomers

Heptachlorodibenzo-p-dioxin isomers

Heptachlorodibenzo-p-furan isomers

Heptachlorodibenzo-p-furan isomers

Hexachlorodibenzo-p-dioxin isomers

Hexachlorodibenzo-p-furan isomers

Lindane

Methoxychlor

Octachlorodibenzo-p-dioxin.

Octachlorodibenzo-p-furan

PCB 1016

PCB 1221

PCB 1232

PCB 1242

PCB 1248

PCB 1254

PCB 1260

Pentachlorodibenzo-p-dioxin isomers

Pentachlorodibenzo-p-furan isomers

Pentachlorodibenzo-p-dioxin isomers

Pentachlorodibenzo-p-furan isomers

2,3,7,8-TCDD

2,3,7,8-TCDF

Tetrachlorodibenzo-p-dioxin isomers

Tetrachlorodibenzo-p-furan isomers

Toxaphene

Radionuclide Indicators and Radionuclides

Gross alpha

Nonvolatile beta

Radium, total alpha-emitting

Tritium
Sample ID: 100411

RQ AQ B Result Unit D. Limit Method
U

U

U

$\mathrm{U}$

$\mathrm{U}$

U

U

$\mathrm{U}$

$\mathrm{U}$

U

$\mathrm{J}$

U

U

$\mathrm{U}$

U

J

$\mathrm{U}$

$\mathrm{U}$

U

U

U

U

U

U

U

U

U

UI $\mathrm{H}$

UJ $\mathrm{H}$

UJ $\mathrm{H}$

UJ $\mathrm{H}$

v

I

4.0

2.0

4.0

4.0

4.0

4.0

2.0

2.0

0.20

0.20

0.10

0.70

0.20

0.10

2.0

20

L

40

0.30

40

81

40

40

40

40

40

0.30

0.20

0.20

0.20

0.10

0.10

0.20

0.10

200

$1.6 \pm 12$

$22 \pm 18$

$2.1 \pm 0.90$

$5.1 \pm 1.1$

$\mu \mathrm{g} / \mathrm{kg}$
$\mu \mathrm{g} / \mathrm{kg}$
$\mu \mathrm{g} / \mathrm{kg}$
$\mu \mathrm{g} / \mathrm{kg}$
$\mu \mathrm{g} / \mathrm{kg}$
$\mu \mathrm{g} / \mathrm{kg}$
$\mu \mathrm{g} / \mathrm{kg}$
$\mu \mathrm{g} / \mathrm{kg}$
$\mathrm{ng} / \mathrm{g}$
$\mathrm{ng} / \mathrm{g}$
$\mathrm{ng} / \mathrm{g}$
$\mathrm{ng} / \mathrm{g}$
$\mathrm{ng} / \mathrm{g}$
$\mathrm{ng} / \mathrm{g}$
$\mu \mathrm{g} / \mathrm{kg}$
$\mu \mathrm{g} / \mathrm{kg}$
$\mathrm{ng} / \mathrm{g}$
$\mathrm{ng} / \mathrm{g}$
$\mu \mathrm{g} / \mathrm{kg}$
$\mu \mathrm{g} / \mathrm{kg}$
$\mu \mathrm{g} / \mathrm{kg}$
$\mu \mathrm{g} / \mathrm{kg}$
$\mu \mathrm{g} / \mathrm{kg}$
$\mu \mathrm{g} / \mathrm{kg}$
$\mu \mathrm{k} / \mathrm{kg}$
$\mathrm{ng} / \mathrm{g}$
$\mathrm{ng} / \mathrm{g}$
$\mathrm{ng} / \mathrm{g}$
$\mathrm{ng} / \mathrm{g}$
$\mathrm{ng} / \mathrm{g}$
$\mathrm{ng} / \mathrm{g}$
$\mathrm{ng} / \mathrm{g}$
$\mathrm{ng} / \mathrm{g}$.
$\mu \mathrm{g} / \mathrm{kg}$

4.0

2.0

4.0

4.0

$4: 0$

4.0

2.0

2.0

0.20

0.20

0.10

0.10

0.20

0.10

2.0

20

0.20

0.30

40

81

40

40

40

40

40

0.30

0.20

0.20

0.20

0.10

0.10

0.20

0.10

200

EPA8080

EPA8080

EPA8080

EPA8080

EPA8080

EPA8080

EPA8080

EPA8080

EPA8280

EPA8280

EPA8280

EPA8280

EPA8280

EPA8280

EPA8080

EPA8080

EPA8280

EPA8280

EPA8080

EPA8080

EPA8080

EPA8080

EPA8080

EPA8080

EPA8080

EPA8280

EPA8280

EPA8280

EPA8280

EPA8280

EPA8280

EPA8280

EPA8280

EPA8080

4.6

EPA900.0

8.4 EPA900.0

0.080 EPA903.0

1.9

EPA906.0 
SAMPLE NAME: D43111601

Location (SRS Coordinates): 18644.34E 66570.29N

Ground Elevation Above MSL: $134.1 \mathrm{ft}$

Depth of Core Interval: 0.00 to $0.50 \mathrm{ft}$

Sample Type: Normal

Sample Color: 5YR 4/4

Constituent

Physical Parameters

Cation exchange capacity

Cation exchange capacity

$\mathrm{pH}$

Specific conductance

Total organic carbon

Total Metals
Sample ID: 100417
Sample Matrix: Soil

USC Soil Classification: SW
Sample Moisture: Dry

Percent Solids: 93.40

D. Limit Method

\begin{tabular}{|c|c|c|c|c|c|c|}
\hline Arsenic & U & & 1.0 & $\mathrm{mg} / \mathrm{kg}$ & 1.0 & EPA7060 \\
\hline Arsenic & $\mathrm{U}$ & & 1.0 & $\mathrm{mg} / \mathrm{kg}$ & 1.0 & EPA7060 \\
\hline Barium & U & & 19 & $\mathrm{mg} / \mathrm{kg}$ & 19 & EPA6010 \\
\hline Barium & $\mathrm{U}$ & & 19 & $\mathrm{mg} / \mathrm{kg}$ & 19 & EPA6010 \\
\hline Cadmium & $\mathrm{U}$ & & 0.48 & $\mathrm{mg} / \mathrm{kg}$ & 0.48 & EPA6010 \\
\hline Cadmium & $\mathrm{U}$ & & 0.48 & $\mathrm{mg} / \mathrm{kg}$ & 0.48 & EPA6010 \\
\hline Chromium & $\mathrm{J}$ & IX & 9.0 & $\mathrm{mg} / \mathrm{kg}$ & 0.95 & EPA6010 \\
\hline Chromium & $\mathrm{J}$ & IX & 4.6 & $\mathrm{mg} / \mathrm{kg}$ & 0.95 & EPA6010 \\
\hline Copper & U & & 2.4 & $\mathrm{mg} / \mathrm{kg}$ & 2.4 & EPA6010 \\
\hline Copper & U & & 2.4 & $\mathrm{mg} / \mathrm{kg}$ & 2.4 & EPA6010 \\
\hline Lead & & & 2.4 & $\mathrm{mg} / \mathrm{kg}$ & 0.30 & EPA7421 \\
\hline Lead & & & 2.6 & $\mathrm{mg} / \mathrm{kg}$ & 0.30 & EPA7421 \\
\hline Manganese & J & I & 79 & $\mathrm{mg} / \mathrm{kg}$ & 1.4 & EPA6010 \\
\hline Manganese & $\mathrm{J}$ & $I$ & 68 & $\mathrm{mg} / \mathrm{kg}$ & 1.4 & EPA6010 \\
\hline Mercury & $\mathbf{U}$ & & 0.11 & $\mathrm{mg} / \mathrm{kg}$ & 0.11 & EPA7470 \\
\hline Mercury & U & & 0.11 & $\mathrm{mg} / \mathrm{kg}$ & 0.11 & EPA7470 \\
\hline Nickel & $\mathbf{U}$ & & 3.8 & $\mathrm{mg} / \mathrm{kg}$ & 3.8 & EPA6010 \\
\hline Nickel & U & & 3.8 & $\mathrm{mg} / \mathrm{kg}$ & 3.8 & EPA6010 \\
\hline Selenium & UJ & I & 0.50 & $\mathrm{mg} / \mathrm{kg}$ & 0.50 & EPA7740 \\
\hline Selenium & UJ & I & 0.50 & $\mathrm{mg} / \mathrm{kg}$ & 0.50 & EPA7740 \\
\hline Silver & $\mathbf{U}$ & & 0.95 & $\mathrm{mg} / \mathrm{kg}$ & 0.95 & EPA6010 \\
\hline Silver & U & & 0.95 & $\mathrm{mg} / \mathrm{kg}$ & 0.95 & EPA6010 \\
\hline \multicolumn{7}{|c|}{$B / N / A$ Extractables (including $P A H$ and phenols) } \\
\hline Acenaphthene & $\mathbf{U}$ & & 350 & $\mu \mathrm{g} / \mathrm{kg}$ & 350 & EPA8270 \\
\hline Acenaphthylene & U & & 350 & $\mu \mathrm{g} / \mathrm{kg}$ & 350 & EPA8270 \\
\hline Aldol condensate & J & NVK & 400 & $\mu \mathrm{g} / \mathrm{kg}$ & & EPA8270 \\
\hline Aldol condensate & J & NK & 300 & $\mu \mathrm{g} / \mathrm{kg}$ & & EPA8270 \\
\hline Aldol condensate & $\mathbf{J}$ & NK & 100 & $\mu \mathrm{g} / \mathrm{kg}$ & & EPA8270 \\
\hline Anthracene & $\mathbf{U}$ & & 350 & $\mu \mathrm{g} / \mathrm{kg}$ & 350 & EPA8270 \\
\hline Benzo(a)anthracene & $\mathbf{U}$ & & 350 & $\mu \mathrm{g} / \mathrm{kg}$ & 350 & EPA8270 \\
\hline Benzo(b)fluoranthene & $\mathbf{U}$ & & 350 & $\mu \mathrm{g} / \mathrm{kg}$ & 350 & EPA8270 \\
\hline Benzo(k)fluoranthene & $\mathbf{U}$ & & 350 & $\mu \mathrm{g} / \mathrm{kg}$ & 350 & EPA8270 \\
\hline Benzoic acid & $\mathbf{U}$ & & 1,800 & $\mu \mathrm{g} / \mathrm{kg}$ & 1,800 & EPA8270 \\
\hline Benzo(g,h,i)perylene & U & & 350 & $\mu \mathrm{g} / \mathrm{kg}$ & 350 & EPA8270 \\
\hline
\end{tabular}


SAMPLE NAME: D43ï11601 (Continued)

Sample ID: 100417

$\begin{array}{llllll}\text { Constituent } & \text { RQ AQ B Result Unit D. Limit Method }\end{array}$

$B / N / A$ Extractables (including PAH and phenols)

\begin{tabular}{|c|c|c|c|c|c|c|}
\hline Benzo(a)pyrene & $\mathbf{U}$ & & 350 & $\mu g / \mathrm{kg}$ & 350 & EPA8270 \\
\hline Benzyl alcohol & $\mathbf{U}$ & & 350 & $\mu \mathrm{g} / \mathrm{kg}$ & 350 & EPA8270 \\
\hline Bis(2-chloroethoxy) methane & $\mathbf{U}$ & & 350 & $\mu \mathrm{g} / \mathrm{kg}$ & 350 & EPA8270 \\
\hline Bis(2-chloroethyl) ether & $\mathbf{U}$ & & 350 & $\mu \mathrm{g} / \mathrm{kg}$ & 350 & EPA8270 \\
\hline Bis(2-chloroisopropyl) ether & $\mathbf{U}$ & & 350 & $\mu g / \mathrm{kg}$ & 350 & EPA8270 \\
\hline Bis(2-ethylhexyl) phchalate & UJ & $\mathrm{v}$ & 140 & $\mu \mathrm{g} / \mathrm{kg}$ & 350 & EPA8270 \\
\hline 4-Bromophenyl phenyl ther & $\mathbf{U}$ & & 350 & $\mu g / k g$ & 350 & EPA8270 \\
\hline Butylbenzyl phthalate & $\mathbf{U}$ & & 350 & $\mu \mathrm{g} / \mathrm{kg}$ & 350 & EPA8270 \\
\hline 4-Chloroaniline & $\mathbf{U}$ & & 350 & $\mu \mathrm{g} / \mathrm{kg}$ & 350 & EPA8270 \\
\hline 4-Chloro-m-cresol & $U$ & & 350 & $\mu \mathrm{g} / \mathrm{kg}$ & 350 & EPA8270 \\
\hline 2-Chloronaphthalene & $\mathrm{U}$ & & 350 & $\mu \mathrm{g} / \mathrm{kg}$ & 350 & EPA8270 \\
\hline 2-Chlorophenol & $\mathrm{U}$ & & 350 & $\mu \mathrm{g} / \mathrm{kg}$ & 350 & EPA8270 \\
\hline 4-Chlorophenyl phenyl ether & $\mathrm{U}$ & & 350 & $\mu g / k g$ & 350 & EPA8270 \\
\hline Chrysene & U & & 350 & $\mu \mathrm{g} / \mathrm{kg}$ & 350 & EPA8270 \\
\hline o-Cresol (2-Methylphenol) & $\mathbf{U}$ & & 350 & $\mu \mathrm{g} / \mathrm{kg}$ & 350 & EPA8270 \\
\hline p-Cresol (4-Methylphenol) & $\mathrm{U}$ & & 350 & $\mu g / \mathrm{kg}$ & 350 & EPA8270 \\
\hline Dibenz(a,h)anthracene & $\mathrm{U}$ & & 350 & $\mu \mathrm{g} / \mathrm{kg}$ & 350 & EPA8270 \\
\hline Dibenzofuran & 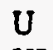 & & 350 & $\mu \mathrm{g} / \mathrm{kg}$ & 350 & EPA8270 \\
\hline Di-n-butyl phthalate & UJ & $\mathrm{V}$ & 88 & $\mu \mathrm{g} / \mathrm{kg}$ & 350 & EPA8270 \\
\hline 1.2-Dichlorobenzene & $\mathbf{U}$ & & 350 & $\mu g / \mathrm{kg}$ & 350 & EPA8270 \\
\hline 1,3-Dichlorobenzene & $\mathrm{U}$ & & 350 & $\mu \mathrm{g} / \mathrm{kg}$ & 350 & EPA8270 \\
\hline 1,4-Dichlorobenzene & $\mathbf{U}$ & & 350 & $\mu \mathrm{g} / \mathrm{kg}$ & 350 & EPA8270 \\
\hline 3.3'-Dichlorobenzidine & $\mathbf{U}$ & & 700 & $\mu \mathrm{g} / \mathrm{kg}$ & 700 & EPA8270 \\
\hline 2,4-Dichlorophenol & $\mathbf{U}$ & & 350 & $\mu \mathrm{g} / \mathrm{kg}$ & 350 & EPA8270 \\
\hline Diethyl phthalate & U & & 350 & $\mu \mathrm{g} / \mathrm{kg}$ & 350 & EPA8270 \\
\hline 2.4-Dimethyl phenol & $\mathbf{U}$ & & 350 & $\mu \mathrm{g} / \mathrm{kg}$ & 350 & EPA8270 \\
\hline Dimethyl phthalate & $\mathrm{U}$ & & 350 & $\mu \mathrm{g} / \mathrm{kg}$ & 350 & EPA8270 \\
\hline 2,4-Dinitrophenol & $\mathbf{U}$ & & 1,800 & $\mu g / \mathrm{kg}$ & 1.800 & EPA8270 \\
\hline 2,4-Dinitrotoluene & $\mathrm{U}$ & & 350 & $\mu \mathrm{g} / \mathrm{kg}$ & 350 & EPA8270 \\
\hline 2,6-Dinitrotoluene & $\mathbf{U}$ & & 350 & $\mu g / \mathrm{kg}$ & 350 & EPA8270 \\
\hline Di-n-octyl phthalate & $\mathbf{U}$ & & 350 & $\mu \mathrm{g} / \mathrm{kg}$ & 350 & EPA8270 \\
\hline Fluoranthene & $\mathbf{U}$ & & 350 & $\mu \mathrm{g} / \mathrm{kg}$ & 350 & EPA8270 \\
\hline Fluorene & $\mathbf{U}$ & & 350 & $\mu \mathrm{g} / \mathrm{kg}$ & 350 & EPA8270 \\
\hline Hexachlorobenzene & $\mathrm{U}$ & & 350 & $\mu \mathrm{g} / \mathrm{kg}$ & 350 & EPA8270 \\
\hline Hexachlorobutadiene & $\mathbf{U}$ & & 350 & $\mu g / \mathrm{kg}$ & 350 & EPA8270 \\
\hline Hexachlorocyclopentadiene & $\mathbf{U}$ & & 350 & $\mu \mathrm{g} / \mathrm{kg}$ & 350 & EPA8270 \\
\hline Hexachloroethane & $\mathbf{U}$ & & 350 & $\mu \mathrm{g} / \mathrm{kg}$ & 350 & EPA8270 \\
\hline Indeno(1,2,3-c,d)pyrene & $\mathrm{U}$ & & 350 & $\mu \mathrm{g} / \mathrm{kg}$ & 350 & EPA8270 \\
\hline Isophorone & $\mathbf{U}$ & & 350 & $\mu \mathrm{g} / \mathrm{kg}$ & 350 & EPA8270 \\
\hline 2-Methyl-4,6-dinitrophenol & $\mathbf{U}$ & & 1,800 & $\mu \mathrm{g} / \mathrm{kg}$ & 1.800 & EPA8270 \\
\hline 2-Methylnaphthalene & $\mathbf{U}$ & & 350 & $\mu \mathrm{g} / \mathrm{kg}$ & 350 & EPA8270 \\
\hline Naphthalene & $\mathbf{U}$ & & 350 & $\mu \mathrm{g} / \mathrm{kg}$ & 350 & EPA8270 \\
\hline o-Nitroaniline & $\mathbf{U}$ & & 1,800 & $\mu \mathrm{g} / \mathrm{kg}$ & 1,800 & EPA8270 \\
\hline m-Nitroaniline & U & & 1,800 & $\mu g / \mathrm{kg}$ & 1,800 & EPA8270 \\
\hline p-Nitroaniline & $\mathbf{U}$ & & 1,800 & $\mu g / \mathrm{kg}$ & 1.800 & EPA8270 \\
\hline Nitrobenzene & $\mathbf{U}$ & & 350 & $\mu \mathrm{g} / \mathrm{kg}$ & 350 & EPA8270 \\
\hline 2-Nitrophenol & $\mathbf{U}$ & & 350 & $\mu \mathrm{g} / \mathrm{kg}$ & 350 & EPA8270 \\
\hline 4-Nitrophenol & $\mathbf{U}$ & & 1,800 & $\mu \mathrm{g} / \mathrm{kg}$ & 1,800 & EPA8270 \\
\hline N-Nitrosodiphenylamine & $\mathbf{U}$ & & 350 & $\mu \mathrm{g} / \mathrm{kg}$ & 350 & EPA8270 \\
\hline N-Nitrosodipropylamine & $\mathbf{U}$ & & 350 & $\mu \mathrm{g} / \mathrm{kg}$ & 350 & EPA8270 \\
\hline
\end{tabular}


SAMPLE NAME: D43I11601 (Continued)

Sample ID: 100417

Constituent

RQ AQ B Result Unit

D. Limit Method

$B / N / A$ Extractables (including $P A H$ and phenols)

Pentachlorophenol
Phenanthrene
Phenol
Pyrene
1.2.4-Trichlorobenzene
2,4.5-Trichlorophenol
2,4,6-Trichlorophenol
Unknown
Unknown
Unknown

Volatile Organic Compounds

Acetone
Benzene
Bromodichloromethane
Bromoform
Bromomethane (Methyl bromide)
Carbon disulfide
Carbon tetrachloride
Chlorobenzene
Chloroethane
Chloroethene (Vinyl chloride)
2-Chloroethyl vinyl ether
Chloroform
Chloromethane (Methyl chloride)
Dibromochloromethane
1,1-Dichloroethane
1,2-Dichloroethane
1,1-Dichloroethylene
1,2-Dichloroethylene
Dichloromethane (Methylene chloride)
1,2-Dichloropropane
cis-1,3-Dichloropropene
trans-1,3-Dichloropropene
Ethylbenzene
2-Hexanone
Methyl ethyl ketone
Methyl isobutyl ketone
Styrene
1,1,2,2-Tetrachloroethane
Tetrachloroethylene
Toluene
1,1,1-Trichloroethane
1,1,2-Trichloroethane
Trichloroethylene
Vinyl acetate
Xylenes

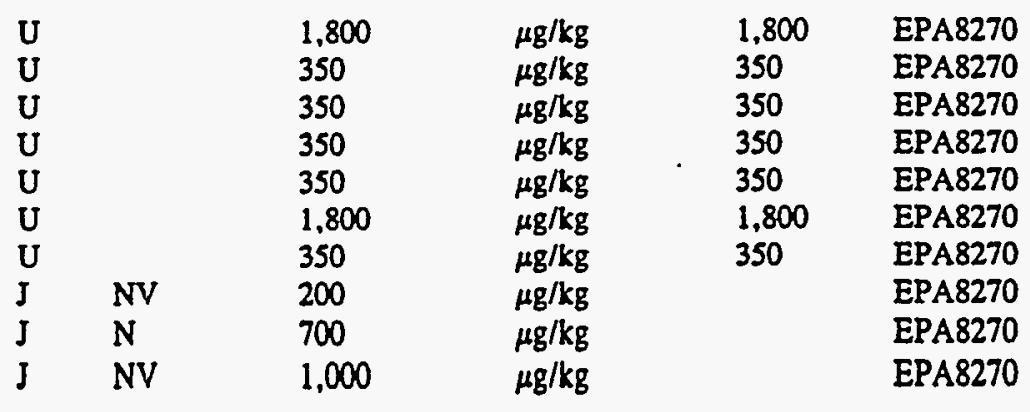

\begin{tabular}{|c|c|c|c|c|}
\hline UJ & 6.2 & $\mu \mathrm{g} / \mathrm{kg}$ & 10 & EPA 8240 \\
\hline U & 5.0 & $\mu g / k g$ & 5.0 & EPA8240 \\
\hline $\mathrm{U}$ & 5.0 & $\mu g / k g$ & 5.0 & EPA 8240 \\
\hline $\mathbf{U}$ & 5.0 & $\mu \mathrm{g} / \mathrm{kg}$ & 5.0 & EPA8240 \\
\hline $\mathrm{U}$ & 10 & $\mu \mathrm{g} / \mathrm{kg}$ & 10 & EPA 8240 \\
\hline U & 5.0 & $\mu g / \mathbf{k g}$ & 5.0 & EPA8240 \\
\hline U & 5.0 & $\mu \mathrm{g} / \mathrm{kg}$ & 5.0 & EPA8240 \\
\hline U & 5.0 & $\mu \mathrm{g} / \mathrm{kg}$ & 5.0 & EPA8240 \\
\hline U & 10 & $\mu \mathrm{g} / \mathrm{kg}$ & 10 & EPA8240 \\
\hline $\mathbf{U}$ & 10 & $\mu \mathrm{g} / \mathrm{kg}$ & 10 & EPA8240 \\
\hline $\mathbf{U}$ & 10 & $\mu g / k g$ & 10 & EPA8240 \\
\hline U & 5.0 & $\mu g / k g$ & 5.0 & EPA8240 \\
\hline U & 10 & $\mu g / \mathrm{kg}$ & 10 & EPA8240 \\
\hline U & 5.0 & $\mu \mathrm{g} / \mathrm{kg}$ & 5.0 & EPA8240 \\
\hline$U$ & 5.0 & $\mu g / k g$ & 5.0 & EPA 8240 \\
\hline $\mathrm{U}$ & 5.0 & $\mu g / k g$ & . $\quad 5.0$ & EPA8240 \\
\hline U & 5.0 & $\mu g / k g$ & 5.0 & EPA8240 \\
\hline $\mathrm{U}$ & 5.0 & $\mu \mathrm{g} / \mathrm{kg}$ & 5.0 & EPA8240 \\
\hline V & 13 & $\mu \mathrm{g} / \mathrm{kg}$ & 5.0 & EPA8240 \\
\hline $\mathrm{U}$ & 5.0 & $\mu g / \mathrm{kg}$ & 5.0 & EPA8240 \\
\hline $\mathbf{U}$ & 5.0 & $\mu \mathrm{g} / \mathrm{kg}$ & 5.0 & EPA 8240 \\
\hline $\mathbf{U}$ & 5.0 & $\mu g / \mathrm{kg}$ & 5.0 & EPA8240 \\
\hline $\mathbf{U}$ & 5.0 & $\mu g / k g$ & 5.0 & EPA8240 \\
\hline $\mathrm{U}$ & 10 & $\mu \mathrm{g} / \mathrm{kg}$ & 10 & EPA8240 \\
\hline $\mathbf{U}$ & 10 & $\mu \mathrm{g} / \mathrm{kg}$ & 10 & EPA8240 \\
\hline $\mathbf{U}$ & 10 & $\mu g / k g$ & 10 & EPA8240 \\
\hline $\mathbf{U}$ & 5.0 & $\mu \mathrm{g} / \mathrm{kg}$ & 5.0 & EPA8240 \\
\hline U & 5.0 & $\mu \mathrm{g} / \mathrm{kg}$ & 5.0 & EPA8240 \\
\hline $\mathbf{U}$ & 5.0 & $\mu g / \mathrm{kg}$ & 5.0 & EPA8240 \\
\hline J & 1.6 & $\mu g / \mathrm{kg}$ & 5.0 & EPA8240 \\
\hline$U$ & 5.0 & $\mu \mathrm{g} / \mathrm{kg}$ & 5.0 & EPA8240 \\
\hline $\mathrm{U}$ & 5.0 & $\mu g / \mathbf{k g}$ & 5.0 & EPA8240 \\
\hline $\mathbf{U}$ & 5.0 & $\mu g / k g$ & 5.0 & EPA8240 \\
\hline $\mathbf{U}$ & 10 & $\mu g / \mathbf{k g}$ & 10 & EPA8240 \\
\hline $\mathbf{U}$ & 5.0 & $\mathrm{\mu g} / \mathrm{kg}$ & 5.0 & EPA 8240 \\
\hline
\end{tabular}


SAMPLE NAME: D431-11601 (Continued)

Constituent

RQ

AQ

Sample ID: 100417

Pesticides/PCBs and Dioxins/Furans

Result
1.8

1.8

1.8

1.8

1.8

1.8

3.5

3.5

3.5

3.5

1.8

3.5

3.5

3.5

3.5

1.8

1.8

0.10

0.20

0.20

0.10

1.8

18

0.50

0.30

35

70

35

35

35

35

35

0.10

0.10

0.10

0.10

0.10

0.10

180

Unit

D. Limit Method

\begin{tabular}{|c|c|c|}
\hline$\mu \mathrm{g} / \mathrm{kg}$ & 1.8 & EPA8080 \\
\hline$\mu g / \mathrm{kg}$ & 1.8 & EPA8080 \\
\hline$\mu \mathrm{g} / \mathrm{kg}$ & 1.8 & EPA8080 \\
\hline$\mu g / \mathrm{kg}$ & 1.8 & EPA8080 \\
\hline$\mu g / k g$ & 1.8 & EPA8080 \\
\hline$\mu \mathrm{g} / \mathrm{kg}$ & 1.8 & EPA8080 \\
\hline$\mu \mathrm{g} / \mathrm{kg}$ & 3.5 & EPA8080 \\
\hline$\mu g / k g$ & 3.5 & EPA8080 \\
\hline$\mu \mathrm{g} / \mathrm{kg}$ & 3.5 & EPA8080 \\
\hline$\mu \mathrm{g} / \mathrm{kg}$ & 3.5 & EPA8080 \\
\hline$\mu \mathrm{g} / \mathrm{kg}$ & 1.8 & EPA8080 \\
\hline$\mu \mathrm{g} / \mathrm{kg}$ & 3.5 & EPA8080 \\
\hline$\mu g / k g$ & 3.5 & EPA8080 \\
\hline$\mu \mathrm{g} / \mathrm{kg}$ & 3.5 & EPA8080 \\
\hline$\mu g / \mathrm{kg}$ & 3.5 & EPA8080 \\
\hline$\mu \mathrm{g} / \mathrm{kg}$ & 1.8 & EPA 8080 \\
\hline$\mu g / \mathrm{kg}$ & 1.8 & EPA8080 \\
\hline $\mathrm{ng} / \mathrm{g}$ & 0.10 & EPA8280 \\
\hline $\mathrm{ng} / \mathrm{g}$ & 0.20 & EPA8280 \\
\hline $\mathrm{ng} / \mathrm{g}$ & 0.20 & EPA 8280 \\
\hline $\mathrm{ng} / \mathrm{g}$ & 0.10 & EPA8280 \\
\hline$\mu \mathrm{g} / \mathrm{kg}$ & 1.8 & EPA8080 \\
\hline$\mu \mathrm{g} / \mathrm{kg}$ & 18 & EPA8080 \\
\hline $\mathrm{ng} / \mathrm{g}$ & 0.50 & EPA8280 \\
\hline $\mathrm{ng} / \mathrm{g}$ & 0.30 & EPA8280 \\
\hline$\mu \mathrm{g} / \mathrm{kg}$ & 35 . & EPA8080 \\
\hline$\mu \mathrm{g} / \mathrm{kg}$ & 70 & EPA8080 \\
\hline$\mu \mathrm{g} / \mathrm{kg}$ & 35 & EPA8080 \\
\hline$\mu g / k g$ & 35 & EPA8080 \\
\hline$\mu \mathrm{g} / \mathrm{kg}$ & 35 & EPA8080 \\
\hline$\mu \mathrm{g} / \mathrm{kg}$ & 35 & EPA8080 \\
\hline$\mu g / \mathrm{kg}$ & 35 & EPA8080 \\
\hline $\mathrm{ng} / \mathrm{g}$ & 0.10 & EPA8280 \\
\hline $\mathrm{ng} / \mathrm{g}$ & 0.10 & EPA8280 \\
\hline $\mathrm{ng} / \mathrm{g}$ & 0.10 & EPA8280 \\
\hline $\mathrm{ng} / \mathrm{g}$ & 0.10 & EPA8280 \\
\hline $\mathrm{ng} / \mathrm{g}$ & 0.10 & EPA8280 \\
\hline $\mathrm{ng} / \mathrm{g}$ & 0.10 & EPA8280 \\
\hline$\mu g / k g$ & 180 & EPA8080 \\
\hline
\end{tabular}

Radionuclide Indicators and Radionuclides

Gross alpha

$\begin{array}{llllll} & & 6.4 \pm 6.8 & \mathrm{pCi} / \mathrm{g} & 4.0 & \text { EPA900.0 } \\ & & 8.3 \pm 6.7 & \mathrm{pCi} / \mathrm{g} & 3.6 & \text { EPA900.0 } \\ & \mathrm{V} & 22 \pm 10 & \mathrm{pCi} / \mathrm{g} & 5.9 & \text { EPA900.0 } \\ & \mathrm{V} & 37 \pm 11 & \mathrm{pCi} / \mathrm{g} & 5.2 & \text { EPA900.0 } \\ \mathrm{J} & \mathrm{C} & 0.75 \pm 0.53 & \mathrm{pCi} / \mathrm{g} & 0.070 & \text { EPA903.0 } \\ \mathrm{J} & \mathrm{C} & 1.0 \pm 0.61 & \mathrm{pCi} / \mathrm{g} & 0.070 & \text { EPA903.0 } \\ \mathrm{U} & & 1.1 & \mathrm{pCi} / \mathrm{g} & 1.1 & \text { EPA906.0 } \\ & & 1.6 \pm 0.33 & \mathrm{pCi} / g & 1.2 & \text { EPA906.0 }\end{array}$

Gross alpha

Nonvolatile beta

Nonvolatile beta

Radium, total alpha-emitting

Radium, total alpha-emitting

Tritium 
SAMPLE NAME: D43111602

Location (SRS Coordinates): 18644.34E 66570.29N

Ground Elevation Above MSL: $134.1 \mathrm{ft}$

Depth of Core Interval: 0.50 to $2.00 \mathrm{ft}$

Sample Type: Normal

Sample Color: 10R 4/6

Constituent

Total Metals

Arsenic

Barium

Cadmium

Chromium

Copper

Lead

Manganese

Mercury

Nickel

Selenium

Silver
Sample ID: 100418

Sample Matrix: Soil

USC Soil Classification: SW

RQ AQ B Result Unit

Sample Moisture: Damp

Percent Solids: 91.80

D. Limit Method

$B / N / A$ Extractables (including PAH and phenols)

Acenaphthene

Acenaphthylene

Aldol condensate

Aldol condensate

Anthracene

Benzo(a)anthracene

Benzo(b)fluoranthene

Benzo(k)fluoranthene

Benzoic acid

Benzo(g,h,i)perylene

Benzo(a)pyrene

Benzyl alcohol

Bis(2-chloroethoxy) methane

Bis(2-chloroethyl) ether

Bis(2-chloroisopropyl) ether

Bis(2-ethylhexyl) phthalate

4-Bromophenyl phenyl ether

Butylbenzyl phthalate

4-Chloroaniline

4-Chloro-m-cresol

2-Chloronaphthalene

2-Chlorophenol

4-Chlorophenyl phenyl ether

Chrysene

o-Cresol (2-Methylphenol)

p-Cresol (4-Methylphenol)

Dibenz(a,h)anthracene

Dibenzofuran

Di-n-butyl phthalate

1,2-Dichlorobenzene
U

J NK $\quad 300$

J NVK 200

U 360

U 360

$\mathrm{U} \quad 360$

U $\quad 360$

$\mathrm{U} \quad 1,800$

U 360

U 360

U 360

U 360

$\mathrm{U}$

$U \quad 360$

UJ ${ }^{\cdot} \mathrm{V} \quad 160$

U 360

U $\quad 360$

U 360

U 360

U 360

U 360

U 360

$\mathrm{U} \quad 360$

U 360

U 360

$\mathrm{U} \quad 360$

U 360

UJ $\quad \mathrm{V} \quad 93$

U. 360

$\mathrm{mg} / \mathrm{kg}$
$\mathrm{mg} / \mathrm{kg}$
$\mathrm{mg} / \mathrm{kg}$
$\mathrm{mg} / \mathrm{kg}$
$\mathrm{mg} / \mathrm{kg}$
$\mathrm{mg} / \mathrm{kg}$
$\mathrm{mg} / \mathrm{kg}$
$\mathrm{mg} / \mathrm{kg}$
$\mathrm{mg} / \mathrm{kg}$
$\mathrm{mg} / \mathrm{kg}$
$\mathrm{mg} / \mathrm{kg}$

0.96

18

0.44

0.89

2.2

0.29

1.3

0.11

3.5

0.48

0.89

EPA7060

EPA6010

EPA6010

EPA6010

EPA6010

EPA7421

EPA 6010

EPA7470

EPA6010

EPA7740

EPA6010

r

$\mu \mathrm{g} / \mathrm{kg}$

$\mu \mathrm{g} / \mathrm{kg}$

$\mu \mathrm{g} / \mathrm{kg}$

$\mu \mathrm{g} / \mathrm{kg}$

$\mu g / k g$

$\mu g / \mathrm{kg}$

$\mu \mathrm{g} / \mathrm{kg}$

$\mu \mathrm{g} / \mathrm{kg}$

$\mu g / \mathrm{kg}$

$\mu \mathrm{g} / \mathrm{kg}$

$\mu \mathrm{g} / \mathrm{kg}$

$\mu \mathrm{g} / \mathrm{kg}$

$\mu \mathrm{g} / \mathrm{kg}$

$\mu \mathrm{g} / \mathrm{kg}$

$\mu \mathrm{g} / \mathrm{kg}$

$\mu \mathrm{g} / \mathrm{kg}$

$\mu g / k g$

$\mu \mathrm{g} / \mathrm{kg}$

$\mu \mathrm{g} / \mathrm{kg}$

$\mu \mathrm{g} / \mathrm{kg}$

$\mu \mathrm{g} / \mathrm{kg}$
EPA8270

EPA8270

EPA8270

EPA8270

EPA8270

EPA8270

EPA 8270

EPA8270

EPA8270

EPA8270

EPA8270

EPA8270

EPA8270

EPA8270

EPA8270

EPA8270

EPA8270

EPA8270

EPA8270

EPA8270

EPA8270

EPA8270

EPA8270

EPA 8270

EPA8270

EPA8270

EPA8270

EPA8270

EPA8270

EPA8270 
SAMPLE NAME: D43111602 (Continued)

Sample ID: 100418

Constituent

RQ AQ B Result

Unit

D. Limit Method

$B / N / A$ Extractables (including $P A H$ and phenols)

1.3-Dichlorobenzene

1,4-Dichlorobenzene

3,3'-Dichlorobenzidine

2.4-Dichlorophenol

Diethyl phthalate

2.4-Dimethyl phenol ...,

Dimethyl phthalate

2.4-Dinitrophenol

2,4-Dinitrotoluene

2,6-Dinitrotoluene

Di-n-octyl phthalate

Fluoranthene

Fluorene

Hexachlorobenzene

Hexachlorobutadiene

Hexachlorocyclopentadiene

Hexachloroethane

Indeno(1,2,3-c,d)pyrene

Isophorone

2-Methyl-4,6-dinitrophenol

2-Methylnaphthalene

Naphthalene

o-Nitroaniline

m-Nitroaniline

p-Nitroaniline

Nitrobenzene

2-Nitrophenol

4-Nitrophenol

$\mathrm{N}$-Nitrosodiphenylamine

N-Nitrosodipropylamine

Pentachlorophenol

Phenanthrene

Phenol

Pyrene

1,2,4-Trichlorobenzene

2,4,5-Trichlorophenol

2,4,6-Trichlorophenol

Unknown

Volatile Organic Compounds

$\begin{array}{ll}U & 360 \\ U & 360 \\ U & 730 \\ U & 360 \\ U & 360 \\ U & 360 \\ U & 360 \\ U & 1,800 \\ U & 360 \\ U & 360 \\ U & 360 \\ U & 360 \\ U & 360 \\ U & 360 \\ U & 360 \\ U & 360 \\ U & 360 \\ U & 360 \\ U & 360 \\ U & 1,800 \\ U & 360 \\ U & 360 \\ U & 1,800 \\ U & 1,800 \\ U & 1,800 \\ U & 360 \\ U & 360 \\ U & 1,800 \\ U & 360 \\ U & 360 \\ U & 1,800 \\ U & 360 \\ U & 360 \\ U & 360 \\ U & 360 \\ U & 1,800 \\ U & 360 \\ J & 1,000\end{array}$

$\mu \mathrm{g} / \mathrm{kg}$
$\mu \mathrm{g} / \mathrm{kg}$
$\mu \mathrm{g} / \mathrm{kg}$
$\mu \mathrm{g} / \mathrm{kg}$
$\mu \mathrm{g} / \mathrm{kg}$
$\mu \mathrm{g} / \mathrm{kg}$
$\mu \mathrm{g} / \mathrm{kg}$
$\mu \mathrm{g} / \mathrm{kg}$
$\mu \mathrm{g} / \mathrm{kg}$
$\mu \mathrm{g} / \mathrm{kg}$
$\mu \mathrm{g} / \mathrm{kg}$
$\mu \mathrm{g} / \mathrm{kg}$
$\mu \mathrm{g} / \mathrm{kg}$
$\mu \mathrm{g} / \mathrm{kg}$
$\mu \mathrm{g} / \mathrm{kg}$
$\mu \mathrm{g} / \mathrm{kg}$
$\mu \mathrm{g} / \mathrm{kg}$
$\mu \mathrm{g} / \mathrm{kg}$
$\mu \mathrm{g} / \mathrm{kg}$
$\mu \mathrm{g} / \mathrm{kg}$
$\mu \mathrm{g} / \mathrm{kg}$
$\mu \mathrm{g} / \mathrm{kg}$
$\mu \mathrm{g} / \mathrm{kg}$
$\mu \mathrm{g} / \mathrm{kg}$
$\mu \mathrm{g} / \mathrm{kg}$
$\mu \mathrm{g} / \mathrm{kg}$
$\mu \mathrm{g} / \mathrm{kg}$
$\mu \mathrm{g} / \mathrm{kg}$
$\mu \mathrm{g} / \mathrm{kg}$
$\mu \mathrm{g} / \mathrm{kg}$
$\mu \mathrm{g} / \mathrm{kg}$
$\mu \mathrm{g} / \mathrm{kg}$
$\mu \mathrm{g} / \mathrm{kg}$
$\mu \mathrm{g} / \mathrm{kg}$
$\mu \mathrm{g} / \mathrm{kg}$
$\mu \mathrm{g} / \mathrm{kg}$
$\mu \mathrm{g} / \mathrm{kg}$
$\mu \mathrm{g} / \mathrm{kg}$

360

360

1,800

360

360

1,800

1,800

1,800

360

360

1,800

360

360

1,800

360

360

360

360

1,800

360
EPA8270

EPA8270

EPA8270

EPA8270

EPA8270

EPA8270

EPA8270

EPA8270

EPA8270

EPA8270

EPA8270

EPA8270

EPA8270

EPA8270

EPA8270

EPA8270

EPA8270

EPA8270

EPA8270

EPA8270

EPA8270

EPA8270

EPA8270

EPA8270

EPA8270

EPA8270

EPA8270

EPA8270

EPA8270

EPA8270

EPA8270

EPA8270

EPA8270

EPA8270

EPA8270

EPA8270

EPA8270

EPA8270
EPA8240

EPA8240

EPA8240

EPA8240

EPA8240

EPA8240

EPA8240

EPA8240

EPA8240 
SAMPLE NAME: D4311.1602 (Continued)

Sample ID: 100418

\section{Constituent}

Volatile Organic Compounds

Chloroethene (Vinyl chloride)

2-Chloroethyl vinyl ether

Chloroform

Chloromethane (Methyl chloride)

1.1-Dichlorocthane

1,2-Dichloroethane

1.1-Dichloroethylene

1.2-Dichloroethylene

Dichloromethane (Methylene chloride)

1.2-Dichloropropane

cis-1,3-Dichloropropene

trans-1.3-Dichloropropene

Ethylbenzene

2-Hexanone

Methyl ethyl ketone

Methyl isobutyl ketone

Styrene

1,1,2,2-Tetrachloroethane

Toluene

1,1.1-Trichloroethane

1,1,2-Trichloroethane

Trichloroethylene

Vinyl acetate

Xylenes
Dibromochloromethane

Tetrachloroethylene
$\mathbf{R Q}$
AQ
Result
Unit
D. Limit Method

$\begin{array}{llll}11 & \mu g / k g & 11 & \text { EPA8240 } \\ 11 & \mu g / k g & 11 & \text { EPA8240 } \\ 5.5 & \mu g / k g & 5.5 & \text { EPA8240 } \\ 11 & \mu g / k g & 11 & \text { EPA8240 } \\ 5.5 & \mu g / k g & 5.5 & \text { EPA8240 } \\ 5.5 & \mu g / k g & 5.5 & \text { EPA8240 } \\ 5.5 & \mu g / k g & 5.5 & \text { EPA8240 } \\ 5.5 & \mu g / k g & 5.5 & \text { EPA8240 } \\ 5.5 & \mu g / k g & 5.5 & \text { EPA8240 } \\ 14 & \mu g / k g & 5.5 & \text { EPA8240 } \\ 5.5 & \mu g / k g & 5.5 & \text { EPA8240 } \\ 5.5 & \mu g / k g & 5.5 & \text { EPA8240 } \\ 5.5 & \mu g / k g & 5.5 & \text { EPA8240 } \\ 5.5 & \mu g / k g & 5.5 & \text { EPA8240 } \\ 11 & \mu g / k g & 11 & \text { EPA8240 } \\ 11 & \mu g / k g & 11 & \text { EPA8240 } \\ 11 & \mu g / k g & 11 & \text { EPA8240 } \\ 5.5 & \mu g / k g & 5.5 & \text { EPA8240 } \\ 5.5 & \mu g / k g & 5.5 & \text { EPA8240 } \\ 5.5 & \mu g / k g & 5.5 & \text { EPA8240 } \\ 5.5 & \mu g / k g & 5.5 & \text { EPA8240 } \\ 5.5 & \mu g / k g & 5.5 & \text { EPA8240 } \\ 5.5 & \mu g / k g & 5.5 & \text { EPA8240 } \\ 5.5 & \mu g / k g & 5.5 & \text { EPA8240 } \\ 11 & \mu g / k g & 11 & \text { EPA8240 } \\ 5.5 & \mu g / k g & 5.5 & \text { EPA8240 }\end{array}$

Radionuclide Indicators and Radionuclides

Gross alpha

Nonvolatile beta

Radium, total alpha-emitting

Tritium

$\begin{array}{llll} & & 8.6 \pm 6.9 & \mathrm{pCi} / g \\ \mathrm{~J} & \mathrm{C} & 23 \pm 9.6 & \mathrm{pCi} / \mathrm{g} \\ \mathrm{U} & \mathrm{C} & 1.1 \pm 0.64 & \mathrm{pCi} / \mathrm{g} \\ & & 1.3 & \mathrm{pCi} / \mathrm{g}\end{array}$

Sample ID: 100419

SAMPLE NAME: D43111603

Location (SRS Coordinates): 18644.34E 66570.29N

Ground Elevation Above MSL: $134.1 \mathrm{ft}$

Depth of Core Interval: 5.00 to $7.00 \mathrm{ft}$

Sample Type: Normal

Sample Color: 10R 4/6

Sample Matrix: Soil

USC Soil Classification: SC

Samiple Moisture: Dry

Percent Solids: 86.40

Constituent

RQ AQ B Result Unit

D. Limit Method

Total Metals

Arsenic

Barium

Cadmium

U

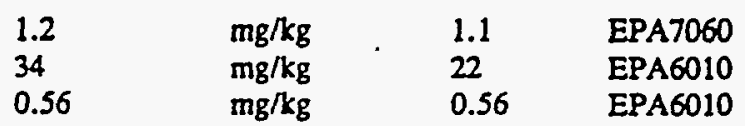


SAMPLE NAME: D43111603 (Continued)

Constituent

RQ AQ B Result

Unit

D. Limit Method

\section{Total Metals}

Chromium

Copper

Lead

Manganese

Mercury

Nickel

Selenium

Silver

Volatile Organic Compounds
U

$\begin{array}{ll} & 5.0 \\ \mathrm{U} & 22 \\ \mathrm{U} & 0.12 \\ \mathrm{U} & 4.4 \\ \mathrm{U} & 0.57\end{array}$

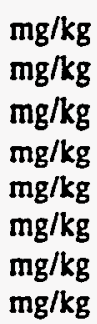

$\mathrm{mg} / \mathrm{kg}$
$\mathrm{mg} / \mathrm{kg}$
$\mathrm{mg} / \mathrm{kg}$
$\mathrm{mg} / \mathrm{kg}$
$\mathrm{mg} / \mathrm{kg}$
$\mathrm{mg} / \mathrm{kg}$
$\mathrm{mg} / \mathrm{kg}$
$\mathrm{mg} / \mathrm{kg}$

$\mu \mathrm{g} / \mathrm{kg}$

$\mu \mathrm{g} / \mathrm{kg}$

$\mu g / k g$

$\mu g / \mathrm{kg}$

$\mu \mathrm{g} / \mathrm{kg}$

$\mu \mathrm{g} / \mathrm{kg}$

$\mu \mathrm{g} / \mathrm{kg}$

$\mu \mathrm{g} / \mathrm{kg}$

$\mu \mathrm{g} / \mathrm{kg}$

$\mu \mathrm{g} / \mathrm{kg}$

$\mu g / k g$

$\mu \mathrm{g} / \mathrm{kg}$

$\mu \mathrm{g} / \mathrm{kg}$

$\mu g / \mathrm{kg}$

$\mu \mathrm{g} / \mathrm{kg}$

$\mu \mathrm{g} / \mathrm{kg}$

$\mu \mathrm{g} / \mathrm{kg}$

$\mu \mathrm{g} / \mathrm{kg}$

$\mu \mathrm{g} / \mathrm{kg}$

$\mu \mathrm{g} / \mathrm{kg}$

$\mu \mathrm{g} / \mathrm{kg}$

$\mu \mathrm{g} / \mathrm{kg}$

$\mu g / \mathrm{kg}$

$\mu g / \mathrm{kg}$

$\mu \mathrm{g} / \mathrm{kg}$

$\mu \mathrm{g} / \mathrm{kg}$

$\mu \mathrm{g} / \mathrm{kg}$

$\mu g / \mathrm{kg}$

$\mu \mathrm{g} / \mathrm{kg}$

$\mu \mathrm{g} / \mathrm{kg}$

$\mu g / k g$

$\mu \mathrm{g} / \mathrm{kg}$

$\mu g / k g$

$\mu \mathrm{g} / \mathrm{kg}$

$\mu g / \mathrm{kg}$

$\begin{array}{ll}1.1 & \text { EPA6010 } \\ 2.8 & \text { EPA6010 } \\ 0.34 & \text { EPA7421 } \\ 1.7 & \text { EPA6010 } \\ 0.12 & \text { EPA7470 } \\ 4.4 & \text { EPA6010 } \\ 0.57 & \text { EPA7740 } \\ 1.1 & \text { EPA6010 }\end{array}$

Trichloroethylene

6.0

6.0

12

Xylenes

6.0

$\begin{array}{ll}12 & \text { EPA8240 } \\ 6.0 & \text { EPA8240 } \\ 6.0 & \text { EPA8240 } \\ 6.0 & \text { EPA8240 } \\ 12 & \text { EPA8240 } \\ 6.0 & \text { EPA8240 } \\ 6.0 & \text { EPA8240 } \\ 6.0 & \text { EPA8240 } \\ 12 & \text { EPA8240 } \\ 12 & \text { EPA8240 } \\ 12 & \text { EPA8240 } \\ 6.0 & \text { EPA8240 } \\ 12 & \text { EPA8240 } \\ 6.0 & \text { EPA8240 } \\ 6.0 & \text { EPA8240 } \\ 6.0 & \text { EPA8240 } \\ 6.0 & \text { EPA8240 } \\ 6.0 & \text { EPA8240 } \\ 6.0 & \text { EPA8240 } \\ 6.0 & \text { EPA8240 } \\ 6.0 & \text { EPA8240 } \\ 6.0 & \text { EPA8240 } \\ 6.0 & \text { EPA8240 } \\ 12 & \text { EPA8240 } \\ 12 & \text { EPA8240 } \\ 12 & \text { EPA8240 } \\ 6.0 & \text { EPA8240 } \\ 6.0 & \text { EPA8240 } \\ 6.0 & \text { EPA8240 } \\ 6.0 & \text { EPA8240 } \\ 6.0 & \text { EPA8240 } \\ 6.0 & \text { EPA8240 } \\ 6.0 & \text { EPA8240 } \\ 12 & \text { EPA8240 } \\ 6.0 & \text { EPA8240 }\end{array}$


SAMPLE NAME: D43111603 (Continued)

Constituent

$\mathbf{R}$

AQ B

Sample D: 100419

Radionuclide Indicators and Radionuclides

Gross alpha

Nonvolatile beta

Radium, total alpha-emitting

Tritium

$\begin{array}{llll}5.3 \pm 6.3 & \mathrm{pCi} / \mathrm{g} & 3.9 & \text { EPA900.0 } \\ 18 \pm 9.7 & \mathrm{pCi} / \mathrm{g} & 5.8 & \text { EPA900.0 } \\ 1.8 \pm 0.84 & \mathrm{pCi} / \mathrm{g} & 0.070 & \text { EPA903.0 } \\ 2.9 \pm 0.57 & \mathrm{pCi} / \mathrm{g} & 1.2 & \text { EPA906.0 }\end{array}$

\section{SAMPLE NAME: D43111604}

Location (SRS Coordinates): 18644.34E 66570.29N

Ground Elevation Above MSL: $134.1 \mathrm{ft}$

Depth of Core Interval: 10.00 to $12.00 \mathrm{ft}$

Sample Type: Normal

Sample Color: 10R 4/6

\section{Constituent}

Total Metals
Sample D: 100421

Sample Matrix: Soil

USC Soil Classification: SW

RQ AQ B Result Unit

Sample Moisture: Dry

Percent Solids: 87.40

D. Limit Method

Arsenic
Barium
Cadmium
Chromium
Copper
Lead
Manganese
Mercury
Nickel
Selenium
Silver

$\begin{array}{lll}\mathrm{U} & 1.2 & \mathrm{mg} / \mathrm{kg} \\ \mathrm{U} & 22 & \mathrm{mg} / \mathrm{kg} \\ \mathrm{U} & 0.56 & \mathrm{mg} / \mathrm{kg} \\ & 16 & \mathrm{mg} / \mathrm{kg} \\ & 2.8 & \mathrm{mg} / \mathrm{kg} \\ \mathrm{U} & 3.0 & \mathrm{mg} / \mathrm{kg} \\ \mathrm{U} & 31 & \mathrm{mg} / \mathrm{kg} \\ \mathrm{U} & 0.11 & \mathrm{mg} / \mathrm{kg} \\ \mathrm{U} & 4.5 & \mathrm{mg} / \mathrm{kg} \\ & 0.56 & \mathrm{mg} / \mathrm{kg} \\ & 1.1 & \mathrm{mg} / \mathrm{kg}\end{array}$

$\begin{array}{ll}1.1 & \text { EPA7060 } \\ 22 & \text { EPA6010 } \\ 0.56 & \text { EPA6010 } \\ 1.1 & \text { EPA6010 } \\ 2.8 & \text { EPA6010 } \\ 0.33 & \text { EPA7421 } \\ 1.7 & \text { EPA6010 } \\ 0.11 & \text { EPA7470 } \\ 4.5 & \text { EPA6010 } \\ 0.56 & \text { EPA7740 } \\ 1.1 & \text { EPA6010 }\end{array}$

$B / N / A$ Extractables (including $P A H$ and phenols)

\begin{tabular}{|c|c|c|c|c|c|c|}
\hline Acenaphthene & $\mathbf{U}$ & & 380 & $\mu \mathrm{g} / \mathrm{kg}$ & 380 & EPA8270 \\
\hline Acenaphthylene & $\mathbf{U}$ & & 380 & $\mu \mathrm{g} / \mathrm{kg}$ & 380 & EPA8270 \\
\hline Aldol condensate & $J$ & NKV & 200 & $\mu g / k g$ & & EPA8270 \\
\hline Anthracene & $\mathbf{U}$ & & 380 & $\mu \mathrm{g} / \mathrm{kg}$ & 380 & EPA8270 \\
\hline Benzo(a)anthracene & $\mathbf{U}$ & & 380 & $\mu g / k g$ & 380 & EPA8270 \\
\hline Benzo(b)fluoranthene & $\mathbf{U}$ & & 380 & $\mu g / \mathrm{kg}$ & 380 & EPA8270 \\
\hline Benzo(k)fluoranthene & $\mathbf{U}$ & & 380 & $\mu \mathrm{g} / \mathrm{kg}$ & 380 & EPA8270 \\
\hline Benzoic acid & $\mathbf{U}$ & & 1,900 & $\mu \mathrm{g} / \mathrm{kg}$ & 1,900 & EPA8270 \\
\hline Benzo(g,h,i)perylene & $\mathbf{U}$ & & 380 & $\mu g / \mathrm{kg}$ & 380 & EPA8270 \\
\hline Benzo(a)pyrene & $\mathbf{U}$ & & 380 & $\mu \mathrm{g} / \mathrm{kg}$ & 380 & EPA 8270 \\
\hline Benzyl alcohol & $\mathbf{U}$ & & 380 & $\mu g / k g$ & 380 & EPA8270 \\
\hline Bis(2-chloroethoxy) methane & $\mathbf{U}$ & & 380 & $\mu \mathrm{g} / \mathrm{kg}$ & 380 & EPA8270 \\
\hline Bis(2-chloroethyl) ether & $\mathbf{U}$ & & 380 & $\mu g / k g$ & 380 & EPA8270 \\
\hline Bis(2-chloroisopropyl) ether & $\mathrm{U}$ & & 380 & $\mu g / k g$ & 380 & EPA8270 \\
\hline Bis(2-ethylhexyl) phthalate & UJ & $\mathbf{V}$ & 150 & $\mu g / k g$ & 380 & EPA8270 \\
\hline 4-Bromophenyl phenyl ether & $\mathbf{U}$ & & 380 & $\mu g / \mathrm{kg}$ & 380 & EPA8270 \\
\hline Butylbenzyl phthalate & $\mathbf{U}$ & & 380 & $\mu g / k g$ & 380 & EPA8270 \\
\hline 4-Chloroaniline & $\mathbf{U}$ & & 380 & $\mu \mathrm{g} / \mathrm{kg}$ & 380 & EPA8270 \\
\hline
\end{tabular}


SAMPLE NAME: D43111604 (Continued)

Constituent
Sample ID: 100421

$B / N / A$ Extractables (including $P A H$ and phenols)

4-Chloro-m-cresol
2-Chloronaphthalene
2-Chlorophenol
4-Chlorophenyl phenyl ether
Chrysene
o-Cresol (2-Methylphenol)
p-Cresol (4-Methylphenol)
Dibenz(a,h)anthracene
Dibenzofuran
Di-n-butyl phthalate
1,2-Dichlorobenzene
1,3-Dichlorobenzene
1,4-Dichlorobenzene
3,3'-Dichlorobenzidine
2,4-Dichlorophenol
Diethyl phthalate
2,4-Dimethyl phenol
Dimethyl phthalate
2,4-Dinitrophenol
2,4-Dinitrotoluene
2,6-Dinitrotoluene
Di-n-octyl phthalate
Fluoranthene
Fluorene
Hexachlorobenzene
Hexachlorobutadiene
Hexachlorocyclopentadiene
Hexachloroethane
Indeno(1,2.3-c,d)pyrene
Isophorone
2-Methyl-4,6-dinitrophenol
2-Methylnaphthalene
Naphthalene
0-Nitroaniline
m-Nitroaniline
p-Nitroaniline
Nitrobenzene
2-Nitrophenol
4-Nitrophenol
N-Nitrosodiphenylamine
N-Nitrosodipropylamine
Pentachlorophenol
Phenanthrene
Phenol
Pyrene
1,2.4-Trichlorobenzene
2,4,5-Trichlorophenol
2,4,6-Trichlorophenol
Unknown

RQ AQ B Result Unit D. Limit Method

\begin{tabular}{|c|c|c|c|c|}
\hline$U$ & 380 & $\mu \mathrm{g} / \mathrm{kg}$ & 380 & EPA8270 \\
\hline$U$ & 380 & $\mu \mathrm{g} / \mathrm{kg}$ & 380 & EPA8270 \\
\hline $\mathbf{U}$ & 380 & $\mu \mathrm{g} / \mathrm{kg}$ & 380 & EPA8270 \\
\hline U & 380 & $\mu \mathrm{g} / \mathrm{kg}$ & 380 & EPA8270 \\
\hline $\mathbf{U}$ & 380 & $\mu g / \mathrm{kg}$ & 380 & EPA8270 \\
\hline $\mathrm{U}$ & 380 & $\mu \mathrm{g} / \mathrm{kg}$ & 380 & EPA8270 \\
\hline$U$ & 380 & $\mu \mathrm{g} / \mathrm{kg}$ & 380 & EPA8270 \\
\hline U & 380 & $\mu g / k g$ & 380 & EPA8270 \\
\hline U & 380 & $\mu \mathrm{g} / \mathrm{kg}$ & 380 & EPA8270 \\
\hline V & 96 & $\mu g / k g$ & 380 & EPA8270 \\
\hline U & 380 & $\mu g / \mathrm{kg}$ & 380 & EPA8270 \\
\hline $\mathrm{U}$ & 380 & $\mu g / \mathrm{kg}$ & 380 & EPA8270 \\
\hline J & 380 & $\mu \mathrm{g} / \mathrm{kg}$ & 380 & EPA8270 \\
\hline U & 760 & $\mu \mathrm{g} / \mathrm{kg}$ & 760 & EPA8270 \\
\hline U & 380 & $\mu \mathrm{g} / \mathrm{kg}$ & 380 & EPA8270 \\
\hline 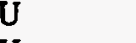 & 380 & $\mu \mathrm{g} / \mathrm{kg}$ & 380 & EPA8270 \\
\hline J & 380 & $\mu g / \mathrm{kg}$ & 380 & EPA8270 \\
\hline$U$ & 380 & $\mu \mathrm{g} / \mathrm{kg}$ & 380 & EPA8270 \\
\hline J & 1,900 & $\mu g / \mathrm{kg}$ & 1.900 & EPA8270 \\
\hline & 380 & $\mu \mathrm{g} / \mathrm{kg}$ & 380 & EPA8270 \\
\hline & 380 & $\mu \mathrm{g} / \mathrm{kg}$ & 380 & EPA8270 \\
\hline & 380 & $\mu \mathrm{g} / \mathrm{kg}$ & 380 & EPA8270 \\
\hline & 380 & $\mu \mathrm{g} / \mathrm{kg}$ & 380 & EPA8270 \\
\hline & 380 & $\mu \mathrm{g} / \mathrm{kg}$ & 380 & EPA8270 \\
\hline 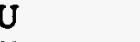 & 380 & $\mu \mathrm{g} / \mathrm{kg}$ & 380 & EPA8270 \\
\hline & 380 & $\mu \mathrm{g} / \mathrm{kg}$ & 380 & EPA8270 \\
\hline & 380 & $\mu \mathrm{g} / \mathrm{kg}$ & 380 & EPA8270 \\
\hline & 380 & $\mu \mathrm{g} / \mathrm{kg}$ & 380 & EPA8270 \\
\hline & 380 & $\mu \mathrm{g} / \mathrm{kg}$ & 380 & EPA8270 \\
\hline & 380 & $\mu \mathrm{g} / \mathrm{kg}$ & 380 & EPA8270 \\
\hline & 1,900 & $\mu \mathrm{g} / \mathrm{kg}$ & 1,900 & EPA8270 \\
\hline & 380 & $\mu g / k g$ & 380 & EPA8270 \\
\hline & 380 & $\mu \mathrm{g} / \mathrm{kg}$ & 380 & EPA8270 \\
\hline & 1,900 & $\mu \mathrm{g} / \mathrm{kg}$ & . 1,900 & EPA8270 \\
\hline & 1,900 & $\mu g / k g$ & 1,900 & EPA8270 \\
\hline & 1,900 & $\mu g / k g$ & 1,900 & EPA8270 \\
\hline & 380 & $\mu \mathrm{g} / \mathrm{kg}$ & 380 & EPA8270 \\
\hline & 380 & $\mu \mathrm{g} / \mathrm{kg}$ & 380 & EPA8270 \\
\hline & 1,900 & $\mu g / \mathrm{kg}$ & 1,900 & EPA8270 \\
\hline & 380 & $\mu \mathrm{g} / \mathrm{kg}$ & 380 & EPA8270 \\
\hline & 380 & $\mu \mathrm{g} / \mathrm{kg}$ & 380 & EPA8270 \\
\hline & 1,900 & $\mu \mathrm{g} / \mathrm{kg}$ & 1,900 & EPA8270 \\
\hline & 380 & $\mu g / k g$ & 380 & EPA8270 \\
\hline & 380 & $\mu g / k g$ & 380 & EPA8270 \\
\hline & 380 & $\mu \mathrm{g} / \mathrm{kg}$ & 380 & EPA8270 \\
\hline & 380 & $\mu \mathrm{g} / \mathrm{kg}$ & 380 & EPA8270 \\
\hline & 1.900 & $\mu \mathrm{g} / \mathrm{kg}$ & 1,900 & EPA8270 \\
\hline & 380 & $\mu \mathrm{g} / \mathrm{kg}$ & 380 & EPA8270 \\
\hline NV & 600 & $\mu \mathrm{g} / \mathrm{kg}$ & & EPA8270 \\
\hline
\end{tabular}


SAMPLE NAME: D43111604 (Continued)

Constituent

$\mathbf{R Q}$

$A Q$

Result

Unit

D. Limit Method

Volatile Organic Compounds

Acetone
Benzene
Bromodichloromethane
Bromoform
Bromomethane (Methyl bromide)
Carbon disulfide
Carbon tetrachloride
Chlorobenzene
Chloroethane
Chloroethene (Vinyl chloride)
2-Chloroethyl vinyl ether
Chloroform
Chioromethane (Methyl chloride)
Dibromochloromethane
1,1-Dichloroethane
1,2-Dichlorothane
1,1-Dichloroethylene
1,2-Dichloroethylene
Dichloromethane (Methylene chloride)
1,2-Dichloropropane
cis-1,3-Dichloropropene
trans-1,3-Dichloropropene
Ethylbenzene
2-Hexanone
Methyl ethyl ketone
Methyl isobutyl ketone
Styrene
1,1,2,2-Tetrachloroethane
Tetrachloroethylene
Toluene
1,1,1-Trichloroethane
1,1,2-Trichloroethane
Trichloroethylene
Vinyl acetate
Xylenes
(n)

Sample ID: 100421

\begin{tabular}{|c|c|c|c|c|}
\hline \multirow[t]{18}{*}{ v } & 13 & $\mu g / \mathrm{kg}$ & 11 & EPA8240 \\
\hline & 5.5 & $\mu g / \mathrm{kg}$ & 5.5 & EPA8240 \\
\hline & 5.5 & $\mu \mathrm{g} / \mathrm{kg}$ & 5.5 & EPA8240 \\
\hline & 5.5 & $\mu \mathrm{g} / \mathrm{kg}$ & 5.5 & EPA8240 \\
\hline & 11 & $\mu g / \mathrm{kg}$ & 11 & EPA8240 \\
\hline & 5.5 & $\mu g / \mathrm{kg}$ & 5.5 & EPA8240 \\
\hline & 5.5 & $\mu \mathrm{g} / \mathrm{kg}$ & 5.5 & EPA8240 \\
\hline & 5.5 & $\mu \mathrm{g} / \mathrm{kg}$ & 5.5 & EPA8240 \\
\hline & 11 & $\mu \mathrm{g} / \mathrm{kg}$ & 11 & EPA8240 \\
\hline & 11 & $\mu \mathrm{g} / \mathrm{kg}$ & 11 & EPA8240 \\
\hline & 11 & $\mu \mathrm{g} / \mathrm{kg}$ & 11 & EPA8240 \\
\hline & 5.5 & $\mu g / \mathrm{kg}$ & 5.5 & EPA8240 \\
\hline & 11 & $\mu g / k g$ & 11 & EPA8240 \\
\hline & 5.5 & $\mu \mathrm{g} / \mathrm{kg}$ & 5.5 & EPA8240 \\
\hline & 5.5 & $\mu \mathrm{g} / \mathrm{kg}$ & 5.5 & EPA8240 \\
\hline & 5.5 & $\mu \mathrm{g} / \mathrm{kg}$ & 5.5 & EPA8240 \\
\hline & 5.5 & $\mu \mathrm{g} / \mathrm{kg}$ & 5.5 & EPA8240 \\
\hline & 5.5 & $\mu \mathrm{g} / \mathrm{kg}$ & 5.5 & EPA8240 \\
\hline \multirow[t]{17}{*}{$\mathrm{v}$} & 12 & $\mu g / \mathrm{kg}$ & 5.5 & EPA8240 \\
\hline & 5.5 & $\mu \mathrm{g} / \mathrm{kg}$ & 5.5 & EPA8240 \\
\hline & 5.5 & $\mu \mathrm{g} / \mathrm{kg}$ & 5.5 & EPA8240 \\
\hline & 5.5 & $\mu \mathrm{g} / \mathrm{kg}$ & 5.5 & EPA8240 \\
\hline & 5.5 & $\mu g / \mathrm{kg}$ & 5.5 & EPA8240 \\
\hline & 11 & $\mu \mathrm{g} / \mathrm{kg}$ & 11 & EPA8240 \\
\hline & 11 & $\mu \mathrm{g} / \mathrm{kg}$ & 11 & EPA8240 \\
\hline & 11 & $\mu \mathrm{g} / \mathrm{kg}$ & 11 & EPA8240 \\
\hline & 5.5 & $\mu \mathrm{g} / \mathrm{kg}$ & 5.5 & EPA8240 \\
\hline & 5.5 & $\mu \mathrm{g} / \mathrm{kg}$ & 5.5 & EPA8240 \\
\hline & 5.5 & $\mu g / \mathrm{kg}$ & 5.5 & EPA8240 \\
\hline & 5.5 & $\mu \mathrm{g} / \mathrm{kg}$ & 5.5 & EPA8240 \\
\hline & 5.5 & $\mu \mathrm{g} / \mathrm{kg}$ & 5.5 & EPA8240 \\
\hline & 5.5 & $\mu g / \mathrm{kg}$ & 5.5 & EPA8240 \\
\hline & 5.5 & $\mu \mathrm{g} / \mathrm{kg}$ & 5.5 & EPA8240 \\
\hline & 11 & $\mu \mathrm{g} / \mathrm{kg}$ & 11 & EPA8240 \\
\hline & 5.5 & $\mu \mathrm{g} / \mathrm{kg}$ & 5.5 & EPA8240 \\
\hline
\end{tabular}

Radionuclide Indicators and Radionuclides

Gross alpha

Nonvolatile beta

Radium, total alpha-emitting

$\begin{array}{ll}8.4 \pm 6.8 & \mathrm{pCi} / \mathrm{g} \\ 19 \pm 9.2 & \mathrm{pCi} / \mathrm{g} \\ 0.83 \pm 0.55 & \mathrm{pCi} / \mathrm{g} \\ 2.9 \pm 0.57 & \mathrm{pCi} / \mathrm{g}\end{array}$

3.6

EPA900.0

$5 . \dot{3} \quad$ EPA900.0

$0.070 \quad$ EPA903.0

1.2 EPA906.0 
SAMPLE NAME: D43111605

Location (SRS Coordinates): 18644.34E 66570.29N

Ground Elevation Above MSL: $134.1 \mathrm{ft}$

Depth of Core Interval: 15.00 to $17.00 \mathrm{ft}$

Sample Type: Normal

Sample Color: 10R $4 / 6$

\section{Constituent}

Sample ID: 100422

Sample Matrix: Soil

USC Soil Classification: SW

RQ AQ B Result Unit
Sample Moisture: Dry

Percent Solids: 89.00

D. Limit Method

Physical Parameters

Cation exchange capacity

$\mathrm{pH}$

Specific conductance

Specific conductance

Total organic carbon

Total Metals

Arsenic

Barium

Cadmium

Chromium

Copper

Lead

Manganese

Mercury

Nickel

Selenium

Silver

17
5.1
16
16
1,100

1,100

meq/100g
pH
$\mu S / \mathrm{cm}$
$\mu \mathrm{S} / \mathrm{cm}$
$\mathrm{mg} / \mathrm{kg}$

2.3

0.010

1.0

1.0

180
EPA9080

EPA9045

EPA9050

EPA9050

EPA415.1

$B / N / A$ Extractables (including $P A H$ and phenols)

Acenaphthene
Acenaphthylene
Aldol condensate
Aldol condensate
Anthracene
Benzo(a)anthracene
Benzo(b)fluoranthene
Benzo(k)fluoranthene
Benzoic acid
Benzo(g,h,i)perylene
Benzo(a)pyrene
Benzyl alcohol
Bis(2-chloroethoxy) methane
Bis(2-chloroethyl) ether
Bis(2-chloroisopropyl) ether
Bis(2-ethylhexyl) phthalate
4-Bromophenyl phenyl ether
Butylbenzyl phthalate
4-Chloroaniline
4-Chloro-m-cresol
2-Chloronaphthalene
2-Chlorophenol

Acenaphthene

Acenaphthylene

Anthracene

Benzo(a)anthracene

Benzo(b)fluoranthene

Benzo(k)fluoranthene

Benzoic acid

Benzo(a)pyrene

Benzyl alcohol

Bis(2-chloroethyl) ether

Bis(2-chloroisopropyl) ether

Bis(2-ethylhexyl) phthalate

Butylbenzyl phthalate

4-Chloroaniline

2-Chloronaphthalene

2-Chlorophenol

$\begin{array}{lll}\mathbf{U} & & 370 \\ \mathbf{J} & & 370 \\ \mathbf{J} & \mathrm{NK} & 300 \\ \mathbf{U} & & 200 \\ \mathbf{U} & & 370 \\ \mathbf{U} & & 370 \\ \mathbf{U} & & 370 \\ \mathbf{U} & & 370 \\ \mathbf{U} & & 1,900 \\ \mathbf{U} & & 370 \\ \mathbf{U} & & 370 \\ \mathbf{U} & & .370 \\ \mathbf{U} & & 370 \\ \mathbf{U} & \cdot & 370 \\ \mathbf{U J} & \mathrm{V} & 370 \\ \mathbf{U} & & 170 \\ \mathbf{U} & & 370 \\ \mathbf{U} & & 370 \\ \mathbf{U} & & 370 \\ \mathbf{U} & & 370 \\ \mathbf{U} & & 370 \\ & & 370\end{array}$

$\begin{array}{llll}4.2 & \mathrm{mg} / \mathrm{kg} & 1.0 & \text { EPA7060 } \\ 22 & \mathrm{mg} / \mathrm{kg} & 22 & \text { EPA6010 } \\ 0.55 & \mathrm{mg} / \mathrm{kg} & 0.55 & \text { EPA6010 } \\ 14 & \mathrm{mg} / \mathrm{kg} & 1.1 & \text { EPA6010 } \\ 2.7 & \mathrm{mg} / \mathrm{kg} & 2.7 & \text { EPA6010 } \\ 3.1 & \mathrm{mg} / \mathrm{kg} & 0.30 & \text { EPA7421 } \\ 24 & \mathrm{mg} / \mathrm{kg} & 1.6 & \text { EPA6010 } \\ 0.11 & \mathrm{mg} / \mathrm{kg} & 0.11 & \text { EPA7470 } \\ 4.4 & \mathrm{mg} / \mathrm{kg} & 4.4 & \text { EPA6010 } \\ 0.50 & \mathrm{mg} / \mathrm{kg} & 0.50 & \text { EPA7740 } \\ 1.1 & \mathrm{mg} / \mathrm{kg} & 1.1 & \text { EPA6010 }\end{array}$

$\begin{array}{lll}\mu \mathrm{g} / \mathrm{kg} & 370 & \text { EPA8270 } \\ \mu \mathrm{g} / \mathrm{kg} & 370 & \text { EPA8270 } \\ \mu \mathrm{g} / \mathrm{kg} & & \text { EPA8270 } \\ \mu \mathrm{g} / \mathrm{kg} & & \text { EPA8270 } \\ \mu \mathrm{g} / \mathrm{kg} & 370 & \text { EPA8270 } \\ \mu \mathrm{g} / \mathrm{kg} & 370 & \text { EPA8270 } \\ \mu \mathrm{g} / \mathrm{kg} & 370 & \text { EPA8270 } \\ \mu \mathrm{g} / \mathrm{kg} & 370 & \text { EPA8270 } \\ \mu \mathrm{g} / \mathrm{kg} & 1,900 & \text { EPA8270 } \\ \mu \mathrm{g} / \mathrm{kg} & 370 & \text { EPA8270 } \\ \mu \mathrm{g} / \mathrm{kg} & 370 & \text { EPA8270 } \\ \mu \mathrm{g} / \mathrm{kg} & 370 & \text { EPA8270 } \\ \mu \mathrm{g} / \mathrm{kg} & 370 & \text { EPA8270 } \\ \mu \mathrm{g} / \mathrm{kg} & 370 & \text { EPA8270 } \\ \mu \mathrm{g} / \mathrm{kg} & 370 & \text { EPA8270 } \\ \mu \mathrm{g} / \mathrm{kg} & 370 & \text { EPA8270 } \\ \mu \mathrm{g} / \mathrm{kg} & 370 & \text { EPA8270 } \\ \mu \mathrm{g} / \mathrm{kg} & 370 & \text { EPA8270 } \\ \mu \mathrm{g} / \mathrm{kg} & 370 & \text { EPA8270 } \\ \mu \mathrm{g} / \mathrm{kg} & 370 & \text { EPA8270 } \\ \mu \mathrm{g} / \mathrm{kg} & 370 & \text { EPA8270 } \\ \mu \mathrm{g} / \mathrm{kg} & 370 & \text { EPA8270 }\end{array}$


SAMPLE NAME: D43111605 (Continued)

Sample ID: 100422

Constituent

RQ AQ B Result

Unit

D. Limit Method

$B / N / A$ Extractables (including $P A H$ and phenols)

4-Chlorophenyl phenyl ether

Chrysene

o-Cresol (2-Methylphenol)

p-Cresol (4-Methylphenol)

Dibenz(a,h)anthracene

Dibenzofuran

Di-n-butyl phthalate

1,2-Dichlorobenzene

1,3-Dichlorobenzene

1,4-Dichlorobenzene

3.3'-Dichlorobenzidine

2,4-Dichlorophenol

Diethyl phthalate

2,4-Dimethyl phenol

Dimethyl phthalate

2.4-Dinitrophenol

2.4-Dinitrotoluene

2.6-Dinitrotoluene

Di-n-octyl phthalate

Fluoranthene

Fluorene

Hexachlorobenzene

Hexachlorobutadiene

Hexachlorocyclopentadiene

Hexachloroethane

Indeno(1,2,3-c,d)pyrene

Isophorone

2-Methyl-4,6-dinitrophenol

2-Methylnaphthalene

Naphthalene

o-Nitroaniline

m-Nitroaniline

p-Nitroaniline

Nitrobenzene

2-Nitrophenol

4-Nitrophenol

$\mathrm{N}$-Nitrosodiphenylamine

N-Nitrosodipropylamine

Pentachlorophenol

Phenanthrene

Phenol

Pyrene

1,2,4-Trichlorobenzene

2,4,5-Trichlorophenol

2,4,6-Trichlorophenol

Unknown
U

U

U

$\mathrm{U}-370$

U 370

$U \quad 370$

UJ $\mathrm{V} \quad 100$

U $\quad 370$

U 370

370

740

370

370

370

370

1,900

370

370

370

370

370

370

370

370

370

370

370

1,900

370

370

1.900

1,900

1,900

370

370

1,900

370

370

1,900

370

370

370

370

1,900

370

NV

900 $\mu \mathrm{g} / \mathrm{kg}$

$\mu g / \mathrm{kg}$

$\mu \mathrm{g} / \mathrm{kg}$

$\mu \mathrm{g} / \mathrm{kg}$

$\mu \mathrm{g} / \mathrm{kg}$

$\mu \mathrm{g} / \mathrm{kg}$

$\mu \mathrm{g} / \mathrm{kg}$

$\mu \mathrm{g} / \mathrm{kg}$

$\mu \mathrm{g} / \mathrm{kg}$

$\mu \mathrm{g} / \mathrm{kg}$

$\mu \mathrm{g} / \mathrm{kg}$

$\mu \mathrm{g} / \mathrm{kg}$

$\mu g / \mathrm{kg}$

$\mu g / \mathrm{kg}$

$\mu \mathrm{g} / \mathrm{kg}$

$\mu \mathrm{g} / \mathrm{kg}$

$\mu \mathrm{g} / \mathrm{kg}$

$\mu g / \mathrm{kg}$

$\mu \mathrm{g} / \mathrm{kg}$

$\mu \mathrm{g} / \mathrm{kg}$

$\mu \mathrm{g} / \mathrm{kg}$

$\mu \mathrm{g} / \mathrm{kg}$

$\mu \mathrm{g} / \mathrm{kg}$

$\mu \mathrm{g} / \mathrm{kg}$

$\mu \mathrm{g} / \mathrm{kg}$

$\mu \mathrm{g} / \mathrm{kg}$

$\mu \mathrm{g} / \mathrm{kg}$

$\mu \mathrm{g} / \mathrm{kg}$

$\mu \mathrm{g} / \mathrm{kg}$

$\mu \mathrm{g} / \mathrm{kg}$

$\mu \mathrm{g} / \mathrm{kg}$

$\mu \mathrm{g} / \mathrm{kg}$

$\mu \mathrm{g} / \mathrm{kg}$

$\mu \mathrm{g} / \mathrm{kg}$

$\mu g / \mathrm{kg}$

$\mu \mathrm{g} / \mathrm{kg}$

$\mu \mathrm{g} / \mathrm{kg}$

$\mu \mathrm{g} / \mathrm{kg}$

$\mu g / k g$

$\mu \mathrm{g} / \mathrm{kg}$

$\mu \mathrm{g} / \mathrm{kg}$

$\mu \mathrm{g} / \mathrm{kg}$

$\mu \mathrm{g} / \mathrm{kg}$

$\mu \mathrm{g} / \mathrm{kg}$

$\mu \mathrm{g} / \mathrm{kg}$
EPA8270

EPA8270

EPA8270

EPA8270

EPA8270

EPA8270

EPA8270

EPA8270

EPA8270

EPA8270

EPA8270

EPA8270

EPA8270

EPA8270

EPA8270

EPA8270

EPA8270

EPA8270

EPA8270

EPA8270

EPA8270

EPA8270

EPA8270

EPA8270

EPA8270

EPA8270

EPA8270

EPA8270

EPA8270

EPA8270

EPA8270

EPA8270

EPA8270

EPA8270

EPA8270

EPA8270

EPA8270

EPA8270

EPA8270

EPA8270

EPA8270

EPA8270

EPA8270

EPA8270

EPA8270

EPA8270 
SAMPLE NAME: D43111605 (Continued)

Sample ID: 100422

Constituent

Volatile Organic Compounds

Acetone
Benzene
Bromodichloromethane
Bromoform
Bromomethane (Methyl bromide)
Carbon disulfide
Carbon tetrachloride
Chlorobenzene
Chloroethane
Chloroethene (Vinyl chloride)
2-Chloroethyl vinyl ether
Chloroform
Chloromethane (Methyl chloride)
Dibromochloromethane
1,1-Dichloroethane
1,2-Dichloroethane
1,1-Dichloroethylene
1,2-Dichloroethylene
Dichloromethane (Methylene chloride)
1,2-Dichloropropane
cis-1,3-Dichloropropene
trans-1,3-Dichloropropene
Ethylbenzene
2-Hexanone
Methyl ethyl ketone
Methyl isobutyl ketone
Styrene
1,1,2,2-Tetrachloroethane
Tetrachloroethylene
Toluene
1,1,1-Trichloroethane
1,1,2-Trichloroethane
Trichloroethylene
Vinyl acetate
Xylenes

$\mathrm{U}$

U

U

$\mathrm{U}$

$\mathrm{U}$

U

U

U

$\mathrm{U}$

U

U

U

U

U

U

U

U

U

U

$\mathrm{U}$

$\mathrm{U}$

U

U

$\mathrm{U}$

U

U

U

$\mathrm{U}$

$\mathrm{U}$

U

U

U

U

Pesticides/PCBs and Dioxins/Furans

Aldrin

alpha-Benzene hexachloride

beta-Benzene hexachloride

delta-Berzene hexachloride

alpha-Chlordane

gamma-Chlordane

$p, p^{\prime}-D D D$

$p, p^{\prime}-D D E$

p.p'-DDT

Dieldrin

Endosulfan I

Endosulfan II
RQ AQ Result * Unit

D. Limit Method
$\mathrm{V} \quad 34$

5.5

5.5

5.5

11

5.5

5.5

5.5

11

11

11

5.5

11

5.5

5.5

5.5

5.5

5.5

$\mathrm{V}$

12

5.5

5.5

5.5

5.5

11

11

11

5.5

5.5

5.5

5.5

5.5

5.5

5.5

11

5.5

1.9

1.9

1.9

1.9

1.9

1.9

3.7

3.7

3.7

3.7

1.9

3.7 $\mu \mathrm{g} / \mathrm{kg}$

$\mu g / \mathrm{kg}$

$\mu g / \mathrm{kg}$

$\mu \mathrm{g} / \mathrm{kg}$

$\mu \mathrm{g} / \mathrm{kg}$

$\mu \mathrm{g} / \mathrm{kg}$

$\mu \mathrm{g} / \mathrm{kg}$

$\mu \mathrm{g} / \mathrm{kg}$

$\mu \mathrm{g} / \mathrm{kg}$

$\mu \mathrm{g} / \mathrm{kg}$

$\mu \mathrm{g} / \mathrm{kg}$

$\mu \mathrm{g} / \mathrm{kg}$

$\mu \mathrm{g} / \mathrm{kg}$

$\mu \mathrm{g} / \mathrm{kg}$

$\mu \mathrm{g} / \mathrm{kg}$

$\mu \mathrm{g} / \mathrm{kg}$

$\mu \mathrm{g} / \mathrm{kg}$

$\mu g / \mathrm{kg}$

$\mu \mathrm{g} / \mathrm{kg}$

$\mu \mathrm{g} / \mathrm{kg}$

$\mu g / \mathrm{kg}$

$\mu \mathrm{g} / \mathrm{kg}$

$\mu \mathrm{g} / \mathrm{kg}$

$\mu \mathrm{g} / \mathrm{kg}$

$\mu \mathrm{g} / \mathrm{kg}$

$\mu \mathrm{g} / \mathrm{kg}$

$\mu \mathrm{g} / \mathrm{kg}$

$\mu \mathrm{g} / \mathrm{kg}$

$\mu \mathrm{g} / \mathrm{kg}$

$\mu \mathrm{g} / \mathrm{kg}$

$\mu \mathrm{g} / \mathrm{kg}$

$\mu \mathrm{g} / \mathrm{kg}$

$\mu \mathrm{g} / \mathrm{kg}$

$\mu \mathrm{g} / \mathrm{kg}$

$\mu \mathrm{g} / \mathrm{kg}$

$\mu \mathrm{g} / \mathrm{kg}$

$\mu \mathrm{g} / \mathrm{kg}$

$\mu \mathrm{g} / \mathrm{kg}$

$\mu g / \mathrm{kg}$

$\mu \mathrm{g} / \mathrm{kg}$

$\mu \mathrm{g} / \mathrm{kg}$

$\mu \mathrm{g} / \mathrm{kg}$

$\mu \mathrm{g} / \mathrm{kg}$

$\mu \mathrm{g} / \mathrm{kg}$

$\mu \mathrm{g} / \mathrm{kg}$

$\mu g / \mathrm{kg}$

$\mu \mathrm{g} / \mathrm{kg}$
11

EPA8240

EPA8240

EPA8240

EPA8240

EPA8240

EPA8240

EPA8240

EPA8240

EPA8240

EPA8240

EPA8240

EPA8240

EPA8240

EPA8240

EPA8240

EPA8240

EPA8240

EPA8240

EPA8240

EPA8240

EPA8240

EPA8240

EPA8240

EPA8240

EPA8240

EPA8240

EPA8240

EPA8240

EPA8240

EPA8240

EPA8240

EPA8240

EPA8240

EPA8240

EPA8240

$\because$

1.9 EPA8080

1.9 EPA8080

1.9 EPA8080

1.9 EPA8080

1.9 EPA8080

1.9 EPA8080

3.7 EPA8080

3.7 EPA8080

3.7 EPA8080

3.7 EPA8080

1.9 EPA8080

3.7 EPA8080 
SAMPLE NAME: D431111605 (Continued)

Constituent
Sample ID: 100422

RQ AQ B Result Unit D. Limit Method

Pesticides/PCBs and Dioxins/Furans

Endosulfan sulfate
Endrin
Endrin ketone
Heptachlor
Heptachlor epoxide
Heptachlorodibenzo-p-dioxin isomers
Heptachlorodibenzo-p-furan isomers
Hexachlorodibenzo-p-dioxin isomers
Hexachlorodibenzo-p-furan isomers
Lindane
Methoxychlor
Octachlorodibenzo-p-dioxin
Octachlorodibenzo-p-furan
PCB 1016
PCB 1221
PCB 1232
PCB 1242
PCB 1248
PCB 1254
PCB 1260
Pentachlorodibenzo-p-dioxin isomers
Pentachlorodiberzo-p-furan isomers
2,3.7,8-TCDD
2,3,7,8-TCDF
Tetrachlorodibenzo-p-dioxin isomers
Tetrachlorodibenzo-p-furan isomers
Toxaphene

$U$
$U$
$U$
$U$
$U$
$U$
$U$
$U$
$U$
$U$
$U$

3.7
3.7
3.7

$\mu \mathrm{g} / \mathrm{kg}$
$\mu \mathrm{g} / \mathrm{kg}$
$\mu \mathrm{g} / \mathrm{kg}$
$\mu \mathrm{g} / \mathrm{kg}$

EPA8080

1.9

1.9

0.20

0.50

$\mu g / k g$

$\mathrm{ng} / \mathrm{g}$

$\mathrm{ng} / \mathrm{g}$

0.10

$\mathrm{ng} / \mathrm{g}$

0.10

1.9

19

13

0.10

37

74

37

37

37

37

37

0.10

0.10

0.10

0.10

0.10

0.10

190

$\mathrm{ng} / \mathrm{g}$

$\mu \mathrm{g} / \mathrm{kg}$

$\mu g / \mathrm{kg}$

$\mathrm{ng} / \mathrm{g}$

$\mathrm{ng} / \mathrm{g}$

$\mu \mathrm{g} / \mathrm{kg}$

$\mu \mathrm{g} / \mathrm{kg}$

$\mu \mathrm{g} / \mathrm{kg}$

$\mu \mathrm{g} / \mathrm{kg}$

$\mu \mathrm{g} / \mathrm{kg}$

$\mu \mathrm{g} / \mathrm{kg}$

$\mu \mathrm{g} / \mathrm{kg}$

$\mathrm{ng} / \mathrm{g}$

$\mathrm{ng} / \mathrm{g}$

$\mathrm{ng} / \mathrm{g}$

$\mathrm{ng} / \mathrm{g}$

$\mathrm{ng} / \mathrm{g}$

$\mathrm{ng} / \mathrm{g}$

$\mu \mathrm{g} / \mathrm{kg}$

EPA8080

EPA8080

1.9 EPA8080

0.20 EPA8280

0.50 EPA8280

0.10 EPA8280

0.10 EPA8280

1.9 EPA8080

19 EPA8080

$0.10 \quad$ EPA8280

$0.10 \quad$ EPA8280

37 EPA8080

$74 \quad$ EPA8080

$37 \quad$ EPA8080

37 EPA8080

37 EPA8080

37 EPA8080

$37 \quad$ EPA8080

$0.10 \quad$ EPA8280

$0.10 \quad$ EPA8280

$0.10 \quad$ EPA8280

$0.10 \quad$ EPA8280

$0.10 \quad$ EPA8280

$0.10 \quad$ EPA8280

190 EPA8080

Radionuclide Indicators and Radionuclides

Gross alpha

Nonvolatile beta

Radium, total alpha-emitting

Tritium
J $\quad$ C

$\begin{array}{ll}8.6 \pm 8.3 & \mathrm{pCi} / \mathrm{g} \\ 16 \pm 11 & \mathrm{pCi} / \mathrm{g} \\ 0.73 \pm 0.52 & \mathrm{pCi} / \mathrm{g} \\ 3.1 \pm 0.58 & \mathrm{pCi} / \mathrm{g}\end{array}$

4.7

7.2

0.070

1.2

EPA900.0

EPA900.0

EPA903.0

EPA906.0

SAMPLE NAME: D43111605D

Sample Type: Split

Constituent

Physical Parameters

Cation exchange capacity

$\mathrm{pH}$

Specific conductance

Total organic carbon
Sample D: 100423

Associated Sample: 100422
RQ AQ B Result
Unit
D. Limit Method

-

$\begin{array}{llll}14 & \text { meq } / 100 \mathrm{~g} & 2.2 & \text { EPA9080 } \\ 5.1 & \mathrm{pH} & 0.010 & \text { EPA9045 } \\ 17 & \mu \mathrm{S} / \mathrm{cm} & 1.0 & \text { EPA9050 } \\ 1.500 & \mathrm{mg} / \mathrm{kg} & 240 & \text { EPA415.1 }\end{array}$


SAMPLE NAME: D431̈11605D (Continued)

Sample ID: 100423

Constituent

RQ AQ B Result

Total Metals

$\begin{array}{lllll} & 4.3 & \mathrm{mg} / \mathrm{kg} . & 1.1 & \text { EPA7060 } \\ \mathrm{U} & 20 & \mathrm{mg} / \mathrm{kg} & 18 & \text { EPA6010 } \\ & 0.44 & \mathrm{mg} / \mathrm{kg} & 0.44 & \text { EPA6010 } \\ & 13 & \mathrm{mg} / \mathrm{kg} & 0.88 & \text { EPA6010 } \\ & 2.5 & \mathrm{mg} / \mathrm{kg} & 2.2 & \text { EPA6010 } \\ & 2.8 & \mathrm{mg} / \mathrm{kg} & 0.33 & \text { EPA7421 } \\ \mathrm{U} & 30 & \mathrm{mg} / \mathrm{kg} & 1.3 & \text { EPA6010 } \\ \mathrm{U} & 0.11 & \mathrm{mg} / \mathrm{kg} & 0.11 & \text { EPA7470 } \\ \mathrm{U} & 3.5 & \mathrm{mg} / \mathrm{kg} & 3.5 & \text { EPA6010 } \\ \mathrm{U} & 0.55 & \mathrm{mg} / \mathrm{kg} & 0.55 & \text { EPA7740 } \\ & 0.88 & \mathrm{mg} / \mathrm{kg} & 0.88 & \text { EPA6010 }\end{array}$

$B / N / A$ Extractables (including $P A H$ and phenols)

Acenaphthene
Acenaphthylene
Aldol condensate
Anthracene
Benzo(a)anthracene
Benzo(b)fluoranthene
Benzo(k)fluoranthene
Benzoic acid
Benzo(g,h,i)perylene
Benzo(a)pyrene
Benzyl alcohol
Bis(2-chloroethoxy) methane
Bis(2-chloroethyl) ether
Bis(2-chloroisopropyl) ether
Bis(2-ethylhexyl) phthalate
4-Bromophenyl phenyl ether
Butylbenzyl phthalate
4-Chloroaniline
4-Chloro-m-cresol
2-Chloronaphthalene
2-Chlorophenol
4-Chlorophenyl phenyl ether
Chrysene
0-Cresol (2-Methylphenol)
p-Cresol (4-Methylphenol)
Dibenz(a,h)anthracene
Dibenzofuran
Di-n-butyl phthalate
1,2-Dichlorobenzene
1,3-Dichlorobenzene
1,4-Dichlorobenzene
3,3'-Dichlorobenzidine
2,4-Dichlorophenol
Diethyl phthalate
2,4-Dimethyl phenol
Dimethyl phthalate

\begin{tabular}{|c|c|c|}
\hline $\mathbf{U}$ & & 370 \\
\hline $\mathbf{U}$ & & 370 \\
\hline$J$ & \multirow[t]{12}{*}{ NVK } & 200 \\
\hline U & & 370 \\
\hline $\mathbf{U}$ & & 370 \\
\hline $\mathbf{U}$ & & 370 \\
\hline $\mathbf{U}$ & & 370 \\
\hline $\mathbf{U}$ & & 1,800 \\
\hline $\mathbf{U}$ & & 370 \\
\hline $\mathrm{U}$ & & 370 \\
\hline $\mathbf{U}$ & & 370 \\
\hline $\mathbf{U}$ & & 370 \\
\hline $\mathrm{U}$ & & 370 \\
\hline $\mathrm{U}$ & & 370 \\
\hline U\} & \multirow[t]{13}{*}{ V } & 210 \\
\hline $\mathbf{U}$ & & 370 \\
\hline U & & 370 \\
\hline $\mathrm{U}$ & & 370 \\
\hline $\mathrm{U}$ & & 370 \\
\hline $\mathrm{U}$ & & 370 \\
\hline $\mathbf{U}$ & & 370 \\
\hline $\mathbf{U}$ & & 370 \\
\hline $\mathbf{U}$ & & 370 \\
\hline $\mathbf{U}$ & & 370 \\
\hline $\mathbf{U}$ & & 370 \\
\hline $\mathbf{U}$ & & 370 \\
\hline $\mathbf{U}$ & & 370 \\
\hline UJ & \multirow[t]{9}{*}{$\mathrm{V}$} & 110 \\
\hline $\mathbf{U}$ & & 370 \\
\hline $\mathbf{U}$ & & 370 \\
\hline $\mathbf{U}$ & & 370 \\
\hline $\mathbf{U}$ & & 740 \\
\hline $\mathbf{U}$ & & 370 \\
\hline $\mathbf{U}$ & & 370 \\
\hline $\mathbf{U}$ & & 370 \\
\hline $\mathrm{U}$ & & 370 \\
\hline
\end{tabular}

\begin{tabular}{lll}
$\mu \mathrm{g} / \mathrm{kg}$ & 370 & EPA8270 \\
$\mu \mathrm{g} / \mathrm{kg}$ & 370 & EPA8270 \\
$\mu \mathrm{g} / \mathrm{kg}$ & & EPA8270 \\
$\mu \mathrm{g} / \mathrm{kg}$ & 370 & EPA8270 \\
$\mu \mathrm{g} / \mathrm{kg}$ & 370 & EPA8270 \\
$\mu \mathrm{g} / \mathrm{kg}$ & 370 & EPA8270 \\
$\mu \mathrm{g} / \mathrm{kg}$ & 370 & EPA8270 \\
$\mu \mathrm{g} / \mathrm{kg}$ & 1,800 & EPA8270 \\
$\mu \mathrm{g} / \mathrm{kg}$ & 370 & EPA8270 \\
$\mu \mathrm{g} / \mathrm{kg}$ & 370 & EPA8270 \\
$\mu \mathrm{g} / \mathrm{kg}$ & 370 & EPA8270 \\
$\mu \mathrm{g} / \mathrm{kg}$ & 370 & EPA8270 \\
$\mu \mathrm{g} / \mathrm{kg}$ & 370 & EPA8270 \\
$\mu \mathrm{g} / \mathrm{kg}$ & 370 & EPA8270 \\
$\mu \mathrm{g} / \mathrm{kg}$ & 370 & EPA8270 \\
$\mu \mathrm{g} / \mathrm{kg}$ & 370 & EPA8270 \\
$\mu \mathrm{g} / \mathrm{kg}$ & 370 & EPA8270 \\
$\mu \mathrm{g} / \mathrm{kg}$ & 370 & EPA8270 \\
$\mu \mathrm{g} / \mathrm{kg}$ & 370 & EPA8270 \\
$\mu \mathrm{g} / \mathrm{kg}$ & 370 & EPA8270 \\
$\mu \mathrm{g} / \mathrm{kg}$ & 370 & EPA8270 \\
$\mu \mathrm{g} / \mathrm{kg}$ & 370 & EPA8270 \\
$\mu \mathrm{g} / \mathrm{kg}$ & 370 & EPA8270 \\
$\mu \mathrm{g} / \mathrm{kg}$ & 370 & EPA8270 \\
$\mu \mathrm{g} / \mathrm{kg}$ & 370 & EPA8270 \\
$\mu \mathrm{g} / \mathrm{kg}$ & 370 & EPA8270 \\
$\mu \mathrm{g} / \mathrm{kg}$ & 370 & EPA8270 \\
$\mu \mathrm{g} / \mathrm{kg}$ & 370 & EPA8270 \\
$\mu \mathrm{g} / \mathrm{kg}$ & 370 & EPA8270 \\
$\mu \mathrm{g} / \mathrm{kg}$ & 370 & EPA8270 \\
$\mu \mathrm{g} / \mathrm{kg}$ & 370 & EPA8270 \\
$\mu \mathrm{g} / \mathrm{kg}$ & 740 & EPA8270 \\
$\mu \mathrm{g} / \mathrm{kg}$ & 370 & EPA8270 \\
$\mu \mathrm{g} / \mathrm{kg}$ & 370 & EPA8270 \\
$\mu \mathrm{g} / \mathrm{kg}$ & 370 & EPA8270 \\
$\mu \mathrm{g} / \mathrm{kg}$ & 370 & EPA8270 \\
& & \\
\hline
\end{tabular}


Constituent

RQ AQ B Result

Unit

D. Limit Method

$B / N / A$ Extractables (including $P A H$ and phenols)

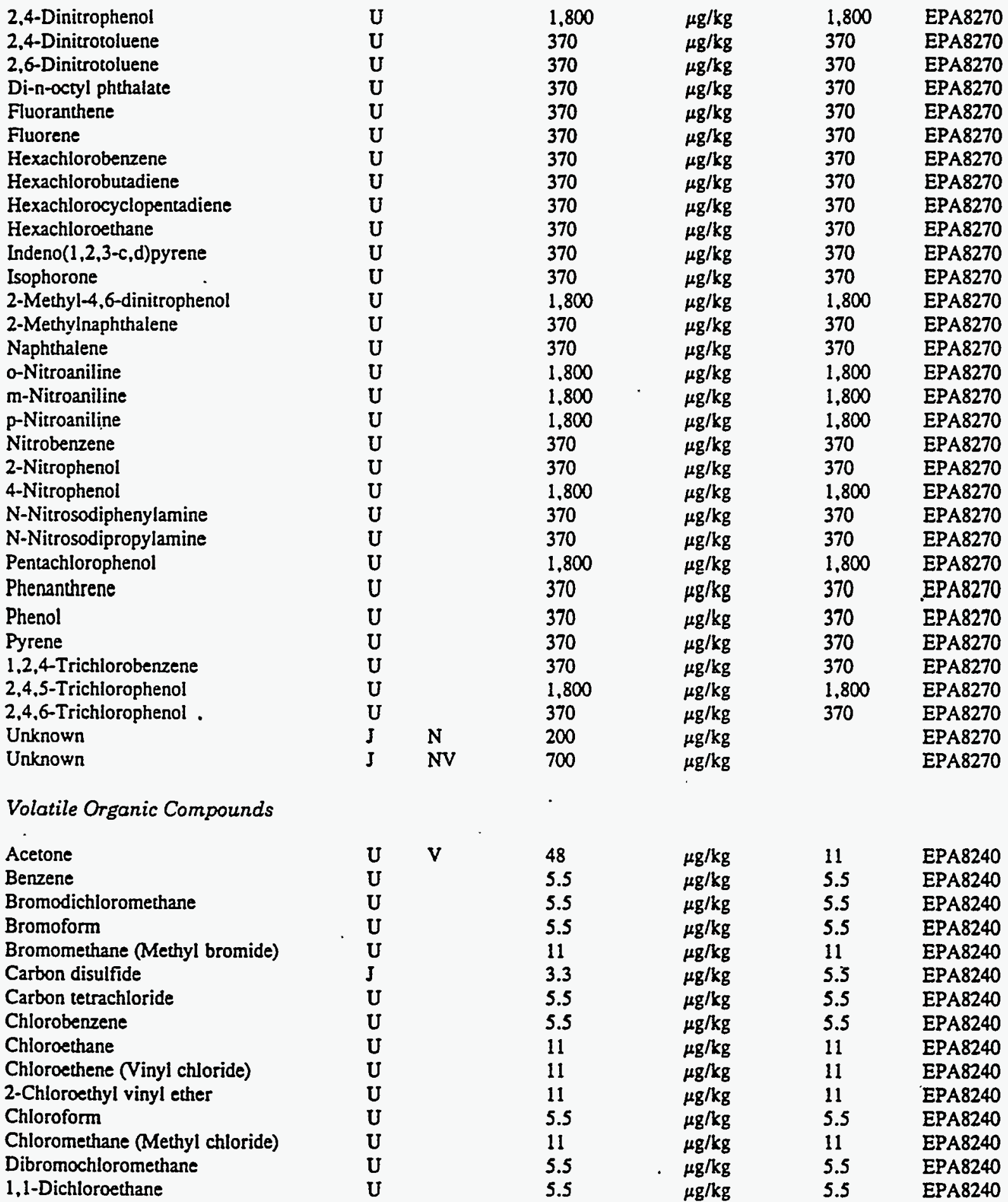


SAMPLE NAME: D43111605D (Continued)

Constituent

Volatile Organic Compounds
RQ

AQ

Sample ID: 100423

1,2-Dichloroethane
1,1-Dichloroethylene
1,2-Dichloroethylene
Dichloromethane (Methylene chloride)
1,2-Dichloropropane
cis-1,3-Dichloropropene
trans-1,3-Dichloropropene
Ethylbenzene
2-Hexanone
Methyl ethyl ketone
Methyl isobutyl ketone
Styrene
1,1,2,2-Tetrachloroethane
Tetrachloroethylene
Toluene
1,1,1-Trichloroethane
1,1,2-Trichloroethane
Trichloroethylene
Vinyl acetate
Xylenes

Pesticides/PCBs and Dioxins/Furans

$U$
$U$
$U$
$U$
$U$
$U$
$U$
$U$
$U$
$U$
$U$
$U$
$U$
$U$
$U$
$U$
$U$
$U$
$U$
$U$

Unit

D. Limit Method

5.5
5.5
5.5
13
5.5
5.5
5.5
5.5
11
11
11
5.5
5.5
5.5
5.5
5.5
5.5
5.5
11
5.5

$\mu g / k g$
$\mu g / k g$
$\mu g / k g$
$\mu g / k g$
$\mu g / k g$
$\mu g / k g$
$\mu g / k g$
$\mu g / k g$
$\mu g / k g$
$\mu g / k g$
$\mu g / k g$
$\mu g / k g$
$\mu g / k g$
$\mu g / k g$
$\mu g / k g$
$\mu g / k g$
$\mu g / k g$
$\mu g / k g$
$\mu g / k g$
$\mu g / k g$

$\mu g / \mathrm{kg}$

$\mu g / k g$

$\mu \mathrm{g} / \mathrm{kg}$

$\mu \mathrm{g} / \mathrm{kg}$

$\mu \mathrm{g} / \mathrm{kg}$

$\mu \mathrm{g} / \mathrm{kg}$

$\mu \mathrm{g} / \mathrm{kg}$

$\mu \mathrm{g} / \mathrm{kg}$

$\mu \mathrm{g} / \mathrm{kg}$

$\mu \mathrm{g} / \mathrm{kg}$

$\mu \mathrm{g} / \mathrm{kg}$

$\mu \mathrm{g} / \mathrm{kg}$

$\mu \mathrm{g} / \mathrm{kg}$

$\mu g / \mathbf{k g}$

$\mu g / \mathrm{kg}$

$\mu \mathrm{g} / \mathrm{kg}$

$\mu \mathrm{g} / \mathrm{kg}$

$\mathrm{ng} / \mathrm{g}$

$\mathrm{ng} / \mathrm{g}$

$\mathrm{ng} / \mathrm{g}$

$\mathrm{ng} / \mathrm{g}$

$\mathrm{ng} / \mathrm{g}$

$\mathrm{ng} / \mathrm{g}$

$\mathrm{ng} / \mathrm{g}$

$\mathrm{ng} / \mathrm{g}$

$\mu \mathrm{g} / \mathrm{kg}$

$\mu g / \mathrm{kg}$
5.5

5.5

5.5

5.5

5.5

5.5

5.5

5.5

11

11

11

5.5

5.5

5.5

5.5

5.5

5.5

5.5

11

5.5

EPA8240

EPA8240

EPA8240

EPA8240

EPA8240

EPA8240

EPA8240

EPA8240

EPA8240

EPA8240

EPA8240

EPA8240

EPA8240

EPA8240

EPA8240

EPA8240

EPA8240

EPA8240

EPA8240

EPA8240

EPA8080

EPA8080

EPA8080

EPA8080

EPA8080

EPA8080

EPA8080

EPA8080

EPA8080

EPA8080

EPA8080

EPA8080

EPA8080

EPA8080

EPA8080

EPA8080

EPA8080

EPA8280

EPA8280

EPA8280

EPA8280

EPA8280

EPA8280

EPA8280

EPA8280

EPA8080

EPA8080 
SAMPLE NAME: D43111605D (Continued)

Constituent

RQ AQ B Result

Unit

D. Limit Method

Pesticides/PCBs and Dioxins/Furans

Octachlorodibenzo-p-dioxin

Octachlorodibenzo-p-dioxin

Octachlorodibenzo-p-furan

Octachlorodibenzo-p-furan

PCB 1016

PCB 1221

PCB 1232

PCB 1242

PCB 1248

PCB 1254

PCB 1260

Pentachlorodibenzo-p-dioxin isomers

Pentachlorodibenzo-p-furan isomers

Pentachlorodibenzo-p-dioxin isomers

Pentachlorodibenzo-p-furan isomers

2.3.7,8-TCDD

2,3,7,8-TCDD

2.3.7,8-TCDF

$2,3,7,8-\mathrm{TCDF}$

Tetrachlorodibenzo-p-dioxin isomers

Tetrachlorodibenzo-p-dioxin isomers

Tetrachlorodibenzo-p-furan isomers

Tetrachlorodibenzo-p-furan isomers

Toxaphene

Radionuclide Indicators and Radionuclides

Sample ID: 100423

B Result Unit D. Limit Method

\begin{tabular}{|c|c|c|c|}
\hline 15 & $\mathrm{ng} / \mathrm{g}$ & 0.10 & EPA 8280 \\
\hline 17 & $\mathrm{ng} / \mathrm{g}$ & 0.30 & EPA8280 \\
\hline 0.10 & $\mathrm{ng} / \mathrm{g}$ & 0.10 & EPA8280 \\
\hline 0.40 & $\mathrm{ng} / \mathrm{g}$ & 0.40 & EPA8280 \\
\hline 37 & $\mu \mathrm{g} / \mathrm{kg}$ & 37 & EPA8080 \\
\hline 74 & $\mu g / \mathrm{kg}$ & 74 & EPA8080 \\
\hline 37 & $\mu g / \mathrm{kg}$ & 37 & EPA8080 \\
\hline 37 & $\mu g / k g$ & 37 & EPA8080 \\
\hline 37 & $\mu g / k g$ & 37 & EPA8080 \\
\hline 37 & $\mu \mathrm{g} / \mathrm{kg}$ & 37 & EPA8080 \\
\hline 37 & $\mu g / \mathrm{kg}$ & 37 & EPA8080 \\
\hline 0.10 & $\mathrm{ng} / \mathrm{g}$ & 0.10 & EPA8280 \\
\hline 0.10 & $\mathrm{ng} / \mathrm{g}$ & 0.10 & EPA8280 \\
\hline 0.30 & $\mathrm{ng} / \mathrm{g}$ & 0.30 & EPA8280 \\
\hline 0.20 & $\mathrm{ng} / \mathrm{g}$ & 0.20 & EPA8280 \\
\hline 0.10 & $\mathrm{ng} / \mathrm{g}$ & 0.10 & EPA8280 \\
\hline 0.10 & $\mathrm{ng} / \mathrm{g}$ & 0.10 & EPA8280 \\
\hline 0.10 & $\mathrm{ng} / \mathrm{g}$ & 0.10 & EPA8280 \\
\hline 0.10 & $\mathrm{ng} / \mathrm{g}$ & 0.10 & EPA8280 \\
\hline 0.10 & $\mathrm{ng} / \mathrm{g}$ & 0.10 & EPA8280 \\
\hline 0.30 & $\mathrm{ng} / \mathrm{g}$ & 0.30 & EPA8280 \\
\hline 0.10 & $\mathrm{ng} / \mathrm{g}$ & 0.10 & EPA8280 \\
\hline 0.20 & $\mathrm{ng} / \mathrm{g}$ & 0.20 & EPA8280 \\
\hline 180 & $\mu g / \mathrm{kg}$ & 180 & EPA8080 \\
\hline
\end{tabular}

$\begin{array}{lcc}\text { Gross alpha } & \text { U } & \\ \text { Nonvolatile beta } & & \text { V } \\ \text { Radium, total alpha-emitting } & \text { J } & \text { C }\end{array}$

SAMPLE NAME: D43111606

Location (SRS Coordinates): 18644.34E 66570.29N

Ground Elevation Above MSL: $134.1 \mathrm{ft}$

Depth of Core Interval: 20.00 to $22.00 \mathrm{ft}$

Sample Type: Normal

Sample Color: 10R 4/6

\section{Constituent}

Physical Parameters

Cation exchange capacity

$\mathrm{pH}$

Specific conductance

Total organic carbon

$\begin{array}{llll}4.8 & \mathrm{pCi} / \mathrm{g} & 4.8 & \text { EPA900.0 } \\ 15 \pm 11 & \mathrm{pCi} / \mathrm{g} & 7.3 & \text { EPA900.0 } \\ 0.75 \pm 0.57 & \mathrm{pCi} / \mathrm{g} & 0.080 & \text { EPA903.0 }\end{array}$

Sample ID: 100424
Sample Matrix: Soil USC Soil Classification: SW

RQ AQ

B Result Unit
Sample Moisture: Wet

Percent Solids: 85.40
D. Limit Method

$\begin{array}{llll}10 & \mathrm{meq} / 100 \mathrm{~g} & 2.3 & \text { EPA9080 } \\ 5.2 & \mathrm{pH} & 0.010 & \text { EPA9045 } \\ 15 & \mu \mathrm{S} / \mathrm{cm} & 1.0 & \text { EPA9050 } \\ 760 & \mathrm{mg} / \mathrm{kg} & 190 & \text { EPA415.1 }\end{array}$


SAMPLE NAME: D43\$11606 (Continued)

\section{Constituent}

Total Metals

Arsenic

Barium

Cadmium

Chromium

Copper

Lead

Manganese

Mercury

Nickel

Selenium

Silver
Sample ID: 100424

RQ AQ B Result Unit D. Limit Method

$B / N / A$ Extractables (including $P A H$ and phenols)

$\begin{array}{lll}\text { Acenaphthene } & \mathrm{U} & 390 \\ \text { Acenaphthylene } & \mathrm{U} & 390 \\ \text { Acenaphthylene } & \mathrm{U} & 390 \\ \text { Acenaphthylene } & \mathrm{U} & 380 \\ \text { Anthracene } & \mathrm{U} & 390 \\ \text { Anthracene } & \mathrm{U} & 390 \\ \text { Anthracene } & \mathrm{U} & 380 \\ \text { Benzo(a)anthracene } & \mathrm{U} & 390 \\ \text { Benzo(a)anthracene } & \mathrm{U} & 390 \\ \text { Benzo(a)anthracene } & \mathrm{U} & 380 \\ \text { Benzo(b)fluoranthene } & \mathrm{U} & 390 \\ \text { Benzo(b)fluoranthene } & \mathrm{U} & 390 \\ \text { Benzo(b)fluoranthene } & \mathrm{U} & 380 \\ \text { Benzo(k)fluoranthene } & \mathrm{U} & 390 \\ \text { Benzo(k)fluoranthene } & \mathrm{U} & 390 \\ \text { Benzo(k)fluoranthene } & \mathrm{U} & 380 \\ \text { Benzoic acid } & \mathrm{U} & 1,900 \\ \text { Benzoic acid } & \mathrm{U} & 1,900 \\ \text { Benzoic acid } & \mathrm{U} & 1,900 \\ \text { Benzo(g,h,i)perylene } & \mathrm{U} & 390 \\ \text { Benzo(g,h,i)perylene } & \mathrm{U} & 390 \\ \text { Benzo(g,h,i)perylene } & \mathrm{U} & 380 \\ \text { Benzo(a)pyrene } & \mathrm{U} & 390 \\ \text { Benzo(a)pyrene } & \mathrm{U} & 390 \\ \text { Benzo(a)pyrene } & \mathrm{U} & 380 \\ \text { Benzyl alcohol } & \mathrm{U} & 390 \\ \text { Benzyl alcohol } & \mathrm{U} & 390 \\ \text { Benzyl alcohol } & \mathrm{U} & 380 \\ \text { Bis(2-chloroethoxy) methane } & \mathrm{U} & 390 \\ \text { Bis(2-chloroethoxy) methane } & \mathrm{U} & 390 \\ \text { Bis(2-chloroethoxy) methane } & \mathrm{U} & 380 \\ \text { Bis(2-chloroethyl) ether } & \mathrm{U} & 390 \\ \text { Bis(2-chloroisopropyl) ether } & \mathrm{U} & 390 \\ \text { Bis(2-chloroethyl) ether } & \mathrm{U} & 390 \\ \text { Bis(2-chloroisopropyl) echer } & \mathrm{U} & \\ \text { Bis(2-chloroethyl) ether } & \mathrm{U} & \end{array}$

3.3
47
0.58
11
2.9
2.1
5.5
0.12
4.7
0.44
1.2

$\mathrm{mg} / \mathrm{kg}$
$\mathrm{mg} / \mathrm{kg}$
$\mathrm{mg} / \mathrm{kg}$
$\mathrm{mg} / \mathrm{kg}$
$\mathrm{mg} / \mathrm{kg}$
$\mathrm{mg} / \mathrm{kg}$
$\mathrm{mg} / \mathrm{kg}$
$\mathrm{mg} / \mathrm{kg}$
$\mathrm{mg} / \mathrm{kg}$
$\mathrm{mg} / \mathrm{kg}$
$\mathrm{mg} / \mathrm{kg}$

0.89

EPA7060

$23 \quad$ EPA6010

$0.58 \quad$ EPA6010

$1.2 \quad$ EPA6010

$2.9 \quad$ EPA6010

0.27 EPA7421

$1.8 \quad$ EPA6010

$0.12 \quad$ EPA7470

4.7 EPA6010

$0.44 \quad$ EPA7740

$1.2 \quad$ EPA6010

\begin{abstract}
Acenaphthylene
Anthracene

Benzo(a)anthracene

Benzo(b)fluoranthene

Benzo(b)fluoranthene

Benzo(k)furoranthene

Benzo(k)fluoranthene

Benzo(k)fluoranthene

Benzoic acid

Benzo(g,h,i)perylene

Benzo(g,h,i)perylene

Benzo(a)pyrene

Benzyl alcohol

Benzyl alcohol

Bis(2-chloroethoxy) methane

Bis(2-chloroethoxy) methan

Bis(2-chloroethyl) ether

Bis(2-chloroethyl) ether
\end{abstract}

EPA8270

EPA8270

EPA8270

EPA8270

EPA8270

EPA8270

EPA8270

EPA8270

EPA8270

EPA8270

EPA8270

EPA8270

EPA8270

EPA8270

EPA8270

EPA8270

EPA8270

EPA8270

EPA8270

EPA8270

EPA8270

EPA8270

EPA8270

EPA8270

EPA8270

EPA8270

EPA8270

EPA8270

EPA8270

EPA8270

EPA8270

EPA8270

EPA8270

EPA8270

EPA8270

EPA8270 


Constituent RQ AQ B Result Unit D. Limit Method

$B / N / A$ Extractables (including $P A H$ and phenols)

Bis(2-chloroisopropyi) ether Bis(2-ethylhexyl) phthalate Bis(2-ethylhexyl) phthalate Bis(2-ethylhexyl) phthalate 4-Bromophenyl phenyl ether 4-Bromophenyl phenyl ether 4-Bromophenyl phenyl ether Butylbenzyl phthalate Butylbenzyl phthalate Butylbenzyl phthalate 4-Chloroaniline 4-Chloroaniline

4-Chloroaniline

4-Chloro-m-cresol

2-Chloronaphthalene

2-Chioronaphthalene

2-Chloronaphthalene

2-Chlorophenol

4-Chlorophenyl phenyl ether

4-Chlorophenyl phenyl ether

4-Chlorophenyl phenyl ether

Chrysene

Chrysene

Chrysene

o-Cresol (2-Methylphenol)

o-Cresol (2-Methylphenol)

o-Cresol (2-Methylphenol)

p-Cresol (4-Methylphenol)

p-Cresol (4-Methylphenol)

p-Cresol (4-Methylphenol)

Dibenz $(\mathrm{a}, \mathrm{h})$ anthracene

Dibenz $(\mathrm{a}, \mathrm{h})$ anthracene

Dibenz $(\mathrm{a}, \mathrm{h})$ anthracene

Dibenzofuran

Dibenzofuran

Dibenzofuran

Di-n-butyl phthalate

Di-n-butyl phthalate

Di-n-butyl phthalate

1,2-Dichlorobenzene

1,2-Dichlorobenzene

1,2-Dichlorobenzene

1.3-Dichlorobenzene

1,3-Dichlorobenzene

1,3-Dichlorobenzene

1,4-Dichlorobenzene

3,3'-Dichlorobenzidine

3,3'-Dichlorobenzidine

3,3'-Dichlorobenzidine

2,4-Dichlorophenol

\begin{tabular}{|c|c|c|c|}
\hline $\mathrm{U}$ & & & 380 \\
\hline UJ & V & & 140 \\
\hline נU & $v$ & & 220 \\
\hline נט & v & & 190 \\
\hline $\mathbf{U}$ & & & 390 \\
\hline $\mathbf{U}$ & & & 390 \\
\hline $\mathrm{U}$ & & & 380 \\
\hline$U$ & & & 390 \\
\hline $\mathbf{U}$ & & & 390 \\
\hline $\mathrm{U}$ & & & 380 \\
\hline U & & & 390 \\
\hline U & & & 390 \\
\hline$U$ & & & 380 \\
\hline U & & & 390 \\
\hline $\mathrm{U}$ & & & 390 \\
\hline $\mathrm{U}$ & & & 390 \\
\hline U & & & 380 \\
\hline UJ & I & $\mathrm{H}$ & 390 \\
\hline$U$ & & & 390 \\
\hline $\mathbf{U}$ & & & 390 \\
\hline $\mathrm{U}$ & & & 380 \\
\hline U & & & 390 \\
\hline U & & & 390 \\
\hline $\mathbf{U}$ & & & 380 \\
\hline $\mathrm{U}$ & & & 390 \\
\hline $\mathbf{U}$ & & & 390 \\
\hline $\mathbf{U}$ & & & 380 \\
\hline $\mathrm{U}$ & & & 390 \\
\hline $\mathbf{U}$ & & & 390 \\
\hline U & & & 380 \\
\hline $\mathbf{U}$ & & & 390 \\
\hline $\mathbf{U}$ & & & 390 \\
\hline $\mathbf{U}$ & & & 380 \\
\hline $\mathbf{U}$ & & & 390 \\
\hline $\mathrm{U}$ & & & 390 \\
\hline $\mathbf{U}$ & & & 380 \\
\hline UJ & V & & 100 \\
\hline UJ & V & & 150 \\
\hline UI & V & & 110 \\
\hline $\mathbf{U}$ & & & 390 \\
\hline $\mathbf{U}$ & & & 390 \\
\hline $\mathbf{U}$ & & & 380 \\
\hline $\mathbf{U}$ & & & 390 \\
\hline $\mathbf{U}$ & & & 390 \\
\hline $\mathrm{U}$ & & & 380 \\
\hline $\mathbf{U}$ & & & 390 \\
\hline $\mathbf{U}$ & & & 780 \\
\hline $\mathbf{U}$ & & & 780 \\
\hline U & & & 770 \\
\hline $\mathbf{U}$ & & & 390 \\
\hline
\end{tabular}

\begin{tabular}{|c|c|c|}
\hline$\mu g / \mathrm{kg}$ & 380 & EPA8270 \\
\hline$\mu \mathrm{g} / \mathrm{kg}$ & 390 & EPA8270 \\
\hline$\mu \mathrm{g} / \mathrm{kg}$ & 390 & EPA8270 \\
\hline$\mu g / \mathrm{kg}$ & 380 & EPA8270 \\
\hline$\mu \mathrm{g} / \mathrm{kg}$ & 390 & EPA8270 \\
\hline$\mu g / \mathrm{kg}$ & 390 & EPA8270 \\
\hline$\mu g / \mathrm{kg}$ & 380 & EPA8270 \\
\hline$\mu \mathrm{g} / \mathrm{kg}$ & 390 & EPA8270 \\
\hline$\mu \mathrm{g} / \mathrm{kg}$ & 390 & EPA8270 \\
\hline$\mu g / \mathrm{kg}$ & 380 & EPA8270 \\
\hline$\mu \mathrm{g} / \mathrm{kg}$ & 390 & EPA8270 \\
\hline$\mu \mathrm{g} / \mathrm{kg}$ & 390 & EPA8270 \\
\hline$\mu \mathrm{g} / \mathrm{kg}$ & 380 & EPA8270 \\
\hline$\mu \mathrm{g} / \mathrm{kg}$ & 390 & EPA8270 \\
\hline$\mu g / \mathrm{kg}$ & 390 & EPA8270 \\
\hline$\mu \mathrm{g} / \mathrm{kg}$ & 390 & EPA8270 \\
\hline$\mu \mathrm{g} / \mathrm{kg}$ & 380 & EPA8270 \\
\hline$\mu \mathrm{g} / \mathrm{kg}$ & 390 & EPA8270 \\
\hline$\mu \mathrm{g} / \mathrm{kg}$ & 390 & EPA8270 \\
\hline$\mu g / k g$ & 390 & EPA8270 \\
\hline$\mu \mathrm{g} / \mathrm{kg}$ & 380 & EPA8270 \\
\hline$\mu \mathrm{g} / \mathrm{kg}$ & 390 & EPA8270 \\
\hline$\mu \mathrm{g} / \mathrm{kg}$ & 390 & EPA8270 \\
\hline$\mu \mathrm{g} / \mathrm{kg}$ & 380 & EPA8270 \\
\hline$\mu \mathrm{g} / \mathrm{kg}$ & 390 & EPA8270 \\
\hline$\mu \mathrm{g} / \mathrm{kg}$ & 390 & EPA8270 \\
\hline$\mu \mathrm{g} / \mathrm{kg}$ & 380 & EPA8270 \\
\hline$\mu \mathrm{g} / \mathrm{kg}$ & 390 & EPA8270 \\
\hline$\mu \mathrm{g} / \mathrm{kg}$ & 390 & EPA8270 \\
\hline$\mu \mathrm{g} / \mathrm{kg}$ & 380 & EPA8270 \\
\hline$\mu \mathrm{g} / \mathrm{kg}$. & 390 & EPA8270 \\
\hline$\mu \mathrm{g} / \mathrm{kg}$ & 390 & EPA8270 \\
\hline$\mu \mathrm{g} / \mathrm{kg}$ & 380 & EPA8270 \\
\hline$\mu \mathrm{g} / \mathrm{kg}$ & 390 & EPA8270 \\
\hline$\mu \mathrm{g} / \mathrm{kg}$ & 390 & EPA8270 \\
\hline$\mu \mathrm{g} / \mathrm{kg}$ & 380 & EPA8270 \\
\hline$\mu \mathrm{g} / \mathrm{kg}$ & 390 & EPA8270 \\
\hline$\mu \mathrm{g} / \mathrm{kg}$ & 390 & EPA8270 \\
\hline$\mu \mathrm{g} / \mathrm{kg}$ & 380 & EPA8270 \\
\hline$\mu \mathrm{g} / \mathrm{kg}$ & 390 & EPA8270 \\
\hline$\mu g / k g$ & 390 & EPA8270 \\
\hline$\mu \mathrm{g} / \mathrm{kg}$ & 380 & EPA8270 \\
\hline$\mu \mathrm{g} / \mathrm{kg}$ & 390 & EPA8270 \\
\hline$\mu \mathrm{g} / \mathrm{kg}$ & 390 & EPA8270 \\
\hline$\mu g / \mathrm{kg}$ & 380 & EPA8270 \\
\hline$\mu \mathrm{g} / \mathrm{kg}$ & 390 & EPA8270 \\
\hline$\mu \mathrm{g} / \mathrm{kg}$ & 780 & EPA8270 \\
\hline$\mu \mathrm{g} / \mathrm{kg}$ & 780 & EPA8270 \\
\hline$\mu \mathrm{g} / \mathrm{kg}$ & 770 & EPA8270 \\
\hline$\mu \mathrm{g} / \mathrm{kg}$ & 390 & EPA8270 \\
\hline
\end{tabular}


SAMPLE NAME: D43ï11606 (Continued)

Constituent

RQ AQ B Result

$B / N / A$ Extractables (including PAH and phenols)
Sample ID: 100424

Unit

D. Limit Method
2.4-Dichlorophenol

2,4-Dichlorophenol

Diethyl phthalate

Diethyl phthalate

Diethyl phthalate

2,4-Dimethyl phenol

2,4-Dimethyl phenol

2.4-Dimethyl phenol

Dimethyl phthalate

Dimethyl phthalate

Dimethyl phthalate

2,4-Dinitrophenol

2.4-Dinitrophenol

2,4-Dinitrophenol

2.4-Dinitrotoluene

2,6-Dinitrotoluene

2,6-Dinitrotoluene

2,6-Dinitrotoluene

Di-n-octyl phthalate

Di-n-octyl phthalate

Di-n-octyl phthalate

Fluoranthene

Fluoranthene

Fluoranthene

Fluorene

Fluorene

Fluorene

Hexachlorobenzene

Hexachlorobenzene

Hexachlorobenzene

Hexachlorobutadiene

Hexachlorobutadiene

Hexachlorobutadiene

Hexachlorocyclopentadiene

Hexachlorocyclopentadiene

Hexachlorocyclopentadiene

Hexachloroethane

Hexachloroethane

Hexachloroethane

Indeno(1,2,3-c,d)pyrene

Indeno(1,2,3-c,d)pyrene

Indeno(1,2,3-c,d)pyrene

Isophorone

Isophorone

Isophorone

2-Methyl-4,6-dinitrophenol

2-Methyl-4,6-dinitrophenol

2-Methyl-4,6-dinitrophenol

2-Methylnaphthalene

2-Methylnaphthalene
U $\quad 380$

U. 390

$\mathrm{U} \quad 390$

U $\quad 380$

390

390

380

390

390

380

1,900

1,900

1,900

390

390

390

380

390

390

380

390

390

380

390

390

380

390

390

380

390

390

$380^{*}$

390

390

380

390

390

380

390

390

380

390

390

380

1,900

1,900

1,900

390

390

$\mu g / k g$
$\mu g / k g$
$\mu g / k g$
$\mu g / k g$
$\mu g / k g$
$\mu g / k g$
$\mu g / k g$
$\mu g / k g$
$\mu g / k g$
$\mu g / k g$
$\mu g / k g$
$\mu g / k g$
$\mu g / k g$
$\mu g / k g$
$\mu g / k g$
$\mu g / k g$
$\mu g / k g$
$\mu g / k g$
$\mu g / k g$
$\mu g / k g$
$\mu g / k g$
$\mu g / k g$
$\mu g / k g$
$\mu g / k g$
$\mu g / k g$
$\mu g / k g$
$\mu g / k g$
$\mu g / k g$
$\mu g / k g$
$\mu g / k g$
$\mu g / k g$
$\mu g / k g$
$\mu g / k g$
$\mu g / k g$
$\mu g / k g$
$\mu g / k g$
$\mu g / k g$
$\mu g / k g$
$\mu g / k g$
$\mu g / k g$
$\mu g / k g$
$\mu g / k g$
$\mu g / k g$
$\mu g / k g$
$\mu g / k g$
$\mu g / k g$
$\mu g / k g$
$\mu g / k g$
$\mu g / k g$
$\mu g / k g$
$g / k$

$\mu \mathrm{g} / \mathrm{kg}$

$\mu \mathrm{g} / \mathrm{kg}$

$\mu g / \mathrm{kg}$

$\mu \mathrm{g} / \mathrm{kg}$

$\mu \mathrm{g} / \mathrm{kg}$

$\mu \mathrm{g} / \mathrm{kg}$

$\mu \mathrm{g} / \mathrm{kg}$

$\mu \mathrm{g} / \mathrm{kg}$

$\mu \mathrm{g} / \mathrm{kg}$

$\mu \mathrm{g} / \mathrm{kg}$

$\mu \mathrm{g} / \mathrm{kg}$

$\mu \mathrm{g} / \mathrm{kg}$

$\mu \mathrm{g} / \mathrm{kg}$

$\mu \mathrm{g} / \mathrm{kg}$

$\mu \mathrm{g} / \mathrm{kg}$

$\mu \mathrm{g} / \mathrm{kg}$

$\mu \mathrm{g} / \mathrm{kg}$

$\mu \mathrm{g} / \mathrm{kg}$

$\mu \mathrm{g} / \mathrm{kg}$

$\mu g / k g$

$\mu g / k g$

$\mu \mathrm{g} / \mathrm{kg}$

$\mu \mathrm{g} / \mathrm{kg}$

$\mu g / k g$

$\mu \mathrm{g} / \mathrm{kg}$

$\mu g / k g$

$\mu \mathrm{g} / \mathrm{kg}$

$\mu g / \mathrm{kg}$

$\mu g / \mathrm{kg}$

$\mu \mathrm{g} / \mathrm{kg}$

$\mu \mathrm{g} / \mathrm{kg}$

$\mu g / \mathrm{kg}$

$\mu g / k g$

$\mu \mathrm{g} / \mathrm{kg}$

$\mu \mathrm{g} / \mathrm{kg}$

$\mu \mathrm{g} / \mathrm{kg}$

$\mu g / \mathrm{kg}$

$\mu g / \mathrm{kg}$

$\mu \mathrm{g} / \mathrm{kg}$

$\mu \mathrm{g} / \mathrm{kg}$

$\mu \mathrm{g} / \mathrm{kg}$

$\mu \mathrm{g} / \mathrm{kg}$

$\mu g / k g$

$\mu \mathrm{g} / \mathrm{kg}$

$\begin{array}{ll}390 & \text { EPA8270 } \\ 380 & \text { EPA8270 } \\ 390 & \text { EPA8270 } \\ 390 & \text { EPA8270 } \\ 380 & \text { EPA8270 } \\ 390 & \text { EPA8270 } \\ 390 & \text { EPA8270 } \\ 380 & \text { EPA8270 } \\ 390 & \text { EPA8270 } \\ 390 & \text { EPA8270 } \\ 380 & \text { EPA8270 } \\ 1,900 & \text { EPA8270 } \\ 1,900 & \text { EPA8270 } \\ 1,900 & \text { EPA8270 } \\ 390 & \text { EPA8270 } \\ 390 & \text { EPA8270 } \\ 390 & \text { EPA8270 } \\ 380 & \text { EPA8270 } \\ 390 & \text { EPA8270 } \\ 390 & \text { EPA8270 } \\ 380 & \text { EPA8270 } \\ 390 & \text { EPA8270 } \\ 390 & \text { EPA8270 } \\ 380 & \text { EPA8270 } \\ 390 & \text { EPA8270 } \\ 390 & \text { EPA8270 } \\ 380 & \text { EPA8270 } \\ 390 & \text { EPA8270 } \\ 390 & \text { EPA8270 } \\ 380 & \text { EPA8270 } \\ 390 & \text { EPA8270 } \\ 390 & \text { EPA8270 } \\ 380 & \text { EPA8270 } \\ 390 & \text { EPA8270 } \\ 390 & \text { EPA8270 } \\ 380 & \text { EPA8270 } \\ 390 & \text { EPA8270 } \\ 390 & \text { EPA8270 } \\ 380 & \text { EPA8270 } \\ 390 & \text { EPA8270 } \\ 390 & \text { EPA8270 } \\ 380 & \text { EPA8270 } \\ 390 & \text { EPA8270 } \\ 390 & \text { EPA8270 } \\ 380 & \text { EPA8270 } \\ 1,900 & \text { EPA8270 } \\ 1,900 & \text { EPA8270 } \\ 1,900 & \text { EPA8270 } \\ 390 & \text { EPA8270 } \\ 390 & \text { EPA8270 }\end{array}$


SAMPLE NAME: D43111606 (Continued) Sample ID: 100424

Constituent

RQ AQ B Result Unit

D. Limit Method

$B / N / A$ Extractables (including $P A H$ and phenols)

2-Methylnaphthalene
Naphthalene
Naphthalene
Naphthalene
o-Nitroaniline
o-Nitroaniline
o-Nitroaniline
m-Nitroaniline
m-Nitroaniline
m-Nitroaniline
p-Nitroaniline
p-Nitroaniline
p-Nitroaniline
Nitrobenzene
Nitrobenzene
Nitrobenzene
2-Nitrophenol
2-Nitrophenol
2-Nitrophenol
4-Nitrophenol
N-Nitrosodiphenylamine
N-Nitrosodiphenylamine
N-Nitrosodiphenylamine
N-Nitrosodipropylamine
Pentachlorophenol
Phenanthrene
Phenanthrene
Phenanthrene
Phenol
Pyrene
1,2,4-Trichlorobenzene
2,4,5-Trichlorophenol
2,4,5-Trichlorophenol
2,4,5-Trichlorophenol
2,4,6-Trichlorophenol
2,4,6-Trichlorophenol
2,4,6-Trichlorophenol
Unknown

\begin{tabular}{|c|c|}
\hline U & 380 \\
\hline U & 390 \\
\hline $\mathbf{U}$ & 390 \\
\hline U & 380 \\
\hline U & 1,900 \\
\hline U & 1,900 \\
\hline U & 1,900 \\
\hline $\mathrm{U}$ & 1,900 \\
\hline $\mathrm{U}$ & 1,900 \\
\hline U & 1.900 \\
\hline $\mathbf{U}$ & 1.900 \\
\hline U & 1,900 \\
\hline U & 1,900 \\
\hline U & 390 \\
\hline U & 390 \\
\hline $\mathrm{U}$ & 380 \\
\hline U & 390 \\
\hline $\mathrm{U}$ & 390 \\
\hline $\mathrm{U}$ & 380 \\
\hline U & 1,900 \\
\hline $\mathrm{U}$ & 390 \\
\hline$U$ & 390 \\
\hline U & 380 \\
\hline U & 390 \\
\hline $\mathbf{U}$ & 1,900 \\
\hline U & 390 \\
\hline$\pi$ & 390 \\
\hline $\mathrm{U}$ & 380 \\
\hline U & 390 \\
\hline 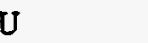 & 390 \\
\hline $\mathrm{U}$ & 390 \\
\hline$U$ & 1,900 \\
\hline 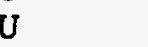 & 1,900 \\
\hline$U$ & 1,900 \\
\hline זי & 390 \\
\hline & 390 \\
\hline & 380 \\
\hline NV & 900 \\
\hline
\end{tabular}

Volatile Organic Compounds

Acetone
Benzene
Bromodichloromethane
Bromoform
Bromomethane (Methyl bromide)
Carbon disulfide
Carbon tetrachloride
Chlorobenzene
Chloroethane

$\begin{array}{lll}\mathrm{U} & \mathrm{V} & 25 \\ \mathrm{U} & & 6.0 \\ \mathrm{U} & & 6.0 \\ \mathrm{U} & & 6.0 \\ \mathrm{U} & & 12 \\ \mathrm{U} & & 6.0 \\ \mathrm{U} & & 6.0 \\ \mathrm{U} & & 6.0 \\ \mathrm{U} & & 12\end{array}$

$\begin{array}{lll}\mu \mathrm{g} / \mathrm{kg} & 380 & \text { EPA8270 } \\ \mu \mathrm{g} / \mathrm{kg} & 390 & \text { EPA8270 } \\ \mu \mathrm{g} / \mathrm{kg} & 390 & \text { EPA8270 } \\ \mu \mathrm{g} / \mathrm{kg} & 380 & \text { EPA8270 } \\ \mu \mathrm{g} / \mathrm{kg} & 1,900 & \text { EPA8270 } \\ \mu \mathrm{g} / \mathrm{kg} & 1,900 & \text { EPA8270 } \\ \mu \mathrm{g} / \mathrm{kg} & 1,900 & \text { EPA8270 } \\ \mu \mathrm{g} / \mathrm{kg} & 1,900 & \text { EPA8270 } \\ \mu \mathrm{g} / \mathrm{kg} & 1,900 & \text { EPA8270 } \\ \mu \mathrm{g} / \mathrm{kg} & 1,900 & \text { EPA8270 } \\ \mu \mathrm{g} / \mathrm{kg} & 1.900 & \text { EPA8270 } \\ \mu \mathrm{g} / \mathrm{kg} & 1.900 & \text { EPA8270 } \\ \mu \mathrm{g} / \mathrm{kg} & 1.900 & \text { EPA8270 } \\ \mu \mathrm{g} / \mathrm{kg} & 390 & \text { EPA8270 } \\ \mu \mathrm{g} / \mathrm{kg} & 390 & \text { EPA8270 } \\ \mu \mathrm{g} / \mathrm{kg} & 380 & \text { EPA8270 } \\ \mu \mathrm{g} / \mathrm{kg} & 390 & \text { EPA8270 } \\ \mu \mathrm{g} / \mathrm{kg} & 390 & \text { EPA8270 } \\ \mu \mathrm{gg} / \mathrm{kg} & 380 & \text { EPA8270 } \\ \mu \mathrm{g} / \mathrm{kg} & 1,900 & \text { EPA8270 } \\ \mu \mathrm{g} / \mathrm{kg} & 390 & \text { EPA8270 } \\ \mu \mathrm{g} / \mathrm{kg} & 390 & \text { EPA8270 } \\ \mu \mathrm{g} / \mathrm{kg} & 380 & \text { EPA8270 } \\ \mu \mathrm{g} / \mathrm{kg} & 390 & \text { EPA8270 } \\ \mu \mathrm{g} / \mathrm{kg} & 1,900 & \text { EPA8270 } \\ \mu \mathrm{g} / \mathrm{kg} & 390 & \text { EPA8270 } \\ \mu \mathrm{g} / \mathrm{kg} & 390 & \text { EPA8270 } \\ \mu \mathrm{g} / \mathrm{kg} & 380 & \text { EPA8270 } \\ \mu \mathrm{g} / \mathrm{kg} & 390 & \text { EPA8270 } \\ \mu \mathrm{g} / \mathrm{kg} & 390 & \text { EPA8270 } \\ \mu \mathrm{g} / \mathrm{kg} & 390 & \text { EPA8270 } \\ \mu \mathrm{g} / \mathrm{kg} & 1.900 & \text { EPA8270 } \\ \mu \mathrm{g} / \mathrm{kg} & 1,900 & \text { EPA8270 } \\ \mu \mathrm{g} / \mathrm{kg} & 1.900 & \text { EPA8270 } \\ \mu \mathrm{g} / \mathrm{kg} & 390 & \text { EPA8270 } \\ \mu \mathrm{g} / \mathrm{kg} & 390 & \text { EPA8270 } \\ \mu \mathrm{g} / \mathrm{kg} & 380 & \text { EPA8270 } \\ \mu \mathrm{g} / \mathrm{kg} & -\mathrm{H} & \text { EPA8270 } \\ & & \\ & & \end{array}$

$\begin{array}{lll}\mu \mathrm{g} / \mathrm{kg} & 12 & \text { EPA8240 } \\ \mu \mathrm{g} / \mathrm{kg} & 6.0 & \text { EPA8240 } \\ \mu \mathrm{g} / \mathrm{kg} & 6.0 & \text { EPA8240 } \\ \mu \mathrm{g} / \mathrm{kg} & 6.0 & \text { EPA8240 } \\ \mu \mathrm{g} / \mathrm{kg} & 12 & \text { EPA8240 } \\ \mu \mathrm{g} / \mathrm{kg} & 6.0 & \text { EPA8240 } \\ \mu \mathrm{g} / \mathrm{kg} & 6.0 & \text { EPA8240 } \\ \mu \mathrm{g} / \mathrm{kg} & 6.0 & \text { EPA8240 } \\ \mu \mathrm{g} / \mathrm{kg} & 12 & \text { EPA8240 }\end{array}$


SAMPLE NAME: D43111606 (Continued)

Constituent

RQ

AQ B Result

D. Limit Method

Sample ID: 100424

Volatile Organic Compounds

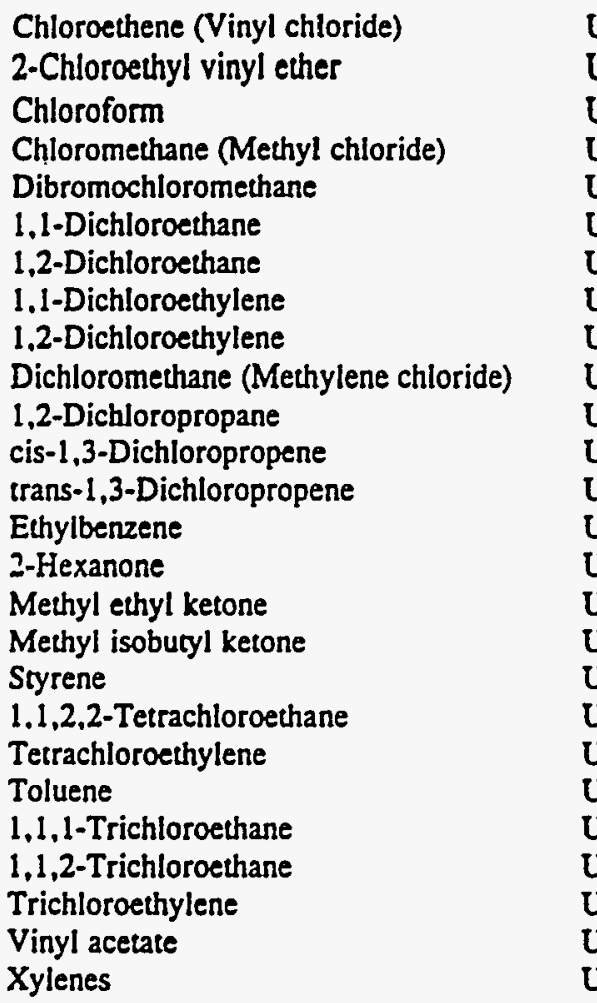

$\mu g / \mathrm{kg}$

$\mu g / \mathrm{kg}$

$\mu \mathrm{g} / \mathrm{kg}$

$\mu g / \mathrm{kg}$

$\mu g / \mathrm{kg}$

$\mu \mathrm{g} / \mathrm{kg}$

$\mu \mathrm{g} / \mathrm{kg}$

$\mu \mathrm{g} / \mathrm{kg}$

$\mu \mathrm{g} / \mathrm{kg}$

$\mu \mathrm{g} / \mathrm{kg}$

$\mu \mathrm{g} / \mathrm{kg}$

$\mu g / \mathrm{kg}$

$\mu \mathrm{g} / \mathrm{kg}$

$\mu g / \mathrm{kg}$

$\mu g / \mathrm{kg}$

$\mu g / \mathrm{kg}$

$\mu \mathrm{g} / \mathrm{kg}$

$\mu \mathrm{g} / \mathrm{kg}$

$\mu \mathrm{g} / \mathrm{kg}$

$\mu \mathrm{g} / \mathrm{kg}$

$\mu \mathrm{g} / \mathrm{kg}$

$\mu \mathrm{g}^{\prime} \mathrm{kg}$

$\mu g ! \mathrm{kg}$

$\mu \mathrm{g} / \mathrm{kg}$

$\mu \mathrm{g} / \mathrm{kg}$

$\mu \mathrm{g} / \mathrm{kg}$

6.0

Pesticides/PCBs and Dioxins/Furans

\begin{abstract}
Aldrin
Aldrin

alpha-Benzene hexachloride

alpha-Benzene hexachloride

alpha-Benzene hexachloride

beta-Benzene hexachloride

beta-Benzene hexachloride

beta-Benzene hexachloride

delta-Benzene hexachloride

delta-Benzene hexachloride

delta-Benzene hexachloride

alpha-Chlordane

alpha-Chlordane

alpha-Chlordane

gamma-Chlordane

gamma-Chlordane

gamma-Chlordane

P.P'-DDD

p. $p^{\prime}-D D D$

p.p'-DDD

$p, p^{\prime}-D D E$
\end{abstract}

$U$
$U$
$U$
$U$
$U$
$\dot{U}$
$U$
$U$
$U$
$U$
$U$
$U$
$U$
$U$
$U$
$U$
$U$
$U$
$U$
$U$
$U$
1.9

1.9

1.9

1.9

1.9

1.9

1.9

1.9

1.9

1.9

1.9

1.9

1.9

1.9

1.9

1.9

1.9

3.9

3.9

3.9

3.9 $\mu \mathrm{g} / \mathrm{kg}$

$\mu g / k g$

$\mu \mathrm{g} / \mathrm{kg}$

$\mu g / \mathrm{kg}$

$\mu \mathrm{g} / \mathrm{kg}$

$\mu \mathrm{g} / \mathrm{kg}$

$\mu \mathrm{g} / \mathrm{kg}$

$\mu \mathrm{g} / \mathrm{kg}$

$\mu \mathrm{g} / \mathrm{kg}$

$\mu \mathrm{g} / \mathrm{kg}$

$\mu \mathrm{g} / \mathrm{kg}$

$\mu \mathrm{g} / \mathrm{kg}$

$\mu \mathrm{g} / \mathrm{kg}$

$\mu \mathrm{g} / \mathrm{kg}$

$\mu \mathrm{g} / \mathrm{kg}$

$\mu \mathrm{g} / \mathrm{kg}$

$\mu \mathrm{g} / \mathrm{kg}$

$\mu \mathrm{g} / \mathrm{kg}$

$\mu \mathrm{g} / \mathrm{kg}$

$\mu \mathrm{g} / \mathrm{kg}$

$\mu g / \mathrm{kg}$
12

12

6.0

12

6.0

6.0

6.0

6.0

6.0

6.0

6.0

6.0

6.0

6.0

12

12

12

6.0

6.0

6.0

6.0

6.0

6.0

6.0

12

6.0
EPA8240

EPA8240

EPA8240

EPA8240

EPA8240

EPA8240

EPA8240

EPA8240

EPA8240

EPA8240

EPA8240

EPA8240

EPA8240

EPA8240

EPA8240

EPA 8240

EPA8240

EPA8240

EPA8240

EPA8240

EPA8240

EPA8240

EPA8240

EPA8240

EPA8240

EPA8240 
SAMPLE NAME: D431H606 (Continued)

\section{Constituent}

RQ AQ B Result
Sample ID: 100424

Pesticides/PCBs and Dioxins/Furans

P.P'-DDE

P.P'-DDE

p.p'-DDT

p.p'-DDT

Dieldrin

Dieldrin

Endosulfan I

Endosulfan I

Endosulfan I

Endosulfan II

Endosulfan II

Endosulfan II

Endosulfan sulfate

Endosulfan sulfate

Endosulfan sulfate

Endrin

Endrin

Endrin ketone

Endrin ketone

Endrin ketone

Heptachlor

Heptachlor

Heptachlor epoxide

Heptachlor epoxide

Heptachlor epoxide

Heptachlorodibenzo-p-dioxin isomers

Heptachlorodibenzo-p-dioxin isomers

Heptachlorodibenzo-p-furan isomers

Heptachlorodibenzo-p-furan isomers

Hexachlorodibenzo-p-dioxin isomers

Hexachlorodibenzo-p-furan isomers

Lindane

Lindane

Methoxychlor

Methoxychlor

Methoxychlor

Octachlorodibenzo-p-dioxin

Octachlorodibenzo-p-furan

PCB 1016

PCB 1016

PCB 1016

PCB 1221

PCB 1221

PCB 1221

PCB 1232

PCB 1232

PCB 1232

PCB 1242

PCB 1242

PCB 1242

\section{9}

\section{9}

\section{9}

3.9

1.9

1.9

\section{9}

3.9

3.9

3.9

\section{9}

3.9

3.9

\section{9}

1.9

1.9

\section{9}

1.9

0.20

0.30

0.20

0.60

0.20

0.10

1.9

\section{9}

19

\section{9}

19

16

39

39

39

77

77

78

39

39

39

39

39

39
Unit .

D. Limit Method

\section{$i$}


SAMPLE NAME: D43111606 (Continued)

\section{Constituent}

Pesticides/PCBs and Dioxins/Furans

PCB 1248

PCB 1248

PCB 1248

PCB 1254

PCB 1254

PCB 1254

PCB 1260

PCB 1260

PCB 1260

Pentachlorodibenzo-p-dioxin isomers

Pentachlorodibenzo-p-furan isomers

Pentachlorodibenzo-p-dioxin isomers

Pentachlorodibenzo-p-furan isomers

2,3,7,8-TCDD

2,3,7,8-TCDF

Tetrachlorodibenzo-p-dioxin isomers

Tetrachlorodibenzo-p-furan isomers

Toxaphene

Toxaphene

Toxaphene
Radionuclide Indicators and Radionuclides

Gross alpha

Nonvolatile beta

Radium, total alpha-emitting

Tritium

$\begin{array}{ll}u & \mathbf{v} \\ \mathrm{J} & \mathbf{C}\end{array}$

\section{RQ AQ B Result}

$\rightarrow$

$\begin{array}{ll}U & 39 \\ U & 39 \\ U & 39 \\ U & 39 \\ U & 39 \\ U & 39 \\ U & 39 \\ U & 39 \\ U & 39 \\ U & 0.20 \\ U & 0.20 \\ U & 0.40 \\ U & 0.30 \\ U & 0.10 \\ U & 0.10 \\ U & 0.10 \\ U & 0.30 \\ U & 190 \\ U & 190 \\ U & 190\end{array}$

Sample ID: 100424

Unit D. Limit Method

$$
\begin{aligned}
& \mu g / k g \\
& \mu g / k g \\
& \mu g / k g \\
& \mu g / k g \\
& \mu g / k g \\
& \mu g / k g \\
& \mu g / k g \\
& \mu g / k g \\
& \mu g / k g \\
& n g g / g \\
& n g / g \\
& n g / g \\
& n g / g \\
& n g / g \\
& n g / g \\
& n g / g \\
& n g g / g \\
& \mu g / k g \\
& \mu g / k g \\
& \mu g / k g
\end{aligned}
$$

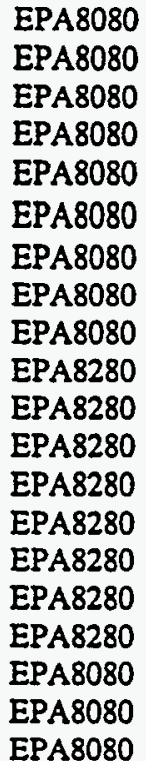

EPA8080

$\begin{array}{ll}6.9 \pm 7.4 & \mathrm{pCi} / \mathrm{g} \\ 6.6 & \mathrm{pCi} / \mathrm{g} \\ 0.25 \pm 0.33 & \mathrm{pCi} / \mathrm{g} \\ 2.0 \pm 0.40 & \mathrm{pCi} / \mathrm{g}\end{array}$

Sample ID: 100304
Sample Matrix: Sediment USC Soil Classification: SM
Location (SRS Coordinates): .E .N

Ground Elevation Above MSL: . $\mathrm{ft}$

Depth of Core Interval: 0.00 to $0.50 \mathrm{ft}$

Sample Type: Normal

Sample Color: 5YR 4/1

Constituent

\section{RQ AQ B Result Unit}

Physical Parameters

$\mathrm{pH}$

Total organic carbon

$\begin{array}{lll}\text { J } & \mathbf{Q} & 5.2 \\ & & 3.300 \\ & & \\ & & \\ \mathbf{U} & & 1.3 \\ & & 24 \\ \mathbf{U} & & 0.56 \\ & & 34\end{array}$

\section{Total Metals}

$\begin{array}{ll}4.4 & \text { EPA900.0 } \\ 6.6 & \text { EPA900.0 } \\ 0.070 & \text { EPA903.0 } \\ 1.0 & \text { EPA906.0 }\end{array}$

$\begin{array}{llllll}\text { Arsenic } & \mathrm{U} & 1.3 & \mathrm{mg} / \mathrm{kg} & 1.3 & \text { EPA7060 } \\ \text { Barium } & & 24 & \mathrm{mg} / \mathrm{kg} & 23 & \text { EPA6010 } \\ \text { Cadmium } & \mathrm{U} & 0.56 & \mathrm{mg} / \mathrm{kg} & 0.56 & \text { EPA6010 } \\ \text { Chromium } & & 34 & \mathrm{mg} / \mathrm{kg} & 1.1 & \text { EPA6010 }\end{array}$

Sample Moisture: Wet Percent Solids: $\mathbf{7 6 . 5 0}$

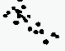

D. Limit Method

0.010 EPA9045 $250 \quad$ EPA415.1 
SAMPLE NAME: D43112102 (Continued)

Constituent

Total Metals
Sample D: 100304

RQ AQ B Result Unit D. Limit Method

\section{Copper \\ Lead \\ Manganese \\ Mercury \\ Nickel \\ Selenium \\ Silver}

\author{
-
}

$B / N / A$ Extractables (including PAH and phenols)

$\begin{array}{lllll} & 3.1 & \mathrm{mg} / \mathrm{kg} & 2.8 & \text { EPA6010 } \\ & 2.0 & \mathrm{mg} / \mathrm{kg} & 0.39 & \text { EPA7421 } \\ \mathrm{U} & 290 & \mathrm{mg} / \mathrm{kg} & 1.7 & \text { EPA6010 } \\ \mathrm{U} & 0.13 & \mathrm{mg} / \mathrm{kg} & 0.13 & \text { EPA7470 } \\ \mathrm{U} & 4.5 & \mathrm{mg} / \mathrm{kg} & 4.5 & \text { EPA6010 } \\ \mathrm{U} & 0.65 & \mathrm{mg} / \mathrm{kg} & 0.65 & \text { EPA7740 } \\ & 1.1 & \mathrm{mg} / \mathrm{kg} & 1.1 & \text { EPA6010 }\end{array}$

\author{
Acenaphthene \\ Acenaphthylene \\ Aldol condensate \\ Alkane \\ Alkane \\ Anthracene \\ Benzo(a)anthracene \\ Benzo(b)fluoranthene \\ Benzo(k)fluoranthene \\ Benzoic acid \\ Benzo(g,h,i)perylene \\ Benzo(a)pyrene \\ Benzyl alcohol \\ Bis(2-chloroethoxy) methane \\ Bis(2-chloroethyl) ether \\ Bis(2-chloroisopropyl) ether \\ Bis (2-ethylhexyl) phthalate \\ 4-Bromophenyl phenyl ether \\ Butylbenzyl phthalate \\ 4-Chloroaniline \\ 4-Chloro-m-cresol \\ 2-Chloronaphthalene \\ 2-Chlorophenol \\ 4-Chlorophenyl phenyl ether \\ Chrysene \\ o-Cresol (2-Methylphenol) \\ p-Cresol (4-Methylphenol) \\ Dibenz $(\mathrm{a}, \mathrm{h})$ anthracene \\ Dibenzofuran \\ Di-n-butyl phthalate \\ 1,2-Dichlorobenzene \\ 1,3-Dichlorobenzene \\ 1,4-Dichlorobenzene \\ 3,3'-Dichlorobenzidine \\ 2,4-Dichlorophenol \\ Diethyl phthalate \\ 2,4-Dimethyl phenol \\ Dimethyl phthalate \\ 2,4-Dinitrophenol \\ 2,4-Dinitrotoluene
}

$\begin{array}{llll}\mathrm{U} & & 430 & \mu \mathrm{g} / \mathrm{kg} \\ \mathrm{U} & 430 & \mu \mathrm{g} / \mathrm{kg} \\ \mathrm{J} & \mathrm{NVK} & 500 & \mu \mathrm{g} / \mathrm{kg} \\ \mathrm{J} & \mathrm{N} & 600 & \mu \mathrm{g} / \mathrm{kg} \\ \mathrm{J} & \mathrm{N} & 600 & \mu \mathrm{g} / \mathrm{kg} \\ \mathrm{U} & & 430 & \mu \mathrm{g} / \mathrm{kg} \\ \mathrm{U} & & 430 & \mu \mathrm{g} / \mathrm{kg} \\ \mathrm{U} & 430 & \mu \mathrm{g} / \mathrm{kg} \\ \mathrm{U} & 430 & \mu \mathrm{g} / \mathrm{kg} \\ \mathrm{U} & & 2.100 & \mu \mathrm{g} / \mathrm{kg} \\ \mathrm{U} & 430 & \mu \mathrm{g} / \mathrm{kg} \\ \mathrm{U} & 430 & \mu \mathrm{g} / \mathrm{kg} \\ \mathrm{U} & 430 & \mu \mathrm{g} / \mathrm{kg} \\ \mathrm{U} & 430 & \mu \mathrm{g} / \mathrm{kg} \\ \mathrm{U} & 430 & \mu \mathrm{g} / \mathrm{kg} \\ \mathrm{U} & 430 & \mu \mathrm{g} / \mathrm{kg} \\ \mathrm{UJ} & \mathrm{V} & 130 & \mu \mathrm{g} / \mathrm{kg} \\ \mathrm{U} & & 430 & \mu \mathrm{g} / \mathrm{kg} \\ \mathrm{U} & 430 & \mu \mathrm{g} / \mathrm{kg} \\ \mathrm{U} & 430 & \mu \mathrm{g} / \mathrm{kg} \\ \mathrm{U} & 430 & \mu \mathrm{g} / \mathrm{kg} \\ \mathrm{U} & 430 & \mu \mathrm{g} / \mathrm{kg} \\ \mathrm{U} & 430 & \mu \mathrm{g} / \mathrm{kg} \\ \mathrm{U} & 430 & \mu \mathrm{g} / \mathrm{kg} \\ \mathrm{U} & 430 & \mu \mathrm{g} / \mathrm{kg} \\ \mathrm{U} & 430 & \mu \mathrm{g} / \mathrm{kg} \\ \mathrm{U} & 430 & \mu \mathrm{g} / \mathrm{kg} \\ \mathrm{U} & 430 & \mu \mathrm{g} / \mathrm{kg} \\ \mathrm{U} & 430 & \mu \mathrm{g} / \mathrm{kg} \\ \mathrm{J} & 50 & \mu \mathrm{g} / \mathrm{kg} \\ \mathrm{U} & 430 & \mu \mathrm{g} / \mathrm{kg} \\ \mathrm{U} & 430 & \mu \mathrm{g} / \mathrm{kg} \\ \mathrm{U} & 430 & \mu \mathrm{g} / \mathrm{kg} \\ \mathrm{U} & 850 & \mu \mathrm{g} / \mathrm{kg} \\ \mathrm{U} & 430 & \mu \mathrm{g} / \mathrm{kg} \\ \mathrm{U} & 430 & \mu \mathrm{g} / \mathrm{kg} \\ \mathrm{U} & 430 & \mu \mathrm{g} / \mathrm{kg} \\ \mathrm{U} & 430 & \mu \mathrm{g} / \mathrm{kg} \\ \mathrm{U} & 2,100 & \mu \mathrm{g} / \mathrm{kg} \\ \mathrm{U} & 430 & \mu \mathrm{g} / \mathrm{kg} \\ & & & \\ & & 4 & \end{array}$

$\begin{array}{ll}430 & \text { EPA8270 } \\ 430 & \text { EPA8270 } \\ & \text { EPA8270 } \\ & \text { EPA8270 } \\ & \text { EPA8270 } \\ 430 & \text { EPA8270 } \\ 430 & \text { EPA8270 } \\ 430 & \text { EPA8270 } \\ 430 & \text { EPA8270 } \\ 2,100 & \text { EPA8270 } \\ 430 & \text { EPA8270 } \\ 430 & \text { EPA8270 } \\ 430 & \text { EPA8270 } \\ 430 & \text { EPA8270 } \\ 430 & \text { EPA8270 } \\ 430 & \text { EPA8270 } \\ 430 & \text { EPA8270 } \\ 430 & \text { EPA8270 } \\ 430 & \text { EPA8270 } \\ 430 & \text { EPA8270 } \\ 430 & \text { EPA8270 } \\ 430 & \text { EPA8270 } \\ 430 & \text { EPA8270 } \\ 430 & \text { EPA8270 } \\ 430 & \text { EPA8270 } \\ 430 & \text { EPA8270 } \\ 430 & \text { EPA8270 } \\ 430 & \text { EPA8270 } \\ 430 & \text { EPA8270 } \\ 430 & \text { EPA8270 } \\ 430 & \text { EPA8270 } \\ 430 & \text { EPA8270 } \\ 430 & \text { EPA8270 } \\ 850 & \text { EPA8270 } \\ 430 & \text { EPA8270 } \\ 430 & \text { EPA8270 } \\ 430 & \text { EPA8270 } \\ 430 & \text { EPA8270 } \\ 2,100 & \text { EPA8270 } \\ 430 & \text { EPA8270 }\end{array}$


$B / N / A$ Extractables (including $P A H$ and phenols)

\author{
2.6-Dinitrotoluene \\ Di-n-octyl phthalate \\ Fluoranthene \\ Fluorene \\ Hexachlorobenzene \\ Hexachlorobutadiene \\ Hexachlorocyclopentadiene \\ Hexachloroethane \\ Indeno(1,2,3-c, d)pyrene \\ Isophorone \\ 2-Methyl-4,6-dinitrophenol \\ 2-Methylnaphthalene \\ Naphthalene \\ o.Nitroaniline \\ m-Nitroaniline \\ p-Nitroaniline \\ Nitrobenzene \\ 2-Nitrophenol \\ 4-Nitrophenol \\ $\mathrm{N}$-Nitrosodiphenylamine \\ N-Nitrosodipropylamine \\ Pentachlorophenol \\ Phenanthrene \\ Phenol \\ Pyrene \\ 1,2,4-Trichlorobenzene \\ 2,4,5-Trichlorophenol \\ 2,4,6-Trichlorophenol \\ Unknown
}

Volatile Organic Compounds

\begin{tabular}{|c|c|}
\hline $\mathbf{U}$ & 430 \\
\hline $\mathbf{U}$ & 430 \\
\hline $\bar{U}$ & 430 \\
\hline $\mathbf{U}$ & 430 \\
\hline $\mathbf{U}$ & 430 \\
\hline U & 430 \\
\hline U & 430 \\
\hline U & 430 \\
\hline U & 430 \\
\hline $\mathrm{U}$ & 430 \\
\hline U & 2,100 \\
\hline U & 430 \\
\hline $\mathrm{U}$ & 430 \\
\hline $\mathrm{U}$ & 2,100 \\
\hline$U$ & 2,100 \\
\hline U & 2.100 \\
\hline U & 430 \\
\hline$U$ & 430 \\
\hline $\mathrm{U}$ & 2,100 \\
\hline $\mathbf{U}$ & 430 \\
\hline U & 430 \\
\hline U & 2,100 \\
\hline $\mathrm{U}$ & 430 \\
\hline $\mathrm{U}$ & 430 \\
\hline $\mathbf{U}$ & 430 \\
\hline U & 430 \\
\hline U & 2,100 \\
\hline $\mathrm{U}$ & 430 \\
\hline
\end{tabular}

$\mu g / k g$
$\mu g / k g$
$\mu g / k g$
$\mu g / k g$
$\mu g / k g$
$\mu g / k g$
$\mu g / k g$
$\mu g / k g$
$\mu g / k g$
$\mu g / k g$
$\mu g / k g$
$\mu g / k g$
$\mu g / k g$
$\mu g / k g$
$\mu g / k g$
$\mu g / k g$
$\mu g / k g$
$\mu g / k g$
$\mu g / k g$
$\mu g / k g$
$\mu g / k g$
$\mu g / k g$
$\mu g / k g$
$\mu g / k g$
$\mu g / k g$
$\mu g / k g$
$\mu g / k g$
$\mu g / k g$
$\mu g / k g$

430

430

430

430

430

430

430

430

430

430

2.100

430

430

2,100

2.100

2.100

430

430

2.100

430

430

2,100

430

430

430

430

2,100

430

EPA8270

EPA8270

EPA8270

EPA8270

EPA8270

EPA8270

EPA8270

EPA8270

EPA8270

EPA8270

EPA8270

EPA8270

EPA8270

EPA8270

EPA8270

EPA8270

EPA8270

EPA8270

EPA8270

EPA8270

EPA8270

EPA8270

EPA8270

EPA8270

EPA8270

EPA8270

EPA8270

EPA8270

EPA8270

Acetone
Benzene
Bromodichloromethane
Bromoform
Bromomethane (Methyl bromide)
Carbon disulfide
Carbon tetrachloride
Chlorobenzene
Chloroethane
Chloroethene (Vinyl chloride)
2-Chloroethyl vinyl ether
Chloroform
Chloromethane (Methyl chloride)
Dibromochloromethane
1,1-Dichloroethane
1,2-Dichloroethane
1,1-Dichloroethylene
1,2-Dichloroethylene

$U$ U
$U$
$U$
$U$
$U$
$U$
$U$
$U$
$U$
$U$
$U$
$U$
$U$
$U$
$U$
$U$
$U$
$U$

$$
\begin{aligned}
& \mu g / k g \\
& \mu g / k g \\
& \mu g / k g \\
& \mu g / k g \\
& \mu g / k g \\
& \mu g / k g \\
& \mu g / k g \\
& \mu g / k g \\
& \mu g / k g \\
& \mu g / k g \\
& \mu g / k g \\
& \mu g / k g \\
& \mu g / k g \\
& \mu g / / k g \\
& \mu g / k g \\
& \mu g / k g \\
& \mu g / k g \\
& \mu g / k g
\end{aligned}
$$

\section{3}

6.5

6.5

6.5

13

6.5

6.5

$6: 5$

13

13

13

6.5

13

6.5

6.5

6.5

6.5

6.5

EPA8240
EPA8240
EPA8240
EPA8240
EPA8240
EPA8240
EPA8240
EPA8240
EPA8240
EPA8240
EPA8240
EPA8240
EPA8240
EPA8240
EPA8240
EPA8240
EPA8240
EPA8240 
SAMPLE NAME: D431.12102 (Continued)

Constituent

Volatile Organic Compounds

Dichloromethane (Methylene chloride)

1,2-Dichloropropane

cis-1,3-Dichloropropene

trans-1,3-Dichloropropene

2-Hexanone

Methyl ethyl ketone

Methyl isobutyl ketone

Styrene

1,1,2,2-Tetrachloroethane

Tetrachloroethylene

Toluene

1.1,1-Trichloroethane

1,1,2-Trichloroethane

Trichloroethylene

Vinyl acetate

Xylenes
Ethylbenzene

RQ AQ B

Result Unit

D. Limit

Method

49
6.5
6.5
6.5
6.5
13
13
13
6.5
6.5
6.5
2.6
6.5
6.5
6.5
13
6.5

$\mu \mathrm{g} / \mathrm{kg}$

$\mu \mathrm{g} / \mathrm{kg}$

$\mu \mathrm{g} / \mathrm{kg}$

$\mu \mathrm{g} / \mathrm{kg}$

$\mu \mathrm{g} / \mathrm{kg}$

$\mu \mathrm{g} / \mathrm{kg}$

$\mu \mathrm{g} / \mathrm{kg}$

$\mu \mathrm{g} / \mathrm{kg}$

$\mu \mathrm{g} / \mathrm{kg}$

$\mu \mathrm{g} / \mathrm{kg}$

$\mu \mathrm{g} / \mathrm{kg}$

$\mu \mathrm{g} / \mathrm{kg}$

$\mu \mathrm{g} / \mathrm{kg}$

$\mu \mathrm{g} / \mathrm{kg}$

$\mu \mathrm{g} / \mathrm{kg}$

$\mu \mathrm{g} / \mathrm{kg}$

$\mu \mathrm{g} / \mathrm{kg}$

6.5

6.5

6.5

6.5

6.5

13

13

13

6.5

6.5

6.5

6.5

6.5

6.5

6.5

13

6.5

EPA8240

EPA8240

EPA8240

EPA8240

EPA8240

EPA8240

EPA8240

EPA8240

EPA8240

EPA8240

EPA8240

EPA8240

EPA8240

EPA8240

EPA8240

EPA8240

EPA8240

Radionuclide Indicators and Radionuclides

Gross alpha

Nonvolatile beta

Radium, total alpha-emitting

$11 \pm 13$

$71 \pm 25$

$0.79 \pm 0.71$

$\mathrm{pCi} / \mathrm{g}$

$\mathrm{pCi} / \mathrm{g}$

$\mathrm{pCi} / \mathrm{g}$

$2.0 \pm 0.44$

$\mathrm{pCi} / \mathrm{g}$

EPA900.0

EPA900.0

EPA903.0

EPA906.0 
SAMPLE NAME: D431 1707

Location (SRS Coordinates): 18492.14E 66454.17N

Ground Elevation Above MSL: $124.9 \mathrm{ft}$

Depth of Core Interval: 13.00 to $14.00 \mathrm{ft}$

Sample Type: Normal

Constituent

Volatile Organic Compounds

Acetone
Benzene
Bromodichloromethane
Bromoform
Bromomethane (Methyl bromide)
Carbon disulfide
Carbon tetrachloride
Chlorobenzene
Chloroethane
Chloroethene (Vinyl chloride)
Chloroform
Chloromethane (Methyl chloride)
Dibromochloromethane
1,1-Dichloroethane
1,2-Dichloroethane
1,1-Dichloroethylene
1,2-Dichloroethylene
Dichloromethane (Methylene chloride)
1,2-Dichloropropane
cis-1,3-Dichloropropene
trans-1,3-Dichloropropene
Ethylbenzene
2-Hexanone
Methyl ethyl ketone
Methyl isoburyl ketone
Styrene
1,1,2,2-Tetrachloroethane
Tetrachloroethylene
Toluene
1,1,1-Trichloroethane
1,1,2-Trichloroethane
Trichloroethylene
Vinyl acetate
Xylenes

Sample ID: 100434

Sample Matrix: Water

RQ AQ B Result Unit
Sample Moisture: Liquid

D. Limit Method

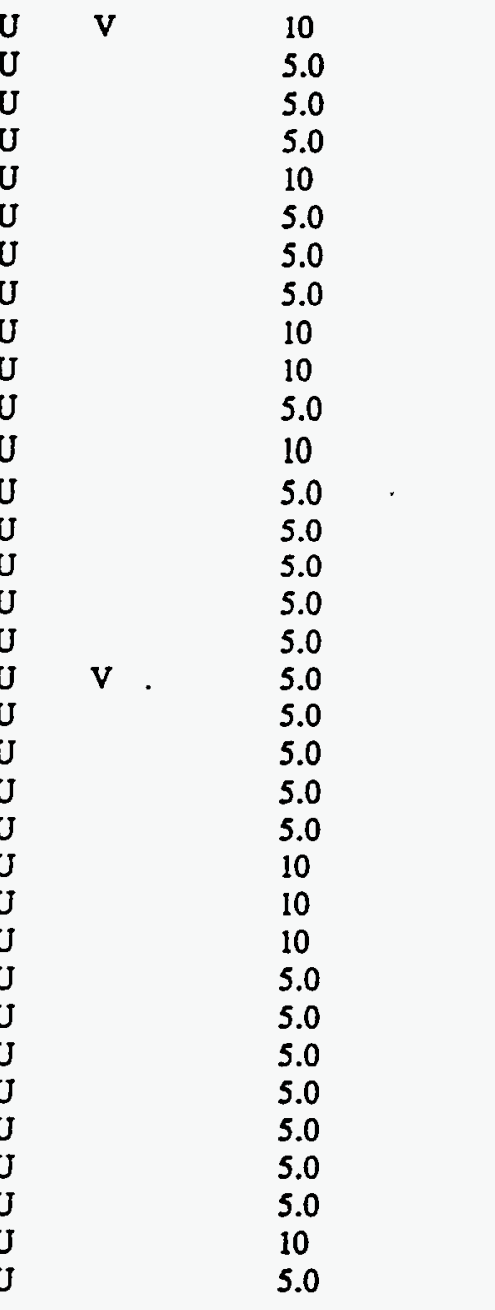

$\mu \mathrm{g} / \mathrm{L}$
$\mu \mathrm{g} / \mathrm{L}$
$\mu \mathrm{g} / \mathrm{L}$
$\mu \mathrm{g} / \mathrm{L}$
$\mu \mathrm{g} / \mathrm{L}$
$\mu \mathrm{g} / \mathrm{L}$
$\mu \mathrm{g} / \mathrm{L}$
$\mu \mathrm{g} / \mathrm{L}$
$\mu \mathrm{g} / \mathrm{L}$
$\mu \mathrm{g} / \mathrm{L}$
$\mu \mathrm{g} / \mathrm{L}$
$\mu \mathrm{g} / \mathrm{L}$
$\mu \mathrm{g} / \mathrm{L}$
$\mu \mathrm{g} / \mathrm{L}$
$\mu \mathrm{g} / \mathrm{L}$
$\mu \mathrm{g} / \mathrm{L}$
$\mu \mathrm{g} / \mathrm{L}$
$\mu \mathrm{g} / \mathrm{L}$
$\mu \mathrm{g} / \mathrm{L}$
$\mu \mathrm{g} / \mathrm{L}$
$\mu \mathrm{g} / \mathrm{L}$
$\mu \mathrm{g} / \mathrm{L}$
$\mu \mathrm{g} / \mathrm{L}$
$\mu \mathrm{g} / \mathrm{L}$
$\mu \mathrm{g} / \mathrm{L}$
$\mu \mathrm{g} / \mathrm{L}$
$\mu \mathrm{g} / \mathrm{L}$
$\mu \mathrm{g} / \mathrm{L}$
$\mu \mathrm{g} / \mathrm{L}$
$\mu \mathrm{g} / \mathrm{L}$
$\mu \mathrm{g} / \mathrm{L}$
$\mu \mathrm{g} / \mathrm{L}$
$\mu \mathrm{g} / \mathrm{L}$
$\mu \mathrm{g} / \mathrm{L}$

10

EPA8240

EPA8240

EPA8240

EPA8240

10 EPA8240

5.0 EPA8240

$5.0 \quad$ EPA8240

5.0 EPA8240

10 EPA8240

10 EPA8240

$5.0 \quad$ EPA8240

10 EPA8240

$5.0 \quad$ EPA8240

$5.0 \quad$ EPA8240

$5.0 \quad$ EPA8240

$5.0 \quad$ EPA8240

5.0 EPA8240

5.0 EPA8240

5.0 EPA8240

5.0 EPA8240

5.0 EPA8240

$5.0 \quad$ EPA8240

10 EPA8240

10 EPA8240

10 EPA8240

$5.0 \quad$ EPA8240

5.0 EPA8240

.5 .0 EPA8240

5.0 EPA8240

5.0 EPA8240

$5.0 \quad$ EPA8240

$5.0 \quad$ EPA8240

10 EPA8240

5.0 EPA8240 
SAMPLE NAME: D431-1707A

Sample Type: Duplicate

Constituent

Volatile Organic Compounds

Acetone
Benzene
Bromodichloromethane
Bromoform
Bromomethane (Methyl bromide)
Carbon disulfide
Carbon retrachloride
Chlorobenzene
Chloroethane
Chloroethene (Vinyl chloride)
Chloroform
Chloromethane (Methyl chloride)
Dibromochloromethane
1,1-Dichloroethane
1,2-Dichloroethane
1,1-Dichloroethylene
1,2-Dichloroethylene
Dichloromethane (Methylene chloride)
1,2-Dichloropropane
cis-1,3-Dichloropropene
trans-1,3-Dichloropropene
Ethylbenzene
2-Hexanone
Methyl ethyl ketone
Methyl isobutyl ketone
Styrene
1,1,2,2-Tetrachloroethane
Tetrachloroethylene
Toluene
1,1,1-Trichloroethane
1,1,2-Trichloroethane
Trichloroethylene
Vinyl acetate
Xylenes

Sample ID: 100435

Associated Sample: 100434

RQ AQ B Result Unit $\quad$ D. Limit Method

$\mathbf{2}$
$\mathrm{V}$
5.0
5.0
5.0
10
5.0
5.0
5.0
10
10
5.0
10
5.0
5.0
5.0
5.0
5.0
16
5.0
5.0
5.0
5.0
10
10
10
5.0
5.0
5.0
5.0
5.0
5.0
5.0
10
5.0

$\mu g / L$

EPA8240

EPA8240

EPA8240

$\mu \mathrm{g} / \mathrm{L}$

EPA8240

EPA8240

$\mu \mathrm{g} / \mathrm{L}$

$\mu \mathrm{g} / \mathrm{L}$

$\mu \mathrm{g} / \mathrm{L}$

$\mu \mathrm{g} / \mathrm{L}$

$\mu \mathrm{g} / \mathrm{L}$

$\mu \mathrm{g} / \mathrm{L}$

$\mu \mathrm{g} / \mathrm{L}$

$\mu \mathrm{g} / \mathrm{L}$

$\mu \mathrm{g} / \mathrm{L}$

$\mu \mathrm{g} / \mathrm{L}$

$\mu \mathrm{g} / \mathrm{L}$

$\mu \mathrm{g} / \mathrm{L}$

$\mu \mathrm{g} / \mathrm{L}$

$\mu \mathrm{g} / \mathrm{L}$

$\mu \mathrm{g} / \mathrm{L}$

$\mu \mathrm{g} / \mathrm{L}$

$\mu g / L$

$\mu \mathrm{g} / \mathrm{L}$

$\mu \mathrm{g} / \mathrm{L}$

$\mu \mathrm{g} / \mathrm{L}$

$\mu \mathrm{g} / \mathrm{L}$

$\mu \mathrm{g} / \mathrm{L}$

$\mu \mathrm{g} / \mathrm{L}$

$\mu \mathrm{g} / \mathrm{L}$

$\mu \mathrm{g} / \mathrm{L}$

$\mu \mathrm{g} / \mathrm{L}$

$\mu \mathrm{g} / \mathrm{L}$

$\mu g / L$

$\mu \mathrm{g} / \mathrm{L}$

$\mu \mathrm{g} / \mathrm{L}$
EPA8240

EPA8240

EPA8240

EPA8240

EPA8240

EPA8240

EPA8240

EPA8240

EPA8240

EPA8240

EPA8240

EPA8240

EPA8240

EPA8240

EPA8240

EPA8240

EPA8240

EPA8240

EPA8240

EPA8240

EPA8240

EPA8240

EPA8240

EPA8240

EPA8240

EPA8240

EPA8240

EPA8240

EPA8240 
SAMPLE NAME: D431 1708

Location (SRS Coordinates): 18492.14E 66454.17N

Ground Elevation Above MSL: $124.9 \mathrm{ft}$

Dépth of Core Interval: 33.00 to $34.00 \mathrm{ft}$

Sample Type: Normal

Constituent

RQ AQ B Result

Volatile Organic Compounds

Acetone
Benzene
Bromodichloromethane
Bromoform
Bromomethane (Methyl bromide)
Carbon disulfide
Carbon tetrachloride
Chlorobenzene
Chloroethane
Chloroethene (Vinyl chloride)
Chloroform
Chloromethane (Methyl chloride)
Dibromochloromethane
1,1-Dichloroethane
1,2-Dichloroethane
1,1-Dichloroethylene
1,2-Dichloroethylene
Dichloromethane (Methylene chloride)
1,2-Dichloropropane
cis-1,3-Dichloropropene
trans-1,3-Dichloropropene
Ethylbenzene
2-Hexanone
Methyl ethyl ketone
Methyl isobutyl ketone
Styrene
1,1,2,2-Tetrachloroethane
Tetrachloroethylene
Toluene
1,1,1-Trichloroethane
1,1,2-Trichloroethane
Trichloroethylene
Vinyl acetate
2-Propanol
Xylenes

Sample ID: 100436

Sample Matrix: Water

Sample Moisture: Liquid

D. Limit Method

Unit

16

5.0

5.0

5.0

10

5.0

5.0

5.0

10

10

5.0

10

5.0

5.0

5.0

5.0

5.0

19

5.0

5.0

5.0

5.0

10

10

10

5.0

5.0

5.0

5.0

5.0

5.0

5.0

10

N

10

5.0

\begin{tabular}{|c|c|c|}
\hline$\mu \mathrm{g} / \mathrm{L}$ & 10 & EPA 8240 \\
\hline$\mu \mathrm{g} / \mathrm{L}$ & 5.0 & EPA8240 \\
\hline$\mu g / L$ & 5.0 & EPA8240 \\
\hline$\mu g / L$ & 5.0 & EPA 8240 \\
\hline$\mu g / L$ & 10 & EPA8240 \\
\hline$\mu \mathrm{g} / \mathrm{L}$ & 5.0 & EPA 8240 \\
\hline$\mu \mathrm{g} / \mathrm{L}$ & 5.0 & EPA 8240 \\
\hline$\mu \mathrm{g} / \mathrm{L}$ & 5.0 & EPA 8240 \\
\hline$\mu \mathrm{g} / \mathrm{L}$ & 10 & EPA 8240 \\
\hline$\mu \mathrm{g} / \mathrm{L}$ & 10 & EPA8240 \\
\hline$\mu g / L$ & 5.0 & EPA 8240 \\
\hline$\mu \mathrm{g} / \mathrm{L}$ & 10 & EPA8240 \\
\hline$\mu \mathrm{g} / \mathrm{L}$ & 5.0 & EPA8240 \\
\hline$\mu \mathrm{g} / \mathrm{L}$ & 5.0 & EPA8240 \\
\hline$\mu \mathrm{g} / \mathrm{L}$ & 5.0 & EPA 8240 \\
\hline$\mu g / L$ & 5.0 & EPA8240 \\
\hline$\mu \mathrm{g} / \mathrm{L}$ & 5.0 & EPA8240 \\
\hline$\mu \mathrm{g} / \mathrm{L}$ & 5.0 & EPA8240 \\
\hline$\mu \mathrm{g} / \mathrm{L}$ & 5.0 & EPA 8240 \\
\hline$\mu g / L$ & 5.0 & EPA 8240 \\
\hline$\mu g / L$ & 5.0 & EPA 8240 \\
\hline$\mu \mathrm{g} / \mathrm{L}$ & 5.0 & EPA8240 \\
\hline$\mu \mathrm{g} / \mathrm{L}$ & 10 & EPA8240 \\
\hline$\mu \mathrm{g} / \mathrm{L}$ & 10 & EPA 8240 \\
\hline$\mu \mathrm{g} / \mathrm{L}$ & 10 & EPA 8240 \\
\hline$\mu \mathrm{g} / \mathrm{L}$ & 5.0 & EPA 8240 \\
\hline$\mu \mathrm{g} / \mathrm{L}$ & 5.0 & EPA 8240 \\
\hline$\mu \mathrm{g} / \mathrm{L}$ & 5.0 & EPA 8240 \\
\hline$\mu g / L$ & 5.0 & EPA8240 \\
\hline$\mu \mathrm{g} / \mathrm{L}$ & 5.0 & EPA 8240 \\
\hline$\mu \mathrm{g} / \mathrm{L}$ & 5.0 & EPA 8240 \\
\hline$\mu \mathrm{g} / \mathrm{L}$ & 5.0 & EPA8240 \\
\hline$\mu \mathrm{g} / \mathrm{L}$ & 10 & EPA8240 \\
\hline$\mu g / L$ & & EPA 8240 \\
\hline$\mu g / L$ & 5.0 & EPA8240 \\
\hline
\end{tabular}


Location (SRS Coordinates): 18160E 66410N

Ground Elevation Above MSL: . $\mathrm{ft}$

Depth of Core Interval: . to . $\mathrm{ft}$

Sample Type: Normal

Sample Matrix: Water

Sample Moisture: Liquid

\section{Constituent}

RQ AQ B Result Unit

D. Limit Method

Physical Parameters

Chloride

Fluoride

Nitrate as nitrogen

$\mathrm{pH}$

Sulfate

Total dissolved solids

Total organic carbon

Total organic halogens

Total suspended solids

Total suspended solids

\section{Total Metals}

Arsenic

Barium

Cadmium

Chromium

Copper

Iron

Lead

Manganese

Mercury

Nickel

Selenium

Silver

Sodium
10

0.14

$\begin{array}{ll}\text { U } & \text { V } \\ \text { J } & \mathbf{Q}\end{array}$

0.020

5.5

180

640

170

370

2.800

2,800

140

$\begin{array}{lll}\text { U } & & 200 \\ \text { UJ } & \text { L } & 5.0\end{array}$

UT 14

UJ L 25

270,000

17

1,200

0.82

40

5.0

10

8,400

$\begin{array}{ll}\mathrm{mg} / \mathrm{L} & 0.50 \\ \mathrm{mg} / \mathrm{L} & 0.10 \\ \mathrm{mg} / \mathrm{L} & 0.020 \\ \mathrm{pH} & 0.010 \\ \mathrm{mg} / \mathrm{L} & 20 \\ \mathrm{mg} / \mathrm{L} & 10 \\ \mathrm{mg} / \mathrm{L} & 5.0 \\ \mu \mathrm{g} / \mathrm{L} & 25 \\ \mathrm{mg} / \mathrm{L} & 20 \\ \mathrm{mg} / \mathrm{L} & 20\end{array}$

EPA300.0

EPA300.0

EPA300.0

EPA9045

EPA 300.0

EPA160.2

EPA415.1

EPA9020

EPA160.2

EPA 160.2

$B / N / A$ Extractables (including PAH and phenols)

Acenaphthene

Acenaphthylene

Anthracene

Benzo(a)anthracene

Benzo(b)fluoranthene

Benzo(k)fluoranthene

Benzoic acid

Benzo(g,h,i)perylene

Benzo(a)pyrene

Benzyl alcohol

Bis(2-chloroethoxy) methane

Bis(2-chloroethyl) ether

Bis(2-chloroisopropyl) ether

Bis(2-ethylhexyl) phthalate

4-Bromophenyl phenyl ether

Butylbenzyl phthalate

$\begin{array}{llll}\text { UJ } & 0 & \text { L } & 11 \\ \text { UJ } & 0 & \text { L } & 11 \\ \text { UJ } & 0 & \text { L } & 11 \\ \text { UJ } & 0 & \text { L } & 11 \\ \text { UJ } & 0 & \text { L } & 11 \\ \text { UJ } & 0 & \text { L } & 11 \\ \text { UJ } & 0 & \text { L } & 55 \\ \text { UJ } & 0 & \text { L } & 11 \\ \text { UJ } & 0 & \text { L } & 11 \\ \text { UJ } & 0 & \text { L } & 11 \\ \text { UJ } & 0 & \text { L } & 11 \\ \text { UJ } & 0 & \text { L } & 11 \\ \text { UJ } & 0 & \text { L } & 11 \\ \text { UJ } & \text { VO } & \text { L } & 2.8 \\ \text { UJ } & 0 & \text { L } & 11 \\ \text { UJ } & 0 & \mathrm{~L} & 11\end{array}$

$\mu g / L$

$\mu g / L$

$\mu g / \mathrm{L}$

$\mu \mathrm{g} / \mathrm{L}$

$\mu g / L$

$\mu \mathrm{g} / \mathrm{L}$

$\mu \mathrm{g} / \mathrm{L}$

$\mu g / L$

$\mu \mathrm{g} / \mathrm{L}$

$\mu \mathrm{g} / \mathrm{L}$

$\mu \mathrm{g} / \mathrm{L}$

$\mu \mathrm{g} / \mathrm{L}$

$\mu g / L$

$\begin{array}{ll}50 & \text { EPA7060 } \\ 200 & \text { EPA6010 } \\ 5.0 & \text { EPA6010 } \\ 10 & \text { EPA6010 } \\ 25 & \text { EPA6010 } \\ 100 & \text { EPA6010 } \\ 3.0 & \text { EPA7421 } \\ 1.5 & \text { EPA6010 } \\ 0.20 & \text { EPA7470 } \\ 40 & \text { EPA6010 } \\ 5.0 & \text { EPA7740 } \\ 10 & \text { EPA6010 } \\ 5.000 & \text { EPA6010 }\end{array}$

$\mu \mathrm{g} / \mathrm{L}$

$\mu \mathrm{g} / \mathrm{L}$

$\mu \mathrm{g} / \mathrm{L}$

$\mu \mathrm{g} / \mathrm{L}$

$\mu g / L$

$\mu \mathrm{g} / \mathrm{L}$

$\mu \mathrm{g} / \mathrm{L}$

$\mu \mathrm{g} / \mathrm{L}$

$\mu g / L$

$\mu g / L$

$\mu \mathrm{g} / \mathrm{L}$

$\mu \mathrm{g} / \mathrm{L}$

$\mu \mathrm{g} / \mathrm{L}$

$\mu \mathrm{g} / \mathrm{L}$

$\mu \mathrm{g} / \mathrm{L}$

$\mu \mathrm{g} / \mathrm{L}$
EPA8270

EPA8270

EPA8270

EPA8270

EPA8270

EPA8270

EPA8270

EPA8270

EPA8270

EPA8270

EPA8270

EPA8270

EPA8270

EPA8270

EPA8270

EPA8270 
SAMPLE NAME: D431 2601 (Continued)

Sample ID: 100301

\begin{tabular}{|c|c|c|c|c|c|c|c|}
\hline Constituent & $\mathbf{R Q}$ & $\mathbf{A Q}$ & $\mathbf{B}$ & Result & Unit & D. Limit & Method \\
\hline \multicolumn{8}{|c|}{$B / N / A$ Extractables (including $P A H$ and phenols) } \\
\hline 4-Chloroaniline & UJ & 0 & L & 11 & $\mu \mathrm{g} / \mathrm{L}$ & 11 & EPA8270 \\
\hline 4-Chloro-m-cresol & UJ & 0 & $\bar{L}$ & 11 & $\mu g / L$ & 11 & EPA8270 \\
\hline 2-Chloronaphthalene & UJ & 0 & $\bar{L}$ & 11 & $\mu \mathrm{g} / \mathrm{L}$ & 11 & EPA8270 \\
\hline 2-Chlorophenol & UJ & 0 & $\mathrm{~L}$ & 11 & $\mu \mathrm{g} / \mathrm{L}$ & 11 & EPA8270 \\
\hline 4-Chlorophenyl phenyl ether & UJ & 0 & $L$ & 11 & $\mu g / L$ & 11 & EPA8270 \\
\hline Chrysene & UJ & 0 & L & 11 & $\mu \mathrm{g} / \mathrm{L}$ & 11 & EPA8270 \\
\hline o-Cresol (2-Methylphenol) & UJ & 0 & $L$ & 11 & $\mu \mathrm{g} / \mathrm{L}$ & 11 & EPA8270 \\
\hline p-Cresol (4-Methylphenol) & UJ & 0 & $L$ & 11 & $\mu \mathrm{g} / \mathrm{L}$ & 11 & EPA8270 \\
\hline Dibenz $(a, h)$ anthracene & UJ & 0 & $L$ & 11 & $\mu \mathrm{g} / \mathrm{L}$ & 11 & EPA8270 \\
\hline Dibenzofuran & UJ & 0 & L & 11 & $\mu \mathrm{g} / \mathrm{L}$ & 11 & EPA8270 \\
\hline Di-n-butyl phthalate & UJ & 0 & $\bar{L}$ & 11 & $\mu \mathrm{g} / \mathrm{L}$ & 11 & EPA8270 \\
\hline 1.2-Dichlorobenzene & UJ & 0 & L & 11 & $\mu \mathrm{g} / \mathrm{L}$ & 11 & EPA8270 \\
\hline 1,3-Dichlorobenzene & UJ & 0 & $\mathrm{~L}$ & 11 & $\mu g / L$ & 11 & EPA8270 \\
\hline 1,4-Dichlorobenzene & UJ & 0 & L & 11 & $\mu \mathrm{g} / \mathrm{L}$ & 11 & EPA8270 \\
\hline 3,3'-Dichlorobenzidine & UJ & 0 & L & 22 & $\mu \mathrm{g} / \mathrm{L}$ & 22 & EPA8270 \\
\hline 2.4-Dichlorophenol & UJ & 0 & $L$ & 11 & $\mu g / L$ & 11 & EPA8270 \\
\hline Diethyl phthalate & UJ & 0 & L & 11 & $\mu \mathrm{g} / \mathrm{L}$ & 11 & EPA8270 \\
\hline 2,4-Dimethyl phenol & UJ & 0 & $\mathrm{~L}$ & 11 & $\mu g / \mathrm{L}$ & 11 & EPA8270 \\
\hline Dimethyl phthalate & UJ & 0 & $\bar{L}$ & 11 & $\mu g / L$ & 11 & EPA8270 \\
\hline 2,4-Dinitrophenol & UJ & 0 & L & 55 & $\mu \mathrm{g} / \mathrm{L}$ & 55 & EPA8270 \\
\hline 2.4-Dinitrotoluene & UJ & 0 & L & 11 & $\mu \mathrm{g} / \mathrm{L}$ & 11 & EPA8270 \\
\hline 2,6-Dinitrotoluene & UJ & 0 & L & 11 & $\mu \mathrm{g} / \mathrm{L}$ & 11 & EPA8270 \\
\hline Di-n-octyl phthalate & UJ & 0 & L & 11 & $\mu \mathrm{g} / \mathrm{L}$ & 11 & EPA8270 \\
\hline Fluoranthene & UJ & 0 & L & 11 & $\mu \mathrm{g} / \mathrm{L}$ & 11 & EPA8270 \\
\hline Fluorene & UJ & 0 & L & 11 & $\mu g / L$ & 11 & EPA8270 \\
\hline Hexachlorobenzene & UJ & 0 & $\bar{L}$ & 11 & $\mu \mathrm{g} / \mathrm{L}$ & 11 & EPA8270 \\
\hline Hexachlorobutadiene & UJ & 0 & $\mathrm{~L}$ & 11 & $\mu g / L$ & 11 & EPA8270 \\
\hline Hexachlorocyclopentadiene & UJ & 0 & $\mathbf{L}$ & 11 & $\mu \mathrm{g} / \mathrm{L}$ & 11 & EPA8270 \\
\hline Hexachloroethane & UJ & 0 & L & 11 & $\mu \mathrm{g} / \mathrm{L}$ & 11 & EPA8270 \\
\hline Indeno(1,2,3-c,d)pyrene & UJ & 0 & $\bar{L}$ & 11 & $\mu \mathrm{g} / \mathrm{L}$ & 11 & EPA8270 \\
\hline Isophorone & UJ & 0 & $\mathrm{~L}$ & 11 & $\mu \mathrm{g} / \mathrm{L}$ & 11 & EPA8270 \\
\hline 2-Methyl-4,6-dinitrophenol & UJ & 0 & L & 55 & $\mu \mathrm{g} / \mathrm{L}$ & 55 & EPA8270 \\
\hline 2-Methylnaphthalene & UJ & 0 & L & 11 & $\mu \mathrm{g} / \mathrm{L}$. & 11 & EPA8270 \\
\hline Methyl pyrrolidinone & J & $\mathbf{N}$ & & 6.0 & $\mu \mathrm{g} / \mathrm{L}$ & & EPA8270 \\
\hline Naphthalene & U] & 0 & L & 11 & $\mu \mathrm{g} / \mathrm{L}$ & 11 & EPA8270 \\
\hline o-Nitroaniline & UI & 0 & L & 55 & $\mu \mathrm{g} / \mathrm{L}$ & s5 & EPA8270 \\
\hline m-Nitroaniline & UJ & 0 & L & 55 & $\mu \mathrm{g} / \mathrm{L}$ & 55 & EPA8270 \\
\hline p-Nitroaniline & UJ & 0 & L & 55 & $\mu g / L$ & 55 & EPA8270 \\
\hline Nitrobenzene & UJ & 0 & $\bar{L}$ & 11 & $\mu g / L$ & 11 & EPA8270 \\
\hline 2-Nitrophenol & UJ & 0 & $\bar{L}$ & 11 & $\mu g / L$ & 11 & EPA8270 \\
\hline 4-Nitrophenol & UJ & 0 & L & 55 & $\mu \mathrm{g} / \mathrm{L}$ & 55 & EPA8270 \\
\hline N-Nitrosodiphenylamine & UJ & 0 & L & 11 & $\mu \mathrm{g} / \mathrm{L}$ & 11 & EPA8270 \\
\hline N-Nitrosodipropylamine & UJ & 0 & L & 11 & $\mu \mathrm{g} / \mathrm{L}$ & 11 & EPA8270 \\
\hline Organic acid & $\mathbf{J}$ & $\mathbf{N}$ & & 50 & $\mu \mathrm{g} / \mathrm{L}$ & & EPA8270 \\
\hline Organic acid & J & $\mathrm{N}$ & & 50 & $\mu g / L$ & & EPA8270 \\
\hline Pentachlorophenol & UJ & 0 & L & 55 & $\mu \mathrm{g} / \mathrm{L}$ & 55 & EPA8270 \\
\hline Phenanthrene & UJ. & 0 & $\bar{L}$ & 11 & $\mu g / L$ & 11 & EPA8270 \\
\hline Phenol & UJ & 0 & L & 11 & $\mu \mathrm{g} / \mathrm{L}$ & 11 & EPA8270 \\
\hline Pyrene & UJ & 0 & L & 11 & $\mu g / L$ & 11 & EPA8270 \\
\hline 1,2,4-Trichlorobenzene & UJ & 0 & $\vec{L}$ & 11 & $\mu g / L$ & 11 & EPA8270 \\
\hline
\end{tabular}


SAMPLE NAME: D431 2601 (Continued)

Sample ID: 100301

Constituent

RQ AQ B Result

Unit

D. Limit Method

$B / N / A$ Extractables (including $P A H$ and phenols)

2.4.5-Trichlorophenol
2,4.6-Trichlorophenol
Unknown
Unknown
Unknown

Volatile Organic Compounds

$\begin{array}{lllllll}\text { UJ } & 0 & \mathrm{~L} & 55 & \mu \mathrm{g} / \mathrm{L} & 55 & \text { EPA8270 } \\ \mathrm{UJ} & \mathrm{O} & \mathrm{L} & 11 & \mu \mathrm{g} / \mathrm{L} & 11 & \text { EPA8270 } \\ \mathrm{J} & \mathrm{N} & & 10 & \mu \mathrm{g} / \mathrm{L} & & \text { EPA8270 } \\ \mathrm{J} & \mathrm{N} & & 20 & \mu \mathrm{g} / \mathrm{L} & & \text { EPA8270 } \\ \mathrm{J} & \mathrm{N} & & 5.0 & \mu \mathrm{g} / \mathrm{L} & & \text { EPA8270 }\end{array}$

21

5.0

5.0

5.0

10

1.7

5.0

5.0

10

10

10

5.0

10

5.0

5.0

5.0

5.0

5.0

5.0

5.0

5.0

5.0

5.0

10

10

10

5.0

5.0

5.0

5.0

5.0

5.0

5.0

10

5.0 $\mu \mathrm{g} / \mathrm{L}$

$\mu \mathrm{g} / \mathrm{L}$

$\mu g / L$

$\mu \mathrm{g} / \mathrm{L}$

$\mu \mathrm{g} / \mathrm{L}$

$\mu g / L$

$\mu g / L$

$\mu \mathrm{g} / \mathrm{L}$

$\mu \mathrm{g} / \mathrm{L}$

$\mu \mathrm{g} / \mathrm{L}$

$\mu \mathrm{g} / \mathrm{L}$

$\mu \mathrm{g} / \mathrm{L}$

$\mu \mathrm{g} / \mathrm{L}$

$\mu \mathrm{g} / \mathrm{L}$

$\mu \mathrm{g} / \mathrm{L}$

$\mu g / L$

$\mu g / L$

$\mu \mathrm{g} / \mathrm{L}$

$\mu \mathrm{g} / \mathrm{L}$

$\mu \mathrm{g} / \mathrm{L}$

$\mu \mathrm{g} / \mathrm{L}$

$\mu \mathrm{g} / \mathrm{L}$

$\mu \mathrm{g} / \mathrm{L}$

$\mu g / L$

$\mu \mathrm{g} / \mathrm{L}$

$\mu g / L$

$\mu \mathrm{g} / \mathrm{L}$

$\mu g / L$

$\mu g / L$

$\mu g / L$

$\mu \mathrm{g} / \mathrm{L}$

$\mu \mathrm{g} / \mathrm{L}$

$\mu g / L$

$\mu \mathrm{g} / \mathrm{L}$

$\mu \mathrm{g} / \mathrm{L}$
EPA8240

EPA8240

EPA8240

EPA8240

EPA8240

EPA8240

EPA8240

EPA8240

EPA8240

EPA8240

EPA8240

EPA8240

EPA8240

EPA8240

EPA8240

EPA8240

EPA8240

EPA8240

EPA8240

EPA8240

EPA8240

EPA8240

EPA8240

EPA8240

EPA8240

EPA8240

EPA8240

EPA8240

EPA8240

EPA8240

EPA8240

EPA8240

EPA8240

EPA8240

EPA8240

Radionuclide Indicators and Radionuclides

Gross alpha

Nonvolatile beta

Radium, total alpha-emitting

Tritium

\begin{tabular}{|c|c|c|c|}
\hline $\begin{array}{l}30 \pm 7.8 \\
54 \pm 12 \\
0.60 \pm 0.50 \\
1,500 \pm 690\end{array}$ & $\begin{array}{l}\mathrm{pCi} / \mathrm{L} \\
\mathrm{pCi} / \mathrm{L} \\
\mathrm{pCi} / \mathrm{L} \\
\mathrm{pCi} / \mathrm{L}\end{array}$ & $\begin{array}{l}1.2 \\
1.1 \\
0.40 \\
420\end{array}$ & $\begin{array}{l}\text { EPA900.0 } \\
\text { EPA900.0 } \\
\text { EPA903.0 } \\
\text { EPA906.0 }\end{array}$ \\
\hline
\end{tabular}




\section{SAMPLE NAME: D43ï11505}

Location (SRS Coordinates): 18533.07E 66779.25N

Ground Elevation Above MSL: $128.5 \mathrm{ft}$

Depth of Core Interval: 16.00 to $17.00 \mathrm{ft}$

Sample Type: Normal

Constituent

Volatile Organic Compounds

Acetone
Benzene
Bromodichloromethane
Bromoform
Bromomethane (Methyl bromide)
Carbon disulfide
Carbon tetrachloride
Chlorobenzene
Chloroethane
Chloroethene (Vinyl chloride)
Chloroform
Chloromethane (Methyl chloride)
Dibromochloromethane
1,1-Dichloroethane
1,2-Dichloroethane
1,1-Dichloroethylene
1,2-Dichloroethylene
Dichloromethane (Methylene chloride)
1,2-Dichloropropane
cis-1,3-Dichloropropene
trans-1,3-Dichloropropene
Ethylbenzene
2-Hexanone
Methyl ethyl ketone
Methyl isobutyl ketone
Styrene
1,1,2,2-Tetrachloroethane
Tetrachloroethylene
Toluene
1,1,1-Trichloroethane
1,1,2-Trichloroethane
Trichloroethylene
Vinyl acetate
Xylenes

Sample Matrix: Water

Sample ID: 100412

RQ AQ B Result Unit

Sample Moisture: Liquid

D. Limit Method

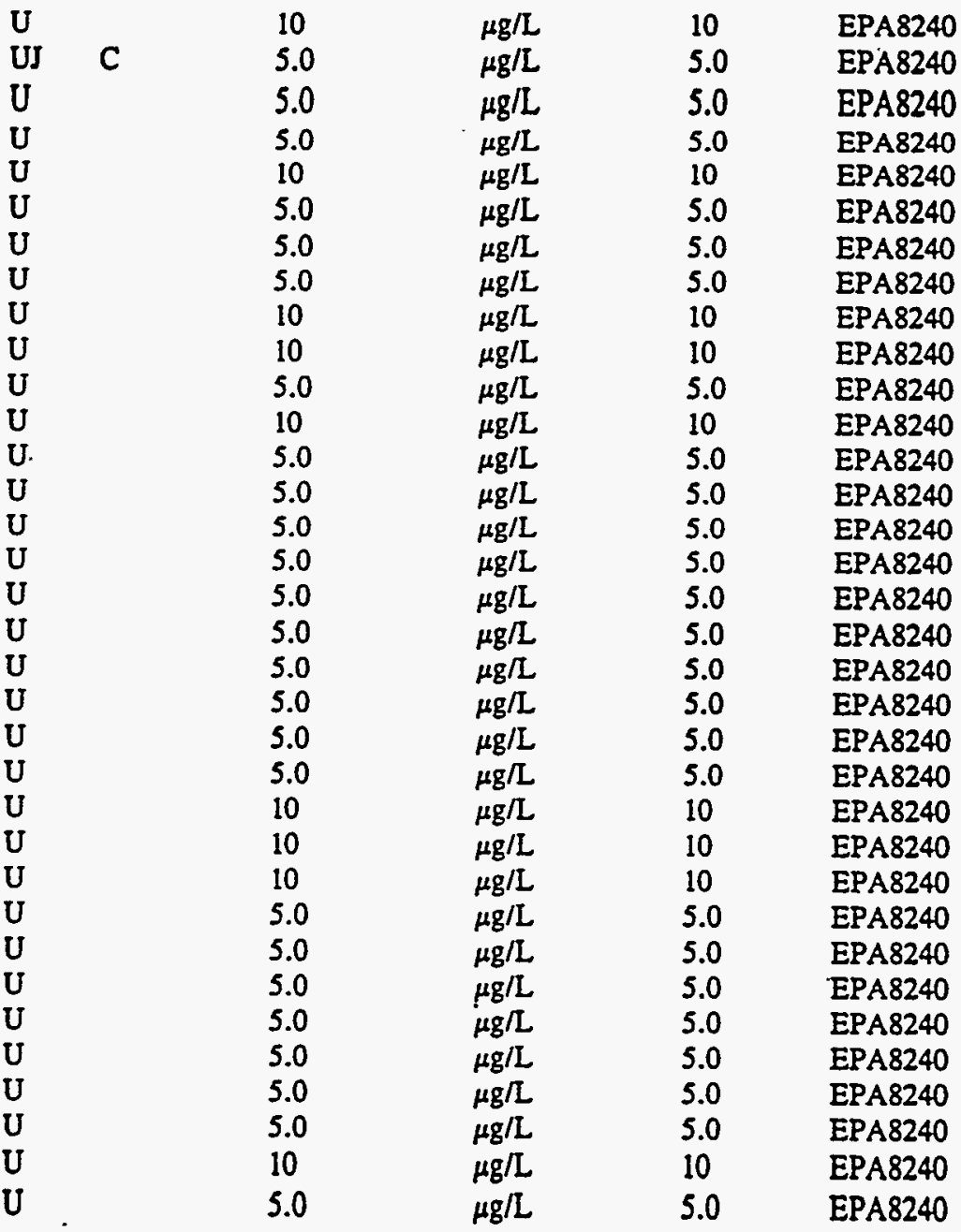




\section{SAMPLE NAME: D43111506}

Location (SRS Coordinates): 18533.07E 66779.25N

Ground Elevation Above MSL: $128.5 \mathrm{ft}$

Depth of Core Interval: 36.00 to $37.00 \mathrm{ft}$

Sample Type: Normal

Constituent

Volatile Organic Compounds

Acetone
Benzene
Bromodichloromethane
Bromoform
Bromomethane (Methyl bromide)
Carbon disulfide
Carbon tetrachloride
Chlorobenzene
Chloroethane
Chloroethene (Vinyl chloride)
2-Chloroethyl vinyl ether
Chloroform
Chloromethane (Methyl chloride)
Dibromochloromethane
1,1-Dichloroethane
1,2-Dichloroethane
1,1-Dichloroethylene
1,2-Dichloroethylene
Dichloromethane (Methylene chloride)
1,2-Dichloropropane
cis-1,3-Dichloropropene
trans-1,3-Dichloropropene
Ethylbenzene
2-Hexanone
Methyl ethyl ketone
Methyl isobutyl ketone
Styrene
1,1,2,2-Tetrachloroethane
Tetrachloroethylene
Toluene
1,1,1-Trichloroethane
1,1,2-Trichloroethane
Trichloroethylene
Unknown
Vinyl acetate
Xylenes -

Sample ID: 100414

Sample Matrix: Water

Sample Moisture: Liquid

RQ AQ

B Result

Unit

D. Limit Method

$\begin{array}{llllll}U & Y & 10 & \mu g / L & 10 & \text { EPA8240 } \\ U & Y & 5.0 & \mu g / L & 5.0 & \text { EPA8240 } \\ U & Y & 5.0 & \mu g / L & 5.0 & \text { EPA8240 } \\ U & Y & 5.0 & \mu g / L & 5.0 & \text { EPA8240 } \\ U & Y & 10 & \mu g / L & 10 & \text { EPA8240 } \\ U & Y & 5.0 & \mu g / L & 5.0 & \text { EPA8240 } \\ U & Y & 5.0 & \mu g / L & 5.0 & \text { EPA8240 } \\ U & Y & 5.0 & \mu g / L & 5.0 & \text { EPA8240 } \\ U & Y & 10 & \mu g / L & 10 & \text { EPA8240 } \\ U & Y & 10 & \mu g / L & 10 & \text { EPA8240 } \\ U & Y & 10 & \mu g / L & 10 & \text { EPA8240 } \\ U & Y & 5.0 & \mu g / L & 5.0 & \text { EPA8240 } \\ U & Y & 10 & \mu g / L & 10 & \text { EPA8240 } \\ U & Y & 5.0 & \mu g / L & 5.0 & \text { EPA8240 } \\ U & Y & 5.0 & \mu g / L & 5.0 & \text { EPA8240 } \\ U & Y & 5.0 & \mu g / L & 5.0 & \text { EPA8240 } \\ U & Y & 5.0 & \mu g / L & 5.0 & \text { EPA8240 } \\ U & Y & 5.0 & \mu g / L & 5.0 & \text { EPA8240 } \\ U & Y V & 4.1 & \mu g / L & 5.0 & \text { EPA8240 } \\ U & Y & 5.0 & \mu g / L & 5.0 & \text { EPA8240 } \\ U & Y & 5.0 & \mu g / L & 5.0 & \text { EPA8240 } \\ U & Y & 5.0 & \mu g / L & 5.0 & \text { EPA8240 } \\ U & Y & 5.0 & \mu g / L & 5.0 & \text { EPA8240 } \\ U & Y & 10 & \mu g / L & 10 & \text { EPA8240 } \\ U & Y & 10 & \mu g / L & 10 & \text { EPA8240 } \\ U & Y & 10 & \mu g / L & 10 & \text { EPA8240 } \\ U & Y & 5.0 & \mu g / L & 5.0 & \text { EPA8240 } \\ U & Y & 5.0 & \mu g / L & 5.0 & \text { EPA8240 } \\ U & Y & 5.0 & \mu g / L & 5.0 & \text { EPA8240 } \\ U & Y & 5.0 & \mu g / L & 5.0 & \text { EPA8240 } \\ U & Y & 5.0 & \mu g / L & 5.0 & \text { EPA8240 } \\ U & Y & 5.0 & \mu g / L & 5.0 & \text { EPA8240 } \\ U & Y & 5.0 & \mu g / L & 5.0 & \text { EPA8240 } \\ J & N & 10 & \mu g / L & & \text { EPA8240 } \\ U & Y & 10 & \mu g / L & 10 & \text { EPA8240 } \\ U & Y & 5.0 & \mu g / L & 5.0 & \text { EPA8240 }\end{array}$


SAMPLE NAME: D43ï11506D

Sample Type: Duplicate

Constituent

Volatile Organic Compounds
Sample DD: 100415

Associated Sample: 100414

RQ AQ B Result Unit D. Limit Method

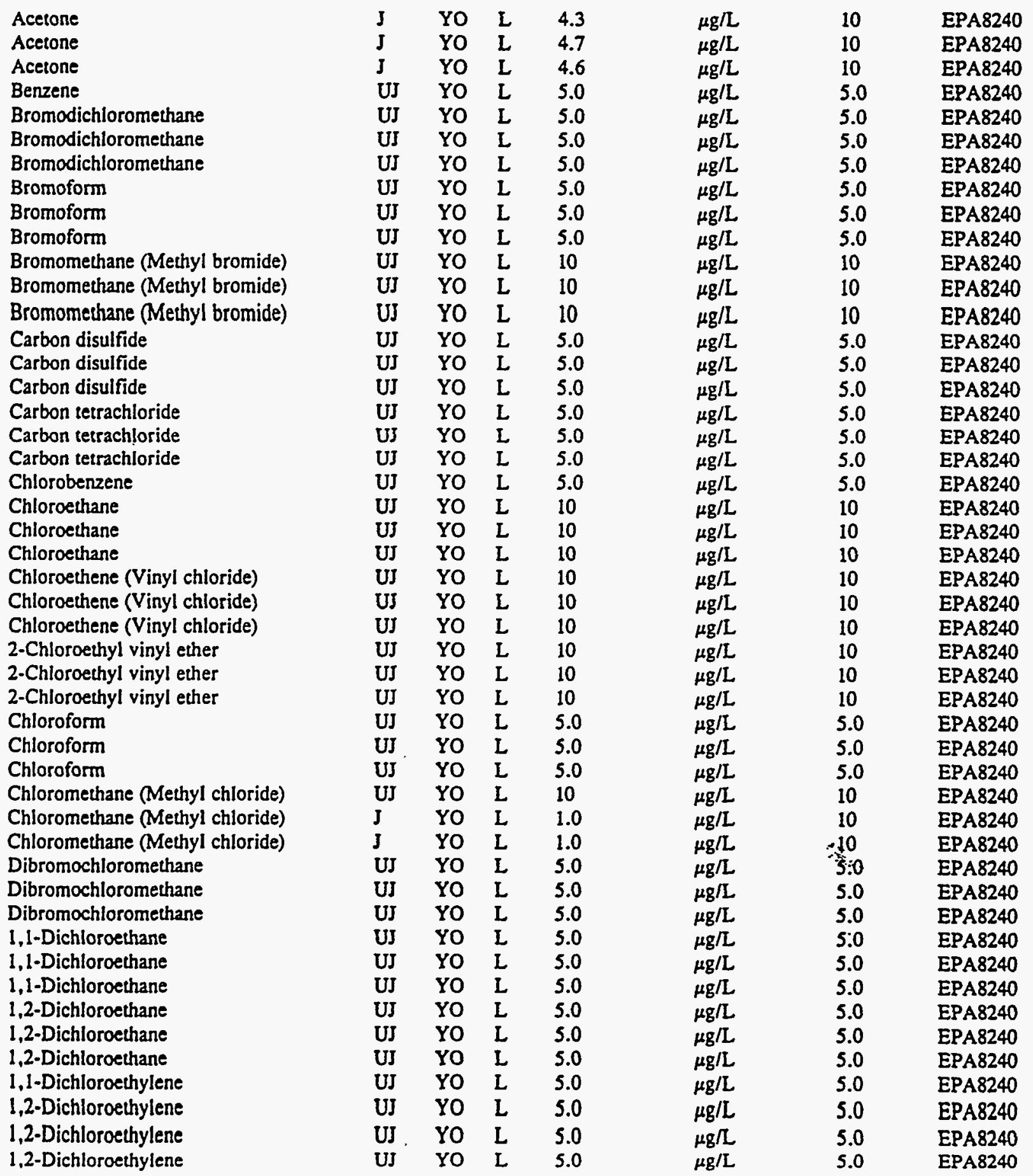


SAMPLE NAME: D43111506D (Continued)

Sample ID: 100415

Constituent

Volatile Organic Compounds

Dichloromethane (Methylene chloride)

Dichloromethane (Methylene chloride)

Dichloromethane (Methylene chloride)

1,2-Dichloropropane

1,2-Dichloropropane

1.2-Dichloropropane

cis-1,3-Dichloropropene

cis-1,3-Dichloropropene

cis-1,3-Dichloropropene

trans-1,3-Dichloropropene

trans-1,3-Dichloropropene

trans-1,3-Dichloropropene

Ethylbenzene

Ethylbenzene

Ethylbenzene

2-Hexanone

2-Hexanone

2-Hexanone

Methyl ethyl ketone

Methyl ethyl ketone

Methyl ethyl ketone

Methyl isobutyl ketone

Methyl isobutyl ketone

Methyl isobutyl ketone

Styrene

Styrene

Styrene

1,1,2,2-Tetrachloroethane

1,1,2,2-Tetrachloroethane

1,1.2,2-Tetrachloroethane

Tetrachloroethylene

Tetrachloroethylene

Tetrachloroethylene

Toluene

1,1,1-Trichloroethane

1,1,1-Trichloroethane

1,1.1-Trichloroethane

1,1,2-Trichloroethane

1,1,2-Trichloroethane

1,1,2-Trichloroethane

Trichloroethylene

Vinyl acetate

Vinyl acetate

Vinyl acetate

Xylenes

Xylenes

Xylenes

$\begin{array}{llll}\mathbf{R} & \mathbf{A Q} & \mathbf{B} & \text { Result }\end{array}$

Unit

D. Limit Method

U

UJ YO L 5.0

UJ YO L 5.0

UJ YO L 5.0

UJ YO $\mathrm{L} \quad 5.0$

UJ YO L 5.0

UJ YO L 5.0

UJ YO L 5.0

UJ YO L 5.0

UJ YO $L \quad 5.0$

UJ YO L 5.0

UJ YO L 5.0

UJ YO L 5.0

UJ YO L 5.0

UJ YO L 5.0

UJ YO L 10

UJ YO L 10

UJ YO L 10

UJ YO L 10

UJ YO L 10

UJ YO L 10

UJ YO L 10

UJ YO L 10

UJ YO L 10

UJ YO L 5.0

UJ YO L 5.0

UJ YO L 5.0

UJ YO L 5.0

UJ YO L 5.0

UJ YO L 5.0

UJ YO L 5.0

UJ YO L 5.0

UJ YO L 5.0

UJ YO L 5.0

UJ YO . L 5.0

UJ YO L 5.0

UJ YO L 5.0

UJ YO L 5.0

UJ YO L 5.0

UJ YO L 5.0

UJ YO L 5.0

UJ YO L 10

UJ YO $L \quad 10$

UJ YO L 10

UJ YO L 5.0

UJ YO L 5.0

UJ YO L 5.0

\begin{tabular}{|c|c|c|}
\hline$\mu \mathrm{g} / \mathrm{L}$ & 5.0 & EPA8240 \\
\hline$\mu g / L$ & 5.0 & EPA8240 \\
\hline$\mu \mathrm{g} / \mathrm{L}$ & 5.0 & EPA8240 \\
\hline$\mu \mathrm{g} / \mathrm{L}$ & 5.0 & EPA8240 \\
\hline$\mu g / L$ & 5.0 & EPA8240 \\
\hline$\mu \mathrm{g} / \mathrm{L}$ & 5.0 & EPA8240 \\
\hline$\mu \mathrm{g} / \mathrm{L}$ & 5.0 & EPA8240 \\
\hline$\mu \mathrm{g} / \mathrm{L}$ & 5.0 & EPA8240 \\
\hline$\mu \mathrm{g} / \mathrm{L}$ & 5.0 & EPA8240 \\
\hline$\mu \mathrm{g} / \mathrm{L}$ & 5.0 & EPA8240 \\
\hline$\mu \mathrm{g} / \mathrm{L}$ & 5.0 & EPA8240 \\
\hline$\mu \mathrm{g} / \mathrm{L}$ & 5.0 & EPA8240 \\
\hline$\mu \mathrm{g} / \mathrm{L}$ & 5.0 & EPA8240 \\
\hline$\mu \mathrm{g} / \mathrm{L}$ & 5.0 & EPA8240 \\
\hline$\mu \mathrm{g} / \mathrm{L}$ & 5.0 & EPA8240 \\
\hline$\mu \mathrm{g} / \mathrm{L}$ & 10 & EPA8240 \\
\hline$\mu \mathrm{g} / \mathrm{L}$ & 10 & EPA8240 \\
\hline$\mu \mathrm{g} / \mathrm{L}$ & 10 & EPA8240 \\
\hline$\mu \mathrm{g} / \mathrm{L}$ & 10 & EPA8240 \\
\hline$\mu \mathrm{g} / \mathrm{L}$ & 10 & EPA8240 \\
\hline$\mu \mathrm{g} / \mathrm{L}$ & 10 & EPA8240 \\
\hline$\mu \mathrm{g} / \mathrm{L}$ & 10 & EPA8240 \\
\hline$\mu \mathrm{g} / \mathrm{L}$ & 10 & EPA8240 \\
\hline$\mu \mathrm{g} / \mathrm{L}$ & 10 & EPA8240 \\
\hline$\mu g / L$ & 5.0 & EPA8240 \\
\hline$\mu \mathrm{g} / \mathrm{L}$ & 5.0 & EPA8240 \\
\hline$\mu \mathrm{g} / \mathrm{L}$ & 5.0 & EPA8240 \\
\hline$\mu \mathrm{g} / \mathrm{L}$ & 5.0 & EPA8240 \\
\hline$\mu \mathrm{g} / \mathrm{L}$ & 5.0 & EPA8240 \\
\hline$\mu \mathrm{g} / \mathrm{L}$ & 5.0 & EPA8240 \\
\hline$\mu \mathrm{g} / \mathrm{L}$ & 5.0 & EPA8240 \\
\hline$\mu \mathrm{g} / \mathrm{L}$ & 5.0 & EPA8240 \\
\hline$\mu g / L$ & 5.0 & EPA8240 \\
\hline$\mu \mathrm{g} / \mathrm{L}$ & 5.0 & EPA8240 \\
\hline$\mu g / L$ & 5.0 & EPA8240 \\
\hline$\mu \mathrm{g} / \mathrm{L}$ & 5.0 & EPA8240 \\
\hline$\mu \mathrm{g} / \mathrm{L}$ & 5.0 & EPA8240 \\
\hline$\mu g / L$ & 5.0 & EPA8240 \\
\hline$\mu \mathrm{g} / \mathrm{L}$ & 5.0 & EPA8240 \\
\hline$\mu \mathrm{g} / \mathrm{L}$ & 5.0 & EPA8240 \\
\hline$\mu \mathrm{g} / \mathrm{L}$ & 5.0 & EPA8240 \\
\hline$\mu g / L$ & 10 & EPA8240 \\
\hline$\mu \mathrm{g} / \mathrm{L}$ & 10 & EPA8240 \\
\hline$\mu \mathrm{g} / \mathrm{L}$ & 10 & EPA8240 \\
\hline$\mu \mathrm{g} / \mathrm{L}$ & 5.0 & EPA8240 \\
\hline$\mu g / L$ & 5.0 & EPA8240 \\
\hline$\mu g / L$ & 5.0 & EPA8240 \\
\hline
\end{tabular}


SAMPLE NAME: D43171607

Location (SRS Coordinates): $18644.34 \mathrm{E}$ 66570.29N

Ground Elevation Above MSL: $134.1 \mathrm{ft}$

Depth of Core Interval: 23.00 to $24.00 \mathrm{ft}$

Sample Type: Normal

Constituent

Volatile Organic Compounds

Acetone
Benzene
Bromodichloromethane
Bromoform
Bromomethane (Methyl bromide)
Carbon disulfide
Carbon tetrachloride
Chlorobenzene
Chloroethane
Chloroethene (Vinyl chloride)
2-Chloroethyl vinyl ether
Chloroform
Chloromethane (Methyl chloride)
Dibromochloromethane
1,1-Dichloroethane
1,2-Dichloroethane
1,1-Dichloroethylene
1,2-Dichloroethylene
Dichloromethane (Methylene chloride)
1,2-Dichloropropane
cis-1,3-Dichloropropene
trans-1,3-Dichloropropene
Ethylbenzene
2-Hexanone
Methyl ethyl ketone
Methyl isobutyl ketone
Styrene
1,1,2,2-Tetrachloroethane
Tetrachloroethylene
Toluene
1,1,1-Trichloroethane
1,1,2-Trichloroethane
Trichloroethylene
Vinyl acetate
Xylenes

Sample ID: 100425

Sample Matrix: Water

RQ AQ B Result Unit

Sample Moisture: Liquid

D. Limit Method

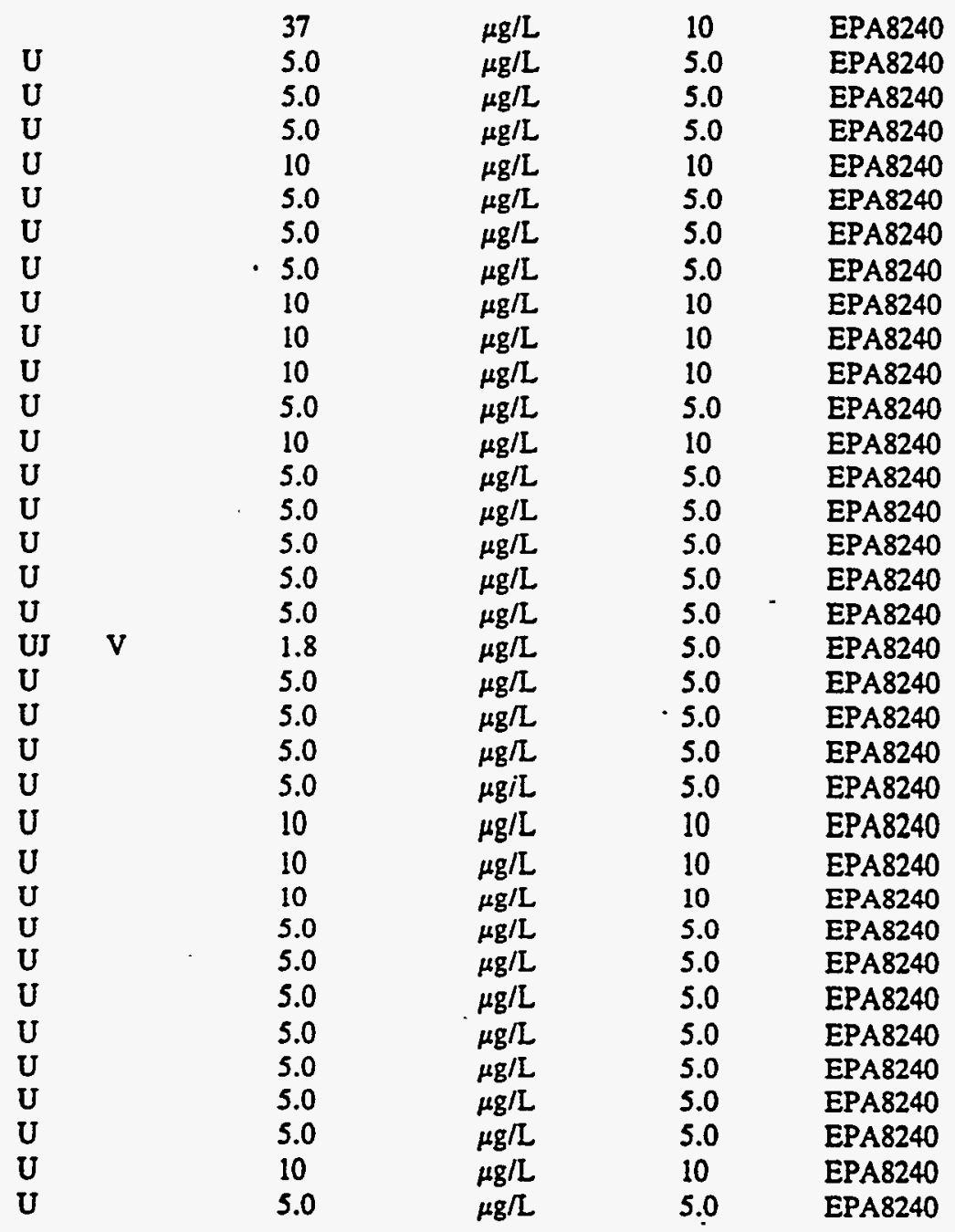


Location (SRS Coordinates): 18644.34E 66570.29N

Ground Elevation Above MSL: $134.1 \mathrm{ft}$

Depth of Core Interval: 39.00 to $40.00 \mathrm{ft}$

Sample Type: Normal

Sample Matrix: Water

Sample Moisture: Liquid

Constituent

RQ AQ B Result Unit

D. Limit Method

Volatile Organic Compounds

Acetone
Acetone
Benzene
Bromodichloromethane
Bromoform
Bromomethane (Methyl bromide)
Carbon disulfide
Carbon tetrachloride
Chlorobenzene
Chloroethane
Chloroethene (Vinyl chloride)
2-Chloroethyl vinyl ether
Chloroform
Chloromethane (Methyl chloride)
Dibromochloromethane
1,1-Dichloroethane
1,2-Dichloroethane
1,1-Dichloroethylene
1,2-Dichloroethylene
Dichloromethane (Methylene chloride)
1,2-Dichloropropane
cis-1,3-Dichloropropene
trans-1,3-Dichloropropene
Ethylbenzene
2-Hexanone
Methyl ethyl ketone
Methyl isobutyl ketone
Styrene
1,1,2,2-Tetrachloroethane
Tetrachloroethylene
Toluene
1,1,1-Trichloroethane
1,1,2-Trichloroethane
Trichloroethylene
Unknown
Vinyl acetate
Xylenes

\begin{tabular}{|c|c|c|}
\hline L & & $\begin{array}{l}1.200 \\
490\end{array}$ \\
\hline $\mathrm{U}$ & & $\begin{array}{l}450 \\
5.0\end{array}$ \\
\hline $\mathrm{U}$ & & 5.0 \\
\hline $\mathrm{U}$ & & 5.0 \\
\hline U & & 10 \\
\hline $\mathbf{U}$ & & 5.0 \\
\hline $\mathrm{U}$ & & 5.0 \\
\hline $\mathrm{U}$ & & 5.0 \\
\hline $\mathrm{U}$ & & 10 \\
\hline$U$ & & 10 \\
\hline $\mathrm{U}$ & & 10 \\
\hline $\mathrm{U}$ & & 5.0 \\
\hline $\mathrm{U}$ & & 10 \\
\hline $\mathrm{U}$ & & 5.0 \\
\hline $\mathrm{U}$ & & 5.0 \\
\hline $\mathrm{U}$ & & 5.0 \\
\hline $\mathrm{U}$ & & 5.0 \\
\hline $\mathrm{U}$ & & 5.0 \\
\hline & V & 1.8 \\
\hline $\mathrm{U}$ & & 5.0 \\
\hline $\mathbf{U}$ & & 5.0 \\
\hline $\mathrm{U}$ & & 5.0 \\
\hline $\mathrm{U}$ & & 5.0 \\
\hline $\mathrm{U}$ & & 10 \\
\hline $\mathrm{U}$ & & 10 \\
\hline $\mathrm{U}$ & & 10 \\
\hline $\mathrm{U}$ & & 5.0 \\
\hline $\mathrm{U}$ & & 5.0 \\
\hline $\mathrm{U}$ & & 5.0 \\
\hline $\mathbf{U}$ & & 5.0 \\
\hline $\mathrm{U}$ & & 5.0 \\
\hline$U$ & & 5.0 \\
\hline $\mathbf{U}$ & & 5.0 \\
\hline $\mathbf{J}$ & $\mathbf{N}$ & 90 \\
\hline $\mathbf{U}$ & & 10 \\
\hline $\mathrm{U}$ & & 5.0 \\
\hline
\end{tabular}

$\begin{array}{lll}\mu \mathrm{g} / \mathrm{L} & 10 & \text { EPA8240 } \\ \mu \mathrm{g} / \mathrm{L} & 50 & \text { EPA8240 } \\ \mu \mathrm{g} / \mathrm{L} & 5.0 & \text { EPA8240 } \\ \mu \mathrm{g} / \mathrm{L} & 5.0 & \text { EPA8240 } \\ \mu \mathrm{g} / \mathrm{L} & 5.0 & \text { EPA8240 } \\ \mu \mathrm{g} / \mathrm{L} & 10 & \text { EPA8240 } \\ \mu \mathrm{g} / \mathrm{L} & 5.0 & \text { EPA8240 } \\ \mu \mathrm{g} / \mathrm{L} & 5.0 & \text { EPA8240 } \\ \mu \mathrm{g} / \mathrm{L} & 5.0 & \text { EPA8240 } \\ \mu \mathrm{g} / \mathrm{L} & 10 & \text { EPA8240 } \\ \mu \mathrm{g} / \mathrm{L} & 10 & \text { EPA8240 } \\ \mu \mathrm{g} / \mathrm{L} & 10 & \text { EPA8240 } \\ \mu \mathrm{g} / \mathrm{L} & 5.0 & \text { EPA8240 } \\ \mu \mathrm{g} / \mathrm{L} & 10 & \text { EPA8240 } \\ \mu \mathrm{g} / \mathrm{L} & 5.0 & \text { EPA8240 } \\ \mu \mathrm{g} / \mathrm{L} & 5.0 & \text { EPA8240 } \\ \mu \mathrm{g} / \mathrm{L} & 5.0 & \text { EPA8240 } \\ \mu \mathrm{g} / \mathrm{L} & 5.0 & \text { EPA8240 } \\ \mu \mathrm{g} / \mathrm{L} & 5.0 & \text { EPA8240 } \\ \mu \mathrm{g} / \mathrm{L} & 5.0 & \text { EPA8240 } \\ \mu \mathrm{g} / \mathrm{L} & 5.0 & \text { EPA8240 } \\ \mu \mathrm{g} / \mathrm{L} & 5.0 & \text { EPA8240 } \\ \mu \mathrm{g} / \mathrm{L} & 5.0 & \text { EPA8240 } \\ \mu \mathrm{g} / \mathrm{L} & 5.0 & \text { EPA8240 } \\ \mu \mathrm{g} / \mathrm{L} & 10 & \text { EPA8240 } \\ \mu \mathrm{g} / \mathrm{L} & 10 & \text { EPA8240 } \\ \mu \mathrm{g} / \mathrm{L} & 10 & \text { EPA8240 } \\ \mu \mathrm{g} / \mathrm{L} & 5.0 & \text { EPA8240 } \\ \mu \mathrm{g} / \mathrm{L} & 5.0 & \text { EPA8240 } \\ \mu \mathrm{g} / \mathrm{L} & 5.0 & \text { EPA8240 } \\ \mu \mathrm{g} / \mathrm{L} & 5.0 & \text { EPA8240 } \\ \mu \mathrm{g} / \mathrm{L} & 5.0 & \text { EPA8240 } \\ \mu \mathrm{g} / \mathrm{L} & 5.0 & \text { EPA8240 } \\ \mu \mathrm{g} / \mathrm{L} & 5.0 & \text { EPA8240 } \\ \mu \mathrm{g} / \mathrm{L} & & \text { EPA8240 } \\ \mu \mathrm{g} / \mathrm{L} & 10 & \text { EPA8240 } \\ \mu \mathrm{g} / \mathrm{L} & 5.0 & \text { EPA8240 }\end{array}$


SAMPLE NAME: D43111901

Location (SRS Coordinates): .E .N

Grouhd Elevation Above MSL: . ft

Depth of Core Interval: . to . ft

Sample Type: Normal

Constituent

Physical Parameters

Chloride

Fluoride

Nitrate as nitrogen

$\mathrm{pH}$

Sulfate

Sulfate

Total dissolved solids

Total dissolved solids

Total organic carbon

Total organic halogens

Total suspended solids

Total suspended solids

Total Metals

Arsenic

Barium

Cadmium

Chromium

Copper

Iron

Lead

Manganese

Mercury

Nickel

Selenium

Silver

Sodium
Sample ID: 100308

Sample Matrix: Water

Sample Moisture: Liquid

RQ AQ B Result Unit

D. Limit Method

$B / N / A$ Extractables (including $P A H$ and phenols)

Acenaphthene

Acenaphthylene

Anthracene

Benzo(a)anthracene

Benzo(b)fluoranthene

Benzo(k)fluoranthene

Benzoic acid

Benzo(g,h,i)perylene

Benzo(a)pyrene

Benzyl alcohol

Bis(2-chloroethoxy) methane

Bis(2-chloroethyl) ether

Bis(2-chloroisopropyl) ether

Bis(2-ethylhexyl) phthalate

$\begin{array}{ccc} & & 5.1 \\ \mathrm{U} & & 0.10 \\ \mathrm{~J} & \mathrm{Q} & 0.21 \\ & & 5.9 \\ & & 6.4 \\ & & 6.5 \\ & & 56 \\ & & 63 \\ \mathrm{~J} & \mathrm{Q} & 1.9 \\ & & 7.2 \\ & & 10 \\ & & 11\end{array}$

$\mathrm{mg} / \mathrm{L}$

$\mathrm{mg} / \mathrm{L}$

$\mathrm{mg} / \mathrm{L}$

$\mathrm{pH}$

$\mathrm{mg} / \mathrm{L}$

$\mathrm{mg} / \mathrm{L}$

$\mathrm{mg} / \mathrm{L}$

$\mathrm{mg} / \mathrm{L}$

$\mathrm{mg} / \mathrm{L}$

$\mu \mathrm{g} / \mathrm{L}$

$\mathrm{mg} / \mathrm{L}$

$\mathrm{mg} / \mathrm{L}$

$\mu \mathrm{g} / \mathrm{L}$

$\mu g / L$

$\mu \mathrm{g} / \mathrm{L}$

$\mu g / L$

$\mu g / L$

$\mu \mathrm{g} / \mathrm{L}$

$\mu g / L$

$\mu \mathrm{g} / \mathrm{L}$

$\mu \mathrm{g} / \mathrm{L}$

$\mu \mathrm{g} / \mathrm{L}$

$\mu \mathrm{g} / \mathrm{L}$

$\mu g / L$

$\mu g / L$
0.25

0.10

0.10

0.010

5.0

5.0

5.0

5.0

0.50

5.0

5.0

5.0
EPA300.0

EPA300.0

EPA300.0

EPA9045

EPA300.0

EPA300.0

EPA160.2

EPA160.2

EPA415.1

EPA9020

EPA160.2

EPA160.2
10

200

5.0

10

25

100

3.0

15

0.20

40

5.0

10

5,000
EPA7060

EPA6010

EPA6010

EPA6010

EPA6010

EPA6010

EPA7421

EPA6010

EPA7470

EPA6010

EPA7740

EPA6010

EPA6010 
SAMPLE NAME: D43111901 (Continued)

Sample ID: 100308

Constituent

RQ AQ B Result

Unit

D. Limit Method

$B / N / A$ Extractables (including $P A H$ and phenols)

4-Bromophenyl phenyl ether

Butylbenzyl phthalate

4-Chloroaniline

4-Chloro-m-cresol

2-Chloronaphthalene

2-Chlorophenol

4-Chlorophenyl phenyl ether

Chrysene

o-Cresol (2-Methylphenol)

p-Cresol (4-Methylphenol)

Dibenz(a,h)anthracene

Dibenzofuran

Di-n-butyl phthalate

1.2-Dichlorobenzene

1,3-Dichlorobenzene

1.4-Dichlorobenzene

3,3'-Dichlorobenzidine

2,4-Dichlorophenol

Diethyl phthalate

2,4-Dimethyl phenol

Dimethyl phthalate

2,4-Dinitrophenol

2,4-Dinitrotoluene

2,6-Dinitrotoluene

Di-n-octyl phthalate

Fluoranthene

Fluorene

Hexachlorobenzene

Hexachlorobutadiene

Hexachlorocyclopentadiene

Hexachloroethane

Indeno(1,2,3-c,d)pyrene

Isophorone

2-Methyl-4,6-dinitrophenol

2-Methylnaphthalene

Naphthalene

o-Nitroaniline

m-Nitroaniline

p-Nitroaniline

Nitrobenzene

2-Nitrophenol

4-Nitrophenol

N-Nitrosodiphenylamine

$\mathrm{N}$-Nitrosodipropylamine

Pentachlorophenol

Phenanthrene

Phenol

Pyrene

1,2,4-Trichlorobenzene

2,4,5-Trichlorophenol
$\mathrm{U} \quad 12$

U 12

U 12

U 12

U 12

U 12

U 12

U 12

U 12

$\mathrm{U}$

U 12

12

UJ $\mathrm{V} \quad 3.5$

U 12

U 12

U 12

$U 24$

U 12

U 12

U 12

$\mathrm{U} \quad 12$

U 60

U 12

U 12

$\mathrm{U} \quad 12$

U 12

U 12

U 12

U 12

$\mathrm{U}$

$U-12$

$\mathrm{U}$

$\mathrm{U} \quad 12$

U 60

$\mathrm{U}$

U 12

U 60

U $\quad 60$

U 60

U 12

U 12

U 60

$\mathrm{U} \quad 12$

U 12

U 60

U 12

U 12

U 12

U 12

U 60 $\mu g / L$

$\mu \mathrm{g} / \mathrm{L}$

$\mu \mathrm{g} / \mathrm{L}$

$\mu g / L$

$\mu \mathrm{g} / \mathrm{L}$

$\mu \mathrm{g} / \mathrm{L}$

$\mu \mathrm{g} / \mathrm{L}$

$\mu \mathrm{g} / \mathrm{L}$

$\mu \mathrm{g} / \mathrm{L}$

$\mu \mathrm{g} / \mathrm{L}$

$\mu \mathrm{g} / \mathrm{L}$

$\mu \mathrm{g} / \mathrm{L}$

$\mu \mathrm{g} / \mathrm{L}$

$\mu \mathrm{g} / \mathrm{L}$

$\mu g / L$

$\mu g / L$

$\mu \mathrm{g} / \mathrm{L}$

$\mu \mathrm{g} / \mathrm{L}$

$\mu g / L$

$\mu \mathrm{g} / \mathrm{L}$

$\mu \mathrm{g} / \mathrm{L}$

$\mu g / L$

$\mu g / L$

$\mu \mathrm{g} / \mathrm{L}$

$\mu g / L$

$\mu \mathrm{g} / \mathrm{L}$

$\mu \mathrm{g} / \mathrm{L}$

$\mu \mathrm{g} / \mathrm{L}$

$\mu \mathrm{g} / \mathrm{L}$

$\mu \mathrm{g} / \mathrm{L}$

$\mu g / L$

$\mu \mathrm{g} / \mathrm{L}$

$\mu \mathrm{g} / \mathrm{L}$

$\mu g / L$

$\mu g / L$

$\mu \mathrm{g} / \mathrm{L}$

$\mu \mathrm{g} / \mathrm{L}$

$\mu g / L$

$\mu \mathrm{g} / \mathrm{L}$

$\mu \mathrm{g} / \mathrm{L}$

$\mu \mathrm{g} / \mathrm{L}$

$\mu \mathrm{g} / \mathrm{L}$

$\mu \mathrm{g} / \mathrm{L}$

$\mu g / L$

$\mu \mathrm{g} / \mathrm{L}$

$\mu \mathrm{g} / \mathrm{L}$

$\mu g / L$

$\mu \mathrm{g} / \mathrm{L}$

$\mu g / L$

$\mu \mathrm{g} / \mathrm{L}$
EPA8270

EPA8270

EPA8270

EPA8270

EPA8270

EPA8270

EPA8270

EPA8270

EPA8270

EPA8270

EPA8270

EPA8270

EPA8270

EPA8270

EPA8270

EPA8270

EPA8270

EPA8270

EPA8270

EPA8270

EPA8270

EPA8270

EPA8270

EPA8270

EPA8270

EPA8270

EPA8270

EPA8270

EPA8270

EPA8270

EPA8270

EPA8270

EPA8270

EPA8270

EPA8270

EPA8270

EPA8270

EPA8270

EPA8270

EPA8270

EPA8270

EPA8270

EPA8270

EPA8270

EPA8270

EPA8270

EPA8270

EPA8270

EPA8270

EPA8270 
SAMPLE NAME: D43111901 (Continued)

Sample D: 100308

Constituent

RQ AQ B Result Unit

D. Limit Method

$B / N / A$ Extractables (including $P A H$ and phenols)

2,4,6-Trichlorophenol

Unknown
J NV
12

20 $\mu g / L$

$\mu \mathrm{g} / \mathrm{L}$
EPA8270

EPA8270

Volatile Organic Compounds

Acetone
Acetone
Acetone
Benzene
Bromodichloromethane
Bromodichloromethane
Bromodichloromethane
Bromoform
Bromoform
Bromoform
Bromomethane (Methyl bromide)
Bromomethane (Methyl bromide)
Bromomethane (Methyl bromide)
Carbon disulfide
Carbon disulfide
Carbon disulfide
Carbon tetrachloride
Carbon tetrachloride
Carbon tetrachloride
Chlorobenzene
Chloroethane
Chloroethane
Chloroethane
Chloroethene (Vinyl chloride)
Chloroethene (Vinyl chloride)
Chloroethene (Vinyl chloride)
2-Chloroethyl vinyl ether
2-Chloroethyl vinyl ether
2-Chlorothyl vinyl ether
Chloroform
Chloroform
Chloroform
Chloromethane (Methyl chloride)
Chloromethane (Methyl chloride)
Chloromethane (Methyl chloride)
Dibromochloromethane
Dibromochloromethane
Dibromochloromethane
1,1-Dichloroethane
1,1-Dichloroethane
1,-Dichloroethane

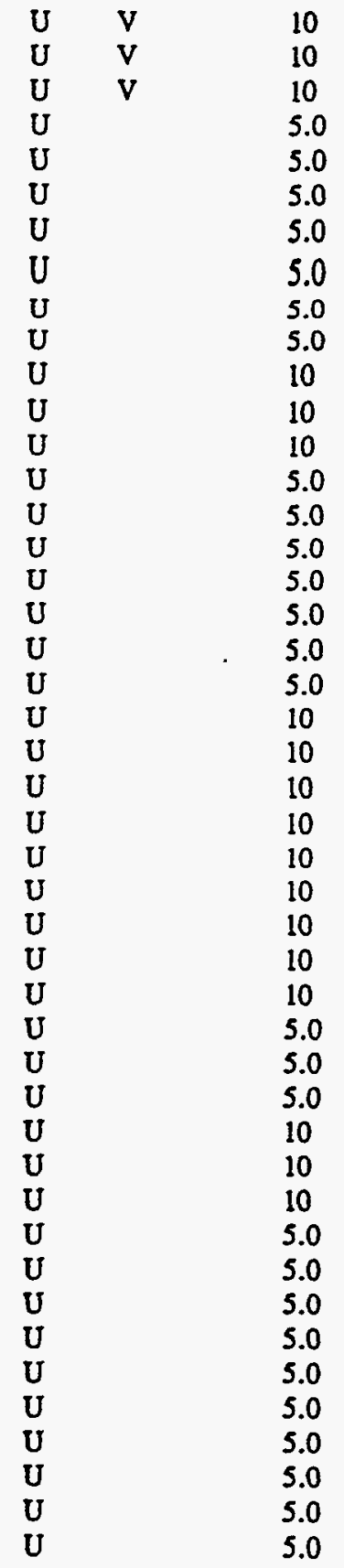

\begin{tabular}{|c|c|c|}
\hline$\mu \mathrm{g} / \mathrm{L}$ & 10 & EPA8240 \\
\hline$\mu \mathrm{g} / \mathrm{L}$ & 10 & EPA8240 \\
\hline$\mu \mathrm{g} / \mathrm{L}$ & 10 & EPA 8240 \\
\hline$\mu \mathrm{g} / \mathrm{L}$ & 5.0 & EPA8240 \\
\hline$\mu g / L$ & 5.0 & EPA8240 \\
\hline$\mu g / L$ & 5.0 & EPA8240 \\
\hline$\mu g / L$ & 5.0 & EPA8240 \\
\hline$\mu g / L$ & 5.0 & EPA8240 \\
\hline$\mu g / L$ & 5.0 & EPA8240 \\
\hline$\mu g / L$ & 5.0 & EPA8240 \\
\hline$\mu g / L$ & 10 & EPA8240 \\
\hline$\mu \mathrm{g} / \mathrm{L}$ & 10 & EPA8240 \\
\hline$\mu \mathrm{g} / \mathrm{L}$ & 10 & EPA8240 \\
\hline$\mu \mathrm{g} / \mathrm{L}$ & 5.0 & EPA8240 \\
\hline$\mu g / L$ & 5.0 & EPA8240 \\
\hline$\mu g / L$ & 5.0 & EPA8240 \\
\hline$\mu \mathrm{g} / \mathrm{L}$ & 5.0 & EPA8240 \\
\hline$\mu \mathrm{g} / \mathrm{L}$ & 5.0 & EPA8240 \\
\hline$\mu \mathrm{g} / \mathrm{L}$ & 5.0 & EPA8240 \\
\hline$\mu \mathrm{g} / \mathrm{L}$ & 5.0 & EPA8240 \\
\hline$\mu g / L$ & 10 & EPA8240 \\
\hline$\mu \mathrm{g} / \mathrm{L}$ & 10 & EPA8240 \\
\hline$\mu \mathrm{g} / \mathrm{L}$ & 10 & EPA8240 \\
\hline$\mu \mathrm{g} / \mathrm{L}$ & 10 & EPA 8240 \\
\hline$\mu \mathrm{g} / \mathrm{L}$ & 10 & EPA8240 \\
\hline$\mu \mathrm{g} / \mathrm{L}$ & 10 & EPA8240 \\
\hline$\mu g / L$ & 10 & EPA8240 \\
\hline$\mu g / L$ & 10 & EPA8240 \\
\hline$\mu g / L$ & 10 & EPA8240 \\
\hline$\mu g / L$ & 5.0 & EPA8240 \\
\hline$\mu g / L$ & 5.0 & EPA8240 \\
\hline$\mu g / L$ & 5.0 & EPA8240 \\
\hline$\mu \mathrm{g} / \mathrm{L}$ & 10 & EPA8240 \\
\hline$\mu g / L$ & 10 & EPA8240 \\
\hline$\mu \mathrm{g} / \mathrm{L}$ & 10 & EPA8240 \\
\hline$\mu g / L$ & 5.0 & EPA8240 \\
\hline$\mu g / L$ & 5.0 & EPA8240 \\
\hline$\mu \mathrm{g} / \mathrm{L}$ & 5.0 & EPA8240 \\
\hline$\mu g / L$ & 5.0 & EPA8240 \\
\hline$\mu g / L$ & 5.0 & EPA8240 \\
\hline$\mu g / L$ & 5.0 & EPA8240 \\
\hline$\mu g / L$ & 5.0 & EPA8240 \\
\hline$\mu g / L$ & 5.0 & EPA 8240 \\
\hline$\mu g / L$ & 5.0 & EPA8240 \\
\hline$\mu \mathrm{g} / \mathrm{L}$ & 5.0 & EPA8240 \\
\hline
\end{tabular}


Constituent

Volatile Organic Compounds
1,2-Dichloroechylene

1,2-Dichloroethylene

1,2-Dichloroethylene

Dichloromethane (Methylene chloride)

Dichloromethane (Methylene chloride)

Dichloromethane (Methylene chloride)

1,2-Dichloropropane

1,2-Dichloropropane

1,2-Dichloropropane

cis-1,3-Dichloropropene

cis-1,3-Dichloropropene

cis-1,3-Dichloropropene

trans-1.3-Dichloropropene

trans-1,3-Dichloropropene

trans-1,3-Dichloropropene

Ethylbenzene

Ethylbenzene

Ethylbenzene

2-Hexanone

2-Hexanone

2-Hexanone

Methyl ethyl ketone

Methyl ethyl ketone

Methyl ethyl ketone

Methyl isobutyl ketone

Methyl isobutyl ketone

Methyl isobutyl ketone

Styrene

Styrene

Styrene

1,1,2,2-Tetrachloroethane

1,1,2,2-Tetrachloroethane

1,1,2,2-Tetrachloroethane

Tetrachloroethylene

Tetrachloroethylene

Tetrachloroethylene

Toluene

1,1,1-Trichloroethane

1,1,1-Trichloroethane

1,1,1-Trichloroethane

1,1,2-Trichloroethane

1,1,2-Trichloroethane

1,1,2-Trichloroethane

Trichloroethylene

Vinyl acetate

Vinyl acetate

Vinyl acetate

Xylenes

Xylenes

Xylenes

\begin{tabular}{|c|c|c|}
\hline $\mathbf{U}$ & & 5.0 \\
\hline $\mathbf{U}$ & & 5.0 \\
\hline$U$ & & 5.0 \\
\hline UJ & V & 2.4 \\
\hline UJ & V & 3.5 \\
\hline UJ & V & 3.0 \\
\hline $\mathbf{U}$ & & 5.0 \\
\hline U & & 5.0 \\
\hline $\mathbf{U}$ & & 5.0 \\
\hline $\mathrm{U}$ & & 5.0 \\
\hline $\mathrm{U}$ & & 5.0 \\
\hline $\mathbf{U}$ & & 5.0 \\
\hline$U$ & & 5.0 \\
\hline $\mathbf{U}$ & & 5.0 \\
\hline $\mathbf{U}$ & & 5.0 \\
\hline $\mathrm{U}$ & & 5.0 \\
\hline $\mathrm{U}$ & & 5.0 \\
\hline $\mathrm{U}$ & & 5.0 \\
\hline$U$ & & 10 \\
\hline $\mathbf{U}$ & & 10 \\
\hline$U$ & & 10 \\
\hline $\mathbf{U}$ & & 10 \\
\hline $\mathrm{U}$ & & 10 \\
\hline $\mathbf{U}$ & & 10 \\
\hline $\mathrm{U}$ & & 10 \\
\hline $\mathrm{U}$ & & 10 \\
\hline $\mathbf{U}$ & & 10 \\
\hline 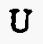 & & 5.0 \\
\hline U & & 5.0 \\
\hline $\mathrm{U}$ & . & 5.0 \\
\hline $\mathbf{U}$ & & 5.0 \\
\hline $\mathrm{U}$ & & 5.0 \\
\hline $\mathrm{U}$ & & 5.0 \\
\hline $\mathrm{U}$ & & 5.0 \\
\hline $\mathbf{U}$ & & 5.0 \\
\hline $\mathbf{U}$ & & 5.0 \\
\hline U & & 5.0 \\
\hline U & & 5.0 \\
\hline $\mathrm{U}$ & & 5.0 \\
\hline $\mathrm{U}$ & & 5.0 \\
\hline $\mathbf{U}$ & & 5.0 \\
\hline $\mathbf{U}$ & & 5.0 \\
\hline $\mathbf{U}$ & & 5.0 \\
\hline $\mathrm{U}$ & & 5.0 \\
\hline $\mathbf{U}$ & & 10 \\
\hline U & & 10 \\
\hline $\mathrm{U}$ & & 10 \\
\hline $\mathbf{U}$ & & 5.0 \\
\hline $\mathbf{U}$ & & 5.0 \\
\hline$U$ & & 5.0 \\
\hline
\end{tabular}

\begin{tabular}{|c|c|c|}
\hline$\mu \mathrm{g} / \mathrm{L}$ & 5.0 & EPA8240 \\
\hline$\mu \mathrm{g} / \mathrm{L}$ & 5.0 & EPA8240 \\
\hline$\mu g / L$ & 5.0 & EPA8240 \\
\hline$\mu \mathrm{g} / \mathrm{L}$ & 5.0 & EPA8240 \\
\hline$\mu \mathrm{g} / \mathrm{L}$ & 5.0 & EPA8240 \\
\hline$\mu g / L$ & 5.0 & EPA8240 \\
\hline$\mu \mathrm{g} / \mathrm{L}$ & 5.0 & EPA8240 \\
\hline$\mu g / L$ & 5.0 & EPA8240 \\
\hline$\mu \mathrm{g} / \mathrm{L}$ & 5.0 & EPA8240 \\
\hline$\mu \mathrm{g} / \mathrm{L}$ & 5.0 & EPA8240 \\
\hline$\mu \mathrm{g} / \mathrm{L}$ & 5.0 & EPA8240 \\
\hline$\mu g / L$ & 5.0 & EPA8240 \\
\hline$\mu g / L$ & 5.0 & EPA8240 \\
\hline$\mu \mathrm{g} / \mathrm{L}$ & 5.0 & EPA8240 \\
\hline$\mu \mathrm{g} / \mathrm{L}$ & 5.0 & EPA8240 \\
\hline$\mu \mathrm{g} / \mathrm{L}$ & 5.0 & EPA8240 \\
\hline$\mu \mathrm{g} / \mathrm{L}$ & 5.0 & EPA8240 \\
\hline$\mu \mathrm{g} / \mathrm{L}$ & 5.0 & EPA8240 \\
\hline$\mu \mathrm{g} / \mathrm{L}$ & 10 & EPA8240 \\
\hline$\mu g / L$ & 10 & EPA8240 \\
\hline$\mu g / L$ & 10 & EPA8240 \\
\hline$\mu \mathrm{g} / \mathrm{L}$ & 10 & EPA8240 \\
\hline$\mu g / L$ & 10 & EPA8240 \\
\hline$\mu \mathrm{g} / \mathrm{L}$ & 10 & EPA8240 \\
\hline$\mu g / L$ & 10 & EPA8240 \\
\hline$\mu \mathrm{g} / \mathrm{L}$ & 10 & EPA8240 \\
\hline$\mu \mathrm{g} / \mathrm{L}$ & 10 & EPA8240 \\
\hline$\mu \mathrm{g} / \mathrm{L}$ & 5.0 & EPA8240 \\
\hline$\mu g / L$ & 5.0 & EPA8240 \\
\hline$\mu \mathrm{g} / \mathrm{L}$ & 5.0 & EPA8240 \\
\hline$\mu \mathrm{g} / \mathrm{L}$ & 5.0 & EPA8240 \\
\hline$\mu \mathrm{g} / \mathrm{L}$ & 5.0 & EPA8240 \\
\hline$\mu g / L$ & 5.0 & EPA8240 \\
\hline$\mu \mathrm{g} / \mathrm{L}$ & 5.0 & EPA8240 \\
\hline$\mu g / L$ & 5.0 & EPA8240 \\
\hline$\mu \mathrm{g} / \mathrm{L}$ & 5.0 & EPA8240 \\
\hline$\mu g / L$ & 5.0 & EPA8240 \\
\hline$\mu \mathrm{g} / \mathrm{L}$ & 5.0 & EPA8240 \\
\hline$\mu g / L$ & 5.0 & EPA8240 \\
\hline$\mu \mathrm{g} / \mathrm{L}$ & 5.0 & EPA8240 \\
\hline$\mu \mathrm{g} / \mathrm{L}$ & $5: 0$ & EPA8240 \\
\hline$\mu \mathrm{g} / \mathrm{L}$ & 5.0 & EPA8240 \\
\hline$\mu g / L$ & 5.0 & EPA8240 \\
\hline$\mu \mathrm{g} / \mathrm{L}$ & 5.0 & EPA8240 \\
\hline$\mu g / L$ & 10 & EPA8240 \\
\hline$\mu g / L$ & 10 & EPA8240 \\
\hline$\mu g / L$ & 10 & EPA8240 \\
\hline$\mu g / L$ & 5.0 & EPA8240 \\
\hline$\mu g / L$ & 5.0 & EPA8240 \\
\hline$\mu \rho / L$ & 5.0 & EPA8240 \\
\hline
\end{tabular}


Radionuclide Indicators and Radionuclides

Gross alpha

Nonvolatile beta

Radium, total alpha-emitting

Tritium

$\begin{array}{ll}\mathbf{U} & \\ \mathbf{U} & \end{array}$

SAMPLE NAME: D43111902

Location (SRS Coordinates): .E .N .

Ground Elevation Above MSL: . ft

Depth of Core Interval: . to . $\mathfrak{f t}$

Sample Type: Normal

\section{Constituent}

Physical Parameters

$\mathrm{pH}$

$\mathrm{pH}$

Total dissolved solids

Total organic carbon

Total suspended solids

Total Metals

Arsenic.

Barium

Cadmium

Chromium

Copper

Iron

Lead

Manganese

Mercury

Nickel

Selenium

Silver

Sodium
Sample Matrix: Water

RQ AQ B Result Unit
Sample Moisture: Liquid

D. Limit Method

$B / N / A$ Extractables (including $P A H$ and phenols)

Acenaphthene

Acenaphthylene

Anthracene

Benzo(a)anthracene

Benzo(b)fluoranthene

Benzo(k)fluoranthene

Benzoic acid

Benzo(g,h,i)perylene

Benzo(a)pyrene

Benzyl alcohol

Bis(2-chlorocthoxy) methane

Bis(2-chloroethyl) ether

$\begin{array}{lll}J & Q & 6.0 \\ J & Q & 6.0 \\ J & Q & 65 \\ & & 1.6 \\ \text { UJ } & Q & 5.0\end{array}$

6.0

6.0

1.6

5.0

pH

$\mathrm{pH}$

$\mathrm{mg} / \mathrm{L}$

$\mathrm{mg} / \mathrm{L}$

$\mathrm{mg} / \mathrm{L}$

$\mu \mathrm{g} / \mathrm{L}$

$\mu \mathrm{g} / \mathrm{L}$

$\mu \mathrm{g} / \mathrm{L}$

$\mu \mathrm{g} / \mathrm{L}$

$\mu \mathrm{g} / \mathrm{L}$

$\mu \mathrm{g} / \mathrm{L}$

$\mu \mathrm{g} / \mathrm{L}$

$\mu g / L$

$\mu \mathrm{g} / \mathrm{L}$

$\mu \mathrm{g} / \mathrm{L}$

$\mu \mathrm{g} / \mathrm{L}$

$\mu g / L$

$\mu \mathrm{g} / \mathrm{L}$
0.80

0.80

0.10

EPA900.0

EPA900.0

EPA903.0

EPA906.0 
SAMPLE NAME: D43r11902 (Continued)

Sample ID: 100309

$\begin{array}{llllll}\text { Constituent } & \text { RQ } & \text { AQ } & \text { Result } & \text { Unit Limit Method }\end{array}$

$B / N / A$ Extractables (including $P A H$ and phenols)

\begin{tabular}{|c|c|c|c|c|c|c|}
\hline Bis(2-chloroisopropyl) ether & $U$ & & 12 & $\mu g / L$ & 12 & EPA8270 \\
\hline Bis(2-ethylhexyl) phthalate & UJ & v & 2.5 & $\mu \mathrm{g} / \mathrm{L}$ & 12 & EPA8270 \\
\hline 4-Bromophenyl phenyl ether & $\mathrm{U}$ & & 12 & $\mu \mathrm{g} / \mathrm{L}$ & 12 & EPA8270 \\
\hline Butylbenzyl phthalate & $\mathbf{U}$ & & 12 & $\mu \mathrm{g} / \mathrm{L}$ & 12 & EPA8270 \\
\hline 4-Chloroaniline & $\mathrm{U}$ & & 12 & $\mu \mathrm{g} / \mathrm{L}$ & 12 & EPA8270 \\
\hline 4-Chloro-m-cresol & $U$ & & 12 & $\mu \mathrm{g} / \mathrm{L}$ & 12 & EPA8270 \\
\hline 2-Chloronaphthalene & $\mathrm{U}$ & & 12 & $\mu \mathrm{g} / \mathrm{L}$ & 12 & EPA8270 \\
\hline 2-Chlorophenol & $\mathrm{U}$ & & 12 & $\mu \mathrm{g} / \mathrm{L}$ & 12 & EPA8270 \\
\hline 4-Chlorophenyl phenyl ether & $\mathrm{U}$ & & 12 & $\mu \mathrm{g} / \mathrm{L}$ & 12 & EPA8270 \\
\hline Chrysene & $\mathrm{U}$ & & 12 & $\mu \mathrm{g} / \mathrm{L}$ & 12 & EPA8270 \\
\hline o-Cresol (2-Methylphenol) & $\mathrm{U}$ & & 12 & $\mu \mathrm{g} / \mathrm{L}$ & 12 & EPA8270 \\
\hline p-Cresol (4-Methylphenol) & U & & 12 & $\mu g / L$ & 12 & EPA8270 \\
\hline Dibenz(a.h)anthracene & $\mathrm{U}$ & & 12 & $\mu \mathrm{g} / \mathrm{L}$ & 12 & EPA8270 \\
\hline Dibenzofuran & $\mathrm{U}$ & & 12 & $\mu \mathrm{g} / \mathrm{L}$ & 12 & EPA8270 \\
\hline Di-n-butyl phthalate & UJ & $\mathrm{v}$ & 3.4 & $\mu \mathrm{g} / \mathrm{L}$ & 12 & EPA8270 \\
\hline 1,2-Dichlorobenzene & $\mathrm{U}$ & & 12 & $\mu \mathrm{g} / \mathrm{L}$ & 12 & EPA8270 \\
\hline 1,3-Dichlorobenzene & $\mathrm{U}$ & & 12 & $\mu \mathrm{g} / \mathrm{L}$ & 12 & EPA8270 \\
\hline 1,4-Dichlorobenzene & U & & 12 & $\mu \mathrm{g} / \mathrm{L}$ & 12 & EPA8270 \\
\hline 3,3'-Dichlorobenzidine & $\mathrm{U}$ & & 24 & $\mu \mathrm{g} / \mathrm{L}$ & 24 & EPA8270 \\
\hline 2,4-Dichlorophenol & $\mathbf{U}$ & & 12 & $\mu \mathrm{g} / \mathrm{L}$ & 12 & EPA8270 \\
\hline Diethyl phthalate & $\mathrm{U}$ & & 12 & $\mu \mathrm{g} / \mathrm{L}$ & 12 & EPA8270 \\
\hline 2,4-Dimethyl phenol & $\mathrm{U}$ & & 12 & $\mu \mathrm{g} / \mathrm{L}$ & 12 & EPA8270 \\
\hline Dimethyl phehalate & $\mathrm{U}$ & & 12 & $\mu \mathrm{g} / \mathrm{L}$ & 12 & EPA8270 \\
\hline 2,4-Dinitrophenol & $\mathbf{U}$ & & 60 & $\mu \mathrm{g} / \mathrm{L}$ & 60 & EPA8270 \\
\hline 2,4-Dinitrotoluene & $\mathrm{U}$ & & 12 & $\mu \mathrm{g} / \mathrm{L}$ & 12 & EPA8270 \\
\hline 2,6-Dinitrotoluene & $\mathrm{U}$ & & 12 & $\mu \mathrm{g} / \mathrm{L}$ & 12 & EPA8270 \\
\hline Di-n-octyl phthalate & $\mathrm{U}$ & & 12 & $\mu \mathrm{g} / \mathrm{L}$ & 12 & EPA8270 \\
\hline Fluoranthene & $\mathrm{U}$ & & 12 & $\mu \mathrm{g} / \mathrm{L}$ & 12 & EPA8270 \\
\hline Fluorene & $\mathrm{U}$ & & 12 & $\mu \mathrm{g} / \mathrm{L}$ & 12 & EPA8270 \\
\hline Hexachlorobenzene & $\mathbf{U}$ & & 12 & $\mu \mathrm{g} / \mathrm{L}$ & 12 & EPA8270 \\
\hline Hexachlorobutadiene & $\mathrm{U}$ & & 12 & $\mu \mathrm{g} / \mathrm{L}$ & 12 & EPA8270 \\
\hline Hexachlorocyclopentadiene & $\mathrm{U}$ & & 12 & $\mu \mathrm{g} / \mathrm{L}$ & 12 & EPA8270 \\
\hline Hexachloroethane & $\mathbf{U}$ & & 12 & $\mu \mathrm{g} / \mathrm{L}$ & 12 & EPA8270 \\
\hline Indeno $(1,2,3-c, d)$ pyrene & $\mathrm{U}$ & & 12 & $\mu \mathrm{g} / \mathrm{L}$ & 12 & EPA8270 \\
\hline Isophorone & $\mathbf{U}$ & & 12 & $\mu \mathrm{g} / \mathrm{L}$ & 12 & EPA8270 \\
\hline 2-Methyl-4,6-dinitrophenol & $\mathrm{U}$ & & 60 & $\mu \mathrm{g} / \mathrm{L}$ & 60 & EPA8270 \\
\hline 2-Methylnaphthalene & $\mathrm{U}$ & & 12 & $\mu \mathrm{g} / \mathrm{L}$ & 12 & EPA8270 \\
\hline Naphthalene & $\mathrm{U}$ & & 12 & $\mu \mathrm{g} / \mathrm{L}$ & 12. & EPA8270 \\
\hline o-Nitroaniline & U & & 60 & $\mu g / L$ & $60^{\circ}$ & EPA8270 \\
\hline m-Nitroaniline & $\mathrm{U}$ & & 60 & $\mu \mathrm{g} / \mathrm{L}$ & 60 & EPA8270 \\
\hline p-Nitroaniline & $\mathbf{U}$ & & 60 & $\mu \mathrm{g} / \mathrm{L}$ & $60^{\circ}$ & EPA8270 \\
\hline Nitrobenzene & U & & 12 & $\mu \mathrm{g} / \mathrm{L}$ & 12 & EPA8270 \\
\hline 2-Nitrophenol & $\mathbf{U}$ & & 12 & $\mu \mathrm{g} / \mathrm{L}$ & 12 & EPA8270 \\
\hline 4-Nitrophenol & U & & 60 & $\mu \mathrm{g} / \mathrm{L}$ & 60 & EPA8270 \\
\hline N-Nitrosodiphenylamine & $\mathrm{U}$ & & 12 & $\mu \mathrm{g} / \mathrm{L}$ & 12 & EPA8270 \\
\hline N-Nitrosodipropylamine & $\mathbf{U}$ & & 12 & $\mu \mathrm{g} / \mathrm{L}$ & 12 & EPA8270 \\
\hline Pentachlorophenol & $\mathbf{U}$ & & 60 & $\mu \mathrm{g} / \mathrm{L}$ & 60 & EPA8270 \\
\hline Phenanthrene & $\mathbf{U}$ & & 12 & $\mu \mathrm{g} / \mathrm{L}$ & 12 & EPA8270 \\
\hline Phenol & U & & 12 & $\mu \mathrm{g} / \mathrm{L}$ & 12 & EPA8270 \\
\hline Pyrene & $\mathbf{U}$ & & 12 & $\mu \mathrm{g} / \mathrm{L}$ & 12 & EPA8270 \\
\hline
\end{tabular}


SAMPLE NAME: D431̈11902 (Continued)

Sample ID: 100309

$\begin{array}{lllll}\text { Constituent } & \text { RQ AQ B Result Unit D. Limit Method }\end{array}$

$B / N / A$ Extractobles (including PAH and phenols)

1.2,4-Trichlorobenzene

2,4,5-Trichlorophenol

2,4,6-Trichlorophenol

Unknown

Unknown

Unknown

Volatile Organic Compounds

\begin{tabular}{lll}
$\mathrm{U}$ & & 12 \\
$\mathrm{U}$ & & 60 \\
$\mathrm{U}$ & & 12 \\
$\mathrm{~J}$ & $\mathrm{NV}$ & 6.0 \\
$\mathrm{~J}$ & $\mathrm{NV}$ & 9.0 \\
\hline & $\mathrm{NV}$ &
\end{tabular}

NV $\quad 10$

12

60

12

6.0

10 $\mu g / L$

$\mu g / L$

$\mu \mathrm{g} / \mathrm{L}$

$\mu \mathrm{g} / \mathrm{L}$

$\mu g / L$

$\mu \mathrm{g} / \mathrm{L}$
EPA8270

EPA8270

EPA8270

EPA8270

EPA8270

EPA8270

$\begin{array}{lll}\mu \mathrm{g} / \mathrm{L} & 10 & \text { EPA8240 } \\ \mu \mathrm{g} / \mathrm{L} & 5.0 & \text { EPA8240 } \\ \mu \mathrm{g} / \mathrm{L} & 5.0 & \text { EPA8240 } \\ \mu \mathrm{g} / \mathrm{L} & 5.0 & \text { EPA8240 } \\ \mu \mathrm{g} / \mathrm{L} & 10 & \text { EPA8240 } \\ \mu \mathrm{g} / \mathrm{L} & 5.0 & \text { EPA8240 } \\ \mu \mathrm{g} / \mathrm{L} & 5.0 & \text { EPA8240 } \\ \mu \mathrm{g} / \mathrm{L} & 5.0 & \text { EPA8240 } \\ \mu \mathrm{g} / \mathrm{L} & 10 & \text { EPA8240 } \\ \mu \mathrm{g} / \mathrm{L} & 10 & \text { EPA8240 } \\ \mu \mathrm{g} / \mathrm{L} & 10 & \text { EPA8240 } \\ \mu \mathrm{g} / \mathrm{L} & 5.0 & \text { EPA8240 } \\ \mu \mathrm{g} / \mathrm{L} & 10 & \text { EPA8240 } \\ \mu \mathrm{g} / \mathrm{L} & 5.0 & \text { EPA8240 } \\ \mu \mathrm{g} / \mathrm{L} & 5.0 & \text { EPA8240 } \\ \mu \mathrm{g} / \mathrm{L} & 5.0 & \text { EPA8240 } \\ \mu \mathrm{g} / \mathrm{L} & 5.0 & \text { EPA8240 } \\ \mu \mathrm{g} / \mathrm{L} & 5.0 & \text { EPA8240 } \\ \mu \mathrm{g} / \mathrm{L} & 5.0 & \text { EPA8240 } \\ \mu \mathrm{g} / \mathrm{L} & 5.0 & \text { EPA8240 } \\ \mu \mathrm{g} / \mathrm{L} & 5.0 & \text { EPA8240 } \\ \mu \mathrm{g} / \mathrm{L} & 5.0 & \text { EPA8240 } \\ \mu \mathrm{g} / \mathrm{L} & 5.0 & \text { EPA8240 } \\ \mu \mathrm{g} / \mathrm{L} & 10 & \text { EPA8240 } \\ \mu \mathrm{g} / \mathrm{L} & 10 & \text { EPA8240 } \\ \mu \mathrm{g} / \mathrm{L} & 10 & \text { EPA8240 } \\ \mu \mathrm{g} / \mathrm{L} & 5.0 & \text { EPA8240 } \\ \mu \mathrm{g} / \mathrm{L} & 5.0 & \text { EPA8240 } \\ \mu \mathrm{g} / \mathrm{L} & 5.0 & \text { EPA8240 } \\ \mu \mathrm{g} / \mathrm{L} & 5.0 & \text { EPA8240 } \\ \mu \mathrm{g} / \mathrm{L} & 5.0 & \text { EPA8240 } \\ \mu \mathrm{g} / \mathrm{L} & 5.0 & \text { EPA8240 } \\ \mu \mathrm{g} / \mathrm{L} & 5.0 & \text { EPA8240 } \\ \mu \mathrm{g} / \mathrm{L} & 10 & \text { EPA8240 } \\ \mu \mathrm{g} / \mathrm{L} & 5.0 & \text { EPA8240 }\end{array}$

Radionuclide Indicators and Radionuclides

$\begin{array}{llll}\text { Gross alpha } & \mathrm{U} & & 0.70 \\ \text { Nonvolatile beta } & \mathrm{U} & \mathrm{V} & 0.80 \\ \text { Radium, total alpha-emitting } & \mathrm{U} & & 0.10\end{array}$

\section{0}

5.0

5.0

5.0

10

5.0

5.0

5.0

10

10

10

5.0

10

5.0

5.0

5.0

5.0

5.0

3.1

5.0

5.0

5.0

5.0

10

10

10

5.0

5.0

5.0

5.0

5.0

5.0

5.0

10

5.0

(1)

0

0

0

.

.

0

.


SAMPLE NAME: D43111902 (Continued)

\section{Constituent}

RQ

AQ

Radionuclide Indicators and Radionuclides

Tritium

SAMPLE NAME: D43112001

Location (SRS Coordinates): .E .N

Ground Elevation Above MSL: . $f \mathrm{t}$

Depth of Core Interval: . to . $\mathrm{ft}$

Sample Type: Normal

RQ AQ B Result Unit

Constituent

Physical Parameters

Chloride

Fluoride

Nitrate as nitrogen

$\mathrm{pH}$

Sulfate

Total dissolved solids

Total organic carbon

Total organic halogens

Total suspended solids

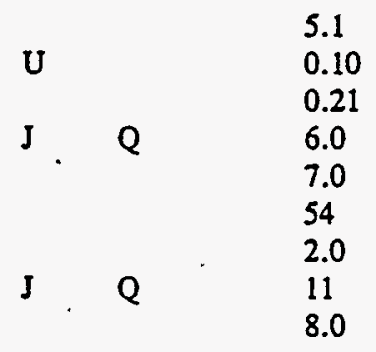

Total Metals

Arsenic

Barium

Cadmium

Chromium

Copper

Iron

Lead

Manganese

Mercury

Nickel

Selenium

Silver

Sodium

$\begin{array}{lll}\mathrm{U} & & 10 \\ \mathrm{U} & & 200 \\ \mathrm{UJ} & \mathrm{L} & 5.0 \\ \mathrm{U} & & 10 \\ \mathrm{UJ} & \mathrm{L} & 25 \\ & & 290 \\ \mathrm{U} & & 3.0 \\ & & 320 \\ \mathrm{U} & & 0.20 \\ \mathrm{U} & & 40 \\ \mathrm{U} & & 5.0 \\ \mathrm{U} & & 10 \\ & & \\ & & 7,900\end{array}$

Sample Matrix: Water

Sample ID: 100309

Sample ID: 100306

Sample Moisture: Liquid

D. Limit Method

\section{$B / N / A$ Extractables (including $P A H$ and phenols)}

Acenaphthene
Acenaphthylene
Acenaphthylene
Acenaphthylene
Anthracene
Anthracene
Anthracene
Benzo(a)anthracene

$$
\begin{aligned}
& \mathrm{mg} / \mathrm{L} \\
& \mathrm{mg} / \mathrm{L} \\
& \mathrm{mg} / \mathrm{L} \\
& \mathrm{pH} \\
& \mathrm{mg} / \mathrm{L} \\
& \mathrm{mg} / \mathrm{L} \\
& \mathrm{mg} / \mathrm{L} \\
& \mu \mathrm{g} / \mathrm{L} \\
& \mathrm{mg} / \mathrm{L}
\end{aligned}
$$

0.25

0.10

0.10

0.010

5.0

5.0

0.50

5.0

5.0

EPA300.0

EPA300.0

EPA300.0

EPA9045

EPA300.0

EPA160.2

EPA415.1

EPA9020

EPA160.2

$\begin{array}{lll}\mu \mathrm{g} / \mathrm{L} & 10 & \text { EPA7060 } \\ \mu \mathrm{g} / \mathrm{L} & 200 & \text { EPA6010 } \\ \mu \mathrm{g} / \mathrm{L} & 5.0 & \text { EPA6010 } \\ \mu \mathrm{g} / \mathrm{L} & 10 & \text { EPA6010 } \\ \mu \mathrm{g} / \mathrm{L} & 25 & \text { EPA6010 } \\ \mu \mathrm{g} / \mathrm{L} & 100 & \text { EPA6010 } \\ \mu \mathrm{g} / \mathrm{L} & 3.0 & \text { EPA7421 } \\ \mu \mathrm{g} / \mathrm{L} & 15 & \text { EPA6010 } \\ \mu \mathrm{g} / \mathrm{L} & 0.20 & \text { EPA7470 } \\ \mu \mathrm{g} / \mathrm{L} & 40 & \text { EPA6010 } \\ \mu \mathrm{g} / \mathrm{L} & 5.0 & \text { EPA7740 } \\ \mu \mathrm{g} / \mathrm{L} & 10 & \text { EPA6010 } \\ \mu \mathrm{g} / \mathrm{L} & 5,000 & \text { EPA6010 }\end{array}$

$\mu \mathrm{g} / \mathrm{L}$

EPA8270

EPA8270

$\begin{array}{lll}\mu \mathrm{g} / \mathrm{L} & 12 & \text { EPA8270 } \\ \mu \mathrm{g} / \mathrm{L} & 23 & \text { EPA8270 } \\ \mu \mathrm{g} & 26 & \text { EPA8270 }\end{array}$

$\mu g / L \quad 12 \quad$ EPA8270

$\mu \mathrm{g} / \mathrm{L} \quad 23 \quad$ EPA8270

$\mu \mathrm{g} / \mathrm{L} \quad 26 \quad$ EPA8270

$\mu \mathrm{g} / \mathrm{L} \quad 12 \quad$ EPA8270 
SAMPLE NAME: D43ï12001 (Continued)

Sample ID: 100306

$\begin{array}{llllll}\text { Constituent } & \text { RQ } & \text { AQ } & \text { Result Unit D. Limit Method }\end{array}$

$B / N / A$ Extractables (including PAH and phenols)

\begin{tabular}{|c|c|c|c|}
\hline Benzo(a)anthracene & $U$ & & 23 \\
\hline Benzo(2)anthracene & $\mathrm{U}$ & & 26 \\
\hline Benzo(b)fluoranthene & $\mathrm{U}$ & & 12 \\
\hline Benzo(b)fluoranchene & $U$ & & 23 \\
\hline Benzo(b)fluoranthene & $\mathbf{U}$ & & 26 \\
\hline Benzo(k)tluoranthene & $U$ & & 12 \\
\hline Benzo(k)fluoranthene & $\mathbf{U}$ & & 23 \\
\hline Benzo(k)fluoranthene & U & & 26 \\
\hline Benzoic acid & $\mathrm{U}$ & & 60 \\
\hline Benzoic acid & U & & 120 \\
\hline Benzoic acid & $U$ & & 130 \\
\hline Benzo $(g, h, i)$ perylene & $\mathrm{U}$ & & 12 \\
\hline Benzo $(g, h, i)$ perylene & $\mathbf{U}$ & & 23 \\
\hline Benzo $(g, h, i)$ perylene & $\mathrm{U}$ & & 26 \\
\hline Benzo(a)pyrene & $\mathrm{U}$ & & 12 \\
\hline Benzo(a)pyrene & $\mathrm{U}$ & & 23 \\
\hline Benzo(a)pyrene & $\mathrm{U}$ & & 26 \\
\hline Benzyl alcohol & $\mathrm{U}$ & & 12 \\
\hline Benzyl alcohol & $\mathrm{U}$ & & 23 \\
\hline Benzyl alcohol & U & & 26 \\
\hline Bis(2-chloroethoxy) methane & $\mathrm{U}$ & & 12 \\
\hline Bis(2-chloroethoxy) methane & $\mathrm{U}$ & & 23 \\
\hline Bis(2-chloroethoxy) methane & $\mathrm{U}$ & & 26 \\
\hline Bis(2-chloroethyl) ether & U & & 12 \\
\hline Bis(2-chloroisopropyl) ether & $\mathbf{U}$ & & 12 \\
\hline Bis(2-chloroethyl) ether & $\mathbf{U}$ & & 23 \\
\hline Bis(2-chloroisopropyl) ether & $\mathbf{U}$ & & 23 \\
\hline Bis(2-chloroethyl) ether & $\mathrm{U}$ & & 26 \\
\hline Bis(2-chloroisopropyl) ether & U & & 26 \\
\hline Bis(2-ethylhexyl) phthalate & UJ & $\mathrm{v}$ & 2.7 \\
\hline Bis(2-ethylhexyl) phthalate & $\mathrm{UJ}$ & $\mathrm{v}$ & 3.5 \\
\hline Bis(2-ethylhexyl) phthalate & & $\mathrm{v}$ & 1.100 \\
\hline 4-Bromophenyl phenyl ether & $U$ & & 12 \\
\hline 4-Bromophenyl phenyl ether & $\mathrm{U}$ & & 23 \\
\hline 4-Bromophenyl phenyl ether & $U$ & & 26 \\
\hline Butylbenzyl phthalate & $U$ & & 12 \\
\hline Butylbenzyl phthalate & $\mathrm{U}$ & & 23 \\
\hline Butylbenzyl phthalate & $\mathrm{U}$ & & 26 \\
\hline 4-Chloroaniline & U & & 12 \\
\hline 4-Chloroaniline & U & & 23 \\
\hline 4-Chloroaniline & U & & 26 \\
\hline 4-Chloro-m-cresol & $\mathbf{U}$ & & 12 \\
\hline 2-Chloronaphthalene & U & & 12 \\
\hline 2-Chloronaphthalene & U & & 23 \\
\hline 2-Chloronaphthalene & $\mathrm{U}$ & & 26 \\
\hline 2-Chlorophenol & $\mathbf{U}$ & & 12 \\
\hline 4-Chlorophenyl phenyl ether & $\mathbf{U}$ & & 12 \\
\hline 4-Chlorophenyl phenyl ether & $\mathbf{U}$ & & 23 \\
\hline 4-Chlorophenyl phenyl cther & $\mathbf{U}$ & & 26 \\
\hline Chrysene & $\mathrm{U}$ & & 12 \\
\hline
\end{tabular}

$\begin{array}{lll}\mu \mathrm{g} / \mathrm{L} & 23 & \text { EPA8270 } \\ \mu \mathrm{g} / \mathrm{L} & 26 & \text { EPA8270 } \\ \mu \mathrm{g} / \mathrm{L} & 12 & \text { EPA8270 } \\ \mu \mathrm{g} / \mathrm{L} & 23 & \text { EPA8270 } \\ \mu \mathrm{g} / \mathrm{L} & 26 & \text { EPA8270 } \\ \mu \mathrm{g} / \mathrm{L} & 12 & \text { EPA8270 } \\ \mu \mathrm{g} / \mathrm{L} & 23 & \text { EPA8270 } \\ \mu \mathrm{g} / \mathrm{L} & 26 & \text { EPA8270 } \\ \mu \mathrm{g} / \mathrm{L} & 60 & \text { EPA8270 } \\ \mu \mathrm{g} / \mathrm{L} & 120 & \text { EPA8270 } \\ \mu \mathrm{g} / \mathrm{L} & 130 & \text { EPA8270 } \\ \mu \mathrm{g} / \mathrm{L} & 12 & \text { EPA8270 } \\ \mu \mathrm{g} / \mathrm{L} & 23 & \text { EPA8270 } \\ \mu \mathrm{g} / \mathrm{L} & 26 & \text { EPA8270 } \\ \mu \mathrm{g} / \mathrm{L} & 12 & \text { EPA8270 } \\ \mu \mathrm{g} / \mathrm{L} & 23 & \text { EPA8270 } \\ \mu \mathrm{g} / \mathrm{L} & 26 & \text { EPA8270 } \\ \mu \mathrm{g} / \mathrm{L} & 12 & \text { EPA8270 } \\ \mu \mathrm{g} / \mathrm{L} & 23 & \text { EPA8270 } \\ \mu \mathrm{g} / \mathrm{L} & 26 & \text { EPA8270 } \\ \mu \mathrm{g} / \mathrm{L} & 12 & \text { EPA8270 } \\ \mu \mathrm{g} / \mathrm{L} & 23 & \text { EPA8270 } \\ \mu \mathrm{g} / \mathrm{L} & 26 & \text { EPA8270 } \\ \mu \mathrm{g} / \mathrm{L} & 12 & \text { EPA8270 } \\ \mu \mathrm{g} / \mathrm{L} & 12 & \text { EPA8270 } \\ \mu \mathrm{g} / \mathrm{L} & 23 & \text { EPA8270 } \\ \mu \mathrm{g} / \mathrm{L} & 23 & \text { EPA8270 } \\ \mu \mathrm{g} / \mathrm{L} & 26 & \text { EPA8270 } \\ \mu \mathrm{g} / \mathrm{L} & 26 & \text { EPA8270 } \\ \mu \mathrm{g} / \mathrm{L} & 12 & \text { EPA8270 } \\ \mu \mathrm{g} / \mathrm{L} & 23 & \text { EPA8270 } \\ \mu \mathrm{g} / \mathrm{L} & 26 & \text { EPA8270 } \\ \mu \mathrm{g} / \mathrm{L} & 12 & \text { EPA8270 } \\ \mu \mathrm{g} / \mathrm{L} & 23 & \text { EPA8270 } \\ \mu \mathrm{g} / \mathrm{L} & 26 & \text { EPA8270 } \\ \mu \mathrm{g} / \mathrm{L} & 12 & \text { EPA8270 } \\ \mu \mathrm{g} / \mathrm{L} & 23 & \text { EPA8270 } \\ \mu \mathrm{g} / \mathrm{L} & 26 & \text { EPA8270 } \\ \mu \mathrm{g} / \mathrm{L} & 12 & \text { EPA8270 } \\ \mu \mathrm{g} / \mathrm{L} & 23 & \text { EPA8270 } \\ \mu \mathrm{g} / \mathrm{L} & 26 & \text { EPA8270 } \\ \mu \mathrm{g} / \mathrm{L} & 12 & \text { EPA8270 } \\ \mu \mathrm{g} / \mathrm{L} & 12 & \text { EPA8270 } \\ \mu \mathrm{g} / \mathrm{L} & 23 & \text { EPA8270 } \\ \mu \mathrm{g} / \mathrm{L} & 26 & \text { EPA8270 } \\ \mu \mathrm{g} / \mathrm{L} & 12 & \text { EPA8270 } \\ \mu \mathrm{g} / \mathrm{L} & 12 & \text { EPA8270 } \\ \mu \mathrm{g} / \mathrm{L} & 23 & \text { EPA8270 } \\ \mu \mathrm{g} / \mathrm{L} & 26 & \text { EPA8270 } \\ & 12 & \text { EPA8270 }\end{array}$


SAMPLE NAME: D431.12001 (Continued)

Sample ID: 100306

Constituent

RQ AQ B Result Unit

D. Limit Method

$B / N / A$ Extractables (including PAH and phenols)

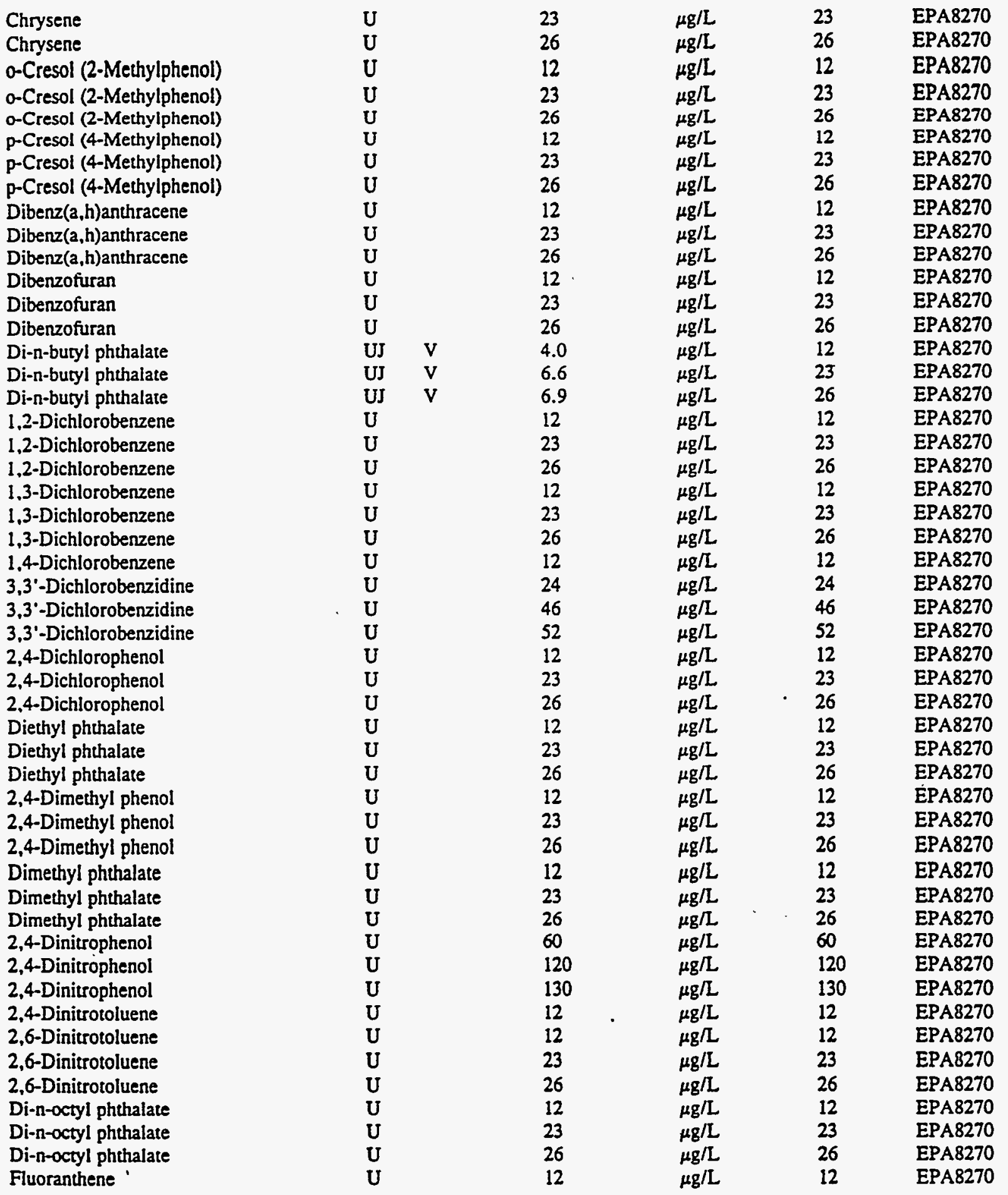


SAMPLE NAME: D43ï12001 (Continued)

Constituent
Sample ID: 100306
$\mathbf{R Q}$
B Result
Unit
D. Limit Method

\section{$B / N / A$ Extractables (including $P A H$ and phenols)}

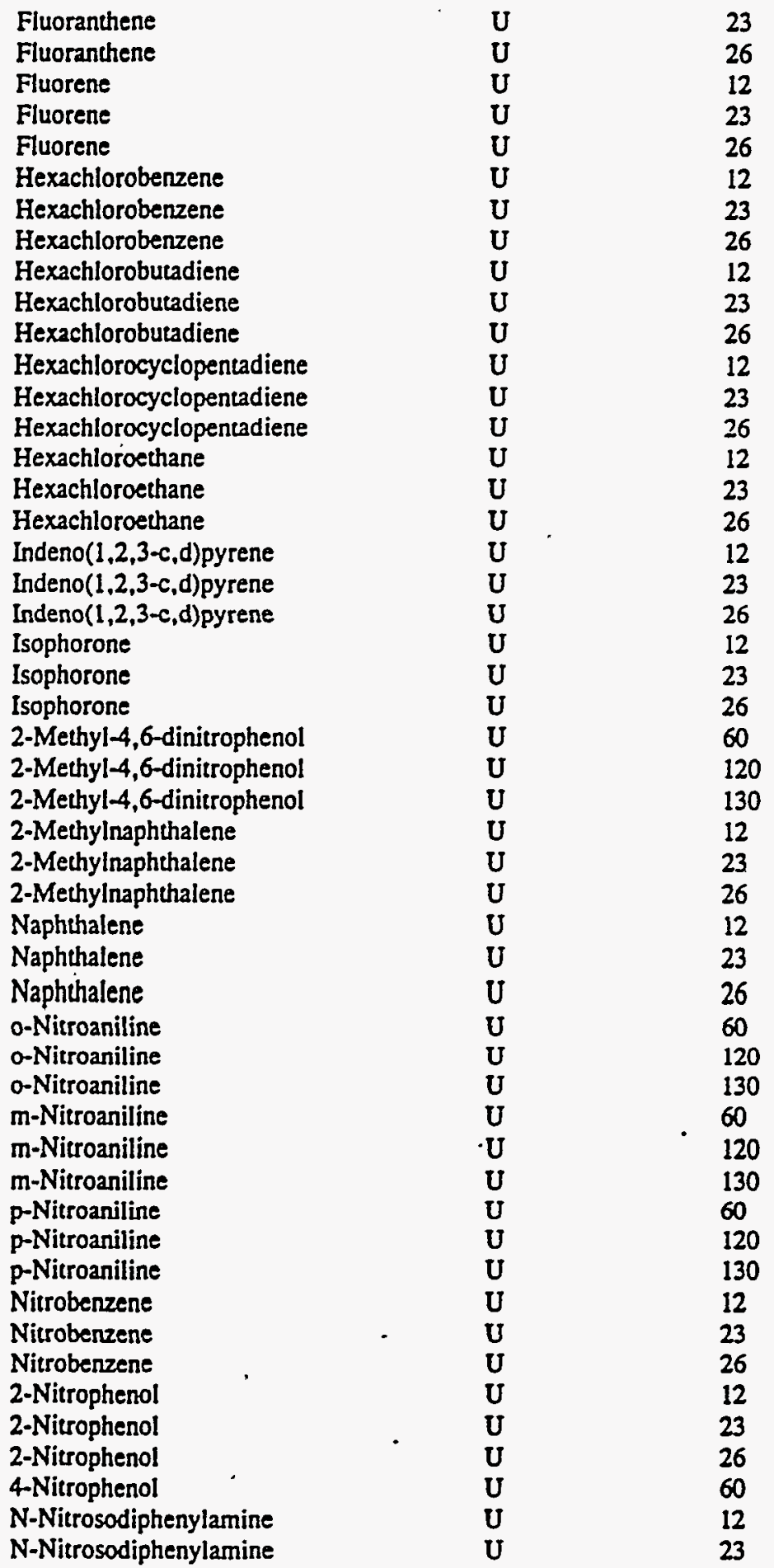

$\begin{array}{lll}\mu \mathrm{g} / \mathrm{L} & 23 & \text { EPA8270 } \\ \mu \mathrm{g} / \mathrm{L} & 26 & \text { EPA8270 } \\ \mu \mathrm{g} / \mathrm{L} & 12 & \text { EPA8270 } \\ \mu \mathrm{g} / \mathrm{L} & 23 & \text { EPA8270 } \\ \mu \mathrm{g} / \mathrm{L} & 26 & \text { EPA8270 } \\ \mu \mathrm{g} / \mathrm{L} & 12 & \text { EPA8270 } \\ \mu \mathrm{g} / \mathrm{L} & 23 & \text { EPA8270 } \\ \mu \mathrm{g} / \mathrm{L} & 26 & \text { EPA8270 } \\ \mu \mathrm{g} / \mathrm{L} & 12 & \text { EPA8270 } \\ \mu \mathrm{g} / \mathrm{L} & 23 & \text { EPA8270 } \\ \mu \mathrm{g} / \mathrm{L} & 26 & \text { EPA8270 } \\ \mu \mathrm{g} / \mathrm{L} & 12 & \text { EPA8270 } \\ \mu \mathrm{g} / \mathrm{L} & 23 & \text { EPA8270 } \\ \mu \mathrm{g} / \mathrm{L} & 26 & \text { EPA8270 } \\ \mu \mathrm{g} / \mathrm{L} & 12 & \text { EPA8270 } \\ \mu \mathrm{g} / \mathrm{L} & 23 & \text { EPA8270 } \\ \mu \mathrm{g} / \mathrm{L} & 26 & \text { EPA8270 } \\ \mu \mathrm{g} / \mathrm{L} & 12 & \text { EPA8270 } \\ \mu \mathrm{g} / \mathrm{L} & 23 & \text { EPA8270 } \\ \mu \mathrm{g} / \mathrm{L} & 26 & \text { EPA8270 } \\ \mu \mathrm{g} / \mathrm{L} & 12 & \text { EPA8270 } \\ \mu \mathrm{g} / \mathrm{L} & 23 & \text { EPA8270 } \\ \mu \mathrm{g} / \mathrm{L} & 26 & \text { EPA8270 } \\ \mu \mathrm{g} / \mathrm{L} & 60 & \text { EPA8270 } \\ \mu \mathrm{g} / \mathrm{L} & 120 & \text { EPA8270 } \\ \mu \mathrm{g} / \mathrm{L} & 130 & \text { EPA8270 } \\ \mu \mathrm{g} / \mathrm{L} & 12 & \text { EPA8270 } \\ \mu \mathrm{g} / \mathrm{L} & 23 & \text { EPA8270 } \\ \mu \mathrm{g} / \mathrm{L} & 26 & \text { EPA8270 } \\ \mu \mathrm{g} / \mathrm{L} & 12 & \text { EPA8270 } \\ \mu \mathrm{g} / \mathrm{L} & 23 & \text { EPA8270 } \\ \mu \mathrm{g} / \mathrm{L} & 26 & \text { EPA8270 } \\ \mu \mathrm{g} / \mathrm{L} & 60 & \text { EPA8270 } \\ \mu \mathrm{g} / \mathrm{L} & 120 & \text { EPA8270 } \\ \mu \mathrm{g} / \mathrm{L} & 130 & \text { EPA8270 } \\ \mu \mathrm{g} / \mathrm{L} & 60 & \text { EPA8270 } \\ \mu \mathrm{g} / \mathrm{L} & 120 & \text { EPA8270 } \\ \mu \mathrm{g} / \mathrm{L} & 130 & \text { EPA8270 } \\ \mu \mathrm{g} / \mathrm{L} & 60 & \text { EPA8270 } \\ \mu \mathrm{g} / \mathrm{L} & 120 & \text { EPA8270 } \\ \mu \mathrm{g} / \mathrm{L} & 130 & \text { EPA8270 } \\ \mu \mathrm{g} / \mathrm{L} & 12 & \text { EPA8270 } \\ \mu \mathrm{g} / \mathrm{L} & 23 & \text { EPA8270 } \\ \mu \mathrm{g} / \mathrm{L} & 26 & \text { EPA8270 } \\ \mu \mathrm{g} / \mathrm{L} & 12 & \text { EPA8270 } \\ \mu \mathrm{g} / \mathrm{L} & 23 & \text { EPA8270 } \\ \mu \mathrm{g} / \mathrm{L} & 26 & \text { EPA8270 } \\ \mu \mathrm{g} / \mathrm{L} & 60 & \text { EPA8270 } \\ \mu \mathrm{g} / \mathrm{L} & 12 & \text { EPA8270 } \\ & 23 & \text { EPA8270 }\end{array}$


SAMPLE NAME: D43112001 (Continued)

Sample ID: 100306

$\begin{array}{llllll}\text { Constituent } & \text { RQ AQ B Result D. Uimit Method }\end{array}$

$B / N / A$ Extractables (including $P A H$ and phenols)

N-Nitrosodiphenylamine

N-Nitrosodipropylamine

Pentachlorophenol

Phenanthrene

Phenanthrene

Phenanthrene

Phenol

Pyrene

1.2.4-Trichlorobenzene

2,4,5-Trichlorophenol

2,4,5-Trichlorophenol

2,4,5-Trichlorophenol

2,4,6-Trichlorophenol

2.4.6-Trichlorophenol

2,4.6-Trichlorophenol

Unknown

Unknown

Volatile Organic Compounds

\begin{tabular}{|c|c|c|}
\hline U & & 26 \\
\hline $\mathbf{U}$ & & 12 \\
\hline $\mathrm{U}$ & & 60 \\
\hline$U$ & & 12 \\
\hline U & & 23 \\
\hline U & & 26 \\
\hline$U$ & & 12 \\
\hline$U$ & & 12 \\
\hline U & & 12 \\
\hline $\mathrm{U}$ & & 60 \\
\hline$U$ & & 120 \\
\hline$U$ & & 130 \\
\hline$U$ & & 12 \\
\hline $\mathrm{U}$ & & 23 \\
\hline $\mathrm{U}$ & & 26 \\
\hline J & NV & 10 \\
\hline J & NV & 10 \\
\hline
\end{tabular}

$$
\begin{aligned}
& \mu \mathrm{g} / \mathrm{L} \\
& \mu \mathrm{g} / \mathrm{L} \\
& \mu \mathrm{g} / \mathrm{L} \\
& \mu \mathrm{g} / \mathrm{L} \\
& \mu \mathrm{g} / \mathrm{L} \\
& \mu \mathrm{g} / \mathrm{L} \\
& \mu \mathrm{g} / \mathrm{L} \\
& \mu \mathrm{g} / \mathrm{L} \\
& \mu \mathrm{g} / \mathrm{L} \\
& \mu \mathrm{g} / \mathrm{L} \\
& \mu \mathrm{g} / \mathrm{L} \\
& \mu \mathrm{g} / \mathrm{L} \\
& \mu \mathrm{g} / \mathrm{L} \\
& \mu \mathrm{g} / \mathrm{L} \\
& \mu \mathrm{g} / \mathrm{L} \\
& \mu \mathrm{g} / \mathrm{L} \\
& \mu \mathrm{g} / \mathrm{L}
\end{aligned}
$$

26
12
60
12
23
26
12
12
12
60
120
130
12
23
26

EPA8270

EPA8270

EPA8270

EPA8270

EPA8270

EPA8270

EPA8270

EPA8270

EPA8270

EPA8270

EPA8270

EPA8270

EPA8270

EPA8270

EPA8270

EPA8270

EPA8270

\section{Acetone}

Benzene

Bromodichloromethane

Bromoform

Bromomethane (Methyl bromide)

Carbon disulfide

Carbon tetrachloride

Chlorobenzene

Chloroethane

Chloroethene (Vinyl chloride)

2-Chloroethyl vinyl echer

Chloroform

Chloromethane (Methyl chloride)

Dibromochloromethane

1,1-Dichloroethane

1,2-Dichloroethane

1,1-Dichloroethylene

1,2-Dichloroethylene

Dichloromethane (Methylene chloride)

1,2-Dichloropropane

cis-1,3-Dichloropropene

trans-1,3-Dichloropropene

Ethylbenzene -

2-Hexanone

Methyl ethyl ketone

Methyl isobutyl ketone

Styrene

1,1,2,2-Tetrachloroethane

Tetrachloroethylene

Toluene

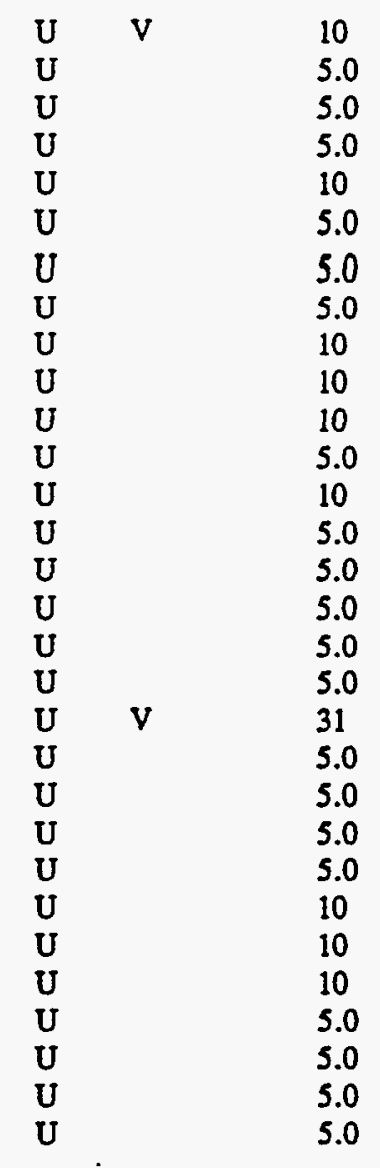

$\begin{array}{lll}\mu \mathrm{g} / \mathrm{L} & 10 & \text { EPA8240 } \\ \mu \mathrm{g} / \mathrm{L} & 5.0 & \text { EPA8240 } \\ \mu \mathrm{g} / \mathrm{L} & 5.0 & \text { EPA8240 } \\ \mu \mathrm{g} / \mathrm{L} & 5.0 & \text { EPA8240 } \\ \mu \mathrm{g} / \mathrm{L} & 10 & \text { EPA8240 } \\ \mu \mathrm{g} / \mathrm{L} & 5.0 & \text { EPA8240 } \\ \mu \mathrm{g} / \mathrm{L} & 5.0 & \text { EPA8240 } \\ \mu \mathrm{g} / \mathrm{L} & 5.0 & \text { EPA8240 } \\ \mu \mathrm{g} / \mathrm{L} & 10 & \text { EPA8240 } \\ \mu \mathrm{g} / \mathrm{L} & 10 & \text { EPA8240 } \\ \mu \mathrm{g} / \mathrm{L} & 10 & \text { EPA8240 } \\ \mu \mathrm{g} / \mathrm{L} & 5.0 & \text { EPA8240 } \\ \mu \mathrm{g} / \mathrm{L} & 10 & \text { EPA8240 } \\ \mu \mathrm{g} / \mathrm{L} & 5.0 & \text { EPA8240 } \\ \mu \mathrm{g} / \mathrm{L} & 5.0 & \text { EPA8240 } \\ \mu \mathrm{g} / \mathrm{L} & 5.0 & \text { EPA8240 } \\ \mu \mathrm{g} / \mathrm{L} & 5.0 & \text { EPA8240 } \\ \mu \mathrm{g} / \mathrm{L} & 5.0 & \text { EPA8240 } \\ \mu \mathrm{g} / \mathrm{L} & 5.0 & \text { EPA8240 } \\ \mu \mathrm{g} / \mathrm{L} & 5.0 & \text { EPA8240 } \\ \mu \mathrm{g} / \mathrm{L} & 5.0 & \text { EPA8240 } \\ \mu \mathrm{g} / \mathrm{L} & 5.0 & \text { EPA8240 } \\ \mu \mathrm{g} / \mathrm{L} & 5.0 & \text { EPA8240 } \\ \mu \mathrm{g} / \mathrm{L} & 10 & \text { EPA8240 } \\ \mu \mathrm{g} / \mathrm{L} & 10 & \text { EPA8240 } \\ \mu \mathrm{g} / \mathrm{L} & 10 & \text { EPA8240 } \\ \mu \mathrm{g} / \mathrm{L} & 5.0 & \text { EPA8240 } \\ \mu \mathrm{g} / \mathrm{L} & 5.0 & \text { EPA8240 } \\ \mu \mathrm{g} / \mathrm{L} & 5.0 & \text { EPA8240 } \\ \mu \mathrm{g} / \mathrm{L} & 5.0 & \text { EPA8240 }\end{array}$


SAMPLE NAME: D43112001 (Continued)

\section{Constituent}

Volátilie Organic Compounds

\section{1,1,1-Trichloroechane}

1,1,2-Trichloroethane

Trichloroethylene

Vinyl acetate

Xylenes

\section{Radionuclide Indicators and Radionuclides}

Gross alpha

Nonvolatile beta

Radium, total alpha-emitting

Tritium
RQ AQ B Result

$\begin{array}{ll}U & 5.0 \\ U & 5.0 \\ U & 5.0 \\ U & 10 \\ U & 5.0\end{array}$

SAMPLE NAME: D43112002

Location (SRS Coordinates): .E .N Ground Elevation Above MSL: . ft Depth of Core Interval: . to . $\mathrm{ft}$ Sample Type: Normal

\section{Constituent}

Physical Parameters

$\mathrm{pH}$

Total dissolved solids

Total organic carbon

Total suspended solids

\section{Total Metals}

\section{Arsenic}

Barium

Cadmium

Chromium

Copper

Iron

Lead

Manganese

Mercury

Nickel

Selenium

Silver

Sodium

$\begin{array}{llllll}\mathrm{U} & & 0.80 & \mathrm{pCi} / \mathrm{L} & 0.80 & \text { EPA990.0 } \\ & \mathrm{V} & 3.6 \pm 1.9 & \mathrm{pCi} / \mathrm{L} & 0.80 & \text { EPA900.0 } \\ \mathrm{U} & & 0.10 & \mathrm{pCi} / \mathrm{L} & 0.10 & \text { EPA903.0 } \\ & & 470 \pm 270 & \mathrm{pCi} / \mathrm{L} & 170 & \text { EPA906.0 }\end{array}$

Sample ID: 100307

Sample Matrix: Water

Sample Moisture: Liquid

RQ AQ B Result Unit D. Limit Method

$\begin{array}{lllll}\mathrm{J} \quad \mathrm{Q} & 6.0 & \mathrm{pH} & 0.010 & \text { EPA9045 } \\ & 53 & \mathrm{mg} / \mathrm{L} & 5.0 & \text { EPA160.2 } \\ & 2.3 & \mathrm{mg} / \mathrm{L} & 0.50 & \text { EPA415.1 } \\ & 9.0 & \mathrm{mg} / \mathrm{L} & 5.0 & \text { EPA160.2 }\end{array}$

$\begin{array}{llllll}\mathrm{U} & & 10 & \mu \mathrm{g} / \mathrm{L} & 10 & \text { EPA7060 } \\ \mathrm{U} & & 200 & \mu \mathrm{g} / \mathrm{L} & 200 & \text { EPA6010 } \\ \mathrm{UJ} & \mathrm{L} & 5.0 & \mu \mathrm{g} / \mathrm{L} & 5.0 & \text { EPA6010 } \\ \mathrm{U} & & 10 & \mu \mathrm{g} / \mathrm{L} & 10 & \text { EPA6010 } \\ \mathrm{UJ} & \mathrm{L} & 25 & \mu \mathrm{g} / \mathrm{L} & 25 & \text { EPA6010 } \\ & & 620 & \mu \mathrm{g} / \mathrm{L} & 100 & \text { EPA6010 } \\ \mathrm{U} & & 3.0 & \mu \mathrm{g} / \mathrm{L} & 3.0 & \text { EPA7421 } \\ & & 760 & \mu \mathrm{g} / \mathrm{L} & 15 & \text { EPA6010 } \\ \mathrm{U} & 0.20 & \mu \mathrm{g} / \mathrm{L} & 0.20 & \text { EPA7470 } \\ \mathrm{U} & & 40 & \mu \mathrm{g} / \mathrm{L} & 40 & \text { EPA6010 } \\ \mathrm{U} & & 5.0 & \mu \mathrm{g} / \mathrm{L} & 5.0 & \text { EPA7740 } \\ \mathrm{U} & & 10 & \mu \mathrm{g} / \mathrm{L} & 10 & \text { EPA6010 } \\ & & 7,900 & \mu \mathrm{g} / \mathrm{L} & 5,000 & \text { EPA6010 }\end{array}$


SAMPLE NAME: D4311.2002 (Continued)

Constituent
Sample ID: 100307

RQ AQ B Result Unit $\quad$ D. Limit Method

\section{$B / N / A$ Extractables (including $P A H$ and phenols)}

\begin{tabular}{|c|c|c|c|}
\hline Acenaphthene & $\mathrm{U}$ & & 9.8 \\
\hline Acenaphthylene & $\mathrm{U}$ & & 9.8 \\
\hline Anthracene & $\mathbf{U}$ & & 9.8 \\
\hline Benzo(a)anthracene & U & & 9.8 \\
\hline Benzo(b)fluoranthene & U & & 9.8 \\
\hline Benzo(k)fluoranthene & $\mathrm{U}$ & & 9.8 \\
\hline Benzoic acid & U & & 49 \\
\hline Benzo(g,h.i)perylene & $\mathrm{U}$ & & 9.8 \\
\hline Benzo(a)pyrene & U & & 9.8 \\
\hline Benzyl alcohol & U & & 9.8 \\
\hline Bis(2-chloroethoxy) metharie & $\mathrm{U}$ & & 9.8 \\
\hline Bis(2-chloroethyl) ether & U & & 9.8 \\
\hline Bis(2-chloroisopropyl) ether & $\mathrm{U}$ & & 9.8 \\
\hline Bis(2-ethylhexyl) phthalate & UJ & V & 2.4 \\
\hline 4-Bromophenyl phenyl ether & U & & 9.8 \\
\hline Butylbenzyl phthalate & $\mathrm{U}$ & & 9.8 \\
\hline 4-Chloroaniline & U & & 9.8 \\
\hline 4-Chioro-m-cresol & $\mathrm{U}$ & & 9.8 \\
\hline 2-Chloronaphthalene & $U$ & & 9.8 \\
\hline 2-Chlorophenol & $\mathbf{U}$ & & 9.8 \\
\hline 4-Chlorophenyl phenyl ether & $\mathbf{U}$ & & 9.8 \\
\hline Chrysene & U & & 9.8 \\
\hline a-Cresol (2-Methylphenol) & U & & 9.8 \\
\hline p-Cresol (4-Methylphenol) & $\mathbf{U}$ & & 9.8 \\
\hline Dibenz(a,h)anthracene & U & & 9.8 \\
\hline Dibenzofuran & $\mathbf{U}$ & & 9.8 \\
\hline Di-n-butyl phthalate & UJ & $\mathrm{v}$ & 2.8 \\
\hline 1,2-Dichlorobenzene & U & & 9.8 \\
\hline 1,3-Dichlorobenzene & $\mathbf{U}$ & & 9.8 \\
\hline 1,4-Dichlorobenzene & $\mathrm{U}$ & & 9.8 \\
\hline 3,3'-Dichlorobenzidine & U & & 20 \\
\hline 2,4-Dichlorophenol & $\mathrm{U}$ & & 9.8 \\
\hline Diethyl phthalate & $\mathrm{U}$ & & 9.8 \\
\hline 2,4-Dimethyl phenol & $\mathrm{U}$ & & 9.8 \\
\hline Dimethyl phthalate & $\mathbf{U}$ & & 9.8 \\
\hline 2,4-Dinitrophenol & $\mathbf{U}$ & & 49 \\
\hline 2,4-Dinitrotoluene & U & & 9.8 \\
\hline 2,6-Dinitrotoluene & $\mathbf{U}$ & & 9.8 \\
\hline Di-n-octyl phthalate & $\mathbf{U}$ & & 9.8 \\
\hline Fluoranthene & $\mathbf{U}$ & & 9.8 \\
\hline Fluorene & $\mathbf{U}$ & & 9.8 \\
\hline Hexachlorobenzene & $\mathbf{U}$ & & 9.8 \\
\hline Hexachlorobutadiene & $\mathbf{U}$ & & 9.8 \\
\hline Hexachlorocyclopentadiene & U & & 9.8 \\
\hline Hexachloroethane & $U$ & & 9.8 \\
\hline Indeno(1,2,3-c,d)pyrene & $\mathbf{U}$ & & 9.8 \\
\hline Isophorone & $\mathbf{U}$ & & 9.8 \\
\hline 2-Methyl-4,6-dinitrophenol & $U$ & & 49 \\
\hline 2-Methylnaphthalene & $\mathbf{U}$ & & 9.8 \\
\hline Naphthalene & $\mathbf{U}$ & & 9.8 \\
\hline
\end{tabular}

$\mu \mathrm{g} / \mathrm{L}$

$\mu \mathrm{g} / \mathrm{L}$

$\mu \mathrm{g} / \mathrm{L}$

$\mu g / L$

$\mu \mathrm{g} / \mathrm{L}$

$\mu \mathrm{g} / \mathrm{L}$

$\mu \mathrm{g} / \mathrm{L}$

$\mu \mathrm{g} / \mathrm{L}$

$\mu \mathrm{g} / \mathrm{L}$

$\mu \mathrm{g} / \mathrm{L}$

$\mu \mathrm{g} / \mathrm{L}$

$\mu \mathrm{g} / \mathrm{L}$

$\mu \mathrm{g} / \mathrm{L}$

$\mu \mathrm{g} / \mathrm{L}$

$\mu \mathrm{g} / \mathrm{L}$

$\mu \mathrm{g} / \mathrm{L}$

$\mu \mathrm{g} / \mathrm{L}$

$\mu \mathrm{g} / \mathrm{L}$

$\mu \mathrm{g} / \mathrm{L}$

$\mu g / L$

$\mu \mathrm{g} / \mathrm{L}$

$\mu \mathrm{g} / \mathrm{L}$

$\mu \mathrm{g} / \mathrm{L}$

$\mu g / L$

$\mu \mathrm{g} / \mathrm{L}$

$\mu \mathrm{g} / \mathrm{L}$

$\mu \mathrm{g} / \mathrm{L}$

$\mu \mathrm{g} / \mathrm{L}$

$\mu g / L$

$\mu \mathrm{g} / \mathrm{L}$

$\mu \mathrm{g} / \mathrm{L}$

$\mu \mathrm{g} / \mathrm{L}$

$\mu g / L$

$\mu \mathrm{g} / \mathrm{L}$

$\mu \mathrm{g} / \mathrm{L}$

$\mu g / L$

$\mu g / L$

$\mu g / L$

$\mu \mathrm{g} / \mathrm{L}$

$\mu g / L$

$\mu g / L$

$\mu \mathrm{g} / \mathrm{L}$

$\mu g / L$.

$\mu g / L$

$\mu \mathrm{g} / \mathrm{L}$

$\mu \mathrm{g} / \mathrm{L}$

$\mu g / L$

$\mu \mathrm{g} / \mathrm{L}$.

$\mu \mathrm{g} / \mathrm{L}$

$\mu \mathrm{g} / \mathrm{L}$
EPA8270

EPA8270

EPA8270

EPA8270

EPA8270

EPA8270

EPA8270

EPA8270

EPA8270

EPA8270

EPA8270

EPA8270

EPA8270

EPA8270

EPA8270

EPA8270

EPA8270

EPA8270

EPA8270

EPA8270

EPA8270

EPA8270

EPA8270

EPA8270

EPA8270

EPA8270

EPA8270

EPA8270

EPA8270

EPA8270

EPA8270

EPA8270

EPA8270

EPA8270

EPA8270

EPA8270

EPA8270

EPA8270

EPA8270

EPA8270

EPA8270

EPA8270

EPA8270

EPA8270

EPA8270

EPA 8270

EPA8270

EPA8270

EPA8270

EPA8270 
SAMPLE NAME: D43112002 (Continued)

Sample ID: 100307

$\begin{array}{llllll}\text { Constituent } & \text { RQ AQ } & \text { U Result Dit Dimit Method }\end{array}$

\section{$B / N / A$ Extractables (including $P A H$ and phenols)}

\begin{abstract}
o-Nitroaniline
m-Nitroaniline

p-Nitroaniline

Nitrobenzene

2-Nitrophenol

4-Nitrophenol

Ṇ-Nitrosodiphenylaminè

$\mathrm{N}$-Nitrosodipropylamine

Pentachlorophenol

Phenanthrene

Phenol

Pyrene

1,2,4-Trichlorobenzene

2,4,5-Trichlorophenol

2.4,6-Trichlorophenol

Unknown

Unknown
\end{abstract}

$\begin{array}{lll}\mathrm{U} & & 49 \\ \mathrm{U} & & 49 \\ \mathrm{U} & & 49 \\ \mathrm{U} & & 9.8 \\ \mathrm{U} & & 9.8 \\ \mathrm{U} & 49 \\ \mathrm{U} & & 9.8 \\ \mathrm{U} & & 9.8 \\ \mathrm{U} & & 49 \\ \mathrm{U} & & 9.8 \\ \mathrm{U} & & 9.8 \\ \mathrm{U} & & 9.8 \\ \mathrm{U} & & 9.8 \\ \mathrm{U} & & 49 \\ \mathrm{U} & & 9.8 \\ \mathrm{~J} & \mathrm{NV} & 4.0 \\ \mathrm{~J} & \mathrm{NV} & 9.0\end{array}$

Volatile Organic Compounds

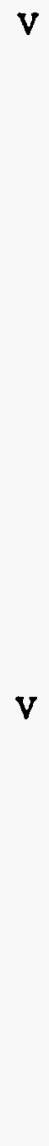

$\mu \mathrm{g} / \mathrm{L}$
$\mu \mathrm{g} / \mathrm{L}$
$\mu \mathrm{g} / \mathrm{L}$
$\mu \mathrm{g} / \mathrm{L}$
$\mu \mathrm{g} / \mathrm{L}$
$\mu \mathrm{g} / \mathrm{L}$
$\mu \mathrm{g} / \mathrm{L}$
$\mu \mathrm{g} / \mathrm{L}$
$\mu \mathrm{g} / \mathrm{L}$
$\mu \mathrm{g} / \mathrm{L}$
$\mu \mathrm{g} / \mathrm{L}$
$\mu \mathrm{g} / \mathrm{L}$
$\mu \mathrm{g} / \mathrm{L}$
$\mu \mathrm{g} / \mathrm{L}$
$\mu \mathrm{g} / \mathrm{L}$
$\mu \mathrm{g} / \mathrm{L}$
$\mu \mathrm{g} / \mathrm{L}$

49

49

49

9.8

9.8

49

9.8

9.8

49

9.8

9.8

9.8

9.8

49

9.8

EPA8270

EPA8270

EPA8270

EPA8270

EPA8270

EPA8270

EPA8270

EPA8270

EPA8270

EPA8270

EPA8270

EPA8270

EPA8270

EPA8270

EPA8270

$\begin{array}{lll}\mu \mathrm{g} / \mathrm{L} & 10 & \text { EPA8240 } \\ \mu \mathrm{g} / \mathrm{L} & 5.0 & \text { EPA8240 } \\ \mu \mathrm{g} / \mathrm{L} & 5.0 & \text { EPA8240 } \\ \mu \mathrm{g} / \mathrm{L} & 5.0 & \text { EPA8240 } \\ \mu \mathrm{g} / \mathrm{L} & 10 & \text { EPA8240 } \\ \mu \mathrm{g} / \mathrm{L} & 5.0 & \text { EPA8240 } \\ \mu \mathrm{g} / \mathrm{L} & 5.0 & \text { EPA8240 } \\ \mu \mathrm{g} / \mathrm{L} & 5.0 & \text { EPA8240 } \\ \mu \mathrm{g} / \mathrm{L} & 10 & \text { EPA8240 } \\ \mu \mathrm{g} / \mathrm{L} & 10 & \text { EPA8240 } \\ \mu \mathrm{g} / \mathrm{L} & 10 & \text { EPA8240 } \\ \mu \mathrm{g} / \mathrm{L} & 5.0 & \text { EPA8240 } \\ \mu \mathrm{g} / \mathrm{L} & 10 & \text { EPA8240 } \\ \mu \mathrm{g} / \mathrm{L} & 5.0 & \text { EPA8240 } \\ \mu \mathrm{g} / \mathrm{L} & 5.0 & \text { EPA8240 } \\ \mu \mathrm{g} / \mathrm{L} & 5.0 & \text { EPA8240 } \\ \mu \mathrm{g} / \mathrm{L} & 5.0 & \text { EPA8240 } \\ \mu \mathrm{g} / \mathrm{L} & 5.0 & \text { EPA8240 } \\ \mu \mathrm{g} / \mathrm{L} & 5.0 & \text { EPA8240 } \\ \mu \mathrm{g} / \mathrm{L} & 5.0 & \text { EPA8240 } \\ \mu \mathrm{g} / \mathrm{L} & 5.0 & \text { EPA8240 } \\ \mu \mathrm{g} / \mathrm{L} & 5.0 & \text { EPA8240 } \\ \mu \mathrm{g} / \mathrm{L} & 5.0 & \text { EPA8240 } \\ \mu \mathrm{g} / \mathrm{L} & 10 & \text { EPA8240 } \\ \mu \mathrm{g} / \mathrm{L} & 10 & \text { EPA8240 } \\ \mu \mathrm{g} / \mathrm{L} & 10 & \text { EPA8240 } \\ \mu \mathrm{g} / \mathrm{L} & 5.0 & \text { EPA8240 } \\ \mu \mathrm{g} / \mathrm{L} & 5.0 & \text { EPA8240 } \\ \mu \mathrm{g} / \mathrm{L} & 5.0 & \text { EPA8240 } \\ \mu \mathrm{g} / \mathrm{L} & 5.0 & \text { EPA8240 }\end{array}$


SAMPLE NAME: D431.12002 (Continued)

Sample ID: 100307

Constituent

RQ AQ B Result Unit

D. Limit Method

Volatile Organic Compounds

1,1,1-Trichloroethane

5.0

5.0

5.0

Trichloroethylene

Vinyl acetate

10

Xylenes

$\begin{array}{ll}U & 5.0 \\ U & 5.0 \\ U & 5.0 \\ U & 10 \\ U & 5.0\end{array}$

$\mu \mathrm{g} / \mathrm{L}$

$\mu \mathrm{g} / \mathrm{L}$

$\mu \mathrm{g} / \mathrm{L}$

$\mu \mathrm{g} / \mathrm{L}$

$\mu \mathrm{g} / \mathrm{L}$

5.0

EPA8240

EPA8240

EPA8240

5.0

EPA8240

Radionuclide Indicators and Radionuclides"

Gross alpha

Nonvolatile beta

Tritium

SAMPLE NAME: D43112101

Location (SRS Coordinates): .E .N

Ground Elevation Above MSL: . $\mathrm{ft}$

Depth of Core Interval: . 10 . ft

Sample Type: Normal

Constituent

Physical Parameters

Chloride

Fluoride

Nitrate as nitrogen

pH

Sulfate

Total dissolved solids

Total organic carbon

Total organic halogens

Total suspended solids

Total Metals

Arsenic
Barium
Cadmium
Chromium
Copper
Iron
Lead
Manganese
Mercury
Nickel
Selenium
Silver
Sodium

Arsenic

Barium

Cadmium

Copper

Iron

Lead

Sodium
V

U

RQ AQ B Result Unit

Sample Matrix: Water

$\mathrm{pCi} / \mathrm{L}$

$\mathrm{pCi} / \mathrm{L}$

$\mathrm{pCi} / \mathrm{L}$

$\mathrm{pCi} / \mathrm{L}$

Sample ID: 100305

\section{1}

0.10

1.1

6.1

7.0

68

2.2

J $Q$

29

9.0

$\mathrm{mg} / \mathrm{L}$

$\mathrm{mg} / \mathrm{L}$

$\mathrm{mg} / \mathrm{L}$

$\mathrm{pH}$

$\mathrm{mg} / \mathrm{L}$

$\mathrm{mg} / \mathrm{L}$

$\mathrm{mg} / \mathrm{L}$

$\mu \mathrm{g} / \mathrm{L}$

$\mathrm{mg} / \mathrm{L}$

0.90

EPA900.0

0.90

EPA900.0

0.10

EPA' 903.0

180

EPA906.0
Sample Moisture: Liquid

D. Limit Method 


\section{$B / N / A$ Extractables (including $P A H$ and phenols)}

\begin{tabular}{|c|c|c|c|c|c|c|}
\hline Acenaphthene & $\mathbf{U}$ & & 14 & $\mu g / L$ & 14 & EPA8270 \\
\hline Acenaphthylene & $\mathbf{U}$ & & 14 & $\mu \mathrm{g} / \mathrm{L}$ & 14 & EPA8270 \\
\hline Anthracene & $\mathbf{U}$ & & 14 & $\mu \mathrm{g} / \mathrm{L}$ & 14 & EPA8270 \\
\hline Benzo(a)anthracene & $U$ & & 14 & $\mu \mathrm{g} / \mathrm{L}$ & 14 & EPA8270 \\
\hline Benzo(b)fluoranthene & $\mathbf{U}$ & & 14 & $\mu \mathrm{g} / \mathrm{L}$ & 14 & EPA8270 \\
\hline Benzo(k)fluoranthene & $\mathbf{U}$ & & 14 & $\mu \mathrm{g} / \mathrm{L}$ & 14 & EPA8270 \\
\hline Benzoic acid & $\mathbf{U}$ & & 70 & $\mu \mathrm{g} / \mathrm{L}$ & 70 & EPA8270 \\
\hline Benzo $(g, h, i)$ perylene & $\mathbf{U}$ & & 14 & $\mu \mathrm{g} / \mathrm{L}$ & 14 & EPA8270 \\
\hline Benzo(a)pyrene & U & & 14 & $\mu g / L$ & 14 & EPA8270 \\
\hline Benzyl alcohol & $\mathbf{U}$ & & 14 & $\mu \mathrm{g} / \mathrm{L}$ & 14 & EPA8270 \\
\hline Bis(2-chloroethoxy) methane & $\mathbf{U}$ & & 14 & $\mu \mathrm{g} / \mathrm{L}$ & 14 & EPA8270 \\
\hline Bis(2-chloroethyl) ether & $\mathbf{U}$ & & 14 & $\mu \mathrm{g} / \mathrm{L}$ & 14 & EPA8270 \\
\hline Bis(2-chloroisopropyl) ether & $\mathrm{U}$ & & 14 & $\mu \mathrm{g} / \mathrm{L}$ & 14 & EPA8270 \\
\hline Bis(2-ethylhexyl) phthalate & UJ & $\mathrm{v}$ & 3.2 & $\mu \mathrm{g} / \mathrm{L}$ & 14 & EPA8270 \\
\hline 4-Bromophenyl phenyl ether & $\mathbf{U}$ & & 14 & $\mu \mathrm{g} / \mathrm{L}$ & 14 & EPA8270 \\
\hline Butylbenzyl phthalate & $\mathrm{U}$ & & 14 & $\mu \mathrm{g} / \mathrm{L}$ & 14 & EPA8270 \\
\hline 4-Chloroaniline & $\mathbf{U}$ & & 14 & $\mu \mathrm{g} / \mathrm{L}$ & 14 & EPA8270 \\
\hline 4-Chloro-m-cresol & $\mathrm{U}$ & & 14 & $\mu \mathrm{g} / \mathrm{L}$ & 14 & EPA8270 \\
\hline 2-Chioronaphthalene & $\mathrm{U}$ & & 14 & $\mu \mathrm{g} / \mathrm{L}$ & 14 & EPA8270 \\
\hline 2-Chlorophenol & $U$ & & 14 & $\mu g / L$ & 14 & EPA8270 \\
\hline 4-Chlorophenyl phenyl ether & $\mathrm{U}$ & & 14 & $\mu \mathrm{g} / \mathrm{L}$ & 14 & EPA8270 \\
\hline Chrysene & $\mathrm{U}$ & & 14 & $\mu \mathrm{g} / \mathrm{L}$ & 14 & EPA8270 \\
\hline o-Cresol (2-Methylphenol) & $\mathbf{U}$ & & 14 & $\mu \mathrm{g} / \mathrm{L}$ & 14 & EPA8270 \\
\hline p-Cresol (4-Methylphenol) & $\mathrm{U}$ & & 14 & $\mu \mathrm{g} / \mathrm{L}$ & 14 & EPA8270 \\
\hline Dibenz(a.h)anthracene & $\mathrm{U}$ & & 14 & $\mu \mathrm{g} / \mathrm{L}$ & 14 & EPA8270 \\
\hline Dibenzofuran & U & & 14 & $\mu g / L$ & 14 & EPA8270 \\
\hline Di-n-butyl phthalate & UJ & v & 5.2 & $\mu \mathrm{g} / \mathrm{L}$ & 14 & EPA8270 \\
\hline 1,2-Dichlorobenzene & U & & 14 & $\mu \mathrm{g} / \mathrm{L}$ & 14 & EPA8270 \\
\hline 1,3-Dichlorobenzene & $\mathbf{U}$ & & 14 & $\mu \mathrm{g} / \mathrm{L}$ & 14 & EPA8270 \\
\hline 1,4-Dichlorobenzene & U & & 14 & $\mu g / L$ & 14 & EPA8270 \\
\hline 3,3'-Dichlorobenzidine & $\mathrm{U}$ & & 28 & $\mu \mathrm{g} / \mathrm{L}$ & 28 & EPA8270 \\
\hline 2,4-Dichlorophenol & $\mathrm{U}$ & & 14 & $\mu \mathrm{g} / \mathrm{L}$ & 14 & EPA 8270 \\
\hline Diethyl phthalate & $\mathrm{U}$ & & 14 & $\mu \mathrm{g} / \mathrm{L}$ & 14 & EPA8270 \\
\hline 2,4-Dimethyl phenol & $\mathbf{U}$ & & 14 & $\mu \mathrm{g} / \mathrm{L}$ & 14 & EPA8270 \\
\hline Dimethyl phthalate & U & & 14 & $\mu \mathrm{g} / \mathrm{L}$ & 14 & EPA8270 \\
\hline 2,4-Dinitrophenol & $\mathbf{U}$ & & 70 & $\mu \mathrm{g} / \mathrm{L}$ & 70 & EPA8270 \\
\hline 2,4-Dinitrotoluene & $\mathbf{U}$ & & 14 & $\mu \mathrm{g} / \mathrm{L}$ & 14 & EPA8270 \\
\hline 2,6-Dinitrotoluene & $\mathbf{U}$ & & 14 & $\mu \mathrm{g} / \mathrm{L}$ & 14 & EPA8270 \\
\hline Di-n-octyl phthalate & $\mathbf{U}$ & & 14 & $\mu \mathrm{g} / \mathrm{L}$ & 14 & EPA8270 \\
\hline Fluoranthene & $U$ & & 14 & $\mu g / L$ & 14 & EPA8270 \\
\hline Fluorene & $\mathbf{U}$ & & 14 & $\mu \mathrm{g} / \mathrm{L}$ & 14 & EPA8270 \\
\hline Hexachlorobenzene & $\mathbf{U}$ & & 14 & $\mu g / L$ & 14 & EPA8270 \\
\hline Hexachlorobutadiene & $\mathbf{U}$ & & 14 & $\mu g / L$ & 14 & EPA8270 \\
\hline Hexachlorocyclopentadiene & $\mathbf{U}$ & & 14 & $\mu g / L$ & 14 & EPA8270 \\
\hline Hexachloroethane & $\mathbf{U}$ & & 14 & $\mu \mathrm{g} / \mathrm{L}$ & 14 & EPA8270 \\
\hline Indeno( $(1,2,3-c, d)$ pyrene & $\mathbf{U}$ & & 14 & $\mu \mathrm{g} / \mathrm{L}$ & 14 & EPA8270 \\
\hline Isophorone & $\mathrm{U}$ & & 14 & $\mu \mathrm{g} / \mathrm{L}$ & 14 & EPA8270 \\
\hline 2-Methyl-4,6-dinitrophenol & $\mathbf{U}$ & & 70 & $\mu \mathrm{g} / \mathrm{L}$ & 70 & EPA8270 \\
\hline 2-Methyinaphthalene & $\mathbf{U}$ & & 14 & $\mu \mathrm{g} / \mathrm{L}$ & 14 & EPA8270 \\
\hline Naphthalene & $\mathbf{U}$ & & 14 & $\mu \mathrm{g} / \mathrm{L}$ & 14 & EPA8270 \\
\hline o-Nitroaniline & $\mathbf{U}$ & & 70 & $\mu g / L$ & 70 & EPA8270 \\
\hline m-Nitroaniline & $\mathbf{U}$ & & 70 & $\mu \mathrm{g} / \mathrm{L}$ & 70 & EPA8270 \\
\hline p-Nitroaniline & $\mathbf{U}$ & & 70 & $\mu \mathrm{g} / \mathrm{L}$ & 70 & EPA8270 \\
\hline
\end{tabular}


SAMPLE NAME: D43112101 (Continued) Sample ID: 100305
Constituent
RQ AQ B Result
Unit
D. Limit Method

$B / N / A$ Extractables (including $P A H$ and phenols)

Nitrobenzene

2-Nitrophenol

4-Nitrophenol

N-Nitrosodiphenylamine

N-Nitrosodipropylamine

Pentachlorophenol

Phenanthrene

Phenol

Pyrene

1.2,4-Trichlorobenzene

2,4,5-Trichlorophenol

2,4,6-Trichlorophenol

Unknown

Unknown

Volatile Organic Compounds

$\begin{array}{lll}U & & 14 \\ U & & 14 \\ U & & 70 \\ U & & 14 \\ U & & 14 \\ U & & 70 \\ U & & 14 \\ U & & 14 \\ U & & 14 \\ U & & 14 \\ U & & 70 \\ U & & 14 \\ J & N V & 10 \\ J & N V & 10\end{array}$

$\mu g / L$

$\mu \mathrm{g} / \mathrm{L}$

$\mu \mathrm{g} / \mathrm{L}$

$\mu \mathrm{g} / \mathrm{L}$

$\mu \mathrm{g} / \mathrm{L}$

$\mu \mathrm{g} / \mathrm{L}$

$\mu \mathrm{g} / \mathrm{L}$

$\mu \mathrm{g} / \mathrm{L}$

$\mu g / L$

$\mu \mathrm{g} / \mathrm{L}$

$\mu \mathrm{g} / \mathrm{L}$

$\mu \mathrm{g} / \mathrm{L}$

$\mu \mathrm{g} / \mathrm{L}$

$\mu \mathrm{g} / \mathrm{L}$

$\begin{array}{ll}14 & \text { EPA8270 } \\ 14 & \text { EPA8270 } \\ 70 & \text { EPA8270 } \\ 14 & \text { EPA8270 } \\ 14 & \text { EPA8270 } \\ 70 & \text { EPA8270 } \\ 14 & \text { EPA8270 } \\ 14 & \text { EPA8270 } \\ 14 & \text { EPA8270 } \\ 14 & \text { EPA8270 } \\ 70 & \text { EPA8270 } \\ 14 & \text { EPA8270 } \\ & \text { EPA8270 } \\ & \text { EPA8270 }\end{array}$

$\mu \mathrm{g} / \mathrm{L}$
$\mu \mathrm{g} / \mathrm{L}$
$\mu \mathrm{g} / \mathrm{L}$
$\mu \mathrm{g} / \mathrm{L}$
$\mu \mathrm{g} / \mathrm{L}$
$\mu \mathrm{g} / \mathrm{L}$
$\mu \mathrm{g} / \mathrm{L}$
$\mu \mathrm{g} / \mathrm{L}$
$\mu \mathrm{g} / \mathrm{L}$
$\mu \mathrm{g} / \mathrm{L}$
$\mu \mathrm{g} / \mathrm{L}$
$\mu \mathrm{g} / \mathrm{L}$
$\mu \mathrm{g} / \mathrm{L}$
$\mu \mathrm{g} / \mathrm{L}$
$\mu \mathrm{g} / \mathrm{L}$
$\mu \mathrm{g} / \mathrm{L}$
$\mu \mathrm{g} / \mathrm{L}$
$\mu \mathrm{g} / \mathrm{L}$
$\mu \mathrm{g} / \mathrm{L}$
$\mu \mathrm{g} / \mathrm{L}$
$\mu \mathrm{g} / \mathrm{L}$
$\mu \mathrm{g} / \mathrm{L}$
$\mu \mathrm{g} / \mathrm{L}$
$\mu \mathrm{g} / \mathrm{L}$
$\mu \mathrm{g} / \mathrm{L}$
$\mu \mathrm{g} / \mathrm{L}$
$\mu \mathrm{g} / \mathrm{L}$
$\mu \mathrm{g} / \mathrm{L}$
$\mu \mathrm{g} / \mathrm{L}$
$\mu \mathrm{g} / \mathrm{L}$
$\mu \mathrm{g} / \mathrm{L}$
$\mu \mathrm{g} / \mathrm{L}$
$\mu \mathrm{g} / \mathrm{L}$
10

5.0

5.0

5.0

10

5.0

5.0

5.0

10

10

10

5.0

10

5.0

5.0

5.0

5.0

5.0

5.0

5.0

5.0

5:0.

5.0

10

10

10

5.0

5.0

5.0

5.0

5.0

5.0

5.0
EPA8240

EPA8240

EPA8240

EPA8240

EPA8240

EPA8240

EPA8240

EPA8240

EPA8240

EPA8240

EPA8240

EPA8240

EPA8240

EPA8240

EPA8240

EPA8240

EPA8240

EPA8240

EPA8240

EPA8240

EPA8240

EPA8240

EPA8240

EPA8240

EPA8240

EPA8240

EPA8240

EPA8240

EPA8240

EPA8240

EPA8240

EPA8240

EPA8240 
SAMPLE NAME: D43112101 (Contnued)

Sample ID: 100305

\section{Constituent}

Vólatile Organic Compounds

Vinyl acetate

Xylenes

U

$\mathbf{U}$

$\mathbf{R Q}$
Result

10

5.0
Unit

D. Limit

Method

Radionuclide Indicators and Radionuclides
Gross alpha

Nonvolatile beta

Radium. total alpha-emitting

Radium, total alpha-emitting

Tritium

\begin{tabular}{|c|c|c|c|c|}
\hline \multirow{5}{*}{ V } & $2.4 \pm 1.4$ & $\mathrm{pCi} / \mathrm{L}$ & 0.90 & EPA900.0 \\
\hline & $1.1 \pm 1.7$ & $\mathrm{pCi} / \mathrm{L}$ & 0.90 & EPA900.0 \\
\hline & $0.40 \pm 0.20$ & $\mathrm{pCi} / \mathrm{L}$ & 0.20 & EPA903.0 \\
\hline & $0.50 \pm 0.30$ & $\mathrm{pCi} / \mathrm{L}$ & 0.20 & EPA903.0 \\
\hline & $830 \pm 310$ & $\mathrm{pCi} / \mathrm{L}$ & 180 & EPA906.0 \\
\hline
\end{tabular}

EPA 8240

EPA8240

$\begin{array}{lll}\mu \mathrm{g} / \mathrm{L} & 10 & \\ \mu \mathrm{g} / \mathrm{L} & 5.0 & \text { EPA8240 }\end{array}$

EPA906.0 
SAMPLE NAME: D431_ $1204 \mathrm{C}$

Sample Type: Blank

Constituen't

Total Metals
Sample ID: 100374

Associated Sample: 100373

RQ AQ B Result Unit
D. Limit Method

Arsenic
Arsenic
Barium
Barium
Cadmium
Cadmium
Chromium
Chromium
Copper
Copper
Lead
Lead
Manganese
Manganese
Mercury
Mercury
Nickel
Nickel
Selenium
Selenium
Silver
Silver

Volatile Organic Compounds

Acetone
Benzene
Bromodichloromethane
Bromoform
Bromomethane (Methyl bromide)
Carbon disulfide
Carbon tetrachloride
Chlorobenzene
Chloroethane
Chloroethene (Vinyl chloride)
2-Chloroethyl vinyl ether
Chloroform
Chloromethane (Methyl chloride)
Dibromochloromethane
1,1-Dichloroethane
1,2-Dichloroethane
1,1-Dichloroethylene
1,2-Dichloroethylene
Dichloromethane (Methylene chloride)
1,2-Dichloropropane
cis-1,3-Dichloropropene
trans-1,3-Dichloropropene
Ethylbenzene

$U$
$U$
$U$
$U$
$U$
$U$
$U$
$U$
$U$
$U$
$U$
$U$
$U$
$U$
$U$
$U J$
$U$
$U$
$U$
$U$
$U$
$U$

10
10
200
200
5.0
5.0
10
10
25
25
3.0
3.0
15
15
0.20
0.20
40
40
5.0
5.0
10
10

$\mu g / L$

$\mu \mathrm{g} / \mathrm{L}$

$\mu \mathrm{g} / \mathrm{L}$

$\mu \mathrm{g} / \mathrm{L}$

$\mu \mathrm{g} / \mathrm{L}$

$\mu \mathrm{g} / \mathrm{L}$

$\mu \mathrm{g} / \mathrm{L}$

$\mu g / L$

$\mu \mathrm{g} / \mathrm{L}$

$\mu \mathrm{g} / \mathrm{L}$

$\mu \mathrm{g} / \mathrm{L}$

$\mu \mathrm{g} / \mathrm{L}$

$\mu \mathrm{g} / \mathrm{L}$

$\mu \mathrm{g} / \mathrm{L}$

$\mu \mathrm{g} / \mathrm{L}$

$\mu \mathrm{g} / \mathrm{L}$

$\mu \mathrm{g} / \mathrm{L}$

$\mu g / L$

$\mu \mathrm{g} / \mathrm{L}$

$\mu \mathrm{g} / \mathrm{L}$

$\mu \mathrm{g} / \mathrm{L}$

$\mu \mathrm{g} / \mathrm{L}$

$\begin{array}{ll}10 & \text { EPA7060 } \\ 10 & \text { EPA7060 } \\ 200 & \text { EPA6010 } \\ 200 & \text { EPA6010 } \\ 5.0 & \text { EPA6010 } \\ 5.0 & \text { EPA6010 } \\ 10 & \text { EPA6010 } \\ 10 & \text { EPA6010 } \\ 25 & \text { EPA6010 } \\ 25 & \text { EPA6010 } \\ 3.0 & \text { EPA7421 } \\ 3.0 & \text { EPA7421 } \\ 15 & \text { EPA6010 } \\ 15 & \text { EPA6010 } \\ 0.20 & \text { EPA7470 } \\ 0.20 & \text { EPA7470 } \\ 40 & \text { EPA6010 } \\ 40 & \text { EPA6010 } \\ 5.0 & \text { EPA7740 } \\ 5.0 & \text { EPA7740 } \\ 10 & \text { EPA6010 } \\ 10 & \text { EPA6010 }\end{array}$

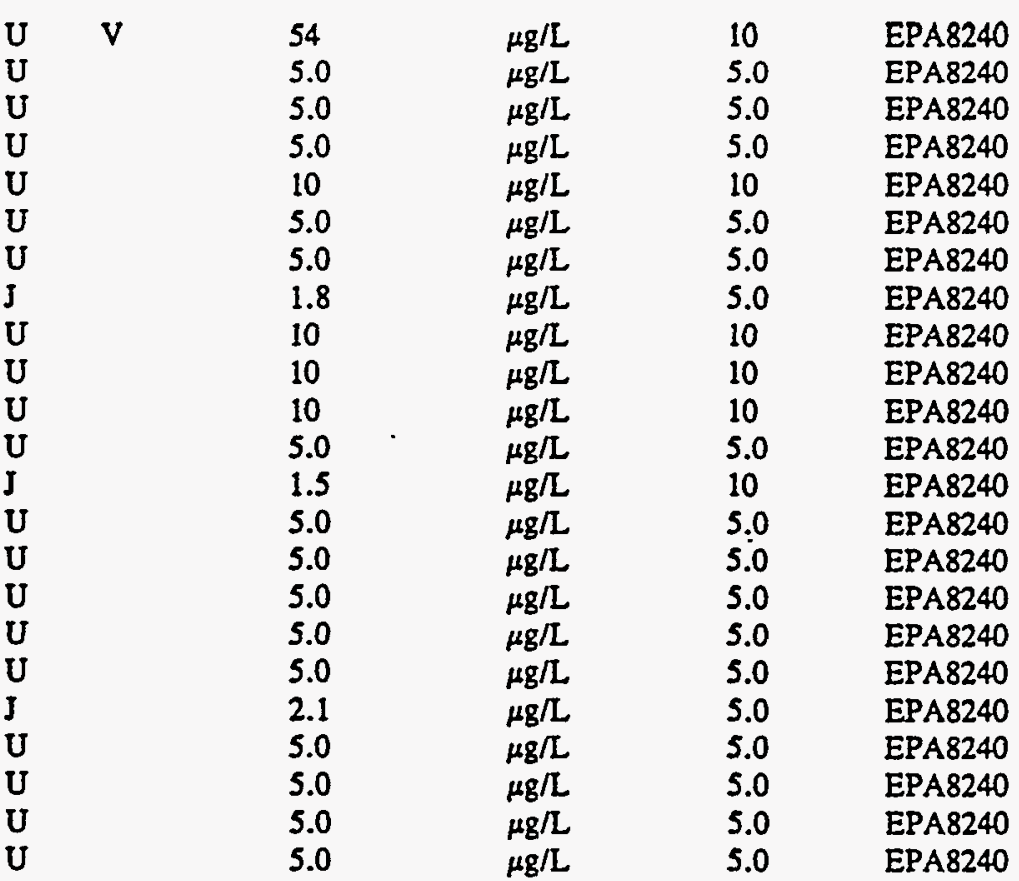


SAMPLE NAME: D431 1204C (Continued)

Sample ID: 100374

Constituent

RQ AQ B Result

Unit

D. Limit Method

Volatile Organic Compounds

2-Hexanone
Methyl ethyl ketone
Methyl isobutyl ketone
Styrene
1,1,2,2-Tetrachloroethane
Tetrachloroethylene
Toluene
1,1,1-Trichloroethane
1,1.2-Trichloroethane
Trichloroethylene
Vinyl acetate
Xylenes

$\mathrm{U}$

U

U

$U$

U

U.

$\mathrm{U}$

U

U

U

Xylenes

U

Radionuclide Indicators and Radionuclides

Gross alpha

Gross alpha

Gross alpha

Gross alpha

Nonvolatile beta

Nonvolatile beta

Nonvolatile beta

Nonvolatile beta

Radium, total alpha-emitting

Radium, total alpha-emitting

Radium, total alpha-emitting

Radium, total alpha-emitting

Tritium

Tritium

Tritium

Tritium
$\mathrm{U}$

U

U

$\mathrm{U}$

J $\quad X$

UJ $X$

U

$\begin{array}{ll}\text { U } & \text { V } \\ \text { U } & \text { V } \\ U & \text { V } \\ U & V \\ U & \\ U & \\ U & \\ U & \end{array}$

SAMPLE NAME: D431 1301B

Sample Type: Blank

Constituent

Volatile Organic Compounds

10
10
10
5.0
5.0
5.0
5.0
5.0
5.0
5.0
10
5.0

$\mu \mathrm{g} / \mathrm{L}$

$\mu \mathrm{g} / \mathrm{L}$

$\mu \mathrm{g} / \mathrm{L}$

$\mu \mathrm{g} / \mathrm{L}$

$\mu g / L$

$\mu \mathrm{g} / \mathrm{L}$

$\mu \mathrm{g} / \mathrm{L}$

$\mu \mathrm{g} / \mathrm{L}$

$\mu \mathrm{g} / \mathrm{L}$

$\mu \mathrm{g} / \mathrm{L}$

$\mu \mathrm{g} / \mathrm{L}$

$\mu g / L$

$\mathrm{pCi} / \mathrm{L}$

$\mathrm{pCi} / \mathrm{L}$

$\mathrm{pCi} / \mathrm{L}$

$\mathrm{pCi} / \mathrm{L}$

$\mathrm{pCi} / \mathrm{L}$

$\mathrm{pCi} / \mathrm{L}$

$\mathrm{pCi} / \mathrm{L}$

$\mathrm{pCi} / \mathrm{L}$

$\mathrm{pCi} / \mathrm{L}$

$\mathrm{pCi} / \mathrm{L}$

$\mathrm{pCi} / \mathrm{L}$

$\mathrm{pCi} / \mathrm{L}$

$\mathrm{pCi} / \mathrm{L}$

$\mathrm{pCi} / \mathrm{L}$

$\mathrm{pCi} / \mathrm{L}$

$\mathrm{pCi} / \mathrm{L}$
0.50

0.50

0.50

0.50

0.70

0.70

0.70

0.70

0.10

0.20

0.20

0.10

140

130

130

140
EPA8240

EPA8240

EPA8240

EPA8240

EPA8240

EPA8240

EPA8240

EPA8240

EPA8240

EPA8240

EPA8240

EPA8240

EPA900.0

EPA900.0

EPA900.0

EPA900.0

EPA900.0

EPA900.0

EPA900.0

EPA900.0

EPA903.0

EPA903.0

EPA903.0

EPA903.0

EPA906.0

EPA906.0

EPA906.0

EPA906.0
Sample ID: 100380

Associated Sample: 100379

RQ $\mathbf{A Q}$ $\therefore$

D. Limit Method

$\mathbf{U}$
$\mathbf{U}$
$\mathbf{U}$
$\mathbf{U}$
$\mathbf{U}$
$\mathbf{U}$
$\mathbf{U}$
$\mathbf{U}$
$\mathbf{U}$

9.1
5.0
5.0
5.0
10
5.0
5.0
5.0
10

$\mu g / L$
$\mu g / L$
$\mu g / L$
$\mu g / L$
$\mu g / L$
$\mu g / L$
$\mu g / L$
$\mu g / L$
$\mu g / L$

\section{0}

5.0

5.0

5.0

10

5.0

5.0

5.0

10
EPA8240

EPA8240

EPA8240

EPA8240

EPA8240

EPA8240

EPA8240

EPA8240

EPA8240 
SAMPLE NAME: D43I_1301B (Continued)

Constituent

RQ

Sample ID: 100380

Volatile Organic Compounds

Chloroethene (Vinyl chloride)

2-Chloroethyl vinyl ether

Chloroform

Chloromethane (Methyl chloride)

Dibromochloromethane

1.1-Dichloroethane

1,2-Dichloroethane

1,1-Dichloroethylene

1,2-Dichloroethylene

Dichloromethane (Methylene chloride)

1.2-Dichloropropane

cis-1,3-Dichloropropene

trans-1,3-Dichloropropene

Ethylbenzene

2-Hexanone

Methyl ethyl ketone

Methyl isobutyl ketone

Styrene

1.1,2,2-Tetrachloroethane

Tetrachloroethylene

Toluene

1,1,1-Trichloroethane

1,1,2-Trichloroethane

Trichloroethylene

Vinyl acetate

Xylenes

$U$
$U$
$U$
$U$
$U$
$U$
$U$
$U$
$U$
$J$
$U$
$U$
$U$
$U$
$U$
$U$
$U$
$U$
$U$
$U$
$U$
$U$
$U$

Radionuclide Indicators and Radionuclides

Gross alpha

Nonvolatile beta

Radium, total alpha-emitting

Tritium

SAMPLE NAME: D431 1304E

Sample Type: Rinsate

Constituent

Total Metals

Arsenic

Barium

Cadmium

Chromium

Copper

Lead

Manganese

$\begin{array}{lll}U & & 0.50 \\ U & X & 0.70 \\ U & V & 0.10 \\ U & & 130\end{array}$

Sample ID: 100384

Associated Sample: 100383

RQ AQ B Result Unit

$\mathrm{pCi} / \mathrm{L} \quad \cdot \quad 0.50$

$\mathrm{pCi} / \mathrm{L} \quad 0.70$

EPA900.0

$\mathrm{pCi} / \mathrm{L}$

0.10

EPA900.0

EPA903.0

EPA906.0
EPA8240

EPA8240

EPA8240

EPA8240

EPA8240

EPA8240

EPA8240

EPA8240

EPA8240

EPA8240

EPA8240

EPA8240

EPA8240

EPA8240

EPA8240

EPA8240

EPA8240

EPA8240

EPA8240

EPA8240

EPA8240

EPA8240

EPA8240

EPA8240

EPA8240

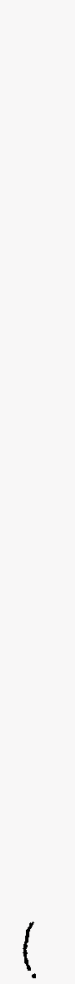


SAMPLE NAME: D431 1304E (Continued)

Constituent

Total Metals

Mercury

Nickel

Selenium

Silver

$\mathrm{U}$
$\mathrm{U}$
$\mathrm{U}$
Sample ID: 100384

RQ AQ B Result Unit

$\begin{array}{lll}\mu \mathrm{g} / \mathrm{L} & 0.20 & \text { EPA7470 } \\ \mu \mathrm{g} / \mathrm{L} & 40 & \text { EPA6010 } \\ \mu \mathrm{g} / \mathrm{L} & 5.0 & \text { EPA7740 } \\ \mu \mathrm{g} / \mathrm{L} & 10 & \text { EPA6010 }\end{array}$

0.20
40
5.0
10

D. Limit Method

Volatile Organic Compounds

Acetone
Benzene
Bromodichloromethane
Bromoform
Bromomethane (Methyl bromide)
Carbon disulfide
Carbon tetrachloride
Chlorobenzene
Chloroethane
Chloroethene (Vinyl chloride)
2-Chloroethyl vinyl ether
Chloroform
Chloromethane (Methyl chloride)
Dibromochloromethane
1,1-Dichloroethane
1,2-Dichloroethane
1,1-Dichloroethylene
1,2-Dichloroethylene
Dichloromethane (Methylene chloride)
1,2-Dichloropropane
cis-1,3-Dichloropropene
trans-1,3-Dichloropropene
Ethylbenzene
2-Hexanone
Methyl ethyl ketone
Methyl isobutyl ketone
Styrene
1,1,2,2-Tetrachloroethane
Tetrachloroethylene
Toluene
1,1,1-Trichloroethane
1,1,2-Trichloroethane
Trichloroethylene
Vinyl acetate
Xylenes

39

5.0

5.0

5.0

10

5.0

5.0

5.0

10

10

10

5.0

10

5.0

5.0

5.0

5.0

5.0

3.1

5.0

5.0

5.0

5.0

10

22

10

5.0

5.0

5.0

5.0

5.0

5.0

5.0

10

$\mu \mathrm{g} / \mathrm{L}$

$\mu g / L$

$\mu \mathrm{g} / \mathrm{L}$

$\mu \mathrm{g} / \mathrm{L}$

$\mu \mathrm{g} / \mathrm{L}$

$\mu \mathrm{g} / \mathrm{L}$

$\mu \mathrm{g} / \mathrm{L}$

$\mu \mathrm{g} / \mathrm{L}$

$\mu \mathrm{g} / \mathrm{L}$

$\mu g / L$

$\mu \mathrm{g} / \mathrm{L}$

$\mu g / L$

$\mu \mathrm{g} / \mathrm{L}$

$\mu g / L$

$\mu g / L$

$\mu \mathrm{g} / \mathrm{L}$

$\mu g / L$

$\mu \mathrm{g} / \mathrm{L}$

$\mu \mathrm{g} / \mathrm{L}$

$\mu \mathrm{g} / \mathrm{L}$

$\mu \mathrm{g} / \mathrm{L}$

$\mu \mathrm{g} / \mathrm{L}$

$\mu \mathrm{g} / \mathrm{L}$

$\mu \mathrm{g} / \mathrm{L}$

$\mu \mathrm{g} / \mathrm{L}$

$\mu g / L$

$\mu \mathrm{g} / \mathrm{L}$

$\mu \mathrm{g} / \mathrm{L}$

$\mu g / L$

$\mu g / L$

$\mu g / L$

$\mu g / L$

$\mu g / L$

$\mu g / L$

$\mu \mathrm{g} / \mathrm{L}$

5.0

Radionuclide Indicators and Radionuclides

Gross alpha

Nonvolatile beta

Radium, total alpha-emitting

Tritium

$\begin{array}{ll}U & X \\ U & V\end{array}$

0.50

$0.80 \pm 1.6$

0.10

140

$\mathrm{pCi} / L$

$\mathrm{pCi} / \mathrm{L}$

$\mathrm{pCi} / \mathrm{L}$

$\mathrm{pCi} / \mathrm{L}$
10

5.0

5.0

5.0

10

5.0

5.0

5.0

10

10

10

5.0

10

5.0

5.0

5.0

5.0

5.0

5.0

5.0

5.0

5.0

5.0

10

10

10

5.0

5.0

5.0

5.0

5.0

5.0

5:0

10

5.0

EPA8240

EPA8240

EPA8240

EPA8240

EPA8240

EPA8240

EPA8240

EPA8240

EPA8240

EPA8240

EPA8240

EPA8240

EPA8240

EPA8240

EPA8240

EPA8240

EPA8240

EPA8240

EPA8240

EPA8240

EPA8240

EPA8240

EPA8240

EPA8240

EPA8240

EPA8240

EPA8240

EPA8240

EPA8240

EPA8240

EPA8240

EPA8240

EPA8240

EPA8240

EPA8240 
SAMPLE NAME: D43L.1406C

Sample Type: Blank

Constituent

Total Metals

Arsenic

Barium

Cadmium

Chromium

Copper

Lead

Manganese

Mercury

Nickel

Selenium

Silver

Volatile Organic Compounds
Sample ID: 100397

Associated Sample: 100396

RQ AQ B Result Unit $\quad$ D. Limit Method

\begin{tabular}{|c|c|c|c|c|c|}
\hline Acetone & & 34 & $\mu \mathrm{g} / \mathrm{L}$ & 10 & EPA8240 \\
\hline Benzene & $\mathrm{U}$ & 5.0 & $\mu g / L$ & 5.0 & EPA8240 \\
\hline Bromodichloromethane & $\mathbf{U}$ & 5.0 & $\mu \mathrm{g} / \mathrm{L}$ & 5.0 & EPA8240 \\
\hline Bromoform & U & 5.0 & $\mu \mathrm{g} / \mathrm{L}$ & 5.0 & EPA8240 \\
\hline Bromomethane (Methyl bromide) & $\mathbf{U}$ & 10 & $\mu \mathrm{g} / \mathrm{L}$ & 10 & EPA8240 \\
\hline Carbon disulfide & $\mathbf{U}$ & 5.0 & $\mu \mathrm{g} / \mathrm{L}$ & 5.0 & EPA8240 \\
\hline Carbon tetrachloride & $\mathrm{U}$ & 5.0 & $\mu \mathrm{g} / \mathrm{L}$ & 3.0 & EPA8240 \\
\hline Chlorobenzene & $\mathrm{U}$ & 5.0 & $\mu \mathrm{g} / \mathrm{L}$ & 5.0 & EPA8240 \\
\hline Chloroethane & $\mathrm{U}$ & 10 & $\mu \mathrm{g} / \mathrm{L}$ & 10 & EPA8240 \\
\hline Chloroethene (Vinyl chloride) & $\mathrm{U}$ & 10 & $\mu \mathrm{g} / \mathrm{L}$ & 10 & EPA8240 \\
\hline Chloroform & $\mathrm{U}$ & 5.0 & $\mu \mathrm{g} / \mathrm{L}$ & $5.0^{\circ}$ & EPA8240 \\
\hline Chloromethane (Methyl chloride) & $U$ & 10 & $\mu g / L$ & 10 & EPA8240 \\
\hline Dibromochloromethane & $\mathrm{U}$ & 5.0 & $\mu \mathrm{g} / \mathrm{L}$ & 5.0 & EPA8240 \\
\hline 1,1-Dichloroethane & $\mathrm{U}$ & 5.0 & $\mu \mathrm{g} / \mathrm{L}$ & 5.0 & EPA8240 \\
\hline 1.2-Dichloroethane & $\mathrm{U}$ & 5.0 & $\mu \mathrm{g} / \mathrm{L}$ & 5.0 & EPA8240 \\
\hline 1,1-Dichloroechylene & $\mathrm{U}$ & 5.0 & $\mu \mathrm{g} / \mathrm{L}$ & 5.0 & EPA8240 \\
\hline 1,2-Dichloroethylene & $\mathrm{U}$ & 5.0 & $\mu \mathrm{g} / \mathrm{L}$ & 5.0 & EPA8240 \\
\hline Dichloromethane (Methylene chloride) & $\mathbf{U}$ & 5.0 & $\mu \mathrm{g} / \mathrm{L}$ & 5.0 & EPA8240 \\
\hline 1,2-Dichloropropane & $\mathbf{U}$ & 5.0 & $\mu \mathrm{g} / \mathrm{L}$ & 5.0 & EPA8240 \\
\hline cis-1,3-Dichloropropene & $\mathrm{U}$ & 5.0 & $\mu \mathrm{g} / \mathrm{L}$ & 5.0 & EPA8240 \\
\hline trans-1,3-Dichloropropene & $\mathrm{U}$ & 5.0 & $\mu \mathrm{g} / \mathrm{L}$ & 5.0 & EPA8240 \\
\hline Ethylberzene & $\mathrm{U}$ & 5.0 & $\mu \mathrm{g} / \mathrm{L}$ & 5.0 & EPA8240 \\
\hline 2-Hexanone & $\mathbf{U}$ & 10 & $\mu \mathrm{g} / \mathrm{L}$ & 10 & EPA8240 \\
\hline Methyl ethyl ketone & & 27 & $\mu \mathrm{g} / \mathrm{L}$ & 10 & EPA8240 \\
\hline Methyl isobutyl ketone & $\mathbf{U}$ & 10 & $\mu \mathrm{g} / \mathrm{L}$ & 10 & EPA8240 \\
\hline Styrene & $\mathbf{U}$ & 5.0 & $\mu \mathrm{g} / \mathrm{L}$ & 5.0 & EPA8240 \\
\hline 1,1,2,2-Tetrachloroethane & $\mathrm{U}$ & 5.0 & $\mu \mathrm{g} / \mathrm{L}$ & 5.0 & EPA8240 \\
\hline Tetrachloroethylene & $\mathbf{U}$ & 5.0 & $\mu g / L$ & 5.0 & EPA8240 \\
\hline Toluene & $\mathrm{U}$ & 5.0 & $\mu \mathrm{g} / \mathrm{L}$ & 5.0 & EPA8240 \\
\hline 1,1,1-Trichloroethane & $\mathrm{U}$ & 5.0 & $\mu \mathrm{g} / \mathrm{L}$ & 5.0 & EPA8240 \\
\hline 1,1,2-Trichloroethane & $\mathbf{U}$ & 5.0 & $\mu g / L$ & 5.0 & EPA8240 \\
\hline Trichloroethylene & $\mathbf{U}$ & 5.0 & $\mu g / L$ & 5.0 & EPA8240 \\
\hline Vinyl acetate & $\mathbf{U}$ & 10 & $\mu g / L$ & 10 & EPA8240 \\
\hline Xylenes & $\mathbf{U}$ & 5.0 & $\mu \mathrm{g} / \mathrm{L}$ & 5.0 & EPA8240 \\
\hline
\end{tabular}

EPA7060

EPA6010

EPA6010

EPA6010

EPA6010

EPA7421

EPA6010

EPA7470

EPA6010

EPA7740

EPA6010

$\mu \mathrm{gg} / \mathrm{L} \quad 10$

EPA8240

EPA8240

EPA8240

EPA8240

EPA8240

EPA8240

EA8240

EPA8240

EPA8240

EPA8240

EPA8240

EPA8240

EPA8240

EPA8240

EPA8240

EPA8240

EPA8240

EPA8240 
Radionuclide Indicators and Radionuclides

Gross alpha

Nonvolatile beta

Radium. total alpha-emitting

Tritium

\section{SAMPLE NAME: D431 1708C}

Sample Type: Blank

Constituent

Volatile Organic Compounds

Acetone
Benzene
Bromodichloromethane
Bromoform
Bromomethane (Methyl bromide)
Carbon-disulfide
Carbon tetrachloride
Chlorobenzene
Chloroethane
Chloroethene (Vinyl chloride)
Chloroform
Chloromethane (Methyl chloride)
Dibromochloromethane
1,1-Dichloroethane
1,2-Dichloroethane
1,1-Dichloroethylene
1,2-Dichloroethylene
Dichloromethane (Methylene chloride)
1,2-Dichloropropane
cis-1,3-Dichloropropene
trans-1,3-Dichloropropene
Ethylbenzene
2-Hexanone
Methyl ethyl ketone
Methyl isobutyl ketone
Styrene
1,1,2,2-Tetrachloroethane
Tetrachloroethylene
Toluene
1,1,1-Trichloroethane
1,1,2-Trichloroethane
Trichloroethylene
Vinyl acetate
Xylenes

$\begin{array}{lll}U & & 0.50 \\ U & X & 0.70 \\ U & V & 0.10 \\ U & & 130\end{array}$

Sample ID: 100437

Associated Sample: 100436

RQ

AQ

Result

Unit

D. Limit Method

$\mathrm{pCi} / \mathrm{L}$

0.50

0.70

EPA900:0

$\mathrm{pCi} / \mathrm{L}$

0.10

$\mathrm{pCi} / \mathrm{L}$

130

EPA900.0

EPA903.0

EPA906.0

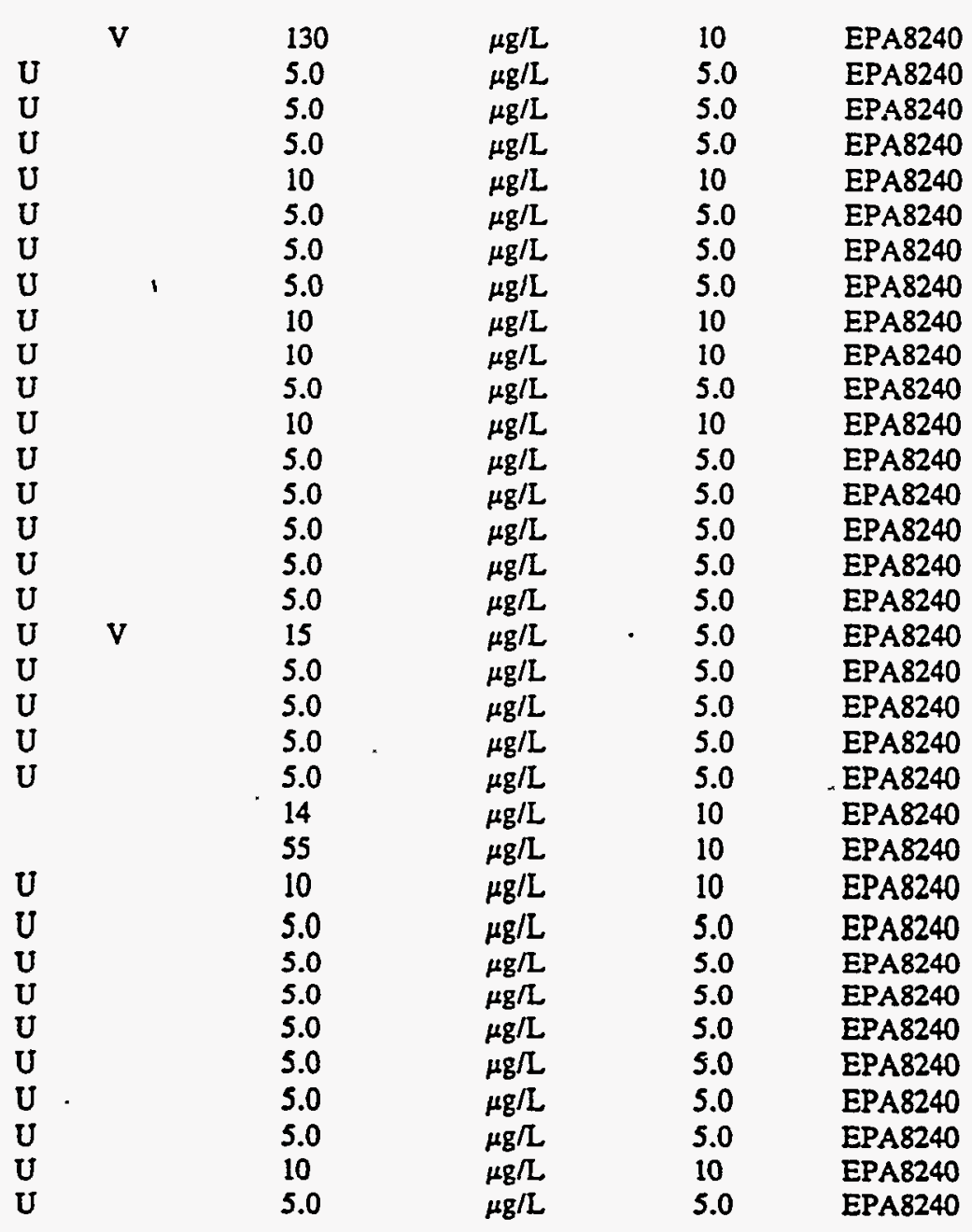


SAMPLE NAME: D431 $1802 \mathrm{C}$

Sample Type: Blank

Constituent

Total Metals

Arsenic

Barium

Cadmium

Chromium

Copper

Lead

Manganese

Mercury

Nickel

Selenium

Silver

Volatile Organic Compounds

Acerone

Acetone

Acetone

Benzene

Bromodichloromethane

Bromodichloromethane

Bromodichloromethane

Bromoform

Bromoform

Bromoform

Bromomethane (Methyi bromide)

Bromomethane (Methyl bromide)

Bromomethane (Methyl bromide)

Carbon disulfide

Carbon disulfide

Carbon disulfide

Carbon tetrachloride

Carbon tetrachloride

Carbon tetrachloride

Chlorobenzene

Chloroethane

Chloroethane

Chloroethane

Chloroethene (Vinyl chloride)

Chloroethene (Vinyl chloride)

Chloroethene (Vinyl chloride)

Chloroform

Chloroform

Chloroform

Chloromethane (Methyl chloride)

Chloromethane (Methyl chloride)

Chloromethane (Methyl chloride)

Dibromochloromethane

Dibromochloromethane
Sample ID: 100403

Associated Sample: 100401

RQ AQ B Result Unit $\quad$ D. Limit Method

$\begin{array}{lllll}U & 10 & \mu \mathrm{g} / \mathrm{L} & 10 & \text { EPA7060 } \\ \mathrm{U} & 200 & \mu \mathrm{g} / \mathrm{L} & 200 & \text { EPA6010 } \\ \mathrm{U} & 5.0 & \mu \mathrm{g} / \mathrm{L} & 5.0 & \text { EPA6010 } \\ \mathrm{U} & 10 & \mu \mathrm{g} / \mathrm{L} & 10 & \text { EPA6010 } \\ \mathrm{U} & 25 & \mu \mathrm{g} / \mathrm{L} & 25 & \text { EPA6010 } \\ \mathrm{U} & 3.0 & \mu \mathrm{g} / \mathrm{L} & 3.0 & \text { EPA7421 } \\ \mathrm{U} & 15 & \mu \mathrm{g} / \mathrm{L} & 15 & \text { EPA6010 } \\ \mathrm{U} & 0.20 & \mu \mathrm{g} / \mathrm{L} & 0.20 & \text { EPA7470 } \\ \mathrm{U} & 40 & \mu \mathrm{g} / \mathrm{L} & 40 & \text { EPA6010 } \\ \mathrm{U} & 5.0 & \mu \mathrm{g} / \mathrm{L} & 5.0 & \text { EPA7740 } \\ \mathrm{U} & 10 & \mu \mathrm{g} / \mathrm{L} & 10 & \text { EPA6010 }\end{array}$

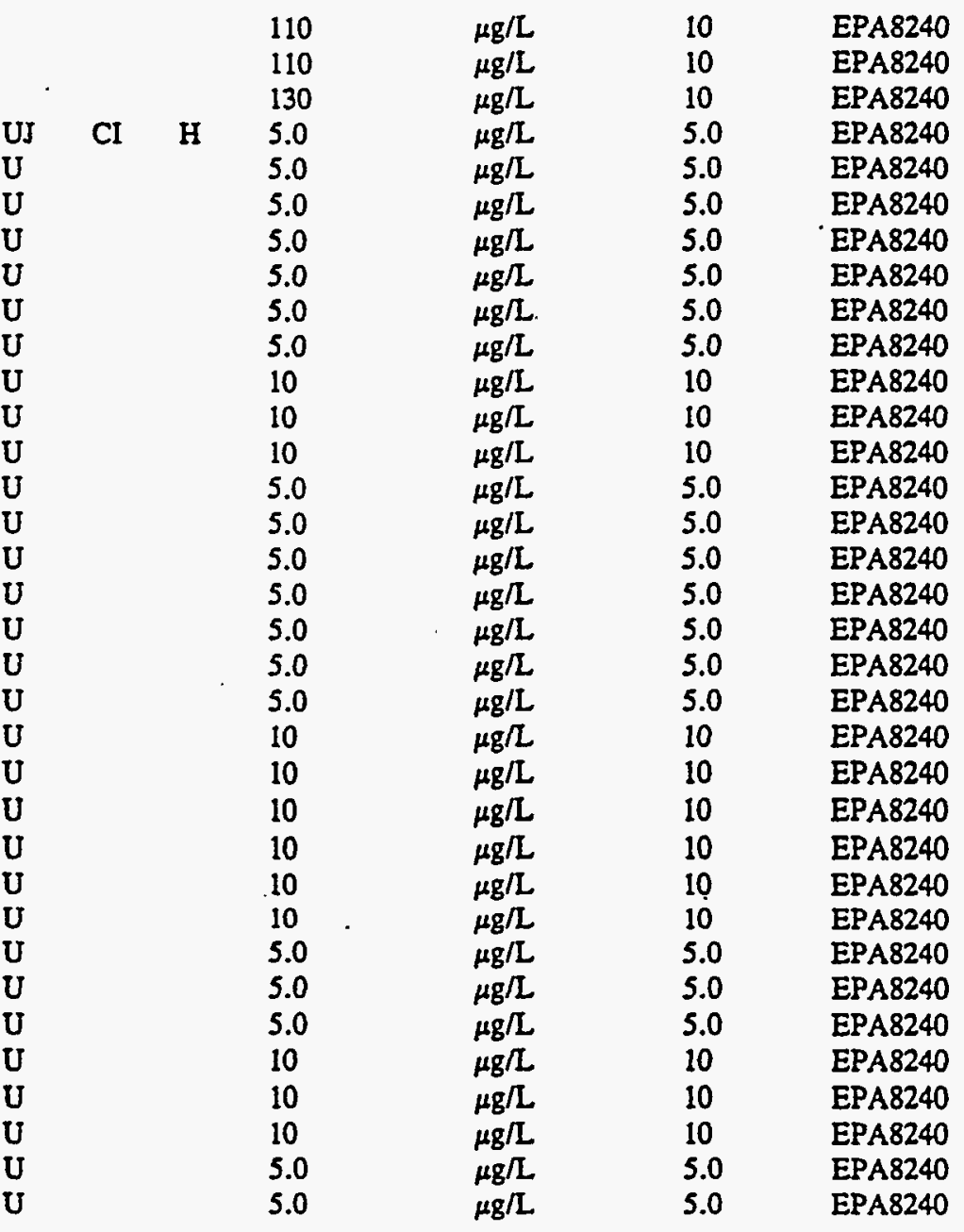


SAMPLE NAME: D431 $1802 C$ (Continued)

Sample ID: 100403

Constituent

Volatile Organic Compounds

Dibromochloromethane

1,1-Dichloroethane

1.1-Dichloroethane

1,1-Dichloroethane

1.2-Dichloroethane

1,2-Dichloroethane

1,2-Dichloroethane

1,1-Dichloroethylene

1,2-Dichloroethylene

1.2-Dichloroethylene

1,2-Dichloroethylene

Dichloromethane (Methylene chloride)

Dichloromethane (Methylene chloride)

Dichloromethane (Methylene chloride)

1,2-Dichloropropane

1,2-Dichloropropane

1,2-Dichloropropane

cis-1,3-Dichloropropene

cis-1,3-Dichloropropene

cis-1,3-Dichloropropene

trans-1,3-Dichloropropene

trans-1,3-Dichloropropene

trans-1,3-Dichloropropene

Ethylbenzene

Ethylbenzene

Ethylbenzene

2-Hexanone

2-Hexanone

2-Hexanone

Methyl ethyl ketone

Methyl ethyl ketone

Methyl ethyl ketone

Methy! isobutyl ketone

Methyl isobutyl ketone

Methyl isobutyl ketone

Styrene

Styrene

Styrene

1,1,2,2-Tetrachloroethane

1,1,2,2-Tetrachloroethane

1,1,2,2-Tetrachloroethane

Tetrachloroethylene

Tetrachloroethylene

Tetrachloroethylene

Toluene

1,1,1-Trichloroethane

1,1,1-Trichloroethane

1,1,1-Trichloroethane

1,1,2-Trichloroethane

1,1,2-Trichloroethane
RQ AQ B Result

Result Unit

D. Limit Method
5.0

5.0

5.0

5.0

5.0

5.0

5.0

5.0

5.0

5.0

5.0

5.0

5.0

5.0

5.0

5.0

5.0

5.0

5.0

5.0

5.0

5.0

5.0

5.0

5.0

5.0

8.1

8.7

9.7

36

35

42

10

10

10

5.0

5.0

5.0

5.0

5.0

5.0

5.0

5.0

5.0

5.0

5.0

5.0

5.0

5.0

5.0 $\mu \mathrm{g} / \mathrm{L}$

$\mu \mathrm{g} / \mathrm{L}$

$\mu \mathrm{g} / \mathrm{L}$

$\mu \mathrm{g} / \mathrm{L}$

$\mu g / L$

$\mu \mathrm{g} / \mathrm{L}$

$\mu \mathrm{g} / \mathrm{L}$

$\mu g / L$

$\mu \mathrm{g} / \mathrm{L}$

$\mu \mathrm{g} / \mathrm{L}$

$\mu \mathrm{g} / \mathrm{L}$

$\mu \mathrm{g} / \mathrm{L}$

$\mu \mathrm{g} / \mathrm{L}$

$\mu \mathrm{g} / \mathrm{L}$

$\mu \mathrm{g} / \mathrm{L}$

$\mu \mathrm{g} / \mathrm{L}$

$\mu \mathrm{g} / \mathrm{L}$

$\mu \mathrm{g} / \mathrm{L}$

$\mu \mathrm{g} / \mathrm{L}$

$\mu \mathrm{g} / \mathrm{L}$

$\mu \mathrm{g} / \mathrm{L}$

$\mu \mathrm{g} / \mathrm{L}$

$\mu \mathrm{g} / \mathrm{L}$

$\mu g / L$

$\mu \mathrm{g} / \mathrm{L}$

$\mu \mathrm{g} / \mathrm{L}$

$\mu \mathrm{g} / \mathrm{L}$

$\mu \mathrm{g} / \mathrm{L}$

$\mu g / L$

$\mu \mathrm{g} / \mathrm{L}$

$\mu \mathrm{g} / \mathrm{L}$

$\mu \mathrm{g} / \mathrm{L}$

$\mu g / L$

$\mu \mathrm{g} / \mathrm{L}$

$\mu \mathrm{g} / \mathrm{L}$

$\mu g / L$

$\mu \mathrm{g} / \mathrm{L}$

$\mu \mathrm{g} / \mathrm{L}$

$\mu g / L$

$\mu \mathrm{g} / \mathrm{L}$

$\mu \mathrm{g} / \mathrm{L}$

$\mu \mathrm{g} / \mathrm{L}$

$\mu \mathrm{g} / \mathrm{L}$

$\mu g / L$

$\mu \mathrm{g} / \mathrm{L}$

$\mu g / L$

$\mu \mathrm{g} / \mathrm{L}$

$\mu \mathrm{g} / \mathrm{L}$

$\mu \mathrm{g} / \mathrm{L}$

$\mu \mathrm{g} / \mathrm{L}$
5.0

5.0

5.0

5.0

5.0

5.0

5.0

5.0

5.0

5.0

5.0

5.0

5.0

5.0

5.0

5.0

5.0

5.0

S.0

5.0

5.0

5.0

5.0

5.0

5.0

5.0

10

10

10

10

10

10

10

10

10

5.0

5.0

5.0

5.0

5.0

5.0

5.0

5.0

5.0

5.0

5.0

5.0

5.0

5.0

5.0
EPA8240

EPA8240

EPA8240

EPA8240

EPA8240

EPA8240

EPA8240

EPA8240

EPA8240

EPA8240

EPA8240

EPA8240

EPA8240

EPA8240

EPA8240

EPA8240

EPA8240

EPA8240

EPA8240

EPA8240

EPA8240

EPA8240

EPA8240

EPA8240

EPA8240

EPA8240

EPA8240

EPA8240

EPA8240

EPA8240

EPA8240

EPA8240

EPA8240

EPA8240

EPA8240

EPA8240

EPA8240

EPA8240

EPA8240

EPA8240

EPA8240

EPA8240

EPA8240

EPA8240

EPA8240

EPA8240

EPA8240

EPA8240

EPA8240

EPA8240 
SAMPLE NAME: D431 1802C (Continued) Sample ID: 100403

Constituent

RQ AQ B Result

Unit

D. Limit Method

Volatile Organic Compounds

1,1.2-Trichloroethane
Trichloroethylene
Vinyl acetate
Vinyl acetate
Vinyl acetate
Xylenes
Xylenes
Xylenes

$\begin{array}{ll}\mathrm{U} & 5.0 \\ \mathrm{U} & 5.0 \\ \mathrm{U} & 10 \\ \mathrm{U} & 10 \\ \mathrm{U} & 10 \\ \mathrm{U} & 5.0 \\ \mathrm{U} & 5.0 \\ \mathrm{U} & 5.0\end{array}$

$\mu \mathrm{g} / \mathrm{L}$
$\mu \mathrm{g} / \mathrm{L}$
$\mu \mathrm{g} / \mathrm{L}$
$\mu \mathrm{g} / \mathrm{L}$
$\mu \mathrm{g} / \mathrm{L}$
$\mu \mathrm{g} / \mathrm{L}$
$\mu \mathrm{g} / \mathrm{L}$
$\mu \mathrm{g} / \mathrm{L}$

$\begin{array}{ll}5.0 & \text { EPA8240 } \\ 5.0 & \text { EPA8240 } \\ 10 & \text { EPA8240 } \\ 10 & \text { EPA8240 } \\ 10 & \text { EPA8240 } \\ 5.0 & \text { EPA8240 } \\ 5.0 & \text { EPA8240 } \\ 5.0 & \text { EPA8240 }\end{array}$

Radionuclide Indicators and Radionuclides

$\begin{array}{lllllll}\text { Gross alpha } & \mathrm{U} & & 0.50 & \mathrm{pCi} / \mathrm{L} & 0.50 & \text { EPA900.0 } \\ \text { Nonvolatile beta } & \mathrm{U} & \mathrm{X} & 0.70 & \mathrm{pCi} / \mathrm{L} & 0.70 & \text { EPA900.0 } \\ \text { Radium, total alpha-emitting } & \mathrm{U} & \mathrm{V} & 1.1 & \mathrm{pCi} / \mathrm{L} & 1.1 & \text { EPA903.0 } \\ \text { Tritium } & \mathrm{U} & & 130 & \mathrm{pCi} / \mathrm{L} & 130 & \text { EPA906.0 }\end{array}$

SAMPLE NAME: D431 2302B

Sample Type: Blank

Constituent

Volatile Organic Compounds
RQ AQ B Result Unit

Sample ID: 100316

Associated Sample: 100313

\section{Y}

$\begin{array}{llllll} & \mathrm{Y} & 20 & \mu \mathrm{g} / \mathrm{L} & 10 & \text { EPA8240 } \\ \mathrm{U} & \mathrm{Y} & 5.0 & \mu \mathrm{g} / \mathrm{L} & 5.0 & \text { EPA8240 } \\ \mathrm{U} & \mathrm{Y} & 5.0 & \mu \mathrm{g} / \mathrm{L} & 5.0 & \text { EPA8240 } \\ \mathrm{U} & \mathrm{Y} & 5.0 & \mu \mathrm{g} / \mathrm{L} & 5.0 & \text { EPA8240 } \\ \mathrm{U} & \mathrm{Y} & 10 & \mu \mathrm{L} / \mathrm{L} & 10 & \text { EPA8240 } \\ \mathrm{U} & \mathrm{Y} & 5.0 & \mu \mathrm{g} / \mathrm{L} & 5.0 & \text { EPA8240 } \\ \mathrm{U} & \mathrm{Y} & 5.0 & \mu \mathrm{L} / \mathrm{L} & 5.0 & \text { EPA8240 } \\ \mathrm{U} & \mathrm{Y} & 5.0 & \mu \mathrm{g} / \mathrm{L} & 5.0 & \text { EPA8240 } \\ \mathrm{U} & \mathrm{Y} & 10 & \mu \mathrm{L} / \mathrm{L} & 10 & \text { EPA8240 } \\ \mathrm{U} & \mathrm{Y} & 10 & \mu \mathrm{g} / \mathrm{L} & 10 & \text { EPA8240 } \\ \mathrm{U} & \mathrm{Y} & 5.0 & \mu \mathrm{g} / \mathrm{L} & 5.0 & \text { EPA8240 } \\ \mathrm{U} & \mathrm{Y} & 10 & \mu \mathrm{g} / \mathrm{L} & 10 & \text { EPA8240 } \\ \mathrm{U} & \mathrm{Y} & 5.0 & \mu \mathrm{L} / \mathrm{L} & 5.0 & \text { EPA8240 } \\ \mathrm{U} & \mathrm{Y} & 5.0 & \mu \mathrm{g} / \mathrm{L} & 5.0 & \text { EPA8240 } \\ \mathrm{U} & \mathrm{Y} & 5.0 & \mu \mathrm{g} / \mathrm{L} & 5.0 & \text { EPA8240 } \\ \mathrm{U} & \mathrm{Y} & 5.0 & \mu \mathrm{g} / \mathrm{L} & 5.0 & \text { EPA8240 } \\ \mathrm{U} & \mathrm{Y} & 5.0 & \mu \mathrm{g} / \mathrm{L} & 5.0 & \text { EPA8240 } \\ \mathrm{U} & \mathrm{Y} & 5.0 & \mu \mathrm{g} / \mathrm{L} & 5.0 & \text { EPA8240 } \\ \mathrm{U} & \mathrm{Y} & 5.0 & \mu \mathrm{g} / \mathrm{L} & 5.0 & \text { EPA8240 } \\ \mathrm{U} & \mathrm{Y} & 5.0 & \mu \mathrm{g} / \mathrm{L} & 5.0 & \text { EPA8240 } \\ \mathrm{U} & \mathrm{Y} & 5.0 & \mu \mathrm{g} / \mathrm{L} & 5.0 & \text { EPA8240 } \\ \mathrm{U} & \mathrm{Y} & 5.0 & \mu \mathrm{L} / \mathrm{L} & 5.0 & \text { EPA8240 } \\ \mathrm{U} & \mathrm{Y} & 10 & \mu \mathrm{g} / \mathrm{L} & 10 & \text { EPA8240 } \\ \mathrm{U} & \mathrm{Y} & 10 & \mu \mathrm{L} / \mathrm{L} & 10 & \text { EPA8240 } \\ \mathrm{U} & \mathrm{Y} & 10 & \mu \mathrm{g} / \mathrm{L} & 10 & \text { EPA8240 }\end{array}$


SAMPLE NAME: D431 2302B (Continued)

Constituent

Volatile Organic Compounds
RQ AQ
Sample ID: 100316

Result

5.0

5.0

5.0

5.0

5.0

5.0

5.0

10

5.0

Unit

D. Limit Method

Vinyl acetate

Xylenes

$\begin{array}{ll}U & Y \\ U & Y\end{array}$

$\mu \mathrm{g} / \mathrm{L}$

$\mu g / L$

$\mu g / L$

$\mu \mathrm{g} / \mathrm{L}$

$\mu g / L$

$\mu g / L$

$\mu g / L$

$\mu g / L$

$\mu \mathrm{g} / \mathrm{L}$

5.0

EPA8240

EPA8240

EPA8240

5.0

5.0

EPA8240

5.0

EPA8240

5.0

EPA8240

5.0

EPA8240

$10 \quad$ EPA8240

5.0

EPA8240

Radionuclide Indicators and Radionuclides

Gross alpha

Nonvolatile beta

Radium, total alpha-emitting

Tritium

$\begin{array}{lll}U & Y & 0.50 \\ U & Y X & 0.50 \\ U & Y & 0.10 \\ U & Y & 160\end{array}$

Sample ID: 100315

Associated Sample: 100313

RQ AQ B Result Unit

pCi/L

$\mathrm{pCi} / \mathrm{L}$

$\mathrm{pCi} / \mathrm{L}$

$\mathrm{pCi} / \mathrm{L}$
0.50

0.50

0.10

160
EPA900.0

EPA900.0

EPA903.0

EPA906.0
SAMPLE NAMEE: D431 2302E

Sample Type: Rinsate

Constituent

Total Metals

Arsenic

Barium

Cadmium

Chromium

Copper

Lead

Manganese

Mercury

Nickel

Selenium

Silver

$U$
$U$
$U$
$U$
$U$
$U$
$U$
$U$
$U$
$U$
$U$

10

200

5.0

10

25

3.0

15

0.20

40

5.0

10 $\mu g / L$
$\mu g / L$
$\mu g / L$
$\mu g / L$
$\mu g / L$
$\mu g / L$
$\mu g / L$
$\mu g / L$
$\mu g / L$
$\mu g / L$
$\mu g / L$
D. Limit Method

Volatile Organic Compounds

$\begin{array}{lllllll}\text { Acetone } & \mathrm{J} & \mathrm{Y} & 64 & \mu \mathrm{g} / \mathrm{L} & 10 & \text { EPA8240 } \\ \text { Acetone } & \mathrm{J} & \mathrm{Y} & 60 & \mu \mathrm{g} / \mathrm{L} & 10 & \text { EPA8240 } \\ \text { Acetone } & \mathrm{J} & \mathrm{Y} & 63 & \mu \mathrm{g} / \mathrm{L} & 10 & \text { EPA8240 } \\ \text { Benzene } & \mathrm{U} & \mathrm{Y} & 5.0 & \mu \mathrm{g} / \mathrm{L} & 5.0 & \text { EPA8240 } \\ \text { Bromodichloromethane } & \mathrm{U} & \mathrm{Y} & 5.0 & \mu \mathrm{g} / \mathrm{L} & 5.0 & \text { EPA8240 } \\ \text { Bromodichloromethane } & \mathrm{U} & \mathrm{Y} & 5.0 & \mu \mathrm{g} / \mathrm{L} & 5.0 & \text { EPA8240 } \\ \text { Bromodichloromethane } & \mathrm{U} & \mathrm{Y} & 5.0 & \mu \mathrm{g} / \mathrm{L} & 5.0 & \text { EPA8240 } \\ \text { Bromoform } & \mathrm{U} & \mathrm{Y} & 5.0 & \mu \mathrm{g} / \mathrm{L} & 5.0 & \text { EPA8240 } \\ \text { Bromoform } & \mathrm{U} & \mathrm{Y} & 5.0 & \mu \mathrm{g} / \mathrm{L} & 5.0 & \text { EPA8240 } \\ \text { Bromoform } & \mathrm{U} & \mathrm{Y} & 5.0 & \mu \mathrm{g} / \mathrm{L} & 5.0 & \text { EPA8240 }\end{array}$


SAMPLE NAME: D431 2302E (Continued)

Constituent

RQ AQ B Result

D. Limit Method

Volatile Organic Compounds

Bromomethane (Methyl bromide)

Bromomethane (Methyl bromide)

Bromomethane (Methyl bromide)

Carbon disulfide

Carbon disulfide

Carbon disulfide

Carbon tetrachloride

Carbon tetrachloride

Carbon tetrachloride

Chlorobenzene

Chloroethane

Chloroethane

Chloroethane

Chloroethene (Vinyl chloride)

Chloroethene (Vinyl chloride)

Chloroethene (Vinyl chloride)

Chloroform

Chloroform

Chloroform

Chloromethane (Methyl chloride)

Chloromethane (Methyl chloride)

Chloromethane (Methyl chloride)

Dibromochloromethane

Dibromochloromethane

Dibromochloromethane

1,1-Dichloroethane

1,1-Dichloroethane

1,1-Dichloroethane

1,2-Dichloroethane

1,2-Dichloroethane

1,2-Dichloroethane

1,1-Dichloroethylene

1,2-Dichloroethylene

1,2-Dichloroethylene

1,2-Dichloroethylene

Dichloromethane (Methylene chloride)

Dichloromethane (Methylene chloride)

Dichloromethane (Methylene chloride)

1,2-Dichloropropane

1,2-Dichloropropane

1,2-Dichloropropane

cis-1,3-Dichloropropene

cis-1,3-Dichloropropene

cis-1,3-Dichloropropene

trans-1,3-Dichloropropene

trans-1,3-Dichloropropene

trans-1,3-Dichloropropene

Ethylbenzene

Ethylbenzene

Ethylbenzene
Unit

Sample ID: 100315

Unit

10
10
10
5.0

$\mu \mathrm{g} / \mathrm{L}$

$\mu g / L$

$\mu \mathrm{g} / \mathrm{L}$

$\mu \mathrm{g} / \mathrm{L}$

$\mu \mathrm{g} / \mathrm{L}$

$\mu \mathrm{g} / \mathrm{L}$

$\mu \mathrm{g} / \mathrm{L}$

$\mu \mathrm{g} / \mathrm{L}$

$\mu \mathrm{g} / \mathrm{L}$

$\mu \mathrm{g} / \mathrm{L}$

$\mu \mathrm{g} / \mathrm{L}$

$\mu \mathrm{g} / \mathrm{L}$

$\mu \mathrm{g} / \mathrm{L}$

$\mu \mathrm{g} / \mathrm{L}$

$\mu \mathrm{g} / \mathrm{L}$

$\mu \mathrm{g} / \mathrm{L}$

$\mu \mathrm{g} / \mathrm{L}$

$\mu \mathrm{g} / \mathrm{L}$

$\mu \mathrm{g} / \mathrm{L}$

$\mu \mathrm{g} / \mathrm{L}$

$\mu \mathrm{g} / \mathrm{L}$

$\mu \mathrm{g} / \mathrm{L}$

$\mu \mathrm{g} / \mathrm{L}$

$\mu \mathrm{g} / \mathrm{L}$

$\mu \mathrm{g} / \mathrm{L}$

$\mu \mathrm{g} / \mathrm{L}$

$\mu \mathrm{g} / \mathrm{L}$

$\mu \mathrm{g} / \mathrm{L}$

$\mu \mathrm{g} / \mathrm{L}$

$\mu \mathrm{g} / \mathrm{L}$

$\mu \mathrm{g} / \mathrm{L}$

$\mu \mathrm{g} / \mathrm{L}$

$\mu \mathrm{g} / \mathrm{L}$

$\mu \mathrm{g} / \mathrm{L}$

$\mu \mathrm{g} / \mathrm{L}$

$\mu g / L$

$\mu \mathrm{g} / \mathrm{L}$

$\mu \mathrm{g} / \mathrm{L}$

$\mu \mathrm{g} / \mathrm{L}$

$\mu g / L$

$\mu \mathrm{g} / \mathrm{L}$

$\mu g / L$

$\mu \mathrm{g} / \mathrm{L}$

$\mu \mathrm{g} / \mathrm{L}$

$\mu g / L$

$\mu \mathrm{g} / \mathrm{L}$

$\mu \mathrm{g} / \mathrm{L}$

$\mu \mathrm{g} / \mathrm{L}$

$\mu g / L$

$\mu \mathrm{g} / \mathrm{L}$
EPA8240

EPA8240

EPA8240

EPA8240

EPA8240

EPA8240

EPA8240

EPA8240

EPA8240

EPA8240

EPA8240

EPA8240

EPA8240

EPA8240

EPA8240

EPA8240

EPA8240

EPA8240

EPA8240

EPA8240

EPA8240

EPA8240

EPA8240

EPA8240

EPA8240

EPA8240

EPA8240

EPA8240

EPA8240

EPA8240

EPA8240

EPA8240

EPA8240

EPA8240

EPA8240

EPA8240

EPA8240

EPA8240

EPA8240

EPA8240

EPA8240

EPA8240

EPA8240

EPA8240

EPA8240

EPA8240

EPA8240

EPA8240

EPA8240

EPA8240 
SAMPLE NAME: D431 2302E (Continued)

Constituent

RQ AQ B Result

Volatile Organic Compounds

2-Hexanone
2-Hexanone
2-Hexanone
Methyl ethyl ketone
Methyl ethyl ketone
Methyl ethyl ketone
Methyl isoburyl ketone
Methyl isobutyl ketone
Methyl isobutyl ketone
Styrene
Styrene
Styrene
1,1,2,2-Tetrachloroethane
1,1,2,2-Tetrachloroethane
1,1,2,2-Tetrachloroethane
Tetrachloroethylene
Tetrachloroethylene
Tetrachloroethylene
Toluene
1,1,1-Trichloroethane
1,1,1-Trichloroethane
1,1,1-Trichloroethane
1,1,2-Trichloroethane
1,1,2-Trichloroethane
1,1,2-Trichloroethane
Trichloroethylene
Vinyl acetate
Vinyl acetate
Vinyl acetate
2-Propanol
Xylenes
Xylenes
Xylenes

$\begin{array}{lll}U & Y & 10 \\ U & Y & 10 \\ U & Y & 10 \\ U & Y & 10 \\ U & Y & 10 \\ U & Y & 10 \\ U & Y & 10 \\ U & Y & 10 \\ U & Y & 10 \\ U & Y & 5.0 \\ U & Y & 5.0 \\ U & Y & 5.0 \\ U & Y & 5.0 \\ U & Y & 5.0 \\ U & Y & 5.0 \\ U & Y & 5.0 \\ U & Y & 5.0 \\ U & Y & 5.0 \\ U & Y & 5.0 \\ U & Y & 5.0 \\ U & Y & 5.0 \\ U & Y & 5.0 \\ U & Y & 5.0 \\ U & Y & 5.0 \\ U & Y & 5.0 \\ U & Y & 5.0 \\ U & Y & 10 \\ U & Y & 10 \\ U & Y & 10 \\ J & Y N & 100 \\ U & Y & 5.0 \\ U & Y & 5.0 \\ U & Y & 5.0\end{array}$

Radionuclide Indicators and Radionuclides

Gross alpha

Gross alpha

Nonvolatile beta

Nonvolatile beta

Radium, total alpha-emitting

Radium, total alpha-emitting

Tritium

Tritium
Unit

D. Limit Method

Sample ID: 100315

10

10

10

10

10

10

5.0

5.0

5.0

5.0

5.0

5.0

5.0

5.0

5.0

5.0

5.0

5.0

5.0

5.0

5.0

10

10

100

5.0

5.0
5.0

$\begin{array}{lll}\mu \mathrm{g} / \mathrm{L} & 10 & \text { EPA8240 } \\ \mu \mathrm{g} / \mathrm{L} & 10 & \text { EPA8240 } \\ \mu \mathrm{g} / \mathrm{L} & 10 & \text { EPA8240 } \\ \mu \mathrm{g} / \mathrm{L} & 10 & \text { EPA8240 } \\ \mu \mathrm{g} / \mathrm{L} & 10 & \text { EPA8240 } \\ \mu \mathrm{g} / \mathrm{L} & 10 & \text { EPA8240 } \\ \mu \mathrm{g} / \mathrm{L} & 10 & \text { EPA8240 } \\ \mu \mathrm{g} / \mathrm{L} & 10 & \text { EPA8240 } \\ \mu \mathrm{g} / \mathrm{L} & 10 & \text { EPA8240 } \\ \mu \mathrm{g} / \mathrm{L} & 5.0 & \text { EPA8240 } \\ \mu \mathrm{g} / \mathrm{L} & 5.0 & \text { EPA8240 } \\ \mu \mathrm{g} / \mathrm{L} & 5.0 & \text { EPA8240 } \\ \mu \mathrm{g} / \mathrm{L} & 5.0 & \text { EPA8240 } \\ \mu \mathrm{g} / \mathrm{L} & 5.0 & \text { EPA8240 } \\ \mu \mathrm{g} / \mathrm{L} & 5.0 & \text { EPA8240 } \\ \mu \mathrm{g} / \mathrm{L} & 5.0 & \text { EPA8240 } \\ \mu \mathrm{g} / \mathrm{L} & 5.0 & \text { EPA8240 } \\ \mu \mathrm{g} / \mathrm{L} & 5.0 & \text { EPA8240 } \\ \mu \mathrm{g} / \mathrm{L} & 5.0 & \text { EPA8240 } \\ \mu \mathrm{g} / \mathrm{L} & 5.0 & \text { EPA8240 } \\ \mu \mathrm{g} / \mathrm{L} & 5.0 & \text { EPA8240 } \\ \mu \mathrm{g} / \mathrm{L} & 5.0 & \text { EPA8240 } \\ \mu \mathrm{g} / \mathrm{L} & 5.0 & \text { EPA8240 } \\ \mu \mathrm{g} / \mathrm{L} & 5.0 & \text { EPA8240 } \\ \mu \mathrm{g} / \mathrm{L} & 5.0 & \text { EPA8240 } \\ \mu \mathrm{g} / \mathrm{L} & 5.0 & \text { EPA8240 } \\ \mu \mathrm{g} / \mathrm{L} & 10 & \text { EPA8240 } \\ \mu \mathrm{g} / \mathrm{L} & 10 & \text { EPA8240 } \\ \mu \mathrm{g} / \mathrm{L} & 10 & \text { EPA8240 } \\ \mu \mathrm{g} / \mathrm{L} & & \text { EPA8240 } \\ \mu \mathrm{g} / \mathrm{L} & 5.0 & \text { EPA8240 } \\ \mu \mathrm{g} / \mathrm{L} & 5.0 & \text { EPA8240 } \\ \mu \mathrm{g} / \mathrm{L} & 5.0 & \text { EPA8240 }\end{array}$

0.50

0.50

$1.0 \pm 1.6$

$\mathrm{pCi} / \mathrm{L}$

$\mathrm{PCi} / \mathrm{L}$

0.50

0.50

EPA900.0

EPA900.0

EPA900.0

$4.2 \pm 2.0 \quad \mathrm{pCi} / \mathrm{L}$

0.50

EPA900.0

$0.10 \quad \mathrm{pCi} / 2$

0.10

EPA903.0

$\mathrm{pCi} / \mathrm{L}$

160

170

$\mathrm{pCi} / \mathrm{L}$

0.10

160

EPA906.0

EPA906.0
EPA903.0 
SAMPLE NAME: D431 2602C

Sample Type: Blank

Constituent

Volatile Organic Compounds

Acetone
Benzene
Bromodichloromethane
Bromoform
Bromomethane (Methyl bromide)
Carbon disulfide
Carbon tetrachloride
Chlorobenzene
Chloroethane
Chloroethene (Vinyl chloride)
2-Chloroethyl vinyl ether
Chloroform
Chloromethane (Methyl chloride)
Dibromochloromethane
1,1-Dichloroethane
1,2-Dichloroethane
1,1-Dichloroethylene
1,2-Dichloroethylene
Dichloromethane (Methylene chloride)
1,2-Dichloropropane
cis-1,3-Dichloropropene
trans-1,3-Dichloropropene
Ethylbenzene
2-Hexanone
Methyl ethyl ketone
Methyl isobutyl ketone
Styrene
1,1,2,2-Tetrachloroethane
Tetrachloroethylene
Toluene
1,1,1-Trichloroethane
1,1,2-Trichloroethane
Trichloroethylene
Vinyl acetate
Xylenes

Sample ID: 100302

Associated Sample: 100301

Unit

D. Limit Method

\begin{tabular}{|c|c|c|c|c|}
\hline \multirow[t]{10}{*}{ V } & 190 & $\mu g / L$ & 10 & EPA8240 \\
\hline & 5.0 & $\mu \mathrm{g} / \mathrm{L}$ & 5.0 & EPA8240 \\
\hline & 5.0 & $\mu \mathrm{g} / \mathrm{L}$ & 5.0 & EPA8240 \\
\hline & 5.0 & $\mu \mathrm{g} / \mathrm{L}$ & 5.0 & EPA8240 \\
\hline & 10 & $\mu g / L$ & 10 & EPA8240 \\
\hline & 5.0 & $\mu g / L$ & 5.0 & EPA8240 \\
\hline & 5.0 & $\mu g / L$ & 5.0 & EPA8240 \\
\hline & 5.0 & $\mu \mathrm{g} / \mathrm{L}$ & 5.0 & EPA8240 \\
\hline & 10 & $\mu g / L$ & 10 & EPA8240 \\
\hline & 10 & $\mu \mathrm{g} / \mathrm{L}$ & 10 & EPA8240 \\
\hline & 10 & $\mu g / L$ & 10 & EPA8240 \\
\hline & 5.0 & $\mu \mathrm{g} / \mathrm{L}$ & 5.0 & EPA8240 \\
\hline & 10 & $\mu \mathrm{g} / \mathrm{L}$ & 10 & EPA8240 \\
\hline & 5.0 & $\mu \mathrm{g} / \mathrm{L}$ & 5.0 & EPA 8240 \\
\hline & 5.0 & $\mu \mathrm{g} / \mathrm{L}$ & 5.0 & EPA8240 \\
\hline & 5.0 & $\mu g / L$ & 5.0 & EPA8240 \\
\hline & 5.0 & $\mu \mathrm{g} / \mathrm{L}$ & 5.0 & EPA 8240 \\
\hline & 5.0 & $\mu \mathrm{g} / \mathrm{L}$ & 5.0 & EPA8240 \\
\hline \multirow[t]{17}{*}{$\mathrm{v}$} & 32 & $\mu \mathrm{g} / \mathrm{L}$ & 5.0 & EPA8240 \\
\hline & 5.0 & $\mu \mathrm{g} / \mathrm{L}$ & 5.0 & EPA8240 \\
\hline & 5.0 & $\mu \mathrm{g} / \mathrm{L}$ & 5.0 & EPA8240 \\
\hline & 5.0 & $\mu \mathrm{g} / \mathrm{L}$ & 5.0 & EPA 8240 \\
\hline & 5.0 & $\mu g / L$ & 5.0 & EPA 8240 \\
\hline & 24 & $\mu g / L$ & 10 & EPA8240 \\
\hline & 99 & $\mu g / L$ & 10 & EPA8240 \\
\hline & 10 & $\mu \mathrm{g} / \mathrm{L}$ & 10 & EPA8240 \\
\hline & 5.0 & $\mu \mathrm{g} / \mathrm{L}$ & 5.0 & EPA8240 \\
\hline & 5.0 & $\mu g / L$ & 5.0 & EPA8240 \\
\hline & 5.0 & $\mu g / L$ & 5.0 & EPA8240 \\
\hline & 5.0 & $\mu \mathrm{g} / \mathrm{L}$ & 5.0 & EPA8240 \\
\hline & 5.0 & $\mu \mathrm{g} / \mathrm{L}$ & 5.0 & EPA8240 \\
\hline & 5.0 & $\mu \mathrm{g} / \mathrm{L}$ & 5.0 & EPA8240 \\
\hline & 5.0 & $\mu \mathrm{g} / \mathrm{L}$ & 5.0 & EPA8240 \\
\hline & 10 & $\mu g / L$ & 10 & EPA8240 \\
\hline & 5.0 & $\mu g / L$ & 5.0 & EPA8240 \\
\hline
\end{tabular}

\section{Radionuclide Indicators and Radionuclides}

\begin{tabular}{|c|c|c|c|c|c|c|}
\hline Gross alpha & $\mathrm{U}$ & & 0.60 & $\mathrm{pCi} / \mathrm{L}$ & 0.60 & EPA900.0 \\
\hline Gross alpha & $\mathbf{U}$ & & 0.70 & $\mathrm{pCi} / \mathrm{L}$ & 0.70 & EPA900.0 \\
\hline Nonvolatile beta & $\mathbf{U}$ & $\mathrm{v}$ & $1.4 \pm 1.6$ & $\mathrm{pCi} / \mathrm{L}$ & 0.80 & EPA900.0 \\
\hline Nonvolatile beta & $\mathrm{U}$ & $\mathbf{v}$ & 0.80 & $\mathrm{pCi} / \mathrm{L}$ & 0.80 & EPA900.0 \\
\hline Radium, total alpha-emitting & $\mathrm{U}$ & & 0.10 & $\mathrm{pCi} / \mathrm{L}$ & 0.10 & EPA903.0 \\
\hline Tritium & $\mathrm{U}$ & & 160 & $\mathrm{pCi} / \mathrm{L}$ & 160 & EPA906.0 \\
\hline
\end{tabular}


SAMPLE NAME:'D43110705E

Sample Type: Rinsate

Constituent

Total Metals
Sample ID: 100325

Associated Sample: 100322

RQ

$\mathbf{A Q}$

B . Result

Unit

D. Limit Method

Arsenic
Barium
Cadmium
Chromium
Copper
Lead
Manganese
Mercury
Nickel
Selenium
Silver

Volatile Organic Compounds

Acetone
Benzene
Bromodichloromethane
Bromoform
Bromomethane (Methyl bromide)
Carbon disulfide
Carbon tetrachloride
Chlorobenzene
Chloroethane
Chloroethene (Vinyl chloride)
Chloroform
Chloromethane (Methyl chloride)
Dibromochloromethane
1,1-Dichloroethane
1,2-Dichloroethane
1,1-Dichloroethylene
1,2-Dichloroethylene
Dichloromethane (Methylene chloride)
1,2-Dichloropropane
cis-1,3-Dichloropropene
trans-1,3-Dichloropropene
Ethylbenzene
2-Hexanone
Methyl ethyl ketone
Methyl isobutyl ketone
Styrene
1,1,2,2-Tetrachloroethane
Tetrachloroethylene
Toluene
1,1,1-Trichloroethane
1,1,2-Trichloroethane
Trichloroethylene
Vinyl acetate
2-Propanol

$\begin{array}{lll}U & Y & 10 \\ U & Y & 200 \\ U & Y & 5.0 \\ U & Y & 10 \\ U & Y & 25 \\ U & Y V & 3.0 \\ U & Y & 15 \\ U & Y & 0.20 \\ U & Y & 40 \\ U & Y & 5.0 \\ U & Y & 10\end{array}$

$\mu \mathrm{g} / \mathrm{L}$
$\mu \mathrm{g} / \mathrm{L}$
$\mu \mathrm{g} / \mathrm{L}$
$\mu \mathrm{g} / \mathrm{L}$
$\mu \mathrm{g} / \mathrm{L}$
$\mu \mathrm{g} / \mathrm{L}$
$\mu \mathrm{g} / \mathrm{L}$
$\mu \mathrm{g} / \mathrm{L}$
$\mu \mathrm{g} / \mathrm{L}$
$\mu \mathrm{g} / \mathrm{L}$
$\mu \mathrm{g} / \mathrm{L}$

10

200

5.0

10

25

3.0

15

0.20

40

5.0

10

EPA7060
EPA6010
EPA6010
EPA6010
EPA6010
EPA7421
EPA6010
EPA7470
EPA6010
EPA7740
EPA6010

\begin{tabular}{|c|c|c|c|c|}
\hline$Y$ & 120 & $\mu g / L$ & 10 & EPA8240 \\
\hline$Y$ & 5.0 & $\mu \mathrm{g} / \mathrm{L}$ & 5.0 & EPA8240 \\
\hline $\mathbf{Y}$ & 5.0 & $\mu \mathrm{g} / \mathrm{L}$ & 5.0 & EPA8240 \\
\hline $\mathbf{U}$ & 5.0 & $\mu g / L$ & 5.0 & EPA8240 \\
\hline$Y$ & 10 & $\mu \mathrm{g} / \mathrm{L}$ & 10 & EPA8240 \\
\hline $\mathbf{Y}$ & 5.0 & $\mu \mathrm{g} / \mathrm{L}$ & 5.0 & EPA8240 \\
\hline $\mathbf{Y}$ & 5.0 & $\mu \mathrm{g} / \mathrm{L}$ & 5.0 & EPA8240 \\
\hline $\mathbf{Y}$ & 5.0 & $\mu \mathrm{g} / \mathrm{L}$ & 5.0 & EPA8240 \\
\hline$Y$ & 10 & $\mu \mathrm{g} / \mathrm{L}$ & 10 & EPA8240 \\
\hline$Y$ & 10 & $\mu \mathrm{g} / \mathrm{L}$ & 10 & EPA8240 \\
\hline$Y$ & 5.0 & $\mu \mathrm{g} / \mathrm{L}$ & 5.0 & EPA8240 \\
\hline $\mathbf{Y}$ & 10 & $\mu \mathrm{g} / \mathrm{L}$ & 10 & EPA8240 \\
\hline$Y$ & 5.0 & $\mu \mathrm{g} / \mathrm{L}$ & 5.0 & EPA8240 \\
\hline$Y$ & 5.0 & $\mu \mathrm{g} / \mathrm{L}$ & 5.0 & EPA8240 \\
\hline$Y$ & 5.0 & $\mu \mathrm{g} / \mathrm{L}$ & 5.0 & EPA8240 \\
\hline $\mathbf{Y}$ & 5.0 & $\mu \mathrm{g} / \mathrm{L}$ & 5.0 & EPA8240 \\
\hline$Y$ & 5.0 & $\mu \mathrm{g} / \mathrm{L}$ & 5.0 & EPA8240 \\
\hline $\mathbf{Y}$ & 5.0 & $\mu \mathrm{g} / \mathrm{L}$ & 5.0 & EPA8240 \\
\hline $\mathbf{U}$ & 5.0 & $\mu g / L$ & 5.0 & EPA8240 \\
\hline $\mathbf{Y}$ & 5.0 & $\mu g / L$ & 5.0 & EPA8240 \\
\hline $\mathbf{U}$ & 5.0 & $\mu g / L$ & 5.0 & EPA8240 \\
\hline $\mathbf{Y}$ & 5.0 & $\mu g / L$ & $\$ 50$ & EPA8240 \\
\hline$Y$ & 10 & $\mu g / L$ & 10 & EPA8240 \\
\hline $\mathbf{U}$ & 10 & $\mu g / L$ & 10 & EPA8240 \\
\hline $\mathbf{Y}$ & 10 & $\mu g / L$ & 10 & EPA8240 \\
\hline $\mathbf{U}$ & 5.0 & $\mu g / L$ & 5.0 & EPA8240 \\
\hline $\mathbf{Y}$ & 5.0 & $\mu g / L$ & 5.0 & EPA8240 \\
\hline $\mathbf{Y}$ & 5.0 & $\mu g / L$ & 5.0 & EPA8240 \\
\hline $\mathbf{U}$ & 5.0 & $\mu \mathrm{g} / \mathrm{L}$ & 5.0 & EPA8240 \\
\hline $\mathbf{Y}$ & 5.0 & $\mu g / L$ & 5.0 & EPA8240 \\
\hline $\mathbf{U}$ & 5.0 & $\mu g / L$ & 5.0 & EPA8240 \\
\hline U & 5.0 & $\mu g / L$ & 5.0 & EPA8240 \\
\hline $\mathbf{U}$ & 10 & $\mu \mathrm{g} / \mathrm{L}$ & 10 & EPA8240 \\
\hline YN & 300 & $\mu g / L$ & & EPA8240 \\
\hline
\end{tabular}


SAMPLE NAME: D431 10705E (Continued)

Sample ID: 100325

Constituent

RQ AQ B Result

U Y

5.0

$\mu \mathrm{g} / \mathrm{L}$

5.0

EPA8240

Radionuclide Indicators and Radionuclides

Gross alpha

Nonvolatile beta

Radium, total alpha-emitzing

Tritium

Unit

D. Limit Method
SAMPLE NAME: D43110706C

Sample Type: Blank

Constituent

Total Metals

\begin{tabular}{ll} 
Arsenic & \\
Barium & \\
Cadmium & \\
Chromium & \\
Copper & \\
Lead & \\
Manganese & \\
Mercury & \\
Nickel & \\
Selenium & $U$ \\
Silver & \\
\hline
\end{tabular}

Volatile Organic Compounds

Acetone

Benzene

Bromodichloromethane

Bromoform

Bromomethane (Methyl bromide)

Carbon disulfide

Carbon tetrachloride

Chlorobenzene

Chloroethane

Chloroethene (Vinyl chloride)

Chloroform

Chloromethane (Methyl chloride)

Dibromochloromethane

1.1-Dichloroethane

1,2-Dichloroethane

1,1-Dichloroethylene

1,2-Dichlorocthylene

Dichloromethane (Methylene chloride)

$\begin{array}{ll}\mathbf{U} & \mathbf{Y} \\ \mathrm{U} & \mathbf{Y X} \\ \mathrm{U} & \mathbf{Y} \\ \mathrm{U} & \mathbf{Y}\end{array}$

0.50

0.50

0.10

170

Sample ID: 100327

Associated Sample: 100326

RQ AQ B Result Unit D. Limit Method

$\begin{array}{lll}\mathrm{pCi} / \mathrm{L} & 0.50 & \text { EPA990.0 } \\ \mathrm{pC} i / \mathrm{L} & 0.50 & \text { EPA900.0 } \\ \mathrm{pCi} / \mathrm{L} & 0.10 & \text { EPA903.0 } \\ \mathrm{pCi} / \mathrm{L} & 170 & \text { EPA906.0 }\end{array}$

$\begin{array}{lll}\mu \mathrm{g} / \mathrm{L} & 10 & \text { EPA7060 } \\ \mu \mathrm{g} / \mathrm{L} & 200 & \text { EPA6010 } \\ \mu \mathrm{g} / \mathrm{L} & 5.0 & \text { EPA6010 } \\ \mu \mathrm{g} / \mathrm{L} & 10 & \text { EPA6010 } \\ \mu \mathrm{g} / \mathrm{L} & 25 & \text { EPA6010 } \\ \mu \mathrm{g} / \mathrm{L} & 3.0 & \text { EPA7421 } \\ \mu \mathrm{g} / \mathrm{L} & 15 & \text { EPA6010 } \\ \mu \mathrm{g} / \mathrm{L} & 0.20 & \text { EPA7470 } \\ \mu \mathrm{g} / \mathrm{L} & 40 & \text { EPA6010 } \\ \mu \mathrm{g} / \mathrm{L} & 5.0 & \text { EPA7740 } \\ \mu \mathrm{g} / \mathrm{L} & .10 & \text { EPA6010 }\end{array}$

$\mu g / L$

$\mu \mathrm{g} / \mathrm{L}$

$\mu \mathrm{g} / \mathrm{L}$

$\mu \mathrm{g} / \mathrm{L}$

$\mu \mathrm{g} / \mathrm{L}$

$\mu \mathrm{g} / \mathrm{L}$

$\mu g / L$

$\mu \mathrm{g} / \mathrm{L}$

$\mu \mathrm{g} / \mathrm{L}$

$\mu \mathrm{g} / \mathrm{L}$

$\mu \mathrm{g} / \mathrm{L}$

$\mu g / L$

$\mu \mathrm{g} / \mathrm{L}$

$\mu g / L$

$\mu g / L$

$\mu \mathrm{g} / \mathrm{L}$

$\mu \mathrm{g} / \mathrm{L}$

$\mu g / L$
EPA8240

EPA8240

EPA8240

EPA8240

EPA8240

EPA8240

EPA8240

EPA8240

EPA8240

EPA8240

EPA8240

EPA8240

EPA8240

EPA8240

EPA8240

EPA8240

EPA8240

EPA8240 
SAMPLE NAME: D431-10706C (Continued)

Constituent

Volatile Organic Compounds

\section{2-Dichloropropane}

cis-1,3-Dichloropropene

trans-1,3-Dichloropropene

Ethylbenzene

2-Hexanone

Methyl ethyl ketone

Methyl isobutyl ketone

Styrene

1,1,2,2-Tetrachloroethane

Tetrachloroethylene

Toluene

1,1.1-Trichloroethane

1,1,2-Trichloroethane

Trichloroethylene

Vinyl acetate

Xylenes
Sample ID: 100327

B Result Unit:

$\mu \mathrm{g} / \mathrm{L} \quad 5.0$

$\mu \mathrm{g} / \mathrm{L} \cdot \quad 5.0$

$\mu \mathrm{g} / \mathrm{L} \quad 5.0$

$\mu \mathrm{g} / \mathrm{L} \quad 5.0$

$\mu \mathrm{g} / \mathrm{L} \quad 10$

$\mu \mathrm{g} / \mathrm{L}$

$\mu \mathrm{g} / \mathrm{L}$

$\mu g / L$

$\mu \mathrm{g} / \mathrm{L}$

$\mu \mathrm{g} / \mathrm{L}$

$\mu \mathrm{g} / \mathrm{L}$

$\mu g / L$

$\mu \mathrm{g} / \mathrm{L}$

$\mu \mathrm{g} / \mathrm{L}$

$\mu g / L$

$\mu \mathrm{g} / \mathrm{L}$

10

$\mathrm{U}-5.0$

Radionuclide Indicators and Radionuclides
Gross alpha

Gross alpha

Nonvolatile beta

Radium, total alpha-emitting

Radium, cotal alpha-emitting

Tritium

Tritium
$\mathbf{U}$
$\mathbf{U}$
$\mathbf{U}$
$\mathbf{U}$
$\mathbf{U}$
$\mathbf{U}$

SAMPLE NAME: D43110804C

Sample Type: Blank

Constituent

Total Metals

Arsenic

Barium

Cadmium

Chromium

Copper

Lead

Manganese

Nickel

Selenium

Silver
Mercury

0.60
0.60
$4.9 \pm 2.0$
0.10
0.20
140
140

$\mathrm{pCi} / \mathrm{L}$

$\mathrm{pCi} / \mathrm{L}$

$\mathrm{pCi} / \mathrm{L}$

$\mathrm{pCi} / \mathrm{L}$

$\mathrm{pCi} / \mathrm{L}$

$\mathrm{pCi} / \mathrm{L}$

$\mathrm{pCi} / \mathrm{L}$
Sample ID: 100333

Associated Sample: 100332
D. Limit Method

EPA8240

EPA8240

EPA8240

EPA8240

EPA8240

EPA8240

EPA8240

EPA8240

EPA8240

EPA8240

EPA8240

EPA8240
RQ
AQ B Result
Unit
D. Limit Method

$\begin{array}{ll}0.60 & \text { EPA900.0 } \\ 0.60 & \text { EPA990.0 } \\ 1.0 & \text { EPA990.0 } \\ 0.10 & \text { EPA903.0 } \\ 0.20 & \text { EPA903.0 } \\ 140 & \text { EPA906.0 } \\ 140 & \text { EPA906.0 }\end{array}$

$\begin{array}{lllll}\mathrm{U} & 10 & \mu \mathrm{g} / \mathrm{L} & 10 & \text { EPA7060 } \\ \mathrm{U} & 200 & \mu \mathrm{g} / \mathrm{L} & 200 & \text { EPA6010 } \\ \mathrm{U} & 5.0 & \mu \mathrm{g} / \mathrm{L} & 5.0 & \text { EPA6010 } \\ \mathrm{U} & 10 & \mu \mathrm{g} / \mathrm{L} & 10 & \text { EPA6010 } \\ \mathrm{U} & 25 & \mu \mathrm{g} / \mathrm{L} & 25 & \text { EPA6010 } \\ \mathrm{U} & 3.0 & \mu \mathrm{g} / \mathrm{L} & 3.0 & \text { EPA7421 } \\ \mathrm{U} & 15 & \mu \mathrm{g} / \mathrm{L} & 15 & \text { EPA6010 } \\ \mathrm{U} & 0.20 & \mu \mathrm{g} / \mathrm{L} & 0.20 & \text { EPA7470 } \\ \mathrm{U} & 40 & \mu \mathrm{g} / \mathrm{L} & 40 & \text { EPA6010 } \\ \mathrm{U} & 5.0 & \mu \mathrm{g} / \mathrm{L} & 5.0 & \text { EPA7740 } \\ \mathrm{U} & 10 & \mu \mathrm{g} / \mathrm{L} & 10 & \text { EPA6010 }\end{array}$


SAMPLE NAME: D43110804C (Continued)

Sample ID: 100333

Constituent

$\mathbf{R Q}$

AQ

B Result

Unit

D. Limit Method

Volatile Organic Compounds

Acetone
Acetone
Benzene
Benzene
Bromodichloromethane
Bromodichloromethane
Bromoform
Bromoform
Bromomethane (Methyl bromide)
Bromomethane (Methyl bromide)
Carbon disulfide
Carbon disulfide
Carbon tetrachloride
Carbon tetrachloride
Chlorobenzene
Chlorobenzene
Chloroethane
Chloroethane
Chloroethene (Vinyl chloride)
Chloroethene (Vinyl chloride)
Chloroform
Chloroform
Chloromethane (Methyl chloride)
Chloromethane (Methyl chloride)
Dibromochloromethane
Dibromochloromethane
1,1-Dichloroethane
1,1-Dichloroethane
1,2-Dichloroethane
1,2-Dichloroethane
1,1-Dichloroethylene
1,1-Dichloroethylene
1,2-Dichloroethylene
1,2-Dichloroethylene
Dichloromethane Methylene chloride)
Dichloromethane (Methylene chloride)
1,2-Dichloropropane
1,2-Dichloropropane
cis-1,3-Dichloropropene
cis-1,3-Dichloropropene
trans-1,3-Dichloropropene
trans-1,3-Dichloropropene
Ethylbenzene
Ethylbenzene
2-Hexanone
2-Hexanone
Methyl ethyl ketone
Methyl ethyl ketone
Methyl isobutyl ketone
Methyl isobutyl ketone

\begin{tabular}{|c|c|c|}
\hline$\mu \mathrm{g} / \mathrm{L}$ & 10 & EPA8240 \\
\hline$\mu \mathrm{g} / \mathrm{L}$ & 10 & EPA8240 \\
\hline$\mu \mathrm{g} / \mathrm{L}$ & 5.0 & EPA8240 \\
\hline$\mu g / L$ & 5.0 & EPA8240 \\
\hline$\mu \mathrm{g} / \mathrm{L}$ & 5.0 & EPA8240 \\
\hline$\mu g / L$ & 5.0 & EPA8240 \\
\hline$\mu \mathrm{g} / \mathrm{L}$ & 5.0 & EPA8240 \\
\hline$\mu \mathrm{g} / \mathrm{L}$ & 5.0 & EPA8240 \\
\hline$\mu \mathrm{g} / \mathrm{L}$ & 10 & EPA8240 \\
\hline$\mu g / L$ & 10 & EPA8240 \\
\hline$\mu \mathrm{g} / \mathrm{L}$ & 5.0 & EPA8240 \\
\hline$\mu \mathrm{g} / \mathrm{L}$ & 5.0 & EPA8240 \\
\hline$\mu \mathrm{g} / \mathrm{L}$ & 5.0 & EPA8240 \\
\hline$\mu \mathrm{g} / \mathrm{L}$ & 5.0 & EPA8240 \\
\hline$\mu \mathrm{g} / \mathrm{L}$ & 5.0 & EPA8240 \\
\hline$\mu \mathrm{g} / \mathrm{L}$ & 5.0 & EPA8240 \\
\hline$\mu \mathrm{g} / \mathrm{L}$ & 10 & EPA8240 \\
\hline$\mu \mathrm{g} / \mathrm{L}$ & 10 & EPA8240 \\
\hline$\mu \mathrm{g} / \mathrm{L}$ & 10 & EPA8240 \\
\hline$\mu \mathrm{g} / \mathrm{L}$ & 10 & EPA8240 \\
\hline$\mu \mathrm{g} / \mathrm{L}$ & 5.0 & EPA8240 \\
\hline$\mu \mathrm{g} / \mathrm{L}$ & 5.0 & EPA8240 \\
\hline$\mu \mathrm{g} / \mathrm{L}$ & 10 & EPA8240 \\
\hline$\mu \mathrm{g} / \mathrm{L}$ & 10 & EPA8240 \\
\hline$\mu \mathrm{g} / \mathrm{L}$ & 5.0 & EPA8240 \\
\hline$\mu \mathrm{g} / \mathrm{L}$ & 5.0 & EPA8240 \\
\hline$\mu g / L$ & 5.0 & EPA8240 \\
\hline$\mu \mathrm{g} / \mathrm{L}$ & 5.0 & EPA8240 \\
\hline$\mu \mathrm{g} / \mathrm{L}$ & 5.0 & EPA8240 \\
\hline$\mu g / L$ & 5.0 & EPA8240 \\
\hline$\mu \mathrm{g} / \mathrm{L}$ & 5.0 & EPA8240 \\
\hline$\mu \mathrm{g} / \mathrm{L}$ & 5.0 & EPA8240 \\
\hline$\mu \mathrm{g} / \mathrm{L}$ & 5.0 & EPA8240 \\
\hline$\mu \mathrm{g} / \mathrm{L}$ & 5.0 & EPA8240 \\
\hline$\mu \mathrm{g} / \mathrm{L}$ & 5.0 & EPA8240 \\
\hline$\mu \mathrm{g} / \mathrm{L}$ & 5.0 & EPA8240 \\
\hline$\mu \mathrm{g} / \mathrm{L}$ & 5.0 & EPA8240 \\
\hline$\mu \mathrm{g} / \mathrm{L}$ & 5.0 & EPA8240 \\
\hline$\mu g / L$ & 5.0 & EPA8240 \\
\hline$\mu \mathrm{g} / \mathrm{L}$ & 5.0 & EPA8240 \\
\hline$\mu \mathrm{g} / \mathrm{L}$ & 5.0 & EPA8240 \\
\hline$\mu g / L$ & 5.0 & EPA8240 \\
\hline$\mu g / L$ & 5.0 & EPA8240 \\
\hline$\mu \mathrm{g} / \mathrm{L}$ & 5.0 & EPA8240 \\
\hline$\mu g / L$ & 10 & EPA8240 \\
\hline$\mu \mathrm{g} / \mathrm{L}$ & 10 & EPA8240 \\
\hline$\mu g / L$ & 10 & EPA8240 \\
\hline$\mu \mathrm{g} / \mathrm{L}$ & 10 & EPA8240 \\
\hline$\mu g / L$ & 10 & EPA8240 \\
\hline$\mu \mathrm{g} / \mathrm{L}$ & 10 & EPA8240 \\
\hline
\end{tabular}


SAMPLE NAME: D43110804C (Continued)

Sample ID: 100333

Constituent

Volatile Organic Compounds
RQ AQ B Result

Unit

D. Limit Method

Styrene
Styrene
1,1,2,2-Tetrachloroethane
1,1,2,2-Tetrachloroethane
Tetrachloroethylene
Tetrachloroethylene
Toluene
Toluene
1,1,1-Trichloroethane
1,1,1-Trichloroethane
1,1,2-Trichloroethane
1,1,2-Trichloroethane
Trichloroethylene
Trichloroethylene
Vinyl acetate
Vinyl acetate
Xylenes
Xylenes

Radionuclide Indicators and Radionuclides
Gross alpha

Nonvolatile beta

Tritium

SAMPLE NAME: D43110807B

Sample Type: Blank

Constituent

Volatile Organic Compounds
Radium, total alpha-emitting
U

U

$\begin{array}{llll}\text { UJ } & \text { O } & \text { L } & 5.0 \\ \text { UJ } & \text { O } & \text { L } & 5.0 \\ \text { UJ } & \text { O } & \text { L } & 5.0 \\ \text { UJ } & \text { O } & \text { L } & 5.0 \\ \text { UJ } & \text { O } & \text { L } & 5.0 \\ \text { UJ } & \text { O } & \text { L } & 5.0 \\ \text { UJ } & \text { O } & \text { L } & 5.0 \\ \text { UJ } & \text { O } & \text { L } & 5.0 \\ \text { UJ } & \text { O } & \text { L } & 5.0 \\ \text { UJ } & \text { O } & \text { L } & 5.0 \\ \text { UJ } & 0 & \text { L } & 5.0 \\ \text { UJ } & \text { O } & \text { L } & 5.0 \\ \text { UJ } & \text { O } & \text { L } & 5.0 \\ \text { UJ } & \text { O } & \text { L } & 5.0 \\ \text { UJ } & 0 & \text { L } & 10 \\ \text { UJ } & \text { O } & \text { L } & 10 \\ \text { UJ } & \text { O } & \text { L } & 5.0 \\ \text { UJ } & \text { O } & \text { L } & 5.0\end{array}$

$\begin{array}{lll}\mu \mathrm{g} / \mathrm{L} & 5.0 & \text { EPA8240 } \\ \mu \mathrm{g} / \mathrm{L} & 5.0 & \text { EPA8240 } \\ \mu \mathrm{g} / \mathrm{L} & 5.0 & \text { EPA8240 } \\ \mu \mathrm{g} / \mathrm{L} & 5.0 & \text { EPA8240 } \\ \mu \mathrm{g} / \mathrm{L} & 5.0 & \text { EPA8240 } \\ \mu \mathrm{g} / \mathrm{L} & 5.0 & \text { EPA8240 } \\ \mu \mathrm{g} / \mathrm{L} & 5.0 & \text { EPA8240 } \\ \mu \mathrm{g} / \mathrm{L} & 5.0 & \text { EPA8240 } \\ \mu \mathrm{g} / \mathrm{L} & 5.0 & \text { EPA8240 } \\ \mu \mathrm{g} / \mathrm{L} & 5.0 & \text { EPA8240 } \\ \mu \mathrm{g} / \mathrm{L} & 5.0 & \text { EPA8240 } \\ \mu \mathrm{g} / \mathrm{L} & 5.0 & \text { EPA8240 } \\ \mu \mathrm{g} / \mathrm{L} & 5.0 & \text { EPA8240 } \\ \mu \mathrm{g} / \mathrm{L} & 5.0 & \text { EPA8240 } \\ \mu \mathrm{g} / \mathrm{L} & 10 & \text { EPA8240 } \\ \mu \mathrm{g} / \mathrm{L} & 10 & \text { EPA8240 } \\ \mu \mathrm{g} / \mathrm{L} & 5.0 & \text { EPA8240 } \\ \mu \mathrm{g} / \mathrm{L} & 5.0 & \text { EPA8240 }\end{array}$

$\begin{array}{llll}0.60 & \mathrm{pCi} / \mathrm{L} & 0.60 & \text { EPA900.0 } \\ 2.3 \pm 1.7 & \mathrm{pCi} / \mathrm{L} & 1.0 & \text { EPA900.0 } \\ 0.10 & \mathrm{pCi} / \mathrm{L} & 0.10 & \text { EPA903.0 } \\ 270 \pm 220 & \mathrm{pCi} / \mathrm{L} & 150 & \text { EPA906.0 }\end{array}$

Sample ID: 100339

Associated Sample: 100337

$\begin{array}{lllll}\text { RQ AQ } & \text { B Result Unit } & \text { D. Limit Method }\end{array}$

$\begin{array}{lllllll}J & 0 & \mathrm{~L} & 11 & \mu \mathrm{g} / \mathrm{L} & 10 & \text { EPA8240 } \\ \mathrm{UJ} & \mathrm{O} & \mathrm{L} & 10 & \mu \mathrm{g} / \mathrm{L} & 10 & \text { EPA8240 } \\ \mathrm{UJ} & 0 & \mathrm{~L} & 5.0 & \mu \mathrm{g} / \mathrm{L} & 5.0 & \text { EPA8240 } \\ \mathrm{UJ} & \mathrm{O} & \mathrm{L} & 5.0 & \mu \mathrm{g} / \mathrm{L} & 5.0 & \text { EPA8240 } \\ \mathrm{UJ} & 0 & \mathrm{~L} & 5.0 & \mu \mathrm{g} / \mathrm{L} & 5.0 & \text { EPA8240 } \\ \mathrm{UJ} & \mathrm{O} & \mathrm{L} & 5.0 & \mu \mathrm{g} / \mathrm{L} & 5.0 & \text { EPA8240 } \\ \mathrm{UJ} & \mathrm{O} & \mathrm{L} & 5.0 & \mu \mathrm{g} / \mathrm{L} & 5.0 & \text { EPA8240 } \\ \mathrm{UJ} & 0 & \mathrm{~L} & 5.0 & \mu \mathrm{g} / \mathrm{L} & 5.0 & \text { EPA8240 } \\ \mathrm{UJ} & 0 & \mathrm{~L} & 10 & \mu \mathrm{g} / \mathrm{L} & 10 & \text { EPA8240 } \\ \mathrm{UJ} & 0 & \mathrm{~L} & 10 & \mu \mathrm{g} / \mathrm{L} & 10 & \text { EPA8240 } \\ \mathrm{UJ} & 0 & \mathrm{~L} & 5.0 & \mu \mathrm{g} / \mathrm{L} & 5.0 & \text { EPA8240 } \\ \mathrm{UJ} & 0 & \mathrm{~L} & 5.0 & \mu \mathrm{g} / \mathrm{L} & 5.0 & \text { EPA8240 } \\ \mathrm{UJ} & 0 & \mathrm{~L} & 5.0 & \mu \mathrm{g} / \mathrm{L} & 5.0 & \text { EPA8240 } \\ \mathrm{UJ} & 0 & \mathrm{~L} & 5.0 & \mu \mathrm{g} / \mathrm{L} & 5.0 & \text { EPA8240 } \\ \mathrm{UJ} & 0 & \mathrm{~L} & 5.0 & \mu \mathrm{g} / \mathrm{L} & 5.0 & \text { EPA8240 }\end{array}$




\section{Constituent}

Volatile Organic Compounds

Chlorobenzene
Chloroethane
Chloroethane
Chloroethene (Vinyl chloride)
Chloroethene (Vinyl chloride)
Chloroform
Chloroform
Chloromethane (Methyl chloride)
Chloromethane (Methyl chloride)
Dibromochloromethane
Dibromochloromethane
1,1-Dichloroethane
1,1-Dichloroethane
1,2-Dichloroethane
1,2-Dichloroethane
1,1-Dichloroethylene
1,1-Dichloroethylene
1,2-Dichloroethylene
1,2-Dichloroethylene

Dichloromethane (Methylene chloride)

Dichloromethane (Methylene chloride)

1,2-Dichloropropane

1,2-Dichloropropane

cis-1,3-Dichloropropene

cis-1,3-Dichloropropene

trans-1,3-Dichloropropene

trans-1,3-Dichloropropene

Ethylbenzene

Ethylbenzene

2-Hexanone

2-Hexanone

Methyl ethyl ketone

Methyl ethyl ketone

Methyl isobutyl ketone

Methyl isobutyl ketone

Styrene

Styrene

1,1,2,2-Tetrachloroethane

1,1,2,2-Tetrachloroethane

Tetrachloroethylene

Tetrachloroethylene

Toluene

Toluene

1,1,1-Trichloroethane

1,1,1-Trichloroethane

1,1,2-Trichloroethane

1,1,2-Trichloroethane

Trichloroethylene

Trichloroethylene

Vinyl acetate
RQ AQ B Result Unit D. Limit Method

\begin{tabular}{|c|c|c|c|c|c|c|}
\hline UJ & o & L & 5.0 & $\mu g / L$ & 5.0 & EPA8240 \\
\hline UJ & 0 & L & 10 & $\mu \mathrm{g} / \mathrm{L}$ & 10 & EPA8240 \\
\hline UJ & 0 & L & 10 & $\mu g / L$ & 10 & EPA8240 \\
\hline UJ & 0 & L & 10 & $\mu g / L$ & 10 & EPA8240 \\
\hline US & 0 & L & 10 & $\mu \mathrm{g} / \mathrm{L}$ & 10 & EPA8240 \\
\hline UJ & 0 & L & 5.0 & $\mu \mathrm{g} / \mathrm{L}$ & 5.0 & EPA 8240 \\
\hline UJ & 0 & L & 5.0 & $\mu \mathrm{g} / \mathrm{L}$ & 5.0 & EPA8240 \\
\hline UJ & 0 & L & 10 & $\mu \mathrm{g} / \mathrm{L}$ & 10 & EPA8240 \\
\hline UJ & 0 & $\mathrm{~L}$ & 10 & $\mu \mathrm{g} / \mathrm{L}$ & 10 & EPA8240 \\
\hline UJ & 0 & L & 5.0 & $\mu \mathrm{g} / \mathrm{L}$ & 5.0 & EPA8240 \\
\hline UJ & 0 & L & 5.0 & $\mu \mathrm{g} / \mathrm{L}$ & 5.0 & EPA8240 \\
\hline UJ & 0 & L & 5.0 & $\mu \mathrm{g} / \mathrm{L}$ & 5.0 & EPA8240 \\
\hline UJ & 0 & L & 5.0 & $\mu \mathrm{g} / \mathrm{L}$ & 5.0 & EPA8240 \\
\hline U & 0 & L & 5.0 & $\mu \mathrm{g} / \mathrm{L}$ & 5.0 & EPA8240 \\
\hline UJ & 0 & L & 5.0 & $\mu g / L$ & 5.0 & EPA8240 \\
\hline UJ & 0 & L & 5.0 & $\mu \mathrm{g} / \mathrm{L}$ & 5.0 & EPA8240 \\
\hline UJ & 0 & L & 5.0 & $\mu \mathrm{g} / \mathrm{L}$ & 5.0 & EPA8240 \\
\hline UJ & 0 & L & 5.0 & $\mu g / L$ & 5.0 & EPA8240 \\
\hline UJ & 0 & L & 5.0 & $\mu \mathrm{g} / \mathrm{L}$ & 5.0 & EPA8240 \\
\hline UJ & 0 & L & 5.0 & $\mu \mathrm{g} / \mathrm{L}$ & 5.0 & EPA8240 \\
\hline UJ & 0 & L & 5.0 & $\mu g / L$ & 5.0 & EPA8240 \\
\hline UJ & 0 & L & 5.0 & $\mu \mathrm{g} / \mathrm{L}$ & 5.0 & EPA8240 \\
\hline UJ & 0 & L & 5.0 & $\mu g / L$ & 5.0 & EPA8240 \\
\hline UJ & 0 & L & 5.0 & $\mu g / L$ & 5.0 & EPA8240 \\
\hline UJ & 0 & L & 5.0 & $\mu \mathrm{g} / \mathrm{L}$ & 5.0 & EPA8240 \\
\hline UJ & 0 & L & 5.0 & $\mu \mathrm{g} / \mathrm{L}$ & 5.0 & EPA8240 \\
\hline UJ & 0 & L & 5.0 & $\mu \mathrm{g} / \mathrm{L}$ & 5.0 & EPA8240 \\
\hline UJ & 0 & L & 5.0 & $\mu \mathrm{g} / \mathrm{L}$ & 5.0 & EPA8240 \\
\hline UJ & 0 & L & 5.0 & $\mu \mathrm{g} / \mathrm{L}$ & 5.0 & EPA8240 \\
\hline US & 0 & L & 10 & $\mu \mathrm{g} / \mathrm{L}$ & 10 & EPA8240 \\
\hline UI & 0 & L & 10 & $\mu \mathrm{g} / \mathrm{L}$ & 10 & EPA8240 \\
\hline UJ & 0 & L & 10 & $\mu \mathrm{g} / \mathrm{L}$ & 10 & EPA8240 \\
\hline UJ & 0 & L & 10 & $\mu \mathrm{g} / \mathrm{L}$ & 10 & EPA8240 \\
\hline UJ & 0 & L & 10 & $\mu \mathrm{g} / \mathrm{L}$ & 10 & EPA8240 \\
\hline UJ & 0 & L & 10 & $\mu \mathrm{g} / \mathrm{L}$ & 10 & EPA8240 \\
\hline UJ & 0 & L & 5.0 & $\mu \mathrm{g} / \mathrm{L}$ & 5.0 & EPA8240 \\
\hline UJ & 0 & L & 5.0 & $\mu \mathrm{g} / \mathrm{L}$ & 5.0 & EPA8240 \\
\hline UJ & 0 & $\bar{L}$ & 5.0 & $\mu \mathrm{g} / \mathrm{L}$ & 5.0 & EPA8240 \\
\hline UJ & 0 & L & 5.0 & $\mu \mathrm{g} / \mathrm{L}$ & 5.0 & EPA8240 \\
\hline UJ & 0 & L & 5.0 & $\mu \mathrm{g} / \mathrm{L}$ & 5.0 & EPA8240 \\
\hline UJ & 0 & L & 5.0 & $\mu \mathrm{g} / \mathrm{L}$ & 5.0 & EPA8240 \\
\hline U & 0 & L & 5.0 & $\mu \mathrm{g} / \mathrm{L}$ & 5.0 & EPA8240 \\
\hline UJ & 0 & L & 5.0 & $\mu \mathrm{g} / \mathrm{L}$ & 5.0 & EPA8240 \\
\hline UJ & 0 & L & 5.0 & $\mu \mathrm{g} / \mathrm{L}$ & 5.0 & EPA8240 \\
\hline UJ & 0 & L & 5.0 & $\mu \mathrm{g} / \mathrm{L}$ & 5.0 & EPA8240 \\
\hline UJ & 0 & L & 5.0 & $\mu \mathrm{g} / \mathrm{L}$ & 5.0 & EPA8240 \\
\hline UJ & 0 & L & 5.0 & $\mu \mathrm{g} / \mathrm{L}$ & 5.0 & EPA8240 \\
\hline UJ & 0 & L & 5.0 & $\mu g / L$ & 5.0 & EPA8240 \\
\hline UJ & 0 & L & 5.0 & $\mu \mathrm{g} / \mathrm{L}$ & 5.0 & EPA8240 \\
\hline UJ & 0 & L & 10 & $\mu \mathrm{g} / \mathrm{L}$ & 10 & EPA8240 \\
\hline
\end{tabular}


SAMPLE NAME: D431 10807B (Continued)

Constituent

RQ AQ B Result

Unit

D. Limit Method

Volatile Organic Compounds

Vinyl acetace
Xylenes
Xylenes

UJ $\quad 0 \quad$ L $\quad 10$

UJ $\quad 0 \quad L \quad 5.0$

UJ 0

L $\quad 5.0$

Radionuclide Indicators and Radionuclides

Gross alpha

Nonvolatile beta

Radium, total alpha-emitting

Tritium

\section{U}

U

$\mathrm{U}$

0.60
1.0
$0.10 \pm 0.10$
140

$\mu g / L$

$\mu g / L$

$\mu \mathrm{g} / \mathrm{L}$
10

5.0

5.0

$\mathrm{pCi} / \mathrm{L}$

$\mathrm{pCi} / \mathrm{L}$

$\mathrm{pCi} / \mathrm{L}$

$\mathrm{pCi} / \mathrm{L}$
0.60

1.0

0.10

140
EPA8240

EPA8240

EPA8240
SAMPLE NAME: D43110901E

Sample Type: Rinsate

Constituent

Total Metals
Arsenic

Barium

Cadmium

Chromium

Copper

Lead

Manganese

Mercury

Nickel

Selenium

Silver

\section{Volatile Organic Compounds}

\section{Acetone}

Benzene

Bromodichloromethane

Bromoform

Bromomethane (Methyl bromide)

Carbon disulfide

Carbon tetrachloride

Chlorobenzene

Chloroethane

Chloroethene (Vinyl chloride)

Chloroform

Chloromethane (Methyl chloride)

Dibromochloromethane

1,1-Dichloroethane

1,2-Dichloroethane

1,1-Dichloroethylene
Sample ID: 100342

Associated Sample: 100340

RQ AQ B Result Unit

$\begin{array}{lll}\mu \mathrm{g} / \mathrm{L} & 10 & \text { EPA7060 } \\ \mu \mathrm{g} / \mathrm{L} & 200 & \text { EPA6010 } \\ \mu \mathrm{g} / \mathrm{L} & 5.0 & \text { EPA6010 } \\ \mu \mathrm{g} / \mathrm{L} & 10 & \text { EPA6010 } \\ \mu \mathrm{g} / \mathrm{L} & 25 & \text { EPA6010 } \\ \mu \mathrm{g} / \mathrm{L} & 3.0 & \text { EPA7421 } \\ \mu \mathrm{g} / \mathrm{L} & 15 & \text { EPA6010 } \\ \mu \mathrm{g} / \mathrm{L} & 0.20 & \text { EPA7470 } \\ \mu \mathrm{g} / \mathrm{L} & 40 & \text { EPA6010 } \\ \mu \mathrm{g} / \mathrm{L} & 5.0 & \text { EPA7740 } \\ \mu \mathrm{g} / \mathrm{L} & 10 & \text { EPA6010 }\end{array}$

\section{$\cdot$}

U

U

U

$\mathrm{U}$

U

$\mathrm{U}$

U

$\mathrm{U}$

$\mathrm{U}$

U

10
200
5.0
10
25
3.0
15
0.20
40
5.0
10

10
D. Limit Method
EPA900.0

EPA900.0

EPA903.0

EPA906.0 
SAMPLE NAME: D43110901E (Continued) Sample ID: 100342

Constituent

Volatile Organic Compounds
RQ AQ B Result Unit D. Limit Method
5.0

5.0

5.0

5.0

5.0

5.0

10

10

10

5.0

5.0

5.0

5.0

5.0

5.0

5.0

10

40 $\mu \mathrm{g} / \mathrm{L}$

$\mu g / L$

$\mu \mathrm{g} / \mathrm{L}$

$\mu \mathrm{g} / \mathrm{L}$

$\mu \mathrm{g} / \mathrm{L}$

$\mu \mathrm{g} / \mathrm{L}$

$\mu \mathrm{g} / \mathrm{L}$

$\mu \mathrm{g} / \mathrm{L}$

$\mu \mathrm{g} / \mathrm{L}$

$\mu \mathrm{g} / \mathrm{L}$

$\mu \mathrm{g} / \mathrm{L}$

$\mu \mathrm{g} / \mathrm{L}$

$\mu g / L$

$\mu \mathrm{g} / \mathrm{L}$

$\mu \mathrm{g} / \mathrm{L}$

$\mu g / L$

$\mu g / L$

$\mu \mathrm{g} / \mathrm{L}$

$\mu g / L$

2-Propanol

Xylenes

$\begin{array}{lll}\mathrm{J} & \mathrm{N} & 40 \\ \mathrm{U} & & 5.0\end{array}$

Radionuclide Indicators and Radionuclides

Gross alpha

Nonvolatile beta

Radium, total alpha-emitting

Tritium

SAMPLE NAME: D43110906C

Sample Type: Blank

Constituent

Total Metals

Arsenic
Barium
Cadmium
Chromium
Copper
Lead
Manganese
Mercury
Nickel
Selenium
Silver

U

U

U

$\mathrm{U}$

0.60
1.1
0.10
140

$\begin{array}{ll}5.0 & \text { EPA8240 } \\ 5.0 & \text { EPA8240 } \\ 5.0 & \text { EPA8240 } \\ 5.0 & \text { EPA8240 } \\ 5.0 & \text { EPA8240 } \\ 5.0 & \text { EPA8240 } \\ 10 & \text { EPA8240 } \\ 10 & \text { EPA8240 } \\ 10 & \text { EPA8240 } \\ 5.0 & \text { EPA8240 } \\ 5.0 & \text { EPA8240 } \\ 5.0 & \text { EPA8240 } \\ 5.0 & \text { EPA8240 } \\ 5.0 & \text { EPA8240 } \\ 5.0 & \text { EPA8240 } \\ 5.0 & \text { EPA8240 } \\ 10 & \text { EPA8240 } \\ & \text { EPA8240 } \\ 5.0 & \text { EPA8240 }\end{array}$

$\begin{array}{ll}0.60 & \text { EPA900.0 } \\ 1.1 & \text { EPA900.0 } \\ 0.10 & \text { EPA903.0 } \\ 140 & \text { EPA906.0 }\end{array}$

Sample ID: 100348

Associated Sample: 100347
$\mathbf{R Q}$
AQ
Result
Unit
D. Limit Method 
SAMPLE NAME: D43110906C (Continued)

\section{Constituent}

Volatile Organic Compounds

Acetone
Benzene
Bromodichloromethane
Bromoform
Bromomethane (Methyl bromide)
Carbon disulfide
Carbon tetrachloride
Chlorobenzene
Chloroethane
Chloroethene (Vinyl chloride)
Chloroform
Chloromethane (Methyl chloride)
Dibromochloromethane
1,1-Dichloroethane
1,2-Dichloroethane

San:. $\because$ ID: 100348

$\begin{array}{lllll}\text { RQ AQ } & \text { B Result Unit } & \text { D. Limit Method }\end{array}$

5.0

5.0

5.0

10

5.0

5.0

5.0

10

10

5.0

10

5.0

5.0

5.0

5.0

5.0

5.0

5.0

5.0

5.0

5.0

10

21

10

5.0

5.0

5.0

5.0

5.0

5.0

5.0

10

.5 .0

$$
\begin{aligned}
& \mu g / L \\
& \mu g / L \\
& \mu g / L \\
& \mu g / L \\
& \mu g / L \\
& \mu g / L \\
& \mu g / L \\
& \mu g / L \\
& \mu g / L \\
& \mu g / L \\
& \mu g / L \\
& \mu g / L \\
& \mu g / L \\
& \mu g / L \\
& \mu g / L \\
& \mu g / L \\
& \mu g / L \\
& \mu g / L \\
& \mu g / L \\
& \mu g / L \\
& \mu g / L \\
& \mu g / L \\
& \mu g / L \\
& \mu g / L \\
& \mu g / L \\
& \mu g / L \\
& \mu g / L \\
& \mu g / L \\
& \mu g / L \\
& \mu g / L \\
& \mu g / L \\
& \mu g / L \\
& \mu g / L \\
& \mu g / L \\
&
\end{aligned}
$$

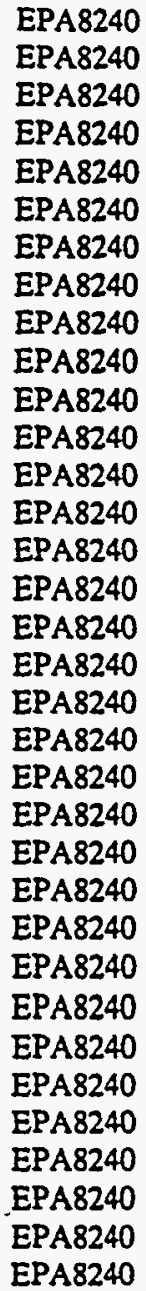

5.0

10

5.0

5.0

5.0

10

5.0

5.0

5.0

10

10

5.0

10

5.0

5.0

5.0

5.0

5.0

5.0

5.0

5.0

5.0

5.0

10

10

10

5.0

5.0

5.0

5.0

5.0

5.0

5.0

10

EPA8240

EPA8240

EPA8240

EPA8240

EPA8240

EPA8240

EPA8240

EPA8240

EPA8240

EPA8240

\begin{abstract}
Nonvolatile beta
Nonvolatile beta

Radium, total alpha-emitting

Radium, total alpha-emitting

Tritium

Tritium

Gross alpha
\end{abstract}

0.50

0.50

0.60

0.60

$\begin{array}{lll}\mathrm{U} & & 0.50 \\ \mathrm{U} & & 0.50 \\ \mathrm{U} & & 0.60 \\ \mathrm{U} & & 0.60 \\ \mathrm{U} & \mathrm{V} & 0.20 \\ \mathrm{U} & \mathrm{V} & 0.40 \pm 0.20 \\ \mathrm{U} & & 150 \\ \mathrm{U} & & 150\end{array}$

150
150

$\begin{array}{lll}\mathrm{pCi} / \mathrm{L} & 0.50 & \text { EPA990.0 } \\ \mathrm{pCi} / \mathrm{L} & 0.50 & \text { EPA990.0 } \\ \mathrm{pCi} / \mathrm{L} & 0.60 & \text { EPA990.0 } \\ \mathrm{pCi} / \mathrm{L} & 0.60 & \text { EPA990.0 } \\ \mathrm{pCi} / \mathrm{L} & 0.20 & \text { EPA903.0 } \\ \mathrm{pCi} / \mathrm{L} & 0.20 & \text { EPA903.0 } \\ \mathrm{pCi} / \mathrm{L} & 150 & \text { EPA906.0 } \\ \mathrm{pCi} / \mathrm{L} & 150 & \text { EPA906.0 }\end{array}$

EPA906.0

Indicators and Radionuclides 
SAMPLE NAME: D43111001B

Sample Type: Blank

Constituent

Volatile Organic Compounds
Sample ID: 100351

Associated Sample: 100350

RQ AQ B Result Unit D. Limit Method

Acetone
Benzene
Bromodichloromethane
Bromoform
Bromomethane (Methyl bromide)
Carbon disulfide
Carbon tetrachloride
Chlorobenzene
Chloroethane
Chloroethene (Vinyl chloride)
Chloroform
Chloromethane (Methyl chloride)
Dibromochloromethane
1.1-Dichloroethane
1,2-Dichloroethane
1,1-Dichloroethylene
1,2-Dichloroethylene
Dichloromethane (Methylene chloride)
1,2-Dichloropropane
cis-1,3-Dichloropropene
trans-1,3-Dichloropropene
Ethylbenzene .
2-Hexanone
Methyl ethyl ketone
Methyl isobutyl ketone
Styrene
1,1,2,2-Tetrachloroethane
Tetrachloroethylene
Toluene
1,1,1-Trichloroethane
1,1,2-Trichloroethane
Trichloroethylene
Vinyl acetate
Xylenes

U

U

U

$\mathrm{U}$

$U$

$\mathrm{U}$

U

U

U

U

U

$\mathrm{U}$

U

U

U

U

U

U

U

U

U

U

U

Radionuclide Indicators and Radionuclides

\section{Gross alpha}

Nonvolatile betz

Radium, total alpha-emitting

Tritium

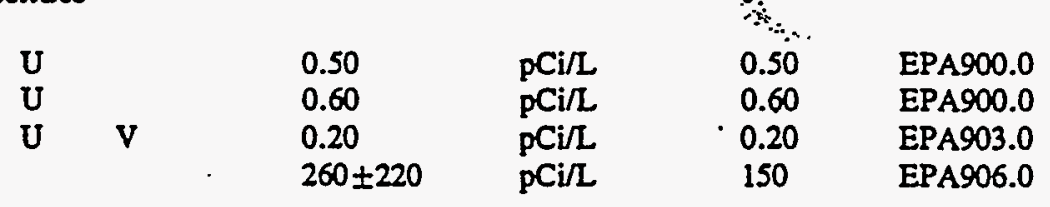

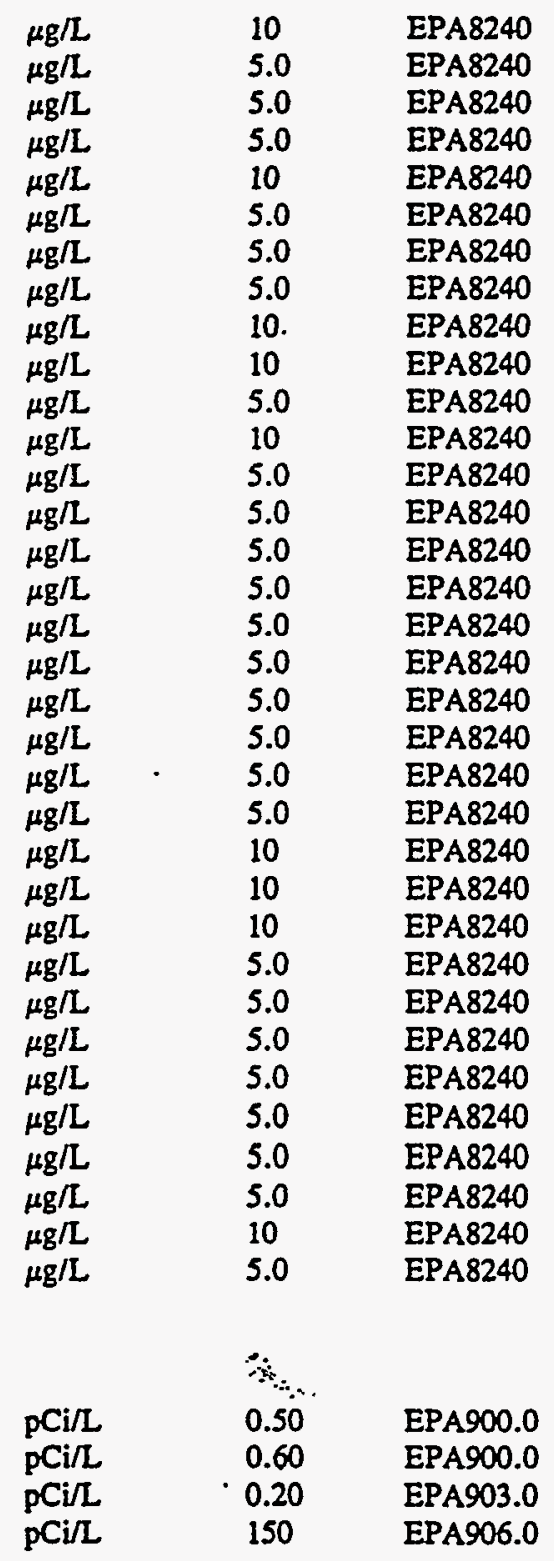




\section{SAMPLE NAME: D43111004E}

Sample Type: Rinsale

Constituent

Total Metals

\section{Arsenic \\ Barium \\ Cadmium \\ Chromium \\ Copper \\ Lead \\ Manganese \\ Mercury \\ Nickel \\ Selenium \\ Silver}

Volatile Organic Compounds
Sample ID: 100355

Associated Sample: 100354
$\mathbf{R Q}$
$\mathbf{A Q}$
B Result
Unit
D. Limit Method

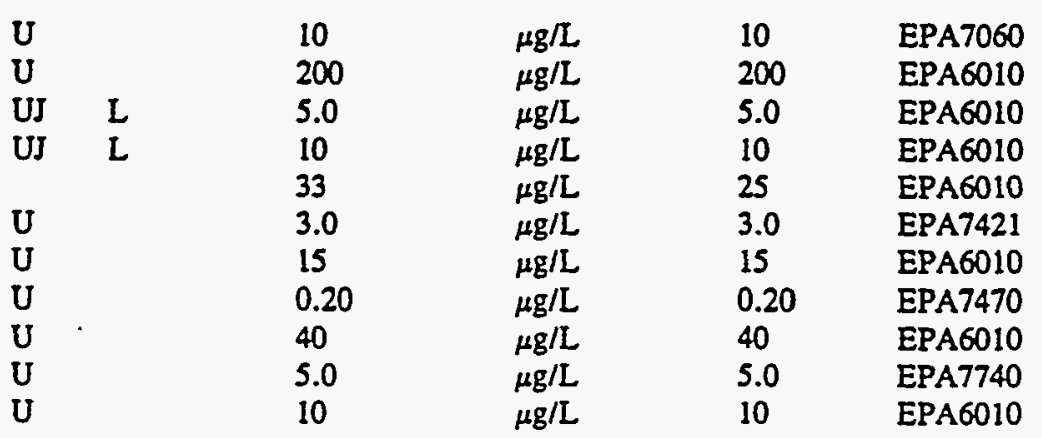

\begin{tabular}{|c|c|c|}
\hline g/L & 10 & EPA8240 \\
\hline$\mu g / L$ & 5.0 & EPA8240 \\
\hline$\mu g / L$ & 5.0 & EPA8240 \\
\hline$\mu \mathrm{g} / \mathrm{L}$ & 5.0 & EPA8240 \\
\hline$\mu \mathrm{g} / \mathrm{L}$ & 10 & EPA8240 \\
\hline$\mu \mathrm{g} / \mathrm{L}$ & 5.0 & EPA8240 \\
\hline$\mu \mathrm{g} / \mathrm{L}$ & 5.0 & EPA8240 \\
\hline$\mu \mathrm{g} / \mathrm{L}$ & 5.0 & EPA8240 \\
\hline$\mu g / L$ & $\vdots:$ & EPA8240 \\
\hline$\mu g / L$ & i: & EPA8240 \\
\hline$\mu \mathrm{g} / \mathrm{L}$ & 5.0 & EPA8240 \\
\hline$\mu \mathrm{g} / \mathrm{L}$ & 10 & EPA 8240 \\
\hline$\alpha \mathrm{g} / \mathrm{L}$ & 5.0 & EPA8240 \\
\hline$\mu g / L$ & 5.0 & EPA8240 \\
\hline$\mu g / L$ & 5.0 & EPA8240 \\
\hline$\mu \mathrm{g} / \mathrm{h}$ & 5.0 & EPA8240 \\
\hline$\mu \mathrm{g} / \mathrm{I}$ & 5.0 & EPA8240 \\
\hline $\mathrm{gg} / \mathrm{L}$ & 5.0 & EPA8240 \\
\hline II & 5.0 & EPA8240 \\
\hline $\mathrm{dg} / \mathrm{L}$ & 5.0 & EPA 8240 \\
\hline$\mu g / L$ & 5.0 & EPA8240 \\
\hline$\mu \mathrm{g} / \mathrm{L}$ & 5.0 & EPA8240 \\
\hline nol & 10 & EPA8240 \\
\hline$g / L$ & 10 & EPA8240 \\
\hline$\mu g / L$ & 10 & EPA8240 \\
\hline$\pi$ & 5.0 & EPA8240 \\
\hline $7 \mathrm{~L}$ & 5.0 & EPA8240 \\
\hline & 5.0 & EPA8240 \\
\hline & 5.0 & EPA8240 \\
\hline is & 5.0 & EPA8240 \\
\hline & 5.0 & EPA8240 \\
\hline & 5.0 & EPA8240 \\
\hline & 10 & EPA8240 \\
\hline & 5.0 & EPA8240 \\
\hline
\end{tabular}


SAMPLE NAME: D43111004E (Continued)

Radionuclide Indicators and Radionuclides

Gross alpha

Nonvolatile beta

Radium, total alpha-emitting

Tritium

SAMPLE NAME: D43111007C

Sample Type: Blank

Constituent

Total Metals

Arsenic

Barium

Cadmium

Chromium

Copper

Lead

Manganese

Mercury

Nickel

Selenium

Silver

Volatile Organic Compounds

Acetone

Benzene

Bromodichloromethane

Bromoform

Bromomethane (Methyl bromide)

Carbon disulfide

Carbon tetrachloride

Chlorobenzene

Chloroethane .

Chloroethene (Vinyl chloride)

Chloroform

Chloromethane (Methyl chloride)

Dibromochloromethane

1.1-Dichloroethane

1,2-Dichloroethane

1.1-Dichloroethylene

1,2-Dichloroethylene

Dichloromethane (Methylene chloride)

1,2-Dichloropropane

cis-1,3-Dichloropropene

trans-1,3-Dichloropropene

Ethylbenzere

2-Hexanone

Methyl ethyl ketone

Methyl isobutyl ketone
Sample ID: 100355

$\mathbf{U}$
$\mathbf{U}$
$\mathbf{U}$

0.50
0.60
$0.30 \pm 0.20$
$160 \pm 210$

$\mathrm{pCi} / \mathrm{L}$

$\mathrm{pCi} / \mathrm{L}$

$\mathrm{pCi} / \mathrm{L}$.

$\mathrm{pCi} / \mathrm{L}$

0.60

EPA900.0

EPA900.0

$\begin{array}{ll}0.20 & \text { EPA903.0 } \\ 150 & \text { EPA906.0 }\end{array}$
Sample ID: 100360

Associated Sample: 100359

RQ AQ B Result Unit

$\begin{array}{llll}10 & \mu \mathrm{g} / \mathrm{L} & 10 & \text { EPA7060 } \\ 200 & \mu \mathrm{g} / \mathrm{L} & 200 & \text { EPA6010 } \\ 5.0 & \mu \mathrm{g} / \mathrm{L} & 5.0 & \text { EPA6010 } \\ 10 & \mu \mathrm{g} / \mathrm{L} & 10 & \text { EPA6010 } \\ 25 & \mu \mathrm{g} / \mathrm{L} & 25 & \text { EPA6010 } \\ 3.0 & \mu \mathrm{g} / \mathrm{L} & 3.0 & \text { EPA7421 } \\ 15 & \mu \mathrm{g} / \mathrm{L} & 15 & \text { EPA6010 } \\ 0.20 & \mu \mathrm{g} / \mathrm{L} & 0.20 & \text { EPA7470 } \\ 40 & \mu \mathrm{g} / \mathrm{L} & 40 & \text { EPA6010 } \\ 5.0 & \mu \mathrm{g} / \mathrm{L} & 5.0 & \text { EPA7740 } \\ 10 & \mu \mathrm{g} / \mathrm{L} & 10 & \text { EPA6010 }\end{array}$

23

5.0

5.0

5.0

10

5.0

5.0

5.0

10

10

5.0

10

5.0

5.0

5.0

5.0

5.0

5.0

5.0

5.0

5.0

5.0

10

12

10 $\mu \mathrm{g} / \mathrm{L}$

$\mu \mathrm{g} / \mathrm{L}$

$\mu \mathrm{g} / \mathrm{L}$

$\mu \mathrm{g} / \mathrm{L}$

$\mu \mathrm{g} / \mathrm{L}$

$\mu \mathrm{g} / \mathrm{L}$

$\mu g / L$

$\mu g / L$

$\mu \mathrm{g} / \mathrm{L}$

$\mu \mathrm{g} / \mathrm{L}$

$\mu \mathrm{g} / \mathrm{L}$

$\mu \mathrm{g} / \mathrm{L}$

$\mu \mathrm{g} / \mathrm{L}$

$\mu g / L$

$\mu \mathrm{g} / \mathrm{L}$

$\mu \mathrm{g} / \mathrm{L}$

$\mu \mathrm{g} / \mathrm{L}$

$\mu g / L$

$\mu \mathrm{g} / \mathrm{L}$

$\mu \mathrm{g} / \mathrm{L}$

$\mu \mathrm{g} / \mathrm{L}$

$\mu \mathrm{g} / \mathrm{L}$

$\mu \mathrm{g} / \mathrm{L}$

$\mu g / L$

$\mu \mathrm{g} / \mathrm{L}$
D. Limit Method 
SAMPLE NAME: D43ī11007C (Continued)

Constituent

$$
\text { RQ }
$$

$\mathbf{R Q}$

$\mathbf{A Q}$

B

Result

Unit

D. Limit Method

Volatile Organic Compounds

$\begin{array}{ll}\mathrm{U} & 5.0 \\ \mathrm{U} & 5.0 \\ \mathrm{U} & 5.0 \\ \mathrm{U} & 5.0 \\ \mathrm{U} & 5.0 \\ \mathrm{U} & 5.0 \\ \mathrm{U} & 5.0 \\ \mathrm{U} & 10 \\ \mathrm{U} & 5.0\end{array}$

Radionuclide Indicators and Radionuclides

Gross alpha

Nonvolatile beta

Radium, total alpha-emitting

Tritium

$\begin{array}{lll}U & & 0.50 \\ U & & 0.60 \\ U & V & 0.20 \\ U & & 150\end{array}$

$\begin{array}{lll}\mu \mathrm{g} / \mathrm{L} & 5.0 & \text { EPA8240 } \\ \mu \mathrm{g} / \mathrm{L} & 5.0 & \text { EPA8240 } \\ \mu \mathrm{g} / \mathrm{L} & 5.0 & \text { EPA8240 } \\ \mu \mathrm{g} / \mathrm{L} & 5.0 & \text { EPA8240 } \\ \mu \mathrm{g} / \mathrm{L} & 5.0 & \text { EPA8240 } \\ \mu \mathrm{g} / \mathrm{L} & 5.0 & \text { EPA8240 } \\ \mu \mathrm{g} / \mathrm{L} & 5.0 & \text { EPA8240 } \\ \mu \mathrm{g} / \mathrm{L} & 10 & \text { EPA8240 } \\ \mu \mathrm{g} / \mathrm{L} & 5.0 & \text { EPA8240 }\end{array}$

$\mathrm{pCi} / \mathrm{L}$

0.50

EPA9O0.0

$\rightarrow \mathrm{Ci} / \mathrm{L}$

$\mathrm{pCi} / \mathrm{L}$

pC: i

0.60

0.20

EPA900.0

EPA903.0

EPA906.0
SAMPLE NAME: D43111502B

Sample Type: Blank

Constituent

Volatile Organic Compounds

Acetone

Benzene

Bromodichloromethane

Bromoform

Bromomethane (Methyl bromide)

Carbon disulfide

Carbon tetrachloride

Chlorobenzene

Chloroethane

Chloroethene (Vinyl chloride)

Chloroform

Chloromethane (Methyl chloride)

Dibromochloromethane

1,1-Dichloroethane

1,2-Dichloroethane

1,1-Dichloroethylene

1,2-Dichloroethylene

Dichloromethane (Methylene chloride)

1,2-Dichloropropane

cis-1,3-Dichloropropene

trans-1,3-Dichloropropene

Ethylbenzene

2-Hexanone

Methyl ethyl ketone
Sample ID: 100409

Associated Sample: 100 :-"
RQ $A Q$
Result
Ziac
D. Limit Method

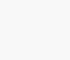


SAMPLE NAME: D43ï11502B (Continued)

Constituent

RQ AQ B Result

Volatile Organic Compounds

Methyl isobutyl ketone

Styrene

1,1,2,2-Tetrachloroethane

Tetrachloroethylene

Toluene

1,1,1-Trichloroethane

1.1.2-Trichloroethane

Trichloroethylene

Vinyl acetate

Xylenes
Sample ID: 100409

Unit D. Limit Method

Radionuclide Indicators and Radionuclides

$\begin{array}{lllllll}\text { Gross alpha } & \mathrm{U} & & 0.50 & \mathrm{pCi} / \mathrm{L} & 0.50 & \text { EPA990.0 } \\ \text { Nonvolatile beta } & \mathrm{U} & \mathrm{X} & 0.70 & \mathrm{pCi} / \mathrm{L} & 0.70 & \text { EPA990.0 } \\ \text { Radium. total alpha-emitting } & \mathrm{U} & \mathrm{V} & 0.10 & \mathrm{pCi} / \mathrm{L} & 0.10 & \text { EPA903.0 } \\ \text { Tritium } & & & 160 \pm 190 & \mathrm{pCi} / \mathrm{L} & 130 & \text { EPA906.0 }\end{array}$

SAMPLE NAME: D43111505B

Sample Type: Blank

Constituent

Volatile Organic Compounds

$\begin{array}{lc}\text { Acetone } & \\ \text { Benzene } & \mathrm{UJ} \\ \text { Bromodichloromethane } & \mathrm{U} \\ \text { Bromoform } & \mathrm{U} \\ \text { Bromomethane (Methyl bromide) } & \mathrm{U} \\ \text { Carbon disulfide } & \mathrm{U} \\ \text { Carbon tetrachloride } & \mathrm{U} \\ \text { Chlorobenzene } & \mathrm{U} \\ \text { Chloroethane } & \mathrm{U} \\ \text { Chloroethene (Vinyl chloride) } & \mathrm{U} \\ \text { Chloroform } & \mathrm{U} \\ \text { Chloromethane (Methyl chloride) } & \mathrm{U} \\ \text { Dibromochloromethane } & \mathrm{U} \\ \text { 1,1-Dichloroethane } & \mathrm{U} \\ \text { 1,2-Dichloroethane } & \mathrm{U} \\ \text { 1,1-Dichloroethylene } & \mathrm{U} \\ \text { 1,2-Dichloroethylene } & \mathrm{U} \\ \text { Dichloromethane (Methylene chloride) } & \mathrm{U} \\ \text { 1,2-Dichloropropane } & \mathrm{U} \\ \text { cis-1,3-Dichloropropene } & \mathrm{U} \\ \text { trans-1,3-Dichloropropene } & \mathrm{U} \\ \text { Ethylbenzene } & \mathrm{U} \\ \text { 2-Hexanone } & \mathrm{U}\end{array}$

Sample ID: 100413

Associated Sample: 100412
RQ AQ B Result
Unit
D. Limit Method

EPA8240

EPA8240

EPA8240

EPA8240

EPA8240

EPA8240

EPA8240

EPA8240

EPA8240

EPA8240

5.0

EPA906.0 
SAMPLE NAME: D43ï11505B (Continued)

\section{Constituent}

Volatile Organic Compounds

Methyl ethyl ketone

Methyl isobutyl ketone

Styrene

1.1,2,2-Tetrachloroethane

Tetrachloroethylene

Toluene

1,1,1-Trichloroethane

1,1,2-Trichloroethane

Trichloroethylene

Vinyl acetate

Xylenes

\section{SAMPLE NAME: D43111506E}

Sample Type: Rinsate

Constituent

Volatile Organic Compounds

Acetone
Acetone
Benzene
Bromodichloromethane
Bromoform
Bromomethane (Methyl bromide)
Carbon disulfide
Carbon tetrachloride
Chlorobenzene
Chloroethane
Chloroethene (Vinyl chloride)
2-Chloroethyl vinyl ether
Chloroform
Chloromethane (Methyl chloride)
Dibromochloromethane
1,1-Dichloroethane
1,2-Dichloroethane
1,1-Dichloroethylene
1,2-Dichloroethylene
Dichloromethane (Methylene chloride)
1,2-Dichloropropane
cis-1,3-Dichloropropene
trans-1,3-Dichloropropene
Ethylbenzene
2-Hexanone
Methyl ethyl ketone
Methyl isobutyl ketone
Styrene

1,1,2,2-Tetrachloroethane
Sample D: 100413

RQ AQ B Result Unit D. Limit Method

$\begin{array}{llll}10 & \mu g / L & 10 & \text { EPA8240 } \\ 10 & \mu g / L & 10 & \text { EPA8240 } \\ 5.0 & \mu g / L & 5.0 & \text { EPA8240 } \\ 5.0 & \mu g / L & 5.0 & \text { EPA8240 } \\ 5.0 & \mu g / L & 5.0 & \text { EPA8240 } \\ 5.0 & \mu g / L & 5.0 & \text { EPA8240 } \\ 5.0 & \mu g / L & 5.0 & \text { EPA8240 } \\ 5.0 & \mu g / L & 5.0 & \text { EPA8240 } \\ 5.0 & \mu g / L & 5.0 & \text { EPA8240 } \\ 10 & \mu g / L & 10 & \text { EPA8240 } \\ 5.0 & \mu g / L & 5.0 & \text { EPA8240 }\end{array}$

Sample ID: 100416

Associated Sample: 100414

$\begin{array}{lllll}\text { RQ AQ B } & \text { Result Unit } & \text { D. Limit Method }\end{array}$

$\begin{array}{llllll}\mathrm{L} & \mathrm{YV} & 6.800 & \mu \mathrm{g} / \mathrm{L} & 10 & \text { EPA8240 } \\ & \mathrm{YV} & 45,000 & \mu \mathrm{g} / \mathrm{L} & 5,000 & \text { EPA8240 } \\ \mathrm{U} & \mathrm{Y} & 5.0 & \mu \mathrm{g} / \mathrm{L} & 5.0 & \text { EPA8240 } \\ \mathrm{U} & \mathrm{Y} & 5.0 & \mu \mathrm{g} / \mathrm{L} & 5.0 & \text { EPA8240 } \\ \mathrm{U} & \mathrm{Y} & 5.0 & \mu \mathrm{g} / \mathrm{L} & 5.0 & \text { EPA8240 } \\ \mathrm{U} & \mathrm{Y} & 10 & \mu \mathrm{g} / \mathrm{L} & 10 & \text { EPA8240 } \\ \mathrm{U} & \mathrm{Y} & 5.0 & \mu \mathrm{g} / \mathrm{L} & 5.0 & \text { EPA8240 } \\ \mathrm{U} & \mathrm{Y} & 5.0 & \mu \mathrm{L} / \mathrm{L} & 5.0 & \text { EPA8240 } \\ \mathrm{U} & \mathrm{Y} & 5.0 & \mu \mathrm{g} / \mathrm{L} & 5.0 & \text { EPA8240 } \\ \mathrm{U} & \mathrm{Y} & 10 & \mu \mathrm{g} / \mathrm{L} & 10 & \text { EPA8240 } \\ \mathrm{U} & \mathrm{Y} & 10 & \mu \mathrm{g} / \mathrm{L} & 10 & \text { EPA8240 } \\ \mathrm{U} & \mathrm{Y} & 10 & \mu \mathrm{g} / \mathrm{L} & 10 & \text { EPA8240 } \\ \mathrm{U} & \mathrm{Y} & 5.0 & \mu \mathrm{g} / \mathrm{L} & 5.0 & \text { EPA8240 } \\ \mathrm{U} & \mathrm{Y} & 10 & \mu g / \mathrm{L} & 10 & \text { EPA8240 } \\ \mathrm{U} & \mathrm{Y} & 5.0 & \mu \mathrm{g} / \mathrm{L} & 5.0 & \text { EPA8240 } \\ \mathrm{U} & \mathrm{Y} & 5.0 & \mu \mathrm{g} / \mathrm{L} & 5.0 & \text { EPA8240 } \\ \mathrm{U} & \mathrm{Y} & 5.0 & \mu \mathrm{L} / \mathrm{L} & 5.0 & \text { EPA8240 } \\ \mathrm{U} & \mathrm{Y} & 5.0 & \mu \mathrm{g} / \mathrm{L} & 5.0 & \text { EPA8240 } \\ \mathrm{U} & \mathrm{Y} & 5.0 & \mu \mathrm{g} / \mathrm{L} & 5.0 & \text { EPA8240 } \\ \mathrm{U} & \mathrm{YV} & 7.0 & \mu \mathrm{g} / \mathrm{L} & 5.0 & \text { EPA8240 } \\ \mathrm{U} & \mathrm{Y} & 5.0 & \mu \mathrm{g} / \mathrm{L} & 5.0 & \text { EPA8240 } \\ \mathrm{U} & \mathrm{Y} & 5.0 & \mu \mathrm{g} / \mathrm{L} & 5.0 & \text { EPA8240 } \\ \mathrm{U} & \mathrm{Y} & 5.0 & \mu \mathrm{g} / \mathrm{L} & 5.0 & \text { EPA8240 } \\ \mathrm{U} & \mathrm{Y} & 5.0 & \mu \mathrm{g} / \mathrm{L} & 5.0 & \text { EPA8240 } \\ \mathrm{U} & \mathrm{Y} & 10 & \mu \mathrm{g} / \mathrm{L} & 10 & \text { EPA8240 } \\ \mathrm{J} & \mathrm{Y} & 9.8 & \mu \mathrm{g} / \mathrm{L} & 10 & \text { EPA8240 } \\ \mathrm{U} & \mathrm{Y} & 10 & \mu \mathrm{g} / \mathrm{L} & 10 & \text { EPA8240 } \\ \mathrm{U} & \mathrm{Y} & 5.0 & \mu \mathrm{g} / \mathrm{L} & 5.0 & \text { EPA8240 } \\ \mathrm{U} & \mathrm{Y} & 5.0 & \mu \mathrm{g} / \mathrm{L} & 5.0 & \text { EPA8240 }\end{array}$


SAMPLE NAME: D43111506E (Continued)

Constituent

RQ

AQ

Volatile Organic Compounds

Tetrachloroethylene

Toluene

1.1.1-Trichloroethane

1.1.2-Trichloroethane

Trichloroethylene

Unknown

Vinyl acetate

Xylenes

SAMPLE NAME: D43111603C

- Samplé Type: Blank

$\therefore$ Constituent

… Totat Metals

Arsenic

Arsenic :

$\therefore$. Barium +...

Bariüm

Cadmium

Cadmium

Chromium

Chromium

Copper

Copper

Lead

Lead

Manganese

Manganese

Mercury

Nickel

Nickel

Selenium

Selenium

Silver

Silver

Volatile Organic Compounds
Sample ID: 100416

B Result Unit D. Limit Method

$\begin{array}{llllll}\mathrm{U} & \mathrm{Y} & 5.0 & \mu \mathrm{g} / \mathrm{L} & 5.0 & \text { EPA8240 } \\ \mathrm{U} & \mathrm{Y} & 5.0 & \mu \mathrm{g} / \mathrm{L} & 5.0 & \text { EPA8240 } \\ \mathrm{U} & \mathrm{Y} & 5.0 & \mu \mathrm{g} / \mathrm{L} & 5.0 & \text { EPA8240 } \\ \mathrm{U} & \mathrm{Y} & 5.0 & \mu \mathrm{g} / \mathrm{L} & 5.0 & \text { EPA8240 } \\ \mathrm{U} & \mathrm{Y} & 5.0 & \mu \mathrm{g} / \mathrm{L} & 5.0 & \text { EPA8240 } \\ \mathrm{J} & \mathrm{N} & 9,000 & \mu \mathrm{g} / \mathrm{L} & & \text { EPA8240 } \\ \mathrm{U} & \mathrm{Y} & 10 & \mu \mathrm{g} / \mathrm{L} & 10 & \text { EPA8240 } \\ \mathrm{U} & \mathrm{Y} & 5.0 & \mu \mathrm{g} / \mathrm{L} & 5.0 & \text { EPA8240 }\end{array}$

Sample ID: 100420

Associated Sample: 100419

RQ AQ B Result Unit D. Limit Method
10

10

200

200

5.0

5.0

10

10

25

25

3.0

3.0

15

15

0.20

40

40

5.0

5.0

10

10

$\begin{array}{lll}\mu \mathrm{g} / \mathrm{L} & 10 & \text { EPA7060 } \\ \mu \mathrm{g} / \mathrm{L} & 10 & \text { EPA7060 } \\ \mu \mathrm{g} / \mathrm{L} & 200 & \text { EPA6010 } \\ \mu \mathrm{g} / \mathrm{L} & 200 & \text { EPA6010 } \\ \mu \mathrm{g} / \mathrm{L} & 5.0 & \text { EPA6010 } \\ \mu \mathrm{g} / \mathrm{L} & 5.0 & \text { EPA6010 } \\ \mu \mathrm{g} / \mathrm{L} & 10 & \text { EPA6010 } \\ \mu \mathrm{g} / \mathrm{L} & 10 & \text { EPA6010 } \\ \mu \mathrm{g} / \mathrm{L} & 25 & \text { EPA6010 } \\ \mu \mathrm{g} / \mathrm{L} & 25 & \text { EPA6010 } \\ \mu \mathrm{g} / \mathrm{L} & 3.0 & \text { EPA7421 } \\ \mu \mathrm{g} / \mathrm{L} & 3.0 & \text { EPA7421 } \\ \mu \mathrm{g} / \mathrm{L} & 15 & \text { EPA6010 } \\ \mu \mathrm{g} / \mathrm{L} & 15 & \text { EPA6010 } \\ \mu \mathrm{g} / \mathrm{L} & 0.20 & \text { EPA7470 } \\ \mu \mathrm{g} / \mathrm{L} & 40 & \text { EPA6010 } \\ \mu \mathrm{g} / \mathrm{L} & 40 & \text { EPA6010 } \\ \mu \mathrm{g} / \mathrm{L} & 5.0 & \text { EPA7740 } \\ \mu \mathrm{g} / \mathrm{L} & 5.0 & \text { EPA7740 } \\ \mu \mathrm{g} / \mathrm{L} & 10 & \text { EPA6010 } \\ \mu \mathrm{g} / \mathrm{L} & 10 & \text { EPA6010 }\end{array}$

Acetone

Benzene

Bromodichloromethane

Bromoform

Bromomethane (Methyl bromide)

Carbon disulfide

Carbon tetrachloride

Chlorobenzene
$\mathrm{U}$

U

U

J

$\mathrm{U}$
140

5.0

5.0

5.0

10

1.9

5.0

5.0 $\mu \mathrm{g} / \mathrm{L}$

$\mu \mathrm{g} / \mathrm{L}$

$\mu \mathrm{g} / \mathrm{L}$

$\mu \mathrm{g} / \mathrm{L}$

$\mu \mathrm{g} / \mathrm{L}$

$\mu \mathrm{g} / \mathrm{L}$

$\mu \mathrm{g} / \mathrm{L}$
10

5.0

5.0

5.0

10

5.0

5.0

5.0
EPA8240

EPA8240

EPA8240

EPA8240

EPA8240

EPA8240

EPA8240

EPA8240 
SAMPLE NAME: D43111603C (Continued)

Constituent

Volatile Organic Compounds

Chlorocthane

Chloroethene (Vinyl chloride)

2-Chloroethyl vinyl ether

Chloroform

Chloromethane (Methyl chloride)

Dibromochloromethane

1,1-Dichloroethane

1,2-Dichloroethane

1,1-Dichloroethylene

1,2-Dichloroethylene

Dichloromethane (Methylene chloride)

1,2-Dichloropropane

cis-1,3-Dichloropropene

trans-1,3-Dichloropropene

Ethylbenzene

2-Hexanone

Methyl ethyl ketone

Methyl isobutyl ketone

Styrene

1,1,2,2-Tetrachloroethane

Tetrachloroethylene

Toluene

1,1,1-Trichloroethane

1,1,2-Trichloroethane

Trichloroethylene

Vinyl acetate

Xylenes
RQ AQ B Result Unit D. Limit Method

Sample ID: 100420
10

10

10

5.0

10

5.0

5.0

5.0

5.0

5.0

2.3

5.0

5.0

5.0

5.0

10

35

10

5.0

5.0

5.0

5.0

5.0

5.0

5.0

10

5.0

$\begin{array}{lll}\mu \mathrm{g} / \mathrm{L} & 10 & \text { EPA8240 } \\ \mu \mathrm{g} / \mathrm{L} & 10 & \text { EPA8240 } \\ \mu \mathrm{g} / \mathrm{L} & 10 & \text { EPA8240 } \\ \mu \mathrm{g} / \mathrm{L} & 5.0 & \text { EPA8240 } \\ \mu \mathrm{g} / \mathrm{L} & 10 & \text { EPA8240 } \\ \mu \mathrm{g} / \mathrm{L} & 5.0 & \text { EPA8240 } \\ \mu \mathrm{g} / \mathrm{L} & 5.0 & \text { EPA8240 } \\ \mu \mathrm{g} / \mathrm{L} & 5.0 & \text { EPA8240 } \\ \mu \mathrm{g} / \mathrm{L} & 5.0 & \text { EPA8240 } \\ \mu \mathrm{g} / \mathrm{L} & 5.0 & \text { EPA8240 } \\ \mu \mathrm{g} / \mathrm{L} & 5.0 & \text { EPA8240 } \\ \mu \mathrm{g} / \mathrm{L} & 5.0 & \text { EPA8240 } \\ \mu \mathrm{g} / \mathrm{L} & 5.0 & \text { EPA8240 } \\ \mu \mathrm{g} / \mathrm{L} & 5.0 & \text { EPA8240 } \\ \mu \mathrm{g} / \mathrm{L} & 5.0 & \text { EPA8240 } \\ \mu \mathrm{g} / \mathrm{L} & 10 & \text { EPA8240 } \\ \mu \mathrm{g} / \mathrm{L} & 10 & \text { EPA8240 } \\ \mu \mathrm{g} / \mathrm{L} & 10 & \text { EPA8240 } \\ \mu \mathrm{g} / \mathrm{L} & 5.0 & \text { EPA8240 } \\ \mu \mathrm{g} / \mathrm{L} & 5.0 & \text { EPA8240 } \\ \mu \mathrm{g} / \mathrm{L} & 5.0 & \text { EPA8240 } \\ \mu \mathrm{g} / \mathrm{L} & 5.0 & \text { EPA8240 } \\ \mu \mathrm{g} / \mathrm{L} & 5.0 & \text { EPA8240 } \\ \mu \mathrm{g} / \mathrm{L} & 5.0 & \text { EPA8240 } \\ \mu \mathrm{g} / \mathrm{L} & 5.0 & \text { EPA8240 } \\ \mu \mathrm{g} / \mathrm{L} & 10 & \text { EPA8240 } \\ \mu \mathrm{g} / \mathrm{L} & 5.0 & \text { EPA8240 }\end{array}$

Radionuclide Indicators and Radionuclides

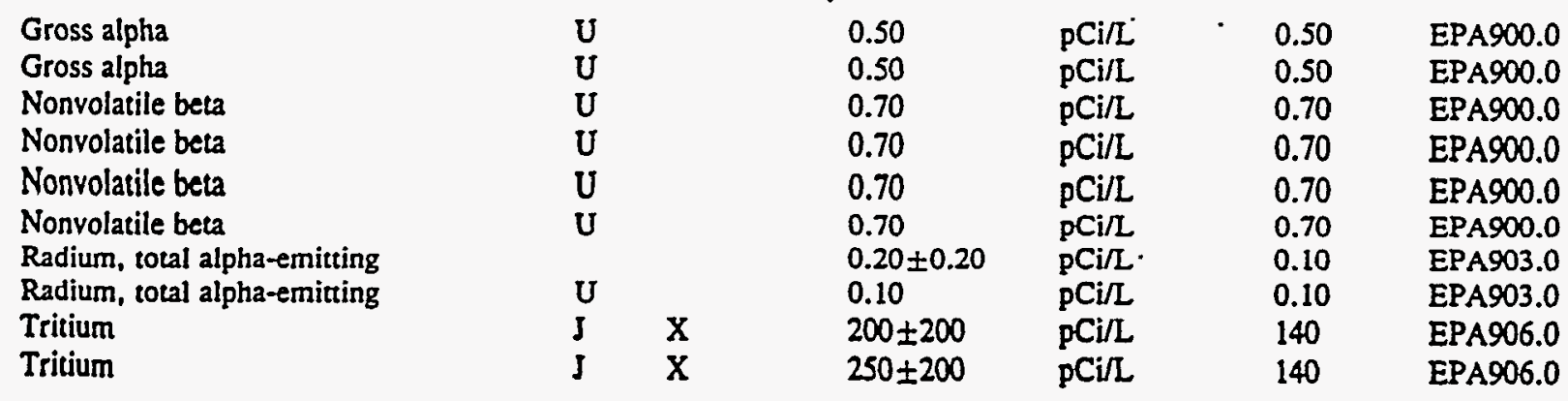


SAMPLE NAME: D43112102B

Sample Type: Blank

Constituent

Volatile Organic Compounds

Acetone
Benzene
Bromodichloromethane
Bromoform
Bromomethane (Methyl bromide)
Carbon disulfide
Carbon tetrachloride
Chlorobenzene
Chloroethane
Chlorothene (Vinyl chloride)
2-Chloroethyl vinyl ether
Chloroform
Chloromethane (Methyl chloride)
Dibromochloromethane
1,1-Dichloroethane
1,2-Dichloroethane
1,1-Dichloroethylene
1,2-Dichloroethylene
Dichloromethane (Methylene chloride)
1.2-Dichloropropane
cis-1,3-Dichloropropene
trans-1,3-Dichloropropene
Ethylbenzene
2-Hexanone
Methyl ethyl ketone
Methyl isobutyl ketone
Styrene
1,1,2,2-Tetrachloroethane
Tetrachloroethylene
Toluene
1,1,1-Trichloroethane
1,1.2-Trichloroethane
Trichloroethylene
Vinyl acetate
Xylenes

Sample ID: 100303

Associated Sample: 100305

RQ AQ B Result Unit D. Limit Method

\section{Radionuclide Indicators and Radionuclides}

Gross alpha

Nonvolatile beta

Radium, total alpha-emitting

Tritium

Tritium

$\begin{array}{llllll} & 1.2 \pm 1.0 & \mathrm{pCi} / \mathrm{L} & 0.60 & \text { EPA900.0 } \\ \mathrm{U} & \mathrm{V} & 2.1 \pm 1.7 & \mathrm{pCi} / \mathrm{L} & 0.80 & \text { EPA900.0 } \\ \mathrm{U} & 0.10 & \mathrm{PCi} / \mathrm{L} & 0.10 & \cdot \text { EPA903.0 } \\ \mathrm{U} & 170 & \mathrm{pCi} / \mathrm{L} & 170 & \text { EPA906.0 } \\ \mathrm{U} & 160 & \mathrm{pCi} / \mathrm{L} & 160 & \text { EPA906.0 } \\ & & & 1 & \end{array}$

EPA8240

EPA8240

EPA 8240

EPA8240

EPA8240

EPA8240

EPA8240

EPA8240

EPA8240

EPA8240

EPA8240

EPA8240

EPA8240

EPA8240

EPA8240

EPA8240

EPA8240

EPA8240

EPA8240

EPA8240

EPA8240

EPA8240

EPA8240

EPA8240

EPA8240

EPA8240

EPA8240

EPA8240

EPA8240

EPA 8240

EPA8240

EPA8240

EPA8240

EPA8240

EPA8240

$\mu g / \mathrm{L} \quad 10$

$\mu g / L \quad 5.0$

EPA8240

EPA900.0

EPA906.0 


\section{APPENDIX C}

QUALITY CONTROL SUMMARY REPORT

FOR SURFACE WATER AND SEDIMENTS COLLECTED

DURING 1994 FOR THE

D-AREA BURNING/RUBBLE PITS RFI/RI UNIT ASSESSMENT

(RUST E \& I Validation) 


\title{
QUALITY CONTROL SUMMARY REPORT
}

\section{FOR SURFACE WATER AND SEDIMENT SAMPLES \\ COLLECTED DURING MAY 1994 \\ FOR THE \\ D-AREA BURNING/RUBBLE PITS \\ RFI/RI UNIT ASSESSMENT}

\author{
Prepared For: \\ Westinghouse Savannah River Company \\ Savannah River Site \\ Aiken, South Carolina \\ Prepared By: \\ RUST Environment \& Infrastructure \\ 955 Millbrook Avenue \\ Aiken, South Carolina
}

October 1994 
Table of Contents

\section{SectionPage}

$1.0 \quad$ Introduction............................................................. 1

2.0 Project Description..................................................... 2

$3.0 \quad$ Analytical Laboratories................................................... 3

4.0 Data Quality ............................................................. 5

5.0 Data Qualification Policy ................................................. 9

6.0 Qualifier Codes........................................................... 13

$7.0 \quad$ Data Validation Results.................................................. 15

8.0 Data Presentation and Conclusions ........................................ 20

References

Tables

Appendix A Surface Water/Sediment Sampling Plan (Addendum)

Appendix B Laboratory Data Records Review 


\section{List of Tables}

1. Analytical Program of Surface Water and Sediment Samples

2. Analytical Program of Laboratory Quality Control Samples

3. Association of Primary and Quality Control Samples

4. Holding Times

5. Detections in Laboratory Blanks

6. Recoveries of Laboratory Control Samples

7. Recoveries of Matrix Spike and Surrogate Samples

8. Detection Summary of Rinsate Blank

9. Detection Summary of Trip Blanks

10. Detection Summary of Field Blanks

11. Evaluation of Surface Water Field Duplicates

12. Evaluation of Sediment Field Duplicates

13. Evaluation of Surface Water Split Samples

14. Evaluation of Sediment Split Samples 


\section{List of Acronyms}

$\mathrm{AQ}$

BS

BQ

CLP

DOE

DQOs

EMSL

EPA

EPD/EMS

LCS

LDDR

PARCC

QA

QC

RPD

RFI/RI

RQ

RUST E\&I

TAL

TCL

TMA

VOCs
Analysis Qualifier

Blank Spike

Bias Qualifier

Contract Laboratory Program

Department of Energy

Data Quality Objectives

Environmental Monitoring Systems Laboratory

Environmental Protection Agency

Environmental Monitoring Section of the Environmental Protection

Department

Laboratory Control Sample

Laboratory Data Record Review

Precision, Accuracy, Representativeness, Completeness, and Comparability

Quality Assurance

Quality Control

Relative Percent Difference

RCRA Facility Investigation / Remedial Investigation

Result Qualifier

RUST Environment \& Infrastructure

Target Analyte List

Target Compound List

TMA/Eberline, Inc.

Volatile Organic Compounds 


\subsection{Introduction}

This report presents a summary of the data validation performed for the surface water and sediment samples collected during May 1994, at the D-Area Burning/Rubble Pits. This work was performed in accordance to the Addendum Surface Water/Sediment Sampling Plan, as presented in Appendix A. The purpose of this report is to verify that all analytical data collected at the D-Area Burning/Rubble Pits for use in developing risk assessment and potential remediation procedures have been validated at the appropriate level. This report documents the data validation procedures performed by RUST Environment \& Infrastructure. 


\subsection{Project Description}

During unit characterization sampling conducted in 1993, dry conditions precluded sampling of six of the planned nine surface water locations. During 1994, it was noted that ephemeral stream flow in the planned surface water drainage areas was dominated by high flows traced back to overflow of two cooling water surge tanks connected to river water intake pump houses. Because of surface water and sediment as a result of river water inflow versus water seepage, runoff, or discharge from the unit, surface water and sediment sampling was conducted during May 1994. This new surface water and sediment sampling was conducted in the drainage channel upstream of the power line right-of-way.

The data collected in this investigation are intended to further characterize the nature and extent of chemical residue in the surface water and sediment. In addition, the data supplement existing geochemical data for the purpose of developing risk evaluation and appropriate remedial activities at the D-Area Burning/Rubble Pits (431-D and 431-1D), if necessary.

\subsection{Site History}

The D-Area Burning/Rubble Pits 431-D and 431-1D are contiguous pits separated by a clay berm. A shallow run-off ditch is located within 50 feet west of the pits. The Savannah River and the associated swamps are approximately 0.5 miles from the rubble pits.

The Burning/Rubble Pits were constructed in 1951 for disposal of spent organic solvents, waste oils, paper, plastics, rubber, wood, rags, cardboard, and degreasers. The waste was burned periodically, typically once a month. The chemical composition and volume of waste disposed of are unknown.

In 1973 , the pits were covered with a soil layer and continued to be used for rubble only. During this time until 1983, these pits were filled with concrete, metal lumber, bricks, tile, asphalt, plastic, empty drums, rubber, telephone poles, and other wood products. When the pits were full, they were covered with a clay layer, compacted, mounded, and closed.

\subsection{Project Objectives}

The purpose of assessment sampling was to further delineate the extent and magnitude of hazardous substance released to surrounding environmental media. 


\subsection{Analytical Laboratories}

Analyses were performed by Roy F. Weston, Inc.-Lionville (PA), TMA/Eberline of Oak Ridge, TN; and General Engineering of Charleston, SC. All the laboratories performing analyses for this project have been certified, or have applied for certification, by SCDHEC.

\subsection{Roy F. Weston, Inc.}

Roy F. Weston, Inc., Laboratories participated in the EPA Water Pollution Laboratory Performance Evaluation Study (WP030), for which results were reported in June 1993. EPA conducts the water pollution study biannually to certify laboratories for specific analyses. EPA's Environmental Monitoring Systems Laboratory (EMSL) of Cincinnati, Ohio, prepares water samples spiked with known concentrations of constituents found in polluted waters and submits them to all laboratories seeking certification to analyze waste water. EMSL evaluates the results, using limits statistically based on the performance of approximately 100 top-rated laboratories that analyze each constituent by the same procedure as the laboratory being evaluated. Results for two samples of mercury and one sample each of beryllium, orthophosphate, total phosphorous, and non-filterable residue were outside the acceptable range. Results for two samples of selenium and one sample each of silver, chloride, orthophosphate, total phosphorous, and non-filterable residue, although within the acceptable range, were near the acceptable limits. The laboratory was instructed to check for error in the latter two cases.

Weston Laboratories also participated in two EPA Water Supply Laboratory Performance Evaluation Studies, WS031 and WS032, for which results were reported in February and August of 1993. In the former study, results for one sample each of cadmium, zinc, bromodichloromethane, chlorodibromomethane, fluorotrichloroethane, and styrene were outside the acceptable range.

\section{Quality Control Standards}

During April and June 1993, the Environmental Monitoring Section of the Environmental Protection Department (EPD/EMS) at the Savannah River Site (SRS) conducted a quality assessment of Weston laboratory by sending them a set of certified environmental quality control (QC) standards from Environmental Resource Associates (ERA) of Arvada, CO (Lot Numbers $418,549,3205,3405,8910$, and 9950). 
Weston's results were compared with the ERA-certified values and advisor ranges. Weston did not analyze alachlor, alkalinity (as $\mathrm{CaCO}_{3}$ ), ammonia nitrogen, atrazine, biological oxygen demand, boron, chemical oxygen demand, dalapon, dinoseb, molybdenum, nitrate-nitrite as nitrogen, nitrogen by Kjeldahl method, pentachlorophenol (by method 8150), pichloram, simarine, or strontium because they are not in the current contract.

Of 96 analyses reported by Weston, 92 , or $95.8 \%$, fell within the advisory range.

\subsection{TMA/Eberline, Inc.}

TMA/Eberline, Inc. (TMA) participated in two types of national performance evaluation programs during 1993. One study, sponsored by EPA's Environmental Monitoring Systems Laboratory (EMSL) of Las Vegas, NV, was conducted from January through August 1993. EMSL provided samples spiked with known concentrations of constituents to laboratories seeking certification to analyze those constituents. TMA submitted results to EPA for certification. One TMA result for gross alpha in water was outside the normalized deviation range of +3.0 to -3.0 ; however, TMA stated that thorium-230, which was used by EPA, has a 50\% lower counting efficiency than americium-241, the EPA-recommended isotope for alpha-absorption efficiency curves. According to TMA, correction for the $50 \%$ difference gives a value within range. TMA's result for nonvolatile beta in air filters also was outside of range.

The other program was conducted by the Department of Energy (DOE) and used water, soil, air, and vegetation matrices. Results were reported for March and September 1993. The DOE program does not include a range of acceptable values; instead, the value obtained by the lab is divided by the DOE's known value to give a ratio. For the water and soil samples, one analysis for strontium90 was outside the ratio range of 0.5 to 1.5 . Results for one sample each of americium-241, cesium-137, potassium-40, strontium-90, and uranium mass concentration, although acceptable, fell within the low advisory range of 0.5-0.8. Results for one sample each of americium-241, cesium-137, and plutonium-239, although acceptable, fell within the high advisory range of $1.2-$ 1.5. 


\subsection{Data Quality}

RUST E\&I has performed independent QC checks of the field and laboratory procedures that were used in collecting and analyzing the data. The QC checks verify that the data collected are of appropriate quality for the intended data use and that the Data Quality Objectives were met. The analytical procedures, where appropriate, were validated with respect to the EPA Contract Laboratory Program (CLP) "National Functional Guidelines for Organic Data Review" (EPA, June, 1991) and "Functional Guidelines for Evaluating Inorganic Analyses" (EPA, July, 1988). The policy for data qualification is provided in Section 5.0. The field activities and laboratory procedures are discussed in the following sections.

\subsection{Assessment of Data Quality Indicators}

The assessment of the data quality indicators determines the data usability. The assessment of data quality indicators for either sampling or analysis involves the evaluation of five indicators: precision, accuracy, representativeness, completeness, and comparability. The indicators are commonly referred to as the PARCC parameters.

Precision measures the reproducibility of measurements under a given set of conditions. Specifically, it is the quantitative measure of the variability of a group of measurements compared to the average value. The overall precision of a measurement data is a mixture of sampling and analytical factors. Analytical precision is much easier to control and quantify than sampling precision. Sampling precision may be determined by collecting and analyzing replicate field . samples. The analytical results from laboratory replicates provide data on analytical precision. Subtracting the analytical precision from the measurement precision defines the sampling precision.

Accuracy measures the bias in a measurement system. Accuracy is difficult to measure for the entire data collection activity. Sources of error are the sampling process, field contamination preservation, sample handling, sample matrix, sample preparation, and analysis techniques. Analytical accuracy is assessed through use of known and unknown QC samples and spike samples. Accuracy determinations by known samples include single control and duplicate control samples, commonly referred to as laboratory control samples. These are samples that consist of reagent grade water that is spiked with known amounts of target compounds. Percent recovery and percent difference parameters are determined from these samples. Analytical accuracy determinations by unknown samples include the evaluation of matrix interferences in the 
environmental samples. These samples also provide percent recovery and percent difference parameters through the use of surrogate and matrix spikes in the environmental samples.

Representativeness expresses the degree to which sample data accurately and precisely represent a characteristic of a population, parameter variations at a sampling point, or an environmental condition. Representativeness is a qualitative parameter which is a function of sampling program design. The representativeness criterion is best satisfied by making certain that sampling locations are selected properly and a sufficient number of samples are collected.

Completeness is defined as the percentage of measurements made which are judged to be valid measurements. The completeness goal is essentially the same for all data uses: that a sufficient amount of valid data be generated. It is important that critical samples are collected and valid data achieved for them.

Comparability is a qualitative parameter expressing the confidence with which one data set can be compared with another. Sample data should be comparable with other measurement data for similar samples and sample conditions. This goal is achieved through using standard techniques to collect and analyze representative samples and reporting analytical results in appropriate units. Comparability is limited to the other PARCC parameters because only when precision and accuracy are known can data sets be compared with confidence.

\subsubsection{Sampling}

The major activity in determining the usability of data based on sampling is assessing the effectiveness of the sampling operations performed.

The measure of completeness is useful for data collection and analysis management. Any decrease in the number of samples from that specified in the Work Plan may affect the final results. The completeness of the field screening and confirmatory samples for this sampling event was determined to be $100 \%$. A sufficient number of samples were completed to meet the projectspecific performance measures.

Comparability issues have little impact on performance measures associated with sampling provided that the sample design is unbiased, and the sample design or analytical methods have not 
changed over time. Comparability was achieved by following the sample design in the Work Plan . and documenting all sample locations.

Representativeness is primarily a planning concern. The solution is in the design of a sampling plan that is representative.

\subsubsection{Analytical}

Determining the usability of analytical results begins with the review of $\mathrm{QC}$ samples and qualifiers. The review is used to determine an overall assessment of analytical performance as determined by the laboratory and method performance.

The completeness for analytical data is defined as the number of chemical-specific data results that are determined acceptable after data review. The off-site laboratories obtained an analytical completeness of $100 \%$.

Comparability is a very important qualitative data indicator for analytical assessment and is a critical parameter when considering the combination of data sets from different analyses for the same chemicals of potential concern. The analytical methods used had common analytical parameters, the same units of measure, and similar detection limits. .

The representativeness of the data is determined by the degree to which the data meet the performance standards of the method and to which analysis represents the sample submitted to the laboratory. Holding times, sample preservation, and results from analyses of blanks all affect the representativeness of analytical data. Analytical data from off-site laboratories are considered representative of the sample since all performance criteria were met during the analyses.

Precision is a measure of the repeatability of a single measurement and is evaluated from the results of duplicate samples and splits. Low precision can be caused by poor instrument performance, inconsistent application of method protocols, or by a difficult, heterogeneous sample matrix. The off-site laborat?. pcitomed the analysis of duplicate laboratory control samples to evaluate the precision of L: LAl us llory. The precision indicator from the duplicate analyses met the performance staridards in all cases. 
Accuracy is a measure of overestimation or underestimation of reported concentrations and is evaluated from the results of spiked samples. Split samples are particularly useful in the analysis of complex sample types to help determine the extent of bias on the sample measurement. Split samples were analyzed by General Engineering.

\subsection{Field Activities}

Sampling activities were conducted in May 1994. Sampling Logs were maintained describing the activities and conditions during the sampling event. Sampling personnel wore gloves, safety glasses, hard-hats, steel-toed boots, and tyvek coveralls. No unusual or problematic situations occurred during the activities.

\subsection{Laboratory Procedures}

Analytical procedures were performed by Roy F. Weston, TMA, and General Engineering. The QC procedures included but were not limited to method blanks, laboratory control samples, matrix spikes, matrix spike duplicates, and surrogate recoveries. Where applicable, data validation procedures were performed in accordance with the EPA "National Functional Guidelines for Organic Data Review" and "Functional Guidelines for Evaluating Inorganic Analyses."

As discussed in the CLP Statement of Work (SOW) for Organics Analysis (EPA, August, 1991) and the National Functional Guidelines for Organic Data Review (EPA, June, 1991), acetone, 2butanone (methyl ethyl ketone), methylene chloride, toluene, and the phthalate esters are considered by EPA to be common laboratory contaminants. In accordance with the EPA "National Functional Guidelines for Organic Data Review" (EPA, June, 1991) and the "Functional Guidelines for Evaluating Inorganic Analyses" (EPA, July, 1988), if the blank contains detectable levels of common laboratory contaminants, then the sample results should be considered as positive results (i.e., site-related) only if the concentrations in the sample exceed ten times the maximum amount detected in any blank. If the blank contains detectable levels of one or more organic or inorganic chemicals that are not considered by EPA to be common laboratory contaminants, then consider site sample results as positive only if the concentration of the chemical in the site sample exceeds five times the maximum amount detected in any blank. Treat samples containing less than five times the amount in any blank as not site-related. 


\subsection{Data Qualification Policy}

This section describes the EPD/EMS data validation policy for assigning qualifiers to RCRA Facility Investigation / Remedial Investigation (RFI/RI) analytical data. Qualification policy is based on the Contract Laboratory Program Statement of Work (CLP SOW), the Functional Guidelines for Organics Analyses, the Functional Guidelines for Inorganics Analyses (Functional Guidelines 7/88), SW-846, and Westinghouse Hanford Company's Data Validation Procedures for Radiochemical Analyses.

Qualification criteria for instrument calibration, analyte identification, and analyte quantitation are given in the EPD/EMS informal "Procedures for Qualifying Data Based on Laboratory Data Record Reviews (LDRRs)."

$\mathrm{RQ}=$ result qualifier $\quad \mathrm{AQ}=$ analysis qualifier $\quad \mathrm{BQ}=$ bias qualifier

\section{Holding Times}

Criteria: EPA holding times for extraction and analysis

Action: Assign $\mathrm{RQ}=\mathrm{J}, \mathrm{AQ}=\mathrm{Q}$ to all analyses for which holding time has been exceeded.

\section{Sample Preservation}

Criteria: Temperature $<8^{\circ} \mathrm{C}$ for organics and inorganics; $\mathrm{pH} \leq 2$ for water samples.

Note: $\mathrm{pH}$ was not recorded by the laboratory, so samples preservation for $\mathrm{pH}$ could not be evaluated.

Action: Assign $\mathrm{AQ}=\mathrm{Y}$ for all exceedances.

Instrument Calibration, Analyte Identification, Analyte Quantitation

Criteria: see EPD/EMS Laboratory Data Record Review (LDRR) checklists for each analytical method.

Action: see "Procedures for Qualifying Data Based on LDRRs."

\section{Trip Blanks}

Criteria: CLP 5x/10x rule

Action: $A d d \mathrm{RQ}=\mathrm{J}$ to the failed analyte(s) in every sample shipped in the same cooler as the trip blank. 
Data Summary Report

D-Area Burning/Rubble Pits

Appendix $\mathrm{C}$

\section{Field Blanks}

No qualification is done on the basis of field blank results.

\section{Rinsate Blanks}

No qualification is done on the basis of rinsate blank results.

\section{Method Blanks}

\section{Criteria: CLP $5 \times / 10 x$ rule}

Action: If an analyte is detected in a method blank, assign $A Q=V$ to that analyte in the blank in and in every sample of the preparation batch. For radiological-samples, if the analyte concentration in a sample is $<5 x$ that in the blank, assign $R Q=U$ and $A Q=V$ to the analyte in the sample. For non radiological samples, if the analyte concentration in a sample is $<5 \mathrm{x}$ that in the blank $(<10 \mathrm{x}$ for common lab contaminants), assign $R Q=J$ and $A Q=V$ to the analyte in the sample.

\section{Field Duplicates}

No qualification is done based on field duplicate results.

\section{Lab Duplicates}

No qualification is done based on lab duplicate results.

\section{Matrix Spikes}

Criteria:

Volatiles: CLP SOW Table 7, p. D-55VOA

Benzene

Chlorobenzene

1,1-Dichloroethene

Toluene

Trichloroethene

\begin{tabular}{ll} 
\% Recovery & \\
\hline Water & Soil \\
\hline $76-127$ & $66-142$ \\
$75-130$ & $60-133$ \\
$61-145$ & $59-172$ \\
$76-125$ & $59-139$ \\
$71-120$ & $62-137$
\end{tabular}


Semivolatiles: CLP SOW Table 7, p. D-59SV

Phenol

2-Chlorophenol

1,4-Dichlorobenzene

N-Nitroso-di-n-propylamine

1,2,4-Trichlorobenzene

4-Chloro-3-methylphenol

Acenaphthene

4-Nitrophenol

2,4-Dinitrotoluene

Pentachlorophenol

Pyrene
\% Recovery

\begin{tabular}{ll}
\hline Water & Soil \\
\hline $12-110$ & $26-90$
\end{tabular}

27-123 25-102

36-97 28-104

41-116 41-126

39-98 38-107

23-97 26-103

46-118 31-137

$10-80 \quad 11-114$

24-96 28-89

9-103 17-109

26-127 35-142

Pesticides: CLP SOW page D-61PEST

gamma-BHC (Lindane)

Heptachlor

Aldrin

Dieldrin

Endrin

4,4'-DDT
\% Recovery

\begin{tabular}{ll}
\hline Water & Soil \\
\hline $56-123$ & $46-127$ \\
$40-131$ & $35-130$ \\
$40-120$ & $34-132$ \\
$52-126$ & $31-134$ \\
$56-121$ & $42-139$ \\
$38-127$ & $23-134$
\end{tabular}

Dioxins: $50-100 \%$

Inorganics: Functional Guidelines

All Parameters

\begin{tabular}{ll} 
\% Recovery \\
\hline Water & Soil \\
\hline $75-125$ & $75-125$
\end{tabular}

Action:

For organics, if an analyte within a matrix spike fails criteria, assign qualifiers only to the failed analyte in the matrix spike itself. For organics, assign $R Q=J, A Q=I$, and $B Q=L$ or $H$.

For inorganics, qualify the sample result and the matrix spike itself; assign RQ according to criteria, $\mathrm{AQ}=\mathrm{I}$, and $\mathrm{BQ}=\mathrm{L}$ or $\mathrm{H}$.

For non-radiological analyses, if the laboratory fails to supply matrix spike data, flag all analytes in . all samples in the appropriate preparation batch. Assign $R Q=P, A Q=I$. 


\section{Laboratory Control Samples \& Blank Spikes (LCS/BS)}

\section{Criteria:}

Organics: same as matrix spike criteria

Inorganics: $80-120 \%$ recovery

Rads: (80-120\% recovery)

Action: Assign $A Q=C$ to the failed analyte(s) in the $L C S / B S$. Assign $R Q=J, A Q=C$ to the failed analyte(s) in every samples in the same preparation batch as the LCS/BS.

\section{Surrogate Recovery}

Criteria:

Volatiles by 8240: SW-846, Table 8, page 8240-38.

Volatiles by 8260 : SW-846, Table 9 , page $8260-44$.

Pesticides by 8080: 60-150\% (from CLP SOW, page D-56PEST).

Semivolatiles by 8270: SW-846, Table 8, page 8270-31.

Dioxins: not applicable

Action:

Volatiles \& Pesticides: Assign $\mathrm{AQ}=\mathrm{O}$ to the failed surrogate(s). If one or more surrogates fail acceptance criteria, assign $\mathrm{RQ}=\mathrm{J}$ or $\mathrm{UJ}, \mathrm{AQ}=\mathrm{O}$ to all analytes in the sample. If all the failed surrogates have low recoveries, assign $B Q=L$. If all the failed surrogates have high recoveries, assign $B Q=H$. If one surrogate is high and another is low, do not assign a bias qualifier.

For pesticides, do not qualify analytes when two surrogates fail if the average of the two surrogate recoveries is within $60-150 \%$. Also, do not qualify when the average recovery is $>150 \%$, but all analytes are non detects.

Action

Semivolatiles: Assign $\mathrm{AQ}=\mathrm{O}$ to the failed surrogate(s). If only one surrogate is bad, and its recovery is $>10 \%$, take no action. If more than one fails, assign $R Q=J$ or $U J, A Q=0$ to all analytes in the sample. If all the failed surrogates have low (or high) recoveries, assign $B Q=L$ or (or $\mathrm{H}$ ). If one surrogate is high and another is low, do not assign a bias qualifier. 


\subsection{Qualifier Codes}

Each analytical record in the computer data files may contain qualifiers in three fields: result qualifiers, analysis qualifiers, and bias qualifiers. The result qualifier describes the analytical result; the analysis qualifier describes why result qualifiers are present; and the bias qualifier describes whether the result is biased high, low, or has poor precision. The qualifiers in the data files may have been added by the laboratory or during data validation. All the qualifiers are described briefly here.

\section{Codes for Result Qualifiers}

Remark Definition

$\mathrm{J}$ The analytical result value is an estimated quantity.

$\mathrm{R} \quad$ Rejected because performance requirements in the sample or associated quality control analyses were not met. The analyte may or may not be present.

U Material analyzed for but not detected. Value reported is the sample quantification limit.

\section{Codes for Analysis Qualifiers}

\section{Code Definition}

B The result is below the contract required detection limit and above or equal to the instrument detection limit. Use with "J".

I Matrix Spike/Matrix Spike Duplicate (VI, Organics FG; VI, Pesticides)

Matrix Spike Sample Analysis (VI, Inorganics FG)

Spike recovery not within control limits. Used alone, or with "J" or "R."

O Surrogate recovery (V, Organics FG; V, Pesticides FG)

Surrogate spike recoveries are out of specification. Use with "J," "UJ," or "R."

Q Holding Time (I, Organics FG; I, Pesticides FG; I, Inorganics FG) Sample held beyond normal holding time. In addition, if the holding time is exceeded by less than 30 days, use a " $\mathrm{J"}$; if the holding time is exceeded by 30 days or more, use a "R."

V Blanks contaminated (IV, Organics FG; IV, Pesticides FG; II, Inorganics FG)

Indicates the analyte was detected in both the sample and associated method blank. Use with " $R$ " or " $U$ " for organics, and with " $U$ " for pesticides. This will follow the $5 \mathrm{x}$ and $10 \mathrm{x}$ rule for organics, and the $5 \mathrm{x}$ rule for pesticides and inorganics. 
X Matrix Spike/Matrix Spike Duplicates(VI, Organics FG; VI, Pesticides FG)

Duplicate Sample analysis(VI, Inorganics FG)

Duplicate analysis relative percent difference (RPD) was not within control limits.

\section{Bias Codes:}

L

Analytical factor causing bias. The associated result may underestimate the true value.

$\mathrm{H}$ Analytical factor causing bias. The associated result may overestimate the true value.

$\mathbf{P}$

Analytical factor causing poor precision. The associated result may be of high variability. 


\subsection{Data Validation Results}

RUST E\&I has performed independent $\mathrm{QC}$ checks of the field and laboratory procedures that were used in collecting and analyzing the data. The $\mathrm{QC}$ checks verify that the data collected are of appropriate quality for the intended data use and that the Data Quality Objectives were met. A summary of all combinations of data qualifiers needed for the surface water and sediment samples are provided below.

\begin{tabular}{|c|c|c|}
\hline $\begin{array}{c}\text { Result } \\
\text { Qualifier }\end{array}$ & $\begin{array}{c}\text { Analysis } \\
\text { Qualifier }\end{array}$ & $\begin{array}{c}\text { Bias } \\
\text { Qualifier }\end{array}$ \\
\hline & O & H \\
J & O & L \\
J & X & P \\
J & B & \\
J & I & P \\
J & Q & L \\
J & V & H \\
R & VI & P \\
R & & \\
R & & P \\
U & & P \\
U & V & \\
UJ & I & P \\
UJ & Q & L \\
UJ & V & \\
UJ & V & H \\
UJ & VB & \\
UJ & X & P \\
\hline
\end{tabular}

The assignment of data qualifiers (presented above) is supported by the following data summaries of quality control information. The definitions of the flags are provided in Section 6.0. The following components of data validation are provided in this section.

- Analytical Program of Primary Samples

- Analytical Program of Laboratory Control Samples

- Association of Primary and Quality Control Samples

- Holding Times 
- Detections in Laboratory Blanks

- Recoveries of Laboratory Control Samples

- Recoveries of Matrix Spike and Surrogate Samples

- Detection Summary of Rinsate Blanks

- Detection Summary of Trip Blanks

- Detection Summary of Field Blanks

- Evaluation of Surface Water Field Duplicates

- Evaluation of Sediment Field Duplicates

- Evaluation of Surface Water Split Samples

- Evaluation of Sediment Split Samples

\section{Analytical Program}

The analytical program excluding the quality control samples is presented in Table 1. The sample identifier, sample name, laboratory, sample type, sample date, extraction / digestion method, analytical method, analytical parameters, preparation batch, and instrument batch are included in Table 1. This table was used to verify that all requested samples were collected and analyzed appropriately. This verification check concluded that all sample and analytical requirements were met.

\section{Analytical Program of Laboratory Quality Control Samples}

The analytical program of laboratory quality control samples is presented in Table 2. The laboratory, sample type, analytical method, analytical parameters, preparation batch, and instrument batch are included in Table 2. Quality control samples include laboratory blanks, spike samples, continuing calibration blanks, continuing calibration verification samples, initial calibration blanks, and initial calibration verification sample blanks. This table was used to verify that the appropriate laboratory quality control samples were analyzed at the appropriate frequency. This verification check concluded that appropriate steps were taken in the quality assurance programs of the primary and quality assurance laboratories.

\section{Association of Primary and Quality Control Samples}

The association of primary and quality control samples is presented in Table 3. This table provides a matrix-type format demonstrating the correlation of primary and quality control samples. Quality control samples include field blanks, splits, rinsates, field duplicates, and trip blanks. This table was used to verify that the appropriate field quality control samples were collected. 


\section{Holding Times}

An evaluation of holding times is presented in Table 4. This table includes the extraction/digestion methods, analytical methods, units, sample identifiers, collection dates, extraction/digestion dates, and analysis dates. Calculation fields are included for the days from collection to extraction and the days from extraction to analysis. This verification check demonstrated that all holding times were met.

\section{Detections in Laboratory Blanks}

A detection summary of laboratory blanks (i.e., method and calibration blanks) is presented in Table 5. The table includes the laboratory, analytical method, analyte, preparation batch, instrument batch, sample type, result, result qualifier, analysis qualifier, bias qualifier, unit, and dilution factor. Detects in method blanks at Weston included total suspended solids; 1,2dichloroethylene; methylene chloride; trichlorofluoromethane; methyl chloride; bis(2ethylhexyl)phthalate, and di-n-butyl phthalate. At Weston, calibration blanks included detects of arsenic, mercury, and selenium. The detects in the method blanks required the "V" and "B" analytical qualifiers and " $\mathrm{U}$ " and " $\mathrm{J}$ " result qualifiers.

\section{Spike Recoveries of Laboratory Control Samples}

The percent recoveries of laboratory control samples (i.e., blank spikes) are presented in Table 6. The table includes the laboratory, sample identifier, sample date, analyte, analytical method, percent recovery, and instrument batch. All recoveries fell within the QC window as prescribed in Section 5.0. No data qualifiers were needed per this evaluation.

\section{Recoveries of Matrix Spike and Surrogate Samples}

The percent recoveries of matrix spike and surrogate samples are presented in Table 7. The table includes the laboratory, sample identifier, analyte, analytical method, and percent recovery. All recoveries fell within the $\mathrm{QC}$ window as prescribed in Section 5.0. No data qualifiers were needed per this evaluation.

\section{Detection Summary of Rinsate Blank}

The detects of rinsate blank "100452" included lead (0.7 ug/l); 1,1,1-trichloroethane (0.34 ug/); methyl chloride ( $0.37 \mathrm{ug} / 1)$; and bis(2-ethylhexyl)phthalate $(2.7 \mathrm{ug} / \mathrm{l})$ as presented in Table 8 . Per Section 5.0, no qualification is done on the basis of rinsate blank results. 


\section{Detection Summary of Trip Blanks}

A detection summary of the trip blanks is presented in Table 9. The table includes the sample identifier, sample date, analytical method, analyte, result, result qualifier, analysis qualifier, bias qualifier, and unit. Detects include toluene, methylene chloride, chloroform, trichlorofluoromethane, 1,1-dichloroethylene, benzene, trichloroethylene, toluene, and chlorobenzene. Toluene and methylene chloride were detected in the associated method blanks. With the exception of the toluene and methylene chloride, all detects of the compounds listed above were assigned a "J" result qualifier for every sample shipped in the same cooler as the trip blank.

\section{Detection Summary of Field Blank}

A detection summary of the field blank is presented in Table 10. The table includes the sample identifier, sample date, analytical method, analyte, result, result qualifier, analysis qualifier, bias qualifier, and unit. Detects include 1,1,1-trichloroethane and methyl chloride. Per Section 5.0, no qualification is done on the basis of field blank results.

\section{Evaluation of Surface Water Field Duplicates}

Table 11 provides a comparison of the primary surface water sample (100454) and its associated field duplicate (100455). Percent differences were calculated for data sets containing at least one detect. Percent differences ranged from 0.0 to 43.1 percent. The 43.1 percent was associated with nitrate. The next highest percent difference was with tritium at 28 percent. The evaluation of the field duplicate concludes that the sample collection was representative of the actual population and that the field and laboratory procedures were in control. Per Section 5.0, no qualification is done on the basis of field duplicate results.

\section{Evaluation of Sediment Field Duplicates}

Table 12 provides a comparison of the primary sediment sample (100462) and its associated field duplicate (100463). Percent differences were calculated for data sets containing at least one detect. Percent differences ranged from 0.0 to 28.0 percent. The 28.0 percent was associated with manganese. The next highest percent difference was with total organic carbon at 24 percent. The evaluation of field duplicates concludes that the sample collection was representative of the actual population and that the field and laboratory procedures were in control. Per Section 5.0, no qualification is done on the basis of field duplicate results. 


\section{Evaluation of Surface Water Split Samples}

Table 13 provides a comparison of the primary surface water sample (100450) and its associated split (100451). The primary laboratories were Weston and TMA while the quality assurance laboratory was General Engineering. Percent differences were calculated for data sets containing at least one detect. Percent differences ranged from 0.0 to 22.0 percent. The 22.0 percent was associated with lead. The next highest percent difference was with gross alpha at 15.0 percent. The evaluation of the split sample concludes that the laboratory procedures of the primary laboratories were in control. Per Section 5.0, no qualification is done on the basis of split results.

\section{Evaluation of Sediment Split Samples}

Table 14 provides a comparison of the primary sediment sample (100458) and its associated split (100459). The primary laboratories were Weston and TMA while the quality assurance laboratory was General Engineering. Percent differences were calculated for data sets containing at least one detect. Percent differences ranged from 1.0 to 24.0 percent. The 24.0 percent was associated with chromium. The next highest percent difference was with gross alpha at 13.0 percent. The evaluation of the split sample concludes that the laboratory procedures of the primary laboratories were in control. Per Section 5.0, no qualification is done on the basis of split results.

\section{Laboratory Data Records Review}

The purpose of this laboratory review was to assess the accuracy, precision, and completeness of the analytical procedures conducted by Roy F. Weston Laboratories Inc. which could not be addressed through review of the electronic data deliverables or report certificates. As part of this review, copies of analytical logbooks and instrument calibration records were requested for organic and radiological analyses. Review of these records were conducted according to the appropriate validation guidelines discussed in the Quality Assurance / Quality Control Guidance for Removal Activities (EPA/540/G-90/004), USEPA CLP National Functional Guidelines for Organic Review, USEPA CLP National Functional Guidelines for Inorganic Review, Data Validation Procedures for Radiochemical Analyses (WHC-SD-EN-SPP-001, Rev. 1), SW-846 Methods, and in accordance with Savannah River Site Procedures. The report of the laboratory data records review is provided in Appendix B. 


\subsection{Data Presentation and Conclusions}

The surface water and sediment sample results are presented in matrix-type summary tables in the Data Summary Report which this Quality Control Summary Report is an Appendix of. Summary tables are presented separately for surface water and sediment by each analytical fraction. The result, analysis, and bias qualifiers are all maintained in the matrix-type summary tables.

Where applicable, the analytical information was validated with respect to the Quality Assurance / Quality Control Guidance for Removal Activities (EPA/540/G-90/004), USEPA CLP National Functional Guidelines for Organic Review, USEPA CLP National Functional Guidelines for Inorganic Review, Data Validation Procedures for Radiochemical Analyses (WHC-SD-EN-SPP001, Rev. 1), SW-846 Methods, and in accordance with Savannah River Site Procedures. All field activities and analytical procedures were performed in accordance with the Sampling Plan (Appendix A).

No non-conformance situations occurred during the field or laboratory activities that prevented the environmental data from meeting the project-specific DQOs. 


\section{References}

U.S. Environmental Protection Agency (EPA), 1991. Test Method for Evaluating_Solid Wastes, 3rd Edition, Office of Solid Waste, Washington, D.C.

U.S. Environmental Protection Agency (EPA), 1988. Functional Guidelines for Evaluating Inorganic Analyses. Hazardous Site Evaluation Division. Contract Laboratory Program. July 1988.

U.S. Environmental Protection Agency (EPA), 1989. Data Evaluation. Risk Assessment Guidance for Superfund: Human Health Evaluation Manual Part A. Interim Final. Office of Solid Waste and Emergency and Remedial Response. EPA/540/1-89/002. (OSWER Directive 9285.701A),

U.S. Environmental Protection Agency (EPA), 1991. EPA Region IV Standard Operating Procedures and Quality Assurance Manual, February 1991.

U.S. Environmental Protection Agency (EPA), 1991. National Functional Guidelines for Organic Data Review. Hazardous Site Evaluation Division. Contract Laboratory Program. June 1991.

U.S. Environmental Protection Agency (EPA), 1991. Statement of Work for Organic Analysis. Hazardous Site Evaluation Division. Contract Laboratory Program. Document Number OLM01.0. August 1991.

U.S. Environmental Protection Agency (EPA), 1991. Statement of Work for Inorganic Analysis. Hazardous Site Evaluation Division. Contract Laboratory Program. Document Number ILM02.1. September 1991. 
Tables 
Tabie 1

Analytical Program of Surface Water and Sediment Samples D-Area Buming/Rubble Pits

Savannah River Site

\begin{tabular}{|c|c|c|c|c|c|c|c|c|c|}
\hline \multicolumn{10}{|c|}{ 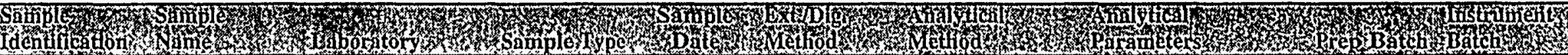 } \\
\hline 100438 & D431-37-01 & Weston & & 50594 & & EPA160.1 & TDS & 94LSSA85 & 94LSSA85 \\
\hline 100438 & D $431-37-01$ & Weston & & 50594 & & EPA 160.2 & TSS & 94LSSA85 & 94LSSA85 \\
\hline 100438 & D431-37-01 & Weston & & 50594 & & EPA300 & $\mathrm{CL} / \mathrm{F} / \mathrm{SO} 4$ & 94LCC066 & 94LCC066 \\
\hline 100438 & D431-37-01 & Weston & Lab Replicate & 50594 & & EPA300 & CL/F/SO4 & 94LCC066 & 94LCC066 \\
\hline 100438 & D $431-37-01$ & Weston & Spike Sample & 50594 & & EPA300 & CL/F/SO4 & $94 \mathrm{LCC} 066$ & 94LCC066 \\
\hline 100438 & D431-37-01 & Weston & & 50594 & & EPA300 & CL/F/SO4 & 94LFC 066 & 94LFC066 \\
\hline 100438 & D431-37-01 & Weston & Lab Replicate & 50594 & & EPA300 & CL/F/SO4 & 94LFC066 & 94LFC066 \\
\hline 100438 & D431-37-01 & Weston & Spike Sample & 50594 & & EPA300 & CL/F/SO4 & 94LFC066 & 94LFC066 \\
\hline 100438 & D431-37-01 & Weston & & 50594 & & EPA300 & CL/F/SO4 & 94LSC066 & 94LSC066 \\
\hline 100438 & D431-37-01 & Weston & Lab Replicate & 50594 & & EPA300 & CL/F/SO4 & 94LSC066 & 94LSC066 \\
\hline 100438 & D431-37-01 & Weston & Spike Sample & 50594 & & EPA300 & CL/F/SO4 & 94LSC066 & 94LSC066 \\
\hline 100438 & D431-37-01 & Weston & & 50594 & & EPA353.2 & $\mathrm{NO} 3$ & $94 \mathrm{LN} 3072$ & 94LN3072 \\
\hline 100438 & D431-37-01 & Weston & Lab Replicate & 50594 & & EPA353.2 & NO3 & $94 L N 3072$ & 94LN3072 \\
\hline 100438 & D431-37-01 & Weston & Spike Sample & 50594 & & EPA353.2 & NO3 & $94 \mathrm{LN} 3072$ & 94LN3072 \\
\hline 100438 & D431-37-01 & Weston & & 50594 & & EPA415.1 & TOC & 94LTC080 & 94LTC080 \\
\hline 100438 & D431-37-01 & Thermo Analytical & & 50594 & & EPA900.0 & GROSS ALPHA/BETA & 9405022 & 5099402 \\
\hline 100438 & D431-37-01 & Thermo Analytical & & 50594 & & EPA900.1 MOD. & TOTAL RAD & 9405022 & 5109402 \\
\hline 100438 & D431-37-01 & Thermo Analytical & & 50594 & & EPA906.0 & TRITIUM & 9405022 & 5099404 \\
\hline 100438 & D $431-37-01$ & Weston & & 50594 & & SW9020A & TOX & $94 \mathrm{LX} 124 \mathrm{X}$ & $94 \operatorname{LX} 124 X$ \\
\hline 100438 & D431-37-01 & Weston & & 50594 & EPA3050 & EPA6010 & METALS & $94 L 5708$ & PS0511F \\
\hline 100438 & $D 431-37-01$ & Weston & & 50594 & EPA3050 & EPA6010 & METALS & 94L5708 & PSOS11Z \\
\hline 100438 & D431-37-01 & Weston & Lab Replicate & 50594 & EPA3050 & EPA6010 & METALS & $94 L 5708$ & PSO511F \\
\hline 100438 & D431-37-01 & Weston & Lab Replicate & 50594 & EPA3050 & EPA6010 & METALS & 94L5708 & PS0511Z \\
\hline 100438 & D431-37-01 & Weston & Spike Sample & 50594 & EPA3050 & EPA6010 & METALS & 94L5708 & PS0511F \\
\hline 100438 & D431-37-01 & Weston & Spike Sample & 50594 & EPA3050 & EPA6010 & METALS & 94L5708 & PSO511Z \\
\hline 100438 & D431-37-01 & Weston & & 50594 & EPA3050 & EPA7060 & ARSENIC & $94 L 5707$ & AS051151 \\
\hline 100438 & D431-37-01 & Weston & Lab Replicate & 50594 & EPA3050 & EPA7060 & ARSENIC & 94L5707 & AS051151 \\
\hline 100438 & D431-37-01 & Weston & Spike Sample & 50594 & EPA3050 & EPA7060 & ARSENIC & 94L5707 & AS051151 \\
\hline 100438 & D431-37-01 & Weston & & 50594 & EPA3050 & EPA7421 & LEAD & $94 L 5707$ & PB051202 \\
\hline 100438 & D431-37-01 & Weston & Lab Replicate & 50594 & EPA3050 & EPA7421 & LEAD & $94 L 5707$ & PB051202 \\
\hline 100438 & D431-37-01 & Weston & Spike Sample & 50594 & EPA3050 & EPA7421 & LEAD & 94L5707 & PB051202 \\
\hline 100438 & D431-37-01 & Weston & & 50594 & EPA3050 & EPA7471 & HG & $94 C 0185$ & HG051004 \\
\hline 100438 & D431-37-01 & Weston & Lab Replicate & 50594 & EPA3050 & EPA7471 & HG & $94 C 0185$ & HG051004 \\
\hline 100438 & D431-37-01 & Weston & Spike Sample & 50594 & EPA3050 & EPA7471 & HG & $94 C 0185$ & HG051004 \\
\hline 100438 & D431-37-01 & Weston & Spike Sample & 50594 & EPA3050 & EPA7471 & HG & $94 C 0190$ & HG051103 \\
\hline 100438 & D431-37-01 & Weston & & 50594 & EPA3050 & EPA7740 & SELENIUM & $94 L 5707$ & SE051104 \\
\hline
\end{tabular}


Table 1

Analytical Program of Surface Water and Sediment Samples

D-Area Buming/Rubble Pits

Savannah River Site

\begin{tabular}{|c|c|c|c|c|c|c|c|c|c|}
\hline 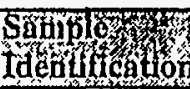 & 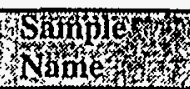 & 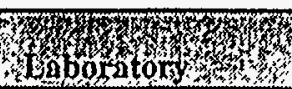 & Samplaty & Shoth & M & 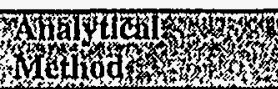 & 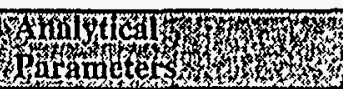 & 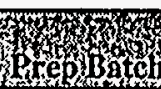 & 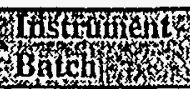 \\
\hline 100438 & $\frac{1}{0431-37-01}$ & Weston & Lab Replicate & $\frac{50594}{5054}$ & EPA3050 & EPA7740 & SELENIUM & $\frac{94 \mathrm{~L} 5707}{4}$ & SE0S1104 \\
\hline 100438 & D431-37-01 & Weston & Spike Sample & 50594 & EPA3050 & EPA7740 & SELENIUM & 94L5707 & SE051104 \\
\hline 100438 & D431-37-01 & Weston & & 50594 & EPA3510/20 & EPA8270 & SEMIVOLATLES & 94LE0899 & 5051001 \\
\hline 100438 & D431-37-01 & Weston & Lab Replicate & 50594 & EPA3510/20 & EPA8270 & SEMIVOLATLES & 94LE0899 & J051001 \\
\hline 100438 & D431-37-01 & Weston & Spike Sample & 50594 & EPA3510/20 & EPA8270 & SEMIVOLATILES & 94LE0899 & J0S1001 \\
\hline 100438 & D431-37-01 & Weston & & 50594 & EPA3510/20 & EPA8270M & SEMIVOLATILES & 94LE0899 & J051001 \\
\hline 100438 & D431-37-01 & Weston & Lab Replicate & 50594 & EPA3510/20 & EPA8270M & SEMIVOLATILES & 94LE0899 & J051001 \\
\hline 100438 & D431-37-01 & Weston & Spike Sample & 50594 & EPA3510/20 & EPA $8270 \mathrm{M}$ & SEMIVOLATLES & 94LE0899 & J051001 \\
\hline 100438 & D431-37-01 & Weston & Spike Sample & 50594 & EPA3540/50 & EPA8270 & SEMTVOLATTLES & 94LE0899 & J051001 \\
\hline 100438 & D431-37-01 & Weston & & 50594 & EPA5030 & EPA8260 & VOLATILES & $94 \mathrm{LVC} 057$ & C051101 \\
\hline 100438 & D431-37-01 & Weston & Spike Sample & 50594 & EPA5030 & EPA8260 & VOLATILES & $94 \mathrm{LVC} 057$ & C051101 \\
\hline 100439 & D431-37-02 & Weston & & 50594 & & ASTMD 497.2 & $\mathrm{pH}$ & 94LPH040 & 94LPH040 \\
\hline 100439 & D431-37-02 & Weston & & 50594 & & EPA415.8 & TOC/TOH & 94LTZ23A & 94LTZ23A \\
\hline 100439 & D431-37-02 & Weston & Lab Replicate & 50594 & & EPA415.8 & TOC/TOH & 94LTZ23A & 94LTZ23A \\
\hline 100439 & D431-37-02 & Weston & Spike Sample & 50594 & & EPA415.8 & тостTOH & 94LTZ23A & 94LTZ23A \\
\hline 100439 & D431-37-02 & Weston & & 50594 & & EPA7471 & HG & $94 \mathrm{C} 0187$ & HG051005 \\
\hline 100439 & D431-37-02 & Weston & Lab Replicate & 50594 & & EPA7471 & HG & $94 C 0187$ & HG051005 \\
\hline 100439 & $D 431-37-02$ & Weston & Spike Sample & 50594 & & EPA7471 & HG & $94 \mathrm{C} 0187$ & HG051005 \\
\hline 100439 & D431-37-02 & Thermo Analytical & & 50594 & & LANLER210 MOD & TRITIU'M & 9405023 & 5109404 \\
\hline 100439 & D431-37-02 & Thermo Analytical & & 50594 & & LANLMLR-100 & GROSS ALPHA/BETA & 9405023 & 5109402 \\
\hline 100439 & D431-37-02 & Thermo Analytical & & 50594 & & SW8469315 MOD & TOTAL RAD & 9405023 & 5109402 \\
\hline 100439 & D431-37-02 & Weston & & 50594 & EPA3050 & EPA6010 & METALS & 94L5706 & PSO511A \\
\hline 100439 & D431-37-02 & Weston & & 50594 & EPA3050 & EPA6010 & METALS & 94L5706 & PS0511B \\
\hline 100439 & D431-37-02 & Weston & & 50594 & EPA3050 & EPA6010 & METALS & 94L5706 & PSOS11E \\
\hline 100439 & D $431-37-02$ & Weston & & 50594 & EPA3050 & EPA6010 & METALS & 94L5706 & PSO511X \\
\hline 100439 & D $431-37-02$ & Weston & & 50594 & EPA3050 & EPA6010 & METALS & 94LS706 & PSO511Y \\
\hline 100439 & D431-37-02 & Weston & Lab Replicate & 50594 & EPA3050 & EPA6010 & METALS & 94L5706 & PS0511A \\
\hline 100439 & D $431-37-02$ & Weston & Lab Replicate & 50594 & EPA3050 & EPA6010 & METALS & 94LS706 & PSO5 $11 \mathrm{~B}$ \\
\hline 100439 & D431-37-02 & Weston & Lab Replicate & 50594 & EPA3050 & EPA6010 & METALS & 94LS706 & PS0511E \\
\hline 100439 & D431-37-02 & Weston & Lab Replicate & 50594 & EPA3050 & EPA6010 & METALS & 94LS706 & PS0511X \\
\hline 100439 & D431-37-02 & Weston & Lab Replicate & 50594 & EPA3050 & EPA6010 & METALS & 94L5706 & PSO511Y \\
\hline 100439 & D $431-37-02$ & Weston & Spike Sample & 50594 & EPA3050 & EPA6010 & METALS & 94L5706 & PS0511A \\
\hline 100439 & D431-37-02 & Weston & Spike Sample & 50594 & EPA3050 & EPA6010 & METALS & 94L5706 & PS0511B \\
\hline 100439 & D431-37-02 & Weston & Spike Sample & 50594 & EPA3050 & EPA6010 & METALS & 94LS706 & PS0511E \\
\hline 100439 & D431-37-02 & Weston & Spike Sample & 50594 & EPA3050 & EPA6010 & METALS & 94L5706 & PSO511X \\
\hline 100439 & D431-37-02 & Weston & Spike Sample & 50594 & EPA3050 & EPA6010 & METALS & 94L5706 & PS0511Y \\
\hline
\end{tabular}


Truse 1

Analytical Program of Surface Water and Sediment Samples

D-Area Buming/Rubble Pits

Savannah River Site

\begin{tabular}{|c|c|c|c|c|c|c|c|c|c|c|}
\hline \multicolumn{2}{|c|}{ 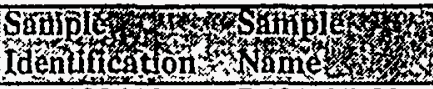 } & \multicolumn{9}{|c|}{ 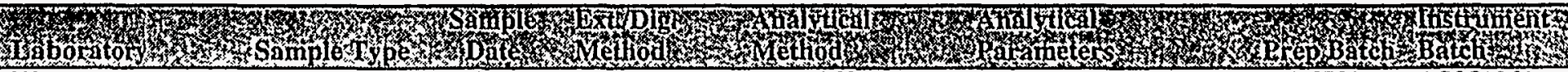 } \\
\hline 100439 & D431-37-02 & Weston & & & 50594 & EPA3050 & EPA7060 & ARSENIC & $94 \mathrm{~L} 5705$ & AS051061 \\
\hline 100439 & D431-37-02 & Weston & & Lab Replicate & 50594 & EPA3050 & EPA7060 & ARSENIC & $94 L 5705$ & AS051061 \\
\hline 100439 & D431-37-02 & Weston & & Spike Sample & 50594 & EPA3050 & EPA7060 & ARSENIC & 94LS705 & AS051061 \\
\hline 100439 & D431-37-02 & Weston & 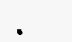 & & 50594 & EPA3050 & EPA7421 & LEAD & 94L5705 & PB051153 \\
\hline 100439 & D431-37-02 & Weston & & Lab Replicate & 50594 & EPA3050 & EPA7421 & LEAD & 94L5705 & PB051153 \\
\hline 100439 & $D 431-37-02$ & Weston & & Spike Sample & 50594 & EPA3050 & EPA7421 & LEAD & 94L5705 & PB051153 \\
\hline 100439 & D431-37-02 & Weston & & & 50594 & EPA3050 & EPA7740 & SELENIUM & 94L5705 & SE051161 \\
\hline 100439 & D431-37-02 & Weston & & Lab Replicate & 50594 & EPA3050 & EPA7740 & SELENIUM & 94L570S & SE051161 \\
\hline 100439 & D431-37-02 & Weston & & Spike Sample & 50594 & EPA3050 & EPA7740 & SELENIUM & 94LS705 & SE051161 \\
\hline 100439 & D431-37-02 & Weston & & & 50594 & EPA3540/50 & EPA8270 & SEMIVOLATILES & 94LE0897 & S051001 \\
\hline 100439 & D431-37-02 & Weston & & Spike Sample & 50594 & EPA3540/50 & EPA8270 & SEMIVOLATILES & 94LE0897 & S051001 \\
\hline 100439 & D431-37-02 & Weston & & & 50594 & EPA3540/50 & EPA8270M & SEMIVOLATILES & 94LE0897 & S051001 \\
\hline 100439 & $D 431-37-02$ & Weston & & & 50594 & EPA5030 & EPA8260 & VOLATILES & 94LVC056 & $\mathrm{C} 051001$ \\
\hline 100439 & D431-37-02 & Weston & & Spike Sample & 50594 & EPA5030 & EPA8260 & VOLATILES & $94 \mathrm{LVC} 056$ & $\mathrm{C} 051001$ \\
\hline 100440 & D431-38-01 & Weston & & & 50594 & & EPA160.1 & TDS & 94LSSA85 & 94LSSA85 \\
\hline 100440 & D431-38-01 & Weston & & & 50594 & • & EPA160.2 & TSS & 94LSSA85 & 94LSSA85 \\
\hline 100440 & D431-38-01 & Weston & & & 50594 & & EPA300 & $\mathrm{CL} / \mathrm{F} / \mathrm{SO} 4$ & 94LCC066 & 94LCC066 \\
\hline 100440 & D431-38-01 & Weston & & & 50594 & & EPA300 & CL/F/SO4 & 94LFC066 & 94LFC066 \\
\hline 100440 . & D431-38-01 & Weston & & & 50594 & & EPA300 & CL/F/SO4 & 94LSC066 & 94LSC066 \\
\hline 100440 & D431-38-01 & Weston & & & 50594 & & EPA353.2 & NO3 & 94LN3072. & 94LN3072 \\
\hline 100440 & D431-38-01 & Weston & & & 50594 & & EPA415.1 & TOC & 94LTC080 & 94LTC080 \\
\hline 100440 & D431-38-01 & Thermo & tical & & 50594 & & EPA900.0 & GROSS ALPHA/BE & 9405022 & 5099402 \\
\hline 100440 & D431-38-01 & Thermo & tical & & 50594 & & EPA900.1 MOD. & TOTAL RAD & 9405022 & 5109402 \\
\hline 100440 & D431-38-01 & Thermo & tical & & 50594 & & EPA906.0 & TRITIUM & 9405022 & 5099404 \\
\hline 100440 & D431-38-01. & Weston & & & 50594 & & SW9020A & TOX & $94 \mathrm{LX} 124 \mathrm{X}$ & 94LX124X \\
\hline 100440 & D431-38-01 & Weston & & Lab Replicate & 50594 & & SW9020A & TOX & 94LX124X & $94 \mathrm{~L} X 124 \mathrm{X}$ \\
\hline 100440 & D431-38-01 & Weston & & & 50594 & EPA3050 & EPA6010 & METALS & 94L5708 & PS0511F \\
\hline 100440 & D431-38-01 & Weston & & & 50594 & EPA3050 & EPA6010 & METALS & $94 L 5708$ & PSO511Z \\
\hline 100440 & D431-38-01 & Weston & & & 50594 & EPA3050 & EPA7060 & ARSENIC & $94 L 5707$ & AS051151 \\
\hline 100440 & D431-38-01 & Weston & & & 50594 & EPA3050 & EPA7421 & LEAD & 94L5707 & PB051202 \\
\hline 100440 & D431-38-01 & Weston & & & 50594 & EPA3050 & EPA7471 & HG & $94 \mathrm{C} 0185$ & HG051004 \\
\hline 100440 & D431-38-01 & Weston & & & 50594 & EPA3050 & EPA7740 & SELENTUM & $94 L 5707$ & SE051104 \\
\hline 100440 & D431-38-01 & Weston & & " & 50594 & EPA3510/20 & EPA8270 & SEMIVOLATILES & 94LE0899 & J051001 \\
\hline 100440 & D431-38-01 & Weston & & & 50594 & EPA3510/20 & EPA8270M & SEMIVOLATILES & 94LE0899 & J051001 \\
\hline 100440 & D431-38-01 & Weston & & Spike Sample & 50594 & EPA3540/50 & EPA8270 & SEMIVOLATILES & 94LE0899 & J051001 \\
\hline 100440 & D431-38-01 & Weston & & & 50594 & EPA5030 & EPA8260 & VOLATILES & $94 \mathrm{LVC} 057$ & C051101 \\
\hline
\end{tabular}


Table 1

Analytical Program of Surface Water and Sediment Samples

D-Area Burning/Rubble Pits

Savannah River Site

\begin{tabular}{|c|c|c|c|c|c|c|c|c|c|}
\hline 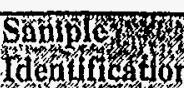 & 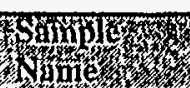 & 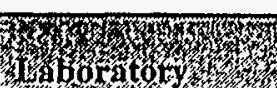 & 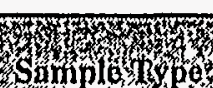 & $b_{0}$ & 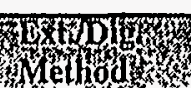 & 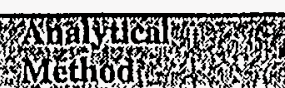 & 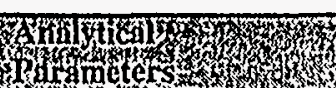 & 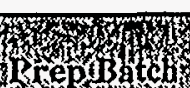 & 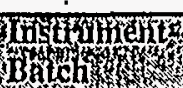 \\
\hline 100440 & D431-38-01 & Weston & Spike Sample & 50594 & EPA5030 & EPA8260 & VOLATILES & $94 \mathrm{LVCOS7}$ & C051101 \\
\hline 100441 & D431-36-01 & Weston & & 50594 & & EPA160.1 & TDS & 94LSSA85 & 94LSSA85 \\
\hline 100441 & D431-36-01 & Weston & & 50594 & & EPA160.2 & TSS & 94LSSA85 & 94LSSA85 \\
\hline 100441 & $D 431-36-01$ & Weston & & 50594 & & EPA300 & $\mathrm{CL} / \mathrm{F} / \mathrm{SO} 4$ & $94 \mathrm{LCC} 066$ & 94LCC066 \\
\hline 100441 & $D 431-36.01$ & Weston & & 50594 & & EPA300 & $\mathrm{CL} / \mathrm{F} / \mathrm{SO} 4$ & 94LFC066 & 94LFC066 \\
\hline 100441 & D431-36-01 & Weston & & 50594 & & EPA300 & CL/F/SO4 & 94LSC066 & 94LSC066 \\
\hline 100441 & D431-36-01 & Weston & & 50594 & & EPA353.2 & NO3 & $94 \mathrm{LN} 3072$ & 94LN3072 \\
\hline 100441 & D431-36-01 & Weston & & 50594 & & EPA415.1 & TOC & 94LTC080 & 94LTC080 \\
\hline 100441 & $D 431-36-01$ & Weston & Lab Replicate & 50594 & & EPA415.1 & TOC & 94LTC080 & 94LTC080 \\
\hline 100441 & D431-36-01 & Weston & Spike Sample & 50594 & & EPA415.1 & TOC & 94LTC080 & 94LTC080 \\
\hline 100441 & D431-36-01 & Thermo Analytical & & 50594 & & EPA900.0 & GROSS ALPHA/BETA & 9405022 & 5099402 \\
\hline 100441 & D431-36-01 & Thermo Analytical & & 50594 & & EPA900.1 MOD. & TOTAL RAD & 9405022 & 5109402 \\
\hline 100441 & D431-36-01 & Thermo Analytical & & 50594 & & EPA906.0 & TRITIUM & 9405022 & 5099404 \\
\hline 100441 & D431-36-01 & Thermo Analytical & Lab Replicate & 50594 & & EPA906.0 & TRITIUM & 9405022 & 5099404 \\
\hline 100441 & D431-36-01 & Weston & & 50594 & & SW9020A & TOX & 94LX124W & $94 \mathrm{LX124W}$ \\
\hline 100441 & D431-36-01 & Weston & & 50594 & EPA3050 & EPA6010 & METALS & 94L 5708 & PS0511F \\
\hline 100441 & D431-36-01 & Weston & & 50594 & EPA3050 & EPA6010 & METALS & 94L5708 & PS0511Z \\
\hline 100441 & D431-36-01 & Weston & & 50594 & EPA3050 & EPA7060 & ARSENIC & $94 \mathrm{~L} 5707$ & AS051151 \\
\hline 100441 & D431-36-01 & Weston & & 50594 & EPA3050 & EPA7421 & LEAD & 94L5707 & PB051202 \\
\hline 100441 & D431-36-01 & Weston & & 50594 & EPA3050 & EPA7471 & HG & $94 C 0185$ & HG051004 \\
\hline 100441 & D431-36-01 & Weston & & 50594 & EPA3050 & EPA7740 & SELENIUM & $94 L 5707$ & SE051104 \\
\hline 100441 & D431-36-01 & Weston & & 50594 & EPA3510/20 & EPA8270 & SEMIVOLATILES & 94LE0899 & J051001 \\
\hline 100441 & D431-36-01 & Weston & & 50594 & EPA3510/20 & EPA8270M & SEMIVOLATILES & 94LE0899 & J051001 \\
\hline 100441 & D431-36-01 & Weston & Spike Sample & 50594 & EPA3540/50 & EPA8270 & SEMTVOLATILES & 94LE0899 & j051001 \\
\hline 100441 & D431-36-01 & Weston & & 50594 & EPA5030 & EPA8260 & VOLATILES & $94 L V C 057$ & C051101 \\
\hline 100441 & $D 431-36-01$ & Weston & Spike Sample & 50594 & EPA.5030 & EPA8260 & VOLATULS & $94 \mathrm{LVC} 057$ & C051101 \\
\hline 100442 & D431-36-02 & Weston & & 50594 & & ASTMD497.2 & $\mathrm{pH}$ & 94LPH040 & 94LPH040 \\
\hline 100442 & D431-36-02 & Weston & & 50594 & & EPA415.8 & TOCTOH & 94LTZ23A & 94LTZ23A \\
\hline 100442 & $D 431-36-02$ & Weston & & 50594 & & EPA7471 & HG & $94 C 0187$ & HG051005 \\
\hline 100442 & D431-36-02 & Thermo Analytical & & 50594 & & LANLER $210 \mathrm{MOD}$ & TRITIUM & 9405023 & 5109404 \\
\hline 100442 & $D 431-36-02$ & Thermo Analytical & Lab Replicate & 50594 & & LANLER210 MOD & TRITIUM & 9405023 & 5109404 \\
\hline 100442 & D431-36-02 & Thermo Analytical & & 50594 & & LANLMLR-100 & GROSS ALPHA/BET & 9405023 & 5099402 \\
\hline 100442 & $D 431-36-02$ & Thermo Analytical & Lab Replicate & $50594^{\circ}$ & & LANLMLR-100 & GROSS ALPHA/BETA & 9405023 & 5099402 \\
\hline 100442 & $D 431-36-02$ & Thermo Analytical & & 50594 & & SW8469315 MOD & TOTAL RAD & 9405023 & 5109402 \\
\hline 100442 & D $431-36-02$ & Thermo Analytical & Lab Replicate & 50594 & & SW8469315 MOD & TOTAL RAD & 9405023 & 5109402 \\
\hline $10044 \dot{2}$ & D431-36-02. & Weston & & 50594 & EPA3050 & EPA6010 & METÁLS & $94 L 5706$ & PS0511A \\
\hline
\end{tabular}


a able 1

Analytical Program of Surface Water and Sediment Samples

D-Area Burning/Rubble Pits

Savannah River Site

\begin{tabular}{|c|c|c|c|c|c|c|c|c|c|}
\hline \multicolumn{10}{|c|}{ 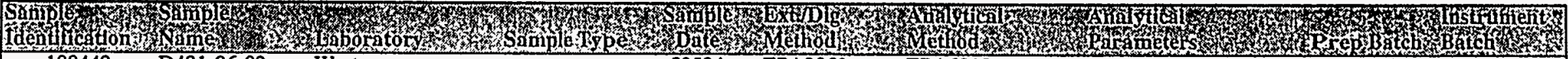 } \\
\hline 100442 & D431-36-02 & Weston & & 50594 & EPA3050 & EPA6010 & METALS & 9415706 & PS0511B \\
\hline 100442 & D431-36-02 & Weston & & 50594 & EPA3050 & EPA6010 & METALS & $94 L 5706$ & PS0511E \\
\hline 100442 & D431-36-02 & Weston & & 50594 & EPA3050 & EPA6010 & METALS & 9415706 & PS0511X \\
\hline 100442 & D431-36-02 & Weston & & 50594 & EPA3050 & EPA6010 & METALS & 94L5706 & PSOS11Y \\
\hline 100442 & D431-36-02 & Weston & & 50594 & EPA3050 & EPA7060 & ARSENIC & 94L5705 & AS051061 \\
\hline 100442 & D $431-36-02$ & Weston & & 50594 & EPA3050 & EPA7421 & LEAD & $94 L 5705$ & PB051153 \\
\hline 100442 & D431-36-02 & Weston & & 50594 & EPA3050 & EPA7740 & SELENIUM & $94 L 5705$ & SE051161 \\
\hline 100442 & D431-36-02 & Weston & & 50594 & EPA3540/50 & EPA 8270 & SEMTVOLATLES & 94LE0897 & S051001 \\
\hline 100442 & D431-36-02 & Weston & Spike Sample & 50594 & EPA3540/50 & EPA8270 & SEMIVOLATILES & 94LE0897 & S051001 \\
\hline 100442 & D431-36-02 & Weston & & 50594 & EPA3540/50 & EPA8270M & SEMIVOLATILES & 94LE0897 & S051001 \\
\hline 100442 & $D 431-36-02$ & Weston & & 50594 & EPA5030 & EPA8260 & VOLATILES & 94LVC056 & $\mathrm{C} 051001$ \\
\hline 100442 & D431-36-02 & Weston & Spike Sample & 50594 & EPA5030 & EPA8260 & VOLATILES & 94LVC056 & Cos1001 \\
\hline 100443 & D431-39-01 & Weston & & 50594 & & EPA160.1 & TDS & 94LSSA85 & 94LSSA 85 \\
\hline 100443 & D431-39-01 & Weston & & 50594 & & EPA160.2 & TSS & 94LSSA85 & 94LSSA85 \\
\hline 100443 & D431-39-01 & Weston & & 50594 & & EPA300 & CL/F/SO4 & 94LCC066 & 94LCCO66 \\
\hline 100443 & D431-39-01 & Weston & . & 50594 & & EPA300 & CL/F/SO4 & 94LFC066 & 94LFC066 \\
\hline 100443 & D431-39-01 & Weston & & 50594 & & EPA300 & $\mathrm{CL} / \mathrm{F} / \mathrm{SO} 4$ & 94LSC066 & 94LSC066 \\
\hline 100443 & D431-39-01 & Weston & & 50594 & & EPA353.2 & NO3 & 94LN3072 & 94LN3072 \\
\hline 100443 & D431-39-01 & Weston & & 50594 & & EPA415.1 & TOC & 94LTC080 & 94LTC080 \\
\hline 100443 & D431-39-01 & Thermo Analytical & & 50594 & & EPA900.0 & GROSS ALPHA/BETA & 9405022 & 5099402 \\
\hline 100443 & D431-39-01 & Thermo Analytical & & 50594 & & EPA900.1 MOD. & TOTAL RAD & 9405022 & 5109402 \\
\hline 100443 & D431-39-01 & Thermo Analytical & & 50594 & & EPA906.0 & TRITIUM & 9405022 & 5099404 \\
\hline 100443 & D431-39-01 & Weston & & 50594 & & SW9020A & TOX & $94 \mathrm{LX} 124 \mathrm{~W}$ & 94LX124W \\
\hline 100443 & D431-39-01 & Weston & & 50594 & EPA3050 & EPA6010 & METALS & $94 L 5708$ & PSOS11F \\
\hline 100443 & D431-39-01 & Weston & & 50594 & EPA3050 & EPA6010 & METALS & 94L5708 & PSO511Z \\
\hline 100443 & D431-39-01 & Weston & & 50594 & EPA3050 & EPA7060. & ARSENIC & 9415707 & AS051151 \\
\hline 100443 & D431-39-01 & Weston & & 50594 & EPA3050 & EPA7421 & LEAD & 94L5707 & PB051202 \\
\hline 100443 & D431-39-01 & Weston & & 50594 & EPA3050 & EPA7471 & HG & $94 \mathrm{C} 0185$ & HG051004 \\
\hline 100443 & D431-39-01 & Weston & & 50594 & EPA3050 & EPA7740 & SELENIUM & $94 L 5707$ & SE051104 \\
\hline 100443 & D431-39-01 & Weston & & 50594 & EPA3510/20 & EPA8270 & SEMIVOLATILES & 94LE0899 & A051007 \\
\hline 100443 & D431-39-01 & Weston & & 50594 & EPA3510/20 & EPA8270M & SEMIVOLATILES & 94LE0899 & A051007 \\
\hline 100443 & D431-39-01 & Weston & Spike Sample & 50594 & EPA3540/50 & EPA8270 & SEMTVOLATILES & 94LE0899 & A.051007 \\
\hline 100443 & D431-39-01 & Weston & & 50594 & EPA5030 & EPA8260 & VOLATILES & 94LVC057 & Cos1101 \\
\hline 100443 & D431-39-01 & Weston & Spike Sample & 50594 & EPA5030 & EPA8260 & VOLATILES & 94LVC057 & Cos1101 \\
\hline 100444 & D431-35-01 & Weston & & 50594 & & EPA160.1 & TDS & 94LSSA85 & 94LSSA85 \\
\hline 100444 & D431-35-01 & Weston & & 50594 & & EPA160.2 & TSS & 94LSSA85 & 94LSSA85 \\
\hline
\end{tabular}


Table 1

Analytical Program of Surface Water and Sediment Samples

D-Area Buming/Rubble Pits

Savannah River Site

\begin{tabular}{|c|c|c|c|c|c|c|c|c|c|}
\hline 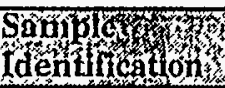 & 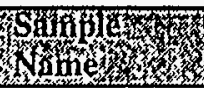 & W & 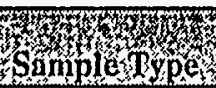 & 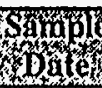 & 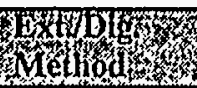 & 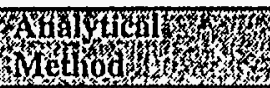 & 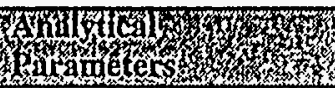 & 准 & 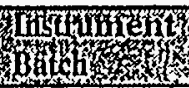 \\
\hline 100444 & D431-35-01 & Weston & & 50594 & & EPA300 & CL/F/SO4 & $94 L C C 066$ & $94 \mathrm{LCC} 066$ \\
\hline 100444 & D431-35-01 & Weston & & 50594 & & EPA300 & $\mathrm{CL} / \mathrm{F} / \mathrm{SO} 4$ & 94LFC066 & 94LFC066 \\
\hline 100444 & D431-35-01 & Weston & & 50594 & & EPA300 & CL/F/SO4 & 94LSC066 & 94LSC066 \\
\hline 100444 & D431-35-01 & Weston & & 50594 & & EPA353.2 & NO3 & 94LN3072 & 94LN3072 \\
\hline 100444 & D431-35-01 & Weston & & 50594 & & EPA415.1 & TOC & 94LTC080 & 94LTC080 \\
\hline $100444^{\circ}$ & $D 431-35-01$ & Thermo Analytical & & 50594 & & EPA900.0 & GROSS ALPHA/BETA & 9405022 & 5109402 \\
\hline 100444 & D431-35-01 & Thermo Analytical & & 50594 & & EPA900.1 MOD. & TOTAL RAD & 9405022 & 5109402 \\
\hline 100444 & D431-35-01 & Thermo Analytical & & 50594 & & EPA906.0 & TRITIUM & 9405022 . & 5099404 \\
\hline 100444 & D431-35-01 & Weston & & 50594 & & SW9020A & TOX & $94 \mathrm{LX} 125 \mathrm{~B}$ & $94 \mathrm{LX125B}$ \\
\hline 100444 & D431-35-01 & Weston & & 50594 & EPA3050 & EPA6010 & METALS & 94L5708 & PS0511F \\
\hline 100444 & D431-35-01 & Weston & & 50594 & EPA3050 & EPA6010 & METALS & 94L5708 & PS0511Z \\
\hline 100444 & D431-35-01 & Weston & & 50594 & EPA3050 & EPA7060 & ARSENIC & 94L5707 & AS051151 \\
\hline 100444 & D431-35-01 & Weston & & 50594 & EPA3050 & EPA7421 & LEAD & 94L5707 & PB051202 \\
\hline 100444 & D431-35-01 & Weston & & 50594 & EPA3050 & EPA7471 & HG & $94 \mathrm{C} 0185$ & HG051004 \\
\hline 100444 & D431-35-01 & Weston & & 50594 & EPA3050 & EPA7740 & SELENIUM & 94L5707 & SE051104 \\
\hline 100444 & $D 431-35-01$ & Weston & & 50594 & EPA3510/20 & EPA8270 & SEMIVOLATULES & 94LE0899 & A051007 \\
\hline 100444 & D431-35-01 & Weston & & 50594 & EPA3510/20 & EPA8270M & SEMIVOLATILES & 94LE0899 & A051007 \\
\hline 100444 & D431-35-01 & Weston & Spike Sample & 50594 & EPA3540/50 & EPA8270 & SEMIVOLATILES & 94LE0899 & A051007 \\
\hline 100444 & D431-35-01 & Weston & & 50594 & EPA5030 & EPA8260 & VOLATILES & 94LVC057 & C051101 \\
\hline 100444 & D431-35-01 & Weston & Spike Sample & 50594 & EPA5030 & EPA8260 & VOLATILES & 94LVC057 & C051101 \\
\hline 100445 & D431-35-02C & Thermo Analytical & & 50594 & & EPA900.0 & GROSS ALPHA/BETA & 9405022 & 5109402 \\
\hline 100445 & D431-35-02C & Thermo Analytical & & 50594 & & EPA900.1 MOD. & TOTAL RAD & 9405022 & 5109402 \\
\hline 100445 & $D 431-35-02 C$ & Thermo Analytical & & 50594 & & EPA906.0 & TRITTUM & 9405022 & 5099404 \\
\hline 100445 & D431-35-02C & Weston & & 50594 & EPA5030 & EPA8260 & VOLATTLES & 94LVC057 & C051101 \\
\hline 100445 & $D 431-35-02 C$ & Weston & Spike Sample & 50594 & EPA5030 & EPA8260 & VOLATILES & 94LVCOS7 & C051101 \\
\hline 100446 & $D 431-35-02$ & Weston & & 50594 & & ASTMD497.2 & $\overline{\mathrm{pH}}$ & 94LPHO40 & 94LPH040 \\
\hline 100446 & $D 431-35-02^{\circ}$ & Weston & & 50594 & & EPA415.8 & TOC/TOH & 94LTZ23A & 94LTZ23A \\
\hline 100446 & D4331-35-02 & Weston & & 50594 & & EPA7471 & HG & $94 C 0187$ & HG051005 \\
\hline 100446 & $D 431-35-02$ & Thermo Analytical & & 50594 & & LANLER210 MOI & TRITIUM & 9405023 & 5109404 \\
\hline 100446 & D431-35-02 & Thermo Analytical & & 50594 & & LANLMLR-100 & GROSS ALPHA/BETA & 9405023 & 5109402 \\
\hline 100446 & D431-35-02 & Thermo Analytical & & 50594 & & SW8469315 MOD & TOTAL RAD & 9405023 & 5109402 \\
\hline $100446^{\circ}$ & D431-35-02 & Weston & & 50594 & EPA3050 & EPA6010 & METALS & 94L5706 & PS0511A \\
\hline 100446 & D431-35-02 & Weston & ' & 50594 & EPA3050 & EPA6010 & METALS & $94 \mathrm{~L} 5706$ & PSO511B \\
\hline 100446 & D431-35-02 & Weston & & 50594 & EPA3050 & EPA6010 & METALS & 9945706 & PS0511E \\
\hline 100446 & D431-35-02 & Weston & & 50594 & EPA3050 & EPA6010 & METALS & $94 L 5706$ & PS0511X \\
\hline 100446 & D431-35-02 & Weston & & 50594 & EPA3050 & EPA6010 & METALS & 94L5706 & PSOS11Y \\
\hline & & & & & & & & 1 & \\
\hline - & & & & & & & & 1 & \\
\hline & & & & & & & & : & \\
\hline
\end{tabular}


laule 1

Analytical Program of Surface Water and Sediment Samples

D-Area Buming/Rubble Pits

Savannah River Site

\begin{tabular}{|c|c|c|c|c|c|c|c|c|c|}
\hline \multicolumn{10}{|c|}{ 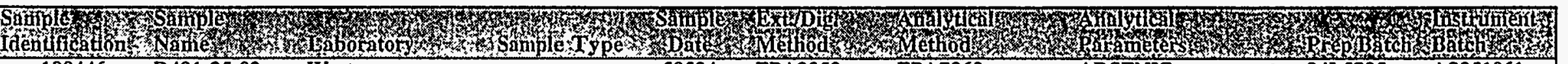 } \\
\hline 100446 & D431-35-02 & Weston & & 50594 & EPA3050 & EPA7060 & ARSENIC & $94 \mathrm{~L} 5705$ & AS051061 \\
\hline 100446 & D431-35-02 & Weston & & 50594 & EPA3050 & EPA7421 & LEAD & 94L570S & PB051153 \\
\hline 100446 & D431-35-02 & Weston & & 50594 & EPA3050 & EPA7740 & SELENTUM & 94L5705 & SE051161 \\
\hline 100446 & D431-35-02 & Weston & & 50594 & EPA3540/50 & EPA8270 & SEMIVOLATRES & 94LE0897 & S051001 \\
\hline 100446 & D431-35-02 & Weston. & Spike Sample & 50594 & EPA3540/50 & EPA 8270 & SEMIVOLATILES & 94LE0897 & S051001: \\
\hline 100446 & D431-35-02 & Weston & & 50594 & EPA3540/50 & EPA8270M & SEMIVOLATILES & 94LE0897 & S051001 \\
\hline 100446 & D431-35-02 & Weston & & 50594 & EPA5030 & EPA8260 & VOLATILES & 94LVC056 & C051001 \\
\hline 100446 & D431-35-02 & Weston & Spike Sample & 50594 & EPA5030 & EPA 8260 & VOLATILES & 94LVC056 & $\cos 1001$ \\
\hline 100447 & D431-34-01 & Weston & & 50594 & & EPA160.1 & TDS & 94LSSA85 & 94LSSA85 \\
\hline 100447 & D431-34-01 & Weston & & 50594 & & EPA160.2 & TSS & 94LSSA85 & 94LSSA85 \\
\hline 100447 & D431-34-01 & Weston & & 50594 & & EPA300 & CL/F/SO 4 & 94LCC066 & 94LCC066 \\
\hline 100447 & D431-34-01 & Weston & & 50594 & & EPA300 & CL/F/SO4 & 94LFC066 & 94LFC066 \\
\hline 100447 & D431-34-01 & Weston & & 50594 & & EPA300 & $\mathrm{CL} / \mathrm{F} / \mathrm{SO} 4$ & 94LSC066 & 94LSC066 \\
\hline 100447 & D431-34-01 & Weston & & 50594 & & EPA353.2 & NO3 & 94LN3072 & 94LN3072 \\
\hline 100447 & D431-34-01 & Weston & & 50594 & & EPA415.1 & TOC & 94LTC080 & 94LTC080 \\
\hline 100447 & D431-34-01 & Thermo Analytical & & 50594 & & EPA900.0 & GROSS ALPHA/BETA & 9405022 & 5109402 \\
\hline 100447 & D431-34-01 & Thermo Analytical & & 50594 & & EPA900.1 MOD. & TOTAL RAD & 9405022 & 5109402 \\
\hline 100447 & D431-34-01 & Thermo Analytical & & 50594 & & EPA906.0 & TRITIUM & 9405022 & 5099404 \\
\hline 100447 & D431-34-01 & Weston & & 50594 & & SW9020A & TOX & $94 \mathrm{LX126W}$ & 94LX126W \\
\hline 100447 & D431-34-01 & Weston & & 50594 & EPA3050 & EPA6010 & METALS & 94L5708 & PSO511F \\
\hline 100447 & D431-34-01 & Weston & & 50594 & EPA3050 & EPA6010 & METALS & 94L5708 & PSOS112 \\
\hline 100447 & D431-34-01 & Weston & & 50594 & EPA3050 & EPA7060 & ARSENIC & 94L5707 & AS051151 \\
\hline 100447 & D431-34-01 & Weston & & 50594 & EPA3050 & EPA7421 & LEAD & $94 L 5707$ & PB051202 \\
\hline 100447 & D431-34-01 & Weston & & 50594 & EPA3050 & EPA7471 & HO & $94 C 0185$ & HG051004 \\
\hline 100447 & D431-34-01 & Weston & & 50594 & EPA3050 & EPA7740 & SELENIUM & 94L5707 & SE051104 \\
\hline 100447 & D431-34-01 & Weston & & 50594 & EPA3510/20 & EPA 8270 & SEMIVOLATUES & 94LE0899 & A051007 \\
\hline 100447 & D431-34-01 & Weston & & 50594 & EPA3510/20 & EPA8270M & SEMIVOLATILES & 94LE0899 & A051007 \\
\hline 100447 & D431-34-01 & Weston & Spike Sample & 50594 & EPA3540/50 & EPA 8270 & SEMTVOLATILES & 94LE0899 & A051007 \\
\hline 100447 & D431-34-01 & Weston & & 50594 & EPA5030 & EPA8260 & VOLATILES & 94LVC057 & C051101 \\
\hline 100447 & D431-34-01 & Weston & Spike Sample & 50594 & EPA5030 & EPA 8260 & VOLATILES & 94LVC057 & $\mathrm{C} 051101$ \\
\hline 100448 & D431-33-01 & Weston & & 50594 & & EPA160.1 & TDS & 94LSSA85 & 94LSSA85 \\
\hline 100448 & D431-33-01 & Weston & & 50594 & & EPA160.2 & TSS & 94LSSA85 & 94LSSA85 \\
\hline 100448 & D431-33-01 & Weston & . & 50594 & & EPA300 & CL/F/SO4 & 94LCC066 & 94LCC066 \\
\hline 100448 & D431-33-01 & Weston & & 50594 & & EPA300 & CL/F/SO4 & 94LFC066. & 94LFC066 \\
\hline 100448 & D431-33-01 & Weston & & 50594 & & EPA300 & CL/F/SO4 & 94LSC066 & 94LSC066 \\
\hline 100448 & D431-33-01 & Weston & & 50594 & & EPA353.2 & NO3 & 94LN3072 & 94LN3072 \\
\hline
\end{tabular}


Table 1

Analytical Program of Surface Water and Sediment Samples

D-Area Burning/Rubble Pits

Savannah River Site

\begin{tabular}{|c|c|c|c|c|c|c|c|c|c|}
\hline \multicolumn{2}{|c|}{ 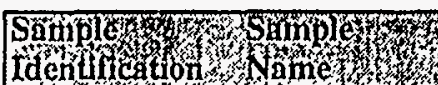 } & Whoratory & 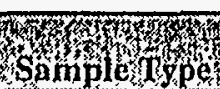 & Spirion & 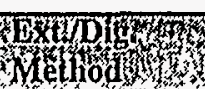 & 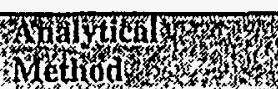 & \multicolumn{3}{|c|}{ 翟 } \\
\hline 100448 & D431-33-01 & Weston & & 50594 & & EPA415.1 & TOC & 94LTC080 & 94LTC080 \\
\hline 100448 & D431-33-01 & Thermo Analytical & & 50594 & & EPA900.0 & GROSS ALPHA/BET & 9405022 & 5099402 \\
\hline 100448 & D431-33-01 & Thermo Analytical & Lab Replicate & 50594 & & EPA900.0 & GROSS ALPHA/BETA & 9405022 & 5099402 \\
\hline 100448 & D431-33-01 & Thermo Analytical & & 50594 & & EPA900.1 MOD. & TOTAL RAD & 9405022 & 5109402 \\
\hline 100448 & D431-33-01 & Thermo Analytical & Lab Replicate & 50594 & & EPA900.1.MOD. & TOTAL RAD & 9405022 & 5109402 \\
\hline 100448 & D431-33-01 & Thermo Analytical & & 50594 & & EPA906.0 & TRITIUM & 9405022 & 5099404 \\
\hline 100448 & D431-33-01 & Weston & & 50594 & & SW9020A & TOX & $94 \mathrm{LX} 124 \mathrm{~W}$ & $94 \mathrm{LX124W}$ \\
\hline 100448 & D431-33-01 & Weston & Lab Replicate & 50594 & & SW9020A & TOX & $94 \mathrm{LX124W}$ & $94 \mathrm{LX124W}$ \\
\hline 100448 & D431-33-01 & Weston & & 50594 & EPA3050 & EPA6010 & METALS & 94L5708 & PS0511F \\
\hline 100448 & D431-33-01 & Weston & & 50594 & EPA3050 & EPA6010 & METALS & 94L5708 & PS0511Z \\
\hline 100448 & D431-33-01 & Weston & & 50594 & EPA3050 & EPA7060 & ARSENIC & 94L5707 & AS051151 \\
\hline 100448 & D431-33-01 & Weston & & 50594 & EPA3050 & EPA7421 & LEAD & 94L5707 & PB051202 \\
\hline 100448 & D431-33-01 & Weston & & 50594 & EPA3050 & EPA7471 & HG & $94 C 0185$ & HG051004 \\
\hline 100448 & D431-33-01 & Weston & & 50594 & EPA3050 & EPA7740 & SELENTUM & 94L5707 & SE051104 \\
\hline 100448 & D431-33-01 & Weston & & 50594 & EPA3510/20 & EPA8270 & SEMTVLATLES & 94LE0899 & A051007 \\
\hline 100448 & D431-33-01 & Weston & & 50594 & EPA3510/20 & EPA8270M & SEMIVOLATILES & 94LE0899 & A051007 \\
\hline 100448 & D431-33-01 & Weston & Spike Sample & 50594 & EPA3540/50 & EPA8270 & SEMTVOLATTLES & 94LE0899 & A051007 \\
\hline 100448 & D431-33-01 & Weston & & 50594 & EPA5030 & EPA8260 & VOLATILES & 94LVC057 & C051101 \\
\hline 100448 & D43i-33-01 & Weston & Spike Sample & 50594 & EPA5030 & EPA8260 & VOLATILES & 94LVC057 & C051101 \\
\hline 100449 & $D 431-34-02$ & Weston & & 50594 & & ASTMD 497.2 & $\mathrm{pH}$ & 94LPH040 & 94LPH040 \\
\hline 100449 & D431-34-02 & Weston & & 50594 & & EPA415.8 & TOCr & 94LTZ23A & 94LTZ23A \\
\hline 100449 & D431-34-02 & Weston & & 50594 & & EPA7471 & HG & $94 C 0187$ & HG051005 \\
\hline 100449 & D431-34-02 & Thermo Analytical & & 50594 & & LANLER210 MOD & TRITIUM & 9405023 & 5109404 \\
\hline 100449 & D431-34-02 & Thermo Analytical & & 50594 & & LANLMLR-100 & GROSS ALPHA/BET & 9405023 & 5109402 \\
\hline 100449 & D431-34-02 & Thermo Analytical & & 50594 & & SW8469315 MOD & TOTAL RAD & 9405023 & 5109402 \\
\hline 100449 & D431-34-02 & Weston & & 50594 & EPA3050 & EPA6010 & METALS & 9415706. & PSO511A \\
\hline 100449 & D431-34-02 & Weston & & 50594 & EPA3050 & EPA6010 & METALS & 94L5706 & PSO511B \\
\hline 100449 & D431-34-02 & Weston & & 50594 & EPA3050 & EPA6010 & METALS & $94 L 5706$ & PSOS11E \\
\hline 100449 & D431-34-02 & Weston & & 50594 & EPA3050 & EPA6010 & METALS & 94L5706 & PS0511X \\
\hline 100449 & D431-34-02 & Weston & & 50594 & EPA3050 & EPA6010 & METALS & 94L5706 & PSOS11Y \\
\hline 100449 & D431-34-02 & Weston & & 50594 & EPA3050 & EPA7060 & ARSENIC & $9.4 \mathrm{~L} 5705$ & AS051061 \\
\hline 100449 & D431-34-02 & Weston & & 50594 & EPA3050 & EPA7421 & LEAD & 94L5705 & PB051153 \\
\hline 100449 & D431-34-02 & Weston & & 50594 & EPA3050 & EPA7740 & SELENIUM & $94 \mathrm{~L} 5705$ & SE051161 \\
\hline 100449 & D431-34-02 & Weston & & 50594 & EPA3540/50 & EPA8270 & SEMIVOLATLLES & 94LE0897 & S051001 \\
\hline 100449 & D431-34-02 & Weston & Lab Replicate & 50594 & EPA3540/50 & EPA8270 & SEMIVOLATILES & 94LE0897 & S051001 \\
\hline 100449 & D431-34-02 & Weston & Spike Sample & 50594 & EPA3540/50 & EPA8270 & SEMTVOLATILES & 94LE0897 & S051001 \\
\hline
\end{tabular}


Tauie 1

Analytical Program of Surface Water and Sediment Samples D-Area Buming/Rubble Pits

Savannah River Site

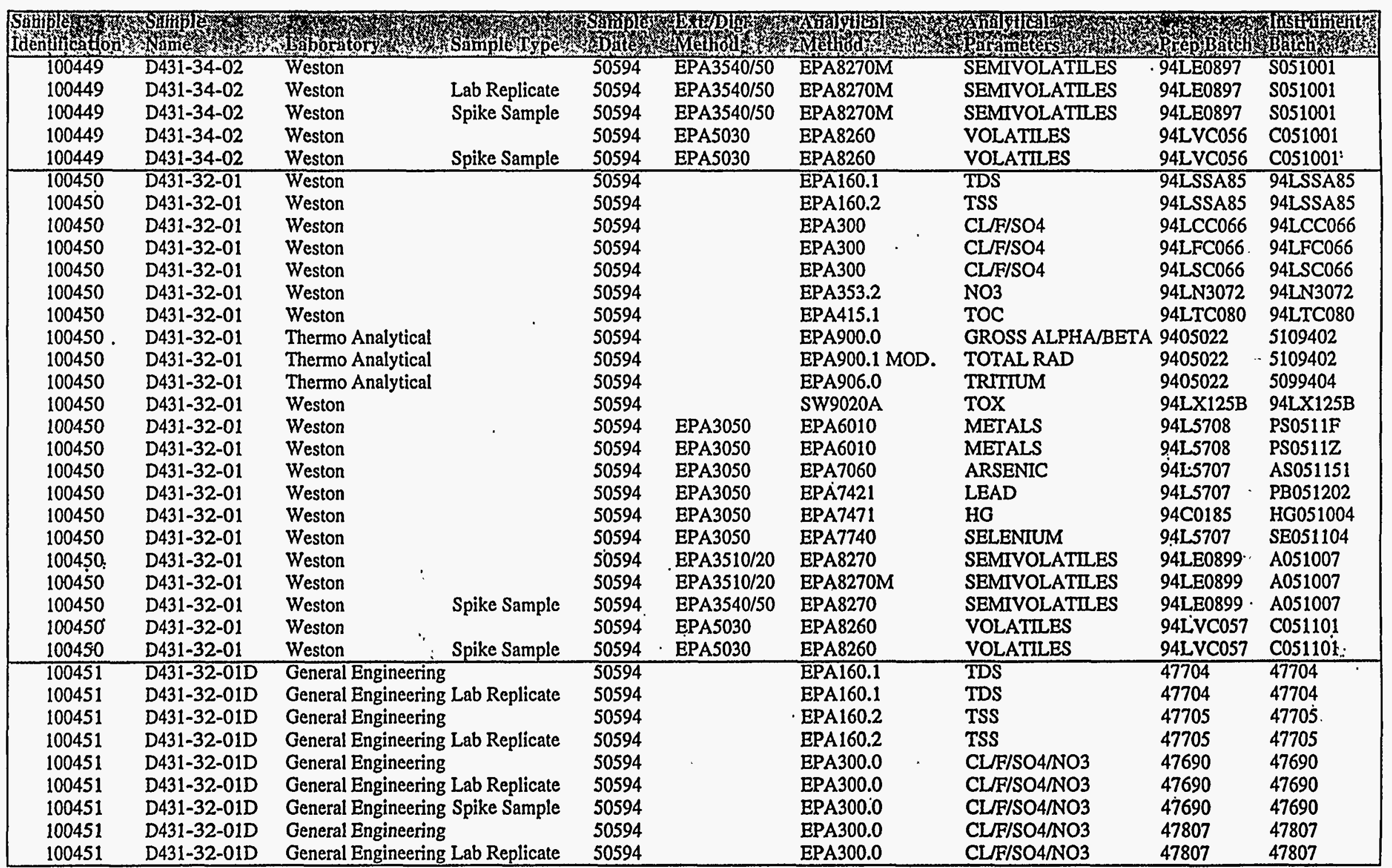

Page 9 of 19 
Table 1

Analytical Program of Surface Water and Sediment Samples

D-Area Burning/Rubble Pits

Savannah River Site

\begin{tabular}{|c|c|c|c|c|c|c|c|c|}
\hline 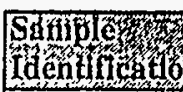 & 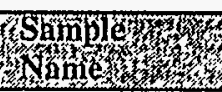 & 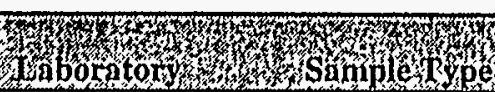 & Sintite & 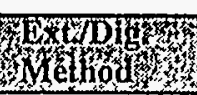 & 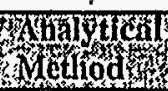 & Why & 3rensat & 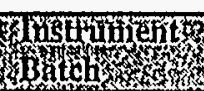 \\
\hline 100451 & D431-32-01D & General Engineering Spike Sample & 50594 & & EPA300.0 & $\mathrm{CL} / \mathrm{F} / \mathrm{SO} 4 / \mathrm{NO} 3$ & 47807 & 47807 \\
\hline 100451 & D431-32-01D & General Engineering & 50594 & & EPA415.1 & TOC & 47767 & 47767 \\
\hline 100451 & D431-32-01D & General Engineering Lab Replicate & 50594 & & EPA415.1 & TOC & 47767 & 47767 \\
\hline 100451 & D431-32-01D & General Engineering Spike Sample & 50594 & & EPA415.1 & TOC & 47767 & 47767 \\
\hline 100451 & D431-32-01D & General Engineering & 50594 & & EPA8260 & VOLATILES & 47729 & 47729 \\
\hline 100451 & D431-32-01D & General Engineering & 50594 & & EPA9020. & TOX & 47737 . & 47737 \\
\hline 100451 & D431-32-01D & GP & 50594 & & EPIA-001 & GROSS ALPHA/BETA & 47823 & 47823 \\
\hline 100451 & D431-32-01D & GP & 50594 & & EPIA-002 & TRITIUM & 47845 & 47845 \\
\hline 100451 & D431-32-01D & Lab Replicate & 50594 & & EPIA-002 & TRITIUM & 47845 & 47845 \\
\hline 100451 & D431-32-01D & Spike Sample & 50594 & & EPIA-002 & TRITTUM & 47845 & 47845 \\
\hline 100451 & D431-32-01D & GP & 50594 & & EPIA-010 & TOTAL RAD & 47708 & 47708 \\
\hline 100451 & D431-32-01D & General Engineering & 50594 & EPA3005 & EPA6010 & METALS & 47711 & 47711 \\
\hline 100451 & D431-32-01D & General Engineering & 50594 & EPA3020 & EPA7060 & ARSENIC & 47710 & 47710 \\
\hline 100451 & D431-32-01D & General Engineering Lab Replicate & 50594 & EPA3020 & EPA7060 & ARSENIC & 47710 & 47710 \\
\hline 100451 & D431-32-01D & General Engineering Spike Sample & 50594 & EPA3020 & EPA7060 & ARSENIC & 47710 & 47710 \\
\hline 100451 & D431-32-01D & General Engineering & 50594 & EPA3020 & EPA742.1 & LEAD & 47710 & 47710 \\
\hline 100451 & D431-32-01D & General Engineering Lab Replicate & 50594 & EPA3020 & EPA7421 & LEAD & 47710 & 47710 \\
\hline 100451 & D431-32-01D & General Engineering Spike Sample & 50594 & EPA3020 & EPA7421 & LEAD & 47710 & 47710 \\
\hline 100451 & D431-32-01D & General Engineering & 50594 & EPA3020 & EPA7740 & SELENTUM & 47710 & 47710 \\
\hline 100451 & D431-32-01D & General Engineering Lab Replicate & 50594 & EPA3020 & EPA7740 & SELENTUM & 47710 & 47710 \\
\hline 100451 & D431-32-01D & General Engineering Spike Sample & 50594 & EPA3020 & EPA7740 & SELENIUM & 47710 & 47710 \\
\hline 100451 & D431-32-01D & General Engineering & 50594 & EPA3510 & EPA8270 & SEMIVOLATILES & 47675 & 47675 \\
\hline 100451 & D431-32-01D & General Engineering Lab Replicate & 50594 & EPA3510 & EPA8270 & SEMIVOLATILES & 47675 & 47675 \\
\hline 100451 & D431-32-01D & General Engineering Spike Sample & 50594 & EPA3510 & EPA8270 & SEMTVOLATILES & 47675 & 47675 \\
\hline 100451 & D431-32-01D & General Engineering & 50594 & EPA7470 & EPA7470 & MERCURY & 47748 & 47748 \\
\hline 100451 & D431-32-01D & General Engineering Lab Replicate & 50594 & EPA7470 & EPA7470 & MERCURY & 47748 & 47748 \\
\hline 100451 & D431-32-01D & General Engineering Spike Sample & 50594 & EPA7470 & EPA7470 & MERCURY & 47748 & 47748 \\
\hline 100452 & $\mathrm{D} 431-32-02 \mathrm{E}$ & Weston & 50594 & EPA3050 & EPA6010 & METALS & 94L5708 & PS0511F \\
\hline 100452 . & D431-32-02E & Weston & 50594 & EPA3050 & EPA6010 & METALS & $94 \mathrm{~L} 5708$ & PSOS11Z \\
\hline 100452 & D431-32-02E & Weston & 50594 & EРA3050 & EPA7060 & ARSENIC & $94 L 5707$ & AS051151 \\
\hline 100452 & D431-32-02E & Weston & 50594 & EPA3050 & EPA7421 & LEAD & 94L5707 & PB051202 \\
\hline 100452 & D431-32-02E & Weston & 50594 & EPA3050 & EPA7471 & HG & $94 C 0185$ & HG051004 \\
\hline 100452 & D431-32-02E & Weston & 50594 & EPA3050 & EPA7740 & SELENTUM & 94L5707 & SE051104 \\
\hline 100452 & D431-32-02E & Weston & 50594 & EPA3510/20 & EPA8270 & SEMIVOLATILES & 94LE0899 & A051007 \\
\hline 100452 & D431-32-02E & Weston & 50594 & EPA3510/20 & EPA8270N & SEMIVOLATILES & 94LË0899 & A051007 \\
\hline 100452 & D431-32-02E & Spike Sample & 50594 & EPA3540/50 & EPA8270 & SEMIVOLATLES & 94LE0899 & A051007 \\
\hline
\end{tabular}


Tabie 1

Analytical Program of Surface Water and Sediment Samples

D-Area Buming/Rubble Pits

Savannah River Site

..

\begin{tabular}{|c|c|c|c|c|c|c|c|c|c|}
\hline \multicolumn{10}{|c|}{ 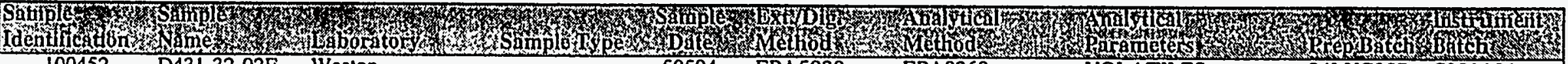 } \\
\hline 100452 & $\mathrm{D} 431-32-02 \mathrm{E}$ & Weston & & 50594 & EPA5030 & EPA8260 & VOLATILES & $94 L V C 057$ & $\cos 1101$ \\
\hline 100452 & $\mathrm{D} 431-32-02 \mathrm{E}$ & Weston & Spike Sample & 50594 & EPA5030 & EPA8260 & VOLATILES & $94 \mathrm{LVC} 057$ & $\mathrm{C} 051101$ \\
\hline 100453 & $D 431-33-02$ & Weston & & 50594 & & ASTMD497.2 & $\mathrm{pH}$ & 94LPH040 & $94 \mathrm{LPHO40}$ \\
\hline 100453 & D431-33-02 & Weston & & 50594 & & EPA415.8 & TOCITOH & 94LTZ23A & 94LTZ23A \\
\hline 100453 & $D 431-33-02$ & Weston & & 50594 & ' & EPA7471 & HG & $94 C 0187$ & HG051005 \\
\hline 100453 & D431-33-02 & Thermo Analytical & & 50594 & & LANLER210 MOD & TRITIUM & 9405023 & 5109404 \\
\hline 100453 & D431-33-02 & Thermo Analytical & & 50594 & & LANLMLR-100 & GROSS ALPHA/BETA & 9405023 & 5099402 \\
\hline 100453 & D431-33-02 & Thermo Analytical & & 50594 & & SW8469315 MOD & TOTAL RAD & 9405023 & 5109402 \\
\hline 100453 & D431-33-02 & Weston & & 50594 & EPA3050 & EPA6010 & METALS & 94L5706 & PSO511A \\
\hline 100453: & D431-33-02 & Weston & & 50594 & EPA3050 & EPA6010 & METALS & 94L5706 & PS0511B \\
\hline 100453 & D431-33-02 & Weston & & 50594 & EPA3050 & EPA6010 & METALS & $94 L 5706$ & PSO511E \\
\hline 100453 & D431-33-02 & Weston & & 50594 & EPA3050 & EPA6010 & METALS & 94L5706 & PS0511X \\
\hline 100453 & D431-33-02 & Weston & & 50594 & EPA3050 & EPA6010 & METALS & 94L5706 & PS0511Y \\
\hline 100453 & D431-33-02 & Weston & & 50594 & EPA3050 & EPA7060 & ARSENIC & $94 L 5705$ & AS051061 \\
\hline 100453 & D431-33-02 & Weston & & 50594 & EPA3050 & EPA7421 & LEAD & $94 L 5705$ & $\mathrm{~PB} 051153$ \\
\hline 100453 & D431-33-02 & Weston & & 50594 & EPA3050 & EPA7740 & SELENIUM & $94 L 5705$ & SE051161 \\
\hline 100453 & D431-33-02 & Weston & & 50594 & EPA3540/50 & EPA8270 & SEMIVOLATILES & 94LE0897 & S051001 \\
\hline 100453 & D431-33-02 & Weston & Spike Sample & 50594 & EPA3540/50 & EPA8270 & SEMIVOLATUES & 94LE0897 & S051001 \\
\hline 100453 & D431-33-02 & Weston & & 50594 & EPA3540/50 & EPA8270M & SEMTVOLATILES & 94LE0897 & S051001 \\
\hline 100453 & D431-33-02 & Weston & & 50594 & EPA5030 & EPA8260 & VOLATILES & $94 L V C 056$ & $\mathrm{C} 051001$ \\
\hline 100453 & D431-33-02 & Weston & Spike Sample & 50594 & EPA5030 & EPA8260 & VOLATILES & $94 L V \operatorname{Cos} 6$ & $\operatorname{Cos} 1001$ \\
\hline 100454 & D431-31-01 & Weston & & 50594 & & EPA160.1 & TDS & 94LSSA85 & 94LSSA 85 \\
\hline 100454 & D431-31-01 & Weston & & 50594 & & EPA160.2 & TSS & 94LSSA85 & 94LSSA85 \\
\hline 100454 & D431-31-01 & Weston & & 50594 & & EPA300 & $\mathrm{CL} / \mathrm{F} / \mathrm{SO} 4$ & 94LCC066 & 94LCC066 \\
\hline 100454 & D431-31-01 & Weston & & 50594 & & EPA300 & CL/F/SO4 & 94LFC066 & 94LFC066 \\
\hline 100454 & D431-31-01 & Weston & & 50594 & & EPA300 & CL/F/SO4 & 94LSC066 & 94LSC066 \\
\hline 100454 . & D431-31-01 & Weston & & 50594 & & EPA353.2 & $\mathrm{NO} 3$ & 94LN3072 & $94 \mathrm{LN} 3072$ \\
\hline 100454 & D431-31-01 & Weston & & 50594 & & EPA415.1 & .TOC & 94LTC080 & 94LTC080 \\
\hline 100454 & D431-31-01 & Thermo Analytical & & 50594 & & EPA900.0 & GROSS ALPHA/BETA & 9405022 & 5099402 \\
\hline 100454 & D431-31-01 & Thermo Analytical & & 50594 & & EPA900.1 MOD. & TOTAL RAD & 9405022 & 5109402 \\
\hline 100454 & D431-31-01 & Thermo Analytical & & 50594 & & EPA906.0 & TRITIUM & 9405022 & 5099404 \\
\hline 100454 & D431-31-01 & Weston & & 50594 & & SW9020A & TOX & $94 \mathrm{LX125B}$ & 94LX125B \\
\hline 100454 & D431-31-01 & Weston & Lab Replicate & 50594 & & SW9020A & TOX & $94 \mathrm{LX} 125 \mathrm{~B}$ & $94 \mathrm{LX} 125 \mathrm{~B}$ \\
\hline 100454 & D431-31-01 & Weston & & 50594 & EPA3050 & EPA6010 & METALS & $94 L 5708$ & PSOS11F \\
\hline 100454 & D431-31-01 & Weston & & 50594 & EPA3050 & EPA6010 & METALS & $94 L 5708$ & PSOS11Z \\
\hline 100454 & D431-31-01 & Weston & & 50594 & EPA3050 & EPA7060 & ARSENIC & 94L5707 & AS051151 \\
\hline
\end{tabular}


Table 1

Analytical Program of Surface Water and Sediment Samples

D-Area Burning/Rubble Pits

Savannah River Site

\begin{tabular}{|c|c|c|c|c|c|c|c|c|c|}
\hline 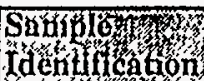 & 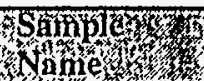 & 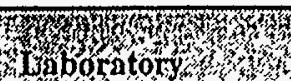 & 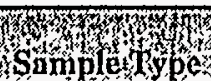 & 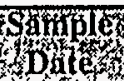 & 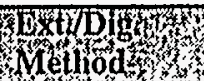 & 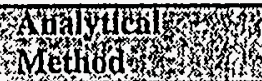 & 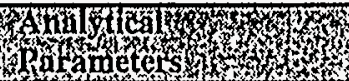 & 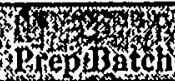 & 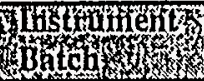 \\
\hline 100454 & D431-31-01 & Weston & & 50594 & EPA3050 & EPA7421 & LEAD & $94 \mathrm{~L} 5707$ & PB051202 \\
\hline 100454 & D431-31-01 & Weston & & 50594 & EPA3050 & EPA7471 & HG & $94 \mathrm{C} 0185$ & HG051004 \\
\hline 100454 & D431-31-01 & Weston & & 50594 & EPA3050 & EPA7740 & SELENIUM & 94L5707 & SE051104 \\
\hline 100454 & D431-31-01 & Weston & & 50594 & EPA3510/20 & EPA8270 & SEMIVOLATLES & 94LE0899 & J051101 \\
\hline 100454 & D431-31-01 & Weston & & 50594 & EPA3510/20 & EPA8270M & SEMIVOLATILES & 94LE0899 & J051101 \\
\hline 100454 & D431-31-01 & Weston & Spike Sample & 50594 & EPA3540/50 & EPA8270 & SEMIVOLATILES & 94LE0899 & $\mathrm{J} 051101$ \\
\hline 100454 & D431-31-01 & Weston & & 50594 & EPA5030 & EPA8260 & VOLATILES & 94LVC057 & C051101 \\
\hline 100454 & D431-31-01 & Weston & Spike Sample & 50594 & EPA5030 & EPA8260 & VOLATILES & 94LVC057 & C051101 \\
\hline 100455 & D431-31-01A & Weston & & 50594 & & EPA160.1 & TDS & 94LSSA85 & 94LSSA85 \\
\hline 100455 & D431-31-01A & Weston & & 50594 & & EPA160.2 & TSS & 94LSSA85 & 94LSSA85 \\
\hline 100455 & D431-31-01A & Weston & & 50594 & & EPA300 & CL/F/SO4 & 94LCC066 & $94 \mathrm{LCC} 066$ \\
\hline 100455 & D431-31-01A & Weston & Lab Replicate & 50594 & & EPA300 & CL/F/SO4 & 94LCC066 & $94 \mathrm{LCC} 066$ \\
\hline 100455 & D431-31-01A & Weston & Spike Sample & 50594 & & EPA300 & $\mathrm{CL} / \mathrm{F} / \mathrm{SO} 4$ & 94LCC066 & 94LCC066 \\
\hline 100455 & D431-31-01A & Weston & & 50594 & & EPA300 & CL/F/SO4 & 94LFC066 & 94LFC066 \\
\hline 100455 & D431-31-01A & Weston & Lab Replicate & 50594 & & EPA300 & CU/F/SO4 & 94LFC066 & 94LFC066 \\
\hline 100455 & D431-31-01A & Weston & Spike Sample & 50594 & & EPA300 & CL/F/SO4 & 94LFC066 & 94LFC066 \\
\hline 100455 & D431-31-01A & Weston & & 50594 & & EPA300 & CL/F/SO4 & 94LSC066 & 94LSC066 \\
\hline 100455 & D431-31-01A & Weston & Lab Replicate & 50594 & & EPA300 & CL/F/SO4 & 94LSC066 & 94LSC066 \\
\hline 100455 & D431-31-01A & Weston & Spike Sample & 50594 & & EPA300 & $\mathrm{CL} / \mathrm{F} / \mathrm{SO} 4$ & 94LSC066 & 94LSC066 \\
\hline 100455 & $\mathrm{D} 431-31.01 \mathrm{~A}$ & Weston & & 50594 & & EPA353.2 & NO3 & 94LN3072 & 94LN3072 \\
\hline 100455 & D431-31-01A & Weston & & 50594 & & EPA415.1 & TOC & 94LTC080 & 94LTC080 \\
\hline 100455. & D431-31-01A & Thermo Analytical & & 50594 & & EPA900.0 & GROSS ALPHA/BETA & 9405022 & 5109402 \\
\hline 100455 & D431-31-01A & Thermo Analytical & & 50594 & & EPA900.1 MOD. & TOTAL RAD & 9405022 & 5109402 \\
\hline 100455 & D431-31-01A & Thermo Analytical & & 50594 & & EPA906.0 & TRITIUM & 9405022 & 5099404 \\
\hline 100455 & D431-31-01A & Weston & & 50594 & & SW9020A & Tox & $94 \mathrm{LX} 125 \mathrm{~B}$ & $94 \mathrm{LX} 125 \mathrm{~B}$ \\
\hline 100455 & D431-31-01A & Weston & & 50594 & EPA3050 & EPA6010 & METALS & 94L5708 & PS0511F \\
\hline 100455 & D431-31-01A & Weston & & 50594 & EPA3050 & EPA6010 & METALS & 94L5708 & PS0511Z \\
\hline 100455 & D431-31-01A & Weston & & 50594 & EPA3050 & EPA7060 & ARSENIC & 94L5707 & AS051151 \\
\hline 100455 & D431-31-01A & Weston & & 50594 & EPA3050 & EPA7421 & LEAD & 94L5707 & PB051202 \\
\hline 100455 & $\mathrm{D} 431-31-01 \mathrm{~A}$ & Weston & & 50594 & EPA3050 & EPA7471 & HG & $94 \mathrm{C} 0194$ & HG051004 \\
\hline 100455 & D431-31-01A & Weston & Lab Replicate & 50594 & EPA3050 & EPA7471 & HG & $94 \mathrm{C} 0194$ & HG051004 \\
\hline 100455 & $D 431-31-01 A$ & Weston & Spike Sample & 50594 & EPA3050 & EPA7471 & HG & $94 C 0194$ & HG051004 \\
\hline 100455 & D431-31-01A & Weston & Spike Sample & 50594 & EPA3050 & EPA7471 & HG & 94C0194 & HG051103 \\
\hline 100455 & D431-31-01A & Weston & & 50594 & EPA3050 & EPA7471 & HG & 94L5708 & PS05i1Z \\
\hline 100455 & D431-31-01A & Weston & & 50594 & EPA3050 & EPA7740 & SELENIUM & 94L5707 & SE051104 \\
\hline 100455 & D431-31-01A & Weston & & 50594 & EPA3510/20 & EPA8270 & SEMIVOLATIES & 94LE0899 & $\mathrm{J} 051101$ \\
\hline
\end{tabular}


Tabie 1

Analytical Program of Surface Water and Sediment Samples

D-Area Buming/Rubble Pits

Savannah River Site

\begin{tabular}{|c|c|c|c|c|c|c|c|c|c|}
\hline \multicolumn{10}{|c|}{ 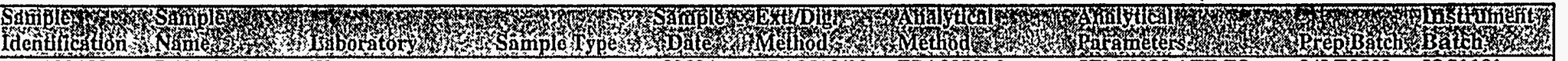 } \\
\hline 100455 & D431-31-01A & Weston & & 50594 & EPA3510/20 & EPA8270M & SEMIVOLATILES & 94LE0899 & $\mathrm{J} 051101$ \\
\hline 100455 & D431-31-01A & Weston & Spike Sample & 50594 & EPA3540/50 & EPA8270 & SEMIVOLATILES & 94LE0899 & J051101 \\
\hline 100455 & D431-31-01A & Weston & & 50594 & EPA5030 & EPA8260 & VOLATILES & 94LVC057 & Co51101 \\
\hline 100455 & D431-31-01A & Weston & Spike Sample & 50594 & EPA5030 & EPA8260 & VOLATILES & 94LVC057 & Cos 1101 \\
\hline 100456 & D431-31-01C & Thermo Analytical & & 50594 & & EPA900.0 & GROSS ALPHA/BE & 9405022 & 5109402 \\
\hline 100456 & D431-31-01C & Thermo Analytical & & 50594 & & EPA900.1 MOD. & TOTAL RAD & 9405022 & 5109402 \\
\hline 100456 & D431-31-01C & Thermo Analytical & & 50594 & & EPA906.0 & TRITIUM & 9405022 & 5099404 \\
\hline 100456 & D431-31-01C & Weston & & 50594 & EPA5030 & EPA8260 & VOLATILES & 94LVC057 & $\cos 1101$ \\
\hline 100456 & $D 431-31-01 C$ & Weston & Spike Sample & 50594 & EPA5030 & EPA8260 & VOLATILES & $94 \mathrm{LVC} 057$ & Cos1101 \\
\hline $100459^{\circ}$ & D431-30-01 & Weston & & 50594 & & EPA160.1 & TDS & 94LSSA85 & 94LSSA85 \\
\hline 100457 & D431-30-01 & Weston & & 50594 & & EPA160.2 & TSS & 94LSSA85 & 94LSSA85 \\
\hline 100457 & D431-30-01 & Weston & & 50594 & & EPA300 & CL/F/SO4 & $94 \mathrm{LCC} 066$ & 94LCC066 \\
\hline 100457 & D431-30-01 & Weston & & 50594 & & EPA300 & CL/F/SO4 & 94LFC066 & 94LFC066 \\
\hline 100457 & D431-30-01 & Weston & & 50594 & & EPA300 & CL/F/SO4 & 94LSC066 & 94LSC066 \\
\hline 100457 & D431-30-01 & Weston & & 50594 & & EPA353.2 & NO3 & 94LN307!2 & 94LN3072 \\
\hline 100457 & D431-30-01 & Weston & & 50594 & & EPA415.1 & TOC & 94LTC080 & 94LTC080 \\
\hline 100457 & D431-30-01 & Thermo Analytical & & 50594 & & EPA900.0 & GROSS ALPHA/BE & 9405022 & 5109402 \\
\hline 100457 & D431-30-01 & Thermo Analytical & & 50594 & & EPA900.1 MOD. & TOTAL RAD & 9405022 & 5109402 \\
\hline 100457 & D431-30-01 & Thermo Analytical & & 50594 & & EPA906.0 & TRITIUM & 9405022 & 5099404 \\
\hline 100457 & D431-30-01 & Weston & & 50594 & & SW9020A & TOX & $94 \mathrm{LX} 126 \mathrm{~W}^{\circ}$ & $94 \mathrm{LX126W}$ \\
\hline 100457 & D431-30-01 & Weston & & 50594 & EPA: & EPA6010 & METALS & 94L5708 & PS0511F \\
\hline 100457 & D431-30-01 & Weston & & 50594 & EPA3050 & EPA6010 & METALS & 94L5708 & PS0511Z \\
\hline 100457 & D431-30-01 & Weston & & 50594 & EPA3050 & EPA7060 & ARSENIC & 94LS707 & AS051151 \\
\hline 100457 & D431-30-01 & Weston & & 50594 & EPA3050 & EPA7421 & LEAD & 94L5707 & PB051202 \\
\hline 100457 & D431-30-01 & Weston & & 50594 & EPA3050 & EPA7471 & HG & $94 \mathrm{C} 0185$ & HG051004 \\
\hline 100457 & D431-30-01 & Weston & & 50594 & EPA3050 & EPA7740 & SELENIUM & 94L5707 & SE051104 \\
\hline 100457 & D431-30-01 & Weston & & 50594 & EPA3510/20 & EPA8270 & SEMIVOLATILES & 94LE0899 & J051101 \\
\hline 100457 & D431-30-01 & Weston & & 50594 & EPA3510/20 & EPA8270M & SEMIVOLATLES & 94LE0899 & J051101 \\
\hline 100457 & D431-30-01 & Weston & Spike Sample & 50594 & EPA3540/50 & EPA 8270 & SEMIVOLATILES & 94LE0899 & J051101 \\
\hline 100457 & D431-30-01 & Weston & & 50594 & EPA5030 & EPA8260 & VOLATILES & 94LVC057 & C051101 \\
\hline 100457 & D431-30-01 & Weston & Spike Sample & 50594 & EPA5030 & EPA 8260 & VOLATILES & $94 L V C 057$ & C051101 \\
\hline 100458 & D431-32-02 & Weston & & 50594 & & ASTMD497.2 & $\mathrm{pH}$ & 94LPH040 & 94LPH040 \\
\hline 100458 & D431-32-02 & Weston & ، & 50594 & & EPA415.8 & TOCITOH & 94LTZ23A & 94LTZ23A \\
\hline 100458 & D431-32-02 & Weston & & 50594 & & EPA7471 & HG & $94 C 0187$ & HG051005 \\
\hline 100458 & D431-32-02 & Thermo Analytical & & 50594 & & LANLER210 N & TRITIUM & 9405023 & 5109404 \\
\hline 100458 & D431-32-02 & Thermo Analytical & & 50594 & & LANLMLR-100 & GROSS ALPHA/BE & 1. 9405023 & 5109402 \\
\hline
\end{tabular}


Table 1

Analytical Program of Surface Water and Sediment Samples

D-Area Burning/Rubble Pits Savannah River Site

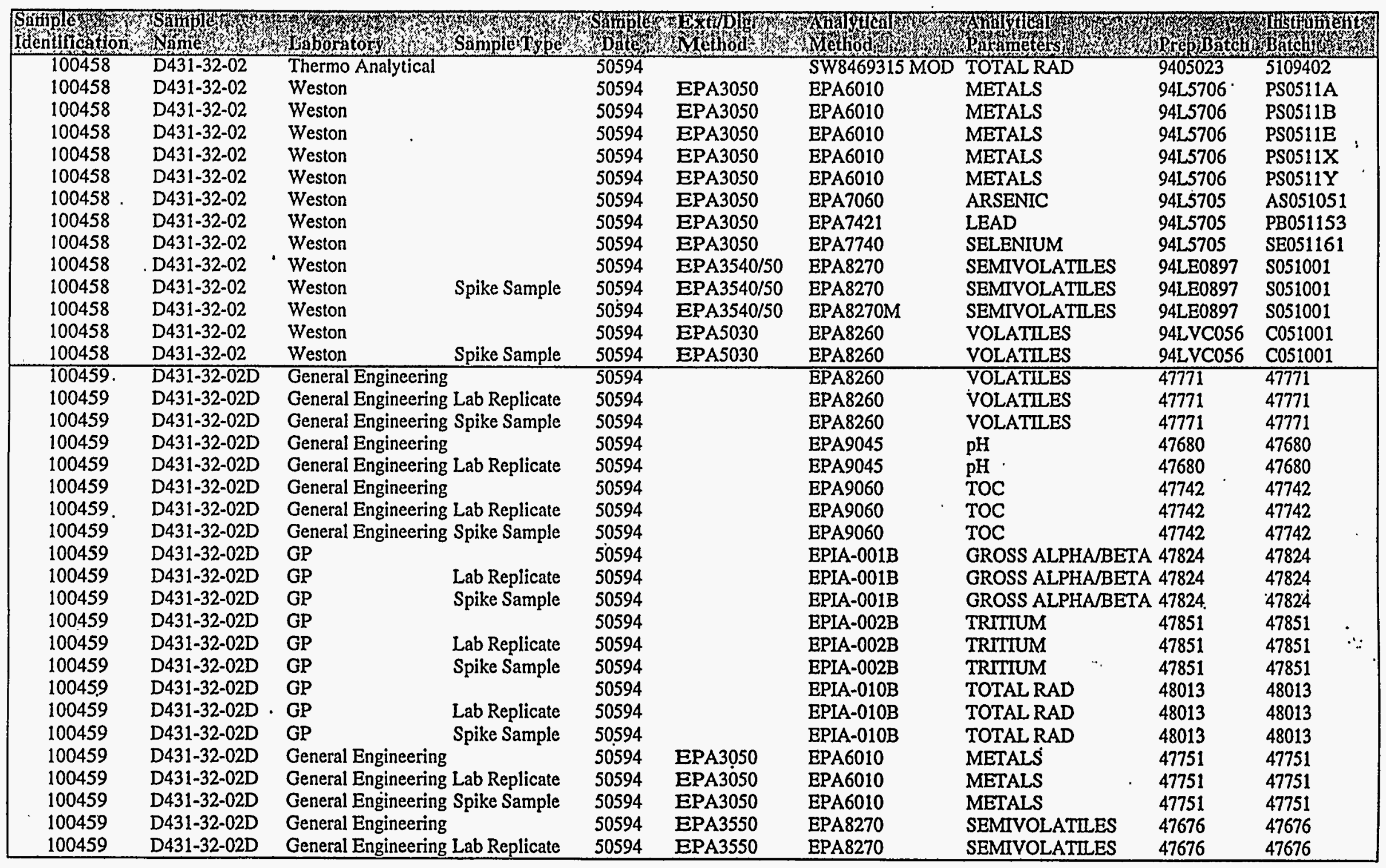


Table 1

Analytical Program of Surface Water and Sediment Samples D-Area Buming/Rubble Pits

Savannah River Site

\begin{tabular}{|c|c|c|c|c|c|c|c|c|c|}
\hline 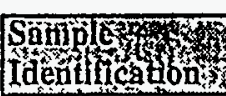 & S & 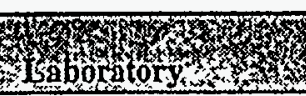 & 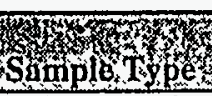 & STingle & 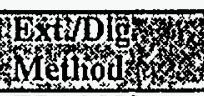 & A & 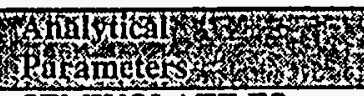 & 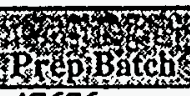 & 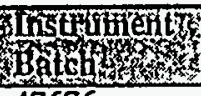 \\
\hline 100459 & D431-32-02D & General Engineering & Spike Sample & 50594 & EPA3550 & EPA8270 & SEMIVOLATILES & $\frac{47676}{47676}$ & 47676 \\
\hline 100459 & $D 431-32-02 D$ & General Engineering & & 50594 & EPA7471 & EPA7471 & HG & 47749 & 47749 \\
\hline 100459 & D431-32-02D & General Engineering & Lab Replicate & 50594 & EPA7471 & EPA7471 & HG & 47749 & 47749 \\
\hline 100459 & D431-32-02D & General Engineering & Spike Sample & 50594 & EPA7471 & EPA7471 & HG & 47749 & 47749 \\
\hline 100460 & D431-29-01 & Weston & & 50594 & & EPA160.1 & TDS & 94LSSA85 & 94LSSA:85 \\
\hline 100460 & D431-29-01 & Weston & & 50594 & & EPA160.2 & TSS & 94LSSA85 & 94LSSA 85 \\
\hline 100460 & D431-29-01 & Weston & & 50594 & & EPA300 & CL/F/SO4 & 94LCC066 & $94 \mathrm{LCC} 066$ \\
\hline 100460 & D431-29-01 & Weston & & 50594 & & EPA300 & CL/F/SO4 & 94LFC066 & 94LFC066 \\
\hline 100460 & D431-29-01 & Weston & & 50594 & & EPA300 & CL/F/SO4 & 94LSC066 & 94LSC066 \\
\hline 100460 & D431-29-01 & Weston & & 50594 & & EPA353.2 & NO3 & 94LN3072 & $94 \mathrm{LN} 3072$ \\
\hline 100460 & D431-29-01 & Weston & & 50594 & & EPA415:1 & TOC & 94LTC080 & 94LTC080 \\
\hline 100460 & D431-29-01 & Thermo Analytical & & 50594 & & EPA900.0 & GROSS ALPHA/BETA & 9405022 & 5109402 \\
\hline 100460 & D431-29-01 & Thermo Analytical & & 50594 & & EPA900.1 MOD. & TOTAL RAD & 9405022 & 5109402 \\
\hline 100460 & D431-29-01 & Thermo Analytical & & 50594 & & EPA906.0 & TRITIUM & 9405022 & 5099404 \\
\hline 100460 & D431-29-01 & Weston & & 50594 & & SW9020A & TOX & $94 \mathrm{~L} \times 125 \mathrm{~B}$ & $94 \mathrm{LX} 1.25 \mathrm{~B}$ \\
\hline 100460 & D431-29-01 & Weston & & 50594 & EPA3050 & EPA6010 & METALS & 94L5708 & PS0511F \\
\hline 100460 & D431-29-01 & Weston & & 50594 & EPA3050 & EPA6010 & METALS & 94L5708 & PS0511Z \\
\hline 100460 & D $431-29-01$ & Weston & & 50594 & EPA3050 & EPA7060 & ARSENIC & 94L5707 & AS051151 \\
\hline 100460 & D431-29-01 & Weston & & 50594 & EPA3050 & EPA7421 & LEAD & 94L5707 & PB051202 \\
\hline 100460 & D431-29-01 & Weston & & 50594 & EPA3050 & EPA7471 & HG & $94 \mathrm{C} 0185^{\circ}$ & HG051004 \\
\hline 100460 & D431-29-01 & Weston & & 50594 & EPA3050 & EPA7740 & SELENIUM & 94L5707 & SE051104 \\
\hline 100460 & D431-29-01 & Weston & & 50594 & EPA3510/20 & EPA8270 & SEMIVOLATUES & 94LE0899 & J051101 \\
\hline 100460 & D431-29-01 & Weston & & 50594 & EPA3510/20 & EPA8270M & SEMIVOLATLES & 94LE0899 & $\mathrm{J} 051101$ \\
\hline 100460 & D431-29-01 & Weston & Spike Sample & 50594 & EPA3540/50 & EPA 8270 & SEMIVOLATILES & 94LE0899 & J051101 \\
\hline 100460 & D431-29-01 & Weston & & 50594 & EPA5030 & EPA8260 & VOLATILES & $94 \mathrm{LVC} 057$ & C051101 \\
\hline 100460 & D431-29-01 & Weston & Spike Sample & 50594 & EPA5030 & EPA8260 & VOLATILES & $94 \mathrm{LVC} 057$ & C051101 \\
\hline 100461 & D431-28-01 & Weston & & 50594 & & EPA160.1 & TDS & 94LSSA85 & 94LSSA85 \\
\hline 100461 & D431-28-01 & Weston & Lab Replicate & 50594 & & EPA160.1 & TDS & 94LSSA85 & 94LSSA85 \\
\hline 100461 & D431-28-01 & Weston & & 50594 & & EPA160.2 & TSS & 94LSSA85 & 94LSSA85 \\
\hline 100461 & D431-28-01 & Weston & Lab Replicate & 50594 & & EPA160.2 & TSS & 94LSSA85 & 94LSSA85 \\
\hline 100461 & D431-28-01 & Weston & & 50594 & & EPA300 & CL/F/SO4 & 94LCC066 & 94LCC066 \\
\hline 100461 & D431-28-01 & Weston & & 50594 & & EPA300 & CL/F/SO4 & 94LFC066 & 94LFC066 \\
\hline 100461 & D431-28-01 & Weston & & 50594 & & EPA300 & CL/F/SO4 & 94LSC066 & 94LSC066 \\
\hline 100461 & D431-28-01 & Weston & & 50594 & & EPA353.2 & NO3 & 94LN3072 & 94LN3072 \\
\hline 100461 & D431-28.01 & Weston & & 50594 & & EPA415.1 & TOC & 94LTC080 & 94LTC080 \\
\hline 100461 & D431-28-01 & Thermo Analytical & & 50594 & & EPA900.0 & GROSS ALPHA/BETA & 9405022 & 5099402 \\
\hline
\end{tabular}


Table 1

Analytical Program of Surface Water and Sediment Samples

Di-Area Burning/Rubble Pits

Savannah River Site

\begin{tabular}{|c|c|c|c|c|c|c|c|c|c|}
\hline 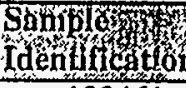 & Shanplex & 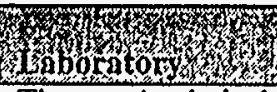 & 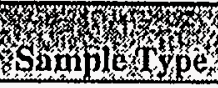 & 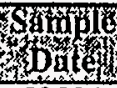 & 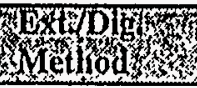 & 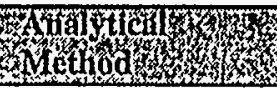 & Wherris & 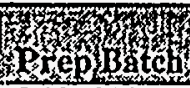 & 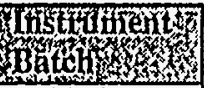 \\
\hline 100461 & D431-28-01 & Thermo Analytical & & 50594 & & EPA900.1 MOD. & TOTAL RAD & 9405022 & 5109402 \\
\hline 100461 & D431-28-01 & Thermo Analytical & & 50594 & & EPA906.0 & TRITIUM & 9405022 & 5099404 \\
\hline 100461 & D431-28-01 & Weston & & 50594 & & SW9020A & TOX & 94LX125X & 94LX125X \\
\hline 100461 & D431-28-01 & Weston & & 50594 & EPA3050 & EPA6010 & METALS & 94L5708 & PSO511F \\
\hline 100461 & D431-28-01 & Weston & & 50594 & EPA3050 & EPA6010 & METALS & 94L5708 & PSO511Z \\
\hline 100461 & D431-28-01 & Weston & & 50594 & EPA3050 & EPA7060 & ARSENIC & 94L5707 & AS051151 \\
\hline 100461 & D431-28-01. & Weston & & 50594 & EPA3050 & EPA7421 & LEAD & 94L5707 & PB051202 \\
\hline 100461 & D431-28-01 & Weston & & 50594 & EPA3050 & EPA7471 & HG & $94 C 0185$ & HG051004 \\
\hline 100461 & D431-28-01 & Weston & & 50594 & EPA3050 & EPA7740 & SELENIUM & 94L5707 & SE051104 \\
\hline 100461 & D431-28-01 & Weston & & 50594 & EPA3510/20 & EPA8270 & SEMTVOLATILES & 94LE0899 & $\mathrm{J} 051101$ \\
\hline 100461 & D431-28-01 & Weston & & 50594 & EPA3510/20 & EPA8270M & SEMIVOLATLES & 94LE0899 & 3051101 \\
\hline 100461 & D431-28-01 & Weston & Spike Sample & 50594 & EPA3540/50 & EPA8270 & SEMTVOLATILES & 94LE0899 & J051101 \\
\hline 100461 & D431-28-01 & Weston & & 50594 & EPA5030 & EPA8260 & VOLATILES & 94LVC057 & C051101 \\
\hline 100461 & D431-28-01 & Weston & Spike Sample & 50594 & EPA5030 & EPA8260 & VOLATILES & 94LVC057 & C051101 \\
\hline 100462 & D431-31-02 & Weston & & 50594 & & ASTMD497.2 & $\mathrm{pH}$ & 94LPH040 & 94LPH040 \\
\hline 100462 & D431-31-02 & Weston & & 50594 & & EPA415.8 & TOC/TOH & 94LTZ23A & 94LTZ23A \\
\hline 100462 & D431-31-02 & Weston & & 50594 & & EPA7471 & HG & $94 C 0187$ & HG051005 \\
\hline 100462 & D431-31-02 & Thermo Analytical & & 50594 & & LANLER210 MOD & TRITIUM & 9405023 & 5109404 \\
\hline 100462; & D431-31-02 & Thermo Analytical & & 50594 & & LANLMLR-100 & GROSS ALPHA/BETA & 9405023 & 5099402 \\
\hline 100462 & $D 431-31-02$ & Thermo Analytical & & 50594 & & SW8469315 MOD & TOTAL RAD & 9405023 & 5109402 \\
\hline 100462 & D431-31-02 & Weston & & 50594 & EPA3050 & EPA6010 & METALS & 94L5706 & PS0511A \\
\hline 100462 & D431-31-02 & Weston & & 50594 & EPA3050 & EPA6010 & METALS & 94L5706 & PS0511B \\
\hline 100462 & D431-31-02 & Weston & & 50594 & EPA3050 & EPA6010 & METALS & 94L5706 & PS0511E \\
\hline 100462 & D431-31-02 & Weston & & 50594 & EPA3050 & EPA6010 & METALS & 94L5706 & PS0511X \\
\hline 100462 & D431-31-02 & Weston & & 50594 & EPA3050 & EPA6010 & METALS & 94L5706 & PS0511Y \\
\hline 100462 & D431-31-02 & Weston & & 50594 & EPA3050 & EPA7060 & ARSENIC & 94L5705 & AS051051 \\
\hline 100462 & D431-31-02 & Weston & & 50594 & EPA3050 & EPA7421 & LEAD & 94L5705 & PB051153 \\
\hline 100462 & D431-31-02 & Weston & & 50594 & EPA3050 & EPA7740 & SELENIUM & 94L5705 & SE051161 \\
\hline 100462 & D431-31-02 & Weston & & 50594 & EPA3540/50 & EPA8270 & SEMIVOLATLLES & 94LE0897 & $S 051001$ \\
\hline 100462 & D431-31-02 & Weston & Spike Sample & 50594 & EPA3540/50 & EPA8270 & SEMIVOLATILES & 94LE0897 & S051001 \\
\hline 100462 & D431-31-02 & Weston & & 50594 & EPA3540/50 & EPA8270M & SEMTVOLATLESS & 94LE0897 & S051001 \\
\hline 100462 & D431-31-02 & Weston & & 50594 & EPA5030 & EPA8260 & VOLATILES & 94LVC056 & C051001 \\
\hline 100462 & D431-31-02 & Weston & Spike Sample & 50594 & EPA5030 & EPA8260 & VOLATILES & 94LVC056 & C051001 \\
\hline 100463 & D431-31:02A & Weston & & 50594 & & ASTMD497.2 & $\mathrm{pH}$ & 94LPH040 & 94LPH040 \\
\hline 100463 & D431-31-02A & Weston & & 50594 & & EPA415.8 & TOC/TOH & 94LTZ23A & 94LTZ23A \\
\hline 100463 & D431-31-02A & Weston & & 50594 & & EPA7471 & HG & $94 \mathrm{C} 0187$ & HG051005 \\
\hline
\end{tabular}

.. 
Taure 1

- Analytical Program of Surface Water and Sediment Samples

D-Area Buming/Rubble Pits Savannah River Site

\begin{tabular}{|c|c|c|c|c|c|c|c|c|c|}
\hline \multicolumn{10}{|c|}{ 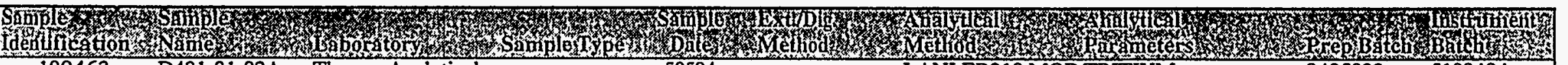 } \\
\hline 100463 & D431-31-02A & Thermo Analytical & & 50594 & & LANLER210 MOD & TRITIUM & 9405023 & 5109404 \\
\hline 100463 & D431-31-02A & Thermo Analytical & & 50594 & & LANLMLR-100 & GROSS ALPHA/BETA & 9405023 & 5099402 \\
\hline 100463 & D431-31-02A & Thermo Analytical & & 50594 & & SW8469315 MOD & TOTAL RAD & 9405023 & 5109402 \\
\hline 100463 & D431-31-02A & Weston & & 50594 & EPA3050 & EPA6010 & METALS & 94L5706 & PSOS11A \\
\hline 100463 & D431-31-02A & Weston & & 50594 & EPA3050 & EPA6010 & METALS & 94L5706 & PSO511B : \\
\hline $100463:$ & D431-31-02A & Weston & & 50594 & EPA3050 & EPA6010 & METALS & $94 L 5706$ & PSOS11E \\
\hline 100463 & D431-31-02A & Weston & & 50594 & EPA3050 & EPA6010 & METALS & $94 L 5706$ & PSOS11X \\
\hline 100463 & D431-31-02A & Weston & & 50594 & EPA3050 & EPA6010 & METALS & $94 L 5706$ & PSOS11Y \\
\hline $100463^{\circ}$ & D431-31-02A & Weston & & 50594 & EPA3050 & EPA7060 & ARSENIC & 94L5705 & AS051051 \\
\hline 100463 & D431-31-02A & Weston & & 50594 & EPA3050 & EPA7421 & LEAD & $94 L 5705$ & PB051153 \\
\hline 100463 & D431-31-02A & Weston & & 50594 & EPA3050 & EPA7740 & SELENTUM & 94L5705 & SE051161 \\
\hline 100463 & D431-31-02A & Weston & & 50594 & EPA3540/50 & EPA 8270 & SEMIVOLATILES & 94LE0897 & S051001 \\
\hline 100463 & D431-31-02A & Weston & Spike Sample & 50594 & EPA3540/50 & EPA8270 & SEMIVOLATILES & 94LE0897 & S051001 \\
\hline 100463 & D431-31-02A & Weston & & 50594 & EPA3540/50 & EPA8270M & SEMIVOLATILES & 94LE0897 & S051001 \\
\hline 100463 & D431-31-02A & Weston & & 50594 & EPA5030 & EPA8260 & VOLATIIES & $94 \mathrm{LVC} 056$ & $\mathrm{C} 051001$ \\
\hline 100463 & D431-31-02A & Weston & Spike Sample & 50594 & EPA5030 & EPA8260 & VOLATILES & 94LVC056 & C051001 \\
\hline 100464 & D431-30-02 & Weston & & 50594 & & ASTMD497.2 & $\mathrm{pH}$ & 94LPH040 & 94LPH0 40 \\
\hline 100464 & D431-30-02 & Weston & Lab Replicate & 50594 & & ASTMD497.2 & $\mathrm{pH}$ & 94LPH040 & 94LPH040 \\
\hline 100464 & D431-30-02 & Weston & & 50594 & & EPA415.8 & TOCTOH & 94LTZ23A & 94LTZ23A \\
\hline 100464 & D431-30-02 & Weston & & 50594 & & EPA7471 & HG & $94 C 0187$ & HG051005 \\
\hline 100464 & D431-30-02 & Thermo Analytical & & 50594 & & LANLER210 MOD & TRITTUM & 9405023 & 5109404 \\
\hline 100464 & $D 431-30-02$ & Thermo Analytical & & 50594 & & LANLMLR-100 & GROSS ALPHA/BETA & 9405023 & 5099402 \\
\hline 100464 & D431-30-02 & Thermo Analytical & & 50594 & & SW8469315 MOD & TOTAL RAD & 9405023 & 5109402 \\
\hline 100464 & D431-30-02 & Weston & & 50594 & EPA3050 & EPA6010 & METALS & 94L5706 & PSO511A \\
\hline 100464 & D $431-30-02$ & Weston & & 50594 & EPA3050 & EPA6010 & METALS & 94L5706 & PS0511B \\
\hline 100464 & $D 431-30-02$ & Weston & & 50594 & EPA3050 & EPA6010 & METALS & 94L5706 & PS0511E \\
\hline 100464 & $D 431-30-02$ & Weston & & 50594 & EPA3050 & EPA6010 & METALS & 94L5706 & PS0511X \\
\hline 100464 & D431-30-02 & Weston & & 50594 & EPA3050 & EPA6010 & METALS & 94L5706 & PSOS11Y \\
\hline 100464 & D431-30-02 & Weston & & 50594 & EPA3050 & EPA7060 & ARSENIC & $94 L 5705$ & AS051051 \\
\hline 100464 & D431-30-02 & Weston & & 50594 & EPA3050 & EPA7421 & LEAD & $94 L 5705$ & PB051153 \\
\hline 100464 & D431-30-02 & Weston & & 50594 & EPA3050 & EPA7740 & SELENIUM & 94L5705 & SE051161 \\
\hline 100464 & D $431-30-02$ & Weston & & 50594 & EPA3540/50 & EPA 8270 & SEMIVOLATILES & 94LE0897 & S051101 \\
\hline 100464 & D431-30-02 & Weston & Spike Sample & 50594 & EPA3540/50 & EPA8270 & SEMIVOLATILES & 94LE0897 & S051101 \\
\hline 100464 & D431-30-02 & Weston & & 50594 & EPA3540/50 & EPA8270M & SEMIVOLATILES & 94LE0897 & S051101 \\
\hline 100464 & D431-30-02 & Weston & & 50594 & EPA5030 & EPA8260 & VOLATILES & 94LVC056 & $\mathrm{C} 051001$ \\
\hline 100464 & $D 431-30-02$ & Weston & Spike Sample & 50594 & EPA5030 & EPA8260 & VOLATILES & $94 \mathrm{LVC} 056$ & $\mathrm{C} 051001$ \\
\hline
\end{tabular}


Table 1

Analytical Program of Surface Water and Sediment Samples

D-Area Burning/Rubble Pits

Savannah River Site

\begin{tabular}{|c|c|c|c|c|c|c|c|c|c|}
\hline S & 等 & 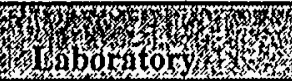 & S & Sying & Mend & 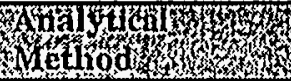 & OHA & 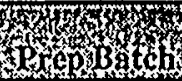 & B o \\
\hline 100465 & D431-29-02 & Weston & & 50594 & & ASTMD 497.2 & $\mathrm{pH}$ & 94LPHO40 & 94LPHO40 \\
\hline 100465 & D431-29-02 & Weston & & 50594 & & EPA415.8 & TOC/TOH & 94LTZ23A & 94LTZ23A \\
\hline 100465 & D431-29-02 & Weston & & 50594 & & EPA7471 & HG & $94 C 0187$ & HG051005 \\
\hline 100465 & D431-29-02 & Thermo Analytical & & 50594 & & LANLER210 MOD & TRITIUM & 9405023 & 5109404 \\
\hline 100465 & D431-29-02 & Thermo Analytical & & 50594 & & LANLMRR-100 & GROSS ALPHA/BETA & 9405023 & 5099402 \\
\hline 100465 & D431-29-02 & Thermo Analytical & & 50594 & & SW8469315 MOD & TOTAL RAD & 9405023 & 5109402 \\
\hline 100465 & D431-29-02 & Weston & & 50594 & EPA3050 & EPA6010 & METALS & $94 L 5706$ & PS0511A \\
\hline 100465 & D431-29-02 & Weston & & 50594 & EPA3050 & EPA6010 & METALS & $94 \mathrm{~L} 5706$ & PS0511B \\
\hline 100465 & D431-29-02 & Weston & & 50594 & EPA3050 & EPA6010 & METALS & $94 L 5706$ & PS0511E \\
\hline 100465 & D431-29-02 & Weston & & 50594 & EPA 3050 & EPA6010 & METALS & 94L5706 & PS0511X \\
\hline 100465 & D431-29-02 & Weston & & 50594 & EPA3050 & EPA6010 & METALS & 94L5706 & PS0511Y \\
\hline 100465 & D431-29-02 & Weston & & 50594 & EPA3050 & EPA7060 & ARSENIC & 94L5705 & AS051051 \\
\hline 100465 & D431-29-02 & Weston & & 50594 & EPA3050 & EPA7421 & LEAD & $94 \mathrm{~L} 5705$ & PB051153 \\
\hline 100465 & D431-29-02 & Weston & & 50594 & EPA3050 & EPA7740 & SELENIUM & 94L5705 & SE051161 \\
\hline 100465 & D431-29-02 & Weston & & 50594 & EPA3540/50 & EPA8270 & SEMTVOLATILES & 94LE0897 & S051101 \\
\hline 100465 & D431-29-02 & Weston & Spike Sample. & 50594 & EPA3540/50 & EPA8270 & SEMIVOLATILES & 94LE0897 & S051101 \\
\hline 100465 & D431-29-02 & Weston & & 50594 & EPA3540/50 & EPA8270M & SEMIVOLATULES & 94LE0897 & S051101 \\
\hline 100465 & D431-29-02 & Weston & & 50594 & EPA5030 & EPA 8260 & VOLATUES & 94LVC056 & $\cos 1001$ \\
\hline 100465 & D431-29-02 & Weston & Spike Sample & 50594 & EPA5030 & EPA8260 & VOLATILES & 94LVC056 & C051001 \\
\hline 100466 & D431-28-02 & Weston & & 50594 & & ASTMD497.2 & $\mathrm{pH}$ & 94LPH040 & 94LPH040 \\
\hline 100466 & $D 431-28-02$ & Weston & & 50594 & & EPA415.8 & тос/TOH & 94LTZ23A & 94LTZ23A \\
\hline 100466 & D431-28-02 & Weston & & 50594 & & EPA7471 & HG & $94 C 0187$ & HG051005 \\
\hline 100466 & D431-28-02 & Thermo Analytical & & 50594 & & LANLER210 MOD & TRITIUM & 9405023 & 5109404 \\
\hline 100466 & D431-28-02 & Thermo Analytical & & 50594 & & LANLMLR-100 & GROSS ALPHA/BETA & 9405023 & 5099402 \\
\hline 100466 & D431-28-02 & Thermo Analytical & & 50594 & & SW8469315 MOD & TOTAL RAD & 9405023 & 5109402 \\
\hline 100466 & D431-28-02 & Weston & & 50594 & EPA3050 & EPA6010 & METALS & 94L5706 & PS0511A \\
\hline 100466 & D431-28-02 & Weston & & 50594 & EPA3050 & EPA6010 & METALS & 94L5706 & PS0511B \\
\hline 100466 & D431-28-02 & Weston & & 50594 & EPA3050 & EPA6010 & METALS & 94L5706 & PS0511E \\
\hline 100466 & D431-28-02 & Weston & & 50594 & EPA3050 & EPA6010 & METALS & 94L5706 & PS0511X \\
\hline 100466 & D431-28-02 & Weston & & 50594 & EPA3050 & EPA6010 & METALS & 94L5706 & PSO511Y \\
\hline 100466 & D431-28-02 & Weston & & 50594 & EPA3050 & EPA7060 & ARSENIC & 94L5705 & AS051051 \\
\hline 100466 & D431-28-02 & Weston & & 50594 & EPA3050 & EPA7421 & LEAD & 94L5705 & PB051153 \\
\hline 100466 & D431-28-02 & Weston & & 50594 . & EPA3050 & EPA7740 & SELENIUM & 94L5705 & SE051161 \\
\hline 100466 & D431-28-02 & Weston & & 50594 & EPA3540/50 & EPA8270 & SEMTVOLATILES & 94LE0897 & S051101 \\
\hline 100466 & D431-28-02 & Weston & Spike Sample & 50594 & EPA3540/50 & EPA8270 & SEMTVOLATILES & 94LE0897 & S051101 \\
\hline 100466 & $D 431-28-02$ & Weston & & 50594 & EPA3540/50 & EPA8270M & SEMTVOLATILES & 94LE0897 & S051101 \\
\hline
\end{tabular}


Tabie 1

Analytical Program of Surface Water and Sediment Samples

D-Area Buming/Rubble Pits Savannah River Site

\begin{tabular}{|c|c|c|c|c|c|c|c|c|c|}
\hline \multicolumn{10}{|c|}{ 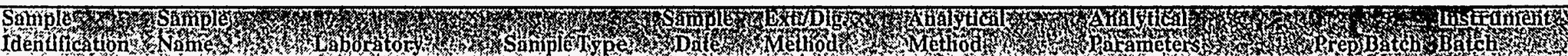 } \\
\hline 100466 & D431-28-02 & Weston & & 50594 & EPA5030 & EPA8260 & VOLATILES & $94 \mathrm{LVC056}$ & $\mathrm{C} 051001$ \\
\hline 100466 & D431-28-02 & Weston & Lab Replicate & 50594 & EPA5030 & EPA8260 & VOLATILES & 94LVC056 & $\cos 1001$ \\
\hline 100466 & D431-28-02 & Weston & Spike Sample & 50594 & EPA5030 & EPA 8260 & VOLATILES & $94 L V C 056$ & $\cos 1001$ \\
\hline 100467 & DBRP-TB-01 & General Engineering & 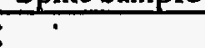 & 50694 & & EPA8260 & VOLATILES & 47729 & 47729 \\
\hline 100468 & TRIP BLANK & Weston & \multirow[b]{2}{*}{ Spike Sample } & 50694 & EPA5030 & EPA8260 & VOLATILES & $94 \mathrm{LVCOS7}$ & $\cos 1101:$ \\
\hline 100468 & TRIP BLANK & Weston & & 50694 & EPA5030 & EPA 8260 & VOLATILES & $94 L V C 057$ & $\mathrm{C} 051101$ \\
\hline 100469 & TRIP BLANK & Weston & \multirow[b]{2}{*}{ Spike Sample } & 50694 & EPA5030 & EPA8260 & VOLATILES & 94LVC057 & C051101 \\
\hline 100469 & TRIP BLANK & Weston & & 50694 & EPA5030 & EPA 8260 & VOLATILES & $94 L V C 057$ & $\cos 1101$ \\
\hline 100470 & TRIP BLANK & Weston & \multirow[b]{2}{*}{ Spike Sample } & 50694 & EPA5030 & EPA8260 & VOLATILES & 94LVC057 & C051101 \\
\hline 100470 & TRIP BLANK & Weston & & 50694 & EPA5030 & EPA 8260 & VOLATILES & 94LVC057 & $\cos 1101$ \\
\hline 100471. & TRIP BLANK & Weston & \multirow[b]{2}{*}{ Spike Sample } & 50694 & EPA5030 & EPA8260 & VOLATILES & 94LVC058 & $\cos 1124$ \\
\hline 100471 & TRIP BLANK & Weston & & 50694 & EPA5030 & EPA 8260 & VOLATILES & 94LVC058 & $\cos 1124$ \\
\hline 100472 & TRIP BLANK & Weston & \multirow{3}{*}{$\begin{array}{l}\text { Lab Replicate } \\
\text { Spike Sample }\end{array}$} & 50694 & EPA5030 & EPA8260 & . VOLATLLES & 94LVC058 & C051124 \\
\hline 100472 & TRIP BLANK & Weston & & 50694 & EPA5030 & EPA 8260 & VOLATILES & 94LVCि 058 & $\cos 1124$ \\
\hline 100472 & TRIP BLANK & Weston & & 50694 & EPA5030 & EPA 8260 & VOLATILES & 94LVC058 & $\cos 1124$ \\
\hline
\end{tabular}

$\cdot$

$\therefore$ 
Table 2

Analytical Program of Laboratory Quality Control Samples D-Area Burning/Rubble Pits Savannah River Site

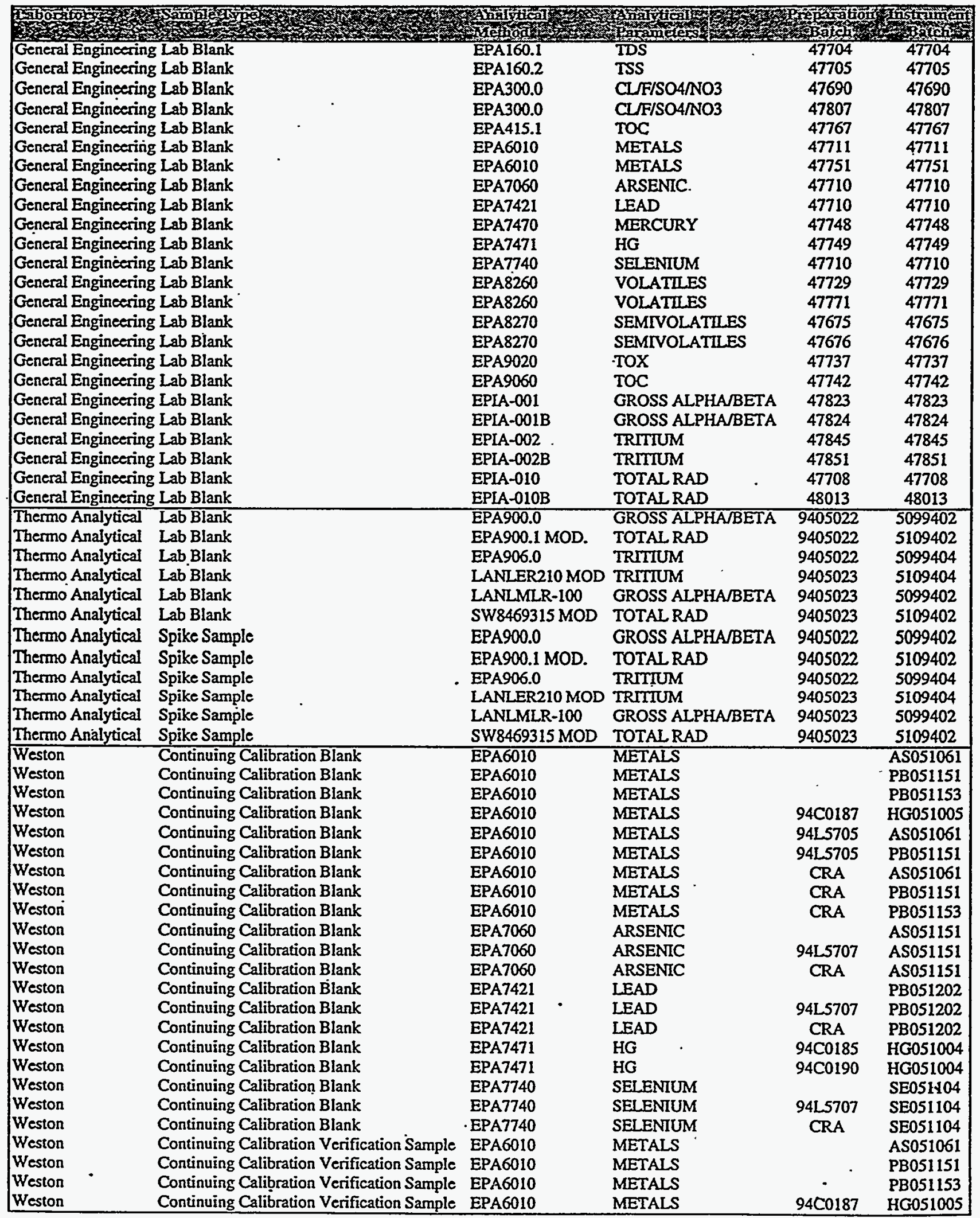


Table 2

Analytical Program of Laboratory Quality Control Samples

D-Area Buming/Rubble Pits

Savannah River Site

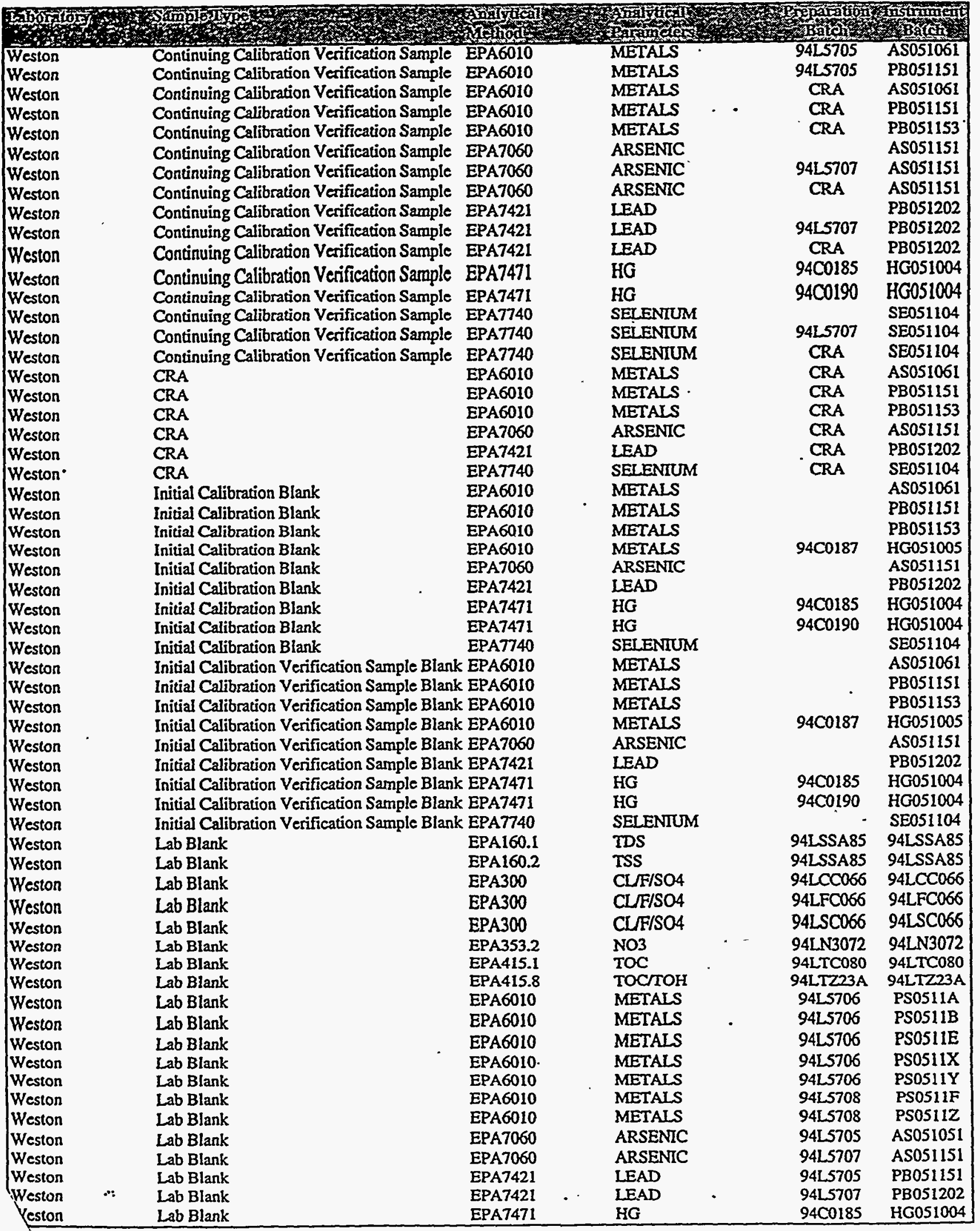

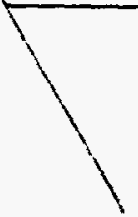


Table 2

Analytical Program of Laboratory Quality Control Samples D-Area Burning/Rubble Pits

Savannah River Site

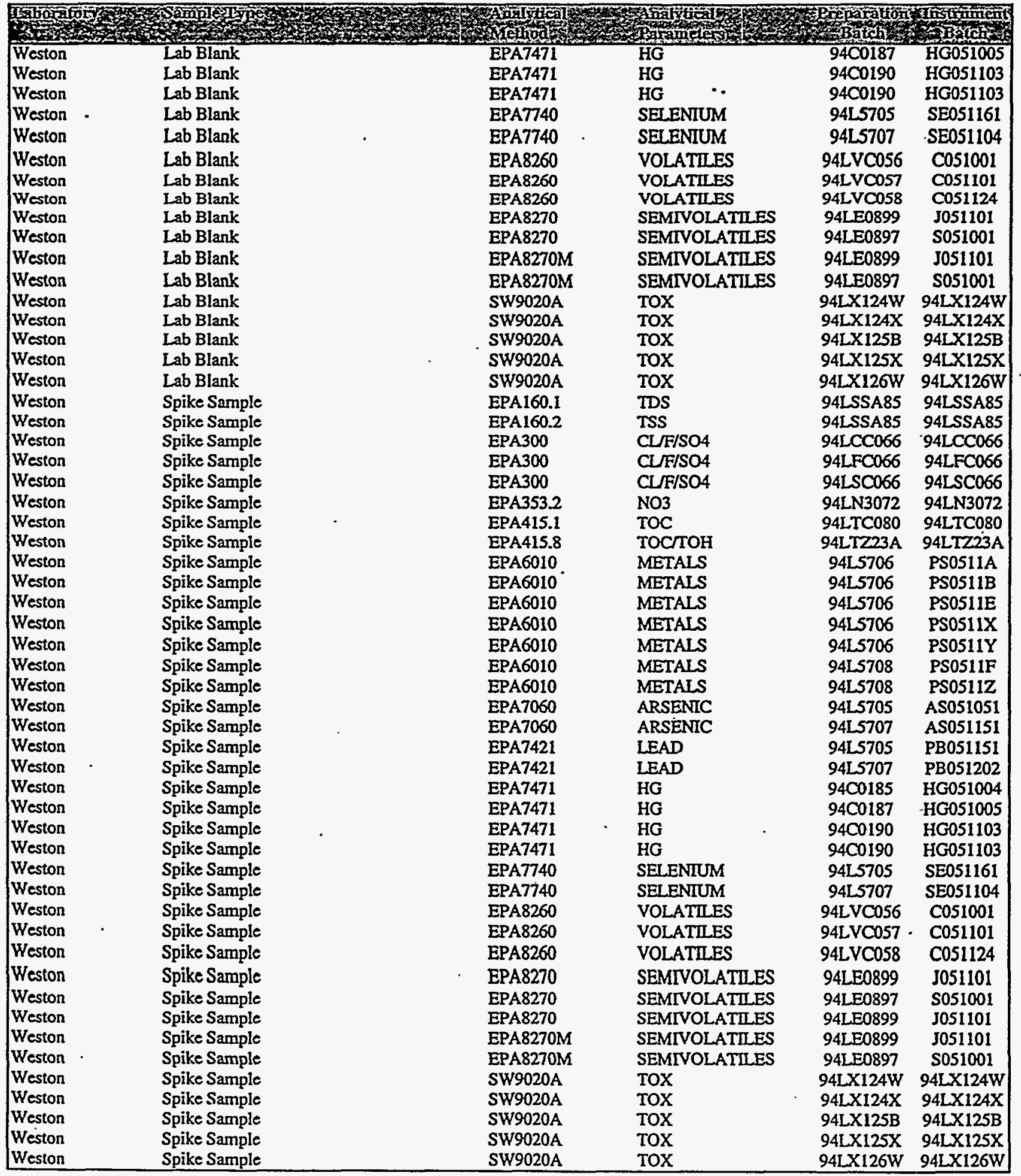


Table 3

Association of Primary and Quality Control Samples

D-Area Burning/Rubble Pits

Savannah River Site

\begin{tabular}{|c|c|c|c|c|c|c|c|c|c|c|c|c|c|c|}
\hline & & \multicolumn{13}{|c|}{ Quality Control Samples } \\
\hline & Sample Name $>$ & 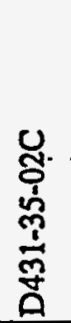 & 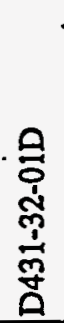 & 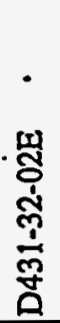 & 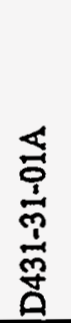 & $\frac{\frac{0}{9}}{\frac{9}{2}}$ & 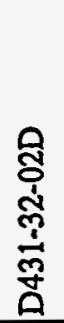 & 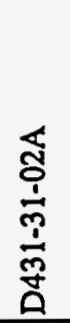 & 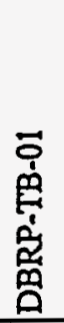 & 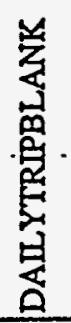 & 悹 & 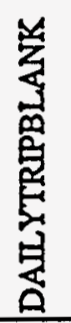 & 萝 & 岕 \\
\hline & Sample Type $>$ & 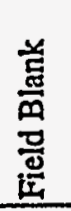 & 总 & 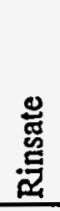 & 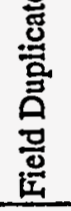 & 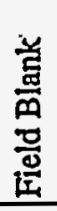 & 蒙 & 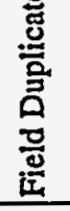 & 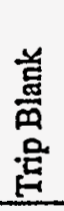 & $\begin{array}{l}\text { 卷 } \\
\text { 品 } \\
\text { 吕 } \\
\end{array}$ & 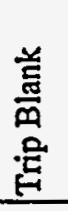 & 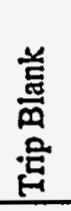 & 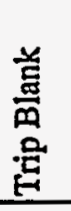 & 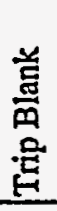 \\
\hline & Sample Identification $>$ & 导 & $\begin{array}{l}\overrightarrow{5} \\
\stackrel{్}{\circ}\end{array}$ & テี & $\begin{array}{l}\text { 年 } \\
\stackrel{8}{8}\end{array}$ & $\begin{array}{l}8 \\
\text { \&े } \\
\end{array}$ & $\begin{array}{l}\stackrel{8}{8} \\
8 \\
\end{array}$ & $\begin{array}{l}\tilde{\delta} \\
\delta \\
\delta\end{array}$ & 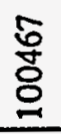 & $\begin{array}{l}\infty \\
\stackrel{\circ}{\circ} \\
\stackrel{0}{0} \\
\end{array}$ & 웅 & 尽 & 突 & 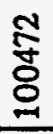 \\
\hline Sample ID & Sample Name & & & & & & & & & & & & & \\
\hline 100438 & D431-37-01 & & & & & & & & & & & & * & \\
\hline 100439 & $\overline{D 431-37-02}$ & & & & & & & & & & & * & & \\
\hline 100440 & D431-38-01 & & & & & & & & & * & & & & \\
\hline 100441 & D431-36-01 & & & & & & & & & & & * & & \\
\hline 100442 & D431-36-02 & & & & & & & & & & * & & & \\
\hline 100443 & D431-39-01 & & & & & & & & & * & & & & \\
\hline 100444 & D431-35-01 & * & & & & & & & & & & & & \\
\hline 100445 & D431-35-02C & & & & & & & & & & & & & \\
\hline 100446 & D431-35-02 & * & & & & & & & & & & & & \\
\hline 100447 & D431-34-01 & & & & & & & & & * & & & & \\
\hline 100448 & D431-33-01 & & & & & & & & & & & & & * \\
\hline 100449 & D431-34-02 & & & & & & & & & * & & & & \\
\hline 100450 & D431-32-01 & & * & & & & & & & & & & & \\
\hline 100451 & D431-32-01D & & & & & & & & * & & & & 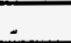 & \\
\hline 100452 & D431-32-02E & & & & & & & & & * & & & & \\
\hline 100453 & $\overline{D 431-33-02}$ & & & & & & & & & & * & & & \\
\hline 100454 & D431-31-01 & & & & * & * & & & & & & * & & \\
\hline 100455 & D431-31-01A & & & & & & & & & & & & & * \\
\hline 100456 & D431-31-01C & & & & & & & & & & & & & * \\
\hline 100457 & D431-30-01 & & & & & & & & & & & & * & \\
\hline 100458 & D431-32-02 & & & $*$ & & & * & & & & & & & \\
\hline 100459 & D431-32-02D & & & & & & & & * & & & & & \\
\hline 100460 & D431-29-01 & & & & & & & & & & & & & * \\
\hline 100461 & D431-28-01 & & & & & & & & & & & & * & \\
\hline 100462 & D431-31-02 & & & & & * & & * & & & * & & & \\
\hline 100463 & D43L-31-02A & & & & & & & & & & * & & & \\
\hline 100464 & $\overline{D 431-30-02}$ & & & & & & & & & & * & & & \\
\hline 100465 & D431-29-02 & & & & & & & & & & * & & & \\
\hline 100466 & D431-28-02 & & & & & & & & & & * & & & \\
\hline 100467 & DBRP-TB-01 & & & & & & & & & & & & & \\
\hline 100468 & DAIIYTRIPBLANK & & & & & & & & & & & & & \\
\hline 100469 & DAII YTRIPBLANK & & & & & & & & & & & & & \\
\hline 100470 & DAIIYTRIPBLANK & & & & & & & & & & & & & \\
\hline 100471 & DAIYYTRPBLANK & & & & & & & & & & & & & \\
\hline 100472 & DAILYTRIPBLANK & & & & & & & & & & & & & \\
\hline
\end{tabular}


Table 4

Holding Times

D-Area Buming/Rubble Pits

Savannah River Site

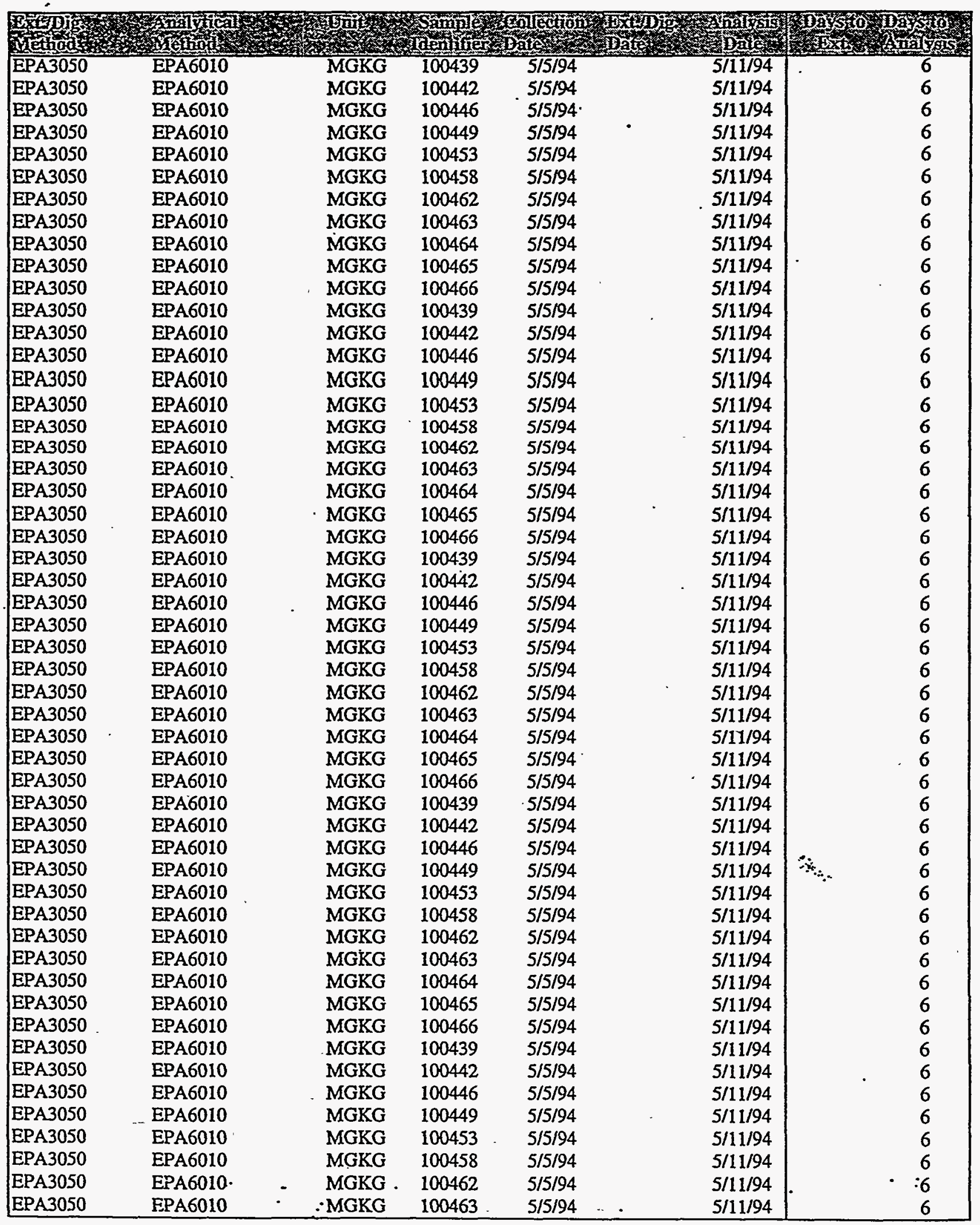




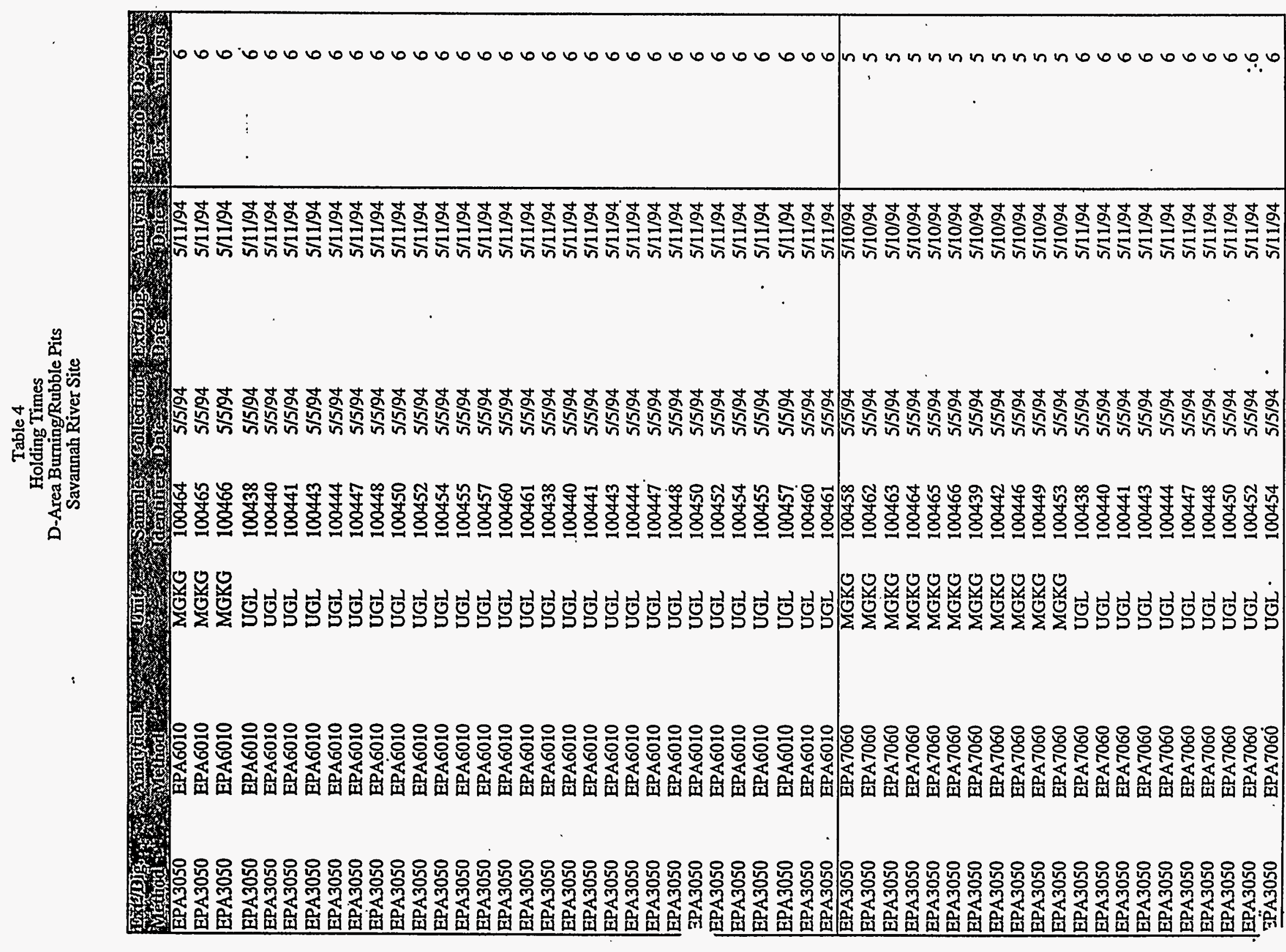


Table 4

Holding Times

D-Area Burning/Rubble Pits

Savannah River Site

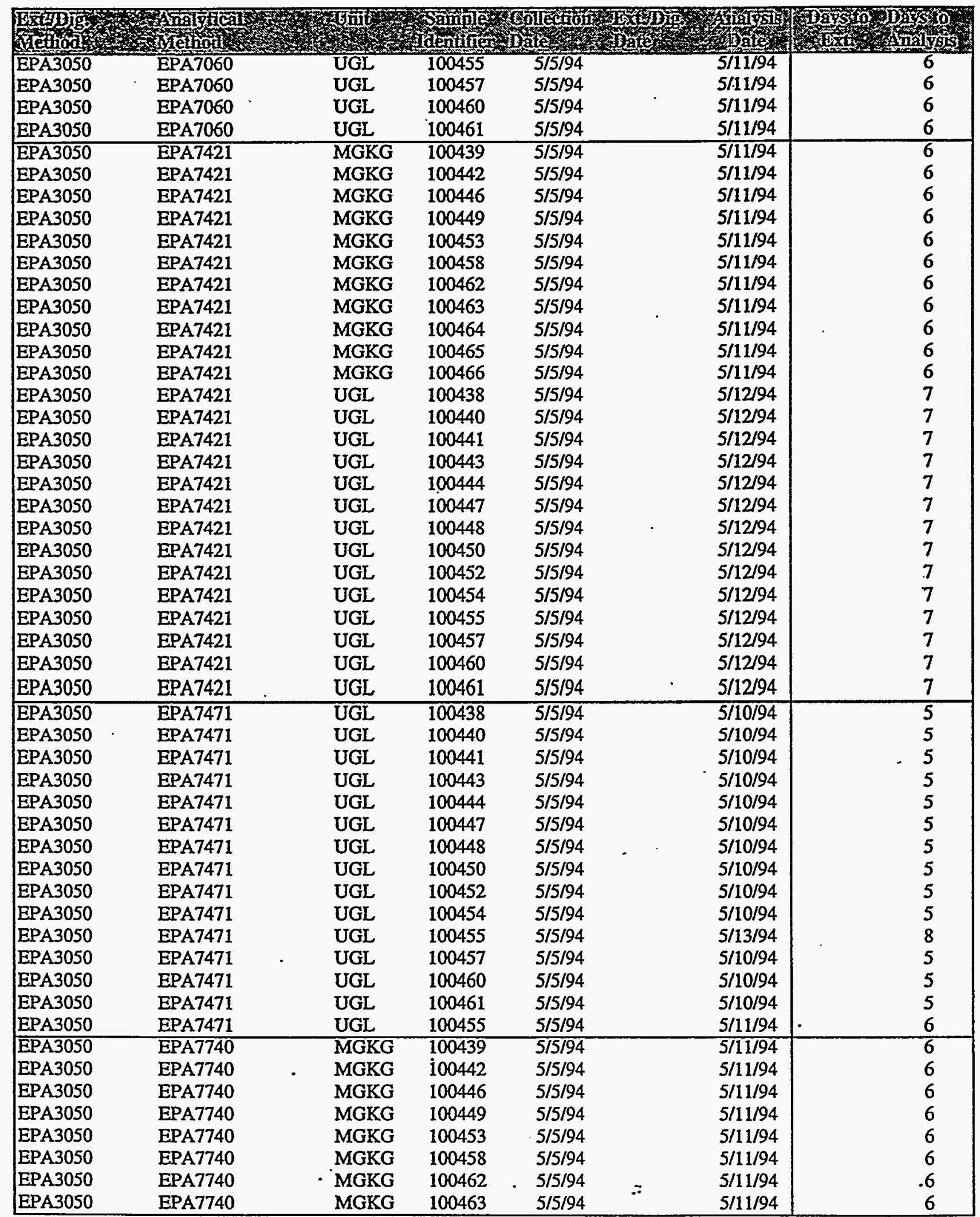


Table 4

Holding Times

D-Area Buming/Rubble Pits

Savannah River Site

\begin{tabular}{|c|c|c|c|c|c|c|c|c|c|}
\hline \multirow[t]{2}{*}{ 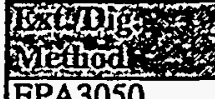 } & 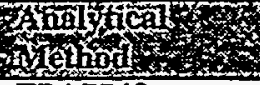 & mont & \multicolumn{2}{|c|}{ 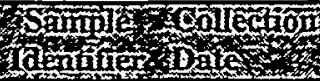 } & \multicolumn{2}{|c|}{ 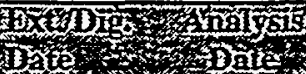 } & \multicolumn{3}{|c|}{ 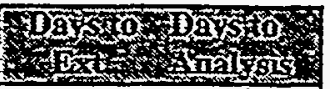 } \\
\hline & EPA7740 & MGKG & 100464 & $5 / 5 / 94$ & & $5 / 11 / 94$ & & & 6 \\
\hline EPA3050 & EPA7740 & MGKG & 100465 & $5 / 5 / 94$ & & $5 / 11 / 94$ & & & 6 \\
\hline EPA3050 & EPA7740 & MGKG & 100466 & $5 / 5 / 94$ & & $5 / 11 / 94$ & & & 6 \\
\hline EPA3050 & EPA7740 & UGL & 100438 & $5 / 5 / 94$ & & $5 / 11 / 94$ & & & 6 \\
\hline EPA3050 & EPA7740 & UGL & 100440 & $5 / 5 / 94$ & & $5 / 11 / 94$ & & & 6 \\
\hline EPA3050 & EPA7740 & UGL & 100441 & $5 / 5 / 94$ & & $5 / 11 / 94$ & & & 6 \\
\hline EPA3050 & EPA7740 & UGL & 100443 & $5 / 5 / 94$ & & $5 / 11 / 94$ & & & 6 \\
\hline EPA3050 & EPA7740 & UGL & 100444 & $5 / 5 / 94$ & & $5 / 11 / 94$ & & & 6 \\
\hline EPA3050 & EPA7740 & UGL & 100447 & $5 / 5 / 94$ & & $5 / 11 / 94$ & & & 6 \\
\hline EPA3050 & EPA7740 & UGL & 100448 & $5 / 5 / 94$ & & $5 / 11 / 94$ & & & 6 \\
\hline EPA 3050 & EPA7740 & UGL & 100450 & $5 / 5 / 94$ & & $5 / 11 / 94$ & & & 6 \\
\hline EPA3050 & EPA7740 & UGL & 100452 & $5 / 5 / 94$ & & $5 / 11 / 94$ & & & 6 \\
\hline EPA3050 & EPA7740 & UGL & 100454 & $5 / 5 / 94$ & & $5 / 11 / 94$ & & & 6 \\
\hline EPA3050 & EPA7740 & UGL & 100455 & $5 / 5 / 94$ & & $5 / 11 / 94$ & & & 6 \\
\hline EPA3050 & EPA7740 & UGL & 100457 & $5 / 5 / 94$ & & $5 / 11 / 94$ & & & 6 \\
\hline EPA3050 & EPA7740 & UGL & 100460 & $5 / 5 / 94$ & & $5 / 11 / 94$ & & & 6 \\
\hline EPA3050 & EPA7740 & UGL & 100461 & $5 / 5 / 94$ & & $5 / 11 / 94$ & & & 6 \\
\hline EPA3510/20 & EPA8270 & UGL & 100443 & $5 / 5 / 94$ & $5 / 9 / 94$ & $5 / 10 / 94$ & 4 & & $\bar{I}$ \\
\hline EPA3510/20 & EPA8270 & UGL & 100444 & $5 / 5 / 94$ & $5 / 9 / 94$ & $5 / 10 / 94$ & 4 & & 1 \\
\hline EPA3510/20 & EPA8270 & UGL & 100447 & $5 / 5 / 94$ & $5 / 9 / 94$ & $5 / 10 / 94$ & 4 & & 1 \\
\hline EPA3510/20 & EPA8270 & UGL & 100448 & $5 / 5 / 94$ & $5 / 9 / 94$ & $5 / 10 / 94$ & 4 & & 1 \\
\hline EPA3510/20 & EPA8270 & UGL & 100450 & $5 / 5 / 94$ & $5 / 9 / 94$ & $5 / 10 / 94$ & 4 & & 1 \\
\hline EPA3510/20 & EPA8270 & UGL & 100452 & $5 / 5 / 94$ & $5 / 9 / 94$ & $5 / 10 / 94$ & 4 & & 1 \\
\hline EPA3510/20 & EPA8270 & UGL & 100438 & $5 / 5 / 94$ & $5 / 9 / 94$ & $5 / 10 / 94$ & -4 & & 1 \\
\hline EPA3510/20 & EPA8270 & UGL & 100440 & $5 / 5 / 94$ & $5 / 9 / 94$ & $5 / 10 / 94$ & 4 & & 1 \\
\hline EPA3510/20 & EPA8270 & UGL & 100441 & $5 / 5 / 94$ & $5 / 9 / 94$ & $5 / 10 / 94$ & 4 & & 1 \\
\hline $\mathrm{EPA} 3510 / 20$ & EPA8270 & UGL & 100454 & $5 / 5 / 94$ & $5 / 9 / 94$ & $5 / 1.1 / 94$ & 4 & & 2 \\
\hline EPA3510/20 & EPA8270 & UGL & 100455 & $5 / 5 / 94$ & $5 / 9 / 94$ & $5 / 11 / 94$ & 4 & & 2 \\
\hline EPA3510/20 & EPA8270 & UGL & 100457 & $5 / 5 / 94$ & $5 / 9 / 94$ & $5 / 11 / 94$ & 4 & & 2 \\
\hline EPA3510/20 & EPA8270 & UGL & 100460 & $5 / 5 / 94$ & $5 / 9 / 94$ & $5 / 11 / 94$ & 4 & & 2 \\
\hline EPA3510/20 & EPA8270 & UGL & 100461 & $5 / 5 / 94$ & $5 / 9 / 94$ & $5 / 11 / 94$ & 4 & & 2 \\
\hline EPA3510/20 & EPA8270M & UGL & 100443 & $5 / 5 / 94$ & $5 / 9 / 94$ & $5 / 10 / 94$ & 4 & & 1 \\
\hline $\mathrm{EPA} 3510 / 20$ & EPA8270M & UGL & 100444 & $5 / 5 / 94$ & $5 / 9 / 94$ & $5 / 10 / 94$ & 4 & & 1 \\
\hline EPA3510/20 & EPA8270M & UGL & 100447 & $5 / 5 / 94$ & $5 / 9 / 94$ & $5 / 10 / 94$ & 4 & & 1 \\
\hline EPA3510/20 & EPA8270M & UGL & 100448 & $5 / 5 / 94$ & $5 / 9 / 94$ & $5 / 10 / 94$ & 4 & & 1 \\
\hline EPA3510/20 & EPA8270M & UGL & 100450 & $5 / 5 / 94$ & $5 / 9 / 94$ & $5 / 10 / 94$ & 4 & & 1 \\
\hline EPA3510/20 & EPA8270M & UGL & 100452 & $5 / 5 / 94$ & $5 / 9 / 94$ & $5 / 10 / 94$ & 4 & & 1 \\
\hline EPA3510/20 & EPA8270M & UGL & 100438 & $5 / 5 / 94$ & $5 / 9 / 94$ & $5 / 10 / 94$ & 4 & & 1 \\
\hline EPA3510/20 & EPA8270M & UGL & 100440 & $5 / 5 / 94$ & $5 / 9 / 94$ & $5 / 10 / 94$ & 4 & & 1 \\
\hline EPA3510/20 & EPA8270M & UGL & 100441 & $5 / 5 / 94$ & $5 / 9 / 94$ & $5 / 10 / 94$ & 4 & & 1 \\
\hline EPA3510/20 & EPA8270M & UGL & 100454 & $5 / 5 / 94$ & $5 / 9 / 94$ & $5 / 11 / 94$ & 4 & & 2 \\
\hline EPA3510/20 & EPA8270M & UGL & 100455 & $5 / 5 / 94$ & $5 / 9 / 94$ & $5 / 11 / 94$ & 4 & & 2 \\
\hline EPA.3510/20 & EPA8270M & UGL & 100457 & $5 / 5 / 94$ & $5 / 9 / 94$ & $5 / 11 / 94$ & 4 & & 2 \\
\hline EPA3510/20 & EPA8270M & UGL & 100460 & $5 / 5 / 94$ & $5 / 9 / 94$ & $5 / 11 / 94$ & 4 & & 2 \\
\hline EPA3510/20 & EPA8270M & UGL & 100461 & $5 / 5 / 94$ & $5 / 9 / 94$ & $5 / 11 / 94$ & 4 & & 2 \\
\hline EPA3540/50 & EPA8270 & UGKG & 100439 & $5 / 5 / 94$ & $5 / 9 / 94$ & $5 / 10 / 94$ & 4 & & 1 \\
\hline EPA3540/50 & EPA 8270 & UGKG & 100442 & $5 / 5 / 94$ & $5 / 9 / 94$ & $5 / 10 / 94$ & 4 & - & 1 \\
\hline EPA3540/50 & EPA8270 & UGKG & 100446 & $5 / 5 / 94$ & $5 / 9 / 94$ & $5 / 10 / 94$ & 4 & & 1 \\
\hline EPA3540/50 & EPA8270 & UGKG & 100449 & $5 / 5 / 94$ & $5 / 9 / 94$ & $5 / 10 / 94$ & 4 & & 1 \\
\hline EPA3540/50 & EPA8270 & UGKG & 100453 & $5 / 5 / 94$ & $5 / 9 / 94$ & $5 / 10 / 94$ & 4 & & 1 \\
\hline EPA3540/50 & EPA8270 & UUGKG & 100458 & $5 / 5 / 94$ & $.519 / 94$ & $5 / 10 / 94$ & 4 & & 1 \\
\hline EPA3540/50 & EPA8270 & UGKG & 100462 & $5 / 5 / 94$ & $5 / 9 / 94$ & $5 / 10 / 94$ & 4 & & 1 \\
\hline
\end{tabular}


Table 4

Holding Times

D-Area Buming/Rubble Pits

Savannah River Site

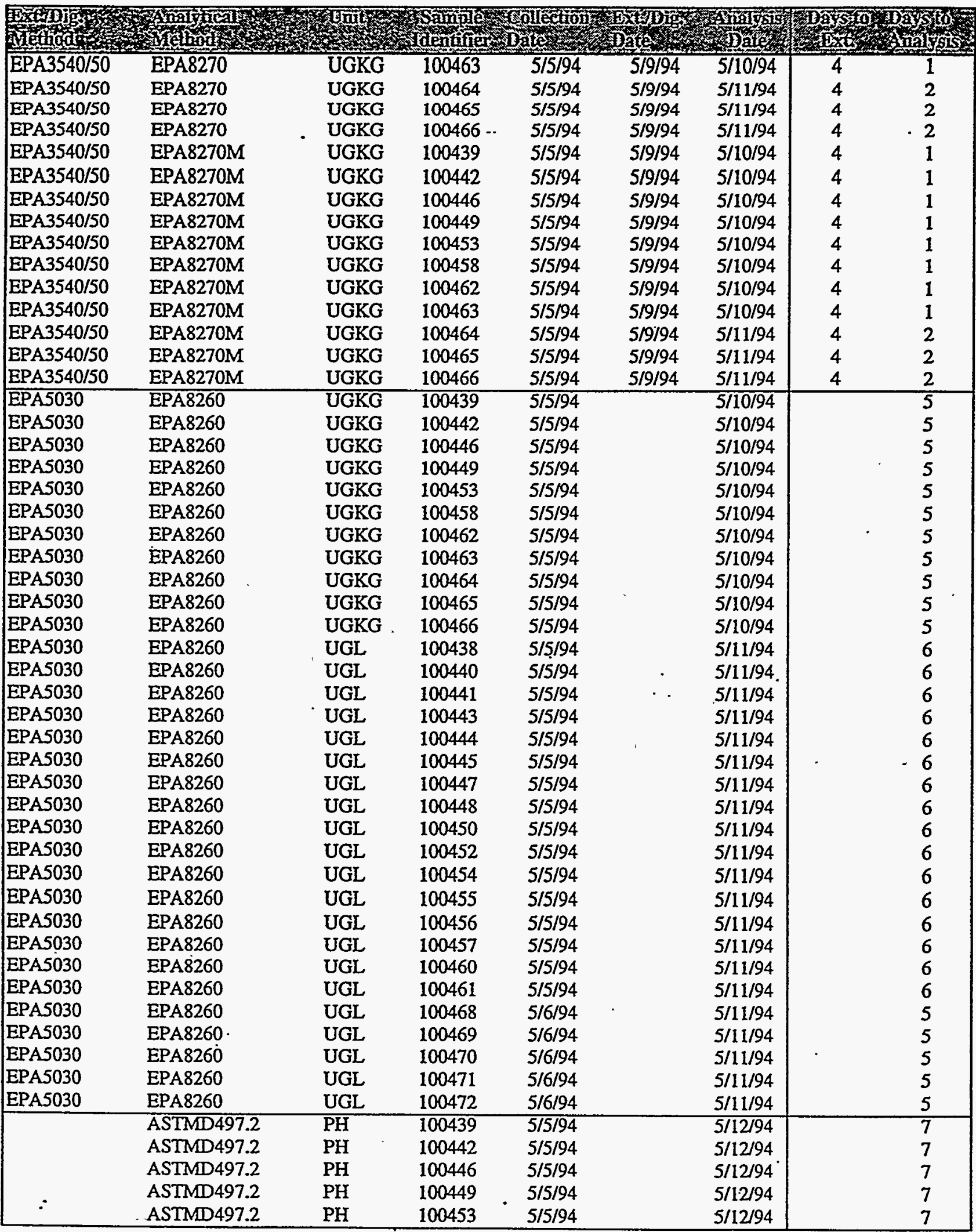


Table 4

Holding Times

D-Area Buming/Rubble Pits

Savannah River Site

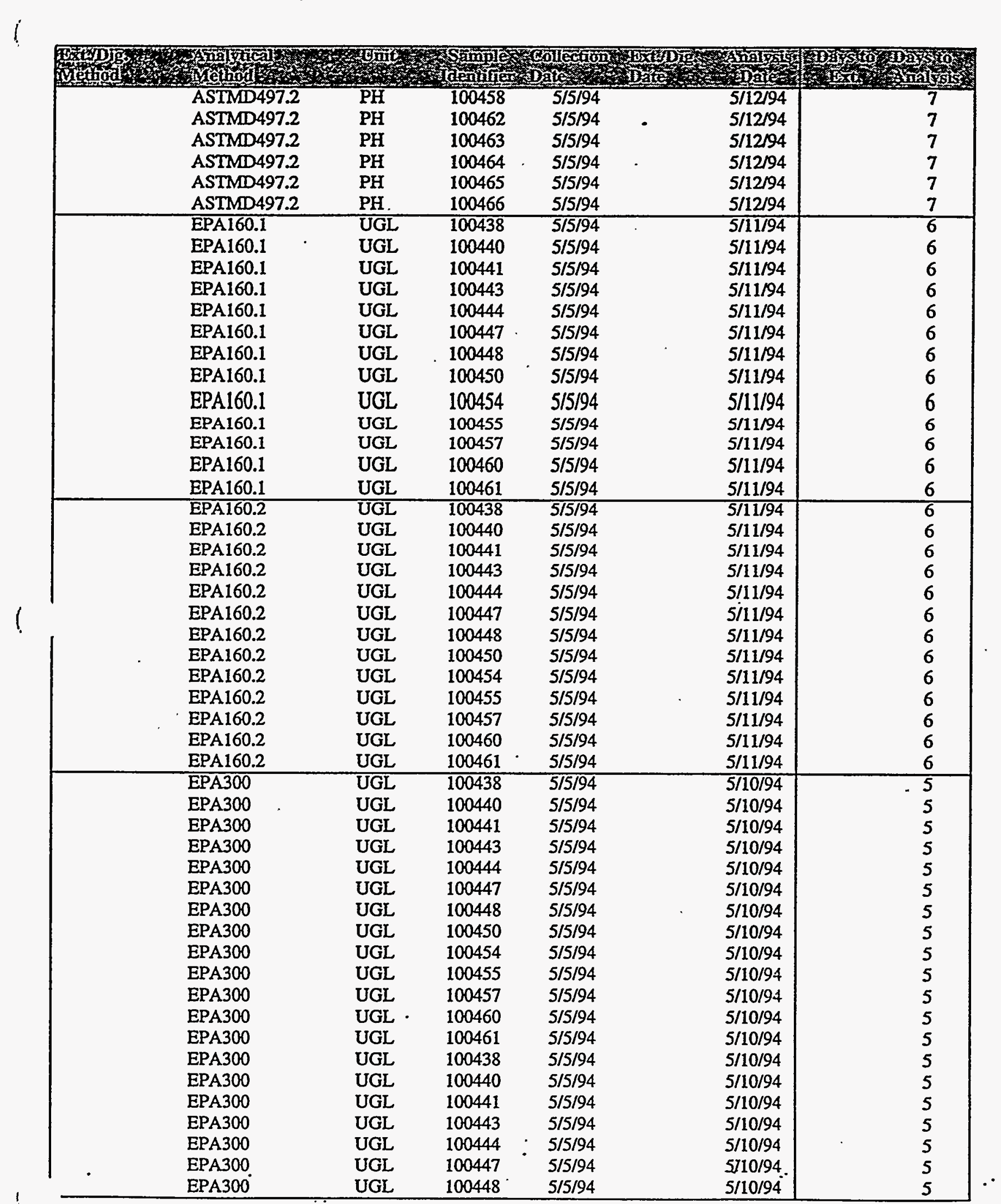


Table 4

Holding Times

D-Area Burning/Rubble Pits

Savannah River Site

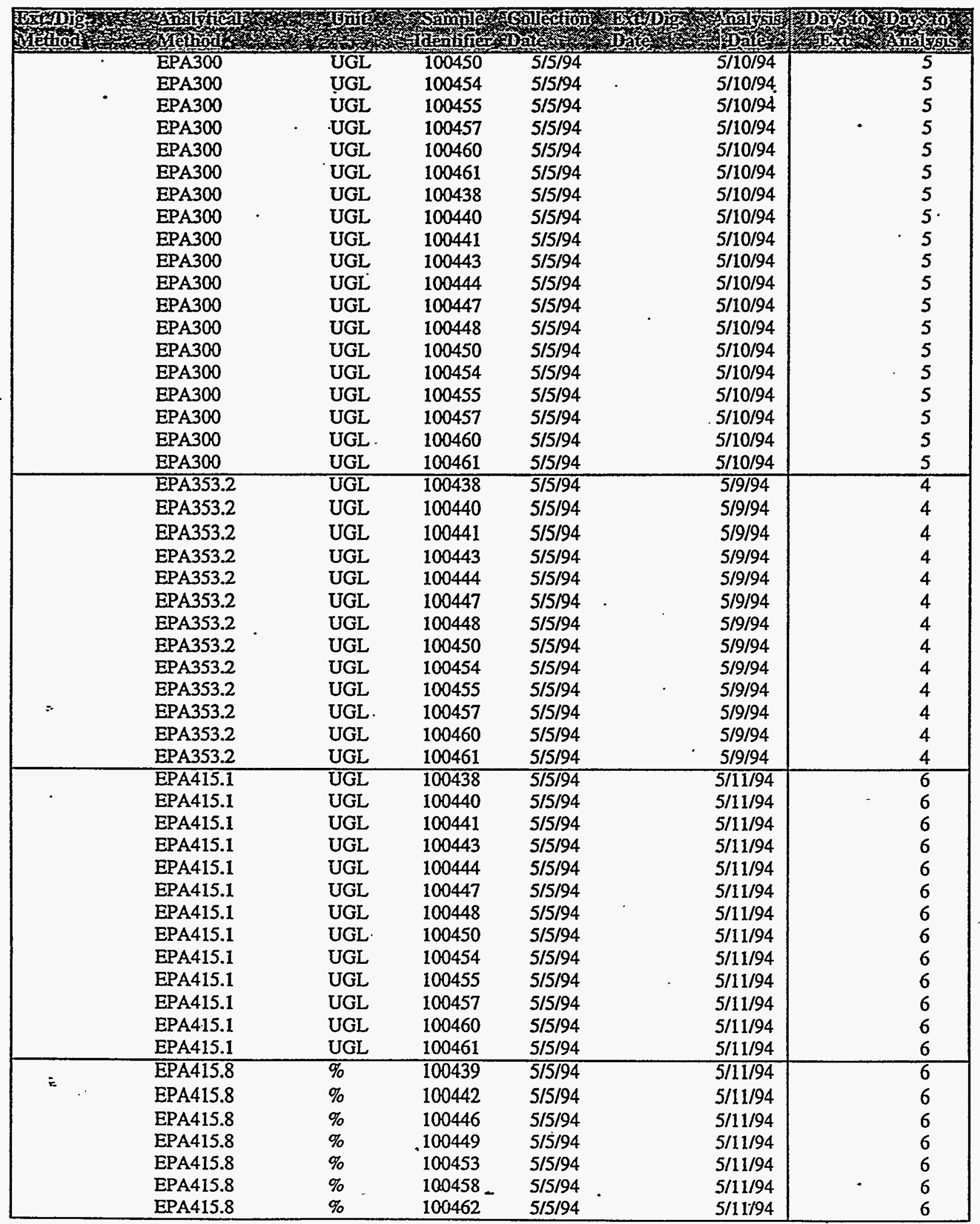


Table 4

Holding Times

D-Area Buming/Rubble Pits

Savannah River Site

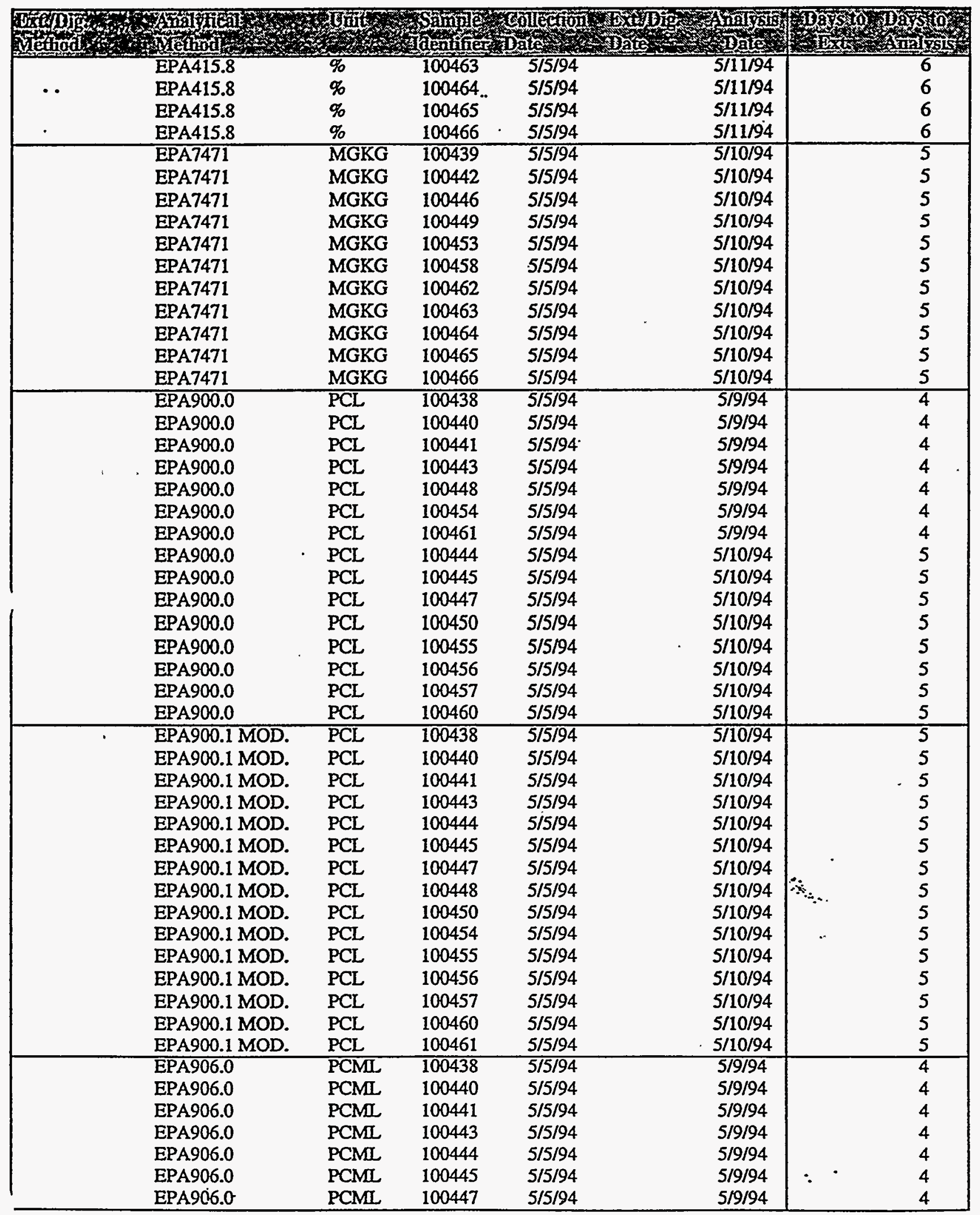


Table 4

Holding Times

D-Area Buming/Rubble Pits

Savannah River Site

\begin{tabular}{|c|c|c|c|c|c|}
\hline 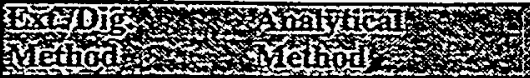 & sing & Shange & Tiection & Won & 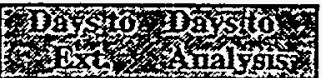 \\
\hline EPA906.0 & PCML & 100448 & $5 / 5 / 94$ & $5 / 9 / 94$ & 4 \\
\hline EPA906.0 & PCML & 100450 & $5 / 5 / 94$ & $5 / 9 / 94$ & 4 \\
\hline EPA906.0 & PCML & 100454 & $5 / 5 / 94$ & $5 / 9 / 94$ & 4 \\
\hline EPA906.0 & PCMO & 100455 & $5 / 5 / 94$ & $5 / 9 / 94$ & 4 \\
\hline EPA906.0 & PCMI & 100456 & $5 / 5 / 94$ & $5 / 9 / 94$ & 4 \\
\hline EPA906.0 & PCML & 100457 & $5 / 5 / 94$ & $5 / 9 / 94$ & 4 \\
\hline EPA906.0 & PCML & 100460 & $5 / 5 / 94$ & $5 / 9 / 94$ & 4 \\
\hline EPA906.0 & PCML & 100461 & $5 / 5 / 94$ & $5 / 9 / 94$ & 4 \\
\hline LANLER210 MOD & PCG & 100439 & $5 / 5 / 94$ & $5 / 10 / 94$ & 5 \\
\hline LANLER210 MOD & PCG & 100442 & $5 / 5 / 94$ & $5 / 10 / 94$ & 5 \\
\hline LANLER210 MOD & PCG & 100446 & $5 / 5 / 94$ & $5 / 10 / 94$ & 5 \\
\hline LANLER210 MOD & PCG & 100449 & $5 / 5 / 94$ & $5 / 10 / 94$ & 5 \\
\hline LANLER210 MOD & PCG & 100453 & $5 / 5 / 94$ & $5 / 10 / 94$ & 5 \\
\hline LANLER 210 MOD & PCG & 100458 & $5 / 5 / 94$ & $5 / 10 / 94$ & 5 \\
\hline LANLER210 MOD & PCG & 100462 & $5 / 5 / 94$ & $5 / 10 / 94$ & 5 \\
\hline LANLER210 MOD & PCG & 100463 & $5 / 5 / 94$ & $5 / 10 / 94$ & 5 \\
\hline LANLER210 MOD & PCG & 100464 & $5 / 5 / 94$ & $5 / 10 / 94$ & 5 \\
\hline LANLER210 MOD & PCG & 100465 & $5 / 5 / 94$ & $5 / 10 / 94$ & 5 \\
\hline LANLER210 MOD & PCG & 100466 & $5 / 5 / 94$ & $5 / 10 / 94$ & 5 \\
\hline LANLMLR-100 & PCG & 100442 & $5 / 5 / 94$ & $5 / 9 / 94$ & $\overline{4}$ \\
\hline LANLMLR-100 & PCG & 100453 & $5 / 5 / 94$ & $5 / 9 / 94$ & 4 \\
\hline LANLMLR-100 & PCG & 100462 & $5 / 5 / 94$ & $5 / 9 / 94$ & 4 \\
\hline LANLMLR-100 & PCG & 100463 & $5 / 5 / 94$ & $5 / 9 / 94$ & 4 \\
\hline LANLMLR-100 & PCG & 100464 & $5 / 5 / 94$ & $5 / 9 / 94$ & 4 \\
\hline LANLMLR-100 & PCG & 100465 & $5 / 5 / 94$ & $5 / 9 / 94$ & 4 \\
\hline LANLMLR-100 & PCG & 100466 & $5 / 5 / 94$ & $5 / 9 / 94$ & 4 \\
\hline LANLMLR-100 & PCG & 100439 & $5 / 5 / 94$ & $5 / 10 / 94$ & 5 \\
\hline LANLMLR-100 & PCG & 100446 & $\cdot 5 / 5 / 94$ & $5 / 10 / 94$ & 5 \\
\hline LANLMLR-100 & PCG & 100449 & $5 / 5 / 94$ & $5 / 10 / 94$ & 5 \\
\hline LANLMLR-100 & PCG & 100458 & $5 / 5 / 94$ & $5 / 10 / 94$ & 5 \\
\hline SW8469315 MOD & $\overline{\mathrm{PCG}}$ & 100439 & $5 / 5 / 94$ & $5 / 10 / 94$ & 5 \\
\hline SW8469315 MOD & PCG & 100442 & $5 / 5 / 94$ & $5 / 10 / 94$ & 5 \\
\hline SW8469315 MOD & PCG & 100446 & $5 / 5 / 94$ & $5 / 10 / 94$ & 5 \\
\hline SW8469315 MOD & PCG & 100449 & $5 / 5 / 94$ & $5 / 10 / 94$ & 5 \\
\hline SW8469315 MOD & PCG & 100453 & $5 / 5 / 94$ & $5 / 10 / 94$ & 5 \\
\hline SW8469315 MOD & PCG & 100458 & $5 / 5 / 94$ & $5 / 10 / 94$ & 5 \\
\hline SW8469315 MOD & PCG & 100462 & $5 / 5 / 94$ & $5 / 10 / 94$ & 5 \\
\hline SW8469315 MOD & PCG & 100463 & $5 / 5 / 94$ & $5 / 10 / 94$ & 5 \\
\hline SW8469315 MOD & PCG & 100464 & $5 / 5 / 94$ & $5 / 10 / 94$ & 5 \\
\hline SW8469315 MOD & PCG & 100465 & $5 / 5 / 94$ & $5 / 10 / 94$ & 5 \\
\hline SW8469315 MOD & PCG & 100466 & $5 / 5 / 94$ & $5 / 10 / 94$ & 5 \\
\hline SW9020A & UGL & 100441 & $5 / 5 / 94$ & $5 / 9 / 94$ & 4 \\
\hline SW9020A & UGL & 100443 & $5 / 5 / 94$ & $5 / 9 / 94$ & 4 \\
\hline SW9020A & UGL & 100448 & $5 / 5 / 94$ & $5 / 9 / 94$ & 4 \\
\hline SW9020A & UGL & 100438 & $5 / 5 / 94$ & $5 / 9 / 94$ & 4 \\
\hline SW9020A & UGL & 100440 & $5 / 5 / 94$ & $5 / 9 / 94$ & 4 \\
\hline SW9020A & UGL & 100444 & $5 / 5 / 94$ & $5 / 10 / 94$ & 5 \\
\hline SW9020A & UGL & 100450 & $5 / 5 / 94$ & $5 / 10 / 94$ & 5 \\
\hline SW9020A & UGL & 100454 & $5 / 5 / 94$ & $5 / 10 / 94$ & 5 \\
\hline SW9020A & UGL & 100455 & $5 / 5 / 94$ & $5 / 10 / 94$ & 5 \\
\hline SW9020A & UGL & 100460 & $5 / 5 / 94$ & $5 / 10 / 94$ & 5 \\
\hline SW9020A & UGL & $100461^{\circ}$ & $5 / 5 / 94$ & $5 / 10 / 94$ & 5. \\
\hline
\end{tabular}


Table 4

Holding Times

D-Area Burning/Rubble Pits

Savannah River Site

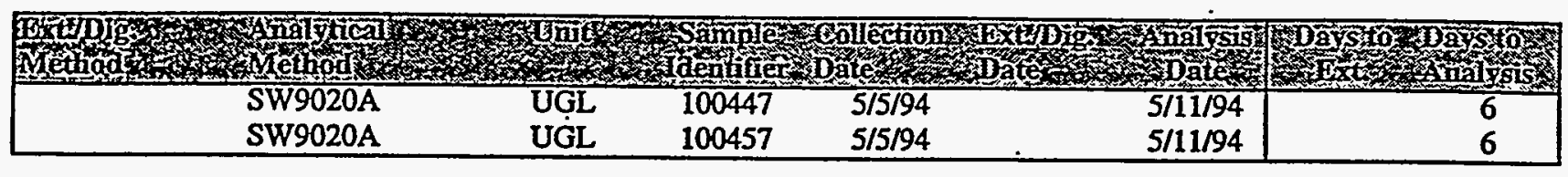


Table 5

Detections in Laboratory Blanks

D-Area Burning/Rubble Pits

Savannah River Site

\begin{tabular}{|c|c|c|c|c|c|c|c|c|c|c|c|}
\hline \multicolumn{4}{|c|}{ 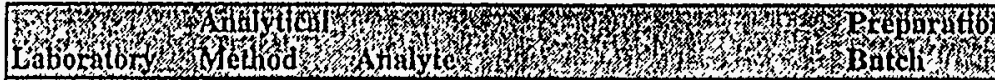 } & \multicolumn{8}{|c|}{ 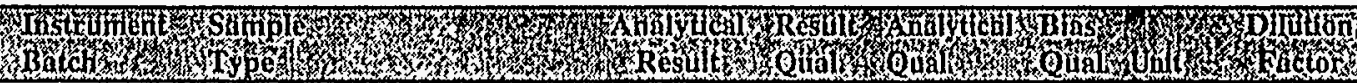 } \\
\hline General Eng. & EPA300.0 & Nitrate as nitrogen & 47690 & 47690 & Laboratory Blank & 0.02 & UJ & Q & L & MGL & 1 \\
\hline General Eng. & EPA6010 & Manganese, total recoverable & 47711 & 47711 & Laboratory Blank & 0.309 & $J$ & & & UGL & 1 \\
\hline General Eng. & EPA6010 & Manganese, total recoverable & 47751 & 47751 & Laboratory Blank & 0.00127 & J & & & MGKG & 1 \\
\hline Gंeneral Eng. & EPA6010 & Barium, total recoverable & 47751 & 47751 & Laboratory Blank & 0.00154 & J & & & MGKG & $i$ \\
\hline General Eng. & EPA7421 & Lead, total recoverable & 47710 & 47710 & Laboratory Blank & 1.21 & J & & & UGL & 1 \\
\hline General Eng. & EPA7470 & Mercury, total & 47748 & 47748 & Laboratory Blank & 0.065 & $\mathrm{~J}$ & & & UGL & 1 \\
\hline General Eng. & EPA8260 & Dichloromethane (Methylene chloride) & 47729 & 47729 & Laboratory Blank & 0.67 & & & & UGL & 1 \\
\hline General Eng. & EPA8260 & Toluene & 47729 & 47729 & Laboratory Blank & 0.07 & $\mathbf{J}$ & & & UGL & 1 \\
\hline General Eng. & EPA8260 & Toluene & 47771 & 47771 & Laboratory Blank & 0.1 & J & & & UGKG & 1 \\
\hline General Eng. & EPA8270 & Bis(2-ethylhexyl) phthalate & 47675 & 47675 & Laboratory Blank & 0.45 & J & & & UGL & 1 \\
\hline General Eng. & EPA8270 & Bis(2-ethylhexyl) phthalate. & 47676 & 47676 & Laboratory Blank & 6.96 & $J$ & & & UGKG & 1 \\
\hline General Eng. & EPA8270 & Di-n-butyl phthalate & 47676 & 47676 & Laboratory Blank & 9.28 & J & & & UGKG & $i$ \\
\hline General Eng. & EPIA-001B & Gross alpha & 47824 & 47824 & Laboratory Blank & 0.87 & $\mathbf{J}$ & & & PCO & 1 \\
\hline General Eng. & EPIA-002 & Tritium & 47845 & 47845 & Laboratory Blank & 930 & J & & & PCL & $i$ \\
\hline General Eng. & EPIA-002B & Tritium & 47851 & 47851 & Laboratory Blank & 2.89 & $\mathrm{~J}$ & & & PCG & 1 \\
\hline Weston & EPA160.2 & Total suspended solids & 94LSSA85 & 94LSSA85 & Laboratory Blank & 2000 & & & - & UGL & 1 \\
\hline Weston & EPA6010 & Arsenic, total recoverable & & AS051061 & Initial Calibration Blank & 0.7 & & & & MGKG & 1 \\
\hline Weston & EPA6010 & Arsenic, total recoverable & 94L5705 & AS051061 & Continuing Calibration Blank & 0.3 & & & & MGKG & 1 \\
\hline Weston & EPA6010 & Arsenic, total recoverable & 94L570S & AS051061 & Continuing Calibration Blank & 0.5 & & & & MGKG & 1 \\
\hline Weston & EPA6010 & Arsenic, total recoverable & CRA & AS051061 & Continuing Calibration Blank & 0.6 & & & & MGKG & 1 \\
\hline Weston & EPA7471 & Mercury, total & $94 \mathrm{C} 0185$ & HG051004 & Continuing Calibration Blank & 0.01 & & & & UGL & 1 \\
\hline Weston & EPA7471 & Mercury, total & $94 C 0190$ & HG051004 & Continuing Calibration Blank & 0.02 & & & & UGL & 1 \\
\hline Weston & EPA7471 & Mercury, total & $94 C 0190$ & HG051004 & Continuing Calibration Blank & 0.03 & & & & UGL & 1 \\
\hline Weston & EPA7471 & Mercury, total & $94 C 0190$ & HG051004 & Initial Calibration Blank & 0.01 & & & & UGL & 1 \\
\hline Weston & EPA7740 & Selenium, total recoverable & & SE051104 & Continuing Calibration Blank & 0.2 & & & & UGL & $i$ \\
\hline Weston & EPA7740 & Selenium, total recoverable & & SE051104 & Initial Calibration Blank & 0.1 & & & & UGL & 1 \\
\hline Weston & EPA7740 & Selenium, total recoverable & 94L5707 & SE051104 & Continuing Calibration Blank & 0.4 & & & & UGL & 1 \\
\hline Weston & EPA7740 & Selenium, total recoverable & 94L.5707 & SE051104 & Continuing Calibration Blank & 0.5 & & & & UGL & 1 \\
\hline Weston & EPA7740 & Selenium, total recoverable & CRA & SE051104 & Continuing Calibration Blank & 0.6 & & & & UGL & 1 \\
\hline Weston & EPA8260 & 1,2-Dichloroethylene & $94 \mathrm{LVC} 056$ & $\mathrm{C} 051001$ & Laboratory Blank & 0.16 & UJ & VB & & UGKG & $i$ \\
\hline Weston & EPA8260 & Dichloromethane (Methylene chloride) & 94LVC056 & C051001 & Laboratory Blank & 0.38 & UJ & VB & & UGKG & 1 \\
\hline Weston & EPA 8260 & Trichlorofluoromethane & 94LVC056 & $\mathrm{Cos} 1001$ & Laboratory Blank & 0.42 & UJ & VB & & UGKG & 1 \\
\hline Weston & EPA8260 & Chloromethane (Methyl chloride) & $94 \mathrm{LVC} 057$ & $\cos 1101$ & Laboratory Blank & 0.2 & UJ & VB & & UGL & 1 \\
\hline Weston & EPA 8260 & Chloromethane (Methyl chloride) & $94 \mathrm{LVC} 058$ & Cosili24 & Laboratory Blank & 0.2 & UJ & VB & & UGL & $i$ \\
\hline Weston & EPA8270 & Bis(2-ethylhexyl) phthalate & 94LE0897 & S051001 & Laboratory Blank & 333 & UJ & VB & & UGKG & 1 \\
\hline Weston & EPA8270M & Di-n-butyl phthalate & 94LE0897 & S051001 & Laboratory Blank & 1010 & & $\mathrm{~V}$ & & UGKG & $i$ \\
\hline Weston & EPA8270M & Di-n-butyl phthalate & 94LE0897 & S051001 & Laboratory Blank & 5050 & UJ & $\mathrm{V}$ & & UGKG & 1 \\
\hline Weston & EPA8270M & Di-n-butyl phthalate & 94LE0899 & J051101 & Laboratory Blank & 0.638 & UJ & VB & & UGL & $i$ \\
\hline
\end{tabular}


Taole 6

Recoveries of Laboratory Control Samples D-Area Burning/Rubble Pits Savannah River Site

\begin{tabular}{|c|c|c|c|c|c|c|}
\hline \multicolumn{7}{|c|}{ 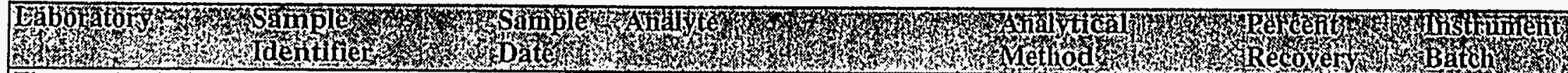 } \\
\hline Thermo Analytical & Laboratory Blank & 50794 & Gross alpha & EPA900.0 & 82.8 & 5099402 \\
\hline Thermo Analytical & Laboratory Blank & 50794 & Gross alpha & LANLMUR-100 & 85.0 & 5099402 \\
\hline Thermo Analytical & Laboratory Blank & 50794 & Nonvolatile beta & EPA900.0 & 101.3 & 5099402 \\
\hline Thermo Analytical & Laboratory Blank & 50794 & Nonvolatile beta & LANLMLR-100 & 106.5 & 5099402 \\
\hline Thermo Analytical & Laboratory Blank & 50794 & Radium, total alpha-emitting & EPA900.1 MOD. & 96.6 & 5109402 \\
\hline Thermo Analytical & Laboratory Blank & 50794 & Radium, total alpha-emitting & SW8469315 MOD & 98.9 & 5109402 \\
\hline Thermo Analytical & Laboratory Blank & 50794 & Tritium & EPA906.0 & 98.9 & 5099404 \\
\hline Thermo Analytical & Laboratory Blank & 50794 & Tritium & LANLER210 MOD & 110.5 & 5109404 \\
\hline Weston & Laboratory Blank & 50994 & 1,2,4-Trichlorobenzene & $\overline{\text { EPA8270M }}$ & 59.5 & J051101 \\
\hline Weston & Laboratory Blank & 50994 & 1,2,4-Trichlorobenzene & EPA.8270M & 76.0 & S051001 \\
\hline Weston & Laboratory Blank & 50994 & 1,4-Dichlorobenzene & EPA8270 & 55.8 & J051101 \\
\hline Weston & Laboratory Blank & 50994 & 1,4-Dichlorobenzene & EPA8270 & 74.4 & S051001 \\
\hline Weston & Laboratory Blank & 50994 & 2,4,6-Tribromophenol (surr) & EPA8270 & 70.8 & J051101 \\
\hline Weston & Laboratory Blank & 50994 & 2,4,6-Tribromophenol (surr) & EPA8270 & 79.2 & J051101 \\
\hline Weston & Laboratory Blank & 50994 & 2,4,6-Tribromophenol (surr) & EPA827.0 & 76.8 & S051001 \\
\hline Weston & Laboratory Blank & 50994 & 2,4,6-Tribromophenol (surr) & EPA8270 & 80.8 & S051001 \\
\hline Weston & Laboratory Blank & 50994 & 2,4-Dinitrotoluene & EPA8270 & 72.6 & J051101 \\
\hline Weston & Laboratory Blank & 50994 & 2,4-Dinitrotoluene & EPA8270 & 80.3 & S051001 \\
\hline Weston & Laboratory Blank & 50994 & 2-Chlorophenol & EPA8270M & 66.9 & J051:101 \\
\hline Weston & Laboratory Blank & 50994 & 2-Chlorophenol & EPA8270M & 74.3 & S051001 \\
\hline Weston & Laboratory Blank & 50994 & 2-Fluorobiphenyl & EPA 8270 & 72.7 & J051101 \\
\hline Weston & Laboratory Blank & 50994 & 2-Fluorobiphenyl & EPA8270 & 75.6 & J051101 \\
\hline Weston & Laboratory Blank & 50994 & 2-Fluorobiphenyl & EPA8270 & 81.9 & S051001 \\
\hline Weston & Laboratory Blank & 50994 & 2-Fluorobiphenyl & EPA8270 & 82.6 & S051001 \\
\hline Weston & Laboratory Blank & 50994 & 2-Fluorophenol & EPA8270 & 49.4 & J051101 \\
\hline Weston & Laboratory Blank & 50994 & 2-Fluorophenol & EPA8270 & 51.2 & J051101 \\
\hline Weston & Laboratory Blank & 50994 & 2-Fluorophenol & EPA8270 & 78.1 & S051001 \\
\hline Weston & Laboratory Blank & 50994 & 2-Fluorophenol & EPA8270 & 79.4 & S051001 \\
\hline Weston & Laboratory Blank & 50994 & 4-Chloro-m-cresol & EPA8270M & 70.8 & J051101 \\
\hline Weston & Laboratory Blank & 50994 & 4-Chloro-m-cresol & EPA8270M & 76.2 & S051001 \\
\hline
\end{tabular}


Table 6

Recoveries of Laboratory Control Samples

D-Area Burning/Rubble Pits

Savannah River Site

\begin{tabular}{|c|c|c|c|c|c|c|}
\hline 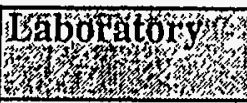 & 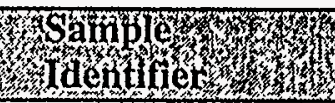 & 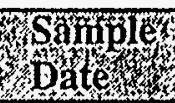 & ${ }^{3}$ & 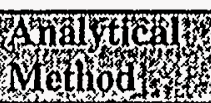 & aching & 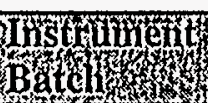 \\
\hline Weston & Laboratory Blank & 50994 & 4-Nitrophenol & EPA8270M & 26.8 & $\mathrm{~J} 051101$ \\
\hline Weston & Laboratory Blank & 50994 & 4-Nitrophenol & EPA8270M & 72.8 & S051001 \\
\hline Weston & Laboratory Blank & 50994 & Acenaphthene & EPA8270 & 74.2 & J051101 \\
\hline Weston & Laboratory Blank & 50994 & Acenaphthene & EPA8270 & 80.4 & S051001 \\
\hline Weston & Laboratory Blank & 50994 & Mercury, total & EPA7471 & 103.0 & HG051004 \\
\hline Weston & Laboratory Blank & 50994 & Mercury, total & EPA7471 & 106.0 & HG051004 \\
\hline Weston & Laboratory Blank & 50994 & N-Nitrosodipropylamine & EPA8270 & 67.2 & J051101 \\
\hline Weston & Laboratory Blank & 50994 & N-Nitrosodipropylamine & EPA 8270 & 72.0 & S051001 \\
\hline Weston & Laboratory Blank & 50994 & Nitrate as nitrogen & EPA353.2 & 102.0 & 94LN3072 \\
\hline Weston & Laboratory Blank & 50994 & Nitrate as nitrogen & EPA353.2 & 103.0 & 94LN3072 \\
\hline Weston & Laboratory Blank & 50994 & Nitrate as nitrogen & EPA353.2 & 105.0 & 94LN3072 \\
\hline Weston & Laboratory Blank & 50994 & Nitrobenzene-d5 & EPA8270 & 74.0 & J051101 \\
\hline Weston & Laboratory Blank & 50994 & Nitrobenzene-d5 & EPA8270 & 78.1 & J051101 \\
\hline Weston & Laboratory Blank & 50994 & Nitrobenzene-d5 & EPA8270 & 78.4 & S051001 \\
\hline Weston & Laboratory Blank & 50994 & Nitrobenzene-d5 & EPA8270 & 86.6 & S051001 \\
\hline Weston & Laboratory Blank & 50994 & p-Terphenyl-d14 & EPA8270 & 78.3 & J051101 \\
\hline Weston & Laboratory Blank & 50994 & p-Terphenyl-d14 & EPA8270 & 82.1 & J051101 \\
\hline Weston & Laboratory Blank & 50994 & p-Terphenyl-d14 & EPA8270 & 99.7 & S051001 \\
\hline Weston & Laboratory Blank & 50994 & p-Terphenyl-d14 & EPA8270 & 112.0 & S051001 \\
\hline Weston & Laboratory Blank & 50994 & Pentachlorophenol & EPA8270 & 76.2 & J051101 \\
\hline Weston & Laboratory Blank & 50994 & Pentachlorophenol & EPA8270M & 85.5 & S051001 \\
\hline Weston & Laboratory Blank & 50994 & Phenol & EPA8270M & 30.6 & J051101 \\
\hline Weston & Laboratory Blank & 50994 & Phenol & EPA8270M & 76.9 & S051001 \\
\hline Weston & Laboratory Blank & 50994 & Phenol-d5 & EPA8270 & 28.8 & J051101 \\
\hline Weston & Laboratory Blank & 50994 & Phenol-d5 & EPA 8270 & 30.8 & J051101 \\
\hline Weston & Laboratory Blank & 50994 & Phenol-d5 & EPA8270 & 73.6 & S051001 \\
\hline Weston & Laboratory Blank & 50994 & Phenol-d5 & EPA8270 & 74.1 & S051001 \\
\hline Weston & Laboratory Blank & 50994 & Pyrene & EPA8270 & 72.4 & J051101 \\
\hline Weston & Laboratory Blank & 50994 & Pyrene & EPA8270 & 100.0 & S051001 \\
\hline Weston & Laboratory Blank & 50994 & Total organic halogens & SW9020A & 96.2 & 94LX124X \\
\hline
\end{tabular}


Table 6

Recoveries of Laboratory Control Samples

D-Area Burning/Rubble Pits

Savannah River Site

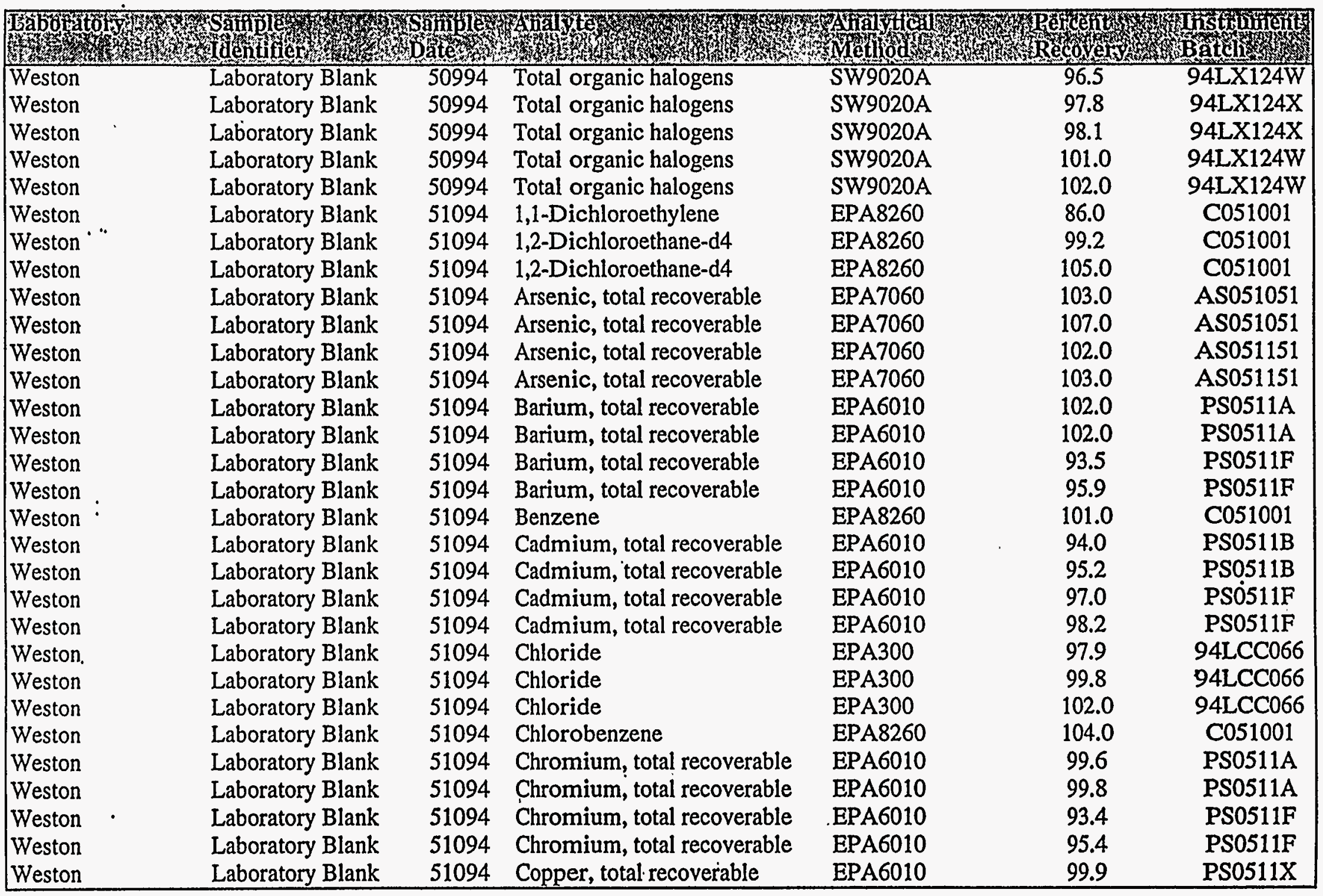


Table 6

Recoveries of Laboratory Control Samples

D-Area Burning/Rubble Pits

Savannah River Site

\begin{tabular}{|c|c|c|c|c|c|c|c|}
\hline 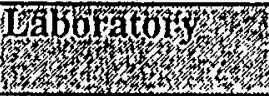 & 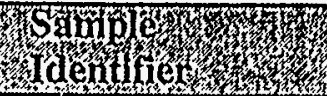 & 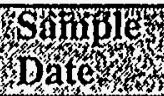 & $\mathrm{H}_{1}$ & 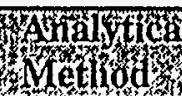 & & Whacher & 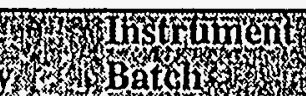 \\
\hline Weston & Laboratory Blank & 51094 & Copper, total recoverable & EPA6010 & & 100.0 & PSO511X \\
\hline Weston & Laboratory Blank & 51094 & Copper, total recoverable & EPA6010 & & 93.3 & PS0511Z \\
\hline Weston & Laboratory Blank & 51094 & Copper, total recoverable & EPA6010 & & 95.1 & PS0511Z \\
\hline Weston & Laboratory Blank & 51094. & Fluoride & EPA300 & & 101.0 & 94LFC066 \\
\hline Weston & Laboratory Blank & 51094 & Fluoride & EPA300 & & 103.0 & $94 \mathrm{LFC} 066$ \\
\hline Weston & Laboratory Blank & 51094 & Fluoride & EPA300 & & 106.0 & 94LFC066 \\
\hline Weston & Laboratory Blank & 51094 & Iron, total recoverable & EPA6010 & & 95.4 & PSO511Z \\
\hline Weston & Laboratory Blank & 51094 & Iron, total recoverable & EPA6010 & & 97.5 & PSO511Z \\
\hline Weston & Laboratory Blank & 51094 & Lead, total recoverable & EPA7421 & & 107.0 & PB051151 \\
\hline Weston & Laboratory Blank & 51094 & Lead, total recoverable & EPA7421 & & 113.0 & PB051151 \\
\hline Weston & Laboratory Blank & 51094 & Lead, total recoverable & EPA7421 & & 96.3 & PB051202 \\
\hline Weston & Laboratory Blank & 51094 & Lead, total recoverable & EPA7421 & & 98.3 & PB051202 \\
\hline Weston & Laboratory Blank & 51094 & Manganese, total recoverable & EPA6010 & . & 99.6 & PSO511X \\
\hline Weston & Laboratory Blank & 51094 & Manganese, total recoverable & EPA6010 & & 101.0 & PS0511X \\
\hline Weston & Laboratory Blank & 51094 & Manganese, total recoverable & EPA6010 & & 93.9 & PSO511Z \\
\hline Weston & Laboratory Blank & 51094 & Manganese, total recoverable & EPA6010 & & 96.0 & PS0511Z \\
\hline Weston & Laboratory Blank & 51094 & Mercury, total & EPA7471 & & 95.1 & HG051005 \\
\hline Weston & Laboratory Blank & 51094 & Mercury, total & EPA7471 & & 109.0 & HG051005 \\
\hline Weston & Laboratory Blank & 51094 & Nickel, total recoverable & EPA6010 & & 90.7 & PS0511Y \\
\hline Weston & Laboratory Blank & 51094 & Nickel, total recoverable & EPA6010 & & 90.8 & PSO511Y. \\
\hline Weston & Laboratory Blank & 51094 & Nickel, total recoverable & EPA6010 & & 94.5 & PSO511Z \\
\hline Weston ${ }^{\circ}$ & Laboratory Blank & 51094 & Nickel, total recoverable & EPA6010 & & 96.5 & PS0511Z \\
\hline Weston & Laboratory Blank & 51094 & p-Bromofluorobenzene & EPA 8260 & & 91.6 & C051001 \\
\hline Weston & Laboratory Blank & 51094 & p-Bromofluorobenzene & EPA8260 & & 93.4 & C051001 \\
\hline Weston & Laboratory Blank & 51094 & Selenium, total recoverable & EPA7740 & & 107.0 & SE051161 \\
\hline Weston & Laboratory Blank & 51094 & Selenium, total recoverable & EPA7740 & & 110.0 & SE051161 \\
\hline Weston & Laboratory Blank & 51094 & Selenium, total recoverable & EPA7740 & & 95.3 & SE051104 \\
\hline Weston & Laboratory Blank & 51094 & Selenium, total recoverable & EPA7740 & & 97.7 & SE051104 \\
\hline Weston & Laboratory Blank & 51094 & Silver, total recoverable & EPA6010 & & 96.0 & PSO511E \\
\hline Weston & Laboratory Blank & 51094 & Silver, total recoverable & EPA6010 & & 97.6 & PS0511E \\
\hline
\end{tabular}


Table 6

Recoveries of Laboratory Control Samples

D-Area Burning/Rubble Pits

Savannah River Site

\begin{tabular}{|c|c|c|c|c|c|c|}
\hline \multicolumn{7}{|c|}{ 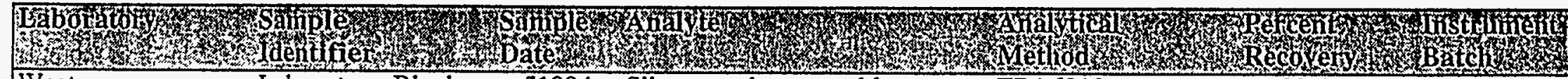 } \\
\hline Weston & Laboratory Blank & 51094 & Silver, total recoverable & EPA6010 & 93.4 & \\
\hline Weston & Laboratory Blank & 51094 & Sodium, total recoverable & EPA6010 & 93.1 & PS0511Z \\
\hline Weston & Laboratory Blank & 51094 & Sodium, total recoverable & EPA6010 & 95.3 & PS0511Z \\
\hline Weston & Laboratory Blank & 51094 & Sulfate & EPA300 & 100.0 & 94LSC066 \\
\hline Weston & Laboratory Blank & 51094 & Sulfate & EPA300 & 102.0 & 94LSCO 066 \\
\hline Weston & Laboratory Blank & 51094 & Toluene & EPA 8260 & 100.0 & C051001 \\
\hline Weston & Laboratory Blank & 51094 & Toluene-d8 & EPA8260 & 94.4 & $\mathrm{C} 051001$ \\
\hline Weston & Laboratory Blank & 51094 & Toluene-d8 & EPA8260 & 97.4 & $\mathrm{C} 051001$ \\
\hline Weston & Laboratory Blank & 51094 & Total dissolved solids & EPA160.1 & 96.0 & 94LSSA85 \\
\hline Weston & Laboratory Blank & 51094 & Total dissolved solids & EPA160.1 & 103.0 & 94LSSA85 \\
\hline Weston & Laboratory Blank & 51094 & Total organic halogens & SW9020A & 95.3 & $94 \mathrm{LX} 125 \mathrm{~B}$ \\
\hline Weston & Laboratory Blank & 51094 & Total organic halogens & SW9020A & 98.4 & $94 \mathrm{~L} \times 125 \mathrm{~B}$ \\
\hline Weston & Laboratory Blank & 51094 & Total organic halogens & SW9020A & 102.0 & $94 \mathrm{~L} \times 125 \mathrm{~B}$ \\
\hline Weston & Laboratory Blank & 51094 & Total organic halogens & SW9020A & 102.0 & $94 \mathrm{LX} 125 \mathrm{X}$ \\
\hline Weston & Laboratory Blank & 51094 & Total organic halogens & SW9020A & 105.0 & $94 \mathrm{LX} 125 \mathrm{X}$ \\
\hline Weston & Laboratory Blank & 51094 & Total organic halogens & SW9020A & 106.0 & 94LX125X \\
\hline Weston & Laboratory Blank & 51094 & Total suspended solids & EPA 160.2 & 102.0 & 94LSSA 85 \\
\hline Weston & Laboratory Blank & 51094 & Trichloroethylene & EPA8260 & 98.8 & $\mathrm{C} 051001$ \\
\hline Weston & Laboratory Blank & 51194 & 1,2-Dichloroethane-d4 & EPA8260 & 98.2 & $\mathrm{C} 051124$ \\
\hline Weston & Laboratory Blank & 51194 & 1,2-Dichloroethane-d4 & EPA8260 & 108.0 & C051101 \\
\hline Weston & Laboratory Blank & 51194 & Mercury, total & EPA7471 & 97.6 & HG051103 \\
\hline Weston $:$ & Laboratory Blank & 51194 & Mercury, total & EPA7471 & 101.0 & HG051103 \\
\hline Weston & Laboratory Blank & 51194 & p-Bromofluorobenzene & EPA $8260^{\circ}$ & 93.6 & $\mathrm{C} 051124$ \\
\hline Weston & Laboratory Blank & 51194 & p-Bromofluorobenzene & EPA8260 & 96.2 & $\mathrm{C} 051101$ \\
\hline Weston & Laboratory Blank & 51194 & Toluene-d8 & EPA8260 & 94.2 & $\mathrm{C} 051124$ \\
\hline Weston & Laboratory Blank & 51194 & Toluene-d8 & EPA8260 & 99.8 & C051101 \\
\hline Weston & Laboratory Blank & 51194 & Total organic carbon & EPA415.1 & 101.0 & 94LTC080 \\
\hline Weston & Laboratory Blank & 51194 & Total organic carbon & EPA415.1 & 103.0 & 94LTC080 \\
\hline Weston & Laboratory Blank & 51194 & Total organic halogens & SW9020A & 96.1 & $94 \mathrm{LX} 126 \mathrm{~W}$ \\
\hline Weston & Laboratory Blank & 51194 & Total organic halogens & SW9020A & 97.8 & 94LX126W \\
\hline
\end{tabular}


Table 6

Recoveries of Laboratory Control Samples

D-Area Burning/Rubble Pits

Savannah River Site

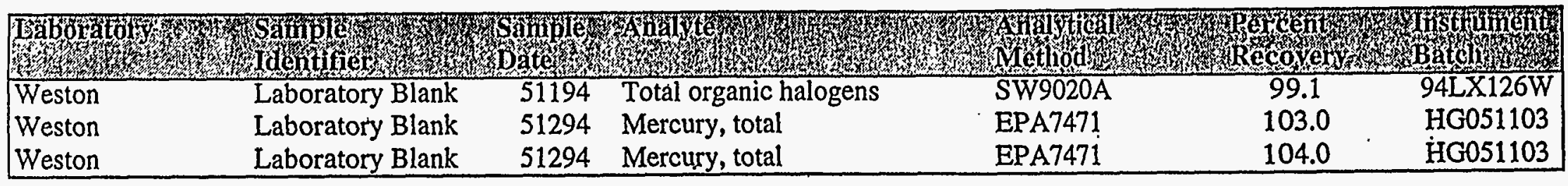


Table 7

Recoveries of Matrix Spike and Surrogate Samples

D-Area Burning/Rubble Pits

Savannah River Site

\begin{tabular}{|c|c|c|c|c|}
\hline \multicolumn{3}{|c|}{ 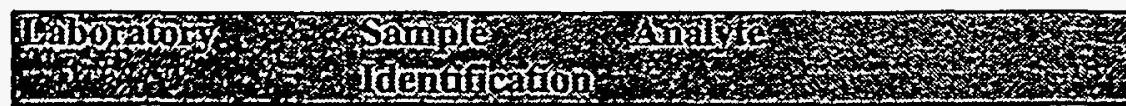 } & \multicolumn{2}{|c|}{ 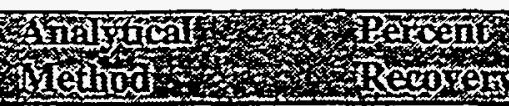 } \\
\hline General Engineering & 100451 & 2,4-Dinitrotoluene & EPA8270 & 80 \\
\hline General Engineering & 100451 & 2-Chlorophenol & EPA8270 & 74 \\
\hline General Engineering & 100451 & 4-Chloro-m-cresol & EPA8270 & 86 \\
\hline General Engineering & 100451 & 4-Nitrophenol & EPA8270 & 19 \\
\hline General Engineering & 100451 & Acenaphthene & EPA8270 & 57 \\
\hline General Engineering & 100451 & Arsenic, total recoverable & EPA7060 & 101 \\
\hline General Engineering & 100451 & Arsenic, total recoverable & EPA7060 & 101 \\
\hline General Engineering & 100451 & Fluoride & EPA300.0 & 112 \\
\hline General Engineering & 100451 & Lead, total recoverable & EPA7421 & 97 \\
\hline General Engineering & 100451 & Lead, total recoverable & EPA7421 & 100 \\
\hline General Engineering & 100451 & Mercury, total & EPA7470 & 105 \\
\hline General Engineering & 100451 & Mercury, total & EPA7470 & 108 \\
\hline General Engineering & 100451 & N-Nitrosodipropylamine & EPA8270 & 83 \\
\hline General Engineering & 100451 & Nitrate as nitrogen & EPA300.0 & $\cdot 107$ \\
\hline General Engineering & 100451 & Pentachlorophenol & EPA8270 & 75 \\
\hline General Engineering & 100451 & Phenol & EPA8270 & 47 \\
\hline General Engineering & 100451 & Pyrene & EPA8270 & 81 \\
\hline General Engineering & 100451 & Selenium, total recoverable & EPA7740 & 96 \\
\hline General Engineering & 100451 & Selenium, total recoverable & EPA7740 & 98 \\
\hline General Engineering & 100451 & Total organic carbon & EPA415.1 & 100 \\
\hline General Engineering & 100451 & Tritium & EPIA-002 & 105 \\
\hline General Engineering & 100459 & 1,1-Dichloroethylene & EPA8260 & 118 \\
\hline General Engineering & 100459 & 1,1-Dichloroethylene & EPA8260 & 175 \\
\hline General Engineering & 100459 & 2,4-Dinitrotoluene & EPA8270 & 95 \\
\hline General Engineering & 100459 & 2-Chlorophenol & EPA8270 & 84 \\
\hline General Engineering & 100459 & 4-Chloro-m-cresol & EPA8270 & 96 \\
\hline General Engineering & 100459 & 4-Nitrophenol & EPA8270 & 38 \\
\hline General Engineering & 100459 & Acenaphthene & EPA8270 & 64 \\
\hline General Engineering & 100459 & Arsenic, total recoverable & EPA6010 & 111 \\
\hline General Engineering & 100459 & Arsenic, total recoverable & EPA6010 & 110 \\
\hline General Engineering & 100459 & Barium, total recoverable & EPA6010 & 99 \\
\hline General Engineering & 100459 & Barium, total recoverable & EPA6010 & 100 \\
\hline General Engineering & 100459 & Benzene & EPA8260 & 97 \\
\hline General Engineering & 100459 & Benzene & EPA8260 & 135 \\
\hline General Engineering & 100459 & Cadmium, total recoverable & EPA6010 & 91 \\
\hline General Engineering & 100459 & Cadmium, total recoverable & EPA6010 & 90 \\
\hline General Engineering & 100459 & Chlorobenzene & EPA8260 & 92 \\
\hline General Engineering & 100459 & Chlorobenzene & EPA8260 & 97 \\
\hline General Engineering & 100459 & Chromium, total recoverable & EPA6010 & 78 \\
\hline General Engineering & 100459 & Chromium, total recoverable & EPA6010 & 79 \\
\hline General Engineering & 100459 & Copper, total recoverable & EPA6010 & 92 \\
\hline General Engineering & 100459 & Copper, total recoverable & EPA6010 & 94 \\
\hline General Engineering & 100459 & Gross alpha & EPIA-001B & 100 \\
\hline General Engineering & 100459 & Lead, total recoverable & EPA6010 & 92 \\
\hline General Engineering & 100459 & Lead, total recoverable & EPA6010 & 90 \\
\hline General Engineering & 100459 & Manganese, total recoverable & EPA6010 & 107 \\
\hline
\end{tabular}


Table 7

Recoveries of Matrix Spike and Surrogate Samples

D-Area Burning/Rubble Pits

Savannah River Site

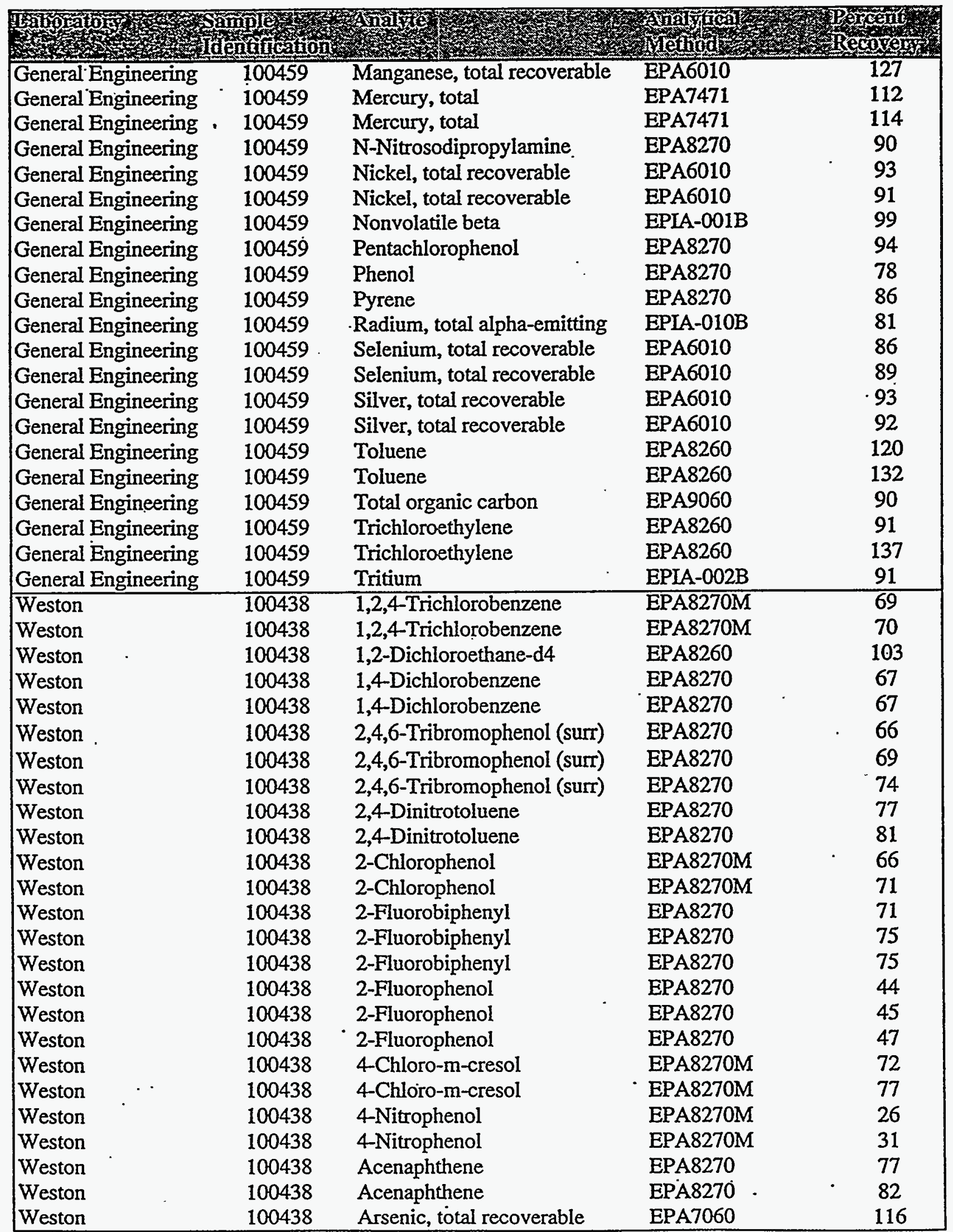


Table 7

Recoveries of Matrix Spike and Surrogate Samples

D-Area Buming/Rubble Pits

Savannah River Site

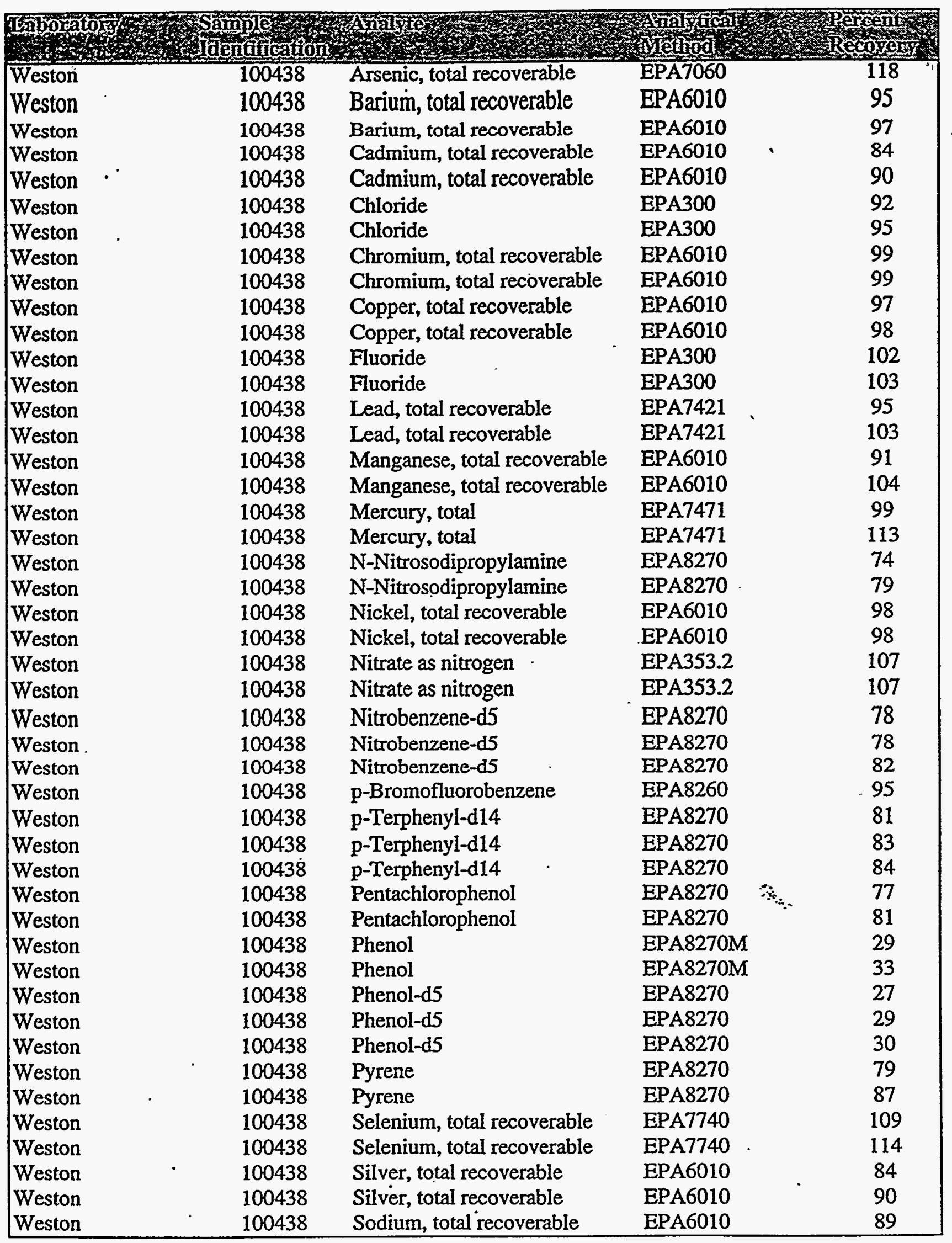


Table 7

Recoveries of Matrix Spike and Surrogate Samples

D-Area Burning/Rubble Pits

Savannah River Site

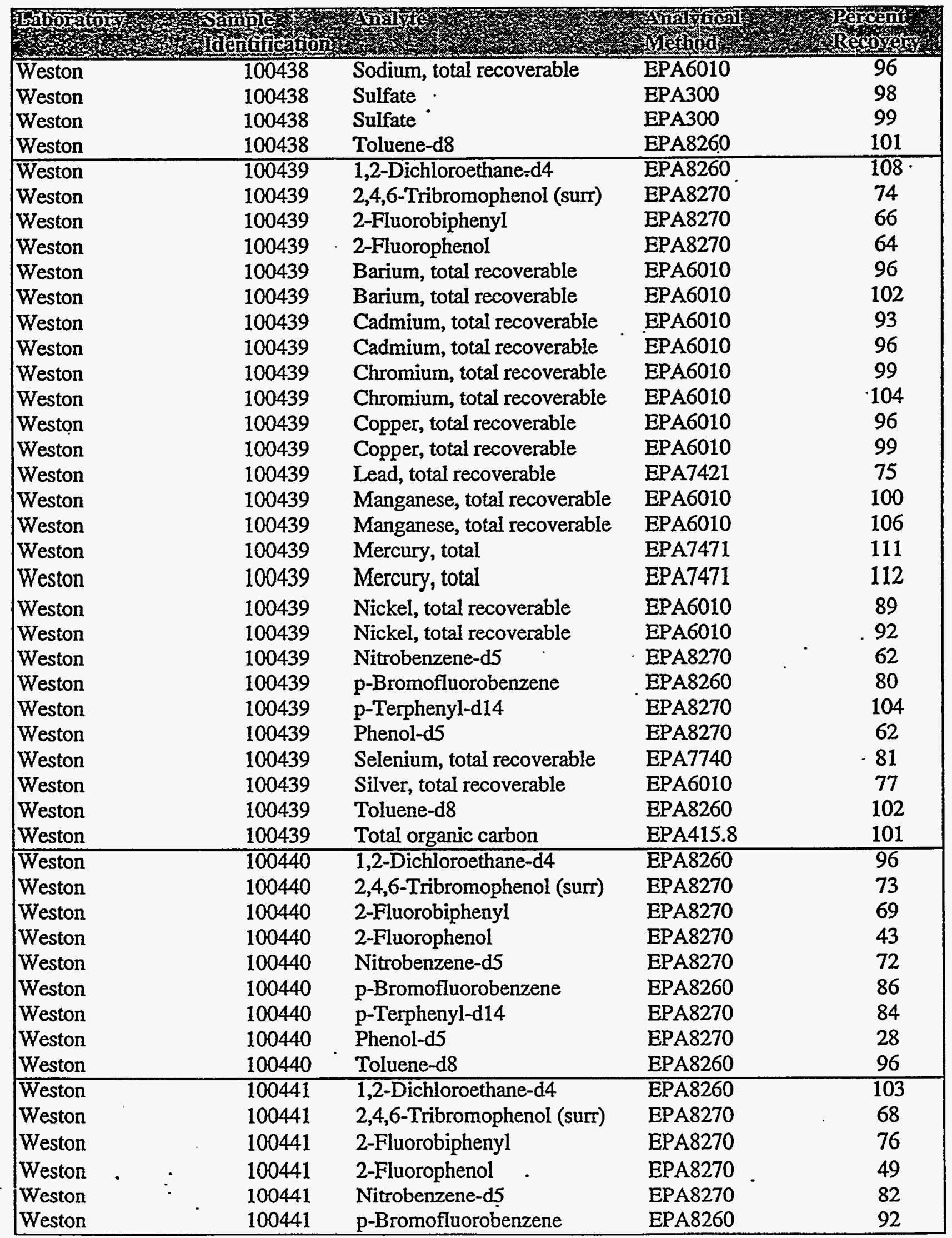


Table 7

Recoveries of Matrix Spike and Surrogate Samples

D-Area Burning/Rubble Pits

Savannah River Site

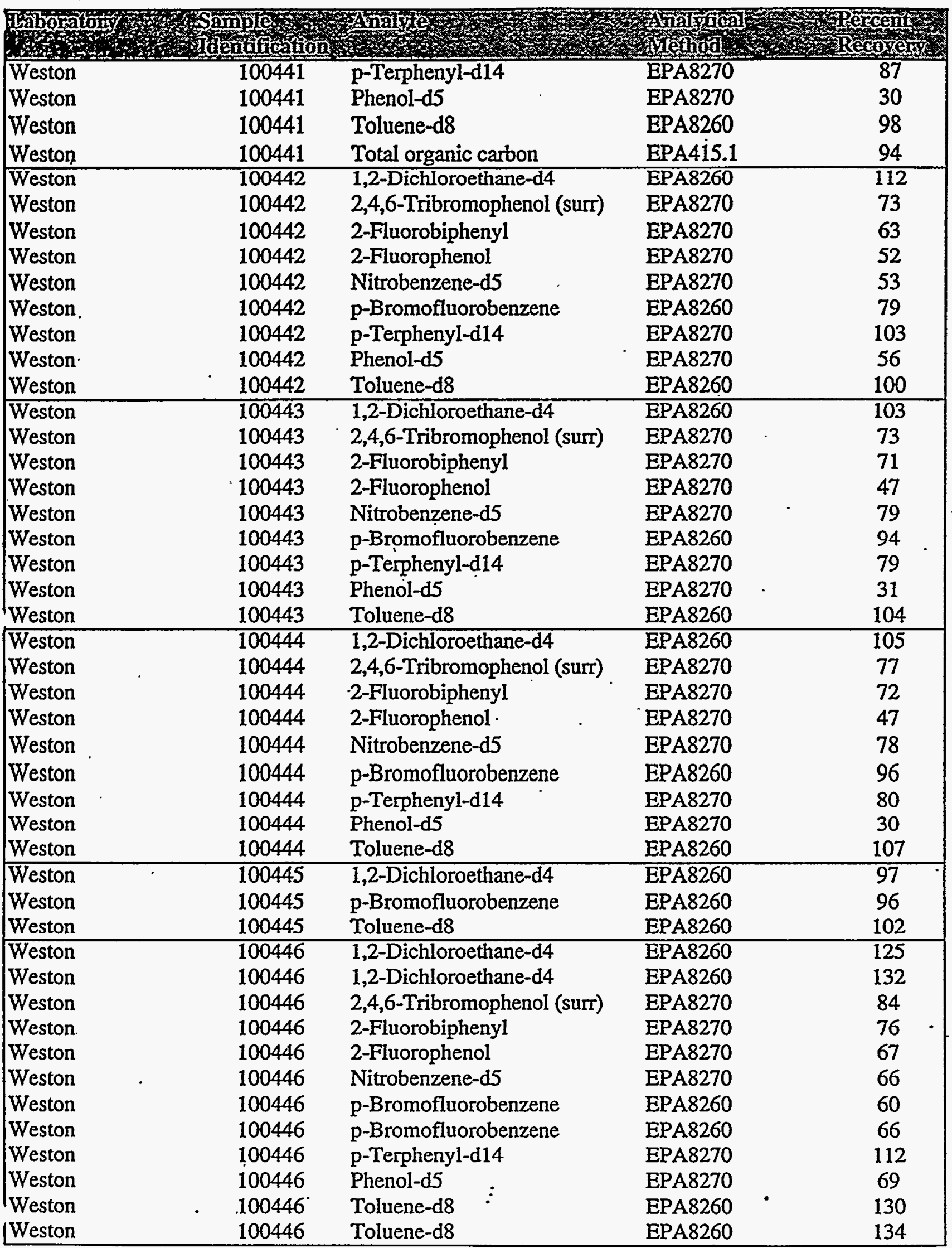


Table 7

Recoveries of Matrix Spike and Surrogate Samples .

D-Area Buming/Rubble Pits

Savannah River Site

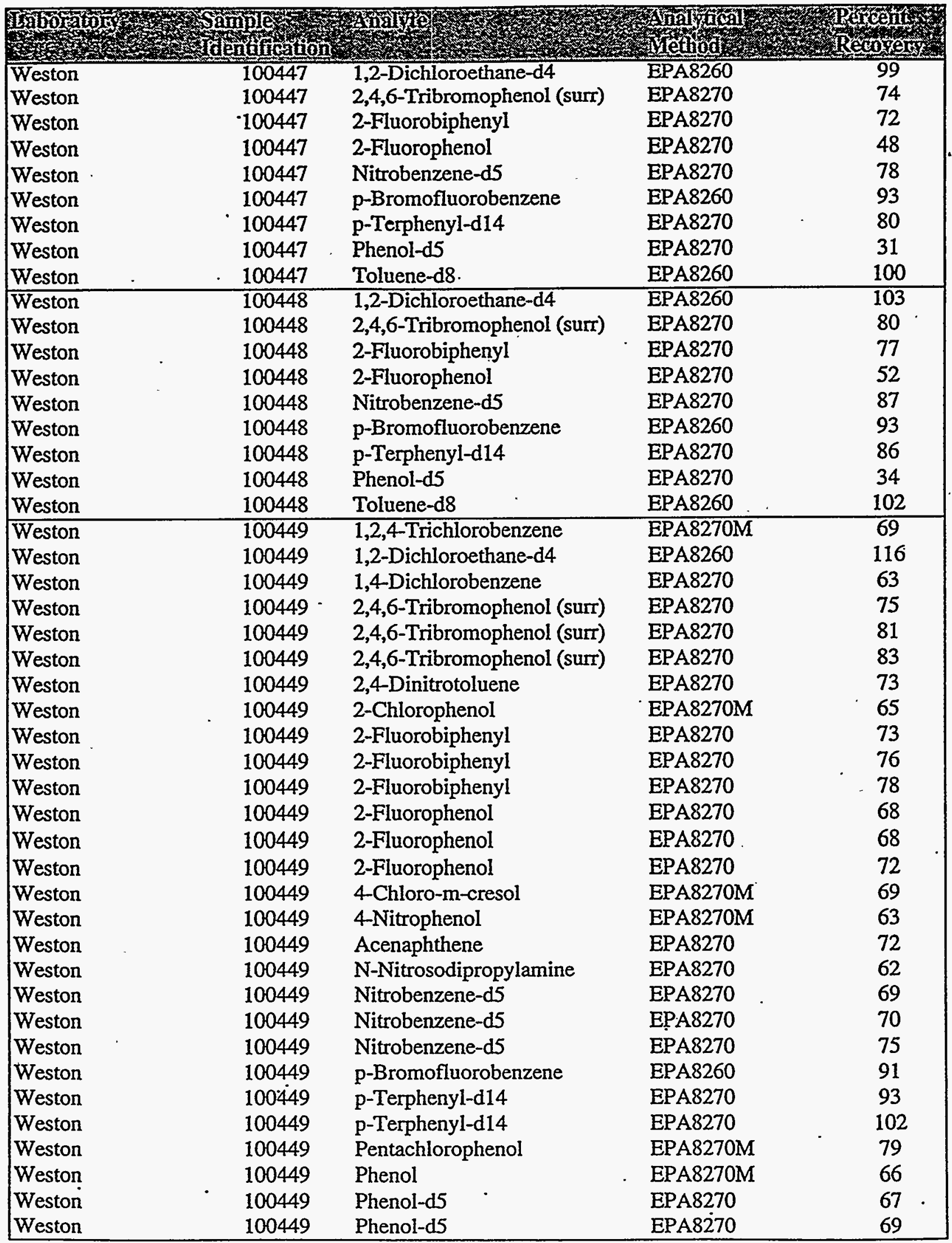


Table 7

Recoveries of Matrix Spike and Surrogate Samples

D-Area Burning/Rubble Pits

Savannah River Site

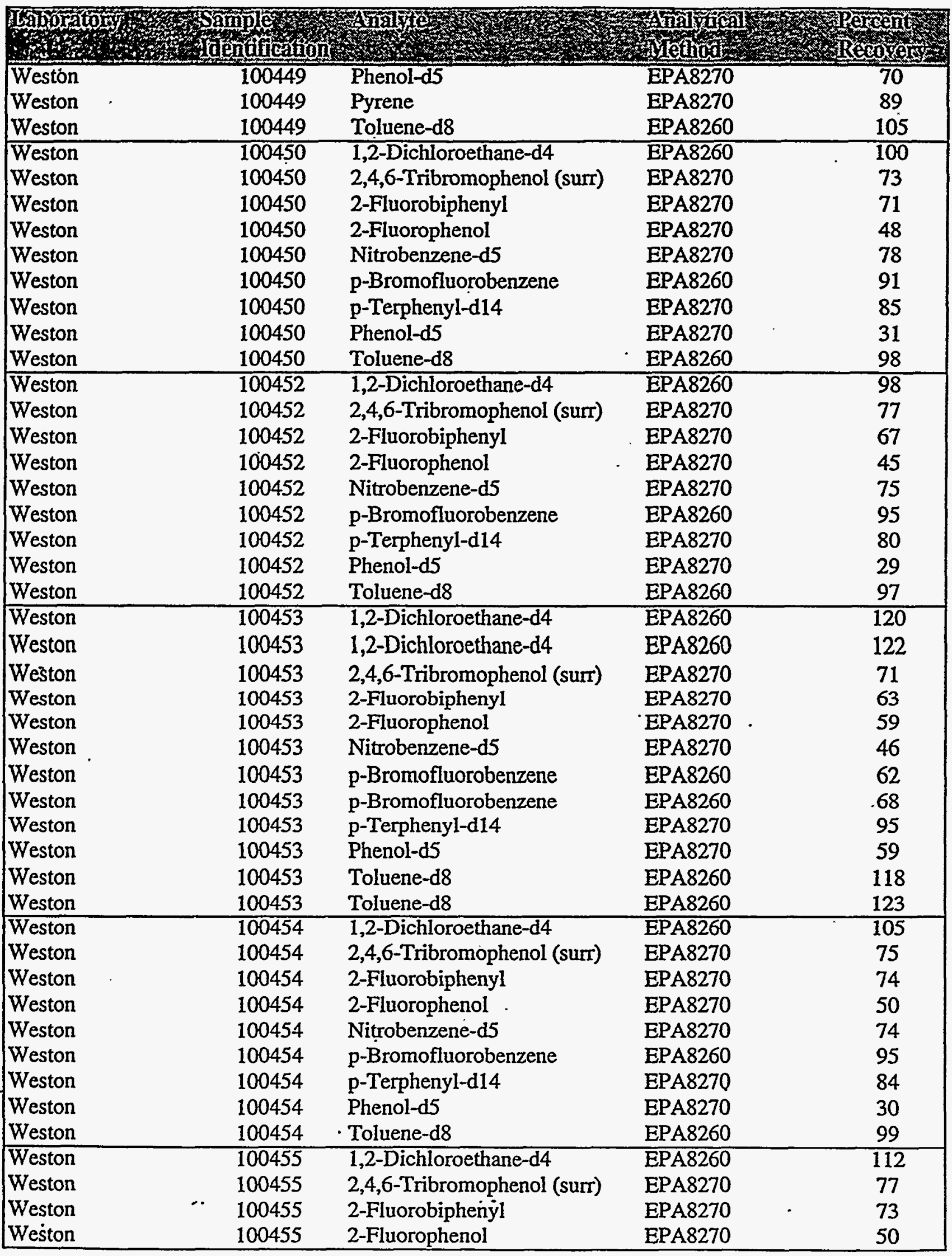


Table 7

Recoveries of Matrix Spike and Surrogate Samples

D-Area Buming/Rubble Pits

Savannah River Site

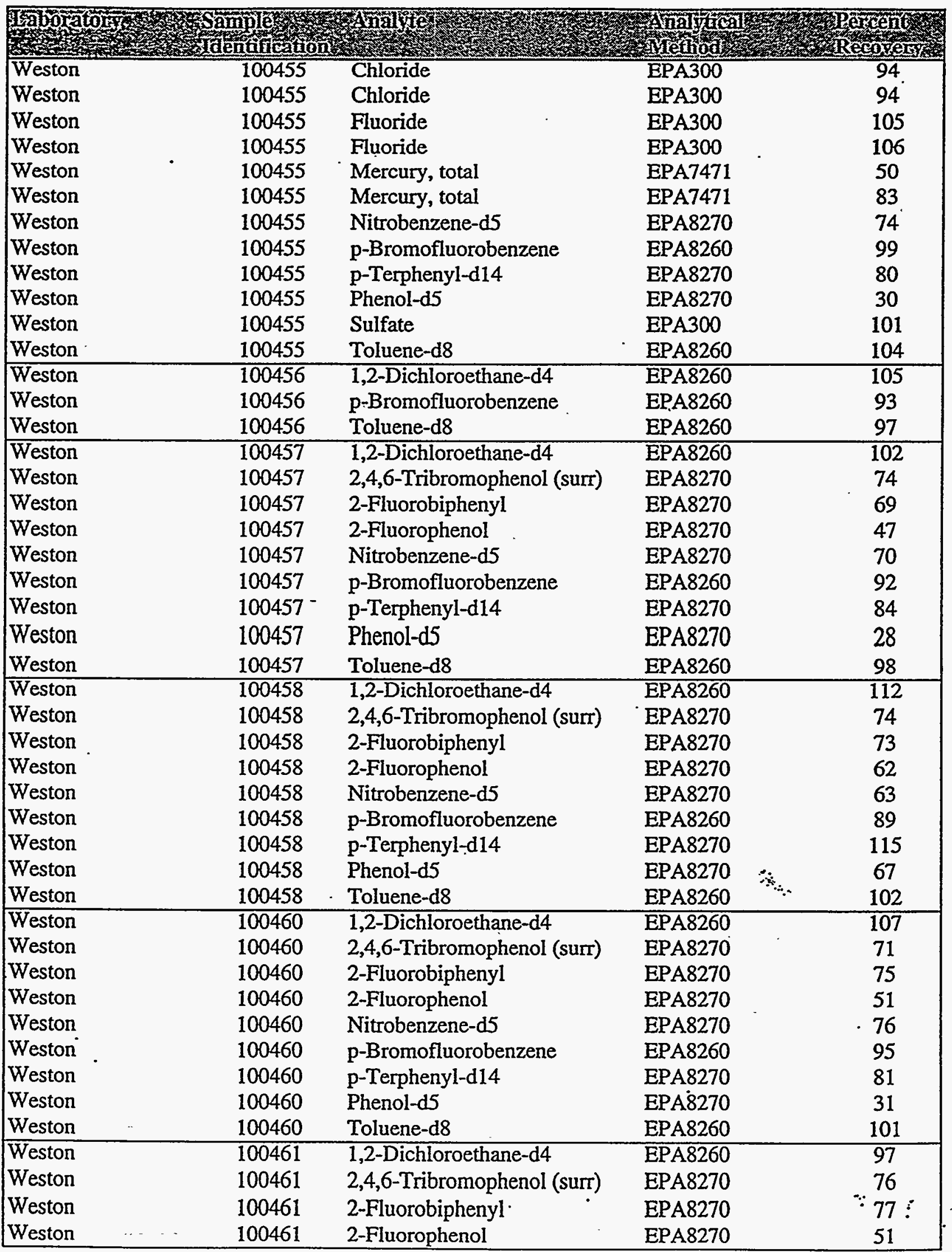


Table 7

Recoveries of Matrix Spike and Surrogate Samples

D-Area Burning/Rubble Pits

Savannah River Site

\begin{tabular}{|c|c|c|c|c|}
\hline \multicolumn{5}{|c|}{ 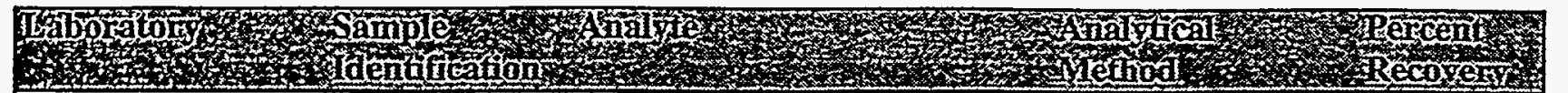 } \\
\hline Weston & 100461 & Nitrobenzene-d5 & EPA8270 & 77 \\
\hline Weston & 100461 & p-Bromofluorobenzene & EPA8260 & 92 \\
\hline Weston & 100461 & p-Terphenyl-d14 & EPA8270 & 83 \\
\hline Weston & 100461 & Phenol-d5 & EPA8270 & 31 \\
\hline Weston & 100461 & Toluene-d8 & EPA8260 & 103 \\
\hline Weston & 100462 & 1,2-Dichloroethane-d4 & EPA8260 & 111 \\
\hline Weston & 100462 & 2,4,6-Tribromophenol (sur) & EPA8270 & 78 \\
\hline Weston & 100462 & 2-Fluorobiphenyl & EPA8270 & 72 \\
\hline Weston & 100462 & 2-Fluorophenol & EPA8270 & 66 \\
\hline Weston & 100462 & Nitrobenzene-d5 & EPA8270 & 64 \\
\hline Weston & 100462 & p-Bromofluorobenzene & EPA8260 & 78 \\
\hline Weston & 100462 & p-Terphenyl-d14 & EPA8270 & 105 \\
\hline Weston & 100462 & Phenol-d5 & EPA8270 & 66 \\
\hline Weston & 100462 & Toluene-d8 & EPA8260 & 101 \\
\hline Weston & 100463 & 1,2-Dichloroethane-d4 & EPA8260 & 109 \\
\hline Weston & 100463 & 2,4,6-Tribromophenol (surr) & EPA8270 & 78 \\
\hline Weston & 100463 & 2-Fluorobiphenyl & EPA8270 & 71 \\
\hline Weston & 100463 & 2-Fluorophenol & EPA8270 & 63 \\
\hline Weston & 100463 & Nitrobenzene-d5 & EPA8270 & 62 \\
\hline Weston & 100463 & p-Bromofluorobenzene & EPA8260 & 79 \\
\hline Weston & 100463 & p-Terphenyl-d14 & EPA8270 & 105 \\
\hline Weston & 100463 & Phenol-d5 & EPA8270 & 63 \\
\hline Weston & 100463 & Toluene-d8 & EPA8260 & 95 \\
\hline Weston & 100464 & 1,2-Dichloroethane-d4 & EPA8260 & 108 \\
\hline Weston & 100464 & 2,4,6-Tribromophenol (surr) & EPA8270 & 64 \\
\hline Weston & 100464 & 2-Fluorobiphenyl & EPA8270 & 58 \\
\hline Weston & 100464 & 2-Fluorophenol & EPA8270 & 78 \\
\hline Weston & 100464 & Nitrobenzene-d5 & EPA8270 & 67 \\
\hline Weston & 100464 & p-Bromofluorobenzene & EPA8260 & 80 \\
\hline Weston & 100464 & p-Terphenyl-d14 & EPA8270 & 114 \\
\hline Weston & 100464 & Phenol-d5 & EPA8270 & 78 \\
\hline Weston & 100464 & Toluene-d8 & EPA8260 & 100 \\
\hline Weston & 100465 & 1,2-Dichloroethane-d4 & EPA8260 & 110 \\
\hline Weston & 100465 & 2,4,6-Tribromophenol (surr) & EPA8270 & 89 \\
\hline Weston & 100465 & 2-Fluorobiphenyl & EPA8270 & 70 \\
\hline Weston & 100465 & 2-Fluorophenol & EPA8270 & 88 \\
\hline Weston & 100465 & Nitrobenzene-d5 & EPA8270 & 76 \\
\hline Weston & 100465 & p-Bromofluorobenzene & EPA8260 & 94 \\
\hline Weston & 100465 & p-Terphenyl-d14 & EPA8270 & 119 \\
\hline Weston & 100465 & Phenol-d5 & EPA8270 & 86 \\
\hline Weston & 100465 & Toluene-d8 & EPA8260 & 92 \\
\hline Weston & 100466 & 1,1-Dichloroethylene & EPA8260 & 88 \\
\hline Weston & 100466 & 1,2-Dichloroethane-d4 & EPA8260 & 112 \\
\hline Weston & 100466 & 1,2-Dichloroethane-d4 & EPA8260 & 112 \\
\hline Weston' & 100466 & 1,2-Dichloroethane-d4 & EPA8260 & 114 \\
\hline Weston & 100466 & 2,4,6-Tribromophenol (surr) & EPA8270 & 30 \\
\hline
\end{tabular}


Table 7

Recoveries of Matrix Spike and Surrogate Samples

D-Area Burning/Rubble Pits

Savannah River Site

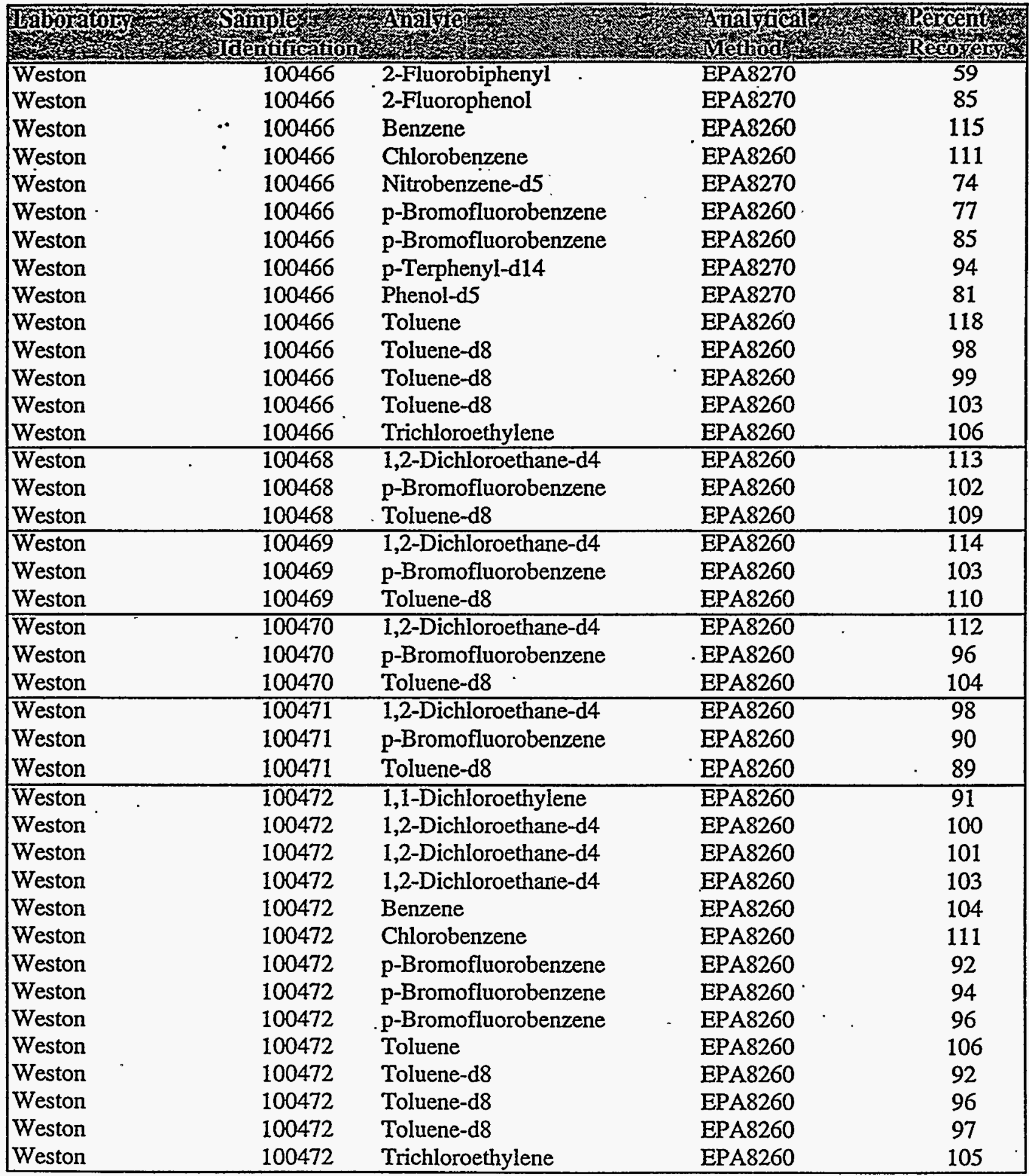


Taule 8

Detection Summary of Rinsate Blank

D-Area Burning/Rubble Pits

Savannah River Site

\begin{tabular}{|c|c|c|c|c|c|c|c|}
\hline \multirow{2}{*}{\multicolumn{8}{|c|}{ 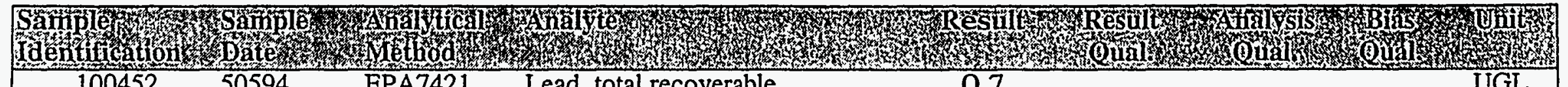 }} \\
\hline 100452 & 50594 & EPA7421 & Lead, total recoverable & & & & \\
\hline 100452 & 50594 & EPA8260 & 1,1,1-Trichloroethane & 0.34 & $\mathbf{J}$ & B & UGL \\
\hline 100452 & 50594 & EPA8270 & Bis(2-ethylhexyl) phthalate & 2.7 & $J$ & $\mathrm{~B}$ & UGL: \\
\hline
\end{tabular}


Table 9

Detection Summary of Trip Blanks

D-Area Burning/Rubble Pits

Savannah River Site

\begin{tabular}{|c|c|c|c|c|c|c|c|c|}
\hline 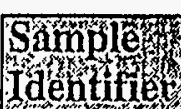 & Miple & Why & Fin & & & & & 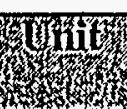 \\
\hline 100467 & 50694 & EPA8260 & Toluene & 0.45 & $\mathrm{~J}$ & $\mathrm{~V}$ & $\mathrm{H}$ & UGL \\
\hline 100467 & 50694 & EPA8260 & Dichloromethane (Methylene chloride) & 0.50 & $\mathrm{~J}$ & $\mathrm{~V}$ & $\mathrm{H}$ & UGL \\
\hline 100468 & 50694 & EPA8260 & Chloroform & 0.39 & & & & UGL \\
\hline 100468 & 50694 & EPA8260 & Trichlorofluoromethane & 0.13 & $\mathrm{~J}$ & B. & & UGL \\
\hline 100469 & 50694 & EPA8260 & Chloroform & 0.40 & & & & UGL \\
\hline 100469 & 50694 & EPA8260 & Trichlorofluoromethane & 0.13 & $\mathrm{~J}$ & B & & UGL \\
\hline 100470 & 50694 & EPA8260 & Chloroform & 0.40 & & & & UGL \\
\hline 100470 & 50694 & EPA8260 & Trichlorofluoromethane & 0.13 & J & B & & UGL \\
\hline 100471 & 50694 & EPA8260 & Chloroform & 0.34 & & & & UGL \\
\hline 100471 & 50694 & EPA8260 & Trichlorofluoromethane & 0.10 & $\mathrm{~J}$ & $\mathrm{~B}$ & & UGL \\
\hline 100472 & 50694 & EPA8260 & Chloroform & 0.34 & & & & UGL \\
\hline 100472 & 50694 & EPA8260 & 1,1-Dichloroethylene & 4.57 & & & & UGL \\
\hline 100472 & 50694 & EPA8260 & Benzene & 5.21 & & & & UGL \\
\hline 100472 & 50694 & EPA8260 & Trichloroethylene & 5.26 & & & & UGL \\
\hline 100472 & 50694 & EPA8260 & Toluene & 5.31 & & & & UGL \\
\hline 100472 & 50694 & EPA8260 & Chlorobenzene & 5.53 & & & & UGL \\
\hline
\end{tabular}


TaL 10

Detection Summary of Field Blanks

D-Area Burning/Rubble Pits

Savannah River Site

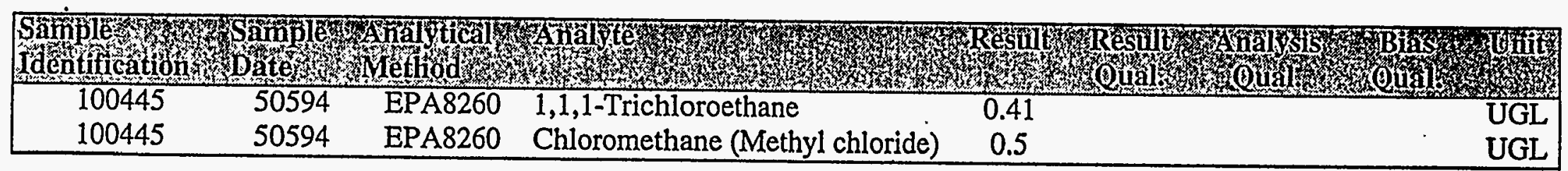

100445

100445

50594 
Table 11

Evaluation of Surface Water Field Duplicates

D-Area Burning/Rubble Pits

Savannah River Site

\begin{tabular}{|c|c|c|c|c|c|}
\hline 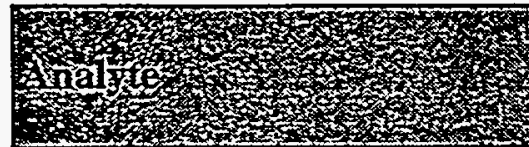 & (x) & Shing & 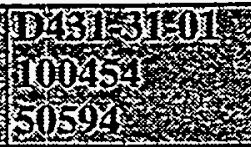 & 145151018 & Pelations \\
\hline $1,1,1,2$-Tetrachloroethane & & UGL & $0.4 \mathrm{U} / /$ & $0.4 \mathrm{U} / 1$ & \\
\hline 1,1,1-Trichloroethane & . & UGL & $0.4 \mathrm{U} / /$ & $0.4 \mathrm{U} / /$ & \\
\hline 1,1,2-Trichloroethane & & UGL & $0.8 \mathrm{U} / /$ & $0.8 \mathrm{U} / /$ & \\
\hline 1,1-Dichloroethane & & UGL & $0.3 \mathrm{U} / /$ & $0.3 \mathrm{U} / /$ & \\
\hline 1,1-Dichloroethylene & & UGL & $0.5 \mathrm{U} / /$ & $0.5 \mathrm{U} / /$ & \\
\hline 1,2,4-Trichlorobenzene & & UGL & $10 \mathrm{U} / /$ & $10 \mathrm{U} / /$ & \\
\hline 1,2-Dichlorobenzene & & UGL & $10 \mathrm{U} / /$ & $10 \mathrm{U} / /$ & \\
\hline 1,2-Dichloroethane & - & UGL & $0.07 \mathrm{U} / /$ & $0.07 \mathrm{U} / /$ & \\
\hline 1,2-Dichloroethylene & & UGL & $0.3 \mathrm{U} / /$ & $0.3 \mathrm{U} / /$ & \\
\hline 1,2-Dichloropropane & & UGL. & $0.2 \mathrm{U} / /$ & $0.2 \mathrm{U} / /$ & \\
\hline 1,3-Dichlorobenzene & & UGL & $10 \mathrm{U} / /$ & $10 \mathrm{U} / /$ & \\
\hline 1,4-Dichlorobenzene & & UGL & $10 \mathrm{U} / /$ & $10 \mathrm{U} / /$ & \\
\hline 2,4,5-Trichlorophenol & & UGL & $50 \mathrm{U} / /$ & $50 \mathrm{U} / /$ & \\
\hline 2,4,6-Trichlorophenol & & UGL & $10 \mathrm{U} / /$ & $10 \mathrm{U} / /$ & \\
\hline 2,4-Dichlorophenol & & UGL & $10 \mathrm{U} / /$ & $10 U / /$ & \\
\hline 2,4-Dimethyl phenol & & UGL & $10 \mathrm{U} / /$ & $10 \mathrm{U} / /$ & \\
\hline 2,4-Dinitrophenol & & UGL & $50 \mathrm{U} / /$ & $50 \mathrm{U} / /$ & \\
\hline 2,4-Dinitrotoluene & & UǴL & $10 \mathrm{U} / /$ & $10 \mathrm{U} / /$ & \\
\hline 2,6-Dinitrotoluene & & UGL & $10 \mathrm{U} / /$ & $10 \mathrm{U} / /$ & \\
\hline 2-Chloroethyl vinyl ether & & UGL & $10 \mathrm{U} / /$ & $10 \mathrm{U} / /$ & \\
\hline 2-Chloronaphthalene & & UGL & $10 \mathrm{U} / /$ & $10 \mathrm{U} / /$ & \\
\hline 2-Chlorophenol & & UGL & $10 \mathrm{U} / /$ & $10 \mathrm{U} / /$ & \\
\hline 2-Methyl-4,6-dinitrophenol & & UGL & $50 \mathrm{U} / /$ & $50 \mathrm{U} / /$ & \\
\hline 2-Methylnaphthalene & & UGL & $10 \mathrm{U} / /$ & $10 \mathrm{U} / /$ & \\
\hline 2-Nitrophenol & & UGL & $10 \mathrm{U} / /$ & $10 \mathrm{U} / /$ & \\
\hline 3,3'-Dichlorobenzidine & & UGL & $20 \mathrm{U} / /$ & $20 \mathrm{U} / /$ & \\
\hline 4-Bromophenyl phenyl ether & & UGL & $10 \mathrm{U} / /$ & $10 \mathrm{U} / /$ & \\
\hline 4-Chloro-m-cresol & & UGL & $10 \mathrm{U} / /$ & $10 \mathrm{U} / /$ & \\
\hline 4-Chloroaniline & & UGL & $10 \mathrm{U} / /$ & $10 \mathrm{U} / /$ & \\
\hline 4-Chlorophenyl phenyl ether & & UGL & $10 \mathrm{U} / /$ & $10 \mathrm{U} / /$ & \\
\hline 4-Nitrophenol & & UGL & $50 \mathrm{U} / /$ & $50 \mathrm{U} / /$ & \\
\hline Acenaphthene & & UGL & $10 \mathrm{U} / /$ & $10 \mathrm{U} / /$ & \\
\hline Acenaphthylene & & UGL & $10 \mathrm{U} / /$ & $10 \mathrm{U} / /$ & \\
\hline Anthracene & - & UGL & $10 \mathrm{U} / /$ & $10 \mathrm{U} / /$ & \\
\hline Arsenic, total recoverable & & UGL & $1.7 \mathrm{U} / /$ & $1.7 \cdot \mathrm{U} / /$ & \\
\hline Barium, total recoverable & & UGL & $13.9 / /$ & $14 / /$ & 0.7 \\
\hline Benzene & & UGL & $0.3 \mathrm{U} / /$ & $0.3 \mathrm{U} / /$ & \\
\hline Benzo(a)anthracene & & UGL & $10 \mathrm{U} / /$ & $10 \mathrm{U} / /$ & \\
\hline Benzo(a)pyrene & & UGL & $10 \mathrm{U} / /$ & $10 \mathrm{U} / /$ & \\
\hline Benzo(b)fluoranthene & & UGL & $10 \mathrm{U} / /$ & $10 \mathrm{U} / /$ & \\
\hline
\end{tabular}


Table 11

Evaluation of Surface Water Field Duplicates

D-Area Burning/Rubble Pits

Savannah River Site

\begin{tabular}{|c|c|c|c|c|}
\hline 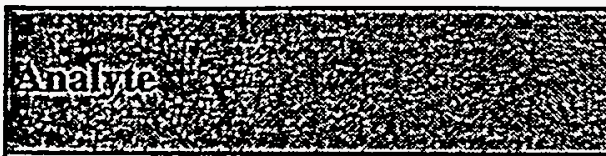 & Him & 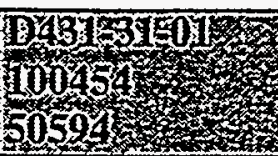 & 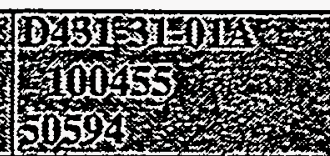 & 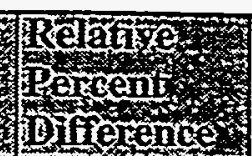 \\
\hline Benzo(g,h,i)perylene & UGL & $10 \mathrm{U} / \mathrm{I}$ & $10 \mathrm{U} / \mathrm{I}$ & \\
\hline Benzo(k)fluoranthene & UGL: & $10 \mathrm{U} / /$ & $10 \mathrm{U} / /$ & \\
\hline Benzoic acid & UGL & $50 \mathrm{U} / /$ & $50 \mathrm{U} / /$ & \\
\hline Benzyl alcohol & UGL & $10 \mathrm{U} / /$ & $10 \mathrm{U} / /$ & \\
\hline Bis(2-chloroethoxy) methane & UGL & $10 \mathrm{U} / /$ & $10 \mathrm{U} / /$ & \\
\hline Bis(2-chloroethyl) ether & UGL & $10 U / /$ & $10 \mathrm{U} / /$ & \\
\hline Bis(2-chloroisopropyl) ether & UGL & $10 \mathrm{U} / /$ & $10 U / /$ & \\
\hline Bis(2-ethylhexyl) phthalate & UGL & $10 \mathrm{U} / /$ & $10 \mathrm{U} / /$ & \\
\hline Bromoform & UGL & $2 U / /$ & $2 U / /$ & \\
\hline Bromomethane (Methyl bromide) & UGL & $0.6 \mathrm{U} / /$ & $0.6 \mathrm{U} / /$ & \\
\hline Butylbenzyl phthalate & UGL & 10.U// & $10 \mathrm{U} / /$ & \\
\hline Cadmium, total recoverable & UGL & $3.3 \mathrm{U} / /$ & $3.3 \mathrm{U} / /$ & \\
\hline Carbon tetrachloride & UGL & $0.2 \mathrm{U} / /$ & $0.2 . U / /$ & \\
\hline Chloride & UGL & $10700 / /$ & $9970 / /$ & 7.1 \\
\hline Chlorobenzene & UGL & $0.2 \mathrm{U} / /$ & $0.2 \mathrm{U} / /$ & \\
\hline Chloroethane & UGL & $0.2 \mathrm{U} / /$ & $0.2 \mathrm{U} / /$ & \\
\hline Chloroethene (Vinyl chloride) & UGL & $0.4 \mathrm{U} / /$ & $0.4 \mathrm{U} / /$ & \\
\hline Chloroform & UGL & $0.3 \mathrm{U} / !$ & $0.3 \mathrm{U} / /$ & \\
\hline Chloromethane (Methyl chloride) & UGL & $0.3 \mathrm{U} / \mathrm{V} /$ & $0.3 \mathrm{U} / \mathrm{V} /$ & \\
\hline Chromium, total recoverable & UGL & $3.3 \mathrm{U} / /$ & $3.3 \mathrm{U} / /$ & \\
\hline Chrysene & UGL & $10 \mathrm{U} / /$ & $10 \mathrm{U} / /$ & \\
\hline cis-1,3-Dichloropropene & UGL & $2 U / /$ & $2 U / /$ & \\
\hline Copper, total recoverable & UGL & $3.3 \mathrm{U} / /$ & $3.3 \mathrm{U} / /$ & \\
\hline Di-n-butyl phthalate & UGL & $10 \mathrm{U} / /$ & $10 \mathrm{U} / /$ & \\
\hline Di-n-octyl phthalate & UGL & $10 \mathrm{U} / /$ & $10 \mathrm{U} / /$ & \\
\hline Dibenz $(\mathrm{a}, \mathrm{h})$ anthracene & UGL & $10 \mathrm{U} / /$ & $10 \mathrm{U} / /$ & \\
\hline Dibenzofuran & UGL & $10 \mathrm{U} / \mathrm{I}$ & $10 \mathrm{U} / /$ & \\
\hline Dibromochloromethane & UGL & $0.5 \mathrm{U} / /$ & $0.5 \mathrm{U} / /$ & \\
\hline Diethyl phthalate & UGL & $10 \mathrm{U} / /$ & $10 \mathrm{U} / /$ & \\
\hline Dimethyl phthalate & UGL & $10 \mathrm{U} / /$ & $10 \mathrm{U} / /$ & \\
\hline Ethylbenzene & UGL & $0.3 \mathrm{U} / /$ & $0.3 \mathrm{U} / /$ & \\
\hline Fluoranthene & UGL & $10 \mathrm{U} / /$ & $10 U / /$ & \\
\hline Fluorene & UGL & $10 \mathrm{U} / /$ & $10 \mathrm{U} / /$ & \\
\hline Fluoride & UGL & $72.7 \mathrm{U} / /$ & $72.7 \mathrm{U} / \mathrm{I}$ & \\
\hline Gross alpha & PCL & $0.33 \mathrm{U} / /$ & $0.32 \mathrm{U} / /$ & \\
\hline Hexachlorobenzene & UGL & $10 \mathrm{U} / /$ & $10 \mathrm{U} / /$ & \\
\hline Hexachlorobutadiene & UGL & $10 \mathrm{U} / /$ & $10 \mathrm{U} / /$ & . \\
\hline Hexachlorocyclopentadiene & UGL & $10 \mathrm{U} / \mathrm{I}$ & $10 \mathrm{U} / /$ & \\
\hline Hexachloroethane & UGL & $10 \mathrm{U} / /$ & $10 \mathrm{U} / /$ & \\
\hline Indeno $(1,2,3-c, d)$ pyrene & UGL & $10 \mathrm{U} / /$ & $10 U / /$ & \\
\hline
\end{tabular}


Table 11

Evaluation of Surface Water Field Duplicates

D-Area Burning/Rubble Pits

Savannah River Site

\begin{tabular}{|c|c|c|c|c|}
\hline 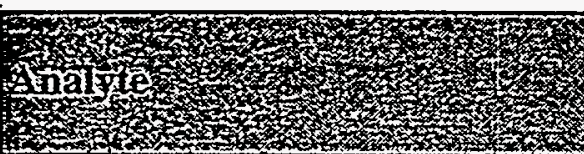 & Hith & $004514-y^{3}$ & 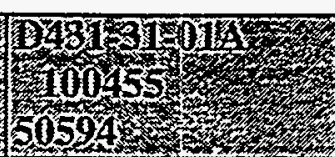 & $\begin{array}{l}\text { Melange } \\
\text { Dercent } \\
\text { Diffexence }\end{array}$ \\
\hline Iron, total recoverable & UGL & $103 / /$ & $93.1 / /$ & .10 .1 \\
\hline Isophorone & UGL: & $10 \mathrm{U} / /$ & $10 \mathrm{U} / /$ & \\
\hline Lead, total recoverable & UGL & $0.6 / /$ & $0.5 \mathrm{U} / /$ & 18.2 \\
\hline m-Nitroaniline & UGL & $50 \mathrm{U} / /$ & $50 \mathrm{U} / /$ & \\
\hline Manganese, total recoverable & UGL & $35.8 / /$ & $35.5 / /$ & 0.8 \\
\hline Mercury, total & UGL & $0.067 \mathrm{U} / /$ & $0.067 \mathrm{~J} / \mathrm{I} / \mathrm{P}$ & 0.0 \\
\hline N-Nitrosodiphenylamine & UGL & $10 \mathrm{U} / /$ & $10 \mathrm{U} / /$ & \\
\hline N-Nitrosodipropylamine & UGL & $10 \mathrm{U} / /$ & $10 \mathrm{U} / \mathrm{I}$ & \\
\hline Naphthalene & UGL & $10 \mathrm{U} / /$ & $10 \mathrm{U} / /$ & \\
\hline Nickel, total recoverable & UGL & $11.7 \mathrm{U} / !$ & $11.7 \mathrm{U} / /$ & \\
\hline Nitrate as nitrogen & UGL & $129 / /$ & $83.3 \mathrm{U} / /$ & 43.1 \\
\hline Nitrobenzene & UGL & $10 \mathrm{U} / /$ & $10 \mathrm{U} / /$ & \\
\hline Nonvolatile beta & PCL & $1.1 \mathrm{U} / /$ & $1.4 \mathrm{~J} / /$ & 24.0 \\
\hline o-Cresol (2-Methylphenol) & UGL & $10 \mathrm{U} / /$ & $10 \mathrm{U} / /$ & \\
\hline o-Nitroaniline & UGL & $50 \mathrm{U} / /$ & $50 \mathrm{U} / /$ & \\
\hline p-Cresol (4-Methylphenol) & ƯGL & $10 \mathrm{U} / /$ & $10 \mathrm{U} / /$ & \\
\hline p-Nitroaniline & UGL & $50 \mathrm{U} / /$ & $50 \mathrm{U} / /$ & \\
\hline Pentachlorophenol & UGL & $50 \mathrm{U} / /$ & $50 \mathrm{U} / /$ & \\
\hline Phenanthrene & UGL & $10 \mathrm{U} / /$ & $10 \mathrm{U} / /$ & \\
\hline Phenol & UGL & $10 \mathrm{U} / /$ & $10 \mathrm{U} / /$ & \\
\hline Pyrene & UGL & $10 \mathrm{U} / /$ & $10 \mathrm{U} / /$ & \\
\hline Radium, total alpha-emitting & $\mathrm{PCL}$ & $0.1 \mathrm{U} / /$ & $0.12 \mathrm{U} / /$ & \\
\hline Selenium, total recoverable & UGL & $1.7 \mathrm{U} / /$ & $1.7 \mathrm{U} / /$ & \\
\hline Silver, total recoverable & UGL & $0.7 \mathrm{U} / /$ & $0.7 \mathrm{U} / /$ & \\
\hline Sodium, total recoverable & UGL & 9840 // & 9950 // & 1.1 \\
\hline Sulfate & UGL & $9600 / /$ & $9090 / /$ & 5.5 \\
\hline Tetrachloroethylene & UGL & $0.5 \mathrm{U} / /$ & $0.5 \mathrm{U} / /$ & \\
\hline Toluene & UGL & $0.8 \mathrm{U} / /$ & $0.8 \mathrm{U} / /$ & \\
\hline Total dissolved solids & UGL & $48000 / /$ & $59000 / /$ & 20.6 \\
\hline Total organic carbon & UGL & $1660 / /$ & $1660 / /$ & 0.0 \\
\hline Total organic halogens & UGL & $3330 \mathrm{U} / /$ & $3330 \mathrm{U} / /$ & \\
\hline Total suspended solids & UGL & $1670 \mathrm{U} / /$ & $1670 \mathrm{U} / /$ & \\
\hline trans-1,3-Dichloropropene & UGL & $2 \mathrm{U} / /$ & $2 U / /$ & \\
\hline Trichloroethylene & UGL & $0.2 \mathrm{U} / /$ & $0.2 \mathrm{U} / /$ & \\
\hline Trichlorofluoromethane & UGL & $0.7 \mathrm{U} / /$ & $0.7 \mathrm{U} / /$ & \\
\hline Tritium & PCML & $0.53 / /$ & $0.4 \mathrm{~J} / /$ & 28.0 \\
\hline
\end{tabular}


Table 12

Evaluation of Sediment Field Duplicates

D-Area Buming/Rubble Pits

Savannah River Site

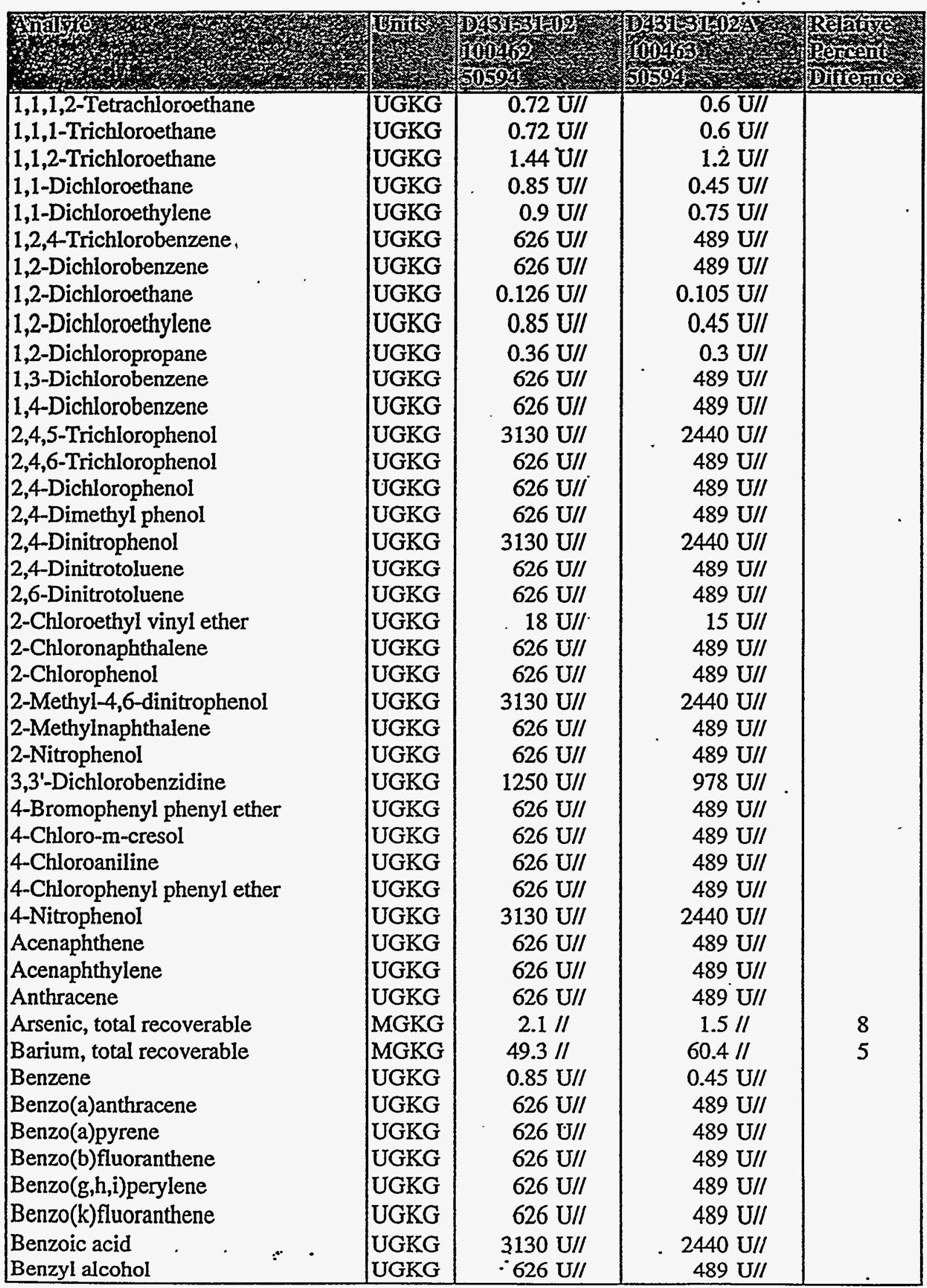


Table 12

Evaluation of Sediment Field Duplicates

D-Area Burning/Rubble Pits

Savannah River Site

\begin{tabular}{|c|c|c|c|c|}
\hline 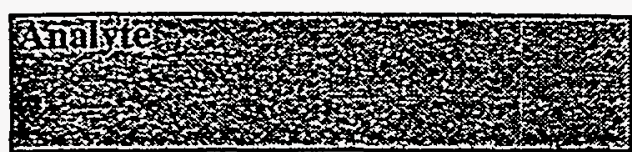 & 7) & 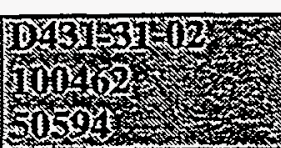 & 1045170 & 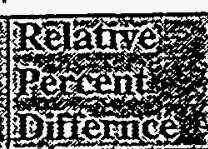 \\
\hline Bis(2-chloroethoxy) methane & UGKG & $626 \mathrm{U} / 1$ & $489 \mathrm{U} / \mathrm{I}$ & \\
\hline Bis(2-chloroethyl) ether & UGKG & $626 \mathrm{U} / /$ & $489 \mathrm{U} / /$ & \\
\hline Bis(2-chloroisopropyl) ether & UGKG & $626 \mathrm{U} / /$ & $489 \mathrm{U} / /$ & \\
\hline Bis(2-ethylhexyl) phthalate & UGKG & $626 \mathrm{U} / /$ & $489 \mathrm{U} / /$ & \\
\hline Bromoform & UGKG & $3.6 \mathrm{U} / /$ & $.3 \mathrm{U} / /$ & \\
\hline Bromomethane (Methyl bromide) & UGKG & $1.08 \mathrm{U} / /$ & $0.9 \mathrm{U} / /$ & \\
\hline Butylbenzyl phthalate & UGKG & $626 \mathrm{U} / /$ & $489 \mathrm{U} / /$ & \\
\hline Cadmium, total recoverable & MGKG & $1.5 \mathrm{U} / /$ & $1.2 \mathrm{U} / /$ & \\
\hline Carbon tetrachloride & UGKG & $0.36 \mathrm{U} / /$ & $0.3 \mathrm{U} / /$ & \\
\hline Chlorobenzene & UGKG & $0.36 \mathrm{U} / /$ & $0.3 \mathrm{U} / /$ & \\
\hline Chloroethane & UGKG & $0.36 \mathrm{U} / /$ & $0.3 \mathrm{U} / /$ & \\
\hline Chloroethene (Vinyl chloride) & UGKG & $0.72 \mathrm{U} / /$ & $0.6 \mathrm{U} / /$ & \\
\hline Chloroform & UGKG & $0.85 \mathrm{U} / \mathrm{I}$ & $0.45 \mathrm{U} / /$ & \\
\hline Chloromethane (Methyl chloride) & UGKG & $0.9 \mathrm{U} / /$ & $0.75 \mathrm{U} / /$ & \\
\hline Chromium, total recoverable & MGKG & $11.3 / /$ & $8.8 / /$ & 6 \\
\hline Chrysene & UGKG & $626 \mathrm{U} / /$ & $489 \mathrm{U} / /$ & \\
\hline cis-1,3-Dichloropropene & UGKG & $3.6 \mathrm{U} / /$ & $3 \mathrm{U} / /$ & \\
\hline Copper, total recoverable & MGKG & $5.2 / /$ & $4.1 / /$ & 6 \\
\hline Di-n-butyl phthalate & UGKG & $626 \mathrm{U} / \mathrm{VI}$ & $489 \mathrm{U} / \mathrm{V} /$ & \\
\hline Di-n-octyl phthalate & UGKG & $626 \mathrm{U} / /$ & $489 \mathrm{U} / /$ & \\
\hline Dibenz(a,h)anthracene & UGKG. & $626 \mathrm{U} / /$ & $489 \mathrm{U} / /$ & \\
\hline Dibenzofuran & UGKG & $626 \mathrm{U} / /$ & $489 \mathrm{U} / /$ & \\
\hline Dibromochloromethane & UGKG & $0.9 \mathrm{U} / /$ & $0.75 \mathrm{U} / /$ & \\
\hline Dichloromethane (Methylene chloride & UGKG & $1.9 \mathrm{UJ} / \mathrm{VJ}$ & $1.9 \mathrm{UJ} / \mathrm{VI}$ & \\
\hline Diethyl phthalate & UGKG & $626 \mathrm{U} / /$ & $489 \mathrm{U} / /$ & \\
\hline Dimethyl phthalate & UGKG & $626 \mathrm{U} / /$ & $489 \mathrm{U} / /$ & \\
\hline Ethylbenzene & UGKG & $0.85 \mathrm{U} / /$ & $0.45 \mathrm{U} / /$ & \\
\hline Fluoranthene & UGKG & $626 \mathrm{U} / /$ & $489 \mathrm{U} / /$ & \\
\hline Fluorene & UGKG & $626 \mathrm{U} / /$ & $489 \mathrm{U} / /$ & \\
\hline Gross alpha & PCG & $3.3 \mathrm{~J} / /$ & $3.5 \mathrm{~J} / /$ & 1 \\
\hline Hexachlorobenzene & UGKG & $626 \mathrm{U} / /$ & $489 \mathrm{U} / /$ & \\
\hline Hexachlorobutadiene & UGKG & $626 \mathrm{U} / /$ & $489 \mathrm{U} / /$ & \\
\hline Hexachlorocyclopentadiene & UGKG & $626 \mathrm{U} / /$ & $489 \mathrm{U} / /$ & \\
\hline Hexachloroethane & UGKG & $626 \mathrm{U} / /$ & $489 \mathrm{U} / /$ & \\
\hline Indeno $(1,2,3-c, d)$ pyrene & UGKG & $626 \mathrm{U} / /$ & $489 \mathrm{U} / /$ & \\
\hline Isophorone & UGKG & $626 \mathrm{U} / /$ & $489 \mathrm{U} / /$ & \\
\hline Lead, total recoverable & MGKG & $8.4 / /$ & $5.3 / /$ & 11 \\
\hline m-Nitroaniline & UGKG & $3130 \mathrm{U} / /$ & $2440 \mathrm{U} / /$ & \\
\hline Manganese, total recoverable & MGKG & $322 / /$ & $1140 / /$ & 28 \\
\hline Mercury, total & MGKG & $0.102 \mathrm{U} / /$ & $0.0903 \mathrm{U} / /$ & \\
\hline N-Nitrosodiphenylamine & UGKG & $626 \mathrm{U} / /$ & $489 \mathrm{U} / /$ & \\
\hline N-Nitrosodipropylamine & UGKG & $626 \mathrm{U} / /$ & $489 \mathrm{U} / /$ & \\
\hline Naphthalene & UGKG & $626 \mathrm{U} / /$ & $489 \mathrm{U} / /$ & \\
\hline Nickel, total recoverable & MGKG & $5 \mathrm{U} / /$ & $4 U / /$ & \\
\hline
\end{tabular}




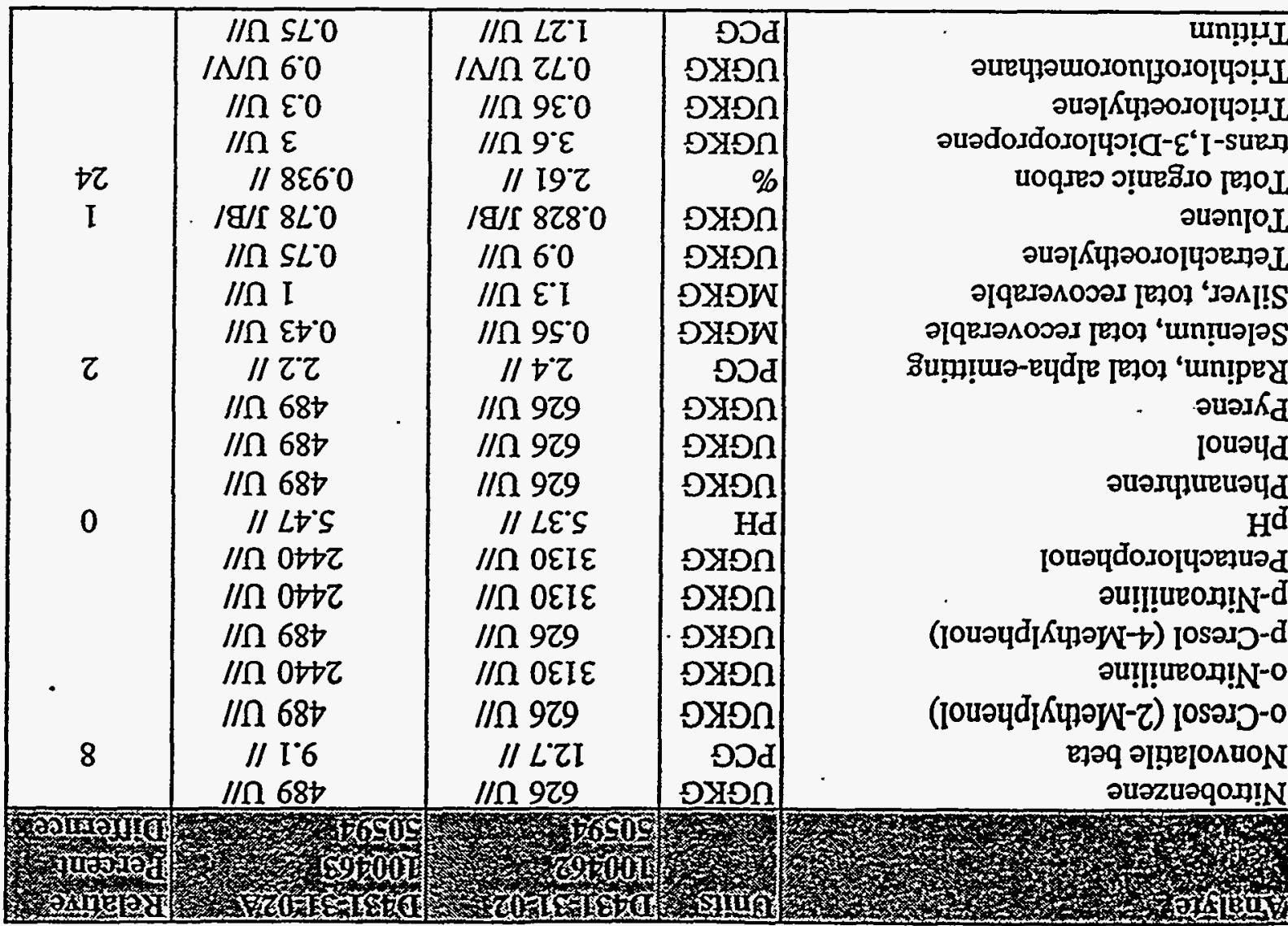

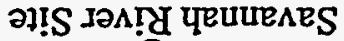

s7!d әlqqny/ofuiung eวr

sə]eว!̣dn

ZI शIQE] 
Table 13

Evaluation of Surface Water Split Samples

D-Area Burning/Rubble Pits

Savannah River Site

\begin{tabular}{|c|c|c|c|c|}
\hline 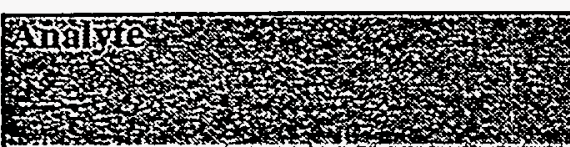 & (1) & 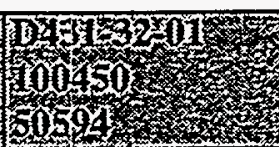 & 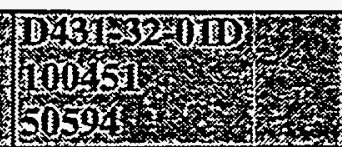 & 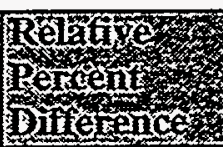 \\
\hline $1,1,1-$ Trichloroethane & UGL & $0.4 \mathrm{U} / /$ & $0.022 \mathrm{U} / /$ & \\
\hline 1,1,2-Trichloroethane & UGL & $0.8 \mathrm{U} / /$ & $0.017 \mathrm{U} / /$ & \\
\hline 1,1-Dichloroethane & UGL & $0.3 \mathrm{U} / /$ & $0.017 \mathrm{U} / /$ & \\
\hline 1,1-Dichloroethylene & UGL & $0.5 U / /$ & $0.018 \mathrm{UIJ}$ & \\
\hline 1,2,4-Trichlorobenzene & UGL & $11 \mathrm{U} / \mathrm{I}$ & $0.075 \mathrm{UJ} / \mathrm{AP}$ & \\
\hline 1,2-Dichlorobenzene & UGL & $11 \mathrm{U} / /$ & $0.017 \mathrm{UJ} / \mathrm{T} / \mathrm{P}$ & \\
\hline 1,2-Dichloroethane & UGL & $0.07 \mathrm{U} / /$ & $0.015 \mathrm{U} / /$ & \\
\hline 1,2-Dichloropropane & UGL & $0.2 \mathrm{U} / /$ & $0.015 \mathrm{U} / /$ & \\
\hline 1,3-Dichlorobenzene & UGL & $11 \mathrm{U} / /$ & $0.117 \mathrm{UJ} / \mathrm{I} / \mathrm{P}$ & \\
\hline 1,4-Dichlorobenzene & UGL & $11 \mathrm{U} / /$ & 0.024 UJ/T/P & \\
\hline 2,4,5-Trichlorophenol & UGL & $55 \mathrm{U} / /$ & $0.051 \mathrm{UJ} / \mathrm{T} / \mathrm{P}$ & \\
\hline 2,4,6-Trichlorophenol & UGL & $11 \mathrm{U} / /$ & $0.012 \mathrm{UJ} / \mathrm{I} / \mathrm{P}$ & \\
\hline 2,4-Dichlorophenol & UGL & $11 \mathrm{U} / /$ & $0.022 \mathrm{UJ} / \mathrm{I} / \mathrm{P}$ & \\
\hline 2,4-Dimethyl phenol & UGL & $11 \mathrm{U} / /$ & $0.104 \mathrm{UJ} / \mathrm{I} / \mathrm{P}$ & \\
\hline 2,4-Dinitrophenol & UGL & $55 \mathrm{U} / /$ & $0.332 \mathrm{UJ} / \mathrm{I} / \mathrm{P}$ & \\
\hline 2,4-Dinitrotoluene & UGL & $11 \mathrm{U} / /$ & 0.092 UJ/I/P & \\
\hline 2,6-Dinitrotoluene & UGL & $11 \mathrm{U} / /$ & $0.11 \mathrm{UJ} / \mathrm{I} / \mathrm{P}$ & \\
\hline 2-Chloroethyl vinyl ether & UGL & $10 \mathrm{U} / /$ & $0.179 \mathrm{U} / /$ & \\
\hline 2-Chloronaphthalene & UGL & $11 \mathrm{U} / /$ & $0.117 \mathrm{UJ} / \mathrm{I} / \mathrm{P}$ & $\cdot$ \\
\hline 2-Chlorophenol & UGL & $11 \mathrm{U} / /$ & $0.031 \mathrm{UJ} / \mathrm{T} / \mathrm{P}$ & \\
\hline 2-Methyl-4,6-dinitrophenol & UGL & $55 \mathrm{U} / /$ & $0.28 \mathrm{UJ} / \mathrm{I} / \mathrm{P}$ & \\
\hline 2-Methylnaphthalene & UGL & $11 \mathrm{U} / /$ & $0.017 \mathrm{UJ} / \mathrm{J} / \mathrm{P}$ & \\
\hline 2-Nitrophenol & UGL & $11 \mathrm{U} / /$ & $0.012 \mathrm{UJ} / \mathrm{T} / \mathrm{P}$ & \\
\hline 3,3'-Dichlorobenzidine & UGL & $22 \mathrm{U} / \mathrm{I}$ & $0.113 \mathrm{UJ} / \mathrm{M} \mathrm{P}$ & \\
\hline 4-Bromophenyl phenyl ether & UGL & $11 \mathrm{U} / /$ & $0.057 \mathrm{UJ} / \mathrm{I} / \mathrm{P}$ & \\
\hline 4-Chloro-m-cresol & UGL & $11 \mathrm{U} / /$ & $0.026 \mathrm{UJ} / \mathrm{T} / \mathrm{P}$ & \\
\hline 4-Chloroaniline & UGL & $11 \mathrm{U} / /$ & 0.015 UJ/I/P & \\
\hline 4-Chlorophenyl phenyl ether & UGL & $11 \mathrm{U} / /$ & $0.015 \mathrm{UJ} / \mathrm{I} / \mathrm{P}$ & \\
\hline 4-Nitrophenol & UGL & $55 \mathrm{U} / /$ & $0.191 \mathrm{UJ} / \mathrm{I} / \mathrm{P}$ & \\
\hline Acenaphthene & UGL & $11 \mathrm{U} / /$ & $0.0168 \mathrm{UJ} / \mathrm{I} / \mathrm{P}$ & \\
\hline Acenaphthylene & UGL & $11 \mathrm{U} / /$ & 0.017 UJ/I/P & $\dot{s}$ \\
\hline Anthracene & UGL & $11 \mathrm{U} / /$ & $0.017 \mathrm{UJ} / \mathrm{I} / \mathrm{P}$ & ; \\
\hline Arsenic, total recoverable & UGL & $1.7 \mathrm{U} / \mathrm{I}$ & $1.5 \mathrm{U} / /$ & \\
\hline Barium, total recoverable & UGL & $14.1 / /$ & $14.1 / /$ & 0 \\
\hline Benzene & UGL & $0.3 \mathrm{U} / \mathrm{I}$ & $0.017 \mathrm{U} / /$ & \\
\hline Benzo(a)anthracene & UGL & $11 \mathrm{U} / /$ & $0.151 \mathrm{UJ} / \mathrm{I} / \mathrm{P}$ & \\
\hline Benzo(a)pyrene & UGL & $11 \mathrm{U} / /$ & $0.076 \mathrm{UJ} / \mathrm{I} / \mathrm{P}$ & \\
\hline Benzo(b)fluoranthene & UGL & $11 \mathrm{U} / /$ & $0.133 \mathrm{UI} / \mathrm{I} / \mathrm{P}$ & \\
\hline Benzo $(g, h, i)$ perylene & UGL & $11 \mathrm{U} / /$ & 0.309 UJ/I/P & \\
\hline Benzo(k)fluoranthene & UGL & $11 \mathrm{U} / /$ & 0.079 UJ/TP & \\
\hline Benzoic acid & UGL & $55 \mathrm{U} / /$ & $0.081 \mathrm{UJ} / \mathrm{JP}$ & \\
\hline Benzyl alcohol & UGL & $11 \mathrm{U} / /$ & $0.025 \mathrm{UJ} / \mathrm{H} / \mathrm{P}$ & \\
\hline Bis(2-chloroethoxy) methane & UGL & $11 \mathrm{U} / /$ & $0.018 \mathrm{UJ} / \mathrm{U} / \mathrm{P}$ & - \\
\hline Bis(2-chloroethyl) ether & UGL & $11 \mathrm{U} / /$ & $0.017 \mathrm{UJ} / \mathrm{J} / \mathrm{P}$ & \\
\hline
\end{tabular}


Table 13

Evaluation of Surface Water Split Samples

D-Area Buming/Rubble Pits

Savannah River Site

\begin{tabular}{|c|c|c|c|c|}
\hline 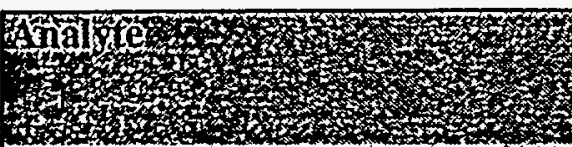 & ginits] & 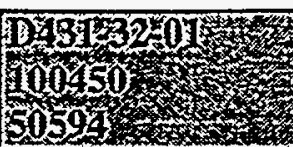 & 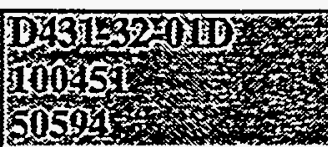 & $\begin{array}{l}\text { Relatide } \\
\text { Percent } \\
\text { Difternce }\end{array}$ \\
\hline Bis(2-chloroisopropyl) ether & UGL & $11 \mathrm{U} / \mathrm{I}$ & 0.042 UJ/I/P & \\
\hline Bis(2-ethylhexyl) phthalate & UGL & $11 \mathrm{U} / /$ & $0.59 \mathrm{~J} / \mathrm{VI} / \mathrm{P}$ & \\
\hline Bromoform & UGL & $2 U / /$ & $0.012 \mathrm{U} / /$ & \\
\hline Bromomethane (Methyl bromide) & UGL & $0.6 \mathrm{U} / /$ & $0.026 \mathrm{U} / /$ & \\
\hline Butylbenzyl phthalate & UGL & $11 \mathrm{U} / /$ & $0.036 \mathrm{UJ} / \mathrm{T} / \mathrm{P}$ & \\
\hline Cadmium, total recoverable & UGL & $3.3 \mathrm{U} / \mathrm{I}$ & $0.48 \mathrm{U} / /$ & \\
\hline Carbon tetrachloride & UGL & $0.2 \mathrm{U} / /$ & $0.012 \mathrm{U} / /$ & \\
\hline Chlorobenzene & UGL & $0.2 \mathrm{U} / /$ & $0.012 \mathrm{U} / /$ & \\
\hline Chloroethane & UGL & $0.2 \mathrm{U} / /$ & $0.025 \mathrm{U} / /$ & \\
\hline Chloroethene (Vinyl chloride) & UGL & $0.4 U / I$ & $0.015 \mathrm{U} / /$ & \\
\hline Chloroform & UGL & $0.3 \mathrm{U} / /$ & $0.017 \mathrm{U} / /$ & \\
\hline Chloromethane (Methyl chloride) & UGL & $0.3 \mathrm{U} / \mathrm{VI}$ & $0.024 \mathrm{U} / /$ & \\
\hline Chromium, total recoverable & UGL & $3.3 \mathrm{U} / /$ & $2.28 \mathrm{U} / /$ & \\
\hline Chrysene & UGL & $11 \mathrm{U} / /$ & 0.012 UJ/I/P & \\
\hline cis-1,3-Dichloropropene & UGL & $2 U / /$ & $0.012 \mathrm{U} / /$ & \\
\hline Copper, total recoverable & UGL & $3.3 \mathrm{U} / /$ & $0.925 \mathrm{~J} / /$ & \\
\hline Di-n-butyl phthalate & UGL & $11 \mathrm{U} / /$ & 0.443 UJ/I/P & \\
\hline Di-n-octyl phthalate & UGL & $11 \mathrm{U} / /$ & $0.07 \mathrm{UJ} / \mathrm{A} / \mathrm{P}$ & \\
\hline Dibenz $(a, h)$ anthracene & UGL & $11 \mathrm{U} / /$ & $0.09 \mathrm{UJ} / \mathrm{T} / \mathrm{P}$ & \\
\hline Dibenzofuran & UGL & $11 \mathrm{U} / /$ & 0.017 UJ/I/P & \\
\hline Dibromochloromethane & UGL & $0.5 \mathrm{U} / /$ & $0.017 \mathrm{U} / /$ & \\
\hline Diethyl phthalate & UGL & $11 \mathrm{U} / /$ & $0.015 \mathrm{UJ} / \mathrm{I} / \mathrm{P}$ & \\
\hline Dimethyl phthalate & UGL & $11 \mathrm{U} / /$ & 0.017 UJ/I/P & \\
\hline Ethylbenzene & UGL & $0.3 \mathrm{U} / /$ & $0.015 \mathrm{U} / /$ & \\
\hline Fluoranthene & UGL & $11 \mathrm{U} / /$ & $0.024 \mathrm{UJ} / \mathrm{J} / \mathrm{P}$ & \\
\hline Fluorene & UGL & $11 \mathrm{U} / /$ & 0.012 UJ/I/P & \\
\hline Gross alpha & PCL & $0.5 \mathrm{U} / /$ & $0.919 \mathrm{~J} / /$ & 15 \\
\hline Hexachlorobenzene & UGL & $11 \mathrm{U} / /$ & $0.028 \mathrm{UJ} / \mathrm{T} / \mathrm{P}$ & \\
\hline Hexachlorobutadiene & UGL & $11 \mathrm{U} / /$ & $0.067 \mathrm{UJ} / \mathrm{I} / \mathrm{P}$ & \\
\hline Hexachlorocyclopentadiene & UGL & $11 \mathrm{U} / /$ & 0.042 UJ/I/P & \\
\hline Hexachloroethane & UGL & $11 \mathrm{U} / /$ & $0.022 \mathrm{UJ} / \mathrm{I} / \mathrm{P}$ & - \\
\hline Indeno(1,2,3-c,d)pyrene & UGL & $11 \mathrm{U} / /$ & $0.035 \mathrm{UJ} / \mathrm{I} / \mathrm{P}$ & \\
\hline Iron, total recoverable & UGL & $101 / /$ & $112 / /$ & 3 \\
\hline Isophorone & UGL & i1 U// & $0.099 \mathrm{UJ} / \mathrm{U} \mathrm{P}$ & \\
\hline Lead, total recoverable & UGL & $2.5 / /$ & $0.95 \mathrm{~J} / \mathrm{V} / \mathrm{H}$ & 22 \\
\hline m-Nitroaniline & UGL & $55 \mathrm{U} / /$ & $0.142 \mathrm{UJ} / \mathrm{I} / \mathrm{P}$ & \\
\hline Manganese, total recoverable & UGL & $27.6 / /$ & $27.8 \mathrm{~J} / \mathrm{V} / \mathrm{H}$ & 0 \\
\hline Mercury, total & UGL & $0.067 \mathrm{U} / /$ & $0.04 \mathrm{UJ} / \mathrm{V} / \mathrm{H}$ & \\
\hline N-Nitrosodiphenylamine & UGL & $11 \mathrm{U} / /$ & $0.018 \mathrm{UJ} / \mathrm{L} / \mathrm{P}$ & \\
\hline N-Nitrosodipropylamine & UGL & $11 \mathrm{U} / /$ & $0.036 \mathrm{UJ} / \mathrm{J} / \mathrm{P}$ & \\
\hline Naphthalene & UGL & $11 \mathrm{U} / /$ & $0.017 \mathrm{UJ} / \mathrm{I} / \mathrm{P}$ & \\
\hline Nickel, total recoverable & UGL & $11.7 \mathrm{U} / /$ & $1.84 \mathrm{U} / /$ & \\
\hline Nitrobenzene & UGL & $.11 \mathrm{U} / /$ & $0.031 \mathrm{UJ} / \mathrm{I} / \mathrm{P}$ & \\
\hline Nonvolatile beta & PCL & $0.8 \mathrm{U} / /$. & $0.643 \mathrm{U} / /$ & \\
\hline
\end{tabular}


Table 13

Evaluation of Surface Water Split Samples

D-Area Burning/Rubble Pits

Savannah River Site

\begin{tabular}{|c|c|c|c|c|}
\hline 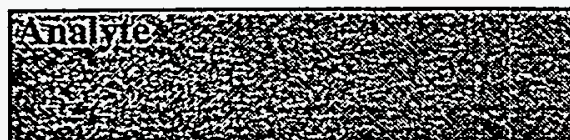 & Gints & $\begin{array}{l}\text { D43432511 } \\
00450 \\
5059\end{array}$ & 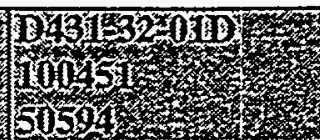 & 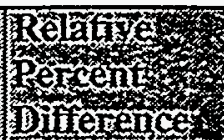 \\
\hline o-Cresol (2-Methylphenol) & UGL & $11 \mathrm{U} / 1$ & $0.048 \mathrm{UJ} / \mathrm{T} / \mathrm{P}$ & \\
\hline o-Nitroaniline & UGL & $55 \mathrm{U} / /$ & $0.031 \mathrm{UJ} / \mathrm{J} / \mathrm{P}$ & \\
\hline p-Nitroaniline & UGL & $55 \mathrm{U} / /$ & $0.102 \mathrm{UJ} / \mathrm{I} / \mathrm{P}$ & \\
\hline Pentachlorophenol & UGL & $55 \mathrm{U} / /$ & $0.192 \mathrm{UJ} / \mathrm{L} / \mathrm{P}$ & \\
\hline Phenanthrene & UGL & $11 \mathrm{U} / /$ & $0.017 \mathrm{UJ} / \mathrm{I} / \mathrm{P}$ & \\
\hline Phenol & UGL & $11 \mathrm{U} / /$ & $0.043 \mathrm{UJ} / \mathrm{I} / \mathrm{P}$ & \\
\hline Pyrene & UGL & $11 \mathrm{U} / /$ & $0.022 \mathrm{UJ} / \mathrm{T} / \mathrm{P}$ & \\
\hline Radium, total alpha-emitting & PCL & $0.12 \mathrm{U} / I$ & $-0.2 \mathrm{U} / /$ & \\
\hline Selenium, total recoverable & UGL & $1.7 \mathrm{U} / /$ & $1.25 \mathrm{U} / /$ & \\
\hline Silver, total recoverable & UGL & $0.7 \mathrm{U} / /$ & $0.512 U / /$ & \\
\hline Sodium, total recoverable & UGL & $10200 / /$ & $9740 / /$ & 1 \\
\hline Tetrachloroethylene & UGL & $0.5 \mathrm{U} / /$ & $0.026 \mathrm{U} / /$ & \\
\hline Toluene & UGL & $0.8 \mathrm{U} / /$ & $0.06 \mathrm{~J} / \mathrm{N} / \mathrm{H}$ & \\
\hline Total dissolved solids & UGL & $54000 / /$ & $53000 / /$ & 0 \\
\hline Total organic carbon & UGL & $1660 / /$ & $1580 / /$ & 1 \\
\hline Total organic halogens & UGL & $3330 \mathrm{U} / /$ & $7.35 \mathrm{U} / /$ & \\
\hline Total suspended solids & UGL & $1670 \mathrm{U} / /$ & $6300 \mathrm{U} / /$ & \\
\hline trans-1,3-Dichloropropene & UGL & $2 U / I$ & $0.015 \mathrm{U} / /$ & \\
\hline Trichloroethylene & UGL & $0.2 \mathrm{U} / \mathrm{I}$ & $0.017 \mathrm{U} / /$ & \\
\hline Trichlorofluoromethane & UGL & $0.7 \mathrm{U} / /$ & $0.036 \mathrm{U} / /$ & \\
\hline
\end{tabular}


Table 14

Evaluation of Sediment Split Samples

D-Area Burning/Rubble Pits

Savannah River Site

\begin{tabular}{|c|c|c|c|c|}
\hline 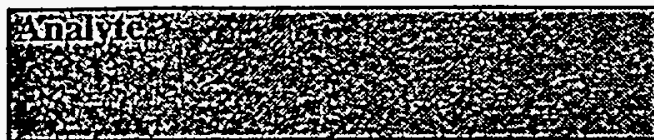 & Thins & 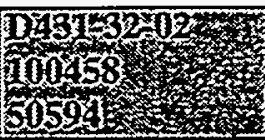 & 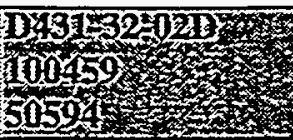 & $\begin{array}{l}\text { Reatie } \\
\text { Perent } \\
\text { Oifferenct }\end{array}$ \\
\hline 1,1,1-Trichloroethane & UGKG & $0.56 \mathrm{U} / \mathrm{I}$ & $0.022 \mathrm{UJ} / \mathrm{T} / \mathrm{P}$ & \\
\hline 1,1,2-Trichloroethane & UGKG & $1.12 \mathrm{U} / /$ & 0.017 UJ/I/P & \\
\hline 1,1-Dichloroethane & UGKG & $0.42 \mathrm{U} / \mathrm{I}$ & $0.017 \mathrm{UJ} / \mathrm{I} / \mathrm{P}$ & \\
\hline 1,1-Dichloroethylene & UGKG & $0.7 \mathrm{U} / /$ & $0.018 \mathrm{UJ} / \mathrm{I} / \mathrm{P}$ & \\
\hline 1,2,4-Trichlorobenzene & UGKG & $483 \mathrm{U} / \mathrm{l}$ & $1.43 \mathrm{UJ} / \mathrm{I} / \mathrm{P}$ & \\
\hline 1,2-Dichlorobenzene & UGKG & $483 \mathrm{U} / /$ & $0.815 \mathrm{UJ} / \mathrm{T} / \mathrm{P}$ & \\
\hline 1,2-Dichloroethane & UGKG & $0.098 U / /$ & $0.015 \mathrm{UJ} / \mathrm{T}$ & \\
\hline 1,2-Dichloropropane & UGKG & $0.28 \mathrm{U} / /$ & $0.015 \mathrm{UJ} / \mathrm{T} / \mathrm{P}$ & \\
\hline 1,3-Dichlorobenzene & UGKG & $483 \mathrm{U} / /$ & 0.932 UJ/I/P & \\
\hline 1,4-Dichlorobenzene & UGKG & $483 \mathrm{U} / /$ & $5.07 \mathrm{UJ} / \mathrm{P}$ & \\
\hline 2,4,5-Trichlorophenol & UGKG & $2420 \mathrm{U} / /$ & $1.39 \mathrm{UJ} / \mathrm{I} / \mathrm{P}$ & \\
\hline 2,4,6-Trichlorophenol & UGKG & $483 \mathrm{U} / /$ & $1.32 \mathrm{UJ} / \mathrm{I} / \mathrm{P}$ & \\
\hline 2,4-Dichlorophenol & UGKG & $483 \mathrm{U} / /$ & $1.17 \mathrm{UJ} / \mathrm{P}$ & \\
\hline 2,4-Dimethyl phenol & UGKG & $483 \mathrm{U} / /$ & $1.2 \mathrm{UJ} / \mathrm{I} / \mathrm{P}$ & \\
\hline 2,4-Dinitrophenol & UGKG & $2420 \mathrm{U} / /$ & $3.67 \mathrm{UJ} / \mathrm{W} / \mathrm{P}$ & \\
\hline 2,4-Dinitrotoluene & UGKG & $483 \mathrm{U} / /$ & 0.554 UJ/I/P & \\
\hline 2,6-Dinitrotoluene & UGKG & $483 \mathrm{U} / /$ & $0.554 \mathrm{UJ} / \mathrm{I} / \mathrm{P}$ & \\
\hline 2-Chloroethyl vinyl ether & UGKG & $14 \mathrm{U} / /$ & $0.179 \mathrm{UJ} / \mathrm{J} / \mathrm{P}$ & \\
\hline 2-Chloronaphthalene & UGKG & $483 \mathrm{U} / /$ & $1.01 \mathrm{UJ} / \mathrm{I} / \mathrm{P}$ & \\
\hline 2-Chlorophenol & UGKG & $483 \mathrm{U} / /$ & $2.42 \mathrm{UJ} / \mathrm{I} / \mathrm{P}$ & \\
\hline 2-Methyl-4,6-dinitrophenol & UGKG & $2420 \mathrm{U} / /$ & $3.69 \mathrm{UJ} / \mathrm{I} / \mathrm{P}$ & \\
\hline 2-Methylnaphthalene & UGKG & $483 \mathrm{U} / /$ & $1.39 \mathrm{UJ} / \mathrm{I} / \mathrm{P}$ & \\
\hline 2-Nitrophenol & UGKG & $483 \mathrm{U} / /$ & $0.815 \mathrm{UJ} / \mathrm{I} / \mathrm{P}$ & \\
\hline 3,3-Dichlorobenzidine & UGKG & $966 \mathrm{U} / /$ & $122 \mathrm{UJ} / \mathrm{I} / \mathrm{P}$ & \\
\hline 4-Bromophenyl phenyl ether & UGKG & $483 \mathrm{U} / /$ & $0.815 \mathrm{UJ} / \mathrm{IP}$ & \\
\hline 4-Chloro-m-cresol & UGKG & $483 \mathrm{U} / /$ & $1.15 \mathrm{UJ} / \mathrm{I} / \mathrm{P}$ & \\
\hline 4-Chloroaniline & UGKG & $483 \mathrm{U} / /$ & $4.5 \mathrm{UJ} / \mathrm{I} / \mathrm{P}$ & \\
\hline 4-Chlorophenyl phenyl ether & UGKG & $483 \mathrm{U} / /$ & $1.04 \mathrm{UJ} / \mathrm{J} / \mathrm{P}$ & \\
\hline 4-Nitrophenol & UGKG & $2420 \mathrm{U} / /$ & $6.52 \mathrm{UJ} / \mathrm{T} / \mathrm{P}$ & \\
\hline Acenaphthene & UGKG & $483 \mathrm{U} / /$ & $1.47 \mathrm{UJ} / \mathrm{I} / \mathrm{P}$ & \\
\hline Acenaphthylene & UGKG & $483 \mathrm{U} / /$ & $0.783 \mathrm{UJ} / \mathrm{I} / \mathrm{P}$ & \\
\hline Anthracene & UGKG & $483 \mathrm{U} / /$ & $0.392 \mathrm{UJ} / \mathrm{T} / \mathrm{P}$ & \\
\hline Arsenic, total recoverable & MGKG & $1.4 / /$ & $1.36 \mathrm{U} / /$ & 1 \\
\hline Barium, total recoverable & MGKG & $22.3 / /$ & $17.8 \mathrm{~J} / \mathrm{V} / \mathrm{H}$ & 6 \\
\hline Benzene & UGKG & $0.42 \mathrm{U} / /$ & $0.017 \mathrm{UJ} / \mathrm{I} / \mathrm{P}$ & \\
\hline Benzo(a)anthracene & UGKG & $483 \mathrm{U} / /$ & $1.17 \mathrm{UJ} / \mathrm{I} / \mathrm{P}$ & \\
\hline Benzo(a)pyrene & UGKG & $483 \mathrm{U} / /$ & $0.986 \mathrm{UJ} / \mathrm{J} / \mathrm{P}$ & \\
\hline Benzo(b)fluoranthene & UGKG & $483 \mathrm{U} / /$ & $0.932 \mathrm{UJ} / \mathrm{J} / \mathrm{P}$ & \\
\hline Benzo(g,h,i)perylene & UGKG & $483 \mathrm{U} / /$ & 0.392 UJ/T/P & \\
\hline Benzo(k)fluoranthene & UGKG & $483 \mathrm{U} / /$ & $0.986 \mathrm{UJ} / \mathrm{I} / \mathrm{P}$ & \\
\hline Benzoic acid & UGKG & $2420 \mathrm{U} / /$ & 4.22 UJ/I/P & \\
\hline Benzyl alcohol & UGKG & $483 \mathrm{U} / /$ & $3.03 \mathrm{UJ} / \mathrm{T} / \mathrm{P}$ & \\
\hline Bis(2-chloroethoxy) methane & UGKG & $483 \mathrm{U} / /$ & $0.783 \mathrm{UJ} / \mathrm{I} / \mathrm{P}$ & \\
\hline Bis(2-chloroethyl) ether & UGKG & $483 \mathrm{U} / /$ & $2.59 \mathrm{UJ} / \mathrm{I} / \mathrm{P}$ & \\
\hline Bis(2-chloroisopropyl) ether & UGKG & $483 \mathrm{U} / /$ & 1.04 UJ//P & \\
\hline Bis(2-ethylhexyl) phthalate & UGKG & -. $483 \mathrm{U} / /$ & 18.1.J/VI/P & - \\
\hline Bromoform & UGKG & $2.8 \mathrm{U} / /$ & 0.012 UJ/I/P & \\
\hline
\end{tabular}


Table 14

Evaluation of Sediment Split Samples

D-Area Burning/Rubble Pits

Savannah River Site

\begin{tabular}{|c|c|c|c|c|}
\hline 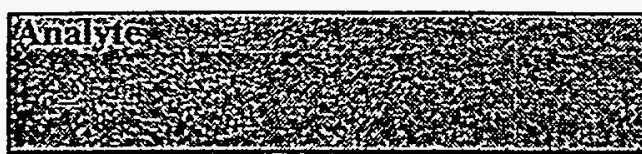 & Fifs, & T00458 & 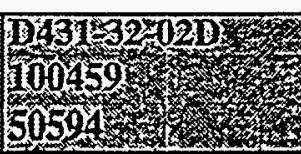 & 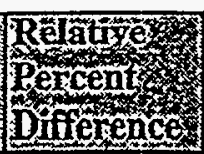 \\
\hline Bromomethane (Methyl bromide) & UGKG & $0.84 U / /$ & $0.026 \mathrm{UJ} / \mathrm{TP}$ & \\
\hline Butylbenzyl phthalate & UGKG & $483 \mathrm{U} / /$ & $1.2 \mathrm{UJ} / \mathrm{I} / \mathrm{P}$ & \\
\hline Cadmium, total recoverable & MGKG & $1.1 \mathrm{U} / /$ & $0.178 \mathrm{U} / /$ & \\
\hline Carbon tetrachloride & UGKG & $0.28 \mathrm{U} / /^{\circ}$ & 0.012 UJ/I/P & \\
\hline Chlorobenzene & UGKG & $0.28 \mathrm{U} / /$ & $0.012 \mathrm{UJ} / \mathrm{T} / \mathrm{P}$ & \\
\hline Chloroethane & UGKG & $0.28 \mathrm{U} / /$ & $0.025 \mathrm{UJ} / \mathrm{I} / \mathrm{P}$ & \\
\hline Chloroethene (Vinyl chloride) & UGKG & $0.56 \mathrm{U} / /$ & $0.015 \mathrm{UJ} / \mathrm{I}$ & \\
\hline Chloroform & UGKG & $0.42 \mathrm{U} / /$ & $0.017 \mathrm{UJ} / \mathrm{I} / \mathrm{P}$ & \\
\hline Chloromethane (Methyl chloride) & UGKG & $0.7 \mathrm{U} / /$ & $0.024 \mathrm{UJ} / \mathrm{I} / \mathrm{P}$ & \\
\hline Chromium, total recoverable & MGKG & $14.9 / /$ & $5.22 \mathrm{~J} /$ & 24 \\
\hline Chrysene & UGKG & $483 \mathrm{U} / /$ & $0.715 \mathrm{UJ} / \mathrm{I} / \mathrm{P}$ & \\
\hline cis-1,3-Dichloropropene & UGKG & $2.8 \mathrm{U} / /$ & 0.012 UJ/I/P & \\
\hline Copper, total recoverable & MGKG & $5.3 / /$ & $4.22 / /$ & 6 \\
\hline Di-n-butyl phthalate & UGKG & $483 \mathrm{U} / \mathrm{N} /$ & $8.9 \mathrm{~J} / \mathrm{VI} / \mathrm{P}$ & \\
\hline Di-n-octyl phthalate & UGKG & $483 \mathrm{U} / /$ & $2.07 \mathrm{UJ} / \mathrm{I} / \mathrm{P}$ & \\
\hline Dibenz(a,h)anthracene & UGKG & $483 U / /$ & $0.505 \mathrm{UJ} / \mathrm{I} / \mathrm{P}$ & \\
\hline Dibenzofuran & UGKG & $483 \mathrm{U} / /$ & $1.32 \mathrm{UJ} / \mathrm{I} / \mathrm{P}$ & \\
\hline Dibromochloromethane & UGKG & $0.7 \mathrm{U} / /$ & $0.017 \mathrm{UJ} / \mathrm{I} / \mathrm{P}$ & \\
\hline Dichloromethane (Methylene chloride) & UGKG & $1.9 \mathrm{UJ} / \mathrm{VI}$ & $0.53 \mathrm{~J} / \mathrm{I} / \mathrm{P}$ & \\
\hline Diethyl phthalate & UGKG & $483 \mathrm{U} / /$ & $1.01 \mathrm{UJ} / \mathrm{I} / \mathrm{P}$ & \\
\hline Dimethyl phthalate & UGKG & $483 \mathrm{U} / /$ & $0.505 \mathrm{UJ} / \mathrm{I} / \mathrm{P}$ & \\
\hline Ethylbenzene & UGKG & $0.42 \mathrm{U} / /$ & $0.015 \mathrm{UJ} / \mathrm{I} / \mathrm{P}$ & \\
\hline Fluoranthene & UGKG & $483 \mathrm{U} / /$ & $0.554 \mathrm{UJ} / \mathrm{I} / \mathrm{P}$ & \\
\hline Fluorene & UGKG & $483 \mathrm{U} / /$ & $1.26 \mathrm{UJ} / \mathrm{J} / \mathrm{P}$ & \\
\hline Gross alpha & PCG & $5.3 / /$ & $9.13 / /$ & 13 \\
\hline Hexachlorobenzene & UGKG & $483 \mathrm{U} / /$ & $0.815 \mathrm{UJ} / \mathrm{L} / \mathrm{P}$ & \\
\hline Hexachlorobutadiene & UGKG & $483 U / I$ & $1.88 \mathrm{UJ} / \mathrm{/P}$ & \\
\hline Hexachlorocyclopentadiene & UGKG & $483 \mathrm{U} / /$ & $3.26 \mathrm{UJ} / \mathrm{T} / \mathrm{P}$ & \\
\hline Hexachloroethane & UGKG & $483 \mathrm{U} / /$ & $1.04 \mathrm{UJ} / \mathrm{/} / \mathrm{P}$ & \\
\hline Indeno(1,2,3-c,d)pyrene & UGKG & $483 \mathrm{U} / /$ & $0.815 \mathrm{UJ} / \mathrm{I} / \mathrm{P}$ & \\
\hline Isophorone & UGKG & $483 \mathrm{U} / \mathrm{I}$ & $1.11 \mathrm{UJ} / \mathrm{I} / \mathrm{P}$ & \\
\hline Lead, total recoverable & MGKG & $6.8 / /$ & $10.5 / /$ & 11 \\
\hline m-Nitroaniline & UGKG & $2420 \mathrm{U} / /$ & $0.845 \mathrm{UJ} / \mathrm{I} / \mathrm{P}$ & \\
\hline Manganese, total recoverable & MGKG & $105 / /$ & $65.1 \mathrm{~J} / \mathrm{VI} / \mathrm{P}$ & 12 \\
\hline N-Nitrosodiphenylamine & UGKG & $483 \mathrm{U} / /$ & $0.932 \mathrm{UJ} / \mathrm{I} / \mathrm{P}$ & \\
\hline N-Nitrosodipropylamine & UGKG & $483 \mathrm{U} / /$ & $0.815 \mathrm{UJ} / \mathrm{T} / \mathrm{P}$ & \\
\hline Naphthalene & UGKG & $483 \mathrm{U} / /$ & $1.39 \mathrm{UJ} / \mathrm{H} / \mathrm{P}$ & \\
\hline Nickel, total recoverable & MGKG & $3.8 \mathrm{U} / /$ & $1.49 \mathrm{~J} / /$ & \\
\hline Nitrobenzene & UGKG. & $483 \mathrm{U} / /$ & $3.72 \mathrm{UJ} / \mathrm{I} / \mathrm{P}$ & \\
\hline Nonvolatile beta & PCG & $9.6 / /$ & $7.77 \mathrm{~J} / /$ & 5 \\
\hline o-Cresol (2-Methylphenol) & UGKG & $483 \mathrm{U} / /$ & $1.74 \mathrm{UJ} / \mathrm{I} / \mathrm{P}$ & \\
\hline o-Nitroaniline & UGKG & $2420 \mathrm{U} / \mathrm{l}$ & $2.8 \mathrm{UJ} / \mathrm{I} / \mathrm{P}$ & \\
\hline p-Nitroaniline & UGKG & $2420 \mathrm{U} / /$ & $3.17 \mathrm{UJ} / \mathrm{I} / \mathrm{P}$ & \\
\hline Pentachlorophenol & UGKG & $2420 \mathrm{U} / /$ & $11.3 \mathrm{UJ} / \mathrm{I} / \mathrm{P}$ & \\
\hline $\mathrm{pH}$ & $\mathrm{PH}$ & $5.24 / /$ & $6.3 \mathrm{~J} / \mathrm{Q} / \mathrm{L}$ & 5 \\
\hline Phenanthrene & UGKG & $483 \mathrm{U} / /$ & $0.554 \mathrm{UJ} / \mathrm{J} / \mathrm{P}$ & \\
\hline Phenol & UGKG & $483 \mathrm{U} / /$ & $1.15 \mathrm{UJ} / / \mathrm{P}$ & \\
\hline
\end{tabular}


Appendix A

Addendum Surface Water/Sediment Sampling Plan 
Table 14

Evaluation of Sediment Split Samples

D-Area Burning/Rubble Pits

Savannah River Site

\begin{tabular}{|c|c|c|c|c|}
\hline 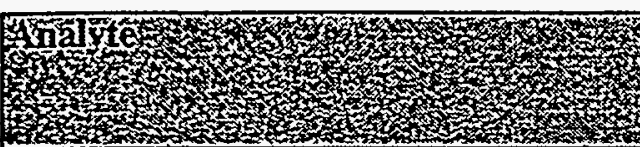 & Ginitis & D431 3202 & 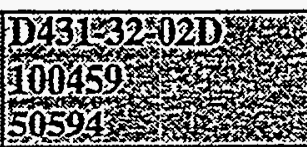 & 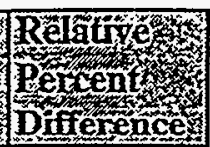 \\
\hline $\begin{array}{ll}\text { Pyrene } \\
\end{array}$ & UGKG & $483 \mathrm{U} / I$ & $0.598 \mathrm{UJ} / \mathrm{I} / \mathrm{P}$ & \\
\hline Radium, total alpha-emitting & PCG & $2.6 / /$ & $2.3 \mathrm{~J} / I$ & 3 \\
\hline Selenium, total recoverable & MGKG & $0.43 \mathrm{U} / /$ & $2.8 \mathrm{U} / \mathrm{I}$ & \\
\hline Silver, total recoverable & MGKG & $0.99 \mathrm{U} / /$ & $0.25 \mathrm{U} / /$ & \\
\hline Tetrachloroethylene & UGKG & $0.7 \mathrm{U} / /$ & 0.026 UJ/ṬP & \\
\hline Toluene & UGKG & $0.854 \mathrm{~J} / \mathrm{B} /$ & $1.2 \mathrm{~J} / \mathrm{VI} / \mathrm{P}$ & 8 \\
\hline trans-1,3-Dichloropropene & UGKG & $2.8 U / /$ & $0.015 \mathrm{UJ} / \mathrm{I} / \mathrm{P}$ & \\
\hline Trichloroethylene & UGKG & $0.28 \mathrm{U} / /$ & $0.017 \mathrm{UJ} / \mathrm{I} / \mathrm{P}$ & \\
\hline Trichlorofluoromethane & UGKG & $0.84 \mathrm{U} / \mathrm{VI}$ & $0.036 \mathrm{UJ} / \mathrm{I} / \mathrm{P}$ & \\
\hline Tritium & PCG & $0.42 \mathrm{U} / /$ & $6.45 \mathrm{~J} / /$ & \\
\hline
\end{tabular}




\section{Mr. Jon Hammock}

Environmental Restoration Department

Westinghouse Savannah River Company

1995 South Centennial Avenue

Aiken, South Carolina 29803

Subject: Subcontract AA46326P - Task Order 26

RCRA/CERCLA Services for the D-Area Burning/Rubble Pits

Revised Addendum Surface Water/Sediment Sampling Plan

Dear Mr. Hammock:

Enclosed is the revised addendum sampling plan for sampling of surface water and sediments at the D-Area Burning/Rubble Pits RCRA/CERCLA unit. The plan was developed to account for sampling that was not possible during the 1993 unit characterization due to dry conditions and lack of surface water flow in the ephemeral stream and other wetland areas. The revised plan incorporates Westinghouse Savannah River Company comments received from you on April 20, 1994.

If you have any questions or comments regarding this plan, please call me at 649-5175.

Sincerely,

RUST Environment \& Infrastructure Inc.
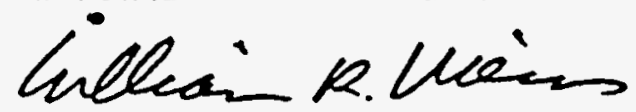

William R. Weiss

Project Manager

cc: C. Perkins, WSRC-Procurement

R. M. Humphrey, WSRC-ERD

J. Beckner, RUST E\&I

L. W. Neal, RUST E\&I

M.T. Scott, RUST E\&I

R. C. Smalley, RUST E\&I

File: $81749 / 3.3$

File: A.1.1.1

940100 


\section{PROPOSED SURFACE WATER AND SEDIMENT SAMPLING D-AREA BURNING/RUBBLE PITS \\ APRII 1994}

Additional RFI/RI unit characterization sampling is proposed for the D-Area Buming/Rubble Pits to more fully characterize potential contamination of surface water and sediment in the area of the unit. During unit characterization sampling conducted in 1993, dry conditions precluded sampling of six of the planned nine surface water locations. During 1994, it was noted that ephemeral stream flow in the planned surface water drainage sampling areas was dominated by high flows traced back to overflow of two cooling water surge tanks connected to river water intake pump houses. Because of the potential difficulties in differentiating chemical constituent concentrations present in surface water and sediment as a result of river water inflow versus water seepage, runoff, or discharge from the unit, SRS proposes to conduct surface water and sediment sampling during April-May 1994 within the surface water drainage depicted in Figure 4-1 (Addendum). A field reconnaissance conducted on April 15, 1994, revealed that vegetation maintenance has recently occurred in the powerline right-of-way south of the unit. The RFI/RI Work Plan had originally included sampling in the low-lying wetland area within this right-of-way. Because of the probability of extensive chemical contamination (e.g., pesticides, hydrocarbons, etc.) as a result of the recent maintenance activities, it is proposed that all new surface water and sediment sampling be conducted in the drainage channel upstream of the powerline right-of-way.

SRS proposes sampling at five locations upgradient and five locations downgradient of the unit. This approach is proposed to allow statistical differentiation of potential contamination to stream surface water and sediment from the unit vs. background environmental concentrations within these media (including contamination resulting from overflow from the two cooling water surge tanks). The upgradient surface water/sediment samples are considered unit background samples [containing naturallyoccurring plus anthropogenic (non-unit) background levels of chemicals: see EPA/540/189/002, Risk Assessment Guidance for Superfund. Vol. I. Human Health Evaluation Manual (Part A). Interim Final, p. 4-5]. Additionally, SRS will sample the water contained in each of the surge tanks to specifically identify chemical constituent concentrations within the cooling water that is discharged to the ephemeral stream. The total number of samples to be collected at each of the surface water/sediment sampling locations is listed in Table 4-2 (Addendum), including an additional 10 percent to be collected for quality assurance/quality control purposes. The proposed analyte list, identical to the analytical program used during 1993 unit characterization sampling, is also detailed in Table 4-2 (Addendum). All sampling and analytical activities will follow the approach outlined in the RFI/RI Work Plan, Revision 2. 


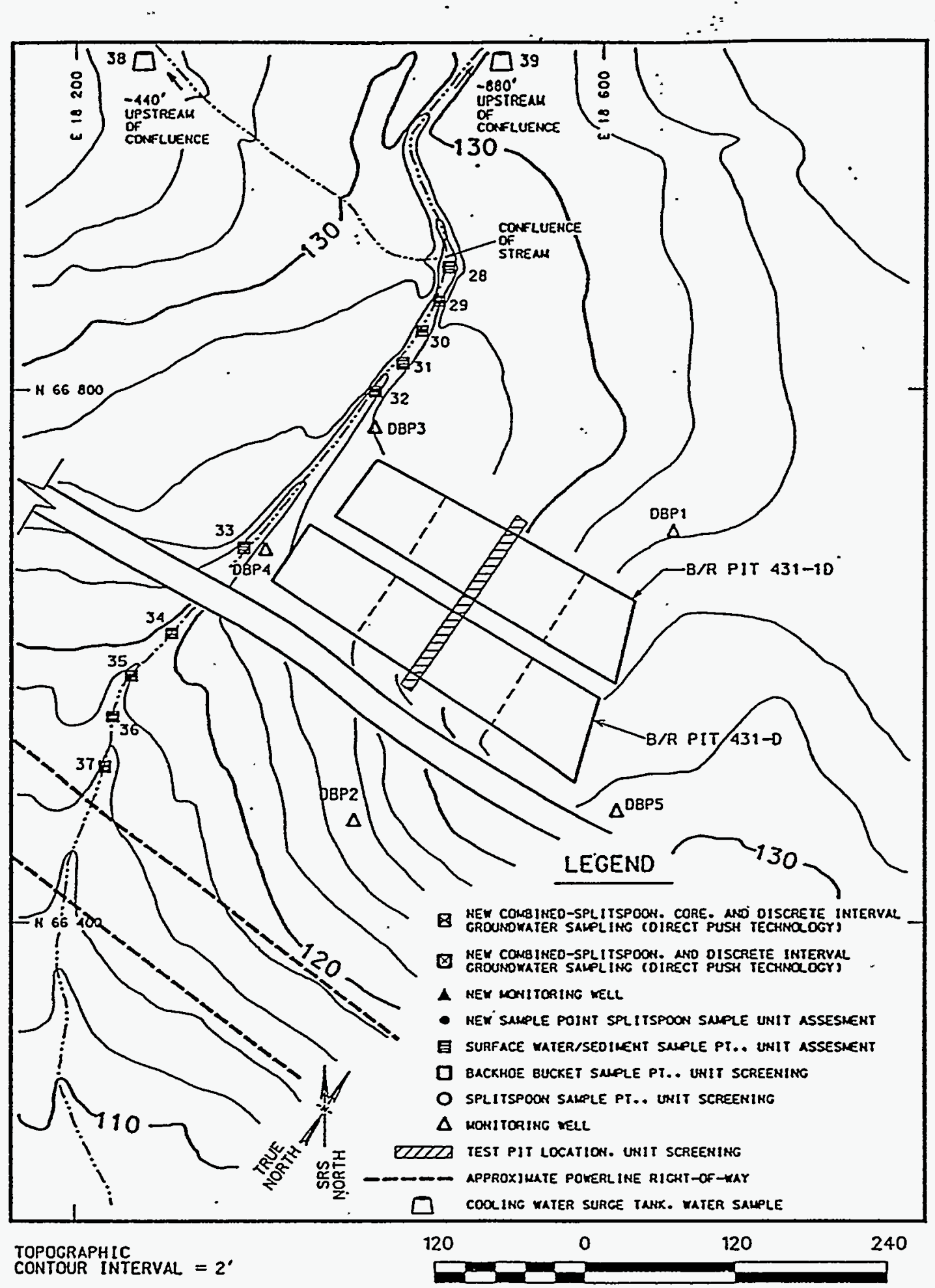

SCALE IN FEET

Figure 4-1 (Addendum)

Proposed Surface Water/Şediment Sampling Locations 
TABLE 4-2 (Addendum)

GROUNDWATER AND SURFACE WATER/SEDIMENT CONSTITUENTS TO BE ANALYZED

\begin{tabular}{|c|c|c|c|c|}
\hline $\begin{array}{l}\text { Facility } \\
\text { Identifier }\end{array}$ & $\begin{array}{l}\text { Sampling Point } \\
\text { Number : }\end{array}$ & $\begin{array}{l}\text { Sample } \\
\text { Number }\end{array}$ & $\begin{array}{l}\text { Location } \\
\text { or Type }\end{array}$ & $\begin{array}{l}\text { Constituents } \\
\text { for Analysis* }\end{array}$ \\
\hline D4311 & 28 & 01 & Surface Water & $1,2,3,4,6,7 ; 9 ; 10$ \\
\hline D4311 & 28 & 02 & Sediment & $2,3,4,6,8$ \\
\hline D4311 & 29 & 01 & Surface Water & $1,2,3,4,6,7,9,10$ \\
\hline D4311 & 29 & 02. & Sediment & $2,3,4,6,8$ \\
\hline D4311 & 30 & 01 & Surface Water & $1,2,3,4,6,7,9,10$ \\
\hline D4311 & 30 & 02 & Sediment & $2,3,4,6,8$ \\
\hline D4311 & 30 & 02B & Trip Blank - Water & 4,6 \\
\hline D431 & 31 & 01 & Surface Water & $\cdot 1,2,3,4,6,7,9,10$ \\
\hline D431 & 31 & $01 \mathrm{~A}$ & Surface Water-Duplicate & $1,2,3,4,6$ \\
\hline D431 & 31 & 010 & $\begin{array}{l}\text { Surface Water - Field } \\
\text { Blank }\end{array}$ & 4,6 \\
\hline D431 & 31 & $01 E$ & $\begin{array}{l}\text { Surface Water Equipment } \\
\text { Rinsate }\end{array}$ & 4,6 \\
\hline D431 & 31 & 02 & Sediment & $2,3,4,6,8$ \\
\hline D431 & 31 & $02 A$ & Sediment - Duplicate & $2,3,6$ \\
\hline D431 & 32 & 01 & Surface Water & $1,2,3,4,6,7,9,10$ \\
\hline D431 & 32 & 010 & Surface Water - Split & $1,2,3,4,6$ \\
\hline D431 & 32 & 02 & Sediment & $2,3,4,6,8$ \\
\hline D431 & 32 & $02 D$ & Sediment - Split & $2,3,6$ \\
\hline D431 & 32 & $02 E$ & $\begin{array}{l}\text { Sediment Sampling } \\
\text { Equipment - Rinsate }\end{array}$ & $2,4,6$ \\
\hline D431 & 32 & $02 B$ & Trip Blank - Water & 4,6 \\
\hline D431 & 33 & .01 & Surface Water & $1,2,3,4,6,7,9,10$ \\
\hline D431 & 33 & 02 & Sediment & $2,3,4,6,8$ \\
\hline D431 & 34 & 01 & Surface Water & $1,2,3,4,6,7,9,10$ \\
\hline D431 & 34 & 02 & Sediment & $2,3,4,6,8$ \\
\hline D431 & 35 & 0.1 & Surface Water & $1,2,3,4,6,7,9,10$ \\
\hline D431 & 35 & 01B & Surface Water-Trip & 4,6 \\
\hline D431 & 35 & 02 & Sediment & $2,3,4,6,8$ \\
\hline D431 & 35 & $02 \mathrm{C}$ & Sediment-Field Blank & 4,6 \\
\hline D431 & 36 & 0.1 & Surface Water- & $1,2,3,4,6,7,9,10$ \\
\hline D431 & 36 & 02 & Sediment & $2,3,4,6,8$ \\
\hline D431 & 37 & 01 & Surface Water & $1,2,3,4,6,7,9,10$ \\
\hline D431 & 37 & 02 & Sediment & $2,3,4,6,8$ \\
\hline D431 & 38 & 01 & Water (Tank) & $1,2,3,4,6,7,9,10$ \\
\hline D431 & 39 & 01 & Water (Tank) & $1,2,3,4,6,7,9,10$ \\
\hline
\end{tabular}


- Sonstituent analytical cödes are as follows:

(1) Total organic halogens

(2) Metals (Ag, As, Ba, Cd, Cr, Cu, Pb, Mn, Hg, Ni, Se)

(3) Semi-volatile organic constituents, including polycyclic aromatic hydrocarbons (PAHs)

(4) Volatile organic constituents (VOA GC/MS)

(6) Radionuclide Indicators (gross alpha, non-volatile beta, total radium) and tritium

(7) Surface water temperature, dissolved oxygen, $\mathrm{pH}$, depth, flow

(8) Sediment $\mathrm{pH}, \mathrm{TOC}$

(9) Inorganics (Cl, $\left.\mathrm{F}, \mathrm{SO}_{4}, \mathrm{NO}_{3}, \mathrm{Na}, \mathrm{Fe}\right)$

(10) TOC, TSS, TDS
A indicates a field blank
B indicates a trip blank
C indicates a field blank
D indicates a split sample
E indicates a rinsate blank 
Appendix B

Laboratory Data Records Review 


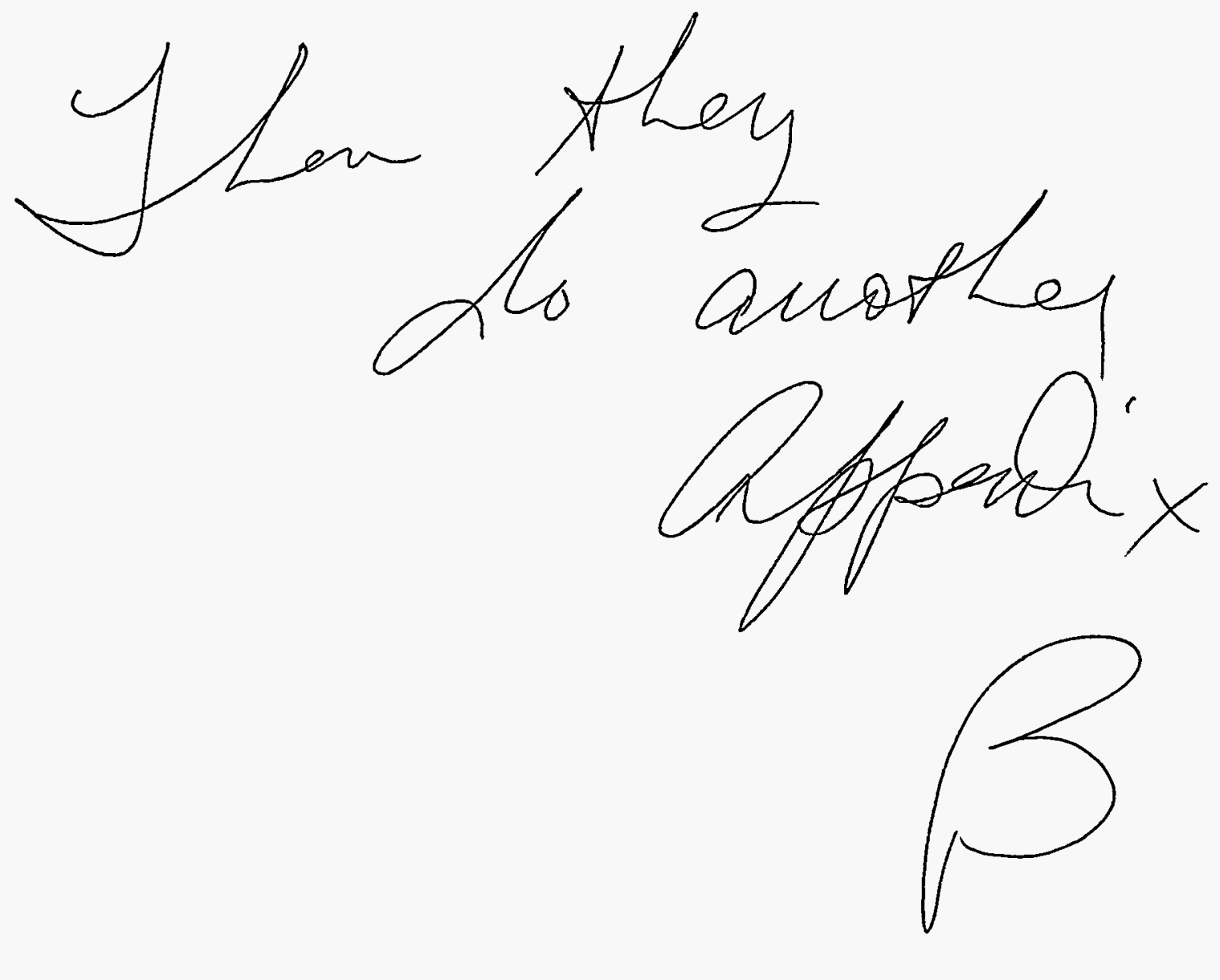




\section{LABORATORY DATA RECORDS REVIEW \\ D AREA BURNING / RUBBLE PIT \\ ROY F. WESTON LABORATORY INC.}

The purpose of this laboratory review was to investigate the analytical procedures conducted by the Roy $F$. Weston Laboratories Inc. (RFW) which can not be addressed through review of Electronic Data Deliverables (EDD) or report certificates. As part of this review, copies of RFW analytical logbooks and instrument calibration records were requested for organic and radiological analyses. Review of these records were conducted according to the appropriate validation guidelines discussed in the Quality Assurance / Quality Control Guidance for Removal Activities (EPA/540/G-90/004), USEPA CLP National Functional Guidelines for Organic Review, USEPA CLP National Functional Guidelines for Inorganic Review, Data Validation Procedures for Radiochemical Analyses (WHC-SD-EN-SPP-001, Rev. 1), SW-846 Methods, and in accordance with Savannah River Site Procedures. The review was completed using Laboratory Data Records Review (LDRR) checklists, prepared by Dr. Kubilius of Westinghouse Savannah River Company (WSRC), for each of the samples identified in the attached List of Samples per Method. Dr. Kubilius of WSRC and Mr. Beckert of RUST Environment and Infrastructure (RUST E\&D) conducted the LDRR.

Samples chosen for the review were selected based on the criteria provided in the WSRC Procedures Manual 3Q1-7 section 6305 and on recommendations from WSRC. These criteria require that $10 \%$ of the samples analyzed for each method be chosen for review. These samples included results with high detects and non-detects. A total of 30 LDRR checklists were completed for the methods listed in the Table of Checklists per Method.

Table of Checklists per Method for

D Area Burning / Rubble Pits

\begin{tabular}{|clc|}
\hline METHOD & ANALYSIS & CHECKLISTS \\
6010 & ICP Metals & 2 \\
7000 & AA Metals & 12 \\
8260 & Volatiles & 3 \\
8270 & Semivolatiles & 3 \\
SW846-9315/EPA 900.1mod & Total Radium-226 & 3 \\
LANL-MLR-100/ EPA 900.0 & Gross Alpha & 2 \\
EPA 900.0 & Nonvolatile Beta & 1 \\
LANL-ER-210/ EPA 906.0 & Tritium & 4 \\
\hline
\end{tabular}

\section{SCORING / QUALIFICATION}

The scoring indicates analytical performances which did not meet criteria recommended in the guidance documents for data validation mentioned above. The score for each method is listed by the sample $\mathrm{ID}$ and includes the data qualifier assigned due to the indicated Method Checklist failure. Result / analysis / bias (R/A/B) qualifiers are assigned based on the guidance documents referenced above, professional judgment, and WSRC procedures.

6010 ICP Metals

1 of 2 samples reviewed did not meet the Method Checklist criterion for Question \#6 of the Method 6010 Checklist because the elements silver and cadmium were below the lower limits of the reporting level for the Instrument Calibration Blank (ICB) and the Continuing Calibration Blanks 
(CCB). All sample results for cadmium and silver analyzed on that instrument on that day will be qualified with $\mathrm{J} / \mathrm{L} /$ if the sample results are 5 times the contract required detection limit (CRDL). Otherwise, the results will receive UJ/L/ for cadmium and silver.

SAMPLE \#

9405L513-001

ANALYTES
Cadmium
Silver

CRITERIA FAILED

Avg. -3.876 limits $(-0.8$ to 0.8$)$

Avg. -4.733 limits (-2.7 to 2.7$)$
QUALIFIERS (R/A/B)

\section{$\mathrm{J}$ or UJ/L}

$\mathrm{J}$ or $\mathrm{UJ} / \mathrm{L}$

\section{Lead by AA}

All Method 7000 Checklists were met for lead in the 3 samples reviewed.

\section{0/7471 Mercury by AA}

1 of 3 samples for mercury failed Question \#1 of the Method 7000 Checklist because the time of analysis did not match the time given in the EDD.

$\begin{array}{lllc}\text { SAMPLE \# } & \text { ANALYTES } & \text { CRITERIA FAIIED } & \text { QUALIFIERS (R/A/B) } \\ \text { 9405L513-012 } & \text { Mercury } & \text { 5/13/94 @ 1:07 LAB } & \text { none } \\ & & 5 / 13 / 94 \text { @ 15:20 EDD } & \end{array}$

1 of 3 samples reviewed failed Question \#14 of the Method 7000 Checklist. This sample did not meet the Method Checklist criteria for matrix spike recovery. The matrix spike recovery was below the required limits of $75 \%$ to $125 \%$. The qualifiers assigned will only be applied to the matrix spike sample and its parent.

\section{SAMPLE \#}

9405L513-012

ANALYTES
Mercury

CRITERIA FAILED

Recovery was $73 \%$

\author{
QUALIFIERS (RA/B) \\ $J / / / L$
}

\section{Arsenic by AA}

2 of 3 samples reviewed failed Question \#14 of the Method 7000 Checklist. This sample did not meet the Method Checklist criteria for matrix spike recovery. The matrix spike recovery was below the required limits of $75 \%$ to $125 \%$. The qualifiers assigned will only be applied to the matrix spike sample and its parent.

$\begin{array}{lllc}\text { SAMPLE \# } & \text { ANALYTES } & \text { CRITERIA FAIIED } & \text { QUALIFIERS (R/A/B) } \\ \text { 9405L514-011 } & \text { Arsenic } & \text { Recovery was 61.5\% } & \mathrm{J} / / \mathrm{L} \\ \text { 9405L514-001 } & \text { Arsenic } & \text { Recovery was 61.5\% } & \mathrm{J} / \mathrm{L}\end{array}$

7740 Selenium by AA

3 of 3 samples reviewed failed Question \#14 of the Method 7000 Checklist. This sample did not meet the Method Checklist criteria for matrix spike recovery. The matrix spike recovery was below the required limits of $75 \%$ to $125 \%$. The qualifiers assigned will only be applied to the matrix spike sample and its parent.

$\begin{array}{lllc}\text { SAMPLE \# } & \text { ANALYTES } & \text { CRITERIA FAILED } & \text { QUALIFIERS (R/A/B) } \\ \text { 9405L514-001 } & \text { Selenium } & \text { Recovery was 69\% } & \text { J/L/L } \\ \text { 9405L514-002 } & \text { Selenium } & \text { Recovery was 69\% } & \text { J/L/L } \\ \text { 9405L514-004 } & \text { Selenium } & \text { Recovery was 69\% } & \mathrm{J} / \mathrm{L} /\end{array}$




\section{$\underline{8270 \quad \text { Semivolatiles }}$}

All Method Checklists were met in the 3 samples reviewed.

$\underline{8260 \text { Volatiles }}$

3 of 3 samples failed Question \#6 of the Method 8260 Checklist because the response factor $(\mathrm{RF})=$ 0 for 2-chloroethylvinyl ether at the lowest level standard. The RF values should be greater than 0 for each element. The other RF values for this compound, however, were greater than zero. No qualifier for this compound is needed unless its result is less than 2 parts per billion (ppb).

$\begin{array}{lccc}\text { SAMPLE \# } & \text { ANALYTES } & \text { CRITERIA FAILED } & \text { QUALIFIERS (R/A/B) } \\ \text { 9405L514-011 } & \text { 2-Chloroethylvinyl ether } & \mathrm{RF}=0 & \mathrm{U} / / \text { if }<2 \mathrm{ppb} \\ \text { 9405L513-021 } & \text { 2-Chloroethylvinyl ether } & \mathrm{RF}=0 & \mathrm{U} / / \text { if }<2 \mathrm{ppb} \\ \text { 9405L514-002 } & \text { 2-Chloroethylvinyl ether } & \mathrm{RF}=0 & \mathrm{U} / / \text { if }<2 \mathrm{ppb}\end{array}$

1 of 3 samples failed Question \#11 because the RFs for the system performance check compounds (SPCCs) for bromoform and 1,1',2,2'-tetrachloroethane were below recommended limits of 0.3 ( 0.25 for bromoform). A laboratory note was included in the case narrative indicating that the RFs were lowered to 0.1 for Bromoform due to the $25 \mathrm{ml}$ volume purged.

$\begin{array}{lccc}\text { SAMPLE \# } & \text { ANALYTES } & \text { CRITERIA FAILED } & \text { QUALIFIERS (R/A/B) } \\ \text { 9405L513-021 } & \text { Bromoform } & 0.127 & \text { none } \\ \text { 9405L513-021 } & 1,1^{\prime}, 2,2^{\prime} \text {-tetrachloroethane } & 0.159 & \text { none }\end{array}$

1 of 3 samples failed Question \#12 because the percent difference $(\% \mathrm{D})$ for one of the Continuing Calibration Compounds (CCCs) was greater than the limits of $+1-20 \%$.

$\begin{array}{llcc}\text { SAMPLE \# } & \text { ANALYTES } & \text { CRITERIA FAILED } & \text { QUALIFIÉRS (R/A/B) } \\ \text { 9405L513-021 } & \text { All detects } & -23.8 \% & \mathrm{~J} / \mathrm{L} /\end{array}$

EPIA-008 Total Radium-226

2 of 3 samples failed Question \#9 of the Radium Checklist because the chemical recovery for the tracer was not between 80 and $105 \%$.

$\begin{array}{lccc}\text { SAMPLE\# } & \text { ANALYTES } & \text { CRITERIA FAILED } & \text { QUALIFIERS (R/A/B) } \\ 94-05-022-011 & \text { Total Radium } & 115.4 \% & \mathrm{~J} / \mathrm{O} / \mathrm{H} \\ 94-05-022-08 & \text { Total Radium } & 113.1 \% & \mathrm{~J} / \mathrm{O} / \mathrm{H}\end{array}$

1 of 3 samples also failed Question \#10 because the chemical recovery for the tracer was not between 60 and $115 \%$.

SAMPLE \#

94-05-022-011

\section{ANALYTES}

Total Radium
CRITERIA FAILED
$115.4 \%$
QUALIFIERS (R/A/B) $\mathrm{J} / \mathrm{O} / \mathrm{H}$

\section{EPA $900.0 \quad$ Gross Alpha}

1 of 2 samples also failed Question \#18 because the relative percent difference between the replicate analyses was greater than $35 \%$.

SAMPLE \#

94-023-03
ANALYTES

Gross Alpha

\section{CRITERIA FAILED \\ $67 \%$}

QUALIFIERS (R/A/B)

$[\mathrm{XJ}$ 
EPA $900.0 \quad$ Nonvolatile Beta

1 of 1 samples met all criteria for the Nonvolatile Beta Checklists, including self absorption curves.

EPA 906.0 Tritium

2 of 4 samples failed Question \#15 because the laboratory control samples (LCS) percent recovery was outside the $90-110 \%$ range.

SAMPLE \#

94-05-023-12

94-05-023-07
ANALYTES

Tritium

Tritium

CRITERIA FAILED
$110.5 \%$
$110.5 \%$

$110.5 \%$

\author{
QUALIFIERS (R/A/B) \\ none \\ none
}

\section{LIST OF SAMPLES PER METHOD}

D AREA BURNING / RUBBLE PITS

\begin{tabular}{|c|c|c|}
\hline $\begin{array}{l}\text { Weston } \\
\text { Sample ID }\end{array}$ & $\begin{array}{l}\text { Method } \\
\text { Number }\end{array}$ & $\begin{array}{l}\text { Parameter } \\
\text { Requested }\end{array}$ \\
\hline 9405L513-001 & EPA 300 & Sulfate \\
\hline 9405L513-002 & EPA 353.2 & Nitrate as N2 \\
\hline 9405L513-001 & EPA 6010 & Manganese, total recoverable \\
\hline 9405L514-002 & EPA 6010 & Chromium \\
\hline 9405L513-001 & EPA 6010 & Cadmium \\
\hline 9405L514-001 & EPA 7060 & Arsenic \\
\hline 9405L513-003 & EPA 7060 & Arsenic \\
\hline 9405L514-011 & EPA 7060 & Arsenic \\
\hline 9405L513-009 & EPA 7421 & Lead \\
\hline 9405L513-004 & EPA 7421 & Lead \\
\hline 9405L513-001 & EPA 7421 & Lead \\
\hline $9405 \mathrm{~L} 513-001$ & EPA 7471 & Mercury \\
\hline $9405 L 514-003$ & EPA 7471 & Mercury \\
\hline 9405L513-012 & EPA 7471 & Mercury \\
\hline 9405L514-001 & EPA 7740 & Selenium \\
\hline 9405L514-002 & EPA 7740 & Selenium \\
\hline 9405L514-004 & EPA 7740 & Selenium \\
\hline 9405L514-002 & EPA 8260 & (1,1,1-Trichloroethane) \\
\hline 9405L514-011 & EPA 8260 & (1,1-Dichloroethane) \\
\hline 9405L513-021 & EPA 8260 & Benzene \\
\hline 9405L514-011 & EPA 8260 & Trichloroethene \\
\hline 9405L514-004 & EPA 8270 & (1,4-Dichlorobenzene) \\
\hline 9405L513-001 & EPA 8270 & Pentachlorophenol \\
\hline 9405L514-002 & EPA 8270 & Phenanthrene \\
\hline $94051513-001$ & EPA 8270M & (1,2,4-trichlorobenzene) \\
\hline 9405L513-001 & EPA $8270 \mathrm{M}$ & (4-nitrophenol) \\
\hline 9405L-514-004 & EPA 8270M & (1,2,4-Trichlorobenzene) \\
\hline
\end{tabular}


TMA

94-05-022-08

94-05-022-04

94-05-022-08

94-05-022-011

94-05-022-05

94-05-022-09

94-05-023-12

94-05-023-07

94-05-023-03

94-05-023-11
Method

Number

EPA 900.0

EPA 900.0

EPA 900.1MOD

EPA 900.1MOD

EPA 906.0

EPA 906.0

LANLER $210 \mathrm{MOD}$

LANLER 210 MOD

LANLMLR-100

SW846 9315
Parameter requested

Gross alpha

Nonvolatile Beta

Radium, total alpha

Radium, total alpha

Tritium

Tritium

Tritium

Tritium

Gross alpha

Radium, total emitting alpha 